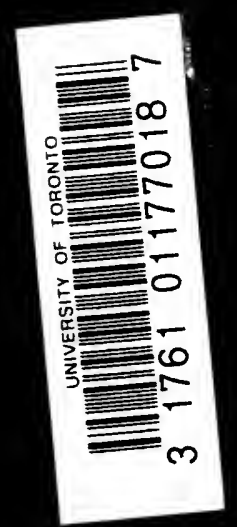




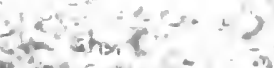

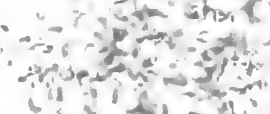

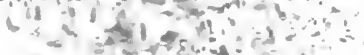

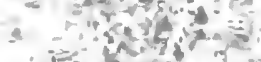

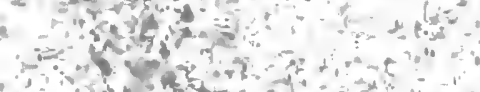

tक

$\Rightarrow$ ar,

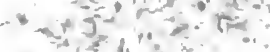

$\therefore \quad \div 0 \div 2$

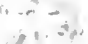




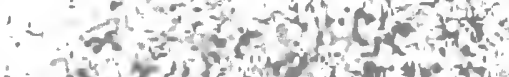

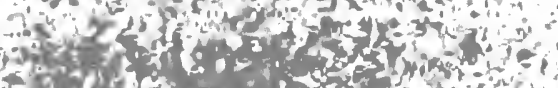
singent.

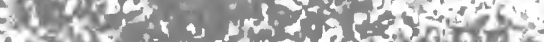

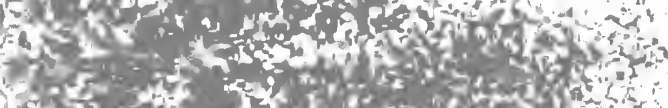

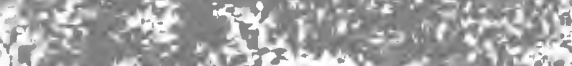

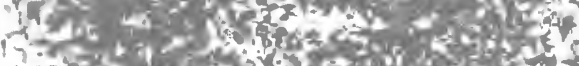

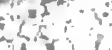

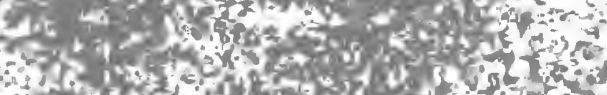

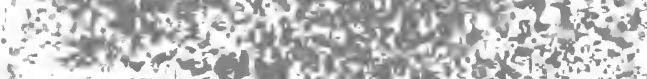

5. $-2+2$ (n)

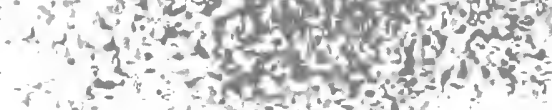

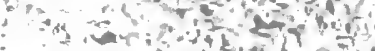

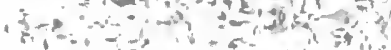

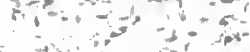




\section{A MANUAL \\ of \\ PHARMACOLOGY \\ and its applications to}

THERAPEUTICS AND TOXICOLOGY

By

TORALD SOLLMANN, M. D.

Professor of Pharmacology and Materia Medica in the School of Medicine of Western Reserve University, Cleveland

ILLUSTRATED

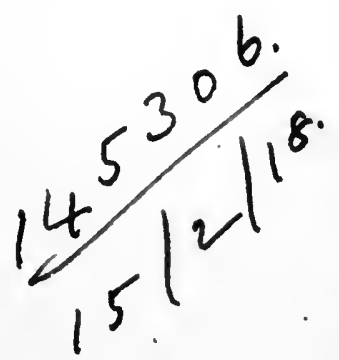

PHILADELPHLA AND LONDON

W. B. SAUNDERS COMPANY 1917 
Copyright, I917, by W. B. Saunders Company

THE USE IN THIS VOLUME OF CERTAIN PORTIONS OF THE TEXT OF THE UNITED STATES PHARMACOPOEIA IS BY VIRTUE OF FERMISSION RECEIVED FROM THE BOARD OFTRUSTEES OF THE UNITEDSTATES PHARMACOPOEIALCONVENTION. THE. SAID BOARD OF TRUSTEES IS NOT RESPONSIBLE FOR ANY INACCURACY OF QUOTATION NOR FOR ANY ERRORS IN THE STATEMENT OF OUANTITIES OR PERCENTAGE STRENGTHS.

\section{PRINTED IN AMERICA}

$$
\text { PREBS OF }
$$




\section{PREFACE}

In the process of revising the "Text-Book of Pharmacology," it became apparent that the extensive development and applications of Pharmacology justified a somewhat more ambitious treatment of the subject.

Pharmacology comprises some broad conceptions and generalizations, and some detailed conclusions, of such great and practical importance that every student and practitioner of medicine should be absolutely familiar with them. It comprises also a large mass of minute details, which would constitute too great a tax on human memory, but which cannot safely be neglected. It is the ambition of this book to present both types of information without confusion. The matter in the larger print aims to give a connected and concise statement of the essentials of pharmacology. The smaller print need not be considered by the reader who wishes to obtain merely a general knowledge of pharmacology. It contains more detailed data for consultation. Side headings have been introduced liberally to facilitate this use. The bibliographic references are intended to put the investigator on the track of further details.

The volume can, therefore, serve both for study and reference. There may be some question as to the general advisability of combining text-books and reference books; but in Pharmacology this appears to be necessary, since every student may need more detailed information at any time.

These objects necessitated a complete rearrangement, and fairly extensive enlargement of the book, and as the work progressed, it appeared plain that it meant not merely a revision, but the making of an entirely new book.

With this enlargement of the text, it became necessary to practice the utmost economy of matter, in order to bring the contents within a single volume. This and other considerations prompted the separation of the Laboratory Guide In Pharmacology, which will be issued as a companion volume. The illustrations have also been omitted wherever the text could be understood without them. A further economy was effected by curtailing the discussion of the drugs of slight importance, thus leaving more space for the relatively few really important drugs. This is in line with the modern movement for the restriction of the "Materia Medica."

I am greatly indebted to my friend, Dr. R. A. Hatcher, for valuable help in reading the proofs. 


\section{CONTENTS}

PAGE

Pharmacognosy

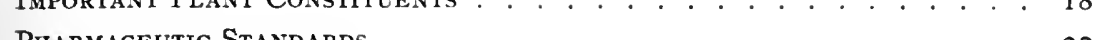

Pharmaceutic Standards . . . . . . . . . . . . . . . . . . . . . . 22

Pharmaceutic Methods . . . . . . . . . . . . . . . . . . . . . 23

Pharmaceutic Assaying, 29.

Classes of Pharmaceutic Preparations . . . . . . . . . . . . . . . 30

Watery Solutions, 30-Alcoholic Solutions, 32-Preparations Made by

Extraction, 32-Mixtures, 35-Solid Preparations, 36-Dosage Forms, 37

Solvbilities

INCOMPATIBILITY

METROLOGY . . . . . . . . . . . . . . . . . .

Coloring and Flayorive

Coloring, 65-Flavoring, 66.

The Treatment of Disease

The Chemical and Physical Basis of Pharmacology, 75 - The Manifestations of Pharmacologic Actions, 78-Classification of Drugs According to Selective Action, 80-The Administration of Drugs, 84-Conditions Influencing Absorption, 88-The Excretion of Drugs, 9Q-Dosage (Posology), 90-Conditions Influencing Drug Actions, 92.

FERMENTS AND Nutrients

Digestive Ferments, 96-Lactic Acid Ferments, 98-Medicinal Foods, 99-Diabetes Foods, Ioo-Cod Liver Oil, IoI-Parenteral Alimentation, 102.

EMOLLIENTS

Olive Oil and Other Bland Fatty Oils (Olea Pingua), 105-Lard and Similar Animal Fats, ro5-Woolfat-lanolin, ro6-Petrolatum and Other Petroleum Products, I06-Waxes, I08-Compound Ointment Bases, I08-Cerates, I08-Glycerin, I09.

Demulcents or Mucilaginous Substances.

Demulcent Teas, I Io-Gelatin, I I I-Starch, i I I-Indifferent Dusting Powders, I I2-Poultice Masses, I I2-Plasters, I I3-Mechanical Protectives, I I 3 .

Phenomena Common to Local Irritants, Corrosives and Astringents Fixed Caustic Alkalies, I25-Ammonia, I26-Alkaline Salts and Soaps, 127-Alkaline Sulphids, I28-Hydrogen Sulphid, I28-Sulphur, I29 -Ichthyol, 130-Thiosinamin, I30-Caustic Acids, I3 I-Dilute Acids, I32-The Haloids, I33-Iodin, I33-Bromin, I34-The Local Actions of Metallic Salts, I 34-Tannins and Vegetable Astringents, I 36-Astringent Styptics, 139-Strength of Most Useful Solutions of Astringents and Antiseptics, 140.

Irritant Volatile Oils and Related Drugs . . . . . . . . . . . . . . Olfactory Stimulants, I42-Hysteric Sedatives or Antispasmodics, 142Oil of Turpentine, I43-Terpin Hydrate, I44-Eucalyptol, I44-Rubefacient Volatile Oils, I45-Iiniments, 146-Juniper Oil, I46-Ecbolic and Toxic Volatile Oils, I46-Volatile Oils and Oleoresins Employed in Ure- 
thritis and Cystitis, 148 -Balsans, I 49 -Insecticides, 150-Mustard Oil, 151 .

NoN-Volatile Organic IRKitant

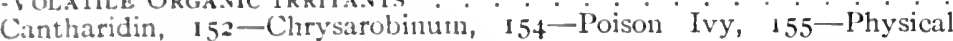
Counterirritants, I 56.

Drugs Employed for Tileir Local Effects on the Alimentary Canal. Stomachics, 157 -Simple Bitters, 158-Astringent Bitters, I59Aromatic Bitters, 160 -Carminatives, 160 -Vegetable Cathartics, I6IThe Anthraquinon or Emodin Cathartics, I 66-Cascara Sagrada, I67Senna, I 68 -Rhubarb, I69-Aloes, I 69 -Phenolphthalein, I I - Bile, 17 I-Castor Oil, I72-Riciu, I73-Abrin and Jequirity, I 74-Cathartic Resins, 174-Jalap, I 75-Resin of Podophyllum, 175-Colocynth, Elaterin, and Bryonia, I 76 -Croton Oil, 177 -Colloid and Emollient Laxatives, $178-$ Evacuant Enemas, 178.

Asthelmintics

Aspidium or Male Fern, 180-Chenopodium Oil, 181-Pelletierin, 182Santonin, I 83 -Vermicides for Thread Worms (Oxyuris), 184.

Convulsant Poisons

Strychnin and Nux Vomica, i 85.

Picrotoxin Group . . . . . . . . . . . . . . . . . . . . . . . I99

Caffein, Theobromin, and Other Methyl-Xanthins . . . . . . . . . 200 Caffein Beverages, 214.

The Morphis Group . . . . . . . . . . . . . . . . . . 217

HYdRASTIS . . . . . . . . . . . . . . . . . 245

Cotarnin . . . . . . . . . . . . . . . . . . . . 247

Canvabis. . . . . . . . . . . . . . . . . . . . . . . 249

Lactucarium; Dried Lettuce Juice, 250-Lupulin and Hops, 250-Loco Disease, 250.

Cocain Grovp; Local Anesthetics

Action of Cocain on the Eye, 253-Systemic Actions, $254^{\circ}$-Acute Cocain Poisoning; Etiology, 257-Chronic Cocain Poisoning, 257-Use of Cocain as a Local Anesthetic in Minor Operations, 258-Catarrh, Hay Fever and Asthma, 258-Use of Cocain in Larger Operations, 258-Use of Cocain as a Cerebral Stimulant, 260-Cocain Substitutes, 260.

Drugs Acting Peripherally on the Autonomic-sympathetic System. .

The Parasympathetic and Sympathetic Systems, 263-Pharmacology of the Sympathetic Division, 264-Pharmacology of the Parasympathetic, Cranio-sacral or Restricted Autonomic Division, 265-Drugs with Partial Autonomic Reactions, 265-Location of Action, 266-The Response of the Uterus to Autonomic Poisons, 270-Gall-bladder, 271 -Ureter, 271 $\rightarrow$ The Nature of Antagonism, 272-Optical Isomers, 273-Autonomic Phenomena of the Intestine, 274.

ATropis

Bronchial Muscles and Glands, 270-Circulation, $280^{\circ}$-Temperature, 282-Gaseous Metabolism, 282-Actions on the Eye, 282-Paralysis of Secretions, 286-Glycogenolysis, 288-Atropin in Diabetes, 288- $\mathrm{He}-$ patic Actions and Coagulability of Blood, 288-Action of Atropin on Peristalsis, 288-Biliary and Renal Colic, 289-Urinary Bladder and Incontinence of Urine, 289-Uterus, 289-Curare Action, 289-Anodyne Action, 289-Atropin Poisoning, 290-Racial and Acquired Tolerance, 291.

Hyoscyamin . . . . . . . . . . . . . . . . . . . . 292

Protocatechyl Tropein. . . . . . . . . . . . . . . . 292

SCOPOLAMIN OR HYOSCIN . . . . . . . . . . . . . . . . 293

Prlocarpin .................... . 297

Prysostigmin. . . . . . . . . . . . . . . . . 30i 
MUSCARIN

Mushroom Poisoning . . . . . . . . . . . . . . . . . . . . . . . . 305

Cholis. . . . . . . . . . . . . . . . . . . . . . . . . . . . . . . 305

NeUrin . . . . . . . . . . . . . . . . . . . . . . . . . . 307

Betain. . . . . . . . . . . . . . . . . . . . . . . . . . . . . . 307

QUaternary Ammonium Bases . . . . . . . . . . . . . . . . . . . . 307 Tetra-methyl Ammonium Chlorid, 307-Tetra-Ethyl Ammonium Chlorid, 308-Methyl-Ethyl Ammonium Compounds, 308.

Nicotrs . . . . . . . . . . . . . . . . . . . . . . . . . 308 Acute Nicotin or Tobacco Poisoning, 3 I I-Habitual Use of Tobacco, 3 I 3 .

LOBELIA

Conium

Gelsemium

SPARTEIN

Curare

Guanidin

EPINEPHRIN

Suprarenal Testing, 338 .

Uzara .

Cotoln(Methyl-Trioxy-BenzophenoNe) . . . . . . . . . . . . . . . 339

Pituitary Extracts. . . . . . . . . . . . . . . . . . . . . . . . . 339 Active Constituents, $340-$ Circulation, $340-$ Clinical Use in Shock and Collapse, 342-Respiratory Center, 342-Uterus, 343-Use in Obstetrics, 343-Urinary Bladder, 343-Intestines, 343-Lacteal Secretion, 344Saliva, 344-Pancreatic Secretion, 344-Cerebrospinal Fluid, 344Pupils, 344-Toxic Effects, 344-Standardization, 345-Anterior $\mathrm{Pi}$ -
tuitary Lobe, 345.

Thyroid Preparations

Parathyroids

Pineal Gland . . . . . . . . . . . . . . . . . . . . . . . . . . . 35 I

Pancreas Preparations in Diabetes . . . . . . . . . . . . . . . . . . . . 352

Secretin . . . . . . . . . . . . . . . . . . . . . . . . . . . $35^{2}$

PhlorhizIN . . . . . . . . . . . . . . . . . . . . . . . . . . . 353

KIDNEYS . . . . . . . . . . . . . . . . . . . . . . . . . . . 354

Red Bone Marrow . . . . . . . . . . . . . . . . . . . . . . . . . 354

Pressor and Depressor Principles in Animal Extracts . . . . . . . . 354

ThyMUS . . . . . . . . . . . . . . . . . . . . . . . . . 355

Nucleoproteins, Nucleins, and Nucleic Acids . . . . . . . . . . . . 355

Yeast ("Fax Medicinalis"). . . . . . . . . . . . . . . . . . . . 355

Sexual Glands. . . . . . . . . . . . . . . . . . . . . . . 356

Orchitic EXTRActs . . . . . . . . . . . . . . . . . . . . . . . . . 356

Ovarian Extracts . . . . . . . . . . . . . . . . . . . 356

Corpus Luteum (Lutein) . . . . . . . . . . . . . . . . . . . . . 356

Mammary Gland . . . . . . . . . . . . . . . . . . . . . . . 357

ERGot . . . . . . . . . . . . . . . . . . . . . . . . . 357

Actions, 359-Actions of Pure Ergotoxin, 361-Histamin, 362-Tyramin, 363 -Phenylethylamin, 364-Indolethylamin, 364-Paraphenylen- 
diamin, 364-Ergotinic Acid, 364-Hordenin, 364-Ptomain Poisoning, 364 -Acite Ergot Poisoning, 365-Chronic Ergot Poisoning, "Ergotism," 365 -Deterioration, 366-Bio-assay, 366.

Echolics (()xytocics) and Emmexagogues. . . . . . . . . . . . . . 367

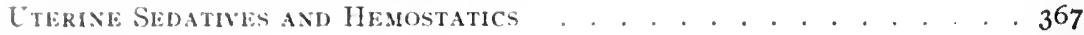

Fiblikncus . . . . . . . . . . . . . . . . . . . 368

Axaplltaxis . . . . . . . . . . . . . . . . 369

Anaphylactic Shock, 369-Anaphylactoid Intoxications, 373.

Nitrites, Nitroglycerix, and otimk Alkyl Nitrates. . . . . . . . 375

Thl: Digitalis Group. . . . . . . . . . . . . . . . . . . . . . . 382

Digitalis Actions on the Circulation, 385-Gastro-intestinal Actions of

Digitaloids, 396-Gall-bladder, 396-Suprarenal Secretion, 396-Local Actions, 396 - Therapentic Uses of Digitalis, 397 - Chronic Digitalis Poisoning in Animals, 403-Toxic Effects in the Clinical Use of Digitalis, 403 Acute Digitalis Poisoning, fo4-Administration, 405-Recapitulation of

Other Digitaloid Drugs, 407-Bio-assay of Digitaloid Drugs, 409.

Cactus

Mescal.

Camphor .. . . . . . . . . . . . . . . . . . . . . . . . 4 43

Toxic Effects, 4I5-Camphor Isomers, 4I6-Camphoric Acid, 416.

MeNThol . . . . . . . . . . . . . . . . . 4I7

SAPONINS (SAPOTONINS; SAPO-Glucosids). . . . . . . . . . . . . 417

Solanix . . . . . . . . . . . . . . . . . . . . . 420

SNAKE VeNOM. . . . . . . . . . . . . . . . . . . . 421

Cholesterol . . . . . . . . . . . . . . . . . 421

Acontte . . . . . . . . . . . . . . . . . . . . 422

VeRATRIN (CEVAdIN). . . . . . . . . . . . . . . . . . . . . . . . . . 428

VERATRUM . . . . . . . . . . . . . . . . . . . . . . . 432

Protoveratrin, 434-Jervin, 435-Rubijervin, 435.

Colchicum . . . . . . . . . . . . . . . . . . . . . . . 435

Therapeutic Use in Gout, 436-Colchicum Poisoning, 436-Colchicin Derivatives, 437

APOMORPHIN

The Emetic Action of Apomorphin is Central, 438-Other Evidence that Emesis is Central, 438- Hypnotic Action and Use in Acute Alcoholism and Hysteria, 440-Phenomena of Nausea, 440-Accidents in the Therapeutic Use of Apomorphin, 44I.

APOCODEIN

Aspidosperma (QUebracho)

IPECACUANHA AND IPECAC . . . . . . . . . . . . . . . . . . . . . 442

Comparative Action of Emetin and Cephaelin, 445-Psychotrin, 445Ipecacuanhic Acid, 445 .

Therapeutic Use of NaUseant Expectorants . . . . . . . . . . . 446

Locally Acting Emetics . . . . . . . . . . . . . . . . . . . . . 446

Cupri Sulphas and Zinci Sulphas, 447-Mustard, 447-Antimony, 447Emesis as an Undesirable Side Action, 447-Therapeutic Use of Emetics, 447.

The Puarmacology of Heat Regulation

Reactions which Raise the Temperature (Pyretics), 45I-Reactions which Lower the Temperature (Antipyretics), 445-The Treatment of Fever, 459.

Quinin

Quinin Derivatives, $467^{\circ}$-Absorption, Fate and Excretion, $468^{\circ}$ 
Antiseptic Benzol Derivatives

Benzol, 477-Toluol, 478-Phenol, 478-Toxicology, 48I-Phenolcamphor, 482-Phenolsulphonates or Sulphocarbolates, 482 -Cresols, 483-Polyatomic Phenols, 484-Resorcinol, 485-Uva Ursi, 485-Pyrogallol, 485-Picric Acid, 486-Nitrobenzol, 486-Anilin, 487-Creosote and Guaiacol, 487-Tar, 489-Thymol, 490-Naphthalin, 490-Naphthol, 49I-Quinolin, 49I-Benzoic Acid and Benzoates, 492-Cinnamic Acid, 495-Salicylic Compounds, 495-Salicyl Derivatives, 502-Acetylsalicylic Acid (Aspirin), 503-Methyl Salicylate, 504-Phenyl Salicylate, 504 -Phenyl-quinolin-carboxylic or Phenyl-cinchoninic Acid (Atophan), 505-Quinic Acid, 507-Saccharin, Benzosulphinid or Glusid, 507Methylthionin Hydrochlorid or Methylene Blue, 508-Other Coal-tar Dyes, 509.

Miscellaneous Antiseptics

Formaldehyd, 5 10-Formaldehyd Compounds, 5i3-Hexamethylenamin, 513-Chlorin, 517-Para-toluene Sodium Sulphochloramid, 519Sulphur, Dioxid (Sulphurous Acid), 519--Sodium Sulphite and Thiosulphate (Hyposulphite), 519-Ozone, 520-Hydrogen Peroxid, 52 I-Peroxids of Metals, $52 \mathrm{I}$-Organic Peroxids, 252-lodoxybenzoic Acid, 522 -Potassium Permanganate, 522-Boric Acid and Borax, 523-Iodoform, 524-General Consideration of Antiseptics, 525.

Serums and Vaccines .

Diphtheria Antitoxin, 528- Normal Horse Serum, 53i-Hemostatic Tissue Extracts; Kephalin, 53I-Tetanus Antitoxin, 53I-Antimeningococcus Serum, 532-Antipneumococcic Serums, 533- Hay Fever Serum, 533-Vaccine Virus, 533-Antirabic Vaccine, 533-Tuberculin Preparations, 534-Typhoid Vaccine, 534-Gonococcus Vaccine, 535.

THEORIES OF NARCosis

ALCOHOL

Food-value of Alcohol, $55^{2}$ - Contra-indications, $553^{\circ}$-Acute Alcohol Poisoning, 553-Antagonism of Alcohol and Caffein, 554-The Habitual but Moderate Use of Alcohol, 554-Chronic Alcoholism, 554-Peculiarities of Alcoholic Beverages, 557-Wines, 558-Higher Alcohols, 559-Isoamyl Alcohol, 559-Methyl Alcohol, 559-Acetone (Dimethyl-ketone), 56I-Denatured Alcohol, 56I

Ether and Chloroform Group ..

Choice of the Anesthetic, 577 - Preparation of Patient for Anesthesia, 581-The Administration of Anesthetics, 582-Causes of Death Under Anesthesia, 585-Poisoning by Swallowing Chloroform, 586-Treatment of Accidents, 586-Idiosyncrasy, 587-Impurities, 588-Origin of Chloroform, 588-Induction of Anesthesia during Sleep, 588-Related Anesthetics, 588.

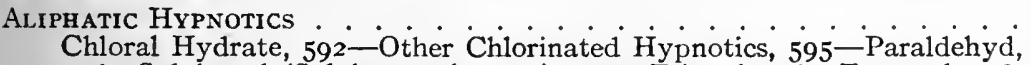
596-Sulphonal (Sulphonmethanum), 597-Trional, 598-Tetronal, 598.

Hypnotic Urea Derivatives . . . . . . . . . . . . . . . . . . . 598 Veronal (Diethyl-Barbituric Acid), $599^{\circ}$-Bromural, 600-Ürethanes, 600 -Ethyl Carbamate, 600.

The Systemic Actions of Gases

Nitrous Oxid, 601-Carbon Dioxid (Carbonic Acid), $60^{\circ}$-Carbonic $^{\circ}$ Oxid (Carbon Monoxid, CO); Illuminating Gas, 608-Oxygen, 610-Oxygen Deficiency, 6I3.

Hydrocyanic Acid and Cyanids

Physical Factors of Salt Actions 
PAGE

Unotic EfFets on Cells. . . . . . . . . . . . . . . . . . . 633 Actions of Electrolytes, 638 -Physiologic or Normal Saline Solutions

(.Irtificial Serums), 641 -Usmotic Exchanges in the Body, 643.

('AtHAKTIC SAlts................... . . 648

Sodium Sulphate, 652 -Sodium Phosphate, 652-Pyrophosphates, 653 -

l'artrates, 653-Magnesium Compounds, 654.

WATER

MINERAl. WATERS

Climate

DILRETICS

Somlum Chlorid

URFA

SACCharum Lactis (MILK-Sugar). . . . . . . . . . . . . . . . . . 675

POtasslum Salts . . . . . . . . . . . . . . . . . . . . . . . 675

I.rThuM SALTS . . . . . . . . . . . . . . . . . . . . . 677

Rubidium AN! Cesium. . . . . . . . . . . . . . . . . . . . . . .678

Ammonium Compounds . . . . . . . . . . . . . . . . . . . . . . . 678

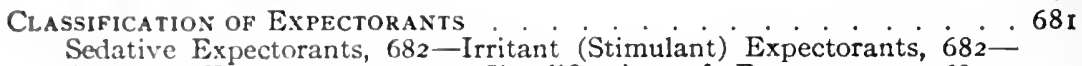
Anodyne Expectorants, 682-Simplification of Expectorants, 683Expectorant Vehicles, 683-Cough Mixtures, 683.

Calcium SAlts . . . . . . . . . . . . . . . . . . . . . . . 683

Strontium . . . . . . . . . . . . . . . . . . . . . . . . . . 690

Oxalic Acid and Oxalates . . . . . . . . . . . . . . . . . . . . . 69I

Fluorid ION . . . . . . . . . . . . . . . . . . . . . . . . . . . . 692

BARIUM . . . . . . . . . . . . . . . . . . . . . . . . . . . . 693

Bromid . . . . . . . . . . . . . . . . . . . . . . . . . . . 694

Organic Bromin Compounds, 699.

IODIDS . . . . . . . . . . . . . . . . . . . . . . . . . . 699

SulphocYanids (RhodANATES) . . . . . . . . . . . . . . . . . 706

Nitrates. . . . . . . . . . . . . . . . . . . . . . . . . . . 707

Potassium Chlorate . . . . . . . . . . . . . . . . . . . . . . . . . . 707

Bromates . . . . . . . . . . . . . . . . . . . . . . . . . . . 709

Methemoglobin Formation . . . . . . . . . . . . . . . . . . . . . 709

The Reaction of the Body (Remote Effects of Acids and Alkalies) 7 io

Dilute Hydrochloric and Other Acids . . . . . . . . . . . . . . . 718

Sodium Bicarbonate and Other Mild Alkalies . . . . . . . . . . . 72 I

URATE SOlvents . . . . . . . . . . . . . . . . . . . 726

Uric Acid and Its Salts, 726.

Organic Acids and Their Salts . . . . . . . . . . . . . . . . . . . 729

Acetates. . . . . . . . . . . . . . . . . . . . . . . . . 730

Citrates . . . . . . . . . . . . . . . . . . . . . . . . . 730

Lactates . . . . . . . . . . . . . . . . . . . . . . . . . 73I

Formates . . . . . . . . . . . . . . . . . . . . . . . . . . 73 I

Metals and Their Salts . . . . . . . . . . . . . . . . . . . . . 732

Colloid Metals . . . . . . . . . . . . . . . . . . . . . . . . . 734

Inorganic Arsenic Compounds. . . . . . . . . . . . . . . . . . . . 735

Organic Arsenic Compounds . . . . . . . . . . . • . . . . . . 743

Sodium Cacodylate, 745 -Sodium Arsanilate (Atoxyl), 746 -Salvarsan and Neosalvarsan, 748 .

Selenium and Tellurium . . . . . . . . . . . . . . . . 755

VANAdiUM . . . . . . . . . . . . . . . . . 755

Antimony ........................ 756 
URANIUM

BISMUTH

IRON . . . . . . . . . . . . . . . . . . . . . . . 762

MANGANESE . . . . . . . . . . . . . . . . . 772

Chromates . . . . . . . . . . . . . . . . . . . . 772

Aluminum . . . . . . . . . . . . . . . . . . . 773

Baking Powders, 774.

Cerrum

YTTRIUM AND ZIRCONIUM . . . . . . . . . . . . . . . . . . . . . . 7775

BerYlliUm SUlPhate . . . . . . . . . . . . . . . . . 775

THORIUM . . . . . . . . . . . . . . . . . . . . . . 775

Radro-Active Metals . . . . . . . . . . . . . . . 775

Silver . . . . . . . . . . . . . . . . . . . . . . 780

Gold and Platinum . . . . . . . . . . . . . . . . . . . . . . . 782

TIN . . . . . . . . . . . . . . . . . . . 782

COPPER ........................ . 782

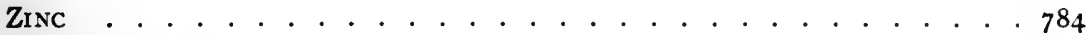

Cadmum . . . . . . . . . . . . . . . . . . . . . . . . 785

Nickel ANd Cobalt . . . . . . . . . . . . . . . . . . . . . . 785

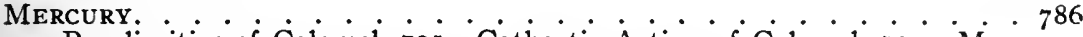
Peculiarities of Calomel, 79i-Cathartic Action of Calomel, 79i-Mercury in Syphilis, 793-Mercury in Tuberculosis, 797.

LEAD . . . . . . . . . . . . . . . . . 799 Acute Lead Poisoning, $80^{\circ}-$ Absorption, $800-$ Retention, 800 -Excretion, 8or-Chronic Lead Poisoning, 801.

Thallium . . . . . . . . . . . . . . . . . . . . . . . 806

Phosphorus . . . . . . . . . . . . . .. . . . . . . . 806 Phosphorus Poisoning, 807-Therapeutic Uses, $8 \mathrm{I}$ I.

Phosphorus Compounds in Nutrition . . . . . . . . . . . . . 812

Lecithin, Glycerophosphates, Hypophosphites, Etc., 812-Lecithin, 814Phytin, 816-Glycerophosphates, 816-Hypophosphites, 816.

APPENDIX.

Appendix A.-Tabulation of Average Doses. . . . . . . . . . . 8 819

Appendix B.-Check-list for the Study of Preparations . . . . . . . . 823

Appendix C.-Bibliography . . . . . . . . . . . . . . . . . . . 826

INDEX ........................ . . . 889 



\section{A MANUAL \\ $\mathrm{OF}$ \\ PHARMACOLOGY}

\section{PHARMACOGNOSY}

Definitions.-Pharmacognosy deals with the origin and the anatomic and chemic structure of crude drugs.

Materia Medica is an older, obsolescent title, which was used to include also the actions, uses and dosage. Organic Materia Medica is limited to the drugs derived directly from the vegetable and animal kingdoms.

Importance of Pharmacognosy.-An accurate knowledge of this branch is necessary to the pharmacist to insure the quality of his wares. It is much less important to the physician. However, it is useful to know the appearance, odor, taste, solubility, and perhaps the origin, at least of the more important drugs.

Vegetable Drugs.-The crude organic drugs are derived mainly from plants, either wild or cultivated; collected at the proper season to insure the maximum activity; and properly dried or otherwise prepared.

Parts Used.-The active principles are often diffused throughout the plant; but they are generally more abundant in one particular part, which is then used. The parts are designated by their usual botanic names. The following are the most important, with some characteristic examples.

Root (Radix).-The underground part of the plant, generally devoid of chlorophyl (green coloring matter), and which does not produce leaves (Dandelion, Belladonna, Ipecac, Rhubarb).

Tuberous Roots.-Roots swollen with accumulation of reserve food material (Sweet Potato, Aconite, Jalap). They are often called tubers; but true tubers (Potato) are similar formations on rhizomes.

Corm.-A thickened underground stem (Colchicum).

Bulb.-Very fleshy, closely crowded leaves, enfolding the underground base of the stem (Onion, Squills).

Rhizome.-Underground stems which bear leaves (Podophyllum, Hydrastis, Ginger, Aspidium).

Wood (Ligmum).-The wood of trees, in fragments, chips or coarse powder (Quassia, Hematoxylon, Sandalwood).

Bark (Cortex). - The outer layer of the stem (Cinchona, Cascara, Wild Cherry), or of the root (Sassafras, Cotton-root). Sometimes only the inner bark is collected (Ceylon Cinnamon, Elm).

Leaves (Folia) -Digitalis, Uva Ursi, Belladonna, Senna.

Herb (Herba or Species).-The smaller leafy and flowering or fruiting stems (Peppermint, Cannabis Indica, Savin). 
Flowers (Flores).-Chamomile, Arnica. Unexpanded flowers are: Cloves, Santonica.

Fruit (Fructus).- The ripened ovary, with any persistent parts of the Hower, and containing the seeds (Pepper, Cardamom, Hops, Colocynth, Anise).

Seed (Scmen).-The essential part of the fruit (Nux Vomica, Strophanthus, Phisostigma, Mustard, Linseed).

Microscopic Structure of Plants.-This differs from the animal cells, especially by the thick cell walls, and by the frequent presence of protoplasmic enclosures, starch, fats, calcium oxalate, protein crystals, etc. The cell walls are chiefly responsible for the shape and physical properties of the cell. 'They consist primarily of cellulose, variously modified by certain allied substances (lignin in wood, suberin in cork). These are all insoluble in ordinary solvents and in the alimentary canal of man. They are therefore so much useless "ballast" in the administration of the drugs. The processes of pharmacy consist largely in isolating the active constituents from this useless material.

Pharmacognostic Importance of Microscopic Structure.-The appearance, size and arrangement of the cells and their inclosures (starch grains, etc.) is of ten the most important aid in identifying a drug, particularly when in the form of powder. The pharmacopeias therefore include microscopic descriptions, whenever these are important.

\section{IMPORTANT PLANT CONSTITUENTS}

Elementary and Proximate Constituents.- The elementary or ultimate, constituents of plants, as of animals, are mainly $\mathrm{C}, \mathrm{H}, \mathrm{O}$, and $\mathrm{N}$. The chemical compounds formed from these are called the froximate principles or constituents of the plant. These belong to the chemical groups of proteins, fats, carbohydrates, tannins, resins, alkaloids, glucosids, acids, terpenes, etc. Plants also contain inorganic salts.

Cellulose.-This is found in the cell walls. It exists in almost pure form in cotton. It is chemically an isomer of starch, having the elementary formula $\left(\mathrm{C}_{6} \mathrm{H}_{10} \mathrm{O}_{5}\right)_{n}$. It is insoluble in all the ordinary solvents, and is not affected by boiling water. It dissolves without change in Schweitzer's reagent (ammoniated solution of copper sulphate). In older cells it is often modified by the introduction of allied molecules: wood (lignin) or cork (suberin). Cork is very resistant to reagents and impermeable to water, and hence protects the plant against evaporation and chemic injury. The cellulose may also undergo a retrograde metamorphosis into gum or pectin.

Starch $\left(\mathrm{C}_{6} \mathrm{H}_{10} \mathrm{O}_{5}\right)_{n}$. - This is produced as one of the first stages in the assimilation of $\mathrm{CO}_{2}$. It occurs in the form of granules, usually showing a laminated structure around a center (hilus). The character of this lamination, as well as the average shape and size of the granules, are important in distinguishing between different plants.

Starch can be easily recognized by the blue color which it gives with iodin. It is insoluble in all the ordinary solvents, but with boiling water swells and forms a peculiar colloidal solution (paste).

Gums.-These are colloidal carbohydrates, swelling or dissolving in water to viscous adhesive fluids (mucilages or pastes). They are precipitated by alcohol. They are formed in the plant by the transformation of cellulose and cell contents, especially as the result of pathologic processes. Pectins are closely related substances, occurring in fruits, and 
causing the boiled fruits to set into jelly. Gums and pectins do not color with iodin; they reduce copper only after inversion.

Sugar.-The various forms of sugars have a wide distribution in the vegetable kingdom. Some reduce copper in alkaline solution; others do so only after inversion. All turn the plane of polarized light. They are soluble in water; much less so in alcohol. The most important types are:

Glucose (Dextrose and Levulose), $\mathrm{C}_{6} \mathrm{H}_{12} \mathrm{O}_{6} ;$. Maltose and Saccharose, $\mathrm{C}_{12} \mathrm{H}_{22} \mathrm{O}_{11} ;$ Mannite $\mathrm{C}_{6} \mathrm{H}_{14} \mathrm{O}_{6}$.

Glucosids. - These are ester-like combinations of sugars with various other substances, frequently with phenols. Acids or appropriate ferments hydrolyze them into sugar and the other substance. Many do not contain nitrogen. Most glucosids are neutral or weakly acid; a few are alkaloidal. They are widely distributed in plants, and include active principles such as the digitalis constituents, saponins, tannins, etc.

Saponins.-These are neutral, non-nitrogenous substances distinguished by foaming with waters, emulsifying oils, and laking red blood corpuscles. Many have the formula $\mathrm{C}_{\mathrm{n}} \mathrm{H}_{2 \mathrm{n}-8} \mathrm{O}_{10}$. Some are glucosids. Those which are markedly toxic are sometimes called sapotoxins. The saponins are also of wide occurrence.

Tannins.-This term is applied to a group of phenol derivatives, distinguished by giving a bluish or greenish color with ferric salts. The greater number also form insoluble compounds with other metallic salts, with alkaloids, proteins, etc. This precipitation leads to an astringent action.

Tannins occur in many plants, especially in the leaves and bark, and in pathologic formations (nut-galls). They are non-nitrogenous; some are glucosids. They are soluble in water and in alcohol; but since they form insoluble compounds with so many substances, they often occur in plants in granular form.

The tannins have been classified by their chemic composition. More convenient is the older classification into:

1. Physiologic Tannins.-Occurring in normal plant tissues; giving a green color with iron; yielding pyrocatechin on dry distillation. Most of these tannins form with connective tissue an extremely insoluble and impermeable compound, and are therefore used in tanning.

2. Pathologic Tannins.-Occurring in pathologic tissues (galls); giving a blue color with iron (changed to green by acid); yielding pyrogallol on dry distillation; unsuited for tanning.

Phlobaphens.-Tannins are easily decomposed into resin-like substances called phlobaphens. These exist naturally in plants, but are usually formed as artificial decomposition products in all extracts. They are dark-colored, soluble in alcohol and in alkaline liquids, insoluble in water.

Tannins and phlobaphens, as well as most other plant constituents, are easily converted into a group of substances called humins. These do not exist in living tissues, and arise on the death of the cells, by the action of air and moisture. They cause the brown color which plants assume on drying; they are also,present in the brown bark.

Tannins, phlobaphens, and humins form a series, without sharp demarcation. They are not subject to the action of bacteria, and in this way protect plants against putrefaction.

Alkaloids.-These comprise many of the most active and important plant constituents. They may be defined as natural nitrogenous organic bases (generally tertiary amins); i.e., they are organic substances, containing nitrogen, of basic character, combining with acids without the elimination of hydrogen, forming well-defined and usually crystalline salts. The 
salts with halogens are called hydrochlorids, hydrobromids, etc. (not chlorids, etc.).

"The" names of alkaloids are often spelled with a final $e$, to distinguish them from neutral principals (thus morphine, an alkaloid; salicin, a glucosid). This distinction is rather arbitrary, and is not followed here.

Discovery of the Alkaloids. - This is an achievement of the roth century (Morphin, W. Serluerner, ISo5 to 1817 ; Strychnin, ISI8; Quinin and Caffein, I820; Nicotin, r829; Atropin, 1833$)$.

Occurrence-Only a few alkaloids occur in the animal kingdom, the most important crample being epinephrin, the active principle of the suprarenal gland. Alkaloidal principles are also formed by the action of bacteria, and are called ptomains. Amongst the higher plants the occurrence of alkaloids is very common, the same plant containing usually several alkaloids, which are often formed from one another. They are often found in all parts of the plants, but sometimes they are strictly localized in certain portions. Amongst seeds, e.g., in aconite, in the central part; in physostigma in the cotyledons; in datura, hyoscyamus and atropa, in the layer beneath the epidermis; in nux vomica, both strychnin and brucin occur in the endosperm, brucin alone in the embryo.

The alkaloidal content of plants varies considerably according to the climate, culture and other conditions. Individual plants also show considerable variations (Sievers, 1913). They can also be improved by selective breeding, although this is technically rather dificult (Sievers, 1916).

Some alkaloids contain oxygen, others do not. Those containing oxygen are solid and comparatively non-volatile, whereas those free from oxygen (nicotin and coniin) are liquid and volatile.

Properties of Alkaloids.-All alkaloids have certain properties in common: bitter taste, alkalinity to litmus, nitrogen reaction; generally profound physiologic action, without postmortem lesions.

Solubility Characters.-Free alkaloids are soluble in ether, chloroform, and oils, much less soluble in alcohol, and comparatively insoluble in water. Alkaloidal salts, on the other hand, have just the opposite solubility: They are soluble in water and alcohol, insoluble in chloroform and ether. With the alkaloidal salts, the combined acid plays a prominent rôle in the solubility.

Precipitation Reactions.-Alkaloidal salts are precipitated by compound solution of iodin, mercuric-potassic iodid (Mayer's reagent); phosphomolybdic acid; picric acid; gold chlorid, platinic chlorid, etc. Many also precipitate mercuric chlorid, potassium bichromate, tannin, etc.

Color Reactions.-Many alkaloids give characteristic colors with concentrated acids, with or without the addition of oxidizing or reducing agents.

Chemic Constitution.-The structure of many alkaloids is well understood; others are still obscure. The greater number are fairly complex derivatives of pyridin (coniin, nicotin); pyrrolidin (cocain, atropin); quinolin (quinin, cinchonin, strychnin, brucin); iso-quinolin (hydrastin, narcotin, cotarnin, berberin); morpholin or phenanthren (morphin, codein, thebain).

Neutral or Bitter Principles. - This class comprises a heterogeneous collection of substances, which have not been assigned to other classes. The principles usually have a bitter or sharp taste, and are usually crystalline and non-nitrogenous. A number are lactones, i.e., derived from alcohol-acids, by the elimination of $\mathrm{H}_{2} \mathrm{O}$.

Resins.-These are also a heterogeneous collection; including solid, non-volatile, amorphous substances of obscure composition, generally nonnitrogenous. The distinguishing character is their insolubility in water, and solubility in alcohol, most fat solvents (except petroleum ether) and 
strong alkalies. The resins are contained in special vessels, from which they are usually obtained as exudations after incising the plant.

Mixed Resins.-When the resins occur mixed with essential oils, they are natural oleoresins; when mixed with gums, gum-resins. If they contain aromatic acids (cinnamomic, benzoic, etc., or essential oils), they are called balsams.

Resinoids.-These are artificially isolated principles soluble in alcohol and insoluble in water. They are generally mixtures, often containing true resins.

Volatile Oils (Essential Oils).--These are odorous principles, of the physical characters of fatty oils, from which they differ by being volatile and soluble in alcohol. They are responsible for the odor of plants. Chemically, they are mixtures of esters, aldehyds, alcohols, ketones, and especially the terpens (Kremers, $\mathrm{r} 898$ ).

The constituents may separate on cooling, the liquid part being called eleopien, the solid stearopten. Camphors are analogous substances, but solid at ordinary temperature. Rubber (caoutchouc and gutta-percha) is also related to the terpens.

Plants also contain many other alcohols, aldehyds, organic acids, aromatic derivatives, etc.

Fats and Fixed Oils.-Typical fats (esters of fatty acids and glycerin) are abundant especially in seeds, occurring within the cells as drops or crystals. The fats are greasy liquids or soft solids; when heated, they undergo decomposition, denoted by acrid acrolein vapors. They are insoluble in water or glycerin, sparingly soluble in alcohol, and freely soluble in ether, chloroform, benzin (petroleum ether), carbon disulphid, turpentine, etc. (fat solvents).

Waxes.-These are more solid substances with the solubility characters of fats. Chemically, they are esters of fatty acids with alcohols other than glycerin.

Proteins.-These are, of course, represented in all vegetable cells, but are especially abundant in seeds, sometimes as crystals (aleuron). Several classes of the proteins are peculiar to plants.

Chlorophyl.- This, the green pigment of plants, is insoluble in water, soluble in alcohol and fat solvents. It is allied to blood pigment, but contains no iron. It is readily changed and discolored by reagents.

The chlorophyl occurs in the cells as granules (chloroplasts), made up of a colorless, spongy, protein groundwork, containing in its meshes the chlorophyl pigment. The latter consists really of a mixture of green and yellow colors (chlorophyl and xanthophyl). These chlorophyl granules are found mainly in the higher plants, and serve in the presence of light to assimilate $\mathrm{CO}_{2}$, and consequently to form starch, etc. During the process of drying, especially if this occurs slowly, the pigment is acted on by acids, etc., developed under these conditions, and undergoes various changes, usually becoming brown.

Other Pigments.-Plants are rich in colors, belonging to various chemic groups, often unidentified. Many alter with changes of reaction. The brown coloring matter of barks, etc., is allied to the tannins and phlobaphens.

Enzymes.-Different plants contain almost every imaginable kind of ferment, a few being used in medicine.

Extractive Matter.-This term is applied to the mixture of unidentified organic constituents; especially to the colloidal smeary mass which remains after evaporating any extract from which the important constituents have been removed. 
Mineral Salts.- These are fairly abundant in plants, constituting the ash which remains on incineration. Ca and $\mathrm{K}$ are especially important.

These salts seem to be combined largely with the protoplasm, and exist partly "lisidicel, party as crystals. (irowing tissues are always richer in salts than those fully developed.

\section{PHARMACEUTIC STANDARDS}

Objects.-Pharmacy deals with the preparation and compounding of drugs for the purpose of administration. It has for its objects the separation of the active principles of drugs, their combination, and the putting of them in a pleasant form.

The Need of Pharmacy.-The necessity for such an art will be readily understood. Drugs may be divided according to their origin into mineral, vegetable, and animal drugs. The last two are often too bulky to be conveniently used, and the substances which determine their action are often in such a condition that they can not readily be separated in the body, and so can not develop their action. Often, one drug alone does not meet all the indications in a disease, and when several are given it is necessary to combine them in such a way that they may not interfere with one another, either chemically or mechanically. Lastly, having chosen and prepared the drugs in a proper manner, and having decided how to combine them, it is highly desirable to give them in such a form as will be least objectionable to the taste, smell, or sight of the patient.

Official Drugs.-A certain degree of uniformity in the strength and preparation of pharmaceutic products is absolutely indispensable. Accordingly, practically all civilized countries have standards established by law, to which drugs and preparations in the shops must conform. The book in which these standards are published is usually called the Pharmacopeia. Preparations listed in these official books are termed "official." A Pharmacopeia, therefore, is an official book of standards for official drugs.

International Uniformity.-The first effective step in this direction was taken by the Brussels Conference of I9o6, the "International Conference for the Unification of the Formulas for Potent Medicines." This conference drew up a list of the potent drugs for which uniformity was most important, and established standards of 'strength for them and their preparations. These have been adopted essentially by most of the civilized nations. These formulas of the Conference are designated as "I.A." (International Agreement) by the B.P.; as "P.I." (Protocol International) by the U.S.P.

Scope of the Pharmacopeias.-These usually aim to include only "standard drugs," i.e., those of established value, including some, however, which have little to recommend them except extensive use. Proprietary drugs are generally excluded, on the plea that it might be difficult to inforce their compliance with the official standard. There is also a healthy tendency to restrict the admission of complex mixtures.

Galenic Preparations.- "Galenicals" are, strictly speaking, medicines prepared after the formulas of Galen. The term is now used to designate standard preparations containing one or several organic ingredients, as contrasted with pure chemic substances.

Proprietary Drugs.-Drugs which are protected by a monopolyby patents, trade marks, secrecy, etc.-are generally not admitted into the pharmacopeias, and are therefore non-official. They are thus not subject to legal control, and are often advertised under extravagant, misleading and false claims. However, some of the most valuable drugs are proprietary, and many are marketed in a strictly ethical manner. 
Patent Medicines.-This term is used to cover proprietary drugs that are advertised, directly or indirectly, to the laity. They are not usually patented, as the name would imply; most manufacturers preferring to rely upon secrecy to impose on the public. The medical profession is generally opposed to patent medicines as a class, and to lay advertising in particular. "The impossibility of controlling the irresponsible claims which are usually made in advertisements to the public, the well-known dangers of suggesting by descriptions of symptoms to the minds of the people that they are suffering from the many diseases described, the dangers of the unconscious and innocent formation of a drug habit, and the evils of harmful self-medication, including the dangers of the spread of many infectious and contagious diseases when hidden from the physician, and similar well-known considerations, are the reasons for discouraging, in the interest, and for the safety, of the public, this reprehensible form of exploitation" (N.N.R.).

The United States Pharmacopeia.-The U.S.P. IX (ninth revision) was issued in September, rgr6. It furnishes mainly the standard definitions, tests and formulas. It is a legal standard.

The U.S.P. was first published in 1820 , and is revised every ten years, by a Pharmacopeial Revision Committee, appointed by the United States Pharmacopeial Convention, which consists of delegates from pharmaceutical and medical organizations. The current committee of revision consists of 50 members, mainly pharmacists, and is divided into 15 subcommittees, and headed by an executive committee.

The British Pharmacopeia.-The B.P., fifth edition, was issued in 1914. It resembles the U.S.P. in scope and arrangement.

The B.P. is published by the General Medical Council, and revised at irregular intervals.

National Formulary (N.F.).-This is a collection of formulas for less important preparations, issued by the American Pharmaceutical Association. It is also a legal standard.

New and Non-official Remedies (N.N.R.).-This contains descriptions of the characters, actions and uses of those proprietary drugs that are marketed in a proper manner. It is issued by the American Medical Association and revised annually by its Council on Pharmacy and Chemistry.

Dispensatories.-These are commentaries which enlarge and explain the official texts, and generally also describe unofficial preparations. The National Dispensatory and the U.S. Dispensatory are principally used in the U.S. The British Pharmaceutical Codex (B.P.C.), and Hager's Praxis are similar works.

Useful Drugs.-This is a small manual issued by the American Medical Association, which contains only the most useful drugs with such information as is of especial interest to physicians.

Physicians' Epitome of U.S.P. and N.F.-This is another A.M.A. publication, giving a brief but critical abstract of all U.S.P. and N.F. preparations.

\section{PHARMACEUTIC METHODS}

IN the making of pharmaceutic products very different methods must be used, depending upon the physical and chemic nature of the crude drug, and upon the character of the desired product.

These may be roughly classified into those used in the making of many different preparations-general methods-and those used in only a very limited number of cases-special methods.

The methods can be best understood when studied in the order in which they are usually applied to the drug. 


\section{PREPARATORY PROCESSES}

Desiccation or Drying. - This is usually the first operation to which the crucle drugs are subjected after their collection. It serves a threefold purpuse: It reduces the bulk, assists preservation, and facilitates comminution.

Formerly the drying was done by spreading or hanging the drugs in airy lofts. At present they are usually placed on perforated trays in special drying closets and heated artificially (steam, etc.). They are often cut into smaller pieces before this drying. The clegree of heat must not be so high as to injure the sometimes very unstable ingredients. The C.S.I". designates 32 to $39^{\circ} \mathrm{C}$. as "gentle heat."

Comminution.- The next step is comminution, or reducing of the substance to smaller pieces.

This is usually done by machinery. Crude vegetable drugs are first sliced or chopped, often before drying. 'They are then bruised by pounding in a mortar and finally ground, the finer grades of powders often several times, the grinding surfaces being brought closer together each time. The mills for this purpose are constructed on the same general principles as flouring mills, employing stones, rollers, etc. The details of the process used depend upon the physical character of the drug. A fibrous material like licorice root requires a different process from a friable substance like gum acacia.

On the small scale, drug mills, constructed more or less on the principle of the coffeemill, are used for fibrous, and mortar and pestle for friable drugs. Mortars are made of iron, wedgewood, porcelain, and glass.

Pulverization is comminution to a powder.

Trituration is the process of rubbing (not pounding) a substance to a powder in a mortar.

Some points deserve special mention. Often a substance will not powder by itself, but will do so when mixed with another substancee.g., sugar of milk. This is called "pulverization by intervention." Sometimes it is well to moisten the drug-e.g., camphor with alcohol, nux vomica with steam, etc.

Fineness of Powder.-In the process of percolation, presently to be described, it is often essential to use a powder of a certain degree of fineness. The powders are therefore sifted, and are classified according to the size of the meshes of the sieve through which they pass, thus:

No. $80=80$ meshes to linear inch, very fine.

No. $60=60$ meshes to linear inch, fine.

No. $50=$ moderately fine.

No. $40=$ moderately coarse.

No. $20=$ coarse.

Since the different structures in a crude drug do not powder with equal readiness, it is essential that the whole of the drug to be powdered should be passed through the sieve, else the different portions will not have the same composition.

Levigation.-This is employed to obtain very fine powders of insoluble substances, by making them into a thick paste with water, and rubbing this between polished slabs.

Elutriation is used to separate fine insoluble powders by suspending them in water and decanting.

\section{PROCESSES OF SEPARATION}

For the separation of the desired ingredients from the inert material three methods are in vogue, depending upon the nature of the active constituents. If volatile constituents are to be separated, this may be readily done by the application of heat-distillation and sublimation. If they 
are not volatile, the separation is usually effected by exposing the drug to the action of some solvent in which the desired principles are soluble, and the rest, as far as may be, are insoluble. In certain cases some mechanical means are sufficient, as in the separation of fixed oils from seeds, etc., by pressure.

Separation by Means of Heat.-This may be done whenever the substances to be separated have a different boiling-point, and are not themselves destroyed by the necessary degree of heat. The methods used must vary according to whether the fixed or the volatile portion is desired, and, if the latter, according to whether it is liquid or solid.

Desiccation, Torrefaction, Carbonization, Ignition.-With all these, the object is to drive off some volatile constituent from a solid, the fixed residue being the portion desired.

When the heat employed is of such degree as not to change the chemic composition, the process is spoken of as desiccation.

Torrefaction.-The process of roasting; the object being to employ such a degree of heat as will alter some of the constituents without affecting others. The roasting of coffee is a familiar example.

Carbonization.- The heating of organic substances under exclusion of air. The object is to destroy the chemic composition without oxidation; carbon results in the process (vegetable or animal charcoal).

Ignition.-This is the process of heating a substance strongly, usually in a crucible, with full access of air, so as to effect complete oxidation; nothing but the ashes remain.

Evaporation consists in vaporizing the solvent from a solution, the object being the concentration of the dissolved substance.

Since the rapidity of the evaporation, aside from the quantity of heat applied, depends upon the extent of the liquid exposed to the air and to the heat, dishes as flat as possible are chosen. For ordinary pharmaceutic and chemic purposes, those made of porcelain are of most frequent service. Vessels made of glass, iron, platinum, etc., find application in special cases. The heat may be applied directly, say by means of a Bunsen flame, only a piece of wire gauze or a plate of asbestos or iron being interposed. This method can be used only when there is no danger of injuring the solution by excessive heat, either the substance being incapable of change, or the solvent sufficient in amount so that the temperature can not rise much beyond its boiling-point. If this is not the case, some method must be used of regulating the amount of heat applied, and this is done by applying the heat indirectly through a bath. This consists of an outer vessel filled with water (steam), oil, sand, or air.

The rapidity of evaporation may be considerably increased by stirring, thus exposing a constantly renewed surface to the air.

In cases where the evaporation must be carried on at a temperature below the boilingpoint of the solvent, this may be done either by evaporation over $\mathrm{H}_{2} \mathrm{SO}_{4}$, or in a vacuum, or by passing a current of dried air through the liquid.

The evaporation which occurs from the surface of a liquid exposed to air at ordinary temperature is called "surface evaporation." It varies in quantity with the amount of surface exposed and with the temperature and dryness of the air.

When very inflammable liquids (ether) are being evaporated, this should be done on a large water-bath, and the Bunsen flame should be protected by wire gauze.

Sublimation.-The process of separating a volatile from a non-volatile solid by heat.

This may be done in a distilling apparatus, provided that the cooling tube has sufficient lumen to prevent its clogging by the condensation of the sublimate. The apparatus is, however, usually modified. A simple illustration of this process is the old method of manufacturing benzoic acid from gum benzoin, a paper hood being used as condenser.

Distillation.- The process of separating a volatile liquid from a less volatile liquid or solid. (The difference between sublimation and distilla- 
tion consists in this, that the product is solid in the former, liquid in the latter.

Nhthugh distillation is such a ready and simple means of separation that one would think it must have been discovered at a very early time, such does not appear to have been the case. We tind the first record of it in the writings of the fourth century alchemists.

The typical apparatus used for distillation consists of three parts.

The still (retort or flask), the vessel in which the vapor is generated.

The condenser (straight or spiral), the apparatus in which the vapor is cooled.

The receiver, for receiving the condensed product.

The alembic is the old-time still, and differs from the retort in having a chamber (helm or hood) where the vapor is partly condensed. By fitting the helm on the body with a flange joint a very wide opening can be secured, which is of use in cleaning.

Fractional distillation is the process of separating a mixture of liquids of different boiling-points by distillation.

Destructive distillation is the name applied to the process of heating a substance so strongly as to decompose it, and collecting the volatile products arising from this decomposition: i.e., in the case of organic bodies, tar.

Solution.- This consists of incorporating a solid into a liquid in a state of "molecular subdivision."

That is, the molecules of the solid diffuse themselves in the liquid and become so widely separated that no solid particles are by any means discernible. In other words, the solid is liquefied and its molecules intermingle with those of the solvent.

A simple solution is one occurring in the manner described, the change in the solid being physical. When a chemic change takes place, the process is called chemic solution (such as the solution of a metal in an acid).

$\Lambda$ solvent is capable, under given conditions, of dissolving but a limited amount of a given solid. A solution which contains as much of the solid as the liquid can dissolve under these conditions is called a saturated solution. The condition which has the greatest influence upon solubility is the temperature. A liquid can usually dissolve the more of the solid, the higher the temperature. There are, however, a few exceptions to this rule.

If a solution saturated at a high temperature is allowed to cool, the originally dissolved substance will be in excess of saturation. Under certain conditions it may still remain in solution at the lower temperature, this being a supersaturated solution. Ordinarily, however, the excess will separate, usually in crystalline form. This process is called crystallization. It is frequently used as a means of purification.

A solution which contains less of the solid than it is capable of dissolving is an unsaturated solution. A solution which is saturated with one substance is still capable of dissolving others, though not as much as if it were the pure solvent.

Solution is effected by placing the solvent in contact with the substance to be dissolved. The process may be hastened by applying heat, or by exposing the largest possible surface to the action of the solvent. The latter may be done by using the substance in a pulverized condition, and by constant stirring. With circulatory solution the substance is suspended near the surface of the solvent. As this takes up the substance, it gains in specific gravity, and hence sinks to the bottom, a new portion of liquid taking its place. The same object may be secured by a process analogous to percolation, the powder being placed in a funnel partly occluded by a pledget of cotton, etc., and the solvent allowed to percolate through it.

The simple solution of a substance always causes a depression of temperature. But if a chemic change occurs, the temperature may be raised.

The process of solution applied to crude drugs has for its purpose the separation of the active ingredients from the insoluble inert material. The object is to dissolve out the greatest possible amount with the least possible menstruum. This accomplishes two results: We obtain a strong extract, and we waste neither drug nor menstruum. There are a 
number of methods of accomplishing this, each with its advocates. They are combinations of two extremes: maceration and percolation. Neither of these is commonly used alone in this country, the practice being to combine the two.

Maceration is by far the simpler process. It consists in simply leaving the solvent in contact with the drug under suitable conditions for a sufficient length of time.

When maceration alone is employed, a given quantity of the drug is put in a bottle or other suitable vessel with a definite proportion of the solvent (called menstruum) and left a certain time, usually a week or two. The liquid is then strained off, the residue (marc) is expressed and the mixed extract filtered. The details of the process are influenced by the fineness of the powder, the time of maceration, and the temperature.

Different names are given to the process according to the temperature at which if is carried out. Maceration proper $=$ room temperature; $30^{\circ}$ to $40^{\circ} \mathrm{C}$. = digestion; boiling $=$ decoction. Possible injury to some constituents by heat, or evaporation of a constituent or of the solvent, are objections to the application of heat in certain cases.

This process of maceration is the one almost exclusively employed in Europe; and it offers certain advantages, not the least being its simplicity and the constant results which it gives. Its main disadvantages are the required time and the loss of the extract retained in the insoluble residue or "marc." Certain drugs are physically unfit for percolation, since the moistening causes them to form into a tough mass, as good as impenetrable to the solvent.

The loss of menstruum does not, of course, weigh when an aqueous solvent is used and only small quantities are prepared. Hence maceration is used in making infusions and decoctions.

Percolation consists in passing a solvent through a thick layer of the powder to be exhausted. This exposes a large surface of the latter; the nearly saturated solvent flows off and fresh unsaturated portions continuously replace it, insuring very rapid solution.

The principle of the method is to pack the powder into a tall vessel, with an opening at the bottom, and to let the solvent trickle through it. Usually the process is combined with a short previous maceration.

The U. S. Pharmacopeia defines percolation as consisting in "subjecting a substance or a mixture of substances, in powder, contained in a vessel called a percolator, to the solvent action of successive portions of a certain menstruum, in such a manner that the liquid, as it traverses the powder in its descent to the receiver, shall be charged with the soluble portion of it, and pass from the percolator free from insoluble matter."

The details are as follows:

The powder (the fineness of which depends upon the nature of the drug and is directed for each case by the Pharmacopeia) is moistened in a jar with some of the menstruum. This moistening is for the purpose oi swelling the drug, for if this took place in the percolator, the drug would become so firmly impacted that the menstruum could not penetrate through it; or it could even burst the percolator. The moistened powder is then passed through a coarse sieve and transferred to the prepared percolator, which it should fill about two-thirds.

The choice of the shape of the percolator depends upon the nature of the drug. Should the drugs have a tendency to swell particularly if they are in fine powder or if weak alcohol menstruum is used, a conical percolator is employed; otherwise, the cylindrical. The size corresponds to the quantity of the powder.

Arrangement of Percolator. - The percolator is prepared in the following manner (Fig. I): Into the small end there is inserted a cork perforated by a short glass tube which projects about $\mathrm{r} \mathrm{cm}$. inside the percolator. The outer end of this tube is attached to a piece of rubber tubing, about one-fourth longer than the percolator, and this to a $\mathbf{U}$-shaped glass tube. This is held to the percolator by a rubber band in such a way that it can be raised and lowered. The percolator is then set in the stand. A pledget of absorbent cotton is loosely packed in the neck of the percolator and this is covered by a layer of clean sand, and over this goes a well-fitting disk of filter-paper. Then the moistened drug is pressed in-and this is the part of the process which requires the greatest skill and judgment, and on it depends the success of the product. It should be done very evenly, else the menstruum will choose the path of least resistance and some 
portions of the powder will be entirely exhausted whilst others are still scarcely affected. The firmness of the packing is also of great importance; if not firm enough, the menstrum will run through too rapilly and the percolate will consequently be weak. If too tirm it can not run at all; and if any swelling occurs the percolator will be broken. Drugs in coarse powder should be packed more firmly than fine powders. An alcoholic menstruum requires firmer packing than a watery one.

The packing being completed, the menstruum is poured on until it stands an inch or two abuve the drug; the percolator is then covered and set aside for maceration for a specified time, the tube being raised so that no liquid flows out. When the time

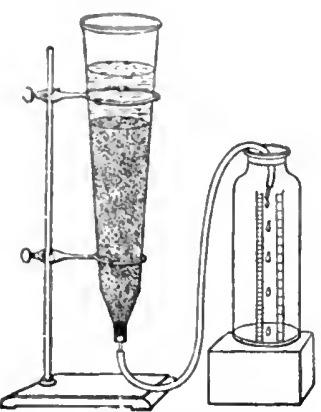

FIG. I.-Method of percolation (Thornton). of maceration is completed, the tube is lowered and fixed at such a level that the outflow occurs at the rate of 2 to I5 drops per minute. New menstruum is poured on in the measure that the old flows out. Care should be taken always to maintain the layer of liquid above the powder; else cracks may appear in the latter, necessitating repacking.

The process is continued, in the case of tinctures, until a certain volume of percolate is obtained. The quality of the percolate will, of course, depend upon the care and skill of the operator, and the product is apt to vary. Maceration would. therefore, be a better process for tinctures.

This difficulty is avoided in the case of extracts, for here the percolation is continued "until the drug is exhausted," i.e., until the active ingredients have become completely dissolved out. This is recognized by testing the last portions of the percolate in the appropriate manner, such as Mayer's reagent for alkaloids, water for resins, etc. It may here be remarked that a drug is usually more rapidly exhausted of its active ingredients than of its coloring-matter, so that the last portions of percolate may be colored and yet devoid of activity.

The choice of a menstruum must be determined by the nature of the constituents. The object is, to extract all the active ingredients and the minimum of inactive. Alkaloids and resins require strong alcohol; gums, weak alcohol; licorice, alkaline alcohol; sanguinaria and ergot, acidified alcohol; gentian and quassia, water plus alcohol enough to keep.

Pharmaceutic Solvents.-The most useful are the following:

Water or Glycerin.-Dissolve salts (including those of alkaloids), sugar, gums, tannin, acids, and alkalies, etc.

Dilute Acetic Acid.-Especially for alkaloids.

Alcohol.-Dissolves alkaloidal salts, neutral principles, resins, volatile oils. Precipitates gums and most inorganic salts.

Ether, Chloroform, Acetone.-Dissolve free alkaloids, neutral principles, resins, volatile and fixed oils, and fats.

Petroleum Benzin.--Solvent properties as the preceding, except resins. Aromatic Spirits of Ammonia.-Dissolves resins and organic acids.

In the case of very volatile menstrua, such as ether, chloroform, or petroleum ether, some means must be employed to collect and return the evaporated solvent. One of the best apparatus of this kind is the familiar Soxhlet extractor.

Expression.-The process of separating a liquid from a solid by pressure.

Its principal employment in pharmacy is for the recovery of tinctures from the "marc," i.e., the liquid retained by the drug residue after maceration and percolation. It is also a process of separating fixed oils.

The drug is put in a coarse strong cloth and subjected to pressure in a press. These are of various patterns; screw, lever, hydraulic, or centrifugal. The pressure must be applied gradually to prevent the bursting of the cloth. Small quantities can of ten be pressed sufficiently by putting them into a cloth and tightly twisting the end.

Straining or Colation.-The process of separating solid coarse particles from a liquid by pouring it through a cloth or strainer. 
Filtration.-The process of separating solid particles (fine or coarse) from a liquid by pouring it through a finely porous material, such as filter-paper.

The usual material for filtration is pure unsized paper, "filter-paper," which is made of various grades - white and gray - and of varying texture and thickness according to the purpose for which it is to be used.

There are two principal methods of folding a filter-plain and plaited. These two filters have different uses. The plaited filter offers a much larger surface for filtration and is therefore more rapid; it is the form usually employed in pharmacy. The plain filter, on the other hand, facilitates washing and removal of the precipitate, and is of more frequent use in chemistry.

Other materials are sometimes employed instead of paper. A very useful filter for large quantities of liquid is made from felt. A plug of glass-wool or asbestos placed in the tube of the funnel is especially useful for strong acids or alkalies. A cell of porous clay is also employed, as in the various forms of the Chamberland filter. With this a vacuum is indispensable.

Dialysis.-This is sometimes used to separate crystalloids (alkaloids, salts, etc.) from colloids (extractives).

Decolorization.-It is often desirable to remove the coloring-matter from a solution. This may sometimes be accomplished by choosing appropriate solvents. More often, however, the solution is filtered through recently calcined animal charcoal. This very often retains some of the active constituents as well as the coloring-matter.

Clarification.- The process of rendering turbid mixtures clear and transparent, by removing the suspended solid. When filtration does not suffice, the object is accomplished by agitating the mixture with insoluble powders (talcum, phosphate of lime, aluminum hydrate) or by adding egg-albumen and boiling; or sometimes by the centrifugal machine.

Sterilization.-The destruction of bacteria and their spores is accomplished by the various customary methods.

Glass and metal utensils may be sterilized by dry heat of 160 to $170^{\circ} \mathrm{C}$. for two hours; by superheated steam of 115 to $120^{\circ} \mathrm{C}$. for fifteen minutes; heating in a current of steam for thirty minutes or boiling for fifteen minutes, especially in 0.I per cent. sodium bicarbonate. Kills all non-spore-bearing organisms.

Solutions that are not readily decomposed by heat may be boiled in a current of steam for one-half or better one hour.

Solutions that are readily decomposed by heat are either prepared aseptically or filtered through porcelain.

Emulsions in glycerin or oil are prepared aseptically, the solvent being first sterilized by heat.

\section{PHARMACEUTIC ASSAYING}

Different samples of vegetable drugs may vary widely in the quantity of active constituents which they contain. These variations are most undesirable from a therapeutic standpoint, especially when they occur in potent, so-called "heroic" drugs. Inorganic drugs or other definite chemic compounds are not subject to this variation; but in many cases the removal of the last traces of innocuous impurities would greatly increase their cost without adding to their therapeutic usefulness. The pharmacopeia has therefore aimed to furnish quantitative methods of estimation, assays, wherever possible, and to establish practical standards to which medicinal substances must conform. With inorganic drugs, it states the largest permissible quantity of innocuous impurities (usually less than 2 per cent.). With crude vegetable drugs, it states the lowest permissible percentage of active ingredient, wherever practical methods for their determination are available. Assayed galenic preparations are directed to be diluted so as to contain a definite proportion of active constituents, corresponding to the minimum permitted in the crude drug. These preparations should therefore be preferred to the crude drug whenever accurate dosage is desired.

The assay of inorganic drugs involves the well-known methods of ordinary quantitative analysis, volumetric processes being preferred. Special tests are furnished for determining the permissible limits of accidental impurities, and the presence of harmful substances, such as metals. 
1 series of volatile oils are atssayed for their most important ingredient. Fats and fixed oils are tested for their "iodin absorption" and "saponification values"" resins for the "acid number" some vegretable products for their ash. These tests serve mainly for identification and the exclusion of willful adlulterations.

Pepsin is atssityed for its digestive power for proteins, pancreatin for starch. In rosinous drugs, such als Jalip, Scammony, or (Guaiac, the proportion of ether-or alcoholsoluble matter is determined.

The must important class of assay's refers to drugs containing alkaloidal principles, utilizing the mothor of immiscible solvents, as in toxicologic analysis. This rests on the fitct thitt free alkaloils are generally soluble in chloroform or ether, whilst their salts are insoluble in these, but soluble in water. In principle, alkaloids are extracted by chloroform or cther, or a mixture of both, in alkaline reaction. This solution is then shaken in a separator with acidulated water, which converts the alkaloids into salts and dissolves them, leaving the impurities behind. For further purification, the watery solution is again rendered alkaline and extracted with chloroform or ether. This, on evaporation, leaves the fairly pure alkaloid which may be weighed; or titrated, by dissolving it in a known amount of acid, and titrating back with alkali. Each cubic centimeter of acid corresponds to a definite quantity of each alkaloid. It is important to observe the directions minutely to obtain reliable results. The process for extracts and fluidextracts difiers merely in details (the percolation being omitted, etc.). Tinctures are first concentrated by evaporation.

This method estimates the sum of all the alkaloids present in the drug, and suffices when the important one predominates very largely over the others. When this is not the case, it is necessary to confine the assay to the particular alkaloid desired, generally a very difficult matter. In Hydrastis, advantage is taken of the comparatively greater solubility of hydrastin in ether; in Opium, of the comparative insolubility of the morphin in this solvent. In Nux Vomica, the brucin is removed by oxidizing it with nitric acid.

Bio-4ssay.-Drugs which depend for their activity on neutral principles are unfortunately unsuitable for chemic assay; there being no reliable chemic method for estimating the constituents of Apocynum, Cannabis Indica, Convallaria, Digitalis, Ergot, Scilla, etc. Attempts have been made to supply this deficiency by physiologic standardization-estimating the strength of a preparation by comparing its effects on animals with those of a standard product. A just fatal dose is generally the best criterion for quantitative work. Individual differences of susceptibility and absorption in different animals can be largely eliminated by using an extensive series of animals. The expense and labor is scarcely warranted by the results, except in a very few cases, for instance with Digitalis. Qualitative physiologic tests of drugs of very uncertain activity, such as Cannabis Indica or Ergot, are more simple. The U.S.P. describes the most important bio-assays, but leaves their employment optional with the manufacturer (except in the case of Cannabis).

\section{CLASSES OF PHARMACEUTIC PREPARATIONS}

The pharmaceuticals are grouped into certain definite classes, established by long usage, such as Waters, Spirits, Tinctures, Extracts, Pills, Plasters, Ointments, etc. The student should learn to distinguish clearly between these preparations, according to the definitions given below.

\section{WATERY SOLUTIONS}

These include the Waters, Liquors, Mucilages and Syrups. Their advantage lies in the fact that water is a cheap solvent of very wide applicability, itself devoid of any therapeutic property. In the case of substances which are insoluble in it, it can not of course be employed. The greatest drawback lies in the fact that watery solutions of organic substances tend to spoil rapidly by the development of bacteria and infusoria. Solutions of chemic substances are less subject to this change, as they do not furnish a pabulum, and are often themselves antiseptic.

Aquæ, Waters (singular, Aqua; abbreviated, Aq.).-Clear aqueous solutions of volatile substances. Two very dissimilar classes of preparations come under this heading: 
Aquæ Aromaticæ, Aromatic Waters, U.S.P. (Flavored Waters).Saturated solutions of volatile oils (Aqua Cinnamomi, etc.). These are alone included under Aquæ in the B.P. They are prepared either by distilling the plant or oil with water (B.P.); or by triturating the oil with an absorbent substance (talc, etc.) until it is finely subdivided, and then extracting this with water.

The quantity of active substance in them is just large enough to give them a pleasant flavor without imparting to them any noticeable therapeutic properties. The absence of alcohol makes them good solvents for salts, which are generally insoluble in this liquid. Hence their main use is as a pleasant vehicle (a vehicle being the substance which serves for the convevance of another substance).

The dose of flavoring waters is practically unlimited ( 15 c.c., 4 drams, U.S.P.); except Aqua Amygdelæ Amaræ, 4 c.c.

Waters, as a rule, do not keep well. Their keeping qualities may be improved by adding to them some of the oil of which they are solutions. This floats on the surface, and in this way excludes air and bacteria, and at the same time insures permanent saturation.

The second class of Waters (included under Liquores by the B.P.) are fairly strong solutions of gases (ammonia, etc.) prepared by passing the gas into water. Their doses are relatively small.

Liquores (Liquor, Liq.); Solutions.-Aqueous solutions of solid chemic salts or hydrates, made either by dissolving the pure salt directly in water by trituration or heat; or, more often, by chemic decomposition (simple and chemic solution). (The British Pharmacopeia includes solutions of gases under this heading.)

The advantage of the solutions consists mainly in the convenience of measuring the relatively large volumes of the solution against weighing the small quantity of salt. Water is chosen as the solvent because salts are but little soluble in alcohol, and because the therapeutic qualities of the latter are not desired.

Solutions, when used for special purposes, receive special names, thus: Injectiones (Hypodermicæ; Urethrales), Collyria (Eyewaters), Lotiones (Washes), Gargarismæ (Gargles), etc.

Injectiones Hypodermicæ (B.P.) (Inject. Hyp.).-These are watery solutions of active drugs, intended for subcutaneous administration, and of such strength as to be used in the dosage of 0.3 to 0.6 c.c., 5 to ro minims. They are sometimes preserved sterile by the addition of phenol or salicylic acid. Manufacturers also market a variety of unofficial hypodermic solutions in aseptic ampouls, i.e., small sealed glass vials containing a single dose of the sterilized solutions.

Mucilagines, (Mucilago, Muc.); Mucilages.-Aqueous solutions of gummy substances. They are used as vehicles and demulcents. Since gums are insoluble in alcohol, mucilages are incompatible with this substance. They should be recently prepared because they are very apt to mold.

Mucilages are made by either hot or cold process: the former being solution by heat, the latter by percolation. Heat should be used only when necessary (tragacanth), as it usually causes discoloration of the product.

Syrupi (Syrupus; Syr.); Syrups.-Dense saccharine solutions of medicinal substances. The syrup serves mainly as a vehicle and preservative. The dose of flavoring syrups is practically unlimited ( 5 C.C., 4 drams, U.S.P.). 
limple syrup is practically a saturated solution of cane-sugar, containing $85 \mathrm{Gm}$. in 100 c.c. L...P. (06.7 (im. of sugar in 100 Gm. of syrup, B.P.). Syrups are usually premat by tirst making an infusion, and dissolving in this the sugar, either by heat or ly perculation, according to whether or not the drug is injured by a high temperature. Iin pecial catsen modifications ma be introduced. Some syrups are prepared by mixing the duidentract and syrup. Honey is sometimes used instead of cane-sugar, making a clas of preparations called melliti. If acetic acid is added to this, we have oxymellita. Confections (Confertio; Conf.) are thick medicated jams.

They were formerly very perpular, but have now been almost abandoned. Electuaries were similar but somewhit thinner preparations.

Elixiria (Elivir; Elix.); Elixirs.-Sweetened aromatic alcoholic liquors, used as flavoring vehicles, similarly to the syrups. Their content in alcohol (about 25 per cent.) is apt to lead to abuses, which must be kept in mind.

The official Aromatic Elixir is flavored with orange peel, lemon, coriander and anise. This, mixed with licorice, constitutes Elixir Adjuvans.

Glycerites (Glycerilum, L.S.P.; Glycerinum, B.P.; Glyc.).-Solutions in glycerin. Glycerin is a good solvent for many substances. It keeps well, and is useful especially for external application on account of its adhesiveness. Glycerin is also less irritant than alcohol and devoid of the pharmacologic action of the latter agent.

\section{ALCOHOLIC SOLUTIONS}

Alcohol is a specific solvent of certain substances (volatile oils, alkaloids, resins). In prescribing, these should not be mixed with an aqueous solution. Alcohol is also a good preservative; but it has distinct therapeutic qualities, which may or may not be useful.

Spirits (Spiritus, genitive Spiritus; $S p$.).-Alcoholic solutions of volatile drugs. They are all fairly strong. They are prepared by simple or chemic solution or by distillation. Flavoring spirits contain ro per cent. of the respective volatile oils. Their dosage is 2 c.c., 30 minims, U.S.P. ( 0.3 to I.2 c.c., 5 to 20 minims, B.P.). Spirits other than flavoring vary in strength and dose.

\section{PREPARATIONS MADE BY EXTRACTION}

In these, only a part of the crude drug is dissolved. They comprise the aqueous Infusions and Decoctions; and the alcoholic Tinctures and Fluid and Solid Extracts.

Infusions (Infusum; Inf.).-Aqueous solutions of the soluble principles of vegetable drugs, obtained by brief maceration in hot or cold water.

Decoctions (Decoctum; Dec.).- Analogous preparations, in which the ingredients have been boiled with water for at least fifteen minutes.

Infusions and decoctions are especially useful when it is wished to extract some principle which is more soluble in water, or when the therapeutic effect of alcohol or the mechanical incompatibility of alcohol with salts is to be avoided. There are some inconveniences connected with their use: They take a long time to prepare. Like all watery solutions, they spoil quickly, and must, therefore, be made fresh. The decoction can only be used if there are no delicate constituents to be destroyed by boiling.

The solvent being so very cheap and having no action, it is usual to make decoctions considerably weaker than tinctures. In prescribing 
infusions or decoctions of potent drugs, the proportion should always be stated by the prescriber. When the strength is not stated, the pharmacist is supposed to use 5 per cent. of the drug. The dose of non-poisonous infusions and decoctions lies between $I_{5}$ and I 20 c.c. ( $1 / 2$ to 4 ounces).

Type Formulas (U.S.P.).-Infusa.-I,, 000 c.c. of boiling water are poured on 50 $\mathrm{Gm}$. of the coarsely comminuted drug, the vessel is covered tightly, and allowed to stand for half an hour in a warm place. It is then strained with expression, and enough water is poured through the strainer to make the infusion measure 1,000 c.c.

Decocta. $-50 \mathrm{Gm}$. of the coarsely comminuted drug and r,000 c.c. of cold water are boiled in a covered vessel for fifteen minutes, cooled to about $40^{\circ} \mathrm{C}$., strained and expressed, and cold water added through the strainer to make the decoction measure I,, 00 c.c.

Preservatives.-The amount of preservative which must be added to a watery solution to insure its keeping qualities must vary with its nature, and in the same direction as the amount of "extractive." The proportions generally necessary are: Alcohol, 20 to 25 per cent.; glycerin, ro per cent.; sugar, $66 \mathrm{Gm}$. to roo c.c. of finished product. Alcohol, 20 per cent. + glycerin, 5 per cent.

A bibliography of the deterioration of drugs and pharmaceutic products is given by Eberhardt and Eldred, I9I4.

Tinctures (Tinctura, Tr.).-Alcoholic or partly alcoholic solutions of the useful constituents of such drugs as are not wholly soluble in the menstruum.

Exceptions of this definition are tincture of iodin, tincture of chlorid of iron, and tincture of tolu, in which the solution is complete.

The drug-strength of the tinctures varies from 0.4 to 50 per cent.; but those containing potent drugs conform to an international standard of ro per cent.

The tinctures are in many respects the most useful therapeutic preparations: The dose is relatively large and uniform (about 2 to 4 c.c. for the non-toxic); the quantity of solvent is sufficiently large to keep the principles in solution; the use of heat in the preparation is avoided.

The greater number of tinctures are prepared by percolation; a few by maceration. The menstruum is alcohol or alcohol and water; acetic acid or glycerin are added in a few cases; aromatic spirit of ammonia is used in the ammoniated tinctures.

Type-process for Percolation Tinctures (U.S.P.).-The powdered drug is moistened with the menstruum, transferred to a percolator without pressing, and allowed to stand covered for six-hours. It is then packed firmly, and menstruum poured on to more than cover the powder. When the liquid begins to drop, the lower orifice of the percolator is closed, and the maceration prolonged for twenty-four hours. The percolation is then allowed to proceed slowly, gradually adding sufficient menstruum to the percolator until the percolate measures the required volume.

Type-process for Maceration Tinctures (U.S.P.). - The drug is macerated in a moderately warm place with the menstruum, using four-fifths of the final volume. This is continued, with frequent shaking, for at least three days. It is then filtered, the filter being washed with menstruum sufficient to make the required volume.

Detannated tinctures are tinctures from which the tannin has been removed (as by powdered skin). They do not precipitate with iron salts.

Ethereal tinciures are made with ethereal spirit, a mixture of 7 parts of ether and 3 parts of alcohol.

Green Tinctures.-Since some drugs are supposed to lose part of their activity by drying and keeping, a class of tinctures made from the freshly collected green drugs has been introduced under the name of Tincturæ Herbarum Recentium. The directions are to macerate $500 \mathrm{Gm}$. of the fresh herb with 1,000 c.c. of alcohol for fourteen days, to express and filter. These preparations are justly unpopular. They are of very inconstant strength, since the natural moisture of plants is variable. Again, they can be prepared only in the localities where the plants are native and where there often 
are no reliable facilities for their manufacture. These remarks apply equally to the Iutces (Succus; Succ.) of the British Pharmacopeia, made by using I part alcohol and 3 parts of the freshly expressed juice.

Wines ( for alcohol. They have a more pleasant taste, but inferior keeping qualities, and are now very little used. acid.

leclis (Acet.) are medicated vinegars, prepared by maceration with dilute acetic

Fluidextracts, U.S.P.; Liquid Extracts, B.P. (Fluidextractum; Fldext.-Extractum Liquidum; Ext. Liq.).-These are liquid alcoholic extracts, roo c.c. representing approximately roo $\mathrm{Gm}$. of the drug. The menstruum is in certain cases modified by the addition of glycerin, acetic acid, etc. They are the most concentrated fluid preparations, and were supposed to possess considerable advantages, but many are of doubtful value.

The simple ratio of drug-strength has no special advantage in therapeutics, and has led to serious abuses such as the practice of preparing tinctures, infusions, etc., by the dilution of the fluidextracts. This is highly reprehensible, since these dilutions often differ materially from the orthodox preparations.

Concentration may sometimes be desirable to reduce the bulk of the dose, but it is often secured at the cost of efficiency. The heat which must necessarily be used in their preparation is never beneficial. Precipitates are apt to form on standing, and while these are often inactive, they may contain the active principles. They are also much more subject to precipitation on mixture with other liquids, and the dose is usually so small that they require some such admixture.

Fluidextracts are usually prepared by percolation, the first four-fifths of the percolate being set aside, the remainder evaporated to the consistency of a soft extract, dissolved in the reserved portion, and menstruum added to make the required volume.

Type-processes for Fluidextracts.-The following are described by the U.S.P.:

Type 1. - The powdered drug is moistened and macerated with the menstruum as described under tinctures, the maceration being prolonged to forty-eight hours. The percolation is then started, and continued by the addition of menstruum, until 850 c.c. of percolate have been collected for $1,000 \mathrm{Gm}$. of drug. This is reserved and percolation continued, with additional menstruum, until the drug is exhausted. These later percolates are evaporated to a soft extract, below $60^{\circ} \mathrm{C}$., dissolved in the reserved portion, and diluted with menstruum to make I,00o c.c.

Type B.-This is employed when two successive menstrua are used, the first containing glycerin, acid, etc. In this case, the powdered drug is moistened and macerated with the first menstruum. The percolation is then started, and continued with the plain alcoholic menstruum. Otherwise, the process is identical with Type $\boldsymbol{A}$.

Type C.-Fractional or Divided Percolation. - This is used for drugs containing constituents that are volatile or injured by heat. It may also be used in place of Type $A$.

The drug is divided into three portions of $500 \mathrm{Gm}$., $300 \mathrm{Gm}$. and $200 \mathrm{Gm}$. The first portion is then moistened and macerated, as usual. The first 200 c.c. of percolate are reserved; and the percolation then continued, collecting five additional portions of 300 c.c. each.

The second $300 \mathrm{Gm}$. portion of drug is moistened with the first 300 c.c. percolate of the preceding percolation; and macerated and percolated, as usual. The first 300 c.c. of percolate are reserved; and the percolation continued with the successive portions of the percolate from the first drug, until exhausted. These weaker percolates are collected in 200 c.c. portions.

The third $200 \mathrm{Gm}$. portion of the drug is moistened with the first 200 c.c. percolate of the second drug, macerated and percolated with the successive weak portions of the percolate of the second drug, until 500 c.c. are obtained. This is mixed with the reserve portions of the two first drugs.

Type D.-In this, the drugs are exhausted with water and preserved by the subsequent addition of alcohol. I,000 $\mathrm{Gm}$. of the ground drugs are mixed with 5,000 c.c. of boiling water and macerated for two hours. They are then transferred to a percolator, gradually adding boiling water until the drug is practically exhausted. The 
percolate is evaporated on a water-bath to the specified volume. It is then cooled, and sufficient alcohol added to make $\mathrm{I}, 000$ c.c.

Solid Extracts (Extractum; Ext., U.S.P. Extractum Siccum; Ext. Sicc.; B.P.).-Must not be confused with the fluidextracts. They are solid or semisolid preparations obtained by evaporation of solutions of the medicinal principles of drugs. Some of the solid extracts are dry, others of a "pilular" consistency, i.e., like a pill-mass. They are convenient for administration in solid form, e.g., in pills, ointments, plasters, etc. Nearly all the potent, and most of the other, extracts are adjusted to four times the strength of the fluidextracts.

"Powdered Extracts" are dried, and mixed with sufficient diluent (milk-sugar) to give them the same strength as the solid extracts. Most extracts are prepared by percolating and subsequent evaporation; the last and weaker portions of the percolate being evaporated first, to avoid the deleterious actions of prolonged heat on the main portion. Some of the extracts are obtained by evaporating the fluidextract. The menstruum is sometimes water, or alcohol, or a mixture of both; acetic acid being added in some cases. The finished product is assayed, if possible, and adjusted to a definite standard by the addition of sugar of milk.

Artificial resins are precipitates obtained by mixing alcoholic solutions with water. Where they constitute the active principle, this is a convenient method of isolating it in a concentrated although somewhat impure form. Their strength is fairly constant. They are practically identical with the eclectic "resinoids."

Oleoresins (Oleoresina, Oleores), are extracts containing the resinous and oily constituents of the drug obtained by evaporating ethereal, acetone or alcoholic percolates.

\section{MIXTURES}

Mixtures, in the wider meaning of the word, are fluids resulting from the mixture of fluids with other fluids or with solids. They comprise: Linimenta, Misturæ, Emulsa.

Liniments (Linimentum, Lin.).-These are solutions of irritant drugs in oily, soapy or alcoholic solutions, intended to be rubbed on the skin as counterirritants.

Mixtures (Mistura; Mist.).- - In the narrow meaning of the term, these are generally suspensions of a solid in a liquid, sometimes by the use of a gummy substance; for heavy powders can not be evenly distributed in a light liquid without this aid. The B.P. also includes some emulsions under this title.

Emulsions (Emulsum, Emul.).-These are mixtures of a milky appearance, made by suspending fats, oils, or resinous substances in aqueous liquids by the intervention of some viscid, usually gummy, substance.

The object of emulsification is to break up the insoluble oil into the finest particles and to envelop each of these in a coating of the emulsifying agent, which will keep them from reuniting. ${ }^{1}$ This allows of dilution, of the admixture of other substances, and it facilitates absorption.

Milk is a natural emulsion in which the butter-fat is kept in emulsion by the casein. It may be taken as a type to which artificial emulsions must conform. The globules must be uniform and of about the same size as those in milk.

Emulsions may be divided into the following classes:

'See also Roon, r916, Physical significance of emulsions. 
Fulural cmulsions, such als are found rearly formed in nature. Instances of these are milk, the yolk of egr, and some plant juices.

Gilm-resin and sed emulsions; that is, emulsions marle from such substances as contain their own emulsifier. Bximples of such gum-resins are ammoniac, asafetida, and myrrh. The drugs atre reduced to a coarse powder and water is added gradually. Seeds which yield such emulsions are poppy, hemp, and almond.

In arlificial cmulsions an emulsifier must be added. Quite a number of substances may be used for this purpose, the principal ones being gum acacia, tragacanth, yolk of egg, Irish moss, soap bark, and extract of malt. The substance most commonly used is gum acacia. This emulsilier is incompatible with large quantities of alcohol, borax, tincture of iron, or glycerin. It may be used by either the Continental method, where a nucleus is first formed from gum, oil, and water, and to which the remainder of the water may then be added; or by the English method, where a mucilage is first made, to which the oil and water are added in alternate small portions.

The Continenlal method deserves the preference. To form a nucleus there should be used for each part of oil $1 / 4$ to $1 / 2$ part of acacia and I part of water. Stir the oil with the acacia in granular powder, then add the water at once. The mixture of the oil and the gum must not be allowed to stand too long before adding the water, otherwise it will cake. In the English method the acacia, the amount of which should be half that of the oil, is rubbed up with an equal volume of water and then small portions of oil and water are added alternately. If this addition should be done too rapidly, there is danger that the emulsion will separate or "crack." This does not necessarily spoil it, for it may be re-emulsionized by adding it to a fresh portion of acacia and repeating the process.

In making medicated emulsions the ingredients should be mixed in the following order: First the nucleus, then the flavoring, then the syrup, and, lastly, the water in which the solids have been dissolved.

In yolk emulsions, the yolk of egg is used in place of the nucleus in the Continental method. The yolk is triturated in a mortar and the oil and water are added alternately in small portions. One yolk suffices for from I to 2 ounces of oil. The yolk emulsions are incompatible with the same substances as gum emulsions and do not keep nearly as well.

Soap bark has saponin for its emulsifying agent. It is not incompatible with any of the above-named substances, but possesses very decided therapeutic properties, which preclude its use in many cases. It is used in the proportion of I part of the tincture to 8 parts of the oil: Place the tincture in a dry bottle, add the oil in portions, and shake after each addition. Finally add the water. Crude saponin (0.3:I oo of oil) can also be employed.

Extract of malt emulsifies its own weight of oil. It is used as the nucleus in the Continental method.

Solutions of alkalies may also be used for emulsification, since they form soaps, but they are usually not desirable.

\section{SOLID PREPARATIONS}

These comprise the Powders and the various dosage forms, Pills, etc., for internal use; and Ointments, Plasters, for external applications.

Powders (Pulvis; Pulv., Gen., Pulveris) are finely powdered drugs intended for either external or internal administration.

When intended for internal use, they are generally folded in papers (chartula; chartul.). It must be borne in mind that hygroscopic (deliquescent) substances, such as potassium acetate or citrate, can not be prescribed as powders, nor such substances as become.a fluid when mixed (e.g., camphor and chloral).

In making compound powders, one should begin with the smallest ingredient and add the others in the order of their amount, triturating thoroughly after each addition. 
In dividing the powder, it is not always necessary to weigh out each powder. The object is often accomplished with sufficient accuracy by flattening the powder on a piece of paper, squaring off the edges, and dividing into a number of equal parts by means of a spatula. In the case of more bulky powders, such as Seidlitz powders, measures are used.

When intended for external use, as for dusting-powders, extreme fineness is the main desideratum. They should in this case be mixed with a spatula and not in a mortar, since the former insures greater smoothness.

Triturations (Trituratio; Trit.). - These are powders obtained by triturating the active substances with some inert material such as sugar of milk. Their advantage lies in the greater ease in weighing out a comparatively large amount of substance. When no special directions are given. triturations are made of a strength of ro per cent. The trituration of elaterin is the only one official.

Eleosacchara are triturations of volatile oils with sugar in the proportion of $\mathrm{I}: 30$. They are used for the purpose of flavoring other powders.

Effervescent (Eff.) Salts.-These are dried salts, mixed with sodium bicarbonate and tartaric acid, etc., so as to evolve carbonic acid when they are dissolved in water. This aids in disguising the taste.

\section{DOSAGE FORMS}

Pills, capsules, tablets, lozenges, cachets, etc., are used to divide the drug into definite doses, to avoid the inconveniences of dry powders, and to reduce the bulk. Care must be used in prescribing the dosage forms so as not to make them too large to be swallowed.

The administration of drugs in compact solid form delays solution and absorption. This is especially true of old pills, in which the excipient may become so impervious that they pass unchanged into the feces; but it applies also to many tablets. A mere delay in solution may be an advantage if an intestinal action is to be procured; but difficultly soluble substances (salol, bismuth, etc.) should not be administered in these compact forms. Corrosive substances are also unsuited for solid administration.

Pills (Pilula; Pil., U.S.P.).-These are defined as spherical or elongated masses of medicinal substances, of such size as to be convenient for swallowing; that is to say, containing up to $5 \mathrm{gr}$. $(0.35 \mathrm{Gm}$.) of active substance. If the pill is of larger size, it is called a bolus. Very small coated pills are spoken of as granules. The quantity of drug in the efficial pills is adjusted so as to make the average dose two pills.

Pill-Masses (Massa; Mass., U.S.P. Pilula; Pil., B.P.).-These are soft solid preparations of such consistency that they can be formed into pills.

A pill consists of the active ingredient and of the excipient (cohesive). In order to make pills, the substance is first made into a mass by means of this excipient. The mass must be sufficiently soft to admit of molding, but on the other hand it should be sufficiently consistent not to lose its shape. It should neither harden nor soften nor crumble on keeping.

The quantity of drug in the B.P. pills is adjusted so as to make their dose, with few exceptions, 0.25 to 0.5 Gm., 4 to 8 gr. (The exceptions are Pil. Phosphori; Pil. Ferri; Pil. Plumbi c. Opio; Pil Saponis Co.).

Excipients.-Syrup, glycerin, acacia, vegetable extracts (Extr. Gentianæ), and a great variety of other substances are used, according to the characters of the active drug. The choice may usually be left to the pharmacist.

Liquid Excipients.-Glycerite of acacia or tragacanth, thick flour paste, glycerin syrup, confections, or extracts. 
Solid Excipients.-Acacia, tragacanth, starch, althea, licorice powder, soap.

For chemicals which are destroyed by organic substances the best excipient is formed by a mixture of petrolatum and kaolin.

Absorption Influenced by Excipients.-These, being colloidal, all delay absorption somewhat. For iodid pills, Rieben, 1907 , found the greatest delay with waxy and oily excipients, the least with vegetable extracts and syrups. Silver-foil coating produced considerable delay.

Preparation of Pills. - The ingredients are first triturated to a fine powder. In case crystalline salts are used, these must be desiccated. The excipient is then added in small portions and thoroughly triturated with the powder until the proper consistency is obtained. If accidentally too much excipient is added this can be remedied by the addition of some inert powder, such as starch, gum acacia, or powdered licorice.

The mass having been formed by thorough trituration, it is placed on a glass or porcelain slab marked with equal divisions. It is well to put a little dusting-powder on the slab to prevent the mass from sticking to it. The best dusting-powders are starch, lycopodium, or licorice, according to the color of the pill-mass. This mass is then rolled out by means of a broad spatula into a cylinder of uniform diameter, and this is cut with a sharp knife into the requisite number of equal parts. These are formed into spherical shape by rolling them between the thumb and the first two fingers. The finish may be rendered more perfect by placing the pills with a liberal amount of dusting-powder in the lid of the pill box and gently rolling them with the ball of the thumb.

Pill-coatings.-Pills are often coated with sugar, gelatin, chocolate, etc., to disguise their taste while being swallowed. The coatings tend to interfere somewhat with absorption.

In coating with sugar, the pills are moistened with a thick syrup and rapidly rotated and dried in a current of warm air until they acquire a sufficiently thick coating and a fine polish. The latter is often enhanced by a little wax. For a gelatin coating they are dipped into a strong hot solution of gelatin, which is allowed to harden in the air. When it is desired to exclude the air from the pills, they are sometimes varnished by dipping them into an ethereal solution of tolu. This is the official process for keeping phosphorus pills. A still finer polish may be given to pills by coating them with silver leaf, which is done by shaking them in a box with silver foil.

Enteric Pills.-These are pills coated with some substance (keratin, salol, stearic acid, or formaldehyd-hardened gelatin), supposed to protect them from the gastric juice, but which dissolves in the intestines. They would be useful when the drug is intended to act only after passing the stomach. Unfortunately, they fail to accomplish their purpose, for soluble drugs diffuse through them rapidly (Linossier, I9I I).

Improved directions for the salol-coating of pills are given by Peacock, 1915; for stearic acid by Toplis, 1915. The latter claims that the stearic coating apparently fulfils its purpose.

Keratin (a protein derived from horn, goose quill, etc.) was tried extensively; but it is totally unsuitable, since commercial specimens are largely soluble in artificial gastric juice (Puckner, I9II).

Formaldehydized Capsules.-These are prepared by immersing the filled capsules in water containing I per cent. of absolute formaldehyd, and drying. The capsules should become insoluble in acid-pepsin solution, but remain soluble in 0.5 per cent. sodium carbonate. The degree of insolubility is governed by the duration of the immersion. If they are to be used on the next day, the immersion should last six minutes; but these will have become quite insoluble in the carbonate after they have been kept a week With immersion of thirty seconds, the capsules will be properly hardened only after storing them for two weeks; but they will preserve their proper solubility in carbonate for one year (Scoville, I9I5).

Capsules.-These are small containers, usually. of gelatin, intended to be filled with the drug and swallowed. Those used for powders are hard, and consist of a body and a cap. In filling these capsules, the powder is divided into the requisite number of parts, forced into the body with a 
spatula, and the caps placed on. Soft capsules are used for oils, etc. These are sealed with a drop of melted gelatin. These soft capsules may be made to hold as much as $4 \mathrm{Gm}$. Hard capsules should not hold more than $0.3 \mathrm{Gm}$. or at most $0.5 \mathrm{Gm}$. A somewhat larger amount may be used by making the powder into a pill-mass. The purpose of capsules is to disguise the taste of the substance.

Capacity of Capsules for Dry Powders.-The capsules are marketed by numbers, which have about the following average capacity (Caldwell, r913); probably differing somewhat, however, for the various makes:

\begin{tabular}{|c|c|c|c|}
\hline \multirow{2}{*}{ Size of capsules } & \multirow{2}{*}{$\begin{array}{l}\text { Capacity in } \\
\text { minims }\end{array}$} & \multicolumn{2}{|c|}{ Average capacity for most powders } \\
\hline & & In grains & In grams \\
\hline 3 & $\cdot 5 \cdot 3$ & 3.0 & 0.20 \\
\hline 2 & -6.5 & 4.0 & 0.25 \\
\hline I & 8.4 & $5 \cdot 5$ & 0.35 \\
\hline 0 & 10.0 & $7 \cdot 5$ & 0.50 \\
\hline$\infty$ & I5.5 & IO. 0 & 0.65 \\
\hline
\end{tabular}

Parke, Davis \& Co. state the capacity of their capsules as: No. ०, I 2 minims; No. 1,9 minims; No. 2, 6 minims; No. $3,4 \frac{1}{2}$ minims; No. 4 , 3 minims; No. 5 , 2 minims.

Wafers are thin sheets formed of a dried flour-paste, in which the powder is enveloped. They are immersed in water and swallowed. They possess the advantage that larger quantities of the drug (up to I Gm.) may be administered.

Cachets are wafers, molded into concave circular forms.

Troches or Lozenges (Trochicus, Troch.).-These are made by punching or cutting out circular or oblong disks from a mass made up from the active substance, sugar, and mucilage or fruit base. These are then dried in the air. They are usually intended for solution in the mouth, and are most popular for throat medication. They are, however, sometimes used instead of pills.

Tablets (Tabella, Tab.).-These are small disks composed of medicated powders. Tablet triturates are prepared from the powdered drug and milksugar or sugar, made into a paste with alcohol, and pressed into molds. Compressed tablets are formed by strong dry compression. These forms are used similarly to pills, and dissolve more readily, especially the triturates. By suitable choice of ingredients, they may be made very acceptable to children (Tabella Dulces, "Candy medication" Fantus, Igr2I9r4).

Variability of Commercial Tablets.-Commercial tablets are often very unreliable. Variations of Io per cent. may be excusable, but Kebber, 1914, found that nearly a fifth of the samples bought varied by 20 per cent. or more; and this even with toxic substances.

Hypodermic tablets are similar to the tablet triturates, especial care being exercised to secure quick solution.

Lamellæ (B.P.) (Lam.) are thin gelatin disks, softened with glycerin, and impregnated with substances acting on the pupil. They are intended to be placed under tho eyelids.

Suppositories (Suppositorium, Supp.).-These are suitably shaped masses of solid, medicated usually fatty substances, intended for introduction into the rectum, vagina, or urethra (Fig. 2). They take the place of ointments for local treatment where these can not be readily applied. 
Suppositories are made by incorporating the medicinal substance into a suitable base, and molding into masses of suitable shape and size 'The ideal base is one which, whilst solid at the ordinary temperature, is melted by the heat of the body. Such is cacao-butter (Oleum Theobromatis). Glycerinated gelatin or a soapy base is also sometimes used, especially with urethral suppositories for which cacao-butter would be too loritile. Rectal suppositories are usually made with sufficient base to weigh $\mathrm{r}$ or $2 \mathrm{Gm}$; ; vaginal, $4 \mathrm{Gm}$.

Urethral suppositories are pencil-shaped, pointed at one end, and measure 7 or 14 $\mathrm{cm}$., weighing 2 or $4 \mathrm{Gm}$.

Theobroma suppositories are made either by the hot or cold process. The hot process consists in melting cacao-butter at a temperature not exceeding $35^{\circ} \mathrm{F}$. and addling the active substances, then pouring the mixture into cold molds. In the cold process the active substances are triturated with grated cacao-butter with the addition of a small amount of castor-oil, sufficient to make it into a suitable mass, which is then rolled out and divided as for pills. They may also be formed by pressure.

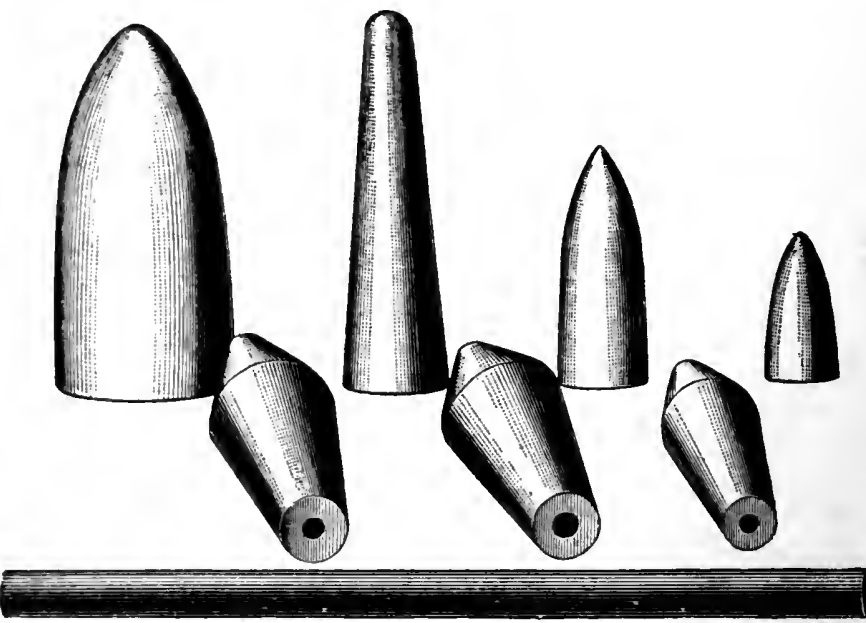

FIG. 2.-Different forms of suppositories (H. Blair). Natural size: The largest is for use in the vagina; the cylinder is a urethral suppository or bougie; the others are various shapes and sizes of recta! suppositories.

Suppository capsulcs, whether of gelatin or of cacao-butter, largely defeat the object for which suppositories are employed. They are, however, much more convenient to prepare than the suppositories, and may suffice when the object is merely internal medication. Glycerin is made into a suppository by means of a very hard soap formed from stearic acid and sodium carbonate.

Ointments (Unguentum, Ung.) are soft fatty masses intended for external application. They consist of the active ingredient and the base.

Ointments are prepared by fusion, mechanical admixture, and chemic reaction. In mixing ointments by fusion, that constituent of the ointment which has the highest melting-point is first melted, and the others are then added in the order of their meltingpoints. The active substance is added last, to obviate the prolonged action of heat upon it. The mechanical admixture is usually done on a slab or in a mortar. It is needless to say that powders must be in the finest stage of subdivision. If the quantity of powder is large, it is usually first mixed with some of the melted ointment.

Ointment Bases.-The base of ointments is formed by lard, by petrolatum, by lanolin, or by various mixtures, of which the "simple ointment," consisting of 4 parts of lard and I part of white wax, is the most important. The base must vary according to the object for which the ointment is employed, whether absorption, protection, or 
local action is desired. Petrolatum (vaselin), which consists of the less volatile parts of petroleum, is simply protective, or useful as a vehicle for substances intended to have a mere local action, since it is not absorbed. Lanolin (Adeps Lanæ Hydrosus) consists of a mixture of cholesterin-like substances obtained from sheep's wool. It is very readily absorbed, does not become rancid, and mixes with its own weight of water. This latter property is of great advantage when it is desired to use crystalline salts in ointment form, since these can be incorporated in the form of solution, making a much smoother ointment. Lard is the cheapest ointment material, It penetrates well; but an important objection to it rests upon the fact that it becomes rancid very rapidly. This tendency can be greatly diminished by the incorporation of antiseptic substances. Official Benzoinated Lard is an attempt in this direction.

Emollient or soothing, and antiseptic ointments, should not be absorbed, and are, therefore, best made with petrolatum. 'Stimulating and absorbent ointments are intended to penetrate, and therefore require lard or lanolin. With astringent ointments, the base should vary according to the intended depth of action (Corlett, roro).

Oleates (Oleatum, Oleat.). - These are solutions of metals or alkaloids in oleic acid. They are used similarly to ointments.

Cerates (Ceratum, Cerat.).- These are preparations similar to ointments but of a firmer consistency. They are generally made from a mixture of wax and petrolatum, in the same manner as ointments.

Plasters (Emplastrum; Emp.). - These are made by spreading on a thin cloth or leather support a mass or base which is hard at an ordinary temperature, but is softened and rendered adhesive at the temperature of the body. They are applied as adhesive or for counterirritation. A rubber composition is the base for most modern plasters.

Plasters are now usually obtained from manufacturers, and are but rarely made to order. The typical old plaster, had for its base diachylon, Burgundy pitch, or other resins. Diachylon is a lead soap made by precipitating soap with lead acetate. This alone, or with the addition of a little rubber and petrolatum forms the base of the other official plasters. The base is fused and the active ingredients incorporated into it by stirring.

To spread the plaster, the cloth, cut to the proper size and shape, is tacked on a board and the mass is heated and spread evenly with a trowel or spatula. A margin $1 / 2$ in. wide must be left to allow of handling. To avoid the rather excessive irritation resulting from the confinement of the secretions of the skin, plasters are now frequently made porous. Isinglass plaster is made by spreading a thick solution of isinglass on silk.

Chartæ are medicated papers, i.e., pieces of paper covered or impregnated with a medicinal substance. Charta Sinapis is used as a plaster, Charta Potassii Nitratis for fumigation.

Collodia (Collodium, Collod.).- - Solutions of pyroxylin (gun-cotton) in a mixture of ether and alcohol, or in acetone. By the evaporation of the solvent they form a film on the skin, and thus act like plasters. Collodia must not be brought near fire.

Poultices (Cataplasma, Catapl.). - These are used mainly for the purpose of supplying heat. It is often necessary to give a patient directions for preparing them. Linseed poultices may be taken as a type: Pour a cup of linseed meal into $2 \frac{1}{2}$ cups of boiling water, stirring constantly. Spread the mush thickly on a piece of flannel, fold so as to form a sack, and apply as hot as can be conveniently borne. Other pasty materials (flour, clay, etc.) are also used.

\section{SOLUBILITIES}

It is well to have a general idea of the solubility of the substances usually prescribed. The subjoined compilations will be found useful in 
this connection. A knowledge of analytic chemistry and of incompatibilities can be utilized here, for a substance appearing as a precipitate is, of course, relatively insoluble.

GENERAL SOLUBILITY OF COMMONLY USED SALTS IN WATER

I. Arranged by Acids.-

Group A: Salts Mostly Soluble.-

I. Acetates and Nitrates: all soluble (except bismuth subnitrate).

2. Il alogen group (=iodids, bromids, and chlorids): Soluble, except $\mathrm{Ag} ; \mathrm{Hg}$ (ous); $\mathrm{Pb} ; \mathrm{Bi}$.

3. Sulphates: Soluble, except $\mathrm{Pb}, \mathrm{Sr}, \mathrm{Ba} ; \mathrm{Ca}$ sparingly soluble.

4. Tartrates and Citrates: mostly soluble.

Group B : Salts Mostly Insoluble.-

I. Arsenates

Arsenites

Carbonates

Hydrates (Ca sparingly soluble)

Oxids

Oxalates

Borates

Phosphates

Insoluble, except those of alkali metals.

II. Arranged by Base.-

The salts considered in this table are: Acetates (Ac), Halogens (H), Oxids (O), Sulphates $\left(\mathrm{SO}_{4}\right)$, Phosphates $\left(\mathrm{PO}_{4}\right)$, Oxalates $(\mathrm{Ox})$, Carbonates $\left(\mathrm{CO}_{3}\right)$, Sulphids $(\mathrm{S})$, Nitrates $\left(\mathrm{NO}_{3}\right)$, Citrates $(\mathrm{Ci})$. Hydrates agree with oxids.

Those of the above salts which are not mentioned with the respective base are insoluble.

I. Alkali Metals $\left(=\mathrm{Na}, \mathrm{K}, \mathrm{NH}_{4}\right)$ : all soluble.

2. Lithium: Soluble, except $\mathrm{O}$ and $\mathrm{CO}_{3}$, sparingly soluble, and $\mathrm{PO}_{4}$, insoluble.

3. $M g, A l$ : Soluble: $\mathrm{NO}_{3}, \mathrm{Ac}, \mathrm{H}, \mathrm{Ci}, \mathrm{SO}_{4}, \mathrm{~S}$.

4. $\mathrm{Ca}, \mathrm{Ba}$, Sr: Soluble: $\mathrm{NO}_{3}, \mathrm{Ac}, \mathrm{H}, \mathrm{Ci}, \mathrm{S}$; sparingly: $\mathrm{O}$.

5. $\mathrm{Zn}, \mathrm{Mn}, \mathrm{Ni}, \mathrm{Co}, \mathrm{Fe}$ Soluble: $\mathrm{NO}_{3}, \mathrm{H}, \mathrm{Ci}, \mathrm{SO}_{4}$ (mercuric iodid $\mathrm{Hg}(\mathrm{ic}), \mathrm{Cu}, \mathrm{Sn}$

6. Ag: Soluble: $\mathrm{NO}_{3}, \mathrm{SO}_{4}$.

7. $\mathrm{Pb}$ : Soluble: $\mathrm{NO}_{3}$, Ac.

8. $B i, S b$ : Only soluble in form of double organic salts, e.g., Bismuth and Ammonium Citrate; Antimony and Potassium Tartrate.

9. Mercurous: Insoluble.

Strength of watery solution, in which commonly used salts may safely be prescribed. (It must be remembered that where several salts are prescribed in the same mixture, the solubility of each is apt to be lowered.)

The following table gives the amount of very commonly used drugs which can be safely prescribed in water enough to make 100 c.c. These should be memorized:

50 per cent., or grams: ( $=3$ iv in water q. s. 3 j) Tannin; Antipyrin; Acetate, Citrate, Salicylate, Iodid or Bromid of Potassium or Sodium; $\mathrm{AgNO}_{3} ; \mathrm{ZnSO}_{4} ;$ Chloral; Cocain Hydrochlorid. 
5 per cent.,or grams: ( $=25$ grains in water q. s. $5 \mathrm{j}$ ) Alum; Carbolic Acid; Borax; $\mathrm{KClO}_{3} ; \mathrm{NaHCO}_{3} ; \mathrm{HgCl}_{2}$; Tartar Emetic; Quinin Bisulphate; Citrated Caffein; the majority of the soluble salts of alkalies, earths, and metals.

smaller quantities: Boric Acid, 4; Morphin Sulphate, 4.5; Quinin 4 to o. I per cent. Hydrochlorid, 3; Quinin Sulphate, o.r3; Strychnin Sulphate, 2.

III. Solubility in Different Media.-As a general rule, inorganic substances are more soluble in water than in alcohol.

Basic alkaloids are insoluble in water, more soluble in alcohol.

Alkaloidal salts are soluble in either alcohol or water.

Gums are soluble in water, insoluble in alcohol. Resins and essential oils are the reverse.

(In making mixtures, it must be remembered that spirits, tinctures, and fluidextracts all contain alcohol.)

Glycerin stands intermediate between alcohol and water as a solvent.

The following substances are:

I. Practically insoluble in water: Iodin, calomel.

2. Soluble in water, but almost insoluble in alcohol: Alum, $\mathrm{NH}_{4} \mathrm{Cl}$, $\mathrm{KClO}_{3}$, tartar emetic, $\mathrm{ZnSO}_{4}$, Borax.

3. Much more soluble in glycerin than in water: Boric acid, alum, carbolic acid, $\mathrm{HgCl}_{2}$.

Terms Used in Defining Solubility.-The following terms (Wilbert, I909) are employed in "Useful Drugs" to describe the approximate solubility, which is often sufficient for prescribing. Substances that are soluble in less than I part of solvent $=$ very soluble. From I to ro parts of solvent $=$ freely soluble. From Io to 100 parts of solvent $=$ soluble. From I00 to I,000 parts of solvent $=$ slightly soluble. From I,000 to I0,000 parts of solvent $=$ very slightly soluble. From I0,000 to I00,000 parts of solvent $=$ nearly insoluble. More than roo,ooo parts of solvent $=$ practically insoluble.

Official Solubilities.-The U.S.P. and B.P. state the solubility of articles, as grams of substance to cubic centimeters of solvent; i.e., a solubility of $\mathrm{I}$ :ro means that I $\mathrm{Gm}$. of substance requires ro c.c. of solvent for solution, at the standard room temperature, $25^{\circ} \mathrm{C}$., $77^{\circ} \mathrm{F}$., U.S.P.; $15.5^{\circ} \mathrm{C} ., 60^{\circ} \mathrm{F}$., B.P. The standards given in this book are those of the U.S.P. In percentage solutions, ro per cent. signifies that roo c.c. of solution contain I Gm. or c.c: of the substance.

\section{INCOMPATIBILITY}

This is a subject usually very confusing to the student, since it consists of what appears at first sight a vast array of details. However, it rests only upon an application of the ordinary chemic reactions, and when the latter have been mastered, the subject is comparatively easy and simple. It may be laid down as a general rule that substances are incompatible if they are used in testing for each other or if they form antidotes.

In the following compilation it has been attempted to arrange the incompatibilities into general groups.

In this generalization, it is not feasible to take account of individual exceptions. Care has been taken to err on the safe side. It is much more important for the physician to remember that mercuric chlorid is generally incompatible with alkaloids, than it is to know that it may be prescribed with some. 
Definitions.-Incompatibility is said to exist when the constituents of a mixhre interfere with one another in a way not intended by the prescriber.

If an interference is intentional, it is called an intentional incompatibility.

If a chemical change occurs, the incompatibility is called chemical.

When it consists in a precipitation of the substance by a change in the solvent, or when a chemic incompatibility does not interfere with the active substance, but produces an unsightly appearance, it is pharmaceutic.

If the interference is with the solubility, or if liquefaction or deliquescence occurs, the incompatibility is mechanic.

When without causing any chemic changes it interferes with the physiologic action of the ingredients, it is therapeutic.

Chemic Incompatibility. - This may result in explosion, precipitation, or the production of new substances with undesired properties.

Explosives.-Powerful oxidizing agents are apt to explode when mixed in concentrated form with easily oxidizable substances.

The most important oxidizing agents are: Chromic acid; Concentrated nitric acid and nitrates; Permanganates; Chlorates; Peroxids.

The most important oxidizable substances are: All organic substances; Sulphur, sulphids, sulphites, and hyposulphites; Iodin and iodids; Phosphorus, phosphites, and hypophosphites; Reduced iron.

Explosions may also result from mixing iodin with ammonia or turpentine; and chlorin with ammonium chlorid.

Explosions will occur only when the substances are dry or at least concentrated, and when they are heated or percussed.

Dilute solutions may be mixed without danger if not heated. Glycerin, phenol, and in some cases alcohol, behave like dry solids.

Permanganate of potash or chromic acid or nitrate of silver will decompose organic substances even in solution, but in this case without explosion.

Powders may be mixed without concussion, but even this should be avoided, since conditions favorable to explosion may arise after they leave the hands of the dispenser.

Pills containing these easily decomposed substances are best made with inorganic excipient (clay and vaselin).

Substances containing loosely combined oxygen may explode on concussion or heating when no reducing substances have been added; this is due to their containing dust or other organic matter. They should therefore be handled with care.

Gradual Evolution of Gas.-This occurs especially on mixing acids with carbonates or sulphids; also when mixing strong acids with alcohols (= esters), or with chlorate $\left(=\mathrm{Cl}\right.$ ); also on mixing strong $\mathrm{HCl}$ and $\mathrm{HNO}_{3}$.

Incompatibility by Precipitation.-Alkalies precipitate earths, metals and alkaloids from their salts. Under alkalies may be included oxids, hydroxids, carbonates, phosphates, borates, arsenates and arsenites.

Chlorids, bromids and iodids precipitate salts of $\mathrm{Ag}, \mathrm{Pb}$, and some alkaloids. Iodid also precipitates mercuric salts, redissolving in an excess.

Sulphates precipitate $\mathrm{Pb}, \mathrm{Ca}$ and the other earthy metals, except $\mathrm{Mg}$.

Salicylates, phenol and tannin precipitate or color iron salts. Tannin also precipitates most other metals, alkaloids, and some glucosids and neutral principles. Salicylates precipitate quinin.

Salts of metals are precipitated by alkalies, sulphids, tannin and proteins. They precipitate some alkaloids and acacia. Mercuric salts are often incompatible with other metals. Silver and lead salts are precipitated by chlorids, bromids, and iodids. Silver salts are also incompatible 
with organic substances. Iron salts are colored by tannin, salicylate and phenol.

Calcium salts are precipitated by alkalies, phosphates and sulphates; Magnesium salts by alkalies and phosphates.

Alkaloidal salts and synthetic bases (antipyrin, etc.) are precipitated by alkalies, tannin, iodin, potassium-mercuric iodid, and some by iodids or metallic salts.

Acacia is precipitated by alcohol, borax, Tr. Ferri Chlor.

Production of Substances with Undesired Qualities.-Iodids liberate iodin on the addition of acid and oxidizing agents, including $\mathrm{H}_{2} \mathrm{O}_{2}$, nitrites, salicylic acid, etc. They oxidize mercurous to the dangerous mercuric salts.

Chlorates liberate chlorin with concentrated acids.

Chloral liberates chloroform with alkalies.

Glucosids ( $f . i$. , glycyrrhizin) are destroyed by acids.

Pharmaceutic Incompatibilities.-Alcohol should not be added to: Acacia, gelatin, proteins, emulsions, strong salt solutions.

Water should not be added to:

Alcoholic liquids in general (such as tinctures, spirits, fluidextracts); or to Ethereal liquids (oleoresins), or oils.

(Phenol should not be mixed with collodion.)

Liquefaction occurs when chloral is rubbed with camphor, menthol, phenol, salol, etc. Any of these, when rubbed together, or with coal-tar antipyretics, are apt to liquefy.

Especially Incompatible Drugs.-The following are most likely to give incompatibilities, and any combinations should therefore be scrutinized with special care: Acids, alkalies and carbonates; permanganates; iodids. Mercury, iron, silver and lead salts; tannin; alkaloids.

\section{METROLOGY}

A ready working knowledge of weights and measures is indispensable in prescribing.

Formerly every country, even every State, and some cities, had their own system of weights and measures, resulting in endless confusion and loss of time. This state of affairs still exists to some extent. In the United States and Great Britain no less than five different systems are in common use. It is a hopeful sign that the United States and British Pharmacopeias and the U. S, government medical services employ the metric system. This system originated in France near the close of the last century. It has been adopted in science to the exclusion of all others, and possesses a number of advantages which should cause all other systems to be discarded. This is the ultimate tendency, and the student is urged to learn the doses and practice prescription-writing in the metric system. Since, however, the common systems are still used in many hospitals and journals, these must also be mastered, and the conversion of the systems practised.

The Metric System.-This is based on the decimal system, and has for the unit the measure of length, the meter (M.).

This was intended to be a natural unit, viz., the ten-millionth part of the distance from the pole to the equator of the earth at a particular meridian. Subsequent measurements have given a slightly different value to this distance. The meter is therefore an arbitrary standard-the length of a platinum bar, the original of which is preserved in Paris.

The meter is divided into I०, I০o, and I, , 
decimeter (dm.), centimeter ( $\mathrm{cm}$.$) , and millimeter (mm.). The thousandth$ part of a millimeter is a micron $(\mu)$

The contents of a cube whose edges measure a decimeter, or $10 \mathrm{~cm}$., form the unit of capacity, the liter (L.). The thousandth part of this is a cubic centimeter (c.cm., or, briefly, c.c.; also termed a "milliliter" or "mil"). The unit of weight is given by the weight of a liter of distilled water at $4^{\circ} \mathrm{C}$. in zacuo: this is the kilogram (Kg.). A thousandth part of this is a gram (Gm.). A quantity ten times the unit is expressed by prefixing the Greek numeral Deca; one hundred times, Hecto; one thousand times, Kilo. The tenth part of the unit is expressed by prefixing the Latin numeral deci; one-hundredth, centi; one-thousandth, milli.

Thus:

$\begin{array}{rll}\text { I,000 } & \text { Gm. }=\text { Kilogram } & \text { (Kg.) } \\ \text { I00 } & \text { Gm. }=\text { Hectogram } & \text { (Hg.) } \\ \text { I0 } & \text { Gm. = Decagram } & \text { (Dg.) } \\ \text { I } & \text { Gm. = Gram } & \text { (Gm.) } \\ \text { 0. I } & \mathrm{Gm} .=\text { decigram } & \text { (dg.) } \\ \text { 0.01 } & \mathrm{Gm} .=\text { centigram } & \text { (cg.) } \\ \text { ०.001 } & \mathrm{Gm} .=\text { milligram } & \text { (mg.) }\end{array}$

In quantities including several denominations only one unit is used: thus, I.234 $\mathrm{Kg}$. would be read as I,234 Gm.; $0.002 \mathrm{Gm}$. as $2 \mathrm{mg}$., etc. The quantities are always denoted by Arabic figures placed before the appellation. Fractional parts are always converted into decimal fractions.

Milliliter.-The U.S.P. and B.P. designate the cubic centimeter as milliliter, and the B.P. further employs the following divisions and abbreviations. Their use does not seem advisable, as they tend to introduce complications and confusions that may retard the final adoption of the metric system.

$\begin{array}{llll}\text { Liter (Lit.) } & =\mathrm{I}, 000 & \mathrm{Gm} \text {. of water at } 4{ }^{\circ} \mathrm{C} . \\ \text { Milliliter, Mil, Ml. } & =\mathrm{I} & \mathrm{Gm} \text {. of water at } 4{ }^{\circ} \mathrm{C} \text {. } \\ \text { Decimil, Dl. } & = & 0 . \mathrm{I} \mathrm{Gm} \text {. of water at } 4{ }^{\circ} \mathrm{C} \text {. } \\ \text { Centimil, Cl. } & = & 0.0 \mathrm{I} \mathrm{Gm} \text {. of water at } 4{ }^{\circ} \mathrm{C} \text {. }\end{array}$

In continental Europe the liquid measure is very little used in pharmacy, liquids being usually weighed.

Common Systems of Weights and Measures. ${ }^{2}$ - The denominations are the following:

APOTHECARIES' OR TROY WEIGHT

(USED IN PRESCRIPTIONS)

Grain (gr.)

[Scruple $(\mathscr{H})=20 \mathrm{gr}$.]

$\operatorname{Dram}^{3}(3) \quad[=3 \mathrm{H}]=60 \mathrm{gr}$.

Troy ounce $(\xi)=83=480 \mathrm{gr}$.

[Troy pound $=\mathrm{I}_{2} \xi=5,760 \mathrm{gr}$.]

( $\mathrm{j}$ of water under standard conditions ${ }^{4}$ measures 504.83 minims.)

AVOIRDUPOIS OR IMPERIAL WEIGHT

(A SYSTEM USED IN COMMERCE)

Grain $=$ same as Troy grain.
$\begin{array}{ll}\text { Ounce (oz.) } & = \\ \text { Pound (lb.) } & =1371 / 2 \text { grains. } \\ \text { Ton } & =2,000 \mathrm{lb} .\end{array} \quad 7,000$ grains.

1 Some authors begin all the abbreviations with capitals; others employ capitals for the units: gram, liter and meter, and their multiples; and small letters for fractions (as in the above lists) the latter plan has some advantages.

Those in square brackets are practically obsolete.

Also spelled drachm.

- At $4^{\circ} \mathrm{C}$. and in vacuo. 


\section{UNITED STATES APOTHECARIES' OR WINE MEASURE}

(USED IN UNITED STATES FOR BOTH PRESCRIPTION AND COMMERCIAL PURPOSES)

Minim ( $m$ or min.) (approximately equal to I drop or to I grain of water-more exactly, 0.95 grain).

Fluidram (fi $)$

Fluidounce (fl亏)

$$
\begin{aligned}
& =60 \mathrm{~m} . \\
& =8 \mathrm{fl} 5=480 \mathrm{~m}\left(\mathrm{fl} \mathrm{j} \mathrm{j} \text { of water under standard conditions }{ }^{1}\right.
\end{aligned}
$$

weighs $4562 / 5$ grains).

Pint (pt., or Octarius, O)

Quart (qt.)

$$
=\mathrm{r} 6 \mathrm{fl} 5=7,680 \mathrm{~m} \text {. }
$$$$
=2 \text { pts. }=32 \mathrm{fl} \text { 多. }
$$

Gallon (gal., or Congius, C) $=8 \mathrm{O}=\mathrm{r} 28 \mathrm{fl} \xi=6 \mathrm{r}, 440 \mathrm{~m}$.

A gallon holds 231 cubic inches.

Another system of liquid measure is in use in Great Britain, and must not be confused with the American system. It is the

\section{IMPERIAL MEASURE}

Minims (min.)

Fluidrachm (fl.dr.)

Fluidounce (fl.oz.)

Pint (O)

Gallon (C)

$$
\begin{aligned}
& = \\
& =60 \text { min. } \\
& =8 \text { drachms } \\
& =20 \text { fluidounces } \\
& =8 \text { pints }
\end{aligned}
$$

In writing the apothecaries' measure in prescriptions, the figures are written in the Roman system and placed after the appellation. Thus, gr. $\mathrm{xx}$, not $20 \mathrm{gr}$. The ones are always dotted, and the last one is formed like a j: thus $3 \mathrm{iij}, \bar{z} \mathrm{vj}$, etc. The fl. before the sign is often omitted with liquids.

Fractions are written as common fractions: gr. $1 / 10$, not gr. O.I.

Popular Measures. - These are formed of utensils commonly found in the household, and are, of course, very inexact. They should be displaced by graduated medicine glasses, which can now be obtained very cheaply. Spoons are supposed to be filled so that the fluid stands level with the rim.

The usually accepted equivalents of these measures are:

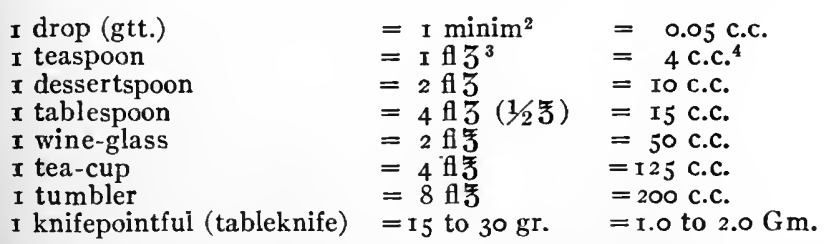

The units of temperature may also be treated in this place.

The scientific scale is the Centigrade or Celsius. In this the range between the freezing-point of water $\left(0^{\circ} \mathrm{C}\right.$.) and its boiling-point $\left(100^{\circ} \mathrm{C}\right.$.) is divided into 100 parts. In the inconvenient and unscientific Fahrenheit scale, in common use, the freezing-point of water is $32^{\circ} \mathrm{F}$., the boiling-point $212^{\circ} \mathrm{F}$., and the range, therefore, $180^{\circ} \mathrm{F}$.

$$
\begin{array}{ll}
\text { Each degree Centigrade therefore } & =180 / 100=9 / 5{ }^{\circ} \mathrm{F} . \\
\text { Each degree Fahrenheit } & =5 \% 9^{\circ} \mathrm{C} .
\end{array}
$$

The conversion of one scale into the other may be done by the following rules:

To convert degrees Centigrade into Fahrenheit: multiply by $9 / 5$ and add 32 .

To convert degrees Fahrenheit into Centigrade: subtract 32 and multiply by $5 \%$.

Normal Temperature.-The U.S.P. has adopted $25^{\circ} \mathrm{C}$., $77^{\circ} \mathrm{F}$., for solubilities, specific

1 At $4^{\circ} \mathrm{C}$. and in vacuo.

As a matter of fact, the size of a drop varies greatly according to the nature of the fluid and of the container; there may be from 50 to 150 to a fuid drachm. The International Protocol authorized a standard dropper, designed to deliver exactly 20 drops per cubic centimeter of water.

Really from $1 / 2$ to 2 fi 3 .

As a matter of fact, the actual capacity of teaspoons averages 5 c.c., when filled level (Wilbert, 1905). 
grasity, calibration, etc., except for the specific gravity of alcohol, which is taken at $15.1^{\circ} \mathrm{C}^{\circ}$. The 13.!'. used $15.5^{\circ} \mathrm{C}$., $60^{\circ} \mathrm{F}$, for metric determinations; $16.7^{\circ} \mathrm{C}$. for imperial measures.

Advantages of the Metric System.-These will now be manifest.

I. 'The metric system is widely used, and is understood throughout the . civilized world.

2. There is a simple relation between linear, solid, and liquid measures.

3. The decimal feature determines great ease in multiplications, since only a change of a decimal point is required to change one denomination into another. It is also much easier to write the quantities. ${ }^{1}$ Calculations involving specific gravity are also much more easily made.

4. The adherents of the common system claim that it is more convenient for dosage. There may be some truth in this, for the grain, minim, and dram happen to be more convenient doses than the gram or cubic centimeter. This advantage, however, is insignificant.

Equivalents of Metric and Common Systems.-Doses and formulas may be calculated from one system into the other. For this, it is only necessary to remember that $\mathrm{I}$ Gm. $=\mathrm{r}_{5} .4 \mathrm{gr}$. However, this always entails the possibility of some error in reckoning, which may have serious consequences. The student is therefore advised to learn the dosage in both systems, and to construct prescriptions directly in whichever system is chosen. If a transposition must be made, the following approximate equivalents (which should be thoroughly memorized and practised) are sufficiently exact for prescription purposes; and being simpler, they are less apt to lead to arithmetical errors, than the exact equivalents.

\section{APPROXIMATE EQUIVALENTS}

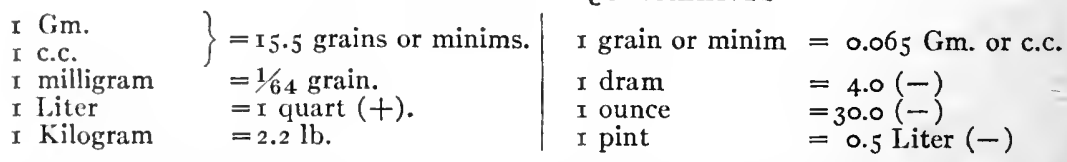

Dosage Equivalents.-In memorizing "average doses" even this degree of approximation is needlessly complicated. The following table gives the most convenient figures:

\begin{tabular}{|c|c|c|c|c|c|c|c|}
\hline Above $1 \mathrm{Gm}$. & $\begin{array}{l}\text { Above } \\
15 \mathrm{gr} \text {. }\end{array}$ & $\begin{array}{c}\text { o. I to } 0.75 \\
\mathrm{Gm} .\end{array}$ & $\begin{array}{c}1 / 2 \text { to } 12 \\
\mathrm{gr} .\end{array}$ & $\begin{array}{l}2 \text { to } 50 \\
\mathrm{mg} \text {. }\end{array}$ & $\begin{array}{c}1 / 30 \text { to } \mathrm{I} \\
\mathrm{gr} \text {. }\end{array}$ & $\begin{array}{c}\text { Below } 2 \\
\text { mg. }\end{array}$ & $\begin{array}{c}\text { Below } 3 / 30 \\
\text { gr. }\end{array}$ \\
\hline $\begin{array}{r}350 \\
120 \\
25 \\
15 \\
10 \\
4 \\
3 \\
2 \\
1\end{array}$ & 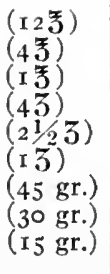 & $\begin{array}{l}0.750 \\
0.500 \\
0.300 \\
0.250 \\
0.200 \\
0.150 \\
0.125 \\
0.100\end{array}$ & $\begin{array}{l}\text { (r } 2 \text { gr.) } \\
(8) \\
(5) \\
(4) \\
(3) \\
(21 / 2) \\
(2) \\
(1 / 2)\end{array}$ & $\begin{array}{l}0.050 \\
0.030 \\
0.015 \\
0.010 \\
0.008 \\
0.005 \\
0.004 \\
0.003 \\
0.002\end{array}$ & $\begin{array}{l}(\mathrm{I}) \\
(1 / 2) \\
(1 / 4) \\
(1 / 6) \\
(1 / 8) \\
(1 / 12) \\
(1 / 15) \\
(1 / 20) \\
(1 / 30)\end{array}$ & $\begin{array}{l}0.0015 \\
0.001 \\
0.00075 \\
0.0005 \\
0.0004 \\
0.0003 \\
0.00025 \\
0.0002 \\
0.00015\end{array}$ & $\begin{array}{l}(1 / 40) \\
(1 / 60) \\
(1 / 80) \\
(1 / 120) \\
(1 / 160) \\
(1 / 200) \\
(1 / 250) \\
(1 / 300) \\
(1 / 400)\end{array}$ \\
\hline
\end{tabular}

1 An example will make this clearer: Take $40 \mathrm{Gm}$.: in the United States system this must be written $3 \mathrm{j} 3 \mathrm{ij}$ gr. xvij. Should it be necessary to calculate five times this quantity, one multiplication will suffice with the metric system: $5 \times 40=200 \mathrm{Gm}$. But with the Apothecaries' system, the operation would be much more complicated:

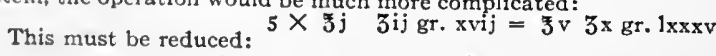

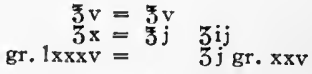

$$
\begin{aligned}
& \text { Answer }=\overline{3 v j ~} 3 \mathrm{iij} \mathrm{gr.} \mathrm{xxv}
\end{aligned}
$$




\section{EXAC'T EQUIVALENTS}

SPACE

I meter $=39.370$ inches.

$=3 \mathrm{ft} .3 .370$ inches.

$=\mathrm{I}$ yd. 3.370 inches.
I inch $=0.0254 \mathrm{M}$. $=2.54 \mathrm{~cm}$.

I ft. $=30.227 \mathrm{~cm}$.

I yd. $=91.440 \mathrm{~cm}$.

CAPACity (United States)

$$
\begin{aligned}
\text { I c.c. } & =16.23 \mathrm{~m} . \\
\text { I L. } & =33.8 \text { I } 5 \mathrm{fl} \xi \\
& =2.113 \mathrm{pts} . \\
& =0.264^{2} \text { gal. }
\end{aligned}
$$

I L. $=1.760$ pints.

$=0.2209$ gallons.

$$
\begin{aligned}
\text { I mg. } & =1 / 64 \quad \text { gr. } \\
\text { I } \mathrm{Gm} . & =15.432 \quad \mathrm{gr} . \\
& =0.03527 \mathrm{oz.} \text { Av. } \\
& =0.03215 \mathrm{~T} \text { Troy. } \\
\text { I } \mathrm{Kg} . & =2.2046 \mathrm{lb} .
\end{aligned}
$$

$$
\begin{aligned}
& \text { CAPACITY (British) } \\
& \qquad \begin{array}{ll}
\text { I pint } & =0.5679 \mathrm{~L} . \\
\text { I gallon } & =4.5435 \mathrm{~L} \text {. }
\end{array}
\end{aligned}
$$

WEIGHT

$$
\begin{array}{ll}
\text { I gr. } & =64.8 \mathrm{mg} .=0.0648 \mathrm{Gm} . \\
\text { I } 5 & =3.888 \mathrm{Gm} . \\
\text { I oz. Av. } & =28.3495 \mathrm{Gm} . \\
\text { I 兮 Troy } & =31.1035 \mathrm{Gm} . \\
\text { I lb. } & =0.4536 \mathrm{Kg} .
\end{array}
$$

\section{GENERAL TOXICOLOGY}

Toxicology is the science of poisoning. The details of the actions and symptoms will be considered in connection with the individual poisons; but certain matters of general application may be discussed here.

Definition of Poison.-A poison (from potion, a draught), by the broadest definition, is a substance the administration of which is injurious to health. However, injurious mechanical, physical, or bacterial agencies are not commonly classed as poisons; nor are the substances which produce injurious effects only in very large doses (say over $50 \mathrm{Gm}$.). It is very difficult to give a definition which will not be ambiguous in some cases (see, f.i., the collection of definitions by Murray and Frame, I9I4). The following covers most of the points which must be considered in classing a substance as a poison:

A poison is any substance which, acting directly through its inherent chemic properties, and by its ordinary action, is capable of destroying life, or of seriously endangering health, when it is applied to the body, externally, or in moderate doses (to $50 \mathrm{Gm}$.) internally.

Etiology of Poisoning.-Poisoning may result through criminal or suicidal intent, or through accident.

The statistics of the relative frequency of the different forms of poisoning vary from year to year, and with each country. A classified list of deaths from poisoning in the U.S. registration area is given by Wilbert, igr6. Suicidal poisoning is probably the most frequent, nearly a half of the suicides in the United States being due to poisoning.

It is calculated that here five-sixths of all suicidal cases are due to phenol. There is, however, a fashion in poisons, as in other things. This is greatly fostered by the notoriety which the newspapers often give to these cases.

Symptoms of Poisoning and Classification of Poisons.-Suspicion of poisoning is aroused if a person, previously in good health, manifests rather. suddenly marked pathologic symptoms, which become rapidly worse. The suspicion becomes more firm, if the phenomena appear a 
short time after swallowing some substance which may perhaps have an unusual odor or taste; if they agree in character with those produced by some group of poisons, and if they do not agree with any other disease.

With reference to these symptoms several classes of poisons are quite well defined.

1. Irritants.-These produce inflammation; if they are taken by the mouth, there is pain throughout the alimentary canal, vomiting, purging, delirium, coma. Most poisons are to some extent irritant, so that these symptoms are almost universally present. The irritants can be subdivided into corrosives, which produce a direct destruction of tissues; and simple irritants, which do not. If corrosives are taken by the stomach, the romit is oiten bloody.

2. Nerve Poisons.- These act on the neuromuscular apparatus, and include most of the poisons which are fatal in minute doses. They are subdivided into: Convulsants, whicl cause spasms; Somnifacients, causing sleep and coma; and Cardiac poisons, which stop the heart.

3. Blood Poisons.-Those which alter the hemoglobin or blood corpuscles. These include the toxic gases, the nitrites, etc. Their action is generally characterized by cyanosis.

It must not be supposed that the action of a poison is confined generally to one class of structures or functions. All functions suffer directly or indirectly, and whatever the class to which the poison belongs, the final cause of death is, in almost all cases, a paralysis of respiration, preceded by the phenomena of asphyxia. In virtue of the latter, or through other causes, the urine of ten contains sugar.

The irritants, and especially the corrosives, produce lesions which can be demonstrated at AUTOPSY. With other poisons the postmortem examination is generally negative. The SPECTRUM OF THE BLOOD shows characteristic changes with some poisons. These are also apt to cause ecchymotic discolorations of the skin. Antiseptic poisons delay the putrefaction of the body, so that mummification may result. Convulsive poisons quicken the onset of rigor mortis.

Duties of the Physician in Cases of Poisoning.-These are twofold: to save life or suffering, and to forward justice. The former object requires the removal of the poison, the administration of chemical antidotes, and the treatment of the symptoms.

For the detection of the poison, and to aid in fixing the guilt on the proper person, the physician must carefully observe the symptoms, take possession of any suspected material, medicine, vomit, etc., and in case of autopsy, preserve the stomach and its contents, the intestines and contents, blood, liver, kidneys, and portions of other organs, separately, without antiseptics, ${ }^{2}$ in clean, hermetically closed, glass vessels, which should be sealed with wax. ${ }^{3}$ An exact written record of all the observations should be made as soon as possible.

The symptoms in cases of suspected poisoning are very rarely sufficient to affirm the presence or nature of a poison, although they may be of great aid to the analyst. The final proof rests generally as the results of the chemic examination.

So much depends on this analysis, that it should never be undertaken by anyone who has not had extensive experience in this class of work, and who has not the necessary facilities. It lies entirely outside of the scope of the practising physician. The latter should, however, be familiar with the general outline of the process used for isolating poisons; and with such chemic tests as may be quickly applied. These tests are of ten valuable for diagnostic and therapeutic purposes. The physician is also expected to give expert testimony on toxicologic questions, and to do this intelligently, he must

1 It may appear strange that poisons which possess a pronounced and disagreeable taste could be used for criminal poisoning, except with infants; but a moment's thought will show that if a liquid is taken unsuspectingly, the taste is not noticed until a large amount has been swallowed.

2 Even glucosid and alkaloid poisons can be isolated for a long time (r60 to 270 days) from putrefying masses. Some poisons, however, disappear rapidly (Phosphorus, Cyanid, Picrotoxin, Phenol, etc). Strychnin and morphin are very persistent.

In emergency, a rare or specially marked coin, or a key, may serve to impress the seal. 
have at least an elementary knowledge of toxicologic analysis, such as is furnished in the following section.

Some poisons can be demonstrated much better by tests on animals than by any chemic tests. For this object, they should be isolated in as pure a form as possible, by the methods laid down in this section. The application of these life tests, which have not hitherto received the attention that they deserve, falls peculiarly into the province of the scientifically trained physician.

\section{OUTLINE OF TOXICOLOGIC ANALYSIS ${ }^{1}$}

Precautions.-The first duty of the analyst is to guard the material confided to him from the wilful or accidental introduction of poisons. For this purpose, precaution must be taken that no other person has access to the material; and every reagent and apparatus must be tested personally.

As a rule, the different organs must be kept strictly separated throughout the analysis. It will depend on circumstances whether the analysis of the individual organs is made at the same time, or successively. If the latter is decided on, the largest organ, or tha: most likely to contain the poison, is examined first. It may be advisable, however, to $\mathrm{mix}$ a weighed quantity (one-fourth or one-third) of the comminuted organs, and to use this mixture for the first analysis.

Since the material to be analyzed is usually limited in amount, and can not be replaced, the examination must be arranged in such a way that as many tests as possible can be made successively on a single sample of the material. An economy of time and material is also secured by obtaining, as quickly as possible, some idea of what poisons may be present. This may be done by some easy preliminary tests, or by using socalled "group-reactions" which, if negative, will exclude a number of substances. The. symptoms may also have furnished some important hints, but should never prevent the complete examination of the substance.

During the isolation and the preliminary search for the poison, only the most important tests should be applied. When the poison has been isolated, however, it should be subjected to every known test. A sample of the isolated poison should be preserved, in stable form.

Preliminary Examination.-The systematic examination is begun by a careful inspection of the portions of the alimentary canal. These are opened, and extended on an inverted evaporating dish, mucous surface upward. Pathologic lesions are looked for, as also particles of the poison which may be adherent. A magnifying lens should be employed. (Granules of arsenic have often been isolated in this way.) The contents of the alimentary canal, or vomited matter, are subjected to a similar close inspection. The odor should be carefully observed. During this examination, the reaction to litmus paper should be noted (caustic acids or alkalies). jars. $^{2}$

Each organ is then hashed, carefully weighed, and replaced in hermetically sealed

Isolation of the Poison.-No routine schema of analysis will fit all cases, since each presents its own problems. However, the following illustrates the usual procedure.

Division of Material.-Ordinarily, each organ, after comminution, is divided into the following portions carefully weighed: One-third is reserved for control; onetwentieth for preliminary tests. The remainder is divided into four parts, used respectively for the search for volatile, fixed organic and inorganic poisons, and for reserve. If the quantity of material is very scanty, two equal portions will suffice: one is reserved for preliminary tests, easily decomposable poisons, and control; the other is examined successively for easily volatile poisons, for fixed organic poisons, and for metals.

Volatile Poisons. - A portion of the material is acidulated with tartaric acid (adding water if necessary), and distilled from a flask or retort con-

1 This section should be studied in connection with the practical exerciscs. Gadamer's "Lehrbuch der chemischen Toxicologie" is an excellent reference book.

2 As soon as the absence of volatile poisons has been proven, the contents of the jars may be covered with 95 per cent. alcohol. 
nected with a Liebig's condenser. ${ }^{1}$ It is advisable to pass a slow current of live steam through the mass. The distillation is continued until about two-thirds of the liquid have been collected. The distillate is collected in three portions. The ollor is noted (volatile oils, chloroform, ether, etc.). and the characteristic tests applied for phosphorus, phenol, cyanids, alcohol, 2 chloroform, chloral, etc.

Phosphorus. $-\lambda$ preliminary test for this element must be made with silver nitrate and lead acetate papers before the distillation is begun. If this test indicates its presence, the condenser is set vertically downward, and the distillation is carried on in a darkened room. All air is expelled from the apparatus by a stream of carbon dioxid. 'This is then shut off, and replaced by live steam, the flask being heated at the same time. If phosphorus is present, a luminous ring appears in the tubes or condenser, shifting its position when the heat applied to the flask is altered (Mitscherlich's method). The appearance of this phenomenon proves the presence of phosphorus absolutely.

There are, however, quite a number of substances the presence of which interferes with the formation of this ring. Almost any volatile substance may do so; turpentine, chloroform, ether, alcohol; and alcohol is often present, as it is usually given as an antidote.

The absence of the ring does not, therefore, prove the absence of phosphorus. The distillate will contain phosphorus in the lower stages of oxidation, as phosphorous or hypophosphorous acid. 'The best way to prove phosphorus in this is to add some bromin water to the distillate and to evaporate to dryness. This results in phosphoric acid, which may be demonstrated by magnesia mixture or ammonium molybdate. The quantitative determination of phosphorus is not important; because if it is present at all, it is present as a toxic agent.

Cyanids.- The presence of mere traces of hydrocyanic acid in the distillate is no proof of poisoning, since these may have been introduced in the way of food (almonds or other seeds). A quantitative estimation, by means of silver nitrate, may be necessary. The qualitative proof also requires two further precautions:

With the method which we have given, ferrocyanids might also be decomposed and give rise to hydrocyanic acid; and since ferrocyanids are not toxic, this would lead to wrong conclusions. To eliminate this, the original liquid is filtered and the Prussian blue test applied to it directly. Mercuric cyanid does not yield its hydrocyanic acid in this treatment. If it is suspected, the material must be treated with hydrogen sulphid.

Distillation from Alkaline Solution.- It is sometimes recommended to add water to the residue in the retort, to make it alkaline with sodium carbonate, and to distill again. The distillate contains ammonium, amines, chloroform (if chloral was present), and the volatile alkaloids. This step may generally be omitted, as these poisons are discovered in other parts of the process; or a small sample may be heated in a test-tube with sodium carbonate, and the odor noted.

Extraction of Fixed Organic Poisons.-The extraction, separation and purification of these poisons are based on their special solubility in certain solvents. As a rule, they are all fairly soluble in acidulated water and alcohol. The neutral principles are removed from the acid watery solution by ether or chloroform. The alkaloids are dissolved by these substances only after the addition of an alkali.

In brief outline, the suspected material is first extracted with boiling acidulated water and alcohol. The acid watery extract is then treated with ether, which dissolves neutral principles and various impurities, but which leaves the alkaloids. These are extracted later by fresh ether, after rendering the solution alkaline.

Various methods are in use. The most practical is that of Stas-Otto, slightly modified:

II. The poisons are first brought into solution by boiling the material, if solid, with about 5 parts of water for fifteen minutes (or the residue remaining after the distillation

\footnotetext{
1 See below for special arrangement to be used when phosphorus is present.

Alcohol is only important if it is present in large amounts. Smaller quantities may be present accidentally or as antidote.
} 
of the acid solution may be used). The mixture is cooled and strained. This removes fat, coagulable protcid, fiber, etc. ${ }^{1}$

$\$ 2$. The strained solution is evaporated at a low temperature to a syrupy consistency, and boiled with twice its volume of alcohol, for fifteen minutes. The evaporated alcohol is replaced. It is then filtered, and the filter washed with alcohol. This removes salts, non-coagulable proteids, etc.

\$3. The alcoholic filtrate is diluted with an equal volume of water and filtered. This removes resins, fats, etc. (The residue may be examined for cathartic resins and croton oil.) (For further purification, the filtrate may be again treated by $\$ \$ 2$ and 3. .)

$\$ 4$. The filtrate, which should have an acid reaction, is now shaken in a separatory funnel with ro c.c. of ether. This is drawn off, and the same process is repeated twice. The ether removes neutral principles, picric acid, and salicylic acid. It is filtered and allowed to evaporate in a glass capsule, and the residue is purified and tested for these acids, for caffein, cantharidin, colchicin, digitalin, picrotoxin, and the coal-tar antipyretics.

§5. The watery solution, remaining from the extraction with ether in the last paragraph, is now made fairly alkaline with sodium carbonate (to liberate the alkaloids) and again extracted with ether, as in the last paragraph. From this alkaline olution, the ether removcs all alkaloids except morphin. The ethereal layer is filtered and evaporated, the residue is purified, and tested, first by potassium mercuric iodid, then for physostigmin, apomorphin, nicotin, coniin, veratrin, strychnin, brucin, atropin, cocain, codein, narcotin, emetin, and aconitin.

§6. To isolate the morphin, the watery liquid remaining in the last paragraph, is made acid, then alkaline by ammonia, and shaken at once with acetic ether, or with hot amyl alcohol.

\$7. To test for oxalic acid, the original substance is partly dried, extracted with 5 c.c. of hydrochloric acid and 20 c.c. of boiling alcohol, for half an hour, filtered, evaporated, extracted with, water, and tested by Ex. 10, No. 25. Santonin and Meconic Acid must also be extracted by special methods.

Dragendorff's Method.-In this, a more extensive separation of the poisons is obtained, by multiplying the solvents and operations. The preliminary treatment is as per $\$ \S \mathrm{I}, 2$, and 3. The acid solution is then exhausted successively by petroleum ether, benzol and chloroform; and then in alkaline solution by the same sequence of solvents, and finally by amyl alcohol.

Kippenberger's Method.-This possesses the advantage of separating the poisons in purer form from the start, but it involves technical difficulties. It depends on the insolubility of the tannates of proteins in glycerin, whilst the organic poisons are soluble in this medium. The solid material (say $100 \mathrm{Gm}$.) is digested for two days at $40^{\circ} \mathrm{C}$. with $10 \mathrm{Gm}$. tannin, $1 \mathrm{Gm}$. tartaric acid, and $100 \mathrm{Gm}$. glycerin. The mass is expressed; the residue is washed with tannic glycerin. The fluids are diluted with water, heated to $50^{\circ} \mathrm{C}$., and filtered. The filtrate is then extracted twice with petroleum ether, which removes mainly fats. The glycerin layer is then extracted as in the Stas-Otto method $\left(\$ \S_{4}\right.$ and 5$)$, but using chloroform in place of ether.

For the purification of the alkaloids, the residue, left by the evaporation of the chloroform, is dissolved in acidulated water, neutralized, and at once precipitated by iodin-potassium-iodid. The precipitate is collected on a small filter, washed with cold water dried, and dissolved in hot acetone. The filtered acetone solution is treated first with alkali, then with acid, then with water; the acetone is expelled on a water-bath, and the remaining watery solution is decolorized by sodium hyposulphite. The solution is then made alkaline with sodium carbonate and extracted with ether or chloroform.

Physiologic Tests. - The poisons isolated by these methods are of ten not sufficiently pure to give the typical chemic tests; furthermore, certain of the tests are closely simulated by ptomains. This holds true also of the physiologic tests, i.e., the effects on animals. The only way to distinguish with certainty between these is to use both the chemic and the physiologic tests. For, as far as is known, there are no ptomains which give both the chemic and physiologic tests of one alkaloid.

The physiologic tests can generally be obtained with fairly impure preparations. They are most typical in the case of strychnin, atropin, physostigmin, aconite, digitalis group, picrotoxin, veratrin, cantharidin, croton oil. The subject is elaborated in Fuehner's "Nachweiss von Giften."

Isolation of Fixed Inorganic Poisons.-The residues of the preceding operations may be used for the search of these poisons. The usual

1 It is understood that the remaining mass should be washed, in every instance of filtration or straining. 
methods of inorganic analysis are followed; but it is superfluous to test for non-poisonous constituents.

The absence of many metallic poisons can be quickly shown by Reinsch's test. 'This cloes not, however, dispense from further tests. Only an outline can be given here:

A : Destruction of Organic Matter and Solution.-Method of Fresenius and Babo.()rgans or syrupy residue, plus 300 c.c. to 500 c.c. of arsenic-free $\mathrm{HCl}(\mathrm{r}: 2)$ in liter flask: heat lukewarm. Add $+206 \mathrm{Gm} \mathrm{KClO}_{3}$ in $0.5 \mathrm{Gm}$. portions, till dissolved. Evaporate to about roo c.c. (no free (l). Dilute to 400 c.c. Add 2 c.c. dilute $\mathrm{H}_{2} \mathrm{SO}_{4}$. Stand overnight. Filter. Fillrate $=B$. Residue $=K$.

Filtrate B.-Pass through filter, add water to just 500 c.c. Use 50 c.c. for Marsh's test (see $L$ ). If $A s$ is present, use balance for quantitative (see $C$ ). If not, evaporate small sample, dissolve in Io c.c. water, add $\mathrm{NH}_{4} \mathrm{OH}$ : blue $=\mathrm{Cu}$.

C. - Heat balance of filtrate $\mathrm{B}$ to $80^{\circ} \mathrm{C}$. and pass arsenic-free $\mathrm{H}_{2} \mathrm{~S}^{1}$ for two or three hours, until cool. Heat again, and repeat. Stopper and set aside in warm place for twenty-four hours. Precipitate may contain $\mathrm{As}, \mathrm{Sb}, \mathrm{Hg}, \mathrm{Cu}, \mathrm{Pb}$. It may be used for the quantitative estimation of As, or for further identification by $D$. Filtrate $=I$.

D ( $H_{2} \mathrm{~S}$ precipitale of $\left.\mathrm{C}\right)$. - Wash with $\mathrm{H}_{2} \mathrm{~S}$ water, warm with 4 c.c. ammon. sulphid, 4 c.c. ammonia, 8 c.c. water. Filter. Filtrate $=E$. Insoluble $=F$.

$\mathrm{E}$ (Filtrate of $D$ ).-Evaporate to dry; heat with $\mathrm{HNO}_{3}$ till pure yellow; heat to expel $\mathrm{HNO}_{3}$; add $\mathrm{Na}_{2} \mathrm{CO}_{3}$ and $\mathrm{NaNO}_{3}$; fuse; extract with boiling water; add 2 Gm. $\mathrm{NaHCO}_{3}$. Filter. Fillrate contains As and may be used for quantitative. The insoluble $=\mathrm{Sb}$ : (Apply tests.)

F (Insoluble of D).-Oxidize residue and filter in capsule with $\mathrm{HCl}$ and $\mathrm{KClO}_{3}$; filter; dilute; heat; pass $\mathrm{H}_{2} \mathrm{~S}$; filter; wash precipitate with warm $\mathrm{HNO}_{3}$. Filtrate $=G$. Precipitate $=I I$.

$\mathrm{G}$ (Filtrate of $F$ ).-Add ro drops dilute $\mathrm{H}_{2} \mathrm{SO}_{4}$; evaporate; take up with water. Residue $=\mathrm{Pb} . \quad$ Fillitate $=\mathrm{Cu} . \quad$ (Apply tests.)

$\mathbf{H}$ (Precipitate of $F)$. - Oxidize with aqua regia; evaporate; filter; dilute; test for $\mathbf{H g}$.

I (Filtrate of $C$ ). - Use half for zinc, half for chromium.

$\mathrm{Zn}$ : Neutralize with $\mathrm{KOH}$; acidulate with $\mathrm{H}_{2} \mathrm{C}_{3} \mathrm{O}_{2}$; precipitate with $\mathrm{H}_{2} \mathrm{~S}$; wash precipitate with $\mathrm{H}_{2} \mathrm{C}_{3} \mathrm{O}_{2}$ in $\mathrm{H}_{2} \mathrm{~S}$ water $(\mathrm{I}: 5)$; incinerate, precipitate and filter; dissolve in dilute $\mathrm{H}_{2} \mathrm{SO}_{4}$, plus a little $\mathrm{HNO}_{3}$; evaporate dry; dissolve in $\mathrm{H}_{2} \mathrm{O}$; test for $\mathbf{Z n}$.

Cr: Evaporate to just moist; mix with $\mathrm{KNO}_{3}$; dry; fuse; dissolve; test for chromate.

$\mathrm{K}$ (Residue of $A$ ). - Fuse with $\mathrm{KNO}_{3}, \mathrm{Na}_{2} \mathrm{CO}_{3}$, and $\mathrm{NH}_{4} \mathrm{NO}_{3}$. Suspend in $\mathrm{H}_{2} \mathrm{O}$; pass $\mathrm{CO}_{2}$; boil; filter. Dissolve precipitate in dilute $\mathrm{HNO}_{3}$. Test this solution for $\mathrm{Ag}, \mathrm{Ba}$, and $\mathrm{Pb}$.

Marsh's Test (see B).-Produce hydrogen in flask by acting on pure zinc with arsenic-free $\mathrm{HCl}$; pass through $\mathrm{CaCl}_{2}$, through tubes drawn out at several places. Heat to redness at the thick portion of a segment. (This blank test should be continued for six hours.) If no mirror appears, introduce the suspected solution. Black mirror occurs with arsenic or antimony. They may be distinguished as follows:

ARsenic

Mirror beyond heated portion.

Garlic odor on heating in air.

Dissolves in hypochlorite.

Easily volatilized when heated in hydrogen.

Heated in air, yields easily volatilized white crystals. $\mathrm{HCl}$.

Heated in $\mathrm{H}_{2} \mathrm{~S}$, yellow, insoluble in

Discolved in $\mathrm{HNO}_{3}$, evaporated, plus $\mathrm{AgNO}_{3}$, plus vapor of $\mathrm{NH}_{3}$, red or yellow precipitate.

\section{TREATMENT OF POISONING}

Whilst every case of poisoning demands to be treated by itself, yet certain general principles are all but universally applicable. These are the more important since the nature of the poison is so often unknown.

$1 \mathrm{H}_{2} \mathrm{~S}$ purified by passing through water, calcium chlorid, and a tube, $30 \mathrm{~cm}$. long, filled with alternate layers of glass-wool and iodin crystals. 
The first peculiarity of poisoning to deserve especial attention is the rapid course. This demands that whatever measures are taken, they must be taken quickly. The physician should therefore be thoroughly familiar with the general rules of treatment, so that no hesitation or delay occurs. On this account also, the antidote nearest to hand should be used in preference to one which can only be obtained with delay, even if the latter should be theoretically preferable.

The treatment of poisoning is directed against the cause and the symptoms. The former consists in removing the poison, or in rendering it harmless. Since neither of these objects can usually be attained absolutely, both are generally attempted at once.

Removal of the Poison. - The measures directed to this end must vary with the site to which the poison was applied.

On the skin and on accessible mucous membranes this will be accomplished by a thorough washing with water. This will also be useful by diluting the poison. If the poison is only sparingly soluble in water (as phenol) alcohol may be employed. Soap may be useful, but should be avoided if the poison is an alkali. The appropriate chemical antidote should also be added to the wash water (for acids or bromin: Soap or Linimentum Calcis; for alkalies: vinegar or lemon juice). The poisons which are important in these situations are all irritants, and the further treatment consists in the application of oils or salves.

If the poison has been applied hypodermically or to wounds, the systemic effects may be lessened by preventing or retarding the absorption of the poison. The absorption can often be delayed sufficiently so that the drug is excreted as fast as it is absorbed, and doses which would otherwise be fatal may then cause no effect. The best means for this purpose is a firm ligature applied centrally to the wound. Where this is not feasible, sucking, cautery, or excision may be resorted to.

If an irritant poison has been taken by the mouth, the oral cavity and the pharynx demand the same treatment as would the skin.

With gaseous poisons, the treatment consists in free ventilation of the lung, using artificial respiration, and oxygen, if necessary.

Removal from the Alimentary Canal.- This is always the first step in the treatment of a case of poisoning by mouth, unless the symptoms are already so far advanced as to make it useless. Even if the poison has been administered some time before the treatment is begun, or if it has been given hypodermically, the alimentary canal should be cleansed, since many poisons (notably morphin) are excreted by this channel, and then again reabsorbed. The only contraindications to the emptying of the alimentary canal are: extensive corrosion, and advanced strychnin poisoning. Great caution and careful judgment are required in these cases.

The measures for the removal of poisons from the alimentary canal consist in emptying the stomach and intestine.

$\dot{A}$ cathartic, however, need only be administered after the acute manifestations are past. Oily cathartics should usually be avoided, and enemeta are useless. The best purgatives in poisoning are the cathartic salts ( 15 to $30 \mathrm{Gm}$. of Magnesium sulphate).

Evacuation of the Stomach.-Vomiting often occurs spontaneously, but even in this case, more active measures are generally required. These consist in the administration of emetics, or in lavage.

Lavage always deserves preference, if it can be used. It cleanses 
the stomach much more effectually, particularly of small insoluble adherent particles; it is less depressant to the patient; and it permits the convenient use of chemic antidotes. It is indispensable with depressant poisons which paralyze the vomiting center (such as deep morphin or chloral poisoning).

The complicated stomach pump has been generally abandoned for the more convenient stomach tube. In emergencies, 6 feet of rubber gas tubing, with a funnel attached, answers very well. The tube should not be forced down, but should be gently pushed to the pharynx, where the pharyngeal muscles will grasp it and push it further. Care should be taken to avoid overdistention of the stomach; it is much better to use small quantities of fluid frequently. If there is much pain, 0.05 per cent. to 0.1 per cent. of cocain may be added to the wash water; against organic poisons, a $0.5: 1,000$ solution of potassium permanganate may be used. If there is trismus, or the patient resists, the tube may be passed through the nose.

Emetics have the advantage of greater convenience, and avoid struggling. They should be repeated every fifteen to thirty minutes, if necessary.

A pomorphin.- [5 mg. (grain 1/10 in I per cent. solution $=0.5$ c.c. or $m x$ hypodermically. This is the only emetic which can be given by the skin, and is therefore particularly useful if the patient resists. It is very prompt and certain, but rather more depressing than the following emetics:

Copper Sulphate or Zinc Sulphate.-These act promptly and with a minimum of depression, but should not be given if the poison is a corrosive. Zinc sulphate is less irritant than the copper, and nearly as efficient; $2 \mathrm{Gm}$. may be given in a glass of water. The dose of copper sulphate is $0.5 \mathrm{Gm}$. at once, or three doses of $0.3 \mathrm{Gm}$., fifteen minutes apart. If it is ineffective it must be removed by lavage.

I pecac. - A teaspoonful of the powder in water, or a tablespoon of the wine. Uncertain, and produces considerable depression.

M ustard. A teaspoonful stirred in a tumbler of warm water. This is the most uncertain of these emetics, but useful in emergencies.

Neutralization of the Poison-Chemical Antidotes.-The object of these is, to render the poison incapable of acting, or of being absorbed. They accomplish this by enveloping the poison with an impenetrable coating, or by precipitating it, or by destroying it. Since these antidotes are hindered in their action by the presence of food, and since the precipitates are not perfectly soluble, it is well to combine them with lavage or emesis, adding the antidotes to the wash water, or giving them in the interval between vomiting; they must be repeated frequently.

Demulcents (raw eggs, acacia, boiled starch or flour, milk, ad libitum). -These act by lessening absorption; by allaying inflammation; and in the case of metals, by precipitation.

Precipitants.-The most universally applicable of all precipitants is tannin (a teaspoonful of tannic acid, or preferably, very strong hot tea, ad libitum). The efficiency will be increased by the addition of sodium acetate or bicarbonate, and diminished by alcohol and acids, since the precipitates are soluble in these media.

From the experiments of Kiefer (1892), on tannin, and Sollmann (1901), on tea, it may be deduced that these are useful against: Apomorphin, Cinchona alkaloids, Hydrastin, Strychnin, Veratrin, Digitalis, Antipyrin, Colchicin; and the metals: Pb, $\mathrm{Ag}, \mathrm{Al}, \mathrm{Co}, \mathrm{Cu}, \mathrm{Ni}, \mathrm{Ur}, \mathrm{Zn}, \mathrm{Fe}$. 
Scarcely useful against: Cocain, Brucin, Aconitin, Lobelin, Nicotin, Pilocarpin, Codein, Muscarin, Physostigmin, Solanin.

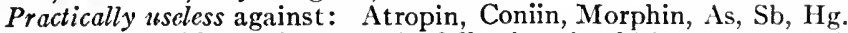

Of other, specific precipitants, the following should be remembered:

For

Alkaloids.-Tincture or compound tincture of iodin, 15 drops in half a glass of water.

Metals (especially mercury).-Raw eggs.

Arsenic.-Arsenic antidote (see Index).

Barium.-Sulphates (Glauber's or Epsom salt).

Oxalates.-Calcium (limewater, chalk, whiting).

Phosphorus. Copper sulphate or Oxidized (old) Turpentine. (The former envelopes the phosphorus with an insoluble coating of metallic copper. Turpentine forms the insoluble turpentine-phosphoric acid.)

Antidotes Which Destroy the Poison in the Alimentary Canal.(These, for the most part, will not be useful after the poison is absorbed.)

Acids.-Alkalies (burnt magnesia, soap, chalk, baking soda).

Alkalies.-Weak acids (vinegar, lemon juice).

Organic Poisons (Alkaloids, Glucosids, etc.) and Phosphorus.-Oxidizing agents, especially Potassium permanganate, I $\mathrm{Gm}$. (one-fourth teaspoonful) in a quart of water. Care must be taken that no undissolved crystals are administered; $\mathrm{H}_{2} \mathrm{O}_{2}$. (In case of snake bite, the powdered permanganate may be rubbed into the wound, after free incision.)

Hydrocyanic Acid.-The above, also arsenic antidote, hydrogen peroxid, and hyposulphite of sodium.

Antidotes Which Destroy Absorbed Poisons.-To be effective, these must be given hypodermically or intravenously. The best known examples of this class are the antitoxic sera. The following are also useful: Sodium hyposulphite against hydrocyanic acid; and sodium carbonate against mineral acids.

Removal of Poisons after Their Absorption.-This can be attempted by increasing the excretions, but is usually not very successful. The elimination by the alimentary canal has been discussed. The principal remaining channels are the urine and sweat, of which the former is by far the more important.

Diuretics.- Water, especially as weak tea or carbonated drinks; theobromin; potassium acetate (irritant diuretics should be avoided).

Diaphoretics.-Hot drinks; heat; pilocarpin (if not contraindicated by pulmonary edema). Diuresis and diaphoresis often fail, partly because they act too slowly, partly because not all poisons are eliminated by these channels.

Infusion of Normal Saline Solution (I Liter of 0.9 per cent. injected under the skin of the subclavicular region) increases elimination by all channels. Bleeding may be resorted to in some cases. Up to a liter of blood may be drawn, a double quantity of salt solution being injected.

Symptomatic Treatment.-The symptoms produced by poisons can of ten be lessened or removed by the use of drugs having an opposite action. It is not to be supposed that these physiologic antidotes really lessen the action of the poison, although they cover its symptoms. But by tiding the patient over the dangerous period, and by preventing exhaustion, they are often potent means of saving life. It must be remembered, however, that large doses of the antidotes often cause effects similar to those of the poison, which may add to the fatality of the latter. They must therefore be used in appropriate small doses.

It is not purposed to enter in this place into the special physiologic antidotes, but we shall take up only those measures which are generally useful.

These measures are directed, in the order of their importance, to: sup- 
porting the respiration, heart, and vasomotor tone; to lessen cooling, pain, convulsions and coma.

Respiration.- This is usually the first function to fail, and accelerates the other actions of the poison through the convulsant and paralytic effects of asphyia, and by preventing the destruction of poisons through oxidation. For these reasons, it is well not to wait with the supporting measures until the respiration has actually failed, but to begin as soon as it shows signs of weakening. The measures consist in direct or reflex stimulation of the respiratory center; in artificial respiration; and in the administration of oxygen.

Reflex stimulation of the respiratory center is the quickest, but can not be kept up as long as the direct stimulation. It may be secured by the administration of ammonia, by inhalation of ammonia water or smelling salts, or by aromatic spirits of ammonia (half a teaspoonful in a glass of water); the alternate application of heat and cold (whipping with wet towels); friction with alcohol, or camphorated oil; hypodermic injection of brandy, whiskey, or ether; mustard plasters.

Direct Stimulation of the Respiratory Center.-By strong hot coffee, strychnin $(0.002 \mathrm{Gm}$.) or atropin $(0.001 \mathrm{Gm}$.). The respiration may also be raised by improving the circulation. Saline infusion is also very effective.

Artificial Respiration. - This should be resorted to whenever the above measures are ineffectual.

Any of the commonly used methods (also rhythmical stimulation of the phrenic nerve) may be used. It should be remembered that very prolonged and violent artificial respiration may injure the lungs. It should also be remembered that artificial respiration is apt to induce apnea, so that the patient does not breathe spontaneously simply because there is not enough $\mathrm{CO}_{2}$ in the blood to stimulate the respiratory center. If the heart is strong, the artificial respiration should therefore be intermitted occasionally for a short time.

Oxygen.-This will be useful in every case of failing respiration, and particularly if an asphyxiant gas is the cause of the poisoning.

Heart.-Attempts to revive or even to support the poisoned heart directly have not been very successful. The best results are obtained by the injection of normal saline, possibly with the addition of some epinephrin ( $\mathrm{r}: 100,000)$. Digitalis is usually unsuccessful. Strong rhythmical pressure (rate of roo per minute) over the cardiac region of the chest, may be helpful. A dilated heart can sometimes be revived by withdrawal of blood.

Vasomotor Stimulation.-This is usually accomplished by the reflex stimulants mentioned under respiration. Lowering of the head and bandaging the extremities is often sufficient.

Cooling.- This is prevented by the application of external heat; pain by morphin, or if local, by cocain; convulsions are controlled by the cautious inhalation of chloroform; coma by reflex stimulants, coffee, and atropin.

Methods of Administering Antidotes.-Physiological antidotes should be given hypodermically or intravenously, if possible. This obviates the loss of the antidote through vomiting, and the action is more prompt and more certain.

If the circulation is very low the absorption of hypodermic injections may also be very slow. It is therefore well thoroughly to massage the site of the injection, and if the circulation has almost stopped, to employ vigorous rapid rhythmical compression 
of the heart (this maintains a fairly efficient artificial circulation even after the heart has stopped beating).

Résumé of the General Treatment of Poisoning.-Promptness is of vital importance. The physician should be familiar with antidotes; he should have these antidotes readily accessible; he should plan his treatment on his way to the patient. If he finds the condition of the patient dangerous, he should at once proceed to relieve the symptoms. Otherwise he should first administer the chemical antidote and evacuate the stomach; apply heat; attend to the respiration, to pain, to any other symptoms; give a diuretic, and, finally, a cathartic.

Antidotes for First Aid.-Every physician (and every drug store) should keep the following antidotes together, in a special satchel ("Antidote-Bag") so that they can be readily transported. The dose should be written on each container. Amyl nitrite pearls; Apomorphin tablets, 2 mg.; Atropin tablets, I mg.; Caffein Citrate; Chloroform; Cocain hydrochlorid tablets, $0.03 \mathrm{Gm}$. (2 to 4 per quart); Tincture iodin; Copper sulphate, powdered; Lime Water; Magnesia, calcined; Potassium permanganate, I per cent. solution (to be diluted ten times); Sodium sulphate; Spir. Ammon. Arom.; Strychnin sulphate tablets, 2 mg.; Whiskey; also a hypodermic syringe in good order, and a stomach-tube with funnel.

The following should be demanded at the house of the patient: Boiled water; Coffee (strong, hot, and black); Eggs; Hot-water bags; Milk; Mustard; Salad oil; Salt; Soap; Starch, boiled; Tea, Vinegar.

\section{PRESCRIPTION WRITING}

Parts of the Prescription.-A prescription is an order for medicine, sent by a physician to a pharmacist. It consists of the following parts (in this order):

I. Superscription.-The heading $(\mathrm{R})$.

The $\mathrm{P}$ stands for recipe, take thou. The stroke is generally considered an ancient invocation to Jupiter; but probably it is simply a sign of abbreviation (McGuigan, I9 3 ).

2. Inscription.-The ingredients and their amounts.

3. Subscription.-The directions to the dispenser.

4. Signature.-The directions to the patient.

The prescription should also bear the signature of the physician, the date, and the name of the patient.

The Inscription may consist of but one ingredient. If several are used, they should be placed in the following order:

Basis: the principal substance.

Adjuvant: the substance which is used to aid the action of the basis.

Corrective: whose purpose it is to modify or correct an undesirable action of the basis.

Vehicle: the indifferent substance used to dilute the active ingredients.

The following is the type of a complete prescription:

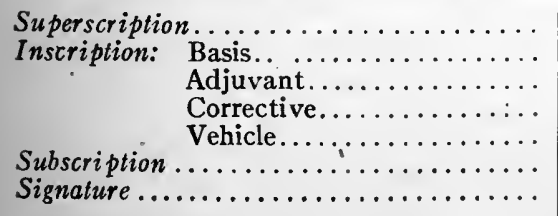

R. Tincturæ Aconiti. ............ of 45

Spiritus Atheris Nitrosi....... I5

Liquoris Ammonii Acetatis...... I 5

Elixiris Aromatici........... 30

Misce

S. Teaspoonful every hour in half a glass of water.

Dr. X.

Jan. I, I905. 
The names of the ingredients and the directions to the pharmacists are generally written in Latin, usually with abbreviations; but they may be written in English. With liquid mixtures, the directions to the pharmacist may often be confined to $\mathrm{M}$. (misce, $\mathrm{mix}$ ).

The directions to the patient (signature abbreviated Sig. or S., for "signa," sign) are always written in English, so that the patient can read them. The directions should be made as complete as possible, and should include everything which it is necessary for the patient to know. The habit of giving verbal instructions to patients and of having the medicine labeled "use as directed" can not be too much discouraged. Aside from the fact that human memory is extremely apt to fail, the patient or relatives, when the prescription is given to them, are usually in a more or less excited frame of mind, and can not be relied upon to remember what is told them. Medicines intended for external application should be plainly labeled to that effect, and when a medicine contains poison, it should be so labeled, except when there is special objection to this.

The custom of having prescriptions "refilled" obtains in many localities. Whereas it is often impossible for the physician to put a stop to this practice, it is absolutely necessary that he should prevent such prescriptions being refilled which contain narcotics or other drugs likely to cause a habit. He can attain this result by writing "non repetatur" under the prescription. The druggist refilling this will do so on his own responsibility. (The law prohibits the refilling of prescriptions containing morphin or cocain, with their derivatives. A prescription must be written each time that these are to be dispensed.)

When the patient is very poor, it is often customary to invite the druggist to charge him the lowest terms by writing $P$. $P$. (Pauperissimus) under the prescription. It is, of course, not just to do this if the physician himself receives a regular fee.

Forms of Administration.-If the medicine is to be used internally, it may be prescribed as:

Solution.-If the ingredients are soluble in water, alcohol or glycerin.

Mixtures or emulsions, i.e., suspended by acacia, if insoluble in water.

Powder.-If the substance does not have a very bad taste. These are preferred to the following preparations if the dose is bulky, or if the substance is to be dissolved in water before swallowing.

Pills, Capsules, Etc.-If the substances are solid or semisolid, and possess a disagreeable taste; or if they are to act locally on the alimentary canal.

If the medicine is intended for external use, it may be prescribed as:

Plaster.-If the action is to be prolonged and superficial.

Ointment.-If the action is to be deeper, or briefer.

Lotions or Injections, Eye-waters, Gargles, Etc.-If it is soluble in water.

Liniment.-If it is soluble in oil or alcohol.

Size of Prescriptions.-Fluids should be prescribed so as approximately to fill the bottles in current use (I5,30,60, I25, I50, 200, 400 c.c.) $(1 / 2, \mathrm{I}, 2 ; 4 ; 6 ; 8$ and $\mathrm{I} 6$ ounces).

For drop doses and eye-waters, the usual amount is $I 5$ to 30 c.c. ( $1 / 2$ to I5) ; for teaspoon doses, 30 to I 25 c.c. ( $I$ to 45 ); for gargles and injections, 60 to I 50 c.c. ( 2 to 65 ); lotions, I 20 to 200 c.c. ( 4 to 85 ); tablespoon doses, $\mathrm{r} 50$ to 400 c.c. (6 to 165 ). Pills are prescribed in numbers of 5 to 25 ; powders, I to I 2 ; ointments, Io to $30 \mathrm{Gm}$. ( $1 / 2$ to I 5$)$. 
The Need of Practice in Prescription Writing. ${ }^{1-}$ The subject of prescription writing seems to possess almost insurmountable difficulties for the student. There is, perhaps, no other subject in the whole course of study which he finds more discouraging and finishes with less satisfaction. Nevertheless the principles upon which it is based are few, simple, and easily memorized. When asked for them, the student usually has no difficulty in answering the questions. The difficulties appear when he tries to put these principles into practice. But it is the same with any other art: nothing but practice will give expertness.

The prescription is usually a fair indication of the physician's training, knowledge and character. The student who would master this subject must not rest content with doing the few exercises which can be given him in class. As he studies each drug, or as he reads up the treatment of diseases, he should himself compile such prescriptions as the subject suggests. This will not only aid him in prescription writing, but in pharmacology and therapeutics as well. It is only in this way that he can acquire the necessary self-confidence and skill. In this home practice, method and detail should be cultivated, for in these lies the secret of the art. The following rules may prove helpful:

Routine Construction of Prescriptions.-When writing a prescription for a given condition, put down, first, the name of the best remedy. Ask yourself whether there is any other drug which may be employed to aid or usefully modify this. Put this down also.

Then consider in which form the medicine should be administered, whether as liquid, powder, salve, etc. This will usually determine which preparation of the ingredient is to be employed. Put this down also.

Then ask yourself what may be added to render the mixture agreeable to the patient. Add the vehicle. When this is written, all the ingredients will be represented.

Now look over these carefully and see that there are no incompatibilities and that the constituents are soluble if the mixture is to be a liquid. Write the directions to the dispenser. Assure yourself that the prescription is grammatically correct (especially the endings).

Decide how many days the mixture is to be taken, and how many doses a day (on the basis of sixteen hours to the day). Decide whether the dose of the total mixture is to be a teaspoonful, tablespoonful, etc. By multiplying the total number of doses with the size of the single dose, ascertain the approximate size of the mixture. Round this off to a convenient figure. Multiply the single dose of each ingredient by the total number of doses (again reducing the quantities to round numbers, unless the constituent is very active). ${ }^{2}$ Check the doses. Write the directions to the patient. Consider whether a non repetatur is advisable. Affix your signature, the date, and the name of the patient. This finishes the prescription. Look over the result carefully in the same order.

It would seem almost superfluous to mention that it is extremely essential in writing prescriptions to use as legible handwriting as possible. It is astonishing, in view of the dire results that may follow, how often this self-evident rule is disregarded. The same remarks apply to abbreviations. While it is justifiable to employ abbreviations even extensively, it is necessary, to make these in such a way that they can not possibly be

1 The author has found it very useful in class practice in prescription writing, to make some of the students in turn work out the prescriptions on the blackboard, and to subject these to the criticism of the class.

One may also write the quantities for a single dose, and state in the subscription the number of doses desired: "Fac tales doses no. XX" (make 20 such doses.) 
misinterpreted. It is, therefore, advisable to employ the official abbreviations which are given under the "Preparations."

An efficient aid in acquiring practice in writing prescriptions is to look over a druggist's prescription file, when this may be done.

The copying of a prescription, ingredient for ingredient and dose for dose, is as much empiricism as the use of any other ready-madecompound. The physician should be well enough educated to devise his own prescriptions and to select such ingredients as will best suit the special needs of the case in hand.

Simplicity in Prescription Writing.-Formerly the ingredients of a prescription were almost numberless. This was in the time of empiricism, and was simply an application of the idea that in a large mixture of substances there would probably be one which would do good. This was the so-called "shot-gun prescription." At present the tendency in prescription writing is to make the prescription as simple as possible. This avoids the chances of incompatibilities, and, what is more important, makes the action of the medicine more easy to watch and control. Especially, one should avoid to combine, in the same prescription, drugs which differ markedly in quickness of action, absorption or elimination; or habitproducing drugs with others that will presumably be used for long periods.

If the prescription includes a number of mixtures, each containing several ingredients, such as the numberless preparations now put on the market by many firms, the result is, of course, as much a "shot-gun" prescription as if the prescriber had enumerated all the ingredients.

Posology (Doses).- The dosage of drugs must be adapted to the individual patient. The Pharmacopeias state "Average Doses" (adult) for each preparation that is likely to be used internally; but these are not intended to be binding on physicians. They are, however, convenient approximations for memorizing. The metric and apothecaries' doses in the U.S.P. are not intended as mathematical equivalents, but represent rather the quantities that would be used by prescribers in these systems.

The doses of crude drugs are given mainly as a basis for calculating the preparations.

Maximal Doses.-The pharmacist is supposed to check the quantities of the ingredients, and not to dispense a prescription containing an unusual dose of a powerful poison without convincing himself that the physician prescribed this intentionally. While this does not in any way lessen the responsibility of the physician, it is a safeguard which deserves all encouragement. To avoid delay, it is customary to mark such large doses in such a way that the pharmacist will have no doubt that they are intentional. Thus: the best.

Tr. aconiti, $3 \mathrm{j}$ or $3 \mathrm{j}$ !; or $3 \mathrm{j} \mathrm{Q}$. R. (quantum rectum), the last being

Most foreign pharmacopeias (but not the U.S.P. or B.P.) give tables of "Maximum Doses;" not with the purpose of restricting the physician, but as a guide to the pharmacist. The latter is not permitted to dispense doses larger than these, unless the physician indicates specifically (as explained above) that the larger doses are intentional. The maximum doses in this book are taken from the compilation of the U.S.P. Digest, I91 2, pp. 40-42. 


\section{PRESCRIPTION LATIN}

It is customary, but not compulsory, to write the inscription, and often the subscription in Latin. A slight knowledge of the rules of grammar of this language is therefore desirable. It is supposed that this is possessed by the student, and the following is intended merely to recall some of the more important facts.

Advantages of Latin.-The use of the Latin names of drugs is generally urged because they are more definite and concise, and less liable to change than common names; and because they serve to keep the patient in ignorance of the nature of the drugs. This is sometimes very desirable, fi.., to guard against drug habits. However, the official English names are equally definite; and as either names are usually abbreviated, there is little difference. As a matter of fact, less than 5 per cent. of the U.S.P. "Latin" names are really Latin (Oldberg, igro).

In the directions to the dispenser, Latin has no advantage, except in the brevity of the customary abbreviations. . Indeed, unusual Latin terms are apt to lead to mistakes. The discarding of Latin in prescription writing is being actively discussed (Fantus, I916).

Grammatic Rules.-The superscription, R (recipe: "take thou"), requires the name of the substance to be in the genitive, if the quantity is given, the quantity itself being in the accusative (the latter is, of course, very rarely written out in full). When the quantity is not given, the name of the substance is to be placed in the accusative. Adjectives agree with their nouns in gender, number, and case.

These rules will generally be understood by translating into English: e.g., Take thou of tincture of aconite I ounce.

The following rules for the formation of the genitive case will be found valuable ("Mann's Manual"):

RULE I.-All nouns ending in a, form the genitive in $æ$; as Quinina, Quininæ. Exceptions: Aspidosperma, Physostigma, and Theobroma form the genitive in atis. Folia is plural; genitive, foliorum.

RULE 2.-All nouns ending in us, um, os, on, form the genitive in $\mathrm{i}$; as Conium, Conii. Exceptions: Rhus, gen. Rhois; Flos, gen. floris; Erigeron, gen. Erigerontis. Fructus, Cornus, Quercus, Spiritus, do not change.

RULE 3.-All other nouns of whatever termination make the genitive in $\mathrm{s}$, or is. Elixir, gen. Elixiris.

Some lengthen the termination thus:

as, genitive
is, "
$0, \quad$ "
$\mathrm{x}$,

\author{
atis; as Acetas, \\ idis; as Anthemis, \\ onis; as Pepo, \\ cis; as Cortex,
}

Acetatis.

Anthemidis.

Peponis.

Corticis.

There are a few exceptions. Asclepias, gen. Asclepiadis; Mas, gen. Maris; Phosphis, Sulphis, etc., gen. itis; Mucilago, gen. Mucilaginis; Solidago, gen. Solidaginis; Pulvis, gen. Pulveris, etc.

The following words do not change in their genitive: Azedarach, Buchu, Cannabis, Catechu, Condurango, Cornus, Curare, Cusso, Fructus, Digitalis, Hydrastis, Jaborandi, Kino, Matico, Quercus, Sassafras, Sago, Sinapis, Spiritus, Gambir, Sumbul.

The accusative is rarely employed. It is formed according to the following rules (Mann):

RULE I.-Nouns singular ending in a, are feminine, and make the accusative singular in am and the plural in as. Example: Drachma, acc. sing. Drachmam, pl. Drachmas.

RULE 2.-Those ending in um or us, make the accusative singular in um. The accusative plural of those in us is os, and of those in um is a. Those in $u s$ are masculine, those in $u m$ are neuter:

Congius, acc. sing. Congium; acc. pl. Congios.

Granum, acc. sing. Granum; acc. pl. Grana.

RULE 3.-Those whose genitive ends in is, form the accusative in em, plural es. 
Attention is also called to the fact that adjectives change their endings, a fact which the student is apt to forget.

The following prepositions are frequently used, and command the following cases:

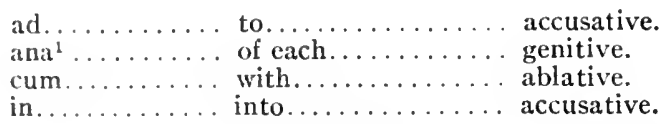

The following Latin words and phrases occur frequently in prescriptions (adapted from Mann):

ad

ad li

ana $(\bar{a} \bar{a})$

aqua bulliens

"fontana.

" fervens.

destillata

bene.

bis in dies.

cape, capiat.

charta.

chartula.

cibus

cochleare magnum

parvum.

cola, colatus

collyrium

congius

cum

(C.).

(C.)..............

dilute, dilutus

dimidius.

divide (Div.)

dividendus.

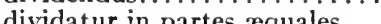

atur in partes aquales

dosis.

extende supra

fac, fiat, fiant $(\mathrm{ft}.) \ldots \ldots \ldots \ldots \ldots \ldots \ldots \ldots \ldots$

fac tales doses.

filtra.

gargarisma

gutta, gutta (gtt.).

haustus.

hora.

hora somni.

hora decubitus

in dies.

instar.

lac.

libra (lib.)

mane primo

magnus.

misce (M.)

more dictu.

non repetatur.

numerus, numero (No.)

octarius (O.)

ovum.

pars.

partes aquales (P. æ.)

parvus.

\footnotetext{
2 From the Greek.
}

to, up to

at pleasure

add (thou)

of each

boiling water

spring water

hot water

distilled water

well

twice daily

take, let him take

a paper (medicated)

a small paper for a powder

food

a tablespoon

a teaspoon

strain, strained

an eye wash

a gallon

with

dilute (thou), diluted

one-half

divide (thou)

to be divided

let it be divided into equal parts

a dose

spread upon

make, let be made, let them be made

make such doses

filter (thou)

a gargle

a drop, drops

a draught

an hour

just before going to sleep

at bed time

daily

like (with genitive)

milk

a Troy pound

very early in the morning:

large

mix

as directed

do not repeat

a number, in number

a pint

an egg

a part

equal parts (governs genitive)

small 
pilula (pil.) ................. a pill

pro re nata (p.r.n.)................ according to circumstances, occasionally

pulvis (gen., pulveris) $\ldots \ldots \ldots \ldots \ldots \ldots$ a powder

quantum sufficit (q. s.) ........... as much as is necessary

quaque hora .................... every hour

(cum) semisse (ss.) .............. and a half

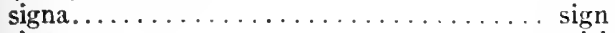

sine......................... without

si opus sit..................... if necessary

solve, solutus................. dissolve, dissolved

solutio...................... a solution

statim...................... immediately

talis........................ such

tritura.......................... triturate

tere simul.................... rub together

tèr in die $(\mathrm{t} . \mathrm{i} . \mathrm{d}.) \ldots \ldots \ldots \ldots \ldots \ldots \ldots \ldots \ldots$ three times a day

vitellus..................... the yolk (of an egg)

\section{COLORING AND FLAVORING}

\section{COLORING}

Liquid prescriptions intended for internal administration should be clear if possible. It might be well to mark all such prescriptions "filtra." The appearance may often be materially improved by the addition of some coloring-matter, and this may also prove useful through its suggestive element and by hiding the nature of the medicine from the patient. The nature of the coloring-matter must vary according to the nature, and especially the reaction of the mixture. The usual colors for internal use are red, brown, and yellow. Anilin dyes should be avoided, except methylen blue and gentian violet for external preparations.

The most useful dyes are the following:

\begin{tabular}{l|l|l|l}
\hline & \multicolumn{1}{c}{ Red } & \multicolumn{1}{c}{ Brown } & \multicolumn{1}{c}{ Yellow } \\
\hline For alkaline or neutral fluids: & $\begin{array}{l}\text { Tr. Cardamomi } \\
\text { Co. }\end{array}$ & $\begin{array}{l}\text { Fldext. Glycyr- } \\
\text { Lhizæ }\end{array}$ & Tr. Hydrastis \\
For acid fluids: & $\begin{array}{l}\text { Tr. Persionis } \\
\text { Elixir Adjuvans }\end{array}$ & $\begin{array}{l}\text { Caramel } \\
\text { For powders: }\end{array}$ & $\begin{array}{l}\text { Tr. Hydrastis } \\
\text { Tr.Curcumæ }\end{array}$ \\
\hline
\end{tabular}

\section{PREPARATIONS-RED COLORS ${ }^{1}$}

Coccus, Cochineal, U.S.P., B.P.-The dried female insect of Coccus Cacti.

Tr. Cocc., B.P. - 10 per cent. Dose, 0.03 to 0.1 c.c., 5 to 15 minims, B.P.

Carminum, Carmine.-A precipitate obtained from decoction of cochineal by alum or cream of tartar. Soluble in alkalies, brightened and precipitated by acids. Also soluble in alcohol. Contains carminic acid. Used in powders, etc. $(r: 500)$.

*Liquor Carmini (Liq. Carm.).-A glycerinated extract of carmine. Pink to deep red in alkaline solutions, precipitated by acids. Used in the proportion of about I per cent.

$\left({ }^{*}\right.$ Tr. Cardamomi Co. (Tr. Card. Co.). - An aromatic alcoholic (50 per cent.) vehicle, colored with cochineal. Used undiluted (see Index).

*Tr. Persionis (Tr. Persion.); Tinct. of Cudbear (a lichen allied to litmus).- - Red in

1 The preparations of each class are generally arranged alphabetically under the crude drugs. The important preparations, which the student is expected to study, are marked. * In addition to these, he should glance over the corresponding crude drugs. 
neutral or acid, purplish in alkaline solutions. Used in the proportion of about $\mathbf{r} .5$ per cent.

Sant:alum Rubrum (Santal. Rub.), U.S.P.; Pterocarp. Lign., B.P., Red Saunders.The heart-wood of Pterocarpus santalinum. Used in Tr. Lavand. Co.

\section{PREPARATIONS-BROWN COLORS}

Caramel is a concentrated aqueous solution of carbonized sugar. Commercial caramel is generally made by heating glucose with a little alkali, until the sweet taste is destroyed and a uniform dark mass results. It is probably a complex mixture. Caramel from cane-sugar according to Stolle, $\mathrm{I} 899$, is a single substance, $\mathrm{C}_{12} \mathrm{H}_{18} \mathrm{O}_{9}$, formed by the elimination of two molecules of water from sucrose. Caramel is being tried in diabetes, being apparently well tolerated (Grafe, I9I4; Umber, I9I5).

*Fldext. Glycyrrhize and *Elix. Glycyrrh. (see Index).

\section{I'REPARATIONS - YELLOW COLORS}

Crocus, Safiron, has been used as abortifacient; but it apparently lacks this action and is practically non-toxic (Cevidalli, I9I4).

${ }_{*}$ Tr. Curcume (Tr. Curcum.) (Turmeric) precipitates with water, but sufficient remains in solution to impart a yellow color. Used in ratio of 1 per cent. Curcuma changes to reddish brown in alkalies.

*Tr. Hydrastis (Golden Seal) is used similarly (see Index).

\section{FLAVORING}

This subject, although often neglected, is quite important. It not only humors the patient and secures more conscientious compliance with the prescription, but also of ten aids absorption and digestion-just as condiments do in foods.

Patients often fail to take a disagreeable medicine, and the physician should always be on the lookout for such cases. It is scarcely necessary to say that he should approximately control the amount taken by judging as to the quantity left in the container, as he should also, in general, control the medicine dispensed by the druggist as to its appearance and taste.

Some patients carry the deceit further and pour away the appropriate amount of the medicine, and if the physician does not obtain the anticipated results, it may be well to prescribe some test medicine, such as salol $(0.3 \mathrm{Gm}$.), or potassium iodid $(0.5$ $\mathrm{Gm}$.), which can be detected in the urine.

Improving the Taste of Liquid Medicines.-This may be done by dilution; sweetening; demulcents; aromatics; alcoholics; acids; bitters; and drugs which paralyze the taste-endings. Several of these means are often combined.

Simple Dilution.-This is often most effective for salts, alkalies, chloral and other hypnotics; and in general, substances with disagreeable but not very marked taste. These should be taken in a half-glass or glass of water, milk, tea, or carbonated water.

The Most Important Flavors.-The following will be found to be generally sufficient:

To disguise bitter taste: Aromatics (Tr. Cardamomi Co.; Elixir Aromatic; Syr. Aurantii); for children, Glycyrrhiza, Aq. Anisi, Syr. Tolutanus.

To disguise acrid taste: Aromatics as above; also syrups, acacia, acid flavors (Syr. Aurant.).

To disguise salty or alkaline taste: Dilution with simple or carbonated or aromatic water or milk.

To disguise oily taste: Aromatics (Oil or Spirit of Peppermint, lemon, orange, wintergreen or almond, according to personal preference).

For insipid drugs: Aromatics and acids (Syr. Aurantii). 


\section{PREPARATIONS-SUGARS AND HONEYS}

*Saccharum (Sacch.), U.S.P., ${ }^{1}$ Saccharum Purificatum (Sacch. Pur.), B.P.; Sugar (Cane or Beet), $\mathrm{C}_{12} \mathrm{H}_{22} \mathrm{O}_{11}$. - Very sol. in water ( $\mathrm{r}: 0.5$ ), slightly in alc. ${ }^{2}$ ( $\mathrm{r}: \mathrm{I}$ 70). Used for sweetening powders, for the administration of volatile oils (eleosacchara), and the preparation of syrups, elixirs, etc.

* Sugar Substitutes.-In cases where sweetening is desired and sugar is excluded, particularly in cases of diabetes, the artificial synthetic product saccharin may be substituted. It is about 300 times as sweet as sugar, but the taste is not exactly the same. The dose for a cup of coffee or tea is about $1 / 2$ to $\mathrm{Igr}$. Glycer in is another sweetening substance which does not contain sugar, and is sometimes employed in place of saccharin. A principle of quite a different kind is the glucosid of glycyrrhiza. This acts only in alkaline liquids. It has no taste if the liquid is made acid.

Many other substances have a sweet taste. Attempts to connect this with the chemic structure (Haycraft, I882; Sternberg, I 898 ) have not been very successful.

*Saccharum Lactis (Sacch. Lact.), U.S.P., B.P.; Sugar of Milk (Iactose); $\mathrm{C}_{12} \mathrm{H}_{22} \mathrm{O}_{11^{-}}$ $\mathrm{H}_{2} \mathrm{O}$. - White, hard, crystalline masses, or white powder, feeling gritty on the tongue; odorless; faintly sweet taste. Freely sol. in water ( $1: 4.9$ ); almost insol. in alc.

Glucosum (Glucos.), U.S.P., B.P., Glucose (Syrupy).- The product obtained by the hydrolysis of starch, consisting chiefly of dextrose and dextrins. Colorless, or slightly colored, thick, syrupy liquid. Very sol. in water, sparingly sol. in alc.

Syr. Glucos, B.P.-Glucose 1, svrup 2.

Mel, U.S.P.-Honey.

Mel Depuratum (Mel Depurat.), U.S.P., B.P.-Clarified Honey.

Oxymel, B.P.-Honey with $1 / 7$ acetic acid. Dose, 2 to 8 c.c., $1 / 2$ to 2 drams.

Rosa Gallica (Rosa Gall.), U.S.P.; (Ros. Gall. Pet.), B.P.-Rose Petals.

Conf. Ros. Gall., B.P.-25 per cent. of fresh Rose Petals.

Mel Rosa, U.S.P.; Honey of Rose.-Fldxt., r; Honey, 7. Dose, 4 c.c., I dram, U.S.P.

Fldext. Ros., U.S.P.-Dose, 2 c.c., 30 minims, U.S.P.

Syr. Rosa (Syr. Ros.), B.P.-Dose, 2 to 4 c.c., $1 / 2$ to I dram, B.P.

Rhæados Pctala, B.P.-Red Poppy Petals.

Syr. Rhead., B.P.-Dose, 2 to 4 C.C., $1 / 2$ to 1 dram, B.P.

\section{PREPARATIONS-FLAVORING SYRUPS}

*Syrupus (Syr.), U.S.P., B.P.; Syrup.-A strong aqueous solution of sugar containing $85 \mathrm{Gm}$. of sugar in 100 c.c. of syrup, U.S.P. (66.7 Gm. in $100 \mathrm{Gm}$. of syrup, B.P.). Miscible with water or alcohol. It is more commonly employed in the form of the flavored syrups. Their dose is ad libitum.

* Syrupus Acidi Citrici (Syr. Acid. Cit.), U.S.P.-A I per cent. solution of citric acid, flavored with sugar and lemon peel.

Limonis Cortex (Limon. Cort.), U.S.P., B.P.-Lemon peel (fresh).

Ol. Limon., U.S.P., B.P. - A volatile oil obtained by expression from the fresh, ripe peel of Citrus medica var. Limonum. Dose, 0.2 c.c., 3 minims, U.S.P.; 0.03 to 0.18 c.c., $1 / 2$ to 3 minims, B.P.

Syr. Limon, B.P.-About 50 per cent. of lemon juice, flavored with lemon peel.

Tr. Limon. Cort., U.S.P.-5o per cent.

Tr. Limon., B.P. -25 per cent. Dose, 2 to 4 c.c., $1 / 2$ to 1 dram, B.P.

Aurantii Amari Corlex (Aur. Amar. Cort.), U.S.P.; A urant. Cort. Sicc., B.P.; Bitter Orange Peel.- The dried rind of the fruit of Citrus Aurantium amara. Dose, I Gm., I 5 gr., U.S.P.

Aurant. Cort. Rec., B.P.-The fresh peel.

Fldext. Aur. Amar., U.S.P.-Dose, I c.c.; 5 minims, U.S.P.

Inf. A urant., B.P. -5 per cent. Dose, 15 to 30 c.c., $1 / 2$ to I ounce, B.P.

Inf. Aurant. Co., B.P. -25 per cent., with lemon and cloves. Dose, i 5 to 30 c.c.;

$1 / 2$ to 1 ounce, B.P.

Ol. Aur., U.S.P. -Dose, 0.2 c.c., 3 minims, U.S.P. anise.

Spir. Aur. Co., U.S.P. - 20 per cent. of orange oil, with oils of lemon, coriander and

1 "U.S.P." stands for preparations official in the United States Pharmacopeia; "B.P.," British Pharmacopeia; "N.N.R." "New and Non-official Remedies; "N.F." National Formulary; "B.P.C." British Pharmaceutic Codex.

2 The following abbreviations will be used in connection with solubilities. Alc. = alcohol: glyc. = glycerin; eth. = ether; chlorf. = chloroform. The figures refer to the parts solvent required to dissolve $\mathrm{I}$ part of drug ( $f . i$., $1: 0.5=1$ part of drug dissolves in 0.5 parts of solvent). The solubilities sefer to $25^{\circ} \mathrm{C}$. (room temperature), unless otherwise stated. 
"Syrup. Aurantii (Syr. Aur.), U.S.P.; (Syr. Aurant.), B.P.; Syrup of Orange.flavored with orange peel. The U.S.P. contains $1 / 2$ per cent. of citric acid. Dose, 2 to + c.c., t. to 1 dram, B.P.

Tr. Iur. Amar., U.S.I', 20 per cent. Tr. Aurant, B.P., 25 per cent.-Dose, 4 c.c., dram, U.S.P.; 2 to + c.c., 1, 2 to I dram, B.P.

lin. Aurunt., B.P.

Auramii Dnlcis Cortex (Aur. Dulc. Cort.), U.S.P.-From Citrus Aurantium sinensis. A urist. Cort. Ind., B.P.

Aq. Aur. Flor., U.S.P.; Aq. Aurant. Flor., B.P.; Orange Flower Water.-A so per cent. U.S.I'. (33 per cent. B.P.) dilution of the Stronger Water.

iq. Aur. Flor. Forl., U.S.P. - The saturated aqueous distillate prepared by distilling the fresh flowers of Citrus Aurantium amara with water.

Syr. Aur. Flor., U.S.P.; Syr. Aurant. Flor., B.P.; Syrup of Orange Flowers.-Dose, 2 to 4 c.c., 1,2 to I dram.

* Elivir Aromaticum (Elix. Arom.), U.S.P. (Simple Elixir).-Syrup containing about 25 per cent. of alcohol and flavored with aromatics (Comp. Spir. Orange).

Hle.t. Arom., U.S.P.-From Pulv. Arom. Dose, I c.c., 15 minims, U.S.P.

Pulv. Arom., U.S.P.-Cinnamon, Ginger, Cardamon and Myristica. Dose, I Gm., 15 gr., U.S.P.

Syr. Aromat., B.P.-Flavored with orange and cinnamon. Dose, 2 to 4 c.c., 1/2 to I dram, B P.

Glycyrrhiza (Glycyrrh.), U.S.P.; (Glycyrrh. Rad.), B.P.-The dried rhizome and roots of Glycyrrhiza glabra typica, Spanish Licorice; or of Glycyrrhiza glabra glandulifera, Russian Licorice. Dose, 2 Gm., 30 gr., U.S.P.

*Elix. Glycyrrh., U.S.P. (Elix. Adjuvans).-Fldext. I; Elix. Arom. 7.

Ext. Glycyrrh., U.S.P.-Commercial Extract of Glycyrrhiza (licorice).

Ext. Glycyrrh. Pur., U.S.P.; Ext. Glycyrrh., B.P.-An evaporated watery, pilular extract.

*Fluidexiractum Glycyrrhize (Fldext. Glycyrrh), U.S.P.; Ext. Glycyrrh. Liq., B.P.A watery extract of Glycyrrhiza (licorice root), preserved with 25 per cent. of alcohol. The U.S.P. contains some ammonia to dissolve the sweet glucosid, Glycyrrhizin. This is precipitated by acids, which are therefore Incompatible. It is miscible with water or alcohol. Mixed in the proportion of $\mathrm{I}: 7$ with syrup or elixir, it is a good vehicle for quinin etc. Dose, 2 c.c., 30 minims, U.S.P.; 2 to 4 c.c., $1 / 2$ to 1 dram, B.P.

Glycyrrhizin belongs to the saponins, although it does not have the typical formula and does not hemolyze (Kobert, 1915; he also describes a new chemic test). The bark likewise contains resins. Composition: Houseman, i9 6.

* Syr. Glycyrr.-Fldext. I; Syrup 7.

Glycyrrhizinum Ammonialum (Glycyrrh. Ammon.), U.S,P.-Dark brown or brownish-red scales, without odor, and having a very sweet taste. Freely sol. in water and sol. in alc. Dose, 0.25 Gm., U.S.P.

*Syr. Tolutanus (Syr. Tolut.), U.S.P.; (Syr. Tolu.), B.P.-Prepared from Tolu Balsam. Dose, I 5 c.c., 4 dram, U.S.P.; 2 to 4 c.c., $1 / 2$ to I dram, B.P.

Vanillinum, U.S.P.; Vanillin, Methylprotocatechuic aldehyde $\left(\mathrm{C}_{6} \mathrm{H}_{3} \cdot \mathrm{OH} \cdot \mathrm{OCH} \cdot \mathrm{COH}\right.$ 4:3:r).-Fine, white, crystalline needles; odor and taste of vanilla. Sol. in water ( $1: 100)$; freely sol. in alc., glyc. or eth. Dose, 0.03 Gm., 1/2 gr., U.S.P.

\section{AROMATIC WATERS, SPIRITS AND OJLS}

Aromatic Waters.-These are miscible with water or alcohol. Their dose is ad libitum.

Aromatic Spirits.-These are used in alcoholic and oily liquids, in the proportion of 2 per cent. They are immiscible with water.

Aromatic Oils.-These may be used for flavoring alcoholic or oily liquids, in the proportion of 0.2 per cent. However, the spirits are generally preferred.

Aromatic Tinctures.-These are used similarly to the Spirits. None of the simple tinctures are especially important.

Some flavors, such as Gaultheria, etc., have also more important actions, and will not be discussed here.

Aq. Anis., U.S.P., B.P.-Anise Water. Dose, Is c.c., 4 drams, U.S.P.

$S p$. Anis., U.S.P., B.P.- ro per cent. Dose, 2 c.c., 30 minims, U.S.P.; 0.3 to 1.2 c.c., 5 to 20 minims, B.P. 
Ol. Anis., U.S.P., B.P.-The volatile oil. Dosc, 0.2 c.c., 3 minims, U.S.P.; 0.03 to 0.18 c.c., $1 / 2$ to 3 minims, B.P.

Anisum (Anis.), U.S.P.; Anis. Fruct., B.P.-The dried ripe fruit of Pimpinella Anisum. Dose, $0.5 \mathrm{Gm} ., 8$ gr., U.S.P.

Faniculum, U.S.P. (Fennel seed).-The dried, ripe fruits of cultivated varieties of Fœniculum vulgare. Dose, I Gm., 15 gr., U.S.P.

Aq. Fanic., U.S.P., B.P.-Fennel Water. Dose, 15 c.c., 4 drams, U.S.P.

Ol. Fanic., U.S.P.-The volatile oil. Dose, 0.2 c.c., 3 minims, U.S.P.

Aq. Aneth., B.P.-Dill Water.

Ol. Aneth., B.P.-Volatile oil. Dose, 0.03 to 0.18 c.c., $1 / 2$ to 3 minims, B.P.

Anethi fructus, B.P.-Dill Fruit. From Peucedanum graveolens.

Aq. Carui, B.P.-Caraway Water.

Ol. Cari, U.S.P.; Ol. Carui, B.P.-Volatile oil. Dose, 0.2 c.c., 3 minims, U.S.P.; 0.03 to 0.18 c.c., $1 / 2$ to 3 minims, B.P.

Cartm, U.S.P.; Carui Fruct., B.P.-The dried fruits of Carum Carvi. Dose, I Gm., I 5 gr., U.S.P.

Ol. Ajowan, B.P.- Volatile oil of fruit of Carum copticum. Dose, 0.03 to 0.18 c.c., $1 / 2$ to 3 minims, B.P.

Cinnamomum Zeylanicum, U.S.P.; Cinnam. Cort., B.P.; Ceylon Cinnamon.-The dried bark of cultivated trees of Cinnamomum zeylanicum. Dose, 0.25 Gm., $4 \mathrm{gr}$., U.S.P.

*Aqua Cinnamomi (Aq. Cinnamom.), U.S.P., B.P.-Cinnamon Water. Dose, I 5 c.c., 4 drams, U.S.P.

ol Cassia, U.S.P., Ol. Cinnam., B.P.-A yellowish or brown volatile oil distilled from the bark of Cinnamomum Cassia, U.S.P.; zeylanicum, B.P. Dose, 0.2 c.c., 3 minims, U.S.P.; 0.03 to 0.18 c.c., $1 / 2$ to 3 minims, B.P.

Pulv. Cinnam. Co., B.P. (Pulvis Aromaticus).-Equal parts of Cinnamon, Cardamon and Ginger. Dose, 0.6 to $4 \mathrm{Gm}$., to to 60 gr., B.P.

Sp. Ciknam., U.S.P., B.P.- Io per cent. of oil. Dose, 2 c.c., 30 minims, U.S.P.; 0.3 to $\mathrm{I} .2$ C.C., 5 to 20 minims, B.P.

Tr. Cinnam., U.S. P., B.P.-20 per cent. of the bark. Dose, 2 c.c., 30 minims, U.S.P.; 2 to 4 c.c., $1 / 2$ to I dram, B.P.

Cinnamomum Saigonicum, U.S.P.-The bark of an undetermined species of Cinnamomum. Dose, 0.25 Gm., 4 gr., U.S.P.

Oliveri Cort., B.P.-The oark of Cinnam. Oliveri.

Tr. Oliver. Cort., B.P.- Io per cent. Dose, 2 to 4 c.c., $1 / 2$ to I dram, B.P.

*Aqua Mentha Piperite (Aq. Menth. Pip.), U.S.P., B.P.-Peppermint Water.

Dose, 15 c.c., 4 drams, U.S.P.

Aqua Menthe Viridis (Aq. Menth. Vir.), U.S.P., B.P.-Spearmint Water. Dose,

I5 c.c., 4 drams, U.S.P.

Sassafras, U.S.P.-The bark of the root of Sassafras variifolium. Dose, ro Gm., $21 / 2$ drams, U.S.P.

Olenm Sassafras (Ol. Sassaf.), U.S.P.-A yellow or reddish yellow volatile oil distilled from Sassafras root. Dose, o.2 c.c., 3 minims, U.S.P.

*Aqua Rosa (Aq. Ros.), U.S.P., B.P.; Rose Water.-A 50 per cent. dilution (33

per cent., B.P.) of Stronger Rose Water.

Aqua Rose Fortior, U.S.P.-Prepared by distilling the fresh flowers of Rosa cen-

tifolia with water.

Ol:Ros., B.P. (Otto of Rose).- The volatile oil of the fresh flowers of Rosa damascena.

Ol. Graminis Citrati, B.P.-Oil of Lemon Grass. Dose, 0.03 to 0.18 c.c., $1 / 2$ to 3 minims, B.P.

Ol. Coriandri (Ol. Coriand.), U.S.P., B.P.-Volatile oil. Dose, 0.2 c.c., 3 minims,

U.S.P.; 0.03 to 0.18 c.C., $1 / 2$ to 3 minims, B.P.

Coriandrum, U.S.P.; Coriand. Fruct., B.P.; Coriander.-The dried ripe fruit of

Coriandrum sativum. Dose, $0.5 \mathrm{Gm} ., 8$ gr., U.S.P.

*Tinctura Cardamomi Composita (Tr. Cardam. Co.), U.S.P., B.P.-2 per cent. of

Cardamom, with Cinnamon, Caraway and Cochineal, in 50 per cent. alcohol, U.S.P.

Dose, 4 c.c., I dram, U.S.P.; 2 to 4 c.c., $1 / 2$ to I dram, B.P.

U.S.P.

Tr. Card., U.S.P.- 5 per cent. in 50 per cent. alcohol. Dose, 2 c.c., 30 minims,

Cardamomi Semen, U.S.P., B.P.; Cardamom Seed.-The dried seeds of Elettaria

Cardamomum. Dose, I Gm., I 5 gr., U.S.P.

Tr. Lavand. Co., U.S.P., B.P. (Compound Spirit of Lavender).-Lavender, Rose.

mary, Cinnamon, Clove, Myristica, Red Saunders, in 75 per cent. Alcohol, U.S.P.

Dose, 2 c.c., 30 minims, U.S.P.; 2 to 4 c.c., $1 / 2$ to 1 dram, B.P. 
Spir. Lasand., 5 per cent. of oil, U.S.P.; ro per cent., B.P. Dose, 2 c.c., 3 minims, L'S.I'; 0.3 to 1.2 c.c., 5 to 20 minims, li.l'.

(1). Latund. L.S.P." B.P. - I volatile oil distilled from the fresh flowering tops of Lavindula vera, Dosé, 0.2 c.c., 3 minims, U.S.P.; 0.03 to 0.18 c.c., $1 / 2$ to 3 minims, B.P.

\section{LRIODICTYON (YERBA SANTA)}

The leaves of this plant disguise bitter taste (not sweet, salt or acid taste). It is, however, therapeutically objectionable. It probably renders alkaloids insoluble, and as for ordinary bitters, it is extremely probable that the therapeutic action is connected with the bitter taste. One c.c. of the fluidextract covers the taste of $0.012 \mathrm{Gm}$. of quinin sulphate or $1.5 \mathrm{Gm}$. of quassia.

Similar properties are found in the following plants: Gymnema sylvestre; Bulmenia dulcifica, and Plirynium Danielli. Gymnema contains gymnemic acid, which destroys bitter and sweet taste, not acid or salt.

\section{PREPARATIONS}

Eriodiclyon, U.S.P. (Yerba Santa).-The dried leaves of Eriodictyon californicum (Composition, Tutin, 1910). Dose, I Gm., I 5 gr., U.S.P.

Fldert. Eriodict., U.S.P. Dose, I c.c., ${ }_{5}$ minims, U.S.P.; precipitated by water.

\section{THE TREATMENT OF DISEASE}

Development of Therapeutics.-If we cast a glance at the history of therapeutics, the treatment of disease, we are met with some very singular facts. Some of these will serve to explain errors which have long adhered and which still adhere to the subject.

We may imagine one of our remotest ancestors brought face to face with disease. How mysterious must have seemed to him the phenomenon that to-day he is strong, active, and full of life, and to-morrow, without any cause apparent to him, he is weak, listless, and about to die! What strong hold it must have taken upon his untutored imagination! How earnestly he must have sought for means to remedy it! Here he happened at once upon two apparently very different methods: a spiritual and a material. On the one hand, overpowered by the mysteriousness of the process, he lost himself in superstition. He deemed the disease due to malevolent spirits which could be appeased by prayers and incantations. This formed the principal materia medica of prehistoric ages, as it does of the modern savage. It still survives in a modified form in "Christian Science."

On the other hand, chance and the observation of animals revealed to him that certain material products were also efficient. As long as he limited himself to actual observation, the results were usually good. However, he soon began to search for more of these remedial agents. In this search we must remember the total ignorance which then existed regarding the nature of the action-both of the disease and remedy. In this darkness he was only too glad to be guided by any ray of light, without having the means to examine whether the light came from a beacon or was only an ignis fatuus. With the fantastic, half logical incongruity so characteristic of the untutored mind he assumed the most extraordinary relations.

It is interesting to observe a little closer the principles which guided the blind savage in his search for remedies. Having found that most active medicines had a bitter or disagreeable taste, he came to regard any such substance as beneficial. Thus arose a host of simples which are now stowed away to molder in the attic of science, and which might well be disregarded were it not that some zealot occasionally disturbs their wellearned repose and attempts to launch them as something new.

Nor did this love of the disgusting die out with the stone age. It was prolonged far into the middle ages. To it we can probably trace the employment of feces and urine, of smoked snake, and of others still worse.

At a later period of the middle ages it was tried to combine the spiritual and the material treatment. It was thought that the Deity alone could cure disease, but that He had given man material remedies, and in His wisdom He had put a seal upon them by which man might know them. Thus arose in due time what is called the doctrine of "signatures." According to this, the use of a remedy was suggested by a fanciful resemblance in shape or color to some organ; c.g., the liverwort, the lungwort, bloodroot, 
etc. These are survivals of this custom. Even a name was sufficient. Silver was used in lunacy because it was dedicated to Luna. ${ }^{1}$

On the other hand, alchemists had arisen with their pertinacious search for the philosopher's stone, which was to convert all metals into gold, and cure all diseases. In this search they gave their nostra extensive trial on sick and well.

The school of spagirists was founded toward the end of the fourteenth century and reached its zenith of power with Paracelsus. They insisted upon the mystical virtues of antimony, arsenic and silver and chemicals in general, and stood opposed to the old Galenists, who used only organic drugs. Notwithstanding their mysticism, which savors of quackery, we must thank them for the discovery of some of our most valuable medicines.

Thus, a mass of materials, rubbish and otherwise, was added to the various simples, etc. Having so well-stocked an armory, the physician of that day felt that he was not doing his duty unless he gave his patient the benefit of it all, and the "shot-gun" prescription flourished at its best.

The Homeopathic Doctrine.-A natural reaction against this excessive drugging set in, and had for one of its first results the establishment of homeopathy by Hahnemann, near the end of the eighteenth century. The Hahnemann system was by no means new. For the most part it had its roots much further back. It was the natural result of the then existing theory of "vitalism."

Hahnemann believed that disease depends upon a perversion of the purely spiritual vital powers and is entirely immaterial in its nature. Logically, a thing spiritual could not be combated by material remedies, and, hence, Hahnemann turned to a spiritual power which he believed to be bound up in plants and liberated by dilution. The activity would therefore increase with the dilution, and be the greater, the smaller the dose (doctrine of potency). This liberation of the principles exactly turned their action around, so that the action of his dilutions was, he stated, exactly the opposite of that of the concentrated drug, and could be used for the relief of such symptoms as the latter produced: Similia similibus curantur. This was the first tenet of Hahnemann. The second was that the nature of the disease being unseizable, it was not subject to treatment, but that only its symptoms can be treated. Hence, homeopathy, in so far as it follows the principles of its founder, has no place for the medical sciences, such as physiology, anatomy, pathology, or chemistry. Any one with an indexed book of symptoms and their remedies would be able to practice it without an elaborate study or preparation.

In marked contrast to the above is the third dictum: that the medicinal treatment must be supported by dietetic and hygienic measures.

The claims of homeopathy as a rational system hinge on the proof of the similia similibus theory and of the doctrine of potentiation by dilution. Most of its advocates seem to deny altogether the relevance of scientific testimony, and to base themselves purely on the slippery ground of empirical experience. Others, however, whilst they carefully neglect the great body of scientific experience which disproves their theory, seem very glad to avail themselves of the few experimental facts, which, through a variety of logical contortions and sophistications, can be twisted into a specious support. Hahnemann himself seems to have started from the observation that large doses of drugs produce the opposite effects from moderate doses. The correctness of this principle may be granted, for most cases. But it is a very unwarranted feat of logic to assume that infinitesimal doses must again cause effects opposite to those of moderate doses!

The more recent discovery of the effect of dilution on electrolytic dissociation, on jonization, has also been seized upon as illustrating homeopathy-quite disregarding the fact that the action depends on the number of ions rather than on the degree of ionization; and the further fact, that the majority of the substances employed in homeopathy are not electrolytes at all! These few examples of inconsistencies may suffice.

Hahnemann's system was the natural outgrowth of his time. At present it is an anachronism, as his pupils are the first to acknowledge in practice, if not in words. But in his time Hahnemann accomplished considerable for medical science. He called attention to the importance of diet, etc., when this was only too much neglected; but perhaps the principal use of homeopathy has been to show to rational medicine the fact that disease tends to recovery without any medical interference.

1 It would appear that the native Chinese materia medica is largely based on the doctrine of signatures. 
Therapeutic Nihilism.-This was, indeed, the next step which medicine took-total emancipation from all drugs. This dates from the establishment of the Vienna school by van Swieten, in 1745 . The strongest advocate of nihilism was Skoda ( $\left.1805^{-188} \mathrm{I}\right)$, the founder of the methods of percussion and auscultation. Such nihilism was absolutely necessary at that time, just as periods of skepticism are necessary in philosophy, and mark steps in progress. The accumulated refuse was so great as to bury the good. The only way was to empty out the whole and begin anew. This was a necessity then, but now consistent nihilism is as obsolete as the "Shot-gun" prescription. He who proclaims it, simply proclaims his own ignorance and want of critical faculty.

The Rise of Rational Therapeutics.-The reëstablishment of therapeutics, founded now upon reason, was thus aided by the very man who had attempted to destroy it. For he established physical methods of diagnosis, and demonstrated the effects of disease as they had never been demonstrated before, making it possible also to demonstrate the effect of remedies.

Then followed the isolation of active principles (led by the discovery of morphin in I8I 7 ), thus substituting the definite for the indefinite drugs. Finally followed animal experimentation, by means of which modern pharmacology has developed.

Services of Pharmacology.--This has made possible a much more exact knowledge of the action of drugs, without which their employment would be a matter of mere chance. It also made available much more accurate methods of observation, and furnished standards by which therapeutic trials must be conducted. It led to the introduction of the great number of synthetic drugs, some of great usefulness, because it pointed out the directions in which new drugs could be sought with most success. It furnished the means of trying their actions and probable usefulness on animals, when their use on man would not be justified without these preliminary trials. It has also contributed to the development of experimental pathology, and has thus laid the foundation of experimental therapeutics - the experimental investigation of the action of drugs in disease.

Psychotherapy.- Rational therapeutics is now on a firm basis. But, at the same time, the mystic has also been further developed, not only in homeopathy, but also in the many forms of suggestion. The value of suggestive therapeutics proper cannot be denied. It is a strictly scientific method of treatment, and is employed in its milder forms by every physician as "the personal influence" and the "faith in doctor and medicine." It often constitutes all there is of merit in those medical fads which have accompanied medical science since the oldest time.

Vis Medicatrix Naturæ.-It is most important to the rational treatment of disease that the physician should understand that he cannot directly make a patient well. That is exclusively nature's task. Physiologic functions tend to run their normal course, or to return to it if they have been disturbed. "Nature," therefore, tends very strongly to bring the organism back to its normal conditions, or life would long since have disappeared from the globe. The task of the physician consists in directing his treatment in such a manner as to remove obstacles from nature's path.

Just as the surgeon can not cause the union of a broken bone, but can only put it in the most favorable condition for nature to perform this union-i.e., set it-so the physician cannot cure heart disease. He may either remove the condition which causes it, if still present, or remove by digitalis, etc., the factors which retard the cure; but in 
any case he must rely upon nature to perform the last, the really important act: viz., the permanent return to normal.

That nature not only puts the final touch upon every reparative process, but that she may take every step as well-i.e., that a patient may get well without any medical interference-is too well known to require further discussion. The ways in which these processes of repair take place constitute one of the departments of pathology and medicine.

The Field of Therapeutics.-In the light of the above, it might well be asked: If nature is thus able to effect cures; if the greater number of diseases tend to spontaneous recovery, what is the function of the physician? Can he do anything but harm if he attempts to meddle with the processes of nature? If he undertakes to aid, does he not really meddle? We must, in examining this question, lay the emphasis in the above sentence on "nature tends to effect a spontaneous cure." But nature is essentially blind in her workings. She works by general laws, which often do not take account of individual cases. Though we must recognize that her processes are generally in the right direction, they may be greatly at fault quantitatively. Nature may do too much or too little. It is now conceded that fever, pain, inflammation, etc., are protective mechanisms; but when the fever becomes so high as to be in itself dangerous to life; when the pain is intolerable and constant, and persists after it is no longer needed; when inflammation spreads; then it is evident that the originally salutary process is becoming the reverse. That the processes of nature are often insufficient is evident from the fact that they do not in all cases effect a cure. Nature may sometimes be absolutely wrong; e.g., in the desire for solid food in typhoid.

It is, then, the duty of the physician judiciously to modify the natural tendency, if he possesses the means of doing so. But he must do so wisely, or it were better not at all. He must understand the diseased condition; he must understand nature's way of meeting the difficulty; he must judge in what ways nature may be supported; and, finally, he must thoroughly understand the means at his command for the purposei.e., pharmacology. As long as he is not clear in regard to these factors, he is merely groping in the dark, as likely at least to do harm as good. In this case expectant treatment is alone justifiable. ${ }^{1}$

We see how from the above we can deduce a number of methods of treatment.

Preventive or Prophylactic Treatment.-This aims to protect the body from disease. It comprises individual and community hygiene.

Etiologic, Causal or Curative Treatment.-The cure of disease is scarcely possible while the original cause persists. The physician must therefore always aim to discover and remove that cause. If this can be done, the conditions will generally return to the healthy normal unless anatomic lesions have already occurred. The chances for complete recovery are therefore the better, the earlier effective treatment is instituted. However, even considerable anatomic deficiencies can be compensated by the fact that the organism has large "factors of safety" (Meltzer).

Symptomatic, Functional or Alleviative Treatment.-When the cause of the diseased condition can not be attacked, it is often possible to remove its functional manifestation or symptoms. This may be useful in some cases, objectionable in others.

In striking the symptoms one very often also strikes the disease. The symptoms in themselves may be so objectionable or lead to such secondary results as to make their removal desirable. Pain, cough, and fever are all purely symptoms, and yet no one would refuse to treat them simply because unable to remove the root of the disorder. The derangement of function may be immediately threatening to life, f.i., cardiac dilation; or it may add to the tax on the diseased organs, f.i., the edemas of valvular disease. The faulty functionation may also be relieved by increasing other compensating functions, $f . i$, diaphoresis in renal disease.

On the other hand, the symptoms may be very deceptive-a chill will not require external heat; a referred pain will not be relieved by local application of iodin to the place where it is felt. In removing the symptoms the physician also deprives himself of the only index to the treatment of the underlying disorder. He must constantly

1 In this connection see S. J. Meltzer's essay on the Therapeutics of Self-Repair; Am. J. Med. Sc., July, Ig08. 
be on his guard against believing himself successful when he has succeeded in removing one or several of the symptoms of the discase. In many cases the symptom may itself be salutary; in which case it would not do to remove it (e.g., cough when there is hypersecretion of mucus; a certain amount of pain when rest is indicated in fracture).

It need scarcely be added that it is not ethical to persuade a patient that he is being cured when he is in fact only being relieved of the symptoms.

Restorative or Roborant (Strengthening or Tonic) Treatment.Disease generally impairs the vitality and resistance of the organs directly involved, and of the body at large. This is corrected by rest and efficient nutrition of the diseased organs and of the entire patient; and by correcting any other abnormal conditions (digestive disturbances, suppurations, etc.) which may be present, and which are added drains on the vitality of the patient.

Expectant Treatment.-This is the absence of any real attempt at treatment beyond hygiene, rest, diet, and other similar general measures; with the object of leaving the powers of nature free play. This should be employed in all cases where no better treatment is known; but, as has been said, it is usually within the province and power of the physician to support nature in her endeavor.

The expectant treatment must also be used when it is desired to let the disease progress to a certain point, if this is necessary for diagnosis.

Empiric and Rational Therapeutics.-The treatment of disease has developed by two different methods, or perhaps more accurately, attitudes of mind. The Empiric method follows merely the dictates of experience, without concerning itself about the reasons of these.

It is typified by the patent medicine slogan; "it has cured others and will cure you."

While in the present state of our science it is still necessary to employ it only too of ten, it requires scarcely a thought to see how of ten it may be not only useless, but even injurious.' Conditions which resemble each other very closely superficially may really be diametrically opposite, and may require very different treatment. The objection to the empiric method is not that it rests upon experience, which is the basis of all science, but that it does not endeavor sufficiently to distinguish whether the experience is real or deceptive. It is, of course, an equally grave error to go to the opposite extreme, and to base treatment on theories and deductions inadequately supported by observation.

Rational or scientific therapeutics should employ observation, experiment and scientific reasoning, checking each against the others.

A real science of therapeutics, by which it would be possible to foretell the exact results is still largely a vision. The numerous physiologic and pathologic variables render predictions uncertain. The personal factor plays a large rôle; and the due appreciation of this-" the art of therapeutics"-is therefore very important. However, the best results can only be secured by the critical application of all the known scientific facts.

Pharmacal and Non-pharmacal Therapeutics.-A distinction is sometimes made between "drugs" and "physiological agents"; including under the latter physical measures, such as heat or cold, bathing or climate, rest, exercise and massage, diet, etc. The distinction is convenient, but not fundamental. The use of drugs is no more "unphysiological" than the use of abnormal heat or cold, or of special diets, etc. The mechanisms of their action are not essentially dissimilar. Each is intended to produce definite changes of functions. One or the other may be better adapted to secure this end; and the physician should not hesitate to employ whichever is best suited.

Physiologic Processes Normally Controlled by Pharmacologic Agents.-The body normally produces a variety of pharmacologically active substances, as direct or by- 
products of its metabolism, and uses them as aids in regulating its own activity. The $\mathrm{CO}_{2}$ control of the respiration and the various other hormones and internal secretions are familiar examples in mammalian physiology (Starling, I908). The use of drugs is therefore not an unphysiologic proceeding.

\section{THE CHEMICAL AND PHYSICAL BASIS OF PHARMACOLOGY}

Scope of Pharmacology.-The term Pharmacology in a general sense covers all scientific knowledge pertaining to drugs, i.e., to substances which may be used in the treatment of disease. Materia Medica, Pharmacognosy, and Pharmacographia have the same meaning when they are used in a general sense.

More commonly, however, the term "pharmacology" is used in a more restricted sense, as a synonym for pharmacodynamics. It concerns itself with the actions of drugs on living structures; or with the reactions occurring between "drugs and living structures. It is therefore in a sense a branch of physiology on the one hand, of chemistry on the other; and is thus essentially a division of biology. It has important practical relations; on the one hand, with toxicology (the science of poisons); on the other hand, with therapeutics (the treatment of disease).

The Nature of Pharmacologic Action.-The processes of life are essentially conditioned on chemical and physical changes in the constituents of the cells. Foreign chemic substances may enter into these reactions, and thus modify them more or less profoundly, with corresponding changes of function. These are termed drugs or poisons, according to whether they are useful or harmful in a given case. Some of the reactions are comparatively simple; analogous to those produced on isolated proteins. Others, however, occur only in living substance. Our limited knowledge of the chemical details of the living cell does not permit any deep insight into the nature of the action of these substances, except in a few directions. They suffice to show that the mechanism of the action of different drugs is not uniform, but is sometimes along chemical, and sometimes along physical lines. No sharp division can be drawn.

Physico-chemical Conditions of Life.-The living cell may be considered as a very complex laboratory, where chemic decompositions and syntheses, reductions and oxidations, etc., are constantly going on. These chemic changes lead to transformations of energy which find their final expression in the phenomena of life. The vital manifestations of the cell are, therefore, inseparably connected with physico-chemic transformations, which require for their occurrence the existence of certain chemic and physical conditions. The chemic essentials are: the presence of substances capable of liberating energy, and the conditions suitable for their reactions, such as a proper temperature, alkalinity, presence of ferments, etc. The physical conditions of life are: A viscid medium, containing colloid proteins, salts, fats, and water.

Ordinary Chemic Reactions. - In the simplest cases the actions occur on dead tissues, and resemble those produced on substances of known composition; this is the case with strong acids, alkalies, metals, etc.

Obscure Chemic Reactions. - In many cases, particularly with alkaloids, glucosids and toxins, the action is confined to the living cells; for most of these, no adequate explanation can be offered. It is very probable that many of these produce chemic changes in the protoplasm; but the evidence is suggestive rather than conclusive.

From the complicated structure of the protein molecules we conclude that it must be capable of a very great number of reactions, a conclusion which is confirmed by the large number of substances which are utilized by the cell. We find an expression of these chemic changes in the final excretory products of the cell; but we are very ignorant of the reactions by which these final changes are produced. When we find, therefore, that a chemic substance possesses actions for which there is no adequate physical explanation, we presume that it enters into chemic reactions with the protoplasm. 
This has been expanded especially in the side-chain theory of Ehrlich. This assumes that the poisons combine with certain groups (receptors) of the cell which are essential for its metiabolism.

Evidences of Chemic Actions. - The theory of chemic combination is confirmed by the fact that most of the poisons (not all) are altered in the body; although it does not follow that the action is due to this particular change. The toxicity is often parallel to the chemical reactivity or lability of the poison. Other indications of chemic reaction are, that these poisons often accumulate in the cells; and that the effects generally increase with the dose. This would be expected with chemic reactions, but could also occur on physical grounds. In any case, the combination is often very loose and unstable, for the poison can often be removed by simple solvents ( $f . i$., antipyretics and fuchsin stain by alcohol, P. Ehrlich). Indeed, the organism recovers promptly from most poisons when these are washed out, unless secondary changes have occurred. In some cases, the action seems to be produced only whilst the poison is passing into or out of the cell (Straub; Kuyer and Wijsenbeck, I9I3). The action is then probably on the plasma membrane. The relation between the action and chemic constitution of poisons also speaks for chemic reactions, but may in some cases be explained by corresponding alterations of the physical properties. Compounds between alkaloids and proteins have been described; but it is not certain whether these are real combinations, or merely adsorption products (Eddy and Gies, 1907).

Modifications of Ferments. - Catalytic reactions, which are so important to the cell, may be modified by chemic reagents, without as well as within the cell. This explains the asphyxiant action of cyanids.

Changes in the Coll Membrane.-Since most cells are in contact with surrounding fluids, they must be protected against changes of composition by a limited permeability of their surface layer, the cell wall or plasma membrane. This apparently contains lipoid and protein constituents. It may, therefore, be altered by fat-solvents, or by agents which either precipitate or liquefy proteins. This explains, at least partly, the effects of lipclytic narcoties; hemolysis by these, by alkalies and saponins; the actions of various ions, especially $\mathrm{Ca}$, etc.

Osmotic Changes.-Cells are generally much more permeable to water than to dissolved substances. This leads to osmotic phenomena-the withdrawal or absorption of water-when the cells come into contact with solutions of higher or lower salt concentration. This explains the actions of many otherwise nearly indifferent salts, e.g., saline catharsis and diuresis.

Surface Forces. - The physical structure of protoplasm is such as to present large surfaces, and therefore gives peculiar opportunity for the development of the surface forces, which are very important for various vital functions. These are greatly modified by a variety of substances, including the lipoid solvents, salts, etc.

Colloid Phenomena.--Many chemical and physical conditions depend on the colloid character of protoplasm. The state of aggregation of colloids, and hence their reactions, are easily modified, especially by salts, acting both by their chemic properties and electric charges. This is another important element in salt-action.

Dependence of the Pharmacologic Action on the Chemic Constitution. - As a general rule, drugs having a similar constitution, possess similar actions; and definite changes in the molecule - as in homologous series, or the introduction of new groups-often produce definite modifications in the pharmacologic effects. This should be expected, since the physical as well as chemical properties change with the constitution. This relation has a wide general application; but its application to details has been disappointing, because we have not sufficient knowledge of the chemic constitution and reactions to take account of the numerous variables.

It must be remembered, for instance, that the action is not so much determined by the elementary composition of the substance, but rather by the manner in which the elements are combined. Isomeric compounds have often very different actions. Apparent exceptions result also from the different penetrability of cells to substances which, could they be introduced into the cell, would cause similar effects. Other manifestations of selective action also come into play.

Attempts to construct a general theory of pharmacologic action on chemical lines have merely shown that this is impossible. The data which we possess had therefore to be gathered empirically without much guidance. 
The Importance of the Chemic Radicals or "Side-chains." - A substance, to produce chemical pharmacologic actions, must be capable of combining with protoplasm and then of altering its properties. These two functions-combination and action (analogous to amboceptor and complement, P. Ehrlich)-may be united in the same group; but more commonly they are distinct ("haptophore" and "toxiphore" groups), so that one may be modified without the other. Often several groups are present which may enter different combinations under different conditions. It is evident that these may introduce great variations in the actions.

Morphin, f.i., produces its hypnotic action by "anchoring" or combining with its phenyl-hydroxyl. When this group is closed, f.i., by the methyl radical, as in codein, it anchors in a different way, to different cells; and the convulsant action becomes more prominent. The introduction of acid radicals into the amido-group renders this more stable and less toxic; but the same acid radicals when introduced in the $\mathrm{OH}$ group ( $f . i$., as in heroin) increases the toxicity, by providing a new combining group. In these cases, the new groups act only by altering the combining properties of the active group. In other cases, the new group may itself be active.

The Construction of Synthetic Compounds. - The recognition of these laws of the modifications produced by the different radicals is of practical importance. It permits the prediction, with considerable probability, of the effects of new drugs; and it points the way to modifications which will emphasize desirable quantities and eliminate those which are undesirable. This has lead to the introduction and improvement of many new remedies. Really new properties can not, of course, be predicted by known properties. Their discovery is generally accidental. Accordingly, synthetic chemistry has introduced relatively few new principles, but endless modifications; some useful in the ways indicated; many others needless and detrimental. It is very easy to introduce minor changes in composition which do not alter the actions of the original drug materially, but which are commercially profitable by evading the patent and trade mark laws. This needless multiplication is confusing and, therefore, undesirable.

Distribution of Drugs Within the Body.--Drugs are not distributed uniformly, but accumulate especially in certain cells, according to their permeability and physical and chemical affinities. This influences their action; either by bringing them in contact with reactive tissues, or by storing them in places where they may be inactive. The distribution has been studied in relatively few cases, especially for inorganic poisons; but the data are not sufficient to permit generalizations. The distribution appears to be altered by disease; e.g., degenerating tissue takes up more iodid, etc.

Storage Places.-The poisons disappear quite rapidly from the blood, the greater part within a few minutes. The chlorids, bromids, and related ions accumulate in all organs, but mainly in the skin and blood. The thyroid stores a relatively high percentage of iodin as iodothyrin. The bones retain the earthy metals and fluorid. The heavy metals are deposited as loose organic compounds, especially in the liver and spleen; mercury as a loose globulin compound; arsenic as a more stable nuclein combination (Vamossy, 1905). The iron deposits serve as a reserve stock of this metal. Little is known about the distribution of organic posions, mainly for want of suitable assay methods.

Chemic Changes in Drugs.-The majority of poisons are more or less altered in the body, and generally rendered less harmful. This is effected by the oxidation, reduction, hydration or dehydration, or other 
decomposition of the poison, or by the storage of the poison in certain organs, or by its combination with other substances which render it harmless. This process of disintoxication is of great practical importance. It makes it necessary to administer the drugs continuously, in order to maintain their effect; it often requires the use of continuously increasing loses as the power of disintoxication becomes more developed; and were it not for the power of the body to destroy or remove poisons, and thereby to recover from their action, all therapeutic use of drugs would be impossible.

Decomposition by Digestion.-The digestive juices destroy many organic poisons by hydrolytic cleavage; especially the proteins, toxins and antitoxins, and the glucosids. On the other hand, they are necessary to saponify and liberate the active constituents of insoluble esters, c.g., phenyl salicylate. The acidity of the gastric juice is important for the solution of bases. It also often splits off acyl groups.

Decomposition in Tissues.-Strychnin and many other poisons are partly oxidized in the body, and the toxic effect is, therefore, reduced if the poisoned animal is placed in an at mosphere of oxygen. Morphin is largely destroyed, especially by those accustomed to its use. Reid Hunt (I005) has shown that feeding with thyroid markedly diminished the toxicity of acetonitril, by preventing its conversion into cyanid. The resistance to morphin is also modified. It has been claimed that excision of the thyroids increases the toxicity of a number of other poisons; but Lerda and Diez, I905, obtained negative results with caffein, strychnin, urine, and tetanus and diphtheria toxin. Organic compounds of metals (cacodyl, etc.) only develop the metal action after oxidation. Many organic acids (citric, etc.), alcohol and formaldehyd, are oxidized, and thus disintoxicated. Hexamethylenamin is activated by the liberation of formaldehyd in acid urines. The benzol ring is very resistant, most of the changes occurring in the side-chains.

Disintoxication by the Liver. - This is believed to be especially active in disintoxication, partly by destroying, but particularly by storing poisons, so that the same dose is much less effective (perhaps one-half) when given by the mesenteric, than by the jugular vein. This has been demonstrated for curare, strychnin, morphin, cocain, veratrin, quinin, atropin, and the metals. Perfusion of alkaloids, glucosids, toxins, barium, etc., through excised liver, also decreases their toxicity (Woronzow, I9I2). Liver emulsions seem to be less active. They did not destroy strychnin, but attacked caffein (Petron, 1905). Experiments with Eck's fistula gave rather negative results (Rothberger and Winterberg, I905).

Other Organs.- Perfusion through excised muscle also produced disintoxication, but weaker than the liver, with chloral, atropin, physostigmin, curare and alcohol, not witb muscarin and ricin (Woronzow, I9I2). Excision of the spleen is said to increase the toxicity of most alkaloids, but not all. The phagocytes accumulate and thus disintoxicate poisons, especially colloids. The serum of atropin-resistant animals destroys atropin.

Disintoxication by Combination.-Phenols and other aromatic compounds are rendered less toxic by combining with sulphates; many metals by the proteins; toxins by antitoxins; acids by alkalies; aldehydes, camphor, chloral and ethereal oils by glycuronic acid; cyanids by sulphur; benzoic and salicylic acid by glycocoll; etc. The extent of the disintoxication will depend upon the activity of the metabolic processes which are concerned, or on the amount of neutralizing substance present in the body.

Quantitative Relation of Concentration and Action.- The ratio is generally not in simple proportion. There is usually a "minimal threshold," all concentrations below this being apparently ineffective. At the other extreme, once the "maximal response" has been reached, further increase of concentration can produce no further effect. Between these points, every increase of concentration increases the action; the increment being the greater the lower the original concentration. For instance, when the concentration of ouabain was doubled from 0.00125 to 0.0025 , the action was increased 4.6 times; when the concentration was doubled from 0.005 to 0.01 , the action increased only I.28 times (Sollmann, Mendenhall and Stingel, I9I 5 ; the paper also discusses the reasons for the phenomenon).

\section{THE MANIFESTATIONS OF PHARMACOLOGIC ACTIONS}

Modifications of Functions.-Since protoplasm is essentially identical in all cells, the action of pharmacologic agents must also be essentially the 
same in all situations; but just as quantitative differences exist in the cell, so does the pharmacologic action show quantitative differences and selective properties. Of these we shall have more to say presently. As a general rule, the most conspicuous changes occur in the most conspicuous function of the cell-partly because these are the most readily appreciated, but partly also because specialized functions are the most complex and hence the most sensitive.

Pharmacologic agents can in no case create new functions in a cell or tissue; they can only modify existing functions, or at most make evident functions which have previously been latent. The functions of a cell may be increased or diminished, resulting in stimulation or depression. Since there are also structures the stimulation of which would lead to an inhibition of other structures, an actual increase of function is sometimes distinguished as an excitation. A very violent stimulation passes usually into injury; such injurious stimulation is called irritation. Míoderate, but prolonged, stimulation also passes into depression, either by the disappearance of food substances (exhaustion), or by actual injury to the structure (fatigue). If the depression is so great that the given function has disappeared, the condition is called paralysis; if all the functions are abolished, there is death.

The greater number of pharmacologic agents produce at first a stimulation, which is followed in larger doses by a depression. In this respect the differences between the different poisons are again mainly quantitative.

The so-called "stimulants" produce a very strong and prolonged stimulation, the depression being produced only by relatively large doses. The "depressants," on the other hand, cause only a slight stimulation, which passes readily into depression. Indeed, with some depressants, no stimulation whatever exists. It is very rare that a depression precedes a stimulation; when this occurs, the action is presumably on different structures.

Selective Action. - The actions of drugs are generally much greater on some tissues than on others; i.e., they are selective. The difference may be almost or quite absolute, so that the drug acts specifically on one class of cells. This "monotrop" action is especially important in parasiticides, and is the ideal of modern "chemo-therapy," the treatment of infections by chemic agents. Antibodies also illustrate a strictly monotropic selective action. With most drugs the differences are merely quantitative or relative; a number of structures being affected with various degrees of predilection, which may sometimes shift with changing conditions. The actions on the central and peripheral nervous systems generally belong to this relative type.

Mechanisms of Selective Actions.-These may consist in differences of penetration; of the chemic affinities of the cell; or of sensitiveness of the functions. The details are known only in a few cases.

Selective Absorption.-A drug may act upon a cell without actually penetrating into it; for instance, by exciting the nerves supplying the cell, or more directly, by withdrawing water from the protoplasm; but as a general rule, the poison must be absorbed into the cell or cell-membrane, before it can produce any action. In order that this absorption may take place, the drug must be soluble in the cell contents, and particularly in the cell envelope. The solubility of a substance in protoplasm is not necessarily the same as in water. Indeed, it varies for each kind of cell, 
and consequently the penetrability of different cells for a given substance may vary greatly.

Whilst the renal cells, for instance, are very permeable for sulphates, the intestinal cells are but slightly permeable. It is in virtue of this peculiarity that cells are capable of preserving their own composition, notwithstanding considerable changes in the fluids in which they are bathed. This fact also explains why a given substance acts much more strongly upon one cell than upon another.

These differences are illustrated strikingly by the distribution of dyes in living and dead tissues; or indeed, in unorganized materials. Their behavior has been extensively studied, but as yet without leading to secure general conclusions.

The differences in absorption may be due partly to the cell envelope, partly to the cell contents. Lipoid solvents generally penetrate better than other agents, because of their affinity for the lipoid constituents of the cell membrane. Other agents probably form "solid solutions" with some of the cell constituents; others are attracted by chemic differences-e.g., the basic stains by the acid character of the nuclear chromatin. Absorption and surface forces probably play an important part.

The distribution is often modified by conditions-reaction, oxygen, the presence of a second substance, etc. Important differences arise also in diseased conditions, P.g., iodids are taken up in higher concentration by degenerating tissues.

Effect of Concentration.-The amount of a drug absorbed into a cell varies generally with the concentration of the drug in the surrounding fluid This concentration is greatest at the place where the poison enters and leaves the body, i.e., in the alimentary canal, liver, and kidneys. Differences of blood supply also come into play. The influence of concentration is most readily seen with locally acting drugs.

Chemic Differences.-The various cells differ in their chemic properties, and therefore in their reactions. This is seen most definitely with the antibodies, precipitins, cytolysins, antitoxins, etc., which are supposed to fit especially into certain cells, "as a key is fitted to a special lock." Something of the same kind doubtless applies at least to some of the ordinary poisons, which can unite only to certain side-chains or certain constituents; but little is known of the details. Similar combining properties may make a poison innocuous to certain cells, by leading to its destruction, or binding it into a harmless form. On the other hand, the decomposition may serve to liberate or form an active substance- $f . i$., formaldehyd from hexamethylenamin in acid urines.

Functional Differences.- The cause of selective action lies often in the cells themselves, the more delicate functions being damaged more readily. Hence a poison which acts indifferently on all tissues produces always the most conspicuous effect on the central nervous system.

The sensory nerve fibers are uniformly more sensitive than the motor fibers. The heart of the king-crab limulus furnishes a good illustration. Its structures fail in the same order (ganglion, motor nerve-plexus, muscle) in respect to all kinds of injurious agencies, alkaloids, anesthetics and other drugs; heat and cold, etc. (Meek, 1908).

Certain cells also accommodate themselves more readily to altered conditions.

\section{CLASSIFICATION OF DRUGS ACCORDING TO SELECTIVE ACTION}

Poisons which alter all tissues, and which therefore produce effects at the place where they are applied, are termed "locally-acting" drugs. They may produce similar chemic changes in dead tissues. These usually cause inflammation when applied to living tissues, and are then called irritants (including simple irritants, corrosives, and astringents). Others lessen inflammation mechanically (emollients and demulcents).

Others act chemically on all living structures, without changing dead tissues; these are termed "protoplasmic poisons." 
Poisons which act selectively on a few structures are called "musclenerve poisons." They may affect end-organs (sensory endings, gland cells, or striped, smooth or cardiac muscle); or the nerve-endings; or ganglia; or any part of the central nervous system.

Nerve trunks are so resistant that they are only affected when the poison is applied to them directly. The access of drugs to them is restricted, in intact animals, by their limited blood supply. The nerve fibers are also protected by the slight permeability of their sheath.

Pharmacologic Groups.-Various systems of classifying nerve-poisons have been devised. None is really satisfactory, because most drugs produce several effects which, by their interaction, complicate the subject so that no simple system will apply. With some drugs, however, one pharmacologic, chemic or physical feature predominates so greatly that they can be separated as fairly distinct groups. However, this is more or less arbitrary and artificial.

Variations in Symptoms.-The selective action of drugs is rarely absolute; even strychnin, a highly selective poison, acts as a general protoplasmic poison when it is directly applied in sufficient concentration. However, some one action often predominates so greatly over the others, that it may be considered as characteristic for the drug. Even in this case, the poison as a rule acts selectively on several structures. This accounts for the great variability which is often seen in the action of the same drug under different conditions. Thus, atropin stimulates the vagus center, but paralyzes the endings: it may therefore cause either a quickening or a slowing of the pulse. Again, through the opposed action of small and large doses, strychnin, e.g., may cause either a stimulation or a paralysis of the vasomotor center. Another frequent cause of variable actions lies in the indirect actions of a drug. A drug which causes convulsions will thereby tend to stimulate the vasomotor center, although its direct action on this center may be depressant.

These variable actions and interactions make the effects of many poisons appear very complicated, although they prove quite simple on analysis. This analysis is very important for a proper understanding of the action, but it is still more important to remember the complex effects which result, for it is with these that the practicing physician has to deal.

Often the pharmacologic action in this sense is not the therapeutic action. A very important pharmacologic action of morphin, for instance, is a stimulation of the spinal cord; but no one would think of employing the drug therapeutically for this purpose, since this action is entirely overshadowed by its other effects.

For this reason it would not be a good plan to classify the drugs strictly according to their pharmacologic actions. On the other hand, a therapeutic classification, although useful in some respects, is not favorable to a study of the underlying actions of the drugs, and tends to empiricism. The best principle of classification yet devised is that of Buchheim, ${ }^{1}$ according to which drugs are grouped according to their principal pharmacologic characters, taking account of all the important actions, as well as of the chemic properties, ${ }^{2}$ and in many cases also of the therapeutic uses of the drugs. This is the general plan adopted in this volume.

Definitions of Pharmacologic Terms.-Some rather loosely used pharmacologic terms may be defined in this place, in the sense in which they are generally employed in this volume:

Local actions: produced at the place where the drug is applied; Remote actions: occurring in distant parts of the body (may be either systemic or reflex); Systemic effects (sometimes called general action): produced

1 Buchheim may be considered the founder of modern pharmacology, by the establishment of the first pharmacologic laboratory, at Dorpat in I 856.

2 Drugs of very different chemic character often appear to have identical actions. Thus the action of strychnin resembles that of tetanus toxin, arsenic that of cholera, barium that of digitalis. In some cases the action may be supposed to be really identical, but in others the resemblance is merely superficial. 
after the absorption of the drug into the circulation; Direct effects (sometimes called primary): produced by the direct action of the drug on the tissue concerned; Indirect effects (sometimes called secondary), are not produced by the action of the drug on the tissue concerned, but by the intervention of some other structures on which the drug acts (e.g., asphyxial convulsions are an indirect effect of asphyxiant poisons); Reflex effects are indirect actions arising from local irritation; Immediate effects (also sometimes called primary) are the effects resulting at once; Late effects are those occurring later; if they are preceded by other (immediate) actions, they are properly called secondary actions. Side actions are actions which are not desired in the therapeutic use of the drug.

Localization of Action.-The effects of drugs on patients, or in cases of poisoning, are by far too complex to furnish any real insight into the actions which are involved. To attain the object of pharmacologythe explanation of the action of drugs-it is indispensable to simplify the conditions as much as possible, and to make the functions, which are to be studied, accessible to measurement, and if feasible, to graphic representation. The methods of experimental physiology are employed for this purpose.

The Study of Isolated Structures.-To eliminate the complications which arise from a simultaneous action on several structures or from indirect actions, the tissues to be studied are generally isolated. The most certain method is the actual anatomic isolation of the structure. Drugs of known antagonistic actions may also be utilized, but they are more apt to lead to erroneous conclusions.

The anatomic isolation may be accomplished by employing unicellular organisms if the action on undifferentiated protoplasm is to be investigated; or by excising the tissue (as muscle, etc.) from the body; or by applying the drug to the exposed tissue (e.g., to a sympathetic ganglion); or by severing the connection with other tissues which might be affected (as by section of a nerve); or by restricting the action of a drug to a given part, by cutting off the circulation. In some cases it suffices to confine the observation to the structure to be studied.

One or the other method of isolation may be employed, according to circumstances; that giving reliable results with the least difficulty of technic being naturally preferred. Complete isolation is in many cases superfluous. If the question is, for instance, whether an observed stimulation is on a central or on a peripheral structure, it suffices to divide the nerve: on an efferent chain, this will abolish the effects of a central stimulation, whilst those of a peripheral stimulation will persist. With an afferent chain the conditions would be reversed. By making sections at various levels of the chain, the location of the action may be accurately determined. When the structures to be investigated are inaccessible to the scalpel, one may substitute drugs which are known to paralyze these structures selectively (curare for the endings in striped muscle; nicotin for ganglia; atropin for endings of the vagi or sympathetic, etc.). The site of a paralysis is similarly located by successive stimulation. The stimulation is accomplished by electricity or by appropriate drugs (epinephrin for sympathetic endings; muscarin for vagus endings; pilocarpin for glands; barium for smooth muscle, etc.).

A few examples will make this general method clear:

I. Strychnin.-It is noted that strychnin produces a tetanus. This implies a motor stimulation somewhere. The sciatic nerve is cut; it is found that the convulsions disappear in the leg but persist in the rest of the body. The action must therefore be 
central. The cerebrum and medulla are successively excised; the convulsions persist, and must, by exclusion, be located in the cord. This is confirmed by destroying the cord, which causes the complete disappearance of the tetanus.

2. Curare.-This produces a complete muscular paralysis. Stimulation of the sciatic elicits no response. The paralysis must therefore be peripheral. Direct stimulation of the muscle is effective. This excludes all the possible structures except the nerve trunk and endings. The nerve of another preparation is laid into the curare solution, and after a time, is stimulated; a contraction results, so that the nerve trunk is not paralyzed. The action must therefore be on the endings.

If a peripheral structure is stimulated or paralyzed, it is impossible to decide by this method whether there is not also a central action, for the peripheral effects would obscure the central. A stimulation of the cord, for instance, could cause no effect if the drug had paralyzed the motor endings. In these instances, it is necessary to confine the action of the drugs to the centers, which requires a more complicated technic.

The Study of the Effects of Drugs on Intact Normal Animals.-A complete conception of the actions of a drug can be obtained only by supplementing the study of its effects on isolated structures, by careful observation and analysis of the symptoms which it produces in intact mammals. The effects on metabolism, and the histologic lesions, etc., can only be studied in this manner. Indeed, these experiments are often undertaken before the more difficult investigations on isolated structures; for they furnish valuable hints of the direction which the latter should take. When circumstances permit, it is advisable to proceed from the lower to the higher classes of animals, and finally to man. Opportunities for observing the effects of drugs on man are frequent in cases of poisoning, and should not be neglected; but intentional experiments with drugs on man are to be undertaken only with the greatest caution, with doses which do not exceed the therapeutic maxima; and, as a rule, only after the effects have been thoroughly studied on animals.

The Effect of Drugs in Disease.-The action of drugs is not always the same in disease as in health. The differences are, however, as a rule quantitative rather than qualitative. Since the drugs are in practice employed most extensively in disease, their action in these conditions is of the greatest importance. As a general rule, it is possible to explain, and even to predict, the action of drugs in disease from their action on normal tissues. However, the actual test must always be made.

Animal experiments are as yet of limited value in this connection, and we are forced to rely mainly on observations on patients. To make these of any value it is in the first place necessary that the observations be made very accurately and that all psychic factors be excluded; it is further necessary that the existing pathological condition be correctly known. These requirements are unfortunately not fulfilled in many cases, which accounts in part for the differences which are occasionally noted between the clinical and the experimental data. These exceptions will be discussed in the text.

In the case of all the older drugs, clinical tests have been made so abundantly that further observations would seem superfluous. This is by no means the case. Accurate observations in the light of our advancing knowledge, and employing the improved methods of diagnosis and observation, are always needed.

It is also highly desirable that every physician should obtain his knowledge of the therapeutic action of drugs at first hand. He should utilize every case under his care for this purpose, and conduct his treatment as if it were a critical experiment, the interests of the patient being, of course, paramount. The conclusions will be greatly simplified if but one drug is used at a time.

Chemic Investigations. - Since the action of drugs depends so largely upon their chemic constilution, the latter is a legitimate subject of pharmacologic inquiry. The study of the fate of the drug in the body, of the mechanism of its absorption, excretion, and storage, is also indispensable. These involve the methods of quantitative chemic analysis.

Transfer of Results of Animal Experimentation to Man.-The utilitarian aim of pharmacology is to supply the science of medicine with a rational and scientific basis 
for the practice of therapeutics and for the study of toxicology. It was shown in the preceding section that these objects can be attained only by experimentation on lower animals. This brings up the fundamental question: To what extent can results observed on animals be transferred to man? The same question applies to all other fields of experimental medical research, and has been abundantly answered by their results. A similar physiology implies a similar pathology and pharmacology. In the great majority of cases, similar structures are affected in the same way by a given drug, no matter in what animal they are studied. Where differences exist, they can usually be explained by differences of physiologic function, as will be discussed under "Racial Idiosyncrasy." 'Those which are not yet explained must be referred to our ignorance. The differences in animals, physiologic and unexplained, are now generally recognized so that suitable species can be chosen for experimentation. They can furthermore be eliminated by using the drug on several species: If a given poison affects all species alike, it may be concluded that its action on man is also the same. If it produces different effects, but if these can be explained by differences in physiology, the effects on man will be similar to those produced on the species the physiology of which resembles most closely that of man.

Value and Limitations of Experimental Pharmacology.-It will be seen that great care must be used in applying the results of experimental pharmacology to man. The neglect of this precaution, the drawing of far-reaching conclusions from a few limited experiments, threw discredit on pharmacology in its earlier days, and is still seen all too frequently. Pharmacology can not be held responsible for this misapplication of its data by half-trained enthusiasts. Its scope is limited primarily to its own results and not to their application, although it may legitimately suggest the latter. It should not be made to replace the science of therapeutics, but should only aim to place well-studied tools in the hands of the latter. If this limitation is realized; if the therapeutist will carefully study the results of pharmacology and will utilize and interpret them in the light of bedside experience, then pharmacology will be of very great value to medicine.

One very important service is rendered by pharmacology through the examination of new remedies. The development of synthetic chemistry especially has resulted in the discovery of a very large number of new substances of some therapeutic value. The number is indeed so large, that all could not be given a thorough trial on patients. Most of these substances possess some value, but many differ from each other by very insignificant details. In this case, pharmacology can select the most promising drugs of a type, and by their thorough study, indicate those which are worthy of trial by the clinician.

\section{THE ADMINISTRATION OF DRUGS}

The effects of a given drug or poison are not always uniform, but vary with conditions; such as the dose; the absorption and elimination; the method and time of administration; the simultaneous presence of other substances; the age, sex and race of the patient; the existence of disease, etc. A knowledge of these variations is very important.

Methods of Administering Drugs.-The channel by which a drug is introduced into the body, or the place to which it is applied, must vary with the object to be secured-whether the action is to be local or systemic; the desired rapidity of absorption; the necessity of avoiding irritation of certain organs, etc.

Local A pplication.-Drugs may be used locally either to protect a surface, or for reflex effect, or as antiseptics, or as stimulants. They may be applied to the skin in various vehicles: If it is desired to secure the absorption of the remedy or its deep penetration, vegetable or animal oil must be used, preferably adeps lanæ hydrosus (lanolin). The influence of the ointment base, however, varies for different substances (Sauerland, r9r2). Where the local effect alone is required, the mineral fats (petrolatum or vaselin) may be employed. The remedy may also be placed in aqueous solutions (washes), especially if intended for an antiseptic; or it can be used in powder form. Caustics may be used either as solids or liquids. Counterirritants are used as liniments, i.e., dissolved in oil, turpentine, or alcohol. 
Absorption from Skin. - Local application to the skin can be used for producing general effects, but it is only employed in those cases (mercury with some patients) where the stomach has to be avoided and subcutaneous administration is not practical. The principal objection to the administration of drugs by the surface of the skin consists in the uncertain absorption, an exact dosage being in consequence impossible. The absorption is greatest where the skin is most delicate; in the axilla, loins, and the inner surfaces of the extremities. Absorption is aided by friction and cleanliness.

Watery solutions are not absorbed from the skin, unless the drug is caustic. The reason for this non-absorption lies in the fact that the stratum corneum of the epidermis is practically non-permeable to solutions. Absorption must take place through the glandular structures of the skin, and these are filled with fatty matter, which prevents the penetration of watery solutions, but not, of course, of other fats. However, when the skin has been macerated for several hours it may absorb some salt. It also absorbs $\mathrm{H}_{2} \mathrm{~S}$ and other gases.

It must be borne in mind that the application of solutions to open wounds or abraded surfaces is practically the same as subcutaneous injection, and absorption occurs in this case very readily.

Local medication may be used also on other surfaces than the skin, if they are accessible; e.g., mucous membranes. They are usually applied as aqueous solutions (injections, washes, and gargles).

Cataphoresis.-This process has been employed in dentistry to facilitate the penetration of cocain, but it has not as yet found very extensive adoption in medicine and surgery. It is a process by which the molecules are carried from the + to the - pole. The solution to be introduced must possess a higher conductivity than the liquid of the tissues.

Sprays.-Vapors and finely "atomized" sprays are inhaled for their local action on the respiratory mucous membranes. They must not be too irritant.

To reach the lower air passages, they should be inhaled deeply with the nostrils closed, the mouth wide open, and the tongue protruded. Even so, the numerous branching surfaces prevent the spray from reaching any but the larger bronchi (Semmola).

Inhalation.-This method is used only for gaseous medicines, such as anesthetics or oxygen.

When giving drugs by inhalation, it must be borne in mind that the effect does not depend upon the quantity given, but the concentration of the gas and the time during which it is administered. The rich capillary area of the alveoli is one of the best absorbing surfaces, so that the action is very rapid. The lungs also absorb fluids and dissolved substances rapidly, if these are introduced through the trachea.

Oral Administration.-This, the most ancient method, is still the standard one. Its advantage lies in its great convenience and in the absence of local irritation. Nevertheless, certain drugs do give rise to disturbances of digestion. This can be avoided by giving them in such a form that they will not be dissolved in the stomach (pills), or by giving them at a time when the stomach is filled with food. Absorption is, of course, delayed in these cases. Pills should never be employed for insoluble powders or for corrosive drugs which require dilution.

Absorption from Alimentary Canal. - The absorbing power of the stomach is relatively low, even for water and soluble substances (Hirsch, I892); so 
that the main absorption does not occur until the drug has reached the small intestines. 'The effects, with gastric administration, are therefore relatively slow. The passage is quickest if the drug is taken fasting in water; somewhat slower with milk, soup or wine; slowest after eating, especially when administered dry (Moritz, i 898 ).

The conditions in the small intestines are very favorable for absorption, being aided by the long sojourn, the extensive surface, the folds and villi, the segmental movements, etc.

The relative rôle of the stomach and inlestine in absorption varies for different drugs and animals. Strychnin, e.g., is absorbed from the stomach with dogs and cats, but not with rabbits and guinea pigs; whereas sodium salicylate and iodid give just the opposite result. Inoye and Kashiwado (1905) have shown that atropin and rhubarb are not absorbed from the dog's stomach, whilst salol is absorbed. The data as to man are insufficient.

Certain drugs are more or less destroyed in the alimentary tract, f.i., toxins and antitoxins, so that oral administration is relatively inefficient. Glucosids are also partly destroyed, so that the oral dose must be larger. On the other hand, drugs which require the action of the digestive juices for their solution (resins, oils, etc.) are more efficient by mouth than by other channels. The oral route is of course the only one for drugs intended to act locally on the upper parts of the digestive tract.

Rectal Administration.-The stomach and small intestine may be avoided by giving the drugs per rectum, either in the form of enema or suppositories. The rectum is a fairly good absorbing surface for many soluble substances; so that the effects are often greater or more prompt than with oral administration ( $f . i$., for salts, narcotics, etc.). The absorbed drug also avoids the passage through the liver, and the consequent destruction.

Enemata, when introduced for the absorption of the medicine, should be as small as possible, but not so strong as to produce local irritant effects. One or 2 ounces is usually the proper quantity, the rectum being first cleansed with warm water. When enemata are employed for their mechanical effects, the amount must, of course, be much greaterI or 2 pints. These should be raised to the body temperature.

Conjunctiva, Urethra and Vagina.-These are very good absorbing surfaces, so that systemic effects of ten follow local application (vagina; Menges, 1906). Iodid is also absorbed from the uterine cavity (Higuchi, I908).

Subcutaneous or Hypodermic Administration.-The injection of solutions through a hollow needle into the loose subcutaneous tissue. It has the advantage of being quicker and more certain in its effects, and the dosage is more exact than can be secured by any other method. The principal objection to it lies in the fact that while it is not very painful with some medicines, it is very much so with any irritating substance. There is also a tendency to abscess formation. This is frequently due to deficient asepsis, but certain substances (protoplasmic poisons) produce abscess formation even with the most rigorous asepsis.

Hypodermic injections are generally made into the forearm, arm, thigh, or nates. For very bulky injections, e.g., for antitoxin or for saline solution, the loose areolar tissue of the subscapular or mammary region is chosen. The subcutaneous dose may often be made somewhat smaller than the oral dose. The rapidity of absorption from hypodermic injections may be hastened by massage, by distributing the injection over several places, and by dissolving the drug in a small amount of fluid. The concentration 
in salts should not exceed that of the blood, or the injection will be painful. Normal saline solution is the least irritant solvent for alkaloids. Subcutaneous injection is naturally inadmissible, if local effects in other parts of the body are desired (e.g., for stomachics, cathartics, locally acting emetics, etc.).

Hypodermic injection was probably first practiced by E. Rynd, 1844 , but was introduced practically by Alex. W. Wood, in 1853 . Pravaz invented his syringe in 1853 , for the injection of aneurisms (Macht, I916).

Intramuscular Injections.-These are made by thrusting the needle through the skin deep into the substance of the gluteal or lumbar muscles. The absorption is more rapid than with subcutaneous administration (Meltzer and Auer, rgo4), and the irritation and tendency to abscess formation are less. Auer and Meltzer, IgII, advise the sacro-spinal muscles in preference to the gluteal, as giving better absorption with less pain and other complications.

Subnasal Injection.-Injection under the mucous membrane of the nasal septum gives extremely rapid absorption, the efficiency approaching intravenous injection (Pilcher, 1914, 1915).

Intraperitoneal and intrapleural injections are used in experimental technic, and resemble subcutaneous injections, the drug being absorbed more rapidly. Intraperitoneal injections in man have been made by Schmidt and Meyer (1905), but are not recommended. The pleura absorbs through the parietal and pulmonary surface; but the absorption is less than from the peritoneum (Naegeli, I9r3). In both membranes, it occurs by the blood rather than lymph. Gravity plays a part, the absorption being rather better with pelvis down position (Dandy and Rowntree, r914).

Intra pericardial Injections. - Solutions are quite rapidly absorbed from the pericardial sac into the heart-muscle. Gunn and Martin, 1915, suggest that this could be utilized in epinephrin-resuscitation.

Injections into the trachea are very rapidly absorbed through the alveolar capillaries, and act more like intravenous injections. They also cause asphyxia, and are not used intentionally. Tracheal sprays are used for local effects.

Intracerebral injections (i.e., into the substance of the brain) have been used experimentally. The injections are at once conveyed to the ventricles and produce local and mechanical effects often different from the systemic action of the drug.

Subdural Injections.-These are used if the drug is to act directly on the spinal cord, especially for spinal anesthesia. The technic is that of lumbar puncture, some cerebro-spinal fluid being withdrawn before the injection is made. The procedure is dangerous, since the poison may produce local effects or be conveyed directly to the medulla.

The absorption of quinin and atropin from the cerebro-spinal fluid is almost as rapid as if they were injected into a vein. It occurs probably by the blood (Dixon and Halliburton, rgr 2). Irritation of the meninges does not modify the absorption (for salvarsan, Stollman and Swift, 1915).

Intravenous Administration.-This is often used in pharmacologic experiments. Clinically, it has been tried in recent years ( $F$. Mendel, I908). Its dangers restrict its application mainly to emergencies when an immediate action is necessary (strophanthin), or where other methods are not applicable (salvarsan).

Intravenous injection was tried rather extensively in the seventeenth century, but its modern application dates from Landerer, I88I (history, Epstein, 1915; Macht, 1916).

While it is the quickest way of securing the action of the substance, the slight operation required is some objection. There are also more serious dangers. Air may be 
introduced into the vein, and while the presence of a small bubble of air in the circulation of a man is not as dangerous as in that of a rabbit, it may lead to very serious results. The action of drugs injected intravenously may be quite different than when taken by other channels. They act upon the heart more directly and with less dilution. Many substances also have the property of clotting the blood, and unless the administration be very skilfully done, the result might be disastrous.

\section{CONDITIONS INFLUENCING ABSORPTION}

The time required for absorption into the body and into the cells varies from a few seconds (e.g., hydrocyanic acid), to several weeks (e.g., lead). The rapidity of absorption depends upon the nature of the drug, the place of administration, and a number of accessory factors.

Place of Administration.-Absorption of drugs may occur from the alimentary canal or from other mucous surfaces; from subcutaneous tissue or serous cavities; from the alveoli of the lungs (gases and volatile substances); a limited number of substances may also be absorbed from the intact skin. The influence of the place of administration has been discussed.

Solubility.--Only soluble drugs can be absorbed (except the small quantities taken up by phagocytosis); but the solubility in the protein containing fluids of the body is not necessarily the same as the solubility in water. The solubility may also be modified by chemic changes in the digestive canal.

Solid Substances.- These are practically unabsorbable from the alimentary tract, although Koellicker and many others, on feeding rabbits with carbon, found particles in the prevertebral and mesenteric ganglia.

There is always considerable uncertainty in the absorption of solid substances, even when they are soluble. They should therefore be avoided unless a very slow action is desired. Free dilution is favorable to absorption.

Influence of Colloids, Etc.-Oil, gums, extractives, kaolin and other colloids hinder absorption, partly by adsorption of the drug, partly by hindering its access to the absorbing surface. The presence of food in the alimentary canal has a similar action. The isolated active constituents (alkaloids, etc.) are preferred if a quick systemic action is desired, whilst the galenic preparations (extracts, tinctures, pills, etc.) are used for local effect. In case a crude drug contains several active ingredients, the employment of the isolated constituents will give more definite results. On the intact skin, absorbable oils facilitate the absorption of other substances dissolved in them; alcohol usually has a similar favorable effect in the stomach.

Fuller's Earth on Alkaloids.-Fuller's earth (hydrous aluminum silicate) has a high adsorbing power, especially for alkaloids - varying, however, in degree with different samples. (Lloyd, 19I6.) The proprietary brand "Alkresta" is being especially investigated. McGuigan, 1914, found that this mixture with strychnin is insoluble in acid fluids, and develops the bitter taste quite slowly; and that it delays and diminishes the tetanic action in frogs. Fantus, I $_{9}{ }_{5}$, finds that the combination is not dissolved in the stomach, but dissociates gradually in the intestines. He also investigated the suitability of the earth as alkaloidal antidote, and found it very limited. It is somewhat improved by the addition of tartaric acid or acid sodium phosphate; it is highest against morphin, cocain, nicotin and ipecac; and least efficient against strychnin, aconitin and colchicin. The effects of Kaolin and related substances were also investigated by Friedberger and Tsuneoka, 1913.

Sabbatani, 1913, found considerable antagonism between strychnin and colloidal carbon (caramel) when mixed in vitro. The intravenous injection of the carbon also furnished some protection against the oral administration of strychnin; but this was too uncertain for practical application. 
v. Paulucci, 1915, showed that fatty substances (including petrolatum) diminish the toxicity of strychnin, both hypodermically and with direct application to the nerve centers.

Concentration.-The absorption varies generally in the same direction as the concentration, especially for hypodermic injections; but the relation is not a simple one. Absorption tends also to increase with the extent of absorbing surface (e.g., by making multiple hypodermic injections). This seems to be less important in the intestines (Sollmann and Hanzlik, IgrI-I9I3).

Changes of the Absorbing Cell.-In the alimentary canal, injury to the absorbing cell may either facilitate or hinder absorption; astringents tend to have the latter effect, corrosives and simple irritants the former.

Sollmann and Hanzlik have found that phenol, alcohol, iodid, and probably other substances tend to inhibit their own absorption; so that the absorption, at first very rapid, is promptly slowed.

Circulation.-The rapidity of absorption is proportional to the rapidity with which the drug is removed; just as a burning lamp-wick absorbs the oil more quickly (Mialhe). It therefore increases with the rapidity of the circulation and of the lymph flow. Active hyperemia (hot air; Klapp, I90I) and hemorrhage hasten absorption; while it is delayed by venous stasis, passive congestion, deficient heart action, vasoconstriction, catarrh, etc. Increase of lymph flow, by moderate distention and massage also quickens absorption. Excessive distention diminishes absorption by slowing the local lymph flow (Hamburger, I907).

Absorption in Absence of Circulation.-Considerable absorption occurs when the circulation has stopped. Thus, strychnin and morphin are absorbed by frogs with ligated hearts, producing interesting modifications in their actions (Meltzer, I9II1913). Abel, I9I 2, and Abel and Turner, 1914, believed that the solutions in cardiectomized frogs are circulated by the lymph hearts; but Meltzer, I9r4, Joseph, r9 4 , and Githens and Meltzer, I915, affirm that general effects from the local injection of strychnin, fuchsin or epinephrin may occur even when all the lymph hearts are definitely excluded, the absorption occurring through the lymph spaces.

The absorption in frogs is delayed by destruction of the spinal cord (Filippi, I9r2). Considerable absorption occurs also in dead animals (Sollmann and Hanzlik, r9r3), probably by diffusion.

Absorption by Blood and Lymph.-Most soluble substances are absorbed from the alimentary canal by the blood, rather than the lymph. This also holds for the serous cavities; methylen blue injected into the peritoneum or pleura, for instance, appears in the urine before it is seen in the thoracic duct (Starling and Tubby). A few drugs, however, take the lymphatic path. Tetanus and diphtheria toxin, and perhaps other toxins, are absorbed from the local injections by the nerve sheaths, and reach the central nervous system along these (Meyer and Ransom, 1903; Meyer, 1915; C. W. Field, 1907). These toxins are more active on hypodermic or intramuscular than on intravenous injection, since apparently they reach the nerve centers in more concentrated form by the lymph than by the blood.

\section{Sojourn in Blood.-This is generally very short.}

It must not be forgotten that a drug, after it has been absorbed into the circulation, needs still to penetrate into the cells. This intracellular absorption depends upon the nature of the poison (selective absorption), and to some degree on its concentration. As a rule, it is a very rapid process: Masoin (1903) found the time which elapses before just toxic doses disappear practically completely from the blood, after intravenous injection, to be for Arsenic, nine-tenths to thirty seconds; Tetanin, twenty seconds; Cyanids, two to six minutes; Diphtheria toxin, four minutes; Antitoxin, several hours. 


\section{THE EXCRETION OF DRUGS}

Channels of Excretion.-This occurs mainly through the urine and feces, and with volatile drugs through the lungs. The sweat and, indeed, all the secretions play a minor part. The relative importance of the different channels varies for each drug, the reasons being but little understood. The rapidity is proportional to the circulation and to the functional activity of the excretory organs, and may be increased by the factors which stimulate these.

The excretion of certain drugs appears to be limited by their existing in the body in the form of combinations. The elimination of these is favored by substances which displace them from the compounds. This is probably the explanation of the increased excretion of jodids on the administration of chlorids, and possibly of the favorable effect of iodids in chronic poisoning by metals.

Excretion by Sweat.-A considerable number of drugs are thus excreted (iodids, bromids, borates, phenol, salicylates, antipyrin, methylen blue, $\mathrm{As}, \mathrm{Hg}$ ). The quantities are too small to make them significant for elimination, but they may help to explain exanthemata.

Excretion by Saliva.-This is rather limited, and is only important for the haloids (iodids) sulphocyanates, $\mathrm{K}$ and $\mathrm{NH}_{4}, \mathrm{Hg}$ and $\mathrm{Pb}$, menthol and guaiacol, hexamethylenamin, and some alkaloids (morphin and quinin). The excretion generally begins within twenty minutes and may last over nine hours (Howe, I912).

Passage into Cerebro-spinal Fluid.-Inorganic substances are excreted into this only in a slight degree; iodids and bromids are generally present. Many organic substances pass into it much more freely: Alcohol, chloroform, acetone and hexamethylenamin occur constantly in about one-third the concentration of serum (Hald, IgII).

Passage to Fetus. - The more important drugs which have been demonstrated are: ferrocyanid (Mayer, I871); chloroform, ether, CO, ethyl bromid, atropin, morphin, scopolamin, chloral, salicyl, quinin, benzoic acid, phlorhizin, alcohol, nitrate, urea, methylen blue, $\mathrm{As}, \mathrm{Hg}, \mathrm{KI}, \mathrm{KBr}$ (Kehrer, I907).

\section{DOSAGE (POSOLOGY)}

Definition.-Drugs are administered to produce a desired effect, and the dose must be sufficient for this purpose, and neither too small, nor too large. Since the effect is influenced by numerous conditions, it is impossible to state the exact doses for any drug; experience is the only safe guide. However, the doses vary under ordinary conditions only within narrow limits, so that it is feasible to state the customary or average dose, as is done in the U.S.P. and in this book. This is convenient as a starting point.

The "maximum dose" signifies the largest dose which can be safely used in ordinary cases; the "minimum dose" is the smallest dose which produces therapeutic effects; a "toxic dose" is one which produces dangerous effects; the "just fatal dose" is that which is just sufficient to cause death; the "physiologic dose" (a rather indefinite term), is used in physiologic experiments on animals.

Variations According to Administration.-The different methods of administration, by altering the rapidity of absorption, produce quantitative differences in the effects, and therefore in the dosage. Since these vary with each drug, they must be determined by observation. General ratios of oral, rectal and hypodermic doses, supposed to apply more or less to all drugs, are dangerous (Hatcher, I9I0; Hatcher and Eggleston, I9I4).

The same dose acts the more profoundly, the more rapidly it is introduced; mainly because there is less excretion and destruction before the full dose has entered; also be. cause it reaches the heart and other centers before being distributed through the body- 
This must be taken into account in fixing the minimal fatal dose. Extremely rapid intravenous injection may simulate a higher fatal dose, if death occurs during the injection; because in this case really more poison may have been administered than was necessary for death (I. Simon, I 905 ).

Action and Dose.- With some drugs, the fatal and active dose are far apart (atropin); with others, they are close together (strychnin). The distance varies with the delicacy of observing the active dose, and for different actions of the same drug.

Daily Dose.-The single doses are usually calculated on the assumption that sufficient time is allowed to elapse between the doses for the greater part of the drug to be excreted. This constitutes a periodic medication. The exact time varies of course with the nature of the drug, but in most cases the drug is administered three or four times a day; so that the daily dose is about three or four times the single dose. If a contimuous action is desired, correspondingly smaller doses are given at shorter intervals.

Repetition of Dose--Cash, 1908, found that doses of indaconitin, repeated at sufficiently close intervals, gave imperfect summation of the temperature action; but if the interval exceeded two hours, with doses less than one-half fatal, the later doses produced actually less effect.

Variation of Dosage.-The doses ordinarily stated in text-books apply only to adults of average size, and to oral administration.

Effect of Weight.-Other things being equal, the effect of a given dose is inversely proportional to the weight of the individual (exclusive of the adipose tissue). It is rarely necessary to make allowance for the weight in adults (twenty to sixty years), but it may be used for calculating the doses for children.

Body-Surface.-This is regarded by some as a more accurate although less convenient index of the dose (Moore, I909; Dreyer \& Walker, I9I4); but the subject is in dispute (Kisskalt, I9I5).

Calculation of Doses for Children.-In most cases, the adult dose is reduced in simple proportion to the weight of the child-either by direct calculation, or by the use of empiric rules based on averages, as described below. The dosage so obtained is generally sufficiently exact. It is inadmissible only with extremely young children; and with drugs the action of which is influenced specifically by age.

Clark's Rule.-Multiply the adult dose with the weight of the child (in pounds) and divide by 150 (the weight of the average adult). This rule gives the most exact results.

Cowling's Rule.-Multiply the adult dose with the age of the child at the next birthday, and divide by the adult age, taken as twenty-four. $F . i$., the dose for a child of three years would be $4 / 24=1 / 6$ of the adult dose. This rule is simple, easily remembered, and gives data agreeing sufficiently well with the average weight curve; more accurately than Young's Rule. The results are rather too low, below four years and above fifteen years, but the error is on the safe side.

Young's Rule.-Multiply the adult dose with the age of the child (in years), and divide by the age plus $I 2$. $F, i$, the dose for a child of three years would be $3 / 15=15$. As compared with Cowling's Rule, it gives somewhat higher doses below twelve years, and somewhat lower above this age.

Fried's Rule for Infants. - Divide the age in months by 150 and multiply it by the adult dose; f.i., for a baby of five months, the dose would be $5 / 150=1 / 30$.

The dose for aged people is generally taken as somewhat less than 
that for adults. Above sixty years, the adult dose is reduced to fourfifths or two-thirds; and in extreme senility, to one-half.

Specific Influence of Age on Drug-action.-These (i.e., apart from the difference due to weight) are known definitely only for a few drugs. Children are especially susceptible to morphin and nicotin, and comparatively tolerant to cathartics, strychnin, iodids, iron, belladonna, calomel, digitalis and spartein (A. Jacobi, I907). Old age is generally less resistant to drugs; purgatives and emetics are especially debilitating. The frequent existence of atheroma makes it dangerous to use drugs which raise the blood pressure, directly or indirectly.

Influence of Sex.-Women usually require somewhat smaller doses than men (one-half to four-fifths). The greater susceptibility is in large part due to the lesser weight, but in part also to the anatomic and functional peculiarities. The influences of sex are of course most pronounced with drugs which act on the generative organs. Pregnancy also modifies the action of drugs, and contraindicates the use of irritant cathartics (because of the danger of inducing abortion) and of irritant diuretics (on account of the tendency to nephritis), etc.

\section{CONDITIONS INFLUENCING DRUG ACTIONS}

The time of administration also influences the action of drugs. Hypnotics and Cathartics, for instance, are most effective when their action coincides with the natural time of sleep and defecation, and if the external conditions are favorable. Stomachics are best given shortly before meals. Drugs which are to be absorbed rapidly are given on an empty stomach, whereas irritants are administered just after meals, when the stomach is protected by food; etc.

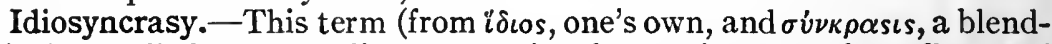
ing) is applied to peculiar, exceptional reactions to the effects of drugs. The differences are generally quantitative, and may concern the main action, or the side actions, especially skin eruptions. They may be inherent in the remedy, or may be due to extraneous causes, or they may be referred to the constitution of the patient. In the latter case, they may be due to anatomic or to functional peculiarities. They may be congenital or acquired, temporary or permanent. Neurotic patients are especially liable to show unusual effects. (Many apparent instances of idiosyncrasy are doubtless due to differences in the strength or constituents of drugs.)

Impurities in Drugs.-Qualitative abnormalities in the effects of drugs are usually due to the exaggeration of a side action; but in some cases they may be referred to the presence of impurities. It is rather doubtful, however, whether these impurities have the importance which is often assigned to them. Whilst it is undoubtedly desirable that drugs should be as pure as it is practical to make them, minimal amounts of foreign substances can not be said to be very objectionable, unless they are particularly poisonous. The pharmacopeias have taken a wise stand in this matter by permitting the presence of small amounts of such innocuous impurities which it would be very difficult and costly to remove.

Increased Susceptibility.- This may be due to very rapid absorption, or slow elimination; to the presence of synergistic agents in the body; or to increased functional susceptibility.

Anaphylaxis.-This is a striking type of highly increased susceptibility; but it seems to be confined to proteins. It has been suggested that the peculiar irritative reactions 
to iodids, volatile oils, etc., are also anaphylactic; but this is not the case (H. N. Cole; Zieher, I9I2; Glueck, I9I3). One striking difference is that the hypersusceptibility to ordinary drugs can not be transferred passively.

Cumulative Action.-This is said to exist when the continuation of a given dose of a drug produces greater effects than the first doses. It may be due to an actual accumulation of the drug, absorption being greater than the capacity for excretion (lead); or it may be due to the summation of effects, when the changes brought about by the first doses persist (digitalis); the drug may use up some substance required for its disintoxication (phenol); or there may be general decrease of resistance.

Inconstant absorption is a frequent cause of apparent cumulative action. Successive doses of the drug may lie unabsorbed in the alimentary canal, to be finally taken into the system in toto when the conditions are favorable to absorption. This explains that the greatest individual variability to toxic doses exists precisely for those drugs which are absorbed with the greatest difficulty.

Irregularities of Excretion (nephritis, etc.) are also favorable to the development of cumulative effects.

"Education."-It is sometimes noted that effects are more easily reproduced after they have once been induced. This is seen particularly in drugs acting upon the central nervous system. It is found, e.g., that the susceptibility to strychnin increases with its administration, and it would seem that this is caused by the central nervous system becoming educated to the stimulating actions and responding to them more readily.

Tolerance.-This may be due to non-absorption, to rapid elimination, to the neutralization or destruction of the poison, or to anatomic peculiarities. Many cases can not be explained in this manner, and must be assumed to be functional (the "histogenetic" immunity of Behring). The tolerance is rarely absolute, so that it is scarcely correct to speak of "immunity." (Critical review of the literature of tolerance, Heger and Zunz, rgr4.)

Habituation.-Tolerance may be congenital, or it may be developed by the repeated administration of the poison. This habituation may be functional (alcohol, caffein, nicotin); or it may be due to diminished absorption (arsenic) or increased elimination (atropin in cats); or to increased destruction of the poison (morphin); or to the production of antibodies (toxins). The tolerance is usually limited, not absolute. With some poisons, it develops a craving and serious abstinence symptoms, if the drug is suddenly withheld.

It is interesting that functional habituation, when acquired for a particular drug, may hold also for other drugs having a similar action. A habitual drunkard, e.g., is resistant to the general anesthetics; morphinists are said to be more tolerant to cocain (Chouppe, r889); etc.

Hausmann, 1907, and Santesson, I91 2, give reviews of the recent data on habituation.

Antitoxin formation is a conspicuous instance of acquired tolerance. It was supposed to be confined to proteins, but seems to exist also toward certain glucosids (toadstools, and poison ivy, Ford, I909; snake venom, Faust, 19II). It does not occur with alkaloids or any of the simpler poisons.

Acquired Immunity in Protozoa.- This has a practical as well as theoretic bearing on chemotherapy, and has been investigated by Ehrlich, 1909, and Neuhaus, 1910. These organisms acquire immunity for several groups of poisons-metals and dyesthe tolerance induced by one drug applying to other chemically related drugs, but not to the other groups. The immunity, when once induced, is transmitted unlimited from generation to generation. It is probably due to diminished affinity of the protoplasm for the poison. With alkaloids, Prowazek, I9 10, found that different individuals, even in the progeny of a single parent, show varying resistance to the same alkaloid. He also found that lecithin protects against the alkaloids.

Racial Idiosyncrasy in Animals.-Different species of animals often vary in their reaction. This can usually be explained by differences in the physiologic functions. 
For instance, the cerebral actions are usually the more pronounced, the more highly the central nervous system is developed; whereas spinal actions predominate in the lower vertebrates. Rodents are incapable of vomiting and are, therefore, not affected by emetics. Atropin quickens the heart of the dog, but not that of the rabbit, because it acts by paralyzing the vagus, which is not tonically active in the rabbit. The dog-fish, which is more or less resistant to nephrotoxic poisons, normally excretes urea and probably other metabolic products mainly by the intestines and bile (Denis, 1913).

Natural resistance is often due to the destruction of the poison, f.i., that of atropin by the serum of rabbits, etc. The destructive mechanism may be greatly increased by habituation; f.i., with morphin. Differences of absorption and excretion also come into play.

There are still a number of differences which can not yet be explained on a physiologic basis. 'These must be referred to our ignorance of the physiologic differences which are involved. The hedgehog has a high unexplained resistance to many poisons; morphin, atropin, nicotin, cyanid, cantharides, arsenite, diphtheria and tetanus toxins; not for strychnin (Strubell, I909; Willberg, 1913).

Even the same organs in different classes of animals may show unexplained differences. Thus, Gunn, I909, claims that apocodein, quinin, and yohimbin, which cause vasodilation in mammals constrict the blood vessels in frogs-both peripherally. Characteristic differences in epinephrin are well known; but the mechanism of these is explained by differences in innervation.

Individual Idiosyncrasy in Animals.-In animals, as well as in man, peculiarities exist in different individuals of the same species. Qualitative differences are seen particularly in the action of Cannabis on dogs.

Instances of quantitative differences are very numerous:

In a large series of experiments with toxic doses of drugs on animals the author has found that there is a fair degree of uniformity in the proportion of animals which die with a given dose. Thus, certain limits can be found inside of which, out of five animals three will always die. These limits vary from 0.5 per cent. (strychnin) to 25 per cent. (ergot), but are usually comprised within from 5 per cent. to Io per cent.

On the other hand, the susceptibility of any one animal is subject to greater possible variations; $e . g$., with a given preparation of digitalis, $0.6 \mathrm{mg}$. per gram will always kill three guinea pigs out of five. But in a large series of experiments, a number of animals will be found which will die of doses as small as $0.4 \mathrm{mg}$., while others will die only when $0.9 \mathrm{mg}$. is reached. Whether these comparatively immune animals always enjoy this immunity, or whether the condition is only temporary, as well as the influence of age, sex, etc., has not been determined.

These individual differences are still more striking if, instead of observing the toxic doses-i.e., the sum total of the effects-we direct our attention upon some one particular action, e.g., the amount of slowing of the heart or the variation of blood pressure. The differences in this respect are so great qualitatively that it is undoubtedly unsafe to draw conclusions from a single experiment, and it is absolutely impossible in these cases to establish any quantitative standard.

Seasonal Variations. - The susceptibility of animals to a number of poisons varies considerably with the season. With cold-blooded animals, this would be referred mainly to differences of temperature; but considerable variations exist also in mammals. Guinea pigs, f.i., are more resistant to ouabain, and less resistant to acetonitrile and diphtheria toxin during November to January than in the spring and summer months (Haskell, I912).

Temperature.-This has a marked effect in cold-blooded animals; digitalis, veratrin, nicotin, strychnin, tetanus toxin, chloral, and alcohol, for instance, are rendered more active, morphin and curare, less active, by raising the temperature The temperature quotient of strychnin was investigated by Schlomovitz \& Chase, I9I6.

The influence is especially marked with poisons that have to undergo a transformation in the body. At $37^{\circ} \mathrm{C}$., the susceptibility of frogs to atoxyl is increased 12 times, that for colchicin 50 times, above ordinary room temperature. With muscles, the activity of drugs increases in a mathematical ratio with the temperature within certain limits (Veley and Waller, I9IO).

The temperature relations of digitaloids are fully discussed by Sollmann, Mendenhall and Stingel, I915. An indication of this influence in man is seen in the modification of the action of antipyretics by fever. (In mammals, cooling generally increases the fatal effect by adding its direct depressant action.)

Diet.-This has a marked effect on the resistance of animals to many poisons (Hunt, 1910; Opie and Alford, 1914); but little is known of this in man. The calcium content is important in influencing cutaneous irritation (Luithlen, IgI I, I9I2). Barium is better absorbed from a Ca-poor diet (Alsberg and Black, I9I2). 
Influence of Pathologic States.-The effects of drugs in disease may differ materially from those on healthy animals. The pathologic conditions may lessen absorption (diarrhea) or increase it (corrosion); they may hasten the destruction of the poison (alcohol produces less intoxication in fever); or they may alter the effects entirely. Some of these modifications are readily explained by the functional or anatomic changes of disease; others are obscure.

The antipyretics reduce the temperature in hyperpyrexia, but do not effect it when it is normal; digitalis is an efficient diuretic in cardiac disease, but not in health; it affects the normal cardiac muscle, but has little action on a fatty heart, etc.; the antiseptics and antitoxins are active only in infections, etc. Numerous other instances may be found in the papers of Salant, IgII, and Wallace, I9I 2.

Pathologic conditions which modify absorption and excretion are of particular practical importance: Suppression of urine, as by nephritis, may lead to toxic symptoms from drugs or doses which are ordinarily harmless. The general reduction of vitality, the "lowered resisting power," which is so common in many diseases is similarly important. As an instance of such modifications, the investigations of Lusini and Sebastiani, I906, may be quoted. They found the absorptions of poisons hastened immediately after hemorrhage but not later. The toxic symptoms were not modified. The resistance was found lowered to a varying degree for different poisons: Most for strychnin, less for veratrin, and not at all for physostigmin.

Inflamed Vessels.--These react abnormally. Eskin, I9I4, found constrictor drugs generally less and dilator drugs more effective. Epinephrin often produced dilation, whilst caffein, which normally dilates, constricted the inflamed vessels of rabbit's ear.

Diseased intestines allow a much more rapid diffusion of proteins, toxins and ferments than healthy intestine (Mayerhofer and Pribram, I909).

The distribution of the drugs in the body may be different in disease and health. This is best illustrated by fluorescein (see Index).

The Combined Action of Drugs.-The effects of drugs are also influenced by the unusual conditions induced by the simultaneous administration or presence of other drugs. A quantitative change may occur either in the sense of increasing the efficiency (synergism), or of diminishing it (antagonism). There may also be qualitative changes. In other cases, new actions are developed by the reaction of the drugs on each other, with the production of new compounds. For instance, the presence of acids renders the basic salts of bismuth soluble and toxic; oxidizing substances may liberate iodin from iodids; the iodids also decompose calomel and render it irritant.

Antagonism of action is employed in the treatment of poisoning; or sometimes to remove undesired side effects. When it interferes with desired effects, it constitutes "therapeutic incompatibility."

Combination Therapy.-Synergism is utilized to secure the summation of the desirable effects of several drugs, whilst the side actions are not increased or may even be neutralized. The efficiency itself may be increased, especially when the drugs attack a given cell or function simultaneously by several different mechanisms. Chemo-therapy has furnished some illustrations of this (Ehrlich, I909). Formerly, combinations of drugs were very popular, but on the empiric principle of the shotgun mixture that of many ingredients, some at least might fit the disease. Such indiscriminate mixtures are not scientific. It is better to employ the fewest possible drugs, until it has actually been shown that combinations give superior results in the particular condition. In giving several drugs of similar actions, the dosage of each must be correspondingly reduced.

Variations of Combined Action.-When several drugs are administered together, each may act independently, as if it were present alone. The 
result would then be a simple algebraic summation of the effects, either synergistic or antagonistic. In many cases, however, the combined action is greater or smaller than would be calculated (potentiated and deficient summation). The relations may differ entirely according to dosage.

$F . i$, stimulants are antagonistic to depressants only in small doses. Large doses often become synergistic, the depression being greater than if the depressant were used alone. Instances of this are atropin and pilocarpin on embryonal growth, Sollmann, I904; caffein and alcohol toxicity, Pilcher, I9I2; atropin and physostigmin toxicity, Fraser; Fothergill gives other interesting examples of antagonism.

Sometimes, an action develops only in the presence of another drug; f.i., pilocarpin provokes the action of minute doses of atropin on the intestine; or the point of attack may be shifted by the second drug. Instances will be found in the chapter on "Autonomic Poisons." The ratio of ions in the medium surrounding the cell influences the effects of drugs very materially.

Instances of Synergism. - The best-studied examples are from the group of narcotics. 'The simple lipolytic narcotics (alcohol, ether, chloroform, etc.) show practically only simple summation; but the addition of the alkaloidal narcotics (morphin and scopolamin) gives considerable potentiation (Knell, r907; Fuehner, 19I r Kochmann and pupils, 1913). Bromids give simple summation with lipolytic and alkaloidal narcotics (Klammer, 1913). The different opium alkaloids potentiate each other in interesting ways (Straub, 1913; Macht). The organic local anesthetics do not usually potentiate (Schmid, 1913), but show marked potentiation with potassium (Zorn, 1913). Fuehner, 1912, has worked out the summation of hemolytic agents.

Mechanisms of Potentiation.-There are several possible explanations, but few positive data. Qne drug could modify the penetration of another. Fuehner, r913, showed that this is true of the solubility coefficient of the narcotics; or it might alter the chemic affinity, either by acting as an amboceptor, or by closing the combining groups of the cell or of the drug (antitoxins); or it might attack the cell from a different point.

Mansfield and Hamburger, I9I5, believe that magnesium potentiates urethane; and that ether potentiates chloral or morphin, by favoring the distribution of these agents in the nervous system; for they find that the augmented action of the combinations persists after the magnesium is neutralized by calcium; or after the ether has been eliminated.

Those interested in summation and antagonism should read Chapter VIII of Verworn's "Erregung und Laehmung."

Buergi's Law.-Buergi, I910-I9I2, proposed a universal explanation of synergism; but his generalization is doubtless too sweeping. He announced that potentiation occurs, always and only, when two drugs have different points of attack in the cell; because two different receptors could take up more poison in a given time than could a single receptor from the double quantity of a single drug, Fuehner, 191 2; Issekutz, 1913; Zorn, I9I3, and others have pointed out numerous important exceptions to this "law." It is certainly not universally applicable.

\section{FERMENTS AND NUTRIENTS}

\section{DIGESTIVE FERMENTS}

Nature of Ferments.-The digestion of food in the alimentary canal is accomplished mainly by the secretion of organic ferments or enzymes; colloid substances of unknown composition, whose presence accelerates chemical changes. Commercial ferments are more or less purified extracts, rich in protein, and dried or preserved by glycerin.

Our knowledge of the nature of ferments is very incomplete. Like colloids, they possess electric charges (Michaelis, 1909). Emil Fischer found their action on sugars to depend on their optic activity. Several workers have shown that the cleavage ferments may, under proper conditions, produce syntheses (Taylor, 1904). The term "fermentation" is derived from fermentum, leaven; and this probably from fervere, to boil. It was originally applied to all effervescence. 
Conditions of Action and Deterioration.-The digestive ferments are specifically adapted to the hydrolytic cleavage of the various food products (amylolytic, proteolytic, lipolytic, etc.). Their activity is greatly influenced by various conditions, especially by temperature $\left(40^{\circ}\right.$ to $45^{\circ} \mathrm{C}$. being optimal for mammalian ferments); by acid or alkaline reaction; by salts, etc. Solutions are quite unstable. They are destroyed rapidly about $50^{\circ} \mathrm{C}$., immediately by boiling and more gradually by any excessive conditions, and by putrefaction. Pepsin is much more resistant to destruction than trypsin, but both deteriorate even in neutral solutions, trypsin often in a few days. Dilute watery solutions deteriorate quickly even at ordinary temperature; and even dry "pancreatin" is unstable (Long and Muhlmann, I9I4). Pepsin can also be completely destroyed by shaking (Shaklee and Meltzer, I909).

Incompatibilities.- Solutions of ferments are therefore incompatible with excess of acid and alkali (Long and Muhlmann, I9I4) or salts; with many antiseptics; with excess of alcohol; and with all protein-precipitants. Pepsin in neutral or alkaline solution is destroyed by sodium chlorid (W. H. Hamburger, I9I 5).

Mixtures of ferments digest each other more or less rapidly according to their reaction. Pepsin, in the presence of weak acids, destroys trypsin and amylopsin completely; but trypsin has little effect on pepsin under ordinary conditions (Long and Muhlman). "Compound Digestive Elixirs," supposed to contain pepsin with pancreatin, etc., are therefore practically worthless (J. A. M. A., I907, v. 48, p. 434).

Administration of Ferments in Digestive Disorders.-This has not realized the early expectations, one reason being that digestive disturbances are rarely due to deficiency of ferments. It is also difficult to administer ferments so that they could develop their actions. This is probably possible only with pepsin and perhaps with diastase. The latter, if thoroughly mixed with food, could act in the fundus of the stomach for an hour or so (Cannon) before the mass becomes acid (Gruetzner, I905). It is almost inconceivable that trypsin should reach the intestines unchanged, since it would certainly be exposed to the digestive action of the gastric juice. However, there are some data which suggest that the administration of fresh pancreas by mouth improves the absorption of fat and proteins in dogs deprived of natural pancreatic secretion (Sandmeyer, I895; Pratt, I909). The action of papain is too feeble at body temperature to deserve consideration.

Pepsin and possibly diastase may therefore have a limited value in a few cases; but little could be expected from the other ferments. Apparently favorable results may of ten be explained by the other treatment with which they are usually combined.

Other Uses. - A more rational field for ferments is in the preparation of predigested foods, especially for rectal alimentation. Rennin and Pepsin are used for curdling milk. Pepsin has been employed to dissolve diphtheritic membranes. Trypsin injections were tried against cancer (J. Beard, I905; Shaw-Mackenzie, ı906), but proved disappointing. They produce local lesions and toxic effects resembling peptone-poisoning (Kirchheim, IgII). Papain and takadiastase act similarly (Kirchheim, I9 3 ).

Intravenous. Injection of Ferments.-This produces toxic symptoms resembling those of Albumoses (Hildebrandt, 1890 ). Smaller doses give rise to the formation of specific antibodies. Normal serum and other native proteins are also markedly re- 
sistant to protolytic ferments (Levene and Stookey, I903). This resistance is destroyed by heating to $70^{\circ} \mathrm{C}$, and is connected especially with the albumen fraction (Cathcart, I90.4).

PREPARATIONS-FERMENTS

* Pcpsinum (Pepsin), U.S.P., B.P.-A proteolytic extract obtained from the mucous membrane of the stomach of the pig (or sheep or calf, B.P.). Capable of digesting not less than 3.000 parts of coagulated egg-albumen (U.S.P.), (2,500 parts, B.P.), when the test is made according to the official directions. Various processes are used in its manufacture. White or yellowish scales or powder, slight odor and taste; acid reaction. $S_{o l}$. in water $(\mathrm{I}: 50)$; nearly insol. in alc. Solutions are incompalible with alkalies, salts, metals, and tannin. Dose, 0.5 Gm., 8 gr., U.S.P.; 0.3 to $0.6 \mathrm{Gm} ., 5$ to 10 gr., B.P. Numberless proprietary preparations are advertised, but without material advantage.

Glycer. Pepsin., B.P.- Io per cent. of pepsin; r.I5 per cent. of hydrochloric acid. Dose, 4 to 8 c.c., I to 2 drams, B.P.

Pepsin. Sacch.- I: 10 of milk-sugar.

Liq. Seriparus, N.F., B.P.C.; Liquid Rennet.-Extracts of the fourth stomach of calves; used to coagulate unboiled milk ( 1 : 100 to $300,38^{\circ} \mathrm{C}$., I00 $\mathrm{F}$.) for the preparation of junket and whey.

* Pancreatinum (Pancrcat.), U.S.P.; Pancreatin.-A mixture containing enzymes, consisting principally of amylopsin, trypsin and steapsin, obtained from the fresh pancreas of the hog or of the beef. It converts not less than 25 times its own weight. of starch into sugars. However, commercial pancreatins generally contain little or no proteolytic ferment, and very uncertain amounts of diastase. The amylopsin acts best in a faintly alkaline $\left(0.025\right.$ per cent. $\left.\mathrm{NaHCO}_{3}\right)$ or neutral medium, and is destroyed by very weak acidity (0.006 per cent. $\mathrm{HCl}$ ) (Long and Muhlman, I9 I4). Trypsin also acts best when alkaline $\left(0.2\right.$ to I per cent. $\left.\mathrm{Na}_{2} \mathrm{CO}_{3}\right)$ but is somewhat active with very weak acidity. Excess in either direction is detrimental. It acts best on fibrin or casein, feebly on cooked albumen, and scarcely at all on raw albumen (Long and Muhlmann; Sugimoto, 1913). Pancreatin occurs as a cream-colored, amorphous powder, having a faint, peculiar odor. Slowly sol. in water; insol. in alc. Dose, $0.5 \mathrm{Gm} ., 8 \mathrm{gr}$., U.S.P. B.P.

Liq. Pancrcat., B.P.-An alcohol-glycerin extract. Dose, 4 to 8 c.c., I to 2 drams,

Pulv. Pancreat. Co.. N.F., B.P.C.-Pancreatin mixed with 4 parts of sodium bicarbonate; is used for the artificial digestion of milk $\left(1.5\right.$ to $500,38^{\circ} \mathrm{C}$., $100^{\circ} \mathrm{F}$., for half an hour).

Papain.-The dried milk-juice of the unripe fruit of Carica Papaya. Extracts are marketed under proprietary names. It is supposed to digest proteins in acid, alkaline and neutral media; its activity, however, is variable, and always much less than pepsin. The digestion is more rapid at $80^{\circ}$ to $95^{\circ} \mathrm{C}$. (Pozerski, r 909 ; some of its peculiarities were studied by Mendel and Blood, roro). Dose, o.I to I Gm.

Extractum Malti (Ext. Malt), U.S.P.-A watery, pilular extract of malt, prepared at $55^{\circ} \mathrm{C}$., and evaporated at this temperature to the consistence of a thick honey. Contains diastase, and therefore digests starch; but the diastatic power of the commercial extracts is very weak. ${ }^{2}$ It is more properly nutrient, being rich in carbohydrates. Dose, I5 c.c., $1 / 2$ ounce. Many liquid malt cxtracts are practically strong beer.

Maltum, U.S P.; Malt.-The grain of barley, Hordeum sativum, partially germinated artificially, and then dried at a temperature not exceeding $55^{\circ} \mathrm{C}$. It is capable of converting not less than five times its weight of starch into sugars.

Diastasum, U.S.P.; Diastase.-A mixture containing amylolytic enzymes obtained from an infusion of malt. It converts not less than fifty times its weight of potato starch into sugars. A yellowish-white, amorphous powder or in translucent scales; odorless and tasteless. Diastase gradually loses its amylolytic power on keeping; is diminished by the presence of acids or alkalies. Sol. in water; almost insol. in alc. Dose, 0.5 Gm., 8 gr., U.S.P.

Diastatic ferments are also obtained from other sources, animal and vegetable; Taka diastase, for instance, from a Japanese mould. Their diastatic action is also weak and unreliable. ${ }^{2}$

\section{LACTIC ACID FERMENTS}

These were introduced by Metchnikoff, with the idea of acidifying the intestines, and thereby preventing intestinal putrefaction, which he held responsible for premature senility. They had only a brief popularity. Even if the theory of old age were ac-

1 Rep. C.P.C., J.A.M.A., July I I, 1908.

2 Rep.C.P.C., J.A.M.A., July II, I908; July 6, I912. 
cepted, there is no evidence that these organisms check intestinal putrefaction. To the contrary, they usually succumb to the putrefactive bacteria. The commercial preparations were of uncertain composition (Heinemann, I909). The more reliable are listed in N.N.R. Metchnikoff's Bacillus bulgaricus is in no way superior to the Streptococcus lacticus which produces the ordinary souring of milk.

\section{MEDICINAL FOODS}

Limitations. - The administration of food in the guise of medicine is sometimes advantageous; but medicinal foods are subject to the ordinary law of dietetics, and therefore can not accomplish the wonders which are often claimed for them. The proprietary foods have been enormously overestimated, and therefore have probably done more harm than good. The ultimate value of any food depends mainly on the amount of calories which it can yield, and on its supplying at least a minimum of proteins. In these respects, the medicinal foods are all inferior, for they can not be administered practically in sufficient quantity to supply the needs of the body. They have a place as adjuvants to other foods, permitting the introduction of more food than the patient could otherwise be induced to take. This may be needed for superalimentation; when digestion is low, as in fevers or convalescence; or to tide the patient over some temporary crisis. Aside from the special diabetes foods and cod liver oil, their value is largely psychic. The better medicinal foods, it is true, are free from indigestible portions, and are pleasantly flavored; those which contain meat extractives would stimulate the flow of gastric juice; but all these properties are possessed by milk, eggs, cereals and soups. A few are more concentrated; but this has no advantage in feeding the sick, since water must be supplied in one form or another.

Predigested Foods. - The value of these is doubtful, even if they are well utilized; for digestive disturbances involve more commonly the motor functions and absorption than the chemical functions. The continued use of predigested foods often produces irritation. Their main field is in rectal alimentation.

Utilization of the Products of Artificial Digestion.-It has been questioned whether this is as effective as that of the products of natural digestion. The work of Abderhalden and others has shown that nitrogen equilibrium can be maintained in animals on a diet of the products of acid-hydrolysis of proteins, if these are sufficiently varied to secure a proper balance of the various amino-acids, etc. (Rep. C.P.C., rgI2).

Animals with their digestive canal excised can be kept alive by intravenous injection of amino-acid mixtures, but not by peptones, proteoses, ammonium or urea (Henriquez and Anderson, rgri).

Dry "Peptones."-The dried products of the artificial digestion of meats, usually consisting mainly of albumoses. Their taste is disagreeable and they often cause irritation. Intravenously they are toxic, lowering the blood pressure and rendering the blood non-coagulable (see Index): In "Erepton," N.N.R., the digestion has been carried to the stage of amino-acids.

Liquid Predigested Foods.-As found on the market, these are flavored solutions containing small amounts of predigested proteins ( $1 / 2$ to 6 per cent.) and of sugars and other carbohydrates ( $1 / 2$ to 15 per cent.), with $\mathrm{I}_{2}$ to $\mathrm{r} 9$ per cent. of alcohol, and of ten with large quantities (up to 30 per cent.) of glycerin (details in Rep. C.P.C., J.A.M.A., May Ir, I907). Their protein content averages less than that of milk (3.5 per cent.), and in energy value, on the basis of calories, they are vastly inferior. Their daily dose ( 50 to 150 c.c.) yields but 55 to 300 calories, including their alcohol; and this is only one-thirtieth to one-fifth of the minimum daily requirement, even of resting patients ( $\mathrm{I}, 500$ calories, yielded by two quarts of milk). To increase their dose to that required to maintain nutrition would mean the ingestion of an amount of alcohol equivalent to about a pint of whisky per day.

No liquid food should be given consideration un!ess it contains, exclusive of alcohol and glycerin, at least as much food value as milk; and at least one-fourth of this should 
be in the form of nitrogenous constituents. Those complying with this requirement are enumerated in N.N.R. Even these can only be considered as adjuvants and vehicles.

Meat and Beef Juices. - When prepared extemporaneously by expression of fresh or slightly broiled meat, these contain from 2.5 to 9 per cent. of coagulable proteins, and 2 to 4 per cent. of other proteins and nitrogenous extractives. They have therefore a moderate value as sources of nitrogen. The commercial preparations are supposed to be concentrated; in fact, however, they are very inferior, containing but 0.2 to 3 per cent. of coagulable proteins; others are reinforced by the addition of defibrinated blood, sugar, glycerin, etc., which are of doubtful utility (Rep. C.P.C., J.A.M.A., Nov. 2o, 1909).

Bouillon and Meat Extracts.-Bouillon (beef-tea) is prepared by boiling meat with water; the extracts by evaporating the solution to a semisolid consistency. The coagulable proteins are removed by this process, the extract containing the salts of the meat, the flavoring substances and meat bases (xanthin and creatin products) and a certain amount of non-coagulable protein, in the form of gelatin, albumoses, etc. In the small amounts in which they are used, these preparations contain a very insignificant quantity of nutriment. They are, however, valuable as stimulants. They owe their action largely to the odorous principles which they contain, and which are excellent stomachics (Wolff, I9I2).

The potassium salts have also been invoked to explain the action, it being claimed that they stimulate the heart in moderate doses and paralyze it in large doses. But the dose required to produce the former effect is very much larger than would be administered in beef-tea.

Beef extracts of the Liebig type (pasty consistency) contain 60 to 80 per cent. of solids, of which 3 to I 8 per cent. are soluble proteins, i to 5 per cent. insoluble proteins, 12 to 25 per cent. meat-bases, and 13 to 30 per cent. ash (McGill, I899). They are used to flavor soups, etc.

Insoluble Meat Powders.-Tropon, Sozon, etc., are employed to thicken soups. Their usefulness is very limited.

Casein Powders.-Include nutrose, N.N.R. (sodium caseinate); and some advertised to the public with most extravagant claims (Sanatogen, J.A.M.A., May 6, I9II, etc.). It is difficult to understand in what ways these could be superior to ordinary cottage-cheese.

Dextrose, N.N.R.-Prepared artificially by the action of dilute acids on starch; is sometimes used as nutrient (to $180 \mathrm{Gm}$., 6 ounces daily); and as an addition to saline infusion liquids ( 0.1 per cent.).

Rapidity of Oxidation of Sugars. - In man, the respiratory quotient shows that saccharose, lactose, levulose and lactose begin to be oxidized within five or ten minutes after their ingestion. With glucose and maltose, oxidation begins only after 20 or 30 minutes (Higgins, 1916).

\section{DIABETES FOODS}

Gluten flours should be practically free from carbohydrates; but many are fraudulent (J.A.M.A., 1913, 60:922; Street; 1913). Those acceptable are enumerated in N.N.R. Other "Diabetes Flours" consist of casein, etc.

Levulose, N.N.R., and inulin are sometimes tolerated better than other sugars. (v. Noorden, 1910; Goudberg, 1913); but the difference is usually not important. In pancreatic diabetes and phosphorus glycosuria the levulose is deposited as glycogen, whilst dextrose is not (E. Neubauer, 1909). H. B. Lewis and Frankel, 1914, conclude that inulin is quite useless. It is not digested, although some is burned by the intestinal bacteria. None is converted into utilizable carbohydrate, even in phlorhizin animals.

Grafe, 1914, finds promising results with caramelized sugar. It is all absorbed and completely tolerated even in severe diabetes. It does not increase the blood-sugar, and the respiratory quotient shows that it is utilized in the diabetic as well as normal organism.

Mannit is also employed as sweetener, being assimilated without causing glycosuria; doses above 20 to $30 \mathrm{Gm}$. are laxative. (For normal assimilation limits of various carbohydrates, see Macleod, I9I3 and I914; Jacobson, 1913.)

Hediosit, N.N.R.-An artificial sugar, the lacton of a glucoheptonic acid; was introduced by Rosenfeld, I9I I, as sweetening agent and food, but has failed to realize the original expectation. When administered by mouth, 25 to 85 per cent. is excreted unchanged by the urine and feces. The fate of the remainder has not been established. Large doses produce diarrhea and other digestive disturbances. Its excretion is diminished in nephritis, resulting in retention (Kohshi, I912; Kramer, 1912; Lenel, I9I4). 
Jambul.-The fruit, leaf, or bark of Syzygium Jambulana, Eastern Asia. It contains an essential oil, tannin, and probably a glucosid. It has been recommended in diabetes mellitus in doses of 0.3 to $0.5 \mathrm{Gm}$. of the fruit ( 5 to 8 grains). Efficiency doubtful.

\section{COD LIVER OIL}

This is employed as nutrient in tuberculosis, rickets, diabetes and other wasting diseases. It has a very high food value, a tablespoonful yielding about I30 calories.

Animal experiments support the clinical conclusion that it favors nutrition and growth more than other fats. The reasons for this superiority are obscure, but it is not shared by fat-free extracts.

The oil may cause distaste, eructations and diarrhea. These are often avoided by starting with small doses, gradually increased. It is generally administered two hours after meals. Indigestion and fever contraindicate its use.

Superiority over Other Fats.-Cod liver oil was adopted into medicine in the early decades of last century from the Norwegian fishermen, who prepared it by very crude methods, involving putrefaction. These oils, therefore, contained free fatty acids (Buchheim, 1874), bile constituents (Naumann, 1865), cholesterin, putrefactive alkaloids (asellin, morrhuin, etc.; Gautier and Mourges), traces of iodin (about as much as oysters), and other impurities. All of these have been credited with the effects; but these substances are eliminated in the modern sanitary processes, without decreasing the usefulness of the oil; whilst "oil-free," "tasteless" extracts, wines, etc., are useless (Rep. C.P.C., J.A.M.A., Oct. 9, r9o9).

The easier emulsification of the oil is probably a peculiarity of its characteristic fats, which are largely glycerids of jecoleic and therapic acids. Lipanin was a mixture of olive oil with 6 per cent. of oleic acid, based on Buchheim's suggestion that free acids aid emulsification. It has not establshed itself clinically. The digestibility of different animal fats is discussed by Langworthy and Holmes, I915. Mottram, I 91 5, attributes the superiority to the high percentage of unsaturated fatty acids, which approaches more closely the form in which fat is supposed to be utilized in metabolism

Specific Influence of Fats on Growth.-Osborne and Mendel, I9I3 and I9I4; McCollum and Davis, I9 3 ; and Mendel, $191_{5}$, investigated this on rats maintained on artificial diets that kept up their body-weight, but did not allow further growth. Growth resumed when butter-fat, beef-fat egg-yolk lipoid or especially cod liver oil were added to the diet; whereas lard and olive or almond oil were ineffective. This fat-soluble growth-promoting substance " $\mathrm{A}$ " is widely distributed in plants, occurring also in leaves (McCollum, Simmonds \& Pitz, 1916). Fat-free extracts of cod liver oil do not share this specific nutrient effect of the oil (Street, I915).

The growth-promoting action of butter-fat is not due to contamination with nitrogen or phosphorus compounds (Osborne and Wakeman, I915). It is confined to the portion of the fat with low melting-point is not destroyed by steam (Osborne and Mendel, 1915), and as it exists in butter, is very stable under ordinary conditions of storage (Mendel and Ooborne, I916). Another, a water-soluble growth-promoting substance " $\mathrm{B}$ " (Funk and Macallum, I9I4), is also of wide occurrence in animal and vegetable foods (McCollum et al., 1916).

Vitamins.-These are somewhat analogous but probably not identical substances, that counteract the harmful effects of certain limited dietaries. Eykman discovered that polyneuritis resembling beri-beri can be induced in fowls by an exclusive diet of polished rice. Funk, I9II, found that this can be prevented by small amounts of a substance separated from rice polishings, which he calls a vitamin. Its exact nature is not yet known, but it is probably a fairly simple substance. Similar principles can be prepared from yeast, etc.

PREPARATIONS-COD LIVER OIL

* Oleum Morrhua (Ol. Morrh.), U.S.P., B.P.; Cod Liver Oil (Ol. Jecoris Aselli).A fixed oil from the fresh livers of Gadus Morrhua and other species. Occurs as a pale yellow thin oil, having a peculiar fishy, but not rancid odor, and a bland fishy taste. It thickens on exposure to air. Dose, 1o c.C., $2 \frac{1}{2}$ drams, U.S.P.; 4 to 15 c.c., 1 to 4 drams, B.P. 
* Emulsum Olei Morrina (Emul. Ol. Morrh.), U.S.P.-A 50 per cent. emulsion, made with acacia, sweetened with syrup and flavored with wintergreen. Dose, I5 c.c., 4 drams, U.S.P.

\section{PARENTERAL ALIMENTATION}

Rectal Feeding.-This becomes necessary when alimentation by mouth is inadmissible. However, only sugars are well absorbed by this channel; fats, undigested proteins, milk and casein poorly.

The absorbability may be increased by pancreatic predigestion (Reach, rgor). Even with peptonized milk, the average daily absorption of nitrogen is only I.I4 Gm., i.e., practically insignificant (Adler, I915). The absorption of fats from enemas is negligible (Nakashima, I9I4). Judged by the urinary nitrogen, practically no protein is absorbed from milk or eggs, unless the peptonization is carried to the a mino-acid stage. Dextrose is well absorbed and utilized, being capable of diminishing acetonuria (Bergmark, I9 I5).

Rectal alimentation suffices for only a limited time, varying with the previous condition of the patient, especially as regards adipose tissue. The food is introduced in to the rectum in the form of enemata. These must be made as non-irritant as possible; i.e., they must not be too concentrated. They must be warmed and injected very slowly in small quantities of 6 to 8 ounces $(200$ c.c.) at each injection, repeated at intervals of six to eight hours. 'The constituents must of course all be in the liquid form. The best medium is milk, pancreatized for twenty-four hours, with 5 per cent. of dextrose added (Bywaters and Short, I913); and in this the sugar probably plays the main part.

Small enemas are carried by antiperistalsis to the cecum, but not into the ileum; with larger injections, some of the material passes into the small intestine (Cannon).

Subcutaneous Feeding.-The hypodermic injection of nutrient solutions has been tried with limited success. Dextrose has given the most promising results. Even large doses are well utilized and have no detrimental effects on nitrogen metabolism (Underhill and Closson, 1906; Berendes, I9Ir); but are capable of diminishing acetonuria (Bergmark, I915). Only a small proportion is excreted into the alimentary tract (Kleiner, I9II). A 5 per cent. solution may be given under the skin, or ro per cent. intravenously, up to $\mathrm{I}, 000$ c.c. per day (Kausch, I9I I).

Saccharose is partly utilized (Mendel and Mitchell, I905; about one-third, Japelli and d'Errico); but mainly excreted unchanged (Mendel and Kleiner, 1910; Heilner, I9II). It is not available for feeding (Mendel, I9I4). Roehmann, I9I5, claims that the blood, after intravenous saccharose injections, sometimes develops ferments that invert it to dextrose and levulose, and even convert these into lactose. Abderhalden and Brohm suggest that the utilization may be explained by partial excretion into the alimentary canal, where it is converted, and reabsorbed in usable form. Lactose, dextrinı and glycogen are also not available. In normal animals, lactose injected into the blood disappears rapidly into the tissues and urine; after nephritis or in nephrectomized animals, it is retained in the blood (O. Schwarz and Pulay, 1915).

Oil can be utilized hypodermically; to to Ioo $\mathrm{Gm}$. per day of olive oil being slowly injected with the same technic as is used for the injection of antitoxic serums. The absorption, however, is so slow that these injections are almost worthless (Winternitz, 1903; Henderson and Crofutt, 1905).

Intravenous Feeding.-This has been accomplished successfully in animals, by a mixture of protein-cleavage products, dextrose, sodium acetate and salts (Hendriquez and Anderson, I913). Serum protein is well utilized (Austin and Eisenbrey, I9I2), but the dangers (anaphylaxis, etc.) preclude its clinical use as nutrient.

Fat introduced in emulsion intravenously is also burned (Murlin and Riche, I915); and so, of course, is dextrose.

\section{EMOLLIENTS}

Definition of Emollients and Demulcents.-These are drugs which soften, "relax," protect, and "soothe" the parts to which they are applied; in other words, drugs which lessen irritation. The term emollient is restricted more to those used on the skin, demulcent to those applied to mucous membranes. No very sharp distinction can be drawn between these, and 
many belong to both classes; but, as a rule, the fats are used as emollients, the gums as demulcents.

These substances lessen the action of all chemic, mechanical or bacterial irritants; diminishing pain, reflexes, catarrh, and all manifestations of irritation. They also delay and diminish absorption, particularly from the stomach, but also from the intestine, subcutaneous tissue, etc. In this way they diminish the systemic effects of absorbable poisons, whilst they prolong their local actions.

Mechanism of Action.-Colloids and fats are practically indifferent and inabsorbable for cells, so that they do not produce any direct effects on them; but through their adhesive character, they form an extra covering to the surfaces to which they are applied, and thus hinder the access of irritant agents. Their action consists, therefore, in mechanical protection, and is strictly local. Fine insoluble adhesive powders (talcum, bismuth) act similarly.

Explanation.-The oils prevent the penetration of water-soluble substances, the gums that of fats and resins. Gums also lessen the effects of crystalloids, although they do not impede their diffusion. They produce this effect by increasing the viscidity of the solution, thereby interfering with its transportation (von Tappeiner, 1902). Adsorption probably also plays a part, for dissolved substances are condensed on the surfaces of fine powders and thus removed from the solution.

One sees a beautiful illustration of these facts in the natural lubricants of bodycoverings. Whilst the skin is normally covered with a thin layer of oil, the membranes of the interior of the body are moistened with mucus, which is a typical gummy demulcent.

Emollient Drugs.- These comprise the true fats and oils (glycerids of fatty acids), woolfat, petrolatum, and glycerin. They are employed mainly on external surfaces, to soften and protect the skin, ulcers, burns, and other superficial wounds; as vehicles (salves) for the application of medicaments to the skin or, more rarely, for systemic effects (mercurial inunctions). Internally, the true fats serve as nutrients. Larger amounts than can be digested stimulate peristalsis and act as intestinal lubricants and laxatives. For external use, the liquid or semifluid fats (oils, lard, woolfat, glycerin) should be chosen if a deep effect is desired for softening the skin or for inunction. The effects of petrolatum and of the harder "cerates" and plasters are more superficial and prolonged.

Penetration of the Skin.-Oils penetrate the epidermis much more readily than watery solutions. Rubbed into the skin, they enter the sebaceous glands and eventually can be demonstrated microscopically in the lymph channels. True fats are gradually oxidized and disappear; but the mineral fats remain for a long time in the subcutaneous tissue, and thus produce chronic irritation and fibrosis. The animal and vegetable fats therefore deserve preference when penetration is desired (Sobieranski; Juckuff; Meyer; see "Ointments" in Index).

Paraffin Prothesis.-The persistence of the mineral fats in the subcutaneous tissue has been utilized for the correction of deformities (e.g., of the nose), by injecting subcutaneously a mixture of paraffins melting at about $40^{\circ} \mathrm{C}$. There is some danger of embolism, and the results are not permanent, the paraffin being gradually replaced by scar-tissue (Heidingfeld, I 9 Io).

Protection of the Skin.-The oily emollients and glycerin soften the stratum corneum, and render the skin more pliable and resistant to injurious agencies. They reinforce in this manner the natural fat of the skin and prevent roughness and "cracks" from wind, cold weather, sunburn, skin diseases, etc. 
Rancidity.-Fats turn "rancid" on keeping, especially if they are exposed to air, light, and heat, and if they contain protein impurities. The change consists in oxidation with the development of free volatile fatty acids. Rancid fats are disagreeable and irritating, and therefore unfit for medicinal use. Lanolin and the petroleum products are free from this objection.

\section{OLIVE OIL AND OTHER BLAND FATTY OILS (OLEA PINGUA)}

Uses.-The expressed oils of olive, cottonseed, almond, sesame, etc., are essentially similar. For internal use, olive oil is preferred because of its more agreeable flavor. These oils are employed externally to soften the skin and crusts in eczema and psoriasis; as dressing for burns; as vehicles for liniments, etc.; and by rectal injection, to soften fecal concretions ( 150 to 500 c.c., 5 to 20 ounces, of the cheaper cottonseed oil, warmed. Hertz claims that oil is less effective than water for this purpose). Internally, olive oil is used as nutrient, laxative, and in hyperchlorhydria ( 15 to 50 c.c., $1 / 2$ to 2 ounces before meals). Kennedy and others have claimed beneficial results in biliary colic, using large doses ( 150 to 500 c.c. in four to eight portions within three hours). Its effects, if any, have not been explained; it does not increase the flow of bile, and the concretions found in the feces when olive oil and epsom salt are given, are not gallstones, but simply soap (magnesium oleate).

These oils are insoluble in water or glycerin, very sparingly in alcohol, freely in chloroform, ether, volatile oils, or fats.

Fats are useful antidotes in corrosive poisoning.

Influence on Digestion.-Neutral fats allay gastric peristalsis, diminish gastric secretion and acidity (Pawlow; Cowie and Munson, I908; Chiari, 1915), and lessen the pyloric tone. They leave the stomach slowly and in small portions; a fatty meal requiring seven hours for complete passage into the duodenum. The digestibility of animal fats is discussed by Langworthy and Holmes, 1915. The length of gastric sojourn increases with the melting-point of the fat, Feyer, I9I3. In the duodenum, fats check the gastric discharge and stimula te the flow of pancreatic juice and the evacuation of the gall-bladder (Klee and Knuepfel, I9I4).

Subcutaneous injections of fats as nutrients were proposed by Leube, 1895 . They can be administered indefinitely without irritation or other disturbance, and are gradually absorbed by the lymph. However, the absorption of plain fats is too slow to be of practical value (Winternitz, I903; Y. Henderson and Crofutt, 1905). Somewhat better results were obtained with fats emulsified by lecithin (Mills and Congdon, I 9 I I). The injection of free fatty acids would be toxic. Fats are also oxidized when emulsions are injected into a vein (Murlin and Riche, I915).

Oleic Acid Anemia. - The continued administration of large doses of oleic acid, by mouth or hypodermically, to animals, produces hemolysis and severe anemia (Faust, I 906 and I908; Polano, I909; Adler, I9II). Similar effects produced by the injection of extracts of tapeworm (bothriocephalus) are also due to oleic acid-cholesterin esters (Faust). The saturated higher fatty acids are also hemolytic (Shimazono, I9II). The hemolysin of bacterium putidum is oxydimethyl thiol-erucic acid, a typical unsaturated fatty acid (Burckhardt, I910).

\section{PREPARATIONS-FATTY OILS}

*Oleum Olive (Ol. Oliv.), U.S.P., B.P.; Olive oil (Sweet Oil).-A pale yellow or greenish-yellow fixed oil, expressed from the ripe fruit of Olea europæa. Slightly sol. in alc. Dose, 30 c.c., I ounce, U.S.P.

Oleum Gossypii Seminis (Ol. Gossyp. Sem.), U.S.P.; Cottonseed Oil.-Fixed oil, expressed from seeds of cultivated varieties of Gossypium. Slightly sol. in alc. On account of its cheapness, it is especially adapted for external use. It is inferior forinternal administration; it can not be readily emulsified or saponified, since the free fatty acids have been removed in the process of purification. 
Gossypol.-Feeding with "cottonseed meal" (not the oil) sometimes produces toxic effects. These are due to the gossypol, a crystalline substance, first isolated by Marchlewski, I899. It is soluble in gasolin. It may be rendered harmless by various methods of oxidation, precipitation or extraction (Withers and Carruth, 1915).

Ol. Sesami, U.S.P., B.P.; Oil of Sesame (Teel Oil, Benne Oil).-Expressed from seeds of Sesamum indicum.

Ol. Arachis, B.P. (Peanut Oil).

Ol. Amygdala Expressum (Ol. Amygd. Exp.); Ol. Amygd., B.P. (Oil of Sweet Almond).-Fixed oil from bitter or sweet almond.

Ol. Lini, U.S.P., B.P.; Linseed Oil.-Expressed from Linseed. Disagreeable odor. Employed mainly in veterinary practice and in liniments. "Boiled" oil must not be used. Dose, 30 c.c., 1 ounce, U.S.P.

Acid. Oleic, U.S.P., B.P.-Mixture of fat acids consisting chiefly of $\mathrm{C}_{17} \mathrm{H}_{33} \mathrm{COOH}$. Yellowish or brownish-yellow, oily liquid; peculiar lard-like odor and taste. Practically insoluble in water; completely miscible with about 85 per cent. alcohol; soluble in chloroform and in fixed and volatile oils. Used as solvent for metallic oxids and alkaloids, intended to be absorbed from the skin.

Acidum Stearicum (Acid. Stear.) U.S.P.-Mixture of fat acids consisting chiefly of $\mathrm{C}_{17} \mathrm{H}_{35} \mathrm{COOH}$ obtained from tallow or other fats. Hard, white, or yellowish-white, somewhat glossy, solid, odorless, having a slight, tallow-like odor, tasteless. Melts above $56^{\circ} \mathrm{C}$. Sol. in alc. ( $\left.1: 2 \mathrm{I}\right)$; almost insol. in water. Used in glycerin suppositories.

*Ol. Theobromatis (Ol. Theobrom.), U.S.P., B.P.; Oil of Theobroma (Cacao Butter).-A yellowish-white solid fat from the roasted seeds of Theobroma Cacao. It melts at the temperature of the body. It is used mainly for making suppositories, and as a lubricant in massage. Not to be confused with:

Oleum Cocos; cocos-oil.-The expressed oil of the cocoanut, the fruit of the palm, Cocos nuifera. It is a whitish fat, soluble in alcohol, used especially in soap-making.

Ol. Chaulmoog., B.P.; Chaulmoogra Oil (Gynocardia Oil).-A fatty oil expressed from seeds of Taraktogenos Kurtzii. Favorable, but temporary results are reported in leprosy. The action, if any, is unexplained. Dose (oral), 0.3 to 0.6 c.c., 5 to ro minims, gradually increased to 2 to 4 c.c., $1 / 2$ to I dram, B.P. As it becomes very nauseating, it has been given hypodermically, 0.3 to $0.8 \mathrm{Gm}$., 5 to Io drops. Heiser, I9 I4, advises a mixture of 60 parts, each, of Chaulmoogra and camphorated oil, with 4 parts of resorcin.

Injections are made weekly, beginning with I c.c. and increasing gradually. He claims very constant improvement and sometimes apparent cure. The oil is buttery, of greenish color, and peculiar odor and taste. It contains i 8 per cent. of gynocardic acid, which has also been used in doses of 0.03 to $0.02 \mathrm{Gm}$. $(1 / 2$ to 3 grains). Power, 1915, finds that the genuine (Taraktogenos) oil consists largely of the glyceryls of a new type of fatty acids, mainly chaulmoogric acid, $\mathrm{C}_{18} \mathrm{H}_{32} \mathrm{O}_{2}$.

Ung. Chaulmoog., B.P.- Io per cent.

\section{LARD AND SIMILAR ANIMAL FATS}

These are employed as soft ointment bases. They all have the inconvenience of rapidly turning rancid. This is delayed by antiseptics, as in Benzoinated Lard.

\section{PREPARATIONS-FATS}

*Adeps Benzoinatus (Adeps Benz.), U.S.P., B.P.; Benzoinated Lard.-Lard digested with Benzoin. In this process it takes up a certain amount of the latter, and acquires the antiseptic and stimulant properties of balsams, besides increasing its own keeping qualities.

Adeps, U.S.P.; Adeps Propp., B.P.; Lard.-The purified internal fat of the abdomen of the hog. Insol. in water; very slightly sol. in alc.; melts between $36^{\circ}$ and $42^{\circ} \mathrm{C}$.

Sevum Praparatum (Sev. Præp.), U.S.P., B.P.; Suet.-The internal fat of the abdomen of the sheep, purified by melting and straining.

Sev. Benz., B.P.

Butyrum, Butter, is sometimes used as an ointment basis. Since it can not be salted for this purpose, it keeps very poorly.

Other Animal Fats.-Other animals also yield fats which are popularly supposed to have special advantages-dog fat, goose grease, etc. There appears to be no reason for preferring them to the more easily obtainable lard. 


\section{WOOLFAT-LANOLIN}

This differs chemically from ordinary fats in that the fatty acids are not united to glycerin but to cholesterin and isocholesterin and smaller quantities of other alcohols.

Crude woolfat or suint was used by the ancients, but was too disagreeable, until a purified product was introduced by Liebreich as "lanolin." Even this has a rather unpleasant odor and disagreeable sticky consistency, but it does not readily turn rancid. Since its composition resembles that of the natural skin-fat, it was believed that it would be more readily absorbed; but this does not seem to be the case. Its chief advantage lies in the property of forming salve-like emulsions with up to two or three times its volume of water, so that watery solutions may be incorporated with it. This is attributed by Liefschuetz and Unna (E. Unna, I9I2) to traces of free isocholesterin and oxycholesterin. It also emulsifies other fats and petrolatum. Anhydrous woolfat is too sticky for use. It is employed after the admixture of 30 per cent. of water (Hydrous Woolfat), or better with the further addition of an equal weight of petrolatum or other fat. It is not absorbed from the intestines (Bloor, I913).

\section{PREPARATIONS-WOOLFATS}

* Adeps Lance Hydrosus (Adeps Lan. Hyd.), U.S.P., B.P.; Hydrous Woolfat (Lanolin).-Contains from 25 to 30 per cent. of water. A yellowish-white, or nearly white, ointment-like mass, having not more than a slight odor. Miscible with about twice its weight of water.

Adeps Lan., U.S.P., B.P.; Woolfat.-The purified fat of the wool of sheep freed from water. A light-yellow, tenacious, unctuous mass, not more than a slight odor. Miscible with about twice its weight of water. Sparingly sol. in alc., readily in chlorof., eth., acetone, etc. Melts between $38^{\circ}$ and $42^{\circ} \mathrm{C}$.

\section{PETROLATUM AND OTHER PETROLEUM PRODUCTS}

Properties.-These were introduced under the name of Vaselin by Chesebrough, 1871. They are obtained from petroleum by distilling off the more volatile products, chilling, purification by filtration through bone-black, etc. They consist of hydrocarbons of the methane and related aliphatic series. According to the process of preparation, they occur of various consistencies, from the waxy "paraffin" (employed for hardening ointments), through the buttery "petrolatum" (a typical emollient), to the "liquid paraffin" (used especially for laxative and sprays). The color, from deep yellow to almost pure white, does not affect the action.

Notwithstanding the chemical difference, they resemble the fats in physical character and therapeutic application. They have the advantage that they are chemically indifferent, do not undergo deterioration, can not become rancid or irritant, and do not react with drugs, such as oxidizing or reducing agents. They are not so well adapted for inunction.

Liquid Petrolatum as Laxative.-Mineral oils are not absorbed (except perhaps doubtful traces; Bradley and Gasser, I912); but are eliminated unchanged by the feces (Randolph, r 885; Hutchison, I899; Bloor, I9I3). They can not, therefore, act as nutrients. By softening the feces, they are mildly laxative, and are used as such in intestinal stasis (A. D. Schmidt, 1905; Lane, I913). Sometimes they do not mix well with the feces, the oil leaking from the anus. Occasionally they are followed by nausea and 
regurgitation (Bastedo, I9I4). Any of the "liquid paraffins" (Petrolatum Liquidum Album) may be employed-there being no therapeutic difference between the light or heavy Russian or American oils (Bastedo, I915). Their composition is perhaps also similar. The usual dosage is from ro to 30 c.c., one-half hour before meals; or a single dose of 30 to 60 c.c. before retiring. The "light" oil is preferred for sprays (Wilbert, I9I4); the more viscid oils for laxatives.

The Russian oil is non-fluorescent, and consists mainly of naphthenes; the American oil contains members of the methane series, and is generally fluorescent. The history and composition is discussed in the J.A.M.A., I914, 62 : 1740; and by Hilton, I914.

Composition of Liquid Petrolatums.-All crude petroleum oils are complex mixtures of hydrocarbons, the composition varying quantitatively according to their origin. These differences are eliminated in refining, so that the composition of the thoroughly refined, colorless products is probably similar, whether the product is derived from Russian or from American oils. The liquid petrolatums consist mainly of naphthenes $\left(\mathrm{C}_{n} \mathrm{H}_{2 n}\right)$ and polynaphthenes $\left(\mathrm{C}_{n} \mathrm{H}_{2 n-2}\right.$ and $\left.\mathrm{C}_{n} \mathrm{H}_{2 n-4}\right)$. They do not contain true paraffins $\left(\mathrm{C}_{n} \mathrm{H}_{2 n+2}\right)$; so that the term "Paraffin Oil" is a misnomer. The irritant olefin hydrocarbons and the benzenes of the crude oils are absent from the refined. Certain ingredients, which give the fluorescence and rancid taste, are more difficult to eliminate from some oils than from others (Brooks, Igr6).

Criteria of Availability.-Brooks considers the physical qualities, the color, odor, taste and viscidity or specific gravity as much superior to all chemic tests.

\section{PREPARATIONS-MINERAL FATS}

* Petrolatum (Petrolat.), U.S.P.; Paraffinum Molle (Paraff. Moll.), B.P.; Petrolatum, Soft Paraffin (Petroleum Jelly, Vaselin).-A purified mixture of semisolid hydrocarbons obtained from petroleum. An unctuous mass, varying in color from yellowish to light amber, having not more than a slight fluorescence; without odor or taste. Insol. in water; scarcely sol. in cold or hot alc.; freely sol. in fats and fat solvents. Melting-point, $38^{\circ}$ to $54^{\circ}$ C., U.S.P.; $40^{\circ}$ to $46^{\circ}$ C.; B.P. The color may be specified by adding "Album" for white, "Flavum" for yellow.

* Petrolatum Liquidum (Petrolat. Liq.), U.S.P.; Paraff. Liq., B.P.; Liquid Petrolatum, Liquid Paraffin.-A mixture of liquid hydrocarbons. A colorless, transparent, oily liquid free from fluorescence, odorless, and tasteless when cold; insol. in water or alc. Dose, I5 c.c., 4 drams, U.S.P.; 4 to 16 c.c., I to 4 drams, B.P. Heavy Liquid Petrolatum (Heavy Liquid Paraffin, Heavy Mineral Oil) is a very viscous liquid. Light Liquid Petrolatum (Light Liquid Paraffin, Light Mineral Oil) is almost free from viscosity.

Paraffinum (Paraff.), U.S.P.; Paraff. Dur., B.P.; Paraffin, Hard Paraffin.-A purified mixture of solid hydrocarbons. Colorless, or white, more or less translucent mass, without odor or taste; insol. in water or in alc. Melts at $50^{\circ}$ to $57^{\circ} \mathrm{C}$., U.S.P.; $50^{\circ}$ to $60^{\circ} \mathrm{C} .$, B.P.

Toxic Effects of Petroleum Products.-Gasolin (Petroleum benzin).-The inhalation of the vapors causes headache, nausea, giddiness, inebriation, unconsciousness, muscular tremors, convulsions, dyspnea, cyanosis, and death (Buegi and Burgl; Wolf, IgI I). Chronic poisoning results in dulness, pain in limbs, trembling, muscular weakness and other nervous disturbances (Rambousek), and pulmonary hemorrhages (Jaffe, I9I4). The symptoms of acute and chronic poisoning, as they occur in the rubber industry, are described by Alice Hamilton, I9I5.

Concentrated vapors cause purely paralytic symptoms in frogs, but in mammals they seem to have only a weak anesthetic action. If they are inhaled to the exclusion of air, they will cause an asphyxial anesthesia. Before this sets in there are very characteristic convulsions (Sollmann, 1904). The animal struggles violently, then falls on its side and claws the air with all fours, as if running. The pupils are widely dilated. Reflexes absent. The spasms are intermittent, and between them the dog is perfectly limp, except that the toes, tail and eyelids continue to twitch. The respiration is first stimulated, then weakened. There is paralysis of the vagus, then a depression of the cardiac muscle, and later of the vasomotor center. Either heart or respiration may stop first. 
Gasolin is, therefore, unsuitable as an anesthetic even for animals; although it has been tried with man (Felix, I888). It is said to be used as an intoxicant. Its oral alministration does not produce gastroenteritis. It is absorbed slowly from the alimentary tract, and excreted mainly by the lungs (Jaffe, I9I4).

Petroleum (Coal oil). - When petroleum is swallowed, it produces narcotic effects similar to those of alcohol, with strong gastroenteritis. It is toxic in proportion to its content of the more volatile products. No fatal case has thus far been reported, although as nuch as a liter has been swallowed. Coal oil applied to the skin or continuous handling of unpurified paraffin is a moderate irritant, and may lead to dermatitis (Joseph, I896), acne and other eruptions, and papillomas (see "Tar").

\section{WAXES}

These are used in pharmacy mainly to stiffen ointments (as in cerates).

\section{PREPARATIONS}

Cera Flava (Cer. Flav.), U.S.P., B.P.; Yellow Wax, Beeswax.-The purified product of the hive bee, Apis mellifera. Mlelts between $62^{\circ}$ and $65^{\circ} \mathrm{C}$. Sparingly sol. in alc.; sol. in eth., chlorof., and oils.

Cera Alba (Cer. Alb.), U.S.P., B.P.; White Wax.-Bleached beeswax.

Celaceum (Cetac.), U.S.P., B.P.; Spermaceti.-A fatty substance, obtained from the head of the sperm whale, Physeter macrocephalus. . Consists mainly of ester of cetylic alcohol and palmitic acid.

Resina (Res.), U.S.P., B.P.; Rosin (Colophony).-The residue left after distilling off the volatile oil from turpentine, the concrete oleoresin obtained from species of Pine.

\section{COMPOUND OINTMENT BASES}

These are survivals of older formulas, without serious advantage over the simpler bases. Typical ointments melt at the temperature of the body.

\section{PREPARATIONS-COMPOUND OINTMENT BASES}

Unguenlum (Ung.), U.S.P.; Ointment (Simple Ointment).-2 parts White Wax, 8 parts Benz. Lard.

Ung. Paraff., B.P.-Hard Paraff., 27 per cent.; Soft Paraff., 7o per cent; Beeswax, 3 per cent.

Ung. Aq. Ros., U.S.P., B.P. (Cold Cream).--Expressed Almond Oil, thickened with Spermaceti and White Wax, flavored with Rose Water, and containing 5 per cent. of Sodium Borate, U.S.P.

Ung. Cela., B.P.-Spermaceti, 20 per cent.; White Beeswax, 8 per cent.; Liq. Paraff., 72 per cent.

Ung. Lana Co., B.P.-Lard, 40 per cent.; Woolfat, 40 per cent.; Paraff. Oint., 20 per cent.

Ung. Res., B.P.-Resin, 26 per cent.; Yellow Beeswax, 26 per cent.; Olive Oil, 26 per cent.; Lard, 22 per cent.

\section{CERATES}

These are stiffer ointments, containing sufficient wax so that they do not melt at body temperature. They are also superfluous survivals.

\section{PREPARATIONS-CERATES}

Ceratum (Cerat.), U.S.P.; (Simple) Cerate.-Three parts of White Wax and 7 parts of Benz. Lard.

Ceral. Res., U.S.P.; Rosin Cerate (Basilicon Ointment).-Rosin, 35 per cent.; Yellow Wax, 15 per cent.; Lard, 50 per cent. 


\section{GLYCERIN}

Therapeutic Uses.- This acts both as emollient and demulcent. It is used to soften the skin, to prevent chapping, internally in coughs, etc. Concentrated glycerin abstracts water, so that the soothing action is preceded by smarting. It should therefore be diluted with two or three volumes of water (rose water for lotions). Roth has shown (with Cataplasma Kaolini), that glycerin does not abstract water from the intact skin, because of the impermeability of the stratum corneum.

Undiluted glycerin injected into the rectum causes evacuation in a few minutes, without pain if there are no abrasions (5 to ro c.c., I to 23 ; or the official suppository). Similarly, it provokes uterine contractions if introduced into the cervical canal. Large doses ( 100 to $5_{50}$ $\mathrm{Gm}$. per day, in divided portions) have been employed to kill intestinal trichinæ.

Pharmaceutic Uses.-Glycerin has many uses in pharmacy, as a vehicle and solvent for salts; sweetening agent, to preserve ferments and vaccines; to keep pill masses or extracts moist, etc. As a sweetening agent in diabetes it has been replaced by saccharin.

Nutrient Value.-Glycerin has been tried as a substitute for cod liver oil. It is transformable into glycogen and sugar; but its nutrient value is rather doubtful, since larger quantities lessen gastric secretion (L. Kast, I910) and provoke diarrhea. Like other intestinal irritants, it produces increased elimination of uric acid (Abl, I9I3).

Toxic Effects.-These have never been reported in man, but they occur in animals when large doses are given, by any channel. The symptoms are convulsant and paralytic. probably through a direct action of glycerin on the central nervous system. The blood corpuscles are laked, especially if the glycerin is injected hypodermically. This is probably an osmotic effect, the glycerin remaining for a time unabsorbed, and in high concentration at the place of injection; and the corpuscles being laked during their passage through this area (Filehne).

On muscle-nerve preparations, glycerin acts similarly to veratrin.

\section{PREPARATIONS - GLYCERIN}

${ }^{*}$ Glycerinum (Glycerin), U.S.P.; (Glycer.), B.P; Glycerin, $\mathrm{C}_{3} \mathrm{H}_{5}(\mathrm{OH})_{3}$ - - A liquid obtained by the hydrolysis of vegetable or animal fats, purified by distillation. Clear, colorless liquid, of a thick, syrupy consistence, sweet to the taste. Miscible with water or alc.; insol. in eth., chlorof. or oils. Dose, 4 c.c., I dram, U.S.P.; 4 to 8 c.c., $\mathrm{r}$ to 2 drams, B.P.

* Suppositoria Glycerini (Supp. Glycerin), U.S.P.; Supp. Glycer., B.P.; Glycerin Suppositories.-Each suppository contains $3 \mathrm{Gm}$. of Glycerin, solidified by sodium stearate, U.S.P.; 70 per cent. of Glycerin, solidified by Gelatin, B.P.

Glycerites are solutions of medicinal substances in glycerin.

\section{DEMULCENTS OR MUCILAGINOUS SUBSTANCES}

These comprise gums and other colloid substances, which form viscid solutions with water, and are insoluble in alcohol. They are used to emulsify oils; to suspend insoluble powders; to mitigate the taste and local effects and to delay the absorption of other drugs; and to allay inflammations of mucous membranes, especially in bronchitis, gastritis, enteritis and diarrhea. The effects are strictly local, but in the respiratory passages they reach far into the bronchi. Gums and proteins (raw eggs, milk, flour) are employed for their protective action against irritant poisons. They are also precipitant chemic antidotes for metals. 
Mucilages and similar drugs (salep, Irish and Iceland moss) were formerly considered efficient carbohydrate nutrients; but it has been shown that they are very imperfectly digested, and for the most part climinated unchanged by the feces (Mendel, I908; Swartz, I9II).

Demulcents as Flavors.-Cums and similar substances markedly diminish the characteristic taste of all substances, acid, salt, and sweet, as well as bitter. They act by enveloping the substance and forming a protective layer over the mucous membrane, and in this way preventing the access of the substance to the taste organs. This, of course, climinishes absorption as well as taste. One can very readily convince himself of this "corrective" action by mixing a I per cent. solution of citric acid with water and with a thin starch paste. The latter will taste very much less sour. Colloid substances of this kind are present in fruits as pectin bodies, and have a very marked influence upon their taste. The raspberry, for instance, actually contains more acid than the currant ind but little more sugar, its less sour taste being due to the greater amount of these pectin substances present in it.

\section{PREPARATIONS - GUMS}

* Acacia (Acac.), U.S.P.; Acacia Gummi (Acac. Gum.), B.P.; (Gum) Acacia (Gum Arabic, Gum Senegal).- The dried, gummy exudation of Acacia Senegal, and of other African species of Acacia. More or less spheroidal tears, or angular fragments; whitish to light amber colored; translucent; very brittle; nearly inodorous; taste insipid, mucilaginous. Insoluble in alc.; slowly soluble in twice its weight of water, forming a mucilaginous liquid, which is acid to litmus. Incompatible with alc., borax or metallic salts.

* Mucilago Acacia (Mucil. Acac.), U.S.P., B.P.; Mucilage of Acacia.-35 per cent., U.S.P., 40 per cent., B.P., dissolved in cold water. Dose, I5 c.c., 4 drams, U.S.P.

Syr. Acac., U.S.P - ro per cent.

Gum Ind., B.P.-A gummy exudation from the stem of Anogeissus latifolia.

Mucil. Gum. Ind., B.P. -25 per cent.

* Tragacantha (Trag.), U.S.P., B.P.; Tragacanth.-The spontaneously dried gummy exudation from the stems of Astragalus species. Swells in water to a gelatinous mass, without dissolving. Used in pill excipients, emulsions, etc.

$M$ ucil. Trag., U.S.P., B.P. 6 per cent. in diluted glycerin, U.S.P.; 1.25 per cent. in water, B.P.

Glycer. Trag., B.P.-2o per cent.

Pulv. Trag. Co., B.P.-Tragacanth, Acacia, Starch and Sugar. Dose, 0.6 to 4 Gm., ro to 60 gr., B.P.

Dextrin.- - Prepared by heating starch with nitric acid. 'Presents all the characters of gum arabic, and forms the principal ingredient of commercial mucilages. A good formula for this is the following (Sykes): Mix I80 Gm. of dextrin with I80 c.c. cold water; add 240 c.c. boiling water and boil five minutes, stirring constantly. Add hot water q.s. 400 c.c. When cold, add 30 c.c. of dilute acetic acid, Io drops of phenol and 30 c.c. of glycerin, previously mixed.

\section{DEMULCENT TEAS}

Drugs containing mucilages are used as decoctions, ad libitum, mainly in folk medicine.

\section{PREPARATIONS-DEMULCENT TEAS}

Althea U.S.P.; Marsh Mallow Root.-The root of Althæa officinalis deprived of the brown, corky layer and small roots.

Amygdala Dulcis (Amygd. Dulc.), U.S.P., B.P.; Sweet Almond.-The ripe seeds of Prunus Amygdalus dulcis.

Emul. A mygd., U.S.P. (Milk of Almond)._An emulsion made by rubbing 6 parts of blanched sweet almonds with I part of acacia, 3 parts of sugar, and enough water to make 100 parts.

Mist. Amygd., B.P. $-\mathrm{I} 2.5$ per cent. Dose, $\mathrm{I} 5$ to 30 c.c., $1 / 2$ to $\mathrm{I}$ ounce, B.P.

Pulv. Amygd. Co.; B.P.-Sweet Almond, Sugar and Gum Acacia.

Cetraria; Iceland Moss, a Lichen.-A gelatinous decoction $(\mathrm{I}: 20)$ is prepared after previous extraction with cold water, to remove a bitter principle.

Chondrus, U.S.P. (Irish Moss, Carrageen). - The dried plant of the seaweeds Chondrus crispus and Gigortina mamillosa. Yields a demulcent jelly with boiling water. 
Cydonium.-Quince Seed. B.P.

Ispaghula, B.P.-Dried seeds of Plantago ovata. Dose, 3 to 10 Gim., 45 to $150 \mathrm{gr}$.,

Dec. Ispagh., B.P.- $-\mathbf{5}$ per cent. Dose, $\mathrm{I}_{5}$ to 00 c.c., $1 / 2$ to 2 ounces, B.P.

Salep.-The tubers of an orchid.

Sassafras Medulla.-Sassafras Pith.

Triticum (Trit.), U.S.P.; Agropyron (Agropyr.), B.P.; Triticum, Couch Grass.The dried rhizome and roots of Agropyron repens. Dose, $8 \mathrm{Gm} ., 2$ drams, U.S.P.

Fldext. Trit., U.S.P.; Ext. Agropyr. Liq., B.P.-Dose, 10 c.c. $21 \frac{1}{2}$ drams, U.S.P.; 4 to 8 c.c., I to 2 drams, B.P.

Dec. Agropyr., B.P. - 5 per cent. Dose, 15 to 60 c.c., $1 / 2$ to 2 ounces, B.P.

Ulmus, U.S.P.; Elm (Slippery Elm).-The bark of Ulmus fulva.

\section{GELATIN}

Uses.-This is employed in pharmacy as a coating for pills, for making gelatin capsules, etc., and in the form of glycerinated gelatin as a base for suppositories. In the form of soups and jellies it is used as a nutrient, being easily digested and capable of replacing from one-fourth to twothirds of the protein of an ordinary diet, especially if the carbohydrates are ample (Murlin, 1907). Subcutaneous injections of gelatin have been given to increase the coagulability of the blood, especially in aneurism. Their efficiency is doubtful. They have the serious objections of severe pain and danger of infection, since ordinary gelatin solutions are of ten not sterile, and may even carry tetanus.

Gelatin as Styptic.-The efficiency of gelatin is affirmed by some and denied by others. Burton-Opitz, I906, found that it increased the viscosity of the blood on intravenous injection. Cmunt, I9I2, claims the same effect hypodermically. Dastre and Floresco, I897; and Grau, I910, describe increased coagulability of the blood, lasting for several days. Zibell, I90I, and others are inclined to attribute the action to $\mathrm{Ca}$, of which gelatin contains about 0.6 per cent. The contradictory data do not permit any definite conclusions. The gelatin was originally used subcutaneously (100 to 200 c.c. of sterile I to 5 per cent. solution, injected slowly into the thigh, not near the aneurism, every ten to fifteen days, until to to 20 injections have been given). The treatment is quite painful, and may raise the temperature to $103^{\circ} \mathrm{F}$. Wood (I9O2) claims that the gelatin is just as effective, only somewhat slower, when given by mouth, and recommends eating 3 or 4 ounces of flavored ro per cent. jelly, three times a day.

\section{PREPARATIONS - GELATIN}

* Gelatinum (Gelat.), U.S.P., B.P.; Gelatin.-The purified, air-dried product obtained by the hydrolysis of animal tissues, as skin, ligaments, and bones, by treatment with boiling water. "Insol. in cold water, but swells and softens when immersed in it, gradually absorbing from five to ten times.its own weight of water; sol. in hot water, acetic acid or glycerin; insol. in alc. Watery solutions of 2 per cent. or over solidify on cooling. Precipitated by phenol or tannin; not by dilute solutions of metallic salts.

Gelatinum Glycerinatum (Gelat. Glycerin.), U.S.P.-Equal parts of Gelatin and of Glycerin. Used for making bougies, etc.

\section{STARCH}

Uses.-Dry starch is used as a dusting powder. By boiling with water, starch is converted to a colloid condition, starch-paste. This is used as an emollient, in the form of the glycerite; in poultices; and to secure the retention of enemas (teaspoonful, rubbed smooth with a little cold water and poured into a cup of boiling water). It is a chemical antidote to iodin. It is also employed as a nutrient, especially as "arrowroot," the starch of Maranta, which has a somewhat finer flavor than the official corn starch. 


\section{PREPARATIONS - STARCH}

* A mylum, U.S.P., B.P.; Starch.-Prepared from maize, U.S.P.; from wheat, rice or maize, B.P.

Glycer. Amyl., U.S.P.-10 per cent. of starch.

Glycerin. Amyli, B.P.-2o per cent. of starch.

* Burley-iitater is used as a demulcent decoction; and for diluting cow's milk for infants, to prevent the precipitation of solid masses of casein in the stomach. It is prepared by boiling for twenty minutes Io parts of washed pearl barley with 150 parts of water and straining. (B.P.C.)

\section{INDIFFERENT DUSTING POWDERS}

These are very fine ("impalpable"), insoluble, non-irritant powders, such as talcum, chalk, starch, lycopodium, etc. They form a protective covering, prevent friction, absorb secretions by capillary action, and are therefore drying. Purified Talcum and Terra Silicea are also used for clarifying turbid solutions.

\section{PREPARATIONS-INDIFFERENT DUSTING POWDERS}

* Talcum Purificalum (Talc. Purif.), U.S.P.; Purified Talcum.-A purified, native, hydrous magnesium silicate. A fine, white or grayish-white powder; odorless and tasteless. Insol. in water and dilute acids or alkalies.

Terra Silicea Purificata (Ter. Sil. Purif.), U.S.P. (Purified Kieselguhr, Purified Infusorial Earth). - A form of silica $\left(\mathrm{SiO}_{2}\right)$ consisting of the frustules and fragments of diatoms, purified by boiling with diluted hydrochloric acid, washing and calcining.

* Lycopodium (Lycopod.), U.S.P.; Lycopodium.-The spores of Lycopodium clavatum. A light yellow, very mobile powder, nearly inodorous and tasteless. Insoluble in water.

\section{POULTICE MASSES}

Poultices or cataplasms are used to convey heat, and to macerate the skin. A variety of pasty materials may be used. The most common are linseed, starch, bread and clay. ' Linseed poultice is prepared by stirring I part of ground linseed into 3 parts of boiling water, to which a little baking soda may be added, to render it fluffy and less irritant. Clay or Kaolin poultice is much heavier, but can be sterilized if desired. The rate of cooling of different poultice masses has been investigated by Pilcher, I908. They do not differ materially. Whole linseed may be made into a demulcent decoction against bronchitis.

\section{PREPARATIONS-POULTICES}

* Linum, U.S.P.; Lini Sem., B.P.; Lini Sem. Contus. (crushed or ground, "meal"), B.P.; Linseed, Flaxseed.-The ripe seeds of Linum usitatissimum.

Cataplasma Kaolini, N.F.-Essentially a thick mass formed of about 2 parts of kaolin and I part of glycerin, together with a little boric acid ( 4.5 per cent.) and flavoring oils. It is spread hot in a thick layer and left in place for twelve to forty-eight hours.

Kaolinum, B.P.; Kaolin.-Native aluminum silicate (china clay; bolus alba), powdered and freed from gritty particles by elutriation. When added directly to blood, kaolin is hemolytic; and toxic when injected intravenously (Friedberger and Tsumeoka, 1913).

Kaolin in Diarrheas.-Bolus alba, willow charcoal and silica are being used as adsorbents in watery diarrheas and dysentery. In acute bacterial dysentery, large doses are given, $45 \mathrm{Gm}$. three times daily, stirred into hot tea (after a preliminary cleansing by calomel). Smaller doses suffice in chronic dysentery (Stoerk, 1915). Hess, r916, reports good results from Fuller's earth in infantile diarrhea. It is easily administered in the food, or by a spoon; giving $I$ to 2 tablespoons three times daily (Charcoal, H. Strauss, I9I6). 
The application of dry Kaolin to the nose and throat is being tried, with apparent success, to remove the bacteria of diphtheria carriers (Rappaport, 1916).

\section{PLASTERS}

These serve similar purposes as emollients; for the slow conveyance of drugs to or through the skin; and for mechanical support. They are somewhat irritant, partly by preventing evaporation from the skin, and partly by the small quantities of volatile oils contained in the resins from which they are often prepared. The official plaster-masses are unimportant, since the commercial plasters are usually made by private formulas from some rubber mixture. "Court plaster" is made of isinglass (Ichthyocolla), a variety of gelatin prepared from the swimming bladder of the sturgeon.

\section{PREPARATIONS-PLASTER MLASSES}

Emplastrum Elasticum (Emp. Elast.), U.S.P.; Rubber (Adhesive) Plaster--A mixture of rubber, pitch, wax and an absorbent powder (starch, etc.), spread on cloth.

Emplastrum Resince (Emp. Res.), U.S.P., B.P. (Rosin Adhesive Plaster).-Lead plaster with rosin and yellow wax, U.S.P.; hard soap instead of wax, B.P.

Emplastrum Plumbi (Emp. Plumb.), U.S.P., B.P.; Lead Plaster (Diachylon Plaster). -A soap made by boiling lead oxid with olive oil and lard, U.S.P.; with olive oil, B.P.

Emp. Saponis (Emp. Sap.), B.P.; Soap Plaster.-Similar to preceding, with greater proportion of soap.

\section{MECHANICAL PROTECTIVES}

Certain substances which are used for this purpose may be mentioned in this place.

\section{PREPARATIONS-MECHANICAL PROTECTIVES}

* Collodium (Collod.), U.S.P., B.P.; Collodion.-A 4 per cent. solution, U.S.P., 2.I per cent. B.P., of Pyroxylin (gun-cotton) in a mixture of 3 vol. of ether and I vol. of alc. Should be kept in a cool place remote from fire. Clear, or slightly opalescent, syrupy liquid; colorless, or slightly yellowish and having the odor of ether. Highly inflammable. Collodion dries rapidly into a firm film, which is used to protect smali wounds. The film cracks easily and contracts considerably, exerting pressure on the tissues. Similar solutions may be prepared with acetone or other solvents.

* Collodium Flexile (Collod. Flex.), U.S.P., B.P.; Flexible Collodion.-Collodion rendered flexible by the addition of a little Castor Oil and Camphor, U.S.P.; Castor Oil and Canada Turpentine, B.P. It does not contract or crack, but does not adhere quite so well.

Pyroxylinum (Pyroxylin), U.S.P., B.P.; Pyroxylin (Soluble Gun-cotton).-Obtained by acting on cotton with a mixture of nitric and sulphuric acids. Consists chiefly of cellulose tetranitrate, $\mathrm{C}_{12} \mathrm{H}_{16}\left(\mathrm{ONO}_{2}\right)_{4} \mathrm{O}_{6} .{ }^{\circ}$ Slowly sol. in 25 parts of a mixture of 3 vol. of ether and I vol. of alc. (equal vol. of 90 per cent. alc. and eth., B.P.); Sol. in acetone. Celloidin, N.N.R., is a purified form. Ordinary gun-cotton is a hexanitrate.

*Gossypium Purificatum (Gossyp. Purif.), U.S.P.; (Gossyp.), B.P.; Purified (Absorbent) Cotton.-The hairs of the seed of Gossypium herbaceum freed from adhering impurities and deprived of fatty matter (so as to make it absorbent) and often sterilized. Consists of almost pure cellulose $\left(\mathrm{C}_{6} \mathrm{H}_{10} \mathrm{O}_{5}\right)$. Used in bandaging, etc., either as such, or as gauze (Tela, Carbasus), lint (Lintum), etc., or impregnated with antiseptics, astringents, etc.

Calcii Sulphas Exsiccahus (Plaster of Paris, Burnt Gypsum), $\mathrm{CaSO}_{4}+1 / 2 \mathrm{H}_{2} \mathrm{O} .-$ A fine white powder, which sets into a stone-like mass when mixed with half its weight of water. Used in bandaging. The "setting" takes place in fifteen to twenty minutes. It may be delayed to an hour by the addition of 5 per cent. of glycerin, or hastened by the addition of sodium silicate. Plaster of Paris must be kept dry.

Liquor Sodii Silicatis (Waterglass).-A colorless syrupy liquid, of alkaline properties, but not corrosive. Contains about 20 per cent. of sodium silicate. Forms a solid glassy mass on exposure. Used in bandaging. 
Actions of Sodium Siliculc.- When given by the mouth or skin, this acts like a typical mild alkali without showing any specific features. It is readily absorbed from the alimentary canal, and excreted by the urine. Injected intravenously it causes agglutination of blood corpuscles, and consequently intravascular clotting. Silicates are normally present in all tissues, but in very small amount (Siegfried, 1901). They are constantly present in hairs and feathers (H. Schulz, I9or).

\section{PHENOMENA COMMON TO LOCAL IRRITANTS, CORROSIVES AND ASTRINGENTS.}

Uniformity of Irritant Phenomena.-The local actions of drugs are, as a rule, simple and uniform, since they can occur ordinarily in only a few situations, especially the skin and mucous membranes. These agree closely in structure and functions. There are therefore many phenomena which hold true of all local irritant poisons. These may be studied once for all, and present only minor modifications in individual cases.

Chemical Basis of Irritation.- The majority of local poisons produce the typical phenomena of inflammation ("irritation"), by causing necrosis of protoplasm through coagulation, liquefaction, etc. Many of these reactions are purely chemic or pisysical, and can be reproduced in the test-tube with proteins. Remembering the extreme sensitiveness of protoplasm to reagents, it will be readily understood that almost all substances - even water-may be irritant under suitable conditions.

Degrees of Cutaneous Irritation.--The general phenomena of irritation can be studied very typically on the skin. They are identical with the familiar changes produced by scalds or burns of increasing severity.

Rubefaction. - The first degree of irritant action is shown in an arterial and capillary hyperemia, at first active, later passive. This constitutes the "dermatitis erythematosa" of the dermatologists. Or, speaking pharmacologically, it constitutes rubefaction, and the agents which produce it are therefore called rubefacients. The dilation involves at first the most superficial vessels, but progressively also those of the deeper subcutaneous tissue, partly directly and partly by reflexes.

The congestion is accompanied by sensory stimulation-by itching, burning or pain. The intensity of the pain depends upon the rapidity, rather than on the degree of the irritation. It is often succeeded by more or less anesthesia.

If the irritant action does not go any further, resolution takes place without leaving any lesions, simply by a return of the vessels to normal. The pain may outlast the congestion. The upper layers of the skin are usually desquamated.

If the action is stronger than rubefaction, it may pass into vesication or pustulation.

Vesication.-This occurs if the inflammatory action results in the formation of an exudate greater in amount than can be carried off by the lymphatics. Every hyperemia is accompanied by an increase of exudation, but up to a certain amount, as in rubefaction, this is readily reabsorbed. When this limit is exceeded, an actual, visible, effusion results. This liquid will accumulate in the parts of the tissues offering the least resistance to distention. In the case of the skin, it penetrates readily through the lower layers of the rete Malpighii, but is arrested by the impermeable stratum corneum. It is therefore confined between the upper and lower layers of the rete Malpighii, and separates them, in this 
way causing blisters or vesicles. The agents which produce these are called vesicants or epispastics.

The vesicles appear as small points which gradually coalesce into a large blister. The fluid is at first clear, but later becomes turbid by leucocytes. The sensory stimulation is stronger than in rubefaction.

Resolution takes place in these cases without loss of tissue, by the formation of a new stratum corneum from the remaining rete Malpighii. The area may remain pigmented for years.

If the overlying and separated layers of epidermis are removed, there is much chance of infection, the lower layers of the rete Malpighii offering but little resistance. In this way there may be a loss of substance from secondary infection.

Suppurative and Inflammatory Necrosis.-If the vesicant is allowed to act after the blister has been formed, the infiltration will become purulent, with deeper destruction of the skin, and healing with scar formation. This degree of action should be avoided.

Aseptic Pus Formation.-A few drugs possess a specific chemotactic power on leucocytes; so that their injection (hypodermic or into serous cavities) leads to the collection of pus, even when asepsis is perfect. Turpentine, croton oil, petroleum, mercury, silver nitrate, digitoxin, cadaverin, aleuron suspensions, etc., are the principal examples. Turpentine also stimulates the chemotactic action of $b$. coli extracts (Hamburger, I913).

Pustulation.-This occurs if the irritant does not penetrate the epidermis, but only the cutaneous glands, especially if the acid secretion of these glands dissolves or liberates the irritant agent. Discrete and later confluent pustules are formed, which heal with more or less "pitting." They produce severe sensory irritation, but are now rarely used. The principal pustulants are croton oil and tartar emetic.

Influence of Systemic Conditions of Cutaneous Irritation.-Luithlen, I9II, found that the local reaction to croton oil is increased by feeding with acids or oxalates and diminished by calcium. Different diets also modify the susceptibility. These systemic influences may be connected with the great variations in the susceptibility of different individuals to cutaneous irritants; and with such skin diseases as eczema,' which do not seem to be explainable by local irritation. There are also, however, local differences in susceptibility, and if an irritative reaction has once been induced, the skin remains hypersensitive for some time.

Dermatitis from Systemic Drugs.-Many drugs cause a dermatitis when they are given by mouth (belladonna, arsenic, iodoform, quinin, salicin, antipyretics, iodids and bromids, digitalis, chloral, chloroform, etc.). This is sometimes due to changes in the cutaneous circulation, sometimes to the excretion of the drug by the skin. It may take the form of scarlatinal, desquamating, urticarial or papular rashes or acne.

Corrosion, cauterization, or direct destruction of tissue results if the agent (cauterizant or escharotic) acts chemically on the protoplasm, as do strong acids or alkalis, iodin or bromin, some metallic salts, etc. 'This leads to the production of a scab or "eschar;" and resolution by scar formation.

Areas of Corrosion.-The chemic destruction of tissue is generally preceded by inflammatory necrosis. Chemic cauterization therefore shows three areas: The first, situated at the depth and periphery of the ulcer, is simply an area of inflammation and hyperemia. Then follows a layer of necrotic tissue, the result of the inflammatory action; and last, a layer in which the chemic cauterization results in solution of the cells which have already been killed by the inflammatory process. These three-hyperemia, inflammatory necrosis, and chemic solution-are to be considered as successive stages in the same action; and by proper dilution, the second or first degree of action may be obtained without the succeeding stages.

The Eschar. - This (scab) is formed after corrosion by the coagulation of the inflammatory exudate and by the chemical products (albuminates, etc.) of the interaction of the corrosive agent with this exudate and with 
the killed cells. According to whether the proteins are dissolved (as by alkalies) or precipitated (as by most metals), the scab will be soft or hard. This has an important bearing on the penetration of the corrosive agent: if the scab is soft, the chemical will penetrate it, and will spread and extend deeply. On this account the alkalies, for instance, are not practical for the purpose of cauterization. If, on the other hand, the scab is solid, it prevents deeper penetration, so that the action can be easily confined to the desired areas.

Astringent Action.-Agents which have a strong precipitant action produce a relatively mild irritation; and if their concentration is not too high, their primary inflammatory effect does not proceed to necrosis; on the contrary, it leads to diminution of any existing inflammatory process. This "astringent" (drawing together) action is characterized by visible contraction of the tissue; blanching and wrinkling of mucous membranes; and diminished exudation, or secretion of mucus, according to the place of application. These agents also possess a peculiar "astringent" taste. The effects are probably due to precipitation and hardening of the cement substance of the capillary endothelium, producing contraction and decreasing the permeability of the capillaries; and thus counteracting the essential phenomena of inflammation (congestion and exudation).

Astringents are used therapeutically to check diarrhea, reduce inflammation of mucous membranes, promote healing, and to arrest hemorrhage (by coagulating the blood).

The principal astringents are: metallic salts, tannins, dilute acids, and strongly hypertonic solutions.

Explanation of Astringent Action.-The manner in which this astringent action is brought about is only imperfectly understood. All astringents produce precipitation of proteins, and this insoluble protein precipitate seems to be the cause of the astringent action. To explain this action it has been assumed that these precipitates form a lining along the capillary walls, and in this way add an additional coat, as it were, to each capillary. It seems, however, much more likely that they act by coagulating the ordinary semifluid cement substance between the endothelial cells, and that this prevents the filtration of fluids, and more especially the emigration of cells. The silver "staining" of endothelia by silver nitrate is a visible illustration of this fixation of the cement substance. The diminished mucus secretion is perhaps due to a similar superficial coagulation of the cell envelope. The blanching and puckering (produced only by the more concentrated solutions) points to a direct stimulation of the arterial and other muscular tissue. The absorption of already formed effusions, following the use of astringents, may possibly be explained by osmostic laws: By precipitating the proteins of these effusions, they lower their molecular concentration, and render them more diffusible. Not all protein precipitants are astringents. The precipitant action must be of a special kind. It must be produced very quickly, and the precipitate must be practically impermeable to the precipitant. Otherwise the precipitant action would extend so deeply as to lead to extensive necrosis, and would thus continue the inflammatory process.

Quantitative Estimation of Astringent Action.-This has been measired by Motolese, I9I0, by noting the degree in which the extensibility of frogs' lungs is reduced after immersion in the astringent solutions (Dreser's method). With I per cent. solutions, the order was: silver nitrate $>$ tannin $>$ lead subacetate $>$ picric acid $>$ lead acetate $>$ alum $>$ zinc sulphate.

Astringent Effects are Strictly Local.-Since astringents are precipitated by proteins, they can not be absorbed nor exist in the blood or tissues, in effective form. It is therefore absolutely irrational to expect a remote action from astringents. The very facts of their action exclude such a possibility. Before this was well understood it was tried to obtain astringent action throughout the body by external application or by 
giving astringents by the mouth. The want of success confirmed what has just been said.

Astringents on Peristalsis.-In the intestinal canal the astringents form a deposit along the lumen of the intestine, and in this way diminish absorption, and also the penetration of other irritant substances, in this way lessening peristalsis.

Irritation of Mucous Membrane.-Differences in detail are seen when irritants are applied to other surfaces than the skin; for instance, to the mucous membranes. These are readily explained by anatomic peculiarities. There will, for instance, be less tendency to vesication, since the epithelium is not impermeable, as it is in the skin. The oral mucous membrane presents a transition; it blisters, but less readily than the skin. Pustulation can not occur since this depends upon the cutaneous glands. On the other hand, the mucous glands will be stimulated to an increased activity, producing "catarrhal" conditions.

Gastroenteritis. - The effects of irritants on the alimentary tract present some special features; the effects being stomachic, carminative, nauseant or emetic; diarrhea, dysentery; abortion; corrosion and perforation; according to the nature and degree of their action.

Respiratory Passages. - If an irritant poison is volatile, its main effects may fall upon the respiratory passages. The general phenomena will be those of acute laryngitis, bronchitis, or pneumonitis. Similar effects follow the aspiration of non-volatile irritants.

Local Irritation of Wounds and Ulcers.-The effects are more marked than on the intact skin. Even the milder irritants destroy the superficial cells, especially if diseased; but the deeper cells multiply more rapidly, partly through direct stimulation and partly through the hyperemia. Mild irritants therefore promote healing. Astringents, balsams, quinin, salts and even water have this therapeutic action.

Hypodermic Injection of Irritants.-These produce more or less pain, and often aseptic abscesses. The danger of infection is also considerable if the tissues are killed by the irritant. With intramuscular injection, the pain is less severe.

Antiseptic Action.-All irritants are somewhat antiseptic for they destroy the protoplasm of the bacteria just as they do that of the tissue cells.

Concentration.-The degree of the local action depends upon the concentration, rather than on the absolute quantity of the irritant.

A small quantity of concentrated acid will do much more damage than much larger quantities diluted; as a gram of $\mathrm{MgSO}_{4}$ in solid form introduced into a solution, may precipitate all its globulin, whilst an unlimited quantity may be added in 5 per cent. solution, without any such effect.

Specific Differences Between the Different Irritants.-These affect the strength of their action in different situations. Some, for instance, seem to act especially on the alimentary canal, and to a very small extent on the skin. This is probably connected with differences of absorbability. A drug which can not penetrate the skin, can not, of course, act upon it. It will be remembered that the epidermis is impermeable to most substances. In order to penetrate, these must be either fatty, like croton oil or turpentine; or they must actually destroy the epidermis, like caustics.

Irritant Effects after Absorption.--None of the irritants acts indiscriminately on the body at large. Many are not absorbed; and even when 
absorption occurs they are too greatly diluted to have much effect, except at the place of their absorption and excretion; i.e., the liver and kidneys. Other tissues are involved only if the administration is longcontinued, as in alcohol or lead poisoning. Such chronic intoxications lead to increase of connective tissue, arteriosclerosis, cirrhosis, etc.

Drug Nephrites.-These are produced by all absorbable irritants and often occur acutely. The inflammation may involve all the renal tissues; or it may be predominantly glomerular or parenchymatous, according to the poison. Continued administration leads to interstitial nephritis.

The inflammation is characterized by albuminuria, casts, and the histologic lesions. The urine flow is increased in the early stages, and decreased later (Schlayer and Hedinger, I907). The excretory efficiency is diminished (Eisenbrey, I9I r, I9I3), as estimated by the phenolsulphonephthalein test of Geraghty and Rowntree, I9II. The changes in nitrogen and chlorid excretions are complex (Austin and Eisenbrey, I9II). The nitrogen excretion is decreased in tubular nephritis (uranium), not in glomerular (arsenic). Retention of nitrogen in the blood occurs, especially in tubular nephritis (Foster, I915). Renal Glycosuria may occur (E. Franck, r9r3; Pollak, r9r I); also hepatic edema (Opie, r9I2), and sometimes slight myocardial changes (Walker, I9II). Hemorrhagic nephritis is seen with cantharides, turpentine, formaldehyd, etc. Edema occurs only with uranium, at least in animals. The restoration of the bloodvolume after saline injections is delayed in uranium, chromate and tartrate nephritis (Bogart, Underhill \& Mendel, I9I6; Boycott, I9I3 and I9I4; Chisholm, I9I4).

Experimental Nephrites. - These have been extensively investigated (bibliography in R. M. Pearce, I9Io).

Tubular nephritis with little or no primary glomerular injury is produced by uranium, chromate, mercuric chlorid and aloin. The anatomic changes in the early stages of uranium and chromate nephritis are confined essentially to the tubules, especially the convoluted; they consist of granular and fatty degeneration and necrosis. Mercury acts similarly but mainly on the ascending loops of Henle, with deposition of lime salts. The glomeruli are at first unaffected but later show thickening of the capillary walls, and vascular disturbances can be shown by physiologic methods.

Glomerular Nephritis. - This, generally with some secondary involvement of the tubules, is produced by arsenic, cantharides and snake venom. Diphtheria toxin acts on all the structures; and many other irritants act diffusely. The anatomic changes vary according to the poisons. Arsenic produces little or no anatomic change, except dilatation of the capillary tufts, filling Bowman's capsules. Physiologic methods, however, show serious vascular injury. The cantharides changes involve both the glomerular tufts and space. The capsules are filled with desquamated cells, whose origin is disputed. There is also functional vascular injury. The functional changes are not proportional to the anatomic.

Chronic Nephritis. - This is difficult to produce experimentally, the acute experimental lesions usually ending in rapid and complete recovery. Positive results have been obtained by Ophüls with lead, by Dickson with uranium, and by Klotz, I9I4, with infections.

Reflex Effects of Irritants.-Stimulation of sensory areas acts reflexly on the vasomotor, cardiac, respiratory and other centers, producing changes in the circulation, respiration and other functions. These vary according to the place, extent, speed, intensity and kind of the stimulation. As "counterirritation" they are used extensively in therapeutics.

The Respiratory Reflexes.-These are of special therapeutic importance. They differ according to the state of the center: if the respiratory center is depressed, as in collapse, morphin poisoning, anesthesia, asphyxia, 
etc., moderate counterirritation produces marked stimulation; and if the collapse is not too deep, natural respiration may be restored.

If the respiration is normal, mild cutaneous irritation may either slow or quicken the rate. If the irritation is strong, the respiration is always slowed. Sudden and extensive stimulation, as by cold douches, produces momentary standstill, followed by deep inspirations.

Consciousness.- This is also often revived by counterirritation, f.i., in fainting or light coma. The usual measures for this purpose are dashing with cold water, slapping with wet towels, or inhalation of dilute ammonia vapor (smelling salts or aromatic ammonia).

Circulation.-Mild cutaneous stimulation (f.i., blowing on the skin) tends to produce widespread vasoconstriction and therefore some rise of blood pressure (Gruetzner and Heidenhain, I877). The vasoconstriction involves mainly the cutaneous vessels, except those which are dilated by direct contact with the irritant. The renal vessels are also constricted, whilst those of the brain are dilated (Wertheimer; Roy and Sherrington). The cutaneous vasoconstriction tends to raise the internal temperature. The heart rate is quickened (Naumann).

Vasomotor Reflexes.-Mild stimulation of sensory nerves generally produces a slight depressor action; stronger stimuli cause a pressor effect, increasing with the intensity of the stimulation (Stiles and Martin, I9 15 ).

With stronger stimulation of the skin, the vasoconstriction is soon followed by dilation, fall of blood pressure and of internal temperature. The effects may be less than with mild irritation.

Gastro-intestinal Irritation.-Sollmann, Brown and Williams (I907) have shown that irritation of the stomach or peritoneum has practically no effect on the blood pressure in anesthetized animals, even when the anesthesia is light. There is generally a marked increase of respiration, and sometimes a slight and momentary rise of blood pressure, but no fall is noticed for several hours. This holds true both for mild irritants, such as peppermint, mustard or moderate heat; and for strong corrosion by formaldehyd, concentrated acids, or actual cautery, even when these measures produce perforation.

Irritation of the Mouth and Larynx.--This produces very much more marked effects (Sollmann); even contact with 5 per cent. acetic acid causes struggling, convulsive rise of blood pressure and dyspneic respiration.

Reflex Shock.- Violent and extensive corrosion may produce immediate "shock," which may be promptly fatal. This appears to consist essentially in profound inhibition of the nervous system. Since it is not produced in anesthetized animals, at least with anything like the same rapidity, it must be due to psychic factors-apprehension, pain and others-rather than to reflexes in the ordinary sense. However, longcontinued irritation of the viscera gradually produces, even in anesthesia, a condition. (surgical shock), which is allied to, if not identical with, acute traumatic shock.

Metabolism.-Mild stimulation of extensive skin areas (salt-water baths) increase metabolism. Paalzow found increased oxygen consumption and carbon dioxid excretion on applying sinapism to animals; Zuntz and others have demonstrated increased nitrogen excretion.

"Derivative" Action.-The application of irritants of ten subdues inflammation in deep-seated organs. This counterirritation.was formerly supposed to act simply by mechanical withdrawal of the blood from the inflamed area to the area of "counterirritation." This explanation is certainly incorrect. It is now believed that the circulation in the distant organs is modified by reflexes along homologous nerve paths-in a sense a reversal of the "referred pain" which occurs in definite skin areas when the corresponding viscera are irritated (Head). This would explain the 
old empirical observations that the counterirritation, to be most effective, must be applied at a definite place for each internal inflammation (Figs. $3^{a}$ and $3^{b}$ ).

Cold applied to one hand constricts the vessels of both hands, and heat dilates them (G. N. Stewart, I9I3; Hewlett, I9I3). Irritation of the hand by mustard does not increase the local blood flow, as would be expected from the rubefaction (Wood and Weisman, I01 2; Hewlett, 1913).

Influence of Pain on Inflammation.--Bardy, 1914, confirms Spiess and Bruce that inflammatory phenomena depend mainly on sensory disturbances (pain, reflex hyperemia, etc.). The reflex, however, is quite peripheral. For instance, mustard conjunctivitis is prevented by local anesthetics or by division of the peripheral nerves, but not

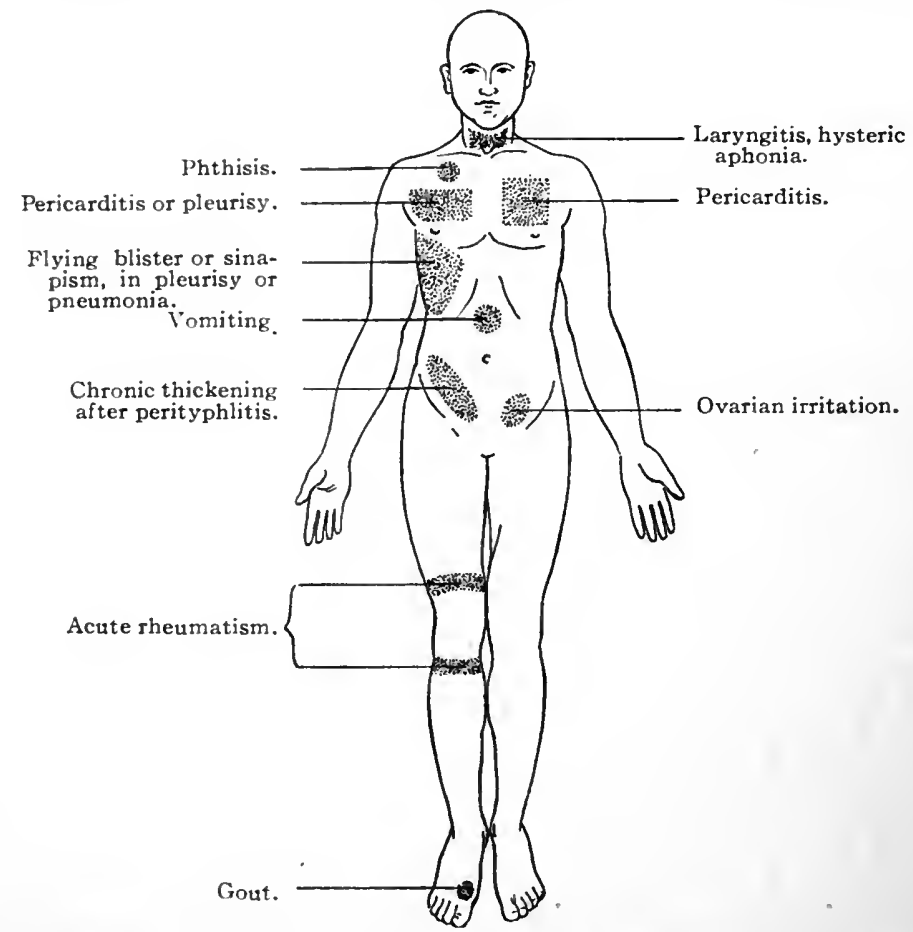

FiG. 3a.-Diagram of the body showing some of the points where blisters or sinapisms are usually. applied. Front view-(Brunton.).

by section of the ramus ophthalmicus. It is prevented by nicotin, so that the reflex must involve the ganglia. Central anesthetics, ether or morphin, also prevent the inflammation. Other substances having a similar effect are: calcium chlorid, antipyrin, quinin, salicylates, atophan, bromids, etc.; and substances yielding available oxygen. (Amberg, Lovenhart and McClure, 1916.)

Changes in the Blood. - Irritation of the skin produces changes in the blood which may have some influence on inflammation: Winternitz, I896, found at first leucopenia, then leucocytosis. Van den Velden, rgr 2, claims that the coagulability of the systemic blood is increased by a number of local irritant and especially vasoconstrictor processes (ice to the skin; astringents or epinephrin to the mouth; cocain to the nose; turpentine inhalation, etc.).

Relief of Pain by Counterirritation.-This is partly explained by homologous reflexes; but largely also by the diversion of the attention of the patient from the disease-pain, to the usually more bearable sensations of the counterirritant. 
Therapeutic Indications of Cutaneous Irritation.-This is employed mainly in the following conditions:

I. In certain skin diseases, for the local effects-alkalies, sulphur, chrysarobin, etc.

2. To promote diaphoresis-alcohol, frictions, heat, etc.

3. For reflex stimulation of the central nervous system, in fainting, collapse, narcotic poisoning, accidents of anesthesia, etc.-ammonia inhalation, heat or cold.

4. To alter the distribution of blood, to prevent colds-mustard; to relieve congestion of viscera-poultices, vesication; or to promote the absorption of exudates-iodin, vesication.

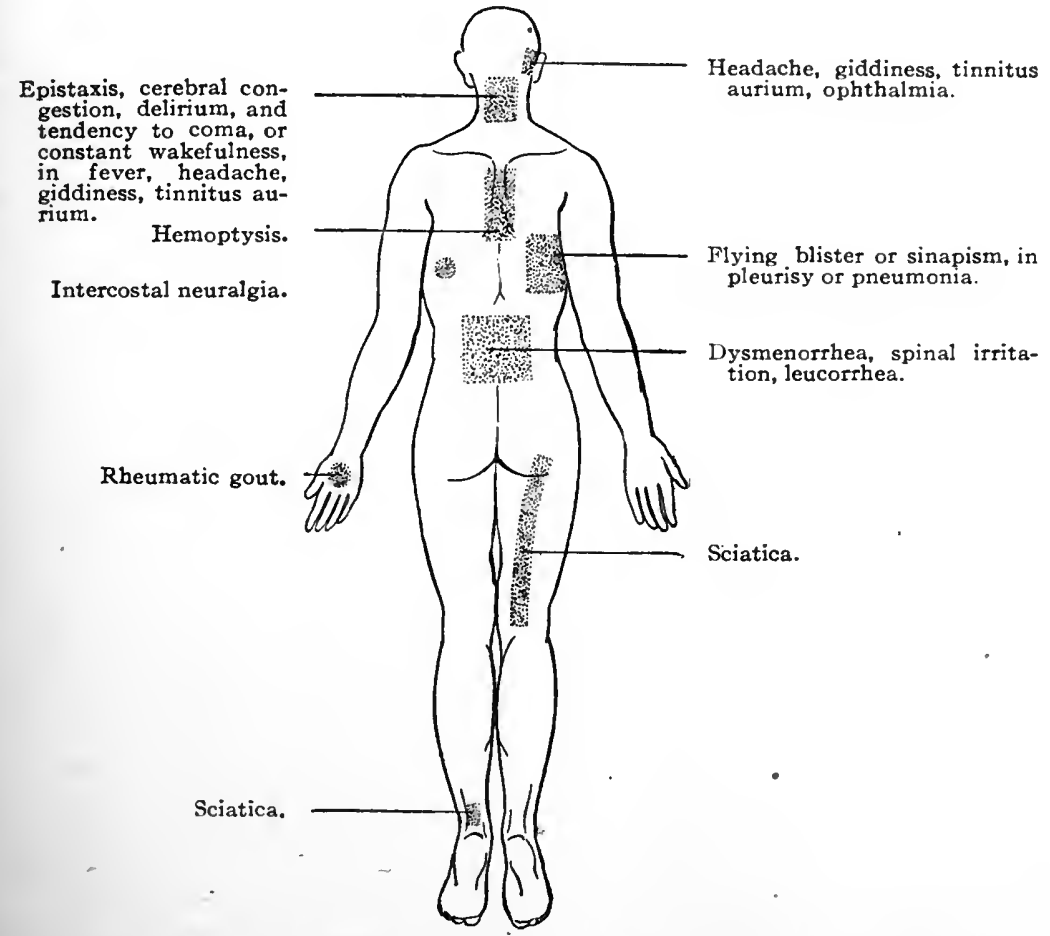

Pig. 3b.-Diagram of the body showing some of the points where blisters or sinapisms are usually applied. Back view.-(Brunton.)

Counterirritation is, as a rule, useful only in chronic inflammation. In acute inflammation there is always a danger of increasing the process or of causing it to extend to neighboring organs.

5. To diminish pain, especially in neuralgic and rheumatic affectionsturpentine and other volatile oils, capsicum, chloroform.

6. As a general tonic, in the form of salt-water baths or alcohol frictions.

Therapeutics of Cauterization.-Cauterization-the destruction of tissue-is sometimes employed for severe counterirritation, but particularly to remove tissue: (I) In cases of poisoning, snake-bite, etc.; (2) for the removal of pathologic tissues, tumors, warts, etc.; (3) indolent granulations, etc.; (4) to cause cicatricial contraction of hypertrophied mucous mem- 
branes (nose, etc.); (5) for removing the nerves of teeth; and (6) to remove superfluous hair.

In many cases the chemic cautery has been replaced by galvano- and thermocautery, which are more prompt and permit a more exact limitation of the cauterized area. On the other hand, the slower effect of chemic caustics is of advantage in permitting a graduation in the strength of the action, or in confining it to certain tissue elements. Pathologic formations, being less stable, are in this way more profoundly altered than normal tissue.

The caustics may be applied in solid form (sticks, or fused at the end of a probe), in paste, or in solution - the first being the most strictly localizable, the last the most diffuse. In the latter case, or when the eschar liquefies, the surrounding tissue should be protected by court-plaster.

General Toxicology of Irritants.-The phenomena produced by irritant poisons depend in the first place upon the part of the body with which they are brought into immediate contact. The most prominent symptoms arise from the skin, alimentary canal, or respiratory organs; the last only in the case of very volatile poisons. Later symptoms may appear in the urinary organs.

The extent of the action depends upon the concentration of the poisons, the time during which they act, and the extent of surface with which they come into contact-less upon their absolute amount. If taken by the alimentary canal, their action will also be modified by the presence of food.

Cauterization of the Skin.-This may be accidental or criminal. In the latter case it is usually by sulphuric acid ("Vitriol"). The results are the same as in the case of extensive burns. The diagnosis offers no difficulty. The character of the stains is that described in the next section. Sufficient of the corrosive can generally be collected from the clothing, etc., to establish its identity by chemic means. The treatment is precisely like that for burns, after previous neutralization and removal of the corrosive agent. Salves and oils are useful-especially the Linimentum Calcis (Carron Oil, i.e., equal parts Linseed Oil and Lime-water).

Caustics in the Eye. - These are best washed away by liberal application of water.

Caustic Poisoning by the Alimentary Canal.-The introduction of caustics by the mouth is generally accidental or suicidal. The effects are so painful, appear so promptly, and the lesions are so persistent, that they would scarcely ever be used in criminal poisoning-except possibly in infanticide. They are sometimes taken by mistake for syrup or other medicine, and may be swallowed before the difference is noticed. However, certain organic irritants, usually insoluble, such as croton oil, do not produce their action for some time.

The phenomena vary according to whether the irritant produces an actual cauterization - a destruction or solution of the tissues; or whether it causes only inflammation.

Irritants Which Do Not Destroy the Tissue.-To this class belong elaterium, croton oil, and most of the other organic irritants, such as volatile oils, formaldehyd, etc.

The symptoms are those of a violent gastroenteritis: nausea, vomiting, and diarrhea. If the poison acts only when dissolved, and is insoluble in the stomach, as is croton oil, the nausea and vomiting may not be present, but only the diarrhea. The symptoms will appear correspondingly late. The abdomen is usually distended and extremely painful, especially upon pressure. As a result of the gastroenteritis, there is extensive dilatation of the splanchnic area, and consequently withdrawal of blood from other parts of the body. This produces marked changes 
in the circulation. The pulse will be soft, small, and quick. The lowered circulation reacts upon other organs, and most conspicuously upon the central nervous system. There is great anxiety, vertigo, delirium, convulsions, then collapse, and finally coma and death. This picture is common to the entire series of irritant poisons.

Abortion.-The hyperemia is not confined to the intestine, but extends to all the abdominal organs, which therefore partake in the inflammation, although they do not come into direct contact with the irritant. The most important organ involved in this is the uterus, and the organic irritants have been used to procure criminal abortion. Oil of savin, of tansy, and of pennyroyal enjoy a special reputation in this connection, but any other irritant produces the same result. The ecbolic effect is only secondary to the gastroenteritis, and the latter is very often fatal without accomplishing the object for which it was produced.

The postmortem appearances are those of gastroenteritis, and in cases of suspected criminal abortion this must be of sufficient extent to explain the fatal issue. The pathologic condition consists in an intense congestion of the entire alimentary canal, often with inflammatory exudate into the lumen of the intestine. The congestion may be so violent as to produce ecchymoses. If these are present, the vomit and stools will be tinged with blood during life. Destruction of tissue is quite rare. It may, however, occur from gangrene due to the interference with the circulation.

The Fixed Caustics.-The most important are the mineral acids; oxalic acid, which, however, stands apart on account of its specific toxic action; the organic acids, which are, generally speaking, corrosive in proportion to their volatility; the alkalies and their carbonates; the haloid substances, bromin, chlorin, and iodin. Phenol and the metals are also to some extent corrosive, but not usually sufficiently so to produce perforation. The alkalies and bromin produce the most extensive destruction of tissue, because of their deep penetration. With them, the scar formation is also the most extensive.

Corrosive effects occur especially in those situations which are in prolonged contact with the caustic: the lips; mouth and pharynx; esophagus at its beginning and end, and where it crosses the left bronchus ${ }^{1}$ and the stomach, especially the pyloric end ${ }^{2}$ (because the caustic follows the lesser curvature and accumulates at the pylorus). The firm closure of the pylorus furnishes more or less protection to the intestines.

Characteristic Appearance of the Corrosions and Stains.-These are of diagnostic importance, since they may be recognized about the mouth during life:

Alkalies cause transparent swelling of the epithelium, which will detach as a gelatinous mass, exposing the scarlet-colored inflamed area beneath. ${ }^{3}$ The other corrosives, which precipitate proteins, produce at first a grayishwhite opaque stain. This persists in the case of the metallic poisons. The acids, however, change the hemoglobin in the neighboring area into the dark acid-hematin, and the color of the stain consequently becomes dark or black. Nitric acid is an exception: its stain takes on a yellow color. This differs from that of picric acid by being changed to orange by alkalies, whilst the picric acid stain remains unaltered. Bromin pro-

1 E. v. Hoffmann, Atlas of Legal Medicine, W. B. Saunders, 1898. Figs. I86 and I87.

ib. Fig. 187 . 
duces a characteristic light brown or orange stain; iodin stains a mahogany color. The silver stain turns black after a time.

Removal of Stains.-The stains of iodin and silver are frequently a source of annoyance in their therapeutic exhibition. They can be readily removed: The iodin by ammonia water or by thiosulphate; the silver by potassium cyanid, or by painting first with iodin and then with ammonia.

Symptoms of Corrosive Poisoning.-These begin in the mouth, with burning pain, dysphagia, and loss of tissue. The taste of many of these substances is characteristic-acid, alkaline, metallic, astringent, etc.

The further symptoms are those of gastroenteritis, similar to those described under non-corrosive irritants, but generally more severe. The vomiting and diarrhea are more frequently bloody. In the case of acids the vomited blood is frequently very dark in color on account of the formation of acid hematin. This is the so-called "coffee-grounds" vomit. The pain is very much more marked with corrosive poisons. The destruction of the tissue gives rise to reflexes which may take the form of "shock." The absorption of the chemic products of corrosion may produce fever; or the temperature may be lowered by collapse. If perforation occurs, the clinical picture turns into that of peritonitis.

Death.-This may occur from shock before the local symptoms have time to develop; or it may follow after one or two days of more gradual collapse; or it may result later from peritonitis; or finally, after recovery from the acute effects, the formation of scar-tissue in the corroded areas may lead to stenosis and thus to gradual starvation.

Postmortem Examination.-This shows the characteristic stains and corrosions in the mouth, esophagus, stomach and duodenum; with the metallic corrosives, the cecum and large intestines may show the principal changes ${ }^{1}$ since many metals are excreted in these situations. The other abdominal organs are also hyperemic.

When the action has not progressed to actual corrosion, there is often very marked hyperemia and ecchymosis. The color is frequently much darker than corresponds to the amount of congestion, especially in the case of acids (due to acid-hematin). ${ }^{2}$

Treatment of Poisoning by Corrosives.-The first measure is dilution, since the action is proportional to the concentration. The drinking of water in abundance and the washing out of the stomach are therefore important. If corrosion is already advanced, it is not advisable to pass the stomach-tube. The further treatment consists in the administration of demulcent substances, as mucilage or boiled starch; or proteins, as white of egg; or milk. The proteins are especially useful against the metallic poisons, since they form rather insoluble albuminates.

The pain usually requires the exhibition of narcotics in free doses. Most of the irritant poisons can be treated by chemic antidotes; in the case of alkalies, by acids (vinegar, lemon juice, or any acid diluted to $I$ to 5 per cent.); in the case of acids, by means of alkalies; preferably magnesium oxid; or in emergencies by soap. (The free alkalies are usually too caustic, and the carbonates involve danger of rupturing the corroded stomach by the evolution of carbon dioxid gas. Potassium compounds are objectionable because of the danger of toxic absorption from the corroded mucosæ. In case of necessity any alkali may be used, such as whitewash or chalk.)

Iodin, chlorin or bromin may be neutralized by sodium bicarbonate.

Volatile Caustics.-These comprise ammonia, chlorin, bromin, the fuming mineral acids, the gaseous acids, such as sulphurous, nitrous, etc.; 
and certain organic acids-acetic, formic, etc. Also other organic substances, such as formaldehyd; and the volatile oils, especially the oil of mustard.

When swallowed, these produce the symptoms already described, the actions being, however, more rapid and extending more deeply. When inhaled, they irritate mainly the respiratory passages and other exposed mucous membranes, causing coryza, conjunctivitis, bronchitis, pulmonary edema, pneumonia, etc. Through the irritation they produce profound nervous effects, at first mainly stimulant. The respiration stops at first in expiration, the glottis closes spasmodically, and the bronchial muscles contract. This is a conservative mechanism, and explains why fatal poisoning is not more common. During this stage there is cardiac inhibition through vagus stimulation, and also dilation of peripheral vessels. The inhalation irritants may prove promptly fatal by spasm or edema of the glottis; or the course may be slower, passing through bronchitis, pneumonia, etc. ( $c f$. L. Lewin, I908). The irritation may also involve the esophagus and stomach, with protracted hemorrhagic gastritis (Loeper et al., I915). The inhalation of irritant vapor is especially deleterious to asthmatic individuals.

Bronchodilator drugs (atropin, etc.) have a limited efficiency against the acute respiratory distress of irritant gases (Symes, I9 I 5).

The effects of small quantities of volatile irritants in the air has an economic importance, in view of their escape from chemical factories, etc. There can be little doubt that even a small proportion tends to produce bronchitis, and to diminish the resistance to infections (Ronzani, r9o8: low concentrations of $\mathrm{Cl}, \mathrm{SO}_{2}, \mathrm{NO}_{2}$ ). They may possibly cause cachectic conditions; but it is difficult to assign the limits between which these poisons are harmless, objectionable, and dangerous.

Nitric Peroxid (Nitrous Acid, $\mathrm{NO}_{2}$ ).-This constitutes the brown vapors arising from the action of nitric acid on metals. It is also produced in large amount in the combustion (not in the explosion) of nitroglycerin and gun-cotton. The acute phenomena are relatively slight, but they are followed after six or eight hours by sudden bronchitic attacks with extreme dyspnea, pulmonary edema, and often death within forty-eight hours (F. C. Wood, I9I2).

This gas is therefore especially dangerous.

\section{FIXED CAUSTIC ALKALIES}

Members.-These comprise $\mathrm{NaOH}$ and $\mathrm{KOH}$, which corrode even the skin; the carbonates of sodium and potassium which are not caustic, but which irritate the mucous membranes violently; and quicklime, which on account of its limited solubility, is caustic only when used in substance.

Manner of Action.-The free alkalies combine with the tissue elements to form alkaline albuminates, or with the fats to form soaps, and in this way produce a destruction of substance. They are also very hygroscopic, and withdraw water from the cells, which contributes to the necrosis. The scab which they produce is very soft, and the compounds which they form are very soluble; consequently the alkalies penetrate very deeply, are very painful, their action continues for several days, and leads to extensive scar formation. It is therefore difficult to circumscribe their effects. This is only partly remedied by mixing them with the non-diffusible quicklime as in the "Vienna Paste," a mixture of equal parts of $\mathrm{KOH}$ and $\mathrm{CaO}$, stirred into a thick paste with alcohol.

Actual and Potential Alkalinity.-The "actual" or immediately effective alkalinity of a solution depends on the dissociated $\mathrm{OH}$ ions; but as these combine with the tissues, further $\mathrm{OH}$ ions are split off ("potential") alkalinity), which continue the action. The total effect, therefore, depends upon the total quantity of $\mathrm{OH}$ ions which can be split off under the conditions of the body (Dreser, I9I0). This can be estimated by appropriate indicators. 
Poisoning by Caustic Alkalies (Lyes).-This is quite common. Half an ounce of potassium carbonate or an ounce of the liquors would generally be fatal. Sodium carbonate is less toxic. On account of the deep action, stricture of the esophagus is a frequent sequel, and the fatality is fairly high.

\section{PREPARATIONS-CAUSTIC ALKALIES}

* Polassii IIydroxidum (Pot. Hydrox.), U.S.P.; Potassa Caustica (Potass. Caust.), B.P.; Potassium Hydroxid (Caustic Potash, Potassium Hydrate); KOH. - White flakes or masses or pencils; odorless; caustic. Very sol. in water ( 1 :0.9), freely sol. in alc. $(1: 3)$. Used as caustic, to remove warts, etc.; and to soften epidermis (2 per cent.).

* Liq. Pot. Hydrox., U.S.P.; Liq. Potass., B.P. -5 per cent. Sometimes used internally as alkali. Dosc, I c.c., 15 minims, U.S.P.; 0.6 to 1.8 c.c., 10 to 30 minims, B.P.; frecly diluted.

Potas. Carbonas (Pot. Carb.), U.S.P., B.P. (Salts of Tartar); $\mathrm{K}_{2} \mathrm{CO}_{3}$ - White granular powder; odorless; strongly alkaline taste. Very deliquescent. Very sol. in water (1 : 0.9 ), practically insol. in alc. Dose, I Gm., 15 gr., U.S.P.; 0.3 to 1.2 Gm., 5 to $20 \mathrm{gr}$., B.P.

Sod. Hydrox., U.S.P. (Caustic Soda); NaOH.-Dry, white flakes, or fused masses or pencils. Very sol. in water $(\mathrm{I}: 0.9)$ and alc. U.S.P.

Liq. Sod. Hydrox., U.S.P. (Solution of Soda).-5 per cent. Dose, I c.c., 15 minims,

* Sodii Carbonas Monohydratus (Sod. Carb. Monohyd.), U.S.P.; $\mathrm{Na}_{2} \mathrm{CO}_{3}+\mathrm{H}_{2} \mathrm{O} .-$ A white, crystalline, granular powder; strongly alkaline taste. Freely sol. in water $(1: 3)$ and in glyc. $(1: 7)$; insol. in alc. Used as lotion $(0.5$ per cent.). Is nearly twice as strong as the ordinary crystallized carbonate. Dose 0.25 Gm., 4 gr., U.S.P.

* Sod. Carb., B.P.; Sodium Carbonate (Washing Soda); $\mathrm{Na}_{2} \mathrm{CO}_{3}+10 \mathrm{H}_{2} \mathrm{O}$ (equivalent to 37 per cent. of $\mathrm{Na}_{2} \mathrm{CO}_{3}$ ). - Freely sol. in water $(\mathrm{I}: 2)$. Dose, 0.3 to $2.0 \mathrm{Gm}$., 5 to 30 gr., B.P.

Sod. Carb. Exsic., B.P.; $\mathrm{Na}_{2} \mathrm{CO}_{3}$. Dose, 0.2 to $0.6 \mathrm{Gm}$., 3 to Io gr., B.P.

Calx, U.S.P., B.P.; Calcium Oxid (Lime, Quicklime); CaO.-Hard, white or grayishwhite masses, or a white powder; odorless; caustic taste. Slightly sol. in water ( $1: 840)$; sol. in glyc.; insol. in alc. When sprinkled with about half its weight of water, calcium oxid becomes heated, and is gradually converted into a white powder (calcium hydroxid or slaked lime). When this is mixed with about 3 or 4 parts of water, it forms a smooth magma (milk of lime).

Calc. IIydr., B.P. (Slaked Lime); $\mathrm{Ca}(\mathrm{OH})_{2}$.-White powder.

* Liquor Calcis (Liq. Calc.), U.S.P., B.P.; Solution of Lime (Lime-water).-A saturated solution of $\mathrm{Ca}(\mathrm{OH})_{2}$, containing about 0.14 per cent. Clear colorless liquid; odorless; alkaline taste. Incompatible with carbonates or "hard" water. Used as antacid, against diarrhea and externally against burns. It is often added to milk $(\mathrm{I}: 4)$. Dose, I5 c.c., 4 drams (about $0.02 \mathrm{Gm}$. of the hydroxid), U.S.P.; 30 to I 20 c.c., I to 4 ounces, B.P.

Liq. Calcis Saccharalus (Liq. Calc. Sacch.), B.P. (Syrup of Lime).-Contains about 2 per cent. of $\mathrm{CaO}$. Antacid, and antidote for phenol poisoning. Dose, 2 c.c., 3 minims.

${ }_{*}^{*}$ Linimentum Calcis (Lin. Calc.), U.S.P., B.P.; Lime Liniment (Carron Oil).-A mixture of equal volumes of lime-water and oil (olive, B.P.; linseed, U.S.P.). Applied to burns, on cloths, frequently renewed.

\section{AMMONIA}

This differs from other alkalies by its volatility and deeper penetration.

Use as Counterirritant.-It passes through the stratum corneum of the epidermis without injuring it, and, acting upon the lower layers of the skin, produces blisters. It is sometimes used for this purpose instead of cantharides, especially in nephritis, where the latter can not be employed. It is, however, more painful.

It is frequently used in more dilute form as liniment for counterirritation, when a deep action is desired. 
The Inhalation of Ammonia.-Free, or in the form of aromatic spirit, or of carbonate, this is used for reflex stimulation in fainting, etc. Care must be used, since too high concentrations of the vapor may produce bronchitis or pneumonia.

Poisoning by Swallowing Ammonia.-This presents the same phenomena as other caustic alkalies except that the respiratory passages are more involved by the inhalation of the vapor.

Five to $10 \mathrm{Gm}$. of the official solution would be toxic; $30 \mathrm{Gm}$. are generally fatal, although recovery has occurred from $60 \mathrm{Gm}$.

Inhalation of Ammonia.- This produces the effects described under volatile irritants. For man 0.5 to $1: 1,000$ become insupportable.

For "Preparations," see Index.

\section{ALKALINE SALTS AND SOAPS}

The soluble bicarbonates, basic phosphates, borates, sulphids and soaps, act as mild alkalies, since their watery solutions undergo hydrolytic dissociation, with the liberation of $\mathrm{OH}$ ions.

Actions and Uses.-By their alkalinity, they dissolve mucus, soften the epidermis and hair, and emulsify and dissolve fats. They are therefore employed in catarrhal conditions; in skin diseases (seborrhea, acne, ichthyosis, psoriasis, and epidermal proliferations); to facilitate the penetration of antiseptic remedies into the skin (in scabies, favus, ringworm); to emulsify cutaneous remedies (mercury, tar, sulphur); as lubricants for liniments (soap liniment); as cleansing agents (detergents), especially in the form of soaps and borax; and as depilatories (especially the sulphids).

Laxative Action.-In the rectum, soap secures prompt evacuation. It is used for this purpose as warm soap-suds enemas; or solid, formed into a suppository.

The Detergent Action of Soaps.-This is due to the mechanical effect of the foam, the emulsification of the grease, and the softening of the epidermis, so that the superficial layers are readily removed with the adherent dirt.

Potassium soaps are the more energetic. They are semiliquid (soft soap); sodium forms hard soaps.

Alkaline Baths.-In skin diseases, these are best administered at bedtime. Any of the alkaline salts can be used; such as sodium carbonate or bicarbonate, potassium carbonate, or borax, in the proportion of about $100 \mathrm{Gm}$. per bath; for lotions, 2 per cent.

Hemolytic Action.-Alkalies, and especially soaps, are hemolytic. These also sensitize pneumococci to serum-lysis (Flexner, I913).

Phagocytosis. - This is stimulated by soaps, even in fairly high concentrations; but excessively strong solutions are detrimental (Hamburger and de Haan, .1913).

\section{PREPARATIONS-SOAP}

* Sapo, U.S.P.; Sapo Durus (Sap. Dur.), B.P.; Soap (White Castile Soap).-Prepared from sodium hydroxid and olive oil. A white solid; sol. in hot water and alc.

Sap. Animal., B.P.; Curd Soap.- Hard soap prepared from animal fat.

* Sapo Mollis (Sapo Moll.), U.S.P.; (Sap. Moll.) B.P.; Soft Soap (Green Soap, Sapo Viridis, Sapo Kalinus).-Made from potassium hydroxid and vegetable oil (cottonseed, U.S.P., olive, B.P.). A soft, unctuous, yellowish mass.

* Linimentum Saponis, (Lin. Sapon.), U.S.P.; Soap Liniment (Opodeldoc).-An alcoholic solution of 6 per cent. of soap, 4.5 per cent. of camphor, and I per cent. of rosemary oil.

* Lin. Sap., B.P.-Similar to the preceding.

* Lin. Sapon. Moll., U.S.P. (Tincture of Green Soap).-2 parts of the soap, I part of alcohol, flavored with lavender oil. 


\section{ALKALINE SULPHIDS}

When applied to the skin, the sulphids ( $\mathrm{K}, \mathrm{Na}, \mathrm{Ca}$ ) soften the stratum corneum, much like the alkalies, and they are used in the same conditions. Their action on hair is even greater, so that they are valuable as depilatories, especially calx sulphurata. When taken by moulh, they act as irritants and corrosives, through the liberation of sulphuretted liydrogen and free alkali.

Toxic Doses.-These produce the systemic effects of hydrogen sulphid. Death has occurred from 12 to $15 \mathrm{Gm}$. Smaller doses have little systemic action, as they are rapidly oxidized. They are excreted in the urine mainly as sulphates; but there seem to be also some organic sulphur compounds.

A small amount of some volatile sulphur compound is also excreted by the lungs, giving the peculiar $\mathrm{H}_{2} \mathrm{~S}$ odor to the breath, and causing some expectorant action.

When injected intravenously, the sulphids produce violent convulsions, followed by depression of the medullary centers. This is due to a direct action. Outside of the body, sulphids form a peculiar compound with hemoglobin, but this does not occur during life, in mammals.

Therapeutic Uses.-The sulphids are only used externally, in skin diseases (psoriasis, acne, etc.); and as parasiticides in scabies (solution of the skin and eggs of the mite).

The sulphids have been proposed as an antidote for hydrocyanic acid, since they would form sulphocyanids, which do not have the hydrocyanic acid action. This has not been tested. It is possible that the reaction goes on too slowly to be of value.

\section{PREPARATIONS-SULPHIDS}

Calcii Sulphidum Crudum (Calc. Sulphid. Crud.), U.S.P.; Calx Sulphurata (Calx Sulphur), B.P.; Crude Calcium Sulphid, Sulphurated Lime.-A mixture containing about 55 per cent. of $\mathrm{CaS}$. A pale gray or yellowish powder; faint odor of hydrogen sulphid; nauseous alkaline taste. Very slightly sol, in cold water; more readily sol. in boiling water with partial decomposition; insol. in alc. Used as depilatory, mixed with an equal quantity of starch and made into a paste. Internally it is used against boils and other suppurations. Its efficiency is doubtful. Dose, $0.06 \mathrm{Gm}$., I gr., U.S.P.; I 6 to $60 \mathrm{mg} ., 1 / 4$ to $\mathrm{r}$ gr., B.P.

Vlemingkx's solution is a solution of calcium polysulphids, used in scabies ( $\mathrm{r}: \mathrm{x}$ ), and as a bath in skin diseases (2 drams per gallon).

Pot. Sulphurat., U.S.P.; (Potass. Sulphur., B.P.); Sulphurated Potash (Liver of Sulphur).-A mixture composed chiefly of potassium polysulphids and potassium thiosulphate. Freely sol. in water $(\mathrm{r}: 2)$. Used in baths $(30$ to $200 \mathrm{Gm}$., I to 6 ounces, per bath).

\section{HYDRO GEN'SULPHID $\left(\mathrm{H}_{2} \mathbf{S}\right)$}

This gas is extremely toxic, much more so than is commonly realized.

Actions.-These are partly local and irritant, due to its acid character; and partly central somewhat resembling asphyxia. In vitro, it forms a firm compound (sulphhemoglobin) with the blood pigment. Thick layers have a brown color. Thin strata appear a dirty green, which is responsible for the postmortem discoloration. Lewin believes that the effects are due to the interference of the compound with oxygenation; however, it is generally believed that only traces are formed during life (E. Meyer, 1898 ) and that the sulphid is directly toxic to the nervous system.

Occurrence.-Hydrogen sulphid is formed in putrefaction, particularly of sewerage. "Sewer gas" consists essentially of $\mathrm{CO}_{2}, \mathrm{NH}_{3}, \mathrm{CH}_{4}$, and 2 to 8 per cent. of $\mathrm{H}_{2} \mathrm{~S}$. Small quantities are also formed in intestinal putrefaction, giving rise to diarrhea and possibly to some of the symptoms of "intestinal autointoxication." It may be removed by the administration of bismuth salts, and its further formation checked by cathartics and intestinal antiseptics.

Concentrations in the Respired Air.- -0.02 per cent. produces symptoms, particularly irritation of the conjunctiva and bronchial mucosa; 0.05 per cent. produces asphyxial symptoms; hyperpnea, nausea, giddiness, headache, cerebral excitement, and finally narcosis; 0.07 per cent. produces death after about an hour under coma, often with convulsions; 0.2 per cent. is fatal to dogs in one and one-half minutes. The aftereffects resemble those of carbon monoxid. Edema of the lungs and pneumonia are common sequels (Lehmann, 1892).

$\mathrm{H}_{2} \mathrm{~S}$ is partly excreted by the lungs. The urine is also said to contain sulphids. 
It is readily detected in air by its odor and by blackening lead or silver-paper. In case of death, it may be recognized by the blackening of a silver coin laid on the skin.

Sulphur Springs.- Hydrogen sulphid is contained in small quantity in a number of mineral waters (sulphur springs). These are recommended for a variety of obscure disorders-rheumatism, gout, diabetes, etc. When these waters are administered for some time, they cause diuresis and diaphoresis, irritation of the urinary passages, intestinal irritation, and muscular pains. Their therapeutic effects are probably to be referred to their laxative, diuretic and diaphoretic actions, although the general hygienic conditions must contribute largely to the results, as with other mineral waters (Boecker, 1895).

\section{SULPHUR}

Uses.-Sulphur is employed as a parasiticide in itch; as a mild cutaneous irritant in skin diseases (sulphur ointment); and internally as a laxative for softening the stools, especially in hemorrhoids (as Compound Licorice Powder, or mixed with an equal amount of Potassium bitartrate). Like other intestinal irritants, sulphur somewhat increases the elimination of uric acid (Abl, I9I3).

Manner of Action.-Sulphur itself is inactive, but by contact with proteins and alkalies it is slowly converted into the active sulphids. This process is so gradual that the action is always mild and protracted, unless the finely divided "precipitated" sulphur is used. Ordinary or "washed" sulphur therefore deserves preference. Sulphur is somewhat soluble in ointment bases, and this facilitates its action (Sabbatani, I913). Sulphur is also burned for fumigation, the disinfectant action being due to the formation of $\mathrm{SO}_{2}$.

Formation of Sulphids.-This is effected on the skin by the cutaneous secretions. No change occurs in the stomach; but a considerable amount (to ro per cent.) is converted into $\mathrm{H}_{2} \mathrm{~S}$ in the intestine, as may be readily seen from the increased secretion of sulphate by the urine. The conversion was formerly referred to the alkalies of the intestine; but Heffter (1904) points out that the alkali exists as bicarbonate, which can not affect the conversion. He showed that it is produced by proteins of all kinds (even after these are boiled). The hydrogen sulphid is formed most abundantly in the large intestines (Taegen, IgI2). (Frankl, I9II, claims that the effects are due to the formation of sulphites).

Colloid Sulphur.-A new modification of sulphur in "colloid solution" produces $\mathrm{H}_{2} \mathrm{~S}$ much more rapidly, also on intravenous injection; and thus causes typical $\mathrm{H}_{2} \mathrm{~S}$ poisoning without other visible effects (Sabbatani, 1908, 1913, 1914).

Alopecia.-Sulphur ( 2 to 4 per cent.) is used in the seborrheic form, as also other irritants (salicylic acid, I to 2 per cent.; mercuric chlorid, 0.1 to 0.4 per cent.; betanaphthol, $\mathrm{I}$ to 2 per cent.; pilocarpin, 0.15 per cent.; cantharides; resorcin (I to 2 per cent., discolors the hair). These agents may be dissolved in water, glycerin or oils, or applied as ointments (Dore, r9I4).

\section{PREPARATIONS-SULPHUR}

* Sulphur Sublimatum (Sulph. Sublim.), U.S.P.; (Sulphur Sublim.), B.P.; Sublimed Sulphur (Flowers of Sulphur). - A fine, gritty, light yellow powder, of slight odor, and faintly acid taste. Insol. in water; nearly insol. in alc.; slightly sol. in fat solvents and oils; freely sol. in carbon disulphid. For fumigation, it is used in the ratio of 2 pounds per $I, \infty 00$ cubic feet. It contains some free acid and other impurities, but is sufficiently pure for medicinal use, although the Washed should be preferred. It enters into all the B.P. preparations, whilst those of the U.S.P. are made from Washed Sulphur. Dose, $4 \mathrm{Gm}$., I dram, U.S.P.; I.2 to $4 \mathrm{Gm}$., 20 to $60 \mathrm{gr}$., B.P. Sulphur also enters into Pulvis Glycyrrhizæ Co. (see Index).

* Sulphur Lotum (Sulph. Lot.), U.S.P.; Washed Sulphur.-This is prepared by washing the preceding with ammonia water to remove the acid. Its properties are similar to the preceding, but it is tasteless and odorless. Dose, $4 \mathrm{Gm}$., I dram, U.S.P.

Sulphur Pracipitalum (Sulph. Præc.), U.S.P.; (Sulphur Præc.), B.P.; Precipitated Sulphur (Lac Sulphuris, Milk of Sulphur).-This is prepared by precipitating sulphur- 
ated lime with acid. An amorphous pale yellow powder. It is a much finer powder than sublimed sulphur and is therefore rather more active; but its dose is the same.

* L'ngucntum Sulphuris (Ung. Sulphur.), U.S.P., B.P.; Sulphur Ointment.-I 5 per cent. of sulphur. U.S.P.; io per cent., B.P.; in benzoinated lard.

Conf. Sulphur., B.P.-45 per cent. of Sulphur, I i per cent. of Potass. Bitart. Dose, 4 to 8 Cim., 60 to I 20 gr., B.P.

Troch. Sulphur., B.P.- $0.3 \mathrm{Gm}$. of Sulphur.

\section{ICHTHYOL}

Uses.-Peculiar value has been attributed to the incompletely oxidized sulphur occurring in organic compounds. The pioneer product of this kind is ichthyol, which contains ro per cent. of sulphur as sulfons, mercaptans and sulphids. It is weakly antiseptic and mildly irritant. Taken internally, it produces gastro-intestinal irritation, with diarrhea. Its influence on metabolism is in dispute. It has been nised locally to cause absorption of swellings and effusions, in contusions, burns, etc., and especially in gynecologic practice. It has also proven useful in skin diseases, acting somewhat like sulphur. Internally, it has been endorsed against the greatest variety of diseases of digestion, respiration, genito-urinary tract, tuberculosis, etc. These claims bear the stamp of exaggeration.

Ichthyol Substitutes. - The disagreeable odor and taste of ichthyol can not be disguised by flavors, but can be removed by combination: Ichthalbin, a compound with albumen, has been recommended for internal administration; Ichthyoform, an almost insoluble compound with formaldehyd, as antiseptic dusting powder.

The commercial success of ichthyol has led to the substitution of a number of synthetic products, formed by saturating mineral oils with sulphur. Thiol and Tumenol are the principal products of this class. They seem to have the same actions as ichthyol, and are tasteless. Linseed oil saturated with sulphur has long been used in domestic medicine under the name of Harlem Oil.

\section{PREPARATIONS-ICHTHYOL}

Ichthyol, N.N.R. (Ammonium Sulpho-ichthyolate).-A mixture prepared by sulphoning the tar-like distillate obtained from bituminous shales found in Tyrol, and which contain the fossil remains of fishes. A dark brown syrupy liquid of characteristic empyreumatic odor and burning taste; of faintly acid reaction. Miscible with glycerin, oil or fats; incompletely soluble in alc. Insoluble in water (Hostmann, I915). Dose, internally, 0.2 to 2 c.c. ( 3 to 30 minims) mostly in simple suspension in water, or peppermint water, sometimes in the form of pills or capsules; locally, in vaginal, uterine or rectal suppositories; in 0.06 to 0.18 c.c. (I to 3 minims) bougies or I to 3 per cent. solution for gonorrheal treatment.

The compounds and substitutes are described in N.N.R.

\section{THIOSINAMIN}

Uses.-This is allyl-sulphocarbamid, prepared by heating volatile oil of mustard (allyl thiocyanate) with alcoholic solution of ammonia. It was introduced by Hebra and Unna, I 892, and has been credited with causing the absorption of exudates, lymphatic swellings, scar-tissue, etc.; and with the cure of lupus. The administration must be continued for weeks, and combined with massage and other adjuvant measures. It is therefore difficult to judge whether it has any value. The clinical opinions are contradictory, and no satisfactory explanation has been offered for its reputed effects. It probably does little, if any good, and it may produce toxic systemic effects, although it is usually well borne, except for the bitter taste and garlic eructations.

Toxic Effects.- In man, I Gm., or $0.2 \mathrm{Gm}$. on repeated use, have caused headache, nausea, vomiting, lassitude and fever (J.A.M.A., $56: 835$, I9II; Charteris, I9Io; Seifert, Nebenwirk., 1915, p. 182). These may set in suddenly after it has been used for a time uneventfully. In animals, Tyrode, I910, found impaired respiration, eventually cessation, and death; sometimes pulmonary edema and hyperemia (Lange, I892). The circulation is not affected in mammals, but high concentrations paralyze the frog's heart. Smaller doses produce severe changes in metabolism, with loss of nitrogen and rapid emaciation; and fatty degenerations of parenchymatous organs, especially the heart and kidneys. Normal connective tissue showed no changes. 
Other Allyl Compounds.-Allyl acetic acid, allyl urca and dimethylallylamin are indifferent; allyl iodid is strongly irritant; allyl formate produces necrosis of the liver and kidneys; allyl amin is highly toxic. The effects are described by Piazza, I9I5.

\section{PREPARATIONS-THIOSINAMIN}

Thiosinamina, N.N.R.; Thiosinamine; $\left(\mathrm{NH}_{2}\right) \cdot \mathrm{CS} \cdot \mathrm{NHCH}_{2} \mathrm{CH}: \mathrm{CH}_{2}-\mathrm{Colorless}$ crystals, of slight garlic odor and bitter taste. Slightly sol. in water, with decomposition; dissolves in alc. or glyc. The oral dose is 0.03 to $0.1 \mathrm{Gm}$. in capsules. It is usually administered by hypodermic injection (which need not be made at the site of the lesion), daily to every third day, 0.05 to $0.2 \mathrm{Gm}$., in i 5 per cent. alcohol or 10 per cent. glycerin. Since the solvents produce local irritation, Fibrolysin may be substituted (Mendel, I905).

Fibrolysin, N.N.R. (Liq. Thiosinam. Sodio-Salicylatis). - 55 per cent. of a double salt of thiosinamin and sod. salicyl. Dose, a 2 c.c. vial, corresponding to $0.2 \mathrm{Gm}$. of Thiosinamin.

\section{CAUSTIC ACIDS}

Members.-The most typical acids in regard to the local action are sulphuric and hydrochloric acid. Nitric acid produces the same effects, but differs from these in its chemic action, producing xanthoproteic acid from the proteins. The sulphurous acid has also a marked corrosive power. Hydrofhuoric acid has a specific toxic action, penetrates very deeply in virtue of its volatility, and is especially strongly corrosive, producing obstinate ulcers. Its inhalation from air containing 0.02 per cent. produces acute catarrhal irritation. Of the organic acids, those of the fatty series act similarly, but are weaker. The trichlor-acetic acid is the most corrosive of these. The volatility of most of the fatty acids makes them more penetrating than the mineral acids. Oxalic acid occupies a place by itself on account of its specific toxic action, produced probably by the precipitation of the calcium.

The compound acids-such as ethyl-sulphuric, etc--act like organic acids. The aromatic acids act partly as acids, but this action is greatly obscured by their collapse action.

The irritant action of the acids is also shared to some extent by the acid salts, acid tartrates, acid sulphates, etc.

Manner of Action.- This varies to some extent with the constituents of the tissues. But, on the whole, it consists, with concentrated acids, in withdrawal of water; in the formation of acid albumins; in softening of the connective tissue and epithelium; and in special situations, in solution of calcareous material.

All the concentrated acids have an affinity for water, and withdraw this from the cells. This affinity is so strong in the case of concentrated $\mathrm{H}_{2} \mathrm{SO}_{4}$ that not only the formed water is withdrawn from the tissues, but the elements $\mathrm{H}$ and $\mathrm{O}$ are split off from their chemic combinations with carbon, leading to carbonization.

All acids convert proteins into acid-albumins, which are insoluble in moderately strong, but soluble in concentrated or very weak acids. Upon this precipitation of proteins depends their astringent and styptic action.

The connective tissue undergoes a rather peculiar change. It is not dissolved, but is softened and rendered more soluble in boiling water. (This explains why meat becomes more tender on keeping.) The concentrated acids have a similar effect upon epithelium. Without actually dissolving it, they soften it in such a manner that it is readily detached. Dilute acids, on the other hand, harden it.

Poisoning by Concentrated Acids.- This presents the usual phenomena of caustic poisoning. It is extremely painful. The "coffee-grounds" vomit is characteristic. Scar formation and stenosis are frequent. ${ }^{1}$ The prognosis and toxic dose depend upon the concentration, and the parts affected. The treatment has been discussed. Sulphuric acid poisoning is the most common.

Continued exposure to the vapors of acids, such as occurs in certain trades, gives rise to chronic bronchitis. They also attack the teeth and from these the necrosis may spread to the jaw, as with phosphorus.

Uses.-Because of the intense pain, deep penetration and extensive scar formation, acids are not desirable cauterizants. Nitric and tri-chlor-acetic acid form relatively firm eschars, so that their action is more circumscribed and less painful. Sulphuric 1 See v. Hoffmann's Atlas of Legal Medicine, Plates 33, 34, 35, 36 and 37 . 
acid has been made into a paste with various inert jowders (charcoal, sawdust, lead sulphate, etc.) to localize its action, but is still painful and difficult to control (Pusey, I913). Lactic acid has a relatively mild action on normal tissues. Chromic acid may cause poisoning when used as a caustic.

Antiseptic Action.- The destruction of proteins makes acids efficient antiseptics Even quite dilute solutions, such as the gastric juice, suffice to limit the growth of bacteria. The concentrated acids destroy them outright.

\section{PREPARATIONS-CAUSTIC ACIDS}

Acidum Hydrochloricum (Acid. Hydrochlor.), U.S.P., B.P.; Hydrochloric Acid (Muriatic Acid).-About 32 per cent. of $\mathrm{HCl}$. Colorless, fuming liquid. Pungent odor. The commercial acid. (strength $=30$ per cent. to 33 per cent.) has a golden yellow color due to $\mathrm{Fe}$ and free $\mathrm{Cl}$. Since it often contains As, it should not be used in internal medicine.

Acidum Lacticum (Acid. Lact.), U.S.P., B.P.; Lactic Acid-A liquid containing Lactic Acid (optically inactive alphahydroxypropionic acid, $\mathrm{CH}_{3} \mathrm{CHOH}$.COOH) and lactic anhydrides. A colorless, or slightly yellow, syrupy liquid, nearly odorless, acid taste, absorbing moisture on exposure to air. Miscible with water, alc. or ether. Dose, 2 c.c., 30 minims, U.S.P.; I to 2 c.c., 30 to 60 minims, B.P. Used as caustic, especially for dissolving diphtheritic membranes.

* Acidum Nitricum (Acid. Nit.), U.S.P., B.P.; Nitric Acid.-About to per cent. of $\mathrm{HNO}_{3}$. Fuming liquid, very caustic and corrosive. Peculiar somewhat suffocating odor. Used as a caustic for warts (glass rod); against hyperhydrosis of feet ( 1 to 2 ounces to pail of water); as disinfectant (will corrode metal vessels or pipes).

Commercial nitric acid contains about 60 to 64 per cent.; the "fuming" acid is almost absolute $\mathrm{HNO}_{3}$, saturated with $\mathrm{NO}_{2}$.

Acid. Nitrohydrochl., U.S.P.; Nitrohydrochloric acid (Nitromuriatic acid, Aqua Regia).-A strong solution containing hydrochloric acid, nitric acid, nitrosyl chlorid and chlorin. Made by mixing I 8 c.c. of nitric acid with 82 c.c. of hydrochloric acid. A golden-yellow, fuming, and very corrosive liquid; strong odor of chlorine. Dose, 0.2 c.c., 3 minims, U.S.P., diluted.

Acid. Nitrohydrochl. Dil., U.S.P.-Nitric acid, ro c.c.; Hydrochloric acid, $45 \cdot 5$ c.c.; Water, I94.5 c.c. Colorless or pale yellowish liquid. Faint odor of chlorine. Dose, I c.c., ${ }_{5}$ minims, U.S.P., diluted. On account of the free chlorin, it may be supposed to have a stronger local irritant action than $\mathrm{HCl}$. It is popularly supposed to "stimulate the liver," but its action does not differ in kind from that of other acids.

Acid. Nitro-hydrochl. Dil., B.P.-Nitric acid, 24 c.c.; Hydrochloric acid, 32 c.c.; Water, 200 c.c.; kept fourteen days before use. Dose, $0.3 \mathrm{I}$ to r.2 c.c., 5 to $20 \mathrm{minims}$, B.P., diluted.

Acidum Osmicum, Osmic Acid, $\mathrm{OsO}_{4}$.- Intraneural injections are used to produce degeneration of nerves for the relief of persistent neuralgias (Billroth and Neuber, 1885). The treatment is best applied by injecting $1 / 2$ to $x$ c.c. of a fresh 1 to 2 per cent. solution directly into the, preferably exposed, nerve. The result is sometimes immediate, but more commonly it is not complete until after one or two weeks, or it may even fail entirely. If successful, the effects persist at least for several months, when it may become necessary to repeat the injection. They are rarely if ever permanent. The local reaction is always painful but not serious. It is probably not advisable to use osmic acid in the presence of renal disease (Eastman, rgo6).

Acid. Sulphuricum (Acid. Sulph.), U.S.P., B.P.; Sulphuric Acid.-About 95 per cent. of $\mathrm{H}_{2} \mathrm{SO}_{4}$. An oily, colorless liquid, acquiring a brown color if exposed to dust. - Very intensely corrosive, charring organic substances. Miscible in all proportions with water or alc. with the evolution of much heat. (Such mixing must be done very cautiously by slowly pouring the acid into the water, under constant stirring.) The Commercial Sulphuric Acid (Oil of Vitriol) is very apt to contain arsenic, and should not be employed in medicine.

Acidum Trichloraceticum (Acid. Trichloracet.), U.S.P.; Trichloracetic Acid.-A monobasic organic acid, $\mathrm{CCl}_{3} \mathrm{COOH}$. Colorless, deliquescent crystals, having a slight, characteristic odor. Very sol. in water or alc. Used in substance or strong solution, especially against warts, less painful than nitric acid.

\section{DILUTE ACIDS}

Local Effects.-Dilute acids produce a mild irritation, and at the same time harden the epithelium, without destroying it. They differ from the volatile organic irritants in that they do not penetrate so deeply, and do not cause nephritis. 
They may be used in the form of baths, in the strength of about 30 c.c. ( 1 ounce) of concentrated acid to the bath ( 30 gallons). Or they may be applied as lotions. In this case the volatile acids are preferred, because their action is deeper. Formic acid has a special reputation. It is used in the strength of about 4 per cent. or 5 per cent. in alcohol.

This stimulation of the skin without destruction of epidermis is used to increase the amount of sweat; and acids, usually in the form of vinegar, are therefore used for sponging in fever. On this account they have received the name of "refrigerants." On the other hand, they are used in excessive secretion of sweat (sweating feet) to harden the epidermis. For this purpose 5 or Io c.c. of concentrated hydrochloric acid are put in a basin of water and the feet placed in this until they become painful. 'This is done about twice a week.

The irritant action of the volatile acids is sometimes employed to produce reflex stimulation of the central nervous system by inhaling vinegar, etc.

Acidum Formicum.-Formic Acid, $\mathrm{HCO}_{2} \mathrm{H}$.

\section{THE HALOIDS}

These comprise bromin, iodin, clilorin, and the hypochlorites.

Their corrosive action is determined by their entering very easily into chemic reaction with all kinds of organic substances, taking from them hydrogen and forming hydrobromic, hydrochloric, and hydriodic acids, which have the ordinary acid actions. If water is present they set free oxygen in the form of ozone, by combining with the hydrogen of the water; this is also a strong irritant. The halogens furthermore enter directly into the protein molecule.

Chlorin is discussed under the disinfectants.

\section{IODIN}

Uses.-The tincture or solution, painted on the skin, produces a mild but deep and persistent irritation which can be easily graduated by successive applications. This irritation is used to secure the correction of chronic inflammations and the absorption of inflammatory exudates (pleura, joints, etc.); also as an antiseptic; and formerly for securing adhesive inflammation of serous cavities and cysts. The internal uses are discussed under "Iodids."

Antiseptic Action.-This is used especially for disinfecting wounds, and for preparing the skin for operations (Woodburg, 1907). For the latter purpose, the official tincture is painted over the operative field on the preceding day, and again just before operating. The action is attributed to the formation of antiseptic compounds with the tissues (Schumacher, I9I5). Bacterial tests from the skin have given equivocal results; clinically, the results are excellent, even dirty wounds healing rapidly. Experimental results with infected wounds were also good, the iodin being effective within five hours after infection, i.e., until the bacteria have spread too deeply (Brunner, I9I5). The stain may be removed by warm concentratèd solution of sodium thiosulphate (Snoy, I9II). Sometimes there is severe irritation (Herzog, I9I4).

Local Phenomena.-Applied to the skin iodin produces a mahogany color, smarting, erythematous inflammation, infiltration of subcutaneous tissue, desquamation of the epidermis in large shreds; if repeated, vesication results. The effects on mucous membranes are more severe and may produce corrosion. Its action is chemical, since it precipitates proteins as easily dissociated compounds. It is absorbed from the skin fairly readily, and excreted mainly in the urine as iodid.

Employment as Counterirritant.-Iodin is used especially in chronic pleural effusions, chronic rheumatism, diseased joints, enlarged lymph glands, lymphangitis, etc. It has also been employed in erysipelas, painting the tincture from the periphery toward the center, five or six times daily (Ferrari, I9I I); but Wood warns that the action must be graded very mildly, or it will produce extensive necrosis. 
In jections into cysts, hydrocele, empyema, etc., produce at first increase of the fluid, then athesive inflammation. The injections are very painful, and have of ten caused serious poisoning. 'They are now displaced by surgical procedures.

Toxic effects from iod in absorption from cysts consist in cyanosis, salivation, vomiting, skin eruptions, high fever, and collapse. The urine is scanty and albuminous. The intoxicition may persist for several days and then end with sudden death.

The toxic effects of swallowing iodin are successively: gastric uneasiness; disagreeable metallic taste; violent vomiting; abdominal pain; and sometimes purging. 'The vomit has the color of iodin, or is blue if starch is present.

The fatal dose corresponds to about 2 or $3 \mathrm{Gm}$.; but recovery is said to have occurred after Io Gm.

The treatment of iodin poisoning consists in evacuation; demulcents (eggs, milk, oils, and cooked flour or starch, which form loose compounds with iodin); dilute alkalies $\left(\mathrm{NaHCO}_{3}\right)$; and 5 per cent. sodium thiosulphate ("Hypo"). The last binds the iodin: $2 \mathrm{Na}_{2} \mathrm{~S}_{2} \mathrm{O}_{3}+2 \mathrm{I}=2 \mathrm{NaI}+$ $\mathrm{Na}_{2} \mathrm{~S}_{4} \mathrm{O}_{6}$ (Sabbatani, I9I3). The further treatment would be symptomatic.

Circulation.-Perfusion of excised frog hearts with dilute iodin solutions produces irregularities, followed by depression. Stronger solutions result in diastolic arrest. Intravenously, it depresses the circulation greatly in cats, not in dogs (Salant and Livingston, I916).

Toxic Effect in Mammals.-Boehm, 1876 , found that the intravenous infection of $0.04 \mathrm{Gm}$. per $\mathrm{Kg}$. is fatal. Symptoms set in only after 6 or 8 hours, with exhaustion, dyspnea, and death in 12 to 24 hours. Necropsy shows bloody pulmonary and pleural exudate and renal congestion and hemorrhages,

\section{PREPARATIONS-IODIN}

Iodum, U.S.P., B.P.; Iodin, r. Bluish black, friable scales, of distinctive odor and acrid taste. Very slightly sol. in water $\left(\mathrm{I}: 295^{\circ}\right)$; sol. in alc. ( $\left.\mathrm{I}: \mathrm{I} 2.5\right)$, or glyc. ( $1: 80$ ); freely sol. in fat solvents or aqueous solutions of iodids. Dose, $5 \mathrm{mg}$., $1 / 12$ gr., U.S.P., diluted; maximal, $30 \mathrm{mg} ., 1 / 2 \mathrm{gr}$.

* Tr. Iodi, U.S.P.; Tincture of Iodine.-7 per cent. iodin; 5 per cent. KI; in alc. Dose, O.I c.c., I $1 / 2$ minims, U.S.P., diluted; maximal, 0.3 c.c., 5 minims. The KI in the tincture makes it miscible with water and insures against loss of strength on keeping (Wetterstroen, 1908). (When iodin is administered in milk, it is largely converted into iodid and albuminates),

*The principal incompatibilities of iodin are: alkaloids; alkalies and carbonates; tannin; turpentine and volatile oils.

* Tr. Iodi Fort., B.P.- ro per cent. iodin, 6 per cent. KI.

Tr. Iodi Mit., B.P.-2.5 iodin, 2.5 per cent. KI. Dose, 0.12 to 0.3 c.c., 2 to 5 minims, B.P., diluted.

Liq. Iodi Co., U.S.P. (Lugol's Solution). -5 per cent. iodin, ro per cent. KI, in water. Dose, 0.2 c.c., 3 minims, U.S.P., diluted.

Ung. Iodi, U.S.P., B.P. - 4 per cent. each of Iodin and KI, in lard base.

\section{BROMIN}

This is a reddish brown, very volatile and intensely corrosive liquid. Only a few instances of swallowing bromin are recorded. In one of these (Snell, r850), death occurred seven and one-half hours after taking an ounce. The antidotes would be eggs and starch.

Inhalation of bromin vapors is very irritant to the respiratory organs. I : $1,000, \infty 00$ of air is already disagreeable; to: $1,000,000$ is said to be dangerous.

\section{THE LOCAL ACTIONS OF METALLIC SALTS}

Mechanism of Action.-Metallic salts combine with proteins to form more or less difficultly soluble compounds containing varying amounts of the metal and proteins. 
The acid of the metallic salt is thus set free. If, for instance, a solution of ferric chlorid is added to egg-albumen, the result is an albuminate of iron, and free hydrochloric acid. This free acid will exert its own irritant action.

The local effects of metallic salts, therefore, rest on two factors: the precipitant action of the metal, and the irritant action of the liberated acid. Both influence the total effect.

Corrosive and Astringent Metallic Salts.-Some of the metal albuminates are almost insoluble in water; some are soluble in excess of protein. especially when neutral salts are present; others are not. If the precipitate is soluble, there is no obstacle to the penetration of the metal, and its action, irritant or caustic, is deep. If, on the other hand, the precipitate is insoluble, as in the case of lead salts, penetration can not take place; the irritation is confined to the surface, and an astringent action results. In regard to this, the metals stand in about the following order: The most astringent is lead; then comes aluminum; then iron; then zinc, copper, silver, and tin, which stand about on a level; the most caustic is mercury. As to the liberated acids, the strongest caustic action appears in hydrochloric acid; then comes nitric acid; then sulphuric; then phosphoric; the weakest of all are the organic acids-acetic, citric, and tartaric.

By proper combination, then, between the metals and the acids, one may obtain any grade of action from pure caustic to pure astringent.

The most typical caustic would be mercuric chlorid, the most typical astringent, lead acetate.

The strength of action will, of course, also depend upon the concentration in which the salt is used, and this is often limited by its solubility. The chlorid of silver would, theoretically, be a stronger caustic than the nitrate, but since it is not soluble, it can not be usedin the same concentration.

It must not be forgotten that the irritant action, the astringent action, and the caustic action, are merely degrees of the same process. The astringent action always precedes the caustic action; and, consequently, by proper dilution, one may obtain astringent effects from salts which are ordinarily purely caustic. For instance, silver nitrate can be so graduated in strength as to have a purely astringent action, without any caustic effect whatever.

It is, therefore, impossible to establish a perfectly definite classification between the metallic salts. An approximation to it is given in the following table:

Mainly Caustic:' All Hg salts; $\mathrm{ZnCl}_{2} ; \mathrm{SnCl}_{4} ; \mathrm{SbCl}_{3}$; tartar emetic; $\mathrm{CuSO}_{4}$.

Both Caustic and Astringent: Fe salts; $\mathrm{ZnSO}_{4} ; \mathrm{ZnAc}_{2}{ }^{*} ; \mathrm{CuAc}_{2} ; \mathrm{AgNO}_{3}$; $\mathrm{Pb}\left(\mathrm{NO}_{3}\right)_{2} ; \mathrm{PbI}_{2}$.

Mainly Astringent: Alum; $\mathrm{PbAc}_{2} ; \mathrm{Pb}_{2} \mathrm{OAc}_{2} ; \mathrm{ZnO}$. Bi subnitrate; white precipitate.

The Caustic Action of Metallic Salts.- This was formerly used quite extensively, but it has now been largely abandoned. Most are not sufficiently powerful for this purpose; others, again, are too toxic, being absorbed in sufficient amount to produce poisoning. To the latter class belong arsenic, antimony, and mercury. Zinc chlorid and antimony chlorid (Butter of Antimony) are very active caustics, but rather too diffluent. Their scab is so soft that their action can not be kept within bounds. In fact, of all the metallic caustics, silver nitrate in the form of sticks (Lunar Caustic), and to a less extent copper sulphate, are alone used to produce a purely caustic action. Arsenic, were it not for its toxicity, would be a very useful corrosive. Its action is so slow that it can be very readily limited. It was believed to destroy only pathologic formations, leaving healthy tissue intact. This would be easily understood, from the fact that the former are much less staple. Silver nitrate is also quite easily controlled, since its action may be stopped at once by washing with $\mathrm{NaCl}$, which converts it into $\mathrm{AgCl}$.

Astringent Efficiency.-The most actively astringent metallic salt, lead acetate, can not be used internally, nor for any length of time externally, on account of the danger of chronic poisoning. Next in activity comes alum, and especially the burnt alum (alum which has been roasted, so as to deprive it of its water of crystallization, and which therefore acts not only as a metallic astringent, but mechanically by withdrawing water). Next to alum, come the soluble zinc salts, the sulphate, the acetate, and the sulphocarbolate. Then, after these, insoluble zinc salts, oxid and carbonate. Of other insoluble metallic salts the subnitrate of bismuth and 
the oxalate of cerium are most commonly used. Then come the caustic salts in proper dilution. The most important is silver nitrate. Then the iron salts in dilute solution; iron sulphate, about 5 per cent.; ferric chlorid, about 3 per cent.

In actual use, these different astringents are frequently combined. Whether this has any advantage is somewhat difficult to say. Better results could perhaps be secured by using only one astringent, since its action could be much more exactly controlled.

Therapeutic Applications.-For use on open wounds, ulcers, abscesses, etc., for the astringency and a mild nutritive stimulation leading to repair, silver nitrate is the most useful. Next to this, the soluble zinc salts; then alum. They are used in strengths of from $1 / 2$ per cent. to 5 per cent. The insoluble astringents may be used as dusting-powders, or in the form of ointments -5 per cent. to 20 per cent. It must not be forgotten that absorption is fairly free from open surfaces, and calomel, bismuth, lead, etc., must be used with caution. Zinc oxid is quite safe, and is one of the most useful.

The mucous membranes which are easily accessible to the local action of astringents are those of the mouth, conjunctiva, nose, genito-urinary tract, and rectum. The same salts as in the case of open wounds can be used, as also tannin. They are employed in somewhat weaker solution, as gargles, washes or injections. The usual strength is from $1 / 2$ to I per cent. For vagina or rectum, double this; in the conjunctiva and nose, perhaps one-fourth of this. The strength, as with all local medication, must be adjusted to the anatomic peculiarities of the surface: It should be very different for the cornea and for the plantar surface of the foot. In the case of the genito-urinary tract, irritation is particularly undesirable. For this reason non-irritant protein compounds of silver have become popular.

Astringents cause actual constriction of the mucous membranes, and may in this way bring about the complete disappearance of small polypi.

In the alimentary canal the astringents are useful mainly in lessening the reflexes resulting from inflammation; i.e., the vomiting and diarrhea.

Against vomiting, especially when caused by ulceration, bismuth subnitrate or subcarbonate seems to be the most useful. These act not only in virtue of their astringency, but also somewhat after the manner of inert dusting-powder, affording an artificial protective covering to the walls of the viscus by adhering to them. Silver nitrate is also sometimes used in doses of about I cg. ( $1 / 6$ grain), dissolved in water and given three times a day.

Their action on diarrhea is entirely similar. Bismuth is again preferred; silver nitrate is often very useful in the summer diarrhea of infants.

Further details will be found under the respective metals.

\section{TANNINS AND VEGETABLE ASTRINGENTS}

Actions and Uses.-The tannins are a group of widely distributed vegetable principles, which percipitate proteins and are therefore astringent; and which give blue or green compounds with iron salts. They act more mildly than the metals; and being practically non-toxic they are especially suited for use in the alimentary canal. They do not decrease peristalsis directly, but do so indirectly, by allaying the underlying inflammation. Their employment in diarrhea dates back beyond the fourth century B. C. 
The official "tannic acid" is derived from nut-galls. Free tannin would be too irritant to the stomach; so that its action must be slowed, by the presence of extractives, as in the crude plant extracts (Gambir, Krameria, Kino); or by delaying its solution through combination with proteins (Tannalbin); or by esters, which are but slowly decomposed in the intestines.

In the course of its absorption, tannin is decomposed into the nonastringent gallic acid, pyrogallol, etc.

Varieties of Tannin.-Ordinary "tannin" or gallo-tannic acid is derived chemically by the elimination of a molecule of $\mathrm{H}_{2} \mathrm{O}$ from 2 molecules of gallic acid (Tri-hydroxybenzoic acid, $\left.\mathrm{C}_{6} \mathrm{H}_{2} .(\mathrm{OH})_{3} \cdot \mathrm{COOH}\right)$. The numerous other varieties are of different composition, in many cases unknown. Some are compounds of gallic acid with sugar or with phlorhizin. Their gross classification has been discussed under the general "Chemistry of Plants."

Constipative Action. - This has been studied on cats with the X-ray method by Hesse, 1913. In the absence of diarrhea, tannalbin has no effect on the intestinal movements beyond a slight delay in the emptying of the stomach; nor had it any influence on the diarrheas produced by milk-feeding or senna; and but little on the catharsis of castor oil. However, it arrested certain dietetic diarrheas (bread), and that produced by the colonaction of colocynth. Its action is therefore not on peristalsis, but on the inflamed mucosa.

Pharmacologic Peculiarities. - Tannins form more or less insoluble compounds with many metals, alkaloids, glucosids, etc., and are therefore useful as antidotes. They also precipita te proteins, gelatin and connective tissue (leather), and thus act as astringents, styptics, and antiseptics (mainly by depriving the bacteria of food). The different tannins are not equivalent in these respects. Some (which are perhaps misnamed), such as those of coffee and ipecac, are practically non-precipitant. Others present differences in the firmness and solubility of the precipitate, which, when better known, may prove of therapeutic importance.

Fate.-In the stomach, tannin precipitates the proteins in the form of tannates, which are decomposed by further digestion, again setting free active tannin. In the intestine, a part of the free tannin combines with the alkali. to form the non-precipitant alkali tannates. The further fate is imperfectly known; but all of the tannin, even if injected intravenously, undergoes decomposition into gallic and pyrogallic acid and other decomposition products, before being excreted. These appear both in the urine and feces (E. Rost, I 897; the older contradictory statements were based on faulty tests for tannin). Only the insoluble tannates (Tannigen, Tannalbin, etc.) pass in part undecomposed into the feces. The decomposition must, therefore, take place to a large extent in the intestines. With oral administration, the urine contains at most I per cent. of the gallate of the tannin.

The use of tannin as antidote has been discussed under "General Toxicology."

The insolubility of tannin compounds has been utilized in securing a more prolonged local action of the kations. It will be remembered that this is one reason for the more lasting local effects of galenics as compared with alkaloids. It has been suggested to prepare such combinations artificially, but these have not come into general use.

The effect of the continued administration of small amounts of tannins has considerable importance, because they are contained in a number of beverages; as tea and certain wines. A mild astringent action may be locally tonic and beneficial; but larger quantities prove actually irritant, and may lead to gastroenteritis.

Even small amounts of tannin interfere somewhat with absorption. This is largely due to their precipitating proteins. But these combinations are again decomposed in the alkaline intestine, so that the interference is not large.

In the actual experiments of Biberfeld ( 1903 ) on the absorption of normal saline solution from a Vella fistula, this was accelerated by 0.01 per cent. of tannin; 0.04 per cent. had no effect; O.I to I per cent. delayed absorption.

On the whole, one may say that the small quantities of tannin ordinarily taken with the food and drink are not injurious; but that large quantities (excessive tea drinking) are certainly deleterious. The tannin of coffee is scarcely astringent, and therefore lacks this action.

Effects of Larger Doses.-These produce irritation and corrosion, especially if the stomach is empty; pain, vomiting, diarrhea or obstipation. 
Incompatibility.-Tannins are incompatible with alkaloids, alkalies, iron and many other metals; also with proteins and gelatin.

\section{PREPARATIONS-TANNIC AND GALLIC ACIDS}

* Acidum Tannicum (Acid. Tann.), U.S.P., B.P.; Tannic Acid (Tannin, Gallotannic Acid, Digallic Acid); $\mathrm{HC}_{14} \mathrm{H}_{9} \mathrm{O}_{9}-\mathrm{A}$ tannin obtained from nut-gall. Very sol. $(\mathrm{r}: \mathrm{I})$, in water glyc. and alc. Almost insol. in eth. or chlorof. Watery solutions gradually spoil on keeping, although they still color iron. Incompatible with alkalies, alkaloids, salts of iron and most other metals, proteins and gelatin. Dose, 0.5 Gm., 8 gr., U.S.P.; 0.3 to 0.6 Gm., 5 to Io gr., B.P. Lacally, for urethra, I to 2 per cent.; vagina, 5 per cent.; enema, I to 2 per cent.; rectal irrigation (cholera) $1 / 4$ to $1 / 2$ per cent.; skin and ulcers, 5 to 20 per cent., or the Glycerite.

* Glycer. Acid. Tann., U.S.P., B.P.-2o per cent.

Ung. Acid. Tann., U.S.P.—-2o per cent.

Supp. Acid. Tann., B.P.-0.2 Gm., 3 gr. (against hemorrhoids).

Troch. Acid. Tann., U.S.P.-0.06 Gm., I gr.

Troch. Acid. Tann., B.P.-0.03 Gm., 1/2 gr.

* Tannalbin (N.N.R.).-Albumin tannate, containing about 50 per cent: of tannic acid (Gottlieb, I896). Light brown, odorless and tasteless, powder, practically insoluble in water and gastric juice. Dilute alkalies (intestinal and pancreatic juice) liberate the tannin slowly. Used in diarrhea, especially of children. Dose, I to $4 \mathrm{Gm}$. (15 to $60 \mathrm{gr}$.) in powder or tablets, followed by water; infants, 0.3 to $0.5 \mathrm{Gm}$. in gruel.

Other Artificial Tannin Compaunds of N.N.R. are: Tannigen, Diacetyltannin ( $\mathrm{H}$. Meyer, 1894); insoluble in stomach, soluble in intestine; also Tannopin, Tannoform, Eugallol, Gallogen, Protan.

Acidum Gallicum (Acid. Gall.), U.S.P.; $\mathrm{C}_{2} \mathrm{H}_{2}(\mathrm{OH})_{3} \mathrm{CO}_{2} \mathrm{H}+\mathrm{H}_{2} \mathrm{O}$.--Occurs in many plants, usually with tannic acid. It does not precipitate alkaloids, albumin, or glue. Sol. in water ( $1: 87$ ); freely sol. in alc. (I : 4.6) or glyc. (I : 10). Dose, I Gm., r5 gr., U.S.P. Externally, I per cent., as weak astringent.

\section{TANNIN-DRUGS}

These are very numerous, and many might well be dispensed with. Gambir (Catechu) would fulfill all indications.

Acacia Cortex, B.P.; Acacia Bark.-The dried bark of Acacia arabica and decurrens. Astringent and demulcent.

Dec. Acac. Cort., B.P. 6 per cent. Dose, 15 to 60 c.c., $1 / 2$ to 2 ounces, B.P.-

Bela Fruct., B.P.-The fresh fruit of Aegle Marmelos.

Ext. Bela Liq., B.P.-Dose, 4 to 8 c.c., 1 to 2 drams, B.P.

Butea Sem., B.P.- The seeds of Butea frondosa.

Butea Gum., B.P. (Bengal Kino).-The inspissated juice of Butea frondosa.

Galla, U.S.P., B.P.; Nut-gall.-An excrescence on the young twigs of Quercus infectoria and other allied species of Quercus (Oak), induced by the punctures on the leafbuds and by the deposited ova of a wasp, Cynips tinctoria. Contains 50 to 60 per cent. of tannin, 2 to 5 per cent. of gallic acid. Dose, $0.5 \mathrm{Gm}$., 8 gr., U.S.P.

Ung. Gall., U.S.P., B.P.-2o per cent.

Ung. Gall. C. Opio, B.P.-The preceding, with 7.5 per cent. of powdered Opium.

Catechu, B.P.; Catechu (Pale); identical with Gambir, U.S.P.-A dried extract of the leaves of young shoots of Uncaria Gambir. B.P.

* Tr. Catechu, B.P. - 20 per cent., with Cinnamon. Dose, 2 to 4 c.c., $1 / 2$ to 1 dram,

Pulv. Catech. Co., B.P.-Catechu, 40 per cent.; Kino and Krameria, each 20 per cent.; Cinnamon and Nutmeg. Dose, 0.6 to $4 \mathrm{Gm}$., ro to $60 \mathrm{gr}$., B.P.

Troch. Catech., B.P. -0.06 Gm., 1 gr.

Catechu Nigrum (Catech. Nigr.) B.P.; Black Catechu.-An extract from the wood of Acacia Catechu.

Embelia (Embel.), B.P.-The dried fruit of Ribes Embelia and robusta. Dose, 4 to $\mathrm{I} 6 \mathrm{Gm}$., 60 to $240 \mathrm{gr}$., B.P.

Gambir, U.S.P.; Gambir (Pale Catechu); identical with Catechu, B.P.-A dried extract prepared from decoctions of the leaves and twigs of Ourouparia Gambir. Contains 33 to 47 per cent. of catechu-tannic acid. Dose, I Gm., 15 gr., U.S.P. 
* Tr. Gambir Co., U.S.P. (Compound Tincture of Catechu). 5 per cent. in jo per cent. alcohol; flavored with cinnamon. Dose, 4 c.c., I dram, U.S.P.

Hamamclidis Cortex, B.P.; Hamamelis Bark (Witch Hazel Bark).-The dried bark of Hamamelis virginiana.

Tr. Hamam., B.P.- - Io per cent. of bark. Dose, 2 to 4 c.c., $1 / 2$ to I dram, B.P.

Hamam. Fol., B.P.; Hamamelis Leaves.-Fresh or dried. 8 per cent. of tannin (Straub, I899).

Ext. Hamam. Liq., B.P.-50 per cent. of dried leaves. Dose, 0.3 to I c.c., 5 to 15 minims, B.P.

Ung. Hamam., B.P.- - Io per cent. of the preceding.

Hamatoxyli Lignum, B.P.; Logwood.- The heart-wood of Hæmatoxylon campechianum. I2 per cent. of Hæmatoxylin.

Dec. Hematox., B.P. -5 per cent. Dose, I5 to 60 c.c., $1 / 2$ to I ounce, B.P.

Kino, U.S.P., B.P.; Kino.-The dried juice from the trunk of Pterocarpus Marsupium. 75 per cent. of Kino-tannic acid. Dose, $0.5 \mathrm{Gm}$., 8 gr., U.S.P.; 0.3 to $1.2 \mathrm{Gm}$., 5 to $20 \mathrm{gr} .$, B.P.

Tr. Kino, U.S.P., B.P.- - Io per cent. Dose, 4 c.c., I dram, U.S.P.; 2 to 4 c.e., $1 / 2$ to I dram, B.P.

Pulv. Kino Co., B.P. - 5 per cent. of opium. Dose, 0.3 to 1.2 Gm., 5 to 20 gr., B.P.

Kino Eucalypti (Kino Eucalyp.), B.P. (Eucalyptus Gum, Red Gum).-An exudation from the stem of Eucalyptus species. Dose, 0.3 to $1.2 \mathrm{Gm} ., 5$ to 20 gr., B.P.

Troch. Kino Eucalypti, B.P.—0.06 Gm., I gr.

Kramer. Rad., B.P.; Krameria (Rhatany).-The dried root of Krameria species.

Ext. Kramer., B.P.-A dry extract. Dose, 0.3 to I Gm., 5 to 15 gr., B.P.

Tr. Kramer., B.P. - 20 per cent. Dose, 2 to 4 cc., $1 / 2$ to I dram, B.P.

Inf. Kramer., B.P. -5 per cent. Dose, I5 to 30 c.c., $1 / 2$ to I ounce, B.P.

Troch. Kramer., B.P. $\rightarrow 0.06 \mathrm{Gm}$., I gr.

Myrobalanum, B.P., Myrobalans.- The dried immature fruit of Terminalia Chebula.

Dose, 2 to $4 \mathrm{Gm}$., 30 to $60 \mathrm{gr}$., B.P.

Ung. Myrobal., B.P.-2o per cent.

Ung. Myrobal C. Opio., B.P.-The preceding with 7.5 per cent. of Opium.

Sappan, B.P.-The heart-wood of Caesalpinia Sappan; similar to Logwood.

Dec. Sappan., B.P. $\rightarrow 5$ per cent. Dose, I 5 to 60 c.c., $1 / 2$ to 2 ounces, B.P.

Diarrhea Mixtures.-These are generally "shot-gun" prescriptions, of which the following are types:

Mistura Contra Diarrhaam, N.F. (Sun Cholera Mixture).-Equal parts of Tincture of Opium, Capsicum, Rhubarb, Camphor, Peppermint. Dose, to one teaspoonful. Or, Tr. Opium, Tr. Catechu, Tr. Rhubarb, Sp. Peppermint, Bismuth subnitrate. (History, Raubenheimer, I9I6).

\section{ASTRINGENT STYPTICS}

All the metallic salts, the irritant as well as the astringent, and also the vegetable astringents, act as local styptics; i.e., lessen local hemorrhage. They do so mainly by the formation of precipitates which occlude the lumen of the small vessels, just as it is occluded ordinarily by fibrin. (Whilst the majority lessen the formation of fibrin, this is offset by the precipitation.) Besides this precipitation, they also act by injuring the vessel walls in such a way as to produce thrombosis. This is claimed especially for zinc chlorid.

It is scarcely needful to mention that astringents will act only at the place to which they are applied. It is necessary that they come into actual contact with the bleeding vessels. They can not act through a large clot of blood, and if such exists, it must first be removed. At one time they were used internally with the idea of producing astringent action in remote places; iron was given by the mouth to check bleeding in the uterus. This was entirely irrational. Their action can not even extend beyond the stomach, since they are precipitated or decomposed in the intestine.

The indications for the use of styptics are to lessen bleeding, especially capillary oozing. They are sometimes injected into hemorrhoids, and have even been injected into aneurisms. Their injection into larger vessels is dangerous, as it may produce embolism. 
The most useful of the metallic styptics are the iron salts, especially the ferric chlorid and ferric sulphate. The ferric chlorid is used either as the solution, or tincture, quite largely diluted with water. Cotton may be steeped in this, forming "styptic cotton." Next comes alum, especially the burnt alum. Then the tannins in any form.

Besides these, any substance which gives a precipitate with proteins will act as a styptic in the same manner; c.g., dilute acids in concentrations which need not be at all caustic (vinegar and lemon juice). Quite a number of purely mechanical measures favor the formation of clot; for instance, ordinary cotton or Pengawhar Djambe (this also contains tannin). Cobwebs also form a popular and very effective measure for producing the same result, but are unfortunately very septic. One may obtain the same effect by fine powders which have a strong attraction for water. In case of emergency powdered or granulated sugar is a good styptic, and at the same time antiseptic. Other styptic measures are position, raising the limb and keeping it quiet so as to reduce the local congestion; local pressure; depression of the vasomotor center by narcotics; direct constriction of the vessels by the application of cold, or by drugs, such as cocain, suprarenal extract, hydrastinin, etc.

\section{STRENGTH OF MOST USEFUL SOLUTIONS OF ASTRINGENTS AND ANTI- SEPTICS ${ }^{1}$}

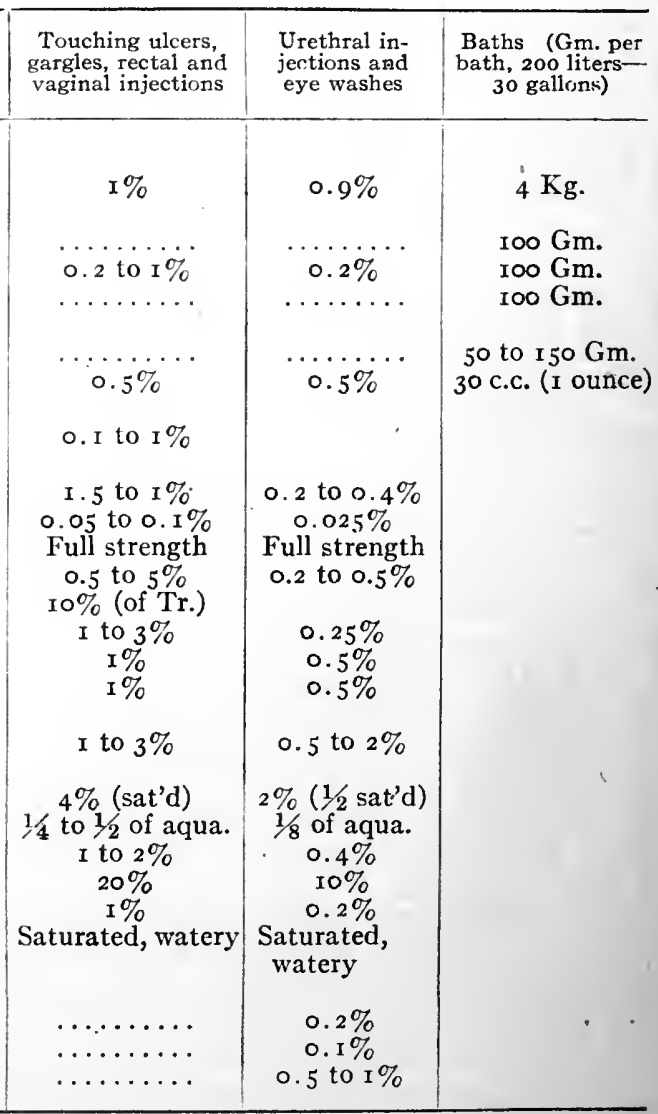

Sodii bicarbon..............

Sodii carbonas.

Potassii carbonas.

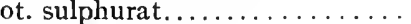

Acids (Mineral)..............

Haloids:

Iodin......................... . .

Zinc sulphate or phenolsulphonate.

Mercuric chlorid.............

Liq. plumbi subacet. dil. . . . . . . .

Silver nitrate. $\ldots \ldots \ldots \ldots \ldots \ldots \ldots$

Tr. ferri chloridi...............

Alumen and alum. salts.........

Cupric sulphate. .............

Lead acetate..............

Tannic Tannins:

Tannins:

Miscellaneous:

Boric acid, or borax...........

$\mathrm{H}_{2} \mathrm{O}_{2} \ldots \ldots \ldots \ldots \ldots \ldots \ldots$

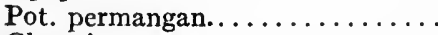

Glycerin.................

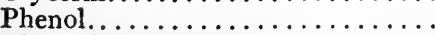

Thymol, essential oils..........

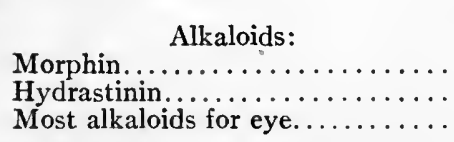

1 ( 1 per cent. $=5$ grains per ounce. $)$ When several are combined, the dose of each must be correspondingly decreased. 
Baths.-Usually taken in the evening before going to bed. Metal-lined tubs must be avoided for medicated baths.

Gargles.-No toxic substance should be lised, especially with children, on account of the danger of swallowing. The metallic salts attack the tecth, so that they can not be employed for a long time.

Urethral Injections. - Always have the patient urinate just before in jecting, to remove bacteria. Let injection remain at least one minute, then let fow out, but patient should not micturate immediately after.

\section{IRRITANT VOLATILE OILS AND RELATED DRUGS}

General Statement.-Most volatile (or "essential") oils are complex mixtures, generally containing terpenes and oxidized aromatic derivatives. The balsams and resins, which contain volatile oils, may be included in the group. These oils occur in numerous plants, and determine their use as flavors, aromatics, carminatives, antiseptics and irritants. Their chief application is for their local effects, consisting in deep irritation with a minimum of tissue destruction. The sensory stimulation is followed by some anesthesia. They exert a favorable influence on chronic inflammations; partly by their mild antiseptic and irritant action, and partly by chemotactic attraction of leucocytes. In acute inflammations they are more apt to do harm. Their antiseptic action is, in practice, relatively weak, since they are but slightly soluble in water.

After their absorption they are excreted mainly in combination with glycuronic acid. They irritate the kidneys and produce diuresis.

Central effects are only seen in poisoning, and agree broadly with those of camphor, which really belongs to this group. These effects are more or less convulsive, followed by narcosis; on direct application, stimulation of cardiac muscle; and curare action on the muscle-nerve endings.

The Flavoring and Aromatic Oils are considered in another place.

Composition.-A convenient tabulation of the constituents of volatile oils is given by Rippetoe and Wise, I9I2. The terpenes are aromatic, cyclic compounds of the general empirical formula, $\mathrm{C}_{10} \mathrm{H}_{16}$. Related to them are the semi-terpenes $\left(\mathrm{C}_{5} \mathrm{H}_{8}\right)$; sesqui-terpenes $\left(\mathrm{C}_{15} \mathrm{H}_{24}\right)$; and di-terpenes $\left(\mathrm{C}_{20} \mathrm{H}_{32}\right)$. Other constituents are oxidation products of these and aromatic phenols, ketones, aldehydes, acids and their compounds.

The Antiseptic Effects.-These have been investigated especially by Kobert, 1906; Bruning, I906; Geinitz, I9 2 ; and Rippetoe and Wise, rg 2.

Chemotactic Action.-The value of volatile oils (particularly those enumerated as urinary antiseptics) in chronic inflammations of all sorts has been abundantly proved by clinical observations and laboratory experiments. They also lessen aseptic inflammations at points remote from the site of their application, by decreasing the formation of exudates and by hastening their absorption. The explanation probably lies in a chemotactic attraction for leucocytes (Lémière, I 891; Borissow; Hamburger, I9I2). In this way they withdraw these cells from the inflamed area into the blood (Winternitz, IgOI).

Excretion.-Terpenes are for the most part excreted in the urine in combination with glycuronic acid (Matzel, 1905), sometimes after partial oxidation.

Systemic Actions of Volatile Oils. - The principal central effects of volatile oils are reflex. With the ordinary doses, no direct action whatever can be observed. Large doses act on the nervous centers, especially the brain and medulla. These are first stimulated, then depressed. The details have been investigated especially by Rimini, 1900; Hildebrandt, I901 and I902; Matzel, I905; and Geinitz, I912. The degree of stimulation varies for the different oils. Turpentine has scarcely any effect, whilst absinthe, thujon, feñchoñ, carvoñ and camphor produce violent epileptic convulsions. The depression is more uniform: The majority (valerian, fennel, chamomile, eucalyptus, mint, rosemary, sabinol, citral, turpentine) diminish the reflex excitability, so that large doses will entirely prevent strychnin convulsions in rabbits. The effective doses are, however, entirely too large to make it possible to employ this action in man. 
The Curare-actions have been studied by Hildebrandt and by Matzel; the cardiac effects by H. Schwalb, IgI 2.

According to d'Ormea (1903), the intravenous injection of volatile oils causes a peculiar dilation of the cerebrul vessels, the volume of the brain increases, whilst the pressure in the circle of Willis falls. This effect is strongest with absinthe, weak with anise or lemon. Camphor has a similar effect. The general blood pressure also falls in most cases, but quite independently of the changes in the cerebral circulation.

If volatile oils are injected hypodermically, they produce at first the reflex action, and in a more marked degree than when they are applied to the surface of the skin. Later their systemic, and still later the renal, actions take place.

Isolated Intestinal Segments are stimulated by oil of anise, turpentine and chenopodium, in concentrations of $1: 50,000$ to $1: 25,000$. Higher concentrations of all volatile oils depress the movements (Mruirhead \& Gerald, I9 16).

Hemolytic Action of Terpenes. - Ishizaka, I9I4, found that this varied mainly with the surface tension; but that the alcohol or ketone character also has an influence. Some form methemoglobin.

\section{OLFACTORY STIMULANTS}

Odorous substances produce pronounced reflex effects. Pungent and aromatic drugs cause in this way a prompt medullary stimulation, increasing the respiration and blood pressure, and slowing the pulse. They are especially useful in fainting. They are employed by inhalation. Tinctura Lavandule Composita (U.S.P., B.P.) and Aromatic Ammonia are used for this purpose.

Any pungent substance will answer in an emergency; the burning of a feather under the nose of the patient is a standard household measure.

Substances which produce sneezing (sternutatoria or errhines) act in a similar manner, but have rather passed out of fashion. They are sometimes useful as local counterirritants in nasal catarrh (snuff).

\section{HYSTERIC SEDATIVES OR ANTISPASMODICS}

Actions and Uses.-Asafetida, valerian, and some other malodorous drugs have been used since antiquity as sedatives in hysterical and similar nervous conditions. They are of ten effective, presumably by olfactory and psychic reflexes. Asafetida is also carminative, and has been used by mouth and enema (emulsion) in tympanites; but it imparts its odor to the excreta and eructations. Very large doses $(1 / 2$ ounce) have been taken without other noticeable effects. Large doses of valerian produce systemic action, but it is very doubtful whether these occur with the ordinary doses. Valerian was formerly used as a perfume, and as a spice for meat, and asafetida is said to be similarly employed in Persia.

\section{PREPARATIONS-HYSTERIC SEDATIVES}

* Asafetida (Asafet.), U.S.P.; Asafetida (Asafet.), B.P.; Asafetida.-The gumresin obtained by incising the rhizome and roots of Ferula Asafetida. Characteristiodor; bitter and acrid taste; forms milk-white emulsion when triturated with water. Constituents: 3 to 9 per cent. volatile oil; 20 to 30 per cent. gum; 45 to 70 per cent. resin. The alcoholic preparations yield turbid mixtures with aqueous liquids. Dose, 0.25 Gm., 4 gr., U.S.P.; 0.3 to I Gm., 5 to 15 gr., B.P.; best as pills.

Pil. Asafet., U.S.P.-0.2 Gm., 3 gr. Dose, 2 pills, U.S.P.

Emul. A safet., U.S.P. (Milk of Asafetida, Asafetida Mixture).-Made by rubbing 4 parts of asafetida with roo parts of water. Dose, I.5 c.c., 4 drams, U.S.P. Used especially as enema against tympanites.

Tr. A safel., U.S.P., B.P.- 20 per cent. Dose, I c.c., 15 minims, U.S.P.; 2 to 4 c.c., $1 / 2$ to I dram, B.P.

Sp. Ammon. Fet., B.P.-An alcoholic distillate of Asafetida, mixed with Ammonia. Dose, 1.2 to 2.5 c.c., 20 to 40 minims, repeated; single, 4 to 6 c.c., 60 to 90 minims, largely diluted, B.P.

Valeriana (Valer.)., U.S.P.; Valer. Rhiz., B.P.; Valerian.-The dried rhizome and roots of Valeriana officinalis. Dose, $2 \mathrm{Gm}$., $30 \mathrm{gr}$., U.S.P. Contains $1 / 2$ to 2 per cent. volatile oil, valeric and other organic acids; tannin and resins. The oil consists of esters 
of valeric (Valerianic) acia, especially with borneol. These are the bearers of the action, free valeric acid and its salts being quite ineffective. The fresh root is also ineffective, the esters being formed only during drying, by the action of oxidases (Kochmann, rgo4). The juice of the fresh plant also produces different effects from the dried valerian (Pouchet and Chevalier, 1905). The esters again deteriorate by oxidation on kecping. Certain synthetic esters are said to be more stable (Kionka, 1902).

The Injection of Valerian.-This produces the usual effects of volatile oils (Pouchet and Chevalier, Kionka, I904); in small doses, psychic exaltation and rise of blood pressure form cardiac and vasomotor stimulation; in larger doses, central sensory and motor depression.

Excised Uterus.-This is stimulated by Valerian oil (Pilcher, Igr6).

Tr. Valer., U.S.P.—2o per cent. Dose, 4 c.c., I dram, U.S.P.

* Tinctura Valeriance Ammoniata ('Tr. Valer. Ammon.), U.S.P., B.P.-2o per cent.; with Sp. Ammon. Arom., U.S.P.; with ammonia and aromatics, B.P. Dose, 2 c.c., 30 minims, U.S.P.; 2 to 4 c.c., $1 / 2$ to I dram, B.P.

Valer. Ind. Rhiz., B.P.-From Valeriana Walachii.

Tr. Valer. Ind. Ammon., B.P.Dos:, 2 to 4 c.c., $1 \frac{1}{2}$ to I dram, B.P.

Synthetic Valerian Esters (N.N.R.).-Bornyval (borneol isovalerate); Validol (mixture of menthol); and Valyl (valeryldiethyl-amin). Side actions, Seifert, Nebenwirk, 1915, p. 99 .

Valerates (Valerianates).-These have very little value. They are given in doses of 0.06 to I Gm. ( $\mathrm{I}$ to I $5 \mathrm{gr}$.), generally in capsules. They are colorless crystals of a valerianic odor, readily soluble in water; Zinci Valeras, U.S.P., R.P.; Ammonii Valeras, U.S.P.

Minor Drugs of this group.-Sumbul (Musk-root), Moschus (Musk), Castoreum, Cataria (Catnip); and Symplocarpus (Skunk-cabbage).

$M: s, k u$ s, U.S.P.; Musk. - The dried secretion from the preputial follicles of Moschus moschiferus. Dose, 0.25 Gm., 4 gr., U.S.P.

Tr. Moschi, U.S.P. 5 per cent. Dose, 4 c.c., I dram, U.S.P.

Sumbul, U.S.P. (Musk-root). -The rhizome and roots of Ferula Sumbul. Dose, 2 Gm., 30 gr., U.S.P.

Ext. Sumbul, U.S.P.-A pilular extract. Dose, 0.25 Gm., 4 gr.

Fldext. Sumbul, U.S.P.-Dose, 2 c.c., 30 minims, U.S.P.

\section{OIL OF TURPENTINE}

Actions and Uses.-Applied to the skin, this produces simple reddening and burning, and is used as a counterirritant; undiluted, or mixed with I to 5 parts of oil in liniments. It is often used as "Stupes," i.e., flannel wrung out of hot water, and then out of warm turpentine, and applied for ten to thirty minutes. In susceptible individuals it may cause severe eruptions, and if left on for several hours, blistering.

Oil of turpentine is used externally in bronchitis, neuralgic and rheumatic pains, and to relieve meteorism. It causes more severe irritation on mucous membranes.

Inhaled or taken internally it diminishes bronchial secretions (Rossbach and Fleischmann). It was used for this purpose (a teaspoonful inhaled from a pint of hot water) in chronic bronchitis. It is now displaced by the more pleasant terpene hydrate and pine-needle oil. Its use as anthelmintic ( 2 to 15 c.c.) and as diuretic and urinary antiseptic has also been discontinued.

Absorption, Fate and Excretion.-Oil of turpentine is readily absorbed from the skin. lungs and intestines. It circulates for a time unchanged in the blood, and is excreted mainly in the urine as terpenals, paired with glycuronic acid. The urine has the odor of violets. Neither turpentine, nor eucalyptol nor menthol, is excreted by the lungs or sputum (Falk, Hofbauer, I915).

Systemic Actions. - These do not occur with small doses.

Turpentine Poisoning.- Half an ounce has been fatal to children, 6 ounces to adults. The symptoms in man are those of gastro-intestinal irritation; nausea and vomiting, colic and diarrhea, wild excitement and delirium, ataxia, painful micturition, hematuria, albuminuria, and glycosuria, skin eruptions, coma. 
The Trealment.-This comprises evacuation, demulcents and coffee.

Inhalation.-Fatal poisoning has been reported from inhalation of varnish in a confined space (Drescher, r906); largely by exclusion of air (Wolf, I9II). There is rapid breathing, palpitations, vertigo, stupor, convulsions, and other nervous disturbances; pain in chest, bronchitis and nephritis. The latter may also occur on chronic exposure.

\section{PREPARATIONS-TURPENTINE}

* Oleum Terebinthina (Ol. Tereb.), U.S.P.; Oil of Turpentine (Spirit of Turpentine) -The volatile oil recently distilled with water, below $100^{\circ} \mathrm{C}$., from the concrete oleoresin (Turpentine) obtained from Pinus palustris and from other species. It consists mainly of pinen, $\mathrm{C}_{10} \mathrm{H}_{16}$, with small quantities of other terpenes and traces of organic acids. A thin, colorless liquid, of characteristic odor and taste, becoming stronger and more unpleasant by age and exposure to air. Practically insol. in water, sol. in 3 vol. of alc. and in all proportions of oils. The rectified oil should be employed for internal use.

* Ol. Tereb. Rect., U.S.P.; Ol. Tereb. Rectif., B.P.-Rectified (by distillation with. NaOH, U.S.P.) Dose, 0.3 c.c., 5 minims, U.S.P.; 0.12 to 0.6 c.c., 2 to to minims, B.P.; anthelmintic dose, $\mathrm{I} 2$ to $\mathrm{I} 5$ c.c., 3 to 4 drams, B.P.

Emul. Ol. Tereb., U.S.P.-I 5 per cent. Dose, 2 c.c., 1/2 dram, U.S.P.

Lin. Tereb., U.S.P.; Turpentine Liniment.-Cerat. Res., 2; Ol. Tereb., r.

Lin. Tereb., B.P.-OI. Tereb., 65 per cent.; Camphor, 5 per cent.; Sap. Moll. and water.

Lin. Tereb. Acct., B.P.-Ol. Tereb., 53 per cent.; Lin. Camph., 45 per cent.; Ac. Acet. Glac., I I per cent.

* Ol. Pini Pumilionis (Ol. Pin. Pumilion.), U.S.P., Oil of Dwarf Pine Needles; Oleum Abietis (Ol. Abiet.), B.P., Oil of Siberian Fir.-Colorless or faintly yellowish volatile oil, of pine-needle odor, distilled with steam from the fresh leaves of Pinus montana, U.S.P.; of Abies sibirica, B.P. Contains 1-pinene, other hydrocarbons, and esters. Resembles oil of turpentine, but has more agreeable odor, and is employed as inhalant in bronchial affections.

Terebinlhina Canadensis (Tereb. Canad.), B.P.; Canada Turpentine.-An oleoresin obtained from Abies balsamea.

\section{TERPIN HYDRATE}

This is used to lessen cough and expectoration in phthisis and in chronic bronchitis, acting similarly to oil of turpentine, and being less irritant, less disagreeable and non-toxic. It is also sometimes employed as diuretic and urinary antiseptic, but is not very effective.

Lepine, 1887 , claimed a diuretic effect from 0.2 to $0.4 \mathrm{Gm}$. Matzel, 1905, took 4 $\mathrm{Gm}$. daily without any effect; the urine was not albuminous. It was excreted as paired glycuronic acid.

\section{PREPARATIONS-TURPENTINE DERTVATIVES}

* Terpini Hydras (Terpin. Hyd.), U.S.P.; Terpin Hydrate, $\mathrm{C}_{10} \mathrm{H}_{18}(\mathrm{OH})_{2}+\mathrm{H}_{2} \mathrm{O}$ ).Prepared by hydrating oil of turpentine with alcohol and nitric acid. Colorless prisms, odorless, of slightly aromatic and somewhat bitter taste. Slightly sol. in water $(\mathrm{I}: 200)$; freely sol. in alc. ( $1: 12.5$ ). Dose, 0.25 Gm., 4 gr., U.S.P.; 0.1 to $1 \mathrm{Gm} ., 11 / 2$ to 15 gr., in powders or capsules. The N.F. Elixir contains $\mathrm{I}$ gr. to the drachm, in a vehicle of alcohol and glycerin.

Terebenum (Tereben.), U.S.P., B.P.; Terebene.-Dipentene and other hydrocarbons, obtained by the action of concentrated sulphuric acid on oil of turpentine. Colorless, thin liquid; rather agreeable odor; aromatic, somewhat terebinthinate taste. Slightly sol. in water; freely sol. in alc. $(\mathrm{I}: 3$ ). Dose, 0.25 c.c., 4 minims, U.S.P.; 0.3 to $x$ c.c., 5 to 15 minims, B.P., on sugar; or by inhalation.

\section{EUCALYPTOL}

Eucalyptol (Cineol, Cajeputol), $\mathrm{C}_{10} \mathrm{H}_{18} \mathrm{O}$, is obtained chiefly from oil of eucalyptus, but occurs also in other volatile oils, such as cajuput. It is a mild local irritant, used especially in bronchitis, as inhalation (teaspoonful in hot water), or internally ( 5 to ro drops on sugar); and in 
coryza (spraying with 3 to 5 per cent. solution in liquid petrolatum). The oil of eucalyptus may be substituted.

Eucalyptol imparts to the urine the same violet odor as does oil of turpentine, which it closely resembles in action. It has been used in malaria but is greatly inferior to quinin.

PREPARATIONS-EUCALYPTUS AND CAJUPUT

Eucalyptus, U.S.P. (Blue Gum Leaves).-The dried leaves of Eucalyptus Globulus. Dose, 2 Gm., 30 gr., U.S.P.

Fldext. Eucalypt., U.S.P.-Dose, 2 c.c., 30 minims, U.S.P.

*Ol. Eucalypt., U.S.P., B.P.-A volatile oil distilled from the fresh leaves of Eucalyptus species, yielding not less than 70 per cent. of eucalyptol. Colorless or pale yellow liquid. Dose, 0.5 c.c., 8 minims, U.S.P.; 0.03 to 0.18 c.c., $1 / 2$ to 3 minims, B.P.

Ung. Eucalypi., B.P.- Io per cent. of the oil.

${ }^{*}$ Eucalyptol, UU.S.P.; Eucalyptol (Cineol), $\mathrm{C}_{10} \mathrm{H}_{18} \mathrm{O}$.-An organic compound obtained from the volatile oil of Eucalyptus Globulus and from other sources. A colorless liquid; characteristic, aromatic and distinctly camphoraceous odor; a pungent spicy taste, and producing a cooling sensation in the mouth. Very sol. in alc. or oils practically insol. in water. Dose, 0.3 c.c., 5 minims, U.S.P.

Oleum Cajuputi (Ol. Cajup.), U.S.P., B.P.-A volatile oil distilled from the fresh leaves and twigs of several varieties of Melaleuca Leucadendron. Used mainly as counterirritant. Dose, 0.5 c.c., 8 minims, U.S.P.; 0.03 to 0.18 c.c., $1 / 2$ to 3 minims, B.P.

Sp. Cajup., B.P.—

\section{RUBEFACIENT VOLATILE OILS}

A large number of drugs containing volatile oils have been used as ingredients of liniments, but without serious advantage. With some of the tinctures (arnica, witch hazel) used in domestic medicine, the alcohol is perhaps the main active ingredient. Thymol, menthol, and camphor, and some of the volatile oils that are discussed in other connections could be classed with the rubefacients.

The oils of cloves, cinnamon, and creosote are employed in dentistry to destroy the nerves and to disinfect the cavities of carious teeth.

\section{PREPARATIONS}

Arnica, U.S.P.; Arnic. Flor., B.P.; Arnica.-The dried flower-heads of Arnica montana. Contains a volatile oil, small quantities of volatile acids, and an acrid bitter principle (Arnicin). Used externally. U.S.P.

Tr. Arnic., U.S.P. - 20 per cent. in 50 per cent. alcohol. Dose, I c.c., 15 minims,

Tr. Arnic. Flor., B.P.- - ro per cent. Dose, 2 to 4 c.c., $1 / 2$ to $\mathrm{I}$ dram, B.P.

Caryophyllus, U.S.P., B.P.; Cloves.-The dried flowerbud of Eugenia aromatica. Dose, 0.25 Gm., 4 gr., U.S.P.

Inf. Caryoph., B.P. -2.5 per cent. Dose, 15 to 30 c.c., $1 / 2$ to 1 ounce, B.P.

* Ol. Caryoph., U.S.P., B.P.; Oil of Clove.-The volatile oil, consisting mainly (82 per cent.) of eugenol." Dose, 0.2 c.c.; 3 minims, U.S.P.; 0.03 to 0.18 c.c., $1 / 2$ to 3 minims, B.P.

Eugenol, U.S.P.-An unsaturated, aromatic phenol $\left(\mathrm{C}_{6} \mathrm{H}_{3} \cdot \mathrm{C}_{3} \mathrm{H}_{5} \cdot \mathrm{OCH}_{3} \mathrm{OH}\right)$ obtained from oil of cloves and from other sources. Dose, 0.2 c.c., 3 minims, U.S.P.

ol. Cedrela.-Cedarwood Oil.

Aqua Hamamelidis (Aq. Hamam.), U.S.P.; Liq. Hamam., B.P.; Hamamelis Water, Solution of Hamamelis (Witch Hazel, Extract of Witch Hazel).-A saturated aqueous distillate obtained by distilling with steam or water the bark, twigs and smaller stems of Hamamelis virginiana and adding about 15 per cent. of alcohol, U.S.P. Clear or colorless or slightly yellowish liquid having a characteristic aroma and taste.

Ol. Origani.-Origanum oil.

Ol. Piment., U.S.P. (Oil of Allspice).-A volatile oil distilled from the nearly ripe IO 
fruit of Pimenta officinalis yielding not less than 65 per cent. of eugenol. Dose, 0.2 c.c., 3 minims, U.S.P.

Ol. Rosmarini (Ol. Rosmar.), U.S.P., B.P.; Oil of Rosemary.-Distilled from the fresh flowers of Rosmarinus officinalis. Dose, 0.2 c.c., 3 minims, U.S.P.

Sp. Rosmarin., B.P.-1o per cent.

Ol."Succini.-Oil of Amber.

Ol. Thymi, U.S.P.; Oil of Thyme.-Distilled from the flowering plant of Thymus vulgaris. Dose, 0.2 c.c., 3 minims, U.S.P.

\section{LINIMENTS}

Cutaneous irritants are usually employed in the form of liniments; i.e., in solution or suspension in oil or alcohol. The proportions in which they are used are about the following:

\begin{tabular}{l|l} 
Ammonia Water & \\
Tr. Belladonna or Opium. & \\
Spirits Chloroform & $1: 5$ \\
Spirits Ether & \\
Spirits Camphor & \\
Tr. Iodin & \\
Tr. Aconite & \\
Tr. Capsicum & \\
Tr. Cantharides & \\
Turpentine & \\
Sp. Sinapis & \\
Essential Oils & \\
Croton Oil & $1: 50$ \\
Creosote &
\end{tabular}

\section{JUNIPER OIL}

This is closely allied to turpentine (containing pinen, $\mathrm{C}_{10} \mathrm{H}_{16}$, and cadinen, $\left.\mathrm{C}_{15} \mathrm{H}_{24}\right)$; but it has a much more agreeable flavor. It is distilled from the "berries," the fruit, of the conifer, Juniperus communis. In the form of "Holland Gin" or its official substitute, the Spir. Juniperis Comp., it is an old-fashioned diuretic (Cow., I9I2) in cardiac and hepatic dropsies. It is supposed to act by mild irritation of the kidneys. Its use in nephritis therefore requires caution. It also has some reputation as emmenagogue. Overdoses produce poisoning, similar to Savin.

\section{PREPARATIONS-JUNIPER}

Ol. Juniperi (Ol. Junip.), U.S.P., B.P.; Juniper Oil.-Distilled from the ripe fruit of Juniperus communis. Dose, o. 2 c.c., 3 minims, U.S.P.; 0.03 to 0.18 c.c., $1 / 2$ to 3 minims, B.P.

Sp. Junip., U.S.P. - 5 per cent. Dose, 2 c.c., 30 minims, U.S.P.

$S$ p. Junip., B.P.-10 per cent. Dose, 0.3 to 1.2 c.c., 5 to 20 minims, B.P.

$S p$. Junip. Co., U.S.P. - 4 per cent. of Juniper oil, with Caraway and Fennel oils, in 70 per cent. alcohol. Similar to "Holland Gin." Dose, ro c.c., $2 \frac{1}{2}$ drams, U.S.P.

\section{ECBOLIC AND TOXIC VOLATILE OILS}

Actions.-Many volatile oils are popularly employed as emmenagogues and ecbolics: turpentine, juniper, savin, rue, pennyroyal, tansy and apiol. They produce pelvic congestion through intestinal irritation. A mild degree of this action may be useful in delayed and painful menstruation, as after exposure to cold. Abortion occurs only with toxic doses; indeed fatal doses are not always effective. Prochnow, I9Ir, also finds a slight direct action on the excised uterus. Macht, I9I3, finds the contraction inhibited, never stimulated. 
Savin (Sabina) and Arbor Vitæ (Thuja) also contain volatile oils similar to turpentine but much more toxic. Savin oil contains pinen, cadinen and sabinol, $\mathrm{C}_{10} \mathrm{H}_{15} \mathrm{OH}$. Thuja contains pinen, fenchon, and thujon (isomers of camphor, and having similar actions (Hildebrandt, 1902; Matzel, 1905). Poisoning has often occurred from their use as abortifacients.

Toxic symptoms from oil of savin are those common to volatile oils: Burning, nausea, vomiting, colic and diarrhea, sometimes bloody; severe congestion of the pelvic organs, increased menstrual flow and sometimes abortion; hematuria and painful micturition; unconsciousness, convulsions and coma. Death may occur in a few hours, but more often after several days. Six drops are said to have produced toxic effects (Lewin).

Fatty degeneration of the liver and of other organs (Heffter, 1895; Lindemann, 1899) is produced by large doses of these oils, especially by pulegon, the active constituent of pennyroyal; and also by the oils of sassafras, rosemary and thyme.

Apiol.- Two drugs are known under this name: "Green Apiol," or Oleoresin of Parsley Seed (N.N.R., Oleores. Apii), a green oily liquid. Dose 0.3 to I c.c., 5 to 15 minims; and "Apiol" proper, N.N.R., "Crystallized Apiol," "Parsley Camphor," a crystalline aromatic derivative extracted from the oleoresin, dose, 0.1 to $0.3 \mathrm{Gm}$., 2 to $5 \mathrm{gr}$. It has been studied by Heffter, 1895 . Overdoses $(0.6$ to $0.8 \mathrm{Gm}$.) produce arythmia, incoördination, digestive disturbances and fever. Fatal poisoning, with intestinal symptoms, icterus and uremia, is reported by Brenot, igr6.

The Apiols are administered in capsules, as emmenagogues and antipyretics. The actions of the different apiols do not seem to be identical (Lutz, 1910).

Tansy.-The leaves and tops of Tanacetum vulgare have been used mainly as anthelmintic against Ascaris and Oxyuris, internally, 0.5 to $2.5 \mathrm{Gm}$. as infusion, twice a day; or as enema of 15:200. Half an ounce to an ounce of the oil (which contains thujon) has produced death after two to four hours, with convulsions and unconsciousness.

Pennyroyal.-The leaves and tops of Hedeoma pulegioides are used as carminative, 5 to ro Gm., I to 2 ounces, as infusion. Ol. Hedeome (U.S.P.) 0.2 c.c., 3 minims; a teaspoonful has produced convulsions. Its active constituent, pulegon, $\mathrm{C}_{10} \mathrm{H}_{16} \mathrm{O}$ has been studied by Lindemann, I 899; Falck; and Matzel. 1905.

Absinthe.-The herb Artemisia Absinthium, contains a volatile oil, whose chief constituent is thujon, $\mathrm{C}_{10} \mathrm{H}_{16} \mathrm{O}$. This produces effects similar to camphor (Hildebrandt, I901 and 1902; Matzel, 1905). The liqueurs "absinthe" and "vermouth" contains this oil and also anise, fennel, and others; it is doubtful what part each of these and the alcohol (50 per cent.) plays in the toxic complex. In mammals, the liqueur produces tremors and epileptiform convulsions (Magnan, 1871). In man overindulgence causes similar effects and is also said to lead to mania.

Nutmeg Poisoning. - This is by no means rare. In man severe symptoms have occurred from one to one and one-half nutmegs, or from a teaspoonful of powdered mace. The effects appear in one to six hours, and are mainly narcotic, varied by excitement and delirium, with some motor stimulation and local irritation; they usually end in recovery in twenty-four hours. Nutmeg is also ecbolic. Similar effects occur in animals. Hepatic necrosis has been demonstrated in cats (Dale, rgog). Cushny and Wallace, I908, showed that the narcotic effect is produced by "myristicin," the high-boiling portion of the volatile oil. The oil has been investigated chemically by Power and Solway,-Igo8.

Senecio.-The alkaloids (senecin and senicionin) of S. Jacobea and vulgaris produce in cattle toxic effects resembling those of volatile oils: Hemorrhages in abdominal organs, especially the liver; fatty changes, necrosis, congestion, and hepatic cirrhosis (Cushny, I911.) The fluidextract (ro to 20 drops) has been used as emmenagogue. It has a slight depressent effect on the excised uterus (Pilcher, 1916).

Helenium Autumnale. - This contains a neutral bitter principle (Reeb, 1910), which is strongly irritant to mucous membranes. Systemically, it stimulates respiration and directly paralyzes cardiac and other muscle. It is not hemolytic (Lamson, r913).

PREPARATIONS-ECBOLIC AND TOXIC VOLATILE OILS

Petroselini Fructus, U.S.P.; Parsley Fruit.-The dried ripe fruit of Petroselinum sativum.

Oleores. Petrosel., U.S.P. (Liquid Apiol).-Evaporated ethereal extract of Parsley Seed. Dose, 0.5 c.C., 8 minims, U.S.P.

Myristica, U.S.P., B.P. (Nutmeg). - The ripe seeds of Myristica fragrans. Dose, 0.5 Gm., 8 gr., U.S.P. 
Ol. Myrist., U.S.P., B.P.-Distilled from the preceding. Dose, 0.2 c.c., 3 minims, L.S.P.; 0.03 to 0.18 c.c., $1 / 2$ to 3 minims, B.P.

Sp. Iy yist., B.P.- 10 per cent. Dose, 0.3 to 1.2 c.c., 5 to 20 minims, B.P.

\section{VOLATILE OILS AND OLEORESINS EMPLOYED IN URETHRITIS AND} CYSTITIS

Members.-The oil of Sandalwood and the oleoresins of Copaiba and Cubeb, and Matico are used mainly as urinary antiseptics in gonorrhea; Buchu as a diuretic in catarrhal cystitis.

Manner of Action.-The oleoresins are natural mixtures of resins and volatile oils rich in terpens and terpen alcohols. The terpens are excreted as glycuronic compounds, which have a slight but distinct antiseptic action on the urine. This is much less than that of hexamethylenamin; but it has the advantage that it is not affected by the reaction of the urine (Jordan, I9I I); and probably they are specifically more effective for certain bacteria. The gonococci are not killed (Bruck, I9I3); but the control of the putrefactive bacteria presumably puts the mucous membrane in a better position for resistance. The resin-acids are mildly irritant. The urinary passages are thus kept under the continuous influence of the antiseptic terpens, and stimulated to repair by the resins. The combination seems to be more effective than either the oils or the resins administered separately.

Side Actions. - The gastro-intestinal tract is also irritated, resulting in anorexia, colic, eructations, and diarrhea. These unpleasant,effects are less with sandal oil, which therefore deserves preference; and they may be further avoided by the use of the santalol esters, which are only dissolved in the intestines. Skin eruptions, scarlatinal rashes, etc., occur in some patients, either directly or from the digestive disturbance. Excessive doses of the oils produce more pronounced irritation of the entire urinary tract, with kidney pain, albuminuria, and vesical tenesmus. Albuminuria may be simulated by the precipitation of the resin-acids with nitric acid; to be distinguished by the addition of alcohol, which dissolves the resins. Glycosuria may also be simulated by the glycuronic acid.

Therapeutic Uses.-These drugs are employed mainly in subacute or chronic gonorrhea, as adjuvants of the local treatment. They should not be used in the acute stage, since they would increase the irritation. They are best administered in capsules, on a full stomach, to avoid gastric irritation. Cubeba is sometimes used in bronchitis; and Buchu as a diuretic.

\section{PREPARATIONS-VOLATILE OILS USED AGAINST GENITO-URINARY INFLAMMATION}

Buchu. U.S.P. (Buchu Fol., B.P.); Buchu.-The dried leaves of Barosma betulina (also of B. serratifolia, U.S.P.). Contains volatile oil, glucosid, bitter principle, etc. Dose, 2 Gm., 30 gr., U.S.P.; best as infusion.

Inf. Buchu., B.P. -5 per cent. Dose, 30 to 60 c.c., $\mathrm{I}$ to 2 ounces, B.P.

Fldext. Buchu, U.S.P.-Dose, 2 c.c., 30 minims, U.S.P.

Tr. Buchu, B.P. -20 per cent. Dose, 2 to 4 c.C., $1 / 2$ to I dram, B.P.

* Copaiba (Copaib.), U.S.P., B.P. (Balsam of Copaiva).-An oleoresin derived from South American species of Copaiba. Contains volatile oil and resin (copaivic acid). Pale yellow to brownish yellow, more or less viscid liquid, either without fluorescence or with only a slightly greenish fluorescence; having a peculiar aromatic odor, and a persistent, bitter and acrid taste. Insol. in water, partly sol. in alc., freely sol. in oils. Dose, I c.c., 15 minims, U.S.P.; 2 to 4 c.c., $1 / 2$ to $1 \mathrm{dram}$, B.P.; in capsules or pills (with 
magnesia). The various "Lafayette," "Chapman" and similar mixtures are undesirable.

Ol. Copaib., B. P.-Distilled from the preceding. Dose, 0.3 to r.2 c.c., 5 to 20 minims, B.P.

Cubeba, U.S.P.; Cubeb. Fruct., B.P.; Cubeb.-The dried, full-grown, unripe fruits of Piper Cubeba. Contains volatile oil and resin (cubebic acid). Dose, I Gm., I 5 gr., U.S.P.; 2 to 4 Gm., 30 to 60 gr., B.P. U.S.P.

Oleores. Cubeb., U.S.P.—An evaporated alcoholic extract. Dose, 0.5 Gm., 8 gr.,

Ol. Cubeb., U.S.P., B.P.-The distilled oil. Dose, 0.5 c.c., 8 minims, U.S.P.; 0.3

to $I .2$ c.c., 5 to 20 minims, B.P.

Tr. Cubeb., B.P. -20 per cent. Dose, 2 to 4 c.c., $3 / 2$ to I dram, B.P.

Troch. Cubeb., U.S.P.- 0.02 Gm., 3 gr.

Kave Rhiz., B. P.-From Piper methysticum.

Ext. Kave Liq., B.P.-Dose, 2 to 4 c.c., $1 / 2$ to I dram, B.P.

Matico. - The leaves of Piper angustifolium.

Sabal, U. S. P. (Saw Palmetto).-The partially dried, ripe fruit of Serenoa serrulata. Statements as to presence of alkaloids and volatile oils are contradictory. Has been used as nutritive tonic, alterative, in respiratory diseases, digestive disturbances, as aphrodisiac, etc. Of very doubtful value. Dose, 1 Gm., 15 gr., U.S.P.

Fldext. Sabal., U.S.P.-Dose, I c.c., 15 minims, U.S.P.

* Oleum Santali (Ol. Santal.), U.S.P., B.P.; Sandal Oil.-Distilled from the wood of Santalum album. A pale yellow, somewhat thick liquid; peculiar aromatic odor, spicy taste. Readily sol. in alc. Dose, 0.5 c.c., 8 minims, U.S.P.; 0.3 to r. 8 c.c., 5 to 30 minims, B.P.

Santal Oil Derivatives. - Sandal oil consists chiefly ( $9 \circ$ per cent.) of santalol, $\mathrm{C}_{15} \mathrm{H}_{26} \mathrm{O}$, a mixture of two sesquiterpen alcohols. It is used in the form of its carbonic ester (Carbosant, Santalolis Carbonas, N.N.R.), and of its salicylic ester (Santyl, N.N.R.). These are oily liquids, almost without odor or taste. Dose, 0.5 c.c., three times daily.

\section{BALSAMS}

Balsams (Peru, benzoin, tolu, storax) are oleoresins containing cinnamic and benzoic acids. The oils act as antiseptics; the oils and resins are mildly irritant, stimulating repair; and the resins furnish local protection and thus allay inflammation. The balsams are therefore employed in chronic inflammations of mucous membranes (bronchitis) and of the skin (eczema and pruritus); and to promote the healing of ulcers and wounds. Myrrh acts similarly. The balsams are also used locally against scabies, destroying the acarus and their ova. Peru balsam is employed locally and internally for tuberculosis (for intravenous use, see "Cinnamic Acid"). Its internal administration is of doubtful utility, as is also the internal use of other balsams in bronchitis. Even large doses of the balsams do not produce albuminuria (Stockman, I89I).

\section{PREPARATIONS-BALSAMS}

Benzoinum, U.S.P., B.P.; Benzoin (Gum Benjamin).-A balsamic resin obtained from Styrax Benzoin and other species growing in the East Indies. Dose, 1 Gm., ${ }_{5}$ gr., U.S.P.

Tr. Benz., U.S.P. - 20 per cent. Dose, I c.c., 15 minims, U.S.P.

* Tinctura Benzoini Composita (Tr. Benz. Co.: Tr. Benzoin. Co.), U.S.P., B.P.; Compound Tincture of Benzoin (Friars' Balsam, Turlington's Balsam).- - Io per cent. of Benzoin, with Aloes, Storax, and Tolu, in alcohol. Dose, 2 c.c., 30 minims, U.S.P.; 2 to 4 c.c., $1 / 2$ to $I$ dram, B.P., as stimulant expectorant; as inhalant, teaspoonful to cup of boiling water; externally, mixed with glycerin or water, as lotion for chapped hands. The original formula was much more complex.

* Balsamum Peruvianum (Bals. Peruv.), U.S.P., B.P.; Balsam of Peru.-A balsam obtained from Toluifera Pereiræ, Central America (not from Peru); often adulterated. Dark brown, viscid liquid of agreeable vanilla odor and persistent bitter acrid taste. Freely sol. in alc. Dose, 0.3 to I Gm., 5 to 15 minims, B.P. Externally on lint; in scabies, inunction of 2 to $3 \mathrm{Gm}$., four to six times in the day. 
Slyrax, U.S.P.; Styrax Pracp., B.P.; Storax.-A balsam obtained from the wood and inner bark of Liquidambar orientalis. Dose, I Gm., 15 gr., U.S.P.

Balsamum Tolutanum (Bals. Tolu.), U. S. P., B. P.; Balsam of Tolu.-A balsam obtained from Toluifera Balsamum. A yellowish-brown or brown, plastic solid, becoming brittle when old, dried, or exposed to cold; pleasing, aromatic odor, resembling that of vanilla; mild, aromatic taste. Sol. in alc. Dose, 0.3 to 1 Gm., 5 to 15 gr.. B.P.

Tr. Tolut, U.S.P.-20 per cent. in alcohol. Dose, 2 c.c., 3 minims, U.S.P.

Tr. Tolut., B.P.-- Io per cent. Dose, 2 to 4 c.c., $1 / 2$ to I dram, B.P.

(*Syr. Tolut., U.S.P., B.P.; see Index.)

\section{PREPARATIONS-AROMATIC RESINS}

Ammoniacum, B.P.-Gum resin exuded from stem of Dorema species. Dose, 0.3 to I Gm., 5 to 15 gr., B.P.

Mist. Ammoniac, B.P.-3 per cent. Dose, 15 to 30 c.c., $1 / 2$ to I ounce, B.P.

Galbanum.-Gum-resin from Ferula galbaniflua. Dose, 0.3 to I Gm., 4 to $15 \mathrm{gr}$., B.P.C.

Mastiche, Mastic.-Resinous exudation from Pistacia Lentiscus. An ethereal solution is used as a skin-varnish (Merck's Rep., 27:324).

Myrrha, U.S.P., B.P.; Myrrh.-Gum-resin from Commiphora species. Dose, 0.5 Gm., 8 gr., U.S.P.; 0.3 to I Gm., 5 to 15 gr., B.P., as carminative. Was used by the ancients as incense in religious ceremonies; and by the Egyptians in embalming, in combination with spices.

*Tr. Myrrh., U.S.P., B.P. - 20 per cent. in alcohol. Dose, I c.c., 15 minims, U.S.P.; 2 to 4 c.c., $1 / 2$ to $\mathrm{I}$ dram, B.P. Used especially as mouth wash (r:25) in stomatitis and pharyngitis; as a lotion, I: 5 or 10.

\section{PREPARATIONS-RESINOUS DRUGS}

Echinacea.-The root of Echinacea angustifolia (Purple Cone Flower). It contains resins, and no alkaloids (Heyl and Staley, 1914). Several proprietary preparations have been praised for sialagogue, diaphoretic, antiseptic, stimulant, and general alterative effects (Lloyd, I904; Madden, 1905); but no good evidence of its value has been published anrl the claims appear extravagant (J. A. M. A., Nov. 27, 1909). Dose, I to $2 \mathrm{Gm}$., I 5 to $30 \mathrm{gr}$.

Grindelia, U.S.P., B.P.-The dried leaves and flowering tops of Grindelia camporum (also Grindelia squarrosa, U.S.P.). Contains an amorphous resin (probably the active principle), sugars, proteins, tannin, and a very small quantity of volatile oil, no saponin or alkaloid (Power and Tutin, 1905). It is said to relax the muscular coats of the bronchi and diminish the excretion of mucus. It is therefore used in asthma and bronchitis. Dose, $2 \mathrm{Gm}$., 30 gr., U.S.P.

Fldext. Grindel., U.S.P.; Ext. Grindel. Liq., B.P.-Dose, 2 c.c., 30 minims, U.S.P.; 0.6 to 1.2 c.c., to to 20 minims, B.P. Also employed locally in ivy poisoning.

Pyrethrum, U.S.P.; Pyreth. Rad., B.P. (Pellitory). - The dried root of Anacyclus Pyrethrum. Chewed as sialogogue and against toothache. Dose, $2 \mathrm{Gm}$., $30 \mathrm{gr}$. U.S.P.

$$
\text { Tr. Pyreth., U.S.P., B.P.-2o per cent. }
$$

\section{INSECTICWES}

Certain volatile oils, or drugs containing them, are used to kill or repel noxious insects, such as Mosquitoes, Roaches, Flies, etc. "Insect Powder" (the powdered flowers of Pyrethrum species-Persian Powder-and of Chrysanthemum cinerariæfolium-Dalmatian Powder); Eucalyptus, Menthol, Erigeron, Cedar, and Lavender may be cited. (Roach powders commonly contain borax; arsenic is efficient, but dangerous; White Hellebore is also poisonous.) Naphthalin and Camphor are used against moths.

Pediculosis. - The experiences of the war have greatly revived the interest in insecticides against these parasites. Mercury, staphisagria, phenol, etc., are effective against limited infections; but they are too toxic and irritant for extensive application. Naphthalin and volatile oils stupefy and paralyze the parasites, but they are likely to recover subsequently. Acids are definitely fatal. Sulphur dioxid is effective (KnaftLenz, 1915). 


\section{MUSTARD OII}

Actions and Uses.-The volatile oil which is developed from black mustard on contact with water, produces a more prompt, more violent, and more penetrating irritation than the other volatile oils. Mustard preparations are extensively used for counterirritation, but care must be taken to stop the application when marked tingling is felt, or there may be vesication and even ulceration. Internally, mustard is used as a condiment; larger doses ( $\mathrm{r}$ to $4 \mathrm{~J}$ in a tumbler of warm water) produce vomiting; still larger doses cause violent gastro-intestinal irritation. Boiling water prevents the development of the oil by destroying the ferment.

Composition.-Volatile oil of mustard (allyl iso-thiocyanate, $\mathrm{C}_{3} \mathrm{H}_{5} \mathrm{NCS}$ ) is derived from black mustard by the action of a ferment, myrosin, on the glucosid sinigrin (potassium myronate). The latter itself is not irritant. Mustard seed also contains about 25 per cent. of bland fixed oil.

White mustard contains a similar ferment and glucosid, sinalbin, which yields an analagous oil, acrinyl iso-thiocyanate. Much less volatile oil is liberated than with black mustard. White mustard seeds have a cathartic action, due to the liberation of $\mathrm{H}_{2} \mathrm{~S}$ on contact with water. Large doses may produce sulphid poisoning, with cyanosis, etc. (van Leesum, I9r6).

Similar sulphur-containing oils are formed in other cruciferous plants, such as horseradish, radish, cress; and in onion, garlic, etc.

Plants containing mustard oil (Bursa pastoris) stimulate the contractions of the excised uterus (Groeber, I9I5).

\section{PREPARATIONS-MUSTARD}

Armoracice Radix, B.P.-Fresh Horseradish Root.

$S p$. Armor. Co., B.P.-Dose, 4 to 8 c.c., I to 2 drams, B.P.

Sinapis Alba (Sinap. Alb.), U.S.P.; White Mustard.-The ripe seeds of Sinapis alba. Dose, as emetic, 10 Gm., $21 / 2$ drams, U.S.P., of the powder, in tepid water.

* Sinapis Nigra (Sinap. Nig.), U.S.P.; Black Mustard.-The ripe seeds of Brassica nigra. Dose, as emetic, $10 \mathrm{Gm} ., 21 / 2$ drams, U.S.P., of the powder, in tepid water. Powdered mustard is applied as a poultice, mixed with 5 to ro parts of flour and enough water to make a paste; it may also be sprinkled on the surface of the ordinary linseed poultice. It is used in footbaths for preventing colds ( $\mathrm{I}$ to 2 ounces to a gallon); and in general baths for infantile coma (large tablespoonful to small bath).

* Emplastrum Sinapsis (Emp. Sinap.), U.S.P.; Mustard Plaster (Charta Sinapis, Mustard Paper).-A mixture of de-oleated black mustard and rubber, uniformly mixed and spread on paper, cotton cloth or other fabric. Mustard plaster is moistened thoroughly with warm (not hot) water, and applied to the skin for fifteen to thirty minutes.

*Oleum Sinapis Volatile (Ol. Sinap. Vol.), U.S.P., B.P.; Oil of Mustard.-A volatile oil produced synthetically or obtained from the seed of Brassica nigra, consisting chiefly of allyl iso-thiocyanate $\left(\mathrm{C}_{3} \mathrm{H}_{5} \mathrm{SCN}\right)$. Colorless or pale yellow, strongly refractive liquid, having a very pungent and acrid odor and taste. Dose, 0.008 c.c., $1 / 8$ minim, U.S.P'. It may be used as counterirritant in $1 / 10$ to 2 per cent. solution in 50 per cent. alcohol (Herzfeld, rgog).

Lin. Sinap., B.P.-3.5 per cent. of Ol. sinap., 5.5 per cent. of Camphor.

\section{NON-VOLATILE ORGANIC IRRITANTS}

A number of fixed organic drugs also act as local irritants. The most important of these is cantharidin. Capsicum, euphorbia, poison ivy, and croton oil, the "acrid principles" of certain fresh plants, especially of the family of Ranunculacex, etc., may be counted in this group. They owe their activity to neutral, resinous, or oily principles. Some benzol derivatives - chrysophanic acid, resorcin, pyrogallol, etc. - have a slimilar action, so also do some of the toxins and the poison of bees (Faust, 1910). 


\section{CANTHARIDIN}

Actions and Uses.-Cantharides (Spanish Flies) are used for vesication and for prolonged counterirritation in pleurisy, neuralgic and rheumatic pains, etc. The irritation is active, but the penetration slow, so that the effects are gradual, with only moderate pain, and without involvment of the deeper layers of the skin. The blisters therefore heal readily and without scar. On mucous membranes and open wounds, the effects are more severe, leading to local necrosis, and slowly healing, suppurating ulcers. Internally, cantharis is highly toxic by gastroenteritis and nephritis; even the absorption from a large blister may cause severe irritation of the kidneys and urinary passages. It is therefore contraindicated in nephritis, and should never be used internally, although it was formerly employed as a diuretic.

Origin of Cantharidin.-Cantharidin is a crystalline principle, the anhydrid of cantharidic acid. It combines readily with alkalies, forming soluble salts. These produce the same local and systemic actions. Cantharidin is contained in a number of insects, especially beetles. A similar substance exists also in some caterpillars. It is present in varying amount, even in the same species. It was isolated by Robiquet in 1812 from the Spanish "Fly" (a beetle-Cantharis or Lytta vesicatoria). It is contained in the soft parts, particularly the blood.

Cantharis was recommended by Hippocrates in dropsy and amenorrhea; it was very popular in the days of heroic medication.

Absorption and Excretion.--Cantharidin is readily absorbed from all surfaces even from the skin. It is excreted mainly by the kidneys (Eliaschoff); but since it irritates the gastro-intestinal tract when injected hypodermically, some must be excreted by this channel.

Symptoms of Poisoning.-When cantharis is taken by mouth, it produces successively: burning in the mouth, thirst, difficulty in swallowing, swelling and blistering of the tongue; salivation, nausea, vomiting, colic, and sometimes bloody diarrhea and tenesmus. The gastro-intestinal symptoms may be less pronounced in rapid poisoning. Kidney pain, burning in urethra, frequent micturition, albuminuria, casts, hematuria, painful erections, abortion. Weak and slow pulse, chill, syncope and collapse. Recovery may occur in about five days, the urine returning to normal. Toxic effects have been produced by $0.6 \mathrm{Gm}$.; death by 1.5 to $3.0 \mathrm{Gm}$., but recovery has also occurred from much larger doses. Of cantharidin, Io $\mathrm{mg}$. is said to be fatal.

Central Actions. - When injected into the circulation, cantharidin affects the central nervous system, producing short stimulation, excitement, and increased reflexes, followed by paralytic symptoms, coma, etc.

This central action is not often seen, being obscured by gastroenteritis or nephritis.

The Treatment of Poisoning.-This would consist of gastric lavage, demulcents, opiates, alkalies and plenty of fluid, heat to the gastric and renal region, and warm baths. No oils or fats should be given.

Cantharidin Nephritis. - The kidneys are extremely sensitive to cantharidin. Small doses act entirely on the glomeruli, which are enormously dilated; numerous leucocytes are found in Bowman's capsule. The urine becomes albuminous within half an hour after subcutaneous injection. The smallest doses increase its amount, while larger doses diminish it. The epithelium of the convoluted tubules is only affected by larger doses, and rather late in the course of the poisoning. The interstitial tissue escapes entirely in the acute intoxication, and is but slightly changed even in the subacute form (Mürset, 1886; Lyon, 1904; Richter and Roth; Schlayer and Hedinger, 1907; Pearce, 1913). The excretion of phthalein is delayed (Eisenbrey, 1913); the non-protein nitrogen of the blood is increased (Folin, Karsner and Denis, I9I2).

Hepatic Changes and Edema.- These are seen in dogs, with fatal doses. Opie, Igr 2, found fibrinous clots in the lymph nodes of the hepatic region, around the injured cells, and considers their obstruction responsible for the edema. In their absence, the lymph flow is increased independent of the renal changes.

Racial Tolerance. - The hedgehog, chicken, and duck are remarkably tolerant to the nephritic action (Ellinger, 1900 ). This is not due to differences in the absorption, nor to destruction of the poison, for the cantharidin is found in the urine, just as it is in susceptible animals. Nor are these animals immune to other nephritic poisons. The immunity to cantharides is also only partial; even a single injection of a large dose 
causes chronic nephritis. But taking the fatal dose for man ( $30 \mathrm{mg}$. by stomach) as the unit, that for the same weight of hedgehog lies about 3,000 . For the dog and cat it is about 2.5 (I mg. per kilo); for the rabbit, about 45 .

This immunity to the nephritic action does not confer immunity to the local action on the skin. In this respect, the hedgehog is even more susceptible than the rabbit, which latter animal is almost immune to the cutaneous action. The nephritic action is lessened by rendering the urine alkaline (Ellinger, 1905). In the rooster, cantharidin causes changes in the comb, analogous to those produced by ergot. Frogs are not susceptible.

Other Vesicants.-When cantharis is contraindicated-e.g., in cases of inflammation of the urinary passages-it is usually replaced by ammonia water or chloroform, which also produce a vesicant action if their evaporation is prevented, as by covering the point of application by a thimble. These are rather more rapid in action, but much more painful than fly blister, and are, therefore, avoided, if possible. Iodin, croton oil, and mustard have also been used, but act too deeply.

Contraindications to Vesication.-Blisters in general are contraindicated in people of feeble condition, since they may then lead to ulceration. When they are employed for counterirritation, they should not be applied directly over the inflamed part, but at some distance from it. They might otherwise render the inflammation more violent.

Alopecia.-Cantharis is used in the treatment of baldness, in the form of tincture, very greatly diluted with alcohol.

Treatment of Impotence.-Many drugs have been employed for this purpose, but our knowledge concerning them is still very meager. Cantharis is one of the most certain, acting through reflex irritation from the urethral mucous membrane. It is, however, quite dangerous, since effective doses are apt to set up considerable nephritis. Many essential oils act in the same manner, and are at once less dangerous and less active. Here belong, e.g., damiana, ginseng, mint, garlic, etc., and possibly camphor. Strychnin is thought to be effective by raising the tone of the spinal centers. Phosphorus and arsenic enjoy some reputation. If they are effective at all, it must be through improvement in the general condition of the patient. Alcohol, morphin, cannabis, and other narcotics act as aphrodisiacs by stimulating the imagination. Yohimbin has a specific action. The best treatment for impotence consists, of course, in the removal of the cause and improvement in the general health of the patient by appropriate hygiene.

\section{PREPARATIONS-CANTHARIDES}

Cantharis (Canthar.), U.S.P.; Cantharides (Spanish Flies, Russian Flies).-The dried beetle, Cantharis vesicatoria. Contains cantharidin, 0.4 to I per cent. (not less than 0.6 per cent., U.S.P.) volatile and fixed oil, etc. Maximal dose, 0.05 Gm., I gr.

Cerat. Canthar., U.S.P. (Blistering Cerate).- 35 per cent. of Cantharides.

* Emplastrum Cantharidis (Emp. Canthar.), U.S.P.; Cantharides Plaster.-Prepared by spreading cantharides cerate upon rosin plaster, leaving a margin around the edges. Each square centimeter of spread plaster should contain $0.1 \mathrm{Gm}$. of cantharides cerate. It requires from six to ten hours to raise a blister, according to the thickness of the skin, its content in fat, and probably also individual susceptibility. Since cantharidin is insoluble in water, it is well that the skin be rather greasy, to facilitate its absorption. The plaster adheres very poorly, and must usually be fixed with adhesive plaster. When the blister has appeared, the plaster should be carefully removed without rupturing the vesicle. The latter is then pierced and dressed with an ointment. This prevents further pain, irritation, or infection. By a "flying blister" is meant a series of small blisters raised along the course of a nerve by the application of successive plasters.

Collod. Canth., U.S.P. (Blistering Collodion, Vesicating Collodium). -6 per cent. Cantharides in Flexible Collodion.

Tr. Canthar., U.S.P. - to per cent. Dose, 0.I c.c., I $1 \frac{1}{2}$ minims. Maximal, 0.5 c.c. 8 minims. May be added to liniments to prolong their local action; in hair lotions, $1: 50$.

Cantharidinum, B.P.; $\mathrm{C}_{10} \mathrm{H}_{12} \mathrm{O}_{4}$.- - Obtained from various species of Cantharis and of Mylabris. Colorless, glistening crystals; no odor. Very slightly sol. in water or alc., 
more sol. in chlorof., ether or oils. Cantharidin, 0.05 to $\mathrm{I} \mathrm{mg}$. in a drop of oil, and covered with simple plaster, may be employed as a blister.

The cantharidal preparations of the B.P. are now made of Cantharidin. The proportions are adjusted on the basis that I part of Cantharidin is equivalent to about 200 parts of Cantharis; so that the activity of the new preparations is about the same as that of the old.

Acet. Cantharidin, B.P.- -0.05 per cent. (equiv. to so per cent. of Cantharis).

Collod. Vesic., B.P.- 0.4 per cent. (equiv. to 50 per cent. of Cantharis).

* Emplastrum Cantharidin (Emp. Cantharidin), B.P.-0.2 per cent. (equiv. to 35 per cent. of Cantharis). See "Emp. Canthar."

Emplastrum Calefaciens (Emp. Calefac.), B.P.; Warming Plaster.- -0.02 per cent. (equiv. to 4 per cent. of Cantharis).

Liquor Epispasticus (Liq. Epispast.), B.P.; Blistering Liquid. -0.4 per cent. (equiv. to 50 per cent. of Cantharis), in acetone menstruum.

Tr. Cantharidin., B.P.-O.or per cent., equiv. to $1 \frac{1}{4}$ per cent. of Cantharis $(1 / 4$ of U.S.P.). Dose, 0.12 to 0.3 c.c., 2 to 5 minims, B.P.

Ung. Cantharidin., B.P. -0.033 per cent., equiv. to 7 per cent. Cantharis $(2 / 3$ of the old preparation).

\section{PREPARATIONS-CAPSICUM AND SIMILAR IRRITANTS}

Capsicum, U.S.P.; Capsic. Fruct., B.P.; Capsicum (Cayenne Pepper, African Pepper). -The dried ripe fruits of Capsicum fastigiatum, U.S.P.; Capsicum minimum, B.P. The action is due to about 0.02 per cent. of a crystalline neutral principle, capsaicin ( $\mathrm{r}$ : 100,000 produces lasting burning of the tongue, Nelson, I910). Capsicum is used internally as a condiment and carminative, and externally to produce prolonged counterirritation for rheumatism, etc. (20 per cent. ointment; or equal parts of tincture and of soap-liniment). Dose, 0.06 Gm. I gr., U.S.P.

Emp. Capsic., U.S.P.; Capsicum Plaster.-Prepared by brushing oleoresin of capsicum upon the surface of rosin plaster so as to form a thin coating, leaving a margin around the edges. U.S.P.

Oleores. Capsic., U.S.P.-Evaporated ethereal extract. Dose, 0.03 Gm., 1/2 gr.,

* Tr. Capsic., U.S.P.--1o per cent. Dose, 0.5 c.c., 8 minims, U.S.P., diluted.

* Tr. Capsic., B.P. -5 per cent. Dose, 0.3 to I c.c., 5 to 15 minims, B.P.

* Ung. Capsic., B.P.-25 per cent.

Euphorbium.-The dried juice of Euphorbia resinifera, consists of a gum-resin. The active constituent is the resinous euphorbon which resembles the active resin of croton oil (Boehm, 1915). The drug is rarely used (in liniments, Io per cent.; plasters, 3 per cent.).

Mezereum, U.S.P.-The dried bark of Daphne species. The active constituent is the anhydrid of mezerinic acid. There is also a glucosid daphnin. The fluidextract is rarely used as an addition to liniments or plasters; also in Fldext. Sarsap. Co.

Piper, U.S.P.; Black Pepper.-The dried unripe fruit of Piper nigrum. (White pepper is the ripe fruit, deprived of its outer-pericarp.) Contains the crystalline alkaloid piperin, a volatile oil, and a pungent resin. Dose, $0.5 \mathrm{Gm}$., 8 gr., U.S.P.

Oleores. Piper, U.S.P.-Evaporated ethereal extract. Dose, $0.03 \mathrm{Gm} ., 1 / 2 \mathrm{gr}$., U.S.P.

Piperin (piperyl-piperidin).- Has been used as an antipyretic and carminative, 0.1 to $0.5 \mathrm{Gm}, 2$ to $8 \mathrm{gr}$., in capsules.

Pulsatilla.-The herb of Anemone Pulsatilla and A. pratensis; Europe. Should not be kept over one year. Active ingredient, Anemonin. The drug is very irritant, and is almost obsolete, but has been used against asthma, hemicrania, etc., in doses of 0.1 to $0.4 \mathrm{Gm}$. It has a depressant action on the excised uterus (Pilcher, 1916).

Arisaemia (Indian Turnip; Jack-in-the-Pulpit).-The fresh corm of this plant is very irritating'or "acrid" when chewed. This is probably due to the mechanical effect of its sharp calcium oxalate crystals (Lazenby, 1915).

\section{CHRYSAROBINUM}

This is strongly irritant to the skin and mucous membranes. It is employed as ointment ( 5 to 25 per cent. daily) against psoriasis and parasitic skin diseases, ringworm, etc. It stains the skin (keratin) and linen 
a brownish-violet color. This can be removed by chlorinated lime. Caution must be used lest it reach the eyes and produce violent conjunctivitis (or rather keratitis; Igersheimer, rgr2) and corneal opacity. It is absorbed from the skin, and excreted partly as chrysophanic acid, which is easily formed by its oxidation, especially in alkaline solutions. The urine is, therefore, colored red by alkalies. A part is excreted unchanged, and causes renal irritation, casts and albuminuria. By mouth, it produces (0.18 Gm.) vomiting, diarrhea, renal pain and hematuria.

Chrysarobin has a strong affinity for oxygen, abstracting it from the tissues. This is supposed to be the basis of its action, the oxidation product, chrysophanic acid, being relatively inactive. The oxidation occurs only in watery suspensions in the presence of alkali. Fats, therefore, diminish its activity (Schamberg, Raiziss and Kolmer, I915).

\section{PREPARATIONS-CHRYSAROBIN}

Araroba, B.P. (Goa Powder, Crude Chrysarobin).-A substance found in cavities of the trunk of Andira (Vouacapoua) Araroba.

* Chrysarobinum (Chrysarob.), U.S.P. B.P.; Chrysarobin.-A mixture of neutral principles extracted from Goa powder; chiefly chrysophanolanthranol, $\mathrm{C}_{15} \mathrm{H}_{12} \mathrm{O}_{3}$. An orange-yellow, microcrystalline powder, tasteless, odorless, and irritating to the mucous membrane. Very slightly sol. in water, slightly sol. in alc. $(1: 385)$; partially sol. in fats. A saturated ethereal solution of chrysarobin is used in the treatment of warts. It is painted on daily, the dead tissue being pared off. Dose, $0.03 \mathrm{Gm} ., 1 / 2 \mathrm{gr}$., U.S.P.

Ung. Chrysarobin, U.S.P. -6 per cent. in Benz. Lard.

Ung. Chrysarobin, B.P. - 4 per cent. in Soft Paraffin.

Scarlet Red, Medicinal, N.N.R. (Rubrum Scarlatinum, toluylazo-naphthol).-This is used as 4 to 8 per cent. ointment, to stimulate the growth of epithelium, on clean granulating wounds, especially burns, and on clean ulcers. (Schmieden, I908; Morawetz, 1909; Dobrowolskaya, I9 2 , report the results as no better than without the dye.) The application is described in N.N.R. Its use is in the experimental stage. The 8 per cent. ointment often produces irritation and even erosion and sometimes systemic effects (violent gastroenteritis; Lyle, I9 I2). O. Sachs, I9II, claims that a number of other anilin dyes also stimulate epithelial growth. A midoazotoluol is being advocated as less staining (Hayward; Beck, 1913). It is used in the same strength.

\section{POISON IVY}

Actions.-Contact with certain species of Rhus, common along roadsides, on fences, in woods and swamps, etc., produces typical dermatitis, passing through the successive stages of hyperemia and itching, to violent vesication, edema, and suppuration, according to the specific sensibility of the individual; many persons are practically immune, although a sufficient quantity of the isolated toxicodendrol has never failed to produce the dermatitis. The active ingredient of all the species is a glucosid toxicodendrol. It is so highly active that $1 / 1,000 \mathrm{mg}$. has caused severe vesication (Pfaff). These small amounts may easily be conveyed by dust, etc., to some distance, although toxicodendrol itself is nonvolatile. (Warren, r9r3, could not find the poison in the pollen, as has been claimed.) Similarly, it may be conveyed by the hands or clothing from one person to another, as if it were contagious. The dermatitis has a latent period of one to nine, usually four to five days, apparently independent of the dose. Internally and hypodermically it produces local irritation and nephritis, but the toxicity is relatively low (critical bibliography, Ford, rgr3).

Toxic Species.-The most important are: Rhus vernix, synon. venenata (Poison Sumach, the most powerful); Rhus Toxicodendron (Poison Oak); Rhus radicans and 
diversiloba (Poison Ivy); Rhus pumila (a Southern species) - all common in North America; the Japanese lacquer tree (Rhus vernicifera). Other toxic species are mentioned by L. E. Warren, I909. The common sumach, Rhus glabra, does not have this action.

Nature of the Toxicodendrol.-Pfaff, I 897 , showed that the toxicity is not due to a volatile alkaloid, as claimed by Khittel, I858; nor to a volatile acid (Maisch, 1866); but that it is caused by a fixed oily substance. Acree and Syme, I907, isolated this as a tar or wax, and showed it to be a glucosid, dissociable into rhamnose, gallic acid and fisetin. It is soluble in alcohol and oils and is precipitated by lead acetate, from which it is dissolved by ether. It is not destroyed by heating an hour at $100^{\circ} \mathrm{C}$. (v. Adelung, 1912, I913). The toxicodendrol occurs in the lacteal vessels, which terminate in fine hairs (Schwalbe, 1902).

Artificial Immunity.-Ford, 1907, found that repeated hypodermic injections of increasing doses of the fluidextract induced resistance to the local necrosis and to the nephritis; and that the serum of these animals conferred passive immunity on others. v. Adelung, I9I3, was not successful in producing immunity to the isolated toxicodendrol.

Congenital Tolerance.-The congenital variations in susceptibility are very marked, many persons being immune to ordinary contact, although the immunity is probably never absolute. The tolerance is sometimes lost, and one attack seems to leave the skin more sensitive to others; but Ford mentions that Syme gradually acquired tolerance. Perspiration seems to facilitate infection.

Treatment of Ivy Poisoning. - This is best accomplished by thorough scrubbing with mild soap, then thoroughly rubbing in a hot solution of potassium permanganate $(2$ per cent. for ordinary skin, I per cent. if the skin is delicate or broken); and applying a paste of castile soap. The permanganate stain may be removed by 5 per cent. oxalic acid, or by sulphurous acid; but these must be used cautiously. Salves or oils must not be used since, by dissolving the toxicodendrol, they would spread the infection. They become indicated only in the later stages, when the poison has become completely removed. Rubber gloves protect against infection.

Lead acetate (saturated alcoholic solution) precipitates the toxicodendrol; but the compound dissociates easily, so that the applications, to be effective, must be made frequently. Other popular remedies are: copper or iron salts; the expressed juice of "Touch-me-not" (Impatiens fulva); and fluidextract of Grindelia Robusta (diluted with 4 to 8 vol. of water and used as a lotion).

Other Plants Producing Dermatitis.-These comprise a number of tropical trees belonging to the same family-Lithrea caustica; Anacardium (active principle, cardol, an oily substance); Semecarpus. Primula, chrysanthemum, arbor vitæ, and many other plants (Vanilla beans, Claveric, I908); the fruit of the ornamental Ginko tree (Starr, I9I3), affect sensitive persons in the same manner.

Primula Dermatitis. - This is produced by direct contact with the common Chinese house plant, Pr. obconica, and a few other species. Nestler describes toxic crystals in the resinous secretion of the leaves. The treatment consists in cleansing with alcohol or ether, and the application of Zinc Ointment (O. H. Foerster, 19ro; Rost, rgr4).

Hypericum.-Ray, I9r4, stated that cattle feeding on a Tunisian species ( $\mathrm{H}$. crispum) developed dermatitis of non-pigmented portions of the skin, and inflammation of the mucous membranes exposed to light. The phenomena are due to a fluorescent substance. Rogers, I9r4, claims analogous effects for the common St. John's wort, Hypericum perforatum. Similar symptoms are also said to be sometimes produced by buckwheat.

The action of fluorescent dyes on plants is discussed by Gicklhorn, rgr4.

\section{PHYSICAL COUNTERIRRITANTS}

Irritation of the skin is easily produced by physical agencies, which are often preferred to the chemical irritants. The effects and indications are similar. They can only be enumerated here: heat and cold, in the form of applications, baths, heated air, or cautery. Friction, generally combined with massage. Electricity, radiotherapy and phototherapy.

Antiquated are: Acupuncture (pushing needles through the skin to the underlying organs, said to be popular with the Chinese); scarification (small incisions with knife or needle); setons (a string carried through a fold of skin and left to suppurate). blood.

Venesection, cupping and leeches also act by altering the distribution of the 
DRUGS EMPLOYED FOR THEIR LOCAL EFFECTS ON THE ALIMENTARY CANAL

\section{STOMACHICS}

Bitter, aromatic and "sharp" drugs have been used since ancient times as "tonics," and in various functional dyspepsias. They were supposed to increase the appetite and improve digestion, and thus favor nutrition.

Classification.--Simple bitters generally contain some "bitter principle" -neutral substances, often crystalline, non-nitrogenous, sometimes glucosidal, and without pronounced systemic actions. In aromatic bitters these are mixed with volatile oils. The astringent bitters contain tannin. Condiments, which also contain aromatic oils or other sharp principles, form the transition to carminatives. These are employed to secure the expulsion of gas from the alimentary canal.

Efficiency in Health and Disease.-About a tenth of the medicines mentioned by Hippocrates belong to this class, and they have always played a prominent rôle in folk medicine.

Modern scientific investigations (Moorhead, I9 I $_{5}$ ) have confirmed that the bitters may improve deficient appetite, and the flow and quality (acidity) of the gastric juice, in conditions of poor health (anemia); although they have no effect on normal individuals (Carlson and co-workers, I9I4 and I9I5).

The aromatics and condiments probably act by mild direct irritation of the gastric mucosa, the beneficial effects being due to the hyperemia and motor stimulation.

Effect in Experimental Cachexia.-In dogs provided with a Pawlow gastric pouch, and rendered cachectic by repeated large hemorrhages, Moorhead, 1915, found that the administration of bitters improved the appetite and the gastric deficiency, although not quite to normal. The animals consumed more food and the gastric juice became more abundant and more acid. The increase of appetite occurred whether the bitters were given by mouth or by stomach; the increase of secretion was confined to oral use. The improvement occurs with the first administration so that it is not due to the estabiishment of a habit. Possibly the bitter taste enhances the taste of food, by contrast.

Experiments on Normal Individuals. - Carlson and co-workers, I9I4 and I9I5, could find no beneficial effects in normal men or dogs. Therapeutic doses of bitters, however given, have no immediate or delayed effect on gastric secretion. When taken by mouth (or large doses introduced directly into the stomach) they inhibit the normal hunger contractions and the sensation of hunger, just as do other irritant or disagreeable substances.

Gastric Secretion.-Carlson, I915, tested the effects on a man with gastric fistula, and on dogs with Pawlow miniature stomachs. The bitters were placed in the mouth, or directly in the stomach, etc., before, with, or without food, etc. However, the experiments were varied, the results were uniformly negative. This disposes of the ráther contradictory results of older investigators as to direct secretion (Buchheim and pupils, 1849; Reichmann, 1888; Korczynski, r9or; Hoppe, 1905) and "psychic juice" (Borisoff, 1904; Bonanni, 1905; Strasheko, 1905).

The introduction of irritant substances into the mouth also fails to provoke gastric secretion in man (Carlson, 1915).

Absence of "Taste" in the Stomach.-Albertoni and Tullio, 1912 , found on a human subject that the direct introduction, through a gastric fistula, of bitter or sweet substances or bouillon, produced only the same immediate sensation as saline; but bouillon, bread or alcohol provoked speedier secretion of acid and, taken with food, afforded a more satisfactory sensation.

Influence on Pancreatic Secretion.- The presence of any irritant in the intestine stimulates the flow of pancreatic juice (Gottlieb, 1894). The effect of bitters is somewhat different: 'They have no effect on the pancreatic or bile flow; the intestinal juice is first diminished, then increased (Reichmann, I888; Jodlbauer).

Effect on Absorption.-Bitters hasten the absorption of sugar and peptone from the 
stomach (Brandl, I893), but seem to delay it somewhat from the intestine (Rieder, 1910).

Sojourn of the Food in the Stomach.-According to Heubner, this is shortened by small, delayed by large, doses.

Effect on Digestive Ferments.-Most of the bitters have no effect on digestion in ritro.

Antiseptic Action.-Bitters and aromatics are antiseptic, and may therefore influence digestion favorably by limiting putrefaction and abnormal fermentation.

Leucocytosis. - A further action of stomachics (both bitters and aromatics) which may be concerned in their therapeutic action, is that they increase the leucocyles of the blood. (Hirt, 1856, Binz, 1875, Pohl, 1888). The theory has been advanced that these leucocytes play a rôle in intestinal absorption. (Hofmeister, 1887).

Therapeutic Uses of Stomachics.-These are employed:

I. To increase appetite, from whatever cause this be deficient.

2. To improve digestion in all kinds of "atonic" dyspepsias, with motor or secretory deficiency. They tend to remove the anorexia, oppression, constipation, hypochondria, etc. In subacute or chronic gastritis, the astringent bitters may be more useful, otherwise they are rather objectionable. Tannin must be especially avoided when the bitter is to be prescribed with iron, since this makes an unsightly mixture. Tanninfree are the simple bitters and the majority of the aromatic bitters.

3. As tonics in anemia, convalescence, phthisis, etc., to hasten reconstruction.

4. As antemetics.

5. To modify or improve the taste of food or medicines; also to obscure a "bad taste in the mouth."

Administration.- - It is believed that stomachics should be administered about a half an hour before meals. They are generally more pleasant, and therefore more effective, if they are judiciously blended, as in the aromatic and compound bitters (Tr. Gentianæ Co.; Tr. Cardamomi Co.). Overdoses produce nausea and so defeat their object.

\section{SIMPLE BITTERS}

These are practically free from aromatic oils or tannin, and can therefore be mixed with water. The tinctures are strongly alcoholic.

Certain pure alkaloids, such as Quinin or Strychnin, may be used as simple bitters.

\section{PREPARATIONS}

Berberis, B.P.-The dried stem of Berberis aristata. The rhizome and roots of B. aquifolium were formerly official in the U.S.P. The pharmacology has been studied by Hildebrandt, 1907 .

Tr. Berber., B.P.- 10 per cent. Dose, 2 to 4 c.c., $1 / 2$ to I dram, B.P.

Calumba, U.S.P.; Calumbæ Radix, B.P.; Calumba (Colombo). -The dried root of Jateorhiza palmata, U.S.P.; Jateorhyza Columba, B.P. Resembles gentian but can be mixed with iron. Contains several alkaloids (Calumbanin, jateorhizin, and palmatin), related to berberin. Biberfeld, rgog, found that these differ only quantitatively in action, paralyzing the central nervous system, especially the respiratory center, and lowering the blood pressure through cardiac and vasomotor depression. Dose, $2 \mathrm{Gm}$., 30 gr., U.S.P.

Inf. Calumb., B.P. -5 per cent. Dose, 15 to 30 c.c., $1 / 2$ to I ounce, B.P. U.S.P.

Tr. Calumb., U.S.P. 20 per cent. in 60 per cent. alcohol. Dose, 4 c.c., I dram,

Tr. Calumb., B.P.- Io per cent. Dose, 2 to 4 c.c., $1 / 2$ to I dram, B.P.

Chirata, B.P.-The dried plant of Swertia Chirata.

Inf. Chirat., B.P. -5 per cent. Dose, 15 to 30 c.c., $1 / 2$ to 1 ounce, B.P.

Tr. Chirat., B.P.- 10 per cent. Dose, 2 to 4 c.c.; $1 / 2$ to 1 dram, B.P. 
Gentiana, U.S.P.; Gentiance Radix, B.P.; Gentian.-The dried rhizome and roots of Gentiana lutea, Switzerland. One of the most useful of the bitters; mentioned by Pliny. The bitter principles are the crystalline glucosid gentiin, and the amorphous gentiamarin. The fresh drug contains the crystalline glucosid gentiopicrin, which disappears in drying. There is also a small amount of tannin, not cnough to be astringent, but sufficient to be incompatible with iron. Dose, x Gm., I 5 gr., U.S.P.

* Extractum Gentiance (Ext. Gent.), U.S.P., B.P.-A watery pilular extract, used largely as pill excipient. Dose, 0.25 Gm., 4 gr., U.S.P.; 0.12 to $0.5 \mathrm{Gm} ., 2$ to 8 gr., B.P. Inf. Gent. Co., B.P.-r.25 per cent., with Bitter Orange and Lemon peels. Dose, 15 to 30 c.c., $1 / 2$ to 1 ounce, B.P.

* Tinctura Gentiana Composita (Tr. Gent. Co.), U.S.P., B.P.- o per cent. of Gentian, with Bitter Orange Peel and Cardamom, in 45 per cent. alcohol. Dose, 4 c.c., I dram, U.S.P.; 2 to 4 c.C., $1 / 2$ to I dram, B.P.

Pareira.-The root of Chondodendron tomentosum. Contains bebeerin, isobebeerin, and other alkaloids (studied by Faltis, r912; Scholtz, 1913). Commercial "Bebeerin sulphate cryst." is isobebeerin. It is isomeric with codein, and like this, has narcotic actions (Scholtz and Koch, r9r5). Dose of Pareira, 2 Gm., $30 \mathrm{gr}$.

Picrorhiza, B.P., -The rhizome of Picrorhiza Kurroa. Dose, 0.6 to $1.2 \mathrm{Gm}$., to to $20 \mathrm{gr}$; antiperiodic dose, 3 to $4 \mathrm{Gm}$., 45 to $60 \mathrm{gr}$., B.P.

Ext. Picrorh. Liq., B.P. -Dose, I to 4 c.c., 15 to 60 minims, B.P.

Tr. Picrorh., B.P. -25 per cent. Dose, 2 to 4 c.c., $1 / 2$ to I dram, B.P.

Quassia, U.S.P.; Quassice Lignum, B.P.; Quassia.-The wood of Picraena excelsa (also of Quassia amara, U.S.P.). Dose, $0.5 \mathrm{Gm} ., 8 \mathrm{gr}$., U.S.P.

Inf. Quass., B.P. -1 per cent. Dose, I 5 to 30 c.c., $1 / 2$ to I ounce, B.P.

Tr. Quass., U.S.P.-2o per cent. Dose, 2 c.c., 30 minims, U.S.P.

Tr. Quass., B.P. - 10 per cent. Dose, 2 to 4 c.c., $1 / 2$ to I dram, B.P.

Taraxacum, U.S.P.; Tarax. Rad., B.P.; Taraxacum (Dandelion).-The dried rhizome and roots of Taraxacum officinalis, U.S.P.; the fresh root, B.P. (Composition, Power and Browning, 1912). Dose, ro Gm., $2 \frac{1}{2}$ drams, U.S.P. . U.S.P.

Ext. Tarax., U.S.P.-A pilular extract; used as pill excipient. Dose, $1 \mathrm{Gm}$., 15 gr.,

Fldext. Tarax., U.S.P.-Dose, ro c.c., $21 / 2$ drams, U.S.P.

Succ. Tarax., B.P.- The juice, preserved with 25 per cent. of alcohol. Dose, 4 to 8 c.c., 1 to 2 drams, B.P.

Xanthoxylum, U.S.P. (Prickly Ash).-The dried bark of Xanthoxylum americanum or X. Clava-Herculis. Contains berberin. Dose, $2 \mathrm{Gm}$., $30 \mathrm{gr}$., U.S.P.

Fldext. Xanthox., U.S.P.-Dose, 2 c.c., 30 minims, U.S.P.

\section{ASTRINGENT BITTERS}

With these, tannin is a prominent ingredient, whilst volatile oils are present only in small quantity, if at all. The preparations can be mixed with water.

\section{PREPARATIONS}

(*Tr. Cinchona Co., U.S.P., B.P., see Index.)

Cascarilla, B.P.-The dried bark of Croton Eluteria.

Inf. Cascarill., B.P. -5 per cent. Dose, I 5 to 30 c.c., $1 / 2$ to I ounce, B.P.

Tr. Cascarill., B.P.- 20 per cent. Dose, 2 to 4 c.c., $1 / 2$ to I dram, B.P.

Cimicifuga, U.S.P. (Black Cohosh, Black Snakeroot; Macrotys).-The dried rhizome and roots of Cimicifuga racemosa (Constituents, Finnemore, 1910). Dose, I Gm., I 5 gr., U.S.P.

Ext. Cimicif., U.S.P.-A powdered extract, I Gm. representing $4 \mathrm{Gm}$. of the drug. Dose, 0.25 Gm., 4 gr., U.S.P.

Fldext. Cimicif., U.S.P.-Dose, I c.c., I5 minims, U.S.P.

Condurango. - The bark of Marsdenia Condurango. Used as astringent bitter especially in gastric ulcer and carcinoma.

Fldext. Condur.-Dose, $\mathrm{I}$ to $\mathrm{r} .5$ c.c., ${ }_{5}$ to 25 minims.

Cusparia (Angostura Bark). -The bark of Cusparia febrifuga. Contains alkaloids, chiefly cusparin (Troeger and Mueller, I915). Dose, 0.6 to $2.5 \mathrm{Gm}$., ro to $40 \mathrm{gr}$.

Serpentaria, U.S.P.; Serpent Rhiz., B.P.; Serpentaria (Virginia Snakeroot). - The dried rhizome and roots of Aristolochia Serpentaria or reticulata. Dose, r Gm., I5 gr., U.S.P.

Tr. Serpent., B.P.-2o per cent. Dose, 2 to 4 c.c., $1 / 2$ to I dram, B.P. 


\section{AROMATIC BITTERS}

These contain both aromatic oils and bitter principles, but no tannin. Their alcoholic preparations can not be mixed with water without turbidity.

\section{PREPARATIONS}

(Aurantii Amari, Cortex, see Index.)

Anthemidis Flores, B.P. (English or Roman) Chamomile Flowers.-The dried flowerheads of Anthemis nobilis.

Ol. Anthem., B.P.-The distilled oil. Dose, 0.03 to 0.18 c.c., $1 / 2$ to 3 minims, B.P.

Betel, B.P.-The dried leaves of Piper Betle.

Calamus (Sweet Flag). - The rhizome of Acorus Calamus.

Matricaria, U.S.P. (German Chamomile). - The dried flower-heads of Matricaria Chamomilla. Volatile oil and bitter principles. Used as infusion, internally as stomachic, carminative, and diaphoretic; externally as counterirritant. Dose, ${ }_{5} \mathrm{Gm}$., 4 drams, U.S.P.

Panax, Ginseng.-The root of Panax quinquefolium, very highly valued by the Chinese, belongs to this series. So do other species of Panax and Aralia.

Numerous other bitters are used in domestic medicine, but they are not worth mentioning.

\section{CARMINATIVES}

This class comprises certain aromatic oils exerting an irritant action upon the stomach and intestine, being somewhat specific in causing the expulsion of gas rather than of fluid or solid contents. Since they are also antiseptic, they are especially useful in abnormal fermentation, removing at once the discomfort caused by the gas, and checking the growth of the bacteria which give rise to it. They also form useful additions to laxative mixtures, since they diminish the "griping." Charcoal is also classed as a carminative.

\section{PREPARATIONS-CARMINATIVES}

* Carbo Ligni (Carb. Lig.), U.S.P., B.P.; Wood Charcoal.-Black, tasteless and odorless, very fine powder. Dose, I Gm., 15 gr., U.S.P., to 4 Gm., $60 \mathrm{gr}$.

When freshly heated, charcoal condenses gases and dissolved substances in its pores, therefore acting somewhat as a catalyst in facilitating oxidations and other chemic reactions. It has been used as a dressing for infected wounds. Internally, it is sometimes employed in flatulence, with the idea that it will absorb the gases. It is probably useless since moist charcoal has this property only to a very slight degree. It is also used as an antidote, especially for alkaloidal poisons (Wiechowski). Animal charcoal, Carbo animalis, U.S.P., Bone-black, is used in chemical industry for decolorizing. It has been employed in dysentery, similar to Kaolin. It adsorbs and therefore hinders digestive ferments in vitro; but this seems to be compensated by increased secretion (Strauss, I9 r6). It also adsorbs and inactivatec toxins ( $R$. Kraus \& Barbara, I915).

Colloidal Carbon (caramel) is harmless, even on intravenous injection. It antagonizes alkaloids (strychnin) when mixed in vitro, and even somewhat when the strychnin is given by mouth, and the carbon by vein; but this antagonism is too uncertain for practical application (Sabbatani, I9I3 and I9I4).

Mentha Piperita, U.S.P.; Peppermint.-The dried leaves and flowering tops of Mentha piperita. Dose, 4 Gm., 60 gr., U.S.P.

(*Aq. Menth. Pip., see Index.)

Ol. Menth. Pip., U.S.P., B.P.--The distilled oil, containing at least 50 per cent. of menthol. Colorless liquid, characteristic odor, pungent aromatic taste. Dose, 0.2 c.c., 3 minims, U.S.P.; 0.03 to 0.18 c.c., $1 / 2$ to 3 minims, B.P.

* Spiritus Menthe Piperite (Sp. Menth. Pip.), U.S.P., B.P.; Spirit (Essence) of Peppermint.- - Io per cent. of the Oil; the U.S.P. is tinged green by maceration with Peppermint. Dose, 2 c.c., 30 minims, U.S.P.; 0.3 to 1.2 c.c., 5 to 20 minims, B.P.; in water. 
Mentha Viridis, U.S.P.; Spearmint.-The dried leaves and the flowering tops of Mentha spicata. Dose, 4 Gm., 60 gr., U.S.P.

(Aq. Menth. Vir., see Index.)

Ol. Menth. Vir., U.S.P., B.P.-The distilled oil, containing at least 40 per cent. of carvone. Dose, 0.2 c.c., 3 minims, U.S.P.; 0.03 to 0.18 c.c., $1 / 2$ to 3 minims, B.P.

Sp. Menth. Vir., U.SP.- - ro per cent. of oil, colored by maceration with spearmint herb. Dose, 2 c.c., 30 minims, U.S.P.; diluted.

Zingiber, U.S.P., B.P.; Ginger.-The dried rhizome of Zingiber officinale. The chief active ingredient is a volatile oil. Dose, I Gm., 15 gr., U.S.P., as powder or in water.

Similar to Ginger are: Galanga, Zedoaria, and Asarum.

Fldext. Zingib., U.S.P.-Strong alcohol. Dose, I c.c., I 5 minims, U.S.P.

Oleores. Zingib., U.S.P.-An evaporated ethereal extract. Dose, 0.03 Gm., 1/2 gr., U.S.P.

Syr. Zingib., U.S.P., B.P.-3 per cent., U.S.P.; 2.5 per cent., B.P. Dose, I 5 c.c., 4 drams, U.S.P.; 2 to 4 c.c., $1 / 2$ to I dram, B.P.

* Tinctura Zingiberis (Tr. Zingib.), U.S.P., Tincture of Ginger.-2o per cent. in alcohol. Dose, 2 c.c., 30 minims, U.S.P.

*Tr. Zingib.-B.P.; ro per cent. Dose, 2 to 4 c.c., $1 / 2$ to I dram., B.P.

\section{VEGETABLE CATHARTICS}

Manner of Action.-Cathartics or evacuants are drugs which produce defecation. They may do so by simply increasing the bulk of the intestinal contents (petrolatum, agar, etc.); or by preventing the absorption of water (salines); or by irritating the small or large intestine, as most of the vegetable cathartics. The cathartics differ from other irritants in that their irritation is relatively mild and is confined more specifically to the intestines. This may be explained by selective action, or more simply by the alkaline reaction of the intestines favoring their solution or decomposition. This solution must not be too rapid or the drugs will be largely absorbed or decomposed before reaching the lower intestine. The isolated principles are therefore less useful, and sometimes less effective than the galenic preparations in which they are protected by the extractives, gums, etc., of the drug.

Liberation and Solution of the Cathartic Constituents.-Castor oil and croton oil become active only when their fatty acids are liberated; croton oil contains some free acid and is, therefore, pustulant also on the skin. The emodin cathartics often contain glucosidal compounds, which must be split before the action occurs. The group of "resins" are insoluble in water, but are decomposed and dissolved by alkalies and bile.

Influence of the Bile on Cathartics.-The bile seems to be necessary for the activation of many of the resinous cathartics, the following being relatively or quite ineffective in its absence: gamboge, podophyllum, convolvulin, jalap, and scammonium (Buchheim and Stadelmann). The bile acts presumably by increasing the solubility. Rhubarb, senna and calomel are active in the absence of bile (Valeri, 1910). Aloe seems to act quite well without bile in animal experiments; but clinically it has been claimed that it is ineffective in biliary obstruction.

Efficiency on Hypodermic Administration.-The anthracene and resinous cathartics also increase peristalsis if they are injected subcutaneously or intravenously; the resingroup is even more active by this channel. The action, however, is even then a local one, the constituents being excreted into the intestine. The cathartics are not administered in this manner, in practice, since the injection would be painfully irritant; the effects are also more easily controlled when the drugs are given by mouth.

Absence of Toxic Effects.-Only very excessive doses of the drastic cathartics produce serious effects through gastroenteritis. A relatively mild irritation suffices for active catharsis. None of the vegetable cathartics are at all corrosive; and even toxic doses cause necrotic changes only as the result of excessive inflammation. The irritation is confined to the intestinal tract; the stronger cathartics escaping absorption, whilst the milder laxatives would scarcely be irritant even if absorbed. There is consequently no danger of nephritis. 
Location of Action.-This is of practical as well as scientific interest, because it often determines the choice of the cathartic. The older investigations have only a limited value, because they generally involve operative procedures which are apt to render the intestinal movements abnormal or to arrest them altogether. Cannon's method of observing these movements in intact animals by means of the Roentgen-rays has opened the way for more reliable observations. This method has been applied to pharmacologic problems especially by Magnus, I909 and by his pupils. Their results have been confirmed in man (f.i. Meyers-Betz and Gebhardt, 1912). They have shown in this way that Senna acts mainly on the large intestine, increasing the defecation-reflex. Castor oil stimulates peristalsis in the small intestine. Colocynth stimulates the movements of the small intestine and leads to intestinal effusion. Magnesium sulphate quickens the passage through the small intestine, and prevents the absorption of fluid and the concentration of the contents of the large intestine. Calomel increases peristalsis both in the small and large intestine.

The Normal Course of the Food through the Alimentary Canal.-The following description (Magnus) applies specifically to cats fed with 25 c.c. of potato mash, mixed with $5 \mathrm{Gm}$. of bismuth subnitrate (to render it opaque to the Roentgen-rays) and observed with the fluoroscope. The phenomena are similar with other food and in other animals, including man (Cannon, I9 I I).

Passage through the Stomach.-Immediately after feeding, the stomach is entirely filled, with a fundus dilated and resting. In the pyloric antrum, regular powerful peristaltic waves are observed, sweeping the contents against the pyloric sphincter. In a quarter of an hour, the food begins to be expelled into the duodenum, small portions at a time, the expulsion being completed in two or three hours; meats and especially fats are discharged more slowly.

Passage through the Small Intestine.-This exhibits three motor processes: (I) The pendulum movements, which produce an alternating "rythmic segmentation" of the contents; kneading and mixing the chyme, and exposing it to a large absorbing surface. (2) True peristaltic waves, appearing from time to time, and propelling the entire contents of the loop slowly for a few centimeters in a descending direction. - (3) Peristaltic "rush," sudden waves of contraction sweeping the contents swiftly through long segments. 'This is seen especially under pathologic conditions, such as poisoning.

In cats the passage of food into the cecum begins in two and one-half to three hours and is completed in seven to eight hours after the feeding. The passage is hastened by cellulose, delayed by fats, and especially by meats (some two hours). In man the usual time of appearance in the colon is four to six hours. The chyme as it reaches the colon is still semifluid.

Passage through the Large Intestine.-The colon is divided functionally into two portions. The proximal portion effects the concentration of the chyme by a churning process; groups of powerful ascending (antiperistaltic) waves sweeping the contents against the ileo-cecal valve. Extensive absorption of fluid occurs during this process, and as the contents becomes solidified, fragments are separated from the lower portion by annular contractions of the intestinal muscle. These masses then wander very slowly toward the rectum. The passage through the large intestine occupies about the same time as that of the small intestine, in man about six hours.

Defecation Reflex.-This is started by the arrival of the mass in the rectum. It is greatly influenced, however, by the sensitiveness of the rectum and by the habits of the patient. It is here that the delay in constipation mainly occurs.

Total Time for the Passage of Food.- In man the journey from the mouth to the rectum is made in about nine hours; the further delay in the rectum depends on the habits of the individual.

Peculiarities of the Cathartics.-These are largely explained by the mechanism, the location and the severity of the action.

Time of Action.-A cathartic which acts on the large intestine can not produce any effect until it reaches this place. Even then the response will depend on the habits of the patient. These become less important 
with the more irritant cathartics. The usual time of response, for the more important cathartics, is as follows:

Salines, one-half to one hour on empty stomach: two to four hours if in bed.

Castor oil, one to two hours on empty stomach.

Jalap, three hours or less.

Senna, four to five hours.

Rhubarb or Phenolphthalein, four to eight hours.

Cascara or Aloes, eight to twelve hours.

Podophyllum, ten to sixteen hours.

Colic and "Griping" is proportional to the severity of the irritation. It is due partly to increased peristalsis, partly to direct irritation, as evidenced by abdominal tenderness. It may be mitigated by belladonna and carminatives.

Consistency of the Stools and Elimination of Fluid.-The consistency varies from the soft but formed stools of the milder laxatives, to the watery stools of the "hydragogue" cathartics, the salines and drastics. The more rapid passage of the intestinal contents leaves less time for the absorption of fluid, so that the stools always contain more water. Some of the cathartics also prevent the absorption of fluid by osmosis (salines); or by imbibition (agar); and some perhaps by inhibiting the absorbing power of the epithelium (castor oil, calomel). The fluid in these cases comes really from the food rather than from the body. Even large doses of the anthracen cathartics (senna, cascara, etc.) do not increase the secretion of intestinal fluid (Thiry, Brieger, Flemming). On the other hand, the irritant cathartics (colocynth, jalap, etc.) actually produce effusion into the small intestine. The practical results, as concerns the water supply of the tissues, is the same in either case; whether these are deprived of fluid by effusion or by non-absorption.

Effects on the Local Circulation.-All catharsis must be accompanied by some hyperemia, were it only by the increased intestinal movement. The irritant cathartics produce a more violent intestinal congestion, which extends also to the pelvic organs, and thus increases the menstrual flow.

The Systemic Circulation.-This is affected indirectly by the splanchnic dilation; and by the withdrawal of fluid from the blood. Both factors tend to lower the blood pressure moderately. The heart rate is slowed reflexly.

C. H. Neilson and Hyland, r $9 \mathrm{r}_{3}$, made a clinical study of these effects after full doses of salts and compound jalap powder. The latter gave somewhat greater results. The average decrease of the systolic pressure was 17 per cent.; of the diastolic pressure, 8 per cent.; of the pulse pressure, 24 per cent.; of the heart rate, i4 per cent. The conditions generally returned to almost normal within a day. Individual cases, especially of arteriosclerosis, gave much greater falls (from 180 to $100 \mathrm{~mm}$.), sometimes with the appearance of arythmias and increased heart rate.

Influence on Dropsies.-Cathartics favor the absorption of dropsies by carrying off liquid from the body; in ascites, the emptying of the intestines also removes some of the pressure from the veins, and thus tends to the removal of the edema by improving the general circulation and the urine flow.

Elimination of Waste Products.-Cathartics are employed in nephritis to relieve the kidneys of a part of their work, by eliminating fluid, and presumably some of the metabolic products, through the stools. 
Uric Acid.-Abl, I913, claimed that cathartics increase the elimination of endogenous uric acid. Stehle, I915, found no change with laxatives, but only with drugs that stimulate the digestive glands.

Effects of Cathartics on Bile; Cholagogues.-The stools, with moderate catharsis, have usually a darker color, suggesting an increased bile content. This is not due to increased secretion of bile, as was supposed by the older therapeutists; but to the more rapid passage of the intestines, leaving less time for the reabsorption and decomposition of the bile. The dark color of calomel stools is due partly to mercurous sulphid. The only really effective stimulant of bile formation is the administration of bile or bile salts; salicylates (5 Gm. per day) and olive oil are perhaps also very slightly effective.

Experimental Investigations of Cholagogues.-The older experiments on animals with biliary fistulas, gave contradictory results, because they did not extend over sufficient time to discount the natural variations in bile flow. Reliable experiments on a human patient were made by Pfaff and Balch, I897; Van Hengel, I9I3; and Ignatowski and Monossohn, 1914. The last-named found the bile secretion increased by feeding animal protein, and especially fat. Sodium salicylate was less effective, and bile-acids least. Weinberg, 191 I, experimented in Pawlow's laboratory with dogs provided with gastric and biliary fistulas. He found the order of efficiency to be: bile $>$ soap $>$ albumose $>$ hydrochloric acid >olive oil. No effect was obtained with glycerin, egg albumen, saccharose, dextrose, water, normal saline or sodium carbonate. Okada, 19.5, found marked increase with salicylate, chloral, salol, potas. bitart., bile salts, acids, and large doses of alcohol. A slight diminution was caused by atropin; no effect by calomel or sod. bicarb. Food increased the secretion, but the nature of the diet was generally unimportant; fats, egg white, albumose and meat extracts produced some increase; starch and sugar were indifferent. Volborth, 1915; reports diminished filling of the gall-bladder after milk or oil; increased filling after acid or bile.

Evacuation of Gall-bladder.-Klee and Knuepfel, $\mathbf{r}_{9} \mathbf{r}_{4}$, found that this is stimulated, in animals, by eating, entrance of the chyme into the intestine, injection of Witte's peptone; and especially by the administration of olive oil. No effect was produced by soap or hydrochloric acid. Weinberg, rgi r, also observed this effect of oil.

Aulonomic Drugs on Gall-bladder.-Lieb and McWhorter, I915, found that the muscles of the gall-bladder receive augmentor impulses from the parasympathetic, inhibitory from the sympathetic. The autonomic drugs act correspondingly: Pilocarpin, barium and strophanthin stimulate; atropin, epinephrin, nitrites and bile salts relax. The rhythmic movements are increased during digestion; or on the introduction of acid into the duodenum (Okada, I9 15).

Sphincter of Biliary Papilla.--Reach, I9r4, states that the tone of this is increased, and the bile flow correspondingly hindered, by morphin, epinephrin, pilocarpin and atropin.

Therapeutic Indications.-Cathartics are used chiefly for the following indications:

To relieve constipation-cascara, rhubarb, phenolphthalein, small doses of salts.

To sof ten the stools, in hemorrhoidal and other rectal diseases; and to prevent straining in aneurism, hernia, and apoplexy-petrolatum, cascara, podophyllum, sulphur.

To cleanse the intestines in digestive disturbances, diarrheas and other intestinal putrefactions-calomel, castor oil, rhubarb, cascara, aloes.

To facilitate the absorption of dropsies; to relieve the kidneys in nephritis; to lower the temperature in fevers; to relieve internal congestions, especially of the brain-calomel, large doses of salines, compound jalap powder; in emergencies, croton oil or elaterin.

Cathartics are perhaps the most ancient method of internal medication, and were for a long time practically the only method. Even the Greeks used the same word,

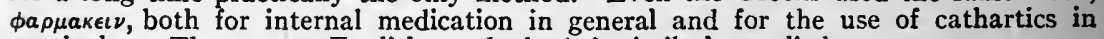
particular. - The present English word physic is similarly applied. 
Contraindications.-All inflammatory conditions of the abdominal organs (peritonitis, gastroenteritis, etc.) preclude the administration of intestinal irritants. Pregnancy and menstruation are contraindications to the use of the stronger cathartics, since the hyperemia may lead to abortion or excessive menstrual flow. General debility, tendency to collapse, threatened intestinal hemorrhage, and toxic spasm of the intestine, are further contraindications. Cathartics should not be used in intestinal obstruction or intussusception.

Habitual Constipation.-In this condition ("chronic intestinal stasis"), cathartics should be used as little as possible; the condition being treated most effectively by the removal of the cause and the cultivation of proper hygienic and dietetic habits; aided by the mechanical laxatives, petrolatum, agar or enemas. Small doses of salts, cascara or similar aperients may be needed. The irritant cathartics should be avoided.

In using cathartics the rule should always be to employ the mildest remedy which will accomplish the result. One reason for this is that soft, not liquid, stools are desired; but, further, a "habit" is quite readily acquired so that the intestines will require stronger and stronger stimuli, and the usual cathartics gradually lose their efficiency. If the treatment has been begun with mild measures, stronger ones are left for later use if necessary. The habitual use of very irritant cathartics is also quite apt to engender a chronic enteritis.

Therapeutic Classification. - The cathartics may be classified according to their clinical characters, i.e., mainly by the degree of their effects. This can not be done rigidly, because the effect varies with the dose; moreover, the names are defined somewhat differently by individual authors.

The following table gives a useful clinical classification:

I. Laxatives or Aperients.-Increase peristalsis only moderately, producing somewhat more frequent stools, of almost normal consistency, and this without causing notable irritation. They are active in doses of ro $\mathrm{Gm}$. or over.

Fruits, Manna, Agar, etc.; mechanical means (massage, enemas, etc.); Sulphur, Magnesia, Carminatives, Bile, Bland Oils (Olive, Cottonseed, Petrolatum, etc.).

(Small doses of Cascara, Senna, Castor Oil, Rhubarb, and Ipecac are also laxative.)

2. Purgatives.--Increase peristalsis actively, causing more frequent semifluid stools.

(A) Simple Purgatives.-Active in doses of 0.2 to $\mathrm{r} \mathrm{Gm}$. These cause more or less colic and irritation:

Aloes, Rhubarb, Senna, Cascara, Phenolphthalein, Castor Oil, Calomel, small doses of drastics.

(B) Saline Purgatives.-Active in doses of about to Gm. Rather profuse, watery stools, with practically no irritation or griping:

Sulphate of Magnesium or Sodium, Sodium Phosphate, Magnesium Citrate, Potassium Bitartrate, Rochelle Salt, etc.

3. Drastics.-Produce watery stools, with much irritation. Large doses are apt to set up an enteritis:

Elaterium, Colocynth, Jalap, Scammony, Gamboge, Podophyllum, Croton Oil, the stronger Mercurials, and Antimony Sulphid.

4. Hydragogues.-All which produce watery stools, i.e., the salines and drastics.

5. Cholagogues.-Were supposed to increase the bile flow: 
Aloes, Rhubarb, Mercurials, Podophyllum, Euonymin, Sod. Salicylate or Phosphate, Acid. Nitrohydrochlor. Dilut., Bile.

Pharmacologic Classification.--The cathartics may also be divided into the following groups:

I. Those which act mainly on the colon and rectum, inhibiting colon antiperistalsis, and provoking the defecation reflex. This includes the drugs containing emodin and other anthraquinon derivatives: Senna, Cascara, Rhubarb, Aloes, and perhaps also Phenolphthalein and Bile.

2. Those which stimulate the peristalsis of the small intestine and inhibit the antiperistalsis of the colon, but without producing much irritation or effusion. This includes Castor Oil and probably Calomel and Sulphur.

3. Those which, in addition to these actions, cause marked intestinal effusion and irritation. This comprises Colocynth, Jalap, and presumably the other drastic resinous cathartics, Podophyllum, Elaterin, Gamboge, Croton Oil, etc.

4. The saline cathartics, which retain water by osmosis.

5. The colloid cathartics, which retain water by imbibition: Agar, Fruits, Manna, etc.

6. Emollient cathartics, such as Petrolatum or Olive Oil.

7. Enemas and similar local measures.

\section{THE ANTHRAQUINON OR EMODIN CATHARTICS}

Active Constituents.-Senna, Rhubarb, Cascara, Aloes and related drugs contain glucosidal compounds which are themselves inactive. In the alkaline intestine they are hydrolyzed or oxidized, yielding various oxymethyl-anthraquinons (Buchheim; Tschirch and pupils, I899), which produce the cathartic action. The pure substances would be too irritant, but their action is graded by their slow liberation, and by the presence of colloid extractives. The most common of these substances are emodin and chrysophanic acid. Numerous isomers of these are possible ( 5 for emodin alone). The special characters of the different drugs is probably due to differences in these isomers; in the stability of their glucosidal combination; and to the presence of other associated substances (tannin in rhubarb). The action of these drugs never progresses to inflammation. They are often more effective when given in divided doses (three times daily), than when given in a single dose.

Emodin is partly absorbed and is excreted into the urine and milk (laxative effects on sucklings).

The structure of these anthraquinons is shown by the following formulas:

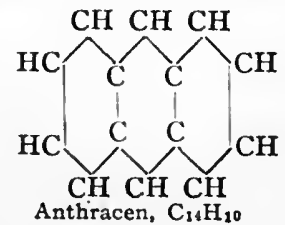

Anthracen, $\mathrm{C}_{14} \mathrm{H}_{10}$<smiles>O=C1C=CCCCCCCCCCCC1</smiles>

Anthraquinon, $\mathrm{C}_{14} \mathrm{H}_{3} \mathrm{O}_{2}$ 


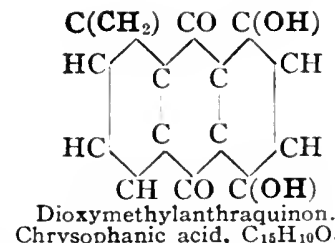

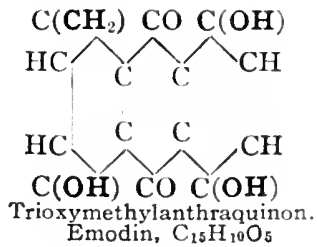

Emodin is probably the most important of these principles. It is present in the amount of 0.8 to $\mathrm{r}$ per cent. in senna; 2.6 per cent. in frangula; 0.6 per cent. in cascara; 0.8 per cent. in Cape aloes; and $\mathrm{I} .5$ per cent. in rhubarb (Tschirch and Hiepe).

Coloration of Urine.-The drugs containing emodin color the urine yellowish-brown when it is acid, reddish or violet when alkaline. It may be advisable to acquaint the patient with this fact.

Other drugs which change the color of the urine are: Logwood (Hematoxylon) does not color acid urine, but produces a reddish or violet color in alkaline urine. Santonin gives a yellow color to acid urine, with a yellow foam; if the urine is made alkaline it gives a very pronounced pink. Picric acid gives reddish-brown color in both acid and alkaline urine. The various coal-tar products give a brownish-black color. Methylen blue imparts a green color.

Manner of Action.-The older work (Buchheim; Tappeiner and Brandl, I889) showed that their action is mainly on the large intestine, which reacts by increased movement also to intravenous injection; and that there is no increase of intestinal secretion. The X-ray experiments of Magnus with senna show that the effects consist chiefly in increase of the local defecation reflex, and inhibition of the colon antiperistalsis.

Experiments of Magnus (rgo9).--Senna does not affect the course of the chyme through the stomach and small intestine. The first eifect occurs when the senna reaches the colon, so that its action must be strictly local. Here it inhibits antiperistalsis and thus prevents the concentration of the feces. At the same time, it starts the defecation reflex. It thus leads to the expulsion, first of the solid contents already present in the rectum, and then of the more fluid contents of the upper colon.

\section{CASCARA SAGRADA}

Cascara is used chiefly in habitual constipation. It softens the stools without noticeable irritation. The susceptibility is not lost with use; on the contrary, the establishment of regular habits usually permits it to be gradually withdrawn. By its bitter taste, it acts also as stomachic. It may be given as the fluidextract, 15 drops three times daily; or in a single dose, a half teaspoonful, in the evening. This causes a single soft stool in the morning. In the Aromatic Fluidextract, which is much more pleasant, some of the bitter and cathartic principles have been destroyed (Wenzell r886) by an alkali (magnesia). It may be given in twice the dose.

It is generally stated that the fresh bark is emetic, through the presence of a ferment, which gradually disappears as the bark is aged. R. H. True, and Klugh, rgog, did not find any emetic effect from the unaged bark.

In the excised intestine, cascara increased the rate, but lessened the intensity of the contractions (Flury, r912). The tone is increased by small, and lowered by large doses, also intravenously (Ott and Scott, rgo8).

When cascara is administered hypodermically (as with the proprietary "Peristaltin"), its constituents are rapidly absorbed and excreted by the kidneys, producing renal irritation, but not catharsis (Chistoni, r914).

Cascara sagrada ("Sacred Bark") was used by the early settlers, but was little known to the profession till introduced by Bundy, 1877. Johnson and Hindman, r9r4, review its history, composition and bibliography. 


\section{PREPARATIONS-CASCARA}

Cascara Sagrada (Casc. Sagr.), U.S.P., B.P.; Cascara Sagrada (Cascara; Rhamnus Purshiana). - The dried bark of the trunk and branches of the shrub Rhamnus Purshiani, stored at least one year. The active principles are: Cascarin, which is a cathartin or emodin principle; isoemedin, bitter resins, etc.; no chrysarobin or chrysophanic acid (Jowett, I904. This author claims that emodin is not the main active constituent). Dose, I Gm., I 5 gr., U.S.P.

* Ext. Cascar. Sagr., U.S.P.; Exi. Casc. Sagr. Sicc., B.P.-A powdered extract, I Gm. representing 4 Gm. of drug, U.S.P. Dose, 0.25 Gm., 4 gr., U.S.P.

* Fldcxt. Cascar. Sagr., U.S.P.; Ext. Cascar. Sagr. Liq., B.P.-Dose, I c.c., I 5 minims, U.S.P.; 2 to 4 c.c., $1 / 2$ to I dram, B.P.

* Fldext. Cascar. Sagr. Arom., U.S.P. (Aromatic Cascara).-A watery extract, with the bitter principles partly destroyed by maceration with magnesia; preserved with 25 per cent. of alcohol, flavored with glycyrrhiza, aromatics and saccharin. Dose, 2 c.c., 30 minims, U.S.P.

* Syr. Casc. Arom., B.P.-40 per cent. of Liquid Extract, flavored with orange, cinnamon and syrup. Dose, 2 to 8 c.c., $1 / 2$ to 2 drams, B.P.

Frangula, U.S.P. (Buckthorn Bark).- The dried bark of Rhamnus Frangula. Dose, I Gm., I5 gr., U.S.P.

Fldext. Frangul., U.S.P.-Dose, I c.c., I 5 minims.

\section{SENNA}

The effects are more extensive and more irritant than with the other drugs of the group, so that it is better suited for acute than for chronic constipation. There is considerable griping, which may be corrected by carminatives, or by the previous extraction of the resins with alcohol (as in the Fluidextract).

\section{PREPARATIONS-SENNA}

* Senna (Senn.), U.S.P.; Sennce Folia (Senn. Fol.), B.P.; Senna.--The dried leaflets of Cassia acutifolia or angustifolia. Introduced by the Arabians. Constituents: senna-emodin, senna-isoemodin, senna-chrysophanic acid, and other anthraquinon derivatives, free and in glucosidal combinations (Tutin, I9I3; Tambach, I913). Dose, ${ }_{4} \mathrm{Gm}$., I dram, U.S.P., as cathartic; as laxative, I to $2 \mathrm{Gm}$., I 5 to $30 \mathrm{gr}$., infused in a cup of hot water. Many of the laxative nostrums ("Castoria;" "Fig Syrup;" various "Teas") owe their activity mainly to senna.

A preparation for hypodermic use is marketed as "Sennatin." Betke, I9I4, claims good effects in postoperative ileus. B.P.

Conf. Senn., B.P.- io per cent. of powdered Senna. Dose, 4 to $8 \mathrm{Gm}$., I to 2 drams,

Inf. Senn., B.P.- - тo per cent., with Ginger. Dose, I 5 to 30 c.c., $1 / 2$ to $\mathrm{I}$ ounce, repeated; single, 60 c.c., 2 ounces, B.P.

Inf. Senn. Co., U.S.P. (Black Draught) - Average dose, 120 c.c., 4 ounces, U.S.P., contains about $7 \mathrm{Gm}$. of Senna; $15 \mathrm{Gm}$. of Magnes. Sulph., with Manna and Fennel. Fldext. Senn., U.S.P.-Dose, 2 c.c., 30 minims, U.S.P.

Mist. Senn. Co., B.P.-25 per cent. of Magnes. Sulph. in Inf. Senn., with aromatics. Dose, 30 to 60 c.c., $I$ to 2 ounces, B.P.

* Syrupus Senne (Syr. Senn.), U.S.P.-25 per cent. Flavored with coriander. Dose, 4 c.c., I dram, U.S.P.

* Syr. Senn., B.P.-About 40 per cent., with coriander. Dose, 2 to 8 c.c., $1 / 2$ to 2 drams, B.P.

Tr. Senn. Co., B.P. -20 per cent., with caraway and coriander. Dose, 2 to 4 c.c., $1 / 2$ to I dram, repeated; single, 8 to I 5 c.c., 2 to 4 drams, B.P.

* Pulvis Glycyrrhize Compositus (Pulv. Glycyrrh. Co.), U.S.P., B.P. (Compound Licorice Powder).- Greenish-yellow to greenish-brown powder; odor of fennel. Average dose, $4 \mathrm{Gm}$., I dram, U.S.P.; contains about $0.7 \mathrm{Gm}$. of Senna, $0.3 \mathrm{Gm}$. of Sulphur and a little Fennel oil. Dose, 4 to $8 \mathrm{Gm}$., I to 2 drams, B.P.

Sennce Fructus (Senn. Fruct.), B.P.; Senna pods. - The dried ripe fruits of Cassia acutifolia or angustifolia.

Cassice Fructuis (Cass. Fruct.), B.P.-The ripe fruits of Cassia Fistula. 
Cassia Pulpa (Cass. Pulp.), B.P.; Cassia Pulp.-An evaporated watery extract of the preceding.

\section{RHUBARB}

This contains a considerable quantity of tannin as well as cathartic principles. The astringent action predominates with smaller doses $(0.05$ to $0.3 \mathrm{Gm}$.) and these are used as astringent bitter in gastric catarrh, and in diarrhea. Larger doses ( $\mathrm{I}$ to $5 \mathrm{Gm}$.) are laxative with little colic. They may be employed in chronic constipation, but the astringent action makes it inferior to cascara. It may cause skin eruptions.

Constituents (Tutin and Clewer, I9II). The cathartic principles are mainly a resin, non-glucosidal, but yielding on hydrolysis various anthraquinon derivatives, which also exist partly free: rhein, emodin, aloe-emodin, chrysophanic acid, etc. The astringent principles are glucosidal compounds of tannin (rheotannic acid), and gallic acid. There is also a large amount of calcium oxalate.

\section{PREPARATIONS-RHUBARB}

Rheum, U.S.P.; Rhei Rhiz., B.P.; Rhubarb.-From Rheum officinale and probably other species growing in China and Thibet. (The species of Rhubarb cultivated in this country are devoid of cathartic properties.) Rhubarb has been used since antiquity in China, and by the Roman physicians. Dose, I Gm., I 5 gr., U.S.P.; 0.2 to 0.6 Gm., 3 to Io gr., repeated; single, I to 2 Gm., I 5 to 30 gr., B.P.

Ext. Rhei., U.S.P., B.P.-A powdered extract, I Gm. representing $2 \mathrm{Gm}$. of drug, U.S.P. Dose, 0.25 Gm., 4 gr., U.S.P.; 0.12 to 0.5 Gm., 2 to 8 gr., B.P.

Fldext. Rhei, U.S.P.-Dose, I c.c., I 5 minims, U.S.P.

Inf. Rhei, B.P. -5 per cent. Dose, I 5 to 30 c.c., $1 / 2$ to I ounce, B.P.

Mist. Rhei et Soda, N.F.-Sod. bicarb., 3.5 per cent.; Fluidext. Rhubarb, I. 5 per cent.; Fluidext. Ipecac, 0.3 per cent.; Sp. Peppermint, Glycerin, and Water. Dose, 4 c.c., I dram.

Pil. Rhei Co., U.S.P.-Each pill contains 0.13 Gm. of Rhubarb, ०.0I Gm. of Aloes. Dose, two pills, U.S.P.

Pil. Rhei Co., B.P.-Pill-mass containing 25 per cent. of Rhubarb, 20 per cent. of Aloes, I4 per cent. of Myrrha. Dose, 0.25 to $0.5 \mathrm{Gm}$., 4 to $8 \mathrm{gr}$., B.P.

Pulv. Rhei. Co., U.S.P., B.P. 25 per cent. of Rhubarb, 65 per cent. Magn. Oxid., to per cent. of Ginger, U.S.P.; the B.P. formula is almost the same. Dose, 2 Gm., 30 gr., U.S.P.; 0.6 to $4 \mathrm{Gm}$., io to 60 gr., B.P.

Syr. Rhei, U.S.P. - Io per cent. of Rhubarb; I per cent. of Pot. Carb.; flavored with Cinnamon. Dose, Io c.c., $21 / 2$ drams, U.S.P.

Syr. Rhei, B.P. - 7 per cent., with Coriander. Dose, 2 to 8 c.c., $1 / 2$ to 2 drams, B.P.

* Syr. Rhei Aromaticus (Syr. Rhei Arom.), U.S.P.; Aromatic Syrup of Rhubarb. I 5 per cent. of Tr. Rhei Arom.; o.I per cent. Pot. Carb. Dose, 10 c.c., $21 / 2$ drams, U.S.P.

Tr. Rhei, U.S.P. -20 per cent.; flavored with Cardamon. Dose, 4 c.c., I dram, U.S.P.

* Tinctura Rhei Aromatica (Tr. Rhei Arom.), U.S.P.-20 per cent. flavored with Cinnamon, Clove and Nutmeg. Dose, 2 c.c., 30 min., U.S.P.

* Tinctura Rhei Composita (Tr. Rhei Co.), B.P.-ro per cent.; flavored with Cardamon and Coriander. Dose, 2 to 4 c.c., $1 / 2$ to I dram, repeated; single, 8 to I6 c.c., 2 to 4 drams, B.P.

\section{ALOES}

General Statement.-The inspissated juice of the leaves of various species of Aloe from the West Indies, Africa, etc., furnishes the different commercial varieties of aloes, which differ somewhat in composition and action. They contain anthraquinon derivatives, especially aloins (Io to 16 per cent.). These are glucosids which are probably inactive themselves; but which yield emodin and other active anthraquinon derivatives on cleavage. This cleavage occurs in the intestine; it is favored by alkalies and perhaps also by bile and by iron salts. Aloin may be used as such, 
but is generally considered less satisfactory than aloes. Aloe is intensely bitter, so that it must be administered in pills. Very small doses may be used as stomachic. Moderate doses are simply laxative, but somewhat apt to gripe, and are therefore commonly combined with belladonna or carminatives (myrrh, etc.). They do not lose their efficiency on continued use and would be suited to habitual constipation, but have no advantage over cascara. Aloes are believed to produce considerable congestion of the pelvic organs and are therefore contraindicated in hemorrhoids, menstruation and pregnancy. On the other hand, they may be used as emmenagogues. They are especially valuable for correcting the constipative action of iron medication.

Hypodermic Administration.- Aloin is also effective subcutaneously, being excreted into the large intestine; but this method is too painful (H. Meyer, $\mathrm{I} 890$ ).

Aloin Nephritis. - In rabbits, hypodermic injection (2 c.c. per kilogram of 5 per cent., repeated on two to three successive days) produces a tubular nephritis. The anatomic lesions have been investigated especially by Mürset (1885); they are practically the same in acute and chronic poisoning, and consist mainly in degeneration of the epithelium of the convoluted tubules. This loses its striations and staining qualities, and the nuclei disappear. The glomerular epithelium is but slightly altered, and the glomerular vessels show no lesions. The urine may be increased or diminished and contains proteins, leucocytes, casts, crystals, and blood.

Metabolism.-There is also an increased protein metabolism, resulting in rise of urea in mammals, of uric acid in birds. The latter may therefore develop gout. Dogs show some fever (Berrar, I9I3).

\section{PREPARATIONS-ALOES}

* Aloes, U.S.P., B.P.; Aloes.-The dried juice of the leaves of Aloe species: Aloe Perryi, yielding Socotrine Aloes; Aloe vera, yielding Curaçoa Aloes; Aloe ferox, yielding Cape Aloes (Natural history of the commercial aloes, Wilbert, r903). Aloe was used by the Greeks and probably by the Egyptians. Brown, opaque, glassy masses; aromatic odor; nauseous bitter taste. Partly sol. in water; completely sol. in alc. Dose, 0.25 Gm., 4 gr., U.S.P.; O.I 2 to $0.3 \mathrm{Gm}$., 2 to 5 gr., B.P.; best as pills.

Dec. Aloes Co., B.P.- I per cent. of Ext. Aloes, with Myrrh, Aromatics and Liquorice. Dose, I 5 to 60 c.c., $3 / 2$ to 2 ounces.

Ext. Aloes, B.P.-A powdered watery extract. Dose, 0.06 to $0.25 \mathrm{Gm}$., I to $4 \mathrm{gr}$.,

Pil. Aloes, U.S.P. -0.13 Gm., 2 gr. of Aloes, with Soap. Dose, two pills, U.S.P.

Pil. Aloes, B.P. -58 per cent., with Soap and Caraway Oil. Dose, 0.25 to $0.5 \mathrm{Gm}$., 4 to 8 gr., B.P.

Pil. Aloes et Asafet., B.P.- 20 per cent., each, of Aloes, Asafet., and Soap. Dose, 0.25 to 0.5 Gm., 4 to 8 gr., B.P.

(Pil. Aloes et Ferr., see Index.)

Pil. Aloes et Myrrh., B.P.-44 per cent. of Aloes, 22 per cent. of Myrrh. Dose, 0.25 to $0.5 \mathrm{Gm} ., 4$ to 8 gr., B.P. U.S.P.

Tr. Aloes, U.S.P.- 10 per cent. of Aloes, with Glycyrrhiza. Dose, 2 c.c., 30 minims,

* Aloinum (Aloin.), U.S.P., B.P.; Aloin.-A pentosid or mixture of pentosids obtained from aloes (usually from Curaçoa, W. Hills, rgo8), varying in chemical composition, physical and chemical properties according to the source. A yellow microcrystalline powder, practically odorless, intensely bitter taste. Sol. in water and alc. Dose, $0.015 \mathrm{Gm}$., $1 / 4$ gr., U.S.P.; 0.03 to $0.12 \mathrm{Gm} ., 1 / 2$ to 2 gr., B.P.

Pil. Aloin. Bellad. et Strychn. (Pil. Laxativæ Co., U.S.P. VIII).-Each contains: Aloin, $13 \mathrm{mg}$. (1/5 gr.); Strychnin, 0.5 mg. (1/128 gr.); Ext. Belladonn., $8 \mathrm{mg}$. (1/8 gr.); Ipecac, $4 \mathrm{mg}$. (1/16 gr.). Dose, Two pills.

Nataloin.- This differs from the other aloins in composition, and is more difficultly hydrolyzed (H. Meyer).

Synthetic anthracen derivatives with laxative action have been prepared, but have little practical importance (purgatin, anthrapurpurin diacetate: exodin, methyl esters of diacetyl-hexaoxy-anthraquinon). 


\section{PHENOLPHTHALEIN}

This is a mild tasteless purgative, acting probably on the large intestine. It is but little absorbed from the alimentary canal, and is ordinarily nontoxic even in large doses. It may be used in habitual constipation, and has been exploited under several proprietary names (purgen, laxans, etc.). Phenoltetrachlorphthalein, $0.4 \mathrm{Gm}$. dissolved in $20 \mathrm{c.c}$. of neutral olive oil, has been suggested as a subcutaneous purgative (Abel and Rowntree, I909; Rowntree, I9I0). Phenolsulphonephihalein is used to test the excretory efficiency of the kidneys (Abel and Rowntree, I909; Rowntree and Geraghty, I9Io; Chesney, Marshall and Rowntree, I9I4).

Action.-The purgative effect of this familiar indicator was discovered by $\mathrm{v}$. Vamossy, 1900. It appears to be connected with the quinon structure. It has been investigated experimentally by v. Vamossy, 1902; Tunnicliffe, 1902; Fleig, 1908; Abel and Rowntree, I909, etc.; and clinically by Dornblueth, 1903, and many others. Animals are rather less susceptible than man. Ott and Scott, I908, found evidence that it acts directly on the muscle of the excised intestine. (Fleig and others attribute the action to increased intestinal secretion, but the evidence is not satisfactory.) It acts also on hypodermic or intravenous administration, reaching the intestine through the bile. The blood pressure is scarcely affected, even when large doses are injected into a vein (Tunnicliffe; Abel and Rowntree, etc.); the kidneys are not irritated, nor are there any other systemic effects. Phenolphtbalein itself does not produce local irritation, but its soluble salts are highly irritant. It is not bactericidal (Abel and Rowntree).

Absorption, Fate and Excretion. - Phenolphthalein is insoluble in acids, but gives a pink'solution with alkalies. It passes the stomarh unchanged. It is partially dissolved in the alkaline intestine, but very little is absorbed, almost the entire quantity (over 85 per cent.) appearing unchanged in the feces (Vamossy). After large doses, traces are found in the urine (Fleig; Elmer, I909), which is then colored violet-red by alkalies (Gruebler, rgo6).

After hypodermic administration, it is excreted by the urine, feces, saliva, tears, etc. The excretion is very rapid, beginning within fifteen minutes and ending in twelve to eighteen hours. In the urine it exists partly free, partly conjugated. The body-fluids dissolve it more readily than water (Fleig). It is excreted into the intestine mainly by the bile, and is to some extent reabsorbed in the large intestine (Abel and Rowntree). (The fate of the phthaleins is also discussed by Kastle, I906.)

Toxic Effects.-Only a few doubtful cases are on record (Holz, I905; Best, 1906; Zabel, I9II; Orland, I9I3, etc.). These presented phenomena of intestinal irritation, always ending in recovery. Hydrick, 1914 , claims that even 0.05 to $0.15 \mathrm{Gm}$. produce quite constantly a slight albuminuria, lasting one to three days. On the other hand, very large doses have often been given without bad effects: to man, several grams by mouth, $0.5 \mathrm{Gm}$. subcutaneously; to dogs, $1.25 \mathrm{Gm}$. per kilogram.

Administration.-Phenolphthalein may be taken in the evening, as powder, capsules, or lozenges, in doses of 0.1 to $0.2 \mathrm{Gm}$., I $1 / 2$ to $3 \mathrm{gr}$.; for bed-ridden patients, $0.5 \mathrm{Gm}$., 8 gr.; for children, $0.03 \mathrm{Gm}$., $1 / 2 \mathrm{gr}$. These smaller doses soften and regulate the stools for several days, with little or no colic. Large doses are hydragogue.

\section{PREPARATIONS-PHENOLPHTHALEIN}

* Phenolphthaleinum (Phenolphthal.), U.S.P., B.P.; Phenolphthalein (Dihydroxyphthalophenone), $\left(\mathrm{C}_{6} \mathrm{H}_{4} \mathrm{OH}\right)_{2} \mathrm{CO} . \mathrm{C}_{6} \mathrm{H}_{4} \mathrm{CO}$. - White crystalline powder, odorless and tasteless. Almost insol. in water, sol. in alc. (r: $\left.\mathrm{I}_{3}\right)$. Dose, o.1 5 Gm., $2 \frac{1}{2}$ gr., U.S.P.; 0.12 to $0.3 \mathrm{Gm} ., 2$ to $5 \mathrm{gr} .$, B.P.

Phenolsulphonephthalein, N.N.R.-The preceding, with $\mathrm{CO}$ replaced by $\mathrm{SO}_{2} . \quad$ Dose, $6 \mathrm{mg}$., as test of kidney function (for details, see N.N.R.).

\section{BILE}

The administration of bile or bile salts (sodium glycocholate and taurocholate) stimulates the secretion of bile (Pfaff and Balch, I897), increases 
peristalsis (Stadelmann, I 896); acts as antiseptic (Limbourg), and facilitates the digestion of fat. They have been advocated in obstructive jaundice and in biliary fistulas; but their effects are not very pronounced. Added to enemas, bile is used as a laxative against impacted feces and against ascaris, but has little advantage over soap.

Peristalsis.-On excised small intestines, or large, bile or bile salts produce mainly depression (Ott and Scott, 1909; d'Errico, 1910); but the large intestines may be stimulated (Scliuepbach, 1910). In man, evacuation is assured especially by enemas, but also by oral administration (Glaessner and Singer, I9I0).

Excised Uterus.-Bile and bile salts, in moderate concentrations, increase the contractions of the pregnant, not of the virgin uterus. High concentrations inhibit (Cantoni, I9I4).

Toxic Actions.-Bilc is non-toxic by mouth, but when injected into the circulation (Meltzer and Salant, I905, I906; Bunting and Brown, I9II) it produces severe nervous and cardiac depression (Brandenburg, 1903; Glur, 1909) and hemolysis. It is also directly toxic to muscle and nerve. J. H. King and H. A. Stewart claim that the pigments are mainly responsible for the circulatory effects. However, bile-acids are highly toxic. Small doses stimulate the vagus center; larger doses depress the heart directly, with slow irregular pulse and low blood pressure. They also produce depression of the medullary centers, coma and paralysis; and hemolysis and cell destruction.

Bile Salts in Gonorrhea.-Loehlein, 1909, introduced the local injection of bile salts in the acute stage for destroying the gonococci. V. Hofmann, I9I2, found that I to 2 per cent. had little effect; 5 to ro per cent. gave better but only temporary resultsThese were improved by following with silver injections, or by using a soluble silverbile acid compound.

\section{PREPARATIONS-BILE}

Fel Bovis, U.S.P.; Oxgall.-The fresh bile of Bos taurus.

Fel Bovinum Purificatum (Fel Bov. Pur.), B.P.; Purified Ox Bile.-An evaporated alcoholic extract.' Dose, 0.3 to I Gm., 5 to 15 gr., B.P.; as enema, I: 50 .

* Extractum Fellis Bovis (Ext. Fel. Bov.) U.S.P.; Extract of Oxgall (Purified Oxgall). - An alcoholic extract of bile, evaporated and mixed with starch; I Gm. representing $8 \mathrm{Gm}$. of Oxgall. Very bitter yellow powder. Dose, $0.1 \mathrm{Gm}$., I1/2 gr., U.S.P., in pills or formaldehyd-hardened capsules. As enama $2 \%$.

Bile Salis, N.N.R.-The various commercial preparations are used as pills, generally 0.05 to $0.5 \mathrm{Gm}$., $1 / 2$ to $1 \mathrm{gr}$; or better as suppositories, in the same dosage.

\section{CASTOR OIL}

This consists mainly of the triglycerid of an unsaturated fatty acid, Ricinoleic acid. The neutral fat is not active, but becomes so when it is saponified in the intestine. The cathartic effect is due mainly to motor stimulation of the small intestine. The intestinal secretion is not increased, the fluid character of the stools being due to the quicker passage of the feces.

With the usual dose, $1 / 2$ to $\mathrm{I}$ ounce, the response is certain and complete, resulting in a few hours in one or two extensive semifluid stools, with but little hyperemia or irritation, there being little or no colic. The evacuation is so complete that there may be some after-constipation. It is especially suited to the treatment of acute constipation or acute diarrhea from dietetic errors, ptomain and other poisoning, etc. It is less useful for continuous use, since this may produce gastric disturbance. There is some pelvic congestion, so that it is avoided during menstruation and pregnancy.

Mechanism of Action. - This has been studied with $x$-ray by Magnus, 1908 , and his observations have been confirmed on man. The pure oil delays slightly the passage through the stomach (as do other neutral oils). Rancid oil hastens the gastric expulsion. In the small intestine, the segmenting movements and peristalsis are greatly increased, 
so that the expulsion may be completed in two hours instead of the normal eight hours. In the colon, the normal antiperistalsis is allayed, so that the feces escape concentration. The propulsive movements of the colon are not much increased, but the passage of the fecal material is hastened by its thinner consistency.

In China it is used as an article of diet. The properties of Castor Oil were known to Herodotus; but Croton Seeds were first described in the middle of the sixteenth century.

Administration. - It is best given on an empty stomach an hour before breakfast. The taste may be somewhat disguised by wetting the mouth with hot coffee or milk, and giving the oil floating in these liquids, so that it will not be so adherent. The after-taste may be mitigated by peppermint or bread. It is sometimes preferred in emulsions: N.F., 33 per cent.; B.P. (Mistura), 40 per cent., flavored with orange flower and cinnamon. The soft gelatin capsules are the only tasteless method; but since the ordinary size contains only I c.c., it is difficult to administer an effective dose. (For other methods, see J.A.MA., 60: II 74.)

\section{PREPARATIONS -CASTOR OIL}

* Oleum Ricini (Ol. Ricin.), U. S. P.; Castor Oil.-A fixed oil expressed from the seed of Ricinus communis. An almost colorless viscid oil, of faint but characteristic odor and taste, extremely disagreeable to many persons. Sol. in an equal volume of alc. Dose, 15 c.c., 4 drams, U.S.P.; 4 to 30 c.c., I to 8 drams, B.P.; for infants, a teaspoonful. Also used as hair tonic, diluted with I to Io vol. of alcohol.

Mist. Ol. Ricin, B.P.-A 37.5 per cent. emulsion. Dose, 30 to 60 cc., I to 2 ounces, B.P

\section{RICIN}

Occurrence, Clinical Symptoms and Treatment.-This toxin is contained in the castor seeds, but does not pass into the oil. Similar phyto-toxins occur in croton seeds (Crotin); and in jequirity seeds (Abrin); in the bark of the locust tree, Robinia pseudacacia (Robin); and in the seeds of some leguminous plants (Phasin). The last is but weakly toxic (Review of Literature, Ford, I9I3). The ricin is responsible for the toxic effects on eating the castor seeds; five or six of these are fatal to a child, $t$ wenty to adults; three or four seeds may cause violent gastroenteritis, with nausea, headache, persistent vomiting, colic, sometimes bloody diarrhea, thirst, emaciation, and great debility. The symptoms usually do not set in until after several days. More severe intoxications cause small frequent pulse, cold sweat, icterus, and convulsions. Death occurs in six to eight days, from the convulsions or from exhaustion. The fatality is about 6 per cent. This small fatality is due to the destruction of the poison in the alimentary canal. The treatment would be evacuant and symptomatic. Three to ten days are required to complete recovery (Critical Review and Bibliography, Ford, IgI3).

Effects on Animals. - The actions can be best studied on rabbits, by hypodermic or intravenous injections. Even with the latter, there is an incubation period of at least twelve to eighteen hours before symptoms appear. These correspond to those described for man. They are partly local-gastroenteritis; and partly central-paralysis of the respiratory and vasomotor centers. The local inflammation also occurs on other mucous membranes to which the poison may be applied, especially the conjunctiva.

The autopsy findings are very characteristic. They consist in swelling and reddening of Peyer's Patches and mesenteric lymph glands, internal hemorrhages and diffuse nephritis. Cruz, Flexner, Mueller and others have shown that these lesions are not due to thrombosis, but to direct action on the tissues. The site of the injection is boggy.

Frogs have a much higher resistance than mammals; but this is weakened by their temperature. The phytotoxins have no direct effect on muscle or nerve.

Action on Blood. - In vitro, ricin hemolyzes and agglutinates the corpuscles of nearly all warm-blooded animals (Stillmark, I 888). The agglutination does not seem to occur in the body, but is of great importance as an immunity phenomenon. Leucocytes, epithelial and other cells (except those with thick membranes, as yeast) are also agglutinated; as likewise the stroma of laked corpuscles (Elfstrand). The presence of serum hinders the effect.

The agglutination has been referred to precipitation of the nucleoalbumins (Stassano) or other proteins, such as those of serum (Kraus, I902). All kinds of colloid precipitates carry down ricin, and it is adsorbed by solid proteins and lipoids.

Nature of Ricin.- This appears to be a true protein; for a preparation of ricin has been obtained, which is a typical albumin, and which is so active that $0.0005 \mathrm{mg}$. is fatal to a kilogram of rabbit; i.e., I part of the ricin is fatal to $2,000,000$ parts of rabbit; 
the fatal dose for man would therefore be about $0.035 \mathrm{mg}$., or I/2,000 grain (Osborne, Mendel and Harris, 1905; Osborne, 1909). The agglutinating action is also very powerful.

The attempt is also being made to separate the agglutinin (which is adsorbed by blood corpuscles) from the cytotoxin, which is destroyed by peptic digestion. Jacoby, I902, believes that they have certain groups in common.

Antiricin.-Injections of the phytotoxins produce typical antitoxins, so that an immunized animal can survive 5,000 ordinary fatal doses of ricin. Some of the basic work of Ehrlich was done with ricin and abrin. He showed (1891) that the immunity starts in five to six days, and lasts six or seven months. The resistance of the corpuscles is unchanged, the antiricin being contained in the pseudoglobulin fraction of the serum (Jacoby, I902). It contains antitoxin, antiagglutinin (probably identical) and precipitin. Madsac and Walbum found that this combination obeys the same laws as diph theria antitoxin. The toxicity of ricin is modified rather complexly by lecithin (Lawrow, I9I3).

\section{ABRIN AND JEQUIRITY}

Abrin, a toxalbumin from Jequirity bean (abrus precatorius), resembles ricin so closely in its action that the difference was established only when it was noticed that immunity against one did not constitute immunity against the other. Hausmann, 1902, has studied it by the methods of Jacoby. The literature was reviewed by Ford, I9I3.

Poisoning.- When the whole beans are swallowed, no toxic effects result, since the shell is so hard that the poison does not dissolve. But if the powder is taken, it produces effects similar to ricin. Its toxicity is greatly increased by heating the animals (Lesne and Dreyfus, 1913).

Use in Ophthalmology.-The action on the eye is much stronger than with ricin, producing ophthalmitis. This has been used therapeutically (Weeker, 1882), to clear corneal opacities. This is dangerous, unless standardized preparations are used, and excessive effects checked bv an antiserum.

\section{CATHARTIC RESINS}

General Statement.-Jalap, Colocynth, Podophyllum, Elaterin and similar drugs contain active principles which are for the most part resinous (i.e., soluble in alcohol, but slightly soluble in water), often glucosids; on chemic manipulation (probably hydration) these yield resinous acids. The latter are much less active.

The effects of these resins are highly irritant, producing considerable colic (sometimes vomiting), and several watery stools, in one or two hours with jalap and most of the others; but with podophyllum only after twelve to twenty-four hours. They are used especially in dropsies (Compound Jalap Powder); small doses of podophyllum also as laxative in habitual constipation. The others tend ultimately to produce constipation. Colocynth, and presumably the others, stimulate peristalsis in the small intestine, inhibit antiperistalsis in the colon, and lead to the effusion of fluid from the whole intestinal canal.

The resins require the presence of bile, presumably to secure their solution. They are therefore inactive in obstructive jaundice. They produce griping, and are therefore often prescribed with belladonna and aromatics. If they are not removed by catharsis, they may produce severe enteritis.

The principles are not as effective when given pure as when they are mixed with some extractive, as in the crude drugs and "resins" (alcoholic extracts precipitated by water), or as preparations of the crude drug. This is due, in some cases, to their being somewhat soluble, and therefore absorbed in the stomach.

Subcutaneous Administration.-This produces diarrhea with podophyllin and colocynthin, but is not available because it also produces nephritis, local irritation and abscesses. 
Mechanism of Action. - This has been studied on colocynth by the X-ray method by Padtberg, I909, under Magnus. Similar observations have been made with jalap on man: the gastric movements may be slightly increased but often remain normal. In the small intestine the peristalsis is so much quickened that the contents may be completely expelled in half an hour instead of the normal eight hours. This is accompanied by the effusion of considerable fluid into the lumen. In the large intestine the propulsion is only slightly increased; but the stools are rendered still more liquid by the inhibition of antiperistalsis, and by the secretion of additional mucus. Padtberg, rgrr, found that the colocynth peristalsis and effusion are arrested by morphin and opium, although these do not ordinarily act directly on the intestine, but on the stomach.

\section{JALAP}

This is perhaps the mildest member of the group. It produces watery stools within three or four hours, with little intestinal irritation, without gastric disturbance or much prostration. It has little taste and is therefore easily administered. It is used in ascites, cerebral congestion, hypertension; and as adjuvant to anthelmintics. Scammony is closely related to jalap.

Constituents. - These have been studied by Power and Rogerson, rgog and Igr 2. The "resin" is a complex substance, which can be separated by solvents into several glucosidal portions: Jalapin (convolvulin, jalapurgin) and Scammonin (orizabin).

\section{PREPARATIONS-JALAP GROUP}

Jalapa U.S.P., B.P.; Jalap.-The dried tuberous root of Exogonium purga. At least 7 per cent. of resin, U.S.P.; ro per cent., B.P. Dose, I Gm., I 5 gr., U.S.P.; 0.3 to $\mathrm{I} .2 \mathrm{Gm} ., 5$ to $20 \mathrm{gr}$. , B.P.

* Pulvis Jalapa Compositus (Pulv. Jalap. Co.), U.S.P., B.P., Compound Jalap Powder.- $-\mathrm{r}$ part of Jalap, 2 parts Pot. Bitart. (flavored with Ginger, B.P.). Dose, 2 Gm., 30 gr., U.S.P.; 0.6 to $4 \mathrm{Gm}$., ro to $60 \mathrm{gr}$., B.P., stirred into water.

Tr. Jalap., B.P.- - 5 per cent. of resin. Dose, 2 to 4 c.c., $1 / 2$ to $\mathrm{r}$ dram, B.P.

Tr. Jalap. Co., B.P.-8 per cent. of Jalap, r.5 per cent. Scammon. Res., r per cent. Turpeth. Dose, 2 to 4 c.c., $1 / 2$ to I dram, B.P.

Resina Jalapa (Res. Jalap.), U.S.P.; Jalap. Res., B.P.-Prepared by precipitating an alcoholic extract of Jalap with water. Dose, 0.x25 Gm., 2 gr., U.S.P.; 0.I 2 to 0.3 Gm., 2 to 5 gr., B.P. Maximum dose, $r$ Gm., 15 gr.

Scammonii Radix (Scam. Rad.), U.S.P.; Stammon. Rad., B.P.; Scammony Root.The dried root of Convolvulus Scammonia, yielding not less than $85 \%$ of resins. Used by the ancients. The constituents are similar to Jalap. They have been investigated by Power and Rogerson, rgr2. Dose, 0.25 Gm., 4 gr., U.S.P.

Pulv. Scammon. Co., B.P.-Scammon. Res., 50; Jalap., 35; Ginger, 15. Dose, 0.6 to $\mathrm{I.2} \mathrm{Gm}$., ro to $20 \mathrm{gr}$., B.P.

Res. Scamm.,-U.S.P., Scammon. Res., B.P.-Prepared by precipitating an alcoholic solution with water. Dose, 0.2 Gm., 3 gr.,.U.S.P.; 0.25 to $0.6 \mathrm{Gm}$., 4 to 8 gr., B.P.

Ipomece Radix (Ipom. Rad.), B.P.; Orizaba Jalap Root (Mexican Scammony Root). - The dried root of Ipomœæ orizabensis.

Kaladana, B.P. (Pharbitis Seeds). - The dried seeds of Ipomœa hederacea. Dose, 2 to $3 \mathrm{Gm}$., 30 to $45 \mathrm{gr}$., B.P.

Pulv. Kalad. Co., B.P.-Similar to Pulv. Jalap. Co. Dose, 0.6 to 4 Gm., ro to 60 gr., B.P.

Tr. Kalad., B.P.-20 per cent. Dose, 2 to 4 c.c., $1 / 2$ to 1 dram, B.P.

Kalad. Res., B.P.-Dose, 0.12 to 0.5 Gm., 2 to 8 gr., B.P.

Turpethum, B.P.; Turpeth.-The dried root and stem of Ipomoea Turpethum. Dose, 0.3 to I.2 Gm., 5 to $20 \mathrm{gr}$., B.P.

\section{RESIN OF PODOPHYLLUM}

This acts very slowly, generally only after twelve to twenty-four hours or longer, producing several soft stools. Larger doses $(20 \mathrm{mg}$., $1 / 3 \mathrm{gr}$. or more) act as a hydragogue cathartic; but it is chiefly employed in small 
doses ( 5 to $10 \mathrm{mg} ., 1 / 10$ to $1 / 6 \mathrm{gr}$.) as a laxative in chronic constipation, often combined with aloes or calomel. 0.3 to $0.5 \mathrm{Gm}$. has been fatal.

\section{PREPARATIONS-PODOPHYLLUM}

Podophyllum, U.S.P., Podoph. Rhiz., B.P.; Podophyllum (Mandrake, May Apple Root). -The dried rhizome and roots of Podophyllum peltatum (yielding not less than 3 per cent. of resin, U.S.P.). Active constituents, resin and a crystalline principle, podophy lotoxin. Podophyllum was used by the Indians.

Fldext. Podoph., U.S.P.-Dose, o.5 c.c., 8 minims, U.S.P.

Tr. Podoph., B.P. -3.65 per cent. of resin. Dose, 0.3 to I c.c., 5 to 5 minims, B.P.

* Resina Podophylli (Res. Podoph.), U.S.P.; Podoph. Res., B.P.; Resin of Podophyllum (Podophyllin).-Prepared by precipitating an alcoholic extract of Podophyllum with acidulated water. Amorphous greenish-yellow powder, of slight peculiar odor and faintly bitter taste. Very irritating to mucous membranes, especially of the eyes. Sol. in alc. A very similar resin is prepared from the East Indian species P. Emodi (literature, Scoville, 1909). Dose, 10 mg., 1/6 gr., U.S.P.; I6 to $60 \mathrm{mg}$., $1 / 4$ to I gr., B.P.; as pill. Maximum dose, o.I Gm., I $1 / 2$ gr.

Podoph. Ind. Rhiz., B.P.-The dried rhizome and roots of Podophyllum Emodi. Corresponds practically to Podophyllum, also in the strength and dose of its preparations.

Tr. Podoph. Ind., B.P.

Podoph. Ind. Res., B.P.

Stillingia, U.S.P. (Queen's Root).-The dried roots of Stillingia sylvatica. Contains pungent resin and volatile oil. Used mainly in domestic merlicine in purgative mixtures, also as nauseant and emetic. Dose, 2 Gm., 30 gr., U.S.P.

Fldext. Stilling., U.S.P.-Dose, 2 c.c., 30 minims, U.S.P.

Leptandra (Culver's Root). - The rhizome and roots of Veronica Virginica. Contains an amorphous bitter principle, and not a crystalline glucosid, as formerly reported (Power and Rogerson, I9IO). "Leptandrin" is a resinoid. The preparations are pharmacologically inert, but were supposed to be cholagogue without producing intestinal irritation. Dose, I Gm., I5 gr.

Juglans (Butternut). - The root bark of Juglans cinerea, collected in autumn. Used as rather mild laxative. Dose of extract, I to $2 \mathrm{Gm}$., I 5 to $30 \mathrm{gr}$.

Iris (Blue Flag).- The rhizome and roots of Iris versicolor. Power and Salway, IgI I, could not find any active principle, although the drug and the resinoid "iridin" were formerly considered cathartic and diuretic. Dose, I Gm., I 5 gr.

Iridis Rhizoma (Orris Root).- The rhizome from several cultivated species of iris, has a violet odor. It is used in sachets and tooth powders.

\section{COLOCYNTH, ELATERIN AND BRYONIA}

A number of plants of the cucumber family (Cucurbitaceæ) yield these drastic drugs. They are of complex composition, each containing several active principles, resinous and alkaloidal. They produce continuous watery stools, with intense irritation and hyperemia (mechanism described above). They are less used than formerly, when they were frequently employed to reinforce the action of other purgatives as in the Compound Cathartic Pills. Colocynth is excreted by the kidneys and milk, and should therefore not be used by nursing women. Elaterin is one of the most powerful cathartics, sometimes used in uremia. Large doses cause dangerous prostration.

The toxic dose of Colycynth is 0.6 to I $\mathrm{Gm}$.; fatal, $4 \mathrm{Gm}$. Of elaterin, the toxic dose is $5 \mathrm{mg}$.; fatal, $0.6 \mathrm{Gm}$.

\section{PREPARATIONS-COLOCYNTH, ETC.}

Bryonia.-The root of Bryonia alba and dioica. Bryonin and Bryonidin are complex mixtures. The purgative action is due to non-glucosidai resins and alkaloids (Power, 1912). Dose, 0.6 to $4.0 \mathrm{Gm}$. (10 to 60 grains).

Cambogia, U.S.P.; Gamboge.-A gum-resin obtained from Garcinia Hanburii. The resin ( 70 to 80 per cent.) contains garanolic acids, which form readily soluble com- 
pounds with alkalies, and thus become active in the intestine. The effects resemble those of colocynth; $4 \mathrm{Gm}$. are fatal. It is rarely used, in pills. Dose, $0.125 \mathrm{Gm} ., 2$ gr., U.S.P., 0.03 to 0.25 Gm., B.P.

Colocynthis, U.S.P.; Colocynthidis Pulpa (Colocyn. Pulp.), B.P.; Colocynth (Bitter Apple).-The dried pulp of the fruit of Citrullus Colocynthis. The action is due to at least two constituents, an alkaloid and a resin. Colocynthin and colocynthidin are merely impure mixtures (Power and Moore, I9ro). Dose, 0.06 Gm., I gr., U.S.P., in pills. Maximum dose, $0.4 \mathrm{Gm}$., $6 \mathrm{gr}$.

Ext. Colocyn., U.S.P.-A powdered extract, I Gm. representing $4 \mathrm{Gm}$. of drug. Dose, 0.03 Gm., $1 / 2$ gr., U.S.P. Maximum dose, 0.06 Gm., I gr.

Ext. Colocyn. Co., U.S.P.-Ext. Colocyn., I6 per cent.; Aloes, 50 per cent.; Scammon.

Res., I4 per cent.; Cardamon, 5 per cent. Dose, 0.25 Gm., 4 gr., U.S.P.

Ext. Colocyn. Co., B.P.-A dry extract from Colocynth, Aloes, Scammony and Cardamon. Dose, 0.12 to $0.5 \mathrm{Gm}$., 2 to 8 gr., B.P.

Pil. Colocyn. Co., B.P.-Colocynth, 20 per cent.; Aloes, 35 per cent.; Scammon. Res., 35 per cent. Dose, 0.25 to $0.5 \mathrm{Gm} ., 4$ to 8 gr., B.P.

Pil. Colocyn. et Hyoscy., B.P.-Pil. Colocyn. Co., 2; Ext. Hyoscy., I. Dose, 0.2 to $0.5 \mathrm{Gm}$., 4 to $8 \mathrm{gr}$., B.P.

Pilula Cathartice Composite (Pil. Cathart. Co.), U.S.P.; Compound Cathartic Pills.-Each pill contains: Comp. Ext. Colocynth, $0.08 \mathrm{Gm}$.; Calomel, $0.06 \mathrm{Gm}$.; Jalap Resin, 0.02 Gm.; Gamboge, $0.015 \mathrm{Gm}$.; a total of seven active ingredients. Dose, two pills, U.S.P.

* Elaterinum (Elaterin.), U.S.P.; Elaterin.-A neutral principle obtained from Elaterium, a substance deposited by the juice of the fruit of Ecballium Elaterium (a substance used in antiquity). White, hexagonal scales or prismatic crystals; odorless, and having a slightly acrid, bitter taste. Insol. in water; slightly sol. in alc. $(1: 325)$. Dose, 3 to $6 \mathrm{mg}$., $1 / 20$ to $1 / 10 \mathrm{gr}$., repeated in five hours if necessary, but not daily (U.S.P., $3 \mathrm{mg}$., $1 / 20 \mathrm{gr}$.). The dose of elaterium is 6 to $30 \mathrm{mg}$., $1 / 6$ to $1 / 2 \mathrm{gr}$.

Elaterin consists of two crystalline substances, the levorotary $\alpha$-elaterin, which is inert; and 60 to 80 per cent. of the active dextrorotary $\beta$-elaţerin. Commercial elaterin varies in composition and activity (Power, I9I2). Elaterin does not exist as such in the fruit, but is formed during the expression by a diastatic ferment, acting on a glucosid (Berg, I 909).

Davis, I913, advises elaterium in hepatic cirrhosis to provoke watery'stools ( 5 to I5 mg. at night; first day, two or three doses). This rarely causes nausea or pain, but may act during the night.

Trit. Elaterin., U.S.P.- 10 per cent. Dose, 0.03 Gm., 1/2 gr., U.S.P.

\section{CROTON OIL}

This contains, besides ordinary fats, about to per cent. of "crotonresin," the active component (Dunstan and Boole, 1895). The old "crotonolic acid" was an impure mixture of this resin with inactive fatty acids. The resin produces the local and systemic effects of the oil (Kobert and Hirscheydt, I89o; Boehm, I915). It is destroyed by boiling with alkalies.

Croton oil is the strongest of all cathartics, $1 / 2$ to I drop producing burning in the mouth and stomach, often vomiting, and after one-half to three hours, several extensive fluid evacuations, with much colic and tenesmus. It is used only in extreme cases of constipation (lead), and in unconscious patients (uremic and apoplectic coma). It should not be given to weak patients, nor if the gastro-intestinal canal is inflamed. Toxic doses produce collapse. Twenty drops have been fatal, but recovery is said to have followed $1 / 2$ ounce-presumably because of vomiting. The treatment would be evacuant, demulcent, opiates and symptomatic (directed against the enteritis and collapse).

Applied externally, croton oil produces irritation, proceeding to pustulation and even sloughing. When diluted with 2 to ro parts of olive oil, it may be used as counterirritant. 


\section{PREPARATIONS-CROTON OIL}

* Oleum Tiglii., U.S.P.; Oleum Crotonis (Ol. Croton.), B.P.; Croton Oil.-A fixed oil expressed from the sceds of Croton Tiglium. A pale yellow or brownish-yellow, somewhat viscid and slightly fluorescent liquid, having a slight, fatty odor. Slightly sol. in alc. Dose, 0.05 c.c., I minim, U.S.P.; 0.03 to 0.06 c.c., $1 / 2$ to I minim, B.P.; on a lump of sugar, slice of bread or lemon. With unconscious patients, a small drop may be placed at the back of the tongue, and repeated every hour to three doses. Maximum dose, 0.05 c.c., I minim.

Lin. Croton., B.P. - I 2 per cent., in equal volume, of OI. Cajup. and alcohol. Produces severe inflammation.

\section{COLLOID AND EMOLLIENT LAXATIVES}

These act mainly by retaining water in the intestine through imbibition, and thus modifying the bulk and consistency of the feces, so that these are more easily expelled. They are useful in habitual constipation, especially if aided by the occasional administration of cascara. Agar is typical of this class. In fruits (prunes, grapes, tamarinds, etc.) this colloid action is supported by the organic acids and sugars. Manna acts through its difficultly absorbable sugar, the action being largely osmotic. Petrolatum is not absorbed and therefore softens and lubricates the feces. Olive oil, in so far as it escapes digestion and absorption, acts in the same manner.

Agar-agar.-This is prepared from various Japanese marine algæ. It consists mainly of the hemicellulose "gelose," which gelatinizes with water. It is tasteless and has been used for culinary jellies, but is not digested ( 8 to 27 per cent. is utilized in man; Saiki, I906; Swartz, I9II; herbivoræ utilize about 50 per cent.; Lohrisch, I908). It was introduced as a laxative by A. Schmidt, I905. J. L. Morse, I9I0, advises the chopped shreds, $1 / 2$ to I ounce for adults; I or 2 teaspoons for children; part with the breakfast cereal, part with sauce or cream at another meal. For children, it may be boiled with oatmeal, or made into a jelly ( $1: 200$ boiling water). It is not very effective, and usually requires the addition of cascara or phenolphthalein (Bastedo, I9 4 ).

\section{PREPARATIONS-AGAR, ETC.}

Agar, U.S.P.; Agar-agar.-The dried mucilaginous substance extracted from Inarine alga growing along the eastern coast of Asia. Thin, translucent, membranous, agglutinated pieces, yellowish-white or brownish-white; odor slight; taste mucilaginous. Dose, ro Gm., $21 / 2$ drams, U.S.P.

Manna, U.S.P.-The dried saccharine exudation of an Ash-tree, Fraxinus Ornus. Contains mannit, about 90 per cent. Dose, ${ }_{5}$ Gm., 4 drams, U.S.P., to 60 Gm., 2 ounces, as infusion.

Tamarindus, B.P.; Tamarind.-The fruit of Tamarindus indicus. It owes its activity to organic acids, especially citric. It is used as a laxative, in doses of 4 to $30 \mathrm{Gm}$. (I to 8 drams).

\section{EVACUANT ENEMAS}

Rectal injections or clysters increase the peristalsis of the large and small intestines in response to the mechanical stimulation. The effect is enhanced by the addition of irritants.

Enemata have an advantage over cathartics taken by mouth, in that they may be made absolutely non-irritant, and may therefore be used in conditions in which the other cathartics are positively contraindicated. If the purpose of the enema be merely to soften hardened scybala, they would be used warm, in copious quantities, preferably with soap; or as decoctions of mucilaginous substances.

To secure the maximum motor effect, the injection must be used cold, and in fairly large quantity: Adults a pint, quart, or more; children 
according to age (at a year, an ounce, and about 1/2 ounce more for each year). Water and bland oils are also often used by high injection.

The irritants which are most commonly used in clysters are: Soap; castor oil; molasses, I :8; salt, I : I6; turpentine, I : 20 (emulsified with egg-yolk); bile, $\mathbf{I}: 50$.

Enemata also have other uses: For local effects on the rectal mucous membrane (astringents, etc.); for the removal of parasites; and as a method of introducing medicine and nourishment.

\section{ANTHELMINTICS}

General Statement.-Anthelmintics (anti, against; helminthos, worm) are remedies used against intestinal parasites.

An active peristalsis will tend to remove intestinal parasites, as well as the other intestinal contents. Active cathartics are therefore necessarily Vermifuges-i.e., drugs which expel parasites. But these parasites, when in good condition, are endowed with remarkable faculties of maintaining their position in the intestines-by traveling in the direction opposite to peristalsis, or by fixing themselves to the intestinal wall by means of suckers or hooks, or by their serrated margins, etc. It therefore becomes necessary to lower their vitality. This may be done to some extent by appropriate diet. But this is rarely sufficient, and it is generally necessary to employ drugs which will paralyze them-Vermicides. Since the latter necessarily present some danger to the host as well, it is desirable that they should be used in the smallest doses. For this reason it is well to give them their maximum efficiency by preceding them with a course of diet which lowers the resistance of the parasite without affecting the host. The vermicides but rarely kill the parasites; these usually recover if they remain in the intestine. It is therefore very necessary to follow the vermicide by an active cathartic, a saline, jalap, or calomel. Oily cathartics must be avoided, since they favor the absorption of most anthelmintics.

Preliminary Dietary Measures.-A limitation of the proteins of the diet is generally regarded as injurious to the parasite, but care must be taken not to carry this so far as to weaken the resistance of the patient. Carbohydrates may be allowed in any amount. Mechanical irritants - vegetables rich in fiber; the seeds of strawberries, blackberries, or figs; the husks of grain, etc.-are very useful. So are "sharp" articles of diet-condiments, especially those of the mustard group, strongly salted meat, etc. At the time when the vermicide is taken, the intestinal canal should be fairly empty, so that the parasite will not be protected by the contents. The remedies are therefore usually given before breakfast, and no food is taken for several hours after. This unfortunately increases the tendency to the absorption of the poison, and to the local irritation. Vomiting may occur and render a repetition of the whole cure necessary. It has been attempted to circumvent this difficulty by combining the principles with tannin, but this lessens their action. The best that can be done is to inclose them in gelatin capsules.

Vermicides.-The substances which are toxic to intestinal parasites are in general toxic to all forms of protoplasm. The class of intestinal antiseptics are all to some extent vermicidal, but can scarcely be introduced in sufficient amount to kill the parasites without injuring the host. Special qualities are necessary for this end: The remedy must be absorbed to the smallest possible extent, since its absorption would not only render it deleterious to the patient, but would also prevent its reaching the lower portions of the intestine and acting on the parasites found there. On the other hand, it must be capable of penetrating the resistant, often chitinous, covering of these worms. This combination can only be secured with volatile poisons, whose vapors permeate the intestinal canal and penetrate into the parasites before there is time for an extensive 
absorption. From this volatility of the active principles, it follows necessarily that these drugs are not very stable; the more so since these principles also undergo chemic changes very readily. This uncertain activity has thrown mistrust on the whole class of anthelmintics. The pharmaceutic extracts or isolated principles share this instability, although to a less degree.

Finalíy, it is more than probable that these parasites, as most other forms of life, show peculiar susceptibility to certain poisons. There is some hope that further research will bring forth specific vermicides. At present, the following data have been gathered largely from empirical observations:

Specific Vermicides.-The most efficient for Tapeworms are Male Fern (especially for Bothriocephalus); Pelletierin (especially for Tænia); and Kosotoxin; for Round Worms, Santonin, Chenopodium and Spigelia. Thread Worms are treated most efficiently from the rectum by enemata. Thymol (see Index) and Chenopodium are used for Hook-worm. Aspidium is also said to be effective against Hook-worm.

It may be well to mention that the vermicides are under no circumstances absolutely safe. They should never be given unless the parasites or their eggs are actually demonstrated in the feces. This is also the time when treatment offers the greatest chance of success.

\section{ASPIDIUM OR MALE FERN}

Composition.-The anthelmintic action resides in several closely related substances, complex methane derivatives of phloroglucin and its homologues, with butyric radicles in ketone combination (Boehm, I897). The most important is probably the amorphous filicic acid. Analogous compounds occur in several other anthelmintic drugs (Rotlerin in Kamala; Ascaridol in Chenopodium; Tanacetin in Tanacetum; Kosotoxin in Cusso).

The Active Constituents of Aspidium (Poulsson, I89r; Boehm, 1897) are: Amorphous filicic acid $\left(\mathrm{C}_{35} \mathrm{H}_{40} \mathrm{O}_{13}\right)$; Aspidinin; Albaspidinin; Flavaspidic acid (weak); Aspidin (probably from other species). The amorphous filmaron $\left(\mathrm{C}_{47} \mathrm{H}_{54} \mathrm{O}_{13}\right.$; Kraft; Jacquet; 1904) is perhaps an impure filicic acid (Schmiedeberg). The inactive constituents are: the crystalline filicic acid or filicin $\left(\mathrm{C}_{35} \mathrm{H}_{38} \mathrm{O}_{11}\right)$, and aspidinol. The crystalline filicic acid is the lacton of the active amorphous acid, the two being readily converted into each other. This probably explains the deterioration on keeping the root. All these substances are so closely related, and change so readily into each other that our knowledge is very confused; even the elementary formula are in dispute. Aspidium also contains volatile and fixed oil, tannin, etc. Filicic acid is decomposed in the body, yielding trimethylphloroglucin. Filicinic acid is an inactive artificial reduction product of filicic acid or aspidin.

Actions.-Active filicic acid probably paralyzes the muscles of the parasites; at least it has this action in rain-worms, without affecting the nerves (W. Straub, I902). This has been utilized for the standardization of filix preparations (Yagi, r9r4). In frogs, it paralyzes the central nervous system, the heart and skeletal muscles; in mammals, it produces local gastro-intestinal irritation; followed by tetanic convulsions with simultaneous central and cardiac paralysis.

Administration.-Aspidium is used against tapeworms and hookworm. Its reputation dates from the ancients. The oleoresin is administered in capsules of $1 / 2$ to 2 c.c., or emulsions, as described. If a cathartic is given, if no oil is taken and if the patient is vigorous, the ordinary doses cause little disturbance. If unsuccessful, several weeks should elapse before another administration.

Phenomena of Poisoning.-This may occur even with moderate doses (Sidler Huguenin, 1898); and larger doses are dangerous. Four grams 
of the oleoresin have produced severe poisoning, but $20 \mathrm{Gm}$. have been given without toxic effects (Drenkhaw, I9II). 'The milder symptoms consist in colic, diarrhea, headache, dizziness, dyspnea, yellow vision, and sometimes temporary blindness. Severe cases showed delirium, violent muscle-cramps, syncope, tonic convulsions and coma; of ten glycosuria, albuminuria and casts, and icterus; death occurs by respiratory paralysis.

Recovery is slow, often with permanent blindness (Stuelp, I904). This occurs also in animals. It is due to spasm of the retinal vessels, with subsequent optic atrophy (Harnack, I9 I 2).

The treatment would be evacuant, demulcent and symptomatic.

\section{PREPARATIONS-ASPIDIUM}

Aspidium, U.S.P.; Filix Mas, B.P. (Male Fern).-The rhizome and stipes of Dryopteris Filix Mas and marginalis; using only such portions as have retained their green color. Dose, 4 Gm., 60 gr., U.S.P.

* Oleoresina Aspidii (Oleores. Aspid.), U.S.P.; Exl. Filicis Liq., B.P. (Oleoresin of Male Fern).-An evaporated ethereal extract (containing at least 20 per cent. of filicin, B.P.). Greenish oily liquid, often containing a crystalline sediment which should be incorporated before dispensing. Disagreeable taste and odor. Dose, $2 \mathrm{Gm}$., $30 \mathrm{gr}$., U.S.P. (Caution; not repeated on same day); 45 to 90 minims, B.P.; in capsules or emulsion. Maximum dose, $\mathrm{r}_{5} \mathrm{Gm} ., 4$ drams.

Amorphous Filicic Acid, N.N.R. -0.5 to I Gm., Filmaron Oil, N.N.R., 10 per cent., Io c.c.

Cusso, B.P.; Kousso (Brayera).-The pistillate flowers of Brayera anthelmintica. (The male flowers are powerfully emetic, and, therefore, useless as vermicides.) Contains kosotoxin, related to filicic acid (Leichsenring, 1894; Lobeck, 1901). Dose, 8 to $16 \mathrm{Gm} ., 2$ to 4 drams, B.P., as infusion.

Kamala (Rottlera).- The glands and hairs from the fruit-capsules of Mallotus Phillipinensis. Contains Rottlerin, related to filicic acid (Telle, r907; Semper, roro. Dose, 10 Gm., $2 \frac{1}{2}$ drams.

\section{CHENOPODIUM OIL}

The oil distilled from American Worm-seed has again come into prominence as an anthelmintic, especially against round worm and hook-worms. It appears to be highly efficient and relatively safe, although large doses are toxic. Its effects on the worms are similar to santonin, viz., peripheral stimulation, followed with large doses by paralysis (Trendelenburg, 1915).

Ascaridol.-This is the principal active constituent. It is a very unstable compound of peroxid type (Alsberg, 1913).

Efficiency.-Bruening, 1906, 1912, found that chenopodium oil has a high toxicity for ascaris, and a low toxicity for mammals. In hook-worm, Schueffner and Verwoort, 1913, found it clinically fully equal to thymol, and superior to beta-naphthol or eucalyptol. Bishop and Brosins, 1915, consider it more efficient than thymol, with the further advantage of easier administration. In the Orient, according to Heiser, I9 5 , the clinical results have been superior to other hook-worm remedies. It was also highly effective against ascaris, tapeworms and whipworms.

Administration.-The oil does not kill, but merely paralyzes the worms, so that it must be given with a cathartic, usually castor oil. Oils do not increase, but rather diminish, the toxicity (Salant and Bengis, 19 5 ).

Dosage against Ascaris.-For children, 5 to ro drops of the oil are given on sugar two or three times a day for two days, and followed by a tablespoon of castor oil (Levy, I9I4).

Dosage against Hook-worm.-The patient is allowed a light supper and no breakfast. The treatment starts in the morning with $50 \mathrm{Gm}$. of magnesium sulphate; two hours later, I6 drops of the oil on a teaspoon of granulated sugar; the same dose is repeated twice with two-hour intervals. The last (third) dose is followed after two hours by 2 tablespoons of castor oil with 50 drops of chloroform. The treatment is repeated weekly until the castor oil stools are free from ova. For children below fifteen years, 
the dosage is approximately I drop per year (Schuefiner and Verwoort, I9I3; Levy, I9I 4 ).

Bishop and Brosins, $1_{9}{ }_{5}$, advise even simpler technic, requiring no dietary preparation. The oil is used in capsules of 0.5 c.c. For adults, three doses, each of I c.c., are given at intervals of two hours. Castor oil, 2 ounces, is administered four hours after the last dose. For convenience in collecting the stools, the treatment is usually started in the evening. The course is repeated every three to five days, until the parasites have disappeared from the feces. The dosage for children is I drop per year, on sugar. The treatment may be contraindicated during fever or acute gastro-intestinal inflammations.

Absorption and Excretion.- The oil is absorbed from the ligated stomach and especially from the small intestine. The excretion occurs partly by the lungs (Salant and Livingston, 1915 ).

Toxic Effects in Man.-These are rare, and generally attributable to gross overdosage. The symptoms consist in nausea, vomiting and abdominal pain; headache, drowsiness, deafness and tinnitus; ataxia and depression. Fatal cases show coma, tachycardia and convulsions (Levy, I9I4; Seifert, Nebenwirk., I9I5, p. r $_{40}$ ). The treatment would consist in purgatives and stimulants (Motter, I9I4).

Toxic Effects on Animals. - These have been studied especially by Salant and his co-workers, r910-r9r6. The toxicity varies greatly, even with intravenous injection. It is increased by starvation, diminished by abundant diet. Cumulative effects have also been observed.

Intact animals first show depression of the higher centers, then convulsions. There is a cardiac fall of blood pressure and organ volume, with slowed heart and decreased vagus irritability. Somewhat larger doses also decrease the rate and amplitude of the respiration, independently of the circulation. Peristalsis is diminished, by intravenous injection, as well as in the excised intestine. The depression is antagonized by barium, but not by pilocarpin. Renal irritation is also evidenced. Caffein antagonizes the respiratory depression, epinephrin and digitalis the cardiac depression (Salant and Livingston, I916.)

\section{PREPARATIONS-CHENOPODIUM}

* Oleum Chenopodii (Ol. Chenopod.), U.S.P.; Oil of Chenopodium (American Worm-seed). - A volatile oil distilled from Chenopodium ambrosioides anthelminticum. Dose, 0.2 c.c., 3 minims, U.S.P.

\section{PELLETIERIN}

This is a mixture of alkaloids obtained from Pomegranate bark. It acts much more powerfully on Tænia than on Ascaris (v. Schroeder, I884; also effects on higher animals). Decoctions of the bark have been used as tapeworm remedy since the Romans; but the large amount of tannin ( 20 or 25 per cent.) often causes vomiting, so that the alkaloids are preferred. These are generally given in the form of the tannates to avoid gastric irritation, employing the usual precautions. The ordinary dose often causes mild toxic symptoms: vertigo, dimmed vision, great weakness and cramps in the legs, formication, convulsive trembling, etc. Toxic doses produce promptly mydriasis, partial blindness, violent headache, vertigo, vomiting and diarrhea, profound prostration, sometimes convulsions (Landis, r 889).

\section{PREPARATIONS-PELLETIERIN, ETC.}

*Pelletierine Tannas (Pellet. Tann.), U.S.P., B.P.; Pelletierin Tannate.-A mixture of the tannates of alkaloids obtained from the bark of the root and stem of the Pomegranate. Tanret, 1878 , found two active, liquid volatile alkaloids, Punicin and Isopunicin. There are, further, two less active alkaloids, Methylpunicin and Pseudopunicin. The commercial alkaloid is of rather variable composition. The tannate occurs as a light yellow, odorless, amorphous powder, of astringent taste. Slightly sol. in water ( $\mathrm{r}: 24 \mathrm{O})$, sol. in alc. ( $\mathrm{r}: \mathrm{I} 6)$. Dose, $0.25 \mathrm{Gm} ., 4$ gr., U.S.P.; 0.12 to $0.5 \mathrm{Gm}$., 2 to 8 gr., B.P.; suspended in water.

Granatum, U.S.P.; Pomegranate.--The dried stem and root of Punica Granatum. Dose, 2 Gm., 30 gr., U.S.P. 
Decoct. Granat.-2o per cent. Dose, $\mathrm{I}_{5}$ to 60 c.c., $1 / 2$ to 2 ounces.

Fldext. Granat., U.S.P.-Dose, 2 c.c., 30 minims, U.S.P.

Areca.-The fruit of Areca Catechu. Contains vermicidal volatile alkaloid (Arecolin). Areca is used only in veterinary medicine. Arecolin stimulates peristalsis (Paetz, I9 10). Arecolin hydrobromid has been used locally, $1 / 4$ to 1 per cent., as miotic (smarting).

Pepo, U.S.P.; Cucurbite Semina Praparata (Cucurb. Sem. Præp., B.P.; Pumpkin Seed, Melon Pumpkin Seed.-The dried (fresh, B.P.) ripe seeds of Cucurbita Pepo (maxima). Dose, $30 \mathrm{Gm}$., I ounce, U.S.P.; Ioo Gm. bruised to a cream with water or milk, B.P.

Pumpkin or Melon Seeds, fresh or at least not over a year old, are eaten in 1/2 ounce doses, on the day preceding the regular treatment. They are not sufficiently active themselves, but serve to support other measures. They were investigated chemically and pharmacologically by Power and Salway, roro; but none of the constituents had any action on the host or parasites. IVeinblum, I9 2 , found neither alkaloids nor glucosids, and also considers the action very unreliable. The fresh "milk" of the cocoanut is also said to be anthelmintic.

Spigelia, U.S.P.; (Pink Root). - The dried rhizome and roots of Spigelia marilandica. It is said to stupefy round worms. It contains a bitter acrid resin; a poisonous alkaloid; volatile oil, etc. Little is known of its action. Toxic effects, which are rare, resemble those of pelletierin. Dose, $4 \mathrm{Gm}$., 60 gr., U.S.P.

Fldext. Spigcl., U.S.P. Dose, 4 c.c., 1 dram, U.S.P.

Butea Semina, B.P.; Butea Seeds.-The seeds of Butea frondosa. Used in India as anthelmintic.

Pulv. Butea Sem., B.P.-Dose, 0.6 to r.2 Gm., to to 20 gr., B.P.

\section{SANTONIN}

General Statement.-Santonica has been a favorite remedy against round worms since Dioscorides. It is also used against pinworms if these have ascended beyond the reach of enemas. It does not seem to kill the ascaris, either in the intestine or in vitro, but they attempt to escape from it and thus flee into the large intestine, whence they are evacuated by a purgative. Santonin is insoluble, non-irritant and almost tasteless, and is therefore easily administered to children. It may be mixed with some sugar, and combined with calomel or castor oil, which does not increase its absorption. To children of two to five years, $0.03 \mathrm{Gm}$., $1 / 2 \mathrm{gr}$., may be given every hour for two or three doses, according to age, and then not repeated for at least three days. The adult dose would be three times as much. It should not be given on an empty stomach, since this would favor absorption.

Santonin is the lacton of santonic acid. It is dissolved in the intestine as sodium santoninate, and the greater part is eliminated unchanged in the feces. A small amount is absorbed, oxidized, and the derivatives (especially the crystalline Santogenin, Jaffé, I890) are excreted by the urine. An unknown derivative imparts a bright yellow color to the urine when acid, changing to pink when alkaline.

Doses but little above the ordinary cause "yellow vision," persisting for some hours, without other bad effects. Overdoses produce serious poisoning. Santonin should therefore only be sold on prescriptions. The Troches, by their resemblance to candy, are especially liable to produce accidental poisoning.

Action on Worms; Relation to Structure.-This has been studied by Trendelenburg, I9 5 , on rain-worms, leeches and ascaris. It consists in strong stimulation (increased tonus and contractions) of the muscle, persisting after removal of the ganglia. The effect is highly specific, since it is not produced in anything like the same degree by other substances (although some alkaloids have a weak action); nor does santonin stimulate vertebrate muscle. It is connected with the lactone structure, as is also a depressant effect on the isolated frog heart. The convulsant effect on mammals, on the other hand, does not depend on the lactone, but on the naphthalin nucleus. 
The Phenomena of Severe Intoxication.-These are vomiting, colic and diarrhea, painful micturition, hematuria, headache and vertigo, weakness, somnolence: finally convulsions (which may be unilateral), and fall of temperature (Binz, I877; Harnack, r9or).

The Treatment.-This would consist in evacuation; chloroform inhalation against the convulsions; and stimulants against collapse.

Fatal Dose.-In children, $0.06 \mathrm{Gm}$., I gr., has produced serious poisoning, and two such doses have been fatal; in other cases, $0.18 \mathrm{Gm}$., 3 gr., caused only light symptoms, and recovery has occurred after $0.72 \mathrm{Gm}$., II gr. By adults, 0.5 to I $\mathrm{Gm}$. and more have been taken without damage (Lewin).

The Color of the Urine.-This may be confused with that of emodin (rhubarb, etc.). The emodin colors can be shaken out with ether, or precipitated by lime or barium water, whilst the santonin color can not.

Uric Acid Elimination.-This is increased by Santonin, as by other intestinal irritants (Abl, I9 I3).

Xanthopsia.-Doses as small as O.I Gm. may cause "yellow vision"-i.e., white light has at first a violet, then a yellowish-green hue, and these colors tint the entire field of vision. The power of seeing in dim light is also lessened. It has been demonstrated that these effects are peripheral (Knies, I894), and the theory is advanced, based on some experimental data, that santonin impairs the reproduction of the visual purple and violet, which are at first used very rapidly. There is no truth in the statement that it discolors the media of the eye (Filehne, I900).

Colored vision also occurs, but more rarely with amyl nitrite, picric or chromic acid, and digitalis (de Schweinitz, I899).

\section{PREPARATIONS-SANTONIN}

* Santoninum (Santonin.), U.S.P., B.P.; Santonin, $\mathrm{C}_{16} \mathrm{H}_{18} \mathrm{O}_{3}-$ The inner anhydrid or lacton of santonic acid obtained from Santonica. Colorless crystals (turning yellow on exposure to light); odorless and nearly tasteless when first placed in mouth, but afterward developing a bitter taste. Very slightly sol. in water, sol. in alc. $(x: 43)$. Dose, $0.06 \mathrm{Gm}$., I gr., U.S.P.; 0.06 to $0.2 \mathrm{Gm}$., I to 3 gr., B.P.; in powder, with a little sugar. Maximum dose, $0.2 \mathrm{Gm} ., 3 \mathrm{gr}$.

Santonin has been used in epilepsy and in diabetes, but with little success.

Santonica, "Levant Worm-seed" (really the unexpanded flowerheads of Artemisia pauciflora), also contains cineol, but this has no action on ascaris (Bruening, I9I2).

Troch. Santonin., B.P.; Santonin Lozenges.-Each contains $0.06 \mathrm{Gm}$., I grain.

\section{VERMICIDES FOR THREAD WORMS (OXYURIS)}

These are usually treated most efficiently by the rectal injection of various irritants. The rectum is first washed with injections of iron, tannin, or bitters (quassia,) to limit the secretion of mucus, and is then irrigated with solutions or emulsions of salt ( 3 ss to $\mathrm{pt}$.), aloes ( $3 \mathrm{j}$ to $\mathrm{pt}$.), or turpentine ( $3 \mathrm{ij}$ to pt.), etc. Mercury salts are sometimes used as injection or suppository, but are dangerous.

\section{CONVULSANT POISONS}

Convulsions may be caused by direct stimulation of the muscles (veratrin); or of efferent motor nerves (physostigmin, aconitin); or by reflexes from strong afferent stimulation; or by stimulation of the motor centers in the cord, medulla or brain, either directly by the drug, or as a result of asphyxia, etc. These produce different types of convulsions. Spinal convulsions, as typified by strychnin and caffein, are generally symmetrical and when fully developed, tetanic (i.e., the contractions are maintained). Medullary convulsions (picrotoxin, camphor, asphyxia) are more irregular, 
asymmetrical and clonic (rapid intermissions). Cerebral convulsions (absinthe) are of the epileptic type, irregular, of ten involving only limited groups of muscles.

Convulsions are usually the result of extreme stimulation of the motor centers, and as such are mainly of toxicologic interest. Smaller doses of these drugs, however, may be used for therapeutic stimulation; their action often extending to other nervous centers, and exciting the respiratory and vasomotor centers.

\section{STRYCHNIN AND NUX VOMICA}

General Statement.-Strychnin, the main alkaloid of Nux Vomica, has considerable therapeutic and toxicologic importance. It increases the reflex excitability of the spinal cord and of the medullary centers. The action is chiefly on the dorsal gray matter of the cord.

Therapeutic doses produce a "bitter" and, therefore, tonic effect on the alimentary canal; improved tone and nutrition of muscle; and a limited amount of respiratory and vasomotor stimulation.

Toxic doses cause characteristic tetanus, violent changes in blood pressure, and spasmodic respiration. Death occurs from asphyxia and from the paralysis which succeeds the stimulations.

Actions closely resembling those of strychnin are produced by a number of other alkaloids, which have little practical importance. The actions of tetanus toxin are very similar.

Description of the Motor Effects.-The principal symptoms of strychnin poisoning in all vertebrate animals are referable to modified and increased reflex excitability of the spinal cord, culminating in symmetrical convulsions and tetanus. The motor reflexes are modified so that smaller stimuli are effective; and the response even to slight stimulation, is maximal, tetanic, and tends to spread to all the muscles. There is also a reversal of inhibitory into augmentory reflexes, so that the reciprocal action of antagonistic muscles is disturbed.

The symptoms in man correspond to those in the lower vertebrates. With small doses, there is merely a greater response to stimulation, and the muscles feel somewhat more taut. As the dose is increased to 6 or $7 \mathrm{mg}$. in men, 5 or $6 \mathrm{mg}$. in women (Hartenberg, 1913), this tightening becomes more sensible, especially in the neck and jaws; the movements become rather abrupt; and slight twitchings may appear in the individual muscles, often in the little finger. (In case of paralysis from a high lesion (apoplexy), the paralyzed muscles may be the first to react since they are removed from the cerebral inhibitory impulses.)

Convulsant Effects.-With toxic doses, these symptoms are promptly succeeded by sudden convulsions, usually following some stimulation; or the premonitory symptoms may be absent. The attack may start with a loud cry, which is usually caused mechanically, by the convulsive movement of the air, rather than by pain. The convulsions involve all the voluntary muscles of the body, including the diaphragm. The movements are at first rapidly intermittent, but become promptly tonic, resulting in a typical tetanus. Since all the muscles are contracted, the convulsions are symmetrical, and the body assumes the position corresponding to the stronger muscles, which in most situations are the extensors. Accordingly, the body is arched backward (opisthotonus), so that the 
patient may touch the ground only with the head and heels. ${ }^{1}$ The legs are adducted and extended, the feet curved inward. (A frog may be held horizontally by the feet.) The arms are either strongly flexed over the chest, or rigidly extended, with the fișts balled. The jaws are fixed, and foam gathers at the mouth. The fixed diaphragm and the tense thoracic and abdominal muscles arrest respiration. The skin and mucosæ are, therefore, cyanotic and congested; the eyes protruded, evolved; and the pupils dilated. The pulse is small and tense, often imperceptible. The patient remains perfectly conscious, and suffers severe pain from the violent contractions. The asphyxia may be fatal in the first attack. More commonly there is a:

Remission.-The tetanus lasts perhaps a minute, then the muscles relax, and the patient passes into a greatly depressed, almost paralytic condition, still perfectly conscious and very anxious and oppressed, with dry throat and thirst, sometimes sweating. This intermission lasts at most ten or fifteen minutes, when there is another attack, usually again following some slight stimulation. If this is not fatal by asphyxia, it is again followed by remission, and so on, the whole sequence being repeated. The remissions, however, become progressively shorter, the spasms become weaker and the paralysis more prominent.

Death occurs usually in the interval after the second to fifth attack, from exhaustion and general depression. It may be preceded by asphyxial coma.

Recovery may occur from sublethal doses, or under appropriate treatment, the convulsions and depression disappearing gradually. Animals, however, have been observed to relapse into fatal convulsions several hours after apparently complete recovery (Sollmann), so that patients should be watched.

Dependence of Convulsions on Reflex Stimulation.--In the lighter grades of strychnin poisoning, the convulsions occur only on reflex stimulation. In the severe grades, they are often apparently spontaneous; but even here they are really reflex, due to slight and accidental sensory stimulations. This is shown by the fact that convulsions do not occur spontaneously if all sensory impressions are prevented from reaching the spinal cord, as by dividing all the posterior roots in frogs (Hermann Meyer, 1846); together with the medulla (Hering, I893; Verworn, I900); or by placing the frog in a very weak cocain solution (Poulsson, I889).

Different Forms of Stimulation.-Practically all kinds of sudden sensory stimulation may provoke the convulsions. The skin is especially sensitive, even to the slight jars produced by heavy walking. Sudden light and sound are also dangerous. Gradual stimulation is much less effective (as with all reflexes), so that a patient may support considerable but deliberate manipulation, as for instance artificial respiration; when a slight, but abrupt or unexpected touch would cause an attack.

Stimulation of the exposed intestines does not cause convulsions, since these are poor in sensory fibers.

Chemical stimulation (the application of dilute acids) not only fails to produce tetanus, but even the normal reflex is greatly diminished (Schlick, I890). The absence of tetanus is easily explained because the slow penetration of the acid leads to a gradual instead of abrupt stimulation. The various explanations of the diminished reaction are mainly hypothetical (Baglioni, I900 and I909; Sano, 1908).

Simple and Crossed Reflexes.-The effects of strychnin are relatively greater (i.e., the threshold is lowered more) for the crossed or leg-arm reflexes than for simple uncrossed reflexes, apparently because the excitability in simple paths is normally

1 The opposite position, body bent forward, constitutes "emprosthotonus." 
already so high that it can not be materially increased by strychnin. Therefore, the strychnin increase of excitability is better marked in the more difficult complex paths, where several synapses interposed; or when the normal threshold has been raised by ether or asphyxia (E. L. Porter, $1_{9} I_{4}, 1_{1} I_{5}$ ). The excitability is increased for single induction shocks, as well as for interrupted stimuli (van Leeuwen, I9 I3).

The Modification of the Short Reaction of Ordinary Reflexes into the Prolonged Contraction of Strychnin Tetanus. - It might be supposed that the strychnin tetanus is due to the summation of repeated stimuli; each muscular contraction, by the movements of the tendons and joints, sets up a fresh reflex, these rapidly succeeding stimuli fusing into a tetanic contraction which persists until the nerve centers are exhausted (Baglioni, I900). However, there is good evidence that this is not necessary, but that the tetanus is in fact a true multiple response to a single stimulus: For it occurs also if all reflexes but the original single stimulus are excluded; $f . i$, tetanus occurs in a leg with a single stimulus of its divided sensory nerves, the remainder of the animal being curarized (Veszi, I9I3; Buchanan, I9I2; Henkel, I9I3). The spinal stimuli, in strychnin tetanus, reach the muscles at the rate of 50 per second (Fahrenkamp, I9I4).

Elrington, I9 I4, concludes that the strychnin action consists in greater sensitiveness of the central receptor cells; not in greater spread of the impulses, or in greater intensity of the afferent impulses.

Reversal of Reciprocal Innervation.--The modification of the reflexes by Strychnin is not merely quantitative, but also qualitative. Sherrington (I905 to I909) showed that in a normal animal, the contraction of a muscle is accompanied by the automatic relaxation of its antagonistic muscles. The reciprocal reflex is destroyed by such substances as strychnin and chloroform. With strychnin, all reflex stimulations result in simultaneous contraction of all the muscles, including the antagonist; whilst with chloroform, they result in simultaneous relaxation. (The vasomotor system presents analogous phenomena, Bayliss.) The work of de Barenne (quoted under "Localization") shows other qualitative changes in the functions of the sensory cells.

Location of the Tetanizing Action.-The distinction between the possible sites of convulsant action furnishes interesting illustrations of the general methods of experimental pharmacology. Most of these experiments can be carried out more conveniently on frogs; but they can also be demonstrated on mammals.

The Action is Localized in the Spinal Cord.- This can be easily shown by exclusion: (a) The peripheral motor apparatus is excluded by section of the nerve trunk, which arrests the convulsions in the corresponding muscles (Johannes Mueller, I844). Conversely, no convulsions will occur if strychnin is injected directly into a muscle-say the leg; provided that the leg is first ligated so that the poison can not reach the general circulation. (b) If this experiment is modified so that the nerve is not included in the ligature, the absence of convulsions will also prove that strychnin does not affect the peripheral sensory apparatus (Magendie, I8I9).

(c) The brain and medulla oblongata are not essential to the convulsions, for these occur in frogs in which these centers have been destroyed. (d) The posterior root ganglia are also not concerned; for typical tetanus can be obtained, by direct stimulation of the cord, when all the posterior roots have been cut in frogs (Hermann, I846; Verworn, I90I); and in dogs even when the afferent fibers from these ganglia have completely degenerated after section (Sherrington, 1898). Nor does the localized application of strychnin to the exposed posterior root ganglia produce any effects (de Barenne, r9Io).

(e) Action on the Spinal Cord.-It is evident, therefore, that the strychnin action is located somewhere within the spinal cord. The 
next question, whether it acts on the sensory or on the motor tracts, or on both, has not been conclusively answered, although it has been the subject of much ingenious investigation. At present, it appears that both the motor and sensory portions of the cord must be poisoned, to obtain the typical strychnin convulsions.

Methods of Anatomical Isolation.-The basic experiments in this direction were made by Hermann Meyer in I 846 : after cutting away the entire posterior columns of the spinal cord, he found that strychnin does not produce tetanus, even if the remaining cord is touched. If the ablation is confined to a part of the cord, the corresponding muscles do not participate in the convulsions. This seems to indicate that the action is located in the posterior and not in the anterior columns. However, as pointed out by Verworn, 1900, the proof is not conclusive, for the operation produces too much shock, and besides, destroys all the physiological relations of the motor cells. Similar objections apply to all anatomical methods, so that these can not furnish conclusive evidence. It was therefore necessary to have recourse to physiological means.

Localized Application of Strychnin to the Spinal Cord.-Houghton and Muirhead, I895, basing themselves on the earlier and imperfect work of A. J. Spence, I866, at-

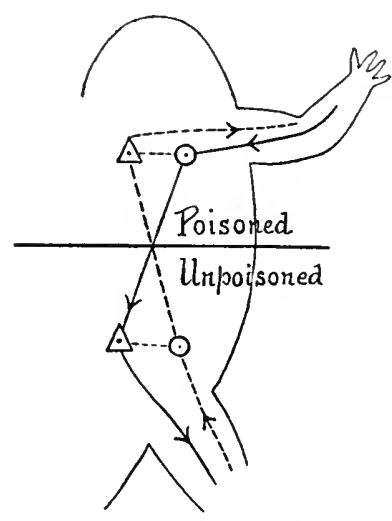

FIG. 4.-Diagram of HoughtonMuirhead experiment. tempted to utilize the fact that impulses from the sensory cells of the cervical segments of the cord to the motor cells of the caudal segments, and vice versa. By destroying the circulation and applying strychnin locally to the exposed cord it is therefore possible to make impulses pass either through a poisoned sensory cell to an unpoisoned motor cell; or through an unpoisoned sensory to a poisoned motor cell. In Fig. 4 the strychnin is supposed to be restricted to the cervical half of the cord.

The path - goes through a poisoned sensory to an unpoisoned motor cell; the path - - through an unpoisoned sensory to a poisoned motor cell. When the foreleg is stimulated in this frog, the hindleg participates in the convulsions.' When the hindleg is stimulated, there is no convulsion.

Analogous observations have been made on mammals (Ryan and McGuigan, I9II); on frogs with the sensory paths paralyzed by phenol (Baglioni, I900); and on the excised nervous system of toads (Baglioni, Igog).

These results were interpreted as proof that strychnin acts essentially on the sensory mechanism of the cord, and that the motor cells were at best unessential: for convulsions appeared whenever the impulse passed through a poisoned sensory cell, even though the motor cell be unpoisoned; whereas no convulsions appeared when the sensory cells were not poisoned, even though the motor cell was poisoned.

However, these results are obtained only when the cervical cord is poisoned. If the strychnin is applied to the lumbar cord, the convulsions do not spread at all to the cervical segments, but remain confined strictly to the poisoned area (McGuigan and Becht, I914). Furthermore, if the entire animal is poisoned by small doses of strychnin, the same phenomena are observed as in the Houghton-Muirhead experiement; namely, minimal stimuli applied to the forelegs produce general convulsions, whereas the same stimuli applied to the hindlegs produce only local contractions. The HoughtonMuirhead phenomenon may therefore be explained by the more easy spreading of impulses in the caudal than in the cephalic direction. (The entire subject is reviewed by. McGuigan, Keeton, and Sloan, I916.)

Barenne, IgI I to I9I3, adduced some new facts. By strictly localized application of strychnin to the exposed spinal cord of frogs and dogs he finds:

r. When the application is restricted to the ventral surface of the cord, no perceptible symptoms are produced.

2. When it is restricted exclusively to the dorsal surface, there is no tetanus; but instead, a characteristic syndrome, comprising sensory disturbances (paresthesias), increased reflexes, and incoördinate asymmetrical twitchings. These "dorsal symptoms" are sharply localized on the skin according to the level and side of application to the cord; the skin areas being identical with the "dermatomata" defined by anatomic-physiologic isolation methods. They occur even after the division and degeneration of the posterior root fibers. 
3. Typical tetanus occurs only if both the sensory and motor tracts are poisoned. $F . i$., if the strychnin is applied dorsally to the arm region, and ventrally to the leg region, then stimulation of the leg causes only normal reflexes (unpoisoned sensory to poisoned motor); stimulation of the arm causes tetanus of the legs (poisoned sensory to poisoned motor) in which the arms do not participate (poisoned sensory to unpoisoned motor). This experiment (which was confirmed by Beritoff, I9 $\mathrm{I}_{3}$ ) would indicate that both thse ensory and motor cells must be poisoned, to obtain the typical tetanus.

Motor Cortex.- The cerebral motor centers react to the direct application of strychnin (and also to picrotoxin) by increased excitability to electric stimulation, and even by rhythmic spontaneous movements, identical with those which would be induced by electric stimulation. The action is not abolished by deep anesthesia (Bickeles and Zbyszewski, I9I3). It is localized in the gray mattter, for it disappears on ablation (Baglinoi and Magnini, I909).

The "silent area" of the brain does not respond to the local application of strychnin (Amantea, ro I $_{3}$ ).

Psychic Centers. - These are evidently not affected by strychnin, since consciousness is not disturbed until the onset of asphyxial coma.

Practical Significance of the Location of Strychnin Action.-The location of the tetanizing action of strychnin in the spinal cord is not only of experimental interest, but also of practical importance. It explains, for instance, why strychnin convulsions are abolished by curare, since curare blocks impulses from the cord to the muscles. Also, that strychnin has a stronger action on a paralyzed limb in those cases of paralysis in which the lesion is above the cord, for cutting off of the spinal cord from the brain always increases its reflex excitability.

The fact that strychnin in small doses increases the tone of muscles is also due to its heightening the reflex excitability of the spinal cord. Not only the convulsive centers, but other spinal centers are put in a condition more favorable to reflexes. It is in this way that strychnin is useful in impotence or in paralysis of the bladder or other sphincters, when these are due to lowered activity of their respective spinal centers.

Indirect Results of the Motor Actions.- The increased activity of the muscles brings about several secondary results, such as pain, asphyxia, increased metabolism, disturbance of temperature, tendency to rise of blood pressure and quickening of pulse; early postmortem rigor. The asphyxia in turn produces its characteristic phenomena.

Pain.-Strychnin convulsions are extremely painful, just as any other form of muscle cramp.

Metabolism.-The increased muscular tone or convulsions result in increased consumption of oxygen and output of carbon dioxid, and increased use of glycogen. The increase of gaseous metabolism is observable even with therapeutic doses (Edsall and Means, I9I4). The asphyxia will also cause hyperglycemia and glycosuria.

Effect of Convulsants on Temperature.-All convulsant poisons (santonin, picrotoxin, strychnin) produce characteristic changes in heat regulation. Small doses cause increased heat loss and a slightly smaller heat production. Larger doses cause increased metabolism, through muscular action, and hence increased heat production, which is accompanied by a further increase of heat loss. Paralytic doses diminish the heat production very greatly. The temperature is accordingly variable: Small doses tend to lower it; moderate convulsive doses would increase it; paralytic doses lower it greatly. The heat loss is particularly conspicuous in small and young animals, whilst larger animals tend to show a rise of temperature, with moderate doses.

Lactic Acid.-Strychnin and other convulsions cause the appearance of lactic acid in the blood, provided that the glycogen store is adequate (Lusk, I9I6).

Nux Vomica.-The preparations of Nux Vomica, and its second alkaloid, Brucin, act essentially like strychnin.

Brucin.-The action of brucin is much weaker than that of strychnin, the ratio varying with different animals. The paralytic and curare effects are relatively stronger 
(resembling methyl-strychnin), so that Brucin is less useful therapeutically ( $c f$. Dixon and Harvey, 1908).

Brom-Strychnins.-The two mono-brom-strychnins act similarly to strychnin, but are only $1 / 8$ or $1 / 9$ as effective. Dibrom-strychnin is also convulsant, but in frogs it produces mainly the curare effect (C. R. Marshall, I9 2 ).

Other Spinal Convulsants.-Spinal tetanus, in every respect similar to that of strychnin, is produced by caffein, thebain (an opium alkaloid), gelsemin, calabarin and hydrastin, etc. It is also a late phenomenon in morphinized frogs. Tetanus toxin likewise increases spinal tetanus, the minor differences being due mainly to peculiarities of absorption (Froehlich and Meyer, I9 I $_{5}$ ).

The Convulsant Action of Sulphonated Dyes; Inhibitory Action of Cerebrum.-Acid Fuchsin (Barbour and Abel, I9I0), and other water-soluble, neurophilic sulphonated dyes (Macht, I9 I2), produce strychnin-like convulsions in frogs. In normal frogs, the tetanus occurs only after a long latent period (up to twenty hours), and requires relatively large doses. If the anterior third of the cerebrum is removed (either before or after the injection), the convulsions occur much more promptly (within thirteen minutes), and very much smaller doses $(1 / 10$ to $1 / 20)$ suffice. The anterior cerebrum therefore exercises a strong inhibitory effect on the convulsant action of these drugs (much less, if any, on other convulsant poisons). With the cerebrum intact, the latent period is also shortened by voluntary fatigue.

Cardiectomized Frogs.- Joseph and Meltzer, I9I I, found that the late convulsant action of acid fuchsin, and of morphin, could be greatly hastened, and that a very much smaller dose would suffice, in frogs in which the heart had been tied, the circulation of the blood being thus arrested. According to Abel, I9 I2, this interesting observation is explained by the peculiar distribution of the poison. The ligation of the heart prevents the dissipation of the poison in the body, and the anterior lymph hearts drive the solution directly into the communicating vessels of the central nervous system. This is therefore exposed to a much more concentrated solution. At the same time the interruption of the normal circulation, and the partial asphyxia, increase the sensitiveness of the nervous system to convulsants; for the outbreak. of these is hastened even if the heart is excised after the drug has been injected. Meltzer, however, does not accept this explanation.

Carbon Dioxid.-This also greatly hastens the convulsions, when its tension in the air exceeds 20 per cent. (Joseph, I915). The gas also lowers the threshold for strychnin convulsions (Pilcher and Sollmann, I9I5).

Therapeutic Use of Strychnin in Paralyses.-Strychnin and nux vomica are used to preserve muscular nutrition in paralysis from functional neurites and high lesions; to raise the tone of the rectal and vesical sphincters in incontinence of urine or feces; to improve the effects of cathartics, especially in atonic constipation. It is also used (but is of doubtful value) in sexual impotence. Most of these uses have been discussed in the text.

Paralytic Disorders.-It should be remembered that strychnin only increases the excitability of existing structures. It must therefore be useless in organic lesions of the cord. In spastic paralysis, and in inflammatory conditions, it would be contraindicated. Moderate doses ( $\mathrm{I}$ to $2 \mathrm{mg}$., three times a day) may be useful in functional depression of the cord (lead poisoning, or sometimes in diphtheritic paralysis); but its main indications are for maintaining the muscular tone, and thereby preventing muscular atrophy whilst awaiting the repair of cerebral lesions (apoplexy); but it must be remembered that large doses of strychnin may bring apoplectic attacks in predisposed patients. The trophic effect should be supported by massage and electricity. Strychnin is also said to lessen the pain of some older paralyses.

Incontinence of Urine.- Strychnin would be useful only if the incontinence is due to atony of the sphincter; if due to overaction of the detrusor urinæ, the indication would be for atropin.

Sexual Impotence.-The spinal stimulation of strychnin would be useful theoretically; in fact, however, the condition requires either suggestion or systematic general tonic treatment. The reputed specific nerve tonics (phosphorus, etc.) act mainly as general tonics; whilst others (damiana, zinc, yohimbin) act mainly by suggestion.

Medullary Actions of Strychnin.-These are analogous to its effects on the spinal cord, consisting in a more or less violent and convulsive 
exaggeration of the reflex excitability, particularıy of the respiratory and vasomotor centers, followed by paralysis with toxic doses. These effects are more or less modified by the coexistence of asphyxia.

These medullary stimulations are very marked and violent with convulsive doses. With therapeutic doses, they are so slight that they can not be demonstrated with certainty, either clinically or experimentally. The therapeutic use of strychnin as a respiratory or circulatory stimulant therefore rests on a very insecure basis. It was doubtless due mainly to the uncritical transfer of the results of convulsive doses to therapeutic doses.

Therapeutic Doses on the Circulation.-These produce either no effect whatever, or a very slight (10 to $20 \mathrm{~mm}$.) rise of blood pressure, fairly well sustained. The rise is rather more marked in the diastolic pressure. Direct observation of the vasomotor center may also, but exceptionally show a slight and doubtful stimulation; but in asphyxia, the same doses $(0.05$ mg. per kg.) cause intense vasomotor stimulation (Pilcher and Sollmann, I9I5). These effects (aside from asphyxia). are so inconstant and small that they could be accidental. The cardiac contractions are not

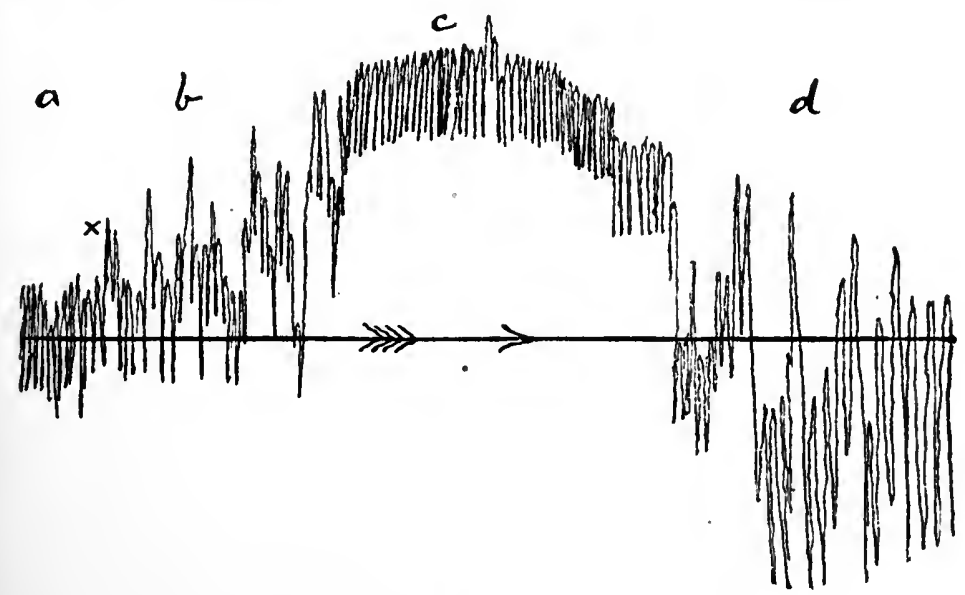

FIG. 5.-Convulsive doses of strychnin on blood pressure.

changed. Clinical observations on the blood pressure and heart rate, in health and in various diseased conditions (cardiac diseases, fevers, etc.), give similarly negative or inconclusive results, even when rather large doses (1/10 gr. every hour) were used (Cabot, 1904; Parkinson and Rowlands, I9 3 and I9 4 ; Neuburgh, I9 5 ; Lucas, I9I4). The positive resultsfof Cook and Briggs, I903, and of Marvin, I9I3, must therefore be viewed with suspicion.

Convulsive Doses.-Whilst small doses of strychnin have but little effect on the circulation, convulsive doses cause very conspicuous changes; especially a large rise of blood pressure, due to central vasomotor stimulation, produced mainly by the direct action of strychnin on the center; but aided by the convulsions and asphyxia. This is followed by vasomotor depression.

These effects are synchronous with the convulsions: the typical course is shown in Fig. 5. With the onset of the irregular spasms at (b) the mean pressure rises (central vasomotor stimulation), the heart being slowed and strengthened (central vagus stimu- 
lation). During the tetanus (c), the pressure is very high (intense stimulation of vasomotor center), the heart beats are faster and smaller (reflex vagus depression, mainly through muscular exertion). As the tetanus disappears (d) the pressure falls below normal (central vasomotor paralysis), and the heart beats become very slow (intense vilgus stimulation, mainly asphyxial). The phenomena $\mathrm{b}$ to $\mathrm{d}$ are repeated during each spasm.

As death approaches, and the respiration stops permanently, the pressure remains low, and the heart beats rapidly but weakly (total paralysis of vasomotor and vagus centers), and finally stops.

This description applies almost equally well to the effects of convulsions produced by any other cause; and particularly to asphyxia (see Fig. 6). These are both present in strychnin poisoning, and must be contributory factors. However, they are not the main cause of the rise of blood pressure; for this occurs when convulsions are excluded by complete curarization; and when asphyxia is prevented by artificial respiration. Only the portion (d) of the tracing is due entirely to asphyxial vagus stimulation.

Vasomotor Center.-Pilcher and Sollmann, $\mathrm{I}_{9} \mathrm{I}_{5}$, found that the vasomotor center is greatly stimulated by convulsive doses, independently of the convulsions or asphyxia. Very large doses (above I mg. per kg.) depress and paralyze the center.

The Vein pressure which is also unchanged by therapeutic doses, rises during tetanus, indicating cardiac insufficiency (Capps and Matthews, I9 13).

Vascular Areas.-During the strychnin rise of pressure, all the splanchnic vessels contract, while those of the skin (Wertheimer and Belezenne, 1897) and cerebrum (Roy

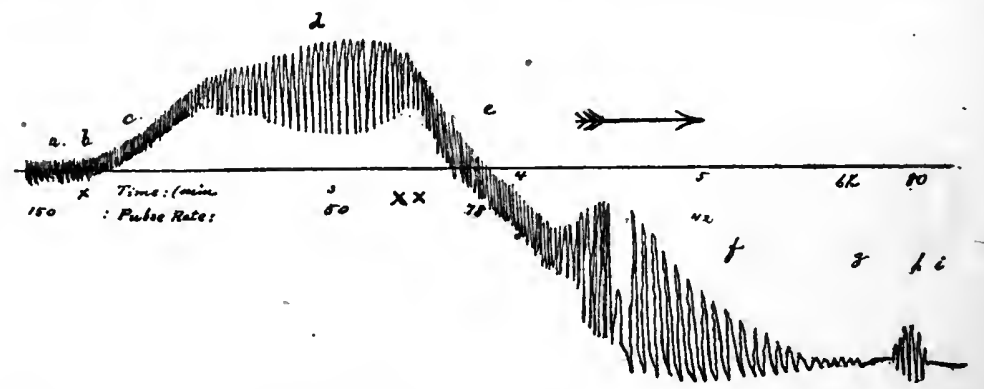

FIG. 6.-Asphyxia on blood pressure (Dog). The trachea was tied at $\mathrm{X}$. The respiratory movements are at first exaggerated, but become shallow, and stop at XX. The heart stops at (g); but it often gives a few isolated beats (h) after having stopped for several minutes.

and Sherrington, 1890 ) dilate. The vessels. of the pia mater and retina are constricted (Hirschfelder, 1915).

Vasomotor Convulsions.-In curarized animals, the rise in blood pressure occurs spasmodically (S. Mayer, I872), and can be brought on by reflex stimulation, just like the convulsions in ordinary animals. The dose required to produce the convulsant and the vasomotor action are also identical. This supports the view that the action of strychnin on the medullary centers is essentially identical with its action on the spinal centers.

Vasomotor Reversal.-According to Bayliss, I908, contraction of the vessels through the vasomotor center is always brought about by stimulation of the vasoconstrictor nerves and simultaneous automatic inhibition of the vasodilators-the reciprocal innervation of Sherrington. Bayliss claimed that this is modified by strychnin and chloroform in exactly the same sense as with skeletal muscle; and there is the same antagonism between the two drugs. The vasoconstrictors are so much more powerful than the dilators, that under strychnin, every reflex stimulation will cause a rise of blood pressure; under chloroform, a fall through inhibition. Langley, I9I2, however, failed to confirm the "reversal" of depressor fibers by strychnin; small doses simply increase the response of the pressor fibers and large doses (Io to $20 \mathrm{mg}$. per rabbit) produce transient paralysis of the depressor response, but never "reversal."

Heart.-Amongst clinicians, the opinion prevails very widely, that strychnin is 
a "cardiac stimulant." This clinical term does not necessarily imply that the drug stimulates the heart directly; but merely that it improves the pulse. (The expression is used so loosely, that the student is advised to discard it altogether.)

As a matter of fact, strychnin has no effect whatever on the heart in therapeutic doses with living animals. Convulsant doses cause some stimulation of the ventricles (Wiggers, r916). (Cameron claims increase of cardiac tone in animals with $0.03 \mathrm{mg}$. per kilogram; but this lacks confirmation.)

When perfused directly through the excised heart, the effects are first stimulant, then depressant (Hedborn, Igersheimer, I905); but the necessary concentration could not be maintained in vivo.

Respiration.-In animals, therapeutic doses of strychnin increase respiration mainly by acceleration (Cushny, I9I3). This is due to increased excitability of the center, and indircctly to the increased movements. The increase of respiration is not always conspicuous, on account of the variable excitability of the respiratory center. Clinically, it is practically absent (Newburgh, I9I4; Edsall and Means, I9 44; Higgins and Means, I9I 5, 4.5 mg. hypodermically). It is presumably greater when the excitability is partly depressed, as in anesthesia or morphin poisoning (Biberfeld, r904).

With toxic doses, the respiration is spasmodic, arrested during the tetanus; accelerated immediately after; and depressed during the intermissions.

Therapeutic Use of Medullary Stimulation.-The supposed circulatory and respiratory actions of strychnin led to its use in collapse (fainting, trauma and hemorrhage; fevers; depressant poisons, alcohol, anesthetics, coal-tar derivatives, snake venom, etc.) and in exhaustion of the respiratory center (pneumonia, phthisis). It is probably inefficient. In any case, it should be remembered that it would be only a temporary remedy, useful to tide the patient over a crisis; it could not in itself produce any permanent improvement in the central nervous system. It would merely raise the reflex excitability; and it is doubtful whether the permanent maintenance of this artificially raised irritability is ever of benefit.

Traumatic Shock.-Profound surgical shock involves a complete paralysis of the vasomotor center, associated with depression of the heart, and presumably of the vessels themselves. The poor circulation causes the prompt degeneration of the nervous centers, in which they can not respond to strychnin or any other stimulant (Sollmann and Pilcher, I9I4). Even epinephrin, transfusion or other mechanical means, whilst they may temporarily restore the blood pressure, can not restore the nervous centers (Crile). Excessive hemorrhage presents the same conditions.

Minor Degrees of Collapse and Especially in Hemorrhage, in Fevers, and in Intoxications. - In these, the increased excitability of the vasomotor center might somewhat counteract the lowered blood pressure, and enhance the effect of other stimulant measures (saline infusion, etc.). Moderate doses of strychnin ( $2 \mathrm{mg}$.) are used, preferably hypodermically. However, the clinical studies of Newburgh, I914, and Lucas, I9I4, were entirèly negative. Its effects are certainly much less pronounced than those of the reflex vasomotor stimulants (ammonia, alcohol, ether, camphor). These reflex stimulants are more useful especially for fainting and temporary myocardial insufficiency.

In Heari Disease proper strychnin is generally contraindicated, unless it be to ease the dyspnea; even this effect could be secured better by caffein. Newburgh, I $_{9} \mathrm{r}_{4}$, found no effect from $1 / 30$ to $1 / 10$ grain in broken compensation.

Depressant Effects of Toxic Doses of Strychnin.-The medullary and spinal functions all show a curious mixture of stimulant and depressant response to strychnin; the stimulation predominating during the convulsions, the depression during the intermissions. The paralytic effects gradually increase as death approaches, the respiratory center, vasomotor center, vagus center, and the cardiac muscle failing in about this order. Exhaustion and asphyxia as well as the direct depressant action of strychnin, are important factors in this paralysis. 
Contributory Factors in Strychnin Paralysis.-That exhaustion is an important contributory factor is shown by the fact that life may be greatly prolonged by preventing the convulsions through chloral or curare. The prolongation of life by artificial respiration shows the contributory action of asphyxia. However, animals die from large doses of strychnin, when exhaustion and asphyxia are both excluded, in the manner indicated. Indeed, the direct depression seems generally to be the main factor (Heubner and Loewe, I9 $\left.I_{3}\right)$.

There scems considerable reason to believe that in the frog the paralysis of the central nervous system is caused largely by the failure of the circulation through cardiac paralysis; but this is not the sole cause, for the heart is often still beating when the reflexes have disappeared. In the case of mammals deatil usually occurs before the heart has stopped.

Importance of Trealing the Paralysis.-It is important to bear in mind that the convulsions are not in themselves the dangerous element in strychnin poisoning. Death is rather due to paralysis. Remedial measures must therefore be directed not only against the convulsions, but also against the subsequent paralysis.

Tctanus alone is not such a dangerous condition. Thus, tetanus quite as violent as that of strychnin has been produced, e.g., by camphor, without being fatal, and the very severe convulsions of traumatic tetanus may last for weeks, whereas large doses of strychnin may kill after a single twitch or even without any signs of convulsions (death may then, however, be due to cardiac paralysis).

Special Senses. - Strychnin (2 to $3 \mathrm{mg}$.) increases the sharpness and field of vision for all colors (Dreser, 1894), and also the olfactory sense. The sense of touch is little, if at all, affected. These actions are central. In the eye it acts probably also on the retinal ganglion cells, the effects being obtainable unilaterally by injection into the temples or under the conjunctiva. It has been employed to arrest the progress of optic atrophy amaurosis $\left(\mathrm{Nagel}^{1}\right)$, sometimes with temporary improvement; $1 / 2$ to I c.c. of a $1 / 2$ per cent. solution $(2.5$ to $5 \mathrm{mg}$.) being injected into the temple.

Bitter and Tonic Actions.-Strychnin is extremely bitter (perceptible in dilution of $I: 400,000$ to $I: 100,000$, according to the sensitiveness of the observer). It shares the stomachic effect of other bitters, and is therefore used to improve appetite and digestion in nervous dyspepsias, chronic gastric and intestinal catarrhs, hyperemesis, seasickness, etc., and thus favor nutrition. The increased tone of the muscles creates a feeling of vigor which contributes to the "tonic" effect. To enhance the local action, the tincture of nux vomica ( $\mathrm{I}$ c.c. diluted, before meals) is often preferred to the alkaloid ( $\mathrm{I}$ to $2 \mathrm{mg}$ ). It is often combined with other tonics (Elixir Ferri, Quininæ et Strychninæ Phosphatum). If the bitter effect alone is desired, the dose of the tincture may be reduced to I or 3 drops, which is too small to produce any central effects.

In scasickness it has been employed as a prophylactic in conjunction with atropin (Skinner, N. Y. Med. Jour., December, 1893; Girard, 1906).

Drug Habit. - The bitter and tonic effects of strychnin make it a useful adjuvant in the treatment of chronic alcoholism and other drug habits.

Diabetes Insipidus.--Strychnin is said to be occasionally effective in reducing the polyuria. There is no pharmacologic explanation for this action, if it exists.

Peristalsis.--Strychnin is often used as an addition to other cathartics ("Aloin, Belladonna and Strychnin") with the idea of improving their action, especially in atonic constipation. It stimulates the Auerbach ganglia on direct application (Langley and Magnus, $x_{907}$ ). It has not been shown whether this action would occur in its therapeutic use. Hypodermically, it probably has no direct effect in ordinary doses, whilst toxic doses weaken or arrest peristalsis (Pollak, I9I0). Any results may perhaps be explained by increased tone of the spinal centers which influence intestinal movements and defecation. For this purpose, the extract of Nux Vomica is generally used ( $15 \mathrm{mg}$. in pills).

Other Peripheral Actions.-Strychnin, when used systemically, has practically no peripheral action on muscle, nerve, or glands except the bitter effect.

${ }^{1} C f$. Derby, 1902, Boston Med. \& Surg. Jour., 20, 508. 
The local application of strong solutions paralyzes most nervous and muscular tissues; for instance, the heart muscle, superior cervical ganglion, striped muscle endings. The last effect (curare action, Lapicque, I913) also occurs weakly in living frogs. Small doses seem to sensitize the neuro-muscular junction, lowering the threshold to indirect stimulation, and delaying fatigue (Hammett, I9I6). The curare effects are stronger in brucin; and methyl-strychnin may be counted in the curare group. After death, strychninized forgs cease to respond to sciatic stimulation much earlier than normally (Githens and Meltzer, Igr 2).

Action on Invertebrates.- Strychnin acts as a weak protoplasmic poison on ameboid cells, yeast, etc. Higher invertebrate animals are also relatively insusceptible. Many insects are quite immune, others are only slightly injured (Juchenack and Griebel, I9IO).

Absorption.-Strychnin is promptly absorbed, mainly from the intestine. In rabbits, none is absorbed from the stomach proper; it is not known whether this is also true for man.

Gastric Absorption.-Rather variable results have been obtained by different investigators when strychnin was placed in ligated stomachs. Generally, alcoholic solutions are absorbed readily, aqueous solutions scarcely at all. These results seem to depend partly on the ligation; for from unligated stomachs (Pawlow's pouch) aqueous solutions are well absorbed, better than alcoholic (Ryan, Igr 2).

Excretion.-A part of the strychnin is excreted unchanged, mainly by the urine. Small quantities occur also in the saliva, milk, sweat, bile, and feces. The excretion starts within five minutes after absorption (Ipsen, I892); and is practically completed within forty-eight to seventy-two hours (Bukunin and Majone, Igo6), although traces may be found even after five days (Plugge, I885; Hale, I909; Kuenzer, I9 4 ). In the remarkable case of a patient who had swallowed $\mathbf{r}_{5}$ grains and recovered, Hewlett, $I_{9} I_{3}$, recovered $4 \frac{1}{2}$ grains from the first stomach washings, and a total of $\mathrm{r}^{3} / 5$ grains from the urine, mainly between the first and tenth hour. Traces were present on the fourth, none on the fifth day.

Destruction.-At least a fourth, and often much more, of the administered strychnin can not be recovered from the excreta (Kobert), and is presumably destroyed in the tissues (perhaps mainly in the liver; not in the alimentary canal, Hatcher, 1904). Kratter, 1882 , however, believes that none of the alkaloid is destroyed in mammals.

Influence of Absorption and Channel of Administration on Effect.Since the capacity for the excretion and perhaps the destruction of strychnin is relatively great, the effect of the same dose will vary considerably with the rapidity of its absorption. Hypodermic injections are therefore two to eight times more active than oral administration; intravenous injections are two to three times more active than hypodermic. The effects of rectal administration approach more closely to hypodermic than to oral administration. The toxicity of strychnin is slightly reduced by the administration of large quantities of fluid (Kleiner and Meltzer, I9I2). Colloids have a marked retarding effect (Hatcher, I904.)

Even very large doses may produce no symptoms, if the experiment is arranged so as to make their absorption very gradual; for instance, by injecting it into the ligatured limb of a guinea pig. A considerable quantity will be gradually absorbed through the ligatured tissue; and if the ligature is released, after several hours, the effects will be correspondingly small.

Distribution in Body.-In fatal cases, the strychnin is found mainly in the blood, liver and kidneys, in frogs especially in the spinal cord, but there is no evidence that strychnin is bound by spina! cord emulsions (Dixon and Ransom, I912).

W. Koch and Mostrom, 19r I, found that phosphatids increase the solubility of basic strychnin; but this is probably a simple solution phenomenon, and has nothing to do with its selective action on the nervous system. Lombardi, I9 ${ }_{3}$, also finds that the toxicity of strychnin is somewhat diminished when it is first digested with brain substance; but not if the brain of a strychnin-poisoned dog is used.

Influence of Age.-Children are said to be comparatively unsusceptible to strychnin. In old people with atheromatous arteries, large doses might lead to apoplexy. 
Increased Susceptibility on Continued Administration.- The continuous use of strychnin does not lead to tolerance; on the contrary, the repetition of its action "educates" the nervous system to respond more readily, so that the effects are apparently slightly increased.

After the repeated administration of equal tetanic doses to frogs, the spasms occur earlier and last longer (Mostrom and McGuigan, I912). In mammals, the experiments of $W$. Hale, r909, gave rather inconclusive results, some indicating increased susceptibility, others slight tolerance.

Susceptibility of Different Animals.-This varies greatly. With subcutaneous administration, man, cats, and dogs require about the same dose, $0.75 \mathrm{mg}$. per kilogram, to produce a fatal effect; rabbits are slightly more susceptible. Guinea pigs and frogs require about six times, and snakes eighteen times, this dose. With the frog, the spasms appear with one-sixth the fatal dose; with the guinea pig they only set in when at least 95 per cent. of the fatal dose has been given. The differences for oral administration a'e even greater. Some birds are almost insusceptible to oral poisoning, the fatal dose for chickens by mouth being 30 to $40 \mathrm{mg}$. per kilogram; subcutaneously, 3 to $5 \mathrm{mg}$.

Temperature on Convulsions in Frogs.-Smaller doses suffice to produce tetanus when the temperature is either below or above the ordinary $\left(55^{\circ}\right.$ to $75^{\circ} \mathrm{F}$.). In the cold, the tetanus appears later, but lasts longer (Githens, I9 13 ). The rapidity of onset, with large doses, rises with the temperature according to van't Hoff's law (Schlomovitz and Chase, 1916). This, however, may depend on absorption.

Toxicology of Strychnin.-The symptoms and course of strychnin poisoning have been sufficiently described under the convulsant effects.

Toxic Dose.-In man, 5 to Io $\mathrm{mg}$. may exceptionally produce convulsive effects, more pronounced with 20 to $30 \mathrm{mg}$. These doses may even be fatal. The ordinarily fatal dose, by mouth, would probably be about roo mg. With efficient treatment, patients $\mathrm{ma}_{\mathrm{y}}$ be saved after $250 \mathrm{mg}$; ; and even doses of a gram and over are said to have been treated successfully (Hewlett). The fatal dose of Nux Vomica lies about 0.75 to $3 \mathrm{Gm}$.

Time of Appearance of Symptoms and of Death.-These depend upon the mode of administration, the condition of the stomach, and other factors influencing absorption. With oral administration, the symptoms generally appear in fifteen to thirty minutes; the convulsions sometimes only after an hour or later. Death occurs usually in one to three hours, but the time may be as short as ten minutes, or as late as nine to twenty hours, or even later.

Differential Diagnosis of Strychnin Poisoning.-Strychnin tetanus may be confused with traumatic tetanus, spinal meningitis, epilepsy, or hysteria. Traumatic tetanus is characterized by previous malaise and slow development. The convulsions begin in the jaw. The muscles remain rigid in the intermission. The course is comparatively slow. Strychnin tetanus may also begin in the jaw, but this is not so conspicuous. In rare cases of strychnin poisoning the muscles also preserve their rigidity during the interval, so that the diagnosis is sometimes difficult. When in doubt, strychnin treatment should be used, as it is beneficial in all similar conditions. The course of the case will clear up the diagnosis.

In spinal meningitis the diagnosis may be made by the fever and history. Epilepsy differs by the loss of consciousness; the refiexes are normal. In certain cases of hysteria the diagnosis may be impossible. Such cases should also be treated as for strychnin.

Postmortem Phenomena.-Death by strychnin is characterized by early and often persistent rigor. This, however, is common to all forms of convulsions, and is presumably due to the increased production of acid. The anatomical appearances are those of asphyxia and violent convulsions: venous congestion, often hyperemia of the central nervous system, and small hemorrhages; in a few cases hyperemia of the alimentary tract. 
Identification of Strychnin.--Strychnin may be identified in the suspected material, vomitus, or tissues (after isolation and purification) by its taste, convulsant effects on frogs, and chemical tests (violet and cherry color with bichromate-sulphuric acid). It is very resistant to putrefaction, and may be demonstrated in putrefying organic matter after a year or longer (e.g., Sundrick, I884).

Treatment of Strychnin Poisoning. 2-This comprises the prompt administration of chemic antidotes (permanganate, iodin, tannin); evacuation by emetics or stomach-tube (under chloroform); suppression of the convulsions by chloroform and chloral or ether; and artificial respiration. Absolute quiet of the patient is imperative.

Chemic Antidotes.-Potassium Permanganate is probably the most effective, if it is given sufficiently early, since it destroys the strychnin. One gram (1/4 teaspoonful) should be dissolved in a quart of warm water, carefully decanted, and administered in tumbler doses at short intervals. Iodin ( 15 drops of tincture in $1 / 2$ glass of water) or tannin (teaspoon in $1 / 2$ glass of hot water) merely delay absorption, but this is distinctly useful. Tea or coffee should be avoided, since the caffein is synergistic. Charcoal, or better caramel or Fuller's earth, are useful by absorbing the strychnin and delaying its absorption (Sabbatani, I9I4; Fantus, $\mathrm{r}_{9} \mathrm{r}_{5}$ ); but their efficiency is limited.

Evacuation.-Emesis by zinc or copper sulphate or mustard may be tried, or apomorphin (although the depression is therapeutically objectionable). Gastric lavage should be done if the patient is thoroughly under chloroform-otherwise it may start a fatal convulsion. Diuresis and catharsis are probably of little use. Hatcher, r9 5 , obtained fair results in animals with large quantities of hot water, or the intravenous injection of 2 per cent. sod. sulphate.

Physiologic Antidotes.-Chloroform, chloral, paraldehyd, ether, etc., suppress the strychnin convulsions. By preventing their painful and exhaustive effects, they conserve the energy of the patient so that he may more successfully resist the strychnin depression. They also lessen the danger of tetanic asphyxia, and are therefore the most useful antidotes.

Chloral is given in doses of $2 \mathrm{Gm}$. ( $1 / 2 \mathrm{dram}$ ), with the addition of another gram (I5 grains) after half an hour or longer, as necessary. There is always the danger in giving this, that its paralytic effects may coincide with those of the strychnin, and thus increase the danger. For this reason chloroform or ether is preferred, since their action can be better controlled. Ether has the advantage that it is not liable to the dangers of late chloroform poisoning. Githens and Meltzer, rgrr, advise the intratracheal insufflation of air and ether, combined with intravenous injection of Ringers' solution. Bickeles and Zbyszewski, I9 $_{3}$, found differences in the antispasmodic action of the various narcotics; amylen hydrate being especially effective, veronal very little, and chloral between.

Morphin is theoretically objectionable, since its action on the spinal cord is synergistic to strychnin. It has been employed, however, and its analgesic action at least would be useful; but chloral and chloroform should be preferred. Other depressants which have been used, but are of doubtful efficiency, are bromid, nicotin, and pilocarpin.

Epinephrin counteracts the cardiac depression, on local application (Januschke, rgro); but systemically, it increases the convulsive effects, so that there is no therapeutic antagonism.

Artificial Respiration.-This prevents or suppresses the convulsions, and may save life if it is carefully maintained until the patient is out of danger. The effect is probably due mainly to the removal of the synergistic effects of asphyxia.

There are, however, some conflicting theories and data: Osterwald, rgoo, claimed that strychnin spasms are diminished by excess of oxygen in the air, and vice versa.

1 The student should review the general "Treatment of Poisoning" and the "Exercises on Chemical and Physiological Antidotes." 
This might suggest that the result is due to destruction of the strychnin by oxygen; but there is no other evidence for this view. Meltzer and Gies, 1903, found that the mere rythmic movements of the chest were also important, for these prolonged life even when the animals were in an atmosphere of pure hydrogen. They suggest that this is due to a reflex inhibitory effect on the convulsive centers. It is also conceivable, that it might be due to removal of carbon dioxid. Paradoxically, however, Ryan and Guthrie, I908, find that the inhalation of carbon dioxid also arrests strychnin convulsions, in frogs and mammals.

It is claimed as a result of a nimal experiments that the application of external heat also decreases the strychnin mortality.

\section{PREPARATIONS-STRYCHNIN}

Strychnina, U.S.P., B.P.; $\mathrm{C}_{21} \mathrm{H}_{22} \mathrm{~N}_{2} \mathrm{O}_{2}$.-An alkaloid obtained from nux vomica and other plants of the Loganiaceæ. Colorless, transparent crystals or white, crystalline powder. Very slightly sol. in water (I: 6420); slightly sol. in alc. (I : I36). Dose, I.5 mg., $1 / 40$ gr., U.S.P.; I to 4 mg., $1 / 64$ to $1 / 15$ gr., B.P.

* Strychnina Nitras (Strych. Nit.), U.S.P.; $\mathrm{C}_{21} \mathrm{H}_{22} \mathrm{~N}_{2} \mathrm{O}_{2} . \mathrm{HNO}_{3}$.-Sol. in water (I : 42); slightly sol. in alc. ( $\mathrm{I}: \mathrm{I}_{50}$ ). All strychnin salts occur as colorless crystals or white powder; intensely bitter, even in dilute solutions. They are incompatible with alkalies, iodids and bromids (slow precipitation), tannin, arsenates and arsenites. Dose (of all Strychnin Salts), I.5 mg., $1 / 40$ gr., U.S.P.; 1 to $4 \mathrm{mg}$., $1 / 64$ to $1 / 16 \mathrm{gr}$., B.P. Maximum dose, $0.01 \mathrm{Gm} ., 1 / 6 \mathrm{gr}$.

Strych. Sulph., U.S.P. $\left(\mathrm{C}_{21} \mathrm{H}_{22} \mathrm{~N}_{2} \mathrm{O}_{2}\right)_{2} \cdot \mathrm{H}_{2} \mathrm{SO}_{4}+\mathrm{H}_{2} \mathrm{O}$. - Sol. in water (I: 32) and in alc. (I:8I). Dose, etc., see "Strych. Nit."

* Strych. Hydrcchl., B.P.; $\mathrm{C}_{21} \mathrm{H}_{22} \mathrm{~N}_{2} \mathrm{O}_{2} . \mathrm{HCl}+{ }_{2} \mathrm{H}_{2} \mathrm{O}$. - Sol. in water ( $\mathrm{I}: 60$ ). Dose, etc., see "Strych. Nit."

Inject. Strych. Hyp., B.P. -0.75 per cent. Dose, 0.3 to 0.6 c.c., 5 to to minims, B.P. Liq. Strych. Hydrochl., B.P. - I per cent. Dose, O.I 2 to 0.5 c.c., 2 to 8 minims, B.P. Elix. Ferri, Quin. et Strychn. Phosph., N.F.-The dose (4 c.c., I dram) contains (about): Strychnin, 11/3 mg., $1 / 40$ gr.; Quinin, 45 mg., 3/4 gr.; Ferric Phosphate, $90 \mathrm{mg}$., $\mathrm{r} 1 / 2 \mathrm{gr}$.

Syr. Ferr. Phosph. c. Quin. et Sirych., B.P. (Easton's Syrup).-Ferrous Phosphate, I.7\% per cent.; Quin. Sulph., I.5 per cent.; Strych., 0.57 per cent. Dose, 2 to 4 c.c., $1 / 2$ to I dram, B.P.

Nux Vomica, U.S.P., B.P. (Strychni semen, P.I.). - The dried, ripe seeds of Strychnos Nux Vomica, East Indies; yielding not less than 2.5 per cent. of total alkaloids, U.S.P.; not less than 1.25 per cent. of strychnin, B.P. Dose, $0.06 \mathrm{Gm}$., I gr., U.S.P., 0.06 to $0.25 \mathrm{Gm}$., I to 4 gr., B.P. Maximum dose, $0.25 \mathrm{Gm}$., $4 \mathrm{gr}$.

The constituents are strychnin, brucin, tannin, fat, etc.

The drug was unknown to the ancients, and was probably introduced by the Arabs. The first good description occurs in 1540. Strychnin was discovered in 1818.

The bark contains the same principles in less amount, but relatively more brucin. It was formerly found in commerce under the name of "false angostura." Several arrow poisons are also derived from the genus Strychnos, especially the Upas Tieute from Java. Some of the strychnos species do not contain any active principle.

Nux vomica contains a small quantity of a third alkaloid, igasurin, which has not been very greatly studied, but which seems similar to strychnin.

Ext. Nuc. Vom. Liq., B.P.-I.5 per cent. of strychnin. Dose, 0.06 to 0.18 c.c., I to 3 minims, B.P.

* Extractum Nucis Vomica (Ext. Nuc. Vom.), U.S.P.; Ext. Nuc. Vom. Sicc., B.P.A powdered extract; I $\mathrm{Gm}$. representing about $4 \mathrm{Gm}$. of drug, Io per cent. of total alkaloids, 5 per cent. of strychnin. Dose, $15 \mathrm{mg}$., 1/4 gr., U.S.P.; I6 to $60 \mathrm{mg}$., $1 / 4$ to I gr., B.P., as pills. Maximum dose, $60 \mathrm{mg}$., I gr.

* Tinctura Nucis Vomice (Tr. Nuc. Vom.), U.S.P., B.P.; Tincture of Nux Vomica.Io per cent. of drug, 0.25 per cent. of alkaloids, 0.125 per cent. strychnin, in about 75 per cent. alcohol. Miscible with water and alc. Incompatibilities as for Strych. Nit. Dose, 0.5 c.c., 8 minims, U.S.P.; 0.3 to I c.c., 5 to 15 minims, B.P. Maximum dose, 2.5 c.c., 40 minims. 


\section{PICROTOXIN GROUP}

General Statement.-The pharmacologic group of which picrotoxin is the main representative produces a conspicuous stimulation of the medullary centers, with characteristic convulsions, slowed pulse, etc. There is also some stimulation of the spinal cord. These effects are followed by paralysis. The actions are not utilized therapeutically, but are of some toxicologic as well as scientific interest, since the members of the group have often caused poisoning.

The Principal Members of the Group.-These comprise picrotoxin from Cucculus indicus (the seed of Anamirta) paniculata ("Fish-berries;" used for poisoning fish, the flesh of which becomes toxic; also for adulterating beer; and in "knockout drops." The external use against pediculi has given rise to toxic effects); Cicutoxin from the rhizome Cicuta virosa "Water Hemlock," an umbeliferous plant eaten by mistake for parsley, etc. (the American species, Cicuta maculata and bulbifera, are also toxic). Cicutoxin is a complex pyrone'derivative, occurring as an unstable resinous substance (C. A. Jacobson, I9 $\mathrm{I}_{5}$ ). Some of the decomposition products of digitalis (digitaliresin, toxiresin) are also counted in this group.

Less important members are: Coriamyrtin (from Coriaria myrtifolia); and Tutin (from Coriaria species of New Zealand) (C. R. Marshall, ro ro). W. W. Ford, rgro, found that tutin combines with nervous structures, thereby losing its toxicity; but no tolerance is acquired.

Chemic Nature.-Most of the substances are non-alkaloidal, non-nitrogenous "neutral principles," more soluble in alcohol than in water. Some are glucosids. Picrotoxin itself consists of a mixture (or perhaps a feeble combination; Sielisch, I9 12) of nearly equal parts of picrotoxinin and picrotin (Cervello, IgIr; structural formula, Angelico, ror2). These are closely allied chemically and have identical actions, but picrotoxinin acts far stronger. Coriamyrtin seems to vary. That first isolated by Riban was very soluble, while Merck's is almost insoluble, but probably more active (Marshall, Igr2).

Picrotoxin Convulsions.-The characteristic effects on the frog, and the localization of the convulsions in the medulla, are described in the "Laboratory Exercises." In mammals, also, the convulsions are not modified by destruction of the hemispheres

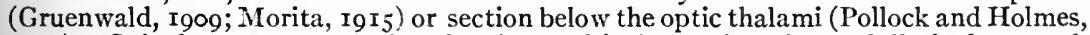
I915). Spinal 'Tetanus may often be observed in frogs after the medulla is destroyed, the spinal stimulation having previously been masked by the more intense stimulation of the higher centers (Luchsinger, I878). The convulsions are not so much dependent upon reflex stimulation so that they are probably in part due to a direct stimulation. According to Baglioni, rgog, the action is located in the sensory cells.

Symptoms of Picrotoxin Poisoning in Mammals. - The effects of convulsive but non-fatal doses were described by J. Crichton Brown, 1875, as: Salivation and vomiting (central; Eggleston and Hatcher, I9I5); then quiet and apathetic; then restless and apprehensive; then-trembling of legs; lies on side, with efforts to stand; progressively stronger twitching of face and neck muscles; head drawn back. Then, suddenly, generalized clonic convulsions; clonic champing of jaws; closing of eyelids; marked frothing at mouth; involuntary urination; pupils dilated during the convulsions. The clonic spasms change imperceptibly to running movements, become slower, and finally cease. The animal remains quietly on its side, and then, after some efforts, regains the erect position. After a time, varying with the dose, the convulsions may recur.

Respiration.-This is stimulated before the convulsions; becomes irregular during the initial twitching and arrested during the clonic spasms (Pollock and Holmes, $1_{9} r_{5}$ ). These authors point out the similarity of the respiratory, convulsive and circulatory changes with those of epileptic attacks (Pollak and Treadway, r913). The threshold of the respiratory center to $\mathrm{CO}_{2}$ is lowered by Coriamyrtin (Wieland, $\mathrm{r}_{9} \mathrm{I}_{5}$ ).

Spasms of the laryngeal muscles lead, with the frog, to distention of the body with air and to a characteristic cry similar to that sometimes heard with strychnin. With digitaliresin, toxiresin, and oleandresin, the convulsions are preceded by immobility.

Circulation.-In the early stages, the blood pressure may rise from stimulation of the vasomotor center. About three to eight seconds before the convulsions, the blood pressure falls, remaining low until asphyxia sets in (Pollock and Holmes, r9 5 ).

Vagus Center. - The heart is greatly slowed, and may even cease for a time. After division of the vagi, the heart will return almost to normal. There is, however, some 
depression of the cardiac muscle involved in this slowing. Later there may be a quickening, partly due to stimulation of the accelerator center and partly to paralysis of the vagus center and to fatigue of its endings.

The romiting, salivary, and sweating centers are also excited. The sweat may, however, be suppressed by vasomotor constriction.

Aulonomic Centers.-Ciruenwald, I909, pointed out that picrotoxin produces the symptoms of parasympathetic stimulation: Contraction of pupils, salivation, vomiting, slowed pulse, contraction of urinary bladder, erection, etc. These effects are central, for they do not occur after section of the nerves. They are not hindered by decerebration.

Uterine Spasms.-These lave been observed, and depend probably on stimulation of the spinal cord, since they cease upon destruction of this organ.

Paralysis. - With larger doses, all the stimulant effects give way to paralysis.

Hemolytic Action.-Picrotoxin, cicutoxin, and phytolacca decandra are said to act similarly to saponin.

Excretion.-This occurs by the urine; picrotin is partly unchanged; the picrotoxinin is decomposed (Chistoni, xy 12 ).

Toxic Dose.-According to Lewin, 0.03 to $0.24 \mathrm{Gm}$. of cocculus is toxic, $2.4 \mathrm{Gm}$ fatal. Of picrotoxin, $20 \mathrm{mg}$. are toxic. The fatal dose is not known.

The Symptoms of Poisoning in Man.-These consist in burning sensation, nausea, salivation, cold sweat, pallor, colic, vomiting, diarrhea; pulse slowed or quickened; palpitation; shallow respiration. These are rapidly followed by confusion, stupor and unconsciousness. After one-half to three hours, trembling, clonic and tonic convulsions. These generally pass into paralysis and death by asphyxia, after several hours.

The postmortem findings are those of asphyxia. The poison disappears rapidly during putrefaction (within one or two weeks); so that the toxicologic analysis must be made promptly. The characteristic effect of the isolated poison on the frog constitutes the best test. The intensely bitter taste (discernible in dilutions of $\mathrm{x}: 80,000$ ) may arouse suspicion.

Treatment.-The chemic alkaloidal precipitants would not be efficient. The best treatment would be emetics (if vomiting has not occurred), permanganate, chloral, chloroform, and external heat. The combined administration of chloral, morphin, and minimal doses of atropin has recently been recommended as the result of animal experiments.

Therapeutic Uses.-The medullary stimulation produced by the group might be expected to have many therapeutic uses; in fact, however, they have not been found available, perhaps because the effective doses are too near to the toxic. Picrotoxin ( 0.5 to $2 \mathrm{mg}$.) has been used in the night sweats of phthisis, and in epilepsy, but with very doubtful results. Powdered cocculus has been used against pediculi, but is dangerous.

Solubility.-Picrotoxin is soluble in about 8 parts of alcohol, or 240 parts of water.

\section{CAFFEIN, THEOBROMIN, AND OTHER METHYL-XANTHINS}

Members.-This group comprises the various methyl derivatives of xanthin (dioxypurin). These occur in several plants; as products of metabolism in animals; and may also be obtained synthetically. The most important are caffein (trimethylxanthin), the principal alkaloid of coffee, tea, kola, guarana, maté, etc.; theobromin ( 3.7 dimethylxanthin) from cacao; and theophyllin (I.3 dimethylxanthin), which is usually prepared synthetically. Xanthin itself, and all its other methyl derivatives, produce the principal actions of the group.

Nature of Caffein.-Caffein is capable of forming salts, and is, therefore, an alkaloid; but it is so feeble a base that it does not turn litmus, and the salts are dissociated by water. Its solubility is also peculiar. It was isolated from coffee in 1820 by Runge, Pelletier and Caventou, and Robiquet. Its identity with the alkaloid of tea (sometimes called Thein) was surmised by Berzelius, and confirmed by Jobst and Mulder in 1838. Commercial caffein is now produced almost exclusively from tea.

Occurrence.-Caffein occurs in plants of at least six families, which are scattered over many portions of the globe. It is rather remarkable that these plants have nearly all been discovered and consumed by the natives as stimulants. These plants of ten 
contain volatile products or tannin, which slightly modify their effects. Theobromin is of ten associated with the caffein; in cacao, the theobromin predominates.

The chemical structure and derivation of the purin derivatives have been elucidated by Emil Fischer. 'The most important are as follows:

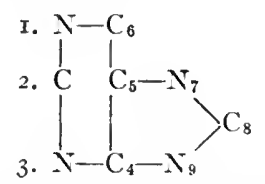

Purin (the numbers indicate the positions in which radicles may be introduced).

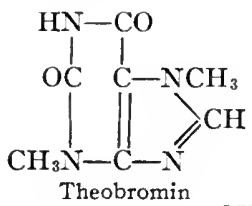

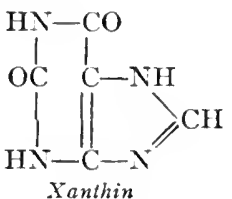

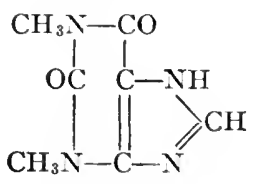

Theophyllin

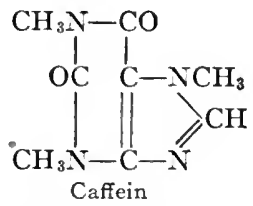

Principal Actions.-I. Increase of the reflex irritability of the central nervous system from above downward; leading to stimulation of the psychic areas (insomnia, etc.); of the medullary centers (respiratory, vasomotor and vagus); and with large doses to heightened reflexes and tetanic convulsions. With toxic doses, the stimulation is accompanied by paralysis.

2. Increased ease of muscular contraction, progressing to loss of elasticity and to rigor; this affects all forms of muscle to a varying degree. The heart rate is thereby quickened.

3. Vasodilation by a direct action on the vessels with moderate doses; this combines with the cardiac stimulation to quicken the circulation.

4. Diuresis results through the interaction of several factors.

Caffein is used as a psychic, muscular and respiratory stimulant; and as a cardiac tonic and diuretic. Theobromin and theophyllin are probably more effective when the peripheral actions are desired.

Psychic Functions.- In man, moderate doses of caffein (to $0.3 \mathrm{Gm}$.) produce a quicker and clearer flow of thought; disappearance of drowsiness and fatigue; more sustained intellectual effort; more efficient appreciation of sensory impressions, and more perfect association of ideas (Kraepelin, 1872; Dietl and Vintschgau, I878; Ach, I900; Hollingsworth, I912). The central actions of theobromin are relatively much weaker than those of caffein (Filehne, r886). Theophyllin is intermediate (Dreser, Pouchet, Thomas).

Wilh larger doses, these effects pass into wakefulness, excitement, and the other unpleasant reactions described under the toxicology.

The lower mammals react by restlessness and excitement. The cat is said often to become frantic. Frogs do not exhibit any symptoms referable to the brain.

Use as Antidote in Narcotic Poisoning.-Caffein is often used as an antidote against acute poisoning by morphin (Bennett, 1874); alcohol 
(Binz, I 878 ); paraldehyd (Schroeder, I887); chloral (Airila, r9r3, Sollmann). The psychical actions are directly antagonistic, the mental and muscular coördination being immediately improved, although the effect is not lasting. The respiration and circulation may also be somewhat stimulated, but the effects easily become additive, so that with fatal alcohol poisoning, caffein is actually deleterious. With morphin, the conditions are probably more favorable.

As a stimulant antidote, the caffein is usually administered as a very strong, hot, black coffee, to secure the synergistic stimulant action of the heat and oils. Strongly boiled tea, through its tannin, would be a chemical antidote in poisoning by corrosive metals. Coffee has not this precipitant effect.

Mutual Antagonism of Alcohol and Caffein.-This has been studied especially by Pilcher, I9 I2. He found that with the smaller doses, each drug tends to act qualitatively as if present alone. In alcohol sleep, small doses of caffein cause awakening, restore the reflexes, and diminish the fall of temperature. With larger doses, there is a qualitative change in the direction of greater depression. Light alcohol coma is intensified by moderate or large doses of caffein; the reflexes are further depressed. With toxic doses, the fatality is greatly increased if both drugs are present, mainly by their deleterious action on the heart.

Medullary Centers.-These are moderately stimulated by caffein, similarly to strychnin. There are, however, some important differences: With caffein the stimulation is on the whole weaker for toxic doses, but rather stronger and much more prolonged with therapeutic doses; and there is little or no subsequent depression, even with toxic doses. The most conspicuous effect is on the respiration. The stimulation of the vasomotor and vagus centers is interfered with by other factors, as described under "Circulation."

Respiration.-In man, the respiration is only moderately increased (Edsall and Means, I9I4), or scarcely affected by oral therapeutic doses (Newburgh, I9I4; Taylor, I9I4); unless cardiac dyspnea is improved by the circulatory effect. With hypodermic injection of 0.15 to $0.25 \mathrm{Gm}$., there is definite evidence of respiratory stimulation. The $\mathrm{CO}_{2}$ tension is markedly lowered, inducing a lowered threshold of the respiratory center. The rate and depth of the respiration, and the minute-volume are increased. There is also increased gas metabolism, and sometimes bronchial dilation (Higgins and Means, I915).

Anesthetized animals respond by increased rate (Binz, r878; and generally also increased depth of the respiration (Heinz, r890; Impens, r899). The latter is probably due to partial awakening from the anesthesia; for with decerebrated animals, the increase is confined to the rate, the depth being unchanged or more shallow (Cushny, x9x3). Inhibitory reflexes are less effective.

Use in Asthma.-Coffee somtimes gives relief in asthmatic attacks. The action is probably partly psychic, partly bronchial; for in guinea pigs, it relaxes the bronchial spasm produced by peptone or muscarin ( $\mathrm{Pal}, \mathrm{I}$ (12). Bronchial dilation is also sometimes observed in man (Higgins and Means, 1915). F. Meyer, 1915, concludes that caffein acts mainly by stimulating the respiratory center to greater effort. He considers it less efficient than atropin, epinephrin or nicotin.

Spinal Cord.-Ordinary doses of caffein heighten the reflexes (Wood, I9r 2; Leeuwen, 1913). Toxic doses produce convulsions of spinal origin (Bing, 1901), which agree in all essential respects with those of strychnin; except that the tetanus is neither as violent nor as prolonged. The exhaustion in the intervals is also less pronounced. The tetanus may produce death by fixation of the respiratory muscles. The convulsant dose is relatively large, and it is therefore doubtful whether it has ever been observed in man. Ordinary toxic doses produce only tremor. In frogs, the tetanus is not in- 
creased or hastened by removal of the cerebrum (difference from morphin; Githens, I9I4).

The paralytic action of large doses can be shown in frogs by protecting the muscles from rigor; i.e., by ligating a leg exclusive of the nerve. Caffein will then destroy its reflexes.

Skeletal Muscle.-Ordinary doses of caffein increase the functional activity of skeletal muscle so that it contracts more readily and more completely and powerfully, and is less easily fatigued (Wood, I9I2). This improvement can be demonstrated by the ergograph, as well as in the excised or curarized frogs' muscle (Fig. 7). It is, therefore, at least in large part, peripheral. Larger doses depress the muscle, and eventually cause it to go into rigor, even in the living animal.

The Stimulant Phase.-A smaller stimulus suffices to produce contraction (Paschkis and Pal, 1886). The latent period is shortened. The height and rapidity of the contraction are greater, and a larger weight can be lifted (Kobert, I 88I). Fatigue is lessened, and a greater amount of work can be performed (Dreser, I 890), so long as the load and stimulation are optimal; but with excessive load or stimulation, caffein diminishes the total work (Golowinsky, 1915). The other methyl-xanthins affect muscle qualitatively like caffein (Golowinsky).

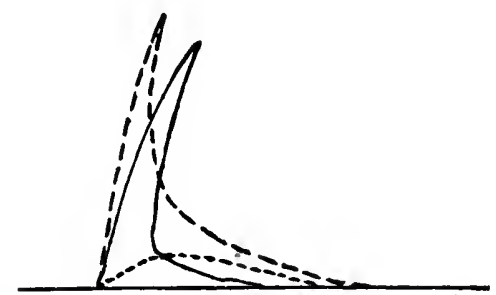

Fig. 7.-Effect of caffein on gastrocnemius muscle of frog, - normal tracing; - after five minutes in $\mathrm{I}$ : 10,000 caffein solution; . . . in $\mathrm{I}: \mathrm{I}, 000$ solution.

The Depressant Phase.-As the dose or concentration is increased, these effects are reversed. The contraction becomes less powerful, and the curve resembles that of a fatigued muscle; i.e., it is lower and more drawn out. This lengthening shows first in the relaxation (Buchheim and Eisenmenger; Golowinsky), eventually also in the contraction. The elasticity is lessened. The muscle is exhausted more rapidly by tetanus.

Similar muscular effects are produced by xanthin (Paschkis) and creatin (Dreser).

Resemblance to Fatigue.-The successive increase and diminution of the muscular functions by caffein resembles the successive phenomena of work and fatigue; and suggests that a part of these may be due to the accumulation of the xanthin products which are formed in metabolism; but other products which accumulate during work also influence the muscle similarly to fatigue (Lee, I 906).

Caffein Rigcr. - This was observed by Voit, 1866 , and then investigated by Johannsen, and by Schmiedeberg, $\times 872$. Its occurrence in the living body can be demonstrated by hypodermic injection in certain species of frog (the European Rana temporaria, and the ordinary American laboratory frog, R. pipiens); while another European frog (R. esculenta) is less susceptible to the rigor, and therefore succumbs to spinal convulsions. In both cases, the frog becomes rigidly extended; but the rigor (as distinguished from tetanus) persists after division of the sciatic nerve (Johannsen) and occurs after curare. The heart may continue to beat strongly for some time. Mammals also show some stiffness after large doses of caffein, but actually rigor results only if the alkaloid is injected directly into an artery. The coagulant action is possessed by other xanthins, being strongest with xanthin itself, and progressively weaker with theobromin, theophyllin and caffein (Filehne, r886; Dreser).

The muscle in caffein rigor is inexcitable and appears white, bloodless, stiff and considerably swollen and shortened, and is acid to litmus (Ransom, Igri).

Microscopic Changes. - If the caffein is applied to a teased muscle under the microscope, the contents of the fiber are seen to move; the cross-striations disappear, and the longitudinal striae become more prominent. The sarcolemma becomes detached. The fibers shorten by half (Johannsen). 
These changes have been studied by Secher, I9I4, through perfusion with dilute solutions of xanthin and the various methyl-xanthins, which all produce similar effects. The histologic changes increase with the concentration, to disorganization (caffein, $1: 2,000$ ). Short of disorganization, the changes are reversible. The disorganized fibers also eventually regenerate, provided their nuclei are intact. Hardening and true rigor is confined to the destroyed fibers. Similar changes are produced by chloroform and other poisons, and in other vertebrates, al though mammals require higher concentrations.

Perfusion of the muscle with saline or blood, if undertaken soon after the onset of the rigor, restores the plasticity but not the excitability (v. Fürth).

Coagulation of I uscle Extracts.-Rigor may also be produced in living animals by the injection of a number of other substances in sufficient concentration; for instance, by chloroform. All of these, as well as caffein, favor the coagulation of muscle extracts (v. Fürth, I896). However, there need not be any relation between rigor and this coagulation; for certain substances coagulate muscle extracts even more actively, but are incapable of producing rigor during life.

Muscular Effects in Man.-Morse showed by the ergcgraph that moderate doses of caffein (0.I to $0.6 \mathrm{gm}$.) increase the muscular work. In the first hour this may be raised four or five times; the effect then lessened, but extended over two to seven hours. Practically all subsequent experimenters have confirmed the increase, although it was not always as great (Hellesen, I904). Sometimes the increase was mainly in the height of contraction (Kraepelin); with others, in the number (Rossi). This indicates that the action is both on the muscle substance (height) and on the motor centers (fatigue). Rivers and Weber, I907, found it quite variable, and sometimes followed by a decrease. Schumburg showed that the effect is less when the experiment is made fasting on an exhausted muscle. The increase of efficiency is due partly to a lesser feeling of fatigue (Rivers and Weber), but it is at least in part peripheral, since the efficiency is also increased when the muscle itself is stimulated electrically.

Use of Caffein Against Fatigue.-The psychic and muscular action of caffein are popularly used against fatigue. This will be discussed under the "Caffein Beverages."

Use in Headache. - Fairly large doses of caffein ( $0.5 \mathrm{Gm} .=7 \frac{1}{2}$ grains) are rather effective against certain forms of headache, such as migraine, neuralgia, fatigue, etc. It may be combined with acetanilid. It does not prevent the depressant effects of the latter; but rather increases its toxicity (Hale, r909; Salant, I9I2); it also increases the toxicity of alcohol and barium chlorid by its circulatory action. Caffein is also useful in some cases of nervous dyspepsia.

Smooth Muscle.-The effect of caffein on smooth muscle consists in a longer and more persistent contraction curve. Larger doses prolong the period of relaxation. The tone seems to be affected differently in various muscles. The arteries are relaxed by relatively small doses; whereas the caffein has a tonic effect on the pelvic portion of the ureter (Lucas, I907). It has very little effect on the uterus (Röhrig); and none on peristalsis (Nasse, I866).

Other Peripheral Actions.-Caffein has no action on peripheral nervous structures when it is used systematically (Aubert, r872); nor does it cause any local irritation.

It has a paralyzing action only when it is applied in a very strong paste directly to the nerve (Pratt). It does not act on any ganglia, nor on any glands other than the kidney; nor on red corpuscles, nor on fibrin formation. Small doses increase the movements of ?eucocytes in shed blood, whilst higher concentrations kill them.

Lower Organisms. - The actions of caffein on these are not characteristic (Brunton, I882: oxidation of guaiac; Haskins, I903: gas formation by yeast; Bokorny, 1894, and Korentschewsky, 1903: amebae; Romanes, 1867: medusae; Sollmann, 1906: adult and embryonic fish; Pickering, I893: embryonic chick's heart; Ransom, I912: germination of seeds).

Metabolism.-The results of different investigators have been contradictory, but it appears that caffein causes a rather small and variable increase in urea and $\mathrm{CO}_{2}$. Both effects are probably indirect; the urea 
being explained by the diuresis, the $\mathrm{CO}_{2}$ by the increased movements. This also explains the slight rise of temperature.

The earlier investigators claimed diminished urea excretions (J. Lehmann, I 853 ; Böcker, $\mathrm{I}_{54}$, and others), with varying results as to $\mathrm{CO}_{2}$. The earlier work has not been confirmed, and may possibly be explained by imperfect methods. (However, Farr and Welker, 1912, describe decreased $N$ elimination after theophyllin in health and renal disease; whilst Endilyi claims an increase of $V$ after theophyllin or theobromin. The contradictions probably depend on differences of diuresis.) C. J. Lehmann, Voit, Roux and others have demonstratedincreased urea excretion after caffein; Reichert, increased heat production; Heerlein, I892, Edsall and Means, I9I4, and Higgins and Means, $\mathrm{I}_{9} \mathrm{I}_{5}$, found increased $\mathrm{CO}_{2}$ and $\mathrm{O}_{2}$ metabolism, with therapeutic doses. Salant and Phelps, I9ro, and Salant and Rieger, 1914, found slight changes in the excretion of creatin and creatinin.

Temperature.-The rise was demonstrated by Binz and Peretti, I878. It is very slight in normal animals $\left({ }^{\circ}\right.$ to $\mathrm{I} .5^{\circ} \mathrm{C}$.). It is perhaps rather more effective in preventing the fall of temperature which occurs in light narcosis, but is ineffective in deep narcosis (Pilcher, I I I 2). The rise is much greater in thyroidectomized dogs (Karelkin, I9I4).

Glycosuria.-During the caffein or theobromin diuresis, there is commonly a slight hyperglycemia and glycosuria, especially in rabbits fed on carbohydrate food. However, it occurs also in fasting animals (E. Hirsch, I9I5). It may be induced by oral administration, and occurs also in cats, but not in dogs (Salant and Knight, I 909 ). The phenomenon, which occurs also with other diuretics, has not received a satisfactory explanation (Miculicich, 1912). It is not renal, for sugar appears in the urine only when there is a considerable rise of blood sugar (Hirsch). The hyperglycemia indicates increased glycogenolysis, which resembles that produced by piqûre (Nishi, 1909). Like this, it is generally (but not always; Jarisch, I9I4) prevented by section of the splanchnics, excision of the suprarenals (A. Mayer, I906) and by nicotin (Hirayama, I9II). It must therefore be usually central, perhaps through increased reflex irritability; but under certain conditions it is also peripheral (Jarisch).

Digestion.-The effects will be discussed under the beverages.

Circulation.-Caffein acts on the heart and blood vessels, peripherally and centrally. The effects are therefore quite complex and vary with the dose and other conditions. The predominant action of therapeutic and moderate physiologic doses consists in vasodilation, combined with sufficient cardiac stimulation to maintain the blood pressure, or even to raise it somewhat. Both actions should combine to favor the blood flow, and would therefore be useful in circulatory diseases; the diuretic action being a further desirable feature. On the other hand, the effects of caffein are not so powerful nor so lasting as those of digitalis; and its side actions often interfere with its use. The other methyl-xanthins act very similarly to caffein, and the same description probably applies to all.

Stages of Action.-The effects in animals may be divided into the immediate effects of intravenous injection, the more persistent effects of moderate (stimulant) doses; of larger depressant doses; and fatalities (Sollmann and Pilcher, I9II; Wood, I912; Salant, I9I3; Pilcher, I912).

Intravenous Injection of Caffein.-This produces a fairly severe fall of blood pressure, with prompt recovery. The acute fall of pressure is presumably due to depression of the myocardium by the concentrated drug. It does not occur with other methods. of administration, and therefore has little practical importance. It is not characteristic for caffein, but occurs with many other drugs. The organ volume (oncometer) falls with the pressure (indicating that the action is cardiac). Direct observation of the heart shows dilation with diminished excursions. The heart rate is quickened and the vasomotor and respiratory center are stimulated by the low pressure.

Stimulant Doses.-Small and moderate doses (to $20 \mathrm{mg}$. per kilogram) generally produce a slight ( 10 to $20 \mathrm{~mm}$.) rise of blood pressure, usually with more or less increase of the heart rate. The tone of the heart is increased and the amplitude of its excursions may be greater. This, 
and the faster rate, raise the output of the heart. The vein pressure is not changed (Capps and Matthews, I9I3). The blood flow is increased (Landgren and Tigersted, I 892; Loewi, I905). The volume of the splanchnic organs (Phillips and Bradford, I887; Albanese, I88I; Gottlieb and Magnus, I90r; Loewi, r905) and of the cerebrum (Roy and Sherrington, $\mathrm{r}_{887}$ ) increases. This oncometric change is more marked and more persistent than that of the blood pressure, and therefore indicates a marked vasodilation. It has been claimed that the dilation is more powerful in the kidney than in other organs (Phillips and Bradford; Loewi); but in fact it seems to be about equal at least in the splanchnic area (Sollmann and Pilcher). The vasodilation is peripheral, since the perfusion method shows that the vasomotor center is actually stimulated. Ordinarily, the peripheral dilation is more powerful than the central constriction; but under some conditions the constriction may predominate, so that the oncometer falls with the rising pressure. This, however, is very exceptional. The older conception of a powerful and constant constriction was based on slender and indirect evidence (mainly on the experiments of Wagner, I885, and Schroeder, I886). Vinci, I 895, found that caffein gave a much larger rise of blood pressure (presumably cardiac) in animals which had been weakened by anemia or starvation.

Depressant Doses.-Doses of caffein above $40 \mathrm{mg}$. per kilogram progressively lower the blood pressure, until a level of 60 to $85 \mathrm{~mm}$. is reached, with doses of about $140 \mathrm{mg}$. per kilogram. This level is then maintained until death approaches. If the original pressure was low, it may not be further lowered. The tone of the heart is decreased, so that it gradually dilates, and the amplitude of the excursions and the output are diminished. The oncometer falls with the blood pressure, so that cardiac depression is the dominant element. It is accompanied, however, by a progressive and finally complete vasomotor paralysis, when epinephrin, splanchnic, and other forms of vasomotor stimulation become less and less effective. (Epinephrin still causes a relatively small rise through cardiac stimulation.) The vasomotor center can be shown (by the perfusion method) to be stimulated even after very large doses; but this stimulation can not become effective against the peripheral paralysis.

Fatal Doses.-Death occurs by acute cardiac failure. Caffein serves to lower the resistance of the heart, so that the fatal dose may vary greatly in different animals.

Factors Involved in the Circulatory Actions of Caffein.-These may be summarized as follows: Cardiac stimulation or depression, according to the dose and rapidity of injection.

Increased heart rate, not due to vagus depression. (If the vagus is intact, there may be slowing through central stimulation, but with large doses the heart escapes from the vagus tone.)

Vasodilation, through peripheral depression of the vasoconstrictor mechanism. tion.

Central vasoconstrictor stimulation generally ineffectual, also convulsive stimula-

Cardiac irregularities, with large doses.

Direct Action on Blood Vessels. - These tend to dilate when excised organs are perfused with caffein (Beco and Plumier; Cow, IgrI). The effect is not very great with caffein alone, since the vessels are already dilated after death; but very little caffein suffices to counteract the constrictor effect of epinephrin (Sollmann and Pilcher). The caffein must therefore act on or beyond the structure stimulated by epinephrin; i.e., on the receptive or muscle substance.

Special Vessels.- The Coronary Vessels are not affected directly by caffein or theobromin (Bond, I9II; Rabe, I9I 2); but in intact animals there is increased blood flow and some passive dilation with therapeutic doses when the blood pressure rises ( $F$. Meyer, 1912). Toxic doses generally dilate (Sakai and Saneyoshi, I915; Macht, I915). The Lung Vessels are first constricted, soon ceding to dilation (Berezin, I914). Macht, I9I4, observed dilation of excised pulmonary artery. Theobromin was practically negative. Baehr and Pick, 1913, found no effect with caffein on lung perfusion. The Liver veins are dilated (Berezin, I9I4). The vessels of the pia mater and retina are dilated during life (Hirschfelder, r9r5). The dilation of the eye-vessels may raise the intra-ocular tension even with falling systemic blood pressure (Wessely, 1915). 
The Heart Rate.- When the ragi are intact, small doses of cafiein may cause some slowing through stimulation of the vagus center (Swirski, r904) and increased vagus excitability (Fredericq, I9 3 ). This slowing does not occur in all animals. With larger doses, or always if the vagi are paralyzed, the rate is markedly quickened. With the vagi divided, $2 \mathrm{mg}$. per kilogram increases the rate by 25 per cent.; $20 \mathrm{mg}$. per kilogram by 33 per cent.; 30 to roo mg. by 50 per cent. Beyond this there is no further increase, but the heart shows arythmia and irregularity. The quickening is evidently not due to vagus depression, since it occurs after section of the vagi or atropin (Johannsen, i 869 , and others). The vagus tone is indeed lost after large doses (Aubert, 1872, and others), and the response to vagus stimulation is uncertain; but direct central vagus stimulation may still cause marked slowing.

Nor is the quickening due to stimulation of the acclerator mechanism. The effect occurs after excision of the stellate ganglia and in the excised mammalian heart. Swirski, I904, found that when the caffein quickening had reached its maximum, a further increase would be obtained by stimulating the accelerator. (Large doses destroy the response to accelerator stimulation, Fredericq, I913.) Cushny showed that the details of the cardiac curve are different in caffein and accelerator stimulation. Dixon, $x 903$, found that caffein quickens the heart after apocodein, which paralyzes the accelerator endings. Pickering, I 893, observed it in the embryonic chick's heart before the nervous structures had become developed. The quickening must therefore be due to increased contractibility of the muscle substance itself.

Frog's Heart.- -Small doses increase the rate (Voit, I860), excursions (IVagner, I885), pulse volume, and especially the absolute force or maximal resistance (Dreser, I887). The diastole is shortened more than the systole.

Large doses cause slowing and weakening (Maki), with diastolic tendency (Faval). The heart beats for some time after the skeletal muscles are in rigor (WVagner). The final arrest may be either in systole (Johannsen, I869) or diastole (Faval).

With hypodermic injection, the stimulant dose is $5 \mathrm{mg}$; the depressant $25 \mathrm{mg}$. (Wagner). Dropped on the exposed heart, I:I0,000 is stimulant, and I: roo depressant (Sollmann).

The terrapin's heart responds similarly to the frog's (Beyer, I 885).

Excised Mammalian Heart. - When studied by modifications of the Langendorff method, caffein and the other xanthins produce increased rate, amplitude, and force of the cardiac contraction, and generally quickened coronary flow (Hedbom, i $\$ 99 ;$ O. Loeb, 1904; Beco and Plumier, 1906; Lifschitz, 1907; Plavec, r 909). The increased amplitude is first in the systole, then also in the diastole; but the cardiac tone is not increased (Plavec). The stimulation is greatest with theophyllin, then theobromin, then caffein. The coronary vasodilation is also greatest with theophyllin, but least with theobromin. This indicates that the increased flow is not simply due to the increased contractions.

These experiments seem to refer only to stimulant concentrations, and they may be considerably modified by changing the conditions (Lifschitz, I907; Plavec, I909; Bock, I900).

Mammalian Heart in Situ.--The effects of the various doses were studied by Pilcher, I9I2, with the results described under the general discussion of the circulatory effects.

Experimental Heart-block.-In complete block, caffein increases the strength of the ventricular contraction. Larger doses produce ventricular extrasystoles and tachycardia. The strength of the auricles is temporarily quickened (Egmond, I9I3).

Circulatory Effects in Man.-These correspond with what would be expected from animal experiments. With doses to I. $5 \mathrm{Gm}$., the acute effects are inconstant and generally slight. The heart rate may be slowed or quickened or unchanged (literature, Sollmann and Pilcher; Wood, Igr 2). Palpitation is common, but apparently largely subjective. The blood pressure may rise (Wood, I9I3); or fall (Mirano, I906); or remain unchanged (Lucas, I9I4; Newburgh, I9I4). The volume of the human arm is diminished (Hewlett, I9I3). The blood flow tends to improve by increased systolic output.

Human Blood Flow.-Hypodermic doses of 0.3 to $0.5 \mathrm{Gm}$. in resting men, produce a definite increase of the total blood flow (Keogh-Lindhard method). The improvement occurs by increased systolic output. It does not occur during work. Means and Newburgh, 1915, attribute the rest effect to increased venous return to the heart, by some action outside of the heart. During work, the venous return is adequate even without caffein, and is not materially increased by the drug. 
Blood Pressure.-In advanced myocardilis, full and continued doses of caffein (about I $\mathrm{Gm}$. per day) cause a progressive and permanent lowering of blood pressure, reaching a total of 10 to $50 \mathrm{~mm}$. 'This is parallel to the diuresis, and to the relief of the edema and other symptoms (L. Taylor, rgr4). Theobromin does not lower the pressure, although it is otherwise equally or more efficient.

Therapeutic Effects on Circulation. - The methyl-xanthins are used as cardiac stimulants in syncope, and especially in cardiac dropsies. They lack the permanent, tonic action of digitalis, but are useful as adjuvants or when a quick effect is desired. In Taylor's myocarditis series, the results were excellent in one-half, moderate in one-fourth, and unsuccessful in one-fifth. Theobromin is effective on the first day, if at all, so that its usefulness can be determined more quickly than with caffein. Furthermore, the side actions of caffein interfere with its employment, especially in excitable patients. Theobromin therefore deserves preference.

In hypotension and infectious diseases, caffein fails to improve the circulation (Newburgh, r9r4; Lucas, rgr4).

Clinical Side Actions.-Effective doses of caffein, especially in cardiac cases, produce palpitation, insomnia, nausea, vomiting, headache, vertigo, restlessness, anxiety and sometimes delirium. Theobromin is relatively free from these objections, but they may arise (Seifert, Nebenwirkungen, I9I5, p. I28). They are also produced by theophyllin (Widmer, I9I4; Seifert, l.c., p. r3I). The side actions occur much more violently, and with much smaller doses $(0.3 \mathrm{Gm}$. of caffein per day, or even less) in patients with interstitial nephritis (L. Taylor).

Effects on the Urine.-The most important therapeutic effect of caffein consists in a considerable diuresis. The increase concerns mainly the water of the urine. The absolute quantity of solids is also increased, but less than the water, so that their percentage is lowered. The efficiency varies with conditions, especially with the amount of water in the body (Widmer, r9 4 ); the diuresis is most abundant in dropsical conditions, and fails entirely with dry food. It is much greater in wet-fed rabbits than in dogs. It fails also in glomerular nephritis, but is effective in tubular nephritis unless the vessels are involved. The albumin in nephritis may be increased. Theobromin and especially theophyllin are more effective. This difference is explained in part by the relatively larger doses which can be taken without prohibitive side actions.

Mechanism of the Diuresis. - No agreement has been reached on this subject. The increased renal circulation resulting from the simultaneous cardiac stimulation and vasodilatation must be a powerful factor, and may in itself suffice to explain the diuresis. This does not exclude the possibility of stimulation of the secretory epithelium; but the direct evidence for this is weak. This theory of secretory stimulation, which is still widely accepted, was advanced by von Schroeder, mainly because he could not explain the diuresis by the blood-pressure changes; but he was ignorant of the dual and complementary action of caffein on the heart and vessels. At present, its main supports are the occasional failure of complete agreement, in minor details, of the circulatory and urinary changes; and the perfusion experiments of Richards and Plant, ror 5.

Relation of Caffein Diuresis to Circulation.-Schroeder, 1886 and 1887 (and independently, Langaard) found that morphinized rabbits often fail to respond to caffein. Assuming this to be due to vasoconstriction, he administered chloral to paralyze the center, or divided the renal nerves, and now found caffein uniformly effective. This appeared to show that the diuretic effect was opposed by the circulatory effect of caffein, 
namely, vasoconstriction. However, it is now known that caffein ordinarily causes vasodilation, and not vasoconstriction; and von Schroeder's results have not always been confirmed by later observers (Loewi, I905). It appears that chloral heightens the response to caffein, but von Schroeder himself showed that the same result is attained by paraldehyde in doses which certainly did not paralyze the vasomotor center. The synergism may perhaps be explained as a simple summation of the vasodilator effect of the two drugs (Albanese, I900). The combination of caffein with salines also gives a greater diuresis than either alone; whilst a mixture of the several methyl-xanthins only gives simple addition (Schlosser, I9I3).

When the diuresis is compared with the changes in the oncometer (Phillips and Bradford, I887; Gottlieb and Magnus, r901; Loewi, Fletcher and Henderson, I905), they are found to exhibit a remarkable parallelism. During the intravenous fall, the urine flow ceases; as the oncometer recovers, the urine resumes, to reach its maximum with the rise of the oncometer, after which both shade gradually back to the normal. After large doses of caffein, there is neither dilation nor diuresis.

If the conditions are otherwise unfavorable to diuresis, as in dry-fed animals or when the kidneys have been injured, the caffein dilation may fail to result in diuresis (Loewi; Phillips and Bradford). Other discrepancies have occasionally been noted (Gottlieb and Magnus, IgOr), but they are exceptional and usually insignificant; especially in view of the statement of Loewi that the blood flow may be increased (as judged by the arterial color of the blood in the renal vein) even when the kidney volume is kept constant by encasing it in plaster.

The restitution of blood after hemorrhage is not modified by the caffein diuresis, so that there is no sign of altered permeability of the vessels (Gaisboeck, $19 x \mathrm{x}$ ). The viscosity of the blood is said to be diminished (Loria, I9 12).

Perfusion of Excised Mammalian Kidneys.-Munk, I887, adding caffein to the perfusing blood, found a large increase of ureter flow and a moderate increase of vein flow. This result is certainly exceptional (von Schroeder, 1887; Kobert, I886; Sollmann and Hatcher, 1907). It would naturally be explained by the vasodilator action; but Richards and Plant, r9I5, report increased urine flow even when the perfusion flow is kept constant. This would mean that a diuretic effect occurs apart from circulatory changes. Further observations are desirable.

Changes in the Size of Excised Kidney Cells and Absorption of Saline Solutions.Sections of kidney treated with caffein absorb less fluid from hypotonic salt solutions than do control sections (Filehne and Biberfeld, r902); but the change is small and inconstant (Sollmann and Hatcher, I907). Histologically, the epithelium of the convoluted tubules tends to swell slightly in caffein solution (W. W. Williams, 1907).

Intra-vitam Staining.- Sobieransky found that caffein (and saline) diuresis prevents the intra-vitam staining of the tubular nuclei by indigocarmin. Assuming that the staining is due to reabsorption, he argued that this reabsorption was paralyzed by caffein; and extending this, he argued that the diuresis is due to failure to reabsorb the glomerular fluid. There is no secure basis for either assumption, and the absence of pigment would be the natural result of the flushing of the kidneys by the diuresis.

Ureter Peristalsis.-Lucas, I907, found that caffein has a tonic effect on the pelvic portion of the ureter. This would have but little influence on the urine flow, since this is not influenced even by a considerable negative ureter pressure (Sollmann).

Perfusion of Frogs' Kidneys.-Cullis claims that if the perfusion is arranged so that the caffein acts only on the glomeruli, the effects are unsatisfactory; if it is confined to the tubules, it increases the perfusion flow and starts the urine. This result would indicate that the action of caffein is on the tubules; but it is opposed to the findings in mammalian nephritis; and the conditions are too complex to be satisfactory.

Renal Irritation by Caffein.-Ordinary doses of caffein give no indications of injury to the kidney cells; in nephritic patients, however, large doses may be followed by marked albuminuria. Animals also respond to large doses (40 mg. per kilogram), especially when long continued, by definite nephritic changes: albuminuria of 1.4 per r,oo; leucocytes, epithelium and casts in the urine. The kidneys show dilated vessels, cloudy swelling, to vacuolar degeneration and necrosis of the epithelium, and slight interstitial swelling. These histologic changes may be present even when the urine appears normal. Dogs are more subject than rabbits, but the lesions are usually only transitory, disappearing a few days after discontinuance. No lesions are perceptible with doses of 5 to ro mg. per kilogram, corresponding to 5 to ro grains in man (Vinci, I9I4).

Caffein in Experimental Nephritis.-Hellin and Spiro, 1897 , found caffein ineffective in glomerular and general nephritis, but quite effective in pure tubular nephritis. This has been confirmed by Schlayer and Hedinger, r907; and by Pearce, Hill and Eisenbrey, I910; and these further showed that the failure of diuresis is accompanied by failure 
of the oncometric reaction. This is important evidence thal the diuretic action is exerted on the glomerular circulation and not on the tubular epithelium.

In uranium nephritis, diuretics, including caffein, are effective during the early polyuric stage; but they become ineffective when the anuric stage is reached, although the circulatory response may be well preserved (MacNider; 1912; Boycott and Ryffel, 1913). This depends on the intactness of the renal epithelium (MacNider, I9I4). In the subacutely fatal form. caffein and the other methyl-xanthins did not prolong life (Christian, 1914). The phenolphthalein excretion was rather improved, but the nitrogen retention in the blood was not materially affected (Christian, I913; Christian and O'Hare, I913; Fitz, 1914).

For chromate nephritis, on the other hand, theobromin and theocin were distinctly harmful, since they diminished the phthalein output and the animals died more promptly (O'Hare, I9I 5), although the nitrogen excretion was unchanged, and the elimination of urine and chlorid was increased (Fitz, I9I4). Finally, the kidneys ceased to react (fatigue?). This is especially marked if the caffein has been preceded by several injections of saline (Mosenthan and Schlayer, I913).

Failure of Caffein Diuresis.-Aside from glomerular and general nephritis, there are a number of other factors which may interfere with diuresis. The quantity of water in the tissues is very important; and also the water in the food, which is the ultimate source of the water of the urine. There is, consequently, a conspicuous difference between dry-fed and wet-fed rabbits (von Sobieranski, I 895; Loewi, 1905); and presumably the limited water intake of dogs accounts in part for their lesser diuresis response (Sobieranski). Schroeder, I887, found the water content of the rabbits' blood decreased by ro per cent. after an effective diuresis. The same factor is active in the poor diuresis of hemorrhage (Michaud, 1904), and in the superior diuresis in dropsy (Hofmann, I880); but other factors may coöperate. On the other hand, when an effective water diuresis has been secured by excessive continued water administration (Sollmann and Hofmann, I905) or in diabetes mellitus (Meyer, 1905), there is no further increase when caffein is added.

The Diuretic Response in the Normal Human Subject.-This is comparatively small. Raphael, I904, found the daily urine increased (under otherwise constant conditions) by $0.5 \mathrm{Gm}$. caffein sodium salicylate, 42 per cent.; $0.5 \mathrm{Gm}$. theobromin sodium salicylate, 2 per cent.; $1.5 \mathrm{Gm}$., I 4 per cent.; $3 \mathrm{Gm}$., 53 per cent. This was much less than the increase secured by the consumption of an extra liter of water or beer (Ioo per cent.); whilst a liter of milk increased the urine by $\mathrm{r}_{53}$ per cent.

Interference by Vasoconstriction and Vagus.- Whilst the vasomotor stimulation has not ordinarily the importance attributed to it by von Schroeder, it may sometimes be sufficiently powerful to overcome the peripheral dilator effect. At all events, the diuresis is aided by the hydrocarbon narcotics. Curare is also said to aid the diuresis (Cervello and LoMonaco).

Corin, I886, claimed that the vagi exert an inhibitory influence, which Anten, rgor, attributed to a direct inhibitory influence of these nerves on the secreting cells. Atropin would therefore increase the diuresis. These claims need confirmation, before they can be accepted.

Failure in Repeated Administration.-It is a common clinical experience that caffein often becomes ineffective after a time (LeNoir and Camus, 1903). This is not surprising when it is remembered that the factors which favor and impair the diuretic response are apt to vary from time to time. The disappearance of the edema, for instance, would decrease the response.

In experiments, it is also noted that repeated injections become less and less effective (Phillips and Bradford, 1887; Loewi, 1905). This, however, is simply due to the fact that the depressing dosage is gradually attained. 
Relative Efficiency of the Xanthin Derivatives.-Caffein has now been largely supplanted, as a diuretic, by theobromin (diuretin), and more lately by theophyllin (theocin), which are credited with a much more powerful action. With theobromin, this is not surprising, since the customary dose is four times as large as with caffein; with theophyllin, the superiority is genuine. Both are superior to caffein in producing less of the nervous side actions. Theobromin also acts much more promptly, i.e., within a day, whilst caffein requires four days to reach its full efficiency. Exceptionally, caffein succeeds where theobromin fails (Christian, I9I 5).

No reliable quantitative experiments on the relative diuretic action of the various xanthin derivatives have been made - the much-quoted experiments of Ach being based on too few animals to have much value. The attempt to correlate the diuretic and muscular action (Schmiedeberg) is therefore premature. Mendel and Kahn, I9I3, found that most of the newer methylated xanthins are not diuretic.

Differences in the Members of the Group.-Caffein has the most powerful central nervous action, with relatively weak peripheral effects. The strongest diuretic action is produced by theophyllin (theocin), which also causes the most marked cardiac stimulation and coronary dilation. Its central effects and its action on muscle approach those of caffein. Theobromin (diuretin) acts most powerfully on muscle; its effect on the heart and urine are intermediate. It causes very little central stimulation. (The toxicity of theobromin to isolated muscle is $1 / 2$ that of caffein (Veley and Walker, r9ro).)

The pharmacology of theobromin was first investigated by Mitcherlich, 1859, and further by Filehne, I886. It was used experimentally as a diuretic by von Schroeder, I887, to avoid the supposed vasoconstriction of caffein. He found it effective without chloral; and the action on oral administration extended over twenty hours-presumably because its insolubility caused slow absorption. This led to its clinical introduction by Gram.

The Composition of the Urine.--von Schroeder, r886, showed that the urine in caffein diuresis is more diluted (its molecular concentration may fall below that of the blood; Dreser, I 892); but that the absolute quantity of the solids-urea as well as salts-is increased. Entirely similar changes occur in other forms of diuresis, so that they are not characteristic of caffein, and throw no light on its actions. The urinary protein in nephritis is ordinarily not increased.

Different investigators disagree as to which of the solids is most increased, the salts (LeNoir and Camus, 1903), or the urea (Anten). Presumably this varies with circumstances. In rabbits, the salts may be increased without diuresis (Katsuyama, I 898 and I90I); and this may also occur when caffein is given in human diabetes (E. Meyer, 1905). On the other hand, Sellei, I9I 2, describes theophyllin diuresis with little change in the specific gravity or solids. In rabbits, theophyllin increased the $\mathrm{K}$ and $\mathrm{Na}$, but not parallel with each other or with the diuresis (Bock, I9II). The chlorid-retaining mechanism is broken down in rabbits (Pototsky, 1902; Loewi, 1902), but not in dogs or man (Sollmann, I903; Haskins, I904; Sollmann and Hofmann, 1905; Saccone, I911).

Sollmann and McComb, I899, found that caffein did not increase a physiologic albuminuria and Emerson, I902, saw no increase of albumin in acute or chronic nephritis under theobromin. Pouchet and Chevalier, I903, claim that large doses of theophyllin injure the glomerular and tubular epithelium.

Astolfani, 1905, states that caffein, in common with other diuretics, increases hippuric acid synthesis, when benzoic acid is administered.

The secretion of milk is not affected by caffein (Ott and Scott, I9I2).

Therapeutic Use of Diuretic Action.-The methyl-xanthins are employed mainly for the removal of cardiac effusions. The diuresis is pro- 
portional to the effusion, sets in promptly, and ceases when the drug is withdrawn. It is therefore especially useful for two or three days at the beginning of the more slowly acting but more permanent digitalis treatment. Caffein is the least active of the series. It may be given in the doses of $0.3 \mathrm{Gm}$. (5 grains) of citrated caffein, in watery solutions, three times a day, but Taylor found at least $0.5 \mathrm{Gm}$., four times daily, necessary for a full effect.

Theobromin (I Gm. or I5 grains of theobromin-sodium-salicylate, or diuretin, in water, four times a day) has the advantage of producing a better diuretic effect with the least side effects. Taylor found that a daily dose of 30 grains produced very little effect; 40 grains a moderate diuresis; but 80 grains $(5 \mathrm{Gm}$.) were required to secure the maximum efficiency. This quantity, when divided into four doses, was well tolerated. It has also been used intravenously, 20 c.c. of 5 per cent., Neuhof, I9 3 ; but it is doubtful whether this is justified. 'Theophyllin (theophyllinsodium-acetate, or theocin) is the most powerful, but has been charged with sometimes causing renal irritation. Its dose is $0.3 \mathrm{Gm} .=5$ grains, three times per day, administered in hot tea.

In renal or hepatic effusions, the efficiency is less certain. In acute or chronic parenchymatous nephritis, there is often no response whatever, while chronic interstitial nephritis usually responds (J. Miller, I9 I 2); but unless the dosage is very small and carefully watched, the toxic side actions may set in, and the renal condition grow worse. Theophyllin and theobromin may cause renal irritation; it would therefore be better to avoid the methyl xanthins when the kidneys are acutely irritated. In anuria, the methyl xanthins are generally ineffective. Christian et al., I9 5 , state that diuretics are generally ineffective in chronic nephritis, unless there is edema. They would therefore be of little use against nephritic toxemia.

Absorption, Fate and Excretion.-Caffein is readily and completely absorbed: none appears in the feces even after large doses. Only a small fraction is excreted unchanged in the urine. This excretion starts and is mainly completed promptly, but traces may continue for two or three days (Salant and Rieger, I9I2). A somewhat larger fraction loses a part of its methyl groups, and appears in the urine as di- and mono-methylxanthins. It is not known whether this is effected by oxidation or hydrolysis. The remainder-up to 80 per cent.-is completely oxidized to urea. Practically none of the caffein is converted into uric acid (which is largely the fate of hypoxanthin and of xanthin).

Theobromin undergoes changes similar to caffein, but a larger fraction (32 per cent.) escapes decomposition. The greater part leaves as heteroxanthin.

Quantitative Excretion.-The quantity of caffein which is excreted unchanged varies with the dose and with different animals, being only $x$ per cent. in carnivora, less than 8 per cent. in man, and 6 to 20 per cent. in rabbits (Rost, 1895; Salant and Rieger, rgr2). The relative quantity of the various mono- and dimethyl-xanthins also varies in different animals, representing Io to 40 per cent. of the caffein (Albanese, I 895 ; Bondzynski and Gottlieb, I895, Krueger and Schmidt). The literature has been summarized by Bloch, rgo6. The demethylation is retarded by alcohol (Salant and Phelps, r912). Emory and Salant, rgog, report experiments on the decomposition of caffein in the liver. A trace appears in the stomach, intestines and in the bile (Salant and Rieger). The gastro-intestinal excretion is higher when the kidneys are excised (Salant and Rieger, 19r3). The loss of methyl groups occurs in pretty much all organs (J. Schmidt, 1910). 
Toxicology.-The fatal dose of caffein is so large (presumably about Io $\mathrm{Gm}.){ }^{1}$ that no fatal case of poisoning is on record; but doses above I Gm. may produce alarming symptoms, and even therapeutic doses may cause unpleasant side actions.

With larger doses, the pulse is full and hard, quickened or slowed, with palpitation and precordial distress (sometimes anginal attacks). The head is heavy, throbbing, confused; often intense headache and great anxiety. Restlessness and excitement, insomnia (sometimes mild delirium, aphasia, fever). Vertigo, nausea, general discomfort, fatigue, weakness (sometimes burning in the throat, gastric distress, heat flashes, perspiration, insensible pharynx, swollen tongue, ringing in the ears, flashes in eyes). Tremors of jaws, hands and feet, and muscular stiffness. Quickened, embarrassed respiration. Increased micturition (sometimes ardor urinæ and erection).

In very severe cases, there is vomiting (sometimes violent diarrhea and tenesmus); violent choreic tremors. Collapse, with small, irregular arrhy thmic pulse, cold extremities, dilated pupils. Consciousness is usually intact, but there may be delirium.

Toxic Effects on Animals. - The symptoms in animals correspond fairly to those observed in man: restlessness and increased reflexes; increased respiration, vomiting and diarrhea; muscular weakness. Then: clonic or tetanic convulsions, during which respiration may stop, with or without resumption; exhaustion and increasing paralysis; death in one to four hours. The fatal dose lies about $0.15 \mathrm{Gm}$. per kilogram. Dogs and cats are somewhat more susceptible than rabbits and guinea pigs. The dose by mouth is but little larger than the intravenous dose, except in rabbits (Salant and Rieger, I9I0, I9I2). Young mice are more resistant than old. The effects in birds are similar (Brill); in frogs they are modified by the onset of rigor. The toxicity is diminished by temperature above $98^{\circ} \mathrm{F}$. At $45^{\circ} \mathrm{F}$. the muscular effects predominate; while the convulsions become more prominent as the temperature is increased (Salant, I9r3).

Recovery is usually complete within a day, even when the symptoms were violent. Some restlessness and weakness may remain for a time. It is claimed (Kunkel) that large therapeutic doses have been followed by nephritic urine, but on the other hand, daily doses of $1.25 \mathrm{Gm}$. have often been given without detriment (Becher, 1884 ). Idiosyncrasy toward caffein is marked, as every one knows. Children and nervous and weakened individuals appear to be relatively more susceptible. Overdoses should be avoided, especially in myocarditis.

Treatment.-This would consist in evacuation and narcotics-bromid, alcohol, chloral, or morphin. The principal indication would be to reassure the patient.

Theobromin and theophyllin, in excessive doses or in susceptible individuals, may produce toxic effects similar to caffein: headache, nausea, vomiting, epileptic spasms, albuminuria, gastric hemorrhage, etc. (Kobert, Schmiedeberg, Seifert). These manifestations are, however, rare. In epileptic patients, large doses of theophyllin are said to have produced attacks (Schlesinger, I905). Chevalier, I9r4, claims that some commercial theobromins contain loxic impurities; this requires more confirmation.

\section{PREPARATIONS-CAFFEIN GROUP}

* Caffeina, U.S.P., B.P.; Caffein (Thein); $\mathrm{C}_{8} \mathrm{H}_{10} \mathrm{~N}_{4} \mathrm{O}_{2}+\mathrm{H}_{2} \mathrm{O}$. - A feebly basic alkaloid, usually prepared from tea. White, silky, needle-crystals; odorless; bitter taste. Sol. in water $(x: 46)$ and in alc. $(x: 66)$. Its solubility in water is greatly increased by heat, citric acid, benzoates or salicylates, bromids, antipyrin and a number of other substances. Incompatible with tannin. Dose, 0.I5 Gm., $2 \frac{1}{2}$ gr., U.S.P.; 0.06 to 0.3 Gm., I to 5 gr., B.P.; in capsules. Maximum dose, 0.5 Gm., 8 gr.

* Caffeina Citrata (Caffein. Cit.), U.S.P.; (Caffeina Citras.), B.P.; Citrated Caffein; Caffein Citrate.-An unstable compound of uncombined caffein and citric acid, containing

1 The doses refer to pure caffein; the corresponding doses of citrated caffein would be twice as
large. 
not less than 48 per cent. of caffein. White powder, odorless; slightly bitter, acid taste. Citrated Caffein gives a clear syrupy solution with a little water, but precipitates on dilution. The precipitate redissolves on the further addition of water, the solubility being about $1: 32$. Incompatible with carbonates. Dose, 0.3 Gm., 5 gr., U.S.P.; 0.12 to $0.6 \mathrm{Gm} ., 2$ to $10 \mathrm{gr} ., \mathrm{B} . \mathrm{P}$., in water. The free acid renders this unsuitable for hypodermic use. Maximum dose, I Gm., I5 gr.

Caffeina Citrata Effervescens (Caff. Cit. Eff.), U.S.P.; Caffeine Citras Effervescens (Caffein. Cit. Eff.), B.P.; Effervescent Caffein Citrate.-A granulated mixture of Sodium Bicarbonate and Tartaric and Citric Acid, with about 2 per cent. of Caffein. Dose, 4 Gm., I dram, U.S.P.; 4 to 8 Gm., I to 2 drams, B.P.

* Caffeina Sodio-benzoas (Caff. Sod. Benz.), U.S.P.-A mixture of about 50 per cent. caffein with sodium benzoate. A white powder; odorless and of a bitter, aromatic taste. Freely sol. in water ( $\mathrm{I}: \mathrm{I} . \mathrm{I})$, sol. in alc. ( $\mathrm{I}: 30$ ). Dose, $0.3 \mathrm{Gm}$., 5 gr., by mouth; hypodermic, 0.2 Gm., 3 gr., U.S.P.; the most suitable compound for hypodermic use. Maximum dose, I Gm., I5 gr.

Theobromina, Theobromin.-A dimethylxanthin prepared from Cacao seeds (Theobroma Cacao) or synthetically. Very slightly sol. in water.

* Theobromine Sodio-salicylas (Theobrom. Sodio-Sal.), U.S.P.; Theobrom. et Sod. Salicyl., B. P.; Theobromin-sodium-salicylate (Diuretin).-Sodium Theobromin $\left(\mathrm{C}_{7} \mathrm{H}_{7} \mathrm{~N}_{4} \mathrm{O}_{2} \mathrm{Na}\right)$ and Sodium Salicylate, in approximately molecular proportions. Not less than 46.5 per cent. of theobromin. White, odorless powder; sweetish saline and somewhat alkaline taste. Freely sol. in water ( $I: I)$; slightly sol. in alc. It gradually absorbs carbon dioxid from the air with the liberation of theobromin, becoming partially insoluble in water. It must thérefore be protected against exposure to air, and is incompatible with acids; also with chloral. It should not be given with meals, to avoid precipitation by the gastric acid. Dose, I Gm., I 5 gr., U.S.P.; 0.6 to I.2 Gm., ro to 20 gr., B.P.; well diluted. Solutions do not keep.

Other Theobromin Salts.-The following are listed in N.N.R., but have no special advantage: Sodio-acetate (Agurin); Sodio-formate (Theophorin).

* Theophyllina (Theophyll.), U.S.P.; Theophyllin (Dimethylxanthin, Theocin); $\mathrm{C}_{7} \mathrm{H}_{8} \mathrm{~N}_{4} \mathrm{O}_{2}+\mathrm{H}_{2} \mathrm{O}$.- White crystalline powder; odorless; bitter taste. Sol. in water ( $\mathrm{I}: \mathrm{IO0})$ and alc. $(\mathrm{I}: 80)$. Dose, $0.25 \mathrm{Gm}$., 4 gr., U.S.P., three times a day, in warm tea. After two or three days, it should be replaced by theobromin.

Theo phylline Sodio-acetas, N.N.R. (Soluble Theocin).-Contains 60 per cent. of the alkaloid. It dissolves in 23 parts of water.

Guarana, U.S.P.-A dried paste consisting chiefly of the crushed seeds of Paullinia Cupana, yielding not less than 4 per cent. of caffein. Dose, $2 \mathrm{Gm}$., 30 gr., U.S.P. -

Fldext. Guaran., U.S.P. -4 per cent. of Caffein. Dose, 2 c.c., 30 minims, U.S.P.

\section{CAFFEIN BEVERAGES}

Caffein is an important article of popular consumption, in the form of coffee, tea, and other beverages. The usual per capita consumption in the United States is about ro pounds of coffee and I pound of tea (Graham, I9I2). A cup of coffee or strong tea contains about $0.1 \mathrm{Gm}$. (I $1 / 2$ grains) of caffein. Its effects are somewhat modified by the associated products (the volatile products causing more psychic stimulation, the tea-tannin and coffee oil deranging digestion); but essentially the actions are those of the alkaloids. They consist in increased mental and physical efficiency, psychical stimulation, comfort, and relief from muscular and mental fatigue and from their attendant unpleasant sensations. These effects may be very useful in certain conditions, as in those exposed to severe hardship, hunger, fatigue, etc., but ordinarily they are mere luxuries-pleasant, but superfluous. They may do no harm if the consumption is kept within bounds; but nervous individuals, who are the most apt to be injured by caffein, are most likely to exceed these bounds. The bad effects are usually not very serious, and disappear promptly if the habit is discontinued. They consist in nervousness, tremor, palpitation, insomnia, headache, and digestive disturbances.

The habitual consumption of caffein confers a very limited tolerance. Its withdrawal does not produce any marked abstinence symptoms. 
Coffee and chocolate are useful flavors and extemporaneous vehicles, especially for castor oil and cod liver oil.

Coffee (Caffea) consists of the dried seed of Coffea Arabica, Rubiacex. The constituents of the "green bean" are caffein, fat, coffalic and chlorogenic acid, saccharose, etc. The chlorogenic acid gives a green color with iron, similar to tannin, and in its impure form it was formerly called "Caffeotannic acid." Gorter, I9Io, has found it in about roo plants. On oxidation it yields caffeic acid. It differs from tannins in that it does not precipitate proteins, and is therefore not astringent. The percentage of caffein varies in different specimens from $2 / 3$ to $21 / 2$ per cent., usually about 1.2 per cent. (Koenig). It exists mainly as caffein-potassium chlorogenate (Gorter, I908).

In the process of roasting (i.e., heating the seed to $200^{\circ}$ to $250^{\circ} \mathrm{C}$.) a small amount of caffein is volatilized; but since about Io per cent. of water is driven off, the percentage of caffein is actually a trifle higher ( 1.25 per cent.). The main change in roasting consists in the production of aromatic, brown and oily products. This oil ( $\mathrm{Caffeol}$ or Caffeon), according to Erdmann, I902, and Grafe, Ig I 2, consists of 50 per cent. of furfurol alcohol, and small quantities of valerianic acid, phenol, pyridin, and a nitrogenous aromatic substance. It is probably derived mainly from the hemicellulose.

The beverage, coffee, is a decoction made with 6 to ro per cent. of the drug. Practically the entire caffein (perhaps $9 / 10$ ) is thus extracted; so that a cup of strong coffee, prepared from 15 to $17 \mathrm{gm}$., contains about 0.1 to $0.12 \mathrm{gm}$. of the alk aloid.

The use of coffee arose in Arabia and Egypt about the middle of the fifteenth century. Coffee and tea were introduced into Europe about the last quarter of the seventeenth century, about the same time as the potato, cinchona, tobacco and chocolate.

Action of Coffee Oil.-The volatile aromatic constituents produce local irritation and reflex stimulation, in the same manner as the condiments. The hot water contributes to this effect; and possibly the greater reactivity induced by the caffein heightens the reflex response (Schmiedeberg). The local irritation stimulates peristalsis, and with excessive use, tends to nervous dyspepsia. It is doubtful whether the quantities taken in the beverage cause any direct central stimulation.

Wilhelm, Rorer, and Reichert could not obtain any effects with coffee distillates. J. Lehmann, Archangelsky, Hare and Marshall, and Erdmann found, with small doses: Pleasant stimulation; increased respiration; increased heart rate, but fall of blood pressure; muscular restlessness; insomnia; perspiration; congestion. Large doses: increased peristalsis and defecation; depression of respiration and heart; fall of blood pressure and temperature; paralytic phenomena.

The intravenous injection of coffee is more toxic than the corresponding caffein. This is presumably due to the potassium (Aubert). By mouth, however, the potassium $(0.44 \mathrm{Gm} . \mathrm{KCl}$ in $20 \mathrm{Gm}$. coffee) is not sufficient to produce any effect.

Decaffeinated coffee does not produce the cardiac or diuretic effects of coffee or caffein (Busquet and Tiffeneau, I912). The caffein-content of commercial decaffeinated coffees and other coffee substitutes is given by Street, I916.

Tea (Thea) consists of the dried leaves of Thea sinensis, Theacea. Constituents: Caffein (I.4 to 3.5 per cent., usually 2 to 3 per cent.); tannin (Io to 30 per cent.); traces of theobromin, theophyllin, xanthin, adenin, and volatile oil. The black and green teas differ only in the treatment to which the leaves are subjected-a fermentation with the black variety, which mainly alters the color and flavor; the green tea is rather more rich in volatile oil. The beverage is a I to 4 per cent. infusion. A cup of strong tea prepared from $5 \mathrm{Gm}$. of leaves, contains about 0.1 Gm. of caffein (Aubert). A quick infusion extracts practically all the caffein, but only a part of the tannin (which is especially abundant in the finer sorts). The customary brief infusion is therefore commendable, since the tannin is deleterious to digestion, by precipitating proteins and albumoses, by lessening absorption, and by irritating the gastric mucosa.

The tea-distillate is credited by Archangelsky with actions similar to those of caffeol. Lehmann and Tendlau found it inactive.

Cola (Kola, the seed in Cola acuminata, Sterculiaceæ).-This is used extensively by the natives of Africa, the fresh and often germinated seed being chewed. The dried seed, which has been introduced as a popular "tonic," contains caffein ( $11 \frac{2}{2}$ to $31 / 2$ per cent., usually $2 \frac{1}{3}$ per cent.); theobromin ( $1 / 40$ per cent.), tannin (Kola red); fat, sugar, etc.

In the freșh nut, the caffein is combined with a glucosidal tannin ("Kolatin," Goris, 
1907). This compound, which was formerly termed "Kolanin," is partly decomposed in drying, by an oxidase, into free caffein, glucose, and Kola red (Chevrotier and Vigne, 1907).

Guarana.-A dried paste, prepared by the Indians of South America, consists chiefly of the dried and pounded seed of Paullinia cupana, Sapindaceæ. It contains 4 to 5 per cent. of caffein, tannin, etc. Maté (Paraguay tea) consists of the leaves of Ilex paraguayensis. It contains 0.13 to 1.85 per cent. caffein ( 2 per cent., Bertrand and Devuyst, I9I0), Io to 16 per cent. of tannin, etc. It is used in South America for preparing a beverage.

Cacao is consumed extensively as a beverage and in the form of chocolate. (The latter is a mixture of melted cacao and sugar, often with the addition of flavors, starch, etc.). It contains theobromin, instead of caffein, and therefore acts less on the nervous system. It is also rich in fat, which is nutrient. A part (about half) of this fat (Oleum Theobromatis or Cacao Butter) has been removed from the commercial powdered cacao.

The name Cacao should not be confused with Cocoa (the palm yielding the cocoa nut), nor with Coca, the source of cocain.

Cacao consists of the fermented, dried and often roasted seeds of Theobroma Cacao, Sterculiaceæ. Constituents: Theobromin ( $1 \frac{1}{2}$ to $41 / 2$ per cent., usually $\mathrm{I} 1 / 2$ per cent.); a trace of caffein; 50 per cent. of fat; starch, tannin, etc. The theobromin is liberated from a glucosid during the fermentation.

The fat is generally considered rather indigestible, but Neumann, 1906, showed that it is digested as well as other fats.

Chicory.-The roasted root of this plant is used as an adulterant and substitute for coffee. It probably contains similar empyreumatic substances, but no caffein. Schmiedeberg, I9I2, considers it a harmless stimulant, stomachic and antiseptic: while Horwith, I908, believes it liable to disturb digestion. Still less is known about roasted malt and other cereals which are used as coffee substitutes.

Phenomena of Chronic Caffein Poisoning.-These have been described most commonly in excessive tea drinkers. A good analysis of the effects is given by Bullard, I886. The first symptoms are usually dyspepticepigastric uneasiness after meals, and a general lowering of the mental and physical tone. This is succeeded by restlessness and nervous excitability, tremors, and disturbed sleep, soon followed by anorexia and by headache, vertigo, confusion. Constipation is very common, but not more so than in individuals who are not addicted to tea. Palpitation, generally associated with irregularity, becomes distressing, and is often accompanied by dyspnea. In the severer cases, these symptoms become aggravated and continuous. Neuralgias, sensory and hysterical disturbances are common, but it is not easy to say whether these are the cause or effects of the caffein habit. Children who drank coffee were found by Taylor, I912, to average below the normal weight, height and strength.

The quantity of tea required to develop these symptoms varies greatly with the individual. The daily average consumption of Bullard's patients was five cups, which corresponds to about $0.6 \mathrm{Gm}$. or 8 grains of caffein. The susceptibility is much greater in youth, anemia, weakness, insufficient food, exhaustion, and in "nervous" individuals.

Differences between Tea, Coffee and Cacao.- The effects of excessive coffee consumption differ only in minor details from tea; whilst tea tends to constipation, coffee is laxative. Both interfere with digestion, but in different ways: the coffee through the irritant effects of its volatile oil; the tea through the coagulant action of the tannin. It may therefore be observed that different individuals are more injured by one or the other. The caffein itself probably contributes to the digestive derangement, through its vasodilator action. This may account for the common tendency to hemorrhoids. Cacao may disturb the digestion through its richness in fat, but the other symptoms are not common; partly because there is less inclination to its excessive use.

The acute effects on digestion deserve special mention. Cushny states that coffee and tea retard the action of the ferments considerably_(in test-tube experiment), whilst 
caffein slightly accelerates the action. The secretion of gastric juice has been investigated by Pincussohn, I906, using dogs with the Pawlow gastric pouch. Coffec gave a considerable but brief increase of quantity and acidity. "Malt coffee" (the type of the coffee substitutes prepared by roasting cereals) has a similar, but weaker action. Tea and ordinary cacao decrease the secretion; but cacao in which the fat has been reduced to I 5 per cent. causes some increase.

Chronic Caffein Poisoning in Animals.-Salant and Rieger, rgro, found that the continued administration of doses which did not cause acute effects, eventually produced emaciation, often ending in death. The stomach and, to a less degree, the intestine showed inflammatory changes.

On the other hand, they found that habituation increased the tolerance to acute poisoning, the fatal dose being i 5 to 80 per cent. larger than ordinary. Gourewitsch, 1907 , also claims a "histogenetic" habituation.

Mice kept continuously under caffein were found to exhibit less than the normal activity, but showed a normal gain in weight (Nice, I9I2).

\section{THE MORPHIN GROUP}

Members.-The morphin group comprises opium and most of its alkaloids: Morphin, codein, narcotin, papaverin; also the esters formed by replacing the $\mathrm{H}$ of one or both of the hydroxyls of morphin: Methyl morphin (codein); diacetyl morphin (heroin); ethyl morphin (dionin), etc. These resemble each other in their pharmacologic actions, but present minor differences which are of practical significance. Since morphin is the most important member, it will be considered first.

Central Nervous System. - The most important actions of morphin are on the central nervous system. It depresses the brain, especially its higher functions. The medullary centers are first stimulated, then depressed. The reflexes and the spinal functions are mainly stimulated. The effects are broadly identical in all vertebrate animals, although the dosage varies greatly, and the symptoms show considerable differences in the relative prominence of certain phenomena and in the time required to produce the successive actions.

Narcotic Action.-The descending depressant action of morphin is well illustrated by the frog, in which the development of the symptoms corresponds very closely to those produced by progressive removal of the brain. This is also the general course in mammals; but with these the symptoms are more confused. The difference is explained by the more rapid action of the drug, and by the more intimate correlation of the nervous centers.

Effects of Morphin on Frogs.-After doses of 20 to $50 \mathrm{mg}$. the symptoms start with diminution, and then absence, of voluntary movements, but the animal reacts normally when stimulated. (This corresponds to ablation of the hemispheres.) The frog sits quietly in the normal position; he will climb up an inclined plane and give the croaking refiex when stroked. When placed in a tumbler filled with water and inverted in a large vessel of water, it will leave the glass to seek the air. As the narcosis deepens, it will remain under the glass. Placed on the table, its leaps become more and more clumsy, and it does not avoid obstacles in jumping (corresponding to excision of the corpora quadrigemina). Then the animal fails to leap even when stimulated (paralysis of cerebellum); but when turned on its back, it will resume its normal sitting position. This reaction also becomes more and more delayed, and can be obtained only by reinforcing the stimulation through pinching, etc., until finally it fails altogether (paralysis of medulla oblongata). The reflexes are weakened, but present. The respiration is decreased.

Morphin Tetanus in Frogs.-After the frog has remained in this depressed condition for some time (a few minutes to several hours; the shorter, the larger the dose), the respiration becomes spasmodic, resembling superficially the Cheyne-Stokes type; then the spinal reflexes return; and the frog passes gradually into a tetanus of the strychnin type, located in the spinal cord. The delayed appearance of this tetanus is due to the 
slow absorption of the effective dose, for it appears at once on intravenous injection (Stockman, 1891). The convulsions occur more promptly if the heart is tied off (Githens and Meltzer, 1911), presumably because thus more of the morphin reaches the nervous axis (Abel, I9I2). The onset is also hastened, and the necessary dose reduced, by cold, and by removal of the cerebrum, which inhibits convulsions. The results are more prompt if the medulla is not destroyed (Githens, 1913).

McGuigan and Ross, 1915, find that the previous injection of morphin sensitizes frogs to strychinin, so that strychnin tetanus develops more quickly-provided that a latent period of one-half to two hours elapses between the morphin and strychnin injections. They believe that the tetanizing action is due to an oxidation product of morphin, since an artificially oxidized morphin tetanizes more rapidly. Along the same lines, they explain the usual absence of tetanus in mammals by assuming rapid destruction of the tetanizing substance. The explanation involves some unproven assumptions.

Dealh. - The tetanus is mixed with paralytic phenomena, the animal lying lax between the spasms. Eventually the paralysis becomes permanent and ends in death. The heart is still beating at this stage and is indeed little affected directly. Death may also occur during a spasm. The convulsive stage may be absent with very large or very small doses.

Recovery.-If recovery occurs, the depressed functions recover in inverse order to their appearance (Witkowski, I 877 . This paper also gives the older bibliography of the experimental work with morphin).

Invertebrates do not show typical morphin effects.

Narcotic Symptoms in Human Subjects.-The first effect, produced by doses too small to elicit any other symptoms ( $5 \mathrm{mg}$.), consists in diminished sensibility to lasting impressions; especially such as give rise to pain, cough, fatigue, hunger, discomfort, and other disagreeable sensations.

Somewhat larger doses (ro mg. or more) depress the attention and thus weaken the appreciation of other external impressions. A sudden stimulus may evoke a normal or exaggerated reflex response; there is even increased acuteness of perception of external impressions (Kraepelin, I892); but persistent or gradual stimuli are neglected. The stimuli are evidently transmitted to the brain, but do not fix the attention. The faculties of concentration, of judgment and of memorizing (Weygandt, I003) are all seriously disturbed, constituting a narcosis. ${ }^{1}$ Through this exclusion of external stimuli, the patient is quieted, and then passes through a dreamy, apathetic, drowsy state, into natural sleep. Exceptionally, the disturbed balance of the brain leads to excitement (especially in cats) and even to delirium.

Larger doses cause a much more extensive depression, the sleep becoming so profound that the patient can be aroused only incompletely and with difficulty. Eventually the coma and insensibility are complete.

Relative Analgesic Action of Opium Alkaloids.-Morphin is decidedly the strongest hypnotic and analgesic member of the group.

Analgesic Experiments on Man.-Macht, Herman and Levy, I916, have investigated the influence on the sensory threshold by a quantitative method. They find the efficiency highest with morphin; then comes papaverin; then codein; narcotin, narcein and thebain are practically not analgesic. However, narcotin has a considerable potentiating action on morphin.

With hypodermic injection, $5 \mathrm{mg}$. of morphin produced no measurable analgesic effect; with Io mg., the analgesia was marked in two subjects; the third responded by hyperesthesia, especially to larger doses, although all the other narcotic effects were present. With codein, 20 to $25 \mathrm{mg}$., the analgesia was only slight; with papaverin, $40 \mathrm{mg}$., it was as strong as with $10 \mathrm{mg}$. of morphin, but slower and shorter; with narcotin, $8 \mathrm{mg}$. was ineffective; 20 to $40 \mathrm{mg}$. produced first some hyperexcitability; then slight analgesia. Narcein and thebain, ro mg., were ineffective. With a mixture of equal parts of morphin and narcotin, containing $31 / 3 \mathrm{mg}$., the analgesia was almost as great

\footnotetext{
1 Narcotics are substances which have the property of stupefying: Opium, alcohol, cannabis, atropin and similar drugs.
} 
as with ro mg. of morphin alone. This combination was also effective in the subject who resisted morphin.

Excitant Effects in Man.-Some Eastern races, especially the Malays, as also some individuals of other races, particularly women, are apparently more excited than depressed by morphin. This also can be explained by diminished restraint, rather than by direct stimulation. The same explanation probably holds true also for the flights of imagination which fill the period preceding the sleep.

Effects of the Other Members of the Group.-Codein and diacetylmorphin are less quieting, and perhaps more excitant and convulsant than morphin. Codein does not inhibit, but rather increases the convulsions of camphor, etc. (Januschke and Masslow, I9I5).

Imagination.-It is self-evident that an unrestrained imagination will take very different directions, according to the individual. In some it will tend directly to sleep; in others, it may even prevent sleep; in some it will be highly unpleasant, whilst others will seek to repeat the experience and thus fall easy victims to the morphin habit. The aphrodisiac effects, which occur in some, and the anaphrodisiac action in others, depend upon this action on the imagination.

Special Senses.-These are not affected directly. They may appear disturbed through the impaired attention, or through the heightened reflex irritability.

Symptoms in the Lower Mammals. - The effects are almost purely depressant (apathy, stupor, analgesia, sleep and coma) in the dog, rabbit, guinea pig, white rat, mouse and sparrow. The acute reflexes are heightened, but firm pressure, which would be much more painful in normal animals, elicits no response. Dogs exhibit a peculiar clumsiness and incoördination in their voluntary movements. They stand and walk unsteadily, and often drag their hind legs ("Hyenoid walk" Cl. Bernard). These motor symptoms resemble those of ablation of the motor areas. On the other hand, one often sees convulsions, tremors or choreiform twitchings of the limbs. The $e x-$ citability of the motor areas to electric stimulation is not altered even by the largest doses (Hitzig, I873). The respiration may at first be very rapid, but is eventually depressed. Vomiting and defecation occur early.

In the cat (and also with the horse, ass, beef, sheep, pig and goat and sometimes in dogs) the coma is preceded by more or less excitement. The cat particularly exhibits a violent mania, races about as if in extreme terror, with prominent eyes and widely dilated pupils, scratching and clawing wildly. Sometimes there are even convulsions. These symptoms have been interpreted as stimulation. In fact, however, the psychic functions are depressed; the animal does not avoid obstacles in its career; marked analgesia is present. The excitement is apparently due mainly to terror, to which the nausea contributes greatly, and which is 'not inhibited by the normal restraining impulses (since these are dulled by morphin). The excitant effect on cats is also produced by codein and thebain; whilst papaverin and narcein are mainly depressant (G. H. Müller, r9o8).

Relative Toxicity for Different Animals.-The tolerance for morphin differs remarkably. Man is by far the most susceptible, the fatal dose man for being no greater than that required for a frog of but 1,2000 the weight. Pigeons, goats, rabbits and pigs require nearly roo times the human dose, per kilogram; dogs and cats about I5 times; horse and cow two to four times.

Respiratory Center.-Morphin and its derivatives depress the excitability of the respiratory center. The spontaneous rhythm and the response to asphyxia, and to reflexes are all lowered. There is also a marked decrease of the cough reflex; this action probably involving a somewhat different mechanism and depending partly on the analgesic effect.

Very minute doses ( $2 \mathrm{mg}$. of morphin for man) lessen the cough reflex without producing any other noticeable change. Somewhat larger, but still non-narcotic doses ( 3 to $15 \mathrm{mg}$. of morphin), diminish the excitability for carbon dioxid, and to a lesser degree for other afferent impulses ( $Y$. Henderson and Scarbrough, I9IO). The threshold to $\mathrm{CO}_{2}$ is raised (Hig- 
gins and Means, I9I5). The respiratory rate may be slowed (Gscheidlen, r 869), or slightly quickened (Higgins and Means, r9 5 ). With the smaller of these doses, the depth and efficiency of the respiration is somewhat increased, especially if it has been superficial. Dyspneic respiration is quieted, and "air hunger" relieved. Toxic doses cause very marked decrease of excitability, rate, depth, and efficiency; periodic respiration (Filehne; Barbour, I9r4), resembling the "Cheyne-Stokes type" (Fig. 8); and finally arrest of respiration, before the heart is seriously injured.

Codein, heroin, and all other morphin derivatives produce the same effects (Issekutz, IgII).

Codein has the material advantage of relative freedom from undesirable side action and is therefore preferable in cough. Heroin has no real advantage; it is at least as liable as morphin to induce habit formation; and it is much more toxic (Harnack, r899). Morphin is much more effective than its derivatives in quieting dyspnea.

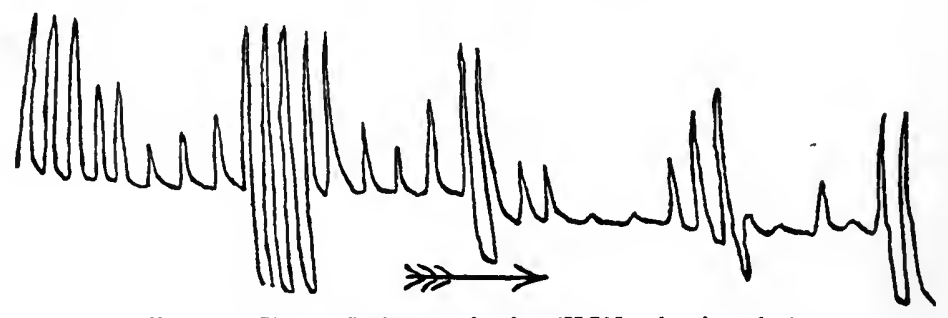

FIG. 8.-Cheyne-Stokes respiration (HCN poisoning, dog).

Some of the minor opium alkaloids stimulate the respiratory center; so that opium is rather less depressant to the respiration than would be expected from its morphin content (Macht, I9I5).

Rate and Depth of Respiration.-The effect of morphin, as of most drugs acting on the respiratory center, is mainly on the rate. With small doses, the depth is affected only secondarily, namely, increased in the attempt to compensate for the slowed rate, especially if the respiration was previously superficial (Issekutz, I9II). The effects, therefore, resemble those of section of both vagi: this suggests that the effect might be due to blocking of the normal augmentor vagus impulses; but this is not the correct explanation, for morphin does not block the response to electric stimulation of the vagi, and it also causes a further slowing after both vagi have been divided (Cushny, I9I3).

In the dog, the hypodermic injection of morphin first causes a quickening of respiration (perhaps through nausea) followed in fifteen minutes by the typical slowing (Mayor, 1908). In the frogs, the slowing is succeeded by quickening, just prior to the convulsive stage. A secondary quickening is also seen in rabbits (Filehne, 1879 ; Cerña, 1892) but never in man.

Decreased Excitability of Respiratory Center.-This is shown by the fact that apnea is more easily produced, and more lasting (Filehne, 1879; Cushny and Lieb, 1915). The blood becomes more venous, the oxygen content being decreased and the carbon dioxid increased (Filehne and Kionka, 1895). Asphyxia produced by the inhalation of carbon dioxid or deprivation of oxygen in normal animals increases the depth and rate of respiration. Under morphin, this response is of the same type, but very much decreased (A. Loewy, I89o; Lindhard, I9II; Cushny, I9I3; Cushny and Lieb, I915). Afferent inhibitory respiratory reflexes are rendered more effective; but acute augmentor reflexes are not materially altered (Cushny). The response to persistent irritation (cough) is of course diminished.

Since the reactions of the center to reflexes and $\mathrm{CO}_{2}$ stimulation preserve the normal type, Cushny and Lieb ascribe the action of morphin to slowing of the intrinsic rhythm of the respiratory center. The hydrocarbon narcotics (urethane), on the other hand, depress mainly the asphyxial reflex.

Morphin Derivatives on Respiration.-Heroin was introduced by Dreser, 1898 , with the claim that therapeutic doses lessen the cough reflex and slow the respiration; but 
that the inspirations are deepened and more powerful, so that the alveolar air is more effectively ventilated. Independent workers, however, have shown that there is no real difference from morphin (Cushny, I9 13 ). The deepening of the respiration is not constant (Santesson; Lewandowsky; Harnack, I899), but is seen only with small doses, when the slowing is excessive or when the respiration has been shallow (Fraenkel, i 899). Appropriate doses of codein, dionin, and even morphin produce practically the same effect (Fraenkel; Issekutz, I9I I). In man, the respiratory effects of $5 \mathrm{mg}$. of heroin correspond almost quantitatively to those of $15 \mathrm{mg}$. of morphin (Higgins and Mcans, I9 15 ).

Dreser also claimed that heroin did not alter the excitability of the center to carbon dioxid, but lessened the reflex excitability (to stretching of the lungs). Impens, under Dreser, likewise claimed the carbon dioxid threshold unaltered by heroin (and dionin), but raised by codein, and, of course, still more by morphin. Winternitz, 1899, found just the opposite result, the threshold being raised by heroin, but not by codein or dionin. The difference is probably insignificant: none of the derivatives raise the threshold as much as morphin, and they are, therefore, less effective in relieving dyspnea.

Separate and Combined Opium Alkaloids on Respiration.-This subject has been investigated by Macht, I9I5. He finds the effects of morphin on rabbits and dogs essentially as described. Small doses (o.I mg. per kilogram) markedly slow the rate, but somewhat increase the minute-volume and alveolar ventilation; but the response of the center to $\mathrm{CO}_{2}$ is somewhat decreased. Full doses ( $\mathrm{I}$ to $5 \mathrm{mg}$. per kilogram) diminish the rate, minute-volume, alveolar ventilation, and response to $\mathrm{CO}_{2}$

$S$ mall Doses of Codein (I to $2 \mathrm{mg}$. per kilogram) generally slow the rate, without much change in the minute-volume, alveolar ventilation, or $\mathrm{CO}_{2}$ response. Convulsive doses (20 mg. per kilogram) increase the ventilation and $\mathrm{CO}_{2}$ response. Narcotin and papaverin increase the $\mathrm{CO}_{2}$ response, although the rate may be slightly slowed. $N a r$ cein, thebain and cryptopin produce either no effect, or slight stimulation of the respiratory center.

Combination of the stimulant alkaloids with morphin gives simple summation of their respiratory action; i.e., their stimulation partly counteracts the morphin depression. This is true for opium as well as for artificial mixtures. It also holds for the perfused-respiratory center.

Bronchial Muscles.-These are slightly relaxed by therapeutic doses of morphin, and more powerfully by papaverin and narcotin ( $\mathrm{Pal}$, I9I3; Macht, I915); whereas large doses of morphin, codein, dionin and especially heroin, narcotin and thebain, produce marked constriction. Higgins and Means, I915, observed bronchial constriction in man with I5 mg. of morphin or $5 \mathrm{mg}$. of heroin. The relaxation may play a part in the relief of bronchial spasm (asthma); whilst the constriction may contribute to the asphyxia of toxic doses.

The effects are peripheral, for they occur on excised bronchial muscle (relaxation, Trendelenburg, r912) as well as in pithed (constriction, Jackson, I9r4 and I9 5 ) and in intact animals (relaxation with small, constriction with large doses; Brodie and Dixon, r903). The constrictor action is not antagonized by atropin, and must therefore be on the muscle or myoneural junction. It can be overcome by powerful dilator (sympathetic) stimulation (epinephrin, hordenin, Jackson, I9 44 and I9 5 ).

The combination of morphin and narcotin in dilator concentration gives less dilation than either alone (Macht, I9I5). This is similar to the behavior of the coronary artery.

Bronchial Secretion.-Unless there is nausea, the secretion of mucus appears diminished; particularly in bronchitis. This may be explained largely by the suppression of cough, permitting a longer sojourn of the mucus in the bronchi, with consequent removal of its water by absorption and drying; but there may also be a central depression, analogous to that of the salivary secretion, for a decrease of tracheal secretion has been demonstrated in animals (Rossbach, I882).

Circulation.-Therapeutic doses of morphin or its derivatives do not affect the circulation seriously. In man, there is generally an (indirect) increase of pulse rate immediately after an injection; this is quickly fol- 
lowed by persistent moderate slowing, with increased fulness and force (stimulation of vagus center). The blood pressure, after a short rise, may remain level (Anderes, I913), or falls slightly (Guinard, I895), through the slowing, and perhaps through incipient depression of the vasomotor center. The cutaneous vessels are markedly flushed.

With toric doses, the fall of blood pressure is more marked (central vasomotor depression), and the pulse becomes weak, varying in rate, and often arythmic (stimulation of vagus center and disturbed cardiac conduction).

The heart muscle suffers indirectly, by the low blood pressure and asphyxia (hence danger in cardiac insufficiency, especially when combined with dyspnea). Ordinarily the circulation remains fairly good, until death occurs by paralysis of respiration. Direct depression of the heart is produced only when morphin, or especially its derivatives, are perfused directly through the heart.

Hering, I9I5, believes that the increased vagus tone resulting from large doses may indirectly produce ventricular automatism, or even fibrillation, especially in pathologic hearts tending in that direction.

Vagus Center and Cardiac Conduction.-Dogs show the same phenomena in more marked degree. The preliminary acceleration is due to nausea. The typical slowing does not occur after section of the vagi, and is therefore central. It occurs with very small doses, increases with larger doses, and passes into arythmia (van Egmond, I9I I; Anderes, I913). Electrocardiograms also show central vagus stimulation (Einthoven; Cohn, I9I3); and sinus-auricular and auriculo-ventricular heart-block, separate or combined, removable by atropin (Eyster and Meek, I9I2, I9I3). The reflex excitability of the vagus center is also increased (Jackson and Ewing, I9I4).

The effect on the pulse rate is inconstant in cats, absent in rabbits (Egmond). Anderes found constant slowing in rabbits, the vagus stimulation being partly central, partly peripheral through decrease of the negative thoracic pressure.

Cardiac Muscle.-On the Langendorff excised mammalian heart, morphin and all its derivatives first increase the rate and amplitude; this is followed by decrease, and finally systolic standstill (Vinci, 1907). The rate of the perfused, atropinized frog's heart is quickened by dilute, slowed by more concentrated solutions of all the papaveraceous alkaloids; morphin being the least deleterious (Hale, I909). Lippens, I908, found peronin highly toxic for the turtle's heart. Narcotin is the most toxic of the opium alkaloids, for the heart (Macht, I915). Papaverin lowers the cardiac tone (Pal); this is followed by considerable tachycardia (Renon and Desbouis, 19r4).

Cardiac Ganglia.- These show degenerative histologic lesions, similar to those of alcohol or chloroform (Lissauer, I9I4).

Coronary Circulation.-This is quickened, by dilation of the coronary vessels, by most of the opium alkaloids. The action is most marked with narcotin and papaverin, slight with morphin, very slight with codein, heroin or pantopium, and absent with narcein and thebain (Macht, I9 5 ).

Vasomotor Effects. - These have not been satisfactorily investigated. Gscheidlen, I869, found that the center is not completely paralyzed even in advanced poisoning; stimulation of the spinal cord still gives a rise. The venous pressure is not changed by moderate doses, slightly lowered by toxic doses, indicating vasodilation (Capps and Matthews, I9I3).

Cerebral Circulation.-The effects are small and inconstant with ordinary doses. Huerthle, I 889, found sometimes an increase, sometimes a decrease, of the flow. Aliprandi, r 905 , describes a brief constriction, followed by lasting dilation. Frankfurther and Hirschfeld, r9ro, describe an initial increase of brain volume, followed by decrease, the latter probably due to the fall in general blood pressure.

Circulation of the Skin; Diaphoretic Action.--The cutaneous vessels are specifically dilated (through the vasomotor center) even by small doses. The skin is reddened, warm, and the perspiration increased. This action is utilized, in the form of Dover's powder (Pulvis Ipecac et Opii, $0.5 \mathrm{Gm}$. at night) for the "abortion" of colds. In susceptible individuals, 
the cutaneous dilation may give rise to exanthemata. With toxic doses the skin becomes pale (vasodilation of the splanchnic area), and cyanotic (asphyxia).

Other Poisons which may Produce Erythema on Systemic Administration are: atropin, quinin, chloral, veronal, benzol derivatives (antipyrin, atophan, salicylates, aspirin, etc.) iodids, bromids, sera, etc.

Wheals.-The local application of solutions of morphin or any of its esters to the scarified skin results in the production of an urticarial wheal. Similar wheals are produced by histamin, peptone, emetin, and calcium chlorid (Sollmann and Pilcher).

Effect on Pupils. - In man (not in animals) even small, scarcely analgesic doses constrict the pupils. The miosis is due to a stronger constrictor (oculomotor) tone; for it disappears when the oculomotor endings are paralyzed by atropin; whilst it is scarcely affected by cocain, which stimulates the sympathetic endings. The action is central, for it is not produced by local application. It has been suggested that morphin depresses the inhibitory impulses which normally keep the oculomotor tone in abeyance (Meyer and Gottlieb). There is a fairly lasting rise of intraocular pressure (Myashita, r9r3). Ethyl-morphin produces a shorter rise.

The oculomotor center (pupillo-constrictor) appears to be normally in a state of partial inhibition through a variety of sensory impulses. When these are diminished-as by sleep, narcosis, and in a specific manner by morphin - the oculomotor tone increases, and the pupils contract. When the inhibitory impulses are reinforced-as by emotion, electric stimulation of the cerebral cortex, asphyxia, etc.-the oculomotor tone is diminished, and the pupils dilate. That this type of mydriasis is not due to stimulation of the sympathetic (dilator) center, is shown by the fact that it occurs after division of the cervical sympathetic nerve (Braunstein, I894).

Pupil Changes in Animals.--In cats, the pupils are dilated, in conformity with the general excitement. In $d o g s$, the effect is variable, but generally dilator.

Conjunctival Reaction to Ethylmorphin (Dionin).- The direct application of $\mathrm{a} I$ to 5 per cent. solution or ointment produces burning pain, hyperemia, and acute edema of the lids and conjunctiva, which disappears without any ill effects. This reaction is used in ophthalmology for the removal of old inflammatory products.

The edema, which is ascribed to vascular congestion, appears in a few minutes and lasts for several hours. There is also some more persistent local anesthesia. The pupils are at first constricted, and the intraocular tension is raised (Toczyski, ror 2). The edema does not occur in rabbits, but readily in dogs or cats (Axenfeld, 1905).

Benzoly-morphin (peronin) is said to act in the same way; but the effect does not occur with morphin, codein, heroin, or any of the natural opium alkaloids.

Spinal Cord.-Morphin and all the members of the group increase the spinal reflexes.

Large doses, in frogs, produce convulsions which are indistinguishable from those caused by strychnin. Mammals generally die from the respiratory depression before the convulsant stage can develop; but the smaller mammals may exhibit muscular stiffness and tremors. Convulsions are sometimes seen, even in man, when toxic doses (3 grains) are taken (A. Gordon, I9 5 ; Keen). In dogs, the intraspinal (subarachnoid) injection of morphin produces marked convulsions (McGuigan and Ross, ror 5).

The convulsant action is especially strong in one of the minor opium alkaloids, thebain, so that this is generally placed in the strychnin group. 
The spinal stimulation would seem to contraindicate the use of morphin in convulsant conditions of spinal origin, as in strychnin poisoning. Practically, however, the analgesic effect may offset this disadvantage; nor does the theoretical objection hold against convulsions that are not spinal.

Sensory Nerves.-Opium and its alkaloids have no action whatever on the sensory endings, or on the peripheral sensory nerves, for the site of application is no more affected than other regions of the body (Jolly and Hilsmann, I874). Their local use is therefore irrational. Any apparent success is largely psychical; although there may be some central analgesia, since morphin is readily absorbed from wounds and mucous membranes, and to a slight extent even from the unbroken skin. With the timehonored "Lead and Opium Wash," the precipitate formed by the lead with the gums and meconic acid furnishes a soothing protection. (The morphin is not precipitated by the lead.)

Curare Action.-All the members of the group, when applied directly to a muscle preparation, exert a weak and slow curare action: If the motor nerve is stimulated with a tetanizing current, the muscle responds with a series of rapid separate contractions, instead of a fused tetanus. Larger doses paralyze the muscle-nerve endings completely. Diacetyl morphin acts more powerfully than morphin or codein; but the action is too weak to be observed in intact mammals (Hale, 1909).

Protopin Group.- The curare and muscular actions were first described by v. Engel, r89o, for the protopin group-certain unimportant alkaloids (protopin, crytopin, chelidonin, homochelidonin, and chelerythrin) which occur in opium, but especially in other Papaveraceous plants (Sanguinaria or Bloodroot; and Chelidonium or Celandine; Bocconia, etc.). These alkaloids share also the depressant morphin action on the heart (Hale). They are said to paralyze the sensory endings like cocain. The respiration is stimulated.

Sanguinaria also contains sanguinarin (Reports, Council Pharm. and Chem., rgrr, 60) and other alkaloids (Kozniewski, I910). Chelidonium contains berberin. Both drugs are irritant. Sanguinaria has been used as a nauseant (0.I $\mathrm{Gm}$.). The fresh juice of chelidonium is applied in folk medicine to remove warts.

Chelidonin.-Ceniral Actions.-H. Meyer, 1892 , found that it produces, in frogs, a descending depression of the central nervous system, similar to morphin, but without spinal stimulation. Mammals also respond by analgesia and sleep, without marked depression of reflexes. Larger doses cause coma, without nausea or vomiting; finally, the vasomotor center becomes paralyzed.

Peripheral Actions of Chelidonin.-These have been investigated especially by Hanzlik, 1915. Like other isoquinolin alkaloids (narcotin, papaverin), it depresses all kinds of smooth muscle. The effect appears to be directly on the muscle substance, since it antagonizes not only pilocarpin, histamin and epinephrin, but even barium; whilst these do not remove chelidonin depression. It is relatively non-toxic; and when its therapeutic dosage has been ascertained, it may have a clinical field in asthma, colic, and other spasms of smooth muscle.

Meyer also found that it paralyzes skeletal muscle and sensory and motor endings.

Morphin on Urinary Bladder.-Morphin, in toxic doses, produces spasm of the vesical sphincter (either through depressed inhibition or analogous to pyloric spasm), leading to distention of the bladder and constant but ineffective desire for micturition. This sometimes occurs in the therapeutic use (Czapek and Wassermann, I9I4). In guinea pigs the distention may rupture the bladder (Tappeiner, 1899).

Uterus.-Morphin probably tends to delay the progress of labor, by its psychic sedative action, largely by preventing the reinforcement of labor pains by the contraction of the abdominal muscles (Barbour and Copenhaver, rgr6). It has practically no effect on the normal uterine contractions in animals, in situ (Barbour, 1915); when applied directly to the excised uterus, the tone is somewhat increased (Barbour and Copenhaver, I915); but this would have no clinical bearing.

Effects on the Digestive System.-Morphin tends to produce nausea and vomiting, and thus disturbs digestion. The effect is independent of the mode of administration, the action being central. The severity varies greatly in different individuals. In some cases it is so serious, and accom- 
panied by so much depression, as to preclude the use of the drug. It may sometimes be lessened by bromid or by atropin (I. Simon, I907).

Larger doses are antemetic.

Morphin is one of the most efficient drugs for checking peristalsis. Small doses suffice for this purpose. The action is evidently peripheral, but its mechanism is complex, involving several factors, one or the other of which may predominate under certain conditions. The known factors are closure of the gastric sphincters, delaying the entrance of food into the intestines; decreased responsiveness to defecation reflex; and a direct sedative action on irritated (not on normal) intestine. With opium, there is also the direct depression of intestinal tone by the isoquinolin alkaloids (papaverin, etc.).

Of the individual opium alkaloids, morphin acts most powerfully on the alimentary canal. However, the minor alkaloids, especially papaverin, narcotin and codein, are also constipating, with less narcotic action (Gottlieb and v. d. Eeckhout, r9o8; Zehbe, r9r3; Hess and Neukirch, r9I3; Macht, Herman and Levy, I9I6). These differences, and the delayed absorption, may explain the clinical preference for opium when the constipative effect is desired.

Nauseant and Emetic Action.-This is essentially central (Eggleston and Hatcher, I9I5). It may conceivably be aided by reflexes from the gastric spasm; for atropin, appears to diminish the emetic tendency. There is no evidence of local gastric irritation, although the alkaloid is rapidly excreted into the stomach. Vomiting occurs more readily with hypodermic than with intravenous injection; probably because the latter depresses the vomiting center too rapidly.

The emesis is produced by absolutely pure samples, and is therefore not due to contamination with apomorphin (even prolonged boiling does not convert morphin into apomorphin; Feinberg, I9 3 ).

Relative Emetic Action of Opium Alkaloids.-Morphin is more nauseant and emetic than the other isolated alkaloids. Opinions differ as to the position of the combined alkaloids. Eggleston and Hatcher, 1915, believe that the emetic effect of pantopon and narcophin is more powerful than would correspond to their morphin content. As narcotin alone is not emetic, it would be conceived as sensitizing the center to morphin. Macht, Herman and Levy, I9 16 , incline to the opposite conclusion.

Salivary Secretion.-The nausea, as usual, is accompanied by salivation. In the absence of nausea, the mouth is dry and the patient thirsty, from depression of the salivary center (V. E. Henderson, I9ro).

The appetite is diminished, through the lesser perception of hunger, and through the gastric derangement.

Gastric Secretion.-Morphin starts the gastric secretion in the empty stomach (therefore contraindicated in gastric ulcer), but is said to diminish the secretion when the stomach contains food (Chiari, I915). It also increases the pancreatic secretion. Opium increases gastric secretion, but causes a lasting decrease of pancreatic flow (Bickel and Pincussohn, I907; O. Cohnheim and Modrakowski, I9II). In chronic morphinism, gastric secretion is said to be abolished (Hitzig).

Antemetic Action.-Dogs, after a primary emesis, do not respond to irritant emetics or apomorphin. This has been attributed to paralysis of the vomiting center; but it could also be explained by the firm contraction of the cardial sphincter (Magnus, 1 908); for morphin, codein, or heroin are antemetic in doses which are not narcotic, whilst chloral or urethane, to prevent vomiting, must be exhibited to full narcotic action (v. Issekutz, I91 2; Ruth, I913). Papaverin is not antemetic (Ruth).

Constipation Action in Milk Diarrhea.-A new light has been shown on morphinconstipation by R. Magnus, 1906 and I 1908 , who tested its effect on experimental diarrhea. A very suitable quasi-physiological form of diarrhea can be maintained in cats by an exclusive milk diet. This diarrhea is very effectively stopped by morphin. The remedial action is equally pronounced when the intestinal nerves have been divided so that the action is certainly peripheral. On the excised intestine, however, morphin increases peristalsis (except with enormous doses), indicating that its constipating effect is not due to peripheral intestinal depression. (The excised intestine has also been studied by Popper, I913.) 
Gastric Tonus.-To solve this riddle, Magnus investigated the passage of the alimentary contents by the Bismuth $x$-ray method of Cannon. He now observed that morphin acts exclusively on the stomach, producing prolonged contracture of the sphincter antri pylori, as also of the pyloric and cardiac sphincters. As a result, the food remains for hours in the fundus, the complete evacuation occupying twenty-five hours instead of the normal three hours. The intestines thereby are granted a very large amount of physiological rest, the food entering very slowly and gradually, being more perfectly macerated and digested, and presumably relatively aseptic. This furnishes the whole explanation of the constipating action in milk-diarrhea; for the same method shows that the movements of the small intestine are scarcely affected by the morphin, whilst those of the large intestine, as also the defecation of enemata, are quite unaffected by the drug. (Schwenter, Igr2, using a similar method, describes relaxation of the large, and probably also of the small, intestines.) Nor has morphin any constipating action in experimental diarrhea from certain irritant cathartics, viz., senna, castor oil, or magnesia.

Entirely analogous phenomena are observed in the dog (Magnus; E. Zunz, I909), and in the human subject. They are probably a highly important factor in the therapeutic effect. Magnus himself, however, suggested that morphin may possibly have further indirect actions on peristalsis, by modifying intestinal secretion or absorption.

Colocynth Diarrhea.- This surmise was confirmed by the results of his pupil, Padtberg, I9I $\mathrm{I}$, in colocynth diarrhea. This drug produces exudation and increased movements throughout the alimentary tract. Both effects are antagonized by morphin (and much more by opium), even when the food is already in the intestine. The action of morphin in colocynth diarrhea is therefore different from its action in milk, senna, castor oil or magnesia diarrhea, in which morphin would not affect the progress of the food after this has reached the intestine.

Other Opium Alkaloids on Colocynth Diarrhea.-Takahashi, I9I4, found that codein acts similarly to morphin, but weaker. Mixtures of morphin and codein act stronger than either alone (potentiation), so that constipation occurs by combining one-fourth of the minimal active dose of morphin with one-fortieth to one-four hundredth of the minimal active dose of codein. Equal parts of the two alkaloids constitute the best ratio. The other alkaloids do not seem to contribute, but rather to interfere; so that Pantopium, for instance, is inferior. Codein also potentiates the gastric spasm, but only with much larger doses than are needed in colocynth. When used alone, the codein does not produce gastric spasm.

Observations on Man.-The results are rather inconstant; but those of Zehbe, I9I3, with the $x$-ray method are fairly representative for normal subjects. The time of the total sojourn of the food was nearly doubled by the opiates; more by opium and pantopium than by morphin and narcophin. That in the stomach and small intestines was each delayed by about a third. The main effect, however, was on the colon and rectum. The defecation-reflex is delayed or suppressed (Stierlin and Schapiro, I9I2; Schapiro, I9 3 ; Mahlo, I9I3).

On the other hand, Pancoast and Hopkins, I9I5, observed the main delay in the small intestines; but little in the colon; the defecation-reflex was not observed. The effects, however, are extremely inconstant in different individuals. Small doses were more effective than large. Hypodermic and oral administration produced the same results.

Older Explanations.--Prior to the conclusive experiments of Magnus, the constipating action was explaincd either by a peripheral depression of the local reflex (the cells of Auerbach's and Meissner's plexus); or central stimulation of the inhibitory splanchnic fibers (Nothnagel, 1882). Neither theory was satisfactory. The apparent results of Nothnagel were probably accidental (Magnus, I9ro) and need not be discussed.

Other Opium Alkaloids on Peristalsis. - Whilst the phenanthren alkaloids (morphin, thebain and codein) increase the pendulum movements of excised intestine, these are said to be decreased by the isoquinolin alkaloids (papaverin, narcotin and narcein) (Popper and Frankl, 1912 and I913). Pal, 19r3, claims that the latter also remove spastic contractions of the stomach and intestines clinically.

Cathartic Action.- - Large doses produce catharsis in dogs and some other animals, but not commonly in man. The action is presumably due to local irritation. 
Bile Flow.-Large doses of morphin increase the tone of the sphincter of the biliary papilla, and thus hinder the flow of bile (Reach, igr4). The musculature of the bilebladder is not affected (Lieb and McWhorter, I9I5).

Metabolism.-The output of $\mathrm{CO}_{2}$, and to a lesser degree of urinary nitrogen, are materially decreased, mainly by the muscular quiet; in prolonged experiments also by the digestive derangement. The $\mathrm{CO}_{2}$ content of the blood rises, through the defective respiration. The urine of ten contains reducing substances, partly morphin-glycuronic acid (P. Mayer, I899), partly glucose from the asphyxia. The flow of urine is said to be decreased (Ginsberg, I9I2). The body temperature is lowered through lessened heat production.

Lessened $\mathrm{CO}_{2}$ Output.-That this is due to muscular depression is shown by the fact that cats exhibit an increase (Boeck and Bauer, 1874), and this is also the result in other animals with the more stimulant members of the group, as codein. In man, however, the oxygen consumption is not altered, whilst the $\mathrm{CO}_{2}$ production is decreased. The paradox is not explained (Higgins and Means, I9I5).

Ratio of Urea to Total Nitrogen.-This is not altered.

Morphin Glycosuria.-A peculiar reducing substance in human urine is described by Spitta, I 908 .

Temperature.-The fall (which is sometimes preceded by a slight rise) may be as great as $2^{\circ} \mathrm{C}$. with large doses; the extent depending, however, more upon idiosyncrasy than upon the dose. The cooling is particularly great if the animal is kept in cold surroundings. With small doses, the fall may be explained by increased heat loss from cutaneous vasodilation; but with large doses it is due mainly to diminished heat production, and finally to circulatory collapse. The heat production may be reduced by 80 per cent. in extreme cases, reaching its minimum in the third hour. The heat loss is diminished (up to 20 per cent.), partly through the effort of the heat centers to compensate for the diminished production, partly through the depressed circulation (Reichert, Igor).

Leucocytes. - These are diminished in acute and chronic morphin poisoning (Cloetta, 1903). The injection of morphin inhibits the migration of leucocytes' in inflamed frog's mesentery (Ikeda, I9I6).

Regulation of Blood Volume.-Morphin hinders the transfer of injected saline solution from the blood to the tissues (Bogert, Underhill and Mendel, 19r6).

Absorption, Fate and Excretion.-Morphin is rapidly absorbed from all mucous surfaces, and from the abraded skin; but scarcely, if at all, from the intact skin. It disappears from the blood, being partly fixed in the organs, and largely destroyed, so that only a fraction can be recovered at autopsy. A part is excreted unchanged, especially by the alimentary tract. The saliva and gastric juice show its presence in a few minutes. Some of this excreted morphin is reabsorbed-hence the indication of emptying the stomach in morphin poisoning, even after subcutaneous administration. The intestinal secretion proceeds somewhat more slowly. In acute poisoning with large doses, administered hypodermically to dogs, one to two-thirds of the morphin can be recovered from the feces. The urine participates in the excretion to a smaller degree. The destruction is much more complete in habituated animals, thus accounting partly for the increased tolerance for morphin.

Some of the morphin is said to be excreted by the milk and to cause morphinism in sucklings. It also passes through the placental circulation to the fetus, sometimes killing the latter in utero (Jung, I9I4).

The investigation of the fate of morphin is of great scientific, as well as of practical toxicologic interest. Unfortunately it is beset with considerable difficulties. The methods of isolation are complicated, and the more or less firm combination and partial alteration of the morphin in the body render its quantitative isolation in pure form practically impossible. Marquis, 1896 , believes that it exists in the tissues in three different forms. 
Disappearance from the Blood.-The morphin usually ceases to be demonstrable within twenty minutes, although it is not destroyed by the blood (Cloetta, r903). It must therefore have passed into the tissues.

Presence in the Tissues. - These contain only a fraction of the morphin-for the most part mere traces. The largest quantity is usually found in the liver and in the contents of the alimentary canal (Kauzmann, I868; Marquis, I896). The brain of poisoned animals is very poor in morphin, although in vitro, brain substance fixes the alkaloid much better than liver pulp (Cloetta, I903) (Localization in rabbits; van Rijn, I908).

Excretion by the Urine.-The statements in regard to this are very contradictory. Kauzmann found it qualitatively in less than two up to thirty-six hours; negative in fifty hours. Recent investigators agreed that it can be demonstrated only after large doses, and then merely in traces. However, Kaufmann-Asser, 1913, finds that dogs and rabbits excreted by urine 3 to 23 per cent. of the morphin in acute poisoning; and to 39 per cent. in chronic poisoning.

Excretion by Milk.-Contrary to the usual statement, Koldewijn, I9ro, found that this does not occur (in cows and goats).

Excretion by Saliva.-Rosenthal, 1893, showed its presence in man, after 0.05 Gm. per day.

Gastric Excretion.-This was discovered by Marmé, 1883 . Alt, I889, demonstrated morphin in the stomach (by lavage) in two and one-fourth minutes after hypodermic injection; the excretion rose for five minutes, then remained steady for twenty-five to thirty minutes, and was arrested in an hour. He also showed the benefit of gastric lavage after hypodermic injection. This indicates that part of the excreted morphin is ordinarily reabsorbed; Leineweber, $188_{3}$, and Bongers, 1895 , also observed the gastric excretion. This is increased by the presence of hypertonic solutions (ro per cent. $\mathrm{NaCl}$ ) in the stomach. Heroin behaves similarly (Langer, I9r 2; Kleiner, I913). The gastro-intestinal excretion of morphin is greatly diminished by vegetarian diet in dogs (Valenti, 1914).

Excretion by Intestine.-Marquis found morphin in the large intestine one hour after intravenous injection; in the small intestine only after two hours. The large intestine contains the larger amount. This indicates that morphin is excreted by the mucosa of the whole alimentary tract. With oral administration, the morphin may reach the intestine directly, within half an hour (Kauzmann). The bile may contain traces in twenty-five minutes, but is generally negative (Kauzmann). The intestinal excretion is increased by intestinal irritants (quillaja, senega, alcohol), even when the morphin has been administered hypodermically (McCrudden, I9IO).

Quantity Excreted in Feces.-Faust, 1900, found that non-habituated dogs excreted by the feces 66 per cent. of the morphin which had been injected hypodermically (Cloetta, 1903, recovered 23 to 32 per cent.) With repeated injection, as the animals became habituated, the feces contained less and less morphin (twenty-six per cent. after twenty-one to twenty-four days; 8 per cent. after twenty-nine to thirty-two days; none after forty days); although the later doses were up to 50 times as large as the earlier doses. It is evident, therefore, that the power of the organism to destroy morphin is greatly enhanced by habituation.

Quantity of Morphin Recovered from the Tissues.-Cloetta, I903, succeeded in recovering about one-third of the injected morphin from the entire bodies of non-habituated rats and pigeons killed in five to twenty-four hours after the injection of large doses of morphin. The same quantity was recovered from animals which had been habituated, but left without morphin for two days prior to the final injection (to permit the complete elimination of retained morphin). This indicates that the destruction is not a rapid process, even in habituated animals.

Destruction of Morphin in the Body. - The facts described in the preceding section leave no doubt that the morphin is largely destroyed; but as to the place and manner of this destruction, the evidence is incomplete. It indicates that the morphin may be destroyed in various organs, and more actively in habituated animals.

Tauber, 1890, could not demonstrate any destruction on perfusing morphin through excised liver or kidney. Cloetta, I903, claims the disappearance of one-sixth to onethird of the morphin digested with oxygenated emulsions of lung, liver, and especially brain; no destruction with filtered extracts; so that the action is not due to soluble ferments, but presumably oxidative. The destruction is increased in habituated animals. Albanese, r 909 , found practically no destruction by liver pulp, from either normal or habituated animals; but marked destruction during abstinence following habituation. Other organs showed similar but weaker destruction. Dorlencourt, 1913, again claims that morphin is destroyed by liver in vitro, and that this is increased by habituation. (Theories of decomposition, Spitta, 1908). 
Fatc in Chick-embryos.-If morphin is injected into fertilized eggs, which are then incubated, no destruction of morphin or its derivatives occurs if the embryo ries before it is half developed. If development is completed, heroin is destroyed absolutely; morphin very largely, codein and dionin not at all. The destruction therefore takes place only after a certain development, perhaps of the nervous system, has been reached. The alkyl groups protect the morphin against oxidation, just as they do in the adult organism, while the acetyl groups are evidently easily separated (Grueter, I9I6).

Fate in Frogs.-In these, the destruction is very small, and no tolerance is acquired. The excretion is very slow, extending over eight days, and occurs exclusively through the alimentary tract (B. Frenkel, I9IO).

Fate of Codein.-This behaves quite differently from morphin: about 80 per cent. is excreted unchanged, mainly in the urine, but somewhat also in the feces (Tauber, I892). Repeated administration does not increase its destruction, nor does it appear to produce tolerance in animals (Bouma, r9o3).

Heroin.-The greater part is excreted unchanged in the urine; some also in the feces. A part is destroyed and this destruction is greatly increased in habituation (Langer, I9I2).

Odorous Substances.-The substances which give the characteristic odor to opium are excreted largely by the urine, and also in the breath, sweat and milk.

Toxicology.-Acute Symptoms of Morphin and Opium Poisoning.Suicidal and accidental poisoning by morphin or opium is common, and familiarity with its successive symptoms is therefore important. The following description of the morphin effects applies equally to opium (with ten times the dose); except that the symptoms do not appear quite (but almost) as promptly.

Moderate Therapeutic Doses ( 5 to $\mathrm{I}_{5} \mathrm{mg}$; $1 / 12$ to $1 / 4 \mathrm{gr}$. of morphin).The symptoms set in promptly (five to ten minutes after hypodermic, fifteen to thirty minutes after oral administration). They begin with slight flushing of face, sensation of warmth and comfort; lassitude, giddiness, dreamy state, with free imagination but confused intelligence; mouth dry and thirsty.

The patient becomes sleepy; skin pale; respiration and pulse slowed; pupils constricted. Nausea and vomiting are exceptional with this dosage.

In a short time-the sooner, the larger the dose-a natural sleep sets in, lasting six to eight hours or more; the patient commonly awakens refreshed.

Intravenous Injection.-If the injection accidentally enters a vein, there is tinnitus, tachycardia, fainting, but usually rapid recovery.

Larger Therapeutic Doses ( 20 to $30 \mathrm{mg}$.; $1 / 3$ to $1 / 2 \mathrm{gr}$.).- The initial symptoms are the same. Sleep occurs more promptly and is more profound. On awakening there is usually some confusion, nausea, anorexia, and constipation.

Toxic Doses ( 100 to $300 \mathrm{mg}$.; $1 \frac{1}{2} 2$ to $5 \mathrm{gr}$.).-The initial symptoms are as described. The somnolence deepens rapidly into sleep and coma; severe symptoms being generally conspicuous in five to ten minutes after hypodermic, or fifteen to forty minutes after oral administration; rarely delayed for one or two hours.

In the earlier stages of the coma, the patient can be partially aroused to a confused consciousness, but relapses promptly into lethargy.

As the coma deepens (i.e. almost always within two hours, and often 
in half an hour after taking the poison), the patient can not be roused, the reflexes disappear, the muscles relax, the jaw drops. The miosis becomes extreme ("pin-point pupil"); the skin pale, with cold sweat, finally cyanotic; fall of temperature; respiration very slow, irregular, often Cherne-Stokes; pulse slow, feeble and often irregular. Convulsions are rare in arlults, more common in infants; they are sometimes violent, but rarely if ever tetanic.

The respiration stops before the heart. The pupils almost always dilate as the terminal asphyxia sets in. (With heroin, death is due to the convulsions; Langer, I9I 2.)

The time of death is usually seven to twelve hours; sometimes in two hours or even less. Survival over twelve hours indicates a good prognosis.

Autopsy findings are not characteristic. There are only the common asphyxial lesions: Cerebral and meningeal congestion, sometimes with serous effusion; lungs hyperemic and sometimes edematous; general venous congestion; rarely intestinal or cerebral ecchymoses.

Ordinary Fatal Dose. - With morphin, this is 0.2 to $0.4 \mathrm{Gm}$. (3 to $6 \mathrm{gr}$.); with opium, $3 \mathrm{Gm}$. (45 gr.). There is, however, considerable idiosyncrasy, and $60 \mathrm{mg}$. (I gr.) of morphin must be regarded as dangerous. With $250 \mathrm{mg}$. (4 gr.) the prognosis is unfavorable, even under treatment, although patients have been saved after $4 \mathrm{Gm}$. (6o gr.) of morphin.

Recovery.-This may occur even after asphyxial convulsions. The respiration and pulse improve, the coma becomes lighter, and passes into a very prolonged sleep, often lasting for one or two days. On awakening, the patient is troubled with headache, constipation and digestive disturbances, often vomiting, itching, impotence of the bladder; exceptionally excessive depression. Sometimes the recovery is interrupted by a serious relapse, perhaps through the reabsorption of morphin from the intestine.

Idiosyncrasies.-Infants are highly susceptible to morphin; as to older children, there is difference of opinion. The female sex, and patients weakened by disease, succumb relatively easily. On the other hand, large doses are well tolerated in diabetes, in delirium tremens and in tetanus. In fever, morphin is sometimes exciting; in neurotic patients it may cause insomnia and delirium, instead of sleep. Nausea and vomiting are very pronounced in some individuals. Urticaria has been observed. Itching of the skin, especially of the nose, is a rather common symptom in poisoning. These idiosyncrasies should be remembered in the therapeutic use of the drug.

Death has been reported from almost incredibly small doses. Lewin cites two fatalities in children from $0.3 \mathrm{mg}$. $(1 / 200 \mathrm{gr}$.) of opium. In adults, $0.2 \mathrm{Gm}$. (3 gr.) of opium ( $1 / 2 \mathrm{gr}$. of morphin), is credited with a death. The great variation in the susceptibility of different animals has been described, man being by far the most susceptible.

Hypersusceptibility of Infants. - Young animals are said to be more susceptible than adults, with the exception of cats. Doebeli, rgr r, from a clinical and experimental investigation, concludes that the hypersensitiveness, both in the human and in animals, applies only to the age before weaning, older children following the ordinary rule. This, he finds, is also the opinion of most pediatrists. The special susceptibility of sucklings is perhaps explained by the observation of Hunt, rgro, that a milk diet greatly increases the toxicity of morphin (for mice). The hypersusceptibility exists only for the narcotic action, and is, therefore, insignificant for codein. 
Infuence of Thyroid and Iodids.-Hunt, I905 and 1907, found that feeding with thyroid or iodids increases the susceptibility of mice, rats and guinea pigs to morphin. Githens and Meltzer, 1913, find in rabbits that iodid increases the morphin depression but lessens the tetanic effect, and therefore the fatality.

Diagnosis of Morphin or Opium Poisoning.-A prompt diagnosis is very important for proper treatment. The coma is the most conspicuous symptom, and its gradual development is characteristic. In advanced poisoning, it may be difficult to distinguish the morphin coma from other forms. The symmetrical pin-point pupils are important, but it must be remembered that these dilate as death approaches (asphyxia). The odor is characteristic of opium poisoning.

Differential Diagnosis of the Origin of a Coma. ${ }^{1-}$ Those forms of coma which might be confounded with morphin are mainly alcoholic (chloral), uremic and diabetic, epileptic and apoplectic.

One of the most important points is furnished by the pupils. If these are dilated, the coma is probably alcoholic, but may be diabetic. With pin-point pupils the coma is either from opium or pontine apoplexy. If the latter, they are very often unequal, and on lifting the arms, one may often detect a paralysis.

The pupils respond readily to light in epileptic coma, not in the others.

The smell of the breath furnishes presumptive evidence of alcohol-poisoning, but it is not a definite proof, since this substance is often given as an antidote, and is so often present in quantities which would not cause a coma. The smell is, however, usually characteristic of opium, uremia and diabetes, but not of morphin. Uremia would also be characterized by albumin in the urine.

There are yet other forms of coma which may be confused with these, but no rules can be laid down for them. The history is often of the greatest importance.

Treatment of Acute Morphin and Opium Poisoning.- The first indication is to empty the stomach, and this no matter whether the drug has been taken by the mouth or hypodermically. If narcosis has already set in, emetics may act too slowly, and it may be necessary to employ a stomach-pump. The best chemic antidote is potassium permanganate, about $0.5 \mathrm{Gm}$. per liter ( 5 gr. per quart) by lavage; orally, a o.I Gm. ( $1 / 2$ gr.) in a tumbler of water. Tannin is not very effective. The patient should be kept awake as far as possible and in constant movement, since this contributes to the better tone of the medullary center. Reflex stimulants may be employed, such as cold ablutions, the inhalation of ammonia in the form of smelling salts, hypodermic injections of ether, etc. Caffein, especially in the form of strong, black, hot coffee, is the best physiologic antidote. Atropin may be valuable if the dose of $1.5 \mathrm{mg}$. (1/40 gr.) is not exceeded. The patient should be kept warm. If the breathing shows signs of failing, artificial respiration should be supplied.

When the danger is over, the constipation which usually follows should be relieved by cathartics and enemata.

Permanganate.-De Buscher (1904, I905) has found this effective in rabbits, even when it was administered three hours after the morphin; it was much less successful with dogs. It was quite ineffective if the morphin was given subcutaneously. Nothing could be expected from the hypodermic injection of permanganate, since this would be destroyed before entering the circulation.

Atropin.-This has been used extensively and somewhat indiscriminately in the treatment of morphin poisoning. It is a very dangerous remedy in this condition. The most conspicuous antagonistic actions of these two poisons are on the pupil, heart-rate, psychic processes, secretions, etc.-in short, upon functions which are of very subordinate importance in dangerous cases of poisoning. Any useful antagonism must be sought in their actions on the circulation, respiration, and metabolism. A careful study will show that the effects of morphin and atropin on these functions are antagonistic only with certain stages; whilst more severe grades are actually synergistic.

1 Coma: A condition of insensibility from which the patient can not be aroused. 
The later paralytic effects of atropin coincide with those of morphin; whilst in the last stages of morphin poisoning the centers are too greatly depressed to respond to the slow and weak stimulation of atropin. The usefulness of this antidote exists therefore only if moderate doses of atropin are given in moderate morphin poisoning (or vice versa). This conclusion is supported by several series of experiments on animals. As a practical deduction, the atropin should be given hypodermically in the dose of $\mathrm{r} .5 \mathrm{mg}$. $(1 / 40 \mathrm{gr}$.) and this should not be repeated. It is fair to state, however, that this is not the universal opinion. For instance, Roch, 1907, advises 2 mg., frequently repeated, until the pupils begin to dilate.

Cocain.-A general antagonism exists also between morphin and small doses of cocain, especially as regards the effects on temperature and metabolism. Larger doses are synergistic, and as the susceptibility to cocain is variable, the hypodermic dose of o.or Gm. (1/6 gr.) should not be exceeded.

Acute Heroin Poisoning.--Animals are killed by the convulsions; if these are prevented by etherization, almost double the fatal dose may be survived (Langer, rgr 2).

Chronic Opiumism.-Etiology; Early Effects.-Opium, Morphin and Heroin habits are unfortunately very prevalent and together with the Cocain habit (and to a much lesser extent of "Hashish") they constitute one of the terrible scourges of modern times. All these drugs produce at first a feeling of well-being, relief and contentment, of stimulation and freedom of imagination, and of ease and facilitation of mental effort. These agreeable actions vary in degree in different individuals, and therefore all do not fall their victims with equal readiness; women and neurotic and dissipated individuals are especially in danger, if they become acs quainted with the effects of these drugs. The majority of the heroin and cocain addicts begin the use through curiosity or dissipation (Drysdale, I9I 5, McIver and Price, I9I6); but "Patent medicines" (cough, consumption, diarrhea, and catarrh "cures," etc.) are responsible for many cases. Physicians must also be on their guard in prescribing these drugs, even in acute conditions. McIver and Price found that most cases of morphinism arise from the medicinal use. The continued use of opiates will lead to the habit, or disease, in practically every one, and is therefore excusable only in exceptional conditions, such as hopeless, painful diseases (cancer; etc.). Petteys believes that the habit becomes firmly established when the drug has been used daily for a month.

Historical.-Whilst the therapeutic use of opium dates back of Hippocrates, its habitual use seems to be of more recent origin. The first authentic records fall in the beginning of the sixteenth century. It would seem that its use is much older in India than in Turkey, and that the Mohammedans learned it through the conquest of the former country. Their acquaintance with cannabis indica is of much earlier date. The existence of the opium habit was at first confined to the Orient; its introduction into Europe and America is of very modern date-it began in the United States about 1860 .

Prevalence of Opium and Cocain Habit.-It is impossible to secure reliable statistics; but some idea may be gained from the fact that of about 600,000 pounds of opium, or its equivalent in morphin, and of coca leaves corresponding to perhaps 150,000 ounces of cocain, which are imported annually into the United States, at least half, and more probably nine-tenths, are used by habitués. The alarming increase of the habit may be judged by this, that whilst the population in the past fifty years has increased $\mathrm{I} 33$ per cent., the importation of opium has increased 350 per cent. (H. Wright, roro); and in addition, the cocain and heroin habits took their rise only within this period. In Tennessee, registration showed o.r per cent. of the population to be afflicted with drug habits (Wilbert, I915). From this and other data, he estimates the number of drug addicts in the United States as not over 200,000 . The applications to the city institutions of Cleveland for relief of opium, morphin, heroin and cocain habits in consequence of the Harrison Law, amounted to about o.or per cent. of the population. Of the sixty-six cases, forty-eight used opium or morphin; twenty-nine heroin, seven cocain (Drysdale, I915). 
Opium Habit in Children.-This is unfortunately not at all rare. It is usually started by the indiscriminate employment of paregoric and other soothing syrups. They present the typical symptoms. Withdrawal of the medicine is followed by restlessness, wakefulness, and every indication of suffering and distress. The treatment would be mainly hygienic.

Forms of Opium Habit.-The drugs are introduced by all possible channels: by the mouth, hypodermically, by smoking, by suppositories; and heroin, like cocain, by snuffing. The effects are very similar in all cases.

Smoking.-This is mainly practised in the Orient; but the International Opium Commission estimates that there are in the United States probably over 100,000 opium smokers, outside of the Chinese.

Smoking opium (chandoe) is prepared by the Chinese, by a complicated process of roasting, and repeated extraction and evaporation. Its morphin content is about the same as that of ordinary opium. It has been claimed that the opium smoke does not contain morphin (e.g., Hartwich and Simon, 1903); but Pott, 1912, has proved that morphin is indeed the active constituent. It has been said that Orientals are less injured by opium; but this is mainly because, in smoking, smaller quantities are consumed, with longer intermissions. The hypodermic method is now becoming the more popular, and with it, the Chinese present the same phenomena as Europeans (A. S. Rochester, 1909). The Chinese government has been making serious and successful efforts to suppress the opium evil.

Heroin Habit.-This has become very common, especially in the form of "snuffing" (J. Phillips, I9I2; bibliography, Farr, I915). It exhibits all the phenomena of the morphin habit, and its cure presents similar difficulties and dangers (Manguat and Blondel, I903; Sollier, I905; Fauntleroy, I907; Duhem, I907; McIver and Price, I9I6). Fatalities from overdosage are also more common. The quantities consumed daily average $0.6 \mathrm{Gm}$. (McIver and Price), may reach I Gm. (Wholey, I9 2 ) or even $2.8 \mathrm{Gm}$. (Comar and Buvat, I904); so that considerable tolerance is acquired. Snuffing causes rhinitis.

Codein Habit.-Codein is not nearly so apt to induce a habit, although this has been reported (Pelz, r905; Petteys). Little is known about habit from the other morphin derivatives.

Later Phenomena; Tolerance; Degeneration.-The more or less unpleasant immediate after-effects of morphin have been described under the toxicology. These also vary in intensity in different individuals; in many, the famed dreams of the opium eater degenerate into frightful nightmares. As the use of the drug is continued, an increased tolerance to it becomes gradually established. The desired effects are weaker and evanescent, unless the dosage is progressively raised. This, however, exaggerates the unpleasant after-effects. Most habitués use the drugs as stimulants, rather than for pleasurable effects (Bishop, I9I3). Moreover, any attempt to stop the drug results, not only in violent craving, but also in more serious and even dangerous "abstinence symptoms." The patient now takes the drug, not because he desires it, but because he can not do without it. The organism, accustomed to working under the influence of morphin, revolts against its withdrawal. When these drug habits have taken a thorough hold, they cease to be mere vices, and become real diseases, with physical, as well as mental and moral degenerations.

The Acquired Tolerance.- This may reach a remarkable degree, so that extraordinarily large doses can be taken daily without producing acute poisoning, and are even necessary to prevent the withdrawal symptoms. This immunity to morphin is, however, never absolute, and death from 
overdoses forms the most frequent "excitus letalis" of the morphinists. The tolerance is lost if the drug is discontinued.

The tolerance is explained partly by a very considerable increase in the power of the organism to destroy morphin; but since the destruction proceeds more slowly than the absorption, there must also be an acquired tolerance to the action of morphin. No "antitoxin" is formed.

The daily consumption of morphin: This is generally about I Gm. (McIver and Price, I9I6); but as much as $5.5 \mathrm{Gm}$. (85 gr., or one and two-thirds of the ordinary bottles) has been reported. Even larger doses are claimed by patients; but their statements are generally unreliable, since they are made in the hope that the drug will be withdrawn more gradually.

Bishop, I9I3, states that the tolerance persists unimpaired after seven months of abstinence. In animals, at least, it disappears in a few days.

Tolerance in Animals.-This can be induced in dogs (Faust), rabbits, goats, rats, and pigeons (Cloetta, I903). Frogs seem to become more susceptible.

The Explanation of Acquired Tolerance to Morphin.-This has been the subject of many investigations and theories. Marmé, $\mathrm{I} 88_{3}$, aimed to show that morphin is transformed in the body into oxydimorphin, to which he attributed actions precisely the reverse of morphin. It would thus explain both the tolerance, and the abstinence symptoms. This theory has only historical interest, since Donath, I886, and Marquis, I896, have shown that oxydimorphin is not formed; and moreover, the properties of this substance do not agree with the assumption of Marmé.

Another theory which may be dismissed is the formation of an antitoxin. Hirschlaff, 1902 , claimed that the serum of habituated animals is protective; but his experiments are discredited by the negative results of Morgenroth, 1903, and Cloetta, 1903.

The increased destruction of morphin (Faust) and of heroin (Langer) in habituated animals must be a factor in the protection, but can not explain it completely. The experiments of Cloetta and of Ruebsamen, I908, incidate that this destruction is a relatively slow process, so that the tissues must in the meantime be exposed to a large amount of morphin. van Egmond, I9I I, found that habituation to the toxic effect does not diminish the susceptibility to the central vagus stimulation. In further contrast to the vagus, the respiratory center and the pupil acquire marked tolerance in dogs (not in rabbits, van Dongen, 1915). Langer, I9I2, also states that the acquired tolerance of dogs to the narcotic action of heroin does not extend to its convulsive effects. This persistence of some of the effects proves that the disappearance of others can not be due to destruction of the alkaloid.

Biberfeld, I9I 5 , found no difference in the lipoid content of the brains of normal and habituatęd animals.

Crossed Tolerance.-Dogs habituated to morphin also show marked tolerance toward heroin and codein, as regards the respiratory effects; slight as to equilibrium; none as to heart or peristalsis. They have not acquired toleration toward cannabis or chloral (H. B. Myers, Igi 5 ).

The later consequences of opium habit are insidious, but none the less dangerous. For years, victims of the habit may appear quite normal to superficial observers, but closer attention would even then reveal signs of the disease. Especially characteristic is the variability of moods-depressed or stimulated-according to the interval since the last dose.

The physical phenomena relate at first to the digestive tract: obstinate constipation, alternating later with equally obstinate diarrhea; loss of appetite alternating with voracious hunger and thirst, and polyuria. These disturbances of digestion, as well as the more direct action of the drug, are not long in showing their effects upon the rest of the body. The patient loses weight rapidly and suffers from marasmus and cachexia. ${ }^{1}$

The skin becomes hard, pale, dry and rough. The nails, teeth and hair are also diseased. The condition of the integument is rendered still

1 These are two ill-defined terms. The former, marasmus, signifying a continued low condition of the nutrition and a wasting of the flesh without apparent organic cause. Cachexia also indicates a wasting of the body, with some striking change in the features, which are usually pinched and yellow. 
worse by the local effects of the injection when the drug is used hypodermically. The whole skin may be mottled with scars and marks of recent or older injections, and abscesses are often produced through want of cleanliness. Even when the drug is used by the mouth, the entire skin often acquires a peculiar waxy appearance.

The pupils are almost invariably contracted; the eyes lose luster; so that an opium user may often be recognized from his appearance. The pupillary and accommodation movements are affected. The heart is irregular. Albuminuria, glycosuria, and amenorrhea are frequent. The sexual functions are lessened (functional impotence, sterility), but usually return on withdrawal (Wholey, I912). Fevers resembling simplex, intermittent, and typhoid, are often seen. The motor-nervous system shows considerable change: nervous tremors, increased reflex irritability, etc. These conditions sooner or later weaken the resisting powers of the patient, so that he falls an easy prey to some other ailment, and thus rarely reaches old age. However, the habit may persist for over thirty years (McIver and Price).

The effects on the character of the patient are even more deplorable. This sinks progressively to the lowest level of unscrupulous cunning and cowardice. It is very doubtful whether the testimony of an opium user can ever be accepted, even in instances which do not affect him. He becomes absolutely incapable of any effort. Duty no longer appeals to him, and in order to escape it, or, still more, in order to obtain his drug, he will resort to any lie or any trick, no matter how dishonest. He will promise everything and fulfil nothing. Were he not so cowardly and disinclined to, or rather incapable of, any effort, he would be fit for any crime. His condition is all the more unhappy since he fully realizes it and sees himself in his true colors. He makes grand plans, and, at the same time, knows that he can never summon the energy even to begin them. Add to this the fact that he is a social outcast, and it is difficult to imagine a more unhappy condition. To the physician he should appeal as a sufferer, as one afflicted with a form of insanity; one who, like any other insane patient, should be treated with unflinching firmness, but with the most considerate kindness. Only in this way will it be possible to help him. He is himself devoid of the necessary will power, and this must to some extent be supplied by his physician and attendants.

Abstinence Symptoms. - The withdrawal of morphin from those accustomed to its use leads to a train of very severe effects, the severity being proportional to the rapidity with which the drug is withdrawn and to the dose which has been used. Prominent throughout is uneasiness and restlessness, with almost uncontrollable craving for the drug, passing sometimes into a true mania. Besides this, the first symptoms consist in spasmodic yawning and sneezing; coryza and lachrymation; and hoarseness. The pupils dilate again. Most conspicuous are muscular twitchings and violent pains or "cramps," sometimes in the abdomen, more commonly in the legs; also headache and other neuralgias. The extremities are cold, the head congested. Insomnia is a very constant symptom; the patients are very irritable and excitable, and this condition may culminate in delirium or acute mania, often suicidal. Women often have hysteric attacks. The digestion is profoundly disturbed, presenting the symptoms of a violent functional gastroenteritis. The most dangerous phenomenon is sudden collapse, ushered in by irregular and weak pulse, alternating between extremes of rapidity and slowness, cold sweat, and general pros- 
tration; and often ending fatally by heart-failure. 'This collapse, if severe, demands the prompt injection of a moderate dose of morphin, which generally causes the symptoms to disappear.

The abstinence symptoms are practically absent if the patient is rendered delirious by scopolamin (Wagner and Riewel, I905). This suggests that they are mainly of psychic origin.

Abstinence Symptoms in Animals.-According to Valento, I9I4, these show circulatory disturbances, arrhythmia, tachycardia, low blood pressure, etc.; and he states that these effects are produced acutely when abstinence blood is injected into normal animals. He finds, further, that the blood retains this property long after the morphin has been stopped, which speaks rather against an analogy with the human abstinence symptoms.

Treatment of Opium and Other Drug Habits.-The prime indication, as in all diseases, is the removal of the cause, the withdrawal of the drug. This must be done under medical supervision, best in special institutions, for the patients have neither the will power, nor the physical resistance to carry it through unaided; attempts at self-treatment are generally unsuccessful, and always dangerous. In fact, the closest surveillance of the patient is indispensable, both to prevent clandestine continuance of the habit, and to control the abstinence symptoms. The main object, therefore, is to withdraw the drug without danger or too great discomfort to the patient. When the drug has been completely stopped, even for a few days, the desire for it disappears, and the patient may be considered cured, although still in need of tonic treatment. Unfortunately, the seductive memories remain, and when occasion offers, sooner or later, relapses are all too common, often within a few weeks. These remarks apply equally to cocain and other serious drug habits; and the treatment of these is identical.

Methods of Withdrawal.1-Attempts at "insensible withdrawal," by very gradual diminution of the dose, amounts to useless and protracted agony; the sensations of the patient gauge the dose with surprising accuracy, and their patience reaches its end long before the drug.

Rapid withdrawal is much better, and generally succeeds if the patient can be supervised. With this method, the drug is reduced as rapidly as can be borne by the patient without producing very violent reaction.

It is better to begin by reducing the number, rather than the size of the doses. Profuse catharsis is a very effective adjuvant. The withdrawal may be completed within ten to fourteen days. Very hot baths relieve the restlessness and insomnia; salicylates the "pains" (McIver and Price, I9I6). The treatment is effective and not excessively difficult (Drysdale, I9I5).

Sudden complete withdrawal is both cruel and dangerous. It can be made more pleasant by substituting another habit-drug, but this accomplishes nothing more than the deceit of the patient, and is no better than the many advertised "Morphin Cures" which are thinly disguised solutions of morphin.

The Scopolamin Treatment of Drug Habits. - This permits the sudden and complete withdrawal of morphin, cocain, or alcohol from habitues, without the usual unpleasant results. It depends; apparently, on the maintenance of a mild delirium or "twilight sleep" which renders the patient more or less unconscious of the withdrawal. According to the experience of Wagner and Riewel, I905, the cure can be completed in a

1 Fuller descriptions of the standard methods are found in the J.A.M.A., 64: I022, I9I5. 
very few days, and is as complete as with any of the more difficult methods.

Details.- The treatment consists in absolute withdrawal of the drug, and in administering hypodermically a mixture of scopolamin hydrobromid, $0.3 \mathrm{mg}$. (1/200 $\mathrm{gr}$.); atropin sulphate, $0.1 \mathrm{mg}$. (1/600 gr.), strychnin sulphate, $0.4 \mathrm{mg}$. (1/150 gr.), and water I.5 c.c. ( 25 minims). The first dose, which is usually without results, is repeated in one-half to two hours, and again in one and one-half hours, and then every one-half to two hours, until the full scopolamin effect is indicated by dry throat, flushed face, dilated pupils, restlessness, and mild delirium. The patient is maintained continuously in this state for three or four days, by repeating the injection every two to six hours, reducing the individual dose to half, if possible. Constant, careful and skilled watching of the patient is indispensable. If the pulse (which remains ordinarily of normal character, with a rate of 60 to 70 ) should fall below 50 ; or rise above 110 , the treatment is intermitted, and some strychnin and a very little morphin are given.

The second day usually produces some abstinence symptoms (violent attacks of sneezing and some gastro-intestinal phenomena), but these are rarely of sufficient severity to require special attention.

The patient can be about the room throughout the treatment, and receives "fever diet." The delirium is usually mild and busy, the patient being mainly engaged in picking imaginary insects, etc.

At the end of the third day the injections are stopped, to see whether craving for the drug will return. Should this occur, the treatment is continued for another day. This is rarely required.

The Lambert-Towns Method, described by A. Lambert, I909 and 1913 , seems to involve the same principles, since it uses essentially Hyoscyamus and Belladonna. (Details in J.A.M.A., I9II, v, 56, p. 503; v. 60, p. I933.) Collapse sometimes occurs (Wholey, I915).

Pilocarpin has also been employed.

Sceleth, r9r6, advises a method including scopolamin, pilocarpin and ethylmorphin.

The After-treatment. - This is important with all methods. The patient should receive tonics, nutritious food, and rest for several weeks. Insomnia is rather troublesome at first, and should be relieved by giving chloral and bromid for two or three nights. The bowels should be kept clear, both before and after the treatment.

The "recovered" patient must be protected as much as possible against pain and other circumstances which would tempt him to the resumption of the drug. Psychotherapy may be useful to prevent recurrence.

Legislation Against Drug Habit.-The real cure of drug habits lies in the enforcement of appropriate legislation. The Harrison Law, if properly enforced, should sufficc to suppress the opium and cocain habits almost completely. It is asserted that it has already reduced the sales of the affected drugs to half (Wilbert, 1916).

Therapeutic Uses.-Morphin and Opium are used mainly to lessen pain, to procure sleep, to check peristalsis, to suppress cough, to ease dyspnea, to facilitate anesthesia, and to secure muscular quiet. These indications arise in a great variety of diseases; and since the action of the opiates is certain and highly effective, their field of usefulness is extensive and important. However, they must be used with discretion. It should be remembered that the relief is generally only symptomatic and may sometimes interfere with the natural processes of repair, or obscure the observation of the progress of the disease. ${ }^{1}$ The danger of habit formation restricts their employment in neurotic individuals and in chronic diseases (except when these are hopeless). The habit danger can be somewhat avoided if the morphin is administered only by the physician, with somewhat impressive formalities of asepsis, and not intrusted to the patient

\footnotetext{
1 Cushny, I9r4, aptly points out that the avoidance of opiates for fear of obscuring the diagnosis is a confession of deficient diagnostic technic; it should not be necessary to make the diagnosis at the expense of exhausting pain.
} 
himself. The side actions (constipation, nausea, gastric disturbance, and occasional excitement) may also interfere with the usefulness. Even with these restrictions, however, the range of usefulness of the opiates is still very wide.

Pain.-Morphin surpasses in efficiency all other analgesics, particularly for persistent pain. It is generally aimed to dull, rather than to abolish the sensibility; and for this purpose relatively small doses suffice. Morphin by hypodermic injection is to be preferred, 5 to $\mathrm{I}_{5} \mathrm{mg}$. ( $1 / 12$ to $1 / 4$ gr.) according to severity, and repeated as necessary. Local application is irrational, although it is still somewhat used. (H. C. Wood, Jr., I9I6).

Insomnia.-Morplin should be avoided, if possible, especially in nervous insomnia and in psychic excitement. It may be needed if the insomnia is caused by pain, cough, or dyspnea; and small doses (5 mg. or $1 / 12$ gr.) may be employed to reduce the dosage, and thus the side actions, of other hypnotics ( $f . i$., the circulatory depression of chloral).

Diarrhea.-O Opiates are very effective in arresting the excessive peristalsis in acute intestinal catarrh. If the intestines are first cleared of toxic and irritant material by a cathartic, the rest afforded by opiates favors the subsidence of the inflammation. Tincture of Opium by mouth ( 0.5 c.c. or 1o drops) deserves the preference. If the opiates are given while the materies morbi are still in the intestine, they may do harm, even when they give symptomatic relief.

Opium is also useful in the constipation and colic of lead poisoning, by relaxing the intestinal spasm. In peritonitis it relieves the pain, both directly and by lessening peristalsis; but the disguise of the symptoms may be objectionable surgically. In biliary and renal colic, Morphin ( $5 \mathrm{mg}$. or $1 / 4 \mathrm{gr}$.) is given hypodermically, to lessen the suffering (usually with I mg. atropin, which relaxes the ducts).

Cough. - The opiates check cough by lowering the reflex irritability of the respiratory center. Small doses suffice for this purpose-by mouth, Morphin $5 \mathrm{mg}$. or $1 / 12$ gr.; Tr. Opii $1 / 2$ c.c. or Io drops; Codein $30 \mathrm{mg}$. or $1 / 2$ gr.; Heroin $5 \mathrm{mg}$. or $1 / 12 \mathrm{gr}$. (children above one year, $1 / 2 \mathrm{mg}$. or $1 / 120$ gr.; below one year, $1 / 4 \mathrm{mg}$. or $1 / 240 \mathrm{gr}$.) ; Dionin I $5 \mathrm{mg}$. or $1 / 4 \mathrm{gr}$. Codein deserves preference, since it produces the desired effect with less side actions, and without habit formation.

The use of opiates for this purpose, as in bronchitis and phthisis, requires judgment. They are indicated when the cough is mainly irritative; and contraindicated when it is required for the expulsion of excessive mucus. Even then, however, they may be needed to produce sleep.

Asthma and Dyspnea.-Morphin (I $5 \mathrm{mg}$. or $1 / 4$ gr., hypodermically) often relieves this condition, by quieting the patient, allaying the "air hunger" and perhaps by lessening reflex irritability. It should be avoided if possible. In nervous asthma, it tends to form a habit. In respiratory dyspnea, the reduction of respiration may be dangerous. In cardiac asthma, the increase of carbon dioxid in the blood may injure the cardiac function and establish a vicious circle. The use of caffein or atropin would be preferable; but it may be justifiable to resort to morphin, especially for relieving the anxiety.

Colds.-The diaphoretic action is utilized for aborting colds; generally as Pulv. Ipecac et Opii, $0.5 \mathrm{gm}$. or $7 \frac{1}{2} \mathrm{gr}$.

Anesthesia.-Morphin in safe doses does not !nduce complete insensibility, but it is an efficient synergistic to other ansthetics (Io mg. or $1 / 6$ gr., hypodermically, see under "Ether," also under "Scopolamin.") 
Morphin is similarly useful for distracting the patient's attention from operations under local anesthesia.

Hemorrhage.-Morphin favors the arrest of hemorrhage by quieting the patient, thus keeping the blood pressure low, and permitting the formation of the clot. It is especially valuable when the hemorrhage is in inaccessible situation, f.i., the pulmonary hemorrhage of phthisis. The depressant effect on the respiration enjoins caution in the use of larger doses, especially in severe hemorrhage, cardiac disease, and other debilitating conditions.

Convulsions.-Since morphin increases reflex excitability, it can not compare with ether, chloral or chloroform for suppressing tetanus or other spinal convulsions; but its analgesic effect may justify its use as an adjuvant. The depressant action on the respiratory center must be borne in mind (Dreyfus, I9r4).

Psychic and motor exaltations, e.g., delirium tremens or atropin poisoning, are controlled only by very large doses, the danger of which offsets the problematic benefit.

Diabetes. - Large doses of opium, morphin or codein appear to benefit some otherwise intractable cases; but the ultimate results are not brilliant. The effects are probably indirect, by slowing the absorption of sugar from the alimentary tract.

Experimental Glycosurias.-The opium alkaloids have no effect on the hyperglycemia resulting from the conversion of hepatic glycogen (epinephrin or piqure); but they have an inhibitory action on alimentary hyperglycemia (Klercker, I914). This indicates that the action is on the alimentary canal, presumably by the gastric delay slowing the digestion and absorption of the carbohydrates, and thus preventing the sudden flooding of the organism with sugar (Macleod, I9I4). Morphin delays the disappearance of injected dextrose from the circulation of dogs, although it facilitates its excretion by the urine (Kleiner and Meltzer, 19I6). It must therefore hinder the passage of sugar from the blood into the tissues.

Clinical Evidence.-Good clinical observers claim that the thirst, polyuria, glycosuria, and itching of the skin are all markedly diminished. Part of this action must be attributed to the analgesic effect, while the influence on the glycosuria is probably due to its action on digestion, and is produced in the same way as by a limitation of the diet or by nauseants. As a matter of fact, opiophagic diabetics die faster than others. Codein has been used instead, but without any marked advantages.

Emesis.-Morphin is employed as an ant-emetic, but the doses which can be safely prescribed are often ineffective.

Dosage of Opiates for Children.-This is best adjusted to the weight: with children above ten months, the weight (in pounds) is divided by 150 ; below ten months, by 300 . The adult dose is multiplied by the factors thus obtained (Doebeli, I9I2).

Recapitulation of the Chief Differences of the Morphin Derivatives. -The action of all morphin derivatives are quantitatively identical, so long as they involve only changes in the outlying side-chains (R. Stockman, I89r). Even the quantitative differences are not of very great practical importance.

Morphin.-Produces the strongest narcotic, analgesic, hypnotic and intestinal effects, and the weakest stimulation. It causes the greatest derangement of digestion; and, with heroin, is most apt to induce a habit.

Codein (Methyl-morphin) is less narcotic, less constipating, and less apt to induce a habit or tolerance. It has some advantage in the treatment of cough. Bastedo uses I 5 to $30 \mathrm{mg}$., but Fraenkel, r899, claims that at least 40 to $60 \mathrm{mg}$. are required to affect cough. This dosage is some- 
what quieting, but not narcotic, and scarcely analgetic. Larger dosage produces restlessness instead of quieting. The susceptibility is the same for all ages (Doebeli, I9I I).

It increases the spinal reflexes more than morphin (v. Schroeder, r883). Frankfurther and Hirschfeld, 1910, claim a greater dilation of the cerebral vessels (without rise of blood pressure).

Dionin (Ethyl Morphin) seems to stand intermediate between morphin and codein, in all respects. Mering advises it in cough. Lindenmayr, I 9 I 2, uses it in colds, $0.05 \mathrm{Gm}$. before retiring. It sometimes causes itching of the skin (Seifert, Nebenwirk., I9I5, p. 6r). It has a special action on the conjunctiva (edema).

Heroin (Diacetylmorphin) approaches still more to morphin, of which it shares all the disadvantages, and over which it has no serious advantage. Bastedo finds it inferior to codein (Side actions, Seifert, Nebenwirk., roI 5, p. 69):

Thebain is so markedly convulsant that it may be placed in the strychnin group (Cl. Bernard; Stockman and Dott).

Structure of Opium Alkaloids. - These belong to two groups, those containing a phenanthren nucleus (morphin and its esters); and those derived from isoquinolin (papaverin, narcotin, narcein). The difference in the nuclei is shown in the diagram.

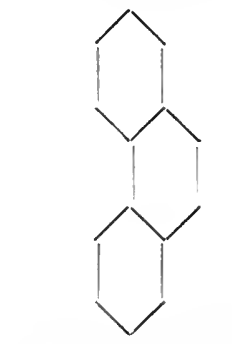

Phenanthren nucleus

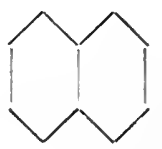

Isoquinolin nucleus

Structure of Morphin and its Derivatives.-Morphin is a complex derivative of phenanthren. It contains two $\mathrm{OH}$ groups (one phenolic, the other alcoholic) in which substitutions can be made, either by alkyl or acid radicals.

The more important alkyl esters are the monomethyl (Codein), dimethyl (Thebain) and ethyl (Dionin). Heroin is the di-acetyl derivative.

The nature of the radicals-whether acid or alcoholic, aromatic or aliphatic-is not of great importance. Replacement of a single $\mathrm{H}$ (codein, dionin) diminishes the narcotic actions and increases the respiratory and tetanic action, and the toxicity for animals. When both $\mathrm{OH}$ groups are replaced by acids (heroin) the narcotic action is stronger than for codein, the tetanic action is weaker than with morphin.

Comparative Activity of the Two Groups.-The alkaloids of both the phenanthren and isoquinolin are more or less narcotic and convulsant. The essential differences are in the peripheral actions: whilst the phenanthren alkaloids produce but few peripheral actions, the isoquinolin group causes extensive depression, as described under "Papaverin."

Convulsant-narcotic Series. - In general, the narcotic actions on the brain, on the one hand, and the stimulant action on the cord and medulla on the other, are in inverse ratio; morphin being the most narcotic and least convulsant, and thebain the most 
convulsant and least narcotic; the other morphin esters and the isoquinolin alkaioids being intermediate. Claude Bernard arranged them in the order of: Morphin, corlein, narcotin, papaverin, laudanosin, thebain. Buergi, r9I4, places them as: Morphin, papaverin, codein, narcotin, narcein, thebain.

Meconic Acid.-This has no effect in the doses which would be administered in opium. Larger doses produce narcosis, muscular fibrillation; medullary convulsions; diarrhea; curare action (Barth, $\mathrm{r} 9 \mathrm{I} 2$ ).

Papavcrin.-This was recommended as an analgesic by Baxt, r869, and Macht, I9I5; but its central effects are insignificant (Pal). Its toxicity is low. Bouchet claims that $\mathrm{I} \mathrm{Gm}$. produced no effects on man. Its important peripheral actions have been investigated mainly by Pal, I9I3, I9I4. He finds that it relaxes the tonus of all smooth muscle, especially when this has been spasmodically contracted, and this without. paralyzing the contractility of the muscle; $f . i$., he claims that it inhibits intestinal tonus without interfering with normal peristalsis or producing constipation; that small doses lower abnormally high blood pressure, whilst it requires much larger doses to lower the normal pressure. Macht, rgr6, claims that it lowers blood pressure, mainly by peripheral vasodilator action, especially in the splanchinic area. The coronary circulation is increased. Small doses slow the heart, with tendency to stronger contractions. Popper claims that the isoquinolin alkaloids relax the longitudinal coat of the intestines; the phenanthrens the circular coat. Macht, Herman and Levy, I 16 , find that $40 \mathrm{mg}$. hypodermically in man, produces marked analgesia with some vasodilation, fall of blood pressure and slight constipation.

Papaverin and narcotin are toxic to protozoa; Pick and Wasicky, I915, have therefore suggested the trial of papaverin against amebic dysentery.

Therapeutic Use of Papaverin.-Pal advises its use in gastric and intestinal spasms and colics, and in spastic constipation ( 30 to $80 \mathrm{mg}$. by mouth or hypodermic; 5 to 30 mg. by vein several times). Stoerk, 1915 , employs it against the tenesmus of bacterial dysentery (0.06 Gm., three times daily, continued as long as required). It has also been tried with less reason, in hypertension, angina pectoris; vomiting, gastric crises; asthma and pertussis (Pal; Popper, I9I4; L. Levy, I9I4). It is a local anesthetic, and has been used on the cornea as solution of 4 to ro per cent. Macht, 19r6, finds that papaverin relaxes the tone of the ureter, without inhibiting the peristalsis. Gerathy and Macht, rgr6, use its injection by ureteral catheter, against the colic of ureteral calculi.

Commercial papaverin contains considerable cryptopin, to which most of its chemical tests are due (Pictet and Kramers, I 910 ). In general, however, the quality of commercial opium alkaloids is satisfactory (Warren, I9 15 ).

Fate of Papaverin.- - It is probably extensively destroyed in the body, since neither papaverin nor any immediate decomposition products have been recovered from the organs or excreta after its hypodermic administration (Zahn, I9I5).

Tetra-Hydro-Papaverolin.-Laidlaw, I9Io, found that this inhibits the tone of unstriped muscle, dilates the bronchioles and blood vessels, and lowers blood pressure, whilst at the same time stimulating the heart. It is relatively non-toxic. Marshall, 1912 , tried it clinically in arteriosclerosis, but found it practically inefficient-probably therefore it is too easily oxidized.

Narcotin.-The peripheral actions appear to be similar to papaverin, but weaker (Pal, I9I4). Macht, I9I 5 , found, contrary to Straub, that it is quite toxic to the heart, respiration and other functions.

Narcein.-The peripheral actions resemble narcotin (Pal, I9I4).

Differences between Morphin and Opium; Opium Alkaloid Mixtures. -Whilst the actions of opium are essentially those of its principal alkaloid, morphin, there are various differences, especially quantitative. These are due partly to the mechanical action of the gums and resins, which must delay absorption, and thus increase the local and diminish the central actions. In the main, however, the differences are due to the presence of the minor alkaloids, which modify the effects of morphin; for similar differences exist in mixtures of the alkaloids, natural (Pantopium) or artificial (Narcophin, Laudanons).

The precise differences between the actions of morphin, opium and the mixed alkaloids are still under dispute. The statements of different (and sometimes of the same) observers are so contradictory that it is of ten impossible to judge them (Literature, E. Buergi, IgI4). 
Claimed Therapeutic Advantages.-It is asserted that opium and the alkaloid mixtures, for a given degree of analgesic and cough sedative action, cause less depression of respiration and less nausea; that their action, though slower, is more prolonged; and that they are sometimes effective in patients in whom morphin has failed. It is also believed that opium and pantopium are more efficient in checking diarrhea; whilst at the same time, it is stated that pantopium is not as constipating for normal individuals.

Before considering these actions in detail, it is necessary to describe the nature of the commercial products which have been largely used in the investigations.

Pantopon (Pantopium Hydrochloricum), N.N.R.-This consists of the isolated alkaloids of opium in their natural proportions. It was introduced by Sahli, as an improvement over the opiates. Discounting the early extravagant claims, it has the advantage that the absence of gums and resins insures prompter absorption and makes it much more suitable for hypodermic injection. The pharmacologic data on pantopium are reviewed by Barth, I9I2; Schwentner, IgI2; Watkyn-Thomas, I9I3. Severe poisoning from $0.04 \mathrm{Gm}$. is reported by Voigt, IgII. The side actions are quoted by Seifert, Nebenwirk., I9I 5, p. 8I.

Artificial Mixtures.-Since it is unlikely that all the alkaloids are therapeutically useful, and that they occur naturally in the optimal proportions, there exists a field for artificial mixtures. Faust, I9I2, introduced mixtures of the principal opium alkaloids under the name of "Laudanons." The proportions appear to be merely arbitrary. A more scientific attempt has been made by W. Straub in the introduction of Narcophin.

Narcophin.-W. Straub and Caesar, I $9 \mathrm{I} 2$, investigated the modifications produced by a large variety of mixtures of opium alkaloids. They found narcotin the most important alkaloid in the combination; claiming that doses of narcotin, which were ineffective alone, modified the actions of morphin, so as to increase the toxicity and narcotic effect, and at the same time diminish the respiratory depression; that a mixture of equal parts of the two alkaloids was the optimal proportion and fully represented the advantages of opium; that the other alkaloids of opium had less effect and introduced complex modifications which render them undesirable. These claims have been both confirmed and contradicted, clinically and experimentally. Meissner, I9I3 and I9I4, failed to confirm the synergism in the most important effects, viz. the respiratory and psychic; Straub's reply, 1913, is not quite convincing. Under the circumstances, judgment must be suspended.

Potentiation of Toxic Action.-Issekutz, 1912, found that the toxicity of mixtures of morphin and its esters corresponds to simple addition, but that mixtures of these with the isoquinolin alkaloids shows marked potentiation. This has been confirmed by W. Straub and Caesar, I9I2, who claim that doses of narcotin, which are in themselves inactive, double the toxicity of morphin for mice. This synergism may be considered as established; but it has little bearing on the therapeutic use.

Analgesic Action.-The statements are contradictory, as might be expected from the difficulty of making quantitative observations in a subjective condition. As yet, the claims of superior analgesic or narcotic action are not fully established. The sedative action on cough comes under the same category.

Straub claims a marked increase of narcotic action by narcotin, in that the morphin excitement of cats is prevented. (This, however, is a very variable condition, which is greatly influenced by external conditions.) Macht, Herman and Levy, 1916, also claim a synergistic decreased sensitiveness to cutaneous pain in human subjects. On the other hand, it is very suggestive that the clinical doses of narcophin and pantopium are strictly equivalent to their morphin content. Zeelen, rgro and I9Ir, found only simple summation in the narcotic (and tetanizing) action of the various opium alkaloids. Meissner also failed to confirm Straub.

Respiratory Action.-Straub asserts that the respiratory center in rabbits (as judged by response to $\mathrm{CO}_{2}$ stimulation) is much less depressed by narcophin than by morphin. This is contradicted by Meissner. Straub's statements appear self-contradictory, since he also claims that the toxicity, which generally depends on respiratory depression, is increased. However, Macht, 1915, confirms that the minor opium alkaloids stimulate respiration, and thus antagonize somewhat the morphin depression.

Circulation.-The data are scanty. Macht, I915, finds that morphin-narcotin mixtures dilate the coronary vessels much less than either alkaloid alone; and that Pantopon and Laudanon have practically no effect on the coronary circulation.

Gastric Disturbance. - It is quite generally asserted that the combinations are less liable to produce nausea and vomiting; but quantitative differences of this kind are diff- 
cult to judge, clinically. It is possible, though not proven, that there may be a slight difference, since morphin itself stimulates the vomiting center more than any other opium alkaloid.

Constipating Action.-This has been discussed under morphin.

The synergism of morphin with scopolamin and with the anesthetic and hypnotics will be discussed in connection with those drugs.

\section{PREPARATIONS-OPIUM}

* Opium, U.S.P., B.P. (Meconium, Thebaicum).-The dried milky juice exuding from the incised unripe seed-capsules of the poppy, Papaver somniferum. Brown masses or powder, of peculiar odor and bitter taste. Contains a number of alkaloids, the most important being morphin (about ro per cent.). Dose, 0.06 Gm., I gr., U.S.P. (equivalent to $6 \mathrm{mg}$. or $1 / 10 \mathrm{gr}$. morphin); 0.03 to $0.12 \mathrm{Gm} ., 1 / 2$ to $2 \mathrm{gr} ., \mathrm{B} . \mathrm{P}$. Maximum dose, 0.2 Gm., 3 gr.

Opium was mentioned by Theophrastus, third century B.C. An interesting account of its history is given by Macht, I9I5. The history of the opium preparations is also interesting (Wilbert, I9I6). Laudanum is said to have been originated by Paracelsus (1493-I 54I) but as a solid aqueous extract; paregoric by Le Mort of Leyden, early in the eighteenth century. Sydenham's laudanum (Vinum Opii) is derived from a formula of Sydenham (r624-r689). Tr. Opii Deodorati was originated by Robiquet ( 1780 1840), and Robt Hare, I827; Mist. Glycyrrh. Co. by Dr. B. J. Barton of Philadelphia, about I 8I4.

The opium poppy is cultivated in Asia and Egypt; it can also be raised in the United States, but the cost of labor renders the production of opium unprofitable. The capsules (especially before ripening, Caesar and Loretz, I902) and seed also contain the alkaloids. The seeds yield 50 per cent. of a bland fixed oil which may be used like olive oil.

The usual percentage of the other alkaloids is: Codein, 0.2 to 0.7 per cent.; Thebain, 0.15 per cent.; Narcein, 0.02 to 0.7 per cent.; Narcotin, I.3 to ro per cent.; Papaverin, I per cent. (absent in some sorts; Van Italie and Kerbosch, I9 Io). The development of these alkaloids in the growth of the plants is interesting (Kerbosch, r9IO).

Opium also contains a number of other minor alkaloids; meconic acid $(2.5$ to 5.5 per cent.), lactic acid; gums, resins, fats, odorous principles. No starch or tannin.

* Opii Pulvis (Opii Pulv.), U.S.P.; Powdered Opium.-Light brown powder, yielding Io per cent. of anhydrous morphin. Dose, $0.06 \mathrm{Gm} .$, I gr., U.S.P.

Opium Deodoratum (Opium Deod.), U.S.P.-Opium exhausted with petroleum ether, to remove some of the odorous principles. It is similar to the secret "improved" opiums.

Opium Granulatum, U.S.P.-This is employed in manufacturing.

Ext. Opii Liq., B. P.-0.75 per cent. of morphin, 3.75 per cent. of the Dry Extract, in 20 per cent. alcohol. Dose, 0.3 to I.8 c.c., 5 to 30 minims, B.P.

Ext. Opii, U.S.P.; Ext. Opii Sicc., B.P.-A powdered extract, I Gm. representing ${ }_{2} \mathrm{Gm}$. of the drug, or 20 per cent. of morphin. Dose, $0.03 \mathrm{Gm}$., $1 / 2 \mathrm{gr}$., U.S.P.; 16 to 60 mg., $1 / 4$ to I gr., B.P. Maximum dose, o.1 5 Gm., $21 / 2$ gr.

Lin. Opii, B.P.-Equal parts of Tr. Opii and Lin. Sapon.

Lotio Opii Et Plumbi, N.F.; Lead and Opium Wash.-Lead acetate, 4.5 Gm.; Tinct. opium, 9 c.c.; Water, q.s., 250 c.c.

*Mistura Glycyrrhiza Composita (Mist. Glycyrrh. Co.), U.S.P. (Brown Mixture).The adult dose, Io c.c., $21 / 2$ drams, of this mixture contains 1.2 c.c. of Paregoric or $5 \mathrm{mg}$., $1 / 12$ gr. of opium; and $2.5 \mathrm{mg}$., $1 / 24 \mathrm{gr}$., of Tartar Emetic; also a little Sp. Aether. Nit.

It is a popular expectorant, but needlessly complex.

(Pil. Plumb. C. Opio, see Index.)

Pil. Sap. Co., B.P. - 20 per cent. of Opium. Dose, 0.12 to $0.25 \mathrm{Gm} ., 2$ to 4 gr., B.P.

Pulv. Cret. Arom. C. Opio, B.P.-25 per cent. of Opium. Dose, 0.6 to 4 Gm., ro to $60 \mathrm{gr}$., B.P.

* Pulvis I pecacuanhe Et Opii (Pulv. Ipecac et Opii), U.S.P.; Pulv. Ipecac. Co., B.P. (Dover's Powder; Opii et Ipecac. Pulv. Co., P.I.).-Contains Io per cent. each of Opium and Ipecac with Milk-sugar, U.S.P., or Potass. Sulphate, B.P. (This was introduced by Thomas Dover, I 742 , as a diaphoretic in gout.) Dose, $0.5 \mathrm{Gm} ., 8 \mathrm{gr}$., U.S.P.; 0.3 to I Gm., 5 to I 5 gr., B.P. Maximum dose, I.5 Gm., $20 \mathrm{gr}$.

(Pulv. Kino Co., see Index.)

Pulv. Opii Co., B.P. - ro per cent. of Opium, with aromatics. Dose, 0.3 to I Gm., 5 to 5 gr., B.P.

(Supp.Plumbi Co., B.P., see Index.)

*Tr. Opii,.U.S.P., B.P.; Tincture of Opium (Laudanum).-- ro per cent. of opium, or I per cent. of morphin, in diluted alcohol. Miscible with water or alcohol. Dose, 
0.5 c.c., 8 minims, U.S.P.; 0.3 to I c.c., 5 to 15 minims, repeated; single, I. 2 to r. 8 c.c, 20 to 30 , B.P. Maximum dose, 2 c.c., 30 minims.

Tr. Opii Ammon., B.P.- - o. per cent. of Morphin, with Anise oil, Benzoic acid and Ammonia. Dose, 2 to 4 c.c.; $1 / 2$ to 1 dram, B.P.

* Tinctura Opii Camphorata (Tr. Opii Camph.), U.S.P.; Tr. Camphor. Co., B.P. (Paregoric; Opii Tinctura Benzoici, P.I.).- 0.4 per cent., each, of opium, benzoic acid, camphor, and anise oil, U.S.P. The B.P. contains the same ingredients with 0.5 per cent. of opium. The preparation is used especially for children. Dose, 4 c.c., I dram, U.S.P.; 2 to 4 c.c., $1 / 2$ to I dram, B.P. 4 c.c. contains $16 \mathrm{mg}$., $1 / 4 \mathrm{gr}$, of opium or 1.6 $\mathrm{mg.,} 1 / 40 \mathrm{gr}$., of morphin, U.S.P. $(20 \mathrm{mg}$., $1 / 3 \mathrm{gr}$. of opium or $2 \mathrm{mg}$., 1/20 gr. of morphin B.P).

* Tinct. Opii Deodorati, U.S.P.-Same strength and dose as the Tincture; has less odor, having been extracted by petroleum ether. Similar to McMunn's Elixir and other nostra.

(Ung. Gall. C. Opio, B.P., see Index.)

(Ung. Myrrh. C. Opio, B.P., see Index.)

Pantopon (Pantopizm Hydrochloricum), N.N.R.-A purified mixture of opium alkaloids, containing 50 per cent. of morphin. Sol. in water. Dose, 5 to $20 \mathrm{mg}$., $1 / 2$ to $1 / 3$ gr., by mouth or hypodermically.

Narcophin; Morphin-Narcotin meconate.-About 33 per cent. of morphin. Sol. in water. Dose, three times that of Morphin.

Sanguinaria, U.S.P. (Blood Root). -The dried rhizome and roots of Sanguinaria canadensis. Dose, 0.125 Gm., 2 gr., U.S.P.

Tr. Sanguin., U.S.P. - Io per cent., in acidulated 60 per cent. alcohol. Dose, I c.c., I 5 minims, U.S.P.

\section{PREPARATIONS-MORPHIN}

*Morphin and its salts occur as odorless, bitter white powders or colorless crystals. They are incompatible with alkalies, tannins, iodids and other precipitants of alkaloids. Dose, $8 \mathrm{mg}$., 1/8 gr., U.S.P.; 8 to $30 \mathrm{mg}$., $1 / 8$ to $1 / 2 \mathrm{gr}$., B.P. The rectal dose is the same; the hypodermic about two-thirds of the oral. Maximal single dose, $30 \mathrm{mg}$., $1 / 2$ gr. The actions begin in about five minutes on hypodermic administration. Solutions are said to decompose rather readily when heated above $60^{\circ} \mathrm{C}$. (Welmans, I908). Morphina, U.S.P., $\mathrm{C}_{17} \mathrm{H}_{19} \mathrm{NO}_{3}+\mathrm{H}_{2} \mathrm{O}$.-Very slightly sol. in water ( $\mathrm{I}: 3,340$ ); slightly sol. in alc. ( $\mathrm{I}: 2 \mathrm{IO})$; sol. in lime-water $(\mathrm{I}: \mathrm{I} O 0)$.

Morph. A cet., B.P., $\mathrm{C}_{17} \mathrm{H}_{19} \mathrm{NO}_{3} \cdot \mathrm{C}_{2} \mathrm{H}_{3} \mathrm{O}_{2}+{ }_{3} \mathrm{H}_{2} \mathrm{O}$.- Freely sol. in water ( $\left.\mathrm{I}: 2.5\right)$. Loses acetic acid on exposure to air.

* Morphina Hydrochloridum (Morph. Hydrochl.), U.S.P., B.P., $\mathrm{C}_{17} \mathrm{H}_{19} \mathrm{NO}_{3} . \mathrm{HCl}+$ ${ }_{3} \mathrm{H}_{2} \mathrm{O}$. - Sol. in water $(\mathrm{I}: \mathrm{I} 7.5)$ or alc. $(1: 52)$.

*Morph. Sulph., U.S.P., $\left(\mathrm{C}_{17} \mathrm{H}_{19} \mathrm{NO}_{3}\right)_{2} \cdot \mathrm{H}_{2} \mathrm{SO}_{4}+{ }_{5} \mathrm{H}_{2} \mathrm{O}$.-Sol. in water (I : $15 \cdot 5$ ); slightly sol. in alc. $(\mathrm{r}: 565)$.

Morph. Tart., B.P., $\left(\mathrm{C}_{17} \mathrm{H}_{19} \mathrm{NO}_{3}\right)_{2} \cdot \mathrm{C}_{4} \mathrm{H}_{6} \mathrm{O}_{6}+{ }_{3} \mathrm{H}_{2} \mathrm{O}$.- -Sol. in water (I : I I).

Inj. Morph. Hyp., B.P.-2.5 per cent. of tartrate. Dose, 0.3 to 0.6 c.c., 5 to ro minims, B.P.

Liq. Morph. Acet., B.P.-I per cent. Dose, 0.6 to 3.6 c.c., ro to 60 minims, B.P.

Liq. Morph. Tart., B.P.- I per cent. Dose, 0.6 to 3.6 c.c., Io to 60 minims, B.P.

Supp. Morph., B.P.-0.017 Gm., $1 / 4$ gr.

Tr. Chlorof. Et Morph. Co., B.P.-Chlorof., 7.5 per cent.; Morph., I per cent.; Acid. Hydrocyan. Dil., 5 per cent.; with Capsicum, Cannabis Indica and Peppermint. Dose, 0.3 to I c.c., 5 to 15 minims, B.P.- Used in colic.

Troch. Morph., B.P.-2 mg., $1 / 30$ gr.

Troch. Morph. Ei I pecac., B.P. -2 mg., 1/30 gr., of Morphin; 6 mg., 1/10 gr., of Ipecac.

\section{PREPARATIONS-MORPHIN DERIVATIVES}

These resemble the morphin salts in physical characters and incompatibilities; they are further incompatible with strong acids or alkalies.

Aethylmorph. Hydrochlor., U.S.P. (Dionin), $\mathrm{C}_{17} \mathrm{H}_{17} \mathrm{NO}(\mathrm{OH})\left(\mathrm{OC}_{2} \mathrm{H}_{5}\right) \cdot \mathrm{HCl}+{ }_{2} \mathrm{H}_{2} \mathrm{O}$. -White or yellowish, odorless, microcrystalline powder. Freely sol. in water $(\mathbf{I}: 8)$; sol. in alc. ( $\mathrm{I}: 22)$. Dose, $15 \mathrm{mg} ., 1 / 4 \mathrm{gr}$., U.S.P.; Locally, to to 20 per cent. Maximum dose, $50 \mathrm{mg} ., 3 / 4 \mathrm{gr}$.

Diacetylmorphina, U.S.P. (Heroin), $\mathrm{C}_{17} \mathrm{H}_{17}\left(\mathrm{C}_{2} \mathrm{H}_{3} \mathrm{O}_{2}\right)_{2} \mathrm{NO}$.-Very slightly sol. in water (I : I,700); sol. in alc. (I :3 I). Dose, $3 \mathrm{mg} .1 / 20$ gr., U.S.P.

* Diacelylmorph. Hydrochl., U.S.P., Diamorph. Hydrochl., B.P. (Heroin Hydrochlorid), 
$\mathrm{C}_{17} \mathrm{H}_{17}\left(\mathrm{C}_{2} \mathrm{H}_{3} \mathrm{O}_{2}\right)_{2} \mathrm{NO} . \mathrm{HCl}+\mathrm{H}_{2} \mathrm{O}$. Freely sol. in water ( $\left.\mathrm{I}: 2\right)$; sol. in alc. Dose, 3 mg., $1 / 20$ gr., U.S.P.; 2.5 to $8 \mathrm{mg}$., $1 / 25$ to $1 / 8$ gr., B.P. Maximum dose, Io $\mathrm{mg}$., $1 / 6 \mathrm{gr}$.

Codeina, U.S.P., B.P., $\mathrm{C}_{17} \mathrm{H}_{18}\left(\mathrm{CH}_{3}\right) \mathrm{NO}_{3}+\mathrm{H}_{2} \mathrm{O}$. Slightly sol. in water (I : 1 20); freely sol. in alc. (1 : 2), Dose, $30 \mathrm{mg} ., 1 / 2$ gr., U.S.P.; 16 to $60 \mathrm{mg} ., 1 / 4$ to I gr., B.P.

*Codein. Phosph., U.S.P., B.P., $\mathrm{C}_{18} \mathrm{H}_{21} \mathrm{NO}_{3} \cdot \mathrm{H}_{3} \mathrm{PO}_{4}+2 \mathrm{H}_{2} \mathrm{O}$. 67 per cent. of anhydrous codein. Freely sol. in water $(\mathrm{r}: 2.3)$; slightly sol. in alc. $(\mathrm{x}: 325)$. Dose, $30 \mathrm{mg} ., 1 / 2$ gr., U.S.P.; I 6 to $60 \mathrm{mg}$., $1 / 4$ to I gr., B.P. Naximum dose, $0.1 \mathrm{Gm}$., $11 / 2 \mathrm{gr}$.

Syr. Codein. Phosph., B.P. 0.5 per cent. Dose, 2 to 8 c.c., $1 / 2$ to 2 drams, B.P.

Codein Sulph., U.S.P., $\left.\left(\mathrm{C}_{18} \mathrm{H}_{21} \mathrm{NO}_{3}\right)_{2} \cdot \mathrm{H}_{2} \mathrm{SO}_{4}+{ }_{5} \mathrm{H}_{2} \mathrm{O}\right)$. - Sol. in water $(\mathrm{I}: 3 \mathrm{O})$; very slightly sol. in alc. ( $\mathrm{I}: \mathrm{I}, 280$ ). Dose, $30 \mathrm{mg} . ; 1 / 2$ gr., U.S.P.

Papaverin. Sulph., N.N.R., $\left(\mathrm{C}_{20} \mathrm{H}_{21} \mathrm{O}_{4} \mathrm{~N}\right)_{2} \cdot \mathrm{H}_{2} \mathrm{SO}_{4} .-$ Sol. in water or alc. Dose, 30 to $80 \mathrm{mg}$., $1 / 2$ to $\mathrm{I} / \frac{1}{4} \mathrm{gr}$.

\section{HYDRASTIS}

Hydrastis (Golden Seal) has few, if any, rational indications. It is employed empirically as a bitter stomachic; to check internal hemorrhage; and locally in catarrhal conditions, especially of the genito-urinary tract.

Constituents.-Hydrastis contains at least three alkaloids: Berberin, 1.5 to 4 per cent.; Hydrastin, at least 2.5 per cent. (U.S.P.), and Canadin, a derivative of berberin. There is also some resinous matter, etc.

Hydrastin.-This is an isoquinolin derivative, closely related to some of the minor opium alkaloids, Narcotin, Laudanosin and Papaverin. Narcotin is methoxy-hydrastin, an $\mathrm{H}$ of hydrastin being replaced by $\mathrm{OCH}_{3}$.

$$
\begin{gathered}
\text { Hydrastin - } \mathrm{C}_{19} \mathrm{H}_{14} \mathrm{NO}_{4} \cdot \mathrm{H}\left(\mathrm{OCH}_{3}\right)_{2} \\
\text { Narcotin - } \mathrm{C}_{19} \mathrm{H}_{14} \mathrm{NO}_{4} \cdot\left(\mathrm{OCH}_{3}\right)_{3}
\end{gathered}
$$

By oxidation both give analogous artificial alkaloids, which are somewhat important:

$$
\begin{gathered}
\mathrm{C}_{21} \mathrm{H}_{21} \mathrm{NO}_{6}+\mathrm{H}_{2} \mathrm{O}+\mathrm{O}=\mathrm{C}_{10} \mathrm{H}_{10} \mathrm{O}_{5}+\mathrm{C}_{11} \mathrm{H}_{12} \mathrm{NO}_{3} \mathrm{H} \\
\mathrm{Opianic}^{\mathrm{Hydrastin}} \\
\mathrm{C}_{22} \mathrm{H}_{23} \mathrm{NO}_{7}+\mathrm{H}_{2} \mathrm{O}+\mathrm{O}=\underset{\mathrm{O}}{=}=\mathrm{C}_{10} \mathrm{H}_{10} \mathrm{O}_{5}+\mathrm{C}_{11} \mathrm{H}_{12} \mathrm{NO}_{3} \mathrm{OCH}_{3} \\
\text { Narcotin } \\
\text { Opianic acid }
\end{gathered}
$$

Berberin, $\mathrm{C}_{20} \mathrm{H}_{17} \mathrm{NO}_{4}$ (structure, Faltis, 19ro) is an intensely yellow and bitter alkaloid, of wide occurrence, which gives the color and taste to a large number of "golden" roots and barks (Berberis, Xanthoxylon, Coptis, etc.).

Actions of Hydrastis. - An extensive study of hydrastis and its alkaloids was made by Bunge, 1895. This gives the literature to that time. Of later papers, mention may be made of those of Fellner, I906; Williams, I908; and Laidlaw, I910. The effects of the drug and of its different alkaloids are qualitively similar.

Central Nervous System.- Hydrastin produces convulsive effects analogous to those of strychnin. These are also obtained with the fluidextract. The derivatives hydrastinin and cotarnin have a purely depressant action, producing death by respiratory paralysis (Laidlaw). There is no narcosis.

Circulation.-From the experimental results, it seems that the effects of hydrastis and its alkaloids and derivatives on the circulation are too uncertain to be therapeutically useful. The clinical evidence also appears insufficient to establish their value. Frey, 1909, found that hydrastinin had no effect on pulmonary hemorrhage. It is possible that these drugs may have a more pronounced influence on uterine hemorrhage, through their action on the uterine muscle.

Very discordant results have been described. Williams obtained no effects with hypodermic or oral administration, even with very large doses. Intravenously the fluidextract, hydrastin and berberin produced the same results; considerable fall of blood pressure, followed with small doses by a slight and short rise. With large doses, the pressure remained low. These effects were predominantly cardiac (myocardiograms and oncometer); this overshadowed any possible vasomotor changes. 
- The vasomotor center is not directly affected by moderate doses of hydrastis, hydrastin, berberin, hydrastinin or cotarnin; but may be stimulated indirectly through respiratory depression, convulsive phenomena or cardiac arrest (Pilcher and Sollmann, $1915)$.

With toxic doses, there is vasomotor paralysis, and paralysis of vagus endings. Excised organs show some vasodilation.

On the scarified skin, hydrastin ( $\mathrm{r}: \mathrm{roo}$ ) produces some blanching (Sollmann and P'ilcher).

Hydrastin is relaterl to the protopin group, and therefore depresses the cardiac and skeletal muscle, and has a weak local anesthetic action.

Iydrastinin also causes some primary fall but a larger rise (about $15 \mathrm{~mm}$.) maintained for over fifteen minutes; the heart rate is slowed. This rise is also predominantly cardiac, but there is a stimulation of the arterial muscle, not prevented by paralyzing the nerve endings with ergotoxin. Kurdinowski, I904, interprets its actions rather differently.

Uterine Actions.-Fellner, 1906; Kehrer, I907, and Lieb, I9r4, showed that hydrastis, hydrastin, hydrastinin and cotarnin increase the tonus and excite rhythmic contractions of the uterus, both in the excised organ and in living animals. This has been confirmed by the subsequent workers; also for the surviving human uterus (Ruebsamen and Kligermann, I9I2). High concentrations relax (Wendling, 1915). Berberin has similar effects (Marek, IgII). La Torre, IgI2, examined histologically the uteri of animals treated with hydrastinin. The muscular layers were contracted and thickened, compressing the larger vessels, and thereby probably decreasing the blood supply of the mucosa. Laidlaw found the stimulant action of hydrastinin and cotarnin constant in the excised organ, but varying with conditions during life. These actions resemble those of the pituitary and ergot prificiples; but hydrastis does not act on the intestine, and very slightly on the bladder. Synthetic hydrastinin appears to be rather more stimulant than the natural alkaloid (Wendling, I9 I5).

The bronchioles are not markedly affected in pithed animals (D. E. Jackson, 1914).

Action of Berberin and Canadin.-Berberin acts as a simple bitter in small doses. Its bitter taste and the yellow stain which it produces on linen, render it objectionable in the therapeutic use of hydrastis. Berberin is about seven times as active as hydrastin on the circulation; and as hydrastis contains about one and one-half times as much berberin, about 85 per cent. of the circulatory effect is due to this alkaloid. Large doses lower the temperature, increase peristalsis, and kill by central paralysis (Curci $\mathrm{r} 89_{2}^{2}$ ). It is not excreted, being probably oxidized completely (Berg).

Canadin resembles morphin somewhat in its action. It is present in too small a quantity to be of practical importance. It occurs also in xanthoxylum, where its actions have been studied by Laidlaw, I913.

\section{PREPARATIONS-HYDRASTIS}

Hydrastis, U.S.P.; Hydrast. Rhiz., B.P. (Golden Seal).--The dried rhizome and roots of Hydrastis canadensis, yielding not less than 2.5 per cent. of hydrastin, U.S.P. Dose, 2 gm., 30 gr., U.S.P.

Hydrastis was employed by the Indians, mainly as a pigment, but also as medicine. It was introduced by the eclectics about the end of the eighteenth century. The plant is becoming rare, but is easily cultivated (Henkel and Klugh, I 908).

Ext. Hydrast., U.S.P. - A powdered extract with ro per cent. of hydrastin, $\mathrm{I} \mathrm{Gm}$. representing $4 \mathrm{Gm}$. of drug. Dose, $0.5 \mathrm{Gm}$., 8 gr., U.S.P.

* Fldext. Hydrastis, U.S.P.; Ext. Hydrast. Liq., B.P.-2 per cent. of hydrastin; $2 / 3$ alcohol, $1 / 10$ glycerin, U.S.P. Dose, 2 c.c., 30 minims, U.S.P.; 0.3 to I c.c., 5 to 15 minims, B.P. U.S.P.

Glycer. I ydrast., U.S.P.-About 1.25 per cent. of hydrastin. Dose, 2 c.c., 30 minims,

Tr. Hydrast., U.S.P.-2o per cent. Dose, 4 c.c., I dram, U.S.P.

Tr. Hydrast., B.P.- ro per cent. Dose, 2 to 4 c.c., $1 / 2$ to I dram, B.P.

Indrastina, U.S.P., $\mathrm{C}_{21} \mathrm{H}_{21} \mathrm{NO}_{6}$.-White prisms. Almost insol. in water. Dose, to mg., $1 / 6$ gr., U.S.P.

IIydrastin. IIydrochl., U.S.P., $\mathrm{C}_{21} \mathrm{H}_{21} \mathrm{NO}_{6} . \mathrm{HCl}$.-White powder. Very sol, in water or alc. Dose, 10 mg., 1/6 gr., U.S.P. Maximum dose, 0.I Gm., I $1 / 2$ gr.

Hydrastinin. IIydrochl., U.S.P., $\mathrm{C}_{11} \mathrm{H}_{11} \mathrm{NO}_{2} \cdot \mathrm{HCl}$.-Light yellowish needles or powder. Very sol. in water or alc. Its aqueous solution, especially when highly diluted, shows a blue efflorescence. Dose, $30 \mathrm{mg}$., $1 / 2 \mathrm{gr}$., U.S.P. 


\section{COTARNIN}

Cotarnin Salts (marketed under the names of "Stypticin" and "Styptol") are used to arrest bleeding, especially in menstrual disorders.

The clinical reports, on which their employment rests, are often superficial and sometimes contradictory. The mechanism of the reputed actions has not been cleared by experimental investigations. This obscurity necessitates a more extensive discussion than the importance of the subject would otherwise justify.

Cotarnin was introduced by M. Freund, I 893 , on the basis of its chemical similarity with the more expensive hydrastinin. This chemical relation was discussed under hydrastis.

Clinical Uses.-Cotarnin has been employed systemically in various forms of uterine hemorrhage; in other internal hemorrhages; and locally in superficial bleeding.

Uterine Hemorrhage.-The most favorable results are reported in menstrual menorrhagia, endometritis and congestive hemorrhage resulting from malposition (Gottschalk, r895 and r899; Katz, r903; Vieth, 1903; Boldt, r904; K. Abel, r905; Aarons, r907; Offergeld, I915). Cotarnin is said to shorten the duration of the periods and to lengthen the intervals (Elischer, r904). The response is prompt if adequate doses are injected (Offergeld, I9r 5). Some observers claim that it also acts as a "uterine sedative," meaning that it lessens the pain of dysmenorrhea (Gottschalk; Katz; K. Abel; Jacoby, 1906; Lock yer, 1907); but the claim rests mainly on loose statements of patients, and others have failed to observe any anodyne effect (Handfield-Jones, 1907; Offergeld, I915). All agree that it does not provoke uterine pain or contraction. It is conceded to be ineffective against postpartum hemorrhage, and probably also against bleeding resulting from abortion, tumors and other gross anatomic lesions.

Other Internal Hemorrhage.- Positive resul ts have been reported in bronchial hemorrhage and tubercular hemoptysis (Lavialle and Ruyssen, r898; Hussa, I912); in hematuria of urethritis, catheter injuries, etc. (Kaufmann, r905); in intestinal hemorrhage, etc.

These statements, however, are not sufficiently substantiated, in view of the difficulty of predicting the spontaneous cessation of such hemorrhages, and the history of other "internal hemostatics." The claims are flatly contradicted by at least one of the warmest advocates of Cotarnin (Vieth).

Local Hemorrhage.- The topical application of cotarnin in substance or strong solution (ro to so per cent.) to superficial wounds apparently produces a local vasoconstriction with prompt arrest of bleeding. This has been used successfully especially in tooth extraction (Munk, r899; Marcus; Sigrist, r913), epistaxis; and in nasal and genitourinary operations. Its effects would be more lasting than those of epinephrin, and devoid of the coagulant action of the astringents. It is said to be effective even in hemophilia (Hulisch, r899). This local effect of strong solutions is quite distinct from the systemic administration; for Marcus states specifically that the latter was ineffective, either prophylactically or remedially, in tooth extraction.

The local styptic effect has also been confirmed experimentally (K. Abel, rgo5). Ammonium phthalate likewise acts as a local styptic, and this may contribute to the local effects of Styptol; although it can play no part in the systemic action (Offergeld, rors).

Experiments on Mechanism of Action.-The numerous investigations so far made have yielded little of practical value. They show that cotarnin has a low toxicity. Very large doses produce some lassitude, ataxia, general depression, and finally death by paralysis of respiration. The narcotic action is not pronounced. Intravenous injections produce brief, inconstant and usually slight changes in the circulation, which can play no part in the therapeutic action. The contractions of the excised uterus are stimulated, but the clinical data show that this does not occur with therapeutic doses. Perfusion of excised organs have failed to show any peripheral or central effects on the systemic vessels. The local styptic action is obtained with high concentrations, and can not therefore apply to the systemic administration. There remains the possibility of a specific constrictor effect confined to the uterine vessels. The only direct evidence for this consists in the histologic observations of la Torre, which require confirmation on living tissue.

General Symptoms.-K. Mohr, I905, found that o.r to $0.2 \mathrm{Gm}$. per kilogram, in rabbits, dogs or cats produced only some lassitude and drowsiness. Larger doses had a pronounced sedative action, especially in cats and dogs. Ataxia was also pronounced. With fatal doses, these effects were followed by some excitement, then progressive 
paralysis till death. Toward the end, the respiration became very slow and shallow, sometimes interrupted by asphyxial convulsions.

Laidlaw, I910, on guinea pigs, describes indefinite depression of the central nervous system and death by paralysis of the respiratory center. Relatively small doses slowed the respiration, without change of depth.

Fatal Dose.-This ranges from about 0.I Gm. per kilogram for guinea pigs (Laidlaw) to 0.3 to $0.4 \mathrm{Gm}$. per kilogram for rabbits, and even higher for dogs and cats (Mohr).

Uterine Contractions.-Kehrer, I907, found that cotarnin stimulates the uterus, both excised and in situ, pregnant or non-pregnant; increasing the contractions and tonus. This has been confirmed for the excised organ by all subsequent workers (Laidlaw, I9I0; Ruebsamen and Kligermann, I9I2; Lieb, I9I4). The results in situ are contradictory. Kehrer's stimulant effects were obtained with cats. With a pregnant rabbit, Mohr, I905, obtained no stimulation. Laidlaw, I910, also found the results on living rabbits negative, whilst the excised organ responded. La Torre, I9 2 , observed no histologic evidence of contraction, such as is seen after ergot and hydrastinin. Chiappe and Ravano found that the pregnancy of guinea pigs was not affected by toxic doses; and that even fatal doses produced no abnormalities.

The clinical data are in harmony with these negative results. All reporters agree that cotarnin never provokes uterine pains, even in late pregnancy; that it does not hasten the involution of the uterus; and that it has no influence on postpartum hemorrhage.

Mohr claimed that cotarnin depresses the excitability of the uterus to hypogastric stimulation; but his conclusion appears to be based on a single very imperfect experiment with enormous doses, and must therefore be rejected.

It appears, from all this, that cotarnin stimulates the excised uterus, on local contact; but that, with systemic administration, the uterus does not respond, at least in rabbits; and certainly not with therapeutic doses in the human.

Other Smooth Muscles. - Offergeld quotes contraction of the intestines and bladder; but Laidlaw found the bladder irresponsive.

Systemic Circulation.-Intravenous injection produces variable changes in the blood pressure; sometimes a fall; or a momentary fall followed by a slight rise (Kehrer, 1907; Laidlaw, I910; Lieb, I9I4). If the vagi were divided, the rise is more prominent (Pilcher and Sollmann, I915). The vasomotor center is not affected directly, but may be stimulated by the slowed respiration (Pilcher and Sollmann); and this probably accounts for the rise. The fall of pressure appears to be cardiac; for the Langendorff heart is slowed and weakened (Laidlaw). Kehrer and Heinz also consider the fall cardiac.

This diminished output of the heart has been credited with being the cause of the hemostatic effect; but this is most improbable. There is no evidence that it occurs with therapeutic doses; and even with the experimental doses, it is too slight and especially too evanescent, to influence a persistent hemorrhage.

Direct Action on Systemic Arterioles. - All the earlier investigators (Falk, Marfori and Ronsse) affirm that cotarnin has no effect on the arterioles, with systemic administration. This has been confirmed by the negative results of perfusion experiments (Laidlaw, leg). The positive results of direct contact with strong solutions have no bearing on this. Cotarnin does not produce the gangrene changes characteristic of ergot (Mohr).

Coagulation of Blood.-This is not affected, whether the cotarnin is added directly to the blood, or injected into the animal (Laidlaw; Marfori).

Uterine Vessels. - Many of the clinical authors explain the action of cotarnin by a special action on the uterine vessels, a stimulation of their vasomotor nerves, etc. (K. Abel, 1905). This appears to be pure assumption. More recently, however, La Torre, I9I2, reports histologic evidence of contraction of the larger uterine vessels in animals treated with cotarnin. Since postmortem appearances usually give a very imperfect picture of the changes during life, this evidence requires confirmation by physiologic methods.

Dosage and Administration. - The cotarnin salts may be used by mouth or hypodermically, the latter being much more efficient. The intramuscular dose is 0.25 to $0.5 \mathrm{Gm}$., as Io or 20 per cent. solution, repeated daily as needed. The injections are not irritant. By mouth, the dosage is from 0.05 to $0 . x \mathrm{Gm}$. four to five times daily. Smaller doses are inefficient. In menorrhagia, it may be started several days before the expected period.

Side Actions. - None have been reported from oral administration or from hypodermic injections of $0.5 \mathrm{Gm}$. Offergeld found some lassitude with $0.7 \mathrm{Gm}$. and a single case of temporary collapse with $0.65 \mathrm{Gm}$. by intramuscular injection. 


\section{PREPARATIONS-COTARNIN}

Cotarnine Hydrochloricum (Cotarn. Hydrochl.), U.S.P. (Stypticin), $\mathrm{C}_{12} \mathrm{H}_{13} \mathrm{O}_{6}$ N.HCl. -Odorless, bitter, yellow, crystalline powder. Very sol. in water or alc., yielding yellow solutions. Dose, o.06 Gm., I gr., U.S.P.

\section{CANNABIS}

The tops of the hemp plant are somewhat of a pharmacologic curiosity. They contain resinous constituents (cannabinol) which produce a peculiar psychic exaltation. It is employed as an intoxicant in the Orient. Therapeutically, it is used empirically (Extract $0.01 \mathrm{Gm} ., 1 / 6 \mathrm{gr}$.) as an adjuvant to other drugs in nervous headaches, and as a coloring agent for corn remedies. The drug is of uncertain activity, and has no rational indication.

Intoxicant Action in Man.-The oriental use of Cannabis ("Hashish," Bhang, Charas, etc.) antedates history. It was introduced in Western medicine about the middle of the nineteenth century. When used as an intoxicant, the tops or resins are made into a confection or smoked with tobacco. Effective doses (2 gr. of solid extract of the Indian or American drug) produce after about an hour a state of partial inebriety and confusion, with happy and humorous tendencies. Ideas pass rapidly but disconnectedly, so that the time seems very long. The subject often has hallucinations of double personality. The exaltation, which seems to be much more marked in orientals, is succeeded by some irritability, dozing, and complete recovery in five or six hours. The effects are modified by individual disposition and surroundings (Hamilton, Lescohier and Perkins, I9I3). It is stated that the drug is not fatal even in very large doses, but experience on dogs certainly shows that it presents some danger. The habit to which it gives rise shows less effect upon the alimentary canal and less marasmus than does morphin, but more often psychic alterations, dulness, or mania. The clinical side actions are described by Seifert, Nebenwirk., I915, p. I49.

Effects on Animals. - Dogs (Dixon, I899) also show a decided narcotic effect. After a preliminary ataxia, excitement, and nausea, the animals usually fall into a deep and prolonged sleep, during which the sensation of pain is much diminished, whilst the reflexes persist. Some animals show acute mania; a fatal ending is not rare from doses which are ineffective in other animals, the same preparation being used. Considerable idiosyncrasy exists, and the action is fairly independent of the dose. The effects can not be obtained by hypodermic administration (on account of the non-absorption of the resin).

Rabbits seem absolutely insusceptible to the narcotic action of cannabis.

\section{PREPARATIONS $\longrightarrow$ CANNABIS}

Cannabis, U.S.P.; Cannabis Indica, B.P.; Cannabis, Indian Hemp (Hashish, Bhang, Guaza, Gangja, Charas, Momeka, etc.).-Dose, 0.06 Gm., I gr., U.S.P.

The dried flowering or fruiting tops of the pistillate plant of Cannabis sativa. The B.P. requires that it be grown in India; the U.S.P. permits any origin, but provides that the activity must be confirmed by testing its narcotic effects on dogs (Houghton and Hamilton, I907; U.S.P. IX). This is necessary since different samples vary greatly, and are liable to deteriorate rapidly. If the Indian drug is preferred, Cannabis Indica may be specified; if the American, Cannabis Americana. American grown Cannabis produces identical effects (Houghton and Hamilton, 1907 and 1908), but is generally only half as active (Eckler and Miller, 1912 ). 'The preparations, however, are brought to the same degree of activity.

The active ingredient is a resin, cannabinol (Fraenkel, 1903), $\mathrm{C}_{21} \mathrm{H}_{30} \mathrm{O}_{2}$, which presents the appearance of a thick reddish yellow oil, soluble in petroleum ether, etc. (not identical with the commercial "cannabinol"). This changes by oxidation to an inactive black pitch. The change accounts for the deterioration which the drug and its preparations undergo in keeping. The deterioration can be prevented by excluding the air (Marshall, 1909). Cannabis also contains a volatile oil (terpenes), paraffin, pitch, etc., which are 
not concerned in its action. There is no specific alkaloid, but the extracts may give alkaloid reaction from the formation of cholin and triamethylamin. These are also not connected with the action. The fresh extract has a beautiful green color (if prepared without excessive heat), due to chlorophyl. The active principle is completely extracted by alcohol. but is insoluble in water.

The pretended Isolated Principles on the market under various names are generally quite inactive (S. A. Matthews, 1908).

*Ext. Cannabis, U.S.P.; Ext. Cannab. Ind., B.P.-A pilular alcoholic extract. Dose, Io mg., $1 / 6$ gr., U.S.P.; I6 to $60 \mathrm{mg}$., $1 / 4$ to $1 \mathrm{gr.,} \mathrm{B.P.} \mathrm{Maximum} \mathrm{dose,} \mathrm{0.I} \mathrm{Gm.,}$ $1 / 2 \mathrm{gr}$.

Fldext. Cannab., U.S.P.-Dose, 0.05 c.c., I minim, U.S.P.

Tr. Cannab., U.S.P.- ro per cent. of drug. Dose, 0.5 c.c., 8 minims, U.S.P. Maximum dose, 1.25 c.c., 20 minims. B.P.

Tr. Cannab. Ind., B.P. -5 per cent. of extract. Dose, 0.3 to $x$ c.c., 5 to 15 minims,

\section{LACTUCARIUM-DRIED LETTUCE JUICE}

This was introduced by Dr. Coxe of Philadelphia in I 799 as a sedative. Lettuce had some reputation as a hypnotic in ancient times, but it is probably undeserved. Kelterborn took $12 \mathrm{Gm}$. of Lactucarium without any effects. No active principle has been determined.

\section{PREPARATIONS-LACTUCARIUM}

Lactucarium, U.S.P.-The dried milk juice of wild lettuce, Lactuca virosa. Dose, I Gm., I5 gr., U.S.P.

Syr. Lactucar., U.S.P. -5 per cent. Dose, Io c.c., $21 / 2$ drams, U.S.P.

Tr. Lachucar., U.S.P.-5o per cent. Dose, 2 c.c., 30 minims, U.S.P.

\section{LUPULIN AND HOPS}

These act as bitters, and have some popular but exaggerated reputation as hypnotics. Hops are the dried pistillate flowers of Humulus Lupulus. The active principles are contained in small glands, which can be separated as a powder, and constitute the Lupulin.

Two acids ( $\alpha$ - and $\beta$-lupulinic acids) are present. They stimulate the respiratory and vagus centers; this is followed by depression. They also depress the cardiac muscle. Lupulin contains a further, unknown, constituent which is insoluble in water and a strong cardiac poison. Power, Tutin and Rogerson, r913, also found a trace of alkaloid. All these, however, are not active when administered by the stomach, and are therefore not concerned in the action of the drug.

\section{PREPARATIONS-HOPS}

Humulus (Humul.), U.S.P.; Hops.-The dried strobiles of Humulus Lupulus. Dose, 2 Gm., $30 \mathrm{gr} .$, U.S.P.

Lupulin.-Dose, $0.5 \mathrm{Gm} ., 8 \mathrm{gr}$.

\section{LOCO DISEASE}

Horses, cattle and sheep on the Western stock ranches are subject to this peculiar disease, which bears some resemblance to drug habits. The symptoms consist in motor incoördination, forced movements, misjudgment of distance, stupidity, apparently hallucinations. In the chronic form there is emaciation. Death is preceded by coma and convulsions.

The cause of these conditions is obscure. It is generally attributed to feeding on certain leguminous plants ("loco weeds"-especially Astragalus). C. D. Marsh, 1909, claims to have reproduced the condition (neryous symptoms, etc., with exudative meningitis, anemia, etc.) experimentally by very long-continued feeding with these plants; but H. T. Marshall, I9r4, considers the results inconclusive, and believes that there is no distinct loco disease; and that the name covers a variety of conditions-malnutrition, parasitism, etc. - unconnected with the loco weed. 


\section{COCAIN GROUP; LOCAL ANESTHETICS}

Actions and Uses. - The alkaloid cocain is important mainly as a local anesthetic, paralyzing the sensory nerve fibrils on direct application. With dilute solutions, its action may be confined to these; but higher concentrations paralyze all other nerve fibers, and, indeed, they kill all protoplasm. Dilute solutions also produce local vasoconstriction and dilate the pupil through stimulation of the sympathetic pupillo-dilator mechanism, both central and peripheral.

When cocain is absorbed in sufficient quantity, it produces complex systemic actions, involving stimulation and paralysis of various parts of the central nervous system. These are mainly of toxicologic and scientific interest. Its continued use leads to the formation of a habit, resembling morphinism.

Analogous local anesthesias are produced by a variety of synthetic bases, which may be used as substitutes for cocain.

Historical. - Cocain is derived from the leaves of Erythroxylon Coca, a tree indigenous to Peru, Chili and Bolivia. The leaves were chewed from time immemorial by the natives to relieve hunger and fatigue, and to produce a psychic stimulation, somewhat after the manner of caffein. It is now cultivated in other tropical countries.

In Europe, trials with the leaves gave disappointing results. The discoverers of cocain (Woehler; Niemann and Lossen, I860) incidentally described its anesthetic action on the tongue, but without realizing its significance. As late as I880, a British medical commission reported on the drug as merely a poor substitute for caffein (further details, J. U. Lloyd, I9II, "History of Vegetable Drugs," and I9I3, J. Am. Pharm. Assoc., 2: г 242). In the same year, Anrep subjected cocain to a thorough pharmacologic investigation; and in I884, the Vienna oculist Koller introduced it as a practical local anesthetic. Its importance was then at once recognized.

Peripheral Nerves.-Local Anesthesia.-Local contact with cocain paralyzes all forms of nervous tissue, without preceding stimulation. The susceptibility of the various nerve fibers presents marked and characteristic quantitative differences. Sensory fibers are especially easily attacked; and by using appropriate dilutions, the paralysis can be confined strictly to them. With sufficient concentration, the paralysis is as complete as if the nerve fibers had been severed with a knife. If the alkaloid is washed away, or absorbed, the nerve recovers its functions promptly and completely (but very strong solutions may produce neuritis and permanent paralysis).

Since these effects are strictly local it follows that the cocain must be applied in such a way that an effective concentration will reach the nerve supply of the part which it is desired to affect. This may be accomplished, according to circumstances, by painting a solution on mucous membranes (from which it is very readily absorbed); or by injecting it into or under the skin (infiltration and subcutaneous anesthesia); or around or into the nerve trunk (peri- or intraneural anesthesia); or around the spinal nerve roots (subdural or spinal anesthesia). The intact skin is practically impermeable to cocain.

Duration of Local Action.-The onset and duration of the anesthesia varies with the concentration of the solution, and the method of its application. Roughly speaking, it starts in a few minutes and lasts perhaps ten to thirty minutes, with a single application.

Local Anemia. - As the cocain is absorbed and thus removed from the site of application, its local action ceases and its systemic and toxic effects 
start. Since cocain is rapidly destroyed in the body, the systemic toxicity increases with the rapidity of absorption. It is therefore desirable and of ten necessary to delay the absorption. This may be done by restricting the local circulation. Cocain itself tends to do this by producing a local vasoconstriction - an advantage which is not shared by its substitutes. This vasoconstriction should be reinforced by the addition of epinephrin. In suitable situations, the circulation may be slowed by bandages, or arrested by temporarily clamping the arterial blood supply. The anemia also favors anesthesia directly; ligation of a large artery in itself produces anesthesia of the nervous end structures.

When a large quantity of dilute cocain solution is injected under pressure, as in the infiltration anesthesia, the cocain action is reinforced by anemia, pressure, and edema.

The Selective Action of Cocain on Motor and Sensory Fiber.-When a $1 / 2$ to 2 per cent. solution of cocain is injected into a mixed nerve, or into the subdural canal, the selective action is very marked, so that there is complete anesthesia, without motor impairment. If the contact is proionged, by stopping the circulation, or if 'stronger solutions are employed, the motor fibers also become paralyzed, so that the difference is merely quantitative. A similar difference in the susceptibility of motor and sensory structures exists also for the alcohol group, aconitin, phenol, and hydrocyanic acid; and even for the centrally acting narcotin, ether, etc. It is therefore a characteriscic of nervous tissue rather than of cocain.

Cocain is Less Effective in Inflamed Tissues. - This may be explained by the hypere mia, resulting in more rapid absorption.

Peripheral Paralysis of Special Sense Nerves.-Cocain abolishes not only the sensation of pain, but other special sensations, if it is suitably applied. Here also there is some selection. In the skin, it paralyzes the sense of pain and touch, but has little or no effect on temperature sensation. In the nose, it abolishes the olfactory sense. On the tongue, it destroys the taste for bitter substances, but has less effect on sweet and sour taste, and none on salty taste.

Selective Action on Other Nerves. - When cocain is applied to the appropriate nerves, it is found that the centrifugal vagus fibers are paralyzed before the centripetal; vasoconstrictor fibers before vasodilator; bronchial constrictors before the dilators, etc. (Dixon, 1904).

Visceral Pain.-The abdominal viscera are sensitive to pain in normal animals; but the sensation is abolished by cocain, even when small doses ( $0.7 \mathrm{mg}$. per kilogram) are injected at a distance; for instance, into the pectoral muscles (Kast and Meltzer, r906I909). This special anesthesia lasts about half an hour. The insensitiveness of viscera in surgical operations under cocain anesthesia is thus explained. The effect is probably central, for the animals are greatly quieted, although general sensation is not impaired.

Motor Endings.- - Large doses of cocain paralyze these in frogs. This curare action would not be seen in intact mammals.

Smooth Muscle.-This is generally first stimulated, then depressed, regardless of innervation (constituting a fundamental distinction from epinephrin). Cardiac muscle and the salivary glands are not stimulated (Kuroda, I915).

Site of Action.- Cocain can paralyze the nerve fibers anywhere in their course, wherever it is brought into contact with them. When it is applied to mucous membranes or hypodermically, it doubtless selects the portions peripheral to the main trunks, the thinner sheath of the terminal fibrils facilitating its penetration. It is therefore unnecessary to assume a selective action on the histological sensory endings.

Penetration. - It is quite possible that its selective action on different nerve fibers may also depend, in part, on differences in penetration. On this account, the free cocain base is more effective than its salts, in equal concentration, since the base is more soluble in the lipoids of the nerve sheath. Free cocain, however, is not sufficiently soluble in water for practical use. O. Gros, I910, therefore advises an extemporaneous mixture of molecular quantities of novocain hydrochlorid and sodium bicarbonate.

Nature of Action.- Of this, little is known, except that it is purely functional. No structural changes are discoverable in the nerve fibers.

Synergisms. - The mixture of cocain with its related bases (hovocain, etc.), or with antipyrin or nitrites, gives simple summation of the anesthetic effect (A. Schmid, I913); mixtures with potassium salts give potentiation by 75 per cent.; potassium also potentiates novocain, but not stovain or alypin. Kochmann, 1914, therefore advises to make 
cocain solutions in a medium of 0.5 per cent. potas. sulphate and 0.9 per cent. $\mathrm{NaCl}$. Epinephrin also increases the anesthetic power of cocain.(H. Braun) by direct synergism (Esch). Conversely, cocain increases the epinephrin constriction, even in very small concentrations (Fischel, I915). The Tropacocain, however, deprives epinephrin of its synergistic and constrictor action (Kochmann, I9 I4).

Local Vasoconstrictor Action.-The application of cocain to mucous membranes, particularly the inflamed conjunctiva, produces distinct local vasoconstriction, with blanching, astringent sensation, and actual contraction of vascular formations, such as polypi. The cocain substitutes do not possess this action; many of them dilate the vessels.

The analysis of the constrictor action of cocain has given contradictory results. Since the action is local, it must be peripheral. However, direct perfusion of the vessels is either ineffective or produces dilation, never constriction; nor does cocain counteract the dilator effect of nitrites (Kuroda, I9I5). The constriction can not be explained by anesthesia, since other local anesthetics do not constrict.

General Toxicity to Protoplasm.-Cocain is a typical, if rather weak, protoplasmic poison, paralyzing all sorts of cells, without producing any gross chemical changes. It is toxic to the lower forms of animal life (infusoria, etc.); but has little effect on bacteria, or on ferments.

\section{ACTIONS OF COCAIN ON THE EYE}

When cocain is applied to the conjunctiva, it produces local anesthesia (which may not extend to the iris); local anemia (which extends to the iris, but not to the retina); and submaximal dilation of the pupil by peripheral sympathetic stimulation. The mydriasis occurs also on systemic administration. The accommodation is impaired, but the light reflex is preserved; there is some exophthalmos. The intraocular pressure is usually lowered, but may be increased. The mydriasis and its associated phenomena are not produced by most of the cocain substitutes.

Injury.-Cloudiness and even ulcers of the cornea sometimes follow the application of cocain; they are explainable by the drying, and the irritation of dust and other foreign matters which are not perceived on account of the anesthesia and the abolition of the winking reflex. The protoplasmic toxicity may have a part in their production.

The Cocain Mydriasis.-This differs in important respects from the atropin mydriasis; with atropin, the dilation is greater; the light reflex is lost; the intraocular tension is always raised; exophthalmos is absent. The pupil does not react readily to pilocarpin or muscarin, whilst these constrict the cocain pupil easily.

These differences point to a different mode of action. In effect, atropin paralyzes the oculomotor (constrictor) endings, whilst cocain stimulates the sympathetic (dilator) mechanism; for when the sympathetic fibers have degenerated (eight days after extirpation of the superior cervical ganglion) ordinary doses of cocain cease to be effective (Limbourg, 1892; Schultz, 1899). Dilation may still occur, however, with large doses or under special conditions. Simple (postganglionic) division of the sympathetic does not immediately abolish the cocain reaction; the stimulation must therefore involve the endings; but since the dilation is not as strong, it would seem that, ordinarily, the pupillo-dilator center is also concerned (Schultz, 1898).

Under ordinary conditions, the cocain pupil continues to respond to oculomotor stimulation, showing that the constrictor mechanism is not paralyzed. Prolonged application of a 5 per cent. solution, however, also paralyzes the oculomotor endings. In birds' eyes cocain produces no dilatation, whereas in frogs it is very marked.

Cycloplegia. - This begins in five or ten minutes. The maximum is reached in thirty minutes and lasts ten to fifteen minutes; the normal is restored in one and onequarter to two hours. The accommodation is merely disturbed by a drop of 6 per cent. solution; high concentrations paralyze it completely. The mydriasis develops somewhat more slowlyland is more lasting (Horovitz, I912). C. Wood, 1893, advises the addition of cocain to homatropin, to paralyze accommodation.

Intraocular Pressure. - This may be unchanged, or raised or lowered (Tourriere, 1913). The reduction is probably due to the local vasoconstriction, which would tend to lessen the lymph flow into the chambers. This is partly counteracted by the mydriasis, which leads to obstruction of the lymph channels by the relaxed ciliary 
muscles (see "Atropin"). This obstruction may predominate, and the pressure may thus be increased, especially in glaucoma (Myashita, I9r3).

Eucain and alypin act similarly. Holocain is said to have provoked acute glaucoma (Gjessing, 1915). Tropacocain and acoin are probably inactive (Bollet and Curtil, I91 I).

Retinal Vessels.- These are strongly constricted (Hirschfelder, I915). This may be responsible for the occasional occurrence of optic atrophy (Harnack, I912).

\section{SYSTEMIC ACTIONS}

'These are rather variable and complex, depending largely upon the dose. Whilst all structures are first stimulated and then paralyzed, the susceptibility to the poison is not uniform. Indeed, some portions of the nervous system show only stimulation, death occurring before the paralysis of these structures is reached.

Stimulation of Higher Functions.-The first effect is a well-marked stimulation of the higher parts of the brain (caffein action). This is shown in animals by increased movement (sometimes "circus movements"). In man there is some psychic stimulation and wakefulness. A greater endurance against fatigue and hunger is also noticed.

How far this may be due to a stimulation after the manner of caffein, or to a narcosis' after the manner of morphin, is impossible to state. It is not at all unlikely that both play a part. In regard to the sensation of hunger, it is also probable that local anesthetization of the stomach aids in the effect.

The resistance to fatigue can be demonstrated with the ergograph.

Another evidence of the stimulating action of cocain is furnished by the fact that animals to which it has been administered are more difficult to put and to keep under chloroform or other anesthetic. Mosso, I887, and Airila, I9r 3 , find that ro to $40 \mathrm{mg}$. of cocain, hypodermically, wakens dogs or rabbits from deep chloral sleep. The stimulation is greatest with excitable individuals, and may seriously interfere with operations.

Incoördination, Narcosis, Convulsions.-This stage of stimulation may be very short or even absent. With somewhat larger doses it may be succeeded by depression, first of the coördinating functions. The movements lose their purposive type and become choreic. There is then a general narcosis after the manner of morphin.

This is followed by convulsions. If the paralysis is rapid, the convulsive stage may not appear.

The Seat of the Convulsions.-This has not been exactly determined. They, like the other effects, are probably descending, and the different convulsive centers may be affected in succession. In some stages at least they seem to reside exclusively in the hind brain. In dogs, they appear to be confined to the cerebral cortex, for Feinberg and Blumenthal, 1887 , claim that they do not occur after ablation, nor in new-born animals, in which the cortical motor centers are not yet excitable. Rabbits deprived of the hemispheres do not show the clonic convulsions or respiratory effects; but they do exhibit tonic convulsions and "running movements." Their resistance to toxic doses is increased (Morita, rgr 5 ).

Central Phenomena in Frogs. - These show at first symptoms of stimulation by increase of the voluntary movements and exaggeration of the reflexes, sometimes leading to convulsions. This is followed by paralysis of the whole central nervous system.

Spinal Cord.- In frogs from which the brain has been removed, cocain causes at first an increase of the reflexes, then convulsions, and finally total paralysis. In intact animals this effect is obscured by the action on the higher centers of the nervous system.

The results of applying cocain directly to the cord will be considered later.

Respiration.-This is at first accelerated. During the spasms it is irregular. The volume then diminishes. It may assume the CheyneStokes type. Respiratory paralysis is the usual cause of death. This is also the first center to fail when the cocain is applied locally to the fourth, 
ventricle. Excised bronchial muscle is somewhat dilated (Trendelenburg, I9I2).

Emesis.-The vomiting which frequently occurs in cocain poisoning is perhaps due to the medullary stimulation, but its mechanism has not been fully investigated.

Circulation. - The effects of cocain on the general circulation are partly central, partly peripheral. They vary according to the dose, as shown diagrammatically in Fig. 9. They are also influenced by individual susceptibility.

The typical effects are as follows:

Very small doses diminish the pulse rate, by stimulation of the vagus center (Vulpian, I884). There is a quick rise of blood pressure from stimu-

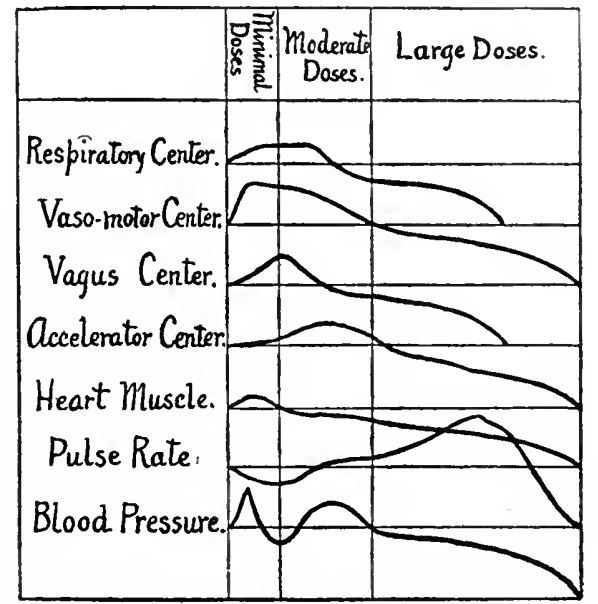

F1G. 9.-Diagram of the Actions of Cocain on Respiration and Circulation. (A rise of the curve signifies an increase or stimulation; a fall, the reverse.)

lation of the vasomotor center; this is followed by a temporary fall due to the slowing.

Moderate doses quicken the pulse, mainly by central and peripheral depression of the vagus (v. Anrep, I880) with some stimulation of the accelerator center (Mosso, I887). The pressure rises (v. Anrep) from stimulation of the vasomotor center (Berthold, I885), aided by the faster heart rate. The vasomotor factor is predominant, for the rise is practically absent if the cord is cut.

Large doses cause a great fall of pressure and slow and weak pulse ( $v$. Anrep), from the depression of the medullary centers (collapse) and of the cardiac muscle.

Actions on Heart Rate.-This demands some further discussion: The slowing from small doses does not occur if the vagi have been cut, so that it is of central origin. The quickening from moderate doses is also less marked if the vagi have been divided, so that it is probably due in part to a depression of the vagus center. The vagus ganglia are also depressed, for electrical stimulation of the vagus trunk is only partly successful (v. Anrep, I880). In the frog these ganglia can be paralyzed completely by the local application of cocain, but in the intact mammal the paralysis is not complete. Some quickening occurs, however, even when the vagi have been divided, but none is seen if the accelerators have also been cut. The final slowing is due to a direct paralysis of the muscle, for it is accompanied by weakening of the contraction, and it occurs after atropin, and in the excised heart. 
The Excised (Langendorff) Mammalian Heart.-This has been investigated by Ifedbom, 1899; Kochmann and Dacls, I908; and Prus, I913. Low concentrations slow and strengthen the contractions; higher concentrations cause slowing and weakening; fatal concentrations give diastolic arrest. Kuroda, 1915, observed no stimulation in excised hearts of mammals or frogs.

The excised frog's heart is stimulated by small concentrations (Mosso, 189o), and depressed by higl concentrations. The terrapin's heart is similarly affected (H. G. Beyer, I885).

The effects on blood pressure have been described mainly by Ott, I874; v. Anrep, I880; Vulpian; Mosso, 1890, and Reichert, I891. The comparative effects of cocain and novocain have been investigated on dogs by Kamenzove, IgII.

Splanchnic Circulation; Influence on "Shock."-The intravenous injection of cocain has a very pronounced effect on the splanchnic circulation. The intestines appear unusually palc. Handling of the viscera and other measures of "shock" which cause a splanchnic dilation and consequent fall of blood pressure in normal animals, have less or no effect after cocain. Burning and stimulation of the sciatic, which cause a rise of . pressure normally, are also ineffective (Crile, I901). Féré, I906, on the other hand, claims that local anesthesia (by cocain, its substitutes, or freezing) does not abolish the depressant reflexes (decrease of muscular work, of sharpness of vision, etc.); these occur from procedures which would ordinarily be painful, even when the sensation of pain is eliminated.

Cerebral Circulation.-This is increased by cocain, and still more by novocain (Frankfurther and Hirschfeld, 1910). The vessels of the retina and pia mater are constricted (Hirschfelder, I915).

The urine flow is generally diminished, but sometimes increased, depending mainly on the vasomotor effect.

Temperature.-Cocain causes a rise of temperature, due essentially to increased heat production, and this largely from muscular excitement (see chapter on "Heat Regulation").

Effect on Metabolism.-In rabbits, large doses of cocain cause rapid loss of weight. The quantity of urine, its specific gravity, and particularly the urea, are diminished, whilst the incompletely oxidized (extractive) nitrogen is increased. During recovery, the quantity of urine returns promptly to normal or above; the disturbances of the nitrogen metabolism persist for some time (Maestro, I904).

The effects of repeated injection on dogs have been investigated by Underhill and Black, I912: Doses of ro mg. (per kilogram) produce no change in fat or nitrogen metabolism; I5 mg. gave a slight impairment of fat utilization, and considerable decrease of body weight; $20 \mathrm{mg}$. markedly decreased the nitrogen and fat utilization. The nitrogen balance may become negative. Lactic acid excretion was markedly increased in wellfed dogs, but not in starvation-the increase being probably due to the muscular activity. The ammonia output bore little relation to the lactic acid.

Blood-sugar.-This is not changed (cats, $\mathrm{I}_{5}$ to $35 \mathrm{mg}$. per kilogram), except as a result of excitement (Schaer, 1915).

Fate of Cocain.-This is partly destroyed in the organism, but mainly excreted unchanged in the urine (contrary to the results of Wiechowski, I90I).

Rifatwachdini, I9I3, recovered 42 to 85 per cent. from the urine of rabbits after acute poisoning, and all in chronic poisoning. None was present as ecgonin; but if ecgonin itself was injected, 25 per cent. was recovered from the urine. Cocain left for several hours in a ligatured limb was not destroyed.

Destruction of Cocain in Vitro; Sterilization.-Long-continued boiling decomposes cocain into benzoyl-ecgonin and methyl alcohol. It was therefore believed that solutions could not be sterilized by boiling. In fact, however, the decomposition on boiling half an hour, is insignificant (Merck, r907; Holbrook, I91 2); and even longer boiling only decreases the activity, for the decomposition products are merely inactive and not toxic.

Benzoyl Ecgonin.-This was found by Stockman, I886, to resemble caffein in its central and muscular action. It had no effect on sensory nerves or the pupil. 


\section{ACUTE COCAIN POISONING; ETIOLOGY}

This is of special importance, since cocain poisoning is not a very uncommon occurrence in the therapeutic employment of the drug. Very large amounts are sometimes used in a most careless manner for local anesthesia, and since the absorption is fairly rapid, serious and even fatal results may follow. The susceptibility varies enormously, due partly to the uncertainty of the absorption; partly to the rapidity of destruction; and partly to the varying intensity of the effects. In some cases 2 drops of a 4 per cent. solution $(=0.005 \mathrm{gm}$.) in the conjunctival sac caused serious collapse, whilst very much larger doses produced no effect in other cases. The ordinary fatal dose is considered by Kunkel to be about I.2 gm. (I 8 gr.); but death has been reported from as little as $0.08 \mathrm{gm}$. ( $1 / 1 / 4$ gr.). The toxicity is greatly lessened by the addition of epinephrin.

Symptoms with Slow Absorption.-The symptoms vary according to the rapidity of absorption. ${ }^{1}$ If this occurs relatively slowly, there is confusion, laughter, vertigo, motor excitement; quickened pulse and palpitation; irregular respiration; pallor, chill, with sweat and rise of internal temperature; dilated pupils and exophthalmos; nausea, vomiting and abdominal pain; great anxiety; disturbance of cutaneous sensation (worms under skin, etc.), finally delirium, dyspnea and Cheyne-Stokes respiration, convulsions, unconsciousness; and death by collapse and asphyxia. Recovery may be followed by more lasting psychic depression and melancholia.

With rapid absorption of large doses (especially from mucous membranes) severe symptoms may develop without warning: fainting, extreme pallor, brief convulsions, and death, sometimes in a few minutes.

Treatment.-If the drug has been taken by mouth, one should promptly 'resort to evacuation and chemic antidotes. If the symptoms have developed, the head should be lowered, and the collapse treated by sinapism to chest and abdomen; and by caffein, or aromatic ammonia. The convulsions should be allayed with chloroform, chloral, and artificial respiration. Amyl Nitrite has been recommended.

\section{CHRONIC COCAIN POISONING}

This bears considerable resemblance to the morphin habit. It is unfortunately on the increase, especially amongst negroes, prostitutes, and the criminal classes (see under "Morphin Habit").

The immediate effects consist in a sense of elation and increased mental and physical vigor. The excitement may rise to delusions and mania. These stimulant effects are succeeded by depression, tremors, with pale face, and sunken and unsteady eyes (Owens, I9r2).

The chronic effects are similar to those of the morphin habit, with more severe psychic disturbance-insomnia, hallucinations, apathy, melancholia, suicidal mania. (For Cocain Psychoses, see Vallon and Bessiere, I9I4.) The pupils are inconstant. A very considerable tolerance is acquired, so that the daily hypodermic consumption often reaches $2.5 \mathrm{Gm}$., sometimes even ro $\mathrm{Gm}$. (Grode, r9r2). Animals do not become tolerant (Grode, I9 3 ). Chouppe, I889, claims that morphinists are relatively tolerant to cocain. Sudden withdrawal leads to abstinence symptoms very similar to those of morphin. 
With cocain snuffing, where smaller amounts are used in intermittent debauches, the chronic effects, the craving and the abstinence symptoms are proportionately less marked. Such patients show a characteristic ulceration of the nasal fossæ (Owens; also present in heroin snuffing, Phillips).

The treatment of cocain habit coincides exactly with that of the morphin habit (which see); it is said to be more difficult to cure.

\section{USE OF COCAIN AS A LOCAL ANESTHETIC IN MINOR OPERATIONS}

The direct application of cocain abolishes the sensation of pain more or less completely in about five minutes, the anesthesia lasting for about half an hour; the duration increases with the concentration. Solutions of 2 to 5 per cent., or the trace of the powder on the point of a probe, are applied to the surface of mucous membranes, or injected under the skin. Stronger solutions are dangerous and offer no advantage. Application to the surface of the skin is useless, since the drug is not absorbed by this channel. Mucous surfaces, on the other hand, absorb it readily. It is desirable to limit the local circulation, e.g., by a constricting rubber band. The vasoconstrictor action of the cocain may be heightened by the addition of epinephrin ( 1 : 10000 to I : I00000).

In eye, nose, and larynx operations the abolition of reflexes and the diminution of hemorrhage are very useful side actions.

In connection with its action on the eye, it must be remembered that it does not ordinarily anesthetize the iris when applied to the cornea. However, the iris may also be anesthetized by applying one or two drops of a ro per cent. solution to the cornea every two or three minutes, for ten minutes; keeping the conjunctiva moist by frequent winking, to prevent epithelial sloughing (Gifford).

\section{CATARRH, HAY FEVER AND ASTHMA}

Cocain is also useful in the treatment of diseases which appear to be due to heightened irritability of the peripheral endings, such as hay fever and asthma. In these the cocainization of the nasal mucous membrane is often specific. The astringent action renders it effective in acute coryza.

One per cent. cocain ointment has been recommended in herpes zoster; it is said not only to relieve the pain, but to put a stop to the disease.

Cocain is also useful locally in hemorrhoids, producing contraction and diminishing pain. It has been taken by the stomach to prevent vomiting and dyspeptic pain.

The danger of the formation of the habit interferes with its use in all these conditions; indeed many cases of cocain habit were started by proprietary catarrh "cures."

\section{USE OF COCAIN IN LARGER OPERATIONS}

This demands the most economical utilization of the alkaloid, to avoid systemic poisoning, by securing the maximum effect from the smallest dose.

The infiltration method (Schleich, I892) involves the injection of very dilute solutions (1/100 to $1 / 5$ per cent.) under considerable pressure, directly into the place where the next incision is to be made. It is especially useful for anesthetizing the skin for extensive incisions. It is rather too painful for inflamed tissues. 
The object is to produce a local edema, which supports the action of the cocain by causing local anemia and by compressing the nerve filaments. The injections can be made with an antitoxin syringe with a long needle, by means of which the solution is first injected into (not under) the epidermis, so as to raise a blister. The needle being left in place and gradually pushed deeper, the entire field of operation is saturated with the solution, 30 to 500 c.c. being used. The usual solution (Sol. II) contains $1 / 10$ per cent. of cocain and $1 / 5$ per cent. of sodium chlorid. In inflamed tissue, the cocain may be doubled (Sol. I); or if the field is extensive, it may be reduced to 1/100 per cent. (Sol. III). Bevan, I9I5, speaks highly of novocain infiltration. He employs up to 100 or 200 c.c. of a solution with 0.25 to 0.5 per cent. of novocain, to which $1 / 2$ to I c.c. of epinephrin, I: 1000, has been added.

In the paraneural method the solution, of a strength of $1 / 2$ to 2 per cent., is injected in the neighborhood of the nerve trunk. The results are uncertain.

Intraneural Method (Nerve Blocking).-In this, a $1 / 2$ to 2 per cent. solution is injected directly into the nerve trunk.

If the injection is made quickly and directly into the nerve tissue, the procedure is quite painful. A few drops should first be injected under the nerve sheath. When these have caused a local anesthesia, the needle should be pushed deeper and more of the solution injected, until the anesthesia is complete. In this way the pain is relatively slight. Anesthesia requires fifteen to twenty minutes, and is not always completely successful.

The combination of all the above methods is perhaps most useful. The skin and superficial muscles are anesthetized by infiltration. The deeper structures are exposed, and the smaller nerves are treated by the paraneural, the larger by the intraneural methods. In this way, surprisingly small quantities of cocain suffice $(8 \mathrm{mg}$. for amputation of shoulder joint!).

Influence on Shock.-The complete blocking of nerve impulses obtained in this way tends to prevent surgical shock. No method of local anesthesia can, however, prevent the psychic shock and pain, the nervous dread of the patient, the removal of which is one of the most valuable features of general anesthesia; but it may at least belessened by morphin (0.015 $\mathrm{Gm}$. hypodermically) half an hour before the operation. It may at times be justifiable to operate without the knowledge of the patient, which is quite feasible by the use of cocain (Crile).

Spinal Anesthesia.-The subdural injection of cocain, or other local anesthetics, anesthetizes the sensory nerve roots at their emergence from the spinal cord. This abolishes sensation in their entire peripheral distribution for about one and one-half hours (one-half to two hours), without loss of consciousness or motor functions. The method was demonstrated by Corning of New York in 1885 , and introduced into practice by Bier, I 899 , but it was temporarily abandoned, and did not come into vogue until about r904. The injection is performed by lumbar puncture, withdrawing a little cerebro-spinal fluid, and introducing $1 / 2$ to I c.c. of 2 per cent. cocain solution; or more commonly, corresponding quantities of its substitutes: Tropocain, 5 per cent., novocain (not above $0.15 \mathrm{Gm}$.); stovain (not above o.I Gm.; beginning with one-half or two-thirds of these doses). Epinephrin and strychnin may be added.

The anesthesia extends to the level of the nerve roots reached by the cocain, the aim being to confine it to the lower half of the body. If it should extend to the fourth ventricle, it will result in paralysis of the respiration. Alarming symptoms and fatalities from this cause are not infrequent (Seifert, Nebenwirk., I9I5, p. I05), and the method is more dangerous even than chloroform. The fatality is probably over $\mathrm{I}: 500$ (Bevan). It has also been fatal to the fetus in utero (Jung, I914). Minor. accidents are not uncommon. Cord injuries are sometimes produced; severe headache may last for days or weeks. Further, the technic is 
difficult, disagreeable to the patient, and the anesthesia is often unsuccessful. Spinal anesthesia should therefore not be employed (Hohmeier and Koenig, I910), unless inhalation anesthesia is directly contraindicated (Anesthesia Commission, Journ. Amer. Med. Assoc., r908). It is especially useful in pulmonary disease, arteriosclerosis, bladder and rectal cases, impending uremia, and diabetics; emergencies after a full meal. It is not justifiable to use it for operations above the costal margin (F. L. Richardson, I9I3). Bevan, I9I5, can see no justification for spinal anesthesia under any conditions.

Phenomena of Spinal Anesthesia.-These occur in the following order: Loss of kneejerk; of plantar and cremasteric reflex; ascending analgesia; later, ascending loss of motor power. The upper limit is usually quite abrupt. Subjectively, there is an ascending feeling of warmth, swelling and heaviness. Occasional toxic effects are pallor, nausea, vomiting, sweating, feeble pulse, relaxation of sphincters, dyspnea. The most frequent after-effects are headache and insomnia.

Fall of Blood Pressure.-This is a very common phenomenon of spinal anesthesia. Smith and Porter, I915, found that it is due to paralysis of the splanchnic fibers in the cord, not in the medulla. Epinephrin with tropacocain or novocain caused even a greater fall. Measures to restore the pressure were only temporarily successful. As the effects of the anesthetic began to wear off, sciatic stimulation produced a fall, instead of the normal rise.

To prevent the spreading of the solution E. Erhardt, r908 and r9r2, has proposed to increase its viscidity by gum arabic. Bulk of solution (dilution) favors spreading (Smith and Porter, I9I 5 ).

The combined action of stovain and strychnin on the cord has been investigated by Aron and Rothman, I909; and Simon, 1915. The two drugs are not antagonistic; and strychnin does not render stovain any safer.

Intravenous Injection of Cocain.- The action of cocain is sufficiently selective so that a marked, though incomplete general analgesia, without disturbance of consciousness or motor functions, can be produced by intravenous (Ritter, rg09; Harrison, r9I r) or intra-arterial (Ransohoff, I 909) injection. However, these methods are both unsatisfactory and dangerous (Bevan, 1915).

\section{USE OF COCAIN AS A CEREBRAL STIMULANT}

Cocain has been employed to combat fatigue, and as a general tonic. It has no advantages and many disadvantages as compared with other stimulants, especially cafiein and strychnin. The danger of habit formation should suffice to condemn it. That the harm has not been greater may be attributed to the unreliability of the coca preparations which are generally employed.

\section{COCAIN SUBSTITUTES}

A large number of substances have been introduced as substitutes for cocain. Some of these (Novocain, Stovain, Eucain) may have a slight advantage by combining an equal anesthetic activity with somewhat lower toxicity and much greater stability; but practically these advantages are not very serious and scarcely justify the introduction of these numerous products. Moreover, Stovain and Eucain produce far more irritation than cocain, and stovain may permanently injure the nerves. Orthoform or Anesthesin, on account of their limited solubility, are only slightly toxic and produce a slow and prolonged anesthesia when applied in substance to superficial or gastric ulcers, etc.

The substitutes have in general the same incompatibilities as cocain. They resemble it more or less in their chemic structure.

Structure of Cocain.-Cocain is the methyl-benzoyl ester of ecgonin, a base closely related to tropin, the corresponding base of atropin, both being pyrrolidin derivatives. 
The relation is shown by the structural formulas:

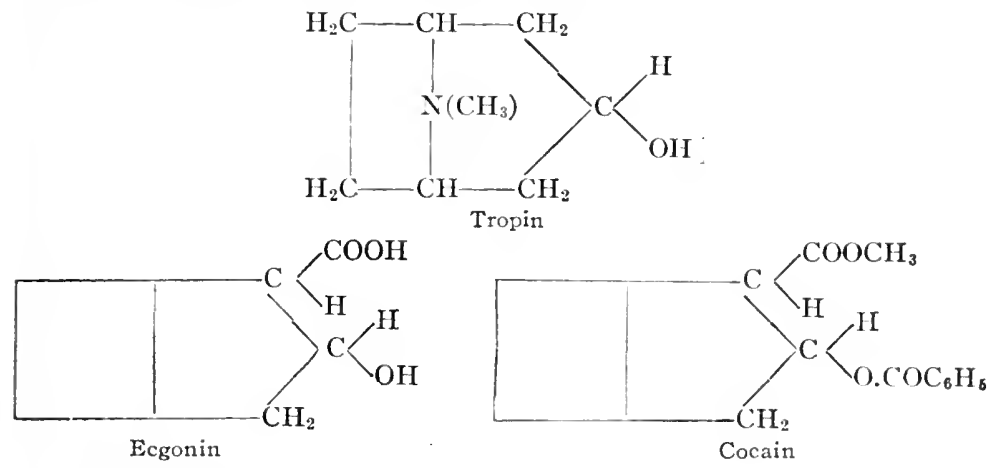

Relation of Structure to Actions.-Ecgonin itself produces only the least important of the actions of cocain, viz., the hepatic degeneration. The other actions are only developed by the entrance of both radicals. The methyl may be replaced by any other alkyl radical, without changing the actions of cocain. The benzoyl radical, however, can not be replaced by other aromatic acids (possibly with a few exceptions) nor by fatty acids without great impairment of the local anesthetic action. Indeed, the presence of the benzoyl group gives the anesthetic properties to other alkaloids, and may be considered as the hook by which the molecule attaches itself to the protoplasm of the sensory cell. It is present in most of the cocain substitutes. The required conditions for. the development of a local anesthetic action seem therefore to be: a base with a structure analogous to ecgonin, containing a benzoyl and an alkyl radicle in certain relations.

Advantages and Disadvantages of Cocain.-The valuable features of cacain consist in its strong, prompt and comparatively certain action; it is the best studied and the most familiar product of its class; the vasoconstriction lessens hemorrhage (but may be objectionable in snaring polypi); the dilation of the pupil may be a convenience in ophthalmic operations; it is practically free from local irritation; it acts through intact mucous membranes. Some of these features, viz., vasoconstrictor and mydriatic actions may be given to the substitutes by the addition of epinephrin or atropin.

The Disadvantages of Cocain.-These consist in the instability of its solutions; in the occasional collapse; and in the habit formation.

The Relative Advantages of the Substitutes.- These have been the subject of much discussion-which indicates that they are not very striking. The comparative experiments of.Le Brocq, 1909, and of Dreyfuss, 1910, favor novocain; but it is only one-third as strong. Stovain, holocain and alypin cause some local injury. Quinin is weaker than cocain, but much more lasting. Extensive compilations of the anesthetic efficiency and toxicity of the cocain substitutes are given by Closson, I9r4, and especially by Kochmann, rgr4. The latter found the "value factor" anesthetic power $\div$ toxicity greatest for a combination of novocain and potas. sulphate.

\section{PREPARATIONS-COCANN}

Coca Folia.-The dried leaves of Erythroxylum Coca and E. truxillense, shrubs indigenous to Peru and Bolivia, and cultivated in tropical countries. Ceylon coca contains mainly cocain; Java coca contains only other ecogonin derivatives (Jong, Igri).

Cocaina, U.S.P., B.P., $\mathrm{C}_{17} \mathrm{H}_{21} \mathrm{NO}_{4 .}$-Colorless prisms or crystalline powder, of slightly bitter taste followed by anesthesia. Slightly sol. in water $(\mathrm{r}: 600)$; freely sol. in alc. ( $x: 6.5)$, chlorof., eth.; sol. in oils ( $x: r_{2}$ olive oil). Used especially in ointments. Dose, $15 \mathrm{mg} ., 1 / 4 \mathrm{gr}$.

*Cocain. Hydrochl., U.S.P.; B.P., $\mathrm{C}_{17} \mathrm{H}_{21} \mathrm{NO}_{4} \cdot \mathrm{HCl}$.-Colorless, transparent prisms; or fiaky, lustrous leaflets, or white, crystalline powder. Very sol. in water ( $1: 0.4)$; freely sol. in alc. ( $\mathrm{r}: 3.2)$; sol. in glyc.; practically insol. in oils. Incompatible with alkalies, sodium borate (not boric acid), silver nitrate and calomel. Solutions decompose on keeping (a 15 per cent. solution in Dilute Alcohol is said to be permanent; Wintersteiner, Ig06). Contrary to older statements, they are not materially decom- 
posed by boiling. Dose, 15 mg., 1/4 gr., U.S.P.; 6 to 16 mg., 1/10 to 1/4 gr., B.P. Maximum dose, $50 \mathrm{mg} ., 3 / 4 \mathrm{gr}$.

Inject. Cocain. II yp. -5 per cent. Dose, 0.3 to o.6 c.c., 5 to ro minims, B.P.

Lam. Cocain., 13.P. $-1.3 \mathrm{mg} ., 1 / 50 \mathrm{gr}$.

Ung. Cocain., B.P. -4 per cent.

Troch. Kramer. el Cocain., B.P.-6o mg. of krameria; $3 \mathrm{mg}$. of cocain.

\section{PREPARATIONS-COCAIN SUBSTITUTES}

Bctaencaine IIydrochloridum (Eucain. Hydrochl.), U.S.P. (trimethyl-benzoxypiperidin), $\mathrm{C}_{15} \mathrm{H}_{21} \mathrm{NO}_{2} \cdot \mathrm{HCl}$. - White, crystalline powder; odorless. Sol. in water ( $\mathrm{r}: 30$ and alc. $(1: 3.5)$. Dosage, locally, one to two times that of cocain. The anesthetic effect is perhaps slightly slower and weaker, and the irritation considerably greater, than with the same concentration of cocain. The pupils are not dilated; the tissues are rendered hyperemic. The toxicity is four-tenths that of cocain. Toxic doses cause convulsions, central vagus stimulation, and direct cardiac depression, with fall of blood pressure (Seifert, Nebenwirk., I915, p. 64; case and bibliography, Orr, I916).

Bcnzamin. Lact., B.P. (Beta-eucain Lactate), $\mathrm{C}_{15} \mathrm{~N}_{21} \mathrm{NO}_{2} \cdot \mathrm{C}_{3} \mathrm{H}_{6} \mathrm{O}_{3}$. - Freely sol. in water $(\mathrm{I}: 5)$ and alc. $(\mathrm{r}: 8)$. Dose, 8 to $30 \mathrm{mg} ., 1 / 8$ to $1 / 2 \mathrm{gr} .$, B.P.

Orthoform-New, N.N.R. (amino-oxybenzoate of methyl). White tasteless powder, scarcely soluble in water (the hydrochlorid is soluble). Not absorbed from skin or mucosæ. It is said to have repeatedly caused local inflammatory reaction (Seifert, Nebenwirk., I 9I 5, p. 79; McCleave, I9I4). Dose, 0.5 to I Gm., 8 to 15 gr.; locally as dusting powder or oinment.

Anesthesin, N.N.R. (ethyl-aminobenzoate) is very similar (side actions, Seifert, Nebenwirk., I9I5, p. 44).

*Novocain, N.N.R. (aminobenzoyl-diethyl-amino-ethanol hydrochlorid).-Colorless needles, sol. in equal weight of water, or 30 parts alcohol. The nitrate is compatible with silver salts. Dosage, locally, one to three times that of cocain. Anesthetic and irritant action perhaps slightly less. No effect on pupil.

Novocain is probably safer than cocain. Occasionally, however, toxic effects occur even with small doses (0.01 to $0.13 \mathrm{Gm}$.); while in other cases up to $3 \mathrm{Gm}$. have been used safely. There is no definite ratio between the toxicity of novocain and cocain, since this depends enormously on the rate with which they enter the circulation; especially in the case of novocain. This is due to the great rapidity with which novocain leaves the circuation, being fixed and probably destroyed in the liver. Epinephrin diminishes the toxicity, even by intravenous injection, probably by cardiac stimulation (Hatcher and Eggleston, I916).

The details of the clinical toxic effects are described by Seifert, Nebenwirk., I 915 , p. 78; lumbar, p. 107. Renal irritation is not uncommon after local use of 5 to ro per cent; but disappears in a few days (Morian, 1915).

Stovain, N.N.R. (benzoyl-ethyldimethyl-aminopropanol hydrochlorid).-Colorless scales, extremely soluble in water, or in 5 parts alcohol. Dosage, locally, as for cocain. Anesthetic action slightly stronger, irritant action (hyperemia) much greater, toxicity two-thirds that of cocain. No effect on pupil.

It has been used especially for spinal anesthesia, in combination with strychnin (T. Jonnescu, 1909). The immediate danger may be somewhat less than with cocain, although it is not negligible. The local injury is much greater. Spiller and Leopold, I9I0, and Consoli, I9I3, have observed degenerations in the nerve roots and cells, after subdural injection of stovain. The immediate paralysis is more extensive than with cocain, involving muscular movements (Santesson, 1906) and spinal reflexes (Adler, 1906). On the circulation, it causes in dogs, quickened pulse and fall of blood pressure (Kamenzove, $19 \mathrm{II}$ ). The kidneys are also rather irritated, especially if albuminuria was previously present (Schwartz, 1907; Chiaie, I913). The liver is not affected (side actions, Seifert, Nebenwirk., I9r5, p. 91; lumbar, p. Iog). A comparative study of the local anesthetic actions of stovain and its homologues has been made by Symes and Veley, rgri.

Alypin, N.N.R., which resembles stovain in structure, is used especially in the urethra. It is more irritant than cocain. Several cases of sudden collapse and asphyxia, after its injection, have been reported (Ritter, I91 2; Lichtenstein, 1914; lumbar, Seifert, Nebenwirk., I9I5, p. IO4).

Tropacocain Hydrochlorid, N.N.R., (benzoyl-pseudo-tropein); occurs in Javanese coca, but the commercial is synthetic. Colorless needles, readily soluble in water. Dosage, locally, one or two times that of cocain.. Anesthetic action about the same; irritation greater; toxicity one-half that of cocain; less mydriatic; more stable (side 
actions, Seifert, Nebenwirk., I915, p. 97; lumbar, p. Ir 2). It is used especially for spinal anesthesia (Stanley, I9I6).

Holocain, N.N.R., (phenetidyl-acetphenetidin hydrochlorid).-Colorless crystals, soluble in 50 parts water. Dosage, for eye, I per cent. Anesthetic action about as strong as cocain, but more rapid (anesthesia in half a minute); more toxic, but staterl not to injure or anesthetize cornea. No effect on vessels has been reported (Gjessing, 1915 ).

Yohimbin, $\mathrm{C}_{22} \mathrm{H}_{28} \mathrm{~N}_{2} \mathrm{O}_{3} \cdot \mathrm{HCl}$. - In alkaloid isolated by Spiegel, I896, from the bark of Yohimbehe tree (Apocynaceæ; West Africa). Fourneau and Page, I9r4, consider it identical with quebrachin. Local anesthetic, about same strength but more lasting than cocain. Less toxic, but much more irritant. Mydriasis, without loss of accommodation. Vessels dilated. Intestines excised or in silu, and bladder stimulated by small, depressed by large, doses (A. Loewy and Rosenberg, r914). Solutions are unstable.

When it is given by the mouth or hypodermically in moderate doses, it produces a general vasodilation in the skin, mucous membranes, and particularly in the sexual organs. In consequence of the latter, and perhaps by a direct action of the spinal centers, it produces erection. It does not seem to stimulate the production of spermatozoa or sexual desire. The effect is not produced by therapeutic doses in man ( $5 \mathrm{mg}$. or $1 / 12 \mathrm{gr}$.), although it has been promoted as an aphrodisiac. The reports of clinical improvement after several weeks are probably explainable by suggestion. Yohimbin has also been proposed for lowering abnormally high blood pressure; but Lawrence, I9 I2, found that it may produce a further rise, with dangerous symptoms.

Larger doses produce psychic excitement, cerebral congestion, vertigo, gastric disturbance; and in rabbits, marked injury of the renal epithelium (Huebner, Igr2). Toxic doses produce general stimulation and subsequent paralysis of the nervous centers, particularly in the medulla; and complex effects on the cardiac muscle (F. Mueller, I907; J. Tait, I9IO). The coronary vessels are not affected (Rabe, I9I2); or they are dilated (F. Meyer, I9I2). Death occurs by respiratory paralysis (for literature, see Strubell, rgo6).

\section{DRUGS ACTING PERIPHERALLY ON THE AUTONOMIC-SYM- PATHETIC SYSTEM}

A variety of poisons act peripherally upon the several portions of the involuntary or "vegetative" nervous system, in a more or less specific manner. It appears advisable to premise their individual consideration by a more general exposition, so as to obtain an intelligent grasp upon their various relations, and an insight into what is known of their action. Much light has been thrown upon these in recent years, and our conceptions have been largely revolutionized.

Anatomic and Physiologic Relations.-The "autonomic" system (in the wider sense in which the term was introduced by Langley) has certain physiologic and anatomic peculiarities. Physiologically, it includes all the functions which are subject to involuntary nervous control-the smooth and cardiac muscles and the glands. Their relation to the central nervous system is peculiar, in that they are capable of functionating automatically, and by local neuro-muscular reflexes, when all connections with the cerebrospinal axis are severed (hence "Autonomic"). On the other hand, their functions are normally regulated, augmented or inhibited, by central tonic impulses of varying intensity.

The chief anatomic peculiarity of these autonomic nerves is, that they all pass through a relay ganglionic cell, situated outside of the central nervous axis. These cells are partly scattered but mostly collected into the "sympathetic ganglia." A given nerve fiber has but a single relay cell, although it may pass through a number of ganglia.

\section{THE PARASYMPATHETIC AND SYMPATHETIC SYSTEMS}

Physiologically, and especially pharmacologically, as also in its embryologic development, the autonomic system as a whole has two fairly sharply defined divisions, which correspond, anatomically, on the one hand, to the nerves having their origin in the roots of the midbrain, medulla oblongata, and sacral cord (the cranio-sacral or parasympa- 
thetic system, also often called the autonomic system in the restricted sense); and on the other hand, those arising from the dorsal and down to the fourth or fifth lumbar nerve roots (the sympathetic system). The relay cells of the sympathetic system are situated in the sympathetic ganglion-chain (including the ccrvical and splanchnic ganglia); those of the cranio-sacral system are scattered more peripherally, near their end organs.

Distribution and Function.- The two divisions are antagonistic, a given organ usually receiving its innervation from both divisions, one being augmentor, the other inhibitory.

The parasympathetic division, for instance, supplies the vagus (cardio-inhibitory, intestinal and bronchial augmentor), oculomotor (pupillo-constrictor), chorda tympani, etc.

The sympathetic division supplies the vasoconstrictors, pupillo-dilator, intestinal inhibitory, cardiac accelerator, etc.

The innervation of the sweat glands occupies a peculiar position; anatomically, it is purely sympathetic, but it reacts pharmacologically as if it were purely parasympathetic. 'The uterus is also exceptional; its sympathetic innervation responding to both classes of poisons. It must be remembered, however, that the anatomic evidence of the origin of the autonomic nerves is in some respects inconclusive.

Pharmacologic Reactions: Nicotin.-Pharmacologically, the most prominent characteristic of the whole autonomic system is its reaction to nicotin: this alkaloid paralyzes all the ganglionic synapses of both the parasympathetic and the sympathetic nerves (often after a fleeting primary stimulation). This paralysis is so specific, that it has proved of great value for locating the site of these synapses, especially in the hands of Langley. Nicotin, whether it is applied locally or systemically, abolishes the response to stimulation of the preganglionic fibers, whereas the postganglionic fibers respond normally.

Deprivation of calcium increases the excitability to the augmentor impulses of both divisions (see "Calcium Ions"); most of the other drugs which affect the autonomic system restrict their action exclusively to one or the other of the two divisions: Epinephrin, ergotoxin and cocain act exclusively on the sympathetic divisions; atropin, pilocarpin, muscarin, cholin, and physostigmin act exclusively on the parasympathetic division. Still others act only on certain parts of one or of both divisions.

Anisotonic Solutions.-Hypertonic solutions (Ringer's) inhibit the tone of smooth muscle, increase the response to inhibitory drugs, and lessen the response to stimulants. Hypotonic solutions have the opposite effect (Dale, I9I3).

\section{PHARMACOLOGY OF THE SYMPATHETIC DIVISION}

Epinephrin, the alkaloid of the suprarenal gland, produces a pure peripheral stimulation, restricted exclusively to the sympathetic system, but involving the entire distribution of this system (with the exception of the sweat glands). In other words, the effects of typical doses of epinephrin on a given organ correspond precisely to the stimulation of its sympathetic nerve supply (Levandowsky, I899; Langley, I90r; Elliott, I905). This has been aptly described by the term "sympathomimetic action" (Barger and Dale, I910). The action of epinephrin is therefore augmentory or inhibitory, according to whether the sympathetic impulses are augmentory or inhibitory, as follows:

The Principal Sympathetic Reactions (e.g., the Effects of Epinephrin). - The augmentory reactions consist in constriction of most of the arteries and consequent rise of blood pressure; stimulation of the cardiac accelerator; dilation of the pupil; contraction of the plain muscle of the orbit; flow of sympathetic saliva and of tears, not readily abolished by atropin; erection of hair, etc. 
Inhibitory Reactions concern the tone and rhythm of the contractions of the stomach, intestine, biliary and urinary bladder (cat), and of the virgin uterus of the cat.

Peculiarities of Species.-Certain of the sympathetic reactions vary with the species of animals, being augmentory in some, and inhibitory in others (urinary bladder, anal sphincter). The sympathetic reaction of the uterus varies according to its physiological state; in the cat, the virgin uterus is inhibited, whilst the pregnant uterus is stimulated.

All these peculiarities are reproduced exactly in the reaction to epinephrin, thus furnishing the most striking illustrations of its sympathomimetic action.

Other Drugs with Sympathomimetic Action.- Similar actions are possessed by a large number of primary and secondary amins, the simplest being the primary fatty amins, amylamin, etc.). There are quantitative differences between these. The relative effect on the different sympathetic distributions does not go parallel; nor is there any parallelism between augmentory or inhibitory innervations. The intensity and speci. ficity increase as the amins approach the structure of epinephrin (Barger and Dale, 1910).

Cocain increases the peripheral excitability of the sympathetic system without being directly stimulant.

Peripheral Sympathetic Paralysis : Ergotoxin.-The active alkaloid of ergot (in large doses) destroys the response of the augmentory sympathetic impulses, but does not affect the inhibitory sympathetic impulses (Dale, Ig06). It therefore prevents, $f . i$., the vasoconstrictor action of epinephrin, but does not abolish the inhibitory action of epinephrin on the intestine or uterus.

\section{PHARMACOLOGY OF THE PARASYMPATHETIC CRANIO-SACRAL AND RESTRICTED AUTONOMIC DIVISION}

The action of drugs on this system is confined mainly to its augmentory functions (with the important exception of the vagus).. These, in their entire peripheral distribution, are paralyzed by atropin; and stimulated by pilocarpin, muscarin, cholin and anaphylotoxin. Physostigmin heightens their irritability, perhaps without being directly stimulant. (These statements must be restricted to typical doses.)

Atropin is therefore antagonistic to all the other drugs named. If a sacro-cranial function is exposed simultaneously to the depressant action of atropin and to the stimulant action of one of the other drugs named, the end result depends on the nature and quantity of the drug, and upon other conditions. Physostigmin, for instance, is a more powerful antagonist of atropin than is pilocarpin; however, larger doses of the stimulants tend to become depressant, so that the atropin action tends to appear predominant. toxin:

The centers of the parasympathetic system are stimulated by picro-

Principal Results of Parasympathetic Stimulation (Pilocarpin, etc.).These consist in: slowing of the heart; constriction of the pupil; bronchial spasm; contraction of intestine and uterus; increase of practically all centrally innervated secretions (sweat, saliva, mucus, gastric, pancreatic, etc; not urine, bile, milk, nor the internal secretions). These are the main effects of pilocarpin, muscarin and physostigmin.

Craniosacral depression (atropin) produces precisely the opposite results.

\section{DRUGS WITH PARTIAL AUTONOMIC REACTIONS}

The preceding presentation is somewhat schematic, and includes only the typical effects, as they are currently conceived. When the doses and 
other conditions are varied, many of these drugs depart more or less from the schema, as will be discussed later. This indicates that the physiological divisions, which are the basis of the pharmacologic divisions, are not as absolute as the schema would imply. In accordance with this, there are a number of drugs (for instance, apocodein and pituitary extract; the latter, however, is probably a mixture), which do not fit anywhere in the schema, but whose actions involve isolated functions of one or both divisions.

\section{LOCATION OF ACTION}

The precise site of action of the autonomic drugs is not yet satisfactorily decided. It is currently assumed that they act on the "myoneural junction," or upon a "receptive substance" (Langley, r905), which is believed to be interpolated between the anatomical nerve-endings and the reactive substance of the cell (the contractile elements of the muscle, or the secretory elements of the gland cells). It would perhaps be equally or more exact to say that they act upon that property of the cells which excites or initiates their peculiar functions.

The essential evidence for this view is the fact that these poisons preserve their action after severance and anatomic degeneration of the postganglionic nerve fibers, showing that their action is peripheral to the nervous structure. On the other hand, the specificity of the reactions, and their correspondence with the physiologic innervation, indicates that the action is not directly on the contractile or secretory substance. If epinephrin, for instance, acted directly upon the contractile elements of smooth muscle, it would be difficult to understand why it should throw them into contraction in some situations, relax them in others, or in others again, leave them entirely unaffected. Anatomically, these muscles are all alike; their physiologic properties are also identical, they respond alike to muscular poisons, such as barium; the only difference between them lies in their innervation. This difference of innervation is reproduced exactly by epinephrin; and since the degeneration-experiments show that the poison does not act upon the nervous elements themselves, it follows that joth the nervous impulse and epinephrin must act upon the same substance or property of the muscle.

It is idle to speculate upon the nature of this receptive substance, or receptive property, until we know somewhat more of the real nature of the nervous impulse, of stimulation, and of excitability. The term "myo-neural junction" has the advantage that it does not commit one to any unproven hypothesis.

Anatomic Localization of Depressant Effects. - The anatomic methods of localization are quite simple, since the problem concerns a purely efferent path. The nerve would be stimulated in various sites in its course; the paralysis would be located just central to the highest point which gave a positive response.

For instance, after nicotin, stimulation of the preganglionic fibers of the vagus (say in the neck), does not slow the heart; stimulation of the postganglionic fibers (in the sinus) causes slowing. Nicotin has therefore paralyzed the ganglia.

After atropin, stimulation of the sinus is also ineffective; the paralysis must therefore be peripheral; the heart-muscle is evidently not paralyzed, since it is beating vigorously. The paralysis must therefore be just central to the muscle, i.e., in the endings or myo-neural junction. It must be 
remembered, however, that this does not exclude the possibility of an additional more centrally located paralysis; for instance, so far as this evidence goes, atropin might have paralyzed the vagus ganglia in addition to the "myo-neural" junction. Further, the precaution must be taken to employ a sufficient dose of the poison, for a slight paralysis may of ten be overcome by a powerful stimulation.

Anatomic Localization of Stimulant Effects.- This would also be very simple, involving the division of the nerve (or its equivalent, the application of the drug to excised organs or structures). For instance, pilocarpin and muscarin contract the pupil of the excised eye, and physostigmin increases its excitability to stimulation of the postganglionic oculomotor. Their action is therefore postganglionic. It is again possible that the drug may stimulate an additional, more centrally located structure.

To distinguish between anatomic endings, myo-neural junction, and muscle substance, the nerve is allowed to degenerate, as just described.

Pilocarpin, f.i., remains effective after the anatomic endings are degenerated, and when therefore electric stimulation of the nerve is ineffective. However, if a still longer time elapses after the division of the nerve, pilocarpin also becomes ineffective. It is therefore generally assumed that the myo-neural junction also degenerates, but more slowly than the anatomic endings. However, it is also conceivable that pilocarpin acts on the anatomic nerve-endings, and that the degenerating endings remain excitable to pilocarpin longer than they do to electric stimulation. This proof of myoneural action is therefore not conclusive.

The evidence from degeneration may also mislead if the drug, instead of stimulating, raises the excitability of the reacting cell. In this case, the drug would produce no visible effect after degeneration of the endings, for this would exclude extraneous stimulation, and the increased excitability would therefore not be apparent. This increased excitability of the myo-neural junction is supposed to be the action of physostigmin on the oculomotor mechanism.

Localization by Antagonistic Action.-The anatomical localization, although simple in theory, is often technically difficult, and it is beset with the limitations which have just been pointed out. It has therefore been attempted to supplement and extend it by pharmacologic evidence, based on antagonistic actions. The matter was at first conceived as very simple, namely as strictly analogous to anatomic localization; a drug paralysis being considered as equivalent to anatomic division or excision; and a drug stimulation as the equivalent of electric stimulation. 'The action of a few drugs having been localized (with more or less probability) by anatomic means, it was thought that the actions of others could be localized by simply "matching" them against these standards. In localizing action by antagonism, a stimulant poison was paired with a depressant poison of known action, $f . i$, atropin. If stimulation resulted (as with physostigmin) it was concluded that the action must be peripheral to that of the atropin; if there was no stimulation (as with pilocarpin), it was assumed that it must be central to that of atropin.

Unfortunately, there has been a good deal of cumulative evidence (well summarized by Magnus, I908) that matters are not so simple, and that localization by antagonism has limitations so serious that it can be utilized only in relatively few cases. The difficulty is that the pharmacologic stimulation and paralysis are not strictly equivalent to the anatomic. In pharmacologic antagonism, the tissue is still in the body and exposed to both poisons; and the resultant effect is rarely, if ever, the simple algebraic sum of their separate actions. One drug may modify the effects of another qualitatively, as well as quantitatively. Moreover, a drug 
paralysis is not an irreversible process, as is anatomic division or excision. The numerous instances of mutual antagonism prove that a function which has been completely abolished (paralyzed) by one drug may be revived by another. For instance atropin can abolish the physostigmin miosis; but physostigmin can also remove an atropin mydriasis- the result depending upon the relative dosage.

Localization by antagonism is therefore conclusive only if the antagonism is not mutual and if it involves absolutely distinct structures; for instance, atropin abolishes the action of cevadin (veratrin) on the cardiac vagus; whilst cevadin does not interfere with the atropin action. Evidently, the cevadin action must be central to that of atropin.

Mutual Antagonism.-When two drugs are mutually antagonistic, it is natural to assume that they act on the same structure. This is doubtless of ten the case; but there are instances in which this simple assumption offers difficulties; for in some cases, the point of attack of a drug appears to be different in the presence of an antagonist. This is practically important; for it is probable that disease may produce similar modifications. The following instances are adduced by Magnus, I908:

Physostigmin and Curare on Striped Voluntary Muscle.-Physostigmin produces fibrillary twitchings of the voluntary muscles. These persist after section of the motor nerve (sciatic). They are therefore peripheral (Harnack and Witkowski, I876). They are suppressed by curare, which paralyzes the endings; on the other hand, physostigmin removes the curare paralysis (Pal and Rothberger). Magnus found that the physostigmin fibrillations can not be produced when the motor nerve has been cut and allowed to degenerate completely (but whilst the muscle fibers still respond to direct stimulation). This would seem to localize the physostigmin action strictly in the endings; and the case would be a typical instance of mutual antagonism, both poisons acting on the nerve-endings.

This conclusion appeared to be opposed by the antagonism of nicotin and curare on striped muscle. Langley, I906, showed that certain muscles of the fowl are thrown into tonic contraction by nicotin, and that this is removed by curare. This peculiar nicotin action, however, persists after degeneration of the nerve; hence nicotin, and therefore also curare, must act upon the intramuscular "receptive substance." But if curare paralyzes an intramuscular structure, its action could not be removed by physostigmin, which stimulates an extramuscular structure.

The two phenomena are reconcilable by the assumption that the curare attacks the receptive substance in the presence of nicotin, and that it attacks the nerve-endings in the presence of physostigmin; in other words, that the point of attack of curare is determined by its antagonist; in other words, that we are dealing with two distinct and unrelated paralytic actions of curare, manifested under different conditions, one on the extramuscular, the other on the intramuscular structure. The nicotin-tonus effect appears to be a distinctive reaction, which has probably nothing to do with ordinary muscular contraction; so that the curare block, $f . i$. , with ordinary sciatic stimulation, is probably the same in all animals (i.e., in the nerve-endings).

Langley, I $1 \mathrm{I}_{3}$ and I $19 \mathrm{I}_{4}$, investigated the nicotin effects on frogs' muscle. He found the nicotin-tonus action quite distinct from the nicotin-twitchings. The twitchings, $f . i$., are prevented by a definite concentration of curare, whatever the concentration of the nicotin; whereas, to prevent the nicotin-tonus, the concentrations of the two drugs must be kept proportional.

Peculiarities of Fowls' Muscle.-The tonus action is particularly characteristic in fowls' muscle, which also shows other related peculiarities (Edmunds and Roth, 1908). In these animals, not only the nicotin, but also the physostigmin actions persist after degeneration of the nerve; the physostigmin action is not removed by atropin, whilst it is so removed in mammals. Barium produces only an increase of muscular tone in a normal fowl's leg; but in a denervated leg, or after nicotin, barium causes a strong contraction.

Atropin-Pilocarpin-Physostigmin on the Pupil.-When the pupil has been dilated by atropin, stimulation of the short ciliary, i.e., of the postganglionic fibers of the oculomotor nerve, is ineffective, whilst direct 
electric stimulation of the iris sphincter contracts the pupil. Atropin therefore paralyzes either the nerve-endings or the myoneural junction. Pilocarpin and physostigmin both constrict the normal pupil. When the endings of the short ciliary are made to degenerate, pilocarpin is still effective (Anderson), but physostigmin is now ineffective. The pilocarpin therefore acts on the receptive substance or muscle; the physostigmin would be supposed to act on the extracellular nerve-endings.

From this, one might suppose that pilocarpin should be a more powerful antagonist of atropin than is physostigmin. In fact, however, the opposite is the case, so that the point of attack of atropin seems to be shifted more centrally by the physostigmin, as if it were determined by its antagonist.

However, in this case also another explanation is possible, perhaps more probable. The weak point in the argument is the localization of the physostigmin action. There is some evidence that physostigmin acts by increasing the excitability of the oculomotor mechanism, and not by direct stimulation; for it lowers the threshold for stimulation when it does not stimulate directly. If this be true, the failure of physostigmin after nerve degeneration would not argue against its action being on the receptive substance; for degeneration of the nerve endings might interfere with the physostigmin action simply by eliminating the possibility of extraneous stimulation. (If this view is correct, physostigmin should be rendered active through the presence of pilocarpin even after nerve degeneration; but the experiment has not been tried.)

Conflict of Sympathetic and Parasympathetic Actions.-Langley and Dickinson, I 89o, showed that the effects of nicotin on the pupil and nictitating membrane are the resultant of simultaneous stimulation of the sympathetic and cranial innervation. In cats, the sympathetic usually predominates (dilation of pupil and retraction of membrane). Pilocarpin, on the other hand, causes constriction (oculomotor stimulation), which is also unaffected by complete denervation (Anderson). However, it acts also on the sympathetic, for the nictitating membrane retracts. Both drugs therefore act on both systems.

Both drugs act after degeneration of the nerves, in the body; but with the excised iris, only the augmentor (oculomotor) response persists. To explain this contradiction, Dale and Laidlaw, 1912, assume that the sympathetic stimulation in denervated animals is brought about by increased secretion of epinephrin, to which the denervated sympathetic endings are especially susceptible.

The other examples adduced by Magnus appear more conclusive:

Pilocarpin and Atropin on Intestine.-Magnus has worked out an anatomic method for localizing the action of poisons on the intestinal movements, as will be explained presently. He could thus show that the ordinary atropin action is located in Auerbach's plexus, whilst the pilocarpin stimulation is peripheral to this plexus. Nevertheless, the two drugs are mutual antagonists. Moreover, the pilocarpin stimulation is prevented by doses of atropin but one-hundredth as large as the minimum dose which has any effect on the unpoisoned gut.

Apparently therefore the point of attack of atropin is shifted by the presence of pilocarpin. There is another possibility, however; for it is conceivable that atropin might inhibit the pilocarpin action, not by depressing the cells, but by preventing their combination with pilocarpin in some way. This seems to be nearer the truth, for Bayliss and Starling, rgor, showed that atropin, whilst it completely inhibits the pilocarpin stimulation, does not prevent the response to stimulation of the extrinsic motor or inhibitory nerves of the intestine, or to the pinching reflex. 
Threshold of Sympathetic Stimulation.- It is also possible, however, that the differences are only quantitative; for there exist considerable differences in the threshold of the different functional fibers of sympathetic nerves (Gruber, I9I5).

Atropin and Pilocarpin on the Vasomotor Mechanism.-This furnishes a strictly analogous example: Brodic and Dixon found that atropin removes the pilocarpin vasoconstriction, without impairing the response to electric or other stimulation of the vasoconstrictor nerves.

Bladder and Uterus.-Similar conditions are found in the Urinary Bladder (the response of which to autonomic poisons has been studied by Langley, I9I2) and in the uterus of the rabbit. In the latter, pilocarpin produces violent contractions, which are arrested by atropin, whilst hypogastric stimulation continues effective.

\section{THE RESPONSE OF THE UTERUS TO AUTONOMIC POISONS}

This presents some very interesting problems of antagonism, especially in the cat (Cushny, roro).

Spontaneous Movements.-The surviving uterus placed in oxygenated Ringer's fluid continues to perform rhythmic movements for hours (Kehrer, I908). These vary with the physiologic condition of the organ, being most lively in early pregnancy. In advanced pregnancy they are slower and separated by longer pauses; but they are extensive. They can be influenced through the uterine nerves and by the drugs which act upon these (Cushny, r9o6).

The nerve supply of the uterus is derived from the sympathetic, chiefly through the hypogastric; and from the parasympathetic through the pelvic nerve. Of these, the hypogastric is by far the more important. The response to hypogastric stimulation differs according to the animal and pregnancy. Rabbits respond by contraction; so also do pregnant or recently pregnant cats; in non-pregnant cats, the same stimulation inhibits contraction and lessens the muscular tone. There are occasional exceptions to this; but in any case, it may be assumed that the augmentor or inhibitory response is due to the different functional condition of the muscle. In guinea pigs, the uterine effects of poisons resemble their intestinal actions (Sugimoto, I913).

Action of Autonomic Poisons on the Uterus.-Pilocarpin, Nicotin, and Epinephrin.-These always produce the same effect on the cat's uterus as hypogastric stimulation: augmentation in the pregnant; inhibition in the non-pregnant. They would therefore seem to act on the same neuromuscular path. Their reaction to atropin, however, is different:

Atropin by itself stimulates the movements in small doses; whilst larger doses cause arrest. Its main interest lies in the antagonisms: Atropin abolishes the pilocarpin effect, whether this be augmentory or inhibitory; but it does not alter the reaction to nicotin, to epinephrin, or to electric stimulation of the hypogastric nerve. This is in harmony with the specific predilection of atropin against pilocarpin, of which several instances have just been given.

Ergotoxin, on the other hand, abolishes the augmentory effects, both of pilocarpin and of epinephrin; it therefore appears to differentiate between the effects, rather than between the drugs.

Still another phenomenon is presented by the physostigmin and atropin antagonism: Physostigmin has a purely augmentor action, whatever the state of the muscle; this is abolished by atropin, but the antagonism is one-sided (contrary to the experience in other organs): further quantities of physostigmin remain ineffective.

The uterus is the only instance in which physostigmin, pilocarpin, and atropin act on the same nervous (sympathetic) path as epinephrin, and is thus a transition to the sweat glands in which the sympathetic innervation responds to the craniosacral poisons, and apparently not to the sympathomimetic epinephrin.

Reaction of Different Uteri to Epinephrin.-The response to this drug, and to sympathetic stimulation, varies in different animals and functional states, as shown in the following table (adapted from Gunn and Gunn, 1914). 


\author{
By contraction Responds
}

Rabbit......... Pregnant or non-pregnant

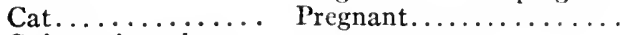

Guinea pig and rat. . .................

Monkey.......... Non-pregnant..........

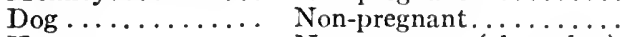

Human .......... Non-pregnant (also tubes).

Non-pregnant

Pregnant or non-pregnant

Other Drugs Acting Peripherally on the Uterus.-Infundibular extract produces maximal contraction (Frankl-Hochwart and Froehlich, 1909), in all animals, pregnant or non-pregnant; also in human uterus, but not in the tubes (although these are contracted by epinephrin (Gunn, I9 4 ).

Quinin increases the contractions (Kurdinowski-Kehrer, 1906). Cinchonin, cinchonidin and quinidin contract even more powerfully than quinin (Hale, I9I5).

Morphin in small doses is stimulant, in larger doses depressant (Kehrer).

Scopolamin has no effect.

Histamin or beta-imidazolylethylamin (a constituent of ergot, also formed by the action of bacteria on histidin) is powerfully stimulant (Barger and Dale, r9Io). Other related amins, peptone, and anaphylotoxin act similarly. IIydrastin, hydrastinin and cotarnin are stimulant (Kehrer, I90 7 ). The actions of a number of other drugs are described by Pilcher, I9r6.

Purified Organ Extracts.-Fawcett, et al., ro15, examined aqueous extracts, after removal of the nucleo-proteins and coagulable proteins. Pituitary, pineal, thyroid, parathyroid, thymus, adrenal glands, pancreas and spleen were found to contain substances that stimulate cat's uterus. The stimulation was inhibited by epinephrin. The effects on intestinal muscle differed; when stimulated, it was also inhibited by epinephrin.

Difference between Uterus in Situ and Excised.-With non-pregnant cats, the uterus in situ is inhibited by nicotin, cytisin, hydrastinin, indolethylamin and pilocarpin. Excised uteri, on the other hand, are generally stimulated by these drugs. It seems from this that there is both sympathetic (inhibitory) and parasympathetic (augmentory) stimulation. The sympathetic effect predominates during life, but does not survive excision. Dale and Laidlaw, I9 I2, believe that the sympathetic stimulation is not direct, but through increased epinephrin secretion.

Practical Importance of the Uterine Actions.-The stimulation of uterine contractions is useful for arresting postpartum hemorrhage. Ergot particularly is employed for this purpose, hydrastis and cotarnin have also been used, but they seem to be less effective. Pituitary extract is used to initiate or strengthen uterine contraction.

Nicotin (tobacco infusion), pilocarpin and quinin are sometimes used by the laity as abortifacients; effective doses, however, are dangerous.

Reflex Increase of the Movements.-This is secured by irritation of the intestine (Kehrer, rgro), especially, by drastic cathartics and irritant volatile oils. These should therefore be avoided in pregnancy. They also have a reputation as abortifacients, but are highly dangerous.

Central Action on the Uterus.-These are relatively unimportant. The motor center in the lumbar cord is stimulated by anemia and asphyxia.

\title{
GALL-BLADDER
}

Lieb and McWhorter, r9r5, found that this is augmented by vagus and inhibited by sympathetic stimulation. Epinephrin therefore relaxes, whilst physostigmin and pilocarpin stimulate, and this stimulation is removed by atropin. It is also stimulated by strophanthin and barium; relaxed by bile salts and nitrites. Okada, I9r5, states that the normal rhythmic movements are greatly increased during digestion, and by the introduction of acid into the duodenum.

\section{URETER}

The movements and tone of the excised ureter are stimulated by the parasympathetic stimulants and paralyzed by atropin; they are also stimulated by epinephrin. Ergo- 
toxin reverses the epinephrin stimulation, but has no effect on the parasympathetic stimulants. Nicotin produces an initial stimulation and subsequent paralysis. Papaverin relaxes the tone, without inhibiting the peristalsis (Macht, I9r6).

\section{THE NATURE OF ANTAGONISM}

Until there is a clearer understanding of the chemical and physical changes which condition the actions of the antagonistic poisons, we can conceive the nature of their antagonism only in terms of crude analogies:

I. We may suppose that the stimulant and depressant drugs each act upon different chemic constituents. In this case, the combined effect would be a simple summation of their separate actions. This might be the explanation of the action of atropin and physostigmin on the oculomotor junction.

2. Both poisons may act upon the same constituent. In this case the end result would depend upon the relative affinity of the two poisons for this constituent. This offers several possibilities.

(a) The two drugs may have approximately the same affinity-in this case the end effect would depend purely upon the relative quantity.

(b) One drug may have a much greater affinity than the other; this might apply, for instance, in the case of atropin and pilocarpin on the pupil, where a small quantity of atropin suffices to abolish the effects of a large quantity of pilocarpin; whilst a large quantity of pilocarpin is needed to overcome even a trace of atropin. The case may be somewhat analogous to the combination of oxygen and carbon monoxid with hemoglobin.

(c) The efficiency of the one may be so much greater than that of the other, that the antagonism is practically irreversible. Atropin, f.i., completely prevents the action of physostigmin on the uterus.

3. One poison might influence the penetration of another, and in this way alter its distribution, and thus again entirely change its point of attack. This has been shown by Ehrlich for certain dyes; and similar conditions have been shown to prevail for muscarin and atropin, by Straub; and for morphin and chloroform by Fuehner.

With these possibilities in mind and remembering that they are based merely on crude analogies, it is clear, at least, that antagonism is a highly complex subject. We must be content, for the present, with the empiric facts: That the point of attack of the poisons is not absolutely specific: that it may shift according to conditions, dose, time of action, etc.; that the effect of the same drug on different structures, and even on the same structure, may be augmentory or inhibitory, or both; that a poison may present mutual antagonism to two poisons which act on different structures; that the antagonistic dose may be much smaller than the minimal effective dose under ordinary conditions; that the removal of a drug (pilocarpin) stimulation by a depressant (atropin) need not be accompanied by paralysis to electric stimulation, whilst in other cases they go hand in hand. The schematic presentation which has been given may serve as the thread of Ariadne through this labyrinth; but after all, it is still more important to grasp the individual facts which have a practical application.

Quantitative Antagonism of Atropin and Pilocarpin Salivary Secretion.-This has been investigated by Cushny, I9 5 , on dogs with salivary fistulas. It was found that the antagonistic dose was the same for different dogs. The ratio also remained the same (about I part of atropin to 9.5 of pilocarpin) whether the two drugs were injected simultaneouslyor some time apart; and that it remained unaltered whatever total dose was injected. This agrees with the laws of mass action, rather than with those of chemical combination.

Relation of Action to Intracellular Penetration, Storage, and Destruction.-According to W. Straub, 1907, the effect of most alkaloids is proportional to their concentration within the cell. With muscarin, however, he found the relation quite different: Here the effect appears to depend upon the concentration outside the cell; or rather, on there being a higher concentration outside than inside the cell.

The experiments were made by placing the frog's heart into a muscarin solution. The quantity of poison remaining in solution, and the quantity which could be extracted 
from the heart were then tested on aplysia-hearts. Hearts laid in a muscarin solution show a primary diastolic standstill, followed by spontaneous recovery in a few hours. The maximal diastolic action occurs when very little muscarin has passed into the muscle; whilst at the time of "recovery," all the muscarin has passed into the heart. If now the recovered heart is placed into a fresh muscarin solution, it is again arrested, with spontaneous recovery. This may be done repeatedly; but the action becomes shorter and shorter, and finally nil. That is, the heart becomes completely refractory to even the highest concentrations of muscarin. If the muscarin concentration is increased very slowly, the heart does not respond. The presence of atropin appears to delay the penetration somewhat. Any explanation of these phenomena would have to be too hypothetical. (O. Loewi, IgI2, considers the resumption of the beat under muscarin as analogous to escape from vagus stimulation, and attributes both to increased excitability of the cardiac muscle.)

Incidentally, this furnishes an interesting example of antagonism. The effects of muscarin are exactly the same in animals which have no vagus mechanism; but atropin is only antagonistic in animals in which a vagus mechanism is developed.

With Epinephrin the action is also proportional to the concentration outside the cells. A still more striking example is described by Trendelenburg, I9I2, for bronchial muscle. This constricts strongly in solutions of muscarin, pilocarpin or arecolin. Washing the poisoned solution with pure Ringer's fluid does not remove the constriction; on the contrary, this often causes a further contraction. The same phenomenon was observed by Kuyer and Wijenbeck, I9r3, on excised intestine and uterus. Transferring the poisoned tissues into pure saline again brought on the typical augmentory or inhibitory effect of the poison; whilst transfer from the old to a fresh solution of the poison produced no result. This is taken to indicate that the effect occurs only during the passage of the poison through the cell membrane, into or out of the cell.

Cell Permeability.-Asher and Gannus, I9I I, found that the gland cells of the nictitating membrane of frogs take up much more of vital stains when they are stimulated by pilocarpin than when depressed by atropin.

\section{OPTICAL ISOMERS}

Most if not all the autonomic poisons are optically active (or racemic); and the chemic reactions which determine their specificity depend largely on their stereochemic configuration. The stereoisomers have an identical composition and radicals; but owing to the presence of one or more asymmetric carbon atoms, these groups are arranged differently in space (Van't Hoff and Le Bel, I874). This is indicated by the optical activity, so that these isomers are levorotary, dextrorotary, or by the combination of the two, racemic. Our knowledge of these phenomena dates from the classic discovery of Pasteur, that racemic tartaric acid is separated into its optical constituents by mould fungi. Since then, very numerous instances have been worked out on higher animals; such as atropin and hyoscyamin; atroscin and scopolamin; the epinephrins, muscarins, nicotins, cocains, cinchona alkaloids, camphors, etc. (Fraenkel, Arzneimittelsynthese). Cells and ferments distinguish sharply between these stereoisomers, so that they invariably differ, at least quantitatively, in their pharmacologic effects, the racemic being intermediate between the active forms. This indicates that the reactive substance of the cells must also be optically active; and it is suggestive that the effects of the l-active hyoscyamin and l-epinephrin are antagonistic to those of the d-pilocarpin and ergotoxin (Cushny, I908). In higher animals, the l-alkaloids usually have more powerful peripheral effects. It is probable that the cells can either attack or destroy the one isomer more readily than the other (Poulsson, I9I0). The asymmetric nitrogen atom also modifies the pharmacologic actions, as shown by Hildebrandt, I905, for coniins; and Laidlaw, I9I3, for canadins. The curare action of the ammonium bases (Brown and Fraser, I869) also depends upon the peculiar orientation of the pentavalent atom of nitrogen or similar elements. 


\section{AUTONOMIC PHENOMENA OF THE INTESTINE}

The innervation of the intestines differs from the general scheme of autonomic innervation so that Langley, I9I3, proposes to differentiate them as the "enteric system." A second set of nerve cells (Auerbach's and Meissner's plexus) is interpolated between the ordinary (extrinsic) autonomic innervation and the muscle. The intestinal reflexes (segmentation and peristalsis) are determined by the local nerve centers of Auerbach's plexus; but the extrinsic nerves can also initiate, augment or abolish these movements.

These peripheral nerve centers and their connections react to the usual autonomic poisons; but the nature of the reaction is distinctive and can not be deduced from the ordinary schema. The extrinsic nerve ganglia responds to nicotin in the usual manner. The effects on the Auerbach ganglia can also be located; these are stimulated by atropin and nicotin; these and the other autonomic poisons also act more peripherally.

Peripheral Reflexes in Other Organs.-The intestines are the most prominent, but by no means the only, example of reflex response in the absence of central innervation. In fishes and amphibians, for instance, the iris of the excised eye responds to light, even after removal of the retina (Steinach, I892); blood vessels regain their tone when their nervous connections degenerate, etc. These reflexes do not even involve the presence of peripheral nerve cells. They may be considered as survivals of the reflex mechanism of the lower invertebrates (Parker, I909). This conception may help to elucidate some obscure pharmacologic phenomena.

Extrinsic Innervation of the Intestine.-Sympathetic nerves, running through the superior and inferior splanchnic and mesenteric ganglia, supply the whole tract, from the cardia to the anus, mainly with inhibitory fibers.

The parasympathetic supply consists of the augmentory erigens and pelvic nerves to the anus, rectum and colon; and the vagus fibers, distributed from the esophagus to the end of the ileum. The vagus innervation causes a short primary inhibition, followed by marked stimulation. It is not known whether the extrinsic nerves connect with the local nerve cells; or whether they act independently on the muscle; or both. The bulbosacral innervation seems useful mainly for initiating tone, which is then maintained by the local mechanism. The initial inhibition fayors the ingress of the chyme (Cannon, I9II). The "roll movements" which propel the contents for long distances, are of extrinsic origin, due to vagus stimulation with simultaneous depression of sympathetic inhibition (Meltzer and Auer, 1907).

Differences of Rhythm at Various Levels.-In intact animals, the rhythm is faster toward the two ends of the intestine than toward the middle. In excised intestine, the rate decreases with the distance from the pylorus, the rate of the duodenum in rabbits being about 50 per cent. faster than in the lower ileum. The oral end is more irritable and therefore acts as pacemaker. It is also less subject to epinephrin relaxation. Its tone is also higher. On the other hand, the slower rate of the aboral end is accompanied by greater excursions (Alvarez, I914, I915).

The Intrinsic Neuro-muscular Mechanism.-The segmentation (pendulum) movements and the ordinary peristaltic movements are entirely of peripheral origin, since they are observed also in the excised intestine even when the mucosa and submucosa are removed. They are originated peripheral to the ganglia; for they persist, under suitable conditions, for five days after death, when the ganglia must be dead; and they can also be evoked in preparations from which the ganglia have been removed anatomically (Gunn and Underhill, I9I4). The rhythmic segmentation movements are automatic, the peristaltic movements are evoked reflexly (as in the Bayliss-Starling reflex; a pinch causes the intestine to constrict for I or $2 \mathrm{~cm}$. above, and to relax for some $30 \mathrm{~cm}$. below the stimulus). The local nerve-muscle mechanism for these reflexes consists of the longitudinal and circular muscular coats, innervated by Auerbach's plexus of nerve cells and fibers, which lies between them. This communicates also with Meissner's plexus in the submucosa, which innervates the glands, muscularis mucosæ, etc., but which is not concerned in the intestinal movements.

Isolation of Intestinal Coats.-Magnus, 1904, found that the muscular coats of the cat's intestine could be separated into layers, so as either to retain or eliminate Auerbach's plexus. 
The "plexus preparations" contain the peripheral nerve cells, and respond to continued stimulation by rhythmic contractions.

The plexus-free preparations (which contain only the nerve-endings and muscle) ordinarily respond to continued stimulation by a single tetanic contraction, like ordinary smooth muscle; but if the preparations are carefully protected against injury, they also resume spontaneous rhythmic movements after a time (Gunn and Underhill, 1914).

With the disappearance of spontaneous movements by exposure, the reaction to all drugs, even barium, is abolished. The response to pilocarpin and 2 tropin disappears before that for epinephrin or barium, indicating that the parasympathetic innervation dies before the sympathetic (Gunn and Underhill).

The muscularis mucosa also contracts, especially in the diagonal direction. Its rhythm is slow, rather similar to that of the spleen. Its tone and rhythm are increased by epinephrin. Barium causes marked tonic contraction, abolishing the rhythm (Gunn and Underhill).

Localization of the Action of Poisons on the Intestine.-The use of excised intestine, and of plexus and plexus-free preparations, aids in the localization of the action of poisons.

If a given action occurs in the excised intestine, the point of attack must be local. If the action occurs in plexus preparations, and not in plexus-free strips, it must be on the ganglion cells. If it occurs in the plexus-free preparations, it must be on the endings or muscle. No reliable method has been found to distinguish between the nerve-endings and muscle; or between the endings of the extrinsic and intrinsic system, if the two be distinct. All conclusions as to these are based on the insecure ground of analogy.

This general method as outlined only discovers the most peripheral point of attack, which is practically the most important; but it does not exclude that the poison may also affect more central structures at the same time. This possibility is not merely theoretical; for even this method shows that the same drug may attack different structures, according to the dose and other conditions. The conditions have not been fully analyzed, and until this has been done, the localization of the intestinal phenomena must be rather unsatisfactory. The following exposition is mainly along the lines laid down by Magnus (the Ergebnisse der Physiologie, vol. 7, 1908, and vol. 2, 1903), and by Hans Meyer (Meyer and Gottlieb's Lehrbuch).

Action of Drugs on the Intestinal Movements.-Barium salts provoke very violent contraction; the effect occurs in plexus-free preparations and is presumably (from analogy) exerted directly on the contractile substance. It is abolished only by the very large doses of atropin which paralyze the muscle substance.

Strophanthin (and presumably the other Digitaloid drugs) stimulate in small doses and inhibit in large doses. The effects occur in plexus-free preparations, and hence involve the endings or muscle, presumably the latter. The stimulation is abolished only by the extreme doses of atropin.

Physostigmin, Pilocarpin, Muscarin, Cholin and Colchicin produce violent irregular contractions, and also tetanic contraction of plexus-free: preparations. Their action must be postganglionic; presumably stimulation of the vagus endings.

The stimulation of these drugs is abolished by small doses of atropin; pilocarpin is antagonized by extremely minute doses; physostigmin requires relatively the largest amount.

Atropin.-The effects and their localization differ according to conditions: Very small doses have no effect on the normal intestine, but remove intestinal spasm, due to peripheral vagus stimulation (physostigmin, pilocarpin, etc.; lead colic, anemia of intestine, etc.). The motor response to stimulation of the vagus trunk is lessened, but not abolished. This dosage does not affect the contraction on direct muscular irritation (barium, strophanthin, cathartics).

The action consists presumably in depression of the augmentory vagus endings. 
Intermediate doses of atropin (from therapeutic to fatal), seem to act differently, according to conditions and species. In man (Katsch, I9I3), in rabbits, and in some cats (Hirz, I913),'they cause further depression and relaxation of the intestinal tone.

In typical cats (Magnus) they increase the movements of the normal intestines (i.e., unless there is vagus-spasm). This occurs also in the excised intestine, but not in plexus-free preparations. The segmentation movements also become more regular. The action consists apparently in stimulation of the Auerbach ganglion cells. Larger doses also paralyze the inhibitory splanchnic endings.

Extreme doses of atropin (so large that they can not be brought into action by systemic application, but only locally and in the excised intestine) paralyze successively the ganglion cells, the endings, and the muscle, thus destroying the response to the muscular poisons such as barium.

In the excised intestine, the relative doses of atropin are as follows: 0.1 per cent. is required to produce depression; 0.025 to produce stimulation; and 0.0005 to antagonize pilocarpin:

Nicotin also has complex actions: In the first place, it paralyzes the extrinsic ganglia (both the vagus and splanchnic) rendering ineffective the stimulation of their preganglionic fibers. The nicotin paralysis is preceded by a short stimulation, which occurs after degeneration of the preganglionic fibers and must therefore involve the ganglion cells. These effects on the extrinsic ganglia are practically overshadowed by the peripheral actions.

Nicotin produces violent peristalsis, proceeding to intense spasmodic contracture. This occurs in the excised intestine, but not in the plexusfree preparation. It is therefore due to stimulation of the Auerbach cells. This stimulation is preceded by a temporary inhibition, which appears to be in the endings.

Very large doses (local application) abolish the segmentation movements, but the peristaltic reflex persists. Large doses of atropin abolish the nicotin contraction by muscular paralysis.

Epinephrin.-This inhibits the normal intestinal movements; as also those produced by atropin, nicotin, pilocarpin, and strophanthin, but not those of barium. This inhibition occurs in plexus-free preparations, and after degeneration of the postganglionic fibers, and is therefore located in the myoneural junction.

A pocodein paralyzes the sympathetic ganglia, thus abolishing inhibition on splanchnic stimulation. In the isolated intestine, it first stimulates the Auerbach cells, and then paralyzes the endings or muscle.

Autonomic Poisons on White Cells.-The proportion of these cells is changed, the significance being obscure. Pilocarpin and cholin increase, atropin diminishes the eosinophiles and lymphocytes. Epinephrin increases the lymphocytes but diminishes the eosinophiles. Histamin also diminishes the eosinophiles (Port and Brunow, 19I4)

\section{ATROPIN}

Summary of Actions.-Atropin and other closely related alkaloids (hyoscyamin, scopolamin, etc.) produce a variety of peripheral and central effects, namely:

I. Complete paralysis of the peripheral distribution (myoneural junction) of the parasympathetic (cranio-sacral autonomic) nerves (oculomotor, 
secretory, bronchial musculature, cardiac and intestinal vagus, uterus, etc.). etc.

2. Consequently, antagonism to pilocarpin, muscarin, physostigmin,

3. Slight stimulation and in large doses, subsequent paralysis of smooth and cardiac muscle and other cells.

4. Slight peripheral sensory paralysis, on local application.

5. Excitation and then paralysis of certain cerebral and medullary centers.

These actions find varied application in therapeutics, and are of great scientific interest.

Specificity of the Autonomic Actions.-The peripheral actions of atropin are confined mainly to the augmentory nerves of the cranio-sacral division of the autonomic system. To this there are a few exceptions; for it affects also inhibitory cranial nerves (cardiac vagus) and a few augmentory and inhibitory sympathetic nerves (uterus and sweat glands) (Cushny, rgro).

Occurrence.-Atropin occurs in a number of drugs (Belladonna, Stramonium, Hyoscyamus, Scopola, Mandragora, Duboisia, etc.) derived from plants belonging to the order Solanaceæ (the potato family). These also contain varying proportions of other alkaloids (hyoscyamin, scopolamin, etc.) which are closely related to atropin in structure and in action.

The alkaloids, as first isolated, were mixtures; consequently, their properties appeared to vary with the origin, and these mixtures were given specific names (Daturin, Duboisin, etc.). The pretended difference between scopolamin and "hyoscin" is a survival of this tradition.

Historical.-Belladonna received but little medical notice, and seems to have been first introduced into practice, as an anodyne, about 1860.

The first indubitable notice of belladonna occurs in ${ }^{5} 504$, but it then came quickly into use for poisoning and cosmetic purposes. The name Atropos, which Linné gave to the plant, is from the oldest of the Three Fates, who cuts the thread of life. Belladonna comes from the Italian "handsome woman," as it was used to give luster to the eyes. On account of the slow course and the obscure symptoms, belladonna was a favorite with professional poisoners in the middle ages, and this abuse in regard to several species of Datura (Stramonium) has existed in India since remote ages. The name itself is Sanskrit (Dhatoora).

Hyoscyamus was mentioned by Dioscorides in the first century, and has been used continuously in domestic medicine. It was introduced into practice by Stoerck in the middle of the eighteenth century. He also introduced Stramonium. Mandragora (Mandrake) was the subject of some interesting medieval myths. The "herbarium" of Apuleius (eleventh century), quotes its use, externally, for headache and insomnia, and internally for mania.

Chemical Structure and Relations.-These alkaloids are ester-like combinations of certain bases with organic acids of the aromatic series.

Atropin, $\left(\mathrm{C}_{17} \mathrm{H}_{23} \mathrm{NO}_{3}\right)$, is the tropeic acid ester of a base, tropin, and is isomeric with Hyoscyamin; hyoscyamin being levorotary, whilst atropin is racemic. The esters of tropin are called tropeins. Homotropin is the mandelic ester of tropin; and other tropeins occur naturally or may be prepared synthetically. Tropin itself is closely related to Ecgonin, the basic constituent of cocain. Its effects and those of a series of its synthetic esters have been investigated comparatively by Gottlieb, I 896. The osceins are analogous esters of the base oscin. The tropeic acid ester constitutes the important alkaloid Scopolamin or hyoscin, $\left(\mathrm{C}_{17} \mathrm{H}_{21} \mathrm{NO}_{4}\right)$. This also occurs in isomeric forms: Atroscin (racemic, corresponding to Atropin); and Scopolamin proper (levorotary corresponding to Hyos- 
cyamin). Atropin and Atroscin are the more stable products: Alkalies and many other agents convert hyoscyamin into atropin, scopolamin into atroscin.

Chemic Constitution.-Tropin has the formula<smiles>CN1[C]CC[C@H]1O</smiles>

Ecgonin (the base of cocain) is the beta carbo-acid of tropin, one $\mathrm{H}$ at (a) being replaced by $\mathrm{COOH}$.

Atropin is the tropcic ester of tropin, the $\mathrm{H}$ at $(b)$ being replaced by the radical

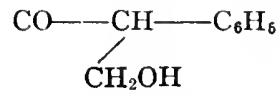

The sulphuric ester of atropin is nearly devoid of peripheral actions, while the central effects, especially the respiratory stimulation, are preserved. Scopolamin-sulphuric ester does not share this respiratory action (Trendelenburg, 19r3).

Belladonnin and Atropamin are isomeric alkaloids (belladonnic acid tropeins). Their formula differs from atropin by the absence of a molecule of $\mathrm{H}_{2} \mathrm{O}$. They are present in the plants in very small amount, and it is possible that they are only formed during the extraction.

Distribution of Alkaloids.-Belladonna and Stramonium contain mainly atropin and hyoscyamin; one or the other alkaloid may predominate. The hyoscyamin is the most widely distributed of these alkaloids, and is especially abundant in young parts of the plants, whereas older parts contain more atropin. It appears therefore that the hyoscyamin is the original alkaloid, and that it is partly transformed into atropin in the plant itself, and also during extraction. These plants also contain small traces of scopolamin and atroscin, but the latter alkaloids are relatively more abundant in Hyoscyamus and Scopola, taking the principal part in the actions of these plants. The distribution and variation of the alkaloids in individual plants has been studied by Sievers, rgr 3 and I9I4. This suggests the feasibility of improving the alkaloidal content by selective breeding (Sievers, 1915).

Effects on Psychic Functions.-Therapeutic doses of atropin have no perceptible effects on the psychic functions; but overdoses produce symptoms (see "Toxicology") superficially resembling alcoholic inebriation. However, the exaltation and stimulation are more prominent, passing from mere restlessness to hallucinations and delirium, and even mania; and the effects are much more lasting. Similar symptoms are seen in animals. With toxic doses the stimulation passes into coma, convulsions, and asphyxia.

The convulsions are not entirely asphyxial, for they are not abolished by artificial respiration.

The vision is disturbed more than can be explained by loss of accommodation.

The motor areas in dogs are stated by some observers to be more excitable, but others deny this.

In frogs, atropin first increases the reflexes; this is followed by general paralysis, and this again by late tetanus.

Respiration.-Therapeutic doses, in man (I mg. hypodermically), stimulate respiration, increasing somewhat the rate and minute volume, and sometimes the depth of the respiration. The excitability of the respiratory center is not increased directly, however; for the alveolar $\mathrm{CO}_{2}$ tension is unchanged, whereas it would be found lower if the threshold of the center were lowered. The stimulation is therefore indirect; being due partly to increased $\mathrm{CO}_{2}$ production (increased gaseous metabolism); partly to increased "dead space" in the lungs by bronchial dilation (Edsall 
and Means, I9r4; Higgins and Means, I915). Toxic doses produce a late depression of the center (Binz, I896), which is the usual cause of death in atropin poisoning.

Respiration in Animal Experiments.- The results have been rather contradictory; Heinz, I 890 , found no appreciable change in the respiratory volume of rabbits; Wood and Cerna, r892, increased rate and minute volume in dogs. According to most observers, it contenteracts respiratory depression from chloral (Husemann, I877) or morphin (Henbach, I 878; Binz, I 887; Vollmer, I 892); but others found that it augments the morphin depression (Orlowski, I89I; Unverricht, I892 and I896). With larger doses, the psychic excitement and muscular activity would increase the respiration indirectly. Toxic doses depress respiration, producing slowing, Cheyne-Stokes type, and finally cessation. Artificial respiration may enable an animal to survive six times the ordinary fatal dose.

Therapeutic Uses of Respiratory Stimulation.-The mechanism of this respiratory action in man would render its usefulness doubtful. On the basis of the animal experiments (which, however, involve larger doses) it has been used against poisoning by morphin, and as a preliminary to anesthesia, or even as a restorative in anesthetic fatalities (Webster, I908). It is also often used in whooping cough, but is of doubtful efficiency (child of one year, $1 / 10$ to $1 / 12$ gr. of Extr. Belladonna, or 8 to Io drops of Tr. Bellad. three times daily, doubling the dose in a week if tolerance is acquired (A. Jacobi, 1907).

\section{BRONCHIAL MUSCLES AND GLANDS}

Atropin paralyzes the innervation of these structures, and therefore dilates and dries the bronchial tubes. The dilation is obtained in excised rings of bronchial musculature (Trendelenburg, I9I2), and is therefore peripheral. These effects are especially important for relieving attacks of bronchial asthma.

Pharmacology of Bronchial Muscle.-This also has an important bearing on the explanation and treatment of asthma. The bronchi are innervated by the vagi, which carry mainly constrictor, but also dilator fibers. The response to drugs ha's been studied in intact animals by Dixon and Brodie, 1903, and by Sanoz, I914, by recording the excursions of the lungs under uniform artificial respiration. D. E. Jackson, rgr 3 , has controlled their work with the extra precaution of keeping the blood pressure level. Trendelenburg, I91 2, Baehr and Pick, I913, and Titone, I9r3, have also checked it on excised bronchial muscle.

Bronchial Constriction is produced by the drugs which stimulate the vagus endings, such as physostigmin, pilocarpin, muscarin, cholin, anaphylatoxin, peptone, ergotoxin, tyramin, histamin, pituitary, aspidospermin, etc.; by those which stimulate smooth muscle, such as barium, veratrin, the salts of many metals (vanadium), quinin and several other alkaloids, bromin vapor, etc. It may also be produced by direct stimulation of the medullary centers, as by carbon dioxid; or reflexly by irritation of the mucosa of the nose, larynx or bronchioles. Nicotin, lobelia, and curare and small doses of pilocarpin produce an initial constriction, followed by dilation. Jackson attributes this secondary dilation to stimulation of the. suprarenals. Muscarin and pilocarpin also contract the excised muscle; physostigmin and veratrin do not. (E. Weber, rgr4, claims that the bronchoconstrictor action of muscarin, histamin, etc., is largely central.)

Bronchial Dilation. - This is produced by drugs which paralyze the vagus endings, especially atropin and its related alkaloids (Dreser, I890; Einthoven, 1892; Beer, 1892) (large doses of atropin also depress the dilator endings); by epinephrin, which stimulates these dilator endings; and by cholin, agaricin, chloroform and ether. Morphin dilates in small doses but constricts in large doses. The excised muscle is also relaxed by atropin (after a brief constriction), and by epinephrin. The vapors of ether and chloroform, in concentrations even smaller than those used in anesthesia, affect the excised bronchial muscle, ether ( 0.3 per cent.) producing dilation, and chloroform ( 0.6 per cent.) constriction. Amyl nitrite and sodium nitrite gives a temporary dilation; ammonium salts produce a slight dilation. Sodium iodid and camphoric acid have no effect in living animals. In man, with $\mathrm{r}$ mg. of atropin, the increase of dead space amounts to 7 to $r_{5}$ per cent. (Higgins and Means, rgI5).

Therapeutic Application to Asthma.-Bronchial asthma is commonly due to spasmodic constriction of the bronchioles, produced in some cases 
reflexly, in others probably analogous to anaphylactic reaction. The lumen of the bronchi may be further narrowed by catarrhal swelling of their mucosa.

The constricted passages offer an insurmountable resistance to expiration. This is easily overcome by the more powerful inspiratory muscles (Cloetta, I9I3), so that the lungs become overdistended; but the respiratory exchange is greatly hindered.

Such cases may be promptly relieved by atropin or epinephrin. The atropin acts on both the constriction and the hypersecretion. It is also effective against experimental bronchial constriction (Dixon and Lee, I9I2); and with proper dosage against anaphylaxis (Auer, I9I2; Kersner and Nutt, I9II). It could not be effective in cardiac, renal, or emphysematous "asthma." Unfortunately, it seems to lose its effects, even in what would appear to be suitable cases. Epinephrin is often much more active.

Administration.-Atropin ( $1 / 2$ to I mg., $1 / 20$ to $1 / 60 \mathrm{gr}$.) or the drugs containing it may be administered by any channel; but inhalation is most effective since it brings the drug directly to the site of its action. This is accomplished by inhaling the smoke of burning stramonium leaves.

Asthma Powders consist of atropin-containing drugs, mixed with sufficient saltpeter to insure their combustion; e.g., Powdered Stramonium, 3 Gm.; Lobelia, I; Anise, 2; Potas. Nitrate, 2. This dose is burned on a plate, and the smoke inhaled. Stramonium cigarettes are also used; the smoke of I to I.25 Gm. of stramonium contains 0.3 to 0.5 mg. atropin (Guenther, I9II).

\section{CIRCULATION}

Single therapeutic doses of atropin do not usually affect the pulse rate materially; but ordinary experimental doses greatly increase the heart rate by paralyzing the vagus endings. All vagus inhibitions are thus abolished. The blood pressure rises moderately, mainly through the quickened rate, but partly through central vasomotor stimulation. If the blood pressure has been abnormally lowered by excessive vagus stimulation, the atropin rise will appear correspondingly greater. The cutaneous vessels are actively dilated by atropin, the skin assuming a more or less scarlet color. Toxic doses paralyze the vasomotor center and eventually also the arterial and cardiac muscle, so that the blood pressure falls profoundly.

Action on the Vagus.-Atropin stimulates the vagus center in common with the other medullary centers. It may therefore cause a slight primary slowing; but this is soon overcome by the peripheral paralysis. This vagus paralysis results in quickened heart rate in all animals in which the vagus is tonically active (in man, the cat, dog, etc.). In completely atropinized dogs and cats, the rate is about 140 to 240 per minute. The vagus tone being small in rabbits and in frogs, these animals show little or no quickening; but in these the vagus is also paralyzed, for its stimulation does not slow the heart. In man, the vagus tone is greatest in middle life, less in old age, and least in infancy; and the response of the heart rate to atropin varies accordingly.

The duration of the paralysis varies with the dose, and also with the species. It lasts only a few minutes in rabbits (Rossbach, 1874); longer in goiterous rabbits (Fleischmann, I9II). In dogs and cats, a partial effect persists for hours (Pilcher and Sollmann).

The dosage required to paralyze the vagus has been studied by Pilcher and Sollmann, 1914. It is always small, but varies somewhat in different animals, even of the same species, and even for the two vagi of the same animals; the left vagus being more susceptible. Low blood pressure also increases the susceptibility. In every respect, the susceptibility to atropin is inverse to the natural excitability of the vagi. In the dog, about o.or mg. per kilogram lessens the response to vagus stimulation perceptibly; and about two to four times this dose abolishes the response completely. When the paralysis is incomplete, a slight further slowing may be secured by strengthening the stimulus; but with the larger dosage even the strongest stimulus remains ineffective. 
The vagus paralysis is located in the termination of the vagus (or in the receptive substance); for stimulation of the postganglionic fibers (at the sinus-auricular junction of the frog) fails to slow the heart.

Atropin abolishes cardiac inhibition by all drugs which act through the vagus mechanism, whether they stimulate this reflexly (ammonia or chloroform inhalation); centrally (picrotoxin or veratrum), or peripherally in the ganglia (nicotin), or in the endings (pilocarpin, muscarin, physostigmin).

Results of Vagus Stimulation After Atropin.-In dogs, when the atropin action has gone just so far that stimulation of the divided vagus trunk causes no perceptible slowing, this still produces a slight fall of blood pressure; larger doses abolish this response also, so that the inotropic properties of the vagus terminations seem to be somewhat more resistant than the chronotropic.

When the intact vagus is stimulated in atropinized mammals, the blood pressure may fall or rise by stimulation of the afferent vasodilator or constrictor fibers which run in the vagus.

In atropinized frogs, stimulation of the vagus trunk quickens the heart, since this carries the accelerator fibers, which are not affected by atropin.

Atrio-ventricular Rhythm. - This may occur in man from doses of I mg. It sets in within 8 to ro minutes, and soon reaches a maximum, and then disappears. Wilson, I915, assumes that atropin releases the $\mathrm{A}-\mathrm{V}$ tissue from vagus control somewhat before it releases the sinus.

Influence of Heart Rate on the Output of the Heart.-The output of the heart for a given time is the product of the amplitude and rate of its excursions. The removal of the normal vagus inhibition greatly increases the rate; but when the rate exceeds 80 per minute, this tends to lessen the amplitude of the excursions; for a faster rate does not permit time for the complete diastolic filling of the heart. The output is therefore not proportional to the rate, if this exceeds 80 per minute (Y. Henderson, rgog). If the minute-volume with the rate of 90 be taken as roo, it would be about 40 per cent. at the rate of 30 ; 75 per cent. at 60 beats; I 20 to I 40 per cent. between 120 to 240 beats; and 100 per cent. at 300 beats. The approximate influence of the atropin quickening on the output, and through it on the blood-pressure can be easily deduced from this table; but it must not be forgotten that atropin also has other actions on the circulation.

Therapeutic Use of Vagus Action.-The paralysis of the vagus endings indicates atropin ( $\mathrm{I}$ to $2 \mathrm{mg}$., $1 / 60$ to $1 / 30 \mathrm{gr}$., hypodermically, repeated as needed) in conditions of excessive vagus stimulation, such as cerebral pressure; the reflex vagus inhibition of chloroform anesthesia; operations about the vagus trunks, etc. Atropin also abolishes such cardiac irregularities as are due to heightened vagus excitability (pulsus respiratorius), and it may be used to diagnose whether a cardiac slowing is due to vagus stimulation. (It has no effect in complete heart-block; van Egmond, I9I3).

Action on Cardiac Muscle.-Atropin has a slight direct effect on the cardiac muscle, which can be observed in the isolated heart, and also on the nerve-free heart of the embryonal chick. Small doses stimulate the muscle, causing an increased tonus, greater excursions, and some slowing. An exhausted frog's heart may be made to Deat again. Large doses diminish the tonus and may slow or quicken the rate. Finally the heart is paralyzed, but only after the vasomotor center. Indeed, the effects on the heart muscle are not of any importance in the sytemic action of the drug, being overshadowed by the other effects.

In the excised mammalian heart (Langendorff method) atropin provokes rhythmic contractions of the apex after the base is clamped. This requires larger doses than are needed for paralyzing the vagus. These large doses may also restore the beat when it has been arrested by muscular poisons (Luchsinger, r881).

In intact animals, atropin increases the auricular, but decreases the ventricular efficiency (Wiggers, rg16).

Vasomotor Center.-Sollmann and Pilcher, 1910, have shown by their perfusion method, that this is moderately stimulated. The stimulation is not sufficiently marked to produce a rise of pressure when ordinary doses are injected after dividing the vagi.

Large doses of atropin prevent'the depressor action of cholin (q.v.). 
Pulmonary Vessels. - Atropin has no effect on the normal vessels, but removes the constriction of pilocarpin or histamin (Berezin, I9I4).

Cerebral Vessels. - These are dilated (Berezin, 1916).

Cutaneous Circulation and Skin Eruptions.-Atropin produces intense scarlet flushing of the skin, particularly of the face and chest, occurring as extensive ery thematous spots, or as a continuous erythema (which has sometimes been mistaken for scarlatina). This effect is much greater in some patients than in others; it may occur with therapeutic doses. It is due to dilatation of the cutaneous vessels (vasodilator stimulation), with increased general blood pressure. This hyperemia may be so intense as to lead to desquamation.

\section{TEMPERATURE}

Atropin suppresses perspiration, and thus causes a rise of temperature with moderate doses, notwithstanding the cutaneous vasodilatation. Animals which do not possess sweat glands ( $\operatorname{dog}$ ) do not show this rise. Larger doses produce a fall of temperature from a lessened heat production, the result of the general depression. The final convulsions may again cause a rise.

\section{GASEOUS METABOLISM}

This is increased by therapeutic doses (r mg.) in man (Edsall and Means, rgr4; Higgins and Means, I9 15 ).

\section{ACTIONS ON THE EYE}

Atropin dilates the pupil (mydriasis) and renders it insensitive to light. It also paralyzes accommodation (cycloplegia), the lens being ad-

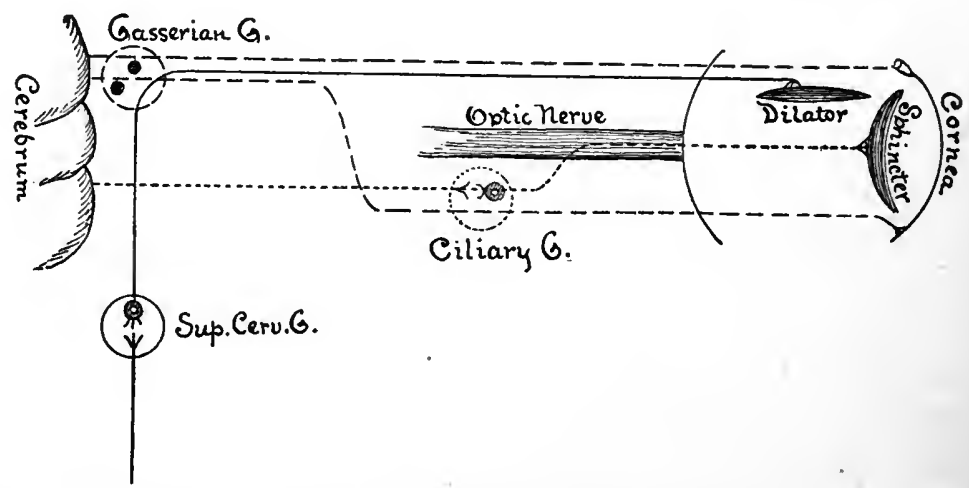

Fig. 10.-Innervation of iris (adapted from P. Schultz): Solid line = sympathetic (dilator); fine dotted line $=$ oculomotor (constrictor); coarse dotted line $=$ trigeminal.

justed only for far vision. These actions are entirely peripheral and consist in paralysis of the oculomotor endings. The intraocular tension in glaucoma is increased through the mydriasis.

The atropin effects may be removed by drugs which stimulate the oculomotor endings (pilocarpin, physostigmin, muscarin); and vice versa.

The atropin mydriasis and cycloplegia are very persistent; the mydriasis especially may persist for several days. The ocular actions are produced to a varying degree by all the atropinoid alkaloids.

Localization of Actions on the Pupil.-To understand the pharmacology of the interesting pupillary reactions, it is necessary to recall the anatomical relations of the iris mechanism (see Fig. $\mathrm{ro}$ ). 
The iris contains two sets of smooth muscle fibers, the sphincters and dilators.

The sphincters are innervated by fibers contained in the oculomotor. These terminate around the cells of the ciliary ganglia. From here the fibers pass as the short ciliary nerve.

The nerve fibers for the dilators run in the cervical sympathetic and terminate in the superior cervical ganglion. The fibers which arise from here go directly to the dilator muscle without passing through any other cells. They run to the Gasserian ganglion, where they join the first branch of the trigeminal, and go from here as the long ciliary to the muscle.

Stimulation of the Sympathetic Mechanism, in any of its parts, results in (submaximal) dilation of the pupil, with little disturbance of accommodation; in exophthalmos and separation of the eyelids; and in constriction of the ocular vessels. These are the effects produced by the drugs which stimulate the sympathetic division; typically by cocain; less typically by epinephrin, for this can produce mydriasis only if the oculomotor tone is low, or if the sympathetic endings are hyperexcitable.

Stimulation of the Oculomotor Mechanism, in any of its parts, results in constriction of the pupil (miosis), spasm of accommodation, and lowering of intraocular pressure. These are the typical effects of the drugs which stimulate the cranio-sacral divisionpilocarpin and physostigmin, etc.

Paralysis of the Oculomotor Mechanism would have the opposite effect; namely those just described for "Atropin." (The asphyxial dilation of the pupils is probably due to paralysis of the oculomotor center.)

Oculomotor Tone.-The iris appears to be normally controlled chiefly by variations in the oculomotor tone, the sympathetic playing mainly the part of a reciprocal antagonist-that is, it is stimulated when the oculomotor is inhibited, and vice versa. The strong oculomotor (constrictor) tone is normally held in check by a great variety of sensory and psychic impulses. When these inhibitory impulses are abolished ( $f . i$., by sleep, narcosis, and in a specific manner by morphin), the pupils are constricted by the unchecked oculomotor tone. On the other hand, when the inhibitory impulses are reinforced, the pupils dilate. This occurs, in excitement.

Causes of Mydriasis.-Mydriasis can be due to any of the following causes:

Stimulation of the sympathetic mechanism (cocain).

Paralysis of the oculomotor mechanism, peripheral (atropin) or central (asphyxia).

Increased inhibition of the oculomotor center (excitement).

Causes of Miosis.-These may consist in:

Stimulation of the oculomotor mechanism, peripheral (pilocarpin, physostigmin), or central (picrotoxin).

Lessened inhibition of the oculomotor center (sleep, narcosis, morphin).

Paralysis of the sympathetic mechanism (practically unimportant.)

Experimental Localization of the Atropin Mydriasis.-Atropin dilates the pupil on local, as well as on systemic application. The mydriasis remains confined to the eye, and even to that side of the eye, to which it is applied. The dilation is produced in the excised eye of the frog, and even in the isolated iris. ${ }^{1}$ Its action must therefore be peripheral.

The constrictor response to stimulation of the oculomotor nerve is abolished by atropin. Stimulation of the postganglionic fiber (short ciliary) is also ineffective. The oculomotor mechanism is therefore paralyzed peripherally to its ganglia. The iris, however, still contracts to direct electric stimulation, so that the muscle fibers are shown to be unaffected. The paralysis must therefore be located in the oculomotor endings or in the receptive substance. This is as far as the analysis can be safely carried.

The Behavior of the Sympathetic Dilator Mechanism.-There has been considerable discussion as to whether atropin in addition to paralyzing the oculomotor, also stimu-

1 Slight effects may be obtained from atropin and physostigmin for three or four hours after death, in the human eye (Groenow, 1910). 
lates the sympathetic mechanism. The question may now be safely answered in the sense that there is no direct sympathetic stimulation by atropin; for stimulation of the sympathetic, electrically or by cocain causes a further dilation of the pupil, even after maximal doses of atropin. 'The atropinized pupil constricts somewhat when the sympathetic is divided; but this is the natural result of cutting off the tonic sympathetic impulses. It has been argued that the atropin dilation can not be a passive process, since it is of sufficient force to tear firm adhesions of the iris. This merely shows that the dilator muscles contract actively; but their contraction is simply the result of the withdrawal of the constrictor tone, and need not involve a direct stimulation of the sympathetic mechanism. It is quite probable, however, that the natural sympathetic tone is heightened when the constrictor tone is withdrawn, for this would harmonize with the general law of reciprocal innervation.

Direct Actions on the Iris Muscle and Ciliary Ganglia.-The statement that the muscular fibers are not affected by atropin must be limited to moderate doses. In greater concentration (according to most observers) it at first stimulates them, producing a temporary narrowing of the pupil; later it paralyzes them.

Direct application of very strong solutions to the ciliary ganglion also depresses this structure.

The iris of birds is not dilated by atropin, but by curare, since it consists of striped muscle.

Changes in Intraocular Tension.-All mydriatics tend to increase intraocular tension in glaucoma, although not in the same degree. Conversely, the tension is lowered by miotics. Neither has much effect on the normal intraocular tension (Wessely, I905; Bollet and Curtil, I9ri; Luebs, I9I3; Myashita, I9I3).

Mechanism of the Change in Tension.-It is commonly assumed that the rise of pressure in mydriasis is produced mainly by lessened filtration of the aqueous humor into the venous sinuses of the channels of Fontana and canal of Schlemm. These lie in the angle of attachment of the iris, and would be compressed by the contraction of the ciliary muscle, and by the crowding back of the iris. Miosis would have the opposite effect (especially in glaucoma; in the normal eye miotics are said to lower the pressure very little). The intraocular tension is also controlled by the blood pressure in the vessels of the iris (Leber), and would be lowered whenever these are ischemic, either through fall of the general blood pressure (relatively little influence; Kochmann and Roemer, I9I4); or through local vasoconstriction. The vasoconstricting cocain, therefore, generally lowers intraocular tension, although it produces mydriasis; and it is claimed that atropin also tends to lower the pressure in the normal eye. Exceptionally, however, glaucoma has been reported after cocain, holocain, and even after epinephrin (Gifford, I9I6).

Glaucoma.-The phenomena of this very serious eye disease depend mainly upon excessive intraocular tension; due, presumably, to blocked drainage (Schoenberg, I9I3). It is therefore treated by miotics (see physostigmin); and it contraindicates the employment of mydriatics. If their use is indispensable, the choice should fall upon cocain; and if cycloplegia is necessary, the briefly acting homatropin would be preferred to atropin.

Practical Application of Atropin to the Eye.-The effects of atropin are utilized mainly for paralyzing the ciliary muscle to facilitate ophthalmoscopic examination; and in the treatment of iritis.

For diagnostic work, the persistent cycloplegia is of ten objectionable, but in other cases the enforced rest of the eye may be desirable. The atropin can be temporarily antagonized by physostigmin; but if a short action is preferred the less persistent homatropin may be substituted. This is also much less liable to provoke glaucoma. However, homatropin should be used as dilute as possible (I or 2 per cent.), and followed by physostigmin, 0.2 per cent., a drop three times daily until the pupil and accommodation have returned to normal (Gifford, I9r6).

In iritis; the atropin mydriasis is used to secure rest, to prevent adhe- 
sions of the iris to the lens, or break them if already formed; and to effect assumed favorable changes in the circulation of the iris. It is also a weak anesthetic. The more prolonged action of atropin causes it to be preferred for this purpose.

Administration to Eye.-The atropin is always applied locally, by dropping an aqueous solution of the sulphate, or an oily solution of the free alkaloid, on the cornea; or by placing impregnated gelatin discs (Lamelle Atropina, B.P., 1/5,000 gr.) in the conjunctival sac. Watery solutions tend to flow into the lachrymal duct, thereby lessening the local action, and tending to systemic poisoning. To avoid this, but a single drop of a r per cent. solution should be applied at a time, and the lachrymal sac should be compressed with the finger. For continued use, in iritis,

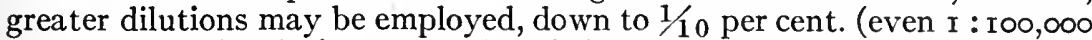
is somewhat effective). The effect is increased by the addition of cocain.

Duration of Action of Atropin.-With a single drop of I per cent. solution, the mydriasis reaches its maximum in about twenty-five minutes, the cycloplegia in about two hours. The cycloplegia disappears in two or three days (seven to nine days if the application has been continuous); the mydriasis endures even longer, ten to twelve days.

Side Actions.-Besides the increase of tension, atropin causes photophobia (because of the irresponsive pupil); and micropia, from the loss of accommodation.

In sensitive individuals, atropin produces some conjunctivitis, and rarely edema of the eyelids. If it is used carelessly, it may give rise to systemic poisoning, headache, dryness of the mouth, palpitation, etc. A species of chronic poisoning may occur in this way (Tyrrell, I906).

If atropin causes the local irritation, scopolamin should. be substituted, since this may be used in one-fifth the concentration.

Substitutes for Atropin.- These fall into two classes: (I) Those which produce mydriasis without cycloplegia (Euphthalmin, Ephedrin); and (2) those which produce both mydriasis and cycloplegia (Atropin, Hyoscyamin, Scopolamin, Eumydrin, and Homatropin-in the order of the persistence of the cycloplegia). Of these, atropin is the standard, the others being used only when it is contraindicated.

The action of hyoscyamin is similar to atropin, but it is effective in about one-half the concentration. Scopolamin is effective in one-fifth the concentration.

Quantitative measurements of the relative efficiency of mydriatics and miotics, alone and combined, are given by Jackson, r895; by Grube, 1905; by Horovitz, 1912; and by Joachimoglu, rgr 5 .

The following table shows the strength of solutions usually employed for ophthalmoscopic examinations; and the ordinary duration of their actions.

\begin{tabular}{|c|c|c|c|c|c|}
\hline \multirow[b]{2}{*}{ Drug } & \multirow[b]{2}{*}{$\begin{array}{c}\text { Percentage } \\
\text { Used }\end{array}$} & \multirow{2}{*}{$\begin{array}{l}\text { Action } \\
\text { Complete, } \\
\text { Hours }\end{array}$} & Mydriasis & \multirow{2}{*}{$\begin{array}{c}\text { Pairalysis } \\
\text { Complete, } \\
\text { Hours }\end{array}$} & \multirow{2}{*}{$\begin{array}{c}\text { Cycloplegia } \\
\text { Recovery } \\
\begin{array}{c}\text { Complete, } \\
\text { Days }\end{array}\end{array}$} \\
\hline & & & $\begin{array}{c}\text { Recovery } \\
\text { Complete, } \\
\text { Days }\end{array}$ & & \\
\hline 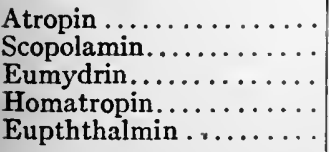 & $\begin{array}{l}1 \\
1 / 5 \\
\text { I to } 5 \\
2 \\
5 \text { to } 10\end{array}$ & $\begin{array}{l}1 / 2 \\
1 / 2 \\
1 / 2 \\
1 / 2 \\
1\end{array}$ & $\begin{array}{c}5 \text { to } 7 \\
5 \\
2 \\
\text { to } 1 / 2 \\
I\end{array}$ & $\begin{array}{l}2 \\
\mathbf{I} \\
\mathbf{I} \\
\mathbf{I}\end{array}$ & $\begin{array}{c}3 \text { to } 4 \\
3 \\
2 \\
1\end{array}$ \\
\hline
\end{tabular}




\section{PREPARATIONS-SYNTHETIC MYDRIATICS}

*IIomatropina IIydrobromidum (Homotrop. Hydrobr.), U.S.P., B.P.; $\mathrm{C}_{16} \mathrm{H}_{21} \mathrm{O}_{3-}$ N.HBr.-Mandelic ester of tropin. Freely sol. in water ( $1: 6)$; sol. in alc. ( $I: 40$ ) Instilled as I to 2 per cent. solution. Dose, $0.5 \mathrm{mg}$., $1 / 120$ gr., U.S.P.; to $2 \mathrm{mg}$., $1 / 64$ to $1 / 32$ gr., B.P. Maximum dose, 2 mg., $1 / 30$ gr.

Systemic actions similar to atropin, less toxic. Its action on the vagus is purely paralytic, but weaker than atropin (Zulick, I9I5).

Lam. Homatrop., B.P.-0.65 mg., 1/100 gr.

Eumydrin, N.N.R. (Methylatropin Nitrate.)-Readily sol. in water. It agrees very closely with atropin in systemic actions, and may be substituted for it, in the same dosage; in eye, $1 / 2$ per cent. Its toxicity is about one-fiftieth, and its mydriatic strength one-tenth that of atropin. Continued use often leads to tolerance (Seifert, Nebenwirk., x 915 , p. 66).

Euphthalmin Hydrochlorid, N.N.R.-Closely related to betaeucain in composition. It is readily soluble in water; 2 to 3 drops of 5 to ro per cent. solution are instilled. Side actions, Seifert, Nebenwirk., 1915, p. 66.

\section{PARALYSIS OF SECRETIONS}

Atropin and the related alkaloids arrest all secretions which depend upon central innervation: This includes the sweat, saliva, tears, mucus, gastric juice and ordinary pancreatic secretion; but not the urine, milk, bile or secretin pancreatic secretion. The action is localized in the nerveendings or receptive substance, and is mutually antagonistic with the pilocarpin action.

Suppression of Saliva (Antisialogogue Action).--The submaxillary glands furnish a classical object for localizing the action of Atropin. This suppresses the secretory response to chorda-tympanic stimulation, even when this is applied at the hilus of the gland, i.e., to the postganglionic fibers. The paralysis must therefore be peripheral to these. The cells, however, still respond to sympathetic stimulation, so that the paralysis must be in an intermediary substance, in the nerve-endings.

This argument involves the assumption that the chorda tympani and the sympathetic act upon the same cells in the salivary gland. This is doubted by A. P. Mathews, and by Gaskell, IgI6. The evidence is, therefore, not as conclusive as in the case of smooth and cardiac muscle, where the excitability of the contractile substance can be confirmed by direct stimulation; but the analogy is otherwise so complete, that the conclusion is fairly safe.

Large doses of atropin inhibit also the response to sympathetic stimulation (Cannon and Cattell, r9I6); perhaps by paralyzing the secretory cells directly.

In man, Ewing, I9I $\mathrm{x}$, found that 0.5 to I mg., by mouth, caused a marked decrease in the volume of saliva secreted. The percentage of organic solids was somewhat diminished; the percentage of inorganic solids showed only a trifling decrease. The percentage of ptyalin fell markedly, but recovered, or was even somewhat increased, long before the volume or solids had returned to'normal.

Vasodilator Fibers of the Chorda Tympani Nerve.-These are not affected, so that stimulation of the atropinized nerve still dilates the vessels and produces an increased venous outflow from the gland. (The effects of atropin and pilocarpin on the submaxillary circulation are discussed by Henderson and Loewi, r905.)

Antagonism.-Atropin completely suppresses the nicotin salivation (since this stimulates the ganglion cells); it is mutually antagonistic with pilocarpin, etc., but the atropin action, as usual, tends to be the stronger. It is also said to suppress the mercurial salivation, but not the "paralytic secretion" which sets in when the divided nerve degenerates.

Practical Significance of the Antisialogogue Action.-This is mainly a rather unpleasant side effect in the therapeutic use of the drug. Small doses (0.5 mg.) may be employed to suppress practically any form of pytalism. It is not much used, since the benefits scarcely offset the inconveniences attending the use of the drug. 
Suppression of Mucous Secretion.-This may be employed in the hypersecretion of coryza, bronchorrhea, ether anesthesia, etc. Minimal doses of atropin are used to abort incipient colds $(0.5 \mathrm{mg} ., 1 / 120 \mathrm{gr}$. or less, in a glass of water, sipped slowly in the course of an hour-Kohnstamm, I906). The cutaneous vasodilation may play a part in this abortive action.

Suppression of Sweat (Anhydrotic Action).-This is one of the earliest actions, and can be secured with small doses ( 0.3 to I mg., 1/200 to 1/60 gr., hypodermically). It is the most effective means of suppressing the nightsweats of phthisis, but the relief is only symptomatic, and scarcely justifies the inconveniences.

Other Anhydrotics.-The nightsweats may also be suppressed by scopolamin or eumydrin. Agaric acid also causes a peripheral paralysis of the sweat glands, but is uncertain. Camphoric acid has been employed, but it does not act upon the sweat glands. If it has any effect (which is doubtful), it must be indirect, through improvement of the respiration.

Local measures are often preferred, especially for local hyperhydrosis: Sponging with cold lotions containing alcohol, dilute acids, tannin, salicylic acid, formaldehyd, etc. Dusting-powders are likewise useful (talcum with 5 per cent. of salicylic acid or ro per cent. of boric acid).

Acidum Agaricum, N.N.R., and the impure mixture Agaricinum, is derived from a fungus of the larch tree. It is a white, odorless and tasteless crystalline powder, but slightly soluble in cold water and alcohol.

The anhydrotic action of agaric acid is peripheral, and probably resembles atropin, but is much weaker. The effect appears in a few hours, and is not lasting. It does not act on any other glands, and has none of the other actions of atropin. It is a local irritant (and therefore cannot be given hypodermically). Large doses produce vomiting, diarrhea, and death through central paralysis (Hofmeister, I 888). The maximum single dose should not exceed $0.03 \mathrm{Gm}$. (1/2 gr.); and the daily dose should be below $0.1 \mathrm{Gm}$. ( $11 / 2 \mathrm{gr}$.). As habituation occurs, it is better to start with $0.5 \mathrm{mg}$. $(1 / 120 \mathrm{gr}$.$) . Clinical opinions differ as to its value.$

Sodium Tellurate appears to act similarly, but is objectionable on account of the persistent garlic odor which it gives to the breath.

Gastric Actions.-The secretion of gastric juice, and especially of hydrochloric acid, is diminished or suppressed, through paralysis of the secretory vagus endings (Chiari, I9I5). The motor activity (pyloric spasm, etc.) is also depressed by the vagus paralysis (Auer and Meltzer, I906). Both actions make atropin useful in hyperchlorhydria, gastric ulcer, tumors, etc. It is especially indicated (0.5 to I mg., $1 / 120$ to $1 / 60$ gr., before meals) in acute conditions. with severe pain and vomiting (Mathieu and Girard, I9I3; A. Schmidt, I9I4). It has been used in seasickness (atropin, $0.5 \mathrm{mg}$.; strychnin, I mg., hypodermically-Girard, I906). The sojourn of meat in the stomach is delayed by large doses of atropin (Tysebaert, I9I2).

Antagonism to Emetics.-Eggleston, r916, finds that very small doses of atropin (0.oI mg. per kilogram) prevent the emetic action of pilocarpin and nicotin, but not of apomorphin, morphin, ouabain or emetin. The ant-emetic dose being smaller than is required against peripheral actions of pilocarpin, suggests that atropin depresses an emetic center.

Pancreatic Juice.-Atropin suppresses the increase of pancreatic secretion which ordinarily follows stimulation of the vagus or the administration of pilocarpin, cholin, etc. Large doses, however, provoke a pancreatic flow in the dog (Popielski, 1896; Modrakowski, 1906); the mechanism of this peculiar action is not understood; it may possibly be connected with the actions on the liver.

On the secretin flow, atropin has no direct effect (Chiari, 1915), but since it diminishes the secretion of acid in the stomach, it probably lessens the production of secretin indirectly, and hence interferes with the physiologic pancreatic secretion. 
The pancreatic stimulants which are antagonized by atropin yield active juice, whereas those which are not affected by atropin require the addition of erepsin (Camus and Gley, 1905).

Uric Acid.-Atropin is said to prevent the increase of uric acid that normally follows the ingestion of food; presumably by preventing the secretion of the digestive juices (Mendel and Stehle, 1915). It also prevents the atophan increase (Abl, I9I3). $^{2}$.

Lacteal Secretion.-Ott and Scott, Igr2, confirm Roehrig, that this is somewhat diminished by atropin.

\section{GLYCOGENOLYSIS}

The old statement that atropin prevents the transformation of the hepatic glycogen into sugar, appears to be erroneous. It originated with Morat, 1883, who concluded that pilocarpin stimulates, and atropin inhibits, the conversion of glycogen into sugar. Cavazzani and Soldaini, 1896, also stated that atropin paralyzes glycosecretory nerves in the liver. The evidence, however, was unsatisfactory and has been disproven by the results of Mosenthal, I913, and of Macleod, I908. The latter showed that splanchic stimulation produces the same hyperglycemia after atropin as normally. McGuigan, I916, also finds no direct effect of atropin or pilocarpin. Large doses of atropin may increase the blood sugar (also observed by Pitini, r908). This is due simply to the excitement.

\section{ATROPIN IN DIABETES}

It has been asserted that atropin increases the tolerance for carbohydrates (Rudisch, Forchheimer); but the statement needs confirmation (Mosenthal, I9 2 ).

\section{HEPATIC ACTIONS AND COAGULABILITY OF BLOOD}

The secretion of bile is sometimes slightly diminished by atropin (Okada, I9r5).

The injection of large doses of atropin into the portal vein lowers the coagulability of blood, first that of the hepatic vein. This effect can not be produced by injection into the jugular vein, nor by addition of atropin to shed blood (Doyon and Kareff, I 904 and 1906), and is due to an action on the liver. The anticoagulant substance is also produced by the perfusion of atropinized blood through an excised liver (Doyon, rgro).

The leucocytes are not changed by ordinary doses. Toxic doses, especially when often repeated, diminish the eosinophiles. The action is probably indirect (Herrick, 1914; Port and Brunow, 19I4). The emigration of leucocytes in exposed mesentery is not inhibited by atropin or scopolamin (Ikeda, I9I6).

\section{ACTION OF ATROPIN ON PERISTALSIS}

These vary according to the dose and conditions: Minimal doses quiet excessive peristalsis provoked by toxic peripheral vagus stimulation, as by pilocarpin, cholin, physostigmin, etc. These doses do not affect normal peristalsis.

Ordinary therapeutic doses relax the intestines in man and rabbits (Katsch, r913; Hirz, I9I3). In cats, they may increase peristalsis (Magnus). It is conceivable that this also occurs in man in some conditions.

Excessive doses, such as can be brought into play only by local application, paralyze the intestine. All these actions are peripheral, but affect different structures in the intestine. The details are described on pages 269,275 .

Therapeutic Use of the Peristaltic Actions.-The inhibitory action of atropin is utilized against toxic spasms of the intestine, which presumably depend upon peripheral vagus stimulation: the colic pains of indigestion, lead colic, ptomain diarrhea, etc. The dose for this purpose is usually I mg. ( $1 / 60$ gr.).

It also lessens, in this way, the "griping" effect of purgatives, at the same time enhancing their laxative effect by its own stimulant action. The galenic preparations would be preferred, to prolong the local action: For pills, Extractum Belladonnæ, I 5 mg. or $1 / 4$ gr.; for liquids, Tr. Belladonnæ, 0.5 c.c. or Io minims. 


\section{BILIARY AND RENAL COLIC}

Atropin paralyzes the innervation of the smooth muscle of the bile ducts and ureters, and therefore relaxes the painful and obstructive spasm which results from the passage of calculi through these tubes. The vagus paralysis is also useful in this connection by preventing the dangerous cardiac reflex which sometimes occurs. The atropin, I mg., $1 / 60 \mathrm{gr}$, is given hypodermically, usually combined with morphin, $15 \mathrm{mg}$., $1 / 4 \mathrm{gr}$. The gall-bladder is also relaxed (Lieb and McWhorter, 1915).

Excised Ureter.-Small doses of atropin may stimulate the tone and peristaltic movements. Stronger solutions ( $\mathrm{r}: 10,000)$ inhibit them, as also the stimulant effects of pilocarpin, physostigmin and cholin (Macht, 1916).

\section{URINARY BLADDER AND INCONTINENCE OF URINE}

Atropin is employed to relieve incontinence of the urine, due to overaction of the bladder muscle; and retention of urine from overactivity of the vesical sphincter. The details of dosage are described by Ruhraeh, Am. J. Med. Sc., February, rgr2. A. Jacobi advises for children $1 / 2 \mathrm{gr}$. of Extract at night, increasing if necessary, to flush the cheeks.

Urine Flow.-Discordant results have been described by different investigators. They are summarized by Cow, IgI 2 and Igr 3 . He finds that atropin has no effect if the urine is collected directly from the pelvis of the kidney; but that it merely modifies the flow through the ureter, by lessening its tone.

\section{UTERUS}

The effects of atropin correspond to its actions on the intestine: Small doses increase, large doses abolish, the uterine movements (Kehrer, I908). Minimal doses abolish the pilocarpin and physostigmin spasms. Even large doses of physostigmin are ineffective after atropin.

Atropin in Dysmenorrhea.- This was introduced by Drenkham, rgro, and is advocated by Novak, I9r5; especially in the spasmodic, colic type. The administration is started two days before the expected menses, and continued for two or three days during menstruation; usually in doses of $0.6 \mathrm{mg}$. (1/100 gr.) by mouth, three or more times daily. If dry throat, itching skin or disturbed accommodation develop, the dosage is reduced.

\section{CURARE ACTION}

In frogs, large doses of atropin paralyze the motor endings of striped muscle and abolish the physostigmin twicthings, after the manner of curare. This effect would not occur with doses which can be used in mammals.

\section{ANODYNE ACTION}

The local application of atropin dulls, rather than paralyzes, sensory nerves. It is therefore applied in the form of belladonna liniment or plaster, for the relief of pain, especially neuralgic and rheumatic. Belladonna suppositories (extract, 0.I Gm., I1/2 gr.) are also used in painful hemorrhoids.

Dosimetric Table.-The following (Schmiedeberg) may be useful in the therapeutic use of atropin:
MG. GR.
Gr. SyMptoms
0.5 to I $1 / 120$ to $1 / 60$ Dryness in mouth, often with thirst.
$1 / 60$ Dryness in mouth, often with thirst.
$1 / 30$ Pupil dilated, not quite immobile. Increase of pulse rate.
3 to $51 / 20$ to $1 / 12$ Headache. Dysphagia. Alteration of voice. Muscular weakness. Restlessness.

$71 / 10$ Considerable dilatation of pupils. Disturbance of vision.

$81 / 8$ Excitement and muscular incoördination more marked.

ro $1 / 6$ Apathy. Hallucinations or delirium. Unconsciousness. 


\section{ATROPIN POISONING}

Atropin is very readily absorbed, somewhat even from the intact skin, so that toxic symptoms have been observed (though rarely) after the use of belladonna plasters (Doland, r 906). They occur rather commonly in ophthalmic use, through the lachrymal ducts. Where the plant grows wild, the berries are often eaten by children.

Symptoms.-These set in promptly, but usually run a long course; lasting five to fifteen hours, or even several days in fatal cases. The first to be noticed are those arising from dryness of the mouth and throat: difficulty of deglutition and articulation, great thirst, a sense of burning and constriction in the throat. On the eyes, the dilatation of the pupils, impaired vision, absence of reaction to light will be noticed. There is often nausea and sometimes vomiting. Excitement, passing into delirium, is a prominent feature. The delirium may be pleasing, with spectral illusions, or anxious or furious (a graphic description of the psychic experiences is given by L. L. Jepson, 1907). The onset of the paralytic symptoms is ushered in by giddiness, numbness of the limbs, and staggering gait, and passes into drowsiness and stupor. The pulse is quick (to $\mathrm{r} 70$ ) and small. The respiration is stertorous, fast or slowed. The face is flushed scarlet. In fatal cases death is preceded by coldness of the extremities, rapid and intermittent pulse, and deep coma. Convulsions are rare. Glycosuria is sometimes seen and may be attributed to the asphyxia. The postmortem findings are those of asphyxia.

A fatal ending is, however, quite rare (r 2 per cent.). The fatal dose of atropin is probably 0.I Gm. or more for adults; 0.or $\mathrm{Gm}$. for children. Therapeutically, children bear proportionately larger doses than adults, and this holds also for young animals.

Recovery starts within two days, the respiration, pulse and pupils returning gradually to normal. Sometimes violent delirious symptoms may last for days, and it has happened that patients have been consigned to the insane asylum on a mistaken diagnosis.

A psychic "slowness," disturbance of vision, and some other symptoms, may persist for weeks.

Chronic Atropin Poisoning has been reported, especially from ophthalmic use (Tyrrell, Igo6). However, the continued use of small doses establishes partial Tolerance to the toxic action, but not to the effects on the vagus or oculomotor (Anrep and Rossbach, I880; Cloetta, I9II).

Treatment of Atropin Poisoning.-This is usually effective since the prolonged course gives ample time for interference. It resolves itself into chemic neutralization, prompt removal, and meeting the symptoms. The delirium is best treated by the ice-cap, the general symptoms by pilocarpin (Io mg., 1/6 gr. hypodermically, repeated until mouth is moist). Morphin (ro mg.) is also indicated in the early stages, but not after depression has set in. The latter is combated by the usual medullary stimulants (coffee, etc.) Artificial respiration should be kept up persistently, if necessary.

The effects on the eye may be abolished by the local application of physostigmin.

Excretion.-The greater part of the atropin disappears rapidly from the blood (Cloetta, I908). It is excreted mostly unchanged, entirely by the urine, mainly within thirty-six hours.

The application of a drop of this fluid to the eye of a cat is the most convenient test for poisoning. Mydriasis occurs with a single drop of 
I : I 50,000 solution, equivalent to $0.000245 \mathrm{mg}$. of atropin. Of scopolamin, the minimal mydriatic dose is $0.00001855 \mathrm{mg}$. (Joachimoglu, I9I5). The human pupil is about equally sensitive (Metzner, I9I2). The excretion is not complete, more or less of the atropin being destroyed in the body.

A part is excreted as tropin and as another base (Fickewirth and Heffter, I9I2). The variations in this destruction and in the rapidity of excretion explain the racial and individual idiosyncrasies and acquired tolerance.

Atropin resists putrefaction for a long time, and may be found in the cadaver even months after burial. Confusion with ptomatropin (a putrefactive alkaloid) must be guarded against.

\section{RACIAL AND ACQUIRED TOLERANCE}

Rodents and marsupiads are very tolerant, as also, to a less extent, the goat, dog, birds, and some other animals. (The dosage for different animals has been determined by Wilberg, I914.) The immunity is in no case complete. It is perhaps greatest in the case of rabbits, and can be increased by habituation. In rabbits, it is due mainly to destruction of the atropin by the serum and organs.

Heckel, 1875 , found that rabbits and guinea pigs did not only thrive for weeks on an exclusive diet of atropin-containing plants (Schroff, 1852), but that this exclusive diet could be continued for several generations. A further degree of immunity was thus acquired, so that the mydriatic effect was smaller and the fatal dose (hypodermically) higher. The flesh of these animals contained relatively little atropin, although the meat of ordinary rabbits poisoned with atropin is toxic to other animals and man (Koppe, I866; Lewin, 1894).

Cause of the Tolerance.-This has been investigated chiefly in rabbits. It is not due to defective absorption or superior excretion, for the tolerance exists also for hypodermic injection, and after ligation of the renal vessels (Hermann, 1874). The leucocytic theory of Calmette, 1899 , need not be further considered since it was disproved by Ellinger, I901. The correct explanation was furnished by Fleischmann, rgro and IOIx. He found that digestion of atropin with rabbit's serum gradually weakened its activity; $x$ c.c. of the serum may thus destroy o.r mg. in twenty to thirty minutes. The serum of man, dogs, cats and rats does not have this action (except some cases of human goiter: Doeblin and Fleischmann, 1913). These observations have been fully confirmed by independent workers. Cloetta, I9II, and Clark, I9I2, find that the destruction is also effected by emulsions of rabbit's liver. In frogs, the heart and kidneys also have this effect. The blood corpuscles are not concerned in the reaction. Since the property is destroyed by heating to $55^{\circ}$ to $60^{\circ} \mathrm{C}$., it is probably due to a ferment, formed in the liver (Metzner, Igr 2; Clark). Doeblin and Fleischmann, 19r3, find that it has the characters of the albumin fraction. Metzner believes that there is a cleavage of tropeic acid and tropin, which may recombine on very prolonged standing. Homatropin and scopolamin are also destroyed (Doeblin and Fleischmann). Fleischmann found that the destructive power varies greatly in different rabbits, being practically absent in some; he attributed this to goiter. Metzner and Hedinger, I9r 2, confirmed the variation, but showed that it bears no relation to the thyroid; nor does the injection of thyroid confer a destructive power for atropin when this is deficient. Heffter and Fickewirth, 1912 find that the tolerance of rabbits to rapid intravenous injection is relatively small, since this does not permit time for the destruction. The rapid passage of atropin out of the blood indicates that its destruction must take place mainly in the tissues (liver).

Cause of Habituation; Excretion in Animals.-Cloetta, I9xx, found that non-habituated rabbits excrete 15 to 20 per cent. of the atropin, the excretion being completed in two to three days. (Fickewirth and Heffter find that the total excretion of all bases is about 50 per cent. of the administered quantity.) After immunization, the excretion is complete in one day; and as little or none can be found in the tissues, it appears that the rapidity of destruction and excretion has been increased. The destructive power of the serum, however, is not raised.(Doeblin and Fleischmann). Normal cats excrete very little of the atropin; but by habituation, the excretion is markedly raised, 
whilst the capacity for destruction is not increased. With these therefore the tolerance is due mainly to the acquired speed of excretion. Dogs excrete about one-thrd of the atropin, within two days (Wiechowski, Igor).

\section{HYOSCYAMIN}

Actions and Uses.-This is the levo-isomer of the racemic atropin. Cushny, I903, compared these two alkaloids and the dextro-isomer. $\mathrm{He}$ finds that the peripheral actions on the iris, cardiac vagus and glands are I 2 to I 8 times as strong with the 1.-isomer (ordinary hyoscyamin) than with the d.-; and about twice as strong as with atropin. Hyoscyamin, therefore, has some advantage when the peripheral effects are desired, especially in ophthalmic work. Internally, it has no advantage. All the isomers act practically alike on the central nervous system of mammals. The d.- has a greater stimulant effect on the spinal cord of frogs.

\section{PROTOCATECHYL TROPEIN}

This has been investigated by Marshall, I9ro. It paralyzes the vagus endings, but is less powerful than atropin or even homatropin. It produces a curare effect on striped muscle and a temporary central paralysis of respiration. There is also an independent gradual fall of blood pressure.

\section{PREPARATIONS-ATROPIN GROUP}

Atropina, U.S.P., B.P.; $\mathrm{C}_{17} \mathrm{H}_{23} \mathrm{NO}_{3}$.-The alkaloid obtained from Belladonna and other plants of the Solanaceæ family. Slightly sol. in water (I:455); freely sol. in alc. $(\mathrm{x}: 2)$; sol. in fat solvents and oils. Dose, $0.5 \mathrm{mg}$., $1 / 120$ gr., U.S.P.; 0.3 to $0.6 \mathrm{mg} ., 1 / 200$ to $1 / 100 \mathrm{gr}$., B.P.

${ }^{*}$ Atropince Sulphas (Atrop. Sulph.), U.S.P., B.P. $\left(\mathrm{C}_{17} \mathrm{H}_{23} \mathrm{NO}_{3}\right)_{2} \cdot \mathrm{H}_{2} \mathrm{SO}_{4}+\mathrm{H}_{2} \mathrm{O}$.White crystalline powder; odorless. Very sol. in water ( $\mathrm{r}: 0.4)$; freely sol. in alc. $(\mathrm{I}: 5)$ or glyc. ( $\mathrm{I}: 2.5)$. Incompatible with alkalies, tannin and mercuric salts. Atropin solutions deteriorate rather rapidly; a $r: r, 000$ solution losing considerably in activity within three days (I. Simon, I9I5). Sterilized solutions in ampoules, however, preserve their full activity at least four weeks (Joachimoglu, 1915). Dose, 0.5 mg., $1 / 120$ gr., U.S.P.; 0.3 to $0.6 \mathrm{mg}$., $1 / 200$ to $1 / 100$ gr., B.P. Maximum dose, I mg., $1 / 60 \mathrm{gr}$.

Lam Atrop., B.P.-0.0r3 mg., 1/5,000 gr., B.P.

Liq Atrop. Sulph., B.P.- I per cent. Dose, 0.03 to 0.06 c.c., $1 / 2$ to I minim, B.P.

Ung. Atrop., B.P. -2 per cent.

Hyoscyamin. Hydrobr., U.S.P.; $\mathrm{C}_{17} \mathrm{H}_{23} \mathrm{NO}_{3 .} \mathrm{HBr}$.-Strongly levorotary. Very sol. in water; freely sol. in alc. ( $\mathrm{I}: 2.5)$. Dose, $0.3 \mathrm{mg} ., 1 / 200 \mathrm{gr}$.

Hyoscyamin. Sulph., B.P.; $\left(\mathrm{C}_{17} \mathrm{H}_{23} \mathrm{NO}_{3}\right)_{2} \cdot \mathrm{H}_{2} \mathrm{SO}_{4}+{ }_{2} \mathrm{H}_{2} \mathrm{O}$.- -Very sol. in water ( $\mathrm{r}: 0.5$ ). Dose, 0.3 to $0.6 \mathrm{mg}$., $1 / 200$ to $1 / 100$ gr., B.P.

Belludonne Folia, U.S.P., B.P.; Belladonna Leaves (Deadly Nightshade).--The dried leaves and tops of Atropa Belladonna, yielding not less than 0.3 per cent. of mydriatic alkaloids. Dose, $0.06 \mathrm{Gm}$., I gr., U.S.P. Maximum dose, $0.2 \mathrm{Gm}$., $3 \mathrm{gr}$.

Belladonna Radix, U.S.P., B.P.; Belladonna Root.-The dried root of Atropa Belladonna, yielding not less than 0.45 per cent. of mydriatic alkaloids. Dose, $0.045 \mathrm{Gm}$., $3 / 4$ gr., U.S.P.

${ }^{*}$ Emp. Bellad., U.S.P.; Belladonna Plaster.-30 per cent. of Ext. Bellad. Fol.; 0.38 per cent. of alkaloids.

${ }^{*}$ Emp. Bellad., B.P.-Prepared from Liq. Ext. Bellad. Root. 0.25 per cent. of alkaloids.

${ }^{*}$ Exi. Bellad. Fol., U.S.P.—I Gm. represents 4 Gm. of leaves, 1.25 per cent. of alkaloids. Occurs in both pilular and powdered form. Dose, $15 \mathrm{mg} ., 1 / 4 \mathrm{gr}$., U.S.P. Maximum dose, $50 \mathrm{mg} ., 3 / 4 \mathrm{gr}$.

${ }^{*}$ Ext. Bellad. Fol., B.P.-I per cent. of alkaloids of leaf. Dose, 16 to $60 \mathrm{mg}$., $1 / 4$ to I gr., B.P. 
Fldext. Bellad. Rad., U.S.P.- 0.45 per cent. of alkaloids. Dose, 0.05 c.c., I minim, U.S.P.

Ext. Bellad. Liq., B.P.- - 75 per cent. of alkaloids of the root.

Lin. Bellad, U.S.P.-Fldext. Bellad.Fol., with 5 per cent. of Camphor.

Lin. Bellad., B.P.—5o per cent. of Liq. Ext. Bellad. (root); 5 per cent. of Camphor.

${ }^{*}$ Tr. Bellad. Fol., U.S.P.- Io per cent, of the leaves, 0.03 per cent. of alkaloids. Dose, 0.75 c.c., I 2 minims, U.S.P. Maximum dose, I c.c., I 5 minims.

*Tr. Bellad., B.P. - ro per cent. of leaves, 0.035 per cent. alkaloids. Dose, 0.3 to I c.c., 5 to 15 minims, B.P.

Supp. Bellad., B.P.-I mg. of the alkaloids of the root. alkaloids.

*Ung. Bellad., U.S.P.- - o per cent. of Ext. Bellad. Fol., 0.125 per cent. of the

*Ung. Bellad., B.P.- 0.6 per cent. of the alkaloids of the root.

Dature Folia, B.P.- - The dried leaves of Datura fastuosa and Metel.

Datur. Sem., B.P.-The seeds of Datura fastuosa.

Tr. Datur. Sem., B.P. -25 per cent. Dose, 0.3 to I c.c., 5 to 15 minims, B.P.

Hyoscyamus, U.S.P.; Hyoscy. Fol., B.P.-The dried leaves and tops of Hyoscyamus niger, yielding not less than 0.065 per cent. of alkaloids, chiefly hyoscyamin, with some atropin and scopolamin. Dose, 0.25 Gm., 4 gr., U.S.P. Maximum dose, 0.4 Gm., 6 gr.

Ext. Hyoscy., U.S.P.-A pilular extract, I Gm. representing $4 \mathrm{Gm}$. of drug, 0.25 per cent. of alkaloids. Dose, $0.06 \mathrm{Gm}$., I gr., U.S.P. Maximum dose, $0.2 \mathrm{Gm}$., $3 \mathrm{gr}$.

Ext. Hyoscy., B.P.-A dry extract, with 0.3 per cent. of alkaloids. Dose, O.I 2 to $0.5 \mathrm{Gm} ., 2$ to $8 \mathrm{gr}$.

Fldext. Hyoscy., U.S.P.-About 0.065 per cent. of alkaloids. Dose, 0.2 c.c., 3 min ims, U.S.P.

*Tinctura Hyoscyami (Tr. Hyoscy.), U.S.P., B.P.- ro per cent. Miscible with water or alc. Dose, 2 c.c., 30 minims, U.S.P.; 2 to 4 c.c., $1 / 2$ to I dram, B.P. Maximum dose, 4 c.c., I dram.

*Stramonium, U.S.P.; Stramon. Fol., B.P.; Stramonium (Jamestown Weed, Jimson Weed). - The dried leaves of Datura Stramonium (also Datura Tatula, U.S.P.). Contains not less than 0.25 per cent. of alkaloids, chiefly hyoscyamin, with some atropin and scopolamin. Dose, $0.06 \mathrm{Gm}$., I gr., U.S.P. Maximum dose, $0.3 \mathrm{Gm}$., $5 \mathrm{gr}$.

Ext. Stramon., U.S.P.-A pilular or powdered extract, $1 \mathrm{Gm}$. representing $4 \mathrm{Gm}$. of drug, I per cent. of alkaloids. Dose, ro $\mathrm{mg}$., 1/6 gr., U.S.P.

Tr. Stramon., U.S.P.- 10 per cent. Dose, 0.5 c.c., 8 minims, U.S.P.

Tr. Stramon., B.P. -20 per cent. Dose, 0.3 to I c.c., 5 to 15 minims, B.P.

Ung. Stramon, U.S.P.- ro per cent. of the extract.

\section{SCOPOLAMIN OR HYOSCIN}

General Statement.-The commercial samples of scopolamin (also called hyoscin).are usually indefinite, and of ten rather impure mixtures of two isomeric alkaloids (tropeic esters of oscin, see p. 277): scopolamin proper (levorotary, $25.75^{\circ}$ ), and atroscin (optically inactive). They occur in the various atropin-containing plants, but are now prepared mainly from Scopola. The U.S.P. now specifies the optically active alkaloid, i.e., scopolamin proper.

The peripheral actions of scopolamin agree qualitatively with those of atropin; but there are quantative differences; for instance, the actions on the oculomotor and secretions are stronger, whilst the vagus action is relatively weak.

The central actions differ qualitatively; scopolamin being mainly sedative, in all doses; whilst moderate doses of atropin are excitant. However, there are great individual variations in the response to scopolamin, and, therefore, in its toxiticy. The variable toxicity is due mainly to idiosyncrasy, for it occurs with the purest samples; although the uncertain composition of the commercial samples probably plays a part. 
Chemic Differences in Scopolamin.-It is often stated that there are specific differences in the effects according to the origin of the alkaloids; and that "hyoscin" derived from hyoscyamus is therapeutically superior to the "scopolamin" of Scopola. No such difference can exist if the pure alkaloids are employed; for these are identical, whatever their source (E. Schmidt, I 892 and I894). Impure mixtures doubtless produce different effects, but impurities are quite as likely to be present if the alkaloid is prepared from hyoscyamus. A purified "scopolamin" is much more reliable than an impure "hyoscin." Moreover, there is no way of determining from which plant the alkaloid has been prepared. J.A.M.A., I907, 49: 2105). It is, therefore, useless to make this distinction between "scopolamin" and "hyoscin."

Apoatropin $\left(\mathrm{C}_{17} \mathrm{H}_{21} \mathrm{NO}_{2}\right.$, also called belladonnin or atropamin $)$ was demonstrated in a commercial sample by Kobert, 1905 , and has been held responsible for the occasional toxic effects. This isimprobable. It is a rare impurity (Kionka, I008) andits presence is easily shown, by reduction (brown color) on adding a few drops of permanganate solution (Kessel, I906). It exists preformed in the plants and may be derived chemically from atropin by the abstraction of one molecule of water. It causes, in rabbits, a rise of blood pressure, paralysis of cardiac vagus endings, central excitation, and death with relatively small doses, by paralysis of respiration. The oculomotor and secretory endings are not paralyzed (Marcacci, 1885; Kobert, 1905).

Deterioration of Solutions. - This has been suggested as a cause for idiosyncrasy ( $f . i$, by Gauss, I907; Langer, I9 2 and Sachs, I9I2); but according to the seemingly more reliable experiments of Kionka, 1908; Willstatter and Hug, I9I2; and Beck, I9I4; the solutions keep unchanged indefinitely, at least in sealed ampoules.

Pharmacologic Differences between Scopolamin and Atroscin.These play an important part in the peripheral actions, but not in the more important central effects. Cushny and Peebles, I905, found that the 1.- and i.- alkaloids act alike on the central nervous system in mammals, and on the motor endings; but that the left-alkaloid (scopolamin) is twice as active on the salivary and vagus endings as the racemic atroscin.

This has been confirmed, also for the pupil, by Hug, I912. Königsdörfer, I896, claims that there is no difference in the pupil action. Cloetta, 1913, contends that the racemic is less toxic than the 1-. in man, but without good evidence (Cushny, rgr 2). Smith, I9I 5 , found the fatal dose the same for mice.

Psychic Actions.-In man, doses of I to $3 \mathrm{mg}$. ( $1 / 60$ to $1 / 20 \mathrm{gr}$.) generally produce within ten or fifteen minutes, fatigue, drowsiness, and a natural, dreamless sleep, lasting several hours. The response, however, is variable. In some the sleep is preceded by hallucinations, which may be rather pleasant with small doses, but which become violent if the dosage is increased. Animals are also affected differently by scopolamin (Kochmann, I9I3).

Dogs react similarly to man: very small doses produce sleep and hallucinations; larger doses cause more marked hallucinations and restlessness, ataxia, and of ten emesis. The excitability of the motor areas is said to be lowered (Sohrt, I886; Ramm, I893). Narcosis is practically absent in rabbits. Frogs show a central abolition of reflexes (Wood, 1883), preceded by more extensive spreading of the reflexes (Kochmann).

Therapeutic Use of Sedative and Hypnotic Action.-Scopolamin is employed especially in acute maniacal excitement, delirium tremens and tetanus. It has advantages over morphin, by quieting the reflexes and by avoiding habit formations; and over chloral, in that it does not usually depress the medulla. On the other hand, it produces the unpleasant side effects of mydriasis, cycloplegia, and excessive dryness; sometimes muscular 
tremors; and occasionally serious delirium (the latter is said to be promptly controlled by small doses of morphin). The therapeutic dose lies much below the ordinary fatal dose; but small doses (0.2 to $0.75 \mathrm{mg}$.; $1 / 300$ to $1 / 75 \mathrm{gr}$.) have produced serious poisoning, with cardiac and respiratory collapse. On the other hand, $1 / 10$ and even $4 / 5 \mathrm{gr}$. $(6.5$ and $50 \mathrm{mg}$.) have been taken without ill effects. Insane patients generally seem to be tolerant, so that the effective doses (I to $3 \mathrm{mg}$.; $1 / 60$ to $1 / 20 \mathrm{gr}$.) may be given; with ordinary individuals it is not safe to exceed $0.5 \mathrm{mg}$. (1/120 gr.), repeating this smaller dose every six to eight hours, if necessary; and it would be safer to use this dosage also for the insane. Smaller doses (0.2 mg.; $1 / 300$ gr.) may be used to enhance the hypnotic effect of morphin. The use in drug additions has been described under "Morphin."

Respiration and Circulation.-Therapeutic doses ordinarily do not produce any important effects (Kochmann, I903). The respiration is practically unchanged. The pulse may be unchanged or considerably slowed (central vagus stimulation). The blood pressure shows no change, or with somewhat larger doses, a moderate rise (stimulation of vasomotor center). Toxic doses depress the respiration, the heart, and the blood pressure. With fatal doses, the respiration stops before the heart.

The Eye.-The effects of scopolamin correspond qualitatively with those of atropin. They are produced more quickly and by doses only one-fifth or one-tenth as large (Joachimoglu, I9I5), but are not quite as lasting. The intraocular pressure is less affected (Bollet and Curtil, I9II). It is less irritant than atropin, and patients who have an idiosyncrasy against atropin can often tolerate scopolamin. It is used like atropin (iritis, etc., O.I per cent.; cycloplegic, 0.2 per cent.).

The secretion of saliva, sweat, mucus, etc., is paralyzed by therapeutic doses of scopolamin, more actively than by atropin.

The effects on the intestine correspond to those of atropin. The excised uterus is stimulated (Kehrer, r907; Barbour and Copenhaver, I9r 5).

Scopolamin Poisoning.-The symptoms resemble those of atropin poisoning, except for the early tendency to sleep, coma, collapse, and the lesser effect on the pulse rate (Details, Seifert, Nebenwirk., I9I5, p. 87 ). The treatment would be as for atropin. The excretion occurs by the urine.

Scopolamin-morphin Narcosis.-The combination of the two alkaloids results in a deeper narcosis than would correspond to the simple addition of the effects of the individual alkaloid ("potentiated synergism;" Madelung, I9ro; Buergi, r9ro; Kochmann, r913). Complete surgical anesthesia may thus be obtained, permitting of major operations (Scheiderlin, I900; Korff, I901 to I902; Blos, I902). Smaller doses produce a partial anesthesia ("Seminarcosis," "twilight sleep" or "Dämmerschlaf," Gauss, I906 and I907) which has been used in obstetrics. Still smaller doses quiet the patient, and may be employed to facilitate the administration of the ordinary inhalation anesthetics.

Scopolamin alone is not narcotic (for rabbits or mice), even in large doses; nor does it diminish the concentration of ether or chloroform required for light anesthesia; but it does permit a deep anesthesia with relatively lower concentrations. It also deepens the morphin narcosis. The two alkaloids used together produce a deeper anesthesia with a given concentration of inhalation anesthetic (Ludewig, 1913). Fuehner, 1913, suggests that the potentiation may be due to suppression of the glandular secretion of morphin, this insuring a longer retention. 
The advantages of exclusive scopolamin-morphin anesthesia, as compared with ether or gas, are rather minor, while the disadvantages are very serious (see Hatcher, J.A.M.A., r9ıo, liv, p. 446; H. C. Wood, Jr., J.A.M.A., 1906, xii, p. 546; Bevan, ib., r915, 65: 1418; M. Kochmann, I903; A. Mayor, I908). The combination increases not only the narcotic action, but also the depression of respiration and circulation; and this in a very irregular degree. The susceptibility varies so much that the surgical anesthesia is often incomplete and must be supplemented by ether; and on the other hand, serious accidents and fatalities are alarmingly frequent. The anesthesia can not be modified to suit the needs of the moment. Delirium, sometimes serious, is not uncommon. The obstetric anesthesia is especially dangerous to the child, owing to the great susceptibility to morphin. In non-fatal cases, the child may show somnolence and deficient respiration for days after delivery (Jung, I9r4). The use of scopolamin-morphin anesthesia is justified (if at all) only in specially equipped institutions, and not in private practice, or even in ordinary hospitals.

The smaller doses used preliminary to anesthesia would alone appear justifiable; and even these may cause marked fall of blood pressure (A. Mayor, I908). Chloroform is especially dangerous (W. Straub, I9I3). Bevan, I9I5, believes that the use of the alkaloids in ordinary doses (morphin, 1/4 gr.; scopolamin, 1/50 gr.) adds distinctly to the risks of chloroform, ether or gas or local or spinal anesthesia, more than morphin alone; and that it has been responsible for many deaths, especially by masking the danger signals. It also increases the percentage of vomiting and of bladder paresis, and reduces immunity.

Scopolamin-morphin Synergism.-Quantitative experiments were made by M. I. Smith, 1915. In frogs, scopolamin is directly depressant, but increases the excitant and convulsant effects of morphin; while morphin does not greatly increase the paralytic effect of scopolamin. . The fatal effect on frogs and mice is simple summation with morphin and l.- scopolamin; slight potentiation with i.- scopolamin. The perfused frog heart gives potentiation of the depressant action.

Dosage for Surgical Anesthesia.-As a preliminary to inhalation anesthesia, Korff injects hypodermically a mixture of $0.4 \mathrm{mg}$., $1 / 160 \mathrm{gr}$., of scopolamin hydrobromid, and Io mg., $1 / 6$ gr., of morphin hydrochlorid, one and one-half hours before the operation, and repeats the same quantity an hour later. If the scopolamin-morphin is to be the chief anesthetic, to be merely supplemented by ether if necessary, he precedes these injections by another, of the same size, two and one-half hours before the operation. The total dosage would therefore be $1.2 \mathrm{mg}$., $1 / 50 \mathrm{gr}$., scopolamin; and 30 mg., $1 / 2$ gr., morphin.

Results of Scopolamin-morphin Seminarcosis in Obstetrics. - The introduction of the "twilight sleep," intended to deprive labor of its terrors, has led to much discussion and diversity of opinion. Its advocates do not claim that it abolishes pain, but they assert that it successfully removes the memory of the experience, in the majority of cases, and with practically no danger to mother or child; provided it is used by experienced operators, under exactly prescribed conditions, and with proper dosage. The experience of independent observers has been contradictory, but by no means as favorable. The early failures may be attributable to excessive doses of morphin or other faults of technic; but this criticism can not apply to the later complications. The serious objections are: prolonged labor (averaging three to four hours); frequently delayed respiration of child, sometimes requiring vigorous measures; larger number of fetal asphyxias; excessive thirst and intense headaches; difficult control of patients with chance of infection; more frequent postpartum hemorrhage; blurred vision; ghastly deliriums persisting for long time; difficult recognition of onset of second stage; masking of early symptoms of accidents; failure of narcosis in one-seventh to one-third of the cases and uncertainty of the whole treatment (Baer, I915; Libby, I915).

Effects on Uterus.-Morphin and scopolamin slightly increase the tone of the excised uterus, without synergism or antagonism (Barbour and Copenhaver, I9I5). In anesthetized animals, there is little effect of any kind (Barbour, 1915). The clinical delay of labor is therefore probably cerebral (narcotic). 
Dosage for Twilight Sleep.-The minutely defined technic can not be described here with sufficient detail to be useful. The reader is referred to the original articles, or text-books of obstetrics. They involve painstaking exclusion of noise, light and all excitant factors-apparently the coöperation of suggestion is essential. The drugs are not started until the regular pains occur, perhaps every four to five ininutes and lasting half a minute. The first injection is of 0.008 to $0.015 \mathrm{mg}$. of morphin and 0.45 mg. of scopo!amin hydrobrom. The Freiburg school prefers narcophin, $30 \mathrm{mg}$., to the morphin, but this is immaterial. The morphin or narcophin must not be repeated. The scopolamin, however, is repeated; first in three-quarters of an hour with $0.45 \mathrm{mg}$., then every one and one-half hours with $0.3 \mathrm{mg}$., until anamnesia (loss of memory) is sufficiently advanced. This is tested every half hour. When successful, the pains are fully felt, but promptly forgotten. The patient is rather drowsy; the heart slightly quickened (the simplified technic is described by Siegel, I914).

\section{PREPARATIONS-SCOPOLAMIN}

*Scopolamince Hydrobromidum (Scopolamin. Hydrobrom.), U.S.P.; Hyoscin. Hydrobrom., B.P.; $\mathrm{C}_{17} \mathrm{H}_{21} \mathrm{NO}_{4} . \mathrm{HBr}+{ }_{3} \mathrm{H}_{2} \mathrm{O}$.-An alkaloid obtained from various plants of the Solanacea. The U.S.P. specifies the levorotary isomer $\left(22^{\circ}\right.$ to $\left.27.5^{\circ}\right)$. Colorless crystals. Freely sol. in water (I: I.5); sol. in alc. (I:20). Dose, $0.3 \mathrm{mg}$., 1/200 gr., U.S.P.; 0.3 to $0.6 \mathrm{mg}$., $1 / 200$ to $1 / 100 \mathrm{gr}$., B.P. Maximum dose, $0.6 \mathrm{mg}$., $1 / 100 \mathrm{gr}$.

\section{PILOCARPIN}

General Statement.-Pilocarpin, the principal alkaloid of jaborandi leaves (Pilocarpus), stimulates those peripheral structures which are paralyzed by atropin. It therefore increases the secretions, augments the gastro-intestinal movements, constricts the pupils, etc. The stimulation of 'these functions persists even with large doses. The vagus, however, is first stimulated, then depressed. All of these autonomic actions are purely peripheral (on the myoneural junction). The central effects of pilocarpin are unimportant.

Pilocarpin is used as a miotic, but especially as a diaphoretic; in this respect it surpasses all other drugs. Pulmonary disease enjoins caution in its use, to guard against fatal pulmonary edema.

The stimulant effects of pilocarpin are easily inhibited by atropin. Conversely, pilocarpin removes the depressant effects of small doses of atropin, but is ineffective against larger doses.

Other Jaborandi Alkaloids.-Pilocarpus contains two other alkaloids: viz., isopilocarpin and pilocarpidin. These differ merely in the strength of their action, pilocarpin being by far the strongest, and pilocarpidin the weakest. It was formerly believed that pilocarpus contained another alkaloid, jaborin, with atropin actions. This view has been shown to be erroneous (Jowett, I900 and I903; Marshall, 1904).

Arecolin, the alkaloid of Areca or Betel nut, is closely related to pilocarpin in its actions (Marmé, I889, Leepin, I89I). It stimulates peristalsis (Paetz, I9Io) and produces marked bronchial constriction by peripheral action, but is overcome by atropin or epinephrin (D. E. Jackson, 1914).

Glands.-Pilocarpin produces an increase in the secretion of saliva, sweat, tears, mucus, and of the gasstric, pancreatic, and possibly of the intestinal juice and of the milk, not of the urine or bile. A gallon of water may be removed by these secretions, after a single injection.

Pilocarpin continues effective after degeneration of the nerves, but is easily inhibited by atropin. Its action is therefore located in the myoneural junction.

Acceleration of the blood current through the glands occurs as a secondary effect of their increased action. A common effect of pilocarpin, a hyperemia of the skin (resulting in an increase of its temperature), may possibly be due to the increased activity of the sweat glands. 
The increased circulation of the skin is said to increase the growth of hair, and to darken its color. The statement may be doubted.

Gastric Secretion.-The quantity and the ferment content of the gastric juice are increased (Chiari, I9I3).

Pancreatic Secretion.-Lannoy, 1905, claimed that this is not increased if the pylorus is ligated. He therefore believed that the ordinary increase is indirect, due to the increased secretion of acid in the stomach, and therefore increased production of secretin. Camas and Gley, I913, find, however, that it is effective even when the small intestine has been removed, so that it must act directly on the pancreas.

Lacteal Secretion.- Ott and Scott, I9I2, confirm Roehrig that this is somewhat increased, the effect being abolished by atropin.

Urine and Bile.-Pilocarpin has no direct action on glycogenolysis (McGuigan, 1916). Repeated injection of large doses produces diuresis and glycosuria, which Waterman, I9I I, attributes to increased permeability of the kidneys. Ordinarily, there is no direct action on the urine or bile (MacCallum, I905); however, the great loss of fluid by other channels usually diminishes the water and chlorid of the urine (Asher, I905; Cow, I912). Cow, and Macht, I916, also describe stimulant effect on the ureter. This is paralyzed by high concentrations.

Drugs on Bile Duct.-Vater's papilla is closed by intravenous injection of morphin, epinephrin, histamin, caffein, large doses of atropin, and especially by pilocarpin. It is relaxed by papaverin, yohimbin and nitrites (Reach, I9I4).

Cerebro-spinal Fluid. - This is increased by the respiratory embarrassment (Dixon and Halliburton, 1913).

Sialagogue Action in Man.-Five to to $\mathrm{mg}$. of pilocarpin, by mouth, increases the volume of saliva considerably. The percentage of inorganic solids is unchanged; that of organic solids shows an insignificant increase. The proportion of ptyalin is markedly diminished, but its total quantity is considerably increased (Ewing, I9II). The composition of pilocarpin saliva is not changed by the injection of sodium phosphates, sulphate or carbonate, or of glucose, unless toxic doses are given (Asher, I908). The submaxillary gland, when perfused with Locke's solution, can be provoked to secretion by pilocarpin, but not by chorda stimulation (Demoor, I9I2).

Therapeutic Use.-The sialagogue action is employed (ro mg., 1/6 gr., by mouth, three or four times a day) against poisons which suppress this secretion (atropin, meat-poisons, etc.). The increased secretion of mucus may be used in dry cough. This also results in loosening croupous membranes. The increase in biliary mucus is said to facilitate the passage of gall-stones. Its utility is doubtful.

Diaphoretic Action.-To produce this effect, pilocarpin should be injected hypodermically ( $5 \mathrm{mg} ., 1 / 12 \mathrm{gr}$.), the patient being kept warm. This dose may be repeated in half an hour if necessary. Several liters of alkaline sweat may be excreted. There may be considerable nausea and depression, and with larger doses even serious collapse, especially in children. As has been said, pilocarpin should be avoided in pulmonary disease, since the increase of mucus and the action on the circulation may result in dangerous pulmonary edema. ${ }^{1}$

The diaphoretic action is used especially in nephritis, to relieve the kidneys and to remove the toxic metabolites.

In paralyses of the cortical psychomotor region, the corresponding parts of the skin are said to perspire especially strongly in response to pilocarpin (Bikeles and Gerstmann, 1915).

Other Diaphoretics (Sudorifics or Hydrotics).- The secretion of sweat may be increased (I) by stimulating the secretory structures, (a) peripherally (pilocarpin) or (b) the sweating center (camphor, ammonium acetate); or (2) by increasing the cutaneous circulation, (a) locally (mustard; external heat) or $(b)$ through the vasomotor center (internal heat; alcohol, salicylates, acetanilid, nauseants, etc.).

1 The edema produced $\ddot{b y}$ the drugs of this series consists rather in the aspiration of the bronchial effusion, than in a true serous effusion. Injury to the walls of the capillaries is a necessary factor for the latter. 
Children sweat more, old people less easily, than adults. Amongst animals, horses sweat most profusely; pigs and beef, not very readily; dogs, rabbits, and sheep not at at all; cats only on the paws.

Indications for Diaphoretics.-The various sudorific measures specially adapted to the different indications, are as follows:

I. For the Absorption of Edemas and Exudates. - A powerful action is needed: pilocarpin combined with external heat (hot baths or packs).

2. To Relieve Inflamed and Overtaxed Kidneys.-The large amount of fluid and excrementitious material removed by a thorough sweating secures functional rest to the kidneys and lowers blood pressure. Here, also, pilocarpin and hot baths are preferred.

Ordinary sweat (Hoelscher, I904) contains 0.22 to $0.8 \mathrm{I}$ per cent. of ash, and 0.043 to 0.084 per cent. of nitrogen, mainly in the form of urea. ( 0.285 per cent. of $\mathrm{NaCl}$ and $0.2 \mathrm{I}$ per cent. of urea; Kovesi). The daily cutaneous excretion of nitrogen is from 0.3 to $1.8 \mathrm{Gm}$. (Benedict, 1905; Schwenkenbacher and Spitta, 1907). The sweat excreted under the influence of pilocarpin contains in normal individuals $0.05 \mathrm{I}$ to 0.085 per cent. of nitrogen, and 0.26 to $0.3^{\mathrm{I}}$ per cent. of ash. Three liters of sweat-a not unusual quantity after pilocarpin-would therefore remove about $2.5 \mathrm{Gm}$. of nitrogen. In nephritis, the nitrogen content may be much higher, to 0.288 per cent.; so that the 3 liters could remove to $8 \mathrm{Gm}$. of nitrogen. This indicates how efficiently the kidneys may be relieved by diaphoresis. This is also shown by the examination of the blood; the abnormally great depression of the freezing point of the blood of uremic patients may be reduced to normal by diaphoresis (Bendix, 1904). The freezing point of the blood of normal animals is not affected. Alimentary glycosuria could also be prevented by free diuresis, the excess of sugar being excreted by the skin. However, Austin and Miller, I9I4, could find no evidence that sweat baths materially diminish the nitrogen retention in nephritis.

3. For the removal of poisons, especially in chronic intoxication by the metals. The efficiency is rather doubtful. Hot baths and drinks would be used.

4. To reestablish disturbed circulation in the skin, and thereby to relieve congestion of internal organs: this determines their use in colds, rheumatism, etc.; in cold skin from whatever cause; in inflammations of the lungs, pleura, etc. Relatively mild diaphoretics suffice for this purpose: External heat; hot drinks (teas, lemonade or alcohol); Sweet Spirits of Niter; Dover's powder; salicylate, etc.

5. The increased vascularity of the skin is also used to hasten the outbreak of febrile exanthemata, to promote the absorption of salves, etc. Further, in certain diseases of the skin where its nutrit:on is defective.

6. In obesity, withholding carbohydrates at the same time, to oblige the body to form the water which it requires, by the combustion of its adipose tissue.

7. To increase the alkalinity of the tissues, in gout, oxybutyric acid coma (diabetes), etc. Drugs which stimulate the glandular activity directly, such as pilocarpin, must be employed here, since the sweat is acid only when produced in this manner. This removal of acid is so marked that the urine of healthy individuals may be made markedly alkaline by an injection of pilocarpin. The acidity of the gastric juice may also be diminished.

8. In ophthalmology, diaphoresis has been found useful in congestive and exudative lesions of the uveal tract, in retinal detachment and in toxic blindness; it is useless in atrophic and cicatricial lesions.

Pilocarpin on Unstriped Muscle.-The stimulation of the myoneural junction of the cranio-sacral autonomic nerves results in contraction of most unstriped muscles (not of the blood vessels). The intestines are especially stimulated, with diarrhea and colic. The stimulation extends also to the muscles of the bronchi, uterus, ureter, bladder, spleen, stomach, etc. However, the nausea and emetic action are essentially central (Eggleston and Hatcher, I9I5). They are antagonized by atropin (Eggleston, I916). 
The location of the intestinal action was described on p. 269; the action on the uterus on p. 270 . The uterine stimulation is rather weak and inconstant (Gunn and Gunn, I914). Neukirch, I9I2, finds that the action on the excised intestine is purely stimulant, even with 5,000 times the effective dose. The action can be removed by washing; but during the passage of the pilocarpin out of the cells, there seems to be a supplementary stinulation, the reverse of Straub's muscarin phenomenon (see p. 272). The bronchial muscle constricts (Dixon and Brodie), even outside of the body (Trendelenburg, 1912); see p. 279. Leucocytosis results from the contraction of the spleen and lymphs (Harvey, I906). The gall-bladder is stimulated (Lieb and McWhorter, I9I5).

Development of Ova.-Sollmann, I904, has described the phenomena of antagonism with atropin.

Eye-Effects.-The application of pilocarpin produces miosis and spasm of accommodation, by peripheral stimulation of the oculomotor (p. 268). The intraocular tension is affected as with physostigmin, first raised, followed by more persistent fall. Pilocarpin hydrochlorid in $1 / 2$ to 4 per cent. solution is applied as a milder substitute for physostigmin in glaucoma, corneal ulcer, etc.

The miosis begins in fifteen minutes (independently of the concentration); reaches its maximum in thirty to fifty minutes, and disappears in twenty to twenty-four hours. The spasm of accommodation also begins in fifteen minutes, and lasts about two and one-half hours.

Suprarenal Stimulation.-Dale and Laidlaw, I9I2, describe evidences of oculomotor stimulation even if the cervical ganglia have been excised, but not if the suprarenal has been removed. They ascribe this to stimulation of the suprarenals to increased production of epinephrin. There is a similar condition as regards the uterus. Jackson, I9I3, accepts the same explanation for a dilator effect on bronchial muscle.

Circulation.-Pilocarpin first stimulates, and then paralyzes the vagi peripherally, and therefore produces slowing (in frogs, diastolic standstill, Harnack and H. Meyer, I880), followed by quickening of the heart rate.. In mammals, the stage of stimulation and slowing is very short; it may be entirely absent in dogs, and often in man. In these the blood pressure rises, partly from the faster heart, and perhaps partly through stimulation of the vasomotor center.

Perfused blood vessels are dilated (frogs, Froelich and Pick, I913; lung, Baehr and Pick, I913). Berezin, I914, claims constriction of the lung vessels.

Frog's Heart.-The slowing occurs after nicotin (Gaisboeck, I9II) and is therefore probably postganglionic. The vagus excitability is increased. Combinations with muscarin give additive effects; physostigmin does not (Loewi, 1912). In the secondary vagus depression, stimulation of the sinus produces but little slowing, showing that this action is also largely postganglionic. High concentrations paralyze the cardiac muscle. Small doses of chloral by depressing the cardiac muscle, delay the escape from vagus inhibition, whether by pilocarpin, muscarin, or electric stimulation (Loewi, I912).

Excised Mammalian Heart (Hedbom-Langendorff). - The effects are similar to the frog heart, but the stage of vagus stimulation is short: the rate is suddenly slowed; this lasts but a short time, then there is marked quickening with increased tonus (peripheral paralysis of vagus). If the auriculo-ventricular bundle is divided, pilocarpin or muscarin does not produce inhibition (Cullis and Tribe, 1913); nor do they inhibit isolated ventricular strips (Leetham, I913). Their action must therefore be on the auricular vagus terminations. Large doses paralyze the muscle.

Central Nervous System.- The action is weak and appears late, so that it is entirely overshadowed by the peripheral actions. The effects are mainly depressing. Vasomotor paralysis is a rather early and prominent symptom; it leads to dyspnea. Later, the respiratory center is also depressed. The motor centers, especially those of the cord, show some stimulation (increased reflexes, tremors, convulsions) and later paralysis. The emetic center is stimulated.

Metabolism.-Pilocarpin somewhat increases $\mathrm{CO}_{2}$ production and tends to raise the temperature, even in curarized animals, i.e., by direct stimulation of the glands and unstriped muscle. Mendel and Stehle, 1915, claim that pilocarpin and other stimulants of digestive glands increase the excretion of uric acid. 
Fate and Excretion.-A large part is excreted unchanged, but in combination, by the urine (Curci).

Symptoms of Poisoning.-The toxicology of pilocarpin is not very important. The symptoms, which apply also to muscarin, begin with a greatly increased secretion of saliva, sweat, and tears; then nausea, profuse vomiting, and painful diarrhea; pupillary contraction and spasm of accommodation; pulse variable in rate, tense, and arythmic; palpitation; dyspnea with râles; sometimes confusion of ideas, vertigo, tremors, and feeble convulsions. Death occurs either by paralysis of the heart or edema of the lungs.

Untoward effects from the alimentary canal are seen most frequently if the drug is given by mouth, but occur also on hypodermic administration. They consist in very prolonged and depressing nausea and vomiting, sometimes leading tocollapse. The disturbance of accommodation causes misty vision. A burning sensation in the urethra, with sudden and irresistible desire to urinate, is often observed.

Treatment.-Atropin is a physiologic antidote. Otherwise, the the general treatment of alkaloidal poisoning would be employed.

\section{PREPARATIONS-PILOCARPIN}

*Pilocarp. Hydrochl., U.S.P.; $\mathrm{C}_{11} \mathrm{H}_{16} \mathrm{~N}_{2} \mathrm{O}_{2} . \mathrm{HCl}$.-Very sol. in water ( $\mathrm{I}: 0.3$ ). Dose, same as the Nitrate.

*Pilocarpine Nitras (Pilocarp. Nit.), U.S.P., B.P.; $\mathrm{C}_{11} \mathrm{H}_{10} \mathrm{~N}_{2} \mathrm{O}_{2} . \mathrm{HNO}_{3} .-$ Colorless, faintly bitter crystals. Freely sol. in water (I:4). Dose, ro mg., $1 / 6$ gr., by mouth; hypodermic, 5 mg., $1 / 12$ gr., U.S.P.; 3 to 12 mg., $1 / 20$ to $1 / 5$ gr., B.P. Maximum dose, $30 \mathrm{mg}$., $1 / 2 \mathrm{gr}$.

Pilocarpus, U.S.P. (Jaborandi).-The dried leaflets of Pilocarpus species, yielding not less than 0.6 per cent. of alkaloids. U.S.P.

Fldext. Pilocarp., U.S.P.—About 0.6 per cent. of alkaloids. Dose, 2 c.c., 30 minims,

\section{PHYSOSTIGMIN}

General Statement.-Physostigmin (also called Eserin), the alkaloid of Physostigma or "Calabar bean," stimulates the peripheral structures which are paralyzed by atropin. Its most important actions consist in powerful contraction of the smooth muscle of the eye (miosis) and intestine. It is employed in eye diseases (glaucoma, etc.) and in intestinal paresis. The glandular stimulation is less marked than with pilocarpin. It also causes fascicular twitching of striped muscle and is mutually antagonistic with curare and substances having a curare action. On the central nervous sytem, the effects are mainly paralyzing. They have a toxicologic importance and limit its internal use.

Actions on the Eye.-Physostigmin causes constriction of the pupils (discovered by Fraser of Edinburgh, 1863); spasm of accommodation (near vision); twitching of the eyelid and lowering of the intraocular pressure. These actions are due mainly to increased excitability of the oculomotor endings or myoneural junction. The effects of physostigmin can be removed by the application of atropin, and vice versa; but this requires much larger doses than does a normal eye.

The action on the pupil appears somewhat earlier, and lasts somewhat longer, than that on accommodation; on local application of a I per cent. solution, the miosis begins often within ten minutes, reaches its maximum in thirty to fifty minutes, stays maximal for three or four hours, and then disappears gradually in three or four days. The physostigmin contraction is greater than can be produced by the strongest light,although the physostigmin pupil contracts further even to weak light. With the maximal contraction, the pupil is generally irregular in shape. Physostigmin is much more effective in counteracting the mydriasis of cocain than that of atropin.

The spasm of accommodation begins in about twenty minutes, and passes off in about two and one-half hours, leaving the excitability somewhat increased. Through the contraction of the ciliary muscle, the lense becomes more spherical, and objects appear enlarged and more distant. 
Localization of Action.-Paralysis of the sympathetic may be excluded, for stimulation of this nerve dilates the pupil somewhat, even when the physostigmin effect is complete. The muscle may also be excluded, for no miosis occurs if the oculomotor endings have degenerated (after section of the short ciliary). The action is therefore a stimulation or increased excitability of the oculomotor; and since it occurs on the excised eye, the effect must be on the endings. The antagonism with atropin, etc., was discussed on p. 268.

If the experiment is arranged so that the sympathetic is left intact, while the short ciliary is divided, physostigmin will not constrict the pupils, (even if the oculomotor endings are intact), because the physostigmin stimulation is evidently unable to overcome the sympathetic tone. Since the physostigmin easily overcomes the sympathetic tone if the oculomotor tone is intact, it has been argued that the physostigmin action does not consist in direct stimulation, but in increase of excitability to the normal oculomotor impulses (Meyer and Gottlieb). It is possible that this phenomenon is related to synergism (Dixon and Ransom, I912).

Intraocular Tension. - In the normal eye, physostigmin produces a slight fall (3 to $8 \mathrm{~mm}$.) lasting some twelve hours (Luebs, I9I3; Myashita, I9I3). The fall is more marked in glaucoma (Bollet and Curtil, I9II).

Therapeutic Use of Oculomotor Actions.-Physostigmin may be used to counteract the effects of atropin; to break up adhesions of the iris and lens (in alternation with atropin); in the treatment of peripheral corneal ulcer and to lower the intraocular pressure in glaucoma (see p. 284). The continued use of physostigmin or pilocarpin produces distinct amelioration of the glaucoma symptoms, of ten more than iridectomy; but they are, of course, not curative (Posey, rgr4). The mechanism of its action in ulcer is not understood; it is perhaps explained by contraction of the iris vessels.

Application.-Physostigmin is always used locally, in $1 / 10$ to I per cent. solution of the sulphate or salicylate (in glaucoma 0.1 to 0.4 per cent., two to six times a day). Solutions of 0.5 per cent. and higher sometimes cause considerable irritation and pain. This is lessened by employing an oily solution of the free alkaloid. Casey Wood advises the addition of of $1 / 5$ per cent. of cocain to $1 / 10$ per cent. of physostigmin; or pilocarpin or arecolin may be substituted.

Intestine.-Physostigmin greatly increases the gastric and intestinal movements, acting directly on the nerve endings (see p. 275). With therapeutic doses the peristaltic movements are more active. Toxic doses produce tetanic contraction of the gut. The action is utilized in intestinal paresis (postoperative ileus, etc.), $2 \mathrm{mg}$. (1/30 gr.) hypodermically (larger doses would be dangerous). Cannon and Murphy, r907, have shown that it has a marked effect, lasting, however, only a few hours.

Other unstriped muscles contracted by physostigmin are those of the bladder, spleen, ureter, uterus, arterioles, and bronchial muscles.

Glandular Secretions. - Physostigmin stimulates the same glands as pilocarpin (except milk; Ott and Scott, I912); but the increase of secretion is much less marked, being counteracted by the vasoconstriction. Like other intestinal stimulants, physostigmin increases the elimination of uric acid (Abl, I9 13 ).

Striped Muscle.-The fascicular twitchings, and the antagonism with curare, were discussed on p. 268. A similar antagonism exists between physostigmin and magnesium salts (Meltzer and Auer, I906; Joseph, I908; Joseph and Meltzer, I909).

Circulation. - In mammals, ordinary doses slow the heart by vagus stimulation, and raise the blood pressure by vasoconstriction. Large doses produce further slowing and fall of pressure. In man, however, serious dizziness and faintness occur before the heart rate is affected (Robinson, I915). In frogs, the heart muscle is stimulated directly, 
so that it contracts more strongly, and can not be stopped by vagus stimulation. The action of cardiac depressants is antagonized. (This stimulant action is not noted in mammals.)

The slowing in mammals is due to peripheral vagus stimulation, sufficiently powerful to counteract even atropin (Winterberg, I907).

The rise of pressure must be vascular, since the cardiac output is not increased. It occurs after destruction of the spinal cord and must therefore be peripheral. It is due partly to the contraction of the intestines, and partly to direct stimulation of the arterial nerve-endings. It does not occur after apocodein (Dixon, r903). The fall of pressure is due to central vasomotor paralysis and to cardiac depression.

Central Effects. - The action on the central nervous system is first tetanizing (Heubner, r905); then rapidly paralyzing, beginning, at least in man in the lower portions, so that conscionsness is preserved to the end. The respiraticn is at first increased; this is due largely to stimulation of the afferent endings of the vagi; for the quickening is much less after the vagi have been cut.

There are also other evidences of stimulation-excitement, etc.-but these have been considered secondary to respiratory paralysis, the central actions of the alkaloid being mainly depressing. Dyspnea is a marked symptom, due to paralysis of the respiratory center and spasm of the bronchial muscles. Asphyxia forms the cause of death (Harnack and Witkowski, 1876 ).

The depressant action of physostigmin has led to its therapeutic trial in epilepsy, chorea, tetanus, etc., without much success.

Piscidia or Jamaica dogwood is a similarly acting drug which was introduced as a narcotic but is entirely useless for this purpose (Tyrode and Nelson, 1905).

Physostigmin Poisoning.-The symptoms consist in restlessness, weakness, nausea, vomiting, sometimes purging; epigastric pain; rarely miosis; salivation, lachrymation and sweating, palpitation with slowed pulse; dyspnea; muscular twitchings; convulsions. Death occurs by paralysis of respiration, under general collapse, with the reflexes preserved to the end. The fatal dose is not known.

The location of the emetic action has not been definitely determined, because of the difficulty of fixing the emetic dose (Eggleston and Hatcher, 1915).

The Treatment of Physostigmin Poisoning.-This consists in evacuation, stimulants and atropin ( $1 / 2$ to $\mathrm{I} \mathrm{mg}$.).

Frazer found that small doses of atropin saved animals from three times the fatal dose of physostigmin; but that larger doses are synergistic; Curare and Magnesium are effective in the laboratory, but are too dangerous for man.

Fate.-Physostigmin is not destroyed by Frog's liver (Clark, rg 2).

\section{PREPARATIONS-PHYSOSTIGMIN}

${ }^{*}$ Physostigmina Salicylas (Physostigmin. Sal.), U.S.P. (Eserin Salicylate); $\mathrm{C}_{15} \mathrm{H}_{21}$ $\mathrm{N}_{8} \mathrm{O}_{2} \cdot \mathrm{C}_{9} \mathrm{H}_{6} \mathrm{O}_{3}$. - Colorless or faintly yellow crystals; of slightly bitter taste. Soluble in water (r:75) and in alc. Dose, r mg., 1/60 gr., U.S.P. Maximum dose, r mg., 1/60 gr.

Watery solutions of Physostigmin salts (not the dry salts) oxidize under the influence of light, air, and the alkali of glass vessels, acquiring a pink color (due to "rubreserin," Woelffin, r913). Such colored solutions should not be employed; Hallauer, 1899, states that they are more irritant, although the miotic action is not impaired. The decomposition is retarded by the addition of a little boric acid.

Physostig. Sulph., B.P.-More freely soluble, but less stable. Dose, I to 2 mg., $1 / 64$ to $1 / 32$ gr., B.P.

Lam. Physostig., B.P.-0.065 mg., 1/1,000 gr.

Physostigma, U.S.P. (Calabar Bean). -The dried ripe seeds of Physostigma venenosum, yielding not less than 0.I5 per cent. of alkaloids; mainly physostigmin; also small quantities of the related eseridin, and of eseramin. (Calabarin, a strychnin-like alkaloid, was formerly stated as a constituent, but has not been confirmed.) The drug was introduced into England about 1840 . Dose, o.I Gm., $11 / 2$ gr., U.S.P.

Ext. Physostigmatis, U.S.P.-A powdered extract, I Gm. representing ${ }_{3} \mathrm{Gm}$. of drug, 2 per cent. of alkaloids. Dose, $8 \mathrm{mg}$., $1 / 8 \mathrm{gr} .$, U.S.P.

Tr. Physostig., U.S.P.- ro per cent. Dose, I c.c., 15 minims, U.S.P.

\section{MUSCARIN}

Muscarin, an organic ammonium base, found in a mushroom, and a similar base, prepared synthetically from cholin, produce effects closely resembling those of pilocarpin; namely, typical peripheral stimulation 
of the parasympathetic system, i.e., ail the effects of pilocarpin; and also a nicotin and curare action.

The most characteristic action of muscarin is a vagus standstill of the frog's heart. Its interest is mainly scientific, and to some extent toxicologic, since it occurs in the most poisonous fly-mushroom.

Constitution.-M Muscarin, as isolated by Schmiedeberg and Koppe, I869, from the fly agaric ( 0.016 per cent. in the fresh fungus; Harmsen, r903), is a substituted ammonium base:

$$
\mathrm{CH}_{3} \equiv \mathrm{N}\left\langle\begin{array}{l}
\mathrm{CH}_{2} \mathrm{CH}(\mathrm{OH})_{2} \\
\mathrm{OH}
\end{array}\right.
$$

It is closely related to cholin (trimethyl-oxyethyl-ammonium hydroxid:

$$
\mathrm{CH}_{3} \equiv \mathrm{N}\left\langle\begin{array}{l}
\mathrm{CH}_{2} \cdot \mathrm{CH}_{2} \mathrm{OH} \\
\mathrm{OH}
\end{array}\right.
$$

Synthetic Muscarin.- "Synthetic Muscarin" is prepared artificially by the oxidation of cholin ("cholin-muscarin") and was supposed to have the same composition: later investigation, however, showed that it is the nitrous ester of cholin (Dale and Ewins, I9I4). Its actions agree qualitatively with those of the natural, but exhibit quantitative differences; the natural being ten times stronger on the heart, whilst the synthetic produces more pronounced nicotin and curare actions (Schmiedeberg and Harnack, $1876 ;$ Boehm, I885; H. Meyer, 1893 ; Honda, I 111 ). The antagonism to atropin is also less complete with the synthetic base.

Muscarin Action on Frog's Heart.-Muscarin causes slowing, with lessened systolic and increased diastolic excursions, and finally stoppage in diastole. These effects resemble those of electric vagus stimulation (also the electrocardiogram; Samojloff, 1913); and the results of submaximal vagus stimulation and muscarin are strictly additive, in frogs (Honda; O. Loewi), in turtles (Fleischhauer, I9I 2) and in mammals (Schott, rgII). Muscarin also produces additive results with pilocarpin. Large doses of both paralyze the vagus (Loewi) and the ganglia (Schott). The muscarin effect is more persistent than electric vagus stimulation, and occurs even in calcium-poor hearts, in which ordinary vagus stimulation is ineffective (Loewi, I9r2).

The refractory period of the heart to artificial stimulation is shortened by weak muscarin, as by vagus stimulation (Raaflaub, 19I4). The effects of muscarin on the electric response of the heart was investigated by Mines, 19I4.

Perfusion of the excised frog heart with very dilute muscarin in Ringer's solution produces simultaneous weakening and slowing. If it is perfused in blood, it may also slow without weakening; whilst perfusion of muscarin blood through the isolated ventricle may give weakening without slowing (Raaflaub, 1914).

Location of Action.-In mammalian hearts, muscarin causes no inhibition if the auriculo-ventricular bundle is cut (Cullis and Tribe, 1913), or in ventricular strips (Leetham, 1913); but in frogs, the muscarin standstill occurs also in the isolated apex, showing that the stimulation is peripheral to the ganglia; and since it can be abolished by atropin (Schmiedeberg and Koppe), the action can not be on the muscle. It is therefore assumed that it stimulates those endings which atropin paralyzes. If muscarin and atropin are exhibited at the same time or successively, their respective quanities will determine which predominates, but atropin is much the stronger. Drugs which act upon the ganglia-e.g., nicotin - will be ineffectual; but the standstill may be raised by substances effecting a direct stimulation of the muscle fibers-e.g., physos:igmin, veratrin, digitalin, anilin, camphor, guanidin. It should be added, however, that the type of contractions under muscarin differs in some respects from those of vagus stimulation (Rhodius and Straub, 1905). This may possibly be due to the action of muscarin being exerted on the myoneural junction, rather than on the vagus nerve mechanism proper. It is interesting that muscarin causes an acceleration of the crab's heart, although a well-defined inhibitory mechanism exists in these animals. The explanation undoubtedly lies in some structural peculiarity.

The observations of Straub on the penetration of muscarin were discussed on p. 272; its action on the intestines on p. 275 ; on the ureter, p. 271 ; on the bronchial muscle, p. 279. In guinea pigs, it may cause bronchial spasm, similar to that of anaphylaxis (Modrakewski, 1912). It has no effect on lacteal secretion (Ott and Scott, rg12).

Excretion.-In toads, this occurs quantitatively by the urine (Fuehner, I908 and Igog). 


\section{MUSHROOM POISONING}

Active Constituents.-Many fungi contain poisonous constituents, varying in the different species. Ford, I9I I, I9I3, has studied a large number of species, and finds that the principles toxic to man belong mainly to three classes: (I) muscarin, which is confined practically to the "fly-mushroom," Amanita muscaria; (2) substances producing degenerative changes in internal organs (especially Amanita phalloides); (3) substances producing gastroenteritis.

Glucosidal hemolysins are also present in Amanita phalloides and several related varieties, especially in Helvella esculenta (Ford, I9I3). They are easily decomposed by heat and digestion, and are therefore not concerned in poisonous effects. Agglutinins, perhaps glucosidal, are also widely distributed; some of these are not destroyed by boiling, but they are probably not pathogenic (Ford and Rockwood, I9I3). Practically all species of Amanita slow or stop the frog heart on direct application. In the case of A. phalloides, this is not due to muscarin, but to inorganic salts (Ford and Brush, 1914).

From Amanita phalloides, two interesting principles were isolated in pure form, one a hemolysin, the other a toxin. Both are antigens, a high-valence serum being obtainable against the hemolysin, a much weaker one against the toxin. Nevertheless, the hemolysin is a typical glucosid (Abel and Ford, 1907), identical with the "phallin" of Kobert, which was formerly considered a toxalbumin. It is readily destroyed by cooking. The toxin (which was considered an alkaloid by Kobert) is alone responsible for the symptoms of poisoning (widespread fatty degenerations of important organs; Ford, I906 and 1909; Abel and Ford, 1908). Rabe, I9I I, claims to have also isolated an alkaloid of muscarin action.

Symptoms of Mushroom Poisoning.-These must vary with the species. More or less common features are: Abdominal pain, nausea, vomiting, and violent diarrhea; variable pulse; labored respiration; consciousness unaffected, or delirium; coma or convulsions. Some cause fatty degeneration of the liver and kidneys. Many mushrooms produce abdominal symptoms simply by being indigestible.

The treatment, would be by evacuation, and symptomatic. The chance of poisoning may be somewhat diminished by prolonged boiling, as some of these substances are decomposed in this manner, but reliance on this would be dangerous. Drying does not diminish the toxicity. Verrill, I9I4, reports symptoms of inebriation (not dangerous) from eating Panæolus papilionacens (Maine).

Symptoms of Amanita Phalloides Poisoning.-These set in after a latent period of six to fifteen hours; sudden abdominal pain, with vomiting and diarrhea, containing blood and mucus; rapid loss of strength; cyanosis; death in two or three days. Histologic examination shows extensive neurosis and fatty degeneration of visceral organs (Ford, I9I3). Uremia, from greatly impaired kidney function, may be responsible for part of the symptoms (Clark, Marshall and Rowntree, I9I5).

Edible Mushrooms. - The food value of mushrooms is popularly supposed to lie in a large percentage of assimilable nitrogen. This is not borne out by analyses; they contain 0.1 per cent. in the fresh condition, or 0.3 to 1.6 when dried; i.e., no more than potatoes, and only one-tenth as much as, wheat bread, or one-thirtieth of that in lean meat. As the fiber of mushrooms is also quite indigestible, their main dietary value is as a relish rather than as food.

\section{CHOLIN}

General Statements.-Cholin (trimethyl-oxyethyl-ammonium) ${ }^{1}$ occurs as a constituent of lecithin probably in all animal and vegetable tissues, although often merely in traces. It is probably never free in the living tissues, in detectable quantity, but may be liberated by putrefaction or autolysis, not by pepsin or trypsin (Coriat, I904). It therefore occurs in organ extracts, in many vegetables (Schulze and Trier, I9I2) and in<smiles>CN(O)CCO</smiles> 
certain drugs (especially ergot). It may be liberated in intestinal putrefaction and thus contribute to the systemic phenomena of constipation; but it is readily destroyed by the intestinal bacteria and is less toxic than the analogous neurin and various amins which are also found in putrefaction. Cholin is also but slightly toxic on hypodermic injection.

The peripheral actions comprise two independent elements: a muscarin effect (stimulation of parasympathetic endings, similar to physostigmin); and a nicotin effect (stimulation and paralysis of autonomic ganglia; curare action on muscle). These two sets of actions are produced, in varying degree, by all quaternary ammonium bases (Dale, I9I4).

The most marked effects of cholin are on the blood pressure, which it usually lowers, often with a secondary rise. After atropin, it produces a rise instead of fall. This curious phenomenon has received no satisfactory explanation.

Action on Blood Pressure.-Cholin generally produces a marked fall of blood pressure (Mott and Halliburton, 1897); but sometimes there is instead a rise of pressure. In cats, the rise generally follows the fall (Pal, I9II). The variable response does not depend upon impurities (Renshaw, Underhill and Mendel, 1912). The result is influenced by the dose, anesthetic, and other conditions (Abderhalden and Mueller, rgrr). The fall predominates if the blood pressure has been high (Benelli, I9I4); in protracted ether narcosis, and after some sorts of curare. It counteracts the epinephrin rise (Guatrelet, I909). The pressor effect is more prominent in light anesthesia, or after section of the medulla (Pal, rgri).

After large doses of atropin, cholin gives only a rise; and it also constricts the vessels of excised and perfused organs, after atropin (Mueller, I9ro). Nicotin does not act like atropin (Hunt and Taveau, IgII).

Mechanism of Circulatory Actions. - These peculiar effects have not been sufficiently analyzed. Pilcher and Sollmann, 1915 , showed that cholin produces a marked direct depression of the vasomotor center, which seems to play an important part in the fall of blood pressure, and which is abolished by atropin. However, other causes contribute to the fall; and when these predominate they may overcome the vasomotor depression, and may even stimulate the center by anemia.

Mueller attributes the fall to cardiac stasis, and to vasodilation in the extremities, intestines, kidneys and brain. The atropin reversal he interprets as an atropin paralysis of the dilator elements. The vessels of frogs are peripherally dilated by cholin (Handovsky and Pick, I9I3). Dale, 1914, claimed a dilator effect on perfused vessels, but this is denied by Hunt, r9r5. Pal found the effects on the heart and vagus inconstant. Patta and Varisco, I915, refer the main effect to vagus stimulation, both central and peripheral. Golowinski, I9 $\mathrm{r}_{4}$, claims stimulation of intracardiac vagus and accelerator, in mammals. Hunt finds the heart generally slowed-the slowing being sometimes relieved by vagotomy, but not always. He inclines to consider both the fall and the rise as mainly cardiac. Lausana, I9 I3, finds that it slows and strengthens the heart of the turtle, while that of the frog is slowed and weakened, and its irritability to electric stimulation is diminished.

Organ Extracts.-The fall of blood pressure produced by the extracts of many organs and of nerve tissue may be due partly to their cholin content; but some characteristic differences have been described (Osborne and Vincent, r9oo; Busquet and Pachon, 1910).

Cholin Derivatives.-Hunt and Taveau, I906, I909, Igrr; Hunt, I9 5, and Dale, Igr4, have investigated the relative toxicity and circulatory effects of a large series of cholin derivatives, with very interesting results. For instance, the substitution of $\mathrm{OH}$ in cholin, in almost any manner, generally increases its activity, often very greatly (Dale). The introduction of the acetyl radical increases the depressor effect roo,000 times, the toxicity only three times (Hunt). Ewins, 1914, has found acetyl-cholin in ergot, and considers it responsible for the cardiac inhibition.

Other Peripheral Actions.- The effects on intestine and other smooth muscle agree with physostigmin. The secretion of saliva and tears is increased. Gastric secretion is diminished. Pancreatic secretion is diminished by small doses through inhibitory vagus stimulation; increased by large doses through peripheral stimulation (Chiari, r9I5). The output of uric acid is increased (Abl, I9r3).

Sterility. - Werner and Lichtenberg, r906, claim that cholin injections, in rabbits 
produce sterility or lessened fertility, analogous to the effects of $\mathrm{X}$-rays or thorium $\mathrm{X}$. Werner and Asher, I9I2, find similar blood and skin changes.

Distribution.-Normal serum contains about I part of cholin in 400,000 ; about the same concentration exists in amniotic fluid; somewhat more in the urine, and in cardiac and skeletal muscle; much less in cerebro-spinal fluid and saliva. The proportion in the blood or cerebro-spinal fluid has not been found increased in any pathologic conditions examined in man. It has been reported increased by removal of the suprarenals, but the evidence is not decisive and does not prove any causative connection (Hunt, 1015).

Fate.-Cholin is largely destroyed in the body (v. Hocsslin, 1906); a part of the methyl being probably oxidized to formic acid, the remainder to $\mathrm{CO}_{2}$ and water. After oral administration, only 3 per cent. of the cholin can be recovered from the tissues; with intravenous, about 50 per cent., the greater proportion from the skin (Ellinger, 1914). It disappears rapidly from the blood stream (Hunt, 1915).

\section{NEURIN}

Trimethylvinylammonium; $\left(\mathrm{CH}_{3}\right)_{3} \mathrm{~N} .<\mathrm{OH} \cdot \mathrm{CH}_{2}$ may be formed from cholin by bacteria. Its actions are similar to cholin, but much stronger (Pal, rgrI); and it is far more toxic (Lohmann, r9ir).

\section{BETAIN}

$\left(\mathrm{CH}_{3}\right)_{3} \mathrm{~N}<\mathrm{CH}_{2} \cdot \mathrm{COOH}$ is nearly inactive by mouth; hypodermically, it acts similarly to cholin. It is quite non-toxic (Velich, ror 4 ); even $2.4 \mathrm{Gm}$. per kilogram by vein, rat, are harmless. It is excreted unchanged, and is therefore not a food (Andrlick, Velich and Stanek, I902). The preparation and properties of its salts are described by Itolzenberg, I 914 .

Betain Hydrochlorid dissociates in solution, and has been utilized as a means of administering loosely-bound hydrochloric acid. The dose, 0.5 gm., 8 gr., corresponds to about I.I c.c. of Acid Hydrochloric Dilute.

\section{QUATERNARY AMMONIUM BASES}

General Statement.-The organic derivatives of ammonium have a striking similarity in their action. They produce a curare effect, i.e., paralysis of the nerve endings of striped muscle (Brown and Fraser, I 869); some also muscular tremors in frogs; and those possessing three or four methyl groups-cause a muscarin effect, i.e., peripheral stimulation of the cardiac vagus; and a nicotin action, i.e., paralysis of sympathetic ganglia. The curare effect evidently depends on 5-valent nitrogen; or rather, on the stereochemic configuration; for the same characteristic is shown by the corresponding phosphorus, arsenic and antimony compounds. The various actions differ in degree with the various derivatives-i.e., the methyl and ethyl ammoniums-in ways which are not easily explainable by their constitution (Burn and Dale, 1915).

\section{TETRA-METHYL AMMONIUM CHLORDD}

This produces a typical curare effect in mammals and frogs. Ammonium convulsions occur in mammals, but not in frogs, being prevented in the latter by the more powerful curare paralysis. The circulation shows a fall of blood pressure, generally with slowing of the heart, by peripheral vagus stimulation and peripheral vasomotor depression. The respiration is temporarily paralyzed by intravenous injection; in decerebrated animals, this is preceded by respiratory stimulation. The paralysis is 
due to a curare effect on the phrenic nerves (Marshall, I913). Loevenhart, 1914, however, believes that the respiratory action is entirely central; i.e., primary stimulation and later depression of the medullary centers.

\section{TETRA-ETHYL AMMONIUM CHLORID}

In frogs, this produces irregular muscular contractions and tremors; first fibrillary, then tonic. With larger doses, these are succeeded by paralysis. Both actions are seated in the muscle-nerve endings. Mammals also show increased excitability and depression of the endings. Rabbits exhibit dyspnea, tremors, convulsions, and arrest of respiration. In distinction to tetra-methyl ammonium, there is no direct action on the circulation; the respiratory effects are also smaller (Marhsall, 1914). Burn and Dale, 1915, found no curare or muscarin action, but a good nicotin effect.

\section{METHYL-ETHYL AMMONIUM COMPOUNDS}

The actions of these are, on the whole, intermediate; but without definite ratio to the alkyl radicals (Marshall, 1915; Burn and Dale, 1915).

\section{NICOTIN}

General Statement.-Nicotin, the chief alkaloid of tobacco has no therapeutic importance, but considerable scientific and toxicologic interest. Both the central and peripheral actions are important; both consist in short stimulation, followed by depression or paralysis. Centrally, small doses cause chiefly a respiratory stimulation; larger doses produce convulsion of the medullary type. The action is enormously rapid, approaching hydrocyanic acid. Peripherally, nicotin paralyzes all autonomic ganglia, parasympathetic as well as sympathetic. The paralysis may be preceded by stimulation. The most important effects are on the circulatory and gastro-intestinal systems. It has also a curare action on muscle-nerve endings.

The effects of tobacco are due practically solely to its nicotin. Limited tolerance to the acute toxic effects is soon acquired, after which it acts chiefly as a central sedative. Overindulgence produces chronic nicotin poisoning, with digestive disturbances, functional arythmias, optic changes, etc. The differences in the various forms of tobacco habit are probably explainable by differences in absorption.

Related Drugs. - Very similar in their actions to nicotin are piturin, the alkaloid of Duboisia Hopwoodii, which is chewed by the natives of Australia as a substitute for tobacco; and cytisin (Dale and Laidlaw, I9I 2), the alkaloid of the ornamental Laburnum tree (Cytisus Laburnum). Caulophyllum also contains methylcytisin, with similar actions; besides volatile oil, a saponin (Lloyd's Leontin), and caulophyllosaponin (Power and Salway, 1913). Lobelia is less perfectly related.

Central Actions of Nicotin.-These consist in stimulation, followed by depression, of the whole cerebro-spinal axis, from above downward. The symptoms from large doses resemble those of asphyxia or hydrocyanic acid.

The stimulation may be entirely absent, especially in large doses, so that the animal may drop dead almost instantaneously, without any other symptom. But this is not common.

The effects of small doses, such as are noticed in the first attempt at smoking, will be discussed later.

In mammals, moderate doses act mainly on the hemispheres, producing transitory excitement, followed by lasting depression, with violent headache. (In frogs, the action on the cerebral centers seems to be very small, for the effects are the same, whether the hemispheres are intact or removed.) 
Medullary Centers. - The action on these is marked and violent. Respiration is at first increased, and then markedly depressed; paralysis of the respiratory center being the cause of death. The vagus and vasomotor centers are also first stimulated, and then paralyzed. 'The salivation and vomiting, which are so prominent with moderate nicotin intoxication are probably also due to medullary stimulation (Eggleston and Hatcher, I9I5). The emetic action is antagonized by atropin (Eggleston, I916).

Bronchial Muscle.-This is constricted in the surviving lungs (Baehr and Pick, 1913).

Spinal Cord.-The motor cells are strongly stimulated, resulting in convulsions, passage of feces and urine, etc. If the nicotin is applied to one part of the cord, the convulsions remain confined to the muscles innervated from this area (difference from strychnin, which acts on the sensory cells). The stimulation is followed by paralysis. The nicotin convulsions are not seated exclusively in the spinal cord, but involve the hind-brain and medulla as well. They are much weakened by anesthesia.

The cutaneous reflexes of frogs are depressed. Partial tolerance to this action is acquired by repeated administration (Howat, I916).

The spinal ganglia (posterior roots) are not affected, even when a i per cent. solution of nicotin is applied directly to them.

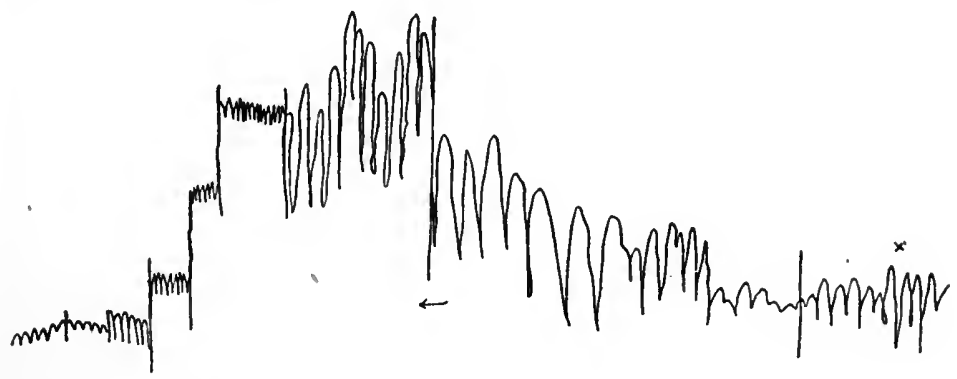

FIG. 11.-Nicotin Carotid pressure, dog. The effect begins at $x$. The beats first become weaker; then very slow and strong (vagus stimulation), with progressive rise of blood pressure (vasoconstrictor stimulation). Suddenly they become very rapid and consequently smaller. The output of the heart is unchanged and consequently the pressure remains high; but the vasoconstrictors becoming paralyzed, the pressure soon falls. Later the heart-muscle also becomes weakened, as shown by the small beats.

Location of Peripheral Actions.-The important work of Langley, 1890 , on the localization of nicotin in the autonomic ganglia was mentioned on p. 264 .

If the preganglionic fibers have degenerated (seven to twenty-six days after section of the nerve), nicotin is still active, so that it must act upon the ganglion cells themselves. The effects are more complex in the intestines (p. 276). Dogs are relatively insusceptible to the peripheral actions.

Antagonisms. - From the situation of the nicotin actions, it follows that they can be removed by atropin or muscarin, but that the latter are quite unaffected by nicotin. The antagonisms for striped muscle are described on p. 268 .

Circulation.-The effects are shown in Fig. II, and explained in Fig. 12. They are complex, involving stimulation and paralysis of the heart-muscle, vagus and vessels, centrally and peripherally. The total effects, are fairly constant and characteristic.

Initial Effects. - The heart is first greatly slowed, from central and ganglionic vagus stimulation. At the same time the blood pressure begins to rise, from strong stimulation of the vasomotor center (Pilcher and Sollmann, I9I5), and ganglia (Hoskins and Ranson, 1915); the central and peripheral stimulation playing about an equal rôle.

The blood pressure may be raised by nicotin (and anagyrin) even in the absence of the vasomotor centep, but not if the suprarenals are also excised (Gley, 1914). A part of the blood-pressure rise seems therefore attributable to increased output of epinephrin. 
Smoning of tobacco increases the coronary pressure (Bond, I9II). In novices, there is generally a rise, then fall of systemic blood pressure (Lee, 1908). John, I913, found in typicil cases, after smoking eight to ten cigarettes or two medium cigars, that the diastolic pressure rises, and often remains high for two hours. The effect is proportionate to the nicotin content, and is perhaps due to the nausea. The effects on the pulse rate were variable.

Carotid Reflex.-The reflex fall of pressure which ordinarily results from traction of the cephalic end of the carotid artery, is abolished by nicotin (Sollmann and Brown, I9I2).

Aryt/mia. - With larger experimental doses, the slow heart beat is replaced quite suddenly by great quickening through paralysis of the vagus ganglia. The nicotinized heart may therefore be slowed by muscarin, or stimulation of the sinus, but not by stimulation of the vagus trunk. According to Kose, $190_{5}$, the secondary acceleration also involves a ganglionic accelerator stimulation, which passes finally into paralysis. Electrocardiograms (dog) often show a nodal rhythm (synchronous action of auricles and ventricles), interrupted by extrasystoles; and other irregularities identical with those of stimulation of the vagi and accelerators, separately or together (Pozzi and Clerc, I9I3; Bull, Clerc and Pozzi, I9I4).

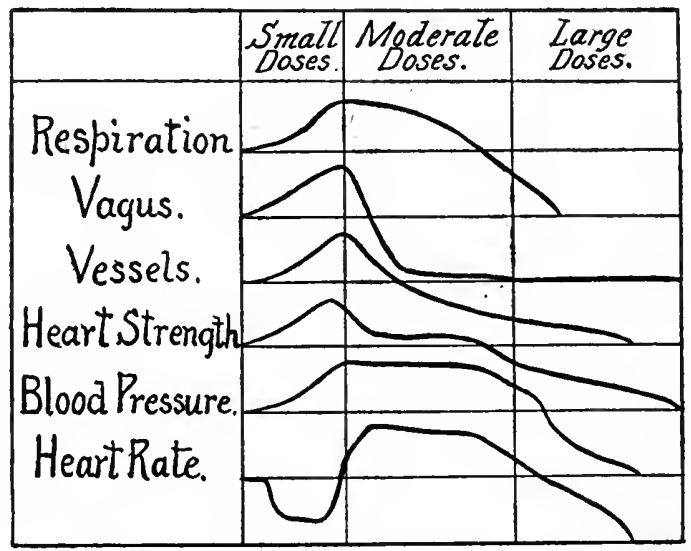

FIG. 12.-Diagram of the actions of nicotin.

Blood Pressure in Toxic Stage.-This may be very high and the central vasomotor stimulation persist into death (Pilcher and Sollmann, 1915), but as the peripheral vasomotor mechanism becomes more and more depressed, the vessels become dilated and the pressure falls. The dilation can be plainly seen in the intestinal vessels or the rabbit's ear. The vasomotor paralysis is rarely complete, so that direct or reflex stimulation, or a second injection of nicotin, again causes a short constriction. The heartmuscle eventually wears out, mainly because of the lowered coronary pressure.

Coronary Circulation.- This is slowed by the constriction of the coronary vessels, notwithstanding the raised blood pressure (Morawitz and Zahn, I9I4).

Pulmonary and Hepatic Vessels.-These are constricted (Berezin, 1914).

Excised Mammalian Heart.- When perfused with nicotin, this shows typical vagus effects; first, diastolic standstill; then gradually resumption and finally acceleration above normal, with increased amplitude. The primary standstill is abolished by atropin. The secondary acceleration is probably due to accelerator stimulation (Clerc and Pozzi, 1912).

Peripheral Actions on Frogs' Vessels.-By perfusion through decerebrated frogs, nicotin produces immediate vasoconstriction and muscular fibrillation. These effects disappear in a few minutes, during the perfusion, and can not be restored by further nicotin. Epinephrin is still effective. Tyramin suppresses the nicotin constriction almost completely (Handovsky and Pick, I9r3).

Frog Heart. - The application of nicotin produces a brief diastolic standstill, (stimulation of vagus ganglia). The heart then resumes its beat, and stimulation of the vagus trunk becomes ineffective, whereas sinus-stimulation or muscarin slows or arrests the heart. The nicotin has therefore paralyzed the vagus ganglia. 
Heat and Nicotin on Frog IIeart.- The typical effects of heat are opposite to those of nicotin; nevertheless, a heated heart responds more readily to the drug (Zanda, I915).

Intestinal Actions.-Nicotin provokes violent contractions, due mainly to stimulation of the Auerbach cells, but also involving other structures (see p. 276). These peripheral actions explain the diarrhea, and are perhaps also concerned in the nausea and vomiting; but these are essentially central.

Pupils.-These show both contraction and dilatation at different times, nicotin acting upon the ganglia of both the oculomotor and sympathetic fibers. There may even be a direct action upon the iris muscle. The effect is different in different animals: The dog and cat usually show dilatation; the rabbit first constriction and then dilatation.

The effects on the bronchial muscles have been described on p. 279; those on the uterus on p. 270. Excised ureter shows primary stimulation and secondary depression (Macht, I9I6).

Effects on Glands.-Secretion is generally stimulated through the ganglia. This is particularly prominent with the saliva. Small doses, however, change the response to stimulation of the chorda nerve, so that this diminishes secretion (Dale and Laidlaw, I9rI). Lacteal secretion is not increased (Ott and Scott, I9I2).

Glycosuria.-Nicotin prevents the glycosuria which ordinarily follows the injection of caffein and epinephrin; or on piqure (Macleod and Dolley, r905). This could be due to paralysis of the ganglia, or to the lowered blood pressure.

Increased Secretion of Epinephrin.-Cannon, Aub and Binger, I9r 2, believe that nicotin has this effect in cats; and Jackson, I9I3, invokes it to explain the peculiarities in the response of bronchial muscle.

Cerebro-spinal Fluid. - The pressure simply follows the circulatory changes (Dixon and Halliburton, I914).

Skeletal Muscles.-In frogs, injections of nicotin produce fibrillary twitchings. These disappear after the section of the nerves, but are started again by short stimulation of the nerve or muscle. They are abolished by curare; and conversely, nicotin counteracts the curare paralysis. The stimulant effect must therefore be on the same structures, namely, the nerve-endings. Mammals also show fibrillar contractions, but these are of central origin. The peculiar muscular phenomena in fowls (Langley) were discussed on p. 268.

Curare Action.-Nicotin also has a curare-like effect, which becomes prominent later. In the milder stages this is shown by an equally strong, or stronger, current being required to obtain contraction when the stimulus is applied to the nerve than when it is placed on the muscle. In the normal preparation the opposite is the case. The excitability and irritability of the muscle cells is, also diminished by nicotin on direct application.

Local Actions.-Free nicotin is caustic by its alkalinity. Strong solutions paralyze the nerve fibers.

Lower Organisms.-Nicotin is one of the most efficient insecticides (McClintock, Hamilton and Lowe, I9II). Tobacco juice and smoke are distinctly antiseptic, and Fullerton, rgr2, claims that they tend to preserve the teeth.

Fate.-Nicotin is absorbed extremely rapidly from mucous membranes, and especially from the lungs; more slowly from hypodermic injections and from the intact skin. It is excreted only in small part, mainly by the kidneys, but also by the lungs and sweat.

Dixon and Lee, ror 2 , confirm the older statement that it is partly destroyed in the liver, and claim that this destruction is increased by habituation; but Clark, I 9 I 2 , found no destruction by frog's liver.

\section{ACUTE NICOTIN OR TOBACCO POISONING}

Most cases of poisoning-outside of the slight ones from first attempts at smoking-have been produced by its medical application, especially by the laity; and since this has been largely abandoned, serious acute nicotin poisoning has become rare. 
The application of tobacco to wounds or bruises is not without danger. E. G. Garvin, 1913, has reported the death of a girl of six and one-half years, forty-five minutes after the administration of less than $1.5 \mathrm{Gm}$. of tobacco by enema, against pinworms. In other cases, the time of death has varied from three minutes to seven hours. Prompt death has also occurred from swallowing nicotin plant spray (Feil, I916).

Toxicity.-Nicotin is one of the most fatal and rapid of poisons; the vapor arising from a glass rod moistened with it and brought near the beak of a small bird causes it to drop dead at once, and 2 drops placed on the gums of a dog may cause a similar result. The fatal dose for a man is about $60 \mathrm{mg}$.; of tobacco, about $2 \mathrm{Gm}$. It acts with a swiftness equaled only by hydrocyanic acid. In view of the high nicotin content of tobacco (one cigar contains a quantity of nicotin which would prove fatal to two persons, if directly injected into the circulation), also because of its popular distribution, it appears astonishing that fatal nicotin poisoning is not more common; but just this wide distribution and knowledge of the drug form the safeguard, as also the marked taste.
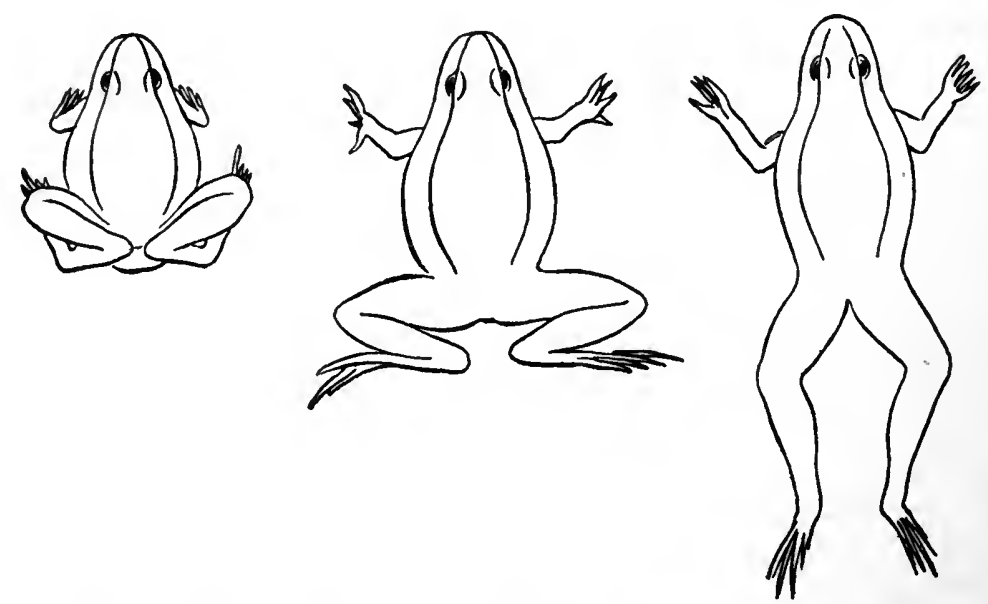

FIa. 13.-Nicotin. Successive positions of frog poisoned with $25 \mathrm{mg}$. nicotin.

Symptoms.-In lighter cases, such as commonly occur in smoking, the peripheral and nauseant actions predominate. There is first an increased flow of saliva, partly reflexly through the mechanical irritation of smoke, but mainly by direct stimulation of the ganglia through the nicotin. Nausea, vomiting, and diarrhea soon appear. The sweat glands are also affected in a peculiar manner: There is a sensation of oncoming sweat, which does not actually break out. A sensation of exhaustion appears very early-partly as the result of nausea, but mainly as the first indication of central collapse action. Palpitation is also noted.

Severe cases show immediate faintness, prostration and muscular weakness; severe nausea, vomiting and diarrhea; followed quickly by convulsions and collapse (Garvin).

The effects of poisoning with pure nicotin, which have been very carefully studied experimentally on man, bear the greatest resemblance to the above. After $\mathrm{I}$ to $4 \mathrm{mg}$. there were burning in the mouth, a scratching sensation in the pharynx, increased salivation, a sensation of heat spreading from the region of the stomach over the whole body; excitement with headache now appeared, then vertigo, confusion, disturbed vision and hearing, photophobia, dryness in mouth, cold extremities, nausea, vomiting, and diarrhea. Respiration quickened, but difficult. Pulse at first increased, then irregular. After fortynfive minutes there was syncope, with clonic spasms. Recovery occurred, but a general depression persisted for three days. 
The treatment, aside from the chemic, consists of coffee and other stimulants and in meeting the symptoms. Emetics will usually not be necessary.

The postmortem appearances are not characteristic, although large doses cause, in animals, anemia of the meninges and peculiar anatomic changes in the cortical nervecells. When taken by the mouth, there may be gastric and intestinal hyperemia, since nicotin is sufficiently alkaline to be somewhat caustic. The odor may furnish a valuable indication.

The proof of the poison after its separation may be had by its odor and by obtaining its physiologic actions on frogs. The muscular tremors and the position which a frog assumes after nicotin are highly characteristic (Fig. ${ }^{3} 3$ ). A control animal should, of course, be used.

The chemic tests are of no practical importance, since very similar reactions are given by coniin and by a ptomain.

Nicotin is very resistant to putrefaction, and has been isolated from the decomposed bodies of animals three months after death.

\section{HABITUAL USE OF TOBACCO}

The question of the effects of smoking has been largely discussed with a rather unscientific extremeness, some contending that it is entirely harmless when moderately used; whereas, on the other hand, an enthusiastic French writer has gone so far as to attribute the defeat of his nation in the war of 1870 to the prevalence of cigarette smoking. Of the two views, the former would seem to come nearest the truth if the stress is laid upon the word "moderate."

Since, next to caffein, nicotin is the alkaloid most widely used, an impartial discussion of this question is important.

Composition of Tobacco Smoke.-The effects of the smoke are due practically entirely to the nicotin contained in it. There are other toxic constituents, but their quantity is too small to be practically important. This has been proved both by chemical considerations and by animal experiments (Lee, I908; K. B. Lehmann).

Nicotin exists in tobacco mainly in the form of salts of organic acids (Garner, r9o8). By the heat of smoking, these are largely dissociated, a part of the nicotin being burned, and a part passing into the smoke as free nicotin (Lehmann, I909). The drier the tobacco and the greater the heat, the less nicotin will escape destruction. Moist tobacco, especially cigars, therefore, produce severer effects. This is more important than the nicotin content of the original tobacco (Lee, Igo8). In experiments, between 15 and 90 per cent. of the nicotin present in the tobacco are recovered from the smoke; but a greater part of this is exhaled or expectorated; in natural (intermittent) smoking, the aspirated smoke contains only a sixth of the nicotin. About I to I.4 mg. of nicotin would actually be absorbed from a cigar (Lehmann, 1907). In cigarette smoking, where inhalation is commonly practised, the percentage absorbed would be relatively high, thus accounting for its reputed deleterious effects. The average nicotin content of cigarettes (r.69 per cent.) is rather lower than that of other forms of tobacco (leaf, 3.04, cigars, $\mathrm{x} .52$; chewing, $\mathrm{x} . \mathrm{I}_{4}$ ); nor is there anything in the popular notion that cigarettes contain opium or other added narcotics (Thurston, 1915).

Other Toxic Constituents.- These are formed by combustion, and vary greatly according to conditions. As an illustration, Lee found in the smoke of $100 \mathrm{Gm}$. of tobacco, besides $x .165 \mathrm{Gm}$. of nicotin, the following: Pyridin bases, 0.146 Gm., chiefly pyridin and collidin; hydrocyanic acid, $0.08 \mathrm{Gm}$.; ammonia, $0.36 \mathrm{Gm}$.; CO, 4 ro c.c. The pyridin bases act somewhat similarly to nicotin, but with important differences (Lee). Pyridin is a strong local irritant with low toxicity, a ro per cent. solution has been used by atomizer in asthma and fetid bronchitis. It produces first dyspneic then shallow respiration. Brunton and Tunnicliffe (J. Physiol., $17: 292$ ) refer the effects mainly to sensory paralysis. Large doses arrest the heart. The hydrocyanic acid in the smoke of a cigar amounts only to 0.002 to $0.5 \mathrm{mg}$., and would have no significance (Lehmann and Gundermann, I9I2). If a large number of persons smoke in a confined atmosphere, the 
carbon monoxid in the air of the room may rise to a dangerous degree. The aromatic essential oils, which give the flavor to tobacco, are practically inactive.

Arsenic is sometimes present in harmful quantities when Paris green has been used on the plant as an insecticide.

Tolerance and Habituation.-The quantitative effects of nicotin vary considerably in different persons. Similar variations are observable in animals. Fonng individuals are more susceptible than adults. Considerable tolerance is acquired by continued use, so that moderate doses lose all the usual unpleasant acute effects. The rapidity and completeness of the habituation also varies with individuals; dependent partly on individual susceptibility; partly on the manner in which the drug is used, whether the smoke is inhaled, the saliva expectorated, etc. The tolerance is never absolute; it seems to be higher against sublethal than against lethal doses.

Habituation in Animals.-These acquire only a limited tolerance (Hatcher, I904; Edmunds, I909; Adler and Hensel, I905, could not produce any tolerance). Hatcher showed that the serum of habituated animals does not protect others against intoxication. Dixon and Lee, I9 I 2 , claim that the liver of habituated animals destroys nicotin somewhat more rapidly. Edmunds, I916, failed to confirm this. At best, since the destruction is slow, it would protect only when small doses are introduced gradually.

Effects of Moderate Use of Tobacco.-When the tolerance has been acquired, the continued use of tobacco within a certain individual limit produces absolutely no unpleasant symptoms; but if the limit be at any time sufficiently exceeded, the symptoms of chronic poisoning, presently to be discussed, arise. After a long time, some twenty years, these symptoms may also, but rarely, occur in those who have always kept within bounds.

With habituated individuals, the use of tobacco by smoking, chewing, or snuffing brings with it a certain pleasant sensation, which is somewhat difficult to define. There appears to be a certain repose, which, whilst it neither directly aids nor hinders the psychic processes, leaves the mind free, and in general raises the user's enjoyment of other pleasures, or lessens his annoyance at the opposite.

The experience of military campaigns appears to show that the use of tobacco enables soldiers to endure greater hardship and hunger. With the ergograph, Palmèn, I9II, found that cigarette smoke at first increased the muscular work, but that fatigue set in sooner, and the total efficiency was decreased. Nice, I9 I2, found that the muscular activity of mice in a revolving cage was not diminished by fairly high and long-continued doses of nicotin.

The blood pressure during smoking rises slightly and slowly in moderate smokers; in inexperienced smokers, it shows a sharp rise at first, and considerable fall when collapse sets in (Lee).

Nicolai and Staehelin, I9ro, found that in tobacco users the heart rate averaged somewhat higher, especially in work; no definite changes could be made out in the systolic and diastolic blood pressure or electrocardiograms. Aliprandi "and Fornaroli ( 1905 ) claim that tobacco chewing causes a distinct constriction of the cerebral vessels.

How much of the psychic effects is due 10 nicotin, how much to other factors, we can not say. It is certain that the nicotin strength of the tobacco is not the determining feature of this action-rather the aroma. Smoking in the dark does not give as much enjoyment; and simply holding an unlighted cigar in the mouth, the chewing of other objects, etc., give similar, though much weaker, sensations. The truth would seem to be that it depends upon a reflex stimulation, from the mucous membrane of the mouth, nose, etc., in which the nicotin plays a part; and with this may be associated a direct action of the nicotin upon the central nervous system, at once stimulating and depressing. 
Chronic Intoxication.- The phenomena resulting from the excessive use of tobacco take many forms; especially local irritation of the respiratory tract; digestive and nutritional disturbances; functional cardiac arythmias; depression of various parts of the central nervous system; neuralgias; more rarely impairment of vision.

Local Effects.-The nicotin and other basic constituents of the smoke act as fairly strong irritants, as shown by the biting sensation on the tongue, noted especially when the smoke is concentrated on one point, as in pipe smoking. This constant local irritation seems to favor the development of epithelioma of the lip and tongue, if the quantity consumed is very large or the smoker specially disposed. There is also a more general irritation of the mucous membrane of the mouth, throat, and pharynx, leading to catarrh and hoarseness.

Circulatory Disturbances.-The first warning symptoms are occasional palpitation, quickened heart rate and arythmia. These become persistent if the nicotin is continued, but disappear on withdrawal. In advanced cases, the abstinence must be continued for months. The arythmia is purely functional, due to extrasystoles (premature beats with long pause), pointing to increased irritability of the cardiac structures. It is similar to that of caffein, hysteria and neurasthenia. In graver cases, the cardiac irregularity may become extreme and lead to sudden fainting (syncope). Respiratory distress naturally accompanies the marked cardiac phenomena. Angina pectoris is so rare in these subjects that it must be attributed to causes other than the nicotin. On the other hand, arteriosclerosis appears to be favored by it.

The extrasystoles are usually auricular. Auricular fibrillation and "flutter," and sino-auricular block have also been encountered (Neuhof, I9I6). The block disappeared within three days after stopping the tobacco.

In rabbits, chronic nicotin poisoning produces anatomic arterial and cardiac changes, similar to those of epinephrin (Adler and Hensel, 1905; Baylac, I906; Otto, I91 I; Adler, I9I4). These results can be only cautiously transferred to man. Pawinski, I9I4, claims that 42 per cent. of I,O00 cases of coronary sclerosis had histories of immoderate smoking; and that nicotin produces marked changes in the coronary vessels and heart-muscle in animals. Lissauer, 1914, also describes histologic changes in the cardiac and other ganglion cells.

Digestive and Nutritional Disturbances.-The symptoms next in order are probably those arising from the alimentary canal, and depending upon the continued irritant action of the nicotin. These are: Loss of appetite, then dyspepsia and chronic intestinal catarrh, shown by alternating constipation and diarrhea. (With moderate smoking the nicotin seems rather to have a tendency to keep the bowels regular.) These conditions lead to emaciation and anemia.

Smoking inhibits the hunger contraction of the stomach, the effect being proportiona to the local stimulation of the mouth, etc. (Carlson and Lewis, r9r4). The digestive movements of the stomach are presumably not arrested. A direct action upon the blood may also have a part in the anemia; the continued administration of nicotin to animals leading to diminution of red corpuscles, and increase of leucocytes. It is also claimed that it diminishes the oxygenating power of hemoglobin. The nitrogen excretion is rather more diminished than the assimilation, so that there may be a gain in body-nitrogen. Transitory albuminuria may occur.

Nervous Disturbances.-These are rarely serious. The psychic functions show a slowness and want of energy. Anxiousness and insomnia are quite frequent. There is a general muscular debility, tremors, and want of control over movements. 
The reflexes are heightened. Vertigo and a tabetic condition may set in. There is then an increase of excitability in the sensory and pain areas, and consequently headache and neuralgias; but the latter are in part due to referred pain from the cardiac disturbances. They are of ten early and quite characteristic, and take the form of pain and hyperesthesia in the precordial region, left nipple and ulnar surface of left arm.

Nicotin Blindness. - This is relatively rare. It seems to be favored by alcoholism. The sight gradually becomes dim, with central scotoma, impaired accommodation, and often miosis. It is always bilateral. The anatomic changes consist in retrobulbar optic neuritis, with subsequent atrophy. Until degeneration has set in, a complete cure can usually be effected by abstaining from nicotin; later, this will only arrest the further progress (de Schweinitz, I896).

Other Toxic Amblyopias. - Many poisons may produce blindness, temporary or permanent, in susceptible individuals, especially on continued administration. This has been recorded for stramonium, cannabis, chloroform, opiates, carbon disulphid, arsenic, lead, iodoform, aspidium, quinin, salicyl, methyl alcohol, etc. For most of these the changes are analogous to those of nicotin. With methyl alcohol, quinin and salicyl, the retinal ganglion cells are also involved. The metals and aspidium may produce primary optic atrophy without preceding neuritis (de Schweinitz, 1896 and I902).

Of other effects which have been attributed to nicotin, but with insufficient cause, may be mentioned: impotence, epilepsy, and insanity (G. W. Jacoby, I898; Naecke, I909).

Treatment.- - It will be seen that tne catalogue of injurious actions to be charged against the abuse of this drug is sufficiently large; but on the other hand, it must be noted that these are absent with moderate use, and can be abolished if the use of the drug is promptly limited on their first appearance. Actual withdrawal is not always necessary. Limitation in quantity, the use of tobacco poor in nicotin, sufficient expectoration, and the avoidance of deep inhalation of the smoke, are often sufficient. Quick total withdrawal does not lead to abstinence symptoms, as with morphin (except possibly in some especially neurotic subjects), although it may disturb the function of the bowels for a few days. The principal point in the treatment is to keep the thought of the patient off the topic of tobacco, and to supply the accustomed stimulus to the mouth in some other manner, as by chewing ginger or gentian.

The use of tobacco must, of course, be avoided in pathologic conditions in which there are special contraindications to it-in heart disease, dyspepsia, inflammation of the respiratory tract, etc.

\section{PREPARATIONS-NICOTIN}

Tabacum (Tobacco).- "The commercial dried leaves of Nicotiana Tabacum, Linné (N. O. Solanaceæ)." An annual plant, probably indigenous in tropical America, and now cultivated in most parts of the world. The annual production of the world is estimated at $1,000,000$ tons $(1,000,000,000 \mathrm{~kg})$. Other species also contain the nicotin. The plant was introduced into Europe shortly after the discovery of America. Its use by smoking was practised by the natives at the time of Columbus.

The important constituents are nicotin, which is also present to a less extent in all other parts of the plant, and a volatile oil developed in drying and "sweating." The percentage of nicotin varies between $I$ and 8 per cent.: In Havana and Maryland, $I .5$ to 3 ; Virginia and Kentucky, 6 to 8 ; South American, 2 to 6; German, 1.5 to 3. A number of other alkaloids have also been announced to exist in small amount in tobacco (Pictet and Rotschy, I90I).

The dried leaves were formerly official, but are now obsolete in medicine; if used, an infusion may be made. The dose was given as $0.5 \mathrm{Gm}$.

Nicotin, $\mathrm{C}_{10} \mathrm{H}_{14} \mathrm{~N}_{2}$, is a fluid, volatile, oxygen-free alkaloid, of strongly basic characters. It forms salts, most of which are soluble. It is colorless and almost odorless when freshly prepared; but it partly decomposes on keeping, acquiring a characteristic odor and a brown color. The dose would be to $0.00 \mathrm{rm}$. 


\section{LOBELIA}

The herb Lobelia inflata was used by the American Inclians as a substitute for tobacco. It was introduced into medicine by Samuel Thompson about the beginning of the nineteenth century as the main basis of his peculiar system of treatment. The chief constituent is the alkaloid Lobelin with actions resembling nicotin (Edmunds, 1904). Small doses lower the $\mathrm{CO}_{2}$ threshold of respiration (Wieland, 1913). Large doses paralyze the respiratory center and cardiac muscle (Muto and Iwakawa, I9Io). Relatively small doses paralyze the bronchial muscles (Dresser, I8S9), which may explain its use in asthma. It is also somewhat employed in other respiratory disorders as expectorant, emetic and cathartic; but it is an unreliable, depressant and dangerous drug. The nausea and emetic action are central (Eggleston and Hatcher, I915).

Poisoning is treated by evacuation and stimulants.

\section{PREPARATIONS-LOBELIA}

Lobelia, U.S.P., B.P. (Indian Tobacco).-The dried leaves and flowering tops of Lobelia inflata. Dose, O.I5 Gm., $21 / 2$ gr., U.S.P.

Fldext. Lobel., U.S.P.-Dose, O.15 c.C., $2 \frac{1}{2}$ minims, U.S.P.

*Tr. Lobel., U.S.P.- Io per cent. Dose, I c.c., I5 minims, U.S.P. Maximum dose, 2 c.c., 30 minims.

Tr. Lobel. Aeth., B.P. -30 per cent. in Spir. Aeth. Dose, 0.3 to I c.c., 5 to $\mathrm{I}_{5}$ minims, B.P.

\section{CONIUM}

Conium or "Water-Hemlock," an Umbelliferous herb, contains a number of alkaloids, of which coniin is the most important. This is a simple derivative of Piperidin, and was the first alkaloid synthetized. Children are sometimes poisoned by eating the root. The juice is believed to have been the poison administered to Socrates. It was introduced into medicine by Stoerck, I770, but is now practically obsolete. It was formerly used as a sedative and antispasmodic in chorea, mania, convulsions, whooping cough and asthma; and locally in hemorrhoids and other anal lesions. Its peripheral actions are similar to those of nicotin, but it produces more pronounced paralysis of the central nervous system and of the skeletal muscle-nerve endings. Poisoning is treated by tannin, evacuation and stimulants. A bibliography is given in Cushny's "Pharmacology."

\section{GELSEMIUM}

This, the root of Yellow Jasmine, acts similarly to nicotin and coniin. Its central depressant action is relatively more marked (Cushny, I892). It contains a crystalline alkaloid, Gelsemin, with weak strychnin action; and several amorphous alkaloids, Gelseminin and Gelsemoidin (C. W. Moore, 1910; Sayre, 1910 and 1912; Sayre and Chillingworth, 1914), which are chiefly responsible for the effects of the drug. "Gelseminin" Merck has been shown to consist of Gelsemin. Stevenson and Sayre, 1915, describe a further alkaloid, "sempervirin."

Uses.-Gelsemium is employed against trigeminal and other neuralgias. This has no pharmacologic explanation. Even small doses sometimes cause toxic symptoms: giddiness, double vision, and slowing and arrest of respiration. Its local application to the eye dilates the pupil, with considerable irritation (Putzeys and Romie, 1887). It is no longer used as a mydriatic. Poisoning is treated by evacuation, atropin and stimulants.

\section{PREPARATIONS-GELSEMIUM}

Gelsemium, U.S.P.; Gelsem. Rad., B.P. (Yellow Jasmine Root).-The dried rhizome and root of Gelsemium sempervirens (nitidum, B.P.) Dase, $0.03 \mathrm{Gm} ., 1 / 2 \mathrm{gr}$.

Ext. Gelsem., U.S.P. - A powdered extract, $\mathrm{I} \mathrm{Gm}$ representing $4 \mathrm{Gm}$. of the drug. Dose ro mg., $1 / 6 \mathrm{gr}^{\text {, U.S.P. }}$

Fldext.Gelsem., U.S.P. -Dose, 0.03 c.c., $1 / 2$ minim, U.S.P.

Tr. Gelsem., U.S.P., B.P. - ro per cent. Dase, 0.25 c.c., 4 minim, U.S.P.; 0.3 to I c.c., 5 to 15 minims, B.P. 


\section{SPARTEIN}

General Statement.-This alkaloid has been used to slow the pulse in cardiac disturbances; but its effects are uncertain, it weakens the heart, and can not take the place of digitalis.

Spartein is a pyridin derivative, like nicotin and coniin, and has similar actions (Fick, 1873 ). Its central effects are slight and its toxicity relatively low (Griffe, 1886 , took $0.4 \mathrm{Gm}$., $6 \mathrm{gr}$., without bad effects, except slight confusion). It has no direct action on the vasomotor center; but the depression of respiration tends to produce asphyxial stimulation (Pilcher and Sollmann, 1915). Peripherally, it has a fairly strong curare action, arresting respiration by paralyzing the phrenic endings (Cushny and Matthews, 1895). Larger doses also paralyze the respiratory center (Muto and Ishizaka, I903). It has a weak local anesthetic effect (Guinard and Geley, I894). No habituation could be induced in guinea pigs (Dorlencourt, I913).

Circulation.- T he experimental data give a confused picture of its actions. Small doses produce peripheral vagus paralysis (Fick and all subsequent workers). This may sometimes result in preliminary quickening of the pulse (Gluzinski, I889) and removal of any vagus arythmias (Masius, I887). The most constant feature is slowing of the pulse rate, which is not prevented by atropin, and is therefore due to direct depression of the cardiac muscle. Because of the slowing, the pulse waves appearlarge; but in contrast to digitalis, the tendency of the heart is diastolic instead of systolic (Cushny and Matthews). Frogs' hearts also show decrease of rate, strength and tone. With intravenous injections, the slowing becomes so extreme that it may be fatal; but this extreme action is of short duration, does not occur on oral administration, and is probably due to direct irritation of the endocardium. The blood pressure shows a progressive fall, explainable by the cardiac depression. Excised arteries are dilated (Cow, I9II). Rise of blood pressure has been reported but is of inconstant occurrence (Cerna, 1894), and is perhaps due to the mechanical effects of the injection and to asphyxia.

Diuretic Effect.- This has been affirmed and denied, clinically and experimentally (MacNider, I9ro; Griffé, 1886). Its existence is very doubtful. Fronmueller, 1878, contended that the reputed diuretic effect of the parent plant (Scoparius) is due mainly to scoparin, a neutral principle.

Uses.-Spartein was recommended by Sée, $188_{5}$; Laborde and Legris, I886; and many others, in cardiac disease, valvular and functional, nervous palpitation, Graves' disease, asthma, etc. Clarke, I887, claimed that it produces marked slowing and strengthening of the pulse, the effect appearing in thirty minutes and lasting for five to six hours. Perhaps more clinicians consider it useless (Leo, I887; H. C. Wood). It is certainly devoid of the cardiac tonic action of digitalis; and without this the slowing would be of little value. The influence of drugs on functional cardiac neuroses is difficult to judge. Atropin would be more efficient for paralyzing the vagi.

Dosage.-ro mg., 1/6 gr., gradually increased to o.I3 Gm., 2 gr., every six to eight hours. A. Jacobi, I907, advises not less than I gr. per day, even for babies. Overdoses produce nausea and vomiting.

\section{PREPARATIONS-SPARTEIN}

${ }^{*}$ Sparteine Sulphas (Spart. Sulph.), U.S.P.; $\mathrm{C}_{16} \mathrm{H}_{26} \mathrm{~N}_{2} \cdot \mathrm{H}_{2} \mathrm{SO}_{4}+{ }_{5} \mathrm{H}_{2} \mathrm{O}$.-Hygroscopic colorless crystals or powder; odorless; slightly bitter. Freely sol. in water (I:I.I) or alc. (1 : 3). Dose, ro mg., 1/6 gr., U.S.P. Maximum dose, $0.2 \mathrm{Gm} ., 3 \mathrm{gr}$.

Scoparii Cacumina, B.P.; Broom Tops. -The fresh or dried tops of Cytisus scoparius.

Inf. Scopar., B.P.- - io per cent. Dose, 30 to 60 c.c., 1 to 2 ounces, B.P.

Succus Scoparii (Succ. Scopar.), B.P.; Juice of Broom.-The juice expressed from the fresh plant and preserved with one-third its volume of alcohol. Dose, 4 to 8 c.c., I to 2 drams, B.P. 


\section{CURARE}

General Statement.-This is practically important in laboratory technic, for paralyzing the skeletal muscles through a selective depression of their nerve-endings, as shown in the classical researches of $\mathrm{Cl}$. Bernard, 1857. It has no effect on sensory nerves and is not an anesthetic. It produces a number of side effects, resembling nicotin in depressing autonomic ganglia (vagus); and strychnin in stimulating the spinal cord. It may produce glycosuria. Intravenously, it causes a temporary fall of blood pressure, returning rapidly to normal with moderate doses. Death occurs by paralysis of the respiratory muscles; animals may be saved by artificial respiration, the drug being rather rapidly excreted by the urine. Curare is mutually antagonistic with physostigmin. It is much less toxic by the stomach, on account of slow absorption and rapid destruction and excretion. It has been used therapeutically against tetanus and other convulsions, but only obviates the fatigue and exhaustion, and is quite dangerous because of the uncertain dosage.

Other Drugs Producing the "Curare Effect."-These are quite numerous.

Among the most important poisons possessing this action are the following:

Certain ammonium bases, amids and amins, cholin, muscarin, etc.

Methyl-strychnin, guanidin (Brown and Fraser, 1869).

Delphocurarin, Heyl (Kahn, I909).

Aromatic series: Pyridin, quinolin, thallin.

Nicotin series, piperidin.

Morphin and related alkaloids.

Cocain.

Camphor in frogs, but not in warm-blooded animals.

Certain putrefactive ptomains.

Products of muscle metabolism.

The curare effect of many of these drugs' is an unimportant side-action, which can only be demonstrated in frogs, being obscured by other actions in mammals.

$\mathrm{S}$. Frænkel, 1904, attributes the curare action of the ammonium bases and related drugs to the stereochemic orientation of the valences.

Origin.-Curare, Woorara, Urari or Tiennas, comprises several varieties of extracts, prepared by South American Indians as arrow poisons, from the root-bark of several Strychnos plants. It was first brought to Europe from Guyana by Sir W. Raleigh, 1595. Little is known of their preparation. They vary greatly in composition (certain varieties were supposed, probably erroneously, to contain snake venom); and they deteriorate on keeping, especially if moist, so that commercial curare is a very unreliable drug. The naming of the active alkaloids is so confused that they are not much better.

Varieties.-These are designated by the kind of containers in which they reach commerce: Calabash (Gourd) curare is obtained principally from Strychnos toxifera and contains curarin. Tube curare, in bamboo.tubes, is of undetermined source, and contains tubocurarin and curin. It is the least desirable but the most common variety. Pot or jar (Topf) curare, comes from Strychnos Castelnæi and Cocculus toxiferus, and contains protocurarin and protocurin. All the varieties consist of brown masses, intensely bitter, not wholly soluble in water, more soluble in dilute acid.

Active Principles. - All the above-named constituents are alkaloids. Curarin and protocurarin have the most typical effects, and the latter is probably stronger. Tubocurarin has a.much weaker muscle-nerve action, a stronger strychnin effect, and increases the heart rate. Curin is devoid of the muscle-nerve action, but first stimulates and depresses the heart. It can be converted into curarin (Boehm).

Curarin is related chemically and in actions to strychnin. Its preparation from calabash curare by the method of Boehm, 1887, is described in the Reports, Counc. Pharm. Chem., I9ro. Tubocurarin is not a proper synonym although it has been so used.

Paralysis of Muscle-nerve Endings.-Ordinarily the only symptoms of "curare" poisoning consist in this paralysis. When the curare is introduced under the skin, it causes a total loss of motion, first of the voluntary and then of the respiratory muscles. 
Sequence.-The order in which this disturbance appears is the following: Short muscles of the toes, ears, and eyes; then the limbs, head and neck; finally the respiratory muscles, first the thoracic, then the diaphragmatic, and lastly the abdominal (Chid, rgr 2).

The heart is not affected except with very much larger doses.

The muscles which are least affected by curare are those which contain the largest amount of utilizable oxygen, and survive longest after the death of the animal. This supply of oxygen accounts, probably, for the lesser susceptibility to the depressing action of curare.

Development of Paralysis. - The first sign of curare action consists in incapacity for sustained effort on repeated stimulation of the nerve; i.e., whereas a single contraction is normal, fatigue sets in more readily than usual (Boehm, 1894). Then the height of contraction is somewhat lowered. Then the current must be strengthened to obtain any response; and finally even the strongest stimulation-of the nerve-is ineffectual.

Location.-This was investigated by Fontana and established by the work of $\mathrm{Cl}$. Bernard, 1857 . It is evident that some structure is paralyzed. The paralysis might have its seat in any part of the central nervous system or it might be peripheral. Stimulation of the sciatic does not produce a contraction if the dose has been sufficient. The point of attack must, therefore, be peripheral to the sciatic nerve. This leaves the nerve trunk itself, the nerve-endings, and the muscle fibers. Stimulation of the muscle directly is effective, so that this is excluded. To decide between the nerve trunk and nerve-endings, he placed a ligature around the body of a frog, with the exception of the sciatic nerves, and tightened the ligature so as to entirely exclude the lower extremities from the circulation. He then injected the curare. In this manner the peripheral portions of the sciatic nerves and the endings did not come into contact with the curare and the nerve trunk was alone exposed to the poison. He found that stimulation of the trunk caused normal contraction, consequently that curare had no action on it, thus leaving only the endings.

$\mathrm{He}$ also proved the point in a simpler manner by ligaturing one leg exclusive of the nerve, or by placing the muscle of one and the nerve of another muscle-nerve preparation into the solution. Garten, I9I2, has shown by careful methods that even prolonged exposure to strong curare solutions does not affect the nerve trunk.

The "muscle-nerve" endings affected by curare need not necessarily be identical with the anatomic end plates, although this is possible (Edmunds and Roth, rgo8). Kuehne, I886, described some observations which indicate that the action is not always strictly localized. The peculiar antagonism with nicotin was discussed on p. 268 . The nerve-endings of invertebrates are not paralyzed (Straub).

Direct stimulation of the muscle (in cats) requires a somewhat stronger current under curare, but not if the nerve has degenerated. Epinephrin also restores the threshold of muscular stimulation. Fatigue increases the threshold (Gruber, I9I4).

Sensory Nerves. - These are not affected by curare. The reports of early travelers who describe poisoning by curare arrows mention that sensation is not impaired when motion is entirely impossible. Bernard also studied this action directly on the frog. He ligatured one leg with the exception of the sciatic nerve, injected the poison, and applied the stimulus to one of the upper extremities. This caused a reflex movement of the ligatured leg, which would not have been the case had the sensory endings of the foreleg been paralyzed.

Paralysis of Respiration as Cause of Death. - In cold-blooded animals in which the respiratory exchange takes place largely through the skin, and respiratory movements are unnecessary, the poison is gradually eliminated if the animal be kept in a moist atmosphere. Complete recovery occurs after eight to ten days, except when the dose is extremely large, in which case other factors come into play.

Warm-blooded animals die of paralysis of the respiratory muscles. If artificial respiration be kept up and the dose has been only just large enough to produce a paralysis they may also recover.

The recovery of the respiratory muscle begins immediately, whilst the sciatic endings require several hours. The effect of the curare increases progressively with the dose: it is as if an increasing resistance were gradually introduced between the nerve and the muscle.

The seat of the respiratory paralysis is also peripheral, for stimulation of the phrenic nerve does not cause contraction of the diaphragm.

The respiratory paralysis, if it is not too profound, is promptly removed by physostigmin; stimulation of the sciatic also becomes effective again.

Bronchial muscle is not affected by Curare (Trendelenburg).

Metabolism. - Curare paralyzes the temperature nerves of the muscles, as well as the motor nerves, so that cocain, e.g., can not raise the temperature. 
The muscular paralysis lowers the metabolism. It is claimed that this diminution is far more conspicuous in the nitrogen, than in the carbon-metabolism.

Glycosuria is a frequent but not constant phenomena (Morishima, I899). It is not wholly due to asphyxia, for it can not be completely prevented by artificial respiration (Macleod, 1909). The earlier literature is reviewed by S. Weber, 1904.

Peristalsis. - This is often increased, partly by asphyxia, but also with artificial respiration. Bidder, $186_{5}$, found no direct effect on intestinal or other smooth muscle, even with the largest doses.

Autonomic Ganglia.-The vagus, vasomotor, salivary, pupillary, etc., ganglia are depressed, but usually not as strongly as with nicotin. 'The heart is quickened after slight primary slowing. Electric stimulation of the vagus does not arrest the heart, but it may be slowed, or beat faster (Dale and Laidlaw, I9I I).

Effects on the Circulation.- If artificial respiration is maintained, the first effect of curare on the circulation consists in a fall of blood pressure, due to peripheral vasomotor depression (Tillie, I89o; Sollmann and Pilcher, I9ro). This is soon accompanied by quickened heart beat, from depression of the vagus ganglia. The depression does not readily pass into paralysis, so that stimulation of the vagus or sciatic is still cffective; indeed, the vasomotor reflexes may be increased, through the central action of the curare. Martin and Stiles, I9I4, also found that the threshold of the vasomotor center is not raised, even by large doses. The reflex depressor fibers are rendered more sensitive (Langley, I9I 2), or rather, the depressor response is more nearly proportional to the strength of the stimulus, than in normal animals (Martin and Stiles, I9I4). Porter, 1915, finds that the response to sciatic and depressor stimulation may be doubled whilst the level of blood pressure remains practically unchanged; and interprets this as evidence that the vasotonic and vasoreflex centers are distinct and independent. These effects on the circulation pass off within fifteen minutes, and the circulation returns to normal, whilst the muscular paralysis persists. They therefore do not seriously interfere with the employment of the drug in experiments. The main objection which may be urged against the latter is, that it does not produce sensory paralysis, whilst the absence of struggling, etc., might cause the inexperienced operator to neglect a proper enforcement of other means of anesthesia.

Perfusion of frog's vessels slows vasoconstriction, which is not modified by absence of calcium (Pearce, I913). $^{2}$.

The lymph hearts of frogs are arrested (Bernard).

Central Nervous System.- When curare is applied directly to the spinal cord of frogs, it causes typical strychnin convulsions. With ordinary methods of administration these are masked by paralysis of the nerve-endings. Certain samples, however, cause strychnin convulsions before the typical curare action appears.

In $d o g s$, the convulsions produced by injecting curare into the spinal canal or cerebral ventricles differ from those of strychnin, being irregular and asymmetric(McGuigan, I9I6).

Inactivity by Gastro-intestinal Canal.-The effects of curare are obtained only if it is introduced under the skin or into the circulation, but usually not if introduced into the stomach. The experiments on the administration of curare by the stomach (mainly by Bernard) have shown that:

I. It is not destroyed by the gastric juice, pancreatic juice, or saliva.

2. It passes very slowly through the walls of the stomach when the epithelium has been killed, and not at all if the epithelium is still living. (It will be remembered also that strychnin is not absorbed by the stomach in rabbits.)

3. It is to some extent fixed or destroyed by the liver, for it is much less active when injected into the portal than into the jugular vein. However, the discrepancy between the gastric and hypodermic dose persists, even when the liver is largely excluded by Eck's fistula; so that the liver destruction can not be very important (Polimanti, r9I4). It is also destroyed in vitro by ox-bile, and by bacteria.

In frogs the liver is the main agent in the disintoxication: in normal animals 50 times as much curare is required by mouth as hypodermically; the difference disappears completely if the liver is excised; digestion of curare with liver substance destroys its activity.

4. It is very rapidly excreted unchanged in the urine.

In mammals the inactivity of curare by the mouth is due partly to its destruction by the bile and bacteria; but mainly to the capacity for absorption being less than the capacity for its destruction or excretion. If the renal vessels are tied, poisoning occurs quite readily even when it is taken by the stomach. If very large doses are taken on an empty stomach, sufficient may be absorbed to cause symptoms.

Use of Curare in Convulsions. - This is still largely experimental and not very promising. It has been suggested to combat the convulsions of strychnin, tetanus, and 
hydrophobia (E. Collins, 1905). It is certainly quite possible to suppress the spasmodic condition by sufficiently large doses. Unfortunately, however, it is impossible to secure this without at the same time paralyzing respiration. This latter may, theoretically, be counteracted by artificial respiration, but this prolonged manipulation is in itself injurious. On the other hand, minimal doses may be considered useless, and, indeed, as has been pointed out, even if the spasms could be suppressed without affecting the respiration, this would not be an ideal treatment for strychnin. In well-chosen cases, however, curare may be the means of saving life. Convulsions certainly tend to heighten fatigue and paralysis of the medullary centers, and if in a case in which the degree of poisoning just exceeded the lethal limit by a very little, a minimal amount of curare were injected, this might, perhaps, reduce the spasm sufficiently to turn the scale, or somewhat larger doses might be given which would require some, but not very much, artificial respiration. This has actually been done, and in desperate cases curare is worthy of a trial; but in addition to the other objections come the very uncertain quantitative effects. It would be justified only to work with tested samples, and these are very rarely accessible when needed.

Administration. - The drug should be given hypodermically, using per kilogram of patient one-tenth the fatal dose determined per kilogram of dog; and being prepared for artificial respiration and physostigmin. Curarin, if pure, would give an exact substance, in the dose of $\mathrm{I}$ to I $2 \mathrm{mg}$. hypodermically. The reliability of the alkaloid is, however, open to doubt.

\section{GUANIDIN}

This and methyl-guanidin produce peripheral stimulation of striped muscle (fibrillary contractions), then central paralysis and finally a curare action.

Guanidin is imido-urea, $\mathbf{N H}=\mathrm{C}=\left(\mathrm{NH}_{2}\right)_{2}$. It is a constituent of creatin and arginin. Methylguanidin occurs in urine and in protein putrefaction.

The muscular twitchings are especially interesting. Thoir peripheral origin was shown by Gergens and Baumann, 1876 . They occur if the isolated muscle is laid in I : 2000 to I : 4000 solution of the chlorid. In living animals they persist if the motor nerve is divided, but fail when it has degenerated; so that the action is located in anatomic endings. They are suppressed by calcium or magnesium (Fuehner, I 907 and I9I I). In cats, the symptoms resemble chorea (Fuchs, 19I4).

Other Substances Producing Fibrillary Twitchings are: Physostigmin, aconitin, nicotin, $\mathrm{NaCl}$, and the salts of $\mathrm{Ba}, \mathrm{Zn}, \mathrm{Cu}, \mathrm{Ni}$, and $\mathrm{Pb}$.

\section{EPINEPHRIN}

General Statement.-Epinephrin (variously called Adrenalin, Suprarenin and numerous similar names) is the active principle of the medulla of the suprarenal gland. It is a relatively simple amin derivative of catechol, and can be prepared synthetically. It behaves as a feeble base and might be classed with the alkaloids. Its solutions are very unstable, unless a preservative is added.

Its typical actions consist in a highly specific stimulation of the physiologic endings (receptive substance) of the entire sympathetic system. The effects upon any given organ-whether augmentory, inhibitory, or indifferent-therefore correspond with the effects of stimulation of its sympathetic innervation. Very dilute concentrations may have opposite effects.

The most important practical manifestation of this action consists in a high rise of blood pressure, from peripheral stimulation of the vasoconstrictor mechanism of the systemic vessels, and of the accelerator mechanism of the heart. 
Epinephrin is used locally for arresting hemorrhage and enhancing the anesthetic effects of cocain; hypodermically in bronchial asthma; intravenously, for tiding the patient over acute circulatory collapse.

Its systemic action is very brief. It is entirely ineffective by oral administration; but little effective subcutaneously, and of moderate efficiency intramuscularly.

Overdoses, intravenously, kill by acute dilation of the heart.

Historical.-Epinephrin is identical with the "chromaffin substance" (giving a green color with ferric chlorid, and pink with alkalies, iodin or chlorin, and taking up chromium salts; Henle, I 865 ), which was discovered by Vulpian, I 856 , in the medulla of the adrenal bodies, and also in association with sympathetic cells elsewhere. The suprarenal medulla, as shown by Balfour, is a direct outgrowth of the sympathetic system, and consists at first of neuroblasts. The cortex is of mesoblastic origin, and does not contain epinephrin, but cholin, whose action is essentially antagonistic to epinephrin (Lohmann, I907).

The pressor action of the medullary substance was discovered by Oliver and Schaefer, 1895, and by Czybulski and Symonovicz, I895. This was followed by many attempts to separate the active principle. The researches of v. Fuerth, 1898 , and particularly those of Abel, paved the way to its isolation in crystalline form, which was first accomplished by Takamine, rgor, Aldrich, 1901, and Abel, 1903. Its structural constitution was determined by Jowett, I904, and its synthesis accomplished by Stolz, 1904, Dakin, 1905, and Flächer, 1908. An extensive series of related amins, with similar sympathomimetic actions has also been prepared (Barger and Jowett, 1905; Barger and Dale, I910), and some of these, as well as the synthetic epinephrin itself, are marketed. I914.

A good discussion of epinephrin is contained in Barger's "Simpler Natural Bases,"

Abel and Macht, rgr 2, have also found considerable epinephrin (along with the digitaloid Bufagin) in the "parotid glands" of the Tropical Toad, Bufa Agua.

Crawford and Watanabe, I915, believe that it (or a similar substance) exists in pituitary extracts and is partly responsible for their pressor effect.

Extracts of the retro-peritoneal chromaffin tissue, of man and mammals, also contain epinephrin or a closely related substance, having the typical action on intestinal and uterine muscle (Fulk and Macleod, I9I6).

Chemic Structure.-Epinephrin is dioxyphenyl (pyrocatechin) ethanol-methylamin, $\mathrm{C}_{6} \mathrm{H}_{3}(\mathrm{OH})_{2}-\mathrm{CHOH}-\mathrm{CH}_{2}-\mathrm{NHCH}_{3}$, corresponding to the empirical

formula $\mathrm{C}_{9} \mathrm{H}_{13} \mathrm{NO}_{3}$ (first established by Aldrich, I902),

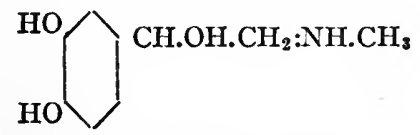

Its structure is rather closely related to that of tyrosin; and it may perhaps be derived from this or similar amins in the body.

Action of Isomers. - The natural epinephrin is levorotary. The synthetic base is racemic, and has been split into an 1 . and d.component by Flächer, I908. The 1. component is identical with the natural base. The dextrorotary base produces qualitatively the same actions, but has only one-twelfth to one-eighteenth the activity; and the racemic mixture therefore has about half the activity of the natural base (Loewi and $H$. Meyer, 1905; Cushny, r908 and 1909; Abderhalden and Mueller, I908; W. H. Schultz, I909). Froehlich, I909, claims that the $\mathrm{d}$-base has a much more persistent action.

Related Sympathetic Substances.-Barger and Dale, I9ro, found that the universal peripheral sympathetic stimulation (which they aptly call "sympathomimetic action") is not peculiar to epinephrin, but is shared by a large number of primary and secondary amins, the simplest being the primary fatty amins, such as amylamin. The catechol nucleus is a rather unessential constituent, and the action of catechol itself is not related to that of epinephrin. However, the intensity and specificity of action increases as the amins approach the composition of epinephrin itself. It is noteworthy that the various sympathetic effects, and the augmentory and inhibitory stimulations, vary independently in these various amins. Nor is there any parallelism between instability and activity. 
Sympathomimetic Action of Epinephrin.-The correspondence between epinephrin and sympathetic stimulation was described on page 264 ; the organs and functions augmented and inhibited, on pages 264, 265; and the localization of the effects in the receptive substance, on page 266.

Effects on Circulation.-The intravenous injection of epinephrin in to a normal animal, with intact vagi, produces the striking phenomena depicted in Fig. 14. The blood pressure rises sharply; as it approaches its maximum, the heart beats are greatly slowed and strengthened. The pressure is not sustained, but returns quickly to normal. The heart beats also return to their former rate; but, as may be seen from the tracing, more slowly than the pressure.

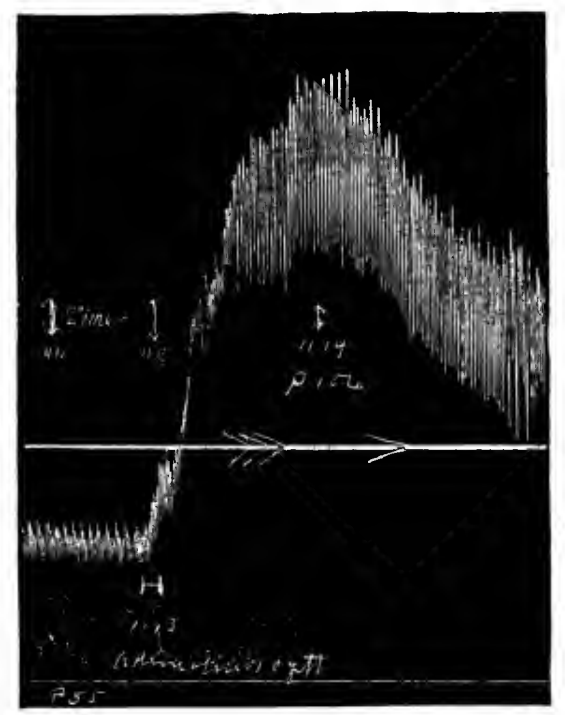

FIG. 14.-Suprarenal on Blood-pressure, dog. I1/2 drops per Kg. of I:I000 epinephrin injected intravenously at $\mathrm{H}$. P.= Blood-pressure in $\mathrm{mm}$. of $\mathrm{Hg}$.

These phenomena are due to the interaction of three factors: vasoconstriction, vagus stimulation, and stimulation of the cardiac accelerator mechanism.

The blood pressure may rise to a very high level, especially if the vagi have been divided or paralyzed. In dogs, it may exceed $300 \mathrm{~mm}$. of mercury. The extent of the rise increases with the dose, but not in simple proportion. The main element in the rise is ordinarily the intense vasoconstriction; for the volume of the organs diminishes as the blood pressure rises. The cardiac stimulation, however, is also an important factor, and sometimes it is the more important.

The cardiac slowing is indirect, due mainly to stimulation of the vagus center by the rise of blood pressure. The direct action of epinephrin would be to quicken the heart rate by stimulation of the accelerator endings; this is the result with small doses (Hoskins and Lovelette, rigr4); or in excised hearts; or if the vagi have been divided or paralyzed; or if the heart - escapes temporarily from the vagus control, as often happens. With the vagi cut, epinephrin stimulates the auricular, and still more the ventricular efficiency (Wiggers, 1916). 
Effects with Different Methods of Administration.-Practically no systemic effect can be secured by administering epinephrin by mouth, and relatively little by hypodermic injection. This is explained by the limitation of absorption through vasoconstriction (for instance, frog's skin, which readily absorbs most alkaloids, but does not absorb epinephrin, Rieder, r909). The slow absorption permits the destruction of the epinephrin before it reaches the circulation. The response is considerably' greater with intramuscular and peritoneal injection (Meltzer and Auer, 1905; Lapponi, I915); but it is still much less, though more lasting, than with intravenous injection. Injection under pressure into the nasal submucosa is almost equivalent to intravenous injection (Pilcher, rgr4). Intraspinal injection gives a considerable but slow rise (Auer and Meltzer, i9r2). None is absorbed through nerves (Meltzer, r909).

In man, the blood pressure response is also practically absent on oral administration. Hypodermically, 0.5 to $\mathrm{I} .5 \mathrm{mg}$. ( $1 / 100$ to $1 / 40 \mathrm{gr}$.) gives uncertain results, usually a rise of ro to $12 \mathrm{~mm}$., rarely higher (to $45 \mathrm{~mm}$.). The same doses intramuscularly give more constant and higher rise, usually 8 to $25 \mathrm{~mm}$.; but sometimes much higher. over $90 \mathrm{~mm}$., so as to produce alarming symptoms (Halsey, r909).

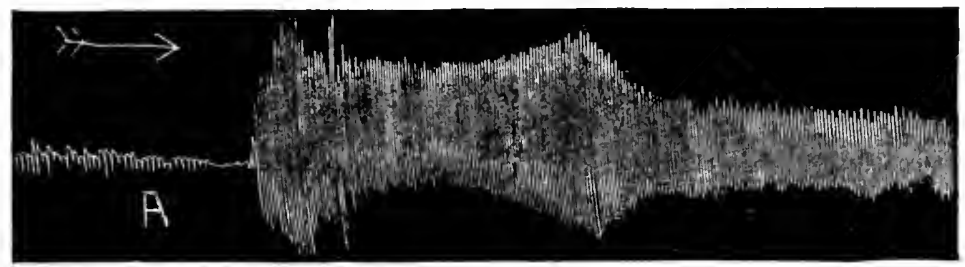

Fig. 15.-Revival of Langendorf heart by epinephrin. The drug is added at A.

Relation of Dosage to Rise of Blood Pressure. - With intravenous injection and atropinized dogs, this is somewhat as follows (from the data of Hunt, I9or, and others): $0.00008 \mathrm{mg}$. per kilogram $=$ rise of $5 \mathrm{~mm}$.; $0.00025=7 \mathrm{~mm}$.; $0.0005=15 \mathrm{~mm}$; $0.0007=$ $20 \mathrm{~mm}$. $0.0017=25 \mathrm{~mm}$.; $0.004=45 \mathrm{~mm}$. $0.006=66 \mathrm{~mm} . ; 0.03=150 \mathrm{~mm}$.

Influence of Dosage on the General Effects.-The effects of intravenous injection vary with the dose. The results of increasing doses may be tabulated as follows (Langley, I90I):

(a) Rise of blood pressure.

(b) Inhibition of bladder, mydriasis.

(c) Contraction of uterus, vas deferens, and seminal vesicles; salivation and lachrymation; inhibition of stomach and gall-bladder; increased bile secretion; inhibition or stimulation of internal anal sphincter.

(d) Contraction of erectores pilorum.

(e) Uncertain effect on tunica dartos and on sweat.

Cardiac Stimulation.-The perfusion of epinephrin through the excised mammalian heart (Hedbom, Cleghorn, Gottlieb, Boruttau), or its perfusion or direct application to a frog's or turtle's heart (Lussana, I9I3), quickens the rate, and increases the amplitude of the contraction. The tone is very markedly increased, of ten doubled. The oxygen metabolism of the heart is increased in proportion to its activity and rate (Barcroft and Dixon, r907; Rohde and Ogawa, I912; Evans and Ogawa, 1914). Existing irregularities are removed, and the effects of muscular depressants are counteracted (aconite, MacNider; strychnin, Januschke, r9ro; phenol, chloral and chloroform, Gunn, I913, I915; potassium, etc.). A heart which has ceased to beat may often be revived by this drug (Fig. 15). 
The fact that epinephrin may start a heart many hours after excision, or when it has been depressed by chloral, goes to show that the heart may contract independently of ganglia; for the epinephrin action is pretty certainly not ganglionic (Gunn, I915).

The cardiac acceleration is not due to vagus paralysis, for it occurs after atropin, and also in isolated strips of the cat's ventricle (Leetham, I9I3), or after the auriculo-ventricular bundle is cut. In complete heart-block, it quickens and strengthens the ventricles and auricles (van Egmond, I913). From analogy, it may be assumed that the action is exerted on the receptive substance connected with the accelerator mechanism.

Influence of IIeat. - With the isolated frog heart, the amplitude is but little affected by epinephrin between 8 and $13^{\circ} \mathrm{C}$., but increases from 13 to $33^{\circ}$. The rate is decreased between 8 and $18^{\circ}$; fairly constant between 18 and $28^{\circ}$, and rapidly decreased between 28 to $30^{\circ}$ (Sprinceanu, 1915).

Effect of Serum and Ions. - These have considerable influence on the response of the frog heart to epinephrin. The stimulant effects of epinephrin and serum are synergistic (potentiation, Moog, r914); whilst in the absence of calcium, epinephrin produces depression and diastolic arrest (Burridge, I9 I4).

In birds, the cardiac effects of epinephrin, pituitrin and barium differ considerably from the mammals (Paton and Watson, IgI2).

Use in Cardiac Failure.-The cardiac stimulant effects of epinephrin have been utilized in therapeutics to counteract acute cardiac failure in asphyxia, etc. It would be useless against persistent cardiac weakness, since its action is too fleeting, and can be obtained effectively only by intravascular injection.

Rcsuscitation of the arrested heart is most effectively accomplished by injecting epinephrin into the pericardial sac (Gunn and Martin, 1915); or more conveniently into the cardiac end of the carotid artery, flushing it toward the heart by a stream of warm saline solution, and at the same time performing cardiac massage and arificial respiration. The chances of success diminish rapidly with the lapse of time after cardiac arrest; and after fifteen to thirty minutes, intracardiac clotting sets an end to all hope.

Cardiac Dilation.-In using epinephrin for cardiac stimulation, the dose--or rather the rapidity of injection-must be carefully controlled by the state of the heart. Overdoses of epinephrin kill by cardiac dilation, with pulmonary edema. The tendency to this increases with cardiac weakness. It may sometimes be relieved by artificial respiration (Emerson, I907). Patients with cardiac disease generally react to I mg. hypodermically, by extrasystoles (D. Roth, I9I4). Fatalities have occurred, especially in cardiac disease, from $0.4 \mathrm{mg}$. hypodermically (Fridericia, I915).

In poisoning by cardiac depressants ( $f . i$., chloroform, phenol or caffein) the intravenous injection of epinephrin will temporarily restore blood pressure, and this may give the heart a chance to recover. But if the blood pressure is raised much above the normal, the heart may stop suddenly, and usually irrecoverably; and this may occur from doses of epinephrin which would be quite safe in healthy individuals (phenol, Sollmann; chloroform, Levy and Lewis, I 12 ; Nobel and Rothberger, 1914).

Epinephrin on Heart Rate of Intact Animals. - Epinephrin tends to have a twofold action on the heart rate: acceleration through direct cardiac stimulation; and slowing through reflex vagus stimulation. When the vagi are intact and in good tone, ordinary doses of epinephrin invariably slow the rate (Meek and Eyster, 1915). When the vagus tone is low, quickening may result (Hoskins and Lovelette).

Vagus Effects. - The vagus stimulation is purely central, since no slowing occurs when the vagi are cut. It is due mainly to the rise of pressure, for it is absent if the pressure is kept from rising by hemorrhage, or by paralyzing the vasomotor endings. However, there is some evidence that the vagus center is also stimulated directly (Biedl and Reiner, 1898 ; Verworn, 1903). This is confirmed by the slowing which may occur when epinephrin is perfused through the medullary centers (E. D. Brown, IgI6).

The degree of slowing varies much in different individuals, but it always tends to lessen the rise of pressure, and this mounts much higher if the vagi are cut.

A secondary increase of the pulse rate is sometimes seen, and is attributed to central and peripheral stimulation of the accelerator nerves (Neujean, I905). 
The Vasoconstriction.-Epinephrin is the most powerful vasoconstricting drug known. It can be readily shown that this is the main factor in the rise of blood pressure, for the organ volume and the outflow of blood from veins are diminished. The site of action is entirely peripheral, for the rise occurs if the central nervous system has been destroyed; the perfusion flow through excised organs is slowed or arrested (Bier, 1897); and even rings of excised artery contract if placed in epinephrin solutions (A. B. Meyer, I907). The vasomotor center is not usually affected directly; but it may respond indirectly, generally by constriction to the increased pressure; occasionally by dilation, when the increased blood supply relieves a preëxisting asphyxial stimulation (Pilcher and Sollmann, I9I 5). (Occasionally, there are doubtful indications of direct stimulation of the vasomotor center; E. D. Brown, rgr6).

The constriction is most conspicuous in the smaller arterioles; but even large arteries (v. Frey, I905) and veins participate. The maximum constriction occurs in the splanchnic vessels, especially the kidneys; and in the vessels supplying the voluntary muscles and mucous membranes. The constrictor action is weak in the coronary, cerebral and pulmonary vessels; and in the intact animal these vessels are passively dilated by the displacement of blood from the more powerfully constricted areas. Epinephrin therefore would be harmful in cerebral or pulmonary hemorrhage.

The arterial muscle remains stimulated, in the susceptible vessels, as long as effective concentrations of epinephrin are present. Even very large doses of epinephrin (7,000 times the effective quantity) do not paralyze the vasomotor mechanism.

Dilator Action of Epinephrin.-This is the predominant response of certain vessels (Cow), but may also occur in others under special conditions, especially with very dilute concentrations; late in the course of perfusions; and particularly when the vascular tone is already high.

Brodie and Dixon often observed that the vessels were dilated by epinephrin. In the freshly excised kidney, suprarenal causes a powerful constriction; but some time after the excision, it dilates the vessels (Sollmann, 1905). The same phenomenon was observed in the postmortem perfusion of the dog's leg. In the ear of the living rabbit, the constriction is also followed by dilation (Meltzer and Auer, 1904). The vessels of the de-nervated ear dilate with doses that are constrictor for the normal ear (Engeloch, I915).

Ogawa, 1912 , found that when very dilute solutions are perfused continuously through kidneys or legs, the constriction is eventually replaced by dilation; increasing the concentration again produced constriction. Intestinal vessels dilated directly when the solution was sufficiently dilute, whilst in kidney vessels the preliminary constriction was relatively prolonged. Relaxed vessels showed a greater tendency to constriction than those with good tone.

Cannon and Lyman, I9I3, find that in living animals, small doses will either raise or lower the blood pressure, according to its previous level; and adduce evidence that the lowering is due to a direct dilator action on constricted arterial muscle. The fall occurs after ergotoxin. The dilator reaction is confined mainly to the peripheral vessels, for the fall of pressure which occurs with normal cats ( $0.002 \mathrm{mg}$.) occurs also if the splanch. nic vessels are ligated, but is replaced by a rise if the splanchnic vessels are intact and the important peripheral trunks ligated (Hartmann, r9I5). He therefore supposes that vasodilator nerves exist in the peripheral vessels, but not in the splanchnics. The dilator reaction fails if the vessels are already relaxed, as by hemorrhage or depressor stimulation. However, Swetschnikoff, rg r 4 , on isolated rabbit's ear, observed the same constriction, whether the pressure was high or low.

Increase of temperature diminished the contractor response, and at $4 \mathrm{I}^{\circ}$ to $45^{\circ} \mathrm{C}$, reversed into dilation. Frog's vessels do not show dilation with dilute solutions, nor with increase of temperature, but only in the absence of calcium or after degeneration of the nerves (Pearce, I9I3). 
Effect on Venous Pressure.-Capps, I9I I and 1913 , found in animals that this is not altered by small doses; it rises with large doses which cmbarrass the heart.

Direct Effect on Veins.--Gunn and Chavasse, 1913, showed on excised veins that these also react by constriction, although this does not apply to all veins (Crawford and Twombly, I913). Segments of the superior vena cava, near the heart, enter into rhythmic pulsations.

Portal Vessels. - These are markedly constricted on perfusion, in mammals and frogs (Berezin, I9I4), as also in intact animals (Burton-Opitz, I9I3). The response is much greater than that to electric stimulation of the nerves (Macleod and Pearce, I914).

Edmunds, I915, confirmed the constrictor effect on excised portal vein; but attributes the changes in living animals to changes in the general venous and cardiac pressure, since the volume changes of the liver vary in different animals and with other conditions.

Antagonistic Phenomena.-The epinephrin constriction is prevented by drugs which paralyze the receptive substance, namely apocodein (Brodie and Dixon, I904), and ergotoxin (Dale, I905; Sollmann and Brown, I905). Mutual antagonism exists with caffein (Sollmann and Pilcher, I9II) and with nitrites. The pressor effect of $0.0075 \mathrm{mg}$. of epinephrin is neutralized by $0.6 \mathrm{mg}$. of nitroglycerin (Cameron, 1906).

Differences in Special Vessels.-There is no question as to the typical response of the vessels which react strongly to epinephrin; but there is some difference of opinion as to whether the cerebral, pulmonary and coronary vessels respond by slight constriction, dilation, or not at all. In intact animals they are certainly dilated, but this might be, and in the main is, passive. The question can be solved only on excised organs and on excised arterial rings by the method of A. B. Meyer. The literature is reviewed by Barbour, I9I2. The histologic structure does not affect the qualitative response (Barbour, I912).

The Coronary Vessels.-Arterial rings respond by dilation in most mammals, but by constriction in man and monkeys (Barbour, I912; Barbour and Prince, I915; Park and Janeway, I912; and others). Perfusion of the arrested heart may cause either dilation (Elliott, I905) or weak constriction (IViggers, 1909).

In the beating heart, the coronary flow is certainly increased in most species of animals (excised hearts, Wiggers; Rabe, I9r 2; Markwalder and Starling, 1913; Morawitz and Zahn, 1914; not in monkeys, Barbour and Prince, I915) on account of the pumping action of the contracting cardiac muscle. In the intact animal, this occurs even if the blood pressure is kept level by hemorrhage (F. Meyer, I9I3); and the increase would be still greater, if the blood pressure, and therefore the coronary pressure are allowed to rise (Bond, IgII). The heart-muscle would therefore be better nourished and thus better able to work against the high resistance. A vasoconstrictor effect on the coronary vessels can be discerned in the excised heart only with doses which are too small to affect the cardiac muscle (Brodie and Cullis, I9II).

In the monkey, however, and therefore presumably in man, the conditions are different. All doses slow the coronary flow of the excised monkey heart, whether this is beating or resting (Barbour and Prince, IgI5).

Pulmonary Vessels.-These show a weak vasoconstriction (Plumier, I904; Wiggers, I909; Plumier and Clermont, I912; Tribe, I9I2). According to Tribe; negative effects (Brodie and Dixon, I904; Burton-Opitz, I907; Heger and Phillipson, I9r 2; Baehr and Pick, I9I3) are attributable to the dilator effect of the chloretone in certain commercial solutions. Cow, I9I I, and Barbour, I9I 2, found that the reaction of arterial rings varied according to their situation; the intravisceral portion of the artery did not react; the extravisceral portion was constricted.

Macht, IgI I, describes powerful constriction; Berezin, I9I4, no action or dilation.

In intact animals, the weak pulmonary vasoconstriction is overcome mechanically by the rise in aortic and intraventricular pressure, so that the pulmonary vessels are passively dilated (Cloetta and Anderes, I914; Anderes and Cloetta, I9I6); and the volume of the lung increases (Anderes, I9I5). Pulmonary hemorrhage is therefore increased by epinephrin (E. Frey, rgog; Wiggers, 1909). The pressure in the pulmonary arteries rises greatly (Wood, Jr., I9II), especially when cardiac dilation sets in(Hallion and Nepper, I9II). This results in pulmonary edema. The circulation time in the lungs is quickened by small, increased by large doses (Langlois and Desbouis, I9I 2 ).

The Cerebral Vessels.-Wiggers, I907 and I9I4, found constriction; Berezin, I9I6, observed constriction, but requiring higher concentration than for peripheral vessels; Dixon and Halliburton claim dilation. Cow observed slight relaxation of arterial rings. Here, again, there is undoubtedly passive dilation in the intact animal (Biedl and Reiner, I905; Neujean, I905). The vessels of the pia and retina are constricted (Hirschfelder, IQI 5 ). 
The Renal Vessels and Urine Flow.-The renal vessels appear to be especially sensitive to epinephrin, being constricted by doses which are too small to affect the general blood pressure; and with ordinary doses, the renal constriction outlasts the pressure rise (D. Jonescu, I908). This results in anuria. It is claimed, however, that very large doses dilate the renal vessels and thus increase the urine flow (Bardier and Fraenkel, r899; Houghton and Merrill, 1908). Freshly excised kidneys certainly do not show any dilation (Wiggers, 1907), until the nerve endings are killed by exposure (Sollmann, 1905). Hypodermic injections of epinephrin into rabbits ( 1.5 to $2.5 \mathrm{mg}$. per kilogram) produces a strong diuresis, lasting several hours (Bilberfeld, 1907). This is independent of the glycosuria (Konschegg, I912; Gramenitzki, I912).

Cow, 19I4, finds a direct vascular connection between the suprarenal medulla and the kidneys. Through this, epinephrin could reach the kidneys directly, and thus diminish the urine flow, without its existing in discoverable quantity in the general blood streams.

Epinephrin as a Local Hemostatic.-The powerful vasoconstriction makes epinephrin a most effective agent for arresting capillary hemorrhage in any situation to which it can be applied locally; especially in nasal and laryngeal operations. Solutions of $I: I_{5}, 000$ to $I: I, 000$ are used for this purpose. The dilutions must be freshly prepared, since they lose their activity in a few hours. The action persists as long as the epinephrin is present; but when this has disappeared, there is often secondary hemorrhage.

Hemorrhoids.-Epinephrin may be used in suppositories; I $\mathrm{mg}$. (1/60 gr.) of the alkaloid in I Gm. of Theobroma oil.

Use on Inflamed Mucosæ.-Epinephrin gives symptomatic relief to inflamed surfaces, by contracting the dilated vessels. It is thus used in catarrhal conditions, e.g., in conjunctivitis, and especially in hay fever. It is best applied in oily solution or ointments, containing $1 / 10$ per cent. of the free alkaloid; oil prolongs the action. Epinephrin is. non-irritant, but its action is too brief and it is claimed that its continued use has caused chronic turgescence of the mucous membrane (Potts, I906; J. N. Hall, IgI I).

When applied to the scarified skin, epinephrin produces an area of blanching and "goose" flesh (Sollmann and Pilcher, 19I6).

Infantile Paralysis.-Meltzer, 1916, urges the intradural injection of epinephrin, to arrest and cause the absorption of the spinal edema, especially when the respiratory center is threatened. He advises 2 c.c. of 1 : 1000 solution, every 4 to 6 hours. The suggestion has been tried on experimental poliomyelitis, with beneficial but not curative results (P. F. Clark, I I I 2). Clinical trials by Fraser, I9I4, have so far given equivocal results, but they were confined to advanced cases.

Marie, $\mathrm{rgI}_{3}$, claims that epinephrin neutralizes the antitoxins of tetanus and diphtheria in vitro, but not in the presence of hemoglobin. It has been tried against tumors, but without certain benefit. Engel, I9I2, claims marked effects on rat tumors, not entirely explainable by ischemia.

Cocain-epinephrin Anesthesia.-The addition of epinephrin, in the usual concentration, enhances the anesthetic action and lessens the toxicity of cocain (Braun), by delaying its systemic absorption, by the anesthetic effect of anemia, and seemingly by a direct synergistic action.

Epinephrin and Cocain Synergism.-It is claimed by Esch, Igro, that the cocain anesthesia is intensified by epinephrin even when the local circulation has been previously completely arrested. Epinephrin alone has no anesthetic action under these conditions. It must therefore facilitate the penetration of the cocain, or otherwise sensitize the nerve fiber. A similar sensitization occurs for Novocain and Alypin, but not for Tropocain.

Conversely, cocain sensitizes to epinephrin, so that the mydriasis, vasoconstriction, etc., are more pronounced; and this with doses of cocain which alone have no sensible effect (Fröhlich and Loewi; v. Fischel, I915). 
Epinephrin on Absorption.-Meltzer and Auer, I904, claim that intravenous injections retard absorption and transudation. Van Lier, 1907, showed that the absorption of sodium iodid is markedly retarded, unless very hypertonic (20 per cent.) solutions are injected. The local effect on intestinal absorption is inconstant for iodid, but more marked on phenol and especially alcohol (Hanzlik, I9 Io, I9I2, I9I3).

In non-corrosive poisoning (cyanid, strychnin, aconite, etc.) Jona, I9I3, suggests the administration of epinephrin by mouth (IO to I5 c.c. of $r: 1, \infty 00$ ) to retard absorption. 'This would need to be supplemented by evacuation and chemic antidotes.

The Effects of Epinephrin in Internal Hemorrhage.-Epinephrin is practically useless in internal hemorrhage; for although it constricts the vessels when it is injected intravenously, it also raises the blood pressure. The end result would not be reliable, and often harmful; especially in pulmonary hemorrhage (Frey, I909; Wiggers, r909) and in cerebral hemorrhages.

In intestinal hemorrhage, the results of Wiggers, I909, were somewhat more promising: after a momentary increase, the hemorrhage was reduced, and this effect outlasted the rise of pressure.

Epinephrin in Circulatory Collapse.-The epinephrin constriction is able to restore the blood pressure in all forms of vasomotor depression; but its action is too fleeting to make it of much use in any but purely temporary emergencies. If it is used, it must be injected intravenously, drop by drop, carefully watching the heart. The dangers are probably greater than the benefits.

Epinephrin Atheroma.--If rabbits receive frequently repeated injections of large doses of epinephrin, atheromatous changes and calcareous patches are often observed in the aorta (Josuè, I903) and other large vessels (d'Amato, 1905). These degenerations are never seen in dogs (Pearce and Stanton, 1906), or in monkeys, and presumably not in man. Dogs also give negative results with nicotin, lead, or toxins (Adler, Igi 4 ).

Identical lesions often occur spontaneously in rabbits which have never received epinephrin (Miles, 1907, Benneke, 1908, Hill, 1910, etc.). The frequency of these spontaneous lesions varies in different series from 3 to 3 o per cent.; but they are so markedly more frequent after epinephrin treatment, that this must at least exaggerate the tendency to the degeneration (I. Levin and Larkin, 19ro). Success depends on frequent repetition of the epinephrin rather than on the size of the dose (Fleisher, I909).

The injury has been attributed to high blood pressure, and to direct toxicity by epinephrin. The older experiments undertaken to decide between these views (summarized by I. Adler, I908), did not take account of the spontaneous occurrence of the lesions (M. K. Meyers, I909; M. C. Hill, I9II) and the question can not be considered settled. The toxic theory seems the more probable; although Dixon, I913, still states that all pressor agencies, when repeatedly applied, produce degenerations of the middle coat. It is highly doubtful whether these atheromas of rabbits have any relation to human atheroma.

Atheroma is also produced by feeding cholesterin, cottonseed oil, the aldehydes of fatty acids, hydrochloric acid and by paracresol (Adler, I9I4; Denney and Frothingham, I9I4).

Epinephrin Myocarditis.-Rabbits also often show a chronic myo-, endo-, and pericarditis, after repeated epinephrin injections. This lesion-which is apparently independent of the atheroma $\rightarrow$ is much more frequent $(60$ per cent.) if a single intravenous injection of epinephrin ( $0.2 \mathrm{mg}$.) is combined with spartein ( $12 \mathrm{mg}$.), caffein (25 mg.), or strophanthin (these drugs never cause the lesion if they are used without the epinephrin). The changes in the heart-muscle start at once, progress for some seven days, persist about six weeks, and then retrogress completely by sixteen weeks (Fleisher and Leo Loeb, 1909; Strickler and Fleisher, 1910; Christian and Walker, I911).

Fleisher and Loeb, 1910, attribute the lesions to "excessive mechanical strain," from excessive contraction of the muscle fibers: For the lesions are situated mainly at the base of the left ventricle, where the strain is greatest; the changes are analogous to those shown by striated muscle subjected to overexertion; and the lesions do not appear in the relatively stronger and more resistant hearts of dogs. H. A. Stewart, I9I2, prefers a toxic theory. 
Hearts which show the microscopic changes are physiologically inferior to the normal; they are less able to cope with extra demands; and the animals appear more disposed to edemas (uranium) and to bacterial infections.

Bronchial Muscle and Use in Asthma.-The bronchi are relaxed, especially when spasmodically contracted, by stimulation of the sympathetic bronchodilator endings. Epinephrin is therefore often very effective in aslhma (I c.c., I: r,000 hypodermically, Kaplan, I905; Matthews, I9ro; Hoover and Taylor, I9I5; v. Jagic, I9I6).

The bronchodilatation has been confirmed in intact animals by Januschke and Pollak, I9II, and others; on excised lung by Baehr and Pick, I9I3; on excised tracheal muscle by Trendelenburg, I912; on excised bronchial muscle by Titone, I9r3. Golla and Symes, rgr3, find differences between the bronchial and tracheal muscle, and claim that normal bronchioles are constricted by epinephrin, and that it dilates only when the bronchioles are spasmodically contracted by pilocarpin, etc.

Respiration.-This changes with the blood pressure (Nice, Rock and Courtright, 19r4). It becomes deeper but irregular during the rise of blood pressure, and is generally arrested at its height (Langlois and Garrelon, I9I0). In man, I mg. hypodermically is said (Fuchs and Roth, I9I2) to increase the respiration volume by 8 to 94 , mean 50 per cent.; the rate being unchanged or also increased. The effects on the perfused respiratory center are variable (E. D. Brown, I9I6).

Direct Central Actions.-Lumbar injection of epinephrin may cause muscular spasm and tremor. Injection under the dura or into the substance of the cerebrum produces an apparently natural sleep, lasting two to five hours, with regular respiration and reflexes, and fall of temperature (Bass, 1914). Bass does not believe that these effects can be explained by vasoconstriction.

Intratracheal In jection.--Auer and Gates, 1916 , found that the injection of epinephrin into the lower trachea produces a prompt rise of blood pressure; presumably by aspiration and absorption from the alveoli. If the vagi have been divided, fatal pulmonary edema often follows. This may be prevented by atropin.

Action on Pupil.-Epinephrin has a mydriatic effect. This occurs readily in frogs; but only under specially favorable conditions in mammals.

Conditions for the Occurrence of Epinephrin Mydriasis in Mammals.-This may be procured by large doses intravenously. With local application, the mydriatic effect may be obtained if the oculomotor tone is low (weak illumination, W. H. Schultz, 1908); if the absorption is hastened (conjunctivitis, H. Straub, r910); or if the excitability of the sympathetic is increased.

Such an increase is supposed to occur in Basedow's disease and in pancreatic insuffciency. O. Loewi, I907 and 1908, accordingly proposed a mydriatic response to epinephrin as a diagnostic sign in these diseases (with certain limitations). Later work, however, showed that the increased mydriatic reaction occurs in all types of hyperglycemia; and that it can therefore be prevented in pancreatectomized dogs by lowering the blood-sugar through phlorhizin (A. Loewy and Rosenberg, I9I4).

The mydriatic response to epinephrin is also obtained a few days after excision of the superior cervical ganglion (Meltzer and Auer, r904; Joseph, I91 2; Githens and Meltzer, 1916), or after removal of the ciliary ganglion (Joseph, 1915). This may likewise be attributed to increased excitability of the sympathetic distribution, accompanying the degenerative changes which follow the excision (H. Straub, I9IO). An analogous increase of excitability in degenerating structures to epinephrin occurs in the ear vessels (Meltzer and Auer, I903), and in other organs (Elliot, 1905); also in skeletal muscle on degeneration of the sciatic nerve (Langley, 1905; Edmunds and Roth, I909).

G. N. Stewart, Rogoff and Gibson, I916, find the mydriatic responses of the denervated pupil the most sensitive and reliable test for the discharge of epinephrin; $f . i$. on stimulation of the peripheral end of the divided splanchnic nerves.

Rabbits respond with mydriasis more readily than other mammals. Epinephrin also relaxes the excised sphincter of the iris (Joseph, I915).

Localization of Action.-Lewandowsky, 1898 , showed that the mydriatic effect occurs after degeneration of the sympathetic, so that the action must be peripheral to the endings (i.e., on the receptive substance).

Other Evidences of Ocular Sympathetic Stimulation. - The mydriasis is accompanied by separation of the lids, protrusion of the eyeball, and withdrawal of the nictitating membrane. 
Intraocular Tension.-- Iccording to Rupert, I009, this is first lowered, then increased.

Mydriatic Reaction in Frogs.-Frogs are especially susceptible to the mydriatic action; and this may be produced by systemic application ( I c.c., I : 100,000 into lymph sac) as well as in the enucleated eye ( $1: 5,000 ;$ Meltzer and Auer, I 904; Meltzer, I909). 'The dilation is prompt and persistent, submaximal, indifferent to light.

Action on the Uterus. - The effects correspond to stimulation of the hypogastric nerve (see page 270); in pregnant cats it produces contraction; in non-pregnant cats relaxation (Cushny, Kehrer, Dale); in dogs, contraction followed by relaxation; in rabbits, increased tonus (Biagi, Igo5). Surviving human uterus is stimulated (Ruebsamen and Kligermann, I9 I $^{2}$, whether pregnant or not (Lieb, I9 I $_{5}$ ). The Fallopian tubes are also stimulated to contraction (Gunn, I9I4).

Epinephrin could be used locally to arrest postpartum hemorrhage; not intravenously, since the increased blood pressure would increase the bleeding.

Action on Intestines.-Peristalsis is inhibited and the tone relaxed by an action on the myoneural junction (see page 276 ). Very dilute solutions increase the contractions (Hoskins, I9 I2).

Gall-bladder.-This is relaxed (Lieb and McWhorter, I9 I5).

Excised Ureter.-Epinephrin $(\mathrm{I}: 500,000)$ increases the rate and tone, or starts the contractions. The effect is reversed by ergotoxin (Macht, I9I6).

Effect on Secretions.-Epinephrin may provoke secretion of the salivary and lachrymal glands (Langley, I90I); and of the skin glands of frogs (Ehrmann, I905). These would not be suppressed by atropin. The increased secretion of the submaxillary glands is accompanied by increased blood flow (Solem and Lommen, 19I 5 ). In most glands, however, secretion is diminished or arrested through the vasconstriction. This is notably the case with pancreatic secretion (Benedicenti, I906; Edmunds, I909).

Sweat.-This is increased in animals (Muto, I9I6. He finds that the sweat glands are innervated by both the sympathetic and parasympathetic. The sympathetic predominates in horse and sheep; whereas man, cattle and cats are more subject to the parasympathetic).

Cerebro-spinal Fluid.-The secretion is not affected, the pressure varying merely with the blood-pressure changes (Dixon and Halliburton, I9 5 ).

Skeletal Muscle.-Oliver and Schaefer, I895, found that the gastrocnemius, excised after hypodermic injection of suprarenal, shows increased efficiency and some veratrin action. Cannon and Nice, I9I3, explain this by the improved circulation. This is only a partial explanation, for Gruber, I9I4, observed that epinephrin injection could cause some improvement in the excitability in fatigued cats (to direct or nerve stimulation) even when there was no rise of blood-pressure. Splanchnic stimulation, with the suprarenals ligated, causes some improvement, but not as much. However, Kuno, I9I5, did not observe any effect on the contraction of frog's skeletal muscle, even in concentration of $6: 100,000$. Excision of the suprarenal glands produces great muscular weakness (Albanese, 1892).

Nerve trunks are not affected. The pigment cells in the frog's skin are contracted into spheres (Lieben, I906).

Epinephrin Glycosuria.-Large doses of epinephrin (much beyond the therapeutic) produce hyperglycemia and glycosuria by converting the hepatic glycogen into sugar. The effect is analogous to asphyxial glycosuria, diabetic puncture, or stimulation of the hepatic, splanchnic or sensory nerves; all acting probably on sympathetic nerves controlling the glycogenic functions. The presence of epinephrin seems necessary for the production of glycosuria by nerve stimulation. The continued administration of epinephrin to rabbits abolishes the glycosuria, but the hyperglycemia persists (Pollak, I9rI). In man, epinephrin does not produce glycosuria, but increases the sensitiveness to alimentary glycosuria (Landau, I9I4).

The occurrence of glycosuria after hypodermic injection of large doses of epinephrin was discovered by F. Blum, IgOI. Intravenous and intramuscular administration are less effective, even if larger doses are used (Pollak, I909; Underhill, I9II). The phenomenon was investigated by Herter and Wakeman, 1902, Noel Paton, I903, and many later observers. The glycosuric action of other sympathomimetic drugs is described by Morita, I9I 5 . 
Mechanism of the Glycosuria.--The glycosuria is generally proportional to the hyperglycemia (Gramenitzki, I9I2). It is not asphyxial. The hyperglycemia occurs even after excision of the kidneys (Zuelzer, Igor; Metzger, r902), showing that it is not renal.

There is conclusive evidence that the glycosuria is due to increased glycogenolysis in the liver. The degree of hyperglycemia and glycosuria depends upon the glycogen content of the animal; they are more intense in well-fed animals (Vorburgh and Richards, 1903), or in animals rich in glycogen; and conversely there is no glycosuria in animals whose glycogen has been completely removed by phlorhizin; and an existing phlorhizin glycosuria is not increased by epinephrin (Ringer, s gro). Hence, also, the epinephrin glycosuria fails after repeated injections, as the glycogen becomes exhausted. The glycemia persists somewhat longer than the glycosuria (Pollak, 1909) presumably because the permeability of the kidney is somewhat diminished by vasoconstriction. Konschegg, I9I2, also believes that repeated injections lessen the permeability. In glycogen-free animals, epinephrin causes deposition of glycogen in the liver, but not in the muscles (Pollak).

The evidence for the hepatic origin of the sugar is also convincing. The blood of the hepatic vein contains more sugar than that of the portal; sugar formation is increased on perfusion of the excised liver, and conversely, there is no glycosuria when the functions of the liver have been injured by phosphorus (E. Frank and Isaac, rgI I). Wilenko, I9 3 , claims that epinephrin increased the consumption of glucose in the perfused heart; and believes that the glycosuria is due to some indirect disturbance of the consumption of sugar. This conclusion would require more experimental evidence. Lusk, I9I4, finds that it does not influence the oxidation of ingested glucose, nor protein metabolism.

Epinephrin Necessary for Splanchnic and Puncture Glycosuria.-In support of this view, Macleod, Pearce and Christie, IgII and I9I2, found that stimulation of the left splanchnic nerve (which furnishes the nerve supply of both adrenals; Kahn and Nishi, 1909) does not produce hyperglycemia, if the left suprarenal gland has been excised; and according to A. Mayer, diabetic puncture is ineffective after removal of the glands. Theobromin glycosuria is also prevented (Miculicich, I9I2). However, these measures do not act simply by increasing the secretion of epinephrin, for the glycosuric response to splanchnic stimulation is also prevented by section of the hepatic plexus, which does not prevent epinephrin glycosuria (Macleod and Pearce, I 91 2).

Parallelism of Vasoconstriction and Glycosuria.-The results of Straub, 1909, and Ritzmann, I909, show that both effects are strictly parallel, and proportional to the rate of injection (intravenous). Smaller doses suffice for the glycosuria than are required for the rise of blood pressure (Gramenitzki, I912); and Ransom believes that the pressor and glycosuric action are connected with different groups in the molecule. This is supported by the observation, that with partial oxidation of epinephrin, the glycosuric action is lost first (McGuigan and Mostrom, I913).

Interfering Factors.- Epinephrin glycosuria is prevented by ergotoxin (Loewi) which lessens the hyperglycemia and also the permeability of the kidney for sugar (but not for chlorid; Miculicich, I912). It is also prevented by nicotin (Hirayama, I9II); by lymphagogues (peptone, hirudin; Miculicich); and by repeated hemorrhage (Wilenko, I9I 2). Urethane increases the activity (Underhill, I91 I).

Epinephrin glycosuria is suppressed by antipyretics (Starkenstein). Since the hyperglycemia is not diminished, the action of the antipyretics must be on renal permeability (Mansfield and Purjesz, 1915).

Coagulability of Blood.-This is increased by intraperitoneal injection, simultaneously with the hyperglycemia (Vorburgh and Richards, I9I3). Cannon and Mendenhall, 1914, found that the intravenous injection of small doses (0.002 mg. per kilogram) hastens coagulation, and that larger doses ( $0.05 \mathrm{mg}$.) retard it. Howell, 1914, considers the delay due to decreased prothrombin, analogous to hemophilia.

Polycythemia. - Intravenous epinephrin injection raises the blood-count, by diminishing the volume of plasma (Lamson and Keith, 1916).

Hemolytic Action.- Epinephrin is strongly hemolytic for beef-blood and to a less degree for other animals. It also produces methemoglobin. Serum weakens the action (Kariga and Tanaka, I9I 2 and I9I3).

Heat Regulation.-Excision of the suprarenals lowers temperature (Hultgren and Andersson, 1898); whilst the injection of epinephrin (and other internal secretions) produces fever (Freund and Marchand, I913). Hirsch, 1913, found, presumably under different conditions, that the injection of epinephrin or pancreas lowers temperature by decreasing heat production. Cloetta and Waser, 1915, describe rise of brain temperature and cooling of skin, with $0.2 \mathrm{mg}$. in rabbits, intravenously. Intracerebral injection acted similarly. The rise was not prevented by ergotoxin.

Nitrogen Metabolism.-Epinephrin, by vein or hypodermically, has little or no 
Influence on the nitrogen excretion (Kraus and Hirsch; Quest, I908; Lusk, 1914). in dogs, large doses, hypodermically, increase the excretion of uric acid and especially of allantoin (Falta, 1914).

Interrelation with Other Internal Secretions.-The epinephrin glycosuria is not related to pancreatic glycosuria. The ability to use sugar is not impaired; the ureaammonium ratio is not altered (Underhill and Closson, 1906), even on intraperitoneal injection (Rosenbloom and Weinberger, I9I2); nor are there any acetone-bodies in the urine. Neither is there any other good evidence for the theory of Eppinger, Falta and Rüdinger, r9o9, that cpinephrin inhibits the internal secretion of the pancreas. The observation of Zuelzer, that injection of pancreas abolishes the epinephrin glycosuria, can be explained simply by irritation (presumably reflex spasm of the renal vessels); for the same results follow the intraperitoneal injection of the simplest irritants, such as turpentine (Fuerth and Schwartz, I9II). Removal of the pancreas has no definite effect on vasomotor irritability; nor is there any other good evidence that the pancreas normally exerts a depressing influence on the sympathetic system (Hoskins and Gunning, 1916).

Removal of the adrenals results in changes of the pancreas; but these are attributable to the low blood pressure and other adynamic phenomena (Mann and Drips, 1915).

Thyroidectomy, also, has no influence on the glycosuria. Some of the apparent results can be explained by the natural variations in the intensity of the glycosuria, which occur even in the same animal under apparently identical conditions (Underhill, 19II). Pituitrin does not increase, but rather diminishes the epinephrin hyperglycemia (Stenstroem, r9r4).

Suprarenal, Thyroid and Depressor Stimulation.-Asher and Flack, rgro and I9ri, have shown that there is a true interrelation between thyroid and epinephrin as regards the blood pressure action; so that increase of thyroid secretion, provoked by stimulation of the superior laryngeal nerve, sensitizes the blood vessels to the epinephrin rise, as well as to the fall on depressor stimulation. (After the thyroids have been excised, the superior laryngeal does not have these effects, showing that it must act through the thyroids.) Injection of thyroid extract, but not of iodothyrin, has a similar effect. (It does not increase the epinephrin content of the adrenal vein; Gley and Quinquand, I9r3.) The pupillo-dilator response is not sensitized by thyroid.

Conversely, epinephrin stimulates the thyroid, producing an action current. This occurs whether the epinephrin is injected directly, or liberated by splanchnic stimulation (Cannon and Cattell, I9r6).

By the (unreliable) intestine test, less epinephrin appears to be present in the suprarenal vein during central depressor stimulation, independently of alteration of the blood flow through the gland (Richards and Wood, 1915).

Epinephrin diminishes or abolishes the response to depressor stimulation (Oliver and Schaefer, and others), and indeed to all kinds of vasomotor stimulation, pressor or depressor; electric, nicotin, etc.; and this independently of the level of blood pressure (Hoskins, I915). This depression of vasomotor irritability occurs more slowly than the direct pressor effect of epinephrin; so that, if both measures are started simultaneously, the results are strictly additive; but if epinephrin is started first, the addition is imperfect. Curiously, a similar diminished reaction to nicotin occurs in from one to eight days after the excision of one-half to seven-tenths of the adrenal tissue (Hoskins, I915).

Sensitization to Pituitary.-Kepinow, IgI2, claimed that doses of pituitary which are themselves inactive, increase the vasoconstrictor effect of epinephrin. Rischbieter, I913, and Hoskins, I9r5, were unable to confirm this, finding only simple summation.

Boerner, I915, found some increase, but attributes this to more prolonged sojourn of the epinephrin in the circulation, due to cardiac depression; for the "potentiation" occurred only in rabbits, in which pituitary depressed the heart; and not in cats, in which depression is absent. A similar "potentiation" was produced by other cardiac depressants, histamin, nicotin, etc.

Toxic Actions. - Excessive doses of epinephrin produce vomiting, excitement, debility, bloody diarrhea, hematuria, ascending central paralysis, great fall of temperature, occasionally convulsions, complete prostration, and death by respiratory (cat) or cardiac (dog) paralysis. The fatal dose varies considerably (usually 0.1 to 0.6 mg. per kilogram intravenously, or 2.5 to $10 \mathrm{mg}$. per kilogram hypodermically), but s about 500 times the therapeutic dose. A noticeable rise of blood pressure can be seen after $1 / 1800$ of the fatal dose. The clinical side actions are described by Seifert, Nebenwirk, 1915, page 234 .

Guanin is said to lessen the toxicity and glycosuria (Desgrez and Dorleans, 19. 3 ).

The toxic effects are the result of the peripheral actions: for as Cushny, ro०9, has pointed out, the ratio of activity of the different isomers is the same for the toxic as for the blood-pressure effects. 
Cause of the Brief Action of Epinephrin.-When epinephrin is injected intravenously, the effects are of very short duration, disappearing within a few minutes after the injection ceases (Fig. I4). This goes parallel with the disappearance of epinephrin from the blood, and is due to the rapid destruction of the substance. Epinephrin is readily oxidized and thus rendered inactive. The oxidation occurs presumably in all tissues, including the blood, but with very different velocity. The blood vessels and liver probably have the largest share.

On the other hand, the vessels continue to respond practically uniformly and indefinitely to whatever epinephrin may be present in the blood. Its effects may therefore be repeated by repeating the intravenous injection, and they may be rendered continuous by continuous infusion. A uniform infusion will maintain a uniform moderate rise of blood pressure for a considerable time.

Persistence of Action if the Concentration of Epinephrin is Maintained.-In excised kidneys, the perfusion flow may be arrested for a long time, sometimes for hours, by a single injection of epinephrin. This illustrates that epinephrin is not destroyed (at least in the absence of oxygen) in the tissue (blood vessels) on which it exerts its main action. It also shows that the stimulation can be continued indefinitely, and that it does not give way to "fatigue," or to depression, no matter how much epinephrin is used.

This corresponds exactly to what is observed in the living animals: Repeated injections of the same dose, on the same animal, give quantitatively the same pressor effect (Bayac, I905), for at least 20 successive injections (Kretschmer, 1907). Sufficient time must elapse between injections for the circulation to return to normal; otherwise, there will be superposition.

Kretschmer also found that if the injection be made continuous, the pressure rise is maintained uniformly so long as the rate of injection is uniform. The blood-pressure level rises as the rate of injection is increased, from the minimal effective dose until a maximum is attained. In rabbits, this occurs when the rate of injection corresponds to about $0.01 \mathrm{mg}$. per kilogram per minute. Up to this point, the destruction must keep step with the injection; else the rise would be progressive. Further injection produces no further rise, but, when the injection is arrested, the return to normal is inverse to the rate at which the epinephrin has been introduced, since it will take a longer time to destroy the excessive epinephrin.

The Uniformity of Response is not Unlimited: Froehlich and Pick, 1912, found that if large doses are given in rapid succession, the rise becomes less pronounced and finally disappears, although the blood pressure may remain normal. This is perhaps due to an altered distribution of the blood; for Froehlich, I910, found that a single dose of d-epinephrin, which has a much more prolonged action, may abolish the response to ordinary l-epinephrin.

Epinephrin is not an Antigen: No antibody is produced, even if the (hypodermic) injections are continued over three months (Elliott and Durham, rgo6); nor is there any habituation (Pollak, I910). Halpern, I913, claims that intraperitoneal injections of suprarenal extracts (not of epinephrin) leads to the production of a dilator substance.

The Return to Normal Pressure Coincides with the Disappearance of the Epinephrin from the Blood.- The direct investigation of this question yielded contradictory results in the hands of the earlier investigators (Weiss and Harris, r904), who worked with methods which at present would not be considered adequate for the purpose. The recent work (De Vos and Kochmann, I905; D. E. Jackson, I909; Trendelenburg, 1910) shows that the disappearance of epinephrin from the blood goes perfectly parallel with the return of the blood pressure.

The Disappearance of Epinephrin is not Due to Excretion.-Fuerth found that the urine contains at most traces, even after large injections; and the pressor effect disappears even when access to the excretory organs is excluded by clamping the aorta (Jackson, rgog). This experiment also shows that the visceral organs are not necessary for the destruction of epinephrin.

Artificial Destruction of Epinephrin.-Epinephrin is readily oxidized, especially in dilute and alkaline solutions (B. Moore, 1895), with complete loss of activity. Through its amido-group, it is also promptly inactivated by formaldehyd (Cramer, I9II). Artificial oxidation does not destroy all the activities at the same rate (McGuigan and Mostrom, 1913). Blood in vitro has a curiously slow destructive effect, so that the 
activity is preserved for many hours (Oliver and Schaefer, 1895; Emden and Fuerth, I903); indeed, small quantities of serum retard the destruction materially (Schwetschnikoff, r913). The oxidation proceeds much faster in the presence of blood vessels (Tatum, r9r2). Cerebro-spinal fluid destroys epinephrin much more rapidly than does serum (Meltzer, 19i I).

Destruction in Tissues.-Gioffredi, 1907 , found that when epinephrin was perfused through the liver, it was rapidly destroyed; more slowly in skeletal muscle; not appreciably in the kidney, lung and brain. The destruction is therefore a special vital function of the liver. This explains the vastly more lasting effects of epinephrin when it does not come into contact with the liver, as in perfusion of the excised kidney. It also explains the observation of Meltzer that epinephrin retains its activity for several hours when it is confined in the tissues by a ligature. Kretschmer, 1907, found that the destruction is hastened (i.e., the action is shortened) by lessening the alkalinity of the blood by the injection of acids; and vice versa.

Physiologic Functions of Epinephrin.-The striking actions of epinephrin invite the theory that it plays an important rôle as a hormone for maintaining the tone of the sympathetic system, particularly the blood pressure; and that it is concerned in pathologic conditions of the sympathetic system, Basedow's disease, diabetes, arterial hypertension, etc. These views seemed supported by the apparent presence of epinephrin in the blood. Subsequently, however, it was shown that the tests were misleading, and that normally, not enough epinephrin passes into the blood to exert any effect on blood pressure, or even on the much more sensitive intestine. There is some evidence that its secretion may be increased by stimulating the sympathetic nerve supply of the adrenal glands (f.i., through stress, fear and other emotions), so as to obtain effects on some of the more sensitive functions, but not sufficiently to influence the blood pressure. Even under these most favorable conditions, the response is so small that it may be doubted whether it has much physiologic significance; nor has the causation of any pathologic condition been definitely connected with altered epinephrin content of the blood. It is possible, but scarcely probable, that epinephrin is merely a waste product, temporarily stored and perhaps destroyed in the adrenal glands. ${ }^{1}$

Chemic Control of Body Functions.-The organism of animals as well as plants produces as direct or by-products of its activities, chemic substances of decided pharmacologic actions. Some of these are probably waste products; but others serve to regulate important functions. In this way, the body is under the constant influence of "drug therapy," and the correction of faulty functions by "drugs" is not an artificial but a natural process of the body. A striking instance of such regulation is the carbon dioxid (Bayliss and Starling, 1906), with its controlling effect on the respiratory and vasomotor centers, etc.; and there are numerous other instances of the importance of the "hormones" of internal secretion, such as the regulation of the pancreatic secretion by secretin; the chemic control of the sexual functions (Starling, Harvey Lectures, r908).

Absence of Epinephrin from the Normal Blood.-Trendelenburg, I9rr, found that blood has a constricting effect on perfusing the vessels of a frog, and believed that this demonstrated the presence of epinephrin. Changes in this reaction were described for various diseases (Brockius and Trendelenburg, rgrr; also by A. Fraenkel, r909, using the uterus as test object). However, G. N. Stewart, IgIr, O'Connor, rgIr, and Janeway and Park, 1912, showed that the consirictor substance of serum is not epinephrin, for it fails to give the inhibitory reactions ( $f . i$., on the intestines). It is not sympathomimetic, but stimulates all smooth muscles, probably directly. It is formed mainly in the process of clotting (O'Connor, rgr2) and increases with the age of the serum (Handovsky and Pick, I9r2).

With decisive methods, no epinephrin has been demonstrated in the serum, even in pathological conditions (Stewart, x 9 r 2), or when the blood pressure is lowered by hemorrhage (Trendelenburg, 19rI). It may, however, be shown in the blood of the adrenal vein (O'Connor, I912), especially on splanchnic stimulation (Stewart; Asher, 1912), or if the gland is massaged; but the quantity is so small that its effects are practically 
destroyed by the time it reaches the general circulation. However, the mydriatic and blood pressure reactions become distinctly discernible if a somewhat larger supply is suddenly admitted to the circulation, by temporarily clamping and then releasing the vena cava. This requires intactness of the splanchnics. By this method, the normal discharge of epinephrin may be calculated as 0.0005 to $0.0009 \mathrm{mg}$. per minute, per kilogram of body weight (G. N. Stewart and Rogoff, I9I6).

Hoskins and McClure, I9I2, point out that if epinephrin persisted normally in the circulation, it would result in inhibition of peristalsis, since the intestines are mucli more sensitive than the blood vessels. Trendelenburg, 191 $_{5}$, also finds that the concentration in normal blood could not be over I im 1 or 2 billions, and is probably smaller-too small to affect the blood pressure or intestine. Further, Hoskins and McPeek, 1913, emphasize that the small quantity which could possibly reach the circulation would dilate instead of constricting the blood vessels.

The fact that the adrenal glands do not normally contribute to the maintenance of vascular tone is also shown by temporarily clamping the adrenal vessels. The blood pressure does not fall whilst the clamp is applied (within one-half hour), but only after a day or so, as death approaches (Trendelenburg, 1914); nor does it rise when the clamp is removed (Hoskins and McClure, I9I2. The positive results of Strehl and Weiss are attributed to obstruction of the vena cava or stimulation of afferent nerves; however, Hoskins and McPeek, I913, also found positive results in pregnant cats).

Response to Splanchnic Stimulation. - Whilst it is thus certain that the amount of epinephrin ordinarily secreted is below the physiologically effective limit, there is evidence of some secretion, especially on splanchnic stimulation. Asher, I910 and I9I2, found that splanchnic stimulation in eviscerated animals does not raise the blood pressure if the suprarenal vessels are clamped. The splanchnic rise under these conditions must therefore be due to secretion of epinephrin. The rise can be made continuous, showing that the epinephrin is secreted and not merely washed out. The secretory influence of the splanchnic is probably continuous, for O'Connor, I912, and Stewart and Rogoff, 1916, find that their section lessens the secretion greatly. Stewart, Rogoff and Gibson, 1916, showed the epinephrin discharge on splanchnic stimulation by the mydriatic and blood-pressure reaction. It occurs very promptly, and is not prevented by attempts to exhaust the epinephrin store through fright or morphin.

Epinephrin discharge is also concerned in the blood-pressure response to splanchnic stimulation under ordinary conditions. The rise generally shows two șummits. The first is due to direct response of the arterial muscle to the nerve-impulse. The second is due to epinephrin discharge, for it does not occur if the suprarenals have been ligated. The cardiac acceleration after splanchnic stimulation may be due to the same cause (v. Anrep, rgr2; Burton-Opitz and Edwards, 1916). However, the relation between the adrenals and the hypertensive function of the splanchnic is not constant (Gley and Quinquand, 1913).

The secretion of epinephrin may be increased by stimulating the splanchnic directly or reflexly.

\section{INFLUENCE OF FUNCTIONAL STATES AND DRUGS ON EPINEPHRIN SECRETION}

Cannon and de la Paz, rgr I, describe increased output of epinephrin by emotion and fright in cats. They tested this by the relaxing action on excised intestine; this is probably the best single-object test, but not as conclusive as the use of several test objects. Cannon, Aug and Binger, 1912 , also find increase of epinephrin after the injection of nicotin $(3.5$ to $7.5 \mathrm{mg}$. per cat). Their conclusions receive partial confirmation from Elliott, 1912, who estimated the amount of epinephrin in the glands. The two glands normally contain the same amount; this is rapidly decreased by excitement (morphin, betatetranaphthylamin); narcosis (chloroform, ether, urethane, morphin); and by stimulation of the afferent sciatic or of the cerebral cortex (tetra-naphthylamin). The action of all these appears to be central, for it does not occur if the splanchnics have been cut. Elliott, 1914, further found similar diminution of epinephrin after fright and bacterial intoxication, etc., in animals, and after worry and infectious diseases in man. Dale and Laidlaw, I9I2, and Jackson, I913, also believe that stimulation of epinephrin secretion by nicotin and pilocarpin is responsible for the differences in the effects of the drugs in intact animals and on excised organs (uterus, bronchi, ocular sympathetic).

The results of Stewart and Rogoff, 1916, throw doubt on some of the interpretations of Elliott. They confirm that morphin diminishes the epinephrin content of the adrenals; but this occurs in dogs as well as in cats, so that it cannot well be attributed to excitement or fright. These emotions, when produced by more direct means, did not 
diminish the epincphrin store. "Tetra" did produce cxtreme exhaustion of epinephrin store in cats with intact vagi; rabbits responded less completely.

Corbett, $\mathrm{I}_{915}$, found the epinephrin stock greatly lowered in traumatic shock. Bedford and Jackson, 1916, show that this exhaustion occurs whenever, by any means, the blood pressure is kept below $50 \mathrm{~mm}$. for at least one or two hours.

The diminished epinephrin content of the gland after prolonged anesthesia has been confirmed by Oliva, I914, and Corbett, 1915. Chloroform discharges the gland more rapidly than smooth ether anesthesia; and the recovery of the normal epinephrin content is slower. However, if the ether produces much fright and excitement, it may diminish the epinephrin content very severely. Marchetti, I915, arrives at opposite results with ether; he reports a marked immediate increase of epinephrin in the gland, lasting for several hours, and returning to normal after twenty-four hours.

Total Amount of Epinephrin in Glands. - In man, this is normally about 4 to $5 \mathrm{mg}$. per gland (Goldzieher, I9ro; Elliott, I9r4); in dogs, 2 mg.; in cats, o.I9 mg. (Marchetti, I915); in sheep, to $7 \mathrm{mg}$. (Folin, Cannon and Denis, I9I3). The percentage is about o.I per cent. of the moist weight, in all animals. The content does not vary with the seasons (Seidell and Fenger, I914).

Epinephrin is present very early in fetal animals (Fenger, r9r2; McCord, I915). In the human fetus, it is absent at six months, but present at full term (J.H. Lewis, I916; Moore and Purinton, r9o0).

Elliott (I905) believes that epinephrin is also present in special cells in other situations, but always closely associated with sympathetic ganglia ( $f . i$., in the intercarotid body). This has been taken to indicate that sympathetic stimulation is affected by both a chemic and a nervous mechanism. The adrenal cortex contains no epinephrin, and is supposed to have relations to growth and sexual development. Voegtlin and Macht, I9 3 , announced a crystalline, lipo-soluble constrictor principle.

Addison's Disease. - This is associated with destruction of the suprarenal glands. The therapeutic administration of preparations of the glands has therefore been tried; also in rickets, diabetes insipidus, etc. The results have not been very good, which is scarcely surprising when the rapid destruction of the substance is considered.

Excision of both suprarenal glands produces death in a few days (BrownSequard, 1856 , page 293). There is fall of blood pressure, muscular weakness, decrease of metabolism, fall of temperature, central paralysis, death by failure of respiration. The blood pressure changes are the effect rather than the cause of the asthenia; for the pressure and vascular response do not begin to fail until the asthenia is far advanced. The asthenia probably depends on the failure of the cortical rather than of the medullary function of the glands. Hoskins and Wheelon, I914).

The injection of the blood of the moribund animals into a normal specimen is said to cause analogous symptoms. Unilateral excision has usually little effect. Transplantation of suprarenal tissue has been accomplished by Busch and co-workers, 1906 and 1908 .

Feeding of Suprarenal.-With normal white rats, the ingestion of suprarenal for 2 to 9 weeks resulted in some hypertrophy of the testicles. The growth of other organs was not affected (Hoskins and Hoskins, I9I6).

\section{SUPRARENAL TESTING}

Commercial preparations of epinephrin vary greatly in activity (Hunt, r906; Sollmann, rgo6; W. H. Schultz, I910). This is partly due to the ready decomposition of solutions; but even the dry commercial base is often far from pure (Schultz, 1909). Assay is therefore desirable. Quantitative methods are also needed for scientific problems. Gravimetric and volumetric methods are not applicable and recourse must be had to colorimetric and physiologic methods. For fairly large quantities, the effect on the blood pressure of mammals is commonly used. A standard procedure is described in the U.S.P. IX and in the Reports, Council of Pharm. and Chem., I9II, page 22. The Trendelenburg method of frog perfusion has also been widely employed. Various other methods are discussed in the papers of Crawford, I907; Schultz, I909; G. N. Stewart, rgIr; Fiffeneau, I9I5.

Colorimetric methods also give good results. A process is described in the U.S.P., IX, and by Seidell, I913 (with critical discussion). Folin, Cannon and Denis, 1913, describe another very sensitive test, which gives good agreement with the blood-pressure method. The method of Krauss is unsatisfactory (Hale and Seidell, I913). The various color reactions are described by Barger, Simpler Natural Bases, I9I4, page 89.

\section{PREPARATIONS-EPINEPHRIN}

*Epinephrina, N.N.R.; Adrenalinum (Adrenal.), B.P.; Epinephrin, Adrenalin (L-Suprarenin, etc.); $\mathrm{C}_{9} \mathrm{H}_{13} \mathrm{NO}_{3}$.-Microcrystalline powder. The free base is prac- 
tically insoluble, and is therefore marketed as an aqueous I : I,000 solution of one of its salts, generally the hydrochlorid. The solutions usually contain a small quantity of preservative (often sulphite) and thus keep fairly well. On dilution they deteriorate in a few hours, by oxidation, acquiring a pink to brown color. This is favored by alkalies, delayed by acids. The alkaloid is a reducing agent and gives a pink or red color with oxidizing substances such as iodin (Ewins, I9ro). Ferric chlorid produces an emerald green color, which turns purple and carmin on adding an alkali. Dose, 0.5 c.c., 8 minims, of the I : 1,000 solution. Maximum dose, $2 \mathrm{mg} ., 1 / 30 \mathrm{gr}$.

*Liquor Adrenalini Hydrochloricus (Liq. Adrenal. Hydrochl.), B.P.- - I per cent., preserved with chloroform. Dose, 0.6 to 1.8 c.c., ro to $30 \mathrm{minims}, \mathrm{B} . \mathrm{P}$.

Suprarenalum Siccum (Supraren. Sicc.), U.S.P.; Dried Suprarenals.- "The suprarenal glands of such animals as are used for food by man, cleaned, dried, freed from fat, and powdered, and containing not less than 0.4 per cent. nor more than 0.6 per cent. of epinephrin, the active principle of the suprarenal gland. One part of Dried Suprarenals represents approximately 6 parts of fresh glands, free from fat." The epinephrin is determined colorimetrically. Dose, 0.25 Gm., 4 gr., U.S.P.

\section{UZARA}

This African drug-the root of an Asclepiadaceous plant-resembles epinephrin closely in its peripheral effects, except that these are more gradual and lasting. It is used by the natives against dysentery, and seems to be effective in amebic dysentery and other diarrheas (Merck's Report, $26: 464$ ). Guerber, I9II and I9I4, found a variety of actions: Central stimulation, proceeding to convulsions; a peripheral sympathomimetic action (dilation of pupils; inhibition of intestines, uterus, stomach, bladder; vasoconstriction); and a digitalis effect on the heart. Tigerstedt and Airila, 1915, find that it slows the heart and blood-stream, but raises the blood-pressure through vasoconstriction. The cardiac slowing is prevented by nicotin. In intact animals, the systemic actions are much less with oral than with hypodermic or intravenous administration. It may be amebicidal in dysentery (Justi, I9I3).

The Intestinal Movements are inhibited by the sympathetic stimulation (Hirz, 19r3), both in situ or when excised. It is very effective against the physostigmin or pilocarpin movements. The diminished peristalsis lessens the excretion of uric acid (Abl, 1913).

The active principle is glucosidal. Uzaron is a purified extract.

\section{COTOIN (METHYL-TRIOXY-BENZOPHENONE)}

This has been used in diarrhea. It is not antiseptic nor astringent, but decreases the tone of excised intestines and uterus (Impens, 1913). It is used in cholera; also to check the night sweats of phthisis. Dose, 0.06 to $0.2 \mathrm{Gm}$., I to $3 \mathrm{gr}$. It is a glucosid, derived from Coto, the bark of an unknown Bolivian plant. Dose, 0.06 to $0.6 \mathrm{Gm}$. $x$ to to $\mathrm{gr}$.

\section{PITUITARY EXTRACTS}

General Statement.-Extracts of the posterior (infundibular) lobe of the pituitary gland have a strong stimulant effect on smooth muscle, leading to considerable and rather persistent vasoconstrictor rise of blood pressure; reflex slowing of the heart; diuresis; augmented contractions of the intestine, uterus and bladder, etc. The effects resemble those of histamin (Fuehner, rgr2) and are quite distinct from those of epinephrin (Dale, rgro, Bayer and Peter, I9r r). They have no relation to the nerve supply, and are probably exerted directly on the muscle. The actions are related to digitalis, but are relatively slight as to cardiac and intense as to smooth muscle. Their complexity is probably partly due to the presence of several active constituents. 
Therapeutically, extracts have been used hypodermically with success to accelerate labor, to check uterine hemorrhage and in paresis of the bladder; and with more doubtful results in intestinal paresis and in collapse conditions. Oral administration is practically ineffective.

A review of the literature of pituitary is given by Simpson, I9I4.

Different Portions of the Gland.-The hypophyseal portion (which has a structure resembling the thyroid) produces a distinct fall of blood pressure, usually accompanied by acceleration and weakening of the heart (W. W. Hamburger, I9O4 and I9IO). The Pars Intermedia also produces a fall preceded and followed by rise (Lewis, Miller and Matthews, I9II). The pituitary gland does not contain iodin, even after excision of the thyroid (Simpson and Hunter, I9II). The active principles appear very early in fetal life (McCord, I9I 5 ).

\section{ACTIVE CONSTITUENTS}

These are soluble in water, are not affected by boiling or by peptic digestion, but are easily destroyed by trypsin, by incipient putrefaction and by hydrolytic agents in general (Dale, 1910). This explains the inefficiency of oral administration.

The nature of the active constituents is still unknown; it is even undecided whether there is but one, or several. Schaefer and Herring, Igo6, believed that the various activities are due to separate constituents. Fuehner, I9I3, has isolated four basic fractions, which are concerned to a varying degree in the blood pressure, respiratory and uterine effects. A o.I per cent. solution of this mixture is marketed as Hypophysin. The dosage of this solution is the same as that of the usual 20 per cent. solution of the fresh gland. Bandonin, I9I3, also claims to have isolated a crystalline active principle. Most of the other workers doubt whether any of these really represent the active substances in pure form (Barger, "Simpler Natural Bases," I9I4, p. I08). There is a growing belief, but without real evidence, that they are related to, but probably not identical with, histamin; this is based mainly on the resemblance of their actions (Fuehner), and on the probable presence of histidin in the gland (Aldrich, 1915). Guggenheim, I9I5, suggests a similarity to acetylcholin. Crawford and Watanabe, 1916, also find evidence of the presence of some substance related to epinephrin.

\section{CIRCULATION}

The most conspicuous effect of intravenous injection is a rise of blood pressure (Oliver and Schaefer, 1895 ; Howell, I898, showed that the effect is confined to the posterior portion). The rise is not as high, but is more sustained than that of epinephrin, lasting ten minutes to one-half hour. It is due mainly to widespread vasoconstriction, from direct stimulation of the arterial muscle. The kidney vessels, however, are passively dilated in the intact animal. The heart is generally depressed and this produces usually a primary fall of pressure before it is overcome by the vasoconstriction. The heart rate is usually slowed, at first from reflex vagus stimulation, and later by direct cardiac depression. Repeated injections of large doses produce less and less rise, and finally a fall.

Method of Administration.-Intravenous or peritoneal injection is much more effective and typical than hypodermic (Pankow, I9I2) or intramuscular; but the last acts longer (Klotz, I9II). When given by mouth, there is practically no effect.

Cardiac Effects.-In intact animals, these are complicated by the interference of the vagus and muscular actions. The total result is generally slowing and weakening of the beat.

Rate.-This may be unchanged or even quickened, but is slowed in most cases. The slowing is much less if the vagi have been divided, and is due largely to stimulation of the center by the rise of blood pressure (Beco and Plumier, 1913). Some slowing occurs even after atropin and in excised hearts (Cleghorn and others) and this part must be ascribed to direct muscular depression. Werschinin, I9I3, believes that the peripheral slowing is due to increased vagus tone; and Claude, Porak and Routier, I913, attribute it to slowed auriculo-ventricular conduction by an action on the His bundle. 
Strength.-The excursions of the mercury manometer are increased, but this is due simply to the slower rate. Direct cardiac tracings show decreased excursions and increased tone. These and the slowing diminish the output, occasionally with a preliminary increase (Wiggers, I9II). The decrease occurs even when the blood pressure rises, showing that the rise is purely vascular. The ventricular efficiency is diminished (Wiggers, r916).

The pressure in the pulmonary artery falls, by weakening of the right ventricle (Wiggers, I9II); or it may be unchanged (Anderes, 1915). The vein pressure is not affected by moderate doses; it rises with large doses by cardiac dilation (Capps and Matthews, r913).

Oft-repeated injections are said to produce cardiac hypertrophy, with lasting rise of pressure (Etienne and Parisot, 1908).

Excised Mammalian Heart.-This also reacts with decreased rate and amplitude (Wiggers; McCord, I9II). Einis, I913, however, attributes this to the chloretone in the solutions; with pure solutions he describes primary slowing and weakening with subsequent quickening and increased excursions.

Isolated Frog's Heart.-The excursions are generally increased; small doses quicken, large doses slow to final standstill (Einis).

Evidence of Vascular Action.--Oliver and Schaefer, and subsequent workers, have shown that the rise of pressure is accompanied by diminished organ volume (except in the kidneys), so that it can not be cardiac and must be vascular. Tigerstedt and Airila, I9I3, also found that the aortic blood flow decreases with the rise of pressure (and still more in the fall). The vasomotor center is not affected directly; but may be somewhat depressed or more often moderately stimulated, by the rise of blood pressure or respiratory embarrassment (Pilcher and Sollmann, I9I5; McCord, I9I I). The vasomotor stimulation must therefore be peripheral; and accordingly a rise of pressure occurs after the cord has been cut; and perfusion of excised organs shows direct constriction (Rischbieter, 1913). Segments of excised arteries and veins are also constricted (de Bonis and Susanna). Unlike epinephrin, the effect is not abolished by ergotoxin, so that it is probably directly on the arterial muscle.

Application of pituitary solution to the scarified skin produces a local blanching (Sollmann and Pilcher, 19I6).

Specific Differences in Vessels.-These are quite unlike those of epinephrin. Pituitary contracts the pulmonary, cerebral, and coronary areas which are scarcely affected by epinephrin. The coronary circulation is therefore slowed, notwithstanding the rise of blood pressure (Morawitz and Zahn, I9I4).

When rings of surviving excised artery are exposed to the pituitary, Cow, I9I I, found the following: The carotid and its branches constricted. The intercostal and cerebral showed no response. The gastric and renal dilated, the dilation of the renal increasing, the nearer the kidney the ring was taken. The hepatic and splenic constricted near their origin, but dilated near the organ. The coronary was sometimes constricted, sometimes dilated. The vessels of frogs are dilated (Froehlich and Pick, I9I3).

Renal Circulation.-Magnus and Schäfer, I9OI, observed that the kidneys in intact animals dilate during the rise of pressure, whilst other organs generally are contracted. The kidney volume diminishes during the fall. Hallion and Carrion described vasoconstriction followed by dilation. Both effects are probably mainly passive; for perfusion of excised kidneys generally produces constriction (McCord, IgII), sometimes followed by dilation. The ureter flow follows the volume-curve (Sollmann). The larger renal arteries are dilated (Cow).

Diuresis.-This occurs generally (not always; Beco and Plumier, I9I3) in intact animals. v. Konschegg and Schuster, 1915, find that small doses cause temporary diuresis, followed within ten minutes by diminished urine flow. Larger doses diminish the flow from the start. Injection during the inhibition again starts diuresis. Harrion and Carrion, and King and Stoland, $19 \mathrm{I}_{3}$, believe that the diuresis occurs only during the renal vasodilation; but it does not seem to bear a constant relation to the changes of blood pressure or kidney volume, and is therefore perhaps a direct action on the renal cells; possibly due to a distinct constituent (Schaefer and Herring, I908; Hoskins and Means, I913). The evidence, however, is inconclusive. In man, with ordinary doses, the diuretic response seems to be small and uncertain; the chlorid excretion is increased (Frey and Kumpiess, 1914). Konschegg and Schuster, 1915, find that 
I to 2 c.c. hypodermically, in normal individuals, diminish the volume and solids of the urine, the effect lasting sixteen hours.

In Diabctcs Insipidus, pituitary injections are said to reduce materially the volume of urine and the thirst. The output of solids is variable (Velden, r913; Graul, I915; Kleeblatt; Konschegg and Schuster, 1915; G. Hoppe-Seyler, I915; Motzfeldt, 1916).

The transformation of benzoic into hippuric acid, in nephritis, is reported to be increased by pituitary, independent of any diuresis (Farini and Ceccaroni, 1915).

Duration and Repetition of Action.-The rise of blood pressure lasts for about half an hour after intravenous injection. (Mummery and Symes, r9o8, state that it persists for several hours if the brain and cord have been destroyed.) The effect may be repeated after one-half to three-fourths of an hour, or more frequently if small doses are used (o.o6 to o.I c.c. of pituitrin for dog; Hoskins and McPeek, r9r3). In this way, the blood pressure rise may be utilized for bio-assay (Hamilton, I9I 2).

Frequently repeated injections of large doses, however, become less and less effective (Howell, I898) and may finally lower the pressure (Schäfer and Vincent, I908). The secondary depressor action does not occur as easily in dogs as in cats or rabbits (Hamilton, I9r2). The mechanism of this action has not been established.

It is not due to cardiac effects, for these remain constant; nor is it due to fatigue of the vessels, for it does not occur in perfusion experiments (McCord, I9II). This observer believes that a new dilator substance is formed by the action of blood on the pituitary principles; for he claims that mixtures of blood and pituitary have a dilator effect when injected or perfused.

Action on Depressor.-Pituitary injection decreases the efficiency of depressor stimulation, especially in rabbits, less in cats. The action is stronger than that of epinephrin, and occurs even when the blood pressure does not rise (Auer and Meltzer, 1913).

Synergism with Epinephrin.-'This has been reported by Kepinow (Gottlieb, I9 I I). The pituitary rise is not due to stimulation of epinephrin secretion; for it is not modified by ligation of the adrenal vessels (Hoskins and McPeek, 1913).

Primary Fall of Blood Pressure.-This is cardiac, but not vagal, for it occurs after section of these nerves (Paukow, 1912). The fall is more pronounced with lairge doses (Sollmann; Auer and Meltzer, rgr3). Schäfer and Vincent, 1899, believe that it is due to a distinct depressor constituent.

Circulatory Effects in Birds.-These are different than in mammals. There is a marked fall of blood pressure by peripheral vasodilation, antagonized by epinephrin or barium. The ventricular and auricular contractions are stimulated (Patos and Watson, 19I2).

\section{CLINICAL USE IN SHOCK AND COLLAPSE}

Mummery and Symes found experimentally that the pituitary rise of pressure is more pronounced and sustained if the pressure has previously been lowered, as by hemorrhage, traumatic shock, cholin, peritonitis, etc. Blair Bell, Igr r, claimed similar beneficial results in patients. The usual dose (I c.c. of the commercial solution $=$ about $0.2 \mathrm{Gm}$. of the moist gland, intramuscularly) may raise the blood pressure 10 to $40 \mathrm{~mm}$. for an hour, with slightly slowed heart rate (Beck and O'Mally, Igog; Klotz, I9II); but the response is uncertain. This may explain why the drug has not commanded confidence. The slowing might be useful in cardiac irregularities (Werschinin, 1913). Hewlett, I9I4, finds that the intramuscular injection of I.5 c.c. converts the dicrotic febrile pulse into a normal pulse, with diminished pulse volume, but without definite change in blood pressure or blood flow in the arm; these changes corresponding to vasoconstriction. Wiggers, I9II, believes that it may be of some use in pulmonary homorrhage by depressing the heart.

\section{RESPIRATORY CENTER}

This is affected, both directly and through the circulatory changes. With intravenous injection, Paukow, 1912, describes a primary arrest during the fall of pressure, then an increase and a second stoppage during the rise. Nice, Rock and Courtright, I914, describe the characteristic effects as primary increase of depth, followed by shallow and generally slow, but sometimes quickened respiration. These disturbances 
set in with the circulatory changes, but are not as lasting. They fail to occur after a few injections have been maae. In frogs, tadpoles and fish, the center is paralyzed (Hepner, I9I 5 ).

Bronchial Muscle and Asthma.-Froehlich and Pick, r913, find a fatal bronchial spasm in guinea pigs. This is peripheral, i.e., preventable by atropin, but not by section of vagi. It occurs also in the surviving lung of dead animals (Baehr and Pick, I9 3 ). Titone, I913, observed no effect on isolated bronchial muscle.

Pituitary has been tried clinically in asthma (Brochardt, r9r3); but the results are not promising. Lanari, I9I5, even reports a patient in whom pituitary provoked asthmatic attacks.

\section{UTERUS}

In the body and on the excised organ, even very small doses of pituitary have a marked augmentary effect, and increases the excitability to hypogastric stimulation (Dale, I907; Frankl-Hochwart and Froehlich, I9I0). This occurs in all animals, pregnant or non-pregnant (Fuehner, I9I3; Gunn and Gunn, I9I4). The action has been used for a bio-assay (Dale and Laidlaw, I912; Roth, I914; U.S.P. IX).

Excised Human Uterus.-Lieb, I9I4 and I915, reports that the non-pregnant human uterus is not contracted, and may even be slightly depressed, by pituitary, the contraction reactions appearing six weeks after impregnation and persisting throughout pregnancy. He believes that the change is due to appearance of some substance that sensitizes the uterus specifically to pituitary. This difference between pregnant and non-pregnant uterus does not apply to histamin which produces contraction in both states.

These results of Lieb must be viewed with some suspicion. Gunn, I9I4, describes contraction response of the ordinary human uterus. The tubes did not respond (in distinction to epinephrin, which contracts both). Injection of pituitary into the virgin human uterus in situ certainly produced extreme contraction (Clarence Webster). The round ligament does not respond.

\section{USE IN OBSTETRICS}

This was introduced by Bell, Igog. Its efficiency for hastening delivery and checking postpartum hemorrhage has been generally confirmed (also in animals; Lescohier and Closson, I9I2). It fails in some cases (Boight, I9II). Excessive doses are dangerous, the powerful contractions closing the cervix, preventing delivery and producing local necrosis (Grumann, I9I3), and sometimes even rupture of the viscus (Espeut, r913; Zuellig, r915; also J.A.M.A., I914, 62:I42I). It may also prevent labor if injected during the first stage of labor. After this, and in proper doses (I c.c., repeated in an hour if necessary) it is not dangerous to mother or child. It does not produce abortion before term, and is most effective when the os begins to dilate (Malinowsky, I9r4).

Clarence Webster injects a few drops of pituitary extract directly into the uterine muscle, to secure a firm and bloodless field for incision, $f . i$. in Cesarean section.

\section{URINARY BLADDER}

The vesical muscle is stimulated and the excitability increased to pelvic, but not to hypogastric stimulation (Frankl-Hochwart and. Froehlich, I9ro). This has been utilized in postoperative paralysis of the bladder (Hoffstätter, I9ı I; Fellenberg, I9II).

\section{INTESTINES}

These show primary inhibition of contractions through sympathetic stimulation central to epinephrin; followed by increased contraction through stimulation of Auerbach's plexus and its postganglionic fibers 
(Bayer and Peters, I9II). The stimulant effect has been used clinically in paralytic distention of the intestines, especially after operations (Bell and Hick, I909). Its efficiency is still under judgment; Bidwell, I9I I, claims a limited usefulness.

Shamoff, 1916 , and Hoskins, 1916 , found that the intestinal action varies greatly in different preparations, some even relaxing, like epinephrin. "Hypophysin" was especially relaxing, but extracts of fresh glands also gave this result. The blood-pressure tests of these preparations gave the normal response.

\section{LACTEAL SECRETION}

Ott and Scott, rgro and I9r2, discovered a marked galactagogue effect in goats; a number of other gland extracts, especially of the sexual apparatus, had a similar but weaker action. The observations were confirmed by Mackenzie, and by all subsequent workers. The increase occurs within fifteen minutes after the intravenous or hypodermic injection; but there is a corresponding decrease at the next milking, so that the daily output of milk is not changed (Hammond, rgr3; Hill and Simpson, rgr4). The fat content is increased at the first milking, without decrease at the next. The non-fat solids are not affected (Hill and Simpson, rgr4). If the injections are repeated over long periods, the effect is lost (Simpson and Hill, I915). No increase of milk is said to occur in cows (Gadin, 1913) or human (Schaefer, 1913); but Hill and Simpson, 19 14, found that intramuscular injection of $x$ c.c. of pituitary solution in the fifth month of human lactation was followed by marked increase of milk and fat.

Mechanism of Action on Milk. - The fact that the response (in goats) is perceptib!e within half a minute, as also the secondary decrease, suggest that the increase is not due to secretion, but merely to the more complete expression of stored milk by contraction of the smooth muscle of the gland (Simpson and Hill, x 914 ). The increase of milkfat would agree with this. Gaines, 19I 5 (full discussion of the subject), presents manometric evidence of such contraction in lactating glands (non-lactating glands do not respond). Simpson and Hill, 1915, point out that barium does not increase the milk, although it stimulates the muscle powerfully; but this would be counteracted by the intense vasoconstriction.

\section{SALIVA}

Intravenous injection into dogs diminishes the flow of saliva, partly by decreased blood flow, partly by direct depression of secretion. The response to pilocarpin is also diminished (Solem and Lommen, I915).

\section{PANCREATIC SECRETION}

This is decreased (Pemberton and Sweet), through vasoconstriction (Edmunds)

\section{CEREBROSPINAL FLUID}

Pituitary affects the pressure merely through its circulatory changes (Dixon and Halliburton, 1913 and 1914).

\section{PUPILS}

These are not affected in normal animals; but according to Ott and Scott, 1909, they are dilated after removal of the superior cervical ganglion (perhaps by sensitization to epinephrin). Meltzer and Auer, 1912 , found different results, dilation in frogs, constriction after removal of the ganglion (ovary, thymus, and mammary have no effect on the pupil).

\section{TOXIC EFFECTS}

The toxic side actions are described by Seifert, Nebenwirk., 1915, p. 206. Excessive and long-continued doses are said to produce ataxic and motor symptoms, emaciation and disturbances of carbohydrate metabolism. Weed, Cushing and Jacobson, 1913, believe that this is influenced by the posterior lobe. Injections of pituitary lower the assimilation limits for carbohydrates, and cause glycogenolysis and glycosuria. Stimulation of the hypophysis in dogs produces hyperglycemia, even when proper precautions are taken against asphyxia, neighboring structures, etc. No result is obtained after section of the splanchnics (Keeton and Becht, 1915). 
STANDARDIZATION

Some extracts are seven or eight times as active as others. In view of the powerful effects, they should therefore be standarized. Various methods have been proposed. The blood-pressure method (Hamilton), although easier, is not as reliable as the use of the excised uterus (technic described by Roth, I9r4; and U.S.P. IX).

\section{PREPARATIONS-PITUITARY}

Hypophysis sicc., U.S.P.; Desiccated Hypophysis.-The posterior lobe of the pituitary body of the ox, cleaned, dried, and powdered. Dose, $0.03 \mathrm{Gm} ., 1 / 2$ gr., U.S.P.

${ }^{*}$ Liq. Hypophysis, U.S.P.; Solution of Hypophysis (Pituitary Solution).-Prepared by extracting the fresh posterior lobe of bovine pituitary with slightly acidulated water, boiling, filtering and sterilizing. Standardized on excised uterus against histamin. Dose, I c.c., I5 minims, U.S.P., hypodermic.

\section{ANTERIOR PITUITARY IOBE}

This has important relations to metabolism, growth, and sexual development. Total removal is followed by death in a short time (Paulesco, r907; Reford and Cushing, r909; Crowe, Cushing \& Homans, r9ro. Partial removal or disease leads to retarded growth or infantilism, obesity, and other nutritional derangements. Hyperactivity (as in acromegaly; P. Marie, I886) is characterized by accelerated and abnormal growth (gigantism).

Feeding of the anterior lobe, fresh or dried, produces different effects before and after adolescence. In the pre-adolescent period, it retards growth (as judged by weight and length of bones), but stimulates sexual development. In fully-grown animals, it accelerates growth, including the replacement of tissue lost by inanition; the healing of granulating wounds, etc. T. B. Robertson, I9I6, has prepared an active principle, "Tethelin."

The experimental results are often rather confusing; probably because the effects depend so much on the state of growth, and perhaps on other conditions. The following authors may be consulted:

Isolation of Tethelin and effects on mice: T. B. Robertson, rgr6.

Rats: Goetsch, rgr6 (excessive doses produce digestive and nutritional disturbances; and if the posterior lobe is also fed, nervous derangements). Surface.

Chicks and egg production: S. S. Maxwell, 1916; L. N. Clark, 1915; Pearl and

Therapeutic Uses.-These are in the experimental stage. The administration of the dried anterior lobe ( 0.05 to $0.25 \mathrm{Gm}$., I to $4 \mathrm{gr}$., in powder or tablets) has given some favorable results in the later stages of acromegaly; in dystrophia adiposo-genitalis; and in certain cases of obesity. It is contraindicated in early stages of acromegaly.

\section{THYROW PREPARATIONS}

General Statement.-The administration of thyroid gland is highly successful in combatting the phenomena of thyroid deficiency-myxedema, cretinism, etc.- whether the deficiency be congenital or acquired by disease or operations. The benefit persists practically only so long as the administration is continued. Metabolism is increased also in normal individuals, first the nitrogen, then fats. Thyroid is therefore used against obesity. However, it is a dangerous remedy, excessive doses causing nervous symptoms. Thyroid administration alters the susceptibility to certain poisons. Acute effects are slight and inconstant, even on intravenous injection.

The activity resides in the colloid material of the gland, and is proportional to its iodin content.

Active Constituents. - Thyroid glands contain a relatively large proportion of iodin. The best-known actions of thyroid preparations-the therapeutic efficiency, increased nitrogen excretion, acetonitrile test, etc.are parallel to their iodin content (Marine, I9r3). These effects are not 
produced by inorganic and most organic iodin compounds, not even by iodized proteins (except after they are assimilated by the thyroid). The active principle must therefore be a characteristic organic iodin compound, contained in the colloid globulin, "thyreoglobin" (Oswald, I899, I908 and I909).

The iodin content of this globulin varies from o to $\mathrm{I} .3$ per cent. or more; usually about 0.35 per cent., the physiologic activity being proportional to the percentage of iodin. However, glands which are practically devoid of iodin still exhibit a very slight activity. An iodin-free thyreoglobulin is also found in thyroids which contain no colloid. Oswald, therefore, suggests that two globulins occur in the gland, differing only by the presence of iodin, and not separable by reagents. The alternative, that the same protein may take up different proportions of iodin (crudely analogous to oxygen and hemoglobin) has at least equal probability (Hunt, 1907). Hunt, r9rr, appears to consider that there is but a single iodized protein, since the activity is in simple ratio to the iodin content.

The iodized thyreoglobulin has been further fractionated. F. C. Koch, r9r 3 , finds that the full activity is contained in the metaprotein fraction of the thyreoglobulin; the simpler cleavage products showing less and less activity per unit of thyroid. Pick and Pineles, I 909, also claim that completely digested thyroid is less efficient in myxedema. Both statements appear to conflict with the universal experience that oral administration is effective, although it may be assumed that complete cleavage occurs before the tissues are reached. Fawcett, Beebe, et al., I915, find an organic iodin compound in extracts freed from nucleoproteins, globulins and other coagulable proteins. Beebe, I $9 \mathrm{I}$ I, recommends a special globulin preparation as the bearer of the therapeutic actions, and as devoid of undesirable and toxic iodized protein. His claims have not yet been accepted.

Kendall, I9r3 and I915, claims to have separated two iodin substances of distinct actions; a crystalline compound producing the "toxic" effects; and an unisolated substance responsible for the therapeutic actions. Wilson and Kendall, r9r6, find that the quantity of the toxic iodin compound varies with the total iodin content of the glands.

It is noteworthy that organic iodin compounds can not be demonstrated in any other mammalian tissues than the thyroid (Blum and Gruetzner, r914).

Thyroid also contains nucleo-proteins, mainly in the nuclei, and therefore in inverse ratio to the colloid (Beebe, I907).

Iodothyrin.-This is a cleavage product prepared by Baumann, 1896, containing from 4.5 to 15.3 per cent. of iodin (Oswald; Koch, 1903). It does not give the protein reactions, and is very resistant to heat and reagents. Baumann and Roos, r 896, and Hildebrandt, $x 896$, claimed that it has the therapeutic actions of thyroid; but most later observers have obtained rather negative results. The commercial product contains much less iodin (as little as o.0r4 per cent.; Hunt and Seidell, I9ro), sometimes in loose combinations.

Iodin Content of the Glands. -This averages about 0.15 to 0.3 per cent. for dry, and 0.03 to 0.06 per cent. for moist glands. (The average percentage for moist glands is given by Aldrich, I9I3, as 0.025 for sheep; 0.036 for beef; 0.047 for hogs.) It is subject to great variations, particularly in goiter. In hyperplastic goiters, the percentage and total iodin are both greatly diminished, proportional to the histologic changes. In colloid goiters the percentage is diminished to a less degree (Marine and Williams, I908). Iodin is said to be absent in wild carnivorous animals, and sometimes in domestic (Roos, r899), but this must be rare. Contrary to older statements, Fenger, r9r 2 and r $9 \mathrm{I}_{3}$, found it in the fetus of domestic animals long before birth. Its asserted absence from the human fetus therefore seems to require further confirmation.

The iodin undergoes marked seasonal variations, the percentage being three times greater (with smaller glands) in June to November, than in December to May. It diminishes gradually from about September till March or April, and then increases abruptly until September. The changes appear to be connected mainly with the prevailing temperature; although diet and the reproductive season are not definitely excluded (Seidell and Fenger, I9I3 and I9I4). The iodin is also decreased by stimulation of the nerves going to the gland (Rahe, Rogers, Fawcett and Beebe, r914), but this is due simply to vasoconstriction (Watts, 1915).

Effect of Administration of Iodin Compounds. - These, whether inorganic, or organic, materially increase the iodin content of the gland, with corresponding increase of its activity, and characteristic anatomic changes (involution of hyperplasia (Marine)). The selective affinity of the thy- 
roids is very high, relative to the other tissues (Marine, I9I 5). Hunt, I907, points out that part of the pharmacologic action of ordinary iodids may be explained by their conversion into thyroid secretion; and this is obviously their effect in thyroid disease.

When iodin compounds are fed, iodin is taken up by the thyroid tissue to the point of saturation, which averages about 5 to $6 \mathrm{mg}$. of iodin per gram of dried thyroid. The selective affinity is so strong that as high as 18.5 per cent. of a given intake (oral) of iodin was recovered from a thyroid whose ratio to the body weight was I:687 (Marine, 1015).

The iodin content can also be increased by the injection of blood of hyperthyroid, and decreased by that of thyroidectomized animals (Jones and Tatum, I9I3).

Perfusion of Thyroid.- Freshly excised thyroid takes up and fixes potassium iodid from the perfusion fluid, presumably in an organic form, so that it can not be washed out by Ringer's solution. The amount fixed is independent of the iodid concentration of the perfusion fluid, but is larger for hyperplastic than for colloid glands, and is about equal to the amount fixed in the living animal, in the same time (an hour), with intravenous injection. In neither case does the iodin absorbed by the thyroid produce the specific effect on tadpoles; so that a further elaboration in the gland is necessary. Activity is acquired in living animals within thirty-six hours. The iodin fixation of perfusion does not occur when the gland becomes disorganized by autolysis; nor does it occur on perfusing other organs than the thyroid (Marine and Feiss, I9I5).

Thyroid Deficiency.- Complete excision of the thyroid apparatus (Cachexia strumipriva) causes a series of acute effects, motor, nervous and metabolic, which are more or less rapidly fatal in most animals, especially in carnivoræ. (These results were first described by Schiff, I856; Reverdin, I882; and especially Kocher, I $88_{3}$; the literature is reviewed by Vincent, I9II.) If the parathyroids are spared, the motor symptoms and fatality are much less; and the metabolic disturbance, though no less severe, runs a more chronic course. In man, thyroid deficiency when occurring in adults, results in Myxedema, with the conspicuous skin changes and nervous disturbances ending in mental deterioration. Congenital deficiency produces the condition of Cretinism.

Experimental Cretinism. - Complete thyroidectomy in young rabbits is said to be followed by typical cretin phenomena, such as,dry, rough hair, retarded growth, skin changes, etc. These are perfectly controlled by feeding dried thyroid or its cleavage products if the hydrolysis has not gone too far. Kendall's " $B$ " compound was ineffective; so was transfusion of normal blood; whereas the serum of thyroid-fed animals was moderately efficient (H. R. Basinger, 1916).

Therapeutic Results of Thyroid Feeding.-The deficiency symptoms are more or less completely relieved by the administration of thyroid by mouth (Howitz, Mackenzie, Fox, 1892 ), as well as by intravenous injection (Colzi, I884; Murrey, I89r), or by implantation of thyroid tissue (Schiff, I884).

Administration.-The dried gland is the most convenient form, either as powder or tablets. The latter ordinarily contain 0.13 Gm., 2 gr. of dried gland. The treatment must be practically continuous, but overdoses must be avoided.

It may be begun with $0.15 \mathrm{Gm}$. per day, increasing by this amount every few days to a maximum of $0.25 \mathrm{Gm}$., $4 \mathrm{gr}$., three times daily, best on an empty stomach. If "toxic" effects appear, the dosage must be temporarily reduced. When the full therapeutic action has been reached, the dose may be reduced to $0.5 \mathrm{Gm}$., $71 / 2 \mathrm{gr}$. daily, and then every second day for a month. The administration may then be intermitted for a time and resumed in a similar way when the indications again arise. It is claimed that arsenic (ro to I5 drops of Fowler's Solution daily) increases the tolerance.

Overdosage.-This is denoted by circulatory, nervous, digestive, and metabolic disturbances, resembling superficially those of Graves' disease.

Symptoms. - These are palpitation; rapid and of ten irregular pulse; vascular congestions. Headache and muscle pains; tremors of extremities; nervousness; insomnia; delirium; diaphoresis; irritations of skin; hyperpyrexia. Polyphagia, or loss of appetite, nausea, vomiting and diarrhea. Sometimes albuminuria and glycosuria. Scotoma has been described (Snydacher, Igri). In fatal cases, uncontrollable vomiting, collapse and coma. .Cretins are said to be more susceptible to the toxic effects (Basinger, I9I6). 
Experimental Administration.-This produces similar effects, but the resistance varies greatly in different animals, carnivoræ being less susceptible. The most constant features are emaciation, gastroenteritis and diarrhea; but no tachycardia, nervousness or exophthalmos. Death may occur with convulsant or depressant symptoms. Fresh, dricd, or stale glands produce the same effect (Carlson, Rooks and McKie, I9r 2; French, I9I 2). Hypertrophic thyroid is less toxic than normal, in accordance with its lower iodin content (Stoland, I9I 2).

Gudernatsch, I9I5, found that small doses fed to rats tend to prevent conception. If pregnancy occurs, the young either abort, or die early, or grow less than normals. The detrimental results are the same whether the feeding has been to the mother or father alone.

"Experimental Hyperthyroidism."-Cannon, Binger and Fitz, rgr5 and r 916 , aimed to produce this condition by fusion of the phrenic with the cervical sympathetic nerve, believing that this would result in continued stimulation of the sympathetic structures, including the thyroid. The cats showed tachycardia (to 260), diarrhea, falling of hair, scleroderma, pruritus, excitable temper, greatly increased metabolism, enlarged pupils, exophthalmos and hypertrophy of the adrenal glands. Removal of the thyroid on the fused side arrests the progress of the disturbances, and restores the metabolism to normal. The interpretation of the results requires further investigation. Troell, 19r6, states that he was unable to obtain Cannon's results.

Innervation of Thyroid. - The gland is abundantly supplied with non-medullated fibers, probably derived from the cervical sympathetic, and going to the cells as well as to the blood vessels. An action-current develops in the gland on stimulation of the sympathetic, high in the thorax, even when the superior and recurrent laryngeals have been cut. Vagus stimulation or pilocarpin does not generate the action-current (Cannon and Cattell, r916).

On the other hand, autografts of thyroid show evidence of growth and function, so that the nervous connections are not indispensable (Manley and Marine, rgr6).

Sensitization to Autonomic Stimulation.-Injection of thyroid, or increased secretion secured by stimulation of the superior laryngeal nerve, does not produce any characteristic autonomic stimulation directly; but it increases the response to epinephrin, splanchnic, vagus and depressor stimulation (Asher and Flack, I9I0; Asher and Rodt, I912). Complete excision of the thyroid-parathyroid apparatus lowers the vagus excitability (Coronedi, I9I3).

Epinephrin on Thyroid Secretion.-Injection of epinephrin, $0.00 \mathrm{r}$ to $0.002 \mathrm{mg}$., evokes an action-current in the thyroid. The same result follows stimulation of the cut splanchnic; but it is absent if the adrenal blood is prevented from reaching the circulation (Cannon and Cattell, rgi6).

Intravenous Injections. - This produces fall of blood pressure and slowing of the heart, probably due to its cholin content (Lohmann; Fuerth and Schwarz, 1908; Fuerth, 1 909; Schoenborn, 1909). Pressure juice produces little effect in dogs and cats, but marked collapse in rabbits (Caldwell, rgr 2). These effects are not specific but due to proteins, embolism, etc. Blackford and Sanford, I913, claim that the pressure fall is much more marked with extracts from toxic goiter; and that the sera of such patients also produce marked cardiovascular depression. These claims await confirmation. Fawcett, Rogers, Rahe and Beebe, rgr $_{5}$, find that the coagulable portions of thyroid extracts (which contain most of the iodin) have no effect on blood pressure. The fall is confined to intravenous injection of the non-coagulable portion, and has no relation to the iodin content. It therefore appears unconnected with the specific constituents of thyroid (they also review the contradictory literature).

Secretion of Cerebro-spinal Fluid. - This is said to be inhibited by thyroid and increased by brain extract, independent of blood-pressure changes. Other organ extracts act only mechanically, through the circulation (Frazier and Peet, I9I5).

Thyroid in Exophthalmic and Other Goiters.-The administration of thyroid is often beneficial in goiter; but the active "toxic" stages are especially susceptible to the toxic effects of thyroid, so that only very small doses should be used. The same effects can be produced by iodids, which are elaborated into the active substance by the thyroid of the patient. Caution should therefore be used in prescribing iodin compounds 
in goiter, especially where nervous symptoms exist (Oswald, 1915; history; description of symptoms and cases).

Nature of Goiter.-Anatomically, this may consist in overgrowth of the epithelium (hyperplasia), or of the colloid. The investigations of Marine, 1907 to 1913 , indicate that the first change is hyperplastic, the epithelium proliferating to meet some physiologic deficiencies, such as an inadequate supply of iodin; or experimentally the excision of a part of the gland. When the deficiency is met, f.i., by the administration of iodin, the glandular changes revert into the colloid type. Transplanted thyroid tissue reacts in exactly the same way, by hyperplasia or involution, to removal of thyroid tissue or iodin; so that the changes are evidently independent of the nerve supply (Manley and Marine, I915).

The relation of these changes to the "toxic" symptoms of Basedow's disease is not understood; for the symptoms do not occur typically in animals. It is commonly assumed that these "toxic" symptoms are due to excessive or perverted secretion of the thyroid (hyperthyroidism, dysthyroidism). The evidence for this theory is not conclusive, for it is based mainly on the resemblance of the clinical nervous symptoms to those of thyroid feeding; and on the beneficial results of partial thyroidectomy. These may have other explanations, and are opposed by the fact that improvement also follows the graduated administration of iodid. (Blackford and Sanford, I913, assert the presence of a powerful depressor substance in the blood and thyroids of Basedow patients.)

Antithyroid Serums.-These were introduced on the hyperthyroidism theory of Graves' disease as substitutes for operative removal, but had only a short popularity.

On the theory that Basedow's disease consists in a thyroid intoxication, Ballet and Enriquex (1894) attempted its treatment by the administration of the serum of thyroidectomized dogs, supposing that these produced substances antagonistic to thyroid secretions. Moebius Serum, Rodagen and Thyroidectin are prepared on this principle, generally from sheep. Their oral administration sometimes has a palliative effect on the nervous symptoms in mild cases, but probably no more than simple rest and diet. Beebe, 1906 and I9I 5 , introduced a cytolytic serum. Encouraging results were reported at first, but these reports have become less frequent.

Increased Metabolism.-This is one of the most constant effects of thyroid administration, occurring in normal animals, but much more marked in the low metabolism of thyroid deficiency (Magnus-Levy, 1897 ; DuBois, rgr4. The heat production in Basedow's disease is also much above the normal). The metabolic effects of thyroid administration are shown by increased excretion of nitrogen (Ord and White, r893; Mendel, r893), and carbon dioxid and increased consumption of oxygen; with improvement of appetite and rapid loss of weight (Leichtenstern, 1893). The first effect is on fat, the proteins being drawn upon when the fat has been reduced to a certain minimum. On a meat-free diet, the nitrogen increase concerns especially the urea, ammonia and creatin, whilst the uric acid and creatinin are very little changed (Krause and Cramer, I912).

Studzinsky and Kaminsky, I915, state that thyroid administration increases the urate excretion in hypo-thyroidism, but not in normal subjects.

Carbohydrate Metabolism. - This is probably not influenced directly by the thyroids (Macleod); although it is claimed that thyroidectomized animals do not utilize sugar on parenteral administration as well as normal animals (Underhill and Saiki, 1908); and that they do not react with glycosuria to some of the procedures which are ordinarily effective (King, I 909 ).

Increased Metabolism in Basedow's Disease.-Du Bois, Igr6, considers this the most definite feature of the disease. Heat production is 50 per cent. above the normal in severe, and 75 per cent. in very severe cases. The excessive heat production accounts largely for the tachycardia, cardiac enlargement, emaciation and mental irritability.

This feature also throws light on the efficiency of treatment: Rest in bed for a week diminishes, the excessive heat production by ro per cent. or more; psychotherapy was 
also of some value. Negative results were obtained with thyroidectin, $\mathrm{X}$-ray, thyroid "residue," ergot, quinin, and Bcebe serum.

The high metabolism means that the patients need $1 / 2$ to 2 times as much food as normal individuals.

In cretins, the heat production was found 20 per cent. below normal. This was corrected in a few days by thyroid administration.

Use in Obesity.-Thyroid administration is often strikingly effective in reducing the fat in constitutional obesity (menopause, etc.), much less so in superalimentation. In any case, the result is not permanent and is connected with the sometimes dangerous effects described under overdosage. Only small doses should be used, under strict medical supervision. The protein income should be kept high to compensate the loss (v. Noorden, I9I5).

Other Therapeutic Uses.--Thyroid has been tried in the following conditions, but the inconstant results indicate that it is probably useless: Skin diseases; psoriasis, eczema, lupus, chronic rheumatism, gout, arteriosclerosis, rickets, infantile cachexias, hemophilia, etc.

Larval Growth and Development.-Gudernatsch, IgI2, made the important discovery that feeding thyroid to tadpoles checks their growth, but at the same time hastens their development into frogs. This has been abundantly confirmed. The activity is proportional to the iodin content (Lenhart, I9I5). Morse, I9I4, finds that the effect is produced by all iodin-containing fractions of the gland, whilst the nucleoproteins, lipoids, etc., are ineffective. Positive results are also produced by iodothyrin, iodated tyrosin, iodized Witte's peptone and iodized serum proteins; not by inorganic iodin or iodids, iodized starch, iodized lecithin, or marine algæ. The activity therefore appears to be due to iodized amino-acid derivatives. The action probably consists in increased autolysis and phagocytosis.

The presence of thyroid tissue also increases very markedly the multiplication of paramecium (Nowikoff and Shumway; Budington, I915).

Susceptibility to Poisons.-The metabolic disturbances alter the destruction of poisons in the body. In mice, f.i., the toxicity of acetonitrile is greatly diminished, whilst that of morphin is increased.

It has been suggested that many symptoms of Graves' disease may be secondary, due to lowered resistance against metabolic; intestinal, or other accidental poisoning.

Acetonitrile Test.-Hunt (1905, I90\%, I908) found that the administration of thyroid to white mice, for a few days, markedly increases their resistance to acetonitrile. The toxic effects of the latter are due to the liberation of HCN. Thyroid feeding does not affect the toxicity of HCN when administered as such; so that its protective influence must be due to a retardation of the metabolic processes which convert acetonitrile into $\mathrm{HCN}$. In rats and guinea pigs it has the opposite effect, increasing the toxicity of the nitriles.

The acetonitrile response is a delicate and nearly specific test by which a milligram of active thyroid tissue can be readily detected. It may also be used for comparing the relative activity of different thyroid products. This is found to go parallel with their iodin content. The administration of iodin therefore renders dogs' thyroid more active. The prompt increase argues that the storage of iodin is the cause, rather than the result, of the activity. Thyroid in which no iodin can be detected by the ordinary chemical tests, as well as certain other organs, show a very feeble activity (Hunt and Seidell, I908), and this may be due to traces of iodin compound, which are not revealed by the ordinary test.

Trendelenburg, I9Io, and Lussky, I9I2, claim that the blood of cats gives the test even after removal of the thyroids. This removal does not seem to alter the response of the mice. Application of the test to the blood of Basedow's disease has not given conclusive results.

Effect of Diet.-Certain diets, dextrose, oatmeal, liver, or kidney also increase the resistance of the mice to the nitrile, as compared with cracker, milk, cheese or egg diet. This seems to be due to an action on the thyroid; for the difference is diminished if the thyroids are excised; and these glands present anatomic changes (Hunt, I9IO, I9II).

Thyreotropic Iodin Compounds.-Other iodin compounds also have some protective action, presumably by being converted into the thyroid compound. None reach the same activity, but they present interesting differences. The iodin of the seaweed, 
bladderwrack, is about 100 times as active as the iodin of potassium iodid (Hunt and Seidell, 1910); and a diet of seaweeds increases the iodin content of the glands (Hunter and Simpson, I015). Iodothyrin has a varying activity (none, Trendelenburg, 1910).

Resistance to Morphin.- Thyroid feeding lowers the resistance of mice, rats and guinea pigs to morphin (Hunt). Gottlieb, IgII, claims to have demonstrated that this is due to lessened destruction of the morphin; and that the serum of a Basedow patient had a similar inhibitory effect, whilst normal serum hastens its destruction. According to Olds, I910, the resistance to morphin is not increased by excision of the thyroids, as might perhaps be expected.

\section{PREPARATIONS-THYROID}

*Thyroideum Siccum ('Thyroid. Sicc.), U.S.P., B.P.; Dried Thyroid. The dried and powdered healthy thyroid glands of sheep (and other animals used for food, U.S.P.). One part of the dried corresponds approximately to 5 parts of the fresh gland. The U.S.P. requires 0.17 to 0.23 per cent. of thyroid iodin. Dose, $0.1 \mathrm{Gm}$., $11 / 2 \mathrm{gr}$., U.S.P., 0.03 to $0.25 \mathrm{Gm}$., $1 / 2$ to 4 gr., B.P. Raw or boiled glands ( $1 / 8$ to $1 / 3$ gland) have also been used, but have no advantage.

\section{PARATHYROIDS}

These small glands were often excised with the thyroid, and their removal was responsible mainly for the convulsive symptoms and the fatality. Their functions are apparently quite distinct from the thyroid; $f . i$., they are usually quite normal in cretins (Rousay and Clunet, I9I0). They were discovered by Sandstroem, 1880, and their physiologic importance pointed out by Gley, I892.

Actions.-Excision of the parathyroids from dogs produces tetany. This is temporarily relieved by the injection of parathyroid extract or nucleo-protein (Beebe, I907), and also by calcium. This suggested that the glands are concerned with calcium metabolism (MacCallum and Voegtlin, I909); but this does not seem to be the case (Cooke, rgIo). The antagonism is merely due to the depressant action of calcium. Strontium and sodium chlorid are similarly protective. The ammonia of the blood is increased by depression of the functions of the liver (Carlson and Jacobson, IOII). It is doubtful whether the ammonia increase is an adequate explanation of the symptoms.

The carbohydrate tolerance is lowered by excision of the parathyroid (Underhill and Hilsditch, I909). Injection of the nucleo-protein produces polyuria and dilation of the pupils (Ott and Scott, I909 and I910).

Destruction of the parathyroid also increases the vasoconstrictor response to nicotin, epinephrin and pituitary, involving therefore the whole peripheral vasomotor mechanism. This effect is also counteracted by calcium (Hoskins and Wheelon, 19r4).

Parathyroid hyperplasia is produced in fowls by feeding with maize or wheat. This is hindered by calcium, increased by sulphuric acid and neutral sulphur (Marine, 1914).

W. F. Koch, I913, finds that the removal of the parathyroid leads to the appearance of toxic bases (guanidin, histamin, etc.) in the urine, which may be partly responsible for the symptoms.

Therapeutic-Uses.-Parathyroid has been administered in tetany resulting from removal or disease of the gland, perhaps with some benefit. It has also been tried in paralysis agitans (Berkeley, I909), in infantile and gastric tetany, eclampsia and chorea, but the results are so inconstant that it is probably useless.

Toxic Actions.-Morris, I91 5 , describes mental symptoms in a patient which he is inclined to attribute to the prolonged administration of large doses of parathyroid.

Desiccated parathyroid gland consists of the dried external parathyroids of the ox, representing about 6 parts of the fresh tissue. Average Dose, $6 \mathrm{mg}$., $1 / 10$ gr., four times daily. Fresh glands or hypodermic injections of fresh glands have sometimes given better results.

\section{PINEAL GLAND}

This is a vestigial remnant of a primitive dorsal eye, but presents some evidence of glandular structure, with sympathetic innervation. It begins to involute early, about the seventh year in homo; but its degeneration is never complete. 
Extirpation experiments have not yielded conclusive results. The early effects are inseparable from traumatisn. There are no late changes in adults; in young animals, ablation is said to lead to precocious development (Sarteschi, Foa), but the effects, at best, are not very striking.

Intravenous injection of extracts does not have any pronounced action on the circulation (Dixon and Halliburton, 1909). It increases the milk secretion in goats (Ott and Scott, 1912).

Continued Oral or Hypodermic Administration.-This is also said to produce precocious development, more definite than with ablation (Dana and Berkeley; McCord, I 9 I 4 ). The growtl of young guinea pigs is hastened, but does not proceed beyond the normal size. Dana and Berkeley used it ( 3 to $6 \mathrm{mg}$., $1 / 20$ to $1 / 10 \mathrm{gr}$., three times daily) in backward young children (to twelve or fifteen years). It had no effect on growth, but they claim improvement of the mental development. This needs independent confirmation.

Neoplasms of the pineal gland are said to be often associated with precocious physical and sexual development. Only a few cases are on record, and the reliability of the connection is not established.

\section{PANCREAS PREPARATIONS IN DIABETES}

The important relation of the pancreas to carbohydrate metabolism encouraged the hope that its administration would be beneficial in diabetes mellitus. The results, however, have been essentially negative, both in human diabetes and experimental glycosurias, however much the experiments, preparations, channels of administration, etc., have been modified (Murlin and Kramer, I 913). The exceptional positive results may be fairly attributed to the natural variations in the clinical course; whilst the experimental results (such as those of Zuelzer, 1908) fall within the limits of ordinary variation (Jacoby, Exp. Ther., p. I35). The hypothetical internal secretion of the pancreas can not therefore be utilized therapeutically.

Intravenous Injection of Dextrose.-Kleiner and Meltzer, I915, find that the restoration of the normal level of blood-sugar requires twice as long as normal in an animal whose pancreas has been removed. They claim that this delay is prevented by pancreas-emulsion, and that this also diminishes the spontaneous hyperglycemia of pancreatectomy.

\section{SECRETIN}

Pawlow showed that the presence of acid chyme in the duodenum is the normal stimulus to the secretion of pancreatic juice. It was later shown that this is not due to a nervous reflex, but to the production of a peculiar substance "secretin," which may be prepared artificially by macerating the duodenal or jejunal mucosa with dilute acids, soaps, etc. (Bayliss and Starling, I 902 ); or by contact of these or of irritants with the living mucosa. Secretin does not exist as such in the mucosa, but is formed from an insoluble "prosecretin." Secretin is identified by causing a marked flow of normal pancreatic juice, when the neutralized and boiled filtrate is injected intravenously. (An extensive review of secretin is given by Carlson, Lebensohn and Pearlman, I916.)

Nature and Properties. - Secretin is soluble in water and dilute alcohol (7o per cent.); insoluble in absolute alcohol, ether, etc. It is fairly readily destroyed by acids, alkalies or oxidation; and by the digestive ferments (Carlson et al, 19I6). It has not been isolated pure; none of the known constituents of the extracts produce the characteristic effect. Barger believes that it is probably an organic base.

Actions.- Intravenous injection of secretin stimulates the flow of pancreatic juice, bile and succus entericus, but not of the other digestive secretions; nor do extracts of other portions than the small intestines produce the secretory effect. The secretion is accompanied by, but not caused by, vasodilation. The pancreas is not readily fatigued, the flow being undiminished after eight hours of continuous injection. Similarly, repeated injections of equal doses give an identical response. The flow is not affected by anesthesia.

Clinical Inefficiency.-Moore, Edie and Abram, 1906, suggested the administration of secretin against diabetes. Subsequent workers have shown that it has no effect (Bainbridge and Beddard, I906; O. Loeb and Stadler, 1914). It was also tried against digestive disorders, with equally inconclusive results. This agrees with the scientific data: In the first place, it has not been shown that prosecretin is deficient in any clinical condition; secondly, the oral administration even of enormous doses, is quite ineffective, 
since secretin is rapidly destroyed by the digestive juices (Carlson). Even the hy"podermic administration is inactive (Matuso, I913; Hallion, r912). Finally, the commercial preparations do not contain secretin, presumably because it is too unstable (Carlson).

\section{PHLORHIZIN}

General Statement.-This glucosid produces a unique glycosuria by stimulating the kidneys to the excretion of dextrose, with polyuria and acetonuria, leading to loss of sugar, and thus to the production of sugar from proteins, greatly increased excretion of nitrogen, etc. The phenomena are of great scientific but of no therapeutic importance (Critical Review, G. Lusk, I9ז2).

Phlorhizin was isolated by Koninck, 1835 , from the root-bark of apple, pear and similar trees. Stas, I839, showed it to be a glucosid, decomposed by acid hydrolysis in to dextrose and phloretin; and the latter again into phloretinic acid and phloroglucin. The characteristic glycosuria was discovered by Mering, I 886 . It has been produced in all vertebrates tried: mammals, birds, frogs and reptiles. The phlorhizin is excreted for the most part unchanged (Pietkiewicz) within two days (Prausnitz). 'The glycosuria outlasts the excretion.

The amount of glucose in the urine may be very high, above I 2 per cent. It persists for several hours after a single injection, but may be prolonged indefinitely at its highest degree, by administering $\mathrm{I} \mathrm{Gm}$. of the glucosid per kilo of body-weight, by mouth, three times a day. Larger doses do not increase the glycosuria.

The Glycosuria is not Pancreatic.-The symptoms after phlorhizin resemble those of pancreatic diabetes, but their mechanism is in fact totally unrelated. The most. striking difference is the absence of hyperglycemia. With phlorhizin, the sugar of the blood is diminished (Mering); if the kidneys are excised, or the ureters tied, the bloodsugar remains normal (Minkowski; Schabad, r892. According to Underhill, r9 1 2, it may rise, indicating overproduction of sugar, but this plays at most a minor part).

The Action is Renal.-It is evident from these experiments that the glycosuria is due essentially to more ready elimination of sugar by the kidneys. The effect is peripheral, for it occurs after destruction of the medulla and cord, and after section of all renal nerves (Frey). If the phlorhizin is injected into one renal artery, glycosuria occurs in this kidney and not in the other (Zuntz). Mosberg found by the Nussbaum arrangement that the sugar excretion occurs in the tubules. Nishi found that the cortex contains more sugar than the medulla, whilst the reverse holds norma!ly. Histologic changes have also been described in the renal epithelium. Carmin and methylen blue, which are also excreted by the tubules, may suppress the glycosuria.

The excretion of sugar is proportional to the activity of the kidneys; it is diminished, for instance, when one kidney is excised, and increases again as the remaining kidney hypertrophies (Schilling, r9o4). The glycosuria is said to be diminished in parenchymatous nephritis, and the injection of phlorhizin (phlorhizin test, $5 \mathrm{mg}$. in warm water, hypodermically) has been proposed as a means of diagnosis. The result, however, is not decisive in either sense.

Other Renal Functions.-Diuresis occurs (Frey, 1906; Loewi and Neubauer, 1908), presumably as a result of the glycosuria. The excretion of the other solid constituents, salts, protein, etc., shows no constant abnormalities. The milk after phlorhizin contains the normal amount of lactose, and no dextrose. Traces of sugar appear also in the saliva and gastric and pancreatic juice (R. G. Pearce, I9I6).

Nature of the Action.-This is not understood. Mering assumed a mere increase of the permeability of the renal cells; but this is not the whole explanation: Levene, $f . i$., found more sugar in the blood of the renal vein than in that of the renal artery. Minkowski suggested that the phlorhizin acts as a glucosidal vehicle, splitting off its sugar in the kidneys, and again taking up sugar from the tissues; but the quantity of sugar excreted when phlorhizin is injected into the renal artery speaks against this view.

Pavy, Brodie, and Siau (1903) have shown that the quantity of sugar which is excreted when the other abdominal viscera are excised, exceeds the quantity of sugar which disappears from the blood, and that which could be derived from the limited glycogen depots of the muscle.

This indicates that phlorhizin causes the kidneys to assume the function of forming sugar from the proteins of the blood, and of secreting this into the urine, i.e., a function analogous to that of the mammary glands. They supposed that the sugar of the blood ex- 
ists in loose and firm combination with the blood proteins; the phlorhizin causes the kidney to secrete first the loosely combined sugar; and when the supply of this fails, the firmer combination is attacked. The stock of carbohydrates in the body therefore disappears first, although some glycogen is retained very persistently (Mering), even in fasting (Sansum and Woodyatt, 1915). With persistent administration, the sugar is derived from the carbohydrates of the food; and if these are excluded, from the proteins. Excision of the liver (Eck's fistula) does not influence the glycosuria (Foster, I 9I 2).

In complete phlorhizin poisoning the ability to oxidize sugar is also impaired, so that dextrose, when injected hypodermically, is completely excreted (Stiles and Lusk).

Liver-glycogen.-Phlorhizin does not mobilize the glycogen directly, but only as a result of the hypoglycemia. On the contrary, when the kidneys are excluded, phlorhizin leads to a re-accumulation of hepatic glycogen: Since there is no hyperglycemia, it appears that phlorhizin stimulates directly the synthesis of glycogen. When the kidncys are intact, this action is overcome by the glycosuria (Eptein and Baehr, I916).

$\mathrm{D} \div \mathrm{N}$ Ratio.-In the urines of depancreated dogs, fasting or fed on meat or fat, Minkowski found that the weight of dextrose divided by the weight of nitrogen gave a constant ratio of 2.8. This signifies that 45 per cent. of the protein molecule is excreted as dextrose. A ratio greater than this shows that some of the sugar ("extra-sugar") is derived from other sources. Phlorhizin produces the 2.8 ratio in fasting rabbits, cats and goats, after the "extra-sugar" has been flushed out. In dogs, the ratio is 3.67 , so that 58.7 per cent. of the protein is excreted as dextrose. This ratio is also found in acute human diabetes.

Sources of the Sugar.-Feeding with meat or gelatin increases the dextrose excretion, but leaves the ratio unchanged, showing that their conversion into sugar takes the ordinary course. Glycocoll, alanin, leucin, asparaginic acid and glutaminic acid are more or less completely converted into dextrose. The following are also converted and excreted as dextrose, and therefore raise the $\mathrm{D} \div \mathrm{N}$ quotient: levulose, lactose, d-lactic acid, glycerin, glyccinic acid, propyl alcohol. Feeding with fat, ethyl or butyl alcohol and tyrosin have no influence, so that no dextrose can be formed from these sources.

Total Metabolism. - The calories lost by the glycosuria are compensated by increased nitrogen metabolism, the fat metabolism remaining unchanged. In fasting animals the $\mathrm{N}$ excretion may be increased to four or five times above hunger alone (Reilly, Nolan and Lusk).

Acidosis.-In starving animals phlorhizin produces fatty changes in the liver, with increased excretion of ammonia, beta-oxybutyric acid and acetone substances (Mering). This is prevented if plenty of carbohydrates is given (Moritz and Prausnitz). It is increased by the ingestion of butyric acid or butter-fat. These changes are analogous to those caused by other agents producing inordinate protein metabolism.

\section{KIDNEYS}

There is no good evidence for or against the formation of an internal secretion by the kidney (Critical Review, Pearce, 1908). Injections of kidney extracts produce blood-pressure changes (Pearce) corresponding to the urine in them. Therapeutically, the administration of kidney preparations has been tried in chronic nephritis, without convincing results. A few apparently favorable cases are described by Formánek and Eiselt, 1907 .

Foster, I9I 5 , claims to have isolated a crystallizable toxic substance from the blood of uremic patients, which is not present in normal blood.

\section{RED BONE MARROW}

This contains lecithin, hemoglobin and other organic iron compounds. Glycerin extracts have been tried in anemias, to stimulate the production of corpuscles. The results are not convincing. The extracts also contain pressor and depressor principles (O. H. Brown and Guthrie, I905; Brown and Joseph, 1906).

\section{PRESSOR AND DEPRESSOR PRINCIPLES IN ANIMAL EXTRACTS}

All glandular organs, and perhaps all animal tissues, contain both pressor and depressor substances. The pressor principles predominate in saline extracts made at ordinary temperature, but are often destroyed by boiling. The depressor substances are soluble in saline solutions and in alcohol or ether. They are not identical with cholin (Swale 
Vincent and Sheen, 1903; Fawcett, Beebe, et al, 1915) and are probably moire nearly allied to the "peptone" and similar effects. Inorganic constituents may also play a part: expressed heart-juice, f.i., depresses cardiac activity, simply by its potassium content (Macleod, 1907).

Choroid Extract.-This produces a specific increase in the flow of cerebro-spinal fluid (Dixon and Halliburton, 1914).

\section{THYMUS}

As this gland is believed to have some relation to growtn and to the thyroid apparatus, it has been used, but with very doubtful results, in Graves' disease, rickets, tuberculosis, hemophilia, infantile atrophy and marasmus. Boiled extracts increase the milk secretion in goats (Ott and Scott, I912). Other lymph glands, spleen, etc., have also been employed in obscure conditions.

\section{NUCLEOPROTEINS, NUCLEINS, AND NUCLEIC ACIDS}

Chemistry.-These terms are often applied so loosely and incorrectly to the commercial produc s, that it may not be amiss to review briefly their meaning. Nucleoproteinswhich occur mainly in nuclear material-are natural compounds of nucleic acids and proteins. By peptic digestion or dilute acids, a fraction of the protein may be split off, resulting in modified nucleo-proteins, correspondingly richer in nucleic acid. These are termed mucleins. They contain about 5 per cent. of organic phosphorus. The remainder of the protein component can be removed by tryptic digestion or alkalies, yielding the true nucleic acids. These are definite chemic substances, their composition varying according to the tissues from which they have been obtained (as does also the protein constituent; so that the different products are not identical). Their composition approximates: $\mathrm{C}_{40} \mathrm{H}_{52} \quad \mathrm{~N}_{14} \mathrm{O}_{25} \mathrm{P}_{4}$; the atomic ratio of $\mathrm{P}: \mathrm{N}$ lying between $4: \mathrm{I}_{4}$ to 20. Commercial mucleic acids are often contaminated with more or less nuclein, as may be readily shown by the biuret or Millon's reactions. They are usually prepared from yeast, but also from thymus, pancreas, spleen, the sperm of certain fishes, and the embryo of grain. Nucleins and nucleic acids have fairly strongly acid characters. They are insoluble in water, but form soluble salts with dilute alkalies.

Digestion and Absorption.-Nucleic acid passes the stomach unchanged. It is partly split in the intestines, with the liberation of phosphorid acid, but not of purins. A further part is presumably split during absorption (bibliography, Forbes and Keith, I9I4).

Actions and Uses.-On hypodermic injection, they increase the number of all varieties of circulating leucocytes; and it is now claimed that they also raise the opsonic index. They have therefore been used in tuberculosis and other infectious diseases (Chantemesse, 1907), but their value is very problematical. They have even been administered by mouth. The benefits to be expected can be judged by the fact that 100 gm. of ordinary moist meat contains about $0.02 \mathrm{gm}$. of phosphorus (Kossel, 1882) corresponding to $0.2 \mathrm{gm}$. of pu:e nucleis acid; the same quantity of moist liver, spleen, or pancreas corresponds to about $2 \mathrm{gm}$. of the pure acid; whilst the oral dose of the impure nucleins is stated at only 0.06 to $0.3 \mathrm{gm}$. (Bibliography, Merck's Rep., I9I4, $27:$ I).

The nucleins are excreted as purin bases and uric acid; so that they are contraindicated in gouty and rheumatic conditions, etc. The phosphate elimination is also increased. The acceptable commercial preparations are listed in N.N.R.

\section{YEAST ("F EX MEDICINALIS")}

This has been recommended as a bactericide, etc., against furunculosis (by mouth and locally); in gastroenteritis ( 1 to $3 \mathrm{gm}$. p.d.); tonsillitis (10 to $20 \mathrm{gm}$. p.d.); gonorrheal vaginitis (Abraham, r9ro); in skin diseases, burns, etc. More extended observations are needed to establish its value. Tsuru, 1909, maintains that the bactericidal effects depend upon the products of fermentation, acids, alcohol, etc. Abraham, rgro, rejects this explanation.

Yeast Vitamin.-Ordinary yeast is a convenient source of "Vitamin," or of the "Water-soluble B" (McCollum, Simmonds and Pitz, I9I6). The preparation of a concentrated adsorption product is described by Seidell, 1916.

Yeast is efficient against beri-beri, and against experimental rice polyneuritis; but it has no effect on infantile scurvy (Hess, 1916). 


\section{SEXUAL GLANDS}

Specific internal secretions of these glands are concerned in the production of the "secondary" sexual characters; and in the cycles of menstruation, pregnancy and lactation. For many of these phenomena, however, the continuous functionation of the glands in situ is necessary; the therapeutic administration of the glands in substance or extracts has therefore been only of limited value.

\section{ORCHITIC EXTRACTS}

Brown-Sequard, 1889 , announced that hypodermic injections had remarkable stimulant qualities. Sensational exploitation brought his claims into rapid discredit, and the extract is now scarcely employed. The most marked effect is said to be an increase of $I_{5}$ to 20 per cent. in the capacity for voluntary muscular work (ergograph).

This action has been attributed by Poehl to the base spermin $\left(\mathrm{C}_{5} \mathrm{H}_{14} \mathrm{~N}_{2}\right)$. While this is especially abundant in this extract, it also exists in many other tissues. Its actions on the heart and circulation resemble those of cholin, which is also present in the orchitic extract. Its phosphate forms the "Charcot-Leyden" crystals found in sputa, etc. The action of these substances is supported by a nucleoprotein, which causes a slowed heart and fall of blood pressure through stimulation of the vagus center, and dilatation of the splanchnic vessels, also through the center. The orchitic extract applied to the isolated heart (Porter and Langendorff method) shows increase in force and frequency. The excised uterus shows slight stimulation. Prostate acts more energetically (Ott and Scott, I $9 \circ 9$ ). The metabolism is increased, but not as much as by ovarian extract.

\section{OVARIAN EXTRACTS}

This has been given for the relief of the symptoms which follow the natural or artificial menopause. It is difficult to say how far the rather inconstant benefits are psychic. The most favorable results have been reported in postoperative menopause in young women.

The so-called "endometritis glandularis" is really a menstrual exaggeration, probably of ovarian origin, due to an atypical course of the luteum production; most likely to occur at puberty and the climacteric (Wagner, I915).

Beneficial results are also claimed in some cases of amenorrhea with chlorosis. Experimentally, it has little effect on normal animals; but when oxidation has been depressed by castration, in bitches and somewhat in dogs, it is restored to the normal level or above by the administration of ovarian extract.

The average daily dose of the dried ovary (hog) is $0.5 \mathrm{gm} ., 71 / 2 \mathrm{gr}$.

\section{CORPUS LUTEUM (LUTEIN) ${ }^{1}$}

This is used similarly to Ovarian Extract (Fraenkel, I903; McDonald, I9ro; Burnam, I9I2) and is supposed to produce more certain effects.

The specific effects of the corpus luteum on the sexual cycle are not secured by extracts (L. Loeb, IgIo and I9II). Injections are slightly galactogogue in goats (Ott and Scott, I9IO; Schaefer and Mackenzie, I9II). They start hypertrophy of the mammary gland and lactation even in virgin rabbits (Ott and Scott, I9I4). Intravenous injection lowers the blood pressure (Villemin, r9ro).

The average daily dose of the dried substance is $0 . x \mathrm{gm} ., \mathrm{x} 1 / 2 \mathrm{gr}$.

Dannreuther, I9I4, uses the blood pressure as a guide: this should fall, not to exceed $15 \mathrm{~mm}$. This usually requires $0.3 \mathrm{gm}$. of the dried substance, three times daily. He believes that the corpora of pregnant ovaries are much more effective; but McCord, I9I4, claims that under slaughter-house conditions, at least 90 per cent. of the weight of indiscriminately collected material would be from pregnant animals.

1 This name is peculiarly unfortunate, since it had previously been applied to the yellow coloring matter of eggs. 


\section{MAMMARY GLAND}

This has been used against excessive menstruation (daily dose, 1 gm., 15 gr., of the dried gland). Extracts increase the movements and the tone of the excised uterus (Ott and Scott, I909) and are somewhat galactagogue, but not in human (Schaefer and Mackenzie, I910).

\section{ERGOT}

General Statement.-Ergot (Secale cornutum) is a fungus (Claviceps purpurea) growing in the grain of cereals, the official being obtained from rye. The fungus goes through several stages of development, the resting stage (sclerotium) constituting the drug. It is used mainly to contract the uterus (by peripheral action) and thus check postpartum hemorrhage. It also stimulates other forms of unstriped muscle, especially the intestine and blood vessels. The consumption of grain infested with ergot leads to gangrene and spasmodic effects. Epidemics of this "ergotism" were formerly frequent and must have occurred in antiquity, although the causal connection was not recognized. It is now very rare in civilized countries, the ergot being removed by the modern methods of treating the grain. The uterine effect was learned from midwives and is first mentioned by Lonicer, $\mathrm{I}_{5} 65$. Its use in regular medicine dates practically from Stearns, I807 (N. Y. Med. Repository).

Active Constituents. - The composition of ergot is complex; several active constituents have now been isolated in pure form. The most important are the alkaloid Ergotoxin, several aromatic amins, especially Tyramin and Histamin, and cholin derivatives. The numerous names applied to these and to impure mixtures render the literature very confusing.

Ergotoxin or Hydroergotinin, $\mathrm{C}_{35} \mathrm{H}_{41} \mathrm{~N}_{5} \mathrm{O}_{6}$, an amorphous alkaloid occurring in alcoholic extracts. It is responsible for the characteristic action on the cock's comb and is concerned in the uterine and vascular effects. It is rather unstable and by loss of $\mathrm{H}_{2} \mathrm{O}$ changes into its lacton: Ergotinin or Ergotin, $\mathrm{C}_{35} \mathrm{H}_{39} \mathrm{~N}_{5} \mathrm{O}_{5}$, a crystalline alkaloid with very weak actions.

Both alkaloids exist ready-formed in ergot, but can be easily converted into each other by chemical means (Barger and Ewins, rgro). Both are insoluble in water and petroleum ether, sparingly soluble in ether, and readliy soluble in most other organic solvents. Ergotoxin is easily soluble in cold alcohol; ergotinin but sparingly. They dissolve in dilute $\mathrm{NaOH}$, but the ergotinin is partly converted. Their salts form colloidal solutions with water, but these are precipitated by electrolytes (salts or mineral acids).

Tyramin, Ergotamin, uteramin, or para-hydroxyphenylethylamin: this is closely related to epinephrin in structure and action. It is mainly responsible for the pressor effect, and is not concerned in the uterine action.

Histamin, Ergotidin, Ergamin, beta-iminazolyl-ethylamin, 4-betaaminoethylglyoxalin: lowers the blood pressure, and stimulates the excised uterus powerfully.

A number of other aromatic amins occur casually; such as agmatin, guanidobutylamin (Engeland and Kutscher, 1910), which has a weak stimulant action on the excised uterus; isoamylamin, etc. 
These aromatic amins are also produced in the putrefaction of meat, and in the intestinal tract, and have been prepared synthetically. They are generally insoluble in ether or chloroform, readily soluble in water; precipitated by phosphotungstic acid. Chemically, they are derived from the amido-acids by the elimination of $\mathrm{CO}_{2}$.

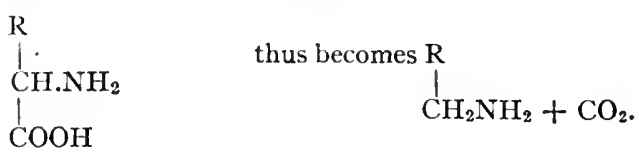

In this way, Tyramin is prepared from Tyrosin; Histamin from Histidin; Agmatin from Arginin; Isoamylamin from Leucin. An authorative discussion is contained in Barger's "Simpler Natural Bases," I 9I4.

Acetyl Cholin.-This was found in ergot by Ewins, I9I4. It is responsible for the cardiac inhibition.

Investigations of the Composition of Ergot.-A large number of constituents has been described in ergot: Alkaloids, amins, resinous acids, glucosids, betain, and cholin, fat (35 per cent.), some peculiar sugars, coloring matter, etc. The red pigment (Sclererythrin) is important for discovering the presence of ergot in bread.

There has been much animated discussion as to the rôle of the several constituents in the actions of the drug, and the matter is not yet altogether decided. . The confusion of the older literature is explained partly by the instability of the principles, but mainly by the difficulty of isolating them pure. The impurities alter their characters suffciently so that successive investigators have repeatedly described the same principle, somewhat differently contaminated, as different substances. Physiologic actions have been ascribed to substances which these owed solely to their contaminations.

The modern conceptions date mainly from the work of Barger, Carr and Dale, I906 and 1907 , on the isolation of the ergot alkaloids. Their papers also give the historical details. By the discovery of the crystalline phosphate of ergotoxin, they succeeded for the first time in preparing this principle uncontaminated; and in determining its properties and its relation to ergotinin, which Tanret had isolated in 1875 . Kraft, I906, arrived independently at the same results. Dale found that the ergotoxin produces the characteristic effe:ts of ergot. Barger and Dale also showed that the alkaloidal and resinous principles (sphacelinic acid, sphacelotoxin, etc.) isolated by earlier investigators, contain large proportions of this alkaloid; and that their apparent activity can be fairly attributed to the latter. On the other hand, they pointed out that the methods of preparing extracts of ergot tend to eliminate this alkaloid; and that it is therefore necessary to assume the presence of other, water-soluble principles. In I909, Barger, Dale and Dixon found these to consist of tyramin and histamin (I910).

Impure Principles.-These are still important for understanding the older literature They are classified by Barger and Dale as follows, in chronological order:

Wenzell, I864: Ecbolline and Ergotine $=$ mixtures of the alkaloids, containing cholin (Meulenhoff, I899).

Tanret, I875: Amorphous Ergotinine = impure mixture of ergotinin and ergotoxin. Dragendorff, 1877 : Picrosclerotine $=$ ergotinin, possibly mixed with some ergotoxin. Podwyssotski, I883: Sclerocrystalline $=$ ergotinin.

Kobert, r 884: Sphacelinic Acid = inactive resin with adherent alkaloids.

Cormutin = alkaloidal resin, probably containing some ergotoxin, and perhaps some active alkaloidal decomposition products.

Ergotinic Acid = a sapotoxin.

Keller, I 896: Cornufin = impure mixture of ergotinin with ergotoxin.

Jacobj, r 897: Chrysotoxin = inactive yellow coloring-matter with small proportion of adherent alkaloid.

Secalintoxin $=$ mixture of ergotinin and ergotoxin.

Sphacelotoxin $=$ impure ergotoxin.

Secalin = impure ergotinin.

Kraft, I906: Hydroergotinin = synonym for ergotoxin.

Vahlen, 1906 and 1908: Clavin = inactive mixture, consisting of nearly equal parts of leucin, isoleucin and valin (Van Slyke, I909).

Marino-Zuco and Pasquero, I9 I : Clavisepsin; claimed to be an active glucosid; not yet confirmed. 
Ergotin is a name applied to a variety of pharmaceutic extracts, generally prepared in such a way that they must contain mainly the amins, and relatively little of the alkaloids. A number are listed in N.N.R.

\section{ACTIONS OF ERGOT}

The effects of the various ergot constituents are so much modified when they are present together, that it is advisable to describe first the actions produced by ergot itself.

Uterine Actions.- The most important use of ergot is in obstetrics. Ergot preparations stimulate the uterus, especially when pregnant. The clinical effects are well described by Sharp, igri. In labor, the ordinary doses improve the uterine tone, and collect small irregular and noneffective pains into larger effective contractions. Small doses may inhibit the contractions. Excessive doses produce prolonged tonic constriction.

When proper doses are used during labor, with the os fully dilated and no obstruction, they tend to hasten normal delivery. Really effective doses, however, are rather dangerous to mother and child. The firm contraction may hinder delivery, and compress and asphyxiate the fetus; even rupture of the uterus has been reported; but these accidents are very rare.

The most common use is after the delivery, to hasten and sustain the normal retraction; thus obliterating the open sinuses, and checking postpartum hemorrhage. In early pregnancy, its effects are much less certain. Contrary to popular opinion it is not abortifacient unless dangerous doses are long continued.

The uterine effects are due to ergotoxin and histamin. Ergotoxin has been used therapeutically and acts more promptly (four minutes after hypodermic injection, Sharp). Pituitrin is now being employed considerably. Tyramin has been tried clinically with varying results; it is not effective in animal experiments.

Ergot has also been used in other forms of uterine hemorrhage and in chronic metritis.

Administration.-The usual dosage is 2 to 5 c.c., $1 / 2$ to I 3 of the Fluidextract, or the equivalent of the solid extract, which is eight times as strong. They may also be used hypodermically or intramuscularly. The response to ergot is quite prompt even when taken by mouth; it appears in fifteen to thirty minutes and lasts half an hour (Brady; I9IO). Opium delays but prolongs the effects; moderate chloroform anesthesia does not modify them (Sharp).

Mechanism of Uterine Action.-The action is peripheral, since it occurs also in the excised uterus (Kurdinowski, I006; Fellner, r906; human, Ruebsamen and Kligermann, rgr2). Moderate concentrations increase the tone and the automatic contraction; high concentrations produce tetanus of the uterus. (François, I9I2, cautions about the interpretation of the phenomena.)

La Torre, I I I 2, found interesting histologic changes in the uteri of dogs treated with ergot. They consist in contraction of all the muscular uterine layers and of the arterial muscle; being especially marked in pregnancy when the muscle is hypertrophied. The retraction of the muscle is then so great that muscle seems almost absent. The smaller vessels going through the muscular layer to the mucosa are greatly compressed, whilst the larger vessels are dilated. Paradoxically, it appears as if the volume of the muscle were diminished, and the lumen of the uterus increased. The interpretation is difficult. 
Blood Pressure.-This is practically unchanged, in man (Sharp, I9 I I), or animals, unless the ergot is injected into a vein. This produces considerable, but inconstant and complex changes. The variability is largely explained by the conflicting actions of the different constituents, and by dosage and other conditions.

Sometimes there is a large but brief fall from direct cardiac depression, followed by a. slight but prolonged rise from direct cardiac and vascular stimulation (Sollmann and Brown, I905). The fall is presumably due to histamin and acetyl-cholin; the rise to ergotoxin and tyramin. Other experiments show very little fall and somewhat greater rise (Cronyn and Henderson, 1909; Edmunds and Hale, I9rr). This rise is greater if the vasomotor center has been destroyed, and is therefore due to a direct peripheral action on the vessels (Jacobj, r897; Wood and Hofer, r9ro). Excised arteries (except the pulmonary) are also constricted (Cow, IgII). The vasoconstriction occurs also in calcium-free solutions (Pearce, ror 3 ), and is due to ergotoxin and tyramin. The vasomotor center is not affected by ergot or ergotoxin (Pilcher and Sollmann, r9i5).

The pressure in the pulmonary artery is increased, whether the carotid pressure is rising or falling (Wood, I9II). Mellin, r904, Plumier, r905, and Anderes, I9I 5, attribute the rise to constriction of the pulmonary vessels; Dixon to increased output of the right ventricle. Wiggers, Igr I, showed that ergotoxin produces both effects.

Very large doses of ergot paralyze the vagus center, and the vasomotor endings (epinephrin becoming ineffective, whilst barium is still active) (Sollmann and Brown, 1905). This "vasomotor reversal" is due solely to the ergotoxin (Dale, r905).

Ergot as General Styptic.-The efficiency of ergot in postpartum hemorrhage, and the vasoconstriction observed in animal experiments, have led to its use against hemorrhage in other situations. However, the conditions here are altogether different; for, as ordinarily administered, it does not affect the blood vessels or blood pressure. Its use is therefore irrational (Bradford and Dean, I894). It is also useless in pulmonary hemorrhage (Frey, 1909). It has been praised rather uncritically in a great variety of other conditions, which it would be useless to enumerate.

Cyanosis of the Comb of the Fowl.-When ergot preparations are administered to roosters, in about an hour the tips of the comb and wattles. become blue and cold, the effect lasting several hours. It may be preceded by temporary blanching. This reaction is used for testing the quality of ergot (see below). It is due to ergotoxin; histamin produces. a similar but much prompter effect (Crawford, I9r3); tyramin is ineffective. The mechanism of this cyanosis is not understood.

It has been attributed to vasoconstriction; but the cyanosis does not occur after other vasoconstrictor drugs; whilst it is produced by paraldehyd which lowers blood pressure (Crawford), by cantharidin (Ellinger, 1908) which injures capillaries; and by other drugs which cause vascular injury (large hypodermic doses of cane-sugar, piperazin, chromic acid, etc.).

Gangrene Action.-This is a characteristic feature of chronic ergot poisoning in man and in many animals. It is most readily studied in roosters.

If ergot is given repeatedly to roosters, the bluing persists in the tips of the comb, and gradually involves the entire comb and the wattles. Eventually, these appendages undergo gangrene and may drop off. The extremities may also be involved; in one experiment, the entire wing fell off after a few days.

Histologic Changes.- - v. Recklimghausen, 1883 , showed that the larger arteries, but not the veins, of the gangrened comb are occluded by hyaline thrombi. The capillaries are congested with red corpuscles. These results were confirmed by Gruenfeld, 1890. They are generally referred to very violent constriction of the small arteries, interrupting the blood stream. This explanation is entirely hypothetical. Krysynski observed formation of thrombi in a variety of conditions; and it is probably a result of a toxic effect on vascular endothelium. No histologic changes are found in the acute cyanosis. 
Susceptibility of Different Animals to Gangrene.-Pigs are quite susceptible to the gangrene action of ergot. They react especially by pustular eruptions on the skin; the ears are particularly susceptible. IIorses and cattle show similar changes. Man is quite subject to the gangrene, which begins in the extremities. The fingers, toes, or an entire member may slough off. Dogs and rabbits do not seem to be susceptible to this action.

The cause of the varying susceptibility has not been demonstrated. It may be due to physiologic peculiarities, or merely to anatomic differences. The gangrene always begins in those situations in which the circulation is weakest. It differs in no essential from gangrene produced by any other cause. It may be dry or wet, according to whether liquefying bacteria are absent or present. Salant and Hecht, I9I4, describe a habituation tolerance to the action of ergot on the cock's comb and to the fatal dose.

Alimentary Canal.-Injection of ergot or ergotoxin (10 mg. for rabbits, intravenously) results in marked increase of peristalsis through the whole alimentary tract; and in increased intestinal motor response to vagus stimulation (Meltzer and Githens, I9 I6). This produces violent diarrhea and vomiting. The emesis, however, is largely central (Eggleston and Hatcher, 1915).

In chronic poisoning, these direct effects are aggravated by vascular stasis, advanced cases showing ecchymosis, ulcer formation, etc. The ulcers involve particularly the lymph follicles, in which the blood supply is poorest. The stasis may lead to eff usion of blood into the lumen of the intestine. The appetite is lost. This action on the alimentary canal is also most marked in the animals mentioned, but occurs in others. The liver of roosters shows peculiar degenerations, resembling amyloid.

Isolated Bronchial Muscle.-Ergot has no effect (Titone, I9I3).

Central Nervous System. - This is affected both directly and indirectly, and the effects are correspondingly variable. With moderate doses, the flow through the cerebral vessels is increased. Small doses may cause medullary stimulation (vagus slowing; increased respiration, convulsions, etc.). Large doses of ergot cause death by medullary paralysis. It has a narcotic effect for roosters.

\section{ACTIONS OF PURE ERGOTOXIN}

This is concerned in the stimulation of the uterus; the vasoconstriction; the bronchial constriction; the intestinal irritation; the gangrene; and the vasomotor reversal. It produces a powerful and prolonged peripheral stimulation of smooth muscle, particularly in the arteries and uterus. Large doses paralyze the augmentary sympathetic endings (myoneural junction) which are stimulated by epinephrin (Dale, I905, I906 and I9I3). Very massive doses paralyze the muscle.

Human Uterus.-This is contracted, but requires higher concentrations than can be used clinically (Lieb, I9I5); Kehrer, I9II, found I or $2 \mathrm{mg}$. hypodermically quite ineffective. Sharp, rgro, however, claims that $0.6 \mathrm{mg}$. contracts the uterus more promptly and powerfully than ergot extracts, but that the action is too brief.

Vascular Effects.-The rise of blood pressure with ergotoxin is purely vascular (diminished organ volume). The constrictor action is exerted directly on the arteries, for it occurs after the medulla has been divided, and the vascular ganglia excised or paralyzed by nicotin.

If now, during this rise, the vasomotor nerves be stimulated-either electrically, or by epinephrin or nicotin-a fall of pressure results, instead of the customary rise. The ergotoxin has therefore paralyzed the peripheral sympathetic vasoconstrictor mechanism, leaving the dilator mechanism active. This vasomotor reversal is obtained in pithed cats by $0.5 \mathrm{mg}$. per kilogram, intravenously. Since ergot itself and barium are still capable of producing a vasoconstriction, the arterial muscle can not be paralyzed; and the constrictor paralysis would be referred to the myoneural junction. This also proves that the constrictor action of ergotoxin is more peripheral, i.e., directly on the muscle fiber. Very large doses finally paralyze this also. The antagonism of ergotoxin and epinephrin can also be demonstrated on excised arteries (Macht, 1915). Other actions of epinephrin, f.i., the fever, are not antagonized (Cloetta and Waser, 1915).

Cardiac Effects.- These are slight. In the excised mammalian heart, Wiggers, I9I r, observed diminished systolic contraction and increased tone with decreased amplitude and output. In intact animals, the heart is mainly affected indirectly by the vascular changes; the amplitude being first diminished and then increased.

Bronchioles.-These are scarcely affected (D. E. Jackson, r9r4). 
Ureter.-Ergotoxin inhibits or reverses the epinephrin stimulation, but does not interfere with the parasympathetic stimulants (Macht, r916).

Sugar.-Ergotoxin arrests the epinephrin and theobromin glycosurias, diminishing the hyperglycemia, and rendering the kidneys less permeable to sugar; the permeability to chlorids is not affected. The epincphrin diuresis is somewhat decreased by it, but not the theobromin diuresis (Miculicich, I9r2).

\section{HISTAMIN}

This is concerned in the uterine effect (stimulation) of ergot, especially with aqueous extracts; in the cyanosis of the fowl; and in the fall of blood pressure observed with some preparations. Its main effect is a peripheral (direct) stimulation of the rhythm or tone of smooth muscle, varying quantitatively in different organs. The phenomena resemble those of peptone, anaphylaxis and pituitrin.

Structure.-Its derivation from histidin is shown by the formulas,
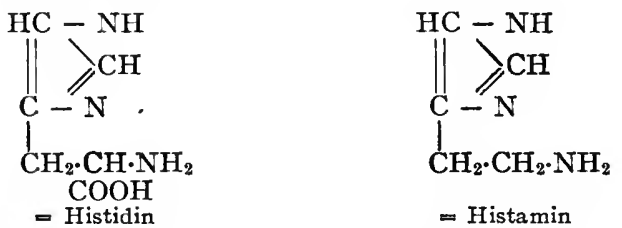

General Action.-The stimulation is most marked in the uterus; the bronchioles are also highly sensitive; the intestine, arteries and spleen are less susceptible; the bladder and iris do not respond. The action is directly on the muscle (Quagliariello, r9r4). Cardiac muscle is mildly stimulated; skeletal muscle is not affected. It has a weak pilocarpin action on glands (Barger and Dale, rgro; Dale and Laidlaw, I9ıо; Kutcher, I9I0; Ackermann, I910).

Uterus.-This is contracted in situ in virgin guinea pigs and non-gravid cats. It increases especially the tonus of the excised uterus of cats, rabbits and guinea pigs (Barger and Dale, I910); but relaxes that of rats (Fuehner, Igr3). The experiments of Kehrer, 1910, and Guggenheim, 1914, indicate that the tone of the human uterus is also increased; but it would have to be used cautiously on account of its toxic effects (respiratory disturbances and convulsions). C. Koch, I9I3, reports successful results in uterine inertia from the intramuscular injection of I mg.; Jaeger, I913, found $8 \mathrm{mg}$. necessary. Even I mg. produces some unpleasant side actions; with effective doses there may be headache and violent vomiting. Its use is therefore not advisable. It has little effect by mouth.

Toleration.-As with muscarin, histamin produces its effects only when a given concentration is applied suddenly; if it is added very slowly ( $f . i$., to the excised uterus), it is inactive. Again, a given concentration soon loses its effect, but raising the concentration again induces contractions. Similarly, rapid intravenous injections are much more toxic than slow injections (Oehme, I9r3).

Blood Vessels.- - Histamin raises the blood pressure in rabbits and guinea pigs, but lowers it in dogs, cats, monkeys and fowls. The cause of these changes is obscure, but probably analogous to the phenomena of anaphylaxis (see Index). The vasomotor center is not affected; but the fall of blood pressure and the respiratory disturbances usually produce moderate stimulation (Pilcher and Sollmann, 1915). Excised arteries are contracted, even those of dogs and cats, which respond by fall of blood pressure. The constriction is shown not only by the systemic arteries, but also by the coronary (Barbour, I913) and pulmonary (Berezin, I914; Cloetta and Anderes, 1914; Anderes and Cloetta, I9I6); but not the liver veins (Berezin, I914). The constriction of the lung vessels is removed by atropin (Berezin). Living animals show increased pressure in the pulmonary artery, with diminished volume of the lung, and usually unchanged pressure in the systemic arteries (Cloetta and Anderes). This indicates constriction of the pulmonary vessels (Anderes, 1915). Baehr and Pick, I913, found no effect on the vessels of perfused lung.

The paradoxical behavior of the blood pressure and excised arteries has not been explained (Dale and Laidlaw, I9Io and IgIr). Similar inconsistencies occur in the blood-pressure changes of peptone injection and anaphylaxis (which see). The fall is probably a part of the general collapse, with accumulation of the blood in the large 
veins of the liver (Pearce and Eisenbrey; Edmunds, r913). Mautner and Pick, 1915, consider the lung and liver vessels as unimportant elements in the "shock," since their reaction varies in different species. They attribute the fall of pressure to extreme spasmodic contraction of the intestinal arterioles, resulting in impeded return of the blood to the heart.

Heart.-The isolated frog's heart is slowed; the perfused mammalian heart shows a short and slight slowing, followed by considerable quickening with increased excursions (Eines, 19r3).

Coagulability of the Blood.-This is not affected, in distinction to peptone (Dale and Laidlaw).

Cerebrospinal Fluid.-This is under higher tension, due simply to the asphyxial effect of the bronchoconstriction (Dixon and Halliburton, Igr4).

Bronchioles. - These are strongly constricted by peripheral stimulation (D. E. Jackson, I914), so that the lungs bloat as in anaphylaxis (Baehr and Pick, I913).

Intestines.-Histamin produces intense stimulation. This is intensified by calcium salts (Vanysek, 1914).

Skin.-Applied to the scarified skin, a few drops of $r: 1, \infty 00$ histamin produces a local pallor, then a spreading urticaria and ery thema (Eppinger, 1913). The urticaria is also obtained by the hypodermic injection of 0.5 to $\mathrm{r}$ c.c. of $\mathrm{I}: \mathrm{z}, 000$ solution. A very similar reaction is produced by morphin; tyramin is ineffective (Sollmann and Pilcher, I9I6).

\section{TYRAMIN}

This is concerned in the vasoconstriction but probably not in the other effects of ergot. It stimulates the sympathetic (Quagliariello, I9I4) very similarly to epinephrin, except that its effects are more prolonged.

Chemistry.-Its relation to tyrosin and epinephrin are shown by the formulas:

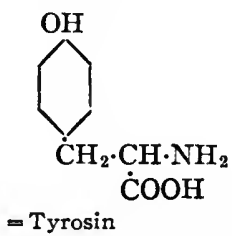

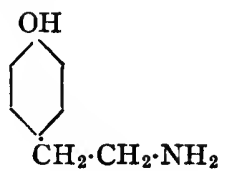

$=$ Tyramin

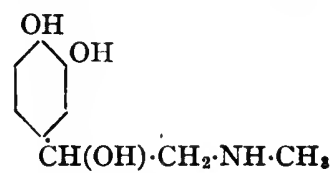

$=$ Epinephrin

It occurs commonly in the products of putrefaction, autolysis, and fermentation; and must therefore be present in the alimentary tract. It was isolated from ergot, and prepared synthetically from hordenin (its dimethyl derivative) by Barger, r 909 . Further syntheses were made by Barger and Wampole, rgro. It occurs also in the common American and European mistletoes (Phoradendron flavescens and Viscum album). It has not been demonstrated in other species of Phoradendron (Crawford, 1914; Ostenberg, r915). It constitutes the "salivary" poison of certain cephalopods (Henge, roI3). In the body a part is converted into para-hydroxyphenylacetic acid. This change is also effected by surviving liver and uterus; it is completely destroyed by the isolated heart (Ewins and Laidlaw, 1910).

Actions.-The resemblance to epinephrin was shown by Dale and Dixon, rgog. It contracts certain smooth muscle-arteries (also the excised coronary, Barbour, $19 \mathrm{r}_{3}$ ), spleen, frog's stomach, rabbit's uterus, gravid uterus of cat; and bronchioles (Baehr and Pick, I9r3; Golla and Symes, r9r3). It inhibits others-mammalian intestine, non-gravid uterus of cat, etc. (Knaff-Lenz and Pick). The intestines are stimulated in rabbits; relaxed in dogs; first stimulated and then relaxed in cats (Vanysek, r914). There is still considerable confusion as to the uterine action (Barger and Dale, rgro, find no effect; Lieb, 1914, some stimulation); and as to whether the effects are central or peripheral (Lenz and Pick). The blood pressure rises and the heart is slowed reflexly. The rise lasts two or three minutes. It is purely vascular (diminished kidney and intestinal volume and decreased vein flow; Bickel and Pawlow, 1912). It does not blue the comb of the rooster acutely (Crawford, r913); nor produce gangrene on repeated injection (Bickel and Pawlow). Harvey, rgrr, asserts that long-continued administration to rabbits produces arterial sclerosis and chronic nephritis. The cerebro-spinal pressure varies with the circulatory changes (Dixon and Halliburton, 19r3, 1914).

Tyramin antagonizes the respiratory depression, but not the other effects, of morphin. In man, $16 \mathrm{mg}$. of morphin require 40 to $50 \mathrm{mg}$. of tyramin (Barbour, Maurer and Glahn, 1916). 
Therapeutic Uses.-Tyramin (N.N.R.) has been tried 5 to $20 \mathrm{mg}$. $(1 / 12$ to $1 / 3 \mathrm{gr}$. hypodermically) against collapsc conditions. Oral administration in man raises the blood pressure very little (Sharp, I9II); but with hypodermic injections of 20 to 40 mg. the rise is considerable and prolonged (A. Clark, I910; Hoyt, I912). Haimann, 1912, regards it as a complete substitute for crgot in labor; this would not be expected from the cxperimental data and is denied by Guggenheim, I9 I 2; Sharp, I9II, and Kehrer. It is certainly not a substitute for ergot. Bickel and Pawlow suggest that it might lessen postpartum hemorrhage by vasoconstriction.

It is not an effective local hemostatic.

\section{PHENYLETHYLAMIN}

This occurs in putrid meat. Its formula, $\mathrm{C}_{6} \mathrm{H}_{5} \cdot \mathrm{C}_{2} \mathrm{H}_{4} \mathrm{NH}_{2}$, is related to epinephrin. Small doses stimulate the cardiac muscle, large doses depress. All doses contract the coronary vessels; this is followed by relaxation with large doses (Barbour and Frankel, 1915).

Barbour, I9I6, has made a comparative study of the sympathomimetic and other actions of a series of phenylethylamin derivatives.

\section{INDOLETHYLAMIN}

This stimulates plain muscles, especially the arteries, iris and uterus. It also has stimulant central effects (Laidlaw, I9II). Perfusion through excised liver converts it into indolacetic acid. Dogs excrete 30 per cent. as indolaceturic acid (Ewins and Laidlaw, I9I3).

\section{PARAPHENYLENDIAMIN}

This is extensively used for dyeing brown and black furs. The wearing of these often causes dermatitis, probably by the formation of an irritant intermediate oxidation product, such as chinon-dichlor-diimin (Olson, I 9 I6).

Hypodermic injection of $75 \mathrm{mg}$. per kilogram body weight produces chemosis, conjunctival irritation, increased ocular pressure and exophthalmos. The effects occur when the cervical sympathetic has been divided, and are probably caused by circulatory disturbances (Troell, IgI6).

\section{ERGOTINIC ACID}

This has a typical sapotoxin action. Taken by the mouth it produces no effects beyond local irritation, as sapotoxins are not absorbed. If injected subcutaneously or intravenously, it will produce paralysis of the central nervous system and of protoplasm generally. It causes a peripheral paralysis of blood vessels, and thereby lowers the blood pressure.

\section{HORDENIN}

This is an alkaloidal amin derivative ( $p$-hydroxy-phenylethyl-dimethylamin) obtained by Leger, 1906, from germinated barley. Its actions were studied especially by Camus, 1906. Small doses stimulate the vagus. Larger doses paralyze the vagus center, quickening the heart and raising the blood pressure. The bronchioles are dilated by stimulation of the sympathetic endings, sufficiently powerful to antagonize most constrictor drugs (D. E. Jackson, 1914). Fatal doses paralyze respiration. The substance is not very toxic. It has been used $(0.5 \mathrm{Gm}$. per day) in heart disease and digestive derangements (Sarbazes and Guerine, 1909). It arrests diarrhea in dogs (30 to $40 \mathrm{mg}$. per kg., hypodermically; Charmoy, I9I 2).

\section{PTOMAIN POISONING}

The cases of poisoning observed as a result of partaking of more or less tainted articles of fpod-sausages (botulismus and allantiasis), meat, milk, ice-cream, cheese, corned beef, etc., and with some specimens of mussels and oysters-are due to the development of ptomain products. In the former cases these are developed by putrefaction; in the latter, probably by disease.

Many of these ptomains have been isolated in crystalline form, and are well-defined compounds belonging to the amin series (Barger and Walpole, 1909; Barger and Dale, 1909; Dixon, 1913; Hanzlik, I916). Their pharmacologic actions are on the autonomic system (Blankenhorn, Harmon and Hanzlik, I9I6).

Uremic Bases.-Foster, 1915 , reports that the methods of isolating organic bases, when applied to uremic blood, yields toxic substances that are not present in normal blood. 


\section{ACUTE ERGOT POISONING}

This is rare in man, but may occur by attempts at abortion. The symptoms are vomiting, diarrhea, vertigo and confusion, precordial distress, dyspnea, muscular pains, paresthesias and anesthesias, disturbances of vision and speech, epileptoid convulsions, small pulse, loss of consciousness. In pregnancy, uterine hemorrhage and abortion of the dead fetus are frequent. Recovery af ter severe poisoning may take several days.

Rosenbloom and Schildecker, I9I4, have isolated ergotinin crystals from a patient dead of acute ergot poisoning.

\section{CHRONIC ERGOT POISONING, "ERGOTISM"}

This is now mainly of historic interest. The initial symptoms are fairly uniform but these develop into two distinct types, the gangrenous and convulsive. These are so dissimilar that they were long classed as separate diseases. Toward the end the symptoms become similar.

Historical.-Ergotism was formerly very frequent endemically in consequence of the presence of ergot in flour, and the population of large tracts of country have been poisoned. The last large epidemic in the United States occurred in New York in I 825 . Since the cause has been recognized these epidemics have been less frequent, but in Russia they still play quite an important rôle (Schmack, I897). A comprehensive discussion of the history of ergot is given by Kobert, I889.

Symptoms.-The initial stage is ushered in by disiurbance of the peripheral sensory apparatus. There is formication in the skin and various other disturbances of cutaneous sensation. Hyperesthesia and anesthesia exist at the same time in different parts, or even in the same part, the skin being hyperesthetic to some forms of stimulation and anesthetic to other forms.

These disturbances of sensation begin at the extremities and spread upward. This distribution favors the view that they are manifestations of changes in the circulation, since these would make themselves felt first of all in the extremities. The disturbance in sensations also involves the alimentary canal. There is apt to be at once voracious hunger and loss of appetite. Digestion is much impaired on account of the disturbed circulation. Diarrhea and vomiting are very common. The vomiting is partly due to the disturbed circulation and partly to the action on the center. The central sensory apparatus also shows changes at this time. There is violent and persistent headache and central disturbances of the special senses. The motor system also begins to show abnormal symptoms, such as twitchings and tremors, most marked in the extremities and in the tongue.

In all these effects of ergot it is extremely difficult to say to what extent the phenomena are caused by the central, and to what extent by peripheral, actions. They are probably largely dependent on disturbances in circulation, and these may in some cases be more prominent in the central nervous system; in others, peripherally.

At this point the sensory symptoms have reached their acme, and do not become any worse, but persist as they have been described. But the motor phenomena go on increasing; the twitchings pass into spasms, and then into permanent and often very painful contractures. Always beginning at the extremities, they involve the terminal phalanges of the fingers and ascend to the other joints. The facial muscles also participate. The type of these contractures shows that their origin is central. They are not absolutely persistent, but last for about half an hour; then pass off for a time, and reappear. This is very different from spasms of peripheral origin, such as those of lead-poisoning. The smooth muscles may also participate in these contractures; especially that of the bladder; so that there may be involuntary evacuation of the urine, tenesmus, etc.

The pulse is always hard and small, pointing to a high blood pressure. Its frequency, however, is variable. It is usually slow, due probably largely to the high pressure.

So far the symptoms, the initial stage, are common to all the different forms of ergotism; they can be accounted for partly by the changes in the circulation and partly by the direct action of the ergot principles themselves. In the second stage the circulatory disturbances become more marked. The phenomena already seen-the disturbances of sensation and the contractures-persist; but to these are added secondary effects due to the prolonged slowing of the circulation. These may be most marked in the central nervous system or in the extremities. The former give rise to the so-called spasmodic form of ergotism. The predominance of stasis in the extremities produces the gangrenous form. 
Why one action should predominate in some individuals, and another action in others, cannot be explained. Perhaps there may be some differences in the anatomic arrangement of the blood vessels or extent of the innervation.

(A) The gangrenous form may have been indicated somewhat earlier. Pustules may have formed in the skin, which are due to this defect of circulation. In more marked degrees it affects the extremities. The entire member may be involved in the gangrene, which differs in no respect from any other gangrene; it has its line of demarcation, and may be wet or dry according to bacterial infection. The finger, toe, or limb may slough away without bleeding.

(B) In the spasmodic form the contractures pass into tonic and clonic convulsions or epileptiform spasms. Since any part of the central nervous system may be affected, the exact symptoms may be extremely variable. It may be repeated that they are due to a defective circulation in the central nervous system, which leads to stimulation and then to paralysis. Both forms, the gangrenous as well as the spasmodic, are therefore due to changes in circulation, and are determined by the ergotoxin.

The treatment of ergot poisoning can be only symptomatic.

\section{DETERIORATION}

The ergot alkaloids and amins are rather unstable substances; and it has long been observed that ergot loses its activity with age. Ergotism is said to have occurred only during the first four months after harvest (Poulsson). Many pharmacopeias therefore direct that ergot must not be older than one year; but the fluidextracts on which the reputation of ergot is mainly based, have probably all been aged for a year before being sold (Eldred, I9I3). The alcoholic preparations probably keep better, but no exact data are available. The activity of ergot should therefore be ascertained, at least qualitatively, by bio-assay.

Using the cock's-comb test, Haskell and Eckler, I9r2, found that the activity of the fluidextract remained practically unchanged for two and one-half years; then deteriorates gradually, so that it has fallen to about one-half in three and one-half years. Special proprietary preparations are very unreliable.

\section{BIO-ASSAY}

The complex composition of ergot makes chemical assay practically out of the question. The same objection, but to a lesser degree, holds for the bio-assay (Edmunds and Hale, IgII). The ideal would be to measure the therapeutically desirable effects; but it is not certain that any of the present methods accomplish this result. The cock's-comb test is the most widely employed and probably the most satisfactory. This reaction was described by Lorinser, I824; used experimentally by Gruenfeld, I802 and I 895, under Kobert; and applied commercially by Houghton, I898. The details are described by Edmunds and Hale, IgII, and in U.S.P. IX. It estimates mainly the ergotoxin, but is also affected by the histamin. Its results agree very closely with the more complex uterine method of Edmunds, I908. Another uterine method is described by F. E. Stewart and Pittenger, I9r3. Dixon, I906, and Wood and Hofer, I9 10, propose the blood-pressure rise as a test; but this gives conflicting results, as might be expected from the opposing actions of the constituents.

\section{PREPARATIONS-ERGOT}

Ergota, U.S.P., B.P.; Ergot ${ }^{1}$ (Secale cornutum).-A fungous growth, Claviceps purpurea, from rye. Generally collected in Russia or Spain. Should not be older than one year.-Dose, 2 Gm., 30 gr., U.S.P.; I to 4 Gm., I 5 to 60 gr., B.P.

Ext. Ergot., U.S.P. - A pilular extract, prepared by defatting ergot with benzin, extracting with acidulated alcohol, and evaporating. Dose, 0.25 Gm., 4 gr., U.S.P

Ext. Ergot., B.P.; Ext. Ergot. Purif. (Ergotin Bonjean).-A soft watery extract, purified by partial precipitation with alcohol. Dose, 0.12 to $0.5 \mathrm{Gm} ., 2$ to $8 \mathrm{gr}$.

Ergotin.-This name has been applied to very different preparations. They are defined in N.N.R.

Haskell, r914, found that "ergotin" and powdered extract produced all the typical

1 From the French, "ergot,". a cock's spur, which it resembles in appearance. 
effects of ergot, but that the effects on the uterus are weakened much more than those on blood pressure.

${ }^{*}$ Fluidextractum Ergota (Fldext. Ergot.), U.S.P.-Prepared with acidulated dilute alcohol. Dose, 2 c.c., 30 minims, U.S.P.

${ }^{*}$ Ext. Ergot. Lig., B.P.-An aqueous solution, preserved with alcohol. Dose, 0.6 to $\mathrm{r} .8$ c.c., ro to 30 minims, B.P.

Inf. Ergot., B.P. -5 per cent. Dose, 30 to 60 c.c., I to 2 ounces, B.P.

*Inject. Ergot. Hyp., B.P.-33 per cent. of Ext. Ergot., B.P., preserved with I per cent. of phenol. Dose, 0.3 to 0.6 c.c., 5 to ro minims, B.P.

Tr. Ergot. Ammon., B.P. -25 per cent., with dilute alcohol and ammonia. Dose, 2 to 4 c.c., $1 / 2$ to I dram, B.P.

Ergotinine Citras, N.N.R.-The weaker alkaloid of ergot. Dose, 0.3 to $0.6 \mathrm{mg}$, $1 / 200$ gr., hypodermically, N.N.R:

Ustilago Maydis.-Corn Smut.-A fungus analogous to Ergot, found on the cornplant. Little is known about its composition or action, although it has been employed by the negroes for abortion, and stock-raisers have also observed that it has an ecbolic effect. Kobert states that it does not produce the ergot action on the cock's comb. $\mathrm{He}$ must have worked with old samples, since the author obtained the typical darkening from several different samples. However, the action is quite weak, and the drug does not deserve much attention unless it should be possible to isolate the principles in more active form. It possesses resins, such as form the active principles of ergot.

Gossypii Radicis Cortex, B.P.; Cotton Root-bark.-The dried root-bark of Gossypium herbaceum and other species. This also was used as abortifacient by the negroes. It contains resins, but no alkaloids nor tannin (Power and Browning, I914, Zellner, I9ro). Clinical and experimental reports are not favorable; the perfused uterus, however, is stimulated (Scott, IgII).

Dec. Gossyp. Rad. Cort., B.P. -20 per cent. Dose, 15 to 60 c.c., $1 / 2$ to 2 ounces, B.P.

Ext. Gossyp. Rad. Cort. Liq., B.P.-Dose, 2 to 4 c.c., $1 / 2$ to I dram, B.P.

\section{ECBOLICS (OXYTOCICS) AND EMMENAGOGUES}

Ecbolics are remedies which stimulate the gravid uterus to the expulsion of the fetus. When used in the non-gravid condition, they of ten increase the menstrual flow, and are then called Emmenagogues. Besides the Ergot group, one may count here:

I. All drugs which produce congestion of the abdominal organs. This generally extends to the pelvic organs as well.

r. All Drastic Purgatives.-Aloes, Myrrh, etc.

2. Irritant Volatile Oils, especially Savin, Thyme, Pennyroyal, and Turpentine.

3. All other Intestinal Irritants: Cantharides, Quinin, Digitalis, Metals, etc. A similar action is also claimed for Borax, but may be considered doubtful.

It must be remembered that these irritants produce their ecbolic effect only second. arily to a gastroenteritis. The latter may be so violent as to be fatal without accomplishing the desired result. They should not be employed for ecbolics, but at most as emmenagogues.

4. Application of heat or counterirritants to pelvic region or feet: hot foot- or hipbaths, mustard plasters, hot irrigations of the vagina, etc. These milder measures are only emmenagogue. When nutrition is low, they may be greatly aided by tonics.

II. All drugs which stimulate unstriped muscle-Muscarin, Nicotin, Pilocarpin, Physostigmin, Hydrastis, etc.-may act as ecbolics, but can not be used in practice.

\section{UTERINE SEDATIVES AND HEMOSTATICS}

A number of drugs of ill-defined actions are popularly credited with "quieting the excitability of the uterus," especially the pains of prema- 
ture labor; with contracting os uteri; with arresting uterine hemorrhage; with regulating dysmenorrhea, particularly at puberty and the climacteric; and even with correcting uterine malpositions.

It is difficult to conceive a mechanism by which a drug could produce all of these actions, except through the imagination. Many of these complaints are so uncertain in their course; and indeed so largely nervous, that it is easy to imagine any results that are desired. The use of these remedies was mostly taken from uncritical folk medicine and promulgated by obscure and ill-trained practitioners during the most uncritical period of therapeutics. They owe their continuance in the pharmacopeia mainly to their extensive use in proprietary "female weakness" remedies. These in turn rely for their sale on the liberal use of alcohol and exaggerated suggestive advertising.

Under these circumstances, there is practically no serious evidence for the clinical usefulness of these drugs. Excepting hydrastis and cotarnin, which belong to a somewhat different class, there is no record of any competent clinical investigation by experienced critical observers under hospital conditions. The clinical cases that are published are generally very superficially recorded; they generally show evidence of prejudice and exaggeration; and are often vitiated by the simultaneous use of other measures of more evident value.

Experimental Investigations.-Pharmacologically, most of the drugs of this class are practically inert, especially as concerns the uterus. Since the excised uterus reacts essentially like the uterus in vivo, it may be fairly taken as an index of uterine activity. Pilcher, 1916, has investigated in this way the drugs which are used most commonly in these female remedies, employing the relatively very high concentrations of $r: 1,000$ to $x: 500$.

He obtained no definite effects with: Chamaelirium luteum (Helonias dioica); Leonurus cardiaca; Passiflora dentata; Mitchella repens, Chestnut bark.

The following gave more or less pronounced effects (but not sufficient to confirm any therapeutic value):

Slight Depression.-Valerian; Dioscorea villosa; Aletris farinosa; Ichthyomethia piscipula; Pulsatilla pratensis; Cypripedium pubescens; Scutellaria laterifolia; Scrophularia marylandica; Senecio aureus; Viburnum opulus.

Inconstant Stimulation.- Acer spicatum; Cnicus benedictus; Viburnum prunifolium. Constant and Marked Increase of Tone.-Caulophyllum thalictroides.

\section{VIBURNUMS}

Viburnum prunifolium and Viburnum opulus enjoy the widest reputation amongst the uterine sedatives, and for that reason deserve more detailed discussion, although their usefulness is no more demonstrated. Illustrative of this is the fact that the drug sold as Viburnum opulus has, for the last twenty years, consisted of the bark of a maple, Acer spicatum (Farwell, rgi3, and others).

Viburnum prunifolium or Black Haw, a rather common shrub, was used somewhat in domestic medicine (Lloyd). It was introduced into medical practice mainly by the clinical papers of Phares, I866, and Jenks, I876. Both praise it with extravagant enthusiasm. The next ten years were the high tide of its popularity, as judged by an output of 75 articles on the subject. The interest then abated equally suddenly, for the next thirty years have produced only some 15 papers.

The whole early literature is highly typical of the loosest class of clinical "evidence," and does not merit serious discussion. The mechanism of its reputed action is at most a matter of speculation. The only attempts at investigation date from R. L. Payne, $\mathrm{r} 888$, and Sherman, $\mathrm{r} 896$, and those did not include the uterus. They showed at most that very large doses may produce depression of the whole nervous system, without practical significance.

La Torre, 19r2, interprets the histologic picture as contraction of the uterus, compressing the uterine vessels.

The first real search for uterine actions was made by Lieb, Igr4. He believed that he observed stimulation of the excised uterus. However, more extensive investigations by Pilcher, 19r6; by A. P. Matthews and Hager; and by Hale, gave generally negative results under all conditions (uterus pregnant and non-pregnant; in situ or excised). 


\section{PREPARATIONS-VIBURNUM}

Viburnum Prunifolium, U.S.P.; Viburnum, B.P. (Black Haw).-The dried bark of Viburnum prunifolium contains resins (viburnin), valeric and tannic acids, etc. Dose, 2 Gm., 30 gr., U.S.P.

Ext. Viburn. Prunifol., U.S.P.-A powdered extract, I Gm. representing $4 . \mathrm{Gm}$. of drug. Dose, 0.5 Gm., 8 gr., U.S.P.

Fldext. Viburn. Prunif., U.S.P.; Ext. Viburn. Liq., B.P.-Dose, 2 c.c., 30 minims, U.S.P.; 4 to 8 c.c., I to 2 drams, B.P.

\section{ANAPHYLAXIS}

\section{ANAPHYLACTIC SHOCK}

Parenteral injection of native proteins sensitizes animals specifically, after an incubation period, so that they react violently to a further injection of the same protein. This "anaphylactic shock," and the closely allied phenomena produced by the injection of peptone, etc., constitutes an autonomic intoxication, resembling especially the effects of histamin, and consisting chiefly in general contraction of smooth muscle. The fact that the response is immediate indicates analogy with ordinary poisons.

Historical.-Anaphylactic phenomena were described by Magendie as early as 839, and rediscovered by Behring, I892; Richet (who coined the name); Theobald Smith, I903; and Pirquet, I905 ("allergy"). Their modern investigation dates from Otto, I905; and Rosenau and Anderson, I906. Anderson and Rosenau, I908, give an extensive summary of the earlier literature. More recent compilations are those of Hektoen, I9I2; Dixon, I913; and Zinsser, I915.

Production of Anaphylaxis.-This results from the parenteral introduction of all "antigens"; i.e., all true proteins; but not from their cleavage products, proteoses, leucin, etc. (Gay, r9r3); nor from gelatin; nor from lipoids, etc. (The native so-called "proteoses" of seeds are antigens and therefore not true proteoses; Wells and Osborne, I915.)

It is necessary to distinguish between the first or "sensitizing" and the later or "provocative" injections. Sensitization occurs with very small doses (as little as $0.000,000,05 \mathrm{mg}$. of egg-white) intravenously or hypodermically, and exceptionally even by stomach or large intestine; or through the nose (Sewall, I9I4). A minimum interval of six to ten days elapses before the sensitiveness is acquired; but then it persists for a long time, weeks or years. Repeated injections during the incubation period may produce immunity. Injection of the serum of sensitized animals confers passive sensitization on the recipient (Otto); immediately, with proper dosage (Doerr and Russ). There is the same specificity as with other antigens. Specific sensitization may thus be secured simultaneously to several proteins. Wells and Osborne, I9I I and I9I3, have studied particularly the specificity for pure proteins. They believe that the specificity is due to certain constituent groups within the protein molecule; but that the whole molecule is involved in the reaction.

The provocative injection must be considerably larger than the sensitizing injection. It is much more effective by vein. The response is immediate. If an animal recovers from the toxic effects, it becomes immediately refractory or immune to further injection, because the anaphylactic antibodies are saturated or exhausted (Weil and Coca, I913). This antianaphylaxis (anergy) persists for a time, but is eventually lost.

General Phenomena.-However induced, anaphylaxis presents fundamentally the same phenomena, differing merely in intensity, according to conditions. The relative predominance of the individual effects in different species may complicate the picture. The actions are essentially peripheral. They consist in: fall of blood pressure (shock); tonic contractions of smooth muscle, especially the bronchioles, resulting in severe respiratory disturbance; and local urticarial reactions. 
Symptoms of Anaphylactic Reaction.-These are mainly as follows: Great restlessness; severe fall of blood pressure, generally attributed to paralysis of the splanchnic vessels and consequent shock; fall of temperature (Pfeiffer and Mita, rgro), etc.; rigidity of the lungs by violent contraction of the bronchial muscles, resulting in respiratory (listress and asphyxial convulsions; stimulation of other smooth muscle (uterus, stomach and intestines); diminished coagulability of the blood; leucopenia; eosinophilia; loss of complement; increased lymph flow (Calvary, IgII); local inflammatory reaction.

Repeated anaphylactic shock results in' cell destruction in several organs, with subsequent chronic inflammatory changes. In the liver, the results resemble portal cirrhosis (Longcope, I9I3).

Analogous but less severe parenchymatous degenerations of the myocardium, liver and kidneys may occur in rabbits after a single injection of a large dose of horse-serum or egg-albumen. They develop in ten to twenty-one days, about the time when the antidodies are produced (Longcope, I9I5).

Susceptibility.-Guinea pigs are especially susceptible, and in them the lung rigidity is most prominent, arresting respiration and producing asphyxial death in a few minutes (Auer and Lewis, 1909). In dogs and rabbits this phenomena is much less marked, the severe and prolonged fall of blood pressure being the most prominent feature. Cats are intermediate (Edmunds, I9I4). Man seems to be relatively insusceptible. The rare acute accidents in the injection of antitoxic sera, serum-disease, and some varieties of asthma and hay fever (Meltzer, I9IO) are probably anaphylactic manifestations.

Lung Rigidity.--Auer and Lewis, I909, showed that in guinea pigs dying acutely of anaphylaxis the lungs are rigidly distended and incollapsible even after death, by tetanic contraction of the bronchial muscles. The effect occurs after destruction of the central nervous system and even after degeneration of the vagi, so that it must be exerted directly on the muscle fiber or myoneural junction (Auer, I9IO). It is abolished by atropin (Auer). Chloral is uncertain (Rosenau and Anderson, r9og). Schultz and Jordan, I9I I, showed that the severity of the bronchial phenomena in guinea pigs is explained by their relatively thick mucosa; so that the contraction occludes absolutely even the larger bronchi.

Blood Pressure.-In guinea pigs, the circulatory changes are mainly asphyxia 1 (Auer and Lewis, I910). In other animals, there is a characteristic fall of blood pressure, often to 20 to $30 \mathrm{~mm}$. Hg. The mechanism of this "anaphylactic shock" appears to be complex. It was commonly attributed to vasodilation (Biedl and Kraus, I909); but the later evidence indicates that it is caused by contraction of the smaller hepatic vessels, preventing the adequate return of blood to the right heart (Mautner and Pick, 1915). The blood accumulates mainly in the large veins, especially of the liver and portal system; so that the smaller vessels of the kidneys, intestines, and other parts of the body are mechanically drained (Pearce and Eisenbray, 1910 and 1912; Edmunds, 1913); indeed, the fall does not occur if the liver has been excluded from the circulation. The liver therefore has a predominant share, directly or indirectly, in the fall, the blood pressure being lowered because a greater part of the blood stagnates in the portal system.

Zucker and R. G. Pearce found evidence that the essential process of the anaphylaxis, peptone or histamin "shock" does not consist in vasodilation, but in constriction of the liver vessels, resulting in deficient blood supply of the right heart, and therefore in venous stasis and fall of arterial pressure. This has been confirmed by Mautner and Pick, 1915. In carnivorous animals, in which the circulatory changes are prominent, they find swelling of the liver volume, from spasm of the smaller hepatic vessels. Immunity to repeated injection is explained by paralysis of these vessels. These effects do not occur in herbivoræ, frogs and turtles, whose liver vessels also fail to respond to epinephrin and barium, and which therefore probably lack constrictor innervation. The intestinal arteries are actively constricted, during life, in all animals; the veins become congested later. The arteries of the lung are also constricted, constituting a further factor in the venous congestion.

The vasomotor center is not directly involved in the fall; nor is there any good evidence of a peripheral action on the blood vessels. These continue to contract to epinephrin or barium; they do not contract to splanchnic stimulation, but this could be explained simply by the altered distribution of the blood. It is conceivable that the 
anaphylaxis directly lowers the tone of the hepatic vessels; but this has not been proven. Airila, I9I4, attributes the fall in rabbits to increased resistance of the fulmonary vessels; but Edmunds, I9I4, found no evidence of deficient blood supply of the left heart in cats; to the contrary, the pressure in the puimonary veins often rose. $v$. Behring, I914, attributes the shock to clumping of the platelets and thrombosis of the cerebral vessels.

The heart suffers by the low blood pressure, and is also affected directly, although this usually plays a minor part in the "shock." The changes are peripheral. As shown by electrocardiograms, on rabbits and dogs, they consist in disturbances of conduction and abnormalities of ventricular contraction, affecting especially the right heart. These may progress to final rigor (Robinson and Auer, I9I3; Auer and Robinson, 1913). The heart-muscle and diaphragm show histologic changes; also with other anaphylactoid agents (Kundratitz, I9I3). The isolated heart of sensitized animals is directly poisoned by the antigen (Cesaris-Demel, 1910-1912). Acute heart failure is also sometimes seen in intact cats (Edmunds, I9 I4).

Treatment of Anaphylactic Shock. - The continued low blood pressure furnishes an opportunity for investigating the treatment; this is of practical importance because the phenomena are very similar to those of surgical shock. The indications are evidently to relieve the splanchnic congestion and to increase the volume of blood to the heart and medulla. Pearce and Eisenbrey, rgro, found that venous transfusion with blood or saline only increases the splanchnic congestion without aiding the heart or medulla. Arterial transfusion is more successful; but the best results are obtained by combining transfusion into an artery with bleeding from a vein. Cardiac or vascular stimulants alone did not produce permanent improvement; but the combination of slow epinephrin infusion with digitoxin gave good results. Mautner and Pick, r9 15, obtained good results from the vasodilator action of nitrites or caffein.

Relation of Anaphylaxis and Immunity Reactions.-The relation of anaphylaxis to other "immunity" reactions indicates identity of the underlying process; anaphylaxis being merely a special manifestation of the defensive mechanism against foreign proteins. It refers simply to those phenomena of the "immunity reactions" which happen to be injurious to the animal. As with the other immunity reactions, it involves the reaction of the antigen with its antibody, under proper conditions; so that the antigen is rendered amenable to the action of the complement (alexin), leucocytes, etc.

In the case of anaphylaxis, the product of this reaction is poisonous, and is therefore called "anaphylatoxin." An "antigen" is any substance that induces the formation of a specific antibody. "Antibodies" are the specific substances produced by the organisms under the influence of antigens. They are supposed to act as amboceptors; i.e., as linking the antigen to the complement. The "complement" is an unknown substance (or substances), normally present in the blood, and which acts on the antigenantibody combination.

Nature of Anaphylatoxin.-The similarity of the anaphylactic phenomena indicates that whatever the nature of the antigen, the resulting poison (the "anaphylatoxin" of Friedberger, Igro; "proteotoxin" of Zinsser; "serotoxin" of Jobling and Peterson, r9r4), is a single (or closely related) substance. It has not been isolated, but is probably a relatively simple protein-cleavage product. This opinion is based mainly on the identity of its effects with those of "peptones" (Biedl and Kraus), and with the toxic protein-fraction prepared by Vaughan $(1907$, I913, I9 I6) by heating proteins with alkaline alcohol. This also appears to be identical for practically all proteins (Edmunds, IgI3). The identity of composition can not, of course, be proven until the pure substances have been isolated. Again, on the basis of its toxic effects, it is probable that the poison is closely related to (although probably not identical with) histamin.

Production of Anaphylatoxin.-Since a sensitizing injection and a following incubation period are necessary to anaphylaxis, it is evident that a definite change must occur in the body before the anaphylatoxin 
is formed or can become effective. Several possible explanations of this are tenable. The view most widely held is that the sensitizing injection leads to the production of "protective ferments," or rather of the specific antibodies by the cells; that these combine either in the tissues or blood with the protein of the provocative injection and thus sensitize it to the digestant action of the complement; and that this "parenteral digestion" results in the sudden liberation of the toxic cleavage product.

The evidence that a toxic cleavage product is actually formed in anaphylaxis is chiefly the following: Zuntz, 1912, found an increase of the proteoclastic power of the blood for the sensitizing protein. Friedberger, Igro, actually demonstrated the formation of anaphylactic poison by the interaction of antigen, antibody and complement (serum) in vitro. Keysser and Wassermann, I9II; and Bordet, 19I4, found that anaphylatoxin may also be produced without antigen, by the action of antibody and complement (serum) in the presence of certain colloids. This suggests that the serum proteins rather than the antigen may be the source of the poison; perhaps both are digested to a varying degree under different conditions.

R. W eil, 1916, confirms that complement is necessary for the production of anaphylatoxin from antigen and antibody in vitro. However, passive immunization is secured by the antibody, even when its complement has been destroyed by heat. From this, he argues that anaphylactic shock does not require complement, and is therefore different from anaphylatoxin.

Site of Production of Anaphylatoxin.-Further considerations show that the direct formation of anaphylatoxin in the blood has really a minor share. In passive anaphylaxis, namely, the degree of reaction is usually quite small or even absent if the antigen and antibody are injected simultaneously, and when they would therefore seem to have the best chance of interacting in the blood; and that the severest reactions occur if the antigen is injected twelve to twenty-four hours after the antibodies -when, in fact, the antibodies have largely disappeared from the blood, and are presumably fixed in the tissues. This makes it probable that the reaction occurs more effectively in the tissues than in the blood. This is further supported by the observations, to be described presently, that blood-free organs of sensitized animals react with the specific antigen. It leads to the view that both in active and passive anaphylaxis, the antibodies are localized mainly in the cells (as "sessile receptors"); and that it is here that the interaction of antibody and antigen, and the formulation of anaphylatoxin mainly occurs (Friedberger). A part of the antibody, however, is given off to the blood, and some anaphylatoxin may therefore be liberated in the blood stream (Friedemann, r909; Biedl and Kraus, 1910). Manwaring, rgro, and Voegtlin and Bernheim, IgIr, believe that the anaphylatoxin is produced mainly in the liver.

There is also direct evidence that the union of antibody and antigen is relatively slow in the blood stream; for they may exist there simultaneously and ununited, for days, combining slowly in vitro if the blood is removed. This suggests the presence in the blood stream of some inhibiting substance or conditions, such as are common in colloid reactions. For instance, it is known that antireactions are inhibited by excess of either antibody or antigen.

It will be seen that, whilst there is direct evidence that antibody, complement and antigen are somehow connected with anaphylaxis, the evidence for the production of anaphylatoxin by proteolytic cleavage is mainly indirect. Against it may be placed the observation that no increase of cleavage products has been actually demonstrated, f.i., in the anaphylactic lung (Auer and Van Slyke). Further, that the reaction of excised tissues of sensitized animals to antigen are more rapid than is customary in ferment processes (Dale, I913). Neither of these facts actually disprove the cleavage theory; for the increase of the products might be too small to be demonstrable; and the conditions for ferment action in the tissues are probably much more favorable than in the test-tube (Dixon, r913). However, they suggest the possibility of other explanations. It is quite conceivable that anaphylaxis consists in a special sensitiveness of the tissues to the antigen itself, rather than in the production of cleavage products; or that the toxicity is developed by some physical change of the serum colloids-perhaps in their surface tension-rather than by their cleavage. However, the cleavage theory at present holds the field as being much more probable.

Share of Tissues in Anaphylaxis.-Anaphylactic sensitization is manifested mainly in the tissues, rather than in the circulating blood. It need not be further discussed whether this is because the cleavage occurs 
in the tissues, or because the tissues have acquired a hypersensitiveness to the antigen itself. The fact is shown conclusively by the transfision experiments of Pearce and Eisenbrey, rgı. They found that a sensitized dog transfused with normal blood exhibits immediate anaphylactic shock when the antigen is injected; whereas a non-sensitized dog transfused with sensitized blood shows no immediate effect when the antigen is injected. It is also proven by the fact that excised and blood-free organs of sensitized animals exhibit a high specific response to the sensitizing protein.

Response of Excised Sensitized Tissue.-Schultz, 1910, first showed that washed smooth muscle of sensitized animals reacts specifically to the sensitizing protein. Foreign serums generally stimulate the smooth muscle of normal (non-sensitized) animals; but the muscles of sensitized animals respond to very much smaller doses of the sensitizing serum, whilst their reaction to other serums remains unchanged.

The further experiments of Dale, 1913, showed that the excised uterus of sensitized guinea pigs is contracted by extreme dilutions of the specific antigen; the production of the hypothetical toxic substance must therefore occur directly in the sensitized tissue. The response is almost immediate, so that the hypothetical cleavage, if such occurs, must be immediate, and not by ordinary ferment action.

In guinea pigs, which have been rendered immune (desensitized) by repeated injections, the excised uterus is still sensitive to the antigen. This form of antianaphylaxis must therefore be due to an inhibiting substance in the blood of the immune animal.

However, in the excised uterus, one dose of the antigen desensitizes the uterus to further doses (but not to normal serum). The antigen therefore destroys the hypersusceptibility of the sensitized muscle (perhaps by saturating the receptors). The persistence of the normal reaction to normal serum is a further proof that this is quite distinct from the anaphylactic response.

Prolonged contact with serum of a sensitive animal again renders the uterus sensitive (passive sensitization); but a normal uterus can not be passively sensitized.

Weil, IgI 4, has confirmed the results of Schultz and Dale.

W. H. Moore, Igr 5 , finds that the susceptibility of the sensitized uterus is increased if all blood has been removed by transfusion with Locke's solution; indicating that the blood has an inhibiting influence.

If the muscle is further freed from tissue lymph and other diffusible elements by repeated centrifugation in Locke's solution, the sensitiveness is greatly decreased; the sensitivemess being quantitatively restored if these muscles are treated with normal guinea-pig serum. This indicates that some constituent of normal serum is essential to anaphylactic stimulation.

Manwaring, Meinhard and Denhart, 1916, observed that the isolated rabbit heart is poisoned by goat-serum. If the rabbit had previously been sensitized to the serum, the heart is more resistant. On the other hand, the addition of the serum of a sensitized rabbit to the goat-serum increases the toxicity.

\section{ANAPHYLACTOID INTOXICATIONS}

Phenomena resembling more or less closely those of anaphylaxis are produced by a large number of substances, ranging from peptone to dialyzed iron. The correspondence is probably never complete (Loewit, I9I3).

Analogous Actions of Blood Serum.-This has a stimulant effect on smooth muscle (Schultz, I9I2), although there are specific differences (Dale, I913). A specific constrictor substance is formed in shed blood, probably by changes in the formed elements rather than by clotting (G. N. Stewart and Zucker, I9I3). This may play a part in hemostasis. The principle which stimulates the intestine and uterus preexists. In the perfused heart (rabbits), Cushny and Gunn, I9I3, find that the addition of serum produces a preliminary stimulation, with stronger and usually faster contractions; and a subsequent depression with weakened and slowed contractions, sometimes heart-block, and eventually arrest. The coronary vessels are constricted. Similar effects are produced by the plasma of the operated animal, by starch solution, etc. The authors attribute them to an alteration in the heart produced by excision, and not to the formation of any poison.

The coronary vasoconstriction has been confirmed by Yanagawa, 1916. Of a con- 
siderable scries of colloids tried, this effect was produced only by serum and by eggwhite. It resides in the proteins.

Manwaring, Meinhard and Denhart, I916, find that the addition of 7 to ro per cent. of goat serum in perfusion of rabbit's heart produces tachycardia, followed by depression and irregularity". The tachycardia is due exclusively to diffusible substances in the serum; the depression to non-diffusible.

The fatal effects of intravenous injections of foreign blood serums are often connected with hemolysis and thrombosis (L. Loeb, Strickler and 'Tuttle, rgro). Organ extracts intravenously also often produce thrombosis (L. Locb, I9r2).

Effects of Albumoses.-Intravenous injections of "Witte's Peptone" ( $0.5 \mathrm{Gm}$. per kilogram) produce typical anaphylactoid phenomena (Biedl and Kraus); temporary but considerable fall of blood pressure (Thompson, ${ }^{1} 896$ and 1899 ) by peripheral action, in dogs (but not in rabbits, Pissemsky, rgr5); stimulation of the isolated uterus (Dale and Laidlaw, r9ro); bronchial constriction, peripheral (Baehr and Pick, I9I3; no effect, Trendelenburg); increased flow of lymph and secretions (Popielski, r 908 and r 1909); of milk (Ott and Scott, I91 2); discharge of the gall-bladder (Klee and Knuepfel, I9I4); diuresis independently of the blood pressure (Thompson; Gizelt, I908; Froehlich and Pick, Igr 2); fever on hypodermic injection, etc. In most animals (but not in rabbits) appropriate doses render the blood non-coagulable, and produce hyperglycemia and glycosuria (Y. Henderson and Underhill, I9II). McGuigan and Ross, I9I 5 , however, state that the usual result is hypoglycemia, which they attribute to circulatory changes in the liver. The secretion of bile is increased (OKada, 1915). Application of peptone to the scarified skin produces a wheal (Sollmann and Pilcher, I9 I 6).

The actions are due to the proteoses themselves and not to foreign toxic admixtures (Underhill, 1903). Protamins, histones, etc., have similar actions (Thompson, 1900); Popielski, rgI3, attributes all the effects to "vasodilatin."

Whipple, I9!6, attributes to a primary proteose the toxic effects of intestinal obstruction, peritonitis, and acute pancreatitis.

The non-coagulability of the blood is due to an antithrombin, the blood containing all the necessary elements for coagulation, so that it clots, e.g., on simple dilution with water. This antithrombin seems to be a nucleoprotein formed in the liver (Doyon, Morel and Policard, r9II; Denny and Minot, r9I5; its production is not increased by bile, bile salts, secretin or electric stimulation). R. J. Lee and Vincent, 1915, attribute the non-coagulability of the anaphylactic blood mainly to an anomalous behavior of the blood-platelets.

The mechanism of the fall of blcod pressure is identical with that of anaphylaxis (Thompson, 1896 and 1899; Pearce and Eisenbrey, 1910; Froelich and Pick, I91 2). The excised mammalian heart is weakened by concentrations of $x: 1,000$ to $\mathrm{r}: \mathrm{ro}, 000$; stimulated by $\mathrm{r}: 200$ (Pissemsky). Excised vessels are generally constricted (Dale and Laidlaw, IgII); the ear vessels are constricted by all concentrations; those of the perfused lung are somewhat dilated (Baehr and Pick, 19r3; also the liver-veins (Berezin, I9r4); the intestinal, renal and coronary vessels are unaffected by dilute solutions, constricted by strong solutions (Pissemsky, 1915; Mautner and Pick, 1915). The effects on blood pressure and respiration have also been investigated by Zuntz, igrr.

Notwithstanding these similarities, there are sufficient differences to indicate that anaphylaxis is not due to the formation of ordinary albumoses (Manwaring, rgro; Loewit, IgII and I912).

Injections of Witte's peptone have been proposed for the treatment of hemophilia, but have caused fatal hemorrhage (Leceboullet and Vaucher, 19r4).

Resemblance to Histamin.-Dale and Laidlaw, IgIr, call attention to the striking similarity of the anaphylactic and peptone effects to those of histamin; and suggested that the active principle may be similar. Certain minor differences exist; f.i., epinephrin removes the bronchial spasm of peptone and not of histamin (Januschke and Pollak, rgrI); histamin does not affect the coagulation of the blood (Modrakewski, rgr2), etc. It is suggestive that histamin and similar amins are among the products of the more complete digestion of peptones.

Other substances producing anaıgous or identical effects are the "Vasodilatin" of organ extracts (Popielski, I9I0, I9I I and I9I2); Secretin (Barger and Dale, I9II; Dale and Laidlaw, I9I2); and Hormonal.

These substances also have not been isolated, but some at least are probably also simple bases. Dale and Laidlaw suggest that vasodilatin may be impure histamin. Normal urine contains pressor substances (the urinary hypertensin of Abelous), which consist mainly of iso-amylamin (Bain, 1914). 
Hormonal.-Zuelzer, I908, believed that various organs, removed at the height of digestion, contain a hormone, which stimulates peristalsis. The commercial "Hormonal-Zuelzer" is extracted with dilute hydrochloric acid from the spleen, removed during digestion. It is injected intravenously and intramuscularly, in intestinal paresis and post-operative ileus. Its injection has often caused serious and even fatal collapse (Rosenkranz, rgr 2; Seifert, Nebenwirkung., I915, p. I38). Mohr, I9I2; Sabatowski. I9 2 , and others, believe that the active principle is albumose or a similar anaphy lactoid substance, which would explain the fall of blood pressure, and by this the peristaltic effect. Zuelzer, I9I 2, attributes the accidents to contamination, and now prepares what he believes to be a purer product, by removing the proteins through boiling and lead acetate. However, fatal accidents are still reported, whilst the therapeutic results are very inconstant (Burianek, I9I3). It seems an uncertain and dangerous remedy (Hesse, 1913).

An experimental investigation of Hormonal is published by Schlaginweit, rgiz. Weiland, I9I2, states that extracts of the intestinal mucosa or muscularis stimulate the intestine, excised or intravenously, much more than other organ extracts; that the effect is located in Auerbach's plexus and is antagonized by weak atropin; and he believes that this hypothetic substance is the cause of the automatic movements of the excised intestine.

Hirudin.-The cervical glands of the common leech (Hirudo) secrete a substance (hirudin, hemophilin) which delays or hinders the coagulation of blood inside and outside of the body. This substance is not destroyed by boiling or by alcohol. It appears to be a deuteroalbumose.

Therapeutic Use.-Leeches were at one time used extensively for the abstraction of blood in local inflammations. Dry or wet cupping is now usually substituted, but they are still employed occasionally; two to four of the animals are applied. The skin should be well cleansed, and, if necessary, a little milk should be rubbed on to tempt the animals to bite. They should not be removed, but allowed to fall off. They cannot, of course, be used again. Each leech draws from 5 to 7 c.c. of blood. The quantity may be somewhat increased by applying hot fomentations after removing the animals. It is sometimes difficult to stop the bleeding.

For experimental use, an extract may be prepared, when needed, by crushing the leech-heads with sand, macerating for two hours, and centrifugalizing. Each kilogram of animal requires the heads of three leeches, extracted with 3 c.c. of normal saline. The solution loses activity on standing, more slowly in the cold.

Immunization.-Daily intravenous injections of hirudin into mice creates an anti-coagulant substance, effective in vivo and in vitro, destroyed at $65^{\circ} \mathrm{C}$. It also inactivates fluorids, but not the calcium-precipitants. The mechanism of its action has not yet been explained (Vera and Leo Loeb, I9I4).

Vessels.-Hirudin dilates these, in frogs, and renders them less sensitive to epinephrin (Kahn, I912; Trendelenburg, 1915).

Toxic Effects. - Fresh preparations may be injected intravenously in sufficient quantity to render the blood non-coagulable, without damage to the animal. Some commercial samples of hirudin, however, are highly toxic, especially to dogs, probably through the presence of putrefactive bases. The symptoms from 20 to $25 \mathrm{mg}$. per kilogram in dogs, consisted in severe bloody diarrhea and continued vomiting, with hemorrhagic lesions of the viscera (Marshall, 1915).

\section{NITRITES, NITROGLYCERIN, AND OTHER ALKYL NITRATES}

General Statement.-The nitrites, the nitrous esters and the organic nitrates, which are reduced to nitrites in the body, lessen the tone of arterial muscle, thus producing a prompt fall of blood pressure. Their other effects are unimportant. They are used for the relief of arterial spasm and other conditions of high blood pressure.

Members. - This group comprises the inorganic nitrites $\left(\mathrm{NaNO}_{2}\right)$; the nitrous esters (amyl nitrite, ethyl nitrite, "Sweet Spirits of Nitre"); and substances which liberate the nitrite ion within the body. Most important amongst the latter is glycerin tri-nitrate (nitroglycerin or glonoin), which is easily broken up by alkalies into a mixture of nitrates and nitrites; a similar change occurs in the body. Erythrol tetranitrate 
and Mannitol hexanitrate undergo analogous decompositions, but much more slowly. Even inorganic nitrates are reduced to a slight extent (Binz, Igor), especially by the putrefactive bacteria of the colon, and may then give rise to the phenomena of nitrite poisoning (but this requires very large doses such as the quantities of Bismuth Subnitrate used in Roentgen diagnosis).

Hydroxylamin, $\left(\mathrm{NH}_{2} \mathrm{OH}\right)$, is decomposed in the body into nitrite and ammonium. It produces the effects of both these substances (methemoglobin formation, convulsions, central paralysis, Lewin, I889).

Diff erences in Activity.- It is generally assumed that the organic nitrates act only after being reduced to nitrites; but this is not proven. The fact that excised vessels react promptly to these nitrates suggests that they may perhaps act directly. It is also remarkable that the effective dose of nitroglycerin is but a hundredth of that of sodium nitrite. Presumably the organic esters penetrate more readily into the cells, and perhaps the reduction occurs only after their penetration or there may be certain peculiarities in the reduction. These may also explain individual differences of susceptibility. C. R. Marshall, I9I2, finds that the introduction of $\mathrm{OH}$ and $\mathrm{CO}_{2} \mathrm{H}$ groups into organic nitrates diminishes their vasodilator action.

The nitrates of monhydric alcohols are lèss effective than the polyhydric, probably because of slower decomposition. Nitroglycerin and erythrol-tetranitrate act quantitatively alike when equimolecular doses are injected into a vein; by mouth the action of the erythrol ester is weaker and more prolonged, because it is much less soluble, and therefore more slowly absorbed (Marshall, I9I3).

Reducing Properties of Tissue.-Many other substances are reduced in the tissues; such are methylene blue, arsenic acid; the oxids of tellurium and selenium; nitrobenzol into anilin; sulphur into hydrogen sulphid, etc. The change is not due to ferments (Heffter, 1907, I908). The reducing power of different organs varies greatly. Many other substances are, of course, oxidized in the tissues, the processes of oxidation and reduction going hand in hand.

Actions on the Circulation.-The most important effect of the nitrites is a prompt fall of blood pressure, due to vasodilation by direct action on the blood vessels.

Course of the Vasodilation.-This is first noticed in the skin of the face, in an area similar to that involved in blushing, but which may extend over the entire trunk to the ilium. The meningeal vessels undergo dilation at the same time. There is consequently redness of the face, heat and throbbing in the head, and headache. The flushing of the skin is brief, but headache is apt to persist, especially with erythrol tetranitrate. The retinal vessels are also dilated.

At the same time the heart is greatly quickened, so much so that a rise of blood pressure is frequently obsèrved at the start (Hewlett, 1906), the dilatation being more than overcome by the quickened beat.

The dilatation does not, however, remain confined to the skin, but spreads to other vessels, and relaxation of the splanchnic area in particular causes a speedy fall of blood pressure (Fig. I6) and a dicrotic pulse (Fig. I7). The fall of blood pressure is due entirely to the extensive vasodilation, produced by the direct action of the nitrite on the arterioles. The vasomotor center is not inhibited, but rather stimulated by the cerebral anemia (Pilcher and Sollmann, I9I4). It is possible, however, that the preliminary blushing is a central effect.

Evidences of Vasodilation.-The fall of pressure occurs equally well when the cardiac changes are excluded by atropin. 'The vasodilatation can be demonstrated directly by inspection (the blushing); by plethysmographic measurements (Dossin, IgII) and by the increased rate of vein flow.

Effects on Vein Flow and Vein Pressure.-Burton-Opitz and Wolf, Igro, on injecting nitrite into the femoral artery, found the vein flow and vein pressure 
increased in the corresponding leg, whilst the general arterial pressure was practically unchanged. If amyl nitrite was inhaled, the vein flow in the leg decreased with the blood pressure, because of the withdrawal of the blood into the more powerfully dilated splanchnic vessels. Capps, IOII-IOI3, also found a fall of vein pressure.

In man, Hewlett, I0I3, found increase of the volume, but scarcely of the blood flow, of the arm. Stewart, I915, showed a quite definite increase of the blood flow of the hand.

The Dilator Action Is Peripheral.-It occurs after ligation of the blood vessels of the brain (Mayer and Friedrich, 1875); after destruction of the spinal cord (Lauder

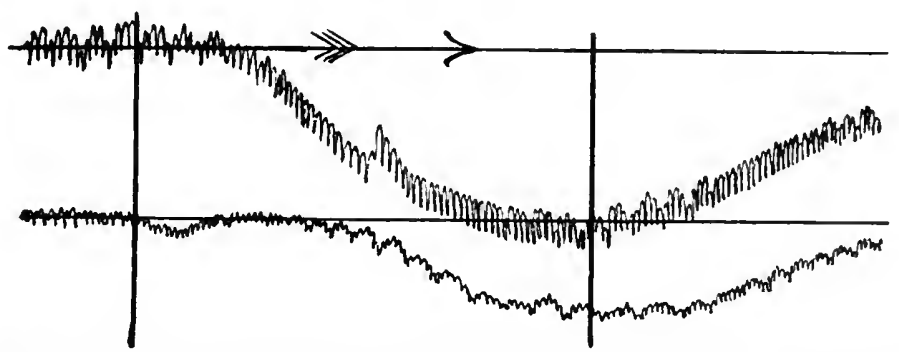

Frg. I6.-Amyl Nitrite on Carotid Pressure, Dog. The nitrite is inhaled between the two vertical lines. The upper tracing is taken from a normal dog, the lower after atropin. The heart was quickened by the nitrite in the upper tracing, but not in the lower.

Brunton, I869); in excised organs (Atkinson, I888; Marshall, I898, etc.); and even in excised arterial segments (Cow, I9II). It is therefore due to depression of the tone of the vascular musculature. There are as yet no convincing data as to the precise site of the action. Strong stimulation of the vasomotor nerves raises the blood pressure even during the nitrite fall (Bernheim; Mayer and Friedrich). The nitrites are said to act after degeneration of the cervical ganglia. It seems probable therefore that they affect the myoneural junction or muscle substance.
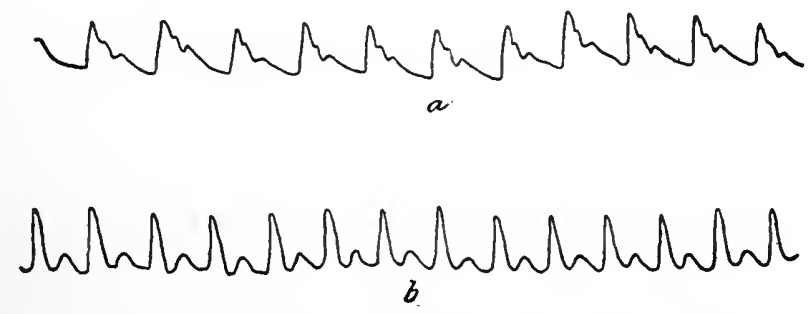

Frg. 17.-Amyl nitrite on sphygmogram: $a$, Normal; $b$, after inhalation of four drops,

Antagonisms.-Nitrites are mutually antagonistic with most vascular constrictors; thus, splanchnic stimulation (Bernheim, I874), epinephrin, strophanthin, etc., raise the blood pressure during the nitrite fall; and conversely, nitrites lessen the rise of these constrictor agencies.

The antagonism is quantitative; $f . i$., sciatic stimulation gives the same millimeter rise during as before the nitrites; and nitrites give the same millimeter fall during as before strophanthin, hydrastinin, pituitary, strychnin or ergot. This suggests that the point of attack of the nitrites is different from that of the pressor agencies (Pilcher and Sollmann, I915).

Cameron, I906, proposed to utilize the antagonism for the bio-assay of epinephrin. He states that $0.6 \mathrm{mg}$. of nitroglycerin exactly neutralizes the pressor effect of 0.0075 mg. of epinephrin.

The Vasomotor Center.-This is not depressed, but rather stimulated by its anemia; the stimulation, however, can not overcome the peripheral depression. This is shown by the perfusion experiments of Pilcher and Sollmann, 1915. Similarly the blood pressure does not fall if a nitrite is introduced into the cerebral circulation, but 
prevented from reaching the systemic vessels. (Filehne, 1874 , had concluded that the vasomotor center is depressed.)

The Pulmonary Circulation.-The pulmonary vessels are not so markedly dilated; incleed, Niacht, IOI4, found constriction in excised pulmonary arteries. The pressure in the pulmonary artery is generally slightly increased (Bradford and Dean, r894; recent literature in Wiggers, I9II). The rise is probably due partly to the engorgement of the large veins.

The Coronary Vessels.-The outflow from the coronary veins is increased in intact animals (F. Meyer, I9I2; Schloss, I9 3 ) and in excised mammalian hearts (O. Loeb, I903). The coronary vessels are therefore dilated (also excised arterial rings; Voegtlin and Macht, I9I3); and the coronary circulation is improved. This explains the beneficial action in angina pectoris.

Intraocular Tension.-This is raised by amyl nitrite, even when the general blood pressure is lowered (Wessely, I9 I 5). This enjoins caution when using nitrites in glaucoma. The retinal vessels are also dilated (Hirschfelder, I9I5).

The Tachycardia.-This is due mainly to depression of the vagus center, for after section of the vagi, or after atropin, nitrites cause but little further acceleration (Mayer and Friedrich, 1875 ). The slight remaining quickening is abolished by dividing the accelerators (Dossin, I9II). The rate of the excised heart is not changed by nitrites (Plumier, I905). The excitability of the vagus trunk to direct electric stimulation persists.

The depression of the vagus center is due to the fall of blood pressure, and not to a direct action of the nitrite, for no quickening occurs if the pressure is kept level by partial compression of the aorta (Filehne, I 879 ; Sollmann). The vagus center may still respond to strong reflex stimulation (Mayer and Friedrich).

In man, the acceleration on inhaling amyl nitrite is considerable, often to double. No acceleration occurs in heart-block. This has a diagnostic value, similar to atropin (Belloir and DuBos, I913).

Character of Pulse.-As the result of the quickening, the excursions of the heart are smaller. The blood-pressure waves appear particularly low, because the pressure wave is more readily dissipated, on account of the dilatation. The pulse; on the other hand, may be bounding, because the arteries are emptied more completely during diastole.

The heart-muscle shows no important direct change; however, the better coronary circulation and the lowered peripheral resistance may improve and relieve a weakened heart (MacNider, I 908).

The excised mammalian heart is scarcely affected by moderate doses, perhaps slightly stimulated, then depressed (Loeb, 1903; Plumier, 1905; Dossin, I9II).

The excised frog's heart may be quickened by low concentrations ( $1: 10,000)$; higher concentrations weaken $(\mathrm{r}: 5,000)$, or stop $(\mathrm{x}: \mathrm{x}, \infty \circ 0)$ the contraction.

Methemoglobinemia.-Excessive doses of nitrites form methemoglobin (Gamgee, I868) and this causes cyanosis and asphyxia. However, since the blood corpuscles are not destroyed, the animals generally recover.

Respiration.-This is accelerated (Mayer and Friedrich), probably by the changes in the blood and circulation.

The Bronchial Muscles.-These are relaxed by nitrites (Doyon, I 906 ; excised, Titone, 19г3; surviving lungs, Baehr and Pick, I9I3). This may explain their use in asthma. The smoke of the "Charta Potassi Nitratis" (paper impregnated with saltpeter) probably owes a part of its effect to nitrite compounds.

Gall-bladder. - This is relaxed by nitrites (Lieb and McWhorter, 1915).

Intestinal Muscle.- Hirschfelder, 1915, finds that the systemic administration of nitrites relaxes the intestinal constriction produced by lead salts. It has been used clinically in lead colic, but with limited success. 
Digestion.-Nitrites cause some slight interference with the digestive ferments (Hale, I910); but probably not enough to be important. Sodium nitrite is supposed to produce some gastric irritation, by the liberation of nitrous acid-this is not sufficient to interfere with its therapeutic use.

Urine.-The variations produced in the secretion of urine appear to be purely secondary, and will depend upon whether the renal arterioles or those of the general circulation are relatively more dilated; the former causing an increase, the latter a diminution, of urine.

In patients with excessively high blood pressure, the nitrites generally increase, sometimes quite considerably, the excretion of urine, nitrogen, total solids and phenolsulphonephthalein (Lawrence, I9 2 ).

Pressure of the Cerebrospinal Fluid.-This is increased by amyl nitrites, presumably through the methemoglobin asphyxia (Dixon and Halliburton, IgI3 and I9I4).

The effects on metabolism have been studied by Surveyor, I906.

Temperature.-Small doses have no effect on normal temperature, but are antipyretic in fever, by dilating the skin vessels (Jacobj, I9 I3; Krauss, I913). Ethyl Nitrite, as "Sweet Spirits of Niter," has been used popularly as a diaphoretic and diuretic. Its action is not very energetic. Large doses of nitrites also lower the normal temperature by depressing the circulation.

Fate.-Some 60 to 70 per cent. of the nitrites disappear in the body, their fate being unknown.

Rapidity and Duration of Action.-This constitutes the principal difference between the members of the group. Amyl nitrite, when inhaled, acts in ten to fifteen seconds, the action disappearing again within seven minutes. Glyceryl trinitrate, ethyl nitrite, and sodium nitrite act in two to ten minutes, the action persisting a half to two hours. With erythrol nitrate, the effects appear only after half an hour, and last five hours (Wallace and Ringer, r909; E. Matthew, r909; Rzenkowski, I909; J. L. Miller, I9ro).

The following table from Matthew shows in detail the average relation for oral administration in man. (The fall in diastolic pressure is about half of that in the systolic pressure; Lawrence, 1912.)

\begin{tabular}{|c|c|c|c|c|c|c|}
\hline Drug & Dosage & $\begin{array}{l}\text { Fall } \\
\text { begins, } \\
\text { minutes }\end{array}$ & $\begin{array}{l}\text { Maximal } \\
\text { fall } \\
\text { reached }\end{array}$ & $\underset{\substack{\text { Maximum } \\
\text { mained }}}{\text { tained }}$ & $\begin{array}{l}\text { Return } \\
\text { to } \\
\text { normal }\end{array}$ & $\begin{array}{l}\text { Extent.1 } \\
\text { of fal! } \\
\text { (mm. } \\
\text { Hg.) }\end{array}$ \\
\hline $\begin{array}{l}\text { Spir. Glyceryl. Nitratis.... } \\
\text { Sodium Nitrite........... } \\
\text { Erythrol Tetranitrate...... } \\
\text { Mannitol Hexanitrate...... } \\
\text { Amyl Nitrite by inhalation } \\
\text { (Wallace and Ringer).... }\end{array}$ & $\begin{array}{l}2-3 \text { minims } \\
2-3 \text { grains } \\
16 \text { to } T \\
\text { grain } \\
\text { I grain } \\
3 \text { minims }\end{array}$ & $\begin{array}{r}5 \\
15 \\
1\end{array}$ & $\begin{array}{l}22 \text { minutes } \\
21 / 2-3 \text { hours } \\
3 \text { minutes }\end{array}$ & $\begin{array}{c}2 \text { minutes } \\
20 \text { to } 60 \\
\text { minutes } \\
2 \text { hours } \\
3 \text { hours } \\
\ldots \ldots\end{array}$ & $\begin{array}{l}5-6 \text { hours } \\
6 \text { hours } \\
7 \text { minutes }\end{array}$ & $\begin{array}{c}25-30 \\
32\end{array}$ \\
\hline
\end{tabular}

1 Systolic blood pressure.

Influence of Dosage.-The extent of the fall increases with the dosage, the increase becoming progressively smaller. The duration of the action is not so much influenced by the dosage.

Influence of Pathologic Conditions. - The average effect is about the same in disease as in health. In cases of abnormally high blood pressure, the absolute fall is greater, but the percentile fall and the duration are the same (Wallace and Ringer).

Idiosyncrasy.-Different patients vary considerably in their reaction, and some fail entirely to respond to the nitrites, especially those with 
chronic Bright's disease and edema, but also in other conditions. Sometimes one member of the group will succeed when another fails. No result need be expected if the arterial fibrosis has destroyed most of the muscular fibers. The tolerance is often very marked. In other patients, even small doses caused alarming syncope and cardiac slowing. The severity of the headache is also variable.

Habituation. - This usually occurs more or less if the administration of nitroglycerin is continued, often in two or three days. The dosage must then be increased or the response disappears. Suspension of the administration for a few days may restore the susceptibility. Matthew claims that the tolerance is not acquired with sodium nitrite or erythrol tetranitrate.

Therapeutic Use in Arterial Hypertonus.-The nitrites are employed mainly for reducing the blood pressure in conditions of arterial hypertonus. The effect is not curative, but the symptomatic relief of the headache, etc., is usually marked, outlasting the blood-pressure effect of the single doses. However, they usually lose their efficiency after a short time. Increasing the dose generally produces unpleasant symptoms-headache, restlessness, sometimes dyspnea. They must therefore generally be alternated with venesection, sweating, nauseants, etc. The effect of these is usually less marked but more lasting (J. L. Miller, I9ro; C. H. Lawrence, rgr 2). Nitroglycerin and sodium nitrite appear to be the most useful; the action of amyl nitrite being too brief, whilst erythrol tetranitrate often causes intense headache. Sodium nitrite is said to give least headache (Hewlett, I9r2).

Administration and Dosage.-The dosage of the nitrites should be kept as low as possible, to prevent the rapid development of tolerance. It is best to give just enough to cause a slight quickening of the pulse, and feeling of fulness in the head; or a fall of systolic pressure of about $30 \mathrm{~mm}$. This dose may be given four times a day. The initial dose may be $0.5 \mathrm{mg}$. $(1 / 100 \mathrm{gr}$.) of nitroglycerin, increasing gradually. The equivalent doses of the other nitrites are shown on page 379. The treatment may be intermitted for a few days in every two or three weeks, starting again with a smaller dose. Hypodermic injections are rarely, needed, the drugs being readily absorbed.

Cardiac Insufficiency.-The relief of the heart from excessive resistance may render nitrites useful in these conditions. Nitroglycerin (I mg.) is used, generally in combination with Digitalis.

Angina Pectoris.- The inhalation of amyl nitrite was introduced by Lauder Brunton in 1867. In some cases (presumably with spasm of the coronary arteries) the relief is complete; in others, probably of different pathology, it is ineffective. One to 3 drops of the promptly acting amyl nitrite are inhaled. It may be carried in thin glass capsules, "pearls," one of which is crushed in a handkerchief, as needed.

Migraine sometimes yields to nitrites, presumably in those cases attributed to spasm of the cerebral arteries. Epilepsy is also said to be sometimes benefited. Relief of Lead colic (Riegel, I 878 ) has been claimed, but the efficiency is at best unreliable.

Internal Hemorrhage.- The lowered blood pressure would tend to arrest hemorrhage but the bleeding would be favored by the direct dilator action. The resultant of these opposite tendencies is difficult to foresee, and probably varies with different conditions. Nitrites would therefore be unreliable remedies. Experimentally, Frey, 19\%9, found a slight increase of pulmonary hemorrhage; Lisin, 1907, claims diminution of intestinal hemorrhage. 
Untoward Effects.-Occasionally, therapeutic doses may cause fainting. Other side actions which may occur are localized edema, excessive sweating, and visual disturbances (Cornwall, I9 3 ).

Toxic Effects.-Nitroglycerin has a toxicologic importance from its use as an explosive. Overdoses produce nausea, vomiting, colic, and sometimes bloody diarrhea. The skin is flushed and perspiring. There is vertigo and persistent throbbing headache; rarely blindness and delirium. The respiration is first hyperpneic, then dyspneic and slowed. The surface becomes cold and cyanotic; the pulse is gradually slowed, dicrotic and intermittent. Paralysis sets in, and is followed by clonic convulsions. The reflexes are lost. Death occurs by failure of respiration, after four to seven hours. The fatal dose varies greatly. D. D. Stewart, 1905, reports a patient in whom $20 \mathrm{gr}$. of absolute nitroglycerin produced only slight effects.

Absorption. - Nitroglycerin is readily absorbed. Even the application of it to the skin causes some effect, and persons engaged in its manufacture suffer severely from headache during the first few days. After this, however, they appear to be quite unaffected, unless they leave work for several weeks and then resume the occupation. The work is said to be not unhealthful. The local action on the hands may result in skin eruptions and ulcerations.

Smokeless gun powders (nitroglycerin, nitrocellulose, etc.), when exploded, liberate gases which produce the combined effects of nitrites and asphyxia. The principal products are $\mathrm{NO}_{2}$ (nitrogen peroxid) and $\mathrm{CO}$ (Kiefer, I905). The vasodilation and tachycardia are followed by persistent headache ("powder-headache"), which does not yeld to acetanilid, etc., but is best treated by strong coffee, and poultices applied to the back of the neck.

Sodium nitrite produces, in animals, symptoms similar to those of nitroglycerin (Dossin, IgIr).

Amyl nitrite also has similar effects (Rosen, I888).

\section{PREPARATIONS-NITRITE-GROUPS}

*Amylis Nitris (Amyl. Nitris), U.S.P.; Amyl Nitris, B.P.; Amyl Nitrite--A liquid containing not less than 80 per cent. of $\mathrm{C}_{5} \mathrm{H}_{11} \mathrm{NO}_{2}$ (chiefly iso-amyl nitrite). A clear, yellowish liquid; peculiar, ethereal fruity odor; pungent aromatic taste. Almost insol. in water, miscible with alc. or eth. Amyl Nitrite is very volatile even at a low temperature, and is inflammable. Dose, by inhalation, 0.2 c.c., 3 minims, U.S.P.; $0 .{ }^{2} 2$ to 0.3 c.c., 2 to 5 minims, B.P. It may be carried in thin glass capsules, "pearls," one of which is crushed in the handkerchief as needed.

Erythrol Tetranitrate, N.N.R. (Tetranitrol), $\mathrm{C}_{4} \mathrm{H}_{6}\left(\mathrm{NO}_{3}\right)_{4}$ - - Colorless and tasteless crystals, sol. in alc., insol. in water. Dangerously explosive. Dose, 0.03 to $0.06 \mathrm{Gm}$., $1 / 2$ to $\mathrm{I}$ gr., N.N.R., in tablets of $0.03 \mathrm{Gm}$.

Liq. Ethyl Nitrit., B.P. - 2.5 to 3 per cent. of $\mathrm{C}_{2} \mathrm{H}_{5} \cdot \mathrm{NO}_{2}$ in alcohol. Dose, $\mathrm{r}$ to 4 c.c., I5 to 60 minims, B.P.

${ }^{*}$ Spiritus Etheris Nitrosi (Spir. Aeth. Nit.), U.S.P., B.P.; Spirit of Nitrous Ether (Sweet Spirit of Niter). - An alcoholic solution of ethyl nitrite, $\mathrm{C}_{2} \mathrm{H}_{5} \mathrm{NO}_{2}$, containing about 4 per cent., U.S.P.; 2 per cent., B.P. (It also contains aldehyd, paraldehyd and ethyl nitrate; but these do not contribute materially to its action; Marshall and Gilchrist, 19r5). A pale yellowish liquid, of ethereal odor and sharp taste. Miscible with water and alcohol. Deteriorates readily. Incompatible with antipyrin, morphin and iodids. Brownish color with salicylates; slight effervescence with uva ursi, buchu, and acetates. The addition of water throws ethyl nitrite out of solution, which then evaporates rapidly, lowering the strength. Dilution should therefore be made shortly before administration (Marshall and Gilchrist, I915). Dose, 2 c.c., 30 minims, U.S.P.; I to 4 c.c., I5 to 60 minims, B.P.

* Spiritus Glycerylis Nitratis (Sp. Glyceryl. Nit.), U.S.P.; Liq. Trinitrini, B.P.; Spirit of Glyceryl Trinitrate; Solution of Trinitrin (Spirit or Solution of Nitroglycerin or Glonoin).-An alcoholic solution of $\mathrm{I}$ per cent. of $\mathrm{C}_{3} \mathrm{H}_{3}\left(\mathrm{NO}_{3}\right)_{3}$. A clear colorless liquid, with odor and taste of alcohol. Miscible with water and alcohol. Dose, 0.05 c.c., I minim, U.S.P.; 0.03 to 0.12 c.c., $1 / 2$ to 2 minims, B.P.

* Tabelle Trinitrini (Tab. Trinitrin.), B.P.-0.5 mg., 1/130 gr. Dose, x or 2 tablets. The commercial tablets are fairly, although not quite uniformly, active. They do not deteriorate very rapidly (Edmunds and Roth, I908; Rippetoe and Smith, 1914).

*Sod. Nitris, U.S.P., B.P.; Sodium Nitrite, $\mathrm{NaNO}_{2}$ - - Freely sol, in water ( $(\mathrm{r}: \mathrm{x} .5)$. Dose, $0.06 \mathrm{Gm}$., I gr., U.S.P.; 0.03 to $0.12 \mathrm{Gm}$., $1 / 2$ to 2 gr., B.P. Maximum dose, 0.3 Gm., 5 gr. 


\section{THE DIGITALIS GROUP}

General Statement.-Digitalis, Strophanthus, Squill, Apocynum, Barium, and a number of other drugs increase the tone, excitability and contractility of cardiac and arterial muscle. The vagus center is also stimulated. With therapeutic doses, these actions result in slower but more powerful beats of the heart, which bring about compensation in valvular cardiac lesions and thus relieve the symptoms-the irregularities, edemas, congestions, dyspnea, etc. Under these conditions, there is often marked diuresis. The blood pressure is generally unchanged in man, usually increased in animals.

The effects set in slowly on oral administration; almost immediately on intravenous injection. They tend to be cumulative, and to outlast the administration for a considerable time.

Toxic doses produce vomiting, cardiac irregularity, and sudden heart failure. The recognition of these toxic effects is important since they often arise from the cumulative action.

The members of the group are local irritants. Their hypodermic injection is painful. Their absorption from the gastro-intestinal canal is slow and often uncertain.

The active principles are generally neutral glucosids, and therefore rather unstable, especially in acid, aqueous solutions. Digitalis contains several distinct glucosids with similar actions, of which the crystalline Digitoxin is the most important. The various "strophanthins" are derived from several plants. They are chemically related, but differ in activity and toxicity according to their origin.

All the characteristic digitalis effects are produced by all the members of the group, so that a single description will suffice. The differences between them are only quantitative. So far as they are known, they will be pointed out as occasion arises.

Drugs Producing Digitalis Effects.-A considerable number of drugs containing glucosids with "digitalis" action are used in medical practice; such as Digitalis, Strophanthus, Squill, and Apocynum. Of less practical importance are Helleborus, Adonis, Convallaria, Oleander, Cheiranthus, etc. Digitaloid glucosids are important constituents of arrow-poisons; viz., strophanthin, ouabain, antiarin, acocantherin, etc. (Trendelenburg, 1909). The toxic principles found in the skin and blood of the common toad (Bufanin and Bufotalin) produce typical digitalis effects. They appear to be non-nitrogenous derivatives of cholesterin (review, Faust, I9I0). A very similar principle (Bafagin) is contained in the "parotid" glands of the tropical toad, Bufa agua (Abel and Macht, IgII). Frogs' skin also has a similar but much weaker action (Fuehner, rgro). Erythrophlein, a glucosidal alkaloid, can be counted in the group. Other alkaloids-veratrin, caffein, curin, epinephrin-remind somewhat of digitalis, but are not typical. Barium salts, alkaline hydrates, and even normal saline solutions produce cardiac actions which resemble digitalis, but the resemblance may only be superficial.

Active Constituents of Digitalis.-These comprise a number of glucosidal principles, of which digitoxin is the most important.

Well-defined chemic principles were first isolated by Schmiedeberg, 1875 ; his results were generally confirmed and extended by Kiliani, 1890 to 1899 ; but our knowledge is still incomplete and the nomenclature confusing. Some if not all of the amorphous products are probably mixtures. The different glucosids yield characteristic decomposition products. Merck, I912, in his "Reports," vol. 25, Kraft, 1912 and Kiliani, 1913, I914, give a summary of our present knowledge.

Pure Principles.-These can be conveniently divided into four classes: 
I. Crystalline; alcohol-soluble: Digitoxin, Schm., Kil. ${ }^{1 *} \mathrm{C}_{34} \mathrm{H}_{45} \mathrm{O}_{11}$. This is the best defined, most abundant, most active, and therefore the most important of the constituents of the leaves ( 0.22 to 0.4 per cent.), but does not occur in the seeds. It forms crystals, readily soluble in alcohol or chloroform, difficultly soluble in water or ether. It can be split into a hexose (digitoxose) and the crystalline digitoxigenin Kil. It is easily destroyed by ferments. It produces all the characteristic digitalis effects, but some of the undesirable side actions are too prominent.

Digitophyllin, Kil., is a similar crystalline principle of the leaves. Anhydrogitalin, Kraft, is also a closely related constituent of Digitalis infusions.

2. Amorphous; alcohol-soluble: Digitalin Verum, Schm., Kil.: $\mathrm{C}_{35^{-}}$ $\mathrm{H}_{36} \mathrm{O}_{14}$. Occurs in the leaves and seed. Slightly soluble in water, chloroform and ether. Splits into dextrose, digitaligenin and digitalose Kil. Produces typical digitalis effects, being about half as active as digitoxin. The name "digitalin" is often misapplied to other products and mixtures.

3. Amorphous; water-soluble: Digitalëin, Schm., Kil.; Gitalin, Kraft, I9ı1, I9г2; and Digalen, Cloetta, are probably mixtures (Kiliani, r9ı3, I9r4) of related substances. They occur in the leaves and seed. Insoluble in chloroform. They produce digitalis effects. (The name "digitalëin" was formerly also applied to other products.)

4. Water-soluble saponins: Digitonin (Digitsaponin); this occurs in a crystalline and amorphous form. The crystalline is less readily soluble in water. Both are only slightly soluble in alcohol and lipoid solvents. The crystalline Digitonin Kil. was considered a definite substance, which can be split into a hexose and digitogenin; but Windaus and Schneckenberger, r913, consider it a mixture, from which they have isolated Gitonin. The amorphous Digitonin Schm. (Digitsaponin, Kraft) is probably a mixture of the crystalline with several amorphous saponins (Kraft). The digitonins are precipitated by tannin; they combine with many alcohols and phenols to crystalline compounds. Digitonin does not produce the digitalis effects but has the irritant and hemolytic properties of the saponin group. The digitsaponins of the leaves are not hemolytic (Kobert, I9I2).

Inactive Principles.-A number of these have been isolated, such as the crystalline glucosid gilin and digin, Kraft, 1912, from the leaves.

Commercial Digitalins.-These are impure, often variable, mixtures of digitalis principles. The following are the most important varieties:

Digitalin "Amorphous;" generally means French or Belgian digitalin (digitaline chloroformique); uncertain composition; insoluble in water (perhaps mainly Digitalin Schm.).

Digitalin "Crystallized;" objectionable synonym for Digitonin; for Nativelle (French); and for Digitoxin.

Digitalin "Homolle;" mixture of glucosids; mainly true Digitalin; practically insoluble in water.

Digitalin "Nativelle;" crystalline, probably mainly Digitoxin or Digitophyllin.

Digitalin "German;" amorphous mixture from seed; contains principally Digitonin (5o to 60 per cent.) and some Digitalin verum ( 5 to 6 per cent)., and Digitalëin. Soluble in water or alcohol.

Galenic Digitalis Preparations.-The marked differences in the solubility of the pure principles is much less important in the galenic preparations. Even digitoxin is largely extracted from the leaves by water, presumably by the solvent action of the digitonin; so that a r:Io 
infusion contains two-thirds of the digitoxin of the leaf. The effects of the infusion and tincture are therefore nearly equivalent. The infusion is practically inferior because of its rapid deterioration.

Variations in the alcoholic strength of the menstruum between 35 and 95 per cent. make little difference. The fluidextract is unreliable, being only three times as active as the tincture, instead of ten times, as it should be theoretically (Hale, I9II). Glycerin extracts are inferior (Rippetoe, I910). Acidified extracts are weak and unreliable (Pearson, I9I3).

Powdered digitalis is often used, but is rather more irritant. All things considered, a good standardized Tincture is still the best and most reliable preparation.

Deterioration of Digitalis.-The principles are stable when dry or in alcoholic solutions; but like all glucosids, they are subject to decomposition in the presence of water, especially by bacteria, moulds or acids. This results in loss of activity; there is little evidence for the statement that the toxicity is increased. J. Loewy, I906, claimed that an infusion lost nearly 25 per cent. of its activity by standing twenty-four hours at room temperature; but Hatcher and Eggleston, I915, found it practically unchanged a week or longer under ordinary conditions. The addition of alcohol is not necessary. Acids are incompatible with the digitaloid glucosids, although they have been employed in some of the preparations, $f . i$., of Squills.

The tinctures and preparations containing at least 50 per cent. of alcohol do not deteriorate materially (Sharp and Lankaster, I9IO; and Hale, I9II, claim a slight deterioration after a year). Symes, I913 $^{1}$, found that the B.P. tinctures begin to deteriorate in three to five months. After a year or two they reach a level after which further deterioration is very slow. The dry leaves keep indefinitely (Sharp and Lankaster; Hatcher and Eggleston, I9I3), so long as they are not mouldy, without the necessity of the elaborate precautions advocated by Fock, I 906 . They are not injured by heat of $120^{\circ} \mathrm{C}$. but suffer above $140^{\circ}$ (Hale, I9II).

Chemistry of Strophanthins.-Great confusion exists as to the identity and relations of the several kinds found on the market and isolated by different investigators. This is due partly to incomplete knowledge of the plants, partly to ignorance of the constitution of the substances; and largely also to careless nomenclature. They are sometimes denoted by prefixing the first letter of the species from which they are obtained; f.i., g-strophanthin from S. gratus, etc. The strophanthins are glucosids, differing quantitatively in their actions (even the similarly named commercial specimens, Heffter, I909).

The numerous species of Strophanthus (Apocynacex) and other plants were used by the natives of Africa for the manufacture of arrow-poisons. The official varieties are the seeds of Strophanthus Kombé (U.S.P., B.P), and S. hispidus (U.S.P.). The strophanthin originally isolated by Fraser, 1870, was the amorphous Kombé variety (Gilg, 1904). The following outline is based mainly on the paper of Heffter and Sachs, I9I 2.

Strophanthin amorphous (Strophanthin-Boehringer) is the official strophanthin. It occurs in the Kombé and hispidus. The two are very similar, probably identical. Kombé further yields a crystalline strophanthin (Houghton, I9 I I Brauns and Closson, I9I3, I9I4) which is very similar. The amorphous is slightly more toxic for rabbits than the crystalline Kombé. All three are hydrolyzed by acids with the production of the crystalline Strophanthidin, $\mathrm{C}_{27} \mathrm{H}_{38} \mathrm{O}_{7}$. This is several times more toxic than strophanthin, and produces similar cardiac action, but with greater tendency to respiratory paralysis (Groeber, 1913 ). The crude drugs Kombé and hispidus are about equally active.

Ouabain, gratus Strophanthin-Thoms; Crystallized Strophanthin-Merck, $\mathrm{C}_{30} \mathrm{H}_{46} \mathrm{O}_{12}+9 \mathrm{H}_{2} \mathrm{O}$. - This is the only "Crystalline strophanthin" on the market. It is the best characterized of the principles. It does not yield Strophanthidin. It was first isolated by Arnaud, I888, from the wood of Acocanthera Ouabaio; and by the same investigator, I898, from S. glaber. Later, I910, Thoms obtained it from S. gratus, which was found to be identical with glaber. This is more readily obtained genuine than the other Strophanthus species. Strophanthin has been described as methylouabain; and acocantherin as dimethyl-ouabain; but the evidence for these views is not 
convincing (Brauns and Closson). Ouabain is about twice as toxic as strophanthin; and this is twice as strong as acocantherin. S. gratus also contains a second amorphous strophanthin (Thoms). The "pseudo-strophanthins" of Feist were probably ouabain, but their identity is now obscure.

\section{DIGITALIS ACTIONS ON THE CIRCULATION}

The effects of digitalis may be divided, especially in mammals, into two well-defined stages, the therapeutic and toxic. The typical phenomena of the two stages are shown in Fig. I8.

Preliminary Fall on Intravenous Injection.-During the intravenous injection of digitalis, the pressure falls sharply $(a)$, probably on account of the local irritant action on the heart. This does not occur in the other methods of administration. The fall is not at all lasting (Blake, I839).

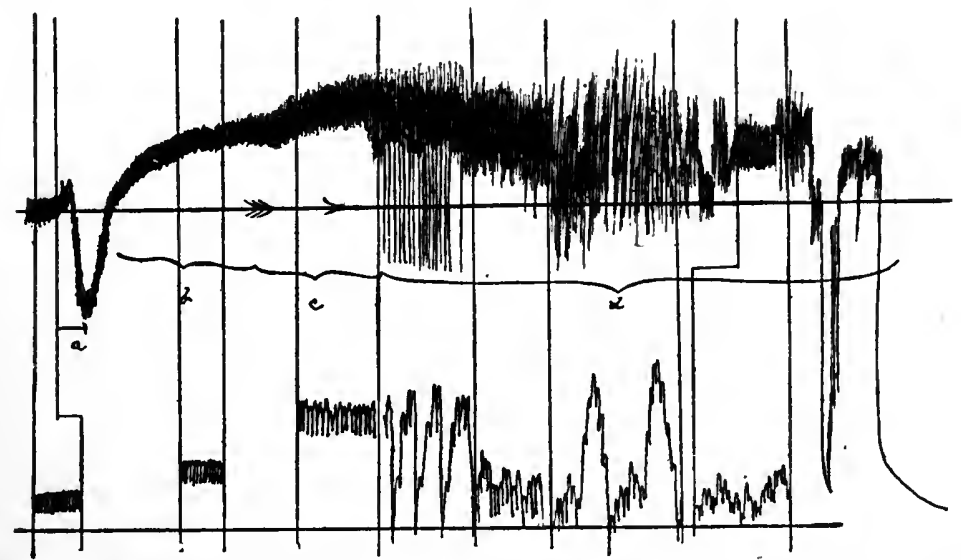

Frg. 18.-Digitalis on blood-pressure, dog. The upper tracing is taken on a very slowly moving drum, to show the successive effects. The lower tracing is taken with a faster speed. The vertical lines indicate the correspondence of the fast and slow tracings. The upper abscissa corresponds to the original mean pressure; the lower abscissa to zero pressure. The digitalis is injected at $a$. (See text.)

The therapeutic stage (Fig. $18, b$ and $c$ ) is characterized by a slower, fuller pulse, due to stimulation of the vagus mechanism (central and peripheral), and of the cardiac muscle. The cardiac efficiency, the output per beat and per minute, are increased. This tends to raise the blood pressure; in animals this tendency is supported by a more or less powerful vasoconstriction, involving particularly the intestines and liver. In man, the blood pressure is not much altered, presumably because of the more efficient vasomotor regulation; nor is the normal human heart usually slowed by ordinary doses, although the conduction of auricular impulses is delayed.

The modifications of these actions in disease will be considered under the therapeutic use. Before taking this up, the important phenomena require a more detailed presentation.

The Digitalis Slowing.-In mammals, this is practically entirely vagal, and involves almost exclusively the vagus center: for it is almost completely eliminated by section of the vagi (Ackermann, r873; Kochmann, rgo6, r909; Etienne, rgog), and atropinized animals show practically no slowing. The slowing produced by digitalis in auricular fibrillation 
is a different phenomenon. It occurs even after atropin, and is therefore quite peripheral (Cushny and Mackenzie; Gottlieb, I9I4).

The different digitalis drugs probably differ quantitatively, in the degree of vagus stimulation (Kochmann; Etienne, 1909; Haynes, 1906). This might be of practical importance were our information more complete.

The slowing is due mainly to direct stimulation of the vagus center, and not merely to the rise of blood pressure (as was supposed by A. B. Meyer); for the slowing occurs in the clinical cases in which the blood pressure is unchanged or even diminished (Fraenkel and Schwartz, 1906). Greene and Peeler, 191 5, found the turtle heart slowed or stopped when digitalis was perfused through the brain only; the slowing being abolished by dividing the vagi. In animals the rise of pressure undoubtedly contributes to the slowing, for the slowing is about twice as great if the pressure rises than if the pressure is kept just level by hemorrhage (Kochmann, I906).

Vagus Stimulation from Rise of Blood Pressure.-It is well known that the tone of the vagus center is governed largely by the blood pressure, the heart being slowed as the pressure rises and quickened when the pressure falls (Bernstein, 1867, Verworn, I903). The slowing on rise of pressure is entirely vagal, for it does not occur if the vagi have been divided. It is also absent if the pressure is raised in the cerebral arteries alone; so that the stimulation is not due to compression of the brain, but to a reflex, mainly from the heart (Kochmann, I906).

Persistence of Vagus Center Stimulation. - This probably persists till near death; but the heightened contractility of the cardiac muscle renders the heart less and less responsive to inhibitory impulses. Vagus stimulation will therefore fail to slow the heart in the later toxic stage (Liagre, 1906); but that this is not due to paralysis of the vagus may be judged by the fact that vagus stimulation still weakens the beats.

Other Phenomena of Vagus Stimulation.-The heart under the influence of digitalis exhibits also the other phenomena of vagus stimulation: greater dilation in diastole and lengthened diastolic pause; tendency to exaggerate the respiratory pulse variations; lessened diastolic tone, lessened conductivity, lengthened interval between auricular and ventricular contraction, tendency to heart-block, etc. Some of these tendencies become important in pathologic conditions and with toxic doses; for clinically the vagus stimulation seems to inhibit most strongly whatever function of the heart is already partly depressed (Mackenzie).

Effect on the Amplitude and Output of the Heart.-In virtue of the vagus stimulation produced by therapeutic doses of digitalis, the diastolic excursions of the normal heart are more nearly maximal, and the diastolic pause is prolonged, thus facilitating the influx of blood. With pure vagus stimulation, the systolic contraction is less complete so that the output per beat is often reduced, and the output per minute is always diminished (unless the rate has been extremely rapid; Y. Henderson, I909). With digitalis, however, the muscular stimulation makes the systolic excursion also more complete, so that the heart is both better filled and better emptied (Cushny, I897). The output per beat is therefore greatly increased; the "pulse pressure" or "pulse volume" is improved. This increase more than offsets the slowing, so that the minute-output is also increased.

The increased output per beat amounted to 50 per cent. in the cardiometer experiments of Cushny, 1897. If the slowing is excluded by atropin, the time-output would be increased proportionately. If the slowing occurs, the increase would be proportionately less; but in the therapeutic stage it is still considerable- to 20 per cent. in Cushny's experiments. Tigersted, 1907 , also showed with the stromuhr that the output is generally increased. Heinz, r9oo, found the flow of blood from the carotid, at a constant pressure, to be increased by 50 to 250 per cent. The effect would be the greater, the weaker the heart was before the digitalis; that is, the more removed the excursions were from the physiologic optimum. 
Heart Rate in Man.-Patients with auricular fibrillation, or with adynamic or unstable hearts, respond constantly by slowed pulse to ordinary doses. Fairly normal hearts are slowed by large doses, $0.4 \mathrm{Gm}$. per day for five to seven days. This, however, is due to blocking of the auricular impulses; the auricular contractions occurring with unreduced or quickened rate. The origination of the sinus rhythm is therefore not slowed by digitalis, except in adynamic hearts (Cohn, I915).

Auriculo-ventricular Conduction in Man.-This is constantly impaired, being an early and positive sign of digitalis action. It may be observed within forty-eight hours, and increases with the administration, from simple lengthening to heart-block. After withdrawal, it persists generally about two days, but sometimes two weeks.

Systolic or Muscular Action of Digitalis.-The increased systolic excursion must be due to a direct action on the cardiac muscle. The study of its fully developed features, as they occur in the toxic stage, shows that digitalis increases the contractility, the systolic tone and the excitability of the cardiac muscle.

Extrasystoles are produced by smaller stimuli in hearts under the influence of the therapeutic and the smaller toxic doses of digitalis (Pletnew).

Relative Duration of Systole and Diastole.-In the digitalis heart, the duration of the actual contraction and relaxation are practically unchanged. The speed of the systolic contraction is somewhat increased (deHeer, I912). The slowing occurs mainly in the diastolic pause; the systolic pause is also lengthened a trifle. Atropin removes the systolic as well as the diastolic slowing; so that the effect is entirely vagal (Cushny, 1897 ). The two phases behave similarly in the excised and perfused mammalian heart (Haynes).

The excised mammalian heart, in the therapeutic stage, shows practically the same phenomena; except that there is much less slowing, since the vagus center is excluded. The slowing is diastolic and can be removed by atropin (Haynes, I906). The excursions of both ventricles are increased especially in systole, so that the energy of each beat may be more than doubled. The time required to produce maximal slowing is an hour for digitalis, one-fourth hour for strophanthin (Haynes). Existing irregularities are removed, and a fibrillating heart may resume regular contractions (Hedbom, i 898; Braun and Mager, I899; Gottlieb and Magnus, I903).

Cardio-electrograms. - These show slowing of the phases and pauses, and changes in the peaks; the latter are increased by small, lowered by large doses (Bickel and P'awlow, I913). Reversal of the T-wave occurs early during digitalis treatment, being probably a sign of the muscular action. This effect is also produced by digitalis in febrile patients (Cohn, I9I5).

Gaseous Metabolism of the Heart.-This is increased in mammals in proportion to the activity (Rohde and Ogawa, I I I 2). In excised frogs' hearts, the oxygen consumption is decreased according to the diminished rate and excursions (Gottschalk, I913).

The Phenomena of the Therapeutic. Stage in the Frog's Heart.-If a digitalis drug is injected into a frog, the exposed heart shows actions which resemble those seen in mammals. The inhibitory effects are more important, whilst the contractility is but little affected in the therapeutic stage. The rate becomes progressively slowed, and the diastolic relaxation is more complete. The slowing occurs even after atropin (Boehm, I872); but otherwise it resembles vagus stimulation. Direct vagus stimulation produces additional slowing. By using the perfused excised heart ( $\mathrm{F}$. Williams, 1880), it can be shown that the diastolic tone is considerably decreased, so that a given pressure distends the heart more than normal; and since it contracts completely in systole, the output per beat is increased. The time-output and the blood pressure may be increased or diminished; but the heart is not more capable of overcoming an excessive aortic resistance. The toxic effects will be described later.

Ventricular Strips of Turtle.-Large doses produce acceleration (even after atropin or ergotoxin), and increase of amplitude and tone (Greene, Boutwell and Peeler, I9 15).

Behavior of the Different Chambers of the Heart.-The two sides of the heart behave very much alike to Digitalis; but the auricles tend to show relatively greater inhibitory effects (since they are more subject to vagus control), whilst the ventricles are more disposed to the muscular action. 
In the therapeutic stage, the rhythm of the auricles and ventricles is synchronous.

The relaxation of the auricles is but little changed; their contraction is more complete; but with slightly larger doses the inhibition predominates so much that the contractions are less complete. The inhibitory effects disappear if the vagi are divided (Cushny, I897).

Influence of the Cardiac Action on the Blood Pressure.-The increased output of the heart should result in a rise of pressure. Accordingly, the pressure rises even when changes in the peripheral vessels are definitely excluded ( $f . i .$, in Bock's method, where the aorta is tied, the circulation being shunted through the carotid directly to the jugular vein-Bock, I898; C. W. Greene, r910).

No Effect on the Maximal Pressure.-Whilst the Digitalis heart can discharge a ıarger volume of blood against a normal pressure, the maximal pressure-i.e., the rise of pressure on clamping the aorta-is not increased (Bode; Heinz, I900).

The Vasoconstrictor Action.-The cardiac actions of digitalis would of themselves tend to raise the blood pressure. The most important element in the rise which is observed in animals, however, is a vasoconstriction, involving mainly the intestinal vessels. This is shown most conclusively by the observation that the volume of the important splanchnic organs decreases as the blood pressure rises (Gottlieb and Magnus, I90I). Further evidence is that the venous outflow is also decreased; and that the rise is not always accompanied by increased output of the heart (Tigerstedt, r907, stromuhr). Small therapeutic doses may produce intestinal constriction even when the blood pressure does not rise. The vascular effects are more lasting than the cardiac (Joseph, I9I3).

The vasoconstrictor effect is peripheral because it occurs in excised organs (Donaldson and Steffens, I883; Kobert, r886; Brunton and Tunnicliffe, 1896 , etc.). It occurs also after the nerve-endings are paralyzed by apocodein (Dixon, 1903). The action is therefore presumably exerted directly on the arterial muscle. The vasomotor center is but little affected.

Vasomotor Center.-Strophanthus produces a moderate stimulation; digitalis much less or none. In no case is the central action sufficient to make itself felt through the other actions of the drugs. Fatal doses result in sudden cardiac arrest and asphyxia, thereby producing intense stimulation of the vasomotor center (Pilcher and Sollmann, I915).

Vasodilator Action.-Very dilute solutions of digitalis produce an opposite effect; i.e., the perfused vessels are dilated (Kasztan, Igro; Fahrenkamp; rgrI). The vascular effects are therefore analogous to the cardiac effects; the smaller doses decrease, and the larger doses increase the tone.

Differences in the Vascular Areas.-The constrictor tendency varies according to the drug and the vessel. It is generally said to be greatest for digitoxin, and relatively weak for strophanthin; but an exact series has not been established.

$F . i$. , de Heer, I9l2, and Joseph, I9r.3, found that ouabain had a strong constrictor effect in mammals; while Moog, I 12 , claims that its constrictor action is but one-tenth that of digitoxin. Stroomann, r9I4, finds strophanthin the weakest constrictor in frogs. Caffein is antagonistic. Vogt, I909, claims that strophanthin does not produce constriction in man, but Straub; I909, finds evidence of constriction with the stringgalvanometer.

The constriction is strongest in the intestinal and splenic vessels, 
less in the renal, and weak in the vessels of the skin, brain, etc. The dilator and constrictor effects vary in degree in different animals; but with therapeutic doses, in animals, the most constant and predominant effect is the slow constriction of the intestinal vessels. The dilator effect occurs earlier, but the constriction lasts longer (Joseph, I913). In man, the volume of the arm is not changed by intravenous digitalis or strophanthin (Hewlett, I9I3).

Altered Distribution of Blood.-The different susceptibility of the vessels leads to alterations in the distribution of the blood in living animals. The intestinal vessels will tend to be constricted; those of the skin, extremities and brain will tend to be dilated (Gottlieb and Magnus, I9or). The renal vessels occupy an intermediate position; with therapeutic doses they tend to dilate (Loewi and Jonescu, rgo8); with toxic doses, they will be constricted. The dilations may be due to the direct dilator action; or to mechanical displacement of blood from the more powerfully constricted areas; or to nervous reflexes.

In the experiments of Gottlieb and Magnus, rgor, the dilation of the vessels of the extremities was shown to be mainly mechanical; for it did not occur (or rather, the leg vessels constricted), if the displacement of blood was prevented by ligating the splanchnic vessels.

The direct dilator effect has been rendered very probably for the kidneys; for it has been shown on the excised organs that certain concentrations dilate the renal vessels, whilst they constrict the intestinal vessels.

Pulmonary Vessels.-These are somewhat constricted in perfusion experiments (Tschistowitch). Excised arterial rings are also constricted by all digitaloids, except digitonin, which dilates (Macht, 1914). In life this action is unimportant; for the slight rise of pressure in the pulmonary artery is mainly cardiac (Plumier, 1905; Wiggers, I9I I). The circulation time is shortened in the pulmonary and in the general circulation (Langlois and Desbouis, I9I2).

Meningeal Vessels.-The filling of the vessels of the pia mater and retina follows the general blood pressure (Hirschfelder, I I I5).

Coronary vessels may be constricted, especially by digitoxin, less by strophanthin (O. Loeb, r903; Haynes, I906; Rabe, I9I2). Excised arterial rings are also constricted (Voegtlin and Macht, I913). In any case, the constriction would be overcome by the improved contraction; so that, with therapeutic doses, the coronary flow in life was found unaltered (Bond, I9I I), or even increased (F. Meyer; Sakai and Saneyoshi, I9I5). J. L. Miller, 1908, found that digitalis enabled the heart to tolerate a greater degree of coronary obsiruction. Large toxic doses contract the coronary vessels even in life, with rising blood pressure; this is sometimes followed by increased flow with falling pressure (Sakai and Saneyoshi, IgI5).

Venous Pressure.- This is not materially altered, even when the arterial pressure rises (Capps and-Matthews, 1913; it has also been studied by Ackermann and by Popper).

Blood Pressure in Man.-The rise of pressure which is so characteristic for most animals is not often seen in man, either in health or disease (Czylharz, I9oo; Schwartz, I906; Cushny, I9II; Mackenzie, Igrr; Price, I9I3; Cohn, I9I5). In heart disease, the improvement under digitalis may even be accompanied by a fall of pressure, if this has been abnormally high (Fraenkel, I905; Price, I9I4; Martinet, I9I4). A rise is very exceptional, occurring only if the pressure has been below the normal level. The diastolic pressure is always lowered when digitalis relieves in compensated lesions, no matter what the changes in the systolic pressure. The pulse pressure is therefore generally increased (Martinet, r9I4).

The difference can not be explained by dosage; for in animals the rise occurs with the smallest doses which have a noticeable effect on the heart. The explanation must be sought in the more perfect blood pressure regulation of man, which compensates the pressor tendency by inhibition 
of the vasomotor center and by stimulation of the vagus center. If the digitalis slowing is prevented by atropin, the pressure rises also in the human subject (Fraenkel, r905).

The compensatory vasomotor regulation has not been investigated in man. From what is known of it in animals, it would be expected that the vessels of the extremities and of the kidneys would probably be dilated, whilst the intestinal and portal vessels would tend toward constriction. The altered distribution of the blood may contribute to the therapeutic result.

Blood Pressure in Rabbits. - These do not show as great or constant a rise as do other animals. This is probably explained by the lesser resistance of their blood vessels, which therefore dilate if the pressure tends to rise above the normal. If the pressure has been lowered by hemorrhage, digitalis produces an effective rise toward the normal level (Hernando, I9I I). Digitalis did not produce much rise after chloral, presumably because the heart was too greatly injured. The effects of small therapeutic doses were studied by Joseph, I9r3.

Diuretic Action.-The digitalis drugs are powerful diuretics in cardiac dropsies; the diuretic effect being proportional to the improvement of the circulation and to the absorption of the edemas. In all other conditions they have little or no effect on the urine. Practically negative results are obtained in normal individuals (Schwartz, r9o6); in heart disease without effusion (Cushny, I9 I I "Cohn, I9 I $_{5}$ ) and in purely nephritic edemas (J. Miller, rgr2). The diuretic effect can not therefore be due to any renal action, but must be ascribed to the improved circulation. There is no diuresis in normal individuals, and in the absence of edema, because the renal circulation is already adequate for the secretion of all the fluid at the disposal of the body. It fails in nephritis, because here the anuria is not due to defective circulation.

The factors involved in the improvement of the renal circulation are several: The dilation of the renal vessels (Jonescu and Loewi, r9o8); the relief of the venous pressure; the increased output of the heart; the increased "pulse pressure;" and the hydremia resulting from the absorption. These mechanical conditions cause corresponding increase of urine filtration even in dead kidneys (Sollmann, r905).

Gesell, I9r3, found that artificial changes in the pulse pressure produced parallel changes in the excretion of urine, chlorids, urea and nitrogen.

Influence of Hydremia. - Martinet, I914, found that the viscidity of the blood and the protein content of the serum remain unchanged so long as the absorption of edema is in progress. When the edema has disappeared, but the diuresis still persists, the viscidity increases. This indicates that the hydremia is not the primary cause of the diuresis. Loria, I91 2, also states that strophanthin or calomel increase the viscidity of the blood even when there is no diuresis.

Excessive doses of digitalis constrict the renal vessels and irritate the kidneys, so that the urine becomes scanty, and often blood-tinged. This may conceivably happen if cumulation occurs in the therapeutic use; especially with digitoxin.

Toxic Effect on the Circulation in Mammals. - These are characterized by irregularities of the rhythm and strength of the cardiac pulsations (Fig. I9).

They are exaggerated manifestations of the vagus and cardiac stimulations which were initiated in the therapeutic stage. The vagus effects (slowed rhythm, diastolic tendency, lessened conductivity) reach their acme most rapidly; so that the first indications of overdosage are commonly those of marked inhibition. This inhibition is in turn overcome as 
the muscular excitability becomes greater and greater; so that the final effects are mainly muscular (increased tone, heightened irritability, spontaneous ventricular rhythm, extrasystoles). The irregularities which result from these interactions weaken the circulation and interfere with the nutrition of the heart, until this becomes arrested.

Cushny's Stages.-The successive effects have been studied most carefully by Cushny, I897, who divides them into the inhibitory stage and the stage of muscular irregularity.
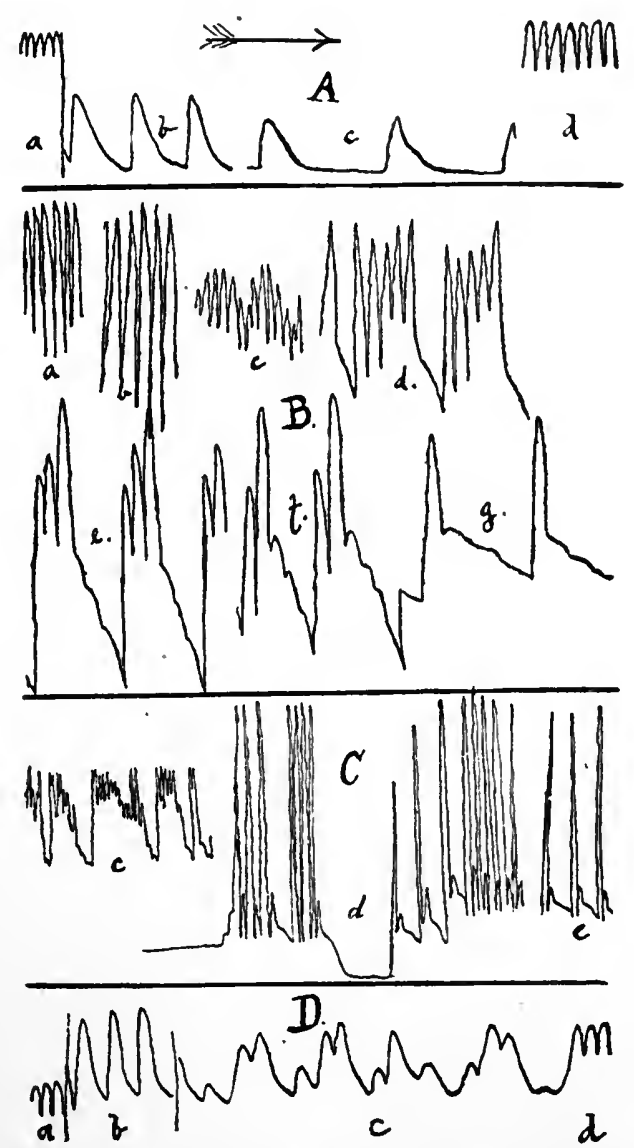

FIG. 19.-Blood-pressure tracings under digitalis, to show some of the types of irregularity in the toxic stages. A, B, C and D represent four different dogs. $(a)$ is the normal tracing; $(b)$ the therapeutic stage; the following letters represent the successive toxic changes.

Traube's Stages.-The effects may also be divided into three stages, according to Traube, I86I : (I) slowed pulse with rise of blood pressure (therapeutic stage); (2) sudden change to rapid pulse, with further rise of blood pressure; and (3) fall of blood pressure with continued rapid but irregular pulse. The inhibitory stage would be inserted between $I$ and 2 .

In the typical course, the effects pass successively through the therapeutic, vagus and muscular stages; but the vagus stage is often omitted in animals. The "toxic effects" in man are generally of the inhibitory type. 
The Inhibitory Stage (Fig. I9, A).-This corresponds to strong vagus stimulation. The rhythm becomes very much slowed-to 40 or 50 beats per minute. It is often irregular; there are scattered diastolic pauses which become more frequent and lasting, particularly in the auricles. These irregularities are partly exaggerations of the normal respiratory variations; but they are often independent. The two sides of the heart contract at the same rate, but the rhythm of the auricles and ventricles is often different. The ventricles are apt to assume a spontaneous rhythm, which may be faster or slower than that of the auricles. This independent rhythm is probably due in part to the blocking of the auricular impulses by the lessened conductivity; the increased excitability of the ventricular muscle also plays a part, for the ventricles may continue to beat during the auricular standstill. The excessive slowing and the irregularity diminish the time-output of the heart in this stage, although the amplitude is increased. The blood pressure falls.

Occurrence and Duration of the Inhibitory Stage.-This stage occurred in about 30 per cent. of Cushny's animals. After lasting a few minutes, it passes quite suddenly into the muscular irregularity. It is more frequent and more persistent in man. The difference may be due to different dosage and administration.

The Stage of Muscular Irregularity.-The rate, rather suddenly, becomes more and more accelerated, as if the heart escaped from the vagus influence. Electric vagus stimulation has less and less effect on the rate; but since it still increases the diastole the vagus can not be paralyzed. The escape is due to the excitability of the heart becoming so greatly increased that the vagus is unable to restrain it. The auricles and ventricles may at first keep the same rate; but the ventricles soon assume a rapid rhythm independent of that of the auricles; for the conduction of the auricular impulses is more and more blocked, whilst the spontaneous excitability of the myocardium is more and more increased. The accelerators usually do not respond to stimulation (Fredericq, I9 33 ).

Periodic Grouped Beats.-This independence of the auricular and ventricular contractions results in curious periodic variations in the volume of the pulse, the rhythm at this time being fairly regular.

One or more enormously strong beats are followed by very much weaker beats, sometimes quite imperceptible in the pulse, which is therefore intermittent (see Fig. 19, $B$, $e$ and $g$ ). These phenomena recur in groups, which remain fairly regular for a short time, but soon change to some other type, as may be seen in Fig. I9, $B$ and $C$. These effects are sometimes due to respiration. In other cases, however, they are cardiac. They are also seen in the atropinized heart, and are mainly due to the arythmia of the auricles and ventricles (Cushny, I 899). It will be recalled that the ventricular contractions are normally originated by the auricular contractions. Toxic doses of digitalis seem to interfere with the transmission of this impulse, and at the same time, they raise the excitability of the cardiac muscle to such a degree that contractions can originate in the ventricles. The auricles and ventricles therefore beat independently; either may be the more rapid. As a consequence, the contractions will sometimes alternate, sometimes coincide, forming groups (see Fig. 20). If the auricular systole occurs during the ventricular diastole, the ventricles will be filled with blood when they contract, and the result will be a large pulse-wave; if the auricular and ventricular systole coincide, no blood will be moved, and there will be no pulse (see Fig. 20, $p$ ). This is the most satisfactory explanation of the group phenomenon. A further factor may come into play: it is probable that the auricular stimulus to the ventricle is not completely blocked by the digitalin. If the auricular and independent stimuli coincide, the ventricular contraction would be strong; if they interfere, the contraction would be weak or absent. This effect would support that previously described and lead to the same kind of grouping.

The two sides of the heart continue to beat synchronously, although the periodic variations of strength have no relation.

After a time, extrasystoles (i.e., premature contractions by which no blood is expelled) 
appear, and the pulse shows corresponding intermissions. These become more numerous. The rate continues to increase, becoming faster than in atropin poisoning. Various complex irregularities are superadded; and the contractions become thoroughly disorganized.

The blood pressure is very high in the earlier periods, through the combination of rapid rate, forceful contraction and vasoconstriction. When the periodic irregilarities set in, the output diminishes materially.

Terminal Changes.-The quickening of the rate soon exceeds the optimal limits. The contractions become superficial, fluttering, much less effective, and more fatiguing. The blood pressure falls and the heart is unable to keep the pace. First the auricles, then the ventricles, pass into fibrillation and the circulation is arrested. The chambers stop in median position, and then gradually become greatly dilated.

Muscular Changes in Rabbits. - In acute death from large doses, all parts of the heart

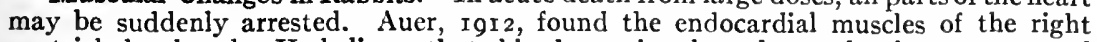
ventricle hardened. He believes that this change involves the conducting system, and that even the earlier actions of digitalis are due to slighter degrees of these chemic changes. Similar abnormalities occur in anaphylaxis.

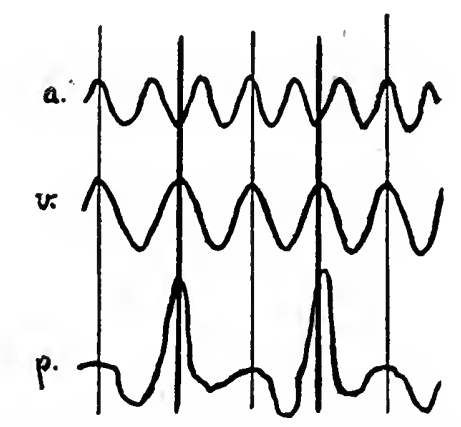

FIG. 20.-Diagram to illustrate the effect of auricular and ventricular arythmia; (a), auricular beat; $(v)$, ventricular beat; $(p)$, pulse-pressure. The auricular systole precedes the ventricular at the heavy lines, and the pulse is consequently strong; it coincides at the light lines, and the pulse is weak. With this difference of rate (I ventricular beat to $I \frac{1}{2} 2$ auricular) each strong contraction will be followed by a weak contraction.

Toxic Effects on the Excised and Perfused Mammalian Heart.-These resemble in the main the muscular irregularity of the intact heart. After the therapeutic stage, the contractions become quicker and irregular. The diastolic relaxation becomes progressively less complete, and the heart has a systolic tendency. It finally passes through delirium cordis into systolic standstill, the tone increasing even after the heart has stopped. Stretching of the muscle (as by increasing the coronary pressure) may temporarily restore the pulsations (Hedbom, 1898; Braun and Mager, I899; Gottlieb and Magnus; 1903; Haynes, I906; Etienne, I909).

The principal difference between the intact and the excised heart is the position of arrest; this is diastolic in the intact, and systolic in the excised. The systolic standstillwhich occurs also in the frog's heart-is the really characteristic effect of digitalis. In the intact mammal, the falling blood pressure impairs the coronary circulation, so that the cardiac muscle can not remain contracted.

Toxic Effects on the Frog's Heart.-This also shows a complex of inhibition and myocardial stimulation, but the manifestations differ materially from those in mammals. They vary somewhat according to the dose and method of application. They are entirely peripheral, and are not modified by excision of the heart or by atropin; but vagus stimulation remains effective until rigor sets in.

Typical Course.-If moderate toxic doses are injected into an intact frog, and the heart is exposed, the phenomena are as follows (Boehm, 1872): After a latent period of five to fifteen minutes, the diastolic tendency becomes conspicuous. The chambers expand more, the diastole is lengthened and the rate is somewhat slowed. The $A-V$ interval is unchanged (Clark and Mines, 1913). The systole is also more complete. With strong hearts, the effect is mainly on the auricles; but if the heart is weak, the ventricles are also stimulated (Clark, I9r4). This corresponds to the therapeutic stage.

The toxic irregularities start soon after. The systolic phase of the ventricle becomes exaggerated, so that at times the apex, at times the base, remains firmly contracted 
during the diastole. These partial diastoles may alternate so that the blood is merely pumped back and forth from one part of the ventricle to the other. This has been called, rather inappropriately, "cardiac peristalsis." Again, a part of the ventricle may remain tirmly contracted for a considerable time, and then may resume rhythmic contraction. (The "peristalsis" was first described by Vulpian, I 855.)

Eventually, the ventricular diastoles become weaker and weaker, until the ventricle finally goes into permanent systolic standstill, and appears as a small white lump, contrasting sharply with the turgid auricles. The apex is often arrested before the base. The auricles continue to pulsate for a time and eventually stop distended.

The ventricular systolic standstill is not paralytic; for if the ventricle is forcibly distended with fluid, it will again start effective beats (Schmiedeberg, I874). The standstill is due to excessive tone, which prevents diastolic relaxation under the ordinary venous pressure. It is not an ordinary but prolonged contraction, for the heart-muscle is not in the refractory phase (Tait and Pringle, I9I6). Schmiedeberg considers it as increased "elasticity."

Other hearts may not show the "peristalsis," but instead the phenomena of partial auriculo-ventricular "block," i.e., the ventricular rate suddenly falls to one-half, then one-third, one-fourth, etc., of the auricular rate (Straub, I90r). The auriculo-ventricular interval is also lengthened (Clark and Mines, I9r3). Eventually the auricular rate, and finally the sinus rate, may also be halved. The block may be explainable by prolongation of the refractory period, for Brandenburg, I 904 , showed that the heart is now less easily excitable to extrasystoles. On the other hand, the spontaneous contractility is increased, for if the sinus-auricular junction is crushed (I-Stannius experiment) the ventricles are not arrested as in the normal frog, but at once initiate a spontaneous rhythm.

In other hearts, the systolic phenomena so far described may be preceded by inhibition. The diastolic relaxation predominates, the heart remains regular and the beats become gradually slower to temporary diastolic standstill. This is witnessed but rarely in intact animals, and is then followed by the usual systolic phase (Boehm).

In excised and perfused frogs' hearts, the effects of dilute solutions correspond to those of vagus stimulation. The rate is slowed, the diastolic excursions increased, the systolic excursions at first unchanged, then diminished. This progresses to diastolic standstill (Jacobj, I g00; Wybauer, I900), from impaired conduction and heart-block (Straub; Clark, I9I4). The heart still responds to mechanical stimulation. Higher concentrations produce the typical systolic phenomena and systolic standstill. Very concentrated solutions paralyze the heart (Werschinin, r9ro). There is always a latent period of five to fifteen minutes, even when strong solutions are used.

The diastolic standstill occurs especially on applying the digitalis to the outer surface of the heart. Werschinin claimed that a given concentration produces the same effect on endocardial and pericardial application; but Holste, I9I2, and Clark, I9I2, attribute the diastolic standstill from intracardial application to technical errors. The diastolic standstill is a regular feature of low concentrations of the various digitaloid substances (Benedicenti, I902), including barium (Poulsson, I910).

The inhibitory phenomena bear a close resemblance to vagus stimulation. Haldschinsky, 1908, demonstrated the summation of submaximal digitalis and vagus inhibition. However, the digitalis inhibition is not influenced by atropin (Benedicenti, $\mathbf{1 9 0 2}^{2}$ ). It must therefore be concluded that the vagus and digitalis act independently on the same function of the muscle. (Schmiedeberg, r9ro, suggests that they stimulate specific "diastolic fibers;" but the existence of these is altogether hypothetical.)

I solated Auricle of the Frog.-The toxic dose of digitalis is at least ten times as great as for the ventricle; the phenomena, however, are similar (W. Straub, I9I5).

Fixation of Digitaloids in Heart.-W. Straub, IgIO to I9I3, investigated the quantitative relations of digitalis on excised frogs' hearts, using the response of "test hearts" as a quantitative method. He found the effects roughly proportional to the concentration of the poison, not to the absolute amount used. From this he argued that the reaction must be confined to the surface (envelope) of the muscle cells; the mechanism being similar to that of saponin action. Further investigation, however, showed that the quantity is also important (Clark, I9I 2; Gruenwald, 1912; Issekutz, 1915), and that the concentration is probably important mainly in determining the rapidity of penetration.

The effects of digitalis outlast its actual application. With small doses, the heart recovers completely on washing; but when immersed anew in digitalis solution, even after a long time, its threshold is found lowered (Gottlieb, 1914; Issekutz, 1915). These facts indicate that digitalis is absorbed and stored in the heart muscle, remaining there in active form for a considerable time. The amount of drug used up, however, is very small (Straub; Gruenwald; Clark; Holste, I9I2); somewhat greater with digitalin than 
with strophanthin (Weizsaecker, I9 I3). Fuehner, I9I2, finds that methyl-violet, which has a typical digitalis effect, actually accumulates in the cardiac muscle of frogs, the effects corresponding to the quantity of dye so fixed.

There is reason to believe that an actual storage of digitaloids occurs in the heart cells of mammals (Hatcher, I9I2), although the evidence is not conclusive. Lhota, I9I2, states that the storage occurs particularly in the heart and blood vessels, but also in the liver and other organs.

Wilbur, I9I1, reports that irritants (iodin) produce effects similar to digitalis on terrapin's heart.

Concentration Coefficient.-Sollmann, Mendenhall and Stingel, I915, determined the ratio of concentration and activity. They found that the feebler the actions of ouabain, the more is its activity increased by doubling the concentration. The reasons for this are explained in their paper.

Vigor of the Heart.-Holste, I9 2, and Weizsaecker, I913, find that this influences the response quantitatively. The greater the intensity of the cardiac functions, the prompter the strophanthin effect.

Temperature. - The cardiac effects of the digitaloids on frogs increase with the temperature (Trendelenburg, I909), the increase being greater for low than for high temperatures. The activity is increased by heat not only directly, but also through the more rapid heart rate (Sollmann, Mendenhall and Stingel, 19.5). In the perfused mammalian heart, the effects are also quickened by heat; in this case the more rapid coronary flow is a contributing factor (Gunn, I914).

Influence of Serum. - Werschinin found the systolic action much weaker in Ringer's solution than in serum-solutions of the drugs. Oppenheimer, I9I3, observed that serum inhibits the action of the less soluble digitaloids, but not of strophanthin. Cholesterin and egg-albumin did not have equivalent effects.

Oxygen increases the digitalis effect (Gruenwald, I9I2).

Influence of Ions. - The systolic digitalis action occurs only in the presence of $\mathrm{Ca}$ ions in the perfused solution. Excess of alkali or absence of potassium rather increases the systolic action; excess of $\mathrm{Ca}$ has little effect (Clark, rgi 2). On the other hand, strophanthin delays the standstill of the heart when this is perfused with $\mathrm{Ca}$-free solutions or with pure $\mathrm{K}$ solutions; but it hastens the arrest when added to a solution from which $\mathrm{Ca}$ and $\mathrm{K}$ are both absent (O. Loewi, I9I 2; Konschegg, 19I3). Strophanthin may therefore to a certain extent replace $\mathrm{Ca}$, and thereby antagonize the $\mathrm{K}$ ions. The effects of the ions on the vagus action are quite different (Clark, 1914).

Antagonism with Alkaloids. - Lhota, I909, claims that cocain diminishes the toxicity of strophanthin by lessening the cardiac excitability. Wilbur, I9II, found that strychnin often removed the ouabain heart-block in terrapin's heart.

Central Effects of Digitalis.-The peripheral effects dominate the symptom-complex so greatly that of the direct central effects only the emetic action is important. The cardiac action is so selective that frogs whose heart has been stopped by digitalis may hop about as if normal. The medullary centers are probably stimulated directly. This is proven for the vomiting and vagus centers, probable for the vasomotor center, and perhaps also for the respiratory center; but the indirect effects from the altered circulation are much more important than any possible direct actions.

Central Symptoms. - When digitalis is injected into the ear vein of unanesthetized rabbits, or subdurally, the symptoms occur immediately, and appear to be largely central. They consist in dyspnea; tremors and convulsions; miosis; debility and paralysis of certain muscle groups, especially the neck; abolition of certain reflexes. These effects are followed by asphyxial convulsions with mydriasis and death by paralysis of the respiration or heart. The later symptoms are, at least partly, due to the circulatory changes; but the earlier symptoms are probably due to direct central actions (Santesson and Strindberg, IgI6.)

Respiration.-Hypodermic or intravenous injection produces a primary increase of the respiration in rabbits. Larger doses depress (Gross, 1914).

Excised bronchial muscle is not affected by strophanthin (Trendelenburg, 1912). 


\section{GASTRO-INTESTINAL ACTIONS OF DIGITALOIDS}

Vomiting and diarrhea occur with overdoses of all digitaloids. Until recently they were erroneously attributed mainly to local action, especially of the digitonin. Hatcher and Bailey, I9II, pointed out that they occur more promptly on intravenous than on oral administration; and Hatcher and Eggleston, I9I 2, proved that the vomiting movements occur even when the whole alimentary tract has been excised. The action is therefore central, and it is useless to look for "non-emetic" digitalis preparations. The differences are merely quantitative. These conclusions are supported by the clinical data; for the nausea or emesis is always preceded by cardiac effects (Eggleston, I9I3). The gastro-intestinal symptoms generally disappear within two or three days after withdrawal (Cohn, I915).

Very sensitive individuals may, of course, be nauseated by the acrid taste of even small doses, but local irritation sufficient to produce emesis could only occur with doses much larger than the clinical. The digitonin must play a very insignificant part; for it is not emetic in doses very much larger than occur in the usual administration. The fat of the leaf is also non-emetic (Eggleston and Hatcher, 1913). The oil of strophanthus seed is emetic, but only in quantities 10,000 times as high as are present in a therapeutic dose of the tincture (Hatcher, I9I6).

Ratio of Emetic Action. - This may be expressed as the relation of the emetic to the fatal dose, intravenously. Eggleston and Hatcher found True Digitalin the most emetic (emetic dose $=22$ per cent. of the fatal); then come strophanthus, ouabain, digitalis, digipuratum, digalen and adonis (ratio $=40$ to 50 per cent.); then digitoxin and amorphous strophanthin ( 58 to 65 per cent.); least emetic were Squill and Apocynum ( 75 to 88 per cent.).

Klocmann, rg1 2, states that digitalis diminishes gastric secretion in fistula-dogs.

Squill was formerly used as nauseant expectorant; Euonymus (wahoo) as cathartic.

\section{GALL-BLADDER}

This is stimulated (Lieb and McWhorter, 1915).

\section{SUPRARENAL SECRETION}

Richards and Wood, I $\mathrm{I}_{5}$, find that the blood, after the intravenous injection of strophanthin or digitoxin, contains substances that inhibit intestinal tonus, and which they interpret as epinephrin. This inbibiting substance is absent if the splanchnics have been cut.

\section{LOCAL ACTIONS}

Digitalis and the allied drugs, when injected hypodermically, cause fairly severe irritation, pain and abscess formation, which practically preclude their use by this channel. Intramuscular injection is feasible, although not painless. The irritant action varies in different drugs.

Digitalis (particularly digitoxin) and squill are more irritant than strophanthin and ouabain (Haynes, I905).

Relative Irritant Action.-Holste, I9I3, from hypodermic and conjunctival application to rabbits, establishes a series for the isolated principles as: Most irritant $=$ digitoxin; then digitonin, then digitalein and cymarin (from apocynum). Practically nonirritant $=$ digitalin, ouabain and strophanthin. Digalen was much more irritant than digipuratum. On the skin of young pigs, O. Loeb and Loewe, I916, also found digitoxin and digalen the most irritant. Digipuratum, strophanthin and ouabain were about as irritant as digitalis infusion.

Strophanthin does not inhibit the emigrations of leucocytes from inflamed mesentery (Ikeda, 1916).

Anesthetic Action.-Steinach, I888, and Panas, I89o, found that application of strophanthin to rabbit's conjunctiva produces anesthesia lasting several hours. In man, however, there is considerable inflammation. Other digitaloids are also anesthetic. 


\section{THERAPEUTIC USES OF DIGITALIS}

Historical.-The professional use of Digitalis is relatively recent. It was recognizer] by Wm. Withering of Birmingham (Cushny, I9 15 ; Alpers, I9 15 ) as an ingredient of an old woman's secret formula. After ten years of experimentation, he published his results in 1785 . He was chiefly interested in the diuretic effect, and mentions the cardiac action only incidentally. The evidence for the connection of the circulatory and diuretic effect was first emphasized by John Ferriar in 1799 . The drug quickly assumed an important place, though its proper field was not recognized. The cardiac slowing particularly attracted attention, and as late as $185 \mathrm{I}$ Traube referred the clinical effects entirely to this "depressant" action; hence, Digitalis was used in aneurysm, internal hemorrhage, etc., for which it would be entirely unsuitable. Ten years later, Traube accepted the rise of blood pressure, which had been discovered in animals, as the basis of the therapeutic effect. This has been disproven only quite recently.

Strophanthus was brought to Europe in the early sixties, and its cardiac actions were promptly discovered. Its introduction into medicine is due mainly to Th. $R$. Fraser, 1885 .

The use of Scilla dates from the ancient Greeks, although its cardiac action was not recognized.

A pocynum was used by the early settlers as a hydragogue cathartic and diuretic; presumably they learned its use from the Indians.

Convallaria has also been used for several centuries in domestic medicine, against cardiac symptoms.

Explanation of Clinical Effects.-Digitalis, and the allied drugs, are used mainly to restore broken compensation in heart lesions. They do this by increasing the tone of the cardiac muscle, thus lessening the dilation; narrowing the leaking valvular ring; and increasing the amplitude of the systolic contraction and the time-output. They slow and regulate the generally rapid rhythm toward the normal optimum. They tend to remove irregularities, particularly in auricular fibrillation. In all of these ways they increase the efficiency of the circulation, insuring a better filling of the arteries, improving nutrition and oxygenation, and relieving the congested veins. The heart is among the first to feel the effects of this improvement. The vicious circle of weaker heart, poorer circulation is reversed in to the beneficial circle of stronger heart, improved circulation; so that the effects outlast the administration of the drug, and the improvement is perpetuated. At the same time, the circulation in the kidneys is improved, by the faster blood flow, the increase of pulse pressure, and the relief of the venous pressure. The kidneys are thus placed in the best condition for filtration or secretion. The anuria is relieved. If there has been edema, the improved kidney action results in extensive diuresis and thus in the absorption of the edematous fluid. This in turn improves the heart action and the whole circulation, by relieving them of the compression of the ascites. The vasoconstrictor action may also play some part; since it is strongest in the intestines and liver, it helps to relieve the congestion of these organs.

All of the effects of the therapeutic stage of Digitalis action may therefore contribute to its clinical usefulness. The increased tone, by diminishing the cardiac dilation; the increased amplitude, by increasing the output and thus balancing the leakage or obstruction of defective valves; the inhibitory action, by restoring the rhythm toward the optimal, and by partially blocking the response of the ventricle to excessively numerous auricular impulses (in auricular fibrillation); and the vasoconstriction, by shifting the blood from the congested venous areas, into the depleted arterial system. Rise of blood pressure as such, has no important share in the action; for it rarely occurs clinically, even with intraven- 
ous injection, or in cases which are greatly benefited (Fraenkel and Schwartz, I907).

Differences in Health and Disease.-The actions of digitalis are the same whether the heart is normal or abnormal; but the results of these actions are somewhat different. In health, the results of digitalis constitute departures from the normal, which are antagonized by compensatory mechanisms; in disease, the results constitute a return toward normal, and are therefore not antagonized in the same way. Consequently, the effects of digitalis are generally greater on those circulatory functions which have departed most widely from the normal. The removal of the cardiac abnormalities also entails consequences which are not witnessed in the normal individuals; such as more efficient regulation of the rate, the removal of irregularities, of dyspnea, of edema, the diuretic effect, etc. These differences could almost be predicted from a proper understanding of the pharmacologic data; but it is important that they be taken into consideration when the results of animal experiments are transferred to the clinic.

Efficiency of Digitalis in Cardiac Dilatation.-The clinical value of digitalis may be best appreciated if it is conceived as restoring the tone and contractions of an exhausted, fatigued, but otherwise normal heart muscle, to healthy efficiency. Its usefulness therefore depends upon the physiologic conditions of the heart, rather than upon the anatomical lesions. It will be most useful in those conditions in which the ventricular muscle is called upon to do an amount of work which exceeds its capacity, and which therefore induces dilation. This occurs particularly in valvular lesions, where the incomplete emptying of the heart places the muscles under an ever-increasing strain. The response will depend upon the quantity of muscular tissue on which the digitalis can act: it is therefore generally more effective in ventricular dilation (mitral and tricuspid insufficiency) than in auricular dilation (mitral and tricuspid stenosis). The diastolic expansion of a normal heart is increased by the vagus action of digitalis; but the excessive dilation of a weakened heart is reduced by the muscular effect. The difference in size may not be demonstrable by clinical methods.

If the cardiac muscle is degenerated, as in cardiosclerosis, digitalis will be useless or even harmful, by imposing too great a task on the few weakened muscle fibers. It will be of little use in acute failure of a previously healthy heart (simple collapse), since this is not due to muscular exhaustion; nor is it very effective against the toxic cardiac dilation of acute fevers. On the other hand, most striking results are produced by intravenous strophanthin injections in threatened acute failure from pure myocardial exhaustion.

Efficiency of Digitalis in Cardiac Irregularities.-The second important factor in the usefulness of digitalis is the regulation of arythmias. It is especially effective in the "absolute irregularity" of auricular fibrillation. Other irregularities are not so much improved or may even be exaggerated. Digitalis is rather dangerous in partial heart-block. It must not be forgotten that overdosage of Digitalis may provoke any of the clinical types of irregularity even in a healthy heart, and this will occur more readily when the heart is disposed to them by disease. It is therefore necessary to watch carefully for the appearance of any irregularities. 
Auricular fibrillation (formerly miscalled "nodal rhythm" by Mackenzie) is apt to occur when the auricles are excessively dilated, as in advanced myocardial and valvular lesions. The overstretched auricular muscles are unable to make concerted contractions, and instead enter into a state of continual tremulation or fibrillation. These numberless irregular impulses are transmitted to the ventricle, and thus produce a very rapid confused rhythm. The output of the heart is greatly decreased, and the results are very serious.

This is the condition in which digitalis produces the most striking and rapid improvement (Cushny, I9Ir; Mackenzie, I9II): The rhythm does not become normal but the rate is reduced to about normal, the irregularity becomes less and the dyspnea and other symptoms of heart failure are relieved. The improvement of the heart is better reflected in the apex-beat (auscultation) than in the pulse (Christian, 1915).

The auricular fibrillation is not changed, although it may be presumed that the distention is lessened. The main factor in the improvement is probably the lessened conductivity, by which many of the superfluous auricular impulses are blocked from the ventricle; for somewhat similar results may be obtained from simple vagus stimulation. (In auricular fibrillation, mechanical stimulation of the right vagus usually slows the ventricular rhythm without affecting the auricular rate (Robinson and Draper, Igr I). The direct regulating action of digitalis on the cardiac muscle may play some part; for it has been found that digitalis tends to remove irregularities in excised hearts.

Effect on Blood Flow.-The improvement under digitalis in auricular fibrillation is accompanied by a prompt and decided increase in the blood flow through the hand. Since there is no evidence of purely local vasodilation, this means improved output of the heart. In a case in which there was no clinical improvement there was also no increase of the blood flow (Stewart and Scott, 1915).

To obtain the best results in auricular fibrillation, the digitalis must be started with full doses. Smaller doses must then be continued for a long time, giving just enough to keep the rate between 60 and 70 per minute. Overdosage is often indicated by occasional reduplication of the pulse wave. This is an indication to reduce the dosage.

These "coupled beats" (pulsus bigeminus) are not produced by digitalis in any other condition (Mackenzie). Each ventricular beat originating from the auricles is followed by another arising in the ventricle (Lewis, I9II).

If the auricular fibrillation occurs in cardiosclerosis or when the myocardium is excessively weakened, digitalis is much less effective and much more dangerous.

Excessive doses of digitalis (also of barium, aconite, etc.) may of themselves produce auricular fibrillation, in man as well as in animals, by increasing the excitability of the auricles (Cushny, I9II).

Deficient Vagus Tone.-In simple quickening of the cardiac rate, by diminished vagus tone, the efficiency of digitalis depends upon the cause. In the milder grades of cardiac dilation, the vagus tone is automatically inhibited in the effort to keep the blood pressure to the normal. Digitalis, by strengthening the beats, removes the need for these rapid contractions, and they naturally return to the normal rate. The return is doubtless hastened by the direct stimulant effect of digitalis on the vagus mechanism. In some cases, it fails to slow a fast but regular rate; $f . i$., the rapid heart rate of fever is not much affected.

Paroxysmal Tachycardia.-The response varies. In some cases, digitalis is practically ineffective; in others, intravenous strophanthin reduces the rate very strikingly.

Periodic changes in sinus rhythm (such as the exaggerated respiratory variations, which occur in general nervous exhaustion, rather than in cardiac disease) are not benefited by digitalis. On the contrary, the vagus stimulation would tend to render them more marked.

Extrasystoles. - These would not be removed by digitalis since they are probably due to heightened irritability of the myocardium - a condition which could only be exaggerated by digitalis. Extrasystoles due to nervous exhaustion, indigestion, nicotin, etc., do not require medicinal treatment; they tend to disappear under suitable hygiene, and in any case, they have no serious significance. (The influence of various drugs on extrasystoles has been studied by McCord, ror 2.)

Partial Heart-block (diminished conductivity of the auriculo-ventricular bundle).-This is always exaggerated by digitalis: a slight retardation between the auricular and ventricular contraction may thus be converted into a partial block, so that the ventricles respond only to every 
second or third beat of the auricles; or a partial block of this kind may be converted into a complete block, the ventricles assuming their characteristic slow independent rhythm. In partial heart-block (even when the $\mathrm{a}-\mathrm{c}$ interval in the jugular tracing is merely lengthened) digitalis should be avoided, and employed only with caution when it is otherwise urgently needed. If the need for it exists, however, it may relieve the symptoms even though it exaggerates the partial block.

Complete Heart-block.-In this, digitalis can do no further harm; for it does not slow the spontaneous ventricular rhythm. On the contrary, it would tend to quicken the beat, at all events it would tend to render it more effective. It might therefore be of some use (Bachmann).

In complete experimental heart-block (dogs) strophanthin first increased the rate and strength of the ventricular contraction. This was followed by sudden ventricular arythmia and tachycardia, increasing to fibrillation. The auricles were strengthened, their rate sometimes quickened or slowed. The blood pressure rose in the first, and still more in the second stage (van Egmond, 1913).

Irregularities in the Strength of the Cardiac Contractions (Pulsus alternans).These, as contrasted with irregularities of rhythm, are currently attributed to exhaustion of the myocardium. They should therefore be improved by digitalis. It must be employed with caution, however, for similar irregularities are prominent in digitalis poisoning, and an exhausted heart is more subject to these effects.

Use of Digitalis in the Special Cardiac Lesions.-No cardiac lesion, as such, either indicates or contraindicates digitalis. Whatever the lesion, digitalis would be superfluous so long as compensation is fairly perfect. The indications for its use arise only when compensation fails; and then its possibilities for good or harm are determined by the physiologic conditions, as they have been discussed, rather than by the location of the anatomic changes. The two are, of course, often associated; and it may therefore not be amiss to review the use of digitalis from the standpoint of the usual anatomical classification of cardiac diseases.

Mitral or Tricuspid Insufficiency.-The leakage of the mitral or tricuspid valves tends to produce excessive dilation, particularly of the auricles, often associated with the more serious arhythmias (auricular extrasystoles and auricular fibrillation). Digitalis would be particularly useful.

Mitral Stencsis. -Digitalis would be less efficient in this lesion. It will be remembered that digitalis acts comparatively weakly upon the auricles, much more strongly on the ventricles. Consequently a mitral stenosis can not be affected by action on the left auricle, but only through the right ventricle. This increased work of the right ventricle, combined with the stenosis of the mitral valve, will tend to produce congestion of the pulmonary vessels, consequently to lessen the oxygenation of the blood, and in this way may interfere with the nutrition of the heart. Then, again, the systolic tendency of the digitalis will render the stenosis more marked, just as it counteracts insufficiency by approaching the valvules. On the other hand, the cardiac slowing will give the lungs more time to empty into the heart. Some conditions of the action of digitalis are therefore favorable, others unfavorable; and the effect upon patients is, in consequence, variable. Some cases of mitral stenosis are benefited by digitalis, others are even made worse. The digitalis must therefore be carefully watched, and if it is seen that the symptoms are not improved, it should be omitted and replaced by other remedies: If the symptoms arising from low blood pressure predominate, it would be well to employ vasoconstrictors; if those from a weakened heart, vasodilators.

Tricuspid Stenosis.--In this also, digitalis would often fail; but it is sometime valuable to restore the tone of the auricle.

Aortic Stenosis.-The difficulty which this lesion offers to the emptying of the ventricle tends to dilation, and this frequently produces irregularities, particularly pulsus alternans and ventricular extrasystoles. Digitalis would be valuable so long as the heart-muscle is able to respond to it.

Aortic Insufficiency. - The usefulness of digitalis in this lesion has been much disputed. The older clinicians, since Corrigan, held it to be detrimental, fearing especially that the prolonged diastole would give the blood in the brain a chance to gravitate 
back into the heart, and thus produce syncope. The danger is doubtless exaggerated, especially if the patient is kept in bed. The improved tone of the ventricle may more than offset the hypothetical objection. At present, digitalis is generally used, at least if mitral insufticiency is also present; but it should be employed more carefully, and it would be well to combine it with nitrite.

The occurrence of syncope on changing suddenly from the prostrate to the upright position is not rare, under the influence of digitalis, even with other lesions; the patient should be cautioned as to this.

Gebhart, I9II, has tried the effect of digitalis on experimental aortic insufficiency in rabbits: He found that it lessened the dropsy and mortality; but did not prevent cardiac hypertrophy.

Chronic Myocarditis.-Digitalis would generally be efficient against the dilation and irregularity; but it must be used cautiously if the muscle is degenerated.

Cardiosclcrosis. - It must be remembered that the most useful effects of digitalis are produced by a stimulation of the cardiac muscle. As a result of the better nutrition produced in this manner, the muscular fibers may afterward hypertrophy, or new fibers may possibly be formed; but the primary action of digitalis itself is confined to the already existing muscle; consequently it will be of no use if there is practically no muscle left to respond to it. It is therefore useless with a heart which has undergone marked fatty or fibrous degeneration. In this condition it is often contraindicated, because the overworked muscle is more readily poisoned.

High Blood Pressure.-This has little bearing on the usefulness of digitalis. Its existence does not contraindicate the administration.

Acute Myocarditis and Endocarditis.-The use of digitalis is debatable: it can easily lead to dangerous overstrain of the hypersensitive muscle.

Circulatory Failure of Fevers.- The usefulness of digitalis is rather limited. It may improve the pulse (making it fuller and more regular), early in the disease, when the heart is relatively inefficient, but when the blood pressure is still maintained. Patients developing auricular fibrillation are very strikingly benefited (Cohn, I9 15). The action of strophanthin is identical in normal and in pneumonia-infected animals (Jamieson, 1915). When collapse (vasomotor paralysis) has set in, digitalis would be practically ineffective (G. Schwartz, 1906).

Aneurysm. - The sudden distention of the aneurysmal sac by a larger mass of blood, which would be the result of digitalis, is exactly contraindicated. The only reason why digitalis has not done more harm in this way is that it has not generally been used in large enough doses to have a marked effect.

Hemorrhage.-This would similarly contraindicate digitalis.

Absorption.-The slow onset of the symptoms shows that absorption from the alimentary canal is very gradual; it is more rapid with rectal, and especially with intramuscular administration.

The dose influences the rate of absorption materially. With oral administration, the rate of absorption increases with the dose (Hatcher, I909); but is always quantitatively uncertain. The variation is especially great with Strophanthus (Hatcher, I909). This accounts for the unsatisfactory clinical results from the oral use of this drug. The safe dose would be so small as to be generally ineffective; a really effective dose could be highly dangerous according to the individual absorption. Helleborein is probably not absorbed at all from the alimentary canal, since it commonly produces only diarrhea, without cardiac effects (Cushny, etc., I912). Digitoxin appears to be better absorbed than digitalis (Eggleston, I9r 5). The tincture of digitalis is absorbed more rapidly than the infusion. Digipuratum and digalen are not decidedly superior (Haskell, McCants and Gardner, I9I6).

Different Portions of the Digestive Tract.-Quantitative studies by Ogawa, I9I2, indicate that digitalis is not absorbed from the stomach. From the intestines, 34 to 45 per cent. were absorbed in two hours from digitalis leaves; with digipuratum, the absorption was 52 to $6 \mathrm{I}$ per cent. in two hours; 65 per cent. in three hours.

Destruction in Alimentary Canal.-The digitaloids are practically unaffected by pepsin or trypsin (Goodall and Reid, I914); but a small quantity of the glucosids is probably destroyed by the acidity of the gastric juice (Hale, I9 Ir; Holste, Igr 2; Segond, I9I2). Johannessohn, I9I4, found that alkalies weakened only crystalline K-strophan- 
thin; acids, this and amorphous $\mathrm{K}$-strophanthin and strophanthus tincture. The amorphous strophanthin is changed mostly to strophanthidin, and partly to an inactive resin-ouabain is unaltered by either acids or alkalies, which is an advantage in its administration. However, with the usual short contact, the destruction of the other principles is probably also unimportant (Hatcher, I 909 ).

Ihotal, I9I 2 and 1913 , invokes gastric destruction to explain the habituation which he believes to occur in rabbits.

Disappearance in the Body. - The digitaloids disappear from the blood almost immediately after the injection of even much more than fatal doses (Hatcher and Eggleston, I9I 2; Lhota, I9I2). Their further fate is not definitely known. From the duration of their action Cloetta and Fischer, 1906, and Hatcher, 1912, conclude that a part must be stored in the cardiac tissue. Lhota, 1912, claims that digitoxin is found chiefly in the heart and blood vessels, less in the liver and other organs. According to Clark, 1913, there is no evidence that the digitaloids are either fixed or destroyed in the tissues, so that their distribution would be rather one of selective solution-affinity. In proportion to their slow excretion, they would gradually diffuse back into the blood.

Excretion.-This also has not been definitely determined because it is difficult to demonstrate the small quantities of the drugs. It probably occurs partly by the urine; but in rats (ouabain) mainly by the feces (Hatcher, 1909). Lhota, 1912, could find none in the urine or feces of rabbits. He has also studied (I9I3) the excretion and fate in the toad.

Onset of Action.-When therapeutic doses of the Digitalis drugs are administered by the alimentary canal, the effects develop very slowly. It may be several hours before any action can be noted. The full effect is reached much later; twenty-four to thirty-six hours for the circulatory actions; seventy-two hours for the diuresis. With a large therapeutic dose $(0.3 \mathrm{Gm}$.) the latent period may be shortened by one-half (Fraenkel, 1907). The delay in starting is evidently due to slow absorption, for when strophanthin is given intravenously, the circulatory action begins at once, and the diuresis starts in a few hours (Fraenkel and Schwartz, I907). Intravenously, digitalis and its principles act almost as promptly (Hatcher and Bailey, I9I0). In any case, however, the reaction continues to increase for some time.

Duration of Action.-The digitalis effects endure for a long time after the administration has stopped, whether the drug was given by the mouth or vein. It is difficult to establish a time for the clinical reaction, because when compensation has been established, it tends to persist, independently of the drug (except in auricular fibrillation), until some extra demand causes a fresh break of compensation. This probably holds true to some degree of the other cardiac changes. The persistence of the drugeffect depends also upon the total dose. One can therefore only deal in approximations. The effects of a "full" dose of digitalis or digitoxin seem to persist, with gradual decline, for one to three weeks, according to the total dosage; but for shorter periods with some of the other digitaloids, especially strophanthin and ouabain.

The duration of the digitalis-efiect can be measured accurately in animals by Hatcher's method (differential fatal dose): In cats, one-half of the original effect of an intravenous injection persists for the following number of days: digitalis, five to twelve days; digitoxin, fourteen (dogs, two days; rabbits less than one day); digitalein, three; digitalin German, three; digitalin true, less than two; ouabain and strophanthin less than one (Hatcher, I9I2). Klein, I9I4, believes that the action of strophanthin may persist for a month in cats.

The duration is much shorter in dogs and especially in rabbits, as quoted for digitoxin. Apparently, the more resistant a species is to digitalis, the shorter is the action. Both facts perhaps depend on the rate of elimination. The clinical data indicate that the duration in man approaches that in cats (Eggleston, 1912). 
Mechanism of the Prolonged Action.-This might be explained either by the persistence of the digitaloid in the tissues; or by persistence of the cardiac changes after the drug has disappeared. The briefer duration after ouabain favors the first rather than the second explanation (Hatcher), but the evidence is not decisive.

Racial Tolerance.-Great differences exist in the susceptibility of different species to the digitalis poisons. Rodents, toads (Vulpian, I 856) and the grass-snake are especially resistant but not immune. With mammals the difference is perhaps partly a matter of excretion; but the resistance seems to be mainly in the tissues.

Curiously, the resistance of the toad and snake does not extend to the related action of barium or saponin (Heuser, I902; Clark, I9I3). The toad resistance is generally attributed to habituation for its own poison; and Filippi, r9 3 , claims that toads deprived of their poison are very sensitive to digitalis.

The fatal dose for rats is nearly a thousand times greater (per kilogram) than that for cats, even hypodermically (Hatcher, r 909); and thirty times as much as for rabbits. Gunn, I9r3, found that the concentration required to arrest the isolated heart in a given time is also forty times greater for rats than for rabbits; showing that the difference lies in the heart itself. Similar relations hold for the toad and snake (Clark).

Lhota, I912, asserts that rabbits acquire an added tolerance by habituation; this needs confirmation. Klein, I9 I4, describes a similar induced tolerance in cats. Salvisberg, r907, claims that ruminants are more resistant to oral administration, but not to intravenous, indicating that in these animals the drugs are partly destroyed in the digestive canal. Lhota, rgog, states that young animals are more resistant than adults.

Seasonal Variations in Susceptibility.-These occur in frogs and guinea pigs (Haskell, I9I 2 and r9I4; Vanderkleed and Pittenger, I9I3; Baker, I9I3). It is interesting that they run reverse to the seasonal variations of acetonitrile and diphtheria toxin (Haskell, I9r3).

Cumulative Action.-The effects of digitalis tend to be cumulative; that is, the same doses, continued day by day, tend to produce ever-increasing effects; or more commonly, toxic effects develop suddenly, without warning, after small doses have been continued for some time. In the therapeutic use, the dose must therefore either be diminished, or toxic phenomena result, often quite suddenly. This cumulative action may be explained in part by the slow and uncertain absorption and excretion. The principal factor, however, is probably the slow, persistent and progressive action: Since the effects of a single dose may persist over several days or weeks, the effects of all the doses which are given in the meantime are more or less summed up; and the effects of digitalis are determined by the total dose, and not by the single or even by the daily dose.

\section{CHRONIC DIGITALIS POISONING IN ANIMALS}

von Lhota, I910, has studied this in dogs which were poisoned for several months by slowly increasing doses of Digitalis. The phenomena correspond closely to those observed in man: The pulse after nine or ten days shows occasional short periods of slowing, but with the heart regular and strong. If the administration is continued, the slowing becomes more persistent and more marked and arhythmic. Later, the pulse rate again increases to normal or somewhat above. The pulse changes outlast the administration for a long time; in severe acute poisoning, they may persist for several weeks; after chronic poisoning, they may last over a month. Lhota believes that the effects are at first cumulative, but that later some tolerance is acquired especially in rabbits (Lhota, I9 2), but the evidence for habituation is not convincing.

Typical cumulative poisoning has also been observed in cats (Klein, 1914).

\section{TOXIC EFFECTS IN THE CLINICAL USE OF DIGITALIS}

These are not at all uncommon. ${ }^{1}$ The effects of the drug vary so much according to the condition of the heart, according to absorption and cumulation, efc., that the dosage is very uncertain. The drug must be given

1 Bailey, I9II, claims that in a series of ninety cases at Bellevuc Hospital, 25 per cent. showed some form of toxic action, although the administration was conducted with the greatest care. 
until the desired therapeutic effect is obtained; and the zone between the really efficient therapeutic action and the toxic action is so narrow, that the minor toxic manifestations are apt to occur at any time. Indeed, it is often necessary to "push" the drug intentionally to this point, for the full therapcutic effect practically coincides with the minor toxic manifestations (Eggleston, I915). These do little permanent harm, if the dosage is reduced immediately on their occurrence; but if they are disregarded the results will be most serious.

Digestive Disturbances. - These occur early and are easily recognized, so that they are very important. They consist in nausea, great malaise, and often headache which may be very distressing. If the drug is continued, vomiting will occur. Diarrhea is not very common with digitalis, more frequent with strophanthus, squill and helleborein. When any of these digestive symptoms occur the dosage should be reduced or stopped. They will then subside in one or two days.

Cardiac Irregularities.-Digitalis may simulate any of the clinical irregularities; so that it is sometimes difficult to distinguish between them, particularly in advanced poisoning. To establish their causation by digitalis, it must be shown that they were absent before the drug was given, and that they disappear in a reasonable time after its discontinuance. The early effects, however, are fairly characteristic. The most common is excessive vagus stimulation (sinus irregularity). It is best seen when the heart is slowing after a little exercise. The rate may fall to 50 or even lower; so that syncope may occur between the contractions. The rhythm may remain regular; but ventricular extrasystoles are common. This stage would not occur in auricular fibrillation, nor in absolute heart-block.

The next stage is usually that of partial heart-block, especially if the conductivity was naturally low. It may occur with relatively small doses (Windle, I9r r), and may be permanent (Taussig, I9r2); Christian, r9 5 , records transient auriculoventricular dissociation.

The final stage is that of muscular irritability, first indicated by extrasystoles. These are particularly common in auricular fibrillation, giving the "coupled beats." With more advanced toxic action, there occur the same effects as in animals: high blood pressure (vasoconstriction); very rapid rhythm, with periodic changes in amplitude, pulsus alternans (rarely).

Dosage.-Eggleston, I912, finds that the toxic effects depend mainly on the tota ${ }^{1}$ dose, modified by the weight of the patient, the activity of the preparation, absorption, individual susceptibility and the nature of the heart lesion. With the ordinary energetic administration, the characteristic coupled beats occur when the total quantity of digitalis has reached 2.25 to $6.25 \mathrm{Gm}$. Since the action persists partly from one to three weeks, these irregularities will recur with a smaller dosage.

Duration of Irregularities. - This depends on the total dosage, and therefore on the severity of the phenomena. Eggleston, I912, found that coupled beats generally outlasted the administration by four to twelve days; heart-block, three to six; combined phenomena, six; auricular fibrillation, three; muscular irritability, two; sinus irregularity, two days. The delayed conduction generally lasts about two days, but may extend over two weeks (Cohn, I915).

\section{ACUTE DIGITALIS POISONING}

The symptoms set in relatively late (one to twenty-four hours); and they generally have a long course, so that a week or two may elapse before death. The symptoms are those of cumulation-gastro-intestinal disturbances, slow and arhythmic pulse, 'etc., lassitude, muscular and sensory derangements. Death generally occurs suddenly, with dyspneic convulsions. Consciousness persists late. 
The treatment would consist in evacuation, quietness, possibly diffusible stimulants, and other symptomatic treatment. A cathartic should be given. (The claim of Krause, I 906 , that diastase is an effective antidote to strophanthus is doubtful.)

The fatal dose varies greatly, on account of the variable absorption, the occurrence of vomiting, etc.: $2.5 \mathrm{Gm}$. of digitalis has been fatal, whilst $4 \mathrm{Gm}$. has been followed by recovery (Lewin).

The symptoms in mammals are very similar. The symptoms of fatal intravenous injection of strophanthin include vomiting and diarrhea (both central), and convulsions (Hatcher, I909).

Contraindications.-Digitalis should be avoided in partial heart-block, and in advanced myocardial degeneration. Its administration requires even more than ordinary care in coronary sclerosis, in aortic insufficiency and in the presence of any irregularities, particularly auricular. Renal diseases emphasize the tendency to cumulation.

\section{ADMINISTRATION}

The administration of digitalis drugs must aim to induce the therapeutic stage as promptly as is feasible, and to maintain it as long as may be needed. The intravenous or intramuscular injection of strophanthin or ouabain would be the ideal method to induce the effect; but it has several disadvantages which restrict its employment largely to emergencies.

The oral administration should be started with fairly large doses. These may be continued, if necessary for two to four days, until a definite response is observed in either the pulse, the urine, or the nausea. The dosage should then be reduced to a third or a fourth; being increased or diminished from this point as occasion arises; aiming to maintain compensation and keeping the pulse about 8o, with the smallest possible dose. The quantity for steady use can best be adjusted by the feelings of the patients, instructing them to recognize the effects of overdosage. The administration should be stopped when compensation is fairly reëstablished. It should be suspended temporarily if nausea sets in; or if the pulse falls below 50; or if any of the characteristic digitalis irregularities arise. The patients should therefore be observed at least daily. The suspension must extend over several days, at least, and the resumption of the drug must be very gradual. Even in the absence of toxic symptoms, it is well to intermit the administration for four days at the end of every ten days, meanwhile giving a cathartic to remove any. unabsorbed drug from the intestine.

The digitalis drugs should be diluted with at least half a tumbler of water, to lessen local irritation. They may be flavored with Compound Tincture of Gentian. If they are not tolerated by the stomach, they can be given by enema; the daily dose being diluted with Ioo c.c. of normal saline, containing a little starch. The absorption is perhaps rather more rapid than by oral administration.

When it is advisable to give nitrites with the Digitalis, it would be better to administer the two drugs separately, since the onset and duration of their action is so different.

Dosage. - With the digitalis drugs, the daily dose is far more important than the single dose. The daily dose, for the start, should be about $0.3 \mathrm{Gm}$. (5 gr.) of Digitalis (Mackenzie and Cushny give as much as 60 to 90 minims, 4 to 6 c.c., of the Tincture). For continuous use, the daily dose should not exceed $0.1 \mathrm{Gm}$., I 1/2 gr. (A. Fraenkel, 1907). The daily dose is usually divided into three or four portions. The following shows 
the doses of the commonly used preparations which would yield approximately equivalent effects.

$0.3 \mathrm{Gm}$. Digitalis $=3$ c.c. $(45$ minims $)$ of Tincture $=30$ c.c. $(3 \mathrm{j})$ of Infusion.

O. I Gm. Digitalis = I c.c. ( 15 minims) of 'Tincture $=$ Io c.c. $(3 \mathrm{ij}$ of Iniusion.

Cushny, I9I I, considers that I part of Digitalis is clinically equivalent to $1 / 2$ part of Strophanthus, or 3 to 4 parts of Squills. For babies of $I$ to 2 years (pneumonia), Jacoby advises 5 drops of the tincture every two hours.

Choice of Digitalis Preparations.-Of the various preparations of Digitalis, a good tincture, physiologically standardized, has many advantages. The infusion is uncertain and unstable. Fluidextracts are unreliable (Haskell and Eckler, 1913). The powder may be used, but has no advantage, and is apt to be more irritant. The pill of Digitalis, Squill and Calomel ("Guy's Hospital Pill") seems needlessly complex. Digipuratum seems to be a good preparation (Boos, Newburgh and Marks, I9II); but as has been stated, a good Tincture is as satisfactory as any.

It may often seem that one preparation, or one digitaloid drug, gives results where another has failed. This is scarcely a conclusive demonstration of its superiority, even in that particular patient, since the cardiac condition, and therefore the response to digitalis, is apt to vary from day to day. Differences in clinical results are often due to differences in the disease, rather than in the drug. The same is true of the gastric effects.

Intensive Oral Digitalis Medication.-Eggleston, $\mathrm{I}_{91} \mathrm{I}_{5}$, points out that, if the dosage of 'digitalis is sufficiently high, the full therapeutic effects may be produced within twelve to twenty-four hours, even with oral administration. This offers manifest advantages, but is only safe where the patient can be under constant observation by modern methods, and by using drugs of known activity.

The drugs are best standardized by "cat-units," i.e., the milligrams of drug fatal to a kilogram of cat, intravenously. Eggleston reports that the relation of effect to dose (cat-units) is remarkably uniform, for a given drug, regardless of the presence or nature of cardiac disease. Whatever the condition, the total dose of digitalis by mouth, to the appearance of the first improvement averages 0.09 cat-units per pound of bodyweight (about $0.9 \mathrm{Gm}$. of good digitalis per roo pounds); for full therapeutic or minor toxic effects, 0.146 cat-units per pound (about $1.5 \mathrm{Gm}$. per roo pounds). With digitoxin, the corresponding doses are: first improvement, 0.04 cat-units per pound (1.4 mg. per roo pounds); full therapeutic effect, 0.066 cat-units per pound ( $2.3 \mathrm{mg}$. per roo pounds). The other digitaloids give variable results, by mouth, because of uncertain absorption. They are therefore unsuited for the intensive method.

Under the above conditions, Eggleston finds it safe to give as much as one-half of the total calculated dose at once (i.e., about 7.5 c.c. of the Tincture); followed in about six hours by one-fourth of the total dose, then smaller fractions every four to six hours. The unskillful use of the method, however, presents the greatest dangers.

Intravenous and Intramuscular Injection of Strophanthin.-Intravenous Digitalis therapy was tried clinically by Kottman and by Mendel in I905; but the preparations which they employed were not satisfactory. The method has been put on a sound basis by the introduction of strophanthin (A. Fraenkel and Schwartz, I907; Krehl, I907). This is soluble in water and has the advantage of a more uniform composition-a very important matter when so potent a procedure is employed. It also acts more promptly than digitalis, and has less tendency to cumulation and vasoconstriction.

The therapeutic events correspond with those of digitalis administration, but occur much more rapidly: The first change is the increased amplitude of the pulse wave, which can be observed in three or four minutes. The pulse then becomes fuller and less irregular, and the symptoms of cardiac failure (dyspnea, cardiac distress, etc.) are improved. The slowing of the rhythm sets in somewhat later: its maximum is only reached after several hours, when the rate may become slower than that of the normal heart. The blood pressure is not altered-or rather it tends to 
remain or return to the normal. The diuresis also begins in a few hours. The increased amplitude outlasts the slowing.

The intravenous administration is especially indicated in the acute muscular failure of advanced heart disease-it is immaterial whether the failure is of the right or left heart. It sometimes gives good results in collapse, e.g., of typhoid fever (Hoepffner; Liebermeister). It is also of advantage in starting digitalis therapy in somewhat less urgent cases, following it in twenty-four hours by oral administration of digitalis. The intravenous method is not very suitable for continuous digitalis therapypartly on account of the inconvenience and discomfort, and partly because the smoother action secured by gradual absorption should be more beneficial to the heart than the daily repetition of the stormy effect of the intravenous injection.

Use after Digitalis.- The previous use of digitalis renders the intravenous injection very dangerous, since the effects of the two drugs will be immediately added. At least two or three days free from Digitalis should intervene, and the dose of Strophanthin should be divided in half and injected in three or four fractions several hours apart. In advanced cases, this fractionation should be practiced even if the patient is not under digitalis.

Intravenous dose: With the amorphous strophanthin (Boehringer) this is I mg. or less per day. Hatcher and Bailey, Igro, have used ouabain in the same way. It is about two and one-half times as active, and its dose would therefore be $1 / 2 \mathrm{mg}$. or less, as $\mathrm{r}: 6,000$ to $\mathrm{r}: 8,000$ solution in normal saline. Bailey, rgo9, prefers the intramuscular injection of the same dose (as I:4000). This is almost as prompt and effective, but is slightly painful. Intravenous injection sometimes produces cyanosis, chills and slight fever (Seifert, r9o9). Digipuratum may be used, o.I Gm., I $1 / 2$ gr., per dose. The intravenous dose of other digitaloids (according to their toxicity for cats, Hatcher and Bailey, I9ro) would be: digitoxin, I.5 mg.; tincture digitalis, 4 C.C.

\section{RECAPITULATION OF OTHER DIGITALOID DRUGS}

These present quantitative differences, which might be of some therapeutic value if they were better understood. At present they possess no serious advantage over digitalis for oral, and the strophanthins for venous administration. - These accomplish all that can be done by any digitaloid drug. Many of the substitutes are inferior in solubility and absorbability.

Isolated Digitalis Principles.-These have proven disappointing, since the only one which can be obtained pure, digitoxin, is undesirable.

Digitoxin.-This has the undesirable features of producing excessive irritation, vasoconstriction, and cumulative action, and of being nearly insoluble in water. It may be dissolved in 25 per cent. of alcohol. It has been used clinically, especially by Masius, 1894, Corin, 1895, and Eggleston, I915 ( $c f$. Intensive Oral Digitalis Medication). It is better absorbed than Digitalis. The usual daily dose by mouth is I to $3 \mathrm{mg}$., largely diluted; hypodermically, $0.5 \mathrm{mg}$. ( $\mathrm{r}$ c.c. of 0.05 per cent. in 25 per cent. alcohol; Unverricht, 1895 ; it produces irritation).

Digitalin "True."-This is also insoluble and emetic, but non-irritant and not greatly cumulative. Clinically, it has proved too feeble, a variable quantity being probably decomposed in the stomach (Deucher, 1896 ). It has been used in doses of 1 to $3 \mathrm{mg}$. to 8 to $\mathrm{x} 6 \mathrm{mg}$. per day; even $50 \mathrm{mg}$. have been given without toxic effects (Pfaff, I892). It is administered in pills or dissolved in dilute alcohol. 
Commercial Digitalins.-These have no advantage over the galenic preparations. The uncertain composition of these mixtures, and the danger of confusing the similarly named but very different preparations, should preclude their use. At least, "digitalin" should never be prescribed without specifying the variety (verum, German, French, etc.).

Digitalin German.-This contains considerable digitonin. Soluble in water or alcohol. Daily dose, 6 to $30 \mathrm{mg}$., $1 / 10$ to $1 / 2$ gr. (Beates, 1907).

Digitalin French (Homolle's).- This consists largely of true digitalin. Daily dose, to $6 \mathrm{mg}$., $1 / 10 \mathrm{gr}$.

Commercial Specialties.-These are also indefinite mixtures of the impure principles. The most important are:

Digipuratum, N.N.R. (Gottlieb).-This is a purified solid extract, freed from digitonin, and standardized to the same activity as digitalis leaves. It contains all the cardiac glucosids, is uniform, reliable and stable (Hale, 1910) and is relatively non-irritant; but all these features are possessed by a good tincture (Dixon, I9I 2; Cow, I9I2). It is insoluble in cold water, but dissolves on the addition of a little alkali. Its dosage is that of digitalis leaves.

Digalen ("Digitoxin soluble," Cloetta, I004).-This has been widely advertised as an amorphous and soluble isomer of digitoxin. Kiliani, 1907, states that it consists mainly of digitalein Schm.; his opinion should carry weight. It was said to be free from the disadvantages of digitoxin; but it is of variable activity, unstable (Hale, I910): produces considerable irritation, is not suitable for hypodermic injection, and causes cumulative effects (Fraenkel, I907; Westenrijk, I908). It therefore possesses no real advantage over a good tincture (Dixon, Cow, I9I 2), except for venous injections, and these cause pain and sometimes thrombosis. It is marketed as a solution (I c.c. $=$ $0.3 \mathrm{mg}$. of the glucosids). The intravenous dose is I c.c., repeated in one-half to one hour if necessary.

Strophanthins.- These have the advantages of being less irritant and less cumulative than digitalis; but the absorption is so uncertain that their use by mouth is not advisable (Hatcher and Bailey, I909). This is reflected in the very different doses of strophanthus used by various clinicians. It is also liable to produce diarrhea. The main field of the strophanthins is for intravenous injection. Ouabain has the advantage that its identity is assured.

Apocynum (Canadian Hemp) is an effective member of the group, but without serious advantages.

Active Constituents.-These were isolated independently by Finnemore, 1909, and Moore, Igog. The principal constituent of Apoc. cannabinum is a neutral crystalline bitter principle, Cynotoxin; that of Apoc. androsaemifolium is Apocynamarin. They are identical pharmacologically and perhaps chemically. They are destroyed by boiling with acids or alkalies, although they are not glucosidal. They are believed to be dilactones of Kiliani's digitogenic acid. Impens, I9I3, affirms that the active principle (which he calls "Cymarin") is the same for both species, and that apocynamarin" is probably a decomposition product. The chemistry and cleavage products of cymarin are described by Windaus and Hermann, 1915. Apocynin and apocynein of Schmiedeberg were impure mixtures. Commercial apocynin is acetovanillin (Finnemore, 1908), and is practically inactive (Wood, I904).

Actions. - These agree with digitalis (Dale and Laidlaw, I909, who studied the pure principles). The cardiac action, therapeutic and toxic, of cymarin (frog, Kuroda, I9I4; electrocardiograph, v. Hecht, I915) is somewhat weaker than that of strophanthin. It produces practically no irritation, but relatively strong vasoconstrictor effect. Kuroda claims that the cumulation and emetic effects are weak; but Taviani, r9I5, finds the serious disadvantages of uncertain absorption, and variable strength of the commercial cymarins. Large doses are also nephritic. The drug could probably be spared.

Cymarin has been used clinically like strophanthin, by vein or muscle, 0.1 to 0.5 mg.; hypodermic injection is too irritant. It is difficultly soluble in water, easily in alcohol. It has also been given by mouth, $0.3 \mathrm{mg}$., three to five times daily (von Noorden, I9I6; bibliography). Gaisböck, I9I 5, on the basis of his clinical and experimental studies, emphasizes the need of caution in its use. 
Scilla (Squills).-Haynes, I906, claims this to be a more efficient cardiac stimulant than digitalis or strophanthus. Dixon, r9o6, points out that any superiority is outweighed by its disadvantages: uncertain absorption; strong gastro-intestinal irritation; and greater vasoconstriction, especially coronary. It was formerly used as expectorant and diuretic. The activity was attributed to two amorphous glucosids, scillipicrin and scillitoxin; but Ewins, I9I I, found these to be impure mixtures. Kopaczewski, r9r4, claims to have isolated two active principles: Scillitin, the toxic constituent, a nitrogen-free glucosid; and Scillidiuretin, watersoluble, markedly diuretic; but of low toxicity.

Adonis resembles scilla more closely than digitalis (Chevalier, I9I3). Roch and Cramer, 1914, find even large doses inefficient in cardiac diseases. Tuckelmann, r913, claims that "adonidin" is a mixture of an acid and neutral glucosid, both possessing digitaloid effects, the acid being also hemolytic.

Convallaria (Root of Lily of the Valley); active constituent, the glucosid convallamarin, is considered rather uncertain (Roch and Cramer, I9I4).

Helleborein is a glucosid from the rhizome of Helleborus niger (Hellebore). Its digitalis action depends on the presence of an acetyl group (Sieburg, 19I3). It is not absorbed from the human intestinal tract and therefore produces only diarrhea clinically (Cushny, etc., Igr2).

Erythrophloein is an alkaloid with digitalis action, obtained from "Sassy Bark" (Chemistry, Power and Salway, 1912).

Barium Salts-(See Index). - These have been used in veterinary medicine, but are too dangerous for man (Report, Counc. Pharm. Chem., I9I2).

\section{BIO-ASSAY OF DIGITALOID DRUGS}

The potent effects of these drugs makes variations in their activity very serious. Considerable variations occur in the crude drugs, and still more in their preparations. It is therefore essential that they be stand. ardized. Since their activity is not due to a single principle, chemic assay is out of the question; and recourse must be had to bio-assays. It has been objected to these assays that they standardize the toxic and not the therapeutic effect. The objection is not well founded, since both actions are produced by the same substances and must therefore bear a definite ratio to each other. Clinical experience has borne out the great practical value of the bio-assay (Rowntree and Macht, I9i6).

The U.S.P. has adopted the frog-methods as official, but optional. Several methods are in actual use (Gottlieb, I9r4; Santesson, I9r5).

Fatal-dose Methods.-These determine the fatal dose, i.e., the smallest dose which kills a given weight of animal in a given time. The most convenient is the twelve- or twenty-four-hour frog method (suggested by Cushny, and elaborated by Houghton, 1898; being the first commercial bio-assay process). Guinea pigs may also be used for determining the fatal dose, but have no serious advantage. The chief objection to the lethal dose methods is that the results are not characteristic for digitalis, but might be due to any toxic constituent.

The average m.f.d. (minimum fatal dose) of some of the drugs is as follows (expressed as milligrams of drug per gram of frog-weight; the data are taken from Houghton and others):

Crude Drugs

Digitalis...... I.5

Digitalis...... I:5

Strophanthus... 0.007

Convallaria... 0.25
Principles

Strophanthin cryst. Kombé..... o.oor

Strophanthin amorph. Merck..... 0.00094
Commercial Preparations

Digitalin German..... 0.05

Houghton, I909 and I I 2 ; and Hamilton, suggest expressing the strength as Heart Tonic Units (H.T.U.), based on the toxicity of crystalline Kombe strophanthin.

For guinea pigs, the M.F.D. of digitalis is 0.06 to $0.1 \mathrm{Gm}$. per $240 \mathrm{Gm}$. pig (Reed).

Pittenger and Vanderkleed, I915, propose the use of gold-fish. 
The Frog-standstill Methods.-These determine the smallest dose which produces the typical systolic arrest of the heart. They are thus theoretically superior to the fatal-dose method; practically, the results are fairly parallel. Various modifications are in use. The simplest of all the assay methods is the one-hour frog method of Famulener and Lyon.

The M.S.D. (minimum systolic dose) is as follows (milligram per gram; Hale and others):

Digitalis...

Strophanthus.. 0.0075

Digitoxin.......... 0.0085

Strophanthin amorph.,. $0.00 \mathrm{II}$ Ouabain...... $0.0003^{-0} 0.001$
Digipuratum..... 0.6

Digitalin German . 0.07

Digitalin French .. 0.013

Digitalein........ 0.024

Modifications.-Gottlieb, I908, I9I4, allows only thirty to forty-five minutes for the systolic standstill. This was used by Lehnert and Loeb, I9I4. Focke, I906, uses a fixed dose, and judges the strength by the time required to produce systolic arrest. The method is described by Fuehner, I9II, and Heinz, I9I3. The short time does not suffice to secure complete absorption (Schmiedeberg). Objections have also been raised by Lutzkaja, 1908, and Hartung, I912.

Excised or perfused hearts have been used mainly in scientific investigations (Schmiedeberg, I9I0; Hartung, I9I 2; Gros, I9r3; Straub, Santesson, I9I 5).

Fallacies. - All frog methods, and to a somewhat smaller extent the guinea-pig method, are open to certain errors, depending on the inconstant absorption and inconstant susceptibility of different animals (Vanderkleed and Pittenger, I9I3). These must be met by using a sufficient number of test-animals. In addition, there are considerable seasonal and other variations. Each lot of frogs must therefore be standardized by determining their susceptibility to a standard preparation. This opens another fallacy, namely, that a given change of conditions may modify the susceptibility in a different degree for each drug, as has been shown for the influence of temperature (Baker, I9I2 and I9I3). Alcohol (25 per cent.) is not disturbing for frogs (Haskell, I913), but is protective for guinea pigs. With guinea pigs, age, sex and weight are unimportant; but there are considerable individual and marked seasonable variations, and the food also has an influence (Vanderkleed and Pittenger, I9I3; Haskell, I913). Vanderkleed and Pittenger, I914, believe that the variations of guinea pigs are not great enough to necessitate control animals.

Even with these limitations, practical experience shows that the intact-frog methods, when properly and critically performed, permit the estimation of the relative activity of similar preparations (such as a series of tinctures) with a probable error not exceeding 25 per cent.- a sufficiently close approximation for practical purposes. On the other hand, they are unreliable for comparing the relative activity of dissimilar drugs, $f . i$., strophanthin and digitoxin. The greater toxicity of strophanthin could be due, at least in part, to more rapid absorption-a factor which might be very different in the human subject. This could be excluded by using the excised heart; even then, however, it would be doubtful whether the same quantitative relations hold for the human heart as for the frog's.

The intravenous cat method of Hatcher avoids most of these objections (Eggleston, I9I3). Differences in absorption are excluded by the method of administration; the action is purely cardiac, and thus measures the characteristic digitalis-effect, which is not easily simulated by other toxic principles; and the cat heart must resemble the human in its reactions much more closely than that of the cold-blooded animals. Indeed, experience indicates that its resemblance is closer than that of other mammals. Even unrelated digitaloid principles may therefore be compared. Another advantage is, that the response of individual cats is remarkably uniform, so that the probable error is much smaller, and the standardization of the individual cats is usually not necessary.

The method is sufficiently simple: it merely determines the fatal $\mathrm{Kg}$.-dose; the solution being injected into the femoral vein at such speed that death occurs in about one and one-half hours. This quantity, expressed as milligram of drug per kilogram of cat, constitutes a "cat-unit" (Hatcher and Brody, I9I0). The intravenous therapeutic dose for man is about 5 cat-units; the daily dose by mouth averages about 50 cat-units.

With the slower acting digitalis preparations, the death-point can be hastened by using the rapidly acting ouabain to complete the reaction: about 75 per cent. of the fatal dose of the digitaloid are injected in fifteen minutes, and after an interval of ten minutes the reaction is completed with $1: 10,000$ of ouabain (Hatcher and Brody, I910). This expedient had also been very useful in ascertaining the absorption and persistence of digitaloids.

The cat-method is probably the best for the investigation of fundamental clinical problems; but it is not necessary for the commercial standardization. The results of 
Eckler, rgi I, enjoin caution against its use without special expcrience. The following table gives the cat units (milligram ner kilogram) for a number of drugs (Hatcher, rgr 2):

Crude Drugs

Principles

Commercial Preparations

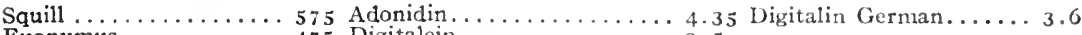

Euonymus ......... 475 Digitalein............ 3.5

Helleborus ........... I oo Convallamarin............

Digitalis ............ I oo Helleborein............ I.

Apocynum.......... 7o Digitalin true........... I.5

Convallaria.......... so Seillitoxin............... 4

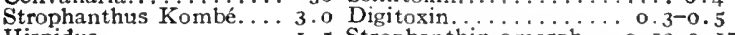

Hispidus............ I.5 Strophanthin amorph... o.13-0.17

Ouabain.................

Attempts to utilize the blood pressure and pulse reactions have been unsuccessful, since these are too variable.

\section{PREPARATIONS-DIGITALIS GROUP}

*Digitalis (Digit.), U.S.P.; Digitalis Folia (Digit. Fol.), B.P.; Digitalis (Fox Glove Leaves).-The dried leaves of Digitalis purpurea. The B.P. specifies that they must be collected when the plant is beginning to flower. Dose, 0.06 Gm., I gr., U.S.P.; 0.03 to $0.12 \mathrm{Gm}$., $1 / 2$ to 2 gr., B.P.; as pills. Maximum dose, $0.2 \mathrm{Gm}$., 3 gr. ${ }^{1}$

The activity of different samples of digitalis varies considerably, so that they should be standardized by bio-assay. This applies also to the Tincture. If preserved reasonably dry, the leaves keep indefinitely. The leaves of the first-year plants are as good as the second (Sharp and Lankaster; Caesar and Loretz, roro). Plants cultivated in America (Newcomb, rgr2) are as good as the wild European (Hale, rgro; Miller and Baker, rgr3; Rowntree and Macht, 19r6). Those grown during a wet season are less active (Caesar and Loretz, r907).

* Infusum Digitalis (Inf. Digit.), U.S.P.; Infusion of Digitalis.-r.5 per cent.; flavored with cinnamon (U.S.P. VIII also contained alcohol). Must be freshly prepared from the leaves. Dose, 4 c.c., I dram, U.S.P.

* Inf. Digitalis, B.P. - 0.7 per cent.; note that this is only haif the strength of the U.S.P. Dose, 7 to 15 c.c., 2 to 4 drams, B.P.

Fldext. Digit., U.S.P.- Made with 5o per cent. alcohol. Dose, 0.05 c.c., I minim, U.S.P. A rather variable preparation.

* Tinctura Digitalis (Tr. Digit.), U.S.P., B.P.-ro per cent. of drug in 70 per cent. alcohol. Miscible with alcohol or water (with slight turbidity due to the fat; certain proprietary tinctures deprived of fat do not become turbid, but have no other advantage). Incompatible with acids or tannin. Dose, 0.5 c.c., 8 minims, U.S.P.; 0.3 to I c.c., 5 to r5 minims, B.P. Maximum dose, 2 c.c., 30 minims. Unstandardized tinctures generally vary in activity by 200 to 400 per cent. (Fraenkel; Edmunds, etc.).

Digitalis Principles and Proprietary Preparations.-These were discussed on pages $383 ; 408$.

Strophanthus, U.S.P.; Strophanth. Sem., B.P.-The dried ripe seeds of Strophanthus Kombé (also of Strophanthus hispidus, U.S.P.); obtained from central and western Africa. Dose, $0.06 \mathrm{Gm}$., I gr., U.S.P.

Ext. Strophanth., B.P.-A powdered extract, representing 50 per cent. of drug. Dose, 16 to $60 \mathrm{mg} ., 1 / 4$ to I gr., B.P.

*'Tinctura Strophanthi, U.S.P., B.P.-ro per cent. (This is the same strength as the U.S.P. VIII, but four times as strong as the old B.P.) Dose, 0.5 c.c., 8 minims, U.S.P.; 0.12 to 0.5 c.c., 2 to 8 minims, B.P. Maximum dose, 0.5 c.c., 8 minims. However, the absorption is so uncertain that no reliable dose can be stated. Unstandardized tinctures are about as variable as digitalis.

* Strophanthinum (Strophanthin.), U.S.P.; (Amorphous) Strophanthin.-A glucosid or-mixture of glucosids from Strophanthus White or yellowish powder. Very sol. in water or diluted alc.; nearly insol. in lipoiđ solvents. Dose, $\mathrm{r}$ mg., 1/6 o gr., by mouth; intravenous (or muscular), $0.75 \mathrm{mg} ., 1 / 80 \mathrm{gr}$., U.S.P. Maximum dose, I mg., 1/60 gr.

Ouabain.-g-Strophanthin-Crystalline; sol. in water (to $x$ per cent.), more sol. in hot; sol. in alc.; difficultly in lipoid solvents. Dose, one-half that of the official Strophanthin.

Scilla, U.S.P., B.P.; Squill.-The dried fleshy scales of the bulb of Urginea maritima (Urginea Scilla, B.P.). Dose, 0.1 Gm., I 1/2 gr., U.S.P.; 0.06 to 0.2 Gm., I to 3 gr. B.P. Maximum dose, 0.5 Gm., 8 gr.

Acetum Scilla (Acet. Scill.), U.S.P.; Vinegar of Squill.- ro per cent., in Acid. Acet. Dil. Dose, I c.c., $\mathrm{I}_{5}$ minims, U.S.P.

1 Under proper precautions, the "maximum doses" of the drugs of this group can be temporarily exceeded. 
1cet. Scill., B.P.-30 per cent. (twice the strength of the old B.P.). Dose, 0.3 to I c.c., 5 to 15 minims, B.P.

Fldext. Scill., U.S.P.-Dose, o.I c.c., $\mathrm{x} \frac{1}{2}$ minims, U.S.P. This formerly contained acetic acid, but Houghton, 1906, showed that the acid diminished the activity by half. For the same reason, the Vinegars of Squill are unscientific.

Oxymel Scill., B.P.-33 per cent. of Acet. Scill. Dose, 2 to 4 c.c., $1 / 2$ to I dram, B.P.

Pil. I pecac. c. Scill., B.P.-Contains about 5 per cent. of Opium as Pulv. Ipecac. Co.; Squill and Ammoniac. Dose, 0.25 to $0.5 \mathrm{Gm} ., 4$ to 8 gr., B.P.

Pil. Scill. Co., B.P.-25 per cent. of Squill, with Ginger, Ammoniac and Soap. Dose, 0.25 to $0.5 \mathrm{Gm} ., 4$ to 8 gr., B.P.

* Syr. Scill., U.S.P., B.P.-Prepared from Acet. Scill., representing about 5 per cent. of Squill. Dose, 2 c.c., 30 minims, U.S.P.; 2 to 4 c.c., $1 / 2$ to I dram, B.P.

Syr. Scill. Co., U.S.P. (Coxe's Hive Syrup).-An antiquated cough mixture; the average dose, 2 c.c., 30 minims, U.S.P., representing $0.16 \mathrm{Gm} .\left(2 \frac{1}{2} \mathrm{gr}\right.$.) each of Squill and Senega, and $4 \mathrm{mg}$. (1/15 gr.) of Tartar Emetic.

${ }^{*} T r$. Scill., U.S.P.- - o per cent. in 75 per cent. alc. Dose, I c.c., 15 minims, U.S.P. Maximum dose, 2 c.c., 30 minims.

${ }^{*}$ Tr. Scill., B.P. - 20 per cent. Dose, 0.3 to I c.c., 5 to 15 minims, B.P.

Urginea (Urgin.), B.P.; (Indian Squill). - The dried young bulbs of Urginea indica. The preparations correspond in title, strength and dose to the B.P. preparations of Squill. bium.

A pacynum (Canadian Hemp).-The dried rhizome and roots of Apocynum canna-

Fldext. A pocyn.-Average dose, 0.75 c.c., 12 minims.

Convallaria; Lily of the Valley.-The root of Convallaria majalis.

Euonymi Cortex, B.P.; (Wahoo).-The dried root-bark of Euonymus atropurpurens. Contains an amorphous bitter principle. Used mainly as cathartic, similar to podophyllum. Dose, $0.5 \mathrm{Gm}$., $8 \mathrm{gr}$.

Ext. Euonym., B.P.-A powdered extract. Dose, 0.06 to 0.12 Gm., I to 2 gr., B.P.

\section{CACTUS}

General Statement.-Preparations from Cactus (Cereus) grandiflorus have been used as substitutes for digitalis, particularly in functional cardiac neuroses. Proprietary preparations have been widely advertised. Experiments on animals and men have shown that these are devoid of digitalis and strychnin actions and are indeed absolutely inert. The clinical testimony is not conclusive. Careful observers have failed to find any clinical effects; those who describe positive results have perhaps undervalued the element of suggestion in the treatment of functional neuroses (Report, Coun. Pharm. Chem., I910; J.A.M.A., 54:888).

Uncertain Origin.-Some of the discordant statements are probably due to confusion with other cacti, some of which contain alkaloids.with marked narcotic, tetanic, and possibly some cardiac actions. No alkaloid or other definite principle has been certainly isolated from the grandiflorus.

Experimental Results.- The drug was introduced in 1864 by Rubini, a homeopathic physician. Boinet and Teissier, 1891, described a weak digitalis action on the frog's heart, produced by an alkaloid, which they considered derived from Cactus grandiforus. They were evidently working with some other substance. No serious attempts were made to check the extreme claims for the cactus preparations, until Hatcher, I907; Matthews, 1908; Lyon, I910; and Hatcher and Bailey, I911, showed that even enormous doses of the best-known preparations are practically inert-entirely so when given by mouth - in animals and in man, in health and in disease. Groeber, 1913, again affirms the presence of a glucosid with digitaloid action, but so weak that it would be therapeutically inactive. He does not state whether his drug was properly identified. This precaution was taken by Hatcher and Bailey. Gordon Sharp, 1894, also found the clinical results negative when the genuine drug was used. The favorable clinical literature (summarized by Hatcher and Bailey, IgII) does not bear stringent criticism.

Other Cactus Varieties.-Alkaloids have been isolated from a number of cacti (Heffter, I898): mescalin, anhalonidin, pellotin, pectenin, pilocerein, etc. Mogilewa, I 903 , investigated their action on the heart, and found that they produce slowing, but without increase of pulse volume (in contrast to digitalis). 


\section{MESCAL}

This plays an important part in the ceremonial of Indian tribes. It is a preparation of the cactus Anhalonium Lewinii, in which Lewin, I888, demonstrated principles with narcotic and strychnin actions. It contains four alkaloids which agree qualitatively in their actions. The latter are quite numerous: a slowing of the heart, a curare action on striped muscles, a specific depression of the respiratory center, and a stimulation of other parts of the central nervous system. The stimulation is shown mainly in certain special senses, most conspicuously in vision, the effect appearing as a loss of coördination. It produces hallucination of all the special senses, but particularly of sight. There are flashes and lines of ever-changing colors. Since they are the same in both eyes, they must be central (Dixon, 1899). A psychologic study of mescalin hallucinations has been made by Knauer and Maloney, 1913. Waeber, I9 1 2, failed to find the described visual and auditory effects and double personality, but observed other psychic and physical disturbances. Bresler, I905, experimented with its action on mentally affected patients. Pellotin, one of the alkaloids, has been investigated by Pincussohn, 1907. Mescal is not used therapeutically.

\section{CAMPHOR}

General Statement.-Camphor, $\mathrm{C}_{10} \mathrm{H}_{16} \mathrm{O}$, is a solid derivative of terpene, $\mathrm{C}_{10} \mathrm{H}_{16}$, allied to the volatile oils. It is prepared by the distillation of the wood of the camphor-tree. This natural camphor polarizes to the right. A synthetic camphor has been prepared, which is racemic. A levorotary camphor is also known. The three isomers agree qualitatively in their actions. The l-form is somewhat more active on the nervous system. Therapeutically, they seem to be equivalent; but only the natural is used.

Camphor is a mild irritant and antiseptic, often employed as a counterirritant in the form of camphor liniment or spirits. It is also used as a protective against moths. Systemically, the effects are very inconstant, owing perhaps to its uncertain absorption and rapid destruction. It is used intramuscularly to stimulate the circulation in collapse conditions, apparently with good results; the mechanism has not been satisfactorily explained. Toxic doses produce convulsions. In frogs it exerts a curare action.

Local Actions.-Externally camphor acts similarly to the volatile oils, producing rubefaction, with little sensory irritation and mild analgesia. It is applied in neuralgia, inflammations, etc.

The irritant action of small doses of camphor on the digestive canal is used against dyspepsia. Large doses produce vomiting.

Camphor has a slight antiseptic action which determines its use in mouth washes and gargles. It has also been employed as intestinal antiseptic ( $0.3 \mathrm{Gm}$. three times daily). Intraperitoneal injections of camphorated oil have been tried in diffused peritonitis (Vignard and Arnaud, I9I 2; 200 to 300 c.c. are said to be absolutely harmless). Seibert, I9I 2 , uses camphor (I 2 c.c. of 20 per cent. oily solution, hypodermically, every twelve hours) in pneumonia, claiming that it reduces toxemia and prevents the crisis. This use is still experimental. Leo, I $9 \mathrm{I}_{3}$, prefers intravenous injection (saturated solution $=$ about I : 5000), but Weintraud, I9I3, finds the results no better than with hypodermic administration.

Applied directly to frog's skeletal muscle, it produces a curare-like action. This is not seen in mammals. Other camphors derived from various volatile oils also produce this effect (Hildebrandt, 1902).

Absorption, Fate and Excretion.-Camphor is quite readily absorbed from the mucous membranes and subcutaneous tissue. Hatcher and Eggleston, 1914, find the absorption more rapid from oral than from intramuscular administration. It is rapidly oxidized to campherol, 
$\mathrm{C}_{10} \mathrm{H}_{15} \mathrm{O} \cdot \mathrm{OH}$; and this excreted in combination with glycuronic acid, $\mathrm{C}_{10} \mathrm{H}_{15} \mathrm{O} \cdot \mathrm{O} \cdot \mathrm{C}_{6} \mathrm{H}_{9} \mathrm{O}_{6} \cdot$ (Schmiedeberg and Meyer, 1879 ). This destroys the activity of the camphor; and accordingly, Happich, IO I2, claims that the toxicity is higher when glycosuric acid formation is disturbed through starvation or deprivation of oxygen. Similarly, he states that intraarterial injections are more than twice as toxic as intravenous.

Experimental Effects on Circulation.-These are especially complex and inconstant so that different investigators have obtained varying results.

According to Liebmann, I9r 2 , the most frequent result of moderate doses is a peripheral dilator effect, first on the pulmonary, then on the systemic blood vessels. The blood pressure therefore generally falls; but this may be preceded by brief central vasoconstriction. Large doses occasionally produce periodic variations in the blood pressure, through the vasomotor center. Large rises of blood pressure may occur during convulsions. If the cardiac muscle is depressed or fibrillating, it may sometimes be revived by camphor, but the response is uncertain. Heard and Brooks, I913, obtained generally negative results. The experimental evidence therefore gives no explanation of the claimed therapeutic results.

Frog's Heart.-The effects of applying camphor solutions were studied by Heubner, I870; and Oehm, I870; those of perfusion by Plant, I9I4 (bibliography). They are slight and inconstant with normal hearts, but camphor restores the beat of frog hearts arrested by muscarin (Harnack and Witkowski, I876) or by chloral (Boehm, I905). The restoration is only transient (Plant, I9r4). It is produced by the various camphor derivatives on turtles (Lippens, I II I); camphoric acid being somewhat more stimulant. With ventricular strips of turtle hearts, Plant observed slight quickening, not modified by chloral. The ultimate effects of strong solutions of camphor are depressant, weakening and slowing the beats.

Mammalian Heart.-No definite effects have been demonstrated in normal hearts, in sitw or isolated. Winterberg, I903; Klemperer, I907; Plant, I914; and Richards, I9I4, obtained purely negative results. Seligman, I905, sometimes observed strengthening, but by no means always. He claimed, however, that camphor tends to prevent or recover fibrillation; and Gottlieb, 1905 and I906, found that the heart of intact animals is also rendered more resistant to fibrillation. This was contradicted by Winterberg. Richards found no definite effect of camphor on the isolated heart in fibrillation or asphyxia; nor Plant, after chloral or phosphorus. Heard, I915, worked with the heart in sitn, but isolated from its nerves, and the pressure kept constant by a compensator; camphor had no effect. Isaak, I9I3, claims a rise of blood pressure from intravenous injection of camphor, in animals with their hearts weakened by phosphorus, but no effect after chloral. During complete heart-block, in dogs, van Egmond, 1913, could generally observe no effect from camphor. The coronary blood flow increases if the pressure rises (F. Meyer, IgI 2). The coronary vessels (as also the earvessels) of rabbits are also dilated directly by perfusion with camphor ( $\mathrm{I}: 2,500$ to 5,000); still more by borneol and menthol. With a tenth of these concentrations, the dilation is preceded by constriction (Likhatcheva, I9I6).

Blood Pressure and Vascular System. - The earlier investigators (Pellacani, 1883; Alexander-Lewin, I 890; Paessler, I 899) generally described a rise of blood pressure from vasomotor stimulation. Wiedemann, 1877, and Stockman, 1888, observed rhythmic rises of blood pressure, attributable to heightened reflex irritability. Stimulation of the vasomotor center, however, does not occur with therapeutic doses, but only as the convulsant effects are approached; and especially during the convulsions proper (Pilcher and Sollmann, I9I5). Often the blood pressure is not changed by moderate doses. The most frequent result, according to Winterberg, I903, and Liebmann, I9 I 2, consists in persistent fall of blood pressure, occasionally preceded by a slight and short rise. The rise is attributed to central vasomotor stimulation; but the fall is produced by peripheral action on the vessels. Heard and Brooks, I913, obtained quite negative results. Liebmann finds that the pulmonary vessels dilate before the systemic. The widespread vasodilation results in lowering the pressure in the right heart. Frey, 1909, found no effect on pulmonary hemorrhage. The cerebral vessels are also dilated (Berezin, I916).

Clinical Effects on Circulation.-Camphor is used clinically as a temporary cardiac stimulant. Opinions are divided as to its usefulness. The effects, if they occur, are inconstant and unreliable. However, its employment is harmless. 
In normal individuals and in cardiovascular disease, camphor (even up to $3 \mathrm{Gm}$. hypodermically-Heard and Brooks, I9 3 ) does not alter the blood pressure, or the rate or quality of the pulse. In collapse conditions, especially in febrile toxemias, most clinicians believe that hypodermic injections improve the volume, tension and regularity of the pulse temporarily. G. Schwartz, I906, generally found a rise of blood pressure of ro to $20 \mathrm{~mm}$., too slight to explain the improvement. It is possible but not certain that the weakened human heart responds better to camphor than experiments on animals would indicate. It is also conceivable that the stimulation is reflex, since the injections produce considerable local reaction (Heard and Brooks). 'The regulating influence on delirium cordis might be beneficial in auricular fibrillation, but the action would probably be too brief.

Administration.-The camphor is employed intramuscularly, dissolved in sterile olive oil, I to 2 c.c. of the ro per cent. solution being repeated in ten-to fifteen-minute intervals as needed. In babies of one year, A. Jacobi advises $1 / 10$ to $1 / 4$ grain every two hours. Intravenous injection ( 250 to $35^{\circ}$ c.c. of 0.1 per cent. in saline) is safe, but less effective (Weintraud, I9r3).

Other Uses. - Camphor Spirit is a household remedy in colds, bronchitis, etc. It is probably somewhat expectorant, diaphoretic and antipyretic (Binz, I875 and I877). The skin vessels are dilated. It was also advised in tuberculosis, but has not found much support. Camphor has also been used as a nervous depressant in hysteria, epilepsy, chorea, and convulsions, and as an anaphrodisiac. This rests on no rational basis.

Respiration.-The effects of non-convulsant doses are very slight and inconstant, practically negative.

Edsall and Means, I9I4, and Higgins and Means, I9I 5, studied the effects on man, using 0.4 to $0.5 \mathrm{Gm}$. hypodermically. They found the center sometimes slightly stimulated by bronchodilation and slight increase of carbon metabolism. The rate was often slowed; the volume, single or minute, was unchanged.

In animals, the respiration is somewhat slowed but deepened, so that the minute volume is increased. Wieland, IgI5, claims lowering of the threshold for $\mathrm{CO}_{2}$. Isaac found it especially effective after morphin.

\section{TOXIC EFFECTS}

These are similar in man and animals, consisting in stimulation and then depression and paralysis of the central nervous system.

The treatment would consist in evacuation and stimulation. Alcohol and oils should be avoided since they favor absorption.

Symptoms in Man.-These consist in nausea, vomiting, colic, vertigo, disturbed vision, tipsiness, excitement, impulsive movements, delirium, loss of consciousness, face flushed, then pale; epileptiform convulsions are a constant feature in animals, and sometimes occur in man (after I.5 Gm.; Austragesillo, I915); often anuria (illustrative reports, Stanley, 1903; F. Barker, I910; Koppang, 1912). Death is rare in man, occurring by asphyxia and collapse. Convalescence may last several days or weeks; the gastric disturbance is especially persistent.

Convulsions.- In mammals, these are located mainly in the hemispheres; for removal of the hemispheres with section below the optic thalami diminishes (Albertoni, I882) or abolishes (Morita, I9I5) the convulsions.

The spinal cord also shows some stimulation, and finally paralysis, but this does not come on until late, and is not important in mammals.

In frogs, on the other hand, the action on the spinal cord is very pronounced, and consists in a paralysis. This entirely obscures any action which the drug may have higher up in the nervous system in these animals. It appears that the path for reflex impulses is blocked before that for impulses coming from the higher brain (Wiedemann, 1877; Binz, 1878). The symptoms are described by Grove, 1910.

Toxic Dose.-This varies greatly: 0.7 to $1 \mathrm{Gm}$. has been fatal to children and toxic to adults; $2 \mathrm{Gm}$. generally produced dangerous effects; but $15 \mathrm{Gm}$. have been survived (Koppang); and in an infant of eighteen months, I $\mathrm{Gm}$. produced little if any symptoms (Miller, I9I4). 
Solvent and Place of Administration on Toxicity.-Cairis, 1914, and Carnot and Cairis, 1914, have studied these on guinea pigs. With oral administration, the toxicity of camphor is greatly decreased by oil. Hypodermically, oily solutions are also less toxic than alcoholic, ethereal or even watery solutions. Intraperitoneal are more toxic than hypodermic injections. Oral administration is more effective than intramuscular (Hatcher and Eggleston, 1914).

\section{CAMPHOR ISOMERS}

The synthesis of artificial camphor is described by Kebler, r907; Houseman, 1915. Langgaard, and Maass, I907, showed that the actions of the three isomers are qualitatively identical. Grawitz, 1907, claimed that the therapeutic effects, internal and external, are indistinguishable. Haemaelaeinen, I908, states that the levorotary is less effective on the heart; but its central actions are more powerful (Grove, I9IO). Heffter, I9I4, concluded that artificial camphor is probably more toxic and often contains impurities; that it could be substituted externally, but more experience would be needed to justify its internal use. The war has furnished the occasion for this experience; and Luetz, I9I 5, concludes that its hypodermic use, clinically, has given no bad effects.

Borneol (Borneo-Camphor, $\mathrm{C}_{10} \mathrm{H}_{18} \mathrm{O}$ ), is used in the orient. It acts similarly to camphor.

\section{CAMPHORIC ACID}

This was introduced by Fuerbinger, r888, as anhydrotic against the night-sweats of phthisis. It aroused some enthusiasm at first (literature, Fujitani, r906); but experimental investigations have given entirely negative results (Dreesmann, I889; Fujitani; Tyrode, I908; Roth, I9I I); and the clinical effects were also disappointing (Tyrode). The drug is therefore going out of use.

Other Actions.-Tyrode, I908, could discover no effects of any kind. Fujitani and Roth described central and peripheral stimulant and depressant effects, somewhat different from camphor. It produces a rather marked stimulation of the respiratory center in mammals. Kobert, 1897 , ascribes to this the alleged benefits in tuberculosis.

\section{PREPARATIONS-CAMPHOR}

${ }^{*}$ Camphor (Camph.), U.S.P., B.P.; Camphor.-A ketone, $\mathrm{C}_{9} \mathrm{H}_{16} \mathrm{CO}$, obtained from Cinnamomum Camphora. White translucent masses of crystalline structure. Its pulverization requires the addition of a little alcohol. It has a characteristic odor and warm aromatic taste. It is slightly sol. in water, but freely in alc., fixed and volatile oils and fat-solvents. Dry camphor volatilizes readily, but its solutions are practically stable, even in open bottles (LaWall, I909). Dose, $0.2 \mathrm{Gm}$., 3 gr. by mouth; 0.1 Gm., I $1 \frac{2}{2}$ gr., hypodermically, U.S.P.; 0.12 to $0.3 \mathrm{Gm}$., 2 to 5 gr., B.P.

Its use in the orient antedates history; but it does not seem to have been known by the Greeks and came into use in Europe in the Mediaeval period. At present, 70 per cent. of all the camphor produced is used in the manufacture of celluloid.

${ }^{*} A q$. Camph., U.S.P.-A saturated watery solution containing less than 0.8 per cent. Very little therapeutic action. Dose, 10 c.c., $21 / 2$ drams, U.S.P.

${ }^{*}$ Aq. Camph., B.P.-O.I per cent.

${ }^{*}$ Linimentum Camphore (Lin. Camph.), U.S.P., B.P.; Camphor Liniment (Camphorated Oil). -20 per cent. of camphor in Cottonseed oil, U.S.P.; in Olive oil, B.P.

${ }^{*}$ Spiritus Camphore (Sp. Camph.), U.S.P., B.P.- - o per cent. Dose, I c.c., I5 minims, U.S.P.; 0.3 to 1.2 c.c., 5 to 20 minims, B.P.

$\left({ }^{*} T r\right.$. Camph. Co., B.P.-See Index).

Camphora Monobromata (Camph. Monobrom.), U.S.P.; Monobromated Camphor, $\mathrm{C}_{9} \mathrm{H}_{16} \mathrm{Br}$.CO.-Colorless prismatic needles or scales, or as powder having a mild but characteristic, camphoraceous odor and taste. Freely sol. in alc. ( $1: 6.5$ ); almost insol. in water. Dose, 0.125 Gm., 2 gr., U.S.P. Action similar to Camphor.

Acidum Camphoricum; Camphoric Acid, $\mathrm{C}_{8} \mathrm{H}_{14}\left(\mathrm{CO}_{2} \mathrm{H}\right)_{2}$.- Obtained by oxidation of camphor. Colorless crystals. Slightly sol. in water (I:125); readily sol. in alc., fats and alkalies. Used with doubtful results as anhydrotic. Dose, 0.6 to $2 \mathrm{Gm}$., 8 to $30 \mathrm{gr}$.

Oxycamphor (camphor with $\mathrm{H}$ replaced by $\mathrm{OH}$ ) has been recommended as respiratory sedative in cough. The aqueous solution decomposes, and it is marketed as a 50 per cent. alcoholic solution (Oxaphor, N.N.R.), of which the dose is 2 c.c. (3 drop). 


\section{MENTHOL}

This is prepared from the volatile oils of various mints. It acts similarly to camphor, and is used especially locally against headache, neuralgia, itching, etc. Applied to the skin, it produces at first a cool sensation, followed by slight prickling and burning. The cooling effect is not physical, the temperature of the skin being normal or even increased. It is explained by specific stimulation of the nerve-endings for cold (Goldscheider). Alcoholic solutions are antiseptic.

PREPARATIONS-MENTHOL

${ }^{*}$ Menthol, U.S.P., B.P., $\mathrm{C}_{10} \mathrm{H}_{19} \mathrm{OH}$ - - A secondary alcohol obtained from peppermint oil. Colorless crystals of peculiar odor and warm aromatic taste. Only slightly sol. in water, freely in alc. Dose, 0.05 Gm., I gr., U.S.P.; 0.03 to $0.12 \mathrm{Gm} ., 1 / 2$ to 2 gr., B.P.; as carminative, etc. Externally, in alcohol, oils or ointments, I to 5 per cent. on mucous membranes, Io to 25 per cent. on skin.

Emp. Menthol, B.P., Menthol Plaster.-I5 per cent.

\section{SAPONINS (SAPOTOXINS; SAPO-GLUCOSIDS)}

General Statement.-These comprise a series of related compounds, generally non-nitrogenous glucosids, which occur in many plants. The actions are somewhat related to the bacterial and other toxins, and to the soaps and bile salts. Like the latter, they lower surface tension, and therefore emulsify or dissolve fats, lipoids and fine powders. This emulsification is characteristic. Through the same property they form an excessive and persistent foam when even very dilute $(\mathrm{I}: \mathrm{I0}, 000)$ watery solutions are shaken; they hemolyze blood, even in isotonic salt solutions; they increase the permeability of other cells and are therefore general protoplasmic poisons. Fish are killed by high dilutions $(\mathrm{I}: 200,000)$. In higher animals, they are but little absorbed, so that they act mainly as local irritants to the mucous membranes. A few, however, are absorbable and toxic. Their interest is mainly scientific. Senega is somewhat used as nauseant and emetic. Sarsaparilla and guaiac were formerly employed as alteratives in syphilis. Quillaja (soap bark) and saponaria (soap root) are used technically for cleansing.

Chemic Properties.-Many of the typical members of the group respond to the formula, $\mathrm{C}_{n} \mathrm{H}_{2 n-8} \mathrm{O}_{10}$. They are glucosids and give a purple color with concentrated sulphuric acid. They are generally not attacked by animal ferments. Physically, they possess the characters of colloids: They do not dialyze; most are soluble in water, and are not precipitated by moderate amounts of alcohol; but they do not dissolve in pure alcohol or in fat-solvents. Many are precipitated by saturation with neutral salts, especially ammonium sulphate; by basic lead acetate, etc. Some behave as acids, others are neutral. When heated with dilute mineral acids they are decomposed into sugars (differing for the individual saponins) and a sapogenin. Schmiedeberg calls these decomposition products "saponins," and all the original substances "sapotoxins." Others apply this name to the more toxic saponins (Review, Kobert, I9I4).

Occurrence.-Sapo-glucosids are very widely distributed through the vegetable kingdom, occurring in at least 400 plants, belonging to 50 different families. They are probably formed in the leaves, but are found in all parts of the plants. The foaming is so conspicuous that many of these drugs have received characteristic popular names, soap-bark, soap-root, etc. They are employed, even by non-civilized peoples, for cleaning, and on account of their high toxicity to fish, as fish-poisons. Ophiotoxin, a definite principle of isolated snake-venom, is believed to be closely related to the saponins (Faust). 
Local Actions.-Most of the sapo-glucosids are markedly irritant to mucous membranes. They have an acrid taste and provoke a flow of saliva (sialogogue action), nausea, vomiting and diarrhea; if inhaled, they cause sneezing; if injected hypodermically, they cause subcutaneous inflammation.

Therapeutic Uses. - The local actions resemble those of ipecac and are similarly employed. Senega is used as nauseant expectorant, especially in chronic cough, and as emetic. Its non-absorption constitutes an advantage. The emulsifying action determines the use of quillaja as detergent, especially for the cleansing of articles which are injured by alkalies. The use of quillaja in medicinal emulsions, or for producing foam in soda water, etc., is not admissible.

Some of the less toxic saponins might be used for this purpose (Kobert, I904), but they have not been sufficiently studied to be entirely safe.

A certain number of these drugs have some importance on account of stock-poisoning (Vaccaria, Raw Potatoes); others have been used as fish poisons, as soap and as counterirritants, etc., by the aborigines.

Sarsaparilla, which owes its activity entirely to the sapotoxin or saponin which it contains, formerly enjoyed considerable reputation as an alterative. This is no longer accepted at the present time (H. C. Wood, Jr., I9I6); and if it possesses any action at all it is simply that of a very mild nauseant and cathartic.

Systemic Actions. - If sapo-glucosids are injected directly into the circulation they produce hemolysis, diuresis (B. MacCallum, I905; Asher, 1908); and direct actions, especially on the central nervous system, which may be rapidly fatal. At first there are violent convulsions; then paralysis, especially of the respiratory center. The effect on fish is also paralytic. Rapid absorption through the gills makes even small doses effective.

Smaller doses given by the blood cause especially intestinal symptoms, and death after several days by collapse. Why the symptoms are so largely intestinal is unexplained. Given subcutaneously, they produce these same symptoms, only, of course, much more slowly. If they are applied directly to skeletal or cardiac musile or to nervetrunks, these lose their irritability at once, and if the solution is fairly strong (I per cent.), there is rigor. The addition of saponin increases the toxicity of digitoxin for the frog's heart (Postojeff, I9II). Saponins act on amebæ, but apparently not on bacteria (Bacon and Marshall, Igo6).

Effects of Hemolysis.- Hemolysis liberates potassium and other toxic substances from the corpuscles (Gottlieb and Lefmann, I907; W. H. Brown, and Loevenhart, I913); but these are not concerned in the systemic saponin actions: The latter occur with doses which are not hemolytic; nor are the systemic saponin effects avoided by cholesterin which prevents the hemolysis. Presumably the permeability of the nerve cells is directly affected by the saponin.

Poisoning.-Most sapo-glucosids are absorbed so poorly that they produce only local effects: gastroenteritis, vomiting, persistent diarrhea, etc. However, the violent local action may lead to corrosion and this to absorption ( $f . i$., with quillaja). Agrostemma, the common corn-cockle (chemistry, Brandt, I 906 ) is absorbed fairly readily and thus produces systemic poisoning. This is important since its seed may be admixed with grain. The systemic symptoms agree with those of intravenous injection. On the other hand, certain vegetables, f.i., spinach, contain saponins which seem harmless to man. Incidentally, some saponins show a different toxicity for different species (Kobert, I9I4). The toxicity is not simply proportional to the hemolytic power.

Hemolytic Action.-Saponins lake blood even in an isotonic medium. The phenomenon depends on their affinity for the lipoids of the cell envelope and stroma. It is prevented by the addition of cholesterin or other lipoids which combines or binds the saponin.

Hemoly tic Concentration.-The limital concentrations, I : 400,000 to I : Io, vary enormously but are characteristic for the different saponins (Kobert, I9I4).

Phenomena of Laking.-Saponin hemolysis occurs in two stages, which are practically distinct: The liberation of hemoglobin; and increased permeability of the envelope (G. N. Stewart, I899 to I909; Woelfel, I908). The latter occurs even in formalde- 
hyd-fixed corpuscles. The action is probably both on the stroma and on the envelope; dissociating the former from its combination with hemoglobin, and rendering the latter more permeable, thus leading to the entrance of water, swelling, and "water-laking." Saponin does not cause the complete disappearance of the stromata; bile salts do (Neuberg and Haendel, 1908).

Solution of Lipoids. - The integrity of the corpuscles is intimately connected with their lipoid content. The stroma and envelope consist to about one-third of lipoids, chiefly cholesterin and lecithin, and two-thirds of protein (Pascucci 1905). Many hemolytic agents are lipoid solvents; such as the saponins, bile salts, soaps and fatty acids, aliphatic narcotics, heat, etc. Their action is generally proportional to their lipoidaffinity. Saponins also increase the permeability of artificial cholesterin-lecithin membranes (Pascucci, 1905). The hemolytic serums and toxins probably also act on these lipoids, but perhaps likewise on the protein. It is perhaps not necessary that the lipoids are actually dissolved; they need merely be altered. Hypoisotonic solutions probably disorganize the entire structure of the corpuscles.

The hemolytic activity of different saponins has no relation to their effect on surface tension (Woodward and Alsberg, I916).

Specific Resistance of the Cerpuscles.-The blood of different animals shows specific differences. It is noteworthy that the resistance of species varies inversely for saponin and water-laking (Rywosch, 1907 and 1911 ). Normal serum is somewhat protective, perhaps by its cholesterin; and this protection is slightly increased by habituation.

Acquired Resistance.-Repeated injections of saponin and other hemolytic agents raise the general resistance of the corpuscles (Kobert; $R$. Weil, I gio; contradicted by Kagan, I9I3). No true antibodies occur. Prolonged saponin feeding increases the resistance, both of the corpuscles proper and of the serum (Alsberg and Smith, I9I4). A similar increase of resistance occurs also in certain diseases in which hemolytic poisons are probably produced, f.i., in syphilis, advanced cancer, etc. (Weil; McNeil, I 910 ).

Mixed Hemolysis. - Fuehner and Greb, ig1 2, found that the combination of various hemolytic agents (aliphatic, alkaloidal and glucosidal) gave complex results; the summation of the effects being sometimes simple, excessive or deficient.

Protective Action of Cholesterol.--Ransom, I90I, showed that the addition of cholesterol prevents the hemolytic effect of saponins (also of toluidin compounds, Hildebrandt, I9II; of cobra lysin, and of some bacterial lysins, f.i., staphylo; tetanus; of sodium oleate; against the immune-serum hemolysins); but not of some other hemolytic agents. Serum, which contains cholesterol, also exerts this protective action. The phenomenon is explained by a chemic combination between cholesterol and saponin. The protection does not extend to the central saponin actions. The $\mathrm{OH}$ group seems essential to all these protections. Lecithin and other lipoids, which do not form chemical compounds, afford a much weaker protection, probably by binding the saponin physically. Their effects extend also to other lipoid-solvent hemolytic agents (Pilcher, 1913). Cholesterol also protects the heart against digitoxin, but not against the other digitaloids (Karaulow, I9I2). It prevents not only the hemolytic but also the systemic toxicity of sodium oleate (Klotz and Bothwell, i915).

Combination of Cholesterol with Saponins.-Cholesterol precipitates saponins from their solutions. Windaus, I909, showed that definite, crystalline, non-hemolytic compounds are formed. Those with digitonin and cyclamin have been especially studied (Yagi, 1910).

Protective Action of Serum.-Washed corpuscles are laked by saponins in one-fourth the concentrations required in defibrinated blood (Laube, I9II). This is probably due to the cholesterol content. The injection of saponin into animals lessens this protective action for two or three days, by binding the cholesterol (Wacker and Heuck, I913).

The hemolytic action of watery solution of saponin is rapidly destroyed by the addition of fuorescent substances, eosin and erythrosin (Noguchi, I906).

Infusion of tea also has an inhibiting effect on saponin hemolysis; this has been proposed as a test for genuine tea (Maggiora and Ferron, 1914). 


\section{PREPARATIONS - SAPONINS}

Caulophyllum; Blue Cohosh; the rhizome and roots of Caulophyllum thalictroides. (Chemistry, Power and Salway, r913.) Used as diuretic, emmenagogue and anthelmintic. (See article under Nicotin and Ergot.) Dose, 0.3 to $2 \mathrm{Gm}$., 5 to $30 \mathrm{gr}$.

Guaiac Lignum, B.P.; Guaiac Wood.-The heart-wood of Guaiacum officinale or sanctum. Contains 20 to 25 per cent. of the Resin and some saponin (a trace of the latter is also contained in the resin). There is some reason to believe that the saponin is the bearer of the action, which is nauseant and purgative. Guaiac is an almost obsolete remedy for syphilis, gout, rheumatism, tuberculosis, etc. It was introduced soon after the discovery of America.

Guaiacum, U.S.P.; Guaiac. Res., B.P.; Guaiac Resin (Gum Guaiac).-The resin obtained from Guaiac Wood. It contains a number of resinous acids, especially guaiaconic acid, which is colored blue by oxidation. Dose, I Gm., I 5.gr., U.S.P.; 0.3 to I Gm., 5 to 5 gr., B.P.

Guaiac resin is commonly used as a reagent for oxidative ferments. Schaer, 1913, finds the best results with resin extracted from the wood by chloroform; then the natural resin; then alcoholic extract of the wood. Solutions lose their sensitiveness with age.

Mist. Guaiac., B.P.; Guaiacum Mixture.-2.5 per cent. suspension of the resin. Dose, 15 to 30 c.c., $1 / 2$ to $\mathrm{I}$ ounce, B.P.

Tr. Guaiac., U.S.P. - 20 per cent. of the resin. Dose, 4 c.c., I dram, U.S.P.

Tr. Guaiac. Ammon., U.S.P., B.P.; Ammoniated Tincture of Guaiac. 20 per cent. with aromatics and ammonia. Dose, 2 c.c., 30 minims, U.S.P.; 2 to 4 c.c., $1 / 2$ to I dram., B.P.

Troch. Guaiac Res., B.P.-0.2 Gm., $3 \mathrm{gr}$.

Hemidesmi Rad.-The root of the Hemidesmus indicus.

Quillaia Cortex, B.P.; Quillaia (Soap Bark).-The dried inner bark of Quillaja Saponaria.

Tr. Quill., B.P.-5 per cent. Dose, 2 to 4 c.c., $1 / 2$ to I dram, B.P.

Saponaria; Soap-root.-The root or leaves of Saponaria officinalis.

Sarsaparilla, U.S.P.-The dried root of Smilax species. Dose, 2 Gm., 30 gr., U.S.P.

Fldext. Sarsap., U.S.P.-Dose, 2 c.c., 30 minims, U.S.P.

Fldext. Sarsap. Co., U.S.P.- Ioo c.c. represents $75 \mathrm{Gm}$. of Sarsaparilla and $3 \mathrm{Gm}$. of Mezereum, flavored with Glycyrrhiza and Sassafras: 1/2 alcohol; 1/10 glycerin. Dose, 2 c.c., 30 minims, U.S.P.

*Syr. Sarsap. Co., U.S.P.-20 per cent. Sarsaparilla; I.5 per cent. Senna; flavored with licorice, sassafras, anise and wintergreen. Dose, I 5 c.c., 4 drams, U.S.P.

Senega, U.S.P.; Seneg. Rad., B.P. (Seneca Snakeroot).- The dried roots of Polygala Senega. Dose, I Gm., I 5 gr., U.S.P.

Fldext. Seneg., U.S.P.-Contains some ammonia. Dose, I c.c., 15 minims, U.S.P.

Inf. Seneg., B.P. -5 per cent. Dose, 15 to 30 c.c., $1 / 2$ to 1 ounce, B.P.

Syr. Seneg., U.S.P. -20 per cent. Dose, 4 c.c., I dram, U.S.P.

Tr. Seneg., B.P. -20 per cent. Dose, 2 to 4 c.c., $1 / 2$ to I dram, B.P.

\section{SOLANIN}

The glucosidal alkaloids, solanin, solanein, and solanidin, which occur in plants of the potato-genus (Solanum nigrum, dulcamara, tuberosum (Potato), Carolinense, and Tomato), possess a typical sapotoxin action. In certain species, particularly in S. dulcamara, they are associated with a small quantity of atropin-like alkaloid and with a saponin (dulcamarin). The latter plant is sometimes used as a nauseant. Solanin has the formula, $\mathrm{C}_{52} \mathrm{H}_{93} \mathrm{NO}_{18}$; it splits into sugar and solanidin, $\mathrm{C}_{40} \mathrm{H}_{61} \mathrm{NO}_{2}$. The solanin literature is reviewed by Meyer and Schmiedeberg, 1895.

Solanum Dulcamara.-Bittersweet.-The young branches are used. Dose, 4 c.c. of the fluidextract.

Potato (Solanum Tuberosum). - The toxic principles are distributed throughout the plant, but only very small quantities are found in the pulp of the tuber-too small to produce any action. The quantity is larger in the peel, and especially in very young and in sprouting potatoes, but it is not increased by storage. The amount, however, is always so small that "potato-poisoning" is probably never due to solanin (Droste, 1915).

Solanum Carolinense.-The berries have been recommended in epilepsy. They are always given with bromids, and the therapeutic effects of the mixture are probably due entirely to the latter. Dose, 4 to 5 c.c. of the fluidextract. 


\section{SNAKE VENOM}

Actions.-The effects of snake venom are partly local, partly central. The local effects consist especially in an intense cellulitis at the site of the bite. This is accompanied by swelling and progresses to gangrene. The blood is also deeply affected. Its coagulability is altered, as with albumoses. The red blood corpuscles are dissolved. There is considerable tendency to extravasation and thrombosis. The leucocytes sink to 25 per cent. of their normal number.

Snake venoms have no effect on infusoria, bacteria, or on plants, but they are toxic to all animals above the hydra.

The systemic actions are nervous, and mainly central. The most conspicuous is a bulbar paralysis. There is a period of incubation (in man, of about four hours' duration); the patient then becomes drowsy, and then presents an ascending paralysis of the cord-i.e., in the order of legs, arms, larynx, and tongue. This may be preceded by convulsive movements. There is also salivation and vomiting. The respiration becomes dyspneic, giving rise to asphyxial convulsions and forming the cause of death. The heart beats for some time after the respiration stops. A paralysis of the vasomotor center is frequent with these poisons, leading to a fall of blood pressure. The latter is in part due to peripheral effects on the heart and on the endings of the splanchnics. In the heart the paralysis involves both ganglia and muscle. There is also some curare action.

These actions are common to all snake venoms; with certain snakes, however, the local actions predominate, with others the systemic.

The danger depends on many conditions. For rattlesnake venom, the fatal hypodermic dose would perhaps be 0.15 to $0.3 \mathrm{Gm}$. The actual mortality in America is probably about 10.5 per cent. for all snakes; II.7 per cent. for rattlesnakes (Willson, I908).

Hemolytic Principle.-This is probably a toxalbumin (Kyes and Sachs). Cobra venom lakes washed human and guinea-pig corpuscles directly, in the absence of serum; whereas washed sheep and ox corpuscles are highly resistant, but are rendered susceptible by the addition of serum or lecithin. Kyes, 1910, therefore considered the venom as amboceptor and lecithin as complement. The existence of this toxic "cobralecithid" was discredited by Manwaring, 1910, and others. Von Dungern and Coca showed that venom contains a lipoid-splitting ferment, which liberates hemolytic cleavage products. Noguchi believes that soaps of unsaturated fatty acids may act as "complement."

Ophiotoxin and Crotalotoxin.-These, according to Faust, rgro and IgII, are the chief toxic constituents of cobra and rattlesnake venom respectively, being responsible for the central actions. They are definite, non-nitrogenous principles, not glucosidal, but otherwise very closely related in their physical, chemical and pharmacological characters to the saponins; and probably related to the toad poison, and hence to cholesterin. They produce practically all the effects of the whole snake venom, except agglutination.

Rattlesnake venom is not toxic to rattlesnakes. The serum of these snakes, however, is not antitoxic. It, as also the bile, has only a low toxicity (Walker and Marshall, I9I 5).

Treatment of Snake Bite.-This must be very prompt. The wound should be ligated, expressed, excised or cauterized. Crystals of potassium permanganate should be rubbed in, or a solution of this, of chlorinated lime or of iodin, should be freely injected into or about the wound. Medullary stimulants, and large doses of strychnin, are useful. The popular treatment with large doses of alcohol is empirical. Immunization by antivenins has been tried extensively.

Other Animal Poisons. - Various animals contain toxic substances belonging to different groups. They are reviewed by Faust, I9Io; and the animal hemolytic poisons by Sachs, I906. The toxins of Intestinal Parasites are discussed by Paulian, I9r4.

\section{CHOLESTEROL}

The protective action of cholesterol against saponin hemolysis has led to therapeutic trials in hemolytic diseases. The results have not been decisive. 
Characters.-Cholesterol is a mon-atomic alcohol, $\mathrm{C}_{27} \mathrm{H}_{45} \mathrm{OH}$, with the solubility characters of fats. It is therefore but poorly absorbed (Stadelmann, I 898; Klein, I IO). Its oxidation products produce effects (digitalis and saponin) resembling those of bile acids and of the poisons of toads and snakes. Indeed all these are probably derivatives of cholesterol (Faust, I9I ; Flury, r9гі). Cholesterol itself is somewhat stimulating to the heart-muscle, but without slowing (Danilewsky). Cholesterol and its fatty esters lessen phagocytosis; lecithin removes this effect for cholesterol, but not with the esters (Steuber, 1913).

Occurrence.-Cholesterol is a common cell constituent, and therefore occurs in most foods. Egg-yolk is especially rich, one yolk containing about $0.25 \mathrm{Gm}$. (Klein, rgro). It is generally prepared from gall-stones. Its esters and homologs constitute Woolfat (see Index). The cholesterol content of the blood plasma is influenced by disease; increased in diabetes mellitus, icterus, and some other diseases; decreased in infectious fevers (Klinkert, I9I3; Buerger and Beumer, I913). It is also increased by partial or complete excision of the suprarenals; at the same time, increased quantities are excreted by the bile (Rothschild, 1914).

Cholesterol is present, partly free and partly as esters, in the blood plasma, red corpuscles, and especially in the leucocytes, in fairly constant quantity under normal conditions. Cholesterol plates may be found in any tissue when the cells have undergone slow destruction and where absorption is poor. It is increased by feeding cholesterol (Lehmann, I9r4; Wacker and Hueck, I9r3). Mice can synthetize cholesterol when it is absent from their food (Degani, I9I4); but it is now believed that if is not ordinarily formed in higher animals, but depends upon the food and therefore eventually on plants. Ordinary food contains adequate amounts; but when the diet is restricted, or when there are extraordinary demands, as in growing children, it may conceivably be deficient (Mendel, I9I4).

Fate.-Cholesterol is absorbed from the alimentary tract into the lymph, being demonstrable in the thoracic ducts. A part is converted into esters before absorption (Mueller, I9I5). The excretion in man, with various diets, was studied by Ellis and Gardner, I9I 2. It is first oxidized into oxycholesterin, and a part probably into the cholic acid of the bile (Liefschuetz, I9 I4).

Hypodermic injection into rabbits produces marked inflammation and necrosis of the subcutaneous tissue. This gradually subsides with the disappearance of the cholesterin, effected mainly through the leucocytes (Basten, I9I5).

With intravenous injection, it leaves the blood rapidly, being deposited in various organs, and partly excreted by the bile and urine. The kidneys show degenerative changes and focal infiltration with cholesterol (K. Dewey, I9r6).

Phagocytosis and Antibodies.-In rabbits and guinea pigs, injections lower phagocytosis by direct action on the leucocytes. They also delay antibody formation (Dewey and Nuzum, I9I4). Burnett, I9I3, states that its injection accelerates the growth of experimental cancer. The division-rate of paramecium is increased (Browder, I9r 5 ).

Atheroma.-Feeding or injections of cholesterol produce arterial lesions in rabbits (Walker and Hueck, I9I3; Ignatowski and Chalatow; Adler, I9I4; Denny and Frothingham, I9I4); also degenerative changes in the suprarenal cortex (Knack, I9I5). Less marked arterial changes are produced in guinea pigs (Bailey, 19 I5).

Therapeutic Use.-In paroxysmal hemoglobinuria, Pringsheim, I9I2 and I9I3, reports partial success from six intramuscular injections, on alternate days, each of 5 c.c. of ro per cent. cholesterol emulsion. The action did not outlast the administration. Reicher, 1908 , in pernicious anemia, administered it by mouth, $3 \mathrm{Gm}$. in 100 c.c. of olive oil, daily, in tablespoon doses. The preparation of a watery emulsion for intravenous injection is described by Dewey, I9 6 .

\section{ACONITE}

General Statement.-Aconite owes its activity to the rather unstable alkaloid aconitin. This is a peripheral and central stimulant and depressant. Locally, the peculiar stimulation (tingling) and anesthesia (numbness) of sensory nerves, without inflammatory phenomena, is used against neuralgic and rheumatic pains. Systemically, therapeutic doses produce mainly a slowing of the heart (central vagus stimulation) which is employed to some extent in sthenic fevers. Larger doses are highly 
toxic; producing great general depression, extreme irregularity of the heart (mainly by direct action on the cardiac muscle), and death by arrest of the respiration or heart.

The tincture, the most commonly used preparation, now contains ro per cent. of aconite, in both the U.S.P. and B.P. (In the older editions, the strength was 35 per cent. U.S.P., 5 per cent. B.P. These differences must be remembered in connection with the older clinical papers.)

Historical.-The toxic properties of aconite were known to the ancients; however, there is considerable confusion, since the Roman poets used "Aconite" to denote vegetable poisons generally (Fleming). Various species, most of which are toxic, grow in different parts of the world. They were used as arrow-poisons by the ancient Chinese and Gauls. The therapeutic use dates from Anton Stoerck ("Observations on the Use of Conium, Stramonium, Aconite, and Colchicum," I 763), who described the tingling without reddening or corrosion, the salivation and sweating, and proposed its use against rheumatic and neuralgic pain, and for "dissipating" swellings and healing ulcers. Alexander Fleming, of Edinburgh, 1845 , published a comprehensive experimental and clinical "inquiry" of Aconitum Napellus, which is still a good model for therapeutic observation. Aconite is a favorite remedy of the homeopathic school.

Chemistry of Aconitin.-The chief active constituent of Aconitum Napellus is the alkaloid Aconitin, which occurs in a crystalline and amorphous form. It is chemically an acetyl-benzoyl-ester of aconin, a base derived from quinolin (Ehrenberg and Purfürst). It decomposes readily, even on boiling, with water, into benzoyl aconin (Picroaconitin, Iso-aconitin) and this again into aconin (Freund and Beck, 1894). They have the same actions as aconitin, but picroaconitin is only about $1 / 50$ as strong as aconitin, aconin $1 / 2000$. These and probably other decomposition products preëxist in the drug, and are probably formed in its extractions and especially in the manufacture of the alkaloid.

Commercial "Aconitins" therefore usually represent mixtures of varying strength, some being a hundred times more active than others. This enormous variability is most unfortunate in a substance of so great a toxicity; and several fatal accidents, to which it gave rise, have led to the practical abandonment of the internal use of aconitin in therapeutics.

Variability of Tincture.-The tincture is also subject to variation, even when it is chemically assayed for total alkaloids. Robinson, I9I 5 , for instance, found a chemically assayed sample practically inactive. Haskell and pupils, I9I5, find the activity to vary nearly rooo per cent. The chemical assay is therefore useless, or rather harmful, and should be replaced by the bio-assay described in the U. S. P., IX.

Other species of aconite contain related alkaloids, with similar actions: Pseudaconitin or Nepalin; Indaconitin; Bikhaconitin; Japaconitin, etc.

Local Actions. - In the mouth, aconite, even a small quantity of dilute solutions, produces a peculiar warm and tingling sensation, a sour rather than bitter taste, followed by numbness and anesthesia. The lips, tongue, and mucous membrane of the mouth and throat feel as if swollen. The flow of saliva is increased reflexly. The apparent irritation is not accompanied by redness, pain, objective swelling, or other sign of inflammation. It is therefore purely sensory.

Analogous effects-heat, tingling and succeeding numbness, without inflammation, are produced in any other situation to which the drug may be applied; and even on the systemic administration of large doses.

From the skin, watery solutions are but slightly absorbed, and friction must be employed to secure the local effect. Oily solutions and ointments are absorbed readily.

Bio-assay.-The tingling sensation in the mouth was proposed by Squibb for the identification and estimation of aconite, and is still one of the most satisfactory methods. Perfusion of the isolated frog's heart is sensitive to aconitin in dilution of two in a million (G. B. Roth, x913). The fatal dose for guinea pigs has been adopted by the U.S.P. as the most practical test.

Eye.-Fleming claims that the application to one the temples or one side of the forehead may produce more or less (temporary) blindness on that side. The pupils 
are then dilated, presumably to admit more light. The application to the conjunctiva constricts the pupils. In systemic poisoning, they are also constricted until the final asphyxial dilation.

Therapeutic Use of the Local Action.-This is used for the relief of toothache, neuralgia, migraine, rheumatic and other similar pain. For this purpose it may be employed by thorough friction of the tincture, undiluted or with the addition of $\mathrm{I}$ to ro parts of soap liniment. This is probably as effective as the expensive Unguentum Aconitinæ (2 per cent.).

Fleming claims that the internal use of the tincture is of ten more effective, increasing the dosage from about I c.c. of the U.S.P. tincture, three times daily, until the full therapeutic effect (pulse of 54 , lethargy, etc.) is obtained.

Systemic Effects of Therapeutic Doses.-These consist mainly in a more or less pronounced slowing of the heart rate, to 60 or 54 beats per minute, according to the dose and the susceptibility of the individual. The pulse is also softer and weaker, but remains regular as lon as the dosage of slowing is not surpassed. The blood pressure falls slightly.

The therapeutic slowing (which is often absent in anesthetized dogs) is due mainly to stimulation of the vagus center, for it is practically absent if the vagi have been divided (S. A. Matthews, I897).

The typical slowing, which occurs in one and one-half to two hours if the tincture has been taken by the mouth, is preceded by subjective sensations of evanescent warmth, and persistent numbness and tingling, beginning in the finger tips, and extending along the arm, as the dosage is increased. The respiration is moderately slowed, and more or less labored. With the full therapeutic dosage, the patient is weak, giddy, confused, lethargic and cold. These phenomena last for three to five hours, and are as far as the therapeutic action should be carried. They are produced by doses corresponding to 0.15 to $0.3 \mathrm{gm}$. of aconite (Fleming, corresponding to I.5 to 3 c.c. ( 20 to 45 minims) of the Io per cent. U.S.P. tincture.

Sweating may occur but is not constant. The urine is not increased. The temperature is not markedly affected by the therapeutic doses. Digestion and peristalsis are not disturbed. There is no cumulative action.

Vasomotor Center.-This is not affected by non-toxic doses. Toxic doses, however, produce a maximal stimulation, more intense than can be obtained from stimulation of sensory nerves) Pilcher and Sollmann, I915).

Inactive Tinctures. - Several recent observers (Price, rgr1; Rudolph and Cole, 1912) have been unable to obtain effects on the pulse and blood pressure, from small and fairly large doses, in a variety of clinical conditions.- These negative results are probably ascribable to inactive preparations of the drug, as with Robinson, 1915.

Therapeutic Uses.-The slowing of the circulation has been utilized in heart disease, in hypertension, and in sthenic fevers, but is of doubtful value, especially in view of the total inefficiency of the doses ordinarily given.

Sthenic Fevers.-In these, it is supposed to "calm" the overactive heart, and to promote the action of mild diaphoretic agents (spirits of niter, ammonium acetate, etc.). It is used in this way during the sthenic stage of acute fevers, especially in children, for the "abortion" of colds. According to Ringer, it is administered in small and frequent doses ( 3 to 5 drops of the ro per cent. tincture for adults, at first every fifteen minutes for two hours, then every hour, until the pulse returns to normal), and only for the first day of the fever, to avoid depression. It is difficult to judge its usefulness under such conditions.

Cardiac Conditions.-Aconite is plainly contraindicated in incompensated valvular lesions, since it would reduce the already defective mass-movement of the blood. Theoretically, it might be indicated in functional overactivity of the heart, in excessive hyper- 
trophy, and in acute inflammatory conditions of the heart. In all of these, a slowing of the beats is desirable. In fact, however, the same effect can be obtained by the more desirable expedient of simple rest in bed. The use of "cardiac depressants" is now almost obsolete. Other drugs which have been employed for this purpose are: Spartein, Veratrum, Colchicin and Potassium salts.

Hypertension. - Fairly large doses of the tincture ( 15 to 25 drops, four times daily) have been tried, but with doubtful results. Thomson, I9I4, claims that it lowers blood pressure and increases the elimination of urea in interstitial nephritis. Pierson, I913, obtained very little result.

\section{EFFECTS OF LARGER (TOXIC) DOSES OF ACONITE}

Mammalian Circulation.-If the therapeutic dosage is exceeded, the primary slowing of the heart rate turns rather suddenly into quickening, accompanied by arhythmia, which finally becomes extreme.

Toxic doses greatly increase the force and especially the rate of the heart, at the same time rendering it extremely arhythmic. The beats may occur in groups, as with digitalis. The heart finally goes quite suddenly into delirium cordis, and stops. During the earlier stages of this action the blood pressure is extremely variable, according to the varying force of the heart, and the successive stimulation and depression of the vasomotor center. Toward the later stages, the pressure drops severely (Matthews, 1897; for further details of the interesting cardiac irregularities consult Fleming, page 3r; and Cushny, I909).

The cardiac effects are due partly to stimulation and depression of the vagus and accelerator mechanisms, but mainly to direct action on the cardiac muscle; the excitability of the latter is greatly increased, somewhat as with digitalis (which see). The quickening occurs even in the nerve-free heart of the embryonal chick; in mammals also it is seen after all the nerve endings in the heart have been paralyzed by apocodein.

The isolated mammalian heart (Hedbom-Langendorff) shows the following: The first effects are inconstant; the frequency is then enormously increased and amplitude lessened below what is accounted for by the quickening (increased irritability and weakened muscle). Then follows a transient increase of amplitude (muscular stimulation), and then sudden stoppage of left ventricle (paralysis of automatic property). The right ventricle and the auricles make a few more contractions. Caffein may start a few beats.

Action of Aconite on the Frog's Heart.-These are practically identical in the intact frog and in perfused excised heart. They are therefore peripheral. They consist essentially in heightened irritability of the auricular, and especially of the ventricular muscle; disturbed conduction; lessened contraction intensity, and finally paralysis. The successive effects are described as follows (Boehm, I87I; Hartung, I9II; Fuehner, 19I1):

I. Preliminary slowing is sometimes observed. It is probably due to stimulation of the vagus endings.

2. Acceleration.- Rather suddenly the beats become quickened and at first strengthened. The acceleration is attributed to heightened irritability of the cardiac muscle. The time of contraction and the refractory phase are shortened.

3. Incoördination. - Ventricular extra-systoles appear (even when the auricles are removed). . The rate of the ventricle increases above that of the auricle, at first in a regular ratio. The combination of the two rates results in "periodic dissociation" or grouped beats. As the rate continues to increase, the contractions can not be maintained regular, and become extremely incoördinated, resulting in the characteristic "peristalsis" of the heart-muscle. This begins in the auricles, but soon extends to the ventricle. It consists in vermicular movements, in which the blood, instead of being propelled, is merely moved about in the heart. The localized contractions may lead to a mulberry appearance, etc. Sometimes the contraction sequence is reversed so that the ventricular contraction initiates the auricular beat. These irregularities may alternate with periods of quite normal beats. The regular rhythm can often be restored by stimulating the accelerator nerve.

4. Standstill.--The extreme irregularity always passes off after a time, and is succeeded by slow and weak, but fairly regular contractions, except that the ventricles beat much slower than the auricles. Eventually the ventricles stop relaxed, and then the auricles, usually rather suddenly. Atropin, accelerator stimulation, or direct stimulation may start a few rhythmic beats, but the contractility is soon lost completely.

Heart-test for Aconitin.--The peculiar heart "peristalsis" is the most delicate and characteristic test for aconitin. Fuehner, $19 \mathrm{ir}$, could thereby detect $1 / 100 \mathrm{mg}$. in intact 
frogs and 1,1000 mg. in excised hearts. Similar effects are produced by the related Delphinin. The Digitalis group also produces peristalsis, but this is of short duration and followed by systolic standstill.

Skeletal Muscles and Motor Nerves.-Strong concentrations of aconitin at first stimulate and then paralyze the motor nerves peripherally. The stimulation is manifested by fubrillary twitching, occurring in muscle-nerve preparation, spontaneously or especially after stimulation of the nerve. The threshold of stimulation is lowered. The twitchings do not occur after curare, and must therefore be in the endings. They may be scen in living animals. The subsequent paralysis of the nerve, and eventually of the muscle substance, can not occur during life, since the dose and the time required are greater than what would paralyze the heart (Hartung, I9I I). The allied Delphinin, however, has been recommended as a substitute for curare in frog work (Schiller, I904).

Infusoria and plants are also killed by aconite (Fleming).

Respiration.- This is progressively slowed in proportion to the dose (Fleming). It is also labored and the later stages become irregular. The slowing bears a superficial resemblance to that of vagus stimulation; but since it occurs also after section of the vagi (Boehm and Wartmann, I 873), it must be due to direct depression of the respiratory center. It is characterized by longer expiratory pauses. Primary increase of respiration has been claimed (Cash and Dunstan; Anrep); but it is small and inconstant, and probably due to other factors (Hartung, rgr 2).

Temperature.-This falls in severe poisoning, because of the collapse and consequent paralysis of heat regulation. The temperature of the animal falls and rises with the external temperature (Brunton and Cash, i886).

Summation of Dosage.-The influence of this on temperature has been studied by Cash, 1906. He found an imperfect summation of effect if the dose is repeated at suffciently close interval. But if this exceeds two hours, the later doses may produce actually less effect, i.e., the temperature may not fall as low as with the first dose. Repeated administration produces some tolerance in rabbits (Hartung, 1912).

Reflexes.-These are diminished, on account of the sensory paralysis.

\section{SYMPTOMS OF ACONITE POISONING IN MAN}

With very large doses death may occur almost instantly-probably from paralysis of the heart. With moderately toxic doses the following picture is seen: Tingling in mouth, stomach, and skin (the most important diagnostic feature); excessive salivation; nausea, retching, vomiting, and diarrhea (the mechanism of the emesis is central; Eggleston and Hatcher, I9I 5). The burning passes into anesthesia. There is great restlessness. The pulse is slow and feeble, later arythmic and very rapid. Respiration is dyspneic. There are muscular weakness, incoördination, vertigo. The skin is cold and livid. The pupils are first constricted, then dilated from the asphyxia and convulsions. The intelligence does not usually suffer, but there may be stupor and even unconsciousness. The special senses and speech may be impaired. Convulsions are common. Death may occur by heart paralysis or by paralysis of the respiratory center (the heart beating after death; Fleming). The symptoms may appear almost instantly, and are rarely delayed beyond an hour. In fatal poisoning death occurs usually in two to six hours. There are no constant postmortem changes.

Etiology.-Accidental poisoning from the fresh plants-which as monk's-hood and the related larkspur are quite common in gardens-as also from liniments, etc., is quite frequent. On account of the small dose, the absence of postmortem signs, and the difficulty of chemic proof, aconitin has recently become quite a favorite for suicidal purposes. The use of the plant for criminal poisoning is widely spread in the East. The symptoms are so unmistakable-especially the tingling - that it has not found many users in civilized countries.

Subtoxic Effects in Man.-According to Fleming, 0.5 Gm. of Aconite produces aggravation of the therapeutic effects, of the subjective symptoms and lethargy with anxiety. The pulse may either be very slow (to 36 ) and regular, or more of ten increases suddenly to 70 or 80 , and becomes small, weak, and more or less irregular. The quickening may occur without any preliminary slowing. Robinson, I9I5, observed extra- 
systoles without other effects. The respiration is also irregular. The temperature falls further. There is generally vomiting. These symptoms do not entirely subside for one or two days.

Dangerous Effects. - With doses of $0.67 \mathrm{Gm}$. of Aconite, the collapse becomes pronounced, with pale sunken face, froth at mouth, and extreme prostration. Consciousness remains, perhaps with slight delirium. The voice is very weak or lost. The pulse is very small, weak, and irregular; the respiration very imperfect. The skin is cold and clammy. The patient may recover with treatment.

Fatal Effects.-The patient is either conscious or mildly delirious, and becomes entirely blind, deaf and speechless. The pupils are dilated. There are general muscular tremors or slight convulsions; the heart beat becomes imperceptible. The temperature continues to fall. Finally, the heart stops, more or less abruptly.

The fatal dose is uncertain: According to Lewin, death has occurred from 2 to $4 \mathrm{Gm}$. of Aconite; recovery from $7 \mathrm{Gm}$.; with aconitin, death has occurred with $4 \mathrm{mg}$., recovery with 12 mg. Fothergill states that it is more fatal for young rabbits than for adults.

Absorption and Excretion.-The absorption occurs readily from mucous membranes; alcoholic and oily solutions are absorbed from the skin. Lewin states that it is excreted promptly by the urine, feces, saliva and intestinal mucosa.

Treatment of Aconite Poisoning.-The common alkaloidal antidotes and evacuation should be employed. The symptomatic treatment should be mainly stimulating: warmth, ammonia, brandy, strychnin, atropin. Artificial respiration is often life-saving (Lewin, I875). In extremis, cardiac stimulants (epinephrin or strophanthin) should be used intravenously.

Antagonism of Aconite and Digitalis.-This was proven by Fothergill, I87 I, but he also showed that the effects of digitalis with ordinary administration are too slow to be of practical value.

\section{PREPARATIONS-ACONITE GROUP}

Aconitum, U.S.P.; Acon. Rad., B.P.; Aconite (Wolfsbane, Monkshood).-The dried tuberous root of Aconitum Napellus, yielding not less than 0.5 per cent., U.S.P., 0.4 B.P., of alkaloids. Frequently cultivated in gardens. All parts of the plant are poisonous.

Ext. A conit., U.S.P.-A powdered extract, representing about 2 per cent. of alkaloids. Dose, io mg., $1 / 6$ gr., U.S.P. U.S.P.

Fldext. Aconit., U.S.P.-About 0.5 per cent. of alkaloids. Dose, 0.03 c.c., 1/2 minim,

*Tinctura Aconiti, Tr. Aconit., U.S.P.; Tr. Acon., B.P.-ro per cent. of drug, 0.05 per cent. of alkaloid, U.S.P.; 0.04 per cent., B.P. Miscible with water or alc. Dose, 0.3 c.c., 5 minims, U.S.P.; 0.12 to 0.3 c.c., 2 to 5 minims, B.P. Maximum dose, 0.5 c.c., 8 minims. The tinctures of the older pharmacopeias were of different strength: 35 per cent. in U.S.P. until I890; 5 per cent. in B.P. until I 915.

Lin. Acon., B.P. - 0.2 per cent. of alkaloids, 3 per cent. of camphor, in alcohol.

Aconitina, U.S.P., B.P.; Aconitin, $\mathrm{C}_{34} \mathrm{H}_{47} \mathrm{NO}_{4 .}$-The most active "crystalline" aconitin. Very slightly sol. in water, sol. in alc. ( $\mathrm{x}: 28)$. Dose, $0.15 \mathrm{mg} ., 1 / 400 \mathrm{gr}$., U.S.P.; maximal dose, $0.3 \mathrm{mg} ., 1 / 200 \mathrm{gr}$. Its internal ase is not advisable.

Ung. Aconitin, B.P. -2 per cent.

Delphiniam.-The various species of Larkspurs contain alkaloids, which are closely related to Aconitin, chemically and pharmacologically. They have of ten caused poisoning in children and in cattle.

Staphisagria, U.S.P.; Staphisag. Sem., B.P. (Stavesacre).-The ripe seeds of Delphinium Staphisagria. Dose, $0.06 \mathrm{Gm}$., I gr., U.S.P. It is used locally against pediculosis capitis or pubis, in the form of the ro per cent. Ointment (B.P.), or as the Fluidextract (U.S.P.) diluted with 8 to ro parts of soap liniment. It causes considerable local irritation, and sometimes eczema (Lewin).

Fldext. Staphisag., U.S.P.

Ung. Staphisag., B.P.-20 per cent. 


\section{VERATRIN (CEVADIN)}

General Statement.-Various species of Veratrum (Green and White Hellebore), Cevadilla, Zygadenus, and other Liliaceous plants contain mixtures of related alkaloids. These are esters of definite bases and acids, related to Aconitin both in structure and action.

"Veratrin" is a mixture of alkaloids, extracted from "cevadilla" seeds, which owes its activity mainly to Cevadin. It must not be confused with Veratrum and its active constituent, Protoveratrin, since their actions differ in important particulars.

Veratrin produces a characteristic prolongation of muscular contraction of considerable physiologic interest. Therapeutically it is rarely used as a counterirritant in neuralgic conditions, and as a parasiticide against pediculi capitis.

Composition of Veratrin.-This has been studied especially by Wright and Luff, I878; and Allen, i 896 .

The principal constituent, both in quantity and action, is the crystalline Cevadin, $\mathrm{C}_{32} \mathrm{H}_{49} \mathrm{NO}_{9}$, the methylcrotonic ester of the base cevin. It is not present in Veratrum album; traces are said to occur in Veratrum viride, but this is doubtful.

The second important alkaloid of "veratrin" is the amorphous Veratridin, $\mathrm{C}_{37} \mathrm{H}_{43} \mathrm{NO}_{11}$, the veratric ester of verin. This is the "veratrin of Wright and Luff." Its effects agree qualitatively with those of cevadin (Boehm and Lissauer).

The commercial "veratrin" contains small quantities of at least three other alkaloids: Cevadillin, Sabadin, and Sabadinin. All these are but weakly active.

Zygadenus (Death Camas), Liliaceæ, a poison plant of Western stock-ranges, owes its toxicity to veratrin bases. It contains at least sabadin, sabadinin and veratralbin (Slade, I905). The actions of the Zygadenus alkaloids have been studied by Mitchell and Smith, Igri. A comprehensive literature is given by Marsh and Clawson, I915.

Action on Striped Muscle.-This consists in a characteristic modification of the muscle substance, so that it responds by a prolonged tonic contraction, even to a single stimulus.

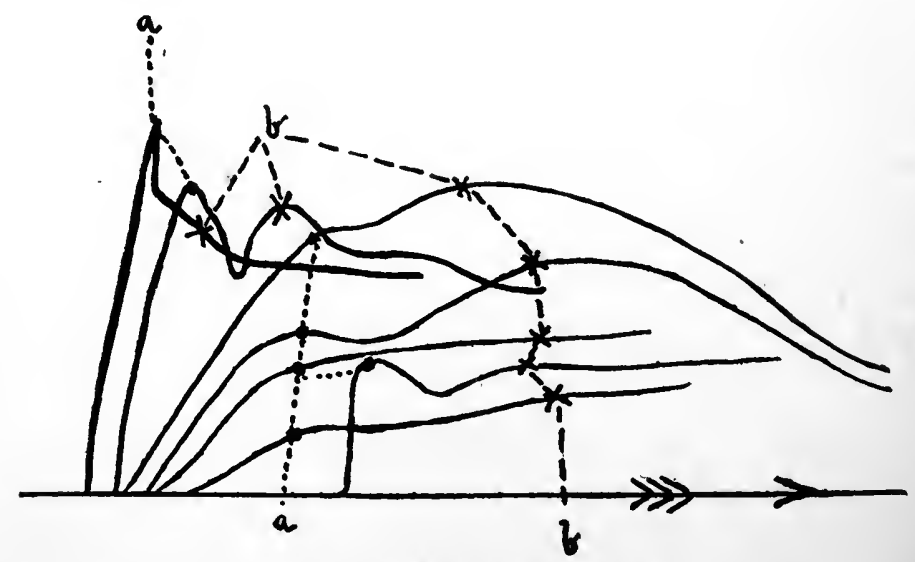

FIG. 21 . - Normal veratrin curves, showing primary $(a \ldots \ldots)$ and secondary $(b . .$.$) contractions.$ Gastrocnemii of different frogs, air temperature, natural size.

Phenomena in Intact Frogs.-When an animal, and especially a frog, has been poisoned with veratrin, it shows very striking peculiarities in its movements. It can contract its muscles with ordinary quickness, but it can not recover its former position for some little time (v. Bezold, I866). 
Form of the Muscle Curve.-The reason for this behavior can be demonstrated on isolated muscle. Frogs are most convenient for this purpose, but identical phenomena occur in mammalian muscle (Quagiariello, I9I3): If a veratrinized muscle is stimulated with a single shock, the ascent of the muscle curve is as abrupt as usual, but the relaxation is enormously prolonged (Fig. 2I). The muscle remains in what appears to be complete tetanus, although it is really a single contraction.

The Contracture is not a Tetanus. - If the nerve of a normal muscle-nerve preparation is laid on the veratrin muscle, a single shock sent into the latter causes a single twitch of the normal muscle, and not a tetanus as would be the case if the veratrin muscle were really tetanized (Fick and Boehm, 1872 ).

The Contracture is Active.-The muscle is able to sustain a considerable weight throughout its contraction; the prolongation of the curve is therefore not due to a mere loss of elasticity, but is an active process. This is also shown by the fact that the formation of heat and the use of material is increased (Fick and Boehm). The tension, the height and quickness of contraction, the irritability, the lifting and sustaining power of the muscle are all raised by veratrin (Dreser, r89o), so that we must look upon its action as an increase of functional activity. It also lessens the effects of fatigue.

Influence of Conditions. - The effect of veratrin is enhanced by agencies which stimulate the muscle, such as moderate heat.

It is diminished by depression of the muscle, produced by cold or excessive heat; by fatigue (Fig. 22); or by muscular depressants, such as potassium or ether.

(If the veratrin action is weak, cold increases the contracture, probably by adding its own contracture to that of the veratrin.--Sollmann.)

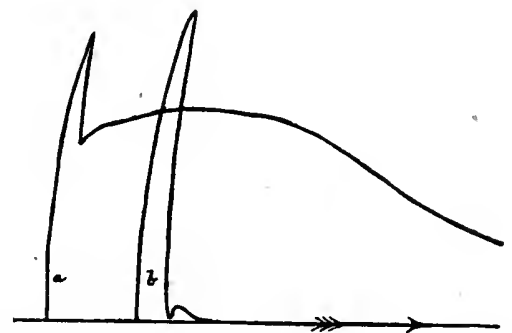
FIG. 2 2.-Effect of fatigue on veratrin curve: $a$, normal veratrin tracing; $b$, after partial fatigue;

Double Peak Curve.-A careful inspection of veratrin-curves generally reveals two contractions (Fig. 2r): a primary contraction (a), which occurs with the normal rapidity, but is somewhat higher than normal, and which tends to relax quickly; and a secondary contraction $(b)$, which occurs more slowly and lasts longer. The two contractions are more or less fused (v. Bezold), but the form varies with conditions (Mostinski, r904). Overend, r889, attributed this phenomenon to the different contraction velocity of the white and red muscle fibers. Cervello and Weiss, r899, showed, however, that uniform muscles exhibit the same phenomenon.

Bottazzi, 1901, suggested that the veratrin curve is due to the fusion of two contractions; the first peak representing the normal contraction of the fibrils, and the further portion of the curve the contraction of the sarcoplasmic substance, which he assumes has the properties of smooth muscle. According to this the veratrin action would consist essentially in raising the excitability of the sarcoplasmic substance above the threshold. There is, however, no direct evidence that the sarcoplasm has contractile functions (Mueller, 1908); and the modern theories of muscle contraction render this very improbable. Woebbecke, r9r3, has modified the theory somewhat. He believes that muscle has two distinct functions, but that these are not specifically connected with the histologic constituents. Verzar and Felter, rgI4, attribute the action to increased sensitiveness to normal stimuli.

Veratrin acts directly on the muscle substance, for the effects occur after curare (Prevost, 1867), and in the nerve-free end of the sartorius muscle. When a muscle is immersed in a dilute solution, a single stimulus produces at first a normal contraction, then fibrillary twitching (Lamm, 1912). The excitability of curarized muscle to direct electric stimulation is not increased; it is diminished by large doses (Boehm, 1913). Large doses of veratrin, however, paralyze the motor endings without previous stimulation.

Spontaneous Contraction of Muscle.-The immersion of a muscle in veratrin causes a slowly developing contracture, even in the absence of spontaneous stimulation. High concentrations paralyze all forms of muscle completely (Koellicker, 1856). 
Other Substances Producing Veratrin Effects.-The prolonged contracture which is so characteristic of veratrin, is by no means confined to this alkaloid, but is also produced by Digitalin, Helleborein, Muscarin, Strychnin; by aldehyds (Verzár and Felter, I915), etc., by cold, and by very strong electric stimulation. This wide occurrence signifies that the phenomenon is of fundamental physiologic importance. These measures also prolong the contraction of cardiac and smooth muscles.

The Action of Glycerin on MI uscle.-The muscles of frogs poisoned with glycerin respond to single stimuli by prolonged contraction, the curve having a superficial resemblance to that of veratrin, but being rather more irregular. The glycerin contraction is, however, a true tetanus, due to greater irritability of the muscle substance; the action-current of each contraction sufficing to start another contraction. The phenomenon occurs also after curarization (Santesson, 1903).

Nerve Trunks.-Direct application of veratrin lessens their excitability (Boehm, I9I3).

Circulation.-The effects are complex. Those on excised hearts resemble aconite and digitalis (slowing with increased systole). In intact animals, stimulation of the vagus center (Fig. 23) predominates at first (marked slowing); later, the fall of pressure from vasomotor paralysis becomes prominent.

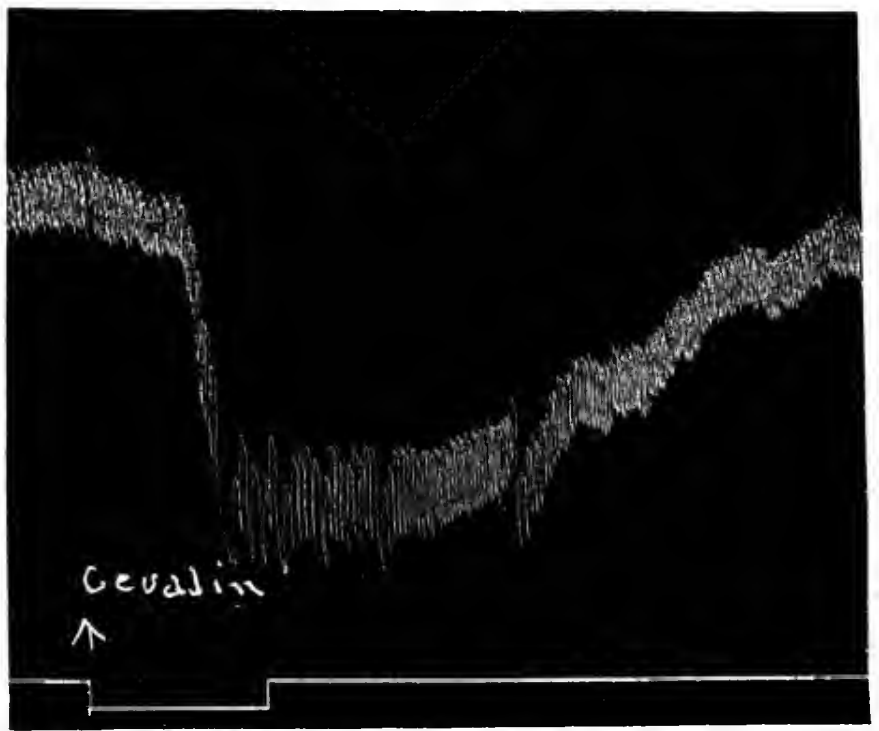

Frg. 23.-Cevadin (Veratrin) on Blood pressure; showing the stimulation of the vagus center.

Intravenous injection of $\mathbf{0 . 0 2} \mathrm{mg}$. per $\mathrm{Kg}$. into anesthetized dog; artificial respiration.

Mammalian Circulation.-Small doses sometimes cause primary quickening of the heart. With larger doses $(0.025-0.05 \mathrm{mg}$. per $\mathrm{Kg}$.) there is sudden and marked slowing with corresponding fall of blood pressure (Bezold and Hirt, 1874; Lissauer, 1887; Pilcher and Sollmann, 1915). The slowing is greatest soon after the injection, often with marked arythmia or temporary arrest. This acute effect is due to stimulation of the vagus center (see, however, under Veratrum), for section of the vagi largely abolishes it, and stimulation of the vagus trunk does not produce further slowing (Busquet, I907). With divided vagi, large doses may cause primary quickening and generally moderate rise of blood pressure (Bezold; Pilcher and Sollmann). The fall of blood pressure is mainly due to the slowing, although some fall may occur independently (Lissauer); The vasomotor center is moderately stimulated by the respiratory embarrassment, even when the vagi are cut. The stimulation does not occur if artificial respiration is maintained (Pilcher and Sollmann, I9 I $_{5}$ ). Reflex stimulation causes vasoconstriction. 
Late Effects.-The acute effect soon passes off. The heart then beats with a steady, moderately slowed rate. This slowing is not removed by atropin and is therefore cardiac.

The blood pressure falls progressively, occasionally interrupted by spasmodic rise from central vasomotor stimulation. The strength of the heart is not impaired, for it reacts well to compression of the aorta, and beats efficiently just before death. The fall must therefore be vasomotor; and accordingly, sciatic stimulation or asphyxia fails to raise the pressure (Lissauer, 1887 ). The vagus remains excitable. The cerebral vessels are constricted (Dixon and Halliburton, I9I0).

The isolated mammalian heart (Hedbom-Langendorfi) shows a primary slowing from stimulation of the peripheral vagus mechanism; then irregularity, and finally paralysis of the cardiac muscle. The tone is increased.

Frog's Heart.-The effects resemble aconite and digitalis. They involve mainly the ventricle and consist in slowing (no further slowing on vagus stimulation; Busquet, 1907); prolonged systole and increased tonus; sudden change in the rate to one-half; "peristaltic" contractions; and final median standstill. High concentrations cause prompt systolic standstill, with continued shortening. The auricles continue much longer. The same effects occur on the excised and perfused heart (Lissauer, 1887; Boehm, I913).

Collapse Action.-The impairment of circulation, combined with the emetic action, result in varying degrees of collapse, and thereby fall of temperature, analogous to veratrum.

Respiration.-This is influenced by the circulatory and convulsive actions, and also directly. It is temporarily arrested during the acute fall of blood pressure, but may be depressed without any change of blood pressure. The respiratory embarrassment is generally diminished by atropin or division of the vagi (Pilcher and Sollmann, I915).

Bronchial Muscles.-These constrict in intact animals (Dixon and Brodie), but not in the excised trachea (Trendelenburg, I912). Titone, I913, observed that veratrin relaxed isolated bronchial muscle.

Convulsions.-These are commonly present in mammals and frogs, and are probably medullary. Rabbits and guinea pigs show convulsive "bucking."

Cerebral Functions.- These are scarcely affected.

Local Action.-Cevadin is a strong irritant, resembling aconite. Applied to the skin, it produces burning, later a cold sensation and partial anesthesia, with reddening and sometimes discrete vesication. On mucous membranes it causes acute catarrh-sneezing, coryza, gastroenteritis, vomiting, diarrhea, etc.; sometimes with corrosive lesions. The vomiting, however, is mainly central (Eggleston and Hatcher, I915). In frogs, the secretion of mucus from the skin is greatly increased.

The irritation is especially strong on the abraded skin; $\mathrm{I}:$ I00,000 solution producing tingling and "shooting" pain (Sollmann and Pilcher, 1916).

Therapeutic Uses.-Veratrin is used locally as counterirritant, in neuralgias and similar conditions, as 2 to 4 per cent. ointment. Sabadilla seeds are employed against pediculosis capitis (Salve, I :4; Decoction, 5:200). The irritation is severe, and absorption has caused serious symptoms. The internal use of veratrin (against fever), even in $\mathrm{mg}$. doses, has produced gastroenteritis.

Absorption is rapid; little is known about the excretion, but it probably occurs by the kidneys.

Symptoms of Poisoning in Man.-These resemble aconite poisoning.

They consist in: Burning in mouth, spreading to stomach; increased salivation, vomiting, diarrhea, abdominal pain; anxiety, headache, giddiness; pupils dilated; pulse slow and feeble, later irregular; weakness, twitchings in muscles. Death by respiratory and circulatory collapse. Consciousness preserved till the end. Postmortem, often intestinal ecchymoses.

In non-fatal doses, the symptoms are very slow in disappearing, and a double case of slow poisoning by continued small doses is on record: The two patients became very weak and thin, suffered from bloody diarrhea, insomnia, disturbance of the intellect, and delirium.

The treatment is the same as in aconite-poisoning. As the veratrin is believed to be rapidly excreted through the urine, it is well to administer hot tea as a diuretic. 
Veratrina, U.S.P. - A mixture of alkaloids obtained from the seeds of Asagræa officinalis. Very slightly sol. in water $(1: 1 ; 60)$; freely sol. in alc. $(1: 2.8)$. Its internal use is not advisable, but it has been given in doses of $2 \mathrm{mg}$., $1 / 30 \mathrm{gr}$. Externally, it is applied as ointinents of 2 to 4 per cent. Maximum dose, $5 \mathrm{mg} ., 1 / 12 \mathrm{gr}$.

Insect Powder.-The flowers of Chrysanthemum Cinnerariafolium contain as active principle Pyrethron (Fujitani, I909), a neutral N-free syrupy substance, with actions apparently resembling veratrin.

Andromedotoxin.-This is an N-free neutral principle, isolated by Plugge from rhododendron and similar plants. It seems to belong to this group (Mountain Laurel-Kalmiapoisoning; Crawford, I908).

\section{VERATRUM}

General Statement.-Veratrum viride and album (Green and White Hellebore) seem to be mere varieties (the green American, the white European), with practically identical actions and composition (Salzberger, I $885^{-1}$ - 890). They owe their activity mainly to the alkaloid, protoveratrin. A number of other related but less active alkaloids are present. There are, however, wide differences of opinion as to the constituents of Veratrum viride (Cramer, I9I5).

The effects of protoveratrin, and therefore of veratrum, are qualitatively similar to those of cevadin; but they differ very materially in their relative significance: The characteristic muscular and irritant actions of cevadin are insignificant in protoveratrin. The most important effects of veratrum are on the medulla, and especially on the circulation, resulting in vagus stimulation, slowed pulse, fall of blood pressure, sweating, "collapse-action," and reduction of temperature. It is therefore employed as a "cardiac depressant." Protoveratrin is more toxic than cevadin, approaching aconitin; but the prompt evacuation of overdoses by emesis (central) renders veratrum practically safe.

Constituents.-The most important alkaloid of Veratrum album, although not the most abundant ( 0.03 to 0.08 per cent.), is Protoveratrin, $\mathrm{C}_{32} \mathrm{H}_{51} \mathrm{NO}_{11}$. It occurs in a crystalline and amorphous form (Salzberger, I890). The other alkaloids are: Jervin, o.I per cent., weakly active; traces of Rubijervin (almost inactive); the inactive pseudojervin or protoveratridin, which is probably a decomposition product; the glycosid veratramin; and veratroidin and veratralbin, which are probably mixtures of amorphous bases (Bull. Am. Pharm. Assoc., r9I I, p. I62; Kobert). Traces of cevadin have been reported in V. viride, but are doubtful.

Black Hellebore, Helleborus niger, is in no way related to the above veratrum species. Its active principle, Helleborein, belongs to the Digitalis group.

Experimental Effects on Respiration and Circulation.-The typical effects of intravenous injection of veratrum viride consist in brief but marked depression of the respiration, slowing of the pulse, and lowering of blood pressure. These effects are largely prevented by section of the vagi, and do not recur if further doses of the veratrum are injected. The repeated injections generally produce marked rise of blood pressure, especially if the vagi are intact.

The effects are probably due to protoveratrin.

Cramer, I915, believes that the effects are produced by the stimulation of the afferent vagus endings, producing reflex inhibition of the respiratory center, and radiating from there to stimulate the vasodilator and the cardioinhibitory centers; these afferent endings being then paralyzed, so that subsequent injections become ineffective. Cramer's doses are so different from those found necessary by other investigators, that comparison of the results is difficult. His explanation is probably correct as to the 
respiration; but requires confirmation as to the circulatory changes. At present, it is simpler to suppose direct stimulation of the vagus center, slowing the heart rate and thus lowering the blood pressure. At least Pilcher and Sollmann, 19I 5, did not observe any significant changes in the vasomotor center. The rise of blood pressure on repeated injection is probably due to paralysis of the cardiac vagus endings, resulting from large doses.

Respiration.- Moderate doses of veratrum viride $(2.5$ to $5 \mathrm{mg}$. per kilogram) injected intravenously into dogs produce brief but marked slowing or arrest of the respiration. This effect is generally absent if the vagi have been divided, and may therefore be attributed to stimulation of the afferent vagus endings (Cramer, I9 5 ; Pilcher and SolImann, 1915). Cramer finds that this does not occur on repeating the injection, and therefore believes that the stimulation of these endings is followed by their paralysis. $\mathrm{He}$ also finds veratrum ineffective after atropin, which would indicate that atropin also paralyzes the afferent vagus endings. Very large doses of veratrum depress the respiration after any of these condicions, presumably by a direct action on the respiratory center.

Moderate Doses on Mammalian Circulation.-In dogs with intact vagi, the intravenous injection of veratrum viride, 2.5 to $10 \mathrm{mg}$. per kilogram, produces a prompt sharp fall of blood pressure and marked slowing of the heart, with prompt return to normal. The fall of blood pressure is due essentially to the cardiac slowing through stimulation of the vagus center, for if the vagi have been divided, the pressure falls but slightly. The efferent vagus endings are not stimulated. The vasomotor center is not directly affected, but is sometimes moderately stimulated by the fall of blood pressure and especially by the respiratory embarrassment (Pilcher and Sollmann, I9 I5).

Wood, Jr., I9o6, also describes moderate fall of pressure and distinct slowing, but apparently more persistent. Both disappear on section of the vagi. Cramer, I9I5, does not believe that the fall of pressure depends chiefly on the slowing, but rather on vasodilation; for he states that the fall starts first; that cats may show the fall without the slowing; that the fall occurs after atropin; and that it is accompanied by intestinal vasodilation. He confirms that the depression of the circulation and respiration are prevented by section of the vagi; and assumes that veratrum acts by stimulating the afferent depressant vagus endings. 'The effect does not involve the depressor nerve, for the vasodilation occurs in rabbits after section of the depressor trunks, but not after the vagi were divided.

Cavazzani, I909, observed that epinephrin prevents the fall of pressure after veratrum viride (just as it does that of depressor stimulation). On the contrary, there results usually a rise of pressure.

Repeated Doses on the Circulation.-These produce considerable rise of pressure, especially if the vagi are intact; less if they are divided (Pilcher and Sollmann). Wood found that this occurs also after section of the cord. It is partly explained by quickened heart rate, from paralysis of the vagus endings. Cramer obtained no effect whatever from the second injection, and believes that the first dose, after first stimulating, paralyzed the vagus endings.

Toxic Doses on the Circulation.-These produce at first exaggeration of the vagus stimulation (marked slowing, irregularity, arrest) with corresponding fall of blood pressure. This is followed by sudden extreme acceleration and rise of pressure (partly asphyxial, partly spasmodic). The rise may last for several minutes, and is succeeded by rapid progressive fall and death. In man, excessive doses also quicken the pulse rate.

Cramer found the results inconstant; due, as hè believes, to an intermixture of the stimulant and depressant affects on the afferent vagus endings. The blood pressure may fall to zero, remain normal, or rise; or follow the convulsive respiration. The heart rate may be slowed, but is more of ten quickened. The inhibitory vagus endings are paralyzed. Subsequent injections have but little effect.

Sensory Actions.-Dried veratrum or protoveratrin are irritant, provoking sneezing, etc.; but in solutions; the irritant effects are very slight. These have a marked anesthetic action, lasting forty-eight hours, on the cornea (Eden).

General Symptoms in Mammals.-The first effects are nauseant and emetic, often so severe as to obscure other symptoms. Of these, the respiratory changes are characteristic, especially in rabbits. They consist in periodic dyspneic attacks, culminating in convulsions of a medullary type. The respiratory disturbance is probably due to the combined influence on the respiratory and other medullary centers, circulatory changes and muscular exhaustion. In the intervals the respiration is regular. Death occurs late, with the circulation fairly preserved and consciousness present to the end (Eden). 
Collapse Action.-The combination of the central, circulatory and muscular depression, nausea and dyspnea, results in more or less collapse. 'This and the profuse sweating lowers the temperature, proportional to the general symptoms (Eden).

Effects of Therapeutic Doses on Man.-Collins, I9I5, I9I6, has made an extensive clinical study of a ro per cent. tincture of veratrum album with single doses of I to 2 c.c. or divided doses of I c.c. every hour, to a total of 4 c.c. Full doses produce constantly a slowing of the pulse to about 62 per minute (irrespective of the original rate); and very generally a fall of systolic and diastolic blood pressure of about $35 \mathrm{~mm}$. Respiration is not disturbed. Headache, gastric burning, nausea and vomiting may set in, but come after the depression of the circulation, and are therefore not its cause. The effects last for several hours. Large doses (4 c.c. within half an hour) may produce more serious and lasting collapse, with a pulse rate of 40 or 30 , suddenly returning to normal after eight or nine hours.

Veratrum Poisoning.-Although protoveratrin is very highly toxic, veratrum is vomited so promptly that death is rare even with large doses (Wood). It has, however, been reported after $\mathrm{x}$ to $2 \mathrm{Gm}$. of the powdered White Hellebore (which is used as insect powder), and in enfeebled patients from a teaspoon of the Tincture.

Symptoms.- These consist in burning, vomiting and diarrhea, dysphagia, collapse, paralysis or light convulsions.

Treatment.-The stomach should be emptied by warm water or lavage, and the collapse treated along the usual lines.

Therapeutic Uses.-Veratrum is probably the most active and reliable cardiac depressant, and was formerly much used to slow and soften the pulse, and lower the blood pressure, especially in eclampsia and other conditions of high blood pressure. Pesci, I906, f.i., claims that it lowers the blood pressure by 7 to $50 \mathrm{~mm}$., slows the pulse, increases diuresis, and lessens uremia. Haultain, I9I4, makes similar claims. It was used for the same purposes, and to lower the temperature, in the early stages of sthenic fevers (pneumonia) and other acute infections. The effects are probably mainly due to the cardiac slowing from vagus stimulation and to the nausea. Veratrum with other cardiac depressants has rather gone out of fashion-perhaps undeservedly. It is contraindicated in asthenic conditions (cardiac weakness, general debility, typhoid, etc.).

Response in Pathologic Conditions.-Collins, I916, observed the most marked effects in cases of hypertonus, the systolic pressure falling more than the diastolic. Little or no response was obtained in arteriosclerosis, paroxysmal tachycardia, or heart-block.

Diuretic Action.-This is not produced in animals (Cramer, I9I5). If it occurs in eclampsia, as claimed, it is probably due to the correction of the circulatory abnormalities.

Administration.-Veratrum is best given in repeated doses-I c.c., I5 minims, of the Tincture (U.S.P.), every half hour or hour-till the skin becomes moist, the pulse slower and slight nausea is present. It may then be stopped. Caution is necessary if a total of 4 c.c. is exceeded. If vomiting should appear before the other effects, 5 to ro drops of Tr. Opii are administered with each dose of veratrum. Repetition of the dosage does not inhibit the action (as occurs with intravenous injections in animals).

\section{PROTOVERATRIN}

The effects, as described by Eden, I892, are similar to veratrum, the reported differences being perhaps explainable by dosage and interpretation. In mammals, moderate doses by vein produce temporary fall of pressure, absent if the vagi were cut. Eden attributes this to reflex depressor stimulation, but vagus stimulation is a more probable 
explanation. This action is short, the pressure rising rather suddenly 40 to $50 \mathrm{~mm}$. above normal, with increase of pulse rate, and inexcitability of the vagus. The rise is probably due to vagus paralysis. This is also of short duration. With repeated in jections, the vagus paralysis becomes permanent, and the primary fall of pressure is absent, there being instead a small rise. Toxic doses produce cardiac arythmia, with periods of stoppage and corresponding changes of blood pressure. The rate in the intervals is at first rapid, then falls with the blood pressure. The vasomotor center becomes paralyzed.

Frog's Heart.-The effects are mainly depressant: the systoles are somewhat prolonged, but the tone is scarcely increased. Soon the ventricular rate is reduced to half, as with cevadin, aconite or digitalis; but this is interrupted by short periods of total arrest of the ventricle or auricles, apparently due to exhaustion of the muscle. Finally the heart stops in diastole, with relatively small doses (Boehm, rgr3).

Motor System.- In frogs poisoned by protoveratrin, the reflexes disappear early. This paralysis is largely central, for it occurs also if the poison is excluded from the muscles by ligature. However, there are also peripheral effects: the muscles may respond normally to a single stimulus or contract even somewhat more quickly and efficiently; but they fatigue very rapidly (Eden, I892). The prolonged contraction characteristic of cevadin is indicated very faintly, if at all, under ordinary conditions; but it may be elicited in excised muscles if precautions are taken against fatigue (Boehm, I9I3). The nerve trunks are paralyzed only by concentrated solutions; the negative variation is said to be prolonged.

\section{JERVIN}

The effects also resemble veratrum, but the quantity of this alkaloid in the drug is too small to have any noticeable effect on circulation (Wood, Jr., 1906). With effective doses, the heart rate is first slowed then rapid. The blood pressure falls progressively, presumably by depression of the cardiac muscle and vasomotor center. The respiration fails simultaneously (Wood, 1874 ).

\section{RUBIJERVIN}

Wood, I874, found that "veratroidin," which was probably an impure rubijervin, acted mainly on the respiratory center, with some cardiac depression. Its quantity is also too small to be important.

\section{PREPARATIONS - VERATRUM}

Veratrum Viride, U.S.P. (Green Hellebore, American Hellebore).-The dried rhizome and roots of Veratrum viride. Dose, 0.06 Gm., I gr., U.S.P.

Veratrum Album, U.S.P. (White Hellebore). - This is probably a mere variety of the former, growing in Europe. It is used in the same doses.

Fldext. Verat. Vir., U.S.P.-Dose, o.I c.c., I $1 / 2$ minims, U.S.P.

*Tinctura Veratri Viridis (Tr. Verat. Vir.), U.S.P.- - per cent. Dose, o.5 c.c., 8 minims, U.S.P. Till I900, the Tincture was of 40 per cent. strength. Norwood's Tincture was supposed to be a saturated solution of the fresh root.

\section{COLCHICUM}

Colchicum (Meadow Saffron), and its active alkaloid Colchicin, are believed to have a specific effect on acute attacks of gout. Their use is empiric and has no pharmacologic explanation. Toxic doses produce a characteristic late gastro-intestinal irritation, even on hypodermic administration. The action is probably due to conversion into oxydi-colchicin. The vomiting, purging, and intestinal congestion may be so violent as to lead to fatal collapse, so that the plant (all parts) is of toxicologic importance. The other actions are relatively insignificant, but are related to aconitin. 


\section{THERAPEUTIC USE IN GOUT}

Colchicum is credited with prompt effects on acute gouty attacks; relieving the pain in one or two hours, the heat and swelling subsiding soon after, and the attack ending in a few days instead of weeks. It must be used early and rather freely. It does not prevent recurrence, soon loses its efficiency, and is of ten unsuccessful; so that many clinicians are altogether skeptical.

Historical.-The toxic properties of colchicum are mentioned by Dioscorides; it was used against gout or rheumatism by the Arabian school (sixteenth century), and since then in domestic practice; but was abandoned in regular practice on account of its poisonous action, till it was reintroduced by Stoerck, 1763 .

Attempted Explanations of Its Use in Gout.-'These are all unsatisfactory. It has no definite effect on the elimination of urine, uric acid or urea. The purgation may contribute to the relief; but it is not essential and other purgatives lack the special effect. The changes in the leucocytes have recently been invoked, but are not convincing. Circulatory and analgesic actions are also unsatisfactory suggestions, since they have not been demonstrated directly, and since colchicum has no effect on other inflammations, or on acute or chronic rheumatic pain, neuralgia, etc.

Administration.-Tincture or Wine of Colchicum Seed or Corm, have been given in divided doses, 0.5 c.c., 8 minims, every three hours (advantageously with an equal quantity of magnesium carbonate or sodium salicylate), until a therapeutic response or slight gastro-intestinal disturbance and weakness occur. Some disturbance of digestion and soft stools are the rule; and in susceptible patients there may be considerable purging and faintness. This may be lessened by atropin, but if possible should be avoided by proper dosage. A single large dose, 2 to 5 c.c., $1 / 2$ to I 3 of tincture acts more promptly, but may cause severe nausea, diarrhea and great muscular weakness. Prolonged administration is generally harmful and therefore inadvisable. Colchicin, which is readily obtainable, permits more definite dosage. It may be given, 0.5 to I mg. $1 / 120$ to $1 / 60$ gr., three times daily, for one or at most two days. This usually causes considerable diarrhea, beginning on the second day, and with its appearance the drug should be discontinued. The stools will remain loose for another day.

Metabolism.-The urine secretion is sometimes slightly increased, sometimes greatly diminished, probably depending on the catharsis. The statements as to uric acid and urea (e.g., Noel Paton, r886; Maurel and Arnaud, I9ro; Abl, 1913) are so contradictory, that they can have little importance. With the modern methods, Denis, I915, found neither increased elimination of urates in the urine, nor diminished concentration in the blood. The bile flow is slightly increased (Kionka, 1906). For about an hour after administration, the leucocyles in the blood are decreased; this is followed by a remarkable increase, especially of the polymorphous, which reach two to five times the normal in twelve hours (Dixon and Malden, 1908).

\section{COLCHICUM POISONING}

Symptoms.- - These set in only after three to six hours, even with large doses. They begin with abdominal discomfort, followed by violent and uncontrollable vomiting and purging and great prostration. The stools are at first normal, but become dysenteric and bloody. They are not especially painful but exhausting; so that the patient passes into collapse, with the nervous and muscular symptoms of cholera. Death occurs in seven to thirty-six hours from exhaustion, consciousness being preserved to the end.

The fatal dose varies; $1 / 2$ ounce of the Tincture has been fatal. This would correspond to about $8 \mathrm{mg}$. of colchicin; but recovery has occurred from much larger doses, presumably with imperfect absorption.

Treatment.- This involves evacuation by emesis and catharsis, if necessary, and diuresis. Tannin is a chemic antidote, but not very effective. Opium and belladonna are given against the gastroenteritis, and the collapse is treated as usual. 
Autopsy reveals intense and of ten hemorrhagic congestion of the intestines, especially the lower portion.

Gastro-intestinal Irritation.-The meclianism of the intense congestion and violent peristalsis has been variously explained. They occur even on parenteral administration, but this has no special significance, since the colchicin is excreted into the intestines (Speyer, 1870; Laborde and Houdé, 1887). The action is not directly on the muscle or its innervation, for excised intestine is depressed in tone and excursions by colchicin or oxydicolchicin (Fuehner and Rehbein, 1915). Roosbach, 1876, stated that the motor response to the intestinal vagus or splanchnic is not affected. The persistence of vagus response has been confirmed by Fuehner and Rehbein. Jacobj, I 89o, conducted experiments with intestines exposed under a saline bath. These showed increased peristalsis of a normal type, inhibited by atropin. He concluded that the action consists in enormously heightened response to the normal peristaltic stimuli. Dixon and Malden, 1908, also described immediate peristalsis, which was arrested by atropin, and which they therefore attribute to parasympathetic stimulation. Fuehner and Rehbein, I9I5, repeated these experiments, but failed to confirm the results. Increased peristalsis occurred only exceptionally. It appears therefore that the peristaltic increase observed by Jacobj and Dixon was accidental, due to causes other than the colchicum.

Fuehner and Rehbein refer the entire action to an irritative capillary vasodilation analogous to the local actions elsewhere, and produced by contact with the drug, reaching the intestine directly or by excretion. They find this hyperemia of the stomach and intestines even with small doses.

Local Action.- Strong solutions applied locally to the conjunctiva or subcutaneous tissue, produce hyperemia, irritation and anesthesia.

Central Nervous System.-This is gradually paralyzed from below upward, partly directly and partly by the collapse.

No appreciable direct effect is exerted on the circulation (therapeutically, it is somewhat slowed by the relief of the symptoms); nor on the glands, striped muscle, or motor nerves (Rossbach; Dixon and Malden).

Explanation of the Delayed Action.-The long period before the symptoms appear can not be materially shortened by increasing the dose (Schorff and Rossbach, 1896), or by intravenous injection. Colchicin itself has relatively little effect on frogs; but its toxicity for these can be increased 50 times by raising the temperature to $37^{\circ} \mathrm{C}$. (Sanno, I9II). The reason for this is, according to Jacobj, I89o, that it is not the colchicin itself which produces the symptoms, but an oxidation product-oxy-di-colchicin - which is formed from it in the mammalian organism - even by circulating it through excised organs-but does not seem to be capable of formation in the frog's at a low temperature. Once formed, whether in the above manner or artificially by the action of ozone, it is toxic to frogs also.

Bio-assay.- The toxicity for mice or heated frogs may be used (Fuehner, I9ro).

\section{COLCHICIN DERIVATIVES}

The structure of colchicin has been established by Zeisel, r888, and Windaus (Fuehner, I913). By treatment with acids, a methyl group is easily split off as methy alcohol and replaced by $\mathrm{OH}$, yielding colchicein. This and various other derivatives have been studied pharmacologically by Fuehner, 1913. The relative toxicity for various animals shows interesting differences.

\section{PREPARATIONS-COLCHICUM}

Colchici Cormus, U.S.P., B.P.; Colchicum Corm (Root).-The dried corm of Colchicum Autumnale (yielding not less than 0.35 per cent. of colchicin, U.S.P.). Dose, 0.25 Gm., 4 gr., U.S.P.

Ext. Colch. Corm., U.S.P.-A powdered extract, I Gm. representing $4 \mathrm{Gm}$. of drug, or about $\mathrm{r} .35$ per cent. of colchicin. Dose, $0.06 \mathrm{Gm}$., I gr., U.S.P.

Ext. Colch., B.P.-The juice expressed from the corm and evaporated to a soft extract. Dose, $\mathrm{x} 6$ to $60 \mathrm{mg} ., 1 / 4$ to $\mathrm{I}$ gr., B.P.

Vin. Colch., B.P.; Wine of Colchicum.-2o per cent. of corm. Dose, 0.6 to I.8 c.c., io to 30 minims, B.P.

Colchici Semen, U.S.P.; Colchici Semina, B.P.; Colchicum Seed. The dried ripe seed of Colchicum autumnale (yielding not less than 0.45 per cent. of colchicin), U.S.P. Dose, 0.2 Gm., 3 gr., U.S.P.

Fldext. Colch. Sem., U.S.P. -Dose, o.2 c.c., 3 minims, U.S.P.

*Tinctura Colchici Seminis (Tr. Colch. Sem.), U.S.P.; Tr. Colch., B.P.; Tincture of Colchicum (Seed).- - o per cent. Dose, 2 c.c., 30 minims, U.S.P.; 0.3 to I c.c., 5 to 15 minims, B.P. Maximum dose, 2 c.c., 30 minims. 
Colchicina, U.S.P.; $\mathrm{C}_{22} \mathrm{H}_{25} \mathrm{NO}_{6}$.-Pale yellow amorphous scales or pale yellow amorphous powder, turning, darker on exposure to light; odorless or nearly so. Sol. in water $(1: 22)$; frecly sol. in alc. Dose, $0.5 \mathrm{mg} ., 1 / 120 \mathrm{gr} .$, U.S.P.

\section{APOMORPHIN}

General Statement.--Apomorphin is an artificial alkaloid, formed by dehydrating morphin through the action of concentrated mineral acids:

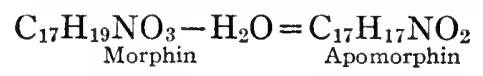

This causes profound changes in both the structure and actions. The peculiar narcotic effect of morphin is almost completely lost. In its place there appears an excitant effect on the nervous centers. In therapeutic doses, this is confined to the vomiting center, apomorphin being the preëminent centrally acting emetic, useful especially for hypodermic administration, in poisoning, etc. It is not so useful as a nauseant.

The emesis is accompanied by the usual side effects of vomiting. It often causes considerable general depression, and sometimes dangerous cardiac depression.

Smaller doses produce a prompt hypnotic effect under certain conditions (acute alcoholism).

Larger doses produce excitement, convulsions, and death by asphyxia.

On direct application it paralyzes the heart and skeletal muscles.

\section{THE EMETIC ACTION OF APOMORPHIN IS CENTRAL}

The specific emetic action of apomorphin was announced by Gee, I869, in the same year as the discovery of the substance by Matthieson and Wright. The emesis is produced by very small doses and is very prompt, with but little preliminary nausea. The fact that there is no evidence of gastric irritation (Gee, I869), and that apomorphin is more effective hypodermically than by mouth (Siebert, I87 I) suggested strongly that its emetic action is not a reflex from irritation of the pharyngeal or gastric mucosa, as is the action of most emetics; but that the apomorphin emesis must result from a central action, a direct stimulation of the hypothetical vomiting center in the medulla. Other evidence also tended strongly to the same conclusion. The absolute proof has been brought by Eggleston and Hatcher, I9I2, who found that the typical phenomena of vomiting could be produced by the injection of apomorphin into animals from which the whole alimentary canal had been removed, from the cardia to the anus. These eviscerated animals show the retching, the vomiting movements, and the expulsion of mucus from the mouth and esophagus. Direct application of the apomorphin to the esophagus or pharynx does not produce vomiting.

\section{OTHER EVIDENCE THAT EMESIS IS CENTRAL}

Absence of Irritant Action.-Siebert, 1871, gave daily emetic doses of apomorphin to dogs for six weeks, and found no signs of any.inflammation at autopsy. Reichert, I879, showed that solutions do not produce irritation even on the sensitive conjunctiva.

Greater Efficiency on Hypodermic Administration.-Siebert and all subsequent workers shuwed that administration by mouth requires larger doses and a longer time to produce vomiting. Eggleston and Hatcher found that dogs require by vein only $1 / 125$ of the oral dose, and vomiting occurs in one-half the time. The effective doses are (milligrams per kilo): by mouth, 5.7 in four to ten minutes; hypodermic, 0.2 in two and 
one-half to ten minutes; intramuscular, 0.05 to 0.075 in three to seren minutes; intravenous (without anesthesia), 0.05 .

Division of Both Vagi.- These constitute the sole afferent paths for the reflex of local emetics (Openchowski, r889). With locally acting emetics therefore vomiting can not occur if both vagi are divided, but this does not prevent the apomorphin emesis (Siebert, Harnack, and others; the contrary results of some investigations are explained by experimental errors-constrained positions of animals). The splanchnics do not carry sensory fibers.

Apomorphin is Not Reëxcreted into Stomach.-Many locally acting emetics produce vomiting even when injected hypodermically or intravenously, by being excreted into the saliva or stomach, and there producing the local action, although this naturally requires larger doses than oral administration (Tartar emetic, etc.; Kleimann and Simonowitsch, I872). Reichert believed that apomorphin is also reëxcreted; but this could not be confirmed by subsequent workers, and has been disproved by Eggleston and Hatcher.

Movements of Stomach in Emesis. - This has been investigated in cats by the X-ray method (Cannon, r898). The same events follow apomorphin and mustard; the first effect is total inhibition of the tone of the cardial portion, which becomes a flaccid bag. The pyloric end is shut off by a deep contraction ring. The contents are then expelled by sudden contraction of the diaphragm and abdominal muscles. As the expulsions are repeated, the gastric walls tighten around the remaining contents.

Replacing Stomach by Pig's Bladder.-Magendie found apomorphin effective under this condition (Magnus). This would not exclude the possibility of intestinal irritation.

Direct Application to the Vomiting Center.-Thumas, r89r, found that the application of apomorphin to a limited area of the medulla produces vomiting.

Location of the Vomiting Center--Openchowski, I889, believes that the center actually stimulated by apomorphin lies in the corpora quadrigemina, and that Thumas stimulated merely the efferent fibers on their way to the splanchnics. He also believes that the quadrigemina are not concerned in reflex vomiting. The nature of the central connections of the vomiting reflex is too obscure for further discussion.

Movements of Excised Stomach.-Schultz, I 886, found that apomorphin and other emetics produce strong reversed peristalsis in excised stomach; but Batelli, 1896 , finds the same movements without giving any drugs, and they may be attributed to asphyxia.

Cocainization of Pharyngeal Region.-Valenti, I9I I, asserts that emesis, by apomorphin as 'well as by local agents, is prevented by cocainizing a certain pharyngeal area which controls the cardial sphincter. He suggests that apomorphin increases the excitability of the center so that it responds by vomiting even to the normal impulses which emanate from this pharyngeal region. However, this may merely mean that the relaxation of the cardial sphincter, which is controlled from this region, is indispensable to successful emesis (Mellinger, Openchowski, Cannon). Magnus, r9r4, discredits the existence of a pharyngeal vomiting center.

Susceptibility of Different Animals to Emetic Action.-Animals which are incapable of vomiting (rabbit, rat, guinea pig, horse, cattle, etc.) do not do so under apomorphin; but many animals which vomit more or less readily in response to local irritants, are also completely insusceptible (pigs, birds, frog) or relatively insusceptible to apomorphin emesis (the cat requires about 5o times as much per body-weight, hypodermically, as the dog). Man and dog are the most susceptible. Continued administration does not establish tolerance (Siebert). Insusceptibility to the emetic actions does not imply tolerance of the other central actions of apomorphin, to which all animals are susceptible.

Recalcitrant Stage.--Excessive doses of apomorphin are not as effective as moderate doses in producing emesis, and may render the animal completely recalcitrant to the emetic action (Reichert, Eggleston and Hatcher).

Ant-emetic Drugs.- Apomorphin vomiting is not affected by local sedatives (bismuth, etc.), but is prevented by narcotics. With chloroform and chloral, full narcotic doses are needed; but with morphin and its esters, very small, non-narcotic doses suffice. Papaverin is ineffective (Ruth, $19 \mathrm{I}_{3}$ ); also atropin.

Other Central Actions.- If apomorphin is given in larger doses it produces a series of excitant effects, especially in animals which do not vomit (Harnack, I874). At first there is great restlessness, gnawing, excitement and terror. These are perhaps due to the nausea and to the unaccustomed sensations. The gnawing is not seen with rabbits, from which the hemispheres have been removed, so that it must be of cerebral origin (Morita, r915). The respirations are quickened (even under chloral narcosis), but regular. Apomorphin also disturbs heat regulation, so that animals do not respond to fever by polypnea and shivering (Magne, I9r4). With larger doses (larger than are ever used in man), there are ataxia and then violent irregular convulsions. These interfere with respiration, and the animal dies in asphyxia. Death oncurs also if the 
convulsions are suppressed by chloral, but then requires a dosage twenty times as high. Frogs exhibit the same phenomena. Hattori, I9I0, finds that the reflexes from the spinal cord and brain are increased.

Local Application.-Harnack, I 874 , showed that this paralyzes cardiac and skeletal muscles in frogs.

\section{HYPNOTIC ACTION OF APOMORPHIN AND USE IN ACUTE ALCO- HOLISM AND HYSTERIA}

C. J. Douglas, I 899 , claims that apomorphin, in small, non-emetic doses ( $2 \mathrm{mg}$.$) ,$ acts as a safe and certain hypnotic, effective even in delirium; natural and refreshing slecp occurring usually in five to twenty-five minutes. Coleman and Polk, I902, experimenting on an extensive series of acute alcoholic patients, confirmed the claim. They employed hypodermic doses of 2 to $6 \mathrm{mg}$. Other observers (Rosebrugh, 1908) report similar results; the tendency, however, has been to use doses ( $5 \mathrm{mg}$.) which produce an emetic action (Rosenwasser, I907). 'This perhaps aids the therapeutic effect by breaking into the fixed ideas of such patients. The sleep occurs in a few minutes after emesis. Hypodermic administration secures by far the best results.

Velocity of Action.-In man, the hypodermic injection of 5 to Io $\mathrm{mg}$. ( $1 / 12$ to $1 / 6$ gr.) of apomorphin produces severe nausea and vomiting within ten or fifteen minutes, generally without other effects. By mouth, twice this dose is uncertain. The emesis may be repeated several times. The nausea is intense and is apt to persist longer than with the reflex emetics, since the apomorphin is not removed.

\section{PHENOMENA OF NAUSEA}

These are common to all emetics. They consist in a feeling of sickness, lassitude and weakness; pale skin; increased secretion of sweat, saliva, mucus and tears; sensation of excessive heat; increase of respiration and pulse rate; fall of blood pressure. During the emesis proper, the contraction of the abdomen will raise the blood pressure temporarily-a fact which contraindicates emesis in apoplectic patients, etc.

Circulation and Respiration in Nausea and Vomiting.-Traube, 1867 , described an initial fall of pressure, with slow vagus pulse, succeeded by quickened pulse toward the end of the emetic action. The details have been studied by Brooks and Luckhardt, I915, on unanesthetized animals. Their results are confirmed by Miller, I915.

They find emesis accompanied by marked disturbances of the circulation and respiration. There is sometimes a period of elevated pressure; but commonly, at the moment of emesis, the heart is inhibited, with sudden severe fall of blood pressure. This is interrupted by periods of great oscillations. These great and sudden changes may rupture blood vessels, which would resist more gradual changes of equal degree.

They find the changes somewhat different in the two types of vomiting.

Rapid projectile type, with short nausea: During the nausea, the blood pressure is slightly raised, the pulse rate increased, its amplitude diminished. During the vomiting, the pressure falls sharply with practically complete arrest of the heart, or arythmia. The respiration is suspended. After the expulsion, rapid recovery occurs to normal.

Slow, labored type, with more marked nausea, etc. During the nausea, there is deep breathing and salivation. During violent retching, the blood pressure and the intrathoracic pressure oscillate greatly. The pulse is slow and irregular. During the vomiting, the changes are as described.

The slowing and fall of blood pressure are due to inhibiting vagus stimulation, since they are prevented by atropin. The oscillations are indirect results of the muscular efforts, and are generally eliminated by curare.

The Acceleration of the Heart in Emesis.-Ackermann, 1856 , showed that all kinds of emesis are accompanied by this acceleration. Harnack, I874, observed some acceleration even after atropin, so that there must be a reflex stimulation of the accelerator mechanism; the vagus mechanism is probably also reciprocally inhibited.

The stimulation of the salivary centers has been studied by V. E. Henderson, igio. 


\section{ACCIDENTS IN THE THERAPEUTIC USE OF APOMORPHIN}

The apomorphin nausea may produce considerable depression, varying in degree in different individuals. It is characterized by muscular debility, and rarely collapse (Harnack, I908). These may precede or follow the emesis, and are especially liable to occur in the rare cases in which the emesis fails; in pulmonary and cardiac disease; in debilitated patients; and in children. The collapse is preceded by irregular respiration, anxiety, vertigo, and fainting. During the collapse, the patient may be unconscious, and pass into early death. These cases are quite exceptional, but their possibility should be borne in mind. It is believed by some that the accidents are often due to impurities, so that only freshly made solutions of the crystallized alkaloid should be used, never of the amorphous.

Old solutions of apomorphin acquire a deep green color, indicating some decomposition. The emetic action may not be affected by this change in some samples, but it is lost in others (Harnack, I874).

Chloromorphids. - These are formed in the manufacture of apomorphin, if the action of the hydrochloric acid on the morphin is not carried sufficiently far. They are, therefore, a frequent contamination of commercial apomorphin. They are chemically analogous to acetyl-morphin, being formed by the substitution of $\mathrm{Cl}$ for the $\mathrm{OH}$ group of morphin. Their pharmacologic actions are also similar to those of acetyl-morphin (heroin), namely exaggerated morphin effects, with greatly increased toxicity, paralysis of respiration, and an antiemetic action (Harnack and Hildebrandt, I9II). These investigators hold these impurities responsible for the failure of emesis and the collapse. They may perhaps also explain the hypnotic action of Douglas.

Dosage.-For emetic effect, $5 \mathrm{mg}$. ( $1 / 12 \mathrm{gr}$.) or at most $10 \mathrm{mg}$. (1/6 gr.) of the crystallized hydrochlorid, in $1 / 2$ to $\mathrm{I}$ c.c. of water, should be given hypodermically, and repeated in fifteen minutes if necessary. If the danger of collapse is apprehended, $2 \mathrm{mg}$. of strychnin sulphate may be added. As a nauseant expectorant, it is inferior to ipecac, but may be used by mouth, in syrup of citric acid, in doses of 2 to 10 $\mathrm{mg}$. (1/30 to to $1 / 6 \mathrm{gr}$.) for adults; $1 / 2$ to $2 \mathrm{mg}$. $(1 / 120$ to $1 / 30 \mathrm{gr}$.) for children. This dosage does not cause depression.

The therapeutic use of emetics will be discussed later.

\section{PREPARATIONS-APOMORPHIN}

*A pomorphina Hydrochloricum (Apomorph. Hydrochl.), U.S.P., B.P.; $\mathrm{C}_{17} \mathrm{H}_{21} \mathrm{NO}_{2}$-$\mathrm{HCl}-1 / 2 \mathrm{H}_{2} \mathrm{O}$, U.S.P.; $\left(\mathrm{C}_{17} \mathrm{H}_{17} \mathrm{NO}_{2} \cdot \mathrm{HCl}\right)_{2}+\mathrm{H}_{2} \mathrm{O}$, B.P.-Prepared from morphin by the abstraction of one molecule of water. The preparations of different manufacturers differ more or less in composition and activity (Harnack, I 909 ). Minute, white or grayishwhite, glistening prisms; odorless, acquiring a greenish tint upon exposure to light and air. Sol. in water $(\mathrm{r}: 50)$ and in alc. ( $\mathrm{r}: 50)$ : Dose, Expectorant, $3 \mathrm{mg} ., 1 / 20 \mathrm{gr}$; ; emetic, ro $\mathrm{mg}$., $1 / 6$ gr., by mouth; hypodermic, $5 \mathrm{mg}$., $1 / 12$ gr., U.S.P.; 3 to $6 \mathrm{mg}$., $1 / 20$ to $1 / 10$ gr., hypodermic; by mouth, 6 to $16 \mathrm{mg}$., $1 / 10$ to $1 / 4 \mathrm{gr.}_{2}$ B.P. Maximum dose, $20 \mathrm{mg}$., $1 / 3 \mathrm{gr}$. B.P.

Inject. A pomorph. Hyp., B.P.-I per cent. Dose, 0.3 to 0.6 c.c., 5 to ro minims,

\section{APOCODEIN}

This is an analogous derivative of codein, apparently variable in composition and actions. Kobert finds that it acts qualitatively like apomorphin. Dixon, 1903, claims that it paralyzes successively the nerve-endings of the vagus, vasomotors, other smooth muscle, striped muscle, and cardiac accelerator, as well as the nerve-centers, but its action does not seem to be absolutely specific. Magnus, 1905, found both stimulant and paralyzant peripheral effects on peristalsis. It has been used as expectorant, emetic, hypnotic, and cathartic (Fraenkel). 


\section{ASPIDOSPERMA (QUEBRACHO)}

The bark of Aspidosperma Quebracho-blanco, contains six or more alkaloids with very similar actions: fall of blood pressure, increased heart rate, clonic convulsions with muscular weakness, and a very great and persistent increase of the rate and depth of respiration. The lungs are markedly hyperemic (Harnack and Hoffmann, i 884 ; IVood and IIoyt, 1903; Wood, r910). These actions are partly central, partly peripheral. Wood, igos, attributes the respiratory effect to intense irritation of local areas of the lung, which is observable at autopsy. The alkaloid quebrachin is mainly responsible for this effect. One of the alkaloids, aspidosamin, produces emesis; the others only nausea, with its usual accompaniments.

Cow, I9I4, finds that larger doses paralyze the nerve cells of the autonomic system, brain and spinal cord. Still larger doses paralyze the vagus, sympathetic and motor endings, similarly to nicotin and curare. Death occurs by paralysis of the respiratory center when the motor nerves still respond to stimulation.

Aspidosperma has been used as a respiratory stimulant in all forms of dyspnea and asthma. Its irritant action on the lungs, and the great circulatory depression should be borne in mind. The dose of the fluidextract is I to 4 c.c.; of the amorphous aspidospermin (a crude mixture), 0.06 to $0.1 \mathrm{Gm}$.; of the crystalline (true) aspidospermin chlorid, 5 to $30 \mathrm{mg}$., at first every hour or two, then less frequently (S. Solis Cohen).

\section{PREPARATIONS-ASPIDOSPERMA}

Aspidosperma, U.S.P. (Quebracho).-The dried bark of Aspidosperma Quebrachoblanco. Dose, 4 Gm., 60 gr., U.S.P.

Fldext. Aspidosp., U.S.P.-Dose, 4 c.c., I dram, U.S.P.

\section{IPECACUANHA AND EMETIN}

General Statement.-Ipecac, the root of a Brazilian plant, contains several alkaloids, of which two, Emetin and Cephaelin, produce nausea and emesis, by central and local action, without danger of side effects. The drug is therefore very useful therapeutically. Large doses produce specific effects in amebic dysentery. Systemic actions (central and cardiac depression; stimulation of smooth muscle) do not occur when the drug is administered by mouth, since there is but little absorbed.

Nauseant and Emetic Actions. - These are produced much more slowly than with apomorphin. In man, vomiting occurs only after one-half to one hour. The drug is therefore more useful as a nauseant expectorant than as an emetic. As expectorant, the Syrup is used (I c.c.). An infusion, used as a gargle, has been recommended to avoid irritation of the stomach. For emesis, the powdered ipecac may be used, $\mathrm{x}$ to $2 \mathrm{Gm}$. in warm water, repeated in half an hour if necessary; or the Syrup (tablespoon for adults, teaspoon for infants, especially in spasmodic croup). It has some advantage over the mineral emetics in that it causes less gastric irritation and less general depression.

Location of Emetic Action.-It has generally been stated that this is purely local, mainly because emesis occurs more promptly on gastric than on hypodermic administration; the efficiency of hypodermic administration being explained by excretion of the alkaloids into the stomach (Valenti, I9II) and intestines (d'Ornella, I873).

However, Eggleston and Hatcher, I9I5, found that the emetic dose is nearly the same by either channel; moreover, they observed the occurrence of vomiting movements in eviscerated animals. It is clear therefore that the vomiting is of central origin; but because of the prompter action of the gastric injection, which was confirmed by these observers, it follows that the local action facilitates the emetic response. Emetin and cephaelin act alike, except that cephaelin is twice as active.

Irritant Action.-Ipecac irritates all mucous surfaces, sometimes to an exaggerated degree. It may therefore produce conjunctivitis, bronchitis, skin eruptions, asthma, diarrhea, rectal irritation, etc. Some persons are extremely sensitive to these effects. 
On the abraded skin, I per cent. solution of emetin raises a wheal (Sollmann and Pilcher, I9I6). 'The subcutaneous tissue is but little subject to irritation by ipecac (Zepf, r 903 ), perhaps because the drug is too rapidly absorbed. Renal irritation occurs rarely.

Use in Dyspepsias.-The gastric hyperemia, produced by small doses, is sometimes utilized in dyspepsias (a drop or two of the syrup in a teaspoon of water, every fifteen minutes).

Action in Amebic Dysentery.--Ipecac or emetin, in adequate doses, cause the prompt, complete and usually permanent disappearance of the Endameba histolytica from the stools, and the removal of the clinical symptoms. The effect is doubtless due to direct amebocidal action. The best results are usually obtained with hypodermic administration of emetin.

Formerly, the opinions of the value of ipecac treatment were divided; but the later clinical investigators are practically in agreement. The older failures are probably attributable largely to faulty administration and inadequate dosage.

Amebocidal Action. - This was demonstrated in vitro for the fluidextract ( $1: 10,000$ ) by Vedder, I9II; and for emetin ( $1: 100,000)$ by Wherry, I9II, and Rogers, I9I 2. Emetin is probably an isochinolin derivative (Windaus) and therefore related to papaverin and narcotin. Pick and Wasicky, I9I 5 , find that these opium alkaloids also kill protozoa.

Harris, r89o, claimed that ipecac more or less freed from its alkaloids ("de-emetinized") is equally effective and less disagreeable. This is now generally denied. Vedder found it quite inert.

Musgrave, 1912, and some others, have referred the clinical results to the intestinal irritation; and believe ipecac effective in all forms of dysentery, whatever the etiology. This view has not obtained acceptance.

Oral Administration in Amebic Dysentery.-A careful technic is required to prevent vomiting, and to secure the retention of the large doses of ipecac which appear to be essential. This was originally attempted by a.preliminary full dose of opium, followed by I $\mathrm{Gm}$. of ipecac every four hours, or by $0.5 \mathrm{Gm}$. every half hour for six doses. The present practice is to protect the stomach by giving the ipecac in pills coated with a heavy ( $1 / 8$ inch) layer of salol, as in the modification of Manson's technic proposed by S. K. Simon.

Simon's Method of Administering Ipecac (Jour. Amer. Med. Assoc., Nov. 6, rgog, p. 1526). -The patient is directed to bed during the treatment (which takes about two weeks), and is kept on a light, mainly liquid diet. After an initial Castor oil purge, he receives a single daily dose of ipecac, as salol-coated pills, in the evening, three hours after the last meal. The first dose is of 3 or $4 \mathrm{Gm}$. This is reduced by $0.3 \mathrm{Gm}$. daily, until $0.65 \mathrm{Gm}$. is reached. This last dose is then continued for two weeks, and is supplemented with daily colon irrigations with salt or borax solution. If ulceration has been extensive, the colon is later washed with a dilute silver nitrate solution. Tonic measures are always necessary.

In normal individuals, $3 \mathrm{Gm}$. of ipecac, given by this method, produces generally diarrhea, lasting some days; of ten considerable abdominal pain and slight tenesmus (Musgrave, I9I 2); no depression and rarely vomiting. A patient took $300 \mathrm{gr}$. in three days without apparent discomfort (Dock and Lyons, I9Io).

Ipecac has also been administered by the duodenal catheter (Beck, I9r2), by rectum, and through the stump of an operated appendix. It may also be administered with Fuller's Earth ("Alcresta Ipecac") which protects it against solution in the stomach. This, because of its slow absorption, does not readily produce vomiting (Eggleston and Hatcher, I915).

Hypodermic Treatment with Emetin.-This was introduced by Rogers, I9ז2, and has been found more successful and less disagreeable than oral administration (review by Vedder, I914).

Efficiency.-Even the first injection causes the disappearance of blood and mucus from the stools, decrease of diarrhea, and relief of pain. The symptoms often disappear completely within a week, but other cases are more resistant. Practically all patients, unless moribund, are clinically cured. This holds also for the hepatitis. - Some retain the amebr in the stools, probably because the emetin does not reach the lumen of the intestines; but they suffer no symptoms or recurrence. These carriers may be treated by local irritation with quinin or silver nitrate. 
Dosage.-Vedder advises $20 \mathrm{mg} ., 1 / 3 \mathrm{gr}$, of the Emetin Hydrochlorid, hypodermically, three times daily for about ten days. It would be suspended with the cure of the clinical symptoms, and resumed on recurrence. The dose may be increased, if necessary, to 2 or 3 gr. per day. Care must be used that the solution is not introduced into the eye, as it is very irritant (Blue, I9 I 5).

Side Effects.-The ordinary dose does not produce any gastro-intestinal or general disturbance, and practically no local reaction if the solution is neutral. The larger doses cause some nausea, vomiting, depression and pain-more with intramuscular than hypodermic. Even $0.25 \mathrm{Gm}$. at one dose produced only persistent nausea (Allan). 0.05 to $0.1 \mathrm{Gm}$. daily for twelve days has caused paralytic and edemic symptoms (Spehl and Collard, I9I 4). Cephaelin would not be used, because of its greatèr emetic action.

Intravenous injection has also been tried, but should be condemned. Vedder rightly cautions against it. Baehrmann and Heinemann, I9 3 , had no bad results with 60 to $200 \mathrm{mg}$; whilst 300 to $400 \mathrm{mg}$. gave severe effects in a few minutes: vascular paralysis, marked dyspnea, unconsciousness, vomiting and diarrhea, very slow pulse. This exceeds the fatal intravenous dose for rabbits $(2.5 \mathrm{mg}$. per kilogram. The fatal hypodermic dose is much higher (20 mg. for rabbits).

Variability of Commercial Emetin Hydrochlorid.-The occurrence of two fatal human cases led R. L. Levy and Rowntree, I916, to investigate the toxic effects. They found wide differences in the toxicity of commercial samples. They describe the toxic phenomena in animals, and the clinical toxic cases.

Emetin in Pyorrhea Alveolaris.-The supposed causation of Rigg's disease by endamebæ prompted the trial of emetin. Many observers report good results; but neither these, nor the etiology, are beyond dispute (Price and Bensing, I9 5 ).

Smith and Barrett, and others, found endameba buccalis constantly present in the lesions, and introduced the local use of ipecac. Bass and Jones, I9I4 and I915, are especially enthusiastic. They report the amebx destroyed by hypodermic injections of emetin, 0.03 daily for three to six days; or 2 or 3 Alcresta ipecac tablets (each of 0.65 $\mathrm{Gm}$. powdered ipecac) two or three times daily by mouth, for the same period. This does not produce nausea, but often some abdominal discomfort or diarrhea. The lesions require a long time to heal, and may become reinfected, when the treatment must be repeated. As prophylactic, they recommend a daily mouth wash of I or 2 drops of fluidextract ipecac in a half-glass of water. Evans and Middleton, 1915, also report success.

Use in Other Parasitic Conditions.-Emetin has been unsuccessful in syphilis, rabies, trypanosomes and bacillary dysentery. Marked improvement was reported for sprue by Schmitter, I9I 5 .

Tuberculosis.- Raeburn, I9I4, suggests its use ( $1.5 \mathrm{mg}$. hypodermically) to lessen pulmonary congestion, expectoration and congestive hemorrhage. The rationale is not clear and it requires confirmation.

Hemoptysis.-Emetin has been tried in tubercular hemoptysis, the reports being generally favorable, but requiring critical confirmation. The mechanism is not explained.

Flandin, I9I3, claims immediate arrest after the hypodermic injection of $0.04 \mathrm{Gm}$. without unpleasant symptoms. The injection may be repeated in intervals of twelve to twenty-four hours. Valassopoulos, 1913, used $0.02 \mathrm{Gm}$. (also in rectal carcinoma). Nicola, I9I4, employing the same dosage as Flandin in hemoptysis, obtained variable results. Apparent success (arrest in forty-eight hours) was obtained mainly in the early stages, with scanty hemorrhage or high blood pressure. Bleeding from venous congestion or with low blood pressure was not influenced.

Systemic Effects.-These occur only after very large hypodermic or intravenous doses. They consist in paralytic symptoms, among the earliest in mammals being vasomotor paralysis with fall of blood pressure. This is further aided by weakening of the heart muscle due to its direct muscle-action (it produces diastolic arrest of the isolated frog heart; Moulinier, I9I4). Death occurs mainly by central paralysis.

If the action has lasted any time, the autopsy will show a marked gastro-enteritis, with ecchymoses and even ulcers. Edema of the lungs from the hypersecretion of mucus 
and the weakened heart is sometimes seen. Trendelenburg finds a dilator action on excised tracheal muscle.

Smooth Muscle.-The effects have not been extensively investigated. Maurel, I9I4, describes for the intestines, stomach and blood vessels a primary increase of tonus, large doses paralyzing.

Uric Acid Excretion.-This is increased by ipecac, as by other gastro-intestinal irritants (Abl, I9I3).

\section{COMPARATIVE ACTION OF EMETIN AND CEPHAELIN}

The composition of the alkaloids differs merely by a $\mathrm{CH}_{2}$ radical $\left(\right.$ Emetin $=\mathrm{C}_{20}$ $\mathrm{H}_{40} \mathrm{~N}_{2} \mathrm{O}_{4}$; Cephaelin $=\mathrm{C}_{28} \mathrm{H}_{38} \mathrm{~N}_{2} \mathrm{O}_{4}$; Carr and Ryman, 1913. They also describe the chemical properties). Their action is similar, but some minor differences are of therapeutic importance: Emetic effect: Cephaelin is by far more emetic (about twice; Eggleston and Hatcher, I9I5); whilst emetin is the better nauseant. Cardiac effect: Emetin is much more depressing. Kidneys: Cephaelin is more irritant. Blood corpuscles: Cephaelin is somewhat hemolytic (Paul and Cownley, rgor; Lowin, I902; Wild, I895; of the older papers, Podwyssotzki, Arch. exp. Path., I I:23I, I879).

\section{PSYCHOTRIN}

Psychotrin, the third alkoloid of Ipecacuanha, is present in too small amount to be important.

\section{IPECACUANHIC ACID}

A further constituent, ipecacuanhic acid was, until recently, considered a bearer of the action of ipecac in dysentery. It has been shown, however, that this tannin is inactive. The ipecacuanhic acid possesses all the chemic characters of caffeotannic acid (see Index), from which it differs only by one atom of oxygen. The pharmacologic actions of these two tannins also agree perfectly (and differ from ordinary tannin) as they are neither antiseptic nor astringent (Kimura, 1903 ).

\section{IPECACUANHIN} I9I2).

Ipecacuanhin is a crystalline glucosidal constituent (Finnemore and Braithwaite,

PREPARATIONS - IPECAC

*Ipecacuanha (Ipecac.), U.S.P.; I pecac. Rad., B.P.-The dried root of Cephaelis Ipecacuanha (Psychotria Ipecacuanha, B.P.), Rio Ipecac. The U.S.P. also recognizes Carthagena Ipecac, from Cephaelis acuminata. Ipecac must yield not less than 1.75 per cent. of alkaloids, U.S.P.; 2 per cent., B.P. The drug was introduced into Europe about the end of the seventeenth century. Dose, as Emetic, I Gm., I 5 gr., U.S.P.; I to $2 \mathrm{Gm}$., I5 to $30 \mathrm{gr}$., B.P., of the powder, stirred into warm water. As Expectorant, etc., 0.03 to $0.12 \mathrm{Gm}$., $1 / 2$ to $2 \mathrm{gr}$., B.P.

Recent researches have shown that the Carthagena variety contains more of both alkaloids, but particularly of Cephaelin. The ratio of emetin to cephaelin varies from 3 emetin: I cephaelin in Rio to $2 / 3: I$ in Carthagena.

*Fldext. I pecac., U.S.P.; Ext. I pecac. Liq., B.P.; about 2 per cent. of alkaloids; the U.S.P. also contains I per cent. of $\mathrm{HCl}$. Dose, as Expectorant, 0.05 c.c., I minim, U.S.P.; 0.03 to 0.12 c.c., $1 / 2$ to 2 minims, B.P. Index.)

*Pulv. Ipecac. et Opii, U.S.P.; Pulv. Ipecac. Co., B.P.; Dover's Powder--(See

${ }^{*}$ Syr. I pecac., U.S.P. $\rightarrow 7$ per cent., acidulated with Acid. Acet. Dose, as Expectorant, I c.c., 15 minims; as Emetic, 15 c.c., 4 drams, U.S.P.

Ipecac Syrup of the International Protocol is only i/7 the strength of the U.S.P.

Troch. I pecac., B.P.-0.015 Gm., 1/4 gr.

Vin. I pecac., B.P.-5 per cent. Dose, 0.06 to I. 8 c.c., to to 30 minims; as Emetic, 16 to 20 c.c., 4 to 6 drams, B.P.

${ }^{*}$ Emetine Hydrochloricum (Emet. Hydrochl.), U.S.P., $\mathrm{C}_{30} \mathrm{H}_{44} \mathrm{~N}_{2} \mathrm{O}_{4} \cdot{ }_{2} \mathrm{HCl}$. - Contains variable amounts of water of crystallization. White or yellowish crystalline powder. Freely sol. in water or alc. Dose, $20 \mathrm{mg}$., 1/3 gr., U.S.P., hypodermic; by mouth, as Expectorant, 5 to ro mg., $1 / 2$ to $1 / 6$ gr.; as Emetic, ro to $20 \mathrm{mg}$., $1 / 6$ to $1 / 3$ gr. Care must be used to keep it from contact with the eyes, as it is highly irritant. 
Cephaelina, N.Y.R., $\mathrm{C}_{14} \mathrm{H}_{2 v} \mathrm{O}_{2 \mathrm{~N}} \mathrm{~N}-$ Dose, 5 to $10 \mathrm{mg} ., 1 / 2$ to $1 / 6 \mathrm{gr}$., in pills or as powder with sugar.

Chipurro Amargosa (Simarubarex), a Mexican plant, has been tried in amebic dysentery, with apparently successful results, and without bad effects. The bitter infusion is used. It kills endamebas (Nixon, 1914, 1916).

\section{THERAPEUTIC USE OF NAUSEANT EXPECTORANTS}

Small doses of emetics produce only some increase of the bronchial and salivary secretion and sweat, which is useful in the early, congestive ("dry") stage of colds and in "dry" coughs. The dose should be sufficiently small to minimize general depression and noticeable gastric disturbance, although this sometimes can not be avoided. The nauseants should be given every two to four hours.

For the "abortion" of colds, the ipecac is most useful in the form of "Dover's Powder," $0.5 \mathrm{Gm}$. at night. In the congestive stage, the syrup of ipecac may be used. Tartar Emetic may be substituted, but is more depressant, and its continued use may give rise to poisoning. Its expectorant dose is $5 \mathrm{mg}$. $(1 / 12$ gr. $)$; or I c.c., 15 minims of the Vinum Antimonii $(=4 \mathrm{mg} ., 1 / 15 \mathrm{gr}$.). This is also one of the constituents of the Mistura Glycyrrhizæ Composita.

Viscid, adherent mucus may be thinned by Ammonium Chlorid or Carbonate $(0.2 \mathrm{Gm}$.); or Potassium Iodide $(0.2 \mathrm{Gm}$.); or by the irritant saponin of Syrupus Senege (2 c.c.). The Compound Syrup of Squill (I c.c.) savors of "shot-gun" prescribing.

The reactions of the salivary center have been studied by V. E. Henderson, Igro. He finds that this is readily depressed by anesthetics and by morphin, even in small doses; Iodids and other salts do not cause acute stimulation, but may act reflexly. Emetin stimulates both directly and reflexly, also anmonium salts, but with these the reflex stimulation is the more important. Antimony acts only reflexly, A pomorphin only centrally.

Bronchial Secretion.-J. L. Miller, I9I4, could not demonstrate any effect with therapeutic doses of emetin, ammonium chlorid or carbonate. Large doses produced an increase, even when administered by duodenum.

Site of Action of Miscellaneous Emetics. - This has been investigated by Eggleston and Hatcher, I9I5. A drug may be considered to act directly on the center if emesis occurs with smaller doses and more rapidly when the drug is given by vein than by mouth; and if the phenomena of nausea and the motions of emesis occur after the whole alimentary tract has been removed. According to these criteria, they found that the emetic action of most alkaloids is central; namely, apomorphin, morphin, nicotin, lobelin, aconitin, veratrin, pilocarpin, quinin and ergot; as also picrotoxin, digitalis, and sodium salicylate. With ipecac, the local action probably contributes to the result.

\section{LOCALLY ACTING EMETICS}

The afferent paths which may reflexly stimulate the vomiting centers are very numerous; but for practical therapeutic purposes only those of the pharynx and gastric mucosa are available. Not every form of gastric irritation results in vomiting - the kind, rather than the degree, of irritation being important. This, and the necessity of avoiding severe local and systemic effects, limits the number of irritants which may be used as therapeutic emetics. However, there is still a wide choice, adapted to the various indications. 


\section{CUPRI SULPHAS AND ZINCI SULPHAS}

These act practically alike, provoking the vomiting reflex before there is time for any other local irritant action. They are not absorbed (unless the stomach is corroded), and therefore do not lead to any systemic effects. Vomiting occurs with certainty in a very few minutes. The nausea is therefore short, and the after-depression is small, constituting an advantage over apomorphin. They are especially useful to empty the stomach of indigestible food or poisons (provided that these are not corrosive). Copper sulphate is of especial value in phosphorus poisoning if any of the poison is still in the stomach, since the metallic copper is precipitated and forms an impermeable coating over the unabsorbed phosphorus particles.

The emetic dose of copper sulphate is about $0.25 \mathrm{Gm}$. in I per cent. solution; that of zinc sulphate is about $\mathrm{I}$ to $2 \mathrm{Gm}$. They may be repeated in fifteen minutes if necessary.

\section{MUSTARD}

A teaspoonful of powdered mustard in a cup of warm-not hotwater, repeated every fifteen minutes is a rather uncertain emetic, but a useful emergency remedy in poisoning, when the patient is already disposed to vomit. Salt water, or even plain warm water, or tickling of the fauces, may be similarly useful.

\section{ANTIMONY}

In the form of Antimony and Potassium Tartrate (Tartar Emetic) antimony acts similar to Copper, by a purely local effect, but its use should be avoided, since it is very depressing. It is fairly easily absorbed, so that $0.2 \mathrm{Gm}$. has repeatedly proven fatal. The ordinary emetic dose is $30 \mathrm{mg}$.; and if this is not effective in an hour, it would be well to give tannin as an antidote.

\section{EMESIS AS AN UNDESIRABLE SIDE ACTION}

This occurs with Digitalis, Squill, Apocynum, the Salicylates, Senega and a very large number of other drugs. The action may be local or central, often both.

\section{THERAPEUTIC USE OF EMETICS}

Emetics are employed especially for the following purposes:

In Acute Poisoning. - The need for evacuation of the stomach, and the relative advantages of gastric lavage, has been discussed under the General Treatment of Poisoning (see Index). It must be remembered that emetics are ineffective in profound narcosis (by chloral or chloroform, Harnack, I874; by morphin, bromid, etc.).

In Acute Indigestion.-Here also lavage would deserve the preference.

Apomorphin is often very effective in exposing malingering.

To remove solid bodies from esophagus, pharynx, or upper air-passages. However, according to de la Paz and Garcia, I9I6, emesis does not dislodge solid bodies from the trachea or bronchi, but may drive them deeper. Plugs of mucus, however, may be displaced by increased secretion of mucus. Emesis may cut short an attack of asthma (Hoover and Taylor, I9I 5 ).

To cause compression of the liver, for the removal of bile and small gall-stones from the gall-bladder and ducts: The usefulness of this measure is perhaps doubtful; since the intestines are also compressed, the added vis a tergo can not be very effectual, and on the other hand it might rupture a distended gall-bladder. 
Contraindications to Emetics.-These are mainly due to increase of pressure and to debilitation, and are as follows:

I. Severe heart-defects, or aneurism of the aorta, since the sudden and violent increase of intrathoracic and intraabdominal pressure may result in the rupture of these organs.

2. Atheroma. The sudden changes in blood pressure are apt to burst a vessel and produce apoplexy.

3. Similarly, they may lead to hemorrhages in phthisis.

4. Abortion may result, in advanced pregnancy.

5. Tendency to hernia.

6. In all debilitated conditions there is danger of collapse.

7. Caustic poisoning.

\section{THE PHARMACOLOGY OF HEAT REGULATION ${ }^{1}$}

General Statement.-In warm-blooded animals, any departure from the normal temperature disturbs a variety of functions more or less profoundly. These disturbances tend automatically to restore the normal temperature. Heat production and heat dissipation both have a share in this regulation, and may be brought into play either directly, or through reflexes. The automatic compensation or regulation is very perfect under ordinary conditions and with normal animals. It may become inadequate if any of the factors entering into it are powerfully altered, whether this alteration be central or peripheral. The temperature may thus rise or fall. The changes usually involve several factors, at least secondarily. These vary with conditions, which are often difficult to analyze. Many of our data are therefore incomplete, confusing and often contradictory.

It is attractively convenient to assume a special heat regulating mechanism-a physiologic, if not anatomic "heat center." This would be located particularly in the corpora quadrigemina, but diffused more or less throughout the whole midbrain. The center could be conceived as a sort of thermo-regulator which is ordinarily "pitched" for the normal temperature, but which may be adjusted, by stimulation, to maintain a higher temperature; then brought back by depressants to its normal pitch, or perhaps even adjusted lower. However, there is no binding evidence for such a specialized "center," and its acceptance is not necessary for the explanation of the phenomena. Indeed, it may rather interfere with a clear understanding of the facts. On the contrary, the mechanism of heat regulation may be conceived as analogous to blood-pressure regulation: Any departures from the normal will alter the physiological conditions, and therefore the functionation of a number of organs and centers; and these alterations generally tend to restore the normal level of the temperature.

Many of these facts belong rather to the domain of physiology and pathology; but it may not be amiss to summarize those which are necessary for presenting the pharmacologic phenomena.

Heat Production.-Heat is evolved in the destructive metabolism of the tissues, particularly the muscles and glands, and this furnishes the "chemical factor" in heat regulation. The production of heat is thus a part of ordinary metabolism. It may be

1 Comprehensiye bibliographies can be found in the review of O. Loewi, Ergeb. Physiol., I904; W. G. MacCallum, Harvey Lect., 1908; and Krehi, Pathol. Physiol. 
increased by greater activity of the organs; as by digestion, by muscular work, or even by increased muscular "tone." It may also be increased by overfeeding and by injury and other destructive changes, as, for instance, the increased tissue destruction of infectious fevers.

Conversely, the production of heat may be diminished by reducing metalolism; by quieting the muscular movements with morphin; by lowering their tone with ether; or paralyzing them with curare; by starvation or by depriving an animal of its glycogen; by reducing nitrogen-metabolism with quinin; by reducing the circulation by vasomotor or cardiac depression (collapse), etc.

Importance of the Chemic Regulation.-This may be judged by the fact that animals become practically poikothermal when they are paralyzed by curare (Pflueger, 1878 ) or by high section of the cord; that glycogen free animals are not rendered febrile by "heat puncture," etc.

The "specific" heat production (as distinguished from that incidental to ordinary physiological activity) is doubtless under the control of the central nervous system; the muscular tone, for instance, depends on the spinal cord. The special relation of the corpora quadrigemina to heat production is seen in "heat puncture."

Heat Dissipation.- Heat is lost mainly by the respiration and skin-partly by heating the air, and partly through the aqueous vapor of the lungs and perspiration. The relative importance of these channels of heat loss differs in different animals and in the same individuals, according to circumstances. In man, the skin is the chief mcans of regulating the heat loss; this would be increased by cutaneous vasodilation and by diaphoresis. These are therefore the "physical factors" of heat regulation. On account of their quick response, and lesser interference with the body at large, they naturally constitute the main means of regulation under ordinary conditions. Vasodilation of the cutaneous vessels may be brought about directly by the local application of heat and cold; but this also acts reflexly - so that the entire skin responds to strictly local variation of temperature. The regulation of sweat is predominantly central.

Regulation of the Heat Loss Through the Skin.-This generally involves a specific action, central or peripheral, on the tone of the cutaneous vessels, of ten combinsd with the opposite change in the tone of the systemic vessels. Such changes are a eonspicuous feature of the various fevers, and may take either direction. The pyretic agent may conceivably act either directly on the controlling centers; or indirectly, by calling for compensation against altered heat production, etc. The cutaneous circulation may also be influenced by a specific central action of certain drugs, especially in the sense of vasodilation. This is seen most typically with antipyrin and the related antipyretics.

The Hypothetical Thermoregulating Centers. - The heat regulation is disturbed by operative interference with certain parts of the central nervous system. High section of the spinal cord destroys heat regulation completely. Ablation of the cerebral hemispheres has little effect. Traumatic or electric irritation of various parts of the midbrain (particularly the corpus striatum and the ponto-bulbar junction) produces a rise of temperature.

Direct Application to Heal Centers.- - Heat regulation is markedly affected by the direct application of heat, cold or drugs to the region of the caudate nucleus. The temperature is thus raised by cold, corrosive poisons, caffein or "tetra;" it is lowered by heat, chloral, antipyrin or quinin applied to this region (Barbour and Wing, 1913; Cloetta and Waser, I9I4; Hashimoto, 1915). Indeed, the drugs are more effective in this way than intravenously.

Local heat to the corpus striatum of rabbits diminishes the oxidative metabolism and the respiratory volume; local cooling of this region produces the opposite effects, also shivering and fall of body temperature. The oxidative changes are contributory causes, rather than effects of the change of body temperature (Barbour and Prince, 1914).

Direct Heating and Cooling of Center on Response to Drugs.-This has been studied by Hashimoto, I915. He found that it has no influence on the febrile reaction to tetra, epinephrin or large doses of cocain (sympathetic stimulants); but that it modifies toxin, salt and anaphylactic fever.

The antipyretic action of antipyrin and salicylate is markedly increased by heat, removed by cold. After quinin, heat is effective, cold not. Morphin removed the response to both heat and cold.

Heat Centers of Rabbit. - Isenschmidt and Schnitzler, I9r4, find the tuber cinereum the most important. The efferent fibers lie in the caudal portion of the midbrain, widely scattered over the ventral and median parts of the cross-section.

The tuber cinereum is not the only center concerned in temperature regulation; but this fails if this portion is destroyed, whilst the preservation of a part of the fibers suffices for the function. 
Direct or Incidental Regulation.-The fact that the corpus striatum or tuber cinereum is intimately concerned in heat regulation has been taken as evidence that the regulation of heat is centralized in these divisions of the midbrain. Strictly speaking, however, it proves only that these lie in the nervous path of some of the factors concerned in heat regulation; the heat changes might be an incidental, rather than a specific part, of their function.

Apparently, the strongest argument for a center, or set of centers, having for their specific function the regulation of the temperature, lies in the surprising accuracy and adaptability of this regulation. The maintenance of a constant temperature seems to call for a thermo-regulator of some sort. Even more impressive is the remarkably efficient, one might say intelligent, adaptation of the means to the end. Of the numerous possible ways of compensating any departure from the normal heat production or dissipation, the organism seems to choose that which will effectively and conveniently accomplish the purpose, under the given set of conditions. This holds not only for the maintenance of the normal temperature, but whenever there is a departure from normal, there is a similar efficiency; when the temperature is to rise in fever, the rise is accomplished, on the whole, by a judicious selection of the most effective or convenient means; at the acme, the interplay is readjusted, most intelligently, to preserve this higher temperature; and when it comes time for the temperature to fall, the choice of means is again made with similar wisdom.

In brief, we appear to have a thermo-regulator, adjustable to various temperaturesto $37^{\circ}$ in health; to $40^{\circ}$ in fever; to $37^{\circ}$ again by antipyretics; and this regulator works with a discriminating judgment and choice of means which seems to demand a superior order of intelligence and "free will." These psychologic attributes being scarcely conceivable in a physiological function, the very perfection of the mechanism tends to become a refutation of a specific heat-regulating center.

Analogy of Blood-pressure Regulation:-One must turn to a simpler explanation; and here a pretty fair analogy presents itself in the maintenance of the normal blood pressure. This also involves a number of factors-the work of the heart and the tone of the vessels - which may be altered in a number of ways. When one of these factors is altered, compensatory changes occur in the other factors, so that the resultant bloodpressure is generally fairly level, just as is the temperature; and like the temperature, its height or "pitch" can be "attuned" to various levels. The essential features of blood-pressure regulation therefore appear quite analogous to those of heat regulation, but no one speaks of a specific "blood-pressure center." The difference lies in this, that in the case of the blood-pressure regulation, we have a fair knowledge of the factors involved, whereas with the heat regulation, our knowledge is much less. One difference may be urged between the two functions, namely, that the temperature runs much more constant than does the blood pressure. It is dangerous, however, to institute quantitative comparisons, between two things whose nature is so dissimilar. But even if this difference be important, it need only mean that the compensating functions are affected more powerfully by changes in temperature than they are by changes in blood pressure, which is likely enough. The essential thing in either case is this: that the regulation is not effected by a specific presiding center; but that changes in any one factor alter the physiologic state of all the other factors, central and peripheral, and thus bring about changes in their function, which generally result in compensation; and these changes will continue until the original physiologic state of all the factors is restored; that is, until the whole condition has returned to the normal.

It is scarcely necessary to add that the share, and therefore the practical importance, of the different factors is unequal. In this sense, we may speak of the corpora striata as one of the important "heat centers," meaning thereby only that they have a very important relation to heat production; but in the same sense, the central station for the cutaneous vessels, and for muscular tone, or for convulsions, are also important "heat centers."

It is one advantage of this conception, that it emphasizes the impartial study of the actual phenomena of heat regulation. These are complex enough, but one may hope to trace, eventually, the primary cause, the starting point, of a given change, and the chief secondary changes to which this gives rise. These will vary under different conditions, each of which will need to be investigated by itself. This accounts for the many imperfections of our knowledge and for the many apparent contradictions. It will therefore be wisest to restrict ourselves to those data which are pretty definitely established. 
Methods of Studying the Mechanism of Heat Regulation.-Calorimetric measurements give the most comprehensive information of the relation of heat loss and heat production. The calorimeter gives directly the quantity of heat lost; and from this, and the changes in internal temperature of the animal, the heat production can be readily calculated. The share of the nitrogen and carbon metabolism in the heat production may be deduced from the nitrogen and carbon dioxid excretion, and from the consumption of oxygen. The changes of the cutaneous vessels and their share in the heat loss may be deduced from direct inspection of the skin, from comparison of the dermal and internal temperature, and from plethysmographic observations. The means for localizing the effects centrally or peripherally will be discussed as occasion arises.

\section{REACTIONS WHICH RAISE THE TEMPERATURE (PYRETICS)}

Any increase of heat production or diminution of heat loss would tend to raise the temperature; but ordinarily, the tendency is offset by compensatory reactions. A significant rise of temperature, i.e., fever, will occur only if the change exceeds the capacity of compensation, either because it is extraordinarily great or because it involves more than one factor. This break in compensation may be caused by a large number of agencies, acting through different mechanisms; only a few of these need to be considered here.

Heat Puncture.-The essential element of "puncture fever" seems to be increased heat production, by increased carbon metabolism, especially of hepatic glycogen. The compensatory heat loss is not adequate; but it is not known whether this disturbance is direct or indirect.

Heat Puncture is important mainly because it is so easily subject to experiment that it is perhaps best adapted for elucidating the nature of febrile and antipyretic processes.

I. Ott; Richet; and Aronsohn and Sachs, 1884 to 1885 , nearly simultaneously discovered that a stab into the corpora striata of rabbits causes a rise of temperature, starting in a few hours, reaching a considerable height, and lasting for some days (Technic in Aronsohn and Sachs). The effect can then be repeated by a fresh puncture but fails if the destruction is extensive. It can also be evoked by electric stimulation of this region, so that it consists evidently in the stimulation of a function concerned in heat regulation.

Relation of Heat Production and Dissipation.-Calorimetric experiments show that the rise is due to increased heat production (Richet, 1895), with partial compensation by increased heat loss. During the ascent, the heat formation and loss are both increased, but the loss lags behind. When the high level is reached, the heat formation lessens somewhat, and the dissipation increases, so that the temperature runs constant. In the descent, both decrease, but especially the heat formation (Schultze, 1899).

Mechanism of the Increased Heat Production.-This is due to increased carbon metabolism (increased $\mathrm{CO}_{2}$ excretion). The nitrogen excretion may also be increased, but this is not essential, and is probably secondary to the hyperpyrexia.

The rise occurs in curarized animals (Hirsch and Mueller, I903) so that the muscular metabolism is not essential, though it may play a part. Glycogen-free animals do not show a rise (Rolly, r903). The liver temperature is high. These facts indicate that heat puncture acts mainly by increasing the hepatic metabolism. Walbaum, I9I3, however, believes that the main factor is central stimulation of the cutaneous vessels.

Regulation. - The uniform course of the temperature shows that the disturbed functions have struck a balance-in other words, that there is some "regulation." When the temperature is lowered artificially, by external cold or by antipyretics, it soon recovers its former high level (Schultze).

The Ponto-bulbar "Center."-Tschedschichin, 1866, observed that the temperature rises when a section is made at the junction of the pons and medulla; the same result follows electric stimulation of this region (Heidenhain, 1870) and irritation of various other parts of the midbrain. 
The Hyperpyrexia of Infection.-The essential element of infective fever appears to be a clisturbance of the central tone of the cutaneous vessels, making the heat loss absolutely or relatively inadequate. The heat production, and particularly the nitrogen metabolism, are generally increased; but this is not essential, and does not exceed the limits which are easily compensated in normal animals.

IIeat Production.- In the ascent, this is generally increased; but it may be normal or even diminished. The same holds true for the plateau. In the descent, it is generally diminished, particularly if the fall occurs by lysis.

Increased heat production is therefore a characteristic, though not a constant feature of the rise; but this does not prove that it is the essential cause of the rise. The greatest increase is witnessed in the initial chill, and is largely due to the shivering movements. This cause disappears as the temperature rises; but now the increased activity of the heart and respiration furnish further causes for increased heat production. The higher temperature of the body must also accelerate oxidation. 'The toxins of the infections undoubtedly cause more or less cell destruction, and this material must be oxidized. A large share - possibly all - of the increased heat production is thus purely incidental; and whilst it doubtless facilitates the rise of temperature, it can not be considered as the essential element.

The site of the heat production is the same as in normal animals; for the temperature of the internal organs show the same relation as in health - the liver, for instance, being the warmest. From this it can be affirmed that the glands and muscles both participate in the increase, but not necessarily in the normal ratio-presumably this would differ according to circumstances.

Oxidation.- - The energy for the increased heat production is furnished by oxidative changes; and with adequate food, the main source consists of non-nitrogenous material. The glycogen in particular tends to disappear. However, the nitrogen metabolism shows a characteristic relative increase from a number of causes.

Total Nitrogen Excretion.-Increased nitrogen metabolism is a nearly constant feature of infective fevers. The increase, however, does not run parallel to the fever; it may start before or after the rise; and it generally reaches its maximum after the fever has started to decline. It must therefore be an incidental, rather than an essential feature of the process.

Explanation of the Febrile Increase of Nitrogen Metabolism.-The increased nitrogen excretion denotes increased tissue destruction. Simple overheating also increases the nitrogen excretion, but relatively much less than in fever. A part may be accounted for by the partial inanition, which is so commonly enforced in fever: Indeed, the nitrogen loss can be entirely prevented by an ample diet; but to do this requires more food than in health. It may therefore be accepted that the nitrogen excretion in fever is always greater than that for the same diet in health. This leaves as the only explanation, direct toxic effects of the infective agents, leading to cell destruction.

Intermediate Nitrogen Metabolism.-Very little is known as to whether this takes the same course as in the normal. Presumably it is somewhat different; for the urea excretion is relatively low (though the absolute value may be high); whilst ammonia, uric acid, purin bases and creatinin are relatively high. The urine often contains creatin, albumen, acetone bodies, oxyacids, etc.

The Course of Heat Dissipation.- The heat loss is always deficient in infective fevers, absolutely or at least relatively; and this deficiency or failure of the heat loss to compensate the heat production, must be considered the essential factor in the high temperature. In man, these changes run parallel with the cutaneous circulation-a pale, dry cold skin meaning a diminished heat loss; and the converse (Kraus, 1894; Maragliano, I 888).

In the ascent, the heat loss is conspicuously decreased, leading to the characteristic "chill" (the vasoconstriction cooling the skin, whilst the internal temperature is high). During the continuance of the fever, the loss is generally increased, absolutely, so as partly to balance the increased heat production-but the increase of heat dissipation is small as compared with what would occur if a normal animal were producing the same amount of heat. One may therefore say that the dissipation remains relatively decreased. In the descent of temperature, the heat loss is increased relatively, as well as absolutely. This is the main element of the "fall by crisis."

Altered Reaction of the Cutaneous Vessels. - The altered behavior of the cutaneous vessels, which seems to be the essential element of fever, finds expression in other directions. They are more easily affected by chemical and mechanical irritation 
(Baeumler, I873); they constrict more strongly in response to reflexes (Heidenhain); they are apt to undergo alternate contraction and dilation, with little apparent cause; a slight degree of cooling produces chilliness, etc. These phenomena all indicate that the innervation of these vessels is disturbed.

Heat Regulation in Fever.-The fact that heat regulation is disturbedbecause one or more of its factors are disturbed-does not mean that all regulation is abolished. The disturbed functions will eventually find a new level, so that fevers generally run a more or less regular coursewhich implies an adjustment. Any adventitious change will alter this adjustment, generally in the same direction as in normal animals.

Fevers therefore show the normal daily variations, the rise during the day, and the fall during the night; although these variations may be smaller or larger than normal. Artificial cooling and heating and the administration of antipyretics provokes the same compensatory reactions as in health, though these are less efiective. The febrile temperature can therefore be more easily lowered or raised, the changes are more extensive and more lasting; but when left to itself, the temperature will tend to assume its original febrile level. It might therefore be said that the temperature of fever is adjusted to a higher level (Liebermeister); which means nothing more, however, than this: that when the altered functions become constant, the resulting temperature must also become constant.

Localizing of the Febrile Process.-Considering fever as being essentially due to disturbed innervation of the cutaneous vessels, the site of action would presumably be in the'centers controlling the tone of these vessels. In accordance with this it is found that fever does not occur when these centers are cut off by high section of the cord, or if they are paralyzed by deep narcosis, or even if they are depressed by the antipyretics.

Heat Centers in Anaphylaxis.-In sensitized animals, the injection of the specific serum into the heat-puncture region produces fever. This does not occur in normal or immunized animals. The centers therefore are specifically sensitized in anaphylaxis (Hashimoto, 1915).

Comparison of Heat Puncture and Infective Fevers.- These two forms of fever have considerable resemblance. Both may show increased heat production with inadequate heat loss; but in puncture, the production seems to preponderate; in infections, the disturbance of heat loss. Both forms are central; both react similarly to antipyretic drugs.

Indirect Hyperpyretics.-Quite a large number of substances are apt to set up fever, particularly on subcutaneous injection in rabbits; and in infants, in which the temperature is more labile. To this class belong plain saline solution or plain water, albumose, egg-white and other proteins, irritants, etc. Many of these cause albumosuria (Krehl) and they probably act by causing tissue decomposition, with the formation of products (albumoses, etc.) which act similarly to infections.

Tetra-hydro-Beta-Naphthylamin.-This substance produces a very considerable rise of temperature, with increased heat production and diminished heat loss. It is not known which is the essential element. 'The hyperthermia differs from infective fever and heat puncture in that it is not reduced by the ordinary antipyretics, but only by profound narcosis.

Composition, Dosage and Efficiency.-The substance has the structural formula<smiles>NC1CCCCC1</smiles>

The subcutaneous injection of 0.04 to $0.05 \mathrm{Gm}$. raises the temperature of rabbits several degrees, the rise starting in an hour and persisting over four hours; but it fails completely in some animals. Dogs react similarly. In man, non-toxic doses do not produce a rise. 
The phennmena have been extensively studied by R. Stern, I889 and I890; Hale White, I 892 , Sacharoff, $1909 ; \mathrm{A}$. Black, $19 \mathrm{Ir}$; Feri, $19 \mathrm{II}$, and others, but without clearing up the mechanism of its action. Cloetta and Waser, I913, have investigated its derivatives to compare the structure and actions.

Other Actions.-Aside from the rise of temperature, it produces dyspnea and rest. lessness, sometimes convulsions. It also revives rabbits from light chloral sleep (Airila, I9r3); it also has important sympathomimetic actions (Jonescu, I909). Stimulation of the cervical sympathetic (mydriasis, exophthalmos); a general vasoconstriction involving both cutaneous and systemic vessels (rise of blood pressure), mainly of peripheral origin. It has a peripheral moderate dilator effect on bronchioles (Jackson, I9I4). Elliott, I91 4, describes the rapid production of gastric ulcers in rabbits from hypodermic injection. It wakens even decerebrated animals from chloral sleep-whilst cocain and caffein have this effect only on normal animals (Morita, 1915).

Mechanism of Pyresis.-Calorimetric observations show increased heat production and diminished heat loss. The latter is perhaps explained by the vasoconstriction; and the increased heat production may possibly be ascribed entirely to the increased muscular movements. In accordance with this, the carbon dioxid excretion is greatly increased, whilst the nitrogen excretion is but little affected. The heat production is apparently the main element in the rise, for curarized or deeply morphinized or etherized animals do not respond to the drug (Mutsch and Pembrey, I9II). The action must be above the medulla, for no fever occurs if the carotids have been blocked by paraffin (Ott and Scott, I908).

To this extent therefore the effects resemble those of heat puncture; but whilst the puncture fever is readily lowered by antipyretics, none of these, i.e., quinin, antipyrin, etc. or small doses of Morphin, have any marked effect on the tetra-fever (Feri, I9I r).

Cocain Hyperthermia. - The effects of cocain, so far as they have been analyzed, appear to agree with those of Tetra-hydro-naphthylamin.

The rise of temperature is due essentially to increased heat production, mainly from increased movement, for these go parallel (Richet and Langlois); and the rise can be prevented by curare or chloral, but not by the ordinary antipyretics (Mosso). The heat dissipation is slightly increased.

The cocain fever does not occur after excision of the caudate nuclei, suggesting that its action is at least in part upon the same nervous centers which are concerned in "heat puncture."

Atropin Hyperthermia.-This may fairly be attributed to the muscular excitement, and to the suppression of sweat.

Caffein Hyperthermia.-Large doses of caffein produce a slight degree of fever (Binz, I878, Pilcher, I9II). This could be explained by the increased muscular tone and by the dilation of the intestinal and consequent constriction of the superficial vessels.

Muscular Exercise.-All muscular movement increases the production of heat. In health, this is ncarly compensated by increased heat loss (sweating, etc.), so that the temperature is but little increased.

Convulsants.-All convulsant poisons (santonin, picrotoxin, strychnin) produce characteristic changes in heat regulation. Small doses cause increased heat loss and a slightly smaller heat production. Larger doses cause increased metabolism, through muscular action, and hence increased heat production, which is accompanied by a further increase of heat loss. Paralytic doses diminish the heat production very greatly. The temperature is accordingly variable: Small doses tend to lower it; moderate convulsive doses would increase it; paralytic doses lower it greatly. The heat loss is particularly conspicuous in small and young animals, whilst larger animals tend to show a rise of temperature, with moderate doses (Harnack, I9oI). In traumatic tetanus, thermic fever, etc., the temperature may rise greatly, usually when there is a general disturbance of heat regulation.

Excessive External Heat.-This diminishes the heat dissipation, and thereby tends to raise the temperature. This calls forth the compensatory reactions-sweating, cutaneous hyperemia, quickened respiration, etc., mainly through the direct action of the heated blood on the centers (e.g., Moorehouse, I9II). This sets in when the internal temperature departs but a fraction of a degree from the normal; about as readily in fevers as in health. The compensation is more or less effective for a time. When it breaks, there results the phenomena of "thermic fever." 


\section{REACTIONS WHICH LOWER THE TEMPERATURE (ANTIPYRETICS)}

The temperature may be lowered by anything which lessens heat production or increases heat loss. Here again, as the temperature tends to fall below normal, compensatory reactions are set up, which ordinarily suffice to keep the temperature at the normal level. Indeed, it is highly difficult to depress the temperature materially below the normal, except by means which effect profound and extensive changes, such as deep narcosis, general vasomotor paralysis, or other collapse conditions.

On the other hand, it is relatively easy to lower an abnormally high temperature; for this coincides with the natural tendency to return to the normal physiological state-a tendency which must be always present, even in fever, although it is not effective unless supported by extraneous means.

Accordingly, the practical antipyretics become such only when the temperature is excessive. They have very little influence on normal temperature, partly because their effects would result in more powerful compensations and partly also because the primary effects themselves are less powerful when they have to overcome a normal state, than when they are assisting an abnormal state to the normal.

Action of Therapeutic Antipyretics.-The therapeutically available antipyretics act in several distinct ways: The application of cold (baths, etc.) results in the direct abstraction of heat; antipyrin, acetanilid, phenace$t i n$, etc., increase heat dissipation by dilating the cutaneous vessels; their action is central and consists presumably in a specific depression of the central tone of these vessels. Quinin diminishes heat production by a direct (peripheral) depression of nitrogenous metabolism. Aconite and veratrum probably lessen heat production by slowing the circulation.

Relation to Heat Centers. - All the antipyretics depress temperature very effectively when applied directly to the caudate nuclei (see page 449). When the central control is excluded, quinin is still antipyretic, while antipyrin and salicyl tend to raise the temperature (Isenschmidt, I9I3; Barbour and Denning, I913).

External Cold.-Regulation against external temperature is effected, in the first place, by reflex constriction of all the cutaneous vessels. This in itself produces the sensation of chilliness. If this physical regulation is not effective, the chemical regulation sets in, the heat production being increased by involuntary shivering. Indeed, the carbon dioxid and heat production is increased before it comes to visible movements, by a mere increase of "tone."

Vigorous individuals, when the external cold is withdrawn in time, usually present a "reaction," with cutaneous hyperemia, etc.

Febrile patients compensate against cold by the same mechanisms as the normal; but the compensation is not as effective, so that abnormally high temperatures are reduced more readily.

Quinin.-Therapeutic doses have little or no effect on normal temperature. The same doses lower febrile temperature (although not quite as effectively as the antipyrin group). The mechanism of action consistprimarily in diminished heat production, through limitation of the nitrogenous metabolism. This is especially marked in fever. This action of quinin is peripheral, i.e., directly on the heat-producing foci.

The diminished heat production tends to set up secondary changes, particularly a limitation of the heat loss. These are adequate to preserve the normal temperature; but in fever they respond very imperfectly, thus accounting for the fall of temperature (Literature, S. Weber, I904).

In malaria, and possibly in some other infections, the superior efficiency of quinin is due to its specific destructive effect on the infectious agent. 
Large doses of quinin (not necessarily toxic) lower even the normal temperature, sometimes with a preliminary rise. Toxic doses lower the temperature proportional to the collapse which they induce.

The relation of heat production and heat loss with normal and febrile temperature is well shown in the following quantitative data from the calorimetric experiments of Gottlicb, I89I :

\begin{tabular}{|c|c|c|c|c|}
\hline Quinin, o.I Gm. & $\begin{array}{l}\text { Fall of tem- } \\
\text { perature }\end{array}$ & $\begin{array}{l}\text { Level of } \\
\text { temperature }\end{array}$ & $\begin{array}{l}\text { Diminution } \\
\text { of heat } \\
\text { production }\end{array}$ & $\begin{array}{l}\text { Diminution } \\
\text { of heat loss }\end{array}$ \\
\hline $\begin{array}{l}\text { Normal rabbit............. } \\
\text { Puncture fever............ }\end{array}$ & $\begin{array}{l}0.2-0.5^{\circ} \\
\quad \mathrm{I}-\mathrm{I} .4^{\circ}\end{array}$ & $\begin{array}{l}38.6^{\circ} \\
39 \cdot 7-40^{\circ}\end{array}$ & $\begin{array}{l}8-15 \% \\
28.9-40 \%\end{array}$ & $\begin{array}{l}10 \% \\
0 \%\end{array}$ \\
\hline
\end{tabular}

Heat Production.-This is diminished in normal animals and much more in fever. The decrease concerns practically exclusively the nitrogen metabolism.

Nitrogen Metabolism. - This is diminished quite materially by small doses, even in normal animals; with large doses the reduction may reach 39 per cent. The reduction is often preceded by a primary rise. The decrease is markedly greater in fever. The ratio of urea to total nitrogen is not altered by moderate doses (Prior, I884). One would be tempted to ascribe this diminution of nitrogen to the diminished utilization of food. Although this undoubtedly plays a part, the diminished excretion is out of all proportion, so that the nitrogen content of the body increases. Large doses of cinchonidin have a similar action.

Oxidation.-This marked influence upon nitrogen metabolism is in conspicuous contrast to its want of influence upon oxidation. The quantity of $\mathrm{O}$ absorbed and $\mathrm{CO}_{2}$ given off is practically unaffected by medicinal doses (up to I.5 Gm.). There is a slight increase, but not more than can be accounted for by the excitement, chilliness, etc. The abnormally high gaseous metabolism of fever is reduced to normal by quinin; but this is probably the result of the antipyretic effect (Strassburg, 1874).

Nitrogen Mi etabolism as a Source of Energy.- -The fact that quinin lessens heat production without lessening oxidation is interesting. We are accustomed to look upon the excretion of $\mathrm{CO}_{2}$ as an index of chemic changes resulting in the liberation of energy and consequently of heat; and the excretion of $\mathrm{CO}_{2}$ is not affected by quinin. But since the calorimeter shows conclusively that the production of heat $i s$ diminished by it, especially in fever, this interesting fact merely forces us to the conclusion that oxidation is not the only source of heat; that heat may also be liberated by other changes -by the splitting or hydration of nitrogenous molecules, in the course of which the nitrogen is converted into urea; and that these changes are those which are hindered by quinin. The reduction by quinin would naturally be greater when this nitrogenous metabolism is excessively high, as is the case in fever.

Heat Loss.- In normal animals, this is variable, generally slightly increased by cutaneous vasodilation. In fever, the heat loss generally undergoes very little change.

The essential share of the diminished heat production is also demonstrated in several other ways: The quinin fall occurs even when the factor of heat loss is practically eliminated by high external temperature (Lewitsky, I869); it occurs when variations in the cutaneous circulation are excluded by high section of the spinal cord (Naunyn and Quincke, I869); indeed, it is here even more marked, because it can not be compensated by diminished heat loss.

The Action of Quinin is Peripheral.-This is shown by the observation of Binz, 1870, that quinin prevents the postmortal rise of temperature which ordinarily occurs when the spinal cord is divided and heat loss is prevented. This conclusion is also in harmony with the fact that quinin is a general protoplasmic poison, reducing the metabolism (for instance the hippuric acid synthesis) in perfused organs, lessening the oxidation in excised muscle (Senta, I908), and retarding the action of ferments generally-which of course play an important role in the chemic changes by which heat is liberated.

Effect on Compensatory Mechanism. - The fact that the normal temperature is not lowered by quinin shows that it does not impair the normal compensation. This is effected by two means: increased carbon metabolism and diminished heat loss.

Efficiency in Heat Puncture. - It is often affirmed that quinin is relatively ineffective in heat puncture; but the table shows that it is fairly effective. Not much weight can be attached to slight quantitative differences.

Acetanilid, Phenacetin and Antipyrin.-These, as also their numerous derivatives, and the whole series of "coal-tar antipyretics," act essen- 
tially alike, so far as is known. Their gross effect on temperature is similar to that of quinin: Therapeutic doses have little or no effect on normal temperature; in fever, the same doses reduce the temperature toward or to normal. The mechanism of their action, however, is quite different from that of quinin. Their primary effect consists in dilating the cutaneous vessels and thus increasing heat loss; the action being central, presumably a specific depression of the centers which control the tone of the cutaneous vessels. This entails secondary changes in heat production, etc., which suffice to prevent the temperature from falling below the normal level, but which are not sufficient to prevent the fall to the normal.

Large doses cause considerable fall, even of the normal temperature, with evidences of collapse.

The relation of heat production and heat loss may again be illustrated by the calorimetric data of Gottlieb, I891. A comparison of this table with that for quinin (page 456) shows at once the striking differences which were emphasized in the preceding paragraph.

\begin{tabular}{|c|c|c|c|c|}
\hline Antipyrin, $0.5 \mathrm{Gm}$. & $\begin{array}{l}\text { Fall of } \\
\text { temperature }\end{array}$ & $\begin{array}{l}\text { Level of } \\
\text { temperature }\end{array}$ & $\begin{array}{c}\text { Heat } \\
\text { production }\end{array}$ & $\begin{array}{c}\text { Heat } \\
\text { dissipation }\end{array}$ \\
\hline $\begin{array}{l}\text { Normal rabbit. ......... } \\
\text { Puncture fever....... }\end{array}$ & $\begin{array}{l}0.2-0.4^{\circ} \\
0.8-1.1^{\circ}\end{array}$ & $\begin{array}{l}3^{8} .6-3^{8} .9 \\
39 \cdot 3-4 I .6\end{array}$ & $\begin{array}{l}+5-9 \% \\
+2-10 \%\end{array}$ & $\begin{array}{l}+10-20 \% \\
+21-55 \%\end{array}$ \\
\hline
\end{tabular}

Heat Loss.-This is considerably increased in normal animals, and the increase is - several times greater in fever (see the preceding table).

Heat Production.-The calorimetric data show a moderate compensatory increase, more marked at normal temperature than in fever. The metabolism data are contradictory (Review, S. Weber, I904). Excessive doses (0.75 Gm. in rabbits) diminish heat production, presumably by incipient collapse.

Nitrogen Metabolism. - With therapeutic doses, and normal temperature, the results are variable, generally negative or some fall. Large doses of ten increase the nitrogen excretion, especially in man. In fever, therapeutic doses decrease the excessive nitrogen metabolism.

Carbon Dioxid Metabolism.-Therapeutic doses with normal temperature produce no change or some decrease. Larger doses ( 2 to $3 \mathrm{Gm}$. of antipyrin for man) produce a small but unmistakable decrease in both gaseous exchange and nitrogen excretion (incipient collapse?). The effect on carbon dioxid metabolism is also inconstant in fever: Therapeutic doses may somewhat increase or diminish the $\mathrm{CO}_{2}$ excretion, or leave it practically unchanged.

After the fall of temperature has set in, the carbon and nitrogen metabolism are commonly lowered. This may be ascribed to the removal of the hyperpyrexia, for metabolism is naturally less active at the lower temperature.

Antipyrin, and especially Phenacetin and Acetanilid, decrease the arterial oxygen; the $\mathrm{CO}_{2}$ is less changed (Piccini, I9ז2).

The respiration of excised muscle is not affected by antipyrin (in contrast to quinin and salicylate) unless very high concentrations are used (Senta, I908).

Mechanism of the Fall of Temperature.-The constant feature in the action of the antipyrin group is the increased heat loss; and this must therefore be considered the specific element in their antipyretic action. The inconstancy of the variations of the heat production indicates that these are secondary results.

Mechanism of the Increased Heat Loss. - This might occur through an increased production of sweat, or by exposing a larger amount of blood to the cooling influence of the surroundings by dilatation of the cutaneous vessels. In the case of this group it is accomplished by the latter means.

The dilatation may be deduced from the hot and reddened skin; it can be plainly shown by the plethysmograph (Maragliano, 1880). And since the reduction occurs even after atropin, which suppresses the secretion of sweat, the latter is not essential. The vasodilatation is confined to the cutaneous vessels, and this is important, since, if the blood vessels in the remainder of the body were also dilated, the circulation through the skin would be diminished rather than increased. This limitation of the vasodilatation to the 
region concerned with the regulation of temperature also indicates that the action is central.

Localization of Action.-Antipyrin does not lower the temperature after high section of the spinal cord (Sawadowski, I888), even if the temperature is artificially raised by external heat. This constitutes a conspicuous difference from Quinin, which latter is effective under these conditions. The result shows that the vasodilator action of antipyrin is located above the spinal cord.

Antipyrin also does not increase the natural rate of fall which occurs when the brain is divided at the posterior margin of the corpora striata. This might mean that its normal action is above this region; but we know too little about the results of this operation to make conclusions safe.

Antipyrin does not interfere with the normal compensation against increase of external temperature; in fact, it renders the compensation rather more efficient. This goes to show that its action does not consist in a depression or narcosis of heat regulation as such. (Toxic doses, however, render the compensation less perfect-a natural result of extensive toxic action.)

Nature of Action. $-A$ priori there are several possibilities:

I. A direct action (presumably depressant) on the center presiding over the tone of the cutaneous vessels. This would be more marked in fever, because it would coincide with a spontaneous tendency.

2. Depression of the hypothetical specific heat regulating centers. This would be more marked in fever, because the "excited" centers are more subject to fatigue and hence to depression.

3. Restoration of the abnormally high "pitch" of the specific regulating centers to their normal pitch (Richter).

The first explanation accounts for all the facts so far established; the others would fall with the superfluous conception of specific heat-regulating centers.

Salicyl.-Aside from the specific effect in rheumatic fever, the antipyretic action is relatively feeble and has not been satisfactorily analyzed. Presumably, it is similar to that of acetanilid group, i.e., increased heat loss. It is certainly not analogous to that of quinin, for the nitrogen excretion is primarily increased (Weber); although there is often a later decrease. The oxidation of excised muscle is retarded by small concentrations.

Morphin, Opium and Codein.-Small doses, according to Gottlieb, I8go, act analogous to acetanilid, i.e., by increased heat loss (cutaneous hyperemia). There is, however, at least one important difference; the fall is as great from normal as from febrile temperature.

Narcotic Doses.-These cause a much more marked lowering of the temperature (sometimes this may be preceded by a slight rise). The fall may be as large as $2^{\circ} \mathrm{C}$. with large doses, the extent depending, however, more upon idiosyncrasy than upon the dose. The cooling is particularly great if the animal is kept in cold surroundings. The cause of the fall is a diminished heat production, which may be reduced by 80 per cent. in extreme cases, reaching its minimum in the third hour. The heat loss is diminished (up to 20 per cent.), partly through the effort of the heat centers to compensate for the diminished production, partly through the depressed circulation.

Apomorphin.-This disturbs heat regulation, inhibiting the polypnea and shivering reactions to artificial fever (Magne, 1914).

Alcohol.- This also increases heat dissipation by dilating the cutaneous vessels. This warms the skin, and hence produces the sensation of heat; but the internal temperature tends to fall. Even small doses therefore 
lower the resistance to cold. With larger doses, the compensatory heat production is also impaired, and the temperature falls rapidly. This is seen more conspicuously in:

Ether or Chloroform Anesthesia.-In these, some fall occurs even when the patient is protected against loss of heat, showing that diminished heat production is an important factor (Scheinesson). This is reflected in the lessened carbon dioxid production, and is due to the muscular paralysis and decreased muscular tone. In the final stages, the dissipation is also lessened by the failure of the circulation, but this is not sufficient to offset the lessened production. If the animal is not artificially heated, and if the coma is of long duration, the temperature may reach a very low level.

Circulatory Collapse.-Any reduction of the circulation-whether cardiac or vasomotor-lowers heat production, and also tends to lower heat dissipation, since less blood circulates through the skin. The advanced grades of this action correspond to what has just been described for anesthetic coma. The slighter grades are produced therapeutically by Aconite, Veratrum, Colchicum, Digitalis, etc., but practically they are not very effective unless the dosage exceeds the safe limit.

The collapse phenomena are particularly important, because febrile patients-especially when weakened by a prolonged infection, with their circulation impaired and poor heat production-are very subject to them. Here collapse may result spontaneously, or by the withdrawal of the stimulant effects of the high temperature, or by antipyretics in doses which would not be dangerous in normal individuals.

\section{THE TREATMENT OF FEVER}

The treatment of fever has always been tinctured by the views which have successively prevailed concerning its nature. When fever was considered mainly as a subjective condition, attention was directed principally to the sensations of heat and thirst, and there arose the class of refrigerants, including the dilute mineral acids. They are useful even now, especially carbonated drinks, in conjunction with other treatment.

On the other hand, alkalies and organic acids have been used to counteract the tendency to acidosis.

As physical observation came more into fashion, the quickened pulse of fever fixed the attention of the clinicians, and it was attempted to combat all the conditions of fever by slowing the pulse. The so-called class of cardiac depressants came into vogue. They include substances acting in various ways:

Aconite and veratrum, producing vagus stimulation and general fall of blood pressure. Digitalis, producing a slowed heart, but increased blood flow. Nanseants, as tartar emetic, acting secondarily through the nausea. Potassium salts, which have a direct action on the heart.

It does not need great acumen to perceive that remedies with actions so diverse could not be successful if employed indiscriminately in all cases of fever. Some have still a place in rational therapeutics, but only when used for special indications: Aconite offers advantages over the antipyretics if the fever is of a short high type; digitalis, if the heart is weak and irregular. Whether nauseants and potassium are ever indicated against fever, as such, is very doubtful.

When the thermometer was introduced into medicine, and it was recognized that an elevation of temperature was the best index to febrile conditions, antipyretic measures came into prominence.

In regard to the application of antipyretic measures in fever, it is essential to bear in mind that they have no direct effects on fever, except upon the temperature.

Exceptions to this general statement are the actions of quinin in malaria, of salicylates in acute articular rheumatism, and the analgesic action of the coal-tar products.

For the rest, they strike neither at the cause of the fever nor at any symptoms other than those resulting from the hyperpyrexia. They make the type of the disease neither less severe nor shorter.

In malaria, e.g., antipyretics may prevent the development of a paroxysm of fever; 
but they do not attack the cause of the disease as does quinin; for their effect is not lasting, nor does their continued administration lead to a reduction in the size of the spleen.

They are a symptomatic and not a specific mode of treatment. And symptomatic treatment must always be carried on with great care, lest more harm than good should result. But where it is not possible to attack the cause, it is often advisable to remove objectionable symptoms. 'The question then is, May a reduction of fever temperature be useful? It must be borne in mind that hyperpyrexia is often a protective mechanism. This is slown by the onset of collapse in certain cases, if the stimulus of the high temperature be removed. Also, bacteriologic research has shown that with most bacteria the optimum temperature for development is confined within very narrow limits, which are exceeded by the temperature of fever. The tendency of fever may perhaps be said to be useful in all cases, but as a matter of fact it usually leads to more damage than good. The cells of the mammalian organism are not adjusted to work under the conditions of so high a temperature. It is detrimental to them as well as to the bacteria, and sometimes more so. Its effects consist in lassitude and enervation, in general discomfort, restlessness, irritability, and delirium. The respiration and heart are quickened. The metabolism, and especially the elimination of nitrogen, is greatly increased. This leads to emaciation, diminished alkalinity of blood, degeneration of important organs, etc. All these, joined perhaps to a deleterious action of the increased metabolic waste-products, produce a condition highly detrimental to the patient. These conditions, in so far as they have not already passed into permanent anatomic changes, are promptly and totally removed by restoring the normal temperature. Measures for this purpose are therefore indicated whenever these symptoms arising from hyperpyrexia become very pronounced, and unless there are special contraindications. They should not be used, for

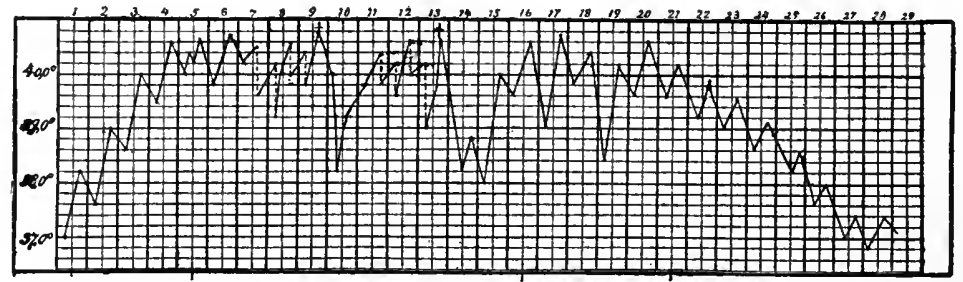

Initial period. Fastigium. Amphibolic stage. Defervescence.

FIG. 24.-Antipyretics in typhoid fever. Dotted lines = cold baths; $+=$ antipyretics (Strūmpell).

instance, if there is ground for suspecting a masked collapse. Nor are they of any use in high continued fevers.

Antipyretic measures of this kind are mainly cold baths and the cold-tar antipyretics, and these have their respective drawbacks and advantages. The chief advantage of cold baths lies in the fact that there is no danger of a direct collapse action; but there is no need for this danger with antipyretics if their dose be properly adjusted. The latter save the patient the exertion, discomfort, and shock of a cold bath. As, in the case of baths, the temperature-regulating mechanism is not adjusted to normal, the patient experiences all the ordinary effects of an attempt to reduce the temperature below normal: chills, cyanosis, etc.; and the metabolism is increased rather than diminished. The action of the chemic antipyretics is also more pronounced and lasting (Fig. 24), and their analgesic action is of marked value in influencing the subjective condition of the patient. In regard to this narcolic action, antipyrin and acetanilid are about on a level; they are surpassed by phenacetin. This action is especially useful when the fever is associated with delirium.

The objections to cold baths are very greatly lessened if tepid baths are used. These are not so much antipyretic as stimulant, improving the blood pressure, digestion, and sleep. They increase the nitrogen excretion. It has been shown that cold baths cause a considerable rise of blood pressure in acute fever, whereas, camphor, caffein, strophanthus, ether, or alcohol is generally ineffectual in this condition. As to the chemical antipyretics, the theory has been advanced that they interfere with the natural protective bodies of the serum. It has been shown, however, that this is not the case, at least as far as the agglutinin of typhoid serum is concerned. In conclusion, it may be said that the best general treatment of fever consists in a combination of antipyretics and graduated baths. 
There are peculiar differences in the susceptibility of different fevers to antipyretic drugs: High continuous fevers react least, those of an intermittent type being most amenable; and with these, again, the greatest effect is produced when the action falls into the period of the natural decline of temperature.

Sometimes antipyretics may even produce a "paradoxical action" a rise in temperature. Graul, I899, has collecterl a number of such cases for antipyrin. They usually showed romiting and severe exanthemata.

\section{QUININ}

General Statement.-Quinin, the principal alkaloid of cinchona bark, is a general protoplasmic poison. It is therefore toxic to all cells, and even to unorganized ferments; but it has a specially high toxicity for ameboid cells, and is relatively non-toxic for higher organisms. It exerts a very selective action on the malarial parasites, and is therefore a specific against malarial diseases, provided sufficiently large doses are administered. It is also a fairly effective antipyretic in other fevers, reducing heat production by diminishing nitrogen metabolism. It is useful as an analgesic in colds; and in the form of quinin-urea hydrochlorid as a local anesthetic. Through its bitter qualities, it acts as a stomachic tonic. It stimulates the uterus. Full doses produce "cinchonism," characterized especially by auditory and visual disturbances, which may assume grave proportions with excessive doses; but very large quantities have been consumed without serious poisoning.

Cinchona and Its Constituents. - The bark of the cinchona tree ( $\mathrm{Pe}$ ruvian or Jesuits' bark) contains a number of alkaloids (cinchonin, cinchonidin, etc.) resembling quinin, but endowed with less of the desired activity, and with more of the undesired side effects (convulsant, etc., Albertoni, r882). It also contains considerable tannin, which interferes with the systemic actions. (Cinchonin is much more depressant to the heart, and more toxic than cinchonidin.)

The trees yielding cinchona are indigenous to the mountainous districts of the Andes in South America, at a height of 3,400 to $x, 200$ meters. Trees growing below this level contain but little alkaloid. The natives were probably acquainted with the medicinal value of this "tree of health," and the bark was brought to Europe by the early explorers. It received its name, cinchona, from the Countess Chinchon, who was one of the first Europeans to receive its benefits (I638).

At present practically all the barks of commerce are from cultivated trees, the original forests having been largely depleted. This cinchona cultivation is carried on in a number of subtropical mountainous countries possessing a rather moist climate-especially in India.

The U. S. Pharmacopœia does not discriminate between the different species. Between thirty and thirty-six of these are recognized; but many are probably mere variations and hybrids, and a recent author has attempted to reduce the species to four. The most important are: C. Calisaya (yellow bark); succirubra (red bark); officinalis; lancifolia; micrantha; scrobiculata.

The alkaloidal content of the different barks varies greatly. It has been considerably increased by cultivation, some samples yielding to 13 per cent. of quinin. The alkaloids are contained in all parts of the plant, in the parenchymal cells. The root bark contains the most, then comes the ordinary bark.

The constituents are: Certain acids (quinic, quinovic, etc.); Tannin, as cinchotannic acid ( 2 to 4 per cent.), which gives a green color with iron; cinchona red, a derivative of the preceding (both are glucosids); gum, wax, resin, etc.

A very large number of alkaloids have been isolated; many of these undoubtedly arise in the course of the manipulations. The most important are in italics: 
Quinin, ${ }^{1}$ Quinidin, Quinicin.

Quinamin, Conquinamin, Quinamidin, Quinamicin.

Cinchonin, Cinchonidin, Cinchonicin, Homocinchonicin.

(Those on a line are isomeric.)

When the crystallizable alkaloids have been separated from cinchona extracts, evaporation of the mother liquor yields a brown extract, Chinoidin (Quinoidin), which contains amorphous alkaloids, mainly Di-cinchonicin and Di-quinidin.

Cuprea Bark, from Remija pedunculata, Rubiaceæ, contains for the most part the same alkaloids, but no cinchonidin. It is used in the manufacture of quinin.

Toxic Action on Protoplasm.-This may be seen on lower organisms and isolated cells of all kinds. It acts most strongly on cells possessed of ameboid and similar movement: on infusoria, white blood cells, ciliated epithelium, spermatozoa, insectivorous plants, muscle, etc.

Action on Leucocytes.-A solution of 0.5 to $\mathrm{I}$ in 1000 is sufficient to inhibit the movements of leucocyles on the warmed slide, and a somewhat larger dose causes their disintegration. Phagocytosis is hindered by 0.00 I per cent. (Hamburger and Hekma, I908), but stimulated by very low concentrations (Manwaring and Ruh, 1907).

The depressant action is also observed in intact frogs. When the mesentery of this animal is exposed, leucocytes in active motion are seen inside and outside of the vessel. If quinin is now applied, the movement of the cells outside of the blood will be arrested, whilst those in the blood stream still emigrate. The result is an accumulation of cells about the vessel wall. If the quinin is injected into the vessel, the reverse takes place. The movement of the cells in the blood is arrested, preventing emigration, whilst those outside do not come into contact with the poison, continue to move away, and leave a clear zone about the vessel (Binz, I867; Ikeda, I916). This action does not occur in mammals, since the necessary dose would kill the animal.

In man and mammals, it causes at first an increase of the lymphocytes, these being pressed into the circulation by the muscular contraction of the spleen and lymph glands. This is followed after an hour by a decrease, and this is again succeeded by rather marked leucocytosis. Continuous administration leads to leucopenia followed by leucocytosis (G. B. Roth, IgI2). The opsonic index is slightly increased in man by doses of $15 \mathrm{gr}$. (T. B. Wilson, I907).

Antiseptic Action.-Bacteria and yeasts are also killed by quinin, but require a higher concentration ( 2 to $8: 1000$ ) than do the protozoa.

McDonald, I9I5, considers its germicidal efficiency about half that of phenol; whilst as an antiseptic it approaches bladder irrigation, especially as prophylactic in cystoscopy. This concentration does not cause noticeable irritation.

Skeletal Muscle.--The effects on frog muscle are depressant. Dilute solutions do not change the form or duration of the single twitch, or the latent period, nor do they cause histologic changes; but they diminish the load which the muscle can lift and the total work, and hasten fatigue. These phenomena are also observed in curarized muscle, so that the actions must be directly on the muscle substance. High concentrations coagulate and destroy the muscle structure (Secher, I9I 5; Santesson, I892).

Cardiac Muscle.- In the frog, this is slowed by I :50,000 solution and weakened in its contraction; its effectiveness is consequently diminished. The slowing occurs after atropin, and is therefore muscular. A I :5,000 solution kills the heart in a few minutes.

The turtle's heart is slowed by all doses; the excursions are increased by small and diminished by higher concentrations (de Arric, I 12 2).

Mammalian Circulation. - In mammals and man small doses cause first a quickened pulse with rise of blood pressure (Literature, de Arric, 1912; Biberfeld, I916).

The cause of the former is still under dispute, but is perhaps central. The rise of pressure depends mainly upon a vasoconstriction, but the cause of this is also still undetermined. It may possibly be due to a direct stimulation of the unstriped muscles of the blood vessels.

Larger doses (in man, from I Gm. upward) cause a fall of blood pressure and slowing and weakening of the heart from the outset.

The fall is due in part to vasodilation. The slowing and weakening of the heart are analogous to those observed in the frog. It is seen in the excised organ (Langendorff

${ }^{1}$ Name from quina, bark. The structural formula of quinin is discussed by Ad. Kaufmann, 1913. 
method). In this it often causes irregularity, but may regulate the heart if it is already irregular.

Perfused mammalian vessels are dilated by quinin, whilst those of the frog are constricted. Apocodein and yohimbin act similarly (Gunn, 1009).

The brain-vessels are dilated in rabbits and fish (Berezin, 1916).

Uterus.-The contractions and tone of the excised uterus are strongly stimulated (Kurdinowski; Kehrer, I906) by concentrations as low as I : 100,000 (Lieb, I9I4).

Cinchonin and cinchonidin acts similarly; quinidin produces only relaxation. All these effects are the stronger, the more active the uterine movements (Zanda, igro and rgr 2). The relative efficiency of the cinchona alkaloids has been determined by Hall, I9I6.

This effect may explain the use of toxic doses of quinin for abortion, although the intestinal irritation would play a part. Maurer, 1907 , uses it as an ecbolic ( 0.5 to $\mathrm{I}$ Gm., repeated in an hour if necessary), claiming marked effects, usually in forty minutes. The action is not used clinically; but toxic doses are credited with producing abortion (probably partly by intestinal irritation).

Contraction of the spleen and intestines may also occur. Excised tracheal muscle is relaxed (Trendelenburg).

The peripheral nerves are not markedly affected, except the poison be applied directly, when it will irritate, depress and finally kill them just as other protoplasm.

Local Action.-Quinin produces some local irritation and anesthesia.

When given hypodermically it occasions severe pain, and may lead to abscess formation. When given by the stomach, large doses cause gastralgia, nausea, vomiting, and diarrhea. It also retards the absorption of salts, and probably of food. Its excretion through the kidneys may give rise, with large doses, to albuminuria and hemoglobinuria. The hemoglobinuria of severe malaria (black-water fever) may be partly due to increased sensitiveness to quinin. It seems not unlikely that a skin eruption which is sometimes observed, results from the irritant effects of its excretion through this channel.

Ulcers. - The sulphate has been used as antiseptic, styptic and stimulant dusting powder in ulcers.

Quinin and Urea Hydrochlorid as a Local Anesthetic.-This and other double salts of quinin are much more soluble and produce little or no irritation, but fairly complete anesthesia, sometimes lasting for several days (Hertzler, Brewster and Rogers, I 909 ). It is used hypodermically, generally as 0.25 per cent. sometimes as I to 3 per cent. Solutions stronger than 0.25 per cent. may produce fibrous indurations and above I per cent. it may cause sloughing. Solutions of to to 20 per cent. can be used on mucous membranes.

Crile uses a $1 / 2$ per cent. solution for infiltrating the peritoneum, etc., to block afferent impulses. This causes some local edema, persisting for a time after the wound has healed.

Double Quinin Salts. - The increased solubility of quinin hydrochlorid (3 parts) by antipyrin (2 parts) was announced by Triulzi, I892, and Santesson, 1897. Gaglio, $\mathbf{1 8 9 8}$, and Giemsa, 1908 , advise the urethane compound as being less acid and less readily decomposed by boiling, than is the urea salt (Filippi, rgr2). It is made with I Gm. of qininin hydrochlorid, $0.5 \mathrm{Gm}$. urethane, and 2 c.c. of water.

Ferments.-Interference with ferment action may be seen in many directions. The most important are: the oxydases of blood and of protoplasm autolysis (Lagneur, I906); slowed acidification of shed blood; lessening of the amount of hippuric acid formed when benzoic acid is circulated through the excised kidney. The various digestive ferments are hindered, but not to the same degree. It also lessens the glycogenetic function of the liver; i.e., the postmortem transformation of the glycogen into sugar (Hoffmann, I877).

Hemoglobin. - This shows a characteristic change with strong quinin solutions (Marx, I906); no methemoglobin is formed (Lewin, 1909). 
Effect on Digestion.--Small doses of quinin ( $0.05 \mathrm{Gm}$., I gr.), taken before meals, act as a stomachic and tonic; but they are probably inferior to other bitters. Larger doses diminish gastric secretion (Klocman, I9 I 2), and interfere with the ferments and with absorption; even small doses when long continued lessen the utilization of food. Toxic doses stimulate the romiting center (Eggleston and Hatcher, I915). Tinct. Cinchonæ Comp. (5 c.c., I3) adds the stomachic effect of aromatics and alcohol, and the astringent action of tannin. It may be useful in chronic gastric catarrh.

Metabolism and Temperature.-The effects were discussed on page 455 .

Use in Fever.-The antipyretic action of quinin may be utilized in any fever. It has several theoretical advantages over the anilin antipyretics. It conserves energy by lessening metabolism, instead of dissipating it by increased heat loss; there is less risk of collapse; and its effects are more prolonged. On the other hand, the annoying cinchonism and occasional idiosyncrasies, eruptions, etc., limit its usefulness. It is employed mainly where the depressant action of the anilin derivatives is especially contraindicated, as in influenza; or where only small doses are required, as in colds. The full antipyretic dosage is from 0.3 to I Gm., 5 to I 5 gr. Like other antipyretics, it is most efficient when the temperature has a natural tendency to fall. It takes about two hours to act, and should therefore be given about that time before an expected fall of temperature.

It has been suggested that its efficiency in fever is due to an antiseptic action on the blood. This is not probable since bacteria are very resistant to it, and would scarcely be affected by it in the concentration in which it could exist in the blood.

Use in Asiatic Cholera.-Quinin has been especially recommended in this disease, in which conditions would be favorable for a direct bactericidal action (Fullerton, I 896 , Ussher, I906). The dry sulphate, $0.6 \mathrm{Gm}$., ro gr., is administered every hour for four to eight doses until bile reappears in the feces. Further confirmation is required.

Use in Pneumonia. - This also is claimed to be especially benefited by quinin; but the natural course of this disease makes judgment very difficult. O. H. Brown, I9I2, states that pneumococci cultures are killed in one-third to five hours by 0.1 per cent. of quinin (especially basic), whilst other organisms (strepto- and staphylococci) require much longer. S. S. Cohen, I9II and I9I3, recommends massive doses, I to I.6 Gm., 15 to 25 gr., of quinin-urea-chlorid, dissolved in 2 parts of hot water, and injected into the muscles; followed by further injections of $x \mathrm{Gm}$. doses every third hour until the temperature remains below $102^{\circ} \mathrm{F}$.-rarely beyond seventy-two hours. He claims that this does not produce cinchonism, gives great symptomatic relief (respiration, cough, pulse), and lowers the temperature at the ordinary time, but by lysis instead of crisis. The anatomic lung changes are not affected, indicating that the action is probably not on the local process, but rather antipyretic and analgesic.

Use of Quinin in Colds, Headaches and Neuralgias.-Like other antipyretics, quinin has an analgesic effect in these conditions. It is used in doses of 0.05 to $0.2 \mathrm{Gm}$., I to 3 gr.

Central Actions.-The effects are not important, except with toxic doses. They consist in a rather slow general paralysis, probably preceded by stimulation; the latter is somewhat difficult to make out in mammals.

In frogs there is first an increased reflex irritability. This is followed by loss of spontaneous movement, then paralysis of respiration, and lastly of the cord.

The depressing effects upon the hemispheres are much less marked in mammals, but a diminished appreciation of pain can be distinctly made out, and upon it rests the employment of quinin against neuralgic and rheumatic pains.

Its addition to morphin or urethane does not increase their narcotic effect. 
Cinchonidin and Cinchonin Convulsions.- These alkaloids, which otherwise agree qualitatively with quinin, produce convulsions of the medullary type. The action is below the cerebrum; for after full operative recovery from hemisection below the optic thalami, with ensuing hemiplegia, cinchonidin still causes bilateral convulsions (Albertoni, I882).

Toxic Actions of Quinin.--With very high doses of quinin, the symptoms of cinchonism (see below) are accompanied by difficulty of speech, confusion of ideas, somnolence. Then loss of consciousness, alternating with delirium, coma, and at times convulsions.

It has been doubted whether the latter are really due to quinin or to the accidental presence of some of the convulsant cinchona alkaloids.

General paresis may appear, preceded by general depression and muscular weakness. The final symptoms are those of collapse, due to general paralysis of the central nervous system, and in part also of the heart. The respiratory center shows a short primary stimulation with following more marked paralysis. The latter is the usual cause of death. But since the medullary centers are not markedly affected until very late, quite large doses are often survived.

The fatal dose is usually given as $8 \mathrm{Gm}$., but $30 \mathrm{Gm}$. have been recovered from. It is doubtful how much of this really entered the circulation, since the sulphate is very insoluble, so that a large amount may not have becn absorbed.

Cinchonism.--Early symptoms of larger doses of quinin are headache, ringing in the ears, and disturbed vision, a complex of symptoms grouped together under the name of cinchonism. These are probably due to a selective action on the vessels of the eye and ear, which can also be demonstrated experimentally on animals. Toxic doses produce photophobia, deafness, and blindness, at first partial, later complete. These are probably partly central. Similar phenomena occur with salicylates and related drugs. Cinchonism is very subject to idiosyncrasy, being much more easily produced in some individuals. It is said that the minor symptoms may be somewhat diminished by bromids.

Anatomic Changes in Eye and Ear.-The retina is said to be anemic, whilst the internal ear is congested, postmortem (Kirchner). Harnack, I9 I 2, also ascribes the eye change to spasm of the retinal vessels with subsequent optic atrophy. Wittmaak $(1903)$ claims that this congestion is not due to quinin, but to death, and points out that changes of blood pressure do not produce deafness. On the other hand, he demonstrates changes in the cells of the spiral ganglion, by the Nissl stain. He is therefore inclined to attribute the auditory effect to a specific toxicity of quinin to these cells. A corresponding origin is assigned by Altland, I 904 , to the visual changes, the retinal cells being altered. This might be secondary to the ischemia.

Skin Eruptions.-These are quite common in the therapeutic use of quinin. They may be scarlatinal or urticarial. Atropin is said to diminish their occurrence.

Quinin in Malaria.-The specific action of quinin in this disease is due to its toxic effects upon the plasmodia causing the disorder.

This organism is especially susceptible to it. On a slide a I : 10,000 solution immediately arrests the movements of the parasite, and similar phenomena occur in the body. About three hours after the administration of quinin by the mouth, the endoglobular forms of tertian and quartan fever become immobile, granular, lose their nucleoli and their affinity for certain stains. Several hours later they may be seen deformed and fragmented (Lo Monaco and Panichi, I899).

The other cinchona alkaloids have a similar, but much weaker, action on the protozoa.

Bass and Johns, I9I2, who have cultivated the plasmodia, find that they are readily destroyed by normal serum. They suggest that quinin acts by rendęring the red blood corpuscles more permeable to the toxic constituents of the serum; but there is no other evidence for this vicw.

Actions of Hematin.-W. H. Brown, I9r2; and Brown and Loevenhart, 19r3, find that the injection of small doses of hematin into rabbits produces paroxysms similar 
to those of human malaria: A "cold stage" of cutaneous vasoconstriction with falling surface temperature and rise of blood pressure; succeeded by cutaneous vasodilation with hot skin. Larger doses cause splanchnic dilation (central) and central vagus stimulation with falling blood pressure. The respiration is depressed, ending in death. Butterfield and Benedict, I 9 I 4, failed to confirm these effects, and claim that large doses kill by intravascular precipitation of hematin.

Different Susceptibility.-Quinin is most effective in the tertian, less in the quartan, and least in the pernicious malaria. It does not act equally on the parasite in all the stages of its development. The sexual forms are more resistant than the usual asexual. Its strongest action is upon the forms which are just breaking into spores and upon the free-swimming organisms; it is much weaker upon the older segmenting bodies, and least upon the young endoglobular forms.

Administration in Malaria.-Since the resistant endoglobular forms exist in the blood just before the paroxysm, and their sporulation gives rise to the characteristic chill and fever, and since quinin does not act well upon them, it will not be effectual against the oncoming paroxysm. But if it is given at this time it will be present in the blood when the spores are liberated, and as these are most susceptible to its action, it will kill them and thus prevent the development of the new cycle (providing that the dose has been sufficiently large). It should therefore be given several hours before the expected paroxysm so as to allow time for its absorption. The dose should then be quite large: $\mathrm{I}$ to $2 \mathrm{Gm}$. given (fasting, if possible) two to three hours before the expected attack (which will in all probability occur), or $0.3 \mathrm{Gm}$. four to five times a day in the interval; $0.5 \mathrm{Gm}$. per day should be continued for three months after the last chill.

To be effective, the quinin must be administered in solution: Large doses of quinin sulphate in pills or powder are probably largely wasted, since they are not dissolved or absorbed; the sulphate requires 720 parts of water for solution. The hydrochlorid is more soluble ( $\mathrm{I}: \mathrm{I} 8)$, but the bisulphate ( $(1: 8.5)$ or the dihydrochlorid is the best.

It is not customary to prescribe the bisulphate directly, but to bring the sulphate into solution by the addition of a sufficient quantity of dilute sulphuric acid. The quantity of acid should be rather in excess. If it is only just sufficient, some sulphate will be precipitated on the tongue by the alkaline saliva, and give rise to a very persistent after taste. There will be very little of this if an excess of acid is used and the mouth rinsed afterward.

Urgent cases may demand intramuscular injections of quinin dihydrochlorid ( $\mathrm{I} \mathrm{Gm}$. in Io c.c.) or of quinin-urea hydrochlorid ( $2 \mathrm{Gm}$. in 5 to Io c.c.). Intravenous injections of the hydrochlorid have also been used. Concentrations of $I: I 5 \circ$ are dangerous (MacGilchrist); and even lower concentrations should be used with caution, for fear of direct cardiac effects.

Ehrlich suggests using methylen blue with quinin.

Use in Malignant Malaria. - Practically all cases of malignant malaria are due to the estivo-autumnal plasmodium (P. falciparium). This spends only a fourth of its life cycle in the circulating blood; the remainder in the capillaries, where it is not easily reached by the quinin. The parasites enter the circulation after they segment. Since this process is continuous, a sufficient concentration of quinin must be maintained in the blood continuously.

The absorption from the intestinal tract is too slow for these severe cases, and hypodermic injection may not be any better, since the local irritation of ten delays absorption. Intravenous injection is therefore preferable in serious cases. Quinin, when administered by vein, appears in the urine in a few minutes; often a half of the dose is eliminated in twelve hours, and practically all in a day. The injections must 
therefore be repeated every few hours, until all the parasites are killed (generally in thirtysix hours). The quantity should never exceed $0.65 \mathrm{Gm}$. per dose, nor $2 \mathrm{Gm}$. per day. Fatalities have occurred from $3 \mathrm{Gm}$. (Bass, I9 15).

Hemoglobinuria. - This sometimes follows the use of quinin in pernicious anemia ("black-water fever"). It is not clear whether the drug has a causative relation; for quinin does not produce hemoglobinuria in the absence of malaria, whereas the urine may become bloody in malarial cases that have not received quinin. On the other hand, it is recorded that the symptom was aggravated by the drug, or appeared only after the drug was started. Brem, 1912, states that extracts of malarial parasites are strongly hemolytic; and it is quite conceivable that quinin aids in the liberation of this hemolytic poison.

Quinin as Prophylactic against Malaria.-Most authorities consider quinin as useful, although not invariably successful (Thayer, I912). Koch advised I Gm. once a week; Mariani, $0.5 \mathrm{Gm}$. daily in divided doses. A minority consider quinin prophylaxis of slight value; and Stitt, I907, claims that it renders active quinin treatment much less effective.

The question has been raised whether it is possible to eradicate malaria in a given region, by killing the plasmodia in the human hosts. The answer appears to be in the negative: Orenstein, I 192 , after subjecting a malarial community to systematic quininization by the Koch-Ollwig method, found the incidence of malaria practically unchanged.

Administration of Small Doses.-Small doses of quinin sulphate or hydrochlorid may be easily administered in the forms of pills or capsules; or in solution, the taste being disguised by glycyrrhiza. (No acid must be prescribed with the lacter!) The free alkaloid is almost tasteless, and the tannate quite so, because of their slight solubility. Their absorption will not suffice for large, but is probably adequate for small doses. They may be placed dry on the tongue and swallowed with a draught of water.

Antimalarial Efficiency of Cinchona Alkaloids.-MacGilchrist states this to be in the order of: Hydroquinin >cinchonin, quinin, quinidin >ethylhydroxycuprein $>$ cinchonidin > quinoidin; the first being the most, the last the least active. The same order holds for the different forms of the parasites. Confirmation would be desirable.

Amebic Dysentery.-Quinin is used for rectal irrigation. Its administration by mouth $(2 \mathrm{Gm}$. per day) is sometimes effective, but less certain than emetin (Brooks, 1914).

Rabies.-V. H. Moon, I9I 3 reports apparently successful results with quinin on dogs. In I915, he reports them only very slightly positive and practically negligible. All subsequent observers report negative results (Krumwiede and Mann, I915; Fielder, $1914,1916)$.

\section{QUININ DERIVATIVES}

The esters of quinin with various acids are insoluble and therefore tasteless. They pass the stomach unchanged, but are slowly saponified in the intestines; in this way exhibiting a very gradual and presumably weak action. They are more easily administered, and produce less side actions, but are not always devoid of cinchonism (Seifert, Nebenwirk., I9I5, pp. II5-II9).

They are used in the same doses as quinin, in powder or suspended in liquids. To this type belong:

Aristochin (diquinin carbonic ester); euquinin (quinin ethyl carbonic ester) and saloquinin (quinin salicylic ester); the latter may be used for a weak salicylic action; and chinaphenin (phenetidin quinin carbonic ester) for exhibiting simultaneously the action of phenacetin. As to the last two mentioned, there is no advantage in a chemic combination of two therapeutic agents; on the contrary, it prevents the necessary elasticity of dosage. 


\section{ABSORPTION, FATE AND EXCRETION}

Quinin is absorbed mainly from the small intestine (MacGilchrist). In the blood it is contained chiefly in the corpuscles (Baldoni, I9I2, I9I3). The greater part (70 to 90 per cent., Merkel, I902; Schmitz, I907; Biberfeld, rgI6) is destroyed in the body (also in the perfused liver; Grosser, I908). The remainder is excreted unchanged by the urine, and traces by other secretions (milk, Koldewjin, Igro). The excretion begins within onehalf hour and is practically completed in twenty-four hours (Nishi, r9o9; Baldoni). With medicinal doses, it can not be demonstrated in the urine.

\section{PREPARATIONS-QUININ GROUP}

The ordinary salts of quinin are white, odorless, and intensely bitter. They are incompatible with alkalies and other alkaloidal precipitants, and with salicylates, benzoates and tartrates. Average dose of all quinin salts, tonic, o.I Gm., I $1 / 2$ gr.; malarial, at least I Gm., 15 gr. daily, U.S.P.; ordinary dose, B.P., 0.06 to $0.6 \mathrm{Gm}$., I to Io gr. The solubilities are shown in the following table.,

\begin{tabular}{|c|c|c|c|}
\hline . & $\begin{array}{l}\text { One part dis- } \\
\text { solves in water }\end{array}$ & In alcohol & $\begin{array}{c}\text { Reaction of } \\
\text { solutions }\end{array}$ \\
\hline 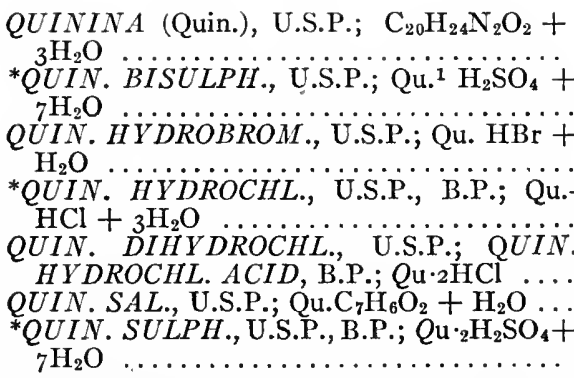 & $\begin{array}{c}\text { I,560 } \\
9 \\
40 \\
\text { I8 } \\
\text { o.6 } \\
\text { slightly } \\
725\end{array}$ & $\begin{array}{r}0.8 \\
23.0 \\
0.9 \\
0.8 \\
12.0 \\
14.0 \\
107\end{array}$ & $\begin{array}{l}\text { Alk. } \\
\text { Acid } \\
\text { Neutr. } \\
\text { Neutr. } \\
\text { Acid } \\
\text { Alk. } \\
\text { Neutr. }\end{array}$ \\
\hline
\end{tabular}

${ }^{*}$ Quinina Tannas (Quin. Tan.), U.S.P.-A compound of quinin and of tannic acid of somewhat varying composition and containing about 33 per cent. of anhydrous quinin. Pale yellow, amorphous powder; tasteless, or having not more than a slightly bitter and astringent taste. Slightly sol. in water; somewhat more sol. in alc. Dose, 0.2 Gm., 3 gr., U.S.P.

*Quinina et Urea Hydrochloricum (Quin. et Urea. Hydrochl.), U.S.P.; Qu.HCl-$\mathrm{CO}\left(\mathrm{NH}_{2}\right)_{2} \cdot \mathrm{HCl}+{ }_{5} \mathrm{H}_{2} \mathrm{O}$ - - Colorless prisms or white granular powder, bitter taste. Very sol. in water $(\mathrm{I}: 0.9)$, freely sol. in alc. $(\mathrm{I}: 2.4)$. Solutions are strongly acid. Dose, I Gm., I5 gr., once daily, hypodermic, U.S.P.

(Ferr. et. Quin. Cit.-See Index.)

(El. Ferr. Quin. et Strych. Phosph.-See Index.)

Pil. Quin. Sulph., B.P. -82 per cent. Dose, o.1 2 to $0.5 \mathrm{Gm} ., 2$ to 8 gr., B.P.

Tr. Quin., B.P. -2 per cent. in Tr. of Orange. Dose, 2 to 4 c.c., $1 / 2$ to I dram, B.P.

Tr. Quin. Ammon., B.P. -2 per cent., in ammoniated alcohol. Dose, 2 to 4 c.c., $1 / 2$ to I dram, B.P.

Vin. Quin., B.P.-0.23 per cent. Dose, 16 to 30 c.c., $1 / 2$ to I ounce, B.P.

Tinctura Antiperiodoca, N.F. (Warburg's Tincture). - This is used in India for administering quinin against malaria. It contains 2 per cent. of quinin sulphate, $0.3 \mathrm{Gm}$. per tablespoon, with rhubarb, aloes, camphor, and aromatic drugs.

Quinina Ethylcarbonas, N.N.R.; Euquinin; $\mathrm{C}_{20} \mathrm{H}_{23} \mathrm{~N}_{2} \mathrm{O} \cdot \mathrm{O} \cdot \mathrm{CO} \cdot \mathrm{OC}_{2} \mathrm{H}_{5}$ - - Nearly tasteless and insoluble. Dose, as for Quinin. 
Aristochin, N.N.R.; Diquinin carbonic ester; $\left(\mathrm{C}_{20} \mathrm{H}_{23} \mathrm{~N}_{2} \mathrm{O}\right) \mathrm{O} \cdot \mathrm{CO} \cdot \mathrm{O}\left(\mathrm{C}_{20} \mathrm{HI}_{23} \mathrm{~N}_{2} \mathrm{O}\right)$.Nearly tasteless and insoluble. Dose, as for Quinin.

Cinchonidince Sulphas (Cinchonid. Sulph.), U.S.P.; $\left(\mathrm{C}_{19} \mathrm{H}_{22} \mathrm{~N}_{2} \mathrm{O}\right)_{2} \cdot \mathrm{H}_{2} \mathrm{SO}_{4}+{ }_{3} \mathrm{Ir}_{2} \mathrm{O}$.White, glistening, silky needles or prisms; odorless and having a very bitter taste. Sol. in water $(1: 65)$ or alc. $(1: 95)$. Dose, 0.15 Gm., $21 / 2$ gr., U.S.P.

Cinchonine Sulphas (Cinchonin. Sulph.), U.S.P.; $\left(\mathrm{C}_{19} \mathrm{H}_{22} \mathrm{~N}_{2} \mathrm{O}\right)_{2} \cdot \mathrm{H}_{2} \mathrm{SO}_{4}+{ }_{2} \mathrm{H}_{2} \mathrm{O}$.White, lustrous, prismatic crystals; odorless; very bitter taste. Sol. in water ( $\mathrm{r}: 60$ ) or alc. (1:12.5). Dose, 0.15 Gm., $21 / 2$ gr., U.S.P.

Cinchona, U.S.P. (Yellow Cinchona; Calisaya Bark; Yellow Peruvian Bark).The dried bark of Cinchona Ledgeriana, Cinchona Calisaya, and of hybrids of these and other species of Cinchona, yielding not less than 6 per cent. of the total alkaloids. Dose, I Gm., I 5 gr., U.S.P.

Cinchona Rubra, U.S.P.; Cinch. Rubr. Cort., B.P.; Red Cinchona.-The dried bark of Cinchona succirubra or of its hybrids, yielding not less than 6 per cent. of the total alkaloids of Red Cinchona; 5 to 6 per cent., B.P. Dose, I Gm., I 5 gr., U.S.P.

Inf. Cinch. Acid., B.P.- 5 per cent. of Cinchona, with 1.25 per cent. of Acid Sulph. Arom. Dose, 15 to 30 c.c., $1 / 2$ to I ounce, B.P.

Fldext. Cinch., U.S.P.; Ext. Cinch. Liq., B.P.-5 per cent. of alkaloids. Dose, I c.c., I5 minims, U.S.P.; 0.3 to I C.c., 5 to I5 minims, B.P.

*Tinctura Cinchone (Tr. Cinch.), U.S.P., B.P.-2o per cent. of Cinchona, with $2 / 3$ alcohol. Incompatible with water, iron and alkaloidal precipitants. Dose, 4 c.c., I dram, U.S.P.; 2 to 4 c.c., $1 / 2$ to I dram, B.P.

*Tr. Cincli. Co., U.S.P., B.P.- Io per cent. of Cinchona, with 2.5 per cent. of Serpentaria, together with Bitter Orange. 'The B.P. is colored with Cochineal; strongly alcoholic. Dose, 4 c.c., I dram, U.S.P., 2 to 4 c.c., $1 / 2$ to $I$ dram, B.P.

Alstonia, B.P.-The dried bark of Alstonia scholaris and constricta. Contains alkaloids and is employed in India against malaria; also as a bitter tonic, anthelmintic and against diarrhea.

Inf. Alston., B.P. -5 per cent. Dose, $\mathrm{I} 5$ to 30 c.c., $1 / 2$ to I ounce, B.P.

Tr. Alston., B.P.-I 2.5 per cent. Dose, 2 to 4 c.c., $1 / 2$ to I dram, B.P.

\section{ETHYL-HYDROXYCUPREIN}

Action in Pneumonia.-This drug (also marketed as "Optochin") has a specific toxicity for pneumococci (Morgenroth and Halberstaedter). It is highly successful against experimental pneumonia in animals, and promising results have been reported in man; but its application is limited by the fact that efficient doses are liable to produce blindness.

Experimental Pneumonia.- In vitro, the drug inhibits the growth of pneumococci with a concentration of $I: 800,000$ in serums; it kills them with $I: 400,000$ (Wright, I9I 2 ). In mice, it is effectively prophylactic in 90 per cent., and curative in $5 \circ$ per cent. of the experimental infections (Morgenroth and Levy; Engwer, 1912). The serum of rabbits is bactericidal after hypodermic doses of o. I Gm. per kilogram, which are well tolerated. Larger doses are usually toxic. Oral administration is not as effective (Moore, 1915).

Specificity.- All strains of pneumococci, as also streptococci, are inhibited in vitro by concentrations of $\mathrm{I}: \mathrm{I00}, 000$; whereas other streptococci, meningococci and gonococci require I: I0, $000-5,000$. However, transition races are occasionally found. Quinin requires much higher concentrations. Optochin also increases greatly (potentiates) the efficiency of anti-pneumococcus serum (Nachmann, 1915; H. F, Moore, 1915).

Human Pneumonia.-Doses of $0.5 \mathrm{Gm}$., three to at most five times daily, by mouth, to a total of 1 to $6 \mathrm{Gm}$., render the serum bactericidal. Its clinical efficiency is difficult to judge, but evidently less striking than in animals. Lapinski, I9I5 (who also gives a review of the subject), and A. Fraenkel, I9I5, consider the results good in the first two or three days of the disease. The success is indicated by shortened fever, greater comfort, diminished dyspnea and decreased mortality. Quinin does not produce this improvement. When started later, the response is very limited, perhaps because the pneumonic coagulation prevents its access to the bacteria. Vetlesen, 1913, reports that it shortens the course. Lenne, I 9 I $_{3}$, claims positive results, especially from intravenous administration. 'Parkinson, I9I3, could see no therapeutic effects.

Side Actions. - The susceptibility to these varies greatly in different individuals. Temporary cinchonism and vomiting are of frequent occurrence; but the most serious accident is the tendency to produce blindness by retinal and optic nerve changes (A. E. Wright and co-workers, I9I 2; Lapinski, 1915; Seifert, Nebenwirk., 1915, p. I). 
Whilst this does not occur in all cases, and is only temporary, the use of the drug involves serious responsibility.

Fraenkel, I915, obtained some degree of amblyopia in ro per cent of his series. If the patient is watched and the drug stopped with the first sign, the vision generally clears in a few days, but sometimes only after months. Hirschfelder, 1915, observed constriction of the retinal and pia vessels in animals.

Administration.-Fraenkel prefers the less soluble base to the salts, so as not to flood the organism. He gives 0.2 to $0.3 \mathrm{Gm}$. every four hours, not exceeding $1.5 \mathrm{Gm}$. per. day, for at most threc or four days; carefully testing the vision throughout.

Fate.-The alkaloid disappears rapidly from the plasma after intravenous injection, It persists longer and in greater amount in the corpuscles (Boecker, I915).

Other Infections.- Izar and Nicosia, 1914, use it in malaria bectuse the cinchonism is shorter than with quinin. Levy, I9I 2, also claims a curative effect against streptococcus mucosus.

Use in Eye.-Locally, I per cent. solution or salve of Ethyl-hydroxycuprein, applied every hour, has been employed in Ulcus corneal serpens. The first instillation is painful. Above I per cent., it injures the cornea; lower concentrations are ineffective (Seifert).

Pharmacologic Actions.-These correspond qualitatively with quinin, but are more toxic. Intravenously, optochin is more depressant to the heart, the blood pressure, however. falls less than with quinin, since there is also vasoconstriction. The antipyretic efficiency of optochin is smaller (Smith and Fantus, I916). It has but little inhibitory action on the emigration of leucocytes (Ikeda, I916).

\section{CUPREIN}

This is an alkaloid from Cuprea bark, closely related to quinin in action and composition (quinin is regarded as methylcuprein). It is less active and less toxic than cinchonin, but acquires activity by the introduction of alkyl-groups.

\section{ACETANILID, ACETPHENETIDIN AND ANTIPYRIN}

General Statement.-These drugs and their derivatives, the "coal tar antipyretics," lower febrile temperature by dilating the cutaneous vessels through a central action. They are highly effective; but the antipyretic doses are apt to produce undesirable side actions, and are clinically supposed to be depressant to the circulation. Smaller doses are efficient analgesics in headache, neuralgic conditions, etc. Their use is not without danger from acute collapse. Chronic poisoning, with methemoglobin formation, is also not infrequent. Acetphenetidin (Phenacetin) is somewhat less dangerous, but the difference is merely one of degree. Antipyrin is freely soluble, the others are not.

The former high price of quinin caused chemists and pharmacologists to look about for cheaper efficient substitutes. It was attempted to make quinin or similar substances synthetically, departing from quinolin, one of the decomposition products of quinin. Whilst this search did not result in an artificial quinin, nor even of any substance analogous to it in action, it brought to light a very large number of substances, in some respects even more valuable than the alkaloid itself, and served to direct attention to the pharmacologic significance of the derivatives of the aromatic series, or, as they are more commonly called, of coal-tar.

It was found that all the simpler compounds built up from the benzol nucleus (with a very few exceptions) possess certain physiologic actions in common, as follows:

Common Actions of Benzol Derivatives.-Central Actions: Stimulation, followed by more pronounced depression of the entire central nervous system, but particularly: An antipyretic action on the heat-centers, and an analgesic action in neuralgias.

Local Actions: A coagulant, irritant, toxic and antiseptic effect on all forms of protein and protoplasm-on tissue cells, bacteria, and ferments. A quinin action on all forms of muscle. Local anesthesia. The formation of methemoglobin.

The actions are exerted in a different manner and to a different degree by the several members of the series, mainly in two directions, so that these may be classified somewhat arbitrarily as "Antipyretics" and "Antiseptics." 
The antipyretic group is distinguished by the predominance of the action on the heatregulating center.

The antiseptic group is characterized by a much more marked local action on protoplasm, which determines the usefulness of the members as antiseptics. The effect upon the thermic center passes much more readily into general collapse than with the former group.

The distinction is by no means sharp, and many of the substances- $-i . i$, the salicylates - could be classed under either group.

Structure and Antipyretic Action.-The large number of antipyretic benzol derivatives differ only quantitatively in their actions, these differences having definite relations to their chemic structure. Practical experience has shown that acetphenetidin, acetanilid and antipyrin are the most useful representatives of the group, and that all the others may well be spared.

The enterprise of manufacturers of synthetic products has overwhelmed the profession with almost numberless antipyretics; but it must be confessed at once that the number of really new and valuable products is comparatively small. The great mass of this material consists simply of insignificant variations in the established products, introduced for the purpose of evading patents; and amounts to little more than do the various fla vorings in the elixirs of the older materia medica. This multiplication can only do harm by creating confusion, and should be abolished as speedily as possible. The selection of the worthy products from this collection would be an almost hopeless task, were it not that certain definite laws have been deduced from the experimental evidence, according to which we may foretell the action of a coal-tar derivative from its composition, with almost absolute certainty. In this way many new compounds can be adjudged as unworthy of extended trial. A presentation of some of these laws will therefore be of practical value, aside from their scientific interest.

The antipyretic action really resides in the benzol ring. Yet benzol $\left(\mathrm{C}_{6} \mathrm{H}_{6}\right)$ itself is not antipyretic, because it can not readily enter into reaction with the body cells; this capacity of reacting may be given to it by substituting for one of its $[\mathrm{H}]$ atoms an $[\mathrm{OH}]$ group, as in phenol, $\mathrm{C}_{6} \mathrm{H}_{5} \mathrm{OH}$; or still more strongly by an $\mathrm{NH}_{2}$ group, as in anilin, $\mathrm{C}_{6} \mathrm{H}_{5} \cdot \mathrm{NH}_{2}$ (Phenylhydrazin, $\mathrm{C}_{6} \mathrm{H}_{5} \mathrm{NH} \cdot \mathrm{NH}_{2}$ being yet stronger); or by both as in paramidophenol, $\mathrm{C}_{6} \mathrm{H}_{4} \mathrm{OH} \cdot \mathrm{NH}_{2}$.
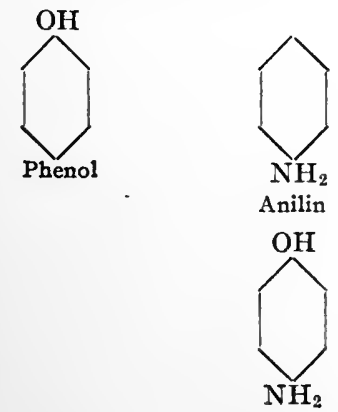

Paramido-phenol
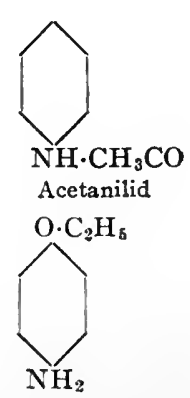

Phenetidin

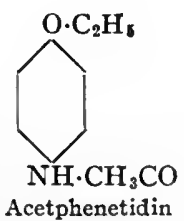

Acetphenetidin

In a few modifications of the benzol ring, the action is quite wanting; thus in Pyridin, $\mathrm{C}_{6} \mathrm{H}_{6} \mathrm{~N}$; in Naphthalin, $\mathrm{C}_{5} \mathrm{H}_{4}$; in Phenanthren, $\mathrm{C}_{6} \mathrm{H}_{4} \cdot \mathrm{CH}$. But it reappears in Quinolin,

$$
\stackrel{\mathrm{C}}{6}_{6} \mathrm{H}_{4}
$$

$$
\mathrm{C}_{6} \mathrm{H}_{4} \cdot \mathrm{CH}
$$

$\mathrm{C}_{8} \mathrm{H}_{4}$, and is also present in the pyrazol ring,

$$
\mathrm{C}_{4} \mathrm{H}_{2} \mathrm{~N}
$$
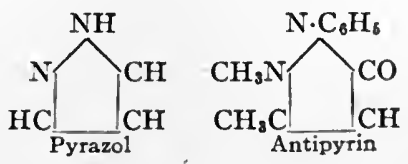
We repeat that the power of acting is given to the benzol or other chain, by replacing $\mathrm{H}$ through $\mathrm{OH}$, or $\mathrm{NH}_{2}$, or both. The substances so produced are the so-called "mother-substances." They are strongly antipyretic, but they also produce collapse. Before they can be used in practice, this collapse action must be diminished. This may be done by replacing the $[\mathrm{H}]$, either of the $[\mathrm{OH}]$ or of the $\left[\mathrm{NH}_{2}\right]$, by other radicals; the reduction of toxicity being greatest if the substitution is made in the $\mathrm{NH}_{2}$.

These substituted compounds act only after the mother-substance is liberated from them in the body. Their action is therefore more gradual, less violent, and more easy of control.

The influence of the substituted radical is as follows:

The reduction of toxicity is greatest if the substituted radical is an alkyl $\left(\mathrm{CnH}_{2} \mathrm{n}\right.$ $+\mathrm{I}$ or $\left.\mathrm{CO}_{2} \cdot \mathrm{CnH}_{\mathrm{i}} \mathrm{n}+\mathrm{I}\right)$; less if it is an acidyl $\left(\mathrm{CO} \cdot \mathrm{CnH}_{2} \mathrm{n}+\mathrm{I}\right)$. If several $\mathrm{H}$ atoms are substituted, the toxicity will be less if both an acidyl and an alkyl radical are introduced, than if both radicals be of the same kind.

The toxicity and the therapeutic qualities both diminish with the size of the introduced radical. The most useful is the ethyl $\left(\mathrm{C}_{2} \mathrm{H}_{5}\right)$ or acetyl $\left(\mathrm{C}_{2} \mathrm{H}_{3} \mathrm{O}\right)$ radical; with the methyl or formyl group, the toxic action is too great; with larger groups, and particularly with aromatic groups, the compound becomes so resistant that the therapeutic action is reduced almost to nothing. This is true of the citrate and salicylate radicals. Such compounds are of course correspondingly harmless.

The above laws must be modified if the compound is decomposed in the stomach with liberation of the mother-substance. It must also be remembered that the radicals must be introduced into the ring by substitution; the mere addition of acid radical, forming salts, does not modify the properties of the original substance. Therefore Anilin Acetate $\left(\mathrm{C}_{6} \mathrm{H}_{5} \cdot \mathrm{NH}_{2} \cdot \mathrm{HC}_{2} \mathrm{H}_{3} \mathrm{O}_{2}\right)$ preserves the original toxic action of anilin; whereas this is greatly weakened in Acetanilid $\left(\mathrm{C}_{6} \mathrm{H}_{5} \cdot \mathrm{NH} \cdot \mathrm{C}_{2} \mathrm{H}_{3} \mathrm{O}\right)$.

The substitution of an $\mathrm{H}$ by $\mathrm{I}, \mathrm{Br}$, or $\mathrm{Cl}$, does not modify the antipyretic or toxic action of the original substance

Absorption, Fate and Excretion.-The antipyretics are rapidly absorbed and excreted. Acetanilid, acetphenetidin and their derivatives are mainly oxidized to paramidophenol, which is probably the form in which they produce their actions. This is excreted in the urine, chiefly as paired compounds with sulphuric and glycuronic acids. This gives the "indophenol reaction" in the urine. Antipyrin is eliminated largely unchanged by the urine (Jonescu, I906), traces also by the sweat (Tachau, I9II), and sputum (Hofbauer, IOI5). The urine quantity is generally decreased. Occasionally the urine is blood red, and green in reflected light (Penzoldt).

The antiseptic action of the antipyretics is not very strong. They are, however, sometimes used as antiseptic, astringent, and hemostatic dusting powders (acetanilid; or antipyrin in 5 per cent. sterilized solution). They are less poisonous and less irritating than iodoform; although toxic effects have been reported from the application of acetanilid to wounds.

Leucocytosis. - It has been believed that this is produced by antipyretics; but Gehrig, 19r5, found no effect. The migration of leucocytes from the inflamed mesentery of frogs is inhibited by antipyrin (Ikeda, IgI6).

Antipyretic Action and Effects on Metabolism.-These were described on page 456 .

Antipyretic Dosage.-On account of the possibility of a direct collapse action if the dose is relatively large, the antipyretics must be administered with care. 
In general, it may be said that, from the smallest effective close $(0.2 \mathrm{Gm}$. for acetanilid, 0.5 to $0.7 \mathrm{Gm}$. for antipyrin or phenacetin), the extent of the antipyretic action increases with the dose of the drug, until the normal temperature has been reached. Up to this point there is practically no danger of a direct collapse action. But if the dose necessary to secure this result be exceeded, the toxic effects will set in.

The average antipyretic dose may be stated as $0.5 \mathrm{Gm}$. (7 gr.) of acetanilid; $0.8 \mathrm{Gm}$. (Io gr.) of phenacetin; I.2 Gm. (20 gr.) of antipyrin. In beginning the treatment, this dose has to be repeated once or twice, with an hour's interval, until the desired fall is secured. After this, the single dose is repeated whenever the temperature begins to rise. Four or five doses are required per day to keep the patient practically fever-free The action does not persist after the drug is excreted. With single doses the antipyretic effect reaches its maximum in about two hours, and then declines gradually, so that the febrile level is practically restored in eight hours (Gottlieb, I89o). Consequently the administration must be continuous, in the manner indicated. Those antipyretics which are rapidly excreted (as is phenocoll) are therefore less useful.

In regard to details, and in determining the choice of the particular substance to be used, experience is the best guide. The same holds here as in other cases: Very much more can be accomplished by any one drug that is thoroughly understood by the user, than can be done with a number of drugs with which he has had only limited experience.

-Features Desired in an Antipyretic.-The ideal antipyretic must be reliable in securing a fall of febrile temperature. This fall must be prompt, but not too abrupt; it must be lasting; and when the effect passes off, the temperature must not rise too suddenly. The analgesic effect should be strong. The drug should not be decomposed in the stomach (or by 0.2 per cent. $\mathrm{HCl}$ ). The toxic dose should be considerably higher than the therapeutic dose. In the latter, the drug must not of itself cause collapse conditions, nor methemoglobinemia, nor profuse sweating, nor skin eruptions. It should not have a bad taste, nor should it irritate the stomach. If it is to be used hypodermically, it must be soluble in water; otherwise great solubility is of no advantage.

Of the compounds which have been proposed so far, Para-acetphenetidin (Phenacetin) seems to fulfil these demands most satisfactorily.

Analgesic Action.-Like quinin and the salicylates, all the antipyretics have an analgesic effect against neuralgic and muscular pains, headaches, migraine, colds, rheumatic conditions, etc.; but are ineffective against other forms of pain, such as trauma. The mechanism of the analgesic action is obscure. It has been suggested that it consists in changes of the meningeal vessels. There is little if any evidence of true narcosis; nor do the antipyretics reinforce the action of the ordinary narcotics to a marked degree (Lomonosoff, I9I I).

The analgesic action is extensively used. Small doses (acetanilid, $0.2 \mathrm{Gm}$., 3 gr.; acetphenetidin, $0.3 \mathrm{Gm}$., 5 gr.) may be repeated a few times with three-hour intervals, if necessary. If these small doses fail to give relief, larger doses are usually also ineffective.

The analgesia has been investigated in man by Martin, Grace and McGuire, 1915. They found that 0.3 to $\mathrm{I} \mathrm{Gm}$. of acetphenetidin distinctly raises the threshold of cutaneous sensibility.

Proprietary Headache Powders.-These usually contain from 50 to 70 per cent. of acetanilid or acetphenetidin (Report C.P.C., J.A.M.A., I905, 44 : I79I), generally mixed with caffein, salicylates, bromids, ammonium carbonate or sodium bicarbonate. The caffein, salicylate and bromid would reinforce the analgesic action. The alkalies were supposed to increase the solubility, but do not do so (Puckner, 1906). It was also claimed that all these drugs diminish the toxicity, but this is contradicted by the available evidence (Hale). The indiscriminate use of the powders by the laity has repeatedly produced acute and chronic poisoning. Authoritative statistics of this are presented in the report of Kebler, Morgan and Rupp, rgog. 
Chronic Poisoning.--The continued use of acetanilid ("headache-powders") has occasionally caused the development of a drug habit, with craving and withdrawal symptoms. An individual predisposition appears to be necessary, for the condition is relatively rare; but it should be guarded against in the administration of the drug. The symptoms of chronic acetanilid poisoning consist in methemoglobinemia (chocolatecolored blood) with the histologic blood changes of pernicious anemia; marked leucocytosis; cyanosis; cardiac weakness; progressive mental and physical debility. Methemoglohin may also appear in the urine. (D. D. Stewart, I905.)

Diabetes Insipidus. - Beneficial results have been claimd for the antipyretics, $f . i$, by Hirschfeld, I904. They require confirmation.

Side Actions.-Undesirable effects are rather common, especially when antipyretic doses are used. Some of these are the indirect results of the heat regulation-sweating and chills, indirect collapse; some are due to irritation-gastric disturbances, skin eruptions, renal irritation; others to the direct systemic actions of the drugs-cinchonism, cyanosis, true collapse. The susceptibility to them varies greatly in different individuals.

The literature of the side actions is given by Seifert, Nebenwirk., I9I5: Acetanilid,' page 2; Antipyrin, page 4; Acetphenetidin, page 26 .

The sweating is due to the increased circulation through the skin, and is produced in the same manner, and has the same significance, as the critical sweat of fever. It must be looked upon as beneficial rather than otherwise, since it aids the reduction of temperature. But should it become too troublesome, it can be suppressed by small doses of atropin.

The cutaneous hyperemia is perhaps also responsible for the skin eruptions. They are particularly frequent after antipyrin.

The tendency to this eruption, as also to a stomatitis which is often seen, increases with the repeated use of the drug.

Phenylhydrazin produces very similar local actions on the skin (eczema erythematosum and papulosum) and on the alimentary canal (gastroenteritis).

The chills occur when the temperature begins to rise again, and are due to a diminished circulation through the skin, just as the chills of malaria. They are not therefore to be attributed to the drugs, but are rather a sign that the action of the antipyretic has worn off.

Gastric symptoms are due to local irritation, but are not frequent. Most of these drugs have a sharp, warm or bitter taste.

Renal irritation, varying from slight diuresis without histologic change, to violent, desquamative, hemorrhagic, parenchymatous nephritis, may be produced by all coaltar derivatives. There is not, however, any danger of nephritic lesions from ordinary doses of the antipyretics. Thallin produces a peculiar papillary nephritis.

Cinchonism symptoms are rare, but have been reported. The cyanosis is due to the methemoglobinemia.

Lactophenin produces icterus in over half of the patients, usually on the ninth to twelfth day of its use. There is no relation to dose. It appears to be harmless, and is probably of the retention type rather than hemolytic (Hanssen, rgi6).

Methemoglobin Cyanosis.-All drugs of the coal-tar series act more or less upon the blood, inside of the body, producing methemoglobin, and in larger doses causing a disintegration of the corpuscles. This action is much more pronounced in the mother-substances-anilin, phenylhydrazin, etc.-and in the antiseptic group it is stronger with acetanilid than with the other antipyretics. It leads to a peculiar cyanosis, and contributes to the collapse.

The methemoglobin formation proceeds much more weakly in shed blood than it does in the body. The relation of the methemoglobin formation to the chemic constitution of benzol derivatives has been investigated by W. Heubner, I913.

Collapse Action.-Excessive, and sometimes therapeutic doses, produce serious collapse, with weak and irregular pulse, dyspneic or shallow respiration, pale or cyanotic skin, cold sweat, disturbed consciousness, 
subnormal temperature, and death by circulatory failure. This collapse is sometimes a direct effect of the drugs (cardiac and vasomotor depression); in other cases it is due to the reduction of the febrile temperature, "unmasking" a preëxisting febrile collapse. Circulatory failure is a frequent feature of fevers. It may be partly masked by the stimulant effects of high temperature, and will then reveal itself when the normal temperature is restored, whether by antipyretic drugs or by baths. Caution is therefore necessary in the use of all antipyretic measures in asthenic fevers. Menstruation also favors the development of collapse.

Of the more usual antipyretics, acetanilid produces probably the strongest direct collapse effects; then come antipyrin and then phenacetin.

The collapse action is strongest in the mother substances-anilin, quinolin, phenetidin, phenylhydrazin, and carbolic acid, and generally in the members of the antiseptic group; so strong, indeed, that the action of these can not be controlled, and they are hence unfit for internal administration.

The treatment of poisoning by overdoses of the antipyretics is the same as for collapse from other causes-stimulants, heat, etc. (see Aconite).

Circulation.- The general circulation is but little affected by ordinary doses. They will slow the heart indirectly by reducing the temperature; this has probably. sometimes been misinterpreted as cardiac depression.

The cutaneous vessels are dilated; the intracranial vessels, after a short dilation, show a more persistent constriction, similar to caffein or alcohol (E. Weber, rgog). Berezin, r9r6, reports dilation in rabbits and fish. Hirschfelder, r9r 5, describes dilation of the pia and retinal vessels, from acetanilid, antipyrin, amyl nitrite, caffein and alcohol. This tends to raise intraocular pressure, en joining caution in glaucoma (Wessely, r9 I 5 ).

With toxic doses, the heart is first accelerated, and later slowed. This is due to direct action upon the heart muscle. Strips of terrapin's ventricle are slowed and arrested by acetanilid (Carr, rgro).

The vasomotor center is not affected by moderate doses (with the exception of the part controlling the cutaneous vessels. In consequence, the blood pressure depends solely upon the cardiac action, being at first increased and later diminished. In doses producing collapse there is paralysis of the vasomotor system, and consequent fall of blood pressure.

Striped muscle shows a somewhat increased efficiency on direct stimulation, and a weak curare action.

\section{PREPARATIONS-ANTIPYRETIC GROUP}

Only the most important preparations are given: others may be found in N.N.R.

${ }^{*}$ Acetanilidum (Acetanil.), U.S.P., B.P. (Antifebrin, Phenylacetamid, etc.), $\mathrm{C}_{5} \mathrm{H}_{5} \mathrm{NH}$. $\mathrm{CH}_{3} \mathrm{CO}$. - White crystalline powder; odorless; warm taste. Slightly sol. in water ( $\mathrm{r}: \mathrm{r} 80$ ), freely sol. in alc. $(\mathrm{r}: 3.4)$ or glycerin $(\mathrm{r}: 5)$. Liquefies with chloral or antipyrin. Dose, $0.2 \mathrm{Gm}$., 3 gr., U.S.P.; 0.12 to $0.3 \mathrm{Gm}$., 2 to $5 \mathrm{gr}$., B.P.; in powders or capsules. Maximum dose, $0.5 \mathrm{Gm}$., $8 \mathrm{gr}$.

*Acetphenetidinum (Acetphen.), U.S.P.; Phenacetinum (Phenacet.), B.P. (Paraacet-phenetidin), $\mathrm{C}_{6} \mathrm{H}_{4}\left(\mathrm{OC}_{2} \mathrm{H}_{5}\right) \cdot \mathrm{NH} \cdot \mathrm{CH}_{3} \mathrm{CO}$. - White crystalline powder or scales, odorless and nearly tasteless. Very slightly sol. in water $(\mathrm{r}: \mathrm{r} 3 \mathrm{ro})$; sol. in alc. $\left(\mathrm{r}: \mathrm{I}_{5}\right)$. Liquefies with chloral or antipyrin. Dose, $0.3 \mathrm{Gm}$., $5 \mathrm{gr}$., U.S.P.; 0.3 to I Gm., 5 to I5 gr., B.P.; in powders or capsules. Maximum dose, r Gm., I $5 \mathrm{gr}$.

*Antipyrina (Antipyr.), U.S.P.; Phenazonum, B.P. (Phenyldimethyl-pyrazolon), $\mathrm{C}_{3} \mathrm{HN}_{2} \mathrm{O}\left(\mathrm{CH}_{3}\right)_{2} \cdot \mathrm{C}_{6} \mathrm{H}_{5}$. - White crystalline powder; almost odorless; slightly bitter taste. Very sol. in water ( $\mathrm{r}: \mathrm{I})$, freely sol. in alc. ( $\mathrm{r}: \mathrm{r} .3$ ). Incompatible with nitrites (Sp. Aether. Nitros.), with tannin, and with mixtures of calomel and sodium bicarbonate (immediate blackening; J.A.M.A., 56:287). Liquefies with sodium salicylate, etc. Dose, 0.3 Gm., 5 gr., U.S.P.; 0.3 to I Gm., 5 to 15 gr., B.P.; in solution, powders or capsules. Maximum dose, I Gm., I5 gr.

Melubrin, N.N.R.-This is another pyrazolon derivative, introduced as analgesic. Dose, I to $2 \mathrm{Gm}$. In rheumatic fever, Hahn, I I I3, uses it intravenously, 5 c.c. of $5^{\circ}$ per cent. solution, three times daily. Riedel, I913, prefers intramuscular or hypodermic, $3 \mathrm{Gm}$. in $5 \circ$ per cent. solution, two or three times daily. Side actions, similar to Antipyrin (Seifert, Nebenw., I915, p. 22). 


\section{ANTISEPTIC BENZOL DERIVATIVES}

General Statement.-These differ from the antipyretic derivatives mainly by greater toxicity. On local contact they coagulate proteins, thus determining the death of cells, resulting in irritation and inflammatory changes, and in antiseptic effects. After absorption they produce some excitement and more pronounced depression of the central nervous system. Many alter the blood pigment to methemoglobin. Their effects are similar in principle, but differ in important details. The high toxicity is due to various side-chains, such as the hydroxyl and carboxyl groups, by which they can react with protoplasm.

Historical.-In the form of the natural mixtures, the antiseptic properties of this group have long been known empirically, as in the smoking of meat. One of the methods of embalming practised by the Egyptians utilized largely balsams and products rich in essential oils- the latter being very closely related to the aromatic series. The isolated substances, however, belong to the achievements of the nineteenth century. Creosote was first made in 1832 , phenol in I 834 ; but almost thirty years elapsed before they were used in surgery.

Influence of Structure on Action.-Benzol, $\mathrm{C}_{6} \mathrm{H}_{6}$, the mother substance of the coaltar series, has a low toxicity, because it can not react with protoplasm. This capacity is given to it by replacing part of the $\mathrm{H}$ atoms by other groups, especially by $\mathrm{OH}$ (forming phenols) or by $\mathrm{CO}_{2} \mathrm{H}$ (forming acids); or by both. The OH radical is the most active, the antiseptic and toxic actions increasing with the number of $\mathrm{OH}$ groups (i.e., progressively from phenol, $\mathrm{C}_{6} \mathrm{H}_{5} \mathrm{OH}$, to resorcin, $\mathrm{C}_{6} \mathrm{H}_{4}(\mathrm{OH})_{2}$, and pyrogallol, $\left.\mathrm{C}_{6} \mathrm{H}_{3}(\mathrm{OH})_{3}\right)$, but it is also influenced by the position; thus, phloroglucin, the isomer of pyrogallol, is not active. The introduction of the $\mathrm{CO}_{2} \mathrm{H}$ group alone (i.e., benzoic acid, $\mathrm{C}_{6} \mathrm{H}_{5} \cdot \mathrm{CO}_{2} \mathrm{H}$ ), does not render the substance very active. The introduction of both $\mathrm{OH}$ and $\mathrm{CO}_{2} \mathrm{H}$ in the ortho-position' (i.e., salicylic acid, $\mathrm{C}_{6} \mathrm{H}_{4}<\mathrm{CO}_{2} \mathrm{H}$ ) results in a compound which is<smiles>C1CCCCC1</smiles>

1 When the substitution occurs in the position 1.2, the ortho-compound results; I: 3 gives the meta-compound; and $I: 4$ the para-compound.

4

less toxic and less antiseptic than phenol, but which has peculiar antirheumatic properties. The antiseptic quality may also be brought out by other acidyl groups $\left(\mathrm{COCH}_{3}\right.$, etc.), the activity being proportional to the size of the introduced molecule. $\mathrm{NO}_{2}$ also develops the antiseptic action, but adds its own toxicity to that of the phenols. The substitution of an $\mathrm{H}$ of the $\mathrm{C}_{6} \mathrm{H}_{5}$ in phenol by alkyls (cresols, $\mathrm{C}_{6} \mathrm{H}_{4}<\mathrm{CH}_{3}$ ) leads to an increase of the antiseptic power, and diminishes at the same time the toxicity to tissues. Higher homologues introduce no important modifications. The union of two phenols directly or by $\mathrm{CH}_{2}$, etc., increases the antiseptic action, but if united by $\mathrm{CO}$ or $\mathrm{SO}_{2}$, it is diminished. The substitution of halogens increases the antiseptic action, according to the number of atoms. The toxicity is diminished by the lower, increased by the higher numbers (Bechhold and Ehrlich, I906).

The group which gives the valuable antiseptic, but also the undesirable toxic and caustic, qualities to these compounds, is the $\mathrm{OH}$.

The violence of its action may be moderated, as in the salicylic acid, or in the cresols; or it may be masked by replacing its $\mathrm{H}$ by other radicals (the "Nencki's salol principle"). These esters (e.g., salol, $\mathrm{C}_{6} \mathrm{H}_{4} \cdot \mathrm{OH} \cdot \mathrm{CO}_{2} \mathrm{C}_{6} \mathrm{H}_{5}$ ) will develop their action when decomposed into their phenol. Combinations which are so firm as to prevent this liberation (e.g., the sulpho-carbolates) are neither antiseptic nor toxic: in fact, it is by the formation of such compounds that the organism protects itself against these poisons.

The antiseptic and local actions of the salicylates or other salts are also much less than those of the corresponding acids.

Fate of the Benzol Derivatives in the Body.-The six-carbon ring is relatively resistant to decomposition, and for the most part passes through 
the body unbroken, although a little is burned. The hydrogen atoms and side-chains undergo more or less oxidation, resulting in new hydroxyl and carboxyl groups (phenols and acids) in considerable variety. These again enter combinations before being excreted by the urine; the phenols forming esters, chiefly with sulphuric acid (Baumann and Herter, I876; O. Neubauer, I901); and to a lesser extent with glycuronic acid. The aromatic acids combine with glycocoll to form hippuric acid, etc.

The formation of the esters is generally proportional to the toxicity of the phenols (S. Fraenkel). Since the esters are much less toxic than the phenols, it constitutes an important protection of the organism against poisoning, especially against the phenols arising in intestinal putrefaction. The normal phenol excretion (largely paracresol) tends to vary directly with the protein intake (Folin and Denis, r915). In phenol poisoning, the urine becomes poor in free and rich in ethereal sulphates, and has a smoky color, especially on standing, from the oxidation of the hydroquinon, pyrocatechin, etc. The glycuronic compounds may be mistaken for sugar, since they reduce copper, although more slowly.

Glycuronic Acid.-This corresponds to dextrose, with the end-chain $\left(\mathrm{CH}_{2} \mathrm{OH}\right)$ oxidized to $(\mathrm{COOH})$. It is probably an intermediate stage in the oxidation of glucose, for very little can be formed in glycogen-poor animals (Dakin). Glycuronic acid is probably not a normal stage of carbohydrate metabolism; for if it is administered parenterally, it appears almost quantitatively unchanged in the urine (Biberfeld, I9I4): It is never excreted free, but always in combination with aromatic alcohols, phenols, ketones and acids. Haemaelaeinen, 1913, supposes that the alcohols first combine with glucose, and that this glucosid is then oxidized to the glycuronate.

The principal drugs which cause the appearance of Glycuronic acid in the urine are: Turpentine, chloroform, chloral, camphor, phenacetin, saccharin, salicylic acid, balsams, morphin; and others are enumerated by S. Fraenkel, 3rd. ed., p. I94.

The Hippuric Acid Formation.-This occurs in some animals exclusively in the kidneys; in some it may also be found in other organs. Dogs, for instance, synthetize hippuric acid even after excision of their kidneys (Kingsbury, I9I 5).

The place of formation of the ethereal sulphates is not known, but it has been shown to be independent of the liver (Lade, I9I2).

\section{BENZOL}

Benzol, $\mathrm{C}_{6} \mathrm{H}_{6}$, has come into some prominence for the treatment of leukemia. It of ten causes temporary improvement, but is rather unreliable and sometimes dangerous. Industrial acute and chronic poisoning is also common, from its use in the arts (as solvent for rubber). It is somewhat antiseptic.

Acute Benzol Poisoning.-Fairly large doses of benzol are tolerated by mouth; 9 to I $2 \mathrm{Gm}$. have caused serious collapse (Perrin, I86I; Averill, I889). The acute symptoms are restlessness, excitement, high rise of temperature, narcosis, and death by paralysis of respiration. Sublethal doses are followed by good recovery. The technical article is somewhat more toxic than the pure (Lehmann, IgII).

Distribution of Benzol after Poisoning.-The brain and spinal cord contain more than other organs (Joachimoglu, I 915 ).

Benzol Vapor.-This causes intoxication by absorption through the lungs. Dilute vapors produce headache, vertigo, ataxia, twitchings, convulsions and coma. Fuehner, I913, finds benzol about as narcotic as chloroform. Concentrated vapor is markedly toxic, sometimes producing acute death. There are no characteristic anatomic lesions, except that the blood remains fluid for a long time; there are often small hemorrhages in various organs. Patients who recover sometimes develop skin changes (Lewin, 1907; Wolf, I9Ir; Heffter, 1915). The entire symptoms are produced, also on animals, by pure as well as impure benzol.

Chronic Poisoning by Vapor.-This causes hemorrhages (purpura), leucopenia, anemia, and finally death under high fever (Santesson, 1897; Selling, I9I2). These effects are attributed to destruction of leucocytes in the circulating blood, and to damage of the bone-marrow, lymph glands, spleen, and the whole hematopœtic system. The formation of blood platelets is first stimulated, then depressed (Duke, 1913). 
Blood Changes in Animals.- Subcutaneous injections of benzol (2 to 40 c.c.) into rabbits or dogs produce death in three to twenty days, with marked destructive changes in the hematopetic organs, especially the myeloid tissue. There is a corresponding diminution of the leucocytes and blood platelets. With large doses, the platelets show a preliminary increase in rabbits, not in dogs (Duke, 1915). There is little change in the red cells (Neumann, I915). The prothrombin is also decreased (Hurwitz and Drinker, I015). The production of antibodies is diminished (Hektoen, rgI6). The changes vary quantitatively in different individuals ( $v$. Neumann, I915).

Use in Leukemia.- The destructive action of benzol on leucocytes led to its introduction by v. Koranyi, IgI 2. Numerous reporters (Kiralyfi, I9 12; Stein, I9I 2; Stern, 1913; Billings, 1913; Morawitz, I9I4; Hahn, I9I4, etc.) have described more or less favorable, but rather inconstant results. The changes are noticed only after continued administration, often requiring some weeks. The leucocyte count falls, more profoundly and persistently than with arsenic, but never to normal, as in X-ray treatment. The general condition is also improved. The best results are obtained when benzol is preceded by or continued with ray-treatment. In a considerable number of cases, especially the advanced, the benzol is unsuccessful. In others, toxic effects supervene.

Mechanism of Action.-Urinary changes indicate metabolic disturbances: increase of neutral sulphur; decrease of Urea $\div \mathrm{N}$, slight increase of ammonia, in most cases albuminuria (Sohn, 1913). The uric acid is not increased, and this speaks against destruction of leucocytes. Pappenheim, I9 I3, believes the decrease to be only apparent, asserting that the leucocytes accumulate in the dilated capillaries of the liver and other internal organs. He found marked hepatic and renal lesions in rabbits after repeated large doses. Duke, I9I5, attributes the platelet changes to irritant, then toxic action on the bone-marrow.

Toxic Effects.--Ordinary doses cause eructations, but the appetite is not disturbed. There may be some dizziness, sometimes bronchitis. Occasionally, late symptoms of chronic benzol poisoning develop, which may be fatal (Neumann, I913; Klemperer and Hirschfeld). It is therefore advised to discontinue the benzol as soon as the leucocytes start to decline (Kiralyfi, I913); or at least when they have fallen to 20,000 . Liver, kidney and intestinal disturbances contraindicate benzol (Hahn, 1914).

Administration.-Billings uses the benzol in extemporaneous capsules, 5 minims, increasing to 15 minims, after meals and at bedtime.

Fate.-Benzol is partly oxidized in the body to phenol ( 15 to 30 per cent.), catechol and quinol and excreted as phenol-sulphates (Baumann and Herter, 1877); a part is completely destroyed to muconic acid (Jaffe, 1909), and a considerable portion is excreted unchanged by the lungs.

\section{PREPARATIONS-BENZOL}

Benzenum, B.P.; Benzene (Benzol); $\mathrm{C}_{6} \mathrm{H}_{6} .-$ Not to be confused with Benzinum Purificatum, U.S.P., Benzin. The latter is Petroleum Ether, a gasoline fraction.

\section{TOLUOL}

Toluol, $\mathrm{C}_{6} \mathrm{H}_{5} \cdot \mathrm{CH}_{3}$, is used as a laboratory antiseptic, especially to preserve ferments. Its toxicity is low (Baumann and Herter, x877; Schultzen and Naunyn, I867). Its effects and those of xylol are similar to benzol (Lehmann, rgrI).

\section{PHENOL}

Phenol (carbolic acid, $\mathrm{C}_{6} \mathrm{H}_{5} \cdot \mathrm{OH}$ ) illustrates the characteristic properties of the series, and is the most widely known and used of these antiseptics. It owes this popularity to its being the pioneer of the group, through its introduction by Lister, 1867. Some of its derivatives are superior, at least for special purposes.

Antiseptic Action.- Phenol is a general protoplasmic poison and a fairly active disinfectant, although not nearly as powerful as mercuric chlorid, or even as the cresols. It precipitates proteins, but the combination is so loose that it readily diffuses further, and therefore penetrates more deeply into the tissues than the metallic antiseptics; and its action is not so much hindered by the presence of organic matter. "The disin- 
fectant power varies greatly for different microörganisms; spores are especially resistant. Tubercle bacilli are killed by exposure to 5 per cent. for twenty-four hours. It is much more effective as an antiseptic, i.e., for checking bacterial growth; $1 / 2$ to I per cent. suffices for this purpose. These are the concentrations used on the body, for here it is not necessary to kill the bacteria if only their development is effectively prevented. These solutions do not ordinarily injure the tissues.

Antiseptic Uses.-Five per cent. solutions are used for the disinfection of the utensils, instruments, the hands, etc. The penetration and therefore the antiseptic efficiency are increased by the addition of salt. "Crude Carbolic Acid," which contains the more active cresols and is cheaper, is preferred for the disinfection of excreta. As antiseptic for wounds and mucous membranes, gargles, lotions or injections $1 / 2$ to I per cent. of phenol are used. A drop of undiluted phenol is useful for disinfecting and cauterizing small wounds, bites, etc. Injections of phenol have been tried against local inflammations, tubercular bones and lymph glands, boils, erysipelas ( 5 per cent. to scarified surface), etc. Lotions are used as parasiticide in scabies, sycosis, pityriasis, and against pediculosis capitis. Inhalations have been advocated in fetid bronchitis and pulmonary gangrene.

Solutions in Fatty Oils.--In these phenol is not antiseptic (Koch, I886), because its solubility in fats is so much greater than its solubility in protoplasm that it prevents its transference to the bacteria or tissues. "Carbolated Oil" is merely a mild local anesthetic and dermal irritant, useful for promoting the healing of small wounds. Alcohol and glycerin have a similar restraining action. The solubility in petrolatum is very small, so that the U.S.P. ointment made with this base should be very actively antiseptic and irritant.

As intestinal antiseptic, phenol has little value because of its prompt absorption and marked toxicity.

Solubility of Phenol in Mineral Oils.-Pilcher, I9r4, finds that I part of crystallized phenol dissolves in 8 to 9 parts of petroleum; 20 to 2 I of gasoline; 23 to 24 of solid petrolatum; 45 to $5 \circ$ of liquid petrolatum.

Phenol Injections in Tetanus.-These have been introduced by Baccelli. Rather large doses are used, a 2 to 3 per cent. solution being injected intravenously or hypodermically along the spine every two or three hours, the daily dose starting with 0.2 to $0.5 \mathrm{Gm}$., and increasing rapidly to 0.8 to $1.5 \mathrm{Gm}$. of absolute phenol in divided doses, according to severity (J.A.M.A., 53:2140, I909). No bad effects have been reported, and it is said to cause prompt improvement and to lessen the mortality, even in severe cases (Baccelli, I9Ir; Ascoli, I9I3), perhaps by its analgesic and antipyretic actions. The treatment has not been tried adequately by independent observers, and final judgment can not be passed on it (Dreyfus, I9r4). McClintock and Hutchings, I9I3, state that it has only a limited value, as a simple sedative.

Fielder, I9r4, obtained negative results in rabies.

Pediculosis Capitis.-Whitfield, I9I2, advises soaking the scalp in a 2.5 per cent. phenol solution for ten minutes and leaving the solution on the hair for an hour.

Actions on Ferments.-Phenol, salicylic acid, and other coal-tar antiseptics have a retarding effect upon ferment action-especially carbolic and salicylic acid. A 5 per cent. solution of phenol suffices to weaken materially the action of most ferments.

Local Actions.-Concentrated phenol applied to the skin or mucous membranes produces prickling, burning and pain, then numbness and anesthesia, wrinkling and softening of the epidermis, the color of the skin becoming first white, then red, and finally brown. There is no direct corrosion, but a local necrosis. A dry scab forms, which separates without pus. Short applications of weaker solutions determine merely some 
wrinkling and blanching of the epidermis and the sensory phenomena, but even a 5 per cent. solution of the acid may cause necrosis, especially when applied continuously to the extremities. This enjoins caution in the use of carbolic dressings.

'The analgesic effect of dilute phenol solution and ointments is used against itching.

Absorption.--Phenol is absorbed from all mucous membranes and wounds, and even from the intact skin. In the intestinal canal its absorption is at first very rapid, but soon becomes almost arrested, apparently by interference with the local circulation (Sollmann, Hanzlik and Pilcher, I910). It is distributed through all the tissues.

Fate and Excretion.-A part of the phenol $(1 / 4$ to $2 / 3)$ is completely burned in the body (Tauber, I878; Schaffer, I878; Jonge, I897). The remainder is excreted in the urine, largely as ethereal sulphates and glycuronates of phenol, hydroquinon and pyrocatechin. However, Folin and Denis, I9I5, find that 30 to 90 per cent. of the urinary phenol is not conjugated. The oxidation products impart the smoky color to the urine. A trace of phenols is found in the sweat (Tachau).

Central Actions.-Very small doses of phenol, as of all the coal-tar antiseptics, produce at first an analgesic and antipyretic action. This is so fleeting, and passes so readily into the graver effects, that it can not be used in therapeutics. This preliminary stage is followed by more pronounced symptoms resembling the "toxic" effects of salicylates. Dangerous doses produce prompt collapse and coma. Frogs and mammals exhibit characteristic muscular tremors, twitchings and convulsions, persisting until the blood pressure falls very low. These are absent, or at most very faint in man (Husemann, I87 I).

Phenol Convulsions.- Salkowski, I872, showed that these are located in the spinal cord (division of sciatic; decapitation). Morita, I9I 5 , also found that the convulsions occur in rabbits after ablation of the hemispheres. Baglioni, rgoo and rgog, by the methods explained under strychnin, concludes that the convulsions depend on increased excitability of the spinal motor cells. Direct application of dilute phenol solutions to the exposed spinal cord produces anesthesia. The excitability of the motor cortex is lowered by its local application (Baglioni and Magnini, rgro), without convulsions (Amantea, I9I3). Muscle and nerve fibers are also paralyzed by local contact with strong solutions, but do not seem to suffer if the drug acts systemically.

Phenol does not awaken animals from chloral sleep (Airila, I9I3).

Symptoms in Frogs.-Phenol produces successively local reflex irritation, short stupor, a medullary cry, then gradually the twitchings and irregular and incoordinated contractions. The reflexes are preserved, but naturally ineffective because of the interference and interruptions of the convulsions (Baglioni).

Circulation.- Intravenous injections of phenol, even with small doses, cause marked fall of blood pressure, usually with rapid recovery until a certain dosage is exceeded. The fall appears to be both cardiac and vasomotor. When phenol is given by stomach, the blood pressure remains low because of the continuous absorption (Sollmann and Brown, I906).

Vasomotor Center.-This is usually depressed, probably directly. This may, however, be modified by indirect stimulation through the convulsions or ischemia. The center may therefore be stimulated; or a period of stimulation may precede or follow the depression period (Pilcher and Sollmann, I9r5).

Respiration, Temperature and Collapse.-The respiration becomes slow and shallow, and finally stops. The temperature falls. Since the collapse affects all the medullary centers, and the cardiac muscle as well, it can not, of course, be removed by artificial respiration. 
This constitutes an important difference from the collapse produced by the drugs of the alcohol series. Another difference consists in the fact that with the antiseptic group the sensibility to pain is often preserved far into the collapse. The local effects of the phenol play no important part in the production of the collapse (Sollmann and Brown, I 906).

Phenol causes an increase of secretions, especially of saliva, sweat, and tears, not yet accounted for.

\section{TOXICOLOGY}

Suicidal poisoning by carbolic acid is very common, particularly in the United States. Accidental poisoning is also common; it may result even when the phenol is applied to the intact skin; its liberal application to open surfaces has frequently led to toxic symptoms. The fatal dose, by mouth, varies between 8.5 and $60 \mathrm{Gm}$. (Lewin); $0.8 \mathrm{Gm}$. has been given therapeutically without bad effects. The toxicity is about the same by mouth and hypodermically (Hale, I9 13 ).

The course of carbolic acid poisoning is very rapid. In almost all fatal cases death ensues inside of twenty-four hours.

Symptoms.- The local symptoms consist in burning of the mouth and throat, nausea and vomiting, abdominal pain. The carbolic burns generally heal promptly, with small tendency to scar formation.

The systemic symptoms are those of a very speedy collapse, starting in a few minutes, even when the drug is taken by mouth. It is manifested by faintness and muscular weakness; rarely twitching and convulsions; pulse small, weak, and slow; face livid; cold sweat; respiration slow and shallow; unconsciousness; coma; death by stoppage of respiration.

The urine is scanty and often contains albumin and casts, sometimes hemoglobin or bile pigments. It has a dark smoky color, due to phenol compounds, and gives little or no sulphate-precipitate with barium chlorid. These points are of diagnostic importance. The urine rarely contains traces of uncombined phenol.

Chronic Phenol-poisoning. - In the days of the Lister spray, chronic phenol poisoning was not at all uncommon amongst surgeons. It presented the general symptoms of marasmus. The quantity of the acid entering the system under these circumstances is quite phenomenal: $2 \mathrm{Gm}$. of phenol were recovered from the urine of a surgeon who had assisted for two and a half hours at an operation under a 2 per cent. spray. Minor grades may occur from carbolic applications. They of ten show skin eruptions (Lewin) and renal irritation (kidney changes, Uyeno, (909).

Treatment of the Local Effects.-These are removed by promptly washing with alcohol (or whiskey) and applying an oil dressing. Alcohol, fats, glycerin, turpentine and ether act simply by their solvent-affinity for phenol, preventing its penetration into the tissues or dissolving it from them (Sollmann, I906).

Treatment of Internal Poisoning.-The best success is obtained by lavage of the stomach with warm water, or first with Io per cent. alcohol; taking care that the fluid does not enter the trachea. The washing must be continued till the phenol odor disappears. It should be attempted even if an hour or more has elapsed since the poison was swallowed (D'Hotel, I907), because a large part of the phenol remains for a long time unabsorbed in the stomach (Sollmann, Hanzlik, and Pilcher, I9I0). Chemic antidotes are of less service. Syrup of lime or potassium permanganate is the most promising. Alcohol and sulphates are practically useless. Intravenous saline infusions have some value against the circulatory depression, and heat against the collapse. 
Lime forms an insoluble compound and is fairly effective (Husemann and Ummethun, IS 70$)$. Syrup of lime may be given freely; lime-water is too weak. Permanganate destroys phenol, but it has not been ascertained whether the ordinary doses are efficient. Tinct ure of iodin was suggested by Maberly, I909. Scoville, I913, claims that charcoal is useful. Alcohol was formerly considered a chemic antidote, supposedly effective even when left in the stomach. Both the clinical and the experimental evidence disprove this view (Clarke and Brown, I906). Its solvent action is useless if it is left in the stomach to be absorbed. Macht, 1915, asserts that alcohol given after phenol increases its toxicity; but that it is protective when taken before the phenol.

Sulphates have been given with the object of forming the non-toxic phenolsulphonates. This combination does not occur in the alimentary canal, and the sulphates are very slowly absorbed, so that the oral administration is useless except in chronic poisoning. 'The combination is so slow, that it is practically not available in acute poisoning, even if the sulphate is injected intravenously (Brown and Sollmann, Ig06).

The antidote-fallacies are reviewed by Wilbert, I9r 6.

\section{PREPARATIONS-PHENOI}

${ }^{*}$ Phenol, U.S.P.; Acidum Carbolicum (Acid. Carbol.), B.P., $\mathrm{C}_{6} \mathrm{H}_{5} \mathrm{OH}$.-Should contain not less than 96 per cent. of hydroxybenzene, $\mathrm{C}_{6} \mathrm{H}_{5} \cdot \mathrm{OH}$, obtained either from coaltar by fractional distillation and subsequent purification, or prepared synthetically. Occurs as needle-shaped crystals, colorless but acquiring a reddish tinge by oxidation of traces of the phenol to quinon and other derivatives (Gibbs, 1909). Characteristic odor and sweetish caustic taste. Melts about $38^{\circ} \mathrm{C}$.; sol. in water (I:I5); miscible in all proportions with alcohol, glycerin and fats; dissolved by alkalies. The solubility in water diminishes with the temperature, and can be increased by the addition of alcohol or glycerin. Phenol is sometimes very faintly acid to litmus, but this is due to impurities (E. Schmidt, I9II). It is not a true acid, either chemically or physiologically. It is incompatible with iron and collodion. Dose, $0.06 \mathrm{Gm}$., I gr., U.S.P.; 0.06 to $0.2 \mathrm{Gm}$., I to $3 \mathrm{gr}$., B.P.; diluted. Maximum dose, $0.2 \mathrm{Gm} ., 3 \mathrm{gr}$.

*Phenol Liquefactum (Phenol.Liq.), U.S.P.; Acid. Carbol.Liq., B.P.-This is obtained by liquefying phenol with 10 per cent. U.S.P., 15 per cent. B.P., of water. It is more convenient for dispensing. When it is diluted with an equal volume of glycerin, the mixture is miscible with water, Dose, 0.05 c.c., I minim, U.S.P.; 0.06 to 0.18 c.c., I to 3 minims, B.P.; diluted.

Glycer. Phenol., U.S.P.; Glycer. Acid. Carbol., B.P.-2o per cent. Useful for making strong solutions. Dose, 0.3 c.c., 5 minims, U.S.P.

Supp. Acid. Carbol., B.P.-0.03 Gm., $1 / 2$ gr.

*Unguenium Phenolis (Ung. Phenol), U.S.P.-21/4 per cent., in wax and lard.

*Ung. Acid. Carbol., B.P.-3 per cent. in White Paraffin ointment.

Carbolized oil is usually made of 5 per cent. strength.

Troch. Acid Carbol., B.P. -0.03 Gm., $1 / 2$ gr.

Liq. Sod. Bor. Comp., N.F. (Dobell's Solution).-This is often used on mucous membranes (nose, etc.). "It contains 0.3 per cent. phenol, I.5 per cent. each of sodium bicarbonate and borate, with glycerin and water.

\section{PHENOL-CAMPHOR}

When camphor and phenol are rubbed together they liquefy. Mixtures of this kind (phenol 30 , camphor 60 , alcohol 10 ) have been employed on suppurating wounds and abscesses, with the claim that the caustic action is diminished and the antiseptic efficiency increased. The latter is doubtful; but the decrease of irritation agrees with the effects of all good phenol solvents. Chlumsky, 1905 and 1913, recommends phenolcamphor in superficial inflammatory conditions, especially erysipelas; painting it on and around the affected area. There is no chemic combination ( $Y$ von, I 876 ; Lemberger, 1906).

\section{PHENOLSULPHONATES OR SULPHOCARBOLATES}

These are less toxic and irritant, but also much less antiseptic than phenol. They have little value. 
Sodii Phenolsulphonas (Sod. Phenolsulph.), U.S.P.; Sodium Phenolsulphonate (Sulphocarbolate), $\mathrm{C}_{6} \mathrm{H}_{4}(\mathrm{OH}) \mathrm{SO}_{3} \mathrm{Na}+{ }_{2} \mathrm{H}_{2} \mathrm{O}$. Colorless, transparent prisms or crystalline granules, odorless, cooling, saline, bitter taste. Sol. in water (1: 4.2). Dose, 0.25 Gm., 4 gr., U.S.P.; as intestinal antiseptic, but inefficient.

(Zinc Phenolsulph.-See Index.)

\section{CRESOLS}

The introduction of alkyl groups into phenol greatly increases the germicidal efficiency. The most important of these derivatives are the cresols, $\mathrm{C}_{6} \mathrm{H}_{4} \mathrm{OH} \cdot \mathrm{CH}_{3}$, obtained from "crude carbolic acid." Attention was directed to their valuable properties by Fraenkel, r889. The three isomers, ortho-, para-, and metacresol, differ somewhat quantitatively, the meta being the most antiseptic and least toxic, and para being the most toxic; but they are all contained in variable proportions in the commercial products usually employed.

The germicidal power averages three or more times that of phenol; preparations containing higher homologues (N.N.R.) may be I 5 times as active as phenol.

The toxicity of the cresols averages somewhat less than phenol (Tollens, 1905; Hale, I9I3; toxicity and fate, Jonescu and Bukarest, I906), but they produce identical effects and lesions (Wandel, I906; Hale, I9r3). They are excreted for the most part "combined," but otherwise unchanged. The treatment would be similar. In practice, they are less toxic than phenol, because they are used more diluted; but they are far from being " nonpoisonous." Another advantage over phenol is their lower cost. Their disadvantages are the disagreeable odor, which depends mainly on impurities; their limited solubility in water; and their variable composition and activity. Organic matter slows their action rather more than with phenol. Acid and hard and salty water hinder them by precipitating the soap with which they are usually combined (Hamilton, r914).

Solutions and Application.-The cresols may be rendered soluble by the addition of soap, as in the official Compound Cresol Solution; and in several other ways. The soap-mixtures partake of the slippery nature of soap and are therefore less useful in operating; but they are well adapted to cleansing the hands (brushing for two or three minutes with $1 / 2$ to $I$ per cent. solution) and for other purposes. A dusting powder containing 3 per cent. of tricresol is being recommended for pediculosis. (Herxheimer and Nathan, I9I5).

Serum Preservatives.-Tricresol, 0.25 per cent., is of ten added to secure the preservation of serums. This is harmless with the usual hypodermic injections, more serious in spinal injections, as with antimeningitis serum. Voegtlin, I9I4, believes that it plays a part in the serious collapse which is sometimes seen, although with this concentration the effect is only temporary. With higher concentrations, the collapse is serious. Phenol acts similarly. Chloroform, even in saturated solution, is harmless. Auer, 1914, however, finds monkeys much more resistant to cresol than the dogs used by Voegtlin. He considers the ordinary concentration quite harmless, and that other antiseptics have no advantage.

Arterial Changes.-Paracresol, in common with many other substances, is said to produce atheromatous lesions in rabbits (Denny and Frothingham, I914).

Phenol Coefficient.-The variability is best discounted by the determination of the "phenol coefficient," i.e., the ratio of the germicidal power of the disinfectant to the germicidal power of phenol, both being tested under identical conditions. A disinfectant three times as active as phenol. would have the coefficient 3 , etc. 
The phenol-coefficient is valuable only for the disinfectants related to phenol; not for metals, iodin, chlorin, etc.

Most disinfectants are now sold with the statement of their coefficient, energally determined cither by the "Rideal-Walker" (I904) or preferably by the "Hygienic Laboratory" method (Anderson and McClintic, Hyg. Lab. Bul., 72, 1912) with or without the presence of organic matter ( 2 per cent. peptone and I per cent. gelatin). The degree of dilution for disinfection is obtained simply by multiplying by 50 the phenol coefficient (with or without organic matter, according to the material to be disinfected); f.i., a disinfectant having the coefficient 3 would be diluted $3 \times 50=150$ times.

\section{PREPARATIONS-CRESOLS}

${ }^{*}$ Cresol, U.S.P., B.P.; Cresol (Tricresol, Cresylic Acid), $\mathrm{C}_{6} \mathrm{H}_{4} \mathrm{CH}_{3} \mathrm{OH}$.-A mixture of the three isomeric cresols obtained from coal-tar, and freed from phenol, hydrocarbons and water. Straw-colored refractive liquid, of phenol-like odor, darkening on exposure to air. Sol. in water $(\mathrm{r}: 50$ ), usually forming a cloudy solution; miscible in all proportions with alcohol, ether or glycerin, and dissolving in alkalies. Dose, 0.05 c.c., I minim, U.S.P.; 0.06 to o.r 8 c.c., I to 3 minims, B.P.; diluted.

For local use, the following concentrations are employed:

In surgery, 1/4 to I per cent.; gargles or cystitis, 1/4 per cent.; cuspidors (tubercle bacilli) or stools, I to I $1 / 2$ per cent.; for sponging rooms or soaking clothes, $1 / 2$ to I per cent. To make a 1 per cent. solution, add 2 teaspoonfuls to a quart of water.

Cresol is generally used in the form of:

${ }^{*}$ Liquor Cresolis Compositus, U.S.P.- $5 \circ$ per cent. of cresol, brought into clear solution by a potash-linseed soap. It has a phenol coefficient of about 3 , and is in every respect equal to most of the proprietary solutions, with the advantage that it is not advertised under exaggerated and dangerous claims, such as being "non-poisonous."

${ }^{*}$ Liq. Cresol. Sap., B.P.—50 per cent. in castor oil - potash soap.

Proprietary preparations may be found in N.N.R.

Metacresyl acetate is very little soluble in water, and therefore less toxic (Greenwald, I9I I). It is being tried as a mild antiseptic for mucous membranes.

Commercial Cresol Preparations.-These contain usually 25 to 50 per cent. of cresol. They may be grouped according to the dissolving agent:

I. Emulsions: turbid, brownish liquids, yielding turbid suspensions with water. Prepared with:

I. Resin-soap: Creolin-Pearson; Izal; Cresolin. Saprol.

2. Petroleum Oils (the mixture lowering the specific gravity to that of water):

3. Coal-tar Products (Carbolic Acid, Naphthalin, Pyridin).

II. Solntions: brownish liquids, yielding clear solutions with sufficient water: Prepared with:

r. Potash Soaps and Alcohol: Lysol, Lysitol; Lysosolveol; Sapokresol, Phenolin. (Hard water will precipitate the soap, and cause a turbid solution.)

2. Creosotinate of Sodium: Solveol, Solutol (Salicylate of sodium may also be used); Kresooxyacetate of sodium: Kresin.

3. Sulfo-acids (Made by heating crude carbolic with equal parts of concentrated $\mathrm{H}_{2} \mathrm{SO}_{4}$ ): Sanatol; Creolin-Artmann (sulfo-acids of resin and mineral oils may be employed).

4. Ethylen-diamin: Kresamin.

Phenoloid Disinfectants. - Cresol mixtures containing the higher homologs are described in N.N.R.

\section{POLYATOMIC PHENOLS}

The introduction of further $\mathrm{OH}$ groups tends to raise the antiseptic, but also the irritant and toxic effects; however, the modifications depend largely on the position of the groups: Of the three isomers with two $\mathrm{OH}$ groups, Resorcin, $1: 3$, is perhaps somewhat more antiseptic than phenol, and rather less toxic and irritant. Hydroquinon, $1: 4$, is more toxic; and Pyrocatechin, $1: 2$, is much more so. They act as reducing agents, especially in alkaline solutions. Pyrocatechin is not used in medicine. Hydroquinon is not used directly, but is formed from arbutin, contained in Uva ursi. Pyrogallol, containing three $\mathrm{OH}$ groups, is highly toxic; whilst its isomer, phloroglucin, is practically inactive. 


\section{RESORCINOL}

Resorcin coagulates albumin. Strong solutions soften and slowly cauterize the skin; weak solutions are merely antiseptic. It is employed in various skin diseases. Internally, it is used to check gastric fermentation, and in diarrhea. After absorption, it produces the same effects as phenol, but with more prominent convulsions, occurring even in man; $8 \mathrm{Gm}$. have caused severe poisoning but with recovery (Murrel, I88I). Smaller doses are antipyretic, but the action is too short to be useful. They produce "toxic" effects resembling those of salicylates. The fate and excretion are similar to phenol.

\section{PREPARATIONS-RESORCIN}

*Resorcinol (Resorcin), U.S.P.; Resorcinum, B.P.; (meta-dihydroxy-benzene), $\mathrm{C}_{6} \mathrm{H}_{4}(\mathrm{OH})_{2}$. - Colorless needle-shaped crystals, of peculiar faint odor, and sweetish, then bitter taste. Acquires pinkish tint on exposure to light and air. Very sol. in water (r:0.9) or alc. ( $\mathrm{r}: 0.9)$, freely sol. in glyc. or eth. Dose, $0.125 \mathrm{Gm} ., 2$ gr., U.S.P.; 0.06 to $0.3 \mathrm{Gm}$., I to $5 \mathrm{gr}$., B.P. Maximum dose, I Gm., I $5 \mathrm{gr}$. On the skin, 5 to 50 per cent. ointment, or io to 20 per cent. solution in glycerin. For urethral or vesical irrigation, $1 / 2$ to 2 per cent. In alopecia, I to 2 per cent. (but discolors hair).

\section{UVA URSI}

This plant has been used for its diuretic and antiseptic effects in vesical catarrh. It contains arbutin, tannin and other constituents.

Arbutin is a crystalline glucosid, yielding glucose and hydroquinon on decomposition. It is decomposed completely in the body (Lewin, I883; Grisson, I888); with large doses the hydroquinon appears in the urine (Bass, IgI2) and is supposed to be the active agent. Lewin and others found it beneficial in cystitis, etc.; Paschkis and Feibes, 1884, doubted its efficiency; Bass found no action on healthy men, even with $20 \mathrm{Gm}$. The usual dose is I Gm., three to six times daily.

\section{PREPARATIONS-UVA URSI}

Uva Ursi, U.S.P.; Uv. Urs. Fol., B.P. (Bearberry).-The dried leaves of Arctostaphylos Uva-ursi. Dose, 2 Gm., 30 gr., U.S.P.

Fldext. Uva Ursi, U.S.P. Dose, 2 c.c., 30 minims, U.S.P.

Inf. Uv. Urs., B.P. -5 per cent. Dose, 15 to 30 c.C., $1 / 2$ to $\mathrm{I}$ ounce, B.P.

\section{PYROGALLOL}

The powerful reducing action overshadows the phenol effects. Locally, it is a mild caustic for wounds and mucous membranes; it produces but slight irritation of the intact skin, passing into erythemas on continued use. Absorbed into the blood it forms methemoglobin, disrupts the corpuscles and leads to intense acute nephritis. Concentrated solutions acting on blood in vitro (not in the body) produce a peculiar insoluble substance, hemogallol. Fatal poisoning may occur even when the drug is applied to the intact skin, and may set in suddenly after it has been used for some time without effects. The symptoms consist in diarrhea, and vomiting, chills, prostration, feeble pulse; nephritis with scant dark urine containing blood or hemoglobin derivatives (Neisser, I88I); sometimes icterus and glycosuria. The rapid cases show cyanosis, dyspnea, convulsions and collapse. The treatment would be symptomatic.

Uses.- The danger of poisoning and the black staining limit its employment. It is used similarly to chrysarobin in psoriasis, lupus, ringworm and other parasitic skin diseases; and in hair dyes.

\section{PREPARATIONS-PYROGALLOL}

Pyrogallol (Pyrogall.), U.S.P.; (Pyrogallic Acid; Tri-hydroxy-benzene), $\mathrm{C}_{6} \mathrm{H}_{3}(\mathrm{OH})_{3}$. -Freely sol. in water ( $1: 1.7)$, alc. ( $\mathrm{I}: 1.3)$, or eth. Solutions turn brown, especially in 
the light; very rapidly if alkaline. Used externally (but dangerous) as I to Io per cent. ointment in skin diseases; and as $\mathrm{I}$ to 3 per cent. solution to deodorize ozena and discharging wounds.

\section{PICRIC ACID}

The saturated aqueous solution is used as a dressing for burns and superficial wounds, weeping eczemas, etc.; exerting anesthetic, antiseptic, astringent and protective actions, and stimulating the reproduction of epithelium, in contrast to most other antiseptics (Ehrenfried, I9II). Stronger alcoholic solutions are irritant, and being absorbed, become toxic. By mouth, I to $2 \mathrm{Gm}$. produce severe poisoning, generally with recovery under treatment (Lewin). It stains the skin, conjunctiva and tissues yellow, even on systemic administration, simulating icterus. This may persist for two weeks after taking I $\mathrm{Gm}$. The urine is colored a peculiar red (Walko, I9or).

Picric acid is trinitrophenol, $\mathrm{C}_{6} \mathrm{H}_{2} \cdot\left(\mathrm{NO}_{2}\right)_{3} \cdot \mathrm{OH}$. Its toxic effects were investigated on dogs by Rapp and Foehr, I 827. Braconnet, I830, introduced it against intermittent fever; it was also used as anthelmintic; but its internal administration was soon considered too dangerous. It was employed in surgery by Chéron, 1876 . Toxic doses destroy the red corpuscles and produce gastroenteritis and hemorrhagic nephritis. The toxic effects on rabbits and dogs are described by Koizumi, I914. It is excreted chiefly in the urine as picramic acid produced by reduction in the liver. A part of the picric acid may be excreted unchanged. Handling the substance, in the arts, may produce eczema. A measle rash also occurs sometimes in poisoning. The common belief of its use in beer seems to be unfounded (Gadamer).

Application.- Picric acid is soluble to Io per cent. in alcohol, to 20 per cent. in ether, to I.2 per cent. in water. In burns, etc., it is used as a saturated aqueous solution, whose antiseptic efficiency is about seven times that of I per cent. phenol. (Picric acid has a phenol coefficient $=6$; so that 0.165 per cent. picric acid would equal $\mathrm{I}$ per cent. phenol; Tidy, I9 I5.) Stronger alcoholic solutions should not be used. An ointment would be illogical. 'The watery solution is said to be free from danger, but should not be applied over large surfaces. It is prepared by dissolving $I 1 / 23$ in a pint of hot water and filtering when cold. Cloths saturated with this solution are applied, covered with dry cotton, and left in place for several days.

\section{PREPARATIONS-PICRIC ACID}

*Trinitrophenol (Trinitrophen.), U.S.P.; Acidum Picricum (Acid. Picr.), B.P.; Picric Acid, $\mathrm{C}_{6} \mathrm{H}_{2}\left(\mathrm{NO}_{2}\right)_{3} \mathrm{OH}$. - Yellow crystalline powder, odorless, of very bitter taste and intense staining power. Sol. in water $(\mathrm{r}: 78)$ or alc. ( $\mathrm{I}: \mathrm{I} 2)$. Dose, $0.03 \mathrm{Gm}$., 1/2 gr., U.S.P.

\section{NITROBENZOL}

Nitrobenzol, $\mathrm{C}_{6} \mathrm{H}_{6} \cdot \mathrm{NO}_{2}$, is used extensively in the manufacture of anilin. It has been tried as abortifacient. Its odor resembles that of hydrocyanic acid, and it has therefore been used in cheap perfumery and soaps, under the name of Artificial Oil of Almonds or Oil of Mirbane; but it is highly toxic; I Gm. may be fatal. It may be absorbed from the skin. The effects depend on asphyxia from methemoglobin formation, and on central paralysis. They resemble anilin poisoning.

The symptoms usually begin only after some hours, and consist in nausea and prostration, burning, headache, intense cyanosis, vomiting with characteristic odor, convulsions and coma, ending in death after a few hours. The mortality is high. In protracted cases, temporary improvement and relapses occur. The treatment demands evacuation and stimulants. No fat should be given.

Subacute and chronic poisoning shows icterus and cyanosis, the clinical picture resembling pernicious anemia.

The reported cases of nitro-benzol poisoning are reviewed by S. S. Adams, rg 2. 


\section{ANILIN}

Anilin, $\mathrm{C}_{6} \mathrm{H}_{5} \mathrm{NH}_{2}$, is used extensively in the arts and therefore gives rise to industrial poisoning, with methemoglobin formation, headache, weakness, dyspnea, cyanosis, convulsions, and psychic disturbances. Its excretion has been studied by Rambousek, I9I3.

Bibliography and history of industrial anilin poisoning, Alice Hamilton, I9 ${ }_{5}$; Luce and Hamilton, IgI 6.

\section{CREOSOTE AND GUAIACOL}

Composition. - The empyreumatic products of the distillation of wood - the smoke, and the creosote and tar which may be condensed from itpossess very considerable antiseptic power. They consist indeed of a mixture of coal-tar derivatives. The most important of these products from a therapeutic standpoint is creosote, the variety which is obtained from beech-wood (first by Reichenbach, I830) being particularly valued. Its principal constituents are

guaiacol, $\mathrm{C}_{6} \mathrm{H}_{4}<\mathrm{OH}$. these compounds could be largely deduced from their composition, which is similar to the cresols. They are more strongly antiseptic and antipyretic, and rather less irritant and toxic, than is phenol. Creosol should be superior theoretically, but practically does not differ sufficiently from guaiacol, to offset its higher cost. Guaiacol constitutes from 60 to 90 per cent. of the creosote, and shares all its properties, with the advantage of constant composition.

Uses.-Creosote and guaiacol are employed mainly in tuberculosis, having been introduced especially by Sommerbrodt, I887. Clinical opinions are divided as to their value. They are generally believed to increase the appetite, nutrition and weight, and to lessen the cough, the fever and the night-sweats. The benefits are seen only in early cases, if the use is long-continued, and if it does not cause gastro-intestinal disturbances. This explains the difficulty of arriving at a fair judgment. It would be still more difficult to explain the effects. The tubercle bacilli are certainly not killed in their foci; it is even doubtful whether their growth is restrained. Possibly the benefits may be referred to the local effect on the alimentary canal and on the bronchitis; and to the antipyretic action. Guaiacol is considered an intestinal antiseptic, although its action is difficult to demonstrate (Harris, I9I2). Creosote-water is sometimes given to check gastric fermentation.

Other Pulmonary Diseases.-These (bronchitis, pneumonia) are also benefited, especially by inhalation, where the local antiseptic and stimulant action can come into play. Absolutely none is excreted by the lungs (Bufalini, r904). When guaiacol is injected hypodermically, the major part is contained in the blood, liver and spleen; much less in the lungs and kidneys (Hofbauer, ror5). It is therefore difficult to conceive of an antiseptic action in the lungs. However, it has been proven that creosote increases the secretion of mucus even when taken by the mouth. In pleuritic effusions, it hastens the absorption of the exudate when it is rubbed into the chest.

As an antipyretic guaiacol (ro to 30 drops, dissolved in an equal volume of oil or alcohol) may be rubbed into the clean and dry skin of the abdomen for ten to fifteen minutes, thus avoiding intestinal irritation. It causes considerable diaphoresis, and has no advantage over other antipyretics.

The local anesthetic action is utilized especially in dentistry.

Administration.-In tuberculosis, creosote or guaiacol is given in the largest doses which can be tolerated without too much gastro-intestinal 
irritation, beginning with I drop and increasing to 5 or to drops, in extemporaneous capsules, three times daily after meals. If they are not tolerated, guaiacol carbonate should be substituted.

Poisoning by Creosote.-This resembles that by phenol, but the effects are less convulsive. 'The local actions are also similar. 'The side actions of therapeutic doses are detailed by Siefert, Nebenwirk., 1915, p. 196. Death has occurred after $7 \mathrm{Gm}$. (Schulze, I894). Hypodermically, guaiacol produces dangerous collapse and cardiac depression. 'The excretion, as with phenol, is mainly in combination with glycuronic and sulphuric acid (Knapp, IgII). Guaiacol (but not its carbonate) causes, in very large doses, the appearance of an undetermined, very viscous, substance in the urine, which is supposed to be capable of obstructing the uriniferous tubules.

Guaiacol Carbonate.-The undesirable local effects of guaiacol, the taste and the toxicity, are largely reduced by converting it into esters, on the "salol-principle," i.e., by replacing the $\mathrm{H}$ of the $\mathrm{OH}$ group by acyl groups. These esters are insoluble and inactive; but in the intestine they are decomposed (saponified), into their constituents, and the $\mathrm{OH}$ is thus regenerated. In this way the antiseptic is liberated but slowly; its action extends over the entire intestine; but the amount which can be liberated at any time is too small to be either irritant or toxic. The creosote esters are decomposed even more slowly than salol, perhaps only through putrefactive bacteria. An excess will pass the intestine unabsorbed. As much as $6 \mathrm{Gm}$. of Guaiacol carbonate has been given to phthisical patients, as much as $75 \mathrm{Gm}$. (21/2 ounces!) to dogs, without producing toxic symptoms. With these large doses, a great deal of the substance is passed in the feces, without having undergone decomposition.

Other Guaiacol Compounds. - Since the liberated creosote or guaiacol is the active portion of these compounds, the acid with which they are combined is absolutely immaterial. 'The carbonic acid esters are the cheapest and contain the largest percentage of active principles, and therefore deserve the preference which has been universally accorded to them. Similar compounds have been prepared from all other phenolsfrom thymol, menthol, etc.

As there are no good reasons for the use of these other guaiacol compounds, they need only be mentioned. They are more fully described in N.N.R.

Compounds formed by replacing the OH by acyl groups (analogous to the carbonate): Benzoate (Benzosol); Cinnamate (Styracol); Salicylate (Guaiacol-salol); Methylglycolate (Monotal).

Replacement of the $\mathrm{OH}$ by alkyl group: The methyl derivative, Veratrol, is practically inactive because the $\mathrm{OH}$ is not regenerated. The glycerin derivative, Guaimar, is soluble and active, but has a bitter taste. Guaiasanol is the chlorid of diethyl-glycocollguaiacol.

Thiocol is potassium guaiacolsulphonate, analogous to sodium phenolsulphonate. Like the latter, it has very little effect.

\section{PREPARATIONS-CREOSOTE}

${ }^{*}$ Creosotum (Creosot.), U.S.P., B.P.-A mixture of phenols and phenol derivatives, chiefly guaiacol and cresol, obtained during the distillation of wood-tar, preferably that derived from beech. Almost colorless or yellowish, highly refractive, oily liquid, having a penetrating, smoky odor; burning, caustic taste. Slightly sol. in water. Miscible with alc. and fixed volatile oils. Dose, 0.25 c.c., 4 minims, U.S.P.; 0.06 to 0.3 c.c., I to 5 minims, B.P. Maximum dose, 0.5 c.c., 8 minims.

Aq. Cresot., U.S.P.-A saturated watery solution, recently prepared, containing less than I per cent. Dose, Io c.c., $21 / 2$ drams, U.S.P.

Ung. Creosot., B.P.- ro per cent.

Creosoli Carbonas (Creosot. Carb.), U.S.P.-A mixture of the carbonates of various constituents of creosote, chiefly guaiacol and creosol. A clear, colorless or yellowish, viscid liquid, odorless and tasteless, or having a slight odor and taste of cresote. Insol. in water, freely sol. in alc. or fixed oils. Dose, 1 Gm., 15 gr., U.S.P. 
${ }^{*}$ Guaiacol, U.S.P., B.P., $\mathrm{C}_{6} \mathrm{H}_{4}(\mathrm{OH})\left(\mathrm{OCH}_{3}\right)$ - - Nearly colorless refractive liquid, crystalline at low temperatures. Odor aromatic; sharp taste. Sol. in water $(r: 53)$; freely miscible with glyc., alc. or oils. Dose, 0.5 c.c., 8 minims, U.S.P.; 0.06 to 0.3 c.c.; I to 5 minims, B.P. Maximum dose, 0.5 c.c., 8 minims.

${ }^{*}$ Guaiacolis Carbonas (Guaicol. Carb.), U.S.P., B.P.; (Duotol), $\left(\mathrm{C}_{6} \mathrm{H}_{4}\left(\mathrm{OCH}_{3}\right) \mathrm{O}\right)_{2} \mathrm{CO}_{3}$. -White crystalline powder, almost without odor or taste. Practically insol. in water, sol. in alc. (r:60). Dose, I Gm., I 5 gr., U.S.P.; 0.3 to I Gm., 5 to 15 gr., B.P.; in powder or capsules. Maximum dose, I Gm., I5 gr.

\section{TAR}

This is a complex product obtained by the destructive distillation of pine woods, especially Pinus palustris. It contains phenols and other aromatic derivatives, resin acids, acetic acid, etc. Tar is strongly irritant to the skin; it is used in scabies and other parasitic skin diseases, chronic dry eczema, itching, etc.; but has now been displaced largely by the less disagreeable naphthol and resorcin. Internally, it has been employed in chronic bronchitis and cystitis, acting similarly to turpentine and copaiba. The official syrup is little more than a flavoring vehicle. Toxic doses of tar produce effects similar to phenol: vomiting, colic, diarrhea and collapse.

Oil of Tar.-When tar is subjected to redistillation, it can be separated into a fixed portion-pitch-consisting mainly of rosin; and a volatile portion which separates into Oil of Tar (Oleum Picis Liquida, U.S.P.) and pyroligneous (crude acetic) acid. The oil of tar consists of various coal-tar derivatives, mainly Cresols, Guaiacol, Phenol, Xylol, Toluol, and Pyrocatechin. It also contains methyl alcohol and acetone.

\section{PREPARATIONS-TAR}

*Pix Liquida (Pix. Liq.), U.S.P., B.P.; Pine Tar.-A viscid blackish-brown substance, obtained by the destructive distillation of Pine wood. Empyreumatic odor and sharp taste. Miscible with alc. and fixed or volatile oils, only partly sol. in water. Dose, $0.5 \mathrm{Gm} ., 8$ gr., U.S.P.

Ol. Pic. Liq., U.S.P.; Oil of Tar.-A volatile oil distilled from Tar. An almost colorless liquid when freshly distilled, but soon acquires a dark reddish-brown color, and has a strong, tarry odor and taste. Dose, 0.2 c.c., 3 minims, U.S.P.

Syr.Pic.Liq., U.S.P.- -5 per cent. Dose, 4 c.c., I dram, U.S.P. Used as flavor for cough mixtures.

${ }^{*}$ Unguentum Picis Liquida (Ung. Pic. Liq.), U.S.P., B.P.; Tar Ointment.-50 per cent. U.S.P.; 70 per cent., B.P.

Oleum Cadinum (Ol. Cadin.), U.S.P., B.P.; Oil of Cade (Oil of Juniper Tar; Ol. Junip. Empyreumaticum).- An empyreumatic oil obtained by the dry distillation of the wood of Juniperus Oxycedrus. A dark brown, clear, thick liquid, having a tarry, empyreumatic odor and a warm, faintly aromatic, and bitter taste. Used externally like tar, having a less unpleasant odor.

Pix Carbonis Praparata (Pix Carbon. Præp.), B.P.-Prepared Coal-tar.-Commercial Coal-tar is heated for an hour at $50^{\circ} \mathrm{C}$. to expel ammonia. It is employed as antiseptic and antipruritic in the form of Liq. Picis Carb.

Liq. Pic. Carbon., B.P. -20 per cent. in alcoholic solution of Quillaja.

Tar Eczema and Cancer.-Workers with coal-tar often develop eczema, which may terminate in epithelioma. "Gas-works tar" is especially liable to produce these affections. The constituents responsible for its "auxetic" action have not been identified; but the anthracen fraction is the most active (Norris, I9r4). . It stimulates cell division also in vitro (Ross, Cropper and Ross, IgI 2).

Paraffin Dermatitis and Cancer.-Workers in crude paraffin are also very often afflicted in the same manner. Pure paraffin, however, is harmless.

The effects were first described by Volkmann, 1875. The acute stage begins with eruptions and abscesses, "wax boils." These last several months, and then pass into a chronic dermatitis, with seborrhea resembling psoriasis, pigmentation, dry fissures, horny thickening, resembling ichthyosis, etc. In the terminal stage, multiple warts or papillomas develop, turning finally to true epithelioma (Davis, Igr5). 


\section{THYMOL}

This is contained in a number of aromatic oils, for instance Thyme. Its chemical structure, $\mathrm{C}_{6} \mathrm{H}_{3} \cdot \mathrm{CH}_{3} \cdot \mathrm{OH} \cdot \mathrm{C}_{3} \mathrm{H}_{7}, \mathrm{x}: 3: 4$, is allied to that of the creosote constituents. Its actions are also similar to those of phenol. Its antiseptic efficiency is higher, restraining the growth of pus organisms in 1 : 3,000; it is not a very active germicide. The local effects and the toxicity are relatively slight, because of its slight solubility and slow absorption. The absorption from the alimentary canal is, however, practically complete (Seidell, I9 5 ). The solution and absorbability are favored by alcohol or oil (Schultz, r9 5 ); these must be avoided in the therapeutic use. Even after absorption its toxicity is but a fourth of that of phenol. About half of the thymol is completely destroyed in the body (Seidell, I915). The remainder is excreted paired with sulphuric and glycuronic acid (Blum). The symptoms are similar to phenol (W. H. Schultz, I9I I), except that there are no convulsions, the central nervous system being primarily depressed. It is rapidly absorbed from hypodermic injections of oily solutions (Schultz and Seidell, rgI2).

Uses.-Thymol has a rather pleasant clean taste; and a saturated watery solution makes a rather agreeable and fairly efficient antiseptic and deodorant mouth wash or gargle; lotion for discharging wounds, etc. Internally, it is occasionally employed as antipyretic and intestinal antiseptic. Its main use is as an anthelmintic in the treatment of hook-worm disease (Bozzolo, I88I).

Administration in Uncinariasis. - It is given fasting, in the total dose of $2 \mathrm{Gm}$. for adults, and the usual modifications for age, divided into three portions, taken an hour apart, best as fine powder in 5-gr. capsules. This is preceded and followed by a saline cathartic (not by castor oil). A rather detailed technic, established by Stiles (described in J.A.M.A., 60: $\mathrm{r}_{546}$, I913) should be used to insure success. The treatment is repeated at weekly intervals until successful.

Although highly effective against hook-worm disease, thymol is not ideal. It sometimes fails; produces some disagreeable after-effects in nearly half the patients; in a few, it causes severe poisoning; and its cost is high (Levy, r914).

\section{PREPARATIONS-THYMOL}

${ }^{*}$ Thymol, U.S.P., B.P., $\mathrm{C}_{6} \mathrm{H}_{3}\left(\mathrm{CH}_{3}\right)(\mathrm{OH})\left(\mathrm{C}_{3} \mathrm{H}_{7}\right)$.-Occurs in the volatile oil of Thymus vulgaris and in some other volatile oils. Large, colorless prisms; aromatic, thyme-like odor; pungent, aromatic taste. Very slightly sol. in water $(r: 1,0 r o)$; freely sol. in alc. (I: I) or olive oil ( $\mathrm{I}: \mathrm{I} .7$ ); sol. in other fixed oils and in vol. oils. Liquefies when triturated with camphor, menthol or chloral. Dose, $0.125 \mathrm{Gm}$. , 2 gr., U.S.P.; 0.03 to $0.12 \mathrm{Gm}$., $1 / 2$ to 2 gr., B.P., as antiseptic; I Gm., I5 gr. (caution!) per day, as anthelmintic, U.S.P.; 1 to 2 Gm., I 5 to 30 gr., B.P.

\section{NAPHTHALIN}

This represents the union of two benzol rings: It is practically insoluble in water; and is employed mainly as insecticide (moth-balls); as antiseptic dusting powder in skin diseases and as intestinal antiseptic and anthelmintic. especially against oxyuris.

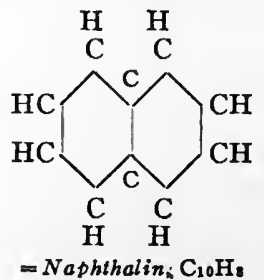


Fumigations have also been tried against pertussis. It has usually a relatively low toxicity for mammals because but little is absorbed, the greater part being eliminated unchanged by the feces. That absorbed is excreted as naphthol, naphthoquinon, etc., and produces renal and vesical irritation and hemorrhage. However, the absorption is sometimes greater, especially in the presence of oil: $2 \mathrm{Gm}$., administered in the course of two days, has proven fatal to a six year old child (Prochownik, I9II). Heine, IgI3, reports poisoning in a woman after $2.8 \mathrm{Gm}$., distributed over three days. Retinal changes may occur similar to those produced by naphthol (Takamura, I9I2). Further references to the side actions are given by Seifert, Nebenwirk., I9I5, p. I40.

\section{PREPARATIONS-NAPHTHALEN}

Naphthalenum, $\mathrm{C}_{10} \mathrm{H}_{8}$ - - Colorless, crystalline powder; coal-tar odor; hot taste. Insol. in water; sol. in alc. ( $\mathrm{I}: \mathrm{I}_{3}$ ). Dose, $0 . \mathrm{I}$ to $0.5 \mathrm{Gm}$. (2 to $8 \mathrm{gr}$.). For children, $0.05 \mathrm{Gm}$., 1 gr., at $1 \frac{1}{2}$ years; to $0.2 \mathrm{Gm}$., $3 \mathrm{gr}$., at twelve years, three times daily.

\section{NAPHTHOL}

The naphthols are compounds analogous to phenol, formed by the introduction of an $\mathrm{OH}$ group into naphthalin: Alpha-naphthol is the more
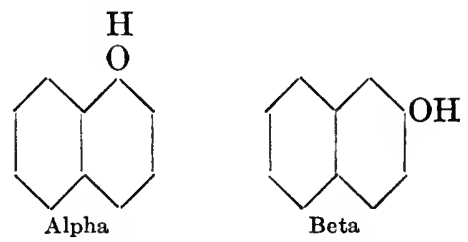

toxic of the isomers and is not employed in medicine. Beta-naphthol acts similarly to phenol, but is more germicidal. It has a special irritant action on the kidneys, and severe nephritis has been caused by its absorption from the intact skin in the therapeutic use (Koposi, I88I; Baatz, 1894). It is therefore especially contraindicated in the presence of renal disease. Changes in the retina and opacity of the lens have also been described after its use (e.g., v.d., Howe, I9I3). Further references to the side actions are given by Seifert, Nebenwirk., I9I5, p. 186.

It is almost insoluble, and is employed as an ointment in psoriasis, acne, parasitic skin diseases, pruritus; and to deodorize ozena. Internally, it is used as an intestinal antiseptic and anthelmintic. It is effective in hook-worm disease but inferior to thymol (Freeman, I908). The local irritation and toxicity may be lessened on the salol-principle, by transforming it into esters. The benzoate, salicylate (Betol) and oxytoluate (Epicarin) are described in N.N.R.

\section{PREPARATIONS-NAPHTHOL}

${ }^{*}$ Betanaphthol, U.S.P.; Naphthol, B.P., $\mathrm{C}_{10} \mathrm{H}_{7} \mathrm{OH}$.-Colorless or buff-colored, crystalline laminæ or powder; faint phenol odor; pungent taste. Very slightly sol. in water $(\mathrm{r}: \mathrm{r}, 000)$; very sol. in alc. $(\mathrm{r}: 0.8)$ and in oils, glycerin and alkalies. Dose, $0.25 \mathrm{Gm} ., 4$ gr., U.S.P.; 0.2 to $0.6 \mathrm{Gm} ., 3$ to Io gr., B.P.; against hook-worm, $\mathrm{x}$ to $4 \mathrm{Gm}$., by special technic (Freeman, I908). Externally, $\mathrm{I}$ to Io per cent. ointment or 2 to 5 per cent. solution in oil or alcohol; in alopecia, I to 2 per cent.

\section{QUINOLIN}

Quinolin, $\mathrm{C}_{9} \mathrm{H}_{7} \mathrm{~N}$, is derived from naphthalin by the substitution of a $\mathrm{N}$ for a $\mathrm{CH}$ group. It is highly toxic and strongly antiseptic, but does not inhibit the growth of 
yeast (Donath, I88I). In rabbits, the hypodermic or intravenous injection of $0.2 \mathrm{Gm}$. per kilogram produces retinitis, similar to naphthalin; the lens, however, remaining clear (Jess, I916).

Oxyquinolin Sulphate, $\left(\mathrm{C}_{9} \mathrm{H}_{7} \mathrm{ON}\right)_{2} \cdot \mathrm{H}_{2} \mathrm{SO}_{4}$, is very strongly antiseptic, but feebly germicidal. It seems to be non-toxic and is used as an antiseptic lotion for mucous membranes, etc., under the name of chinosol (N.N.R.). Its fate was studied by Brahm, I899.

\section{BENZOIC ACID AND BENZOATES}

Benzoic acid, $\mathrm{C}_{6} \mathrm{H}_{5} \cdot \mathrm{CO}_{2} \mathrm{H}$, occurs in the balsams (Benzoin, Tolu, etc.), in cranberries and in other vegetables; and in the decomposed urine of herbivora. It is now prepared synthetically. It is a fairly strong antiseptic of about the same rank as phenol; the salts are somewhat less effcient. The free acid in concentrations above o.I per cent. produces moderate local irritation; the salts are not irritant. The systemic toxicity is very low, even large doses producing mainly digestive derangement, sometimes with further effects, resembling those of salicylates. There is also a similar, but weaker therapeutic action in rheumatic fever. Fatalities are unknown. Benzoic acid combines in the kidneys with glycocoll to form the inactive hippuric acid.

Use as Food Preservative.- The low toxicity and the absence of odor or marked taste has led to the use of benzoates for the preservation of foods, especially fruits and vegetable products. Sodium benzoate is generally employed for this purpose in the concentration of o.I per cent.; in acid foods, especially fruits, most of the benzoic acid would be liberated. This concentration suffices to restrain bacterial growth considerably, but may. not prevent it completely.

Antiseptic Efficiency.-Buchholz, I875, found the free acid to be germicidal in 0.4 per cent., and antiseptic in 0.1 per cent. Salkowski, 1899 , found that 0.25 per cent. kept meat sterile for five months; with 0 .I per cent. there were only a few colonies. Sodium benzoate in neutral media inhibits growth in 0.3 to 0.5 per cent. (Th. Smith).

Action on Digestive Ferments.-In test tubes, benzoic acid up to 0.1 per cent. does not interfere markedly; but higher concentrations are detrimental, especially to pepsin! (Leffmann, 1899). However, peptic digestion proceeds fairly rapidly, even in a saturated solution (Dakin). These actions have no practical importance (Long, I9 12).

Actions of Moderate Doses.-These are especially important in view of the use of benzoates for the preservation of food. In a very careful investigation, Chittenden, Long and Herter, I9I I (Report 88, U.S. Dept. Agr.), could not demonstrate any effects on healthy individuals, if larger quantities than are likely to be used in foods, $0.5 \mathrm{Gm}$. per day, were continued for weeks. Even still larger doses, to $4 \mathrm{Gm}$., were not generally injurious. They were sometimes followed by indications of slight digestive disturbance (Herter); but it is doubtful whether these had any significance. Excessive concentrations (above o.I per cent.) of the free acid, consumed too freely (as in cider or fruit juices) probably cause more pronounced gastro-intestinal irritation (Lucas, I9ro), but not albuminuria (Herter, I9I0; Gerlach, I909). These concentrations have a noticeable taste which might serve as a warning. There is no reason to assume that the benzoate has a cumulative action, since it is promptly excreted as hippuric acid; nor is there any evidence that the formation of this acid is in any way detrimental.

Investigations of the Referee Board.-Chittenden, Long and Herter each experimented on four to six healthy young men, administering sodium benzoate, dissolved in food or drink, $0.3 \mathrm{Gm}$. per day for two months, then 0.6 to I Gm. for one to two weeks, and 4 to $6 \mathrm{Gm}$. for shorter periods. 
Clinical observations and extensive analyses of food and excreta were made. Chittenden and Long observed no effects whatever on the general feeling of the subjects; on pulse or blood pressure; red or white blood corpuscles or hemoglobin; no loss of appetite, nausea, headache, intestinal disturbance and no change in the intestinal flora. The body-weight did not diminish; the digestion, assimilation and utilization of fat or protein food; the composition of the feces; the N-balance; the quantitative composition of the urine; and the distribution of nitrogen, remained absolutely normal. There was no indication of damage to the kidneys.

Herter agreed with these negative results; but with doses above I $\mathrm{Gm}$., he observed a slight increase of indican, of coecal bacteria and of free gastric hydrochloric acid. These effects could mean a slight irritant action from the use of these doses, which, however, are larger than would ordinarily be introduced with foods.

The Prussian Scientific Deputation of Medical Affairs, I9Ir, accepted the conclusion that $0.5 \mathrm{Gm}$. distributed in small doses during the day, are innocuous; but they do not consider the experiments with doses of several grams as affording proof of absolute harmlessness.

The conclusions of Wiley and his collaborators (Bull. 84, I908), were less favorable. Their experiments were also made on healthy young men with doses of I to $2.5 \mathrm{Gm}$.; but these were given dry in capsules, offering opportunity for more violent local actions than when the benzoate is dissolved, as in food. This may in part explain the claimed deleterious results (gastric pain, vomiting, digestive and metabolic disturbances, reduction of body-weight, etc.). However, these results were not very striking, nor their causal connection with the benzoate administration at all conclusive (K. B. Lehmann, Igrr).

Effects of Large Doses.- Sodium benzoate is tolerated without serious symptoms even in much larger doses, at least up to $60 \mathrm{Gm}$. per day (f.i., Jaksch, 1897). Such doses were formerly used in therapeutics. There are generally symptoms resembling those of salicylates (Lewinski, I908; Rost, Franz and Heitzel, I9I3); as with the latter, the dose required to elicit these symptoms varies greatly: Nausea and vomiting were obtained in some cases with 6 to $8 \mathrm{Gm}$. (Meissner and Shepard, 1866); whilst in others, $25 \mathrm{Gm}$. had no effect. The urine sometimes contains reducing subtances, presumably glycuronic acid, especially if very large doses $(25 \mathrm{Gm}$.) are taken (Lewinski).

Toxic Effects on Animals. - These are analogous to phenol; gastric irritation, vomiting, dyspnea, decrease of reflexes, narcosis, sometimes convulsions (Kobert and Schulte, I880). Dogs tolerate I.7 Gm. per kilogram by vein without danger. This produces short vagus stimulation and prolonged rise of blood pressure (Naumann, r879).

Permissibility of Benzoated Foods.-These investigations justify the conclusion that healthy individuals may safely consume reasonable quantities of benzoated foods $(0.5 \mathrm{Gm}$. of benzoate per day, in concentration of o.I per cent.), if these foods are not otherwise spoiled or unhealthy; but that patients with gastro-intestinal or renal diseases would do better to avoid them. Benzoate is probably the least harmful of food preservatives, and where these are needed, its use appears permissible; provided that its presence is stated so that it may be avoided by patients to whom it could be harmful. However, it is very difficult to pass a final judgment on such questions.

Possible Objections.-The evidence in favor of benzoates is really negative, viz., that no deleterious effects have been proven. Such negative evidence is always open to the objection that our methods of investigation are not sufficiently delicate or comprehensive, or otherwise are not adapted to reveal the possible deleterious effects. More serious probably is the objection that the investigations have so far been confined 
almost cxchusircly to healthy individuals. It is quite conceivable that irritant actions which would be too feeble to influence normal organs, might become deleterious to these organs, cspecially the gastro-intestinal tract or kidneys, if these are already diseased, or when the general resistance is lowered. Herter, 1910, advises against the free use of benzoates in dyspepsias. There is also evidence that the formation of hippuric acid, which is probably in part responsible for the harmlessness of benzoic acid, is interfered with in at least some types of kidney diseases.

Hippuric Acid Synthesis. - Benzoic acid and its homologs (cinnamic acid, quinic acid, etc.) combine in the body with glycocoll to form hippuric acid, which is excreted in the urine. The normal hippuric acid (averaging $0.7 \mathrm{Gm}$. per day in man) arises in this manner from the benzoic acid occurring naturally in vegetable foods; and from its homologs which are formed in protein putrefaction in the intestines (Salkowski, I885; Baumann, I886). The synthesis of hippuric acid was discovered by Woehler. Meissner and Shepard, I866, showed that this occurs in the kidneys. Bunge and Schmiedeberg, I 877 , demonstrated the process in excised kidneys, and believed that this is the only organ concerned in dogs. However, considerable hippuric acid is formed in nephrectomized dogs (Kingsbury and Bell, I9I 5). In herbivorous animals, the synthesis also takes place partly in other organs (Salomon, 1877 ), in the small intestines and liver (Jaarsveld and Stokvis, I 879). Chickens do not form hippuric acid (Yoshikawa, I9II). In man, benzoic acid up to ro $\mathrm{Gm}$. per day is excreted almost quantitatively as hippuric acid (Dakin, I910) (85 to 90 per cent. within five or six hours; H. B. Lewis, I9r4). With very large doses $(25 \mathrm{Gm}$., Lewinski) a part escapes synthesis. After the administration of benzoic acid, a fifth of the total nitrogen of the urine may be in the form of hippuric acid. Injected hippuric acid is excreted rapidly, i.e., within nine hours (Raiziss, Raiziss and Ringer, I914).

In parenchymatous nephritis, even smaller quantities are excreted in part as benzoic acid (Jaarsveld and Stokvis, I879; Kronecker, 1883 ).

Uric Acid.-Large doses of benzoates $(8 \mathrm{Gm}$. per day, in man) increase the elimination of urates in the urine, and decrease the urate content of the blood. The effect is therefore similar to salicyl. The creatinin of the blood is not lowered (Denis, I915).

H. B. Lewis and Karr, Igr6, observed a marked decrease in uric acid excretion during the period of maximal hippurate excretion, after 7 to $8 \mathrm{Gm}$. of benzoate. This effect is not produced by administering hippuric acid directly. Creatinin excretion was not affected by the benzoate.

Therapeutic Uses.-The slightly irritant and stimulant action of free benzoic acid is sometimes used to promote expectoration in bronchitis with abundant thin secretion; and in the form of benzoin, to promote the healing of small wounds, chapped hands, etc.

Benzoates were formerly employed in uric acid diseases (Ure, I84I); in arthritis, where it acts similarly to salicylates, but weaker; in tuberculosis; and as a diuretic and antiseptic in cystitis. It is not diuretic, and its antiseptic action on the urine is even weaker than that of the salicylates (Sollmann, rgo8).

\section{PREPARATIONS-BENZOATE}

*Acidum Benzoicum (Acid. Benz.), U.S.P., B.P., $\mathrm{C}_{6} \mathrm{H}_{6} \cdot \mathrm{COOH}$-O Obtained from benzoin by sublimation, or prepared synthetically. Colorless, lustrous scales or needles; odor resembling benzoin, acid pungent taste. Slightly sol. in water ( $\mathrm{r}: 275)$, freely sol. in alc. ( $1: 2.3$ ). Dose, 0.5 Gm., 8 gr., U.S.P.; 0.3 to 1 Gm., 5 to 15 gr., B.P.

Troch. Acid. Benz., B.P. -0.03 Gm., $1 / 2$ gr.

Ammon. Benz., U.S.P., B.P., $\mathrm{NH}_{4} \cdot \mathrm{C}_{7} \mathrm{H}_{5} \mathrm{O}_{2}$ - -White, laminar crystalline powder; odorless, or a slight odor of benzoic acid, saline, bitter, afterward slightly acrid, gradually losing ammonia on exposure to air. Sol. in water ( $\mathrm{r}: \mathrm{IO})$ or alc. ( $\mathrm{r}: 35.5)$; freely sol. in glyc. ( $\mathrm{I}: 8)$. Dose, I Gm., I 5 gr., U.S.P.; 0.3 to I Gm., 5 to 15 gr., B.P.

${ }^{*}$ Sodii Benzoas (Sod. Benz.), U.S.P., B.P., $\mathrm{NaH}_{7} \mathrm{C}_{5} \mathrm{O}_{2}$.-White powder; odorless; sweetish taste. Freely sol. in water (r: 1.8$)$; sol. in alc. ( $(: 6 \mathrm{r})$. Incompatible with acids and iron. Dose, I Gm., I 5 gr., U.S.P.; 0.3 to $2 \mathrm{Gm}$., 5 to $30 \mathrm{gr}$., B.P.

Liquor Antisepticus Alkalimus, N.F.-A mildly antiseptic, alkaline and demulcent mouth wash, containing about 25 per cent. of glycerin; 3 per cent. each of sodium benzoate and potas. bicarbonate; 0.8 per cent. of sodium borate; flavored with thymol and aromatic oils, and colored purplish red with persionis. Applied with 4 to 5 volumes of warm water. 


\section{CINNAMIC ACID}

Cinnamic acid, $\mathrm{C}_{6} \mathrm{H}_{5} \cdot \mathrm{CH}: \mathrm{CH} \cdot \mathrm{COOH}$; its soluble sodium salt and Balsam of Peru (which contains large quantities of cinnamic-benzyl ester and free cinnamic acid) produce leucocy tosis. They were administered intravenously by Landerer against tuberculosis, with the idea of setting up a localized inflammatory reaction about the tubercular foci, leading to cicatrization. The results have been disappointing and the treatment has already gone out of fashion. Sodium Cinnamate (Hetol) and its administration are described in Rep. Counc. Pharm. Chem., I9II, p. 6o.

Not much is known about the pharmacologic actions of cinnamic acid. Erdmann and Marschand, 1842 , showed that it is largely converted into benzoic and hippuric acid. It has no effect on urate metabolism (Denis, I9 15). Cinnamic acid has been proposed as a food preservative; but the German commission decided that whilst its toxicity is low, its harmlessness has not yet been sufficiently demonstrated to make its use permissible (Heffter and Rubner, I9I4).

\section{SALICYLIC COMPOUNDS}

Salicylic acid (ortho-oxybenzoic acid, $\mathrm{C}_{6} \mathrm{H}_{4} \cdot \mathrm{OH} \cdot \mathrm{CO}_{2} \mathrm{H}, \mathrm{I}: 2$ ) and its compounds are less antiseptic and much less toxic than phenol; but they produce marked antipyretic and analgesic effects and a practically specific relief of the phenomena of acute articular rheumatism. Large therapeutic doses produce the so-called "toxic" symptoms resembling cinchonism. The different compounds act qualitatively similar but present quantitative differences. The salicylates are excreted by the urine, partly free and partly combined.

Historical.-An excellent review of the introduction of salicylic acid is furnished by W. O. Moore, I879. The entire literature to date is presented by Hanzlik, I9I4. Salicylic acid was first prepared in 1838 by Piria from salicin, which had been discovered in 1827 by Leroux. Cahours, in 1844, and Procter, prepared the acid from gaultheria oil. Kolbe and Lautemann produced salicylic acid synthetically from phenol in 1860 ; and Kolbe devised a commercially practical synthetic process in 1874. He also investigated its antifermentative action. It soon became thoroughly established as a surgical antiseptic (Thiersch, 1875); antipyretic (Buess, 1875); and antirheumatic (Strickler, $1876)$.

Antiseptic Action.-Free salicylic acid is about equivalent to phenol and benzoic acid; its salts, however, are scarcely antiseptic (Kolbe, I874). The irritant effects disqualify salicylic acid as a surgical antiseptic. It has been used as a food preservative, but it is doubtful whether this should be permitted. Salicylates have a moderate antiseptic effect on the urine (Sollmann, I908; Jordan, I9Ir), and may be used for this purpose.

Action on Leucocytes.-Binz, 1875 , found that these are paralyzed and killed, similar to quinin and many other aromatic derivatives. Ikeda, 1916, observed no effect on the emigration of white cells from inflamed mesentery. In mammals, the leucocytes of the blood are doubled an hour after the administration, but return to normal in two hours.

Local Actions on Skin.-The application of salicylic acid in substance or strong solution, produces a slow and painless destruction of the epithelium. It is used as ointment, 2 to ro per cent., in itching skin diseases (urticaria); as lotion, I to 2 per cent., in alopecia; as powder, 3 per cent. in talc, against sweating feet; and in collodion, ro to 20 per cent. and plasters to soften corns and warts.

Berkenbusch, 19 5 , recommends a ro per cent. ointment (spread thickly) against skin inflammation, boils and eczema.

Hypersensitiveness of Skin to Salicylates.-Sauerland, I9г 2, found that the external application (not the systemic administration) of salicyl preparations cause a gradual 
development of persistent sensitiveness, leading to eruptions after an incubation period. 'This involved all parts of the skin, not only' that to which the salicylate had been appliecl. The skin was not hypersensitive to other irritants.

Local Actions on Mucous Membranes.-Strong solutions cause considerable irritation and superficial corrosion. The salts are free from this objection, and the free acid is therefore little employed internally. It also softens the enamel of the teeth and should not be used in mouth washes.

Salicylates have practically no effect on muscle or nerie.

Absorption, Fate and Excretion.-The salicyl compounds are rapidly absorbed from mucous membranes and even from the intact skin. The insoluble esters are saponified and absorbed in the intestines; a part is probably absorbed without decomposition. In the tissues and blood the salicyl exists probably as sodium salicylate; high tensions of $\mathrm{CO}_{2}$ may liberate free salicylic acid. 'It is excreted to some extent in all the secretions (sweat, Tachau; only traces in the feces, Hanzlik); but by far the larger part appears in the urine; partly free and partly conjugated. The excretion begins within fifteen minutes, and is practically completed in six to forty-eight hours. The rapid excretion explains the need of large and frequent doses. Larger doses may color the urine green.

Conjugated Salicylic Acids and Salicyluric Acid.-The forms in which salicylates are excreted by the urine are still in question. U. Mosso, 1890, estimates that more than half is conjugated; but his methods are open to doubt. It is generally stated that the greater part combines with glycocoll, forming a compound analogous to hippuric acid and named salicyluric acid (Bertagnini, I856). This is supposed to be inactive in rheumatism (Stockmann, I906). However, Hanzlik, I916, failed to find such a compound in human salicyl urines.

A part of the salicyl is excreted conjugated with sulphuric and glycuronic acids,' also as oxysalicylic acid (C. Neuberg, I 1 I I), and perhaps in other forms.

Absorption from the Alimentary Canal. - Sodium salicylate remains in part for a considerable time in the stomach. The insoluble esters pass the stomach rapidly without absorption and are slowly decomposed and absorbed in the intestines (Burow, I9I I dogs with duodenal fistula). Sodium salicylate is also absorbed from the rectum (Ziemsen; Massol and Minet, I908); although not quite as readily as by mouth (Fiedler, $1905)$.

Absorption from the Skin.-Free salicylic acid, sodium salicylate, methyl salicylate and some of the other esters, in ointments or alcoholic solutions, are absorbed by the skin, but less rapidly than by mouth (Franceschini, I906; Schumacher, I908; Sauerland, I91 2). 'The absorption by various channels has been studied by Levin, I9I 2 .

Diffusion through Colloid Membranes.-This is hastened by salicylic acid or sodium salicylate (Oswald, I9ro; Hanzlik, I9I2). Salicylic acid also enters living cells which are generally impermeable to other acids (Harvey, 1914).

Distribution in Organs. - Blanchier, 1879 , investigated this in dogs and found the salicyl in the saliva, bile, cerebro-spinal fluid, articulations; etc. Nencki, 1895, found it in the bile but not in the gastric juice. Filippi, I900, demonstrated it in the synoviæ, in the brain and in the spinal cord. Vinci, I905, again confirmed its presence in all organs and secretions, but found more in the secreting organs (kidney, liver, gastric mucosa) and in blood than in other organs (spleen and muscles). The nervous -system showed the salicyl reaction only after large doses. Olmer and Totiau, I910, confirmed its presence in cerebro-spinal fluid and found its quantity there independent of the dose. Stockman, 1906, found none in the saliva, traces in sweat, and considerable in the jointfluid, blisters, bronchial, mucus and pleural effusions. Scott, Thoburn and Hanzlik, I916, found the percentage in joint fluids of rheumatic patients a trifle smaller thas in the blood. Hofbauer, I9I5, concludes that it appears in the sputum only under certain conditions.

Distribution in Normal and Infected Animals.-Bondi and Jacoby, I906, found the concentration of the salicyl highest in the serum, and relatively high in the joints, especially in rabbits infected with staphylococcus; so that infected joints would appear to have a higher elective affinity for salicylates. However, the evidence is not 
altogether convincing, as the data were obtained with very rough methods and the authors point out that the apparently larger quantity might be due simply to the serous effusion.

Liberation of Salicylic Acid in the Tissues.-It is generally assumed, but without convincing proof, that the salicyl exists in the blood and tissues as sodium salicylate. Binz, 1876 and 1879 , showed that free carbonic acid, if in sufficient concentration, liberates salicylic acid. The $\mathrm{CO}_{2}$ tension of normal blood (about 6 per cent.) is too low for this; but when it rises, as in asphyxia, free salicylic acid can be demonstrated. Hanzlik, I9I6, failed to confirm this liberation. In inflamed tissues the $\mathrm{CO}_{2}$ tension may be even higher locally, to 17.5 per cent. Other acids are also produced in cells and would aid the liberation. Since free salicylic acid is more active than its salts, at least as an antiseptic, its liberation could explain the greater efficiency of salicyl treatment on the joints than on the cardiac lesions of rheumatic fever. However, Scott, Thoburn and Hanzlik, Igr6, failed to find any free salicylic acid in the joint fluids of rheumatic patients,

Bondi and Jacoby, I905, found that the salicyl is precipitated from the serum with the albumin by ammonium sulphate, and they believe that it forms labile compounds with serum albumin and polypeptids. More likely, however, the precipitation is simply an adsorption phenomenon.

Time of Excretion in Different Conditions. - This varies with the preparation, the dose, and also in different patients. It is possible that these differences may partly explain the variations in the efficiency and toxicity (Fraser, r885).

Normal Time of Excretion.-Blanchier, I879, investigated this on sodium salicylate, administered by mouth. It appeared in the urine in fifteen to eighteen minutes; with doses of $\mathrm{I}$ to $2 \mathrm{Gm}$. the excretion was completed in twenty-two hours; with 4 to $5 \mathrm{Gm}$. in forty-four hours. After continued administration, the excretion persisted from three to eight days. In dogs, the excretion was relatively slow; in herbivora, somewhat quicker than in man. Ehrmann, I907, found the excretion in man completed in three to twelve hours. He believes that the excretion is hastened by alkalies. Gaulier, rgr 3 , reports the excretion as completed in eighteen to twenty-six hours. The excretion was not influenced by giving sodium bicarbonate. It was not parallel to the excretion of methylen blue. After application of a salicyl ointment to the skin, the excretion lasted two days (Schumacher, r909).

The elimination after intravenous injection has been studied by F. Mandel, r908.

Excretion and Fate in Rheumatic Fever. - This averages about ro per cent. less than in normal individuals; the deficit being greatest in the first ten to twenty hours of administration. It is not due to retention, or to differences of diuresis, but partly to excretion in the sweat, and chiefly to increased destruction. The percentage of salicyl in the blood and urine at this time is little more than half of that in normal subjects (Scott, Thoburn and Hanzlik, I9I6). Ehrmann, I907, claimed no difference between the normal and rheumatic.

Purpus and Chelchowski report delayed excretion in renal and circulatory diseases.

Time of Excretion of Salicyl Esters. - The excretion of Aspirin, Novaspirin and Aspirophen is about as rapid as that of sodium salicylate, beginning in one-half hour and being completed in two or three days; Salol and Salipyrin are excreted more slowly (Block, r909; Pinczower, r9ro).

Quantity of Salicyl Recovered.-This was investigated for sodium salicylate by $U$. Mosso, I89o, and for ethyl salicylate by Bondzynski, r897. In each case over 90 per cent. of the salicyl was recovered from the urine. However, the methods give high results. More recent investigations indicate that about 20 per cent. is not excreted, but destroyed (Hanzlik).

Metabolism.-Salicylates increase the elimination of nitrogen (Laqueur, Bruenecke and Crampe, I9I2), especially in the form of uric acid; the latter is followed by a compensatory decrease (Kumagawa, I898; Ulrici, I901; Schreiber and Zandy, I899). The increase of uric acid is probably due partly to lessened destruction, but the uric acid concentration in the blood is also decreased, in the same way as with atophan (Denis, rgr5).

The normal non-protein nitrogen of the blood is not lowered; but when abnormally high it may be decreased. The creatin of the blood is not affected (Denis, I915). Gaulier, I9r3, claims that the chlorid excretion is diminished. Phosphate excretion is slightly increased (Denis and Means, 1916$)$. There are few definite data on the respiratory metabolism 
(review, S. Weber, I904). Denis and Means found uncertain changes in the basal metabolism in man.

The gastric secretion is somewhat decreased (Klocman, I9I2); but the absorption of food is not materially affected. The secretion and flow of bile are somewhat increased.

Cric Acid.-The increased elimination is due (as with atophan) to increased permeability of the kidneys to uric acid; for the uric acid content of the blood falls during the salicyl administration, to resume its original level when the salicyl is discontinued. In man, $6 \mathrm{Gm}$. of sodium salicylate may reduce the blood uric acid to one-half or onethird. The efficiency may be greater even than with atophan (Fine and Chace, I9I5; Frank and Pietrulla, ror4; Denis, rgr5). The increase of urate excretion may reach 30 per cent. (Denis and Means).

By less direct methods, Hall, I904, and Rockwood, r9o9, also reached the conclusion that the increased excretion of uric acid is not due to increased production; but they could not exclude diminished destruction of uric acid as a factor. Excised organs indeed destroy uric acid more rapidly in the presence of salicylic acid (Stookey and Morris, I907). Waucomont, I9II, concluded that salicylates decrease the urate and purin excretion in experimental gout. Gehrig, I I $_{5}$, finds that salicylate and other antipyretics do not produce leucocytosis, as was commonly believed.

The para- and meta-isomers do not affect the uric acid excretion (Denis, I915).

Postmortem Autolysis of the Liver is increased by small, and decreased by large concentrations (Laqueur, Igr2).

Cholagogue Action.-Salicylates are among the few drugs which cause a genuine increase of the secretion of bile (Pfaff and Balch, 1897; Okeda, I915). Other aromatic products which conjugate with sulphuric acid act similarly (Petrowa, I9r2). Salicylates have been used in obstructive jaundice and biliary colic, but it is doubtful whether they are of much value.

Diabetes Mellitus.-Salicylates have been credited with decreasing the output of sugar and the consumption of water (Ebstein and Mueller, r876; von Noorden, 1912); but this efficiency is at best inconstant (Kaufmann, I903).

Continued Administration. - The effects of continued moderate doses are important because of the use of salicylate as food preservative. They have been investigated by Wiley, rgo6, on healthy individuals. The drugs were administered in capsules or tablets of 0.2 to $2 \mathrm{Gm}$. per day. The appetite, digestion and utilization of food was at first increased. This effect became less apparent after a time, but the food consumption generally remained above normal. Notwithstanding this increased intake, there was; a slight loss of body-weight and of nitrogen. The excretion of phosphates was dimin. ished. The quantity of urine was not affected; there was some tendency to albuminuri; .

Antipyretic Action.-Salicylates lower febrile temperature energetically and promptly. The mechanism is probably similar to that of acetanilid, i.e., increased heat dissipation by cutaneous dilatation, aided by perspiration. In healthy individuals this is compensated by increased heat production so that the normal temperature is not easily affected. Salicylates may take the place of other antipyretics, but their side actions are more disagreeable.

Kidneys.- Salicylates may have a slight and inconstant diuretic effect (Blanchier, I879). Full doses, however, decrease the output of urine, partly by diaphoresis, partly by water retention. This is accompanied by evidences of renal irritation (slight albumin and casts) and diminished renal permeability (phthalein test and non-protein nitrogen of blood). With the ordinary doses, these changes disappear rapidly, and apparently without permanent damage, on discontinuing the administration (Scott, Thoburn and Hanzlik, I916; Luethje, I902; Knecht, I904; Quenstedt, I905; Ehrmann, I907).

E. Frey, 1905, and Glaesgens, I9I I, found that the irritant action could be prevented by keeping the urine alkaline; Ehrmann, I907, could not confirm this; but it would be well to administer alkalies with the salicylates in the presence of nephritis. 
Highly toxic doses produce anatomic parenchymatous lesions (Vinci, I905).

Therapeutic "Toxic" Actions.-When the dosage of salicylates is raised to the full therapeutic effect, there occur usually a series of side actions resembling cinchonism. They consist in nausea and vomiting, ringing in the ears and deafness, vertigo and headache, mental dulness and confusion, diaphoresis, quickened pulse and deepened respiration; rarely skin eruptions, albuminuria, impaired vision (de Schweinitz, I895; Claiborne, I9o6), and delirium, the latter especially in alcoholic patients. All these effects are usually without danger, disappearing promptly when the drug is discontinued. Abortion has sometimes occurred, but it is doubtful whether this was due to the drug or to the disease. Mattos, I905, claims an emmenagogue action.

The nausea and vomiting are not due to local irritation, except if free salicylic acid is given. The salicylates and esters are not especially irritant; and if fractional doses are given, the gastric phenomena set in only after some hours, about the same time as the ear and other systemic effects. They are therefore probably central. This has been confirmed by the direct method of Eggleston and Hatcher, I9I5. To guard against the liberation of salicylic acid, it is advisable to neutralize the gastric acidity by giving one to two grams of sodium bicarbonate with the salicylate.

The ear symptoms are perhaps connected with congestion of the auditory apparatus (Kirchner, I88I; but Haike, I904, believes these lesions to be asphyxial artefacts). The brain vessels are dilated (Berezin, I9I6).

Toxic Dose.-This has been investigated by Hanzlik, I9I3, in over 500 administrations. If the salicylates are administered in repeated doses of about I Gm., one or two hours apart, the "toxic" effects appear usually when the total dose of sodium salicylate attains between 7 and ${ }^{3} 3 \mathrm{Gm}$., Ioo to 200 gr.; the mean being about I $2 \mathrm{Gm}$., I $80 \mathrm{gr}$. Women require about four-fifths of this dose. There are no differences between sixteen and seventy-five years of age. Children have a relatively high tolerance, i.e., the toxic dose is much higher than would be calculated from their age (Archambault, I878). The dose is the same for the white and the colored race. It is not affected by the presence or nature of disease. Occasionally there are unexplained idiosyncrasies or tolerance (toxic doses as low as 20 and as high as I, 300 gr.). These are usually only temporary. The toxic dose of acetylsalicylic acid and methyl salicylate is about two-thirds that of salicyl-salicylic acid (MacLachlan, I9I3) about one-half that of sodium salicylate. The toxic dose and effects of strontium salicylate are the same as for the sodium salt (Blankenhorn, I9I6).

Dangerous poisoning by sodium salicylate is rare; and it is often difficult to judge whether the effects attributed to the salicylate were not really due to the disease.

The symptoms are described as vomiting, unconsciousness, delirium, dyspnea and deepened respiration (Quincke, I882), fall of temperature, arythmia, albuminuria, paresis, collapse, asphyxial convulsions (Peterson, 1877; Weckerling, r877; Allaire, r879; Vinci, 1905). Sicard and Meara, I915, attribute a case of sino-auricular block, with standstill of the entire heart, to salicylate. The fatal dose is stated as 5 to $12 \mathrm{Gm}$.; but must be very variable, even severe cases ending generally in recovery. The autopsy in Quincke's case showed hyperemia of the brain and its membranes, of the kidneys and lungs, and pericardial ecchymoses.

Acidosis.-Quincke called attention to the resemblance of serious salicylate poisoning to diabetic coma. Langmead, 1906, and Lees, I908, emphasize this resemblance in children, and describe the occurrence of acetone even after relatively small doses of salicylates. The acetonuria is probably often due to the associated conditions of the 
disease rather than to the salicylate; but it is advisable to follow their suggestion to administer sodium bicarbonate for prophylaxis and treatment of any acidosis.

Toxic Effects in Animals. - These are similar to those in man. The respiration is deepened and labored, first quickened, then slowed to final asphyxia with convulsions. The blood pressure rises at first, then falls severely; the pulse is inconstant. The intracranial vessels are constricted (Wiechowski, 1902). There is general collapse and sometimes paralyses. The heart stops with the respiration. The autopsy shows the same congestions as in man (Koehler, I876; Deseille, 1879; Blanchier, 1879; Quincke, 1882). The toxicity is low, the fatal dose per vein for dogs being about I Gm. per kilogram. Methyl and ethyl salicylates intravenously produce pulmonary edema by injuring the capillary walls.

Therapeutic Effects in Rheumatic Fever and Rheumatic Tonsillitis.The proper administration of salicylates controls the manifestations of this disease in a most remarkable manner in nearly all typical casesso uniformly that failure throws suspicion on the diagnosis: the pain and fever disappear; the local inflammation, the immobility, swelling and redness subside; the general condition of the patient is vastly improved; and the disease is under complete control in one to at most five days. These benefits last only so long as the patient is kept salicylated and relapses occur when this is discontinued. According to statistics, the total duration of the disease, as judged by the stay in the hospital, is not affected (J. L. Miller, I9I4).

The rheumatic cardiac lesions are not relieved directly (Vulpian, I880). Indirectly the salicylate treatment doubtless has an important tendency in this direction by favoring rest and avoiding the extra tax of fever, pain and insomnia.

Efficiency in Related Conditions.-Salicylates are much less certain in atypical subacute, chronic, and muscular rheumatism, lumbago, etc., although they usually have some analgesic effect. In acute gout they are often quite effective (Vulpian, I880). They are notably inefficient in scarlatinal and gonorrheal arthritis (G. Sée, I377; Stockman, I906). They are also used in headache, neuralgias, sciatica, tabes and other similar pains, but with inconstant results. In chorea it is difficult to prove any benefits.

Response in Different Affections.-In the routine statistics of Hanzlik, I9I3, in I 32 cases of rheumatic fever, salicylates gave complete relief in 82 per cent., partial relief in 16.5 per cent., and failed in only 1.5 per cent. In subacute rheumatism, thirty cases, it gave complete relief in 53 per cent.; partial in 30 per cent.; failures in I 7 per cent. In sciatica, eleven cases, it did not give complete relief in any, failed completely in 27 per cent., and afforded partial relief in 73 per cent.

Mechanism of Action.-This has been attributed to the general analgesic effect; and to a specific germicidal action on the hypothetic organisms of rheumatic fever. The present data do not suffice to establish either of these views.

The enormous doses which are required; the small efficiency of other antipyretics and analgesics; the relative inefficiency of the salicylates in other forms of arthritis-all these facts point to a specific action on the infecting agent.

Against this view speak the prompt recurrence of the symptoms if the salicylate is discontinued and the failure to prevent the cardiac infection. In rabbits, the prophylactic use of salicyl does not prevent or even influence arthritis from intravenous injection of rheumatic hemolytic streptococci (Miller, I9I4; Davis, I9I5); but this is not conclusive since the etiology has not been established, and the administration is very different. The analgesic explanation is based on the limited efficiency of 
salicylates in other painful conditions (Laborde, I877; Vulpian, I880); and on the effect of other analgesics in rheumatic fever.

Administration.-For internal use, the free salicylic acid is too irritant. The sodium salicylate is the most commonly used, notwithstanding its mawkish, nauseant taste. This may be partly disguised by giving the salt in carbonated water, or flavoring with peppermint or wintergreen water. The direct gastric irritation is avoided by adding an equal or double quantity of sodium bicarbonate.

To be effective in acute rheumatism, large doses must be administered: I to I.3 Gm. ( $I_{5}$ to $20 \mathrm{gr}$.) should be given every hour until "toxic" symptoms (tinnitus, nausea, etc.) set in. The administration should then be stopped for twelve hours. After this, I Gm. ( 5 gr.) should be given every four hours for several weeks, keeping the patient in bed as long as possible. As a prophylactic treatment, I Gm. ( ${ }_{5}$ gr.) may be given three times a day for a week in every month. If the sodium salicylate is not tolerated, oil of gaultheria or aspirin may be substituted. Oil of gaultheria also gives some relief when applied externally to the affected joints.

Hypodermic injections are used by Seibert, I9II, Io c.c. of 20 per cent. sodium salicylate per roo pounds of body-weight, every twelve hours; to avoid pain, cocain may

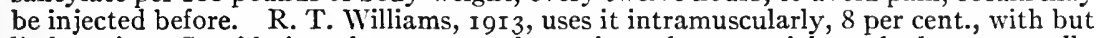
little pain. Considering the prompt absorption, these special methods are usually superfluous.

Rectal Administration.-This is preferred by Heyn, I914, as being more prompt and less disagreeable. He gives a cleansing soap-sud enema. When it has taken effect, it is followed by the salicylate through a rectal tube, inserted 6 to 8 inches. The first dose is of 8 to I I Gm. for men, $6 \mathrm{Gm}$. for women, in I 50 c.c. of water or thin starch, with I c.c. of Tr. Opii. It may be repeated in twelve hours, but usually one enema per day suffices. Salicyl appears in the urine within fifteen to thirty minutes.

In very severe cases, the intravenous injection of sodium salicylate may be justified (Mendel, I905, 1908). Conner, 1914, uses it, with a small needle to avoid thrombosis, in doses of $\mathrm{I}$ to $2 \mathrm{Gm}$., every eight to twelve hours. Cernadas, I $_{9}$ I $_{5}$, advises a solution containing 20 per cent. of sodium salicylate and I per cent. caffein; injecting this daily, at first 6 c.c., increasing to to c.c. The relief is more prompt, and no bad effects have been reported; but it would not be justifiable as a routine measure.

"Natural" and Synthetic Salicylates.-It is often stated that the synthetic sodium salicylate, owing to the presence of supposed impurities, is more toxic and less efficient than that prepared from oil of wintergreen or birch. The evidence for this view, which has been fostered mainly by interested manufacturers, can not stand critical examination. There need be no hesitation in employing the much more economical synthetic product (Rep. Counc. Pharm. Chem., I9r3; J.A.M.A., 61 : 979).

The literature of the subject and its uncritical status have been fully discussed by Eggleston, Igr 2. Various impurities have been announced in synthetic salicylates, especially cresotic acids, phenol, para-hydroxybenzoic acid, and hydroxy-isophthalic acid; but the evidence for their presence is not convincing. However this may be, Hilpert, I9I3, showed that at the present time even the cheapest commercial samples are quite as pure as the most expensive. Waddell, IgII, proved that the effects of natural and synthetic salicylates on animals are quantitatively alike; and the coöperative clinical tests, instituted by the Counc. Pharm. and Chem., arranged so as to exclude all bias, showed that their effects on patients are indistinguishable (Hewlett, 1913). In practice, it is almost impossible to be certain of their source, since the oils of wintergreen or birch are very often adulterated, beginning at the still.

Relation of Constitution to Specific Anti-rheumatic Action.- Phenol, $\mathrm{C}_{6} \mathrm{H}_{5} \cdot \mathrm{OH}$, is quite inactive. Benzoic acid, $\mathrm{C}_{6} \mathrm{H}_{5} \cdot \mathrm{CO}_{2} \mathrm{H}$, has a limited efficiency. The $\mathrm{OH}$ and $\mathrm{CO}_{2} \mathrm{H}$ groups are both necessary, and must be in the ortho position to obtain the full efficiency. Derivatives which can be converted into salicylic acid in the organism are effective, in proportion to the ease of their conversion; but if the $\mathrm{H}$ of either group is firmly combined (as in methyl salicylic acid, $\mathrm{C}_{6} \mathrm{H}_{4} \cdot \mathrm{CO}_{2} \mathrm{H} \cdot \mathrm{OCH}_{3}$, and in salicyluric acid), 
they are inactive. The introduction of additional groups into the benzol nucleus, as in the cresotinic acids, does not disturb the action (May, r9o9; Stockman, I9I3).

Cresotinic Acids.--These were at one time blamed for the reputed higher toxicity of synthetic silicylates. In fact, however, they act very similarly and have about the same toxicity as salicylic acid (R. May, I909; Stockman, I89o, I909 and I9r2; Waddell, IOII). They have a similar specific effect in rheumatic fever, although they are somewhat inferior. They have even been used therapeutically in doses up to $8 \mathrm{Gm}$. (Buss, I 876 ; Catti, I 879; Demme, I888). The resemblance is explained by their chemical structure, $\mathrm{C}_{6} \mathrm{H}_{4} \cdot \mathrm{OH} \cdot \mathrm{CO}_{2} \mathrm{H} \cdot \mathrm{CH}_{3}$; with the $\mathrm{OH}$ and $\mathrm{CO}_{2} \mathrm{H}$ in ortho position.

Isomers of Salicylic Acid.-Meta- and para-hydroxybenzoic acids, which differ from salicylic acid only in the position of the side-chains, are practically inactive and nontoxic. The efficiency therefore depends on the adjacent position of the $\mathrm{OH}$ and $\mathrm{CO}_{2} \mathrm{H}$ groups. The cause of this has not been explained. Another difference is, that these siomers are excreted in combination with sulphuric acid instead of glycocoll.

\section{PREPARATIONS-SALICYLATES}

* Acidum Salicylicum (Acid. Salicyl)., U.S.P., B.P.; Salicylic Acid (Ortho-hydroxybenzoic acid), $\mathrm{C}_{6} \mathrm{H}_{4} \cdot \mathrm{OH} \cdot \mathrm{COOH}$. Prepared synthetically from phenol; or by saponification of methyl salicylate (oil of birch). White, light crystalline powder or needles, odorless or with faint odor of wintergreen, of sweetish, then acrid taste. Slightly sol. in water $(\mathrm{I}: 460)$; freely sol. in alc. $(\mathrm{I}: 2.7)$ or eth. Dose, $0.75 \mathrm{Gm} .$, I2 gr., U.S.P., 0.3 to I. 2 Gm., 5 to $20 \mathrm{gr}$., B.P.

Ung. Acid. Salicyl., B.P.-2 per cent. in Paraffin Ointment.

Ammon. Salicyl., U.S.P., $\mathrm{NH}_{4} \cdot \mathrm{C}_{7} \mathrm{H}_{5} \mathrm{O}_{3}$ - - Resembles Sod. Salicyl. Freely sol. in water ( $\mathrm{r}: \mathrm{I})$ or alc. $(\mathrm{I}: 3)$. Dose, 0.5 Gm., 8 gr., U.S.P.

* Sodii Salicylas (Sod. Salicyl.), U.S.P., B.P.; Sodium Salicylate, $\mathrm{NaC}_{7} \mathrm{H}_{5} \mathrm{O}_{3}$.-White amorphous or crystalline powder or scales, or not more than faint pink tinge, odorless, or disagreeably sweet saline taste. Very sol. in water ( $1: 0.9$ ), freely sol. in alc. ( $1: 9.2$ ). Incompatible with acids, iron salts, iodids, spirit of nitrous ether and quinin. Dose, I Gm., I 5 gr., U.S.P.; 0.6 to 2 Gm., Io to 30 gr., B.P.

Stront. Salicyl., U.S.P., $\mathrm{Sr}\left(\mathrm{C}_{7} \mathrm{H}_{5} \mathrm{O}_{3}\right)_{2}+2 \mathrm{H}_{2} \mathrm{O}$. - Resembles Sod. Salicyl. Sol. in water (r:I9) or alc. (r:6r). Dose, r Gm., I5 gr., U.S.P.

\section{SALICYL DERIVATIVES}

To avoid the disagreeable taste and gastric symptoms of salicylates, esters and similar compounds have been introduced, which are more or less insoluble, and in which the salicyl radical is bound so as to be liberated only in the intestine or after absorption. These compounds have little or no direct action on the stomach, but they do not avoid the nausea and vomiting (Hanzlik, I9 3 ; MacLachlan, I9 3 ), since these are probably central. In this respect they are therefore not superior to sodium salicylate, which also does not produce direct gastric irritation, if properly guarded by bicarbonate. The taste is materially improved, but this advantage scarcely balances their high cost. The alkyl esters (methyl salicylate) are more efficiently absorbed from the skin and are therefore better for external use. The acyl derivatives (acetyl salicylic acid) possess a higher analgesic and antipyretic action and have therefore a special field. The salols contain active phenols which adapt them to intestinal antisepsis. These compounds may be arranged under three types:

1. Replacement of the $\mathrm{H}$ of the Hydroxyl by Acyl Radicals.--To this type belong: Acetyl salicylic acid (aspirin), $\mathrm{C}_{6} \mathrm{H}_{4} \cdot \mathrm{O}\left(\mathrm{COCH}_{3}\right) \cdot \mathrm{COOH},{ }^{1}$ and the corresponding com-

1 Krannichfeldt, 1914, considers the correct formula of acetosalicylic acid as:<smiles>CCOC(CCCO)C(=O)O</smiles> 
pounds of salicylic acid with methylene-citric acid (Novaspirin); with salicylic acid (Diplosal); and with succinic acid (Diaspirin).

2. Replacement of the $\mathbf{H}$ of the Carboxyl by Alkyl Radicals.--Methyl salicylate, $\mathrm{C}_{6} \mathrm{H}_{4} \cdot \mathrm{OH} \cdot \mathrm{CO}_{2}\left(\mathrm{CH}_{3}\right)$, and the corresponding salicyl esters of ethyl (Sal. Ethyl); of methoxymethyl (mesotan); and of monoglycol (spirosal).

3. Replacement of the $\mathbf{H}$ of the Carboxyl by Phenyl Radicals. - Phenyl salicylate (salol), $\mathrm{C}_{6} \mathrm{H}_{4} \cdot \mathrm{OH} \cdot \mathrm{CO}_{2}\left(\mathrm{C}_{6} \mathrm{H}_{5}\right)$, and the corresponding salicyl esters of naphthol (betol); of guaiacol; and of acetparamidophenol (salophen).

To these may be added salicin, from which salicylic acid is formed in the organism.

Only a few of these derivatives need be considered in any detail; the others which are acceptable are described in N.N.R.

Percentage of Salicyl in Various Derivatives.-roo parts of sodium salicylate $\overrightarrow{ }$ 85.6 parts of salicylic acid $=$ I0o of sodium salicylate; Ioo parts of methyl salicylate $=$ 90.7 parts of salicylic acid $=106$ of sodium salicylate; 100 parts of acetyl salicylic acid $=77$ parts of salicylic acid $=89$ of sodium salicylate; roo parts of salicyl salicylic acid $=$ I06.2 parts of salicylic acid $=\mathrm{I} 24$ of sodium salicylate.

\section{PREPARATIONS-SALICIN}

Salicinum (Salicin.), U.S.P., B.P., $\mathrm{C}_{13} \mathrm{H}_{18} \mathrm{O}_{7}$ - - Colorless, silky, shining, crystalline needles or a white, crystalline powder; odorless, and having a very bitter taste. Sol. in water $(\mathrm{r}: 23.5)$ or alc. ( $\mathrm{r}: 88.5)$. Dose, $\mathrm{I}$ Gm., I 5 gr., U.S.P.; 0.3 to I.2 Gm.; 5 to 20 gr., B.P., in solution or wafers.

Salicin occurs in willow bark, which was used as antipyretic in antiquity. It also occurs in poplar bark. It was introduced against rheumatic fever by Maclagan, I876, on the basis of the fancied resemblance to malaria. It is inferior to the salicylates, because its conversion is of ten incomplete. Salicin is a glucosid, yielding on hydrolysis saligenin, $\mathrm{C}_{6} \mathrm{H}_{4}(\mathrm{OH}) \mathrm{CH}_{2} \mathrm{OH}$; this in turn is oxidized to salicylic acid (Nencki, I870), but a part is excreted unchanged. Salicin yields 45 per cent. of salicylic acid, and is therefore at most half as effective.

\section{ACETYLSALICYLIC ACID (ASPIRIN)}

This was introduced by Dreser, I899. It was supposed that it would pass unchanged through the stomach, thus avoiding gastric irritation; and that the salicylic acid would be liberated in the intestine. In fact, however, it produces some gastric irritation (Roch) and the intestinal decomposition is slow and incomplete, a part being absorbed unchanged; but the salicylic acid is completely liberated before excretion (Bondi and Katz, I9II). Acetyl salicylic acid is about one and one-half times as toxic as sodium salicylate in man (Hanzlik, I9I3); about twice as toxic in animals (Chisteni and Lapresa). In man even small doses ( 0.3 to 0.65 Gm., 5 to ro gr.) occasionally produce alarming symptoms, especially acute local or general edema of the skin and mucous membranes, urticaria, erythema, pruritus, etc. (Graham, Morgan, Gilbert, I9I I; Krause, I9I4; literature of side actions, Seifert, Nebenwirk., I9I5, p. I2). The effects of poisonous doses in animals are similar to other salicylates (Block, I 909). These side effects make this drug inferior to sodium salicylate in rheumatic fever. It has, however, stronger analgesic and antipyretic actions, even in non-rheumatic affections, perhaps because the undecomposed acetyl compound enters the nerve cells more readily. It is used extensively in colds, influenza and other fevers; and in neuralgias, headaches, tabes, and other pains, in tablets or capsules of $0.3 \mathrm{Gm}$., $5 \mathrm{gr}$.

\section{PREPARATIONS-ACETYLSALICYLIC ACID}

*Acidum Acetylsalicylicum (Acid. Acetylsal.), B.P., N.N.R.; Aspirin; C6 H.O. $\left(\mathrm{CH}_{3} \mathrm{CO}\right) \cdot \mathrm{COOH}$.-White, crystalline, odorless powder of sour taste. Slightly sol. 
$\mathrm{i}_{11}$ walier $(\mathrm{I}: 100)$, freely sol. in alc. $(\mathrm{I}: 5)$. Incompatible with moisture and alkalies. Dose, 0.3 to I Gm., 5 to I 5 gr., B.P., in powder, tablets or capsules. Maximum dose, 1 Gm., $15 \mathrm{gr}$. It forms soluble salts with calcium (Bercke, 19 2 ; Lewin, I9I 2) and with hexamethylenamin (Puckner, 1913); but these are unstable.

Diplosal, N.N.R., is tasteless and yields one-half more salicylic acid than aspirin and one-fourth more than sodium salicylate-a matter which is easily compensated by the dose. Its toxicity in man is twice that of sodium salicylate (MacLachlan, 1913). It is very resistant to gastric juice, but is rapidly saponified by the duodenal secretion (Tocco, I91 2).

Noraspirin, N.N.R., is said to be less powerful than aspirin, and less liable to produce unpleasant side effects (Koerner, 1910), perhaps because it has not been used as extensively. The literature of its side actions is given by Seifert, Nebenwirk., $19^{15}$, P. 25.

\section{METHYL SALICYLATE}

This acts somewhat more slowly, but otherwise as efficiently as sodium salicylate; the "toxic" dose being about two-thirds of that of the sodium salt. It is for the most part saponified in the intestine, but some may be absorbed unchanged (Baas). It avoids the disagreeable taste, but is more irritant to the stomach. The synthetic ester is said to cause less irritation than the natural oils. It may be given internally in extemporaneous capsules, in the same way as' sodium salicylate; or externally, generally diluted with I or 2 parts of olive oil and painted over the affected region. This is sometimes more effective than the internal use, especially in muscular rheumatism. Fatal poisoning by methyl salicylate is rare but has been reported (Nerthney, I903), especially from its use as abortifacient. An ounce is usually fatal, with convulsions, coma, gastritis and congestion of the kidneys (Pinkham, I887).

Ethyl salicylate is less irritant and half as toxic (Houghton, I905). Spirosal is used externally being non-irritant and easily absorbed (Impens, I907; literature, Seifert, Nebenwirk., 1915, p. 32).

Mesotan was introduced by Floret, I902. It is decomposed by water into salicylic acid, formaldehyd and methyl alcohol. It is used externally, but has caused dermatitis.

\section{PREPARATIONS-METHYL SALICYLATE}

*Methyl Salicylas (Methyl. Salicyl.), U.S.P., B.P.; Oleum Betula (Ol. Betul.), U.S.P.; Oleum Gaultheria (Ol. Gaulth.), U.S.P., B.P.; Methyl Salicylate; Oil of Sweet Birch; Oil of Gaultheria (Wintergreen), $\mathrm{CH}_{3} \cdot \mathrm{C}_{7} \mathrm{H}_{5} \mathrm{O}_{3}$.-Produced synthetically or obtained by distillation from Gaultheria procumbens or from Betula Lenta. A colorless, yellowish or reddish liquid; characteristic odor and taste of gaultheria. It is sparingly sol. in water; sol. in all proportions in alc. Dose, 0.75 c.c., I 2 minims, U.S.P.; 0.3 to I c.c., 5 to 5 minims, B.P. Maximum dose, 2 c.c., 30 minims.

\section{PHENYL SALICYLATE}

Salol was introduced by Nencki, r886. Being insoluble, it passes the stomach practically unchanged; but is gradually decomposed into its components, 40 per cent. of phenol and 60 per cent. of salicylic acid, in the intestine, by the saponifying action of the alkali, pancreatic lipase and putrefactive bacteria. This avoids the taste of the components and the local gastric action; and since the decomposition is gradual, the local intestinal action is less violent but more prolonged, and the toxicity is materially lessened. This "Nencki's salol principle" has been extensively applied in the form of other phenolic esters, $f . i$., guaiacol carbonate, to mitigate the taste, local actions and toxicity of other active phenols or acids. 
Therapeutic Uses.-Salol proper has scarcely fulfilled the original expectations. The toxicity of the phenol limits the dosage, and its absorption is so slow that it is practically inferior to the other salicyl compounds in rheumatism or as urinary antiseptic (Sollmann, I908). The phenol component was thought to be valuable as intestinal antiseptic, especially in infantile diarrhea; but the decomposition is so slow and the absorption of the phenol so rapid that effective quantities could scarcely come into action. The clinical and experimental evidence is not conclusive (Harris, I9I2). As a wound antiseptic it would be valueless because it is not decomposed by wound secretions and only very slowly by bacteria. Its use in tooth powders has caused eczema of the lips (Hugenschmidt, I907). Perhaps its main value is as a coating for "enteric pills," i.e., pill masses which are intended to pass the stomach unchanged ( $f . i$., ipecac in dysentery). It must be remembered that a coating sufficiently heavy to be effective introduces large doses of salol.

Decomposition and Antiseptic Action.-Salol is also decomposed to a slight extent in the mouth and stomach by bacteria (Lesnik, I 887). It is split by human milk, so that its administration to nursing infants may cause gastric irritation (Usener, Ig I 2). Its saponification by pancreatic ferments and alkalies is very slow and incomplete, insufficient to prevent putrefaction even in test-tube experiments; it is inferior to sodium salicylate or bismuth subnitrate (Sollmann, I908). Its antiseptic action on the blood is also slight. A part escapes decomposition in the intestines and passes unchanged into the feces -5 to 8 per cent. in the experiments of Bondzynski and Humnicki, I909; but doubtless often much more. If given in capsules, it may be found in the feces as crystals, "salol calculi" (Brossard, I 897), formed by the partial fusion and recrystallization of the salol (C. R. Marshall, I 897). It should therefore be administered subdivided as emulsion or with indifferent powders.

\section{PREPARATIONS-SALOL}

*Phenylis Salicylas (Phenyl. Sal.), U.S.P.; Salol, B.P., $\mathrm{C}_{6} \mathrm{H}_{4}(\mathrm{OH}) \mathrm{COOC}_{6} \mathrm{H}_{5 .-}$ White crystalline powder, of faint aromatic odor and taste. Very slightly sol. in water $(\mathrm{r}: 6,670)$; freely sol. in alc. ( $\mathrm{I}: 6)$; very sol. in fixed or volatile oils. Dose, $0.3 \mathrm{Gm}$., 5 gr., U.S.P.; 0.3 to I.2 Gm., 5 to 20 gr., B.P., in powders, generally with bismuth. Externally, as 5 per cent. ointment. Maximum dose, $2 \mathrm{Gm}$., $30 \mathrm{gr}$.

\section{PHENYL-QUINOLIN-CARBOXYLIC OR PHENYL-CINCHONINIC ACID (ATOPHAN)}

The formula of this substance $\left(\mathrm{C}_{9} \mathrm{H}_{5} \mathrm{~N} \cdot \mathrm{C}_{6} \mathrm{H}_{5} \cdot \mathrm{COOH}, 2: 4\right)$ shows its relation to quinolin and salicylic acid. It has the antipyretic actions of quinolin, and like salicylic acid it is analgesic, antirheumatic, and increases the excretion of uric acid. In the last respect it is much more efficient than any other drug. It was prepared by Doebner and Giesecke; I887; and introduced by Nicolaier and Dohrn, I908, for the treatment of gout.

Effect on Uric Acid Elimination.-The excretion of uric acid is greatly increased, sometimes more than doubled by therapeutic doses, even on a purin-free diet. The increased excretion begins within an hour (maximum in three hours) and lasts only so long as the drug is continued; and is followed by a compensatory decrease (Haskins, I9I3). With continued administration, the uric acid returns to normal after one or two days, unless the diet contains purins. The mechanism of this action is probably renal. The increase of uric acid is often so great that it precipitates in the urine before it is passed. Indeed, atophan apparently interferes with the urate-solvent action of the urine (Haskins, I9I6). The urine is of a dark color, due to urochrom (Skorczewski, I9I2). 
In other respects, its composition and quantity are not notably changed. The leucocytes of the blood are also unaltered. The uric acid of the blood is diminished by the increased excretion (Folin and Lyman, I9I3; McLester, I9I3), but soon reaches an irreducible minimum; there being no further lowering even if the administration is continued forty-five days. When the administration is stopped, the initial concentration is restored within two to four days (Fine and Chace, r9i4; Frank and Pietrulla, I9I4). The effects on the blood were also studied by Base, IgI4.

Other Changes in the Urine.-Skorczewski and Sohn, I912, found an increase of purin bases and of neutral sulphur (parallel to the urochrom; Skorczewski, 1913); retention of chlorids and the diazo-reaction. Fromherz, I9I2, did not find the uric acid increased in dogs; but Starkenstein, I9I I, reports increase in rabbits and dogs, with diminished allantoin. Schittenhelm and Ullmann, I9I3, found no increase of allantoin, but higher total $\hat{N}$ excretion in dogs. Biberfeld, I I $_{3}$, found no increase in purin bases, but increase of phosphates in dogs. McLester, I913, finds an increase in the quantity of urine and of total nitrogen. Eisner, I915, reports the excretion of chlorid and total nitrogen as often diminished. Renal-function tests gave inconstant results.

The creatin content of the blood is not decreased (Denis, I $_{95}$ ). In birds, the uric acid is decreased. The atophan itself is oxidized in the benzol nucleus (Dohrn, I912).

Mechanism of Action.- This has been attributed to increased destruction of nucleoproteins (Starkenstein, I9II; Schittenhelm and Ullmann; Retzlaff, I913); to increased eliminative activity of the kidneys for uric acid (Weintraud; Bauch, r9Ir; Folin and Lyman, I913; Fine and Chace, I914); to conversion of adsorbed into filtrable uric acid (Frank and Pietrulla, I9I4); to an intestinal action (Abl); and to increased formation of uric acid by lessened oxidation of its purin and nuclein precursors; perhaps as the result of its more rapid elimination (Dohrn; Frank and Prozedborski; Skorczewski, I912). The curve of excretion-temporary increase followed by compensatory decrease-and the lowering of the uric acid concentration of the blood indicate increased elimination of uric acid already present in the body. Weintraud, and Frank and Pietrulla, I9I4, find that uric acid injected into the blood is excreted more rapidly. The urate excretion is not increased by atophan in kidney disease (Fine and Chace, 1915). The absence of increased phosphate elimination speaks against destruction of nucleoproteins. The decrease of allantoin indicates diminished destruction of uric acid in animals, but this might be different in man. In surviving organs, atophan does not lessen the destruction of uric acid (Starkenstein).

Other Actions.--Large doses produce convulsions, paralysis and coma. Intravenous injection, in rabbits, is followed by stimulation of the vagus and vasomotor centers; these, as also the respiratory center, are finally depressed. The pupils are constricted, even after atropin. Atophan also prevents asphyxial and puncture glycosuria, and diminishes mustard chemosis. It lowers temperature, even in normal rabbits. These actions resemble calcium (Starkenstein and Wiechowski, I914).

Atophan inhibits completely the emigration of lewcocytes in the inflamed frog's mesentery, and exhibits in this respect a potentiated synergism with quinin (Ikeda, IgI6).

Therapeutic Uses. - The mobilization of uric acid was supposed to be useful in gout by removing it from the blood and by dissolving the tophi. The uric acid and the non-protein nitrogen of the blood are in fact lowered by it (Folin and Lyman, I9I3). Zuelzer, I9II, states that the increase of urate excretion in gout is more marked and prolonged than in health. Feulgen, I912, claims that the tophi are actually decreased, at least in some cases. Clinically, however, atophan is much less efficient in chronic gout than in the acute attacks and in non-uratic joint affections. This throws some doubt on the uric acid explanation, and makes it probable that atophan acts similarly to salicyl, perhaps as a specific analgesic. In agreement with this, Klemperer, I9 3 , found derivatives which lack the uratic action equally efficient clinically. In acute gout, it causes prompt subsidence of the pain and swelling. In rheumatic fever it also acts promptly, similar but somewhat inferior to salicyl; but it is sometimes 
effective when salicylates fail (Oeller, I9I2). The same applies to other joint affections.

Side Actions.-The precipitation of uric acid contraindicates its use in urate calculism. It is generally free from other unpleasant effects, even in large doses; but occasionally it produces vertigo, complete anorexia, cardiac distress (Loening, I913) and purpural; urticarial and scarlatiniform skin eruptions and fevers (Mueller; Phillips; Herrick, I9I3; Marañon, I9I4).

Administration. - The dosage of atophan is from 0.5 to I Gm., 8 to I5 gr., three or four times a day; or $0.5 \mathrm{Gm}$. every hour for six doses; suspended in large quantities of water. To prevent the precipitation of urates in the urine, Weintraud advises the administration of $15 \mathrm{Gm}$., $1 / 2$ ounce, of sodium bicarbonate (or its equivalent of acetate or citrate) on the first day, and 5 to Io $\mathrm{Gm}$. on the succeeding days. In gout, the diet should be purin-free.

\section{PREPARATIONS-ATOPHAN}

*Acidum Phenylcinchoninicum (Acid. Phenylcinch.), U.S.P. (Atophan; Phenylquinolin carboxylic acid), $\mathrm{C}_{6} \mathrm{H}_{5} \cdot \mathrm{C}_{9} \mathrm{H}_{5} \mathrm{~N} \cdot(\mathrm{COOH})$. - Small colorless needles or white powder; odorless; bitter taste. Almost insol. in water; slightly sol. in alc. (1:125); sol. in alkalies. Dose, $0.5 \mathrm{Gm} ., 8 \mathrm{gr}$., U.S.P.

Novatophan.-This is an ethyl-methyl ester of atophan. It has the trifling advantage of being practically tasteless, but seems to be slightly less effective (Haskins, 1913). Other related compounds have been investigated by Impens, rgr 2 .

\section{QUINIC ACID}

This is hexahydro-tetraoxy-benzoic acid. ${ }^{1}$ It occurs in cinchona bark, coffee and many other plants. In man (also in rabbits, not in dogs; Stadelmann, I879) it is largely transformed into benzoic acid and excreted as hippuric acid (Lautemann, 1863). Weiss, I 899, claimed that it lessened the formation of uric acid and that it had a beneficial effect upon gout. Decreased urate elimination was also claimed by Foerster, 1900. Several observers reported clinical benefits, and various compounds were placed on the market (urosin, sidonal, chinotropin, etc.). Further observations, however, showed the effects to be inconstant (Delacamp, Igor) or rather negative (Hupfer, I 903 ; Fatavall and Gies, 1903; Waucomont, I9II). Denis, 1915, found neither increase of urate elimination nor decrease of the urate content of the blood.

\section{SACCHARIN, BENZOSULPHINID OR GLUSID}

General Statement.-This substance, also sold under many other trade names, has the formula, $\mathrm{C}_{6} \mathrm{H}_{4} \cdot \mathrm{SO}_{2} \cdot \mathrm{CO} \cdot \mathrm{NH}$, and is the anhydrid of ortho-sulphamide-benzoic acid (the para-isomer is not sweet). In common with a number of other benzol derivatives, it has an intensely sweet taste, even in very dilute solutions. It passes through the body almost quantitatively unchanged, 75 to $9 \circ$ per cent. being excreted by the urine within twenty-four hours, and the remainder in the feces. Consequently it has a very low toxicity. Small doses, up to $0.3 \mathrm{Gm}$. per day, continued for months, produced no deleterious effects, so far as ascertainable by available methods of investigation. Large doses, 0.75 to I. $5 \mathrm{Gm}$. per day, are also practically harmless, except for occasional minor disturbances of digestion (Herter and Folin, I9II). Single doses of $5 \mathrm{Gm}$. have been given without much effect (Adduco and Mosso, I887).

Uses. - Saccharin is used as a substitute for sugar in diabetes (tablets of $0.03 \mathrm{Gm} ., 1 / 2 \mathrm{gr}$. = an ordinary lump of sugar); as flavoring agent and

\footnotetext{
${ }_{1}$ Quinic acid $=\mathrm{C}_{6} \mathrm{H}_{7}(\mathrm{OH})_{4} \mathrm{COOH}+\mathrm{H}_{2} \mathrm{O}$.
} 
adulterant of sweet foods; and as a mild antiseptic in fermentative dyspepsia (0.2 Gm., 3 gr., several times daily). Saccharin itself is but little soluble in water, but its sodium salt "soluble saccharin" dissolves readily. It may be prepared by adding $1 / 2$ part of sodium bicarbonate.

Sweetening Power.-This is about 500 times as great as that of cane sugar. It is perceptible in dilutions of $I: 100,000 ; \mathrm{I}: 10,000$ is agreeably sweet; with $\mathrm{r}: \mathrm{I}, 000$ it becomes bitter and disagreeable. Even with the more dilute solutions the quality of the taste is somewhat different from sugar and on continued use the persistent sweetness becomes very objectionable.

Effects of Continued Use.-Long-continued and detailed investigations were made for the Referee Board by Herter and Folin, I9I $x$; Herter administered the sodium salt, Folin the free saccharin, in "small" doses to $0.3 \mathrm{Gm}$. per day, and "large" doses of 0.75 to $\mathrm{I} .5 \mathrm{Gm}$., corresponding in sweetening power to $15^{\circ} \mathrm{Gm}$. and to 375 to $75^{\circ} \mathrm{Gm}$. of cane sugar respectively. The small doses produced no discoverable effects whatever. The large doses were also negative as to metabolism (total nitrogen, ammonia, uric acid, creatinin, sulphur-except that of the saccharin-chlorids, acidity and phosphates). The kidneys were not affected (no diuresis or sediments). There were no general symptoms or blood changes. The digestive functions were also generally unaffected (motor and secretory functions of the stomach, bulk of feces, etc.) even in patients with hyperor hypo-chlorhydria; but occasionally there was slight hyperacidity and indications of slight increase of intestinal putrefaction (urinary oxy-acids, indican, fecal $\mathrm{H}_{2} \mathrm{~S}$, skatol, indol and acidity. The fecal gas flora remained unchanged). In vitro, the efficiency of all the digestive ferments was not altered by the concentrations which might occur in the body (except by the acidity of free saccharin, a factor which had been neglected in some of the earlier investigations; Riegler, 1895; Matthews and McGuigan, 1905). The antiseptic action was very slight.

The older literature contains reports of digestive and other disorders (Bornstein, Igoo), especially after continued use (v. Jaksch); but these observations were not made with the same degree of accuracy.

The toxicity for animals is very low (Adduco and Mosso, 1887). Matthews and McGuigan observed depression, stupor, convulsions and nephritis. Herter, administering soluble saccharin to rabbits by stomach, found no effects with 4 to $5 \mathrm{Gm}$. per kilogram; double this dose was fatal in two hours, the autopsy showing violent gastrointestinal irritation.

Use of Saccharin in Foods.-The preceding investigations show that no serious damage need be apprehended from the quantities of saccharin which would ordinarily be used. Larger quantities, however, tend to produce minor derangements, which could perhaps become more accentuated in pathologic conditions. For this reason, and because saccharin has no food value, its indiscriminate use as a mere sweetening agent should be condemned, except in cases in which sugar is inadmissible.

\section{PREPARATIONS-SACCHARIN}

* Benzosulphinidum (Benzosulphinid.), U.S P.; Glusidum, B.P. (Saccharin), $\mathrm{C}_{6} \mathrm{H}_{4} \cdot \mathrm{SO}_{2} \cdot \mathrm{CONH}$, the anhydrid of orthosulphamido-benzoic acid.-White, crystalline powder; odorless, or having a faint, aromatic odor, and intensely sweet taste in dilute solution. Slightly sol. in water $(\mathrm{I}: 235)$; sol. in alc. ( $\mathrm{I}: 3 \mathrm{I})$; easily sol. in alkalies. Dose, 0.2 Gm., 3 gr., U.S.P.

Sodii Benzosulphinidum (Sod. Benzosulphin.), U.S.P. (Sodium-Saccharin), $\mathrm{NaC}_{7} \mathrm{H}_{4}-$ $\mathrm{NSO}_{3}+{ }_{2} \mathrm{H}_{2} \mathrm{O}$--Colorless prisms, or white, crystalline powder; odorless; intensely sweet taste. Dose, 0.2 Gm., 3 gr., U.S.P.

\section{METHYLTHIONIN HYDROCHLORID OR METHYLENE BLUE}

This dye (tetramethyl-thionin hydrochlorid) was introduced on the basis of its selective coloring power, under the belief that this implied equally selective actions. The expectations were disappointing. It is of rather doubtful value as an analgesic in neuralgia, neurites, sciatica; 
as antizymotic and antipyretic in malaria; as antiseptic in cystitis, urethritis, conjunctivitis and skin diseases; and as a test for kidney function. It colors the urine green or blue (also the sweat; Tachau, Igri).

Urinary Antisepsis.-Methylene blue $(I: 150,000)$ is antiseptic for pure cultures; but it is a slow and weak germicide and practically inactive in the leucoform, in which it is excreted in the urine. In doses of 0.4 to $0.6 \mathrm{Gm}$, four times daily for several days, it perhaps is of some use in staphylococcus cystitis; but useless in urethritis (Hinman, I915).

Toxic Actions.-Therapeutic doses sometimes cause slight vesical or gastro-intestinal irritation (Seifert, Nebenwirk., Igr5, p. 24); but its toxicity is very low, the fatal dose for dogs exceeding I $\mathrm{Gm}$. per kilogram. Its continued use is also harmless (Tanfiljeff, 1907). Hypodermically, it may produce ulcers and abscesses. Intravenously, it raises the blood pressure by a cardiac action (Garfounkel and Gautrelet, I913).

Fate.-Methylene blue is distributed through all the tissues (localization in kidneys, G. D. Shafer, r9o8), but it is rapidly reduced to colorless leuco-compounds. These are oxidized when the tissues are exposed to air (Herter, 1904) or in contact with hydrogen peroxid, thus restoring the blue color. It is excreted in the urine and feces (traces in the sweat and sputum), partly unchanged and partly as "methylene azure." The latter is also present in medicinal methylene blue, being formed from it by alkalies. Both exist in a colored and colorless (reduced) form in the urine (Underhill and Closson, 1905). This is therefore rendered more blue by oxidizing agents, especially in acid reaction; and paler by reducing agents (dextrose, etc.).

Test of Kidney Function.- -0.02 to $0.5 \mathrm{Gm}$. are injected hypodermically. The excretion begins normally between fifteen to twenty-five minutes. Indigocarmin (4 c.c. of 4 per cent., hypodermically) has also been used; as well as phlorhizin. The usefulness of these tests is much more limited than that of the phenolsulphonephthalein test.

\section{PREPARATIONS - METHYLENE BLUE}

Methylthionine Chloridum (Methylthionin. Chlor.), U.S.P. (Methylene Blue; Tetramethylthionin Chlorid). - Not to be confused with methyl bluet A dark green, crystalline powder, or prismatic crystals having a bronze-like luster. Freely sol. in water and alc. Dose, 0.I 5 Gm., $21 / 2$ gr., U.S.P. Externally, 0.5 per cent. solution on mucous membranes, 2 per cent. on skin.

Sodii Indigotindisulphonas (Sod. Indigotin.), U.S.P. (Indigo carmin).-Blue powder or paste, sparingly sol. in water; insol. in alc. Used for coloring Bichlorid Poison Tablets.

\section{OTHER COAL-TAR DYES}

These share the general properties of the aromatic group. A number are discussed in other connections, e.g., fuchsin, scarlet red, trypan red, etc. Basic dyestuffs may become valuable urinary antiseptics, but have not been sufficiently worked out (Hinman, I9I5). Methyl violet (Pyoktaninum Coeruleum) is used as an antiseptic (Stilling, I890) in I to $4: 10,000$ solution or 2 per cent. ointment. Martius yellow resembles cocain in toxicity and in some of its actions (A. P. Mathews and Longfellow, 1910). Acid dyes are generally much less toxic than basic. The penetration of. dyes into cells presents very interesting problems (A. P. Mathews, 19ro). Basic dyes diminish, acid dyes increase the imbibition of gelatin. This may be a factor in the penetration, but is not a complete explanation (Traube and Koehler, 1915). The vital staining and the toxicity of acid and basic dyes are not parallel (Hoeber, 19I4). The addition of dyes to other poisons of ten modifies their toxicity (Sellei, r9I3)

Dyes generally are excreted more rapidly by the bile than by the urine, no matter how administered (Salant and Bengis, 1916).

Fluorescein, N.N.R. (Resorcinolphthalein), the anhydrid of fluoresceinic acid, in the form of its soluble potassium or sodium salt (Uranin) is used for its peculiar staining reaction of the cornea and conjunctiva, to reveal epithelial injury. This was discovered by Ehrlich, 1882, and introduced by Hamberger, 1909. A 2 per cent. solution dropped into the eye does not stain normal tissues, but produces beautiful yellow and green tints on any abraded parts or ulcerations about foreign bodies, etc. Given by mouth, the whole body becomes of a jaundice color, which disappears in twenty-four hours. Here also the normal eye is not colored, the dye being retained in the posterior chamber; but in the presence of intraocular disease, glaucoma, iritis, etc., the aqueous humor is colored bright green in about twenty minutes, thus differentiating these diseases from 
conjunctivitis in which there is no coloration. The dose by mouth for adults is 3 to 6 Gm.. for children 1.5 to $2 \mathrm{Gm}$., in water. This presents no danger.

Kenal Funclion Test.-This is described by Strauss, I913, Sod. Fluorescein, I Gm. in a cup of teal, is given on an empty stomach. Normally, it appears in the urine (best seen when alkaline) in ten to twenty minutes, and lasts thirty-five to forty hours. In renal disease, it appears later and lasts longer. The skin may be stained slightly yellow, but there are no other side effects.

Eosin.-Rost, I915, found its oral administration harmless in man and animals.

Basic Fuchsin (Gruebler), para-rosanilin acetate, was advised by May, I9I 2, and Donnelly, I I I , as a lotion or ointment (I per cent.) for ulcers and burns. It is antiseptic. non-irritant, non-toxic, and seems to encourage epithelial growth and granulation. The color may be removed from the skin by alcohol.

Gentian Violet.-This combines a relatively high toxicity to bacteria with a low toxicity to higher tissues. Russel, I914, for instance, found that tissue cultures are not injured by concentrations which kill bacteria. It is practically ineffective against tetanus, however (Hall and Taber, I9I4); rabies, etc., also showed poor success (Churchman, I914).

Fluorescent Substances.-V. Tappeiner and Raab (1903) have found the fluorescent substances are very destructive to all forms of life, and also to ferments and toxins; but that the effects appear only in the presence of the specific light rays which induce the fluorescence (Review, Tappeiner, I909). Toxins are very susceptible to fluorescence (Noguchi, I906).

\section{MISCELLANEOUS ANTISEPTICS}

\section{FORMALDEHYD}

General Statement.-The aldehyds react chemically with an exceptionally large number and variety of organic substances, amongst others with proteins. This probably explains their actions (Liebreich, I893). Formaldehyd, $\mathrm{HCHO}$, especially is very strongly germicidal and is largely used as a disinfectant. It hardens the tissues on local contact and thus produces irritation, especially of mucous membranes. Its systemic toxicity is relatively low.

Formaldehyd is a colorless, irritant gas, prepared by the oxidation of methyl alcohol. It is freely soluble in water, and is found on the market in the form of a 40 per cent. solution (Formalin). ${ }^{1}$ The solutions tend gradually to become inactive by the formation of the insoluble, polymeric, trioxymethylen or paraform, ( $\mathrm{HCHO})_{3}$, especially on heating, but somewhat also on standing. The latter may be prevented by chemic means (calcium salts, methyl alcohol). Trioxymethylen again yields formaldehyd by dry heat.

Formaldehyd is converted into hexamethylenamin by ammonia or ammonium salts.

Other Aldehyds.-Aldehyds contain the CHO group linked with organic radicals. Acetaldehyd and paraldehyd are somewhat antiseptic, but more markedly narcotic. This narcotic action is especially prominent in trichloraldehyd (chloral).

Acrclein, $\mathrm{CH}: \mathrm{CH} \cdot \mathrm{CHO}$, resulting from the decomposition of glycerin by excessive heat, is highly irritating and but little antiseptic (Fraenkel, Arzneimittel-synth., p. 628).

Historical.-Formaldehyd was discovered by von Hoffmann, r867; its antiseptic action by Loew and Fischer, 1886. Aronson, 1894, showed that it also destroys toxins.

Reaction of Formaldehyd with Proteins.-Formaldehyd enters into rather peculiar compounds, which are precipitated under suitable conditions, from which the formaldehyd and the original proteins may be completely regenerated under other conditions. The literature to 1897 is given by Benedicenti. With albumoses (Sollmann, 1902) and presumably with other proteins the compound contains definite proportions of formaldehyd, albumose and alkali. It is precipitated in weakly alkaline solutions,

1 In making solutions of a given strength, it is therefore necessary to take 213 times as much of the commercial solution as would be required of absolute formaldehyd. 
dissolved without decomposition by stronger alkalies, and is decomposed, or its formation prevented, by weak acids and by excess of neutral salts. Even under the most favorable conditions the combination is completed slowly. It is hastened by increased concentration of formaldehyd, protein or alkalinity, and by heat. ('This combination has also been investigated by Schryver, I grr). The precipitate also contains a further quantity of formaldehyd mechanically.

Other Chemic Actions.-Formaldehyd retards or prevents the coagulation and spontaneous laking of shed blood, when added in the proportion of $\mathrm{I}: 200$ to 400 . Uric acid and urates are dissolved, or their precipitation is prevented, by the formation of soluble compounds. Urea, on the other hand, forms an insoluble compound with formaldehyd.

Digestive Ferments. - The digestibility of fibrin and the coagulability of casein are diminished. Formaldehyd also lessens the activity of certain ferments, namely, papain, trypsin, ptyalin, and amylopsin; whilst pepsin, rennin, and malt diastase are but little altered.

Antiseptic Action.-Formaldehyd is one of the most powerful disinfectants, of relatively high penetrating power, harmless to metals, fabrics and colors and of relatively low toxicity. On account of its irritant effects, its use on the body is inadvisable. It is employed particularly for the disinfection of rooms and excreta.

Antiseptic Efficiency.-Formaldehyd is very much more effective as an antiseptic than as a germicide. Its activity varies for different organisms. Typhoid bacilli, with exposure of $t$ wenty-four hours, are killed by $1: 6000$, completely restrained by $I: I 6,000$; partly restrained by I : 30,000 . I : 40,000 is practically ineffective (Hyman, I9I 4); all the concentrations refer to absolute HCHO. Solutions of $1 / 2$ to $21 / 2$ per cent. destroy all bacteria, spores and toxins, in six to twenty-four hours. The gas is similarly antiseptic, especially when it is moist. The activity is increased by acids, presumably by preventing combination with the proteins of the medium. (Further details, J. F. Anderson, 1897, Hyg. Bull., 39; Hinman, 1913.)

Practical Application.-Feces are mixed with ro per cent. of the commercial solution. This destroys the odor almost immediately, but to insure complete disinfection, they should be left in contact for an hour. Tubercular sputum should be disinfected with 5 per cent. of the commercial solution for an hour. Instruments and other articles may be placed in $x 1 / 2$ to 5 per cent. solutions of formalin.

The Disinfection of Rooms. - Formaldehyd vapor is the most effective disinfectant against infectious diseases. It has little penetrative power; and does not destroy mosquitoes or other insects, and is therefore inferior to sulphur dioxid against malaria or yellow fever (McClintock, etc., IgII). The formaldehyd vapor may be generated by special apparatus involving the incomplete combustion of methyl alcohol or the vaporization of trioxymethylen.

Perhaps the most convenient effective method of evolving the gas is by permanganate (Doerr and Raubitschek, 1907); for a room of 2,000 cubic feet, 2 pounds of potassium permanganate are placed in a 3-gallon pail and this is set in a large tin can containing water. When everything is ready, 2 quarts of 40 per cent. formaldehyd are poured on the permanganate, the room is vacated immediately and left closed for at least twelve hours, then thoroughly aired (Hoxie).

Following a suggestion of Rosengarten, S. G. Dixon, I9I4, advises a chromatic mixture for vaporizing the formaldehyd. A solution of $\mathrm{I} 1 / 2$ fluidounces of concentrated sulphuric acid and I pint of U.S.P. Formaldehyd Sol. is prepared in advance and keeps perfectly. When needed, it is poured on ro ounces of sod. dichromate crystals, which have been spread in a thin layer in a vessel of ten times the capacity of the ingredients. The gas is liberated more rapidly than with permanganate; the operator must therefore withdraw promptly.

Simple spraying with formaldehyd solution (8 c.c. per cubic meter) is also used. Evaporation of formaldehyd solution by heat is wasteful, but may be employed ( $5_{5}$ c.c. for 1,000 cubic feet). A I : 20 dilution, allowed to stand about a room in saucers, is said to kill flies.

Applications to the Body.-This is not recommended on account of the local irritant action. It has been employed, however, for the disinfection of hands (I per cent.); as a mouth wash and gargle ( $1 / 2$ per cent.); in skin diseases and hyperidrosis (2.5 per cent.); for painting the throat in diphtheria or tonsillitis ( $21 / 2$ to 5 per cent.). The evaporation of a small amount of the solution in the room has also been used in tuberculosis. Maguire, 1900, suggested intravenous injections of $I: 20,000$. They were quickly abandoned. 
Formaldehyd as Food Preservative.-Concentrations of $I: 33,000$ to $\mathrm{I}: \mathrm{I0}, 000$ have been used, especially for milk. It is both ineffective and dangerous, and is therefore properly prohibited. The ordinary concentration for milk ( $\mathrm{I}: 10, \infty 00)$ checks lactic fermentation, but does not kill the tubercle bacilli and is therefore of questionable utility. Whilst it may not interfere with digestion in vitro (Price, I904), in life it may produce intestinal ulcers (Schaps, 1905). It probably causes cumulative effects. Even the local application is said to lead to inflammatory changes in the liver and kilneys (Fischer, 1905). The effects on digestion and general health are described by Wiley, r 008 .

Salkowski, 1915, states that formaldehyd, 0.6 to I $\mathrm{Gm}$. per day, added to the food of a I 2 kilogram dog, was well tolerated and did not impair the utilization of protein.

Local Actions.-Formaldehyd solutions or vapor, even when very dilute, produce very strong irritation of all mucous membranes. The susceptibility to this irritation is diminished by habituation. Applied to the unbroken skin, it hardens the epidermis, renders it rough and whitish, and produces anesthesia. Repeated application of strong solutions leads to superficial necrosis of the skin and nails and to persistent eczema (Galewsky, 1905). Its application to broken surfaces is very painful. Isolated tissues are promptly hardened. Formaldehyd is therefore used in histologic technic in strengths of $1 / 2$ to ro per cent. These effects are produced by the coagulation of proteins. They are influenced by the concentration and by the time of contact (Meneguzzi, I9I3).

Red Corpuscles.-These are fixed by dilute solutions similarly to other tissues; strong solutions of commercial (not of pure) formaldehyd may produce laking, by their formic acid (Franchi, I9I3).

Systemic Actions. - The inhalation of formaldehyd vapors, even for a long time, produces but small effects, beyond the local actions, causing bronchitis and pneumonia. The intravenous injection of solutions in normal saline of a strength of $1: 5,000$ also produce no immediate effects, even in man. The fatal dose for mammals intravenously, is about 0.07 to $0.09 \mathrm{Gm}$. per kilogram; the hypodermic dose is approximately ten times as much.

When applied directly to the mucosa of the upper intestine (not the lower), the irritation of the formaldehyd produces an increased flow of pancreatic juice. The most favorable concentration is I to 2 per cent., 8 to Io per cent. being ineffective (Delezenne and Pozerski, I9I5).

Poisoning by Swallowing Formaldehyd.-A number of such cases have been reported. As summarized by MacLachlan, I909, the symptoms consist of immediate and severe abdominal pain; often sudden and prolonged loss of consciousness, and collapse. Death occurs in twenty-four to forty-eight hours. The autopsy shows severe gastritis with erosions. In non-fatal cases, the urine is suppressed for twelve to twentyfour hours, and then generally contains blood and casts. There is of ten diarrhea and tenesmus, sore mouth and dysphagia. The recovery is curiously rapid, the patients being well within a week. The fatal cases all took over 3 ounces of the commercial solution. The largest quantity from which recovery is reported is 60 c.c.

The trealment should consist in the administration of dilute ammonia and ammonium salts, and in lavage.

Fate of the Formaldehyd.-Formaldehyd is absorbed from all parts of the alimentary tract and lungs (McGuigan, I9I4). It is not excreted as such (Blum; Pohl, I893), even with intravenous injection; unless very large doses are given (McGuigan, I9I 2 ; Impens and Rosenberg; Salkowski, I9I5). It is mainly oxidized in the tissues, especially the liver (Pohl), to formic acid. The oxidation of aldehyds is not direct, but through the "Cannizzaro reaction," commonly observed in vilro when aldehyds are treated with alkalies, $2 \mathrm{HCHO}+\mathrm{H}_{2} \mathrm{O}=\mathrm{CH}_{3} \mathrm{OH}+\mathrm{HCOOH}$. A part of the formic acid may appear in the urine (Pohl; Klueber and Gerlasch); but small quantities of formic acid are also completely destroyed (Sollmann, 1908). Some of the formaldehyd may be transformed into hexamethylenamin (McGuigan).

\section{PREPARATIONS-FORMALDEHYD}

*Liquor Formaldehydi (Liq. Formaldehyd), U.S.P., B.P.; Solution of Formaldehyd (Formalin).-A watery solution containing about 37 per cent. of HCOH. Clear, 
colorless liquid; pungent odor and caustic taste. Miscible with water and alcohol. A tablespoon in a quart of water makes about I per cent. of absolute formaldehyd.

Liquor Formaldehydi Saponatus (Lia. Formaldehyd. Sap.), B.P.-2o per cent. of Liq. Formaldehyd; 40 per cent. of Soft Soap; 30 per cent. of Alcohol.

Paraformaldehydum (Paraform.), U.S.P. (Paraform, 'Trioxymethylen), $(\mathrm{HCOH})_{3} \cdot-$ White friable masses, or as a powder, having a slight odor of formaldehyd. Slowly scl. in cold water; insol. in alc. or eth. Dose, 0.5 Gm., 8 gr., U.S.P.

It sublimes slowly at $100^{\circ} \mathrm{C}$. and melts at $17 \mathrm{I}^{\circ} \mathrm{C}$. Heated under proper conditions, it is decomposed into formaldehyd and is used for the disinfection of rooms. Two Gm. of paraform are required per cubic meter ( 35 cubic feet of space). Formaldehyd is also slowly liberated at body temperature: paraform has therefore been suggested as an intestinal antiseptic ( 0.05 to $0.1 \mathrm{Gm}$. every two hours, for children), but its irritant action renders it objectionable. Larger doses (3 to $4 \mathrm{Gm}$.) are cathartic, small doses are rather constipating. It will not cause serious poisoning even in large doses.

\section{FORMALDEHYD COMPOUNDS}

These are generally inactive and non-irritant in themselves, but regenerate formaldehyd under suitable conditions. It was therefore thought that they would prove valuable antiseptics. However, most of them liberate it either too slowly or too readily. Hexamethylenamin alone has proved really valuable.

Glutol (Schleich, r 896) is an insoluble compound of formaldehyd and gelatin, which holds the greater part of the formaldehyd so firmly that it is practically useless (Sollmann, I908). Other protein compounds were also disappointing. Classen introduced compounds with carbohydrates. Starch (amyloform), dextrin, milk-sugar, etc. Their fate was studied by Jacobson, 1906. There were also combinations with phenols, urea (Cohn, 1897), etc. Anhydromethylencitrates (citarin) splits off $\mathrm{CH}_{2} \mathrm{O}$ too readily (Nicolaier, I906); whilst methylendisalicylic acid is not decomposed in its passage through the body (Sollmann, rgo8).

\section{HEXAMETHYLENAMIN}

General Statement.-Hexamethylen-tetramin, $\mathrm{N}_{4}\left(\mathrm{CH}_{2}\right)_{6}$, is formed by the action of ammonia on formaldehyd. It is itself quite devoid of antiseptic, irritant or toxic actions. Its solutions, however, dissociate slowly with the evolution of formaldehyd. This decomposition is favored by the presence of free acid, and arrested by alkali.

Hexamethylenamin is rapidly absorbed, distributed through all the tissues and secretions, and excreted mainly in the urine. By far the greater part is unchanged, but a small fraction is changed into formaldehyd wherever it meets an acid reaction; especially during the secretion of acid urine, and perhaps exceptionally elsewhere in the body. The degree of decomposition depends entirely on the acidity. The presence of free ammonia prevents the formaldehyd liberation completely.

The liberated formaldehyd exerts a more or less marked antiseptic effect. The concentration is rarely sufficiently high to cause sensible irritation, except in specially susceptible individuals. It never causes systemic poisoning, even with very large doses.

This antiseptic action renders the drug an effective prophylactic and sometimes curative remedy against inflammations and suppurations of the genito-urinary tract. By misconception, it has also been used in other conditions, where it is doubtful whether effective quantities of formaldehyd can be liberated: In typhoid bacilluria, infections of the bile-passages, meninges, respiratory tract, and accessory sinuses. It is the most effective of urinary antiseptics, provided that the urine is acid. In alkaline urine 
its effects are slight, and in ammoniacal urine it is inactive. The tendency to gastric and vesical irritation (painful micturition, hematuria) is lessened by liberal dilution.

Historical.-The substance was first prepared by Butlerow in $\mathrm{r} 860$. It was introluced into practice as a urinary antiseptic by Nicolaier, 1895 and 1899 . Richardson, I 898 , popularized its use against typhoid bacilluria. Crowe, 1908, demonstrated it in bile and other body-fluids, and suggested its administration in this connection.

Formation of Formaldehyd.- The reaction between ammonia and formaldehyd is somewhat reversible, ${ }_{4} \mathrm{NH}_{3}+6 \mathrm{HCHO} \rightleftarrows \mathrm{N}_{4}\left(\mathrm{CH}_{2}\right)_{6}{ }^{1}+6 \mathrm{H}_{2} \mathrm{O}$. In watery solution, the reverse reaction, resulting in the liberation of formaldehyd occurs very slowly in the cold, somewhat more readily on heating, especially in the presence of acids (which combine with the ammonia). It increases with the degree of acidity (free hydrogen ions). little if any formaldehyd is liberated in neutral, and none in alkaline liquids. In body-fluids and in tissue-extracts, the liberation proceeds as in aqueous solutions (Hanzlik and Collins, 1913). Exact estimations of the proportion of free formaldehyd have not been made for want of quantitative methods which would distinguish between this and hexamethylenamin. The presence of free ammonia prevents the reaction, and it is perhaps also diminished by the presence of salts. It would be proportional to the concentration of the hexamethylenamin.

An approximate estimation of the liberation of formaldehyd was made by Jordan, I $9 \mathrm{rI}$. Incubating a I : 1,000 hexamethylenamin solution at body temperature for twenty-four hours, he found that the concentration of free formaldehyd reached $x:$ ro,, 00 if the reaction was slightly acid; $1: 200,000$ if neutral. None could be demonstrated with alkaline reaction. Hanzlik, r 3 , found similar results.

Absorption, Distribution and Excretion of Hexamethylenamin.-The absorption from the alimentary canal is rapid and complete: The excretion by the urine - the main channel of its elimination-starts within eleven minutes after oral, rectal or hypodermic administration, reaches its maximum in one to three hours, and practically ceases in four to eight hours, according to the dose. After twelve hours only very small amounts are excreted, although the blood may contain faint traces after twenty-four hours (Sollmann, I908; Crowe, I908; Burnam, I912; Falk and Sugiura, I916). Approximately the same time relations obtain for its concentration and disappearance from the other secretions and tissues. Its distribution is universal, since it has been demonstrated in all parts of the body in which it was sought. By far the greater part is excreted unchanged, only a small fraction being converted into formaldehyd in the urine. A variable proportion is completely destroyed in the body.

The Concentration of Hexamethylenamin.-This depends on the dose, the time, and in the case of urine at least, very largely on the ingestion of fluid.

Quantitative Excretion.-The total recovered in the urine varies from I 2 to 80 per cent. of the dose administered ( $1 \mathrm{Gm}$.), the higher figures corresponding to greater water intake. With continued administration, three times daily, the concentration in the urine ranged from $I: I, 400$ to 2,800 for doses of $0.67 \mathrm{Gm}$; between $\mathrm{I}: 2,400$ to 14,000 for $0.33 \mathrm{Gm}$. The excretion is diminished in renal impermeability (Falk and Sugiura, I9I6). In other secretions and in the tissues, the concentration is probably lower.

Presence of Free Formaldehyd in the Tissues.-Tests for free formaldehyd (as distinguished from hexamethylenamin) have been applied only by a few investigators. It could occur only in situations where there is free acid; i.e., normally only in the gastric juice and urine. Accordingly,

1 The following structural formula has been suggested:

$$
\begin{aligned}
/ \mathrm{CH}_{2}-\mathrm{N} & =\mathrm{CH}_{2} \\
\mathrm{~N}-\mathrm{CH}_{2}-\mathrm{N} & =\mathrm{CH}_{2} \\
\backslash \mathrm{CH}_{2}-\mathrm{N} & =\mathrm{CH}_{2}
\end{aligned}
$$


the results with other normal fluids have been uniformly negative (milk, Schmid and Schroeter; saliva, Hanzlik; bile, cerebro-spinal fluid and sputum, Burnam, Igr2; various situations, McGuigan, I9r2). It is conceivable, however, that acid reaction may obtain strictly locally in other situations under pathologic conditions. Indications of this were found by Hanzlik and Collins, I9I3. However, the formaldehyd liberation was so small as to be ineffective (Hanzlik, I9I4; Hyman, I9I4).

Presence of Free Formaldehyd in the Urine.- The amount of formaldehyd liberated depends upon the acidity, the concentration, the time of standing, and perhaps on other unknown conditions. The liberation may occur in the kidney (Burnam) and increases in the bladder (Sollmann; Hinman, I9I3). Burnam found it often absent with small doses, but believes that with larger doses the concentration of free formaldehyd may exceed I : 5,000. Smith found that $0.5 \mathrm{Gm}$. always gave formaldehyd in acid urines, while $0.3 \mathrm{Gm}$. was inconstant. Alkaline urines do not liberate formaldehyd; but the formaldehyd liberated in the acid kidney may persist for a short time in the alkaline bladder. Kidney disease does not modify the liberation (Hinman).

L'Esperance and H. Cabot, I9r2, found free formaldehyd in about half the hexamethylenamin urines examined. They believe that the liberation is not affected by the dosage or by the reaction of the urine. This conclusion can only have been based on faulty methods of estimating the acidity; for G. G. Smith, r913, and Hanzlik and Collins showed definitely that all acid urines liberate formaldehyd. Smith and Jennes, I9I3, also found it in about half the cases.

Concentration of Formaldehyd in Urine.- With the present rather unreliable methods, Hinman, I9I3, claims that this is below $I: 40,000$ in half the cases; $I: I 6,000$ in 25 per cent.; and over $\mathrm{I}: 7,000$ in 2 per cent. of the urines.

Antiseptic Action.-Hexamethylenamin itself is not at all antiseptic, if the liberation of formaldehyd is prevented by alkalies (Hanzlik, I913). A concentration of 5 per cent. is required to check the growth of Staphylococcus in neutral broth cultures; and this may be attributed to the free formaldehyd (Jordan). The antiseptic action is always parallel to the concentration of free formaldehyd (Hanzlik and Collins). Formaldehyd in concentration of $I: 50,000$ to 30,000 has a distinct though limited antiseptic effect (Mosso and Paleotti).

Antiseptic Effects after Administration.-These are especially marked in acid urines, as might be expected. However, Crowe, I908, claims that the administration of 5 Gm. per day also renders the bile and other body-fluids antiseptic. Exact observations have not confirmed this (Hanzlik and Collins), and the idea of producing a "tissueantisepsis" by its use must be abandoned.

Therapeutic Use as Urinary Antiseptic.-Hexamethylenamin is by far the most effective urinary antiseptic, provided that the reaction of the urine is acid. In neutral or alkaline urine, its efficiency is practically nil (Jordan, IgIr). In ammoniacal urines it would be useless. It would therefore be especially valuable as a prophylactic, $f . i$., in catheterization (Hinman, I9I 5). It has also been advised to prevent the spread of typhoid bacilli (Richardson, I898); but its efficiency is doubtful. It may be used in inflammation or suppuration of the renal pelvis, bladder or urethra. The dosage should be from 0.25 to $0.5 \mathrm{Gm}$. (4 to $8 \mathrm{gr}$.) in half a glass of water, four or five times a day. Burnam, I912, recommends increasing the dosage until free formaldehyd appears in the urine. In typhoid fever, the same dosage should be employed continuously, from the time when the diagnosis is made. If the urine is alkaline, acid sodium phosphate 
$\left(\mathrm{NaH}_{2} \mathrm{PO}_{4}\right)$ should be administered, I to $2 \mathrm{Gm}$. every one to four hours, until the urine is acid. This often causes diarrhea.

Acidulation of Urine.-The official sodium phosphate, $\mathrm{Na}_{2} \mathrm{HPO}_{4}$, does not alter the reaction of the urine. 'The acid salt may be prepared extemporaneously: R. Sodii Phosphat., U.S.P., ro Gm.; Ac. Phosphor. Dil., 25 c.c.; Water, add 150 c.c. A tablespoon represents about I Gm. of acid phosphate. It should be diluted in a tumbler of water. Benzoates have a tendency to render the urine acid (Jordan, I913); also boric acid, 2 Gm. daily (G. G. Smith, I9I3).

Relative Value of Other Urinary Antiseptics.-Using the ordinary therapeutic doses in a normal subject, Sollmann, r905, found that urinary decomposition was prevented, at incubator temperature, with hexamethylenamin, for three days to three weeks; after salicylates for a day; benzoate, phenolsulphonate and boric acid had no discernible effect. Churchman, I906, concludes that hexamethylenamin surpasses methylene blue. Jordan, I9I I, found it much superior to salicylic acid and santal oil. The difference is of course most marked in acid urines, although this increases the efficiency of all the antiseptics. The greatest effect of Santal oil was on Staphylococcus. He found boric acid the best antiseptic for alkaline urines (19r3).

Solvent Action on Uric Acid.--Hexamethylenamin has been proposed as a remedy for gout and urate calculism. It, as well as formaldehyd, dissolves uric acid, with the formation of a series of paired compounds (Nicolaier, r9o6). However, the solvent action is really very slight (Stevens and May, rgII), and the experimental and clinical results have been disappointing (Hanzlik, r916).

Haskins, 1915 , found that the administration of hexamethylenamin in doses of $\mathrm{I}$ to $2 \mathrm{Gm}$. does not confer any solvent action on the urine. Doses of $4 \mathrm{Gm}$. made the urine distinctly more solvent; but equal results are secured by making the urine slightly alkaline (p. H. = 7.r to 7.4) as may be easily done by administering sodium bicarbonate or acetate. These results were also confirmed by adding hexamethylenamin directly to water, urine and phosphate mixtures.

Antiseptic Action on Bile.-Crowe, I908, claimed that the administration of at least $5 \mathrm{Gm}$. ( $75 \mathrm{gr}$.) per day renders the bile unsuitable for the growth of bacteria, and completely antiseptic to typhoid bacilli. It was therefore advised as a prophylactic against biliary infection after operation on the gall-bladder or ducts. Recent observations (Burnam, Hanzlik) have not confirmed these claims, and it may be remarked that there is ordinarily no antiseptic effect on the bile.

Meningeal Infections.--The excretion of hexamethylenamin into the cerebrospinal fluid (Crowe, 1908; it reaches the concentration of r: 20,000 even after a single dose of I Gm., Hald, I9II) was claimed by Crowe, 1908, to restrain the development of bacteria, although the concentration is not sufficient for disinfection. Clinically, early and large doses are said to afford at least a considerable degree of protection against infective diseases of the meninges (poliomyelitis, Flexner and Clark, 19II); epidemic and postoperative meningitis (Crowe, rgr2), etc. Here again, the recent exact observations are negative (infantile paralysis, Fraser, 1914). Usener, I913, claims that diminished excretion into the cerebro-spinal fluid is diagnostic of tubercular meningitis.

Administration for Systemic Disinfection.-To obtain the claimed effects on the bile, meninges or respiratory passages, relatively large doses were employed: at least I Gm. ( $15 \mathrm{gr}$.) in a glass of water, five times per day; in meningeal infections even to $20 \mathrm{Gm}$. per day. The dosage should be reduced if irritant effects are noted. The administration of sodium acetate or citrate would probably lessen the liability to urinary irritation, without hindering the systemic antisepsis. Crowe, 1912, advocates the addition of $1 / 2$ per cent. of the drug to all liquids taken; or in very ill patients, the rectal injection, a drop at a time, of a liter of saline solution containing 3 to $6 \mathrm{Gm}$. of the drug.

Middle Ear.-The excretion of hexamethylenamin in this situation was demonstrated by Barton, 19ro; it appeared to cut short suppurative injection. It was therefore recommended as a prophylactic in scarlet fever, etc. Similarly, it was claimed to be useful against acute and chronic simus infections (Burton, I9ro); and in coryza (A. Miller, 1911), bronchitis (Vanderhoff, 1912) and influenza (Eisenberg, r912), and even as a pulmonary disinfectant (Zak, I9I2). The observations of Shattuck, Igrr, on pneumococcus empyema were negative, although hexamethylenamin was present in the pus. Crowe, 1912, doubts its efficiency in lobar pneumonia and in tuberculosis. 
Excretion in Eye.-Gradle, I9II, found it in the anterior chamber, its excretion being hastened by mydriatics; however, its concentration is not sufficient to be antiseptic. (Witham, I9I3, thinks that there is a slight antiseptic action.) In cats Remelé, I9I6, found it in the aqueous humor shortly after the intravenous injection of $2 \mathrm{Gm}$. He also claims that traces of formaldehyd are present within one-half hour and persist for nineteen hours.

Excretion in Milk.- This was announced by Bucura, I905, and confirmed by Crowe, I908, Woldewijn, I9I0, and by J. Schmid and Schroeter, I9I0, who found no free formaldehyd. Reider, I9I2, found that the highest concentration was reached after an hour, but that this was not sufficient to be antiseptic.

Excretion in Saliva.- This contains no free formaldehyd, but only the unchanged drug. The excretion reaches its maximum in half an hour (Hanzlik).

Undesirable Effects.-Hexamethylenamin does not cause any systemic effects, even on the intravenous or hypodermic injection of enormous doses (Crowe, I908; Burnam, I912). Nor does it have any local action, so that it may be given hypodermically (Gundrum, I9II) or injected into the bladder in 50 per cent. solution (Burnam), with practically noirritation. After the liberation of formaldehyd, this becomesirritant, but only with large doses and in specially susceptible individuals. This causes painful micturition and eventually cystitis and hematuria. In Crowe's cases the hemorrhage was from the bladder. The kidneys may probably also be irritated. The cystoscope shows hemorrhagic cystitis; and postmortem examination also hyperemia of the pelvis (D. L. Simon, I9I4). Other occasional symptoms (Coleman, I903) are: rubeolar skin rashes, catarrhs of various mucosæ, and digestive disturbances. These irritant effects are quite exceptional if the drug is given in sufficient dilution. They disappear promptly and without any permanent damage, if it is discontinued.

In dogs, Wiggs, r9ro, obtained marked nephritis from relatively'small doses. Rabbits show hematuria only with $8 \mathrm{Gm}$. per day (D. L. Simon, I9I4). In guinea pigs, hypodermic injections (Frothingham, I909) produce local necrosis, and gastric congestion, hemorrhage and ulceration.

Incompatibilities.- Hexamethylenamin is a fairly strong base, gradually displacing ammonia from its salts. It is decomposed by acids (even acetyl-salicylic acid) and by acid salts with the liberation of the irritant formaldehyd. It is also incompatible with tannin, mercuric chlorid and oxidizing agents (J.A.M.A., Feb. 8, '1913, p. 465).

\section{PREPARATIONS-HEXAMETHYLENAMIN}

*Hexamethylenamina (Hexam.), U.S.P.; Hexamina, B.P. (Hexamethylentetramin), $\left(\mathrm{CH}_{2}\right)_{6} \mathrm{~N}_{4}$. - It was introduced as urotropin and is also marketed under other numerous and confusing trade names. These commercial brands are in no way superior to the official substance (Base, 1907). It occurs as colorless, lustrous, odorless crystals, of sweet, then bitter taste. Solutions react alkaline to litmus. It is freely sol: in water ( $\mathrm{r}: \mathrm{r} .5$ ), and alc. (I: Io). Dose, 0.25 Gm., 4 gr., U.S.P.; 0.3 to $\mathrm{I}$ Gm., 5 to I gr., B.P.

Hexamethylenamin Salts.-A number of these are described in N.N.R. but they have no serious advantage. Boruttau, I9I4, claims that the urinary antiseptic action is somewhat greater with hezamethylen salicylate or camphorate; and that the urate solvency is higher with the citrate, borate or sulphosalicylate.

Jacobs and Heildelberger, I915, report experiments with some quaternary derivatives that appear promising. The activity of these does not seem to depend on the liberation of formaldehyd, so that they are not inhibited by protein and do not require acid.

\section{CHIORIN}

Disinfectant Action.-The gas, solutions and hypochlorites are efficient germicides by oxidizing and destroying all organic matter. This, 
however, also limits their employment; for chlorin bleaches organic colors, disintegrates fabrics, corrodes metals, and acts as a strong irritant to the tissues. It is used, especially in the form of Chlorinated Lime and of Labarraque's Solution, for the disinfection of excreta: 6 ounces of chlorinated lime are mixed with a gallon of water; a quart of this is added to each discharge and allowed to stand an hour. It is also employed to sterilize drinking water, especially for temporary emergencies.

Five to $\mathrm{r}_{5}$ pounds of chlorinated lime are used per million gallons according to the impurities and other conditions. This imparts a slight taste, but no other bad effects have been demonstrated. On a small scale a level teaspoonful is rubbed with 4 cups of water and a teaspoon of this solution is added to 2 gallons of the drinking water, and left for at least ten minutes.

For Snake Bites, ro c.c. of a I per cent. solution is injected hypodermically about the wound (Calmette, I907).

For the disinfection of the hands, these are moistened and rubbed with a little dry chlorinated lime and washing-soda.

Labarraque's solution is used as a deodorant on fetid ulcers, being diluted to or 20 times. Chlorinated lime itself has been used on wounds; 2 tablespoons of the compound being dissolved in a basin of warm water and used as a lotion, twice daily (Muench, 1915). Internally, it has been tried as a gastro-intestinal antiseptic, but would be useless in the presence of organic matter. Liq. Chlori Co. is employed as a gargle in infections of the throat, being diluted with 6 or 8 parts of water.

The history of the hypochlorites is discussed by Dakin, I9I5. He attributes their action to the formation of chloramido groups in the protein.

Hypochlorous Acid as Antiseptic.-This has been brought into prominence by the war, being especially endorsed by the English workers. It is said to be highly effective, practically non-corrosive; and not being absorbed, it is non-toxic.

It is made by shaking $\mathrm{r} 2.5 \mathrm{Gm}$. of chlorinated lime with a liter of water, and adding $12.5 \mathrm{Gm}$. of boric acid. After shaking, it is set aside over night and filtered. This solution containing about $1 / 2$ per cent. of hydrochlorous acid, is applied directly (Dakin, I9r5).

Another modification (Carrel-Dakin Antiseptic) consists in adding $20 \mathrm{Gm}$. of chlorinated lime to a solution of $\mathrm{I} 4 \mathrm{Gm}$. of anhydrous sodium carbonate in a liter of water. This is shaken, allowed to stand for half an hour, decanted and filtered. Boric acid is then added until the solution does not redden phenolphthalein.

A dry mixture of equal parts of chlorinated lime and boric acid is also used as a dusting powder (Lorain Smith, et al, I915).

C. Mayer, Igı6, prefers magnesium hypochlorite to the Labarraque solution, as being less caustic. It is easily prepared.

Minor Toxic Effects of Chlorin.-These have arisen mainly from its use in the arts. Air contaminated with chlorin produces irritation of the conjunctiva and respiratory mucous membranes, etc.; 0.001 to 0.002 per thousand of air is said to be harmless; 0.003 is disagreeable, and 0.004 irrespirable (Matt, 1889 ). Continuous exposure produces cachexia. The foliage of plants is destroyed by concentrations which are not noticeable for man.

Acute Toxic Effects of High Chlorin Concentrations.-These have been studied in connection with the use of this gas in the war. They consist in violent irritation and rapid asphyxial syncope with pulmonary edema. Schaefer attributes the asphyxia to extreme congestion and obstruction of the pulmonary vessels.

The experiments of Schaefer, I $\mathrm{I}_{5}$, showed that I per cent. of chlorin in the air may be ineffective for several minutes; then, often suddenly, the respiration becomes deep, convulsive, and arrested. The blood pressure falls. Rapid recovery occurs in fresh air.

With 5 per cent., death is rapid and inevitable. The respiration becomes slowed and is arrested in one to three minutes. The blood pressure falls to zero in three to four minutes, the heart still beating well. Artificial respiration does not cause recovery.

The bronchioles are not constricted, air passing at least as well as normally. Secre- 
tion of mucus is also unimportant, for the course is not modified by atropin. The systemic circulation is also unaffected directly, the changes being secondary to the asphyxia. Even the right heart is not especially congested.

The essential changes are seen in the pulmonary circulation. The lungs are intensely hyperemic, microscopic examination showing engorgement of the capillaries and edema of the interstitial tissue and alveoli. The epithelium proper is normal. Perfusion of the pulmonary vessels with Ringer's solution shows marked obstruction of the flow when chlorin is injected into the trachea or perfusion fluid. Schaefer assumed that this phenomenon occurs during life and that this obstruction is the cause of all the symptoms. (It is probable that chlorin coagulates the endotheliv:m, as do other astringents.) Bromin vapor is more irritant, but less fatal.

Bleaching Powder, when swallowed, produces gastro-intestinal irritation. This would be treated by evacuation and demulcents.

\section{PREPARATIONS-CHLORIN}

* Calx chlorinata (Calx Chlorin), U.S.P., B.P.; Chlorinated Lime (Chlorid of Lime, Bleaching Powder).-A product resulting from the action of chlorin upon calcium hydroxid and containing 30 per cent. of available chlorin. Consists mainly of a mixture or weak compound of calcium hypochlorite and calcium chlorid. It occurs as a grayishwhite granular powder of chlorin odor and repulsive salty taste. It is only partially sol. in water or alc.

Liq. Calcis Chlorin., B.P.- ro per cent. of Calx Chlorin, representing 3 per cent. of available chlorin.

${ }^{*}$ Liquor Soda chlorinate (Liq. Sod. Chlorin), U.S.P., B.P. (Labarraque's Solution). -2.5 per cent. of available chlorin. Made by decomposing Calx Chlorin. with Sod. Carb.

Javelle Water is a similar preparation of chlorinated potash.

\section{PARA-TOLUENE SODIUM SULPHOCHLORAMID}

This is marketed under the names of Chlorazene, Chloramine $\mathrm{T}$, and Tolamine. It was introduced by Dakin, ror6, as a stable, non-toxic, non-irritant and non-corrosive surgical antiseptic. It is used as I to 4 per cent. solution (see N.N.R.).

\section{SULPHUR DIOXID (SULPHUROUS ACID)}

This was formerly used extensively for the disinfection of rooms, but is not reliable against bacteria (Koch), and has the objection of bleaching colors. However, it is effective against insects (McClintock, etc., I9II), and therefore against insect-borne diseases, malaria, yellow fever, etc.

It is generated by burning 3 pounds of sulphur.for each $1, \infty 00$ cubic feet of space. To avoid danger of fire, the sulphur is placed in tin pans raised from the floor by bricks. Its action is materially greater when the air is saturated with moisture.

Toxic Effects. - These arise from its use in disinfecting and bleaching. Large quantities occur also in coal smoke, and become particularly objectionable in foggy weather. It is eventually oxidized in the air to sulphuric acid (T. W. Schaefer, I907). Its inhalation produces irritation of the respiratory passages, etc. and extreme discomfort in dilutions of $0.00 \mathrm{r}$ per cent. by volume; and $0 . \mathrm{I}$ per cent. is promptly fatal by paralysis of the respiratory center (Ogata, I884; K. B. Lehmann, 1893).

\section{PREPARATIONS-SULPHUROUS ACID}

Acidum Sulphurosum (Acid. Sulphuros), B.P.-An aqueous solution corresponding to 5 per cent. of $\mathrm{SO}_{2}$. Dose, 2 to 4 c.c., $1 / 2$ to $\mathrm{I}$ dram, B.P.

\section{SODIUM SULPHITE AND THIOSULPHATE (HYPOSULPHITE)}

These are rarely used in medicine, as mouth wash in aphthæ, and as ro per cent. lotion in ringworm and other parasitic skin diseases (pity- 
riasis versicolor). The thiosulphate is the more powerful, and is sometimes used also as a cathartic.

The principal importance of the sulphites arises from their use in preserving foods. The quantities ordinarily used do not seem to be directly harmful. Even much larger quantities do not act systemically, being rapidly oxidized to sulphates; but they may produce local gastric irritation by the liberation of sulphurous acid. Their harm is mainly indirect in that they improve the odor and appearance of spoiled meat so that it is sold as fresh. They are therefore properly prohibited.

Toxic Effects.-The harmlessness of ordinary quantities even when continued for very long periods has been shown particularly by Rost and his co-workers (1904, I 191 2) and by Lehmann and Trentlein, Igog (Long, I9I 2). The metabolism is not deranged. With larger doses by mouth, there are signs of gastro-intestinal irritation, due to the liberation of sulphurous acid (Jacobj and Walbaum, 1906). Kionka and Ebstein, I902, and Harrington, 1904, described hemorrhagic and inflammatory lesions in the kidneys and other organs after long-continued use; but these have not been confirmed by later observers and were probably accidental. The absorbed sulphite is rapidly oxidized to sulphate (Pfeiffer, I 883; Rost and Sonntag, 1904) and it is only when this capacity is exceeded by very large doses or intravenous injection that toxic effects appear. These lower the blood pressure, depressing the central nervous system, especially the medulla, and the cardiac and arterial muscle. Death occurs by paralysis of respiration (Rost, I9I4). In man, I Gm. produced no effects; 4 to $6 \mathrm{Gm}$. caused violent colic, diarrhea and circulatory disturbances (Rost; Franz, 1912).

\section{PREPARATIONS-SULPHITES}

Sodii Sulphis, B.P.; Sodium Sulphite, $\mathrm{Na}_{2} \mathrm{SO}_{3}+{ }_{7} \mathrm{H}_{2} \mathrm{O}$.-Freely sol. in water (I:2). Dose, 0.3 to I. 2 Gm., 5 to 20 gr., B.P. Oxidizes in air to sulphate.

Sod. Sulphis Exsic., U.S.P., $\mathrm{Na}_{2} \mathrm{SO}_{3}$. -Freely sol. in water $(\mathrm{I}: 3.2)$. Dose, 1 Gm., 15 gr., U.S.P.

Sodii Thiosulphas (Sod. Thiosulph.), U.S.P. (Sodium Hyposulphite), $\mathrm{Na}_{2} \mathrm{~S}_{2} \mathrm{O}_{3}+$ ${ }_{5} \mathrm{H}_{2} \mathrm{O}$.- Very sol. in water $(\mathrm{r}: 0.5)$. Dose, I Gm., I 5 gr., U.S.P.; as cathartic, every two hours if necessary (Petteys).

\section{OXIDIZING AGENTS}

\section{OZONE}

This is a polymeric form of oxygen $\left(\mathrm{O}_{3}\right)$, which decomposes very readily with the liberation of active oxygen. It is therefore strongly oxidizing, deodorant and antiseptic; but is too easily destroyed in the presence of organic or other oxidizable matter. Efficient concentrations are also highly irritant and produce toxic depressant systemic effects. It is being used for the sterilization of water. Traces of ozone are contained in the air, especially after thunder storms, but the quantity is much too small to have any effect whatever; in fact, concentrations far beyond human tolerance are of uncertain efficiency (J.A.M.A., 57 : I370, I9II). At most, its persistent presence is an indicator of the absence of organic matter in the air. The odor of ozone is characteristic and disguises other odors.

Effects of Inhalation.-The older methods of preparing ozone developed irritant nitrogen oxids. The modern machines are free from this defect. By their use, concentrations of 5 to ro per million, inhaled for a short time, produce in man increased amplitude of the pulse, drowsiness, and persistent headache - the latter probably by irritation of the sinuses. Ten per million inhaled for fifteen minutes produces sore throat. One per million diminishes the oxygen intake and the $\mathrm{CO}_{2}$ output. Prolonged exposure to concentrations below I per million does not produce noticeable disturbance; but with higher concentrations there is irritation of the respiratory tract and other 
mucous membranes, leading to fatal pulmonary edema; or in acute toxic concentrations, to initial restlessness followed by depression and coma (Jordan and Carlson, I9I3; Hill and Flack, I911; Sigmund, 1905; DuMont, I891). Exposure for two hours to io or 15 per million is dangerous to life, but the irritant effects, cough, headache, etc., give ample warning to avoid the danger. For an antiseptic action, at least i 3 per million would be necessary (L. Hill and Flack, 1911). The air contains at most 0.00015 to o. $5^{8}$ per million. Its seasonal occurrence is discussed by Holmes, I91 2 .

\section{HYDROGEN PEROXID}

The peroxids are fairly stable in pure solutions, but on contact with catalyzers, inorganic or organic (catalase), tissues, etc., they split off active oxygen and are therefore strongly antiseptic and deodorant. The action lasts only so long as oxygen is being evolved, and is therefore brief and superficial. In deep and closed cavities, the rapid evolution of gas presents danger of mechanical injury and embolism.

Uses.-Hydrogen peroxid is employed for washing fetid wounds and ulcers; as injection for gonorrhea; as mouth wash, etc. It is non-toxic and scarcely irritant, but is best diluted with 2 to ro parts of water. A stronger (30 per cent.) preparation is caustic. In contact with pus it foams, and this aids mechanically in cleansing the wound. Hydrogen peroxid is also a chemic antidote for cyanid and phosphorus poisoning. It is often used for bleaching the hair and fabrics. The concentration of 0.35 per cent. (absolute) in milk inhibits the development of pathogenic bacteria (Budde, I903) but it also destroys the ferments of milk and is of questionable utility (Heinemann, r913). Sieber, I90I, claims that it destroys toxins.

Phagocytosis.-This is stimulated in vitro by hydrogen peroxid; whereas free oxygen is generally depressant (H. J. Hamburger, I915). Potassium chlorate and ferricyanid proved inactive.

\section{PREPARATIONS-PEROXIDS}

*Liquor Hydrogenii Dioxidi (Liq. Hydrog. Diox.), U.S.P.; Liq. Hydrog. Perox., B.P.; Solution of Hydrogen Dioxid (Peroxid). - A slightly acid aqueous solution of $\mathrm{H}_{2} \mathrm{O}_{2}$ (approximately 3 per cent.) corresponding to about to per cent. (volumes) of available oxygen. Stronger solutions are also marketed. It occurs as a colorless liquid, odorless, of slightly acid taste, producing a peculiar sensation and soapy froth in the mouth. It keeps best in bottles stoppered merely with a plug of cotton, and standing in a cool place. It is.incompatible with easily oxidizable substances. It decomposes gradually. This is not due to impurities (Clover, I9I3). The decomposition is retarded by small quantities of acid or acetanilid. The latter is used in most brands (Kebler, 1910), but in time these acquire a yellow tint and an odor of nitro-benzol, and should then not be used. Dose, 4 c.c., I dram, U.S.P.; 2 to 8 c.c., $1 / 2$ to 2 drams, B.P.

"Solid Peroxid" consists of easily soluble compounds with urea, containing about 35 per cent. of absolute peroxid.

\section{PEROXIDS OF METALS}

These may be conceived as compounds in which the $\mathrm{H}$ of $\mathrm{H}_{2} \mathrm{O}_{2}$ has been replaced by metals, and which are readily decomposed with the formation of $\mathrm{H}_{2} \mathrm{O}_{2}$ or of active oxygen. They have been suggested as substitutes for hydrogen peroxid. The commercial products are usually mixtures containing 15 to 75 per cent. of the real peroxid. The compounds of the different metals vary considerably in their stability and solubility, and in the ease with which their solutions are decomposed. Acids convert them rapidly into hydrogen peroxid, f.i., $\mathrm{MgO}_{2}+\mathrm{H}_{2} \mathrm{SO}_{4}=\mathrm{MgSO}_{4}+\mathrm{H}_{2} \mathrm{O}_{2}$. In this way the oxygen is made promptly available. Water decomposes them more slowly, inversely to their solubility; f.i., $\mathrm{ZnO}_{2}+\mathrm{H}_{2} \mathrm{O}=\mathrm{Zn}(\mathrm{OH})_{2}+\mathrm{H}_{2} \mathrm{O}_{2}$. Their action is therefore more prolonged. The therapeutic effect is also modified by the nature of the base; thus sodium peroxid would yield a solution sufficiently alkaline to be caustic. Magnesium or zinc peroxid is therefore generally preferred. 
Uses.-The antiseptic effects are utilized in skin diseases $\left(\mathrm{ZnO}_{2}\right)$; dentifrices $\left(\mathrm{MgO}_{2}\right)$; and against gastro-intestinal fermentation $\left(\mathrm{CaO}_{2}\right)$. These compounds are described in N.N.R.

Sodium Perborate.-This also yields hydrogen peroxid on contact with water, being transformed into metaborate which acts as a mild alkali, $\mathrm{NaBO}_{3}+\mathrm{H}_{2} \mathrm{O}=\mathrm{H}_{2} \mathrm{O}_{2}+$ $\mathrm{NaBO}$. . It is used as a dusting powder or 2 per cent. solution, as a substitute for peroxid. The substance is described in N.N.R.

\section{ORGANIC PEROXIDS}

These were introduced by Novy and Freer, 1902. Acetyl-benzoyl peroxid (Acetozone) and succinic dioxid (Alphozone) are described in N.N.R. They may be considered as $\mathrm{H}_{2} \mathrm{O}_{2}$ in which the $\mathrm{H}$ has been replaced by organic radicals. In the presence of water they are slowly hydrolyzed, forming organic per-acids, with very active oxidizing and germicidal properties, $f . i$.

The acetyl benzoyl peroxid decomposes into acetyl peracid, acetic acid, and the insoluble dibenzoyl peroxid, according to the equation ${ }_{2} \mathrm{C}_{6} \mathrm{H}_{5} \mathrm{CO} \cdot \mathrm{OO} \cdot \mathrm{COCH}_{2}+$ $\mathrm{H}_{2} \mathrm{O}=\mathrm{C}_{6} \mathrm{H}_{5} \mathrm{CO} \cdot \mathrm{OO} \cdot \mathrm{COC}_{6} \mathrm{H}_{6}+\mathrm{CH}_{3} \mathrm{CO}_{2} \mathrm{H}+\mathrm{CH}_{3} \mathrm{CO} \cdot \mathrm{OOH}$.

They. are somewhat used as local and intestinal antiseptics, but are of doubtful efficiency for the latter purpose. They should not be mixed with organic liquids, nor kept in a warm place, as they tend to explode. They are described in N.N.R.

\section{IODOXYBENZOIC ACID}

This has a strong oxidizing action, even in the body, and is therefore of considerable scientific interest. Loevenhart and Grove, I9I I, have investigated the sodium salts of the three compounds: (I) Iodbenzoic acid, $\mathrm{C}_{6} \mathrm{H}_{4} \cdot \mathrm{CO}_{2} \mathrm{H} \cdot \mathrm{I}$.

(2) Iodosobenzoic acid, $\mathrm{C}_{6} \mathrm{H}_{4} \cdot \mathrm{CO}_{2} \mathrm{H} \cdot \mathrm{I}=\mathrm{O}$.

(3) Iodoxybenzoic acid, $\mathrm{C}_{6} \mathrm{H}_{4} \cdot \mathrm{CO}_{2} \mathrm{H} \cdot \mathrm{I} / \mathrm{O}$.

(2) and (3) have a metallic taste, resembling hydrogen peroxid, presumably due to the active oxygen. They oxidize hemoglobin to oxyhemoglobin. (2) is irritating, (1) and (3) very mildly. The systemic effects were studied by intravenous injection. (I) has very little action. The oxygenated compounds (2) and (3) produce the phenomena of apnea, marked and continued fall of blood pressure by depression of the vasomotor center; brief arrest of respiration, also by depression of the medullary center. The vagus center is not affected. These actions are due to the active oxygen. (This can not replace ordinary molecular oxygen for maintaining the cardiac activity; Loevenhart and Eyster, I913.)

Antiseptic Action.-The oxidizing property promises an antiseptic effect, perhaps even in the tissues, since it is not inhibited by serum. (2) and (3) are much more bactericidal than (I) (Arkin, IgII). Iodoxybenzoate increased the production of antibodies in dogs (Hektoen, IgII); also the production of hemolysin and agglutinin in rabbits, when injected intravenously shortly after immunization. It stimulates phagocytosis (Arkin, 1915). Its injection also lessens inflammation by mustard oil or allergy, whilst this is increased by cyanid, indicating that edema is lessened by oxidation and increased by asphyxia (Amberg and Knox, I9I2; Amberg, I9I3. Iodosobenzoic acid has also been studied by Jahn, I9I4). It reduces the toxicity of the tuberculin in vitro (Arkin, 1916).

\section{POTASSIUM PERMANGANATE}

In the presence of organic matter, this splits very readily into $\mathrm{MnO}_{2}$ (forming a brown precipitate), and into oxygen. It is therefore an effective oxidizing agent and disinfectant; but its efficiency is limited by any excess of organic matter. Its action is only superficial, and it is markedly irritant. It is used as an antiseptic for mucous membranes, especially the urethra (I: 4,000 to I : I,000). A I : 5,000 solution is said to be a satisfactory anesthetic for introducing urethral sounds (Barton, I9I3). Beck, I9 3 , recommends it against burns ( $I: 4,000$ to I $: 3,000$ on gauze and as lotion). 
It is an antidote to organic poisons, especially morphin, hydrocyanic acid, and phosphorus (I : I, 000 to I : 500). A saturated solution may be employed for disinfecting the hands, the color being removed by oxalic acid. Large doses by mouth cause death by gastroenteritis.

\section{PREPARATIONS-PERMIANGANATE}

*Potassii Permanganas (Pot. Permang.), U.S.P., B.P.; KMnO $\mathrm{K}_{\text {- }}$-Slender, dark purple prisms; odorless; astringent taste. Sol. in water ( $1: \mathrm{I}_{3.5}$ ); decomposed by alc. and all organic matter; explosive. Dose, 0.06 Gm., I gr., U.S.P.; 0.06 to 0.2 Gm., I to 3 gr., B.P.; diluted.

Liq. Pot. Permang., B.P.- - per cent. Dose, 7 to I 5 c.c., 2 to 4 drams, B.P.

\section{BORIC ACID AND BORAX}

These combine a fair antiseptic power with a low toxicity to higher animals. They are also less irritant than most other antiseptics. Boric acid is employed in surgery as dusting powder, as saturated (4 per cent.) aqueous solution, as boroglycerite, and in collyria ( $\mathrm{I}$ to 2 per cent. of boric acid or sodium borate). Boric acid is a mild acid, and increases the acidity of the urine; borax acts as a weak alkali. It dissolves mucus, lessens the urinary acidity, and is used for cleansing. The acid and alkali characters are very weak. The borate ion has no other therapeutic uses.

Use as Food Preservative.-Boric acid and borax have been extensively employed for this purpose. Single doses are generally innocuous; but their continued use produces slight but cumulative effects, so that their employment is not advisable.

Antiseptic Efficiency.-Boric acid is not a disinfectant. Even saturated solutions do not kill bacteria, but solutions of 0.3 per cent. check putrefaction and decomposition (but not the growth of pathogenic organisms, Bernstein, I9Io). Borax is somewhat less efficient. Their popularity as food preservatives is attributable to the absence of distinctive odor and taste, and to their relatively low toxicity. The acid is used in meats in the concentration of 0.5 per cent.; in milk of 0.2 per cent.

Action.-The question of their toxicity has been investigated extensively. It may be premised that boric acid and borax behave exactly alike. A man living largely on preserved food may ingest as much as $0.5 \mathrm{Gm}$. of boric acid (or its equivalent in borax) per day; the consumption would generally fall below this figure, but would rise to several grams. All investigators agree that quantities to $0.5 \mathrm{Gm}$. have no immediate action of any kind in healthy individuals. When, however, the consumption is continued for a long time, quite marked effects are produced (Forster, I883; Heffter, 1902; Rubner, 1902; Rost, I902, 1903, I905; Wiley, 1904). The digestion becomes somewhat deranged; the body weight lessens; the feces become more watery (but there is no diarrhea even with larger doses). The absorption of fat and of nitrogen is somewhat diminished. The urinary nitrogen is slightly decreased, whilst the phosphates rise distinctly. The quantity of urine is scarcely altered. If a slight albuminuria was present, this is increased (Wiley). Boric acid therefore seems to have a cumulative action on digestion and absorption, metabolism, and the kidneys. The cumulative effect may be explained by the observation that the excretion of boric acid is slow, requiring several days. (However, it begins within ten minutes, and reaches its maximum in the second hour.) On the average, Is per cent. is retained, the other 85 per cent. being excreted mainly by the urine, to a slight extent by sweat and milk, and practically none by the feces (Wiley). The excretion is not hastened by diuresis (Rost, 1905). Traces occur normally in milk and eggs (Bertrand and Agulhon, I013).

The effects of such doses as are actually consumed would therefore not be very serious, even if the consumption of preserved food were continued for a long time. However, the results are somewhat deleterious, even in normal individuals; and they would probably be very undesirable in patients with digestive or renal disturbance.

Somewhat larger doses, I to $3 \mathrm{Gm}$. per day, produce the described effects more rapidly and.more severely. Headaches appear. With $5 \mathrm{Gm}$., the subject soon becomes 
unable to do any work. Quantities above $2 \mathrm{Gm}$. per day may be condemned as distinetly harmful, without any hesitation. (Some investigators hold the opposite opinion, for instance Liebreich, I903 and 1904. Their results may perhaps be explained by individual differences of susceptibility, which were also noticed by Wiley.) Harrington, 1904. hats observed that the continued administration of borax to cats leads to very severe kilney lesions. Very large doses cause acute poisoning; this may also occur from local administration (van Dort Krom, r906); especially from flushing serous cavities and from the use of powdered boric acid on extensive burns (Savariaud, rgr4). The symptoms consist ordinarily in gastroenteritis, congestion of the abdominal viscera, nephritis (granular degeneration), scarlatinal skin eruptions; very commonly visual disturbances, muscular debility and incoördination, fall of temperature, collapse. The autopsy shows fatty degeneration (Rinehart, I90I; Best, I904).

With plants, even low concentrations in the soil or culture solution causes spotting of the leaves (Haselhofi, I9I3).

Minute traces of boron occur in all animals, most abundantly in the oceanic (Bertrand and Agulhon, 1912). Plants also take it up readily from the soil (Cook, I916).

\section{PREPARATIONS-BORATES}

*Acidum Boricum (Acid. Bor.), U.S.P., B.P.; Boric Acid (Boracic Acid), $\mathrm{H}_{3} \mathrm{BO}_{3}$.Translucent colorless scales, or light very fine bulky powder, unctuous to the touch; odorless; of slightly saline taste. Slowly sol. in water (I:18); sol. in alc. (I: I8); freely sol. in glyc. $(\mathrm{I}: 4)$. Dose, 0.5 Gm., 8 gr., U.S.P.; 0.3 to I Gm., 5 to I5 gr., B.P.; as urinary antiseptic. Locally, in lotions, injections, gargles, etc., 2 to 4 per cent. (I $1 / 2$ to 3 teaspoons per pint). A saturated watery solution contains about 5 per cent., or 25 gr. per ounce.

${ }^{*}$ Glyceritum Boroglycerini (Glycer. Boroglyc.), U.S.P.; Glycer. Acid. Bor., B.P.30 per cent. For external use, diluted ten times.

${ }^{*}$ Unguentum Acidi Borici (Ung. Acid. Bor.), U.S.P., B.P.--ro per cent.; in White Petrolatum, U.S.P.; in Parafin Ointment, B.P.

Liquor Antisepticus, N.F.-A mild antiseptic, containing in 1,000 : 20. Boric acid; I. Benzoic acid; I. Thymol; 0.25 Eucalyptol; 0.5 Oil Peppermint; 0.25 Oil Gaultheria; O.I Oil Thyme; $25^{\circ}$ Alcohol. A number of proprietary mixtures, e.g." "listerin," have a similar composition. Their antiseptic efficiency is very slight (Verhoeff and Ellis, I907).

${ }^{*}$ Sodii Boras (Sod. Bor.), U.S.P.; Borax Pur., B.P.; Sodium Borate (biborate or tetraborate), Borax, $\mathrm{Na}_{2} \mathrm{~B}_{4} \mathrm{O}_{7}+\mathrm{IOH}_{2} \mathrm{O}$.-Colorless, transparent crystals, or white powder, odorless, of sweetish alkaline taste. Sol. in water ( $1: \mathrm{I}_{5}$ ), very sol. in glyc. ( $: 1)$; practically insol. in alc. Incompatible with alkaloids, acids, mucilage of acacia. (Free boric acid is not incompatible with most alkaloids.) Dose, $0.75 \mathrm{Gm}$., I 2 gr., U.S.P.; 0.3 to I Gm., 5 to 15 gr., B.P.

Glycer. Borac., B.P.-2o per cent.

Mel. Borac., B.P.- 10 per cent.

Sódii Perboras (Sod. Perbor.), U.S.P.; $\mathrm{NaBO}_{3}+{ }_{4} \mathrm{H}_{2} \mathrm{O}$. - Sol. in water. Used for making oxygen.

\section{IODOFORM}

Uses.-This is obtained by the action of iodin on alcohol, acetone, etc., in the presence of alkalies. It has been known since 1832 and was introduced as antiseptic about I879. It acts probably by liberating iodin; but this shows some peculiarities, due to the manner in which it is produced. Iodoform itself is practically insoluble and probably inactive. It is also quite stable outside of the body; but in contact with tissues or their extracts, and particularly with diseased tissues and with bacteria, it slowly evolves iodin (Altenburg, Schmidt, I90I). This occurs so slowly that the effects are mild, but quite sufficient to be markedly antiseptic to bacteria, and stimulant to the cells. It also dries the wound secretions, and is mildly hemostatic and analgesic. Hamburger found that it stimulates phagocytosis. Its injection into dogs leads to persistent leucocytosis (W. Weil, I9I3). 
These actions make it valuable in treating open wounds, since it promotes healing by stimulating granulation and lessening or preventing infection. It is the most widely used dusting powder; it is also injected into tubercular joints, usually as an emulsion with glycerin (iodoform ro, alcohol and glycerin each 45).

Iodoform has, however, a number of disadvantages: The most objectionable feature is the persistent odor, which can not be disguised by any perfume. Iodoform is also apt to develop iodism and irritant phenomena in susceptible individuals, producing particularly eczemas, even in minute amounts. It may also cause severe poisoning with narcotic symptoms, different from those of other iodids.

General Intoxication.-(P. Mulzer, 1905). This may occur when iodoform is applied over a large area, or when it is injected. The symptoms consist in diuresis, lassitude and somnolence, hallucinations, diminished reflexes, light convulsions, and paralyses. The temperature falls. Death occurs through general paralysis of the central nervous system. Sodium bicarbonate is said to be antidotal. Chronic poisoning is also characterized by paralytic phenomena. The heart, liver and kidneys exhibit fatty changes. Iodoform dermatitis is sometimes seen. It resembles ivy poisoning (Bryan, 1903). The idiosyncrasies are not analogous to anaphylaxis, for they can not be transferred positively (Stropeni, 1912). Fatal poisoning from intrapleural injection-is reported by v. Bonsdorff, t916.

Excretion.-Iodoform is not excreted as such, either by the kidneys or lungs. It appears in the urine as iodid and iodate, and as unknown organic compounds (Angliolani, I903; Harnack and Gründler, I883).

Iodoform Substitutes.-The disagreeable qualities of iodoform justify the search for efficient substitutes. A number of odorless iodin derivatives were tried, especially thymol iodid (aristol), but their popularity was shortlived, since they do not seem to possess the peculiar activity of iodoform.

\section{PREPARATIONS-IODOFORM}

*Iodoformum (Iodof.), U.S.P., B.P. (Triiodo-methane), $\mathrm{CHI}_{3 .}$-Contains 97 per cent. of iodin. Usually obtained by the action of iodin upon alcohol or acetone, in the presence of an alkali. Fine, lemon-yellow powder, or crystals; peculiar, very penetrating and persistent odor; unpleasant, slightly sweetish taste suggestive of iodin. Nearly insol. in water; sol. in alc. $(1: 60)$, in glyc. ( $1: 80)$, or olive oil $(1: 34)$; freely sol. in eth. (r: 7.5 ). Dose, 0.25 Gm., 4 gr., U.S.P.; 0.03 to 0.2 Gm., $1 / 2$ to 3 gr., B.P.; in pills or capsules. Maximum dose, $0.2 \mathrm{Gm} ., 3 \mathrm{gr}$.

Supp. Iodof., B.P.-0.2 Gm., 3 gr.

Ung. Iodof., U.S.P., B.P.- Io per cent., in lard.

Iodoform (and other loose iodin derivatives) are incompatible with starch. They are generally decomposed by heat, and can therefore not be sterilized in this manner. Since they become antiseptic when applied to wounds, sterilization is usually superfluous.

The Iodoform Substitutes are also injured by heat and light. They are all insol. in water and are used like iodoform.

*Thymolis Iodidum (Thymol. Iod.), U.S.P. (Dithymol-diiodid; Aristol); $\left(\mathrm{C}_{6} \mathrm{H}_{2}{ }^{--}\right.$ $\left.\mathrm{CH}_{3} \cdot \mathrm{C}_{3} \mathrm{H}_{7} \cdot \mathrm{OI}\right)_{2}$ - - Contains at least 43 per cent. of iodin. Chocolate-colored powder with slight aromatic odor. Insol. in water or glyc.; slightly sol. in alc., readily in oils, eth., etc.

\section{GENERAL CONSIDERATION OF ANTISEPTICS}

Toxicity and Resistance.-The protoplasm of bacteria is fairly readily injured by many substances and by physical changes. The conditions under which it exists are, however, somewhat peculiar: By virtue of a highly impenetrable cell wall, and by the formation of very resistant spores, bacteria are able to survive conditions which would kill other cells. Under these adverse conditions the microörganisms are indeed unable to grow and multiply; but by passing into a dormant state, particularly by spore formation, they are able to preserve their vitality for a considerable time, and to recover their power of growth as soon as the conditions become more favorable. Substances which 
merely suspend the vitality of bacteria are called antiseptics; whilst those which kill them outright are germicides. A third class of disinfectants is formed by the deodorants, which obscure or destroy the odorous bacterial products, but which have little action on the bacteria themselves.

Factors Determining Usefulness.-The nature of the antiseptic substance, or the strength in which it is used, are by no means the only factors determining its efficiency and usefulness. Among the other more important factors may be mentioned:

The nature, peculiarities and number of microörganisms; the time of exposure; the infuence of organic matter; the penetration, toxic and corrosive actions of the agent; and in some cases, its cost. Widely advertised antiseptics especially present a very striking disproportion between cost and efficiency.

The Influence of Proteins and Other Organic Matter.-Many substances which are strongly germicidal when acting on the bacteria alone are much weakened by entering into chemic reactions with the medium.

Thus, potassium permanganate is destroyed by all organic matter; mercuric chlorid is precipilated by proteins; silver nitrate by chlorids, etc. These insoluble combinations are no longer germicidal. Further, they hinder the penetration of the antiseptic, a condition of considerable surgical importance. The antiseptics of the aromatic series are relatively little affected. From the standpoint of antiseptic efficiency and penetration, the phenols stand highest, experimentally (Keilty and Packer, I9 5 ; for relative place of other antiseptics, the original paper must be consulted). However, even these are markedly impeded by serum, even when there is no precipitation (Bechhold and Ehrlich, I 906). Indeed, scrum and body fluids in general interfere very much with the activity of all antiseptics (Verhoeff and Ellis, I907).

The Disinfecting Power of the Commonly Used Antiseptics.-On account of the different resisting power of different bacteria, and the ease with which the antiseptic action is modified by circumstances, the results of experiments directed to comparing the different antiseptics can not be generalized. Tables of antiseptic values are therefore unsatisfactory. In a general way, it may be said that mercuric chlorid and silver nitrate are the most powerful. Then come copper and zinc salts; then formaldehyd, chlorin, and hydrogen peroxid; then the cresols, then carbolic acid. Salicylic acid, boric acid, sulphurous acid, and the essential oils are antiseptics rather than germicides. Iodoform manifests its action only under special conditions. Ferrous sulphate is merely deodordant. Lepine, I886, claims that mixtures of antiseptics are often more efficient than would be expected from their ingredients.

The Preservation of Food.- The liability of organic food-stuffis, especially meat and milk, to bacterial decomposition, often renders their chemical preservation a matter of economic necessity. Asepsis, refrigeration, or sterilization by heat, are not always applicable. That the use of chemic preservatives is theoretically undesirable, on account of their possible deleterious action, is universally granted; in practice, however, this evil may be less than those resulting from the consumption of partly decomposed foods. The time-honored use of sugar or salt-cured, corned, and smoked meats, of vinegar, spices, etc., is a sufficient proof of the comparative innocuousness of some of the preservative measures. More recently there have been introduced a number of preservatives which are more powerful, and which can not be detected by the taste. These offer a special interest, and they have accordingly given rise to much discussion.

It is self-evident that markedly toxic and irritant substances, such as formaldehyd, should be entirely excluded. The principal difference of opinion is in regard to substances, such as benzoates, borates, sulphites, saccharin, etc., which do not produce marked effects in the quantities ordinarily used. Their opponents rest their objections mainly on their action on weak or diseased individuals, on infants, nephritics, and dyspeptics; and to the cumulative effects of their continued administration. There is, however, no evidence as to the degree of danger from their intermittent use, under the actual conditions. At all events, the consumer should be made aware of the addition of the preservative, and of any possible dangers.

Urinary Antiseptics. - The urine and the bladder may be disinfected to a considerable degree by drugs administered through the mouth. The efficiency of urinary antiseptics. is naturally limited by the brief contact. It is therefore not necessarily reflected in the phenol-coefficient (Hinman, I915). The antiseptics of the aromatic series are sufficiently concentrated, in the course of their excretion, to be effective, e.g., in gonorrhea. Sodium salicylate or benzoate or salol are used for this purpose, as also copaiba, cubeba, sandal-wood, methylene blue, etc. Santal is especially useful against staphylococci; but none of these antiseptics are very powerful (Hinman, I915). The most useful product, however, is hexamethylenamin, which yields formaldehyd. This is used in pyelitis, inflamed bladder, and for rendering the urine aseptic (as in typhoid fever). Increased acidity of the urine also destroys bacteria. Dilute mineral acids, or acid 
sodium phosphate, may be used (Jordan, I9II). Alkalinity interferes greatly with the activity of benzoates, salicylates and hexamethylenamin; not so much with borates or sandal (Jordan, I913).

Intestinal Antiseptics. - The disinfection of the alimentary canal would be useful in various dyspepsias, intestinal putrefaction, in typhoid fever, cholera, etc. Many expcriments have been directed to this end. As a result, it may be concluded that complete asepsis is impossible in this situation.

This is easily comprehended if one stops to consider the large number of bacteria present, especially in the colon; the large mass of material in the intestine, tending to weaken the antiseptic and to prevent its access; the ready absorption and consequent danger of general poisoning; the sensitiveness of the intestinal canal to irritating agencies; the fact that ferment action is diminished by all antiseptics, etc.

When the antiseptics are used in such an amount as to injure the mucosa, they may even increase the number of bacteria. It was also argued, at one time, that antiseptics would be injurious by checking the saprophytic bacteria, which were supposed to be necessary for digestion. This theory has been disproved; nor do they appear to hinder the digestive ferments materially, although they tend in this direction (Heineber: and Bachmann, rgog).

Although a complete intestinal asepsis is an impossibility, a relative asepsis, a limitation of an abnormally increased bacterial action, is rather probable; it has not, however, been satisfactorily demonstrated (Harris, rgr2).

The bacteria in the lumen of the intestine would be much more readily acted upon than those which have already obtained a nidus in the intestinal walls, and antiseptic measures will be of greatest benefit against the unprotected bacteria.

The antiseptics, at best, seem to be inferior to suitable modifications of diet and evacuation of the intestines (Friedenwald and Leitz, 1909). For antiseptics, more strictly speaking, the preference is given to those which are only sparingly soluble, so that they will not be absorbed before reaching the colon. The most useful intestinal antiseptics are naphthalin, naphthol, the cresols, and guaiacol, thymol, camphor, salol, etc. Comparative experiments with these and others were made by Hirata, I9Io.

\section{SERUMS AND VACCINES}

Antitoxic Serums.-These contain antitoxic substances, produced by the injection of bacterial toxins into living animals, in practice usually horses. Definite globulins of the serum of such immunised animals contain specific antibodies, which combine with the corresponding toxins into harmless compounds. The injection of these serums into patients therefore confers a passive immunity to the disease, without other direct effects. According to the time and place of injection, rapidity of absorption, accessibility of the toxins and bacteria, the effect may be curative or merely prophylactic. The protection persists only for a few weeks, until the antitoxin is destroyed or excreted. The serum proteins often cause the slight irritative phenomena of "serum disease," and sometimes severe anaphylaxis.

Antitoxin therapy is limited to a few diseases, especially diphtheria and tetanus. Most other bacteria, especially those with endocellular toxins, do not provoke the formation of soluble antibodies, at least in sufficient amount to be effective.

Vaccines.-These provoke the formation of specific antibodies by the patient himself, by inducing a mild or modified harmless attack of the disease. They therefore confer active immunity, which is much more lasting than the passive. Their field is much wider than that of the antitoxic serums, since probably all bacteria and their products can act as antigens. The bacterial vaccines are usually suspensions, extracts or filtrates of killed cultures of the specific bacteria. Those prepared from the organisms obtained from the patient himself (autogenous) are usually 
much more effective than "stock" vaccines. As vaccines do not contain serum, they do not produce "serum disease"; but their toxins usually cause more or less febrile and local reaction. Their value is most striking in prophylaxis; in this way they may be effective even after exposure, but before the disease has developed. They may also be curative, especially in stationary localized infections.

Mechanism of Action.-The vaccines act largely by provoking the production of specific antibodies; but partly: also by stimulation of the leucocytes and other activities (Hektoen, I916). Increase of protease and lipase may be important factors (Jobling and Petersen, I9I6). These mechanisms are invoked especially to explain the activity of non-specific factors such as the injection of non-specific bacteria and toxins, protein, cleavage products, kaolin, etc.

Sensitized Virus Vaccines.-Living bacteria treated with specific antisera are claimed by Besredka to be powerful and harmless vaccines. More extensive experience is needed (Swift and Kinsella, I9I6).

Limitations of the Text.- The theories of the nature and formation of toxins and antibodies are usually assigned to bacteriology and pathology, and need not be considered here; nor will it be profitable to discuss the numerous agents whose value is not yet established. Those which are more or less promising are described in N.N.R.

\section{DIPHTHERIA ANTITOXIN}

This was discovered by Behring in $\mathrm{r} 890$, and applied therapeutically to man by Roux in I89I. The results are strikingly successful. It arrests the local process and the systemic symptoms, and reduces the mortality of the disease to about one-fourth. It should be administered in adequate doses, as early as possible; for the mortality increases from 4 or 5 per cent. if it is given on the first day, to over 20 per cent. if delayed beyond four days (Park, I906).

Production.-The antitoxin is produced by injecting healthy horses hypodermically, at four-day intervals with diphtheria toxin, i.e., with filtered sterile broth cultures, beginning with very small doses, which are increased at varying intervals, according to the amount of reaction. This treatment is continued for from four to six months, until a high antitoxic power has been reached (as determined on small samples of blood, withdrawn from time to time). The horse is then bled from the external jugular vein, with strict asepsis. This can be easily done without anesthesia. Seven to I 2 liters are collected in sterile bottles. When the serum or plasma has separated, it is filtered through Berkefeld filters, and some antiseptic (phenol or cresol), is added. The antitoxic strength is determined, and the appropriate doses are marketed in aseptic glass syringes, to obviate contamination during administration. Weaker serums may be reduced in bulk by partial evaporation in a vacuo.

Standardization. - The strength of the antitoxic sera has to be determined by testing its protective power on guinea pigs. The original immunity unit was ten times that quantity of antitoxin which would completely protect a 250-gram guinea pig against ten times the fatal dose of toxin; in other words, each immunity unit neutralized a hundred fatal doses of toxin.

It was found, however, that a given serum seemed to have a different antitoxic value when tested with different samples of toxin. This was explained by Ehrlich by the assumption that the filtered broth culture contains several constituents capable of neutralizing antitoxin; and that only one of these is toxic. The proportion of toxic to non-toxic neutralizing elements varied in the several cultures, so that the neutralizing and toxic qualities would not go parallel. To obviate this uncertainty a standard serum was prepared from a certain standard toxin.

This standard antitoxin is issued by the U. S. Public Health Service to manufacturers, and is in turn used for determining the "test dose" of the toxin to be employed in the actual standardization. This is the largest quantity of toxin which can be completely neutralized by the unit of standard antitoxin, so that a 250 -gram guinea pig will survive for four days. This toxin is then used in standardizing the serum to be tested; the smullest quantity of serum which neutralizes a test-dose of the toxin contains one unit of antitoxin. This new unit, which is now employed universally, is approximately equivalent to the old unit, but is more uniform. 
"Purified Antitoxin."-If the proteins of antitoxic serum are separated by fractional precipitation with salts, the antitoxin is found practically completely in the pseudoglobulin portion (Gibson and Collins, 1907; Banzhaf and Gibson, 1907). The non-antitoxin proteins may thus be largely eliminated. These "refined" serums permit reducing the bulk of the injection and lessen the liability to rashes but not to anaphylactic shock (Park, r9r3).

The Gibson process of purification consists in precipitating all the globulins by an equal volume of saturated ammonium sulphate solution (other sulphates may be used); the precipitate is treated with saturated solution of sodium chlorid, which dissolves the pseudoglobulin. This is again precipitated by acetic acid, and this precipitate is dialyzed to reduce the salt to 0.8 per cent. The pseudoglobulin fraction of normal serum is about $4 \circ$ per cent. of the total proteins; in immunized serum it is about 78 per cent.; so that this process eliminates only about one-fifth of the total proteins. The proportion can be raised by heating the serum for several hours to $57^{\circ} \mathrm{C}$., before proceeding to Gibson's process (Banzhaf, I9II). This Banzhaf process eliminates about 50 per cent. of the proteins, without material loss of antitoxin (Park, I9ro).

Deterioration.-The antitoxic power of serum, plain or purified, declines gradually, the loss averaging between ro and 30 per cent. per year. If kept on ice, the yearly loss is only about 6 per cent. (Anderson, r909). If aged serums are used, allowance must be made for the deterioration; otherwise their use is quite safe. Dried serum does not deteriorate, but is inconvenient to dissolve.

Clinical Efficiency.-The antitoxin is not directly bactericidal; but by inactivating the toxin, it arrests promptly both the systemic and local phenomena of the disease, the more strikingly and promptly the earlier it is used. The exudation ceases to spread and clears more rapidly; it rarely extends to the larynx; the swelling of the cervical glands subsides; the fever falls promptly, and the general condition improves quickly. The occurrence of paralysis is not affected.

The very much superior results of early administration make it incumbent to employ the antitoxin just as soon as a well-founded suspicion of diphtheria exists; i.e., without waiting for a positive diagnosis. The possible dangers of the treatment are so slight as to be negligible in view of its great benefits. It is even advisable to employ immunizing doses in case of exposure, before the disease develops.

Failure to Prevent Paralysis.-H. Meyer, I905, attributes this to the absorption of part of the toxin through the nerve sheaths, where it would be protected against the antitoxin.

Circulatory Collapse.-This is prevented if the antitoxin is administered before the blood pressure has started to fall; after this, antitoxin is useless (H. Meyer, I909), the toxin being presumably fixed in the cells.

Administration.-All immunizing sera are practically inactive when taken by the alimentary canal (McClintock and King, I909, p. 394). They are therefore injected into the loose subcutaneous tissue of the abdomen, back or flank. The skin and needle should be cleansed with alcohol. The injection is made slowly. Intravenous injection has been advised in urgent cases. It does not seem.to be dangerous if proper precautions are observed (Levin, r909; Park, r9r2).

Absorption.-This is quite slow, the maximum concentration of antitoxin in the blood being reached only after a day with intramuscular, and in three days with hypodermic injection (Levin, Park). The distribution has been studied by Hektoen and Carlson, I9ro.

The concentration of antibodies generally, both in active and passive immunity, 
decrease in the order of serum (highest), thoracic lymph, neck lymph, pericardial fluid, aqueous humor, cerebro-spinal fluid (least). This indicates that they pass from the blood into the lymph (Becht and Luckhardt, 1916).

Dosage.-This varies with the stage and severity of the disease; the age of the patient need not be considered. The immunizing dose is from 500 to I, 500 units. The curative dose is usually 5,000 units in mild cases; 10,000 in severe, and 20,000 in malignant cases. Larger doses do no further good (Park, I9I4). Half the initial dose may be repeated two or three times at intervals of about twelve hours, according to the progress of the disease; but it is better to make the initial dose sufficiently large, since experiments have shown that this insures a higher concentration in the blood (Park, r9r3).

Side Actions and Accidents.- In perhaps a third of the cases, the administration of serum is followed by the insignificant phenomena of "serum disease." Very rarely severe or fatal collapse, presumably analogous to anaphylaxis, occurs within fifteen minutes. The two phenomena are quite distinct, but both are due to the serum proteins and not specifically to the antitoxin. They are produced by all serums. The serious cases are so exceptional that they should not restrict the use of antitoxin; but they should enjoin caution in sensitive patients.

Serum Disease.-After an incubation of eight to twelve days, or a somewhat shorter time if the injections are repeated (Goodall, I9II) a rash develops on the skin, with swelling of lymph glands, a slight fever, and sometimes ( 3 or 4 per cent. of all injections) pain in the joints. The rash varies but is commonly urticarial or erythematous. It is sometimes preceded by prodromal sensitiveness and slight redness of the skin at the site of injection. The serum phenomena are less common with purified antitoxins; with serums of other animals they are more severe than with horse serum.

Statistics of serum sickness are presented by Sturtevant, I916.

Netter, I906, claims that the rashes are less frequent if calcium chlorid is administered: I Gm. daily by mouth, on the day of injection and on the two following days.

Relation of Serum Disease to Antibodies. - In man, anaphylactic antibodies are not present during the early period of serum sickness, but they reach their maximum toward the close. This indicates that the antibodies are concerned in the recovery, rather than in the causation of serum disease (Longcope and Rackemann, I9I6).

Anaphylactic Phenomena.-These occur, but very rarely, in patients disposed to asthma and hay fever, or those having idiosyncrasies against horses; they generally have a status lymphaticus. Serious symptoms occur in something like I : ro,000, death in I : roo,000 injections (Park, I9I3). The effects set in very suddenly with swelling of the respiratory mucosa, intense dyspnea, failure of respiration, collapse, and asphyxial death within ten or twenty minutes. These phenomena resemble very closely those seen in experimental anaphylaxis, but they occur generally with the first injection of serum. The most promising treatment of such cases is atropin and epinephrin, to paralyze the bronchial muscles. If there is cause for apprehension before giving the serum, the susceptibility of the patient may be tested by injecting a small dose (0.5 c.c.) of the serum an hour before giving the full quantity. Atropin may also be used as prophylactic.

Active Immunization.-v. Behring, rgr3, attempted this with overneutralized mixtures of diphtheria toxin and an excess of antitoxin (Hornemann, I9 3 ), with or without vaccines from killed diphtheria bacilli. Park, et al., 1914 and 1915. have checked 
the results with the Schick test. Natural immunity is greatly increased by the treatment, but this would be superfluous. In susceptible individuals, the immunity is produced very slowly; but little has developed in four to six weeks, but it continues to increase for a year or longer. The method is therefore of little value for immediate immunization, but promising for permanent protection.

\section{PREPARATIONS-ANTIDIPITTIERIA SERUM}

* Serum Antidiphthericum (Ser. Antidiph.), U.S.P. (Diphtheria Antitoxin).-The serum of horses properly immunized against diphtheria toxin. It must be kept in sealed glass containers in a dark place at a temperature between $4.5^{\circ}$ and $15^{\circ} \mathrm{C}$. A yellowish transparent or slightly turbid liquid, of at most slight odor. A tube of antitoxin once opened should not be used again. The serum must be prepared in establishments licensed and supervised by the U. S. Public Health Service; it must come from healthy animals; it must be sterile, free from toxin and bacterial products, and must not contain more than 0.5 per cent. of phenol or 0.4 per cent. of cresol; the total solids must not exceed 20 per cent.; and the potency must be at least 250 units per cubic centimeter. The standard of strength, expressed in units of antitoxic power, shall be that established by the United States Public Health Service. Antidiphtheric Serum gradually loses in potency. The label must state the date beyond which the contents can not be expected to yield its specific results. Average dose, Curative ro,000 units; Protective, 1,000 units, U.S.P.

*Serum Antidiphthericum Purificatum (Ser. Antidiph. Purif.), U.S.P.; (Antidiphtheric Globulins; Refined and concentrated Diphtheria Antitoxin). $-A$ solution in 0.8 per cent. $\mathrm{NaCl}$ solution of antitoxic globulins, prepared by Gibson's method, and complying with the regulations for the plain serum.

Ser. Antidiph. Sicc., U.S.P.; Dried Antidiphtheric serum.-Obtained by evaporation of either of the preceding at a low temperature. It must be readily soluble in 9 parts of water.

\section{NORMAL HORSE SERUM}

The injection of normal serum of man, horses, or other animals is sometimes used to arrest persistent hemorrhage in hemophilia, severe anemias, purpura, etc. (Bienwald, r897; Weil, 1907). Its value is not constant, but sometimes it appears to be effective. Trember, roro, attributes the results to leucocytosis. It has the same side actions and dangers as antitoxic serum; and these dangers must be taken more seriously, since its benefits are only problematical. It is administered hypodermically, ro to 30 c.c.; the results are said to appear in twelve to twenty-four hours and last one to three months (Richardson, I908).

\section{HEMOSTATIC-TISSUE EXTRACTS; KEPHALIN}

Extracts of various tissue cells hasten the coagulation of the blood, due to their content of the lipoid kephalin, which is supposed to constitute the factor of bloodcoagulation that has been variously termed zymoplastic or thromboplastic substance, thrombokinase and thromboplastin. Kephalin is the principal lipoid of the brain. A partly purified "Brain lipoid" (N.N.R.) is therefore used as hemostatic (Hess, 19 15; Hirschfelder, I916; J, McLean, 1916). It is used locally on bleeding surfaces, and subcutaneously or intravenously against hemorrhagic conditions. The details of the preparation and application are described in N.N.R. An extract of blood-platelets is marketed as a hemostatic under the name of "Coagulen" (N.N.R.).

\section{TETANUS ANTITOXIN}

This is prepared in the same manner as Diphtheria Antitoxin, except that tetanus toxin is used for the immunization. It has a high protective value if used early; and it would be desirable to administer it in every suspicious case, but without neglecting thorough disinfection. Its 
curative value, after the symptoms have set in, is doubtful and at best limited. It does not affect the toxin which is fixed in the nerve cells, and could only reach that which is still free or being formed. The latter can generally be prevented more effectively by surgical procedures.

Immunizing Dose.-This consists of I,500 units, hypodermically, repeated in a week if the wound continues to suppurate. The wound may also be dressed with dry serum. The protection lasts two or three weeks.

Curative Dosage.- In view of the slow absorption, and the importance of gaining time, hypodermic administration alone is insufficient. Large doses must be introduced by several channels. Park, I9IO, I9I 2 and I9I4, advocates the subdural injection of 3,000 to 5,000 units, followed by 10,000 to $I_{5}, \infty 00$ units intravenously; the spinal injection is repeated in twenty-four hours, and a hypodermic injection of Io,ooo units is given three days later (Park and Nicoll, r9I4; Nicoll, r9 5 ). Dreyfus, I9I4, urges daily injections, combining vein, lumbar, endoneural and local.

Intraneural Injection was advised by Meyer and Ransom, I903, since they found that the toxin travels largely by nerve sheaths. It has not maintained itself in practice, but is again advocated by v. Behring, Igr4.

Subdural Injection, according to Park and Nicoll, is more effective than intravenous, and vastly better than hypodermic. The antitoxin disappears in twenty-four hours, so that the administration must be daily if this channel is used alone (Dreyfus).

Official Unit (U.S.P.H.S.).-This is ten times the least quantity of serum necessary to save the life of a $35^{\circ}-\mathrm{Gm}$. guinea pig for ninety-six hours against the official test-dose of standard toxin furnished by the U.S.P.H.S. (Rosenau and Anderson).

\section{PREPARATIONS-ANTITETANIC SERUM}

*Serum Antitetanicum (Ser. Antitetan.), U.S.P. (Tetanus Antitoxin).-The serum of horses highly actively immunized against tetanus toxin. It resembles Diphtheria Antitoxin and must meet analogous requirements. Average dose, Curative, 10,000 units; Protective, I, 500 units, U.S.P.

*Ser. Antitetan. Purif., U.S.P. (Antitetanic Globulins; Refined and Concentrated Tetanus Antitoxin). - Analogous to Ser. Antidiph. Purif.

${ }^{*}$ Ser. Antitetan. Sicc., U.S.P.—Analogous to Ser. Antidiph. Sicc.

\section{ANTIMENINGOCOCCUS SERUM}

This is markedly successful against epidemic cerebro-spinal meningitis due to Diplococcus intracellularis, especially if used early. It reduces all the symptoms, clears the cerebro-spinal fluid, lessens the duration and sequels, and diminishes the mortality (Dunn, I908; Ladd, I909; Flexner, 19I3). The mechanism of its action has not been decided.

Preparation.-The serum is obtained by the method of Flexner and Jobling, 1908, from horses actively immunized by injections of killed meningococci, autolyzed cultures, and finally living bacteria, successively and in alternation, the treatment being continued for months until the desired efficiency is obtained.

Action. - The serum does not kill the bacteria directly, but causes their disappearance, perhaps by stimulating phagocytosis and intraleucocytic digestion.

Administration.- The action being mainly local, the serum must be injected into the subdural canal, early, and in efficient dosage. As it is absorbed, several injections are generally needed. The usual dose is I 5 to 30 c.c.; for young infants ro c.c. Cerebrospinal fluid is first withdrawn, if possible the same bulk as the serum to be injected. The blood pressure should be watched during injection to guard against excessive pressure. - The administration should be repeated every twelve to twenty-four hours, for at least four injections; and longer if the fluid and symptoms have not cleared. The occurrence of accidents is discussed by Flexner, I913. They consist in sudden collapse with stoppage of respiration and fall of blood pressure. The effects have been attrib- 
uted to the preservative (Voegtlin, I9I $q$ ); but this is probably not the usual cause (Auer, I9I4).

\section{ANTIPNEUMOCOCCIC SERUMS}

These have been developed against certain strains of pneumococci (Cole, 1915). Bull, I9I 5 , refers their action to agglutination.

\section{HAY FEVER SERUM}

This is obtained from horses injected with the pollen-toxin of various plants which are supposed to cause hay fever (Blackley, 1873; Dunbar, I9II). It is used locally in the eyes and nose. Its value is limited; it is not curative, but often gives some relief. "Fall Pollantin" or "Dunbar's Serum" is prepared from ragweed pollen. It is marketed as serum and dried. Other serums are obtained from goldenrod, etc.

Hay fever vaccines are also being tried. Oppenheimer and Gottlieb, 1915, warn against their use.

\section{VACCINE VIRUS}

This consists of the scrapings from the pustules or vesicles of vaccinia or cowpox, of vaccinated, but otherwise healthy calves. It must be removed aseptically, is further sterilized by standing with glycerin, tested for the absence of bacteria, and marketed in tubes or "points." It is used by inoculation (vaccination) to produce active immunity against smallpox, the protection lasting four to ten years.

Preparation.-The "pulp" is curetted from the typical vaccinia vesicles. The "lymph" which flows afterward was formerly used for making "dry points;" but as it is less potent and generally septic, it should not be used. The pulp is ground with 50 to 60 per cent. of glycerin, and allowed to "ripen." This preserves the virus, but kills most of the bacteria. The establishments must be conducted under federal supervision and license.

Vaccine Virus gradually loses potency, most rapidly at high temperatures. It must therefore be preserved at a temperature between $4.5^{\circ}$ and $15^{\circ} \mathrm{C}$.

\section{PREPARATIONS-VACCINE VIRUS}

*Virus Vaccinicum ((Virus Vaccin.), U.S.P.; Vaccine Virus (Glycerinated Vaccine Virus; Jennerian Vaccine).-The pustules of vaccinia or cowpox removed, under aseptic conditions, from vaccinated animals of the bovine species. Preserved at a temperature between $4.5^{\circ}$ and $15^{\circ} \mathrm{C}$., protected from light. The vaccine pulp is thoroughly rubbed up in a mortar or passed through a special grinder, strained to remove coarse particles, and made into a smooth emulsion with a glycerin solution. Vaccine Virus gradually loses in potency, the loss being more rapid at high temperatures than at low temperatures.

\section{ANTIRABIC VACCINE}

This consists of emulsions of the spinal cords of rabbits, which have died as the result of subdural injections of "fixed" rabies virus. If the disease is not too far advanced, so that at least fifteen days of treatment are possible, the mortality is less than I per cent., as against I 5 or 20 per cent. in untreated cases (Stimson, I9ro).

Preparation.-Fixed virus is obtained by the passage of rabies virus through a long series of rabbits, till it acquires a fixed incubation period. This fixed virus is less capable of producing rabies on subcutaneous injection. The spinal cords of the infected rabbits are further attenuated by graduated drying. In the treatment, emulsions of increasing virulence are daily injected hypodermically, the scheme of dosage varying according to circumstances; but generally beginning with cord dried for eight to twelve days and gradually increasing till "two-day" cord is used. 


\section{TUBERCULIN PREPARATIONS}

These represent the toxic products of the tubercle bacilli; consisting either of solutions, i.e., filtered germ-free cultures (e.g., old tuberculin); or of súspensions of killed and ground tubercle bacilli (tubercle vaccine, new tuberculin, emulsion). They are used for the diagnosis and treatment of tuberculosis. The two classes of preparations are practically identical in action, differing only quantitatively and in absorbability. The filtered cultures are more evenly absorbed and therefore permit a better control of dosage, and are thus safer for diagnostic tests and early treatment.

Diagnostic Use.-Relatively large doses of tuberculin have no effect on normal individuals; but in tuberculous patients, there is a definite inflammatory reaction about each localized tuberculous lesion, with slight febrile symptoms. The test is applied in various ways. It requires special knowledge and experience for its proper performance and interpretation; otherwise it may do much harm (Baldwin, I9Io).

Therapeutic Use.-It is questionable whether tuberculin ever produces actual immunization; but a course of carefully graduated doses diminishes the sensitiveness to the toxin, and therefore the constitutional phenomena of the disease. The local reaction about the tuberculous foci also tends to their cicatrization and absorption. The artificial resistance is gradually lost after the treatment is discontinued.

For therapeutic purposes, the tuberculins must be administered hypodermically, The proper dosage requires experience and judgment. Ready-made "serial dilutions" deteriorate rapidly and are not recommended. Various preparations of tuberculin are listed in N.N.R.; the following are important:

*Old Tuberculin, "O.T.", Koch's Tuberculin (N.N.R.).-Prepared by concentrating a six to eight weeks' glycerin-bouillon culture of tubercle bacilli by evaporating to one-tenth its volume, and filtering; contains 50 per cent. of glycerin.

Tuberculin "Denys", "B.F.", Bouillon Filtrate.-A simply filtered culture, not boiled.

New Tuberculin, "T.R.", Tuberculin Residuc, Koch.-This represents a complex extract of virulent human tubercle bacilli; the dried bacteria are subjected to prolonged grinding and thereby killed. They are then suspended in normal saline, centrifugalized, and the first supernatant fluid is rejected. The sediment is again ground with saline and centrifugalized. This fluid is saved. The process is repeated until almost no sediment remains. The supernatant fluids are united, mixed with 20 per cent. of glycerin and a little formaldehyd and adjusted so that each cubic centimeter represents Io mg. of dry bacilli.

Bacilli Emulsion, "B.E.", Koch.-A suspension of ground bacteria in 50 per cent. glycerin, $5 \mathrm{mg}$. in I c.c. Any surviving bacteria are killed by heating for an hour to $60^{\circ} \mathrm{C}$.

\section{TYPHOID VACCINE}

This has given completely successful results for immunization (Russell, I9I3 and I9I4), lasting at least two years (Harris and Ogan, I9I4). It consists of a suspension of avirulent typhoid bacilli, killed by heating for an hour at $55^{\circ} \mathrm{C}$., and preserved by 0.25 per cent. cresol. It should be injected subcutaneously (not intramuscularly) in the arm, giving 500 million bacteria at the first injection; and two further injections, each of one billion bacteria, at ten-day intervals. It generally causes some local reaction, and sometimes more or less severe but harmless febrile symptoms (Russell, I9I2). It is safer to avoid its administration to any but healthy individuals; not to inject it into a vein or intramuscularly; to avoid exposure of the patients to sun, alcohol or exertion; and not to give it during menstruation or pregnancy (Harris and Ozan, I914).

The intravenous injection of typhoid vaccines is being used in the developed fever (E. Fraenkel, 1876; Gay and Chickering, 1916; Hektoen, 1916). It of ten induces a crisis and prompt recovery. The action is probably not due to specific antibodies, for similar effects are produced by other vaccines, albumoses, etc. (J.L. Miller and Lusk, I9 I6). 


\section{GONOCOCCUS VACCINE}

This is being used with fair results in subacute and chronic gonococcus infections involving the joints. It is less useful in other situations. It is also marketed as "Neisser Bacterin."

\section{THEORIES OF NARCOSIS}

General Statement.-The aliphatic compounds or hydrocarbons, like the benzol compounds, resemble each other closely in their main actions. Their most conspicuous effect on the higher animals consists in stupefaction, passing with larger doses into insensibility and motor paralysis; i.e., into profound narcosis or anesthesia of central origin. Analogous narcotic effects are produced on all forms of protoplasm. Hemolysis is also a conspicuous effect.

Narcosis is one of the most common and striking pharmacologic phenomena. Many attempts have been made to gain a clearer conception of its intimate nature, especially through the study of the aliphatic narcotics. The relations between the chemic structure and the pharmacologic efficiency were extensively investigated. More recently, a deeper insight has been gained by the discovery that the narcotic and hemolytic effects of this series are intimately related to the solvent action on lipoids; or rather, to their "partition coefficient," i.e., their solubility in oils, divided by their solubility in water. The discovery of this law by Meyer and Overton was an important step in the solution of the problem, and opened the way for the intelligent investigation of further theories. Several of these are in the field at present, as will be explained later.

Definition of Narcosis.-This is defined by R. S. Lillie, r9 6 , as synonymous with anesthesia: "A physiological condition in which the normal responsiveness or automatic activity of the living system-organism, tissue or cell-is temporarily decreased or abolished." The definition is perhaps too comprehensive, but it emphasizes the two essential features, the depression and the reversibility or possibility of recovery. This may be lost if the action is made too energetic.

The definition also brings out the essential similarity of sleep, fatigue, cold, electric inhibition (anelectrotonus), to the narcotic effects of the aliphatic series, and of certain ions.

Influence of Chemic Structure.-An historical review of the older theories of narcosis is given in Overton's book. The earlier studies indicated that the actions of this series appear to be influenced quantitatively by the chemic structure; and much labor has been expended on determining the share of various side-chains (Binz, r88I; Baumann and Kast, $x 890$ ). The results (summarized in Schmiedeberg, Grundriss d. Pharmacologie, I909, p. I9) seemed to show certain relations, but the generalizations have not stood the test of wider experience (Diehl, I894; Meyer, I899; Kionka, 1900). In fact, it has been pretty definitely shown that the chemic structure affects the narcotic action only indirectly, namely by modifying the partition coefficient, which varies in a definite manner with changes in composition. However, special side-chains may be' responsible for side actions. Thus, chlorin compounds are peculiarly toxic to the heart.

Empiric Relations. - These are practically interesting and important, although they have lost all theoretical significance. The following conclusions appear justified: Other things being equal, the strength of action increases with the length of the chain. A limit is soon reached, however, for the drugs become less volatile, less soluble and less absorbable the higher they stand in the series; so that the higher paraffins are entirely insoluble and inactive. The ethyl radical, $\mathrm{C}_{2} \mathrm{H}_{5}$, seems to be best adapted to therapeutic purposes. The action may be modified by replacing the $\mathbf{H}$ of the group by other elements or groups. The compounds so formed all possess the typical action of the hydrocarbon group; but this may be so overshadowed by other actions as to be scarcely appreciable. A good example of this is the introduction of the group $\mathrm{NO}_{2}$. This acts 
very mucl more strongly than the hydrocarbon part of the molecule, and consequently the nitrite action is obtained long before there can be any hydrocarbon action. The same is true if we substitute an aromatic radical. The introduction of certain other rirlicals weakens the hydrocarbon action to a very great extent. We may mention especially the acid-forming radical $\mathrm{CO}_{2} \mathrm{H}$. The introduction of hydrocarbon radicals into an amin molecule destroys their action entirely. The introduction of more than one OH group also weakens or destroys the action. Aldehyds and ketones are more active than the corresponding alcohols. The introduction of the halogens, and especially of $\mathrm{Cl}$, often enhances the action, but this is not proportional to the number $\mathrm{Cl}$ molecules introduced. The introduction of $\mathrm{O}$ (ethers) also increases the action. The introduction of acids (esters) weakens it.

Narcosis and Sleep.-The analogies of narcosis in the higher animals, with the phenomena of natural sleep suggested a common origin to the earlier investigators. Since sleep was then explained by Cerebral Anemia (Durham, 1860) the same explanation was adopted for narcosis (Bedford-Brown, 1860). However, it was subsequently found that changes in the cerebral circulation are small and variable ( $\mathrm{Cl}$. Bernard, I 875$)$.

All the theories of deficient mutrition of the brain-whether through anemia or otherwise-are definitely disproved by the fact that narcosis can be induced in frogs in which all the blood has been displaced by saline solution, or in which the cerebral circulation has been arrested; in excised nerve-muscle preparations; in animals which do not possess blood; in plants; and even in unicellular organisms.

Coagulation Theories.-The aliphatic narcotics coagulate proteins, and this suggested to $\mathrm{Cl}$. Bernard, 1853 and 1875 , that narcosis might be due to a semicoagulation of the protoplasm, analogous to the muscle-rigor produced by chloroform. This theory had no direct experimental foundation; but it was elaborated by Binz (Lectures, 189r), who showed histologically that the cells of fresh brain sections are coagulated by solutions of chloroform or morphin; but he had to employ relatively enormous concentrations, nor could it be supposed that the coagulated cells could ever resume their activity. The theory was therefore untenable at least in this form.

Removal of Lipoids.-Bibra and Harless, I 847 , immediately after the discovery of the anesthetics, drew attention to the fact that practically all fat-solvents are narcotics, and suggested that they dissolve the fats out of the nerve cells. The difficulty of explaining the rapid recovery proves that this theory can not be correct. It was accordingly abandoned; but later workers continued to point out more or less quantitative relations. Hermann, r866, f.i., showed that all the aliphatic narcotics dissolve red blood corpuscles, connected this with their lipoid solubility, and first suggested the modern theory. Conversely, Richet, I895, argued that the narcotic efficiency is inversely proportional to the solubility in water.

Meyer-Overton Law.-The relations observed by Hermann and by Richet were striking but not conclusive. It was only by their combination that an exact quantitative relation could be demonstrated. This was done, independently and practically simultaneously by $\mathbf{H}$. Meyer and by Overton, I899 and I901. They showed a very close parallelism between narcotic efficiency and the "partition coefficient:"

Solubility in fat $\left(\mathrm{S}_{F}\right)$

Solubility in water $\left(\mathrm{S}_{W}\right)$

The higher this coefficient, the more powerful the narcotic action. The quantitative relation is so constant and the facts in its support so numerous, that it can scarcely be doubted that the narcotic effects of the aliphatic series depend in some way on their lipoidsolvent action, as expressed by this coefficient.

Partition Coefficient.-The experimental determination of this factor is described in Hoeber (p. 196). Berthelot and Jungfleisch, 1872 , showed that it is constant, independent of the absolute quantity, provided there is no dissociation. For convenience, it has so far been determined mainly with olive oil. Objection has been made to this (Moore and Roaf, I906), since this oil differs from the cell-lipoids. The fact that the law holds also for hemolysis, practically disposes of this objection. 
Lipoids. - This term is applied to a chemically heterogeneous group of substances, including phosphatids (W. Koch, I9II); especially lecithin and kephalin, cerebrin, cerebrosids, cholesterin, and others. They are waxy substances, which dissolve readily in lipolytic solvents, and take up more water slowly, but not solutions of sugars or salts. They occur in all cells, particularly in the plasma membrane, probably in an emulsifed condition. Nervous tissue is rich in these lipoids (Stewart's Physiology). The functions of the individual lipoids have certain features in common, but in some respects they behave very differently, $f . i$, cholesterin and lecithin have opposite effects on hemolysis and phagocytosis (Stuber, r913).

The term lipins has been introduced to cover all substances that dissolve in fatsolvents; i.e., it covers fats and fatty acids as well as the lipoids.

Evidence for the Meyer-Overton Law.-The narcotic concentrations have been determined mainly on tadpoles; but the law has also been demonstrated on other animals, plants and tissues. According to the theory every absorbable substance which is a fat-solvent should have an anesthetic action; this should be seen in all cells (since every cell contains lipoids); it should occur in the same concentration of solution; the anesthetic action of a mixture should equal the simple sum of the actions of its constituents; the effect should be proportional to the concentration, and not to the absolute dose, etc. These postulates agree very well with the actual facts. The relation of concentration to action is especially interesting. Precisely the same concentration of chloroform or ether produces the same degree of anesthesia in all cells (excepting plants and a few invertebrates). For instance, ordinary anesthesia requires in all animals a concentration of anesthetic to serum, of ether, $\mathrm{I}: 400$; of chloroform, $\mathrm{I}: 4,500$ to 6,000 ; but cold-blooded animals reach the same concentration in the blood with a lesser concentration in the air, on account of their lower temperature. Again, in those cases in which the coefficient changes with temperature, etc., the narcotic power changes in the required direction: Meyer, rgor, showed that rise of temperature increases the coefficient and the narcotic action for hydrated chloral; and depresses both for salicylamid. Fuehner, 1909, investigated the influence of mixed narcotics, and found that the coefficient and narcosis were always modified in the same direction.

The Meyer-Overton Law Applies Only to the Aliphatic Narcotics.-Cushny has pointed out that many benzol derivatives which are not narcotic have a high partition coefficient. In this case, however, the coefficient modifies the antiseptic action. The peripheral nervous system, although rich in lipoids, is not easily attacked by the aliphatic narcotics. Morphin and other basic and saline narcotics do not obey the law, and must act by entirely different mechanisms. This does not of course impair the validity of the law as applied to the aliphatic narcotics.

Influence of Extraneous Fats on Narcosis.-It might be expected that narcosis would be affected by fatty tissue, deflecting a certain amount of the anesthetic; but this does not seem to be the case to a notable degree.

Mansfeld, I905, suggested the low resistance of starved animals to be due to this. The fact is not convincing, for in starvation the resistance is lowered in many other directions. Indeed, Mansfeld's experiments contradict his explanation; for starvation did not increase the susceptibility to the typical lipolytic narcotics alcohol and urethane; whilst the susceptibility was increased for morphin, which probably does not act as a lipoid solvent. Cloetta, 1907, tried the converse experiment, the influence of increasing the fatty tissue; but his results scarcely justified general conclusions.

Nerking, 1908, claimed that anesthesia can be cut short by intravenous injection of lecithin. This has been contradicted by Kramer, rgr3, who took the necessary precaution to keep the concentration of the anesthetic constant by intravenous injection. Lattes, I9II, stated that fatty food, or the injection of fat-emulsions into the circulation, increases the narcotic action of chloroform, perhaps by lessening the pulmonary excretion. Hanschmidt, I913, supports Nerking, and affirms that lecithin has a protective action against narcotics, but also against a number of alkaloids (curare, strychnin, morphin). The results have therefore been contradictory and have little bearing on the lipolytic theory. Salzmann, 1912, reports that cream and other fats delay slightly the absorption and therefore the action of alcohol and other lipolytic narcotics. Murlin and Riche, 1915, find that the percentage of fat in the blood is lowered proportional to the degree of narcosis; but since this occurs also with morphin, it has no bearing on the lipoid theory.

Changes Effected by Lipoid Solvents.-The partition coefficient does not explain. the nature of narcosis, and it is necessary to resort to further 
theories. The rapid recovery after removal of the anesthetics proves that they can not actually dissolve the lipoids out of the cells. Meyer therefore assumed that they merely liquefy these constituents in the cells and thereby alter their functions. Overton had shown that the selective permeability of cells depends largely on the lipoids of the plasma membrane. Any change in these would necessarily alter the permeability; and it has been demonstrated that narcosis is actually characterized by such alterations.

Polarization Theory of Stimulation.-Narcosis consists in suppression of irritability; and it will be impossible to understand it fully until the nature of stimulation is understood. There is now considerable evidence that stimulation depends upon a change in the electric polarization of the cells, due to a change in the concentration of the ions at the cell membrane (Nernst, I 899), and brought about by a sudden increase of the permeability of the plasma membrane. According to this view, the actions of the lipolytic narcotics would be explained quite simply by their modification of the lipoids of the plasma membrane, rendering impossible the changes in permeability which are necessary for stimulation. Changes in the plasma membrane could also be effected by other means, and the narcotic effects of salts can be explained in this manner. A plausible explanation of the actions of morphin and most of the local anesthetics is still lacking.

Permeability of the Plasma Membranes.-Overton found that cells are generally impermeable to neutral salts, sugars, etc.; but easily permeable to lipolytic substances. This rule is not absolute - there are important exceptions, f.i., for metabolic substances and for some of the dyes, but it is sufficiently general to indicate that cells behave as if they were surrounded by a lipoid film. The depattures from the rule are probably explained by the intermixture of other colloid constituents (A. P. Mathews, I9I0). This protective film, the "plasma membrane," is probably formed by the surface condensation-according to the Gibbs-Thomson principle-of substances, which lower surface tension, as do the lipoids.

Relation of the Membrane to Stimulation.-This subject is well summarized by R. S. Lilly, I9I3. The semipermeability serves to maintain the differences of electrical potential (as shown by the demarkation current) which is essential to life. This potential disappears with death, and is impaired by all poisons which injure the semipermeability; and amongst these by the anesthetics (Waller and Alcock, 1906). Acids, alkalies and certain ions also disturb the potential; but these act presumably on the other colloids of the membrane. Analogous phenomena are observable in artificial systems of phosphatid and salt solutions (J. Loeb and Beutner, I913).

Excitability and Conductivity of Nerve.-Narcosis of a nerve, however produced, always lowers both these functions in strict parallel (Verworn, Erregung und Laehmung, p. I2I). While a normal nerve conducts without loss of stimulus, a narcotized nerve shows therefore a decrement during conduction (ibid., p. 127).

Relation of Permeability and Stimulation.-This is illustrated particularly by the experiments of R. S. Lillie, I9r2, on Arenicola larvæ. He found that pure solutions of $\mathrm{NaCl}$ produce strong stimulations and at the same time increase the permeability, as shown by the diffusion of pigment and the dissolution of cilia. $\mathrm{Mg}, \mathrm{Ca}$ and aliphatic anesthetics produce depression and lessen the permeability, so that they prevent the effects of $\mathrm{NaCl}$. The $\mathrm{Mg}$ ions and the aliphatic anesthetics probably act on different constituents of the envelop. It is therefore all the more striking that in both cases the narcosis is characterized by lessened permeability. Similar phenomena are observed in the effects of anesthetics on the sensitive plant. In nerve fibers and in muscle, Hoeber, I9I5, found that low concentrations of anesthetics decrease permeability, for they prevent the swelling which normally occurs in solutions of potassium salts. High concentrations increase the permeability; this would correspond to a toxic effect. Both phenomena have been confirmed on fish-eggs by McClendon, 1915. Osterhout, I9I2 and $19 I_{3}$, showed that anesthetics lessen the electric conductivity, and therefore the permeability, of plants. (Fatal concentrations again increase the permeability.)

The mechanism by which the lipolytic agents interfere with the permeability of the lipoid 
membrane is unexplained. It consists probably in a physical change of the colloids. Suggestive, but not altogether consistent, parallels have been drawn with colloidal suspensions of lipoids. f.i., Hoeber and Gordon, 19r4, found an interference of anesthetics with the precipitation of lipoid suspensions by calcium; others observed increased viscosity of lecithin suspension (Handowsky and Wagner, I9II; A. Thomas, I915). Clowes describes a change in emulsions, so as to promote the continuity of the fatty phase. The various lipolytic anesthetics differ in their effects on the state of aggregation of the phosphatids (W. Koch, I9IO); so that this can not be the essential factor in narcosis. S. Loewe, I9I3, found that lipoid membranes are rendered less permeable to ions by lipolytic agents, and suggests that the adsorption of the narcotics converts the colloids of the plasma membrane from a hydrophilic to a hydrophobic modification. This would lessen the permeability, and therefore the functions, potential, etc.

The diminished permeability obtains only for the anesthetic concentrations. Toxic concentrations increase the permeability, as seen for instance in hemolysis (Hoeber, I9 5 ; Joel, r9 I5). Autolysis is similarly increased by high concentrations (Chiari). H. Meyer, I909, attempted to explain narcosis by increased permeability through liquefaction of the lipoids; but there is no satisfactory evidence for this view. The lipolytic anesthetics do not change the viscidity of lecithin emulsions (Handovsky and Wagner, I9I I).

Surface-action Theory.-J. Traube, I904, I9I0, I9I3 and I9 5 , contends that the action of the aliphatic narcotics is not due to solution of the cell-lipoids, but to condensation or adsorption of the narcotic agent at the surface of the gross or colloid particles of the protoplasm, both lipoid and protein. The catalytic activity of these surfaces would thereby be diminished, resulting in the slowing or destruction of the chemic processes, oxidations, etc., and thus of the functions of the cells. Surface condensation is proportional to lowering of surface tension (or inversely, to "solution affinity" or "Haftdruck," the tendency of a substance to remain in solution). This proportionality is the basis and test of Traube's theory. Since the surface tension and the partition coefficient are generally parallel, it is difficult to decide between the two views; but there appear to be more exceptions to Traube's rule than to Overton's (Lillie, I9r6). Fuehner, I9I2, has investigated the question with homologous alcohol and finds that for hemolysis, and for animals devoid of a nervous system, the efficiency is parallel to the surface tension; but in proportion as the nervous system develops the activity of the higher alcohols becomes relatively greater, and agrees better with the partition coefficient. Ishizaka, I9I4, also finds a correspondence between hemolysis and surface tension in a series of terpen alcohols and ketones; but the chemic characters also had an influence.

Stimulation of Phagocytosis.-Hamburger and de Haan, and Bubanovic, I9II, showed that this is stimulated by minute doses of lipolytic agents, parallel to their partition coefficient. This is probably explained by a lowering of surface tension. Narcotic concentrations paralyze the movements of leucocytes.

Mobilization of Potassium.-Menten, I9I2, states that the quantity of potassium. ions in medullated nerve fibers is increased by local lipolytic anesthetics and by injury. This is probably an expression of the disturbed permeability; but since potassium depresses the conductivity of the nerves, it is conceivable that this plays a part in narcosis.

Dehydration.-Dubois, I 894 , observed exudation of water in narcotized plant cells, and suggested that narcosis consists in dehydration. Overton, however, showed that the phenomenon is a manifestation of irreparable injury, and not of narcosis.

Verworn's Theory of Oxygen Deprivation.-Verworn, I909 (Harvey Lectures, I9I I; "Narkose," Jena, I9I 2), and his pupils have pointed out that narcosis is generally accompanied by diminished oxidation; and that deficiency of oxygen produces anesthetic phenomena. They therefore believe that narcosis is due to interference with oxygenation; an incapacity of the cell substance to undergo oxidation, even in the presence of oxygen, whilst non-oxidative destruction proceeds unchecked.

Verworn suggests that the transmission of oxygen may be a function of the lipoid substances, thus accounting for the Meyer-Overton law, and harmonizing the two theories. Mansfield, r 909 , also assumes that narcotics lessen the solvent power of lipoids for oxygen and so prevent its penetration. This is contradicted by Winterstein, I913. A. P. Matthews, I9r 4, believes that the anesthetics fix the oxygen receptors of the protoplasm into a non-irritable anesthetic-protoplasm combination. Traube believes that the oxygen catalyzers are hindered by surface condensation. Warburg and Wiesel find a precipitant action of narcotics on yeast press-juice, parallel to anti- 
ferment activity; Batelli and Stern describe precipitation of nucleo-proteins in cell extracts, parallel with checking of oxidases. On the other hand, Buercker, 1910, and Baglioni believe that the narcotics combine with the oxygen.

Verworn concedes that high concentrations cause other changes. such as coagulation; but states that these are non-reversible, and therefore not concerned in true narcosis.

Verworn's facts do not prove his theory; for since narcosis suppresses irritability, it would naturally depress oxidation; this would be the effect instead of the cause of the narcosis. Conversely, the suppressed irritability by oxygen deprivation does not prove that every narcosis must be due to oxygen deprivation. This could be accepted only if interference with oxidation and narcosis were always strictly parallel. This is not the case, and the theory is therefore not tenable.

Anesthetic Effects of Oxygen Deprivation.-The anesthetic effects of asphyxia have long been familiar. As samples of the evidence for Verworn's theory may be cited the fact that frogs perfused with saline solution become inexcitable; but that the excitability is preserved or reappears if the solution is oxygenated (Verworn, I900). Similarly, excised nerve loses its excitability in an atmosphere free from oxygen (Froelich, I904). Mansfeld, I 909, showed that the results are additive; the paraldehyd narcosis in tadpoles being increased by degrees of oxygen deficiency which are in themselves harmless. He also pointed out, rgro, that oxygen deficiency, like narcosis, decreases the current of rest in frogs' skin. Froelich and Heaton, and Ishikawa showed that oxygen deprivation delays recovery from anesthesia. Anaerobic organisms are also subject to narcosis, showing at least that this has nothing to do with free oxygen or its penetration into the cells.

On the other hand, there are instances (nerve trunks, vorticella, cilia) in which oxygen deprivation acts very slowly, while anesthetics act very promptly. HCN, which is a strong asphyxiant, is a weak anesthetic. Anaerobic organisms are also subject to narcosis, showing at least that this has nothing to do with free oxygen or its penetration into the cells.

Diminished Oxidation in Narcosis.-Herter found that the oxidizing capacity of tissues for methylene blue is greatly diminished in anesthesia. Vernon, I909, claims a quantitative relation between the hemolytic effect of alcohols and their influence on the respiration of excised kidneys. Tashiro, Igr 3 , found that anesthetics greatly diminish the $\mathrm{CO}_{2}$ output of nerves. Alexander and Cserna, rgr 3 , claim that anesthetics and morphin diminish the oxygen consumption and $\mathrm{CO}_{2}$ formation of the brain.

Verworn, I9r2, and others have shown that various anesthetics inhibit oxidases parallel to their anesthetic activity. However, the required concentrations are of the, toxic rather than of the anesthetic magnitude.

Interference of narcosis with oxidation is the general rule; but there are important exceptions. Winterstein, 1915 , points out that anesthesia, even when continued for days, need not increase the acidity of the central nervous system; while asphyxia (oxygen deprivation) always increases the acidity. Anesthesia therefore does not always diminish oxidation. Warburg, Igro, and Loeb and Wasteneys, r9 3 , finds the oxygen consumption normal in narcotized sea-urchin eggs.

Chemic Theory of Moore and Roaf.-The evidence for the extension of the lipolytic theories to the permeability of the plasma membrane is not as complete as that for the parallelism of the narcotic action and the partition coefficient. It has been suggested (Kochmann, I906) that the lipoid solubility is merely the necessary condition for the entrance of the narcotics; and that the real action occurring within the cell may be altogether different, f.i., chemic (Heymans and De Buck, 1905). It is most unlikely, however, that the quantitative parallelism between narcotic effect and partition coefficient would obtain if the intracellular action were based on different properties. Nor is it plausible that the chemic effects of the chemically dissimilar lipolytic substances should all result in the identical narcotic phenomena.

Interest in the chemic theories was revived by the observations of Moore and Roaf, I905, which indicate the possible existence of loose reversible chemic combinations between certain anesthetics and proteins. $F . i$., with high concentrations of chloroform, its solubility in serum and tissue extracts does not obey the ordinary laws of solution; but this is probably an adsorption phenomenon, which need not have anything to do with narcosis. Cushny, rgro, finds that the exhalation of the volatile members of the alkyl series is exactly analogous to their evaporation from watery solutions, indicating that anesthetic concentrations exist in the body in purely physical solutions. The 
precipitation of proteins by lipolytic agents requires much higher concentrations than exist in anesthesia.

Morphologic Changes.-It may well be supposed that the changes in the physical composition of the cell will produce corresponding changes in their histologic structure. Most of these would probably disappear in the process of fixation, but certain of them appear to persist. A diminution and fusion of the Nissl granules have been described by good observers after deep anesthesia (Fig. 25); other observers have not been able to confirm this, and the changes may be only artifacts. Kocher, 1916, emphasizes the numerous sources of error and the inconstant results of such observations.

It is also stated that the dendrites of many pyramidal cells show moniliform enlargements; the alterations disappearing completely within forty-eight hours, even when caused by chloroform anesthesia of nine hours' duration. Lépine and Duval attributed anesthesia to retraction of the dendritic processes.
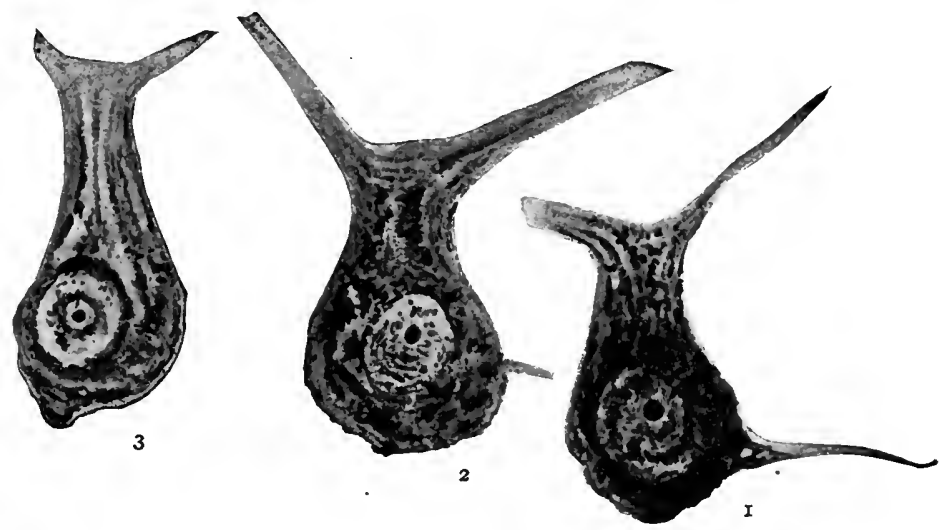

FrG. 25.-Alcohol on Purkinje cells, cat-Nissl stain (after C. C. Stewart): I, Normal; 2, alcohol for fifty minutes; 3 , for fifty-four hours.

\section{ALCOHOL}

Ethyl alcohol is a local irritant. After absorption, it depresses the central nervous system, especially the "higher functions." It simulates stimulation, chiefly by lowering the normal restraining functions; but there may be some direct facilitation of motor processes and respiration.

Local Action of Alcohol.-Concentrated alcohol "hardens" tissues by abstracting their water and precipitating the proteins. It is therefore irritant and astringent. The effect is proportional to the concentration.

Applied to the skin, it causes some redness and burning, followed by slight anesthesia. It is therefore employed as a mild rubefacient and counterirritant in sprains and other minor pains. It may be applied in Io to 50 per cent. concentration, by friction or embrocation. It is often combined with other mild irritants, as in the Linimentum Saponis, Spiritus Camphoræ, Aqua Hamamelidis ("Witchhazel") and Tinctura Arnicæ. The presence of considerable proportions of alcohol in other spirits, tinctures, fluidextracts, wines and elixirs should be borne in mind, both in their external and internal use.

If the alcohol is sponged on the skin and permitted to evaporate it produces a cooling effect. This is utilized in fever, and in excessive perspiration ("night-sweats" of phthisis). The cleansing and hardening of 
the skin is also useful in this connection, and to prevent the development of bed sores. The astringent and stimulant action promotes the healing of ulcers ( 25 to 50 per cent.). Breitmann, I9I3, recommends its liberal use as an astringent lotion ( 70 per cent.) in first- and second-degree burns.

On mucous membranes, the local action produces hyperemia or inflammation according to the concentration (see the "Effects on Digestion"). Even the minor grades of this irritation produce chronic inflammation on continued use, and this tends to extend beyond the local surface, producing fatty and fibrous changes in the internal organs (see "Habitual Use"). The diuretic action of alcohol indicates that it irritates the renal cells.

Subcutaneous Injections of alcohol in concentrations above 50 per cent. are very painful; especial care should be used not to inject it near a nerve.

Intraneural Injections of alcohol were introduced by Schoesser for the relief of neuralgia and spasms (Ostwald, I906; Harris, 1914). They are generally effective; but are frequently followed by toxic degeneration of the nerve. They should therefore be avoided for motor or mixed nerves (sciatica), and find their best field in trigeminal neuralgia, as a substitute for the extirpation of the Gasserian ganglion (Fischler, r907).

Cadwalader, $\mathrm{I}_{915}$, has studied the results of various concentrations: 80 per cent. alcohol produces immediate and complete paralysis, followed by absolute histologic degeneration. The paralysis may persist for a year, or until regeneration is complete; in case of scar formation, there may be no regeneration. $5^{\circ}$ per cent. causes less severe paralysis and degeneration; and 25 per cent. has but little effect in either respect.

Bactericidal Action of Alcohol.-7o per cent. alcohol is a fairly effective germicide. Concentrations above 80 per cent. and below 60 per cent., however, are almost inactive (A. Beyer, I9II; Sottile, I9I2), because they do not penetrate proteins as readily (Tijmstra, I9I3).

"Stimulant" Effects of Alcohol.-These are observed in most individuals, after taking "moderate" doses of alcohol-a quantity not easily expressed in cubic centimeters. The phenomena are only too well known. They are, at first view, typical of stimulation. There is an increase in the rate of the respiration and of the heart; the blood pressure rises. The skin is reddened, with a grateful sensation of warmth and comfort. There is an increased vivacity of motion, action, and speech, which latter may acquire a stamp of brilliancy, even of inspiration. The subjective condition of the individual also undergoes a peculiar change.

Shyness, if it ordinarily exists, is replaced by self-confidence. The person under the influence of alcohol feels an unlimited confidence in his own powers and accomplishments, both intellectual and physical. He will attempt very difficult, even impossible, tasks, and feel that he accomplishes them. And he similarly overestimates the productions of others. ${ }^{1}$

These manifestations are so conspicuous and apparent, that alcohol in proper doses has been considered a typical stimulant; this opinion is still held by some eminent pharmacologists. However, the critical study of its actions tends to the conclusion that most of these actions do not depend upon true direct stimulation of the nerve-centers; but that they are the indirect result of incoördination, of accessory factors of environment, and of reflex stimulations.

If the phenomena be somewhat more closely inquired into, this will become apparent at once. Of the physical phenomena, the flushing of the cutaneous vessels - to which the sensation of warmth must be attributed-occurs as the result of a vasomotor paralysis, restricted, it is true, to this area. 'The quickened pulse results partly from reflexes caused by swallowing and by irritation of the mucous membranes, partly from the

1 No better description of these effects can be given than that of Horace: "What wonders does not wine! It discloses secrets; ratifies and confirms our hopes; thrusts the coward forth to battle; eases the anxious mind of its burthen; instructs in arts. Whom has not a cheerful glass made eloquent I Whom not quite free and easy from pinching poverty!" 
increased excitement and motion; not at all from a direct action on the cardiac nervous or muscular mechanism-for it is entirely absent in animals, and very brief in man if the factor of excitement be eliminated. The quickening of the respiration is also largely due to similar causes.

The psychic phenomena find their explanation in a dulling of certain mental faculties whilst others are still practically unaffected-in a disturbance of what has been called the "normal balance of the brain." The first functions to be lost are the finer grades of judgment, reflection, observation, and attention-the faculties which have largely been acquired through education, and which constitute the elements of the restraint and prudence which man usually imposes on his actions.

Thus, to quote a well-known example: The orator no longer considers that he may be called to account for his utterances; he allows himself to be carried by the impulse of the moment, without reflecting on ultimate consequences, and, as his expressions become freer, they acquire an appearance of warmth, of feeling, of inspiration. And not a little of this inspiration is contributed by the audience, who are usually in a similar condition of increased appreciation.

The view that alcohol increases the intellectual and physical powers of the individual is shown by actual experiment to be erroneous, and based almost entirely upon the subjective conditions of the individual, his weakened faculty of judgment.

Experience soon teaches this lesson: that alcohol does not really stimulate. Persons who have to undergo severe exertion, either physical or intellectual, very rarely take alcohol before or during their labor, but only when this is finished. And then not for any stimulating, but really for its depressing, effect; for the feeling of comfort and general relaxation which it induces. The continued use of large doses of alcohol greatly diminishes the activity of the individual, and even moderate doses tend to have the same effect.

Another very characteristic feature, evidently resulting from this paralysis of the higher functions is the loss of the power to control moods. There may be causeless merriment or sadness, friendliness or the opposite. It is interesting to note how often alcohol brings out the true character of an individual by this abolition of restraint: "There is truth in wine." On the other hand, the habits of self-control, where they have been cultivated, persist to some extent even in intoxication.

Experimental Analysis of Psychic Phenomena.-The extensive studies of Kraepelin, I892, led him to the conclusion that the effects of alcohol are purely depressant on sensory and intellectual processes, while the generation of motor impulses is at first stimulated, but later also depressed. Increase of dosage prolongs the psychic depression, and shortens the motor stimulation. The "stimulant" stage of alcohol therefore favors mechanical performances-the "simpler reactions" of memorizing, mechanical reading, repetition, at the expense of the more intellectual processesassociation, arithmetical, etc. It favors motor impulsiveness, but produces intellectual dulness and silliness, lessens thought and judgment, and resistance to temptation, and thus leads to its abuse. . The euphoria probably depends on the deceptive feeling of facilitation and capability.

Psychic Depression.-With doses of 30 to $45 \mathrm{Gm}$. of alcohol, this lasts thirty to fortyfive minutes; with $60 \mathrm{Gm}$., one to two hours. The phenomena are somewhat analogous to fatigue. In both, mechanical associations predominate. However, the lighter grades of alcohol "fatigue" disappear with exercise, while genuine fatigue is relieved only by rest.

Motor Processes.-The facilitation begins at once and lasts at most twenty to thirty minutes, less with larger doses. It is followed by depression even with the small dose of $7.5 \mathrm{Gm}$. With 45 to $60 \mathrm{Gm}$., the initial facilitation is seen only exceptionally. However, the results depend somewhat on circumstances; in excitement, even large doses produce facilitation; and in fatigue, small doses may depress. The more mechanical the function, the more conspicuous is the facilitation. The force measured by the dynamometer is not increased, so that the rate rather than the force of motor impulses is favored. There is also evidence that the muscular contraction proper is not quickened, but that the motor impulses are started more easily.

Perception of Sensory Impressions. - This is impaired for hours, even by $1 / 4 \mathrm{oz}$. of alcohol (Kraepelin, I9r6).

The reflexes from the frog's skin are depressed, even with the smallest effective doses (Hyde and pupils, I9I3), before there are any grossly visible symptoms. 
F. G. Benedict, I9 16 , finds that 30 to 45 c.c. of alcohol does not even facilitate movements, but that all sorts of reflexes are depressed, especially inhibitions. The sensory threshold is raised. Memory and association are unaffected. The effects are maximal in one and one-half hours.

Use as Stimulant.-Even the "stimulant" doses do not increase intellectual efficiency; but they could be employed for facilitating motor functions of short duration; not so much to increase their force, but for overcoming inhibition or hesitancy; to promote decision or resolution, f.i.. in speaking, in psychic dreads, etc. It would be unfavorable to attention, watchfulness or ready judgment. The stimulant dose should generally not be more than I 5 to $20 \mathrm{Gm}$. of alcohol; or if a longer effect is needed, 5 to $8 \mathrm{Gm}$. in twenty-minute intervals.

Alcohol on Muscular Work.-In man, the ergographic experiments of Lombard, 1892 , showed that the muscular force in voluntary contractions is not increased; but the appreciation of fatigue is lessened (Joteyko, I9O4, Foerster, I9I 2), so that the total work may be increased. When a finger is tetanized, by electric stimulation, the total work is rather decreased (Lombard); the favorable effect must therefore be at least partly central (facilitation of cerebral motor processes). However, the voluntary response is modified by the previous state of the muscles as well as by the dosage of alcohol: Hellsten, 1904 , found that moderate doses ( $10 \mathrm{Gm}$.) at first increase the power of voluntary muscular work (to 9 per cent.), but in one-half hour diminish it to 6 per cent. below normal. Myers, I912, reports no effects from 5 to 20 c.c.; 30 to 40 c.c. gave irregular results, most commonly a primary increase and secondary decrease of the work. The increase is seen only when the muscle is not too much exhausted, but it is favored by moderate fatigue. The favorable effect therefore appears to be due partly to the direct food-value of the alcohol, somewhat as with sugar; the food effect of alcohol is more prompt, but not as efficient in the long run. 'The depression of the larger doses is purely central.

On frog's muscle, minute quantities have no effect; moderate doses were said to exert a favorable action on all the features of muscular activity; larger quantities are very unfavorable (Lee and Salant, I902). Crider and Robinson, I916, attribute the reported favorable effect to experimental error.

The effects on invertebrates and lower vertebrates have been studied by Fühner, I9I2.

The Effects on the Respiration.- "Stimulant" doses of alcohol increase the rate and depth of the respirations, by reflex and central stimulation. This may aid in the therapeutic use of alcohol as a stimulant. With narcotic doses, the respiratory center is depressed.

The increase is quite large; the amount of respired air may be doubled. The effect is especially large in fatigue, and is greater with wines and liquors rich in "bouquet," than with dilutions of pure alcohol (Binz, I903). Wendelstadt, I899, obtained similar results in man.

The increased respiration is partly due to the general excitement and partly to reflexes from the local irritation (Jacquet, I895) and from the odor, etc. However, some increase occurs even in sleep, so that the factors of environment are not essential. Nor can it be affirmed that it is due solely to reflex irritation, for other irritants do not produce an equivalent effect (Binz, I903). Moreover, an immediate increase of respiration occurs if alcohol is injected into the brain through the carotid artery (Wilmanns, I897). It is therefore certain that the respiratory center is stimulated directly. A. Fonteyne, 1906, finds that large doses lessen at first the rate, and later also the volume. In cats, Pilcher, I912, found the rate usually increased by all except fatal doses. In decerebrated cats, doses of 0.5 to 5 c.c. per kilogram, dilute or concentrated, caused no change in the respiration (Lieb, I9I5).

The Effects on the Circulation.-These are rather inconstant, varying not only with the dose and concentration and mode of administration, but also with the condition of the individual. 
Small Therapeutic Doses (in man, to perhaps 5 to 25 c.c. of absolute alcohol, diluted at least to 25 per cent.) produce generally (but not always) an insignificant quickening of the pulse rate, due mainly to excitement.

The cutaneous vessels are dilated, and the splanchnic vessels are somewhat constricted leading to a rise of the systolic blood pressure and a fall of the diastolic pressure. The mean aortic pressure rises slightly in most cases, but not always. The normal heart muscle is probably not affected by these doses; but the exhausted heart may be stimulated by the nutrient action of the alcohol.

The actions, taken together, alter the distribution of the blood, so that more will be contained in the surface vessels and in the arteries, and less in the splanchnic veins. The amplitude and force of the pulse; the "mass-movement" of the blood; the output of the heart; and the efficiency of the circulation in general, will be improved. The effects are not very great and not altogether constant in normal individuals; but they suffice to produce decided therapeutic effects, especially in conditions of exhaustion.

The actions reach their maximum in about half an hour, and disappear in an hour or two.

Somewhat Larger Doses ( 45 to 90 c.c. of alcohol for man, diluted) produce at first the same effects, followed in an hour or less by decreased amplitude and force of the pulse, with progressive fall of the systolic pressure, and unaltered or rising diastolic pressure. If the alcohol is taken in more concentrated form on an empty stomach, this stage may occur at once, without the preliminary improvement of the pulse. The fall of the pressure is attributable to splanchnic dilation, replacing the constriction of the first stage. The action is therefore comparable to that of the nitrites. The effects reach their maximum in one to one and onehalf hours, and last one-half to one hour.

Toxic Doses depress the vasomotor center and perhaps also the cardiac muscle, so that the blood pressure falls severely. The heart rate may be slowed through central vagus stimulation, or through the depressant ac. tion of the coma; or it may be quickened by the fall of blood pressure.

Heart Rate.-The effect is greatly modified by the condition of the individual. It is generally increased, mainly by the psychic and reflex actions (Dixon, 1907); the increase is often absent, especially if the patient keeps quiet (Zimmermann, I 896; Jacquet, I89 I, Dixon); but with larger doses it may occur even then (John, I908), presumably to offset the falling blood pressure. Animals give similar inconstant results (Pilcher, I 1 1 12 ). The intravenous injection into non-anesthetized animals generally causes some slowing (Brooks, I910).

General Bload Pressure. - In man, Kochmann, I905, and Bachem, I905, found a rise of systolic pressure with small doses. Dixon, I907 (dose not stated) found the systolic pressure unchanged or slightly raised, the diastolic pressure lowered. This was confirmed by John, I908. Lieb, I91 5 , observed practically no effect from diluted whiskey (usually 5 to ro c.c.). When given concentrated, this dosage usually produced a slight refiex rise (about $5 \mathrm{~mm}$.) of the systolic and diastolic pressure, returning to nor$\mathrm{mal}$ in less than one-half hour. The return of the diastolic pressure is somewhat slower, so that the pulse pressure is decreased. The Tigerstedt formula indicates a very slight decrease of cardiac efficiency. These effects are entirely reflex, and are most pronounced when the reflexes are hyperactive; least in apathetic patients and toxemias. Moderate users are more susceptible to them than drinkers. With larger doses John found a progressive fall of systolic pressure, with unchanged or rising diastolic pressure.

Anesthetized animals are obviously not suited for determining the effects of small doses of alcohol. In unanesthetized animals, Brooks, I9Io, found that the oral administration produced a slight rise, lasting five to ten minutes; administration through a gastric fistula had no effect; intravenously, there was the sharp drop, common to many drugs, followed by rapid recovery. 
In decerebrated cats, Licb, $\mathrm{r}^{\mathrm{r}} \mathrm{5}$, found no marked change in the blood pressure or heart rate, after 0.5 to 5 c.c. per kilogram, dilute or concentrated.

The lein pressure is not changed by small doses, but increased by large doses which injure the heart (Capps and Matthews, Ig 3 ).

Blood Flow-Alcoholic beverages of the strength of wine, administered to normal men in moderate doses, produce a brief preliminary decrease, followed by a more lasting increase of the blood flow in the hand. Both effects are vascular. Alcohol also favors vasodilator reflexes, $f . i$., the contralateral heat reflex (G. N. Stewart, 1915). Hanzlik and Collins, I913, found the circulation time in intestinal vessels distinctly slowed by relatively" small doses of alcohol, intravenously, even when the carotid pressure was unchanged.

Vasomotor Changes. - The cutaneous vasodilation is obvious on mere inspection, and may be confirmed by plethysmograms of the limbs, and by the blood flow. Since the blood pressure does not fall in the early stages, it is evident that this dilation of the surface vessels is compensated, either by the constriction of other vascular areas, or by increased output of the heart. Both factors appear to contribute to the result, according to circumstances.

Splanchnic vasoconstriction in the early stage has been demonstrated by Dixon, 1907 , who found that the volume of the intestine decreases while the blood pressure rises.

General vasodilation, presumably in the more advanced stages of the action, is shown by the fact that the blood pressure is practically not increased, notwithstanding considerable increase of the output of the heart, as measured by the stromuhr (Hemmeter, 1889; Wood and Hoyt, I905), or by the cardiometer (Dixon). If the vasomotor center is excluded by section, the injection of the alcohol causes a more marked rise of pressure (Kochmann; Wood and Hoyt), showing that the vasodilator action of alcohol must be central. (Bachem, however, found less rise after section of the vasomotor center.)

Vasomoter Reflexps:-Porter and Turner, I91 5, find that alcohol may suspend vasomotor reflexes even when the general level of the blood pressure is not altered. They conclude from this that separate centers exist for vasomotor tone and reflexes.

Direct Action on Vessels. - These appear to be insignificant. On perfusion, Dixon found in mammals slight dilation with 0.1 to 0.2 per cent.; higher concentrations gave constriction. Perfusion of the frog showed moderate constriction. In surviving arterial rings, Cow, I9II, found very slight response; transient constriction followed by dilation. Plumier, I905, believes that alcohol has a peripheral constrictor effect.

Improved Nutrition of the Heart. -The increased output of the heart in situ is attested by the stromuhr, cardiometer, rise of pressure after destruction of vasomotor center, etc. Since this improvement is not usually noted in excised hearts, it can not be due to a direct action of alcohol on the cardiac muscle. Kochmann, 1905, attributes it to improvement of the coronary circulation by the general vascular changes. Dixon, 1907, brings good evidence that the food-value of alcohol plays the most important role; for he finds it most effective, in excised hearts, when the nutrient supply has been previously exhausted. Hamill, IgIo, then showed directly that perfused hearts are able to metabolize alcohol.

Excised Mammalian Heart.- The effects have been studied by perfusion with Langendorff's method, especially by Kochmann, I904; O. Loeb, 1905; Backmann, I906; Kuno, I9 I3; Barry, I9r 5 .

Concentrations up to 0.5 per cent. are absolutely without action, according to most observers. Loeb, however, describes occasional slight stimulation; and Dixon finds increased excursions constantly if the heart has been exhausted (see preceding section). Concentrations to 0.5 per cent. also give no result, or at most insignificant and slight slowing, or weakening or arhythmia. With concentration of I percent. and higher marked depression appears; the systoles are weakened, but the diastolic excursions are not increased. The coronary vessels appear somewhat dilated. 2 per cent. of alcohol causes severe damage to the muscle, especially of the auricles, which are slowed and weakened, while the ventricles may be merely slowed (Barry, I915). Four to 5 per cent. arrests the heart in diastole; the auricles stopping before the ventricle. If the alcohol is removed, the heart recovers rapidly, even from severe depression. Repeated perfusion gives rapid habituation (Kuno).

Brandini, 1913, found the depressant effect on perfused rabbit's heart increased with rise of temperature.

The concentration of alcohol in the blood of living animals is said to be 0.12 per cent. in "slight confusion," and 0.7 per cent. in deep narcosis. It is therefore evident that the direct cardiac effects of alcohol play no part in either the therapeutic or toxic phenomena. 
Pressure in Pulmonary Artery.-Dixon, I907, and Wood, I9I I, finds this slightly increased, even when the carotid pressure is unchanged. Cloetta and Anderes, I9I4, found the effect on the pulmonary vessels inconstant; generally dilation, rarely constriction.

Cerebral Circulation.-Contradictory results were obtained by Aliprandi and Formaroli, 1905; and by E. Weber, 1909. Hirschfelder, 1915, describes dilation of the vessels of the pia mater and retina. Berezin, ig 16 , also observed dilation.

The frog's heart is stimulated by small, and depressed by higher concentrations (Wood and Hoyt, I905); according to others, even the smaller doses are depressant (Dreser, i 887 ).

Hemolytic Action.-Alcohol added in sufficient concentration directly to blood causes hemolysis (C. H. Schulz, $184 \mathrm{I}$ ). This concentration is not reached in living animals; but the resistance to laking agents is noticeably lowered (Liebermann; Fillinger, I912). Continued administration increases the resistance of the corpuscles (Orban, I9I3). If the relative hemolytic effect of different alcohols is compared, it is found to be proportional to their effect on surface tension, rather than to their lipoid solvent action (Fuhner, IgI2). J. N. Schultz, I9r2, has investigated the influence of various substances (including sera in different diseases) on the alcohol hemolysis.

Blood Viscosity.-This is somewhat increased by the administration or inhalation of alcohol or methyl alcohol (Burton-Opitz, rgo4, 1914).

Use of Alcohol in Collapse.-The effects of alcohol on the general circulation are utilized in the treatment of collapse and in exhausting fevers.

Its usefulness as a quickly acting ("diffusible") stimulant can scarcely be doubted in the various forms of sudden circulatory collapse-syncope, exhaustion, hemorrhage, traumatic shock, snake venom, strychnin, aconite, veratrum poisoning, etc. The main element in its action is the reflex stimulation, increasing the pulse rate, the blood pressure, and the respiration. The narcotic action is also useful; the psychic centers are less easily frightened, and the narcotized medullary centers are less subject to dangerous shock. The subsequent vasodilation is theoretically inadvisable, but it is not sufficiently pronounced to have any practical significance.

The reflex action being brief, alcohol acts mainly as a temporary emergency remedy, to tide the patient over the immediate dangers. To secure these reflex effects, 25 c.c. of whiskey or brandy should be given undiluted, and preferably hot, repeated every ten to fifteen minutes, according to effect.

Other Diffusible Stimulants.-A similar reflex stimulation may be secured by ether, aromatic oils, camphor, or ammonia. These may be administered by mouth (Spiritus Aetheris; Spiritus Camphoræ; Spiritus Ammoniæ Aromaticus); or by smelling (aromatic ammonia, smelling salts); or hypodermically (brandy, ether, camphorated oil).

Cardiac Disease.-The actions of alcohol are scarcely a vailable except for temporary conditions, such as acute myocardial insufficiency. In chronic lesions, small doses may be valuable to lessen the worries of the patient, especially if he has been accustomed to its use. The vasodilator effect may be useful in Angina Pectoris, but it is inferior to the nitrites. Alcohol does not influence Pulmonary Hemorrhage (E. Frey, I 9o9).

Use in Exhausting Fevers.-(Typhoid, Pneumonia, etc.). The intelligent and discriminating employment of alcohol should be useful in these conditions; its indiscriminate use would doubtless do more harm than good. The beneficial effects are probably mainly nutrient, due to the direct food-value of the alcohol, and to the stimulation of the digestion and absorption of other foods (see below). This not only conserves the general nutrition of the patient, but also increases the output of the exhausted heart. The pulse becomes stronger and more regular. The altered distribution of the blood of the mildest degrees of alcohol action would also be beneficial. 
'The dilation of the cutancous ressels removes the blood from the atonic and therefore congested internal organs, and lessens the venous distention of the heart. It would also tend to lower the temperature, although the antipretic effect is but small. The narcotic action of alcohol is useful by quieting the febrile excitement, thus reducing the demands on the strength of the patient.

On the other hand, if the action of the alcohol proceeds to the point of inducing splanchnic dilation and lowering blood pressure, it will exaggerate the serious circulatory abnormalities of fever. Again, in the acute, stimulant stages of the "sthenic" fevers, alcohol is apt to increase the circulatory excitement, and should be avoided.

The effects of alcohol must therefore be carefully watched, to secure its stimulant effects, when needed, while avoiding both the depressant and excitant stages. The individual observations on each patient can alone guide its employment: The dose may be increased or maintained so long as it improves the prominent symptoms, quiets the patient, regulates and strengthens the pulse, and lowers the temperature. When it ceases to have these effects-particularly when the pulse becomes "excited," or when the odor of alcohol remains on the breath-it should be diminished or stopped.

The amount must be governed by the previous habits of the patient; but astonishingly large quantities can often be given to fever patients without producing "intoxication" even if they are unaccustomed to its use; the febrile organism probably oxidizing the alcohol more rapidly.

Ordinarily, one may begin with $1 / 2$ to 2 tablespoons of brandy in half a glass of milk, every three hours, increasing the frequency as needed.

Diuresis.-Dilute alcohol beverages (wine, and especially beer) increase the flow of urine; but the effect is mainly that of the excessive ingestion of fluid.

In man, Raphael, 1894 , found that a liter of wine or beer had no more effect than the same quantity of water, and less than that of milk. John, I908, found that the average quantity of urine in four to six hours after taking $1 \frac{1}{2}$ liters of water to be 1,500 c.c.; with $1 \frac{1}{2}$ liters of 3 per cent. alcohol, he averaged 1,800 c.c.; with the same quantity of 6 per cent. alcohol he averaged 2,000 c.c. of urine. The effect was relatively greater in a case of renal insufficiency from hypertonus; in this $x 1 / 2$ liters of water gave only 625 c.c. of urine, while the same quantity of 3 per cent. alcohol yielded $x, 600$ c.c.

This suggests that the greater diuretic action may be due to a dilator effect on the renal vessels. Moreover, alcohol irritates the urinary passages (and is therefore contraindicated in cystitis and urethritis); this may increase the desire to micturate, and make the diuretic effect appear greater than it really is. "Gin," which contains juniper, has a more direct diuretic effect, from the renal irritation produced by the oil. Dogs fail to show more than the water-diuresis, on the oral administration of any dosage of alcohol (Januskiewicz, I9II). (Intravenous injection would arrest the urine flow, as do other hypotonic solutions.)

Temperature.-The ingestion of alcohol causes a sensation of warmth, due to the increased blood flow to the temperature-nerves of the stomach and skin. This sensation, however, is deceptive; for this increased blood flow to the surface and the diaphoresis increase the heat loss, so that the internal temperature tends to fall.

With small doses, this tendency may be overcome by the increased movements of the patient; but with larger doses the heat loss becomes pronounced (Pilcher, r912), and is exaggerated.by the depressant action on the temperature-regulating centers. The fall of temperature is especially great if the external temperature is cold, and also if the heat dissipation was previously low, as in fevers. 
Use of Alcohol in Chills and Exposure.-It is evident that this would be detrimental during exposure to cold; the temporary relief and feeling of warmth is obtained at the expense of an increased loss of heat, and consequently diminished power of resistance. But if taken after the exposure, the dilatation of the cutaneous vessels favors the absorption of external heat, and also prevents the tendency to congestion of internal organs, and thereby the tendency to "catch cold."

Influence of Alcohol on Digestion.-This varies with the quantity and concentration; with the state of the digestive functions; with the habits of the individual, and probably also with the flavor and extractives of the beverage. In general, moderate doses act as "condiment" or stimulant to digestion. In this the flavor and the hyperemia coöperate to increase the appetite, the flow of digestive juices, the movements of the stomach, and absorption. The action of ferments is also accelerated.

Small quantities of alcohol, taken with meals, therefore, tend to have a favorable action on digestion. If digestion is perfectly normal, these effects are quite superfluous; the utilization of food, and the total time of digestion, is scarcely influenced by these doses of alcohol. In pathological conditions, however, and perhaps in overeating, the effects may be beneficial. The best results would usually be secured with wine. The alcohol should not be taken in strength greater than perhaps 20 per cent: even this would be too strong did it remain for any length of time; but it is absorbed so rapidly that this strength would very soon reach the favorable limit.

Large quantities of alcohol, however-and especially when in concentrated form or on the empty stomach-produce an irritation which surpasses the physiologic limit and interferes with the functions. This is seen most markedly in the habitual use, when the inflammatory changes become chronic, and extend to the portal system.

Hunger Contractions.-These are inhibited by alcohol, as by many other chemic and mechanic stimuli (Carlson, I9I3).

Excised Intestine of Rabbit.- The movements are mainly depressed by monovalent alcohols; very dilute solutions produce a primary stimulation (Kuno, 19I4).

Action on Ferments.- Since alcohol is very readily absorbed, and no great amount of it reaches the intestine, it can only influence the ferments of the stomach, and its action on pepsin is alone of practical interest. It is found that in vitro-and there is no reason to suppose that it acts any differently intra vitam-1 to 2 per cent. of alcohol increases the rapidity of peptic digestion. Up to $r_{5}$ per cent., it causes no perceptible retardation. With $I_{5}$ to I 8 per cent., the digestion is reduced by one-fourth to one-third. With 20 per cent., the digestion is strongly inhibited (Chittenden, Mendel, and Jackson, $\mathrm{r} 898$ ).

Beers and wines have a slightly more unfavorable effect on account of the extractive matter contained in them.

Secretion of Saliva.-The presence of alcohol, strong or dilute, in the mouth increases reflexly the amount and the solids of the saliva, just as do many other substances (acetic acid, ether, etc.). This increased secretion does not take place if the alcohol is introduced directly into the stomach through a fistula or if it is given subcutaneously.

Secretion of Gastric Juice. - The quantity, the acidity, the absolute quantity of ferments, and the total solids (but not the mucus) are markedly increased (Chittenden, Mendel and Jackson, I898; Chiari, r915). The increase occurs also if the local action of the alcohol on the stomach is excluded, as in the "Pawlow stomach" (Zitowitsch, I905); or if the alcohol is placed into the intestine (Chittenden) or rectum (Kast, 1906).

With oral administration, in man as well as in animals, the favorable effect obtains with concentrations up to ro per cent.; with ro to 20 per cent., the mucus is increased, as well as the juice. Above 20 per cent., the mucus is further increased, but the secretion is decreased.

Pancreatic Juice.-Gizelt, 1906, claims that the pancreatic secretion is stimulated when alcohol is administered by mouth, intestine, or subcutaneously; but only if the vagi are intact. 
Bile. Salant, I 906 , found that alcohol by mouth (but not intravenously) increases the flow of bile by 50 to 350 per cent.; the solids are also increased. Increased bile secretion was confirmed for large doses by Okada, I915.

Elfects on Absorption. - Alcohol itself is rapidly absorbed (see below).

It also increases the absorption of other substances under certain conditions (Riemschneider, Strychnin, 1900), but not under some other conditions (Ryan, Strychnin, IOI 2). Brequet, I9I 2 , found only a slight effect on the gastric absorption of strychnin, salicylate or iodid. 'Tschekounow, I9I4, observed no increase for saccharose, iodin or $\mathrm{iaCl}_{\mathrm{a}}$ IIanzlik found the absorption of phenol and iodid diminished by strong alcohol.

Alcohol intended as antidote may therefore even increase the toxicity of poisons, if both are taken by the stomach, and this is not evacuated.

Use of Alcohol in Indigestion.-Alcohol, in the form of wine or diluted spirits, taken with meals, is clinically of value in some forms, and harmful in others. The precise conditions have not been sufficiently defined. It would be useful as a "counterirritant" in functional "colic" and gastralgia, and harmful in the presence of an acute gastritis. In chronic conditions, good results might be expected in "adynamic states," where the circulation or tone are defective-in the course or convalescence from fevers or exhausting illness, phthisis, etc. It would be harmful in hyperacidity, and therefore in most "nervous dyspepsias." Certain wines become injurious on account of their acidity. The tannin, which is present particularly in red wines, may be detrimental to digestion, but valuable in diarrhea. Brandy is also used against diarrhea. This probably rests upon the beneficial effects of an increased circulation.

Champagne is also used as an antemetic. Its action in this case depends perhaps more on the carbon dioxid and cold and on the psychical effect, than on the direct action of the alcohol.

The Absorption of Alcohol.-This occurs rapidly, mainly from the small intestine, and is practically independent of the quantity.

Chittenden, Mendel and Jackson, I898, found that 50 c.c. of a 20 per cent. alcohol disappear from the stomach of a dog in less than half an hour; and with the duodenum ligated, 200 c.c. of a 37 per cent. alcohol are completely absorbed from the stomach in three to three and a half hours. Vollmering, I9I2, found absorption practically completed in an hour. Voeltz and Dietrich, I9I5, found the absorption materially faster in habituated animals, but in either case practically completed in two and one-half hours. Nemser, I907, investigated the relative rôle of the successive portions of the alimentary tract in the absorption of alcohol, under normal conditions. Administering 20 per cent. alcohol to dogs with fistulæ at different levels, he finds that the amount absorbed at each level depends mainly upon the time during which the fluid naturally sojourns at this level: Very little of the alcohol is therefore absorbed from the mouth; about a fif th from the stomach; very little from the duodenum; the greatest quantity from the jejunum, and the remaining fifth from the ileum.

Hanzlik and Collins, I9 3 , have studied the absorption from intestinal loops in living animals. It was rather better from the colon than from the stomach or small intestines; the length of the loop had little influence; ro per cent. solutions were absorbed slightly better than stronger or weaker concentrations. The intestinal wall retains a certain amount of alcohol very firmly, thus apparently arresting its absorption. Voeltz; Baudrexel and Dietrich, I9I 2, found rather similar phenomena in the absorption of alcohol by the urinary bladder, which is fairly extensive (Nicloux and Nowicka, 1913).

Excretion of Alcohol.-Only a very small proportion of the ingested alcohol is excreted by the kidneys and lungs, generally less than 2 per cent., and probably never more than ro per cent. (Strassmann, r 89I; Benedicenti, I896). The remainder is completely oxidized in the tissues.

The proportion excreted depends upon the dose and other conditions. It is greater if the respiration is increased as by muscular work; or if the absorption is more rapid, as when the alcohol is taken on an empty stomach (Voeltz, Baudrexel and Dietrich, I9I I, I9I2). 
Channel of Excretion.-This occurs mainly by the kidneys and lungs; a small amount is excreted and reabsorbed from the urinary bladder. The pulmonary excretion is fairly constant; that by the urine varies between one and one-half and 13 per cent., depending largely on the sojourn and reabsorption in the bladder (Nicloux and Nowicka, I913). The milk may contain a small a mount if large quantities are ingested, but not with ordinary doses (Klingemann; Koldewijn, I910; Schottmueller and Schumm, I912). The maximum amount would be too small to affect sucklings (Voeltz and Paechtner, I913). It is also excreted into the cercbro-spinal fluid (also acetone and chloroform, Hald, I 9 I I). This has been proposed as a test for alcoholic psychoses (Selig, I9I3; Vorkastner and Neue, I9r3, caution against hasty conclusions). It is not excreted in the sweat or feces (slight traces by intestinal loops, Hanzlik and Collins, I913).

The excretions do not contain an appreciable amount of acetone or other intermediate decomposition products (Masing, 1854 ).

Percentage of Alcohol in Blood.- According to the old work of Schulinus, 1866 , the blood is said to contain 0.12 per cent. in the stage of slight confusion, and 0.72 per cent. in deep narcosis.

According to Widmark, 1915 , the concentration, in man, is about the same in the blood and urine. It is not affected by the concentration in which the alcohol is administered, but it is higher if the alcohol is taken fasting than after meals.

Existence of Alcohol in Normal Tissues.-A trifling quantity of alcohol appears to be produced in the tissues in the course of normal metabolism. Its presence was claimed by W. H. Ford, 1872, and confirmed qualitatively by Landsberg, 1904. Reach, 1907, finds that fresh rabbit's muscle contains to 0.0017 per cent. of alcohol; the liver may contain 0.02 to 0.14 per cent. Voeltz and Dietrich, I9I 5 , recovered a total of about o.I c.c. from normal dogs. Dakin believed that this "normal" alcohol is not formed in the tissues, but by intestinal fermentation; but Taylor, 1913, finds it even after the removal of the entire digestive tract. Schweisheimer, I9I3, claims that normal human blood contains about 0.03 per cent. of alcohol. In drunkards, the concentration may rise to $21 / 4$ per cent.; but after the ingestion of alcohol the excess disappears more rapidly from the blood of drunkards than from non-habituated individuals.

Distribution of Ingested Alcohol in Tissues.-Vollmering, I912, found that soon after absorption the ratio was blood $>$ liver $>$ muscle $>$ brain. Later, the brain contained the most. Fatty tissue was slow to take it up, but retained it longer. Voeltz and Dietrich, I I I 2 , found 3 to I 2 per cent. still present in the body fifteen hours after doses of 2 c.c. per kilogram; 2 to 4 per cent. had been excreted by the respiration, 0.4 to 4 per cent. by the urine, and 90 per cent. had been oxidized. Messner, 1913, could not demonstrate destruction of alcohol by emulsions of tissues, or by blood in vitro.

The Fate of Alcohol in the Tissues; Effect on Metabolism.-Over 98 per cent. of the ingested alcohol disappears in the body, being completely oxidized to carbon dioxid and water (Atwater and Benedict, I902); probably with acetic acid as an intermediate stage. By the chemical energy thus liberated (about 7 calories for I Gm. of alcohol, or 4 calories for I c.c. of whiskey) alcohol can perfectly replace carbohydrates and fats in the diet, and is a typical non-nitrogenous food. It is even superior to most other foods in one particular, viz., in that it does not require digestion. By its poisonous side actions, however, it may at first cause an increased nitrogen excretion in individuals not accustomed to its use; but this action disappears in a few days, and it then saves nitrogen like any other food.

When added to an ordinary diet, the $\mathrm{CO}_{2}$ excretion and the output of heat are not materially changed. The alcohol therefore saves the other constituents of the diet from decomposition, and the body shows a corresponding gain in weight. If it is added to a diet deficient in carbohydrates or fats, the metabolism is the same as if an isodynamic quantity of these foods were added. It spares carbohydrates in man even when large amounts of sugar are supplied (Toegel and coworkers, r913). It can be used up in muscular work (Krieger, I9I3). In dogs receiving 2 c.c. of alcohol per kilogram, this supplies about 40 per cent. of the energy (Voeltz and Dietrich, I9I 5). (The literature of this subject is summarized by Rosemann, 1901, Pflueger's Archiv, 86:307).

By observing the respiratory quotient, Higgins, I9I6, showed that in man, with doses of 30 c.c. of alcohol, appreciable quantities begin to be burned in five to eleven minutes after administration. Sucrose, lactose and levulose gave similar results: whereas glucose and maltose began to be oxidized only after twenty to thirty minutes. 
The oxidation of alcohol is completed rather slowly; with 2 c.c. per kilogram, it requires about eighteen hours (Voeltz and Dietrich, 19I5). It is not materially faster in habituated individuals, as claimed by Pringsheim, 1908. It is said to be hastened by fever. Increase of external temperature is said to lessen its oxidation and to promote its excretion (thus 96 per cent. oxidized at $16^{\circ} \mathrm{C} . ; 92$ per cent. at $26^{\circ}$; Voeltz and Baudrexel, I9I3).

Effect on Nitrogen Metabolism.-Mendel and Hilditch, rgro, have investigated the effects of moderate quantities on man and dogs. Giving, in man, quantities corresponding to 96 c.c. of 95 per cent. alcohol ( -500 calories), divided into six doses per day, with constant diet, they found no pronounced disturbance in the alimentary utilization of food These doses spared protein; the partition of the urinary nitrogen was unchanged, except that the purin excretion, both endogenous and exogenous, was increased. Larger doses caused a loss of nitrogen and a relative increase of urinary ammonia.

Stehle, 19r5, found no increase in the excretion of endogenous uric acid. Winterstein, I9I4, claims that alcohol increases the gas-metabolism of the spinal cord of pigs.

Subacute Alcoholic Intoxication.-Salant and Hinkel, rgro, found that this caused, in well-fed dogs, a moderate diminution of the total urinary nitrogen and sulphur; a much greater decrease of the inorganic sulphates and phosphates; a tendency to retention of chlorids; a diminution of indican; and increase of neutral and ethereal sulphur.

\section{FOOD-VALUE OF ALCOHOL}

Whilst there can be no doubt that alcohol is an excellent food, in the sense of being a source of energy, other factors must be taken into consideration. The increased nutrition may itself be detrimental to the body, either by preventing the complete combustion of metabolites (which may possibly be connected with the origin of gout), or by leading to an abnormal deposition of fat. A still more potent objection to considering alcohol as a generally useful food lies in its toxic action, especially its psychical effects. Alcohol should therefore be employed as a food only when a sufficient supply of energy can not be obtained from an ordinary diet; as, for instance, in digestive disturbances, or when the demands on the organism are unusually large, as in fever.

The proverbial obesity of persons addicted to the overuse of weak alcoholic liquids (in which the nutrient effect is less obscured by the toxic actions), is a striking illustration that too good a nutrition is not necessarily beneficial. That alcohol lessens the oxidation of metabolites is shown by an increased excretion of uric acid and ammonia nitrogen at the expense of urea (Paton and Eason, 190r; Mendel and Hilditch, rgro). This points to a modification of the functions of the liver.

Excessive doses of alcohol are always detrimental to nutrition, lessening both assimilation and disassimilation. The effects resemble those of the anesthetics.

Alcohol in Diabetes Mellitus.-Alcohol may be expected to act favorably as an easily digestible food, supplying the place of the sugar, and diminishing the excessive draft on proteins, and thus lessening the risk of acidosis. This expectation has been verified: Benedict and Förök, r906, found that the replacement of 50 to $80 \mathrm{Gm}$. of food-fat by isodynamic quantities of alcohol lessened the excretion of sugar, acetone, and nitrogen. O. Neubauer, 1906, also reports it as useful. On the other hand, Higgins, Peabody and Fitz, rgr6; found that alcohol did not prevent acidosis in normal persons on carbohydrate-free diet.

Excessive quantities, however, can not be expected to exert this favorable action, since the toxic effects will predominate. Salant, r.9o6, fed rabbits on alcohol alone, and found no increase of hepatic glycogen; nor did large doses retard the disappearance of stored glycogen.

Use of Alcohol in Convalescence and Debility as an "Analeptic," "Restorative" or "Stimulant."-The value of alcohol in these conditions is supported by long experience. Its action must be quite complicated. In the absence of organic lesions a great deal may be expected from improving the symptoms. This, like nursing and hygiene, increases the com- 
fort and well-being of the patient, and starts him on the way to improvement. Alcohol meets these indications in an excellent manner: The feeling of well-being caused by it, the sense of capability, the removal of worry, the enjoyment in the act of taking it, the rest and sleep induced by its narcotic action, its food-value and its beneficial effects upon digestion, all concur in its action. To this may be added its slight but certain effects upon the vascular system-the altered distribution of blood, the diminished resistance to the heart-which may be of benefit in some cases. For these purposes, the alcohol should be taken well-diluted, as light wines or beers.

Use of Alcohol in Depressed Psychical States.-(Hypochondria, Melancholia, Neuralgia, and other obscure nervous diseases.) This relieves the patient, by its mild narcotic and euphoristic action-but the temporary relief is generally obtained at the expense of a permanent alcohol habit, and is therefore not to be advised.

Use of Alcohol as a Hypnotic.-Alcohol, especially in the form of beer, taken at night and in the absence of excitement, is a fairly efficient hypnotic, producing less derangement than most other hypnotics; but its usefulness is limited by the danger of chronic alcoholism. The hypnotic dosage corresponds to 45 to $60 \mathrm{Gm}$. of alcohol.

\section{CONTRAINDICATIONS TO ALCOHOL}

These may be briefly resummarized as: Danger of habit; states of excitement; sthenic stage of fevers; irritation of the alimentary or genitourinary tract.

\section{ACUTE ALCOHOL POISONING}

With excessive doses, the "stimulant stage" passes progressively into the narcotic, anesthetic, and paralytic stages. The narcotic stage may be said to exist when the symptoms of lessened psychic activity assume prominence. Sensation and motion become impaired. Speech is thick and muttering, the gait uncertain, the special senses are blunted. Drowsiness sets in. The face, which was flushed, may become pale. Vomiting occurs.

Consciousness and sensation and muscular tone are gradually lost completely. This constitutes the anesthetic stage.

- In the paralytic stage proper, the symptoms are those of beginning medullary paralysis: The respiration is slow and stertorous; the pulse scarcely discernible. Skin cold and cyanotic. Pupils generally dilated. Reflexes abolished. The temperature falls severely. The odor of the breath is diagnostic.

If very large doses have been taken on an empty stomach, these paralytic symptoms may set in at once.

Death is relatively rare. It may occur within half an hour, or the coma may grow deeper and terminate in paralysis of the respiration or of the heart, or in pulmonary edema; generally within twenty-four hours. If the coma lasts beyond thirteen hours, recovery is exceptional. Death may also occur later, either from prolonged coma; or after a protracted debauch, as the result of gastric irritation and debilitation.

The acute fatal dose is probably variable. In analogy with animals, it would require about a pint of whisky; and cases of death from such quantities are recorded (Lewin).

Recovery.-The coma-if the intoxication has progressed so farpasses into natural sleep, often with sweating. On awakening there follows a series of symptoms pointing mainly to acute gastric catarrh, and 
perhaps to functional neuritis; and grouped by the Germans under the name of "Katzenjammer": headache, coated tongue, loss of appetite, irritable stomach, diarrhea, muscular pains, etc. These show curious and unexplained peculiarities for the various alcoholic liquids.

The skin, after severe alcoholic poisoning, may show effects resembling those of contusions or burns: edema, blisters, extravasation of blood, and gangrene. Bedsores are especially common. 'These effects may perhaps be due to injury to the capillaries, or ncurites.

The treatment of acute alcoholism consists in evacuation of the stomach preferably by apomorphin, which acts also as a hypnotic; and the administration of stimulants, caffein or strychnin. The subsequent headache and nervousness are met by bromids and caffein. Sodium bicarbonate lessens the gastritis.

\section{THE ANTAGONISM OF ALCOHOL AND CAFFEIN}

This has been studied by Pilcher, I9r2, on cats. The combination of small doses of alcohol and caffein result in an antagonistic effect, but the combination of larger (halffatal) doses results in greater depression and greater fatality than if either drug were used alone. The ordinary doses of caffein would tend somewhat to decrease the narcosis and hasten the recovery from severe, but not fatal alcohol poisoning. With fatal doses of alcohol ordinary doses of caffein would have no effect, and larger doses would be detrimental.

\section{THE HABITUAL BUT MODERATE USE OF ALCOHOL}

It may be considered as probable-some authorities to the contrary notwithstanding - that a.certain amount of alcohol (variable in individual cases) may be taken daily without any demonstrable permanently injurious effect. But it stands equally certain that it is as dispensable to the organism as nicotin or caffein, and that it must be looked upon purely as a luxury. The injury done by such use of alcohol lies chiefly in the fact that it is so apt to lead to the use of immoderate amounts.

Lessened Resistance.-Perhaps the only positive evidence of injury from small doses of alcohol is in this direction: Hunt, 1907, found that the long-continued administration of small quantities of alcohol to mice or guinea pigs increases their susceptibility to the fatal action of acetonitrile. This is attributed to a derangement of metabolism which hastens the decomposition of the non-toxic nitrile into the cyanid. It is not produced by methyl alcohol, chloral, etc. Laitiner, 1907, also claims that very small doses (o.I c.c. per kilogram, daily) suffice to render the erythrocytes less resistant to hemolysis; and also lessen the resistance to infectious diseases. He asserts that this lessened vitality is transmitted to the offspring.

Mueller, I904, Wirgin, 1905, and others have found that the formation of antibodies is lessened, if rabbits are kept mildly intoxicated for several days after the injection of an antigen.

For human subjects, Reich, I915, also claims diminished resistance of erythrocytes to hypotonic solutions, and slight decrease of the bactericidal action of serum against typhoid bacilli. The effects, however, varied greatly, so that it is difficult to estimate their importance. Phagocytosis was not affected.

\section{CHRONIC ALCOHOLISM}

The excessive habitual use of alcohol leads to effects which may be grouped together under the name of chronic alcoholism, and which depend in part upon the irritant action of the alcohol, in part upon specific injury to the neurons.

Gastro-intestinal Irritation.-The first effects are local, and depend largely upon the concentration of the spirits. They consist of a catarrh 
of the whole alimentary canal, progressing from the pharynx downward. They are characterized by the usual symptoms of catarrhal gastroenteritis: loss of appetite, gastric distress, irregularity of stools, craving for spices, etc. The chronic catarrh leads to malnutrition and emaciation when strong spirits are used. It also appears to constitute a predisposing factor to carcinoma of these organs. In the case of excessive beer-drinking the habitual overdistention of the stomach leads to chronic dilatation.

Degenerative Changes in Internal Organs.- The continued presence of alcohol in the body sets up a series of irritant and degenerative phenomena in various other organs with which it comes into contact. These changes consist in fatty infiltrations, cellular degenerations, and hypertrophy of connective tissue.

The necrotic changes in the tissue cells must be attributed to the continued irritation from the constant presence of the alcohol; and to this must be added the interference with circulation due to the changes which alcohol causes in the blood vessels. These two-the direct irritant action of alcohol on the cells, and the impaired circulationare inseparably connected in the production of the degenerations. Of these, the fatty are the most common, since alcohol, by its combustion, prevents the normal consumption of fat. Connective-tissue formation results as the ordinary consequence of necrosis of the parenchyma.

These changes are proportional to the concentration of the alcohol. Since this is naturally greatest in the liver, kidneys and blood vessels, these organs show the action first and most prominently. In the liver, again, the periphery of the lobules is mainly affected, on account of the anatomic relation to the portal vein. Chronic alcoholism is probably the most common cause of hepatic cirrhosis (perhaps half the cases; but only 5 or 6 per cent. of the alcoholics develop cirrhosis; Baumgarten, r908).

Hepatic cirrhosis, preceded by degeneration of the liver-cells, can be produced experimentally in animals (A. L. Grover, I9I6, literature). It is claimed that the degeneration can be avoided by the administration of cane sugar (L. Hill).

Next in point of time comes the action on the blood vessels. This is of especial import, since it contributes materially to the degenerations in other organs: The principal changes are in the intima; there are fatty degenerations, loss of elasticity and atheroma. 1 These may lead to ruptures (apoplexy, etc.).

The degenerative changes in the kidneys lead to nephritis, with cirrhosis, albuminuria, diminished secretion of urine, secondary weakening of the heart, etc. The heart itself, however, in common with skeletal muscle, shows primary fatty degeneration. This, together with the atheroma, etc., leads to hypertrophy and dilatation of the viscus, and later to dropsies, etc. The heart rate is generally rapid. The fatty changes in voluntary muscle lead to muscular debility, especially in beer-drinkers, in whom there is more material for fat formation. Gout is a common sequence of moderate alcoholism.

The respiratory organs show a chronic catarrhal inflammation of the passages, and a disposition to fatal pneumonia. Changes in the skin-vascular ecchymoses, acne rosacea, disposition to furuncles and carbuncles-may be counted among the earlier actions.

Experimental chronic alcoholism, in rabbits, produced fatty changes in the heart, liver and kidneys, and cellular infiltration of the pia (Lissauer, Igr3). Hepatic changes occur also on intravenous injection (Schafir, I9 I3). In the heart, Otto, 1914, found degenerations of ganglion cells, atrophy of muscle, and scar tissue. Large single doses (4 c.c. per kilogram) may also cause changes. 'Single small doses do not leave any lesions (v. Otto, I9I4).

These various anatomic lesions of importan't organs result in a pronounced lowering of the "powers of resistance," and a high mortality with infectious diseases, operations, etc. It appears that the amount of antitoxic complement is lessened.

Nervous Phenomena.-These differ from the preceding in that they are partly functional.

Ten to 30 per cent. of the cases of insanity are attributed directly to alcoholism; but Heron, I9r2, found the proportion of mental defects as

1 As a result of an extensive study of autopsy material, Cabot, I904, disagrees with the common opinion that alcoholism is a cause of arteriosclerosis. 
high in the recent, as in old cases of alcoholism. Epilepsy, which is a hereditary nervous disease, is 70 times as frequent in alcoholics as in the general population. These facts indicate that alcoholism is the effect, rather than the causc, of mental degeneration.

On the part of the motor system there are tremors and later convulsions and paralyses, the latter partly the result of peripheral neurites.

It is difficult to exaggerate the importance of habit and repeated impressions on the psychic activities. The constant repetition of the features of alcoholic excess could not but produce in this manner a permanent moral degeneration. But associated with this functional feature are marked anatomic changes, due to the same causes as similar changes in other organs; and, lastly, it must be remembered that alcohol has a specific action on the nerve cells.

Among the anatomic lesions which have been observed are: Chronic meningitis with thickening; serous effusions into ventricles; softening; tendency to hemorrhages and apoplexy. Histologically, shrinkage and alterations in the staining properties of the cells (Fig. 25) and changes in the dendritic processes have been averred.

Clinically, the first effects are shown by diminished activity of the individual. This occurs even with very moderate doses. (Nice, Igr 2, found the activity of white mice, as recorded by a revolving cage, to be reduced by one-fourth through chronic alcoholism.) Later there is a diminished acumen of the special senses and of the reasoning powers, leading, the former to disturbances of vision, the latter to degeneracy and dementia, often suicidal. It is a noteworthy fact that by far the greater proportion of inmates of insane asylums and prisons were addicted to the excessive use of alcohol.

Children of Alcoholic Parents.-The influence of alcoholism of the parents on their offspring is still an unsolved problem, the data as well as opinions being so contradictory that no definite conclusions can be drawn.

Miss Eldeston and Karl Pearson, I9ro, in a statistical study covering 3,600 school children, could find no real evidence of either physical or mental deterioration (death rate, mean weight and height, general health, mental and visual defects'). They conclude that the danger of alcoholic parents lies in the transmission of the hereditary defects which caused the parental alcoholism. At the other extreme, von Bunge, 1904, claims that chronic alcoholism on the part of the father renders the daughter incapable of efficient lactation, and that this incapacity, as well as a tendency to tuberculosis and to caries of the teeth is transmitted to subsequent generations. But if this danger is really as great as it is painted, it is difficult to account for the comparative vigor of populations among whom chronic alcoholism has been fairly common for many centuries, or for the fact that some mothers still nurse their children.

Bertholet, I909, finds that the testicles of chronic alcoholics generally show atrophy of the parenchyma, and increase of interstitial tissue, with corresponding decrease and abnormalities of the spermatozoa. This would lead to more or less impotence. Ivanow, r913, reports that the addition of 2 per cent. alcohol in vitro has practically no effect on the motility of spermatozoa; 12 per cent. has little effect; with 15 per cent. there is marked impairment. Contact for thirty minutes with ro per cent. alcohol does not hinder their fertilizing action, and the feti are normal. Stockard, I9I2, finds that chronic alcoholism, induced in guinea pigs, either parent, diminishes fertility and causes most of the embryos to die before or shortly after birth. The offspring is of ten defective, sometimes with gross deformities. This degeneration is transmitted for at least three generations; in fact, it rather increases (I9I4). Stockard also found (1909) that alcohol and other anesthetics cause various morphologic defects in fish embryos, but this of course is a very different matter. Whitney, rgr 2 , found that cultivation of rotifers in $1 / 4$ to $1 / 2$ per cent. alcohol lessens their resistance to copper, the injury increasing with successive cultivations; but if the alcohol was withdrawn, the normal resistance was recovered in the second generation. With these animals therefore the alcohol has no direct influence on the germ-plasm. With female pigeons, the daily inhalation for one to two hours of nearly paralytic doses of alcohol, for two to four months, reduced the size of the egg-yolk quite materially (Riddle and Bassett, Igr6).

While it is not proven that ordinary alcoholism has a direct influence on the germ-plasm, it is certain that the embryo is exposed to the influence of the alcohol, since this passes readily through the placenta, and exists 
in the same concentration in the fetal blood as in that of the mother (Nicloux, r905). This may be supposed to cause a more or less persistent lowering of the resistance, and account for the high mortality among the children of alcoholics; the usually poor hygienic surroundings would contribute to this result. Moreover, the children will inherit the degeneracy which induces alcoholism in the parents. The statistics of prisons and asylums indicate a tendency to psychic and moral degeneracy amongst the descendants-epilepsy, idiocy, insanity, disposition to crime and to alcoholism; but it is not decided whether the defective inheritance concerns a degeneration induced in the parent by alcohol, or the degeneration underlying the abuse of alcohol. The latter is more probable.

Other Intoxicants.-Alcohol is not the only member of the hydrocarbon series which has been abused as an intoxicant. Ether, chloroform, chloral, paraldehyd and even turpentine and gasoline have their devotees. Their effects, in so far as they have been studied, correspond closely to those of alcohol.

Treatment of Chronic Alcoholism.-This follows the same principles as with other drug habits. The first essential is the complete withdrawal of the drug. This may be facilitated by scopolamin (see under "Morphin Habit"). A distaste may be created by the addition of some nauseant (apomorphin), but the effect of this deception is usually not lasting. Suggestion may be very useful. The local action of the alcohol may be replaced by capsicum or ginger, the stimulation by beef-tea; the depression may be met by caffein, the insomnia by bromid. The prospects of cure are better than with morphin or cocain; but the patients will often relapse.

Delirium Tremens.- This peculiar manifestation occurs in drunkards when their forces are unusually weakened-by extraordinary excess, or by the suppression of their usual allowance of alcohol; by absence of food; exposure; overexertion; trauma; hemorrhage; operations; psychic shock; or in any serious illness, especially in pneumonia. Premonitory symptoms of restlessness, tremor, insomnia, and anorexia last usually a couple of days. They pass into excessive tremor, persistent insomnia, violent and talkative delirium and terrifying specific hallucinations of sight (often small animals), touch, and sometimes hearing. It usually runs its course in a few days, ending in prolonged sleep. Acute amblyopia has been reported (Kaiser, Igr2). The treatment aims to support the strength of the patient by allaying the gastritis and giving light but nourishing food. The insomnia, restlessness and excitement should be treated on their first appearance, by moderate doses of sedatives and hypnotics; such as bromid (I Gm. every one to four hours); chloral at night (not over I Gm.. since large doses have caused death); veronal ( $1 \mathrm{Gm}$.); paraldehyd (3 Gm.); morphin; or scopolamin (0.5 mg.). Ergot has been advocated, and the statistical study of Ransom, r909, seems to be in its favor, although it is not conclusive.

Petechial hemorrhages in the gastric mucosa are very common lesions in necropsies of delirium tremens patients. They exist without any evidence of acute inflammation, and must be due to a direct toxic action on the vessels (E. F. Hirsch, 19I6).

\section{PECULIARITIES OF ALCOHOLIC BEVERAGES}

Common experience teaches that the effects of the various alcoholic beverages differ in minor details. These differences are due to the concentration of the alcohol, extractive matter, and to the presence of esters and higher alcohols. However, the scientific data are scanty and unsatisfactory. 
Beer owes its marked hypnotic qualities to the lupulin of the hops as well as to the alcohol. Some wines are also hypnotic, while others-the majority-are exalting. The diuretic action of gin is largely due to the essential oils contained in it.

Partially fermented wines produce particularly often a disturbance of the equilibrium sometimes seen with other forms of spirit - the individual becomes ' $k$ nee drunk," i.e., incapable of maintaining the upright position, before the paralysis of the mental functions has progressed to a great extent. Absinthe produces hallucinations and finally epilepsy. The Mexican drink "pulque," produced by the fermentation of the juice of the maguey plant, produces an alcoholic intoxication in which the thought and language are low, the patient is boisterous and quarrelsome, and, it is said, generally unhappy.

The Stronger Spirits contain, besides alcohol, substances which may pharmacologically be divided into two groups: the "oenanthic ethers" that give the bouquet (flavor), which add to the stimulation and have no other marked action; and those that have a deleterious effect. The latter-which are commonly called impurities-are largely destroyed by age. The most abundant is amyl alcohol (fusel oil).

The action of these "impurities" as far as studied is, upon the whole, similar to that of alcohol itself, but more toxic. However, the whole subject is in dispute. (The literature is abstracted by R. Foerster, I9Io.)

Friedenwald, I9I 1 , found that, with intravenous injections into rabbits, the toxicity of alcoholic liquors, wines and beers was higher than would correspond to their alcohol content. The small value of these results is illustrated by the fact that they would place the toxicity of pure ro per cent. alcohol considerably above that of $3^{8}$ per cent.

The aldehyds have a strongly irritant action on mucous membrane-as shown by formaldehyd or acrolein (allyl-aldehyd - the vapors of overheated fatty oil). Furfurol, which was formerly believed to modify the intoxication, does not appear to do so. Larger doses are convulsant (Laborde, I888).

Artificial liquors are made by the admixture of ethers and essential oils to alcohol. Their action is not uniform, but it is generally believed to be more irritating locally, and more injurious to the brain.

\section{PREPARATIONS-ALCOHOL}

Alcohol Dehydratum (Alcohol Dehyd.), U.S.P.; Alcohol Absolutum (Alcohol Absol.), B.P. - Not less than 99 per cent. by weight of $\mathrm{C}_{2} \mathrm{H}_{5} \mathrm{OH}$. Very hygroscopic.

*Alcohol, U.S.P.; Spiritus Rectificatus (Sp. Rectif.), B.P.-Not less than 92.3 per cent. by weight, 94.9 per cent. by volume, U.S.P.; 90 per cent. by volume, B.P., of $\mathrm{C}_{2} \mathrm{H}_{5} \mathrm{OH}$. Colorless, volatile liquid, of characteristic odor and burning taste. Freely miscible with water, ether, chloroform, etc.

Dilution.- Since a condensation occurs on mixing alcohol and water, the percentage of the resulting product can not be deduced by the formula per cent. $\div(\mathrm{V}+\mathrm{V})$.

Tables for making the commonly needed percentages by the dilution from the official alcohols are given in the U.S.P. (page 522) and B.P. (pages 363 and 530).

Alcohol Dilutum (Alcohol Dil.), U.S.P.-A mixture of equal volumes of official alcohol and water, containing $4 \mathrm{I}$ to 42 per cent. by weight or from 48.5 to 49.5 per cent. by volume of $\mathrm{C}_{2} \mathrm{H}_{5} \mathrm{OH}$.

*Distilled Spirits.-These include whisky (Spir. Frumenti), distilled from the mash of fermented grain and aged for at least two years; 44 to 55 per cent. of alcohol by volume; average dose, I5 c.c., 43. Brandy or Cognac (Spir. Vini Gallici), same per cent. Of about the same strength are the other spirits, which are less used in medicine. Holland Gin, distilled over juniper berries; Common Gin, often containing turpentine; Rum from molasses; Arrack from rice, etc.

Mistura Spiritus Vini Gallici.-Four ounces each of brandy and cinnamon water, two yolks of eggs, and $1 / 2$ ounce of sugar.

\section{WINES}

These are made by fermenting the expressed juice (must) of the grape. If this contains the skins of dark grapes, the wine will be red; if made from light grapes, or from the juice of dark grapes without skins, it is "white," i.e., an amber color. 
A wine which contains much alcohol ( 15 to 20 per cent.) is "generous"; one poor in alcohol, "light"; one containing much sugar, "sweet"; poor in sugar, "dry." If it contains $\mathrm{CO}_{2}$, it is "sparkling"; if tannin, "rough" or "astringent"; if acid tartrates, "acidulous." The last two ingredients will interfere with digestion if the wine is habitually used.

The most important wines are:

Sherry (Vinum Xericum): Dark amber, dry, little acid-
ity (Madeira, Marsala, Tokay, Malaga, are similar, ity (Madeira, Marsala, Tokay, Malaga, are similar,

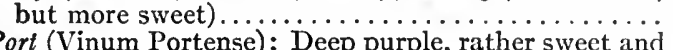

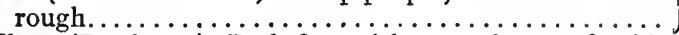

Claret (Bordeaux): Red, dry, with some degree of acid-

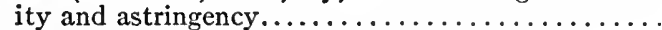

Champagne: Pale amber, sweet, sparkling...............

Hock and Moselle: Pale amber, dry, slightly acid......

Catawba: Amber, dry, rather acid (or sweet)........

$$
\begin{array}{ll}
\begin{array}{c}
\text { Alcohol } \\
\text { Per Cent. } \\
\text { Weight }
\end{array} & \begin{array}{c}
\text { Per Cent. } \\
\text { Volume }
\end{array} \\
\text { I5 to I9 } & \text { I8 to } 23 \\
& \\
8 \text { to I4 } & \text { Io to I } 7 \\
8 \text { to IO } & \text { I0 to I } 3 \\
\text { I2 } & \text { I } 5 \\
\text { IO to I2 } & \text { I3 to I5 }
\end{array}
$$

Unfermented Grape Juice (i.e., must, preserved by heating or an antiseptic) can scarcely be considered a medicinal agent.

Other Fermented Liquors:

From Apple: Cider,

From Pear: Perry,
From other fruits, 5 to ro per cent. (by weight).

Malt Liquors. - These contain alcohol, $\mathrm{CO}_{2}$, sugar, and usually hops. The color varies from pale amber to dark brown, the difference being due mainly to charring of the malt. Lager beer is made by slow fermentation at a low temperature; porter, ale, and stout by rapid fermentation at a higher heat.

Their alcoholic strength is as follows:

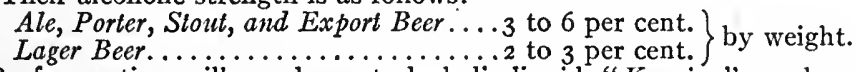

By fermenting milk, a pleasant alcoholic liquid, "Kumiss," can be obtained, which contains to 3 per cent. of alcohol.

\section{HIGHER ALCOHOLS}

In acute intoxication, the toxicity of the alcohols increases with the size of the molecule, as shown in the following table (Baer):

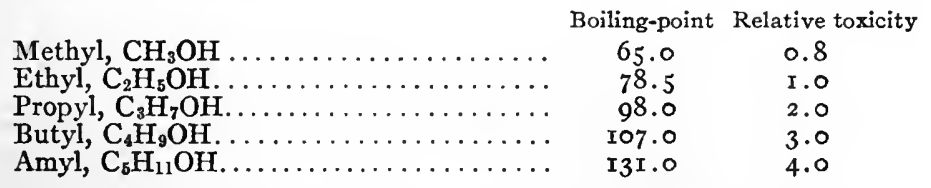

This (Richardson's law) applies also to the individual tissues; sensory and motor nerves, ciliated epithelium, muscle, ova, heart (Kuno; I9 I3).

It is quite conceivable that the late actions may differ more markedly from those of ethyl alcohol; as they do, f.i., in the case of methyl alcohol.

\section{ISO-AMYL ALCOHOL}

The main constituent of "fusel-oil," is more violent than ethyl alcohol in its acute and subacute actions (Salant, 1909 ) and the after-effects are more pronounced; but its admixture up to I per cent. produces very little difference in acute alcoholic intoxication (Baer, 1898 ).

\section{METHYL ALCOHOL}

General Statement.-The extensive use of this substance (Wood Alcohol, Columbian Spirits) in the arts (as a combustible, solvent for shellac, etc.) and as an adulterant of alcoholic beverages and medicines has given rise to numerous cases of poisoning. The acute symptoms resemble ordinary alcoholic intoxication. The main differences are in the stronger 
local irritation and in the more protracted and more serious after-effects, the coma lasting several days and often terminating in death. A very important feature is the frequent occurrence of incurable blindness from retrobulbar neuritis and retinitis. This may occur after taking Io c.c., but the individual susceptibility varies greatly. The effects are produced by the purest samples, and are therefore due chiefly to the methyl alcohol itself, although they may be exaggerated by the impurities (acetone, etc.) which are usually present. Methyl alcohol should never be applied to the body, externally or internally. Its presence in foods and drugs is properly prohibited by law. Protection against the inhalation of its vapors should be enforced in the trades. The treatment of poisoning by methyl alcohol includes evacuation, stimulants, heat; and morphin to allay suffering.

Etiology.- The practical seriousness of methyl alcohol poisoning is illustrated by the fact that Buller and Wood found I 53 cases of blindness and 122 deaths recorded up to 1904 , although the existence of the danger had only been recently recognized. A wholesale poisoning occurred among the inmates of a Berlin municipal lodging house (Stadelmann and IIagnus Levy, I9I2; Review of series of papers, Year Book, Am. Pharm. Assoc., I9I 2, I: $364-367$; Baskerville, I9I3, has abstracted the cases in the literature). About $8 \mathrm{r}$ million gallons are produced annually, and 2 million people are engaged in trades where it is employed (Wilbert, 1915). Adulterated cologne and bay rum, etc., have often proved toxic when used externally. 'The vapor of methyl alcohol (varnish) is dangerous if ventilation is insufficient (recent Bibliography, J.A.M.A., I9r3, $60: 1237)$. The inhalation is also highly toxic to animals, the effects agreeing with those in man (Tyson and Schoenberg, I I I4). Considerable absorption occurs when the air contains 0.2 per cent. (A. Loewy and Heide, I9I4).

General Symptoms. - These begin with gastro-intestinal disturbance, abdominal pain, general weakness, nausea, vomiting, vertigo, headache. The symptoms of ordinary alcoholic intoxication supervene, wtih delirium, restlessness, unconsciousness, collapse and coma. The pupils are usually dilated and irresponsive; nystagmus may be present. Dyspnea and cyanosis are marked. The temperature is subnormal. Death is very much more frequent than with ethyl alcohol. It occurs in coma, from respiratory paralysis, in a few hours to three days. The fatal dose is probably from roo to 250 c.c. The postmortem lesions show irritation of the stomach, duodenum, kidneys and bladder. Continued use leads to fatty degeneration of the liver.

Late Symptoms.-Sometimes the symptoms are deferred for several days and then prove rapidly fatal. Delirium may set in after the acute symptoms have subsided. Cystitis is a frequent sequel. The most important sequel, however, is the:

Optic In jury.- The symptoms are bilateral and consist in scotoma, dimness or total blindness; the pupils are dilated and insensitive to light. The loss of vision may start in a few hours, or be delayed several days; it generally becomes complete; subsequently there is some improvement, which in most cases, however, finally relapses into total blindness (Buller and Wood, r9o4; other reports summarized by Woods, I9r3). The anatomic changes consist chiefly in hyperemia and edema of the fundus and destructive inflammation of the optic nerve or retinal elements or both, followed by their atrophy (Tyson and Schoenberg, I9I4). The effective dose varies greatly: ro c.c. has caused blindness in some cases while 90 c.c. has failed to do so in others. Permanent impairment of vision occurs in something like half of all cases of poisoning (for other toxic amblyopias, see "Nicotin"). The pathologic changes in rabbits are described by Kasava, r9i3.

The cause of the peculiarities of methyl alcohol poisoning has not been satisfactorily explained. In animals, the acute toxicity of methyl alcohol is about ro per cent. less than that of ethyl alcohol (also for rotifers, Whitney, 1912), but the subacute toxicity is markedly greater. The symptoms develop more slowly, even on intravenous administration, and are much more protracted: While the ethyl alcohol coma lasts only six to twenty-four hours, that of methyl alcohol lasts three to four days. This protracted action leads to cumulative effects: Pohl, I893; Reid Hunt, I902; and Langaard, r 913 , found that the daily repetition of sublethal doses is more fatal with methyl than with ethyl alcohol. Birch Hirschfeld demonstrated a similar cumulative action on monkeys. "Tippling" has also been found especially dangerous in man.

Excretion and Fate.-The protracted and cumulative action is connected with the slow excretion. Voeltz and Dietrich, I9 12 , found after 2 c.c. per kilogram that during two 
days about 2 I per cent. were excreted unchanged by the lungs, 3 per cent. by the urine, 39 per cent. had been burned, and 37 per cent. were still in the tissues. Nicloux and Placet, I 9 I 2, found it present in the blood and brain after five days. This would explain the cumulation; but there may be an additional factor: While ethyl alcohol is oxidized completely to $\mathrm{CO}_{2}$ and $\mathrm{H}_{2} \mathrm{O}$, methyl alcohol is oxidized mainly to formic acid (Pohl, I 893). This is excreted rapidly by the urine, but gives rise to acidosis (increased ammonia excretion, Król, I9I3), and this may be responsible for part of the symptoms.

v. Fellenberg, I915, claims that methyl alcohol is readily formed from the pectin of fruit and vegetables, pectin yielding from 9 to 12 per cent. of methyl alcohol. $\mathrm{He}$ asserts that this is not harmful, being practically completely burned in the body; whereas if the methyl is mixed with ethyl alcohol, as in liquors, the combustion is incomplete, and harm results from relatively small doses. Confirmation should be awaited.

Impurities. - The best commercial wood spirit contains about 95 per cent. of methyl alcohol; the common varieties 75 to 90 per cent.; some samples only 35 to 40 per cent. The impurities consist of acetone, methyl acetate, allyl alcohol, aldehyd, empyreumatic products, etc. (Baskerville, I9I3). It has been suggested that these are responsible for the peculiar toxicity (Mueller, I9I0; Kobert, I9I0). However, most investigators have found no essential difference between the ordinary and the purest obtainable articles (Hunt, I902; Buller and Wood, I904; Ahrends, I9Io); nor do any of the impurities produce the characteristic effects (Baskerville, I9I3).

\section{ACETONE (DIMETHYL-KETONE)}

Acetone, U.S.P., $\mathrm{CH}_{3} \cdot \mathrm{CO} \cdot \mathrm{CH}_{3}$, is used as a solvent for fats, resins, rubber, etc.; and for the preparation of oleoresins. It is a rather feeble narcotic, causing considerable dyspnea (Albertoni, I884). Hypodermically (guinea pigs) it is much more toxic than methyl alcohol (Rhamy, I9I2). It is excreted mainly by the lungs. The urinary excretion in fasting dogs is diminished by caffein and cocain (Cervello and Girgenti, I914).

\section{DENATURED ALCOHOL}

Alcohol for industrial uses is mixed with various nauseous substances, to unfit it for consumption, and thus secure exemption from the internal revenue tax. The standard formulas contain 85 to 95 per cent. of grain alcohol, 2 to Io per cent. of wood alcohol, and $1 / 2$ per cent. of benzine or of pyridin bases. A few other formulas are authorized for special technical purposes.

Poisoning by denatured alcohol (containing $I$ per cent. of wood alcohol) has become quite common in Russia since the prohibition of grain alcohol. Visual disorders occur, generally after three to four months, or with large doses (Eleonskaia, I9I6).

\section{ETHER AND CHLOROFORM GROUP}

General Statement.-Ether, chloroform and other volatile hydrocarbons are employed mainly for producing surgical anesthesia. They depress successively the psychic centers, then probably the basal ganglia and cerebellum; then the spinal reflexes, first the sensory, and then the motor functions of the cord, probably from below upward; and finally the medullary centers. The most highly evolved functions are affected first. Chloroform also has a direct depressant effect on the cardiac muscle and is generally more dangerous. Locally, they act as irritants.

Discovery of Surgical Anesthesia.-The use of narcotics for lessening the pain of operations dates back to antiquity; however, the discovery of the means to achieve complete and safe anesthesia is an accomplishment of the nineteenth century ( 1842 to $\mathrm{I} 847$ ). The credit for this discovery must be divided between several investigators: Humphrey Davy for the definite suggestion; C. W. Long for the independent discovery but inadequate publication of ether anesthesia; Horace Wells for the successful use and publication of nitrous oxid; Jackson and Morton for the successful public demonstration of etherization; Flourens and Simpson for the introduction of chloroform. 
The Egyptians probably employed narcotics. The Assyrians are said to have strangled the children before circumcision, producing anesthesia by the aid of $\mathrm{CO}_{2}$. This method was revived by Valverdi in Italy early in the seventeenth century. The Chinese used hashish. All kinds of narcotics were given during the middle ages, especially opium and drugs containing hyoscin (such as the legendary "mandrake," Mandragora officinalis). The recent "Scopolamin-Morphin-Anesthesia" is an interesting reversion to this ancient method, which became obsolete when the vastly superior inhalation anesthesia was discovered.

Sir Humphrey Davy in I 799 announced the anesthetic properties of nitrous oxid (laughing gas) and suggested: "As nitrous oxyd, in its extensive operation, appears capable of destroying physical pain, it may probably be used with advantage during surgical operations in which no great effusion of blood takes place." Unfortunately, the suggestion was not acted upon at the time.

Dr. Crawford W. Long, of Jefferson, Ga., administered ether as a surgical anesthetic in $184^{2}$, but neglected the adequate publication of his discovery.

Dr. Horace Wells, a dentist of Hartford, Conn., witnessed an exhibition of "laughing gas" in a public lecture by Colton in December, 1844. He noticed that one of the subjects was insensible to pain; acting on this hint, he had a tooth extracted under its. influence on the next day. The operation was a complete success. He introduced the gas into general dental practice in Hartford, and gave the matter wide publicity, without reservation. The intoxicant action of ether being well known, he tried this with Dr. Marcy of New York, early in 1845 , but thought it inferior to the gas.

There can be no question that Wells discovered anesthesia independently, and that he introduced it successfully into practice; and he seems to have been the first to realize fully the importance of the discovery. Unfortunately, an attempted public demonstration in Boston in January, 1845 , failed partly and was met with ridicule. The successful demonstration of ether by Morton threw nitrous oxid completely in the shade for many years. Wells was greatly disappointed, became insane and committed suicide (Truman Smith, 1858, "An Examination of the Question of Anesthesia"). The use of the gas was revived about $\mathrm{I} 86_{3}$ by Colton (whose demonstration had started Wells), and by I 868 it was extensively used in dentistry. It has since been reintroduced into major surgery, and has thus vindicated the claims of Wells.

Chas. T. Jackson, a chemist of Boston, etherized himself into unconsciousness, in the winter of $184 \mathrm{I}$ to I842; and thereby conceived the idea of ether-anesthesia. He did not apply it further, but discussed it with various persons, among others with Morton.

Wm. T. G. Morton, a dentist of Boston, was a former friend of Wells and Jackson, from both of whom he seems to have received his inspiration. After the discovery of Wells, he is said to have consulted Jackson about the manufacture of nitrous oxid, and that Jackson suggested ether as more convenient. Morton seems to have started successful experiments with ether-anesthesia in September, 1846. In October, 1846, he gave a perfect demonstration at an operation by Dr. J. C. Warren, at Massachusetts General Hospital. The complete success of this operation achieved the introduction of anesthesia into surgery. Morton obtained a patent in November, 1846. This step, and the methods which he was said to have employed to obtain it, raised much opposition. His life was embittered by the controversies, and he died of apoplexy. Jackson, the third party to the unfortunate contention for priority, also became insane.

The use of anesthetics spread rapidly, and related substances were tried: Chloroform was used on animals by Flourens in 1847 , and in the same year, on the independent suggestion of McWaldie by Simpson of Edinburgh, on man. Flourens in the same year also called attention to ethyl chlorid. It was used surgically by Heyfelder in the next year, but then neglected till 1895 .

The first fundamental scientific work on the action of anesthetics was published by Snow, I858.

Ether was discovered by Valerius Cordus, 1540; chloroform independently by Liebig, Soubeiran, and Guthrie, I831.

Stages of Anesthesia.-The main actions of alcohol, ether, chloroform, chloral, and the numerous other hydrocarbon or lipolytic narcotics are qualitatively similar; but they show practically important differences in severity, rapidity, duration and sequels. The successive effects may be divided into "stages." Hewitt, f.i., classifies these as (I) analgesia; (2) light anesthesia; (3) deep anesthesia; (4) bulbar paralysis. Since the effects pass without abrupt transition from one stage to the other, any classification is arbitrary. In the following, they will be divided merely 
into the pre-anesthetic, anesthetic and paralytic stages. In surgical practice, the normal course of anesthesia is generally "complicated" by asphyxial phenomena, traumatic reflexes, hemorrhage, etc.

Pre-anesthetic Stage.- This sets in with a comfortable feeling of warmth, spreading over the whole body; but soon associated with a distressing sensation of suffocation, especially if ether is used and the access of air is limited, as in the "closed" methods. The local effects make themselves felt by prickling and smarting of the nose, throat and conjunctiva, with hypersecretion of mucus, tears and saliva. The suffocation, as well as the direct effects of the anesthetics, result in more or less excitement and struggling, and sometimes convulsions, especially in hysterical patients. The reflexes are at first rather exaggerated, later somewhat depressed and less purposive. Tonic muscular spasm, general, or of the jaws, glottis or respiration may occur. The sensation becomes disturbed: the sensibility is impaired after the first few inhalations. Stimulation evokes responses, but without pain. This "primary analgesia," while the patient is still conscious, may be used for short operations, especially under nitrous oxid.

The thoughts become confused, with hallucinations (noises, etc.), sensation of stiffness, and want of muscular control. The patient loses self-control and indulges in loud incoherent talking, laughing, singing, etc.; passing gradually into unintelligent muttering. These initial excitement symptoms vary greatly in duration and intensity in different patients. Alcoholics may exhibit violent delirium and even mania. Vomiting (probably central), retching, and coughing may occur. The face is flushed; the pupils at first dilated, then gradually constricted; the pulse rapid; the blood pressure increased; the respiration somewhat quickened and at times irregular, sometimes suspended if the vapor is too strong.

The Anesthetic Stage.-This is the stage which it is aimed to produce and maintain. It is characterized by complete paralysis of the brain and of the motor reflex centers of the cord, and usually some depression of the medullary centers. Consciousness, sensation, and most reflexes are lostthe corneal reflex being among the last. Consequently the muscles are lax. The smooth muscles are not usually affected, but there is sometimes a relaxation of the sphincters. The pulse is full and soft, the rate rather slow with chloroform, slightly faster than normal with ether; the blood pressure being lowered by chloroform, normal or rather raised by ether. The respiration is regular and deep, often softly snoring or stertorous; rather rapid with ether. The temperature falls in consequence of the lessened muscular activity and increased heat loss. The pupils are somewhat small and respond sluggishly to light. The face is pale with chloroform, often cyanotic with ether.

The medullary depression does notr each a dangerous degree if the administration is carefully done. But with prolonged anesthesia the pulse tends to become progressively weaker, the respiration more shallow, and the temperature lower-it may fall as much as $5{ }^{\circ} \mathrm{C}$. This is due to paralysis of the function of temperature-regulation.

The paralytic stage is characterized by progressive paralysis of the medulla. This stage must be carefully guarded against.

All reflexes disappear. The respiration becomes shallow, irregular, labored, and then ceases. The skin is cold and pale, and covered with the clammy sweat of the "agony." The pupils are widely dilated. The pulse becomes slow and weak, and ceases normally after the respiration. 
Recovery of Patient from Normal Anesthesia.-The promptness of the recovery is inverse to the duration of the administration. The first change after the withdrawal is usually in the respiration, which becomes quicter and less stertorous. Then the lid reflex and deglutition movements reappear. The pupils generally grow smaller, but dilate easily. 'The breathing is disturbed by swallowing, coughing, retching and actual vomiting, usually just before consciousness returns. This is commonly succeeded by sleep lasting several hours. Thirst and gastritis persist for some time.

Individual Functions. - The proper administration of anesthetics requires a detailed knowledge of their effects on the important individual functions in the successive stages.

Respiration.-This is influenced, not only by the direct action of the anesthetic, but also by the patency of the respiratory channels; by local and distant reflexes; the state of the circulation, etc. In the early stages, if local irritation is avoided, the respiration is generally somewhat deeper and quicker from stimulation of the respiratory center. Most commonly it is apt to be irregular, especially if the administration is started abruptly on account of the struggling, choking sensation and local irritation. The latter may produce temporary expiratory stoppage by a trigeminal reflex (Kratschmer, I870); but in man, this reflex is not usually so strong or lasting. The "choking" is especially pronounced with ether. Reflex closure of the larynx may produce asphyxia under chloroform (Bernard). With chloroform also spasmodic arrest of respiration may sometimes occur, especially in vigorous subjects, and is probably a common cause of the early deaths. This is less likely to occur if dilute vapors are employed.

As the patient passes into anesthesia, the breathing becomes regular and noisy; with ether, the depth and rate may at first be increased; with chloroform they are decreased, partly by the low blood pressure. The respiration is mainly diaphragmatic. The snoring and the louder "stertor" are generally produced by the vibration of the tongue against the pharyngeal wall. "Stridor" is laryngeal, due to paralysis or spasm of the vocal cords or obstruction of the glottis by mucus. As anesthesia deepens, increase of concentration usually makes the respiration temporarily deeper, quicker and more stertorous, and vice versa. Cheyne-Stokes respiration is not uncommon with chloroform, but exceptional with ether, because the $\mathrm{CO}_{2}$ tension is high.

Reflex Respiratory Changes.-These occur even during full anesthesia but are most marked if the anesthesia is light. The respiration is usually stimulated by the operative procedures, and these may provoke coughing, deglutition, retching, stertor, or spasmodic inspiratory or expiratory arrest. Operations during partial anesthesia are therefore dangerous.

Laryngeal Reflex.-When a fresh portion of ether is poured upon the cone, the irritation of the more concentrated vapor causes the patient, so long as he is partly conscious, to hold the breath momentarily. The disappearance of this reflex is a valuable index of surgical anesthesia (Briggs).

With the approach of the paralytic stage, the respiration becomes more stertorous (softly snoring with chloroform), irregular, dyspneic, and stops, paralysis of the respiratory center being the usual mode of death in deep anesthesia. Chloroform has a more pronounced paralytic action than ether; the paralysis may also be due to very low blood pressure. 
Factors in the Respiratory Effects.-The understanding of the several factors concerned in the respiratory effects is essential to the intelligent conduct of anesthesia. The factors are classified by Y. Henderson and Scarbrough, Igro, as follows:

I. The Threshold of Excitability for Carbon Dioxid.-This is elevated (i.e., the excitability is depressed) by morphin, chloroform, and deep etherization. It is lowered by light etherization; by the acidosis of deficient oxygenation; and by fear.

2. The Carbon Dioxid Content of the Blood.-This acts as a stimulant to respiration. It varies inversely as the respiratory ventilation.

3. The Threshold of Excitability for Afferent Impulses. - This is raised (i.e., respiration is depressed) by deficiency of carbon dioxid (acapnia); and by morphin, chloroform, and ether. It is lowered by fear.

4. The intensity of afferent impulses, especially pain and psychic impressions.

5. Inhibitory cerebral impulses, such as the fortitude of the patient.

The several anesthetics act differently on these factors: Morphin raises the threshold for carbon dioxid; it has less effect on the threshold for afferent impulses. It quiets excitement.

Chloroform raises the threshold more for afferent impulses than forcarbon dioxid.

Ether may lower the carbon dioxid threshold, whilst the afferent threshold may be elevated. Hence, ether tends to increase respiration (Fonteyne, 1906), so much so that in dogs it may cause fatal aca pnia (Henderson). Most ether fatalities occur in the pre-anesthetic stage, the respiration almost always failing before the heart (Cunningham and Anderson, I g08). This tendency may be neutralized by morphin. The respiratory excitement during the early stages of anesthesia has a similar tendency; it lowers the carbon dioxid content of the blood and thus tends to subsequent failure of respiration. Good surgical anesthesia obviates the development of acapnia by preventing painhyperpnea

Excessively deep anesthesia paralyzes respiration by raising the threshold of the respiratory center, and by diminishing the production of $\mathrm{CO}_{2}$. The paralysis is essentially central, although a curare action on the phrenic nerves may play a minor part (Githens and Meltzer, I9I4).

Anesthesia before pain prevents acapnia by lessening the afferent hyperpneic impulses. The induction of anesthesia after pain is more dangerous, because the lowered excitability during hyperpnea is apt to lead to acapnia (Henderson, I gro). The acapnia also damages the heart, so that it succumbs more easily to chloroform (Cathcart and Clark, I9r3).

Stoppage of Respiration is Therefore Most Apt to Occur With Uneven Anesthesia (Henderson, I9II).

Irritation of Respiratory Passages.-Both anesthetics, but especially ether, produce irritation, with reflex phenomena, increased flow of saliva and mucus, and sometimes inflammatory after-effects. The hypersecretion is especially marked with ether; chloroform produces only a slight increase during induction, and practical arrest during full anesthesia. Emphysema, bronchitis and advanced tuberculosis are therefore special contraindications to ether. The increased flow of saliva and the anesthesia of the larynx are apt to lead to aspiration pneumonia (Hoelscher, r898). Pneumonia may also be due to direct irritation; it is not known which has the larger share. It is much more common with ether than with the other anesthetics. It has been attributed to impurities, especially peroxids (Bruns, 1895; Smith, I9I3). Excessive mucous secretion may also produce acute suffocation by "inundation." The tongue during anesthesia is apt to fall against and close the glottis. These accidents are largely obviated by proper position of the head.

Coughing, retching and vomiting sometimes occur immediately after starting the administration; or later, during imperfect full anesthesia, especially with ether, being partly due to the swallowing of "mucus. Their occurrence indicates that the anesthesia should be deepened. The approach of vomiting is heralded by swallowing movements. If it can not be a verted, the head should be turned to the side and the opposite shoulder raised. 
X-ray examinations on dogs reveals marked diminution of gastric tone under chloroform or ether (Baron and Barsony, I9r4).

Bronchial Muscle.-Excised tracheal muscle is relaxed by ether, constricted by chloroform, in vapor-concentrations such as occur in anesthesia (Trendelenburg, 1912). In intact animals, however, chloroform produces slight dilation (Dixon and Brodie). In excised lung (guinea pig), temporary dilation is produced by ether, chloroform and urethane (Baehr and Pick, I9I3).

Blood-gases in Chloroform Anesthesia.-The gas exchange is decreased; in the excitement stage by hyperpneic acapnia and mechanical obstruction; in the anesthetic stage by depression of the center (Y. Henderson; Buckmaster and Gardner, I9I I). The production of $\mathrm{CO}_{2}$ during anesthesia is only one-half to two-thirds of the normal. The $\mathrm{CO}_{2}$ content of the blood was increased from 25 to 36 per cent., the oxygen content reduced from 13.6 to 8.I per cent. (Gardner and Buckmaster, 1910). The blood is therefore darkened. Chloroform seems to diminish the oxygen capacity of the blood; for the diminution of oxygen occurs even with artificial respiration. Gangitano claims that this lasts for twenty to twenty-five days.

The gas-metabolism of the brain is depressed by 60 to $9 \circ$ per cent., proportional to the depth of the narcosis. With ether, the $\mathrm{CO}_{2}$ falls less than the oxygen; the reverse is true of morphin (Alexander and Cerna, I9I3). In nerve fibers, direct exposure to low (stimulant) concentrations increase $\mathrm{CO}_{2}$ production, while anesthetic concentrations depress it (Tashiro and Adams, I914).

Ether on Circulation.-In the early stages, the pulse rate and blood pressure are increased by the reflexes, excitement and incipient asphyxia.

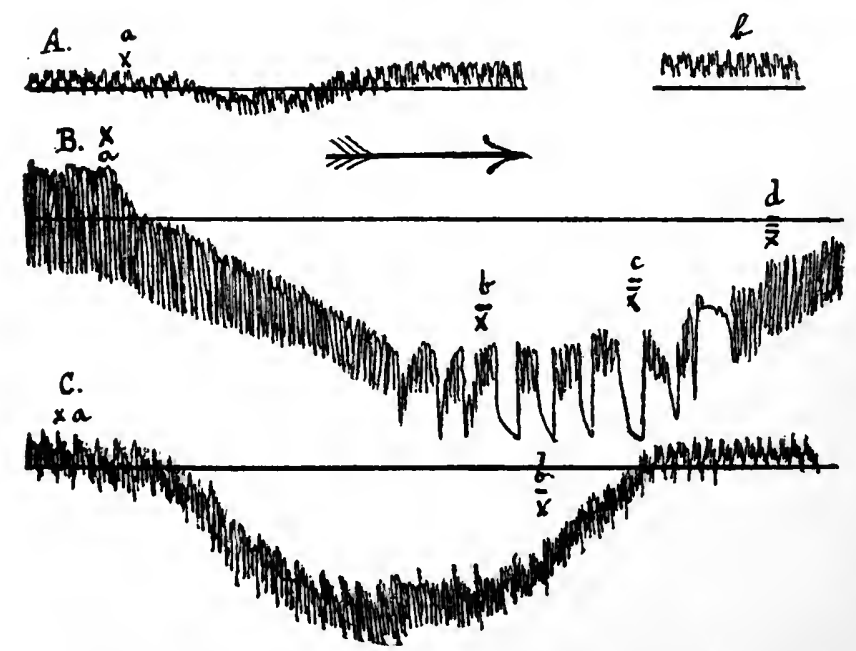

F1G. 26.-Anesthetics on circulation. A. Ether on blood pressure (dog). The animal is very lightly anesthetized at the beginning of the tracing. The ether is pushed at $(a)$. There is practically no effect on the blood pressure, even after ten minutes $(b) . B$. Chloroform on blood pressure $(\mathrm{dog})$. The inhalation is begun at $(a)$ and pushed. The pressure falls, without any material change in the heart (central vasomotor depression). The anesthetic is withdrawn at (b). The respiration is very shallow. Artificial respiration is started at $(c)$ and stopped at $(d)$. The original pressure is gradually recovered. $C$. Ethyl chlorid on blood pressure (dog). The inhalation is continued from $(a)$ to $(b)$. The effects are very similar to those of chloroform.

During anesthesia, the effects are similar to those of alcohol, stimulant rather than depressant: the cutaneous and cerebral vessels are dilated (also with chloroform, Berezin, I9r6), the intestinal constricted (Derouaux: I909), the pulse rate is increased to 80 to IIO, and the blood pressure (Fig. 26A) is practically unchanged, or somewhat above normal in man (Blauel, rgor). The face is reddened. The pressure in the pulmonary artery rises even if the carotid pressure remains unchanged (Wood, I9II). There is generally some venous congestion, especially with the "closed" 
methods (asphyxia). The threshold to vagus stimulation is raised (Jackson and Ewing, r9r4).

These indifferent or favorable actions on the circulation persist through the stage of surgical anesthesia, even if this is prolonged for hours. In the paralytic stage, the vasomotor center is gradually depressed: the pressure falls very slowly, the face becomes pale, while the heart remains practically perfect even when the respiration ceases. It can therefore usually be revived effectively by prompt artificial respiration. Fatty feeble hearts, however, may be affected more severely.

The vascular effects are central; the cardiac changes are mainly the indirect results of the vascular disturbance; the heart-muscle (Bock) and the vagus excitability remaining unchanged. Concentrations of ether which injure the heart directly can be reached only in perfusions.

Asphyxial Effects on the Circulation.-Slight mechanical interference with the respiration rather improves the respiratory movements and the circulation by stimulating the medullary centers. Severer grades produce venous congestion, extensive bleeding from severed vessels, etc. Profound asphyxia leads to extreme cardiac dilation by paralysis of the vasomotor center and heart.

In light ether anesthesia, $\mathrm{r} 2$ to $\mathrm{r} 6$ per cent. of $\mathrm{CO}_{2}$ in the air raises the blood pressure, but slows and weakens the heart. These actions are much less pronounced in deep anesthesia (Cathcart and Clark, r9 3 ). The secretion of cerebro-spinal fluid is increased by the anesthetics and $\mathrm{CO}_{2}$ (Dixon and Halliburton, rgr3).

Vasomotor Center.-Anesthetic doses of ether produce no direct effect, or moderate stimulation. The stimulation is probably largely asphyxial, from respiratory depression (Pilcher and Sollmann, 1915).

Effects of Chlorof orm on the Circulation.-These are much more serious than those of ether. Even in smooth anesthesia, the pressure falls markedly (Fig. 26B) by central vasomotor depression. Slight faults, especially early in the administration, may produce sudden permanent arrest of the heart. "A.C.E. Mixture" and Ethyl Chlorid (Fig. 26C) act similarly.

In normal chloroform anesthesia,' with carefully graduated administration, the pulse and blood pressure in the pre-anesthetic stage are somewhat irregular, but generally increased and the face flushed, similarly to ether. As anesthesia is reached, or shortly after, the blood pressure declines progressively (Lenz, I853; English Chloroform Commission; Blauel in man); in an hour it may have fallen to one-half, in two and one-half hours to one-third the normal level (Rosenfeld, r896). This fall of pressure is primarily due to depression of the vasomotor center: The vessels are dilated, but constrict on stimulation of their nerves (Scheinesson, 1869). The depression of the center can be shown directly by the perfusion method (Sollmann and Pilcher, I9I I). The dilation involves mainly the splanchnic area (oncometer, Embley, I902; increase of blood flow, Pick, I899). The skin becomes pale and cold. The cerebral vessels constrict, the pulse is soft and slow but regular, the heart beating efficiently when the vasomotor paralysis is complete (no response to asphyxia, Knoll, I878). The respiration also remains regular and efficient. The vasomotor depression is not necessarily harmful to the patient, unless it be excessive or prolonged.

With prolonged anesthesia, or with the approach of the paralytic stage, the heart is injured more or less by the direct action of the chloroform, as well as by the low blood pressure and asphyxia. It is also very probable 
that degenerated heart-muscle is more subject to these injuries. Ordinarily, however, the vasomotor paralysis dominates the circulatory phenomena until death, the heart beating after the respiration has stopped (Hyderabad Commission, I889 and 1890), although it may not be able to maintain a circulation.

With faulty administration the events are quite different. Excessive concentration of the chloroform vapor, particularly when the anesthesia is incomplete, stops the circulation before the respiration (Fig. 27). This is the usual mode of chloroform death (Lancet Commission, I893).

If the heart is exposed, it may be contracting feebly, even after the respiration has stopped, so that literally the respiration stops first; but these feeble beats can not propel the blood, so that, in fact, the circulation usually fails before the respiration.

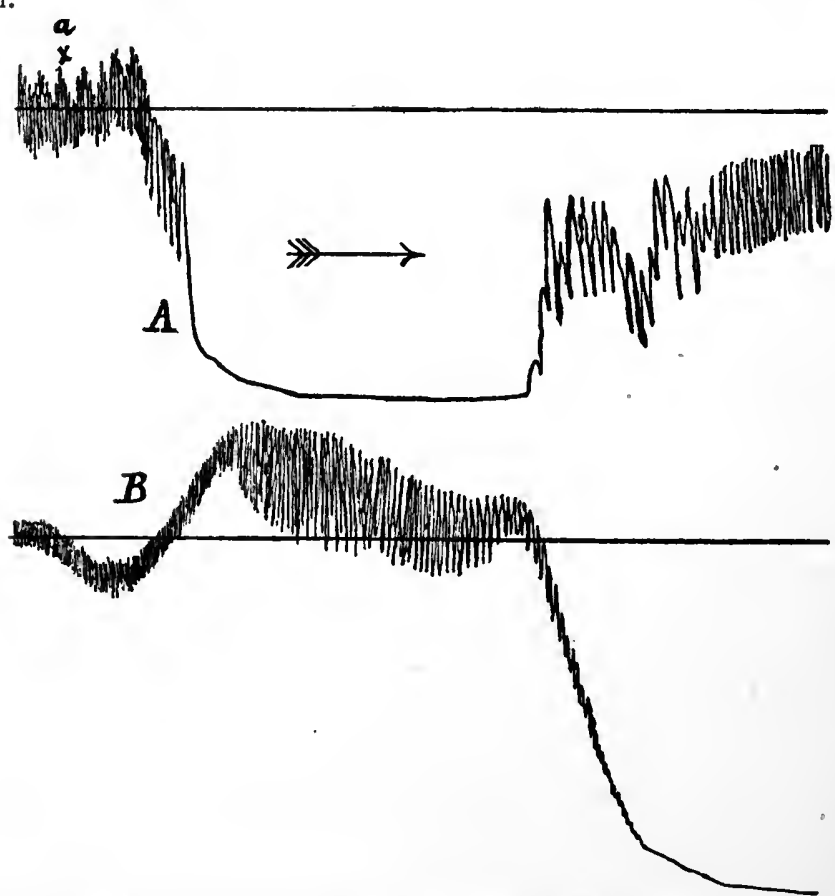

Fig. 27.-Accidents of anesthesia: A. Reflex stoppage of heart; blood pressure, dog. Ethyl chlorid is inhaled at $(a)$. The heart and respiration stop promptly (trigeminus-vagus reflex). They resume spontaneously after two minutes. $B$. Direct paralysis of the heart, preceded by asphyxial rise. Ethyl chlorid inhalation, blood pressure, dog.

The cardiac arrest may be due, in individual cases, to direct or reflex vagus stimulation, to asphyxia, or to direct paralysis of the cardiac muscle; or generally perhaps to a combination of these factors. Reflex circulatory disturbances are most dangerous in incomplete anesthesia where the reflex response is active; and in very deep anesthesia, when the low blood pressure may make even slight reflex changes fatal.

Laryngeal-reflex Syncope.-Desoubry and Magne, r9r4, confirmed that brusque chloroform inhibition produces complete arrest of heart and respiration in dogs and rabbits. However, if absorption of the chloroform is prevented, the arrest is temporary, and followed by rise of blood pressure from splanchnic constriction. In the usual fatal result therefore the toxic action of the absorbed chloroform plays an important part in preventing recovery from the reflex arrest. 
Vasomotor Center.-The preliminary stimulation is partly due to a direct action of the chloroform, for it occurs when this is confined to the cerebral circulation (by perfusing this from the vessels of a second, chloroformed animal; Gaskell and Shore, 1893). In part, the rise of pressure is also reflex and asphyxial. The vasomotor center becomes depressed quite early; f.i., the normal vascular compensation against the head-down position is destroyed (L. Hill, r897). It does not become completely paralyzed until much later (Gaskell and Shore; Embley and Martin).

In their perfusion experiments, Pilcher and Sollmann, 1915 , confirmed that the vasomotor center is depressed directly during chloroform anesthesia. Indirect stimulation may, however, occur when asphyxia is induced by respiratory depression, or if the center becomes suddenly anemic by rapid fall of blood pressure or cardiac arrest. Under such conditions, the center may be violently stimulated, with or without preceding depression.

Chloroform abolishes the effect of pressor reflexes at higher levels of blood pressure than normal (Pilcher and Sollmann).

In an intermediate stage of chloroform anesthesia, stimulation of the vasomotor center lowers blood pressure, since chloroform disturbs the reciprocity of antagonistic nerves so that afferent stimuli produce only inhibitory effects; chloroform causing the inverse effects to strychnin. The chloroform vasodilation may therefore be due to the reversal of the normal pressor impulses of the center. Asphyxia, however, still causes vasoconstriction (Bayliss, I908). When the vasomotor paralysis is complete, asphyxia does not raise the pressure.

The typical fall of blood pressure occurs with all methods of administration.

Direct Action on Vessels. - Perfusion of excised organs with chloroform solutions produces a constrictor effect in some organs, a dilator effect in others, especially the kidneys (Schaefer and Scharlieb, I904; Embley and Martin, I905; Filehne and Biberfeld, I906; Campbell, 1911). The required concentration, however (0.I per cent.), is almost twice as great as that which kills the heart and paralyzes respiration ( 0.06 per cent.); so that this peripheral action can play no part in chloroform anesthesia (Sherrington and Sowton, I904).

Early Stoppage of Heart.-Most fatalities with chloroform occur during the induction of the anesthesia, by sudden arrest of the heart. Experimentally, the heart will often resume if the vagi are promptly divided. In these cases the arrest is due to stimulation of the vagus center. This may be reflex, due to nasal, laryngeal, tracheal or pulmonary irritation by the chloroform vapor (Dastre; Morat; Brodie and Russell); but it may also occur when all reflex paths are eliminated, or on intravenous injection (Embley, 1902); thus indicating direct stimulation of the center. Its excitability is exaggerated by high concentrations of chloroform (about 2 per cent.), by morphin, and by asphyxia. Deep anesthesia dulls this vagus hyperexcitability, thus explaining why these accidents generally occur at the start. The administration of atropin would prevent this form of fatality completely.

There is, however, another factor; for a normal heart can not be stopped permanently by vagus stimulation, direct or reflex; with a chloroformed heart permanent arrest is easily produced (Embley; Desonbry and Magne, I9r4). It follows therefore that the high concentration of chloroform vapor must have injured the heart. This increased sensitiveness of the heart may be fatal apart from the vagus: A. G. Levy, rgr I, finds the cat's heart in early chloroform anesthesia very sensitive to high blood pressure (epinephrin), death in this case being not avoidable by vagotomy or atropin. This condition disappears in deeper anesthesia and was not seen with ether.

Socin, I9I4; showed that chloroform also renders the excised heart less resistant to venous engorgement or increased arterial resistance; i.e., that it dilates more easily. Similarly, excitement may stop the heart of lightly chloralized cats (Sollmann, 1908). Embley, I9I5, finds that early chloroform death with cats is frequently due to ventricular fibrillation. With dogs, this was never witnessed, except as a terminal result of low blood pressure. Henderson, 1914, is inclined to attribute these early fatalities mainly to acapnia.

Direct Cardiac Depression by Chloroform.-This was demonstrated on the excised frogs' heart by Clemens, I850; on the exposed mammalian heart by Snow, I852.

The excised mammalian heart (Langendorff method; Sherrington and Sowton, 1903) shows strikingly the difference between chloroform and ether. The chloroform depression occurs very promptly (within two minutes) and with much lower concentrations than are required for the vessels. It consists in a lessened force of the contractions, the rate being but little altered. The auricles are less affected than the ventricles. The effect is proportional to the cancentration of the chloroform, and independent of the absolute quantity and of the time of perfusion. The heart recovers at once when the chloroform is removed, unless the concentration was excessive (o.15 per cent.), in 
which case fibrillary contractions appear and recovery is impossible. Ether has much less action, and alcohol is practically harmless even when 2 per cent. is present.

The quantitative difference in the cardiac depressant action of these narcotics is illustrated by the results of Dieballa, r 894, on perfused frogs' hearts: If the fatal dose of chloroform be taken as one, that of ethyl bromid would be 12 ; of ether 48; of alcohol r92.

That the heart may be directly depressed by chloroform in the intact animal was shown by Scheinesson, r868, who found that the blood pressure fell even if vasodilation was largely excluded by clamping the aorta. Bock employed the same principle more perfectly, with the same results. This method involves high concentrations of chloroform.

Ordinary concentrations also weaken the heart somewhat (lessened systolic amplitude and rapid dilation; MacWilliam, r89०; Embley, r902); but this may possibly be the result of the fall of blood pressure. However, the concentration of chloroform in the blood which is required for anesthesia ( 0.035 per cent.) is not very far removed from that which paralyzes the heart ( 0.06 per cent.; Pohl, $x 891$ ); so that there is probably some direct cardiac depression even in "safe" anesthesia, and the margin of safety is quite small. Levy and Lewis, Igr 2, also found that low concentrations of chloroform produce marked cardiac irregularity (idiopathic ventricular contractions) in cats. Hecht and Nobel, rgr3, observed disturbed conduction, sometimes to complete auriculoventricular dissociation. In some experiments this could be removed by vagotomy or atropin, but in others it could not, indicating an action on the bundle. In perfused hearts, and doubtless also in living animals, the depressant action of chloroform is greatly increased by $\mathrm{CO}_{2}$, and therefore by asphyxia (Sherrington and Sowton, 1906).

The pressure in the pulmonary artery falls, through the.cardiac and respiratory depression (Wiggers, rgI I).

With frogs' hearts, the depressant effects of anesthetics can be antagonized by calcium (Burridge, 1912).

Actions on Blood.-These are not important in ordinary anesthesia. Ether and chloroform, like all fat-solvents, produce hemolysis when added to shed blood; and an insignificant degree of this action seems to occur during life: examination of the blood shows a polycythemia, due to diminution of the plasma. The hemoglobin is, however, reduced. Some corpuscles are destroyed entirely, others lose only part of their hemoglobin. Since a diminution of hemoglobin to $5 \circ$ per cent. is dangerous, it is never safe to use anesthetics on patients showing only 60 per cent. of hemoglobin (Da Costa and Kalteyer, I9or).

It is conceivable that this hemolysis explains partly the anemia and icterus which are sometimes seen; but these may also be caused by hepatic changes.

Coagulation Time of the Blood.- This is shortened by ether anesthesia. Chloroform and chloral have no effect if the coagulation time is normal; but lengthen it if abnormally short. Mendenhall, ror 5 , suggests that these changes are due to disturbed interaction of the liver and adrenal glands.

When added directly to blood, complex effects are produced on coagulation. Chloroform and ether render antithrombin ineffective. Ether also weakens the thrombin action; chloroform does not, nor does it convert prothrombin into thrombin. The quantities used were much higher than in anesthesia (Minot, r915).

The specific gravity of the blood is somewhat increased during anesthesia and for several days after (Sherrington and Copeman). Buck, I914, describes marked decrease of the globulins after about two hours of chloroform or ether anesthesia. The viscosity of the blood is slightly increased in deep, diminished in light anesthesia, but not to an important degree (Burton-Opitz, IgII). The osmotic pressure is a trifle increased by the molecular concentration of the dissolved anesthetic (Carlson and Luckhardt, I908). The fat-content of the blood is increased by ether; chloroform has this effect only when the animal has been on a fat-rich diet (Bloor, I914).

Disappearance of Fibrinogen.- Profound chloroform narcosis diminishes or abolishes the coagulability of the blood. This change is noticeable after thirty-five minutes of anesthesia. In extreme cases the fibrinogen may be completely absent. This effect is connected with the injury to the liver, similar to that of phosphorus, and is not produced by ether. The coagulability recovers with the repair of the hepatic lesions (M. Doyon, I913; Whipple, 19r4).

The cholesterin content of the blood does not show any significant changes in surgical ether anesthesia (F. C. Mann, rgr6).

Temperature.-Chloroform and ether cause about an equal fall of temperature.

In ordinary operations on man the fall is from $\mathrm{I}$ to $4{ }^{\circ} \mathrm{F}$., averaging about $2.5^{\circ} \mathrm{F}$. (H. A. Hare, I888); much more in prolonged operations 
on animals. It is not markedly increased by the ordinary dosage of morphin. The fall is due mainly to increased heat loss, from the narcosis of the temperature regulation, aided by the cooling action of the vapor and by exposure. It can be prevented by external heat (the room temperature should be above $26.5^{\circ} \mathrm{C} .=80^{\circ} \mathrm{F}$.) and by keeping the skin dry (S. G. Davis, I909). These precautions are important, since chilling favors the occurrence of shock and of postanesthetic pneumonia. Warming the vapor has been advocated (Gwathmey, rgo8); but the loss of heat from this source would be insignificant (Boothby, r9r3).

Reflexes.-The gradual abolition of all spinal reflexes produces complete muscular relaxation in the anesthetic stage-an important surgical advantage over nitrous oxid or cocain anesthesia. The relaxation and reflexes are also valuable for judging the depth of the anesthesia; but different patients vary considerably in the degree to which reflexes in general are depressed.

The reflexes disappear in a rather definite order. Among the last to disappear are the corneal, laryngeal, pharyngeal, rectal, vesical, peritoneal and genital. The patellar reflex persists very late. The movements of paralysis agitans cease during anesthesia as they do in sleep. 'With chloroform, the depression of the reflexes is strikingly parallel to the chloroform content of the blood. No initial stimulation of reflex excitability is noticeable (van Leeuwen, I9 3 ).

The Sensory Component is Depressed Before the Motor Component.-In the early stages, sensation is blunted while motion is unimpaired or indeed increased. The psychical condition is similar to alcohol, but the facilitation of motor processes is early obscured by the sensory depression (Kraepelin, I892). In the deeper stages, the sensory cells of the spinal cord are paralyzed before the motor cells: If chloroform is restricted to the upper half of the cord, so that this will not react to reflexes from the upper extremities, it will still respond to impulses from the unpoisoned sensory cells of the lower cord (Bernstein). The response to direct stimulation of the cord disappears first from the sensory posterior columns (Flourens, 1847). Eventually, however, the motor cells also become inexcitable.

Forbes and Miller, I9I6, conclude (from the action-currents) that the efferent impulses are blocked before they reach the cerebral cortex.

The cerebral motor cortex is also depressed, but loses its excitability only in very deep anesthesia (Hitzig, 1873).

Ether as an Intoxicant.-The resemblance of the earlier effects of ether to those of alcohol has led to its use as an intoxicant. It is said that ether drinking is a common form of dissipation in Ireland. The effects are produced, and pass off, more promptly than with alcohol (Discussion and literature, J.A.M.A., 64: 168, I915).

General Protoplasmic Poisons and Local Irritants.-The anesthetics, on direct application, narcotize all cells, of the lower organisms, as well as of the higher. This is preceded, at least in some cases, by evidences of stimulation. The concentration required for irritation is greater than is reached from systemic administration. At the place of application, however, the irritation is quite marked. Chloroform is more irritant than ether, with equal concentrations, and can be employed as a rubefacient and even vesicant.

The irritation is due largely to coagulation of the protoplasm. The cause of this differs in the va rious members. In the case of alcohol it rests upon withdrawal of water, and consequently all proteins are affected; chloroform has a more marked action upon globulins (especially myosin), and ether upon albumins (especially egg-albumen).

Peripheral Action on Nerves. - Direct application of solutions or vapors of the volatile anesthetics to a nerve lowers its conductivity and excitability, changes the polarization (Waller, 1896; Alcock, 1906; Bethe, r 908), and abolishes the action-current (Forbes, McIntosh and Sefton, I9I6). The sensory fibers succumb before the motor (Longet). The conductivity of the nerve is lowered, as well as its excitability; for if a nerve is ex- 
posed to ether vapor, stimulation at the proximal end ceases to be effectual much sooner than at the distal end. The excitability is recovered if the ether is washed off in time. With chloroform, recovery is more difficult. The concentrations required for these peripheral effects are greater than can exist in living animals; the motor and sensory nerves remaining excitable even in profound anesthesia; nor is the conductivity lowered during life (Forbes, MIcIntosh and Sefton, 19i6).

Narcosis of a nerve diminishes its $\mathrm{CO}_{2}$ production materially. Lower concentrations increase the $\mathrm{CO}_{2}$ ('Tashiro and Adams, I9 $\mathrm{I}_{4}$ ).

The undiluted anesthetics when iniected directly into a nerve produce a circum. scribed neuritis (caution in subcutaneous or intramuscular injections).

Special Senses.-The odor and sweet taste of chloroform and ether show a peripheral stimulation of these sense organs.

Curare Action.-Auer and Meltzer, $\mathrm{I}_{9} \mathrm{I}_{4}$, found that deep ether anesthesia produces a moderate depression of the motor endings. Chloroform does not have this effect (Githens and Meltzer, I9I4).

Muscle.-All forms of muscle are depressed. Chloroform further causes a specific rigor of the muscle when applied directly or injected into an artery (Kussmaul, 1858; Cl. Bernard). It also coagulates the extracted muscle proteins (Fuerth, I 896).

Ureter.- The contractions are depressed by chloroform in living animals, even by doses which do not affect the respiration or circulation (Lucas, I906 and I907). The inhalation of chloroform therefore relieves the ureteral spasm of renal colic. Ether stimulates the contractions.

Ciliary movement (as in the frog's pharynx) is arrested.

The narcotic effects on infusoria were observed by $\mathrm{Cl}$. Bernard, $\mathrm{x} 876$.

Phagocytosis. - This is stimulated by small concentrations, arrested by higher (H. J. Hamburger, Igri). The stimulation is probably due to asphyxia, since cyanid or carbon dioxid produce the same effect (Hamburger, I915).

During surgical ether anesthesia, a leucocytosis sets in slowly, usually after one to four hours. It concerns mainly the polymorphous, but the other forms are also increased. It appears to be due to stimulation of the bone-marrow. The phagocytic activity is not decreased, even after four to six hours of anesthesia (F. C. Mann, I9I6).

In plants the narcotics abolish protoplasmic movements; cause plasmolysis; suppress the gross movements of the Sensitive Plant (Mimosa pudica), etc. Small amounts increase metabolism. Exposure to chloroform and especially to ether vapor hastens blooming (Keeble, 1906).

Vermifuge.-Chloroform has been employed for the expulsion of tapeworms; but the effective doses are too irritant and toxic to make its use advisable.

Antiseptic Action.-Chloroform is fairly antiseptic (Strassmann, I888; Salkowski, $\mathrm{I} 89 \mathrm{I})$, and may be used as a preservative.

Ether has come into some prominence as a surgical antiseptic. It is applied on compresses; and for flushing the peritoneum after perforation (Souligoux, I9 3 ). Saliba, 1916, reports it as satisfactory and generally without untoward after-effects or complications. He injects the liquid ether before closing the sutures, about Ioo c.c. for adults; 30 c.c. for children above one year.

Cutaneous Irritation.-The evaporation of the volatile anesthetics cools the skin. Local anesthesia by freezing may thus be produced (see "Ethyl Chlorid"). With ether and especially chloroform, the cooling is succeeded by a burning sensation and rubefaction. If evaporation is prevented, this may proceed to vesication. Chloroform is therefore used as an active counterirritant (linimentum chloroformi, U.S.P.: Chloroform 30, Soap Liniment 70; B.P.: Chloroform 50, Camphor Liniment 50). The irritant action on mucous membranes is even more violent.

Gastro-intestinal Tract.-The inhalation of the anesthetics generally leads to nausea and vomiting, probably partly central, partly due to local irritation and to the swallowing of mucus. Etherization somewhat slows the passage of food through the alimentary canal (Cannon, IgIr). Paresis of the intestines occurs commonly after laparotomies; but this is mainly due to the operative procedures (Cannon), although Y. Henderson, I909, states that ether acapnia may also produce this result. 
The oral administration of ether and cspecially chloroform causes considerable gastric irritation. In milder degree this produces a carminative and anodyne effect, which is useful in colic and dyspepsia (Spiritus Aetheris, 5 c.c., r 3 ; Spirits Chloroformi, 2 c.c., 1/25; Aqua Chloroformi, $\mathrm{r}_{5}$ c.c., 45$)$. Spiritus Aetheris is also employed as a reflex "diffusible stimulant."

If ether is taken into the stomach, it is rapidly volatilized and larger quantities distend the organ, sometimes so much as to interfere with respiration; it may even produce rupture of the organ. It also causes considerable local irritation, and consequently a temporary leucocytosis.

Pupils. - These furnish valuable information of the efficiency of the reflexes and asphyxia; and therefore of the depth and course of the anesthesia. They are dilated in the excitement stage, with the light reaction moderately decreased. This dilation cedes with the approach of anesthesia. In man there is an actual constriction; the light-reaction is diminished or abolished; the eyes are turned inward and upward as in sleep. The constriction persists throughout the anesthetic stage and into recovery. With ether, the diameter averages $3 \frac{1}{2}$ to $4 \frac{1}{2} \mathrm{~mm}$.; with chloroform, 2 to $3 \mathrm{~mm}$. If the anesthesia deepens they usually dilate; with lighter anesthesia they become smaller. However, their behavior is easily modified by reflexes, respiratory embarrassment, etc.; and should be occasionally ascertained by intentionally changing the depth of anesthesia. Constricted pupils usually mean that the anesthesia is light. Dilation may either mean deep anesthesia, asphyxia, or reflexes with light anesthesia. If the corneal reflex is present, dilation should lead to more rapid administration; in the absence of reflexes, to lighter administration. Sudden and extreme dilation generally signifies dangerous asphyxia. Morphin and atropin interfere with these reactions.

The effect of anesthesia is identical with that of sleep and is due to the abolition of the cerebral impulses which normally inhibit the constrictor tone of the oculomotor center. Asphyxia, on the other hand, increases this inhibition (Braunstein, I894).

The Pupillary Reactions of Animals.-These are somewhat different: all show the dilation in the excitement and asphyxial stages, and the abolition of the light-reflex during anesthesia; but the anesthetic miosis is witnessed only occasionally in dogs, never in cats or rabbits. In these animals the pupils assume a median position. The frog's pupil is not affected by anesthesia (Nakazawa, Igro).

The Corneal and Coniunctival Reflex.-These disappear very late, as in all modes of death. Cl. Bernard found that the conjunctival reflex disappears before the corneal in ether anesthesia and in asphyxia; whilst the corneal reflex disappears first in death by cold or strychnin.

Absorption and Excretion.-Chloroform and ether, like most other chemically indifferent vapors and gases, are absorbed almost instantly from the lungs, and excreted promptly and almost quantitatively by the same channel. A small part is probably excreted by the urine. Absorption from the stomach and rectum is also very prompt, and may be utilized for anesthesia.

Retention.-The percentage of the anesthetic in the expired air is at first much less than in the inspired. It begins to rise after two minutes, but remains considerably below the inspired even after ten minutes (Brodie and Widdows, 1906). This shows an actual retention of the anesthetic, which is reflected by the percentage in the blood Buckmaster and Gardner, I907). Harcourt, I906, states that at the end of half an hour of anesthesia, about one-third of the administered chloroform has remained in the body; after this time but little further retention occurs.

The excretion of volatile anesthetics through the lungs is a merely physical process, analogous to their evaporation from aqueous solutions. It depends more upon their solubility and affinity for water than on their boiling-point (Cushny, I910). 
The ccrcbro-spinal fluid also contains small quantities of chloroform when this is inhaled (Hald, igis).

Destruction of Chloroform in the Body.-A small amount of chloroform is decomposed in the tissues, with the production of chlorid (excreted by the urine; Zeller, 1883 ), and carbon monoxid. The quantity of the latter is too small to produce any effects. This decomposition occurs also in shed blood (Nicloux, rgro).

Concentration of Anesthetic in Inspired Air.-The anesthetics exist in the blood and tissues mainly, if not solely in physical solution: they are therefore absorbed and excreted by the alveolar blood in proportion to their concentration in the alveolar air, until the whole system of air-bloodtissues is in equilibrium. Since the exchange is rapid, the quantity of anesthetic in the tissues at any given moment depends essentially on the concentration (partial pressure) of the anesthetic vapor in the inspired air (P. Bert). The action is determined essentially by this concentration, and only in a minor degree by the absolute dose of the anesthetic. A knowledge of the concentrations is therefore important.

The effective concentrations for the respired air have been determined experimentally, mainly on animals; but they agree for all mammals; and according to available data, also for man (Bert, I 884; Dresser, 1893 ).

Boothby, I9I6, finds the "anesthetic tension" (the partial pressure of ether vapor which maintains anesthesia indefinitely, after it has been established) as $47 \mathrm{~mm}$., equivalent to about 6.7 volume per cent.

With ether, light operative anesthesia can be maintained with about 6 per cent. (volume) of ether vapor (I8.5 mg. per Ioo c.c. of air); deep narcosis (abolition of reflexes) requires 10.5 per cent. volume. The fatal concentration is I I per cent. For chloroform, the corresponding concentrations are, for light anesthesia, I. 35 volume per cent. $(6.8 \mathrm{mg}$. in roo c.c. of air); deep narcosis, I.65 per cent.; fatal, 2 per cent. (Kochmann, I9I4). The anesthetic zone between the minimal anesthetic and the fatal dose is much wider for ether (viz., 5 per cent.) than for chloroform ( 0.65 per cent.). This is one reason for the greater safety of ether. Another difference is that the deep anesthesia can be maintained almost indefinitely with ether, whilst chloroform kills within one or two hours (Rosenfeld, 1896). The time factor therefore plays a part especially with chloroform (also on account of the postanesthetic degenerations). On the other hand, the concentration may rise momentarily above the fatal limit, since the absorption, although rapid, is not instantaneous.

The above figures are those of Kochmann and his pupils. The older experiments generally gave lower numbers, probably due to analytical errors. Thus, Spenzer, I894, stated the effective volume per cent. of ether as 3.5 per cent.; fatal, 6 per cent.; for chloroform, Rosenfeld, I896, named I per cent. as anesthetic, I.65 per cent. as fatal. The various results are collated by Ritschel and Stange, 1893.

Concentration of Chloroform in Practical Anesthesia.-For inducing anesthesia, I per cent. chloroform vapor is insufficient: 2 per cent. is generally effective; less than this must be used in pathologic conditions, but requires a longer time to establish narcosis. Even 3 per cent. may be necessary in exceptional cases. The maximum limit of safety is placed at 2 per cent. by Waller; at I to I.5 per cent. by Embley. Therefore, as soon as anesthesia is reached, the concentration must be gradually reduced to I per cent., and after half an hour even lower (Alcock, I909; Levy, 19ro).

Even perfectly adjusted mixtures are not absolutely safe. In practice the inconveniences of exact vapor mixtures are generally held to outweigh their advantages. However, the above figures are fairly approximated in the ordinary methods of administration. The air under the ordinary mask has been found to contain generally from I to 2 per cent. of chloroform, and with rapid application, up to 4 per cent.

Concentration of Anesthetics in Blood and Tissues.- This has been investigated especially by Nicloux, 1906 to I908; Tissot; Mansion and Tissot, 1906; Sicard, I906. According to these, the arterial blood contains, with complete anesthesia of chloroform, 
0.032 to 0.05 per cent. (o.014 to 0.028 according to Buckmaster and Gardner); of ether, 0.13 to 0.4 per cent. At death, the chloroform concentration averages 0.08 per cent. ( 0.06 per cent., Pohl; 0.04 per cent., Buckmaster and Gardner); ether, 0.16 to 0.17 per cent. The concentration of the venous blood is somewhat lower, since the anesthetic is given off to the tissues. This difference is of course especially large before equilibrium is reached. The left heart is therefore exposed to a higher concentration than the right, especially if the anesthetic is "pushed" (Pohl, I $89 \mathrm{I}$ ).

The distribution of the chloroform in the tissues is related to their content of lipoid matter (Frison and Nicloux, 1907); especially of cholesterin and lecithin, in which chloroform is much more soluble than in water. Blood therefore absorbs considerably more chloroform than does salt solution; and the percentage is two and one-half to eight times greater in the red corpuscles than in the plasma (Pohl, I89r; Nicloux, I906; Buckmaster and Gardner, 1907); twice as great in brain (0.057 per cent. at death) than in liver or muscle ( 0.026 per cent.). Adipose tissue is a curious exception, since it contains no more than muscle. Waller, i 904 , states that only i 5 per cent. of the chloroform when added to blood can be recovered. With ether, which has a relatively smaller affinity for lipoids, the percentages in all the tissues are practically equal (Nicloux). It should be added that Guenter and Hoelscher, I906, contradict these statements of the French observers, and claim that there is no difference in the percentage of chloroform in the blood, brain, liver and kidney; provided that the inhalation of a given concentration is kept uniform until equilibrium becomes established.

Elimination.- When the anesthetic is discontinued, the greater part disappears very rapidly from the blcod: Within five minutes, the percentage of ether or chloroform has fallen to one-half. The final elimination is more gradual: With ether, there are traces in two hours but not in four hours. With chloroform, the concentration falls to only a seventh in three hours, and traces are present even in seven hours (Nicloux).

Ether on Urine Flow.-This is diminished during the anesthesia (e.g., Hawk, Igr I Ginsberg, I9 2 ; MacNider, I9I3), presumably by the altered circulation and perhaps also by the irritation. (Kemp, I899, described contraction of the renal vessles with diminished kidney volume; but according to Buxton and Levy, this occurs only in overdosage, and then as a part of the general circulatory disturbance.) As soon as the anesthesia is terminated, the flow increases, the output being above normal for several days. The specific gravity is also increased. This diuresis is proportional to the duration of the anesthesia (W. H. Thompson; Hawk, I908, r9II, dogs; Pringle, Maunsell and Pringle, human, I905). In surgical patients, the urine output is often diminished by restriction of the water-intake (Epstein, Reiss and Branower, rg 6 ).

Ether on Nitrogen Excretion.-This is diminished during the anesthesia (Pringle, I905); it is then increased for twenty-eight to forty-eight hours, depending but slightly on the duration of the anesthesia. The total increase may amount to 45 per cent.; but is generally smaller, and therefore much less than with chloroform (Hawk, r 908 ).

Ether Glycosuria.-Ether anesthesia sometimes causes temporary hyperglycemia and glycosuria (animals, Hawk, I904; Shaffer, r914; human, Roehricht, r9o5). They occur also on intravenous injection (King, Moyle and Haupt, I9I3). They are due to increased hepatic glycogenolysis, for they do not occur if the liver is excluded from the circulation (King and co-workers, I9I2); and are more intensive in well-fed dogs than in fasting (Hawk, Igri). They occur after division of the splanchnics (King, etc., IgII). Ether and. nitrous oxid also increase the glycosuria and $\mathrm{D} \div \mathrm{N}$ ratio in phlorhizin diabetes, the extra sugar being derived from glycogen, probably through tissue-asphyxia (Sansum and Woodyatt, I9 5 ).

Hawk, IgI I, attributes the ether glycosuria to dyspnea. Grube, rgrr, believed that the cooling was a factor; but Ross and Hawk, 1914, find that the glycosuria is not due to fall of temperature. Ross and McGuigan, 19 $_{5}$, claim a direct action of the ether, and that asphyxia and excitement are not essential.

Renal Irritation.-Postanesthetic albuminuria occurs in about onefourth of ether anesthesias, and about one-third of chloroform (Wunderlich). Tube casts are also common. With previously sound kidneys, the renal irritation usually disappears in a short time. In patients with chronic nephritis there may be fatal suppression of urine; but patients with some albuminuria are often etherized without bad effects. Chloroform is apparently somewhat more injurious than ether, but the difference is not very marked.

Acetonuria is also very frequent after ether or chloorform anesthesia (E. Becker, I895; Beesly, I906; Marshall and Rowntree, I916; ethyl chlorid, Telrofd, I9o6; even 
after local cocain, Gundrum). With ether, it is not much influenced by the duration of the anesthesia; fasting may be a factor (Chalfant, Igr2). It is more common with the clused than with the open methods of administration. Children are more susceptible. Bicarbonate has been advised as prophylactic.

Acidosis.- Ether, chloroform or nitrous oxid anesthesia may reduce the alkaline hyodrogen ion concentration of the blood to neutral $\left({ }_{p} \mathrm{H}=7\right)$, proportional to the depth and duration of the anesthesia (Menten and Crile, 1915).

Phenylphthalein Output.-The "functional capacity" of the kidney, as measured by this test, is generally somewhat decreased by ether anesthesia. The effect is the greater, the longer the anesthesia. The output is lowest soon after the operation, returning to normal in twenty-four to forty-eight hours. Exceptionally, there may be no decrease, but even an increase; or the decrease may be very large. It has no relation to albuminuria. All shock and collapse conditions lower the phthalein output (R. $\mathrm{H}_{\mathrm{e}}$ Miller and Cabot, I915; Epstein, Reiss and Branower, I916). Whipple and Speed, I9 5 , also found the phthalein output lowered after ether, paraldehyd, chloral, chloroform or alcohol anesthesia. They observed that Eck's fistula produced lowered output, as also phosphorus, and therefore attribute the effect to disturbance of liver function.

Anesthetics in Experimental Nephritis.-In dogs rendered polyuric and glycosuric by tubular uranium nephritis, MacNider, $\mathrm{I}_{91} \mathrm{I}_{3}$, found chloroform anesthesia much more damaging than ether. In full-grown animals, chloroform promptly arrested the urine flow, so that it would not respond to diuretics; it made the histologic lesions much more marked. The damage was much less in young animals. Ether produced but little effect even in adults. (Young puppies are remarkably tolerant also to the other dangers of chloroform; Whipple, Igr 2.)

Chloroform on Urine Flow.-In man this diminishes steadily during anesthesia (Bovee, 1909). Animals also usually show this decrease, due to the low blood pressure with diminished kidney volume (Thomson and Kemp, I899). Thompson, I906, often observed increased diuresis in the early stages, and invariably after recovery.

Chloroform on Nitrogen Metabolism. - Chloroform anesthesia increases the total nitrogen excretion (especially in carnivorous animals), and the ammonia, allantoin, diamino-acids, polypeptids, creatinin and organic sulphur. It decreases the excretion of urea and of monamino-acids (Strassmann, I889; Salkowski, I889, Noel Paton, I894; Vidal, I 897; Rostoki, I90I; Howland and Richards, I909; Lindsay, I9I I). 'The increase persists for some days and may amount to 300 per cent. These changes indicate an increased destruction but incomplete oxidation of the proteins. They are similar to those of phosphorus poisoning, and probably depend on the hepatic lesions.

The chlorid excretion is increased during and after the anesthesia.

Chloroform Glycosuria.-This is quite common in ordinary anesthesia. The disturbance is probably analogous to ether (hyperglycemia; E. L. Scott, I9I4) but more profound. There is some dispute as to whether other reducing substances besides dextrose are present (Hawk, I9II). Chloroform diminishes the tolerance to galactose and levulose (Marshall and Rowntree, I9 5 ).

Delayed Chloroform Poisoning (Postanesthetic Toxemia).-The prolonged administration of chloroform is often followed by fatty degeneration of the liver, heart, kidneys; leading to profound toxemia, vomiting, prostration, icterus, and coma, which is often fatal. These results are very exceptional with ether. Postanesthetic toxemia is especially common with children; and in patients with acetonuria, diabetes, hepatic disorders (Hildebrandt, r913), cyclic vomiting, rickets, or wasting diseases.

Symptoms.- The importance of late chloroform poisoning seems to have been first recognized by Bevan and Favill, I905; although cases had been reported previously. The symptoms start after ten to one hundred and fifty, generally twenty-four to fortyeight hours; the intervening time being indistinguishable from the normal course. They consist in profuse, sometimes incessant vomiting, first billious, then blood-stained; excitement and delirium, alternating with apathy and prostration; dilated pupils; rapid pulse; icterus; unconsciousness deepening into coma. Death occurs in four to five days (Herb, I9II).

The blood generally shows retention of non-protein nitrogen, urea and amino acids, similar to phosphorus poisoning, though less intensive (E. K. Marshall and Rowntree, I915).

Anatomic Lesions in the Liver.- These consist in extensive vacuolization, fatty degeneration, swelling and necrosis of the cells, especially about the central veins (Nothnagel, 1866; Ungar and Junker; Wells, I906; Howland and Richards, I909; Whipple and 
Sperry, I909; Herb, I9II). The icterus, the disappearance of fibrinogen from the blood; the diminution of liver lipase with increase of plasma; kidney and muscle lipase (Quinan, 1015); the delay of the biliary excretion of phenoltetrachlorphthalein (Whipple and pupils, 1913); the changes in metabolism; and the occasional occurrence of leucin and tyrosin,must be referred largely to the hepatic lesions, and are analogous to acute yellow atrophy and to phosphorus poisoning. H. G. Wells suggests that they are due to checking of the synthetic processes, whilst the autolytic activity is unimpaired (Quinan). (Large doses of other anesthetics (urethane, Sollmann) cause similar degeneration.) In animals which recover, the liver changes tend to return to normal, even when there has been extensive necrosis (Whipple and Sperry, 1900). Mosiman and Whipple, 1912, found that puppies and feti are less subject to the hepatic necrosis; and that pigeons, frogs and terrapin seem to be immune.

Histologic Changes in Other Organs.-These are also important. Fatty and degenerative changes are seen in the epithelium of the straight and convoluted tubules of the kidneys (Howland and Richards; Apperly, I912); in the pancreas (Whipple and Sperry); in the suprarenal cortex and other glands of internal secretion (Scaglione, Igr5). Involvement of the heart-muscle sometimes dominates the symptoms. Degeneration of the cardiac ganglia has been described (Strassmann, 1898). Small hemorrhagic foci exist in the gastro-intestinal tract, mesentery, pleura and pericardium (Herb). These histologic lesions can be produced, especially in young dogs, by chloroform anesthesia lasting two hours (Whipple and Sperry, 1909; Whipple and Hurwitz, I9Ir). Repeated chloroform anesthesia, with rabbits, produces degenerative changes also in the central nerve cells and those of Auerbach and Meissner plexus. So do many other poisons, but not ether (Lissauer, 1914).

Cragin and Hull, ror r, point out the similarity of the late chloroform phenomena to eclampsia, and suggest that chloroform would be much more injurious than ether in this condition.

McCrudden, Igr 2, finds that chloroform is more apt to produce fatty changes of the rabbit's heart after nephrectomy. Ether does not do this.

Relation to Acidosis.-Lesions resembling delayed chloroform poisoning occur in many intoxications, among others in acidosis. Graham, I 1 I $_{5}$, assumes a causative relation. He points out that the oxidation of chloroform and other analogous halogen compounds may lead to the production of $\mathrm{HCl}$, and believes that this would produce acidosis, and thus the degenerative changes. It has never been shown, however, that such oxidation actually takes place in the body, especially to a degree that would overcome the very efficient defense mechanisms neutralizing acidity. It is also difficult to explain away the observations of similar degenerations after chloral, urethane, ether, phosphorus, etc., which certainly are not oxidized to acids.

Experimental Hepatic Cirrhosis. - This occurs after repeated administrations of chloroform (Mertens, 1896; Herter and Williams, 1905). It is favored by the presence of bacterial infections and may then proceed to acute yellow atrophy (Opie, rgro).

Intravenous injection of ether (large doses in rabbits) causes destruction of liver tissue, with extensive thrombus formation (prevented by leech extract). The mechanism is evidently altogether different from ordinary chloroform degeneration (L. Loeb, r9o6; Loeb and Meyers, I9ro).

Repeated Anesthesia on Growth.-Schapiro, 1906, found marked retardation in young cats anesthetized every morning and evening for forty-eight days. When this was discontinued the animals caught up, at least partly with the controls. The effect therefore has no practical importance.

Other After-effects of Anesthetics.-These are mainly due to the irritant action, and may therefore occur with either anesthetic. They may be recapitulated as:

Gastric Irritation, Nausea and Vomiting.-Almost universal; varies with duration of anesthesia; less with open methods.

Tympanites.-Especially after abdominal operations; the anesthetics are only contributory.

Renal Irritation.-About equally frequent with either anesthetic. Aspiration Pneumonia.-More frequent after ether.

CHOICE OF THE ANESTHETIC

This is determined by the particular conditions of the patient and of the operation. Safety should be the first consideration; but the depth and 
duration of anesthesia, the comfort of the patient, and the convenience of the operator are also very important. The principal anesthetics which deserve consideration are: General Anesthesia by ether, chloroform, nitrous oxid, or ethyl chlorid, with or without morphin and scopolamin; Spinal Anesthesia; and Local Anesthesia.

For short operations, local anesthesia by cocain or similar drugs, or by freezing should be preferred. Where these are not practicable, nitrous oxid or ethyl chlorid are used. Nitrous oxid is the safer. It is practically devoid of danger in short anesthesia.

For longer operations, the advantages of general anesthesia outweigh the disadvantages, as compared with spinal anesthesia. Chloroform is far more dangerous than ether or nitrous oxid; although with practical experience the acute dangers may be greatly reduced. The chief immediate risks are in the induction period. The danger of postanesthetic toxemia makes its employment in prolonged operations almost criminal.

Advantages of Chloroform.- - Its continuance in practice is due to its convenience for patient and operator: Its action is much more rapid than that of ether; it induces the most profound anesthesia and relaxation; it does not have the disagreeable taste, causes less suffocation, less excitement, and less postoperative nausea and vomiting. These disagreeable features of ether can be largely avoided by proper administration (preliminary gas, and open-drop method). At their worst, they are not enough to offset the dangers of chloroform. These should only be risked in emergencies; when prompt anesthesia is imperative; in brain surgery, atheroma and aneurism, where the lowered blood pressure is advantageous; and possibly on the battlefield to increase speed and lessen bulk. It has no advantage over ether in renal disease, and but little in respiratory diseases.

Specific Contraindications to Chloroform are: advanced heart disease, Graves' disease, hemorrhage, prolonged operations, and the conditions which favor acidosis.

Chloroform in Obstetrics.- It is quite generally believed that the use of chloroform in childbirth is relatively safe (J. Hill, I9I6). This is explained partly by the lesser apprehension of the patients, and partly by the light degree of anesthesia or rather analgesia, which ordinarily suffices. For deep anesthesia, it is probably quite as dangerous as in other patients, and presents the added disadvantage of delaying labor and thus favoring postpartum hemorrhage. The analgesic dosage does not cause serious delay. The anesthetics pass into the fetal circulation (Jung, I9r4); and prolonged anesthesia is doubtless as injurious to the child as to the mother.

Chloroform Analgesia.-A light chloroform anesthesia is used to dull the excitability of the central nervous system, in pain or insomnia; and to check convulsions, as those of strychnin, tetanus, or eclampsia. It is especially useful in the onset of these conditions: when the convulsions are fully developed it must be used cautiously, to avoid asphyxia.

For the production of the light anesthesia, chloroform is preferred to ether, since it can be more easily regulated, and its effects are more lasting.

A very deep degree of anesthesia is employed in reducing dislocations, to relax the tone of the opposing muscles.

Ether is probably safer.

Ether and nitrous oxid are practically equally safe in odinary cases. Ether has several advantages: it is more easily administered; it gives more complete relaxation, less asphyxia, and less rise of blood pressure; so that the condition of the respiration and circulation are more nearly normal. Its administration is simple and does not require as much experience; 
ether being perhaps the safest anesthetic for long operations. The disagreeable features of its administration can be practically avoided by skillful administration. The advantages of nitrous oxid appear especially in the cases in which ether is specifically contraindicated.

Contraindications to Ether.-The irritant action contraindicates its use (and also that of chloroform) in renal disease, and in acute respiratory disease (colds, bronchitis). In these, nitrous oxid is preferred, although ether is often employed without damage. Chronic respiratory disease is not a contraindication, unless there is actual dyspnea (emphysema).

Children are especially subject to ether irritation, resulting in respiratory embarrassment and choking. Ether favors bleeding, especially in parts in contact with the vapor; it is therefore not popular for operations of the mouth and larynx. In brain tumor or atheroma, ether and especially nitrous oxide may produce apoplexy; local or spinal anesthesia should be preferred. Advanced heart disease makes all anesthetics more dangerous. In this respect, ether and nitrous oxid are about equal; both should be preferred to chloroform. The danger is proportional to the myocardial degeneration, rather than to the valvular lesion: With fair compensation, ether can be used safely; indeed, the circulation may be rather improved during the anesthesia. In advanced fatty degeneration, it is better to employ local or spinal anesthesia. In hot climates, above $93^{\circ} \mathrm{F}$., ether is vaporized so rapidly that its use by the ordinary methods is difficult.

Nitrous Oxid.-This is the safest anesthetic for short operations. If the anesthesia lasts longer than an hour, it becomes somewhat more dangerous than ether even in the hands of experts; and much more dangerous if experience is limited (Bevan, I9I5). It is more pleasant to take; it causes no irritation, and but little excitement or gastric disturbance. It has little or no influence on immunity. There are practically no after-effects. According to Crile, it lessens the tendency to shock. It is especially preferred for short operations, and for excitable and neurotic patients. Old individuals are good subjects.

Disadvantages.-On the other hand, the muscular relaxation is less complete; it causes more or less asphyxia and abnormal rise of blood pressure; it is expensive; and it requires special apparatus and special experience for its successful administration. It is not appropriate for children under three years, since their thorax is too feeble to withstand the asphyxia. Obese or anemic patients are also unsuited to all anesthetic methods which interfere with respiration.

Statistics of Anesthetic Mortality.-No reliable statistics of sufficient extent can be available until the reporting of all anesthesias, good and bad, is compulsory. Even then, the ratio of "bad risks" will be indefinite. The existing statistics therefore give only a rough idea of the relative danger; but since they agree fairly well, they are worth considering. Modern American statistics are given by Gwathmey, I9I2. According to the various compilations, the acute mortality for chloroform is given as $r: r, 000$, I : $2,075,2,500,3,000,3,000,3,500,3,700,5,900$; a fair estimate would seem to be $I: 3,500$. This does not take account of the delayed deaths. For ether, the figures run I : $5,100,14,000,14,600,16,500,16,800,23,200$; the average would be perhaps I: 16,000 . For A.C.E. mixture, the ratio is $\mathrm{I}: 3,370$; for ethyl bromid, I : 5,400; for ethyl chlorid, I : $3,000,7,000$; for nitron oxid in short operations (dentistry), less than $I: 5,000,000$.

Anesthetic Mixtures.-The attempts to blend the actions of anesthetics by mixing them have been unsuccessful, mainly because the ingredients do not volatilize with equal rapidity (Snow). The composition of the inspired anesthetic is therefore absolutely uncertain. As a rule, the concentration of the chloroform increases during the administration. These 
mixtures are therefore at least as dangerous as chloroform, and should be condemned (Kochmann, I903).

The principal mixtures of this type are the English A.C.E. mixture, introduced by Harley $\left(A_{1} ; \mathrm{C}_{2} ; \mathrm{E}_{3}\right) ;{ }^{1}$ Billroth mixture $\left(\mathrm{Ar}_{\mathrm{r}} \mathrm{C}_{3} ; \mathrm{E}_{1}\right) ; C$. E. mixture of Ilewitt $\left(\mathrm{C}_{2} ; \mathrm{E}_{3}\right) ;$ Schlcich mixture (benzine $\mathrm{I}: \mathrm{C}_{3}: \mathrm{E}_{10}$ ). Other mixtures containing ethyl chlorid or bromid, etc., have also found adrocates. The alcohol and benzine of these mixtures were intended mainly to raise or lower the boiling-point, but benzine is not indifferent. The statement of Schaefer and Scharlieb, that the addition of ro per cent. of alcohol to chloroform practically prevents the fall of blood pressure, needs confirmation. However, there seems to be some combination between ether and chloroform; for on mixing equal volumes $\left(25\right.$ c.c.) the temperature is said to rise $14^{\circ} \mathrm{C}$. (Marcelet, I9I2).

Synergism of Ether and Chloroform.-Honigmann, I899, and Kionka and Kroenig, 1905, believed that there is considerable potentiation when the vapors of ether and chloroform are used together in definite proportions; while Madelung, I910, and Buergi, I9ro, found only simple summation (Fuehner, I9I3, also for mixtures of ether with methyl alcohol or ethyl alcohol). The difference, according to Damkoehler, 1913, is due partly to unreliable methods, partly to the depth of anesthesia. He finds (on rabbits) that there is only simple summation for light anesthesia and for fatal concentrations; but a slight potentiation for deep anesthesia. This is greatest when the ratio of the vapors (by weight) is $\mathrm{C}: \mathrm{E}: \mathrm{I}: \mathrm{I}: 6$ or 7 , and disappears with $\mathrm{I}: 2$ or $\mathrm{I}: 8$. The safety zone is widened a trifle by $1: 6$.

Combination with Aliphatic Hypnotics. - This gives rather complex results (Barten, I9I3; ordinary hypnotic doses, combined with inhalation, rabbits): with ether, chloral and veronal do not materially lower the vapor concentration required for light anesthesia; but reduce that for deep anesthesia by 30 per cent., and paraldehyd even by 50 per cent. The zone of safety is normal. With chloroform, chloral and veronal also reduce the deep-anesthesia concentration, but still more the fatal concentration; so that the danger is greater. Paraldehyd acts as with ether, so that its use would be advantageous.

Mansfeld and Hamburger, 1915, believe that ether actually potentiates with chloral or morphin, by favoring their distribution into the nervous system.

Sequence Anesthesia.-A more rational method of combining the advantages of anesthetics is to employ them in sequence; particularly to induce anesthesia by the pleasant and promptly acting nitrous-oxidoxygen inhalation, and to continue it with ether. This probably presents the nearest approach to an ideal anesthesia.

Chloroform was formerly used for the preliminary anesthetic, but presents considerable danger. Ethyl chlorid may be employed successfully. Nitrous oxid without oxygen is not so satisfactory, because the ether must be pushed rapidly, to avoid asphyxia or return of consciousness, and the concentrated vapor is apt to cause sudden laryngeal spasm (Cunningham and Anderson, 1908). Hewitt endorses the sequence of C. E. mixture ( to two to six minutes), with the gradual addition of ether, by the open method. Changing from full ether anesthesia to chloroform is especially dangerous (Hewitt).

Morphin, Scopolamin, Atropin and Strychnin Preliminary to Anesthesia.-One or several of these drugs are often injected hypodermically, half an hour before starting the operation. They lessen the anxiety of the patient and avoid some of the dangers of anesthesia. The doses as given below may be combined.

Morphin (Io to I $5 \mathrm{mg}$., $1 / 6$ to $1 / 4 \mathrm{gr}$.) is most effective in lessening apprehension, excitement and struggling. Labbé and Guyon, 1872, were the first to use it clinically. It makes anesthesia prompter and smoother. It reduces materially the quantity of anesthetics, and thereby the gastric, pulmonary and toxic after-effects. The patient does not awaken so early. Since the morphin narcosis involves an entirely different mechanism, the side effects of both are probably relatively smaller. On the other hand,

\footnotetext{
2 The proportions of all the mixtures are by volume. $A$, stands for alcohol; $C$, for chloroform; $E$, for ether.
} 
morphin depresses the excitability of the respiratory center, and the miosis interferes with the pupil danger signal; morphin is contraindicated in pulmonary disease, in semicomatose patients, or when the operation would involve bleeding into the air passages. On the whole, the marked advantages appear to outweigh the disadvantages, although many anesthetists condemn its routine use.

Morphin reduces the concentration of ether or chloroform vapor required for light anesthesia, but not that for deep anesthesia or death. The margin of safety is therefore widened considerably - 60 per cent. for chloroform, 50 per cent. for ether (Stange, I9 I 3 ; Kochmann, I914). However, the respiration must be carefully watched with chloroform, for this also shows dangerous potentiation (Wolff, I9I3; W. Straub, I9I3).

Atropin ( $0.3 \mathrm{mg} ., 1 / 200 \mathrm{gr}$.) stimulates respiration and is therefore of ten added as a corrective to the morphin. It also diminishes the reflex vagus stoppage of the heart by chloroform and ether salivation and bronchorrhea, thereby lessening the occurrence of ether pneumonia. The atropin mydriasis and tachycardia interfere with the use of the pupil and pulse as danger signals. On the whole, its use is advisable; but the above dose should not be exceeded on account of occasional idiosyncrasies.

Scopolamin ( $0.5 \mathrm{mg} ., 1 / 120 \mathrm{gr}$.) is often substituted for the atropin. It has the same peripheral actions on the pupil and glands, but greatly intensifies the narcotic effects of morphin. On the other hand, it disposes to pronounced vasodilation, so that the pressure falls as deeply in ether anesthesia as with chloroform (Mayor, I908). Moreover, it sometimes intensifies the respiratory depression of morphin to a very dangerous degree. This danger is so serious that its use is not advised. Bevan, I9I 5, who opposes it strongly, believes that it also increases the percentage of vomiting and of bladder paresis.

The combined action of morphin or scopolamin with the hydrocarbon narcotics is far more than simple addition (Knell, I907; Hauckold, I910; Lindemann, I910; Hammerschmidt, r910). Madelung, 19 10, and Rappaport, rgir, find that one-half to twothirds of the ordinary concentration of anesthetics suffices. Buergi, I9I I, proposed a receptor theory in explanation; but this is not generally accepted. Fuehner, rgrr, found that the lipolytic narcotics change the lipoid solubility of morphin, and thus presumably favor its penetration and action.

Scopolamin alone does not reduce the concentration of chloroform or ether required for light anesthesia, but lessens somewhat that for deep anesthesia. Scopolamin and morphin together resemble morphin; except that they do not increase the zone of safety. The anesthesia is deeper, however; and this may be practically important (Ludewig, r9r3; Jochmann, r9r4).

Bromids.-These give practically only simple summation with morphin or the aliphatic narcotics (Klammer, 1913).

Strychnin (I to $2 \mathrm{mg} ., 1 / 30$ to $1 / 60 \mathrm{gr}$.) has been suggested when respiratory failure is feared (Fothergill). Its usefulness is doubtful. reflex.

Cocainization of the nasal mucosa has been employed to avoid the trigeminal vagus

\section{PREPARATION OF PATIENT FOR ANESTHESIA}

The patient should receive a cathartic (castor oil) twenty-four or thirty-six hours before the operation, and an enema on the morning. An interval of at least five hours should have elapsed since the last meal, which should not have been too heavy. These precautions are to prevent the discharge of the contents of the alimentary canal during the anesthesia. He should be carefully examined for cardiac, renal, and pulmonary disease. He should then be placed in such a position as to interfere to the smallest possible extent with respiration; the head preferably low and turned to the side. The clothing should be loosened and all foreign bodies-false teeth, etc.- removed from the mouth.

To prevent the local action of the anesthetic on the face, it is well to anoint the mouth and nose with petrolatum. Care should be taken to have the patient close his eyes. 


\section{THE ADMINISTRATION OF ANESTHETICS}

Inhalation is superior to all other methods in the convenience of its application, and in the facility with which the depth of anesthesia can be regulated to suit the needs of the moment. It must be remembered that this depends solely on the concentration of the anesthetic vapor. This could be regulated either by using exactly dosed mixtures of anesthetic and air, or more simply, and on the whole more efficiently, by watching closely the condition of the patient. The response of the respiration, pupils and other reflexes to changes in the concentration of the vapor must be studied. The concentration can be increased either by pouring the anesthetic more rapidly; by applying the mask closer to the face; or by limiting the supply of air. With either anesthetic the mask should at first be kept fairly away from the mouth, until some narcotic effect is obtained, to lessen the feeling of choking from the concentrated vapor. The patient should be encouraged to breathe quietly and regularly. Counting is a good expedient for this purpose. If the patient holds his breath, struggles or coughs during the induction of the anesthesia, the concentration should be reduced, never increased. Rapid and deep breathing lessens the concentration of the anesthetic by dilution and cooling. Slow and shallow breathing gives more concentrated vapor. Warming the anesthetic liquid also increases its evaporation and concentration (Snow).

Management during Recovery from Anesthesia.-The patient should not be left until he is fairly conscious, since vomiting, inspiration of mucus, etc., might cause asphyxia. No nourishment should be given for at least four hours. If vomiting is persistent, a tumbler of hot water, with a teaspoon of bicarbonate may be given. Recovery is usually more speedy and satisfactory if the patient is placed on his side.

Special apparatus for supplying measured concentrations can be constructed (Dreser, r 896; Geppert, I899; Kionka, I 895 and I899; Kochmann, I9 3 ; Jackson, I91 5, etc.), but they are complicated and cumbersome. (The simplest form is that of $A$. V. Harcourt, Suppl. Brit. Med. Jour., r9o3, p. I43.) Another objection to standardized mixtures is the slowness with which they produce anesthesia. It is certain that much stronger mixtures may be borne for a short time, and are quite safe in starting the anesthetic.

Regulation of Anesthesia.-Most important is the fact that no mechanical device can replace the sense of responsibility, the constant watchfulness, and the quick reasoning of the experienced anesthetist. Anesthetization is not a physical experiment where the factors can all be foreseen, but the condition of the patient is apt to vary from moment to moment, and must be taken into account. The state of the respiration must be carefully watched: if the patient holds his breath, the mask must be held farther away, since the next respiration will be an especially deep one. When the respiration becomes slow and shallow, this signifies that a sufficient amount has been taken, and that the quantity may be lessened. The aim is to give no more than is necessary to just keep the patient anesthetized. On the other hand, care must be taken to keep him thoroughly under the influence, for excitement, struggling, stoppage of respiration, shock and overdosage are much more common under partial or uneven anesthesia. Since the respiration and circulation react one upon the other, so that no change could occur in the latter without being noticed in the former, and since most accidents occur from stoppage of the respiration, it may be sufficient to watch this alone, as is advised by some. But as it is of the highest importance to discover beginning failure of the one or the other at the earliest possible moment, the anesthetist can not be considered as doing his duty unless he carefully observes both. The argument that watching the circulation distracts the attention from the respiration should not hold: the anesthetist should be able to keep his attention fixed upon both.

Closed and Open Methods of Administration.-The anesthetics may be administered with (open) or without (closed) free access of air. In the closed methods, the patient rebreathes more or less from a permanent mixture of vapor through a fairly air-tight mask or cone. In the open 
methods, he breathes air freely through gauze (the usual chloroform mask), on which the anesthetic is poured. The closed methods are more rapid and economize the anesthetic; but from other standpoints they are rather objectionable. The open methods avoid the asphyxial effects, and are therefore pleasanter, safer, and less subject to after-effects (mucorrhea, vomiting, etc.). The apparatus is also simpler and more easily sterilized. The open methods deserve preference, except for very short operations, or when rapid anesthesia is important. The closed method, however,

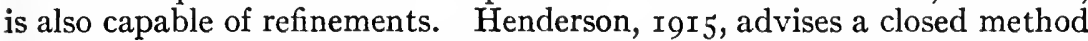
with ether vapor, claiming that this is scarcely more unpleasant than nitrous oxid, and produces less nausea, vomiting, gas pains, etc., than other methods. The skin should be kept pink, neither pale (acapnia) nor dusky (asphyxia).

Administration of Ether by the "Cone" Method.-A special mask is used, which admits only a limited amount of air. About a tablespoonful is poured in the mask, and repeated as needed. The fact that the required concentration of ether is much greater than with chloroform, leads to the temptation not to admit sufficient air. This must be carefully guarded against, or asphyxial symptoms may result simply from a deficient supply of oxygen. Concentrations of ether above 7 per cent. irritate the mucosa so strongly that in imperfect anesthesia, they cause reflex coughing, closure of the glottis and suffocation (Dreser, I893).

To obviate the asphyxia it has been advised to administer oxygen with the ether (Guy, Goodall and Reid, rorr); but this is superfluous, especially if the drop method is used.

Ether by the "Drop" ("Open" or "Perhalation") Method.-This avoids to a very great extent the irritation, suffocation and excitement of ordinary ether anesthesia. This advantage more than compensates for the disadvantages, the relative slowness, waste of ether, saturation of the room, and refrigeration of the inspired air. In this method, the ether is dropped rapidly (about 150 drops per minute), over the entire surface of an open (chloroform) inhaler, covered with some eight layers of gauze, and held an inch from the face. If the mask is closely applied, the vapor concentration may rise to 9.3 to ro.8 per cent.; never above I2 per cent. (The method was originated by L. H. Prince, I 895.)

Danger of Open Lights. - The vapor of ether being highly inflammable and explosive, this anesthetic should not be used with the actual cautery or by an open light. The flame may leap over considerable distance, especially if there are drafts. If the use of an open light is unavoidable, it should be placed high above the patient, since ether vapor is heavier than air.

Chloroform is not explosive; but if used with an open light, it is partly oxidized into phosgen gas ( $\mathrm{COCl}_{2}$, carbonyl chlorid), chlorin and hydrochloric acid, which cause considerable irritation-cough, dyspnea, collapse (Langenbeck, 1888). Fatality to the spectators has been reported (see Gerlinger, I902).

The decomposition of chloroform may be illustrated by the equations:

$$
\begin{gathered}
{ }_{4} \mathrm{CHCl}_{3}+{ }_{3} \mathrm{O}_{2}={ }_{4} \mathrm{COCl}_{2}+{ }_{2} \mathrm{H}_{2} \mathrm{O}+{ }_{2} \mathrm{Cl} \\
\mathrm{COCl}_{2}+\mathrm{H}_{2} \mathrm{O}=\mathrm{CO}_{2}+{ }_{2} \mathrm{HCl} .
\end{gathered}
$$

The inhalation of the burned chloroform vapor by rabbits produces dyspnea, pulmonary edema, and sometimes death (Stobwasser). The toxicity of phosgen was shown 
by R. Mueller, I910: The inhalation by rats of a 0.025 per cent. volume vapor caused death in twenty minutes; half this concentration was severely toxic. Anatomic changes were found in the lungs, kidneys, and heart.

Administration of Chloroform.-This is dropped on a few layers of gauze, supported by a frame, and held some little distance from the face. The rate should under no circumstances exceed 60 per minute, and usually should not be over $\mathrm{r}$ 2. After the anesthetic stage has been induced 6 drops per minute will usually suffice. This will be found better than to remove the mask altogether and reapply it with a larger dose when the patient shows signs of recovery.

Self-inhalation of Chloroform.- It is often necessary to keep patients lightly under the influence of an anesthetic when no skilled assistant is available, as in obstetric practice. Here a method suggested by Brunton is useful:

The inside of a tumbler is covered with blotting-paper. A few drops of chloroform are poured on, and this is given to the patient, with directions to hold it an inch from her mouth and inhale. This works automatically, for as the patient becomes narcotized she naturally allows her hand to drop, and so removes the tumbler; and as soon as she becomes conscious and sensitive to pain, she will replace it.

The quantity of anesthetic which is required for an ordinary operation is very variable. Some 40 c.c. of chloroform, or I 50 c.c. of ether, by the ordinary method, or $25^{\circ}$ c.c. by the drop method, are about the smallest quantities for an hour's anesthesia.

"One-minute Anesthesia." - Short operations can be done under the analgesia of the preanesthetic stage: A teaspoonful of ether is poured into the cone, and the patient directed to count and take deep inspirations. The operation is done as soon as the counting becomes irregular.

Other Methods of Administration.-The anesthetic may also be administered by other methods, but these demand special experience, and generally special apparatus, and therefore have a more limited field.

In operations about the face, the anesthetic may be inhaled through a nasal catheter; in operations on the thorax, it may be administered by tracheal insufflation.

Intratracheal Insufflation.-The insufflation of air passed over the anesthetic has been elaborated experimentally by Githens and Meltzer, I9I3. It eliminates respiratory paralysis as a fatal factor. Ether is especially safe: Excessive doses first stop the spontaneous respiration, but this is immaterial since the insufflation constitutes an efficient artificial respiration. The blood pressure declines very slowly, and recovery is possible during an hour after the respiration is arrested, by simply stopping the ether. With chloroform, the respiration and circulation fail together, so that there is no reliable danger sign, and the zone of safety is much narrower.

Intrapharyngeal Insuffation.- - In this, the ether is driven by bellows through a nasal catheter; usually after anesthesia has been induced by the open method. It is about as simple and safe as the open method (Bevan, I9r5).

Rectal insufflation of the warmed ether vapor is especially useful in operations about the head; it also avoids excitement, struggling, and gastric disturbance. It acts promptly (within six to fifteen minutes) and can be maintained indefinitely. However, is is uncomfortable; the dosage is more difficult; it is apt to be followed by severe, ometimes fatal, irritation of the colon, with gas distention, collicky pains and bloody diarrhea (Cunningham, Igro; Morel, I910; Bevan, I915). The fatality is fairly high.

Intravenous administration of saline solutions of the anesthetics (ether, 5 per cent., Burckhardt, I9I0; 7.5 per cent., Rood, I9II; chloroform, 0.6 per cent., Giani, I910), produce anesthesia; but a priori, one would consider the method both unsatisfactory and dangerous; and this seems to agree with the practical experience (Tyrode, 1910).

Solubility of Ether in Normal Saline. - This varies with the temperature as follows (Bennett, I912):

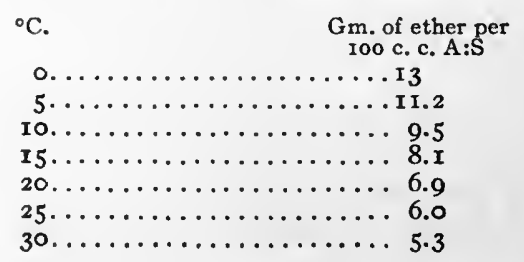


Intramuscular injection of undiluted ether has also been tried. The method is uncertain, painful, and dangerous (see J.A.M.A., I91 2, vol. 59, p. 288, Guibé, 1912).

Gastric administration of an alcoholic solution of chloroform,is used in animal experimentation (see index, "Gréhant Anesthesia"), but is too uncertain and dangerous for man.

\section{CAUSES OF DEATH UNDER ANESTHESIA}

These must be thoroughly understood in order that accidents may be avoided. They may therefore be recapitulated. They differ according to the anesthetic, the stage of anesthesia, the character of the operations, diseases of the patient, and other conditions. On this account, the clinical accidents are rather different from the usual laboratory experiments.

Death not Always Due to Anesthetic.-It is incorrect to attribute every death upon the operating table to the effects of the anesthetic; for patients died upon the table when anesthetics were yet unknown.

One such case was of considerable importance in the history of anesthesia. When Simpson was about to try chloroform on a patient for the first time, the orderly who was carrying the bottle fell and spilled the chloroform. No other being obtainable Simpson proceeded to the operation, which was for hernia, without anesthesia. The patient died with the first cut. Had the chloroform been given in this case and the same accident had happened, its introduction into practice would have suffered a long delay. Other similar cases are not unknown: A patient was to be operated and demanded chloroform. His condition, however, was so low that the surgeon feared to grant his wish, and to calm him held a cloth without chloroform before his face. Scarcely had the patient made four inhalations - of air - when he was dead. In preanesthetic days, the French surgeon Desault drew his fingernail over the perineum of a patient to mark the line of incision, when the patient suddenly gave a cry and was dead. And many similar cases of sudden death from the violent mental impression might be mentioned, besides deaths undoubtedly due to traumatic shock. Even at the present day patients often exhibit "psychic shock" when operated under local anesthesia, so that it is a general practice to precede this by morphin (Strassmann, 1898 ).

It is true that the public nowadays has largely lost the great fear for operations and that anesthetics lessen the danger of traumatic shock. But neither is excluded, and there is no doubt that many deaths attributed to anesthetics have their cause elsewhere.

State of Patient.-Healthy, vigorous patients are not the safest risks in anesthesia, but rather the relatively feeble and phlegmatic: These are less subject to excitement, struggling and muscular obstruction of respiration, and they require less anesthetic. Nervous, apprehensive patients and alcoholic subjects are always difficult to anesthetize. "Status lymphaticus" and Graves' disease predispose to acute circulatory failure ; from trivial causes, fright, excitement, etc. Respiratory catarrh tends to obstruction of respiration by mucus, especially if ether is used. Acute pulmonary diseases dispose to asphyxia; advanced heart disease to circulatory failure.

Death During the Induction of Anesthesia.-This is common with chloroform, rarer with ether. The usual sequence of events is sudden suspension of the respiration from acapnia or muscular spasm; the asphyxia leads to cardiac dilation, vagus stimulation, and finally asphyxial failure of the heart. The failure is especially rapid with chloroform, because this adds the direct cardiac action of the drug. In light chloroform narcosis, the cardiac muscle is hyperexcitable; and any excitement is apt to induce ventricular fibrillation and death (G. Levy, 1913; this excitability disappears in deep anesthesia). The respiration may resume temporarily after the heart has stopped, but the heart itself can not usually be revived. Failure of the.circulation therefore commonly precedes the final arrest of respiration and is the real cause of death.

In the case of ether, similar fatalities are rarer, but may occur early or somewhat later-in either case due to faulty administration during incomplete anesthesia. The error may have been committed an hour before actual death: In light ether anesthesia, the excitability of the respiratory center is increased. If this state is prolonged by excessively slow, 
incomplete or intermittent anesthesia ( $f . i$. , in throat operations), acapnia results. When the anesthesia deepens and the center becomes depressed, the acapnia (deficiency of $\mathrm{CO}_{2}$ ) leads to arrest of respiration and injury to the heart (Henderson, I9II).

Stoppage of respiration by ether is less dangerous, because the more vigorous heart generally beats until the respiration has resumed. Experimentally, the heart may also be stopped reflexly by vagus stimulation, but clinically this seems to be exceptional. In any case, the phenomena occur with little warning. The danger signs are sudden dilation of the pupils, pallor, and change of facial expression.

Too high concentration of the vapor is the immediate cause of these actions. Fright, excitement, struggling, irregular respiration and idiosyncrasy are contributing factors. Crying in children is especially dangerous. These accidents are avoided by beginning the administration with very dilute vapor, and increasing the concentration gradually. Morphin helps to prevent acapnia and fear. Atropin eliminates the vagus reflex.

Death in deep anesthesia is usually due to paralysis of respiration, generally with more or less involvement of the circulation. With ether, the circulation is usually fairly good when the respiration stops. With chloroform, there is a progressive fall of bood pressure, then failure of the respiration, and finally cessation of the heart; but the pulse is generally imperceptible before the respiration stops. These late accidents are caused by maintaining the concentration of the vapor too high; and since the paralytic phenomena develop gradually, with ample warning, these deaths are generally due to inexperience of the anesthetist. The danger signals are: increasingly shallow or irregular respiration; very slow or very rapid pulse; cyanosis; dilation of pupil. These accidents may be avoided by carefully watching for these signs, by maintaining the anesthesia evenly (see "Report of Anesthesia Committee," J.A.M.A., I9 2, vol. 58, p. I908), and no deeper than complete muscular relaxation and absence of the sensitive reflexes.

The autopsy in acute chloroform or ether deaths shows nothing beyond the ordinary phenomena of death by asphyxia - heart distended, veins congested, etc.

\section{POISONING BY SWALLOWING CHLOROFORM}

This usually produces immediate vomiting and sometimes defecation, followed by narcosis (Schoenhof, I914). The acutely fatal dose is not known; an ounce or more would be dangerous. Recovery may be succeeded by fatal gastritis or icterus and the other phenomena of late chloroform poisoning. The treatment would be as for inhalation. Gastric lavage with oil has been advised (Wirth, I909).

\section{TREATMENT OF ACCIDENTS}

Minor Accidents.-The tongue may fall back and interfere with respiration, as denoted by noisy breathing. If this occurs, "place the index finger of each hand upon the corresponding cornua of the hyoid bone, while the middle fingers rest upon the angle of the jaw, and then press forward and upward, the same force serving to extend the head upon the neck; if this fails to open the glottis, by means of a tenaculum, thrust far back into the base of the tongue, draw it forward" (H. C. Wood).

If much mucus accumulates, it should be removed with a cloth (Mayor suggests the dental saliva siphon).

Serious Accidents. - These should be treated with the first warning of danger: The anesthetic should be stopped at once. The head of the patient should be lowered to give the medullary centers and heart the full 
benefit of any remaining circulation. A few rhythmic compressions of the epigastrium may be tried, but if these do not succeed quickly, artificial respiration by any of the methods should be begun at once. This prevents asphyxia and eliminates the poison. The cardiac region should also be compressed strongly at the rate of 40 times per minute, since this aids the action of the heart and supplies a mechanical stimulus. A liter of saline solution, and I c.c. of I : I,Ooo solution of epinephrin may be at the same time injected into the cardiac end of an artery. The combination of these measures often succeeds when the heart has actually stopped beating (Crile and Dolley). The chances of success are proportional to the promptness with which they are applied. Gunn and Martin, 1915, recommend the combination of intrapericardial injection of epinephrin with cardiac massage. This is generally successful even when the heart is arrested and either measure alone would fail.

Of drugs, strychnin, or caffein, by virtue of the stimulation of the respiratory and vasomotor centers, is useful if given in time-i.e., while it may still be absorbed. Digitalis is too slow in its action. Oxygen is of uncertain advantage. Reflex stimulation by the inhalation of ammonia is sometimes remarkably effective; as is sciatic stimulation. Hypodermic injections of ether which have been used in man, might be supposed to act in the same manner. But clinical observers condemn their use, and experiments on animals show that the stimulus is too weak to produce much effect. On the other hand, it would tend to deepen the narcosis. The administration of brandy or nitroglycerin is similarly useless, though not quite as harmful. Faradization of the phrenic nerve or of the heart has also been advocated, but appears to be prompted more by the desire to do something than by any rational view of the object to be accomplished. Stimulation of the phrenic, to be sure, causes contraction of the diaphragm and inspiration, and if done intermittently, would take the place of artificial respiration. But it possesses no advantage over the latter, and besides the fact that the time required to adjust the apparatus might be much better utilized, there is apt to be stimulation of the vagus-a most undesirable feature.

With regard to faradization of the heart, there is no more effectual way known of killing this organ than electric stimulation (by the production of delirium cordis), and the only reason why more harm has not been done by this senseless procedure is that the electricity, as it is ordinarily applied, does not penetrate through the chest walls.

Epinephrin in Light Chloroform Narcosis.-While epinephrin may be very valuable in deep narcosis, it is apt to cause dangerous arythmia in light chloroform anesthesia. This may be prevented by atropin, or section of the vagi; but if the arlythmia has already started, atropin only adds to the danger (Nobel and Rothberger, 1914).

\section{IDIOSYNCRASY}

This doubtless occurs, in analogy to alcohol; however, the actual concentration of ether required to induce and maintain anesthesia is about the same, whether the patient is refractory or otherwise (Boothby, I9I4). The great practical difference in the reaction of patients consist mainly in the manner of "taking" the anesthetic. They are therefore most conspicuous in the induction of anesthesia, which is for instance peculiarly difficult.in alcoholic patients. (Boothby, I9I3, attributes their resistance to deep breathing, blowing the ether off the mask.) Morphinists are said to be relatively insusceptible. Patients with Graves' disease may react badly. A patient may tolerate one anesthetic better than another.

After the anesthesia is established, there is but little evidence of "peculiar" reactions, except such as may be foreseen in heart disease. Fatal accidents should therefore not be lightly ascribed to idiosyncrasy. The postanesthetic toxemias, however, are undoubtedly influenced by the disposition of the patient.

Mansfeld (I905) claims that starved animals are much more susceptible to chloral, paraldehyd, and morphin, but not to alcohol, amylen hydrate, or urethane. 


\section{IMPURITIES}

The impurities which may occur in ether and chloroform, mainly by decomposition, are rather unimportant. They contribute to the local irritation, but probably not to the dangerous effects. Chloroform fatalities seem to be as frequent with the "pure" chloroform as with that not specially treated.

Henderson, I9 4 , considers poor ether as more excitant, disposing to acapnia.

The impurities of ether are due to tne presence of moisture, which leads to complex oxidations. Among the products are hydrogen peroxid, and most commonly the irritant aldehyd. Bruns, I895, concluded from clinical observation that the ether impurities are mainly responsible for the bronchitis (R. B. Smith, I9I3). Ether should therefore be anhydrous, and stored in sealed tin containers. Partly filled glass bottles are especially detrimental (Baskerville, IgI I, IgI 2).

The impurities of chloroform are also due to oxidation, accelerated by light and acids. The products vary with the conditions. The most constant are the irritant phosgen (carbonyl chlorid) and hydrochloric acid. Chloroform should be preserved in sealed, dark glass bottles and should contain $x$ per cent. of alcohol, which acts as a reducing substance.

\section{ORIGIN OF CHLOROFORM}

It has been claimed that the effects of chloroform differ somewhat according to whether it is prepared from acetone or alcohol. Wade showed that the acetone product is purer; the alcohol product contains a little ethyl chlorid, which might increase the anesthetic effects (Regnault, I909). Hewitt, however, could discover no difference in the immediate or after-effects. Hale, I9I 5, also could detect no constant difference in the fatal concentration for mice and fish, for chloroform samples of different age and origin.

\section{INDUCTION OF ANESTHESIA DURING SLEEP}

This question is of some medico-legal importance. Cases are reported, but it must be difficult, and consequently rare. False accusations of criminal assault are often based on the mental confusion of the patient while in the narcotic stage.

\section{RELATED ANESTHETICS}

The production of narcosis is a universal action of all members of the aliphatic series which are fat-solvents, and which are absorbable into the tissues. Accordingly, a large number of the more volatile "lipophilic" hydrocarbons may be used to produce anesthesia; whilst the less volatile are employed as "hypnotics," to produce sleep.

Ethyl Chlorid.-This is the only one of the related anesthetics which is practically useful. On account of its great volatility, the anesthesia appears and disappears very promptly, and is therefore especially adapted for minor surgery. It has the same dangers of chloroform: Relatively small doses may cause sudden stoppage of the heart, though less frequently than chloroform. Larger doses paralyze the heart-muscle directly; and are even more dangerous than chloroform, because of the extreme suddenness and rather indefinite signals (E. D. Brown and Large, I905; Embley, rgo6; Maas, r907; Wood, r910; Seifert, Nebenwirk., r915, p. 36).

Its main advantages consist in the rapidity with which anesthesia is induced; in the consequent absence of struggling, excitement, and unpleasant sensation; in the very prompt and complete recovery. Its administration is often followed by headache, nausea, vomiting, and sometimes collapse. The muscular relaxation and abolition of reflexes are incomplete, and a little experience is required to recognize the proper time for operating. It has occasionally failed in alcoholic patients.

It may be administered by spraying it on a chloroform mask. This method is so wasteful that a special mask is to be preferred, allowing its administration in gas form, and restricting the admission of air. It has also been used by pouring a small amount into a closed mask. These methods utilize a partial asphyxia; they consume from 5 to Io c.c. for short operations (Large, 1906). 
Administering ethyl chlorid by his special closed-circulation apparatus, D. E. Jackson, I916, finds its effects to be intermediate between nitrous oxid and ether.

The concentration of ethyl chlorid in the blood and tissues during narcosis has been investigated by Camus and Nicloux, 1908; and E. Frey, I9r2.

The main use of ethyl chlorid is for local anesthesia, freezing the tissues by a fine spray of the liquid; the tube being held about 3 feet away. The impossibility of dissecting the frozen tissues restricts its use to simple incisions (opening boils); the analgesia is imperfect, and the thawing rather painful.

Ethyl Bromid and Ethyl Iodid.-These resemble ethyl chlorid closely, differing only quantitatively, according to their volatility (Weber, 1906). The more common ethyl bromid is used for short anesthesia or rather analgesia. It must not be pushed to the disappearance of reflexes, since the respiration is paralyzed about the same time. The zone of safety is therefore very narrow. Pain is abolished before consciousness, and the operation must be made before consciousness is entirely gone. The proper time for operating is somewhat difficult to choose, and the drug is very dangerous in unskilled hands. With experience, the danger seems to be slight. The proper amount (for adults, 8 c.c.; for children not more than I c.c. per year) is poured on a folded towel or cone, and administered to the exclusion of air, until the proper degree of anesthesia is reached. It is then removed. It is only suitable for short operations (tonsillotomy, etc.). The administration requires twenty to forty seconds; the anesthesia lasts about two minutes. Recovery occurs at once.

After a bottle has been opened, the contents can not be used again, as they deteriorate rapidly. The drug must not be confused with Ethylen bromid $\left(\mathrm{C}_{2} \mathrm{H}_{4} \mathrm{Cl}_{2}\right)$, which is very poisonous.

Bromoform $\left(\mathrm{CHBr}_{3}\right)$ is not sufficiently volatile to be of use as an anesthetic. It is employed as an antispasmodic in whooping cough, but is of very doubtful value ( 0.2 c.c. -3 minims, dropped on sugar. Practically insoluble in water, soluble in alcohol).

Carbon tetrachlorid ("Tetra," $\mathrm{CCl}_{4}$ ) is used as a non-explosive fat-solvent, and for drying the hair in shampooing. The vapor has given rise to serious poisoning (J.A.M.A., r909, vol., 53, p. 726). Waller and Velay, I909, state that it is more poisonous than chloroform. Lehmann, igI 1 (who also investigated other aliphatic chlorin derivatives) found the vapor narcotic, but not excessively toxic. Industrial poisoning in rubber factories is described by Hamilton, I 915 .

Carbon Disulphid $\left(\mathrm{CS}_{2}\right)$.- This very volatile fluid has a toxicologic importance, from its extensive use in the arts, particularly in the rubber industry (Stadelmann, I896; A. Hamilton, I915).

Acute Poisoning is quite rare and produces effects similar to those of chloroform.

Chronic Poisoning is the more common. The symptoms may not appear for several weeks and then develop quite slowly so that several stages may be distinguished. The effects have a pathological basis in irritative changes throughout the body. The symptoms begin with disturbed sensation (headache, formication, vertigo, etc.) and gastro-intestinal catarrh. In the second stage there is irritability, excitement, hysterical manifestations, etc., and signs of marasmus. The third stage shows central paralytic, or epileptic features, and, peripheral neurites. Ataxia has been reported. The marasmus is pronounced. Degenerative histologic changes are found in blood (hemolysis, leucocytosis, anemia; no methemoglobin formation), nerve cells and dendrites, liver (vacuolization), kidneys, and lungs. Death does not occur until very late. (A more extensive description is given on page $46 \mathrm{r}$ of the first edition.)

The fluid or vapor causes conjunctivitis and other local inflammatory changes in the eye. Continuous exposure, even to small quantities, may produce a toxic blindness, similar to that produced by tobacco (Haas and Heim, I9II).

\section{PREPARATIONS-ANESTHETICS}

*Chloroformum (Chlorof.), U.S.P., B.P.; Chloroform.-Contains at least 99 per cent. $\mathrm{CHCl}_{3}$; and 0.6 to $\mathrm{r}$ per cent. of alcohol. Heavy, colorless volatile liquid; boils at 60 to $61^{\circ} \mathrm{C}$. Characteristic odor, sweet hot taste. Not inflammable. Slightly sol. in water (I : 2ro); freely miscible with alc., eth., etc. Dose, 0.3 c.c., 5 minims, U.S.P.; 0.06 to 0.3 c.c., I to 5 minims, B.P. Maximal dose, 0.5 c.c., 8 minims.

*Aq. Chlorof., U.S.P., B.P.-A saturated watery solution; 0.25 per cent., B.P. Used as hypnotic and flavor. Dose, 15 c.c., 4 drams, U.S.P.

*Linimentum Chloroformi (Lin. Chlorof.), U.S.P.-30 per cent. of Chloroform in soap liniment. 
${ }^{*}$ Lin. Chlorof., B.P.--Equal parts of chloroform and camphor liniment.

*Sp. Chlorof., U.S.P., B.P.-6 per cent., U.S.P.; 5 per cent., B.P. Dose, 2 c.c., 30 minims, U.S.P.; 0.3 to I. 2 c.c., 5 to 20 minims, repeated; single, 2 to 2.5 c.c., 30 to 40 minims, B.P.

(Tr. Chlorof. ct Morph. Co., B.P.-See Index.)

Bromoformum (Bromof.), U.S.P., $\mathrm{CHBr}_{3}$ - A heavy liquid, resembling chloroform. Slightly sol. in water; miscible with alc., chlorof., eth., and fxed or volatile oils. Slightly volatile, not inflammable. Dose, 0.2 c.c., 3 minims, U.S.P. (maximal, 0.5 c.c., 8 minims), on sugar or dissolved in alcohol and glycerin. Used in pertussis, but probably without value. Side actions, Seifert, Nebenwirk., 19r5, p. I16.

Carbon Disulphidum, B.P., $\mathrm{CS}_{2}$.

* Ether, U.S.P., B.P.; Ether Pur., B.P.; Ether (Sulphuric Ether).-Contains about 96 per cent. of ethyl oxid, $\left(\mathrm{C}_{2} \mathrm{H}_{5}\right)_{2} \mathrm{O}$, and about 4 per cent. of alcohol. Light volatile liquid. Sp. gr., 0.713 to 0.716 , U.S.P.; 0.720 B.P. Boils at $35^{\circ} \mathrm{C}$. Characteristic odor. Highly inflammable; vapors explosive. Sol. in water ( $\mathrm{I}: \mathrm{I} 2)$ and in all proportions of alc. Dose, I c.c., I 5 minims, U.S.P.; I to 2 c.c., I 5 to 30 minims repeated; single, 3 to 4 c.c., 45 to 60 minims, B.P.

Ether for anesthesia is to be dispensed only in small, well-closed containers and is not to be used as an anesthetic after the original container has been opened twenty-four hours.

${ }^{*}$ Spiritus Etheris (Sp. Ether.), U.S.P., B.P. (Hoffmann's Drops).-33 per cent. in alcohol. Dose, 4 c.c., I dram, U.S.P.; I. 2 to 2.5 C.C., 20 to 40 minims repeated; single, 4 to 6 c.c., 60 to 90 minims, B.P.

$S p$. AEtheris Co., "Hoffmann's Anodyne," is an antiquated preparation of about the same strength and dose as the spiritus, containing additional ethereal products ("ethereal oil").

Ether Aceticus (Æth. Acet.), B.P., $\mathrm{C}_{2} \mathrm{H}_{5} \mathrm{C}_{2} \mathrm{H}_{3} \mathrm{O}_{2}$.-Dose, $\mathrm{I}$ to 2 c.c., 15 to 30 minims, repeated; single, 3 to 4 c.c., 45 to 60 minims, B.P.

* Ethylis Chloridum (Æthyl. Chlor.), U.S.P.; Ethyl Chlor., B.P. (Monochlor-ethane), $\mathrm{C}_{2} \mathrm{H}_{5} \mathrm{Cl}$ - - Colorless, extremely volatile liquid, of sharp, sweet taste, and peculiar odor. Inflammable. Boiling-point, 12 to $13^{\circ} \mathrm{C}$. Sparingly sol. in water, readily in alc. or eth. Dispensed in special glass or metal tubes. Used for freezing and for general anesthesia.

Ethyl Bromid, $\mathrm{C}_{2} \mathrm{H}_{5} \mathrm{Br}$.-General properties resemble the preceding. Boilingpoint, 38 to $40^{\circ} \mathrm{C}$. Should be protected from light and heat. Used for short general anesthesia.

\section{ALIPHATIC HYPNOTICS}

Sleep is one of the earlier phenomena of light narcosis, and can therefore be induced by small doses of any of the aliphatic narcotics. With many of these, however, the hypnotic stage is too brief, passes too easily into general anesthesia, or is complicated by undesirable side actions, stimulation, local irritation, etc.

Desirable Features.- The usefulness of a hypnotic depends upon the following qualities : Its action must be fairly prompt and lasting, so that the hypnotic effect will persist for some hours. Very volatile or easily decomposable substances are therefore excluded. A good somnifacient must produce the maximum hypnotic action, with the least depression of the medullary centers. It must not possess an odor or taste which would preclude its employment; and it must not irritate the stomach. Preparations which are insoluble in water, but which are nevertheless absorbed, are valuable because they are nearly tasteless, and because their action is apt to be more lasting. A soluble compound which could be used hypodermically would be very useful, but all the aliphatic narcotics are either too irritant or insoluble.

Manner of Action.--Small doses of these drugs depress the sensory and intellectual processes, so that the perception of external stimuli is lowered (Kraepelin, 1892). The subject is therefore put into condition favorable to natural sleep. This should be the aim in their therapeutic use. Larger doses produce an actual stupor; and eventually coma. The stronger hypnotics (chloral) also depress the motor functions. Of other 
functions, the blood pressure and pulse are somewhat lowered, as in natural sleep. The skin vessels are moderately dilated. The brain vessels are also dilated (Berezin, r9r6). The chlorinated hypnotics (chloral) in large doses, have a direct depressant effect on the medullary centers, especially the respiration; and on the heart-muscle. All the members are hemolytic when added directly to blood (L. Herrmann).

Respiratory Center.-Cushny and Lieb, I9 5 , studied the behavior of decerebrated rabbits under urethane. The other lipolytic hypnotics presumably act similarly. They found but little effect on ordinary respiration (in intact animals, the respiration would be diminished by the general narcosis). The respiratory response to reflexes was also normal; the response to oxygen deprivation or $\mathrm{CO}_{2}$ was, however, lessened or even reversed.

Hypnotic Response of Decerebrated Rabbits.-Chloral and urethane have a much more intensive action than in normal animals. Further, while normal animals are awakened from chloral sleep by cocain or caffein, decerebrated animals are not awakened. Tetrahydronaphthylamin awakens either (Morita, I9I5).

Therapeutic Indications.-Hypnotics (Soporifics, Somnifacients) are used to procure sleep in insomnia, especially when more physiologic measures (removal of the cause, etc.) are not applicable. Their continued use generally leads to tolerance and drug-habits and should be avoided if possible. Full doses often produce acute poisoning. Doses only large enough to induce sleep indirectly by quieting the patient, are rarely dangerous, and can be used temporarily without apprehension.

Limitations.- In the treatment of insomnia it must be remembered that the drugs of this class act purely symptomatically; that they soon lose their effect; that none of them are entirely free from objection, be it through the tendency to the formation of drughabit, through an irritant effect, or through the danger of overdosage. They should not therefore be resorted to except in case of necessity. The dose at the beginning should be very small-it must be remembered that in many cases the action of the hypnotic itself need not be very lasting, for sleep once induced tends of itself to continue. And this small dosage presents the opportunity of enlarging the dose when the patient becomes accustomed to it. When the hypnotics need to be continued for a long time, it is well to change frequently to a hypnotic of another type, to return to the first later. This obviates to a great extent the irritant effects and also the difficulty of the patient becoming accustomed to the drug.

Distribution of Hypnotics in Brain.-This has been investigated by Gensler, I9I4 and I915; utilizing the bromin hypnotics, neuronal, bromural and adalin. During the narcotic sleep, the absolute quantity of bromin found in the brain (all in organic form) represents only about $\mathrm{I} .4$ per cent. of the absorbed hypnotics; but the percentage of bromin is about $\mathrm{x} .8$ times greater than that in the remainder of the tissues, including the blood. The percentage in excited conditions is about the same; so that the known resistance of excitement to hypnotics, as would be expected, is due to the altered physiologic state, and not to altered distribution. The distribution is also unaffected by injection of magnesium, although the physiologic effects are additive.

Blood Lipoids. - The influence of continued administration of aliphatic hypnotics was investigated by Waser, I9I5.

Inflammation.- Chloral, urethane, and presumably the other aliphatic narcotics inhibit the migration of leucocytes from the inflamed frog's mesentery (Ikeda, Igr6).

Comparison of the Aliphatic Hypnotics.-Chloral, the oldest member of the group, is still the standard by which the numerous recent drugs are judged. Its principal disadvantages are the unpleasant taste and gastric irritation, if taken too concentrated, and the frequent formation of a habit. The fear of respiratory and cardiac depression is probably unfounded for ordinary doses. These disadvantages, at most, apply mainly to large doses, which are rarely necessary. For equivalent effects, the disadvantages of chloral are probably little if any greater than those of its substitutes. On the other hand, its therapeutic efficiency is probably superior 
(1) most: its action is certain, prompt, sufficiently lasting, and easily graduated by the dosage. The various compounds of chloral have no idluntage, since they act only after being transformed into chloral. Contraindications: Chloral should be used with some caution in depression of the medullary centers; and in the presence of vascular, cardiac, renal, pulmonary or gastric disease. Its efficiency is very slight against pain.

Paraldehyd is milder than chloral, but acts very promptly, and does not affect the respiration and heart. It is quite unpleasant to take.

Bromural and urethane act very weakly, and, like bromids, they are suitable only for mild nervous insomnias.

Sulphonal and trional are fairly powerful, but act very slowly, and produce considerable after-depression; they also irritate the kidneys and cause dangerous cumulative effects.

Veronal is as effective as chloral, and has less side actions. Full doses have produced severe and fatal poisoning, and therapeutic doses may cause cumulative effects.

A critical review of the modern hypnotics is furnished by Gregor, rgr3. This also describes the rather insignificant clinical effects on blood pressure (Siegfried, I9r 3 ).

Comparison with Other Groups.-Morphin is ordinarily less effective than chloral, but is superior when insomnia is due to pain. Scopolamin is useful especially in psychic insomnia and insanity. A pomorphin is said to be superior in acute alcoholism. Bromids are the least objectionable, but are effective only in mild nervous cases, worry, etc.

Composition and Action.-Certain relations have been found to exist between the chemical structure and action, although the connection is probably not a direct one, as explained in the discussion of the narcosis theories. The ethyl group and the next higher homologs seem to be especially valuable in conferring hypnotic action; and these are the most effective in the presence of aldehyd or ketone radicals. Alcohol radicals weaken the action, so that glycerin, e.g., is not narcotic. The compounds must resist oxidation sufficiently, in order that their action may be lasting.

Rôle of Chlorin.-The narcotic effect is greatly enhanced by the introduction of chlorin, as shown by the comparison of acetaldehyd and chloral; and by the methane derivatives, the efficiency of which increases for each additional chlorin atom from methane to carbon tetrachlorid. This peculiar property of the chlorin compounds has not been explained. Binz, I877, assumed the liberation of free chlorin; but there is good evidence that this can not occur (Fraenkel).

\section{CHLORAL HYDRATE}

General Statement.-This is the hydrate of trichloraldehyd, obtained by the action of chlorin on alcohol:

$\begin{array}{lr}\mathrm{CH}_{3} \cdot \mathrm{CHO} & \mathrm{CCl}_{3} \cdot \mathrm{CHO} \\ =\text { Acetaldehyd } & =\text { Chloral }\end{array}$

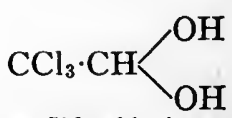

= Chloral hydrate

It was discovered by Liebig in 1832 , and was introduced as a hypnotic by Liebreich in 1869 . Morphin was the only active hypnotic known to that time. Acetaldehyd is also narcotic; the entrance of chlorin greatly increases its efficiency, but also introduces damaging effects on the respiration, heart and metabolism, similar to those of chloroform.

Fate.-Chloral is readily absorbed from all mucous membranes. In the body it is mainly reduced to trichlorethyl alcohol, $\mathrm{CCl}_{3} \cdot \mathrm{CH}_{2} \cdot \mathrm{OH}$, which is also hypnotic but less powerful. This is excreted by the urine, in one and one-half to eighteen hours, in combination with glycuronic acid (V. Mering, I88I), as the non-toxic trichlorethyl-glycuronic acid (urochloralic or chloraluric acid). This reduces Fehling's solution, and thus gave rise to the erroneous belief that chloral causes glycosuria. It 
polarizes to the left, but does not ferment. A true hyperglycemia may occur in profound narcosis, analogous to chloroform (Jacobsen, I913).

A small part of the chloral is excreted unchanged, and at most minimum traces as inorganic chlorids and chloroform.

Liebreich's Theory.- - Liebreich, I 869, was led to try chloral by the assumption that it would be converted into chloroform in the organism, as it is by alkalies in the testtube:

$$
\underset{\text { Chloral }}{\mathrm{CCl}_{3} \cdot \mathrm{COH}}+\mathrm{KOH}=\underset{\substack{\text { Chloro- } \\ \text { form }}}{\mathrm{CHCl}_{3}}+\underset{\substack{\text { Pot. } \\ \text { formate }}}{\mathrm{KCO}_{2} \mathrm{H}}
$$

The theory was disproved: The blood and expired air contained no chloroform, or at most insignificant traces (Nicloux, I906); and the greater part of the chloral is excreted in the conjugated form. Liebreich also stated that a fraction is excreted as chlorid; this is contradicted by Kast, I887.

Hypnotic Action of Ordinary Doses ( 1 or $2 \mathrm{Gm}$. for man).-These act very promptly, producing drowsiness in ten or fifteen minutes, and quiet, sound sleep within an hour, mainly by diminishing attention to external stimuli. The sleep lasts at least several hours; but the patient can be easily and completely aroused, and awakens refreshed, generally without depressant after-effects. The sleep is therefore strictly physiological. Very exceptionally, chloral may produce excitement and even delirium.

Other Functions.- The respiration and pulse are somewhat slowed and the blood pressure and temperature slightly lowered, but little more than in ordinary sleep. In respiratory and especially in circulatory diseases, these depressant effects may be somewhat more pronounced, and may perhaps lead to some cyanosis with the larger doses. In fact, however, the fear of respiratory and cardiac depression is based essentially on faulty deductions from animal experiments. There is no good clinical evidence of cardiac depression; while the danger is greater in cardiac diseases, it has often been given without bad effects (Cushny, I9I4). The experimental evidence also indicates that the depressant dose is considerably larger than the therapeutic dose (Gunn, r9 13 , I9r4).

Effects of Full Doses.-In man, 2 or $3 \mathrm{Gm}$. of chloral produce very deep sleep; and as the dosage is increased, this passes into stupor and coma, with complete muscular relaxation. The other depressant effects become much more important.

The depression of the medulla makes itself felt by slowing of the respiration and fall of blood pressure. The vasomotor paralysis is so prominent that chloral is often used in the laboratory to secure paralysis of this center. The pulse is also slowed through direct depression of the cardiac muscle.

As in the case of chloroform, it is impossible to state to what extent the vasomotor and the cardiac paralysis respectively are concerned in the fall of blood pressure. A dilatation of the cutaneous vessels is quite a marked feature and may lead to the appearance of skin eruptions. Larger doses always cause a marked fall of temperature on account of this cutaneous vasodilatation coupled with the diminished production of heat from muscular relaxation and probably lessened irritability of the heat-regulating centers. The temperature regulation in deep chloral-coma resembles that of cold-blooded animals (Sollmann and Hatcher, I908). In fatal doses death is ordinarily caused by paralysis of the respiratory center, although it may take place by paralysis of a weakened heart, just as in the case of chloroform.

Respiratory Effects.-Cushny, I913, found the respiratory center depressed, in decerebrated rabbits. With ordinary doses, this depression was masked by the accumulation 
of $\mathrm{CO}_{2}$, so that the respiratory movements were almost normal; but with larger doses, the depression predominated, with diminution of rate and depth. Wieland, 1915, also found the $\mathrm{CO}_{2}$ threshold raised.

Cardiac Effects. - These are similar to chloroform. Applied to the exposed heart of frogs or turtles, chloral produces progressive slowing; diminished contractility; lessened sy'stole; and diastolic standstill. The arrested heart at first responds easily to stimuli, but can not be started by atropin. Further details are described by W. H. Schultz, 1906. Similar depressant effects are seen in the mammalian heart (Bock, I 898; Gunn, 19I5). The depressant concentration is at least I : I, 000 ; the fatal, I : 4,000. The oxygen consumption of the heart is decreased, but not as much as its activity (Rohde and Ogawa, I9I 2).

Sudden stoppage of the heart may occur from relatively small doses, in cats, under the influence of excitement (Sollmann, I908). This resembles some of the human cases.

Vagus Phenomena.-In rabbits, doses too small to affect blood pressure or heart rate, lessen the irritability of the heart-muscle, so as to delay markedly the resumption of the beat under continued vagus stimulation (O. Loewi, 1912). Similar phenomena occur in frogs. The accelerator mechanism is generally depressed by chloral (Frederica, 19I3).

Excised Intestines.-The movements are increased by 0.05 per cent., diminished by stronger concentrations. This may be preceded by stimulation: Later, there may be occasional slow but strong contractions (Sembdner, 1913).

Action on Metabolism. - This consists in an increased destruction of protein, the waste products being excreted in a less completely oxidized condition than is the case normally.

The urine flow is diminished during the narcosis (Ginsberg, I912), as also the phenylphthalein output (Whipple and Speed, 1915). The uric acid excretion is somewhat increased (Abl, I913).

The output of nitrogen and neutral sulphur is increased; but in distinction to chloroform, this is delayed for several days after administration (Harnack and Remertz, r893); nor is the disturbance as great as with chloroform. Doses so large as to maintain anesthesia over twenty-four hours may not produce the characteristic effects of prolonged chloroform administration on the liver and kidneys, or only occasionally, and to a slight degree (Hopkins, 1912). Severe chloral coma, however, is followed by prolonged anorexia, marasmus and loss of weight (Sollmann and Hatcher, 1908). Lissauer, 1914, also found changes in the cardiac ganglia in rabbits (which are specially susceptible).

The Local Action of Chloral.-This is so pronounced as to allow of its use as a rubefacient. Its action on the stomach is consequently very prominent, and it must be largely diluted with water before administration; else it may produce vomiting. In any case, large doses are apt to show after-effects, referable to a gastritis.

Acute Chloral Poisoning.-The lighter grades show the narcotic phenomena, together with gastric irritation and vomiting, sometimes followed by icterus and albuminuria, skin lesions, and ataxia. In severe cases (as by "knock-out drops") there is prompt collapse, or delirium; pulse scarcely perceptible; severe fall of temperature; cyanosis; dyspnea or very slow respiration. In fatal cases, death is rarely delayed beyond five hours and may be almost immediate. Fatal relapses, after apparent recovery, have been reported (Purdy, r889). Large doses (as in the Stroganoff treatment of eclampsia) may kill the fetus in utero (Jung, r9r4).

The fatal dose averages about ro Gm., but varies greatly. Death has been reported after I to $3 \mathrm{Gm}$., and recovery after ro to $28 \mathrm{Gm}$. (Lewin). Doses above $3 \mathrm{Gm}$. must be used cautiously. (The dosage for cats was studied by Sollmann and Hatcher, rgo8.)

The treatment consists in gastric lavage; emetics would often be ineffective. The patient should be kept warm. Caffein, strychnin and atropin are useful. The heart may be stimulated by hypodermic injections of camphor. Artificial respiration is given as needed.

Efficiency of Cerebral Stimulants.-These are of little avail against toxic doses. Light chloral sleep, however, is easily broken by caffein, cocain or tetrahydronaphthya min; not by phenol, epinephrin or pituitary (Airila, 1913). 
Chronic Chloral Poisoning.-Chloral habit is not induced as readily as that of morphin or alcohol; but it is not rare. It presents the symptoms and degenerations of chronic alcoholism, with more severe gastric and cutaneous manifestations. The tolerance is only moderately increased and sudden death is not infrequent. Chronic chloral poisoning has been studied in dogs by Wallace, rgr2. Habituation to morphin does not confer tolerance for chloral (H. B. Myers, I9I6). habits.

Chloral habit is treated in the same general manner as other drug-

Therapeutic Uses.-Chloral, as all the aliphatic hypnotics, is used especially in insomnia of psychic origin. In ordinary nervous insomnia it should be given in small doses of 0.3 to I Gm. (5 to I $5 \mathrm{gr}$.), usefully combined with I Gm. of sodium bromid. Its taste is not easily disguised; it may be given in sherry or Syr. Aurantii. Larger doses are needed in mania and in delirium tremens. In the latter condition, the large doses have often caused collapse. It is not very effective against febrile delirium or pain. The addition of morphin secures a stronger effect than if either were used alone (Knell, I907).

Chloral is one of the most useful sedatives against the convulsions of strychnin, tetanus (Dreyfus, I914), eclampsia, etc. Fairly large doses are needed; but it is safer to use moderate doses ( 2 to $3 \mathrm{Gm}$.) repeated at fairly long intervals (to five or six times per day), and supplemented by chloroform inhalation. Chloral may be given by rectum; and is thus rather more effective than by mouth. It can not be used hypodermically, because of its irritant action.

Chloral is also used in asthma and seasickness, but the benefits are uncertain.

Locally, it is sometimes employed against neuralgia and pruritus, as an ointment ( 5 per cent.), or as chloral camphoratum, N.F. (the liquid resulting by triturating equal parts of chloral and camphor.

\section{PREPARATIONS-CHLORINATED HYPNOTICS}

${ }^{*}$ Chloralum Hydratum, U.S.P.; Chloral Hydras, B.P. (Chloral Hydr).; Hydrated Chloral, $\mathrm{CCl}_{3} \mathrm{COH}+\mathrm{H}_{2} \mathrm{O}$.- Colorless transparent crystals, of penetrating aromatic odor and bitterish caustic taste. Slowly volatilized when exposed to air. Very sol. in water (I : 0.25 ); freely sol. in alc. ( $1: \mathrm{I} .3$ ) or eth. and oils, fixed or volatile. Incompatible with alkalies, carbonates, and compounds of $\mathrm{Hg}$., I, or $\mathrm{Pb}$. Dose, $0.5 \mathrm{Gm} ., 8 \mathrm{gr}$., U.S.P.; 0.3 to r. 2 Gm., 5 to 20 gr., B.P.; largely diluted. Maximal dose, 4 Gm., 60 gr.

Syr. Chloral, B.P.-2o per cent. Dose, 2 to 8 c.c., $1 / 2$ to 2 drams, B.P. One dram contains is gr. of chloral.

\section{OTHER CHLORINATED HYPNOTICS}

Many of these have been suggested as substitutes for chloral, but none are seriously superior. Several are compounds which owe their activity merely to the liberation of chloral. Since promptness of action is generally desirable, the slow and uncertain liberation is a disadvantage.

Butyl-chloral Hydras, B.P., N.N.R.; Croton-Chloral Hydrate, $\mathrm{CH}_{3} \cdot \mathrm{CHCl}: \mathrm{CCl}_{2} \cdot-$ $\mathrm{CH}(\mathrm{OH})_{3}$. - This was also introduced by Liebreich, $187 \mathrm{I}$. Its action is more powerful but shorter than that of chloral (Mering, 1875). It has been used especially in facial neuralgia. Sol. in water $(\mathrm{I}: 40)$; very sol. in alc. or glyc. Dose, 03 to $1.2 \mathrm{Gm} ., 5$ to 20 gr., B.P.

Chlorbutanol, N.N.R.; Chloroform-acetone; Methaform; Chloretone, $\mathrm{CCl}_{3} \cdot \mathrm{C}$ $(\mathrm{OH}) \mathrm{CH}_{3} \cdot \mathrm{CH}_{3}$. - This is produced by the action of caustic alkalies on a mixture of 
chloroform and acetone. It acts similarly to chloral, and is probably rather more toxic (Impens, I90I); roo gr. have caused severe poisoning, but recovery occurred (for further details, Seifert, Nebenwirk., I9I, p. 44). It is used mainly as a mild local anesthetic, antiseptic and preservative for hypodermic solutions. Dose, 0.3 to $\mathrm{I} .5 \mathrm{Gm}$., 5 to 20 gr., as powder. Locally, as saturated watery solution, $8: 1,000$. It is sol. in oils, and easily sol. in alc. and fat-solvents.

Chlorbutanol is used as an anesthetic for laboratory animals, as it allows of long operations without requiring any attention. The dogs are given the usual dose of morphin. As soon as vomiting has occurred, $0.2 \mathrm{Gm}$. of chlorbutanol per kilogram of animal, is administered by stomach-tube. The chlorbutanol is previously dissolved in the smallest possible amount of alcohol. The anesthesia is complete in fifteen to twenty minutes, and lasts several hours. The blood pressure falls rapidly in rabbits, slowly in dogs. Chlorbutanol is inadvisable when it is wished to have the animal recover.

Chloraformamidum (Chloral Formam.), B.P. (Chloralamid), $\mathrm{CCl}_{3} \cdot \mathrm{CH}(\mathrm{OH}) \cdot \mathrm{NH} \cdot-$ $\mathrm{COH}$.- This was introduced by $\mathrm{v}$. Mering, $\mathrm{I} 889$, with the idea that the formamid $\left.(\mathrm{CHONH})_{2}\right)$ would counteract the medullary depression of the chloral. This was not realized; it is merely a less active hypnotic, being probably slowly decomposed into chloral. Sol. in water, freely in alc. Dose, I to $3 \mathrm{Gm}$., 15 to $45 \mathrm{gr}$., B.P.

Chloralose.-This compound of chloral and glucose was introduced by Heffter, $\mathrm{r} 888$ and $\mathrm{r} 893$. It is often contaminated with the non-hypnotic but toxic parachloralose (Mosso, 1893 ).

Isopral, trichlorisopropyl alcohol, is over twice as active as chloral, and its action is more prompt. Impens, I903, claimed that it is safer than chloral; but Sollmann and Hatcher, 1908, have shown this claim to be totally unjustified. The clinical side actions are described by Seifert, Nebenwirk., 1915, p. $7 \mathrm{x}$.

\section{PARALDEHYD}

General Statement.-This was introduced by Cervello, r882. Its actions are similar to those of alcohol (Kraepelin, I892); but the hypnotic effect is more powerful, and sets in very promptly, so that the patient may be asleep in ten to fifteen minutes. He should therefore be in bed before the drug is given. The sleep is normal; and with ordinary doses, without after-effects. The drug is less certain and less powerful than chloral; but therapeutic doses do not tend to depress the heart and respiration. It is not analgesic. Its principal disadvantages are the disagreeable pungent taste; fusel-oil odor of the breath, persisting over a day; irritation of the throat and stomach; dizziness and faintness (Seifert, Nebenwirk., I9I5, p. 64). Tolerance is generally acquired, and a habit is not infrequent. This presents the phenomena of chronic alcoholism, including delirium tremens (Fornaca and Querelli, I9r2). Its acute toxicity is low, so that $100 \mathrm{Gm}$. produced only very prolonged sleep.

Indications and Administration.-Paraldehyd is used when chloral is contraindicated, particularly in delirium tremens (Theunisan, I9I5); in the insane; in alternation with other hypnotics, etc. The ordinary dose is 3 c.c., 45 minims, raised in severe cases to 5 to $I_{5}$ c.c. ( $I$ to 43 ). It is best given in iced water, tea or claret.

Metabolism.-Hypnotic doses (in dogs) lower the blood-sugar, and have no effect on the nitrogen excretion. Anesthetic doses produce considerable hyperglycemia and glycosuria, and a considerable decrease of nitrogen excretion (Powell, 19r4).

Other aldehyds of fatty acids are said to produce arterial disease in rabbits (Denny and Frothingham, 1914).

${ }^{*}$ Paraldehydum (Paraldehyd.), U.S.P., B.P., $\left(\mathrm{CH}_{3} \mathrm{CHO}\right)_{3 .}-\mathrm{A}$ polymer of paraldehyd. Colorless transparent liquid, of strong characteristic odor and burning taste. Sol. in water ( 1 : 8), freely miscible with alc. Dose, 2 c.c., 30 minims, U.S.P.; 2 to 8 c.c., $1 / 2$ to 2 drams, B.P. Maximal dose, $5 \mathrm{Gm}$., $75 \mathrm{gr}$. 
Amylen Hydrate (Harnack and Meyer, 1894$)$. - Tertiary amyl alcohol, $\left(\mathrm{CH}_{3}\right)_{2}$. C. $\mathrm{C}_{2} \mathrm{H}_{6} \mathrm{OH}$. It ranks between chloral and paraldehyd. It is said to lessen the polydipsia and polyuria of diabetes insipidus. Colorless fluid; sol. in 8 parts of water, readily in alc. Dose, 1 to $2 \mathrm{Gm}$. ( 15 to $30 \mathrm{gr}$.), in glycerin.

\section{SULPHONAL (SULPHONMETHANUM)}

General Statement.-The ethyl-sulphones were introduced by Baumann and Kast, I888. Sulphonal has the structure:<smiles>CCO[SH](=O)(OCC)C(C)(C)C</smiles>

The hypnotic action is connected with the ethyl radicals, and is further enhanced by replacing the remaining methyl radicals with ethyl (trional, one replaced; tetronal, both replaced).

Sulphonal is a rather powerful hypnotic, but a weak analgesic. Its absorption and therefore its effects are slow, sleep occurring only after one to five hours. Its administration must therefore be properly timed. The sleep lasts six to eight hours, and the effect persists somewhat during the next day, with vertigo, lassitude, drowsiness, and depression. This is due to the delayed excretion, which is even slower than the absorption; thus tending to cumulative effects and very dangerous poisoning, if the administration is continued over longer periods. Sulphonal is therefore unsuitable for prolonged use. In acute cases, or in alternation with other hypnotics, it has several advantages. Its taste is not unpleasant; it does not produce gastric irritation; ordinary doses do not affect repiration and circulation; and the fatal dose is generally much higher than the therapeutic. It does not induce tolerance, but may probably cause a habit.

Acute Poisoning.-The symptoms consist in prolonged and profound sleep, with gradual failure of the circulation and respiration. Death occurs usually after several days. It has been reported from 5 to $30 \mathrm{Gm}$. (Knaggs, I890); while I00 Gm. have been survived (Neisser, I89r).

Chronic Poisoning.-This has been produced even by ordinary therapeutic doses, when continued for some weeks. The symptoms develop slowly and consist in nervous depression, gastro-intestinal disturbances, and urinary changes. The appearance of hematoporphyrin in the urine (dark cherry color) is particularly characteristic. Skin eruptions are sometimes seen. If the drug is stopped when the symptoms are light, recovery occurs, but is very slow. After the urine changes have set in, the prognosis is discouraging; death occurring after some weeks by general exhaustion. The autopsy is generally negative (Taylor and Sailer, I900).

The principal symptoms of chronic poisoning are the following: Nervous: confusion, pareses, ataxia. Digestive: Almost continuous vomiting, abdominal pain, particularly about umbilicus, with tympanites and obstinate diarrhea or constipation (A. W. Rogers, Ig1 2). Urine: Hematoporphyrin, methemoglobin and red corpuscles, albumin, casts, high acidity. The literature of the side actions is given by Seifert, Nebenwirk., I9I 5 , p. 92; Trional, p. 94 .

The occurrence of hematoporphyrin was described by Stokvis, 1889. It is rarely seen in acute poisoning, but is very frequent and fairly early in chronic poisoning. It can be produced in rabbits, but not in dogs (Neubauer, 1900). The hematoporphyrin is also found in the bile (Pfoertner, 1914).

Excretion.-Sulphonal is excreted partly as ethyl-sulphonic acid, partly unchanged by the urine and intestines. 
Administration.- Sulphonal is used in nervous insomnia, especially when chloral is contraindicated. The ordinary doses, 0.75 to I Gm., ro to $\mathrm{I} 5 \mathrm{gr}$., or in severe cases $2 \mathrm{Gm}$., are taken in hot milk at least four hours before bedtime. A smaller dose may be taken the second night. The administration should not be continued beyond a week, when an intermission must be made. It is generally advisable to prescribe cathartics, alkalies and plenty of water.

\section{TRIONAL}

Trional is more effective than sulphonal; and being more soluble, its absorption and action are more prompt, producing sleep in one-half to one hour. It has the same dangers as sulphonal, and the latter is much more commonly used.

\section{TETRONAL}

Tetronal is less soluble, and therefore too slow.

\section{PREPARATIONS-SULPHONALS}

*Sulphonmethanum (Sulphonmeth.), U.S.P. Sulphonal, B.P. (Diethylsulphonedimethyl-methane); $\left(\mathrm{CH}_{3}\right)_{2}$. C. $\left(\mathrm{SO}_{2} \mathrm{C}_{2} \mathrm{H}_{5}\right)_{2 .}$-Colorless, odorless and nearly tasteless crystalline powder, slightly sol. in water $(\mathrm{I}: 365)$; sol. in boiling water $\left(\mathrm{I}: \mathrm{I}_{5}\right)$ and in alc. (1:60). Dose, $0.75 \mathrm{Gm}$., 12 gr., U.S.P., 0.6 to $2 \mathrm{Gm}$., ro to 30 gr., B.P., as powder. Maximal dose, 2 Gm., $30 \mathrm{gr}$.

Sulphonethylmethanum (Sulphonethylmeth.), U.S.P.; Methylsulphonal, B.P. (Tr1onal; Diethylsulphone-methylethyl-methane); $\left(\mathrm{CH}_{3}\right)\left(\mathrm{C}_{2} \mathrm{H}_{5}\right) \mathrm{C}\left(\mathrm{SO}_{2} \mathrm{C}_{2} \mathrm{H}_{5}\right)_{2}$.-Colorless, odorless, crystalline scales; bitter taste. Slightly sol. in water (r:200); sol. in alc. Dose, 0.75 Gm., I 2 gr., U.S.P.; 0.6 to 1.2 Gm., Io to $20 \mathrm{gr}$., B.P., as powder. Maximal dose, 2 Gm., $30 \mathrm{gr}$.

\section{HYPNOTIC UREA DERIVATIVES}

These furnish a convenient means of exhibiting the alkyl groups, the efficiency being apparently enhanced by the urea combination. The three most important types of compounds are represented by Bromural, Veronal and Urethane, as may be seen from the following formulas:

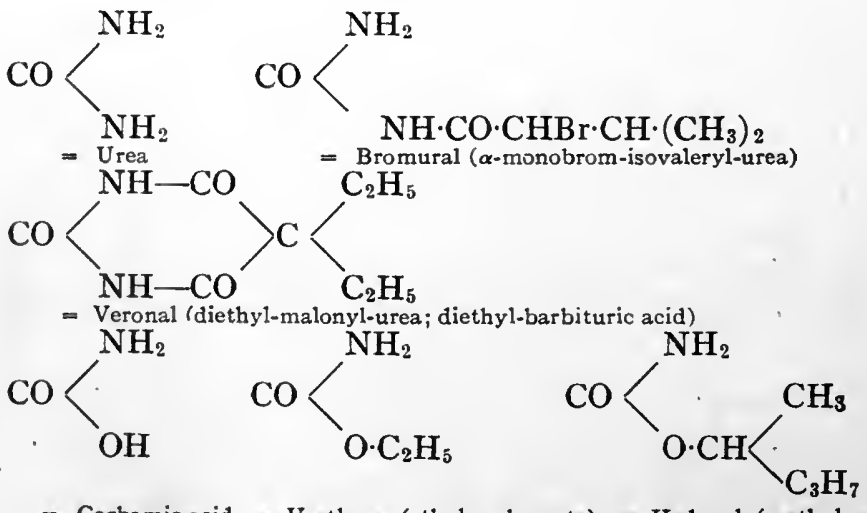

- Carbamic acid = Urethane (ethyl-carbamate) = Hedonal (methyl-propyl- 


\section{VERONAL (DIETHYL-BARBITURIC ACID)}

General Statement.-This was introduced by E. Fischer and Mering, I903 and 1905 . It has a number of advantages over the other hypnotics: It is even more powerful than chloral and somewhat more analgesic; it does not produce local irritation, and the taste is not so disagreeable. The margin between the ordinary therapeutic dose $(0.5 \mathrm{Gm}$.) and the toxic dose (usually 8 to ro $\mathrm{Gm}$.) is somewhat wider; and the ordinary doses have little effect on the blood pressure and respiration. However, fatal collapse (by peripheral paralysis of the blood vessels) has occurred in several cases from relatively small doses (Io to I 5 gr., 0.65 to I Gm., J.A.M.A., 53, I833, r909). On the other hand, recovery has resulted after Io $\mathrm{Gm}$. Small doses are somewhat diuretic (A. Hoffman, 1906). The action is rather slower than with chloral, but more rapid than with sulphonal. After-depression is exceptional, but more common than with chloral. The usual dose, $0.5 \mathrm{Gm}$., 8 gr., induces a deep, dreamless sleep in one-half hour; or even more promptly if the soluble sodium salt is used. The patient generally awakens refreshed, but exceptionally suffers from lassitude, vertigo, headache, nausea, diarrhea, and skin eruptions.

Veronal is excreted slowly, so that ordinary doses may produce serious cumulative effects similar to those of sulphonal, but rather more slowly. Nervous symptoms are especially prominent (Glaser, r9r4). It must therefore be intermitted after a week. Habit has been reported (Krep, I905), but tolerance is not easily acquired.

Acute Veronal Poisoning.-This has been rather frequent; a number of cases are collected by Heffter and Loeb, I006. They present the phenomena of coma, fall of blood pressure, feeble heart, vasodilation, marked fall of temperature even with moderate doses, increased reflexes, sometimes convulsions; slower and especially shallow respiration, asphyxia, but with constricted pupils; renal irritation, diminished urine or anuria; exanthemata (circumscribed dermatitis reported by Zeisler, rgr2). Rommel, 1912, describes epithelial necrosis of the kidneys in two fatal cases, without clinical signs of nephritis. The general effects have been investigated by Groeber, I9II; Roemer, IgI 2; and by Jacobj, I9I2.

The literature of the clinical side actions is given by Seifert, Nebenwirk., 1915, pp. 75 and 99.

Vasodilator Action.-Jacobj and Roemer, I9I I, refer the toxic peculiarities of veronal to a direct action on the blood vessels, perhaps analogous to an arsenic effect. They therefore advise against its use when the circulation and respiration are depressed.

The treatment of acute poisoning would be along the same lines as for chloral: Lavage, cathartics and stimulants. Bachem found caffein the best antidote. Tholl, I 12 , also recommends acetic ether.

Fate and Excretion.-Veronal is excreted almost exclusively by the urine, only traces appearing in the feces. When given by mouth, about 63 per cent. are eliminated unchanged. This proportion is not altered on continued use, so that the remainder must be destroyed (A. Hoffman, rgo6). The proportion excreted may rise to 90 per cent. with small doses hypodermically; and fall to one-half with large doses (Bachem, 19ro). The excretion is very slow, requiring several days even after $0.5 \mathrm{Gm}$. This explains the cumulative effects.

Chronic Veronal Poisoning.-This shows confusion, ataxia, debility, gastro-intestinal irritation, anemia and hematoporphyrin.

Indications.--Veronal is used in nervous insomnia, mania and delirium. It appears to be highly effective in delirium tremens, $0.5 \mathrm{Gm}$., three times daily (Dreyfus, I9r4). It has also been employed against vomiting, seasickness, migraine, and for combination anesthesias (Wolfram, r906). It is taken dry or in hot liquids. If a more rapid action is desired, the 
soluble sodium salt may be given (Katzenstein, r909); but its taste is more disagrecable.

\section{PREPARATIONS-VERONALS}

*Acidum Diacthyl-barbituricum (Acid Diaethylbarb.), N.N.R.; Barbitonum, B.P. (Veronal; Diethyl-malonyl-urea.-White, crystalline powder, odorless, faintly bitter.

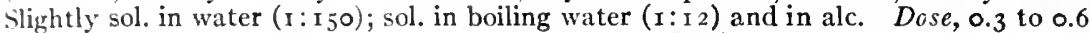
Gm., 5 to $10 \mathrm{gr}$., B.P., in hot milk or hot lemonade. Maximal dose, I Gm., I5 gr.

Sodii Diaethyl-barbituras, N.N.R. (Veronal-Sodium; Medinal).-The monosodium salt. Freely sol. in water, the solution having a bitter alkaline taste. May be used by rectum and perhaps hypodermically.

Dipropyl-barbituric Acid, Proponal, was also introduced by Fischer and Mering, 1904 and I905. It is more powerful than veronal, but more dangerous and less reliable.

Phenylethyl-barbituric Acid, N.N.R.; Luminal.-Resembles Proponal (Gregor, I9I3). Its sodium salt has been used hypodermically, especially in epilepsy (M. Klotz, 1915). The hypnotic and fatal dose are close together, and the circulatory depression is severe. Taddei, I9I3, protests against its use as too dangerous. The literature of the clinical side actions is given by Seifert, Nebenwirk., I915, p. 73. Dial (Diallylmalonylurea) has no advantage over other ureid hypnotics (Castaldi, I9I 5).

\section{BROMURAL, N.N.R.}

This is a prompt but very mild hypnotic, effective only in slight nervous insomnias. It is not analgesic, and is rendered ineffective by cough or delirium. Ordinary doses have no side effects (v.d. Eeckhout, 1907; Sollmann and Hatcher, I908; literature, Seifert, Nebenwirk., I 191 5, p. 48). In man, 0.3 to $0.9 \mathrm{Gm}$. are effective in five to twentyfive minutes, producing a natural sleep lasting three to five hours, without excitement (Krieger and v.d. Velden). It dissolves in hot water, but not in cold. Adalin is a closely related substance.

\section{URETHANES}

These have been disappointing in man; the hypnotic action is feeble. and patients soon become immune. The efficiency increases with the magnitude of the alkyl radicals (Binet). There is also a decided diuretic effect.

\section{ETHYL CARBAMATE}

This acts more satisfactorily on animals than on man; producing a rapid and profound narcosis, with little change in the circulation or respiration (Schmiedeberg, I885). It is therefore employed as a laboratory anesthetic. The $\mathrm{CO}_{2}$ threshold of the respiratory center is, however, somewhat raised (Wieland, I915). It decreases the oxygen consumption (Winterstein, I914). Large doses cause in rabbits a vacuolar degeneration of the hepatic epithelium (Sollmann).

It also lowers the phenolphthalein output (Whipple and Speed, I915). Large doses produce hyperglycemia, independent of the quantity of liver-glycogen and epinephrin, and unaffected by pituitary. Bang, 1914, believes that it acts through pancreatic degeneration. It is excreted as urea. Side actions, Seifert, Nebenwirk., 1915, p. 97.

\section{PREPARATIONS-URETHANES}

Ethylis Carbamas (Ethyl. Carbam.), U.S.P. (Urethane); $\mathrm{CO}\left(\mathrm{OC}_{2} \mathrm{H}_{6}\right) \mathrm{NH}_{2}$ - - Colorless, odorless crystals of saline taste. Very sol. in water $(\mathbf{I}: 0.45)$ and in alc. (I:08). Dose, I Gm., I 5 gr., U.S.P.; to $4 \mathrm{Gm}$.

Hedonal, N.N.R., is twice as active as urethane (Dreser, 1899). It has the same general effects, its absorption is relatively slow and it is more toxic. Its dose is about double that of chloral. It has been used intravenously for anesthesia (Jeremisch; Page, I 19 2); a 0.75 per cent. solution in normal saline being injected at the rate of 100 c.c. per five minutes, reduced to 50 c.c. when anesthesia is attained. Its use is dangerous. Literature, Seifert, Nebenwirk., 1915, p. 67. 


\section{THE SYSTEMIC ACTIONS OF GASES}

General Statement.-The respiration of oxygen is essential to life. A deficient supply of this gas leads to the phenomena of asphyxia. A number of gases $\left(\mathrm{N}, \mathrm{H}, \mathrm{CH}_{4}\right)$ act merely by excluding oxygen mechanically. This also forms an important element in the action of nitrous oxid, as ordinarily used. Carbon monoxid also acts by excluding oxygen, but it does so chemically. Other gases have specific, direct actions, especially on the central nervous system. Oxygen itself, when at a pressure of several atmospheres, is toxic. Carbonic acid and nitrous oxid produce their peculiar actions at lower pressures, but the air must contain considerable proportions. Sulphuretted and arseniuretted hydrogen are toxic in very small proportions. The gaseous acids (sulphurous, nitrous, etc.), alkalies (ammonia) and halogens (bromin and chlorid) act mainly as local irritants. Compressed air acts mechanically.

Rapidity of Action.-The effects of gases appear very promptly after beginning the inhalation, and disappear as rapidly when the animal is made to respire pure air. This is due to the large surface and large quantity of blood exposed to them in the lungs. As these factors are relatively greater in small mammals, these succumb and recover very much more rapidly than man, and may indeed be used as a test of the safety of a suspected air. Whilst the recovery from the direct effects of gases is rapid, indirect after-effects may be very persistent.

The quantitative effect of gases depends on their partial pressure in the air. At ordinary barometric pressure, this is equal to the volume-percentage of the gas. But it is important to remember that the effect of the normal percentage of oxygen at half an atmosphere pressure is the same as that of half the percentage at ordinary pressure; and conversely, that I per cent. of a gas at 3 atmospheres produces the same effect as 3 per cent. at I atmosphere.

The symptoms produced by most toxic gases resemble those of asphyxia, and this whether the effect is produced by the absence of oxygen; or whether the gas has a specific toxicity. They are closely related to those of anesthetics.

\section{NITROUS OXID}

General Statement.-Nitrous oxid, $\mathrm{N}_{2} \mathrm{O}$, nitrogen monoxid or protoxid, "Laughing Gas," was the first of the inhalation anesthetics and is still the safest. When inhaled undiluted, it produces very rapidly unconsciousness and anesthesia, partly by a direct narcotic action, and partly by exclusion of oxygen. The asphyxia limits its use to very short operations, such as the extraction of teeth. It has the advantages of prompt action and recovery, absence of irritation and of after-effects, and is practically devoid of danger. By the addition of oxygen, the asphyxial factor may be removed. The anesthesia also becomes slower and lighter; but by carefully adjusting the percentage of oxygen ( 5 to ro per cent.), a satisfactory degree of surgical anesthesia can be maintained for long periods, without letting the asphyxia obtain dangerous proportions. Nitrous oxid is especially valuable in the "Gas-Ether Sequence," by avoiding the unpleasant features of the induction of ether anesthesia. 
Historical.-Nitrous oxid was first prepared by Priestley about 1776 . The discovery of its anesthetic properties by Humphry Davy and by Wells is described under "Fther" and "Chloroform." Elsworth (Boston Med. and Surg. Jour., 37) in Januars, $18+3$, amputated a thigh under successful nitrous oxid anesthesia. Its administration with oxygen was introduced by E. Andrews of Chicago in I868; and the use of such mixtures under pressure by Paul Bert, I 880 . The technic of its use in surgical operations has been extensively elaborated in recent years.

Inhalation of Undiluted $\mathrm{N}_{2} \mathrm{O}$.-For short operations, as in dentistry, the gas is administered through a tight-fitting mask, so that all air is excluded. The symptoms run a very rapid course: the preliminary effects consist in a sweetish taste; numbness; exhilaration (laughter); confusion; deeper and quicker respiration; and fuller pulse. Partial anesthesia, with loss of consciousness, ensues in twenty to thirty seconds. The patient is subject to dreams and the anesthesia is imperfect. The reflexes are preserved and excitement may be present, especially if the patient is disturbed. The respiration is still regular, deep and quickened; the pulse full and rapid; the pupils enlarged; the face dusky, livid or pallid; the eyelids twitching and slightly separated. Complete anesthesia occurs in fifty to one hundred and twenty seconds, averaging fifty-six. Its onset is denoted by a change in the respiration, which becomes slightly irregular and noisy. The pulse is quickened by about 30 per cent. and is small. The blood pressure shows a large asphyxial rise (Kemp, I897). The vasoconstriction diminishes the urine flow. The limbs are relaxed, but individual muscles exhibit clonic or epileptiform contractions. The pupils are almost invariably dilated. The face is cyanosed. Relaxation of the sphincters occurs rarely.

Operative Period.-As soon as this stage is reached, the gas is removed. The pulse at once becomes slower and fuller; the respiration also recovers rapidly; and the asphyxial symptoms disappear. The anesthesia lasts some twenty-two to thirty seconds after the gas is removed; and it is during this period that the operation must be performed. The total time between the beginning of the administration and complete recovery is therefore one hundred to one hundred and twenty seconds. The duration of the after-anesthesia increases with the duration of the administration. This may be prolonged to several minutes by giving the patient an occasional breath of pure air (every fifth inhalation); or by admitting a little air into the mask. The admission of air, however, makes the anesthesia uneven and unsatisfactory.

After-effects are generally absent with short administrations. Sometimes the patients complain of giddiness, headache, lassitude and drowsiness. Nausea is exceptional.

Accidents. - With short administrations, these are very rare, because of the wide margin between the first danger sign (noisy and irregular respiration) and death. Only about seventeen deaths have been reported, making the fatality less than $I$ in $5,000,000$. In patients with grave cardiac lesions, the pulse may become feeble and the respiration shallow before anesthesia is established. If the administration is continued too long in healthy individuals, the asphyxial phenomena become emphasized, with violent inspiratory, then forced expiratory movements; then shallow and intermittent and finally arrested respiration, in two to four minutes (Haggard, rgro). The violence of ordinary asphyxial convulsions is mitigated by the narcosis. Cyanosis is intense. The heart continues to beat for some time, so that restorative measures are generally successful. They should consist in artificial respiration, preferably with oxygen. 
Contraindications. - Nitrous oxid should not be used in cardiac lesions, valvular or myocardial; in elderly patients especially with advanced arteriosclerosis or high cerebral pressure; and in operations on the brain. It is often ineffective in hysterical, obese, muscular or alcoholic patients (S. G. Davis, I909).

Mechanism of Action. $-\mathrm{N}_{2} \mathrm{O}$ is very soluble in blood, which takes up 45 per cent. by volume (Bert). Davy believed that it acted like oxygen, producing hyperoxidation of the blood; but Frankland showed that it is not decomposed. It was then believed that it acted merely as an asphyxiant. The phenomena produced by the undiluted gas are in fact partly asphyxial. This factor can be removed by the addition of oxygen, when it is seen that narcosis results from the $\mathrm{N}_{2} \mathrm{O}$ itself. This may also be demonstrated on frogs, which preserve their reflexes for hours when placed in pure hydrogen; but lose them promptly in $\mathrm{N}_{2} \mathrm{O}$. Evidently the nitrous oxid has a direct action on the central nervous system; first stimulating the psychic areas, then depressing these to insensibility. The motor reflexes are also abolished. The medullary centers are not affected, and the circulation remains unchanged, in the absence of asphyxia. Nitrous oxid also has no action on other tissues and is therefore absolutely harmless. Dogs anesthetized continuously for three days with a mixture of $\mathrm{N}_{2} \mathrm{O}$ and oxygen, recovered without any damage. The mechanism of its central action is probably the same as with the hydrocarbon narcotics-viz., a physical change in the lipoids. It probably does not combine with hemoglobin. On account of its feeble chemic affinities, the gas is practically free from side actions, and herein lies its chief value.

High Pressures of Nitrous Oxid.- These are fatal above 3 atmospheres, even when inhaled with adequate oxygen. The symptoms consist in narcosis, convulsions, and finally depressed respiration. The heart remains strong (Bock, 19I3).

Nitrous Oxid with Air or Oxygen.-The respiratory disturbances, convulsive features and cyanosis produced by undiluted $\mathrm{N}_{2} \mathrm{O}$ are purely asphyxial. If the gas is mixed with a sufficient proportion of oxygen ( 5 to 20 per cent.), these asphyxial phenomena are entirely absent, and the anesthesia can therefore be prolonged indefinitely. With smaller percentages of oxygen, the symptoms are intermediate.

The dilution of the gas with oxygen lowers its concentration in the blood to a corresponding degree, so that the narcotic action is not as prompt or deep. It is generally stated that a mixture of 80 per cent. of nitrous oxid and 20 per cent. of oxygen, inhaled at atmospheric pressure, produces only confusion and analgesia, with incomplete loss of consciousness.

However, Hewitt claims to produce deep anesthes a in this way within four minutes; and that the anesthesia becomes incomplete only if the concentration falls below 66 per cent. Paul Bert, I880, obtained perfect results by a mixture of 85 parts $\mathrm{N}_{2} \mathrm{O}$ and 15 parts $\mathrm{O}_{2}$, condensed by an excess-pressure of $1 / 6$ atmosphere.

D. E. Jackson, 1916 , describes an apparatus that involves the continuous rebreathing of the nitrous oxid, with removal of the $\mathrm{CO}_{2}$ and renewal of the oxygen. This permits of prolonged anesthesia at a minimum cost. Under these conditions, the effects have considerable resemblance to morphin. Man appears sensitive to lower concentrations than are needed for dogs. Careful administration inducesan apparently natural sleep.

These methods are not very feasible in practice; but a satisfactory anesthesia may be maintained sufficiently long for ordinary surgical operations by adding only enough oxygen to the gas to prevent the major manifestations of asphyxia.

Hewitt found that 6 per cent. of oxygen are required to prevent the convulsive features and noisy respiration; II per cent. to prevent lividity. Above Io per cent., the reflexes and movements of the patient were more pronounced.

Morphin-scopolamin greatly increases the efficiency of nitrous oxid; so that with these alkaloids, a mixture of 80 per cent. and 20 per cent. oxygen even at atmospheric pressure induces an operative anesthesia (Kochmann, I9I4). 
Administration of the Mixed Gases.-This requires a special apparatus for adjusting the two gases in any proportion. This is connected with a close-fitting mask. It is advisable to warm the gas mixture before it is inhaled.

To hasten the anesthesia, the administration is begun with a mixture containing 2 per cent. of oxygen. This is gradually increased to Io per cent., but varying for different patients.

Phenomena.-The primary symptoms are the same as with pure gas. The anesthetic stage is usually reached in a few minutes. It is characterized by regular, calm, moderately snoring respiration; slightly dusky face; and medium pupils. The pulse is moderately quickened; the blood pressure somewhat high; the blood rather venous. Convulsive phenomena are absent. The reflexes and muscular tone are greatly decreased, but not abolished as with ether; and the anesthesia is not so profound.

After-effects. - When the administration is stopped, the patient awakens fully within two or three minutes. Nausea is exceptional, but more common than after short inhalations.

Advantages.- These consist mainly in the greater comfort of the patient, the smaller danger, and the absence of irritant side effects. . The gas would therefore be especially indicated in renal and acute pulmonary diseases; or when the patient dreads the other anesthetics. Crile, r9ro, claims that it lessens the danger of shock and infection.

Disadvantages.- The contraindications and limitations were discussed under the undiluted gas. Its application is further limited by the rather shallow anesthesia; incomplete relaxation; high blood pressure and venosity of the blood; the expense of the gas and apparatus; inconvenience; and the need for very specialized experience.

Influence of Impurities. - Warner, I9I5, finds that ordinary nitrous oxid often produces severe symptoms, especially cyanosis, which does not occur with proper purification. The toxic effects are due partly to nitric oxid, but especially to unidentified basic impurities, perhaps hydroxylamin and hydrazin. The gas deprived of these (as is now generally done) can be given with less oxygen, without cyanosis.

Gas-ether Sequence.-This was suggested by Clover, I876; but has also come into extensive use only recently. It seems to combine the chief advantages of the two anesthetics, and is perhaps the nearest approach to an ideal method.

Nitrous Oxid Analgesia in Obstetrics.-This is being advocated as much safer than scopolamin-morphin "twilight sleep." It does not weaken the uterine contractions and has no bad effects on mother or child (Webster; Lynch; Davis, I9 5 ).

\section{PREPARATIONS-NITROUS OXID}

* Nitrogenii Monoxidum (Nitrogen. Monox), U.S.P. (Nitrous Oxide), $\mathrm{N}_{2} \mathrm{O}$.-Colorless gas; possesses a slight characteristic odor; somewhat sweetish taste. It is marketed, compressed into a liquid, in steel cylinders. It returns to the gaseous state when the pressure is released.

\section{CARBON DIOXID (CARBONIC ACID)}

General Statement.-This acts as a weak acid, producing locally a rather mild irritation; and after absorption, strong medullary stimulation, and in higher concentrations narcosis. These actions play the principal rôle in ordinary asphyxia. Deficiency of $\mathrm{CO}_{2}$ (Acapnia) leads 
to corresponding depressant phenomena. The carbonic acid therefore acts as a hormone (Bayliss and Starling, I906), which regulates the activities of various functions, especially of the respiration. In this way, the $\mathrm{CO}_{2}$ tension of the blood and tissues is normally kept automatically at a practically constant level.

Local Actions. - $\mathrm{CO}_{2}$ as gas or solution produces a prickling sensation and reddening of the skin and mucous membrane, followed by numbness and local anesthesia. The sensory irritation leads to reflex stimulations (Quincke, I877). The cutaneous stimulation is utilized in the form of carbonated baths (Nauheim baths), especially in cardiac diseases. Frozen $\mathrm{CO}_{2}$ (carbon dioxid snow) is employed as a superficial cauterizant. In the stomach, carbonated water ("soda water") hastens absorption, increases the secretion of acid gastric juice (Chiari, I9I5), and acts as a carminative. It is useful against nausea, and valuable for disguising the taste of medicines.

Effects of Inhaled $\mathrm{CO}_{2 .}$ - If oxygen deficiency is excluded by inhaling gas mixtures containing 20 per cent. of oxygen, no effects occur until the concentration of 3 per cent., volume, of $\mathrm{CO}_{2}$ is reached (Zuntz). With this concentration, there is some hyperpnea and discomfort: $81 / 2$ per cent. in man produces in a few minutes distinct dyspnea, rise of blood pressure and congestion, which become insupportable in fifteen or twenty minutes, but disappear promptly in fresh air. The symptoms increase to 15 per cent.; but even 20 per cent. is not dangerous in an hour to animals, and probably not to man. With 25 to 30 per cent., the stimulant phenomena pass into depression, with diminished respiration, fall of blood pressure, coma (generally without convulsions), loss of reflexes, anesthesia, and gradual death after some hours; the heart outlasting the respiration. With higher concentrations, the stimulation is still briefer. With pure $\mathrm{CO}_{2}$, death may occur in a few minutes as a mixed effect of carbon dioxid and anoxemia.

Ordinary Asphyxia.-This involves the two factors of deficiency of oxygen or excess of carbon dioxid in the blood. The phenomena in both cases are almost, though not quite, identical. When asphyxia results from breathing in a confined space, both causative factors are intermingled, but the $\mathrm{CO}_{2}$ factor predominates.

The symptoms of asphyxia vary somewhat according to the rapidity with which it is produced. When it occurs rapidly, three typical stages may be distinguished: During the first stage, the respirations (particularly the inspirations) are increased. In the second stage, the respirations are irregular and convulsive, the inspirations being shallow and weak, the expirations powerful and prolonged. Consciousness is lost. The skin, especially the face, becomes cyanotic. The third slage is characterized by collapse and convulsions. The respiratory centers are depressed. The respiratory movements are shallow and infrequent. The convulsive, vagus, vasomotor and pupillo-dilator centers are stimulated. "The extremities and the muscles of the face and neck twitch convulsively, there are gasping movements; the body of the animal is rigid and arched backward. Urine and feces are expelled. The pulse is very slow and soft, at first strong, then progressively weaker. The pupils are at first constricted, then widely dilated until death. The heart continues to beat weakly for several minutes after the respiration has stopped. Artificial respiration during this interval will generally result in recovery. Death occurs within six or eight minutes, if the trachea is tied.

The autopsy shows a very venous color of the blood. The veins of the upper part of the body, and the right heart, are greatly distended.

If the asphyxia is produced slowly, the first symptoms are cyanosis and dyspnea on exertion. The individual becomes stupefied, but so gradually that he may not be aware of his condition. The stupor passes into unconsciousness and this into collapse. The motor symptoms may be entirely absent.

Medullary Centers in Asphyxia.-The effects are alike whether they result-from $\mathrm{CO}_{2}$, acidosis, or oxygen deprivation. 'The stimulation occurs in the order of respiratory, then vasomotor and then vagus centers. It is followed by depression of these centers. The relative preponderance of 
stimulation and depression depend on the degree and suddenness of the asphyia, and on the prevailing condition of the centers (Loevenhart, I 015 ).

Role of $\mathrm{CO}_{2}$ in Asphyxia.-C. Bernard showed that the deprivation of oxygen ordinarily play's a minor part; inclosing the head of an animal in a bladder, he found that the intravascular injection of oxygen does not retard death. Temporary revival from asphyxia may be secured by artificial respiration in an atmosphere of hydrogen or nitrogen; and according to some experiments of the author, even in $\mathrm{CO}$. The investigations of Loevenhart, I II 3 , however, suggest that the $\mathrm{CO}_{2}$ itself acts by interfering with oxidation.

Role of $\mathrm{CO}_{2}$ in Impure Air.-The popular idea of this is greatly exaggerated. "Pure" air contains 0.03 per cent. of $\mathrm{CO}_{2}$; in city streets it rarely rises above 0.04 , or at most 0.15 per cent. In badly ventilated rooms it may reach 0.3 per cent., which is far below the effective concentration. Neither the presence of $\mathrm{CO}_{2}$ nor the absence of oxygen explains the harmful effect of sojourn in ill-ventilated rooms; nor does the expired air contain any poisonous ingredients. It is supposed that the acute discomfort and the suffocating sensation experienced in these places is largely psychic, being produced by the heat, humidity, odors, noise, etc. Whether this will also account for the severely detrimental effect on health and resistance, the increased tendency to tuberculosis, etc., which are associated with habitually deficient ventilation, is an open question. Winslow and Palmer, I915, conclude that the air of unventilated ronms (with controlled temperature and humidity) contains substances which diminish the appetite for food, without producing discomfort or other detectable symptoms. It may be remarked that the continued or repeated exposure to gases-particularly those acting upon the blood, and indeed to any poison which alters the blood, such as acetaniliddoes not generally cause tolerance, but increase of susceptibility.

$\mathrm{CO}_{2}$ on Circulation.-Increase of carbon dioxid up to a certain point causes a great rise of blood pressure (Fig. 6, p. 192) mainly by stimulation of the vasomotor center; moderate increase of $\mathrm{CO}_{2}$ tension perhaps also increases the output of the heart. The peripheral action on blood vessels results in their dilation. High concentrations paralyze the vasomotor center and heart.

Vasomotor Center.-The violent stimulation has been shown by Sollmann and Pilcher, IgII, and by Mathison, IgrI. The latter demonstrated that identical effects are produced by other acids and by lack of oxygen; and that all these act probably by increasing the hydrogen-ion concentration of the blood. The spinal vasomotor centers are much less sensitive than the medullary (Mathison, I9I0).

Direct Action on Vessels. - This results in relaxation (Bayliss, I9OI); it is also seen in excised arterial or venous rings, with all concentrations (Cow, IgII; Hooker, I912). A similar dilation is also produced by other weak acids (Schwarz and Lemberger, I9II). Barcroft, $\mathrm{I}_{9} \mathrm{I}_{4}$, considers this an important factor for the increase of the local circulation in active organs.

Pulmonary Circulation.-The pressure in the pulmonary arteries rlses in asphyxia, even if the rise of the systemic blood pressure is prevented by destroying the medulla (Wood, Jr., IgII). The pulmonary circulation time is slowed (Langlois and Desbouis, I $91_{2}$ ), indicating pulmonary vasoconstriction.

Heart Rate. - This is ordinarily slowed by stimulation of the vagus center; but if the vagi are divided, it is quickened by stimulation of the accelerator center (Dastre and Morat, $\mathrm{r} 885$ ).

Efficiency of the Heart.-In intact animals, the cardiac effects are greatly complicated by the other actions. Jerusalem and Starling, rgro, claim that increase of $\mathrm{CO}_{2}$ in the coronary blood lessens the tonus both in systole and diastole. The coronary vessels are also dilated (Markwalder and Starling, Igr3). With higher concentrations, the systolic tone is lowered more, so that the excursions are diminished; with moderate concentrations, the diastolic tone is more relaxed and the output increased. A certain concentration would therefore be necessary for the optimum efficiency of the heart; this is insufficient in acapnia. Itami, 1913, claims that the rise of blood pressure with 5 per cent. of $\mathrm{CO}_{2}$ in the inspired air is chiefly due to increased output of the heart; and that above 8 per cent. the vasoconstriction becomes the more important. These results have been contradicted: Ketcham, King and Hooker, 1912, attribute the increased output to dilation of the pulmonary vessels, for they found that the isolated heart is 
depressed by all concentrations. In the limulus heart, $\mathrm{CO}_{2}$ produces depression, acting much more powerfully on the ganglion cells than on the muscle (Newmann, I906). Perfusion of the coronary vessels of a mammalian heart with $\mathrm{CO}_{2}$ gas causes prompt fibrillation and arrest, whereas the heart continues to beat for a considerable time if the perfusion is made with oxygen, or even hydrogen (Magnus, I902).

Blood Viscosity.-This is somewhat increased, but not to an important degree (Burton-Opitz, I91 I).

Oxygen on $\mathrm{CO}_{2}$ of Blood.-Oxygen (and carbon monoxid) tends to expel $\mathrm{CO}_{2}$ from the blood; for when exposed to a given $\mathrm{CO}_{2}$ tension, blood that has been deprived of oxygen takes up considerably more $\mathrm{CO}_{2}$ than oxygenated blood (Christiansen, Douglas and Haldane, (914).

Peristalsis.- In intact animals, this is stimulated by asphyxia. Excised intestine is started to rhythmic contraction if it was quiescent; but is relaxed if it was already rhythmically active (Hooker, I9 I 2 ).

Oculomotor Center.-The pupils are ordinarily widely dilated by asphyxia, after primary constriction (Guthrie and Ryan, I9IO). The mydriasis is not due to symphathetic stimulation, for it occurs after division of the cervical sympathetic (Braunstein, I894). It must therefore be ascribed to inhibition of the oculomotor center. Peripherally, $\mathrm{CO}_{2}$ acts as a miotic on frogs' pupil (Auer, I909).

Respiration.-The $\mathrm{CO}_{2}$ content of the blood is the chief regulating factor in ordinary respiration (Zuntz, I897), the respiratory center being stimulated (mainly the rate) even by very slight increase of the $\mathrm{CO}_{2}$ tension. A rise of 0.2 per cent. of $\mathrm{CO}_{2}(1.6 \mathrm{~mm}$.) in the alveolar air doubles the respiration, and a slight reduction of the $\mathrm{CO}_{2}$ produces apnea. The sensitiveness to oxygen is very much less, the respiration being unaffected by variations of the oxygen in the air between 13 and roo per cent. (Haldane and Priestley, I905; Fitzgerald and Haldane, I905). The effective increase of $\mathrm{CO}_{2}$ also produces a measurable change in the ion concentration of the blood (Campbell and coworkers, I9I3). Other acids are similarly stimulant (Winterstein, I9II; Laquer and Verzar, I9II). Loevenhart, I9I3, refers the effects ultimately to interference with oxygenation. The response to $\mathrm{CO}_{2}$ is used experimentally as a test of the excitability of the respiratory center (A. Loewy, 1890 ).

Bronchial Muscle (Excised).-This is constricted by moderate, relaxed by high concentrations (Trendelenburg, I912).

Strychnin Convulsions. - These may be suppressed by $\mathrm{CO}_{2}$ inhalation (Winterstein, I900; Ryan and Guthrie, 1908). On the other hand, moderate asphyxia increases the convulsant effect (Pilcher). Concentrations above 20 per cent. also heighten the susceptibility to acid fuchsin convulsions (Joseph, 1915).

Temperature. $-\mathrm{CO}_{2}$ dyspnea lowers the temperature in health, and especially in fever. It also reduces metabolism (v. Magyary-Kossa, I9ro).

Asphyxial Glycosuria.-All forms of asphyxia-whether from $\mathrm{CO}_{2}$ or deficiency of oxygen-lead to hyperglycemia and glycosuria. All asphyxiant drugs produce this effect, although there may be additional factors. The glycosuria does not occur if the liver is excluded, nor in poorly nourished animals (Auel, I9I4). It seems therefore that the glycogenolysis is stimulated in the liver, but not in the muscles. This effect is probably due to the increased $\mathrm{H}$-ion concentration of the blood, acting through the nervous centers, but requiring the coöperation of the adrenals, for it does not occur if these have been excised (Macleod, I913).

Drugs Leading to the Appearance of Reducing Substances in the Urine.-The following may be enumerated as among the most important (review, S. Weber, 1904): CO, $\mathrm{N}_{2} \mathrm{O}, \mathrm{H}_{2}, \mathrm{H}_{2} \mathrm{~S}, \mathrm{HCN}$, amyl nitrite, nitrobenzol, nitroglycerin, chlorate, chloral, urethane, chloroform, acetone, strychnin, morphin, atropin, curare, phlorhizin, caffein and other methyl xanthins, $\mathrm{H}_{2} \mathrm{O}_{2}$, many acids, $\mathrm{Pb}, \mathrm{As}, \mathrm{Hg}, \mathrm{Ur}, \mathrm{Cr}$, cantharidin, $\mathrm{P}$, epinephrin, thyroid, saline.

Deficiency of $\mathrm{CO}_{2}$ (Acapnia). - The carbon dioxid tension regulates the heart rate, vascular tone, peristalsis, mental condition, and a number of other functions. A decrease of this tension in the blood and tissues produces depressant effects. 
Phenoment of Acapniat.-These have been emphasized especially by Y. Henderson, 1908 to I9Io. Even a slight reduction of the $\mathrm{CO}_{2}$ causes marked increase of the heart rate and slowing of respiration. Further relluction produces tachycardia, venous stasis, arrest of peristalsis, failure of many reflexes, coma and stoppage of respiration. Extreme reduction causes an almost tetanic condition of the heart, abolishing its output, lowering blood pressure and resulting in death. In the lighter grades, recovery occurs promptly if the $\mathrm{CO}_{2}$ tension is increased, as by breathing through a long tube.

Acapnia and Respiration.- - The respiratory center is readily depressed by deficiency of $\mathrm{CO}_{2}$ in the blood. This is the main factor in the apnea following hyperpnea, and one factor in Cheyne-Stokes respiration (Haldane, 1905 and 1909). Weil, 1906, f.i., showed that the apnea is the shorter, the more $\mathrm{CO}_{2}$ is in the air; and that it can be prolonged by fixing the $\mathrm{CO}_{2}$ with $\mathrm{NaOH}$. The increased oxygen content, to which it was formerly attributed, seems to be unessential although excessive oxygen concentration is also depressant (Loevenhart, I9I3).

The direct depressant effect of $\mathrm{CO}_{2}$ deficiency on the respiratory: center was also demonstrated by Winterstein's (I9II) perfusion experiments. The respiration was not restored by oxygen, but by carbonic or other acids, and is therefore an hydrogen-ion effect In intact animals, a persistently low oxygen tension leads to the accumulation of lactic and other acids, and hence to stimulation of the respiration.

Acapria and Shock.-Y. Henderson, Ig08 and I909, pointed out the analogies between the phenomena of acapnia and surgical shock. He believed that the latter may be explained by loss of $\mathrm{CO}_{2}$, through hyperpnea, exposure of intestines, etc. This may be one of the factors but it is unlikely that it is the only one. Henderson's contentions are contradicted by Janeway and Ewing, r 913.

Apnea. - This suspension of the respiratory movements is usually due to depression of the respiratory center by excessively low $\mathrm{CO}_{2}$ content of the blood (acapnia); for instance, as a consequence of hyperpnea. However, acapnia is not essential; for excessive distention of the lungs may produce apnea even when the air contains 5 per cent. of $\mathrm{CO}_{2}$. This phenomenon does not occur if the vagi were divided (Githens and Meltzer, I914). In fowls, $\mathrm{CO}_{2}$ deficiency also stops the respiratory movements, but in this case the effect consists in tonic spasm of the respiratory muscles, and not in heightened threshold. The function of $\mathrm{CO}_{2}$ would therefore consist in the maintenance of the respiratory rhythm (Meyer and Meltzer, I9r5).

Normal $\mathrm{CO}_{2}$ Percentages and Tensions.-These are as follows:

\section{$\mathrm{CO}_{2}$}

Expired air

Alveolar air

Arterial blood

Venous blood

Tissues
Per cent. (Volume)

About 4

4.6 to 6.2

Avge. 5.5

40

45 to 50
Tension

$30 \mathrm{~mm}$.

$42 \mathrm{~mm}$.

ro to $40 \mathrm{~mm}$.

30 to $50 \mathrm{~mm}$.

50 to $70 \mathrm{~mm}$.

\section{CARBONIC OXID (CARBON MONOXID, CO); ILLUMINATING GAS}

General Statement.-Carbonic oxid gas has considerable toxicologic importance, through its occurrence in illuminating gas, especially watergas, and as a product of incomplete combustion. Accidental and suicidal poisoning by it are very common. It forms a firm compound with hemoglobin, thereby preventing it from carrying oxygen, and thus produces asphyxia. This agrees with ordinary asphyxia, except that the skin and mucous membranes are not cyanotic but bright red, due to the color of CO-hemoglobin.

Occurrence.-Carbonic oxid constitutes 4 to ro per cent. of ordinary coal-gas, and $3 \circ$ to $4 \circ$ per cent. of water-gas (which is consequently more dangerous, Sedgwick, IgIr). It is formed during incomplete combustion and is therefore present in the smoke of damped stoves, the vapor of burning charcoal, after mine explosions, etc. It is the principal toxic ingredient of these gases. Coal-gas, however, is said by Vahlen (1903) to possess more than double the toxicity which would correspond to its $\mathrm{CO}$. This is due to coal-tar products (which may be removed by passing the gas through oil). The disagreeable odor of gas is not due to the carbonic oxid (which is practically odorless), but to sulphur compounds. These are not toxic, but serve as a useful warning of the 
escape of gas. This odor can be detected in air containing 0.01 to 0.02 per cent. of gas; i.e., much below the danger limit. They are removed by filtration through soil; so that underground breaks in gas mains are especially dangerous.

Manner of Action.-Carbonic oxid acts only by displacing oxygen and has no direct action of any kind; for if an animal is made to breathe oxygen under 2 atmospheres pressure (which renders it independent of the hemoglobin) the addition of carbonic oxid in any amount produces no symptoms. However, Geppert, 1892 , believes that there is a direct but insignificant action on the central nervous system; and Giacosa, 1905, even claims that the blood changes do not play the principal rôle in CO-poisoning.

Action on Plants and Lower Organisms. - Carbon monoxid (or illuminating gas) slightly retards the growth of bacterial cultures, but is not germicidal. Infusoria and ciliated epithelia are also checked, but not killed. The germination of the seeds of higher plants is not affected. The gas kills plants when applied to the leaves, but not to the roots (Heider, 1914).

Action on Blood.-Carbon monoxid combines with hemoglobin to form carbonic oxid hemoglobin. The compound is very firm; it is distinguished spectroscopically from hemoglobin, and it is not reduced by ammonium sulphid (Hoppe-Seyler, I864 and 1865). (See Fig. 29.) It has a cherry red color (Piorry, 1826 ), which is quite different from that of oxyhemoglobin, especially when it is diluted. This forms one of the best tests for its presence.

The affinity of hemoglobin for carbonic oxid is about 2 ro times as great as that of oxygen; even very small proportions therefore prevent the complete oxygenation of blood and produce asphyxia.

Fate.-The $\mathrm{CO}$ is excreted unchanged, and is not oxidized to $\mathrm{CO}_{2}$. In fatal cases, a part persists for a long time (over a month) in the blood, and to a less extent in the muscles (Wachholz and Lemberger, I905). It may penetrate postmortem into a cadaver, but so slowly that it can not be demonstrated in the internal organs (Stoll, r 1909). $\mathrm{Cl}$. Bernard observed that $\mathrm{CO}$ delays the postmortem degeneration of blood corpuscles, the serum separating very clear.

Toxic Concentrations. - The absorption of $\mathrm{CO}$ is proportional to its concentration in the air. Gruber, 1881 , found that 0.024 per cent. is harmless even after several hours. The toxicity begins with about 0.05 per cent.; is severe with 0.07 per cent.; and dangerous with 0.16 per cent.; 0.2 to 0.4 per cent. is generally fatal. Dizziness and hyperpnea occur when about 20 per cent. of the hemoglobin is saturated; unconsciousness with 50 per cent.; death with 60 to 80 per cent. of saturation (Dreser, 1891 ; Balthazard and Nicloux, 19II).

Conrse.-The phenomena agree with those of ordinary asphyxia, except by the absence of the cyanosis; the skin being pink or pale, and the lips bright red (i.e., the color of CO-hemoglobin). This is of diagnostic importance. The effects are insidious, the person becoming unconscious of ten with no premonitory symptoms.

The clinical cases may be classified as acute, chronic and relapsing (McConnell and Spiller, Igr2).

Acute Type.-Nausea, malaijse, headache, vertigo, muscular weakness; then drowsiness, coma, relaxation of sphincters; sometimes muscular twitchings and convulsions. There may be cyanosis. Death sometimes occurs acutely through aspiration of vomited matter during unconsciousness. Sequels: aspiration pneumonia, of ten fatal; palpitation, localized hyperemias, gastro-intestinal disturbances, transient asphyxial glycosuria (review, S. Weber, "1904), cutaneous eruptions, localized edemas, gangrene; paralyses of central and peripheral types, choreiform movements, persistent headache; mental changes, loss of memory, confusional insanities, etc.

Chronic Type.- - Headache, nausea, vertigo, weakness, anemia, slowed intellect and failure of memory, and all the phenomena of the acute type more slowly developed. The memory for recent events is impaired much more than for those of older standing (S. Brown, rgo6). The nervous phenomena have been referred to serous leptomeningitis (Zieler, 1897 ).

Relapsing Type.-The acute symptoms are followed by complete recovery, but after some weeks grave paralytic phenomena appear and are often' fatal. These cases show 
softening of the lenticular nucleus, referred to fatty degeneration of the vessels, proceeding to calcification.

Induced Tolerance.-Guinea pigs can be accustomed to live for months in an atmosphere by which 25 per cent. of their hemoglobin is saturated with $\mathrm{CO}$. This tolerance is attained by an increase of the corpuscles and hemoglobin, so that the oxygen capacity becomes almost normal.

Treatment of $\mathrm{CO}$ and Illuminating Gas Poisoning.-In the presence of a large excess of oxygen, the carbonic oxid hemoglobin is again decomposed, and the $\mathrm{CO}$ eliminated (not burned). The treatment therefore consists in artificial respiration, or still better, the forced inhalation of oxygen. Bleeding with transfusion of healthy blood is very effective (WormMueller; Crile and Lenhart, I907). Patients should not be exposed to cold.

With respiration, natural or artificial, of ordinary air, the CO-hemoglobin is decomposed below the danger limit within an hour, the $\mathrm{CO}$ being practically eliminated in two or three hours. It is therefore superfluous to use oxygen or transfusion after an hour.

If the coma continues longer, this is due to persisting injury of the nerve-centers by the early asphyxia. Once this injury has occurred, it would not be helped by oxygen. The existence of acidosis indicates alkalies; otherwise the late treatment would be expectant (Y. Henderson, I9I6).

The prognosis varies with the duration of the exposure and of the coma. It is bad if the coma has lasted more than forty-eight hours, or if edema or cutaneous blebs have developed.(G. I. Jones, I909). Even in favorable cases, recovery is rather more slow than with most gases; but the acute effects.have generally disappeared within three hours.

\section{OXYGEN}

The Inhalation of Pure Oxygen.-Oxygen constitutes about one-fifth (20.94 per cent.), by volume, of ordinary air. An increase of this proportion, and even the inhalation of undiluted oxygen, produces no noticeable effect under ordinary conditions. The rate of oxidation or the consumption of oxygen is not at all increased (Pflueger); indeed, the output of $\mathrm{CO}_{2}$ is rather lessened. Evidently, the proportion of oxygen in air suffices for all ordinary needs (or, rather, it is greater than necessary); and additional oxygen acts merely as an indifferent gas-as so much nitrogen or hydrogen. In other conditions, however, namely when the supply of oxygen is deficient from any cause, its inhalation will remove the asphyxial symptoms with striking promptness and efficiency.

Condition of the Oxygen in the Blood.-The effects of most gases are strictly proportional to their partial pressure in the respired air. Oxygen departs from this rule, for there are practically no effects when its percentage is varied between Io to $1_{5}$ or roo per cent. This is explained by the fact that only a small part of the oxygen is carried in physical solution in the blood plasma. By far the greater part is in loose chemic combination with hemoglobin, I molecule of hemoglobin combining with I molecule of $\mathrm{O}_{2}$ (Butterfield, I9I3). This combination is only slightly affected by the above changes of pressure. When respiring ordinary air without obstruction, the hemoglobin of the arterial blood is already four-fifths to ninetenths saturated with oxygen; and the amount taken up can therefore be but little increased by raising the oxygen tension (Buckmaster and 
Gardner, I9I3). The oxygen of the plasma, however, increases directly with the oxygen percentage of the alveolar air. While the amount thus carried is numerically small, it becomes important in conditions in which the hemoglobin can not perform its functions properiy, as in anemias or $\mathrm{CO}$ poisoning. The plasma oxygen may also play a part when the demands for oxygen are greater than can be supplied by the hemoglobin; as in very severe muscular exertion or in cardiac disease.

Ordinary arterial blood contains about i 8.5 per cent. of oxygen in chemic combination with hemoglobin; and 0.6 per cent. dissolved in the plasma-a total of ro. I per cent. Inhalation of undiluted oxygen increases the percentage in oxyhemoglobin only to 18.7 per cent.; the dissolved oxygen is increased in proportion to the partial pressure; i.e., to 3 per cent., giving a total of 21.7 per cent., or an increase of 2.6 per cent. This is not really as small as it appears; for it seems that the oxyhemoglobin does not yield all its oxygen to the tissues (for instance, after death by asphyxia the blood still contains considerable oxygen). Haldane considers that the increase of 2.6 per cent. really equals about 40 per cent. of the easily available oxygen of the blood.

Dissociation Curve of Oxyhemoglobin.- This follows the law of mass-action, showing that the reaction is chemic and not absorptive. The curve is identical for pure solutions of hemoglobin, whatever their origin; but it is markedly affected by electrolytes, and therefore differs in the blood of different animals or even individuals. The action of electrolytes is explained by aggregation of the hemoglobin molecules. It is especially great with acids (H-ion concentration). Increased acidity diminishes the affinity, and therefore the effective concentration of oxygen. 'Thus $40 \mathrm{~mm}$. of $\mathrm{CO}_{2}$ cuts the efficiency of oxygen into half. The same laws hold for CO-hemoglobin (Barcroft, I9I4).

Absorption of Oxygen in the Lungs.-There has been much discussion whether this follows purely physical laws. The question is even now not absolutely decided. Ac.cording to Douglas and Haldane, Igro, the absorption is simply physical under ordinary conditions; but if so much $\mathrm{CO}$ is inhaled that the supply of oxygen to the tissues become inadequate, there is an active (secretory) absorption, so that the partial pressure of oxygen in the arterial blood becomes higher than in the alveolar air. This occurs also when the demand for oxygen is increased by muscular work. It is not known how this correlation is brought about.

Mechanism of Oxidations in Tissues. - The oxidations of metabolism are not accomplished by oxygen directly, for they can not be reproduced in vitro at ordinary temperature. There must be some activation of the oxygen. The most commonly accepted theories are modifications of that of M. Traube, i.e. the production of superoxids, which produce in vitro types of oxidation corresponding slowly to those in the body. The oxidases are supposed to consist of organic peroxids and a catalyst (peroxidase). Catalases, which occur in many tissues, act only on hydrogen peroxid; their function is problematic (Bibliography, Kastle, Hyg. Lab., Bul. No. 59). The oxidizing ferments so far identified are apparently not connected with the energy-producing oxidations of the body (Batelli and Stern, I9I2).

Relation to Medullary Centers. - These are stimulated by moderate deficiency of oxygen; paralyzed by extreme deficiency. The respiratory center is the most sensitive, then the vasomotor, and least the vagus center. The intensity of the reaction is determined by the rapidity and degree of the change and by the state of the centers. Excessive oxygen is depressant. Loevenhart, I9I3, attributes ordinary asphyxial stimulation to depression of the oxidative processes of the cells, by actual abstraction of oxygen, or by increase of hydrogen-ion concentration (acidosis, $\mathrm{CO}_{2}$ ). Very similar phenomena are produced very promptly by cyanids which are known to interfere with oxidation. The response occurs so promptly that there would scarcely be time for the accumulation of acid; so that deficiency of oxygen probably acts directly (Gasser and Loevenhart, I9I4).

Two-phase Oxidation Theory.-Loevenhart, I915, suggests that asphyxia may be explained by assuming two phases of oxidation: the "rest process," occurring during rest and recovery, and requiring external oxygen; and the "activity process," during activity, which consumes internal oxygen. Both processes would be constantly in operation; their relative intensity determining the state of activity of the cell. The addition of external oxygen would reinforce the "rest process" and thus cause depression; while oxygen deficiency would have the opposite effect.

Relation of Oxygen to Activity.- Every increase of activity, in any organ, appears to produce an increased consumption of oxygen. This outlasts by far the time of actual 
stimulation and risible response. The same is true of the production of heat. This corresponds to the "rest process," and is evidently concerned in the storage of potential energy.

Minor Effects of Oxygen Inhalation. Melabolism.-Lavoisier and Seguin, I 789 , claimed that pure oxygen has no effect on metabolism. This has been confirmed by the modern investigators. However, the reputed benefits from the inhalation of oxygen in falty degeneration and some olher obscure diseases (if the reports are reliable) would point to some effect on metabolism, perhaps too small to be detected by the usual methods of observation.

Oxygen inhalation also produces some other slight effects: Cl. Bernard claimed that birds placed in pure oxygen are greatly excited; and that the urine of rabbits becomes acid. Bencdict and Higgins, 19 1 1, observed some slowing of the pulse, without change in respiration. There are rather more marked effects after exercise (Cook and Pembrey, I9I3). The efficiency of athletes is increased and forced respiration can be maintained longer (Hill and Flack, I909 and I9ro). Christiansen and co-workers, 1913. attribute this to the smaller solvent power of blood for $\mathrm{CO}_{2}$ in the presence of excess of oxygen.

Oxygen under excessive pressures (above I.7 atmospheres) produces pneumonia; pressures above 3 or 4 atmospheres, cause violent convulsions and ultimately death. These effects are not due to pressure as such, for they are not produced by compressed air until the partial pressure of oxygen reaches the above figures. A. Adams, I9I 2, and A. Schmidt and David, 1912, claimed that prolonged inhalation, even of 70 per cent. oxygen at ordinary pressure would produce fatal pneumonia. Bruening, I9I2, refers these results to the dryness of their gas, since the same effects were produced with dry compressed air, and not if moist oxygen was inhaled. However, harsner, r9r5, found that even when these precautions are taken, rabbits exposed forty-eight hours to 80 per cent. oxygen show congestive or inflammatory changes in the lungs. Exposure for twenty-four hours did not usually produce any damage.

Phagocytosis. - This has been studied in iilro by H. J. Hamburger, I915. Deprivation of oxygen stimulates phagocy tosis for a time; prolonged exposure is depressant. Increase of oxygen is also generally depressant. Hy.drogen peroxid stimulates markedly: Potassium chlorate and ferricyanid produce no effect.

Oxygen in Asphyxial Conditions.-The results are especially striking in those forms of asphyxia, in which the access of oxygen to the blood is hindered, as by vitiated air (anesthesia, mines, wells, etc.); by mechanical interference with respiration (suffocation, drowning, croup, etc.); by depressed respiration (anesthesia, collapse); or by a diminished absorbing surface of the lung (pneumonia). In all these cases the blood is distinctly venous, i.e., the hemoglobin is not saturated with oxygen; and the inhalation of the gas promotes the saturation.

In anemia (whether from hemorrhage or any other cause, such as chlorosis or leucemia) asphyxial conditions result, not from deficient supply of oxygen, but from a deficiency of hemoglobin. The inhalation of oxygen, in these cases, does not increase the quantity of oxygen carried by the hemoglobin, but that carried in solution by the plasma, as has been explained.

The inhalation of oxygen is also useful, in an entirely analogous manner, in advanced cardiac disease, when the circulation is too slow to convey the required oxygen to the tissues.

Another factor may possibly come into play, for G. N. Stewart, I9I , found that oxygen inhalation, while it produces no effect on the blood flow of the hand in normal individuals, produced a marked increase in a cyanotic patient. Artificially perfused vessels, (frogs) are constricted by oxygen (Hooker, Igri).

Administration.-Compressed oxygen is marketed in metal tanks. The gas should be passed through warm water and delivered through a funnel suspended over the face of the patient. The rate and duration of the administration is regulated by the symptoms. 
The funnel method, however, is wasteful, and only enriches the air by 3 to 5 per cent of oxygen. If higher concentrations are desired, a mask with valves must be used. (Y. Henderson, I9I6).

Intravenous Injection of Oxygen-This is described by Tunnicliffe and Stebbing, 1916. It gives rapid relief to the cyanosis and dyspnea. In man, the gas can be injected into a vein at the rate of 600 to 1200 c.c. per lour; usually beginning with the rate of 500. The heart must be watched, the injection being stopped if any irregularity appears.

Subcutaneous Injection of Oxygen.- This was introduced by Derose, I9 I 2. McCrae, I914, believes that it has a limited field as substitute for inhalation.

PREPARATIONS-OXYGEN

*Oxygenium (Oxygen.), U.S.P.

\section{OXYGEN DEFICIENCY}

Asphyxia Through Lack of Oxygen.-This may result from a deficiency of oxygen in the air-by breathing rarefied air, or air diluted with indifferent gases $\left(\mathrm{N}, \mathrm{H}, \mathrm{CH}_{4}\right)$; by the presence of CO; by anemia; etc. Ordinary air may be diluted until it contains only about is per cent. of oxygen (or may be rarefied to the same degree), without producing any immediate effect (Bernstein, I 882). A candle will be extinguished when the oxygen has fallen to about 17.5 per cent., and is therefore a test for the safety of the air, as far as oxygen is concerned. At about ro per cent. slight symptoms are noticeable, especially on exertion, but they are not alarming-dizziness, shortness of breath, deeper and more frequent respiration, quickened pulse, slight cyanosis. With 7 per cent., these symptoms become serious, and stupor sets in. With a slightly lesser percentage, unconsciousness occurs. With 2 to 3 per cent., death occurs within forty-five seconds (the effects of low tensions of oxygen are described by Haldane, I908; the effects on respiration are reported by Schenk, I908). Anoxemia dilates the blood vessels (including the coronary) on direct perfusion (Richards, I9I4).

Chronic Oxygen Deficiency.-As seen in anemias, etc., this causes considerable increased tissue destruction, fatty degeneration, and acidosis (ammonia in urine). Finally there is general paralysis of all organ functions.

Exposure to slightly rarefied air, as on mountains, causes "mountain sickness"nausea, headache, insomnia, etc. This generally disappears after a few days. A curious phenomenon is a prompt and persistent increase in the corpuscle and hemoglobin content of the blood (by 25 to 40 per cent.). This is at first due to a diminution of the plasma, the total quantity of hemoglobin being unaltered. After a time, however, the absolute hemoglobin is also increased; the formation of corpuscles by the bone-marrow being stimulated (Dallwig, Kolls and Loevenhart, I9r5; G. H. Martin, Bunting and Loevenhart, I9I6). There is retention of N., S. and P. The respiratory metabolism is increased. These effects are attributed to oxygen deficiency (Douglas, Haldane, Henderson and Schneider, I9 33 , Loevenhart).

Cheyne-Stokes respiration is also referable to oxygen deficiency (Douglas and Haldane, I909): the apnea lowers the oxygen and leads to accumulation of lactic acid. This increases the excitability of the respiratory center, so that a very low $\mathrm{CO}_{2}$ tension suffices to produce hyperpnea. This lowers the $\mathrm{CO}_{2}$ and increases the oxygen, so that the lactic acid is oxidized, apnea reappears and the whole process is repeated.

\section{HYDROCYANIC ACID AND CYANIDS}

General Statement.-Hydrocyanic (prussic) acid is probably the most rapidly acting poison. It produces death under asphyxial symptoms, by hindering the oxidative processes of the tissues. It also interferes with most other fermentations and is therefore a general protoplasmic poison. Compounds containing the $\mathrm{CN}$ radical are toxic only if they can liberate HCN. Ferro-, ferri-, and sulphocyanids are stable and therefore do not show these effects.

Occurrence.-Hydrocyanic acid exists in glucosidal form as amygdalin in many plants, especially in the seeds and leaves of the cherry, almond, peach, etc. This amygda- 
lin, when pure, is almost entirely harmless, except as it is decomposed in the body. But in most plants it coexists with the ferment emulsin, by which it is split up in the presence of water into hydrocyanic acid, glucose, and benzaldehyd:

$$
\mathrm{C}_{20} \mathrm{II}_{27} \mathrm{NO}_{11}+{ }_{2} \mathrm{H}_{2} \mathrm{O}={ }_{2} \mathrm{C}_{6} \mathrm{H}_{12} \mathrm{O}_{6}+\mathrm{HCN}+\mathrm{C}_{6} \mathrm{H}_{6} \cdot \mathrm{CHO}
$$

One gram of cherry kernels yields $I .7 \mathrm{mg}$. hydrocyanic acid; I gram bitter almond pulp yields $2.5 \mathrm{mg}$. hydrocyanic acid.

The most important cyanogen $(\mathrm{CN})$ compounds are the acid (prussic acid, official as the dilute, 2 per cent. solution) and cyanid of potassium. Nercury cyanid has mainly a mercury action. The cyanid action is also shared by the organic esters (nitrils); these differ from potassium cyanid only in the rapidity with which the cyanogen is liberated (Lang, I894; Heymans and Masoin, I897, 1900, r9or; R. Hunt, r904). Nitroprussids liberate CN readily and agree with cyanids, except that they are less convulsive (L. Hermann).

Effects on Higher Animals. - When given in doses small enough to permit of watching its action, this is seen to consist in a feeting stimulation of cerlain parts of the central nervous system, followed by depression and paralysis. The action begins in the medulla. The vomiting, respiratory, vagus, pupil-dilator, and vasomotor centers are all stimulated. Then comes unconsciousness, and after this convulsions. In man these are probably mainly, medullary in origin. Then follows paralysis of the whole central nervous system. Involuntary evacuations of feces, urine, and semen are frequent.

The Respiration.-This is at first greatly increased, especially in depth; it then becomes slow, shallow, and irregular (Cheyne-Stokes type). It stops considerably before the heart, paralysis of the respiratory center being the immediate cause of death.

The effects on respiration are central, for they are not affected by section of the vagi, or by atropin (Boehm and Knie, 1874). The smallest effective doses increase both the rate and depth of respiration; $\mathrm{HCN}$ being one of the strongest stimulants of the respiratory center. This is more sensitive to cyanids than the vasomotor center, and this again is more sensitive than the vagus center. Larger doses slow the rate, but greatly increase the depths of respiration. Toxic doses depress and paralyze the center. The respiratory effects are antagonized by iodosobenzoate, which yields active oxygen (Grove and Loevenhart, igri).

In frogs, the respiratory center is also stimulated by small and depressed by large doses. Decerebrated animals require larger doses than normal to produce respiratory paralysis (Hepner, I9 I 5).

Convulsions.- Rabbits deprived of their hemispheres exhibit the usual type of convulsions; but the course is more rapid (Morita, I915).

The Effect on the Circulation.-This is entirely analogous to that of asphyxia (see Fig. 6, page 192). As in the latter, the heart may again execute a few movements after it has stopped for a time ( $h$ in the figure); but these do not suffice to maintain the circulation.

Vasomotor Center.-This shows intense stimulation, with corresponding rise of blood pressure. The effects correspond with asphyxia (Pilcher and Sollmann, I9I5).

Peripheral Action on Vessels.-In cxcised kidneys perfused with saline solution, the addition of hydrocyanic acid causes a very marked dilation of the renal vessels. The effect is apparently due to a paralysis of the arterial muscle. It disappears promptly on removing the cyanid (Sollmann, r908). The coronary vessels are also dilated (Loevenhart and Eyster, I9I3). It is quite possible that these dilations are due to the interference with the oxidation of metabolic waste-products.

Heart.-Applied directly to the frog's heart, cyanids cause a standstill, which is not removed by atropin; however, the heart responds strongly to electric or mechanic stimulation. The paralysis evidently involves the automatic property of the cardiac muscle. There is no evidence of this action on the blood-pressure tracings from mammals.

In limulus, weak concentrations stimulate the heart muscle and ganglia (Carlson, 1907).

Cyanid Action Explained by Tissue Asphyxia.-The phenomena of the action of hydrocyanic acid on the central nervous system-the stimulation of the medullary 
centers, the convulsions, the vascular paralysis, etc.-bear a close resemblance to those of asphyxia and it can be easily shown that oxidation is enormously diminished. There is, however, one very marked difference from ordinary asphyxia, for the blood remains of a bright arterial color, indeed, the oxygen content of the venous blood in typical cyanid poisoning approaches that of ordinary arterial blood (Cl. Bernard). ${ }^{2}$ The oxygen is in the ordinary combination with hemoglobin, and can be removed as readily as normally in vitro. It is therefore evident that the oxygen supply to the tissues is not lessened. The cause of the asphyxia, of the lessened oxidation, must lie in the protoplasm of the tissues. This undergoes what might be termed "internal asphyxia," in sight of abundant oxygen (Geppert, I889). The interference with oxidation has also been demonstrated with "salted frogs;" excised kidneys (Vernon, r906); many lower animals; and with ferments. It may therefore be assumed that the cyanid interferes with the fermentative processes by which the utilization of oxygen is accomplished.

Hyman, 1916, has measured the effects on oxygen consumption in sponges. They consist in a primary increase, followed by diminution. The depression increases as the concentration of the cyanid, so that high concentrations produce practically pure depression.

Action on Ferments.-Schoenbein, r 867 , first showed that hydrocyanic acid inhibits oxidizing ferments, preventing the catalytic action of shed blood on $\mathrm{H}_{2} \mathrm{O}_{2}$. The catalase is not destroyed, but only inhibited. (In acute cyanid poisoning, the inhibitory concentration is not reached; Duncker and Jodlbauer, T9II). It has a similar inhibitory effect on many other organic ferments and inorganic catalyzers (spongy platinum). The effect on metals is probably produced by a minute film of cyanid, and its mechanism is therefore probably not analogous to that on organic ferments.

Action on Hemoglobin. - It is doubtful whether HCN combines directly with hemoglobin under ordinary conditions. Zeynek, rgor, states that cyanid does not combine at all with hemoglobin; and that with oxyhemoglobin it does so only after several hours; even at body temperature.

When added to methemoglobin, it causes the formation of cyan-hemoglobin (Kobert, I89 ; v. Zeynek), which differs from ordinary hemoglobin in its bright red color and in a peculiar band (Fig. 29). This compound is responsible for the bright red ecchymotic spots seen after hydrocyanic poisoning. Methemoglobin can be used as a test for hydrocyanic acid.

Lower Organisms. - Cyanid is a violent protoplasmic poison, presumably through its restraint of the fermentations which play a most important rôle in life processes. All forms of life, all tissues and organs, are affected. Infusoria are killed in a concentration of o.I per cent. The growth of yeast and of higher plants, the germination of seeds, the contractility of animal cells, the conductivity of nerves, etc., are inhibited. If the action is sufficiently continued, all vitality is destroyed; but if the cyanid is promptly removed, complete recovery occurs. Its effects agree with those of deprivation of oxygen. J. Loeb, I9ro, found in arbacia ova that this lessens the toxic effects of a number of poisons (salts, saccharose, lipoid-narcotics), and Warburg, rgl 2, claims that the combined inhibitory action of HCN and hydrocarbon narcotics is less than simple summation. In certain fish ova, cyanids also suspend the autolytic processes accompanying death, so that the cells remain in a condition between life and death for several days (Sollmann, I906).

Phagocytosis. - This is stimulated in vitro by traces of $\mathrm{KCN}$; the effect being analogous to oxygen deprivation (H. J. Hamburger, 19 15 ).

Membrane Permeability.-Even very dilute cyanid solutions increase the permeability of Tradescantia cells. This effect is enhanced by very low concentrations of chloroform or alcohol; diminished by higher concentrations (Krehan, I9r4).

Other Effects on Metabolism.--Severe cyanid poisoning increases the total $\mathrm{N}$ excretion, mainly as urea, both in starving and in fed animals.

Local Anesthesia.-Direct application of HCN, even to the intact skin, produces local anesthesia.

Fate.-Hydrocyanic acid is very unstable; it changes very readily, even by mere exposure to light and air; and it undergoes still quicker decomposition in the body. Part of it combines with sulphur-containing molecules to form sulphocyanids. A part is excreted unchanged by the lungs. The fate of the remainder is unknown. Postmortem, Waller, Igro, found the largest quantity in the heart and brain.

Sulphocyanid is found in the human parotid saliva, while it is absent in that of all other animals. The nervous connection of the gland must be intact, for it is absent in middle-ear disease, for instance, Juergens, 1901. Gscheidlen found it constantly in human

1 Chib, I III, claims that the red color of the venous blood is not due to lessened oxidation, but to more rapid circulation from vasodilation and cardiac stimulation. 
urine; and Nencki claims that traces exist normally in the stomach. Its significance is not understood, but it does not result from bacterial decomposition as was formerly supposed. It is suggestive that some proteins with loosely combined sulphur convert $\mathrm{KCN}$ into KCXS in vitro at room temperature (Pascheles). Its amount can be increased by taking $H C N$-even that existing in tobacco smoke causing an increase.

Acute Cyanid Poisoning.-Hydrocyanic acid is not much used in criminal poisoning ( 0.5 per cent. of the recorded cases), because its quick action would soon lead to the detection of the crime. On the other hand, it or the cyanids are favorite methods with suicides. On account of the technical uses of potassium cyanid (in photography, electroplating, cleaning of metals, etc.), it can generally be obtained with little trouble. Some cases are also reported of accidental poisoning by the gaseous acid.

Pure hydrocyanic acid is extremely volatile and very toxic. The famous chemist Scheele, who discovered hydrocyanic acid, was killed by the vapors set free in the breaking of a flask of this fluid. Many fatalities have been produced by the use of cyanid rapors as insecticide.

The strength of the official (dilute) acid should be 2 per cent.; but many samples are much weaker, on account of decomposition. Potassium cyanid is also very variable. The pharmacopoeia requires a strength of 90 per cent.; but the commercial article sometimes contains unly 30 or 40 per cent. This accounts in part for the variability in the appearance and severity of the symptoms. Another variable factor is the rate of absorption, according to whether the stomach is full or empty. In the latter case, there is a much better chance of saving the patient. However, the absorption is always very rapid; it occurs even to some extent from the intact skin. Application to raw surfaces or to cuts has given rise to poisoning. Inhalation of the vapors has proven fatal.

The fatal dose of absolute HCN is about $0.05 \mathrm{Gm}$. ( $\mathrm{I}$ gr.); that of $\mathrm{KCN}$ is 0.2 to 0.3 $\mathrm{Gm}$. (3 to $5 \mathrm{gr}$.); that of dilute hydrocyanic acid would be about $2.5 \mathrm{Gm}$.; 50 or 60 Bitter almond seeds, would be fatal. Correspondingly larger doses are needed if the drugs are impure or decomposed. The impression prevails quite widely that prussic acid is one of the most powerful poisons. This reputation is justified by its extremely rapid action, rather than by its toxic dose.

In the so-called apoplectic form; i.e., when very large doses are taken, the patient may fall unconscious, often with a loud cry, within ten seconds, ${ }^{1}$ and die within two to five minutes, after some convulsions. Ordinarily, the course is rather more prolonged; several minutes may elapse after taking the poison before any effect is noticed; death occurs between fifteen minutes to an hour.

The symptoms consist in vertigo, mental dimness, headache, palpitation; then comes dyspnea, which may become very violent from stimulation of the respiratory center; the patient then becomes totally unconscious and shows very violent convulsions. During this stage the heart may be greatly quickened. The respiration becomes first difficult and then ceases. The heart at this time is very much weakened, but still continues to beat for a short time and then stops.

The mortality is very high: 95 per cent. If the patient survives an hour, recovery is the rule. It is often followed by persistent headache, tremors, etc.

The prompt diagnosis of cyanid poisoning is very important in view of the treatment. The odor is quite characteristic. (The student should familiarize himself with the appearance and odor of potassium cyanid.) The very rapid onset of the symptoms is also characteristic.

At the autopsy, the odor is also noticeable on opening the body. There are no peculiar lesions, except with potassium cyanid, which acts as an alkaline caustic. The blood is generally liquid, and remains so after removal from the body (by interference with the fibrin ferment). Its color is stated to be very dark-but this is not true of all cases: it may be of a very bright arterial color. Bright red ecchymotic spots, due to cyan-hemoglobin, occur during putrefaction. The final proof of cyanid poisoning rests on chemic tests. The poison disappears within two weeks during putrefaction; but remains for a long time if the latter is prevented and if there is no evaporation.

Chronic Poisoning. - The repeated administration of sublethal doses of cyanids leads to chronic cachexia, without altering the susceptibility to the poison (Schlegel, 1892). Peripheral neuritis has been reported (Collins and Martland, 1908).

1 This is the shortest time reported-a fact which may be of some medico-legal importance. 
Treatment of Cyanid Poisoning.--This must be very prompt. It consists in lavage with chemical antidotes, and in symptomatic stimulation. The chemic antidotes are oxidizing agents $\left(\mathrm{H}_{2} \mathrm{O}_{2}\right.$; potassium permanganate, $1 / 4$ to $1 / 2$ per cent.); "arsenic antidote;" or sodium thiosulphate ("Hypo"), I per cent. These form harmless molecular compounds. Unfortunately, the absorption of the poison is so rapid that the antidotes are of ten ineffective.

Chemic Antidotes. $-\mathrm{H}_{2} \mathrm{O}_{2}$ is only effective if the stomach is empty. It forms oxamid: $\left(2 \mathrm{HCN}+\mathrm{H}_{2} \mathrm{O}_{2}=(\mathrm{CO})_{2}\left(\mathrm{NH}_{2}\right)_{2}\right.$. Alkaline ferrous sulphate forms ferrocyanid. It was advocated by Smith, 1884, and is indorsed by Sandwick, I906. It is conveniently prepared by adding $\mathrm{FeSO}_{4}$ to the official "arsenic antidote." Cobaltous nitrate ( 0.5 per cent.) forms cobalto-cyanid; and thiosulphate forms sulphocyanid. Thiosulphate has given especially good results as a prophylatic, and in nitril poisoning in animals (Meurice, 1900), but is of little value in developed cyanid poisoning (Heymans and Masoin, I 897 ). It may be injected intravenously ( 0.5 to I per cent., added to 0.6 per cent. $\mathrm{NaCl}$ ) or hypodermically (3 per cent.), using Ioo to 500 c.c.

It has also been suggested to bleed the patient profusely, replacing the blood by saline solution.

The symptomatic treatment consists essentially in supporting the respiration. Brisk artificial respiration should be begun at once and maintained as long as the heart beats. This acts mainly by aiding the elimination of the poison through the lungs. Counterirritation, and general stimulants (caffein and atropin) may also be used.

Therapeutic Uses.- There seems to be no rational basis for the therapeutic use of the cyanids. They are sometimes used as flavors.

The local anodyne action is used against itching of the skin and pruritus, against cough, and against vomiting. If given internally, it must be in small doses frequently repeated, on account of the rapid decomposition. Hydrocyanic acid has also been given for other purposes, especially in phthisis. The powerful respiratory stimulation has been used experimentally against chloroform accidents (Backus), but it would be a dangerous remedy.

Fumigation with HCN is used for the destruction of insects and larger vermin. Rather high concentrations are required (McClintock, Hamilton and Lowe, I9II), and fatal accidents are frequent.

\section{PREPARATIONS-CYANIDS}

*Acidum Hydrocyan. Dil., U.S.P., B.P.-About 2 per cent. of HCN. Colorless liquid, with odor of bitter almonds. Incompatible with metals. Dose, o. I c.c., I $1 / 2$ minims, U.S.P.; 0.12 to 0.3 c.c., 2 to 5 minims, B.P.; maximum dose, 0.3 c.c., 5 minims.

On standing, it loses strength rapidly, decomposing into ammonium formate, $\mathrm{CO}_{2}$, cyanic acid, and a brown precipitate of paracyanogen (L. A. Brown, 1910). The decomposition is retarded by acid, alcohol, and acetanilid (Coblentz and May, rgo8).

${ }^{*}$ Pot. Cyan., KCN.-Occurs usually as white, opaque masses, with cyanid odor. Dose, $5 \mathrm{mg} ., 1 / 12 \mathrm{gr}$. Commercial samples are often very impure.

Sodii Cyanidum (Sod. Cyanid.), U.S.P.; NaCN.-White, opaque pieces, or granular powder; deliquescent in the air and exhaling the odor of hydrocyanic acid. Freely soluble in cold water.

Amygdala A mara, B.P.; Bitter Almond.-The ripe seed of Prunus Amygdalus amara. Contains amygdalin, fixed and volatile oil, etc.

Aq. Amygd. Amar., U.S.P.-Bitter Almond Water contains a mere trace of hydrocyanic acid and differs from the preparation of the same name, recommended by the Brussels Conference of IgO2, which contains O.I per cent. of hydrocyanic acid. Dose, 4 c.c., I dram, U.S.P.; maximum dose, 4 c.c., I dram.

Ol. Amygd. Amar., U.S.P.-The distilled oil. Yields not less than 85 per cent. of benzaldehyd, and not less than 2 per cent. nor more than 4 per cent. of hydrocyanic acid. Dose, 0.03 c.c., $1 / 2$ minim, U.S.P. 
Sp. Amygd. Amar., U.S.P. - 1 per cent. of oil. Dose, 0.5 c.c., 8 minims, U.S.P.

Binzaldchydum, U.S.P., $\mathrm{C}_{6} \mathrm{H}_{5} \cdot \mathrm{COH}$. - An aldehyd produced synthetically or obtainef from natural oil of bitter almond. A colorless or yellowish, strongly refractive lifjuiıl; bitter-almond-like odor; burning aromatic taste. Slightly sol. in water, miscible with alc., eth., and tixed and volatile oils. Dose, 0.03 c.c., $1 / 2$ minim, U.S.P.

Laurocerasi Folia, B.P.- The fresh leaves of Prunus Laurocerasus (Cherry-Laurel).

1q. Laurocer., B.P.- - I per cent. of HCN. Dose, 2 to 8 c.c., $1 / 2$ to 2 drams, B.P.

Prunus IVirginiana, U.S.P.; Prun. Virgin. Cort., B.P.(Wild Cherry Bark).-The stem-bark of Prunus serotina. Contains tannin, amygdalin, and bitter glucosid. Dose, 2 Gm., 30 gr., U.S.P.

*Syr. Prun. Firg., U.S.P., B.P. (Syrup of Wild Cherry).-I5 per cent. Dose, 4 c.c., I dram, U.S.P.; 2 to 4 c.c., $1 / 2$ to I dram, B.P.; mainly as flavor for cough mixtures.

Tr. Prun. Virgin., B.P.-2o per cent. Dose, 2 to 4 c.c., $1 / 2$ to I dram, B.P.

\section{PHYSICAL FACTORS OF SALT ACTIONS}

General Statement.-The drugs so far studied produce their actions mainly by entering into chemic combination with the protoplasm. Since these chemic changes are distinct for each drug, the effects are also distinctive, differing in kind as well as in degree. Besides the properties which are ordinarily designated as chemical, substances in solution or suspension also possess certain physical properties, depending on their molecular state, and independent of their chemic composition. These properties are possessed both by the drugs and by the cells.

Molecules in solution have qualitatively and quantitatively identical effects on the boiling and freezing-point, osmosis, etc., the so-called "colligative properties." By influencing the osmotic exchanges and the surface tension, these alter mainly the water and salt content and internal pressure of the cells. This produces changes in composition and therefore in function, even when the substance itself does not enter into the cells. These effects are produced by all substances which form true solutions (the "crystalloids"). Since the results are proportional to the molecular concentration (the number of molecules dissolved in the unit volume of the solvent), their importance varies with the quantity of the drug that can be introduced without producing serious chemic changes. These physical actions are therefore physiologically insignificant for those substances which cause pronounced chemic effects. They are practically important mainly for the neutral salts of alkalies, and are therefore commonly called "salt actions." Even these salts, however, or rather their constituent ions, produce in addition specific effects, due partly to their electric charges, partly to their specific chemic properties. These specific effects may be called "ion actions." It must also be remembered that the cells are normally bathed in a saline solution; the salt and ion effects are therefore due to a disturbance in the normal environment of the cell, to the relative diminution of the normal constituents, as well as to the increase of the added constituent.

The "colloids"1 of the cells likewise play a highly important part in these salt actions. Colloid solutions, such as those of the proteins, differ in important particulars from the true solutions, such as those of the salts, although there is a gradual transition. In true solutions, the molecules of the solute are dispersed through the solvent, and behave very much as do gases. In colloid solutions, the solute occurs in smaller or larger groups or "aggregates" of molecules; and according to their size they ap-

${ }^{1}$ An interesting discussion of "Colloid Reactions in Biology" is given in the Harvey Lecture of Hedin, I914. 
proach more or less in their properties to mere suspensions of solids or liquids. They therefore lack (practically) the colligative qualities of the true solutions. On the other hand, the suspensions exhibit the electric and surface phenomena of particles. Because of the relatively high state of subdivision, the "surface phenomena"-surface tension, adsorption, catalytic properties, etc--are very conspicuous, and play a highly important part in the functionation of protoplasm. The consistency of the protoplasm, fluid or gelatinous, and the quantity of water imbibed, also depend on the colloids. These properties are greatly influenced by the physical, electrical and chemical conditions of the medium in which the colloid is suspended. It is in this interaction of the saline medium with the protein and lipoid colloids that the salt actions consist.

\section{COLLOWAL SOLUTIONS}

Relation of Colloids and Crystalloids.-Graham, r86r, divided soluble substances into crystalloids and colloids, according to whether they diffuse through parchment and similar membranes. This criterion is by no means a sharp one, and has been supplemented by others as knowledge has increased; but the division is important and has been retained. The terms "colloid" and "crystalloid" do not refer to any particular substance or class of substances, but to the physical state which may be assumed by any substance in a solution under suitable conditions.

As the size of the aggregates of molecules which constitute the colloid state varies, the transition between the physical properties of the two kinds of solutions is not abrupt but gradual. Solutions containing discrete molecules (i.e., true solutions of crystalloids) approach the colloid state especially when the solution is concentrated and the molecules are large; while colloid solutions approach true solutions when they are dilute and the aggregates are small. It must also be remembered that there is no material difference in the purely chemical properties of the two kinds of solutions. These will be essentially the same for a given substance, whether it be in the colloid or crystalloid state.

Size of Particles. - This determines the character of the solution. If 'the particles are larger than $0.1 \mu$ (i.e, visible with the ordinary microscope), the mixture is a suspension (if the particles are solid), or an emulsion (if liquid). These mixtures are separated more or less rapidly by gravity. When the diameter falls below $0.1 \mu$ sedimentation does not occur, and the solution assumes colloid characters. This holds down to $0.00 \mathrm{r} \mu$, i.e. somewhat below the limits of the ultramicroscope $(0.004 \mu)$. Below this limit, the characters are those of a true solution. It is believed that molecules are but little smaller than this limit (sugar, $0.0007 \mu ; \mathrm{NaCl}, 0.00027 \mu$ ).

Properties of Colloids.-Each aggregate behaves like a single molecule, so that the molecular concentration of colloid solutions is relatively low. Colloids therefore have little influence on the osmotic pressure, or boilingor freezing-point. One of the most distinctive properties of colloid solutions is their turbidity, which may be perceived directly, or only by the Tyndall phenomenon (viz., a beam of light becomes visible when passed through a colloid solution). The colloids do not dialyze through parchment or similar membranes. Diffusion through the solvent is also very slow or practically absent.

The electric properties are characteristic: Colloids are usually electrically charged, the quantity and sign of the charge being variable at will. This is very different from ions, whose charge is fixed in character and quantity. The important surface actions will be discussed later. The colloids easily undergo further aggregation to larger particles, i.e., coagulation. Colloid solutions are often permanently changed by temporary physical conditions, such as boiling, freezing or keeping (hysteresis).

Suspension and Emulsion Colloids.-Colloid solutions again fall into two classes: the suspension colloids, in which the particles behave as solids; and the emulsion colloids, in which they behave as fluids. The 
difference is probably one of affinity for the solvent. The emulsion colloids are hydrophilic, i.e., they seem to attract the solvent into the aggregates which are thereby more or less liquefied; the suspension aggregates are hydrophobic, i.e., they do not take up water, and therefore remain discreet and solid. The emulsoids approach more nearly to true solutions, and may sometimes consist of discreet but very large molecules. They are typified by gelatin and other soluble proteins and are therefore of a special biologic importance. Silicic acid also behaves as an emulsoid. The colloid metals and sulphids, congo red, etc., are suspensoids. The emulsoids form viscid solutions or jellies, lower the surface tension, and are coagulated only by large quantities of electrolytes. The suspensoids are not viscid, have little effect on surface tension, and are easily precipitated by traces of electrolytes.

Permanence of Suspensions. - It is a familiar phenomenon (f.i., in the case of oil or mercury) that small particles suspended in a liquid tend to coalesce by surface attraction, to form larger particles. The molecular particles in colloid solutions possess the same tendency, as is shown by the readiness with which they may be precipitated. They are only kept apart by their molecular motion, and by their electric charges. The more the motion is increased (e.g., by increasing the quantity of solvent, or by raising the temperature within the limits which do not produce chemic changes) the greater will be the tendency of the aggregates to fly apart, and to form smaller aggregates. It is quite conceivable that this motion and separation are really electric phenomena. We know that the colloid particles may be charged with electricity, either positive or negative. Since all the particles in a solution are charged with electricity of the same sign (let us say with negative electricity, since this is the case with most protoplasm), they repel each other, just as do the gold leaves of an electroscope. Any increase of the charge (negative in our example) increases this repulsion, and consequently diminishes the size of the aggregates and the viscidity of the solution. If, on the other hand, a small amount of electricity of the opposite sign (positive) is introduced, some of the charges carried by the particles are neutralized and they tend to coalesce. If this (positive) electricity is increased beyond the point when all the original (negative) charges are neutralized, the particles will become charged with positive electricity, and again fly apart. These modifications may be produced by the passage of an electric current; and also, by the electric charges of the ions (see next chapter) of electrolytes. The latter (salts, acids, and bases) may therefore influence the state of aggregation of colloids by withdrawing water and by altering the electric charges. There is yet another way in which the state of aggregation may be varied, viz., by chemic action. This introduces at once profound changes in the state of aggregation, and in the electric condition.

All these changes which we have just described for colloid solutions may be observed in living cells: Withdrawal of water, lowering of temperature, decrease of the electric charge, and certain chemic changes, render the protoplasm more viscid, opaque, and granular, and diminish its motility. A reversal of these conditions leads to the opposite effects.

Precipitation of Suspension Colloids by Electrolytes.-Since suspensoids have no affinity for the solvent, they are very readily precipitated when their electric charges are removed. This occurs most easily when their electric charge is already small, i.e., when they approach the "isoelectric point." Minute traces of electrolytes are then sufficient. The precipitation of positively charged colloids depends mainly on the anions, that of the negative on the cathions (Hardy's Law); the other ions playing practically no part. The precipitant power of the ions is a function of their valence (Schulze, 1882 and 1884 ), since this determines their electric activity. However ions of the same valence are not equally active. Commonly there are also chemic changes, so that the precipitation is generally irreversible.

Precipitation of Hydrophilic Colloids by Electrolytes.-These, like the suspensoids, are precipitated only by electrolytes, not by non-electrolyzed crystalloids (the precipitation of proteins by alcohol is due simply to insolubility). The process, however, is probably quite different. With the emulsoids, much higher concentrations of the salts are required; the precipitation is generally reversible; the electrolytes may also have solvent actions, the end result being the algebraic sum of the actions of the cathion and anion (Pauli, I905 and 1908). 
Lyotropic Series.--The various ions form a regular series ("lyotropic series"), which is reproduced in many different phenomena. The anion series runs $\mathrm{SCN}, \mathrm{I}<\mathrm{ClO}_{3}<\mathrm{NO}_{3}, \mathrm{Br}<\mathrm{Cl}<\mathrm{CH}_{3} \cdot \mathrm{COO}<\mathrm{SO}_{4}, \mathrm{PO}_{4}<$ tartrate $<$ citrate.

With (negative) proteins, lecithin, etc., the left side of this series is solvent, the right side precipitant. They have a similar influence on heat-coagulation temperature; on the solubility of globulins; on the solubility of gases, alcohols, etc.; on the viscidity and surface tension of water; on the saponification of esters, etc. It has been suggested that all these phenomena can be explained by the progressive affinity of these salts for water, thus competing, e.g., with the emulsion colloids.

The alkali cathions are much less important. Their series runs rather irregularly; generally $\mathrm{Mg}<\mathrm{NH}_{4}<\mathrm{K}<\mathrm{Na}<\mathrm{Li}$ ( $\mathrm{Li}$ being most precipitant). If the electric charge of the colloid is reversed, f.i., by acids, then the anion and cathion series are also reversed.

The earthy metals ( $\mathrm{Ca}$, etc.) form irreversible precipitates. They also reverse the charge and therefore the effects of the anion series (MIg acts like the alkali metals). The tendency to irreversible precipitation is very much greater with metallic salts.

Electric Condition of Proteins. - The amino-acid character of proteins renders them amphoteric electrolytes, in which the acid character predominates. They are generally positively charged in acid solutions, negatively in alkaline. If they are brought to the isoelectric point (H-ion concentration about $\left.2 \times 10^{-5}\right)$ all coagulation reactions reach their maximum, and the viscidity is lowest (Pauli, I9 13).

The colloids of the living cytoplasm are predominantly electro-negative; hemoglobin is positive. Serum proteins are always positively charged in alkaline and neutral, as well as in acid solutions (Field and Teague, 1907).

Protein-ion Compounds. - It must not be forgotten that proteins are also capable of ordinary chemic reactions and combinations. They may form complex salts, not only with acids and alkalies, but also with neutral salts; f.i.. with chlorid ions (Oryng and Pauli, I9I5).

Mutual Precipitation of Colloids.-Colloids of opposite electric charge precipitate each other by neutralization. Emulsoids may also be precipitated by suspensoids, irrespective of the charge, perhaps by an adsorption process. On the other hand, excess of the hydrophilic colloid prevents precipitation, and protects suspensoids against precipitation by other substances. It is conceivable, in these cases, that the emulsoid envelops the suspensoid particles, and thus confers on them hydrophilic properties.

An important illustration of this phenomenon is the inhibitory action of serum on the precipitation of urates, calcium salts, etc. Colloids also modify the solubility of electrolytes (Pauli and Samec, I909).

Electrolytes on Osmotic Pressure of Colloids. -Acids and alkalies increase the osmotic pressure, salts generally cause a decrease, while non-electrolytes are indifferent (R. S. Lillie, 1907).

Gels.--Solutions of hydrophilic colloids, when very viscid, form jellies or "gels," such as gelatin, agar or protoplasm. The high viscidity means that there is but little solvent between the particles; either because the particles are naturally large, or have swollen by taking up the solvent; or because the amount of solvent is relatively small. Gels may arise in any of these ways.

The gel resembles a solid in preserving its form, when it is not subjected to external agencies. But it also resembles fluids in being readily changed by external causes, and in permitting the penetration of water, and the diffusion of crystalloids. The latter penetrate it almost as rapidly as they would water. Chemical reactions may occur in its interior very much as they would in water. Other colloids, however, can not diffuse through a gel.

In fact, gels retain all the characters of hydrophilic colloids, and are influenced in the same way by salts; as seen, $f . i$., in imbibition.

Imbibition.-Plates of gelatin (glue) or other hydrophilic colloids, when laid in water or watery solutions, imbibe a certain quantity of water 
and dissolved substance, and thus swell. The water is attracted and retained with considerable force, according to the affinity of the emulsoid for water. This mechanism is important in the regulation of the fluid content of the cells and tissues. The colloid must compete for the water with the other dissolved substances. The imbibition is therefore influenced by the anion series, in the usual order; iodid to chlorid increasing, acetate to sulphate decreasing the imbibition (Hofmeister, I89I). The cathions have relatively little effect. $\mathrm{OH}$ and still more $\mathrm{H}$ ions greatly increase imbibition (Spiro, I904; Ostwald, I905).

The swelling of gelatin is also influenced by non-electrolytes, being decreased by sugars and glycerin, and increased by alcohol and especially urea (Pauli and Rona, I902). In mixtures, the swelling corresponds to the simple algebraic sum of the effects of the constituents. Entirely analogous results are produced on the coagulability of gelatin solutions; this being increased by the substances which lessen swelling, and vice versa (Pauli, I907).

In agar jellies, the phenomena are exactly reversed. According to Ehrenberg, I9I3, the lyotropic series is reversed for gelatin when the concentration of the salts is low $(\Delta<0.5)$.

Distribution of Salts in Imbibition.-The gelatin also takes up the solutes. Hofmeister found that the quantity taken up increases with the concentration of the solution, to a certain limit, which is reached late with the iodid end of the series, and early with the sulphate group. This influences the imbibition, for enough water must then be taken up to render the salt isotonic; and this would be greater for the large quantity of iodid than for the small quantity of sulphate which the gelatin has imbibed.

Surface Phenomena.-Surfaces - the planes of separation of two phases - give rise to very important phenomena, such as surface tension and adsorption. These are especially prominent in colloids and therefore in protoplasm, the particles of which give rise to very extensive surfaces (A. B. Macallum, I9I 2 and I9I3).

Surface Tension.-This is manifested in the tendency of liquids to assume a spherical form, so as to present the smallest possible surface. It may be explained by the mutual attraction of the molecules (estimated at I0,000 to 25,000 atmospheres). The molecules in the interior of the fluid are attracted equally by the molecules on all sides; but at the surface, the attraction of the molecules from within is not balanced by any molecules outward. Consequently, the surface molecules are subjected to a strong pull from the interior, constituting the surface tension (Segner, I75 r).

The degree of this force depends on the nature of the fluid, on the temperature, and on electric forces: Heat and electric charges decrease the surface tension, because they increase the distance between the molecules and therefore diminish the molecular attraction (by the fourth power of the distance). Surface tension may also be resisted by the attraction of the second phase (the air, glass, etc.) on the surface molecules of the liquid. Surface tension may be measured by the rise of capillary surfaces, or by the number of drops delivered from a given surface (stalagmometer).

Biologic Importance of Surface Tension. - This is concerned in the shape and size of the cells; in the consumption or liberation of energy (for any change in surface tension implies a corresponding change in energy); and in the distribution of solutes. Only the last need be considered here.

Solutes on Surface Tension.-Dissolved substances generally modify the molecular attraction and therefore the surface tension. In watery 
solutions, inorganic substances generally increase, organic generally diminish the surface tension; but there is no simple general law, and the effect is not simply proportional to the concentration.

Distribution of Solutes on Surfaces.--Solutes which alter surface tension are not distributed equally through the fluid, but in such a way that substances which lower surface tension are more concentrated on the surface (positive adsorption) and vice versa; i.e., the solute goes to the point where the tension is lowered. This is explained by the Gibbs (1878)-Thompson (I 886) principle (the concentration throughout a fluid system tends to be so adjusted as to reduce the energy at every point to the minimum).

Distribution of Salts in Cells.-Whenever the surface tension is reduced, the solutes will be concentrated at the surface, irrespective of their distribution under ordinary conditions. Accordingly, the distribution and transportation of substances in cells may be controlled by changes in their surface tension. Macallum has shown this to be true for $\mathrm{K}$ and $\mathrm{Cl}$, and probably for other ions; and has pointed out the important part which this may play in the mechanism of secretion and absorption; ameboid and muscular movements; nerve impulse, narcosis, etc.

Solution-Affinity; "Haft-druck."-The surface condensation is proportional to the lowering of surface tension. A substance that lowers surface tension therefore passes more readily out of the solution and into the surface. In the phraseology of Traube, such a substance has a low solution-affinity or "Haft-druck." He believes that this favors the penetration of the substance into cells or through membranes, and that it is an essential factor in narcosis and osmosis.

Adsorption.-The concentration of solutes on surfaces is illustrated by the adsorption of dissolved substances by powders; $f . i$., by the decolorization of potassium permanganate by filtering through sand; the adsorption of colors or alkaloids by charcoal, etc. This adsorption is a very rapid process.

Sugars are not adsorbed because they have relatively little effect on surface tension. These adsorptions are reversible; i.e., the adsorbed substance may be removed by the addition of another solute which lowers surface tension more powerfully. If several substances are present together, each is adsorbed less than if it is adsorbed alone. Salts may have a marked influence, $f . i$., diphtheria toxin in pure solution is scarcely adsorbed by bone-black, but is perfectly adsorbed from normal saline or Ringer's solution (Jacqué and Zunz, rgog).

Catalytic Actions.-Adsorption, by bringing the dissolved molecules into closer contact, facilitates chemic reactions. Accordingly, suspension colloids with their large surfaces generally act as catalyzers. The application of adsorption to ferment action is discussed by Bayliss, I915, in his paper.on "Phase Boundaries."

Membrane Formation.- If the adsorbed substance increases viscidity, its concentration on the surface may produce resistant membranes, such as occur in the shaking of proteins, saponins, soaps, many dyes, etc. The adsorption of colloids also presents some other peculiarities; $f . i$., it is generally irreversible; crystalloids do not displace each other; and the adsorption is not proportional to the concentration, as it is with crystalloids. Salts may have a marked influence, $f . i$, diphtheria toxin in pure solution is scarcely adsorbed by bone-black, but is perfectly adsorbed from normal saline or Ringer's solution (Jacqué and Zunz, I909).

The Physical Status of Emulsions.-Emulsions consist of fine and permanent suspensions of one liquid in another; immiscible liquid; $f . i$. oil in water, or vice versa. Surface tension tends to make immiscible liquids separate. This tendency may be overcome by lowering the surface tension of the dispersing medium (as by saponin or soap) or by increasing its viscosity, and by adsorbing the water by hydrophilic colloids (as by gums or proteins). Emulsification by colloids, is destroyed if an excess of water is added, or if the affinity of the colloid for salts is diminished. (Fischer and Hooker, I9r6; Room, I916).

The water-soluble alkali soaps secure the suspension of lipoids in water, whilst the oil-soluble calcium soaps change the phases so as to suspend the water in the oil. 
IIith the alkali-soaps, droplets of oil are suspended in a continuous matrix of water; with calcium soaps, droplets of water are suspended in a matrix of oil. The addition of sodium or calcium ions therefore changes the phase of an emulsion. It also thereby influences the permeability; the effects of electrolytes on the permeability of soap-films being similar to their effects on the permeability of living cells (Clowes, I9I4 to 19r6).

IIost protoplasm contains emulsions. Fischer looks upon fatty degeneration as due largely to the breaking up of these emulsions by diminished water-affinity of the cell colloids.

\section{OSMOSIS}

Osmotic Phenomena of Crystalloids.-These belong to the more important effects of the bio-chemically inactive salts and other crystalloids, and of water. They occur whenever two solutions of different (molecular) concentration are separated by a ("semipermeable") membrane that permits the passage of the solvent, but which interposes an obstacle to the free diffusion of the solute. Dissolved molecules always tend to distribute themselves equally throughout a solvent. If their passage is hindered by a membrane, the equality can only be obtained by the passage of the solvent from the more dilute to the more concentrated solution. The more concentrated solution therefore tends to increase in volume. If this increase is prevented (e.g., if the solution is inclosed by rigid walls), a certain "osmotic pressure" is developed. The phenomenon is illustrated by the familiar "egg" experiment; by the swelling of red blood and other cells in water; and conversely, by the shrinking of plant cells, and crenation of red corpuscles in strong salt solutions.

Egg Experiment.-The shell is removed from the broad pole of an egg, but without breaking the inner "skin." The narrow pole is then pierced through the skin with a needle, and over this hole a long narrow glass tube is cemented with sealing wax. When the prepared egg is set in a glass of water, the fluid rises slowly in the tube to a considerable height. The contents of the egg contain salts, and therefore have a higher molecular concentration than the water. The "skin" of the egg acts as a partly semipermeable membrane.

Osmotic Pressure and Molecular Concentration.-If the membrane is "strictly semipermeable"-i.e., freely permeable to the solvent, but absolutely impermeable to the solute-then the amount of the osmotic pressure is strictly proportional to the difference in the molecular concentration of the two liquids, independent of the nature of the molecules. The stronger solution is said to be hypertonic, the weaker hypotonic. If the solutions on the two sides are equimolecular, i.e., have the same molecular concentration, there will be no tendency to movement of the solvent and therefore no pressure; or more accurately, no difference in the pressure. The two solutions will be isotonic.

Strictly and Partly Semipermeable Membranes.-Only colloids, organic or inorganic, can form semipermeable membranes (Morse; Meigs, 1915). The envelope or boundary layer of all cells-and indeed the interior cytoplasm (Kite, I9r3)-offers more or less obstacle to the passage of solutes, and is therefore more or less semipermeable. Membranes produced artificially by precipitating ferrocyanid of copper in the pores of an unglazed porcelain cup are almost ideally semipermeable to sugar (Traube, I867). They are, however, permeable to $\mathrm{NaCl}$ and $\mathrm{KCl}$. The envelopes of certain vegetable and animal cells, $f . i$., the red blood corpuscles, also approach fairly closely to the definition. Most cells, however, and dialyzing membranes in general, are not absolutely impermeable to the solutes, but offer only a partial obstacle ("partly semipermeable membranes"); so that the solutes pass the membranes, but more slowly 
than does the solvent. In this case, the concentration is equalized partly by the passage of the solute, and to a correspondingly smaller degree by the passage of the solvent; so that the osmotic pressure is correspondingly less than with strictly semipermeable membranes.

Differences in Permeability of Solutes.-Semipermeable membranes are not usually equally impermeable to all solutes, but permit some to pass rapidly, others more slowly. This will establish differences in osmotic pressure, because the dialyzable substance will pass across to the nondialyzable. Non-dialyzable solutes are therefore hypertonic to those which are easily dialyzable. It is important to note that the passage of the fluid and the osmotic pressure are governed not only by the molecular concentration, but also by the permeability of the membrane to different substances.

Empiric Laws of Osmosis.-For convenience, the data which have been discussed in the preceding paragraphs may be summed up in the form of laws:

I. Solutions separated by a membrane permeable to water tend to have an identical molecular composition, both in number and kind of molecules.

2. If the membrane is perfectly permeable to both solvent and dissolved substance, the exchange of molecules will take place without change in pressure or volume.

3. If the membrane is less permeable to the dissolved substance than to the solvent, an increase of liquid, or increase of pressure will occur in the stronger solution.

4. If a membrane is differently permeable to one dissolved substance than to another, equimolecular solutions of the less diffusible substance will be hyperisotonic to the more diffusible.

Nature of Osmosis.-Several theories are current for the explanation of osmotic pressure. The most important are the kinetic motion theory of Van't Hoff; the solution-affinity theory of Kahlenberg; the hydration theory of Morse; and the surface-tension theory of Traube. None of these theories is conclusively proven to the exclusion of the others. It is conceivable that all these processes are concerned in the passage of the solution; but that the actual pressure is caused by the motion of the molecules, as formulated by Van't Hoff.

Van't Hoff's Theory of Osmotic Pressure.-This is the theory which has found the widest acceptance among physicists. From the observations of osmotic pressure by means of Pfeffer's ferrocyanid of copper cell, Van't Hoff ( 1885 and 1887 ) arrived at the conclusion that the phenomena of osmotic pressure are governed by the same laws as the pressure of gases, quantitatively as well as qualitatively. This would imply, that the pressure in both cases is produced by the same cause, namely, the kinetic motion of the molecules bombarding the walls of the container. Dissolved molecules would behave precisely like gaseous molecules. The membrane would play a purely passive part; being important only by making the kinetic motion manifest to the senses.

According to the Van't Hoff theory, the molecules of both solvent and solute are in constant motion, moving with the same energy as if they were in the form of a gas. Every molecule, by striking against the walls of the container, or against other molecules, exerts a certain pressure, which is precisely the same whether the substance is in the form of a gas or of a solution. If the same number of molecules per cubic space exist in two solutions separated by a semipermeable membrane, the pressure will be 
ergual on each sidle of the membrane. The nature of the molecules is immaterial in this, as long as they all penetrate through the membrane with equal readiness. If all the molecules can pass through the membrane, an exchange will take place; but since as miny patss to one side as to the other, there will be no difference in the pressure in the two compartments. If, on the other hand, the molecules on the one side can pass through readily, while some of the molecules on the other side are not capable of diffusing through the membrane, then the pressure in the two compartments will become unequal.

In an osmotic cell, the non-diffusible molecules will all rebound from the nembrane, and so remain confined to their own side. The diffusible molecules will, like gases, tend to pass through, irrespective of the other molecules, until they exist in equal concentration on each side, i.e., until the partial pressure of each kind of molecule is the same on both sides.

If a solution consisting of $x$ molecules of salt and $(y-x)$ molecules of water per cubic centimeter were inclosed in a closed vessel and separated from water containing $y$ molecules per cubic centimeter by a semipermeable membrane, then water would pass into the solution until it also contained $y$ molecules of water per cubic centimeter. But it would then also contain $x$ molecules of salt. Its total molecular concentration per cubic centimeter would therefore be $y+x$; higher by $x$ than that of the liquid outside of the vessel. The pressure in the vessel would therefore be increased by $x$ molecules.

The process might be represented as follows:

Partial Molecular concentration: $\quad \mathrm{H}_{2} \mathrm{O} x$ salt

Total Molecular concentration:

$$
\begin{aligned}
& y \mathrm{H}_{2} \mathrm{O}^{\prime} x \text { salt } \quad y \mathrm{H}_{2} \mathrm{O}^{\prime} x \text { salt } \\
& \begin{array}{l|l|l}
y-x \mathrm{H}_{2} \mathrm{O} \\
\hline y \quad \text { y }
\end{array} \text { tends to become } \frac{y \mathrm{H}_{2} \mathrm{O}}{y}
\end{aligned}
$$

This excess of total molecular concentration constitutes the osmotic pressure.

An experiment analogous to osmosis can be performed on gases directly. Palladium is permeable for $\mathrm{H}$ but not for $\mathrm{N}$. Ramsay, I894, found that when a palladium vessel is filled with $\mathrm{N}$, closed, and immersed in an atmosphere of $\mathrm{H}$, the pressure rises within the vessel until it exceeds the outside pressure by the partial pressure of the H. However, this experiment could also be explained by the other theories.

Application of Gas Laws to Osmotic Pressure.-Van't Hoff claimed as chief support of his theory, that the osmotic pressure obeys quantitatively the laws of gas pressure. As applied to osmotic pressure, these would read: Boyle-Mariotte's Law: With constant temperature, the osmotic pressure of a solution is proportional to its concentration. Under standard conditions, a gram-molecule distributed over a liter of space, exerts 22.34 atmospheres of pressure.

Gay-Lussac's Law: With equal concentration, the osmotic pressure grows by $1 / 273$ for each degree $C$.

Avogadro's Law: With equal osmotic pressure and equal temperature, equal volumes of solutions contain the same number of dissolved molecules, and the same number as would be contained in an equal volume of gas of the same temperature and presence.

Experimental Evidence.- The experimental confirmation of these laws is unfortunately very incomplete (Kahlenberg), because of the difficulty of constructing strictly semipermeable membranes which will resist the high pressures. The experiments which form the original basis of the Van't Hoff theory were very inaccurate. Since then, only a few rigorous determinations of absolute osmotic pressure have been attempted (especially by Morse, Holland and co-workers, IgII and IgI 2). These showed a good agreement with the theory; but they were confined to a single type of membrane (ferrocyanid of copper) and to a single substance (cane sugar), within a limited range of concentration and temperature. Outside of these limits, there were considerable discrepancies. The numerous determinations of relative osmotic pressure, which can be easily made, are inconclusive; for while they agree with this theory, they also harmonize with the competing theories. On the other hand, Kahlenberg, using different, but typically semipermeable membranes, finds that the gas laws do not hold at all; and that the phenomena can be explained quite simply on an entirely different basis. The evidence for the Van't Hoff theory is therefore incomplete.

Advantages of the Van't Hoff Theory. - This is very attractive in that it reduces all the physical phenomena of gases and solutions to a common basis, and thereby permits convenient and far-reaching theoretical and practical deductions. For instance, by 
postulating that osmotic pressure is directly proportional to the molecular concentration, it justifies the substitution of an easy calculation for the very difficult experimental determination of the pressure. Since it assumes that the membrane is purely passive, it permits the calculation of osmotic pressure in situations which are inaccessible to direct experimentation. In a word, if it is correct, it makes the difficult method of direct experimentation more or less superfluous, substituting for it an easily applied formula. This would be highly convenient and desirable-but before relying on such deductions, the correctness of the formula must be more securely established.

Kahlenberg's Theory.-This has the advantage that it furnishes the simplest explanation of the nature of osmotic pressure; and that it is in the best accord with these phenomena as exhibited in living structures. The theory refers osmosis to solution-affinity, and may be best understood from an experiment made by L'Hermite in 1854 .

L'Hermite's Experiment.-A layer of chloroform is poured into a vessel, then a layer of water, and finally a layer of ether. Even if any mechanical mixing of the strata is carefully avoided, it will be found that the ether layer gradually diminishes, until finally there are only two layers, the upper containing mainly the water, the lower the chloroform and the ether. In other words, the ether has passed through the water into the chloroform, just as water passes through a semipermeable membrane into a salt solution. The analogy can be carried even further: If a cork saturated with water is firmly wedged between the ether and chloroform layers and the chloroform compartment is connected with a manometer, a very considerable pressure will be developed. Analogous experiments have been devised by Nernst, I 890 (passage of ether into benzol, through pig's bladder), and by Gies, I9 2 (passage of ether into oil, through rubber condom).

It is difficult to see in what essential respect these arrangements differ from an osmotic cell. In both cases two dissimilar fluids are separated by a septum. In both cases, one of the fluids passes into the other; and if this is prevented, a pressure is produced. In L'Hermite's experiment we can easily see the explanation: Ether is soluble in water, and therefore diff uses into the latter; it is, however, much more solublein chloroform, and therefore the ether which is dissolved in the aqueous layer tends to pass into the chloroform. The watery layer therefore would lose some of its ether to the chloroform, and would then take up more from the ethereal layer; and this process would continue until.practically all the ether had passed into the chloroform. In other words, the passage of the ether, and the pressure which may thus be developed, is not due to any gas pressure, but simply to the fact that ether has a solution-affinity for water, and hence passes into the latter; and that it has a still greater solution-affinity for chloroform and hence passes into this from the water. If we substitute the hypothetical "semipermeable" membrane for the water, we may say that "osmotic pressure" measures not the gas pressure, but the solution-affinity of the solvent for the solute, less the affinity for the membrane.

There is one further complication; for, since the chloroform is also somewhat soluble in water, there is also some stream in the contrary direction. This is equivalent to saying that the arrangement is not strictly semipermeable. For our present purpose this may be neglected, except in so far as it emphasizes the fact that osmotic changes generally (if not always) do occur in both directions. This is of considerable importance in biology.

It is another important advantage of the theory that it emphasizes that the membrane plays an active part as well as the solute. Its greatest advantage, however, is the simple explanation which it gives of the nature of the osmotic process, namely as a phenomenon of chemical or solution affinity. Osmotic pressure will result if the solute is relatively insoluble in the membrane, i.e., if it has a greater affinity for the solvent than for the membrane. In the case of two solutions the transfer of fluid will occur in the direction of the solution which has the greater affinity; and osmotic pressure is simply the hydrostatic pressure which is required to prevent this attraction of fluid.

The theory supposes that the solution-affinity increases with the molecular concentration; and since it is a fact that equimolar solutions of all substances are isotonic -at least with strictly semipermeable membranes and aqueous solutions-it follows that the solution-affinity must depend solely upon the molecular concentration; i.e., it must be independent of the nature of the dissolved substances. This agrees entirely with the known effect of molecular concentration on the freezing- and boiling-points. The affinity is also raised by temperature. 
Relation of Van't Hoff and Kahlenberg Theories.-In brief then, the main facts of osmosis are explainable by either theory. The principal difference in their practical application lies in the fact that Van't Hoff's theory gives a definite formula for calculating the osmotic pressure from the molecular concentration; while Kahlenberg's theory does not attempt an exact quantitative formula. Presumably, however, even this difference is not of very fundamental practical importance; for the absolute osmotic pressure may well be that of the gas formula, whether it is produced by kinetic motion or by solution-affinity; indeed, as has been intimated, the pressure proper may be due to kinetic motion in either case.

It will be recalled that Van't Hoff's theory postulates a very definite quantitative value for the osmotic pressure, namely 22.34 atmospheres for a molecular solution. According to Kahlenberg's theory, this value is much less definite. If a membrane were strictly semipermeable, the solution-affinity should undoubtedly give rise, eventually, to a constant and definite osmotic pressure. This would quite likely be something like that of Van't Hoff. In practice, however, and especially in biology, this value is never attained. Living membranes are not strictly semipermeable, so that there is more or less passage of solute out of the solution, thereby reducing the concentration. Further, the passage of fluid becomes slower and slower as the concentrations on the two sides of the membrane approach equality; the full pressure would therefore never be realized in any time which would come into practical consideration

Morse's Hydration Theory.-Morse attributes the passage of the water to unequal hydration of the colloids on the two sides of the membrane. The solutes dehydrate the side of the membrane in contact with them; and this is equalized by the attraction of water from the opposite side. The theory is somewhat related to that of Kahlenberg's; but it helps to explain why the rate of osmosis is not parallel to the semipermeability, or to the final osmotic pressure. Salts generally develop an osmotic pressure more rapidly than sugar, even when the final osmotic pressure of the salt solution is lower.

Differences Between Rate of Osmosis and Osmotic Pressure.-These are often not parallel. For instance, the copper ferrocyanid is impermeable to sucrose, and fairly permeable to salt. A sugar solution will therefore be eventually hypertonic to a salt solution. In the start, however, i.e., before the salt molecules have had time to diffuse through the membrane, water passes from the sugar into the salt; it is only as diffusion proceeds that the process is reversed as would be expected. Other membranes show similar phenomena (Meigs, I915). Indeed, acids actually diminish in volume when diffused through a pig's bladder against water (Graham).

Morse explains these observations by the fact that neutral electrolytes dehydrate colloids more readily than non-electrolytes. The "negative osmosis" of acids is due to their hydrating action (see "Imbibition"). Alkalies are intermediate.

Surface-tension Theory of Osmosis.- J. Traube (r904 and later) refers osmotic phenomena to surface tension. He observed that substances which lower surface tension, and which therefore accumulate at the surface according to the Gibbs-Thomson principle, penetrate through membranes more rapidly. Conversely, salts, etc, which increase surface tension, and which therefore accumulate in the interior, would attract the solvent to equalize the pressure. In other words, the fluid of lower surface tension would be drawn into that of higher surface tension. An attraction pressure would thus be created.

Surface tension may well play a part in osmosis. F.i., Macallum, I9I3, finds that a very low surface tension in a film may retain salts so firmly as to act as a semipermeable membrane. However, it is improbable that surface tension is an adequate explanation of the ordinary osmotic phenomena. Thus, it does not explain why substances which affect surface tension very unequally, e.g., sugar and salt, are nevertheless isotonic. It also takes no account of the differences introduced by the nature of the membrane. Traube has later modified his theory to meet some of these objections; but this only introduces further complications and still fails to explain all the facts.

Direct Determination of Osmotic Pressure.-The absolute osmotic pressure would be measured by the rise of a manometer attached to an 
osmotic cell, i.e., a rigid vessel filled with a solution, surrounded by a semipermeable membrane, and immersed in water. Perfect osmometers are difficult to construct, and perhaps theoretically impossible. However, even imperfect instruments give practically useful data as to the velocity and magnitude of osmotic pressure and these show at least an approximation to the gas laws.

Magnitude of Osmotic Pressure.-According to the gas laws, the pressure exerted by a gram-molecule (the molecular weight expressed in grams) or "mol" of any substance, when comfined to a liter of space, equals 22.34 atmospheres. In a "molecular" solution therefore (one containing a mol dissolved in a liter of solvent) the osmotic pressure would raise a column of water to a height of about 725 feet! The osmotic pressure of the salts in the blood would raise it about 250 feet. This may suffice to indicate the enormous molecular force latent in solutions, even if it may not be absolutely correct.

An actual osmotic pressure will only be developed if the solution is confined; if it is free to expand, the incoming molecules will merely increase the volume of the fluid.

In this way a single molecule of a non-diffusible salt would theoretically be capable of attracting an infinite amount of water across a semipermeable membrane-for it is evident that the number of $\mathrm{H}_{2} \mathrm{O}$ molecules per cubic space would always be less, by the molecules of salt, than they are in pure water.

The rapidity with which water will pass into the salt solution across the same impermeable membrane will, of course, depend upon the concentration of salt; i.e., the partial vacuum of $\mathrm{H}_{2} \mathrm{O}$ molecules in the solution. The rate of osmosis will therefore be slowed as the process progresses.

Determination of Relative Osmotic Pressure.--This can be easily made by using artificial membranes, or more conveniently animal or vegetable cells; diluting the solution under investigation until its osmotic effects on the cells are identical with those of a known solution.

The earliest quantitative studies of osmosis (Historical Review, Hamburger, Igrr) were made in this way by de Vries, I 882, by the use of certain plant cells (Tradescantia) which exhibit Plasmolysis (retraction of the protoplasm from the cellulose envelope) in hypertonic solutions. He was thus able to determine the isotonic concentrations of a long series of substances. H. J. Hamburger, I $88_{3}$, introduced the hemolytic method; i.e. the determination of the concentration which produces laking of red blood corpuscles. This is also isotonic, provided that the corpuscles are semipermeable, i.e., that the substances do not penetrate. This may be ascertained by direct chemic analysis and holds practically for most salts and sugars, but not for urea, alcohols, etc. The changes in the weight of tissues after immersion in the solution may also be used as an index; but with these, partial semipermeability is much more common, and imbibition may come into play; thus, according to Overton, 1906, serum is isotonic with 0.9 per cent. $\mathrm{NaCl}$; but when concentrated four times, it requires 5 per cent. $\mathrm{NaCl}$; and when six times, ro per cent.

Value of the Comparative Methods.-All these methods involve comparison with a known solution; and if the absolute osmotic pressure of the latter were known, and if the membrane were truly semipermeable, then the absolute osmotic pressure of the unknown solution could be easily calculated. This, however, is mainly of theoretical importance, for as has been repeatedly said, strictly semipermeable membranes probably do not exist in nature. The true value of these comparative estimations lies rather in the picture which they furnish of the direction and relative value of the osmotic pressure for the particular solution and membrane (or cell) with which they are made- of ten a matter of great biological importance.

Calculation of Molecular Concentration and Osmotic Pressure.Since the osmotic pressure has a definite relation to the molecular concen- 
tration, the determination of the one quantity permits the calculation of the other.

If a cell-membrane were truly semipermeable, then two solutions which had the same effect on the cell volume (or laking) would need to have an identical molecular concentration. The concentration of any unknown solution could therefore be determined by diluting or concentrating it until it became isotonic with a standard solution of known concentration. The semipermeability of certain cells is sufficiently complete to make this method available for practical purposes; but it is less exact and generally less convenient than the calculation from the freezing- or boiling-point.

Estimation of the Molecular Concentration by the Freezing-point.The depression of the freezing-point (denoted by $\Delta$ ) is easily determined by the Beckmann apparatus. It is strictly proportional to the molecular concentration; every mol of substance per liter of solution depressing the freezing-point of water by $1.85^{\circ} \mathrm{C}$. and this no matter what the nature of the substance (Raoult's Law), except as it is modified by electrolytic dissociation. The molecular concentration is therefore calculated by dividing $\perp$ by 1.85 ; and the osmotic pressure by multiplying the molecular concentration by 22.34 atmospheres.

Molecular Solutions.- -Some confusion exists in the use of this term (abbreviated "MI"). The correct practice is to add the calculated quantity of solute to a liter of solvent (Raoult). It is inadmissible to dissolve the mol in water enough to make a liter (Arrhenius's method), as is commonly done in making chemical solutions.

Molecular solutions are sometimes called "normal." This is objectionable; for the normal solution of chemists differs from the molecular solution, in that the molecular weight is divided by the valence. The normal solutions of physiologists are again quite different from the above; they are saline solutions of a strength of 0.6 to 0.9 per cent.

Departure of Electrolytes from Raoult's Law.-According to this law, the molecular concentration, and therefore the freezing-point, osmotic tension, etc., should be calculable by dividing the per cent. of the solution by one-tenth the molecular weight of the dissolved substance. This is found to hold true for concentrated solutions of all substances, and for dilute solutions of non-electrolytes-substances which do not conduct the electric current, such as the sugars, urea, glycerin, alcohol, etc. Dilute solutions of electrolytes-salts, acids, or bases-however, show a greater concentration than would be deduced from Raoult's law.

A solution of $\mathrm{NaCl}$ containing 0.584 gram of $\mathrm{NaCl}$ per liter (equals $1 / 100 \mathrm{~mol}$ ) should depress the freezing-point by $0.0185^{\circ}$. It is found, however, that its depression is nearer to $0.03^{\circ}$; almost twice as great as would be expected. Very dilute solutions of $\mathrm{Na}_{2} \mathrm{SO}_{4}$ have three times the calculated concentrations; those of $\mathrm{H}_{3} \mathrm{PO}_{4}$, four times.

The dissociation of salts, as revealed by the freezing-point, bears a striking relation to the conductivity of salt solutions for electricity. It has been found that salt solutions conduct electricity proportionately better the more they are diluted. If, e.g., a solution which contains 0.1 mol has a coefficient of conductivity which we will call " $\mathrm{K}$," a solution which contains only $0.0 \mathrm{I}$ mol will have a coefficient higher than $\frac{\mathrm{K}}{\mathrm{IO}}$. This increase is exactly proportional to the increased molecular concentration revealed by the freezing-point. The two phenomena must therefore have the same cause.

\section{ELECTROLYTIC DISSOCIATION}

General Statement.-To account for the fact that electrolytes depress the freezing-point more than would agree with Raoult's law, Arrhenius, I 887, advanced the hypothesis that a certain proportion of the electrolytic molecules, by acquiring an electric charge, separate into their constituent ions: $\mathrm{NaCl}$ into $\mathrm{Na}^{+}$and $\mathrm{Cl}-{ }_{-} ;\left(\mathrm{NH}_{4}\right)_{2} \mathrm{SO}_{4}$ into $\mathrm{NH}_{4}{ }^{+}, \mathrm{NH}_{4}+$ and $\mathrm{SO}_{4}=$; 
$\mathrm{H}_{3} \mathrm{PO}_{4}$ into $\mathrm{H}^{+}, \mathrm{H}^{+}, \mathrm{H}^{+}$and $\mathrm{PO}_{4} \equiv ; \mathrm{Ca}(\mathrm{OH})_{2}$ into $\mathrm{Ca}^{++}, \mathrm{OH}^{-}$and $\mathrm{OH}^{-}$. The ${ }^{+}$and - signs denote the nature and quantity of the electric charge. The metals of salts, and hydrogen of acids, carry a positive charge, and therefore wander to the cathode and thus constitute the cathions. The "acid radicals" of salts, and the hydroxyl of bases, carry the negative charge, go to the positive pole, and are therefore the anions. The conduction of electricity through solutions occurs by the conveyance of these ions.

It is scarcely necessary to insist that the ions are not identical with atoms (from which they differ in that they carry charges), nor with the free elements (which exist as molecules). In a dilute solution of $\mathrm{NaCl}$ there does not exist metallic ( $\mathrm{Na}$ ) nor gaseous chlorin (Cl), but $\mathrm{Na}$ ions $\left(\mathrm{Na}^{+}\right)$and $\mathrm{Cl}$ ions $\left(\mathrm{Cl}^{-}\right)$, whose properties are altogether different. It must also be remembered that this ionization exists in only a limited class of compounds, viz., in all acids, bases, and salts (electrolytes), and not in compounds which do not conduct electricity (non-electrolytes).

The electric charges are further important, since they may leave the ions to combine with the tissue elements, so that the electrolytes cause electric stimulation of the protoplasm.

Degree of Ionization.-This increases with the dilution of the solution until complete dissociation is attained.

In concentrated solutions, the two charges approach sufficiently close to neutralize each other, and the two ions combine into an ordinary molecule, e.g., $\mathrm{NaCl}$. In dilute solutions they are separated so far as to exert little attraction, and act as independent molecules.

With similar ions, the degree of dissociation is fairly constant for the same degree of dilution. In I per cent. solution of $\mathrm{NaCl}$ (and in blood) about 82.8 per cent. of the molecules undergo this process, and about the same percentage in equimolecular solutions of $\mathrm{KCl}$ or $\mathrm{NH}_{4} \mathrm{Cl}$. Dissimilar elements, such as $\mathrm{Mg}, \mathrm{Ca}, \mathrm{SO}_{4}, \mathrm{PO}_{4}$, etc., have a different constant. If several salts are present in a solution-particularly if they have a common ion-the degree of dissociation of each salt will be less than if it were present alone in the same concentration, but greater than if it were present alone in the concentration corresponding to the sum of the several salts.

Effect on Freezing-point, Osmotic Pressure, Etc.-Each of these fractions (ions) has the physical properties of molecules, and thus causes a corresponding depression of freezing-point or increase of osmotic pressure. These quantities would therefore be twice as great if the molecules had dissociated completely into two ions $(\mathrm{NaCl})$; three times as great when dissociated completely into three ions $\left(\mathrm{Na}_{2} \mathrm{SO}_{4}\right)$; four times with $\mathrm{H}_{3} \mathrm{PO}_{4}$, etc. With partial dissociation, intermediate values would be obtained.

The total molecular concentration of a dilute solution of a single salt corresponds to the number of undissociated molecules, plus the number of dissociated molecules multiplied by the number of parts into which they divide. For instance, if 80 per cent. of the $\mathrm{NaCl}$ molecules in a solution are dissociated, the concentration will be $\mathrm{I}-0.8+$ $(0.8 \times 2)=I .8$ times the theoretical; if 80 per cent. of $\mathrm{Na}_{2} \mathrm{SO}_{4}$ is dissociated, it will be $\mathbf{r}-0.8+(0.8 \times 3)=2.6$. (Tables of the dissociation of the various salts are given by A. A. Noyes and Falk; J. A. Ch. S., I910).

Limitation of Terms. -To avoid confusion it may be well to confine the term " mol," or better, "molion," to the molecular concentration actually deduced from the freezingpoint (i.e., r.8 in the preceding example of $\mathrm{NaCl}$ ); and "gram-molecule" to the concentration deduced from the molecular weight (i.e., I on the example) without taking account of the dissociation. The abbreviation " $M$ " is always applied to the latter. Several solutions may be related to each other in the following ways: Equimolecular (same number of gram-molecules); Equiosmotic (same number of molions); and Isotonic (same effect on a given cell). The term isotonic coefficient is applied to the factor by which the molecular concentration must be multiplied to obtain the molionic concentration. 
Ion Actions. - In addition to the osmotic actions which have just been described, electrolytes show effects which are due to their constituent ions. These actions are generally proportional to the degree of ionization:

The comparatively firm mercuric albuminate is much less active than the ionized mercuric chlorid; ferrocyanids, in which the iron does not exist as an ion, act quite differently from ferrous salts, and these again differ from ferric salts. Gaseous chlorin does not behave at all like the chlorin ion of $\mathrm{NaCl}$, etc. The chemic behavior varies in the same way with the degree and state of ionization. The ions may act by entering into chemic combinations with the protoplasm, producing various compounds, syntheses, and decompositions, and thereby lead to functional changes. They may also alter the permeability of the cell wall. Besides these chemic actions, it appears more than probable that the electric charges carried by the ions exert actions which are independent of the chemic nature of the ions. These latter actions may well be termed "electron actions," whilst the sum of the chemic and electric actions produced by the ions may be grouped together as "specific ion actions." It is quite conceivable that the chemic actions are also in the last instance resolvable into electric manifestations. But since the proof for this has not yet been furnished, and since the two classes of actions exhibit striking differences, it.is well to keep them apart, in so far as this is practicable.

Electron Actions.-By this term we understand those actions which are produced by the electric charges carried by ions. These modify the electric condition of the protoplasmic colloids, and hence their aggregation. This leads to important modifications of functions.

The existence of these actions is shown by the fact that all electrolytes produce certain effects in common, which are not produced by non-electrolytes. For instance, an eggalbumin solution can not be coagulated by heating when the electrolytes have been removed by dialysis. The coagulability is restored by the addition of electrolytes, but not by nonelectrolytes (sugar, etc.). These effects are largely independent of the chemic properties of the ions, but vary with their electric properties, such as the sign of their electric charge and the ease with which they part with this. The effects agree with those of positive and negative electric stimulation; anions and cathions tend to produce opposite effects, the action of an electrolyte being determined by the predominating ion; analogous phenomena are exhibited when electrolytes are added to solutions of colloids.

The Relation of the Toxicity of Ions to Their Electric Properties.-The quantitative effects of ions on protoplasm can be estimated most exactly and most conveniently by observing the molecular concentration in which the solution will just cause death. This has been studied by a number of investigators. It was noticed that the toxicity increases generally with the atomic weight of the ion- $\mathrm{Hg}$, for instance, being much more toxic than $\mathrm{Na}$; but, on the other hand, $\mathrm{H}$ was found more toxic than the heavier $\mathrm{Na}$. The toxicity also increases generally with the valency; but here also there are very striking exceptions, the monovalent $\mathrm{Ag}$ being more toxic than the trivalent $\mathrm{Al}$. The general rule is so striking, both as to the atomic weight and valency, that some connection could not be doubted; but it is evidently not a simple one. The subject has been very greatly elucidated by the extensive experiments of A. P. Mathews (I904) on fundulus eggs, which are peculiarly adapted to these investigations. They showed a remarkable agreement between the toxicity and the solution-tension of ions.

The solution-tension signifies the tendency of a metal to go into solution, and thus to acquire an electric charge. This may be estimated by physical methods. The greater the solution-tension, the greater is the affinity of the ion for its charge; the less apt will this ion be to part with its charge, and therefore the less its effect on colloids. This is precisely what Mathews found: the toxicity of an ion varies inversely as its solutiontension; the toxicity of a salt varies inversely as its "dccomposition-tension," i.e., as the sum of the solution-tension of both its ions. (For instance, if the solution-tension of the anion is $\mathrm{I}$ and of the cathion 2, the decomposition-tension of the salt is 3.) The same law applies to the inhibitory action of electrolytes on ferments. Mathews also found that the toxicity increases with the quotient: equivalent weight divided by atomic volume. When we understand the electric condition of ions and colloids better, we shall probably find that there is a common basis to these connected phenomena; and some apparent exceptions, which still exist, will probably be explained.

Other forms of life do not show precisely the same correspondence. This disagreement could be expected from the complicated conditions which prevail in most cells, and does

1 Many authors use the term "ion actions" to designate what is here called electron actions. 
not at all speak against Mathews' law. The latter deals only with the affinity of the ion for its charge; but the colloid is also an active factor in the reaction, some protoplasm showing a greater affinity for positive charges, and others for negative charges. The factor of penetration introduces important complications. Electron actions must occur even when the ions do not penetrate into the cell, the charges being transferred through the intermediation of the colloidal cell wall. This is indeed the most favorable condition for the study of electron actions, since it excludes the possibility of chemic changes. It probably explains the comparatively simple results obtained with funclulus ova, for the experiments of $O$. H. Brown (I905) have demonstrated their comparatively great impermeability to salts and water. With most cells, however, the possibility of strictly chemic modifications of the protoplasm by the ions is more or less important. The action of the added ions will be greatly modified by the ions which are already present in the tissues.

Again, a solution containing a single electrolyte will tend to withdraw all other electrolytes from the cell; and the toxicity may in some cases be due to deficiency of the latter ions, rather than to excess of the former (Osborne, 1905). Even non-electrolytes may affect the proportion of electrolytes, since these diffuse with unequal facility (f.i., $\mathrm{Na}$ and $\mathrm{K}$ more readily than Ca or Mig; W. Denis, I906).

Limitations of the Electron Theory.-The theory of electron actions has furnished new and fruitful conceptions, a deeper insight into the reactions of protoplasm, and new indications of research. It should be remembered, however, that it does not as yet attempt to explain the actions of non-electrolytes; and that it can not be said to explain fully all the actions of electrolytes. It does not, for instance, explain the qualitative differences in the action of the iodids, bromids, and chlorids; of calcium, magnesium, or barium; the specific toxicity of potassium for muscle and nerve; nor the need for the presence of several ions. We must not forget that protoplasm has chemical, as well as physical, properties; and that many, if not all, ions are capable of entering into ordinary chemical reaction with the protoplasm. The specific ion actions, as we can observe them, are therefore the result of a coöperation of physical and chemical changes. In the present state of knowledge, it would be unremunerative to attempt to distinguish between these. A more practical conception of the action of the individual ions can still be obtained by describing the phenomena as they are observed, without trying, as a rule, to penetrate into their meaning.

\section{OSMOTIC EFFECTS ON CELLS}

It may now be attempted to apply these physical processes of salt action to the explanation of the phenomena of life. It will be remembered that the latter may be influenced by the osmotic, electric and chemic properties of the salts. The osmotic effects are independent of the nature of the molecules. The electric effects depend solely on the sign and transferability of the charge. The chemic effects are distinctive. All these effects are influenced by the special permeability of the cell membrane; by the solutes already present in the cell and in its surroundings; and by the special properties of the cell colloids:

Osmotic Actions on Cells.- These are due primarily to the changes in the total water or salt content of the cell. It is therefore necessary to distinguish between hyper-, hypo-, and isotonic'solutions. In effect, however, the final functional results of different concentrations are often very similar.

Changes in the Composition of the Cells.- In a physical sense, a cell may be conceived as a solution, the protoplasm, chiefly of hydrophilic colloids and largely dissociated electrolytes; inclosed in a membrane (the cell wall) of characteristic permeability; and generally containing suspended lipoids or solid granules.

The cell walls (which may be merely a modification of the cytoplasm) are usually easily permeable to water, impermeable to colloids and more or less difficultly permeable to electrolytes and other crystalloids. In this way they permit a certain amount of exchange between the cell and its 
surroundings (the lympl, etc.) which is necessary for metabolism. At the same time, since the permeability is limited, and varies for different substances, it enables the cell to preserve its own composition, which is quite different from that of the lymph. However, this protection is not perfect, especially if artificial changes are made.

The cell walls (and indeed also the interior cytoplasm, Kite, I9I5) behave essentially like what we have defined as a "partly semipermeable" membrane (as suggested by Traube, I 867). If therefore the molecular constitution of the medium surrounding the cells be in any way changed, this will effect, in the first place, a change in the total water or salt content of the cell. A hyperisotonic solution will cause the withdrawal of water, and a hypoisotonic solution the withdrawal of salts. If the ratio of the different salts in the surrounding medium is not the same as that in the cells, their ratio in the latter will also be altered. Again, when salts are drawn out of the cell, certain of its salts will leave more quickly than others. The changes will be the greater, on the whole, the more the composition of the surrounding liquid departs from that of the cell.

Molecular Concentration of the Blood and Tissues.-The total molecular concentration of the living cells is not accurately known. It is probably somewhat higher than that of the body-fluids. The plasma, lymph, and all other body-fluids (except urine and gastric juice) of all mammals, depress the freezing-point by about $0.56^{\circ}$, corresponding to $0.33 \mathrm{~mol}$ per liter. Relatively slight departures from this.are promptly injurious. Its maintenance is therefore very important. Accordingly, the organism resists very powerfully any attempts to alter its osmotic concentration. The fluids of lower animals have a somewhat different concentration, adapted to their environment.

Protoplasm.-The osmotic pressure of cells can not be measured during life. Direet cryoscopy of the organs gives unreliable results. Buchner juices generally depress the freezing-point somewhat more than does blood, but this might be due to chemic changes. The protoplasmic pressure is not solely osmotic, but also due to imbibition, surface tension, and the elasticity of the cell wall (Pfeffer, 1897 ).

Human Blood. - The extreme variations of the freezing-point in health are between $0.49^{\circ}$ and $0.59^{\circ}$. The molecular concentration of the blood is not altered by the quantity of fluid or salt in the food; nor by hydremia produced by hemorrhage; nor by thickening the blood by profuse diaphoresis. The intravenous injection of anisotonic solutions alters it but temporarily. About the only method of raising it permanently consists in interfering with the regulating mechanism, by seriously injuring the kidneys; and then it proves fatal when the $\Delta$ approaches $0.8^{\circ} \mathrm{C}$. The importance of comparatively slight changes in the concentration is also shown strikingly by the red blood corpuscles. These swell in hypoisotonic, and shrink (crenate) in hypertonic solutions. If the serum is diluted to about $\Delta 0.35$, the hemoglobin begins to leave the corpuscles, their structure being destroyed. There is reason to believe that the same holds for most other cells, and that the limits between which cells of mammals can functionate at all, are comprised between $\Delta 0.35$ and 0.6 or somewhat higher.

Osmotic Equilibrium of Lower Animals.-The narrow limits of mammals are considerably extended in the lower forms of life. With marine animals, up to the Selachians, the molecular concentration of the body-fluids approaches that of sea water, and becomes independent only in the Teleosteans. Dog-fish are intermediate (Scott, 1913). In the marine animals, a concentration equal to that of the serum of mammals would liberate the hemoglobin. In other words, the resistance of cells is somewhat adapted to their normal environment. This resistance is curiously developed in the small fish Fundulus, which thrives equally well in sea water and in distilled water. It is due, in this case, to a high degree of impermeability to both water and salts, so that osmotic pressure can not be developed.

Sweet-water animals have a considerable molecular concentration approaching that of mammals, the salts being probably retained by chemical or physical affinity for the colloids. The molecular concentration of plant juices is also considerably higher than 
that of water. Fungi often have a very high resistance against osmotic changes, presumably because their envelopes are relatively impermeable. Torula, e.g., can develop in a saturated solution of sodium or lithium chlorid, the latter having an osmotic pressure of 2,769 atmospheres (Raciborski, 1906).

Structural Changes Resulting from Osmosis. - With most cells, however, any departure from the normal concentration of the liquid in which they are bathed, produces the structural changes which we would expect; these can be best seen in monocellular organisms, red or white blood cells, or ova: these show shrinkage or swelling, condensation or liquefaction, precipitation or solution. These often lead to even more important secondary decompositions, which may cause the cells to swell even more in a hyperisotonic solution, than they do in water (Sollmann, I904).

Osmotic Changes in Red Blood Cells.-Hypertonic solutions produce crenation; if the solutions are hypotonic beyond a certain degree, hemolysis occurs. The cells swell and lose their hemoglobin and salts. In life, these changes could be produced only by intravenous injection of anisotonic solutions.

Specific Resistance to Hemolysis.-This varies in different species. With human corpuscles hemolysis usually begins with 0.457 per cent. $\mathrm{NaCl}$, in beef with 0.58 ; in the horse with 0.68 . These specific differences refer only to hemolysis; the osmotic swelling, measured by the hematocrite, being about equal in the different species. The specific difference to water-laking is generally inverse to that for saponin-laking (Rywosch, I907).

Influence of Disease, Etc.-Changes in the resistance to hemolysis have been found in diseased conditions (tumors, etc.). With normal human corpuscles, hemolysis begins in about 0.457 per cent. $\mathrm{NaCl}$, and is complete in 0.340 per cent. $\mathrm{NaCl}$; these limits being fairly constant. They are also practically the same in pernicious and in secondary anemias. The resistance is said to be increased by therapeutic doses of arsenic (L. W. Hill, 1915).

Laking Power of Various Salts.- - Hemolysis depends not only on the concentration, but also on the nature of ions, following the usual lyotropic series. Hoeber, I 908, found the hemolysis least with the $\mathrm{SO}_{4}$ and $\mathrm{Na}$ end, and most with the $\mathrm{I}$ and $\mathrm{K}$ end of this series. The sequence is reversed by combination with lipolytic agents, saponin or bacterial lysins. The differences are referable to the different permeability of the salts and to their effects on the cell membrane.

Hemolysis by Hypertonic Solutions. - This has been investigated by C. C. Guthrie and Lee, I9I4.

Permeability of Red Blood Cells.-These are practically impermeable to most osmotic substances. However, both anions and cathions pass to a slight degree, even under physiologic conditions (Hamburger and Bubanovic, 1907); and certain substances (ammonium ions, urea) pass very readily. In general, the permeability is proportional to the lipoid solubility. Ammonium salts are an exccption for they are directly hemolytic; they lake even when added to isotonic $\mathrm{NaCl}$ solution. The permeability to other solutes is increased by combination with saponin and other hemolytic agents. In saponin-laking, an increased permeability of the cell membrane can be demonstrated directly, before the salts pass out of the cell (G. N. Stewart; Woelfel, I908).

The permeability of the corpuscles for $\mathrm{Na}$ and $\mathrm{K}$ ions has been denied (Guerber, I895), but has been established by $\mathrm{H}$. J. Hamburger, I9I6. He also finds that the addition, within physiologic limits, of $\mathrm{CO}_{2}$ to the serum drives $\mathrm{K}$ ions into, and $\mathrm{Na}$ ions out of the corpuscles. $\mathrm{NaCl}$ drives $\mathrm{Na}$ in and $\mathrm{K}$ out; and $\mathrm{KCl}$ does the reverse.

Preservation of Living Erythrocytes.-Blood cells, when suspended in saline solution die rather rapidly, partly by mechanical and partly by chemical injury. Rous and Turner, $191_{5}$, found that the addition of gelatin protects them against the mechanical injury, but not against the chemical. The addition of glucose or saccharose, on the other hand, preserves them for a long time, so that they show no morphologic changes, and take up and surrender oxygen in the normal manner.

Selective Permeability of Cell Membranes.-This varies for each class of cells, some being impermeable to one substance, some to another. This · is doubtless important for maintaining the normal differences of composition. It is subject to changes with the functional states of the cell. The permeability is often different in one direction from that in the other. 
Permeability to Different Substances. - With most living cells, lipoidsoluble substances penetrate more readily than those which are only watersoluble. 'The penetration is also influenced by the presence of lipolytic agents (anesthetics). The lipoids of the cell membrane are therefore important elements in determining its permeability. They are not, however, the only factor. The membrane is not absolutely impermeable to salts; and some substances penetrate independently of their lipoid solubility. It is generally assumed that the membrane is a mixture of lipoid and protein colloids, and that both play a part in the penetration.

When discussing the red blood corpuscles, it was stated that they were almost impermeable to most salts and sugars, but permeable to ammonium, urea and lipolytic agents. This applies also to plant cells, in which the phenomena have been most extensively studied. Klebs and de Vries, f.i., found that glycerin and urea do not produce plasmolysis. Overton, I895 and I899, has added many data. Striped muscle is also nearly semipermeable (similarly to a celluloid membrane in which $\mathrm{CaHPO}_{4}$ has been deposited); while smooth muscle is highly permeable to sugars and salts (Meigs, 1912 and $\left.19 \mathrm{I}_{3}\right)$. The changes of weight in striped muscle in various conditions were investigated by J. Loeb.

Lipoid Solubility as Condition for Entrance.-The numerous observations of Overton and others show no direct relation between chemic constitution and permeability. Traube, 1867 , believed that the differences depend on the size of the molecule; but this is untenable. L'Hermite, I855, attributed the penetration to solubility in the cell membrane. This is undoubtedly a prerequisite to absorption. Overton found that all lipoid-soluble substances penetrate readily into all cells; and those which are not lipoid-soluble penetrate with difficulty.

This rule, although not absolute, holds so widely, that it must be a simple passive physical phenomenon. It is not absolute, however, or metabolism would be impossible. Nathansohn, I904, therefore assumed that the cell membranes consist of a mosaic of cholesterin and protoplasm. However, this is not a necessary deduction; for Nathansohn, I904, and Ruhland, 1909, showed that artificial lipoid membranes, when soaked in water, become quite permeable to substances that dissolve in water, even if these are insoluble in lipoids; while water-free lipoid membranes are impermeable to the water as well as to the solutes.

Calcium and magnesium phosphates perhaps also play a part in semipermeability; but the degree of impermeability is so different that they are probably unimportant factors (Meigs, 1915).

Permeability for Alkaloids. - This is easily observed in plants by the precipitation of the tannin within the cells. Only the free (basic) alkaloids penetrate; the alkaloidal salts enter very much more slowly, namely in proportion as they are hydrolyzed. Their toxicity is favored by the addition of alkalies; this applies also to fish and frog larvæ. Harvey, I9I3, found that cell membranes are readily permeable to weakly dissociated alkalies, but remarkably resistant to those which are strongly dissociated.

Absorption ' of Dyes.-Ehrlich, I 887 and 1893, found that vital staining occurs especially with basic dyes. Overton, 1900, referred this to their lipoid solubility, and advanced the theory that all absorption occurs through the lipoids of the cell envelope. However, exceptions have been discovered. Certain lipoid-soluble dyes are not absorbed; while others, which are insoluble in fats, are nevertheless absorbable. Indeed, Asher and Gannus, I9II; Harvey, I911; Kite, I913; and others, have found little relation. Acid dyes stain the protoplasm diffusely, while basic dyes stain mainly the cell granules.

Permeability of Internal Cytoplasm.-Kite, I915, has shown that all portions of the protoplasmic gel are more or less semipermeable. Incised and punctured cells, for instance, offer the same obstacles to the penetration of dyes as do intact cells. The rate of penetration is generally inversely proportional to the concentration of the protoplasmic gel.

Relation of Penetration to Functional States.-Asher observed that the vital staining of gland cells is greatly influenced by their functional activity, being increased by pilocarpin and diminished by atropin. Temperature influences the plasmolysis of plant cells ( $v$. Rysselberghe, 190I); $\mathrm{CO}_{2}$ increases the permeability of erythrocytes (Hoeber, 1904). The permeability is also infuenced by salts and narcotics, and stimulation (Osterhout, 1912; R. S. Lillie, 1912; J. Loeb, 1913). Fertilization or electric stimulation increases the permeability of frog eggs, so that their ions diffuse out at a 
faster rate (McClendon, I $\left._{9} \mathrm{I}_{5}\right)$. It is probable that similar changes in permeability are essential to the metabolism of the cells (Hoeber).

The semipermeability of artificial membranes can also be varied at will, especially by the strictly physical conditions of hydration. The permeability of celloidin membranes, for instance, depends on the degree to which they are dried before water is applied; the less the drying, the greater their permeability (Meigs, 19 5 ).

Influence of Electrolytes on Permeability.- J. Loeb and Cattell, r9 I $_{5}$, observed that electrolytes increase the diffusion of $\mathrm{K}$ and $\mathrm{H}$ ions (Loeb, ror5) out of fundulus ova, but retard their passages into these cells. The degree of the effect is parallel to the lyotropic series of anions. They attribute the diffusion to combination of the $\mathrm{K}$ or $\mathrm{H}$ with the colloid anions of the cell membrane; this combination being diminished by the mass-action of excessive anions in the surrounding solution.

Clowes, r9r6, finds that the influence of electrolytes on cell permeability is similar to their effect on the permeability of soap films.

Semipermeability Persists only During Life.-It is lost when the cells die, so that dead cells merely imbibe solutions, like gelatin or fibrin. Acids also destroy the semipermeability (J. Loeb, I9I3). Living tissues resist the entrance of all acids (except salicylic), while dead tissues are freely permeable to them (Harvey, 1914).

Functional Responses to Osmotic Changes.-The alterations in the composition of the cells entail corresponding changes in their functions, consisting in a mild or strong stimulation, often irritative; passing into depression and finally disorganization of the cell if the changes have been large. The irritant phase tends to predominate with hyperisotonic solutions, whilst hypoisotonic solutions tend more to depression.

With unspecialized cells the main changes fall upon their metabolism. These nutritive changes are most pronounced in the cells of lowest vitality. As a rule, pathologic formations possess less resistance than normal tissue, so that one of the most conspicuous phenomena of the general salt action is the breaking down of pathologic formations, no matter what their origin, and whether they are chemic or anatomic. Other specialized tissues also respond by their proper functions: Abstraction of water from a nerve or muscle causes contraction; the unfertilized eggs of certain marine animals undergo parthenogenetic development, etc. (J. Loeb, I899, I90I).

If a very strong salt solution is injected directly into the circulation, the main symptoms will arise from the central nervous system. They consist in stimulation, with subsequent paralysis, being similar to the effects of asphyxia.

Salt Action on Phagocytosis. - The sensitiveness of cells' to small changes in osmotic concentration is shown by the phagocytic power of leucocytes. According to H. Hamburger and his pupils, 1908 and 1910, the phagocytic power is decreased by very small changes of the osmotic concentration of the serum, such as may occur under physiologic conditions. All anisotonic solutions are injurious, but especially increased concentrations. The leucocytes recover when the normal concentration is restored. $\mathrm{NaCl}$ causes but little injury apart from the osmotic effect. $\mathrm{NaI}$ is more toxic than $\mathrm{NaBr}$. Of other ions, a slight excess of calcium stimulated, while decreased activity resulted from a larger excess, and from reduction of calcium as by fluorids or citrates. $\mathrm{Ba}$ and $\mathrm{Sr}$ do not have the stimulant action, so that it is not due to valence. Diminution of the normal alkalinity was deleterious, and to a less degree also an excessive alkalinity.

Osmotic Response of Muscle.-Striped muscle is semipermeable, smooth muscle is permeable. This different permeability is reflected in their response. Immersion in water, f.i., causes striped muscle to become rigid and to shorten, smooth muscle to lengthen. The effects are counteracted by 2 per cent. $\mathrm{NaCl}$ (Meigs, 1912). Dale, 1913, also found the tone of smooth muscle increased by hypo- and diminished by hypertonic Ringer's solutions. Slightly hypertonic solutions increased the response to inhibitory drugs and decreased the effects of stimulant drugs. Slightly hypotonic solutions had the opposite effect. The muscle of the Venus agrees with non-striated muscle, in being permeable (Meigs, 1914).

Faligue increases the molecular concentration (depression of freezing point) of excised skeletal muscle by $3^{6}$ per cent. by the breaking down of molecules. This does 
not sccur when the circulation is intact, for the difierences are then equalized by exchange with th: blood (A. R. Moore, I9I6).

Ion Effects on Muscle.-The immersion of muscle in various solutions produces marked effects even when osmotic changes are excluded by having the solutions isotonic. These must therefore be referred to ion actions.

'The muscles lengthen or shorten; the excitability may be diminished or increased; spontaneous rhythmic contractions may appear; or the muscle may develop "contact irritability" so that it will react to stimuli which are ordinarily ineffective. Invertebrate and mammalian muscles exhibit similar phenomena if proper conditions are maintained.

\section{ACTIONS OF ELECTROLYTES}

Importance of Electrolytes.-Muscles soon lose their excitability completely in solutions of non-electrolytes; but this returns when they are replaced in saline solutions. A medium containing electrolytes is therefore essential to muscular contraction; indeed, any change in the ions produces alterations in the muscular functions. The different ions are not equally effective. It appears that the nature of the cathions is more important than the anions. The ions arrange themselves in the usual lyotropic series, the $\mathrm{Na}$ and $\mathrm{I}$ end being the most favorable, and the $\mathrm{K}$ and citrate end being the most unfavorable.

Toxic Action of Single Salts.-Immersion in any solution of a single salt also renders muscle inexcitable, but with very different rapidity. These differences follow the lyotropic series in the same sense.

Rhythmic Contractions of Skeletal Muscle.-These generally develop before the irritability is lost, when muscle is immersed in solutions of a single electrolyte (Loeb, I 899). If a certain proportion of a calcium salt is added, the rhythmic property disappears again. The rhythmic contraction of the cardiac muscle or of medusæ also disappears when calcium is added. Rhythmic contractibility is not therefore a property characteristic of cardiac muscle, but is inherent in all muscular tissues. The reason why the contraction of the heart is ordinarily rhythmic, and that of skeleta! muscle not, lies in the fact that the ions in the heart are balanced so as to favor rhythmic contractions, and in the skeletal muscle they are not so balanced.

Ciliary and Muscular Movement.-R. S. Lillie ( 1902 ) has studied the effects of ions on the larvæ of a sea-worm (Arenicola), which executes both ciliary and muscular movements. He found that the presence of magnesium in the mixture favors the contraction of the cilia, calcium of the muscles; potassium hinders both. Parker (1905) has found that potassium reverses the stroke of certain cilia.

Ion Actions on Heart.-These are highly important to the cardiac contractions. The excised hearts of cold-blooded animals soon stop their beat when immersed in solutions of non-electrolytes (Carlson); but they continue to contract rhythmically for a long time in certain salt solutions. Analogous phenomena are observed in the perfusion of mammalian hearts. The solutions must contain $\mathrm{Na}, \mathrm{K}$, and $\mathrm{Ca}$ ions; and these must be in a fairly definite "balanced" ratio, as in Ringer's or Locke's solutions or their modifications. Deficiency or excess of these ions cause derangement of function and are equally fatal. The ratio is much more important than the absolute quantity of the ions. The anions are relatively indifferent.

Rôle of the Individual Cathions. $-\mathrm{NaCl}$ is indispensable to the rhythmic contractions of the heart; but when present alone it produces relaxation and is finally fatal to the heart as to other tissues. The addition of $\mathrm{K}$ ions produces a diastolic tendency and standstill. $\mathrm{Ca}$ and $\mathrm{NaCl}$ produce increased systolic contractions but the diastoles 
become imperfect and deficient. Starting with a balanced Ringer's solution, sudden increase of $\mathrm{K}$ results in slowing and weakening (often halving of the rate), and decreased excitability to vagus stimulation, and with higher concentrations in arrest. This may be removed by the injection of $\mathrm{Ca}$, or by accelerator stimulation. Increase of Ca acts like accelerator stimulation, resulting in increased excursions, prolongation and fusion of systoles, and systolic standstill. Ca is also necessary for the inhibitory response to vagus stimulation; but excessive Ca concentration paralyzes the vagus (Schiff; Cazzola, I9I3; Boehm, I9I4). The $\mathrm{K}$ or Ca actions predominate when the percentage of one predominates about three and one-half times over the other. The effects of $\mathrm{K}$-free Ringer's solution resemble Ca predominance. Ca-free Ringer's solution increases the rate-i.c., the result is the opposite of $\mathrm{K}$ in the presence of $\mathrm{Ca}$. Deficiency of $\mathrm{Ca}, \mathrm{K}$ or $\mathrm{OH}$ ions shortens the survival of the heart (Boehm, I914).

Theories of Cardiac Ion Relations.-In the light of these data attempts have been made to assign definite functions to the individual ions. They are not very satisfactory, except in that they emphasize that $\mathrm{Ca}$ and $\mathrm{K}$ have opposite and complementary effects (Ringer, I882), Ca for promoting contraction and $\mathrm{K}$ relaxation. Loeb, I 899 , attributed the contractions to the $\mathrm{Na}$ ions, but requiring the presence of $\mathrm{Ca}$. Howell, 1898 and 1906 , believes that $\mathrm{Ca}$ is concerned in the contraction and $\mathrm{K}$ and $\mathrm{Na}$ in the relaxation; that these ions enter alternately into relation with the contractile substance; that accelerator stimulation liberates $\mathrm{Ca}$ and vagus stimulation $\mathrm{K}$ ions. Martin, I9I3, assumes with Howell that $\mathrm{Ca}$ promotes the conversion of stable into unstable energyyielding material; that $\mathrm{Na}$ brings about the actual dissociation and thus initiates the beat (Lingle); and that $\mathrm{K}$ antagonizes both the $\mathrm{Na}$ and $\mathrm{Ca}$. The chemic conditions of cardiac activity are reviewed by 'Tigerstedt, Erg. Physiol., I 2: 269.

Abnormal Ions.- These have been studied, e.g., by Lussano, I9 13, by modifying the constituents of Ringer's solution. Sr and Ba can not replace $\mathrm{Ca}$, but their effects correspond to those of excessive $\mathrm{Ca}$. $\mathrm{Li}$ is not toxic, but causes some osmotic slowing. $\mathrm{NH}_{4}$ slows, but increases the irritability and strength.

Arterial Muscle.-Prochnow, I9I I, tried the replacement of $\mathrm{NaCl}$ by other anions. The tone was raised by $\mathrm{F}>\mathrm{I}>\mathrm{Br}$. Hatcher, I906, tested the excitability of the kidney vessels to epinephrin and found this preserved twice as long in Locke's fluid minus glucose, as in 0.9 per cent. $\mathrm{NaCl}$. With glucose, the excitability remained eight times as long. The ability to reduce hemoglobin behaved similarly.

Ion Actions on Nerve.-Nerves also lose their irritability in solutions of non-electrolytes, and recover in salts. The cathion series is similar to muscle, but the anion series is reversed.

The reaction of motor-nerves to electrolytes has also been shown to be an additive function of the two ions: the anions (alkalies or increase of valency) stimulating the sciatic nerve of the frog, while cathions (acids) lower its irritability (A. P. Mathews, 1902). This agrees with the old observation of Galvani (confirmed by Grandis, 1902), that the nerve is stimulated when it is charged by induction with negative electricity, and depressed by a positive charge. Mathews assumes that the stimulation is caused by the precipitation of the (positive) colloids of the nerve by the anions, and vice versa. This demands further proof, especially before it is extended to other forms of protoplasm.

$\mathrm{Ca}$ and $\mathrm{K}$ on the Nervous Centers.-Hooker, I9 I3, perfu'sed solutions of these chlorids through the respiratory center of frogs, and found that increase or omission of $K$ from balanced solutions produces depression; decrease of $\mathrm{K}$ causes stimulation. With $\mathrm{Ca}$, increase and omission produce stimulation; diminution depression. The perfused spinal cord of frogs gave similar results (Hooker and Reese, I9I4). As to the perfusion of the medullary cardiac centers, predominance of $\mathrm{K}$ stimulated the vagus and inhibited the accelerator; while predominance of $\mathrm{Ca}$ stimulated the accelerators (Hooker, I9 I5). The perfused central nervous system of mammals preserves its activity best with a solution of 0.9 per cent. $\mathrm{NaCl}$ and 0.05 per cent. $\mathrm{CaCl}_{2}$. These can not be replaced by related ions. Even traces of potassium are harmful (P. Gerlach, I9r4).

Ion Effects on the Kidneys.-The polyuria and glycosuria which follow the intravenous injection of large quantities of most sodium salts (especially in slightly hyperisotonic solutions) perhaps also involve ion action; at least (W. H. Fischer and O. H. Brown, I903) the addition of a small proportion of $\mathrm{CaCl}_{2}$ stops the diuresis, which may then be started again by injecting sodium acetate. Sr or Ba salts prevent the glycosuria without affecting the polyuria.

Sollmann (1905) has found that calcium also hinders the filtration of urine and impedes the circulation in excised kidneys, while magnesium favors them. These effects may be referred to ion actions on the renal protoplasm. The effects of most other ions which were investigated in this manner are referable to osmotic changes, due to differences of penetrability.

Guthrie, I910, claims that direct perfusion of the kidney vessels with saline solu- 
tions, even for a short period, is followed by hemorrhagic and degenerative changes in the kidneys, leatling to uremia and death after a few weeks or months. Simple anemia uf equal durition is much less detrimental. Carrel claims that he has not observed these results.

Balanced Salt Ratios. - A solution containing a single electrolyte, no matter what the chenic nature of the latter, is fatal to all forms of life. The tuxicity can be greatly lessened by the addition of a second salt. This is called the "antitoxic action" of ions. For perfect functionation, several salts are necessary, in a definite "balanced" ratio (J. Loeb, I900). Departures from this ratio modify the functions of the cell, and larger changes are fatal. The mineral constituents of cells are therefore indispensable to life. The phenomena are probably produced by modifications in the permeability of the cell membrane.

Ions Essential to Life.-The following salt ions are absolutely necessary to plants: $\mathrm{K}, \mathrm{Mg}, \mathrm{PO}_{4}, \mathrm{CO}_{3}$ ( $\mathrm{Na}$ and $\mathrm{Cl}$ are not necessary); $\mathrm{Ca}$ to all but the lower fungi and algæ. In forms an essential constituent of vegetable oxidizing enzymes. $\mathrm{NO}_{3}$ and $\mathrm{SO}_{4}$ act as nutrients.

Animals require $\mathrm{Na}, \mathrm{Cl}, \mathrm{CO}_{3}, \mathrm{Ca}, \mathrm{K}, \mathrm{Mg}, \mathrm{I}, \mathrm{Fe}, \mathrm{PO}_{4}, \mathrm{SO}_{4}$. It is very doubtful whether other elements existing only in traces (such as Fl) are necessary, or merely accidental.

The microchemic investigations of A. B. Macallum (1905) indicate that the electrolytes are localized in certain portions of the cell by surface tension.

Mineral Salts in Nutrition.-McCollum and Davis, I913, found a definite salt mixture in the food to be essential to the growth of rats. Osborne and Mendel, 1912, also found that rats fed on a diet free from inorganic salts declined continuously in weight, and that a very definite mixture of salts must be supplied. An artificial mixture was as effective as the "protein-free milk." The salt content of the milk undoubtedly plays an important part in the nutrition of infants. Cows' milk is considerably richer in all the important salts than is human milk, but the difference is probably compensated by the better utilization of the salts of human milk.

Antitoxic Action ${ }^{1}$ of Ions.-As far as known, no cell can remain alive unless it contains some electrolytes. If these are reduced below a certain minimum, the cell dies rapidly. But in many cases it dies even more promptly if it is placed in a solution containing but a single electrolyte. This is the toxicity which was described in the preceding paragraphs. It would seem therefore that several electrolytes, in a certain ratio, are required in order that the cell may perform its functions. This was confirmed by $\mathrm{J}$. Loeb (190I), who showed that the toxicity of an electrolyte can be very greatly lessened by the addition of another electrolyte, sometimes in very small proportion. (For instance, the addition of $1 / 4,000 \mathrm{~m}$. CaCl $\mathrm{Ca}_{2}$ neutralizes the toxic action of $5 / 8 \mathrm{~m}$. NaCl on Fundulus ova.) The antagonism is mutual, at least to some extent. Instructive examples of antagonism are furnished by J. Loeb, I9I I.

From his observations, Loeb at first concluded that the cathions are responsible both for the toxic and antitoxic actions; and that cathions of different valency neutralize each other. The extensive experiments of A. P. Mathews (1905) modify these theories: It would appear that cathions and anions are both concerned in the toxic and antitoxic actions (although the cathions are probably the more important); and that the valency has no direct connection with either the toxic or antitoxic action (Loeb, I914). The decomposition-tension seems to be of subsidiary importance in the antitoxic action, and it appears to be necessary to invoke several mechanisms for its explanation. The antagonism is evidently a complex phenomenon, involving also the specific ion actions. It may be noticed, for instance, that certain ions $\left(\mathrm{NH}_{4}\right.$ and $\mathrm{Li}$ ) are particularly difficult to neutralize; $\mathrm{Ca}$ is especially antitoxic, $\mathrm{Mg}$ much less so; $\mathrm{Ni}$ scarcely at all. The optimum ratio and the degree of antagonism vary for different classes of cells. Closely related ions sometimes have very different effects, and can not replace each other. Even when the antagonism of two salts is at its best, it is never perfect; it requires the addition of one or more further salts to enable the cell to perform its functions properly. Indeed, a strictly normal condition is not reached until the cell is placed in a solution, the cathions of which are within narrow limits the same qualitatively and quantitatively as those of its normal surroundings.

1 The term "antitoxic action," applied to ions, means something very different from the same term when it is used in connection with bacterial poisons. 
Nature of Ion Action.-There are reasons for believing that the ion actions are exerted only on the surface of the cell, especially by altering the permeability of the envelope (plasma membrane); and that the antitoxic actions are also exerted mainly on the permeability.

The high resistance of cells to the penetration of ions makes any internal effects very improbable. With potassium, f.i., which is highly toxic to muscle cells, it has been shown that it passes neither into nor out of the cells (Urano; Fahr). The same is true of calcium (W. Straub, 1912). The promptness with which the effects can be removed by the addition of a second salt also speaks against absorption. It is very probable that the single-salt solutions disturb the semipermeability of the membrane (J. Loeb, 1905, I914), thus interfering with the maintenance of the electric potential which determines irritability. The increased permeability would also produce chemic changes by permitting the entrance of foreign ions. However, the mechanism is not always the same: f.i. Loeb and Wasteneys, Igro, found that the immediate toxicity of $\mathrm{NaCl}$ for sea-urchin eggs is due mainly to greatly increased oxidation; and Warburg, 1910, found accordingly that the toxicity is diminished by the addition of NaCN. On the other hand, the toxicity of $\mathrm{NaCl}$ for the neuro-muscular mechanism of medusæ is quite different; in these it diminishes oxidation, and cyanid is therefore not antitoxic.

Plasma Membrane and Electric Stimulation.-Nernst, I 899, suggested that electric stimulation changes the ion-concentration on the surface of the semipermeable membrane. Lillie, I9I5, proposes a similar theory for nerve-conduction: The change in permeability would itself set up electric changes, which would stimulate the adjacent portions of the nerve.

Mechanism of "Antitoxic" Action.-G. N. Stewart, 1903, suggested that the antagonism of salts may be explained in some cases by alteration of permeability, preventing osmotic changes or the penetration of harmful ions. This has been confirmed by $\mathrm{J}$. Loeb, I905, I9I I, and I9I2. It is probable that K "loosens" the plasma membrane and thus abolishes excitability; and that $\mathrm{Ca}$ counteracts this effect. The reasons for the necessity of $\mathrm{Na}$ and $\mathrm{Mg}$ have not been explained.

Loeb ( $\left.19 \mathrm{I}_{4}\right)$ now believes that the conservation of the plasma membrane requires a specific mixture of $\mathrm{Na}, \mathrm{K}$ and $\mathrm{Ca}$ chlorids; and that any departure from this balanced ratio disturbs the membrane. A partial preservation may be secured by substituting other ions with similar properties. The whole phenomenon' is rather analogous to the behavior of soaps (Clowes, I9I6). Osterhout, I9II, I9I2 and I9I5, arrives at similar conclusions. He finds that cell permeability is increased by Na, decreased by $\mathrm{Ca}, \mathrm{Sr}$, $\mathbf{M g}, \mathbf{H}$ (in the form of chlorids). In general, substances that increase permeability are antagonistic to those that decrease it. This applies also to non-electrolytes.

Other factors may conceivably coöperate, such as the electric and chemic changes, decrease of dissociation (as in the effect of citrates on Ca; Sabbatani, 190I). Finally, the antagonism may be functional, for it has been noticed that calcium also antagonizes some poisons which are not electrolytes (veratrin, Ringer, 1884; physostigmin, S. A. Mathews and O. H. Brown, r904; cascara, MacCallum, 1904), and to which it is opposed in its functional effects. Similarly, magnesium is mutually antagonistic with physostigmin (Meltzer).

Magnesium and Calcium Antagonism.-This presents several special features. Increase of either $\mathrm{Ca}$ or $\mathrm{Mg}$ ions depresses muscle and nervous structures. Nevertheless, the effects of the $\mathrm{Mg}$ may be removed by addition of $\mathrm{Ca}$. Ca also antagonizes the inhibitory effects of $K$, as well as the stimulant effects of Na (Meltzer and Auer, I908). Strontium does not take the place of $\mathrm{Ca}$ in these reactions.

Even more complex relations have been discovered (Joseph and Meltzer, 1910): in frogs, injection of $\mathrm{Mg}$ into the lymph sac produces a curare action, paralyzing the muscle-nerve endings, but not the muscle. The excitability is restored by Ca. Perfusion of $\mathrm{Mg}$ paralyzes the muscle as well as the endings. Ca has no restorative effect. $\mathrm{Na}$ restores only the muscle; $\mathrm{Na}+\mathrm{Ca}$ restores also the endings.

"PHYSIOLOGIC" OR "NORMAL" SALINE SOLUTIONS (ARTIFICIAL SERUMS)

The deleterious effects of single-salt solutions have led to the introduction of solutions better adjusted to the physiologic requirements. These balanced solutions are necessary when experimenting on excised tissues. They are less important for injections into living animals, in 
which even single-salt solutions are rapidly adjusted by exchange with the tissues.

These solutions have been devised partly empirically, partly by imitating the salts of the blood serum or sea water. Different tissues of ten require slight modifications to secure the optimal effects. The following are the most important:

Isotonic $\mathrm{NaCl}$ Solutions.- Nasse, I 869, determined experimentally that 0.6 per cent. is the best concentration for preserving the irritability of frogs' tissues. Later, it was shown that this solution was not indifferent: Kronecker and Stirling observed stoppage of the heart; Ringer, twitching of muscles; Locke, contractures and increased irritability; Carslaw and Locke, curare action, etc. These effects can only be overcome by balancing the solution with other salts. However, the solution is practically isotonic with frog's blood, and therefore obviates osmotic changes. On the other hand, it is hypotonic to mammalian blood, which requires about 0.9 per cent. NaCl. Solutions of 0.8 to 0.9 per cent. are therefore generally preferred for mammals. The concentration of the U.S.P. is 0.85 per cent. Abel, however, found in vividiffusion that a lower concentration, about 0.6 per cent. prevented edema more effectively.

It will be recalled that the exact composition is of relatively small importance for injections into living animals.

\section{TABLE OF COMMONLY USED BALANCED SOLUTIONS}

Percentages

\begin{tabular}{|c|c|c|c|c|c|c|}
\hline Author & Adapted to & $\mathrm{NaCl}$ & $\mathrm{KCl}$ & $\mathrm{CaCl}_{2}$ & $\mathrm{NaHCO}_{3}$ & Other ingredients \\
\hline Ringer. & Frog's heart & 0.6 & 0.0075 & $\begin{array}{l}0.01 \text { (dried) } \\
0.026 \text { (crystals) }\end{array}$ & $0.0 \mathrm{I}$ & \\
\hline $\begin{array}{l}\text { Howell. ......... } \\
\text { Clark......... }\end{array}$ & $\begin{array}{l}\text { Frog's heart } \\
\text { Frog's heart }\end{array}$ & $\begin{array}{l}0.7 \\
0.7\end{array}$ & $\begin{array}{l}0.03 \\
0.014\end{array}$ & $\begin{array}{l}0.025 \text { (crystals) } \\
0.012 \text { (dried) }\end{array}$ & $\begin{array}{l}0.003 \\
0.02\end{array}$ & \\
\hline Goethlin..... . & Frog's heart & 0.65 & 0.01 & 0.0065 (dried) & 0.01 & $\mathrm{Na}_{2} \mathrm{HPO}_{4}, 0.0009$ \\
\hline $\begin{array}{l}\text { Locke. . . . . . . . } \\
\text { Rusch.... }\end{array}$ & $\begin{array}{l}\text { Mammalian heart } \\
\text { Mammalian heart }\end{array}$ & $\begin{array}{l}0.92 \\
0.8\end{array}$ & $\begin{array}{l}0.042 \\
0.0075\end{array}$ & $\begin{array}{l}0.024 \text { (crystals) } \\
0.01 \text { (dried) }\end{array}$ & $\begin{array}{l}0.015 \\
0.01\end{array}$ & Dextrose, o.1 \\
\hline Tyrode....... & $\underset{\text { tine }}{\text { Mammalian intes- }}$ & 0.8 & 0.02 & 0.02 (crystals) & 0.1 & $\begin{array}{l}\mathrm{MgCl}_{2}, \mathrm{O} .01 \\
\mathrm{Na}_{2} \mathrm{HPO}_{4}, 0.0005 \\
\mathrm{Glucose}^{2}, 1\end{array}$ \\
\hline Hedon and Fleig & Mammals & 0.6 & 0.03 & 0.01 (dried) & 0.15 & $\begin{array}{l}\mathrm{Na}_{2} \mathrm{HPO}_{4}, 0.05 \\
\text { Glucose, o. }\end{array}$ \\
\hline Adler......... & Mammals & 0.59 & 0.04 & 0.04 (crystals) & $0.35 \mathrm{I}$ & $\left\{\begin{array}{l}\mathrm{Na}_{2} \mathrm{HPO}_{4}, \mathrm{O} \text {.01 } 26 \\
\text { Glucose, o, r5 }\end{array}\right.$ \\
\hline
\end{tabular}

Note 1.-In making solutions containing $\mathrm{NaHCO}_{3}$, this must be completely dissolved before the $\mathrm{CaCl}_{2}$ is added.

NoTe 2.-Other solutions are described in "Digests of Comments on Pharmacopœia," r9rr, p. $6 \mathrm{Ir}$.

- The lower $\mathrm{K}$ content gives a more rapid heart rate.

Locke's Solution.-This is intended to be used with a stream of oxgyen. It was originally devised for the mammalian heart; but it also maintains the vitality of arteries (Hatcher, 1906), and of nervous and other tissues (Hedon and Fleig, 1905).

Herlitzka, 1910, found the addition of urea and glycerin to Locke's solution essential for the excitability of the nervous system of frogs. Presumably they influence the permeability of the lipoids. Bombiani, I913, finds that the favorable effect of urea on the selachian heart is not shared by related substances, and therefore considers it chemic. It dilates the perfused vessels of the frog (Hooker, I9II).

Sea Water.-This contains the important ions in about the same ratio as the artificial solution; e.g., for 100 molecules $\mathrm{NaCl}$ there are the following molecules of other salts:

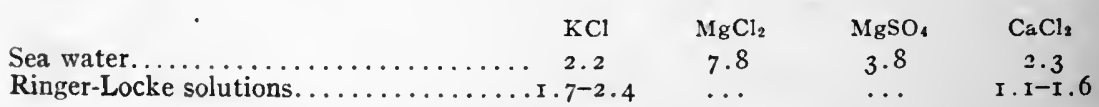

A. B. Macallum, 1910, suggested the interesting theory that the composition of the blood is a survival of the evolutionary adaptation of animals to their original marine environment.

The composition of sea water is somewhat different in different oceans. In the English channel it is as follows: 
I, ooo parts by weight contain:

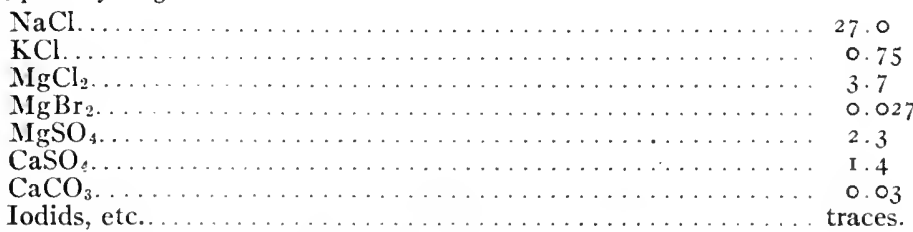

American analyses are furnished by Wheeler, Jour. Amer. Chem. Soc., 32:646, I9 ro.

The freezing-point of sea water is: $-2.29^{\circ} \mathrm{C}$. at Naples (Bottazzi, 1897); $-1.90^{\circ}$ at Pacific Grove, California; and $-1.82^{\circ}$ at Wood's Hole, Massachusetts (Garrey, 1905).

\section{OSMOTIC EXCHANGES IN THE BODY}

General Statement.-Osmosis tends to operate within as without the body, so as to bring all the fluids into osmotic equilibrium, and if possible, to establish an identical composition. It is, however, modified by other forces. If a foreign solution is introduced-f.i., into the intestines, or peritoneal cavity, or blood-the osmotic forces will very rapidly bring its total molecular concentration to that which is normal for the tissues. This exchange could be effected either by the passage of water; or by the passage of the foreign salts out of the solution into the tissues; or by the passage of the normal salts from the tissues into the solution. In fact, all three processes generally participate, their share varying according to the special permeability of the tissues. In general, the exchange occurs mainly in the direction which will favor the normal physiologic functions; i.e., in the direction of absorption.

When the molecular concentration of the blood has been reached, there remains less scope for osmotic exchanges, and the further absorption must occur by other forces (filtration, imbibition, etc.), generally much more slowly. The rôle of osmosis in absorption is therefore important, but limited. Its active share in the secretion of the body-fluids, f.i., the urine or lymph is normally small; it is important mainly by setting limits to the real secretory forces.

The R6le of Osmosis in the Absorption of Solutions from Serous Cavities and Lymph Spaces.-This absorption is almost entirely physical, for it occurs quite well in dead animals, although it is naturally more rapid when the circulation is intact, because the absorbed products are then more rapidly removed. Osmosis plays a very important part in the absorption, especially if the solutions are anisotonic. If a-hypoisotonic solution is introduced, osmosis causes the rapid absorption of water; with hyperisotonic solutions, the volume of the fluid will at first increase, until it has become equimolecular with the plasma. At this time, however, the fluid is really hypoisotonic to the non-diffusible constituents of the cells and blood, so that it would be eventually absorbed, by the operation of osmosis alone. The osmotic absorption, for instance from the peritoneum, is quickened if the body fluids are rendered hyperosmotic, as by nephrectomy, or ligation of the renal vessels, or indeed by operations generally, or by epinephrin. Etherization alone does not affect the osmotic absorption. Caffein lessens it in normal animals, but increases it after nephrectomy (Fleisher and L. Loeb, 1910).

Ordinarily, however, the osmotic absorption is very slow after the solution has become isomolecular; and other factors intervene to hasten the process. Of these filtration and imbibition are the most important. The filtration pressure is furnished especially by muscular, respiratory, and peristaltic movement. The intraperitoneal pressure amounts to 2 to $25 \mathrm{~mm}$. of water (H. Emerson, I907). It may be increased by massage.

Imbibition in Absorption.-The term "imbibition" is applied to the familiar phenomenon of the swelling of pieces of glue, gelatin, agar, tissues, and other colloid gels, when these are placed into solutions ( $\mathrm{F}$. Hofmeister, 1890 and 1891 ). This process is 
quite distinct from osmosis, although both may take place side by side. Imbibition is probably analogous to adsorption.

Hamburger has shown that when a solution is placed in a gelatin cylinder, and this is immersed in it circulating current of the same solution, the stagnant fluid in the cylinder passes gradually through the gelatin into the circulating fluid. The phenomenon is obviously analogous to the absorption of isotonic suitions from serous cavities, and helps to explain the latter. Hamburger considers this process an imbibition effect.

Many physiologists consider that vital forces also coöperate in the absorption; the possibility of this can not be denied; but there is scarcely any positive proof in its support; if it plays any rôle, this must be quite subsidiary.

Absorption of effusions or serum from scrous cavities: In this, the coöperation of osmosis is almost excluded, for these contain non-diffusible protein constituents, as well as the tissues. However, the proteins of these fluids tend to break down constantly into more diffusible molecules (as shown by the fact that exudates generally depress the freezing-point some what more than does the blood). Osmosis therefore plays a part, but this must be relatively small. The existence of proteins also limits the operation of imbibition, for proteins are not imbibed by colloids. This leaves filtration as the main factor in the absorption of protein-containing fluids. The absorption of these liquids is consequently a much slower process than that of salt solutions.

Absorption from the Alimentary Canal.-This is probably even more dependent upon forces other than the osmosis than is absorption from serous cavities.

Filtration aided by the intraintestinal pressure is an important aid (Hamburger). There are also other processes, ill-understood: Thus if an excised and surviving intestine is immersed in a solution, a passage of fluid takes place from thelumen to the surrounding fluid, even when the two fluids are identical (Cohnheim, 1899). Hamburger, 1906, attributes this to imbibition, and finds that even the dead intestine is differently permeable in the two directions. Sodium sulphate, for instance, passes much more readily from the lumen to the exterior than in the reverse direction. He has also demonstrated a "polarity" of absorption in artificial membranes, in which the two surfaces have a different composition. The diffusion through excised intestine is markedly influenced by salicylates and some other substances (Hanzlik, r9i2). The absorption of ions from the excised intestine has been investigated by Benedicenti, I906, and by Hoeber, I 903. The latter found the anion series, $\mathrm{Cl}>\mathrm{Br}>\mathrm{I}>\mathrm{NO}_{3}>\mathrm{SO}_{4}$. Lipolytic substances (short of the narcotic concentration) are absorbed more readily than nonlipoid solutes. From analogy with dyes, Hoeber assumes that the lipolytic are absorbed through the cells, and that the absorption of non-lipoids is confined to the intercellular substance.

Brodie and Vogt, Igro, showed that the oxygen-consumption of the intestines is increased during the absorption of normal saline, water and peptone solutions; indicating that the cells also play an active part in the process.

Absorption Curve.- Hanzlik and Sollmann (1909 to $\mathrm{I}_{913}$ ) have found that the absorption of phenol, alcohol, iodid and presumably other substances from intestinal loops follows a uniform curve, the rate being very rapid for the first ten to thirty minutes, and then very much slowed. Various factors seem to be concerned in this phenomenon. The rate of absorption, under these conditions, is not greatly influenced by the concentration of the solution or by the extent of the intestinal surface.

Importance of Osmotic Exchanges in the Intestine.-Osmosis has at least a modifying influence on intestinal absorption. The time during which the food remains in the alimentary canal is ample to effect osmotic exchanges between the intestinal contents and the blood. An isotonic condition becomes established quite promptly (but is not completed in the stomach; E. Otto, I905).

This equalization, with the absorbable salts, is mainly in the direction to facilitate absorption: hyperisotonic solutions being rendered isotonic rather by the absorption of salt than by the pouring out of fluid; while hypoisotonic solutions are rendered isotonic by the absorption of their water. (In other situations than the alimentary canal, the equalization takes place more uniformly in both directions.)

These changes result in corresponding alterations in the composition 
of the cells of the gastric and intestinal mucosa, which are therefore also subjected to osmotic salt action. This causes irritation. When the irritation is mild, it increases secretion, absorption, and peristalsis. More severe grades of irritation, however, produce emesis and inflammation.

Salts which are not absorbable by the intestine (cathartic salts) are reduced to isotonic conditions by the absorption of fluid. This fluid is retained in the intestine with the salts, increasing the bulk and fluidity of the intestinal contents, and serving as a mechanical stimulus to peristalsis. This explains largely the cathartic effects of these salts.

Molecular Concentration of Gastric Juice.-Carlson, 1915, found the freezing-point of normal human "appetite juice" as -0.55 to 0.62 ; that of hunger juice as -0.47 to 0.52 . Similar results were obtained with dogs.

Osmotic Effects on the Blood and Lymph.-Fate of Salt Solutions Within the Body.- - In whatever concentration and by whatever channels salts are introduced into the body, they will tend to increase the quantity of blood, or rather of plasma; and to render it more watery and less viscid; in other words, to produce hydremic plethora. If isotonic or hypoisotonic solutions are introduced, they will dilute the blood by their own fluid; if hypertonic solutions are injected, the blood will draw fluid from the tissues until it has resumed its original concentration. The result will be hydremia in either case. If anisotonic solutions are introduced, they will temporarily alter the molecular concentration of the plasma.

However, the blood possesses a remarkable power of resuming its normal condition, its total molecular concentration, ratio of individual constituents, ratio of corpuscles to plasma, and total quantity (in about this order). This process of restitution takes place very rapidly; it is far advanced before a quick intravenous injection can be completed; the molecular concentration returns to very near normal within ten minutes; the quantity of blood and individual constituents are restored somewhat more slowly; but they are almost normal in half an hour; after two hours, the blood presents only very slight abnormalities, even when very large injections have been made (Magnus, I90I; Sollmann, rgor).

The total molecular concentration is the first factor to be restored; this is accomplished mainly by the passage of the dissolved molecules: If a hyperisotonic solution is injected, the injected molecules leave the blood very rapidly. Other molecules leave more slowly, and water is taken up into the blood. As soon as the normal molecular concentration is restored, the phenomena become identical with those following the injection of isotonic solutions.

If a hypoisotonic solution is injected, the principal phenomenon at first is the passage of molecules into the blood, the injected molecules leaving the plasma very slowly. The water also leaves only gradually, i.e., mostly after the normal concentration has been reached; and then by the same process as with isotonic solutions.

If an isotonic solution of a single salt (sodium chlorid or sulphate) is injected, both the fluid and the injected molecules leave the circulation; the molecules somewhat more rapidly. At the same time, other molecules enter the circulation. This process continues (for about two hours), until the original quantity of blood and its original composition have been practically restored.

Mechanism of Restoration.-The restitution of the blood consists in a process of exchange with the remainder of the body. There can be no doubt that the tissues (especially the striated muscle cells, Engels, I904; Jappelli, I906; and the liver, Sherrington and Copeman, I893; Uschinsky, I905) play the most important rôle in the rapid exchange which we have so far described.

With intravenous injections of hypertonic solutions, the muscles supply most of the 
water, and take up a large part of the salt; although their salt percentage does not increase as much as that of some other tissues (Wahlgren, I 909). The quantity of material of which tissues can dispose, without visible change, is astonishingly great. In rabbits, the tissues may take up Ringer's solution amounting to four times the quantity of the blood (Bogert, Underhill and Mendel, I916). However, very large injections will give rise to ascites, effusions, etc.

In addition to the tissues, the lymph and urine flow and all the secretions of the body are also increased; and to them falls the task of finally removing the injected substances from the tissues out of the body. The tissues act the part of elastic reservoirs, as it were, quickly relieving the blood of the disturbing element, to again give up the.abnormal substances as rapidly as they can be removed by the more slowly acting excretory mechanisms.

Physical processes, notably osmosis and filtration, play a very important part in the exchange with the tissues, and in the increased lymph flow. Indeed, it is scarcely necessary to invoke any vital mechanism for their explanation.

Conditions Delaying the Restoration of the Blood-volume.-Bogert, Underhill and Mendel, igr6, confirm that in normal dogs and rabbits, the blood-volume returns to normal within half an hour after the intravenous injection of warm Ringer's fluid, equivalent to the total blood-volume. This is delayed by morphin, and by nephritis (uranium, chromate or tartrate), but not by ligation of the kidney vessels. The restoration is not affected by the addition of 0.4 per cent sodium carbonate, either in normal or in nephritic animals. Gelatin solutions (2 per cent.) may leave the blood as rapidly as plain saline, although more commonly from i I to 27 per cent. are still present in the blood after half an hour. The gelatin leaves the blood in the same ratio as the fluid (Boycott, 19r4) and is partly excreted in the urine.

Salt-actions on the Urine.-The hydremic plethora results in an increased elimination of urine. The excretion of water and the injected substance is especially increased. The other uinary constituents are generally decreased, as concerns their percentage; although their absolute quantity may be increased. (The saline diuresis would therefore result in a "flushing of the body.")

The diuretic action of saline solutions is largely physical, and can be reproduced point for point in dead kidneys (Sollmann, I 903 to I905) (except the effect on the composition of the urine). It is explained by the hydremic plethora; the increased glomerular pressure, and the lessened viscidity of the blood. This is aided, in the case of hyperisotonic solutions, by the dehydration and shrinkage of the renal cells. All these processes favor glomerular filtration.

The physical element in the diuresis is especially conspicuous in the early periods of the diuresis, when the changes in the blood are relatively large.

It has been observed, however, that the urinary changes may persist, in a slight degree, after the blood has apparently returned to normal. This suggests the coöperation of a vital stimulation, produced by the (very slightly) altered composition of the blood.

The concentration of the urine varies with the concentration of the solution which has been introduced: it may fall as low as $\Delta$ o.12, or rise as high as $\Delta 3.0^{\circ} \mathrm{C}$. On the whole, the composition of the urine approaches the more closely to that of the blood, the faster the diuresis.

The degree of diuresis (the total quantity exerted) depends largely on the quantity of solution (both of the dissolved substance and of the water) which has been introduced; but the rate of diuresis (the quantity excreted within a short time after injection) depends largely on the concentration of the solution, if this is given intravenously (water, which is so strongly diuretic when taken by mouth, may diminish the urine when injected by a vein).

Fischer and Sykes, I913, claim that the diuretic effect of intravenous saline injections is proportional to their dehydrating action on colloids (fibrin). This would lessen the affinity of the tissues for water, and would therefore place a larger quantity of fluid at the disposal of the kidneys. It may be an additional factor in saline diuresis, but requires further confirmation. 
In the ordinary secretion of urine, osmosis appears to be an obstacle, rather than an aid; for the molecular concentration of urine differs quite widely from that of the blood ( $\Delta$ urine, ordinarily, 0.9 to 2.1 ; extremes, 0.12 to $3.0 ; J$ blood, 0.55 to 0.60 ); and the kidney has to perform a very considerable labor to overcome the difference in osmotic pressure. However, this work seems to be in no way injurious.

Work Performed in Urine-formation.-This cannot be calculated directly from the total molecular concentration, since the departure of every constitutent from its concentration in the blood requires work. Barcroft and his coworkers have investigated the problem qualitatively by the increase of oxygen consumption which accompanies work. They found that the diuresis following the injection of isotonic solutions does not involve work; but that the oxygen consumption increases when hypertonic urines are secreted in response to sulphate injection. Caffeine diuresis also involved work.

Lymph Formation. - This is increased by the hydremic plethora; but beyond this, osmosis can play only a regulating rôle. The hydremic plethora would increase the filtration pressure. This probably plays a large part in the increased lymph formation, although filtration does not seem to be an adequate explanation of normal lymph formation (Carlson and pupils, roo8; Boehm and Asher, r909).

Asher, I913, points out that filtration can not be an important factor in the secretion of saliva, sweat and bile, since the secretion pressure exceeds the blood pressure. The physical-chemical conditions of salivary secretion have been investigated by Japelli, I906; those of tears and collyria by Cerrano, 1909.

Edema.-Salt action, osmosis, and colloid imbibition are important factors in edema, i.e., the excessive accumulation and retention of fluid within and between the cells.

The edema fluid is analogous to lymph, and may therefore be increased by the same agencies which increase lymph flow: hydremic plethora; lymphagogues of the "second class" (peptones, etc.); increased blood pressure; venous obstruction; injury to the vascular endothelium, etc. However, for the retention of fluid it is further necessary that the drainage of the lymph be obstructed; or that there be an increased affinity of the tissues (or colloids) for fluid. In fact, the coöperation of several of these factors is generally necessary for the production of noticeable edemas. Experimental edemas, resembling the clinical, require the simultaneous presence of nephritis, hydremic plethora and vascular injury (Pearce, I909). Extensive intravenous injections of salt solutions produce ascites and edema of the internal organs, but not of the normal skin or subcutaneous tissue. To effect these, the skin must be irritated (Cohnheim and Lichtheim, 1877); or the vessels injured, as by arsenic (Magnus) or uranium.

Normal Variations of the Water Content of Tissues.-These depend mainly on age and nutrition. The average water of human tissues decreases from 94 per cent. in the three-month fetus, to 69 to 66 per cent. at birth, and $5^{8}$ per cent. in adults. It is higher in emaciation, varying inversely to the fat (Albu and Neuberg, 1906).

Salts and Edema.-Effusions of a molecular concentration inferior to that of the blood would be promptly absorbed; or rather, they could not be formed. Since they, like other body fluids, owe their concentration mainly to their content of sodium chlorid, it is evident that a deficiency of this salt in the body will tend to lessen the production of effusions, and hasten their absorption. This is the rational basis of the treatment of nephritic edemas by withholding salt from the food (advocated by Widal and Javal, 1903). The treatment is not always entirely successful (Blooker, 1909), for the salt may not be the cause of the edemas, but only a factor in their production. It is believed, however, that an actual salt retention occurs in some types of nephritis (especially chronic interstitial) and other conditions (Magnus Levy, rgro), owing to diminished permeability of the kidney to salts. These would be most favorably affected by saltwithdrawal. In other cases, the effusions are removed more effectively by administering salts, through their diuretic action. Actual trial alone can decide which form of treatment is needed in a given patient. The salt-poor diets are discussed under "Sodium Chlorid."

Colloids and Edema.-Martin Fischer (I908 to I9I5) believes that the circulation has little if any direct connection with edema; but that this is due practically entirely to changes in the affinity of the tissue colloids for water and salts, and strictly analogous to the swelling of gelatin or fibrin.

This is modified to a varying degree by electrolytes; not by non-electrolytes. It is especially increased by acids; and the production or accumulation of acid, including $\mathrm{CO}_{2}$ in inflammation, venous stasis, etc, would therefore be the principal cause of 
edema (and the concomitant salt retention), and incidentally of nephritis. Fischer is also inclined to deny that osmosis has any part in these or other vital phenomena; and to refer to imbibition all the phenomena which are generally classed as osmotic.

The influence of acids and electrolytes on imbibition can not be doubted. It is therefore very probable that imbibition plays an important rôle along with other factors; but serious objections have been raised to some of Fischer's experiments and deductions. It would require much stronger evidence to place such sweeping statements on a firm foundation.

Fischer's theory fails to explain the close resemblance of some of the vital and ordinary osmotic phenomena; $f . i$. , the equivalent effects of isotonic solutions; the semipermeability of blood cells and striped muscle, etc.; the fact that these phenomena are not modified seriously by the addition of gelatin or serum (Beutner, I9I3), etc. The absorption of water by the skin of the frog is shown by Maxwell, I9 I3, to be mainly osmotic. Beutner, I9I3, and Moore, I9I5, point out the differences between osmotic and colloid swelling of muscle. J. Loeb, I9I3, believes that the osmotic processes predominate so greatly during life that imbibition does not become important until the semipermeability of the cell has been destroyed by death.

The increase of free acid, on which Fischer bases his theory of edema and nephritis, is only hypothetical. Henderson and pupils, I9I4, emphasize that tendency toward acidity in the organism is not usually accompanied by edema; while the acidity of the urine has no relation to albuminuria or nephritis. There is no direct evidence that the free acidity is ever increased to an effective degree during life (Goodridge and Gies, 19r r). Koppel, I913, and L. J. Henderson, Palmer and Newburgh, I9I4, point out that the range of variation of $\mathrm{H}$-ion-concentration, even in the urine, does not affect colloid imbibition. Even if it were granted that this may occur in some cases, there is no evidence that it is a universal or important phenomena; nor would the results of such acidity be uniform; for Pincussohn, 1912, found that acids, while they increase the imbibition of gelatin and muscle, lessen that of kidneys, liver, spleen and lung. Hirschfelder, 1916, finds that the conjunctiva in mustard edema is not acid; nor does the excised eyelid or conjunctiva swell in acid Ringer's fluid. Incidentally, there are agencies other than acids which influence imbibition, $f . i$., proteoses (Tracy and Gies, I912). The effects of inflammation may also be explainable on an entierly different basis. Oswald, I9II, found that inflamed cells become permeable to colloids (proteins), especially in acute inflammation. He explains this by increased "Quellung" of the cell membrane.

The practical application of Fischer's theories to glaucoma and nephritis, has given results so discordant that they are certainly not supports of the theory.

\section{CATHARTIC SALTS}

General Statement.-A number of salts are not absorbed readily from the alimentary canal. These therefore do not produce any marked direct systemic actions, unless they are injected into the circulation. When they are given by the mouth, their direct action is confined to the intestine; they cause catharsis with soft or fluid, painless stools. Through the catharsis they may produce, indirectly, a drying of the tissues and a slight increase of gaseous metabolism. The cathartic action is mainly the result of the retention of fluid in the intestine by the osmotic action of the unabsorbed salt, for this must retain sufficient fluid to preserve itself isotonic with the blood (Wallace and Cushny, I898). It is possible that this action is supported by a direct stimulant effect of the salt ions.

Difficultly Absorbable lons.-The most important cathartic ions are: of the cathions, $\mathrm{Mg}$; of the inorganic anions, $\mathrm{SO}_{4}$ and $\mathrm{PO}_{4}$; of the organic, tartrates, citrates, lactates and malates. Certain sugars, particularly milk-sugar, and mannite, also act as cathartics in the same way.

Many other solutes are difficultly absorbable and would therefore belong to this class; but those named are the principal ones employed in practice. The others gen- 
erally have some interfering factors: The heavy metals and alum produce a precipitation of proteins, and in this way an irritant or astringent effect. The earthy metals, $\mathrm{Ca}, \mathrm{Sr}$, $\mathrm{Ba}$, are converted into insoluble carbonates. Oxalates and Fluorids are specifically toxic to protoplasm.

Easily Absorbable Ions.-Of the Cathions, ammonium is the most readily absorbed; then come the alkali metals. The earthy metals are not easily absorbable. Of the Anions, the chlorids, bromids, iodids, nitrates and acetates are rapidly absorbed.

Cause of Different Absorbability. - Why certain ions should be capable of absorption, others not, can not be satisfactorily explained. Although recent investigations have shown some striking analogies between the absorption of ions by the intestine and by muscle, and the solubility of their soaps and calcium salts, it is not yet clear whether this is a mere coincidence or has a deeper meaning.

Osmotic Factor in Cathartic Action.-Poiseuille, I828, invoked osmosis to explain the cathartic effect, not only of the salts, but also of the vegetable purgatives. This was mainly hypothetical; and Aubert, $185 \mathrm{I}$, showed that there is no direct ratio between the osmotic and purgative strength of the different salts (since the absorbability was not taken into account). Buchheim, 1854 , claimed that the increased water in saline catharsis comes from the alimentary canal, and not from the blood. Colin, I854, showed that concentrated solutions of cathartic salts increase in volume when placed in an intestinal loop. It was clear therefore that the salts could either attract or hold water in the intestines. Wallace and Cushny, 1898 , demonstrated conclusively that saline solutions placed in the intestines become and remain isotonic with the blood; and that therefore the amount of fluid held in the intestines depends strictly on the amount of salt which remains unabsorbed.

Conditions of Absorption.-None of the cathartic salts are altogether unabsorbable; their absorption is favored and their cathartic action correspondingly lessened, if they remain for a long time in the alimentary canal. This is apt to occur if the tissues are very dry. The rate of absorption depends also on the concentration of the salt: Concentrated solutions are generally absorbed more readily than dilute. This is one cause of the somewhat different activity of dilute and strong solutions.

The effect of concentration on absorption has been studied by Merck, rgo6; Weise, I9II; and Cobet, I9r3. Weise showed that the sulphates become isotonic within half an hour. The sulphate ion is absorbed in the same proportion from $\mathrm{Na}_{2}$ and $\mathrm{MgSO}_{4}$. The percentage of $\mathrm{Mg}$ absorbed increases with the dose. The upper and lower intestines do not show essential differences.

Matthew Hay, r883, found the cathartic action of salts less extensive if the income of fluid is restricted; and that the activity is not increased by the injection of water in to the blood.

Source of the Fluid in the Stools. - The ordinary ingestion of water and the quantity of fluid normally secreted into the intestines (perhaps 4 liters daily) would suffice to maintain the salts isotonic; and these are probably the chief source of the water of the stools. Should these be insufficient, especially if the salts are given in strong solutions, fluid could be withdrawn by osmosis directly from the mucous membrane, and hence from the blood. Ordinarily, however, this plays only a small part.

Passage Through Intestines.-This is hastened by the greater fluidity of the stools; but the cathartic salts do not increase peristalsis directly (Legros, I869; Hay, I883).

Studies with the $\mathrm{X}$-ray method (Padtberg, 1909) show that $\mathrm{MgSO}_{4}$ delays the emptying of the stomach, but hastens the passage through the intestines, so that the solution arrives in the colon in one and one-half to two hours. The colon antiperistalsis is not inhibited; but it does not lead to the concentration of the contents, because of the osmotic retention. The fecal matters in the colon are therefore rendered more fluid, and this hastens their expulsion. The saline catharsis is not checked by morphin, unless this is timed so as to secure gastric retention of the salts. 
Other Theories.-In place of osmosis, a number of other factors have been suggested, such as inllammatory irritation, stimulation of intestinal secretion, and muscular stimulation by osmosis, ion action, or by the withdrawal of calcium. None of these rest on adequate evidence, and they probably play at most a minor part.

Secretion Theory.--This was fathered especially by M. Hay, I 883 , who did excellent experimental work on the cathartics. He believed that the salts excite a secretion of fluid into the intestines, which is partly balanced by absorption. However, his experimental results are explainable more simply on the basis of osmosis.

Headland, I 867 , believed that the salts are first absorbed but reëxcreted into the intestines, and that ther stimulate secretion during their elimination; but Thiry, 1864 , had found no secretion in his fistula. The theory of Carl Schmidt, 1850 , that the fluid is a transudate, is without foundation.

Brodie, Cullis and Halliburton, I910, showed that the oxygen consumption of the intestines is not increased by hypertonic magnesium sulphate, so that its dilution must be a purely physical process.

Intravenous and Hypodermic Injection.-It has been claimed by several observers that the cathartic salts stimulate peristalsis even when they are administered parenterally. This has been taken as evidence of direct stimulation of the intestinal muscles. However, these observations seem to have been based on experimental errors; and even if true, they could not explain the ordinary catharfic effect, since this does not involve any marked increase of peristalsis.

Experimental Data on Parenteral Administration.-Catharsis was reported from intravenous, hypodermic or intramuscular injection of sodium or magnesium sulphate by Aubert; Bernard, 1857; Agulhon; and more recently by J. B. MacCallum, 1903 and 1906; and by Bancroft, 1907. Most observers, however, have failed to obtain any increase of peristalsis and catharsis when proper precautions were taken to exclude exposure and asphyxia (Buchheim and Wagner; Donders; Vulpian; Jolezet and Cahours; Rabuteau; Bert; Moreau; Hay; more recently Auer, 1906 and 1908; Meltzer and Auer, 1906; Frankel, 1907; Mendel and Benedict, 1909). Joseph and Meltzer, 1910, found indeed with a careful technic, that intravenous or muscular injection of magnesium inhibits peristalsis.

Hypodermic Iniection is also without result, except when made under the abdominal skin. The success in this case is entirely reflex, for injections of $\mathrm{NaCl}$ or $\mathrm{ZnSO}_{4}$ in this region are also cathartic (Hay).

Excised Intestines.-Tyrode, rgro, found that the application of the purgative salts to the peritoneal surface depresses peristalsis. He also claimed that they stimulate peristalsis when applied to the micous surface. Later observers could not confirm this stimulation (de Heer, Igrr; Sollmann).

Evidences of Irritant Action.- While the main effect of the saline cathartics is osmotic, it is quite possible that a muscular stimulation may occur under special conditions. $F . i$., Herz, Cook and Schlesinger, 1908, state that dram doses of epsom salt may cause watery evacuations within an hour. Their fluid is greater than could be explained by osmosis. Moreover, the stools of the following morning, which contain distinctly more of the salt, had a normal solid consistency. This muscular action may possibly be due to the formation of the irritant sulphids; or to neutralization of calcium.

Relation to Calcium. - Chiari, rgro, suggests that the precipitant effect of most of the cathartic ions for calcium could increase the excitability of the intestine. This may play a part; but at present is only hypothetical.

Fate of the Cathartic Salts.-The portion which is not absorbed is of course eliminated promptly by the feces. The other salts of the feces are also markedly increased; the organic matter only slightly (Hay). The sulphates are in part reduced in the colon to sulphids, which give their characteristic odor to the feces.

After absorption, the cathartic ions are eliminated largely by the urine, producing a diuretic action. The urinary excretion is inverse to the purgative effect. With sodium sulphate, one-third to one-half of the excreted 
salt is in the urine. A considerable portion of the sulphate of magnesium may be retained in the tissues, the excretion extending over several weeks (Hay; Mendel and Benedict, I909).

Diuretic Action.-All the carthartic anions are readily excreted by the urine, and therefore act as saline diuretics; rather more effectively than equivalent amounts of sodium chlorid. However, because of the abstraction of water by the feces, the urine flow is generally decreased in the first twelve hours; it is then increased for about a day (Hay). The diuretic effect is proportional to the quantity of the salt absorbed, and therefore inverse to the catharsis.

Sodium sulphate diuresis increases the gas consumption of the kidney (Bainbridge and Evans, r9r4).

Reaction of Urine.- $\mathrm{MgSO}_{4}$ markedly increases the ammonium and acidity of the urine; $\mathrm{Na}_{2} \mathrm{SO}_{4}$ and $\mathrm{MgO}$ decrease the acidity, with subsequent return to the normal reaction (de Jager, I9I I).

Concentration of the Blood.- - Hay found that this is increased shortly after the administration of concentrated but not of dilute solutions of cathartic salts. The blood count rises from $5,000,000$ to $7,000,000$ by the abstraction or retention of fluid in the intestines. It returns to normal within four hours, even if no fluid has been taken, since the saline solution is gradually absorbed from the intestines. When diuresis sets in, the blood count again rises to $6, \infty 00, \infty 00$; and this even if the salt has been taken diluted.'

Metabolism. - This is not measurably increased by the increased peristalsis of saline cathartics (Benedict, I9I4).

Temperature.-- Saline cathartics have been used in fevers, for their supposed "cooling" effect. Hay found no reduction of the internal temperature, but believes that the skin may be cooled through splanchnic dilation. This is somewhat doubtful.

Therapeutic Uses. - The carthartic salts are used to empty the intestines in constipation, etc.; and to remove fluid from the body in edemas. Their introduction is of comparatively recent date (Glauber, about ${ }_{16} 6$ ). They are useful and effective as simple cathartics, especially in digestive disturbances, and against occasional constipation; but they do not meet the motor deficiency of chronic constipation as efficiently as the cascara group; they are rather unpleasant to the patient; they seem to lose in efficiency on continued use; and they are not very effective in obstinate constipation or mechanical obstruction. As hydragogue cathartic for the removal of edemas, the absence of irritation renders them generally superior to the drastic vegetable cathartics; their diuretic effect is a further aid.

Administration as Laxatives.-For this purpose, the salts should be given in dilute solutions (teaspoon to tumbler; or as the natural mineral waters-Carlsbad, Hunyadi, etc.), since these are less distasteful and act more promptly (one to four hours). The laxative dose of the usual salts is 5 to ro $\mathrm{Gm}$. ( $\mathrm{I}$ to 2 drams). (Larger doses are supposed to accustom the intestines to an abnormal type of peristalsis; Bastedo, I9r4.) They are best administered before breakfast, to hasten their passage through the stomach. If this is delayed, they are less efficient and somewhat irritant. For this reason they should be avoided with bedridden patients, for with these the passage of food from the stomach to the intestine is a rather slow one. Some exercise is always useful after taking salts, and this is one of the reasons why patients receive benefits in watering places which they fail to secure at home.

Administration in Dropsy.-Large doses ( 15 to $30 \mathrm{Gm} ., 1 / 2$ to I ounce) should be given in concentrated solution ( $1 / 2$ tumbler), also before breakfast. The concentrated solutions act much more slowly than the dilute but they remove more fluid since they also increase diuresis. These concentrated solutions are also employed to reduce the' secretion of milk. 
The Choice between the Salt Cathartics.- This is very largely determined by their taste. The sodium sulphate has, perhaps, the most disagreeable taste; the magnesium sulphate somewhat less so. Sodium phosphate and Rochelle salt are perhaps the least disagreeable. MgO and $\mathrm{MgCO}_{3}$ act as alkalies as well as cathartics.

The best corrective for the taste of the salts is ample dilution with water, when this is permissible. Some of the salts are official in effervescent form; i.e., mixed with sodium bicarbonate and citric or tartaric acid.

\section{SODIUM SULPHATE}

This is the most typical of the cathartic salts, being but very little absorbed, and almost free from ion action. It has no action on metabolism. On account of its disagreeable taste, it is not much used, except in veterinary practice, but it forms an important ingredient of many mineral waters, e.g., Carlsbad.

The kidneys are even more permeable to sodium sulphate than to $\mathrm{NaCl}$, so that its intravenous injection produces a copious diuresis. The secretion of gastric juice is not increased.

The stimulant action of hypodermic or intravenous injections of sodium sulphate appear to surpass those of sodium chlorid. An isotonic solution ( 2 per cent. of the dried or 4 per cent. of the crystalline salt) is used in quantities of 500 to 1,000 c.c.

\section{PREPARATIONS-SULPHATES}

*Sodii Sulphas (Sod. Sulph.), U.S.P., B.P. (Glauber's Salt), $\mathrm{Na}_{2} \mathrm{SO}_{4}+\mathrm{roH}_{2} \mathrm{O}$.Large or granular colorless crystals, of bitter saline taste. Freely sol. in water $(\mathrm{r}: 3)$; practically insol. in alc. All sulphates are incompatible with calcium and lead. Dose, I5 Gm., 4 drams, U.S.P.; 2 to $8 \mathrm{Gm}$., $1 / 2$ to 2 drams, repeated; single, to to $16 \mathrm{Gm}$., $2 \frac{1}{2}$ to 4 drams, B.P.; diluted.

Sodii Sulphas Effervescens (Sod. Sulph. Eff.), B.P.-A mixture of dried sodium sulphate, sodium bicarbonate, and tartaric and citric acids; representing 50 per cent. of the ordinary sulphate. Dose, 4 to $8 \mathrm{Gm}$., I to 2 drams, repeated; single, Io to $16 \mathrm{Gm}$., $2 \frac{1}{2}$ to 4 drams, B.P.

Pot. Sulph., B.P., $\mathrm{K}_{2} \mathrm{SO}_{4}$.- -Sol. in water (1: 10 ); insol. in alc. Dose, $\mathrm{I}$ to 3 Gm., 15 to $45 \mathrm{gr}$., B.P.

\section{SODIUM PHOSPHATE}

The phosphates also exhibit merely a local cathartic salt action. The presence of phosphorus in nerve tissue suggested the use of phosphates and phosphoric acid as nerve stimulants. There is no scientific foundation for this. The phosphoric acid of the urine comes almost entirely from the phosphorus of the nucleins of the cells, not from food. The official $\mathrm{Na}_{2} \mathrm{HPO}_{4}$ has a slightly alkaline reaction; but its administration does not influence the true reaction of the urine.

When injected subcutaneously, the phosphates are rapidly excreted by the intestine and kidneys. The feces contain phosphates even in starvation. The urinary phosphates are generally increased by water diuresis (Baetzner, 1913). Milk contains a large proportion of phosphates, and it is possible that the administration of these salts might be useful in lactation, but this has not been demonstrated. The administration of phosphates has no effect on nitrogen metabolism. Intravenously, the phosphates stimulate the vagus endings similarly to thyroid, and they have been claimed to be of benefit in the same conditions. This is doubtful.

Starkenstein, I9I4, claimed that the intravenous injection of phosphates produces muscular twitching, by inactivation of calcium. This is contrary to the results of all previous investigators and is again denied by Greenwald, 1915 . 


\section{PYROPHOSPHATES}

Crawford, I9I0, found these markedly toxic, producing gastro-intestinal irritation, hemorrhages and ulcerations; changes in the liver; with continued administration, nephritis and edema. Metaphosphates acted similarly but weaker; while the ordinary orthophosphate was non-toxic. Crawford believed that the pyrophosphates are responsible for the toxicity of certain cotton-seed meals, but Withers and Ray, I9I3, contradict this conclusion.

\section{PREPARATIONS-PHOSPHATES}

*Sodii Phosphas (Sod. Phosph.), U.S.P., B.P., $\mathrm{Na}_{2} \mathrm{HPO}_{4}+{ }_{1} \mathrm{H}_{2} \mathrm{O}$.- - Large colorless crystals or granular powder, of cooling saline taste. Freely sol. in water ( $1: 2.7)$; practically insol. in alc. Dose, 4 Gm., I dram. U.S.P.; 2 to $8 \mathrm{Gm} ., 1 / 2$ to $2 \mathrm{drams}$, repeated; single, Io to $16 \mathrm{Gm}$., $21 / 2$ to 4 drams, B.P.

${ }^{*}$ Sodii Phosphas Effervescens (Sod. Phosph. Eff.), U.S.P., B.P.-A mixture of exsiccated sodium phosphate, sodium bicarbonate, and tartaric and citric acids, representing about 50 per cent. of the ordinary or 20 per cent. of the dried phosphate. Dose, to Gm., $21 / 2$ drams, U.S.P.; 4 to $8 \mathrm{Gm}$., I to 2 drams, repeated; single, Io to $16 \mathrm{Gm}$., $21 / 2$ to 4 drams, B.P.

Sodii Phosphas Exsiccatus (Sod. Phos. Exs.), U.S.P.; $\mathrm{Na}_{2} \mathrm{HPO}_{4}$. - Sodium phosphate, from which the water of crystallization has been expelled by heating at $100^{\circ} \mathrm{C}$. It is about two and one-half times as strong as the ordinary phosphate. Dose, $2 \mathrm{Gm}$., $30 \mathrm{gr}$., U.S.P.

Liq. Sod. Phosph. Co., N.F.-Sodium phosphate liquefied in its water of crystallization by sodium nitrate and citric acid. rooc.c. represents $100 \mathrm{Gm}$. of the sodium phosphate (about two-thirds in the form of acid phosphate, W. F. Horsa, 1910). Dose, ro c.c., $21 \frac{1}{2}$ drams.

*Sodii. Phosphas Acidus (Sod. Phosph. Acid.), N.N.R., B.P.; Sodium Acid Phosphate (mono-sodium phosphate).-Contains at least 82 per cent. of $\mathrm{NaH}_{2} \mathrm{PO}_{4}$, N.N.R.; 70 per cent. B.P. Large colorless crystals or granular powder, of saline and somewhat acid taste. Very sol. in water; insol. in alc. This salt is used to increase the acidity of the urine (Hanzlik and Collins, I9r3). It is also cathartic, but more irritant than the ordinary phosphate. Dose, I to $\mathrm{I} .5 \mathrm{Gm}$., 15 to $20 \mathrm{gr}$., in water; repeated every one to four hours until the urine becomes acid; 2 to 4 Gm., 30 to $60 \mathrm{gr}$., B.P. It should not be mixed with hexamethylenamin.

A solution may be prepared extemporaneously by adding Dilute Phosphoric Acid to a solution of the official Di-sodium phosphate until it no longer precipitates a solution of $\mathrm{CaCl}_{2}$; or as follows: Sod. Phosph., Io Gm.; Ac. Phosph. Dil., 25 Gm.; Water, q.s. ad I 50 c.c.; a tablespoon of this mixture contains about I $\mathrm{Gm}$. of the acid phosphate.

\section{TARTRATES}

General Statement.-These are difficultly absorbable, and therefore typical saline cathartics, possessing the advantage of a relatively unobjectionable taste. The potassium-sodium tartrate or Rochelle salt is commonly used. Acid potassium tartrate (cream of tartar) acts as a mild acid; and being relatively insoluble, the acid-action extends into the intestines and here stimulates peristalsis. It is therefore more actively cathartic.

Cream of tartar and tartaric acid effervesce with sodium bicarbonate, and are used in baking powders, effervescent drinks, etc.

The tartrates are not readily burned in the body, and therefore do not act as alkalies and diuretics as powerfully as do most other organic anions. When introduced into the circulation, they have a rather high toxicity by immobilizing calcium, and injuring the renal epithelium; but this need not be feared from therapeutic doses (Post, r914).

Toxic Actions of Tartrates.-The effects on calcium are similar to those described under Citrates. Small doses produce only the renal injury; larger doses also muscular and nervous symptoms. The cardiac excursions are decreased, especially in systole, with moderate slowing. The blood pressure is unchanged, the kidney volume slightly 
increased. The effects may be acute or subacute, according to dose (Salant and Smith, 101.t). The four stereochemic isomers behave somewhat differently toward calcium, and therefore have a different toxicity. (Chio, rgi2). Salant and Smith, r9i3, found that the 1 . and 1 . had the same toxicity for frogs and rabbits; but the resistance of different animils varies greatly. In rabbits, the diet has a marked influence, animals fed on oats being much less resistant. This depends probably on differences in the mineral constituents.

Renal Action.-Underhill, Wells and Goldschmidt, r913, and Pearce and Ringer, I 13 , found that Rochelle salts produced marked renal changes when given hypodermicilly in doses of $0.25 \mathrm{Gm}$. per kilogram to phlorhizin rabbits; somewhat larger doses caused the same changes without phlorhizin. Oral administration also produced nephritis, with doses of $3 \mathrm{Gm}$. per kilogram. Five Gm. per kilogram was fatal in fasting animals. The toxicity is not as great in well-fed animals; when diarrhea occurs; or when alkalies are administered. Acetates, citrates and carbonates do not produce these renal changes. Analogous effects are produced on dogs.

'The epithelial changes are confined mainly to the convoluted tubules, with some extension to Henle's loops. The glomeruli and interstitial tissue are intact. The lesions result in retention of the tartrate so that only traces are excreted by the urine even on intravenous injection.

In the acute nephritis of rabbits, the phthalein excretion is materially diminished. In chronic cases, the excretion again improves somewhat with time. The nitrogen excretion is scarcely altered (Underhill and Blatherwick, I9 14). Hippuric acid synthesis is not impaired (Kingsbury and Bell, 1915).

The excretion of injected urea, and the excretion of $\mathrm{N}$ and Dextrose in phlorhizin animals is hindered, but the $\mathrm{NaCl}$ excretion is normal (Baer and Blum; Underhill, $\mathbf{r}_{9} \mathrm{I}_{2}$ ). The restoration of the blood-volume after injection of saline solution is delayed (Bogert, Underhill and Mendel, I9r6).

\section{PREPARATIONS-TARTRATES}

Acidum Tartaricum (Acid. Tart.), U.S.P., B.P., $\mathrm{C}_{2} \mathrm{H}_{2}(\mathrm{OH})_{2}(\mathrm{COOH})_{2}-$-A dibasic organic acid obtained from wine lees or argol. Colorless crystals or white powder of acid taste. Very sol. in water $(\mathrm{x}: 0.75)$; freely sol. in alc. $(\mathrm{x}: 3.3)$. Dose, $0.5 \mathrm{Gm} ., 8$ gr., U.S.P.; 0.3 to I. 2 Gm., 5 to 20 gr., B.P.

*Potassii Bitartras (Pot. Bitart.), U.S.P.; Pot. Tartr. Acid., B.P. (Cream of Tartar), $\mathrm{KHC}_{4} \mathrm{H}_{4} \mathrm{O}_{6 .}$-White, somewhat gritty powder, of pleasant acid taste. Slightly sol. in water, ( 1 : 155); nearly insol. in alc. Dose, 2 Gm., 30 gr., U.S.P.; 1 to 4 Gm., 15 to 60 gr., B.P.; suspended in water.

Pot. Tart., B.P., $\mathrm{K}_{2} \mathrm{C}_{4} \mathrm{H}_{4} \mathrm{O}_{6}+1 / 2 \mathrm{H}_{2}$ O.-Freely sol. in water ( $\left.\mathrm{I}: \mathrm{x}\right)$. Dosé, 2 to $\mathrm{I} 6$ Gm., $1 / 2$ to 4 drams, B.P.

*Pot et Sod. Tart., U.S.P.; Sod. et Pot. Tart., B.P. (Rochelle Salt; Soda Tartarata), $\mathrm{KNaC}_{4} \mathrm{H}_{4} \mathrm{O}_{6}+{ }_{4} \mathrm{H}_{2} \mathrm{O}$. - White powder or colorless crystals of cooling saline taste. Very sol. in water (1:0.9); practically insol. in alc. Dose, ro Gm., $21 / 2$ drams, U.S.P.; 8 to I6 Gm., 2 to 4 drams, B.P.

*Pulvis Effervescens Compositus (Pulv. Eff. Co.), U.S.P.; Pulv. Soda Tart. Eff., B.P. (Seidlitz Powder; Pulv. Sodæ Tart. Co.).-In the U.S.P. the blue paper contains Pot. et Sod. Tart., $8 \mathrm{Gm}$. and Sod. Bicarb., $5 \mathrm{Gm}$.; the white paper, Ac. Tart., $21 / 4 \mathrm{Gm}$. In the B.P., No. I (blue paper) contains Sod. et Pot. Tart., 7.5 Gm.; Sod. Bicarb., 2.5 Gm.; No. 2 (white paper) contains Ac. Tart., $2.5 \mathrm{Gm}$. The contents of the blue paper are first dissolved in a half a tumbler of cold or warm water and the contents of the white paper are then added

\section{MAGNESIUM COMPOUNDS}

General Statement.-The salts of magnesium are relatively little absorbed, and therefore act as osmotic cathartics. With magnesium sulphate, both ions have this effect, so that the salt is particularly effective. Magnesium oxid and carbonate act also as alkalies. Being very sparingly soluble, their antacid action extends into the intestines, with but little interference with the stomach. They are converted in the alimentary tract into the soluble and therefore cathartic magnesium bicarbonate. $\mathrm{MgO}$ (magnesia) is preferred to the usual alkaline carbonates when the stomach must not be burdened with $\mathrm{CO}_{2}$ gas. It is therefore the best antidote against poisoning by acids. 
Magnesium is somewhat absorbed by the alimentary canal, so as to produce some diuresis; but not sufficient to cause toxic effects, unless very large doses of a concentrated solution are taken. When injected hypodermically or into the circulation it is highly toxic by depressing the heart, muscles, and the central and peripheral nervous system. It is antagonized by calcium. Magnesium sulphate has been used as a local and intraspinal anesthetic, and against tetanus; but the effective dose is dangerously close to the fatal (paralysis of respiration), so that its usefulness is limited.

A saturated solution of magnesium sulphate is used, apparently with good results, as a local dressing in erysipelas and similar inflammations.

Administration as Cathartic.-Magnesium sulphate is employed as laxative in doses of 2 to Io Gm., I to 25 , in dilute solution; and as hydragogue cathartic in doses of Io to $5_{5} \mathrm{Gm} ., 2$ to 45 , in concentrated solution. The dilute solution may be mixed with $\mathrm{NaHCO}_{3}$, but not with $\mathrm{Na}_{2} \mathrm{CO}_{3}$. It may also be given as an effervescent salt. Solution of Magnesium Citrate is a pleasantly flavored and effervescent draught, dispensed in bottles containing $\mathrm{I} 2$ ounces. The dose is from $1 / 4$ to I bottle. Magnesium carbonate and the oxid are administered as powder, $1 / 4$ to $2 \mathrm{Gm}$., 5 to $30 \mathrm{gr}$. "Milk of Magnesia" (Magma Magnesiæ) is a suspension of magnesium hydroxid, used as a laxative in doses of I to 4 teaspoons; and as an alkaline dentifrice.

Cathartic Action.--Magnesium salts are converted into the acid carbonate in the small intestine, according to the formula:

$$
\mathrm{MgSO}_{4}+\mathrm{Na}_{2} \mathrm{CO}_{3}+\mathrm{H}_{2} \mathrm{O}+\mathrm{CO}_{2}=\mathrm{Mg}\left(\mathrm{HCO}_{3}\right)_{2}+\mathrm{Na}_{2} \mathrm{SO}_{4}
$$

In this respect it is quite immeterial what particular salt be given. The hydrate, carbonate, chlorid, or sulphate are all converted into bicarbonate. However, in the case of the sulphate, the sodium sulphate which is formed is, of course, also cathartic, so that the effect is doubly large. The hydrate and carbonate, on the other hand, possess also the action of alkalies.

Direct Action on Peristalsis.-This is not seen when magnesium is given by mouth, but only when it is applied by vein or hypodermically. Its direct action then is to depress the excessive peristalsis induced by exposure of the intestines, or by ergot, physostigmin or barium chlorid (Meltzer and Auer, 1906).

Meltzer, 19.4, believes that magnesium also inhibits the intestine with the ordinary intestinal administration; and that this somehow renders the contractions more effective by leaving freer scope to the "contrary innervation."

Normal Occurrence of Magnesium.-Magnesium occurs in the body mainly as phosphate and in association with calcium, in the bones, and in larger absolute quantity in the other tissues. The red corpuscles (Abderhalden, I 898 ) and striped muscle (Katz, I896; Huerthle, I903) contain more $\mathrm{Mg}$ than $\mathrm{Ca}$. The daily requirement is estimated as $0.6 \mathrm{Gm}$. The ordinary diet contains 0.19 to I.2 $\mathrm{Gm}$. (H. C. Sherman, Mettler and Sinclair, rgro).

Absorption.-This occurs mainly from the small intestines. Metallic magnesium is also absorbed from the subcutaneous tissue (Fromherz, 1909).

Excretion.- After absorption, and with parenteral administration, the excretion occurs practically exclusively by the kidneys (Meltzer and Lucas, 1907), only in small part from the large intestine. The toxicity is therefore diminished by inducing diuresis (Meltzer, 1907). A considerable quantity may be retained in the body over two weeks. The urinary excretion of calcium is increased (Mendel and Benedict, 1909). This lessens the lime-deposition in young animals (Malcolm, 1905). The normal urine contains 0.2 to $0.3 \mathrm{Gm}$. of $\mathrm{Mg}$. per day.

Renal Irritation.--The intramuscular or intravenous injection of magnesium salts or sulphates into dogs causes the appearance of hyaline casts in the urine. There are no other casts, albumen or other sign of nephritis (Gates and Meltzer, I9I4). These occur also when toxic doses of magnesium are injected into the ligated small intestine (Gates, 1914). 
Glycosuria.-Parenteral injections produce moderate hyperglycemia and glycosuria. Underhill and Closson ascribed these to asphyxia, E. Hirsch, I9I5, to narcosis and asphyxia; but Kleiner and Meltzer, I9I6, find them even with artificial respiration.

On the other hand, Airila and Bardy, 1915, state that intravenous injections of magnesium inhibit epinephrin gly cosuria and clinical diabetes; presumably by depression of the sympathetic system.

Toxicity by Mouth.- Only the nitrate, which is absorbed much more readily, is apt to produce typical $\mathrm{Mg}$ symptoms (Meltzer, 1907 ); but toxic effects may arise if large doses of the sulphate are given.

Intestinal injection with the stomach tied off produces death by respiratory paralysis in about $t$ wenty-five minutes with a dose of ro c.c. of 20 per cent. magnesium chlorid. When administered to rabbits by mouth, even very large doses are not fatal (Auer and Meltzer, I9I4).

Intravascular Injection.-This produces immediate and severe, often fatal depression of the heart and central nervous system. It resembles potassium, but this acts more on the heart, magnesium on the respiration. The effects are seen in blood-pressure experiments, if the magnesium sulphate solution of the manometer inadvertently enters the artery. There is a sharp fall of blood pressure, and the heart may be arrested promptly. If the effect is not fatal, recovery occurs quickly, since the excretion is quite rapid.

Cardiac Effects. - These consist in slowing with moderate dilation; then incoördination; then sudden dilation and standstill. The heart may be kept beating by electric stimulation, by stimulation of the accelerators, and by epinephrin (Matthews and Brooks, I910).

Respiration.-The cessation is due mainly to the curare action, the center being ordinarily not paralyzed except with considerably larger doses (Matthews and Brooks, I910).

Temperature.-In rabbits, this is lowered by magnesium injection, parallel to the paralytic action, but preceding the latter. It is an independent phenomenon, for it occurs also if the narcosis is prevented by calcium (Schuetz, I915, 1916).

Magnesium Anesthesia.-Meltzer and Auer (1905) found that a general anesthesia, with abolition of reflexes, may be produced by the subcutaneous injection of magnesium salts ( $1.5 \mathrm{Gm}$. of crystalline magnesium sulphate per kilogram of body-weight, used as 25 per cent. solution). The injection causes but little pain, since the magnesium is also a local anesthetic. The anesthesia is complete in half an hour or an hour, and lasts about two hours. The blood pressure is but little lowered; there is some diuresis, but no diarrhea. The recovery is perfect. Somewhat larger doses are fatal, paralyzing the respiration before the heart. The effective dose approaches the fatal so closely that this method is not yet available for man.

Nature of the Anesthetic Action. - There has been considerable discussion whether the effects of magnesium consist in a true abolition of sensation (Meltzer); or whether they consist mainly in a paralysis of muscular response by the curare action (Guthrie), and in asphyxia. There is no doubt that the muscular and possibly the asphyxial factors may play a part; but that magnesium produces direct sensory paralysis, both central and peripheral.

Evidence for Sensory Paralysis.-Guthrie and Ryan, I 9 ro, and Matthews and Brooks, rgro, brought evidence that paralysis may occur by the curare and asphyxial action of magnesium, when the sensation is still preserved. However, Meltzer and Auer claim that, with proper dosage, it is possible to secure anesthesia when the asphyxial factor is entirely excluded and the muscles are still responsive. Indubitable proof of direct central anesthesia is furnished by the results of careful intravenous injection in human subiects, reported by Peck and Meltzer, 1916. This produced unconsciousness and complete surgical anesthesia, with incomplete motor paralysis.

The immediate revival of these animals by the intravenous injection of calcium chlorid (Meltzer, 19\%9) also speaks for a direct central action. Further, they find (I9I3) that small intracerebral injections produce central anesthesia (also V. E. Henderson, I $9 x^{2}$ ); and that the effects of magnesium and ether are additive. Gensler, I9r5, and v. Issekutz, I915, also find additive effects with the aliphatic hypnotics.

Mansfeld and Hamburger, 1915, conclude that magnesium increases the anesthetic action of urethane by altering its distribution; for calcium does not remove the summation if the magnesium has been administered with the urethane; but does remove the narcosis if the magnesium was injected some time after the urethane.

Distribution of Magnesium.-Mansfield and Bosanyi, I913, and Gensler, 1915, find that the magnesium and calcium of the brain substance are not changed during the narcosis or calcium recovery, and therefore conclude that the altered $\mathrm{Mg}$ - Ca ratio acts on the plasma membrane. Stronsky, $\mathrm{IgI}_{4}$, also found that the $\mathrm{Mg}$ content of the 
plasma increased, and the Ca diminished, during the narcosis; while the organs were unchanged. The urinary excretion of both $\mathrm{Mg}$ and $\mathrm{Ca}$ was increased. J. Schütz, I915, reports accumulation of magnesium in the brains of rabbits subjected to repeated magnesium narcosis.

Deglutition.-Graded doses paralyze the central mechanism before the peripheral (Auer and Meltzer, r9i6).

Local Anesthesia.-The effects of direct application of magnesium solutions to exposed nerve are described rather differently. Meltzer and Auer, I906, maintain that concentrated solutions paralyze without previous stimulation, having a selective action on the different funetional fibers somewhat analogous to cocain. Liljestand, r9o9, and Guthrie and Ryan, I9I0, claim that the paralysis is preceded by stimulation and that the $\mathrm{Mg}$ action is not specific, but very similar to that of $\mathrm{NaCl}$, while Ca salts are much more effective. However, Wiki, IgII, found that the hypodermic injection of I $_{5}$ to 25 per cent. $\mathrm{MgSO}_{4}$ into guinea pigs produces much more local anesthesia than equivalent solutions of other salts. It seems therefore that the local anesthetic action of magnesium is relatively, if not absolutely, specific.

Curare Action.-This was discovered by Jolyet and Cahours, r 869; and has been confirmed by Guthrie, Meltzer and others. Meltzer and Auer, 1906, also find that, like curare, it antagonizes the physostigmin twitchings. Further antagonisms to physostigmin were studied by Joseph, I909. Isolated bronchial muscle is slightly dilated (Trendelenburg, I9 2 ).

Rigor Mortis.- Meltzer and Auer, r 908 , found that the hypodermic injection of calcium hastens, and magnesium delays the development of rigor in skeletal muscles and heart. In calcium rigor, the extensor muscles prevail; in magnesium, either the flexors or the position at the time of death.

Subdural Injection of Magnesium.-The hypodermic use of magnesium being too dangerous for man, Meltzer and Auer, I909, introduced its use by lumbar subdural injection, using I c.c. of the 25 per cent. solution. This produces sensory and motor paralysis of the legs and pelvic region, occurring in three to four hours, and lasting eight to fourteen hours. There is also retention of urine for one or two days. Untoward results are cited in Merck's Report, 26:266.

Magnesium in Tetanus.- This has been found highly useful, at least symptomatically and in conjunction with antitoxin. Kocher considers it the best remedy in developed cases. The spasms are temporarily relieved, but return, requiring repeated (at least daily) injections. The dosage presents serious difficulty, the dose that paralyzes the respiratory center being but little above dose required for the therapeutic effect.

Lazarus also reports success in strychnin poisoning.

Administration in Tetanus. - The doses refer to crystalline Magnesium sulphate (Kocher, 1915; Meltzer, I916);

For slight initial symptoms, hypodermic injections may be used, 1.2 c.c. of a 25 per. cent. solution, per kilogram body weight, 3 to 5 times daily. This has been continued for 6 to 18 days without damage. It causes but little irritation. It is safe, but not sufficiently effective for severer cases. In these, the salt may be used intramuscularly, 0.6 c.c. of 25 per cent. per kilogram; six times daily.

In severe cases, however, it is better to proceed at once to the more effective intraspinal injection; using I c.c. of 25 per cent. per 20 lb. of body weight (o. I c.c. of 25 per cent. per kilogram), by lumbar injection. This gives prompt relief, generally lasting 24 hours, when the procedure may be repeated. If the respiration should become depressed, the solution should be washed out of the canal.

Intravenous injection has also been used. It is not generally advised, the effects being short and dangerous, but it may be justified when the spasms threaten the respiration. Straub injects in 2 minutes a total of roo c.c. of 2.5 per cent. solution; repeated every half hour to hour. Meltzer prefers a 6 per cent. solution, injected at the rate of 2 to 3 c.c. per minute, stopping as soon as the respiration becomes depressed. If the respiration does not improve 2.5 per cent. calcium chlorid must be injected slowly.

Accidents. - When using effective doses of magnesium, the physician should be prepared to combat failure of respiration, by artificial respiration, together with physostigmine ( $1 / 2$ to $\mathrm{I} \mathrm{mg}$. hypodermically) or calcium chlorid (50 c.c. of 2 per cent., intramuscular; or 0.02 per cent. in normal saline, intravenous, to 600 c.c.).

In children extra caution must be used. The doses of magnesium should generally be somewhat smaller; supplementing them if necessary by ether or the aliphatic 
hypnotics. Morphin potentiates the respiratory depression, and should therefore not be combined with magnesium (Issekutz, I915).

Lilerature-Cohen, I912; Dodd, Io12; Arnd, Dreyfus, 1914; Th.Kocher, 1915;

II E. Robertson, ion 5 ; Meltzer, rgi6.

Antagonism of Magnesium and Calcium. - This was mentioned on page $6+1$. Oxalates enhance the action of $\mathrm{Mg}$, presumably by removing the antagonistic effect of the tissue-Caicium (Gates and Metz, I9r3).

Magnesium Against Erysipelas and Other Inflammations.-This was introduced by H. Tucker, I907 and I908. He claimed that the local application subdued the inflammation, the pain, swelling and temperature, in cellulitis, arthritis, epididymitis, etc.; and suggested its use particularly for facial erysipelas. It has also been used successfully by others. Its action may be similar to the antiphlogistic effect of $\mathrm{Ca}$.

A pplication.-A thick compress ( 15 layers of gauze) is immersed in a saturated solution of $\mathrm{MgSO}_{4}$, applied to the inflammed region, covered with oiled silk, and renewed every two hours (Choksy, I9I I).

Phagocytosis.-Delbet and Karajanopoulo, I915, claim that the intravenous injection of magnesium chlorid (dogs, about I $_{5}$ c.c. of I.2I $_{\text {I }}$ per cent. anhydrous, per kilogram) greatly increases phagycotosis. It does not inhibit the migration of leucocytes from the inflamed mesentery of frogs (Ikeda, I916).

\section{PREPARATIONS-MAGNESIUM}

* Magnesii Carbonas (Mag. Carb.), U.S.P., B.P.-A mixture of hydrated magnesium carbonate and hydroxid. According to its preparation, it occurs as a more or less bulky powder (Magn. Carb. Levis and Ponderosum), or as white, friable masses, of slightly earthy taste. Practically insol. in water. Dose. 3 Gm., 45 gr., U.S.P.; 0.3 to I. 2 Gm., 5 to 20 gr., repeated; single, 2 to $4 \mathrm{Gm}$., $1 / 2$ to I dram, B.P.

Liq. Mag. Bicarb., B.P. (Fluid Magnesia).-Equivalent to 2 per cent. of Mag. Carb., about Io gr. per ounce. Dose, 30 to 60 c.c., I to 2 ounces, B.P.

*Liquor Magnesii Citralis (Liq. Mag. Cit.), U.S.P.-An effervescent solution containing ro per cent. of a mixture of neutral and acid magnesium citrate; prepared in bottles of $35 \circ$ c.c., I 2 ounces, which may be taken as a single dose or divided.

*.Magna Magnesice (Magma. Mag.), U.S.P.; B.P.C. (Milk of Magensia).-A creamy, smooth suspension of the hydroxid, $\mathrm{Mg}(\mathrm{OH})_{2 \cdot}$ Dose, ro c.c., $21 / 2$ drams, U.S.P.

Mag. Oxid., U.S.P.; Mag. Levis, B.P. (Light Magnesia; Calcined Magnesia); MgO.White, very bulky, and very fine powder, without odor; earthy taste. Almost insol. in water; insol. in alc. Dose, as Mag. Oxid. Pond.

*Magnesii Oxidum Ponderosum (Mag. Oxid. Pond.), U.S.P.; Magnesia Ponderosa (Mag. Pond.), B.P. (Heavy Magnesia); MgO.-White, dense, very fine powder; insol. in water or alc. Dose, 2 Gm., 30 gr., U.S.P.; 0.3 to $1.2 \mathrm{Gm}$., 5 to 20 gr., repeated; single, 2 to $4 \mathrm{Gm}$., 30 to 60 gr., B.P.

${ }^{*}$ Magnesii Sulphas (Mag. Sulph.), U.S.P., B.P. (Epsom Salts), $\mathrm{MgSO}_{4}+7 \mathrm{H}_{2} \mathrm{O} .-$ Small prismatic crystals, of cooling, saline and bitter taste. Very sol. in water $(\mathrm{r}: 0.75)$; practically insol. in alc. It contains about 50 per cent. of water of crystallization. A saturated solution contains about 50 per cent. of the crystals, or 25 per cent. of the absolute $\mathrm{MgSO}_{4}$. Dose, ${ }_{15} \mathrm{Gm}$., 4 drams, U.S.P.; 2 to $6 \mathrm{Gm}$., 30 to $90 \mathrm{gr}$., repeated; single, 8 to $16 \mathrm{Gm} ., 2$ to 4 drams, B.P.

*Mag. Sulph. Eff., U.S.P., B.P.-50 per cent. Dose, 4 to $12 \mathrm{Gm}$., 1 to 3 drams, repeated; single, $\mathrm{I} 6$ to $32 \mathrm{Gm}$., 4 to 8 drams, B.P.

\section{WATER}

General Statement.-The direct application of water ta semipermeable cells produces osmotic changes. If the semipermeability is imperfect or impaired, there results a leaching-out of salts, and with this, a disturbance of the ion-ratio. Instances of these deleterious actions are the waterlaking of blood; the swelling and water-rigor of muscle, etc. The surfaces 
which are normally exposed to contact with water are protected against these actions, either by their impermeability, as the skin and bladder; or by the rapid passage of salts into the water, as in the alimentary tract. Even in these situations, however, the water causes more or less swelling, stimulation or irritation of the cells.

Water is, of course, an essential constituent of all protoplasm. It constitutes about two-thirds of the body-weight, 75 to 90 per cent. of the active tissues, 40 per cent. of bones. Since it is constantly lost to the body by the urine, lungs, perspiration and feces, this would lead to drying and concentration of the tissues; but before this reaches a demonstrable degree, the sensation of thirst prompts the replacement of the water. Any excess of water is promptly eliminated by the excretions, which are correspondingly increased and diluted. However, there is a temporary increase of the water content of the fluids and cells of the body; a removal of their salts; and alterations in the proportions of their ions. These changes in turn produce functional phenomena, characterized especially by increased secretions, and by changes in metabolism. The effects vary according to whether the quantity of water is normal or excessive; and according to the channel by which it is introduced.

Absorption from Alimentary Canal.- Water is rather slowly absorbed from the stomach, where it also delays the absorption of other substances. However, it passes rapidly into the duodenum, and is absorbed readily from the intestine, small and large; and in these it favors the absorption of dissolved substances. In health, the absorption occurs mainly in the upper portions of the small intestine (v. Mering, I893; Moritz, 1893).

Absorption by the Skin.-Water is not appreciably absorbed through the normal skin. Bathing in cold water will contract the cutaneous vessels, and will in this way diminish the loss of water by perspiration; while hot water will increase the loss by favoring diaphoresis.

Digestion and Absorption of Food.-The ingestion of water with meals has decidedly beneficial effects upon all the digestive functions, even when the quantity of water is quite large (a liter with each meal); provided that the food is thoroughly masticated. It aids the digestive action of the saliva; increases the flow of gastric juice, its pepsin and acidity, and thus presumably the flow of pancreatic juice. It secures better absorption of the food, thus leaving less material for the intestinal bacteria, decreasing their number and the putrefactive processes. The same beneficial effects are secured with distilled water, which has no detrimental effects even in prolonged fasting experiments (Hawk, I9I4).

These effects are utilized in the treatment of certain dyspepsias. Water is often useful in nausea. It is also valuable as a diluent for irritant drugs. Excessive water consumption would probably be harmful in gastric atony.

The secretion of acid gastric iuice is increased (Heidenhain, 1879; Pawlow; Foster and Lambert, 1908; Sawitsch and Zeliony, 1913; and others).

Even 50 c.c. increases the acidity and peptic activity quite strongly. The degree of action is not influenced materially by the quantity, temperature, or by the presence of food (Bergheim, Rehfuss and Hawk, I9I4).

The flow of pancreatic juice and bile is also stimulated. The dilution would accelerate the action of the digestive enzymes. The diastatic efficiency of saliva is increased, the optimum being reached with $1: 7$ dilution (Bergheim and Hawk, r913). The discharge of the gastric contents is hastened (at least after a meal of meat, although not with potatoes; Hedbom and Cannon, 1909 ). The water does not actually mix with the gastric contents, but passes along a trough formed on the surface (Cohnheim). The hunger contractions are allayed (Carlson, 1913). Hawk and his pupils (Howe, Mattill, and Hattrem, I91 I and 1912; Blatherwick and Hawk, 1913) found the feces reduced in 
Weight and containing less nitrogen (both food and bacterial). The utilization of fats and carbohydrates is not affected (Blatherwick and Hawk, 19I4). Intestinal pulrefaction (as judged by indican) was lessened (Sherwin and Hawk, I9 I4); the acidity of the feces was increased. The effects are distinct, although they are naturally not very great in healthy subjects (Spiegler, I90I).

The Excretion of Water.-This occurs by the kidneys, lungs, skin, and intestine. The relative importance of these channels varies with conditions. If, for instance, the water is taken hot (so as to favor dilation of the cutaneous vessels), it will act as a diaphoretic. If the conditions are not favorable to diaphoresis, it will be excreted mainly by the kidneys. Excessive quantities of water may also be excreted by the intestine, if the conditions are favorable, and may thus lead to diarrhea.

The Diuretic Effect of Water.-When water is absorbed from the alimentary tract, the diuresis is prompt, strong, and certain so long as the kidneys are normal.

Three to 5 , and even Io liters may be taken and absorbed in the day, in man, without altering the consistency of the feces. If a large quantity is ingested, a half to threefourths may be excreted by the urine within forty-five to ninety minutes (Mattill and Hawk, I9II). The excretion may be forced above ro liters per day, and continued for several weeks, apparently without deleterious effects to the kidneys or otherwise; this does not even create salt-hunger (Sollmann and Hoffmann, 1905). The diuretic action of moderate quantities of water can be greatly enhanced by the addition of other diuretics, but if very large quantities of water are given, the addition of diuretics has no further effect.

Hypodermic and intravenous iniection of water produce little or no diuresis. Hashimoto, 1914, attributes this to deficiency of salts, and not to the production of any diuretic substance in the alimentary canal, as was believed by Cow, 1914.

Composition of the Urine.-The urine in water-diuresis becomes very dilute, although the total or daily excretion of the dissolved constituents is generally increased. However, there is no simple relation between the increase of the different constituents, for instance $\mathrm{N}$ and $\mathrm{Cl}$ (Heilner, I906).

Therapeutic Use of the Diuretic Effect.-This is especially useful for diluting the urine, to lessen its irritation in inflammations of the urinary passages; and to prevent the precipitation of its constituents in urinary calculism.

Theoretically, water-diuresis should also be useful for the removal of toxic substances from the body; but there are few positive data.

Use in Fever.-Cushing (1905) has found the administration of very large quantities of cold water (4 ounces every fifteen minutes while awake) of very marked benefit in typhoid fever. It serves to keep the mouth clean; it must have a distinct antipyretic effect; and it may aid by eliminating toxins and waste products. (The effect of water in fever has also been investigated by Schwenkenbecher and Inagaki, r906.)

Metabolism.- Increased ingestion of water flushes salts and metabolites from the tissues and tends to increase protein metabolism and oxidative changes.

Increased Nitrogen Excretion.-The administration of large quantities of water causes a very considerable temporary increase in the urinary nitrogen, as much as 34 per cent. (Neumann, 1899). The excess is excreted mainly as urea; the uric acid being of ten unchanged. Fairhall and Hawk, 1912 , found the uric acid output in man decreased, while the allantoin was increased. The excretion of the phosphates and sulphates is also increased. The removal of chlorids is so extensive that their percentage in the urine soon falls to a very low figure.

Other Urinary Constituents. - Wilson and Hawk, 1914 , report that the ingestion of additional water, moderate or abundant, causes a temporary decrease in the daily output of chlorid; presumably through the need of equalizing the osmotic pressure of the 
retained water. The ammonium is increased (presumably as a consequence of the heightened gastric acidity), the phosphate unchanged, the acidity variable.

Increased Metabolism.-The excessive excretion of metabolites is mainly due, beyond doubt, to the "flushing out" of the tissues by the diuresis (Haas, 1908). But if this were the only cause, the continued excessive administration of water would soon exhaust the stored waste products, and their excretion would fall rather below the normal figure. Actual results show that this holds for the chlorids, but not for $\mathrm{N}, \mathrm{P}$ or $\mathrm{S}$; their excretion remains permanently increased, although it does not remain at the original high figure (Edsall, I900). There must therefore be an increased production of these metabolites.

Melabolism in Animals.-This conclusion is supported by the results on animals, although these are somewhat different from those obtained on man. Dogs in nitrogen equilibrium do not show any permanent changes in their nitrogen excretion when four times the normal quantity of water is administered, notwithstanding the increased diuresis. In starving dogs, however, it may be increased by 80 per cent. The explanation of these discordant results is probably that the removal of the water by diuresis keeps step with the absorption in the fed animals; so that it never comes to any considerable increase in the water content of the body, and the metabolism is therefore not increased.

"Alterative Effect" and Use in Obesity.-Water, especially in the form of mineral waters, is employed for its metabolic effects in a variety of obscure conditions, particularly in obesity, but the results are uncertain.

It is difficult to predict whether the breaking down of protein molecules will increase or diminish the body fat. If it is only partial, it may result in the formation of fat from the proteins; if carried further, it may lead to the destruction of fat as well.

Local Actions.- Water is mildly stimulant and irritant to sensitive surfaces.

The growth of artificial cultures of frog's tissues is hastened by diluting the serum of the culture fluid (Ruth, I9I I).

The effects of baths and similar hydrotherapeutic measures depend upon reflexes due to the physical action of temperature, etc.

Distilled Water.-This is more injurious than ordinary water to the lower animals and to excised tissues; partly because of the total absence of salts (especially of calcium, thus probably altering the permeability of the plasma membrane; R. H. True, I9I4); and partly because it often contains traces of copper and ammonium compounds. This toxic action can not occur by the drinking of distilled water by higher animals, which obtain their salts from food rather than from water: The salts of the food would in all ordinary cases be more than sufficient to cover the deficiency of salts in the water. The statements, which are occasionally made, that the drinking of distilled water leads to toxic effects, are therefore entirely groundless: Distilled water is often drunk exclusively on ships, without any bad results. Nor is distilled water detrimental in nephritis (Marcus, I907).

Effects of Water by Intravenous Injection.-The immediate effects of small amounts, slowly introduced (and especially of hypodermic iniections) bear a general resemblance to those following the injection of isotonic salt solutions. The changes in metabolism and urine are probably similar to those which occur on taking water by mouth. Larger doses and rapid injection introduce important modifications. Hemoglobin appears in the urine, from the laking of the blood corpuscles. The respiration is slowed and the blood pressure is lowered. The central nervous system shows rather late paralysis and convulsions. The diuresis is quite small, since the hypoisotonic condition of the blood slows the renal circulation by the swelling of the kidney cells. The excretion of water occurs mainly by other channels, so that there is usually a serous diarrhea, increased salivation, etc. The fatal dose of water (by intravenous injection) is some what smaller than that of normal saline solution; distilled water is again somewhat more toxic than well water.

The Withholding of Water.-This affects the metabolism in much the same way as the administration of salts in hyperisotonic solution: The excretion of metabolites is at first lowered, by the lessened diuresis; but as the destruction of proteins is at the same time increased the excretions of metabolites is also increased after a time. When water is given, the stored metabolites are washed out, leading to a very greatly increased excretion (Landauer, 1895; Straub, 1899; Spiegler, 1901). 


\section{PREPARATIONS - WATER}

* Aqma, U.S.P.-The purest natural potable water obtainable.

* Iqua Destillata (Aq. Dest.), U.S.P., B.P.; Distilled water.

* Iqua Destillata Sterilisala (Aq. Dest. Steril.), U.S.P.-Sterilized Distilled Water for injections should, if possible, be used within forty-eight hours after its preparation; for old distilled water is seriously contaminated with bacterial products.

\section{MINERAL WATERS}

These will be discussed more in detail in other places, according to their ingredients, but the whole subject may be very briefly summarized in the following compilation:

The action of natural mineral waters has been known empirically since remote times. Their use came about probably by accidental observation and also through the marked taste which they possess.

Spring and Artificial Mineral Waters.-A very striking observation was early made, namely, that these mineral waters, when used at their source, appear to be more effectual than artificial compounds of practically the same composition, or even than the same water when used at a distance.

The most diverse explana:ions for this have been advanced. Especially in the case of thermal waters, so-called tellurial forces, some mysterious property imparted to them by the earth, have been invoked. Since the discovery of radium, it has been found that some mineral waters are slightly radioactive, and this has been used to explain their action. Others, again, have laid great stress upon the presence of minimal traces of rare elements, somewhat upon the homeopathic principle. Still others believe that the artificial waters-even if they contain the same elements in the same proportion-do not contain them in the same state of combination; for instance, that the artificial water would contain $\mathrm{NaCl}$ and $\mathrm{MgSO}_{4}$, the natural water $\mathrm{MgCl}_{2}$ and $\mathrm{Na}_{2} \mathrm{SO}_{4}$. $\cdot$ This is, of course, contrary to the demonstrated facts; all solutions containing the same proportions of the same ions, will have these ions present in the same state of combination, no matter what particular salts were used to introduce these ions. There is nothing in any of these theories. Mineral waters are simply solutions of the ingredients of the soil, and possess only the action of those ingredients which are present in notable amount.

'The difference between the water of natural sources and the artificial solutions rests upon other causes, which are sufficiently easy to appreciate, but difficult to reproduce.

These factors refer largely to hygiene and climate. A large proportion of the effects must be attributed to the rest, the freedom from care and business pursuits, the exhilarating effect of improved hygienic, atmospheric, and scenic conditions, better regulation of all the habits, of diet, exercise, etc. The mere drinking of large quantities of fluid is also something which it is difficult to enforce at home, but which the patient does regularly in watering-places. Added to these comes finally the specific action of the dissolved salts.

The effect of mineral baths is purely local and reflex. There is practically no absorption of the dissolverl salts through the skin. The effects are in general the same as with hydrotherapy, excepting that they are somewhat stronger.

The waters of mineral springs vary in temperature; those below $25^{\circ} \mathrm{C}$. are called cold; 25 to $37.5^{\circ}$, warm; above this, hot. The higher temperatures favor absorption, and are therefore more favorable for alkaline waters; cold causes motor stimulation of the alimentary tract, and is preferred for cathartics.

The molecular concentration of mineral waters varies considerably; the depression of freezing-point is I.OI 50 for Hunyadi and Apenta; 0.320 for Vichy; 0.275 for Carlsbad; 0.240 for Apollinaris, etc. (Kostkewicz, I899).

Classification.-These waters are usually classified according to the contained salts. Various classifications are current. The following will be used here:

I. Plain Saline $(\mathrm{NaCl})$.

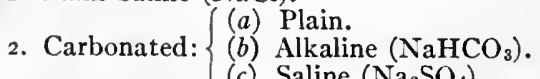

3. Magnesia.

4. Sulphur.

5. Chalybeate.

Occasional constituents of small importance are $\mathrm{Ca}, \mathrm{I}, \mathrm{Br}, \mathrm{Li}$, and $\mathrm{As}$.

The physiologic effects of the majority of these constituents will be discussed under 
their respective headings, and will only be very briefly alluded to in this place. It will also be impossible to take up the composition and special indications of the different mineral waters, in anything like an exhaustive manner. It will be necessary to illustrate these classes on only a few well-known waters. It need scarcely be mentioned that the above classification is not an absolutely sharp one, but that many mineral waters belong partly to several classes.

The composition of American Mineral Waters may be ascertained from the publications of the U. S. Bureau of Chemistry.

I. Plain Saline Waters.- These are used mainly externally for baths. The most typical is sea-water (see Index).

Artificial sea-baths may be made by dissolving 4 per cent. of sea or rock salt in water.

Some of the important saline sources are the following: European:

Kissingen ${ }^{1}$.

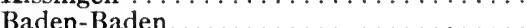

Nauheim.

$\begin{array}{ccc}\text { Per 1.000: } & & \\ \text { Fixed Salts } & \text { NaCl } & \text { Temperature (C.) } \\ \text { I5 } & \text { II } & \text { 18. } 85^{\circ} \\ 28 & 2 \text { I } & 18.5^{\circ} \\ 30 & 25 & 33.0^{\circ}\end{array}$

American: Saratoga Congress, New York, approaches Kissingen.

2. Carbonated Waters. - (a) Plain.-In these the $\mathrm{CO}_{2}$ is the main constituent. This aids digestion, and these waters are used mainly as plain table waters. The most used is the artificial soda-water.

An example of a natural carbonated water is Apollinaris:

Per I,000 Gm.:

$\begin{array}{ccccc}\mathrm{NaCHO}_{3} & \mathrm{NaCl} & \mathrm{Na}_{2} \mathrm{SO}_{4} & \mathrm{CO}_{2} & \text { Temp. } \\ 1.2 & 0.4 & 0.3 & 1.5 & 2 \mathrm{I}^{\circ}\end{array}$

(b) Alkaline.-These contain a notable amount of $\mathrm{NaHCO}_{3}$. They have the alkaline effects and are therefore useful in gastric catarrh, hyperacidity, hypersecretion of mucus, and as diuretics, in obesity, gout, urate stones, and diabetes. They are most useful when taken hot and drunk very slowly, since in this way they cause the least irritation in the stomach.

(c) Alkaline Saline Waters. - These waters usually contain in addition some $\mathrm{Na}_{2} \mathrm{SO}_{4}$. The indications for use are the same as for the preceding. The cold waters contain more $\mathrm{CO}_{2}$ (Marienbad and Franzenbad). The hot alkaline waters are very numerous. The following may be taken as examples:

European Carbonated Alkaline Waters, per 1,000:

\begin{tabular}{|c|c|c|c|c|}
\hline & $\mathrm{NaHCO}_{3}$ & Free $\mathrm{CO}_{2}$ & $\mathrm{NaCl}$ & $\mathrm{Na}_{2} \mathrm{SO}_{4}$ \\
\hline Vich & 5.0 & & & 0.2 \\
\hline & $\begin{array}{l}2.0 \\
1.2\end{array}$ & $\begin{array}{r}500 \\
1200\end{array}$ & 1.0 & $\cdots$ \\
\hline
\end{tabular}

American: Saratoga Selters, Saratoga Vichy, Sweet Springs of Virginia, Gettysburg, etc.

(d) Of the carbonated saline waters the most representative is the Carlsbad. The formula for the artificial salt is:

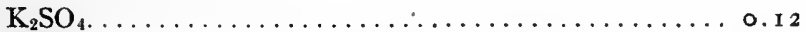

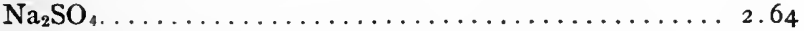

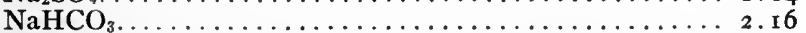

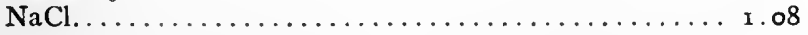

$$
\begin{aligned}
& 6.00 \mathrm{Gm} \text {. }
\end{aligned}
$$

1 Formula for Artificial Kissingen (N.F.):

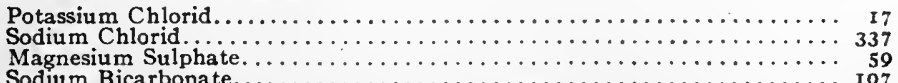

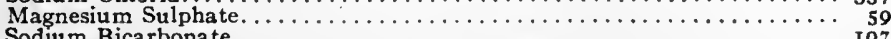

Twenty-four grains of this to 6 ounces of water (1/2 teaspoon to a tumbler) is the usual dose.

2 Formula for Artincial Vichy (N.F.):

Sodium Bicarbonate........................ $846.0 \mathrm{Gm}$.

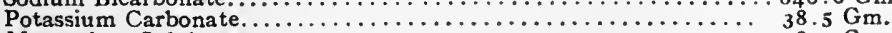

Magnesium Sulphate........................... $38.5 \mathrm{Gm}$.

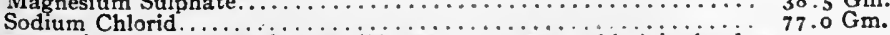

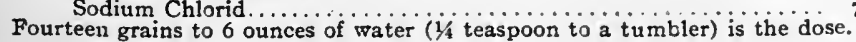


'This quantity (a teaspoonful) is the proper amount for a liter (quart) of water.

3. Magnesia Waters. - These contain $\mathrm{MgSO}_{4}, \mathrm{Na}_{2} \mathrm{SO}_{4}, \mathrm{CaSO}_{4}, \mathrm{MgCl}_{2}$, but no $\mathrm{CO}_{2}$. They are useful as cathartics. If they contain $\mathrm{CaCO}_{3}$, the alkaline action extends deeper into the intestine than in the case of $\mathrm{NaHCO}_{3}$.

European, in $1.000 \mathrm{Gm}$. :

\begin{tabular}{|c|c|c|c|c|c|}
\hline & $\mathrm{MgSO}_{4}$ & $\mathrm{\triangle a}_{2} \mathrm{SO}_{4}$ & $\mathrm{CaSO}_{4}$ & $\mathrm{NaCl}$ & $\mathrm{MgCl}_{2}$ \\
\hline & $22 \cdot 3$ & 22.5 & $\ldots$ & I. 3 & $\ldots$ \\
\hline dlit $z$ & 13.5 & $\ldots$ & I. 4 & $\ldots$ & 0.4 \\
\hline
\end{tabular}

Eipsom and Friedrichshall belong to the same class.

1 mericall: Crab Orchard, Kentucky; and Bedford Springs, Pennsylvania.

4. Sulphur Waters. - These contain free $\mathrm{H}_{2} \mathrm{~S}$ and sulphids. Externally they stimulate the skin and soften the epidermis; internally they act as cathartics. They have considerable reputation in the treatment of chronic rheumatism. Some examples are: tix-la-Chapelle, Harrogate, Virginia Sulphur Springs. The following will give an idea of their composition:

In I,, $00 \mathrm{Gm}$.:

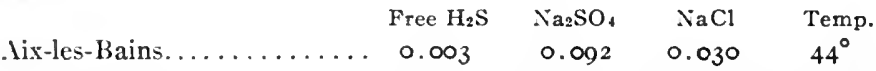

5. Chalybeate Waters.--These contain $\mathrm{Fe}$, usually as bicarbonate, also $\mathrm{NaCl}$ and $\mathrm{Na}_{2} \mathrm{SO}_{4}$. The amount of iron is from 0.01 to 0.12 of $\mathrm{FeO}$ per liter. The most famous springs are: Tunbridge and Brighton in England; Pyrmont, Wiesbaden, and Spa on the Continent; Bedford, Pittsburg, Brandywine, and several Virginia springs, in the United States.

\section{CLIMATE}

This is of such great importance in the action of mineral waters that it may be discussed in this place. Only the roughest sketch can be given, and the student must refer to special text-books for further description.

The principal elements which enter into the climate are the following:

1. Air: $\left\{\begin{array}{l}\text { Purity. }{ }^{1} \\ \text { Density (Altitude) } \\ \text { Moisture. } \\ \text { Temperature. } \\ \text { Special Constituents. }\end{array}\right.$

2. Sunlight.

3. Water, Purity of.

4. Conditions of Life-comfort, hygiene, rest, diversion, pleasant surroundings, out-of-door life, etc.

The subject of climatology is very largely empirical. The work which has been done on the effect of the different climates upon metabolism, nutrition, etc., is as yet too limited to be of great value.

The principal climales used in therapeutics are:
I. Sea.
2. Dry and Warm.
3. Elevated.

1. Sea Climate (Including Islands and Sea Voyage).-The important effects of this are related to constant temperature, humidity, and purity of the atmosphere, and to general surrounding conditions.

This climate, being very restful, is especially useful in cases of overwork. The freedom from atmospheric impurities also renders it valuable in hay fever and phthisis.

2. Dry and warm climate, such as in deserts and Egypt, southwestern Texas, inland Southern California, or in the pine sections of Georgia and the Carolinas

The special advantage of this climate consists in its permitting outdoor life in winter. It is peculiarly valuable in lung disorders which do not stand a northern temperature.

1 Purity: This refers especially to the absence of bacteria, but other fixed impurities may also be of importance; e.g., the causation of hay fever by pollen. In cities, various chemic gases may also be deleterious impurities.

2 Of such special constituents, the aromatic oils given off by needle-trees may be of considerable value as antiseptics and stimulants in phthisis. The presence of ozone, if it is of any importance, serves mainly to indicate the general purity of the atmosphere. 
3. Elevated Climates.-These are generally aseptic, and the air is cool and dry. What importance can be given to the rarefaction of the atmosphere is not at present clear, still less the manner in which this acts.

The rate of the heart and respiration is at first increased, but later it returns to normal.

The quantity of urine is increased, as also the gaseous metabolism and respiratory quotient. The nitrogen excretion is diminished (Jacquet and Stähelin, 1901). The blood becomes rapidly more concentrated in corpuscles, and the serum in proteins. The absolute amount of corpuscles and of hemoglobin is not, however, much increased at first, but notably so later (Jacquet, I904).

These climates cause improvement in a number of conditions, probably in part due to a greater exercise of the organs from climbing, etc. They are useful in dyspepsia, anemia, chlorosis, insomnia, asthma, and consumption.

High climates are unfavorable to feeble conditions of the heart and vessels, which cases should be sent to the seashore.

\section{DIURETICS}

Diuresis (increase of the quantity of urine) is produced by many substances acting by various mechanisms, and belonging to different groups. The whole subject may, however, be summarized in this place as a considerable proportion of the diuretics act, at least in part, by salt action.

The details of the actions of diuretics will not be discussed in this summary. They may be found by referring to the individual drugs.

Diuretics may be divided into the following classes:

I. Acting through changes in the general circulation.

2. Acting purely by local, irritant stimulation of the kidney cells.

3. Acting by non-irritant stimulation of the kidney cells.

4. Acting mainly by salt action.

I. Diuretics Which Act by Influencing the General Circulation.--The secretion of urine is roughly proportional to the glomerular blood pressure. 'This may be raised by increasing the work of the heart, as by Digitalis (which acts also by increasing the pulse wave, lessening venous congestion, and rendering the blood hydremic). Vasoconstrictors generally act very powerfully on the renal arterioles, so that they tend to lower the glomerular pressure, notwithstanding the rise of general blood pressure. Vasodilators also tend to lower the glomerular pressure. They are useful, however, when a vasoconstriction is to be counteracted,

2. Irritant Diuretics.-Moderate irritation raises the glomerular pressure by dilating the renal arterioles. It is conceivable that it also stimulates the vital secretory activity of the cell. Excessive irritation, on the other hand, produces venous congestion, which hinders the filtration of urine by compressing the tubules. The cells are also injured, as shown by the appearance of albuminuria; and their permeability is thereby lessened.

Irritant diuretics are very numerous and only a few of the more important can be mentioned:

(a) Essential Oils.-Especially turpentine, juniper, cubeba, etc.

(b) Hydrocarbons. - Alcohol, ethyl carbamate, hexamethylenamin, etc. These produce only a mild irritation.

(The diuretic action of beer is due mainly to the rapid ingestion of its water; at least, Raphael (1894) failed to discover any difference in the daily excretion, whether a liter of water or of beer was taken.)

(c) Absorbable Metals.-Especially Calomel.

(d) Glucosids. - These are closely related to the aromatic series. Here belong:

Broom Tops (Scoparius). These owe their diuretic action to Scoparin. The alkaloid Spartein (see Index) does not contribute to it.

Asparagus tops seem to act similarly. They are given in 5 c.c. doses of the fluidextract.

Corn Silk, Triticum, Guaiac, etc., may be counted in this group.

Cantharidin, Aloin, etc., are too powerful for therapeutic use.

(e) Acids, Alkalies, and Some Salts (nolably Nitrates) are also mildly irritant.

3. Stimulant Diuretics. - This is the place generally assigned to caffein and theobromin; part of their effect, however, is due to peripheral dilation of the renal arterioles. No irritant action has been described for caffein and theobromin even for large doses; but it has been noted with theocin. 
4. Saline Diuretics. - Water, salts, sugars, urea, etc., act by lessening the viscidity of the blood, thereby increasing its filtrability and raising the glomerular pressure; by shrinking the renal cells; and by preventing the reabsorption of water from the tubules. Excised renal artery is markedly dilated by urea or sodium sulphate, while these have no effect on carotid artery (Cow, ig1r). In addition, it is rather probable that they cause the stimulation of the vital activity of the kidney cells. This is most pronounced in the case of milk-sugar; urea; iodids; nitrates; alkalies; and acids.

Water may be rendered more palatable by carbonic acid, or by giving it as lemonade, or as teas. These additions enhance its diuretic effect.

Normal Saline solution may be injected hypodermically or by rectum.

Milk is a very efficient diuretic, acting partly by its water, partly by its sugar. Milk-sugar and Urea are given in doses of 10 to $30 \mathrm{Gm}$.

Diluted Inorganic Acids (in the form of lemonade) are rather too irritant to the stomach and kidneys, and are to be used only if an acid action is distinctly indicated.

Alkalies are quite effective; they also render the urine alkaline. Free alkalies and carbonates are not used, since they interfere with digestion. The effect is obtained from organic acids and their salts (especially the acetate or citrate of potassium), which are oxidized to carbonates in the body.

Iodids and Nitrates are given as the potassium salts. The iodid acts too powerfully on other functions to be used as a pure diuretic. The reputation of lithium is unmerited.

The comparative diuretic effect of these drugs on normal man was investigated by Raphael (r894), who placed himself on a uniform diet for prolonged periods. He consumed daily $\mathrm{r}, \mathrm{r} 8 \mathrm{0}$ c.c. of fluid; the quantity of urine on this diet amounted to 750 to 960 c.c. Under the influence of drugs, it was increased by the following percentage:
I,, 00 c.c. water- I00 per cent.
r,,$\infty 0$ c.c. carbonated water -73 per cent.
r,, 00 c.c. beer -100 per cent.
r,, 00 c.c. claret- -80 per cent.
r,o00 c.c. milk-r 53 per cent.
$30 \mathrm{Gm}$. milk-sugar-34 per cent.
$0.4 \mathrm{Gm}$. turpentine-r r per cent.
$0.2 \mathrm{Gm}$. Ol. Junip. + I,,$\infty$ c.c. water-I I I
per cent.
$0.5 \mathrm{Gm}$. Caffein-Sod.-Salicyl.-42 per cent.
$0.5 \mathrm{Gm}$. Diuretin- 2 per cent.
I.5 Gm. Diuretin-r 4 per cent.
3.0 Gm. Diuretin-53 per cent.

In disease, the results would probably not be quite the same. The choice of the diuretic must be determined by the condition of the kidneys, and by the object to be accomplished. (In dogs with vesical fistula, Ginsberg, I912, found milk less effective than water.)

Combination of Diuretics.-A combination of several diuretics is often most effective; they are generally administered with plenty of water. Schlosser, rgr 3 , found that the combination of caffein and intravenous sodium sulphate gave a potentiated synergistic effect; while the combination of caffein with theobromin or theophyllin gave only a simple addition of their action.

\section{The indications for diuretics are as follows:}

I. The removal of liquid from the body, in the various forms of dropsy. In this case it is well to employ them with as little fluid as possible.

If the dropsy is of cardiac origin drugs of the digitalis series, combined if necessary with nitroglycerin, are the most efficient diuretics, and salts may also be added. If it is of metabolic origin, benefits may follow salts or arsenic. If, however, it is of renal origin, diuretics should be avoided altogether, and recourse should be had to diaphoresis.

2. To remove toxic substances from the organism, whether these have been introduced from without or formed within the body, a free supply of water, in the form of infusions, supported by the irritants, salts, or theobromin, fulfils the indication. The hypodermic injection of large amounts of normal salt solution is a most effective method. Irritants must be avoided if the kidneys are inflamed, or if the poison is itself irritant.

The efficiency of diuretics for the removal of poisons has been greatly overestimated. They are practically useless when the toxic substance combines in the body, as do the 
toxins, strychnin, etc. They are most effective against saline poisons, such as magnesium (Meltzer, 1907). Irritant diuretics hasten the elimination of Phenolsulphonephthalein; those which act mechanically do not (Rowntree and Geraghty, 191 2). Christian, $19 \mathrm{I}_{3}$, tried a series of diuretics in experimental nephritis, with practically negative results. Clinically, diuretics are generally inefficient in chronic nephritis, in the absence of edemas; and are therefore of little value against the toxemia (Christian et al., 1915).

3. To Dilute the Urine.- This may be useful $(a)$ to render it less irritant to the urinary passages in nephritis or inflammation of the bladder or urethra; it also serves a useful purpose in frequently washing out the pus and bacteria.

(b) To prevent the formation of calculi, or to remove concretions formed in the urinary tubules (as in oxalate-poisoning).

(c) To dilute irritant poisons, whose action on the kidneys is proportional to their concentration.

For the dilution of the urine, water supported if necessary by theobromin is of the greatest service.

Nephritis contraindicates irritant diuretics, and such as will become poisonous if they are not excreted (potassium, digitalis, mercury).

\section{SODIUM CHLORID}

General Statement.-This constitutes the greater part of the electrolytes of the tissues (with certain exceptions), and especially of the fluids, of vertebrates. Its ions are essential for the functionation of most cells. Since the ions are constantly excreted, especially by the urine, they must be constantly replenished, leading to the instinctive use of salt in food. Its administration to mammals, even by intravenous injection, produces practically only osmotic effects; but marked ion-actions may be observed by exposing excised tissues to solutions with or without the sodium and chlorid ions.

The therapeutic uses of salt depend mainly on its osmotic effects. These result in stimulation of the gastric mucous membrane, leading to its use as condiment and in some indigestions; under suitable conditions, a laxative action; irritation of the skin, with attending reflex stimulation, utilized as salt-water baths. After absorption, it leads to diuresis and slight metabolic changes. Isotonic solution is used as vehicle or for cleansing sensitive mucous membranes, $f . i$., the nose, and for hypodermic and intravenous injections. These saline infusions are employed in hemorrhage, collapse, and for the dilution or removal of systemic poisons.

The intravenous injection of strongly hypertonic solutions is fatal, with stimulation and paralysis of the central nervous system.

Retention of chlorid occurs in certain pathologic conditions, especially pneumonia and other fevers, nephritis, cancer, etc. Its mechanism is not explained. It may favor the formation of edemas, against which salt-poor diet is sometimes efficient.

$\mathrm{NaCl}$ Income. - With ordinary diets, this ranges between 10 and ${ }_{5} \mathrm{Gm}$. per day; but its reduction to $5 \mathrm{Gm}$., or even I to $2 \mathrm{Gm}$., causes no disturbance. Carnivorous animals obtain a sufficient supply in their food. But since $K$ predominates over $\mathrm{Na}$ in the ash of plants, herbivora and animals feeding on a mixed diet require additional $\mathrm{NaCl}$. This need has led to the instinctive use of this salt: in regions remote from deposits of this salt, ashes of plants which are rich in $\mathrm{Na}$ have been used by peoples as ignorant as the negroes of the interior of Africa. A table of the salt content of food is given by Leva, Arch. f. Verd, Krankh. $16: 267$. 
Distribution of $\mathrm{NaCl}$ in the Body. - The most exact and comprehensive determinations are those of Wahlgren, I909, and Padtberg, I910, on dogs. Similar relations probably hold for man and other animals. The entire body contains about 0.17 per cent., expressed as Cl (o.r I per cent., Rosemann, r910). Of this, about a third is in the skin; the remainder chiefly in the blood, muscles, bones and intestines. The highest percentage of $\mathrm{Cl}$ exists in the skin ( 0.38 per cent.); then the blood ( $0.3 \mathrm{I}$ per cent.); kidneys ( 0.26 per cent.); and lungs ( 0.24 per cent.). The brain, bones and intestines contain 0.17 to 0.18 per cent.; the liver, 0.13 per cent.; the muscles only 0.07 per cent. According to other determinations, blood serum contains 0.36 to 0.42 per cent. (equivalent to 0.9 to I per cent. $\mathrm{NaCl}$ ); saliva, 0.16 to .024 per ceet. $\mathrm{Cl}$; the human intestinal juice 0.58 to 0.6 ; per cent. $\mathrm{NaCl}$; sweat, 0.285 per cent. (Kovesi); human gastric "appetite" juice 0.49 to $0.5^{6}$ per cent. $\mathrm{Cl}$ (Carlson, I I $^{5}$ ). The red blood corpuscles contain 0.12 to 0.18 per cent. of $\mathrm{Cl}$, and $0.2 \mathrm{I}$ to 0.28 per cent. of $\mathrm{Na}_{2} \mathrm{O}$; except in some animals (horse, pig and rabbit), whose corpuscles contain practically no Na (see Potassium). The chlorid content of the entire human body is estimated at 60 to I $33 \mathrm{Gm}$., equivalent to I 50 to $300 \mathrm{Gm}$. of $\mathrm{NaCl}$ (Mlagnus-Levy, I go6).

Artificial Variations of Chlorid Content.-Padtberg studied the changes following the intravenous injection of $\mathrm{NaCl}$. Here, also, the skin maintains the highest percentage; but because of their greater mass, the muscles and intestines take up the greater absolute a mount. With $\mathrm{Cl}$-poor food, the total $\mathrm{Cl}$ content sinks to o.I 37 per cent., the loss being greatest from the skin. The skin therefore acts as a chlorid depot, storing and giving off most of the excessive or deficient $\mathrm{Cl}$. Rosemann, IgI I, found the entire $\mathrm{Cl}$ content increased by roo per cent. when dogs were given highly salted food. With the return to normal diet, the $\mathrm{Cl}$ content returned to one and one-half times the ordinary level within five days; the further fall to normal occurred much more slowly.

Chlorid Content in Disease.-Biernacki, $\mathrm{I} 894$, found the $\mathrm{Cl}$ of the blood constant in numerous diseases. Javal and Adler, I906, determined the percentage of $\mathrm{Cl}$ and water in the organs after death from various diseases, with a special reference to edema; but the results were not decisive.

Leva, I9I5, states that human organs in renal, cardiac and vascular disease may contain twice, the skin even three times, the normal amount of $\mathrm{Cl}$, without material increase of their fluid. Similar results were obtained on uranium rabbits.

F. C. McLean, I $^{1}{ }_{5}$, found the chlorid of the plasma increased, clinically, in cardiac and renal disturbances, and in pneumonia; diminished in diabetes and with diuretics. The chlorid and urea content of the plasma do not go parallel. In uremia, the diminution of blood-chlorid is concomitant with increased hydrogen ion concentration (McLean, 1916).

Microchemic Distribution.-A. B. Macallum, I905 and 1908, found the chlorid most abundant in the inactive tissues, especially in the intercellular spaces and in degenerating cells. It is absent from the nuclei (as also $\mathrm{PO}_{4}$ and $\mathrm{K}$, and probably all other inorganic ions); but it occurs abundantly in the cytoplasm. Nerve fibers are rich in $\mathrm{Cl}$, especially the axons; but the myeline sheaths are free from it, and nerve cells contain very little (Macallum and Menten, I906).

Excretion of $\mathrm{NaCl}$. - This occurs mainly by the urine, only a small part being lost by the feces and perspiration. The excretion varies quantitatively with the salt income, but generally lags somewhat behind in time. The administration of salt therefore produces a temporary accumulation in the tissues. With a steady income of salt and water, equilibrium is reached, in normal man, in three or four days (Borelli and Girardi, I9 5 ). If the supply of salt is stopped, the excretion falls to a very low level within three days, the tissues retaining their normal salt content with very great avidity. A small excretion ( 0.6 to $0.9 \mathrm{Gm}$. daily in man) continues, however, so that the tissues finally lose salt. This leads to the phenomena of "salt-hunger."

Feces.-The fecal chlorid excretion is normally insignificant, but in nephritis it may reach 3 to $5 \mathrm{Gm}$. NaCl per day (Javal, 1903; Halpern, 1905).

Diurnal Variations.- In man, the urinary chlorid excretion is always greater in the forenoon than in the night, independently of meals. The midday meal has an influence, the excretion being decreased half an hour after eating, due to the gastric excretion of $\mathrm{HCl}$. This lasts an hour, and is followed by marked increase (Herrmannsdorfer 19I2). Time of Excretion.-Giving Io Gm. by mouth, the additional chlorid should be 
excreted within twenty-four to forty-eight hours, either by diuresis, or by increased salt concentration. A longer delay indicates impaired permeability of the kidney. Schlayer and Takayasu, I9I3, consider the salt and iodid excretion as an index of tubular activity; whereas water and lactose excretion indicate the renal vascular function. This classification is based on rather ill-established assumptions.

Influence of Other Salts and Diuretics. - The excretion of $\mathrm{Cl}$ can be hastened by the administration of related salts (bromids, iodids, nitrates, sulphocyanids). Conversely, the administration of $\mathrm{Cl}$ hastens the elimination of these salts, and may be useful against iodism and bromism. Diuretics have little effect: the drinking of large quantities of water reduces the percentage of $\mathrm{Cl}$ in the urine; the absolute quantity may be unchanged or moderately increased (Rulon and Hawk, rgII). With dogs, theobromin and caffein diminish the $\mathrm{Cl}$, both absolute and percentage, independently of any diuretic effect Saccone, I9I $x$ ). In rabbits, however, these and other diuretics tend to increase the $\mathrm{Cl}$ excretion proportional to the diuresis (Bonnamour and Imbert, I9 2.)

Chlorid Retention.-The chlorid excretion is greatly diminished in some cases of nephritis; in fevers, particularly in pneumonia (where much salt is retained in the effusion); and during the rapid development of new tissue (cancer).

Retention in Fever. - This was first described by Redtenbacher, 1850 , and has been extensively investigated, especially by Roehmann, 1889. The low Cl excretion in partly due to the low $\mathrm{Cl}$ income; but even when this is taken into account, there is a considerable deficit in the output, i.e., a true retention (Garratt, I904). This is especially marked in pneumonia; and the $\mathrm{Cl}$ in the exudate is not sufficient to account for the difference. The retained $\mathrm{Cl}$ seems to be distributed indiscriminately through all tissues. The $\mathrm{Na}$ and $\mathrm{Ca}$ are also retained, while $\mathrm{K}$ and $\mathrm{Mg}$ are excreted normally or in excess (Peabody, I9I3). Medigreceanu, I9I I, found a comparatively slight $\mathrm{Cl}$ retention in turpentine pleurisy, and none in pneumococcus septicemia in rabbits. This indicates that the retention is connected with the pulmonary changes. Snapper, I9I3, attributes the retention to altered cell permeability; for he claims that the addition of sodium sulphate to blood causes the passage of $\mathrm{NaCl}$ into the corpuscles in fever; whereas normally it causes the passage of Cl out of the corpuscles. The whole subject is still very obscure.

McLean, 1915, finds that the diminished excretion in pneumonia is associated with lowered $\mathrm{Cl}$ content of the plasma. At the crisis, the $\mathrm{Cl}$ increases both in the plasma and in the urine.

Retention in Cancer. - The diminished excretion is probably explainable entirely by the deficient $\mathrm{Cl}$ income, and its need for the formation of new tissues and transudations (Gaertig, I89o; Laudenheimer, I892).

Retention in Nephritis.-This was mentioned in connection with edema. It is most apt to occur in acute glomerular nephritis (Borchardt, I912). However, in chronic nephritis the ability to excrete chlorid is generally impaired before the nitrogen excretion (O'Hare, rgr6).

The $\mathrm{Cl}$ excretion is increased in the early diuretic stage of uranium and cantharides nephritis; it is usually diminished with large doses of uranium or chromate. The $\mathrm{Cl}$ excretion bears no simple relation to the anatomic changes (Austin and Eisenbrey, I9r I).

Salt Test for Nephritis. - Schlayer, Igri; keeps the patient on a constant diet for several days, then adds io $\mathrm{Gm}$. of $\mathrm{NaCl}$ in one dose. This excess is normally excreted within twenty-four or at most forty-eight hours. A delay beyond this indicates diminished permeability of the kidneys to chlorids. Frothingham, 1915, classifies the excess excretion, within forty-eight hours, of $8.5 \mathrm{Gm}$. as no retention; $4.5 \mathrm{Gm}$. as slight retention; less than $4.5 \mathrm{Gm}$. as considerable retention. Hedinger and Schlayer, I 912 , now employ a "nephritic test meal." A strictly similar test may be made with urea, ro $\mathrm{Gm}$. of which should also be excreted within forty-eight hours (Monakow, I9I4).

Diabetes.-Dogs with pancreatic diabetes excrete only one-tenth to one-seventh of the chlorid of normal dogs on the same diet (Rabens, 1915). With diabetic patients, however, the "chlorid threshold" of the blood is either unchanged or lowered (McLean, 1915).

Mechanism of Chlorid Regulation.-The blood maintains its chlorid concentration remarkably constant, and even the tissues undergo only temporary changes. The regulation is accomplished through the adjustment of the urinary excretion; but the mechanism by which this is controlled 
is not understood, notwithstanding extensive investigations. The problem is probably analogous to that of the regulation of the sugar content of the blood.

Colloid-chlorid Combination.-One theory (Forster, I873) assumes that the greater quantity of the salt exists in the body in a form (perhaps as a combination with the proleins) in which it can not pass through the living kidneys. This salt is therefore retained with great tenacity. Any excess over this, such as is normally present, is free and filters very readily into the urine. This theory agrees with the observation of Sollmann ( $\mathrm{I}_{\mathrm{O}} \mathrm{O}_{2}$ ) that the injection of those ions which are closely related to chlorid (viz., $\mathrm{Br}, \mathrm{I}, \mathrm{NO}_{3}, \mathrm{SCN}$ ) increases the chlorid percentage in the urine, while other ions do not have this effect: it may be assumed that the related ions can displace the chlorid from its protein compound. All attempts to prove by chemical or physical methods the existence of such compounds in the blood have failed. Rona, rgIo, f.i., showed by "compensated osmosis" that the $\mathrm{Cl}$ of the blood diffuses exactly as if it were in the ordinary ionic condition. However, it is still conceivable, that the adsorption of the colloids may modify the filtrability of dissolved substances. M. Fischer, 1915, believes that retention of salt and water are both due to imbibition of body colloids, which is increased by acids, and therefore he thinks by acidosis.

Cushny ( 1902$)$, attributes the chlorid regulation to the absorbing mechanism of the tubules.

Chlorid Threshold.-Ambard and Weil assert that there is a certain threshold of the $\mathrm{NaCl}$ concentration of the plasma-normally $5.62 \mathrm{Gm}$. per liter. If the concentration falls below this, no chlorid is excreted; if it rises above, the excretion varies as the square of the excess (McLean, I9I5).

Limited Salt Income. - The maintenance of a certain salt content is indispensable to the body, sodium chlorid being especially important. Animal food appears to contain a sufficiency of this salt; while a herbivorous and omnivorous diet is insufficient in this respect. Under ordinary conditions, the excretion of salt keeps perfectly parallel with the salt income (Falk, 1848 ), so that a moderate excess of salt income is practically without effect, except on the urine. A very limited salt income (as by an exclusive milk diet) can also be borne for a considerable time, being met by a corresponding decrease of chlorid excretion, so that the percentage of the salt in the blood is scarcely altered, and no general symptoms whatever result. Dogs on a $\mathrm{Cl}$-poor diet retain $\mathrm{Cl}$ or I more efficiently than on ordinary food (Sarvonat and Cremien, IgII). The diminished income of salt in fever seems to have no deleterious effect. Even when the excretion is further increased by liberal water-diuresis, the patients show no particular craving for salt. Complete abstention from food also does not produce any effects referable to the absence of salt. After a ten-day fast, the total quantity of $\mathrm{Cl}$ in the' body is reduced by only 2 per cent.; and the percentage of $\mathrm{Cl}$ is even above the normal, because the tissues disappear more rapidly in starvation than does the $\mathrm{Cl}$ (Rosemann, rg I 2). D'Errico, Igro, found no change in the freezing-point or conductivity of the blood in inanition. The inorganic metabolism in starvation has also been studied by Cathcart and Fawsitt, 1907.

Salt Starvation.-This may be produced by feeding food deprived of all its salts (J. Forster, 1873); or by "deceptive feeding," abstracting the gastric juice through a fistula (Rosemann, I9I 2). Forster found that pigeons died in thirteen to twenty-nine days; dogs in twenty-six to thirty-six days. In man, an abstinence of nine days is serious, but not dangerous. The subject does not return to normal for a week or two af ter resuming the consumption of salt (E. Taylor, 1904).

The animals emaciate rapidly, through anorexia and other interference with digestion and absorption. Great muscular weakness and tremors, with occasional convulsions, are very prominent. The mental faculties are dulled. The urine and breath contain acetone. The loss of the mineral constituents of the body is relatively small. The $\mathrm{N}$ excretion is fairly equal to the income. A conspicuous change is the decrease in the excretion of gastric $\mathrm{HCl}$ (Cahn, 1886). This stops completely when the chlorid stock of the body has fallen by 20 per cent. (Rosemann, I9 I 2). It is not known how far the other changes are due to mere $\mathrm{Cl}$ loss; and how much the absence of the other ions contribute to the result.

Salt-poor Diet.-The use of $\mathrm{NaCl}$-poor food in edemas, etc., was discussed on page 647. Javal, I9 12, suggests the addition of sodium iodid or bromid to improve the taste of the unsalted bread. Cl-poor diet has also been employed to enhance the retention of the bromid ion in epilepsy (Toulouse and Richet, r900). Indeed, the efficiency of the bromid treatment has been referred to de-chloruration; but this is probably erroneous (see Bromids; Januschke, 1913). Tables of the content of the various salts in foods are given by Albu and Neuberg, Mineralstoffwechsel. 
Effects of Excessive Consumption of Salt.-By means of the regulating mechanisms, an excess of these $\mathrm{Na}$ or $\mathrm{Cl}$ ions is eliminated very promptly, and could not produce any very large effect. But even when they are retained, these ions produce only very small actions, for the amounts normally present are so large that the artificial introduction of ordinary amounts will not increase their ratio to a considerable extent. $\mathrm{Na}$ and $\mathrm{Cl}$ are therefore the most indifferent of the ions, and their salts are used when the effects of other ions are to be tested. It is important to remember, however, that they are really very essential to the protoplasm, and that the absence of effects is due only to the fact that the variations which are introduced are relatively small.

Local Actions of Salts.-Salts in isotonic or hypoisotonic solutions have little local action; in substance or in hyperisotonic solutions they act as rather mild irritants, without destroying the tissues.

Action on the Skin; Salt-baths.-Sodium chlorid and other neutral salts, e.g., in the form of sea baths, produce a mild but extensive stimulation of the skin, and thereby reflex tonic effects. The mechanical irritation of the dried salt crystals contributes to the result. They possess an advantage over most other irritants in that they do not injure the epidermis.

Alimentary Canal.-Salt produces a similar stimulation of the mucosa of the alimentary canal, especially in the stomach, i.e., before the salt is diluted.

The action is deep since the salt will stimulate all the cells with which it comes into contact in the course of $i$ ts absorption. Since the salt is quickly removed by further absorption, it does not cause any permanent change. In this way it differs from the majority of gastric irritants, which produce a superficial but persistent action. The stimulation by salts may therefore be continued for a considerable time, and is frequently very useful in the treatment of certain cases of "atonic" dyspepsia. Salt also often aids the digestion of otherwise difficultly digestible articles. Rosenblat, I907, observed markedly increased secretion in the Pawlow miniature stomach, depending on the concentration; I per cent. was indifferent; greater dilutions were slightly stimulant.

Pepsin.-W. W. Hamburger, I915, found that neutral solutions of $\mathrm{NaCl}$, even in the concentration of serum, rapidly destroy pepsin. The inhibition is complete with 2.5 per cent. $\mathrm{NaCl}$; scarcely perceptible with 0.25 per cent. Other inorganic salts act similarly, probably by liberation of $\mathrm{OH}$ ions (Hamburger, and Halpern, 19 5 ). The destruction does not occur in the presence of free acid; but once destroyed, the ferment can not be re-activated by acidulation. This reaction is probably the explanation of the "antipepsin" of the serum. Neutralization of gastric juice likewise destroys its pepsin, since $\mathrm{NaCl}$ is formed. This would contribute to the action of alkalies, for instance in gastric ulcer.

Laxative Action.-A teaspoon of salt in a pint of water, taken before breakfast, is often laxative.

Saline Diuresis on Milk Flow.-In lactating goats, with limited water income, diuretic doses of salt or urea decrease the yield of milk and increase its fat-content, presumably by diminishing the available amount of water. The nitrogen of the milk is not increased (Steenbock, I9 5 ).

Toxic Action.-If salt is given in large amounts and concentrated form, the irritation may lead to a strong inflammation- to severe gastroenteritis, which may be fatal. Poisoning by enema was reported by Brooks, rgro. A pint of saturated solution had been injected, and repeated twice at hour intervals. The symptoms were restlessness, thirst, rapid pulse, coma, fever $\left(109^{\circ} \mathrm{F}\right.$.), frequent micturition, dyspnea. Death occurred eight hours after the first injection, preceded by convulsions and bloody discharge from the rectum.

Action After Absorption.-The ingestion of salts, without sufficient water to render them isotonic, must tend to increase the salt concentration of the body. This increase affects mainly the tissues, the molecular concentration of the blood remaining practically unaltered. This increased salt concentration is counteracted by the stimulation of two mechanisms: a desire for water (thirst); and the elimination of highly hypertonic urine. If water is given freely, the salts are powerfully diuretic. This leads to an increased excretion of metabolites, partly by the flushing out of stored waste products, but also by direct stimulation of metabolism. If the water income is restricted, the poor urine flow decreases the $\mathrm{N}$ excretion temporarily (Desgrez and Guende, IgI2). 
The effect upon the metabolism is uncertain in its outcome, as is the case with all alteratives. It may be useful especially in obesity.

Saline Infusions.-The general effects of hypodermic or intravenous injections of saline solutions on the quantity of blood, lymph and urine, and the fate of these solutions were discussed under "Salt Action." It may be recalled that the quantity of fluid in the blood is temporarily increased. This produces a very moderate rise of blood pressure; and this, perhaps with some direct salt action, results in an increased excitability of the respiratory, vagus (H. C. Jackson and Ewing, I9r4) and vasomotor centers. With normal animals, the rise of capillary pressure is large and persistent; while the rise of arterial pressure and the medullary stimulations pass off quickly, but without noticeable fatigue. In animals whose blood pressure is low-particularly if this is due to hemorrhage, saline infusions bring the pressure toward normal, and maintain it much better (see figure). Excessive injections of saline solutions may cause death from pulmonary edema, or from overdistention of the heart. Even therapeutic injections may produce glycosuria, slight fever, and rarely albuminuria.

These effects are produced by all salts whose ions are not specifically toxic, e.g., by most sodium salts. To have any effect, these must be used in rather large amounts, i.e., from 25 to 50 c.c. of the eighth-molecular solution per kilogram of body-weight. Solutions which contain all the salts of the serum in the proper proportions (e.g., Locke's Fluid) are somewhat better than the normal ( 0.9 per cent.) sodium chlorid solution, but the latter suffices for all practical purposes.

Even small doses of isotonic $\mathrm{NaCl}$ may produce changes in blood pressure, while Ringer's solution or glucose has much less effect (Selig, rorI); so that the action is probably not wholly osmotic. Frogs' vessels are dilated by perfusion with $\mathrm{NaCl}$ (Hooker, I9I I).

Vasomotor Center.-In normal animals, injections of to to 40 c.c. per kilogram produce only slight stimulation or no change. Occasionally, when there is maintained rise of blood pressure, the vasoconstriction is quite marked (Pilcher and Sollmann, I9r4).

Toxic Doses of Different Cathions.-Joseph and Meltzer, I907, found that $\mathrm{MgCl}_{2}$ was fatal for mammals with $0.223 \mathrm{Gm}$. per kilogram (I c.c. $\mathrm{m} / 5$ per kilogram per minute); $\mathrm{Ca}$ and $\mathrm{K}$ required about double, Na twenty times as much.

Therapeutic Uses.--Saline infusions are employed to tide the patient over acute anemia from hemorrhage; in collapse; or in alternation with bleeding for the removal of poisons (uremia, CO, etc.). Direct transfusion of blood is more efficient, but more inconvenient and often inadvisable:

Use in Hemorrhage.-Saline injections are made with the object of restoring the fluid needed for effective work of the heart. The restoration of the blood pressure succeeds, at least temporarily, unless the hemorrhage has reduced the pressure to the level of shock (Pilcher and Sollmann, I9I4; Downs, I9I6). Short of this, the temporary benefits are often striking; but since the solution does not stay long in the vessels, the final fatality is not greatly influenced.

Kwan, I913, f.i., found that rabbits die from acute hemorrhage when 26.7 to 32 c.c. of blood are lost per kilogram. When the blood was replaced at once by simple saline, the fatal limit was 33.5 to 37.5 ; when replaced by Ringer's solution 33.6 to 37.3 c.c. per kilogram.

Colloid Infusions. - The attempt has been made to prolong the stay of the saline in the vessels by the addition of gelatin or other colloids (Hogan, rgI3). These solutions produce less diuresis (Knowlton, I $9 \mathrm{r}_{\mathrm{r}}$ ) and are generally retained much longer; but this prolonged sojourn of the colloid is probably inadvisable, and it is doubtful whether their use is justified. Roger and Garnier, ror3, had very discouraging results.

Hogan, I915, advised the following solution: Gelatin, 1.5 Gm., dissolved in roo c.c. 
of water, and added to $\mathrm{I}, \infty 00$ c.c. of 0.9 per cent. $\mathrm{NaCl}$ with $2 \mathrm{Gm}$. of $\mathrm{Na}_{2} \mathrm{CO}_{3} \mathrm{IOH}_{2} \mathrm{O}$. This is injected (about 500 to 750 c.c.) until the blood pressure is satisfactory.

Colloids on Diuresis. - Knowlton observed that 5 per cent of gelatin or of acacia, added to Ringer's fluid, practically prevented a diuretic response; wt:ereas soluble starch, which is at least equally viscid, did not inhibit diuresis. 'The effect of gelatin is probably due to its water-affinity.

In the experiments of Bogert, Underhill and Mendel, 1916, the urine-flow was as great with 2 per cent. gelatin as with plain saline. 'The gelatin solution generally left the circulation somewhat more slowly than plain saline; but not always. The gelatin was eliminated from the blood at the same rate as the fluid, being partly excreted by the kidneys (Boycott, I9I4).

Plasmaphaeresis. - This term was introduced by Abel to designate the replacement of the plasma with saline solution. The blood is withdrawn, the corpuscles separated and washed by centrifugation, suspended in 0.6 per cent. $\mathrm{NaCl}$, and reinjected. In this way much larger amounts of plasma may be withdrawn without damage, especially if the process is several times repeated. The number of red corpuscles rises at first (i.e., the volume of liquid in the vessels is reduced); but ultimately they decline slowly. The plasma proteins may be reduced to a third. The urea and non-protein nitrogen of the blood are increased. The temperature rises slightly (Abel, Rowntree and Turner, I9I4; Turner, Marshall and Lamson, I9 5 ).

Transfusion of Blood. - This was tried clinically in very early times, but with disastrous results, since foreign species were generally used. Worms-Mueller, I875, investigated the acute and late effects of the transfusion of the blood of the same species, the fate of the blood, the therapeutic field, etc., and practically stated our modern knowledge of the subject, except some technical details (Sollmann, igi5). He showed that changes of the blood volume between 25 per cent., the normal, to 30 or 50 per cent. above normal do not materially alter the normal blood pressure; attributing the adjustment to vasomotor changes. The pressure remains practically unaltered even with extreme plethora; but other acute central symptoms supervene.

Fate of the Transfused Blood.-As to the further fate of the animals, Mueller showed that increasing the normal quantity of blood by 80 per cent., by slow transfusion, does no damage. Of the injected plasma, about half the water has disappeared from the blood within two hours; the remainder leaves more slowly, requiring several days. The plasma protein also disappears promptly; and increased urea excretion indicates that it has been metabolized. The injected corpuscles persist much longer, the normal ratio being restored in three to four weeks. He therefore concluded that the usefulness of transfusion is due solely to the injected corpuscles. No differences were found between fresh or defibrinated blood and blood that had been kept on ice for a day.

These observations have been confirmed by the modern investigators. For instance, Weinberger, r9o8, found in dogs that the water leaves the blood vessels very rapidly, causing diuresis, but not nearly as great as with $\mathrm{NaCl}$ solution. The red corpuscles can thus be raised temporarily to over $13,000,000$; but they gradually decline during the succeeding days. The specific gravity rises still more. Animals invariably die after four or five transfusions, with hemoglobinuria and nephritis. The injected blood probably has no nutritive value, the nitrogen being rapidly eliminated.

Indications for Transfusion. - These were stated by Mueller : excessive hemorrhage; carbon monoxid poisoning; and some chromic amenias, for instance, leukemia These are practically the modern indications, ( $f . i$. Ottenberg and Libman, I9 I 5 ).

Saline Infusion in Collapse.- This may hasten recovery in temporary collapse, as with phenol and other poisons. It is of little use in the lasting collapse of fever or severe shock.

Use in Uremia and Other Intoxications.-Saline solutions were extensively employed as diuretics; but it is now recognized that simple injection does not increase the elimination of the toxic substances sufficiently to be of much value. (Their influence has been studied by Lenhartz, I 899.) The recognition of the impermeability of the kidney to chlorid in some nephrites enjoins special caution. Mosenthal and Schlayer, I9I3, claim that the kidneys of experimental nephritis do not respond well to $\mathrm{NaCl}$. In any case, saline injections must be used cautiously in anuria, and must not be persisted in if a moderate injection does not cause diuresis.

Hypoisotonic Solutions.-These act very much like isotonic solutions, but are somewhat more injurious and may lake the blood.

Hyperisotonic Solutions. - These, by abstracting water from the tissues, produce a much more violent stimulation of the central nervous system, passing on to convulsions, but always followed by depression. Small intravenous in jections raise the blood pressure partly by direct stimulation of the heart (Retzlaff, 1915). With larger injections, the blood pressure shows a progressive fall, with temporary rise during the spasms, 
The respiration is at first quickened, but becomes slow and shallow as the blood pressure falls. The heart is quickened, feeble, and arythmic. In the later stages it may be slowed by stimulation of the vagus center. The temperature rises.

The diuretic effect and the local irritation are also much greater.

It is probable that some of the phenomena of uremia are due to a hyperisotonic condition of the blood, which may reach almost double the normal concentration, in this condition.

Respiratory Metabolism.- This is increased by saline injections, especially if hypertonic (Raeder, I915).

Salt Glycosuria.-Bock and Hoffmann, I87I, observed that intravenous injection of $\mathrm{NaCl}$ solution into rabbits produces glycosuria. This was confirmed by Kuelz, I 872 (who also found that it does not seem to occur in dogs); and later by M. Fischer, O. H. Brown and others. The glycosuria is greater with rapid injection or higher concentrations, and may be abolished by the addition of $\mathrm{Ca}$ ions.

The mechanism of the salt glycosuria is in dispute. Underhill and Closson, Igo6, claim that it is accompanied by hypoglycemia, and therefore attribute it to increased permeability of the kidneys. E. Franck, 1913, failed to find the hypoglycemia, and attributes it to polyuria. Willenko, I9II, states that it does not occur after section of the splanchnics and refers it to stimulation of the central nervous system. Further work is eivdently needed.

E. Hirsch, I915, reports hyperglycemia from intravenous injection of ro per cent. $\mathrm{NaCl}$. The action is central, presumably on the glycosuric center. Solutions of $\mathbf{2 . 5}$ per cent. or more dilute, even in large quantities, do not increase the blood-sugar; nor does sodium carbonate or acetate, nor calcium chlorid. Sodium sulphate also does not produce hyperglycemia (Kleiner and Meltzer, I916). Magnesium sulphate, however, does so.

It is interesting that diabetic puncture and puncture of the funiculus teres in rabbits increases the urine and the percentage of chlorid ("Salt puncture"). The urinary effect is independent of the glycemia, but depends on nervous impulses carried by the splanchnics (Jungmann, I9I4).

Salt Fever.-Finkelstein, 1908, and Schaps, observed that the administration of salt to infants by mouth or hypodermically, may produce fever. A slight rise of temperature is also observed in adults, if at least a liter of 0.9 per cent. $\mathrm{NaCl}$ is injected hypodermically (Bingl, I9IO); but is prevented if Ca is added, as in Ringer's solution (Meyer and Rietschel; Bock, 1912; Burnett and Martin, 1916). The rise is accompanied by an increased $\mathrm{N}$ metabolism and heat production (Freund and Grafe, I9II). Hypertonic salt solution ( 3 per cent. $\mathrm{NaCl}$ ) is much more effective (Rolly and Christjansen, 1914). A similar rise is produced by the injection of isotonic dextrose or lactose, and by indifferent particles (paraffin, colloid metals, etc., Bock); but these may have a different origin. The salt fever is not due to infection, for it occurs with absolutely sterile solutions and with oral administration (Bingl; Freund, 19.3). It is therefore quite distinct from the "water infection," the "wasserfehler" of Wechselmann, IgII; i.e., the fever that occurs after the injection of solutions made with stale distilled water. Finkelstein and Bingl asssumed that the salt fever is caused by irritation of the heat centers by the chemic products resulting from the action of the $\mathrm{Na}$ ions on the tissues.

\section{PREPARATIONS-SODIUM CHLORID}

*Sodii Chloridum (Sod. Chlorid.), U. S. P.; (Sod. Chlor.), B.P.; (Common Salt), $\mathrm{NaCl}$. White, crystalline powder of purely saline taste. Freely sol. in water $(\mathrm{r}: 2.8)$; nearly insol. in alc.; sol. in glyc. ( $1: 10)$. Chlorids are incompatible with salts of silver and lead. Dose, $15 \mathrm{Gm}$., 1/2 ounce, U.S.P.

${ }^{*}$ Liquor Sodii Chloridi Physiologicus (Liq. Sod. Chlor. Physio.), U.S.P. (Normal Saline Solution). -0.85 per cent.

\section{UREA}

Urea, $\mathrm{CO}\left(\mathrm{NH}_{2}\right)_{2}$, is an effective diuretic, acting probably by simple osmosis. It is not toxic. However, Hewlett, I916 states that when taken in sufficient amount to raise the urea concentration in the blood above $160 \mathrm{mg}$. per $100 \mathrm{c.c}$. asthenic symptoms result.

The beneficial effects on perfusions were discussed on page $64^{2}$. It is excreted mainly by the kidneys; a small amount also by the sweat (0.2 I per cent., Kovesi). The 
urea content of the milk is not increased by urea administration (Steenbock, 1915). Urea is not utilized in nutrition (Henriquez and Anderson, I0 I4).

Urea occurs as colorless crystals of cooling, saline taste, freely soluble in water ( 1 : I); soluble in alcohol ( $1: 7$ ). The effective diuretic dose would be 20 to $40 \mathrm{Gm}$. per day:

\section{SACCHARUM LACTIS (MILK-SUGAR)}

This also produces osmotic effects. Nine to $I_{5} \mathrm{Gm}$. ( 2 to $\left.4 \overline{3}\right)$, in warm milk an hour before breakfast, causes a soft stool. Smaller doses (pure or as milk) are efficient for diuresis (the effective dose would be roo to $200 \mathrm{Gm}$. per day). Nilk-sugar is also used to facilitate the comminution of powders, on account of its hardness. It is further employed as sweetening agent, especially for powders; and in infant foods.

\section{POTASSIUM SALTS}

General Statement.-Potassium is widely distributed through the body and is essential to the vital functions. It acts as a depressant to practically all organs, and is mutually antagonistic with $\mathrm{Na}$.

With intravenous or hypodermic injections, the ion-actions manifest themselves by depression of the heart and other muscles, and of the central nervous system. These effects may also be secured from toxic doses by mouth; but not by ordinary doses, since the rapid excretion prevents the accumulation of excessive potassium in the tissues. The therapeutic use of potassium as cardiac and nervous depressant was a faulty deduction from the laboratory experiments. The oral administration of moderate quantities of potassium has practically only osmotic effects-diuresis, slight changes in metabolism, etc. The therapeutic actions of potassium salts are therefore attributable to their anions, and are practically equivalent to the corresponding sodium salts. The potassium salts perhaps produce somewhat more local irritation. They are contraindicated in renal insufficiency, since the deficient excretion could lead to the development of toxic potassium effects.

Income of Potassium.-The daily diet of man contains about 3 to ${ }_{4} \mathrm{Gm}$. of $\mathrm{K}_{2} \mathrm{O}$ and 5 to $8 \mathrm{Gm}$. of $\mathrm{Na}_{2} \mathrm{O}$; but there are great variations. A vegetable diet contains relatively more $\mathrm{K}$.

Distribution of Potassium.-This is in many respects converse to that of sodium. The $\mathrm{K}$ is generally more abundant within the cells; the $\mathrm{Na}$ in the extracellular juices, serum and lymph. The serum of all mammals contains about 0.025 per cent. of $\mathrm{K}_{2} \mathrm{O}$ as against 0.44 per cent. of $\mathrm{Na}_{2} \mathrm{O}$. The percentage in the blood corpuscles differs markedly in different species, being generally inverse to the percentage of $\mathrm{Na}$. The corpuscles of the horse, pig and rabbit contain 0.33 to 0.52 per cent. of $\mathrm{K}_{2} \mathrm{O}$ and no $\mathrm{Na}_{2} \mathrm{O}$; in man, the ratio of $\mathrm{K}_{2} \mathrm{O}: \mathrm{Na}_{2} \mathrm{O}$ is $0.4: 0.075$; in the beef, sheep and goat, it is $0.07: 0.23$; in dogs and cats, $0.3: 0.28$. In the K-rich animals, the dissolution of a fifth of the corpuscles would liberate a fatal quantity of potassium. Muscle fibers also contain $\mathrm{K}$, without $\mathrm{Na}$ (Fahr, I9I2). Macleod, r 907 , found that the juice expressed from the heart or skeletal muscle produces a typical potassium effect on the heart.

The distribution of $\mathrm{K}$ within the cells has been investigated by A. B. Macallum, 1907. It is absent from the nuclei and from the nerve cells; but is present in the cytoplasm of most vegetable and animal cells, including the muscles, the nerve fibers, the granula of the pancreatic cells, etc.

Excretion. - A considerable part of the potassium, $\mathrm{I}_{5}$ to 25 per cent., is reëxcreted in the feces. The urine contains generally $\mathrm{Na}: \mathrm{K}:: 2:$ I to $5: 3$. In starvation, the ratio is reversed to $I: 3$.

- The excretion of $K$ seems to be hastened by the addition of $\mathrm{Na}$ (Biernacki, I9II). A few experiments by Blumenfeldt, 1913, indicate that the excretion of administered potassium is delayed by aortic insufficiency, arteriosclerosis, and interstitial nephritis. 
Influence of Excretion on Effect.-The effect of potassium, as of other cathions, varies greatly according to the channel of introduction, because the excretory capacity for these ions is usually much greater than the rate of their absorption from the alimentary canal. Oral administration is therefore effective only if very large doses are given; if the mucous membranes are corroded, or if the excretion is checked, as by ligation of the kidneys.

The influence of excretion is well illustrated by magnesium: after excision of the kidneys, the toxicity is increased 50 per cent., and the action is more lasting and cumulative. On the other hand, some vicarious channel of excretion becomes established, so that the toxicity is less eighteen hours after operating than it is immediately (Meltzer and Lucas, I 907 ).

The toxicity of intravenous $\mathrm{KCl}$ is diminished by $\mathrm{NaCl}$ (Amberg and Helmholz, 1916).

A summary of the excretion of salts is given by Heffter, I 903 .

Systemic Actions. - The central nervous system is paralyzed in its whole extent. The reflexes suffer, then the medulla. Depression of the respiratory center leads to asphyxial convulsions. Absence or increase of $K$ are both depressant to the perfused respiratory center of the frog (Hooker, 1913). With intravenous injection, the most important effect is the cardiac paralysis.

Cardiac Actions. - The relations of $\mathrm{K}$ to the other ions were discussed on page 638 .

The heart is stimulated by small, fatigued by medium, and paralyzed by large, doses. This is seen even in the nerve-free heart of the chick's embryo, and is therefore muscular. In Langendorff perfusions of the excised mammalian heart, increase of $\mathrm{K}$ produces inhibition, followed by fibrillation. Paralysis occurs when the concentration in the blood reaches 0.08 per cent. (Hald, 1905). Howell and Duke, 1908, found that stimulation of the vagus causes an increased output of $\mathrm{K}$ in the perfused heart, especially the auricles. They attribute the vagus stoppage to the $\mathrm{K}$ which the stimulation of the nerve converts from a non-diffusible into a diffusible form.

In intact mammals, the effects are somewhat different for intravenous and arterial injections (Mathison, I9I I); by the vein, there is a primary fall of blood pressure, with a slight secondary rise. The fall is due to cardiac disturbance, decreased output, heartblock, slowed pulse, tonus changes, sudden systolic standstill, usually before the respiration stops. Slow continuous injections into dogs produce slowing and weakening of the heart by central vagus stimulation; for if the vagi are divided, the heart is quickened and strengthened, and often shows extrasystoles. The direct cardiac action of the small doses is therefore stimulant. Larger doses depress the heart muscle directly, even when the vagi are divided. Fatal doses produce ventricular and eventually auricular fibrillation (Hering, 1915). Arterial injection causes an immediate rise of blood pressure, by stimulation of the vasomotor center, and of the vessels directly (it occurs even when the central nervous system is destroyed). However, the vessels of frogs are dilated by perfusion with $\mathrm{K}$ (Hooker, I9II).

Skeletal Muscles. $-K$ weakens the muscles; diminishes the height of contraction; lengthens the latent period; and decreases the conductivity and excitability (Fahr, I9I0). In this way, potassium counteracts the effects of veratrin.

Meigs and Atwood, 1916, suggest that the changes occurring on the immersion of frog's muscle in 0.9 per cent. $\mathrm{KCl}$ may be explained by the muscle-membrane being more permeable to $\mathrm{KCl}$ than to the $\mathrm{NaCl}$ and $\mathrm{K}_{2} \mathrm{HPO}_{4}$ that are contained in the muscle. $\mathrm{KCl}$ and water would therefore diffuse into the muscle-cell.

Nerve.-Injection of $\mathrm{K}$ suspends the conductivity of nerve and potentiates the anesthetic effect of cocain. $\mathrm{Na}, \mathrm{NH}_{4}$ and $\mathrm{Mg}$ do not have this effect (Zorn, I9I3). Injury, and the local application of lipolytic anesthetics increase the concentration of $\mathrm{K}$ ions in medullated nerve fibers. This may play a part in the anesthetic effect (Menten, 1912).

Potassium Poisoning in Nephritis.-The administration of 5 to $10 \mathrm{Gm}$. of $\mathrm{KCl}$ (diluted) usually produces no symptoms, even when the permeability to $\mathrm{NaCl}$ is impaired, as in chronic nephritis. Occasionally, however, a nephritic patient may develop severe potassium poisoning, as in a case described by Smillie, 1915. The symptoms started after seven hours and reached their maximum in twenty-one hours. They consisted of cyanosis, weak pulse, and vomiting. Rabbits with their kidneys rendered less permeable by uranium nephritis also showed markedly increased susceptibility to potassium. However, with the ordinary potassium income, v. Limbeck, 1892, did not find it increased in the blood of uremic dogs.

Potassium in the Toxicity of Urine and in Uremia.-The toxic action which urine possesses when injected intravenously has been variously attributed to the leucomains, to toxins, etc., and at one time even to the urea. Various theories and methods of diagnosis were built upon the variations in this toxicity. It has, however, been shown that about 85 per cent. of the toxicity of the urine is due simply to its 
potassium salts. The symptoms are also precisely those of the latter. What causes the other I5 per cent. of the toxicity is at the present not known. Hartman, I9r5, claims to have isolated from normal urine a cyclic ketone, "Urinod," which is quite toxic, and produces headache in small doses-perhaps through its disagrecable odor.

If the undiluted urine is injected, its osmotic pressure also contributes to the toxicity. The acidity, volatile products, pigments, and urea have no effect. It is quite probable that the urine contains true toxins and other toxic products in disease, but no method has so far been devised for estimating the importance of these.

The conditions are still more complicated in uremia: it is very probable that the retention of potassium and ammonium salts and the increased osmotic pressure of the blood contribute to the effects, but there seem to be other factors which are not understood. Foster, r 1 I 5, has isolated some unidentified crystallizable toxic substances from the blood of uremic patients, which he failed to find in normal blood. Furtherinvestigation is needed.

\section{PREPARATION}

Potassii Chloridum, $\mathrm{KCl}$.-Readily sol. in water. I to $2 \mathrm{Gm}$. (i 5 to $30 \mathrm{gr}$.). Not to be confused with the toxic chlorate!

\section{LITHIUM SALTS}

General Statement.-Lithium urate is relatively soluble. By a misinterpretation of this chemical fact, the lithium salts were introduced as solvents for uric acid; and hence as remedies against gout, rheumatic conditions, etc. Since the soluble urate can not be formed in the presence of sodium or potassium ions, it can not exist in the body or in the urine. The uses of lithium salts therefore have no rational foundation, and they may be considered inefficient, and in larger doses somewhat dangerous.

The action of this cathion resembles potassium quite closely, but it is somewhat less toxic to the heart, and causes a vagus stimulation. These effects are seen only on intravenous injection. A most important difference from potassium consists in a specific gastroenteritis which is produced even when the salts are given subcutaneously, or if the administration of small doses is continued for some time ( $1 \mathrm{Gm}$. of the carbonate in man). The action is local, the lithium being largely excreted by this channel. It is also excreted by the kidneys, but does not cause nephritis (Krumhoff, r884; Good, I902). It is also excreted in the milk (Koldewijn, I910).

Acute poisoning from larger doses in man (four doses of $2 \mathrm{Gm}$. of $\mathrm{LiCl}$ ) is described by Cleaveland, I913. There were no gastro-intestinal symptoms; but marked muscular and general prostration; vertigo; and eye and ear symptoms resembling cinchonism.

Action on Urates. - Lipowitz, I 84I, found that lithium carbonate is a better solvent for uric acid (about four times) than sodium carbonate. He suggested its therapeutic use, and this was introduced by Ure, 1844, and Binswanger, 1847. Garrod, 186r, found that gouty tophi were dissolved in lithium solution. However, the solvent action occurs only when strong solutions of lithium carbonate act on uric acid. In the presence of $\mathrm{NH}_{4}, \mathrm{Na}$ or $\mathrm{K}$, the less soluble urates of these cathions are formed. The solution can therefore not occur under the conditions of the body or urine (see Index, Urate Solvents). Lithium salts have diuretic actions (Husemann), but no greater than the Na salts. The popular "Lithia waters" have been shown to be practically devoid of lithium. The best contain only 0.01 to 0.02 per cent. "Buffalo Lithia Water" contains only a fifth as much lithium as Potomac River water; 200,000. gallons would be needed to supply the ordinary therapeutic dose of lithium.

\section{PREPARATIONS - LITHIUM}

Lithii Carbonas (Lith. Carb.), U.S.P., B.P., $\mathrm{Li}_{2} \mathrm{CO}_{3}$ - - Light, white powder; odorless; alkaline taste. Sol. in water $(\mathrm{r}: 78)$; almost insol. in alc. Dose, $0.5 \mathrm{Gm}$., $8 \mathrm{gr}$., U.S.P.; 0.12 to $0.3 \mathrm{Gm}$., 2 to 5 gr., B.P.

Lithii Citras (Lith. Cit.), U.S.P., B.P., $\mathrm{Li}_{3} \mathrm{C}_{6} \mathrm{H}_{5} \mathrm{O}_{7}+{ }_{4} \mathrm{H}_{2} \mathrm{O}$. - A white powder, or in the granular form; odorless; cooling, faintly alkaline taste. Deliquescent. Freely sol. in water ( $\mathrm{r}: \mathrm{r} .4$ ); very slightly sol. in alc. Dose, $0.5 \mathrm{Gm}$., $8 \mathrm{gr}$., U.S.P.; 0.3 to $0.6 \mathrm{Gm}$., 5 to ro gr., B.P.

Lith. Cit. Eff., B.P.- 5 per cent. Dose, 4 to $8 \mathrm{Gm}$., I to 2 drams, B.P. 


\section{RUBIDIUM AND CASIUM}

These also resemble potassium. Rubidium iodid and bromid have been introduced into therapeutics, with the claim that they produce less iodism and bromism, although they are quite as soluble, and even more diffusible than the corresponding $\mathrm{K}$ salts. 'The doses are the same as those of the potassium salts.

\section{AMMONIUM COMPOUNDS}

General Statement.-The ammonium ion produces rather strong stimulation and subsequent paralysis of the central nervous system, especially the medulla. The systemic effects are seen only with intravenous administration, because the ammonium ion is rapidly converted into urea and excreted. On excised muscles, it has also a weak curare action.

In intact animals, the effects of the ammonium compounds are strictly local. They diffuse rather rapidly, and thus exert considerable salt action, especially irritation. These local actions increase the flow of mucus, which is also rendered more fluid. Ammonium chlorid is therefore used as an expectorant in the first (dry) stage of acute bronchitis. It is also somewhat diaphoretic (nauseant action?). Large doses are emetic. Ammonium acetate has been employed as a weak diaphoretic and diuretic in fevers, but is probably useless.

Ammonium hydroxid and carbonate act also as alkalies; and by the liberation of the volatile and diffusible $\mathrm{NH}_{3}$, their action extends deeply. Their vapor is used to produce reflex stimulation of the medullary centers (trigeminal reflex), in collapse, fainting, etc. When too concentrated, they produce laryngitis, bronchitis and pneumonia.

Applied to the skin, ammonia produces rubefaction, and if concentrated and confined, vesication, usually without corrosion. It is used in combination with oil (ammonia liniment) for counterirritation in rheumatism, etc. Ammonia is also applied to insect bites.

Expectorant Action. - The increased secretion of mucus and saliva is mainly a reflex from the local irritant action, although there may be some direct central stimulation (V. E. Henderson, I9IO). The liquefaction of the mucus may be due partly to the ammonium ion, partly to the excretion of the alkaline ammonium carbonate by the saliva. This excretion, however, must be quite small, since most of the ammonium is destroyed in the body. Ammonium chlorid is alșo said to have a primary astringent effect on the mucous membrane.

Administration.-Ammonium chlorid is used in acute bronchitis in the dose of $0.3 \mathrm{Gm} ., 5$ gr., repeated as needed; either as troches (0.I Gm., $\mathbf{1} 1 / 2 \mathrm{gr}$.), or in solution. Its disagreeable taste is less noticeable in acid mixtures (syrup of citric acid). Ammonium carbonate (same dose) is more solvent for mucus, but rather nauseant.

When it is desired to extend the direct action to the trachea and bronchi, the ammonium may be administered in the form of inhalation of ammonium chlorid, produced in a finely divided state by bringing together the vapors of ammonia and hydrochloric acid.

Bronchial Secretion.-Ammonium salts, like other emetics, increase the bronchial mucus, even when they are placed in the intestines; but by this method, the dose must be much larger than therapeutic. Ammonium salts produce moderate dilation of excised bronchial muscle (Trendelenburg, 1912).

Absorption and Excretion by the Lungs.-Magnus, 1902, and Brucini, 1907, found that ammonia is neither absorbed nor excreted by the lungs; but Hoeber, 1912, and 
McGuigan, I913, claim that the inhalation increases the percentage of ammonium in the blood. However, this insignificant absorption occurs from the trachea and bronchi, and also from injured alveoli (Magnus, Sorgdrager and Leuwen, I9 I 4).

IV. Coleman, I9I6, states that an ammonium taste can be discerned in the sputum (not in the saliva) twelve hours after discontinuing small doses ( 1,2 grain every two hours).

Disintoxication of Ammonium.-Ammonium is partly excreted as such by the urine; but the greater proportion is rapidly converted into urea, according to the formula:<smiles>NC(=O)NC(N)=O</smiles>

Two molecules of water are split off from the ammonium carbonate and urea remains. This liberates the acid ion with which the $\mathrm{NH}_{4}$ was combined: this will seize upon the free fixed alkali of the body, and lead to a diminished alkali stock of the tissues. In this way any excess of ammonium introduced into the body is very soon eliminated, or at least ceases to act as ammonium.

The transformation of ammonium into urea is much more complete when the ammonium is given combined with organic acids, than in the case of its inorganic salts, for with the latter, a part of the ammonium is needed to help in the neutralization of the anions (Underhill; Caldwell and Clotworthy, Igr6).

Ingested ammonium disappears very rapidly from the circulation, even if the kidneys are ligated (Jacobson, I9r4). The conversion of ammonium carbonate into urea is practically complete in all mammals. Ammonium chlorid is destroyed more efficiently by herbivorous animals than by carnivoræ or man.

Ammonium in Metabolism.-Arterial blood contains normally about $1.5 \mathrm{mg}$. of $\mathrm{NH}_{4}$ per roo c.c. Portal blood contains from 3.5 to $8.5 \mathrm{mg}$. (Carlson and Jacobson, rgro). Folin and Denis, 1912, believe that the excess in the portal blood comes mainly from bacterial changes in the large intestines. The ammonium is probably a normal forerunner of urea. This is formed from ammonium carbamate by the subtraction of $\mathrm{H}_{2} \mathrm{O}$ :

$$
\mathrm{CO}\left\langle\sum_{\mathrm{O}\left(\mathrm{NH}_{4}\right)}^{\mathrm{NH}_{2}}-\mathrm{H}_{2} \mathrm{O}=\mathrm{CO}<\mathrm{NH}_{2}\right.
$$

Place of Urea Formation. - The transformation of ammonium into urea occurs in perfused liver ( $v$. Schroeder, r892; Fiske and Karsner, 1913); the urea may also be considerably higher in the hepatic than in the portal blood (Van Slyke, Cullen and McLean, 1915). The liver must therefore play a prominent part in urea formation. However, probably all organs participate, especially in its formation from amino-acids (Folin, r9r4). For instance, the amount of urea in the tissues or blood, after the intravenous injection of amino-acids, is smaller in animals with the liver and intestines excluded from the circulation (Fiske and Sumner, r914). Jansen, I915, also presents evidence that the liver has a considerable role in the formation of urea from amino-acids.

Function of Ammonium in Neutrality Regulation.-The transforma tion of ammonium into urea is utilized for the control of the reaction of the body: when there is an excessive formation of acid (acidosis), the transformation is less complete, the acid being neutralized by the ammonium, and excreted in the urine. If alkalies are administered, the ammonium is displaced from its salts, and converted more completely, so that none may appear in the urine (Janney, I9 12 ). The ammonium-urea relation of the urine is thus an index of the acid production or alkali income.

Hepatic Lesions.-Rovighi, 1889, and Joannovics, 1903, describe dilation of the hepatic capillaries and protoplasmic changes after the oral or hypodermic administration of ammonium salts. With chronic poisoning, there is also connective tissue proliferation; suggesting the possibility that ammonium may be concerned in the etiology of hepatic cirrhosis. 
Utilization of Ammonium Nitrogen.-Girafe and Schupfer, rg 2, claimed that ammonium mily replace the protein in food. 'This was confirmed by Knoop, 1913; Grafe, 1014 and others. However, it does not prove that ammonium is converted into tissue nitrogen. It is more probable that it is utilized by the intestinal bacteria, and that these are then digested and their nitrogen absorbed. Accordingly, Henriquez and Anderson, 1014. found no retention of nitrogen after the intravenous injection of ammonium or urea. 'Taylor and Ringer, 1913 , did observe considerable $\mathrm{N}$ retention after oral administration of ammonium carbonate. However, the associated changes in metabolism indicate that the retention is due to a toxic action of the ammonium, rather than to its utilization (Underhill and Goldschmidt, I913).

Autointoxication by Ammonia.- The symptoms of uremia, tetany and other convulsive diseases resemble somewhat the picture of ammonium poisoning. It has therefore been suggested that these conditions are due to failure of the liver to transform the ammonium. This appears unfounded. There is no evidence that the ability to form urea is impaired, except perhaps in extreme cases of liver degeneration. Fiske and Karsner, I 14 , found the ammonia conversion by perfused livers unimpaired after acute hepatic injury by chloroform, phosphorus, etc. Even if the ammonium were not destroyed, the excretory mechanism of the kidneys could be expected to remove the excess.

In fact, the ammonium content of the blood has not been found increased in uremia or in acute yellow atrophy of the liver. An increase of 50 per cent. above the normal has been reported after complete excision of the thyroids and parathyroids (MacCallum and Voegtlin, I909; Berkeley and Beebe, 1909); and the ammonium production is increased (Underhill and Saiki, I908), or its destruction diminished (Carlson and Jacobson, 19I0). A similar increase was reported in clinical tetany (Musser and Goodman, I 909). There is also a close resemblance between the symptoms of tetany; those of animals with Eck's fistula on a meat diet (Pawlow, 1892); and ammonium poisoning. Even the concentration of $\mathrm{NH}_{4}$ in the blood required to produce true ammonium tetany (0.008 per cent., Magnus, et a!, I9 I 4) seemed very similar (Jacobson, I9Io). However, the later work of Carlson and Jacobson, I9 I , with improved methods, showed that the increase of ammonium is not constant and falls within the variations of normal animals, so that even these conditions can not yet be classed as ammonium intoxications.

Intravenous Injection of Ammonium Chlorid.-The effects vary with the dose and the rate of injection. Marfori, $\mathrm{I} 893$, described immediate tremors and twitchings, advancing to tetany, violent convulsions and opisthotonus; irregular respiration; salivation and emesis; somnolence and lassitude. 'The respiration shows momentary convulsive inspiratory standstill, followed by increased rate. This stimulation occurs even with small doses. Blood-pressure tracings (Fig. 28) show a temporary fall; then a slow but more lasting rise; with slowed rate and "vagus pulse." The rate may be quickened during the convulsions. These effects indicate stimulation of the respiratory, vasomotor, and vagus centers. If the injection is not promptly fatal the functions recover rapidly (Meneguzzi, I9 I 2 ).

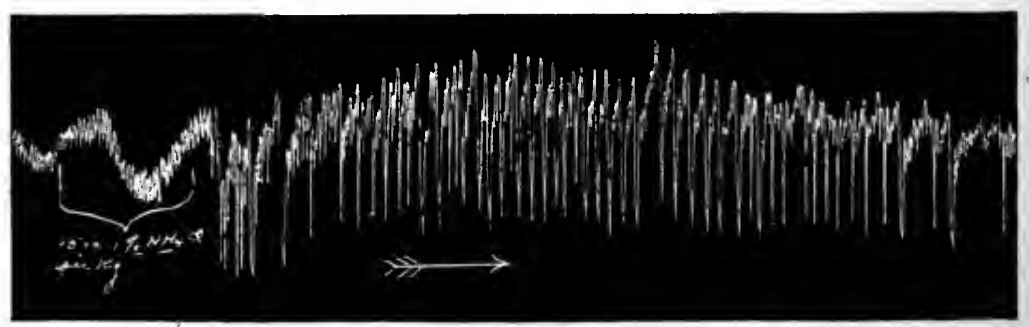

FIG. 28.-Ammonium chlorid, intravenous. Carotid pressure tracing, dog.

Curare Action.-This is much weaker than with the organic ammonium bases (see Muscarin).

Reflexes from the Inhalation of Ammonia Vapors.-Through irritation of the sensory trigeminal endings, these produce momentary standstill, then increase of respiration; stimulation of the vasomotor center (rise of blood pressure); and vagus slowing of the pulse (in rabbits, vagus standstill). 
Use for Medullary Stimulation.-Ammonia is one of the most effective means for stimulating the medulla reflexly. It may be used as the aromatic spirit of ammonia-I 5 to 30 drops in a tumbler of water; or as ammonium carbonate-smelling salts; in headache, fainting, collapse, excessive anesthesia, etc. Care must be used so that the unconscious patient is not made to inhale too concentrated a vapor.

Toxicology.--The neutral ammonium salts may produce gastritis. This would be treated by dilution and evacuation. Ammonia and the carbonate produce more violent irritation, and also inflammation of the respiratory passages. The mortality of ammonia poisoning is therefore high. If the patient recovers from the acute effects, pneumonia may set in; or the hoarseness or aphonia may last for months. The treatment consists in artificial respiration, weak acids and oils.

\section{PREPARATIONS-AMMONIUM}

*Liq. Ammon. Acet., U.S.P., B.P. (Spirit of Mindererus). - 7 per cent. of $\mathrm{NH}_{4} \mathrm{C}_{2} \mathrm{H}_{3} \mathrm{O}_{2}$ with small amounts of acetic and carbonic acids. Should be slightly acid. Must be recently prepared. Dose, ${ }_{5}$ c.c., 4 drams, U.S.P.; 8 to 24 c.c., 2 to 6 drams, B.P.

${ }^{*}$ A mmonii Carbonas (Ammon. Carb.), U.S.P., B.P. (Sal Volatile, Hartshorn, Baker's Ammonia). - Consists of varying proportions of a mixture of acid ammonium carbonate $\left(\mathrm{NH}_{4} \mathrm{HCO}_{3}\right)$ and ammonium carbamate $\left(\mathrm{NH}_{4} \mathrm{NH}_{2} \mathrm{CO}_{2}\right)$. For medicinal purposes, only the translucent portions are used. On exposure to air, it becomes a powder, losing $\mathrm{NH}_{3}$ and $\mathrm{CO}_{2}$; and changing to ammonium bicarbonate, $\mathrm{NH}_{4} \mathrm{HCO}_{3}$. White, hard, translucent, striated masses; strong odor of ammonia, without empyreuma; sharp, saline taste. It is slowly but freely sol. in water $(\mathrm{I}: 4)$, the carbamate changing to normal ammonium carbonate. It is decomposed by hot water. Alcohol dissolves the carbamate and leaves the carbonates. Incompatible with acids and the salts of most metals and alkaloids. Dose, $0.3 \mathrm{Gm}$., 5 gr., U.SP.; 0.2 to $0.6 \mathrm{Gm} ., 3$ to Io gr., B.P.; diluted.

*'Spiritus Ammonic Aromaticus (Sp. Ammon. Arom.), U.S.P., B.P. (Aromatic Ammonia).--A solution of ammonium carbonate ( 3.4 per cent.), anmonia water ( 9 per cent.), and aromatic oils (lemon, lavender and nutmeg) in alcohol of about 60 per cent. (U.S.P.). The composition of the B.P. is similar. A nearly colorless liquid when freshly prepared, but gradually acquiring a yellowish color. Dose, 2 c.c., 30 minims, U.S.P.; 1.2 to 2.5 c.c., 20 to 40 minims, repeated; single, 4 to 6 c.c., 60 to 90 minims, B.P.; largely diluted.

${ }^{*}$ A mmon. Chlor., U.S.P., B.P. (Sal Ammoniac; Salmiac), $\mathrm{NH}_{4} \mathrm{Cl}$.-White crystalline powder, without odor, of cooling saline taste. Freely sol, in water $(\mathrm{r}: 2.6)$; sol. in alc. ( $1: 100)$; freely sol. in glyc. $(\mathrm{r}: 8)$. Neutral or slightly acid to litmus. Incompatible with alkaline hydroxids or carbonates (liberation of ammonia); precipitates with $\mathrm{Ag}$ and $\mathrm{Pb}$ salts. Dose, 0.3 Gm., 5 gr., U.S.P. ( 0.3 to $\mathrm{r} .2$ Gm., 5 to 20 gr., B.P.

Troch. Ammon. Chlor., U.S.P.-O.I Gm., flavored with Licorice and Tolu.

*Aqua Ammonice (Aq. Ammon.), U.S.P.; Liq. Ammon., B.P.; Ammonia Water.Contains about ro per cent. (by weight) of ammonia $\left(\mathrm{NH}_{3}\right)$. It loses strength on standing. Colorless liquid of pungent ammonia odor and caustic soapy taste. Freely miscible with water and alcohol. Incompatible with most metals and alkaloids. Dose, I c.c., I 6 minims, U.S.P.; largely diluted.

Aq. Ammon. Fortior, U.S.P.; contains about 28 per cent. of ammonia; Liq. Ammon. Fortis, B.P., about 32.5 per cent.

${ }^{*}$ Linimentum Ammonice (Lin. Ammon.), U.S.P., B.P.-A mixture of I part ammonia water and 3 parts of fatty oil (sesame, U.S.P.; almond I, olive 2, B.P.).

Lin. Camph. Ammon., B.P. (Compound Liniment of Camphor). - I 2.5 per cent. of Camphor; 25 per cent. of Stronger Ammonia in alcoholic solution of Lavender.

\section{CLASSIFICATION OF EXPECTORANTS}

These are remedies that modify expectoration and are therefore employed in the treatment of coughs. The successive stages of bronchitis present different indications, which are met by the various classes of expectorants. 
The act of conghing is a coördinated reflex involving the respiratory center, and resulting in the sudden and violent expulsion of air from the lungs. It has a physiologic function in the remoral of irritant substances or of accumulation of mucus from the respiratory passages. Like other reflexes, however, it tends to persist after its cause has ceased to be active; or it may become excessive. In either case it will require treatment. It must be borne in mind that cough is merely a symptom, and while it may be treated directly, as such, no treatment can be of permanent benefit unless it is also directed against the original cause, if this is still in operation. This cause lies most commonly in the respiratory passages, but there appear to be other conditions which may give rise to cough, or which at least may help in its production. The changes produced in the general circulation by drugs like digitalis, strychnin, or the nitrites are frequently beneficial. When signs of disease are present in other organs (dyspepsia, etc.), these should receive direct treatment. General measures, such as laxatives, diaphoretics, antipyretics, analgesics, etc., must be used as the indications arise. $R e$ moval to a more suitable climate is always advisable, and sometimes necessary in chronic bronchitis. A dry climate is chosen if the secretion is profuse, and vice versa.

\section{SEDATIVE EXPECTORANTS}

These are used, especially in the early dry stage of bronchitis, to soothe the acute inflammation, mainly by stimulating the secretion of the protective mucus. They comprise the nauseant expectorants, especially ipecac and antimony; more rarely apomorphin, pilocarpin, lobelia, etc. (Senega, although a nauseant, is generally classed as a stimulant expectorant.) With the sedative expectorants may also be classed the demulcent expectorants, acacia, syrup, glycerin, glycyrrhiza, demulcent herbs (species pectorales, breast-tea); the saline expectorants, ammonium chlorid and potassium iodid; and the alkaline expectorants, ammonium carbonate, sodium bicarbonate or borate.

These saline and alkaline expectorants are also used in the other stages of bronchitis, whenever the mucus requires thinning. The'demulcents are useful throughout.

\section{IRRITANT (STIMULANT) EXPECTORANTS}

These are employed in subacute and chronic bronchitis, to irritate the mucous membrane in such a way as to stimulate repair. They tend to diminish the excessive secretion usually present in these later stages. They are represented especially by the aromatic expectorants. The volatile oils, balsams and phenols, especially terpene hydrate, terebene, cubeba, santal, balsam of Peru, tolu, and styrax, creosote and guaiacol, tar, benzoates. The irritant senega and squill are also classed as irritant expectorants. Astringents are used when the inflammation involves the larynx or pharynx.

\section{ANODYNE EXPECTORANTS}

These are represented mainly by the opiates, paregoric, morphin, heroin, and especially codein, all in small doses. These are used to depress the excessive cough reflex, especially if the secretion is scanty, and should not be employed when the mucus is excessive. They appear to diminish the secretion, presumably because the delay in its expulsion permits drying. Hydrocyanic acid was used in coughs to stimulate the respiratory center and perhaps for a local anodyne action. It is ineffective and obsolete. Small doses of chloroform are also employed. 


\section{SIMPLIFICATION OF EXPECTORANTS}

The great number of expectorants is not generally needed. The following suffice to meet the conditions in the most effective manner:

Against dry congestion: Syrup Ipecac, I c.c.; or Tartar Emetic, 5 mg.

Against thick secretion: Ammon. Chlorid, $0.3 \mathrm{Gm}$.; KI, $0.25 \mathrm{Gm}$.

Against excessive thin secretion: Terpene Hydrate, $0.15 \mathrm{Gm}$. in capsules.

Against irritation: Codein, $5 \mathrm{mg}$.; Alkaline gargles.

\section{EXPECTORANT VEHICLES}

The syrups of tolu, of tar, and of wild cherry contain expectorant constituents, but their quantity is so small that they act merely as flavoring vehicles. The syrups of glycyrrhiza and of acacia are also used.

\section{COUGH MIXTURES}

These polypharmacal preparations usually contain morphin and therefore give relief; but since they can not meet the special indications of the different stages, they should be avoided. The "Brown Mixture," whose activity depends on antimony" and opium, is one of the best. This, and a few others, may be mentioned as illustrations.

\section{PREPARATIONS -COUGH MIXTURES}

( ${ }^{*}$ Mist. Glycyrr. Co.-See Index).

(*Syr. Scil. Co. - See Index).

Species Pectorales, N.F.-Althæa, Coltsfoot, Glycyrrhiza, Anise, Mullein, and Orris. Used as infusion $\mathrm{I}$ : ro ad libitum.

Syrupus Pini Strobi Compositus, Compound Syrup of White Pine, N.F.-White Pine Bark, Wild Cherry, Aralia, Gilead, Sanguinaria, Sassafras, Chloroform. Dose, 4 c.c., I dram.

\section{CALCIUM SALTS}

General Statement.-The insoluble calcium phosphate and carbonate serve to give hardness to the bones and teeth. Their deficiency is one of the important phenomena of rickets and osteomalacia. Pathologic calcifications of arteries and other tissues are phenomena of degeneration; their mechanism is not understood.

The soluble $\mathrm{Ca}$ ions have equally important functions. They are present in all tissues, and appear to be essential to the proper permeability of the plasma membrane. Excess of $\mathrm{Ca}$ is depressant to most nervous and muscular functions, and is antagonistic to $\mathrm{Mg}$ and $\mathrm{Na}$. These systemic actions are produced only on excised tissue or with intravenous injection, since the absorption of $\mathrm{Ca}$ is quite slow. It has been tried, with only partial success, in various spasmodic conditions.

Acute deficiency of $\mathrm{Ca}$ produces stimulation of these structures, most conspicuously of the central nervous system. This is seen in poisoning by oxalates, which precipitate $\mathrm{Ca}$. It may also be produced by the intravenous injection of citrates or tartrates which decrease the ionization of Ca.

Calcium is also necessary for the coagulation of blood and for the typical clotting of milk. Calcium salts have therefore been administered in hemorrhagic conditions; but since these are not generally due to deficiency of $\mathrm{Ca}$, it is questionable whether the additional $\mathrm{Ca}$ has any effect. 
Calcium seems to decrease the permeability of blood and lymph vessels, and thereby to lessen exudation. It has been used with some success igainst serum rashes, etc. Calcium carbonate (chalk) is employed in diarrheas as antacid and protective; but it may also decrease the exudation directly. Lime-water (solution of calcium hydroxid) is added to milk to increase its alkalinity. Calcium oxid, quicklime, is a slowly acting caustic, and is used, as also the hydroxid or slaked lime, as disinfectant. Calcium sulphate, plaster of Paris, is important for its mechanical properties.

The calcium salts are also used for the actions of their anions (bromid, etc.). They are less rapidly absorbed, and perhaps less irritant than the corresponding $\mathrm{Na}$ salts.

Distribution of $\mathrm{Ca}$ in Cells.-Calcium is present in practically all cells and tissues. Only a few of the lower fungi are able to live without it. O. Loew, I899, claimed that it occurred only in the nuclei and not in the cytoplasm of the cells. In support of this, Hoerhammer, I9I2, found it absent from the non-nucleated red blood cells (beef), but present in the nucleated corpuscles of chickens. However, Macallum has adduced direct microchemic evidence that it is not in the nuclei proper, but in the nuclear membrane and cytoplasm.

Calcium Income.- The daily requirement for man has been estimated as I.5 to 3 $\mathrm{Gm} . \mathrm{CaO} ; 0.7$ to $1.5 \mathrm{Gm}$. is probably the minimum. Ordinary diets contain 0.3 to 4.2 $\mathrm{Gm}$. (according to Sherman and co-workers, Igro, American diets yield only 0.08 to I.69, mean $0.85,(\mathrm{rm}$.). It is therefore conceivable that the income may sometimes be deficient, $f . i$., in pregnancy. It is easily increased by drinking milk. Only a part of the $\mathrm{Ca}$ is absorbed; Forster, I 885 , estimates the absorption as about 60 per cent. It is stated that its utilization is better from meats than from vegetables.

Calcium Excretion. - This is automatically adjusted to the income; but the minimum quantity required to maintain equilibrium varies with the diet (Kochmann, I910). About 5 to ro per cent. ( 0.15 to $0.5 \mathrm{Gm}$. per day) are excreted by the urine, mainly as phosphate; the remainder leaves by the feces. It is believed (E. Voit, I880) that a part of the fecal $\mathrm{Ca}$ is absorbed by the small intestines and re-excreted by the large. The administration of soluble $\mathrm{Mg}$ salts increases the urinary excretion of $\mathrm{Ca}$, but not if phosphates are present in the alimentary canal (Hart and Steenbock, I9I2). With hypodermic injection of calcium, a large part is excreted promptly by the urine, a small proportion being temporarily retained. The intestines do not always participate in the excretion. The output of $\mathrm{Mg}$ is also increased (Mendel and Benedict, I909).

Calcium Retention and Deficiency.--The administration of Ca (chlorid or lactate, several grams per day) increases the Ca stock of the tissues and blood, even in well-fed adults (Voorhoeve, I9I I and I9I3). An exclusive milk diet is said to reduce the Ca of the blood to one-half (Hirschler and Terray, r905).

Calcium Administration on Urine.-Calcium probably diminishes the permeability of the kidneys, and therefore diuresis. The effect is not great with ordinary doses, and probably varies with conditions, e.g., the ratio of $\mathrm{Ca}$ to other ions. The acidity of the urine is diminished since a part of the $\mathrm{PO}_{4}$ is deflected to the intestines. This has been suggested as possibly beneficial in gout ( $v$. Noorden, I896), but the results are questionable.

The effect of $\mathrm{Ca}$ on diuresis is discussed by Porges and Pribram, I9०8. Perfusion of the excised kidneys with $\mathrm{Ca}$ decreases the vein and ureter flow (Sollmann). The excretion of $\mathrm{Cl}$ is but little affected by $\mathrm{Ca}$ administration. Jacoby and Eisner, 1913, claim some decrease by $\mathrm{Ca}$ in human nephritis; also decrease of water, $\mathrm{Cl}, \mathrm{KI}$ and $\mathrm{N}$ excretion. They state that it lessens greatly the excretion of sugar, acetone and $\mathrm{N}$ in phlorhizin dogs. It is said to prevent the uric acid increase after atophan administration (Abl, I913). It prevents the glycosuria and fever of $\mathrm{NaCl}$ injections; but large doses of $\mathrm{CaCl}_{2}$ may themselves produce glycosuria (Underhill and Kleiner, rgo8). Wright and Ross, I905, claimed that $\mathrm{Ca}$ cures orthostatic, but not renal albuminuria. This would need confirmation. Dünner, 1916, states that intravenous injection of calcium into rabbits converts a subacute uranium nephritis into the acute form. Calcium phosphate has been recommended in diabetes mellitus, but has no effect even in the mildest cases (Bruning, 1898 ). 
Calcium in Bone Diseases.- Rachitis and osteomalacia show deficient $\mathrm{Ca}$ content of the bone. Somewhat similar, but not strictly analogous changes, are produced by deficient $\mathrm{Ca}$ income in young animals. This suggested the treatment of these conditions by the administration of lime salts, particularly calcium phosphate. The results were disappointing; doubtless because the deficiency is not in the income, but in the assimilation of the $\mathrm{Ca}$ by the bone-forming tissues.

Calcium-poor Diet.- - If young animals are fed on such a diet, their bone formation is defective. The bones contain about the normal percentage of lime, but very little bone is laid down, and this is thin, pliable, deformed and fragile (E. Voit, r 889 ). It contains more water, $\mathrm{Na}$ and $\mathrm{K}$, while the $\mathrm{Mg}$ is not materially increased. However, the percentages vary in different parts of the skeleton. The animals are undersized, with poor appetite and defective nutrition (Weiser, I914). In rabbits, the Ca content of the bones is increased by green food, diminished by exclusive oat diet (Luithlen, I9I 2). The administration of sodium oxalate for a week diminishes the $\mathrm{Ca}$ content of the soft tissues before it affects the bones (Sarvonat and Roubier, I9I I). If this diet is rich in magnesium, the bones may accumulate double the normal IIg content, but without improving the skeletal development.

Rachitis.-In this disease, the bone is even excessive, but is very poor in ash, as low as one-half the normal, especially poor in calcium phosphate. The cause of this condition is still in controversy. It can not be due to deficiency of $\mathrm{Ca}$ in the food, for it occurs of ten in bottle-fed infants, who receive plenty of Ca (Stoeltzner, I 899). Zweifel, I 900 , assumed defective absorption of $\mathrm{Ca}$, but without good evidence. In fact, the absorption and excretion of Ca seem about normal (Ruedel, 1893 ). One must therefore assume defective assimilation by the bones. The bone changes are probably a mere incidental phenomenon of the constitutional disturbance. Pfaundler, 1904, suggests a change in the absorption of $\mathrm{Ca}$ salts by the bone-matrix; but this is hypothetical. Sabbatani and Salvioli, I9I2, investigated the precipitation of calcium carbonate in the presence of colloids.

The cau se of rachitis being deficient assimilation and not deficient income of $\mathrm{Ca}$, very little could be expected from the administration of $\mathrm{Ca}$ salts. This is also the verdict of clinical experience (M. Klotz, I915).

Osteomalacia.- In this disease, the bone contains but 20 to 40 per cent., instead of the normal 56 to 60 per cent. of inorganic salts, their place being taken by new formed osteoid tissue. The condition is also not ascribable to deficient $\mathrm{Ca}$ income, but to excessive destruction of bone tissue. Limbeck, 1894 , found the excretion of $\mathrm{Ca}$, especially by the intestines, to exceed the income. Neumann, 1896 , also found the excretion high, especially in the early stages, when the $\mathrm{Ca}$ of the bones is still high. The later stages may show retention.

Moraczewski, 1900 , claims considerable loss of $\mathrm{Ca}$ in pernicious anemia.

Diabetes. - Kahn and Kahn, 1915 and 1916, find that diabetic patients have $a$ negative calcium balance. They claim that the intravenous injection of calcium, in diabetic patients, produces marked decrease of glucose excretion, gradual decline of the glycemia, diminished urine output, and relief of symptoms; and that it appears to prevent the development of acidosis. They inject cautiously an $\mathrm{m} / 8$ solution of $\mathrm{CaCl}_{2}$, in normal saline, from $I_{5}$ c.c. upward.

Calcium Therapy in Phthisis. - This was recommended by Fourcroy, I783. It has been tried repeatedly, on the assumption that phthisis is accompanied by excessive excretion of calcium. This has been aftirmed by some competent observers, but does not appear to be an invariable phenomenon (Kahn, I9I4). The clinical reports are too discordant to justify faith in this treatment.

Reproduction.--Emmerich and Loew, 1915 , observed that administration of calcium to female mice, guinea pigs and rabbits was followed by increased number of pregnancies and higher average of the feti.

Degenerative Calcification. - This is a common pathologic process, especially in connective tissue. Its mechanism is not understood. Klotz, 1905, believed that it starts with fatty degeneration, the fats and proteins forming soaps, and these passing into $\mathrm{Ca}$ soaps, and finally into inorganic Ca compounds. Klotz and Bothwell, 1915 , describe fatty changes and deposition of calcium salts, especially in the capillary endothelium of the lungs of rabbits injected intravenously with olive oil or cholesterin. However, this theory seems disproved by the experiments of Wells, 1906, and especially of Baldauf, 1907. MacCordick, 1913, finds the rigidity of calcified arteries different during life than after death, and therefore suggests that the $\mathrm{Ca}$ is in solution during life, and deposits in the plaques after death. 
Intravenous Injection of Calcium Salts.-This lowers the blood pressure; paralyzes the heart and respiration; arrests diuresis and peristalsis, etc. Hypodermic injections have similar, but nuch weaker and slower actions. Animals die of central paralysis a few days after the hypodermic injection of 0.3 to $0.4 \mathrm{Gm} . \mathrm{CaCl}_{2}$ per kilogram. Oral administration does not produce these systemic effects.

Respiration of Mammals.-Schlick, I9II, described apparent stimulation in anesthetized animals. Haffner, I9I3, finds that this is indirect, due to failure of circulation from cardiac depression. The direct action is respiratory depression, which may be the cause of death.

Perfused Respiratory Center of Frogs.-This was depressed by decrease of $\mathrm{Ca}$, stimulated by absence or excess of $\mathrm{Ca}$ (Hooker, I9r3).

Circulation.- Small doses, intravenously, sometimes increase the blood pressure and heart rate slightly, by brief depression of the vagi; larger doses paralyze the vagi, but diminish the heart rate and blood pressure, by a mixed effect on the cardiac muscles and nerves (Cazzola, I913). The small doses may considerably increase the output of the heart without changing the rate, i.e., by increasing the amplitude (Rothberger and Winterberg, I912).

Calcium on Heart Muscle.-On the excised heart, $\mathrm{Ca}$ has a restraining action, antagonistic to $\mathrm{Na}$ (see page 6.38 ); but complete absence of Ca rapidly abolishes rhythmic contractility. Absence of $\mathrm{Ca}$ also removes the rhythmic activity of other striped and unstriped muscle; and these reappear when $\mathrm{Ca}$ is added (Buglia, I9II). The effects are so rapid as to suggest that they must be exerted on the plasma membrane.

Other Earthy Metals. - $\mathrm{Ba}, \mathrm{Sr}, \mathrm{Ca}$ and radium emanations act identically on the isolated frog heart, the activity decreasing from barium in the above order. Low concentrations increase the diastole, to diastolic standstill. Higher concentrations increase the systole to systolic standstill (Kionka, ro14).

Striped and Smooth Muscle.- The rhythmic contractions, which are started in skcletal muscle when immersed in $\mathrm{NaCl}$ solution, are abolished by $\mathrm{Ca}$ (Sidney Ringer, r883). Calcium also has a curare action (Joseph and Meltzer, I9II). Excised bronchial muscle is constricted by $\mathrm{Ca}$ or $\mathrm{Sr}$, though less than by $\mathrm{Ba}$ (Trendelenburg, 19r2). Calcium arrests pituitary asthma (Kayser, I914).

Cardiac Vagus.-The presence of Ca is necessary for the inhibitory activity of the vagus (Schiff; Busquet and Pachon, I909); but apparently not for muscarin (O. Loewi, I9I 2). A somewhat smaller concentration of Ca suffices in the presence of $\mathrm{K}$ (Hagan and Ormond, I912). A moderate decrease of Ca increases the excitability of the vagus; excess of $\mathrm{Ca}$ diminishes the excitability (Cazzola, I913; see also page 639). Similar phenomena are observed with the pelvic and chorda tympani nerves (O. Loewi, r9I2): Increase of $\mathrm{Ca}$ also restores the irritability of the vagus when this has been depressed by $\mathrm{Mg}$ (Auer and Meltzer, I909).

In complete heart-block injections of calcium (or barium) produce attacks of temporary arythmia and ventricular tachycardia, increasing to fibrillation. These attacks may be preceded by groups of powerful and rapid beats. The auricles are affected in the same sense, but not as greatly. The blood pressure rises (Van Egmond, r913).

Blood Vessels.-Hooker, I9II, found that Ca perfusion dilates the vessels of the frog. R. G. Pearce, I9I3, found Ca necessary to the vasoconstrictor action of epinephrin in perfused frogs; epinephrin in $\mathrm{NaCl}$ producing vasodilation. $\mathrm{Ca}$ was not necessary to the vascular effects of curare, ergot or barium. The conditions seem to be reversed in mammals, for Cow, Igr 1 , found that the sensitiveness of arterial rings to epinephrin and pituitary was increased by absence of $\mathrm{Ca}$; the reaction to barium was again unaffected.

Pupil.- Auer and Meltzer, r9o9, observed that the intravenous injection of Ca produces a maximal miosis, in which the pupil is irresponsive to sympathetic stimulation. The simultaneous exhibition of other drugs shows interesting complex phenomena. The miotic action appears to be due mainly to direct stimulation of the sphincter muscle fibers, and probably also of their nerve-endings, supported by lessened irritability of the peripheral sympathetic mechanism.

Antagonism of $\mathrm{Ca}$ and $\mathrm{Mg}$.- - This was discussed on page $64 \mathrm{I}$. It should be remembered that the actions of $\mathrm{Ca}$ are conditioned on the ratio of other ions. Thus, the intravenous injection of $\mathrm{Ca}$ which is depressant to normal animals, causes recovery of an animal anesthetized with $\mathrm{Mg}$. Strontium does not have this effect (Meltzer, 1909).

The Nature of Calcium Action and Antagonism.-This was discussed on page 641.

Inactivation of Calcium.- The $\mathrm{Ca}$ in the body-fluids, and presumably in the cells, can be rendered inactive by the introduction of precipitant anions, such as the oxalates or fluorids; or by decreasing its ionization with citrates or tartrates (Sabbatani, 19or and 1902). In the -isolated heart, the toxicity is greatest for citrate, then oxalate, then 
tartrate (Salant and Hecht, I9I4). In either case, the inactivation produces the opposite effects from $\mathrm{Ca}$, viz., excessive stimulation, convulsions, hypersensitiveness of the autonomic nerves, and rhythmic contractions. Similar effects are seen in intoxications by acids, $\mathrm{As}, \mathrm{Hg}$ or chlorates, which probably all diminish the $\mathrm{Ca}$ content (Chiari and Froehlich, 19II). 'These effects may be promptly removed by the addition of $\mathrm{Ca}$ and less efficiently by Sr and $\mathrm{Mg}$ (Starkenstein, 1914); but not by other cathions, not even by those ( $\mathrm{Ba}$, etc.) which precipitate the oxalate. The stimulation is therefore not due to the oxalate, etc., as such, but to the inactivation of the Ca. This is confirmed by the observation that equimolecular solutions of the different inactivators have practically equivalent effects (Starkenstein).

Salant and Hecht, rgr4, describe some observations which they consider contradict this explanation. They find in perfusion of the isolated heart that calcium citrate or tartrate solutions act practically the same as equimolecular calcium chlorid. 'This, however, need only mean that the citrate, when already bound to calcium, does not disturb the ionization of the calcium in the tissues.

Use of Calcium in Convulsions.-The depressant action of calcium suggests its use in strychnin poisoning, but Fischer (1904) found it ineffective. Zanda (1902) obtained better results by dural injection; but the effective dose was so large as to be itself dangerous. Januschke and Masslow, I9r 4, conclude that even large doses of calcium have no direct inhibitory effect on experimental convulsions, whether cerebral, medullary or spinal. The apparently successful results of Januschke, 1913, and presumably those of Yogi, 1912, and other workers, are attributed to delayed absorption of the hypodermic injections of the convulsants, which results from previous calcium treatment.

Parathyroid Tetany. - Netter, r907, reported success from calcium-chlorid administration in tetany. This has been confirmed for gastric tetany by Kinnicutt, I909; and for parathyroid tetany by W. G. MacCallum and Voegtlin, rgos; Carlson and Jacobson, rgro, and others. MacCallum and Voegtlin believed that the parathyroids control the retention of $\mathrm{Ca}$; that their loss resulted in a diminished $\mathrm{Ca}$ content, especially of the brain; and that the administration of $\mathrm{Ca}$ replaced this deficiency. However, others could not find any diminution of the $\mathrm{Ca}$ (Cooke, r9ro); and the Ca administration probably acts only by lessening the nervous irritability.

Marine, 1914, found that the parathyroid hyperplasia, which is produced in fowl by a diet of maize and wheat, is retarded by feeding calcium, accelerated by sulphuric acid or neutral sulphur.

Infantile Tetany.-Haskins and Gerstenberger, I9I I, found it ineffective, but Goeppert claims suppression of the frequent convulsions by calcium chlorid, 8 to $14 \mathrm{Gm}$. daily, in doses of $\mathbf{r} .5$ to $2 \mathrm{Gm}$. The administration must be continuous, for spasms reappear if it is discontinued for twelve hours (M. Klotz, I915). Gruenfelder, IgI3, reports that laryngospasm and tetany are controlled more promptly by calcium bromide $(2 \mathrm{Gm}$. per day) than by $\mathrm{NaBr}$.

Control of Inflammations by Calcium.-Ca salts, even when administered systematically, seem to lessen the inflammatory phenomena, especially the tendency to effusion. This is presumably due to decreased permeability of the vessel walls, analogous to the effects of $\mathrm{Ca}$ on other cells.

Effect of Calcium on Effusions.-R. Chiari and Januschke, I9ro and Igri, found that the hypodermic injection of $\mathrm{Ca}$ prevents the pleural effusion which ordinarily occurs after fatal doses of $\mathrm{NaI}$, diphtheria toxin, thiosinamin, etc. It also prevented the inflammatory edema of the conjunctiva after oil of mustard or abrin; and the irritation of the skin by croton oil (Luithlen, $\mathrm{I} \mathrm{I} 3$ ). It has been found similarly useful in various skin rashes. Some of these results were contradicted by Levy, Igr I and Igr4, but confirmed by Leo, rgrr. The effect of a dose lasts about a day. Fleisher, Hoyt and L. Loeb, r9o9, also found that $\mathrm{CaCl}_{2}$, intravenously, decreases the elimination of injected saline fluids, not only by the kidneys, but to a lesser extent also by the intestines. It also causes some depression of intestinal absorption. On the other hand, it increases the tendency to peritoneal and pulmonary transudation. This is only partly due to interference with the kidney function, for the ascitic tendency is exaggerated by $\mathrm{Ca}$ even after nephrectomy. Calcium has also been found unsuccessful in clinical serous effusions. Velden, I9I3, suggests that it lessens the absorption of the ascites more than its formation.

Mechanism of the Antiedemic Action.-Luff, roo9, attributed the effect of Ca to increased coagulability of the blood. However, Chiari and Januschke found it effective even when the blood was kept non-coagulable by hirudin. They suggest that the $\mathrm{Ca}$ has a precipitant effect on the cement substance of the endothelium; but more prob- 
ably, the permeability of all the cells is decreased. Deltjen (rgo4) has observed that small traces of calcium, strontium, barium, and magnesium lessen the solvent action of distilled water on cells. Herbst found that sea-urchin ova swell when $\mathrm{Ca}$ is removed, and shrink when it is added. Osterhout, I9 I I and 19 I 2, showed that $\mathrm{CaCl}_{2} \mathrm{prevents}$ the entrance of $\mathrm{NaCl}$ into spirogyra filaments, and that it renders the plasma membrane of kelp (laminaria) impermeable. Pfeffer, 1877 , stated that Calcium phosphate forms semipermeable membranes. These have been investigated in further detail by Meigs, 1915. The changes in the appearance of fluorescein in the anterior chamber of the eye also indicate decreased permeability (G. Rosenow, r9i6).

Phagocylosis may also play a part. H. J. Hamburger, rgro, found that therapeutic quantities, hypodermically or by mouth, increase the ameboid movements of the leucocytes, and therefore chemotaxis and phagocytosis. On the other hand, Ikeda, ror6, reports the migration of leucocytes from inflamed frog's mesentery, as inhibited by calcium chlorid.

Peripheral Anesthetics on Inflammation.-Januschke, I9 3 , finds that the mustard chemosis can also be checked by all drugs which paralyze the sensory trigeminal endings; and this independently of the central nervous system.

Use of Calcium in Skin Rashes, Catarrhs, Etc.-Wright, I 896, found that the administration of calcium chlorid tends to prevent the occurrence of serum rashes. Netter, 1907, also found it useful in urticaria, acute edema, frost bite, and pruritus. Other similar conditions relieved by $\mathrm{Ca}$ are boils, erythema, lichen planus, hemoglobinuria, etc. (Luff, I909); hay fever and iodid coryza (Chiari and Januschke, rgrr, Emmerich and Loew, I9I5); asthma (Kayser, I9I2, I9I4). The beneficial results are clinically rather unreliable, but have been repeatedly confirmed, provided that the $\mathrm{Ca}$ is given in adequate doses for a sufficient time.

Strong solution of $\mathrm{CaCl}_{2}$ (ro per cent.) applied to the scarified skin, raises an urticarial wheal (Pilcher and Sollmann, rgr6).

Effects of Various Conditions on Skin Reactions.-Luithlen, I9 I to rgr 3, studying the reaction of the skin of cats to croton oil, found that this may be modified by $\mathrm{Ca}$ and oxalates; by different diets (suggesting that eczema may be a constitutional exaggeration of the reaction of the skin to normal irritation); and by the injection of serum, soluble starch and even mineral colloids, i.e., silica. Serum injections have been used clinically against certain skin diseases (A. Mayer and Linser); but are only efficient in those diseases which are based on exudation or transudation.

Calcium on Intestines.-Calcium carbonate, and to a smaller extent the phosphate, have long been used in diarrhea. Their action is generally explained as antacid and protective, but it is possible that the depressant. ion-action comes into play.

Saline Cathartics.-Chiari, rgro, calls attention to the fact that all the cathartic ions are antagonistic to $\mathrm{Ca}$; and that this may explain their action, at least partly.

Excised Intestine.--Vanysek, I914, states that calcium chlorid stimulates the circular and relaxes the longitudinal muscle of cat's intestine.

Calcium in Hemorrhage.- $\mathrm{Ca}$ is necessary for the action of thrombokinase on thrombogen (or for the conversion of prothrombin into thrombinHowell, I9I4) and hence for the coagulation of the blood. It has therefore been thought that the administration of $\mathrm{Ca}$ would be useful when it is desired to increase the coagulability of the blood, as in persistent hemorrhages, aneurism, hemophilia, purpura, etc. However, the normal content of $\mathrm{Ca}$ in the blood is more than is required for coagulation; further increase of $\mathrm{Ca}$ does not increase the coagulability. The addition of calcium to hemophilic blood in vitro delays rather than hastens its coagulation (Hess, I9 5). Nor has it been shown that $\mathrm{Ca}$ is deficient in any of these conditions. Hemophilia is not related to calcium deficiency or any anomaly of calcium metabolism. Rather, it is due to deficiency of 
prothrombin (Howell, I9I4), fibrinogen (Hess, I9I4, I9I6), or thrombokinase (Sahli, I905). Estimations of the coagulation time after administration of $\mathrm{Ca}$ have given contradictory results, due partly to differences of technic, partly to the time when the observations were made. The experiments of Velden, I9I3, indicate that the coagulability is indeed increased for a short time. This increase is also produced by equal quantities of $\mathrm{NaCl}$, and is probably due to a local action on the alimentary canal. It does not last beyond twenty or thirty minutes, and would therefore be useless. However, since the administration of $\mathrm{Ca}$ does no harm, its use may be permissible. Any benefits in purpura (Russel, I907) could perhaps be explained by its action on the permeability of the vessels.

With hirudin plasma the coagulation is further delayed by the addition of calcium (Hess, 1915).

Clinical Data.-The use of $\mathrm{Ca}$ in hemophilia was started by Wright, I893, who claimed a reduction of the coagulation time to 60 to 70 per cent. Coleman, 1907, found no effect on oral administration, but shortened coagulation time from hypodermic injection. Purely negative results were obtained by Robertson, Illmen and Duncan, 1907; Addis, 1909; Rudolph, I910; Dale and Laidlaw, I912; and Hess, I915. In obstructive jaundice, when blood coagulation is delayed by excess of bile in the blood, Lee and Vincent, I9I5, report restoration of the coagulability by calcium lactate ( 7 Gm. per day).

The theories of hemophilia are reviewed by Hurwitz and Lucas, I9I6. Kahn, I9I6, reports one case with deficient calcium content of the blood.

Other Substances Increasing the Coagulability of the Blood.-Gelatin, and kephalin serum are discussed elsewhere (see Index). Velden, I9I I, also found increased coagulability after the digitalis group, suprarenal extract, and amyl nitrite.

Coagulation of Milk.- $\mathrm{Ca}$ is necessary for the precipitation of the caseinogen as casein.

Rigor Mortis.-This is hastened by the systemic administration of $\mathrm{Ca}$, delayed by $\mathrm{Mg}$ (Meltzer and Auer, 1907).

Phlorhizin Diabetes.- Jacoby and Rosenbloom, 1915, state that large doses of calcium diminish the excretion of sugar, nitrogen and acetone. The blood-sugar is also lowered, so that presumably the production of sugar is diminished.

Administration for Systemic Action.-The commonly used salts are calcium chlorid and lactate. The chlorid serves well by mouth, but for hypodermic injections, the less irritant lactate should be preferred; at least, the chlorid should not be used in concentrations above I or 2 per cent. or there will be indurations. The concentration of the lactate should not exceed 5 per cent. since stronger solutions solidify (Hugenholtz, I9I5). The single oral dose of the chlorid is 0.5 to I Gm., 8 to I 5 gr., in solution, with meals. The daily dose against serum rash is 0.75 to $\mathbf{I}$ Gm., beginning on the day of the antitoxin injection, and continuing for three days after the last injection (Netter, I909). Against hay fever, hemorrhage, etc., I to $3 \mathrm{Gm}$. are given daily, commencing as early as possible and continuing for several weeks. This continued use does not seem to cause harm (Emmerich and Loew, I9I3, I9 I5). The lactate may be given in double this dosage. The hypodermic dose of the lactate is 0.5 to $2 \mathrm{Gm}$. in 20 to 50 c.c. of water. Intravenous injections have been employed in spasmodic conditions; 2 to $4 \mathrm{Gm}$. of the chlorid or lactate in a liter of normal saline, injected very slowly.

\section{PREPARATIONS-CALCIUM}

*Calcii Carbonas Pracipitatus (Calc. Carb. Præc.), U.S.P., B.P. (Precipitated Chalk), $\mathrm{CaCO}_{3}$. - Fine white powder, without odor or taste. Practically insol. in water; decomposed by acids. Dose, I Gm., I5 gr., U.S.P., as powder. This will neutralize 
$0.730 \mathrm{Gm}$. absolute $\mathrm{HCl}$. Larger doses are harmless. Dose, B.P., I to $4 \mathrm{Gm}$., I 5 to 60 gr.

${ }^{*}$ Creta Praparata (Cret. Præp.), U.S.P., B.P.; Prepared Chalk (Drop Chalk).Native Calcium Carbonate freed from most of its impurities by elutriation. A white to grayish-white, very fine, amorphous powder, often formed into conical drops; odorless and tasteless. Almost insol. in water; insol. in alc. Dose, I Gm., I 5 gr., U.S.P.; r. to 4 Gm., 15 to $60 \mathrm{gr}$., B.P. Used extensively in tooth-powders.

If ist. Cret., U.S.P., B.P.; Chalk Mixture--The average dose, I5 c.c., 4 drams, contains about $3 \mathrm{Gm}$. of Comp. Chalk Powder suspended in Cinnamon Water, U.S.P.; 3 per cent. of chalk, B.P. Dose, 15 c.c., 4 drams, U.S.P.; I 5 to 30 c.c., $1 / 2$ to I ounce, B.P.

Pulv. Cret. Co., U.S.P.; Compound Chalk Powder.-A mixture of 30 per cent. of Cret. Prep.; 20 per cent. of Acacia; 50 per cent. of Sugar. Dose, 2 Gm., 30 gr., U.S.P.

Pulv. Cret. Arom., B.P.-25 per cent. of Chalk; 50 per cent. of Sugar; with Aromatics. Dose, 0.6 to $4 \mathrm{Gm}$., to to $60 \mathrm{gr}$., B.P.

${ }^{*}$ Calcii Chloridum (Calc. Chlor.), U.S.P., B.P.; Calcium Chlorid (not to be confused with Chlorinated Lime, miscalled "Chlorid of Lime"), $\mathrm{CaCl}_{2}$. - White, translucent, hard fragments, or granular; odorless, of sharp saline taste. Very deliquescent, and should therefore not be dispensed in powders, but as solution. Freely sol. in water ( $1: \mathrm{r} .2)$ also in alc. ( $\mathrm{r}: \mathrm{ro})$. Incompatible with carbonates, phosphates and sulphates. Dose, 0.5 Gm., 8 gr., U.S.P.; 0.3 to I Gm., 5 to 15 gr., B.P., diluted.

Calcium chlorid occurs with varying quantities of water. The content of $\mathrm{CaCl}_{2}$ is roo per cent. in the anhydrous, about 75 per cent. in the fused, and 50 per cent. in the crystals. The U.S.P. specifies a minimum of 75 per cent. The B.P. is dried at $200^{\circ} \mathrm{C}$.

${ }^{*}$ Calcii Lactas (Calc. Lact.), U.S.P., B.P., Ca $\left(\mathrm{C}_{3} \mathrm{H}_{5} \mathrm{O}_{3}\right)_{2}+{ }_{5} \mathrm{H}_{2} \mathrm{O}$.-White, granular. masses of powder, without odor or taste. Sol. in water $(I: 20)$, but very slightly sol. in alc. Dose, o. $5 \mathrm{Gm}$., 8 gr., U.S.P.; 0.6 to $2 \mathrm{Gm}$., 10 to $30 \mathrm{gr}$., B.P.

Syr. Calc. Lactophos., U.S.P., B.P.-2.5 per cent. of calcium carbonate dissolved in syrup with lactic and phosphoric acids, U.S.P.; 7.5 per cent. of Calc. Lact. with phosphoric acid, B.P.; orange-flower flavor. Dose, 10 c.c., $21 / 2$ drams, U.S.P.; 2 to 4 c.c., $1 / 2$ to I dram, B.P.

Calcii Phosphas, B.P., $\mathrm{Ca}_{3}\left(\mathrm{PO}_{4}\right)_{2}$.-White powder, insol. in water, sol. in acids Dose, 0.3 to I Gm., 5 to 15 gr., B.P.

( ${ }^{*}$ Liq. Calc., Syr. Calc., Calx.-See Index).

\section{STRONTIUM}

This cathion resembles calcium, and may take its place in some of its characteristic pharmacologic reactions; but it is much weaker and also less toxic. Strontium possesses no therapeutic indication. It may be useful to moderate the action of anions, since its salts must be decomposed before the anions can be absorbed, but with strontium salicylate at least, this moderation is insignificant (Blankenhorn, r916).

Like $\mathrm{Ca}$, it hastens the coagulation of blood although it is much weaker (Howell, 1914). This action is not shared by $\mathrm{Ba}$ or $\mathrm{Mg}$. In dilute solutions only very small amounts are absorbed from the stomach; none from the intestine, since it is converted into phosphates, in which form it is also generally deposited in the bones. The urine contains only traces of strontium, even if it is given subcutaneously; the excretion occurring mainly by the intestine (Wood, 1898 ). It increases the elimination of uric acid. Its administration to young animals or in intrauterine life disturbs the bone formation. The osteogenetic tissue is stimulated, but the bones are imperfectly calcified, since the $\mathrm{Ca}$ is deficient and the $\mathrm{Sr}$ is deposited very imperfectly (Lehnerdt, 1909 and 1913).

\section{PREPARATIONS}

Stronitum Bromid, Iodid, and Salicylate are official. Their dose is that of the corresponding calcium salts.

Strontii Lactas.-Soluble in 4 parts water. Dose, I to $8 \mathrm{Gm}$. ( $1 / 4$ to 2 drams). 


\section{OXALIC ACID AND OXALATES}

Oxalic acid and the oxalates possess a strong acid action, resulting in local irritation and corrosion. In addition, they produce violent stimulation, and later paralysis, by depriving the tissues of their calcium through precipitation. These actions are completely antagonized by the addition of $\mathrm{Ca}$. The oxalates have some toxicologic importance but no therapeutic interest. The small quantities which occur in many vegetables (rhubarb, etc.) are ineffective.

Lower Organisms.-Oxalates are toxic to all life except to a few lower fungi which do not contain calcium. They are toxic to most plants, but certain species contain soluble oxalates. In algæ the main histologic changes are seen in the portions richest in calcium-nucleus and chlorophyll (O. Loew, I9I2). Oxalic acid is probably a constant product of metabolism, and possibly one of the functions of calcium is to render it harmless.

Actions on Peripheral Autonomic Nervous System.-The oxalates act like deprivation of $\mathrm{Ca} ;$ i.e., they increase the sensitiveness to epinephrin and pilocarpin; but they diminish the excitability to electric vagus stimulation (Chiari and Froehlich, IgII).

Skeletal Muscle.-If dilute solutions of oxalates are applied directly to skeletal muscle, they cause rhythmic contractions. Lessening the proportion of $\mathrm{Ca}$ by any other means has the same effect. Larger doses of oxalates depress the excitability and force of the skeletal and cardiac muscles.

Frog's Heart. - Injections of oxalates produce slowing and weakening; with stronger concentrations prompt diastolic arrest, first of the ventricle, then of the auricles. This can be removed completely by rinsing with Ca solution (Januschke, I909). Washing with $\mathrm{NaCl}$ alone gives much poorer recovery (Gros, 1913). Similar effects are seen with perfused mammalian hearts (Salant and Hecht, r9r5).

Intact Frogs.-These show progressive and descending loss of reflexes; fibrillary twitchings; central paralysis; finally cardiac arrest. This is also promptly removed by $\mathrm{Ca}$ and $\mathrm{Sr}$.

Intravenous Injections in Mammals.-The oxalates affect first the central nervous system in its whole extent, from mental functions to reflexes, producing at first stimulation, with convulsions, and then paralysis, the latter being the more conspicuous with large doses.

The medullary centers are especially affected. In consequence of the asphyxia produced in this manner they cause glycosuria, and possibly through the same cause indicanuria. The temperature rises. Death occurs from respiratory paralysis, excepting when the poison is injected directly into the circulation, in which case it may paralyze the cardiac muscle (slowed heart and fall of blood pressure, v. Vietinghoff-Scheel, 1901; Starkenstein, 19I4). In excised intestines, the calcium precipitants increase the tone and the pendulum movements (Starkenstein).

Oxalates, injected with $\mathrm{Mg}$, increase the anesthetic effect, presumably by inactivating the antagonistic $\mathrm{Ca}$ (Gates and Meltzer, 1914).

Oxalate Poisoning.-When taken by mouth, the oxalates are readily absorbed, and poisoning is not at all infrequent from confusion with other salts.

The symptoms are: first, those of a local caustic, especially when the acid was used; then those of collapse, the latter possibly preceded by convulsions. The pulse is very small and weak.

Death by oxalic acid and oxalates usually occurs very rapidly, much more quickly than by other caustic substances. This is of diagnostic importance. (In one case death took place in ten minutes.) Sometimes fatal collapse may occur after hours of relatively slight symptoms (O. H. Brown and Scott, I912). (A study of seventeen human cases of oxalic acid poisoning is reported by Wichern, I9 12.) The fatal dose varies with the concentration. The smallest recorded amount is $5 \mathrm{Gm}$. Metabolism is markedly depressed in oxalate poisoning, especially the production of carbonic acid. The respiratory quotient falls (Corley, 1902). The urines in Wichern's cases were scanty, with traces of albumin, and rarely calcium oxalate crystals. Polyuria developed later. Hemorrhagic nephritis was not observed.

Fale and Excretion.-Oxalates are very resistant to oxidation in the animal body. Indeed, there has been some question whether any is burned; but it seems certain that a 
limited amount is oxidized. The excretion ( 92 to 95 per cent. after hypodermic injections) occurs almost completely by the kidneys. The fresh urine contains mainly oxaluric acid, CO.NII CONH $\mathrm{N}_{2}$, but on standing, this decomposes into urea and oxalic

\section{COOH}

acid; and this forms calcium oxalate. This is almost insoluble and occurs as cnvelopeshaped crystals, which are diagnostic of oxalate poisoning. 'The crystals may be excreted in such great amounts as to block the urinary tubules, and may thereby possibly lead to nephritis, or retention of urine and uremia. Calcium oxalate, in rod-shaped crystals, may be found in all the organs, but it may be absent from these in very acute poisoning (Mürset, 1885).

Treatment of Oxalate Poisoning.-The chemic antidote would be calcium in any shape, calcium chlorid and lactate, chalk, lime-water, etc. (Husemann). Liberal quantities of water should be given to prevent the deposition of crystals in the kidneys. Calcium lactate could also be given by vein; magnesium sulphate as cathartic; digitalis and strychnin against collapse.

PREPARATIONS-OXALATES

Acidum Oxalicum, $\mathrm{C}_{2} \mathrm{O}_{4} \mathrm{H}_{2}$ - - Colorless crystals, easily sol. in water.

Acid Potassium Oxalate, $\mathrm{KHC}_{2} \mathrm{O}_{4}$.- "Salt of Sorrel."

\section{FLUORID ION}

Sodium fluorid is a general protoplasmic poison. It has a strong local irritant action, a 2 per cent. solution being corrosive to mucous membranes. The systemic action resembles that of the oxalates and is probably produced in the same manner, by the formation of insoluble calcium salts.

In the frog, it produces fibrillary contraction of the muscles. These disappear on section of the nerve. Larger doses produce rigor. The systemic effects are dyspnea, loss of reflexes, stoppage of respiration, cardiac standstill. In mammals it causes salivation, gastroenteritis, dyspnea, muscular weakness and tremors, epileptic convulsions, fall of arterial pressure, and stoppage of respiration and heart. Rigor sets in quickly. The abdominal organs are congested. The intestinal epithelium is destroyed (even when the poison is introduced by other channels), while the cilia of the respiratory epithelium may still be moving (Siegfried, I901). Small doses poison the blood vessels, first increasing their permeability (Asher, I9ro).

Continued administration of small doses ( $\mathrm{I}$ to $2 \mathrm{mg}$. per kilogram) produced cumulative effects: Increased coagulability of blood; tendency to venous thrombosis; irritation of bone marrow; and loss of $\mathrm{Cl}$ and $\mathrm{Ca}$ from the bones. Even dilute solutions irritate mucous membranes (Schwyzer, I9I4).

Deposition in Bone and Normal Occurrence.-When fluorids are given in small amounts, greatly diluted, they are absorbed and deposited for the most part in the bones (Tappeiner and Brandl). The bones become unusually hard, white, and brittle, and contain small crystals, presumably $\mathrm{CaF}_{2} . \quad \mathrm{A}$ small amount of the latter is normally contained in the bones and teeth (Gabriel, 1893); but the precentage ( 0.02 to 0.05 ) is so small that it can not be regarded as essential (Jodlbauer, I90r). Still smaller quantities are found in the epidermis, hair, thymus, testes, blood, milk, etc. (Gautier and Clausmann, I9r3). Zdarek, rgro, found it present in the entire human body, but relatively richest in the liver, kidneys and bones. In the latter, half of the fluorin is in their fat.

Antiseptic Action.-The fluorids are quite markedly antiseptic. In the proportion of $x: 200$ they prevent completely the development of bacteria, and are sometimes used for this purpose. They have no important therapeutic application, and small toxicologic importance.

Ammonium Bifluorid in Dentistry.-This was introduced by J. Head, I9r3, for the treatment of pyorrhea alveolaris. He uses a 23 per cent. solution, injected once or twice weekly around the loose teeth. It is antiseptic and disintegrates the "tartar."

Hydrofluoric Acid is a very violent volatile caustic. The burns suppurate, and heal very slowly. 
Sodii Fluoridum (Sodium fluorid), NaF.-Soluble in 25 parts of water. Used externally as antiseptic. For dressings, 0.5 to $10: 1,000$; injections, 0.25 to $1: 1,000$; against fermentation in food, Io to $15 \mathrm{mg}$. per liter. $0.25 \mathrm{Gm}$. have produced dangerous symptoms in man.

\section{BARIUM}

Unlike the other elements of its group, $\mathrm{Ca}$ and $\mathrm{Sr}$, this element is very toxic. Its effects resemble those of the digitalis group: It produces a similar action on the cardiac muscle; a strong vasoconstriction by direct stimulation of the arterial muscle (occurring therefore after apocodein, Dixon, I903); violent stimulation of peristalsis, even on intravenous injection; and a stimulation and later paralysis of the central nervous system. It also produces local irritation, and is inimical to the lower forms of life, such as yeast cells.

These actions are not utilized therapeutically (except in veterinary practice) on account of the toxicity. The excretion of barium occurs largely by the feces, as with $\mathrm{Ca}$ and $\mathrm{Sr}$.

Its local action results in gastroenteritis and some degree of corrosion, and fairly large absorption. It may, therefore, produce its systemic actions even when given by the mouth. Poisoning is treated by sulphateand evacuation.

Excretion.- The main channel is by the intestine, only traces appearing in the urine even after parenteral administration (G. M. Meyer, Igog). A small amount may be deposited in the bones. Barium lessens the elimination of uric acid (Abl, r9r3).

Cardiac Action.-Ba, like digitalis, increases the excitability of the cardiac muscle, and the force of the contractions (Wertheimer and Boulet, IgII). The rate is slowed, even after atropin, so that this effect is muscular (Fillipi). Intracardiac application causes systolic arrest; dilute solutions, or exocardiac application, produce diastolic arrest, just as does digitalis (Poulsson, rgIo; Werschinin, I9II; Weyland, Igr3). The effects in complete heart-block are similar to those of calcium (van Egmond, I9r3).

Arterial Muscle.--The high rise of blood pressure under barium is due mainly to vasoconstriction. The effect is directly on the arterial muscle, and involves all arteries, regardless of their innervation (Dixon and Halliburton, r9 го Cow, I9 I 1 ). This might suggest its use in pulmonary hemorrhage; the lung vessels being strongly constricted (Baehr and Pick, I9 3 ; Berezin, I9I4). However, the increase of the general blood pressure more than offsets the local constriction, so that the bleeding becomes more profuse (E. Frey, Igog). The liver veins are constricted in the guinea pig, not in the frog (Berezin, I9I4).

Bronchial Muscle.-This is also constricted (Trendelenburg, I9I2; Titone, I9I3; Baehr and Pick, 1913).

Gall-bladder.-The isolated organ is constricted (Lieb and McWhorter, I9I5).

Fowl's Muscle.-Injection of $\mathrm{BaCl}_{2}$ produces only an increase of muscular tonus in the normal leg, but after denervation, it causes a strong contraction. The degenerating muscle has therefore become more susceptible to $\mathrm{Ba}$. A similar result can be secured by preliminary injection of nicotin. Curare does not influence this contraction; indicating that $\mathrm{Ba}$ acts more peripherally than curare, nicotin or physostigmin.

Therapeutic Uses.-Barium chlorid ( $0.03 \mathrm{Gm}$., 1/2 gr., well diluted) has been tried as a substitute for digitalis (Pesci, I904). It is therapeutically inferior; $f . i$., by producing marked coronary vasoconstriction. The maximal daily dose is $0.6 \mathrm{Gm}$., 9 gr.; but this is dangerous (Reports Counc. Pharm. Chem., I9II:54). It has also been tried in various nervous disturbances; and externally, ro per cent. solution, as a wash in scrofulous eye affections. In veterinary medicine, it has been used for acute tympanites and constipation, but even there it was found too dangerous.

Symptoms of Barium Poisoning.-These consist in vomiting, colic, violent diarrhea; convulsive tremors; slow hard pulse and high blood pressure; hemorrhages into the stomach, intestines and kidneys. Death occurs after a few hours or days. The fatal dose is probably 0.8 to $0.9 \mathrm{Gm}$. Barium carbonate is also toxic, but much slower in its action. Crawford, I908, attributes "Loco poisoning" to the Ba contained in the Western plants. This could not be confirmed (Alsberg, Black and Marsh, rgr2). 
$\mathrm{X}$-ray Diagnosis. - The insoluble $\mathrm{BaSO}_{4}$ is used in $x$-ray diagnosis. It is tasteless, incxpensive, and does not delay the gastric movements, as does Bismuth. If pure, roo to $150 \mathrm{Gm}$. (boiled with two and one-half to five times its weight of corn-meal, and flavored with cocoa) may be given without any bad results; but it is essential that it should not be contaminated with soluble barium compounds (Guenther and Bachem, I9I I; Merck's Report, 27:43).

\section{BROMIDS}

General Statement.-Fairly large doses depress the psychic functions and the reflexes; leading to a condition of mental dulness and apathy, which conduces to sleep, particularly in nervous insomnia. The bromids are also employed against other nervous hyperexcitabilities, hysteria, hyperemesis, cinchonism, etc. Their anticonvulsant action is utilized especially against epilepsy. They generally suppress or mitigate the convulsions, at least in the idiopathic form; but they seldom, if ever, cure. The effect occurs only slowly, and is correspondingly lasting. The administration must be fairly continuous. The action seems to be due to the $\mathrm{Br}$ ion as such. It is produced by all the bromids, and to a less degree by the organic bromin compounds. $\mathrm{NaBr}$ is generally preferred. The mechanism is not explained. The excretion of $\mathrm{Br}$ is analogous to that of $\mathrm{Cl}$. A part is retained very persistently. The elimination is hastened by $\mathrm{Cl}$ administration; and conversely, the $\mathrm{Br}$ effect is increased by Cl-poor diet.

Continued administration of large doses often results in bromid acne, and other symptoms analogous to iodism. There may also be considerable psychic depression, malnutrition and lowered resistance. These may contraindicate the use in neurasthenic patients, etc. Acute bromid poisoning is rare, occurring only after enormous doses. Ordinary doses have no effect on the circulation.

Absorption.-The bromids are rapidly absorbed from mucous membranes. It was believed that calcium bromid is absorbed more slowly; but Hales and Fishman, 1908, found it excreted at about the same rate as $\mathrm{NaBr}$. In ligated intestinal segments, the absorption of bromids is not delayed by the presence of chlorids; but bromid somewhat delays chlorid absorption. The lower segments of the small intestine are said to absorb bromid better than the upper (Bolgar, IgIo).

Channels of Excretion.-The excretion occurs as bromid, mainly by the urine. Small quantities are also eliminated by the feces, sweat (Tachau, I9rI) and other secretions; sometimes in the cerebro-spinal fluid (Hald, I9II).

Course of Urinary Excretion.-This starts promptly but proceeds slowly; f.i., in one to one and one-half days only one-tenth may be excreted; and traces are found after twenty days. The rate is only half or less than with iodids (Herzfeld and Gormidor, I912). It depends somewhat on diuresis, but only imperfectly (Hales and Fishman, 1908). The quantity excreted is proportional to that administered, but always with considerable retention. With continued use, the percentage excreted increases until it equals the ingestion, constituting equilibrium or saturation for that dose. When the administration is stopped, the excretion diminishes; at first rapidly, then more slowly, so that traces may be present for a month or longer. The excretion follows the same laws in epilepsy as in normal individuals (v. Wyss, Igo6).

Influence of Chlorids. - Chlorid administration hastens the elimination of both $\mathrm{Br}$ and I (Herzfeld and Gormidor, I912; Wyss, I913); while Cl-poor foods slow the excretion (Honda, r902; Sarvonat and Cremien, x9II). Conversely, the administration of bromids increases the elimination of $\mathrm{Cl}$ (Wolf and Opp, 1912; Wyss, 1913). Iodid also displaces $\mathrm{Br}$ and $\mathrm{Cl}$. Boenniger, I907, claims that bromid administration may save animals from chlorid starvation, and replace the $\mathrm{Cl}$ of the serum completely. This is doubtful.

Bromids on Diuresis.-This is increased by salt action (Wolf and Opp, 1912).

Bromids in Uranium Edema. - Uranium animals become distinctly less edemic when bromids are administered. These cause a greater diuresis; the $\mathrm{Cl}$ excretion is as 
high as normal; and the urine contains a part'of the Br. The body therefore loses both water and salt (Leva, I912). This explains the effect.

Bromids on Metabolism. - The actions are not important. Chittenden, 1884, claimed slight increase of $\mathrm{N}$ and decrease of $\mathrm{PO}_{4}$ excretion. Jappelli, IgI 2 and IgI3, finds little effect on the total N or P; but diminution of the uric acid and increase of the purin bases. He considers that this is due to an inhibitory action on ferments, analogous to the bromid effect on pepsin (Chittenden) and oxidase (Senter). The claim that bromid increases the elimination of phosphates needs confirmation.

Retention of Bromid. - The retained $\mathrm{Br}$ is distributed similarly to the normal $\mathrm{Cl}$, which it partly replaces in the blood, gastric juice, etc. (Nencki and Simonowski, I 893; Ellinger and Kotake, I9I I). Apparently, the tissues and kidneys do not differentiate sharply between the two ions (E. Frey, Igro). The replacement of more than 40 per cent. of the $\mathrm{Cl}$ of the blood by $\mathrm{Br}$ is said to be generally fatal (Bernoulli, I9r3).

Distribution in Different Organs. - This has been studied especially by Wyss, roo6. $\mathrm{He}$ found the largest quantity in the blood. Even after long administration, the brain contained a relatively small proportion. In an epileptic patient, the percentage in the brain was 0.06 ; in the blood, 0.I4. The quantity in the whole brain figures about I Gm. Ellinger and Kotake, I9 I, found the distribution proportional to the normal richness in $\mathrm{Cl}$. The distribution after therapeutic administration is described by Carnot and Coirre, Igr4.

Condition in Blood.-The $\mathrm{Br}$ occurs almost exclusively in the serum (Wyss). However, Boenniger, $\mathrm{rg}_{909}$, states that the corpuscles are permeable to $\mathrm{Br}$ and $\mathrm{Cl}$ in both directions; that a $\mathrm{Br}$ solution may displace all but traces of $\mathrm{Cl}$ from the corpuscles; and that the $\mathrm{Br}$ in turn can be replaced by $\mathrm{Cl}$.

Absence from Normal Tissues.-Baldi claimed the presence of $\mathrm{Br}$ in normal thyroid; but this was not confirmed by Roos, 1898. Pribram, I906, also found absence of notable quantities in the thyroid and other human organs.

Relative Retention of Organic and Inorganic $\mathrm{Br}$.- The organic $\mathrm{Br}$ compounds are apparently retained somewhat more efficiently than the inorganic. This has been cited as a proof of superiority; but it is doubtful whether the retained $\mathrm{Br}$ is the bearer of the therapeutic action (Magnus-Alsleben, I9II). Sabromin was retained relatively more in the adipose tissues, but not in the brain (Ellinger). Bromipin was eliminated by the urine, partly in organic combination, but mainly as inorganic bromid (Bermann, I9ro). $\mathrm{He}$ has also studied the fate of other organic $\mathrm{Br}$ compounds. The lipoid-soluble bromural passes at first mainly into the nervous system; but it is rapidly decomposed into a water-soluble form, presumably bromid; which then follows the course of the ordinary bromids (Takeda, I III).

Historical. - Bromids were first tried as substitutes for iodids. This was unsuccessful, but led to the observation of their sedative action. Their use in epilepsy is said to date from Locock, 1853; as hypnotic, from Behrend, 1864. The older literature is reviewed by Krosz, 1876 .

Sedative Action in Man.-Doses of I to $2 \mathrm{Gm}$. of bromids have no noticeable action on normal individuals. Above $4 \mathrm{Gm}$., depression and decrease of reflexes become apparent. The effects are rather different from those of the ordinary hypnotics. They consist in a condition of lassitude, lack of attention and thought, which inclines toward sleep. Perception, observation, and motor reactions may be normal. With the smaller doses the subject awakens refreshed; but with larger doses, the psychic depression persists. Still larger doses (ro to I5 Gm.) cause, besides some nausea, considerable apathy and confusion of speech, but not sleep (Binz). These doses also lower the reflexes from the skin and mucous membranes; so that irritation of the fauces does not produce retching (Krosz, $\mathrm{r} 876$ ). This was utilized, before the introduction of local anesthetics, for examinations of the throat, etc.

Use as Hypnotic.-Bromids do not enforce sleep, but act rather by permitting sleep through the elimination of excessive worry, etc. They are more efficient in quieting pathologic excitability; and of no value against pain. The dose should be $2 \mathrm{Gm}$. or more, one to two hours before retiring. The bromids may be usefully combined or alternated with the more power- 
ful aliphatic hypnotics or morphin. The effect is simply additive (Klammer, I9I3); or perhaps slightly potentiated (Januschke and Inaba, I9I3).

Use in other Conditions of Nervous Hyper-excitability.-The depressant effect of bromid is largely utilized in hysterical conditions; Baselow's disease; acute cerebral excitement and meningitis; in the nervous romiting of seasickness and pregnancy; to prevent cinchonism; incontinence of urine; as anaphrodisiac in sexual hyperesthesia; and against delirium tremens and convulsions-epileptic, chorea and othets. The dosage for the minor nervous conditions is 0.5 to I $\mathrm{Gm}$. two or three times daily; against cinchonism, 3 parts of bromid to $x$ of quinin; against seasickness, 4 to $5 \mathrm{Gm}$. daily for several days before the voyage; in pertussis, it is said to allay the paroxysms, but without shortening the course. Conrulsions require at least $\mathrm{I}_{5} \mathrm{Gm}$; ; if necessary by rectum. Large doses have been given in acute mania, but probably without much benefit.

Use in Epilepsy.-This is the principal employment of the bromids. They do not seem to be efficient in all cases, probably because epilepsy has different causes. The successful cases amount to something like 90 per cent. in idiopathic epilepsy. ${ }^{1}$ Large doses must be employed and continued for some time. In most cases, the attacks become milder and - less frequent, or may be suppressed entirely; but they usually return soon after the remedy is removed. In some few cases however a permanent effect seems to have been obtained. It is possible that this cure was not due to the bromid, but was spontaneous.

Dosage in Epilepsy.- The effects are secured only when the organism is "saturated" with bromid. The required dose varies for different patients and must be ascertained by trial. The efficiency may be increased by Cl-poor food (Toulouse and Richet, rgoo; Ulrich, I912); or by the administation of calcium (Januschke, I9I3). These modifications may succeed when the simple bromid treatment fails. The daily dose of bromid should be divided into three or four fractions, and freely diluted (never as tablets).

Poulsson recommends the following routine, to be modified as needed: In the first week, $3 \mathrm{Gm}$. daily; second week, $4 \mathrm{Gm}$.; increasing by $1 \mathrm{Gm}$. each week, until 10 $\mathrm{Gm}$. are reached in the eighth week. If well tolerated, and if the attacks persist, the weekly increase may be continued until ${ }_{5} \mathrm{Gm}$. are reached in the thirteenth week. The dosage is then reduced in the same way until the attacks recur; when the treatment is again started in the same way. A few trials ascertain the doses required to reduce the attack to a minimum. This dosage is then continued as long as needed; and renewed at intervals even after complete disappearance of the attacks, to anticipate recurrence. Bromid acne need not interfere with the treatment; but severe depression necessitates reduction, or even discontinuation.

Other Drugs Used in Epilepsy. - Besides the bromids, the following drugs have been used empirically: Opium, Valerian, Belladonna, Zinc Oxid, Chloral, Adonis Vernalis, Solanum Carolinense, Salicylates and Antipyretics. It is very difficult to form an estimate of their value, since epilepsy is temporarily benefited by almost any placebo. These drugs can be recommended only when bromids have failed or are for any reason badly borne. No treatment will be successful except it be joined with a careful regulation of diet and general hygiene, excesses of all kinds being strictly proscribed.

The inhalation of Amyl Nitrite is sometimes useful during the attack.

Central Actions of Bromids on Animals.-These are analogous to man, and can be better utilized for studying the mechanism of $\mathrm{Br}$ action.

'Binz gives the following compilation: Total abolition as long as drug is continued, 12 per cent. Diminished number and violence of attacks, 83 per cent. No influence, $21 / 2$ per cent. Number of attacks, increased $21 / 2$ per cent. 
Albertoni, r882, found that bromids lower the excitability of the motor cortex of dogs, so that a stimulation of the motor areas which, under the conditions of the experiment, gives rise to general epileptiform convulsions, will, after the administration of a bromid, be confined to the area directly stimulated. If a bromid is administered to animalsunder strychnin, the effect of stimulation does not have much tendency to spread, while the primary reflex, say the patellar, will still be exaggerated.

Guinea Pigs.-Large doses of $\mathrm{NaBr}$ (0.1 to I Gm. per pig of 200 to $250 \mathrm{Gm}$.) produce a deep sleep, lasting two or three days. This is preceded by a long latent period, even if the administration is hypodermic or by vein. The hypnotic effect is antagonized by picrotoxin or strychnin (Januschke and Inaba, I9I3).

Frogs.-These do not exhibit acute actions.

Rabbits.-These show both acute and chronic effects closely resembling those observed in man. The symptoms, rapidity and dosage are nearly the same, whether the administration is by mouth, hypodermic or vein (Bernoulli, I $\mathrm{I}^{\mathrm{I}} 3$ ):

Nervous Symptoms. -2.3 to $3.2 \mathrm{Gm}$. per kilogram, vein, generally produce little immediate effect. After several hours to three days, the rabbit becomes apathetic and sluggish. The sensibility and reflexes are somewhat depressed and delayed. Muscular coördination is of ten disturbed and ataxic. Ascending muscular debility and pareses are frequent.

Chronic Poisoning.-Also shows these pareses, but no narcosis or other cerebral depressions (Januschke, $\mathrm{r}_{9} \mathrm{r}_{3}$ ). Both forms prevent camphor and picrotoxin convulsions.

Local Symptoms. - The rabbits often show slowly developing irritant phenomena in the skin and mucous membranes, analogous to bromism: general dermatitis, loss of hair, conjunctival congestion, coryza, etc.

Outcome.-If the nervous symptoms are fully developed, spontaneous improvement is exceptional. The rabbits die in coma after several days.

Efficiency against Experimental Convulsions.-Intravenous injection of sodium bromid suppresses the epileptic convulsions of camphor and the medullary convulsions of cocain, but not the spinal convulsions of strychnin or picrotoxin (Januschke and Inaba, I913). The "Erlenmeyer mixture" of $\mathrm{NaBr}, \mathrm{KBr}$ and $\mathrm{NH}_{4} \mathrm{Br}$, appears to differ in that it does not suppress cocain convulsions with acute, but only with continued administration. This is perhaps explained by the observation that the injection of ammonium bromid is convulsive. $\mathrm{CaBr}_{2}$ is no more efficient than $\mathrm{NaBr}$. (Januschke and Masslow, $19 \mathrm{I}_{4}$ ).

Mechanism of Bromid Action.-Wyss proposed the theory that bromids act merely by displacing $\mathrm{Cl}$ from the body. This explanation is inadequate. The older theories, that their action is due to the liberation of free $\mathrm{Br}$; or to the formation of organic $\mathrm{Br}$ compounds, are also not established. It appears that the action is due directly to the $\mathrm{Br}$ ions.

Chlorid-deprivation Theory.-This was suggested by the increased elimination of $\mathrm{Cl}$ following $\mathrm{Br}$ administration. It was found that the efficiency of bromids was increased by Cl-poor food; and conversely, that the administration of large doses of $\mathrm{NaCl}(20$ Gm.) brought back the epileptic attacks in patients who had been under the control of bromids (Wyss, rgr3). These observations, however, do not prove the contention; for deprivation of $\mathrm{Cl}$ increases the retention of $\mathrm{Br}$; and administration of $\mathrm{Cl}$ increases the elimination of $\mathrm{Br}$. 'The results would therefore equally support the theory of a direct $\mathrm{Br}$ action. On the other hand, a number of facts speak very strongly against the Cl-deprivation theory; Simple dechloration may produce ataxia, but lacks the antispasmodic action of bromids. The bromid action occurs even when $\mathrm{Cl}$ loss is fully compensated by the food. Recovery from $\mathrm{Br}$ may occur even when $\mathrm{Cl}$ is withheld. Injection of $\mathrm{Cl}$ causes some recovery from bromid poisoning; but citrates, sulphates and nitrates have a similar, though weaker saving action (Bernoulli, I913). Januschke and Inaba, I913, found that the bromid depression in animals could not be efficiently antagonized by the administration of $\mathrm{NaCl}$, nor reinforced by $\mathrm{Cl}$-poor food.

The Effects are Due to the Bromid Ion.-The characteristic action is produced in animals by all bromids; but not by organic $\mathrm{Br}$ compounds, nor by bromates (Januschke, I9I3). Bernoulli, 1913, finds that the excised brain and other tissues swell more in bromids than in equimolecular solutions of chlorid. He suggests that this may be the basis of bromid action; but this would require stronger evidence.

Actions on Circulation.-Bromid has no direct effect, except slight depression with single doses above ro $\mathrm{Gm}$. Sedative doses may indirectly 
slow the heart and lower the blood pressure somewhat, especially in cardiac neuroses. Large doses of $\mathrm{KBr}$ may show the depressant potassium action.

In the cat, the intravenous injection of the NaBr, 3 c.c. of I.5 per cent. per kilogram, hat no effect (Lehndorff, 1914).

Perfused Hearts. - The substitution of bromids for chlorids in Ringer's fluid produces practically no effect in cold-blooded hearts, except a slight acceleration in starting. In warm-blooded hearts, there was a slight decrease in rate and amplitude (Greene and Kruse, I913).

Excised Uterus.-Replacement of $\mathrm{Cl}$ by $\mathrm{Br}$ or I produces a slight increase of tonus, without affecting the rhy thmic contractions. Fluorid increases the tonus and rhythmic contractions, without injuring the muscle (Prochnow, I9II).

Secretions.-Bromid somewhat increases the secretion of mucus by salt action. It diminishes the milk. Variable results have been reported as to menstruation.

Gastric Irritation.--Large doses of bromid, especially in concentrated solutions, produce nausea, vomiting and gastralgia, by local salt action.

Acute Bromid Poisoning.-This is rare, but may occur from large doses, especially if the excretion is imperfect in nephritis. There is mild collapse, impaired speech, stupor, and paralytic phenomena. The treatment would be by $\mathrm{NaCl}$ infusion.

Bromid Rashes.- These occur frequently, especially in the face, when the administration of $\mathrm{Br}$ is prolonged. They resemble those of iodism. The mucous membranes may also be irritated. Similar effects occur in animals (Bernoulli, I9I3). The rashes are best prevented or treated by cleanliness. They usually disappear promptly after discontinuation. The irritation has been attributed to liberation of bromin by the acid secretions of the sebaceous glands, etc.; but the evidence is not convincing.

Chronic Bromid Depression and Cachexia.-Long-continued use of the drug generally leads to psychic deterioration, weak memory, apathy, disturbed speech, ataxia, tremors, somnolence, sexual impotence; and to nutritional disturbances, anorexia, malnutrition, anemia, emaciation, diarrhea and lowered resistance. The symptoms disappear rapidly when - the drug is stopped or the dose reduced, and especially if chlorid is given (Schabelitz, I9 I6); but the resistance remains low for some time.

Schabelitz claims that the stoppage of the bromid in his own case caused abstinence symptoms. This does not seem to be generally the case.

Contraindications to Bromids.-Because of these depressant effects, large doses of bromids should be avoided in mental or physical debility, old age, melancholia, neurasthenia, etc.

Choice of Preparations.-The inorganic bromids are most efficient. $\mathrm{NaBr}$ is generally preferred because it is less irritant, and has a less disagreeable taste than $\mathrm{KBr}$, or especially $\mathrm{NH}_{4} \mathrm{Br}$ and $\mathrm{LiBr}$. $\mathrm{NH}_{4} \mathrm{Br}$ liberates bromin on exposure to air, which is a serious objection. A mixture (KBr:NaBr: $\left.\mathrm{NH}_{4} ; \mathrm{Br}:: \mathrm{I}: 2: \mathrm{I}\right)$ has been advocated ("Erlenmeyer Mixture"); but it is difficult to see any advantage.

$\mathrm{CaBr}$ adds the depressant action of $\mathrm{Ca}$ and is said to be more efficient; but the continued administration of the large quantities of $\mathrm{Ca}$ may be detrimental. The same holds probably also for strontium bromid. $\mathrm{HBr}$ is too irritant. 
Organic Br Compounds are said to be less likely to produce toxic $\mathrm{Br}$ effects; but this could be explained entirely by their lower $\mathrm{Br}$ content. No real advantage has been established for them, and they are probably much less efficient.

No effect can be expected from the bromin in salts which are used in small doses$e$. g., bromid of quinin, of arsenic, etc. In these it can be useful only by influencing the solubility or dissociability of the compound.

\section{PREPARATIONS-BROMIDS}

Acidum Hydrobromıcum Dilutum (Ac. Hydrobr. Dil.), U.S.P., B.P.- io per cent. of HBr. Colorless, odorless liquid; strongly acid taste. Dose, I c.c., I5 minims, U.S.P.; I to 4 c.c., 15 to 60 minims, B.P.; diluted.

Ammon. Brom., U.S.P., B.P.; $\mathrm{NH}_{4} \mathrm{Br}$.-Freely sol. in water (1 : r.3); sol. in alc. (I : 12). Other characters. Dose, etc., as Sod. Brom.

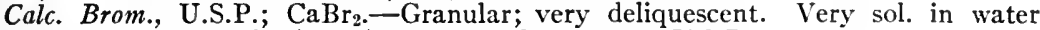
( $\mathrm{I}: 0.7$ ); freely sol. in alc. ( $\mathrm{I}: \mathrm{I} .3$ ). Dose, $\mathrm{r}$ Gm., I 5 gr., U.S.P.

Lith. Brom., U.S.P.; LiBr.-Granular; very deliquescent. Very sol. in water (1:0.6); freely sol. in alc. Dose, I Gm., I5 gr., U.S.P.

* Pot. Brom., U.S.P.; B.P.; KBr.-Freely sol. in water (r: r.5); slightly sol. in alc. ( $1: 250)$; freely sol. in glyc. $(1: 4.6)$. Other characters. Dose, etc., as Sod. Brom.

* Sodii Bromidum (Sod. Brom.); U.S.P., B.P.; NaBr.-Colorless, cubical crystals or white granular powder; odorless; saline taste. Freely sol. in water ( $1: 1.1)$; sol. in alc. (I: I6). All bromids are incompatible with strychnin. Dose, I Gm., I5 gr., U.S.P.; 0.3 to $2 \mathrm{Gm}$., 5 to $30 \mathrm{gr}$., B.P.; in dilute solution.

Stront. Brom., U.S.P., B.P.; $\mathrm{SrBr}_{2}+6 \mathrm{H}_{2} \mathrm{O}$.-Very sol. in water ( $\mathrm{r}: 0.35$ ); sol. in alc. Dose, as for Sod. Brom.

\section{ORGANIC BROMIN COMPOUNDS}

The following are described in N.N.R.: Adalin. = Brom-diethyl-acetyl carbamid. Brometone. $=$ Tribrom-tertiary-butyl alcohol. Bromipin. = Brominized Sesame Oil. Bromural. = Monobrom-isovaleryl urea. Brovalol. = Bornyl-bromvalerate. Sabromin. = Calcium dibrom-behenate.

\section{IODIDS}

General Statement.-Our knowledge of the action of iodids is derived mainly from clinical, rather than from experimental data. They increase the secretion of mucus and urine; rather more powerfully, than other salts, but probably by the same mechanism. They are therefore used as expectorants and diuretics, and to promote the elimination in chronic mercury and lead poisoning. Their continuous use, especially in susceptible individuals, produces inflammatory phenomena in the skin and mucous membranes (iodism). Other iodin compounds act similarly.

Their principal use is in the various manifestations of tertiary syphilisThey do not act on the spirochetes, but lead to the absorption of gummatous exudations and perhaps connective tissue. This action is not explained, but is perhaps analogous to the iodism. The iodids are similarly useful in aortic aneurism, and in arteriosclerosis and angina pectoris. They have no direct effect on the circulation. Their employment in chronic rheumatism, fibroses, asthma, neuralgias, etc., rests perhaps on a similar basis.

The administration of iodids and other iodin compounds increases the formation of iodothyrin, and therefore affects metabolism. Their cautious use is often beneficial in goiters, while excessive doses are dangerous. 
Absorption, Fate and Excretion.--These are similar to the $\mathrm{Cl}, \mathrm{Br}, \mathrm{SCN}$ and $\mathrm{NO}_{3}$ ions. They may mutually displace each other in their excretion. The elimination of iodid is more rapid than that of the related ions. A limited amount of iodin is stored as a special organic compound in the thyroid gland.

Absorption.-The inorganic iodids are rapidly absorbed from mucous membranes; so that, with oral administration, the absorption occurs principally from the stomach (Boruttau, I9ro). The rectum is said to absorb even better (A. Friedmann, 1910).

Rapidity of Absorption.-Administering KI to dogs with duodenal fistulas, iodid could be detected in the duodenum within five minutes; the absorption was completed in about three hours (L. Netzger, iq11).

Intestinal Loops.- Hanzlik, I9 2 , found the absorption of $\mathrm{NaI}$ solutions, placed in ligated loops, nearly equal from all parts of the small intestines; somewhat less from the stomach and colon. The rapidity of absorption was not materially influenced by the extent of surface, or by the concentration of the solution. The absorption is very rapid at first; but becomes slowed when one-half to three-fourths has been absorbed. This inhibition is due to a local change in the epithelium, which is also produced by the local application of dilute $\mathrm{NaCl}$ solution; but not by altering the haloid content of the tissues.

Absorption from the Skin.-The application of iodid ointment is irrational; for although some absorption occurs, it is very imperfect.

The absorption varies with the iodin compound, the ointment base, and the texture of the skin. It is best for the scrotum. Herzfeld and Elin, rgr2 (who also studied several compounds), state that petrolatum secures the best absorption; while Bartenbach, I 909 , found it better from lard than from petrolatum or lanolin.

Channels of Excretion.-This occurs mainly by the urine; but partly also by the saliva, gastric juice, sweat (Tachau, I9II), and other secretions and body fluids and effusions.

Salivary Excretion.-This begins usually in six and one-half to eleven minutes. It is somewhat delayed when the stomach is filled, and much more in gastric diseases. This has been proposed as a diagnostic test $(0.2 \mathrm{Gm}$. KI in gelatin capsules); but it is inconstant and unreliable. The salivary excretion gives rise to a persistent disagreeable melallic taste; best corrected by gargling with bicarbonate.

Other Fluids.-The sweat contains only traces (Kellermann, r905; Tachau, r9ri). The Bile in man excretes about I per cent. of the administered iodid in twenty-four hours, the maximum being in three hours (Frickes, 1908 ). Its presence in effusions and cerebro-spinal fluid, five to fifteen hours after administration, has been confirmed byAchard and Ribot, I 909 . Its passage into the cerebro-spinal fluid is not constant (Hald, I9Ir). It also passes into the milk (Reipt and Scheffer, r 908 ).

Form of Iodin in Urine. - This exists mainly, if not solely, as iodid ions. A small part is probably excreted in organic combination (Crespolani, I909), but the evidence for this is somewhat controversial (Schuerhoff, I905; Harnack, I910).

Rapidity of Urinary Excretion.-The iodid appears in ten to twenty minutes, and reaches its maximum in two hours. About four-fifths is excreted within a few hours; but the remainder very slowly (Heubel, 1865). The excretion is twice as rapid as that of $\mathrm{Br}$ (Heimann, I912); but considerable retention occurs (from one-sixth to one-half after a single dose). With doses of 0.5 to $\mathrm{I} \mathrm{Gm}$., the iodid reaction persists for forty hours; after larger doses or continued administration, traces persist over twenty days. The excretion can be hastened by the administration of chlorids and similar ions, but not by diuresis.

Influence of Related Ions.-This was discussed under Chlorids and Bromids. The administration of chlorids and related ions hastens the elimination of iodids (Herzfeld and Gormidor, rgr2); while Cl-poor food increases the retention (Sarvonat and Crémien, I9II).

Iodid Test of Renal Efficiency.-If a single test-dose of $\mathbf{r} \mathrm{Gm}$. of $\mathrm{KI}$ is taken after a meal, a healthy individual excretes on the average 80 per cent. of the iodid in the first twenty-four hours, and 15 to 20 per cent. between twenty-four and forty-eight hours, independently of quantity of the urine. At the end of this time, the urine fails to respond to the ordinary iodid test. A smaller excretion or more persistent reaction shows diminished permeability of the kidneys. With interstitial nephritis, for instance, the 
excretion may be only i 7 per cent. and extend over five or six days. Tubular nephritis; acute or chronic, shows nearly normal excretion (Sivert and Kaminsky, I9I4).

Considerable delay occurs in experimental chronic passive renal congestion (Rowntree). The iodid delay would therefore seem characteristic of vascular rather than tubular disturbance, contrary to the view of Schlayer and Takayasu, 1913. The elimination of KI, after I Gm. by oral and rectal administration, has been studied in a variety of clinical conditions by Wovschin, I9r 5 .

Differences with Age.-Amstad, 1915, finds the excretion in sucklings the same as in adults ( 64 to 72 per cent.); but older children appear to excrete less effectively; namely, 37 to 50 per cent. between three and five years; $4 \mathrm{I}$ to 48 per cent. between five and ten years. He offers no explanation for this curious observation.

Distribution of the Retained Iodid in Normal Tissues.- When an iodid has been administered, it circulates in ionic form; organically combined iodin having been conclusively demonstrated only in the thyroid (Blum and Gruetzner, I914; Adler and Czapski, IgI 4); although some investigators believe that traces may occur in other tissues, especially the liver (Justus, 1902 and 1908). The distribution has been investigated by Heubel, $1865 ; \mathrm{O}$. Loeb, 1907 and 1913 ; and Boruttau, 1910. The highest percentage is found in the thyroid; then comes the blood; then the secretory and lymph glands, kidneys and lungs; much less in the muscles, intestines, testes and bones; traces in the hair; none in the brain, spinal cord, fat or bone marrow (except traces if the lipoid-soluble organic forms are given). On withdrawal, iodin (organically combined) is retained in the liver and sometimes in the blood.

Partition of Iodin in Cell Constituents.-McLean, I9I4, investigated the proportion of the retained iodin in the water-soluble, protein and lipoid fractions of the liver, both after administration to an animal, and after direct addition to the excised liver tissue. With KI, the results were the same in either case: 68 per cent. in the water-soluble fraction, 32 per cent. in the lipoid, and none in the protein. 'This held also for iodalbin administration. With iodized fat (sajodin) administration, the lipoid fraction showed a larger ( $5^{8}$ per cent.), and the water-soluble a smaller ( 42 per cent.) fraction.

Distribution in Pathologic Tissues.-These generally contain more than normal This is not explained by simple diffusion, since the tissues may contain more than the blood (O. Loeb, 1913).

The increased quantities have been found in tuberculous tissue (Loeb and Michaud, 1907); carcinomatous tissue (Velden, 1908; Takamura); syphilitic tissue (Loeb, 1912); pus (Loeb, 1907); other necrotic tissue and implanted agar (Wells and Hedenburg, I9r3; and organs rendered hyperemic by Bier's stasis (Salomon, 1913). Tuberculous tissue is said to contain iodin even in animals to whom no iodin compound has been administered (P. A. Lewis and Krauss, I9ז4).

Organic Iodin Preparations.-Several different types of these have been introduced to avoid the undesirable actions of KI. This can be accomplished only very imperfectly. Their slight and doubtful advantages do not compensate for the extra cost (McLean, 1912). They are all decomposed in the body, and develop the iodid action only when the iodid ion is regenerated from them (Erlenmeyer and Stein, I909). They do not therefore avoid iodism if active doses are given. The iodinprotein compounds are decomposed before they reach the blood, and behave practically like inorganic iodids. Iodized fats and fatty acids are absorbed largely unchanged and excreted more slowly, and therefore furnish a more steady supply of iodid. Their temporary distribution in the tissues is also different. They are held for a time in lipoid-soluble form by the cells, and therefore enter more readily into nervous and other lipoid tissue. However, this would probably not favor their penetration of syphilitic tissue It is not clear that these features are especially advantageous (Dock, I909); nor are these drugs entirely free from undesirable side actions (Notthaft, r9ro; iodipin, Seifert, Nebenwirk, r9r5, p. 2I4).

Iodin-Protein and Similar Compounds.-Iodo-albuminates, -peptonates, -tannates, -gelatin; iodon, iodolose, iodethylglycin, are so rapidly decomposed that they behave and are excreted practically like inorganic iodids; and have no advantage (Filippi, I9I2; 
McLean, 1914). Even the relatively firm iodalbacid is completely decomposed before it reaches the blood (Fuerth and Friedmann, I908; McLean, I915).

Iodized Fats and Fatty Acids.-Iodipin, sajodin, etc., are absorbed, decomposed and excreted more slowly (Winternitz, I893; Boruttau, I9 I0; Bonanni, I9I I; Abderhalden and Hirsch, I9II). This applies also to Iodostarin (biniodo-taririnic acid) and lipoiodin (ethylester of biniodo-brassidinic acid) (Filippi, I9 I 2; Hassel, I9 I4).

IfcLean found that they are retained mainly in the lipoid-soluble fraction of the cells; and therefore enter the nervous system more freely than inorganic iodids. Their final excretion is in inorganic form (McLean, 1914, 1915).

Secretions and Urine.-Todids have a saline diuretic action. They increase the secretion of bronchial mucus and render this less viscid. They are therefore used as liquefying expectorants. This may also contribute to their action in asthma. The secretion of saliva is not affected (V. E. Henderson, r9ro). Their use in chronic mercury and lead poisoning is probably due to the conversion of the stored metals into more soluble compounds, but this is hypothetical.

Sexual Functions.-Iodids are said to increase menstruation; to decrease the secretion of milk; and cause atrophy of the mammary gland. Free iodin or loose iodin compounds (not KI) produce sterility in both sexes. The spermatoblasts show histclogic changes, and the formed spermatozoa are also affected (L. Adler, 1914).

Metabolism.-Iodids produce no demonstrated, definite, direct effects on normal metabolism. Their continued use, however, leads to malnutrition, as described under iodid cachexia. This may be due partly to their influence on the thyroid gland (see under Thyroid).

There may be some increase of urea, total nitrogen, uric acid, purin bases, and chlorid excretion (Chistoni, I9II).

Hypodermic injection of large doses may produce no effect, or considerable increase or decrease of metabolism (Sgalitzer, 1908).

Normal Occurrence and Relation to Thyroid.-The thyroid gland contains a fairly high percentage of iodin as iodothyrin. It can be increased by the administration of iodin or any of its compounds. There are quantitative differences, the I of bladderwrack being perhaps roo times as active as that of $\mathrm{KI}$; probably because it is more easily assimilated by the colloid (Hunt and Seidell, rgro).

Rapidity of Fixation in Thyroid.-Injecting intravenously $5 \circ \mathrm{mg}$. of $\mathrm{KI}$ into dogs, Marine and Rogoff, 1916 , found the fixation completed in five minutes; i.e., as much had been fixed in the thyroids of the dogs killed after this interval, as in those killed after 30 hours. None was fixed by the liver or spleen. I9I4.

The normal occurrence of iodin in animal and plant tissues was studied by Cameron,

Use in Goiter.-The careful administration of iodids causes a regression of active thryoid hyperplasia into the relatively harmless colloid type of goiter. Marine, I9II and I9I2, advocates the Syrup of Ferrous Iodid, in doses increasing gradually from 0.3 to I. 2 C.C., 5 to 20 drops, per day.

Toxic Effects in Goiter.-Goiterous, especially Basedow patients, often react badly to the large doses of iodid which are commonly used in other diseases; presumably by the overproduction of iodothyrin in the hyperplastic gland. The patients become emaciated, even when the appetite remains good. There are nervous and psychic disturbances, sometimes fever. The symptoms are described in detail by Oswald, r9 15 . The effects do not cease promptly when the administration is suspended (Krehl, rgro). 
Use in Circulatory Diseases.-Iodids are extensively employed in arteriosclerosis, coronary sclerosis, angina pectoris, aneurism (Bouillaud, I858), etc. In the lighter grades of these conditions their continued administration seems to give gradual relief to the functional phenomena, especially the pain; but probably without effecting a cure. The effects are not due to any change in the blood pressure, but probably to structural alterations in the vessel walls, perhaps absorption of cellular exudates; analogous to the effect on syphilis. In many cases, a syphilitic factor is directly concerned.

Direct Action on Circulation.-Potain and others supposed that the iodids lower blood pressure by depressing the heart or blood vessels. This applies to fatal doses of KI, but is then a potassium effect. Ordinary doses have no effect on the blood pressure in normal men or animals (Stockman and Charteris, I 901 ). The slight circulatory changes in intravenous injection, described by Lehndorff, I9I4, and Macht, I9I4, seem practically insignificant. Salant and Livingston, r916, observed no effects on the circulation of frogs or mammals, even with fairly high concentrations of NaI. (The intravenous injection of free iodin markedly depressed the circulation in cats, not in dogs.)

The blood pressure in arterial hypertonus is also unaffected acutely, and probably even with continued administration (Hewlett, I9I2). On the other hand, some clinicians claim a definite fall ( $f . i$. Rutkewitch, $2 \mathrm{Gm}$ daily). There is also a fall in lead poisoning probably indirect (Capps, I9I2). Gurewitsch, I907, frequently observed increase of pulse rate with iodid administration. This may have been a thyroid effect.

Isolated Heart and Arteries.-Macht, I914, investigated the effects of replacing part of the NaCL of Locke's solution by $\mathrm{NaI}$ or $\mathrm{KCl}$. The results can not, of course, be transferred to intact animals, or man, since they involve different concentrations. $\mathrm{He}$ concludes that the iodid and $\mathrm{Na}$ ion stimulate the heart and constrict the vessels; the cardiac stimulation being strong for iodid, weak for $\mathrm{Na}$. The $\mathrm{K}$ ion depresses the heart and relaxes the vessels. Very weak solutions of metallic iodin are also stimulant; stronger concentrations are toxic. Salant and Livingston, 1916 , found that the perfusion of dilute solutions of free iodin through the isolated frog-heart produces cardiac irregularity and depressant after-effects. More concentrated solutions cause greater irregularity and diastolic arrest.

Decreased Viscidity of the Blood.- - Iodids and sulphocyanids decrease the viscosity of colloids (Pauli, I903; the other haloid ions have a similar but weaker effect; Gazzeti, 1913). O. Mueller and Inada, 1904, claimed diminished viscidity of the blood from therapeutic doses, and advanced this as an explanation of the circulatory effect. This could not be confirmed by Determann, rgo8; Determann and Broeking, I91 2; Lanza and Vergano, I912, and Tscheboksarow, I913. Chistoni, rgro, found more positive results. The changes are evidently inconstant, and perhaps even accidental; they are so small that they would have no significance.

Epinephrin Atheroma.-Koranyi, I906, claimed that this could be prevented by iodids; but this has been disproven (Biland, I 906; L. Loeb and Fleisher, 1907; Hedinger and Loeb, 1907; Klieneberger, 1907).

Iodids in Syphilis. - The use of iodids in syphilis was introduced by Wallace, 1836 . Large doses are used in the various tertiary and late secondary manifestations. They give marked relief, largely by leading to the absorption of gummatous formations. They are not curative, and do not affect the Wassermann reaction or spirochetes. The mechanism of their action is not explained, but is perhaps analogous to the irritant phenomena of iodism. It has been suggested that they cause the production of a proteolytic ferment. This is plausible, but requires further proof. Longcope, I9I4, suggests that the breaking down of syphilitic tissue favors the access of mercury or arsenic to the spirochetes.

Analogous Reactions in Tuberculosis and Other Conditions.-Iodids produce a similar resorptive action on the granulations of actinomycosis, sporotrichosis and blastomycosis. In tuberculosis, they soften the connective tissue and the tubercles, and cause a reaction similar to tuberculin. They have an analogous effect in leprosy and produce febrile reactions in a variety of infections. 
Iodids are said to be contraindicated in luberculosis. Sorel, 1909, found that they hasten death in experimental tubercular infection of guinea pigs.

Luetin Reaction. - Administration of potassium iodid or other iodin compounds leads to a positive pustular or nodular luetin reaction, even in non-syphilitics. Moreover, the intradermal injection of agar or starch alone produces similar reactions in iodated patients (Sherrick, 19r5); Kolmer, Matsunami, and Broadwell, 1916).

Older Theories of Mechanism of Antiluetic Action.-Numerous theories have been proposed without adequate evidence (Capps, I I I 2 ). The alterations produced by salt action, and the effect on the thyroid, may play a part; but other salts and thyroid itself lack the antiluetic action. Binz, 1875 and 1894 , suggested the liberation of free iodin; but this is improbable. Heinz, I 899, describes increased leucocy tosis and lymph flow, and assumes increased permeability of the blood vessels. Hirsch, 1914, however, did not find the phagocytic removal of tubercle bacilli hastened by daily KI administration.

In reviewing these various theories, one must be struck by the fact that the results of iodid are altogether too specific to be explained by such general and indefinite explanations.

Ferment Theory.-The resorptive action of iodids on various pathologic tissues, and the similarity of its febrile and inflammatory reactions to anaphylaxis, suggest the possibility that it may cause or favor proteolysis under certain conditions.

All the phenomena would thus be brought under the same heading; the luetic, tubercular and other pathologic tissues merely having an exaggerated susceptibility to the proteolytic and anaphylactic phenomena of iodism. These views, although plausible, have as yet no firm experimental foundation.

Several observers have described a rather doubtful acceleration of autolysis by the addition of iodid, in vilro; and a more definite acceleration after the administration of iodid (Stookey, 1907; Kepinow, I911; Kashiwabara, I912; Morse, 1915).

Dick suggested that iodids lead to the production of a proteolytic ferment. Jobling and Peterson, I9I4 and I9I5, on the other hand, believe that they restrain the antitryptic activity of the serum and tissues, by binding the unsaturated carbon atoms of the antitryptic lipoids, thus permitting the autolytic digestion of the necrotic, luetic or tubercular material, etc. In support of this, they find that the antitryptic power of serum or caseous tubercular material is actually diminished by incubation with iodin or iodids; that full doses of iodid, administered to man, greatly lower the antiferment index of the serum, which, however, recovers with the outbreak of iodism; and that the administration renders caseous tuberculous material digestible, explaining the appearance of tubercle bacilli in the sputum. They assume that gummas would be similarly digested, thus also exposing the spirochetes to the germicidal action of mercury or arsenic. It will be seen that the theory, although attractive, still involves some unproven assumptions.

Use in Other Conditions.-Iodids are also employed to cause the absorption of nonluetic hyperplastic tissue; to reduce chronic inflammatory swellings; against lymphatic swellings; in gout and chronic rheumatism; in neuralgias; in asthma, etc. The mechanism of their action is probably analogous to that in syphilis; in many cases there may also be an undiscovered luetic factor. The action on bronchial muscle is doubtful (Titone, 1913).

Iodism.-The use of iodids is apt to produce irritative phenomena of the skin and mucous membranes, taking the form of various rashes, and of coryza, headache, conjunctivitis, laryngitis, bronchitis, etc.-i.e., the various manifestations of a "cold." Less common are stomatitis, parotitis, anorexia, etc. The skin affections generally occur somewhat later. They may consist in acne, furuncles, erythema, purpura, urticaria, vesication, etc., and these may be accompanied by fever.

The susceptibility to these irritations varies greatly. They may set in a few hours after a relatively small dose or only after the long-continued use of large doses; or again, the symptoms produced by small doses may disappear when the dosage is increased. They are more common when the excretion is interfered with by nephritis (v. Noorden, I908). They disappear in a few days when the drug is discontinued. They may be produced by any iodin compound, e.g., iodoform; but probably only after the dissociation of the iodid ion. They are generally attributed to the liberation of free iodin by carbonic acid, sebaceous secretion, etc. 
There is no good evidence for this view. It is probably incorrect, for it is difficult to conceive how iodin could be liberated under the conditions of the body; and similar symptoms are produced by the related sulphocyanid, from which no irritant element could be liberated.

Bruck, I909, claimed that guinea pigs could be rendered somewhat hypersensitive to iodids by the injection of serum from hypersensitive patients. This was not confirmed by Cole, ror r. Copaiba was also negative.

The catarrhal phenomena can be suppressed in one to three days by the administration of calcium lactate, $4 \mathrm{Gm}$. per day (Velden, I9 3 ); but the continued use of calcium may have drawbacks which probably make it inadvisable.

Iodid Cachexia.- The continued administration of iodid may also lead to anemia, emaciation, lowered tone, mental depression, nervousness, insomnia, nervous tachycardia, sexual impotence, etc. The phenomena result partially from the catarrhs, the thyroid action, etc.

Acute Effects of Intravenous Injection.-Large doses $(0.8 \mathrm{Gm}$. NaI per kilogram produce death in twelve to thirty-six hours, with symptoms of central paralysis. Autopsy shows pleural exudation, of ten with pulmonary edema and pericardial eff usion (Boehm, I876; Chiari and Januschke, 1910). Similar effects are produced with hypodermic but not with oral administration (Sgalitzer,.1908). The symptoms are complex, and the statements regarding central actions are contradictory, and probably untrustworthy (Barbera, I900; Jodlbauer, I902; Heinz, 1898). Intravenous injection is also said to paralyze the vagus endings and to lower the excitability of the depressor. In frogs the iodids produce rigor and acidity of the muscles (Stockman and Charteris, I9OI).

Choice, Administration and Dosage.-KI is generally preferred, although it has probably no real advantage over NaI. It is best administered freely diluted with water or milk, an hour after meals. The dosage for non-syphilitic conditions is from 0.3 to $2 \mathrm{Gm}$., three times daily. In lues, this is often raised to $5 \mathrm{Gm}$. or more. It may be started with 5 drops of the saturated solution, increasing each dose by I drop (I gr.) each day, reducing the dose if iodism becomes severe. In syphilis, one may begin with doses of $\mathrm{I} .5 \mathrm{Gm}$., increasing by $0.3 \mathrm{Gm}$., every second day. Infants tolerate large doses (in meningitis, to 23 per day; $\mathbf{A}$. Jacobi).

Incompatibilities.-Iodids should not be used with calomel or hydrogen peroxid, even in separate prescriptions. They precipitate heavy metals and some alkaloids (strychnin). They are incompatible with mineral acids and oxidizing agents. Salts or solutions which have become yellow by liberation of iodin should not be dispensed.

\section{PREPARATIONS-IODID}

Acid Hydriod. Dil., U.S.P., B.P.; Io per cent. of HI.-Colorless or pale yellow, odorless liquid; strongly acid taste. Should not be used if colored more than pale yellow. Dose, 0.5 c.c., 5 minims, U.S.P.; 0.3 to 0.6 c.c., 5 to 10 minims, B.P.; diluted.

Syr. Acid. Hydriod., U.S.P., B.P.- I per cent. of HI. Dose, 4 c.c., I dram, U.S.P.; 2 to 4 c.c., $1 / 2$ to $\mathrm{I}$ dram, B.P.

Ammon. Iod., U.S.P., NH ${ }_{4}$ I.-Minute, colorless, cubical crystals, or a white, granular powder; odorless; sharp, saline taste. Very hygroscopic. Incompatibilities, as Pot. Iod. Very sol. in water $(\mathrm{r}: 0.6)$; freely sol. in alc. ( $\mathrm{I}: 3.7)$ or glyc. (1 : 1.5$)$. Dose, 0.3 Gm., 5 gr., U.S.P.

('Syr. Ferr. Iod., U.S.P., B.P.-Each cubic centimeter is equivalent to 0.0525 Gm., 0.85 gr., of iodin: see Iron Preparations).

*Potessii Iodidum (Pot. Iod.), U.S.P., B.P.; KI.-Colorless cubical crystals, or white granular powder, faint iodid odor, sharp saline taste. Incompatible with mineral acids, oxidizing agents, and many alkaloids. Very sol. in water ( $: 0.7)$; sol. in alc. $(\mathrm{I}: 22)$; freely sol. in glyc. (I:2). Dose, $0.3 \mathrm{Gm}$., 5 gr., U.S.P.; 0.3 to I.2 Gm., 5 to 20 
Lr., B.P. $\Lambda_{I}$ : I solution ( $\mathrm{I}$ minim $=\mathrm{I}$ grain) is convenient for dispensing. I)issolve I 5 in $5^{1,2} \overline{5}$ of water, and make up to I fld. $\overline{3}$.

Lim. Pot. Iod. C. Sup. B.P.- I 2 per cent. of KI (Irrational).

Lng. Pol. Iod., B.P.- 10 per cent. of KI (Irrational).

* Sodii lodidum, U.S.P., B.P.; NaI.-Properties and dose similar to KI. Very sol. in water $(1: 0.55)$; freely sol. in alc. $(\mathrm{I}: 2)$ and in glyc. $(\mathrm{I}: \mathrm{I})$.

Stront. Iod., U.S.P., $\mathrm{SrI}_{2}+6 \mathrm{H}_{2}$ O.-Very sol. in water (1 :0.2); sol. in alc. Dose, 0.3 Gim., $5 \mathrm{gr}$.

\section{SULPHOCYANIDS (RHODANATES)}

These produce actions resembling the iodids; but their use has not become popular. They are not dissociated into cyanids, and are relatively non-toxic. Their normal occurrence in the saliva is interesting, but without much practical significance.

Iodid Effects.- NaSCN influences the aggregation of colloids in the same way as the iodid ion, being even somewhat more solvent. This analogy suggested a therapeutic trial. In the limited number of cases in which it has been tested, it was found to affect neuroses, arteriosclerosis, and syphilis in a manner analogous to the iodids, being even somewhat stronger (Pauli, I903 and I910). It also hastened the elimination of metals - perhaps by rendering the metal-protein compounds more soluble. It resembles the jodids further in producing coryza, acne, and other symptoms of iodism. Corper, r9r5, observed no results in tuberculosis. Nerking, r9r 3 , reports many disagreeable effects, especially in gastric hyperacidity. A conspicuous difference from iodids lies in the fact that it does not act on the thyroid gland. It was used as the sodium sulfocyanid, in the maximal dose of I Gm. ( 5 gr.) per day. This salt is quite soluble in water. The potassium salt could probably be used in the same dose.

Toxic Actions.- The earlier authors (Cl. Bernard, etc.) considered the sulphocyanids as relatively non-toxic; and as mentioned, they have been used therapeutically in gramdoses. F. Franz, 1912, confirms that no cyanid is split off in the animal organism. Intravenously, very large doses, $0.08 \mathrm{Gm}$. per kilogram, produce no toxic symptoms. Even $0.36 \mathrm{Gm}$. per Kg. intravenously, produces practically no effect on the circulation or respiration or bile and urine flow (Gies, Lieb, and Kahn, 19r4). No effects resulted in man from the continuous administration of $1 / 2$ to $2 \frac{1}{2}$ grains per day. The minimal fatal dose by vein for rabbits is placed by Corper, I 915 , as 0.4 to $0.6 \mathrm{Gm}$. per kilogram. By mouth there were only the local salt actions. Lodholz, I905, described toxic effects from KSCN, which must have been due either to the potassium or to impurities.

Occurrence in Saliva.-Sulphocyanid is found in the human parotid and submaxillary saliva, but not in all individuals; also in the saliva of many animals, not in dogs and horses. It is absent in new-born infants (Rittershain); also in suppurative middle-ear disease (Juergens, r $90 \mathrm{I}$ ). It is found in other mucosæ (nasal, conjunctival, gastric). It is excreted quantitatively in the urine and feces (Pollak, 1902; A. Meyer, I904), generally in the proportion of $\mathrm{I}: 2$ (Gies and Kahn, I9I2). In dogs in which it is absent from the saliva, it occurs in the blood, liver, intestines and urine (Kahn 1912). In normal dogs, it is present in the urine to the extent of 0.001 per cent.; in the blood, 0.003 per cent.; in the bile, from nothing to 0.833 per cent. (Gies, Lieb and Kahn, 1914). No quantity depends on the available supply of $\mathrm{CN}$ radicals. It is formed when $\mathrm{HCN}$ is administered, constituting a chemical disintoxication mechanism. Its quantity is increased by tobacco smoke, which contains HCN. It is also increased by feeding amino-acids (alanin) and acetonitrile; not with sulphur, sulphid, thiourea or taurin. It is probably reduced from the protein in the liver (Kahn), the saliva being merely an excretory channel (Souza, 1907). Its content in the saliva has no relation to tooth decay (Lothrop and Gies, I9II). The quantities present in the mouth could not have

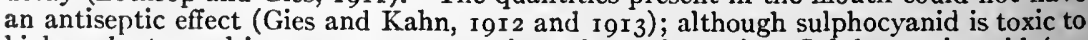
higher plants; and in greater concentrations also to bacteria. Sulphocyanic acid ( 0.3 to 0.4 per cent) inhibits the gastric digestion of fibrin (Cavazzani, 1915). A comprehensive review of the literature is given by Kahn, I91 2. 


\section{NITRATES}

Potassium nitrate, Saltpeter, was formerly used as a cardiac depressant in fevers, etc.; it is now recognized that ordinary doses do not possess this action. Its effects are simply those of a neutral salt; somewhat more irritant to the gastro-intestinal tract, and somewhat more diuretic than $\mathrm{NaCl}$. It has practically fallen into disuse. Accidental poisoning from large doses is not uncommon. It presents the phenomena of gastroenteritis, similar to those of all other soluble indifferent salts. These may be serious, especially if it is swallowed undiluted.

Fate and Excretion.-Nitrates are readily absorbed. Their excretion is similar to that of the halogen ions, and shows the usual mutual relation (Sollmann). Practically the entire quantity is excreted unchanged (Abderhalden and Hirsch, I013); but a small proportion may be reduced to nitrites. This reduction takes place so slowly, in the case of the inorganic nitrates, that no nitrite action can ordinarily be seen; but very large doses may cause methemoglobin formation (Binz and Gerlinger, 1901). Metabolism studies have given contradictory results (Grafe and Wintz, 1913).

Normal urine contains 0.1 to 0.4 per cent. of $\mathrm{KNO}_{3}$. This is considerably more than is ingested with the food, so that a part must be formed as an end-product of metabolism (H. H. Mitchell, Shonley and Grindley, I916).

Toxic Symptoms.-These consist in great abdominal pain, vomiting, frequently bloody stools; irregular pulse, convulsions, and collapse; suppression of urine, or that passed is albuminous and often bloody. The gastroenteritis may be so violent as to lead to an early fatal ending. Of the salts so far studied, the potassium nitrate is by far the most violent; $30 \mathrm{Gm}$. ( $\mathrm{r}$ ) may be fatal if taken in concentrated fcrm.

Smaller doses, long continued, cause a hemorrhagic tendency, edema, and fatty heart (Fackelmann, I898).

The treatment would consist first in dilution, since they act only by virtue of their concentration. Large quantities of water should be drunk and the stomach washed. Demulcents-milk, egg white, acacia-are also useful. The symptoms should be met as they arise.

\section{PREPARATIONS-NITRATES}

Potassii Nitras (Pot. Nit.), U.S.P., B.P. (Niter, Saltpeter), $\mathrm{KNO}_{3}$.-Colorless, transparent prisms, or white, crystalline powder; saline, cooling taste. Freely sol. in water ( $1: 2.8$ ); very slightly sol. in alc. Dose, 0.5 Gm., 8 gr., U.S.P.; 0.3 to $\mathrm{r.2}$ Gm., 5 to 20 gr., B.P.; diluted.

Sodii Nitras (Chili Saltpeter), $\mathrm{NaNO}_{3}$.-Freely sol. in water (I:I.I); sol. in alc. ( $\mathrm{r}: \mathrm{roo}$ ). Dose, as Pot. Nit.

\section{POTASSIUM CHLORATE}

General Statement.-A saturated ( $1: 16$ ) solution is used as an antiseptic and astringent mouth wash in stomatitis, especially mercurial and ulcerative; and as a gargle in pharyngitis and tonsillitis. It often seems to be effective, but its action is unexplained. It was introduced with the idea that it would act as an oxidizer, and thus be antiseptic. However, it does not part with its oxygen in the body, being excreted practically unchanged.

It should not be employed internally, since this has repeatedly led to serious toxic effects (A. Jacobi, I879), especially by the production of methe- 
moglobin and destruction of the corpuscles, thrombosis, etc. Patients should be cautioned against swallowing the gargles. The popular tablets are to be condemned.

Excretion.-Chlorates are excreted mainly by the urine, but partly also by the saliva. The urinary excretion begins promptly and is completed within forty-eight hours, about 90 per cent. of the chlorate being recovered (Rosenbloom). A great deal of this is reduced to chlorid after the urine is voided. It is doubtful whether this reduction occurs ordinarily in the body. Bromates are partly reduced in the tissues, and iodates intensively, when given by mouth, but not when administered hypodermically or by vein (Crespolani, rgog).

The distribution of fatal doses has been studied by Rosenbloom, I9I2.

Blood Changes. - When the chlorates are added to blood, either inside or outside the body, they effect the formation of methemoglobin. The chlorate ion is not used up in this process, so that it may convert an indefinite amount of hemoglobin. They differ in this respect from the nitrites (see index), and their action is in consequence more violent and more prolonged. They may in this way produce an actual asphyxia.

In addition to this formation of methemoglobin, the chlorates break up the blood corpuscles (Huber, I9I 2). This may produce embolism; the proteins, etc., which are liberated by the destruction of the corpuscles are irritant to the kidneys and produce interstitial nephritis, with the usual phenomena-proteins in the urine, casts, sometimes hemoglobin compounds. Possibly the chlorate ion itself irritates the kidneys.

Fat embolism of the lungs and other organs are also of frequent occurrence, at least in animals. They originate from the bone-marrow. The blood contains numerous normoblasts. Fragmentation of the heart muscle is a frequent cause of death (Winogradow, 1907).

Methemoglobin Formation by Chlorates.-The blood of different animals shows a different degree of susceptibility for the methemoglobin formation. These differences are common with all poisons acting on the blood, especially as between carnivorous and herbivorous animals. The cause is not understood, but is perhaps connected with differences in the alkalinity of the body. The conversion occurs fairly readily intra vitam in man, dog, and cat, while rabbits and guinea pigs are almost immune. But in the test-tube chlorates convert rabbit's blood, although more slowly than dog's. The action is much slower outside of the body, of ten requiring several hours.

Iodates and Bromates have a similar action on hemoglobin (Heinz, r898).

Potassium Chlorate Poisoning.-This is fairly common, either by an overdose, since the laity does not generally regard it as a toxic substance; or by the swallowing of some of the solution given for gargling.

Fatal Dose and Course.-The susceptibility varies greatly and this is one of the principal dangers. The variation may be due to differences of absorption and excretion, embolism, etc. In general, ro $\mathrm{Gm}$. may be considered as toxic, 15 to $30 \mathrm{Gm}$. as fatal (Lewin). The mortality is about 70 per cent. The symptoms may start at once or be delayed to six hours; death may occur in six hours to seven days.

Symptoms of Poisoning.- These consist in gastritis (nausea, vomiting, gastralgia); methemoglobinemia (cyanosis, collapse and terminal convulsions); and nephritis (lumbar pain, anuria, albumin, casts, blood, etc.). The postmortem findings correspond with the symptoms.

Treatment.- This consists in gastric lavage, hot drinks, and symptomatic measures.

\section{PREPARATIONS-CHLORATE}

*Potassii Chloras (Pot. Chloras), U.S.P., B.P., $\mathrm{KClO}_{3}$ - Colorless crystals, or white granular powder; odorless; cooling, characteristic taste. Sol. in water ( $\mathrm{r}: \mathrm{r}$ I.5); nearly insol. in alc.; sol. in glyc. Explosive when triturated with organic matter (not when in watery solution). Dose, 0.25 Gm., 4 gr., U.S.P.; 0.3 to I Gm., 5 to 15 gr., B.P.

Troch. Pot. Chlorat., U.S.P. o.1 5 Gm., $21 \frac{2}{2}$ gr.; B.P., 0.2 Gm., 3 gr.

Sod. Chloras., $\mathrm{NaClO}_{3}$.-Freely sol. in water (r:I); sol. in alc. ( $\left.\mathrm{r}: \mathrm{I} \infty\right)$. Dose, as Pot. Chloras. 


\section{BROMATES}

These act similarly to chlorates, but are considerably more toxic, mainly by central paralysis (Santesson and Wickberg, 1913). They form methemoglobin in vitro, but not as actively as chlorates (v. Mehring, I885; Cushny, 1908).

\section{METHEMOGLOBIN FORMATION ${ }^{1}$}

Methemoglobin contains the same amount of oxygen as oxyhemoglobin, but in different combination, so that it is held more firmly (except against certain reducing agents; Haldane). The two hemoglobin compounds therefore differ essentially in certain of their properties: tinge.

I. The spectrum (see Fig. 29). The color of methemoglobin has more of a brownish

Red. Orange. Yellow. Green. Blue.

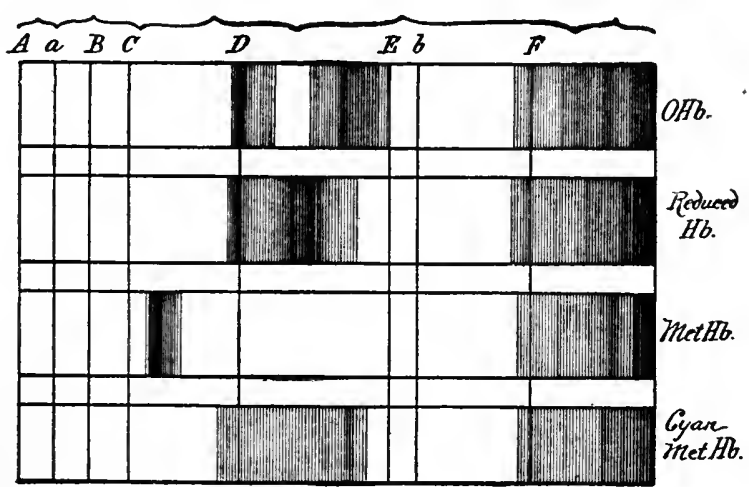

Fig. 29.-Spectroscopic bands of blood pigments.

2. In the readiness with which they give up oxygen. While the oxyhemoglobin is a very unstable compound, giving up its oxygen and taking it again, with great readiness, methemoglobin is a comparatively stable and unchangeable compound.

3. In thei behavior to certain reagents. For instance, HCN does not form any characteristic compound with oxyhemoglobin, but with the methemoglobin it gives cyanmethemoglobin. Similar compounds are formed with $\mathrm{H}_{2} \mathrm{O}_{2}$, sulphocyanids, and many other salts, and also with alkalies.

Methemoglobin may be formed from oxyhemoglobin in quite a number of d.fferent ways:

I. By oxidizing agents: Potassium chlorate, ferricyanid, permanganate, $\mathrm{H}_{2} \mathrm{O}_{2}$, etc.

2. By reducing agents: The nitrites, hydroxylamin, formalin, iodin, chrysarobin, etc.; coal-tar products. (Phenylhydrazin also gives reduced hemoglobin, and destroys some of the hemoglobin absolutely, producing a new compound which, und $\mathrm{r}$ certain conditions, yields a green pigment, hemoverdin.)

3. In the early stages of putrefaction, and by the action of light.

4. By salts and glycerin.

5. By acids (this has recently been denied).

The physiologic significance of this methemoglobin formation rests on the stability of the compound and its consequent inability to carry out the functions of oxyhemoglobin. This produces asphyxia of the tissues. Pure methemoglobin olutions may be injected into the blood, without causing any symptoms. Even he urine remains free from albumin or methemoglobin. The compound is in part secreted by the bile, in part deposited in the hematopoietic organs.

Nor is the temporary conversion of a considerable proportion of the oxyhemoglobin into its isomer of great significance; for methemoglobin is not absolutely stable, and as 
soon as the oxygen starvation of the tissues is carried to a certain degree, they seize upon the methemuglobin and decompose it. The conversion of a third of the hemoglobin in to methemoglobin causes only very slight symptoms; and life is still possible when three-fifths of the hemoglobin has been replaced. Life becomes extinct when the hemoglobin has sunk to one-third. The condition can therefore become dangerous only if the methemoglobin-former continues its action. This does not occur in therapeutic doses of any of these drugs, but may contribute to the fatal ending in cases of poisoning. The symptoms are those of asphyxia. There is a peculiar blue tint about the lips and finger-11ails, etc. The methemoglobin gradually returns to oxy- or reduced hemoglobin after death, so than an examination after everal days may fail to reveal its presence.

Most of these drugs transform the hemoglobin inside of the corpuscles, without injuring the vitality of the latter; but some cause, in addition, a breaking up of the corpuscles, and this greatly increases the danger. Aside from the asphyxia which must be proportioned to it, the proteins and other substances liberated cause injury to the kidney-albuminuria, glycosuria, methemoglobinuria, etc. It is also claimed that it causes the sudden formation of fibrin ferment, which may then cause extensive intravascular clotting. The débris is also credited with causing emboli. But these facts are not admitted by all investigators. A small destruction, such as may be caused by the subcutaneous injection of glycerin, certainly has no permanent injurious effect.

The specific action of the drug is, of course, joined to these methemoglobin effects, and may entirely overshadow them. Thus, rabbits die of $\mathrm{KClO}_{3}$ before it comes to any methemoglobin formation. Herbivorous animals are, as a rule, much less subject to the formation of methemoglobin during life, although their shed blood does not differ in this respect from that of carnivorous animals. The cause lies perhaps in the greater alkalinity of their blood, for it has been found that alkali-methemoglobin is much more easily converted back into oxyhemoglobin. The injection of alkalies has therefore been suggested in the treatment of methemoglobinemia. Other treatment consists in the administration of oxygen, artificial respiration, and shock treatment.

\section{THE REACTION OF THE BODY (REMOTE EFFECTS OF ACIDS} AND ALKALIES) ${ }^{1}$

General Statements.-The $\mathrm{H}^{(+)}$ions of acids and $\mathrm{OH}^{(-)}$ions of bases part readily with their electric charges, and therefore alter the state of colloids. In this way, and also by direct chemic changes, they produce extensive alterations in the functions of cells, which would be rapidly fatal if they exceed a certain degree.

Actual Potential and Total Acidity and Alkalinity. ${ }^{2}$ - These effects are produced only by the dissociated ions, which represent the actual acidity and alkalinity. The activity of an acid or base depends therefore not only on its total concentration, but also on its dissociation. The mineral acids, f.i., which dissociate freely, are much "stronger" than equimolecular solutions of the organic acids or acid salts, which dissociate only slightly. Undissociated molecules do not exert these actions and are practically inert in this respect. They may, however, be drawn upon to neutralize added acid or alkali, in proportion as the free ions become combined, and in this respect are equivalent to the dissociated ions. They may therefore be considered as potential acidity or alkalinity. This relation was formerly not understood, and this has given rise to much confusion. The ordinary methods of titration measure only the total acidity or alkalinity (actual + potential). In complex fluids, like the blood or urine, this bears no relation to the true actual acidity. The actual reaction is measured by the electric potential; or by the use of a series of indicators which are only affected by certain concentrations of free $\mathrm{H}^{(+)}$or $\mathrm{OH}^{(-)}$ions (Soerensen);

"The local actions of concentrated acids and alkalies are discussed under "Local Irritants;" see Index.

"Advanced students are advised to consult the book of Michaelis, "Die Wasserstoffionen-Konzentration," I0I5, for a fuller presentation of the subject and detailed data. 
or by determining the velocity of certain reactions (inversion of cane-sugar, saponification, etc.), which depend on the $\mathrm{H}^{(+)}$or $\mathrm{OH}^{(-)}$concentration.

Hydrogen-ion Concentration.-The actual reaction is also termed the true reaction or $\mathrm{H}$-ion concentration. It is generally expressed in reciprocal logarithms (as the "hydrogen exponent," " $p^{\mathrm{H}}$ " f.i., a solution containing I molecule of dissociated $\mathrm{H}^{(+)}$in $10,000,000 \mathrm{~L}$. ( $1: 10^{7}$ ) is said to have the $\mathrm{H}^{(+)}$concentration of 7.0 (also written $p \mathrm{H}=7$ ), etc. This constitutes neutrality, since water dissociates at $25^{\circ}$ so as to contain $\mathrm{H}^{(+)}$ and $\mathrm{OH}^{(-)}$ions, each in the proportion of $\mathrm{IO}^{-7}$. An increase of $\mathrm{H}$ ions means, of course, a relative diminution of $\mathrm{OH}$ ions and vice versa.

This system of notation, although convenient, is at first somewhat confusing. It must be remembered that $\mathrm{H}^{(+)}=6$ (really ${ }^{10^{-6}}$ ) means a higher acidity than $\mathrm{H}^{(+)}=7$ (really $10^{-7}$ ). The acidity is therefore inverse to the number. Also, since logarithms are used, $\mathrm{H}^{(+)}=3$ means an acidity $\mathrm{r}, 000$ times as great as $\mathrm{H}^{(+)}=6$; not twice as great.

The following may perhaps serve to render the subject clearer:

$$
\begin{aligned}
{ }_{p} \mathrm{HI}_{\mathrm{I}} & =\frac{N}{{ }_{1} 0} \text { acid } \\
{ }_{p} \mathrm{H} 6 & =\frac{\mathrm{N}}{\mathrm{I}, 000,000} \text { acid } \\
{ }_{p} \mathrm{H}_{7} & =\frac{N}{N e u t r a l i t y} \\
{ }_{p} \mathrm{H} 8 & =\frac{N}{1,000,000} \text { alkali } \\
{ }_{p} \mathrm{H}_{4} & =\frac{N}{10} \text { alkali }
\end{aligned}
$$

The older notation is simpler, but less convenient; i.e., $\mathrm{H}^{(+)}=2 \times 10^{-\bar{i}}$ denotes twice as great acidity as $1 \times 10^{-7} ; 0.5 \times 10^{-7}$, one-half as great; etc.

Actual Reaction of the Body-fluids.-The normal reaction of the tissues and fluids within the body proper is very near neutrality, inclining a.trifle to alkalinity $\left(\mathrm{H}^{+}\right)=$about 7.1 to 7.8$)$. The gastric and intestinal juices and urine are the only fluids in higher animals which depart from this rule. The neutrality of the blood is maintained even in acidosisalthough the cells where the pathologic acid is formed, probably develop an increased acidity, which is supposedly responsible for the symptoms. Normal living cells have an alkaline or neutral reaction, judging by intravitam stains (A. P. Mathews).

Reaction of Normal Blood.-The $\mathrm{H}^{(+)}$of fresh defibrinated blood is very weakly alkaline, almost neutral. It is stated, for instance, as 0.3 to $0.7 \times 10^{-7}$ (Friedenthal, I 904 ); ${ }_{p} \mathrm{H}=7.45$ for arterial, 7.3 for venous (Hasselbach, $\mathrm{r} 9 \mathrm{I} 3$ ); 7.6 to 7.8 for human serum, 7.4 to 7.6 for oxalated blood (Levy, Rowntree and Marriott, 1915), at air temperature; at $37^{\circ}$ the alkalinity would be about $0 . x$ higher. Similarly, excessive ventilation of the lungs with air or oxygen lowers the $\mathrm{H}$ concentration of the blood, whereas excessive ventilation with $\mathrm{CO}_{2}$ increases it (Milroy, I9I4). The $\mathrm{CO}_{2}$ tension has some influence, so that venous blood is a little less alkaline than arterial.

Pathologic Blood.-Levy, et al., found the reaction of the serum in sixty miscellaneous medical cases to be normal $\left({ }_{p} \mathrm{H} 7.6\right.$ to 7.8$)$; the whole blood was also nearly unchanged ${ }_{p} \mathrm{H} 7 . \mathrm{I}$ to 7.3$)$. Even when symptoms of acidosis are present, the alkalinity is but little decreased ${ }_{p} \mathrm{H}$ of serum, 7.2 to 7.5 ; Levy et al. Under alkali therapy, this is restored toward normal.

In diabetic coma, Benedict, r9o6, records an extreme change to $1.5 \times 10^{-7}$. Masel, I9I3, and Van Slyke, I9I5, found practically no change, except just before death (7.II, Masel); van Slyke, however, observed that the addition of $\mathrm{HCl}$ to acidosis blood raises its $\mathrm{H}$ concentration much more than when added to normal blood. The essential blood change of acidosis is therefore the diminution of the reserve alkali.

In normal animals, feeding with acids or alkalies has practically no effect on the $\mathrm{H}$ concentration of the blood. Intravenous injection of acid into dogs or rabbits pro- 
duces air hunger when ${ }_{n} \mathrm{H} 7.65$ is reached; death occurs with 6.9 (Levy, et al., $9 \times 10^{-7}$, Szili, 1906). At the other extreme, concentrations above ${ }_{p} \mathrm{H}^{8}$ are probably also fatal (Rowntree).

Other Body Fluids. - With the exceptions noted, these have about the same reaction as the serum (Friedenthal, 1901). Hanzlik, 1914, found cerebro-spinal to range between ${ }_{\text {}} \mathrm{H} 6.98$ and 7.3 ; fresh bile, 6.98 to 7.18 ; edema fluids and pleuritic fluids, about 7.1 ; ascitic fluid, 7.42. On standing outside of the body, many of these fluids become acid.

'The saliva has 6.9 (Michealis and Pechstein, 1914); milk, about 7 ; intestinal juice about 8; gastric juice, about 1.77 (Michaelis, 1914; where further data may be found). The $\mathrm{H}$ concentration of the tissues generally must be somewhat higher than the blood, since they supply the acid that is excreted by urine and lungs.

Hurwite and Tranter, 1916, found the alkalinity of the cerebrospinal fluid constantly somewhat higher than the blood (average ${ }_{p} \mathrm{H}$, fluid, $8.1 \mathrm{I}$; blood, 7.66 ; difference, 0.45 ). This normal reaction was not changed in any stage of syphilis.

Reaction of Urine.- This usually inclines toward actual acidity, the normal range in man being $H^{(+)}=5.12$ to 7.46 ; the average about 6.03 . (The acidity is greater after fasting and perspiration, Haskins, I 9 I4). It exceeds 5.0 in less than I per cent. and 5.3 in less than ro per cent. In most diseases, the acidity averages distinctly higher; in cardiac disease, about 4.91. The highest acidity so far reported is 4.7 (L. J. Henderson and pupils, 1914). The reaction is determined mainly by the ratio of phosphates.

Administration of Acids and Alkalies on Urine.-Acid sodium phosphate raises the acidity, especially if the urine was previously alkaline; but the acidity does not surpass the normal limit $\left(\mathrm{H}^{(+)}\right.$raised from 6.7 to 5.3 ; Henderson and Palmer, 191 3 ; from 7.2 to 4.85 , Hanzlik and Collins, $\mathrm{I}_{91} \mathrm{I}_{3}$. Alkalies increase the alkalinity somewhat beyond

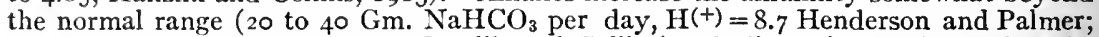
$4 \mathrm{Gm}$., change from 6.5 to 7.4 , Hanzlik and Collins). Sodium citrate also makes the urine somewhat alkaline ( 7.2 to $7 \cdot 4$, Hanzlik and Collins).

Regulating Mechanisms.-Metabolism leads to the production of acids (carbonic, lactic, sulphuric, phosphoric, etc.) which would tend to increase the acidity of the body. However, the maintenance of the normal, nearly neutral reaction is so highly important that it is regulated with the greatest nicety by the use of several mechanisms. The carbonates and alkaline phosphates of the blood and tissues constitute a stock of potential alkali; and the carbonic acid and acid phosphates of potential acid. These are sufficient to neutralize at once all ordinary attempts to change the actual reaction. The amphoteric character of the proteins permits them to neutralize a limited quantity of either acid or alkali. The administration of acid or alkalies by mouth therefore does not change the actual reaction of the blood and tissues, but only the potential acidity or alkalinity. The ammonium formation, and the excretion by the urine and lungs, finally restore the equilibrium of potential alkali.

Neutralization in the Alimentary Canal.- Neither acids nor alkalies are absorbed unchanged from the alimentary canal. The alkalies (including. the carbonates) are neutralized by the $\mathrm{HCl}$ of the gastric juice. Or if given in larger amounts, they enter into loose alkali-protein combinations before they reach the blood. The acids undergo a similar change, or if they are not entirely absorbed before entering the intestine, they are there neutralized by the carbonates. The immediate effects, then, would consist only in altering the reaction of the alimentary canal.

(Concentrated acids and alkalies produce corrosions by direct chemic actions. These local effects were considered under Irritants.)

Neutralization in the Blood.- It is difficult to alter the reaction of blood even in vitro. Friedenthal, I903, showed that serum requires, to secure a given change of reaction, thirty to forty times as much $\mathrm{NaOH}$, and 327 times as much $\mathrm{HCl}$, as would be needed in water. This stability is due to the presence of the salts of weakly dissociating acids, which limit the liberation of $\mathrm{H}^{(+)}$ions to practical neutrality. This property is 
especially marked with phosphates and carbonates, which are important constituents of the serum. The proteins act similarly.

If $\mathrm{H}^{(+)}$ions are added to such a system, they will be expended in liberating the weak acid from its salt; and since the weak acid is not ionized in the presence of its salts, the actual reaction will be practically unaltered. If $\mathrm{OH}^{(-)}$ions are added, they will be expended in neutralizing the free weak acid; and thus will also leave the actual reaction unaltered. A notable change in the true reaction can occur only after enough acid or alkali has been added to neutralize or liberate all of the weak acid-i.e., there will be no material change of reaction until not only the free, but also the potential acidity or alkalinity has been overcome. This would require quantities of strong acids or bases much greater than it would ordinarily be possible to introduce into the living body.

So far as this neutrality regulation is concerned, the blood may be considered as a solution of $\frac{\mathrm{H}_{2} \mathrm{CO}_{3}}{\mathrm{NaHCO}_{3}}$ and $\frac{\mathrm{NaH}_{2} \mathrm{PO}_{4}}{\mathrm{Na}_{2} \mathrm{HPO}_{4}}{ }^{1}$. All these dissociate so weakly that in solutions of the concentration of blood, their $\mathrm{H}^{(+)}$and $\mathrm{OH}^{(-)}$concentration, separately or together, would be practically that of neutrality; i.e., in the close neighborhood of $10^{-7}$.

If an acid $(\mathrm{HCl})$ were added to the mixture, it would react with the salts, producing:

$$
\begin{aligned}
& \mathrm{HCl}+\mathrm{NaHCO}_{3}=\mathrm{NaCl}+\mathrm{H}_{2} \mathrm{CO}_{3} \\
& \mathrm{HCl}+\mathrm{Na}_{2} \mathrm{HPO}_{4}=\mathrm{NaCl}+\mathrm{NaH}_{2} \mathrm{PO}_{4} .
\end{aligned}
$$

This would increase the ratio of $\mathrm{H}_{2} \mathrm{CO}_{3}$ and $\mathrm{NaH}_{2} \mathrm{PO}_{4}$; but these dissociate so weakly that they practically leave the actual reaction unaffected. The $\mathrm{H}^{(+)}$concentration would therefore not be increased until all the $\mathrm{NaHCO}_{3}$ and $\mathrm{Na}_{2} \mathrm{HPO}_{4}$ are used up.

If an alkali $(\mathrm{NaOH})$ were added to the mixture, it would react as follows:

$$
\begin{aligned}
& \mathrm{NaOH}+\mathrm{H}_{2} \mathrm{CO}_{3}=\mathrm{H}_{2} \mathrm{O}+\mathrm{NaHCO}_{3} . \\
& \mathrm{NaOH}+\mathrm{NaH}_{2} \mathrm{PO}_{4}=\mathrm{H}_{2} \mathrm{O}+\mathrm{Na}_{2} \mathrm{HPO}_{4} .
\end{aligned}
$$

This would reduce the ratio of the free acids, $\mathrm{H}_{2} \mathrm{CO}_{3}$ and $\mathrm{Na}_{2} \mathrm{HPO}_{4}$; but since these acids were practically neutral, this does not affect the actual reaction materially, until all the carbonic acid and acid phosphate are used up.

Restoration of the Potential $\mathrm{H}^{(+)}$and $\mathrm{OH}^{(-)}$ions. - The regulating mechanism of the blood is important for protecting the body against sudden changes, for meeting temporary emergencies. However, this impairs the stock of potential $\mathrm{OH}^{(-)}$and $\mathrm{H}^{(+)}$ions, and therefore would not furnish a permanent protection. Other mechanisms are called into play to restore the normal ratio of these potential ions; mainly by regulating the excretion of $\mathrm{CO}_{2}$ through the lungs; by varying the reaction of the urine; and by the more or less complete conversion of $\mathrm{NH}_{4}$ into urea.

Regulation by Excretion of $\mathrm{CO}_{2}$. - Addition of acid to the blood liberates $\mathrm{CO}_{2}$ from the bicarbonate. This increases the $\mathrm{CO}_{2}$ tension, and thus its excretion by the lungs. The increased $\mathrm{CO}_{2}$ tension stimulates the respiratory center, and thus hastens the elimination. This restores the normal ratio of $\mathrm{H}_{2} \mathrm{CO}_{3} \div \mathrm{NaHCO}_{3}$. Conversely, the addition of alkali diminishes the tension of $\mathrm{CO}_{2}$ and thus its excretion.

Regulation by Combustion of Organic Acids. - The organic acids arising in metabolism are normally oxidized to $\mathrm{CO}_{2}$ and $\mathrm{H}_{2} \mathrm{O}$, and excreted by the lungs and urine. This mechanism is disturbed in disease, starvation, etc., and then gives rise to acidosis.

1 Michaelis and Garmendia, 1915, state the normal ratio of the phosphates in bloodplasma as $\frac{\mathrm{NaH}_{2} \mathrm{PO}_{4}}{\mathrm{Na}_{2} \mathrm{HPO}}=\frac{1}{5 \cdot I}$ (molecules). 
Regulation by the Urine.-The urine, like the blood contains a mixture of acid and neutral phosphates (Orth found a mean of 60 per cent. as $\mathrm{MH}_{2} \mathrm{PO}_{4}$ and 40 per cent as $\mathrm{M}_{2} \mathrm{HPO}_{4}$. When acid is introduced, the ratio of acid phosphate is increased; alkalies increase the proportion of alkaline phosphate. This elimination of the excessive acid or.alkali ions rapidly restores the potential acidity or alkalinity of the tissues. The flow of urine is also increased, so that acids and alkalies act as effective diuretics. The administration of acids first increases the $\mathrm{PO}_{4}$ excretion; later, this decreases with the available supply of these ions (Fitz, Alsberg and Henderson, I907). The influence of nephritis was studied by Palmer and Henderson, I9I 5 .

Acid on Alkali Excretion.-Secchi, r9r4, reports that the administration of $\mathrm{HCl}$ to man or dogs on a meat diet increases the elimination of ammonium persistently; that of $\mathrm{K}$ and $\mathrm{Na}$ temporarily. The $\mathrm{Ca}$ and $\mathrm{Mg}$ are not materially affected in the urine, but increased in the feces, especially Ca. The calcium of the tissues may thus be diminished.

With rabbits on a cabbage diet (rich in $\mathrm{Ca}$ ) administration of acid markedly increases the elimination of $\mathrm{Ca}$ and $\mathrm{Mg}$, less that of $\mathrm{K}$ and $\mathrm{Na}$. The Ca excretion is mainly by the urine. The tissue $\mathrm{Ca}$ is greatly decreased.

Feeding with $\mathrm{HCl}$ may cause arterial changes (Adler, I9r4).

Regulation by Ammonia.- $-\mathrm{NH}_{4}$ is a normal precursor of urea. Its conversion is not quite complete and somewhat elastic: If the acidity tends to rise, a larger part of the ammonium is left unconverted, and is used to neutralize the excessive acid. If the alkali tends to rise, more of the ammonium is converted, leaving its anion to neutralize the alkali. The ratio of unconverted ammonia (reflected in the ratio of $\mathrm{NH}_{4}$ to total $\mathrm{N}$ of the urine) is therefore an index of the acid or alkali tendency of the body. It rises if acids are introduced or formed in the organism (acidosis); and is lowered when alkalies are administered. Ammonium is also produced by des-amination of proteins.

Efficiency of the Regulating Mechanism in Different Animals.-The protection against acids is conspicuously more perfect in carnivora, both as concerns the $\mathrm{NH}_{4}$ and the elimination (Spiro, I901) - probably because these are accustomed to ingest a certain amount of acid with their food. It is impossible to lower the alkalinity of the blood of a dog, to such a degree as to produce symptoms, by introducing acid into the alimentary canal, unless corrosion be produced. But if an acid or acid salt be injected into a vein, it will cause very pronounced symptoms. In herbivorous animals, these may also be produced if large quantities are given by the mouth. They will result in death even before the reaction of the blood has become neutral. So that it is not strictly correct to speak of an "acid action," but rather of the effects of diminished alkalinity (Kettner, I902).

Influence of Diet on Ammonium Conversion.-The ammonium regulation is not entirely absent in herbivoræ. Cows and calves use it quite efficiently when acid is fed (Hart and Nelson, I913). Eppinger, 1906, claimed it to be influenced by diet: viz., that a vegetable diet renders dogs susceptible to acid poisoning; and that a meat diet, or injection of amino-acids, evokes the ammonium mechanism even in rabbits, and thus protects them against acid. However, later observers were unable to confirm him (Pohl and Muenger, 1906; G. Bostock, 1913).

Nature of Acidosis.-Acidosis, or Acid-intoxication arises when acids or acid salts are introduced in excessive quantity, or when the organism fails to dispose promptly of the acids that are formed in metabolism. So long as there is an adequate store of potential alkali, the actual reaction of the blood is not materially changed, although it contains less carbonate. The acidity and the ammonium of the urine will also tend to increase. If the acidosis is due to faulty metabolism, oxybutyric acid and acetone substances will appear in the urine. 
Symptoms of Acidosis.-The symptoms up to this point may not exceed a degree of dyspnea, required to eliminate the liberated $\mathrm{CO}_{2}$. When the actual reaction of the blood becomes noticeably changed, the vasomotor and other medullary centers are also stimulated; especially if the change is acute, as with intravenous injection of acid. With further rise of the $\mathrm{H}$-ion concentration, the nervous centers become depressed, as also the cardiac muscle. The effects agree with those of excessive $\mathrm{CO}_{2}$ tension, asphyxia and oxygen deprivation, and diabetic coma, which are really forms of acidosis.

The final symptoms consist of coma, convulsions, depressed respiration and fall of blood pressure, etc. Death takes place by respiratory paralysis, generally before the blood becomes actually acid (see page 7 I 2 ). The symptoms are at once removed-even in the last stages-by injection of $\mathrm{Na}_{2} \mathrm{CO}_{3}$ (F. Walter, I877).

Toxicity of Acids.-This varies greatly according to conditions. In cattle, for instance, Berg, I $9 \mathrm{I}_{5}$, sometimes found considerable tolerance $(0.87 \mathrm{Gm}$. of $\mathrm{HCl}$, as 0.9 per cent., vein, per kilogram).

Respiratory Center.--This has been studied by Douglas and Haldane, I909. It is also stimulated by the direct application of acids to the medulla (in frogs). The action is proportional to the $\mathrm{H}$-ions (Winterberg; T. B. Robertson, I9r2). Winterstein, I915, finds that the injection of acids increases and alkalies diminish the ventilation of the lungs. The change is always parallel to the $\mathrm{H}$-ion concentration of the blood, but not necessarily to its $\mathrm{CO}_{2}$ tension. It is clear, therefore, that the essential factor is the $\mathrm{H}$-ion concentration, and not the $\mathrm{CO}_{2}$. However, Kopaczewski, I9I4, believes that the effects are not due to the $\mathrm{H}$-ion alone, but that the anions or the entire molecule also play some part.

The apnea following on excessive respiration is due to deficient $\mathrm{CO}_{2}$ tension and therefore lowered $\mathrm{H}$-ion concentration. Cheyne-Stokes respiration is attributed to alternate accumulation and oxidation of acids, (Y. Henderson, Igro).

Hemoglobin.-Increased acidity diminishes the affinity of hemoglobin for oxygen (or carbon monoxid). For instance, with a carbon dioxid tension of $40 \mathrm{~mm}$., the oxygen percentage must be doubled to secure the same degree of saturation (Barcroft, 1914).

Dyspnea in Cardiac Disease. - This is probably partly due to acidosis. The actual $\mathrm{H}$ concentration is not much altered, but the diminution of reserve alkali renders every increase of $\mathrm{CO}_{2}$ much more effective; for instance, the respiration is doubled in decompensated patients by 2 to 3 per cent. of $\mathrm{CO}_{2}$ in the air, while normal individuals require 4.5 to 5.5 per cent. Alkali treatment restores the response to normal (Peabody, I915).

Vasomotor Center.-The stimulation (reflected in the rise of blood pressure) has been studied by Mathison, I9Ir. Pilcher and Sollmann, 1915, found moderate and temporary stimulation, independent of blood-pressure changes.

Circulation Rate in Man. - This shows a close parallelism with the $\mathrm{H}$-ion concentration of the arterial blood, and therefore with the oxygen consumption (Boothby, I915, nitrous oxid method of Keough and Lindhard).

Perfused Vessels.-These are temporarily dilated by weak acids, including $\mathrm{CO}_{2}$ (Schwarz and Lemberger, IgIr). Alkalies constrict. This has been generally confirmed. In the frog mesentery, the minimal effective concentrations are: for dilation, ${ }_{p} \mathrm{H}_{2}$; for constriction, ${ }_{p} \mathrm{H}_{9}$ to ir. Excised arterial rings do not respond satisfactorily to acid. They are constricted by ${ }_{p} \mathrm{HI}_{2}$ (I. Adler, I $9 \mathrm{I} 6$ ).

Frog's Heart.- The automatic contractions continue only within certain ranges of $H$ concentrations, which differ for the various portions of the heart (Dale and Thacker, 1913). The sinus requires the highest $\mathrm{H}^{(+)}$concentration $\left(\mathrm{H}^{+}\right)=4$ to 9.5); the ventricles the lowest $(H(+)=6.5$ to II $)$.

Isolated Mammalian Heart. - The effects of oxidizing substances; acids and alkalies, are described by Loevenhart and Eyster, 1913. Yanagawa, 1916, finds the coronary vessels constricted by increase of alkalinity, dilated by acidity.

Penetration.-Living tissues resist the entrance of all acids, except salicylic. Dead cells are permeable to all (Harvey, 1914).

Metabolism. - Acid intoxication lowers gas metabolism and heat production (Choostek, 1893). Small quantities of acids are said to increase protein destruction (increased elimination of $\mathrm{SO}_{4}$ and $\mathrm{PO}_{4}$; Dunlop, 1896 ); perhaps because they increase autolysis.

The effects of lesser changes in the reaction of body tissues must be rather limited, since these are so promptly brought back to normal. While it can not be doubted that such 
changes must have an influence upon metabolism, the nature of this can not be stated, because it is complicated by the actions of these substances on the alimentary canal. The latter is different in the case of acids and alkalies. Some of the other actions also require separate consideration. Organic Acids and their salts are rapidly burned to carbonate after their absorption; so that they act as acids only in the alimentary canal, but as alkalies after they are absorbed (Buchheim, I 888).

Disintoxication of Acids by Salts. - Neutral salts diminish the toxicity of acids for the fish Fundulus. $\mathrm{CaCl}_{2}$ is considerably more efficient (J. Loeb and Wasteneys, I9r I). The effects of electrolytes on the penetration of acid into Fundulus ova are discussed by J. Loeb, I9I5.

Effects of Increased Alkalinity.-Slight increase stimulates many physiologic processes; a few are depressed.

Virchow, I854, showed increased motion of spermatozoa; Gaule, I878, increased cardiac activity; Bethe, I909, diminished rhythmic movements of medusæ. Packard, 1906 , found that sodium bicarbonate increases the resistance of fundulus to lack of oxygen.

Occurrence of Clinical Acidosis.-Accumulation of acid in the body occurs whenever the elimination of acid fails to keep step with its production. This may happen through diminished excretion of acid, as in some nephrites; or by increased production of acid, especially in disturbances of metabolism associated with deficient oxidation. The most striking examples of this form occur in diabetes; but minor degrees are present in fasting, especially with low carbohydrate and high fat income; in poisoning by P, As and other metals; in narcosis, etc. Except in diabetic and uremic coma, the acid does not usually rise sufficiently high to produce symptoms other than dyspnea.

Chemic Criteria of Acidosis.-The hydrogen-ion concentration of the blood has only a limited value, since it is not materially altered until the bicarbonate concentration of the blood has fallen greatly, i.e., only in the most severe cases. The determination of minor degrees of acidosis depends on the direct or indirect estimation of the bicarbonate of the blood; or of the elimination of ammonium or acetone substances by the urine. The urine furnishes only qualitative data at best, since it can indicate only the excretion, and not the retention of acid. In nephritic acidosis it would be entirely deceptive (Peabody, I9r5; Van Slyke, I9r6).

Estimation of the Bicarbonate Content of the Blood.-This furnishes a quantitative index of the potential alkali store, and its diminution is therefore the earliest sign of acidosis and the most accurate gauge of its severity and progress. The bicarbonate can be determined directly by measuring the $\mathrm{CO}_{2}$ liberated by acidulation of the plasma. Normally, this is 55 to 75 per cent. volume; 30 per cent. indicates dangerous acidosis; 20 per cent. is fatal.

Determination of "Buffer-Value" of Blood.-R. L. Levy and Rowntree, I9r6, express this in c.c. of $\frac{N}{50} \mathrm{HCl}$ or $\mathrm{NaOH}$ that can be added to blood without changing its ${ }_{p} \mathrm{H}$ ("simple buffer value"), or without changing it beyond the normal ("reserve buffer value"). The simple buffer value is clinically the more important. The sum of the acid and alkali values gives the "total buffer value." The simple buffer value a verages 0.18 for normal blood; serum averages 0.1 I for acids, 0.05 to 0.3 for alkalies.

Determination of Alkali Reserve of Plasma.-This is stated by Marriott, 1916, as $\mathrm{R}_{p} \mathrm{H}$, i.e. the $\mathrm{H}$-ion concentration after removal of the $\mathrm{CO}_{2}$ (by aeration). It appears to be a very delicate index of acidosis.

The normal value of $\mathrm{R}_{p} \mathrm{H}$, viz. 8.5, corresponds to an alveolar $\mathrm{CO}_{2}$ tension of 38 to $45 \mathrm{~mm}$. Moderate acidosis ranges between 8.0 and 8.3 (28 to $35 \mathrm{~mm}$. $\mathrm{CO}_{2}$ ); dangerous acidosis $=7.7\left(20 \mathrm{~mm}\right.$. $\left.\mathrm{CO}_{2}\right)$; coma $=7.3\left(\right.$ II mm. $\left.\mathrm{CO}_{2}\right)$. An $\mathrm{R}_{p} \mathrm{H} 7.9$ can be controlled by the oral administration of alkali; $R_{p} \mathrm{H} 7.7$ requires alkali intravenously.

Alveolar Carbon Dioxid.-Indirectly, the carbonate content of the blood may be judged by the alveolar $\mathrm{CO}_{2}$ tension, and by the "alkali-tolerance test." The volume percentage of $\mathrm{CO}_{2}$ in the alveolar air is generally about one-tenth of that which can be liberated from the plasma. This relation holds for normal individuals and for diabetic coma, where it has been utilized by Beddard, Pembrey and Spriggs, I915; and Poulton, 1915. It does not apply, however, to conditions in which the respiration or circulation are seriously disturbed (Van Slyke). It is also not very delicate in nephritic acidosis (Peabody). 
Alkali Tolerance Test.-When the bicarbonate of the blood is lowered, a larger dosage of alkali is required to change the action of the urine to alkaline than is the case normally. This increased tolerance for alkali is a delicate and reliable index of decreased potential alkalinity of the body, and therefore of even slight degrees of acidosis, including renal acidosis; and an equally reliable criterion of the progress of the acidosis and of the need of alkali therapy (Sellards, I9r 2; Palmer and Henderson, I9I3; Peabody, I9I5).

The test is carried out by giving orally, between meals, $5 \mathrm{Gm}$. of sodium bicarbonate (in water) every two hours, until the urine becomes alkaline to litmus. Normally, this occurs with the first or at most with the second dose (the $p^{H}$ of the urine being reduced to an average of 7.6 , sometimes to 8.3 , within an hour, Palmer and Henderson).

Renal Acidosis.-Light grades of chronic nephritis, with normal phenolphthalein output, usually show little or no acidosis. In severer grades, the kidneys fail to excrete acid properly, and thus an acidosis (diminished potential alkalinity) develops, which is fairly proportional to the degree of phenolphthalein retention.

The alkali-retention test shows this much earlier than the alveolar $\mathrm{CO}_{2}$; the latter generally diminishing only when the phthalein output falls below ro per cent. in two hours. The actual reaction of the blood is not materially altered except in the extreme stage; so that even in uremia, the symptoms are not commonly attributable to acidosis (Peabody, I915). The acidosis of infancy is frequently connected with diminished output of urine (Howland and Marriott, 1915).

$M$. Fischer, I9I5, refers to acidosis both the edema and the $\mathrm{Cl}$ retention. His theories have been discussed in another place.

The toxicity of uranium is associated with acidosis, and can be diminished by administering alkali. This also protects the uranium kidneys against anesthetics (MacNider, 1916).

Asiatic Cholera.-This disease results in a considerable loss of reserve alkali, with the production of acidosis. It is benefited by the early administration of sodium bicarbonate, $0.5 \mathrm{Gm}$., increasing the dose with the severity of the symptoms (Sellards, I9I4).

Diabetic Acidosis.-Diabetic coma is essentially a manifestation of acidosis (Stadelmann, I883). There is not only a close resemblance in the symptoms; but the increased elimination of beta-oxybutyric acid and ammonium (Boussignault, $185^{\circ}$ ), and the great diminution of carbonates in the blood (to ro per cent.; Minkowski, I888; Kraus, I889) furnish direct evidence of increased acid production. Accordingly, the administration or injection of alkalies has proved successful in preventing and meeting this condition (Magnus-Levy, I899). The success is not so striking as in experimental acid-intoxication; mainly because the acid, in diabetic acidosis, is primarily within the cells, and therefore is not so readily reached by the alkali. The details of this treatment will be discussed under Sodium Bicarbonate.

The alkali could also be furnished by the administration of the salts of organic acids (Folin, I906; Lichtwitz, I9II), or by Ca or $\mathrm{Mg}$; but probably not in sufficient quantity. Eppinger, 1906, suggested increasing the formation of ammonia by the administration of amino-acids; but his results have been challenged by Pohl and Muenzer, and by A. Loewy, roo6.

Ehrmann, Esser and Loewy, r9ı I, claim that the injection of neutral oxybutyric salts also produces coma, so that the diabetic coma would not be a pure acidosis.

Origin of Beta-oxybutyric Acid and Acetone Substances. - These are formed mainly by the oxidation of fatty acids with even number of carbons (the combustion of oddcarbon acids results in glucose; A. I. Ringer, 1913 ).

Marriott, 1914 , believes that oxybutyric acid is normally formed by the successive steps of fatty acid-butyric acid(?)-aceto-acetic acid $>$ D. oxybutyric acid (readily

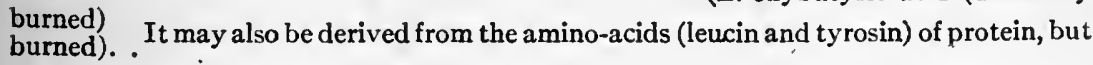


not from carbohydrates. It is a product of ordinary metabolism, but is normally burned completely: In diabetes, this oxidation is defective, because the complete oxidation probably demands the simultaneous oxidation of glucose (perhaps through the formation of a glucosidal compound; Ringer, 1914). The partial oxidation of the oxy-butyric acid leads to aceto-acetic acid and acetone, which are generally associated with it in diabetic urines (G. Lusk, 1909). Traces of oxybutyric acid occur in normal organs (especially in the liver), but are greatly increased in phlorhizin acidosis, and especially in diabetic coma (Sassa, rgr4).

Mottram, I9I5, refers the presence of acetone substances to enforced overactivity of the liver, since he finds them in all conditions that show fatty infiltrations of the liver. In phlorhizin glycosuria, the acidosis may be decreased by feeding proteins (Benedict and Osterberg, I9I4); whereas increased protein intake often increases it in diabetes.

In normal subjects, 100 c.c. of blood contains less than $1.5 \mathrm{mg}$. of aceto-acetic acid, and less than $4 \mathrm{mg}$. of oxybutyric acid. In acidosis, the proportion may rise to $28 \mathrm{mg}$. of acetone and $45 \mathrm{mg}$. of oxybutyric acid (Marriott, I9 4 ).

Lactic Acidosis.- Lactic acid appears in the blood after violent exercise, strychnin spasms, and phosphorus poisoning. It has also been found in the urine after cocain convulsions (Underhill). The lactic acid is derived from glucose, for it does not occur in glycogen-free animals (Lusk, 1916).

Creatin Elimination.- This appears to be connected with acidosis. In rabbits, Underhill, 1916, finds that acid-producing foods (oats and corn) cause the appearance of creatin in the urine, and this is removed by base-producing foods (carrots).

\section{DILUTE HYDROCHLORIC AND OTHER ACIDS}

General Statement.-These are used mainly for their local action on digestion in gastric hypoacidity, etc. The sparingly soluble acid salts (acid potassium tartrate) extend their action into the intestines, and produce catharsis. After absorption, the mineral acids and their acid salts increase the acidity of the urine, and are employed, usually with hexamethylenamin, against ammoniacal fermentation of the urine. They are further used as antidote to alkali poisoning; as local astringents against excessive perspiration; and as local hemostatics.

Action on Digestion.-The reaction of the gastric and intestinal contents modifies the activity of the motor and secretory functions, and of the digestive ferments. These effects may give rise to digestive disturbances, or be utilized for the correction of digestive disorders. The results depend on the condition of the alimentary canal, and therefore on the time of administration, as well as on the dose and concentration of the acid.

Action in the Mouth.-Acids have a characteristic "sour" taste, and are slightly astringent in the mouth. This taste determines their use as flavors. They soften the enamel of the teeth, and should therefore be taken through a glass tube. They also reflexly increase the flow of saliva, but this is of little practical importance.

Use in Fevers.-The addition of acid makes it easier to take larger quantities of cold water than could convenieintly be taken without. They are therefore of therapeutic value in fevers. when one wishes to obtain at the same time the refrigerant action of cold and the diuretic effect of large quantities of fluid. It was believed that the alkalinity of the tissues is raised in febrile conditions, and that this is counteracted by acids. The modern ideas render this doubtful.

Action in the Stomach.-The presence of acid is important for the digestion of proteins and connective tissue; for the control of the pyloric sphincter; and for its antiseptic properties.

Gastric Acidity.-Pure human gastric juice, pathologic as well as normal, is secreted with about 0.4 to 0.5 per cent. of free $\mathrm{HCl}$ (Roeder, 1905; Rehfuss and Hawk, 1914; Carlson, 1915) the same as in dogs or cats. This corresponds to $p^{\mathrm{H}}=0.95$ to I (Menten, I915). 
This acidity, however, is not normally maintained in the stomach, the acid being rapidly. neutralized by the saliva, the mucus and the reflux of duodenal contents, to 0.15 or 0.2 per cent. $\mathrm{HCl}$ (Boldyreff). The breaking down of this mechanism appears to be the basis of "hyperacidity," in which the acid remains at the same concentration ( 0.5 per cent $\mathrm{HCl}$ ) at which it was secreted (Carlson, 1915). The curve of the acidity during normal digestion has been studied in man by McClendon, I9I 5 . He also determined the hydrogen-ion concentration of human duodenal contents, viz., $\mathrm{H}^{(+)}=2$ XI0-8.

Liberation of $\mathrm{HCl}$.-The mechanism by which $\mathrm{HCl}$ is liberated is not fully established. Koeppe, 1896 , supposes that it is formed in the stomacl from $\mathrm{NaCl}$ : assuming that the epithelium is permeable to $\mathrm{Na}$ and not to $\mathrm{Cl}$ ions, the sodium would diffuse out of the stomach, and $\mathrm{H}$ ions would diffuse into it. The theory requires further proof. Hammett, I9r5, confirms Fitzgerald that the acid exists in the bodies and canaliculi of the parietal cells.

Pyloric Control.-Free acid in the stomach increases the pyloric peristalsis; it closes the cardiac and opens the pyloric sphincter. However, the presence of free acid in the duodenum closes the pyloric sphincter until the acid is neutralized by the intestinal juices. Moderate gastric acidity therefore hastens the discharge of food from the stomach, but excessive acidity prolongs the sojourn because it can not be promptly neutralized in the duodenum (Cannon, rgr 1 ).

The controlling influence of acids on the emptying of the stomach appears to be exerted mainly from the duodenal side: clinically, the time of discharge is often normal in achylia; and gastric stasis of ten exists in hyperchlorhydria. In fasting anesthetized dogs, water is discharged from the stomach more promptly than any degree of acidity; acids causing a delay, proportional to their concentration. Duodenal regurgitation begins with 0.2 per cent $\mathrm{HCl}$ (IV. E. Morse, I9I6).

Foods also act on the acid regulation of the pylorus: proteins, by binding the acid, prolong the sojourn; so do fats, by binding the duodenal alkali. Carbohydrates are indifferent.

Relation of Gastric Pain to Acidity.-The pain of gastric ulcer has been attributed to direct irritation by the free acid. However, it appears to be indirect, due to the increase of gastric peristalsis and tone, and pyloric spasm.

In human subjects with normal stomachs, Lowenthal, $189_{2}^{2}$, found no sensation from 250 c.c. of 0.3 per cent. $\mathrm{HCl}$. With 0.5 per cent. there was sometimes a warm sensation, but no pain. Other observers report absence of any sensation with 0.5 per cent. J. E. Schmidt, 1909, found no symptoms with 0.5 to 2 per cent. With 5 per cent., Boring reports a sensation similar to hunger-pains.

The results on patients with gastric ulcer are more variable. Pain was sometimes, but not always present, after 0.1 to 0.4 per cent. $\mathrm{HCl}$. Ginsburg et. al., I916, find that the pain is always accompanied by strong contractions, indicating that it is due to tension. This was relaxed by alkalies or amyl nitrite; strengthened by pituitary.

Digestive Action.-Pepsin acts only in the presence of free acid. While any acid may answer for this purpose, hydrochloric seems to be the best, and is most efficient in the concentration existing in the gastric secretion; it also aids in the solution of the connective tissue of meats; and it determines the antiseptic qualities of gastric juice.

The ingestion of dilute acid also increases the secretion of gastric juice. This effect is partly reflex, for it is observed in Pawlow's separated stomach (Bickel, r905) but only when it is given with food (Heinsheimer, I906).

Salivary Digestion in the Stomach.-Very slight acidity accelerates the action of all diastases, but somewhat stronger concentrations depress. However, the bulk of the food remains for an hour or more in the cardiac pouch, with only the surface layer impregnated by acid (Heyde and Gruetzner). Salivary digestion proceeds practically undisturbed during this time (Cannon, Igr I). 
Pancreatic Juice.-The flow is increased by acids.

This action is perhaps partly reflex, but it has been shown (Bayliss and Starling, 1002) that the presence of acid in the jejunum leads to the production of a chemic substance, "secretin," the intravenous injection of which stimulates the pancreas to increasd secretion.

Bile.-The secretion of bile, and the contractions of the gall-bladder are increased by the introduction of acid into the duodenum (Okada, I9I5).

Use in Indigestion.-Acids are administered in dyspepsias in which the secretion of acid is deficient; in putrefactive fermentation; and in infantile diarrheas due to this cause. All mineral acids act alike. Dilute Hydrochloric Acid (I c.c., diluted, just before or after meals) is generally preferred. It is often combined with bitter stomachics.

Acids are contraindicated in catarrhal conditions, in which there is a hypersecretion of mucus. Even in normal individuals the prolonged administration of large quantities of acids is apt to prove too irritant, and interferes with digestion. This is the explanation of the popular use of vingear to reduce obesity. A direct limitation of diet would seem a more rational means for this purpose.

It has been objected that doses much greater than the ordinary would be required to restore the normal acidity. However, when taken after meals the acid would be confined to the surface of the food-mass and would therefore suffer little dilution. value.

The increased flow of pancreatic juice and of bile is probably too small to be of any

Lactic Acid Organisms.-These were introduced with the object of supplying the antiseptic action of acids to the intestines, in infantile diarrheas, etc.; but they have not fulfilled these expectations very satisfactorily. They are discussed in N.N.R.

Combined Acid.- In order to avoid the irritant effects of free acid, loose acid compounds have been administered to liberate their acid gradually; such are Acidol (betain hydrochlorid) and Oxyntin (acid protein). However, they are therapeutically useless. Long and Hull, I915, showed that the quantity of acid bound by proteins is quite definite and does not depend simply on the $\mathrm{H}$-ions; but it is so small that it barely suffices for the digestion of the combined protein. Betain hydrochlorid is more active, but still below therapeutic efficiency (Long, $191_{5}$ ).

Action on Urine.-The administration of acids increases the acidity of the urine. This leads to an increased irritability of the mucous membranes of the urinary passages, so that inorganic acids are to be avoided in all inflammatory conditions of these organs. They must also be avoided where there is a tendency to the formation of uric acid or oxalate calculi. On the other hand, they are indicated with phosphatic calculi. They are used especially to enhance the action of hexamethylenamin (see Index). It may be repeated that they increase the ammonia of the urine at the expense of the urea.

Effects upon Metabolism.-Outside of this change in the ratio of ammonia and urea, these are quite small, as far as our present means allow us to judge. The excretion of nitrogen seems to be pretty constantly slightly increased.

Applied directly to excised organs-muscle, nerve, etc.-the result is an increase and subsequent diminution of function.

Organic Acids.- The local actions are along the same lines as those of the inorganic acids, but present important differences in detail. They 
are little used in digestive disorders. Their systemic actions are quite different, since they are for the most part burned in the body. They are therefore more conveniently discussed in connection with their salts.

PREPARATIONS-DILUTE MINERAL ACIDS

These have a uniform strength of ro per cent. They are colorless, odorless liquids, of strongly acid taste; miscible with water, alcohol or glycerin. They are incompalible with alkalies and the salts of weak acids (carbonates, salicylates, sulphides, sulphites, etc.). Dose, I c.c., I 5 minims, U.S.P.; 0.3 to 1.2 c.c., 5 to 20 minims, B.P.; in one-half tumbler of water after meals.

*Acidum Hydrochloricum Dilutum (Acid. Hydrochl. Dil.), U.S.P., B.P.-Incompatible also with salts of $\mathrm{Ag}$ and $\mathrm{Pb}$.

Acidum Nitricum Dilutum (Acid. Nit. Dil.), B.P.

(Acid. Nitrohydrochl. Dil.-See Index).

Acidum Phosphoricum (Acid. Phos.), U.S.P.; Acid Phosph. Conc., B.P.-87 per cent. of $\mathrm{H}_{3} \mathrm{PO}_{4}$, U.S.P.; 66 per cent. B.P. Colorless, odorless syrupy liquid.

Acid. Phos. Dil., U.S.P.; Acid. Phosph. Dil., B.P.

Acid. Sulph. Dil., U.S.P., B.P.

Acidum Sulphuricum Aromaticum (Acid. Sulph. Arom.), U.S.P., B.P.-An alcoholic dilution of sulphuric acid, flavored with ginger and cinnamon. Contains free sulphuric acid and ethylsulphuric acid equivalent to about 20 per cent. of $\mathrm{H}_{2} \mathrm{SO}_{4}$, U.S.P.; about I4 per cent., B.P. A clear, reddish-brown liquid; pleasant aromatic odor; intensely acid. Dose, I c.c., I 5 minims, U.S.P.; 0.3 to 1.2 c.c., 5 to 20 minims, B.P., diluted.

It is doubtful whether it possesses any advantage over other acids. Ethyl-sulphuric acid acts simply as a mineral acid (Uyedo, I910).

Inf. Ros. Acid.,B P.- - .25 per cen:. Acid. Sulph. Dil., 2.5 per cent. dried rose petals. Dose, I 5 to 30 c.c., $1 / 2$ to $\mathrm{I}$ ounce., B.P.

\section{SODIUM BICARBONATE AND OTHER MILD ALKAEIES}

General Statement.-Sodium and potassium bicarbonates are used as liquefying expectorants, and to dissolve mucus in other catarrhal conditions. Sodium biçarbonate is particularly useful for neutralizing hyperacidity in digestive disturbances. Magnesium oxid, or magnesium or calcium carbonate, acts more persistently, but less promptly and efficiently. Excessive doses of alkalies disturb digestion and produce diarrhea.

The systemic effects are utilized for combating acidosis. Smaller doses, especially in the form of potassium bicarbonate or citrate, are used in rheumatism and gout. Their action, if they have any, is not understood.

The administration of alkalies increases the flow of urine and reduces its acidity or renders it alkaline. This is useful for decreasing the irritation in cystitis, and to prevent the formation of urate calculi.

Sodium carbonate is too irritant for internal use.

Effect on Mucus.-Mucin is more soluble in alkaline media, so that the alkalies dissolve any accumulations of mucus or render them more fluid. At the same time they increase mucus secretion through a mild irritation.

Use in Catarrhs.-Mild alkalies, especially bicarbonate and borate, are used as a wash or gargle ( $x$ to 4 per cent.) in all kinds of catarrhal conditions; as expectorants; in cystitis, dysentery, etc. 
Removal of False Membranes. - The false membranes of diphtheria, croup, etc., are also composed largely of mucus, and may be broken down by alkalies. They are best employed in this case in the form of a steam saturated with lime-water, commonly prepared by inhaling the vapors produced by slaking lime in the sick-room.

Use on the Skin.-Alkalies are also employed to soften the crusts in eczema; as a soothing lotion in dermatitis and burns; to relieve itching, etc.

Action on Normal Digestion.-Strong alkalies, including sodium carbonate, produce local irritation and gastritis. The weaker alkalies, such as the bicarbonates, would also tend to interfere with normal digestion. They tend to inhibit the secretion of digestive juices; the activity of pepsin; the gastric peristalsis and the opening of the pyloric sphincter (Cannon, I9I I); the protective action of the mucus and the antiseptic action of the gastric acid. They might be conceived to aid the digestion of fats; but the intestines are normally sufficiently alkaline.

In fact, however, these detrimental actions are very slight, and the utilization of the food is practically perfect (unless excessive doses are taken), because moderate quantities are rapidly neutralized and absorbed. ${ }^{1}$

Secretion of Gastric Juice.-It was formerly claimed that this is stimulated; but practically all of the later experiments indicate that it is rather diminished both in quantity and acidity (Pawlow, I897; Bickel, I905; Heinsheimer, I906; Rosenblatt, I907; Wilbrand, I914; Chiari, I91 5).

Pancreatic Secretion.-This is diminished by the oral administration of bicarbonate (Wilbrand, I9I4).

Ferments.-Diastases are hindered by free alkali, being very sensitive. Pepsin is also inhibited or destroyed (Hamburger, I9I 5 ; see under Sodium Chlorid). Trypsin can act in a weakly alkaline medium.

Use of Alkalies in Digestive Disturbances.-While alkalies are either indifferent or disturbing to normal digestion, they are very useful to restore the normal conditions in digestive diseases.

Gastric Catarrh and Dilatation.-The alkalies dissolve the excessive mucus which lessens the permeability of the walls of these organs, preventing at once the pouring out of digestive juices and the absorption of the digestive products. This mucus forms a similar impermeable coating about the masses of food. Further, the mere presence of a large amount of indigestible material-and tenacious mucus must be regarded as such -is in itself irritating. Lavage with I per cent. bicarbonate is the most efficient treatment of these conditions; but the alkali may also be given with an emetic or cathartic.

Intestinal Catarrh and Catarrhal Jaundice.-The alkalies are similarly useful. (They do not increase the secretion of bile; Rutherford, I879.) In intestinal affections, the slowly soluble alkalies, magnesium oxid or magnesium or calcium carbonate, may be preferred, so that they will not be neutralized before reaching the intestines. A saline cathartic enhances their usefulness. This combination exists in several mineral waters (e.g., Carlsbad). For infants, lime-water is generally preferred to the other alkalies.

Gastric Ulcer.-Alkalies are useful by diminishing the quantity and acidity, and therefore the irritation, of the gastric juice. They also permit the destruction of the pepsin through sodium chlorid. Bismuth

\footnotetext{
1 The literature on alkalies to 1800 is covered in the work of Stadelmann.
} 
subcarbonate is preferable, because its action is more prolonged, and because it adds a mechanical protection.

An efficient routine for continuous alkali treatment for gastric ulcer is described by Sippy, 1915: Soft food is given every hour. Midway between the feedings, the patient receives an alkali, alternating between (I) magnesia and sodium bicarbonate, each 0.65 $\mathrm{Gm}$, Io grs., and (2) bismuth subcarbonate, 0.65 Gm, Io grs., with sodium bicarbonate, $2 \mathrm{Gm}$., 30 grs. The progress is controlled by the reaction of the stomach contents, which should not be allowed to become acid.

Gastric Hyperacidity; Functional Dyspepsias, Heart-burn; Gastric Flatulence, Etc.-In these conditions, the soluble alkalies (sodium bicarbonate or aromatic ammonia) are commonly used and effective. They neutralize the excessive acid, relax the spasmodic pylorus (Enriquez, Binet and Gaston Durand, I9I4), and thus empty the stomach and lessen the pain and irritation. The $\mathrm{CO}_{2}$ which is liberated stimulates the gastric movements (Schierbeck, I89I), and promotes the expulsion of the gas. It acts perhaps also as a mild local anesthetic. The bicarbonate is usefully combined with an aromatic carminative (peppermint) and cathartic (rhubarb).

Time of Administration.-This has given rise to much theoretical and unproductive discussion. The alkalies should evidently be given when the digestive conditions call for them. This is better recognized by the symptoms than by rules. The soluble alkalies (bicarbonate, r Gm.; aromatic ammonia, 2 c.c.; for infants, lime-water) should be administered whenever they are indicated by gastric distress, and repeated as often as needed; also at bedtime and on arising. For intestinal effect, they should be given on an empty stomach.

Obesity.-Alkalies are sometimes used. Th ir effects a e probably du' to disturbance of digestion.

Use in Arterial Hypertension.-Full doses of alkalies temporarily lower the arterial pressure, probably as a result of the nausea. The benefit is doubtful. Scheffer, igo8, advocated sodium silicate, but bicarbonate accomplishes the same result.

Use of Alkalies in Acidosis.-Their rationale has been discussed (page 7I7). The alkali must be administered in large quantity. This can best be accomplished by sodium bicarbonate. This has the advantage over the carbonate, and especially the hydroxid, in that its actual alkalinity (OH ions), and therefore its local action, is much lower; while its potential alkalinity, and therefore its neutralizing power is not very much inferior.

Neutralizing Power of Alkalies.-To neutralize I 00 parts of 10 per cent. $\mathrm{HCl}$ requires the following quantities of the official sodium salts: acetate $^{1}=37.3$; citrate $^{1}=32.6$; bicarbonate $=23.3$; carbonate $=17.1$; calcium carbonate $=13.8 ;$ sodium hydroxid $=\mathbf{1 2} 2$; magnesium oxid $=5.5$.

Administration and Dosage in Acidosis.-The use of the bicarbonate should not be delayed until coma has set in, for it may then be too late. It should be begun with the appearance of acetone substances in the urine. The initial daily dose by mouth may be 30 to $45 \mathrm{Gm}$., I to $1 \frac{1}{2}$ ounces, in divided doses and freely diluted, best between meals. This may be raised to $100 \mathrm{Gm}$. or more.

These large doses generally produce a cathartic effect. This purging may not be entirely useless, for it is conceivable that the underlying cause of the diabetes is found in toxins formed in the alimentary canal. At all events, plain purges have been found useful in such cases, and so has pilocarpin, originally used on the theory that it helps the elimination of the "toxins." 
After coma has set in, the oral administration may be continued, and "drop enemas" of a 4 per cent. solution may also be given. However, these can not be relied upon to secure sufficient absorption; and the bicarbonate should be injected slowly by vein ( $r, 000$ c.c. of 4 to 6 per cent. sterilized solution). The heat of sterilization drives off some of the $\mathrm{CO}_{2}$, and thus converts part of the bicarbonate into the carbonate. This is highly corrosive to the subcutaneous tissue. The ordinary solutions must therefore never be injected hypodermically, or even allowed to escape from the intravenous injections; for serious sloughing may result.

IIypodermic Injection of Pure Bi:arbonate Solution.-If the solution is freed from $\mathrm{Na}_{2} \mathrm{CO}_{3}$ by saturating it with $\mathrm{CO}_{2}$ until it is colorless to phenolphthalein, it does not cause necrosis and only very slight i ritation. Magnus-Levy, 1913, and Czapski, $\mathrm{IOI}_{4}$, advocate the hypodermic use of a liter of a 2 per cent. solution of this kind to, follow up the intravenous injection; or if the latter presents special difficulties (sometimes the flow becomes arrested).

Acidosis and Alkalies on Sugar Metabolism.-Aside from the neutralization of the acidosis, alkalies are useful in diabetes by promoting the utilization of sugar. Acidosis tends to produce hyperglycemia.

Pavy and Godden, IgI2, observed that the injection of alkali prevents ether or chloroform glycosuria. Elias, I913, showed that the injection of acid produces hyperglycemia; whereas feeding alkali removes hunger diabetes and diminishes pancreatic diabetes. Underhill, ז916, found that alkali also lowers epinephrin glycosuria. In a case of severe human diabetes, large doses of alkali markedly decreased the sugar excretion and raised the carbohydrate tolerance.

Murlin and Kramer, I9I6, confirmed that the injection of alkali suppresses the glycosuria of depancreated animals, or at leasts lowers the $\mathrm{D} \div \mathrm{N}$ ratio. The respiratory quotient rises, and the blood-sugar and the glycogen are not increased, so that there must be an increased combustion of sugar.

In normal dogs, alkali injection does not increase the respiratory quotient, but tends to inhibit glycogenolysis (Kramer and Marker, I9I6), and increases the deposition of injected dextrose in the liver (Macleod, 1916).

Intravenous Bicarbonate in Shock.-This often gives better results than plain saline infusion; the rise of blood pressure being more sustained and the respiration deeper (Seelig, Tierney and Rodenbaugh, I913). The bicarbonate, 0.5 per cent., may be added to the normal saline (Dawson, I905).

Alkali Injection in Nephritis.-M. H. Fischer, who conceives nephritis and edema as acidosis (page 647), has advocated this treatment. The clinical results in the hands of others have often been unfavorable and sometimes dangerous (Wyss, Weiland; Newburgh, I913). Bogert, Underhill and Mendel, I916, found that the restoration of the blood volume after injection of saline solution was not hastened by the addition of 0.4 per cent. of sodium carbonate, either in normal or in nephritic animals.

Bicarbonate Edema. - Large doses of sodium bicarbonate (about $20 \mathrm{Gm}$. per day may lead to the retention of water and eventually to edema, in proportion to the dose (Hayem, 1890). This may occur in diabetic as well as in normal individuals (Widal, I9II; Levison, Igr5). It is probably analogous to salt edema.

Alkalies with Salicylates.-Large doses of salicylates may cause the appearance of albumin and acetone in the urine. This tendency is believed to be lessened by the addition of equal doses of bicarbonate (Glaesgen, I9II). At least, the bicarbonate prevents the precipitation of the irritant salicylic acid in the stomach.

Metabolism. - The direct effects of alkalies are not important. Perhaps the only certain action is the increase of urea at the expense of ammonia. The indirect effects of digestive disturbance may be more serious. 
It is known that alkalics favor oxidation; and it has been found that they lead to an increased oxygen consumption and $\mathrm{CO}_{2}$ excretion (Lehmann, I884). This effect is not, however, very large-probably because the alterations in the reaction of the body are never very great. Experiments made to determine the effect on nitrogen metabol$i s m$ have given somewhat variable results, and these were certainly not greater than might be expected from the pure salt action, or from the interference with digestion which would result from large doses. (Doses as large as $\mathrm{I}_{3} \mathrm{Gm}$. sodium carbonate, $20 \mathrm{Gm}$. of sodium bicarbonate, or $40 \mathrm{Gm}$. sodium citrate do not influence the total nitrogen excretion.) MacLeod and Haskins, 1906, found increased elimination of endogenous uric acid and purins. Abl, I9I3, attributes this to the intestinal derangement.

The effect upon carbon metabolism is equally small and uncertain.

Increase of alkalinity is said to heighten the bacterial power of the serum and tissues; but further evidence is needed.

Use in Rheumatism, Gout, Etc.-The alkalies, especially potassium bicarbonate, acetate, and citrate, have considerable reputation in acute and chronic rheumatism, gout, etc. It is difficult to estimate their effciency. There is no good foundation for the assumption that they increase the oxidation of uric acid or its solubility in the tissues. Explanations of their actions are purely speculative.

Actions on Urine.-Alkalies increase the flow and alkalinity of the urine (see page 7 14 ). These effects are utilized especially in catarrhs of the urinary passages, and to prevent the formation of urate calculi. To secure this alkalinity requires about $7 \mathrm{Gm}$. of sodium bicarbonate or $\mathrm{I}_{5}$ Gm. of sodium acetate or citrate, per day. The organic salts are preferred, to avoid disturbance of digestion.

Sodium bicarbonate slows the excretion of intravenously injected lactose, and decreases the chlorid output (Hertz and Goldberg, I9I4).

Use in Vesical and Urethral Catarrh.-Alkaline urine is less irritant to the inflamed mucous membranes, and helps to dissolve the mucus and pus. The dilution of the urine, and the flushing of the urinary passages by the diuresis are similarly useful.

Use Against Urate Calculi.-Alkalies and diuretics can not dissolve these calculi; but they prevent the precipitation of uric acid and acid urates, which can occur to a serious extent only in acid urines. The alkalies (potassium or sodium citrate or acetate) must be given in sufficient quantity to keep the urine alkaline to litmus. Care must be taken that this does not lead to the deposition of phosphates, since these might be deposited on the calculus.

In a few instances, it has been observed that alkalies caused the breaking up of large stones into small fragments. This can not be attributed to a solution of the uric acid. The explanation is that probably the calculi were composed originally of small fragments glued together by mucus, and that the alkalies caused the solution of the latter.

The alkalies can exert no effect on the condition of the uric acid in the tissues, since the reaction of these is not altered. This will be discussed further in the next section.

Cystinuria.-Alkali has a solvent action on cystin; its administration in sufficient quantity to keep the urine alkaline prevents the deposition of cystin crystals (Smillie, 1915).

\section{PREPARATIONS-BICARBONATES}

*Potassii Bicarbonas (Pot. Bicarb.), U.S.P., B.P.; $\mathrm{KHCO}_{3 .}$-Colorless crystals; odorless; saline and slightly alkaline taste; permanent in the air. Freely sol. in water $(\mathrm{r}: 2.8)$; practically insol. in alc. Dose, $\mathrm{I}$ Gm., $15 \mathrm{gr}$., U.S.P.; 0.3 to $2 \mathrm{Gm}$., 5 to 30 gr., B.P. 
*Sodii Bicarbonas, U.S.P., B.P. (Baking Soda); $\mathrm{NaHCO}_{3}$.-White powder; odorless; cooling, milely alkaline taste. It is permanent in dry air, but slowly decomposes in moist air. Sol. in water $\left(1:\right.$ I $\left._{2}\right)$ at $15^{\circ} \mathrm{C}$., practically without decomposition. When heated, it loses $\mathrm{CO}_{2}$, and is converted into normal carbonate. Practically insol. in ale. Incompatible with acids and acid salts; and with the salts of most alkaloids, metals and earths; not with magnesium salts in moderately dilute solutions. Dose, $1 \mathrm{Gm}$., I 5 gr., U.S.P.; 0.3 to 2 Gm., 5 to 30 gr., B.P.

Trochisci Sodii Bicarbonatis, U.S.P.--o.18 Gm., 3 gr. "soda mints" generally contain $5 \mathrm{gr}$. , with $1 / 8$ minim oil of peppermint.

\section{URATE SOLVENTS}

\section{URIC ACID AND ITS SALTS}

Acid urates occur in solution in the blood and tissues; and as the peculiar sodium biurate deposits in gouty tophi, etc. In the urine, acid urates also occur in solution. When the urine is scanty or acid they may be deposited as free uric acid or "quadriurate;" i.e., a mixture or compound of free uric acid and acid urate. These deposits may concrete into urinary calculi.

Uric Acid, $\mathrm{C}_{5} \mathrm{H}_{4} \mathrm{~N}_{4} \mathrm{O}_{3}$, is a weak dibasic acid, which, under proper conditions, can form three series of salts, namely:

Neutral Urates.- $-\mathrm{C}_{5} \mathrm{H}_{2} \mathrm{~N}_{4} \mathrm{O}_{3} \cdot \mathrm{Na}_{2}$; also named dibasic, normal or secondary urates. These are formed only by strong alkalies, and can not occur under natural conditions, being decomposed by $\mathrm{CO}_{2}$ and even water. They need not be considered further.

Acid Urates. $-\mathrm{C}_{5} \mathrm{H}_{3} \mathrm{~N}_{4} \mathrm{O}_{3} \cdot \mathrm{Na}$; also named monobasic or primary urate or biurates. They are neutral to litmus.

Quadriurate.- $\mathrm{C}_{5} \mathrm{H}_{3} \mathrm{~N}_{4} \mathrm{O}_{3} \cdot \mathrm{Na}+\mathrm{C}_{5} \mathrm{H}_{4} \mathrm{~N}_{4} \mathrm{O}_{3}$; also named hemibasic urate or tetraurate. It is a mixture or solution of uric acid and acid urate. The quadriurate forms the sedimentum lateritium in cooling acid urines. On standing, it separates uric acid crystals. Water dissolves out the acid urate, and leaves the uric acid.

There has been much discussion as to the nature of the combination. Kohler, I9Ir, considers it a simple mixture. Ringer and Schmutzer, Igr2, regard it as a "solid solution" of uric acid in acid urate; which can be formed at body temperature, but which is unstable on cooling and thus separates the uric acid.

Other Urate Deposits in Urine.-These may consist of uric acid in acid urines; of acid urates in acid or neutral urines; and ammonium urate in neutral or ammonical urine. The acid urate is generally amorphous; but may be crystalline in neutral urine.

Urate Tophi.-These consist always of acid urates (Ritter, r897); so do the uratic deposits in animals, even when free uric acid is injected (Loghem, rgro7).

ictions of Urates.-The importance of these lies in the fact that they may be formed or retained in excessive amounts in pathologic conditions, when they first produce inflammatory necrosis of cells, and are then deposited in insoluble granular form. This, becoming crystalline, acts as a powerful mechanical irritant, producing the phenomena of gout. These can be simulated in animals by the injection of suspensions of acid sodium urate, and in birds by the subcutaneous injection of chromates, or even by ligation of the ureters. The cause of the abnormal appearance of the uratic deposits in gout, etc., is not understood. It seems to be connected with changes in protein metabolism, perhaps also with intestinal putrefaction.

Solubility and Precipitation.-Free uric acid is very little soluble. The acid urates also have a limited solubility. The solution may be favored or hindered by a variety of conditions, some little understood. It would be desirable to increase their solubility, f.i., to prevent urate deposits in the tissues and urine; if possible, to dissolve these deposits when formed; and perhaps to favor the elimination of the urates in gout, etc. Many attempts in these directions have failed, because they were based on imperfect understanding of the chemical data. Alkalies dissolve a limited 
amount of free uric acid, by converting it into the more soluble acid urates. This is the only direction in which some success has been found, and this is confined to the urine.

The study of the solubilities is greatly complicated by the small quantities going into solution, the polyvalence, formation of colloidal solutions, supersaturation, the influence of small quantities of impurities, decompositions, etc. (His and Paul, r900).

Uric Acid.-Pure water dissolves at I $8^{\circ} \mathrm{C}$. only $25 \mathrm{mg}$. of uric acid per liter $=\mathrm{I}: 40,000$ (His and Paul). At $40^{\circ}$ the solubility is probably doubled. Alkalies, even the bicarbonate or phosphate, transform part of the uric acid into acid urate, and therefore increase its solubility. Free acids diminish the solubility, since they decrease the ionization; undissociated uric acid being less soluble (His and Paul). Addition of free uric acid to urine may precipitate a part of the uric acid originally in solution (Voit), presumably by acting as a crystallizing nucleus. The solubility in serum is much greater (500 mg. per liter) than in water (Bechhold and Ziegler, I 909 ). This is probably due to the colloids; and it is probable that the colloid constituents of the urine also contribute to the solubility of the uric acid and urates (Lichtwitz, 19ro). Ruedel, 1892, claimed that urea has an important solvent action; but Ritter, I897, finds this very limited.

Acid Urates.-The solubility of the different urates in water is about as follows (Allan and Bensch, I848, etc.): $\mathrm{Li}, \mathrm{I}: 350 ; \mathrm{K}$, I: $800 ; \mathrm{Na}, \mathrm{I}: \mathrm{I}, 200 ; \mathrm{NH}_{4}, \mathrm{I}: \mathrm{I}, 600$; earths, I $4,0 \infty$ to 6,000 . Excess of the alkali or other salts decreases the solubility, by "saltng out," in proportion to their concentration (Roberts; Ritter). The salts of the plasma, i.i., reduce the solubility to one-tenth of that in water (His and Paul).

Solid sodium acid urate occurs in two forms: when freshly precipitated, it is gelatinous, colloidal or amorphous; later, this becomes crystalline. The colloidal form is more easily soluble (Bensch). Gudzent, 1908 and 1909, claims two forms, the ordinary more soluble lactam, which is gradually converted into the less (two-thirds) soluble lactim.<smiles>O=C1CC2NC(=O)NC2C(=O)N1</smiles><smiles></smiles>

Influence of Phosphates on Acid Urines.-The acid $\mathrm{NaH}_{2} \mathrm{PO}_{4}$ liberates and precipitates uric acid; this is counteracted by the presence of the alkaline $\mathrm{Na}_{2} \mathrm{HPO}_{4}$; but excess of the latter precipitates the acid urate by "salting out" (Ritter).

Alkalies on Urate Deposits in Tissues.-The solvent action of the alkalies extends only to free uric acid. When all the uric acid exists as acid urate, as it does within the body, the addition of alkali can have no further solvent action. On the ontrary, excess of alkali or other salts tends rather to decrease the solubility of the urates. It is evident therefore that no direct solvent action can occur on the urate tophi (Ritter). This inefficiency has been confirmed by clinical experience. If alkalies have any effect, which is doubtful, it would be due solely to their diuretic action.

Loghem, 1907, even claimed that hydrochloric acid is more useful for preventing the formation of urate deposits in animals, presumably by lessening the "salting out." Ritter had a similar idea. This has not been confirmed (Staal, Igo8).

Uric Acid Retention in Disease.-The older notions were based on faulty analytic methods. According to the more reliable determinations of Folin and Denis, I913 and 1915 , and Pratt, 1913 and 1915 , normal human blood contains I to $2.5 \mathrm{mg}$. of uric acid per 100 c.c. The proportion is notably increased $(2.5$ to $4.7 \mathrm{mg}$.) in gout (average $3.7 \mathrm{mg}$.) ; in lead poisoning, leukemia, and some cases of arthritis and nephritis.

Relation of Uric Acid to Gout. - The occurrence of urate tophi is a striking proof of disturbed uric acid conditions in this disease, but need have no causal connection. As has been said, the uric acid of the blood is very generally increased, but this may be even higher in other diseases and non-gouty arthritis. In gout, however, this is not accompanied by retention of non-protein nitrogen (Folin and Denis, 1915). It was formerly believed that definite changes in the uric acid content of the blood occurred during 
the acute gouty attacks. The modern results indicate that this is not correct; but that the urate concentration is the same during as between attacks; and that the atlacks occur when the urate concentration has been lowered by atophan (Daniels and McCrudden, 1015).

Increased Elimination by Drugs.--This has been discussed under Atophan. The climination is also increased by diuresis, but without any constant ratio (J. H. Robertson, I()I 4 ).

Alkalies on Urates in Urine.-The conditions for the solvent efficiency of alkalies are much better in the case of acid urine. This contains not only acid urates, but also free uric acid, often in saturated solution. When the urine cools, becomes more acid, or under other conditions, the solution may become supersaturated. The uric acid will then precipitate alone, or in combination with part of the acid urate (quadriurate). This can be prevented either by making the urine more dilute (diuresis); or by making it less acid. Alkalies act in both directions (Buchheim, r 853). The greatest solvent action can be obtained by administering adequate doses of sodium citrate or bicarbonate, together with plenty of water (Haskins, I9I6). Too high a concentration of alkalies, as of other salts, would render the acid urate less soluble; but in practice, the administration of alkalies does not raise the total concentration of the urine, especially if water is taken freely. Alkaline urines would also dissolve actually precipitated uric acid; for the urine, after the administration of alkalies, is able to dissolve more uric acid than before (Haskins, I9I6). With the practical limits of the alkalinity of the urine, however, this solution is so slight that it has little effect on formed calculi. The injection of alkaline solutions into the bladder has not been more successful (Buchheim).

Solvent Power of Urine for Uric Acid.-Haskins, Igr6, finds that this varies with its reaction and concentration. Urines of acidity ${ }_{p} \mathrm{H}=6 . \mathrm{I}$ and over can not dissolve any added uric acid; those that are less acid or alkaline take up more and more, according to their alkalinity. Concentrated urines dissolve more (though not in strict proportion) than do dilute urines of the same reaction.

Destruction of Uric Acid.-Basham, 1870, claimed that alkalies increase the destruction of uric acid; but further experience showed this to be uncertain and really negative (Severin, I868; lithium, Bosse and Buchheim, I862). The destruction by more concentrated alkalies has been studied by Stevens and May, I9II.

Difference in Bases.-The acid urates of different bases differ somewhat in solubility. The solubility of sodium urate, f.i., is about one-half that of potassium urate, one-third that of lithium urate, etc. It might seem therefore that the solubility could be improved by the administration of $\mathrm{K}$ or $\mathrm{Li}$ salts. This is true of pure solutions (or when sodium urate or urate stones or tophi are treated with lithium carbonate); but it can not occur in the presence of an excess of $\mathrm{Na}$; for according to ordinary chemical laws, when several bases compete for an acid, it is the least soluble salt which is always formed (e.g., $\mathrm{BaSO}_{4}$ in a mixture of $\mathrm{H}_{2} \mathrm{SO}_{4}$, $\mathrm{NaOH}$ and $\left.\mathrm{Ba}(\mathrm{OH})_{2}\right)$. Since there is always an excess of $\mathrm{Na}$ ions in the blood and urine, the $\mathrm{Li}$ and $\mathrm{K}$ would have no chance to exert their solvent action. The very slight superiority of lithium could exist only with very large doses, and these are unsafe (Haskins, I916).

Organic Bases. ${ }^{1}$ - The same reasoning applies to these (ethylenamins, piperazin and piperidin). These also would be unable to compete against the $\mathrm{Na}$. Moreover, they are excreted imperfectly, so that their alkaline efficiency in the urine is probably inferior to that of the ordinary alkalies. However, in some unexplained manner, large doses of piperazin (2 Gm.)

I The literature on organic "urate solvents" is reviewed by Hanzlik. 
do confer a somewhat greater solvent action than exists in normal urines of the same reaction. The difference is not sufficiently great to be significant (Haskins, r9i6).

Piperidin.-This is diethylen-diamin, $\mathrm{HN}\left\langle\mathrm{CH}_{2}-\mathrm{CH}_{2} \mathrm{CH}_{2}\right.$. It produces no, symptoms and is practically non-toxic. The solvent power for uric acid, in pure solution, is said to be higher than that of $\mathrm{Li}_{2} \mathrm{CO}_{3}$ (Mayert and Schmidt, I890; Plugge, I 895; Stevens and May, I91 I), but still insufficient to make much impression on a solid stone (Penzoldt). In the presence of Na salts, and therefore in the blood and urine, it is not superior to other alkalies (Mendelsohn, I 892; Meisels, I 893); and since a part is oxidized, and therefore only a fraction appears in the urine, its effect on the alkalinity, and therefore on the solvent power, is less than that of $\mathrm{NaHCO}_{3}$. The clinical results have been inconstant and indefinite.

These limitations apply also to the derivatives of piperidin (dimethyl-piperazin $=$ Lycetol, Wittzack, I894; ethylen-ethenyldiamin $=$ Lysidin, Ladenburg, I894). Eczema has been reported from lysidin (Ortner). Piperidin was introduced by Tunnicliffe and Rosenheim.

Organic Bases on Elimination and Destruction of Uric Acid.-The attempt to attribute the supposed clinical effects to these mechanisms is also without foundation (Waucomont, I9II), although piperidin does somewhat increase the elimination of uric acid, probably to increasing its formation through intestinal irritation (Abl, I9I3).

Hexamethylenamin on Uric Acid.- This and the related compounds (Citarin, etc.) have a solvent and destructive action on uric acid, even in acid urines (Stevens and May, IgII; Haskins, I9I5). This is due to their formaldehyd. They were therefore advocated as urate solvents (Bardet and Laquers). The action, however, is imperceptible with the ordinary doses (Nicolaier; Haskins, I9I5; review, Hanzlik, 1916). Doses of $4 \mathrm{Gm}$. make the urine distinctly more solvent; but an equal effect can be secured by rendering the urine alkaline to $\mathrm{p}^{\mathrm{H}}$ 7.I to 7.4 , through the administration of bicarbonate, etc. (Haskins).

\section{PREPARATION}

Piperidin and its derivatives are described in N.N.R.

\section{ORGANIC ACIDS AND THEIR SALTS}

Local Action of Organic Acids.-Free citric and tartaric acid are relatively mild acids, used mainly for flavoring and for preducing effervescence with carbonates. Acetic acid is somewhat more active. Lactic acid is employed as a mild caustic for the solution of false membranes. Formic acid is used as a counterirritant. The fatty acids are discussed under the Oils.

Fate and System.c Actions.-The organic acids are mostly oxidized in the tissues to $\mathrm{CO}_{2}$ and $\mathrm{H}_{2} \mathrm{O}$. Their salts therefore are converted into the bicarbonates, potassium acetate into $\mathrm{KHCO}_{3}\left(\mathrm{KC}_{2} \mathrm{H}_{3} \mathrm{O}_{2}+4 \mathrm{O}=\right.$ $\mathrm{KHCO}_{3}+\mathrm{CO}_{2}+\mathrm{H}_{2} \mathrm{O}$ ); sodium citrate, into $\mathrm{NaHCO}_{3}$, etc. and thus exert an alkaline-action after absorption; although before combustion they may be neutral or acid. Their systemic action is thus identical with the administration of alkalies, but the local action is absent. This is sometimes of material advantage, as in rendering the urine alkaline with potassium acetate or citrate. They are also diuretic, and are of ten used in catarrhal cystitis.

Before the oxidation is completed, they may exert ion actions. These are small for acetates; somewhat problematical for formates. Oxalates, citrates and tartrate inactivate calcium (see page 686). The tartrates are but poorly absorbed, and hence act as saline cathartics (see page 653 ). Citrates share this to a less degree. 


\section{ACETATES}

Potassium and sodium acetates are used mainly as alkaline diuretics.

\section{PREPARATIONS-ACETATES}

Acidum Aceticum Glaciale (Acid. Acet. Glac.), U.S.P., B.P.; Glacial Acetic Acid99 per cent. of $\mathrm{C}_{2} \mathrm{H}_{4} \mathrm{O}_{2}$. Congeals about $14.5^{\circ} \mathrm{C}$.

Acid. .1cet., U.S.P., B.P.-36 per cent., U.S.P.; 33 per cent., B.P.

* Acid. Acet. Dil.-6 per cent., U.S.P.; 5 per cent., B.P. Clear, colorless liquid, characteristic vinegar-like odor, acid taste. Miscible with water or alcohol. Dose, 2 c.c., 30 minims, U.S.P.; 2 to 4 c.c., $1 / 2$ to $\mathrm{I}$ dram, B.P.

Actum, Vinegar.-Corresponds in strength to the preceding.

(*Liq. Ammon. Acet.-See Index.)

*Potassii Acetas (Pot. Acet.), U.S.P., B.P., $\mathrm{KC}_{2} \mathrm{H}_{3} \mathrm{O}_{2}$.-White powder or crystalline masses; odorless; saline taste. Deliquesces, and hence should not be dispensed in powders. Very sol. in water ( $\mathrm{I}: 0.5$ ); freely sol. in alc. ( $1: 2.9)$. Dose, I Gm., I 5 gr., U.S.P.; 1 to 4 Gm., 15 to 60 gr., B.P.; diluted.

Sod. Acct, U.S.P., $\mathrm{NaC}_{2} \mathrm{H}_{3} \mathrm{O}_{2}+{ }_{3} \mathrm{H}_{2} \mathrm{O}$.-Colorless crystals or granular powder; odorless; saline taste. Efflorescent. Very sol. in water $(\mathrm{r}: 0.8)$; sol. in alc. $(\mathrm{I}: \mathrm{Ig})$. Dose, I Gm., I5 gr., U.S.P.

\section{CITRATES}

General Statement.-These are used especially as alkaline diuretics. Sodium citrate can be administered, $f . i$., in diabetic coma, in large doses, 30 to $50 \mathrm{Gm}$. per day, without digestive disturbance or diarrhea (Lichtwitz, IgII). Citric acid (about I per cent.) is used as a refrigerant drink in fevers, etc. It is also used as prophylactic against scurvy; often as lemon juice, which contains about 8 per cent. A large lemon contains about $4 \mathrm{Gm}$., ordinary lemonade about 0.5 per cent. The syrup is a useful flavor.

Fate.-When injected into a vein, the citrate disappears rapidly from the circulation. After hypodermic injection in rabbits, about I 2 per cent. is excreted in the urine; with cats, 30 per cent. with dogs, 40 per cent. (Salant and Wise, I916).

Action on Calcium. - The citrates form double salts with calcium, which do not liberate $\mathrm{Ca}$ ions. Their intravenous injection (not oral administration) produces the typical effects of Ca deprivation (Sabbatani, I90I; Vietinghoff-Scheel, I902; Martin, I904; see Calcium). The injection of minute quantities into the brain produces the convulsant effects. Repeated hypodermic injections of increasing doses, create considerable tolerance (Robertson and Burnett, I912).

Coagulability of the Blood.-Citrates, like other $\mathrm{Ca}$ inactivators, render the blood non-coagulable in vitro. When moderate doses (ro to $5 \circ$ c.c. of ro per cent., for man) are injected intravenously, however, the coagulation time of the circulating blood is definitely shortened (Weil, I915). The mechanism has not been explained. With oral administration of $\mathrm{I}$ to $2 \mathrm{Gm}$., Coleman, r 909 , describes a doubtful delay in coagulation.

Use in Transfusion.-Weil, I9r5, introduced the use of "citrated blood," the blood of the donor being kept uncoagulated by the addition of I c.c. of ro per cent. sod. citrate per ro c.c. of blood. This has been kept for two weeks before injection: ${ }_{5} \mathrm{Gm}$. of citrate, introduced in this manner has no other effect on the recipient than the above shortening of coagulation time. 
Preservation of Erythrocytes.-Centrifugation of blood injures the corpuscles mechanically, resulting in immediate or delayed hemolysis. This is expecially marked with dogs, less with rabbits or sheep. Human corpuscles are quite resistant. Protection is afforded by viscid solutions, such as plasma, or better gelatin (1/8 per cent.). Human corpuscles do not require this protection. The resistance to mechanical injury is not related to the osmotic resistance. Drawn blood gradually hemolyses, even on ice. Gelatin does not protect against this. Preservation is best secured by sugars ( 2 per cent. of dextrose or 5 per cent. of dextrose). This preserves blood suitable for injection for over two months (Rous and Turner, I915).

Sodium Citrate in Hemophilia.-Intravenous injection produces an immediate and marked shortening of the coagulation-time. This is followed in one to two days by return to the original, or even by a slower coagulation. Intramuscular injection has no effect (Ottenberg, I9I6).

Other Anticoagulants.-In laboratory experiments, the coagulability of blood can easily be diminished or abolished. Leech extract has this effect, whether it is injected, or added to shed blood; albumoses, only when injected; citrates, oxalates, fluorids, concentrated solutions of other salts, formaldehyd, hydrocyanic acid, etc., only on shed blood (by removing the calcium or inhibiting the fibrin ferments). None of these drugs can be used effectively on patients, except by local application.

Coagulation of Milk-The inactivation of calcium by citrates also prevents the clotting of milk. 'Their addition to cows' milk, produces a looser curd in the stomach. This is utilized in infants ( $\mathrm{r}$ to $3 \mathrm{gr}$. of citrate per ounce of milk), when ordinary milk is not well borne (curds in vomits and stools). It should be reduced or omitted as soon as the need is passed (Cotton, 1906).

\section{PREPARATIONS-CITRATES}

*Acidum Citricum, U.S.P., B.P.; Citric Acid, $\mathrm{C}_{3} \mathrm{H}_{4}(\mathrm{OH})(\mathrm{COOH})_{3}+\mathrm{H}_{2} \mathrm{O}$.-A tribasic organic acid obtained from the juice of limes or lemons. Colorless, translucent prisms or a white powder; odorless; having an acid taste; efflorescent in warm air. Very sol. in water ( $1: 0.5)$; freely sol. in alc. (r:r.8). Dose, 0.5 Gm., 8 gr., U.S.P.; 0.3 to $\mathrm{I.2}$ Gm., 5 to 20 gr., B.P.

(*Syr. Acid. Cit.-See Index.)

Lig. Ammon. Cit., B.P.-Dose, 8 to 24 c.c., 2 to 6 drams, B.P.

${ }^{*}$ Potassii Citras (Pot. Cit.), U.S.P., B.P., $\mathrm{K}_{3} \mathrm{C}_{6} \mathrm{H}_{5} \mathrm{O}_{7}+\mathrm{H}_{2} \mathrm{O}$.- Transparent prisms or white granular powder; odorless; cooling saline taste. Very sol. in water $(\mathrm{r}: 0.6)$; very slightly sol. in alc. Dose, I Gm., I5 gr., U.S.P.; I to 4 Gm., I 5 to 60 gr., B.P.

Liq. Pot. Cit., U.S.P. -8 per cent. of the anhydrous citrate. Must be recently prepared. Dose, I5 c.c., 4 drams, U. S. P.

Sod. Cit., U.S.P., $2 \mathrm{Na}_{3} \mathrm{C}_{6} \mathrm{H}_{5} \mathrm{O}_{7}+{ }_{1} \mathrm{IH}_{2} \mathrm{O}$. - White granular powder; odorless; cooling saline taste. Efflorescent. Freely sol. in water (I:I.I); insol. in al.

Dose, I Gm., I 5 gr., U.S.P.

Sod. Citro., Tart. Eff., B.P.-Dose, 4 to 8 Gm., I to 2 drams, B.P.

\section{LACTATES}

These are but slowly absorbed and therefore act as saline cathartics. They are completely burned in the organism, and have no other action (Faust, I910). It has been claimed that they somewhat limit nitrogen metabolism, after the manner of fats. For Lactic Acid, see Index.

\section{FORMATES}

Local Action.-Free formic acid occurs in several animals (ants, etc., Faust, rgro) and plants (nettles), and is partly responsible for their irritant effects. (Berchart, 1915, concludes that the effects of nettles are not due to formic açid.)

Formic acid resembles acetic acid, but is more volatile, more irritant, more antiseptic and more resistant to oxidation. It has a popular reputation as a counterirritant, but is probably not superior to other irritants. It is usually employed in the form of the Spiritus Formicarum, etc., corresponding to I per cent. of the absolute acid in two-thirds alcohol (this was formerly obtained by macerating ants). Its antiseptic action has been 
utilized for the preservation of food; O.I to 0.15 per cent. of free absolute acid suffices (Lebbin, I go6). The formates are not antiseptic. Daily doses of $0.5 \mathrm{Gm}$. of formic acid are said to cause no injurious efiects; larger doses only local actions, equivalent to acetic acid (Lebbin). Eppinger, 1913, administered sodium formate, 3 to $4 \mathrm{Gm}$. daily, to three patients. Two felt no discomfort; the other developed albuminuria and hematuria, persisting for five days. Further experience is needed.

Fate.-Formates are oxidized less readily than other organic salts, so that a greater proportion (up to two-thirds) appears unchanged in the urine. With smaller doses, the destruction may be more complete (Pohl). The oxidation occurs mainly in the liver (Fleig, 1907).

Normal Occurrence.-A small quantity of formates occurs normally in mammalian tissues. In man, the normal daily urinary excretion is 13 to $120 \mathrm{mg}$. This indicates that considerable quantities are formed in intermediate metabolism, probably of all classes of foods. It is also formed from formaldehyd and methyl alcohol, not from ethyl alcohol (Dakin, Janney and Wakeman, 1913).

Systemic Actions. - Large doses are diuretic, perhaps by renal irritation or vasodilation (Fleig, 1907). They are also said to cause methemoglobinemia (Croner and Seligmann, 1907$)$. The general circulation is not affected, even by large doses; enormous doses depress the heart (Livierato, I906). The toxicity is low, so that 6 to $10 \mathrm{Gm}$. of sodium formate may be taken daily for a limited time (Fleig). Stimulant effects of various kinds have been claimed but shown to be absent by Fleig. Formic acid has been lauded in all sorts of conditions (Garrigue; Clement; Huchard, I902; H. Stern, I 906), but without good grounds (Fleig).

\section{PREPARATION}

Acidum Formicum.-The acid official in foreign countries contains 25 per cent. of $\mathrm{HCO}_{2} \mathrm{H}$. The dose is stated as I to 20 drops.

\section{METALS AND THEIR SALTS}

General Statement.-The different metallic salts have many features in common. They combine with proteins, and therefore act locally as astringents, irritants, corrosives and antiseptics. After absorption, their effects are chiefly circulatory, especially on the blood vessels, but also on the heart. Changes in the capillaries are characteristic, and lead to phenomena analogous to those of inflammation. Their excretion by the kidneys results in nephritis. Being slowly excreted, they readily produce chronic poisoning, resulting in nervous and metabolic disturbances.

The toxicity of the different metals depends largely on their absorbability, which varies greatly. Iron salts, $f . i$., are ordinarily non-toxic because only traces are absorbed.

Metallic poisons are generally more toxic to herbivora than to carnivora-probably because they remain longer in the alimentary canal of herbivorous' animals, allowing greater absorption.

Relative Absorbability of the Metals.-Arsenic, mercury, and uranium are the only metals the absorption of which is of an extent sufficient to cause acute systemic poisoning with non-corrosive doses. Phosphorus, which behaves pharmacologically in many respects like metals, is absorbed still more readily. Other metals are absorbed much more slowly. To this class belong lead, silver, tin, and iron. Any metallic salt, if given in strong solution, will cause corrosion of the mucous membrane of the alimentary canal and will then be absorbed, and would exert its systemic action were it not that the local effects often kill before the systemic can come into play.

Habituation to Local Action.-If the administration of a metallic salt is begun with small doses and gradually increased, it becomes possible 
to administer without local effect doses which would at first have produced corrosion (Hausmann, I906). Habituation also diminishes the absorption of arsenic, but not of antimony (Cloetta, r9o6 and r9i r).

Habituation of Lower Organisms. - Yeasts, bacteria and protozoa readily acquire considerable tolerance to the various metals. This may be transmitted for generations, and may or may not extend to other metals. The experiments of Neuhaus, 19ro, with infusoria; and the trypanosome studies of Ehrlich, rgro, present many interesting facts.

Solubility and Absorption.-To be absorbed, the metal must be in soluble form; but it is to be borne in mind that the solubility in the alimentary canal is not necessarily the same as the solubility in water in a test-tube. The combination of the metal will usually be changed in its passage through the digestive tract. In the stomach it will be converted largely into the chlorid; in the intestines into the carbonate and sulphid. The metallic salts also enter into double combinations with proteins, and these are often soluble in an excess of the latter. In these ways such insoluble compounds as mercuric oxid, mercurous chlorid, lead sulphate, and silver chlorid, may be brought into solution.

Ion-action.- The characteristic effects of the metals are produced only when they are ionized. The uncombined metals and molecular organic compounds (for instance, the organic arsenic derivatives) therefore do not produce the typical actions of their metallic ions directly. They may cause effects of their own, differing from those of the ions; or they may be inactive. Eventually, however, they are usually transformed into ionized compounds, and therefore gradually develop the ordinary actions, but much more slowly. The non-ionized compounds are of ten differently distributed in the body, so that their actions may differ from those of the ordinary salts, even when they are subsequently dissociated. The lead tri-ethyl, $f . i$., simulates chronic lead poisoning (Harnack). The organic arsenic compounds are important examples.

Intravenous Injection of Metallic Salts.-The systemic actions of most metals can only be studied by injecting them intravenously, since the slow absorption and the strong local actions would obscure the remote effects, if the metals were given by the ordinary channels. For the intravenous injection, such metallic salts must be chosen as will not precipitate the blood. Double salts (such as iron-ammonium citrate, etc.) or metallic albuminates may meet this requirement. In some cases it is necessary to have recourse to some non-ionic compounds of the metal, from which the ionic metal is libera ed in the body.

Delayed Action.- When a non-precipitant preparation is injected directly into the blood the symptoms in many cases do not arise at once, but are"developed quite slowly, sometimes only in the course of several days. This is due to the fact that the metals do not act in the blood, but only after they have been absorbed into the cells; and this absorption is so slow that it may take several days to accumulate sufficient metal to produce any action. The metal may by this time have disappeared entirely from the circulating liquids (White, r880).

It would appear also that the symptoms do not arise immediately even after the poison has reached the cells. Tartar emetic, when injected in appropriate doses into a vein, disappears from the blood in ten minutes, but the first symptoms may be seen only after some twelve hours. This corresponds with the behavior of toxins and of many other poisons.

Storage in Cells.- The metals enter into firm combination with the protoplasm, and accumulate in the cells, especially of the liver, until these contain much larger quantities than the surrounding medium (Bokorny, 1905).

Cumulative Action. - The excretion of the metals is usually even slower than their absorption. They have therefore often a cumulative action; doses too small to produce any immediate effect will cause serious poison- 
ing if long continued. $\mathrm{Pb}, \mathrm{Hg}$, and As are prominent examples. single large dose may, on the other hand, produce chronic poisoning.

Excretion. - The metals are excreted partly by the kidneys, but the main part leaves through the intestines, especially the large. The epithelial cells are the channel of this excretion, and not to any extent the bile, as was at one time supposed. A gastroenteritis may result from local action even when the poison has been given intravenously. On this account tartar emetic, for instance, acts largely locally even when administered intravenously or hypodermically.

Metal Nephritis. - The amount of the metals excreted by the kidneys is often sufficient to cause a marked nephritis, characterized by albuminuria, casts, etc. The nephritis begins usually in the epithelium of the convoluted tubules and spreads from here to the glomeruli. If the poisoning is chronic, the nephritis may become interstitial and lead to renal cirrhosis.

Nephritis has been described in poisoning by the following metals: $\mathrm{Ag}, \mathrm{Al}, \mathrm{As}, \mathrm{Au}$, $\mathrm{Be}, \mathrm{Bi}, \mathrm{Cd}, \mathrm{Ce}, \mathrm{Co}, \mathrm{Cr}, \mathrm{Cu}, \mathrm{Hg}, \mathrm{Mn}, \mathrm{Ni}, \mathrm{P}, \mathrm{Pb}, \mathrm{Sb}, \mathrm{U}, \mathrm{W}, \mathrm{Zn}$, . There may also be renal glycosuria, i.e., the appearance of sugar in the urine without hyperglycemia (Luzzato, I9I 4 ).

Circulatory Effects.-All metals produce a very marked fall of blood pressure, which is due partly to a paralysis of the blood vessels and partly to a direct action on the heart.

The metals, aside from silver, may be divided into two principal groups: the first group acting mainly peripherally on the blood vessels, the second group acting peripherally on the heart. To the first group belong arsenic, antimony, uranium, bismuth, iron, manganese, selenium, tellurium, aluminum, tin, nickel, cobalt, gold, and platinum (Wohlwill, I907), to the second group, lead, phosphorus, copper, zinc, cadmium, mercury, vanadium, cerium, and thallium.

In the perfused heart, all metals (in the form of lactates) produce block and diastolic standstill (Voegtlin, 1915).

Nervous Phenomena.-The serious circulatory disturbances lead to central and peripheral nervous symptoms. Direct acute nervous actions are not common in mammals, except perhaps with silver.

Frogs show with most metals a paralysis, preceded by a slight stimulation. The tetanizing action predominates with $\mathrm{Ni}$, Co, and $\mathrm{Pt}$. Whether the metals have any direct effect on metabolism can not be decided with our present methods, because of the disturbances introduced by the intestinal and renal irritation, and by the circulatory changes.

\section{COLLOID METALS}

These occupy a peculiar position. Colloid metals may be obtained by establishing an electric arc between metallic wires under water, very fine particles of the metal being thrown off and forming a colloidal solution. Similar solutions may be obtained from solutions of metallic salts by certain reducing agents. Colloid solutions of Pt, $\mathrm{Ag}, \mathrm{Hg}, \mathrm{Au}, \mathrm{Bi}, \mathrm{Cd}, \mathrm{Pd}$, and Ir have been prepared by these methods. The metal exists in these solutions, not as ions, but in true metallic condition; it is, however, so finely subdivided that it remains permanently and evenly distributed through the solution. It is precipitable by salts, in the same way as other colloidal solutions. The chemic reduction gives equally fine particles and can be used to make more concentrated solution than the electric (Voigt, I914).

Colloid solutions can not produce the ion-actions of the metals directly. The colloid condition, however, gives them catalytic properties, i.e., minute quantities may induce reactions in large quantities of other substances (e.g., conversion of alcohol into acetic acid), the metal taking no part in this transformation. This catalysis is hindered by 
many poisons, just as is the action of true ferments. 'The metals pass quite readily from the colloid into the ionic condition, e.g., by the action of bacteria, and may then exert the ordinary action of the metals. Since this conversion occurs but slowly, these actions will be mainly local, and will produce a minimum of irritation.

Pharmacologic Actions of Colloid Metals.-It is questionable whether the colloid metals are at all absorbed unchanged. If injected intravenously, they sojourn only a few minutes in the blood (Niculescu, I9 I 2), passing first into the liver and more gradually and in smaller quantities into other organs (Voigt, I9 I4). Their gradual transformation into the ionized form will eventually produce the ordinary systemic effects of metals. They have been used, hypodermically and intravenously, for this purpose, but without serious advantage. (Solutions should not be made isotonic with salt, since electrolytes cause precipitation.) They also produce other rather indefinite and inconstant effects, probably attributable to the colloid condition: hyperpyrexia, first hypo-, then hyperleucocytosis, renal and intestinal irritation, and fatal coma (Foà and Aggazotti, I908 and I909; Gros and O'Connor, r9II). They may be followed by increased excretion of nitrogen and uric acid (Ascoli and Izar, 1907).

Therapeutic Use Against Infections.-Colloid silver particularly has been extravagantly lauded as an internal antiseptic in puerperal and other septicemias and infections; but the claims do not stand critical examination (Report, Coun. Pharm. Chem., I9o9; de Arric, I9I 2). Gros and O'Connor found that colloid metals, like charcoal, adsorb poisons, alkaloidal as well as toxins, and therefore decrease their toxicity, but the decrease is insignificant, and does not occur at all unless the poison was treated before being injected. Phagocytosis is perhaps somewhat increased for some bacteria, but not for all (Arric, r9 3 ). Colloid silver or mercury is fairly antiseptic, but not germicidal (Marshall and Killoh, ror 5 ).

Dangers. - Colloid silver preparations are apt to deteriorate rapidly into the insoluble form, and may thus produce embolism (Rittershaus, 1905; H. J. Hamburger, I909). A number of deaths have occurred probably by vascular embolism from the injection of collargol into the renal pelvis for X-ray diagnosis (Eisendrath, 19I4). Buerger, I9I4, finds that these injections may penetrate the renal parenchyma to the surface of the kidney, and lead to necrotic and suppurative foci.

Chemotherapy of Cancer.- This started with the announcement of Wassermann, Keysser and Wassermann, rgr and I9I2, that they had succeeded in causing the disappearance of rat tumors by the intravenous injection of selenium-eosin. compounds. Since then, similar favorable results were reported for colloidal copper, platinum, sulphur and some other colloids and organic metal compounds (Neuberg and Caspari, I91 2; Neuberg, Caspari and Loehe, I91 2; L. Loeb and co-workers, I912 to 19r4). Even human tumors seemed to show some retrogression. However, the results do not with-

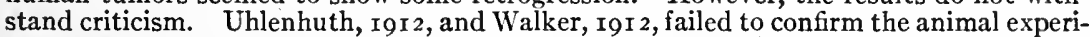
ments with eosin-selenium. Keysser's further experience (rgr4) also convinced him that cure of animal tumors by the various metals occurs in only a small percentage of cases; that they are ineffective against visceral or growing tumors; success being confined to large subcutaneous tumors, which they cause to soften and liquefy. Weil, I9I 5, suggests that they accumulate in the necrotic portions of the tumors, as do iodids and dyes, and there lead to the liquefaction of the necrotic tissue.

As to the human cancer, the reported improvement has been essentially symptomatic only, and this has little significance. Weil, I9I3 and I9I5, points out that the only admissible sign of improvement is reduction of the size of the tumor; and from this standpoint, the clinical reports are negative. Loeb (McClurg, et al., 1915) concedes that a cure can not be expected. Keysser also considers that the effective dose in animals is so near to the fatal, that no results could be expected from a safe dosage.

\section{INORGANIC ARSENIC COMPOUNDS}

General Statement.-Arsenical compounds act locally as mild and slow corrosives and have been employed in superficial cancer. Systemically, they relax the capillaries and increase their permeability, thus simulating inflammation. This change is most conspicuous in the splanchnic area. In acute arsenic poisoning, it results in extreme gastroenteritis, closely resembling cholera. 
This dilatation of capillaries introduces changes in the circulation which cause secondary disturbances in the function of more remote organs, particularly in the nervous system; and further, fatty degeneration of the cells, especially in glands and muscles, with other disturbances of nutrition and metabolism, particularly in chronic poisoning." There may also be a direct paralysis of the heart, both ganglionic and muscular.

Milder clegrees of the same action may stimulate metabolism beneficially. This explains partly the therapeutic uses of arsenic in malnutrition, anemias and skin diseases; but the mechanism of their action, and indeed the results, are somewhat in doubt. There are also characteristic effects on the bones and bone-marrow. Arsenic, especially in the organic forms, is highly effective against protozoan infections.

Arsenous and Arsenic Compounds.-Trivalent As (arsenites) is generally much more toxic to animals and plants than is pentavalent As (arsenates) (Kionka, rgII). This holds also for the different valence in organic compounds, and for the corresponding antimony valencies. It has been assumed that the 5 -valent $A$ s is non-toxic until it has been reduced to the 3-valent (Loew, I887). The occurrence of a partial reduction was demonstrated by Binz and Schulz, 1879 .

Oxidation and Reduction Theory.-Schulz, 1884, and Binz, 1897, attempted to explain the arsenic effects as alternating oxidation and reduction of the cells, due to the easy passage of arsenous and arsenic compounds into each other (which Schulz, I 892 , demonstrated on organ-emulsions). Husemann, I892, pointed out that if this were true, both forms should have the same toxicity. The difference is not great for mammals, but very considerable for lower animals and plants (O. Loew). The explanation is therefore not tenable. It is now generally believed that the arsenicals hinder protoplasmic oxidation in an unknown way; although Schaefer and Boehm, 1872, showed that arsenic has little effect on ordinary ferments.

The ratio of toxicity of arsenous acid: arsenic acid varies for different tissues. With intravenous injection in rabbits, it is as $6: 10$; for the excised frog's heart, as $300: \mathrm{r}$. The arsenous is also more toxic for the excised intestine of rabbits. These different ratios indicate that the toxicity of arsenic acid is due to reduction to arsenous acid, which varies in degree for different tissues (Joachimoglu, I9 15).

Resistance of Lower Animals.-Arsenic is toxic to all animals which possess a central nervous system; also to most of the higher plants, but not to all the lower organisms. Its antiseptic action is comparatively small. It can not therefore be classed as a general protoplasmic poison. New-born salamanders show a very great tolerance. Among mammals, the resistance is greatest in mice, then come the hedgehog, rabbit, dog and cat (Willberg, Igr 3 ).

The toxicity is the same for splenectomized as for normal mice (Towles, 1916).

Local Actions.-The arsenicals are not precipitant and therefore irritate only weakly, even on wounds or mucous membranes; but the cells die slowly after prolonged contact.

This is sometimes used for killing exposed dental nerves; and as salves for superficial epitheliomas. Arsenic is much more destructive to pathologic cells, so that the cancerous tissue may be killed without injury to the surrounding normal tissue. As with all cancer-salves some of the deeper tumor cells generally escape, leading to inoperable recurrences. Their use is therefore not advisable.

Blood Pressure.--Arsenic poisoning is characterized by pronounced and persistent fall of blood pressure; due partly to depression of the arterioles and heart, but mainly to paralysis of the capillaries.

Heart.-In the excised (Langendorff) heart the amplitude is first increased, then diminished; the rate may be slowed, quickened or arythmic. Arsenites are more toxic than arsenates, and these more than the organic. Of the latter, cacodylate is the most potent; salvarsan and its oxidation products are inactive (Archangelski, I9I2). In intact animals, the cardiac action of the arsenicals is relatively unimportant, for the heart is able to maintain a fairly high pressure if the aorta is clamped (Boehm and Unterberger, 1874). 
Vascular Action.-The vasodilation is most conspicuous in the splanchnic area; but the blood pressure falls even when the intestines are tied off, so that the muscular vessets are also dilated. The dilation can be demonstrated by perfusion of excised organs. It is therefore peripheral. The vessels, however, continue to react to splanchnic stimulation till very late; and to epinephrin even after the splanchnic response is lost (A. Loeb, I9II). The arterioles must therefore still be capable of contracting.

Paralysis of Capillaries. - For this reason it is assumed that structures beyond the arterioles-namely, the capillaries-are the seat of the paralysis. This view is favored by the fact that they have become more permeable. Intravenous injections of large quantities of salt solution will cause edema in animals poisoned with As, but not in the normal (Magnus, 1899; other capillary poisons are discussed by W. Heubner, 1907).

These changes in the capillaries explain partly the therapeutic actions, and the whole course of the poisoning. Since increased permeability of the capillaries is one of the essential features of inflammation, the phenomena of arsenic poisoning are similar to those produced by an irritating inflammation, although the primal cause is different.

However, arsenic inhibits the migration of leucocytes in the inflamed mesentery of frogs (Ikeda, I9I6).

Gastroenteritis.-The first and principal symptoms of acute arsenic poisoning are those of violent inflammation of the digestive tract.

This suggested that arsenic acts as a corrosive. However, the autopsy rarely shows extensive corrosion, and it is known that the corrosive action occurs extremely slowly. Furthermore, the gastroenteritis may be obtained with at least equal readiness if the arsenic is injected into the circulation or subcutaneously. This would not definitely exclude all local action, since some arsenic is excreted into the alimentary canal; but the quantity is not nearly enough to account for the symptoms.

The inflammation is really due to the systemic action on the capillaries, which is strongest in the intestines, no matter how the arsenic has been introduced. The capillary paralysis results in the production of an exudation into the connective tissue. This raises the epithelium (just as would a blister on the skin), and causes it to be thrown off in shreds or false membranes. The exudation is then poured into the lumen of the intestine and largely coagulates. This distention, as well as the circulatory changes, causes increased peristalsis and watery diarrhea; and the shreds of mucus and coagulated exudation give to the evacuations the character of "ricewater" stools (Boehm and Pistorius, I882).

The pathology of this condition is exactly the same as that of Asiatic cholera, and without a history it is impossible to distinguish between the two conditions except by chemic examination of the dejections.

The extreme distention of the capillaries may lead to their rupture, to the formation of ecchymoses, or possibly bleeding into the intestine or stomach, and consequently bloody vomiting or diarrhea; but this is by no means always the case.

Kidneys.-Arsenic produces nephritis, at first mainly vascular (Schlayer and Hedinger, I907), but always with more or less involvement of the epithelium (MacNider, I9ı2; O'Hare, I9 5 ).

The vessels of the kidneys participate in the capillary dilation. The general glomerular capillaries are swollen so as to fill the capsule; the urine is albuminous and scanty, and can not be increased by caffein (Hellin and Spiro, I897). The retention of nitrogen in the blood is relatively slight (Karsner and Denis, I9I4).

The types of nephrites from different arsenic compounds, and the actions of arsenicals on the adrenals are described by $L$. Pearce and Brown, igi 5 .

Absorption.-This occurs readily, to some extent even from the intact skin. Poisoning may therefore result from the external use of arsenical cosmetic preparations. With hypodermic injections, the diarrhea often sets in within an hour.

Excretion.-This occurs by all the usual channels-urine, feces, sweat (Tachau, I9II), milk, and epithelium of the skin (Heffter, I905). With 
oral administration, the main part leaves by the feces; with hypodermic by the urine. The excretion is always slow and incomplete, especially with prolonged administration (Croner and Seligmann, I907).

Time of Excretion.- The excretion begins in two to eight hours after the administration, but lasts for a long time - three to ten days, after a single dose; to seventy days when it had been given continuously. In rabbits' urine it was found for I 20 days, in dogs" for 160 days, after the administration was stopped. The elimination and retention in dogs has been studied by Dutcher and Steel, rgru.

Distribution in Tissues. - The arsenic is stored in all the tissues, but especially in the liver, kidneys and heart; less in the skeletal muscles and brain (Blarez and Deniges, I90; Eckeley, human, I9I3). In the liver of dogs it is found as an arsenical nucleoprotein (Slowtzoff, 1902). The cerebro-spinal fluid contains at most traces in normal inclivicluals (Eckeley), more in meningitis (Finel and Leroid, I913). Arsenic also passes across the placerital circulation to the fetus. It is just as toxic when injected into the mesenteric, as by the jugular vein, showing that the liver neither neutralizes nor retains it. It is less toxic on hypodermic injection, since it enters into more slowly dissociated compounds with the tissue elements.

Deposition in Hair.- - After the administration of arsenic, the hair contains detectable quantities, in firm combination. The deposition is slow but persistent. Therefore none is found in acutely fatal poisoning, the earliest demonstration being after two weeks. It may then persist for months or years, long after the metal has disappeared from the liver and bones (Heffter, 1915).

Normal Occurrence of Arsenic.--A. Gautier, I 899, found minute traces of arsenic in normal tissues. Its universal presence was confirmed by Bertrand and all those who worked with sufficiently delicate methods. In man, it occurs especially in the thyroid (0.16 mg.); also in the thymus, brain, and skin. It appears to be in organic combination with the nuclein. None is found in the liver. This arsenic is introduced with the food and especially the drinking water. (According to the Royal Commission, I903, the quantity of arsenic in food should not exceed 1/100 gr. per pound, or in liquids, per gallon.)

The quantity of arsenic normally present in the intestinal canal never exceeds $1 / 10$ mg. Larger quantities point to poisoning (Gautier, I904).

Etiology of Arsenic Poisoning.-Arsenic was at one time used very extensively for criminal poisoning, especially in the seventeenth century. An Italian woman, Toffania, carried this science to its greatest refinement, using under the name of "Acqua Toffana" a mixture of arsenic and ptomains obtained from the putrefied juices of animals poisoned with arsenic.

At the present time arsenic is less used with criminal intent, the most frequent forms of poisoning being suicidal or accidental. This may be attributed to the perfection of chemic means of detection, which allow of the discovery of the minutest trace. Accidental poisoning has been lessened to some extent by requiring the preparations of arsenic sold at retail to be colored either with lamp-black or indigo, so that they do not have the innocent appearance of a white powder. This is also of some importance in diagnosing the poisoning, the color of the vomit calling attention to it. But accidental and suicidal poisoning is still very common, since arsenic is so extensively distributed. It is easy to obtain it as a rat and fly poison. It is frequently used in the arts. Paris green is a preparation whose sale is almost unrestricted. A great many of the cosmetic preparations on the market contain arsenic and have given rise to accidents. The use of arsenic compounds, such as Schweinfurth green, as pigments has been absolutely prohibited; but a great many of the coal-tar dyes, which are popular at the present time, employ arsenic in their preparation, and very frequently this is not entirely removed. Formerly wall paper dyed with arsenic compounds was a common source of arsenic poisoning, but this has now largely disappeared.

The arsenic is given off from these papers as dust. However, undoubted cases of poisoning have also occurred when the arsenical paper was completely covered by a harmless paper; in these, some volatile arsenic compound must be formed.

Most modern wall papers still contain minute, but practically insignificant traces (Schulz, I9I5). The permissible limit of arsenic in papers is placed at 0.I gr. per square yard; but this is probably not quite harmless. The Bureau of Chemistry of the United States Department of Agriculture has analyzed a number of papers on the American market (Haywood and Warner, I904). Over 90 per cent. of these contained less than $0.046 \mathrm{gr}$. per square yard, and are therefore safe. Furs, rugs, and fabrics were less satisfactory. Black stockings particularly of ten contained quantities of arsenic (above 0.0 r gr. per square yard), which must be considered dangerous, considering their intimate contact with the skin. 
Fruits, after the use of arsenical insecticide sprays (Bordeaux mixture, lead arsenate), contain arsenic (and lead), for long periods (Sonntag, 1915). The quantities, however, are probably unimportant.

Course of Acute Arsenic Poisoning.-This may be very rapid: In fulminant cases, the withdrawal of the blood from the general circulation into the splanchnic area may produce prompt collapse, and death before the enteritis has time to develop (corresponding to "dry cholera"). Usually, however, the symptoms do not appear for from one-half to one hour after the arsenic is taken, and then run through eighteen hours to three days sometimes four to fourteen days.

Acute Symptoms.-The suspicion of the patient may have been aroused by the sweetish astringent taste of the substance. Almost the first symptoms are vomiting and profuse and painful diarrhea. The withdrawal of water from the body leads to great thirst, dryness of the mouth and throat, and difficulty in swallowing and articulation. The urine is diminished and often bloody and albuminous. The nervous symptoms consist in vertigo, headache, and pain in the limbs. There will be cyanosis and cold extremities. Toward the end occur syncope, coma, clonic and tonic spasms, and a general paralysis. Death usually occurs by exhaustion as a result of the prolonged gastroenteritis, as in cholera.

In the subacute poisoning the inflammation of the mucous membrane of the alimentary canal is prominent. Inflammation of other mucous membranes also becomes conspicuous, and shows as conjunctivitis, coryza, stomatitis, salivation, and pharyngitis Skin eruptions make their appearance if the arsenic poisoning is at all prolonged. In this case there are also symptoms arising from the central nervous system, as well as neurites.

The diagnosis of acute arsenic poisoning is made by the violent gastroenteritis. This can usually be distinguished quite easily from that produced by acids and alkalies, by the history of the case, absence of corrosion in the mouth, and furthermore by the lesser prominence of the local symptoms. The very quick onset distinguishes it from other metals.

Autopsy Findings. - The patient appears emaciated from the withdrawal of liquid from the body by the profuse diarrhea, and this even if he retains a fair amount of adipose tissue. The appearance of the alimentary canal with its large amount of fluid and the presence of shreds of mucus and false membrane, with usually no pronounced corrosion, are characteristic. ${ }^{1}$ Microscopically gastroadenitis and cell infiltration are often seen.

The body after arsenic poisoning usually putrefies very slowly and may become mummified, which always causes a suspicion, although it is not at all a proof of such poisoning. The positive proof can be furnished only by the chemic examination. It must not be forgotten that arsenic is frequently introduced into the body in the embalming fluid.

Fatal Dose.-This varies, especially with the solubility of the preparation, etc. Of the trioxid, 5 to $5 \circ \mathrm{mg}$. are toxic; 0.1 to $0.3 \mathrm{Gm}$. are usually fatal; but recovery may occur after much larger quantities. The mortality is 50 to 75 per cent. (Lewin).

1 See plates 49 and 50 of E. v. Hof mann: Atlas of Legal Medicine, W. B. Saunders Co. 
Treatment of Acute Poisoning.-Acute arsenic poisoning is best treated by lavage with warm water, emetics, or purgation. The chemic antidotes are of doubtful value.

Ferri IIydroxidum cum Magncsii Oxido (see Index) was long considered the best antidote, the ferric hydrate and arsenic forming a compound which was deemed almost insoluble. De Buscher, 1902, believes, however, that the compound is even more soluble than arsenous acid, and that it is quite ineffective. So also is hydrogen sulphid.

The hypodermic injection of magnesium sulphate has saved animals from fatal doses of arsenic, eren when the latter was also injected hypodermically. Presumably, difficultly soluble arsenic-magnesium compounds are formed (Sieber, 1912). However, the intidote must be used very promptly, and the effective dose of magnesium is itself dangerous.

Arseniuretted Hydrogen $\left(\mathrm{AsH}_{3}\right)$. - This is an extremely toxic gas, so that a few bubbles may be fatal to man. It is produced in lead-burning; by the action of impure sulphuric acid on metals; from ferrosilicon, etc. It may produce the ordinary arsenic phenomena, but the toxic effects are mainly due to hemolysis. It combines with the hemoglobin (Meissner, I913), and destroys the corpuscles. The symptoms are often delayed, and start with malaise, collapse, fainting fits, etc. This is followed by bloody urine; icterus, painful liver and kidneys; eventually by anemia (Stadelmann, I882). Severe cases may die during the earlier stages with convulsions, delirium and coma. The gas has a characteristic garlic odor.

"Ferrosilicon."-This is a mixture of $\mathrm{Fe}$ and Si compounds, used in the manufacture of steel. In the presence of water or moist air (as in shipment) it liberates phosphoretted and arseniuretted hydrogen, which have produced fatal poisoning (J.A.M.A., $55: 78 \mathrm{I}, 1910)$. The grades containing 30 to 70 per cent. of silicon are much more subject to the decomposition, and are therefore more dangerous than those containing a greater or smaller percentage.

Garlic Odor with Arsenic.-This is a rather characteristic feature under certain conditions. It is noted when the metal is burned in air to $\mathrm{As}_{2} \mathrm{O}_{3}$. Moulds growing on damp wall paper and other arsenicals produce it by forming ethyl arsin, $\mathrm{As}\left(\mathrm{C}_{2} \mathrm{H}_{5}\right)_{2} \mathrm{H}$. The odor is sometimes observed in the arsenic vomitus (Gumprecht), and is probably due to reduction products. Garlic breath does not occur, or very rarely, in the therapeutic administration of inorganic arsenic compounds, but is very strong after the use of cacodylates.

Habituation. - The mountaineers of Styria and certain other regions consume arsenic once or twice weekly, as a general stimulant and tonic. There appears to be unimpeachable evidence that they gradually accustom themselves to use with immunity quantities of arsenic (to $0.4 \mathrm{Gm}$.) which would produce very serious toxic, irritant and cumulative effects in ordinary individuals (Schaefer, $186 \mathrm{I}$; Knappe, 1875). This tolerance can also be induced in dogs and rabbits. Cloetta, 1906, attributes it to lessened absorption from the alimentary canal; Hausmann, I906, to increased excretion. The latter also showed that the corrosive action on the intestines is decreased by habituation. Withdrawal does not cause any abstinence symptoms, even if the arsenic has been consumed for years. Joachimoglu, 1916, finds that the absorption of small doses, in dogs is not diminished by habituation; but that it is rather increased in accordance with the larger dose. However, habituation leads to increased resistance of the intestinal mucosa to the inflammatory and necrotic actions of arsenic, which would promote the absorption of toxic doses. For this reason, the absorption of large doses is smaller in habituated animals.

Chronic Arsenic Poisoning.-In this, chronic gastro-intestinal catarrh develops, possibly with ulceration. The less intense but persistent capillary paralysis gives time for widespread fatty and other degenerative changes. These affect first the endothelium of the capillaries, later the intestinal epithelium, and finally the cells of other organs-liver, kidney, heart-muscle, etc. The increased size of the fatty liver may cause pressure on the bile-ducts, consequently reabsorption of bile and icterus. There is a considerable tendency to the development of local effusions; swelling of the eyelids is fairly characteristic.

Impaired nutrition or toxic actions on peripheral nerves give rise to polyneuritis, with atrophy of the muscles, disturbance and paralysis of sensation, and of the special senses (blindness). The voice is frequently 
altered, from the paralysis of the vocal cords. The skin is also particularly affected, perhaps because it takes part in the excretion. This action results in acne-like eruptions, exfoliation, and in falling out of hair and detachment of finger-nails. In a few cases a peculiar cutaneous melanosis can be noticed. This is not due to a deposition of arsenic, but to the formation of organic pigment granules in the epidermis and the exterior layers of cutis (Gans, rgr4). The mucous membranes, especially the conjunctivæ, may also suffer.

The diagnosis of chronic arsenic poisoning may be somewhat difficult because the symptoms are sometimes quite obscure or resemble very closely those produced by chronic lead poisoning. There is some difference in the electric reaction of muscle and the absence of the blue line on the gums, which are characteristic of lead. But in other cases of the acute or chronic form the chemic examination is the only absolute means of making the diagnosis.

Chronic arsenic poisoning is treated by withdrawing the cause; the same measure may also be used as for chronic lead poisoning.

Causation of Nervous Phenomena.-These are not solely due to ischemia, even in acute poisoning, but must be partly direct; for they occur also in frogs, whose nervous functions are not immediately influenced by the blood supply.

Therapeutic Use in Nervous and Rheumatic Conditions.-Arsenic has been used in chorea, neuralgias, and the various rheumatic and arthritic diseases. Its effects are usually not striking, and may probably be referred to the general tonic action.

Effects on Metabolism.-These resemble phosphorus: Therapeutic doses check oxidation, and favor nutrition and growth, even in adults. The bones become larger and more compact. The condition of the skin is especially improved. The blood formation seems stimulated under suitable conditions.

Excessive doses, as with phosphorus, lead to increased excretion of nitrogen, loss of weight, lessened gas metabolism, fatty degeneration of tissues, acidosis, decreased functionation of organs, destruction of blood, icterus, inability of the liver to form glycogen.

Hepatic Degeneration.-A further analogy with phosphorus poisoning consists in fatty degeneration of the liver cells, more marked with inorganic than with organic arsenic compounds. With intravenous injection, this is localized around the central veins; with hypodermic, it is more general (Ullmann, IgI4).

Tissue Degenerations.- Toxic doses produce degenerative changes also in the kidneys, capillaries and blood. Pathologic tissues-malignant lymphomata, syphilitic gummata, etc.-are more susceptible and may be attacked even by therapeutic doses. Experimental tumors may also be liquefied and destroyed (Neuberg, Caspari and Loehe, r. 9 12).

Factors Concerned in the Metabolic Actions. - The metabolic effect is quite variable, as it depends upon a number of mutually opposed causes. Among these are: the capillary dilatation; supposedly a direct action of arsenic upon the cells; and the action on the gastro-intestinal canal and on the kidneys. Small doses of arsenic increase the gastric secretion (Klocman, I9г2); larger doses cause irritation, colic and diarrhea. In common with other intestinal irritants, it increases the output of uric acid ( $\mathrm{Abl}, \mathrm{Ig}_{3}$ ).

The interaction of these factors may produce different results, so that the experimental data must be interpreted cautiously. As with all metabolic effects, only the gross features are known; the detailed actions on special organs would probably be even more important.

Therapeutic Use in Blood Diseases.-Arsenic is employed with limited success in various leucemias and anemias, especially in pernicious anemia; and with iron in chlorosis. The results are generally referred to an action on the bone-marrow, but in some cases may be parasiticidal. This is the basis of its use in malaria and syphilis. 
Irsenic in Lewcemia.- The results are not brilliant, but it usually improves the general conditions somewhat. The early, milder stages are most promising (Hahn, 1014 ).

Irsenic in Syphilis. - This is not entirely new. Arsenic was used in the days of liallopius, and intermittently since. The difficulty lies in the toxicity of efficient doses. Herzfeld, I9I I, advises the painless hypodermic or intramuscular injection of 1 per cent. sodium arsenite.

Actions on Bone-marrow and Blood Formation.-In normal animals, the effects of arsenic on the marrow are mainly destructive: atrophy of the fat cells, dilation and multiplication of the capillaries; increase of the leucoblastic, but not of erythroblastic cells (in contrast to hemorrhage). Larger doses cause hyaline degeneration and anemia. Similar changes are produced by other metals, organ extracts, peptones, etc. (Stockman and Charteris, I 903 ).

Saneyoshi, I9I3, has also brought evidence that ordinary doses of arsenic do not normally stimulate the production of corpuscles. It is conceivable, but not demonstrated, that it acts more positively in anemia. von Hoesslin, I9I3, claims generally restoration of hemoglobin and erythrocytes, decrease of lymphocytes and increase of neutrophiles. Its clinical effects might also be explained by the antihemolytic action, which has been demonstrated by Gunn and Feltham, I9II. The viscosity of the blood is sometimes slightly increased (Farmachidis, 1912).

Resistance of Erythrocytes.-As judged by hypotonic hemolysis, this is increased by therapeutic doses of arsenic in anemias. Hill, I9 15 , suggests this as an explanation of the clinical improvement.

Bones.- Arsenic leads to thickening of the bones and filling up of the Haversian canals (Gies, 1877), which may possibly justify its use in rickets and osteomalacia.

Skin.-Arsenic is extensively used, externally and internally, to improve the nutrition of the skin and hair, and in non-parasitic chronic skin diseases. It should never be used in acute inflammatory conditions. The results are often disappointing.

Hartzell, rgo8, considers it most successful in recent, slowly spreading psoriasis; in chronic stationary lichen; and of limited value in pemphigus. Its injudicious use is often very harmful to the skin (pigmentation, etc.). Arsenic is used extensively in veterinary practice, to improve the appearance of cattle, by brightening the coat and increasing the subcutaneous (also the peritoneal) fat.

Glycosuria.- Arsenic treatment (not salvarsan) lessens alimentary glycosuria in dogs. It has no effect on epinephrin glycosuria (Begemann, I9I2). It has been tried in human diabetes, but without much success. Arsenic poisoning causes rapid loss of glycogen from the liver, and leads to the formation of lactic acid. Small doses increase, larger doses retard autolysis (of the liver; Laquer and Ettinger, 19r2). Small doses increase the catalytic action of the blood; larger doses have little effect (Duncker and Jodlbauer, I9II).

Cachectic Conditions.-Arsenic is employed as a tonic in a variety of these conditions, especially after malaria; in tuberculosis, and in asthma. Although it is easily understood how it may be of benefit, its actual use must be entirely empirical. We do not know sufficient about the nature of these conditions, nor can we predict the action of arsenic with sufficient certainty, to be able to foretell its results.

Dosage. - The ordinary tonic dose of arsenic trioxid is I to $2 \mathrm{mg}$., $1 / 60$ to $1 / 30 \mathrm{gr}$.; of the liquid preparations, 2 to 3 drops, three times daily. For more energetic effects, it should be aimed to establish a tolerance by beginning with small doses, and gradually increasing these until some local manifestation of the arsenic appears-usually diarrhea, colicky pains, or conjunctivitis or swelling of the eyelids. As soon as these are seen, the amount must be diminished. The starting dose may be 3 drops of Fowler's solution, or $5 \mathrm{mg}$. of the oxid, three times daily, increased by I drop, or I mg., every day. All the official liquid arsenic preparations contain I per cent. They may be given in water; the trioxid in pills, often with iron and strychnin. 
*Arsenic Trioxidum (Arsen. Triox.), U.S.P.; Acidum Arseniosum(Acid. Arsen.), B.P., Arsenic Trioxid, Arsenious Anhydride, Arsenous Oxid, Arsenous Acid (White Arsenic), $\mathrm{As}_{2} \mathrm{O}_{3}$.-White powder, or irregular masses of two varieties; one, amorphous, transparent and colorless, like glass; the other, crystalline, opaque and white, resembling porcelain. Both are odorless. Slowly sol. in 30 (glassy) or roo (powdered or porcelain) parts of water; in 15 boiling water; sparingly in alc. Freely sol. in hydrochloric acid and alkalies. Dose, $2 \mathrm{mg}$., $1 / 30$ gr., U.S.P.; I to $4 \mathrm{mg} ., 1 / 6.4$ to $1 / 16 \mathrm{gr} .$, B.P.; maximum dose, $5 \mathrm{mg} ., 1 / 12 \mathrm{gr}$.

*Liquor Acidi Arsenosis (Liq. Acid. Arsen.), U.S.P.; Liq. Arsen. Hydrochl., B.P.I per cent. of $\mathrm{As}_{2} \mathrm{O}_{3}, 0.5$ per cent. $\mathrm{HCl}$. Clear, colorless, acid liquid. Incompatible (of all arsenic solutions): Salts of iron and of magnesium; lime-water; tannins. Dose, 0.2 c.c., 3 minims, U.S.P.; O.I 2 to 0.5 c.c., 2 to 8 minims, B.P.

Arsenic Iodidum (Arsen. Iod.), U.S.P., B.P.; Arsenous Iodid, As $\mathrm{I}_{3}$ - - Orange-red, inodorous, crystalline powder. Sol. in water ( $\left.1: I_{2}\right)$, with partial decomposition Dose, 5 mg., $1 / 12$ gr., U.S.P.; 3 to $12 \mathrm{mg}$., $1 / 20$ to $1 / 5 \mathrm{gr} .$, B.P.

*Liquor Arsenic et Hydrargyri Iodidorm (Liq. Arsen. et Hydrarg. Iod.), U.S.P., BP. (Donovan's Solution).--Represents I per cent. each of $\mathrm{AsI}_{3}$ and $\mathrm{HgI}_{2}$. Dose, O.I c.c., $x 1 \frac{1}{2}$ minims, U.S.P.; 0.3 to $x .2$ c.c., 5 to 20 minims, B.P.

*Liquor Potassii Arsenitis, (Liq. Pot. Arsen.), U.S.P.; liquor Arsenicalis (Liq. Arsen.), B.P. (Fowler's Solution; Liquor Kalii Arsenicosi).-Potassium arsenite, corresponding to I per cent. of $\mathrm{As}_{2} \mathrm{O}_{3}$, colored and flavored with Co. Tr. Lavender. A clear or faintly opalescent liquid with a pink color and a slight odor of lavender. It is slightly alkaline, and therefore incompatible with alkaloidal salts (in addition to the incompatibilities mentioned under Liq. Acid. Arsen.). Dose, 0.2 c.c., 3 minims, U.S.P.; 0.12 to o.5 c.c., 2 to 8 minims, B.P. Maximum dose, 0.5 c.c., 8 minims.

${ }^{*}$ Sodii Arsenas (Sod. Arsen.), U.S.P., Na ${ }_{2} \mathrm{HAsO}_{4}+7 \mathrm{H}_{2} \mathrm{O}$-Colorless, transparent prisms. Freely sol. in water (r:r.5). Dose, 5 mg., 1/12 gr., U.S.P. Maximum dose, Io $\mathrm{mg} ., 1 / 6 \mathrm{gr}$.

Sodii Arsenas Exsiccatus (Sod. Arsen. Exs.), U.S.P.; Sod. Arsen. Anhydr.-Freely sol. in water (r:3.r). Dose, $3 \mathrm{mg} ., 1 / 20$ gr., U.S.P.; $\mathrm{r} .5$ to $6 \mathrm{mg}$.; $1 / 40$ to $1 / 10$ gr., B.P.

Liq. Sod. Arsen., U.S.P., B.P. - I per cent. of $\mathrm{Na}_{2} \mathrm{HAsO}_{4}$. Dose, 0.2 c.c., 3 minims, U.S.P.; o.I 2 to 0.5 c.c., 2 to 8 minims, B.P.

\section{ORGANIC ARSENIC COMPOUNDS}

General Statement.-The organic compounds of arsenic contain the metal in non-ionic form. This is more or less readily liberated in the organism, with the gradual production of the ordinary arsenic effects. In detail, however, these differ considerably from those of inorganic arsenic compounds, partly because the whole organic molecule may act as such, and partly perhaps because the organic side-chains alter the penetration and therefore the selective action of the arsenic, so that this is liberated in the cells which it does not ordinarily reach in effective concentration. At all events, the differences from inorganic arsenic are mainly quantitative rather than qualitative. In the body, the organic compounds are less toxic to mammals and more toxic to protozoan parasites. In this way they become available for combatting trypanosomes, syphilis, spirillosis and other protozoan infections.

However, while arsenic trioxid kills the protozoa in test-tube cultures, the currently used organic arsenic compounds are practically ineffective outside of the body. In order that they may become effective, they must be altered in the tissues. The active decomposition products are not well known, but they must contain trivalent arsenic, which is vastly more toxic to protozoa than is pentavalent arsenic. 
Chemic Structures.-Cacodylic Acid and Arsanilic Acid (Atoxyl) are derivatives of arsenic acid, and therefore contain the pentavalent arsenic. Salvarsan (I)ioxy-diamino-arseno-benzol) contains trivalent arsenic, which acts much more powerfully on the parasites.

'Their structure is illustrated by the formulas:

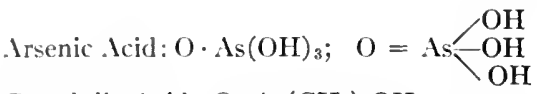

Cacodylic Acid: $\mathrm{O} \cdot \mathrm{As}\left(\mathrm{CH}_{3}\right)_{2} \mathrm{OH}$.

Arsanilic Acid: $\mathrm{O} \cdot \mathrm{As}\left(\mathrm{C}_{6} \mathrm{H}_{4} \mathrm{NH}_{2}\right)(\mathrm{OH})_{2}$ (Atoxyl).

Acetylarsanilic Acid: $\mathrm{O} \cdot \mathrm{As}\left(\mathrm{C}_{6} \mathrm{H}_{4} \mathrm{NH} \cdot \mathrm{CH}_{3} \mathrm{CO}\right)(\mathrm{OH})_{2}$ (Arsacetin).

Dioxy-diamino-arseno-benzol: (Salvarsan).<smiles>NC1CCC(O)CC1</smiles>

Arsenic Content.-Arsenic trioxid contains 75.74 per cent. of arsenic; crystallized sodium arsenate, 24 per cent.; sodium cacodylate, 35.6 per cent.; sodium arsanilate (atoxyl), 22 to 26 per cent.; salvarsan, 31.6 per cent., neosalvarsan, about 20 per cent.

Chemotherapy.-This term has been restricted, somewhat arbitrarily, to the parasiticidal treatment of infections by chemical agents; "Internal Antisepsis" is perhaps a more descriptive title.

Other examples of chemotherapy are the specific effect of quinin in malaria, of mercury in syphilis, and possibly that of the salicylates in rheumatic fever. These were all empirical discoveries; the occasional use of arsenic in syphilis, trypanosomiasis, etc., was also haphazard. The recent development of this field is due mainly to the systematic work of P. Ehrlich and his pupils. ${ }^{1}$

Affinity of Chemic Substances and Living Protoplasm.-The numerous antiseptics which are universally effective against infective agents outside of the body are not generally available for internal therapy, because they have, for the most part, a greater affinity for the protoplasm of the host than for that of the parasites (Bechhold and Ehrlich, I906). They are more "organotropic" than "parasitotropic," and therefore more injurious to the host than to the parasite. The problem of chemotherapy is therefore largely one of specific selective affinity (penetration or otherwise), between the specific living cells and specific chemical substances. These differences are mainly quantitative, but they vary greatly for individual substances (as illustrated, for instance, by the vital staining of tissues; the triple stain for leucocytes; the methylene blue stain for nerves, etc.). While therefore the existence of a harmless universal internal antiseptic is highly improbable, it is quite conceivable that a series of specific substances may exist which combine a relatively high toxicity for a certain parasite with a relatively low toxicity for the cells of the host. This hope has not been realized for most bacteria, which have generally a very high resistance. The protozoan parasites, on the other hand, are much more susceptible, and the efforts of Ehrlich in this direction have been highly successful.

Trypanosome Experiments. ${ }^{2}$-The experimental therapy of protozoan infections was most conveniently investigated on the -Trypanosomes, which by their different species, produce the tropical "sleeping sickness," and similar diseases in animals. Ehrlich and Shiga, I904, studied the influence of a great variety of substances on trypanosome infections of white mice. The search was at first haphazard; but when partly successful results were obtained, it was sought systematically to improve these by altering the side-chains so as to increase their specific affinity for the chemoreceptors of the parasites. The effective agents found by Ehrlich and by independent workers belong to four groups: (I) Benzidine Dyes (Trypan red, etc.); (2) Basic Triphenylamin dyes

'P. Ehrlich; "Beitraege zur exp. Pathol. and Chemother.," r909. A short discussion of Ehrlich's work is given by L. H. Marks, 1910, J. A. M. A., 55: 1974. 2 A synopsis of the experimental therapy of tryanosome infections is given by B. T. Terry, Arch.
Intern. Med., March, I909. 
(Malachite Green, Parafuchsin, etc.); ${ }^{1}$ (3) Arsenic Compounds; and (4) Antimony compounds and also Bismuth.

These investigations were important mainly by elucidating the general principles of chemotherapy; for it was soon found that the detailed results could not be transferred directly to man: Agents which were effective in one species of host were of ten much less effective in another. The dyes in particular were not practically available, since they cause intense coloration of the body, irritation and necrosis of the skin, and severe cachexia. These objections do not hold for the arsenic or antimony compounds; but with these also the efficiency for man can not be deduced from the animal experiments.

Acquired Resistance.-One of the most important results was the discovery that the injection of ineffective doses may gradually render the parasites resistant ("fast") to the agents of the same group, although they preserve their susceptibility to drugs of the other groups, which act upon other receptors (Ehrlich, 1907). In this way the trypanocidal agents may be divided into three principal groups: (I) Arsenicals; (2) Parafuchsin; and (3) Acid azo-dyes of the benzo-purpurin series (trypan red and blue). There are a few significant exceptions to these laws: Pyronin, which contains no arsenic, induces resistance to arsanilic acid. Arsenic trioxid does not render the trypanosomes resistant; bacteria acquire resistance to inorganic arsenic, and are then also resistant to antimony (H. Marks, I9ro). The arsenic resistance of trypanosomes extends only to animals, and can not be demonstrated in vitro. Fhrlich explains this by lessened avidity of the parasites for arsenic, so that they do not compete with the tissue for the arsenic. This diminution of arsenic avidity is a graded process, and the trypanosomes may be made "fast" either against the weaker or the active arsenicals.

This insusceptibility is preserved for many generations, even if the resistant parasite is passed through a succession of hosts. It is therefore worse than useless to employ small doses, since these would only render the organism less susceptible to further treatment. In the phraseology of Ehrlich, one must aim at a Therapia Magna Sterilisans; at killing all the protozoa with a single massive dose of the antiseptic: When this can not be done, the result may sometimes be attained by employing, simultaneously or successively, two or more agents belonging to different groups ( $f . i$., Atoxyl and mercury, Nierenstein and Todd, 1907 ).

Arsenic in Protozoan Infections.-Arsenic has had some reputation in syphilis since the times of Fallopius (Herzfeld, I9rI), and the cacodylate was employed in this disease by Gautier in 1896 . Arsenic was also used in malaria with fair success. These instances were, of course, entirely empirical. Experimentally, Laveran and Mesnil, r9o2, found that arsenous acid caused the temporary disappearance of trypanosomes from the blood of infected mice. It has also been used with fair success in human cases (Merck's Reports, 2r:223) but the really effective dose is too dangerous. A more selectively parasitotropic modification was found by $\mathrm{H}$. W. Thomas, 1905, in Arsanilic acid (Atoxyl). This compound was first prepared by Bechamp in $186_{3}$, and its general actions investigated by $\mathrm{F}$. Blumenthal, 1902 . It was then used as an arsenic substitute in skin diseases, anemias, etc. Its toxicity to mammals is much less than that of arsenic trioxid, its acutely fatal dose being some forty times as great. It proved highly efficacious in mice and was tried extensively in human sleeping sickness, especially by Robert Koch, I907. In man, however, it often causes severe toxic effects, and the clinical results have not come up to expectation.

Spirochete Infections and Spirillosis.-The discovery of the infective agent of syphilis by Schaudinn and Hoffmann, and its transmissibility to monkeys (Metchnikoff and Roux) and to rabbits (Bertarelli, Parodi), opened the way for its experimental therapeutics. After a series of trials, Ehrlich and Hata, r9. ro, found that Dioxy-diaminoarseno-benzol (Salvarsan) was highly specific against spirochete and certain other protozoan infections in animals. In human syphilis, the results have not been quite as absolute, but still more rapid than with the old forms of treatment

\section{SODIUM CACODYLATE}

General Statement.-The effects of cacodylic acid (dimethylarsenic acid) are essentially those of the inorganic arsenic, to which it is partly reduced in the body. Since this reduction occurs but slowly, the action is more prolonged and less toxic, and the local irritant effects are avoided. It has therefore some advantage. On the other hand, the degree of reduction, and therefore of the effect, is variable; and the cacodylate, especially when given by mouth, imparts a garlic odor (from cacodyl

1 The chemistry of these dyes is discussed by H. Schweitzer, Science, Dec. 9, 1910. 
oxid, $\left.\mathrm{As}_{2}\left(\mathrm{CH}_{3}\right)_{4} \mathrm{O}\right)$, to the breath, sweat, urine, etc. To avoid the more rapid reduction in the stomach, intestines and liver (Heffter, I9oI), it is usually administered hypodermically or intramuscularly. Excessive doses produce toric symptoms, due mainly to the ionic arsenic (Schulz, is79; Heffter, I90I; Dawes and Jackson, I907), although the cacodyl molecule may conceivably play a part.

Therapeutically it is used in the same conditions as inorganic arsenic. The dosage is from 0.025 to $0 . \mathrm{I}_{5} \mathrm{Gm} .1 / 2$ to $2 \frac{1}{2} \mathrm{gr}$. per day, as pills; or hypodernically, as a freshly prepared warm sterile solution; also by enema. In syphilis, Oppenheim, r907, and J. B. Murphy claimed some success (single intramuscular injection of 2 to $4 \mathrm{gr}$.), but Cole, I9r6, showed it to be relatively ineffective, even with intravenous injection of large doses.

Fate of Cacodylate. - A part is excreted unchanged in the urine (on hypodermic injection, 6 to ro per cent., Dawes and Jackson); but the greater portion undergoes reduction, first to the volatile cacodylic acid, and a small part finally to inorganic arsenic.

A bibliography of Cacodylate is given by de Biehler, 1907. It was introduced into therapeutics mainly by Gautier, 1899 . The clinical side actions are discussed by Seifert, Nebenwirk., I9I5, page 172 .

\section{PREPARATION-CACODYLATE}

*Sodii Cacodylas (Sod. Cacodyl.), U.S.P.-About 73 per cent. of sodium dimethylarsenate, $\mathrm{Na}\left(\mathrm{CH}_{3}\right)_{2} \mathrm{AsO}_{2}$. White, deliquescent prisms or granular powder, without odor or taste. Very sol. in water ( $\mathrm{r}: 0.5)$; freely sol. in alc. ( $\mathrm{r}: 2.5)$. Dose, o.o6 Gm., I gr., U.S.P. in pills, hypodermically, or by enemas. Maximum dose, $0.2 \mathrm{Gm}$., $3 \mathrm{gr}$.

\section{SODIUM ARSANILATE (ATOXYL)}

General Statement.-This salt, $\mathrm{C}_{6} \mathrm{H}_{4}\left(\mathrm{NH}_{2}\right) \cdot \mathrm{AsO} \cdot \mathrm{OH} \cdot \mathrm{ONa}$ (sodium aminophenyl-arsenate), may be derived from sodium arsenate by replacing one hydroxyl by anilin. (It was at first believed to have a different constitution.)

Under the dangerously misleading name of "Atoxyl" (for the substance is quite dangerous) this salt has received considerable prominence as an organic form of arsenic, effective in all conditions in which arsenic is indicated; especially in sleeping sickness, in which it is superior to other arsenicals; and in syphilis and other protozoan affections, in which it is inferior to Salvarsan (Tomasczewski, r9o8; Greven, I9ro).

In sleeping sickness it causes prompt disappearance of the trypanosomes from the blood, but they may recur, so that the injection must be repeated every seven days (R. Koch, 1907 , Deut. Med: Wochenschr., 33: 1889. This paper discusses also the details of dosage and administration). Kopke's (I906) clinical results were less favorable. The parasites become gradually resistant. B. Moore, Nierenstein and Todd, 1907 , therefore recommended alternation with mercury which acts on another stage of development (Hodges, 1908).

The chief objection to atoxyl" is the fairly frequent occurrence of toxic effects, especially permanent blindness, from therapeutic doses. These, as well as the therapeutic actions, are mainly due to the reduction products which are formed in the organism.

Sodium arsanilate should not be given by mouth, since it is there decomposed and rendered more toxic. The hypodermic dosage is from 0.02 to $0.2 \mathrm{Gm}$. every other day. The injections are said to be nonirritant. 
Toxic Effects of Atoxyl in Man.-Acute symptoms occur especialiy with doses exceeding $0.5 \mathrm{Gm}$. They resemble partly those of ordinary arsenic poisoning, and consist in nausea and vomiting; colic, vertigo, general weakness and somnolence; neurites, pain in limbs; deafness, retention of urine, sometimes nephritis; iritis, and not uncommonly permanent optical atrophy (Hallopeau, I907; Fordyce, I9I I). Chronic poisoning presents the ordinary phenomena of chronic arsenic intoxication. The clinical side actions are discussed by Seifert, Nebenwirk., r915, page r 78 .

Optic Effects of Atoxyl (and Arsacetin). -The eye symptoms usually appear within a few weeks (in Koch's series only when the dosage exceeded $0.5 \mathrm{Gm}$.). They begin with scintillation, cloudiness, diminished vision, and contraction of the visual field (Fordyce, r9II). The anatomic changes in animals as well as in man (Birch-Hirschfeld and Koster, rgro) show degeneration of the optic nerve and retinal ganglia, the retina being the more susceptible. The cerebral cells are also injured.

Inorganic arsenic and cacodyl may also cause optic neuritis (of the nicotin type), but only very rarely (Sulzer, r9I I). Salvarsan does not seem to cause the optic injury.

Protein Metabolism.-Boyd, rgrr, found in two human cases that arsanilate lessens the excretion and causes the retention of nitrogen.

Toxic Effects of Atoxyl on Animals.-The acute toxicity of atoxyl is perhaps onefortieth of that of inorganic arsenic; and injections have no acute effects on blood pressure. However, they produce chronic effects and anatomic lesions, the distribution of which varies quantitatively in different animals (Igersheimer and Itami, r9o9): dogs show mainly characteristic renal hemorrhages with secondary nephritis; cats exhibit mainly nervous symptoms with histologic lesions in the brain and cord. Rats and rabbits are intermediate. The distribution of the arsenic corresponds with the differences in effects. In cats, $f . i$., the arsenic is found mainly in the nervous system; in dogs, in the parenchymatous organs (Igersheimer and Rothmann, rgrr). Mammals also show the optic degeneration, both on local and systemic administration. (The toxicity of atoxyl has also been investigated experimentally by Cianni, I908.) In frogs, Sanno, I9II, found that the toxicity is greatly increased by raising their temperature, presumably by favoring its transformation into more toxic products. (A similar influence is found with colchicin.)

Difference in the Actions of Inorganic Arsenic and Atoxyl.-The atoxyl effects run a slower course, and are devoid of the local actions of inorganic arsenic. This might be due simply to the slow liberation of ionic arsenic. Some of the other differences, such as the optic changes, are more characteristic. They might be attributed to the distinctive localization of the arsenic. It is possible, however, that they are produced by some organic decomposition product. Artificial reduction products are much more toxic than the arsanilate, to mammals as well as to protozoa.

Excretion.-After a single dose of arsanilate, the arsenic is excreted promptly and exclusively by the urine (Croner and Seligmann, r9o7). With continued administration, the excretion is somewhat more prolonged; the greater part leaves within three days (Simonot, I9ro), but traces continue to be excreted for three weeks (Welander, 1909). The effects are therefore somewhat cumulative. A small part leaves by the feces. Only a small proportion is excreted in inorganic form.

A relatively large part of the arsanilate remains for a long time unchanged in the blood and tissues (Simonot, roro), but a part is fixed and changed in the cells. The characteristic distribution in different animals is described by Igersheimer and Rothmann, rgog.

Reduction.- Since arsanilic acid is itself practically non-toxic to trypanosomes, it follows that it must undergo some change in the tissues. The degree of decomposition and therefore the efficiency and toxicity, vary in different animals. The decomposition products have not been isolated, but it is probable that they must contain the arsenic in trivalent form. (Rothermundt and Dale, rgr2, however, believe that it is directly trypanocidal.) The artificial reduction products, which vary according to conditions, are much more active than the arsanilate, and paraminophenyl-arsenous acid kills the parasites in the test-tube. B. T. Terry, rgr2, has found that emulsions of liver and blood reduce arsanilate into directly effective products.

\section{PREPARATIONS-ARSANILATES}

Sodii Arsanilas, N.N.R. (Atoxyl).-Occurs as white, odorless crystals, of faintly salty taste. Readily sol. in water ( $1: 5$ or 6 ). Dose, 0.02 to $0.2 \mathrm{Gm}$., $1 / 3$ to 3 gr. hypodermically every other day, gradually increasing if necessary until the single dose reaches $0.65 \mathrm{Gm}$., ro gr., and $\mathrm{u}$ ntil a total of $6.5 \mathrm{Gm}$. (roo gr.) has been given. It must not be administered by mouth. 
Arsactin.-Sodium acetyl arsanilate, $\mathrm{C}_{6} \mathrm{H}_{4}\left(\mathrm{NH} \cdot \mathrm{CH}_{3} \mathrm{CO}\right) \mathrm{AsO} \cdot \mathrm{OH} \cdot \mathrm{ONa}+{ }_{4} \mathrm{H}_{2} \mathrm{O}$. Is less toxic acutely than arsanilate, and is highly parasiticidal in mice. In man, however, it is no more effective than the original substance, and is equally liable to cause blinclness (J.A.M.A., I910, 54: 1559); and it is even more apt to produce renal irritation (Borchers, I9I0). Its only advantage is the greater stability, so that it can be sterilized without decomposition. The dosage, hypodermically, is 0.1 to $0.5 \mathrm{Gm}$.

Ehrlich, r907, noticed that some mice treated with arsacetin acquire the curious waltzing habit.

\section{SALVARSAN AND NEOSALVARSAN}

Composition.-Salvarsan (Arsenphenol-amin, "606," Arsenobenzol), is diamino-dihydroxy-arseno-benzene hydrochlorid (see Structural Formula, page 744).

Neosalvarsan ("9r4") is a soluble preparation of salvarsan, viz., Sodium diamino-dihydroxy-arseno-benzene-methanal sulphoxylate, $\mathrm{NH}_{2} \cdot \mathrm{OH} \cdot$ $\mathrm{C}_{6} \mathrm{H}_{3} \cdot \mathrm{As}: \mathrm{As} \cdot \mathrm{C}_{6} \mathrm{H}_{3} \cdot \mathrm{OH} \cdot \mathrm{NH}\left(\mathrm{CH}_{2} \mathrm{O}\right) \mathrm{OSNa}$, mixed with inert inorganic salts, so that 3 parts of neosalvarsan approximate in arsenic content 2 parts of salvarsan.

Experimental Results.-The introduction of salvarsan was the direct outcome of the chemo-therapeutic studies of Ehrlich. The substance was prepared by Bertheim, and tested by Ehrlich and Hata, rgro. They found that a single injection of a harmless dose freed animals completely and permanently from spirillosis and certain other protozoan infections, including syphilitic spirochetes. Bacteria are generally not very susceptible, but according to Roos, I9 I2, salvarsan is directly germicidal to anthrax, even in living animals. It is ineffective in rabbits (Mejia, I9 I3). The susceptibility of spirochetes is materially greater in the presence of enzymes from the liver, and especially from the blood (Bronfenbrenner and Noguchi, I913).

Therapeutic Results in Man.-Salvarsan has been completely successful in relapsing fever (Iversen, roro), in frambesia, and in yaws (Nichols, I910). The degree of its usefulness in tertiary malaria, sleeping sickness, and certain other protozoan infections; and in leprosy and pellagra is undecided. Wadhams and Hill, I9I3, have used it successfully in a few cases of amebic dysentery. It has been tried as a substitutefor ordinary arsenic, in skin diseases, pernicious anemia, as a tonic, etc., but without serious advantage.

Results in Human Syphilis.-Salvarsan has proved remarkably successful, although it has not fulfilled the original hope of complete cure by a single injection. The conditions are evidently not as simple as in experimental animals.

Early Lesions.-Complete success, similar to that in animals, occurs only in specially favorable conditions, when it is injected during the primary stage, the initial focus being excised or destroyed by local mercurial treatment. Ordinarily, the spirochetes begin to disappear in a few hours from the chancre and condylomata, and are completely absent in one or two days; however, they generally reappear after a time. In this way, salvarsan abolishes promptly the main dangers of contagion-a very important advantage. Its efficiency is in direct ratio to its early use, and its principal field is in the primary stage, where its use prevents the development of secondary symptoms and of positive Wassermann reaction.

Later Stages.-In these, it still produces very marked but temporary effects, a single injection often equaling months of mercurial treatment. Complete disappearance of the syphilitic symptoms is the rule. Surface lesions and ulcers especially are repaired with astonishing rapidity. Malignant cases and those which have resisted mercury may cede promptly to the salvarsan. However, even these striking results are almost always 
followed, after a time, by relapses, so that the treatment must be repeated, or supplemented by mercury. In a few cases salvarsan fails completely; presumably the spirochetes are in some region which is difficultly accessible to the drug. In general, the older the infection, the less is the efficiency of salvarsan.

In parasyphilitic conditions (tabes, general paresis, etc.) salvarsan is usually ineffective, although it sometimes produces st riking improvement. It is worth trying at the very start of the symptoms. In syphilitic aortitis, salvarsan and mercury rarely give more than temporary relief (Longcope, r9r4).

Hereditary syphilis has been cured through the milk, by injecting the nurse; and congenital syphilis by injection of the mother. Arsenic has been found in the placenta. but it is doubtful whether it is transmitted to the fetus.

Wassermann Reaction. - The efficiency of salvarsan is reflected in the Wassermann reaction: In a few of the most favorable cases, this becomes and remains permanently negative after a single injection. Much more commonly the negative reaction is not lasting, but returns in a short time to positive. It may then be again suppressed by further treatment. Lesser, ror r, considers the cure incomplete until a permanent negative reaction is obtained. In less favorable cases, the Wassermann reaction is not altered, and in a few a temporarily negative reaction may even be roused to a positive one.

Possibility of Permanent Cure.-There is now little doubt that suitable cases are cured by sufficiently large and repeated doses, especially when alternated with mercury.

Advantage over Other Arsenicals. - The curative action of 'salvarsan is also shared by all other arsenic compounds, but it possesses the practical advantage that the effective doses can be introduced with much greater safety.

Advantage over Mercury. - The relative advantages of mercury and salvarsan are rather evenly balanced, so that salvarsan has supplemented, rather than displaced, the older drug. Its main advantage is the much more rapid action, which brings the disfiguring or dangerous lesions (especially of the mucosæ) under prompt control, limits contagion, and is highly important in malignant cases. The second advantage is that, in combination with mercury, it is more efficient than this drug alone or with iodids. It has a special field in cases in which mercury is ineffective or not tolerated.

Disadvantages of Salvarsan.-These involve partly the inconvenience, expense, loss of time, relatively complicated technic, and pain of its administration, certain special contraindications, and the not infrequent occurrence of more or less toxic side effects. These are also produced by Neosalvarsan (Wolff and Mulzer, r9r2). Fatalities and permanent injury, however, are so infrequent, if proper precautions are employed, that they do not seriously interfere with the usefulness of salvarsan.

Comparison of Salvarsan and Neosalvarsan.-It is still doubtful which of these drugs is superior. The neosalvarsan has the advantage of easy solubility, and therefore simpler technic. However, it is more readily decomposed, and the products are more toxic. Some believe that salvarsan is more efficient (Whitehouse and Schuyler, r9r3; Nelson and Haines, r9r4). Opinions differ as to the relative local irritation. 
Direct Local Irritation.-Intramuscular and subcutaneous injections of acid solutions cause very severe local and neuralgic pains, persisting for several hours or days. These are due mainly to the acidity, and are very much less with the neutral suspensions. However, even with the latter, and] with neosalvarsan solutions, there is more or less swelling and infiltration, lasting one or two weeks; and sometimes necrosis, infection, or abscess formation. H. E. Robertson, I9I3, describes severe, slowly healing destructive lesions, often complicated by hemorrhages and sloughing abscesses. Sometimes the injections are not absorbed (due to local thrombi, Loehe r9 22; Takahashi, I9I4), so that the deposit may remain indefinitely. Salvarsan always leaves an insoluble yellow pigment, which acts as a foreign body (Robertson). During the intravenous injection, there is often intense, but fleeting pain in the shoulder (endocardial irritation?).

Systemic Toxic Actions.- The cause of these has not been fully elucidated. There appear to be several distinct varieties (Ehrlich, I9II). Some may be ordinary arsenic phenomena, such as the occasional nephritis (which occurs especially on intramuscular injection). Others are attributed to decomposed solutions, and some to the toxicity of the salvarsan itself. The common febrile reactions are ascribed mainly to impure water. Reactions of the luetic tissues and skin are explainable by the liberation of luetic toxins, while the late "neuro-recurrences" are ascribed to the more vigorous activity of surviving spirochetes. All of these explanations are more or less hypothetical. The mortality is perhaps I : 2,000 (Corlett, r913).

Wechselmann, I9I4, has collected reports of 140 fatalities. He is inclined to attribute these mostly to the salvarsan itself, acting on some pathologic conditions, usually renal. This interpretation is not universally accepted.

Lethal Dose.--According to animal experiments, arsenic in the form of salvarsan is about one-seventh (one-third to one-fourth, Luithlen, I9r3) as toxic as in inorganic form (Kochmann, I9I2). The fatal intravenous dose for rabbits would correspond for man to about 5 or $6 \mathrm{Gm}$., or ten times the therapeutic dose. I Gm. has been given clinically without serious results. The acute toxicity of neosalvarsan is stated as about half as high (Kerstner, I9I2).

Solutions of salvarsan in serum are much less toxic to rabbits than are watery solutions (Roick, IgI5).

Acute Effects in Animals.-These resemble ordinary arsenic poisoning superficially, although the immediate effects are probably molecular. They result in fall of blood pressure (mainly cardiac), renal irritation, ana tomic parenchymatous lesions, and nervous phenomena. Ionic arsenic may play a part in these late phenomena.

Circulation.-The blood pressure falls severely on intravenous injection; but in contrast to ordinary arsenic, the fall is mainly cardiac, and the vessels of frogs are constricted. Excised hearts are depressed. This cardiac action is the common cause of acute fatalities. The cardiometer may show cardiac dilation even when the blood pressure does not fall, the vasoconstriction compensating for the weakening (Luithlen, I I I3). The effects are greatly influenced by the concentration and reaction. Acid solutions are especially fatal to the heart, but alkaline solutions render it more susceptible to strain (Auer, I I I I). The fall is greater when tricuspid insufficiency exists, but not with aortic insufficiency (Alwens, I9I3).

Clinical Effects on Circulation.-Occasionally there are severe reactions (Burzi, I9I 2), but usually doses of 0.3 to $0.6 \mathrm{Gm}$. produce only slight and inconstant changes of blood pressure. The heart rate shows slight but lasting increase. There may be a temporary diuresis. The erythrocytes are temporarily increased. Rolleston, 1915, finds that neosalvarsan injections are generally followed by a fall of systolic (average $16 \mathrm{~mm}$.) and diastolic $(13 \mathrm{~mm}$.) pressure. The formation of white corpuscles (especially 
neutrophiles) is stimulated (Rzentkowski, I9II). Heden, I9I3, describes a decrease of hemoglobin.

Precipitation of Blood.-Joseph, I9I I, observed that acid (not alkaline) solutions, above o.I per cent., precipitate blood, in the vessels as well as in the test-tube. The quantity and coarseness of the precipitate depend upon the concentration of the solution, not upon the quantity. In living animals, the precipitate does not pass the pulmonary circulation. With 0.2 per cent. solution, the precipitate is so fine as to be harmless, but with 2 per cent. it may cause fatal pulmonary embolism.

Hemolysis. - According to Corridi, isotonic solutions of neosalvarsan or acid solutions of salvarsan are not hemolytic, but alkaline solutions dissolve the corpuscles. He prefers neosalvarsan in 0.4 per cent. $\mathrm{NaCl}$ to watery solutions for intravenous injections, but these must be used promptly or oxidation will occur (denoted by brown color and turbidity).

Lcucocytes.-Rabbits show at first leucopenia, followed in several hours by leucocytosis, especially of neutrophile polynuclears (Corridi, I9I2).

M uscle and Nerve.-Joseph, IgII, found that the excitability of frog's muscle is abolished, both in acid and alkaline solutions, but the action occurs only slowly, and requires high concentrations.

Parenchymatous Degeneration.-Fatty and necrotic changes are of inconstant occurrence, clinically and in animals. They resemble ordinary arsenic lesions, involve especially the liver (acute yellow atrophy); then the kidneys, heart, nervous system (encephalitis hemorrhagica) and spleen (Busse and Merian, I9 I 2; Morel, Mouriguand and Policard, rgr2; Schlasberg, kidney, r9r2; Schirogorow, 1913. Mucha and Ketron, I9 12, claim that even small doses damage the renal vessels and glomeruli). The intestines may also show hemorrhagic and inflammatory changes (Saccone, 1912). The lesions occur especially after repeated injection, and are increased by combination with mercury, uranium, epinephrin or Roentgen treatment (Luithlen, I9I3; A. Loewy and Wechselmann, I9I3).

Luithlen emphasizes the greater toxicity of salvarsan to animals which have been subjected to these vascular injuries, and believes them analogous to the Herxheimer reaction; i.e., that this is a specific salvarsan effect on vessels previously injured by the luetic process.

Nervous Phenomena.-v. Marschalko and Veszpremi, IgI2, found multiple hemorrhages and thrombi in the brain, with symptoms corresponding to the encephalitic type of clinical salvarsan poisoning.

Metabolism.-The effects resemble arsenic (Postoiev, 1912). Syphilitic patients under treatment show some nitrogen loss on the day following the injection; otherwise, the changes are inconstant (Cappelli, r9I I).

Acute Clinical Fatalities.-Acute collapse (cardiac?) has sometimes occurred immediately after the injection, generally after the second dose. The collapse usually ends in rapid recovery, very rarely in death (Tomasczewski, I913).

Some patients, after repeated injections of salvarsan, exhibit respiratory and vasomotor symptoms resembling anaphylaxis (Swift, I9I2).

Subacute Clinical Arsenic Fatalities.-These are difficult to distinguish from the indirect fatalities, but may be classed as arsenical by their resemblance to ordinary arsenic and salvarsan poisoning in normal animals. They start a day or two to a week after the administration, often after the first injection, with headache, weakness, vomiting, sometimes icterus, incontinence of sphincters, coma, dyspnea, cyanosis, and death in three or four days. Paralytic symptoms may be observed (Chiari, I913). Identical effects, with fatal ending and autopsy lesions typical for arsenic, have been witnessed on non-luetic patients (Lube, I9I4),

The lighter cases may exhibit nephritis, diarrhea, or exanthemata. The autopsy shows hemorrhages, thrombosis, focal inflammatory softening of the central nervous system, degenerative parenchymatous changes in the kidneys, liver, heart-muscle, spleen, etc. (Busse and Merian, 1912; Tomasczewski, r913; Corlett, 1913). 
Decomposed Solutions.-Salvarsan and neosalvarsan decompose very quickly on contact with air, with the formation of other, much more toxic, arsenic compounds (such as amino-oxyphenyl-arsenoxid, Ehrlich and Bertheim, Iy I 2). They are therefore marketed in sealed air-free tubes. The contents of a tube should be used at once after opening and under no circumstances should the contents of a tube damaged in transportation or any remnants of the powder from previously opened tubes be used.

Acute Febrile Reaction.-Very commonly the injection is followed by more or less fever, chills, rapid pulse, headache, lumbar pains, malaise, nausea and vomiting, and diarrhea. These effects are of short duration and rarely serious.

They are especially marked in the presence of infections, even if slight (Wechselmann, rgro). or if stale distilled water is used (Wechselmann, I9rr; Swift and Ellis, rgr I). Ehrlich assumes that the bacterial products sensitize the body to arsenic. Neisser, 1910, attributes it to the destruction of the spirochetes. Perhaps the most probable explanation is that of Luithlen and Mucha, 1913 , namely a destructive action of salvarsan on pathologic tissues.

Reaction in Luetic Tissue and Skin.-The luetic lesions, especially of the trunk, often show a bright red erythema ("Jarisch-Herxheimer reaction"). These phenomena set in early and last only a few days. Generally they cause little inconvenience; but it is conceivable that analogous reactions in more vital organs (cerebral gumma) might be immediately dangerous. The icterus which is often seen may possibly belong to this class. It might, however, be an arsenic phenomenon. Acute yellow atrophy of the liver has been described (Severin and Heinrichsdorf, I9I2). Fischer, I9I5, attributes this to the syphilis, and not to the drug.

Neuro-recurrences.-Sometimes salvarsan injections are followed, after an interval of weeks or months, by severe nervous manifestationsepileptiform convulsions, acoustic, more rarely optic, facial, and other neurites. These do not appear to be due to the arsenic, for similar effects may occur in mercurial treatment, and they cede on further salvarsan or mercury medication. Ehrlich, I9II, attempted to explain them by the incomplete destruction of the parasites; those which escape being then less exposed to the normal restraining influences, and assuming a greater virulence.

Optic Neuritis.-This is a rare sequel of salvarsan and neosalvarsan. It is generally classed with the neuro-recurrences; but Arganaraz, I9I4, reports a series of cases which he attributes directly to the drug, and not to the spirochetes.

Contraindications to Salvarsan. - It must not be forgotten that salvarsan is an heroic remedy. Ehrlich himself never claimed that effective doses would be free from danger, and always advised against its use in debilitated individuals; specifically in severe disturbances of the circulatory organs, advanced degenerations of the central nervous system, fetid bronchitis, and cachexia, unless these are directly syphilitic; in optic neuritis; in patients hypersusceptible to arsenic, or in those who have recently received a course of other arsenical medication. Diabetes, tuberculosis and nephritis are considered contraindications by some, by others not. Caution must also be used in old or alcoholic patients.

Absorption, Fate and Excretion.-The absorption of salvarsan is slow, even from intramuscular injections, some arsenic generally remaining at the site of injection for months (Schuebel, r914). Neosalvarsan is ab- 
sorbed more rapidly (Ullmann, I9I 2, 75 to 85 per cent. within a week; Swift, I9I3). Very little is absorbed from the rectum. The excretion of arsenic occurs mainly by the urine and feces. In the urine it begins promptly, but proceeds rather slowly especially after muscular, but even after intravenous injection, unknown compounds being present, eapecially in the parenchymatous organs, for weeks. Only a small part is excreted unchanged, the main portion being ionized (Valenti, I91 2; Usuelli, I902). The course of excretion is essentially similar in luetic patients and in healthy rabbits (Burnascheff, I9r2).

Course of Excretion.-Salvarsan may be demonstrated in the urine within a few minutes to two hours after administration (Abelin, I9II). With intravenous injection, it remains in the blood plasma for I to 2 hours (Böcker, $191_{5}$ ). The excretion begins in two or three hours (Usuelli, I9I 2), and is practically completed in four days (Hoppe, I9I0); but traces may be found up to three months (Heuser, I9II).

After intramuscular injection, the excretion is much slower (Greven, I9Io; Fischer and Hoppe, I9го; Irokawa, I914), but varies with the absorption. In man it begins in six hours; the maximum excretion occurs in four to six days; in eight days two-thirds have been excreted by the urine. Traces were found in twenty to forty days (Muto and Sanno, I9II; Usuelli, I9I2; by Heuser up to nine months). The course of excretion has also been studied by Escallon, I9 2 . The excretion is said to be hastened by Potassium iodid (Greven, I9I0).

Hypodermic injections are eliminated more rapidly than the intramuscular (Greven). Relatively large quantities are excreted by the gastro-intestinal canal (Ullmann, I 9 I 2; Stuehmer, I9I4); two to ten times as much as by the urine (Frenkel-Heiden and Navassart, I913). The milk also contains arsenic (Caffarena, I9 2 ).

Excretion of Arsenic into Cerebro-spinal Fluid.- Hall, $191_{5}$, found arsenic absent after intravenous injection of sodium arsenate or cacodylate, and after intravenous neosalvarsan. With intravenous salvarsan, the results were variable. Camp, I9I2, obtained it only with toxic doses of salvarsan. The permeability of the meninges is not increased by the subdural injection of irritants (Stillman and Swift, I9 I 5 ).

Distribution of Salvarsan.- - After intravenous injection, the salvarsan does not remain free in the blood, which contains only traces after twenty-four hours; but it is promptly deposited in the spleen, kidneys, bones, muscles, and especially in the liver (Bornstein, I9II), and exceptionally traces in the brain. It may be demonstrated in these organs for months, as inorganic and organic arsenic (Stuempke and Siegfried, I9II; Ritter, I9II; Ullmann, I9I2). Arsenic also passes into the placenta (Burnascheff, I9I 2).

Administration.-The intravenous method is now preferred to the intramuscular; some operators prefer to follow the intravenous injection in two or eight days, with a second intravenous or with an intramuscular injection of about half the ordinary dose; and a third full dose after three to four weeks. In any case the salvarsan should be followed by vigorous mercurial treatment. The course may be repeated at intervals, until a permanent cure is effected.

Kolmer and Schamberg, I9 2, have tried oral administration in man $(0.6 \mathrm{Gm}$.) and in animals. There were no toxic symptoms other than vomiting and diarrhea, but the therapeutic effect was very feeble.

Intraspinal Injections of Salvarsanized Serum.-The administration of salvarsan by the ordinary methods is not very effective in cerebro-spinal lues, since but little of the salvarsan passes into the cerebro-spinal fluid. Swift and Ellis, 19r3, have therefore introduced the direct spinal injection of the patient's serum, withdrawn an hour after the intravenous injection of salvarsan, and heated for half an hour to $56^{\circ} \mathrm{C}$. Such serum has a distinct effect on the spirochetes, in the test-tube as well as in vivo, and the method has given encouraging results. The pain, psychical disturbances and cell count are usually markedly improved. The best results 
are obtained with early lesions; they are sometimes successful in tabes and beginning paralysis; poor with established progressive paralysis. The injections are safe, and can be repeated as often as needed (Draper, 1915).

The salvarsan may also be added directly to serum, using not over $0.5 \mathrm{mg}$. It is unsafe to give more than two, or at most three, consecutive injections (Draper).

Intraspinal Inicetion of Saldarsan Solutions.-This causes severe irritation of the cord, ind some writers advise that it should not be attempted in man (Ellis and Swift, I II3; Marinesco, I9I3). Others consider it fairly safe when a careful technic is followed. either using a hypertonic (6 per cent.) solution (Ravaut, I914; Wile, 1914); or mixing the drug with cerebro-spinal fluid so as to give a concentration of 0.05 to 0.15 per cent, and a dosage of $1 / 2$ to $12 \mathrm{mg}$. Neosalvarsan is almost always preferred. The injections are generally repeated every week to three weeks, and combined with systemic administration (bibliography, Naegeli, I 15 ).

Gennerich, 1913, dissolves 0.15 Gm. of neosalvarsan in 300 c.c. of normal saline. He withdraws 60 c.c. of cerebro-spinal fluid, mixes this with 60 c.c. of the above solution, and reinjects.

Schubert, 1914, withdraws Io c.c. of cerebro-spinal fluid. In 3 c.c. of this he dissolves $0.045 \mathrm{Gm}$. of dry salvarsan. A reservoir is connected with the spinal puncture and lowered so as to fill with at least 20 c.c. of spinal fluid. Into this he drops 0.1 to 0.2 c.c. of the salvarsan solution, and then raises the reservoir so as to make the fluid flow slowly into the canal. He cąutions that everything must be absolutely dry.

Dosage.-This should be adjusted to the strength and condition of the patient, and the method of administration. For adults, the dose of salvarsan is 0.2 to $0.4 \mathrm{Gm}$. intravenously, or 0.3 to $0.6 \mathrm{Gm}$. intramuscularly. The adult dose of neosalvarsan is from 0.45 to $0.9 \mathrm{Gm}$.

The details may be seen from the following table:

\begin{tabular}{|c|c|c|c|}
\hline & \multicolumn{3}{|c|}{ Dose in grams, of } \\
\hline & Neosalvarsan & $\begin{array}{l}\text { Salvarsan in- } \\
\text { travenously }\end{array}$ & $\begin{array}{l}\text { Salvarsan intra- } \\
\text { muscularly or } \\
\text { hypodermically }\end{array}$ \\
\hline$\ldots \ldots \ldots \ldots$ & 0.6 to 0.75 to 0.9 & 0.4 & 0.6 \\
\hline Women................ & 0.45 to 0.6 to 0.75 & 0.3 & 0.45 to 0.5 \\
\hline Weak adults.............. & $\ldots \ldots \ldots \ldots$ & 0.2 to 0.3 & 0.3 to 0.4 \\
\hline Children. . . . . . . . . . . . & 0.15 to 0.3 & 0.1 to 0.2 & 0.2 to 0.3 \\
\hline 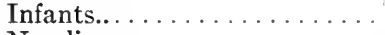 & $\ldots \ldots \ldots$ & 0.02 to 0.1 & 0.02 to 0.1 \\
\hline Nurslings . . . . . . . . . . . . . & $\ldots \ldots \ldots$ & 0.01 to 0.015 & $\ldots \ldots \ldots \ldots$ \\
\hline
\end{tabular}

\section{PREPARATIONS-SALVARSANS}

"Salvarsan ("606") is marketed in tubes containing $0.6 \mathrm{Gm}$. It is a sulphurcolored, crystalline, hygroscopic powder, dissolving readily in water. The solution has an acid reaction (hydrolytic dissociation). It is not precipitated by acids. The addition of sodium hydroxid at first precipitates the free base. The precipitation is complete when 2 molecules of $\mathrm{NaOH}$ have been added for each molecule of salvarsan. This precipitate, suspended in water, is the form used for intramuscular injection. The further addition of $\mathrm{NaOH}$ again forms a soluble salt, the solution being complete when 2 further molecules of $\mathrm{NaOH}$ have been added. This is the form used for intravenous injection. (By actual measurement, the reaction is not completed until 54 per cent. more than the theoretical amount of alkali has been added; Bongrand, 19 r 3.$)$ (Arsenphenolamin may act both as a base, through its amine, or as an acid, through its phenol, radicals.) Sodium carbonate does not dissolve the precipitate.

Salvarsan is also marketed under other names (Arsenobenzol, etc.). When pure, these have the same efficiency and toxicity (Wile, 1916).

* Neosalvarsan.-Marketed in tubes containing the ordinary doses. It is an orangeyellow powder of peculiar odor, dissolving readily and smoothly in water, with neutral reaction. The solutions are precipitated by acids. On standing they acquire a dark brown color and precipitate.

Preparation of Salvarsan Solutions for Intravenous Injections.-These must be perfectly clear, aseptic, and prepared with 0.9 per cent. $\mathrm{NaCl}$ in recently distilled 
water. The saline may be warmed to body temperature. The $0.6 \mathrm{Gm}$. tube of salvarsan requires for complete solution 23 drops of $\mathrm{I}_{5}$ per cent. NaOH, or more accurately, 4.2 c.c. of normal $\mathrm{NaOH}$. The solution may be made by triturating the salvarsan in a mortar with the soda solution, and then adding roo to 300 c.c. of the saline solution; or it may be made in a graduated stoppered cylinder, first shaking the salvarsan with about 40 c.c. of saline until dissolved, then adding the soda, and finally saline to roo to 300 c.c. Should any remain undissolved, a few drops more of the $\mathrm{NaOH}$ may be added. Any excess of alkali can be neutralized with acetic acid. If the solution is not perfectly clear, it must be filtered through a sterile filter. The injection is made from a burette into an arm vein. Dreyfus, $19 \mathrm{I} 5$, recommends sodium salvarsan, which is soluble to 20 per cent., as more convenient than the extemporaneous preparation.

Neutral Salvarsan Suspension for Intramuscular Injection.-This is made aseptically, in a mortar, by triturating $0.6 \mathrm{Gm}$. of the salvarsan with ro drops of I $_{5}$ per cent. $\mathrm{NaOH}$, or with 2.I c.c. of normal $\mathrm{NaOH}$. Water is then added, with constant stirring, at first drop by drop, to a total of 5 or ro c.c. The mixture is finally made exactly neutral to litmus paper, by the addition of further $\mathrm{NaOH}$ or acetic acid. The injections are made with a thick platinum needle, usually in the region of the shoulder blades. Suspensions in neutral oil have also been used.

Neosalvarsan Solutions.- These are prepared by simple solution in sterile, recently distilled water, using for each o. $5 \mathrm{Gm} ., 3$ c.c. of water for intramuscular, or 25 c.c. for intravenous injection. The solution must not be warmed: the temperature must not exceed $22^{\circ} \mathrm{C}$. or $72^{\circ} \mathrm{F}$. Ravaut, 1913, urges the use of concentrated solutions (Merck's Report, $27: 345$ ).

\section{SELENIUM AND TELLURIUM}

Selenium and Tellurium resemble arsenic in the general action on the capillaries of the splanchnic area. They also affect the central nervous system, apparently directly. Tellurates arrest perspiration (after the manner of atropin?), and have been recommended for this purpose (Sodii Telluras, 5 to $50 \mathrm{mg} ., 1 / 4$ to $3 / 4 \mathrm{gr}$.), but they impart a very persistent garlic odor to the breath, tissues, urine, and feces, even in a quantity as small as $0.005 \mathrm{mg}$.! This may last for several months after the administration is stopped. It is caused by methyl-tellurid. Selenium is more toxic than tellurium (Woodruff and Gies, I902).

Potassium tellurite may be used as an indicator of living bacteria, yeasts, molds, etc. When incubated for 24 hours with a concentration of about $1: 50, \infty 00$, living cultures of all the more common microorganisms reduce the tellurite, as indicated by the appearance of a black precipitate. This concentration is practically harmless when injected into animals (W. E. King, and Davis, r914).

The effects of selenium compounds on tumors was discussed on page 735 .

\section{VANADIUM}

The pharmacology of this metal has been studied by Jackson, ror 2. According to Luzzato (1903) poisonous doses of $\mathrm{VCl}_{3}$ or $\mathrm{VBr}_{3}$ cause the usual anatomic lesions of metals in the kidneys, liver, intestine, and stomach. No lesions are found in the central nervous system. The blood pressure is raised, and the excitability of the vagus is depressed. Death occurs through respiratory paralysis.

Circulatory Effects.-Jackson found, with intravenous sodium orthovanadate, that the rise of blood pressure is due to systemic vasoconstriction, by direct action on the arterial muscle or vasomotor endings. The heart is not strengthened. The pressure in the pulmonary artery also rises by the systemic and pulmonary vasoconstriction. Perfused lung vessels are strongly constricted (Baehr and Pick, 1913). Bronchial muscle also shows moderate but prolonged constriction (confirmed by Baehr and Pick, 1913).

Chronic Vanadium Poisoning-This occurs especially through its industrial use as a mordant, and by the wearing of dyed fabrics. The symptoms and lesions are those common to other chronic metallic intoxications. The lungs, kidneys and digestive canal are especially affected (Dutton, rgr 1 ). 
Therapeutic Use.-The pharmacologic actions do not point to any definite therapeutic usefulness. Vanadium has been recommended as an alterative, similar to arsenic, in tuberculosis, diabetes, etc., but the evidence is not convincing.

\section{PREPARATION}

Sodii Meta-ianadas. - White, almost tasteless powder, sol. in water. Dose, I to $8 \mathrm{mg}$. ( $1 / 60$ to $1 / 8 \mathrm{gr}$.). According to Jardin ( 1905$)$ it lessens assimilation and increases protein destruction.

\section{ANTIMONY}

Actions.-The actions of antimony resemble those of arsenic. The difference lies in a greater local irritation and a much lesser absorption. Consequently, when given by the mouth, doses can be chosen whose only action is that of producing nausea, or if somewhat larger, vomiting.

Toxicology.- - If injected into the circulation, or if given in overdoses, it produces precisely the same effects as arsenic, but vomiting is always a prominent phenomenon, the poison being rapidly excreted into the alimentary canal. Small doses long continued lead to a train of symptoms of subacute poisoning, entirely analogous to those produced by arsenic in the same manner (Gaehtgens, I876; Soloweitschyk, I880), also including the metabolic changes. Antimony has not been recorded as giving rise to industrial poisoning, except through contamination with arsenic. However, the pentasulphids that are used in the rubber industries are fairly soluble in human gastric juice (Carlson, I915). Antimony is sometimes used in the glaze of cheap granite ware. This should be prohibited by law (as it is in some European countries), since some samples yield from 3 to I4 mg. of dissolved antimony in a single cooking test (E. W. Miller, Igr6).

In distinction to arseniç, selenium and tellurium, antimony is not converted into volatile compounds by molds (V. Knaffl-Lenz, I9r3).

Treatment of Antimony Poisoning.-The chemic antidotes are eggs, milk and tannin (tea, etc.), or in its absence magnesia.

Potassium hexatantalate precipitates antimony compounds, even in the tissues; but it could be utilized only orally, since its hypodermic injection is too toxic (Rosenthal and Severin, I912).

Habituation.- One difference from arsenic is that the continued administration of antimony to mammals does not lead to tolerance; nor does arsenic habituation confer tolerance to antimony. This difference is explained by the observation that the absorption of antimony is not diminished (Cloetta, I9II); on the contrary, it is rather increased (Brunner, I91 2; he claims some tolerance for 3-valent, but not for 5 -valent). Protozoa also do not become immune as readily as with arsenic (Rowntree and Abel, IGI2). Antimony immunity can be induced in infusoria and does not éxtend to arsenic (Neuhaus, I910). Bacteria immunized to arsenic are also resistant to antimony (Marks, 1910).

Local Action.-Applied to the skin, the chlorid is a strong caustic. The double tartrate of antimony and potash (tartar emetic) when applied over long periods, produces a pustular eruption. This is due to the decomposition of the double salt, which is but slightly irritant, by the acid, secretion of the follicles into more irritant antimonous oxid, $\mathrm{Sb}_{2} \mathrm{O}_{3}$, and other compounds. Such salves were formerly used for counterirritation, but have been abandoned as too destructive and toxic.

Differences in Antimony Compounds. - As with arsenic, the 3-valent is more toxic (to ten times) than the 5 -valent. The organic derivatives show similar relations, the side-chains being relatively unimportant (Brunner, I912). Cloetta found the pyrostibiate much less toxic than tartar emetic.

Mechanism of Emetic Effect.-This is produced by local action; for while it occurs both by gastric and venous administration, the latter requires much larger doses, vomiting occurs much more slowly, and only after the antimony has been excreted in to the 
stomach, as shown by its presence in the vomitus (Kleimann and Simonowitsch, I 872 ). The nauseant action, and the increase of the salivary and bronchial secretion, are also reflex (V. E. Henderson and Taylor, I9ro). Like other irritants, antimony is said to increase uric acid excretion ( $\mathrm{Abl}, \mathrm{19} \mathrm{I} 3$ ).

Therapeutic Use as Nauseant and Emetic.- "Tartar Emetic," the tartrate of antimony and potassium, is the compound usually employed internally. As an emetic it has fallen out of favor, its action being too slow and too depressing. As a nauseant it has advantages over all other metals, since the dose required for this is only about one-tenth of that which produces vomiting. Its continued use, however, is dangerous. It is employed mainly as a depressant expectorant in the first stages of acute bronchitis; often in the form of Wine of Antimony, I c.c. $=4 \mathrm{mg}$., $1 / 15 \mathrm{gr}$; or smaller doses ( $\mathrm{I} \mathrm{mg}$.) may be repeated every hour with precaution against excessive depression. It also enters into a number of compound expectorants, such as Mistura Glycyrrhizæ Co. (8 c.c. $=2 \mathrm{mg}$. of tartar emetic and $x$ c.c. of paregoric): and Compound Syrup of Squill (2 c.c. $=$ $4 \mathrm{mg}$. of tartar emetic).

Antimony has an interesting history. Paracelsus in the sixteenth century praised it as a panacea, and it was used against the plague, etc., perhaps a forecast of its most recent uses. However, numerous deaths caused its formal proscription.

Trypanocidal Action.- Antimony and bismuth, as well as arsenic, are highly toxic to trypanosomes, although not to all protozoa. At the suggestion of Cushny, various investigators (Plimmer and Thomson, I907, Mesnil and Brimont, I908, Thomson and Cushny, Igro, Camac, x gri, and others) have successfully used injections of antimonysodium tartrate in experimental and human tyrpanosome infections. The intravenous dose for man is ro mg., in saline. Rowntree and Abel, I ro, advocate certain organic compounds, such as sodium-antimony thioglycollate. They have the advantages over the organic arsenicals in that the parasites do not become immune as readily. They are also cheaper. The trypanocidal activity of tartar emetic is greater in the presence of serum; this does not hold for arsenicals (Shilling and Goretti, rgI4).

Jemma, x9x6, states that tartar emetic is specific in the Leishmaniosis of children.

\section{PREPARATIONS-ANTIMONY}

* Antimonii et Potassii Tartras (Antim. et Pot. Tart.), U.S.P.; Antimonium Tartaratum (Antim. Tart.), B.P.; Tartarated Antimony (Tartar Emetic), $2 \mathrm{~K}$ (SbO) $\mathrm{C}_{4} \mathrm{H}_{4} \mathrm{O}_{6}$ $+\mathrm{H}_{2} \mathrm{O}$.-Colorless, transparent crystals or white granular powder. Sweet, then metallic taste. Efflorescent. Slightly acid to litmus. Incompatible with alkalies and tannins. Sol. in water ( $1:$ I 2 ) or glyc. (I : I 5). Insol. in alc. Dose, expectorant, 5 mg., $1 / 12$ gr., U.S.P.; 2.5 to 8 mg., $1 / 25$ to $1 / 8$ gr., B.P.; emetic, 30 to $60 \mathrm{mg}$., $1 / 2$ to $\mathrm{rgr}$., B.P.

*Vinum Antimoniale (Vin. Antim.), B.P.-Contains 0.4 per cent. of Tartarated Antimony. Dose, expectorant, 0.6 to r. 8 c.c., ro to 30 minims; emetic, 8 to 16 c.c., 2 to 4 drams, B.P.

Antim. Oxid., B.P., Antimonious Oxid., $\mathrm{Sb}_{2} \mathrm{O}_{3}$.-Grayish powder, insol. in water. Dose, 0.06 to $0.12 \mathrm{Gm}$., $x$ to 2 gr., B.P.

Pulv. Antim., B.P.-Antimonious oxid, I part; calcium phosphate, 2 parts. Dose, 0.2 to $0.4 \mathrm{Gm} ., 3$ to 6 gr., B.P.

Antim. Sulphur., B.P.; Sulphurated Antimony.-A mixture of antimony sulphids and oxids and sulphur. Orange-red powder. Dose, 0.06 to $0.12 \mathrm{Gm}$., I to $2 \mathrm{gr}$., B.P.

\section{URANIUM .}

The salts are very corrosive, consequently readily absorbed, and highly toxic. Their systemic actions are similar to those of other metals, with a special paralytic effect on respiration; this resembles cyanid poisoning superficially. They produce a characteristic nephritis; glycosuria; and a unique edema. 
Systemic Actions.-These have been studied especially by Woroschilsky, 1890, and D. F. Jackson, rgro. In acute poisoning, the respiration is peculiarly affected, first increased, then paralyzed. This results in the usual asphyxial phenomena, stimulation of the vasomotor center and rise of blood pressure. The respiratory paralysis is the cause of death. The blood is rendered non-coagulable by an unknown mechanism.

Sublethal doses are followed in a few days by albuminuria and glycosuria; parenchymatous dlegenerations, gastro-intestinal disturbances; paralytic symptoms; and changes in the blood ressels.

Anatomic Changes in Uranium Nephritis. - These affect both the tubular epithelium and the glomeruli, one or the other predominating according to conditions (Fleckseder, 1907; Ireineke and Myerstein, 1907; Dickson, 1909). The vascular mechanism is involved first, but the tubular changes progress longer and finally predominate (MacNider, I I I 2). The anatomic and functional changes are more severe in old animals, and are increased by anesthetics, especially chloroform (MacNider, I9I3, 1914). Dogs are more resistant than rabbits (O'Hare, I9I3). 'The epithelium shows fatty changes; the glomeruli are congested. Christian, I9I r, describes characteristic hyaline droplets in the vessel-walls of the glomerular tufts. These do not occur in other vessels of the kidneys or in other organs.

Repeated injections of small doses produce a chronic interstitial nephritis with fibrous changes. (These are not as severe as with chromates; arsenic did not produce interstitial changes; R. M. Smith, IgIr.) The histologic lesions are similar to human chronic diffuse nephritis, but not quite identical. They are not associated with arterial lesions (Dickson, I9I2).

Urine Changes.-In the early (vascular) stages, the quantity and specific gravity of the urine are increased. Albumen, glucose, and acetone substances appear, especially in older dogs (MacNider, 1914). A further increase of urine flow may be secured by caffein or salines, although the response is slower than normal (Théohari and Giurea, I9Io; Boycott and Ryffel, I9I3; Mosenthal and Schlayer, I9I3). With the appearance of the epithelial changes, the urine decreases, the tubules being obstructed by the swelling and desquamation of the cells. This is not relieved by diuretics, although the vascular response to the diuretics may be preserved (MacNider, I912, 1914, 1915). The excretion of chlorids and urates is diminished in acute, somewhat increased in the subacute nephritis; i.e., it varies in the same direction as the urine flow.

Pohl described a subacute nephritis in rabbits, with polyuria until death, and with increased excretion of nitrogen and diminished chlorid output. Dünner, 19 15, finds this inconstant. The intravenous injection of calcium or of hypotonic $\mathrm{NaCl}$ converted this subacute nephritis into an acute form. Isotonic saline had no effect.

The excretion of endogenous cretinin is diminished in both stages; but injected creatinin is completely excreted in the subacute, retained in the acute (Kraus, 1913). The phenolphthalein excretion also varies in the same direction as the urine flow (Eisenbrey, I913). It bears no definite relation to the histologic lesions (Smith, I9II). The non-protein nitrogen retention in the blood goes parallel to the phthalein retention; both increase and recover together, except that the phthalein responds more promptly (Frothingham, Fitz, Folin and Denis, I913; Karshner and Denis, I914). However, Mosenthal, I9I4, points out that the increase of non-protein nitrogen in the blood is not necessarily proof of retention, but could be explained by increased protein destruction or by inspissation of the blood through polyuria.

MacNider, I916, finds the toxicity of uranium associated with acidosis, sodium carbonate having a distinct saving effect. It also protects the uranium kidneys against anesthetic, so that these produce much less epithelial degeneration.

Uranium Glycosuria. - This develops on the first or second day. The action consists partly in increased permeability of the kidneys for sugar, for it may occur without hyperglycemia (Franck, I $9^{1} 3$ ), and may even relieve hyperglycemia when this is due to decreased permeability of the kidney cells (Pollak, I9II). The sugar does not bear any definite relation to the other urinary constituents, except albumin, which goes fairly parallel (Wallace and Myers, I9I4). However, uranium may itself cause hyperglycemia, even when the kidneys are excised, so that part of its action must be extrarenal (Fleckseder, r9o6).

Nephritic Glycosuria.-Glycosuria without hyperglycemia occurs with many nephritic poisons. With cantharides, it is attributed to increased permeability of the vessels; with uranium, chromium, tellurium, mercury and aloin, to lesions of the tubular epithelium (Luzzato, I9I4).

Uranium Hydrops. - The hypodermic administration of uranium salts produces general anasarca and ascites (Leconte, 1854; Richter, 1904), a highly albuminous fluid transuding mainly into the serous cavities, but also into the other tissues, and extending even to the fetus in utero (Bibergeil, 1905). 
It seemed natural to explain this by nephritis and anuria, but this explanation does not correspond to the facts. Nephrectomy alone does not produce hydrops, so that the anuria does not furnish the explanation. On the other hand, the uranium-hydrops occurs in nephrectomized animals, so that the kidneys are not concerned in its production (Fleckseder); nor do the histologic kidney lesions bear any relation to the edema (R. M. Smith, I9I I). The fundamental change consists presumably in an altered permeability of the capillary walls (Richter, I904). The injection of the blood of uranium animals into chromate animals also produces edema (Heinecke, 1905; Blanck, 1906). Edema, however, occurs only in animals which are freely supplied with water-otherwise the uranium causes anuria without hydrops (Fleckseder). Retention of salts is not connected with its production (Richter, I905). In acute uranium poisoning, the lymph flow is not increased (Jackson, I010).

Uranium poisoning interferes with the restoration of the blood-volume after saline infusion (Bogert, Underhill and Mendel, i9 16).

Therapeutic Uses.- The soluble uranium nitrate, has been used in diabetes mellitus and in phthisis in doses of $75 \mathrm{mg}$. (1 gr.); largely diluted. The results are too indefinite to justify the further employment of so dangerous an agent.

\section{PREPARATION-URANIUM}

Uranii Nitras (Uran. Nit.), U.S.P., $\mathrm{UO}_{2}\left(\mathrm{NO}_{3}\right)_{2}+6 \mathrm{H}_{2} \mathrm{O}$. -Light yellow prisms; bitter, astringent taste. Freely sol. in water ( $1: 1.2$ ) or in alc. Dose, $0.01 \mathrm{Gm} ., 16$ gr., U.S.P.; diluted. Use with caution.

\section{BISMUTH}

The insoluble basic bismuth salts are used on inflamed surfaces. They act largely mechanically, as protective and drying dusting powders. The use of soluble bismuth preparations is therefore irrational. The insoluble bismuth compounds are also employed in X-ray diagnosis. Even large doses are ordinarily harmless, but may become poisonous under special conditions. The medicinal use of bismuth is said to date from Dr. Odier of Geneva, 1785 .

Local Actions of Insoluble Basic (oxy) Bismuth Salts.-These form fine adhesive powders. The subcarbonate, subsalicylate and magma are insoluble in water. The subnitrate and subgallate are somewhat hydrolyzed and therefore very slightly soluble (Bonček, rgro; Stingel, I9II). Their solubility in the body-fluids is only very slight.

They have a marked healing effect when dusted on wounds and on inflamed mucous surfaces. Applied to wounds, they dry the secretions and form a protective covering, so that the wound heals as under an aseptic scab. This action is partly mechanical, due to the stoppage of the lymph and blood capillaries by the fine particles of the powder $(\mathrm{H}$. H. Meyer, $\mathbf{1 8 8 5}$ ). Other indifferent substances in an equally fine state of subdivision have a similar action. However, a small amount of bismuth undoubtedly goes into solution, and aids by exerting an astringent and mild antiseptic action; indeed, the basic bismuth salts are among the most effective of the non-irritant intestinal antiseptics (Sollmann, I908). In the alimentary canal, the basic bismuth salts act not only in the manner indicated, but also by precipitating hydrogen sulphid (Stingel, I9II). Bismuth stools are therefore black. The subcarbonate (not the subnitrate) is a slowly acting antacid (J.A.M.A., $60: 1175$ ).

Therapeutic Use in Digestive Disorders.-The combination of these actions render the subnitrate or subcarbonate $(0.5$ to $2 \mathrm{Gm}$., 8 to $30 \mathrm{gr}$., every two to four hours) very useful for allaying diarrhea (X-ray observa- 
tions. Hesse, r9I3) and gastritis, and sometimes against vomiting. They may be combined with other intestinal antiseptics, such as salol. The hismuth powders are similarly useful in gastric ulcers ( $\mathrm{r}$ to $2 \mathrm{Gm}$. suspended in fluid, before each meal). They lessen gastric secretion and acidity (Bastedo). In normal individuals, bismuth is not constipating, even with the large doses used in X-ray work (Cannon, r9ir). This indicates that the effects are on the inflammation, and not on the peristalsis.

Hulse, I910, claimed that "bismuth-milk" (a suspension of the hydroxid) does not check diarrhea, the stools remaining light-colored. However, Stingel found that these preparations also bind sulphids.

Like other intestinal sedatives, bismuth subnitrate is said to diminish uric acid excretion (Abl, I9I3).

Use in X-ray Diagnosis. - The opacity of the bismuth salts to X-rays makes them very suitable for outlining the shape and movements of the alimentary canal. About $50 \mathrm{Gm}$. ( 2 ounces) of the subcarbonate (not the subnitrate) are administered, suspended in buttermilk. thick soup, etc. Best and Cohnheim, IgII, believe that the bismuth meal delays the emptying of the stomach by an hour. The use of other substances for radiographic purposes is discussed in Merck's Report, 27:43, 19I4.

Other Uses.-Bismuth is used as dusting powder or ointment (30 per cent.) on ulcers and other open surfaces and mucous membranes. Suspensions containing ro per cent. of bismuth subnitrate may be injected in urethritis. Bismuth poisoning may arise if it is applied too extensively, or if it is confined, as in the injection of bismuth paste into sinuses.

Bismuth Paste.-E. Beck introduced a paste of I part of bismuth subnitrate and 2 parts of petrolatum, in chronic suppurative abscesses and sinuses, often with curative results. The injections are made at first every three or four days, then not oftener than once a week. Acute abscesses do not respond. Stomatitis has followed its use after three weeks; on its occurrence the remaining paste should be washed away with warm oil. Jensen, I9I3, also reports nitrite poisoning from fistulæ infected with $\mathrm{b}$. coli.

The technic and precautions for the use of bismuth paste are described by E. G. Beck, igi6.

Differences Between Bismuth Salts.-The subnitrate and subcarbonate, the oldest of the insoluble bismuth compounds, answer every purpose.

Various organic salts have been introduced as substitutes, such as the subgallate (Dermatol), especially for dusting powders; but it is difficult to see bow they can be superior to the much more economical older products. In other substitutes, antiseptic aromatic radicals have been introduced, but the same or better results can be secured by mixing the ordinary bismuth salts with other insoluble antiseptics, such as salol or betanaphthol; in fact, however, the bismuth alone is sufficiently antiseptic for all purposes for which it is used.

There is little difference between the actions of the subcarbonate and subnitrate; but on the whole, the subcarbonate deserves the preference, since it can not give rise to nitrites. The subnitrate has also been said to be irritant, since it consists of sharp-pointed microscopic crystals, whereas the subcarbonate is amorphous. This supposed irritant action is wholly imaginary, as can be shown by dusting the subnitrate on the conjunctiva.

The use of soluble bismuth salts is irrational, since they lack the soothing qualities which constitute the main value of the insoluble products. On the contrary, they are markedly irritant. Their introduction is due mainly to the extravagant desire for "elegant pharmacy" which was responsible for the oil-free cod-liver oil preparations.

Absorption and Excretion.-A small amount of the basic bismuth salts is dissolved by the acid gastric juice, is absorbed, and excreted by the urine (Dorner and Weingaertner, r9o9), mouth and large intestines. The quantity is not sufficient to produce any systemic effects. The corrosive soluble salts are absorbed more extensively, and even the insoluble salts may be absorbed sufficiently from extensive burns to produce poisoning 
(T. Kocher, I882). In human poisoning the bismuth was found mainly in the stomach and kidneys, and a little in the liver (Barthe, I9I3).

Poisonous Effects of Bismuth Compounds.--Even very large doses of insoluble bismuth salts do not ordinarily produce any harmful effects when taken by mouth. Under other conditions, toxic symptoms may arise.

The phenomena may take several forms: nitrite effects from the reduction of bismuth subnitrate; capillary thrombosis from precipitation of bismuth sulphid in the intestinal vessels; chronic bismuth poisoning; and the acute effects of soluble corrosive bismuth salts.

Cabot, I9r 2, has collected thirty-three human cases, of which thirty-one were from injections into sinuses or wound dressings in extensive burns. He also reviews the experimental work.

\footnotetext{
The literature of bismuth poisoning is also reviewed by W. H. Higgins, I9I6.
}

Nitrite Poisoning.-The use of the relatively enormous doses of bismuth subnitrate for radiodiagnostic purposes has caused several intoxications, with mehemoglobinemia, intense cyanosis, diarrhea, dyspnea and death by arrest of respiration. These effects are not due to the bismuth, but to the production of nitrite by the reducing action of putrefactive fecal bacteria, especially in the large intestines and rectum (Benneke and Hoffman, I906; Boehme, I907; E. G. Beck, r909; Zollinger, I912; Zadek, r914).

Precipitation of Bismuth Sulphid.-Bismuth forms a black and very insoluble sulphid. Since $\mathrm{H}_{2} \mathrm{~S}$ is always present in the large intestine, the feces are always colored black. When the bismuth is in the blood, the precipitation may occur in the vessels of the large intestine, and lead to capillary embolism, and this to ulceration, with the clinical symptoms of vomiting, diarrhea, cramps, colic, and colitis-the latter very rarely as severe as with mercury. The autopsy in bismuth poisoning shows the staining of the cecum, adjoining colon and appendix; less in the descending colon; and none in the small intestines (Cabot, $19 \mathrm{I}_{2}$ ).

Bismuth sulphid is also precipitated in the mouth, and forms a "lead-line" at the junction of the gums and teeth; then patchy discoloration of the adjacent mucosa and tongue, and general stomatitis. These are prominent symptoms in chronic bismuth poisoning (Warfield, I9r 2).

Direct Systemic Actions.-These can be elicited by intravenous injection of the non-precipitant salts. They may also occur from relatively small doses of the soluble bismuth salts when taken by mouth, since these are strongly corrosive.

If $a$ soluble bismuth salt is injected directly into the blood, it produces the arsenic phenomena on blood vessels. There is perhaps also a direct involvement of the central nervous system and depression of the vasomotor center. The heart muscle is also depressed. The blood pressure sinks therefore very rapidly (Meyer and Steinfeld, r885).

Clinical Bismuth Poisoning.-This is characterized by fever, headache, stomatitis, lead-line, gastro-intestinal irritation, colic, and albuminuria. It differs from lead poisoning by the absence of paralytic, circulatory, blood and respiratory changes. Fatal cases may show convulsions and lock-jaw (Zollinger, I912).

If bismuth symptoms develop with Beck's paste, this should be removed with warm olive oil, curetting if necessary. Oxygen and saline may be administered for methemoglobinemia (Zollinger, I91 2).

\section{PREPARATIONS-BISMUTH}

Bismuthi Betanaphtholas (Bism. Betanaph.), U.S.P. (Orphol).-A compound of bismuth and betanaphthol of somewhat varying composition, yielding not less than 73 per cent. of $\mathrm{Bi}_{2} \mathrm{O}_{3}$. Decomposed into its constituents in the intestine. A buffcolored to grayish-brown, amorphous powder; odorless, tasteless, nearly insol. in water and alc. Dose, $0.5 \mathrm{Gm}$., 8 gr., U.S.P. 
Bism. of 4 mmon. Cil., U.S.P.-Of somewhat varying chemical composition; yields not less than to per cent. of $\mathrm{Bi}_{2} \mathrm{O}_{3}$. Very sol. in water and sparingly sol. in alc. Used for making bismuth solutions and elixirs. Dose, o.125 Gm., 2 gr., U.S.P.

Liq. Bism. el 1 mmon. Cil., B.P. Dose, 2 to 4 c.c., $1 / 2$ to I dram, B.P.

Masmu Bismulhi (Magma Bism.), U.S.P. (Milk of Bismuth).-A thick, white, opaque liquid, containing bismuth hydroxid and bismuth subcarbonate in suspension in water. Prepared by precipitating a solution of Bismuth nitrate with Ammonia; corresponding to about 6 per cent. of $\mathrm{Bi}_{2} \mathrm{O}_{3}$. Dose, 4 c.c. (I dram), U.S.P.

* Bismulhi Subcarbonas (Bism. Subcarb.), U.S.P.; Bism. Carb., B.P. (Bismuth Oxycarbonate). - Of varying chemical composition; yields not less than 90 per cent. of $\mathrm{Bi}_{2} \mathrm{O}_{3}$. White powder; odorless and tasteless. Insol. in water or alc. Incompalible with acids, sulphur, sulphids, and iodids. Dose, $0.5 \mathrm{Gm} ., 8 \mathrm{gr}$., U.S.P.; 0.3 to I.2 Gm., 5 to 20 gr., B.P., in powders or suspension.

Troch. Bism. Co., B.P.- Each contains 0.15 Gm. of Bism. Subcarb., o. 5 of Magn. Carb., and 0.5 of Calc. Carb.

* Bism. Subgallas (Bism. Subgall.), U.S.P. (Dermatol).-Of varying chemical composition; yields not less than $\mathrm{s}^{2}$ per cent. of $\mathrm{Bi}_{2} \mathrm{O}_{3}$. An amorphous, bright yellow powder, odorless and tasteless. Insol. in water or alc. Dose, $0.5 \mathrm{Gm} ., 8 \mathrm{gr} ., \mathrm{U} . \mathrm{S} . P$.

* Bismulli Subnitras (Bism. Subnit.),U.S.P., B.P. (Bismuth Oxynitrate).-Of varying chemical composition; yields not less than 79 per cent. of $\mathrm{Bi}_{2} \mathrm{O}_{3}$. A white powder; odorless, almost tasteless, and slightly hygroscopic; almost insol. in water, insol. in alc. Incompatibilities, see Bism. Subcarb.; effervesces with soluble carbonates. Dose, 0.5 Gm., 8 gr., U.S.P.; 0.3 to I. 2 Gm., 5 to 20 gr., B.P.

* Bism. Subsal., U.S.P.; Bism. Sal., B.P.-Of varying chemical composition; yields not less than 62 per cent. of $\mathrm{Bi}_{2} \mathrm{O}_{3}$. A white or nearly white, amorphous or crystalline powder; odorless, tasteless; almost insol. in cold water. Incompalible with acids, alkalies, alcohol, tannin, or iron. Dosc, 0.5 Gm., 8 gr., U.S.P.; 0.3 to I.2 Gm., 5 to 20 gr., B.P.

\section{IRON}

General Statement.-Iron occurs widely distributed in the body, in several more or less firm and non-ionizable combinations. It is found in all chromatin ${ }^{1}$ and therefore in all cells; in the hemoglobin of the blood; and in granular form as "reserve iron" in the blood-forming and destroying organs. It is also a necessary constituent of the oxidases.

Iron is therefore of great importance in metabolism. When administered to normal animals, it has no systemic action, but increases the "reserve iron." In most anemias, the administration of iron tends to restore the hemoglobin to the normal and therefore improves nutrition. The soluble ionizable salts exert local astringent effects.

Direct systemic actions may be secured by the intravenous or hypodermic injection of non-precipitating salts, or by the absorption of corrosive preparations. The effects resemble those of arsenic (H. Meyer and Williams, I88I; Jacobj, I887). Toxic effects occur with $60 \mathrm{mg}$. per kilogram, hypodermic or by vein; but not with therapeutic hypodermic doses (Lépine, I897).

Astringent Action and Uses.-The soluble salts, especially the ferric, precipitate proteins readily and are therefore styptic, astringent, irritant and even corrosive. Their internal administration is apt to produce some gastric irritation and constipation. The tincture of ferric chlorid is employed as an astringent, especially in gargles ( $\mathrm{r}: \mathrm{IO})$; this, and other iron salts, particularly the Liq. Ferri Subsulph., are also used as local styptics.

The administration of iron does not cause renal irritation.

Ringworm.-Garrett, I913, recommends painting the scalp with undiluted Liq. Ferri Chlor. Fortis.

${ }^{1}$ A. P. Mathews states that recent work throws doubt on the presence of iron in all nuclei. 
Income and Output of Iron.-The body of an adult man contains about 3.0 to $3.5 \mathrm{Gm}$. of iron, of which about 2.4 to $2.7 \mathrm{Gm}$. are in the form of hemoglobin. On a normal diet, he excretes daily about $20 \mathrm{mg}$. of iron; even in starvation the excretion averages about $8 \mathrm{mg}$. (F. Mueller). This is normally replaced by the absorption of iron from the food. A daily minimum income of 6 to $12 \mathrm{mg}$. is required to maintain the equilibrium. The iron content of the ordinary daily diet may vary between 7 and $35 \mathrm{mg}$., generally between $\mathrm{I}$ and ig $\mathrm{mg}$., $16 \mathrm{mg}$. being the mean value (Sherman, 1907; extensive bibliography). The iron income is therefore ordinarily more than sufficient for the needs; this even in otherwise poorly nourished individuals, since a vegetable diet is relatively rich in iron.

Iron Content of Foods. - This varies considerably, a fact which may be utilized in the treatment of anemias. It suffices to remember that blood, meat, yolk of eggs, and the green portions of vegetables, carrots, fruits, etc., are especially rich in iron ( 77 to 40 mg. per I00 Gm. of dry substance); while egg-white, milk, and the cereals (especially white flour) are poor in iron. Whole wheat, potatoes and the legumes are intermediate.

Dock (Rumex crispus), according to Sachet, has the highest iron content of any plant, and has therefore been recommended for anemia (Vasseur, I905). It is claimed (Gilbert and Lereboullet,' 1906 ) that this content of organic iron can be considerably raised by cultivating the plant on a soil artificially enriched with ferrous carbonate.

The iron of plants, when once fixed in the leaves, is not transported into new tissue; differing in this respect from $\mathrm{N}, \mathrm{K}$ or $\mathrm{P}$ (Gile and Carrero, 1916).

Metabolism of Iron.-The increase of the iron content of the body in growing animals, and the equilibrium in adults, are sufficient evidence that food-iron is normally absorbed, metabolized and excreted. The controversy, whether inorganic iron compounds can also be metabolized, may now be considered settled in the affirmative: their fate is identical.

All compounds of iron undergo some preliminary and probably unimportant changes during digestion. They are then absorbed, mainly from the duodenum, but also in decreasing order from the succeeding portions of the intestine. A part generally escapes absorption and is eliminated by the feces. The part which is absorbed passes into the intestinal epithelium in a dissolved condition, the organic compounds being at the same time changed into looser combinations. The absorbed iron may then pass directly into the capillaries; or through the stroma into the lymph, reaching the blood via the thoracic duct. The relative rôle of the blood and lymph absorption is not yet clear, but it seems that normally the main absorption is directly into the blood.

From the blood, the iron is deposited as granules in an easily decomposed organic form (ferratin) in the cells of the hematopoetic organs, in the liver and red marrow, and particularly in the spleen, to a less extent in the kidneys. In this form it remains until it is used or excreted. The spleen seems to be important in maintaining the iron reserve and in preventing excessive excretion.

The excretion occurs mainly by the large intestines with traces in the urine, bile and gastric juice. The excreted iron is in firm organic combination.

The utilization of the iron, its transformation into hemoglobin, occurs only as needed; so that the total quantity of hemoglobin does not rise above normal. The same is probably true of its utilization by other cells. An excessive absorption of iron results merely in an increase of the reserve stock. 
The administration of iron is therefore useful only in those conditions in which the normal income or the assimilation of iron is deficient.

Organic and Inorganic Iron.- To understand the following discussions, it is necessary to have a clear conception of the terms "inorganic" and "organic" iron. Inorganic iron preparalions contain the metal in ion-form; i.e., the iron can be separated from them by electrolysis; and they give the ordinary iron reactions (with ammonium sulphid, ferrocyanid, or hemotoxylin) without preliminary treatment of any kind. It is immaterial whether the iron is combined with an organic or inorganic anion. Even the al. buminate of iron is considered as "inorganic" in this sense.

Organic iron (" masked" or "non-ionic") preparations, on the other hand, contain the metal as a constituent part of the molecule, in a non-dissociable condition, so that they do not give the iron tests until the metal has been liberated by the decomposition of this molecule. There are all degrees in the firmness of the organic combination: some are decomposed by dilute acids (ferratin, the granules in which the iron reserve is stored in the spleen, liver, etc.); in others (hemoglobin, and the iron of food) nothing short of combustion or heating with concentrated acids will liberate the iron. In certain inorganic compounds, e.g., the various double salts containing citric and tartaric acid, the iron is also largely non-ionic, but can be easily liberated. In molecular compounds, such as ferrocyanids, the iron is very firmly combined.

Hematoxylin (fresh $1 / 2$ per cent. solution in water) is one of the best reagents, since it retains its yellow color with masked iron, but is blackened by inorganic (Macallum, I897). However, acids interfere with the reaction, so that "dialysed iron," although inorganic, does not react until it is made alkaline. Ammonium sulphid is also a good reagent; but its prolonged contact liberates iron from the less firmly masked compound.

Nature of Food-iron.-Iron occurs in food exclusively in organic form, more or less firmly masked, generally in combination with nucleo-proteins. The first natural iron nucleo-protein was prepared by Bunge, 1885 , from egg-yolk and named Hematogen. Zaleski also found an iron nucleo-protein in the liver. This was later isolated by Marfori and Schmiedeberg, 1804, and named Ferratin. These products are probably not uniform substances, since they contain varying proportions of iron (Salkowski, I909). Schmiedeberg and Marfori also prepared what they considered an Artificial Ferratin, by the interaction of iron and alkaline egg-albumin. It has been shown, however, that this has absolutely no relation to the natural substance, being simply a rather firm ferrialbuminate (De Groot, I895; Battistini, 1895). While true ferratin is not attacked by digestion, the artificial is converted into ferric chlorid. It has therefore no advantage over the ordinary mild iron salts.

Hemoglobin holds the iron much more firmly than most other compounds. It is converted into hematin in the stomach (Rep. Counc. Pharm. Chem., I9II; 64). Its further fate is not known, but at least a part of its iron is evidently absorbed, since its administration can satisfy the iron requirements of the body. It has no serious therapeutic advantage.

Outline of the Iron Controversy.-Even the ancients employed iron in the treatment of anemia; drinking water in which swords had been allowed to rust. Perhaps they were led to do so by an obscure idea that the strength of the steel would in some way pass into the patient. With the discovery of the presence of iron in the blood (Menghinis, r746), the therapeutic results seemed to be easily explained on the assumption that the iron is absorbed and converted into blood, much as the nitrogen of food is converted into muscle. The earlier experiments, however, failed to show any absorption of the medicinal iron preparations. This led some writers to consider the clinical results obtained with iron as largely imaginary; while others (especially Bunge, 1885 ) were inclined to attribute them to the local actions of iron in the alimentary canal.

There could never be any question as to the absorption of food-iron, for the hemoglobin increases with the growth of the animal. The same holds true for certain organic irons, and Macallum (r894) and Hall (r896) studied this absorption by microchemic methods. The absorption of inorganic iron was also proven, but the latter result was criticized, the objection being made that the iron had produced corrosion. This was finally withdrawn, but it was now claimed that inorganic 
iron, while it is absorbed, can not be converted into hemoglobin (Abderhalden, I899; Hoffmann, I900); but that it is nevertheless beneficial in anemia, by stimulating the blood-producing organs to a better use of the organic irons. This objection was also found untenable and abandoned (Abderhalden, I906). It appears therefore that inorganic iron is absorbed, assimilated and converted into hemoglobin, precisely like food-iron.

The long resistance to the acceptance of this explanation was due largely to the fact that the conversion of iron into hemoglobin would have been an unique instance of the synthesis, in the animal organism, of a protein from an inorganic compound. It is now probable that the phosphorus of nucleo-protein, and probably other proteins, may also be derived from inorganic sources.

However, even without prejudice, the problems of iron metabolism presented some peculiar difficulties, which could be only gradually overcome (critical bibliography, Erich Meyer, 1906).

Evidence from Excreta.-If one wishes to show the absorption of nitrogen or of chlorids, it suffices to estimate the quantity of these substances in the food, and in the feces and urine. The same method was applied to iron. The results seemed to show that absolutely no absorption occurs, for the iron of the feces approximates that of the food (Kletzinsky, 1854) and the iron in the urine is not at all increased. It was soon shown, however, that the intestines are the principal channel for the excretion of the iron; for the feces contained this metal even in starvation; and when iron was injected subcutaneously very much more was excreted by the feces than by the urine. It was therefore evidently impossible to decide whether the fecal iron was unabsorbed or excreted. Absorption could be proven, by this method, only if it predominated greatly over the excretion. The estimation of the iron in the bile and in the intestinal juice gave similarly inconclusive results.

Iron Excretion by Urine.- This always contains traces of iron (in man, normally I to $2 \mathrm{mg}$. daily), partly in rather loose, partly in very firm combinations (Wolter, I9ro). There is practically no increase if iron is administered by mouth (E. W. Hamburger, I878; Kobert, I89I), even when organic iron is continued for weeks (Gottlieb, I89o). Nor is there any increase on intravenous injection of ferratin (Cloetta, 1900). Some increase occurs in fever, nephritis, hepatic disease, leucemia, and especially in diabetes. This indicates that the iron of the urine comes from tissue destruction, and not from the iron of the food or drugs (Tartakowsky, 1904).

Intestinal Excretion.--The iron leaves the body mainly by the feces. This is true for starvation (Bidder and Schmidt, $1_{52} ; \mathrm{F}$. Mueller), and for the normal iron income; or when iron is administered orally, hypodermically or intravenously. However, the total excretion of the injected iron requires several weeks (Gottlieb, I89I). It was at first assumed that the excretion occurred mainly by the Bile, but actual observation showed that the quantity of iron in the bile is small (mean, about $40 \mathrm{mg}$. per liter); and that it is not increased by the administration of iron (E. W. Hamburger, I880; Anselm, I892). The iron must therefore be excreted through the Intestinal Epithelium. This is confirmed directly by fistula experiments, in which the access of food to isolated parts of the intestine is prevented (Kobert; Gottlieb, I891; Honigmann, 1896). These show that the excretion occurs mainly in the large intestine; the excreted iron being very firmly combined, so that it gives the iron reactions only after incineration (Tartakowsky). In rabbits, after hypodermic or peritoneal injection of iron salts or hemoglobin, the main excretion is through the epithelium of the upper duodenum (Chevallier, 1914). As with metals in general, the excretion is a slow process; requiring several weeks for the complete elimination of a hypodermic injection (Gottlieb).

Chemic Evidence of Absorption in Small Intestine.-A fistula in the lower ileum would separate fairly sharply the place of the principal absorption, the small intestine, from the principal excretion, the large intestine. Honigmann, 1896 , found in this way that about 80 per cent. of citrated iron was absorbed in the small intestine. Rabe, I9I 2 , by using fistula dogs, found that the greatest absorption of saccharated iron occurs in the duodenum, the lower portions playing a progressively smaller rôle.

Iron Content of Organs.-Changes in the iron content of individual organs, or of the entire body, also give evidence of the absorption of iron. We have seen that injected iron is excreted slowly; and must therefore be temporarily stored in the body. Jacobj, 1887 and 1890 , found that iron preparations, injected into a vein, had disappeared from the blood in two to three hours. At this time, only ro per cent. had passed into the excreta; about 5o per cent. was deposited in the liver, and the remainder in other organs 
(spleen, kidney, intestines). The Liver is also normally very rich in iron (this is true likewise of animals which do not form hemoglobin-molluscs, crayfish, lobster, etc.). The liver iron decreases with iron-poor food, or when there are extra demands for iron, as in pregnancy or after hemorrhage. The iron content of the liver may thus be taken as an index of the iron stock of the body, and therefore of absorption. With small animals, the iron content of the cntire body can be estimated.

Since the animals must be killed to estimate the iron, control experiments are necessiry. These would be impossible if the animals were chosen at random, for the individual differecnes of iron content are often greater than the quantities which could be absorbed. Bunge found, however, that the proporton of iron in new-born animals is very high (increasing very rapidly in the last three months of fetal life; Hugounencq, I 800 ); and that it decreases steadily during the period of lactation (because milk is very poor in iron) until it has reached a practically constant quantity-about the seventeenth day in guinea pigs or rabbits, and the twenty-third or twenty-fourth day in dogs. The animals of the same litter can therefore be used for controls, some being fed, at the end of the lactation period, with iron-poor food, others with the same food to which the iron preparation is added. Rice and milk, which contain a very small proportion of iron, are commonly employed. The animals which do not receive the iron thrive very poorly, remaining small and emaciated. Those which receive iron, in any form, show an unmistakable increase in the total iron content of the body, especially of the liver, spleen and bone-marrow (Hall, I894; Kunkel, I895; Oerum, I906).

Abderhalden, I900, and Haeussermann, I897, objected to the experiments of Hall, that he used corrosive doses of the inorganic preparations, as was necessary with small animals to obtain differences beyond the experimental error. Kunkel, however, who worked also with dogs, had used therapeutic doses. These experiments therefore prove that inorganic, as well as organic iron can be absorbed and deposited in the liver and other organs.

Microchemic Evidence.-Important information was obtained by applying the color reactions for iron directly to the tissues. The macroscopic examination of the depth of stain gives a general idea of the quantitative distribution of the iron, the details being revealed by microscopic study. Since, however, the iron compounds are apt to dissolve in the fixing solutions, many of the earlier experiments gave fallacious results. The best method consists in hardening the tissues in alcoholic ammonium sulphid and converting the ferrous sulphid into the more stable Berlin blue, by treatment with potassic ferrocyanid and dilute hydrochloric acid. Only the inorganic and the loosely combined organic iron is revealed. The experiments are made on animals with different diets, or fed on iron, for varying periods. The method is of value especially for showing the place and manner of absorption, and the course of iron through the system.

Course of Absorption.-Macallum, I894; Quincke, and Hochhaus, I896; and Abderhalden, I906, after the administration of iron preparations, found the reaction especially strong in the epithelial cells of the duodenum. The results below the duodenum were inconstant, depending upon the quantity administered. The gastric epithelium gives no reaction.

The large intestines show a diffused iron reaction of the epithelium, and granules in the submucous leucocytes. It was generally assumed that this iron was in the course of excretion. However, Tartakowsky found that the large intestine shows no sulphid reaction in starvation or with iron-poor food, although the chemic examination of the intestinal contents proves the continued excretion of fixed iron. It would therefore seem that the excreted iron is too firmly combined to give the sulphid reaction; and that the sulphid reaction of the large intestine is also due to iron in the course of absorption. However, the amount absorbed here must be small.

The absorption of iron must therefore take place mainly through the cells of the duodenum. This differs from other metals, which are absorbed between the cells (Hoeber, I907). The iron reaction is essentially identical, whether organic or inorganic iron is fed. Since food-iron does not give the sulphid reaction, even in the intestinal contents, it must be broken down into ionic iron within the epithelium. This does not exclude the possibility that a part of the organic iron may be absorbed unchanged; for this would not be revealed by the microchemic reaction.

Further Course of the Absorbed Iron.-The iron reaction is also seen in the stroma of the duodenal villi; in the central lacteal, and sometimes in the mesenteric lymph glands, partly dissolved in the lymph plasma, often as granules in the lymph corpuscles (ferrocytes) (Quincke; Gaule; Abderhalden). Gaule, 1896 , also found that iron administration does not increase the iron content of the spleen, if the lymph is prevented from reaching the circulation, by a fistula of the thoracic duct. These observations would indicate that the iron reaches the blood through the lymph. Frantz-Mueller obtained just the opposite results: The lymph of the thoracic duct contained no iron; 
and the fistula did not prevent the accumulation of iron in the organs. The difference is perhaps explained by the dosage: Gaule worked with large doses and his histologic results at least could be referred to corrosion; while Mueller used smaller doses. It is therefore probable that the iron normally passes directly into the intestinal capillaries. It is then rapidly deposited in the organs.

Reserve Stock of Iron.- Animals on an ordinary diet lay up a considerable reserve stock of iron, as loose protein compounds, especially in the liver and spleen. This reserve disappears ("asiderosis" of Quincke) gradually when a diet poor in iron is given, or when there is an extraordinary use of iron, as after hemorrhages, or during pregnancy (when considerable iron is transferred to the fetus). In acute hemolytic anemia of rabbits, the reserve iron largely disappears, especially from the liver cells, when the blood is regenerated. Small amounts are still present in the endothelium of the hepatic capillaries, in the spleen and in the renal cortex (Muir and Dunn, 1915). The deposits increase, on the other hand, when the iron of the food is increased.

Relation of Spleen to Iron Metabolism.-The constant occurring of loosely bound iron granules in the spleen, varying in quantity with the iron income and blood destruction, shows that it is one of the important depots for reserve iron (Chevallier, I0I4). Even animals fed on iron-free food from birth, and whose liver is free from reserve iron, contain some in the spleen (Schmidt, I912). However, this function is shared by many other organs, so that splenectomy does not entail any constant or important changes in normal iron metabolism (Austin and Pearce, I9I4). When the iron income is in adequate, the loss of the spleen may perhaps lead to a less effective retention of iron, as is believed by Asher and his pupils, I909 and I9II.' On the other hand, the spleen has real, if not very conspicuous effects on the red blood corpuscles.

Effects of Spleriectomy.-These have been investigated especially by Pearce and pupils, I9I 2 to I9I5. They find that excision of the spleen from normal dogs is followed by moderate anemia, with very slow recovery. The decrease is greater for the hemoglobin than for the blood count. The mechanism of this anemia is not clear. Subsequently, as the anemia recovers, the red cells become abnormally resistant to hemolytic agents and the fatty bone-marrow becomes cellular. The cells of the lymph nodes and perhaps of the liver show a compensatory increase of phagocytic function toward the red cells.

Injection of Spleen Extracts.-Intraperitoneal injection produces for one or two days a distinct increase of hemoglobin and corpuscles in normal, not in splenectomized animals, apparently by stimulating the bone-marrow. Feeding of spleen is ineffective (Krumbhaar and Musser, 1914).

Splenic extract has no hemolytic action in vitro; nor does the blood of the splenic vein contain more free hemoglobin than the splenic artery (ibid).

Assimilation of Iron.- It would be quite conceivable that the absorbed iron, particularly the inorganic preparations, were of as little use to the organism as the particles of solid carbon which are absorbed by the lungs. It had to be shown therefore that the iron can be actually assimilated and converted into hemoglobin. This could not be done on normal animals, because the normal income of iron is amply sufficient for all the needs of the organism; an excess has no effect whatever on the hemoglobin. The animals must be reduced to a condition in which the needs exceed the ordinary income. In young and actively growing animals, this may be secured by a diet poor in iron (milk and rice). This produces a progressive fall of the percentage of hemoglobin, and the animals become emaciated and do not develop. As soon as iron, in any form, is added to the diet, the animals recover very rapidly (Hösslin, 180o; Cloetta; Tartakowsky; Abderhalden). It must therefore be concluded that all forms of iron can be assimilated. The same result is obtained with adult animals. These can not be rendered markedly anemic by an iron-poor diet; by drawing on their reserve stock, and possibly utilizing the iron several times, they can keep up an almost normal percentage of hemoglobin for a considerable period. They can, however, be rendered anemic by hemorrhage. Even a rather severe single hemorrhage is rapidly recovered from on a milk and rice diet; but this is hastened by the administration of organic or inorganic iron (Eger; Mueller, 1900). When several hemorrhages have been made and the iron reserve has become exhausted recovery occurs only when iron (in any form) is added to this diet. (Kunkel, r895; Tartakowsky, r903 and r904).

Abderhalden at first contended that even these experiments did not prove the assimilation of inorganic iron: He concluded from his data that the undernourished animals did not improve as rapidly by the simple addition of inorganic iron, as did the animals which were placed on a normal (iron-containing) diet plus inorganic iron; but that the improvement was more rapid on a full diet plus inorganic iron than on a full diet alone. He interpreted these observations to the effect that inorganic iron was not assimilated as such, but that it increased the assimilation of organic iron, perhaps by stimulat- 
ing the bone matter. Jaquet, however, pointed out that the slower improvement, when the iron was added to the restricted diet, would be explained by the lower protein content of this diet; and Tartakowsky showed that inorganic iron added to a diet rich in iron does not hasten the improvement, as it should if it stimulated the bone-marrow. Abderhalden seems to have withdrawn his objection.

Changes in Bone-marrow. - When iron is administered in anemia, the marrow doubtless shows greater activity, more nuclear erythrocytes and mitotic cells; but this is the natural accompaniment of the increase of hemoglobin. The observation of Schmincke, I 913 , with iron administration in human anemia, that the increase of erythrocytes considerably precedes the increase of hemoglobin, is also no convincing proof of direct bone-narrow stimulation, until we know more about the normal and pathologic sequence of blood regeneration.

A. B. Macallum, I 890 , found in frogs that the hemoglobin arises from the transformation of the iron-containing nuclear chromatin of the hematoblasts.

Disassimilation of Iron.-A part of the reserve iron is doubtless derived from the destruction of the red corpuscles and their hemoglobin; for the injection of hemoglobin or hematin increases markedly the iron content of the liver, spleen and marrow. The transformation of hemoglobin in to the less firmly masked iron may be seen in extravasations of blood, and takes place there in gradual stages. The impossibility of producing severe anemia in adult animals by limiting the iron of the food, indicates that the iren-stock may be used scveral times. But a portion is undoubtedly lost by the feces and urine.

Fate of Hemoglobin.-If free hemoglobin occurs in the plasma, as a result of injection or hemolysis, in concentrations above about 0.12 per cent., the excess is mainly filtered through the kidney and produces hemoglobinuria. A part, however, and with lower concentrations all of the dissolved hemoglobin is taken up and destroyed by the liver, spleen, kidney, etc. In the liver, the hemoglobin is converted into bile-pigment. If the a mount of this exceeds the excretory capacity of the bile, it results in icterus (Pearce, Austin and Eisenbrey, ror2).

With hemblysis so severe as to destroy nearly half of the blood in three days, Muir and Dunn, I9 I5, found nearly all of the iron of the destroyed hemoglobin deposited in the liver, spleen and kidneys. A third of the total iron of the blood may be thus deposited in twenty-four hours.

Local Actions on Alimentary Canal.--These were advanced in explanation of the tlerapeutic effects when the assimilation of inorganic iron preparations was still in doubt. The inorganic salts of iron produce an astringent effect, which may conceivably support their other actions, by improving digestion, (Kobert, 1882). There is always danger, however, that the useful action passes into irritation, and, in fact, iron causes digestive disturbance (especially constipation) more often than improvement.

It was also suggested that the inorganic iron protected organic iron against precipitation by sulphids in the intestines. This would, of course, be superfluous, since foodiron is not precipitated by sulphids. Accordingly, Stockman, 1893, found that iron produced its therapeutic effect even when it was administered as the sulphid. On the other hand, other metals which bind $\mathrm{H}_{2} \mathrm{~S}$ with avidity ( $f . i$., bismuth), are entirely ineffective in chlorosis.

Effects on General Nutrition.-While these are doubtless mainly the indirect result of the increase of hemoglobin, it must not be overlooked that iron forms a constituent of all cells, and some of its effects may be direct; but its importance in this connection is difficult to estimate. The majority of organisms require some iron, even those which do not form hemoglobin. (The blood of some invertebrate animals contains copper or manganese in place of iron).

Therapeutic Use in Anemias.-The administration of iron would be useful in all conditions in which the need for iron exceeds its income; conditions which are characterized by a low percentage of hemoglobin, and by the general clinical picture of anemia.

The plainest indication for iron is furnished by the anemia produced by severe or repeated hemorrhage. Infantile anemias are often traceable to a deficient iron content of the food, and would be greatly benefited by iron. Some cases of simple anemias in adults may also be due to this cause. It is conceivable that deficiencies in the iron income are concerned in the etiology of rickets and the other nutritive diseases of infants. 
Iron Content of Milk.-Anemia is very apt to occur if the infant is kept too long on an exclusive milk diet. It may also be favored by an inferior quality of milk: while normal human milk contains 3.5 to $7.2 \mathrm{mg}$. of iron per liter (mean $5 \mathrm{mg}$.), that of unhealthy mothers is poorer in iron; and artificial infant foods generally contain only I.4 to $2.6 \mathrm{mg}$. Cow's milk is even poorer in iron than is generally believed. It contains 0.4 to $0.7 \mathrm{mg}$., mean $0.5 \mathrm{mg}$. per liter; the higher figures of older determinations were due to contamination from metallic containers (Edelstein and Csonka, I9I I.

Pernicious Anemia.-Iron is useless in those cases of anemia in which there is no deficiency in the income or store of iron; but in which the fault concerns the formation or destruction of the corpuscles. In pernicious anemia, a most severe destruction of the corpuscles (probably through an unknown hemolytic agent) is accompanied by a very considerable increase of the reserve iron of the liver and spleen (Rumpf, I90I) and of the dissolved iron of the spleen (Erben, 1900). The urinary excretion of iron remains normal (Queckenstedt, I9I3). Clinical experience has confirmed that the administration of iron is useless in this condition.

Most instances of anemia, and especially chlorosis, can not be attributed directly to deficient iron income; but nevertheless, the administration of iron often secures brilliant results. It must therefore be supposed that conditions exist in these diseases, which demand an extraordinary income of iron to keep the hemoglobin normal.

Iron in Chlorosis.-A complete explanation of the action of iron in chlorosis can be furnished only when the pathology is understood. This is not the case at present; little is known beyond the fact that the hemoglobin is decreased (Födisch, I832) much more than the number of corpuscles (several authors, I842), and that the excretion of iron by the urine is nearly normal. Even the question, whether the reserve stock of iron is altered, does not seem to be decided. Digestive disturbances are often present, but they may be secondary or accidental; indeed, they disappear spontaneously with the iron treatment.

Our knowledge of the etiology is equally unsatisfactory. It has indeed been shown by Stockmann that the diet of chlorotic girls is often very poor in iron (containing 2.8 to $3.2 \mathrm{mg}$. per day, instead of the normal, 6 to $14 \mathrm{mg}$.) But this, as well as other unhygienic conditions, can be considered only as contributory factors, since many persons are subject to these conditions, while chlorosis is confined to females about the age of puberty. There is no evidence of defective absorption of iron.

The treatment is much more successful than might be expected. The administration of iron holds the first place. This should be supplemented by proper hygiene--good air, rest, suitable diet, stomachics, attention to the bowels (aloes), etc. Arsenic, phosphorus or manganese are often used in addition to the iron: it is difficult to judge of their value.

Administration.-Although both organic and inorganic iron are absorbed and produce therapeutic effects, the ionized iron appears to be more active therapeutically; for reasons not fully understood. On the other hand, inorganic-iron preparations are more apt to set up digestive distress, which may interfere with the prolonged administration that is ordinarily needed. In such cases, it is necessary to begin with the milder organic or insoluble preparations; and to induce a tolerance to the local effects by progressing slowly to the stronger compounds. The ferrous carbonate can be given to most patients without difficulty, and is sufficiently effective. The most suitable form and dosage are governed by individual experience. The daily dose should correspond to about 0.x Gm. of iron. This would be contained in about 5 or 6 Blaud pills, but would require 30 to $250 \mathrm{Gm}$. of the organic preparations (Morawitz, I9I4).

The order of irritation and efficiency is about as follows (in ascending 49 
order): food-iron; other organic irons; reduced iron (in powder or pills); ferrous carbonate (saccharated; Blaud's pill); the double "scale" salts; ferrous sulphate; ferric chlorid.

Reduced iron should be given just before meals, to permit the solvent action of the gastric juice. All other inorganic iron preparations should be taken just after meals, taking care to protect the teeth. The incompatibilities of iron must be remembered. The tendency to constipation may be met by aloes or other laxatives.

The citrate has been used hypodcrmically, $50 \mathrm{mg}$. in 55 solution daily. It is efficient but rather painful. Overdoses cause gastric irritation.

\section{IRON PREPARATIONS}

Only the most important preparations need to be studied. It may be remembered that ferric salts have a reddish, the ferrous a greenish color. The most important incompatibilities are: alkalies, salicylates, phenol.

Organic iron preparations had a brief popularity when it was believed that inorganic preparations were not absorbed. The modern conceptions have caused them to be practically abandoned, except in so far as iron foods are emphasized in the diet in anemia.

\section{PREPARATIONS-INSOLUBLE IRONS}

Ferrum, U.S.P., B.P.; Iron.-Metallic iron in the form of fine, bright, and nonelastic wire. Used only for preparing the salts.

${ }^{*}$ Ferrum Reductum (Ferr. Reduct.), U.S.P.; Ferrum Redactum (Ferr. Redact.), B.P.; Reduced Iron (Iron by Hydrogen; Quevenne's Iron).-Iron reduced to the metallic state by the action of hydrogen upon ferric oxid. Very fine, grayish-black, lusterless powder, without odor or tastc. Dose, $0.06 \mathrm{Gm}$., I gr., U.S.P.; 0.06 to $0.3 \mathrm{Gm}$., I to 5 gr., B.P., as powder.

Troch. Ferr. Redact., B.P.-0.06 Gm., I gr.

*Fcri Carbonas Saccharatus (Ferr. Carb. Sacch.), U.S.P. (Eisen-zucker).-Contains not less than 15 per cent. of $\mathrm{FeCO}_{3}$. Made by precipitating ferrous sulphate with sodium bicarbonate, and diluting with milk-sugar. A greeninh-brown powder, gradually becoming oxidized by contact with air; odorless, and having at first a sweetish afterward slightly ferruginous, taste. Only partially sol. in water. Dose, 0.25 Gm., 4 gr., U.S.P., suspended in water.

Ferrous Carbonate tends to give off $\mathrm{CO}_{2}$ and absorb oxygen, changing rapidly into a basic carbonate and finally into ferric hydroxid. The change may be retarded by the addition of sugar, as in pharmaceutic forms; but these should always be prepared as freshly as possible.

*Ferr. Carb. Sacch., B.P.-Not less than 50 per cent. of Ferrous Carbonate, preserved by Glucose. Dose, 0.6 to $2 \mathrm{Gm}$., Io to 30 gr., B.P.

Mist. Ferr. Co., B.P. (Griffith's Mixture).-About 0.7 per cent. of Ferrous Carbonate in suspension in flavored water. Must be freshly prepared. Dose, $\times 5$ to 30 c.c., $1 / 2$ to I ounce, B.P.

*Massa Ferri Carbonatis (Mass. Ferr. Carb.), U.S.P. (Vallet's Mass).-A pill-mass, with not less than $4 \mathrm{I} .5$ per cent. of $\mathrm{FeCO}_{3}$. Dose, 0.25 Gm., 4 gr., U.S.P.

${ }^{*}$ Pil. Fcrr., B.P.-Pill-mass with 22.5 per cent. of $\mathrm{FeCO}_{3}$. Dose, 0.3 to $1 \mathrm{Gm}, 5$ to 15 gr., B.P.

${ }^{*}$ Pil. Ferr. Carb., U.S.P. (Blaud's, Chalybeate, or Ferruginous Pills) -0.065 Gm., I gr., of $\mathrm{FeCO}_{3}$, made from ferrous sulphate and pot. carb. Dose, 2 pills, U.S.P.

Contrary to the prevailing impression, the U.S.P. Blaud's pills are quite stable, and preferable to the proprietary substitutes (Warren, I9I5).

Ferri Hydroxidum Cum Magnesii Oxido (Ferr. Hydrox. cum Mag. Oxid.), U.S.P. (Arsenic Antidote; Ferric Hydrate with Magnesia). - 40 c.c. of Solution of Ferric Sulphate are diluted with I25 c.c. of water. This is added, when needed, to $10 \mathrm{Gm}$. of Magnesium Oxid or 300 c.c. of Magma Magnesia diluted with water to about 750 c.c.

Always keep the diluted solution of ferric sulphate and magnesia mixture on hand in separate bottles, ready for immediate use, so that this antidote to arsenical poisoning may be quickly prepared.

Dose, 120 c.c., 4 ounces, U.S.P: 
PREPARATIONS-COLLOIDAL IRONS AND " SCALE SALTS"

Liq. Ferri Oxychloridi.-A colloidal solution of ferric hydroxid in ferric chlorid; approximately $\mathrm{FeCl}_{3}+8 \mathrm{Fe}(\mathrm{OH})_{3}$, containing the equivalent of about 3.5 per cent. of metallic iron. It is only mildly astringent. Dose, 0.5 to 2 c.c., 8 to 30 ninims.

Liq. Ferri Dialysatus. (Dialysed Iron) - This is a similar solution from which the excess of acid and chlorid has been removed by dialysis. It has the same iron content and is less astringent. Dose, $1 / 2$ to 2 c.c., 8 to 30 minims.

Liquor Ferri et Ammonii Acetatis (Liq. Ferr. et Ammon. Acet.), U.S.P. (Basham's Mixture).-The average dose, 15 c.c., 4 drams, U.S.P., contains 0.6 c.c. of Tr. Fer. Chlor., 5 c.c. of Liq. Ammon. Acet., Acetic Acid, Elixir, etc. Must be recently prepared.

*Ferri el Ammonii Citras (Ferr. et Ammon. Cit.), U.S.P. (Soluble Ferric Citrate, Ammonio-ferric Citrate).-A mixture of iron citrate and ammonium citrate containing about 7 per cent. of $\mathrm{Fe}$. Thin, transparent, garnet-red scales, without odor and having a saline, mildly ferruginous taste; deliquescent in moist air. Readily and completely sol. in water; insol. in alc. Dose, $0.25 \mathrm{Gm}$., 4 gr., U.S.P.; 0.3 to $0.6 \mathrm{Gm}$., 5 to ro gr., B.P., in solution.

Vin. Ferr. Cit., B.P.-I.8 per cent. of Ferr. et Ammon. Cit. Dose, 4 to I6 c.c. I to 4 drams, B.P.

Vin.Ferr., B.P.-Prepared by macerating iron in Sherry Wine. Contains the equivasent of 0.125 to 0.3 per cent. of Fe. Dose, 4 to 16 c.c., I to 4 drams, B.P.

Ferr. et Pot. Tart., B.P. (Ferrum Tartaratum).-Garnet scales. Dose, 0.3 to 0.6 Gm., 5 to Io gr., B.P.

Ferr. et Quin. Cit., U.S.P., B.P.-Contains about 12.5 per cent. of quinine and $\mathrm{I} 3.5$ per cent. of Fe. Dose, $0.25 \mathrm{Gm} ., 4$ gr., U.S.P.; 0.3 to $0.6 \mathrm{Gm}$., 5 to $10 \mathrm{gr}$., B.P.

*Ferr. Phos., U.S.P.-Ferric phosphate, made soluble by the presence of Sod. Cit.; corresponding to not less than I2 per cent. of Fe. Bright green scales. Freely sol. in water, insol. in alc. Dose, $0.25 \mathrm{Gm} ., 4$ gr., U.S.P.

(El. Ferr. Quini et Strych. Phosph.-See Index.)

(Syr. Ferr. Phos. c. Quin. et Strych.-See Index.)

\section{PREPARATIONS-SOLUBLE FERROUS SALTS}

Pil. Fer. Iod., U.S.P.-Each pill contains $\mathrm{FeI}_{2}$ corresponding to not less than 0.04 Gm. of ferrous Fe. Dose, 2 pills, U.S.P., contain about o.I Gm. ofodin.

*Syrupus Ferri Iodidi (Syr. Ferr. Iod.), U.S.P. -5 per cent. of ferrous iodid, $\mathrm{FeI}_{2}$. Pale green acid liquid of strong iron taste. Very susceptible to oxidation, turning brownish. It combines the therapeutic properties of iron and iodids, and is often prescribed in goiter ( 2 drops, twice daily, gradually increased to 15 drops three times daily; Marine and Lenhart, IgII) and as a general tonic in "scrofulous" conditions. Average dose, x c.c., 15 minim. U.S.P., contains about $52 \mathrm{mg}$., $0.85 \mathrm{gr}$., of iodin.

${ }^{*}$ Syr. Ferr. Iod., B.P. 7 per cent. of $\mathrm{FeI}_{2}$. Dose, 2 to 4 c.c., $1 / 2$ to I dram, B.P.

Ferr. Phosph. Sacch., B.P. -6o per cent. of ferrous phosphate, $\mathrm{Fe}_{3}\left(\mathrm{PO}_{4}\right)_{2}+8 \mathrm{H}_{2} \mathrm{O}$, preserved with glucose. Partially sol. in water; sol. in $\mathrm{HCl}$. Dose, 0.3 to $0.6 \mathrm{Gm}$., 5 to ro gr., B.P.

Syr. Ferr. Phosph., B.P.- I.7 per cent. of ferrous phosphate. Dose, 2 to 4 c.c., $1 / 2$ to I dram, B.P.

Ferri Sulphas (Ferr. Sulph.), U.S.P., B.P.; Ferrous Sulphate (Green Vitriol); Iron Proto-sulphate, $\mathrm{FeSO}_{4}+7 \mathrm{H}_{2} \mathrm{O}$.- Large, pale green crystals; odorless, saline styptic taste; effloresces in dry air. Freely sol. in water ( $1: 1.4$ ), practically insol. in alc. Dose, $0.1 \mathrm{Gm}$., I $1 / 2$ gr., U.S.P.; 0.06 to $0.3 \mathrm{Gm}$., I to $5 \mathrm{gr}$., B.P.-The crude salt is employed as a deodorizer for fecal matter, but is not germicidal.

Ferri Sulphas Granulatus (Ferr. Sulph. Gran.), U.S.P.-The preceding, in granulated form. Dose, o.1 Gm., I $1 / 2$ gr., U.S.P.

${ }^{*}$ Ferri Sulphas Exsiccatus (Ferr. Sulph. Exs.), U.S.P., B.P.-8o per cent. of anhydrous $\mathrm{FeSO}_{4}$, I part being equivalent to about $\mathrm{r} .5$ parts of the crystalline salt. Grayishwhite powder; astringent taste. Dose, $0.06 \mathrm{Gm}$., I gr., U.S.P.; 0.03 to $0.2 \mathrm{Gm}$., $1 / 2$ to $3 \mathrm{gr}$., B.P.; in pills, often with aloes.

Pil. Aloes et Ferr., B.P.- 10 per cent. of Ferr. Sulph. Exs.; 20 per cent. of Aloes. Dose, 0.25 to $0.5 \mathrm{Gm}$., 4 to 8 gr., B.P.

\section{PREPARATIONS-SOLUBLE FERRIC SALTS}

Ferr. Chlor.; U.S.P.; Ferric Chlorid (Iron Perchlorid).-Contains $\mathrm{FeCl}_{3}$ equivalent to 20 per cent. of Fe. Very sol. in water $(\mathrm{r}: 0.2)$ or alc.; sol. in glyc. Dose, $0.06 \mathrm{Gm}$., I gr., U.S.P. 
Liq. Ferr. Chlor., U.S.P.-3o per cent. of $\mathrm{FeCl}_{3}$ or ro per cent. of Fe. Dose, o. I c.c. I 12 minims, U.S.P.

Liquor Ferri Pcrchloridi Fortis (Liq. Ferr. Perchlor. Fort.), B.P. 20 per cent. of Fe.

liq. Ferr. Pcrchl., B.P. - 5 per cent. of Fe. Dose, 0.3 to I c.c., 5 to 15 minims, B.P.

* Tinctura FerriChloridi (Tr. Ferr. Chlor.), U.S.P.; Tr. Ferr. Perchlor., B.P.; Tincture of Ferric Chlorid ('Tincture of Iron).-Nearly I $_{5}$ per cent. of $\mathrm{FeCl}_{3}$, or 5 per cent of $\mathrm{Fe}$. Preparell by mixing the Liquor with Alcohol; the U.S.P. directs it to stand three months before using, to permit the formation of esters. A bright a mber acid liquid, of slightly ethereal odor and very astringent taste. Miscible with water or alcohol. Dose, 0.5 c.c., 8 minims, U.S.P.; 0.3 to I c.c., 5 to 15 minims, B.P., diluted. Often used as local astringent in tonsillitis (equal parts of the tincture, glycerin and water).

Liq.Ferr.Subsulph., U.S.P. (Monsel's Solution; Solution of Basic Ferric Sulphate).13.5 per cent. of basic ferric sulphate. Dose, 0.2 c.c., 3 minims, U.S.P. Used mainly locally, as styptic.

Liq. Ferr. Persulph., U.S.P. (Solution of Ferric Sulphate).-- 0.5 per cent. of Ferric Sulphate, $\mathrm{Fe}_{2}\left(\mathrm{SO}_{4}\right)_{3}$.

Liq. Ferr. Persulph., B.P.; Solution of Ferric Sulphate.

Iron (Chalybeate) Mineral Waters.-These contain the iron most often as ferric bicarbonate; sometimes as sulphate, oxid, and very rarely as chlorid.

\section{MANGANESE}

Occurrence.-Traces of manganese occur naturally in all organs, in man and various animals, its presence being fairly constant in the same species (Bertrand and, Medigreceanu, 1912). The amount in wheat, for instance, is about equal to the iron, and is independent of the manganese percentage of the soil (Headdon, I $\mathrm{r}_{5}$ ). The manganese of animals is derived partly from vegetable, but mainly from animal food, but does not disappear from the body even on manganese-free food.

Actions. - When administered by mouth, the salts of this metal (but this does not apply to the permanganates) produce no noticeable effect. Manganese is absorbed to a slight degree from the alimentary canal, being deposited mainly in the liver and kidneys, and passing also to the fetus in utero. Its excretion begins within three hours, mainly by the intestine (as sulphid in the feces), and the bile; but the urine participates (Bargero, I906, dog; Piccinini, I910). It appears to take about the same course as iron (Harnack and Schreiber, I901). Benedetti, I906, and Piccinini, I910, I912, claim that it increases the reserve iron. Benedetti has investigated its fate by subcutaneous injection (citrate, dog). It is toxic by vein (Bargero), and also causes fatty degeneration of the liver.

Manganous chlorid accelerates autolysis when added to excised liver; perhaps by converting indigestible proteins into forms that can be attacked by the liver enzymes (Bradley and Morse, IgI5).

Manganese Toxicosis.-Chronic poisoning results from exposure to the dust of any of its compounds in the industries, in zinc mines, grinding, etc. (Embden, Casamajor, I913; Jaksch, 1913). The symptoms are nervous, with mental and ataxic disturbances. The prognosis is good as to life, bad as to recovery. The condition has not been produced in animals.

\section{PREPARATIONS-MANGANESE}

Mangani Dioxidum Pracipitatum (Mangan. Diox. Præc.), U.S.P.-Consists chiefly of manganese dioxid $\left(\mathrm{MnO}_{2}\right)$. Prepared by precipitating Manganese Sulphate by Ammonia in the presence of Hydrogen Dioxid. Heavy, fine, black powder, without taste or odor; insol. in water or alc. Dose, $0.25 \mathrm{Gm} ., 4$ gr., U.S.P.

( ${ }^{*}$ Pot. Permang.-See Index.)

\section{CHROMATES}

Uses.-Chromium trioxid (chromic acid) is used externally as a caustic. It and the chromates have some toxicologic importance, the dichromate being used in dyeing and in "battery fluids." They are also interesting by producing a characteristic nephritis and glycosuria. 
Kolipinski, 1902 and 1908 , reported favorable results from chromium sulphate ( $8 \mathrm{gr}$., three to four times daily) in neurasthenia, tabes and other nervous conditions. They have not been adequately confirmed.

Acute chromate poisoning exhibits corrosive effects and nephritis, with death in one to forty-eight hours. The fatal dose of potassium dichromate is below $8 \mathrm{Gm}$.; of chromic acid, $6 \mathrm{Gm}$. The treatment is by evacuation and demulcents. Chronic poisoning produces local irritation (severe dermatitis, ulcers, etc.) and nephritis.

Ulcers are produced by chromate dust, especially where the skin is already injured. They develop slowly, but are very penetrating and heal with difficulty. It also causes ulceration and perforation of the nose.

Fate.-When bichromates are given by the mouth, they are reduced to chromous oxid and partly deposited as such in numerous organs, the rest being excreted by the urine (Kappeler, 1896).

Chromate Nephritis.- This is produced experimentally by hypodermic injection of the chromates. It is mainly tubular, but the glomeruli are involved first. All the phenomena are similar to uranium (including retention, glycosuria, chronic nephritis, etc.); except that edema is not common (Gergens, I876; Hellin and Spiro, 1897; Schlayer and Hedinger, I907; Ophuls, I908; Austin and Eisenbrey, I9 I ; R. M. Smith, rgrı; Folin, Karsner and Denis, r912; MacNider, I912; Eisenbrey, I913; E. Franck, I913; glycosuria, Mosenthal and Schlayer, I913; diuretics, etc.).

\section{PREPARATIONS-CHROMATES}

Chromii Triodidum (Chrom. Triox.), U.S.P.; Acidum Chromicum (Acid. Chrom.), B.P.; Chromium Trioxid, Chromic Acid (Chromic Anbydrid), CrO.- Dark red, $^{-}$ needle-shaped crystals, very deliquescent and very sol. in water(1:0.6). Explosive with all organic matters. Used as a caustic, solid or in solution ( $\mathrm{I}: \mathrm{I}$ ); as astringent ( 2 to 3 per cent.); for urethral injections ( $I: 2, \infty \infty$ to $4, \infty \infty)$; and against sweating feet (5 per cent.).

Liq. Ac. Chromici, B.P., 25 per cent.

Potassii Bichromas, B.P., $\mathrm{K}_{2} \mathrm{Cr}_{2} \mathrm{O}_{7}$.-Large orange-red crystals. Sol. in water (I: I0). Incompatible with alcohol and all reducing agents. Dose, 6 to I $2 \mathrm{mg}$., $1 / 10$ to $1 / 6$ gr., B.P., diluted (against gastric ulcer); locally, I: Io solution against rodent ulcer and cancer.

\section{ALUMINUM}

General Statement.-The soluble salts are strongly precipitant to proteins, and therefore astringent and antiseptic. They are used externally, and sometimes against diarrhea. They are not readily absorbed from the alimentary canal, so that even very large doses cause only local exudative inflammation (vomiting and diarrhea). The precipitates redissolve in an excess of protein.

The quantities which may be dissolved from aluminum cooking vessels, even by dilute acids, are too small to be of any importance. Alum baking powders are perhaps more seriously objectionable.

Absorption, Distribution and Excretion.-It was formerly stated that aluminum salts are not absorbed (Plagge and Lebbin, I893). Gies I9I I, Steel, I9I I, and Kahn, 1912, however, showed that considerable absorption occurs even from biscuits baked with alum baking powder, and that this tends to accumulate in the body. The rile is especially rich; the parenchymatous organs retain considerable; the brain and heart but little. A part is excreted by the urine (Kahn); the remainder by the intestines, even when it was injected into the circulation (Steel).

On the other hand, the Referee Board, I914, found no aluminum in the blood after administering to men I Gm. per day for four days.

Delayed Symptoms.- The aluminum compounds spread very slowly even when injected subcutaneously, and appear to penetrate cells with great difficulty; for the symptoms appear only several days or weeks after the injection (Siem, I886), at a time when the metal has entirely disappeared from the blood, and has become fixed in the cells. The symptoms then resemble those of subacute arsenic poisoning. They never occur 
when aluminum salts are given by mouth, no matter in what doses, nor how long continutel. Roth and Voegtlin, I916, found that prolonged feeding of aluminum salts to animals produced mainly gastro-intestinal irritation.

Astringent Action. - This has been studied by Barinetti, ror4.

\section{PREPARATIONS - ALUMINUM}

Alumen (Alum.), U.S.P.; Alumen Purificatum (Alum. Pur.), B.P.; Potassium Alum (Aluminum Potassium Sulphate), $\mathrm{AlK}\left(\mathrm{SO}_{4}\right)_{2}+\mathrm{I}_{2} \mathrm{H}_{2} \mathrm{O}$; or Ammonium Alum, $\mathrm{AlNH}_{4^{-}}$ $\left(\mathrm{SO}_{4}\right)_{2}+{ }_{12} \mathrm{H}_{2} \mathrm{O}$. - Large colorless crystals; strongly astringent taste; without odor. Freely sol. in water $(\mathrm{I}: 7.2)$, practically insol. in alc.; freely sol. in glyc. Incompatible with alkalies, carbonates and lead salts. Dose, 0.5 Gm., 8 gr., U.S.P.; 0.3 to 0.6 Gm.; 5 to 10 gr., B.P. Used as gargle ( 1 to 5 per cent.; somewhat injurious to teeth); as urethral injection ( 0.5 to $\mathrm{I}$ per cent.); and as skin lotion ( $\mathrm{I}$ per cent.).

*Alumen Exsiccatum (Alum. Exsic.), U.S.P., B.P. (Burnt Alum).-Alum with 45 per cent. of water (of crystallization) expelled. It is used as a mild caustic.

Glycerinum Alwminis (Glycer. Alum.), B.P.-2o per cent. of Alum.

*Liquor Alumini Acelatis (Liq. Alum. Acet.), N.F., (Burow's Solution).-A clear colorless solution containing about 5 per cent. of neutral aluminum acetate, $\mathrm{Al}\left(\mathrm{C}_{2} \mathrm{H}_{3} \mathrm{O}_{i}\right)_{3}$. Used as a mild astringent and antiseptic. A I per cent. dilution is said to be antiseptic and 5 per cent. germicidal (Kuehl, I9r3). For application to the skin it should be diluted five to fifteen times.

Liq. Alum. Subacet., N.F.-Contains nearly 8 per cent. of basic aluminum acetate, $\mathrm{Al}(\mathrm{OH})\left(\mathrm{C}_{2} \mathrm{H}_{3} \mathrm{O}_{2}\right)_{2}$. It is practically equivalent to the preceding and is used in the same dilutions.

Alumini Hydroxidum (Alum. Hydrox.), U.S.P.-Chiefly $\mathrm{Al}(\mathrm{OH})_{3}$. Prepared by precipitating a boiling solution of alum with sodium carbonate. White, bulky, amorphous powder, odorless and tasteless. Insol. in water and alc. Used as mild astringent and desiccant.

\section{BAKING POWDERS}

These are chemicals which serve as substitutes for yeast-fermentation, aerating bread and pastry dough by evolving carbonic acid. They are of course altered in this process. The decomposition products are partly expelled by the heat of baking. The fixed residue is the part which could conceivably produce pharmacologic action.

The baking powders belong to four chemic types (Crampton, r889):

1. Cream of Tartar Powders.-Mixture of acid potassium tartrate and sodium bicarbonate. Reaction: $\mathrm{KHC}_{4} \mathrm{H}_{4} \mathrm{O}_{6}+\mathrm{NaHCO}_{3}=\mathrm{KNaC}_{4} \mathrm{H}_{4} \mathrm{O}_{6}+\mathrm{CO}_{2}+\mathrm{H}_{2} \mathrm{O}$. Residuum: is $\mathrm{Gm}$. of Rochelle Salts per loaf of bread. Innocuous.

2. Phosphate Powders.-Calcium super-phosphate and sodium bicarbonate. Reaction: $\mathrm{CaH}_{4}\left(\mathrm{PO}_{4}\right)_{2}+{ }_{2} \mathrm{NaHCO}_{3}=\mathrm{CaHPO}_{4}+\mathrm{Na}_{2} \mathrm{HPO}_{4}+{ }_{2} \mathrm{CO}_{2}+\mathrm{H}_{2} \mathrm{O}$. Soluble residuum: $3.5 \mathrm{Gm}$. sodium phosphate per loaf of bread. Innocuous.

3. Alum Powders.-Alum (usually ammonic) and sodium bicarbonate. Reaction: $\left(\mathrm{NH}_{4}\right)_{2} \mathrm{Al}_{2}\left(\mathrm{SO}_{4}\right)_{4}+6 \mathrm{NaHCO}_{3}=\mathrm{Al}_{2}(\mathrm{OH})_{6}+3 \mathrm{Na}_{2} \mathrm{SO}_{4}+\left(\mathrm{NH}_{4}\right)_{2} \mathrm{SO}_{4}+6 \mathrm{CO}_{3}$. Residuum, per loaf of bread: I.3 Gm. aluminum hydrate; $3.65 \mathrm{Gm}$. sodium sulphate; 1.I Gm. ammonium sulphate. The aluminum hydrate is insoluble in water; in the stomach, some is dissolved by the hydrochloric acid, and perhaps by the proteins. Gies, I9I I, considers this seriously objectionable. The sulphates are practically without action. (The addition of alum whitens pastry baked from inferior flour. This fraudulent use is prohibited in several countries.)

4. Ammonium Carbonate.-Decomposed and volatilized as $\mathrm{NH}_{3}+\mathrm{CO}_{2}$. Expelled so completely that it is inactive.

Some baking powders are mixtures of several of the above. They are often diluted with indifferent substances (starch, etc.).

\section{CERIUM}

Cerium resembles aluminum. The insoluble Cerium Oxalate is employed against vomiting, especially in pregnancy (Simpson, I854; Mills, 1876), but it is probably useless. It is non-toxic even in large doses. 
Since cerium oxalate is not absorbed (Bachem, 1907), its action must be entirely local and analogous to that of bismuth. This has been demonstrated experimentally by Baehr and Wessler, rgog: They found that it had no effect on central vomiting (apomorphin), but that large doses delayed vomiting from local gastric irritation (ipecac). They also showed that central vomiting was not affected even by the soluble and absorbable cerium nitrate. The systemic effects corresponded to those of Thorium, but the metal is excreted exclusively by the feces.

\section{PREPARATIONS-CERIUM}

Cerii Oxalas (Cerii Oxal.), U.S.P.-A mixture of the oxalates of cerium (about 40 per cent.; Coblentz, I9I0), didymium, lanthanum, and other associated elements. Fine white or slightly pink powder, without odor or taste. Insol. in water, alc. or eth. Dose, 0.2 Gm., 3 gr., U.S.P.; 0.05 to $0.5 \mathrm{Gm}$., I to $8 \mathrm{gr}$., as powder.

Cerium Nitrate (soluble) has been substituted, in doses of $0.05 \mathrm{Gm}$. or less, but is irrational.

\section{YTTRIUM AND ZIRCONIUM}

Neodymium, Praesodymium, Lanthanum, Yttrium and Zirconium (as the chlorids) also precipitate proteins, and the observed effects can be attributed to this reaction (antisepsis, paralysis of muscle, etc.) (Dreyfuss and Wolf, 1905; Bachem; Hobert, 1907; Hoeber and Spaeth, 1914). Their oxalates are all practically non-toxic by mouth, even in enormous doses ( $50 \mathrm{Gm}$. per dog).

\section{BERYLLIUMM SULPHATE}

Seaman, I9I2, found that this salt, when administered to dogs with food, resulted especially in nutritional disturbances: emaciation, with loss of $\mathrm{N}, \mathrm{S}$, and $\mathrm{P}$. Large doses produced vomiting without acute toxic symptoms. Hypodermically, it caused local edema and necrosis. Intravenous injections were markedly toxic, with disturbances of circulation, respiration and temperature; diuresis, bloody diarrhea and vomiting. It inhibits the action of digestive ferments.

\section{THORIUM}

The chemic effects resemble those of aluminum. The nitrate of thorium is markedly astringent, precipitating proteins and coagulating blood. Injected hypodermically, it causes ulceration. The toxicity is slight. It is fairly well tolerated by the stomach, very large doses being required to produce vomiting. It is not absorbed from the alimentary canal. The addition of sodium citrate prevents the coagulant action. When this solution is injected intravenously, it has absolutely no effect on the circulation, or any other function, even when $0.25 \mathrm{Gm}$. are injected per kilogram of dog. However, the animals remain emaciated, and show ulcers of the gums. When killed, after some weeks, an extensive calcification of connective tissue is noticed. The thorium is excreted by the kidneys. The solutions have no effect on the excised muscle or heart of frogs (Chace and Gies, Brown and Sollmann, 1905). The nitrate was tried for stimulating ulcers, but did not prove superior to alum. The oxalate and oxid are practically insoluble.

The thorium-sodium citrate solution is impervious to $\mathrm{X}$-rays, and has been suggested for radiographic work (Burns, 1915).

\section{RADIO-ACTIVE METALS}

General Statement.-Thorium, radium, ùranium, etc., undergo atomic' decay into a series of new elements (emanation, etc.). This decay is accompanied by the generation of rays which produce irritant or destructive effects. Radium itself is thus used to irritate or destroy pathologic 
tissues. The milder emanations are employed especially against chronic joint and rheumatic diseases. Their therapeutic status is still uncertain, but the original expectations were probably too optimistic.

Nature of Radio-activity.-Radio-activity is defined as "the property of spontaneously emitting radiations capable of passing through plates of metal and other substances opaque to ordinary light and having the power of discharging electrified bodies."

The rays may be of several kinds:

Cathode rays are produced when an electric discharge is passed through a highvacuum tube. They are interpreted as negatively charged particles (corpuscles or electrons) sent off from the cathode with very high velocity.

$\mathrm{X}$-rays (Roentgen, 1895) are produced when the cathode rays strike the wall of the tube. They are believed to consist of a series of pulses in the ether, somewhat analogous to light, but sent out at irregular intervals.

Radio-active Metals. - Active rays are also emitted by a number of metals of high atomic weight, and by their compounds, presumably in the course of the disintegration of their atoms.

'The discovery of radio-activity started with the observation of Becquerel, $\mathrm{r} 896$, that uranium salts act on photographic plates through black paper. Similar rays were discovered in thorium in 1898 by Schmidt and Mme. Curie; and in the same year the Curies discovered polonium and radium.

The principal radio-active metals are: thorium, radium, uranium, and some others (ionium, polonium, etc.), and their disintegration products. Four kinds of rays are distinguished.

The alpha-rays have low penetrating power, but are most efficient in ionizing gases. They consist probably of positively charged helium aloms. They represent the main energy of radiation.

The beta-rays are more penetrating, with less ionizing action. They are considered as negative electrons, and therefore identical with the cathode rays, except in their velocity. They are also identical with the more slowly moving della-rays, which are produced when alpha-rays strike solids.

The gamma-rays have the highest penetrating and the lowest ionizing power. They are identical with the $X$-Rays; i.e., they consist of ether vibrations, produced by the impact of the beta particles.

The ratio of these rays varies for the different metals, but is constant for the same metal, independent of its combination or other conditions.

Disintegration of the Radio-active Metals.-A spontaneous disintegration of the atoms characterizes all the radio-active elements, and is the cause of the discharge of the rays. This transmutation is also independent of external conditions. The probable course of the atomic decay for thorium, uranium and radium is as follows:

Uranium minus $\alpha=$ Ur. X; $-\beta$ and $\gamma=$ Ionium; $-\alpha$ and $\beta=$ Radinım; $-\alpha, \beta$ and $\gamma=$ Emanation (Gas); $-\alpha=$ Radium A (Induced Radio-activity; $-\alpha=$ Radium B; little radiation $=$ Radium $\mathrm{C} ;-\alpha, \beta$ and $\gamma=$ Radium D; no radiation $=$ Radium $\mathrm{E} ;-\beta$ and $\gamma=$ Radium $\mathrm{F} ;-\alpha=$ (? Lead ?).

Thorium $\alpha=$ Mesothorium; $-\beta=$ Radiothorium; $-\alpha=$ Thorium X; $-\alpha=$ Emanation; $-\alpha=$ Thor. $\mathrm{A} ;-\beta=$ Thor. $\mathrm{B} ;-\alpha=$ Thor. $\mathrm{C} ;-\alpha=$ ?

Emanation.-This is the first product of the disintegration of radium. It has the properties of a colorless indifferent gas. It diffuses through porous, but not through tight containers; can be condensed into a liquid by low temperature and volatilized by heat, etc. Its chemic properties resemble the argon group. A similar gaseous emanation is produced by thorium $\mathrm{X}$. When the metals are subjected to high temperature, the gas is driven off and their emanating power is lost; but they recover on resting, as their deterioration proceeds.

Radium Emanation.- "Radium emanation is continuously given off from aqueous solutions of radium salts. It can be collected as it escapes from the solution, drawn off through the use of the mercury pump, or by other suitable means, quantitatively determined by either the alpha- or gamma-ray electroscope, brought into solution in water for internal or external use or be set free in an emanatorium for inhalation treatment. It may be collected into small glass containers and this used in place of the applicators described under surgical use"' (N.N.R.).

Decay of Emanation. - The emanations lose their activity rapidly. That of thorium falls to one-half in one minute and to almost nothing in a very few minutes. That from radium persists for some days. The decay is due to the change of the emanation into radium $A$ (induced radio-activity) or thorium $A$, with the production of alpha-rays 
(charged helium). The rate of decay is independent of external conditions, such as temperature.

Induced Radio-activity.-Any substance, liquid or solid, when left in the presence of radium, acquires radio-activity. 'This is due to the deposition of a radio-active substance, "radium A" or "emanation X" on the surface. "This substance is formed by the decay of the emanation. Its quantity is extremely small. The deposit can be removed chemically or mechanically, $f . i$, by sandpaper. It also decays similarly to the emanation into radium $B$, etc.

Properties of Radium.-Radium is a bivalent element, closely related to barium. Because of its chemic reactivity, it can not be kept in the metallic state and is therefore marketed in the form of its salts, especially the soluble bromid or chlorid, and the insoluble sulphate and carbonate. It is generally mixed with more or less of the corresponding barium salts and is sold on the basis of its radium content, as determined by the radio-activity.

Determination of Radio-activity.-This is accomplished by measuring the ionization of gases. "Thus when radium is allowed to act on the air in a charged gold-leaf electroscope the air becomes ionized and therefore a conductor of electricity and allows the charge to leak out causing the leaf in the electroscope to move. By observing therate of movement of the leaf in a calibrated apparatus the radio-activity can be determined " (N.N.R.)

Units.- "Quantities and concentrations of radium emanation are expressed in terms of "curies" and Mache units. A "curie" is the amount of emanation in equilibrium with one gram of radium; a microcurie, one millionth of a "curie," is the amount of emanation in equilibrium with $0.001 \mathrm{mg}$. radium and is equivalent to about 2,500 Mache units"' (N.N.R.). A more detailed description of the measurement of radium emanations is given by Stratton, I9I5.

Radio-active Thorium Compounds.-Ordinary thorium gives off alpha-rays and changes into mesothorium (discovered by $\mathrm{O}$. Hahn, 1907). Its chemic properties resemble those of radium and barium, differing in the intensity and power of radiation. It gives off beta-rays and changes into Radiothorium, and this, by giving off alpha-rays, passes successively into Thorium $\mathrm{X}$ and Emanation. The thorium $X$ is therefore the principal radio-active product. The distribution, excretion and effects are-described by Plesch, Karzag and Keetman, I912. The actions and therapeutic uses are in all respects similar to radium. It is less efficient, but much cheaper (Schwarz and Zehner, I9I2; Falta and Zehner, I9I2; Gudzent, I9I2). Ledoux and Lebard, I9I3, consider mesothorium even better than radium against malignant tumors, and believe that thorium $\mathrm{X}$, because of its richness in alpha-rays, is especially suitable for internal medication.

Biologic Effects of Radium Rays.-Exposure to radium affects all living cells, similarly to X-rays. The tissues differ, however, in their relative susceptibility to the different radiations. According to the dosage, the results are irritant or destructive. In higher animals, the irritation grades from hyperemia to destructive inflammation (Bibliography, Loewenthal, r9r 2; Hussakoff, r907).

Intact Animals.-Continued exposure of mice (London) produces in three or four days, nervous depression terminating in coma. In rabbits, the symptoms begin in two weeks and last many months. They consist in loss of hair, ulceration of the skin, nervous changes similiar to those in mice, and sexual impotence. In man, overexposure has produced persistent diarrhea, vomiting, general weakness, and coma.

The susceptibility of the individual organs, according to Horowitz, is greatest in the central nervous system, lymphatic tissues, sexual glands and liver. It is less in the kidneys, suprarenals, cartilage, muscle, and vessels; least in salivary glands, pancreas and mucous membranes.

Central Nervous System.-Obersteiner found the following symptoms in mice which died after three to five weeks' exposure to radium: convulsions; forced movements; motor restlessness; paralysis of individual extremities; ataxia; paralysis of sphincters; trophic disturbances. The autopsy showed inflammatory changes of the entire nervous axis, with the degenerative lesions in the nerve cells.

Sexual Organs. - The spermatozoa of guinea pigs, when exposed to radium in vitro, soon lose their motility (London and Goldberg). The testes of exposed guinea pigs show atrophy, degeneration of the epithelium, and absence of spermatozoa (AlbersSchoenberg; London; Frieden). The ovaries exhibit analogous changes (Halberstadter; London). 
Lymphatic Tissue.-This reacts especially strongly. Inflammatory changes are found in the spleen, intestinal follicles, mesenteric glands, etc. (Heineke; London). Smaller doses destroy the follicles and increase the connective tissue. The leucocytes are liminished by emanation and by X-rays. It is not known whether there is increased destruction or lessened production.

Blood. - The hypodernic injection of soluble radium salts produced rapid increase of the red cells, persisting for several weeks. The hemoglobin did not rise as much. The leucocytes were also increased by small doses but diminished by large (Brill and Zehner, 1912). In vilro, sheep corpuscles are hemolyzed, and methemoglobin is formed (thorium X, Schwartz and Zehner, 19I 2).

Leukemia.-Treatment with $\mathrm{X}$-rays, radium and thorium $\mathrm{X}$ cause at least a temporary decrease of the leucocytes. However, thorium $\mathrm{X}$ also decreases the red corpuscles and produces parenchymatous changes. It should not be used where such lesions exist (Hahn, 19 4 ).

Skin.- The effects are identical with X-rays. After a quiescent period of about two weeks, the changes begin, and resemble sunburn; with increase of pigment; rubefaction and burning sensation. This may subside in a few days with a little scaling, or it may pass into intense congestion, vesication and loss of hair. The blisters rupture, leaving a raw surface covered with necrotic, diptheritic membrane. This may progress to highly indolent and painful ulceration. Selective destructive changes occur in cutaneous tuberculosis, carcinoma and sarcoma (Pusey, 19II).

The Blood Vessels of the Skin show dilation, emigration of leucocytes; later swelling of endothelium and extravasations (Thies).

The effects of mesothorium on the vessels of the rabbit's ear were studied by Ricker, 1914 .

Embryonal Development.-This is hindered in the ova of lower animals, as also in chicken eggs.

Plants.-The leaves and seeds are killed.

Bacteria.-The rays are rather feebly bactericidal, the alpha-rays being most effective. Toxins are probably not injured (Goldberg).

Ferments.-Autolysis is increased (Neuberg). Other ferments differ in susceptibility.

Decomposition of Lecithin.-Schwartz, 1904, observed discoloration and odor in the yolk of hen's eggs, suggesting decomposition of lecithin. This occurred also in vitro. Wohlgemuth referred this to stimulation of autolytic ferments; but Mesernitzky observed the decomposition even in boiled yolk. Werner and Ascher, 1912, confirmed the decomposition, and showed that the injection of decomposed lecithin or cholin into animals produced degenerative changes analogous to the rays, in the blood, testes, skin, etc. Promising results were also obtained with tumors. The same effects are produced by thorium $X$. This also decomposes proteins and reacts with many other organic substances (Falta and Zehner, 19r2). Fernau and Pauli, 1915, find that the changes of proteins by radium rays resemble those, of heat coagulation.

Surgical Uses of Radium.-These are employed for producing inflammatory reaction or destruction of tissue in skin lesions, birth-marks, scars, epithelioma and other accessible tumors, malignant or benign (pelvic, breast, neck, etc.). Deeper structures may be reached by filtering the gamma-rays through at least $3 \mathrm{~mm}$. of lead. Nearly all pathologic tissues are more sensitive than normal tissue. The efficiency is due to the beta- and gamma-rays, which are proportional to the radium content of the salt.

Surgical Application.-The dosage, etc., is still in an experimental stage. Heavy doses are usually employed for epithelioma and deep work (always filtered); and lighter doses for the other conditions.

Roentgen-rays in Cancer.-They are said to be useful in cutaneous epithelioma and localized, superficial, inoperable cancers; but useless in deep-seated or widely disseminated forms (Pusey, 1913). The use of mesothorium in cancer is discussed by Bumm, I9I3.

Fate of Radium.-After oral administration of insoluble radium salts (sulphates) to mice, the digestive tract is strongly radio-active; the other organs and the urine contain mere traces. With soluble preparations, orally or hypodermically, the excretion also occurs mainly by the small and large intestines, and by the lungs; little by the urine, 
and none by the liver and skin. The excretion of the soluble preparations is prompt; it is very slow after the injection of the insoluble salts (E. Smith and Bellingham, I9 I 2; Brill and Zehner, r9 2). The emanation has a high lipoid coefficient, and is distributed in the organs in proportion to its solubility (Knaffl-Lenz, I9I2). With inhalation, the amount in the blood increased with the time of exposure. The excretion is rapid, especially by the expired air, and partly by the urine.

Biologic Effects of Radium Emanation.-Large doses produce toxic effects essentially identical with those of radium. The toxic dose for man has not been determined. The actions are greater by inhalation than by hypodermic administration. With the smaller (therapeutic) doses, the effects are somewhat different.

Inhalation of Large Doses.-This leads to dyspnea and depression (but no true narcosis), terminating in death. The autopsy shows widespread hyperemia, with histologic changes in the brain cells, and to a less degree in other central nerve cells: The emanation hemolyzes the blood of guinea pigs, but not of rabbits. It decomposes lecithin (Knaffl-Lenz, r91 2; Poulsson, 1908).

Effects of Therapeutic Doses.-The activity of autolytic ferments is increased; diastase is first hindered, then augmented; pepsin and trypsin are augmented. There is little bactericidal action. The embryonal development of lower animals and the growth of plants is hastened. In healthy man, the lencocytes are first increased, then diminished. The coagulability of the blood is promptly and markedly raised. The blood presure is generally lowered 20 to $25 \mathrm{~mm}$. (Loewy and Plesch), so that the work of the heart is diminished. This is attributed to vaso-motor changes. The hypnotic action which is often observed may be due to modifications in the cerebral circulation. The frog's heart, perfused with activated Ringer's solution, is slowed with increased diastole and irregularities; small doses are sometimes stimulant (Maas).

In rheumatic, gouty and chronic inflammatory conditions the emanation treatment often causes a "reaction," consisting in an exacerbation of the inflammatory symptoms. This occurs after a few days of treatment, and subsides spontaneously if the treatment is continued.

Urate Metabolism. - Investigations with modern methods have failed to show any effect on the uric acid of the blood, by radium, intravenously or by inhalation (Fine and Chace, 1914). Less perfect methods led to the general belief that the inhalation of emanation leads to increased elimination of uric acid and purin bases, endogenous as well as exogenous; that this is followed by the disappearance of urate deposits, tophi or artificial (Fofanow); and that in gout, the uric acid disappears from the blood, of ten with subjective improvement. Abl, rgI3, also claims increased elimination of endogenous uric acid by thorium $X$. The mechanism of these effects (if they occur) is not established.

Gudzent claimed that radium D (formed from the emanation but not from the rays), converts in the test-tube the lactim urate into the soluble isomeric lactam, and then into $\mathrm{NH}_{3}$ and $\mathrm{CO}_{2}$. Falta and Zehner, rgr2, claimed that thorium $\mathrm{X}$ also increases the solubility of urates and destroys uric acid. Other observers (Lazarus; Knaffl-Lenz and Wiechowski, I9I2) have been unable to confirm the destruction. Lazarus attributes Gudzent's results to mould fungi.

Kaplan, I9I2, claims that alkaline radium water decreases the uric acid and increases the purin bases; Staehelin and Maase, I9r 2, affirm that these alkaline waters diminish the gas metabolism in health, but not in gout.

Therapeutic Use in Gout.-Favorable effects are claimed. In most cases the treatment brings on an acute attack, generally mild, in the first six to fourteen days of treatment. Improvement sets in after three to four weeks, parallel to the disappearance of urates from the blood. Cases with anatomic lesions are not so favorable.

Therapeutic Use in Arthrites.-Emanation treatment is said to be markedly successful in chronic joint and "rheumatic" lesions, sciatica, tabes, pain, etc. The main effect is on the pain but the inflammatory phenomena are also decreased. The improvement is often, but not always, preceded by the "reaction" a flare-up of the inflammatory symptoms (Loewenthal; Fuerstenberg). The late fibrous and anatomic changes are not removed. The results are therefore better in early cases and in young subjects. Acute arthritis, arthritis deformans, and luetic or tubercular lesions are not affected. Progressive polyarthritis and chronic myalgia react well.

This was the first, and is probably the most successful use of the emanation (Loewenthal; Laqueur). The mechanism of the action is not explained. It may be mainly 
analgesic; but Gudzent assumes a direct effect on the inflammation, activation of iutolytic ferments, and general increase of metabolic processes.

Administration of the Emanation.- This can be carried out properly only in specially equipped hospitals. "It may be administered as baths, by subcutaneous injection in the neighborhood of an involved joint ( 0.05 to 0.5 microcurie in I or 2 c.c. distilled water), by local application as compresses ( 5 to Io microcuries), by mouth as a drink cure (in increasing doses of from I to Io microcuries three or more times a day), by inhalation, the patient for two hours daily remaining in the emanatorium which contains 0.0025 to 0.25 (average 0.I) microcurie per liter of air" (N.N.R.).

\section{SILVER}

General Statement.-The inorganic silver salts, especially the nitrate, are used to produce astringent, caustic and antiseptic effects. They form resistant precipitates with proteins, so that their local action is easily controlled. The toxicity for higher animals is very low, and the antiseptic efficiency is high. In the presence of the tissues, silver surpasses mercury, since the protein compounds of silver are also antiseptic. These may be used directly when antiseptic action without irritation is desired.

Silver is not absorbed in sufficient quantity from the alimentary canal to produce systemic actions. Because of its precipitation by proteins and chlorid, even large doses of silver nitrate rarely produce serious poisoning. Long-continued use results in blackish discoloration of the skin (argyrism) by the deposition of silver particles, probably organic. Traces must therefore be absorbed.

Argyrism.-This develops gradually after prolonged internal or external use of silver when the total dose has reached I 5 to $30 \mathrm{Gm}$. Since silver is now rarely used in this way the condition is infrequent. It has been produced by the application of silver-containing hair dyes. Argyrism consists in a bluish-black discoloration of the skin, especially where exposed to light; and of internal organs and mucous membranes (Frommann, I857; Riemer, I876). The black granules do not consist of metallic silver, for they are easily soluble in KCN, and difficultly in concentrated nitric acid. In the skin, they are situated in the connective tissue of the corium, and remain indefinitely. No silver can be demonstrated in the urine. The argyrism does not give rise to other symptoms. In animals, silver desposition has also been produced in internal organs, but not in the skin.

Systemic Actions. - These are never seen in man but may be produced in animals by the injection of non-precipitant silver solutions $(\mathrm{AgCl}$ in sodium thiosulphate, Gaehtgens, I890; colloid $\mathrm{AgCl}$ or $\mathrm{AgI}, \mathrm{Gros}, \mathrm{Ig}_{\mathrm{I}} 2$ ). The actions are mainly on the central nervous system, consisting of paralyses and spasms, beginning in the lower extremities; disturbances of respiration; and fall of blood pressure (paralysis of vasomotor center?); sometimes diarrhea. The secretion of bronchial mucus is greatly increased, probably by injury to the epithelium, and may lead to asphyxia. Death occurs usually promptly, by paralysis of respiration; the diaphragm being paralyzed before the other respiratory muscles. The heart is but little affected and may beat after death. In frogs, the paralysis is preceded by convulsions (Ball, I865; Jacobj, I877; Gaehtgens).

Chronic systemic poisoning shows changes in the blood corpuscles; disturbances of metabolism; cloudy swelling of the heart and skeletal muscle; and fatty degeneration of the liver and kidney (Bogoslowsky).

Therapeutic Use of Silver in Nervous Diseases.-(Insanity, Epilepsy, Etc.)-This was a survival of the fantastic teaching of the middle ages, when it was based on its dedication to the moon, and the supposed connection of the latter with lunacy. Although it is absolutely proved that silver can not be absorbed in amounts sufficient to have any action whatsoever, it has been tried again and again against all forms of nervous disease, with uniformly negative results.

Local Astringent and Caustic Action.--Silver nitrate acts as astringent, irritant or caustic, according to the strength and duration of its application. The surface first turns white, then gradually gray and black by the 
reduction to metallic silver and oxid. The nitrate, fused into pencils ("Lunar caustic") is used in substance for the removal of warts, etc., and the stimulation of ulcers and granulations. The pencils should be moistened before use. Solutions are employed for their astringent and antiseptic action in acute and chronic catarrhal inflammations of mucous membranes, in the following concentrations:

Ordinary conjunctivitis, 0.2 to 2 per cent. Disinfection of conjunctiva (prevention of ophthalmia neonatorum), a drop of 2 per cent., followed in a few minutes by normal saline to arrest the action. Larynx, 2 to ro per cent. Urethritis (gonorrheal or other), irrigation with $\mathrm{I}: 10,000$ to $\mathrm{I}: 2,000$. Cystitis, irrigation with $\mathrm{I}: 5,000$. Gastric douche (hyperchlorhydria and ulcer), I :2,000 to I : I,000, followed after two minutes by sodium chlorid solution.

Penetration of Silver Nitrate.-Contrary to the prevalent opinion, the action of silver nitrate extends quite deeply, since it forms easily soluble double salts of silver albuminates-sodium chlorid in the tissues (Unna, Schumacher, 1915).

Antiseptic Concentration. $-1: r, 000$ is fatal to bacterial cultures in five minutes; I: 80,000 in blood serum has a distinct inhibitory effect.

A watery solution of silver phosphate with ethylendiamin is said to be more penetrating, which would be an advantage especially in urology; but it seems to be too irritant (Fraenkel). It is marketed as argentamin, N.N.R., corresponding to ro per cent. of silver nitrate.

The mucous membranes should always be cleansed before the silver is applied. The solutions must be made with distilled water. The action may be stopped with sodium chlorid.

Silver Pills.-Pills of silver nitrate, ro to $20 \mathrm{mg}$., $1 / 6$ to $1 / 3 \mathrm{gr}$. (made with kaolin and petrolatum), are sometimes used in gastric ulcer, etc. There is probably a slight local action before the silver is precipitated, and after it is dissolved by protein. The oxid, 0.03 to $0.1 \mathrm{Gm}$., $1 / 2$ to $1 \frac{1}{2} \mathrm{gr}$., may be similarly employed.

Protein-silver Compounds.-A number of these products are on the market, such as Argyrol (with vitellin, 20 to 25 per cent. Ag.); Argonin (with casein, 4 per cent. Ag.); Protargol (with albumose, 8 per cent. Ag.); Largin (with protalbin, i I per cent. Ag.). The compounds retain considerable of the bactericidal properties of silver, but are non-precipitant, penetrate more deeply, and are practically non-astringent and non-irritant. The absence of these qualities may be desirable in some cases, while it is a serious drawback in others.

They are used in gonorrhea ( 0.1 to 2 per cent.); conjunctivitis ( 0.5 to 5 per cent.); nose and throat infections ( 2 to ro per cent.); etc.

The products usually dissolve slowly in cold water. The solutions decompose on exposure to light.

Freshly precipitated silver iodid was recommended by Siter and Uhle, 1905; but has not come into general use. Thum, 1915, gives a formula for concentrated solution of silver iodid. Colloid silver was discussed under "Colloid Metals."

Toxic Actions.-Silver nitrate to 0.0 r or o.r $\mathrm{Gm}$. by mouth produces no symptoms. Swallowing pieces of the pencils up to $2 \frac{1}{2} \mathrm{Gm}$., is also often inoffensive. Larger quantities cause acute gastritis. The fatal dose would be above ro $\mathrm{Gm}$. (Lewin); even $30 \mathrm{Gm}$. have been survived. The treatment would be by sodium chlorid and demulcents.

\section{PREPARATIONS-SILVER}

*Argenti Nitras (Arg. Nit.), U.S.P.; Argent. Nit., B.P.; Silver Nitrate (Lunar Caustic); $\mathrm{AgNO}$. - Colorless crystalline tables, becoming gray to black on exposure to light in the presence of organic matter. Odorless; metallic taste. Very sol. in water ( $1: 0.4)$; sol. in alc. $(\mathrm{r}: 30)$. Incompatible with chlorids, bromids, iodids; carbonates and hydroxids; organic drugs and reducing agents. Dose, ro mg., 1/6 gr., U.S.P.; 16 to $30 \mathrm{mg}$., $1 / 4$ to $1 / 2 \mathrm{gr}$., B.P. Maximum dose, $30 \mathrm{mg}$., $1 / 2 \mathrm{gr}$. 
*.1rgenli Nitras Fusus (.Irg. Nit. Fus.), U.S.P.; Moulded Silver Nitrate (Stick Caustic). - Ig.NO, fused and moulded into pencils. A small quantity of $\mathrm{AgCl}$ is added to foughen the mass.

*. Irgenti Vitras Induratus (Argent. Nit. Indur.), B.P.; Toughened Causlic.-Contains 5 per cent. of $\mathrm{KNO}_{3}$.

Argenti Vitrus llitigatus (Argent. Nit. Mitig.), B.P.; Mitigated Caustic-Contains 1 part $\lg \mathrm{NO}_{3}$ and 2 parts $\mathrm{KNO}_{3}$.

Argenti Oxidum, Arg. Oxid., U.S.P.; $\operatorname{Ag}_{2} \mathrm{O}$.-Heavy, dark brownish-black powder; odorless; metallic taste. Very slightly sol. in water. Dose, $0.06 \mathrm{Gm}$., I gr., U.S.P.

* Argenti Proleinas (Argent. Protein.).-Protargol, etc., are described in N.N.R.

\section{GOLD AND PLATINUM}

Gold and platinum are still more easily reduced to the metallic state than is silver, and are therefore devoid of general action when taken by the mouth. When injected into the circulation they produce an arsenic action. (Gold, Schultz, 1892; Platinum, Kebler, I 878 ; Cohnstein, I892).

Gold cyanid was advised against tuberculosis and scrofula by Chrestien, I853. It fell into disuse, but has again been resuscitated, with doubtful justification. Bruck and Glueck, I9I3, claim successful results from intravenous injection of gold-potassic cyanid (especially when combined with tuberculin!) in lupus; also in syphilis, though inferior to salvarsan. Hauck, I9I3, however, reports a fatal result. Further references in Merck's Report, Vol. XXVII.

\section{PREPARATIONS-GOLD}

Auri et Sodii Chloridum (Aur. et Sod. Chlor.), U.S.P.; Gold and Sodium Chlorid.A mixture of equal parts of anhydrous gold chlorid $\left(\mathrm{AuCl}_{3}\right)$ and anhydrous sodium chlorid. Orange-yellow powder, odorless, saline and metallic taste; deliquescent. Very sol. in water. Dose, 5 mg. $1 / 12$ gr., U.S.P.

\section{TIN}

Actions. - This metal is absorbed in part even from non-corrosive preparations. However, poisoning is rare, the metal not passing very easily into soluble form, and having no pronounced tendency to cumulative action. The symptoms on injection, devolve to some extent on the central nervous system, as stimulation and subsequent paralysis. The arsenic action and paresis of the heart are also prominent. With more chronic poisoning the gastroenteritis is most marked, but there is also an ataxia and motor paralysis, resembling chronic lead-poisoning (White, r 880 ).

Absorption and Fate. - When soluble salts of tin are fed, a small quantity is absorbed and accumulates in the tissues, traces appearing also in the urine. When soluble salts are injected hypodermically elimination occurs slowly, mainly by the alimentary tract, but somewhat by the urine, producing marked injury of the kidneys. Considerable quantities are retained: 20 to 25 per cent. in the skin, 5 per cent. in the liver (Salant and co-workers, I914).

\section{COPPER}

General Statement.-Cupric sulphate irritates the nerve endings in the stomach which give rise to the vomiting reflex, and thus has a rather specific emetic action. It is also used on mucous membranes as an astringent and caustic. It has a relatively high toxicity for fungi and algæ, and has been employed for the purification of water, etc. It is not a reliable bactericide. Its absorption is limited and its toxicity to animals is therefore low. Its use for brightening the green color of vegetables is innocuous under proper conditions, but under others it may be somewhat harmful. 
Emetic Action.-Copper sulphate is effective and prompt, with short nausea. However, it is irritating; and should it not be effective, there is some danger of poisoning, and it must be removed by lavage. It is therefore not recommended, except in phosphorus poisoning, in which it oxidizes the phosphorus and coats the globules with a layer of metallic copper. The emetic dose is $0.5 \mathrm{Gm} ., 8 \mathrm{gr}$. in a single dose; or three doses (if necessary) of $0.25 \mathrm{Gm} ., 4$ gr. each, fifteen minutes apart. It should be dissolved in a glass of water.

Astringent and Caustic Action.-Solid copper sulphate is used as a mild escharotic against granulations and trachoma. The everted lid should be lightly touched with the copper crystal or pencil, and then rinsed with water. The pencils are made by fusing I part of potassium alum with 2 parts of copper sulphate. Solutions of o.I to 3 per cent. may be employed as stimulant astringents in conjunctivitis, urethritis and vaginitis, but have no particular advantage over silver. Copper sulphate would probably be effective against aphthous stomatitis and other fungous diseases. It has been used internally against chronic diarrhea, Io mg., $1 / 5 \mathrm{gr}$.

Antiseptic Action and Use in Water Purification.-Copper is markedly germicidal for fungi and algæ, and fairly effective against bacteria of the coli group. It may be used to purify water from algoid contamination. The traces of copper remaining in the water are probably harmless. Its use against typhoid contamination seems to be unreliable (Clarke and Gage, 1906). Thomas, 1915, describes its use for the disinfection of swimming pools.

The mechanism of the action of copper on lower plants is discussed by Spiro, I9 I 5 .

Toxic Actions. - Large doses of soluble copper salts produce mainly gastroenteritis, and characteristic lesions of the spleen, kidneys and other organs. The toxicity is low. The absorption is very limited.

Even on continued use, quantities of the copper sulphate up to $0.5 \mathrm{Gm}$. per day have been shown to be devoid of bad effects ( $\mathrm{Du}$ Moulin). It is true that these were not continued indefinitely.

Use of Copper in Foods. - Copper is sometimes used to give a bright green color to preserved vegetables, especially peas and beans. These contain 25 to r $20 \mathrm{mg}$. per kilogram. The copper enters into a very firm compound with the chlorophyll. Older vegetables, which are not so rich in chlorophyll, contain it in much looser combinations with proteins. The chlorophyll compound produces no effects of any kind, even when continued for long periods in quantitics corresponding to 12 to $15 \mathrm{mg}$. Cu per day, the maximum amount which would be taken in food. However, the same quantities, when more loosely combined, produce some digestive and metabolic disturbances. The use of copper with young green vegetables would therefore be harmless; but with older vegetables it could become harmful, and its use would better be prohibited (J. H. Long, I9r2).

Ocurrence of Copper in Tissues.-Copper is not usually present in mammalian tissues; but after its administration, it occurs in the liver mainly as cupric nucleinates, and in the blood and bile. Traces are also retained in the kidneys, spleen and lungs; none in tubercular lymph glands or pus. Colloid copper, injected intravenously, shows a similar distribution (Corper, 1914).

It is found naturally in organic combination in the liver and other organs of cephalopods (octopus), snails and mussels; and as a blue blood pigment, hemocyanin, in mollusks and crustaceans. This is somewhat analogous to hemoglobin and contains about $3^{8}$ per cent. of $\mathrm{Cu}$ in masked form (Henze, I90I). Copper is also a constituent of the feather pigments of certain birds.

Systemic Actions. - Direct contact with copper salts depresses skeletal and cardiac muscle. Intravenous injections therefore lower blood pressure.

The effect of colloid copper on tumors was discussed in the general article on "Metals.". 


\section{PREPARATIONS-COPPER}

"Cupri Sulphas (Cupr. Sulphi.), U.S.P., B.P.; Copper Sulphate (Cupric Sulphate, Blue Vitriol, Bluestone); $\mathrm{CuSO}_{4}+{ }_{5} \mathrm{H}_{2} \mathrm{O}$. - Large, deep blue crystals; odorless; metallic taste. Freely sol. in water ( $1: 2.5)$; slightly sol in alc. $(\mathrm{I}: 500)$; freely sol. in glyc. (I: 2.8). Incompatible with fixed alkalies and carbonates; iodids; tannins; lead salts. Dose, Emetic, 0.25 Gm., 4 gr., U.S.P.; 0.3 to 0.6 Gm., 5 to ro gr., B.P.; as Astringent, 0.016 to 0.12 Gm., 1/4 to 2 gr., B.P., diluted. Maximum dose, I Gm., $15 \mathrm{gr}$.

\section{ZINC}

General Statement.-The actions of the soluble zinc salts resemble those of copper, but are milder. Zinc sulphate is employed as emetic, and locally as astringent and antiseptic. Other soluble zinc salts are also used locally. The acetate is milder; the chlorid is escharotic. The insoluble zinc oxid and stearate are used externally as protectives, similarly to bismuth. There are no systemic effects under therapeutic conditions.

Zinc Sulphate as Emetic.-This acts like copper, and is generally preferred, being nearly as efficient and less irritating. The short nausea, slight depression and absence of harmful irritation makes it particularly suitable in gastric disturbance from overeating, etc. I to $2 \mathrm{Gm}$., I 5 to 30 gr. may be given in a glass of water.

Astringent and Antiseptic Action.-Zinc sulphate or acetate is used in conjunctivitis, particularly that caused by the Morax-Axenfeld bacillus; and in chronic gonorrheal urethritis and other catarrhal conditions. The strength of solution for collyria is o.I to I per cent.; for urethral injections, 0.5 to 4 per cent. The phenolsulphonate has no advantage.

Zinc Chlorid as Disinfectant.-This has been extensively exploited and overestimated in proprietary preparations. MacClintic, I905, finds $1: 200$ to $\mathrm{I}: 500$ a very poor germicide and feeble antiseptic, though a fair deodorant.

Zinc Chlorid as Escharotic.-This is used as the Liq. Zinci Chlor. on granulations, ulcers, etc.

Insoluble Zinc Compounds.-Zinc oxid is mildly antiseptic and astringent. It is extensively used as a local sedative application in inflammatory skin diseases and superficial wounds. It may be applied dry or as an ointment, alone or mixed with boric acid, bismuth subnitrate, starch or phenol. Zinc stearate is perhaps somewhat smoother as a dusting powder, but has no other advantage.

Systemic Action.--The effects of intravenous injection are mainly paralytic. The brain is affected first-i.e., consciousness is lost-but the motor areas are not involved. The blood pressure falls rapidly, but this is mainly due to the cardiac depression. The skeletal muscles are but little affected. Zinc salts are not sufficiently absorbed to produce systemic effects on oral administration. Zinc oxid has been used as a nervous sedative, in epilepsy, etc., but is probably useless.

The movements of excised intestines are depressed by very dilute solutions; abolished by medium concentrations (Salant and Mitchell, 1916).

Industrial Poisoning.- "Brass founders ague" is produced by the inhalation of the finely divided zinc oxid formed in the combustion of the metal-even of pure zinc (Lehmann, 1910). It has not been observed in animals, but only in man. After exposure of several minutes or hours-depending on the susceptibility and ventilation-there is slight irritation of the throat; but the main symptoms occur later, often after the patient has been in the air for some hours. The attack resembles a malarial chill, which may last some hours, with ordinary febrile symptoms-lassitude, headache, nausea, muscle cramps and joint pains, constricting sensation over lungs, etc. The attack ends in perspiration and rapid recovery (W. G. Thompson, 1912; Hayhurst, 1013; 
Pfender, I914). Lehmann attributes the symptoms to the absorption of the remains of the respiratory cells that were destroyed by the zinc. The hypodermic injection of zinc salts also produces fever (Kisokalt, I9I2); but so do most irritants.

Repeated exposure to zinc fumes may result in chronic lesions of the respiratory, digestive, excretory and nervous systems (Hayhurst, r9i3).

\section{PREPARATIONS-SOLUBLE ZINC SALTS}

*Zinci Acctas (Zinc. Acet.), U.S.P., B.P.; $\mathrm{Zn}\left(\mathrm{C}_{2} \mathrm{H}_{3} \mathrm{O}_{2}\right)_{2}+\mathrm{H}_{2} \mathrm{O}$.-Soft, white plates; faintly acetous odor; astringent, metallic taste. Freely sol. in water $(1: 2.3)$; sol. in alc. $(\mathrm{r}: 30)$. Dose, $0.125 \mathrm{Gm} ., 2$ gr., U.S.P.; 0.60 to $0.12 \mathrm{Gm}$., I to 2 gr., B.P.

Zinc Chlor., U.S.P., B.P.; $\mathrm{ZnCl}_{2}$ - - White granular powder or porcelain-like masses or in pencils; odorless; highly caustic. Very sol. in water ( $\mathrm{I}: 0.25$ ); freely sol. in alc. (I: I.3) or glyc. $\mathrm{ZnCl}_{2}$.

* Liq Zinc. Chlor., U.S.P., B.P.-An acid solution, containing about 5o per cent. of

Zinci Phenolsulphonas (Zinc. Phenolsulph.), U.S.P. (Zinc Sulphocarbonate); $\mathrm{Zn}\left(\mathrm{C}_{6} \mathrm{H}_{5} \mathrm{O}_{4} \mathrm{~S}\right)_{2}+8 \mathrm{H}_{2} \mathrm{O}$.-Colorless, transparent prisms, or tabular crystals or in granular form; odorless; astringent, metallic taste. Freely sol. in water (r: 1.6). Dose, 0.125 Gm., 2 gr., U.S.P. Locally, similar to Zinc. Sulph.

*Zinci Sulphas (Zinc. Sulph.), U.S.P., B.P.; Zinc Sulphate (White Vitriol); ZnSO 4 ${ }_{7} \mathrm{H}_{2} \mathrm{O}$. - Small colorless crystals; odorless, metallic taste. Very sol. in water ( $\left.1: 0.6\right)$; practically insol. in alc. Incompatible with alkalies, carbonates, sodium borate, tannins, lead salts. Dose, as Emetic, I Gm., I 5 gr., U.S.P.; 0.6 to $2 \mathrm{Gm}$., io to 30 gr., B.P., diluted; as Astringent, 0.06 to $0.2 \mathrm{Gm}$., I to $3 \mathrm{gr}$., B.P. Maximum dose, $2 \mathrm{Gm}$., 30 gr.

Zinci Valeras (Zinc. Valer.), U.S.P., Zinci Valerianas, B.P. (Zinc Valerianate); $\mathrm{Zn}\left(\mathrm{C}_{5} \mathrm{H}_{9} \mathrm{O}_{2}\right)_{2}+{ }_{2} \mathrm{H}_{2} \mathrm{O}$. - White, pearly scales, or white powder; odor of valeric acid; sweetish, astringent, and metallic taste. Dose, $0.125 \mathrm{Gm} ., 2$ gr., U.S.P.; 0.06 to 0.2 Gm., I to 3 gr., B.P.

\section{PREPARATIONS-INSOLUBLE ZINC COMPOUNDS}

Zinci Carbonas Precipitatus (Zinc. Carb. Præc.), U.S.P.; Zinc. Carb., B.P.-Basic Zinc Carbonate is a compound of somewhat variable chemical composition, corresponding to not less than 68 per cent. of $\mathrm{ZnO}$, U.S.P. Impalpable, white powder without odor or taste. Insol. in water or in alc.

*Zinci Oxidum (Zinc. Oxid), U.S.P., B.P.; ZnO.-Very fine amorphous, white or yellowish-white powder, without odor or taste. Practically insol. in water or alc. Incompatible with acids. Dose, 0.2 to $0.6 \mathrm{Gm} ., 3$ to ro gr., B.P.

*Unguentum Zinci Oxidum (Ung. Zinc. Ox.), U.S.P. (Zinc Ointment).—2o per cent., in Benz. Lard.

* Ung. Zinc., B.P.-I5 per cent. of ZnO in Benz. Lard.

Lassar's Paste, which is used in eczema, contains Zinc. Ox. I part, Starch, I part, Petrolat., 2 parts.

Zinci Oleostearas (Zinc. Oleost.), B.P.-A zinc soap. White amorphous, insoluble powder.

Ung. Zinc. Oleat., B.P.-A zinc soap, incorporated with white soft petrolatum.

\section{CADMIUM}

This resembles zinc closely.

\section{NICKEL AND COBALT}

These metals are only absorbed when given in strongest solutions. The local action is that of metals in general, with nothing particularly characteristic. Nickel salts have been used as emetics, but are not be be recommended. Nickel cooking vessels give up a harmless trace of the metal to the food (Dzerzgowski and Schumoff-Sieber, I906 and r908; Lehmann, I909). 
When injected intravenously or hypodermically, these metals affect the central nervous system directly, in addition to the usual metal action on capillaries, heart, and lidists. There are tremors, chorea, and convulsions, followed by paresis. In frogs the niedullat is stimulated before the spinal cord (A. Stuart, I884). Some habituation ocours.

The urine is increased, and always contains sugar, often proteins. Cobalt salts act as antidotes to IICN poisoning, through the formation of cobalt-cyanids. To be ffective they must be introduced subcutaneously in doses which are not devoid of dinger. They are therefore not to be used in man.

IIith excised intestine, dilute solutions of nickel salts produce a primary depression, which may be followed by stimulation. Higher concentrations abolish the movememts. Nickel is much less toxic than zinc (Salant and Mitchell, r9r6).

Nickel Carbonyl. - This gas- $\mathrm{Ni}(\mathrm{CO})_{4}$-is formed by passing $\mathrm{CO}$ over finely divided nickel, as in the Mond process of obtaining pure nickel. It decomposes at $150^{\circ} \mathrm{C}$. into CO and metallic nickel. The toxic effects are probably due to the deposition of the metal in the respiratory tract, for the carbonyl is not absorbed as such. The phenomena following its inhalation are very serious. There is transient malaise, with quick recovery in fresh air; but after twelve to thirty-six hours the patient becomes dyspneic, cyanotic, febrile, and delirious, with a blood-stained cough. Death occurs in four to eleven days, with hemorrhages (due to fatty degeneration of the vessel walls) in the lungs and brain, and pulmonary edema (Mond, Langer and Quincke, I890; Armit, 1907, 1908).

\section{MERCURY}

General Statement.-Mercury, particularly in the mercuric salts, has a strongly toxic action on all protoplasm. In virtue of this action mercurial compounds are active germicides; but their usefulness is limited by precipitation, irritation and general toxic effects. Even the insoluble mercury compounds are rapidly absorbed, distributed through all the tissues, and excreted by all channels, but mainly by the urine and feces. Traces remain in the body for many months. Acute poisoning presents chiefly the phenomena of local corrosion. The characteristic features of systemic poisoning are stomatitis, dysentery, and nephritis. In chronic poisoning, nervous symptoms, and cachexia appear.

Mercury kills the parasites of syphilis, and therefore may have a curative effect when properly used. Calomel is relatively insoluble and mild, so that it may be employed as a cathartic and diuretic.

Antiseptic Actions.-Mercuric chlorid is one of the most effective of the mercurial germicides, since it dissociates readily and therefore gives the maximum concentration of mercuric ions, which produce the antiseptic effect. In pure cultures it inhibits the growth of most microörganisms in dilutions of $\mathrm{I}: 30,000$ to $\mathrm{I}: 20,000$; it is germicidal in $\mathrm{I}: 2,000$ to $I: I, \infty 00$. Spores and tubercle bacilii are more resistant.

Application.-Corrosive sublimate is used outside of the body, on hands, eic., in concentrations of $I: I, 000$. On exposed tissues, as $I: 5,000$ to I $: 2,000$.

In wounds, however, mercuric salts are not so useful; their activity is greatly lessened by the proteins; and they cause much irritation and toxic effects. These last are particularly to be feared when large serous cavities are irrigated.

Mercury compounds produce rapid corrosion of metallic instruments and a more gradual destruction of rubber.

To hasten the solution, to prevent precipitation in the water and to lessen the irritation it is customary to add some sodium or ammonium chlorid, as is done in the commercial bichlorid tablets. These form double salts which are less dissociated and therefore somewhat less active (Sab- 
batani, r906). Hydrochloric, tartaric and citric acids have similar effects.

Mercury compounds are used in various skin diseases, especially those of parasitic origin. The bichlorid in Pityriasis and Sycosis; Blue Ointment against Pediculi; White Precipitate against Impetigo; etc.

Potassium-Mercuric Iodid.-This is at least an equally efficient antiseptic, and has the advantage of being much less precipitant, irritant and toxic. It may be used on catarrhal mucous membranes, boils, etc., in I per cent. solution (Macfarlan, I9I4).

Germicidal Efficiency of Calomel.- This is generally considered much inferior to mercuric chlorid; but Schamberg and Kolmer, IGI4, contend that it and other insoluble mercury salts are equally effective.

Emigration of Leucocytes from Frog's Mesentery.-This is inhibited by the injection of mercuric chlorid (Ikeda, rigr).

Local Irritation.-The soluble mercuric salts are strongly irritant. They coagulate protoplasm and thus give an ashy color and cooked appearance to the tissues, which are slowly corroded. These local changes are especially important in acute poisoning. The intact skin is also irritated; I to 5 per cent., causes vesication and corrosion; and sensitive skins may become inflamed by the strengths used in operations.

Mercurials in Ophthalmology.-Mercury preparations are used in a number of inflammatory eye diseases; conjunctivitis, blepharitis and keratitis.

Hydrargyri Oxidum Flavum is employed most extensively as a 1/10 to $1 / 4$ per cent. ointment (made by diluting the official ro per cent. ointment-Pagenstecher's Salve). Hydrargyrum Ammoniatum is somewhat less irritating and is used as I to ro per cent. ointment.

Calomel is also used as a dusting powder, but especially, on ulcers.

Mercuric Chlorid is antiseptic, but very irritating to the eye. It may be used as $I: 5,000$ to $I: 20,000$, with boric acid. Subconjunctival injections have also been employed, but their usefulness is doubtful (de Schweinitz, I893).

Mercurials and Iodid on Eye--Mercury compcunds should not be applied to the eye when the patient is using iodids; the very irritant mercuric iodid is formed on the conjunctiva and causes severe injury. Iodin compounds that do not ionize readily (iodtropin) naturally produce but little reaction (Grumme-Fohrde, I9I4). Bromids behave like iodids, but chlorids are harmless (Schloms, 1913).

Absorption, Fate and Excretion.-Mercury compounds and metallic mercury are absorbed readily from all surfaces, even from the intact skin. All of these channels are used therapeutically. After their absorption, the mercury disappears rapidly from the blood; being taken up partly by leucocytes, and deposited in various organs, especially the kidney and liver. Here the mercury appears to be fairly firmly bound, forming "depots" from which traces of the metal may be mobilized for six months or longer after discontinuing the administration.

The excretion occurs mainly by the urine and feces (Wellander, I886). These take a variable, but on the whole about equal share. There are reasons to believe that the urine plays the main part in the excretion of the organic, the feces of the inorganic compounds (Müller, Schoeller and Schraut, I9II). The excretion begins in a few hours, and after a single dose lasts for perhaps eight days; after long-continued administration the excretion persists intermittently for many months. 
Form of Absorption. - The compounds undergo complex changes in the course of their absorption. Ne'tullic mercury penetrates mechanically, by its fluidity; it is readily oxidized, and then behaves as the other forms.

Mercuric salts form soluble organic compounds with the proteins, $\mathrm{NaCl}$, and alkalies, (Voit, 1857 ).

Distribution in the Body.-The proportion of mercury is greatest in the kidneys; then in descending order, come the liver, spleen, bile, intestinal wall (Ullmann, 1892; Abelin, IOI 2). None is present in the nervous system. It has been reported in the cerebrospinal fluid (Conti and Zuicola, I906); but Lasarew, I912, found it absent in mercurial treatment and poisoning. If mercurial vapor has been inhaled, the metal may be found in the lungs. The deposition in the liver is said to be lessened by potassium iodid (Blumenthal and Oppenhein, I9ri).

It is sometimes found in the placenta and in the embryo, but probably only after very large doses, which may injure the vessels. It is claimed, however, that congenital syphilis may be treated by mercurial inunction of the mother (Jung, I9r4).

The distribution of mercury in acute poisoning is discussed by Kahn, Andrews and Anderson, 1915 .

The distribution of aromatic mercury compounds is described by Blumenthal and Oppenheim, I9I4.

Minor Channels of Excretion.-Variable traces are generally found in the bile, sweat (Tachau, I9II; Lambert and Paterson, I915), gastric (Lieb and Goodwin, 1915) and intestinal juice and saliva (literature in Almquist, I912).

Rate of Encretion.-Buchtala, I913, found that the excretion, after intramuscular injection of mercury salicylate, was greatest on the following day. With intravenous injection, the greatest excretion (often half) occurred within twenty-four hours.

Potassium iodid, diuretics and sweating are said to hasten excretion; but there is little evidence for this. Buchtala, found that the iodid rather delayed the urinary elimination of mercury.

Excretion of Metals in Cow's Milk.-Lead is excreted into the milk, but not mercury, antimony or zinc (Koldewijn, I910).

Varieties of Mercury Poisoning.- These may be classed under three forms:

(a) Acute, occurring generally from the abuse of mercuric chlorid, chiefly with local phenomena.

(b) Subacute, especially in the therapeutic use of excessive doses.

(c) Chronic.-This may also occur medicinally, but is seen especially in industrial poisoning (workers in mercury, manufacturers of thermometers, mirrors, electric lamp bulbs, felt hats, etc.). A large proportion of those exposed to mercury are affected, because the adherence of the metal to the skin, and the inhalation of its vapor, makes protection difficult.

Phenomena of Acute Poisoning with Mercuric Salts.-The immediate effects are due to coagulation, irritation and superficial corrosion, to which mucous membranes are highly susceptible. There is an astringent metallic taste, salivation; burning, swelling and ashy discoloration of the mouth and pharynx; thirst; abdominal distress and pain, at first epigastric, then diffused; vomiting with white or often bloody mucous shreds; sometimes edema of the glottis.

The primary symptoms generally yield to local treatment, including abstinence of food for thirty-six hours; and the patient may be quite comfortable. Signs of mercurial stomatitis usually develop in twenty-four hours, but are not especially severe.

Somewhat later, after absorption, the large intestines and kidneys are chiefly involved. Within two or three days, the urine shows albumin and casts, and becomes scanty. Anuria may be complete, with death within a week, without uremic convulsions. If the nephritis is not extreme, a membranous colitis develops, with dysentery and tenesmus, ulcerations and hemorrhages and hepatic degenerations. These phenomena may only appear, and prove fatal, several weeks after taking the poison (Lambert and Patterson, I9I5). 
The circulation is affected only in the severest cases, with a fall of blood pressure, probably mainly cardiac (v. Mering, I880), perhaps with some vasomotor involvement. The poisoning then ends with feeble pulse, insensibility of the skin, coma and collapse.

The course is therefore variable; death may occur in a few hours, but is generally postponed for several days.

In case of recovery, the phenomena of subacute poisoning set in: nephritis, stomatitis and colitis, as described.

If the poison has been taken otherwise than by mouth, the symptoms, except the local ones, are strictly similar.

Postmortem Changes.-The mucous membranes are of an ashy color, congested and corroded. The kidneys show acute nephritis, often with calcification of the epithelium. The lesions in the colon are indistinguishable from those of dysentery: coagulation necrosis with hemorrhages and black ulcerations. In experimental poisoning, degenerative changes are found in the kidneys, much less in the liver (Morel, Mouriguand and Policard, I9I 2).

The viscosity of the blood (not of the serum) is doubled in mercurial, lead, and phosphorus poisoning (Farmachidis, I9I2). A neutrophile hypo-leucocytosis is a constant phenomenon (Donzello, I915).

Toxic Doses. - These may be stated as follows:

Mercuric chlorid, for children 0.18 to $0.6 \mathrm{Gm}$.; for adults $0.8 \mathrm{Gm}$. Recovery, however, has occurred, under treatment, after $2.5 \mathrm{Gm}$. Opium is said to lessen the toxicity.

Mercuric cyanid, which produces the same phenomena, has caused severe poisoning with $0.12 \mathrm{Gm}$. and death with 0.6 to $1.2 \mathrm{Gm}$. A case is described by Gettler, I9I6.

Catomel.-Even large doses ordinarily produce only diarrhea; exceptionally, more severe and even fatal effects are recorded; for children with $0.4 \mathrm{Gm}$., for adults with 2 to $3 \mathrm{Gm}$.

Metallic mercury, which was formerly given in ileus in doses of roo to $500 \mathrm{Gm}$., usually passes off by the feces with very little effect; sometimes there is diarrhea and stomatitis; and exceptionally, severe and even fatal poisoning.

Relative Toxicity of Different Compounds. -This is under dispute. It is generally believed that the toxicity is proportional to the ionization, and therefore greatest with the chlorid, succinimid, etc. The aromatic compounds are much less toxic (Abelin, rgr2). With mercurial dyes, the toxicity, and the efficiency against spirillosis, are not proportional to the mercury content or dissociation (Klages and Schreiber, I9I4). Schamberg, Kolmer and Raiziss, I9I5, state that the toxicity for hypodermic and intravenous injection depends practically on the mercury contact; and is essentially alike for inorganic and the different types of organic compounds.

Treatment of Acute Poisoning.-The ordinary antidotes are raw egg, milk or flour, which act as precipitants and demulcents. The resulting compounds must be promptly removed by emetics or lavage to prevent absorption and systemic poisoning. The success is not very encouraging

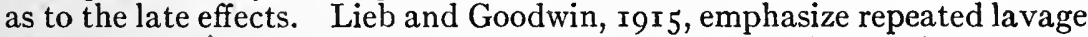
for removing mercury excreted into the stomach. Lambert and Patterson, I9I5, combine this with continuous rectal irrigation.

Fantus, 1916, emphasizes that albumin will only be of value if it is given immediately after the poison. He recommends sodium hypophosphite, I $\mathrm{Gm}$.; water, 1o c.c. and hydrogen peroxid, 5 c.c., per o.I Gm., of mercuric chlorid. This should be followed by lavage with the diluted reagent.

The possible value of hydrogen sulphid has been investigated by Sabbatani, rgo7. Sodium phosphite has also been suggested to reduce mercuric chlorid to calomel. Hall, I9I4, proposed potassium iodid and quinin (a reversal of Meyer's reagent). This might have some effect in the stomach, but naturally not in the blood or tissues (Barbour, I9I5).

Subacute Poisoning.-This (as seen in sequence to acute poisoning or in the ordinary medicinal use) presents especially the phenomena of nephritis, stomatitis and colitis; more rarely skin eruptions. The stomach and small intestines usually escape. 
Mercurial Vephritis.-Some degree of albuminuria is common even in the ordinary therapeutic use of mercurials. It is attributed to the excretion of the mercury. After severe intoxication it may last for years. It is mainly interstitial; although the glomeruli, and with large doses the epithelium, may be involved. Later the kidney becomes cirrhotic. The changes in clinical subacute nephritis are further described by Foster, r9 15. The acute nephritis is chiefly tubular, with polyuria (Schlayer and Hedinger, 1907), and often renal glycosuria (Franck, r9r3). The lumen of the tubules, and sometimes the epithelium, may contain crystals of calcium carbonate (Saikowsky, r866), the origin of which is not explained.

Stomatitis.-This is important as the earliest symptom of excessive therapeutic use or of chronic poisoning. It occurs about as readily when the mercury is administered otherwise than by mouth. It begins with fetor, then metallic taste, soreness of the gums and some salivation (ptyalism). This is as far as the therapeutic use should go. If it is pushed further, the teeth loosen, the edges of the gums blacken; and they, and later the tongue, become swollen and ulcerated. The irritation and infection cause very severe salivation and progressive exhaustion. In very advanced poisoning there may be loss of the teeth and necrosis of the jaw.

The susceptibility to stomatitis varies greatly and is markedly influenced by the hygienic condition of the mouth and teeth.

This conforms to Almkvist's theory that the stomatitis, as well as the colitis, are due to hydrogen sulphid produced by putrefaction, which precipitates mercuric sulphid in the capillary endothelium of these situations. The salivation is perhaps partly due to direct irritation of the salivary mechanism by the mercury. Giani, 1913, reports degenerative histologic changes of the parotid gland in mercurial poisoning.

Treatment of Stomatitis. - The stomatitis is most effectively prevented and treated by hygiene and care of the teeth. The mouth should be in the best possible condition before mercury is started. Hydrogen peroxid or potássium chlorate (a tablespoonful of the saturated solution to a glass of water) should be used as a mouth wash several times a day. The use of tobacco is said to be injurious.

Cause of the Stomatitis and Colitis.-These are generally attributed to irritation during the excretion of the metal. Almkvist, 1906 and I9II, however, points out that they have no relation to the excretion but depend upon the presence of hydrogen sulphid and the precipitation of mercuric sulphid which can be shown chemically in the capillary endothelium of these situations. Similar deposits and irritation can be produced experimentally elsewhere by exposure to hydrogen sulphid. He therefore explains the process as due to bacterial action causing injury and desquamation of the epithelium, and the production and absorption of $\mathrm{H}_{2} \mathrm{~S}$, which precipitates the mercury in the capillaries from where it is deposited in the endothelium. Here it acts as an irritant and disturbs the function and nutrition of the vessels, leading to necrosis. The explanation is largely hypothetical.

Bismuth also gives similar precipitates but they are much less toxic. Lead and copper are not so easily precipitated.

Metabolism. - The effects are imperfectly known but appear to resemble those of arsenic, lead and phosphorus. Small doses, therapeutically and in animals, of ten cause considerable increase of weight (fat). The red corpuscles are also increased (Schlesinger, I884). In chronic cachexia they are diminished. In acute poisoning there is often glycosuria and disappearance of glycogen. On the other hand, mercury is sometimes beneficial in diabeles, presumably of syphilitic origin.

Chronic Mercury Poisoning.-If this takes a slow course, there are, in addition to the subacute symptoms, nervous and nutritional phenomena. 
The most common are tremor; erethismus (psychic irritabiiity and restlessness), and other psychoses; cachexia with anemia, malnutrition and muscular atrophy; bone changes (decalcification) and more rarely neurites or pareses.

Treatment of Chronic Poisoning.-Prophylaxis is of the first importance; it is doubtful whether treatment materially influences the course after the appearance of symptoms. Potassium iodid and hot baths are generally tried, with the intention of hastening elimination.

\section{PECULIARITIES OF CALOMEL}

The mercurous or "mild" chlorid, because of its insolubility in water and limited solubility in the alimentary canal, has very much weaker actions. It is therefore suitable for a cathartic and intestinal antiseptic. In cardiac dropsies it is often markedly diuretic. It may be employed as an antisyphilitic.

Solubility of Calomel in the Alimentary Canal.-Calomel is not dissolved in the gastric juice and none is absorbed from the stomach or rectum (Nemser, I906); but it is attacked in the intestines, especially in the ileum, by the alkaline pancreatic and intestinal juice. These dissociate it into mercury (shown by immediate darkening), and yellow mercuric oxid; the latter dissolves slowly and incompletely in the alkaline intestinal fluid (T. W. Schaefer, rgro).

Interaction of Calomel and Salt.-Mialhe attributed the solution of calomel to interaction with sodium chlorid, forming mercury and mercuric chlorid, and complex double salts. Similar reactions were claimed by Joly to occur with hydrochloric and citric acids, alkalies, carbonates and sugar. These reactions do occur in pure solutions, especially at high temperatures and with concentrated solutions; and to some extent even under conditions resembling those of the body. The amount dissolved would be very small (at most I to $4 \mathrm{mg}$. per $0.5 \mathrm{Gm}$. of Calomel). However, mercuric chlorid could not exist as such in the alkaline intestine, and the chlorid can therefore not be made responsible for the solution of calomel (as was suggested, $f . i$., by Carracido, I 906 ).

Iodids, bromids, sulfocyanids, thiosulphates, etc., dissolve larger quantities of calomel.

\section{CATHARTIC ACTION OF CALOMEL}

This may be referred to the irritant effects of the small quantities of mercury which are progressively dissolved. The irritation is generally so mild that it may be employed even in enteritis. Some patients, however, are hypersusceptible. Ordinarily it produces dark green, semisolid stools, with mild colic, in about ten to twelve hours. Laxative doses diminish intestinal putrefaction (diminished indoxyl in urine). Larger doses probably favor the bacteria by lessening the intestinal resistance.

Dosage.- This is relatively unimportant, because any excess is generally expelled before it is dissolved. It is customary to give either a single dose of 0.125 to $0.5 \mathrm{Gm}$. ( 2 to $7 \mathrm{gr}$.); or fractional doses of $15 \mathrm{mg}$. ( $1 / 4 \mathrm{gr}$.) every hour until $0 . \mathrm{I}_{5} \mathrm{Gm}$. ( $2 \frac{1}{2} \mathrm{gr}$.) have been taken. The divided doses insure better solution. Formerly much larger doses were used but they are superfluous; and while usually harmless, they may become dangerous should conditions become exceptionally favorable to absorption.

Calomel is not suited for long-continued use on account of systemic actions. These may also be produced by large single doses, if they are not expelled in the usual time. It is therefore advisable to follow the calomel in six hours by a saline; or it may be mixed with Resin of Podophyllum (5 mg.; 1/12 gr.).

Calomel should not be prescribed with iodids, bromids, alkalies or organic substances. 
Other mercurial salts are sometimes used as cathartics but without atdrantage.

Bile-flow.--This is not affected by calomel. The older views of cholagogue action were based merely on the color of the stools, which is due partly to mercury sulphid and partly to lessened decomposition of the ordinary bile.

The absence of cholagogue action has been shown with biliary fistula experiments by numerous investigators (Okada, 1915). Doyon and Dufourt, 1897, also showed that the green calomel stools occur even if the bile is prevented from reaching the intestines. The rôle of the antiseptic effect is shown by incubating the bile with calomel, when the green color persists much longer than with pure bile. Jaksch, however, claims that calomel stools do not contain biliverdin.

Mechanism of the Cathartic Action.- This is due to a local effect, but there are differences of opinion as to its mechanism. It is generally at tributed simply to irritation, and $\mathrm{X}$-ray work shows that peristalsis is increased in the small and large intestines (Meyer-Betz and Gebhardt, 1912); but Fleckseder, rg12, believes that calomel also prevents the absorption of water in the small intestine, directly and by quickening the passage of the contents. The catharsis is prevented by atropin or opium. It is not influenced by the presence or absence of bile (Valeri, r909).

Calomel probably acts mainly on the duodenum, and much less on the colon. Small doses may therefore only sweep the duodenal contents into the colon, without producing a stool. Large doses produce griping and tenesmus.

Calomel Diuresis.-Adequate doses are sometimes remarkably diuretic in cardiac dropsies. The effect is uncertain and does not usually occur in health or in other dropsies. The mechanism is not definitely explained. It is a heroic remedy, requiring large doses and generally producing salivation and diarrhea. Caution should be used in nephritic or debilitated patients.

The diuretic effect of calomel in cardiac dropsy is said to have been well known to the physicians in the latter half of the eighteenth century. It was used, f.i., by Alex. Munro. However, it fell into neglect, and was practically rediscovered by Jendrássik in 1886. He found it effective mainly in cardiac dropsies, in which it produced results far greater than could be obtained with digitalis or caffein. The urine was often increased to 7 or 8 L. a day. The absolute amount of urea and chlorids was also greatly increased. To obtain the best effects, $0.2 \mathrm{Gm}$. was given from four to five times a day, until a slight mercurial stomatitis was produced. If this did not prove effective from the start, the remedy was discontinued; it also seems wise to intermit it occasionally. The bowels may be regulated by opium. Jendrássik remarks that the calomel appears relatively or quite ineffective when the heart disease is uncomplicated by dropsies; it was also ineffective in pleuritic exudates, in nephritic effusions, and in healthy individuals. This report gave rise to extensive trials of the drug. The results of these were summarized by Jendrássik in $189 \mathrm{r}$, together with further observations and experiments of his own. His previous conclusions concerning the best method of administration, and the usefulness of calomel in cardiac dropsies seem to have been generally confirmed, as also the relative insufficiencies in non-dropsical heart disease, in pleuritic exudates and in normal individuals. It seemed to be slightly diuretic in the latter but the action can never compare with that seen in cardiac dropsies. It was often found effective in hepatic ascites, but failed frequently. It gave good results in some cases of nephritic edema, but in most instances gave no result; it seemed impossible to predict what it would do.

The theoretical objections to the use of so powerful a renal irritant as mercury in nephritis, were early emphasized, especially by Cohn, I887, and supported by some clinical observations. It was claimed that there is a very marked tendency to mercurial symptoms, and that the nephritis is of ten made worse. The'greater number of observers, however, hold the opposite view, viz., that calomel does not render the nephritis worse, whether it has a diuretic action or not. This is defended by Jendrássik, and by Heuck, 1889. Schild, 1892 , reported three cases, in which he claims that a diuretic effect was obtained, together with a lessened per cent. of albumin; the daily output of protein being unchanged.

Briefly, it seems certain that the doses of calomel which are advocated have never 
produced albuminuria in normal individuals, but the question of their effect on an existing nephritis is not sufficiently investigated to admit of a decisive answer. Great conservatism in its employment is therefore indicated.

The mechanism of this calomel diuresis is not understood, notwithstanding the investigation of Jendrássik, of Cohnstein, 1892, and of Vejux-Tyrode and Nelson, rg03. Fleckseder, 19 I 2 , finds that all mercury compounds, by all methods of administration, produce a diuretic effect in rabbits. He believes that mercury lessens absorption of food-water from the small intestines; the water would therefore reach the large intestines in excessive quantity; and when absorbed from this place would act as a diuretic. The explanation is not altogether satisfactory; f.i., it does not explain the absorption of exudates.

Hydremia in Calomel Dinresis.-Fleckseder found the blood of rabbits hydremic. This would speak against the diuresis being of renal origin. In man, however, the results appear confused; in dropsies, hydremia occurred; but in healthy individuals the blood became concentrated by the diarrhea. Loria, I91 2 , reports the viscosity increased independently of diuresis. The diuretic action of mercuric chlorid has been studied in rabbits by Ferron, I 12 .

Influence of Other Drugs. - The diuresis is surpressed by atropin; this would favor Fleckseder's explanation; but opium which also lessens peristalsis increased the diuresis. Schargorodsky, IgII, found it surpressed by ether or morphin. Excessive doses of mercury also produce anuria.

The constituents of the urine after calomel has been studied by E. Frey, Igo6.

\section{MERCURY IN SYPHILIS}

The antisyphilitic effect of mercury was discovered about I500, very soon after the discovery of the disease; according to Rhaenodaeus, it was first used by one Jacobus Carpensis, "whose use was so successful that he presently became rich thereby." Till the present century, little further advance was made in its employment, which remained entirely empirical. It was not known whether it acts symptomatically, or as a general tonic; or whether it really affects the causative agent directly; whether it merely relieves or actually cures. Its affects had to be judged entirely by the symptoms, which in syphilis are extremely subject to spontaneous variations. The proper dose, as well as the time and continuance of its administration were therefore regulated only by caprice and prejudice. This empiricism was revolutionized by the three important discoveries: of the Treponema pallida as the causative agent; of its transmissibility to monkeys and rabbits; and of its more definite diagnosis by the Wassermann reaction. Through these discoveries, it was possible to establish that mercury acts directly on the Treponemas, and not merely on the symptoms; that like all chemo-therapeutic agents, it must be used as early as possible, energetically and persistently; and that if so used, it can effect a perfect cure.

Antiluetic Agents. - Experiments on monkeys (Neisser, etc.) have shown that mercury eradicates the disease in the presymptomatic, in the active, and in the latent stages. Organic arsenic is similarly successful, and acts more rapidly. Iodids also have some effect, but are much weaker. Their clinical efficiency in tertiary syphilis must be by some other mechanism.

Importance of Early Treatment.-It was formerly taught that mercury should be postponed until the onset of the secondary symptoms, so as not to interfere with the clinical diagnosis. This consideration became superfluous with the introduction of the laboratory methods. It is now recognized that the chances of complete success are the greater, the earlier the treatment. This should be started as soon as the diagnosis is made. If the administration is delayed, or if it is not sufficiently energetic and relapses occur, treatment becomes much more difficult and requires larger 
doses. In syphilitic aortitis, mercury rarely gives more than temporary relief (Longcope, I9I4).

Abortive Treatment.-The site of suspected infection should be disinfecterl and dressed with an ointment containing 33 per cent. of calomel or 0.3 per cent. of sublimate ("Neisser-Siebert Ointment"). This should be followed by systemic treatment.

Systematic Treatment.-Formerly mercury was administered expectantly, intermittently or continuously. It is now recognized that the treatment should not depend on the symptoms, and the expectant treatment is therefore of no use. The intermittent treatment (Fournier's) was based on the premise that the syphilitic virus acquires an immunity to the continued administration of mercury, and that its susceptibility is reëstablished by occasional intermittence of the treatment. There is no proof for this assumption; but the method seems to give the best clinical results (perhaps because it permits a higher temporary concentration of mercury than could be attained with continued administration; in chemotherapy, the concentration generally counts for more than the time of exposure). The interrupted method also lends itself better to combination with Salvarsan. The two metals probably attack the parasite in a different way; at least, they seem more effective together than separately.

In the intermittent method, the mercury is given to slight ptyalism, in courses of five to six weeks' duration, with intermissions of one to six months, for at least one and a half years; or longer if the disease is not eradicated.

Whatever method is used, it should be aimed to introduce the maximum of mercury that the patient can tolerate; something like $2 \mathrm{Gm}$. of $\mathrm{Hg}$ during the first year, and I $\mathrm{Gm}$. in the two succeeding years. The relative failure of the older methods may be attributed to insufficient dosage.

If metasyphilitic lesions have set in, mercury is of little use, but its early energetic use is the best preventative of these lesions.

Oral Administration. - This is probably the most congenial method to the patient; but it is difficult to introduce sufficient mercury on account of the local action on the digestive canal. The patient is also under. less perfect control. It has therefore been largely superseded by the intramuscular method, but may be preferred under special conditions. It is a continuous method; beginning with a small dose, this is increased daily by about ro per cent., to tenderness of the gums. The dose is then reduced to one-third or one-half and continued at this point till the end of the treatment. The usual preparations are, in order of preference:

Hydrargyri Iodidum Flavum ("Proto-iodid")... . 0.or to 0.5 Gm. (1/6 to I gr.). Hydrargyrum cum Creta........................ 05 to $0.2 \quad \mathrm{Gm}$. (I to $3 \mathrm{gr}$.).

Hydrargyri Chloridum Corrosivum.............0.005 to $0.015 \mathrm{Gm}$. (1/12 to $1 / 4 \mathrm{gr}$.).

(These doses three times daily).

Inunction.-Although effective quantities of mercury can be introduced by this method, the dosage is always uncertain. Inunction, moreover, is time-consuming, dirty and disagreeable, and difficult to keep secret. It has a special field in the secondary cutaneous lesions, where it must have a local parasiticide effect.

Inunction is performed by rubbing about $2 \mathrm{Gm}$. of Ung. Hydrargyri into the skin, until it is entirely absorbed. This is repeated every night on a different skin-area (to prevent local irritation, especially of the hair follicles), making the tour of the body in six nights. The seventh night 
a hot bath is substituted, and so on. The course lasts for four to six weeks. Inhalations, fumigations and plasters are obsolete methods of introducing mercury.

Intramuscular Injections.- These permit the introduction of the maximum of mercury, with relatively slight gastro-intestinal irritation (although it may be doubted whether the stomatitis, colitis and nephritis are less severe than with oral administration). One of the chief advantages of injections is, that they keep the patient under more effective observation, and permit a better control of the quantity of mercury introduced. The disadvantages are, that the absorption especially of the insoluble preparations is variable; and that the injections are more or less painful, of ten with destructive lesions in the muscles (Robertson, I9I3). These are unimportant practically. With the soluble preparations the pain lasts some hours or longer; with the insoluble several days. Of these, calomel is the most, Oleum cinereum the least, painful. The pain may be lessened by injecting I c.c. of 2 per cent. novocain from the same syringe. The injections are made deep into the gluteal muscles.

The preparations used for intramuscular injections fall into two classes; according to whether they are absorbed rapidly or slowly. These have distinctive uses.

The Easily Absorbable Injections.-These include all the soluble salts; and the salicylate, which is insoluble in water but is dissolved by sodium chlorid or carbonate.

The soluble preparations act more promptly, the dosage is under better control, and there is less possibility for cumulative action; the effects are correspondingly less persistent, and the injections must be repeated at more frequent intervals-every day to every third day. They are usually employed in I per cent. solution. Mercuric chlorid is quite irritant. The biniodid, dissolved in 3 per cent. NaI is a good form. The cyanid, oxycyanid, oxybenzoate and succinimid are also relatively nonirritant. The average daily dose is about I c.c. of I per cent. ( $=0.0 \mathrm{I}$ Gm., 1/6 gr.), in courses of five to six weeks' duration. The treatment may be intensified by beginning with 2 c.c., increasing slowly to 3 or 4 c.c. $(0.03$ to $0.04 \mathrm{Gm}$.). or until mild ptyalism occurs, when the dosage is reduced so as to keep the patient just short of salivation.

Mercuric salicylate is employed as to per cent. suspension in oil or liquid petrolatum in the dosage of I c.c. It causes very little irritation, since the mercury is not ionized (not precipitated by $\mathrm{H}_{2} \mathrm{~S}$ ). Asurol is a soluble double salt of mercury oxysalicylate and sodium amido-oxy-isobutyrate. It is injected as 5 per cent. solution, 2 c.c. (o.I Gm.) two or three times a week.

The Insoluble Injections. - These are used with the purpose of establishing "depots" from which the mercury may be gradually absorbed, so that injections need only be made at weekly intervals. Unfortunately the absorption of these depots is not controllable: Sometimes "nodes" of unabsorbed mercury remain indefinitely; and again, their absorption may occur suddenly, and give rise to severe poisoning. Schamberg, Kolmer and Raiziss, I 9 I $_{5}$, claim that only about I per cent. of the injected mercury is absorbed per day, and that $5^{\circ}$ per cent. may remain unabsorbed after six or seven weeks.

The convenience of the insoluble injections, however, seems to offset these occasional accidents, which may also be somewhat discounted by 
judgment. The method is at present one of the most important. The chief preparations are:

Calcomel, ro per cent. suspended in oil, I c.c. twice weekly; this is considered one of the most active, but a painful method.

Oleum Cinereum (gray oil), a 40 per cent. suspension of metallic mercury in oil. 'This is the most slowly absorbed of the mercurials. The injections ( $1 / 4$ c.c.) are therefore spaced a week apart, and after five injections an intermission of five or six weeks is made to discount capricious absorption; five further weekly injections are then given. (Semiweekly injections of $1 / 8$ c.c. may be substituted for the weekly injections.)

Absorption from Intramuscular Injections.-The mercury, in whatever form it is introduced, acts on the proteins to form albuminates of mercury. These are strongly chemotactic, and attract leucocytes. Some of these are killed, but others take up the mercury, especially in their nuclei, and distribute it through the body; its final fate being the same, as with other methods of administration (Conti and Zuicola, 1906; Lombardo, IQI 2). The quantitative excretion, however, is very capricious. There is still much uncertainty as to the relative absorbability of the different preparations. Wallfisch, 1912, finds that the solubility of the preparations is of subordinateimportance; but that all inorganic salts are absorbed and excreted much less readily than the organic salts.

Quantity of Mercury Introduced by the Different Preparations.-This may be seen from the following table (adipted from Neisser, rgrI):

\begin{tabular}{|c|c|c|c|c|c|c|}
\hline & $\begin{array}{l}\text { Per cent. } \\
\text { used }\end{array}$ & $\begin{array}{c}\text { Per } \\
\text { cent. } \\
\text { of } \mathrm{Hg} .\end{array}$ & $\begin{array}{l}\text { Usual } \\
\text { single } \\
\text { dose, } \\
\text { c.c. }\end{array}$ & $\begin{array}{l}\mathrm{Hg} . \\
\text { con- } \\
\text { tent } \\
\text { (mg.) }\end{array}$ & $\begin{array}{l}\text { Number } \\
\text { injections } \\
\text { in six- } \\
\text { week } \\
\text { course }\end{array}$ & $\begin{array}{l}\text { Grams } \\
\text { Hg. in } \\
\text { six-week } \\
\text { course }\end{array}$ \\
\hline Oxybenzoate. & I & 44 & $\mathbf{I}$ & $4 \cdot 35$ & 42 & 0.19 \\
\hline Cyanid......... & I & 79 & I & 7.9 & 42 & 0.33 \\
\hline Sublimate............... & $\mathbf{r}$ & 74 & I & $7 \cdot 4$ & 42 & $0.3 \mathrm{I}$ \\
\hline Salicylate........... & ro & 59 & I & 59.0 & II & 0.65 \\
\hline Calomel.................. & ro & 85 & $\mathbf{I}$ & 85.0 & II & 0.94 \\
\hline Asurol..... & 5 & 40 & 2 & 40.0 & $I_{3}$ & $0.6 \mathrm{I}$ \\
\hline Ol. Cinereum. . & 40 & 40 & $1 / 4$ & 140.0 & 10 & $\mathrm{I} .40$ \\
\hline
\end{tabular}

Intravenous Injections. - These were recommended by Bacelli, 1892 , and are used in several clinics. However, they present some danger of intoxication and embolism, at least in inexperienced hands; and a large part of the mercury is excreted so promptly that it probably has little effect. Mercuric chlorid, iodid, or cyanid is used; in I per cent. solution, I to 3 c.c. daily or every second day, injected into a superficial arm vein, with glass syringe and platinum-iridium needle (Crume, 1908). Similar injections have been tried in puerperal infections (Barsony, I 909 ).

Spinal Injections of Mercurialized Serum.-This was introduced by Byrnes, I914, and is endorsed by Ireland and Wilson, I91 5 , against cerebro-spinal syphilis, as being about equally successful and safe as the Swift-Ellis serum. It has the advantage that the serum may be kept on hand. It is prepared by adding $x$ c.c. of 0.13 per cent. mercuric chlorid to $\mathrm{I} 2 \mathrm{c.c}$. of normal human or horse serum, heating to $56^{\circ} \mathrm{C}$. for one-half hour. This gives a clear solution. This dose (double would be safe) is injected by gravity. at body temperature, after withdrawing cerebro-spinal fluid till its pressure is $30 \mathrm{~mm}$. E. L. Hunt, r 916 , also reports encouraging results. Hall, Culbertson and Slaght, 1916, describe the reactions following the intraspinal injection. They are usually not serious.

Inhalation of Mercury or Calomel Vapor.-This has been abandoned, mainly because of the uncertain dosage. It also produces marked pulmonary hyperemia (Ricker and Hesse, 19 4 ).

Organic Mercury Compounds.-On intravenous injection these produce at first characteristic acute "molecular" actions, distinct from those of mercury; and which are absent when they are absorbed more gradually. The mercury effects set in as the mercury is split off; the intensity of action depending on the readiness of this liberation, and on the rapidity with which the molecular form is excreted (F. Mueller, Schoeller and Schraut, rgrx). 
Combined Arsenic and Mercury Treatment.--This is generally considered to give the best results. Its principle is to bring the patient rapidly under the influence of the quickly acting Salvarsan, intravenously; then of the more absorbable mercurials, and then to keep him mercurialized with the less absorbable compounds; repeating the Salvarsan at intervals.

Salvarsan is given on the first day and repeated at the end of a week and again in a month. Intramuscular injections of absorbable mercury (iodid, salicylate or Asurol) are made during the first two weeks; insoluble mercury (calomel or gray oil) from the third to the sixth week.

Szily and Friedenthal, 1915 , describe promising results from intravenous or muscular injections of a mercury-arsenic-iodid combination.

Continuance of Treatment.-When the above treatment is used (or when it is modified by the omission of the Salvarsan, or by using a single mercurial), a Wassermann test may be made at the end of the first course. Even if this is negative, the patient can not be considered cured, for relapse would be the rule. An intermission of six weeks should be given, then another six-weeks' course of mercury; preceded, if desired, by a Salvarsan injection. After a two or three months' intermission, a Wassermann test may be made. If this is positive, the course must be repeated; if negative, the question of cure may be considered. Generally, however, it will be safer to repeat the courses at lengthening intervals, during two years.

Contraindications of Mercury.-Caution should be exercised in weak or anemic individuals, in albuminuria, during digestive derangements, and in diseases of the mouth or teeth. Pregnancy is not considered a contraindication. It is safer to begin treatment with small doses, since some patients are hypersusceptible.

Vegetable Antisyphilitics.- The prejudice against mercury has led to the introduction of a number of vegetable "alteratives"-Echinacea, Phytolacca, Iris, Sarsaparilla, etc., but their uselessness is attested even by modern eclectics (Hilton, rgog).

\section{MERCURY IN TUBERCULOSIS}

This was advised by B. L. Wright, but Hartz, 19ro, found the ultimate results detrimental and never beneficial.

\section{$\therefore$ PREPARATIONS-METALLIC MERCURY}

Hydrargyrum (Hydrarg.), U.S.P., B.P.; Mercury (Quicksilver); Hg.-A fluid, silver-white heavy metal. It is used only in finely divided form, as in the following preparations:

${ }^{*}$ Hydrargyrum cum Creta (Hydrarg. c. Cret.), U.S.P., B.P.; Mercury with Chalk (Gray Powder).-Contains 38 per cent. of Hg., U.S.P.; 33 per cent., B.P., with honey and chalk. A light gray, rather damp, sweetish powder. Used especially to secure the constitutional effect of mercury in infantile syphilis. Dose, $0.25 \mathrm{Gm}$., $4 \mathrm{gr}$., U.S.P., equivalent to about $0.1 \mathrm{Gm}$. of metallic mercury; 0.06 to $0.3 \mathrm{Gm}$., I to $5 \mathrm{gr}$., B.P.; dispensed in powders.

Emp. Hydrarg., B.P. -33 per cent. of $\mathrm{Hg}$. in lead plaster base. Used against glandular enlargements, etc.

Lin Hydrarg., B.P.-50 per cent. of Ung. Hydrarg., with ammonia, in Camphor Liniment.

*Massa Hydrargyri (Mass. Hydrarg.), U.S.P., Pil. Hydrarg., B.P. (Blue Mass, Blue Pill). -33 per cent. of Hg. Dose, 0.25 Gm., $4 \mathrm{gr}$., U.S.P.; 0.25 to $0.5 \mathrm{Gm}$., 4 to $8 \mathrm{gr}$., B.P. Used similarly to Calomel.

*Unguentum Hydrargyri (Ung. Hydrarg.), U.S.P.— 50 per cent. of Hg., in Suet and Lard. Used for inunction. 
* ('ng. II ydrarg. D)il., L.S.P.; Ung. IIydrarg, B.P.; (Blue Ointment).-30 per cent. of 11 . . in Suet and Lard (with petrolatum, U.S.P.). Used especially against cutaneous parasites.

l'ng. II virurg. Co., B.P. (Scott's Dressing).-r2 per cent. of Hg. and of Camphor. Lsed in synovitis, etc.

* Oleum Cinereum, B.P.C. (Gray Oil).-40 per cent.

PREPARATIONS-INSOLUBLE MERCUROUS COMPOUNDS

*II ydrargyri Chloridum Mite (Hydrarg. Chlor. Mit.), U.S.P.; Hydrargyri Subchloridum (Hydrarg. Subchlor.), B.P.; Mild, Mercurous Chlorid (Calomel, Protochlorid of Nercury); $\mathrm{HgCl}$ - - Fine white powder, odorless and tasteless. Practically insol. in water or alc. Incompatible with alkalies; oxidizing acids; iodids and bromids; and with a mixture of sodium bicarbonate and antipyrin (J.A.M.A., 56:287 (I9II). Dose, as laxative, $0.15 \mathrm{Gm}$., $21 / 2 \mathrm{gr}$.; as alterative, $15 \mathrm{mg} ., 1 / 4 \mathrm{gr}$, U.S.P.; 0.03 to $0.3 \mathrm{Gm}$., $1 / 2$ to 5 gr., B.P.; as powder or tablets. Maximum dose, $0.6 \mathrm{Gm}$., Io gr.

Pil. Il ydrarg. Subchlor. Co., B.P. (Plummer's Pill).-22 per cent. of Hydrarg. Subchlor.; 22 per cent. of Antim. Sulphur; 43 per cent. of Guaiac Res. Dcse, 0.25 to 0.5 Gm., t to 8 gr., B.P.

Ung. IIydrarg. Subchlor., B.P. (Calomel Ointment).-2o per cent., in Benz. Lard.

*Hydrargyri Iodidum Flavum (Hydrarg. Iod. Flav.), U.S.P.; Yellow Mercurous Iodid (Mercurous Iodid; Protoiodid of Mercury); Hgl.-Bright, yellow amorphous powder, without odor or taste; almost insol. in water, wholly insol. in alc. On exposure to light, it darkens by decomposition into mercuric iodid and metallic mercury. Incompatible with oxidizing agents, alkalies and carbonates, chlorid, bromids and iodids. Dose, ro mg., 1/6 gr., U.S.P.; maximum dose, $0.05 \mathrm{Gm}$., I gr. "Green Iodid" is an analogous salt prepared by a different method. It is said to be less potent.

Lotio Hydrargyri Nigra (Lot. Hydrarg. Nigr.), B.P.; Black Mercurial Lotion (Black Wash).-A suspension of mercurous oxid, obtained by mixing $0.685 \mathrm{Gm}$. of Calomel with 95 c.c. of Lime-water and 5 c.c. of Glycerin. Used on venereal sores and foul ulcers.

Hall, Culbertson and Slaght, 1916, describe the reactions following the intraspinal injection. 'They are usually not serious.

\section{PREPARATIONS-INSOLUBLE MERCURIC COMPOUNDS}

Hydrargyrum Ammonialum (Hydrarg. Ammon.), U.S.P., B.P.; Ammoniated Mercury (White Precipitate); $\mathrm{NH}_{2} \mathrm{HgCl}$.-White, amorphous, insoluble powder, obtained by precipitating $\mathrm{HgCl}_{2}$ with ammonia. Represents about 80 per cent. $\mathrm{Hg}$. Irritating and not used internally. Employed externally against eczema and parasitic skin diseases, especially pediculi, as dusting powder, but chjefly as:

*Ung. Hydrarg. Ammon., U.S.P., Io per cent. in woolfat and white petrolatum (U.S.P.).

${ }^{*}$ Ung. IIydrarg. Ammon., B.P. -5 per cent. in Benz. Lard.

* Hydrargyri Oxidum Flavum (Hydrarg. Oxid. Flav.), U.S.P., B.P.; Yellow Mercuric Oxid; HgO.-Light orange-yellow amorphous powder, turning darker on exposure to light. Odorless; somewhat metallic taste. Nearly insol. in water or alc. Incompatible with acids. Maximum dose, $0.03 \mathrm{Gm} ., 1 / 2 \mathrm{gr}$. Applied chiefly as:

*Ung IIydrarg. Oxid. Flav.; U.S.P., ro per cent.; B.P., 2 per cent.-It is used in ophthalmology against inflammations of the lids and conjunctiva, being diluted with petrolatum so as to contain I.ro to 2 per cent. of the oxid. "Pagenstecher's Ointment" contains 4 per cent.

Lotio II ydrargyri Flava (Lot. Hydrarg. Flav.), B.P.; Yellow Mercurial Lotion (Yellow Wash). - A suspension of mercuric oxid, obtained by mixing $0.46 \mathrm{HgCl}_{2}$, with 100 lime-water. Used as application to venereal sores.

II ydrargyri Oxidum Rubrum (Hydrarg. Oxid. Rubr.), U.S.P., B.P.; Red Mercuric Oxid (Red Precipitate), HgO.-Orange-red crystalline scales or powder. Because of its gritty character it can not be used on the eye, but its Ointment is employed in skin diseases, etc.

Ung. II ydrarg. Oxid. Rubr., B.P.- - ro per cent.

Oleatum Hydrargyri (Oleat. Hydrarg.), U.S.P.-Made by dissolving 25 per cent. of yellow mercuric oxid in oleic acid.

Hydrarg. Oleat., B.P.-2o per cent. of Mercuric Oxid in Oleic Acid.

Ung. Hydrarg. Oleat., B.P. -25 per cent. of the Oleat. 
PREPARATIONS-SOLUBLE HERCURIC COMPOUNDS

*Hydrargyri Chloridum Corrosivum (Hydrarg. Chlor. Corr.) U.S.P.; Hydrargyri Perchloridum (Hydrarg. Perchlor.), B.P.; Corrosive Mercuric Chlorid (Corrosive Sublimate; Bichlorid or Perchlorid of Mercury); $\mathrm{HgCl}_{2}$.- - Heavy, colorless crystals or white powder of acrid metallic taste. Slowly sol. in water ( $1: 13.5$, freely sol. in alc. ( $1: 3.8$ ), sol. in glyc. ( $\left.1: I_{2}\right)$ or eth. ( $\left.1: 22\right)$. Its solubility is increased by ammonium or sodium chlorid and tartaric or citric acid ("Sal Alembroth"-2 parts $\mathrm{HgCl}_{2}$, I part $\mathrm{NaCl}$ ). Incompatible with alkalies and carbonates; iodids; many alkaloids and other organic compounds and reducing agents. Dose, $3 \mathrm{mg}$., $1 / 20$ gr., U.S.P.; 2 to $4 \mathrm{mg}$., $1 / 32$ to $1 / 16$ gr., B.P. Maximum dose, $30 \mathrm{mg} ., 12 \mathrm{gr}$.

Liq. Hydrarg. Perchlor., B.P.- o.I per cent. Dose, 2 to 4 c.c., I'́2 to I dram, B.P.

*Toxitabella Hydrargyri Chloridi Corrosivi (Toxitab. Hydrarg. Chlor. Corr.) U.S.P.; Poison Tablets of Corrosive Mercuric Chlorid (Corrosive Sublimate Tablets; Bichlorid Tablets). - In order toguard against accidents, the U.S.P. directs these tablets to be of an angular, not disk shape, each having the word "Poison" and the skull and cross bones design distinctly stamped upon it. Each tablet contains not less than $0.45 \mathrm{Gm}$. nor more than $0.55 \mathrm{Gm}$. of $\mathrm{HgCl}_{2}$, and about $0.5 \mathrm{Gm}$. of $\mathrm{NaCl}$, and is to be colored blue, preferably with sodium indigotindisulphonate. Poison Tablets of Corrosive Mercuric Chlorid should be dispensed in securely stoppered glass containers on the exterior of which is placed a red label bearing the word "Poison" and a statement of the amount of corrosive chlorid in each tablet. A tablet to a pint of water makes an approximately I : $\mathrm{r}, 000$ solution.

Hydrarg. Cyan., N.N.R.; $\mathrm{Hg}(\mathrm{CN})_{2} .-$ Sol. in water (I : I 2.8) or alc. (I : I 5). Used Ilke Hydrarg. Chlor. Corr. Maximum dose, $20 \mathrm{mg} ., 1 / 3 \mathrm{gr}$.

Hydrarg. Oxycyan., N.N.R.; $\mathrm{Hg}(\mathrm{NH})_{2} \mathrm{HgO}$.- Sol. in water (I:17). Used like Hydrarg. Chlor. Corr.; said to be less irritating and does not corrode metals.

${ }^{*}$ H.ydrargyri Iodidum Rubrum (Hydrarg. Iod. Rubr.), U.S.P., B.P.; Red Mercuric Iodid (Biniodid); $\mathrm{HgI}_{2}$. - Scarlet red powder, odorless and tasteless. Nearly insol. in water, slightly sol. in alc. ( 1 : I I 5); rendered sol. in water by the presence of four-fifths its weight of KI or NaI. Incompatible with alkalies and carbonates and alkaloids. Dose, $3 \mathrm{mg}$., $1 / 20 \mathrm{gr}$., U.S.P.; 2 to $4 \mathrm{mg}$., $1 / 32$ to $1 / 16 \mathrm{gr}$., B.P. Maximum dose, $20 \mathrm{mg}$, $1 / 3 \mathrm{gr}$.

Ung. Hydrarg. Iod. Rubr., B.P.- 4 per cent.

${ }^{*}$ Potassium Mercuric Iodid, N.N.R.; $\mathrm{K}_{2} \mathrm{HgI}_{4} \cdot-$ This is usually made as extemporaneous solution, by dissolving $\mathrm{HgI}_{2}$ or $\mathrm{HgCl}_{2}$ in excess of $\mathrm{KI}(\mathrm{I}: 4)$. For intramuscular injection, the corresponding sodium salt is preferred $\left(\mathrm{HgI}_{2}=\mathrm{I} ; \mathrm{NaI}=3\right.$; Water $=100$ ).

(*Liq. Arsen. Et. Hydrarg. Iod.-See Index.)

Liq. Hydrarg. Nit. Acid., B.P.

Ung. Hydrarg. Nit., U.S.P., B.P. (Citrine Ointment) -7 per cent. of Mercury, dissolved in Nitric Acid, and diluted with Lard (and olive oil, B.P.). Used as antiseptic, irritant and caustic.

Ung. Hydrarg. Nit. Dil., B.P.-2o per cent. of the Ointment.

*Hydrargyri Salicylas (Hydrarg. Salic.), U.S.P.-A compound of mercury and salicylic acid containing about 57 per cent. $\mathrm{Hg}$. White, slightly yellowish or slightly pinkish powder; odorless and tasteless. Nearly insol. in water or alc.; sol. in alkalies, forming a double salt. Incompatible with alkalies. Dosc, $4 \mathrm{mg}$. 1/15 gr. U.S.P.; intramuscular, 0.6 c.c. of to per cent. suspension in liquid paraffin every four days. Maximum dose, $20 \mathrm{mg}$., $1 / 3 \mathrm{gr}$.

Hydrargyri Succinimidum (Hydrarg. Succinim.), N.N.R.-Sol. in water ( $1: 75$ ); slightly sol. in alc. $(\mathrm{I}: 300)$. Dose, ro to $15 \mathrm{mg}$., $1 / 6$ to $1 / 4 \mathrm{gr}$.; Intramuscular, 0.5 to I c.c. of 2.5 per cent., daily. Relatively non-irritant.

\section{LEAD}

General Statement.-The lead salts precipitate proteins and thereiore act as local astringents. Their acute toxicity, however, is low. They are fairly easily absorbed but very slowly excreted, so that they tend to produce cumulative effects and chronic poisoning. This is a common disease with characteristic symptoms: Nutritional and structural changes (anemia, etc.); paroxysmal colic (stimulation of the nervous mechanism 
of the intestines); "drop-wrist" and other muscular paralyses (direct depression of the muscle); central motor stimulations (tremors, "arthralgia"); psychic changes ("encephalopathia"); etc. On withdrawal, these phenomena generally recede; but the patient is apt to remain in bad health. Prophylaxis is more important than treatment.

Astringent Actions.-Lead acetate and subacetate ("lead water") produce more astringent and less irritant effects than other soluble metallic salts. They are effective in diarrhea and catarrhal inflammations; but they are so liable to produce chronic poisoning that they should never be used internally nor on open surfaces; and even on the skin their use should not be long continued. "Lead and opium wash" is used in superficial contusions. Saturated alcoholic solution of lead acetate is especially effective in Rhus ("Ivy") poisoning. Emplastrum Plumbi"Diachylon plaster" - acts as a mild irritant.

\section{ACUTE LEAD POISONING}

This is a rather rare intoxication, generally produced by the acetate or subacetate, sometimes by the carbonate (white lead). The phenomena are at first local and not immediately dangerous, but they are generally followed by chronic poisoning.

The best treatment is the administration of some soluble sulphate (magnesium or sodium), which may save animals from several times the fatal dose (Dauwe, 1907). Tannin, milk and eggs may also be used. The stomach must then be washed and a cathartic administered. Colic is treated symptomatically, as in chronic poisoning.

Phenomena of Acute Poisoning.- There is first a sweet, then disagreeably metallic and astringent taste. In a few minutes, sometimes in several hours, there is vomiting (which is often milky with lead chlorid), burning, abdominal pain and thirst; then obstinate constipation or diarrhea with black stools (lead sulphid).

Severer cases show collapse with slow pulse; or muscular cramps and colic. Fatal cases pass into coma with or without convulsions. The fatal dose lies above $50 \mathrm{Gm}$. Postmortem, there is little characteristic, except the local astringent changes.

Most patients recover, apparently completely, in two or three days; but after a few weeks they show the phenomena of chronic poisoning (also animals, Dauwe, Igo7). Application to the surface (lead wash, Diachylon plaster) has rarely if ever caused acute poisoning (Paessler, I894). Diachylon pills (as abortifacient) have proven toxic (A. Hall and Ransom, 1906; W. F. Warner, 1907).

\section{ABSORPTION}

All lead compounds are absorbed slowly but unmistakably; chiefly from the alimentary tract, but also from the lungs and tissues; and with longcontinued use even somewhat from the skin (Lewin, r883).

The skin is probably the least important channel. It probably plays no part in industrial poisoning. The lungs are also of subordinate importance, the toxicity of the lead dust being due to swallowing rather than to inhalation (Carlson and Woelfel, I9I3.

Absorbability of Different Compounds (Cf. A. Hamilton, IgI2).-This is independent of their solubility in water. Lead carbonate and even lead sulphate are dissolved fairly readily by the aid of the gastric juice. Alkalies, including milk, diminish the solution (Carlson and Woelfel, I913). Alkalies of the concentration of the pancreatic and intestinal juices convert lead sulphate into carbonate and lead carbonate into a mixture of neutral and basic carbonate (Auerbach and Pick, I9I3). Lead is also rendered soluble on the bronchial mucosa. Presumably it forms soluble protein combinations. The sulphid was supposed to be inabsorbable, but Woelfel and Carlson, 1914 showed that it is absorbed sufficiently, even from ores, to be dangerous. The toxicity of the various lead pigments is reviewed by A Hamilton, 1913.

\section{RETENTION}

Lead disappears rapidly from the blood (the greater part in less than two minutes after intravenous injection; Dauwe, 1907). The main part is deposited in the organs (Kobert); most in the kidneys, then the bones, then the liver and other glands, least in 
the brain, striped and smooth muscle and the blood. It may be retained for years and be demonstrable at times in the urine. In chronic poisoning of dogs, it exists in the liver as a firm globulin combination (Riva, r9 3 ).

\section{EXCRETION}

This occurs mainly by the feces (small and large intestines and bile), and to a lesser extent by the urine; traces also by the saliva, sweat and milk.

All is excreted in organic combination, except the unabsorbed fraction in the feces (Erlenmeyer, I913). The skin is usually credited with some excretion because it gives the black color of lead sulphid when painted with ammonium sulphid; but Miura found that this depends simply on the adherence of lead to the skin.

\section{CHRONIC LEAD POISONING}

This is very common on account of the extensive industrial use of lead and its indefinite storage and cumulative action. The lead acts independently on the blood, nutrition, muscular and nervous structures; and it is difficult to separate the direct and indirect actions. Similar phenomena may be produced in animals.

Sources of Chronic Lead Poisoning.-Over a hundred industrial processes use lead or one of its salts, and the workers in these-painters, dyers, type-setters and typefounders, plumbers, etc.- are the most frequent sufferers after lead miners and the workingmen in white-lead and red-lead factories. Artisans working with lead paints are by far the most common victims. Automobile factories furnish numerous victims.

Comprehensive reviews of industrial lead poisoning are given by A. Hamilton (potteries, I9I2; painters' trades, 1913; lead smelting, 1914).

The occurrence of chronic lead poisoning is by no means confined to these artisans. The metal is so widely distributed that every one is to some extent exposed. Some of the ways in which poisoning has occurred are rather surprising, f.i., by hair.dyes.

The smoke and fumes from lead factories contain quite a large amount of the metal. This is deposited on the soil and on the surface of plants, and is also taken up into the tissues of the latter. Cases of poisoning have been referred to the milk of cows fed on these.

The fruit from orchards that have been sprayed with lead arsenate contains traces of lead (Sonntag, 1915); put probably not sufficient, ordinarily to be harmful.

The danger from lead water pipes has been much controverted.

At present it seems to be accepted that, if perfectly pure water is allowed to flow through bright and clean lead pipes, poisoning results invariably. In this case the surface of the lead is changed into a hydrate (Clowes, 1902), and this is sufficiently soluble to cause the intoxication. But the danger is very much less with old lead pipes as ordinarily used; these have become lined with a layer of lead oxid, $\mathrm{PbO}+\mathrm{Pb}$. This is quite insoluble, and does not readily form a hydrate. The solubility is increased by oxygen and salts, especially by nitrates (Fortner, 1905), but even by sulphates; it is diminished by carbonic acid and carbonates (Paul, etc., 1906).

Ordinarily lead pipes would therefore present but little danger. But even then, if the water is allowed to stand a long time in the pipes, some solution is bound to occur. On account of this danger, lead pipes should be condemned.

The employment of lead vessels for cooking should, of course, be entirely discarded. Tinned vessels, especially tin cans, usually also contain a certain amount of lead in the solder. If the percentage does not exceed a certain limit (Io per cent. for solder, I per cent. for tin plate) the lead seems so firmly combined in the alloy as to prevent its solution even by moderately acid liquids, such as vinegar. If it exceeds this quantity, a certain amount will be dissolved, and will exert its toxic action. The addition of some lead is also necessary in tin-foil, such as is used for wrapping articles of food, to make the tin workable. Here, also, it is harmless, if it does not exceed a certain limit. Lead enters into the glazing of earthenware vessels, and is contained in many varieties of glass; but if it exists entirely in the form of silicate of lead, it presents no danger. All these vessels may be easily tested with sufficient accuracy by allowing vinegar or dilute acetic acid to stand in them for twenty-four hours and then passing a current of sulphuretted hydrogen through the liquid. If there is no precipitate, the amount of lead is below the dangerous limit. 
It is somewhat peculiar that lead bullets in a wound do not usuaily seem to exert the lead action. 'They' probably become oxidized in such a way that none of the metal is alsorberl. However, with small shot, which offers a large surface, several cases of severe and fatal poisoning have been reported (Curtillet and Lombard, I9 2 ).

Individual Susceptibility.- THere is no acquired tolerance to lead, but the natural susceptibility, varies greatly as concerns the time, severity and symptoms of poisoning 'These differences depend partly on personal and factory hygiene, partly on differences of absorption and excretion; on general health and resistance; and other unknown factors. Anemia and alcoholism are believed to increase susceptibility.

The quantily of lead required to produce poisoning is not definitely known and must vary with the susceptibility. Brouardel believes that poisoning may result from I mg. per day; others place it much higher. Gaertner claims that symptoms occur in min only after several months with a daily ingestion of 4 to $7 \mathrm{mg}$., in three to four weeks, if the divily dose is $60 \mathrm{mg}$. (Fluegge and Heffter, I912).

The lingths of exposure varies greatly; from a week or less, upward.

Characteristic Symptoms of Chronic Lead Poisoning (Cf. Alice Hamilton, I912). - These have a slow and vague beginning and run an irregular and intermittent course, with paroxysmal symptoms and recurrences. They may develop only some months after stopping work with lead. They are quite independent of the channel of introduction.

The first signs are rather indefinite manifestations of bad health: Pallor, disproportionate to the anemia (spasm of cutaneous vessels?); lead-line; foul breath, bad taste, caries of the teeth, gingivitis, of ten chronic parotitis. Anorexia and various digestive disturbances: Nausea, vomiting, diarrhea or constipation. Loss of flesh, especially in the cheeks. Recurrent attacks of weakness and trembling; increasing loss of strength; spots before the eyes, itching of the eyelids; rheumatic pains.

There is cachexia, with the skin dry, gray or slightly icteric.

Blood Changes.-The hemoglobin and red corpuscles are diminished. The latter contain basophilic granules, staining with methylene blue. This "stippling" is an important early and almost constant diagnostic sign (van Emden and Kleerekoper, I904); although the basophilic granules may occur also in other anemias, and are sometimes absent in leadpoisoning. Many of the corpuscles are destroyed, producing icterus and widely distributed "hemosiderin." Schnitter, I9I5, attributes the blood changes to injury of the bone-marrow, involving also the leucoblastic tissue.

The Lead-line.-This consists in a slate-colored margin of the gums where they meet the teeth. It is one of the earliest and most constant signs, and occurs also in animals. It is due to the precipitation of lead sulphid (Ruge). Similar lines are given by bismuth, silver, mercury, iron, and perhaps other metals. It may also be simulated by charcoal toothpowders.

Other General Phenomena occur later: Fatty degenerations in the kidneys, liver and other glands; and in the intima, leading to arteriosclerosis. lead.

Gout, with urate deposits similar to those of true gout, but always containing much Nephritis is common in acute, and invariably present in chronic poisoning. It is primarily vascular, followed by epithelial degeneration, deposition of calcium phosphate, and finally cirrhosis (Bright, 1827). The uric acid and non-protein nitrogen of the blood are increased (Folin and Denis, 1913).

The urine flow and chlorids are diminished during the colic, increased after the attack (Nistico, 1913); the urinary nitrogen increases during the acute attacks; the uric acid is said to be diminished during attacks (Goetze, r893).

Pregnancy.-Lead always exerts an unfavorable influence upon pregnancy. The number of miscarriages in women affected by lead poisoning is very large; and of the children born, by far the largest proportion die during the first year of life (Leggs, Igor). 
Plumbism of Father on Offspring.- The clinical evidence is quite slender. Sterility, still-births, and early death of the children are common clinical occurrences. If the children survive, they are particularly liable to various nervous affections (Adami). Experimental feeding of lead acetate to male rabbits and fowl also result in smaller offspring of low vitality (Cole and Bachhuber, I9I4). Weller, I914-I915, confrrmed this on male guinea pigs, finding sterility or reduction of birth-rate, early death of the fetus, and especially retarded development (underweight), which persists through life.

Gradual recovery occurs if the administration of the lead is stopped, indicating that the deleterious action is confined to the maturing cells.

Bone-marrow Changes.- Those correspond to those produced by mercury, iron, phosphorus; and many infective and wasting diseases. They are perhaps secondary to anemia. At first there is marked increase of leucoblastic cells with disappearance of fat, and sometimes with moderate hyperemia; then, gelatinous degeneration and atrophy of the whole marrow tissue (R. Stockman and Charteris, 1903).

Later Special Symptoms.-These are highly characteristic, but may take various forms; in order of frequency: Colic, arthralgia, paralysis, nephritis, cerebral symptoms and anesthesias.

Colic.-This is preceded by continued minor digestive disturbances; pressure sensation in the epigastrium; anorexia; obstipation; and some abdominal tenderness. The acute attacks consist of paroxysms of intense pain, radiating from the umbilicus, and relieved by pressure. The patient therefore instinctively retracts the abdomen so that this often appears boat-shaped (scaphoid); and he may lie on the face with the fists pressed against the painful region. The skin is very pale and dry; the temperature low; the blood pressure high; the pulse hard, wiry and slowed, often to 40 or 50 . The colic is sometimes accompanied by dyspnea (spasm of the bronchial muscles); and sudden severe muscular pains, especially in the flexors of the legs.

After some hours the pain disappears completely; but the constipation lasts some days. Nausea and vomiting are frequent. Relapses are the rule. The colic is very painful but not dangerous. Attacks are more frequent in summer. The action is presumably on the intrinsic nervous mechanism. X-ray examination shows spasmodic contractions of the small intestines (Hertz).

Blood Pressure and Circulation.-It is generally assumed that lead poisoning is accompanied by rise of blood pressure and vasconstriction. This is true for the colic attacks (Riegel and Frank, 1874), where it may be explained by the mechanical displacement of blood from the violent contraction of the intestines; and by pain-reflexes. There are few definite data as to the blood pressure at other times. The pallor of the skin points to vasoconstriction. In advanced stages the arteriosclerosis and nephritis would raise the blood pressure. Quellien, 1905, believes that the pressure is raised even in acute poisoning.

In his animal experiments Harnack (see below) found no rise or other direct change in the circulation, except with fatal doses. These paralyzed the heart and respiratory muscles in common with the skeletal muscles.

Arthralgia.-This also occurs in paroxysms. After some preceding muscular weakness there are sudden attacks, generally at night, of violent remitting pains and disturbed functions of groups of muscles, especially those of the shoulder and the flexors. Pressure gives some relief. The attacks last generally from five to eight days and of ten recur. The phenomena are probably due to central motor stimulation.

Drop-wrist and Other Muscular Paralyses.-Special groups of muscles, most commonly the extensors of the forearm (distribution of musculospiral below supinator longus), or others most exposed to fatigue, become gradually paralyzed. The condition generally begins with weakness, tremors and: disturbed sensation and passes through progressive paraly- 
sis, with the "reaction of degeneration" (muscles respond to galvanic and not to faradic stimulation). Finally there is muscular atrophy and contracture. The contracture begins usually at the metacarpo-carpal articulation of the two middle fingers, then the two outer fingers and the thumb, and then the wrist. The action is peripheral and presumably directly on the muscle (see below). There is no pain. Restoration may occur even from advanced stages.

Encephalopathia Saturnalis.-Cerebral symptoms are relatively rare, but especially liable to occur in alcoholics. They indicate a grave prognosis. The phenomena consist in: Hallucinations, illusions, delirium, melancholia, pseudoparalyses, coma, and epileptiform convulsions. (Convulsions may also occur from uremia). Neurasthenia is very frequent (Hirsch, r914).

Occasional nerious phenomena are: Neuralagia, anesthesias and hyperesthesias occurring in attacks of eight to eleven days' duration; amaurosis, amblyopia, and deafness.

Experimental Chronic Lead Poisoning in Animals.-This has been produced most characteristically by Harnack, 1878 , by using an organic compound, lead triethyl, $\mathrm{Pb}\left(\mathrm{C}_{2} \mathrm{H}_{5}\right)_{3}$. It does not cause lead effects directly but only as its lead is liberated in the body. The rapidity and intensity of the action can thus be regulated by the dose and manner of injection. Effects closely resembling those of lead poisoning are secured sufficiently acutely for pharmacologic study. The nutritional and degenerative changes are thereby excluded. This simplifies the phenomena, but for this very reason some judgment must be used in transferring the results to clinical lead poisoning. W. Straub, I9I I and I9I4, and Erlenmeyer, I9 33 , have also produced chronic lead poisoning by hypodermic injections of the insoluble salts.

The actions may be brought under three groups: Direct depression of muscle; stimulation of the central motor system; and stimulation of the intrinsic nervous mechanism of the intestines.

Muscular Actions.-Lead acts directly on all striped muscle, so that this is much more quickly fatigued (especially if moderately warmed, Cash, I908). Finally the muscle loses its excitability and goes into partial rigor. The phenomena are exhibited by isolated and curarized muscle. This action explains the lead palsy and the reaction of degeneration; i.e., a good response to the single galvanic stimulation, and a poor response to the fatiguing tetanic faradic stimulation. There is no predilection for special muscles, so that the peculiar location of lead paralysis must be determined by fatigue (Mellon, I9I3).

Central Motor System.-Its stimulation results in ataxia, tremors, twitching and convulsions with intact consciousness. The action is presumably located in the mid-and hindbrain. These effects are supposed to represent the clinical encephalopathia and arthralgia; the pain being attributed to muscular twitching. However, the connection is not absolutely clear.

Animals show no sign of pain but this may be because they seem to be much less sensitive to articular pain than man. They also show very little distress if acute arthritis is produced by uric acid injection.

Intestinal Effects.- These consist in spasmodic contraction and increased peristalsis. These result in painful colic and generally diarrhea, but sometimes obstipation, according to whether the peristalsis or the spasm predominates. It occurs in all animals and is easily abolished by atropin. The human colic is not so easily influenced by this drug, but otherwise the phenomena correspond very closely and may be explained by stimulation of the intrinsic motor ganglia. Another theory which attributes the colic to spasm of the mesenteric vessels is not supported by evidence. The vessels are indeed constricted; but this is the effect rather than the cause.

Hirschfelder, 1915, describes acute increase of peristalsis and contraction of the intestinal vessels by intravenous injection of lead acetate. Both phenomena are relieved by local or systemic administration of atropin, nicotin, or nitrites. The action of the nitrites is probably both on the vessels and the intestinal muscle.

Experimental colic was produced by F. Wassermann, I9r6, by keeping cats in cages sprinkled with sawdust and lead carbonate. Feeding a barium meal after the toxic symptoms had set in, he found by X-ray examination that the gastric movements were practically normal. The small intestines showed apparently less segmentation, but more rapid passage of the food. In the large intestines, the passage was delayed, but 
there was lively peristalsis, with strong contraction rings, separated by dilated segments.

During the colic attacks, the small intestine contracted to a thread; the large intestine exhibited nothing special.

Wassermann refers the effects to vagus stimulation.

Chronic Tephritis.-This was described in animals by Charcot and Gombault, I88I; and studied especially by Ophüls, I908 and I9I 2. It is chiefly interstitial, exceptionally with epithelial changes. Many animals showed hemorrhagic serofibrinous inflammation of serous membranes. Lead administration does not produce arteriosclerotic changes in dogs (Adler, I9I4).

Diagnosis of Plumbism.-The chief points may be recapitulated as: The history of exposure; lead-line; ammonium sulphid reaction on the skin; black stools; especially the chemical demonstration of lead in the stools or urine; pallor, anemia, stippling, pinched features, asthenia, rheumatic pains, digestive symptoms, colic, obstipation, drop-wrist, recovery when removed from exposure to lead. The concurrence of most of these signs is highly characteristic; but none of them is absolutely constant, and most may be simulated individually by other conditions (Linenthal, I9I4).

Prophylaxis.-The public and especially the artisans, should be thoroughly educated to the insidious dangers of lead, and the chances of poisoning carefully guarded against.

The sources of danger-lead pipes, etc.-should be eliminated. Tin cans, foil, etc., should be frequently examined by authorized persons. Proper precautions are required in lead factories and in exposed trades. Lead-laden dust is especially dangerous. Since the main channel of poisoning is by the mouth, extreme cleanliness should be encouraged and made possible by liberal facilities for washing. Sulphates are useless (see absorption). Food should not be permitted on the premises, and the clothing should be changed before leaving the works. More stringent laws are greatly needed.

The Treatment.-Consists in resting the patient in hygienic surroundings until the lead has been eliminated and the general health fairly restored. Tonics are valuable.

Symptomatic measures may be used: Against the pain of colic, morphin is most effective. To this may be added atropin or scopolamin, and hot applications or baths. Amyl nitrite (Riegel, 1878) gives slight and only temporary relief.

Constipation is treated with olive oil enemas; or 200 to 500 c.c. of oil by mouth. This is better than castor oil or calomel, which increase the pain if they do not relieve the constipation. The palsies may be treated with strychnin, massage and electricity.

To hasten the elimination of the lead, potassium iodid, baths, sweats, and sulphur internally, are commonly tried; their efficiency is doubtful. The iodid is the most promising: Melsens, 1849 , found that it hastened the elimination at first; but it soon appears to lose this effect.

\section{PREPARATIONS-SOLUBLE LEAD COMPOUNDS}

*Plumbi Acetas (Plumb. Acet.), U.S.P., B.P.; Lead Acetate (Sugar of Lead); $\mathrm{Pb}\left(\mathrm{C}_{2} \mathrm{H}_{3} \mathrm{O}\right)_{2}+{ }_{3} \mathrm{H}_{2} \mathrm{O}$. - Colorless or white granular crystals or masses, of faintly acetous odor and sweetish astringent, afterward metallic taste, efflorescent. Freely sol. in water $(\mathrm{r}: 2)$, sol. in alc. Incompatible with alkalies and carbonates; iodids, chlorids and sulphates. Dose, 0.05 Gm., I gr., U.S.P.; 0.06 to $0.3 \mathrm{Gm}$., I to 5 gr., B. P. Maximum dose, $0.3 \mathrm{Gm}$., $5 \mathrm{gr}$.

Pil. Plumb. c. Opio, B.P.- I 2 per cent. of Opium; 80 per cent. of Plumb. Acet. Dose, 0.12 to $0: 25 \mathrm{Gm} ., 2$ to 4 gr., B.P. 
Supp. Plumb. Co., B.P. -0.2 Gm., 3 gr., of Plumb. Acet.; 0.067 Gm., I gr. of Opium.

Glycer. Plumb. Subacet., B.P.-About 20 per cent. of Subacetate.

${ }^{*}$ Liquor Plumbi Subacctatis (Liq. Plumb. Subacet.), U.S.P.; Solution of Lead Subacetate (Goulard's Extract; Bleiessig).-An aqueous, clear colorless, alkaline solution; containing not less than 25 per cent. of lead subacetate and lead oxid (Litharge) with water. Should be diluted 15 to 30 times before application. Used as astringent and intipruritic in inflammatory conditions of the skin, and in sprains and bruises. It should not be applied to denuded surfaces, and should be used with caution on the face.

${ }^{*}$ Liq. Plumb. Subacet. Fort., B. P. - About 35 per cent. of Subacetate.

Liq. Plumb. Subacet. Dil., U.S.P., B. P. (Goulard's Lotion; Leadwater).-Prepared by diluting the stronger solution, in the ratio of $1: 25$, U.S.P. $I: 80$, B.P. May be applied directly.

Ung. Plumb. Subacet., B. P. - 12.5 per cent, of the Strong Solution.

\section{PREPARATIONS-INSOLUBLE LEAD COMPOUNDS}

Plumbi Carbonas (White Lead); $(\mathrm{Pb} \mathrm{CO})_{2} \mathrm{~Pb}(\mathrm{OH})_{2}$ - Insol. Used as Ointment, Io per cent., against dermatitis, burns, etc.

Plumbi Iodidum (Plumb. Iod.), B.P.: PbI.-An insoluble, bright yellow powder. Used externally as mild counterirritant against glandular swellings, etc., in the form of the Ointment.

Ung. Plumb. Iod., B.P.- - 0 per cent.

Plumbi Oxidum (Plumb. Oxid.), U.S.P., B.P. (Litharge); PbO.-Heavy, yellowish powder, without odor or taste. Almost insol. in water. Used in making the Subacetate.

(Emp. Plumb., U.S.P., B.P.--See Index).

Ung. Diachylon, U.S.P. (Hebra's Ointment).-Equal parts of Lead Plaster and White Petrolatum, flavored with Lavender.

\section{THALLIUM}

This metal is almost as toxic as arsenic. The acetate has been given internally 5 to $10 \mathrm{mg}$. per day) against the night sweats of phthisis, and both internally and externally as a depilatory. The therapeutic use has repeatedly caused severe poisoning and is scarcely justified. It is not anti-syphilitic (Buschke, rgrI)

The toxic phenomena are: stomatitis, diarrhea, dyspnea, pain in the extremities, alopecia (also in animals; Buschke, rgro), depression and other neuroses and neurites, diuresis and persistent albuminuria (Olmer and Tiau, 1909; Swain and Bateman, I910).

\section{PHOSPHORUS}

General Statement.-Phosphorus produces severe acute poisoning, resembling acute yellow atrophy of the liver; characterized by alterations of metabolism and fatty changes of the liver and other organs. Chronic poisoning, as in match factories, results in necrosis of the jaw. Therapeutic doses effect changes in nutrition and bone formation, which are utilized especially in rickets.

Phosphorus acts as such: its actions are not shared by any of its compounds, which are for the most part harmless.

Varieties of Phosphorus.- The element exists in two forms, red and yellow. Red or amorphous phosphorus is non-volatile, insoluble in water or oil, is not absorbed, and is therefore not toxic. The yellow or ordinary phosphorus, while almost insoluble in water, is very volatile and dissolves in fats and bile (Hartmann, I866) ${ }^{1}$ so that it is absorbed, especially when it is finely divided.

\footnotetext{
1 Quoted by Blyth.
} 
Absorption, Fate and Excretion.-Absorption occurs from the intestines; to some extent also by the inhalation of the vapor It is always slow, and the systemic effects are therefore delayed several days. It is finally excreted in oxidized form (hypophosphoric acid, Poulet, 1872 ), but its fate is very imperfectly known. Although it oxidizes so readily in the air, its oxidation in the blood requires days (H. Meyer, 1881 ); perhaps it has an inhibitory action on oxidizing ferments similar to hydrocyanic acid (Schultzen and Riess, I 869). During its action it exists unchanged in the organs and in the expired air.

\section{PHOSPHORUS POISONING}

Etiology.- Phosphorus is quite accessible in the form of matches or phosphorus rat paste, so that poisoning, accidental or suicidal, is not uncommon. It also occurs, rather rarely, in the therapeutic use. Infusions of matches have been taken as abortifacient, with serious results. Chronic poisoning of a somewhat different character is seen from its fumes, in phosphorus and match factories. This, as well as the poisoning by matches, has been very greatly diminished since the introduction of a morphous (red) phosphorus.

Phosphorus Matches. - The first phosphorus friction matches were made by Dérosne, Paris, in 1816 , but they did not become practical until I 833 , when processes for their manufacture were invented in several countries. These all employed yellow phosphorus; each match contains $1 / 3$ to $5 \mathrm{mg}$., so that I 6 would be fatal for an adult. The other ingredients are oxidizing agents (potassium chlorate or dichromate; lead oxid or nitrate; manganese dioxid).

The use of the dangerous yellow phosphorus in matches is now prohibited by law in practically all European countries. In the United States, a prohibitive tax has been placed on their manufacture. Phosphorus sesquisulphid, which is said to be non-toxic, is now used in ordinary matches, which strike anywhere. The safety-matches" have no phosphorus on the sticks, which are usually headed with $\mathrm{Sb}_{2} \mathrm{SO}_{3}, \mathrm{KClO}_{3}$ and glue; the red phosphorus is here on the friction surface coating of the box.

The Symptoms of Poisoning.- These have the peculiarity that they occur in two well-defined stages: At first, there is local gastro-intestinal irritation; then more or less complete recovery; then, after two or three days, the phenomena of the systemic actions appear. These are distinguished by fatty enlargement of the liver, intense icterus, and metabolic changes pointing to diminished oxidation. They extend over several days and generally terminate in death by heart failure. Gastric, syncopal, or cerebral phenomena may predominate.

Acute Symptoms.-These generally start after some hours with gastric pain and burning, eructations with garlic odor, and finally vomiting, which may be luminous in the dark. Later there is of ten diarrhea. If treated, there is more or less remission or even complete recovery for two or three days.

The Later Phenomena.-These begin with nausea; then gradually slight icterus; abdominal pain and tenderness; vomiting and soft stools, both often streaked with blood; coated tongue and fetor oris.

The symptoms become gradually more severe; and within two days the area of liver dulness has rapidly increased; the hepatic region is painful and very tender, and the icterus more intense. If death occurs within the first two days of the second stage, there is usually no icterus (Magnus, rgr4). All foods and liquids are vomited. The patient becomes profoundly prostrated with insomnja, anxiety, headache, muscle pains and feebleness. The temperature is normal, the skin dry, the respiration increased, the pulse soft but of normal rate. The urine is scanty, acid, dark brown from bile and blood, and contains albumin, casts and abnormal nitrogenous substances. Multiple hemorrhages occur as petechiæ or in larger areas, in the skin, mucosæ, lungs and other viscera.

Agonal Stage.-The pulse becomes thready and intermittent; the heart dilated, with systolic murmurs; the respiration dyspneic or Cheyne-Stokes. Consciousness may be undisturbed, or generally there is somnolence, sometimes delirium and disturbances of temperature, finally coma.

Diagnostic Post-mortem Features.-These are: the icterus; phosphorus odor on opening abdomen; blood tarry and difficultly coagulable; multiple hemorrhages; fatty enlargement of the liver and other organs.

The Course.-This runs from two to fifteen, averaging perhaps eight days.

The Fatality. - This is high, about 55 per cent. (Falck); with larger quantities such as are used for abortion, it may reach 95 per cent. (Kobert). 
The Fatal Dose.-This may be as low as $50 \mathrm{mg}$., generally about $100 \mathrm{mg}$.; but $\mathrm{I}_{5}$ me. may be severely toxic. It is much more dangerous in solution or when finely divided, than when larger lumps are taken. A two-year-old child has been severely poisoned by therapeutic doses (1.2 mg. distributed over threc days; Nebelthau). Magnus, I914, states that no dangerous effects have ever occurred (in children up to eight years) from $0.5 \mathrm{mg}$. per day; although there may be some vomiting at first. He states the smallest dose that has cxceptionally produced dangerous effects as $1 \mathrm{mg}$. per day.

Recovery.--This may occur gradually, even from advanced symptoms; but cerebral hemorrhages may leave paralytic after-effects.

Treatment of Acute Poisoning.-Any phosphorus remaining in the alimentary tract should be oxidized and removed. The best antidote is copper sulphate, 0.1 to $0.2 \mathrm{Gm}$. in water, every ten minutes until emesis.

This acts as a prompt emetic, and meanwhile oxidizes the phosphorus and envelops the globules with a coating of reduced copper which retards absorption (Woehler and Vogel, I836; Straub, r9o3). Gastric lavage with other oxidizing agents may be substituted: $\mathrm{KMnO}_{4}$, o.I per cent. (Bokai and Antal; Koranyi, I889); $\mathrm{H}_{2} \mathrm{O}_{2}, 2$ per cent. of the official solution. These should be followed by a saline cathartic. Oils, fats and milk should be avoided while any phosphorus remains in the intestines, since they increase its solubility and absorption. Sodium bicarbonate may be given to prevent acidosis. When the systemic symptoms have set in, these measures are practically useless and the treatment must be mainly symptomatic. Transfusion of blood is said to have given good results.

Oil of Turpentine. $-0.5 \mathrm{Gm}$. several times daily for a week may be tried (Andant, 1868; Koehler, 1872) but its benefits are doubtful, or at best inconstant. They seem to vary with different varieties of the turpentine, the fresh being often as effective as old ozonized samples. The action is not due to simple oxidation of the phosphorus, but to the formation of complex compounds (Busch and Fischer); Kobert believes that it may be effective even after the phosphorus has been absorbed.

Special Phenomena of Phosphorus Poisoning.-Action on Metabolism.-This is the most interesting feature of phosphorus poisoning from a scientific standpoint. It occurs in chronic poisoning, or as a secondary process after a single larger dose. The phenomena consist in increased tissue destruction with disturbances of syntheses, oxidations and dissociations; increased but incomplete nitrogen metabolism; interference with glycogen deposition; increased lactic acid and ammonia excretion; and inhibition of other synthetic processes; deposition of fat in the liver.

The Mechanism. - This is not understood. It appears to involve interference with certain ferment actions, primarily with some of those involved in carbohydrate metabolism.

Nitrogen Metabolism. - This is very greatly increased. The urinary nitrogen may rise by 300 per cent. (Storch, I 865). The excretion of phosphate and sulphur is also increased, while the chlorids are diminished by the inanition (Welsch, r905). All the nitrogenous metabolites may participate in the increase (v. Jaksch, r 1903); but not to the same extent (Muenzer, 1894). The urea is not nearly as much increased as the others and may even be diminished. It is to a large extent replaced by ammonia. This may be attributed to an increased production of sarcolactic and other organic acids. The uric acid excretion does not show characteristic changes; and the creatinin output is scarcely affected (Lusk). The hippuric synthesis of excised kidneys is checked (Hauser, 1895).

The blood of dogs poisoned with phosphorus shows increase of non-protein nitrogen, urea and amino-acids; and generally a terminal acidosis (E. K. Marshall and Rowntree, 1915). The protein cleavage products may play a part in the production of the symptoms (Moriani, 19r6).

A very important phenomenon is the appearance in the urine of amino-acids, leucin, tyrosin, cystin; and peptone-like substances (Schultzen and Riess, r 869). Clinical cases may not show the tyrosin. The peptone-like substances are present especially in the early stages. Their nature is not known (Harnack, 1893). 
Fatty Changes.-Extensive deposition of fat occurs particularly in the liver cells, whose fat-content may reach three or four times the normal; but it is seen also in practically all other cellular organs: muscle, skeletal smooth and cardiac; kidneys; blood vessels; epithelium of stomach and intestine, etc. The fatty changes are preceded by "cloudy swelling," which is probably an intermediate stage. They are followed by proliferation of interstitial connective tissue (cirrhosis).

Similar fatty changes occur in poisoning by: Phlorhizin, arsenic, antimony, many volatile oils, the alcohol group, benzol, phallin, etc., in starvation, etc.

The Mechanism of the Fatty Degeneration.-This has been studied especially in the liver. It was originally assumed that the fat was formed in situ, by true degeneration of the protoplasm of the liver cells (Bauer, 1878 ). However, Lebedeff, 1883, disproved this, finding that fat is deposited in the liver only if the fat store of the body is ample; and that if some foreign fat is introduced into the subcutaneous tissue, the fat deposited in the phosphorus liver agrees in composition with this foreign fat. This suggests that the liver fat is merely transported from the fat depots. These observations have been confirmed by Rosenfeld, 1897; Pflueger, 1898; and others. They apply also to other poisons that cause "fatty degeneration" of the liver. The conclusion was strengthened by the observation of Athanasiu, 1898 , that the total fat of the body is not increased by phosphorus. In fact it is rather diminished (Shibata, I9II). The increased fat of the liver must therefore have been drawn from other parts of the body so that the process is one of infiltration.

Lusk finds that the combustion of fat in phosphorus poisoning is not changed. Some of the "fat" consists partly of other lipoids (Dastre and Morat, I879); Heffter, I890, claims that the percentage of lecithin is decreased. The fat in the heart (studied by Rosenfeld, 1906) is also infiltrated (Leik and Winckler).

Leathes, 1909, showed that the liver effects certain changes in the depot-fats (desaturation, increasing the iodin-number). He believes that this is a necessary preliminary to the utilization of the fats in metabolism. Fatty infiltration of the liver would therefore represent an excessive attempt at such conversion. Accordingly, it is found in all conditions in which there is a high need for fat (starvation, etc.). If such animals are freely fed, the fatty infiltration of the liver may disappear within a day (Mottram, I9I5).

Carbohydrate Metabolism.-Phosphorus causes the disappearance of glycogen from the liver. This is not due to increased glycogenolysis, for the percentage of sugar in the blood is generally not increased, and in the later stages it is even decreased. Phosphorus must therefore inhibit glycogen synthesis, especially from non-carbohydrate sources (E. Franck and Isaak, IgII). This would explain the prevention of epinephrin glycosuria by phosphorus; and the non-combustion of lactic acid. According to v. Fuerth, I914, lactic acid appears in the urine only when the rabbits are fed on an excess of sugar. Dextrose also is not converted into glycogen (E. Neubauer, I 909 ); thus explaining the occurrence of glycosuria when carbohydrates are fed. Glycogen may be still formed from levulose. The tolerance for galactose and levulose is lowered (E. K. Marshall and Rowntree, Igr5).

The increase of nitrogen excretion may be due to carbohydrate disturbance: Franck and Isaak, Igrr, believe that this interference with glycogen synthesis is the primary action of phosphorus; they attribute the increased protein destruction to the necessity of making up for the lessened supply of energy material. The fat transportation to the liver could be similarly explained.

Accordingly Rettig, I914, found that a diet rich in carbohydrates prevents the increased protein catabolism and the fatty changes. Ray, McDermott and Lusk 1899 , also showed that with dogs rendered glycosuric by phlorhizin, phosphorus does not increase the nitrogen excretion; again indicating that the nitrogen excretion is not due to direct destruction of the protoplasm, but is in some way connected with the disturbed carbohydrate metabolism. Lusk, 1907, suggests that the primary action is to inhibit the ferment which burns lactic acid; and that the nitrogen changes are due to the resulting acidosis.

Oxygen Consumption.- The metabolic effects of phosphorus resemble those of oxygen deprivation (e.g., Porges and Pribram, 1908); and it has been suggested that phosphorus renders the cells less capable of utilizing oxygen. However, it is also possible that the metabolic changes in both cases are the secondary result of some other common factor. Indeed, Lusk, 1907, has shown that the oxygen consumption is not diminished but rather somewhat increased, partly by fever, and probably also by the toxic action of increased protein destruction.

Autolysis. - In advanced phosphorus poisoning, the postmortem autolysis of the liver is greatly accelerated (Jacoby, I900; Porges and Pribram, 1908), probably by decreased resistance of the liver cells to the normal autolytic ferments (Wells). It is not clear what, if any, part this plays in the liver and metabolic changes during life. 
Direct addition of phosphorus to excised normal liver does not hasten autolysis (Jacoby). The phenomena are probably analogous to those of late chloroform proisoning.

Bile and Icterus. - The swelling and subsequent cirrhosis of the liver obstruct the bile capillaries, so that the bile secretion is diminished or suppressed; and the gall-bladder contains only a little mucous fluid. The retention of the bile constituents produces the characteristic intense icterus. It was formerly believed that the formation of bile was also increased by the destruction of the blood; but there is no evidence for such destruction.

The Effects on the Liver.-These may therefore be summarized as: disappearance of glycogen; incomplete combustion of lactic acid; increased excretion of ammonia; increased protein destruction with incomplete desamidization of certain amino-acids; fatty infiltration; mechanical interference with bile formation; increased postmorten autolysis; decrease of fibrinogen.

The excretion of phthalein is delayed (Whipple and Sperry, I9r5). The liver, heart, ganglion and other nerve cells show degenerative changes similar to those of chloroform intoxication (Lissauer, I9I4).

Changes in the Blood; Red Corpuscles.-It was formerly believed, on the basis of experiments on birds (Fraenkel and Roehmann), that these were rapidly destroyed; in man and mammals (Taussig, I892; v. Jacksch, I893), however, no destruction occurs even in severe poisoning. In fact the corpuscles are often increased (Ragazzi, I9Io; Leoncini, 1914) and this is the typical effect of therapeutic doses.

The hemoglobin is not altered (Leoncini). The viscosity and electrical resistance of the blood are increased by the polycythemia; and remain so until death approaches, when they suddenly decrease. The osmotic pressure of the blood was found increased by Bottazzi, I896; unchanged by Ragazzi. The alkalinily is diminished. The catalase action is moderately decreased (Duncker and Jodlbauer, I9II). The statements as to the leucocytes are confused; Ragazzi describes increase of the polynuclear, and disappearance of eosinophiles. According to Leoncini, the leucocytes are at first diminshed, then sometimes moderately increased.

The coagulability of the blood is diminished. This is probably due to the destruction of fibrinogen or of fibrin ferment; or possibly to the production of peptone bodies by the destruction of proteins (Cevidalli, I902). Denny and Minot, I915, also find the antithrombin content of the blood diminished.

The hemorrhagic tendency is explained by the lessened coagulability of the blood and by the fatty changes in the capillary endothelium.

The ecbolic action which occurs with fatal doses may be referred to degeneration of the blood vessels. However, phosphorus also passes to the fetus in sufficient amount to produce fatty hepatic degeneration.

Circulation.-Phosphorus appears to have direct toxic effects on the heart-muscle; for with intravenous injection this is paralyzed before anatomic degeneration has set in (Meyer, I88I). In the collapse, splanchnic stimulation is ineffective. In clinical poisoning, the syncopal cases may be due to this cardiac paralysis, but generally the circulation suffers but little until the agonal state.

$M$ uscle and Nerve. - These are not directly affected.

The Central Nervous System. - This plays a subsidiary rôle, but is said to show histologic changes (Rossi, 1897 ).

Local Actions.-Irritation of the mucosæ is shown by the early acute symptoms, but phosphorus is not corrosive.

Chronic Phosphorus Poisoning.-This is seen especially in match factories using yellow phosphorus. The most common manifestation is necrosis of the jaw; sometimes bronchitis, to which tolerance is acquired; and cachexia resembling mild acute poisoning.

Strict prophylaxis should be enforced. The manufacture of yellow phosphorus matches has been prohibited in most civilized countries. 
Exposed workers should attend to ventilation, cleanliness and strict oral hygiene (Ivy, I9I I).

The necrosis of the iaws begins with salivation, suppurative ulceration of the gums, and ends in a very profound periostitis, involving the whole jaw. It usually starts in carious teeth. The lower jaw is more often affected. The only treatment is excision of the diseased bone.

Identical necroses in the teeth and other bones have been produced experimentally

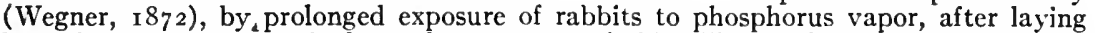
bare the periosteum. The bone changes are probably allied to those seen in the therapeutic use, but intensified by the more direct contact and by secondary infections.

Phosphorus Burns. - These are no more dangerous than other burns of similar extent and do not produce any of the symptoms of phosphorus poisoning.

Bone Changes by Prolonged Administration of Small Doses. These stimulate bone formation (Wegner, I872); in growing animals, the epiphyses form compact instead of spongy bone; in adults, the Haversian and marrow canals are gradually filled with dense bone. This has the normal chemical composition and structure. Larger doses increase the vascularization of the bone (Kassowitz).

\section{THERAPEUTIC USES}

The stimulation of bone formation led to the use of phosphorus in rickets, osteomalacia, and delayed healing of fractures (Kassowitz, I 884). The clinical evidence of its usefulness is contradictory; the best results are reported in rickets, in which it is said to improve not only the bone changes, but all the other symptoms. The improvement should be seen in about four weeks.

The dose is usually about I mg. per day, with meals; best dissolved in olive or cod liver oil (0.01: IOO c.c.). The solution should be freshly made. A pill ( $0.6 \mathrm{mg}$.) is also official. It is coated with Tolu to prevent oxidation.

The administration of phosphorus generally causes some gastraintestinal disturbance; sometimes the jaw necrosis; and rarely typical phosphorus poisoning. It must therefore be carefully watched and stopped if any gastric or intestinal effects appear.

Stability of Phosphorus Preparations.-Engelhardt and Winters, I9I5, find the pills and spirit fairly stable, but not always. Compound elixirs are less stable.

Phosphorus in Nervous Diseases. - It has been advised as a tonic in alcoholism, neurasthenia, sexual exhaustion, neuralgia, tabes, cerebral degeneration, etc.; and in chlorosis and anemias. There is little scientific support, either experimental or clinical, for this use.

Zinc Phosphid.-This has been suggested as a therapeutic substitute (Vigier, I 876); there is very little scientific information about $i$.

Amorphous or Red Phosphorus.-This is non-volatile, inabsorbable and therefore non-toxic when taken by mouth; but since it may contain traces of ordinary phosphorus (to 0.6 per cent.-Blyth), large doses could become toxic. Intravenous injections (Nasse, I885) produced the effects of ordinary phosphorus.

Phosphoretted Hydrogen.-This (Phosphine- $-\mathrm{PH}_{3}$ ) is a violent poison; 0.025 per cent. is harmful after a time; and 0.2 per cent. is quickly fatal, producing dyspnea, fainting, lowered blood pressure, slowed heart; nausea and vomiting; convulsions and paralyses; coma and asphyxia. Postmortem examination shows pulmonary congestion and exudation. Industrial poisoning occurs from its pressure in acetylene and ferrosilicon.

Phosphorus Pentachlorid.-Poisoning is described by Roch, 1914.

\section{PREPARATIONS-PHOSPHORUS}

*Phosphorus (Phosphor.), U.S.P., B.P. - A translucent waxy substance of distinctive odor. Melts about $44^{\circ} \mathrm{C}$. Oxidizes (ignites!) on exposure to air. Practically insol. in water, but slightly sol. in alc. $(I: 400)$. Sparingly sol. in oils. Sol. in fat- 
colvents. Dose, $0.5 \mathrm{mg}$., $1 / 120$ gr., U.S.P., 0.6 to $2.5 \mathrm{mg}$; $1 / 100$ to $1 / 25 \mathrm{gr}$., B.P.; as pill or oily solution. These must be recently prepared. Mi aximum dose, $2.5 \mathrm{mg}$., $1 / 25 \mathrm{gr}$. Olerm Phosphoratum (Ol. Phosphor.), B.P.; I per cent. in olive oil.-Dose, 0.06 to 0.3 c.c., I to 5 minims, B.P., in almond or cod liver oil, or in capsules.

Pil. Phosphor., U.S.P.- $0.6 \mathrm{mg} ., 1 / 100 \mathrm{gr}$; coated with Tolu to retard oxidation.

Pil. Phosphori, B.P.-1 per cent. (formerly 2 per cent' Dose, 0.06 to 0.25 Gm., I to 4 gr., B.P.

\section{PHOSPHORUS COMPOUNDS IN NUTRITION ${ }^{1}$}

\section{LECITHIN, GLYCEROPHOSPHATES, HYPOPHOSPHITES, ETC.}

General Statement.-Phosphorus compounds are important constituents of animal and vegetable cells, and especially of nervous tissue. Preparations of phosphorus have therefore been used as tonics and "brain foods" in all sorts of depressed conditions: Scrofula, incipient phthisis and cachexia; neurasthenia and tabes; neurites and neuralgias, etc.

With the possible exception of lecithin, there is no experimental evidence. of their value; the clinical evidence also is too indefinite and contradictory to establish their position. With lecithin, positive results are claimed by some investigators but even these are denied by critical observers. The ordinary diet furnishes a very free supply of lecithin and other phosphorus compounds, and it is difficult to see how the small quantities added in the form of more or less expensive medicines could have any other than psychic effects.

Occurrence of Phosphorus in Body.-Phosphorus is found as inorganic phosphate, mainly of $\mathrm{Ca}$ and $\mathrm{Mg}$, especially in the bones; and dissolved as sodjum phosphate in the fluids and cells. The cells also contain the organic compounds, nucleins, lecithins, and phosphatids. The organic phosphorus compounds of the serum are almost exclusively phospho-lipins (Greenwald, I916).

Quantitative Distribution.- The total phosphorus content of the body (young dog) is equivalent to about 0.7 per cent. of $P$. Of this, three-fourths is in the bones; the muscles contain more than the combined total of the central nervous system, skin, liver and kidneys (Heubner, Igr4).

Organic Phosphorus Constituents or Tissues.-It may be well to premise the discussion of phosphorus metabolism by some chemical data.

Structure and Occurrence of Lecithins.-These are esters of fatty acids, glycerophosphoric acid, and a nitrogenous base (mainly cholin). Their structure will be understood by the following formula, in which $R$ represents fatty acid-oleic, stearic, palmitic or other. The part to the right of the dotted line represents cholin.
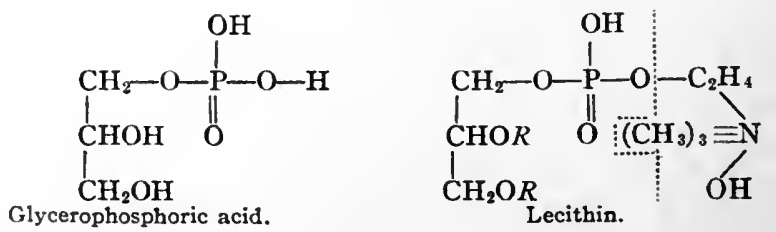

The various lecithins differ according to the fatty acid, etc. They occur in animal (not in vegetable) cells, generally combined with proteins. Their place may be taken by related compounds or "phosphatids" which are but little understood.

Lecithins are conveniently isolated from brain substance or egg-yolk ("vitellin"). The commercial products are quite impure. They contain 3.84 to 4.12 per cent. of phosphorus. The purest are those obtained by Erlandsen's method. (Bibliography of Lecithins, Merck's Report, $26: 50$ ). H. MacLean, 1915, states that ordinary lecithin

'A very full review of these subjects, with bibliography, is given by Forbes and Keith, 1914. 
is really a mixture of cephalin (which tends to become insoluble in alcohol), and true lecithin (which remains alcohol-soluble).

Properties.-Lecithin is a white to orange-colored substance, usually amorphous and waxy when dry. It is very hygroscopic, softening to a semifluid mass in the air. It also oxidizes readily, becoming darker. It dissolves in alcohol or most fat-solvents (not in acetone). It is insoluble in water, but readily takes up water, swelling to a gelatinous mass and finally to a colloidal solution, from which it is precipitated by certain acids and salts; while other salts prevent the precipitation (W. Koch). It is decomposed by prolonged contact with water; alkalies saponify it readily into cholin, fatty acid, glycerophosphoric acid. Further cleavage results in ordinary phosphoric acid. The cleavage with dilute acids is quite slow.

Cephalin. - This is a highly unsaturated monamino-monophosphatid. Its nitrogenous base is probably not cholin (W. Koch, 1902); otherwise it is closely related to lecithin.

Plant Phosphatids.-These were formerly considered identical with lecithin, but are now known to be quite distinct. They usually contain a sugar molecule.

Phytin.-This is a calcium-inosit phosphate, occurring abundantly in plant seeds.

Absorption, Fate and Elimination of Phosphorus Compounds.-The inorganic phosphates are absorbed as such, mainly from the upper portions of the small intestines. Phosphates that are insoluble in water generally dissolve readily in the gastric juice. They are excreted by the urine and feces. Hypophosphites are also absorbed, and for the most part excreted unchanged. Glycerophosphates and all organic phosphorus compounds are almost completely hydrolyzed in the digestive tract (Plimmer, I913), and absorbed and excreted as inorganic phosphate (Oeri, r909). Opinions differ as to the importance of the small fraction (if any) that escapes decomposition. Further details will be given under the individual drugs.

The absorbed phosphate is utilized in the construction of bone and in the synthesis of the various forms of organic phosphorus compounds in the body. These can be constructed-completely from inorganic phosphates.

The greater part of the normally excreted phosphate is derived from the metabolism of the tissues and therefore generally varies in the same direction as the nitrogen excretion, although they are not necessarily proportional. "Phosphaturia," the deposition of phosphates in the urine, is due to alkaline reaction of the urine, and not to increased phosphate excretion; indeed, the excretion is generally diminished.

The path of phosphate excretion is markedly influenced by the calcium of the food. With a high calcium income, the phosphate is excreted mainly by the feces as calcium phosphate; whereas the urinary phosphates increase with a lime-poor diet (Zuelzer, I905).

Phosphorus Compounds of Urine.-Practically all the phosphorus of the urine is normally in the form of inorganic mono- and di-hydrogen phosphates. A variable fraction may exist in organic form, as glycerophosphoric and phosphocarnic acid. The organic phosphorus is increased in fasting, chloroform anesthesia, morphin poisoning, and some pathologic conditions.

Phosphorus Compounds of the Feces.-These are derived partly from unabsorbed phosphates, from intestinal secretions, and from the bacteria. They include various organic forms, nucleins, lecithins, etc;. as well as calcium and other inorganic phosphates.

Rôle of Organic and Inorganic Phosphate Compounds in Nutrition. -Animals which receive an adequate supply of inorganic phosphates require very little, if any, organic phosphorus compounds (Review, Marshall, I9I 5). The presence of some organic phosphorus in the food appears to favor nutrition; but this may be due to the association of some "vitamin" or similar substance. 
Synthesis of Lecithin.- Miescher, 1896 , deduced that the production of roe and milt in the fasting salmons must involve the synthesis of large amounts of phosphorized fat irom inorganic phosphorus compounds. Roehmann, 1908, showed that mice can be maintained for two generations on lecithin-free food; and Fingerling, I912, and McCollum, Halpin and Drescher, 1912, demonstrated that the organic phosphorus of egg-yolk can be formed from inorganic phosphorus compounds, since it is not diminished if birds are kept on a diet free from organic phosphorus. Plimmer, rgr 3 , came to the same conclusion. Nacallum found no phosphate ions in the nuclei, so that the nucleins are probably not formed here.

Daily Phosphorus Requirement.-Under ordinary conditions, the daily phosphorus excretion of normal human adults is equivalent to about 1.5 to $1.75 \mathrm{Gm}$. of $\mathrm{P}$, or about $3.5 \mathrm{Gm}$. of $\mathrm{PO}_{4}$. 'This must be replaced by a corresponding income to preserve a balance. The ordinary diet contains about this amount (from 1.52 to $5.88 \mathrm{Gm}$., average $3 \mathrm{Gm}$., of $\mathrm{PO}_{4}$ (Sherman, Mettler and Sinclair, rgro). Under special conditions of diet equilibrium may be preserved temporarily with $0.9 \mathrm{Gm}$. of $\mathrm{P}$. This is also the average daily excretion in fasting.

Phosphorus Balance.-Increased intake of phosphorus compounds is followed by increased elimination: in carnivora mainly by the urine; in herbivora mainly by the feces; in omnivora by either, according to the diet. A portion of the excess is retained, especially in growing animals or after wasting diseases. The retention is diminished by deficiency of calcium or excess of magnesium in the food. The bones constitute important depots for a phosphate reserve. The deposition of phosphate in them is hindered by inorganic acids, calcium-precipitating ions, or high potassium or low sodium content of the ration.

Excision of the parathyroids is followed by marked retention of phosphorus, increasing its concentration in the blood (Greenwald, r9i6).

Deficient Phosphorus Income.-If this falls below the normal requirement, the excretion, and finally the phosphorus content of the tissues, is diminished. This soon leads to disturbances of growth and nutrition, the spongy tissue of the bones being most affected (Masslow, 1914). The bones become soft and flexible when their phosphorus content has fallen by one-sixth (Heubner, I9I4). In fasting, the loss of phosphorus is considerable.

Phosphorus Compounds as "Tonics."-As was pointed out in the introductory paragraph, the extensive employment of phosphate compounds as tonics, particularly as "nerve foods," was based on mistaken theoretical conceptions. With the doubtful exception of lecithin, it lacks all experimental or reliable clinical foundation.

Phosphorus in Nerve Tissue.-Phosphatids, especially lecithin, are contained in glands, cell membranes (especially of the red corpuscles), and nerve tissue, where they subserve important physical and chemical functions. Nervous tissue is rich in phosphorus ( $1 / 3$ per cent. of moist; $1 \frac{1}{2}$ per cent. of dry); but not more so than glandular organs. Its phosphatid content ( 27 per cent. of dry), however, is three times or more that of glands. Its importance therefore can not be doubted; but the percentage of phosphorus is not decreased even in severe nervous diseases (dementia precox). Nervous diseases are therefore not due to deficiency of lecithin (W. Koch, I909).

Even in growth, when the nervous tissue, and therefore its lecithin, increases rapidly, an abundance of phosphorus compounds is supplied by the food. Moreover, Hart, 1908, showed that even in extreme phosphorus starvation the tissues keep up their phosphorus content by drawing on the phosphates of the bones. The effects of withholding or adding inorganic and organic phosphorus compounds to the food of growing dogs was studied by Durlach, r 913.

The lecithin content of different tissues was studied by Cruikshank, 1913 ; it is also discussed in Merck's Reports, 26:6.

\section{LECITHIN}

General Statement.-This is probably the most promising of the phosphorus tonics. There is at least some experimental evidence that its administration favors growth, although this is open to suspicion. It has not achieved a secure place in therapeutics. The occurrence, composition and properties have been sufficiently discussed in the preceding paragraphs. 
Lecithin Content of Food.-The ordinary mixed dict contains at least $5 \mathrm{Gm}$. of lecithin daily; and this can be doubled by adding a couple of eggs (egg-yolk contains 6.8 per cent. of lecithin); or by liver or sweetbreads, which are also rich in phosphorus. In comparison with this, the phosphorus content of medicines is ridiculously small; for instance, the daily dose of lecithin amounts to only $0.2 \mathrm{Gm}$. Milk, however, is rather poor in lecithin (I pint contains 0.3 to $0.4 \mathrm{Gm}$.) (II. Koch, I905); but the organism is able to synthesize its lecithin from inorganic phosphates.

Fate of Lecithin.-Modern investigators agree that little, if any, lecithin is absorbed as such (contrary to the opinion of Bunge); but that the fat-splitting enzrmes of the intestines split off the glycerophosphoric acid, which is further decomposed into phosphoric acid before it is absorbed (Bokay, 1877; Pasqualis, I893; Plimmer, 1013). Possibly a fraction of the glycerophosphoric acid, and even of undecomposed lecithin, is absorbed before the cleavage is completed. In this case, or if the lecithin is introduced parenterally, the cleavage occurs in the tissues; the excretion being entirely as inorganic phosphates.

Effects on Growth.-Danilewsky, I 895 and I 896 , believed that feeding isolated lecithin stimulates the processes of growth, producing increase of red corpuscles and accelerated physical development as compared with control animals. The growth of plants was also increased. The division-rate of paramecium is hastened by lecithin (to a smaller degree also by cholesterol) (Browder, igi 5 ).

Since the experimental quantities were too small to have much food value, they would have acted as true stimulants. However, a critical attitude toward these statements appears justified. It is rather remarkable that the addition of these small doses of isolated lecithin should have effects which are not produced by the much larger quantities in the ordinary diet. Moreover, several recent investigators (Goldfarb, 1097; Yoshimoto, I910) were unable to confirm the experimental data. They obtained negative results even with long-continued administration.

On the other hand, the lipins of egg-yolk or butter-fat have a positive effect on the growth of rats kept on a uniform artificial diet (McCollum and Davis, I914; Osborne and Mendel); but this is not due to phospho-lipins.

Effects on Metabolism.-The statements are similarly contradictory. Tunnicliffe, 1906, and Slowtzoff, 1906, described retention of nitrogen and phosphoric acid.

The Clinical Data.-These are even more unsatisfactory than the experimental, although apparently successful tonic and hematinic effects have been described (e.g., Serono, r897; Cronheim and Mueller, I903, etc.).

Action on Circulation.--Danilewsky, I906, and Michailowsky, I9Io; and Katznelson, I IIO, state that the heart of frogs and rabbits is stimulated by dilute, depressed by concentrated solutions. Lifschitz, I910, describes rise of blood pressure and cardiac stimulation. Lawrow and Woronzow, IgI 2, produced a marked antagonistic effect to cardiac poisons (chloral and other lipolytic narcotics) in mammals, by the intravenous injection of $0.1 \mathrm{Gm}$. per kilogram, as 5 per cent. emulsion. Much larger doses were harmless. The results were still more marked in excised hearts. They attribute the effects partly to direct cardiac stimulation, partly to disintoxication of the lipolytic poisons. Hauschmidt, I9I3, confirms that intravenous injection is harmless and lessens the toxicity of the lipolytic narcotics and also of curare, strychnin and morphin; but that it increases the toxicity of ricin.

Role in Fat-Assimilation.-Bloor, I915 and 1916 , found that the lecithin content of the blood increases ro to 35 per cent. during the absorption of fats, generally parallel to the increase of blood fat. The accumulation of lecithin is greatest in the corpuscles, which contain about twice as much as the plasma. The cholestel content (which is about equal in the corpuscles and plasma) is not increased by feeding fats.

These data indicate an important rôle of lecithin in fat assimilation. Bloor believes that lecithin is a regular stage in fat metabolism, through which all fats must pass before they are assimilated. The formation of lecithin probably occurs in the liver.

Lecithin on Cutaneous Absorption.-Borshim, I9I 2, claims that small amounts, rubbed into the skin before the application of a potassium iodid solution increases its absorption; while large amounts have the reverse effect. Glucose, salicylic acid and tetanus toxin were not absorbed.

Administration.-Commercial lecithin may be administered as pills, 0.1 to 0.5 $\mathrm{Gm}$., $\mathrm{I} 1 / 2$ to $8 \mathrm{gr}$. per day; for infants, one-third of this dosage. It has also been given hypodermically, I c.c. of 5 per cent. in oil; but in view of the impurities, this is not advisable. It is incompatible with alkalies (Details of administration; Merck's Reports, 26:40). 


\section{PHYTIN}

This consists of the salts (usually calcium) of an organic phosphoric acid, probably containing inosit (presumably inosit-hexaphosphoric acid). It is widely distributed in plants (bibliography, A. R. Rose, I912). It is prepared from plant seeds, and has been proposed as a substitute for lecithin. It is absorbed and metabolized, but must be decomposed before it can be used by the tissues. Abundant amounts are contained in ordinary food; and there is no clear evidence that it is superior to inorganic phosphates.

\section{GLYCEROPHOSPHATES}

These are absorbed in part unchanged, but for the greater part are decomposed in the intestines into ordinary phosphates (Plimmer, r9r3). The excretion is entirely as phosphate.

Since lecithin is split into glycerophosphates in the intestines it was suggested by Pasqualis, 1894, Buelow, 1894, and Robin, 1894, that the direct administration of the cheaper glycerophosphates (especially the calcium salt) should accomplish the same results. However, according to Willstaetter, the synthetic glycerophosphoric acid is not identical with that formed from lecithin. In any event, the evidence of its usefulness is even less convincing than for lecithin (Report, Counc, Pharm, Chem, Igr6, J. A. M. A., 67; 1033).

A proprietary compound of glycerophosphates and casein has been widely and extravagantly advertised as "Sanatogen." It is a very costly food, in no sense superior to ordinary casein, such as cottage cheese (Street, r914).

\section{PREPARATIONS - GLYCEROPHOSPHATES}

Calcii Glycerophosphas (Calc. Glycerophos.), U.S.P.; the normal calcium salt of glycerophosphoric acid $\left(\mathrm{C}_{3} \mathrm{H}_{5}(\mathrm{OH})_{2} \mathrm{PO}_{4} \mathrm{Ca}\right)$. - The most commonly used glycerophosphate. Fine, white powder; odorless and almost tasteless, somewhat hydroscopic. Sol. in water $(1: 50)$; insol. in alc. Solutions of glycerophosphates are decomposed on heating. Dose, $0.25 \mathrm{Gm} ., 4$ gr., U.S.P.

Sod.Glycerophos., U.S.P. - Not less than 68 per cent. of $\mathrm{C}_{3} \mathrm{H}_{5}(\mathrm{OH})_{2} \mathrm{PO}_{4} \mathrm{Na}_{2}$. White plates or scales, or powder; saline taste. Very sol. in water; nearly insol. in alc. Dose, 0.25 Gm., 4 gr., U.S.P.

Liq. Sod. Glyceroplios., U.S.P.- - Not less than 50 per cent. of $\mathrm{Na}_{2} \mathrm{C}_{3} \mathrm{H}_{5}(\mathrm{OH})_{2} \mathrm{PO}$. Dose, 0.35 c.c., 6 minims, U.S.P.

\section{HYPOPHOSPHITES}

General Statement.-These were introduced by Churchill, 1855, against tuberculosis, on the theory that phthisis is caused by lack of oxidation and that this would be stimulated by organic phosphorus; and that the hypophosphites would be converted into this "phosphatide element." On the basis of this fantastic theory, they have been used considerably as tonics in tuberculosis. The clinical evidence is too indefinite and the experimental evidence is generally negative (Marriott, I9I6). Report Counc. Pharm. Chem., 19r6, J. A. MI. A. ,67:760).

The hypophosphites are excreted rapidly and almost completely unchanged ( 85 per cent; Marriott, I9I6) by the urine (Paquelin and Joly; Boddaert, 1895; Massol and Gamel, I90I); a trace may possibly be oxidized (Delaini, I9II). (Patto, I9ro, claims that only a fraction is excreted unchanged.) They are not diuretic.

The unscientific status of the hypophosphites is reflected in the polypharmacal syrups in which they are generally prescribed, such as the Syrupus Hypophosphitum, U.S.P., containing four different hypophosphites, dose ro c.c.; and the Syrupus Hypophosphitum cum Ferro (dose Io c.c.), containing six hypophosphites besides quinin and strychnin; there is also an emulsion of cod liver oil with hypophosphites.

The hypophosphites are easily soluble in water; dose, O.I to I Gm.; I to $15 \mathrm{gr}$. The calcium salt is probably the most important.

\section{PREPARATIONS-HYPOPHOSPHITES}

Acidum Hypophosphorosum (Acid. Hypophos.), U.S.P.-About 31 per cent. of $\mathrm{HPH}_{2} \mathrm{O}_{2}$. Colorless or slightly yellow, odorless liquid. Intensely acid taste.

Acid. Hypophos. Dil., U.S.P.-About 1o per cent. of $\mathrm{HPH}_{2} \mathrm{O}_{2}$. Colorless, odorless liquid; strongly acid taste. Dose, 0.5 c.c., 8 minims, U.S.P.

Calcii Hypophosphis (Calc. Hypophos.), U.S.P., B.P.; $\mathrm{Ca}\left(\mathrm{PH}_{2} \mathrm{O}_{2}\right)_{2}$ - -Colorless, 
cransparent prisms, or small lustrous scales, or a white, crystalline powder; odorless, nauseous and bitter taste. Freely sol. in water ( $1: 6.5$ ); insol. in alc. Dose, $0.5 \mathrm{Gm}$., 8 gr., U.S.P.; 0.2 to $0.6 \mathrm{Gm} ., 3$ to 10 gr., B.P.

Pot. Hypophos., U.S.P.; $\mathrm{KPH}_{2} \mathrm{O}_{2}$. - Very sol. in water (1:0.6). Dose, $0.5 \mathrm{Gm}$., 8 gr., U.S.P.

Sod. Hypophos., U.S.P., B.P.; $\mathrm{NaPH}_{2} \mathrm{O}_{2}+\mathrm{H}_{2} \mathrm{O}$.- Saline taste. Frecly sol. in water ( $\mathrm{r}: \mathrm{I})$; sol. in alc. Dose, I Gm., Is gr., U.S.P.

Syrupus Hy pophosphitum (Syr. Hypophos.), U.S.P.-Contains a mixture of calcium, potassium and sodium hypophosphites (total, 7.5 per cent.), acidulated with hypophosphorous acid. Dose, ro c.c., $2 \frac{1}{2}$ drams, U.S.P.

Syrupus Irypophosphitum Compositum, N.F.-Contains the above, also the hypophosphites of Fe and Mn, with quinin and strychnin. Dose, ro c.c., 2 drams; contains I r mg. (1/6 gr.) quinin; I.I mg. (1/60 gr.) strychnin. 



\section{APPENDIX}

\section{APPENDIX A.-TABULATION OF AVERAGE DOSES}

The following list may aid in memorizing the subject. The drugs have been divided into four groups, according to the order of their practical importance. It is not advisable that medical students should trouble themselves with group D.

\section{AVERAGE DOSES}

Medical Students are not required to memorize Class C

Group A.-Highest Importance

\begin{tabular}{|c|c|c|c|}
\hline Scopolamin. & 0.0003 & Acetanil. & \\
\hline Atropin ...... & 0.0005 & Hexam... & 0.25 \\
\hline Strophanthin.... & 0.001 & Acetphen............ & 0.3 \\
\hline Strychnin Salts. & 0.0015 & Iodids, Sod., Pot., etc. & 0.3 \\
\hline Arsen. Triox.... & 0.002 & Bismuth, Insol. Salts. . & 0.5 \\
\hline Mercuric Salts........ & 0.003 & Chloral Hydr. ........ & 0.5 \\
\hline Apomorph. Hydrochl. ........... & 0.005 & Tr. Digit.... & 0.5 \\
\hline Morphin Salts............ & 0.008 & Tr. Opii... & 0.5 \\
\hline Pilocarpin Salts. $\ldots \ldots \ldots \ldots \ldots$ & 0.01 & Bicarb., Sod. or Pot... & I \\
\hline Hydrarg. Chlor. Mit. . . . . . . & 0.015 & Bromids, Sod., Pot. etc. & I \\
\hline Codein Salts $\ldots \ldots \ldots \ldots \ldots$ & 0.03 & Salicylates, Sod. or Stront. & I \\
\hline Sp. Glyceryl. Nit.... & 0.05 & Inf. Digit. $\ldots \ldots \ldots \ldots \ldots$ & 4 \\
\hline Opium ........... & 0.06 & Tr. Opii Camph............. & 4 \\
\hline Quinin Salts....... & $0 . \mathrm{I}$ & & \\
\hline $\begin{array}{l}\text { Arsenic Solutions............ } \\
\quad \text { (Except Liq. Arsen. et } \mathrm{Hg} . \text { Iod }\end{array}$ & $\begin{array}{l}0.2 \\
0.1)\end{array}$ & & \\
\hline
\end{tabular}

Group B.-Very Important

Phosphor.

Epineph

Physostigmin, Sal ........

Diacetylmorph Hal. $\ldots \ldots$.....

Elaterin. ................

Antim. et Pot. Tart. . . . . . . . .

Res. Podolph...............

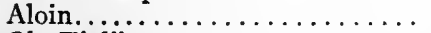

ol. Tiglii.................

Digit. $\ldots \ldots \ldots \ldots \ldots \ldots \ldots \ldots . . . . \ldots 6$

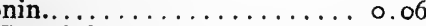

Sod. Cacodyl................. 0.06

Sod. Nitris................. 0.06

Caffein.................... 15

Amyl Nitris................. 0.2

Camph......................

Aloe........................ 0.25

Cupr. Sulph................

Ferr. Carb. Sacch............. 0.25

Mass. Ferr. Carb. . . . . . . . . 0.25

Mass. Hydrarg. . . . . . . . . . . 0.2.

Terpin Hydr................. 0.25

Acid Acetyl Sal................ 0.3

Ammon. Carb. or Chlor....... 0.3
5 Antipyr................ 0.3

Caff. Cit. or Caff. Sod. Benz.... 0.3

Tr. Aconit................ 0.3

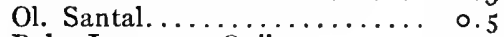

Pulv. Ipecac. et Opii. . . . . . . . . 0.5

Salvarsan.................. 0.5

Tr. Nuc. Vom. . . . . . . . . . 0.5

Tr. Strophanth............ 0.5

Veronal................ 0.5

Methyl Salicyl............. 0.75

Neosalvarsan............. 0.75

Sulphonal or Trional. . . . . . . . 0.75

Acetate, Pot. or Sod........... I

Acid. Hydrochl. Dil.... . . . . . . . 1

Calc. Carb............... I

Casc. Sagr. Fldext. . . . . . . . I I

Citrate, Pot. or Sod............ I

Ipecac.................. I

Liq. Hypophysis. . . . . . . . . . I

Syr. Ipecac............ I

Syr. Ferr. Iod.............. I

Theobrom. Sodio-Sal.......... I

Zinc. Sulph.............. I

Casc. Sagr. Arom. Fldext. . . . . . . 2 
lirgot, liklext.

Marg. Oxid

()leores. Aspid.

Paraldehyd.

l'uly. Jalap. Co.

Sp. Ammon. Arom

Mag. Carb

Pulv. Glycyrrh. Co
Sod. Phos. 4

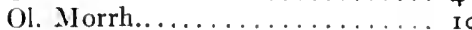

Pot. et Sod. Tart.............. ro

Syr. Rhei Arom.............. Io

Ol. Ricin............... I 5

Petrolat. Liq................ I

Sulphate, Mag. or Sod.......... I5

Liq. Mag. Cit.............. 300

Group C.-Fairly IMPortant

Aconitin.

Hyoscyamin

Colchicin. .

Homatrop. Hydrobr

(Juabain.

Hydrarg. Salic.

Sod. Arsen. .

Arg. Nit.

Ext. Cannab.

Hyolrarg. Iod. Flav

Hydrastin .

Spartein. Sulph.

Ethylmorph. Hydrochl.

Ext. Bellad. Fol.

Cocain Hydrochl.

Emetin. Hydrochl.

Erythr. Tetranit.

Ext. Opii. ...........

Bellad. Rad., Fldext. .

Cannab., Fldext........

Fldext. Ipecac.

Ol. Phosphor.

Papaverin Sulph............. 0.05

Thiosinamin ............. 0.05

Cotarnin. Hydrochl. . . . . . . . 0.06

Ext. Colch................ o. o6

Ext. Hyoscy. ................ 0.06

Ferr. Reduct................ . 0.06

Phenol. .................. 0.06

Plumb. Acet................ 0.06

Pot. Permang.............. 0.06

Acid. Hydrocyan. Dil. . . . . . . . . . o. I

Ext. Fel. Bov............... o. I

Ferr. Sulph. Exsic........... o. I

Tr. Iodi............... o. I

Resorcin.................. o.125

Thymol................ o.125

Zinc. Acet, or Valer............ o. I 25

Cinchonin. or Cinchonidin. Sulph. o. 5

Methylthionin. Chlor.......... o. I5

Phenolphthal................... o. 5

Volatile Oils.................. 0.2

(Except O1. Amygd. Amar..... 0. 0.

(Except O1. Tereb. Rect........ 0.3 )

(Except Ol. Cubeb.............. 0.5 )

(Except O1. Eucalypt........... o.5)

(Except Ol. Santal............... o.5)

(Except Ol. Gaulth.............. 0.75 )

Benzosulphinid (Sod.)............. 0.2

Bromof..................... 0.2

Hyoscy., Fldext.................. 0.2

Liq. Iodi Co..................

Ol. Chenopod..................
1 $_{5}$ Quin. Tann................... 0.2

0.25

0.25

0.25

0.25

0.25

0.25

0.25

0.25

0.25

0.25

0.25

0.25

0.25

0.25

0.25

0.25

0.25

0.3

0.3

0.3

0.5

Acid. Bor...................

Acid Phenylcinch............. 0.5

Acid. Tann................ 0.5

Alum..................... 0.5

Butyl Chloral Hydr............ 0.5

Calc. Chlor. or Lact... . . . . . . . . 0.5

Chlorbutanol.............. 0.5

Guaiacol..................... 0.5

Hypophos. Calc. or Pot........ 0.5

Lith. Carb. and Citr........... 0.5

Oleores. Cubeb.............. 0.5

Pancreat.................... 0.5

Pepsin.................. 0.5

Pot. Nitras................. 0.5

Tr. Cannabis.............. 0.5

Tr. Capsic................. 0.5

Tr. Ferr. Chlor............. 0.5

Tr. Veratr. Vir............. 0.5

Apocyn. Fldext.............. 0.75

Sod. Bor................ 0.75

Tr. Bellad. Fol............... 0.75

Acid. Camph............... I

Acid. Nitrohydrochl. Dil........ I I

Ether............... I

Ethyl Carbam.............. I

Benzoates, Sod. or Ammon...... I

Carbo Lig................. I

Copaib................. I

Ext. Tarax.............. I

Guaiacol. Carb.............. I

Quin. et Ureae Hydrochl......... I

Salicin.................. I 
Sp. Camph .

Sod. Hypophos. . . . . . . . . . . .

Tannalbin............... I

Tr. Lobel. . . . . . . . . . . . . . . I

Tr. Scill.

Vin. Antim

Vin. Colch.

Sod. Phos. Acid.

Spirits .

(Except $\mathrm{Sp}$. Glyceryl Nit ....

(Except Sp. Amygd. Amar. ... .

(Except Sp. Ether...........

(Except Sp. Juniper Co....... Io

Acid. Acet. Dil. .. . . . . . . . . . . . 2

Acid. Phosp. Dil. . . . . . . . . . . . 2

Acid. Sulph. Arom. . . . . . . . . . 2

Fldext. Buchu.................. 2

Fldext. Glycyrrh............. 2

Fldext. Senn. . . . . . . . . . . 2

Fldext. Hydrast. . . . . . . . . . . 2

Pot. Bitart. . . . . . . . . . . . . 2

Sp. Ether. Nitros. . . . . . . . . . 2

Sp. Chlorof. ............. 2

Syr. Scill................. 2

Syr. Scill. Co............... 2

Tr. Card.................. 2

Tr. Colch................ 2

Tr. Guaiac Ammon. . . . . . . . . . . 2

Tr. Hyoscy. .............. 2

Tr. Quass............... 2

Tr. Rhei Arom.............. 2

Tr. Valer. Ammon............ 2

Tr. Zingib. . . . . . . . . . 2

Uvæ Ursi, Fldext. ........... 2

El. Ferr. Quin. et Strych. Phos... 4

Glycerin................ 4

Liq. Hydrog. Dioxid.......... 4
Magma Bism............. . 4

Pot. Cit. Eff............... 4

Senn.................. . 4

Sp. Ether............... . 4

Sulphur................ 4

Syr. Pic. Liq. . . . . . . . . . . . 4

Syr. Seneg............... . 4

Syr. Senn............... 4

Tr. Calumb.............. 4

Tr. Card. Co............... 4

Tr. Cinch. and C

Tr. Gambir Co............. . 4

Tr. Gent. Co............... . 4

Tr. Hydrast. . . . . . . . . . . 4

Tr. Kino. ................ . 4

Agar................... 10

Aq. Camph............... Io

Aq. Creosot................ 10

Magma Mag. .............. 10

Mist. Glycyrrh. Co............. 10

Sinapis................... 10

Syr. Hypophos. . . . . . . . . . . 10

Flavoring Syrups............ I5

(Except Pic. Liq........... . 4)

(Except Pruni. Virg........ . . 4)

Flavoring Waters............. I5

(Except Aq. Amygd. Amar. . . 4)

Aq. Chlorof............... 15

Emuls. Asafœet............... I 5

Emuls. Ol Morrh............. I5

Ext. Malt............... I 5

Liq. Ammon. Acet. . . . . . . . . . I 5

Liq. Calc................. I 5

Mist. Cret............. I5

Mucil. Acac.............. I5

Ol. Oliv................... 30

Ferr. Hydrox. c. Magn. Oxid. . . I 20

Group D.-Slight Importance

Arsen. Iod. . . . . . . . . . . . 0.005

Aur.. et Sod. Chlor. . . . . . . . . 0.005

Iodum... . . . . . . . . . . . . 0.005

Sod. Arsen. Exsic. . . . . . . . . . 0.005

Ext. Physostig. . . . . . . . . . . . 0.008

Ext. Aconit............... o or

Ext. Gelsem................ o. or

Ext. Stramon.............. o. or

Ferr. Sulph.............. o. or

Hydrarg. Succinim. . . . . . . . . o. or

Uran. Nit. . . . . . . . . . . o. or

Aconit. Fldext. . . . . . . . . . . 0.03

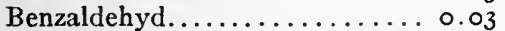

Chrysarobin.................. 0.03

Ext. Colocynth............ 0.03

Gelsem. Fldext............. 0.03

Hypophysis Sicc.............. 0.03

Oleores. Capsic............... 0.03

Oleores. Piper.............. 0.03

Oleores. Zingib............. 0.03

Trinitrophen............... 0.03

Trit. Elaterin............. 0.03

Vanillin..................... 0.03

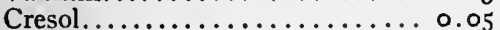

Menthol................. 0.05
Nuc. Vom. Fldext........... 0.05

Sod. Arsanil................ 0.05

Arg. Oxid................. 0.06

Bellad. Fol. . . . . . . . . . . . 0.06

Calc. Sulph. Ven............ 0.06

Capsic.................. 0. 06

Colocynth.................. 0.06

Euonym. Ext............. 0.06

Ferr. Chlor................. 0.06

Staphisag.................. 0.06

Stramon................... 0.06

Strophanthus................... 0.06

Tr. Canthar............... . I

Liq. Ferr. Chlor............... o. I

Scill. Fldext................. o. I

Verat. Vir. Fldext............ o. I

Bism. et. Ammon. Cit. . . . . . . . 0.1 25

Cambog................. 0.125

Camph. Monobrom.......... 0.125

Lobel. Fldext.............. 0.125

Res. Jalap................ 0.1 25

Sanguin................... 0.125

Zinc Phenolsulph........... 0. I 25

Colch. Sem. Fldext. . . . . . . . 0.2

Eugenol................. 0.2 
Liq. Ferr. Subsulpli. .

()1. Pic. liq. . . . . . .
Re. Scammon. . . .

Betisuptliol.

Cirguph.

lixt. Cimicil.

Ciunam. .

Colch. Corm.

Ext. Colocynth. Co.

lixt. Sumbul.

Ferr. et Quin. Cit.

(ilyerrh. Ammon.

Mlangan. Diox. Praec.

Mosch

Scammon. Rad.

Sod. Carb. Monohyd.

Supraren. Sicc.

Zinc. Oxid.

lucalyptol.

Glycer. Phenol.

Liq. Sod. Glycerophos.

Acid. Hydriod. Dil.

Acid. Hypophos. Dil.

Acid. Tart.

Ammon. Salicyl.

Anis. .

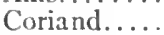

Diastas.

Euonym. Cort.

Ext. Hydrast.

Ext. Viburn. Prunif.

Galla. . . . . . . . . .

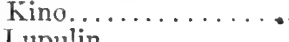

Myrrh

Olcores. Petrosel. . . . . . . . . . . .

Ol. Cajup................. 0.5

Ol. Cubeb................ 0.5

Ol. Eucalypt............... 0.5

Piper................. 0.5

Pix. Liq................ 0.5

Podoph., Fldext............ 0.5

Quass.................... 0.5

Tr. Stramon............. 0.5

Acid. Salicyl................ 0.75

Acet. Scill................

Acids, Dilute: Hydrobr., Nitr., Sulph................ I

Acid. Gall. ................ .

Amylen Hydr. . . . . . . . . . . . I I

Aq. Ammon.............. I

Benzoin............... I

Card. Sem................ I

Carum...............

Chloral Formam........... I

Cimicif., Fledxt. . . . . . . . . . . I I

Cinch., Fledxt............. I

Creosot. Carb............... I

Cubeb................. I

Fldext. Arom................ I

Fldext. Erodict ............ I

Fldext. Sabal. ............... I

Fonic .................. I

Frangul., Fldext............. I

Gambir................ I
Gentian Fldext............. I

Guaiac................. I

Ich thyol............... I

Jalap.................. I

Lactucar.................. I

Liq. Ferr. Dial. ............. I

Liq. Potass. or Sod. Hydrox... . . . I

Pot. Carb.............. I

Pulv. Arom............... I

Rhei, Fldext.............. I

Seneg................. I

Serpent. . . . . . . . . . . . I

Sod. Sulphis Exsic............ I

Sod. Thiosulph............. I

Styrax................. I

Tr. Arnic ................ I

Tr. Asafœet.............. I

Tr. Benzoin............. I

Tr. Myrrh............. I

Tr. Physostig. ............ I

Vin. Ipecac. . . . . . . . . . . . I

Zingib., Fldext........... I

Calumb................ 2

Emul. Ol. Tereb............. 2

Eucalypt., Fldext............ 2

Granat., Fldext............ 2

Gossyp. Rad. Cort. Fldext...... 2

Grindel., Fldext............ 2

Guaran., Fldext............. 2

Pareira................ 2

Pilocarp., Fldext. . . . . . . . 2

Prun. Virg.............. 2

Pulv. Cret. Co.............. 2

Pulv. Rhei Co.............. 2

Pyreth................. 2

Sarsap., Fldext. and Co........ 2

Sumbul., Fldext............. 2

Sod. Phos. Exsic. . . . . . . . . . 2

Stilling. Fldext............. 2

Tr. Aloes................. 2

Tr. Benzoin Co............ 2

Tr. Cinnamon............ 2

Tr. Lactucar.............. 2

Tr. Lavand............... 2

Tr. Tolut.............. 2

Valer................. 2

Viburn. Prunif., Fldext........ 2

Xanthox., Fldext............ 2

Aspid.................. 4

Aspidosp., Fldext........... 4

Mel Rosæ................ 4

Menth., Pip. or Vir.......... 4

Spigel., Fldext. . . . . . . . . 4

Syr. Acid. Hydriod. . . . . . . . . . 4

Tr. Guaiac. .............. 4

Tr. Mosch............... 4

Tr. Rhei............... 4

Tr. Valer.............. 4

Liq. Sod. Phos.............. . Io

Sassafras................. 10

Sod. Phos. Eff. .............. 10

Syr. Calc. Lactophos. . . . . . . . . Io

Syr. Lactucar. . . . . . . . . . . . Io

Syr. Rhei............... 10

Tarax. Fldext............. 10

Trit., Fldext.............. ro 
Manna................ I 5

Matricar..................... 15

Liq. Ferr. et Ammon. Acet . . . . . 15

Liq. Pot. Cit . ........... I 5

Sod. Chlor. . . . . . . . . . . . I 5

Ol. Lini..................

Pepo.................. 30

Inf. Senn. Co............. I 20

\section{APPENDIX B.-CHECK-LIST FOR THE STUDY OF PREPARATIONS}

Introductory.-The student should keep a separate notebook, ruled in columns as illustrated in the schema, ${ }^{1}$ into which the names of the drugs and the relevant data are entered as they are studied. The preparation of these tables is probably the most efficient method of learning the subject. The synonyms, origin, miscibility, dose, and remarks will be found in the text. The appearance, odor, and taste should be studied directly from the specimen. It is highly desirable that the physician should be somewhat familiar with the physical characters of the principal drugs, if for no other reason than that it will give him some confidence, save him some embarrassment, and guarantee the proper dispensing of his prescriptions. This knowledge may be of vital importance for the prompt diagnosis of poisoning.

Those drugs which may be readily recognized by their physical characters are marked with an asterisk. It is expected that the student will learn these so that he can identify the unlabelled specimens. The other specimens should be learned sufficiently, so that the student is able to decide whether or not an unlabelled sample may or may not be whatever is suggested. For instance, whether a white powder may be arsenic trioxid; whether a colorless fluid may be Tincture of Nux Vomica, etc. The drugs which are bracketed in the list are for inspection only. No data need be entered for them.

The Materia Medica Museum should contain the drugs mentioned in the following exercises. These may be duplicated for large classes. It will be found convenient to arrange the drugs in the order of this list.

A card index is almost indispensable. It. should give the case, shelf, and bottle number, and these should also be placed on the containers. The labels should give the name of the drug; whether it is for identification, important, or unimportant; and whether it may be tasted. Separate gummed slips, distinguished by colors, are very handy for this purpose).

White Labels

\section{Strong Poison! $\underset{\substack{\text { Do Not } \\ \text { Taste. }}}{ }$}

(Red Label)

Poison! cautiousty.

(Blue Label)

\section{Practically Harmless.}

(Green Label)

1 See "Schema," page 824.

\section{Identification.}

(Red Print)

Important.

(Blue Print)

Unimportant.

(Black Print) 
()ther drugs especially crude specimens-are valuable for reference, but may be dinfellsed with. Illustrations and herbarium specimens of important and domestic medicinal plints are also profitable.

SCHEMA FOR TIE TABULATION OF MATERIA MEDICA NOTES

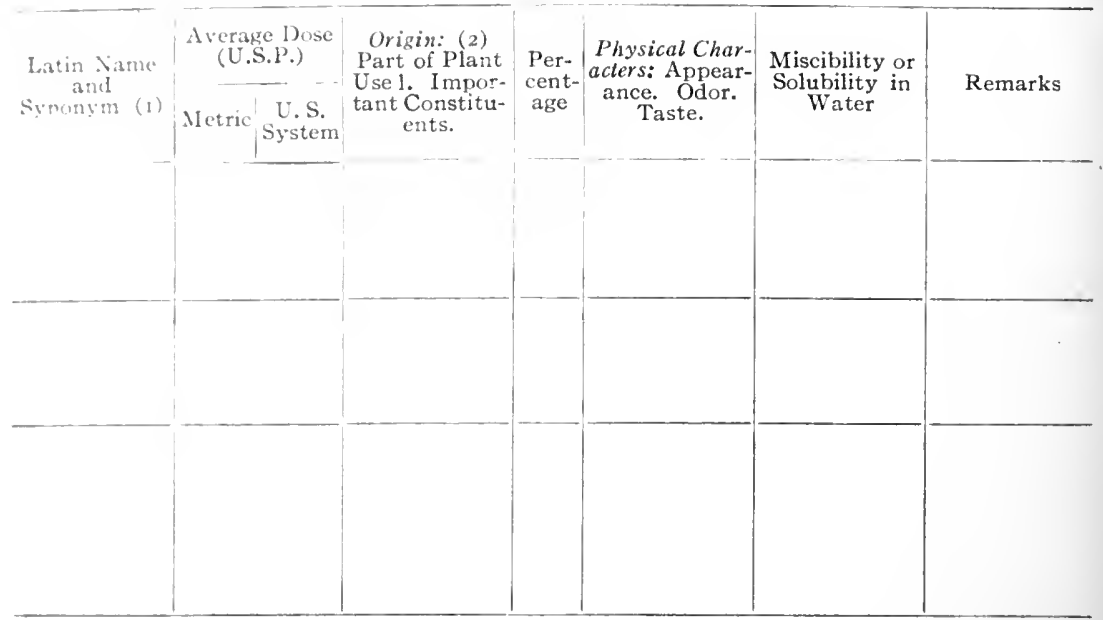

Explanatory:

(I) Enter the full Latin name and the abbreviation. Synonyms or English Names need be entered only if they differ markedly from the Latin name.

(2) The origin need be given only for drugs or preparations derived from the organic kingdom.

\section{CHECK LIST OF IMPORTANT PREPARATIONS}

" $\mathrm{H}$ " means that the drug is harmless.

"C" " that it may be tasted cautiously.

"Dnt.": Do Not Taste!

\section{Colors}

Liq. Carmin................ Dnt. (Coccus).................. Dnt.

Tr. Persion............... Dnt.

Tr. Curcum. ................ Dnt.

\section{Sugars and Syrups}

* Sacch.................. H

* Sacch. Lact. Pulv... . . . . . . . . . H H

(Sacch. Lact. Cryst.)............H

(Syr..................... H

* Syr. Acid. Cit................ H

* Syr. Aur. ... . . . . . . . . . . . . . H H

* Syr. Tolu.................. H

* Elixir Arom................ H

(Glycyrrh. Rad.)............... H

* Syr. Glycyrrh... . . . . . . . . . . H

* Fldext. Glycyrrh............. H

* Elix. Glycyrrh.. . . . . . . . . . . . H H

Aromatic Waters, Spirits and Oils

* Aq. Cinnam............... H

(Ol. Cassia) .................
* Aq. Menth. Pip............ H

* Aq. Ros. . . . . . . . . . . . . . H

*Tr. Card. Co. . . . . . . . . . . . . H

(Card. Sem.)................ H

\section{Ferments and Nutrients}

Pepsin................ H

Pancreatin................ H

* Ol Morrh............... H

* Emul. Ol. Morrh. ............ H

(Ext. Malt.)................H

\section{Emollients}

* Ol. Oliv................ H

* Ol. Theobrom............ H

* Adeps Benz................. . Dnt.

* Adeps Lan. Hyd. . . . . . . . . . . D Dnt.

* Petrolat. . . . . . . . . . . . . . Dnt

* Petrolat. Alb................ Dnt.

* Petrolat. Liq... . . . . . . . . . . . Dnt.

\section{Demulcents}

* Glycer....................H Supp. Glycerin.......... No specimen 
* Acac.

* Mucil. Acac.

* Trag.

* Gelat

\section{Protectives}

* Amylum

* Talc. Purif.

* Lycopod

* Lini Sem. Contus.

(Linum)

* Collod

Collod. Flex

Gossyp. Purif

\section{Caustic Alkalies}

* Pot. Hydrox.

Liq. Pot. Hydrox.

Sod. Carb. Monohyd.

Sod. Carb.

Liq. Calc.

Lin. Calc.

\section{Soap, Sulphids and Sulphur}

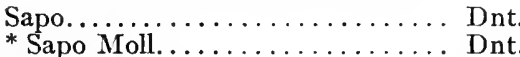

* Lin. Sapon............... Dnt

Lin. Sapon. Moll. ............. Dnt.

Calc. Sulphid. Ven............ Dnt.

* Pot. sulphurat. . . . . . . . . . . . . D Dnt.

Sulphur Sublim............... H

* Sulphur Lot. . . . . . . . . . . . . . . . H

Ung. Sulphur.............. Dnt.

Ichthyol.

\section{Caustic Acids, Iodin, Tannin}

* Acid, Nit.... . . . . . . . . . . . Dnt.

* Tr. Iodi. . . . . . . . . . . . . . . . . Dnt

(Iodum)..................... Dnt.

* Acid. Tann............... C

* Glycer. Acid. Tann. . . . . . . . . . C

(Galla) ................... Dnt.

Tannalbin............... C

* Tr. Gambir. Co............... C

(Gambir)................ H

\section{Volatile Oils}

* Asafet................. Dnt.

* Tr. Valer. Ammon........... . C

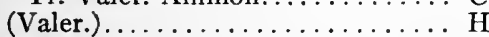

* Ol. Tereb. ................ C

Ol. Tereb. Rect................ C

* ol. Pini Pumil.............. Dnt.

Terpin. Hyd................. C

* Ol. Eucalypt. . . . . . . . . . . . . . C

Eucalyptol................. C

* Ol. Caryoph .............. C

* Copaib................... C

* Ol. Santal ................. C

* Tr. Benzoin. Co.............. C

(Benzoin).............. H

* Bals. Peruv. ................ C

(Bals. Tolut.).............. H

Tr. Myrrh............... C

(Myrrh) ................. H

*Sinap. Nig. Pulv........... C
(Sinap. Nig.).

Emp. Sinap.

C

Ol. Sinap. Vol................. Int.

(Do not smell directly)

\section{Fixed Organic Irritants}

Emp. Canthar.......... No specimen

(Canthar.)................. Dnt.

Tr. Capsic.

(Capsic.)

Chrysarobin.

C

C

Dnt.

\section{Bitters and Carminatives}

Ext. Gent.................. C

Tr. Gent. Co. . . . . . . . . . . . . H

(Gentian)............... H

* Sp. Menth. Pip............. . C

(Ol. Menth. Pip.) . . . . . . . . . . C

* Tr. Zingiber.............. C

(Zingib.)............... H

\section{Emodin Cathartics}

Ext. Casc. Sagr............... Dnt.

* Fldext. Casc. Sagr. . . . . . . . . . C

* Fldext. Casc. Sagr Arom........ H H

* Senna..................... H

Syr. Senn. ............... H

* Pulv. Glycyrrh. Co........... H

Syr. Rhei.................. H

* Syr. Rhei Arom. . . . . . . . . . . H

* Tr. Rhei Arom................. H

(Rheum) ............... H

Aloe..................... C

Aloin...................... Dnt

Phenolphthal.............. C

\section{Other Cathartics}

Ext. Fel. Bov................ C

* Ol. Ricin. . ............. H

(Mist. Ol. Ricin.) . . . . . . . . . H

Pulv. Jalap. Co... . . . . . . . . . . C

(Jalap).................. C

Res. Podoph................ Dnt.

(Podoph. Rhiz.)............... C

Elaterin................... Dnt

Ol. Tiglii................ Dnt.

\section{Anthelmintics}

Oleores. Aspid................ C

(Aspid.)................. C

Ol. Chenopod................ C

Pellet. Tann................. Dnt.

Santonin................. C

\section{Strychnin}

Strych. Nit................. Dnt.

Ext. Nuc. Vom .............. Dnt.

Tr. Nuc. Vom................. C

(Nux Vom)............... Dnt.

\section{Caffein}

Caffein................ C

Caff. Cit................

Caff. Sod. Benz................ C 
lheobrom. Siol. Sal lheophyll.

\section{Opium}

* () pium.

* Mist. Cilycyrrh. Co

l'ul. Ipreace et Opii

*'Tr. (Y)ii.

* Tr. Oprii Camph..

Tr (Opii l)eod.

Morph. Mydrochl.

lithimorph. Ilydrochl.

biacetylmorph. Itydrochl.

Codein Phos.

\section{Hydrastis, Cannabis, Cocain}

Fldext. Hy-drast.

(llydrastis).

Evt. Cannab.

Cocain. Hydrochl.

(Erythroxylon)

\section{Atropin}

Homatrop. Hydrobr.

Atrop. Sulph.

Ext. Bellad. Fol

Tr. Bellad

Ung. Bellad.

Tr. Hyoscy.

Stramon

Scopolamin. Hydrobr.

\section{Pilocarpin to Ergot}

Pilocarp. Nit.

Physostigm. Sal.

(Pliysostigma).

Tr. Lobel.

Spartein. Sulph.

Epineph.

Liq. Hypophysis

Thyroid Sicc.

* Fldext. Ergot.

(Ergot)........

\section{Nitrite}

* Amyl. Nitris. Dnt.

* Sp. Ather. Nitros... . . . . . . . . C Sp. Glyceryl Nit. . . . . . . . . . . D D Sod. Nitris. . . . . . . . . . . . D Dnt.

\section{Digitalis}

Digit.................... Dnt.

Inf. Digit. ...............

Tr. Digit................

Tr. Strophanth..............

(Strophanthus)................ Dnt.

Strophanthin........... No specimen

Syr. Scill. ................... C

Tr. Scill. .....................

(Scill.)

C

\section{Camphor}

* Camph................... C
* Lin Campl.

* Sp. Camph.

* Menthol.

\author{
Dnt. \\ C
}

\section{Saponins to Colchicum}

* Syr. Sarsap. Co............. . H

(Sarsap.)................... H

Tr. Aconit. . . . . . . . . . . . Dnt.

(Aconit.).................. Dnt.

Tr. Verat. Vir... . . . . . . . . . . Dnt.

Tr. Colch. ... . . . . . . . . . . . Dnt.

(Colch. Corm.)................. Dnt.

(Colch. Sem.)............... Dnt.

\section{Apomorphin and Ipecac}

Apomorph. Hydrochl. . . . . . . . . . Dnt.

Ipecac. Pulv................. C

(Ipecac. Rad.). . . . . . . . . . . . . . C

Fldext. Ipecac. . . . . . . . . . . . . C

Syr. Ipecac................ C

Emetin. Hydrochl. ... . . . . No specimen

\section{Quinin}

Quinin. Bisulph............. C

Quinin. Hydrochl. ............ C

* Quinin. Sulph............. C

Quinin. Tann................ C

Quinin. et Urea Hydrochl......... C

Tr. Cinchon............... H

* Tr. Cinchon. Co............. H

(Cinchona).............. H

\section{Antipyretics}

Acetanil................. C

Acetphen $\ldots \ldots \ldots \ldots \ldots \ldots \ldots \ldots \ldots$. C

Antipyr................. C

Coal Tar Antiseptics

* Phenol. . . . . . . . . . . . . . Dnt.

* Phenol. Liq.... . . . . . . . . . Dnt.

* Ung. Phenol. . . . . . . . . . . . D Dnt.

* Cresol. . . . . . . . . . . . . . . . . Dnt.

*Liq. Cresol. . . . . . . . . . . . . . . Dnt.

Resorcin................... C

(Uva Ursi).................. H

*Trinitrophen............. Dnt.

${ }^{*}$ Creosot.................. C

${ }^{*}$ Guaiacol. . . . . . . . . . . . . . C

*Guaiacol.................. C

Guaiacol. Carb.................. C

*Pix Liq. . . . . . . . . . . . . . . D D

(Syr. Pic. Liq.)... . . . . . . . . . . . H

*Ung. Pic. Liq. . . . . . . . . . . . . . Dnt.

*Thymol.................. C

Betanaphthol............... C

Acid. Benz. . . . . . . . . . . . . . . C

Sod. Benz. . . . . . . . . . . . . H

*Liq. Antisept. Alk.............. H

Acid. Salic... . . . . . . . . . . . . C

${ }^{*}$ Sod. Salic. . . . . . . . . . . . . . . H

Acid. Acetylsal................ C

*Methyl Salic................... C

*Phenyl Salic................. C

Acid. Phenylcinch............. C

Benzosulphin................ C 


\section{Miscellaneous Antiseptics}

${ }^{*}$ Liq. Formald. . . . . . . . . . . . . . Dnt.

Hexam.................. H

${ }^{*}$ Calx Chlorin.............. Dnt.

*Liq. Sod. Chlorin............... Dnt.

Sod. Sulphis Exsic........... C

*Liq. Hydrog. Diox............ H

*Pot. Permang................ Dnt.

*Acid. Bor. Pulv............. H

(Acid. Bor. Cryst.) . . . . . . . . . H

Glycer. Boroglyc........... H

Ung. Acid. Bor... . . . . . . . . . Dnt.

*Liq. Antisept............... H

*Sod. Bor. . . . . . . . . . . . . H

*Iodof ................... Dnt.

Thymol. Iod............... Dnt.

\section{Serums and Vaccines}

Ser. Antidiph........... No specimen Ser. Antidiph. Purif...... . No specimen Ser. Antitet............. No specimen Ser. Antitet. Purif... . . . . . No specimen Ser. Antitet. Sicc......... No specimen Virus vaccin............ No specimen Tuberculin Old.......... No specimen

\section{Alcohol and Anesthetics}

*Alcohol. ................. C

*Chlorof................... C

*Aq. Chlorof................ H

Lin. Chlorof.............. Dnt.

*Æther.................. C

*Sp. Æther.............. C

Ethyl Chlor............. No specimèn

\section{Hypnotics}

*Chloral Hydr............... C

*Paraldehyd.................. C

Sulphonmeth............. C

Acid. Diethylbarb............ C

\section{Gases}

Nitrogen Monox.......... No specimen Oxygen............... No specimen

\section{Cyanids}

*Acid Hydrocyan. Dil.......... Dnt. *Pot. Cyan.................. Dnt.

*Syr. Prun. Virg. ........... H

\section{Cathartic Salts}

Sod. Sulph............... H

Sod. Phos.................. H

Sod. Phos. Eff. .............. H

Sod. Phos. Acid............... H

${ }^{*}$ Pot. Bitart................... H

*Pot. et Sod. Tart............. H

*Mag. Carb................ H

Liq. Mag. Cit................ Dnt.

Magma Mag............... H

Mag. Oxid. Pond . . . . . . . . . H

${ }^{*}$ Mag. Sulph.............. H

\section{Water}

Aqua............... No specimen

Aq. Dest.............. No specimen

Aq. Dest. Steril........... No specimen

\section{Salt Cathions}

Sod. Chlor.............. No specimen Liq. Sod Chlor. Physiol.... No specimen Liq. Ammon. Acet............. H

*Ammon. Carb............. C

*Sp. Ammon. Arom . ......... C

*Ammon. Chlor............... C

*Aq. Ammon.................. Dnt.

${ }^{*}$ Lin. Ammon... . . . . . . . . . . . Dnt.

Calc. Carb. Prec. . . . . . . . . . . H

* Cret. Præc..................... H

Calc. Chlor................. C

Calc. Lact... . . . . . . . . . . . . . . C

Calc. Phosph............. H

\section{Salt Anions}

Pot. Brom................ H

*Sod. Brom.................. H

*Pot. Iod................... H

Sod. Iod.................. H

*Pot. Chloras................. C

\section{Dilute Acids and Alkalies}

Acid. Hydrochl. Dil. . . . . . . . . . C

Pot. Bicarb.................. H

*Sod. Bicarb............... H

*Acid. Acet. Dil................. H

Pot. Acet............... H

Acid. Cit................. H

Pot. Cit.................. H

Pot. Cit. Eff.............. H

\section{Arsenicals}

Arsen. Triox.............. Dnt. Liq. Acid. Arsen................ Dnt.

Liq. Arsen. et. Hydrarg. Iod.... . . Dnt.

*Liq. Pot. Arsen . . . . . . . . . . . D Dnt.

Sod. Arsen . . . . . . . . . . . . . Dnt.

Sod. Cacodyl. . . . . . . . . . . D Dnt.

Salvarsan............ No specimen

Neosalvarsan.......... No specimen

\section{Antimony and Bismuth}

Antim. et. Pot. Tart............ Dnt.

Vin. Antim.............. C

*Bism. Subcarb............... H

Bism. Subgall.............. H

Bism. Subnit................ H

Bism. Subsalic.............. H

\section{Iron}

*Ferr. Reduct.............. H

*Ferr. Carb. Sacch. ............. H

Mass. Ferr. Carb............. Dnt.

${ }^{*}$ Ferr. et Ammon. Cit........... . C

Ferr. Phos.................. C

*El. Ferr. Quin. et Strych. Phos.... C

${ }^{*}$ Syr. Ferr. Iod................ C

Ferr. Sulph. Exsic............ C

*Tr. Ferr. Chlor............... C

\section{Aluminum, Silver and Copper}

*Alum.................. C

Alum. Exsic............... Dnt. 
I.ir. . Alum. Acet.

Irg. Nit.....

*.Irg. Nit. Fus

Irg. Protein.

* Cupr. Sulph

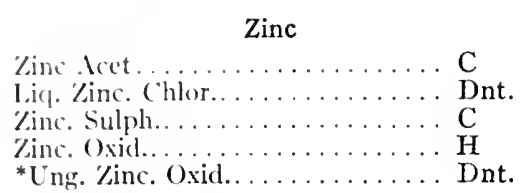

\section{Mercury}

Hydrarg. c. Cret............. C

* Ilasea Hydrarg. . . . . . . . . . . . Dnt.

*Ung. Hydrarg.............. Dnt.

Ung. Hydrarg. Dil............. Dnt.
Ol. Cinereum............ Dnt.

Hydrarg. Chlor. Mit........... C

Hydrarg. Iod. Flav.............. Dnt.

Hydrarg. Ammon.............. Dnt.

Hydrarg. Oxid. Flav........... Dnt.

Ung. Hydrarg. Oxid. Flav....... Dnt.

Hydrarg. Chlor. Corr........... Dnt.

Toxit. Hydrarg. Chlor. Corr. No specimen

Hydrarg. Iod. Rubr. . . . . . . . Dnt.

Hydrarg. Salicyl. . . . . . . . . . Dnt.

\section{Lead}

Plumb. Acet................. Dnt.

Liq. Plumb. Subacet........... Dnt.

\section{Phosphorus}

${ }^{*}$ Phosphor................. Dnt. 


\section{APPENDIX C.-BIBLIOGRAPHY}

Aarons, S. J.: Cotarnin, Brit. Gyn. J., Feb., I907.

Abderhalden, E.: Alcohol, Bibliography, 1904; Iron, Zs. Biol., 39, II3, 193, 483, I899-1900; Med. Klin., No. 24, 1906.

Abderhalden and Hirsch: Iodin, Zs. physiol. Chem., 75, 39, igi i; Nitrate, ib., 84, I89, I913.

ABEr, J. J.: Epincphrin, Zs. physiol. Chem., 28, 318, 1902; J. H. H. Bul., 13, 29, 1902; J. Biol. Chem., I, I, 1905; Morphin, ib., 3, 581, 191 2; Teaching of Pharmacology, Phil. Med. J., Sept. I, I89o.

ABEL and Ford: Amanita, Arch. exp. Path. Pharm., Suppl., 8, igo8.

Abel and Maсhт: Bufa, J. Am. Med. Assoc., 56, I531, I9II.

Abel and Rowntree: Phthaleins, J. Pharmacol., I, 23 I, rgog.

Aber, J. J., Rowntree and Turner: Vici-diffusion, J. Pharmacol., 5, 6II, I9I4.

ABEL, J. J., and TURNer: Lymph Hearts, J. Pharmacol., 6, 9i, I9I4.

ABEL, K.: Cotarnin, Berl. klin. Woch., 34, 1905.

Abelin: Salvarsan, Miuench. med. Woch., 58 , No. 33 , I9I I.

ABL, R.: Uric Acid, Arch. exp. Path. Pharm., 74, I19, I913.

Abraham: I east, Mon. Geb. Gyn., 3i, I9io.

Acr, N.: Caffein, Arch. exp. Path. Pharm., 44, 319, Igoc.

ACree and Syme: Rhus, J. Biol. Chem., 2, 547, I907.

Adams, A.: Oxygen, Bioch. J., 6, 307, i9 г2.

AdDis: Calcium, Quart. J. Med., Jan. I909; Brit. Med. J., I, 997, I909.

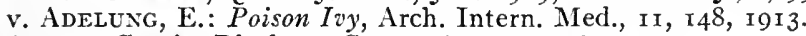

AdLer: Cocain, Biochem. Centr., 6, 299, I906.

Adler, H. M.: Olive Oil, Proc. Soc. Exp. Biol. Med., 9, I, I9гі; Rectal Feeding, An. J. Med. Sci., $\mathrm{r}_{5} \mathrm{O}, 562$, 1915.

Adler, I.: Arteriosclerosis, J. Exp. Med., 20, 93, 1914; Trans. Assoc. Am. Phys., 29, 5I2, Igr4; Blood vessels J. Pharm. Exp. Ther., 8, 297, 1016, Epincphrin, Am. J. Med. Sci., I36, 24I, 1908.

Adler, L.: Iodin, Arch. exp. Path. Pharm., 75, 362, I9I4.

ADler, L., and CzAPSki: Iodid, Bioch. Zs., 65, II7, I9I4.

AIrila, Y.: Anaphylaxis, Skand. Arch. Physiol. 31, 388, I9r4; Chloral, Arch. Int. Pharm., 23, 453, 1913.

Airila, Y., and BARDY: Magnesium, Skand. Arch. Physiol., 32, 246, I9I 5.

AlbaNese: Caffein, Arch. exp. Path. Pharm., 35, 449, 1895; ib., 43, 305, 1900; Morphin, Biochem. Centr., 9, 274, 722, I909.

Albertoni: Acetone, Arch. exp. Path. Pharm., 18, 218, I884; ib., 23, 393, 1887; Convulsants, ib., I5, 272, 1882.

Alcock: Anesthetics, Proc. Roy. Soc., 77B, 267, 1906.

Aldrich, T. B.: Adrenalin, Am. J. Physiol., 7, 359, x902; Pituitary, Am. J. Physiol., 3I, 95, I912; Thyroid, Intern. Congr. Appl. Chem., 19, I913.

Alexander, F. G., and Cserna: Narcosis, Bioch. Zs., 53, 100, I913.

-AlipRAndi: Morphin, Gaz. Med. ital., 56, No. 14, I905.

Aliprandi and Foriraroli: Cerebral Circulation, Bioph. Centr., I, 25, 1905; Gaz. med. ital., 56, No. 14, 1905 .

Allen, F.: Spinal Anesthesia, J. Am. Med. Assoc., 59, I841, 1912.

Almquist, J.: Mercury, Derm. Zs., 19, 949, I9I 2.

Alsberg, C. L.: Chenopdium, Bur. Chem. U. S. Dept. Agr., I9r3.

Alsberg, Black and Marsh: Barium, U. S. Dept. Agr. Bull., 246, I9I2.

AlsBerg, C. L., and Smitr: Saponin, J. Pharmacol., 5, 517, 1914.

Alpers, W. G.: Digitalis, Clev. Med. J., 14, 99, 1915.

Alt: Morphin, Berl. klin. Woch., 560, I889.

AlteNBURG: Iodoform, Arch. int. Pharmacod., 8, I 25, I90r.

Altland: Cinchonism, Klin. Monats. f. Augenh., July, I904.

Alvarez, W. C.: Intestinal Rhythm, Am. J. Physiol., 37, 267, 1915.

Alwens: Salvarsan, Arch. exp. Path. Pharm., 72, I77, 1913.

Amantea: Motor-cortex, Zentr. Bioch. Bioph., 15, 503, I913; Strychnin, ib., 15, 812, I9I3.

AMrBERG, S.: Iod-benzoate, Zb. ges. exp. Med., 2, I9, I9I3. 
Immikg, S., and IIlinmolz: Salls, J. Pharmacol, 8, i 20, igr6.

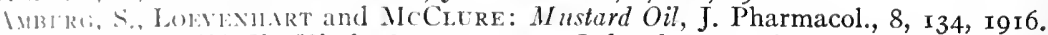

lmst 41, K.: Iodid, Jb. Kind., 8I, 322, I9r5; Iodoxybenzoate, J. Am. Med. Assoc., 59, $150 \mathrm{~s}, 10 \mathrm{r} 2$.

1xbleks: Morphin, Arch. exp. Path. Pharm., 72, 331, rgi3; Uterine Tonies, Arch. f. Givec., I04, 103, 1915.

Axmerie, F., and Cloetta: Lnng Circulalion, Arch. exp. Path. Pharm., 78, 291, Igr6. ANol:rsox, J. F.: Antitoxin, U. S. Hyg. Lab. Bull., 66, I9ro; Formaldehyde, U. S. Hyg. lab. Bull., 30, 1897 .

Anuersox, R. J.: Inosite, J. Biol. Chem., 25, 39r, r9r6.

ANIERsoN, R. J., and boswortir: Inosite, ibid., 25, 399, rgi6.

ANorlik. Velicil and Stanek: Betain, Zs. Zuckerind., 27, I6I, I902.

Axgetico, T.: Picrotoxin, Zentr. Bioch. Bioph., I4, 827, I9I 2.

ANciolaxi: Iodoform, Chem. Zbl., I, 48, I903.

v. A.rrer.: Cocain, Arch. ges. Physiol., 21, 38, 1880; Epinephrin, J. Physiol., 45, 307, I9I 2 .

Antex: Caffein, Arch. intern. Pharmacod., 8, 455, r9or.

Anvescinat: Iodid, Arch. exp. Path. Pharm., I0, 26I, I879.

Apperly: Chloroform, Brit. Med. J., Sept. I4, I9I2.

Arcilangelski, N. M.: Arsenic, Zentr. Bioch. Bioph., I5, 778, г 9 г 2.

Arganaraz, R.: Salvarsan, Ref., J. Am. Med. Assoc., 63, ro64, igr4.

ARkix, A.: Iodoxybenzoate, J. Pharmacol., 3, I45, I9I I; J. Inf. Dis., I6, 349, 360, I9r 5 ; Am. J. Physiol., 40, I48, I916.

Arirr, H. W.: Nickel, J. Hyg.; 526, 1907; 565, 1908.

Aron and Rothmann: Spinal Anesthesia, Z. exp. Path., 7, 94, I909.

Arossonn and Sachs: Heat Puncture, Arch. ges. Physiol., 37, 232, 1884-1885.

Aronson: Formaldehyde, Berl. klin., 75 I, I894.

Arrhenius: Dissociation, Z. physik. Chem., I, 631, 1887.

Ascoli and IzAr: Metals, Ref., Merck's Rep., 21, 172, 1907.

Asier R: Epinephrin, Z. Biol., 58, 274, 1912; Fhiorid, Arch. ges. Physiol., I36, 41 I, I9Io; Permeability, Bioch. Z., I4, I, 1908; Suprarenal, Zentr. Physiol., 24, 927, I910.

Asuer and Bruck: Pilocarpin, Z. Biol., 47, I, I905.

AsHer and FlaCk: Thyroid, Zentr. Physiol., 24, No. 6, I9ro; Z. Biol., 55, 83, I9II.

Asmer and Ganvus: Vital Stains, Zentr. Physiol., 25, 844, I9I I.

ASHER and RODT: Thyroid, Zentr. Physiol., 26, I9I2.

Astolfani: Hippuric Synthesis, Arch. it., 43, 372, 1905.

Atranasiu: Fat, Arch. ges. Physiol., 74, 56r, I 898.

Atkinson: Nitrite, J. Anat. Physiol., 22, 236 , 1888.

Atwater and Benedict: Alcohol, U.' S. Exp. Stat. Bull., No. 69, 1902.

Atwater and Langworthy: Metabolism, Office Exp. Stat. Bull. 45 , U. S. Dept. Agr.

Aubert: Caffein, Arch. ges. Physiol., 5, 589, 1872; ib., 9, I15, 1874.

Auelw: Glycosuria, Zs. exp. Med., 2, 42I, I9I4.

AUer, J.: A naphylaxis, Am. J. Physiol., 26, 439; J. Exp. Med., I2, 638, I9 10; Cathartics, Am. J. Physiol., I 7, I5, I906; J. Biol. Chem., 4, I97, 1908; Salvarsan, Arch. Int. Med., 8, I69; J. Exp. Med., I4, No. 3, I9I I ; Serum Preservatives, Trans. Assoc. Am. Phys., 29, 359, I9I4.

Ader, J., and Gates: Epinephrin, Journ. Exp. Med. 23, No. 6, I916; Respiratory Tract, J. Pharm. Exp. Ther., 6, 608, I915.

AUer, J., and LeWIs: Anaphylaxis, J. Exp. Med., I2, I51, 1910.

Auer, J., and Meltzer: Atropin, Am. J. Physiol., 17, 1906; Calcium, ib., 25, 43, 1909; Ether, J. Pharmacol., 5, 524, I9I4; Intramuscular Absorption, J. Exp. Med., I3, 328, I9II; Magnesium, Soc. Exp. Biol. Med., 11, 95, I9 I4; J. Pharmacol., 5, 521, 1914; Magnesium Sulph., Proc. Soc. Exp. Biol. Med., 13, 106, 1916; Pituitary, J. Pharmacol., 4, 359, I9I3.

Auer and Robixson: Anaphylaxis, J. Exp. Med., I8, 450, I9I3.

AUEr, J., and VAn SLyke: Anaphylaxis, J. Exp. Med., I8, 210 , I913.

Auerbacir, F., and Pick: Lead., Zentr. Bioch. Bioph., i6, 2 16, I9I3.

Austin and EISENBrey: Nephritis, J. Exp. Med., I4, 366, I9II; Serum, Arch. Int. Med., 10, 305, I912.

Austin, J. H., and LEOPOLD: Glucose and Meat, Soc. Exp. Biol. Med., I3, 3, 1915.

Austin and Müller: Sweat Baths, J. Am. Med. Assoc., 63, 944, I9I4.

Austin, J. H., and Pearce: Iron, J. Exp. Med., 20, No. 2, I9I4.

Austregesillo, A.: Camphor Poisoning. J. Am. Med. Assoc., 64, I694, 1915.

BaAs: Salicyl, Z. physiol. Chem., I4, 416, r8go.

Baccelli: Phenol, Berl. klin. Woch., IO2I, I9II. 
BACHeM: Veronal, Arch. exp. Path. Pharm., 63, 228, I9 Iо.

Baches and Guenther: Barium, Deut. med. Woch., 717 , i91

Backmax, E. L.: Alcohol, Skand. Arch. Physiol., i8, 323, ioo6.

Bacon and Marsiall: Saponin, Bioch. Centr., 6, 202, 1006.

Baenr and Pick: Bronchial, Arch. exp. Path. Pharm., 74, 4I, 19I3; Lung Vessels, ib., 74,65 , I9r 3 .

Baehr, G., and Wessler: Cerium, Arch. Int. Med., 2, 5 I 7, r9o9.

BAER: Alcohols, Arch. Physiol., 287, I898; Scopolamin-morphin, J. Im. Med. Assoc., 64,1723 , I9I5.

Batetzer, W.: Phosphate, Arch. exp. Path. Pharm., 72, 309, 1913.

Baglioni: Convulsants, Arch. Physiol. Suppl., I93, I900; Zs. allg. Physiol., 9, 24, I909.

Baglioni and Magnini: Cortex, Bioch. Centr., Io, 97, r9o9.

BAIley, C. H.: Cholesterol, Soc. Exp. Biol. Med., I 2, 68, I9I4; I3, 60, I0I5.

BaIn: Urine, Qu. J. Exp. Physiol., 8, 229, I9I4.

Bainbridge, F. A., and Evaxs: Diuretics, J. Physiol., 48, 278, I9I4.

BAKER, W. F.: Ouabain, Am. J. Pharm., 84, 247, I9I2.

Baldauf, L. K.: Soaps, J. Am. Med. Assoc., 49, 642, r9o7.

Baldonı: Quinin, Zentr. Bioch. Bioph., I4, 315, I912; I7, 837, I913.

BALl: Silver, Gaz. med. Paris, 620, I865.

Ball, Clerc and Pozzi: Nicotin, Bul. Soc. Biol., 77, 213 , I9I4.

Ballet and Enriquez: Thyroid, Med, mod., Paris, Dec. 26 , I894.

Balthazard and Niclocx: Carbon Monoxid, Zentr. Bioch. Bioph., i 2, 326, ig I

BANCroft: Sulphate, J. Biol. Chem., 3, I9I, I907.

BANG, J.: Urethane, Bioch. Zs., 65, 283, 296, I9I4.

BANG: Venom, Zs. Imm., 8, 202, I9io.

Banzhaf and Cribson: Antitoxin, J. Biol. Chem., 3, 253, 1907.

Barbera: Iodid, Arch. ges. Physiol., 79, 31 2, r9oo.

Barbour, H. G.: Epinephrin, Arch. exp. Path. Pharm., 65, 4I, I9I2; IIistamin, J. Pharmacol., 4, 245, I9I3; Mercury, J. Am. Med. Assoc., 64, 736, 1915; Morphin, J. Pharmacol., 5, 393, I914; Morphin-scopolamin, J. Pharm. Exp. Ther., 7, 547, I9I5; Phenylethylamin Derivatives, J. Pharmacol., 8, I 26, I9I6.

BARbour, H. G., and ABel: Fuchsin, J. Pharmacol., 2, r67, т9то.

Barbour, H. G., and Copenhaver: Morphin, Proc. Soc. Exp. Biol. Med., I3, I5\%, 1916; Morphin-scopolamin, J. Pharm. Exp. Ther., 7, 529, I9I5.

Barbour, H. G., and Deming: Antipyrin, J. Pharmacol., 5, I49, I9r3.

Barbour, H. G., and Frankel: Phenylethylamin, J. Pharm. Exp. Ther., 7, 5II, I9I5.

Barbour, H. G., and Kleiner: Caffein and Epinephrin, J. Pharmacol., 7, 54I, I9I5.

Barbour, H. G., Maurer and v. Glalin: Tyramin-morphin, J. Pharmacol., 8, I24, I9I6.

Barbour, H. G., and Prixce: Epinephrin, J. Exp. Med., 21, 330, I9I5; Temperature, J. Pharmacol., 6, I, I9I4.

Barbour, H. G., and Wisg: Fever, J. Pharmacol., 5, 105, I9I3.

Barcroft and Straub, Diuresis, J. Physiol., 41, I45, 1910.

BARdy, H.: Inflammation, Skand. Arch. Phys., 32, I98, r9i4.

BARgER, G.: "Simpler Natural Bases," I0I4.

BARger, CARr and Dale: Erget, J. Chem. Soc., 9I, 337, 1907.

Barger and Dale: Autonomic, J. Physiol., 42, I9, I9IO.

BARger and Ewins: Ergot, Trans. Chem. Soc., 97, 284, I9Io.

BARGER and JowetT: Epinephrin, ib., 967, I905.

BARGER and WALPOLE: Tyramin, ib., 95, I909.

BARgero: Manganese, Bioch. Centr., 5, 504, 1906.

Barinetti, C.: Alum, Arch. Farm., I6, 2 10, 19I4.

BARKER, F.: Camphor, Brit. Med. J., Apr. I6, I9Io.

Baron, A., and Barsoxy: Anesthetics, Arch. ges. Physiol., 158, 464, 1914.

Barry D. T.: Alcohcl, Prac., London, 44, 775, 910 , I9I 5.

Barsony, J.: Mercury, Ref., J. Am. Med. Assoc., 54, 4I5, I9o9.

Barten, O.: Anesthelics, Arch. intern. Pharmacod., 23, 505, I9I3.

BARTENBACH, K.: Iodid, Zentr. Bioch. Bioph., Io, 278 , I909.

Barth: Morphin, Arch. exp. Path. Pharm., 70, 258 , ror 2.

BARTHE, L.: Bismuth, Zentr. Bioch. Bioph., I4, I60, I9 13.

Barton, W. B.: Hexamethylenamin, J. Am. Med. Assoc., 54, 87 r, r910; Permanganale, J. Am. Med. Assoc., 6r, I96, I9i3.

BAsE: Hexamethylenamin, Ref., J. Am. Med. Assoc., 49, I 294, 1907.

BASHFord: Atropin and Morphin, Arch. intern. Pharmacod., 8, 3 I I, 1901.

BASINGer, H. R.: Cretinism, Arch. Int. Med., I7, 260, 19r6.

Baskerville: Anesthetics, J. Am. Med. Assoc., 59, 1837, 19 Í 2. 
B.ıss, 1.: Epinephrin, Zentr. Bioch. Bioph., I7, 639, I9I4.

B.ss, (C. C.: Malignant Melaria, J. Am. Med. Assoc., 65, 577, 1915.

13.1s. ( C., and Jonxs: Emctin, J. Am. Med. Assoc., 64, 553, I915; Malaria, J. Exp.

Med., Oct., igra.

13..ss, R.: Atophan, Arch. exp. Path. Pharm., 77, 40, 1914.

Biso, (i. l.: Pituitury, Zentr. Bioch. Bioph., I7, 43I, I9I4.

B.1steme, W. 1.: Constipation, J. Am. Med. Assoc., 63, 7r5, 1914; Petrolatum, J. Am. Med. Assoc., 64, 808, I915.

Bistix, G.: Cholcstcrin, Arch. Path. (Virch), 220, 176, 1915.

Bsc10oun: II ypophysis, Zentr. Bioch. Bioph., I 5, 574, 1913.

B.ncmax: Plicnol-sulphutes, Arch. ges. Physiol., r 3; Z. physiol. Chem., I, 60, 70; 2, 244. 335, 350, 1876; Thyroid, Z. physiol. Chem., 21, 319 and 481; 22, I, 1896.

B.MWAN and Kast: Narcosis, Z. physiol. Chem., I, 52, 1890.

B.IlMixx and Roos: Thyrcid, ib., 2I, 319, 48I; 22, I; Muench med. Woch., No. 14, 1806 .

Bhyer and Peter: Pituitary, Arch. exp. Path. Pharm., 64, 204, I9II.

Baxt: Papaverin, Arch. Anat.' Physiol., I I9, I869.

B.1Ýliss, W. M.: Phase Boundaries, Sci., 42, 509, I915; Phenol on Vessels, J. Physiol., 20, 224, I902; Vasomctor Reflexes, Proc. Roy. Soc., B., 80, 339, I908.

Bayliss and Starling: Autonomic System, J. Physiol., 26, 107, 125, I901; Hormones, Errgeb. Physiol., 5, 664, 1906; Peristalsis, J. Physiol., 26, 107, 125, 1901; Secretin, Centr. Physiol., 15, 682, 1902.

Beard, J.: Trypsin, Lancet, No. 4249, 28I, I905; Brit. Med. J., No. 235I, I40, 1906. Beates: Digilalis, J. Am. Med. Assoc., 70, I907.

Becht, F. C., and Luckhardt, Antibodics, Am. J. Physiol., 40, 366, I9i6.

BEck, A.: Scopolamin, Muench. med. Woch., I29, I9I4.

Beck, A., Bıkeles and Zbyszewski: Temperature, Zbl. Phys., 29, I, 3, r9i4.

Beck, E. G.: Bismuth, J. Am. Med. Assoc., 52, 14, 1909; Bismuth Paste, ibid 67, 21, 1916.

Веск, H. G.: I pecac, ib., 59, 2 г го, г9г 2.

Beck, S. C.: Pcrmanganate, and Scarlet Red, Therap. Monatsh., 27, 561, 1913.

Beck and O'Mally: Pitnitary, Am. Med., Oct., r9o0.

Beco and Plumer: Caffein, J. de Physiol., 8, Io, I9o6; Pituitary, Zentr. Bioch. Bioph., I5, 64.5, I9I3.

Beddard, A. P., Pembrey and Spriggs: Acidosis, Br. Med. J., Sept. ir, 1915.

Bedford, E. A., and Jackson, Epinephrin, Proc. Soc. Exp. Biol. Med., 13, 85, Igг 6.

Beebe, S. P.: Goiter Serum, J. Am. Med. Assoc., 46, 484, I9o6; Parathyroid, Proc. Soc. Exp. Biol. Med., I, r6r, 1909; Thyroid, J. Am. Med. Assoc., 49, I155, 1907; Am. J. Pharm., 83, 56, I9II; J. Am. Med. Assoc., 64, 4I3, I9I5.

v. Benrivg, E.: Anaphylaxis, Deut. med. Woch., No. 42, I9r 5 ; Diphtheria Immunization, Deut. med. Woch., 39, 873, 1913.

Bell: Pituitary, Bioch. Centr., 9, 63I, Igog.

Bellots and DuBos: Nitrite, Zentr. Bioch. Bioph., 15, 670, 19r3.

Bendix: Diaphoresis, Deut. med. Woch., Feb.; Bioch. Centr., 2, 437, 1904.

Benedetri: Manganese, Bioch. Centr., 5, 504, igo6.

Benedict, F. G.: Alcohol, Journ. Amer. Med. Assoc., 66, 1424, 1916; Sc., 43, 907, Ig16; Carnegie Lab. Investigations, Sci., July I6, I914; Diaphoresis, Proc. Soc. Exp. Biol. Med., 3, 40 1905.

Benedict H., and Török: Alcohol, Bioch. Centr., 5, 77 I, I9o6.

Beniedict, S. R., and Osterburg: Acidosis, Soc. Exp. Biol. Med. I2, 45, 19 I4.

Benepicentr, A.: Absorption, Bioch. Centr., 5, 188, 1906; Digitalis, Arch. exp. Path. Pharm., 47, 360, I902; Methemoglobin, Arch. Physiol., 2 10, 1897.

Beneldi, A.: Cholin, Arch. Farm, Sper., 13, 3, 1914.

BenNeke: Epinephrin, Arch. path. Anat., 19I, 202, 1908.

BENNetT: Caffein, Brit. Med. J., I874.

Bennetr, R. R.: Ether, Ref., Yr. Bk. Am. Phar. As., I, 357, I912.

Berchart, K.: Netlle Poison, Zentr. Bioch. Bioph., I8, 88, I9I5.

BERCKe: Salicyl, Berl. klin. Woch., 49, I378, 1912.

Berendes: Glucose, Zentr. Chirurg., I 217 , I 191.

Berezin, W. J.: Brain Vessels, Ref., J. Am. Med. Asoc., 67, 844, 1916; Liver Vessels, Ref., J. Am. Med. Assoc., 63, 1909, 1914; Lungs, Arch. ges. Physiol., 158, 219, 1914. BerG: Elaterin, Proc. Am. Pharm. Assoc., 57, 392, I909.

BERG, Wr. N.: Acid Tolcrance, Soc. Exp. Biol. Med., I2, 145, I9r5.

BERGHeIM and HAwK: Water, J. Am. Chem. Soc., 35, 46I, I9I 3.

Bergheim, O., Rehfuss and Hawk: Water Drinking, J. Biol. Chem., I9, 345, r9I4.

Beritoff: Strychnin, Fol. Neuro-biol., 7, 187, 1913. 
Berkenbusch: Salicylic Acid, Ther. Mon., 29, 565, I915.

Berkeley: Pineal, Med. Rec., 85, 513 , I914.

Bermany, E.: Bromid, Zentr. Bioch. Bioph., Io, 277 , 19 Iо.

Bernard, Cl.: Les Tissus Vivants, Paris, 1857 ; Legons sur les Anesthetiques, Paris, I875.

Bernhein: Nitrites, Arch. ges. Physiol., 8, 253, I874.

Bernoulli: Bromid, Arch. exp. Path. Pharm., 73, 355, I9I3.

Bernstern, J.: Boric, Brit. Med. J., Apr. i6, igio.

BERRAR, M.: Aloin, Bioch. Z., 49, 426, I9I3.

BERry, E.: Sweat, Bioch. Zs., 72, 295, 1915.

Bertagnini: Salicyl, Ann. Chem., 97, 248, r856.

Bertholet: Alcohol, Ref., J. Am. Med. Assoc., 54, 6I 7, 1909.

Bertrand, G., and Agulhon: Borale, Zentr. Bioch. Bioph., 15, 303, 1913; Boron. Comp. Rend. Acad. Sci., I55, 248, I91 2.

Bertrand and Devuyst: Maté, Zentr. Bioch. Bioph., Io, 547, I9Io.

Bertrand and Medigreceanu: Manganese, Ann. Pasteur., 26, I013, 1912.

BEST: Borate, Trans. Chi. Path. Soc., 6, I6I, 1904; Phenolphthalein, Jahresb. Pharm., $66,257,1906$.

Best, F., and Cohnheim: Bismuth, Muen. med. Woch., 2732, I9II.

Beutner, R.: Edema, Bioch. Zs., 48, 217 , I9I3.

Bethe: Narcotics, Arch. exp. Path. Pharm., Suppl., 75, I908.

BetKe, R.: Sennatin, Ther. Mon., 28, 688, igr4.

Bevan, A. D.: Anesthetics, J. Am. Med. Assoc., 65, r418, 1915.

Bevan and Favill: Chloroform, J. Am. Med. Assoc., 45, 691, 754, 1905.

Beyer: Alcohol, Zs. Hyg. Inf., 70, 225, I9I I Caffein, Am. J. Med. Sci., July, I885.

BIAGI: Epinephrin, Bioch. Centr., 4, 762, 1905.

Biberfeld, J.: Atophan, Z. exp. Path., I3, 30ז, I9r3; Calumbo, ib., 7, 569, I9o9; Epinephrin, Arch. ges. Physiol., II9, 34I, r907; Glycuronic, Bioch. Zs., 65, 479, 1914; Morphin, Bioch. Zs., 70, 158, I915; Quinin, Arch. exp. Path. Pharm., 79, 361, 1916; Tannin, Arch. ges. Physiol., 100, 252, I903.

Bibergeil: Nephritis, Berl. klin. Woch.. 42, 427 , I905.

BICKel: Acids and Alkalies, Berl. klin. Woch., 869, I905.

BICKEL and Pawlow: Digitalis, Bioch. Z., 48, 459, r9I3; Tyramin, ib., 47, 435, 19 I 2.

BICKel and Pincussonn: Morphin, Biochem. Centr., 6, I85, I907.

BIDDER: Curare, Arch. Physiol., 337, 1865.

Bidwell: Pituitary, Ref., J. Am. Med. Assoc., 57, I242, I9Ir.

BIEDL and Kraus: Anaphylaxis, Wien. klin. Woch., 22, 363, 1909.

BIER: Cocain, Münch. med. Woch., Nov. 21, I900.

Brernacki: Potassium, Zs. exp. Path., 8, 685, igi I.

Bikeles, G., and GerstmanN: Pilocarpin, Neurol. Zbl., 34, 770 , 1915.

BIKELES and ZBYSzewSKI: Strychnin, Zentr. Physiol., 27, 433, 533, I9I3.

Biland, J.: Epinephrin, Deut. Arch. klin. Med., 87, Nos. 5 and 6, I906.

Billings: Benzol, J. Am. Med. Assoc., 60, 495, I9I3.

Binz, V. C.: Alcohol, Arch. exp. Path. Pharm., 6, 3I2, I877; ib., I3, I63, I880; Berl. klin. Woch., Nos. 3 and 4, I903; Atropin, Berl. Klin. Woch., 885, 1896; Camphor, Arch. exp. Path. Pharm., 8, 50, 1878; Hypnotics, Arch. exp. Path. Pharm., 6, 3 10, I877; Morphin, Arch. intern. Pharmacod., 8, 449, I901; Quinin, Berlin, r870, Arch. exp. Path. Pharm., I, I8, I873; ib., 5, 39, I876; ib., 7, 275, 1877; Salicyl, Berl. klin. Woch., I3, 385, 1875; Arch. exp. Path. Pharm., 7, 275, I877; ib., Io, 147, 1879; Santonin, ib., 6, 300, 1877; Stomachics, ib., 5, 1 22, 1875.

Binz and Gerlinger: Nitrate, Arch. int. Pharmacod., 9, 44I, I901.

Bingl: Salt, Arch. exp. Path. Pharm., 64, r, rgro.

Birsch-Hrrschfeld and Koster: Atoxyl, Zentr. Bioch. Bioph., ir, 60, I9io.

BISHOP: Morphin, J. Am. Med. Assoc., 60, 431, I9I3.

BIshop, W. A., and Brosiss: Chenopodium, J. Am. Med. Assoc., 65, 1610, 1915.

BLACK, A.: Tetra, Zentr. Bioch. Bioph., I 2, 62, I9Ir.

Blackford, J. M., and SANFord: Basedow's, Trans. Am. Phys., 28, 595, 1913.

BLACKLEY: Hay Fevier (London), i 873.

Blanchier: Salicyl, These, Paris, No. I4I, I879.

BlankenhoRn, M. A.: Strontium Salicylate, J. Am. Med. Assoc., 66, 331, rgr6.

Blankenhors, M. A., Harmon and Hanzili : Ptomaine Poisoning, Arch. Int. Med., $17,140,1916$.

Blatherwick, H. R., and Hawk: Water, Bioch. Bull., 3, 28, I9r3; J. Am. Chem. Soc., 36,152, I 914 .

BlaUEL: Anesthetics, Schmidt's Jahrb., 273, 165, I90I.

BLock: Salicyl, Inaug. Diss. Giessen., Igog. 
BıоктR: Fdema, D. Arch. Klin. Med., 96, 80, 1909.

BLook, W. R.: Emmolliculs, J. Biol. Chem., I5, r05, I913; Ether, J. Biol. Chem., rg, I, I014; Fat, ib., I I, 429, I9I2; 16, 517, I914; Fat Assimilation, J. Biol. Chem., 24, 477, 1016; Lecihhin, J. Biol. Chem., 23, 317, 1915; Lipoids, ib., I5, 105, 1913; distribution in blood, il,., 25, 577, 1916.

Bi.ce, J. B.: Emetin, 65, I297, I9I5.

Buc:M, F.: Epinephrin, Deut. Arch. klin. Med., 7I, I46; Arch. ges. Physiol., 90, 6I 7, I001.

Bium, F., and Gruttzener: Thyroid, Zs. physiol. Chem., 91, 400, igr4; 92, 360, I9I4. Bi.mexthlis, F., and OppeNHeis: Mercury, Bioch. Zs., 65, 460, I9I4.

Boск, A.: Fever, Arch. exp. Path. Pharm., 68, I, I9 2.

Bock, J.: Caffein, Skand. Arch. Physiol., 25, 239, I9I I; Nitrous Oxid, Arch. exp. Path. Pharm., 75. 43, Igr3.

Bоск: Isolated IIeart, ib., 4I, 458, I898.

Bondarert: II y pophosphiles, Arch. int. Pharmacod., 2, I895.

Boecker: Sulphur, Inaug., Greifswald, I89.5.

BoEcker, E.: Oplochin and Salvarsan, Zs. Immun., 24, I48, I9I5.

BoEnм, R.: A conile, Arch. exp. Path. Pharm., I, 385, I871; Aspidium, ib., 35, r, 1895; I 5,38 , 35, I895; Croton Resin, Arch. exp. Path. Pharm., 79, I38, I9I5; Curare,

ib., 4, 351, I887; 35, 16, 1894; Digitalis, ib., 2, I 23, 1872; Iodid, Arch. exp. Path. Pharm., 5, 329, I876; Muscarin, ib., 19, 87, 1885; Salts on Heart, ib., 75, 230, 1914; Verutrin, ib., 7I, 269, I913.

Boein and Pistorius: Arsenic, ib., I6, I88, I882.

BoeinM and UNterberger: Arscnic, ib., 2, 89, I874.

Boenus, A.: Bismuth, ib., 57, 447, I907.

Boexviger: Bromid, Bioch. Centr., 6, 549, r9o7.

Boerner, H.: Epinephrin, Arch. exp. Path. Pharm., 79, 218, I915.

Bogert, L. J., Underhill and Mendel: Blood-volume, Am. J. Physiol., 41, i89, 219, 229 , I9I6.

Bornet and Teissier: Cactus, Bull. gen. Therap., I 2 I, 343, I89i.

Bokorvy, Th.: Caffein, Arch. ges. Physiol., 59, 557, 1894; Metals, Bioch. Centr., 4, 405,1905 .

Boldt, H. J.: Cotarnin, Bost. Med. Surg. J., Jan. I2, 1905.

Bolgar, G.: Bromids, Arch. int. Pharmacod., I9, 3I I, I9 IO.

Bollet and Curtil: Cocain and Ocular Tension, Zentr. Biochem. Biophys., 13, 456, IOI I.

Bosani, A.: Bitters, Bioch. Centr., 5, 779, 1905; Sajodin, Zentr. Bioch. Bioph., I3, I20, I9II.

BoNCEK: Bismuth, ib., II, 60, I910.

Boxp, G. S.: Coronary Flow, J. Med. Res., I 2, 575, I9 Ir.

BoNdi and JACOBY: Salicyl, Beitr. Chem. Physiol., 7, 5 14, 1906.

BoNdi and Katz: Salicyl, Zentr. Bioch. Bioph., 2, 373, I91 I.

Bongers: Morphin, Arch. exp. Path. Pharm., 35, 41 7, 1895.

Bongrand, J. C.: Anaphylaxis, Ref., Jb. Pharm., 73, r75, I913.

$\because$ BonsDORFF: Iodoform, Ref. J. Am. Med. As., 67, ro52, I9 I6.

Boos, Newburgh and Marks: Digipuratum, Arch. Int. Med., 7, 55I, I9II.

Boothiу, W. M.: Anesthetics, J. Pharmacol., 5, 379, r914; Ether, J. Am. Med. Assoc., 61, 830, I913; J. Pharmacol., 8, II5, 1916.

BorchardT: Chlorid, Deut. med. Woch., 38, I723, r912.

BORDET: Anaphylaxis, C. R. Biol., 74, 877, r9I4.

Borelit, L., and Girardi: Sodium Chlorid, Ref., Zentr. Bioch. Bioph., I8, I59, I9I5.

BoRIsoff: Bilters, Arch. exp. Path. Pharm., 51, 362, r904.

Borsstein: Saccharin, Z. klin. Med., 40, 203 , I 900.

Boruttau, H.: Haloids, Zs. exp. Path., 8, 418, I910; Hexamethylenamin, Zs. exp. Path. Ther., I6, 484 , I914.

Botrazzi: Muscle, Arch. Physiol.,.377, r9or; Sea Water, Arch. it. Biol., 28, 77, r897.

Bouma: Codein and Morphin, Arch. exp. Path. Pharm., 50, 353, I903.

Boycorr: Blood-volume, J. Path. Bact., I8, II, 498, I9I3 and I4.

Boycott and Ryfres: Diuretics and Nephritis, J. Path. Bact., I7, 458, 19г3.

BRADFORD and DEAN: Pulmonary Circulation, J. Physiol., 16, 34, 1894.

BRADley and Gasser: Absorption, J. Biol. Chem., II, I9I 2.

BRADLEY, H. C. and MORSE: Manganous Chlorid, J. Biol. Chem., 21, 209, 1915.

BRADY: Ergot, N. Y. Med. J., 9I, 2 I I, rgio.

Brans, C.: Chinosol, Z. physiol. Chem., 28, 439, 1899.

BrandenbURG: Bile, Arch. Physiol. Suppl., 150, r903.

Brandini, G.: Alcohol, Zentr. Bioch. Bioph., I6, 76, Igr3. 
Braxdl: Stomachics, Z. Biochem., 29, 277 , I 893.

Brandl and Tappeiner: Cathartics, Arch. exp. Path. Pharm., 26, I77, i 889.

BrandT: Agrostemma, ib., 54, 245, igo6.

BraUn and MAger: Digitalis, Sitzb. Wien. Akad., I899.

Brauxs and Clossex: Strophanthin, J. Am. Pharm. Assoc., 2, 850, 1913; Arch. Pharm., 252, 294, I9I4.

Breituan, M. J.: Alcohol, Ther. Mon., 27, 847, igI3.

Breu: Malaria, Arch. Int. Med., 9, I 29, I9I 2.

Brenot, M. R.: Apiol, Ther. MIon., 30, 56, 1916.

Brequet: Alcohol, Zentr. Bioch. Bioph., 14, 601, I9r 2.

Bresler: Mescalin, Merck's Rep., 26, 279, I905.

Brieger: Cathartics, Arch. exp. Path. Pharm., 8, 355, 1878.

Brill and ZehNer: Radium, Berl. klin. Woch., 49, I 261, I9I 2.

Brodie and Cullis: Epinephrin, J. Physiol., 43, 31 3 , I9 I I.

Brodie, Cullis and Halliburtox: Magnesium Sulphate, J. Physiol., 40, i73, igio.

Brodie and Dixon: Autonomic System, ib., 30, 476, I904.

Brodie, and Vogt: Intestinal Absorption, J. Physiol., 4 I, I35, I9 10.

Brodie and Widdows: Chloroform, Brit. Med. J., July i 4, I9o6.

Brooke, R.: Quinin, J. Am. Med. Assoc., 62, roo9, 1914.

Brooks, B. J.: Liquid Petrolatum, J. Am. Med. Assoc., 66, 24, r9г6.

Brooxs, C.: Alcohol, ib., 55, 372, I9Io; Epinephrin, J. Exp. Med., I4, 550, I9 I I.

Brooks, C., and Luckhardt: Emetics, Am. J. Physiol., 36, I04, I9I 5.

Brooks, H.: Chlorid, Arch. Int. Med., 6, 576, roro.

Browder, A.: Paramecium, Univ. Cal. Publ. Physiol., 5, I, I9 5.

Brown, E. D. Medullary Centers, J. Pharmacol., 8, 195, 1916.

Brown, L. A.: Cyanid, Dig., U. S. P., 273, 1910.

Brown, O. H.: Glycosuria, Am. J. Physiol., ıo, 378, 1904; Quinin, Proc. Biol. Chem., 2, 70, 1912.

Brown, O. H., and Guthrie: Bone-marrai', Am. J. Physiol., I6, i io, igo5.

Brown, O. H., and Scotr: Oxalic, J. Am. Med. Assoc., 58, I 280, 1912.

Brown, S.: Carbon Monoxid, ib., 46, i 265 , i 906.

Brown, W. H.: Hematin, J. Exp. Med., I5, 579, I91 2.

Brown, W. H., and Loevenhart: Hematin, ib., 18, 1913; J. Pharmacol., 4, 347, 1913.

Brown and Fraser: Autonomic System, Proc. Roy. S. Edinb., 560, I869.

Brown-Sequard: Testicular Extract, C. R. Soc. Biol., 1, 4I 5, 420, 430, 1889.

Browne, J. C.: Picrotoxin, Brit. Med. J., r875.

Brucini, G.: Ammonium, Bioch. Centr., 6, 323, I907.

BrUCK: Anaphylaxis, Berl. klin. Woch., Nos. I2 and 42, I910; Gonorrhea, Ther. Monatsh., 27, I, I913.

Bruck, C., and Glueck: Gold, Muen. med. Woch., 57, r9r3.

Bruening: Anthelminitics, Zs. exp. Path., 3, 564, r9o6; Chenopodium, Deut. med. Woch., No: 50, 2368, I9 I 2; Cineal, Z. exp. Path., II, I55, I9I 2.

Bruning: Calcium, Inaug. Halle, I898; Volatile Oils, Centr. Inn. Med., 27, No. I4, I906.

Brunner, C.: Antimony, Arch. exp. Path. Pharm., 68, rgr 2; Iodin, Zbl. Chir., 62, No. 32 , I9I5.

Brunton and Cash: Caffein, J. Physiol., 9, i I 2, I888.

Brunton and Tunnicliffe: Digitalis, J. Physiol., 20, 354, 1896.

Bryan: Iodoform, J. Am. Med. Assoc., 40, 972 , 1903.

Buchanan, F.: Strychnin, Quart. J. Exp. Physiol., 5, I912.

Buchherm: Cod Liver Oil, Arch. exp. Path. Pharm., 3, I 18, 1874; Organic Acid, Arch. physiol. Heilk., N. F., 1, I38, I 888 .

Buchholz: Antiseptics, Arch. exp. Path. Pharm., 4, I, I875.

Buckmaster and Gardner: Chloroform, Proc. Roy. Soc., 84, B, 347, igi i; Oxygen, ib., $B, 85,56$, 1913 .

Buchtala, H.: Mercury, Zs. physiol. Chem., 83, 249, 266, 1913.

Buck, L. W.: Anesthetics, J. Pharmacol., 5, 553, r914.

Budington, R. A., and HARvey: Thyroid, Sci., 41, 470, 1915.

Buerger, L.: Silver, Surg., Gyn., Obstet., 536, I9I5.

Buergi: Synergism, Deut. med. Woch., 20 and 62, 1910; Corr. Schweiz. Aerz. 43, I345, rg14; Mixed Narcosis, Bioch. Centr., 9, 850, r9ro.

Buess: Salicyl, Deut. Arch. klin. Med., I 5, 526, 1875.

Bufalini: Creosote, Lo Sper., May, r9o4.

Buglia, G.: Calcium, Zs. Biol., 55, 343, rorr.

Bult, C. G: Pneumonia, J. Exp. Med., 22, 457, I9I5.

Bull, Clerc and Pezzi: Nicotin, Soc. Biol., 77, 213 , ror4. 
lilitirn: Tea, Bost. Med. Surg. J., Apr. 8, 314, is86.

B1.M. E.: Jesothorium, Berl. klin. Woch., I00I, r9r3.

lifi:e, G.: Alcohol, Die Alkoholfrage, Leipzig, I887; Hydrastis, Dorp. Arb., Ix, I2,

I1 9, ISo5; Iron, Zs. physiol. Chem., 9, 48, I885; Salt, Zs. Biol., 9, 104, 1873.

Brve and Schmedeberg: Hippuric Acid, Arch. exp. Path. Pharm., 6, 233, 1877.

Bt>Tixg and Bkowx: Bile, J. Exp. Med., I4, 445, I9I.

BrorkilARDT: Either, Muench. med. Woch., No. 7, 36r, rgro.

Bl'RIANE; IIormonal, Ref., Ther. Mon., 27, 585, I913.

BURx, J.H., and DALE: Organic Ammoniums, J. Pharmacol., 6, 4I 7, 19x5.

BiriscinfF, A.: Salvarsan, Zentr. Bioch. Bioph., I5, 778, r9 2.

Burvet, 'T. C.: Cholesterol, Proc. Soc. Exp. Biol. Med., II, 42, r9r3.

Blrinte, T. C. and Martix: Salt Fever, Soc. Exp. Biol. Med., I3, I4I, igr6.

BLRNS, J. E.: Thorium, J. Am. Med. Assoc., 64, 21 26, 1915.

Burow: Salicyl, Med. Klin., 7, 34I, I9Ix.

Burridge, W.: Anesthetics, J. Physiol., 45, v, i9 2 ; Epinephrin, J. Physiol., 48, No. 4, I9 I4.

Burshim, S.: Lecithin, Bioch. Zs., 35, 47 r, r9г 2.

Burton-Opitz: Alcohol, J. Physiol., 32, 8, 385, r904; Am. J. Physiol., 35, 265, r9 14;

Anesthetics, J. Am. Med. Assoc., 57, 353, r9r r; Blood-viscosity, Arch. ges. Physiol.,

I 19, 359, I907; Epinephrin, Quart. J. Exp. Physiol., 5, 1912; ib., 7, 57, 1913.

Burtox-Opitz and Edwards: Splanchnics, Am. J. Physiol., 41, 91, x916

Buschke, A.: Thallium, Deut. med. Woch., 37, x61, r91o.

Busquet: Veratrin, J. de Phys. Path. gen., 9, 50, r907.

Busquet and Pachon: Cholin, Bioch. Centr:, ro, r 5, roro.

Busquet and Tiffeneau: Coffee, Zentr. Bioch. Bioph., 14, 238, x9x 2.

Buss: Salicyl, Berl. klin. Woch., 13, 445, 503, 518, I876.

Busse and Merian: Salvarsan, Muench. med. Woch., 59, 2330, I9 2.

Butterfield, E. E.: Hemoglobin, Proc. Soc. Exp. Biol. Med., ro, 74, r9 3 .

Butterfield and Benedict: Hematin, Proc. Soc. Exp. Biol. Med., I I, 80, r9r4

Byrnes, C. M.: Mercury, J. Am. Med. Assoc., 63, 2182 , I9 4 .

BYwaters and Shert: Rectal Feeding, Arch. exp. Path. Pharm., 71, 42, ror 3.

Cавот, R. C.: Arteriosclerosis, J. Am. Med. Assoc., 43, 774, I904; Bismuth, Trans. Assoc. Am. Physic., 27, 457, I9I 2; Strychnin, J. Am. Med. Assoc., 42, 1378, rgo4.

Cadwallader, W. D.: Intra-neural Alcohol, J. Am. Med. Assoc., 64, 1892, 1915.

Caesar, H.: Morphin, Biochem. Zs., 42, 316, I9 2.

CAesar and Loretz: Digitalis, U. S. P. Digest., 226, r907.

Caffarena: Salvarsan, Zentr. Bioch. Bioph., I5, 576, I9I2.

Cahours: Salicyl, Ann. Chim. Physique., 10, 337 ; 13, 90, 1844.

Calris, V.: Camphor, J. Pharm. Chem., Io, 224 , I9I4.

Caldwell: Capsules, Drug. Circ., 57, r88, r9 33 .

Caldwell W., and Clotworthy: Ammonium, Bioch. J., ro, I4, r9r6.

Calarette: Chlorin, Ref., Merck's Rep., 21, 63, 1987.

Camac: Antimony, Am. J. Med. Sci., Aug., igr I.

Camas and Gley: Pilocarpin, Arch. Intern. Physiol., 13, I02, I913.

Cameron, A. T.: Iodin, J. Biol. Chem., r8, 335, r9r4.

Cameron, I. D.: Epinephrin, U. S. P. Dig., 27 I, 1906.

CAur, C. D.: Salvarsan, Ref., J. Am. Med. Assoc., 60, 241, r912.

Campbeil: Chloroform, Zentr. Bioch. Bioph., I 2, 44I, I IIr.

Campbell, Douglas, Haidane and Holson: Respiration, J. Physiol., 46, 301, ror3.

Cauus: Hordenin, Arch. internat. Pharmacodyn., I6, 43, I906.

Camus and Nicloux: Ethyl Chlorid, J. de Phys. Path., ro: 76, 665, 844, 1908.

Cannon, W. B.: Emetics, Am. J. Physiol., 1, 373, 1898; Mech. Factors of Digestion, Internat. Med. Monogr's, IgI I.

Canvon, Aư and Binger: Nicotin, J. Pharmacol., 3, 379, r9 2.

Cannon, W. B., Binger and Fitz: Hyperthyroidism, Am. J. Physiol., 36, 363, I9x5.

Cannon, W. B., and Cattell: Adrenal on Thyroid, Am. J. Physiol, $4 \dot{1}, 74$, Ig r6; Atropin, ib., 4I, 39, I916; Thyroid Innervation, ib., 4I, 58, 1916.

Cannon, W. B., and Frtz: Sympathetic-phrenic Fusion, ib., 40, 126, 19г6.

Cannon, W. B., and Mendenhall: Coagulation, Am. J. Physiol., 34, 225, rgr4; Epinephrin, Am. J. Physiol., 33; Proc., r914.

Cannon and Murphy: Ileus, J. Am. Med. Assoc., 49, 840, 1907.

CANxion and NICE: Epinephrin, Am. J. Physiol., 32, 44, rol3.

Cantelli, O.: Indol, Zentr. Bioch. Bioph., 18, 59, 1915.

Cañon, V.: Bile, Arch. Farm., i 7, r 78, I9r4.

Cappelia: Salvarsan, Zentr. Bioch. Bioph., r4, 265, rgrr. 
Capps, J. A.: Iodids, J. Am. Med. Assoc., 59, I350, 191 2; Venous Pressure, Ref., J. Am. Med. Assoc., 57, I5I, I9II.

Carlson, A. J.: Antimony, U. S. Bur. Lab. Stat., No. 179, I915; Bitters, J. Am. Med. Assoc., 64, I5, r9r5; Cyanid, Am. J. Physiol., I9, 223, 1907; Gastric Juice, Am. J. Physiol., 37, 50, I915; 38, 248, 1915; Hunger Contractions, Am. J. Physiol., 32, 245, т913; ib., 33, 95, г913; Limulus IIeart, ib., I 2, 67, 1904 .

Carlson, Erve, Lewis and OrR: Bitters, J. Pharmacol., 6, 209, I9I4.

CArlson and Jacobson: Ammonium, Am. J. Physiol., 25, 403, 1910; Parathyroid, ib., 28, I33, I9II.

Carlson, Lebensohn and Pearlaian: Secretin, J. Am. Med. Assoc., 66, i 78 , igr6.

CARLSON and Lewis: Inunger Contractions, Am. J. Physiol., 34, I49, I9I4.

Carlson and Luckhardt: Anesthetics, Am. J. Physiol., 2I, I908.

CARLSON, A. J., and pupils: Lymphagogues, 22 , 104, 1908.

CARLson, Rooks and MCKIE: Thyroid, Am. J. Physiol., 30, I9I 2.

Carlson and Woelfel: Lead, J. Am. Med. Assoc., 6r, I8I, I9I3.

Carnot, P., and CaIris: Bromid, Soc. Biol., 77, 197, I914; Camphor, ib., 77, 200, 19I4.

CARr, F. H., and Pyman: Ipecac, J. Am. Pharm. Assoc., 2, 428, I91 3.

CARR, G.: Acetanilid, J. Pharmacol., 2, 399, I910.

CARRel and Ingebrigsten: Antibodies, C. S. Soc. Biol., 72, 220 , I91 2.

CARTER: Poison Mushrooms, Am. J. Physiol., 5, I38, I901.

Carvalio and Weiss: Veratrin, J. de Physiol., I, I, I889.

CASH: Indaconitin, Brit. Med. J., May 23 , I908.

CAstaldi, L.: Dialylmalonylurea, Archiv Farm. Sper., I9, 289, I91 5.

Cathcart: Ferments, J. Physiol., 3I, 497, I904.

CAthCart and Clark: Anesthcsia, J. Physiol., 47, 392, I9r3.

Cavazzani, E.: Sulphocyanic Acid, Arch. Fisiol., I3, 387, I915; Veratrum, Clin. Med. Ital., 1909 .

Cazzola: Calcium, Zentr. Bioch. Bioph., I5, 777, r9r3.

Cernadas, P. V.: Salicyl, J. Am. Med. Assoc., 66, 780, I9 5.

Cerrano, E.: Collyria, Arch. Farmacol. Sper., 8, I909.

Cenvelio: Paraldehyde, Arch. exp. Path. Pharm., I6, 265, 1882; Picrotoxin, Arch. exp. Path. Pharm., 64, 407, I9II.

Cervelio and Girgenti: Acetone, Arch. exp. Path. Pharm., 76, ir8, igi4.

Cesaris-Demel: Anaphylaxis, Zentr. Bioch. Bioph., I 5, 609, IoI 2.

Cevidalir, A.: Phosphorus, La Riforma Med., r902; Saffron, Zb. Bioch. Bioph., I7, 7 II, I9I4.

Chalfant: Anesthesia, J. Am. Med. Assoc., 59, 852, r912.

Chantemesse: Nuclein, Merck's Rep., 2 I, II, I907.

Charcot and Gombault: Lead, Arch. de Physiol., 8, r26, i88r.

Charmoy: Hordenin, Ref., Merck's Rep., 27, 269, 191 2.

Charteris, F.: Thiosinamin, Glasg. Med. J., Sept., I9Io.

ChASE and Gres: Thorium, Soc. Exp. Biol. and Med., March, I905.

Chesney, Marshall and Rowntree: Liver Function, J. Am. Med. Assoc., 63, 1533, I9I4.

Chevalier, J.: Theobromin, Bul. Gen. Ther., I67, 599, r9i4; Adonis, Zentr. Bioch. Bioph., i 5, 208, 1913.

Chevallier, P.: Iron, Arch. Med. Exp., 26, 277, I914; Spleen, Arch. path. Anat., 217 , 358,1914 .

Chiare, Sd.: Stovain, J. Am. Med. Assoc., 62, 494, г9г3.

Chiappa and Ravano: Colarnin, Arch. It. Gin., No. 2, igog.

Chiari, R.: Cathartics, Arch. exp. Path. Pharm., 63, rgro; Gastric Secretion, Ther. Mon., 29, 202, 1915; Pancreatic Secretion, ib., .29, 204, 1915; Salvarsan, Ref., J. Am. Med. Assoc., 61, 2074, I9I3.

Chiari and Froelich: Oxalate, Arch. exp. Path. Pharm., 64, 214; 66, rio, igir.

Chiari, R., and Januschke: Calcium, Wien. klin. Woch., 23; Arch. exp. Path. Pharm., 65 , I 20 , I910.

Chio, M.: Curare, Zentr. Bioch. Bioph., 13, 598, I9r2; Tartaric Acid, Arch. intern. Pharmacodyn., 22, 473, I912.

Chrsholi: Blood Volume, J. Path. Bact., 18, 552, 1913; 19, 265, 1914.

Chrstoni, A.: Cascara, Arch. Farm., I7, 97, r914; Iodid, Zentr. Bioch. Bioph., 10, 883, I9Io; Iodin, Arch. int. Pharmacod., 21, 339, I 1 I I Picrotoxin, Zentr. Bioch. Bioph., I3, 556 , I912.

Chistoni and Lapresa: Salicyl, Arch. Farmacol., 8, 63.

Chittenden and Cutmbert: Bromid, Stud. Lab. Physiol., Yale, I 52, 1884.

Chittenden, Mendel and Jackson: Alcohol, Am. J. Physiol., I, 164, I898.

Chlumsky, V..: Phenol Camphor, Zbl. Chir., No. 33, I905; Zbl. inn. Med., 2 26, 1912. 
('ulksy: Magnesium, Lancet, 300 , I9 I r.

('Hnt.siles: Gold, Ref., Merck's Report, 27, $25 \mathrm{r}$.

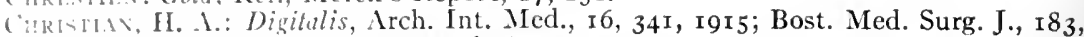
Sol, 1915; Diuretics, J. Am. Med. Assoc., 6I, 267, 1913; Theobromin, Arch. Int.

iled., It, No. 6, igr4; Uranium, ib., 8, No. 4, r9ı.

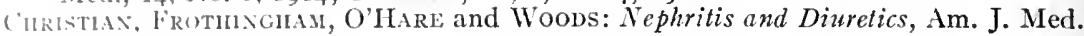
Sci., $150,655,1015$.

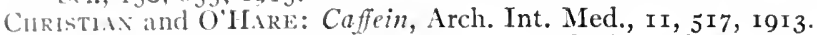

('llmistinsex, J., Dolglas and Haldane: Carbon Dioxid, J. Physiol., 48, 244, i9r4; Oxygen, J. Physiol., 47, Proc.

Cintrcimas, J. IV.: Gentian Violet, Proc. Soc. Exp. Biol. Med., I I, 54, I9 I4.

Ci.tccio, Vox C.: Suprarenal Cortex Functions, Arch. exp. Path. Pharm., 78, 347, 19i5. CIANis: Atoxyl, Sperim., 62, 243, Igos.

Claborve: Salicyl, Trans. Ophth. Soc., io, 3, I906.

Ciskk, 1. J.: Atropin, Quart. J. Exp. Physiol., 5, 386, r912; Digitalis, Proc. Roy. Soc. Med., 5, I8 I, I9I 2; J. Pharmacol., 4, 399, I913; Strophanthus, I. Pharmacol., 5, 215, i914; Tyramin, Bioch. J., 5, 236, 1910.

Clakk, 1. J., and Mines: Strophanthus, J. Physiol., 47; Proc. VII, I9I3.

Clark, L. N.: Pitwitary, J. Biol. Chem., 22, 485, I9 I5.

Clark, M., Marshall and Rowntree: Mushrooms, J. Am. Med. Assoc., 64, I230, I9 I 5 .

Clark, P. F.: Epinephrin, J. Am. Med. Assoc., 59, 367, r9r 2.

Clark, T. W., and Brow's: Alcohol and Phenol, J. Am. Med. Assoc., March r 7, 1906.

Clarke and Gage: Copper, J. Inf. Dis., Feb., Igo6.

Claude, Porak and Routier: Pituitary, Zentr. Bioch. Bioph., I5, 574, i913.

Claveric: Vanilla, Proc. Am. Pharm. Assoc., 57, I60, I908.

Cleaveland, S. A.: Lithium, J. Am. Med. Assoc., 60, 722, I9I3.

Cleghorn: Heart, Am. J. Physiol., 2, 273, I899.

Clerc and Pezzi: Nicotin, J. Phys. Path., I4, 704, I9I 2.

Cloetta, M.: Antimony, Arch. exp. Path. Pharm., 64, 352, r9i ; Arsenic, ib., 54, 196, I906; Zentr. Bioch. Bioph., I2, I 86, I9 I ; Atropin, Arch. exp. Path. Pharm., 64, 427, I9II; Bronchio-constriction, ib., 73, 233, I9I3; Digalen, Muench. med. Woch., No. 33, I904; Iron, Arch. exp. Path. Pharm., 38, 170; 44, 363, 1900; Morphin, ib., 50, 453, I903; Scopolamin, ib., 7 I, 290, I9I3.

Cloetta and Fisciner: Digitoxin, Arch. exp. Path. Pharm., 54, 1906.

Cloetra and Waser: Epinephrin, Arch. exp. Path. Pharm., 79, 30, 19r5; Fever, Arch. exp. Path. Pharm., 73, 398, I913; ib., 75, 406, I914; ib., 77, I6, I914.

Clossox, O. E.: Local Anesthetics, J. Mich. St. Med. Soc., 13, 587, 1914.

Clover, A. M.: Peroxid, Am. J. Pharm., 85, 538, I9 I3.

Clowes: Lead, Chem. News, 86, 168, 1902.

C.runt, E.: Gclatin, Ther. Monatsh., 27, 60, 1912.

Conet, R.: Epsom, Arch. ges. Physiol., I 50, 325, I9I3.

Coblentz: Cerizm, U. S. P. Dig., 383 , I9 Io.

Coblextz and May: Cyanid, Proc. Am. Pharm. Assoc., 879, rgo8.

Conen, S. S.: Quinin, J. Am. Med. Assoc., 6r, ro7, 19 13.

Cohs: Calomel, Diss., Berlin, 1887.

Cons, A. E.: Digitalis, J. Pharm. Exp. Ther., 6, 606, r9г5; J. Am. Med. Assoc., 65, 1527, I915; Morphin, Proc. Soc. Exp. Biol. Med., Io, 93, 1913.

Connheis: Absorption, Zs. Biol., 38, 419, I899.

Connilers and Lichtherm: Edema, Arch. path. Anat., 69, то6, I877.

Cohnheis, O., and Modrakowski: Morphin, Zentr. Biochem. Biophysik., i I, 823. IgII.

Coinstein: Metal-diuresis, Arch. exp. Path. Pharm., 30, I 26, I892.

Cole, H. N.: Anaphylaxis, Clevel. Med. J., 10, 442, I9I I.

Cole, L. J., and Bachnuber: Lead, Soc. Exp. Biol. Med., i 2, 24, I9i4.

Cole, R.: Pneumonia, Harvey's Lectures, p. 85, I915; Trans. Assoc. Am. Phys., 30, 230,1915 .

Coleman, W.: Hexamethylenamin, Med. News, 83, 393, I916; Typhoid Diet, J. Am. Med. Assoc., 53, I I 45, I900.

Collins, J., and Martland: Cyanid, J. Nerv. Ment. Dis., 35, 41 7, I908.

Collins, R. J.: Veratrum, Arch. Int. Med., I6, 54, I915; J. Pharmacol., 8, т 28 , I9r6.

Conner, L. A.: Salicyl, Med. Rec., No. 8, I914.

Consoli, G.: Stovain, Arch. intern. Pharmacodyn., 23, 17, I913.

Contr and Zuicola: Mcrcury, Bioch. Centr., 5, 503, I906.

Cook, F. C.; Boron, J. Agr. Res., 5, 877, I9I6.

Cook and Pembrey: Oxygen, J. Physiol., 45, 429, r9 г3. 
Cooke: Parathyroid, J. Exp. Med., i 2, 45, г910.

Corbetr, J. F.: Suprarenal Gland in Shock, J. Am. Med. Assoc., 65, 380, 1915.

Coriat: Cholin, Am. J. Physiol., I 2, 353, I904.

CoRlett: Ointments, Clevel. Med. J., Iо, 202, igio.

Corley: Oxalate, Arch. intern. Pharmacod., Io, 293, 1902.

Connwall: Nitrite, J. Am. Med: Assoc., 6r, i 18 , I9г3.

Conoved1, G.: Thyroid, Arch. intern. Pharmacod., 23, 353, r9r 3.

Corper, H. J.: Copper, J. Inf. Dis., I5, No. 3, I914; Sulphocyanids, J. Inf. Dis., I6, No. I, I9I5.

Corridi, L.: Salvarsan, Arch. Farmacol., I4, I91 2; Arch. Farm. Sper., I6, 3, I9 I3.

Cotron, A. C.: Citrate, J. Am. Med. Assoc., 47, ro8o, I9o6.

Cow, D.: Adrenals, J. Physiol., 48, 443, I914; Digitalis, Bioch. J., 6, 219, г9г 2; Diuresis, Arch. exp. Path. Pharm., 69, 393, I91 2; Pituitary, Zentr. Bioch. Bioph., I4, I88, I91 2; Quebracho, J. Pharmacol., 5, 341, 1914; Surviving Arteries, J. Physiol., 42, I 25, I9I I; Water Diuresis, J. Phy'siol., 48, I, I9I4.

Cowre and Munson: Emollients, Arch. Intern. Med., Jan., I908.

Cragin and Hull: Chloroform, J. Am. Med. Assoc., 56, 5, I9II.

Cramer, W.: Epinephrin, J. Physiol., 42, No. 5, igi I; Veratrum Viride, J. Pharmacol., 7,63 , I9 5 .

Crampton: Baking Powders, U. S. Div. Chem., Bul. ×3, I889.

Crawford, A. C.: Barium, U. S. Bur. Plant. Ind., Bul. I 29, I908; Epinephrin, ib., Bul. I I2, I907; Ergot, J. Am. Med. Assoc., 61, I9, I9I3; Mottntain Laurel, U. S. Bur. Plant. Ind., Bul. I2 I, 1908; Phosphate, J. Pharmacol., I, 519, I910; Tyramin, J. Biol. Chem., I9, I9I4.

Crawford, A. C., and Trourbly: Epinephrin, N. Y. Med. J., Aug. 16, i913.

Crider, J. O. and Robinson: Fatigue, Am. J. Physiol., 4I, 376, igi6.

Croner and SeligmanN: Formate, W. Hyg. Inf., 56, No. 3, i907.

Cronyn and Henderson: Ergot, J. Pharmacol., I, 203, I909.

Crowe, S. J.: Hexamethylamin, Arch. intern. Pharmacod., I8, 3 I 5, 1908; Johns Hopkins Hosp. Bul., 23, 255, 19 2.

Crowe, Cushing and Homans: Pituitary, Johns Hopkins Hosp. Bul., 21 I, I 27 , 1910.

Cruikschank, J.: Lecithin, J. Path. Bact., r8, I34, I9i 3.

CRUMe, G. P.: Mercury, J. Am. Med. Assoc., 51, 2155 , 1908.

Cullis and TriBe: Autonomic, J. Physiol., 46, I4I, I9I3.

Cunningham: Anesthesia, Bost. Med. Surg. J., I62, 38\%, г910.

Cunningham and Anderson: Ether, J. Am. Med. Assoc., 5 I, I574, 1908.

Curtillet and Lombard: Lead, Ref., J. Am. Med. Assoc., 58, 1646, I9I 2.

Cushing and Clarke: Water, Am. J. Med. Sci., Feb., I905.

Cushny, A. R.: Anesthetics, Zentr. Bioch. Bioph., ro, 494, rgro; Antagonism, J. Pharmacol., 6, 439, I915; Atrcpin, J. Physiol., 30, 176, 1903; Autonomic System, ib., 35, I, I906; ib., 37, 130, 1908; ib., 4I, 233, I910; Digitalis, J. Exp. Med., 2, 234, I897; J. Physiol., 25, 49, r899; Am. J. Med. Sci., Apr., I9I I; Berl. klin. Woch., 50, 717, 1913; Diuresis, J. Physiol., 27, 429; 28, 431, 1902; Epinephrin, ib., 38, 259, I909; Gelseminin, Arch. exp. Path. Pharm., 31, 49, 1892; Hypnotics, XV̈II, Int. Congr. Med., 5. 2, I 27 , I9 14; M yocardiogra ph, Heart, 2, 1, I910; Nutmeg, Proc. Roy. Soc. Med., Feb., I908; Respiration, J. Pharmacol., 4, 363, I913; Senecio, ib., 2, 53 I, I9п. William Withering, $F . R$. S., Proc. Roy. Soc. Med., 8, 85, I9I 5 .

Cushny and Guns: Serum, J. Pharmacol., 5, I, I9ז3.

Cushny and LIEB: Urethane, J. Pharmacol., 6, 45I, I9I5.

Cushny and Matthews: Spartein, Arch. exp. Path. Pharm., 35, I 29, I895.

Cushny and PeEbles: Hyosein, J. Physiol., 32, 501, 1905.

Czapex and Wassermavis: Urine Retention under Morphin, Deut. med. Woch., July 30 , I9I4.

Czapski: Alkali, Arch. exp. Path. Pharm., i7, 226, 1914.

Da Costa and Kalteyer: Anesthctics, Ann. Surg., Sept., r 901.

Dakin, H. D.: Benzoate, J. Biol. Chem., 7, 103, 1910; Hypochlorous Acid, Brit. Med. J., 2866, 1915; Presse med., Aug. 5, I915; Oxidations and Reductions, I912; Paratoluen sodium sulphochloramid, Brit. Med. J., Jan. 29, I9г6; Suprarenal, Proc. Roy. Soc., B 75, $49 \mathrm{I}$ and 498 , I905.

Dakin, JanNey and Wakeman: Formic Acid, J. Biol. Chem., I4, 34I, I9I3.

DALE: A utonomic, J. Physiol., 34, I63, I906; J. Pharmacol., 4, I67, 517, 1913; Cholin, ib., 6, I47, I914; Ergotoxin, Physiol.. Proc., May 20, I905; J. Physiol., 46, I9 13, Pituitary, Bioch. J., 4, 427, 19 ro.

DALE and DrXoN: Tyramin, J. Physiol., 39, 25, 1909. 


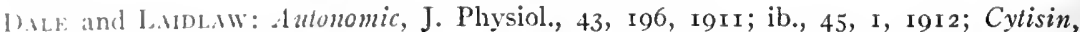

J. I'harmacol., 3, 205, r912; Histamin, J. Physiol., 41, 318, 1910; Pituitary, J.

Pharmacol., 4, 75, 1912; Reversed Chorda, J. Physiol., 43, I96, I9I I.

I) At: and THicker: Acids, J. Physiol., 47, I9I3.

I) Mr.wic, II. C., Kolds and Loevenilart: Oxygen Capacity, Am. J. Physiol., 39, 77.

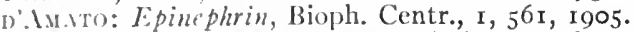

DAmoEILl:R, E.: Anesthetics, Arch. intern. Pharmacod., 23, 229, I9r3.

I)A.i and Berkeley: Pineal, Med. Rec., 83, 835, I9I3.

I).Ning, II. E. and Rowstree: Peritoneal Absorption, Ann. Surg., 59, No. 4, r9I4.

Darikls, 1. 1.., and McCrcdoen: Uric Acid, Arch. Int. Med., 15, 1046, 1915.

I).Niretthis, W. T.: Luteum, J. Am. Med. Assoc., 62, 359, r9I4.

D.ISTRE and l'Loresco: Gclatin, Arch. de Physiol., 8, 402, 1897.

I) AUWE, O.: Lead, Arch. int. Pharmacod., r 7, 387, ro07.

D.W1s, B. IF : Paraffin, J. Am. Med. Assoc., 62, r7r6, r9r4.

D.w1s, C. H.: Vitrous Oxid, J. Am. Med. Assoc., 65, 992, I9I 5.

D.vis, D. J.: Arthritis, Arch. Int. Med., 55, 555, r9I5.

Davis, N. S.: Elaterium, J. Am. Med. Assoc., 6r, 273, r913.

DAvis, S. G.: Ancsthetics, Johns Hopkins Hosp. Bul., 20, 2 I 7 , I909.

DAwes, S. L., and Jackson: Cacedyl, J. Am. Med. Assoc., 48, 2090, r907.

DAwsox: Saline, J. Exp. Med., 7, I, 1905.

DE Arric: Quinin, Proc. Soc. Roy. Brux., May, rgi 2.

DE BARExit: Strychnin, Fol. Neurobiol., 4, 467, 1910; 5, 42, I911; 6, 277, 191 2; 7, 549, I9I3.

De Biehler: Cacody $l$, Arch. internat. Pharmacod., I $7,65,1907$.

DE Busscher: Arsenic, Arch. intern. Pharmacod., 10, 415, I902; Morplin, ib., r3, 309;

I4, 505, r904-5; Permanganate, ib., I3, 309, I904.

DeEtjen: Calcium, Berl. klin. Woch., No. i6, I904.

D'Errico: Bile, Bioch. Centr., Io, 647, igio.

DE HeER: Magnesium, Arch. intern. Pharmacod., 21, 32 I, I9II.

D'Hotel: Phenol, Ref., J. Am. Med. Assoc., 48, r945, r907.

De la PAz and Garcia: Apomorphin, Ref., J. Am. Med. Assoc., 67, 772, ror6.

Delbet, P., and Karajanspoulo: Magnesium, Bul. Acad. Med., Sept. 7, r9r 5 .

Delezerie, C., and Pozerski: Formaldehyd, Soc. Biol., 78, 23, r915.

Demse: Salicyl, Med. Chir. Centr., 23, 243, I 888.

DE.MOOR: Pilocarpin, Zentr. Bioch. Bioph., I4, 52, r9I 2.

Denis, W.: Diffusion, Am. J. Physiol., r 7, 34, r9o6; Fish, J. Biol. Chem., r6, 395, 1913; Gout, J. Pharmacol., 7, 601, I915; Salicyl, J. Pharm. Exp. Ther., 7, 255, r9I 5.

Denis and Means: Salicylate, J. Pharmacol., 8, 273, 1916

Denvy, G. P., and Frothingham: Arteriosclerosis, J. Med. Res., 31, 277 , I914.

Denny, G. P., and Minot: Antithrombin, Am. J. Physiol., 38. 233, 1915.

1)EROUAUX: Ether, Arch. int. Pharmacod., 19, 63, roo9.

DE Schweinitz: Mercury, Ther. Gaz., June 15, 1893; Salicyl, Trans. Am. Ophth. Soc., I895; Santonin, Ophth. Rec., Jan., r899.

Deseille: Salicyl, These, Paris, No. 494, I879.

Desgrez and Dorlfans: Epineplerin, Zentr. Bioch. Bioph., r6, 452, rgr3.

Desoubry, G., and Magne: Anesthesia, Bul. Gen. Ther., r67, 574, r9i4.

Deucher: Digitalis, Arch. klin. Med., 62, 1896.

DeVamossy: Liver on Poisons, Arch. intern. Pharmacod., 13, 156, 1905.

DE Vos and Kocmmans: Epinephrin, Arch. internat. Pharmacod., I 4, 83, r905.

DEwey, K.: Cholesterol, Arch. Int. Med., r 7, 757, r9r6.

DEwey, K., and Nuzum: Cholestercl, $\mathrm{r}_{5}, 472$, $19 \mathrm{I}_{4}$.

Dezani, S.: Cholesterin, Arch. Farm., I 7, 4; I7, 416, r914.

Dieballa: Anesthetics, Arch. exp. Path. Pharm., 34, r37; 45, 367, 1894.

DieIrl: Narcosis, Diss. Marburg, r 894 .

Dietl and Vintschgau: Caffein, Arch. ges. Physiol., r6, 359, r878.

Drxos, S. G.: $\mathrm{CH}_{2} \mathrm{O}$, J. Am. Med. Assoc., 63, ro25, r914.

Dixon, W. E.: Alcohol, J. Physiol., 35, 346, r9o7; Alimentary Toxemia, Proc. Roy. Soc., 6, r29, r9 3; A naphylaxis, Brit. Med. J., Nov. 22, r91 3 ; A pocodein, J. Pysiol., 30, 97, r903; Cactus, ib., 25, 69, I899; Cannabis, Brit. Med. J., Nov. I I, 1899; Cocain, J. Physiol., 32, 87, I904; Digitalis, Quart. J. Med., 297, I9r2; Ergot, Brit. Med. J., 2, 1459, 1906;

Dixon and Brodie: Asihma, Trans. Path. Soc., 54, r9o3.

Dixon and Hallmurton: Cerebral Circulalion, Qu. J. Exp. Physiol., 3, 315, 1910; Cercbro-spinal Fluid, J. Physiol., 47, 2 15;48, I 28,317 , 19r3-r4; Cerebro-spinal Fluid, J. Physiol., 44, r9r 2; Pineal, Qu. J. Physiol., 2, 283 , r9o9.

Dixon and Harvey: Brucin, Ref., Proc. Am. Pharm. Assoc., 57, 375, r9o8. 
Dixon and Lee: Nicotin, Qu. J. Exp. Physiol., 5, 373, I9r 2.

Dixon and Maldex: Colchicin, J. Physiol., 37, 50, 1908.

Dixon and Ransom: Broncho-dilators, J. Physiol., 45, 413, 1912; Selective Action, Erg. Physiol., 12, 768, 19I2.

Dock, G.: Iodid, J. Am. Med. Assoc., 53, I607, 1909.

Dock and Lyoss: I pecac, Trans. Assoc. Am. Physicians, 25, 515, 1910.

Doebelr: Morphin, Therap. Monatsh., 27, 303, 1911.

Doeblin and Fleischmans: Atropin, Z. klin. Med., 77, 145, 1913.

Doebrer and Giesecke: Atophan, Liebig. Ann., 242, 291, I887.

DOERR and Russ: Anaphylaxis, Zs. Immun., 3, I 909.

Dorrs: Atophan, Bioch.Z., 43, No. 3; Z. klin. Med., 74, 445, r9 2.

DolAnd: Belladonna, Am. J. Med. Sci., Apr., 1906.

Donneliy, W. H.: Fuchsin, J. Am. Med. Assoc., 62, 528, 1914.

Donzello, G.: Sublimate, Arch. di Farm., I9, 203, I9 I5.

Dore, S. E.: Alopecia, Clin. J., 43, No. 3, I9I4.

Dorland: Ustilago, Med. News, Phil., 5I, 534, I887.

DorlencourT, H.: Mcrphin and Spartein, Zentr. Bioch. Bioph., 15, 382, 1913.

D'Ormea: Volatile Oils, Arch. it. Biol., 40, I4I, 1903.

Dornblueth: Phenolphthalein, Muench. med. Woch., No. 52, 1903.

Dorner and Weingaertner: Bismuth, Bioch. Centr., 9, 672, Igog.

Dossin: Nitrite, Arch. intern. Pharmacod., 2I, 425, I9II.

Douglas, C. G., and Haldane: Cheyne-Stokes, J. Physiol., 38, 401, r9o9; Oxygen, Proc. Roy. Soc., B, 82, 331, I910.

Douglas, Haldane, Henderson and Schneider: Pikes Peak, Proc. Roy. Soc., 85, $\mathrm{B}, 67$, I9r3.

Downs, A. W.: Hemorrhage, Am. J. Physiol., 40, 522, 19г6.

Doyon: Atropin, Bioch. Centr., 10, 94, I910; Nitrite, ib., 6, 80, I906.

Doyon and KAREFF: Atropin, C. R. Soc. Biol., 56, 588, 1904.

Doyon, Morer and Policard: Peptone, Zentr. Bioch. Bioph., I I, 740, I9II.

DraPER, Z.: Salvarsan, Arch. Int. Med., I5, I6, I9I5.

DRENKHAW: Aspidium, Muench. med. Woch., 2020, r9I r.

DrEscher: Turpentine, Jahreb. Pharm., 66, 297, 1906.

Dreser, H.: Alkalies, Arch. intern. Pharmacodyn., 20, 43r, т91о; Anesthetics, Arch. exp. Path. Pharm., 37, 375, I896; Aspirin, Arch. ges. Physiol., 76, 306, 1899; Atropin, Arch. exp. Path. Pharm., 26, 255, 1890; Caffein, ib., 24, 221, 1887; ib., 29, 303, 1892; Dintresis, ib., 29, 303, 1892; Heart Work, ib., 24, 221, 1887; ib., 27, 50, 189o; Lobelin, ib., 26, 237, I889; Morphin, Arch. ges. Physiol., 72, 485, I898; Muscle, Arch. exp. Path. Pharm., 27, 50, I890; Salicyl, Arch. ges. Physiol., 76, 306,1899 .

DreYer and Walker: Dosage, Bioch. Zs., 6o, II2, Igr4.

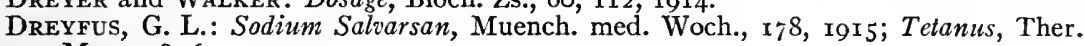
Mon., 28, 692, I914.

Droste: Solanin, Zentr. Bioch. Bioph., r8, 627, r9r 5.

Drysdale, H. H.: Anti-narcolic Law, Clev. Med. J., I4, 353, I9r 5.

Du Bors, E. F.: Goiter, Arch. Int. Med., I7, 915, I9I6.

DUEnNER: Uranium, Zs. klin. Med., 8r, 355, I9I5.

Duhem: Morphin, Merck's Rep., 21 , I 23 , I907.

Duke, W. W.: Benzol, Arch. int. Med., II, Ioo, I9I3; Platelets, J. Am. Med. Assoc., $65,1600,1915$.

Dunbar: Hay-fever Serums, Deut. med. Woch., Nov. 13, I9ı ; Pollantin, Berl. klin. Woch., Nos. 24-26, 1903.

DunCKer and Jodlbauer: Katalase, Bioch. Zs., 33, 253, I9I I.

DurhaM: Sleep, Guy's Hosp. Rep., 6, I49, r86o.

Durlach: Phosphorus, Arch. exp. Path. Pharm., 7I, 210, I913.

DUtCher and SteEl: Arsenic, J. Am. Chem. Soc., I9I4.

Dutron, W. F.: Vanadium, J. Am. Med. Assoc., 56, I648, I9г r.

Dzerzgowski and Schumofr-Sieber: Nickel, Bioch. Zs., 2, I9o, rgo6; Ref.y Proc. Am. Pharm. Assoc., 57, 270, I908.

Eastman, J. R.: Osmic Acid, J. Am. Med. Assoc., 46, 556, 1906.

EBERHARDT and ELDRED: Bibliography of Determination of Drugs and Pharmaceutical Products, J. Am. Pharm. Assoc., 3, 38, I9 I4.

EBstern and Mueller: Salicyl, Berl. klin. Woch., I3, 337, 1876.

ECKLER: Digitalis, Am. J. Pharm., 83, 478, т91 1.

ECKler and Miller: Cannabis, Am. J. Pharm., 84, 488, I9I 2.

EDDY and GIES: Alkaloidal Protein Compounds, Proc. Soc. Exp. Biol. Med., 4, I 45, 1907. 
Fins: Protuceratrin, Arch. exp. Path. Pharm., 29, 440, r892.

Fin vos, C. II.: A muphyluxis, J. Pharmacol., 5, 518 , 1914; Liver, J. Pharm. Exp. Ther., 0. 509, 1915; Lobelin, Am. J. Physiol., II, 79, I904; Nicotin Tolerance, J. Lab. (Clin. II., I, 315, I916; Protein Poisons, Z. Immur., I7, 105, I913.

Eumus and Itite: Ergot, J. Pharmacol., 2, 393, I9I.

Finums and Rotr: A utonomic System, Am. J. Physiol., 23, 28, I908; Tablets, J. Am. Med. Issoc., 5I, 2130, I908.

Ensili., F. L.: W'ater, Conts. Pepper Lab., 368, I900.

Eoside, E. l., and Meass: Respiration, Arch. Int. Med., 15, No. 6, I9I4; Trans. Ass'n Am. Phys., 29, 69, I9I4.

Eicilestox, C.: Atropin, J. Pharmacol., 9, Ir, igr6; Digitalis, J. Am. Med. Assoc., 59, I352, I9I2; ib., 6r, 757, I913; Digitalis Dosage, Arch. Int. Med., I6, I, I915; Salicyl, J. Am. Med. Assoc., 59, 2057, I9 I2.

Fgglestox, C., and Hatcher: A pomorphin, J. Pharmacol., 3, 55I, igr 2; Emelics, J. Pharm. Exp. Ther., 7, 225, 1915.

vax Egurosd, A. A. J.: Ileart Block, Arch. ges. Physiol., 154, 39, 1913; Morphin, Arch. exp. Path. Pharm., 65, 197, I9II.

Enrevfried: Picric Acid, J. Am. Med. Assoc., 56, 41 2; N. Y. Med. J., March 25, 1911. Einticis, P.: Dves, Ther. Monatsh., March, I887; Fluorescein, Deut. med. Woch., ISs2; Ricin, ib., I7, 976, I89r; Trypanosomes, Berl. klin. Woch., Nos. 9-12, I907. Enrmax: Salicyl, Muench. med. Woch., 54, 2595, 1907.

Eirmaxy, Esser and Loewy: Acidosis, Zentr. Bioch. Bioph., ir, 834, I9II.

Ervis: Pituitary and II istamin, Bioch. Z., 52, 96, 1913.

Eisesperg: Ilexamethylenamin, J. Am. Med. Assoc., 58, 2032, I9I2.

Eisexbrey, A. B.: Phthalein Excretion, J. Exp. Med., I4, 462, I913.

Eisexdr.ith, D. N.: Collargol, J. Am. Med. Assoc., 62, I392, I9I4.

EisNer, G.: Atophan, D. Arch. klin. Med., I 18, I25, I915.

Eldred: Ergot, J. Am. Pharm. Assoc., 2, I I09, I9I3.

EliN, J. B.: Iodin, Zentr. Bioch. Bioph., I4, 445, I9I 2.

Elischer, V.: Cotarnin, Wien. med. Woch., No. 32, I904.

Ellixger, 1.: Atropin, Z. Biol., 42, 228, 1901; Cantharidin, Arch. exp. Path. Pharm., 45, 89, 1900; Muench. med. Woch., No. 8, 1905.

Ellinger, A., and Kotake: Bromid, Arch. exp. Path. Pharm., 65, 87, igri.

Ellinger, P.: Cholin, Muench med. Woch., 2336, r9r4.

Elliotr, T. R.: Adrenals, Quart. J. Med., 8, No. 29, 1914; Anesthetics, J. Physiol., 44, 400, 19I 2; A utonomic, J. Physiol., 32, 401, 1905; Tetra, Qu. J. Med., 7, r 20, 1914.

Elliott and DuRhaM: Epinephrin, J. Physiol., 34, 490, I906.

Ellis A. W. M. and Swift: Salvarsan, J. Exp. Med., I8, 428, 19r3.

Ellis, G. W., and Gardner: Cholesterol, Proc. Roy. Soc., 86B, I3, I9I 2.

ElMER: Phenolphthalein, Med. Rec., Nov. 7, I909.

Elrington, G.: Sirychnin, Z. allg. Physiol., I6, II5, I9I4.

Eurbley: Chloroform, Brit. Med. J., Apr. 5, I2, 19, 1902; J. Physiol., 28, I902; Lancet, Aug. 7, I9I 5 .

Esibley and Martin: Chloroform, J. Physiol., 32, I47, 1905.

Emden and Fuertir: Epinephrin, Beitr. chem. Physiol., 4, 421, 1903.

Eyersox: Epincphrin, Proc. Soc. Exp. Biol. Med., 5, I, I907; Nephritis, Johns Hopkins Hosp. Rep., IO, 323 , I902.

EMrmerich, R., and Loew: Calcium, Berl. klin. Woch., 50, no. 26, rgi3; Muench. med. Woch., 62, No. 2, I915; Reproduction, Zentr. Bioch. Bioph., I8, 558, 1915.

Excel: Epinephrin, Z. exp. Path., II, 9, I9I2; Morphin, Arch. exp. Path. Pharm., $27,419,1890$.

Engelihardt, H., and Winters: Phosphorus, J. Am. Pharm. Assoc., 4, I450, I9I5.

ENGeloch, F., Epinephrin, Zs. Biol., 66, 99, I9 15.

E.vGWer, T.: Ethyl Hydrocuprein, Zs. Hyg. Inf., 73, r94, I9I 2.

ENriquez, E., Binet and Gaston-Durand: Bicarbonate, Bul. gen. Ther., I68, 40, r9I4.

EppiNger: Acidosis, Wien. klin. Woch., Feb., No. 5, 1906; Zs. exp. Path., 3, 530, I906; Formate, Wien. klin. Rund., 49, I9I3; Histamin, Wien. med. Woch., 23, $1414,1913$.

Epstein, A. A. and Baenr: Phlorhizin, J. Biol. Chem., 24, I7, igr6.

Epstern, Reiss and Branower: Surgical Procedures, J. Biol. Chem., 26, $25,1916$.

Epstein, E.: Intravenous Therapy, Ther. Mon., 29, $21 \mathrm{I}$, 1915.

ERBEN: Iron, Zs. klin. Med., 40, 266 , 1900.

ERDELYI, P.: Caffein, Zentr. Bioch. Bioph., 14, 562, I9I 2.

ERHARDT, E.: Spinal Anesthesia, Arch. intern. Pharmacod., 21, 213, I9r 2.

Erlenseyer, E.: Lead, Bioch. Zs., 56, 330; Zs. exp. Path., I4, 310, I913.

ERLENMEYer and Stein: Iodids, Jahrb. Pharm., 69, I 20, I909. 
Escallon: Salvarsan, Chem. Abstr., 7, r054, 1912.

Escr: Epinephrin, Arch. exp. Path. Pharm., 64, 84, i910.

Eskin, D. A.: Epinephrin, Ref., J. Am. Med. Assoc., 63, I990, r914.

Espeut, H.: Pituitary, Muench. med. Woch., 60, I774, 1913.

EtienNe: Digilalis, Arch. intern. Pharmacod., I9, I 19, I909; ib., 20, 265, I910.

EtienNe and PARisot: Pituitary, Chem. Abstr., 3, 82, igo8.

Evans, C. L., and Ogawa: Epinephrin, J. Physiol., 47, 446, I9I4.

Evans, J. S., and Middleton: Emetin, J. Am. Med. Assoc., 64, 422, 1915.

Ewing: Saliva, J. Pharmacol., 3, I, I9II.

Ewins, A.: Acetyl-cholin, Bioch. J., 8, 44, I9 4.

Eyster and MEeK: Morphin, Heart, 4, 59, I9I2; J. Pharmacol., 4, 343, 1913.

FaCkelmann: Nitrate, Inaug. Greifswald, i 898.

Fahrenkamp, K.: Digitoxin, Arch. exp. Path. Pharm., 65, 367, r9rr; Strychnin, Zs. Biol., 65, 79, 1914.

Fairhall and HAwK: Water, J. Am. Chem. Soc., 34, 546, I9I 2.

FALK, F.: Pulmonary Excretion, Zs. f. klin. Med., 7 I, 234, 255, I9 5.

Falk, K. G., and Sugiura: Hexamethylenamin, J. Pharm. Exp. Ther., 8, 39, I9r6.

FALdA: Epinephrin, Zs. exp. Path., I5, 356, I914.

FALTA and ZeHNer: Thorium, Berl. klin. Woch., 49, 2444, I9I 2.

Faltis: Pareira, Chem. Abstr., 6, 3420, I9I2.

Fantus, B.: Pharmacy, J. Am. Med. Assoc., 59, 842, I9г 2 ; Fuller's Earth, J. Am. Med. Assoc., 64, I838, I915; Mercuric Antidotes, J. Lab. Clin. Med., I, 879, I916; Prescriplions in English, J. Am. Med. Assoc., 66, I696, I916; Tablets, J. Am. Pharm. Assoc., 3, 657, 1914.

FArini, A., and CECCARONi: Pituitary, Zentr. Bioch. Bioph., I8, 56, I915.

FARMACHIDIS: Metals, Zentr. Bioch. Bioph., I4, 5I9, I9I 2.

FARR: Heroin Habit, N. Y. Med. J., May I, I9I5.

FARR, C. B., and WeLKER: Theophyllin, Arch. Intern. Med., IO, 22.

FARwell, O. A.: Viburnum, Bull. Pharm., Feb., I9I3.

FaUST, E. S.: Animal Poisonts, Abderh. Handb., 2, 815, igro; Rattle-snake Venom, Arch. exp. Path. Pharm., 64, 244, I9I I; Morphin, Arch. exp. Path. Pharm., 44, 2 I 7, I900; Münch. med. Woch., 2489, I9I2; Oleic Acid, Arch. exp. Path. Pharm., Supp., I 7 I, I908; Toad Poisons, ib., 47, 278; 49, I, I902.

Fawcet, G. C., BeEBe, et al: Organ Extracts, Am. J. Physiol., 37, 453; 39, 154, 1915. FEIL, H.: Nicotin, Clevel. Med. J., I5, I74, I9I6.

FeINBERG, M.: A pomorphin, Zs. physiol. Chem., 84, 363, I9I3.

FELIX: Gasolin, Virchow Arch., i I 2, 35, 59, I888.

v. FellenberG, Th.: Methylalcohol, Zentr. Bioch. Bioph., I8, 327, 1915.

FELLNER: Hydrastis, Arch. Gynec., 78, No. 3, 1906.

FENGER: Adrenals, J. Biol. Chem., II, 489, I9I 2; Thyroid, ib., II, 489, I9I 2; ib., I4, 397, I9I3.

FERE: Cocain, Biochem. Centr., 5, 954, rgo6.

Fernan, A., and Pauli: Radium, Zentr. Bioch. Bioph., 70, 246, I9I5.

Ferrari, M.: Iodin, J. Am. Med. Assoc., 56, I372, IgI I.

FERron: Calomel, Arch. Farm. sper., igr 2.

Feulgen: Atcphan, Zentr. Bioch. Bioph., I3, 818, 1912.

FICK: Spartein, Arch. exp. Path. Pharm., I, 397, I873.

Fickewirth and HefFTER:Atropin, Bioch. Z., 40, 26, I912.

FIEDLER: Salicyl, Diss. Halle., r 905 .

FIELD, C. W.: Absorption of Toxins by Nerves, Proc. Soc. Exp. Biol. Med., 4, 149.

Field, C. W., and Teague: Electric Charge, J. Exp. Med., 9, 222, 1907.

FIELdER, F. S.: Rabies, Bur. Lab's., N. Y. City, I914-15; J. Am. Med. Ássoc., 66, 1300, I9 16.

FilehNe: Caffein, Arch. Physiol., 72, r886; Arch. ges. Physiol., 91, 565, I902; Glycerin, Arch. Path. Anat., I I 7, 4I3; Santonin, Arch. ges. Physiol., 80, 96, 1900; Morphin, Arch. exp. Path. Pharm., Io, 442; I1 45, 1879; Nitrite, Arch. Physiol., 386, 1879; Santonin, Arch. ges. Physiol., 80, 96, 1900.

FilehNe and Biberfeld: Chloroform, Z. exp. Path., 3, 1 71 , 1906.

FILIPPI, E.: Digitalis, Arch. Farmacol., 15, 453, I913; Absorption, Sperimentale, 66, 473, I9 I 2; Iodid, Rivist. crit. Clin. Med., I3, Nos. 31, 34, 43, I9 1 2; Quinin, Sperim., 66, 383, I9 1 2; Salicyl, Clin. moderna., 6, No. 7, 1900.

Fillinger: Alcohol, Deut. med. Woch., 999, I9I2.

Fine, M. S., and CHACE: Atophan, Soc. Exp. Biol. Med., I I, IrI, I9r4; J. Pharmacol., 6, 219, I9I4; Arch. Int. Med., I6, 48r, r915; J. Biol. Chem., 21, 223, 509, 19r5; Salicyl, Soc. Exp. Biol. Med.; I2, 95, 1915. 
Finet and Lerolde: Arscnic, Zentr. Bioch. Bioph., $\mathbf{r}_{5}, 583$, I9r3.

Fincerling: Phosphorus, Bioch. Zs., 38, 448, 1912.

Finimore and Bratriwate: I pecacuanhin, Zentr. Bioch. Bioph., I4, I59, I9I 2.

Fiscurl, R.: Cocain, Zs. exp. Med., 4, 362, I915; Cocain and Epinephrin, Ref., Ther. Hon., $4,408,1915$.

Fiscilir, MI. II.: Acidosis, J. Am. Med. Assoc., 64, 325, 19r5; Diuresis, Calif. Publ. Physiol., I, 77, 87, 1903; Edcma, J. Am. Med. Assoc., 51, 830, 1908; Formaldehyde,

J. Exp. Med., 6, 487, 1905; Strychnin, Am. J. Physiol., 10, 345, 1904.

Fiscier, M. II., and Sykes: Diuresis, Coll. Zs., I3, I I 2, I9I3.

Fisciler, P., and Honpe: Salvarsan, Zentr. Bioch. Bioph., ro, 770, I910.

Fiske, C. H., and Karsner: Urea Formation, J. Biol. Chem., r6, I8, 1913-r4.

Fishe, C. H., and Sumer: Urca, J. Biol. Chem., I 8, 285, x914.

lisz, R.: Diureties, Arch. Int. Med., I3, 945, r9r4; Kidney Tests, Am. J. Med. Sci., $148,330,1914$.

Fitz, Alsberg and Henderson: Acidosis, Am. J. Physiol., 18, I I3, i907.

Fla.din: Emetin, Presse med., 779, I913.

Fieckseder, R.: Calomel Dineresis, Arch. exp. Path. Pharm., 67, 409, I9 I 2.

Fleig, M. C.: Formates, Arch. intern. Pharmadym., 17, 147, 1907; Phenolphthalein, Arch. intern. Pharmacod., I8, 327, 1908.

Fleischer: Epincphrin, Centr. allg. Path., 20, I06, 1909.

Fleisier, M. S., and Leo Loeb: Absorption, Proc. Path. Soc. Philad., Feb., rgio; Proc. Soc. 13iol. Chem., I, 232, x91 I; Epinephrin, J. Exp. Med., II, I 2, I909; J. Am. Med. Assoc., 53, 1561, 1910; Arch. Intern. Med., 6, 427, 1910.

Fleischinaver: Mitscarin, Z. Biol., 59, 262, rgr 2.

Fleischmanx: Atropin, Z. klin. Med., 73, I75, I9x I; Arch. exp. Path. Pharm., 62, $5 \mathrm{I} 8,19 \mathrm{Ir}$.

Flexner, S.: Moningitis, J. Exp. Med., I7, 553, 1913; Soaps, J. Am. Med. Assoc., $61,1873,1913$.

FlexNer and Clark: Ilexamethylenamin, J. Am. Med. Assoc., 65, 586, rgir.

Fuury: Cascara, Berl. klin. Woch., 49, Aug., r9x 2; Cholesicrol, Arch. exp. Path. Pharm., 66, 221, I9II; Ascarides, ib., 67, 275, I9I 2.

FoA and Aggazottr: Colloid Metals, Bioch. Centr., 8, 302, r908; Bioch. Zs., x9, Nos. $I$ and 2, I900.

Focke: Digitalis, Jahrb. Pharm., 66, 98, x906.

Foerster, O. H.: Primula, J. Am. Med. Assoc., 55, 642, r9io.

Foerster, R.: Alcohol, Arch. ges. Physiol., I44, 5r, I9I $2 ;$ Fusel Oil, Bioch. Centr., 9, 789, 19 10.

Folin: Acidosis, J. Am. Med. Assoc., 40, 128, 1907; Urea, ib., 63, 823, I914.

Folin, Cannon and Denis: Epinephrin, J. Biol. 'Chem., r3, 477, 1913.

Folin and Denis: Ammonium, J. Biol. Chem., I1, 161; 16, 599, 1912; Blood Uric Acid, Arch. Int. Med., 16, 33, I915; Nitrogen, ib., 14, 29, I913; Phencls, J. Biol. Chem., 22, 305, 1915; Uric Acid, ib., I4, I913.

Folin, Karsner and Denis: Nitrogen Retention, J. Exp. Med., r6, 789, rgr 2.

Folin and Lyman: Atophan, J. Pharmacol., 4, 539, I9r3.

Fonteyne: Alcohul, Arch. int. Pharmacod., 16, 357, x906; Ether, ib., 16, 369, 1906.

Forbes, A., McIntosh and Serton: Ether, Am. J. Physiol., 40, 503, I9I6.

Forbes and Miller: Ether, Am. J. Phys., 40, r 48, 1916.

Forbes, E. B., and KeIth: Phosphorus Compounds, Ohio Agr. Exp. St. Bul. No. 5, x9I4. FORD, W. H.: Alcohol, N. Y. Med. J., 15, 561, 1872.

FORD, W. W.: Plant Poisons, Cbl. Bact., 58, r29, r913; Poisonous Mushrooms, J. Inf. Dis., 3, x91, I906; J. Pharmacol., 2, 285 , I911; Tutu, ib., 2, 73, I910.

FORD, W. W., and BrUSH: Amanitas, J. Pharmacol., 6, I95, x914.

FORD, W. W., and Rockwood: Amanita, J. Pharmacol., 4, 225, 1913.

Fordyce, J. A.: Arsenic, J. Am. Med. Assoc., 56, r86, I9II.

FormaneK and Eiselt: Kidney Extract, Arch. intern. Pharmacod., 17, 231, 1907.

ForNaca and Querelli: Paraldehyde, Berl. Klin. Woch., Dec. 23, 1912.

Forster, J.: Salt Starvation, Z. Biol., 9, 297, 18\%3.

ForTNer: Lead, Arch. Hyg., 4, No. 4, 1905.

Foster, N. B.: Mcrcury, Arch. Int. Med., I 5,754 , $1915 ;$ Phlorhizin, Proc. Soc. Exp. Biol. Med., ro, 34, I912; Uremia, Arch. Int. Med., 15, 356, 1915; Soc. Exp. Biol. Med., 13, 39, 1915; J. Am. Med. Assoc., 65, 50, I915.

Fothergill: Antagonistic Therap. Agents, Phila., 1878.

Fourneau and PAGE: Yohimbin, Zentr. Bioch. Bioph., 16, 5xr, 19r4.

Fourrifre: Cocain, Zentr. Biochem. Biophys., 15, 95, x913.

Fraenkel, A.: Digitalis, Arch. exp. Path. Pharm., 5I, 84, I903; Muench. med. Woch., No. 32, 1905; Arch. exp. Path. Pharm., 57, 79, 123, 131, 1907; Epinephrin, ib., 
60, 395, I909; Morphin, Muench. med. Woch., No. 24, 1899; Pneumonia, Ther. Mon., 29, 533, 1915; Strophanthin, Ther. Gegenw., 48, 56, 1907.

Fraenkel, A., and Schwartz: Digitalis, Arch. exp. Path. Pharm., 57, 79, I907.

Fraenkel, C.: Cresols, Z. Hyg., 6, 521 , i889.

Fraenkel, S.: Cannabis, Arch. exp. Path. Pharm., 49, 266, I903; Stereochemic, Erg. Physiol., III, 1, 290, 1904.

Franceschini: Salicyl, Arch. Farmacol., 5, 539, 1906.

FRANCHI, D.: Formaldehyd, Arch. Farmacol., I 5, 497, I91 3.

FRANCK, E.: Irrilants, Arch. exp. Path. Pharm., 72, 387, I913; Renal Glycosuria, ib., 72,387, I913.

Frank, E., and IsaAC: Diabetes, Arch. exp. Path. Pharm., 64, 293, igr r.

Frank, E., and Pietrulla: Atophan, Arch. exp. Path. Pharm., 77, 361, I9I4

Frankfurther and Hirschfeld: Morphin, Arch. Physiol., 5I5, I9Io.

Frankl, Th.: Sulphur, Arch. exp. Path. Pharm., 65, 302, I9i1.

Frankl-Hochwart and Froenlich: Autonomic System, Arch. exp. Path. Pharm. 63, 347, I 909.

FraNz, F.: Sulphocyanid, Zentr. Bioch. Bioph., I3, 314; I4, 439, I91 2.

Franz, S. I.: Functions of Cerebrum, Psychol. Bull., I I, No. 4 , I9I 5.

Fraser, Th. R.: Physostigmin, Edinb. Med. J., 9, 36, I864; Salicyl, ib., July to Sept., 1885; Strophanthus, Trans. Royal Soc., Edinb., 35, 955, I890.

Fraser: Infantile Paralysis, Am. J. Med. Sci., July, rgi4.

Frazier, C. H., and Peet: Gland Extracts, Am. J. Physiol., 36, 464, I915.

FredericQ, L.: Accelerators, Arch. Intern. Physiol., I3, II5, I9I3; Caffein, Zentr. Bioch. Biophys., I4, 107, 191 2.

Freeman: Naphthol, Merck's Arch., Io, i I, Igo8.

French: Thyroid, Am. J. Physiol., 30, 56, I9I2.

Frenkel, B.: Morphin, Arch. exp. Path. Pharm., 63, 331, r9io.

Frenkel-Heiden and Navassart: Salvarsan, Zs. exp. Path., I3, 531, 1913.

FreUnd, H.: Salt, Arch. exp. Path. Pharm., 74, 311, 1913.

Freund, H., and Grafe: Salt, Arch. exp. Path. Pharm., 67, 55, I9I I.

Freund and Marchand: Epinephrin, Arch. exp. Path. Pharm., 72, 56, 1913.

v. FreY, E.: Bromid, Zs. exp. Path., 8, 29, I910; Diuresis, Arch. ges. Physiol., I I 2, 7 I, 1906; Epinephrin, Bioph. Centr., I, 392, I905; Hydrastis, Z. exp. Path., 7, 63, 1909; Phlorhizin, Arch. ges. Physiol., I15, 204, 1906; Salicyl, Muench. med. Woch., $52,1326,1905$.

Frey, W., and KuMpiess: Pituilary, Z. exp. Med., 2, 380, I9I4.

Fridericia, L. S.: Epinephrin, J. Am. Med. Assoc., 66, 466, I 915.

FrIedBerger: Anaphylaxis, Berl. klin. Woch., Nos. 32 and 42 , 1910.

FrIEDBERGER and TsuNESKA: Kaolin, Zs. Imm. exp. Ther., 20, 405; Zentr. Biochem. Bioph., I6, 413, I913.

Friedenthat, H.: Acidity, Zs. allg. Physiol., 4, I904.

Friedenwald and Leitz: Antiseptics, Am. J. Med. Sci., i 38, 653, 1909.

Friedlander: Paraldehyde, Ther. Mon., I44, I 893.

Friedmann, A.: Anaphylaxis, Zs. Innu. exp. Ther., 2, I909; Iodid, Zentr. Bioch. Bioph., 10, 619, I910; Perfusion, ib., 10, 864, 1910.

Frison and Nicloux: Chloroform, Bioch. Centr., 6, 603, 1907.

Froelich, A., and Meyer: Tetanus, Arch. exp. Path. Pharm., 79, 55, 1915.

Froenlici A., and Pick: Ergot, Arch. exp. Path. Pharm., 74, I07, I I4, I9I 3 ; Hypophysis, Arch. exp. Path. Pharm., 74, 92, 107, II4, I913.

Froendich, F. W.: Epinephrin, Zentr. Physiol., 25, No. I, I910; Oxygen, Zs. allg. Physiol., 3, 1904 .

Fromann: Silver, Arch. path. Anat:, I7, I35, 1859.

Fromerne, K.: Atophan, Bioch. Zs., 35, 494, I912; Magnesium, Arch. exp. Path. Pharm., 6I, 219 1909.

Fromm and HildebrandT: Camphor, Zs. physiol. Chem., 33, 579, 1901.

FrothinghaM: Hexamethylenamin, Bioch. Centr., 9, 882, 1909.

Frothingham, Futz, Folin and Denis: Uranium, Arch. Int. Med., I 2, 245, I9I3.

Fuchs, A.: Guanidin, Zentr. Bioch. Bioph., I7, 943, 1914.

Fuchs and Roth: Epinephrin, Z. exp. Path., I2, 586, I91 2.

Fuenner, H.: Aconitin, Arch. exp. Path. Pharm., 66, I911; Alcohol, ib., 69, 29, I9r 2; Colchicin, Arch. exp. Path. Pharm., 72, 228, I913; Guanidin, ib., 65, I911; Histamin, Ther. Mon., 27, March, I913; Hypophysin, Z. exp. Med., I; 397, 1913; Muscarin, Arch. exp. Path. Pharm., 6r, 283, I909; Narcosis, Z. Biol., 57, 465, 191 2; Muench. med. Woch., No. 4, I9I I; Narcolics, Ber. deut. Chem. Ges., 42, 887, 191 2; Pituitrin, Muench. med. Woch., No. I6, I91 2; Synergism, Arch. exp. Path. Pharm., 75, 53, 1913; Toads, ib., 63, 374, I910. 
Ithiner and Greis: Itemolysis, Arch. exp. Path. Pharm., 69, 348, I9r2.

limerr, H., and Remmis: Colchicin, Arch. exp. Path. Pharm., 78, I, i9 5 .

1. Horti, O.: Muscle Plisma, Arch. exp. Path. Pharm., 37, 389, I896; Phosphorus, Bioch. Zs., 64, I3I, 1914; Suprarcnin, Z. physiol. Chem., 26, I5, I898.

lictertil and Friedmixi: Iodin, Arch. exp. Path. Pharm., Suppl., 2 r4, Igo8.

1. TERT11 and Schwartz: Epinephrin, Zentr. Bioch. Bioph., II, 407, I9II.

Futili: Cumphoric Acid, Arch. intern. Pharmacod., 16,273 , 1906.

FlLK, M. E. and MACl.tod: Chromaphil Tissue, Am. J. Ph., 40, 21, I9I6

Fullertos: Tobacco, Clevel. Med. J., Ir, 585, r9i2.

Fuxk, C., and MAcalder: Fats, Zs. physiol. Chem., 92, I3, I9r4.

G.IEnTGens: Antimony, Centr. med. Wiss., $32 \mathrm{I}, \mathrm{x} 876$.

Gigl.ro: Quinin, Rev. crit. Clin. Med., 4, No. 13, r898.

Garses, IV. L.: Lactation, Am. J. Physiol., 36, 360; 38, 285 , то15.

Garsböck, F.: Caffcin, Arch. exp. Path. Pharm., 66, 387, 19 г ; Cymarin, Zs. exp. Path., I 7. 3II, I9I 5 .

Galewsky: Formaldehyde, Muench. med. Woch., 52, No. 4, I905.

Gass, S.: Arsenic, Beitr. path. Anat., 60, 22, 1914.

Garfol'xkel and Gautrelet: Mfthylen Blue, Zentr. Bioch. Bioph., 16, 475, i913.

Garver: Nicotin, U. S. Dept. Agr. Bull., ro2, I908.

Garratt: Chlorid, Medico-Chis. Trans., 87, I904.

GARRETT, J. H.: Iron, Brit. Med. J., I, 390, I9I3.

GARkEY; Seu Watcr, Biol. Bull., 8, 257 , I905.

Garten: Curarin, Arch. exp. Path. Pharm., 68, 243, I9r2.

Garie, E. G.: Nicotin, Clevel. Med. J., I2, I30, I9I3.

Gaskell, W. H.: "The Involuntary Nervous System," I916.

Gasser, H. S., and Loevexhart: Medullary Centers, J. Pharmacol., 5, 239, I9I4.

Gates, F. L.: Magnesizm, Soc. Exp. Biol. Med., I 2, 59, r914; J. Pharm. Exp. Ther., $6,6 \mathrm{ro}$.

Gates, F. L., and Meltzer: Magnesium, Soc. Exp. Biol. Med., i I, r67, I914; Oxalate, Am. J. Physiol., 33, xxi, I9r4.

Gaule: Iron, Deut. med. Woch., No. 19, I896

Gaulier, A.: Salicylate, These, Paris, r9 3.

Gauss: Scopolamin, Med. Klin., 136, 1906.

GAUTIER: Arsenic, Sci., 20, 443, 1904.

Gautier and Clausmann: Fluorid, Zentr. Bioch. Bioph., 15, 638, rgr3.

Gautrelet: Cholin, Bioch. Centr., 9, ro9, r909.

Gay, F. P.: Anaphylaxis, Trans. Assoc. Am. Phys., 28, I3, r913.

Gazzetti, C.: Haloids, Zentr. Bioch. Bioph., 15, 786, 1913.

GeHRIG, R. P.: Leucocytosis, Zs. exp. Path., I7, I6r, I9I5.

Geinitz: Volatile Oils, Zentr. Bioch. Bioph., I4, 77, r9 2.

GenNerich: Salvarsan, Muench. med. Woch., No. 52, igiz.

Gensler, P.: Magnesium-neuronal, Arch. exp. Path. Pharm., 78, 317, 1915; Neuronal, Arch. exp. Path. Pharm., 77, 161, 1914.

Geppert: Cyanid, Z. klin. Med., 15, 208 and 307, r889; Narcosis, Deut. med. Woch., $25,433,457,476,1899$.

Geraghty and Rowntree: Phenol-sulphonephthalein Test, J. Am. Med. Assoc., 57, 8 II, I9II.

Geraghty, J. T. and Macht: Papaverin, J. H. H. Bul., 27, r19, Igr6.

Gergens: Chromate, Arch. exp. Path. Pharm., 6, 148, 1876.

Gerlach, P.: Saline, Bioch. Zs., 61, 125, 1914.

Gerlinger: Chloroform, Arch. exp. Path. Pharm., 47, 438, 1902.

Getrle. A, A. O.: Mercuric Cyanid, J. Am. Med. Assoc., 66, 1694, 1916.

Grani, E.: Chloroform, Muench. med. Woch., 870, I910; Mercury, Zentr. Bioch. Bioph., 15, $54 \mathrm{I}, \mathrm{rg} 3$.

Gribs, H. D.: Phenol, Jahresb. Pharm., 69, 207 , 1900.

Gicklhorn, J.: Fluorescent Dyes, Zentr. Bioch. Bioph., r8, 37 I, I914.

Gremsa: Quinin-zurea, U. S. P., Dig., 460, 1908.

Gres, TH.: Arsenic, Arch. exp. Path. Pharm., 8, $175,1877$.

Gies, War. J.: Alum, J. Am. Med. Assoc., 5†, 816, rgrr; Membranes, Bioch. Bul., 2, 55, I9I 2.

Gies and Kahn: Sulphocyanate, Dent. Soc. N. Y., May, I9r 2.

GIES, LIEB and KaHN: Sulphocyanate, Dent. Cosmos., 56, r75, r9r4.

Gubert and LeRebouliet: Rumex, Jahrb. Pharm., 66, 83, I906.

Gile, P. L., and CARrero: Iron, J. Agr. Res., 7, 83, 19 16.

Ginsberg, W.: Diuretics, Arch. exp. Path. Pharm., 60, 381, I9г 2. 
Ginsburg, H., Tumpowsky and Hamburger: Gastric Ulcer, J. Am. Med Assoc, 67 , $990,1916$.

Grofredd: Epinephrin, Bioch. Centr., 6, 607, 1907.

GIRARD: Sirychnin, J. Am. Med. Assoc., 46, I925, I906.

Grthens, T. S.: Caffein, Soc. exp. Biol. Med., i I, I66, rgr4; Morphin, J. Pharmacol., 4, 356, 19г3; Strychnin, J. Exp. Med., I8, 300, 19г3.

Githens, T. S., and Meltzer: Anesthetics, J. Pharmacol., 5, 523, i9i4; A pnea, Soc. Exp. Biol. Med., I 2, 64, 19г4; Cardiectomy, ib., I 2, 60, I9I4; J. Pharm. Exp. Ther., 6, 607, I915; Epinephrin Pupil, J. Pharmacol., 8, 133, 1916; Morphin, Proc. Soc. Exp. Biol. Med., 9, 30, I9 I I ; ib., I0, I 28, I91 3; Strychnin, J. Pharmacol., 3, 463, I9 1 2; Strychnin Treatment, ib., 2, 357, 1911.

Gizelt, A.: Alcohol, Centr. Physiol., I9, No. 23, I906; Arch. ges. Physiol., i i r, No. i I, I906.

GJEssing, H.: Holocain, J. Am. Med. Assoc., 65, 988, 1915.

Glaessner and Singer: Bile Acids, Bioch. Centr., 9, 907, I9Io.

Glaser, O.: Veronal, Wien. klin. Woch., 27, No. 44, I9I4.

Gley, E.: Nicotin, C. R. Soc. Biol., I58, 2008, I9I4.

GLEY and QurNQuAUd: Thyroid, Zentr. Bioch. Bioph., I5, 648, I9I3.

Guveck, A .: Hypersensitiveness to Drugs, Wien. klin. Woch., 26, 536, I913.

GLUR: Bile, Z. Biol., 52, 479, I909.

Goetsch, E.: Pituitary, Bull. Johns Hopkins Hosp., 27, 29, I9г6.

Goetze, R.: Lead, Inaug., Berlin, I 893.

Goldfarb: Lecithin, Proc. Soc. Exp. Biol. Med., 4, I 59, I907.

Goldziener: Adrenals, Wien. klin. Woch., 23, 809, г910.

Golla and Symes: Bronchioles, J. Pharmacol., 5, 87, I913; J. Physiol., 46, 38, I9 3.

Golowrnski, J. W.: Cholin, Arch. ges. Physiol., 159, I, I9I4; Xanthins on Muscle, Zentr. Bioch. Bioph., I8, 60, I9I5.

Good: Lithinm, Am. J. Med. Sci., Feb., r9o3.

Goodall, A., and REID: Digitalis, Lancet, I, I679, x914.

Goodall, E. W.: Scrum, Ref., J. Am. Med. Assoc., 56, I 726 , igi r.

Goodridge and Gres: Edema, Proc. Soc. Exp. Med. Biol., 8, ro6, igr I.

Gordon, A.: 'Morphin, J. Am. Med. Assoc., 64, I867, I915.

GoRTER, K.: Chlorogenic Acid, Zentr. Bioch. Bioph., I3, I32, I9IO.

Gotruirb, R.: Antipyretics, Arch. exp. Path. Pharm., 26, 245, 419, I890; Basedows, Deut. med. Woch., No. 47, I9I I; Camphor, Zs. exp. Path., 2, 385, I905; ib., 3, 588, 1906; Digitalis, Int. Congr. Med., 5. 2, 225, I914; D. Congr. Inn. Med., 374, I9I4; Digitalis Frog Assay, Muench. med. Woch., 813, 1914; Iron, Arch. exp. Path. Pharm., 26, I891; Pancreas, ib., 33, 261, 1894; Suprarenal, ib., 43, 286, I900; Tropins, ib., 37, 218 , 1896.

GotTuIEB and LEFMAN: Blood Toxicity, Bioch. Centr., 6, 302, I907.

Gotruieb and Magnus: Digitalis, Arch. exp. Path. Pharm., 47, 135; 48, 262, 1901; ib., 51, 20, 1903; Dinuresis, Arch. exp. Path. Pharm., 45, 223 and 248, I90I.

GotTschalk, G.: Strophanthin, Arch. exp. Path. Pharm., 75, 33, I9I4.

Gotrschalk, S.: Cotarnin, Ther. Mon., No. 9, 646, 1895; Ther. Gegenw., Aug., I 899.

GoudBerg, A.: Inulin, Zs. exp. Path., I3, 310, r9r3.

Gradie: Hexamethylenamin, Zentr. Bioch. Bioph., 12, 764, 19 I I.

Grafe, E.: Ammonium, Zs. Physiol. Chem., 90, 75, I914; Caffeol, Chem. Abstr., I4, 618, I9I 2; Caramel, D. Arch. klin. Med., II6, 437, I914; Muench. med. Woch., June, 30 , r9r4.

Grafe and Schaepfer: Ammonium, Zs. Physiol. Chem., 77, I, I9I 2.

GrAFE and Wintz: Nitrate, Zs. physiol. Chem., 86, 283, I9I3.

GrahaM: Colloids, Liebig's Ann., I 2 I, I, I861.

Graham, E. A.: Chloroform, J. Exp. Med., 22, 48, I9I 5.

GramenITZKI: Epinephrin, Bioch. Zs., 46, I86, I91 2.

GRANDIS: Nerve, Arch. it. Biol., 37, 313, I902.

GRAU: Gelatin, D. med. Woch., 27,1270 , I910.

Graul, G.: Antipyrin, Deut. med. Woch., No. 3, I899.

GrawiTz: Camphor, Merck's Rep., 21, 68, 1907.

GreEne, C. W.: Strophanthin, J. Pharmacol., 2, 399, 1910.

Greene, C. W., Boutwell and Peeler: Digitalis, J. Pharm. Exp. Ther., 6, 598, I915.

Greene and Kruse: Bromid, J. Am. Med. Assoc., 6r, 27x, I9r3.

Greene, C. W., and PeEler: Digitalis, J. Pharmacol., 7, 59I, I9I 5.

Greenwald, I.: Cresol, J. Pharmacol., 2, 513, I9I I; Parathyroidectomy, J. Biol. Chem., 25, 223, 1916; Phosphate, J. Pharmacol., 7, 57, 1915; Phosphorus of Serum, J. Biol. Chem., 25, 431, 1916. 
(iRfior, 1.: IIpnotics, Ther. Mon., 27, 549, I9I3.

Grimar: A mestheria, Bul. Acad. Med., 69, No. 30, r905.

(itivis, K.: Arsenic, Muench. med. Woch., 57, No. 40, r9ro.

(ikubl, J.: Coctin, Irch. exp. Path. Pharm., 57, r72, r9r2; Zentr. Bioch. Bioph., I7, $78,1013$.

Grorivow: Atropin, Zentr. Bioch. Bioph., r г, i т т, roro.

(inos, ().: Cocuin, Arch. exp. Path. Pharm., 62, 380, roro; Oxalates, ib., 71, 395, ror3;

Silier, ib., 70, 375, 1912 ; Strcphanthin, ib., 71, 364, I913.

(iros, O., and O'Connor: Colloid Metals, Arch. exp. Path. Pharm., 64, 456, igi I.

(ikosis, li.: Digitalis, Zs. exp. Med., 4, 210, I9I4.

(iRositer: Quinin, Bioch. Zs., 8, 98, r9o7.

(iRorl: Cumphor, J. Pharmacol., I, 445, r9ı.

Grove and Loevexilart: Cyanid, J. Pharmacol., 3, I3I, I9I I.

(imotir, A. L.: Alcoholic Cirrhosis, Arch. Int. Med., i7, ro3, г916.

(iRvBE: Atropin, Bioch. Centr., 5, 249, I905.

Gruber, C. M.: Epinephrin, Am. J. Physiol., 33, 335; 34, 89, I914; Threshold Stimu-

lation, Am. J. Physiol., 37, 259, r9 I5.

Gruebler: Phenolphthalein, Bioch. Centr., 5, 62, I906.

Grunfeld: Ergot, Arb. Pharmacol. Inst. Dorpat., 7, I08, г891; ib., 8, I I 5, I892; ib., I 2, 205, I 895 .

Grtenfelder, B.: Calcium, Ther. Mon., 4I6, ior3.

Gruenwald: Digitalis, Arch. exp. Path. Pharm., 68, 230, 191 2; Picrotoxin, Arch. exp. Path. Pharm., 60, 249, I909.

Grteter, M.: Morphin Destruction, Arch. exp. Path. Pharm., 79, 337, igi6.

Gruetzner: Ferments, Arch. ges. Physiol., 106, 463, ig05.

Grützinfri and Heidenhain: Corrosion, Arch. ges. Physiol., i6, 56, i877.

Grumme: Iodin, Arch, exp. Path. Pharm., 79, 41 2, r9i6.

Gruma-Fohrde: Iodin, Arch. exp. Path. Pharm., 77, 448, i9i4.

Gudernatsch, J. F.: Thyroid, Arch. Entwickl., 35, 457, I912; Thyroid, Rats, Am. J. Physiol., 36, 370, I9 I 5 .

Gudzent, F.: Thorium, Berl. klin. Woch., 49, r9г2; Uric Acid, Zs. Physiol. Chem., 60,38 , 1909.

Guenther: Atropin, Wien. klin. Woch., 748, г9гі.

Gur.NTHER and BACHeM: Barium, Deut. med. Woch., 7 7 7, I9II.

Guerber: Uzara, Arch. exp. Path. Pharm., 75, 75, 1914; Uzaron, Muench. med. Woch., 40, I9II.

Gugcronerm, M.: Histamin, Ther. Mon., 28, 174, I9r4; Pituitary, Bioch. Zs., 65, r89, I9 14; Tyramin, Ther. Mon., 26, 795, I9I2.

Gu1BE: Ether, J. des practiciens, I9 2.

Gurnard: Morphin, J. Physiol. path. gen., I, 964, I895.

Guinard and Dumarest: Picrotoxin, Arch. intern. Pharmacodyn, 6, 283, 406, 1899.

Gunx, J. A.: Chloral, Brit. Med. J., Sept. I3, I9г3; Epinephrin., Qu. J. Exp. Physiol., 7, 75, I9 13; Fibrillation, Heart, 5, 2-4, I9I3; Blood Vessels, Arch. intern. Pharmacodyn., I9, 319, 1909; Strophanthus, J. Pharmacol., 4, 225, 1913; Uterus, ib., 5, 527, I9I4; Proc. Roy. Soc., 87B, 55I, I9I4.

Gunn and Chavasse: Epinephrin, Proc. Roy. Soc., B86, I92, I913.

Gunn and Feltham: Arsenic, Brit. Med. J., Jan. 2 I, I9I I.

Gunn, J. A., and MARTIN: Intrapericardial Medication, J. Pharmacol.', 7, 31, r9 5.

Gunn, J. A., and Underhill: Surviving Mammatian Intestine, Quart. J. Exp. Physiol., $8,27.5,296$, I 914 .

Gunn, J. W. C.: Strophanthin, J. Pharmacol., 6, 39, I9r4.

GurewitsCh: Iodid, Bioch. Centr., 7, 230, I907.

Gutirie, C. C.: Kidneys, J. Am. Med. Assoc., 54, 831, r9io.

Guthrie, C. C., and LEe: Hypertonic Solutions, Soc. Exp. Biol. Med., II, 149, I9I4.

Gutirie, C. C., and Ryan: Magnesium, Am. J. Physiol., 26, 329, r9io.

GuY, Goodall and ReId: Anesthesia, Zentr. Bioch. Bioph., I2, I53, I9I1.

Givaturey: Anesthetics, J. Am. Med, Assoc., 59, 1844, I9I 2.

HA^s, E.: Diuresis, Bioch. Zs., I 2, I908

HaAs and Heıм: Carbon Disulphid, Zentr. Bioch. Bioph., I 2, I84, г9г 1.

Haemaelaeinen: Glycuronates, Skand. Arch. Physiol., 30, I96, I9r3.

Haeussermann: Iron, Zs. physiol. Chem., 26, No. 6, I897.

HaffNer, F.: Calcium, Arch. int. Pharmacod., 23, 37, I9I3.

Haggard, W. D.: Nitrous Oxid, J. Am. Med. Assoc., 55, 2225 , 19 Io.

HAin, B.: Leucemia, Ther. Mon., 28, 555, I9I4. 
Hain, R.: Melubrin, Muench. med. Woch., 2232, I913.

HaIkE: Salicyl, Arch. Ohrenhlk., 63, 78, I904.

Hald, P. T.: Ccrebrospinal Excretion, Arch. exp. Path. Pharm., 64, 329, I9 I ; Potassium, Arch. exp. Path. Pharm., 53, $227,1905$.

Haldane, J. S.: Carbonic Oxid, J. Physiol., 25, 225, 1900; Dead-space, Am. J. Physiol., 38, 20, I915; Oxygen, J. Physiol., 37, 355, 1908.

Haldane and Prestiey: Carbon Dioxid, ib., 32, 225, 1905.

Hale, Worth: Antipyretics and Caffein, U. S. Hyg. Lab. Bul., No. 53, I9o9; Chloroform, Arch. Int. Med., I5, 945, I015; Cinchona Alkaloids, J. Pharmacol., 8, I 22, I9 6; Digitalis, J. Am. Med. Assoc., 54, 35, r29, г9го; ib., 57, 1515, r9гі; U. S. Hyg. Lab. Bul., No. 74, I9г г; Morphin, Am. J. Physiol., 23, 389; 28, 408, I909; Phenoloids, U. S. Hyg. Lab. Bul., No. 88, I9I3; Strychnin, J. Pharmacol., I, 39, I909; Uterus, J. Pharm. Exp. Ther., 6, 602, I015.

Hale and Seidell: Epinephrin, Chem. Abstr., 7, SO4, I9I3.

Hales and Fishncan: Bromid, Am. J. Physiol., 22, 32 , ioo8.

Hall, A., and Ransom: Lead, Brit. Med. J., I, 428, I906.

Hall, G. W.: Arsenic, J. Am. Med. Assoc., 64, I384.

Hall, G. W., Culbertson and Slaght: Mercury, J. Am. Med. Assoc., 66, 2062 , igi6. Hall, I. C., and Taber: Gentian Violet, J. Inf. Dis., 15, No. 3, I9I 4.

Hall, J. IV.: Salicyl, Brit. Med. J., 2, 744, I904.

Hall, W. S.: Iron, Arch. Physiol., 45., I804; ib., 49, I 896.

Hallion and Nepper: Epinephrin, Zentr. Bioch. Bioph., I3, 886, I9I I.

Hallopeau, H.: Atoxyl, Bioch. Centr., 6, 601, I907.

Halpern, J.: Epinephrin, Arch. exp. Path. Pharm., 73, 347, I913.

Halsey: Epinephrin, Trans. Assoc. Am. Phys., 24, 520, 1909.

Halsted, IV. S.: Thymus, Harvey Lectures, p. 234, IOI4.

Hamburger, H. J.: Blood Corpuscle Method, J. de Phys., 889, rgoo; Cyanid, Int. Zs. physik. chem. Biol., 2, No. 4 and 5, I9I5; Imbibition, Arch. Physiol., 302, I896; Intestinal Absorption, Bioch. Centr., 5, 891, I906; Introduction, Bioch. Zs., 3, 359, I007; Osmosis History, Sci., 34, 583, I9I r; Oxygen, Int. Zs. physik. chem.-Biol., 2, No. 2 and 3 , I9 5 ; Peroxid, ib., 4 and 5, I9 5 ; Permeability, Wien. klin. Woch., I 4 and I5, I9 16; Phagocytosis, review, Brit. Med. J., Jan., I9 I6; Phagocytes and Respiratory Center, Kon. Akad. Wetensch., Amsterdam, I7, I325-1342, I9 15; Silver, Bioch. Centr., 9, 539, r909; Turpentine, Kon. Akad. Wetensch. Amsterd., 16, 609, I9I3.

Hamburger, H. J., and de Hahn: Phagocytosis, Soaps, Kon. Akad. Wetensch., Amst., I 5 , I 290, I9I3.

Hamburger and Hekma: Quinin, Phagocytosis, Bioch. Z., 9, 512, 1908.

Hamburger, K.: Fluorescein, Merck's Rep., 23, 2 I I, I909.

Hamburger, W. W.: Pepsin, Arch. Int. Med., r6, 356, ig I5; Piluitary, Am. J. Physiol., I I, 282, 1904 .

Hamburger, W. W. and Halpern: Pepsin, Arch. Int. Med., r8, 228, igi 6.

Hamiston, A.: Hygicne of Painters' Trade, U. S. Bur. Lab. Bul., r 20, Ic13; Lead, J. Am. Med. Assoc., 59, 777, I9I 2; Lead Smelting, U. S. Bur. Lab. Bull., I4I, I914; Potteries, ib., Bull. I04, I9I 2; Rubber Industry PGisoning, ib., Bull. I79, I9 5 .

Hamrton, H. C.: Phenoloid Disinfectants, Ther. Gaz., 38, 3 I I, I9I4.

Hamilon, H. C., Lescohler and Perkins: Cannabis, J. Am. Pharm. Assoc., 2, 22, I9I3.

Hammerschmid: Narcosis, Zentr. Bioch. Bioph., II, 450, I9io.

Hammetr, F. S.: HCl Formation in Stomach, Anat. Rec., 9, 21, 1915; Strychnin, J. Pharmacol., 8, I 75, I916.

Hammond, J.: Pituitary, Qu. J. Exp. Physiol., 6, 3 I I, I9г3.

Handfield-Jones: Cotarnin, Ann. Gyn. Ped., June, I907.

Handovsky and Prck: Autonomic, Arch. exp. Path. Pharm., 7 I, 89, I9 3.

Hanzlik, P. J.: Alcohol, J. Pharmacol., 5, I85, I9I3; Chelidonin on Smooth Muscle, J. Pharm. Exp. Ther., 7, 99, I915; Irexamethylenamin, J. Am. Med. Assoc., 54, I I40, roro; Hexamethylenamin on Uric Acid, J. Lab. Clin. Med., I, 32 I, I9r6; Iodid, J. Pharmacol., 3, 387, r9 2; Salicyl, J. Pharmacol., 3, 387, 1912; J. Am. Med. Assoc., 60, 957, I9 13; Salicyl Literature, Rep. Ther. Res. Com., A. M. A., 3, I3 I, 1914; Salicyluric Acid, J. Pharmacol., 8, 130, г9 6.

Hanzlik and Collins: Hexamethylenamin, Arch. Intern. Med., i 2, Nov., rgr3; J. Am. Med. Assoc., 62 2, 295, r9r4.

Hanssen, O.: Lactophenin, Ref. Ther. Mon., 30, 55, 19 6.

Harcourt, A. V.: Chloroform, Brit. Med. J., July I4, 1906; Inhaler, Brit. Med. J. Suppl., 143, I903.

HARdy: Colloid Solutions, J. Physiol., 24, 288, I899; J. Physik. Chem., 4, 254, I900. 
Hikik, E.: Amblyopia, Muench. med. Woch., 59, 1941, 1912; A pomorphin, Arch. (x). Path. l'harm., 2, 254, I874; Muench. med. Wod., 20, I745, r9ro; Iodid, Arch. int. Pharmacod., 20, 247, r910; Santonin, Arch. exp. Path. Pharm., 45, 272, 447, 1001.

Il.ik lin and (irïnder: Iodoform, Berl. klin. Woch., I882, No. 20; I883, No. 47.

Hikick and IHLdebrandt: Chloromorphid, Arch. exp. Path. Pharm., 65, 38, r9ir.

HIRNiCk and Hoffraxi: Aspidosperma, Zs. klin. Med., 8, 47 I, I884.

HiRNick and Reslertz: Chloral, Fortsch. Med., i I, 265, i 893.

H.IR.iCK and Schriaber: Manganese, Arch. exp. Path. Pharm., 46, 372, r9or.

II $\mathrm{R}$.ICK and Witkowsk1: Physostigmin, Arch. exp. Path. Pharm., 5, 401, I876.

M.ırminctox: Borax, Am. J. Med. Sci., I 28, 418, I904; Sulphite, Bost. Med. Surg. J., May 26, I904.

Hirris, L. J., and Og.ax: Typhoid, J. Am. Med. Assoc., 64, 3, I9I4.

IHARris, IV.: Alcohol, J. Am. Med. Assoc., 63, 1725, I9I4.

IIArrison: Cocain, Bost. Med. Surg., J., Feb. 2, I9 Ir.

Ifart, E. B., and Nelsox: Acidosis, Pr. Soc. Biol. Chem., 3, 40, rgr3.

If ARteNberg, P.: Strychnin, Presse med., 7I, I9I3.

Hartan, F. A.: Epinephrin, Am. J. Physiol., 38, 438, 1915; Urinod Poisoning, Arch. Int. Med., 16, 98, 19 I5.

Hartuxg: Aconitin, Arch. exp. Path. Pharm., 66, 1, 58, I911; ib., 69, I76, I911; Digitalis, ib., 69, 149, I9I2.

Hartilicil and Simox: Morphin, Apoth. Ztg., 505, 5 I 2, 1903.

Hartz, H. J.: Mercury, J. Am. Med. Assoc., 55, 9 15, 1910.

Ihartzlil, M. B.: Arsenic, J. Am. Med. Assoc., 5I, I482, I908.

Hartey, F. N.: Acids, Int. Zs. Physiol. Chem. Biol., I, 463, I914; Permeability, Bioch. Bul., I, 227 , I9I I Am. J. Physiol., 31, 335, I913.

HARVEY, IV. H.: Lym phocytosis, J. Physiol., 35, I I 5, I 906.

Haselhoff, E.: Borate, Zentr. Bioch. Bioph., I5, I42, I9I3.

Hasmimoto, M.: Heat Center, Arch. exp. Path. Pharm., 78, 370, 394, r9i5; Waterdiuresis, Arch. exp. Path. Pharm., 76, 367, r914.

Haskell, C. C.: Digitalis, J. Am. Pharm. Assoc., 2, 836, r9 13; Am. J. Pharm., 86, 7, I9I4; Ergotin, J. Am. Pharm. Assoc., 3, 786, I914; Seasonal Variations, Am. J. Pharm., 84, 241, I9I2.

Haskell and Eckler: Digitalis, J. Am. Pharm. Assoc., 2, 146, I913; Ergot, ib., I, 41 2, I9I 2 .

Haskell, McCaxts and Gardxer: Digitalis, Arch. Int. Med., 18, 235, i9i6.

Haskell, C. C., and Thomas: Aconite, Am. J. Pharm., 88, 3 , I916.

Haskell, C. C., and Zirkle: Aconite, Am. J. Pharm., 87, 537, 1915.

Haskins, H. D.: Alcohol, Am. J. Med. Sci., Dec., r903; Atophan, J. Pharmacol., 5, 63, I913; Diuretics, Am. J. Physiol., 10, 362, I904; Hexamethylenamin, Arch. Int. Med., 16, 1055, 1915; Uric Acid Solvents, Arch. Int. Med., 17, 405, 1916; Urine Acidity, Sci., 38, 679, I913.

Haskins, H. D., and Gerstenbe RGER: Calcium, J. Exp. Med., I3, Igi I.

Hatciner, R. A.: Colloids, Am. J. Pharm., 74, 283, I902; Digalen, J. Am. Med. Assoc., 58 , 921, I9I 2; Administration, ib., 55, 746, I9 10; Nicotin, Am. J. Physiol., I I, I 7, 1904; Salines, ib., 15, 144, 1906; Strophanthin, ib., 23, 303, 1909; Strophanthus Oil, J. Am. Pharm. Assoc., 5, 157, r916; Strychnin, Am. J. Physiol., 12, 237, 1904; J. Am. Med. Assoc., 64, 244, 1915.

Hatcher and Bailey: Strophanthus, J. Am. Med. Assoc., 55, I697, I91o.

Hatcier and Brody: Digitalis, Am. J. Pharm., 82, 360, 1910.

Hatciler and Eggleston: Absorption, J. Am. Med. Assoc., 63, 469, 19r4; Digitalis, J. Pharmacol., 4, II3, I912; J. Am. Med. Assoc., 60, 499, 1913; Digitalis Infusion, J. Am. Med. Assoc., 65, 1902, I915; Novocain, J. Pharmacol., 8, 385, I916.

Hatrori: A pomorphin, Arch. internat. Pharmacod., 20, 57, 1910.

Hauckold: Scopolamin, Zs. exp. Path., 7, 743, I9ro.

Hausmann: Abrin, Beitr. chem. Physiol., 2, 134, I902; Arsenic, Deut. med. Woch., No. 52, 1903; Arch. ges. Physiol., II3, 327, I906; Habituation, Erg. Physiol., $6,58,1907$.

HauschmidT: Lecithin, Bioch. Zs., 51, I71, г913.

Hawk, P. B.: Anesthetics, Arch. Intern. Med., 8, 39, r9ı r; Ether, J. Biol. Chem., 4, 321, 1908; J. Med. Res., 18, 203, 1908; Arch. Int. Med., 8, 37, 177, I91 I; Water Drinking, Bioch. Bul., 3, 420, I9I4.

Hay: Saline Cathartics, J. Phys. and Anat., 16, 17, 1883-1884.

Hayliurst, E. R.: Brass, Am. J. Med. Sci., 145, 723, I913.

Haynes: Digitalis, Bioch. J., r, 62, 1906.

Head, J.: Fluorid, J. Am. Med. Assoc., 6r, 2233 , г9г3. 
Headden, W. P.: Manganese, J. Agr. Res., 5, 349, I915.

Heard, J. D., and Brooks: Camphor, Am. J. Med. Sci., I45, 238, I9r3; J. Pharm. Exp. Ther., 6, 605, I9I5.

Hecht, A. F.: A pocynum, Ref., Ther. Mon., 29, 402, I9I5; Zs. exp. Med., 4, 264, I9I5. Hechr and NoBel: Chloroform, Z. exp. Med., I, 23 , I9I3.

Heobom: Cocain, Skand. Arch. Physiol., 9, 34, 1899; Excised Heart, ib., 8, I 47

Hedbom and Cannon: Water, Am. J. Med. Sci., I38, 504, I909.

Hedin, S. G.: Colloid Reaclions, Harvey Lectures, p. I62, 1915.

Hedinger and Loeb: Iodid, Arch. exp. Path. Pharm., 56, 314, 1907.

Hedon and Fleig: Salincs, Bioph. Centr., I, 259, I905.

Heffter, A.: Arsenic, Arch. int. Pharmacod., I 5, 399, r905; Viert. ger. Med., 49, 194; 50, 2, 1915; Benzol Vapor, Deut. med. Woch., No. 7, I915; Boric, Arb. Kais. Ges., I9, No. I, I902; Cacodyl, Arch. exp. Path. Pharm., 46, 230, I901; Cactus, ib., 34, 65; 40, 385, I898; Camphor, Artificial, Viert. ges. M., 48, No. 2, I9I4; Excretion, Inorganic, Ergeb. Physiol., III, I, 95, I903; Excretion, Organic, ib., IV, I, I84, I905; Reductions, Sitzber. Marburg, June I2, I907; Arch. exp. Path. Pharm., Suppl., 253, 1908; Strophanthin, Jahrb. Pharm., 69, 304, 1909; Sulphur, Arch. exp. Path. Pharm., 51, 176, 1904; Volatile Oils, ib., 35, 342, 1895.

HeFFTER and Fichewirth: Atropin, Bioch. Z., 40, 48, I9 I 2.

Heffter and Loeb: Toxicologic Review, Schmidt's Jahrb., 294, I, I906.

Heffter, A., and Rabner: Cinnamic Acid, Viert. ges. M., 48, No. 2, igi4.

HeFfTer and SACHs: Strophanthins, Bioch. Z., 40, 83, I9 2.

Heger and Phillipson: Epinephrin, Zentr. Bioch. Bioph., I3, 823, I9 2.

Heger and Zunz: Defense, XVII Intern. Congr. Med. Sect. V, Pt. II, igiu.

Heider, R.: Carbon Monoxid, Zentr. Bioch. Bioph., I8, 372, I9I4.

Heilner, E.: Sugar, Z. Biol., 56, 75, I9I I.

Heimann, P.: Iodid, Zentr. Bioch. Bioph., r3, I24, I91 2.

Heineberg and Bachmann: Antiseptics, J. Am. Med. Assoc., 53, I454, I909.

Heinshemer, F.: Acids, Bioch. Centr., 5, 281, 484, r9o6.

HeInz, R.: Digitalis, Merck's Rep., 26, I913; Iodid, Arch. Path. Anat., 155, 44, I899; Diss. Erlangen, I899; Methemoglobin, Arch. Path. Anat., I 22, 100, 1890.

Heiser, V. G.: Chaulmoogra, Ref., J. Am. Med. Assoc., 63, 2259, I9I4; Chenopodium J. Am. Med. Assoc., 65, 526, I9 5 .

Hektoen, L.: Anaphylaxis, J. Am. Med. Assoc., 58, Io8г, I9 12; Benzen, J. Infect. Dis., I9, No. I, 1916; Iodoxybenz, Trans. Chi. Path. Soc., 8, I9II; Vaccines, J. Am. Med. Assoc., 66, I 591, 1916.

Hektoen and Carlson: Antibodies, J. Am. Med. Assoc., 54, I30, r9io.

Hellesen: Muscular Work, Skand. Arch. Physiol., r6, I39, I904.

Hellin and Sprro: Caffein, Arch. exp. Path. Pharm., 38, 368, r897.

Henderson, L. J., and Newburgh: Colloid Swelling, J. Pharmacol., 5, 449, I914.

Henderson, L. J., and Palmer: Acidity, J. Biol. Chem., I3, 393; I4, 81, I9 I 3.

Henderson, V. E.: Anesthesia, J. Pharmacol., I, I99, I909; Morphin, ib., 2, I, I9Io.

Henderson, V. E., and Loewi: Salivary Gland, Arch. exp. Path. Pharm., 53, 62, I905.

Henderson, V. E., and Taylor: Expectorants, J. Pharmacol., 2, r53, roio.

Henderson, Y.: Acapnia, Am. J. Physiol., 23, 345, 1909; ib., 25, 310, 1910; Anesthesia, Surg., Gyn., Ob., Sept., 386, 389, I9 14; Carbon Dioxid, Am. J. Physiol., 21, I 26, I9o8; ib., 27, 152, 1910; Carbon Monoxid, J. Am. Med. Assoc., 67, 580, I916; Ether, Surg., Gyn., Ob., I6r, I9I ; J. Am. Med. Assoc., 65, r, I915; Resuscitation Apparatus, J. Am. Med. Assoc., 67, I, I916.

Henderson, Y., Chillingsworth and Whitney: Respiratory Dead Space, Am. J. Physiol., 38, I, r9ז 5.

Henderson, Y., and Crofutr: Emollients, Am. J. Physiol., 14, 193, I905.

Henderson, Y., and Scarbrough: Morphin, Am. J. Physiol., 26, 260, 19io.

Henderson, Y., and Underhill: Glycosuria, Am. J. Physiol., 28, 275, r9ir.

Hendriquez, V., and Anderson: Ammonia, Zs. physiol. Chem., 92, 21; r914; Foods, ib., 88, 357, I91 3; Peptones, ib., 94, 194.

Henkel, H.: Strychnin, Zs. allg. Physiol., I 5 , rg 3 .

Henkel and Klugh: Hydrastis, Circ. 6, U. S. Dept. Agr., Igo8.

Henze, M.: Tyramin, Zs. physiol. Chem., 87, 51, I913.

HePner, J.: Dyspnea, Zentr. Bioch. Bioph., 18, 65, I9I 5 ; Pituitary, ib., 18, 55, I9 I5.

Herb: Anesthetics, J. Am. Med. Assoc., 56, I31 2, I9I I.

Hering, H. E.: Morphin, Deut. med. Woch., 6r, No. 39, I I45, I915; Potassium, Arch. ges.Physiol., I6r, 537; ib., I6r, 544, I9r5.

Herlitzka, A.: Salines, Zentr. Bioch. Bioph., ro, 597, 1910.

Hernando: Digitalis, Arch. exp. Path. Pharm., 66, in8, 19 ir. 
Hikn): Hlcohd, Eugen. Lab. Mem., I 7, 1912.

IHRUINSDORER: Chlorid, Arch. ges. Physiol., I44, I69, I9 I 2.

IlFkrik, W: W.: Atophan, J. Am. Med. Assoc., 6r, r376, r9i3; Atropin, Arch. Int. Med., 13, No. 5. I9 I4.

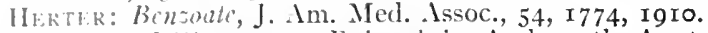

IERTEk and II.ikEuAx: Epinephrin, Arch. path. Anat., r69, 479, 1902.

IIERTz and GoldberG: Alkali, Zentr. Bioch. Bioph., I6, 844, I9 I4.

Ilkexilimer, K., and NathiN: Cresol, Ther. Mon., 29, 87, I9I5.

IItrz, Cook and Schlesinger: Magnesium, Ref., Am. J. Physiol., 25, 4, igo8.

IItrzFeb, 1.: Arsenic, J. Im. Med. Assoc., 56, 588, r9i i Mustard, ib., 52, I35, Igog.

HrRzFlin and Elix: Iodin, Zentr. Bioch. Bioph., I3, 25 I, r9I 2.

HerzFtid and Gormoor: Haloids, Zentr. Bioch. Bioph., I3, 790, I9I 2.

IHERFELD and HALPT: Iodid, Med. Klin., 7, 1426, I9II.

Herzog, W.: Iodin, Muen. med. Woch., 6r, 48, Igr4.

Hess, 1. I.: Calcium, Soc. Exp. Biol. Med., I3, 59, I915; Fuller's Earth, J. Am. Med.

Assoc., 66, ro6, т9г6; II mophilia, Soc. Exp. Biol. Med., I 2, 54, r914; Bull. J.

Hopk. Hosp.; 26, 297, 19г5; Arch. Int. Med., 17, 203, 1916; Tissue Extract, Soc. Exp. Biol. Med., I 2 , I 77 , I9 5 ; J. Am. Med. Assoc., Apr. 24, I9 I5; Yeast, Soc. Exp. Biol. Med., I3, I45, I9 I6.

Hesse, F. A.: Hormonal, Ther. Mon., 27, 698, r913.

Hesse, O.: Astringents, Arch. ges. Physiol., I 51, 363, I9 3.

Hesse, O., and Neukirch: Opium, Arch. ges. Physiol., I5I, 309, I9I3.

Hec'Bel: Iodid, Arch. exp. Path. Pharm., 67, 30, 1865.

Heubner, W.: Arsenic, Arch. exp. Path. Pharm., 56, 370, 1907; Phosphorus, Arch. exp. Path. Pharm., 78, 24, I914; Physostigmin, Arch. exp. Path. Pharm., 53, 313, 1905 .

Hetbrer, W., and Loewe: Strychnin, Arch. exp. Path. Pharm., 7 I, i 74, I9I3.

Heuck: Calomel, Diss. Heidelberg, r 889.

Heuser: Digitalis, Arch. internat. Pharmacol., 10, 483, 1902.

Hewlet, A. W.: Blood-flow, Arch. Intern. Med., I I, 507; Hypertension, Med. Chir. Bull., 4, 2I I, I9I 2; Pituitary, Soc. Exp. Biol. Med., I 2, 6I, r9r4; Salicyl, J. Am. Med. Assoc., 6r, 319, I9г3; Strychnin, Am. J. Med. Sci., Oct., I9I3; Urea, J. Am. Med. Assoc., 67, 939, 1916.

Heyl, F. IW., and Staley: Echinacea, Am. J. Pharm., 86, 450, I9r4.

Heyn, L. G.: Salicylic Acid, J. Am. Med. Assoc., 63, roo4, I9 4 .

Heymans and Masolx: Nitrites, Arch. int. Pharmacod., 3, 77, 359; 7, 297; 8, .

Higgrns, W. H.: Bismuth Poisoning, J. Am. Med. Assoc., 66, 648, 1916.

Higgrxs, II. L.: Alcohol, Am. J. Physiol., 41, '258, 19 I6.

Hrgains, H. L., and Means: Respiration, J. Pharmacol., 7, I, I915.

Higgins, Peabody and Fitz: Alcohol, J. Mied. Res., 34, No. 2, 1916.

Higucui: Vagina, Arch. Gyn., 86, rgo8.

Hildebrandt, W.: Camphor, Arch. exp. Path. Pharm., 48, 451, 1902; Chloroform, Muench. med. Woch., 60, 527, I913; Ferments, Arch. path. Anat., I 21, I, I890; Volatile Oils, Arch. exp. Path. Pharm., 45, I10, I900; ib., 44, 278; 46, 26r ; 48, 45I, I $901-I 902$.

Hill, I : Chloroform, J. Am. Med. Assoc., 67, 559, г9 т6.

Hill, L., and FlaCk: Oxygen, J. Physiol., 40, 347, I909-r9ro; Ozone, Proc. Roy. Soc., $\mathrm{B} 84,404,573$, 19 II.

Hitl, L. IV.: Erythrocyles, Arch. Int. Med., r6, 809, I9 5.

Hill, M. C.: Epinephrin, Arch. Intern. Med., 5, 22, I910.

Hill, R. L., and Simpson: Pituitary, Proc. Soc. Exp. Biol. Med., I1, 82, rgr4.

Hilpert: Salicyl, J. Am. Med. Assoc., 60, II37, I9I3.

Hilton, S. L.: Parafin, J. Am. Pharmacol. Assoc., 3, 577, 1914.

Hinmax, F.: Hexamethylenamin, J. Am. Med. Assoc., 61, 1601, 1913; Urinary Antisepsis, J. Am. Med. Assoc., 65, I769, I915.

Hirata: Antiseptics, Chem. Abstr., 5, 1490, r9io.

Hirayama, K.: Glycosuria, Z. exp. Path., 8, 649, r9r.

Hirscir, E. F.: Delirium Tremens, Arch. Int. Med., I7, 354, I9 16; Epinephrin, Z. exp. Path., I3, 142, I913; Hyperglycemia, Zs. physiol. Chem., 94, 227, 1915; Iodid, J. Inf. Dis., 15, No. 3 , r9I 4 ; Lead, D. med. Woch., 40, No. 8, I9I4.

Hirschf Ld : Diabetes, Festsch. Salkowski., I 87 , r904.

Hirsciffelder, A. D.: Acids, Sci. 44, 250, 1916; Kephalin, ib., 44, 25 1, 1916; Lead Colic, J. Am. Med. Assoc., 65, 516, I915; Pia Mater, J. Pharm. Exp. Ther., 6, 597 , I9 I 5 .

Hurschler and v. Terray: Calcium, Zs. klin. Med., 57, I905.

Hirt: Stomachics, Arch. Physiol., 196, 1856. 
Hirz, O.: Atropin, Arch. exp. Path. Pharm., 74, 318, I913; Uzara, Muench. med. Woch., 2220, I9I3; Uzaron, Arch. exp. Path. Pharm., 74, 318, I9I3.

Hrtzig: Morphin, Arch. Physiol., I 873.

Hodges, A. D. P.: Atoxyl, Ref., J. Am. Med. Assoc., 52, 586, I908.

Hoeber, R.: Ammonia, Arch. ges. Physiol., 149, 87, r912; Dyes, Bioch. Zs., 67, 420, I914; Narcosis Theory, Deut. med. Woch., 273, 1915; Salt Actions, Bioch. Centr., I, 497, I903.

Hoeber, R., and Spaeth: Rare Earths, Arch. ges. Physiol., 159, 433, I9I4.

Hoelscher: Elhor, Arch. klin. Chir., I898; Sweat, Bioch. Centr., 2, 485 , I904.

Hoerhammer, C.: Calcium, Bioch. Zs., 39, 270 , I9I 2.

v. Hoesslin, R.: Arscnic, Ther. Mon., 27, 849, r9r3; Cholin, Beitr. chem. Physiol., $8,27 \mathrm{I}$.

Hofbauer, L.: Therapy, Ther. Mon., 29, 237, I9I5.

v. Hofmand, A.: Bile, Wien. klin. Woch., 25, I74, I9I 2; Iron, Arch. path. Anat., I60, 235, I900; Formaldeliyde, Proc. Roy. Soc., I6, I56, I867; Quinin, Arch. exp. Path. Pharm., 7, 233, I877.

Hofmeister: Leucocytes, Arch. exp. Path. Pharm., 22, 306, I887.

Hogan, J. J.: Gelatin, J. Am. Med. Assoc., 74, 721, r9 5.

Hohmeier and Koenig: Spinal Anesthesia, Ref., J. Am. Med. Assoc., 55, i 507, r9io.

Holbrook: Cocain, J. Am. Med. Assoc., 58, 72 I, I9 2.

Holland and Co-workers: Osmosis, Am. Chem. J., 48, 29, I 9 I I-I 2.

Hollingsworth: Caffein, Ther. Gaz., 36, I, I9I 2.

Holmes, H. N.: Ozone, Sci., Mch. I5, 433, I9 I 2.

Holscher: Sweat, N. Y. Med. J., Feb. I3, I904.

Holste: Digitalis, Arch. exp. Path. Pharm., 68, 323; 70, 435, I91 2; ib., 73, 457, I9 I3.

Honda: Helleborein, Arch. int. Pharmacod., 9, 43, r9o2; Muscarin, Arch. exp. Pith. Pharm., 65, 454, 19I r.

Honigmann: Narcosis, Arch. klin. Chir., 58, 30, 1899.

Hooker, D. R.: Carbonic Acid, Am. J. Physiol., 31, 47, 191 2; Medulla Perfusion, Am. J. Physiol., 38, 200, I915; Respiration, J. Pharmacol., 4, 443, I9r3; Vessels, Am. J. Physiol., 28, 36r, I9I.

Hooker, D. R., and Reese: Spinal Cord, Am. J. Physiol., 33; Proc., xxvii, I9 I4.

Hoover, C. S., and TAylor: Asthma, Arch. Int. Med., I 5, 1, 1915.

HopPe: Bitters, Berl. klin. Woch., ro38, Aug., r905.

Hoppe-Seyler, G.: Pituitary, Muench. med. Woch., 62, 1633, I9 5 .

Horn, W. F.: Phosphate, U. S. P., Dig., 522, I910.

Hornemann: Diphtheria, Ther. Mon., 27, 757, I9I4.

Horovitz: Mydriatics, Zentr. Bioch. Bioph., 15, 94, I912.

Horrax, G.: Pineal, Arch. Int. Med., 17, 607, 627, I9 16.

HoRwith: Chicory, Proc. Am. Pharm. Assoc., 57, I84, I908.

Hoskins, R. G.: Adrenal Deficiency, J. Am. Med. Assoc., 62, 1803, 1914; Am. J. Physiol., 36, 423, 1915; Adrenal Problem, J. Lab. Clin. Med., 1, No. 7, 19 16; Epinephrin, ib., 29, 363, I91 2; Epinephrin-pituitary, Soc. Exp. Biol. Med., I3, I6, I915; Pituitary (Intestines), J. Am. Med. Assoc., 66, 732, r9r6.

Hoskixs and Gunning: Pancreas Deficiency, Am. J. Physiol. 41, 79, 19 6.

Hoskins and Hoskins: Suprarenal Feeding, Arch. Int. Med., I7, 584, I9 6.

Hoskins and Lovelette: Epinephrin, J. Am. Med. Assoc., 63, 3 I6, I9I4.

Hoskins and McClure: Epinephrin, Am. J. Physiol., 3 I, 59, r9 I 2.

Hoskins and McPeek: Adrenal Massage, J. Am. Med. Assoc.,60, 1777, I9I3; Pituitary, Am. J. Physiol., 32, I9I3.

Hoskins and Means: Pituitary, J. Pharmacol., 4, 435, I9г3.

Hoskins, R. G., and Ransom: Nicotin, J. Pharm. Exp. Ther., 7, 375, I9r 5 .

Hoskins, R. G., and Rowley: Epinephrin, Am. J. Physiol., 37, 47 I, I9I5.

Hoskins and Wheelon: Adrenal Deficiency, Am. J. Physiol., 34, 172, 1914; Parathyroid Deficiency, ib., 34, 263, I9I4.

Hostmann, J.: Ichthyol, J. Am. Pharm. Assoc., 4, I490, I9 5.

Houghton, E. M.: Digitalis, J. Am. Med. Assoc., Oct. 22, r898; Lancet, June I9, r9o9; Ergot, Ther. Gaz., I4, 433, I898; Squill, J. Am. Med. Assoc., 46, I4I 7, I906; Strophanthin, Bull. Am. Pharm. Assoc., 6, I56, 1911 .

Houghton and Aldrich: Chloretone, J. Am. Med. Assoc., Sept. 23, r899.

Houghton and Hamrton: Cannabis, Am. J. Pharm., 79, 497; 80, I6, I9o7-8.

Houghton and Merrill: Epinephrin and Pituitary, J. Am. Med. Assoc., 5 I, I849, 1908.

Houghton and Mulrhead: Strychnin, Med. News, 66, 61 2, I895:

Houseman, P. A.: Camphor, Am. J. Pharm., 87, 49, I9I5; Glycyrrhiza, ib., 88, 97, I9I6.

Howat, I.: Nicotin, Am. J. Physiol., 39, 447, I9 I6. 
Howe: Salivary Excretion, Chem. Abstr., 7, I056, I9 2.

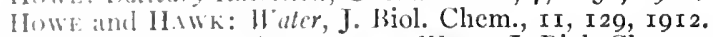

Howr, M.tThi.L and Hattrem: Ilaler, J. Biol. Chem., Io, 4I 7, I9 I I-I 2.

IImel., W. H.: IIcart, Am. J. Physiol., 2, 47, 1898; Hemophilia, ib., 33, xiii, I914; Pituitury, J. Exp. Mled., 3, 245, 1898; Prothrombin, Am. J. Physiol., 35, 474, r9x4.

Howel. and DLkE: Ions, J. Physiol., 35, r 3 I, I9o8.

Howland, J., and Marriott: Acidosis, J. Am. Med. Assoc., 66, r43, r9i 5.

Howland) and Richards: Chloroform, J. Exp. Med., I I, 344, r9o9.

Hoут: Tyramin, Im. J. Med. Sci., I44, 76, I9г 2.

Hic: Scopolamin, Arch. exp. Path. Pharm., 69, 45, г9 2.

Hegenuoltz, C. H.: Calcium Lactatc, Ref., Ther. Mon., 29, 568, I9 5.

Holisch: Cotarnin, Zahn. Rundsch., No. 43 I, I899.

I1t'ise, J. A.: Bismuth, J. Am. Med. Assoc., 55, 236, 1910.

Huxt, E. L.: Mercurialized Sermm, J. Am. Med. Assoc., 66, 404, 19г6.

Hunt, Reid: Alcohol, Hyg. Lab. Bull., No. 33, I907; Cholin, J. Pharm. Exp. Ther., 7. 301, т9г5; Ilomocholins, J. Pharmacol., 6, 477, x9х5; Methyl Alcohol, Johns Hopkins Hosp. Bull., I3, 213, г902; Morphin, Hyg. Lab. Bull., No. 69, I910; Nitrite, Arch. intern. Pharmacod., I 2, 447, I904; Parathyroid, Proc. Soc. Exp. Biol. Med., 4, 64, I907; Thyroid, J. Biol. Chem., I, 33, 1905; J. Am. Med. Assoc., 57 , IO32, I9II.

Hunt and SeIdeli: Thyroid, J. Am. Med. Assoc., 5 I, I385, 1908; J. Pharmacol., 2, I 5 , I 9 Io.

Hunt and Taveau: Cholins, U. S. Hyg. Lab. Bull., No. 73, I9 I .

Hunter, A., and Simpson: Marine Alga, J. Biol. Chem., 20, r9 5.

Hupfer: Quinic Acid, Zs. physiol. Chem., 37, 302, I903.

Hurwitz, S. H., and Drinker: Benzol, J. Exp. Med., 21, 401, 423, I9 5.

Hurwitz, S. H., and Lucas: Hemophilia, Arch. Int. Med., I 7, 543, I9 6.

Hurwitz, S. H. and Tranter: Cerebro-spinal Fluid, Arch. Int. Med., i 7, 828, ig 6.

Huseman; Atropin, Arch. exp. Path. Pharm., 6, 443, 1877.

Hussakoff, L.: Radium, Med. Red., July 20, 1907.

Hutcuison: Petroleum, Brit. Med. J., March 25, I89o.

Hyde, S. H., Spray and Howard: Alcohol, Kans. Univ. Sci. Bull., June, ror3.

Hydrick: Phenolphthalein, J. Biol. Chem., I 7, xxxvi, I9r4.

Hysia, L. H.: Cyanid, Am. J. Physiol., 40, 238, I9 I6.

Hyman, F.: Hexamethylenamin, Arch. Int. Med., I3, 841, I9I4.

IgersheIMer: Chrysarobin, Merck's Rep., 26, r49, I91 2; Strychnin, Arch. exp. Path. Pharm., 54, 73, 1905.

Igershimer and Itami: Atoxyl, Zs. physiol. Chem., 59, 256, igo9.

Ignatowski and Monossonn: Biliary Fistula, Zs. exp. Path. Ther., r6, r9 4.

IkEDA, Y.: Quinin and Atophan, J. Pharmacol., 8, гог, т9г6; Inflammation, ib., 8, I37, I9 16.

Impens, E.: Alypin, Med. Klin., г38, I9г3; Atophan, Arch. intern. Pharmacod., 22, 379, 1912; Chloretone, Arch. int. Pharmacod., 8, 77, 1901; Cotoin, Deut. med. Woch., 38, 1827, 19г3; Cymarin, Arch. ges. Physiol., 153, 239, 1913; Heroin, ib., 78, 527, I899.

Irokawa: Salvarsan, Zentr. Bioch. Bioph., i7, 615, ז914.

IsAak, J.: Camphor, Arch. ges. Physiol., I 53, 491, I913.

Isenschim, R.: Antipyretics, Arch. exp. Path. Pharm., 75, 10, 19 3.

ISEnschmid and Schnitzler: Heat Centers, Arch. exp. Path. Pharm., 76, 202, igr4.

Ishizaka, N.: Terpenes, Arch. exp. Path. Pharm., 75, I 94, I9I4.

Issekutz, B.: Digitaloids, Arch. exp. Path. Pharm., 78, 155, I915; Bürgis Law, Arch. ges. Physiol., I5 1, 456, 1913; Magnesium, Ther. Mon., 29, 379, I915; Morphin, Arch. ges. Physiol., r 42, 255, r9 Ir.

Ivy, R. H.: Phosphorus, J. Am. Med. Assoc., 56, ror8, r9r1.

IwANow: Alcohol, Zentr. Bioch. Bioph., I5, 6, I9г3.

IzAR: Ethylhydrocuprein, Berl. klin. Woch., 385, r9 4 .

Janksveld and Stokvis: Hippuric, Arch. exp. Path. Pharm., Io, 268, I879.

Jackson, D. E.: A nesthesia, J. Lab. Clin. M., I, I, I915; J. Pharmacol., 8, Ir3, rgr6; Bronchi, J. Pharmacol., 4, 291, I913; 5, 479, I914; Epinephrin, Am. J. Physiol., 23, 227, 1909; J. Pharmacol., 4, 59, r912; Nitrous Oxid, J. Lab. Clin. Med., I, No. 9, I9 16; Vanadium, J. Pharmacol., 3, 477, r9 2.

Jackson, H. C., and Ewrng: Vagus Reflex, Am. J. Physiol., 33, Proc. xxx, rgi4.

Jacobi, A.: Doses, Ohio Med. J., 3, r45, r907; Potassium, Tr. Med. Soc., N. Y., p. 365 , I9I5. 
JaсовJ, C.: Digitalis, Arch. exp. Path. Pharm., 44, 368, r9oo; Colchicum, Arch. exp. Path. Pharm., 27, I I9, I890; Ergot, ib., 39, 85, I897; Iron, Inaug., Strassburg, r887; Arch. exp. Path. Pharm., 28, 256, 189г; Nitrite, ib., 72, r29, 1913; Veronal, ib., 66, 296, I9II.

JACoBJ, C., and Roemer: Veronal, Arch. exp. Path. Pharm., 66, 26г, igi I.

JacoвJ, C., and Walbaum: Sulphite, Arch. exp. Path. Pharm., 54, 42I, I 906.

JACOBJ, J.: Silver, Arch. exp. Path. Pharm., 8, I98, I877.

Jacobs, W. A., and Heidelberger: Hexamethylenetetramine, Proc. Nat. Acad. Sci, I, 226, I915.

JAcobson, A. T. B.: Chloral, Bioch. Zs., 51, 443, r9г3; Glycosuria, ib., 56, 471, 19г3.

Jacobson, C.: Ammonium, Am. J. Physiol., 26, 407, 1910; J. Biol. Chem., 63, 605, 1914; Cicutoxin, J. Am. Chem. Soc., 37, 916, I9I5.

Jасову, G. W.: Nicotin, Berl. Klinik., No. I 26, I898.

JACOBY, M.: Cotarnin, Ther. Gegenw., June, I906; Phosphorus, Zs. physiol. Chem., 30, 174, I900; Ricin, Beitr. chem. Physiol., I, 51; 2, 535, 1902.

JACOBY, M., and EISNer: Calcium, Berl. klin. Woch., 50, I339, I913; Deut. med. Woch., I657, I913.

JACOBY, M., and Rosenfeld: Calcium, Bioch. Zs., 69, I55, I9I5.

JACQUE and Zunz: Absorption, Arch. int. Physiol., 8, 277, I909.

JAcQueT: Altitude, Univ. Program, Basel, r904; Aspidium, Ther. Mon., I904.

Jacquet and Stahelin: Altitude, Arch. exp. Path. Pharm., 46, 247, igor.

JAEGER, F.: Histamin, Ref., Ther. Mon., 27, 433, r913; Zbl. Gyn., 265, I913.

JAFFe, R.: Gasoline, Zentr. Biochem. Bioph., I6, 508, I9I4.

v. JAGIC, N.: Bronchospasm, Wien. klin. Woch., 29, No. 4, r9 16.

Jahn: Iodoso-benzoic Acid, Arch. exp. Path. Pharm., 77, I6, I9I4.

v. JAKSCH: Phosphorus, Zs. Physiol. Chem., 40, I23, т903.

JAmieson, R. A.: Strophanthin, J. Exp. Med., 22, 629, I915.

JANNEY: Ammonium, Zs. physiol. Chem., 76, 99, I9 2.

JANSEN, B. C. P.: Urea, J. Biol. Chem., 21, 557, r915.

JANuschke, H.: Epinephrin, Wien. klin. Woch., 23, No. 8, rgro; Bromid, Ther. Mon., 27, 772, 1913; Inflammation, Wien. klin. Woch., 26, 1913.

JanusChKe, H., and InABA: Bromid, Zs. exp. Med., I, I 29, I9г3.

Januschke, H., and Masslow: Bromids, Zs. exp. Med., 4, 30I, I9I5; Calcium, ib., 4 , I49, I9I4.

JANuSchke and Pollak: Epinephrin, Arch. exp. Path. Pharm., 66, 205, I9Ir.

JANUSKIEWICZ: Alcohol, Zs. Biol., 56, 401, I9זr.

JAPELli, A.: Osmosis, Arch. int. Physiol., 3, 369, I906.

JARDIN: Vanadium, C. R. Soc. Biol., 58, 364, 1905.

JARISCH, A.: Theobromin, Arch. ges. Physiol., I 58, 502, I9I4.

JAVAL, M. A.: Chlorid, Sem. med., 23, 224, I903; Zentr. Bioch. Bioph., I4, 526, I9 2.

JAVAL, A., and ADLER: Chlorid, Bioch. Centr., 5, 589, r9o6.

Jemma, R.: Tartar-emetic, J. Am. Med. Assoc., 66, 780, I9I6.

JENDRASSIK: Calomel, Deut. Arch. Klin. Med., 38, 499, r886; ib., 47, 226, r89r.

Jensen, T.: Bismuth, Muench. med. Woch., 60, r202, r913.

Jepson, L. L.: Atropin, W. Va. Med. Assoc., May, r907.

Jerusalem and Starling: Carbon Dioxid, Zentr. Bioch. Bioph., 10, 463, I910.

JoAchimoglv, G.: Arsenic, Bioch. Zs., 70, 144, I915; Arch. exp. Path. Pharm., 79, 4r9, I9r6; Atropin, Berl. klin. Woch:, No. 35, I915; Benzol, Bioch. Zs., 70, 93, I9I5; Elarson, Arch. exp. Path. Pharm., 78, I, I914.

Joannovics: A mmonia, Arch. internat. Pharmacodyn., I2, 36, 1903.

Jobling, J. W., and Petersen: Anaphylaxis, J. Exp. Med., 19, No. 5, 1914; Iodin, J. Am. Med. Assoc., 63, 1930, I914; Arch. Int. Med., I5, 286, I915; Zs. Immun., 24, 292, 1915; Nonspecific Factors, J. Am. Med. Assoc. 66, 1753, 1916.

Jobling, Petersen and Eggstein: Ferment-Action, J. Exp. Med., 22, 568, 1915.

JodlbaUer: Fluorid, Zs. Biol., 4r, 487, r9or; Muench. med. Woch., No. r6, r902.

JoEL, A.: Narcotin, Arch. ges. Physiol., I6I, 5, I9 5.

Johannessonn, F.: Strophanthin, Arch. exp. Path. Pharm., 78, 83, 92, 1914.

JoHN, M.: Alcohol, Zs. exp. Path., 5, 579, r908; Nicotin, Zs. exp. Path., 14, 352, I913.

Johnson and Hindman: Cascara, Am. J. Pharm., 86, 387, r9r4.

Jones, A. P., and TATuM: Thyroid, Arch. Intern. Med., I 2, 225 , r9r 2.

Jonescu, D.: Antipyrin, Digest., U. S. P., 162, 1906; Fever, Arch. exp. Path. Pharm., 6o, 346 , I909.

Jonescu, T.: Stovain, Deut. med. Woch., $2 \mathrm{I}_{55}, \mathrm{I} 909$.

JoNesCu and BUKAREST: Cresols, Bioch. Zentr., I, 399, 1906.

JoNescu and LoewI: Digitalis, Arch. exp. Path. Pharm., 59, 7 I, I 1908.

Jonge: Phenol Excretion, Zs. physiol. Chem., 3, 181, 1897. 


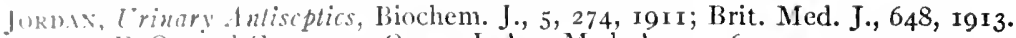

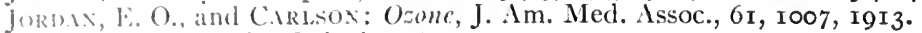

Ju-1.11: (izsolin, Diss. Leepzig, 1896.

Jusin, I). R.: Acid Fuchsin, J. Pharm. Exp. Ther., 6, 83, 1914; 6, 603, 1915; Digitalis, Irch. exp. Path. Iharm., 73. SI, r913; Epinephrin, J. Exp. Med., I2, 212, I915; 15. 1012; Magnesium, \m. J. Physiol., 23, 215, 1909; Physostigmin, Proc. Soc. Exp. Biol. Med., 5, i 18, r908; Saliarsan, J. Exp. Med., I3, 634; I4, 83, I79, I9I I.

Josepil and MrLtzLR: Fuchsin. J. Pharmacol., 3, I 83 , i 9 I ; Hypertonic, J. Pharmacol., 2, 271, 1911 ; Magnesium, Proc. Soc. Exp. Biol. Med., 7, 95, I9ro; Centr. Physiol., 24. 7. 1910; Parathyroid, J. Pharmacol., 2, 361, I9I I; Am. J. Physiol., 29, I, I9I I; Physostigmin, J. Pharmacol., I, 369, 1909 .

Joste: Epincphrin, Presse med., Nov. I8, 1903.

Jowett and M.1rshall: Jaborandi, Brit. Med. J., Oct., I900.

J'CHExAck and Griebel: Strychnin, Zentr. Bioch. Bioph., Io, 626, igio.

J tockuff: Paraffin, Arch. exp. Path. Pharm., 32, I 24, r893.

Julrgexs, E.: Sulphocyanid, Monsch. Ohrenhk., No. 8, I9or.

Juxig, R.: Fetus, Ther. Mon., 28, I04, I9I4.

Joxguns, P.: Salt Puncture, Arch. exp. Path. Pharm., 77, 122, igr4.

Justi, K.: Usara, Muen. med. Woch., 765 , i913.

Justus: Iodid, Arch. path. Anat., I70, 50I, I908.

KAGax, A.: Saponin, Zentr. Bioch. Bioph., I6, 477, I913.

KAIN, M.: Allm, Bioch. Bul., 2, 235, 1912; Hemophilia, Am. J. Dis. Child., r 1, Iо3, 1916; Sulpho-cyunates, Dios. Columb., r9 12; Calcium in Tuberculosis, Med. Rec., May 23 , I9 14.

Kams, if. H., Andrews and Anderson: Mercury, Med. Rec., Aug. 28, I9 I5.

KaHN, MAX and KaHN: Calcium in Diabetes, Med. Rec., Oct. 30, I9 I5; Arch. Int. Med., I8, 2I 2, 19 I 6.

KanN, R. H.: II irudin, Arch. ges. Physiol., I44, 25 I, I9I5.

Kaiser, Alcohol, Muench. med. Woch., 59, 2508 , I9I 2.

Kameszove: Cocain, Arch. intern. Pharmacodyn., 21, 5, I9I I.

KAPPELER: Chromate, Inaug. Bonn, i 896 .

Karelkis, D.: Caffein, Zentr. Bioch. Bioph., r 7, 923, r9r4.

Karsier, H. T.: Oxygen, Clev. Med. J., I4, 305, I9 I5; J. Exp. Med., 23, 149, 19 I6.

Karsater, H. T., and Denis: Nephritis, J. Exp. Med., i9, I9I4.

Kasass: Methyl Alcohol, Ref., Zentr. Bioch. Bioph., I7, 393, r9I3.

KAST: Alcohol, Bioch. Centr., 5, 483, I906; Glycerin, Proc. Soc. Exp. Biol. Med., 7, 36, I9 10; Sulfonal, Berl. klin. Woch., 309, 1888; Arch. exp. Path. Pharm., 31, 69, I 893 .

Kast and Meltzer: Cocain, Med. Rec., Dec. 29, I906.

Kastle: Phthaleins, Bull. Hyg. Lab., No. 26, r9o6.

Kasztax: Strophanthin, Arch. exp. Path. Pharm., 63, 405, I910.

Kaтsch: Intestines, Zentr. Bioch. Bioph., I6, I8I, I9I3.

Katsuyama: Diuretics, Zs. physiol. Chem., 26, 543; 28, 587, r899; ib., 32, 235, r9or.

Katz: Colarnin, Ther. Mon,, June, I903.

Katzenstein: Veronal, Ref., Merck's Rep., 23, 343, I9o9.

Kaufmanx: Cotarnin, Deut. med. Ztg., March, I905.

KaUfMañ, AD.: Quinin, Berl. Ber., 46, I823.

Kaufmann-Asser: Morphin, Bioch. Zs., 54, I6r, I9г3; Salicyl, Zs. klin. Med., 48, 260, I903.

Kayser, C.: Calcium, Ther. Mon., r67, rgr2; Zs. exp. Path., r6, 369, 378, r9i4.

Kebler, F.: Platinum, Arch. exp. Path. Pharm., 9, 137, 1878.

Kebler, L. F.: Camphor, Am. J. Pharm., 79, 349, 551, 579, r907; Peroxid, Proc. Am. Pharm. Assoc., 58, 903, I910; Tablets, J. Am. Pharm. Assoc., 3, 1062, r914.

Kebler, Morgan and Rupp: Antipyretics, U. S. Bur. Chem., Bull. I26, r9o9.

KeEton, R. W., and Becht: Iypophysis, Am. J. Physiol., 39, 109, 1915.

Kenrer: A utonomic System, Arch. Gynec., 81, 160, r907; Arch. exp. Path. Pharm., 58, 366, 1908; Passage of Drugs to Fetus, Ther. Monatsh., 28, 104, 1907.

Keilty, R. A., and PAcker: Antiseptics, J. Am. Med. Assoc., 64, 2 I 23 , I9I 5.

Keiti, N. M., Rowntree and Geraghty: Blood Volume, Arch. Int. Med., 16, 547, 1915 .

Kellermann: Iodid, Zs. exp. Path., I, I89, 686, I904.

Kepinow: Epinephrin, Arch. exp. Path. Pharm., 67, 247, I91 2.

Kendall, E. C.: Thyroid, Proc. Soc. Exp. Biol. Med., 10, 165, 1913; Proc. Am. Soc. Biol. Chem., 3, 2 I, r915; J. Am. Med. Assoc., 64, 2042, 1915.

Kettner: Acids, Árch. exp. Path. Pharm., 47, I78, I902. 
KeysSer: Tumors, Zs. Chemother., 2, I 88, I9I4.

Keysser and Wassermaxx: Anaphylaxis, Fol. serol., 7, igi i; Zs. Hyg. Inf., 68, igir.

Kiefer, C. F.: Powder Gascs, J. Am. Med. Assoc., 44, I359, I905.

Kiliani: Digitalis, Arch. Pharmacie, I 890 to I899; Zentr. Bioch. Bioph., I6, 940, I9I4; Gitalin, Arch. Pharm., 252, 13, I914.

Kimura: Ipccacuanhic Acid, Arch. intern. Pharmacod., II, 405, I903.

Kivg: Thyroid, J. Exp. Med., i 1, 665, igog.

KInG, W. E., and Davis: Tellurite, Am. J. Publ. H., 4, 9 I 7, I9I4.

King, Moyle and Haupt: Ether, J. Exp. Med., I6, I78, i9г3.

King and Stewart: Bile, J. Exp. Med., i I, 673, I909.

KING and Stoland: Pituitary, Am. J. Physiol., 32, 41 2, r913.

Kingsbury, F. B.: Hippuric Synthesis, J. Biol. Chem., 21, 288, I9I5.

Kingsbury, F. B., and Bell: Hippuric Acid, J. Biol. Chem., 20, 73, r9I5.

Kinnicutt, F. P.: Calcium, Trans. Assoc. Am. Phys., 24, 475, I909.

Kionka, H.: Arscnic, Arch. int. Pharmacod., 21, 489, I9I I; Colchicin, Z. exp. Path., 2, I5, I9o6; Metals, Zs. exp. Path., I 7, 108, 1 26, I914; Sulphites, Hab., Breslau, I896; Valerian, Deut. med. Woch., No. 49, I902; Arch. intern. Pharmacodyn., I3, 2 I 5, I904.

KionkA and EBstein: Sulphite, Zs. Hyg. Inf., 41, I23, 1902.

KIONKa and Koenig: Narcosis, Arch. klin. Chir., 75, I3, I905.

Kiralyfi: Benzol, Wien. klin. Woch., 25, I3I I, I912; ib., 26, No. 26, I9 3.

Kirchheim, L.: Trypsin, Arch. exp. Path. Pharm., 74, 374, I91 3.

KIRChNer: Cinchontism, Berl. klin. Woch., I8, 725, I88I.

KIte: Cytoplasm, Biol. Bul., 25, I, I913; Am. J. Physiol., 32, I46, I913; 37, 282, I915.

Klages, A., and Schreiber: Mercury, XVII Int. Congr. Med., IgI4.

Klamure, M. H.: Synergism, Z. exp. Med., I, 575, I913.

KLApp: Hpyodermic Injections, Arch. exp. Path. Pharm., 47, 86, I901.

KleE and Knuepfel: Gall Bladder, Mitt. Grengg., 27, I9I4.

KLeIN, H. A.: Cholesterol, Bioch. Z., 29, 465, I910.

KLEIN, K.: Strcphanthin, Zs. exp. Path., I7, I27, I42, I43, I60, I9I4.

Kleiner, I. S.: Morphin, J. Pharmacol., 4, 357, I913; Sugar, J. Exp. Med., I4, I9I I.

Kleiner, I. S., and Meltzer: Dexirose, Trans. Assoc. Am. Phys., 30, 342, I9 I5; Magnesium Glycosuria, Soc. Exp. Biol. Med., I3, 65, I9I5; Proc. Am. Soc. Biol. Chem., 3, 20, 19 16; Morphin, Soc. Exp. Biol. Med., I3, 142, I9 16; Strychnin, Proc. Soc. Exp. Biol. Med., 9, IOI, I9I 2.

Klemperer, G.: Atophan, Ref., Ther. Mon., 27, 862, i913; Camphor, Z. exp. Path., $4,3^{89}, 1907$.

KLERCKER: Opium, Bioch. Zs., 62, II, I9I4.

Klieneberger: Iodid, Zbl. inn. Med., 28, 273, r907.

KLINkerT: Cholesterol, Berl. klin. Woch., 820 (May), igr3.

Klocman: Gastric Secretion, Z. physiol. Chem., 80, I 7, I9I 2.

Klotz, M.: Calcium, Ther. Mon., 29, I 29, I9I5.

Klotz, O.: Calcification, J. Exp. Med., 7, 633, r905; Nephritis, Trans. Ass'n Am. Phys., 29, 49, I914.

Krotz, O., and Bothwerl: Calcification, Soc. Exp. Biol. Med., I2, I59, I9 5 ; Cholesterin, ib., I2, 199, I9I5.

Klotz, R.: Pituitary, Zentr. Bioch. Bioph., I I, 881, I9I i Arch. exp. Path. Pharm., 65,348 , г9ı I Muench. med. Woch., I9̇i I.

KNACK, A. V.: Cholesterin, Arch. Path. Anat., 220,36 , I9 5.

Knaffl-Lenz: Antimony, Arch. exp. Path. Pharm., 72, 224, 19г3; Pediculosis, Wien. klin. W., 38, No. 26, I9 5; Radium, Wien. klin. Woch., 25, No. I 2, I9 2.

Knauer and Maloney: Mescalin, Ref., J. Am. Med. Assoc., 61, 433, igr3.

KNEcht: Salicyl, Muench. med. Woch., 51, 956, 1904.

KNELL: Combined Action of Morphin and Chloral Hydrate, Merck's Rep., 21, 72 , I907.

Kroop, F.: Ammonium, Harvey Lect., 280, I9I3.

KNowlton: Colloids, J. Physiol., 43, 2 I 9, I9I I.

Kobert, R.: Digitsaponins, Merck's Rep., 26, I66, 191 2; Ergot, Arch. exp. Path. Pharm., 18, 317, 1884; Ergot History, Dorp. Stud., I, I, I889; Glycyrhizin, Ref., Am. J. Pharm., 87, 556, I9 1 5; Iron, Petersb. med. Woch., No. 49, 189 I Kobert's Reaction, Am. J. Phar., 74, Jan., r902; Methyl Alcohol, Chem. Abstr., 5, r9ro; Saponin, Arch. exp. Path. Pharm., 23, 233, I887; Ref., Zb. Bioch. Bioph., I 7, 775, I 914; Ther. Mon., 28, 669, I9I4; Saponin Substanzen, Stuttgart, I904; Volatile Oils, Schimmel's Rep., Oct., I9o6.

Косн, C.: Histamin, Zbl. Gyn., 265, 1913; Ref., Ther. Mon., 27, 605, I913.

Косн, F. C.: Parathyroid, J. Biol. Chem., I5, 43, I913; Thyroid, J. Biol. Chem., I4, IOI, I9I3. 
Kocil, RoBT.: Atovyl, Bioch. Centr., 7, 4r, I907.

Ku(1. W.: Lecithin, Bioch. Centr., 4, 549, 1905; J. Am. Med. Assoc., 52, I381, 1909;

Lipoid, ib., 56, 799, 191 r Phosphatid, J. Pharmacol., 2, 239, 249, r910.

Koch, IV., and Mostrom: Strychnin, J. Pharmacol., 2, 265, I9I.

Kixhir, R. A.: Neree Cells, J. Am. Med. Assoc., 67, 278, I9I6.

Kocnes, Tir.: Tetanus, Korr, Schw. Aerz., I 249, I9r5.

Kocm.n., Mf.: Alcohol, Arch. intern. Pharmacod., I3, 29, r904; Deut. med. Woch., 3 I, No. 24, I905; Anulgesics, Ther. Mon., 28, 641, I914; A nesthetics, Arch. intern. Pharmacod., 10, 347, 1903; Deut. med. Woch., Oct., 1934, I913; Calcium, Bioch. Zs., 27, 85, I910; Digitalis, Arch. intern. Pharmacod., 16, 221, 1906; ib., 19, 327, 1909; Wi.red Nareosis, Zs. exp. Path., I3, I and 328, I913; Narcosis Apparatus. Arch. internat. Pharmacod., 22, 487, I9I 3; Narcosis Theories, Bioch. Centr., 4, 689, 1906; Salvarsan, Muench. med. Woch., 59, I8, 1912; Scopolamin, Arch. intern. Pharmacod., I2, 99, 1903; Therap. Gegenw., May, I903; Valerian, Deut. med. Woch., No. 2, I904.

Kochmane and Daels: Cocain, Arch. intern. Pharmacodyn., I8, 4I, 1908.

Kocmmaxs and pupils: Mixed Narcosis, Zs. exp. Path., I 2, H. 2, 328, I9r3.

Kochuaxi and Roemer: Ocular Pressure, Arch. Ophth., 88, 528, 1914.

Koeliler: Salicyl, Centr. med. Wiss., No. IO, 411, I876.

Koeppe: Gastric Acidity, Arch. ges. Physiol., 62, 567, 1896.

Konlen: Urates, Zs. physiol. Chem., 70, 360, I9II.

Konsuiohta: Hediosit, Biochem. Z., 38, г 2 I, I9т 2.

Koizum, T.: Pieric Aeid, Zentr. Bioch. Bioph., I7, 6I5, r9r4.

KolBE: Salicyl, J. prakt. Chem., I0, 89; I1, 9; I 2, I6 I, I 874 .

Kolbe and Lautemann: Salicyl, Ann. Chem. Pharm., II 5, I57, I860.

KoliplNski: Chromium, Merck's Arch., Io, Nov., I908.

Koller: Cocain, Sitb. Wien. Akad., Math. N. W. Kl., Nov., i 884.

Kolmer, J. A., Matsunami and Broadwell: Iodid on Luetin, J. Am. Med. Assoc., 67, 7 I8, I916.

Kolmer, J. A., and Schamberg: Salvarsan, J. Exp. med., I5, 498, I912.

KoxschegG: Epinephrin, Arch. exp. Path. Pharm., 70, 3 I I, I9I 2; Pituitary Diuresis, D. med. Woch., IO9I, I9I5.

Kosschegg, A., and Schuster: Pituitary, Deut. med. Woch., 4I, 109 I, I9 I5.

Kopaczewski, W.: Acids, Int. Zs. Phys. Chem. Biol., I, 420, I914; Squill, Bioch. Zs., 66, 501, 19r4.

KoppaNG: Camphor, Ref., J. Am. Med. Assoc., 60, 330, 1912.

KOPPEL, M.: Inhibition, D. Arch. klin. Med., II 2, 594, I913.

v. Koranyi, A.: Benzol, Berl. klin. Woch., 49, 1857, 1912; Iodid, Deut. med. Woch., 32, No. I 7 , I906.

KORFF: Morphin-scopolamin, Münch. med. Woch., No. 45, r903.

KOSE: Nicotin, Sbomik. Klinicky, 5, 433, 1905.

KozNIEWSKI: Morphin, Zentr. Biochem. Biophys., 2, 324, 1910.

Kraepelin: Alcohol, Ref. Bioch. Bul., 5, 223, I916; Morphin, "Beeinflussung psych. Vorgänge, etc.," Jena, I892.

Kraft: Digitalis, Year Bk. Am. Pharm. Assoc., I, 437, 1912; Jahrb. Pharm., ir, 201, I9I r; Digitsaponins, Merck's Rep., 26, 165, 1912.

Kramer, B.: Nareosis, J. Exp. Med., I 7, 206, I 913.

Kramer and Marker: Alkali, Am. Soc. Biol. Chem., 3, 24, 19 16.

Kramer, B., and Murlin: Sod. Carb., Proc. Am. Soc. Biol. Chem., 3, 24, I9I 5.

KraNer: Hediosit, Deut. med. Woch., 38, 2416, igi 2.

v. Krannichfeldt, H.: Acetosalicylic, Zentr. Bioch. Bioph., I6, 587, 1914.

Krats: Ricin, Zs. Heilk., 24, Igoz.

Kraus, A. D.: Aspirin, Arch. Derm., I I9, I, I9I4.

KRAUS, P.: Fever, Arch. intern. Pharmacod., 6, 345, 1899.

Kraus, R. and Barbara: Charcoal, Wien. klin. Woch., 524, 1915 .

KRAUS, W. M.: Uranium, Arch. Int. Med., I 1, 612, 1913.

KRAUSE and CraMER: Thyroid, Zentr. Bioch. Bioph., I4, 24, I9I 2

Krauss, E.: Nitrites, Arch. exp. Path. Pharm., 72, 97, I913.

KREHAN, M.: Cyanid, Zentr. Bioch. Bioph., I7, 328, I9I4.

Kremers: Pharmacognosy, Proc. Am. Pharm. Assoc., $214,1898$.

Kretschurer: Epinephrin, Arch. exp. Path. Pharm., 57, 423, I907

KROL: Melhyl Alcohol, Arch. exp. Path. Pharm., 72, 442, 1913.

Krosz, J. G.: Bromid, Arch. exp. Path. Pharm., 6, $x, 1876$.

KrumbiaAr, E. B., and Musser: Spleen, J. Exp. Med., 20, No. 3, rgr4.

Krumbaar, E. B., Musser and Pearce: Spleen, J. Exp. Med., r8, No. 6, r9r 3.

KRUMHOFF: Lithium, Inaug. Goettingen, I884. 
Krumwiede, C., and Manx: Quinin, J. Infec. Dis., I6, No. I, I915.

KuEHL: Aluminum, Zentr. Bioch. Bioph., I5, 697, I913.

Kuenzer, R.: Strychnin, Arch. exp. Path. Pharm., 77, 241, igr4.

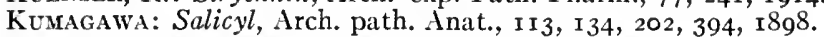

Kundratitz: Anaphylaxis, Arch. exp. Path. Pharm., 73, 33, I9I3.

Kuno, G.: Epincphrin, J. Physiol., 49, I39, I915.

Kuno, Y.: Alcohol, Arch. exp. Path. Pharm., 74, 399, I9r3; ib., 77, 206, I9I4.

KURDINowski: Uterus, Arch. Gynec., 78, No. 3 , I906.

KuRoda, M.: Cocain, J. Pharm. Exp. Ther., 7, 423, I915; Cymarin, Zs. exp. Med., 4, 55 , 1914.

Kuyer, A., and Wijenbeck: Autonomic, Arch. ges. Physiol., I54, i6, I9r3.

Kyes: Lecithin, Berl. klin. Woch., 40, Nos. 2 and 4, I903; Venom, J. Inf. Dis., 7, I8 I, I9IO.

LABORDE: Salicyl, Bull. gen. Ther., 93, 276, 1 877.

LADE: Ethylsulphates, Z. physiol. Chem., 79, 331, г9г 2.

LaIDLAw: Hydrastis, Biochem. J., 5, 243, I9ro; Indolethylamin, Bioch. J., 6, I9I6.

LAITINER: Alcohol, Bioch. Centr., 7, 39, I907.

Lambert, S. W., and Patterson: Mercuric Chlorid, Arch. Int. Med., i6, 865, I9I 5.

LAMM: Veratrin, Z. Biol., 58, 37, r9 2 .

Lasson, P. D.: Helenium, J. Pharmacol., 4, 471, I913.

Lamson, P. D. and Keith: Epinephrin, J. Pharmacol., 8, 247, r916.

Lanari, R.: Pituitary, Ther. Mon., 29, 24I, 1915.

LANDAU, A.: Epinephrin, Z. klin. Med., 79, 201 I I9I4.

Landauer: Thirst, Ung. Arch. Med., 3, 136, 1895.

LandGREN and Tigerstedt: Caffein, Skand. Arch. Physiol., 4, $241,1892$.

LANe, W. A.: Paraffin, Brit. Med. J., 2, I I 26, I913.

LANGgaARD: Caffein, C. Med. Wiss., 513, 1886.

LANGgaARD and MaAss: Camphor, Ther. Mon., Nov., I907.

LANGE, K.: Thiosinamin, Inaug. Dis., Rostock, I 892.

LANGENDORFF: Heart, Arch. ges. Physiol., 61, 292, I 895.

LANGer: Morphin, Biochem. Z., 45, 221 , 239, I91 2; Scopolamin, Ther. Monatsh., 26, Feb., I912.

Langley, J. N.: Autonomic System, J. Physiol., I7, 224; 33, 374, I901; 43, I45, I9I 2; Curare and Nicotin, J. Physiol., 46; 47, I59; 48, 73, I913; Epinephrin, ib., 27, 237, I901; Sympathetic System, Zentr. Physiol., 27, 1913; Vascular Reflexes, J. Physiol., 45, 239, I9I 2.

LANGLEY and DiCkINSON: Nicotin, J. Physiol., II, I23, 265, 509, I89o.

LANGLEY and MAGNUS: Strychnin, ib., 36, I907.

LANGlois and Desbouis:" Pulmonary Circulation, J. Phys. Path. Gen., I4, III3, 1912.

LaNGlois and Garrelson: Epinephrin, Zentr. Bioch. Bioph., Io, 724, I9IO.

Langworthy, C. F., and Holmes: Fats, U. S. Dept. Agr. Bul., 3 IO, I91 5.

LANNoy: Pilocarpin, Bioph. Centr., I, 208, 1905.

LANZA and Vergano: Iodid, Zentr. Bioch. Bioph., I4, 602, I91 2.

LAPICQUe, L. and M.: Strychnin, Zentr. Bioch. Bioph., I5, 581 , I913.

LAPINSKI, J.: Ethylhydrocuprein, Ther. Mon., 29, I03, 1915.

LAPPONI, D.: Epinephrin, Zentr. Bioch. Bioph., I8, I63, I9I 5.

LAQUEUR, E.: Salicyl, Zs. physiol. Chem., 79, 38, I91 2.

LAQUeur, E., and EtTINGer: Arsenic, Zs. physiol. Chem., 79, I, I9I 2.

LAQuevr and Verzar: Carbon Dioxid, Arch. ges. Physiol., I43, 395, I9II.

LARGE and BRown: Ethyl Chlorid, Laryngoscope, Nov., I905.

LASAREw: Mercury, Zentr. Bioch. Bioph., I4, 573, I9I 2.

LA Torre, F.: Uterus, Gyn. Rundsch., 6, 359, 1912.

Laube: Hemolysis, Z. exp. Path., I0, 28, I9I I.

LAVERAn and Mesnil: Arsenic, Ann. Inst. Pasteur, I902.

LaWAlL: Camphor, Am. J. Pharm., 81, 54.5, I909.

LAWRENCE, C. H.: Hypertension, Am. J. Med. Sci., 72, 189, 1912; Arch. Int. Med., 9, 409, I9I2.

LAWROW, D. M.: Ricin, Bioch. Z., 54, I6, r913.

LAWrow and Woronzow: Lecithin, Arch. internat. Pharmacod., 22, 389, 1912.

Lazenby, W. S.: Arisaema, Sci. Mo., I, 272, I915.

Leathes: Phosphorus, Lancet, Feb. 3 , I909.

LebBin, G.: Formic Acid, Bioch. Centr., 6, 83, 1906.

LEBEDEFF: Phosphorus, Arch. ges. Physiol., 31 , II, 1883.

LECONTE: Uranium, Gaz. med. Paris, No. $13,1854$.

LEDoux and LEBARD: Thorium, Ref., Ther. Monatsh., 27, 789, 1913. 
1.1.1 : Viotin. Quart. J. Physiol., I, 355, r9o8.

I.EE, R. J.. and VexceNT: Calcium in Obstructive Jaundice, Arch. Int. Med., I6, 59, 1915: Cougulation. J. Med. Res., 32. No. 3, r9 5 .

L.FF and SALAXT: Alcohol, .Im. J. Physiol., 8, 6I, I902.

l.Fikk, li.: Hordenin, C. R., I42, I08; 143, 34, 916; I44, 488, 1906.

l. ricie: Ledd, J. Hyg., r, 96 , r901.

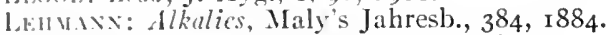

Lkum.1., E. P.: Cholesterul, J. Biol. Chem., I6, 495, i9i4.

Lemman, K. B.: Benzoule, Sci., 35, 576, I91.I; Brass, Arch. Hyg., 72, 358, 19ro;

Vickel, Jahrb. Pharm., 69, 148, r909; Nicotin, Bioch. Centr., 6, 757, 1907; Ref.,

Proc. Im. Pharm. Issoc., 58, 179, 1909.

LehmiNe and Gundermane: Nicotin, Zentr. Bioch. Bioph.; I3, 7 I5, I9I 2.

Leminis, K. B., and Trentleix: Sulphile, Ref., Proc. Am. Pharm. Assoc., 58, 235.

l.mindorff, 1.: Bromid, Irch. exp. Path. Pharm., 76, 236, 1914; Iodid, ib., 76, 224, 1914.

LeinerdT: Strontium, Zs. exp. Med., I, I75, I9I3.

LenNert and O. Loeb: Digitalis, Ther. Monatsch., 28, r64, 19r4.

Lemberger: Phenol-camphor, Ther. Gegenw., No. 5, i9o6.

LFNEl, R.: II diosit, Arch. exp. Path. Pharm., 77, 335, r914.

Lenulint, C. H.: Thyroid (Tadpoles), J. Exp. Med., 22, 739, 19г5.

Lrinartz: Saline, Deut. Arch. klin. Med., 64, 189, I 899.

LENNE: Ethyl-hydrocuprein, Berl. klin. Woch., I984, I9I3.

LENorr and Cames: Diurctics, J. de Physiol., 5, I 17, I903.

LEO, H.: Camphor, Deut. med. Woch., 39, 590, I9I3.

Leoncini, F.: Phosphorus, Zentr. Bioch. Bioph., I7, 391, 1914.

Lereboullet and Vauciner: Pcplone, J. Am. Med. Assoc., 62, 151 I, 1914.

Lescomifr and Closson: Pituitary, J. Mich. St. Med. Soc., r9 2 ; Secretin, J. Physiol., 44, xi; Zentr. Bioch. Bioph., I4, 37, I9 2.

LESNE and DREYFUS: Abrin, C. r. Soc. Biol., 432, I908.

LesNik: Salicyl, Arch. exp. Path. Pharm., 24, 167, i887.

L'Esperaxce and H. Савот: IIexamethylenamin, Bost. Med. Surg. J., Oct. 24, 1912.

Leuwen, W. S. van: Spinal Reflexes, Arch. ges. Physiol., I 54, 306, I913.

Leva, J.: Bromid, Zs. exp. Path. Ther., I0, 522, I9I 2 ; Chlorid, Zs. klin. M., 82, I, I9I5.

Levexe, P., and Stookey: Fcrments, J. Med. Res., 10, 217, 1903.

Levin: Antitoxin, Z. Immun., I, 3, I go9; Salicyl, Deut. med. Woch., 38, 2412, 1912.

Levin, I., and Larkin: Arteriosclerosis, Proc. Soc. Exp. Biol. Med., 7, ro9, rgio.

Levison, L. A.: Bicarbonate, J. Am. Med. Assoc., 64, 326, I9 5.

LEvy, A. G.: Chloroform, J. Physiol., 42, No. 2, I9I I.

LEvY, G.: Chloroform, Heart, 4, 320, I9I3.

I.EVY, G., and LEwis: Chloroform, Heart, 3, 99, r9i2.

Levy, L.: Papazerin, Ref., Ther. Monatsh., 28, 665, I9I4.

LEvy, R.: Calcium, D. med. Woch., 949, I9I4.

Levy, R. L.: Chenopodium, J. Am. Med. Assoc., 63, 1946, 1014.

Levy, R. L., and Rowntree: Buffer-Value, Arch. Int. Med., 17, 525, i 9 16; Emetin, J. Pharmacol., 8, I 20, I016; Arch. Int. Med., I 7, 420, I9 I6.

Levy, R. L., Rowntree and Marriott: Blood Alkalinity, Arch. Int. Med., i6, 389, I9I5.

Lewandowsky: Autonomic, Arch. Physiol., 360, i 899.

LEwin, C.: Acetosalicyl, Ther. Gegenw., 509, I912.

Lewin, L.: Ammonia, Berl. klin. Woch., H, 42, r9o8; Arbutin, Arch. path. Anat., 92, 517, I883; Cactus, Arch. exp. Path. Pharm., 24, 401; 34, 374, 1888; Hydroxylamin, Arch. exp. Path. Pharm., 25, 306, 1889 .

LEwis, A. B., and Frankel: Inulin, J. Biol. Chem., 17, 365, 1914.

LEwIS, H. B.: Hippuric Acid, J. Biol. Chem., 18, No. 2, 1914.

LEwIS, J. H.: Epinephrin, J. Biol. Chem., 24, No. 3, 1916.

Lewis, P. A., and Krauss; Iodin, J. Biol. Chem., I8, 3 I3, I9I4.

Lewis, Miller and Matrhews: Pituitary, Trans. Assoc. Am. Phys., 26, 126 , 1911.

Lнота: Digitalis, Arch. internat. Pharmacod., 20, 369, 451, 1910; Bioch. Z., 48, I44, rgr 2; Arch. intern. Pharmacod., 23, 307, 1913.

Liagre: Strophanthus, J. de Phys. Path., 8, No. 6, 1906.

Liвby, W. E.: Narcosis, Z. exp. Path., 7, 725, 1910; Volatile Oils, Arch. exp. Path. Pharm., 42, 356, I899.

Licutenstein: Alypin, Ther. Gegenw., r6, r9r4.

Lichiwitz, L.: Citrate, Ref., J. Am. Med. Assoc., 56, 785, r9r1; Colloids, Deut. med. Woch., 36, 704, I910 
Lieb, C. C.: Alcohol, J. Am. Med. Assoc., 64, 898, i9i 5; Ecbolics, Am. J. Obst., 69, I, I9 4 ; Pituitary, Soc. Exp. Biol. Med., I 2, 8, I9r4; Uterus (Iuman), Am. J. Obstet., 7 I, No. 2, I9I5.

Lieb, C. C., and Goodwix: Mercury, J. Am. Med. Assoc., 64, 204I, I9 I5.

Lieb, C. C., and McWhorter: Gall Bladder, J. Pharmacol., 7, 83, I9 I 5.

Liebmann: Camphor, Arch. exp. Path. Pharm., 68, I9 2.

Lifschitz, A. J.: Caffin, Bioch. Centr., 7, 45, 1907.

Lifscruetz, J.: Cholesterin, Zs. physiol. Chem., 91, 5, i91 4.

Likhatcheva, N. P. Camphor, Ref., J. Am. Med. Assoc., 67, 843, 19 I6.

Lillie, R. S.: Anesthesia Theory, Biol. Bul., 30, 3 I I, i9 г6; Arenicola, Am. J. Physiol., 7, 25, I902; Narcosis and Salts, Am. J. Physiol., 30, I, I9 1 2; ib., 31, 255, 1913; Sci., 37, 959, I9 13; Nerie Conduction, Am. J. Physiol., 37, 348, I9 I 5.

Limbek, V.: Potassium, Arch. exp. Path., Pham. 30, i 80, I 892.

Lindemanx: Narcosis, Z. exp. Path., 7, 725, I9 10; Volatile Oils, Arch. exp. Path. Pharm., $42,356,1899$.

Lindenmayer: Dionin, Berl. klin. Woch., 796, I9I 2.

LINDHARD: Morphin, J. Physiol., 42, 337, I9I I.

Lindsay: Chloroform, Bioch. J., 5, 407, I9I I.

Linenthal, H.: Lead, J. Am. Med. Assoc., 62, I 796, I9i4.

Linossier: Diffusion, J. Am. Med. Assoc., 56, I 274, 19 I I.

LIPpens: Camphor, Arch. intern. Pharmacod., 2I, II9, I9II; Morphin, ib., I8, 203, I 908.

Lipowitz: Lithium, Ann. Chem. Pharm., 38, 348, I84 I.

Lisix: Nitrice, Arch. internat. Pharmacod., I 7, 484, I907.

Lissauer, M.: Alcchol, Deut. med. Woch., 18, Jan., i9r3; Heart Ganglia, Arch. Path., 218,263 , I914; Veratrun, Arch. exp. Path., Pharm., 23, 36, I887.

Livifrato: Formates, Bioch. Centr., 5, 247, I906.

Lloyd, J. U.: Cocain, History of Vegetable Drugs, Cincinnati, rgr ; Echinacea, Am. J. Pharm., 76, 15, Ig04; Fullers' Earth, J. Am. Phar. Assoc., 5, 381, 19 I6.

Locke: Heart, Centbl. f. Physiol., i 4, 670, г9or.

Lockyer: Cctarnin, Ann. Gyn. Ped., Oct., I907.

Lodholz: Sulphecyanid, U. Pa. Med. Bul., 18, 279, I905.

Loeb, J.: Acids, Diffusion, J. Biol. Chem., 23, 139, I9 15; Cyanid, Bioch. Zs., 29, 80, I910; Ion-antagonism, J. Bioch., I9, 43I, I9I4; Parthenogenesis, Am. J: Physiol., 3, I35, I899; ib., 4, 423, I901; Rythmic Muscles, ib., 3, 329, r900; Salts, Bioch. Zs., $3 \mathrm{I}, 450 ; 32$, I 55, 308, I9II.

Loeb, J., and.BeutNer: Narcosis, Bioch. Zs., 5 I, 300, I9I3.

Loeb, J., and Cattell: Polassium, J. Biol. Chem., 23, 4I, I9 I5.

Loeb, J., and Wasteneys: Disintoxication, Bioch. Zs., 33, 480, I9 I ; $\mathrm{NaCl}$, Bioch. Zs., 28, 350, 1910; Narcosis, Bioch. Zs., 56, 295, 1913.

Loeb, L.: Ether, U. Pa. Med. Bull., Nov., r9o6.

Loeb, L., and Fleisher: Iodid, Deut. med. Woch., 33, 382, I907.

Loeb, L., and Meyers: Ether, Arch. path. Anat., 201, 78, 1910.

Loeb, L., Strickler and Tuttle: Sera, Arch. path. Anat., 201, 5, i9ro.

Loeb, O.: Alcohol, Arch. exp. Path. Pharm., 52, 459, 1905; Iodid, Arch. exp. Path. Pharm., 56, 320, 1907; ib., 69, 108, I91 2; Nitrite, ib., 51, 64, 1903.

Loeb, O., and Michaud: Icdid, Bioch. Zs., 3, 1907.

Loeb, O., and Stadler: Secretin, Arch. exp. Path. Pharm., 77, 326, I9I4.

LoEhlein: Bile Salts, Merck's Rep., 26, I43, I909.

Loening: Atophan, Ther. Monatsh., 27, I23, I9 3.

Loeper, et al: Gastritis from Asphyxiating Gases, J. Am. Med. Assoc., 65, 562, I915.

Loevenhart, A. S.: Oxidation, Arch. Int. Med., 15, 1059, 1915.

Loew, O.: Atoxyl, Arch. ges. Physiol., 40, 437, I887; Calcium, Bioch. Zs., 38, 226, I9I 2; Formaldehyde, Muench. med. Woch., 35, 41 2, I888; Salts, U. S. Agric., Veg. Phys. Bul., I 8 , I 890 .

Loewe, S.: Narcosis, Bioch. Zs., 57, I6r, I9I3.

Loewr, O.: Caffin, Sitzb. Marburg, July, 1904; Chloral, Arch. exp. Path. Pharm., 70, 322, 1912; Diuresis, ib., 48, 410, 1902; Muscarin, ib., 70, 343, 19 2; Strophanthin, Chem. Abstr., 7, 843, r9 12; Vagus, Arch. exp. Path. Pharm., 70, 322, r9 2.

Loewi and Jonescu: Digitalis, Arch. exp. Path. Pharm., 59, I908.

Loewi and H. Meyer: Suprarenal, Arch. exp. Path. Pharm., 53, 2 I3, igo5.

Loewi and Neubauer: Phlorhizin, Arch. exp. Path. Pharm., 59, I908.

Loewrt, M.: Anaphylaxis, Arch. exp. Path. Pharm., 73, 1, I9 3 .

Loewy, A.: Acidosis, Centr. Physiol., 20, No. 10, 1906; Morphin, Arch. ges. Physiol., $47,601,1890$.

Loewy, A., and·v. D. Herde: Methyl-alcohol, Bioch. Zs., 65; 230, I9r4. 
L.okWr, .1., and MÜllek: Yohimbim, Münch. med. Woch., No. I5, I903.

Lolili and MlXzer: Acids, Arch. Physiol., 8I, I90I.

Lomw, .1., and Kosenberg: Epinephrin, Bioch. Zs., 67, 323, 1914; I'ohimbim, Arch. exp. Path. Pharm., 78 , ro8, igr4.

LowY, 1., and Wronselmax: Salvarsan, Berl. klin. Woch., I342, I9r3.

LoEwY, J.: Digitalis, Bioch. Centr., 5, 805, x 906.

Lominiart, I. S.: Ammonium, J. Pharmacol., 5, 5I5, I9I4.

l.olitinist and Eyster: Heart, J. Pharmacol., 5, 21, I9I3.

Lonnax: Neurin, Arch. ges. Physiol., I28, I42, I9I ; Suprarenals, Arch. ges. Physiol., II $8,215.1907$.

Lombard: Alcohol, J. Physiol., i3, i 8ç2.

L.OMBaRd, A.: Strychnin, Zentr. Bioch. Bioph., 16, 625, 19г3.

Lombardo, C.: Mercury, Zentr. Bioch. Bioph., 13, 715, 1912.

Lo Moxaco and PANichi: Quinin, Arch. Farmacol., 157, 1899.

Lomososoff: Antipyretics, Z. exp. Path., 8, 566, I9I I.

Lovg, J. H.: IIydrochioric Acid, J. Am. Chem. Soc., 3i, No. 5, 1915; Preservatives, Trans. Internat. Congr. Hyg., I9I 2.

Loxg, J. H., and Hull: Protein, J. Am. Chem. Soc., 37, No. 6, I9I 5.

Long, J. H., and Muhlman: Ferments, Arch. Intern. Med., 13, 314, I9I4.

Loxgcope, W. T.: A naphylaxis, Trans. Assoc. Am. Phys., 28, 497, I913; J. Exp. Med., 22, 793, 1915; Syphilitic Aortitis, Clev. Med. J., 13, I4I, I914.

Loxgcope, W. T., and Rackemaxn: Serum Disease, Soc. Exp. Biol. Med., I3, ro I, rg I6. LORIA, G.: Diuretics, Zentr. Bioch. Bioph., I3, 174, I912.

Lowiv: I pecac, Arch. intern. Pharmacod., II, 9, I902.

Lu'Be, F.: Salvarsan, Deut. med. Woch., 946, I9I4.

LuCAS, D. R.: Benzoute, J. Am. Med. Assoc., 54, 759, I910; Ureter, Am. J. Physiol., I 7, 302, 1906; N. Y. Med. I., Aug. Io, I907.

LucAs, W. P.: Strychnin, Am. J. Dis. Child., 7, 208, I9I4.

Luce, R. V. and Hamilton: Anilin, J. Am. Med. Assoc., 66, ז44I, I9I6.

Ludewig, H.: Narcosis, Arch. intern. Pharmacod., 23, 479, I913.

Luebs: Ocular Tension, Zentr. Bioch. Bioph., 15, 95, 1913.

Luethje: Salicyl, Deut. Arch. klin. Med., 74, 163, 1902.

LuETz, C: Synthetic Camphor, Berl. klin. Woch., 52, No. 14, 1915.

LUFF: Calcium, Brit. Med. J., I, 26I, I909.

Luitilen, F.: Calcium, Arch. exp. Path. Pharm., 68, 209, I9I2; Cutaneous Reactions, Wien. klin. Woch., No. 20, I9II; Arch. exp. Path. Pharm., 69, 365, I9I2; Salvarsan, Zs. exp. Path., I3, I, 495, I9I3; Skin Reactions, Wien. klin. Woch., 26, No. 17, 19г3.

Lusk, G.: Acidosis, J. Am. Med. Assoc., 66, 144, I9r6; Diabetes, Arch. Int. Med., Feb., I909; Epinephrin, Proc. Soc. Exp. Biol. Med., II, 49, I914; Foods, J. Am. Med. Assoc., 64, 1717, 1915; Phosphoris, Am. J. Physiol., 19, 461, 1907.

Lusini and Sebastiani: Transfusion, Bioch. C., 5, 446, 1906.

Lussky: Thyroid, Am. J. Physiol., 32, 63, r9r2.

Lussana, F.: Cholin, Zentr. Bioch. Bioph., I4, 792, I913; Perfusion, ib., I5, 925, 1913.

Lutz, C.: A piols, Proc. Am. Pharm. Assoc., 58, 380, 1914; Camphor, Berl. klin. Woch., 322, I915.

Luzzato: Nephritis, Zs. exp. Path., 16, I8, 1914; Vanadium, Arch. Farmacol., 1903.

Lyle, H. H. M.: Scarlet Red, Med. Rec., 82, Nov. I6, I9I 2.

LyMAN: Ca on Urine and Fcces, J. Am. Med. Assoc., 65, 556, I9I 5.

Lynch, F. W.: Nitrous Oxide, J. Am. Med. Assoc., 64, 81 3, 1915.

Lyon, E. P., and Qualls: Cactin, J. Am. Med. Assoc., Aug. 6, 455, 1910.

MaAss: Ethyl Chlorid, Ther. Monatsh., 21, 303, 1907.

Macallum, A. B.: Chlorid, Proc. Roy. Soc., B, 76, 217, 1905; Electrolytes, J. Physiol., 32, 95, 1905; Energy, Am. Soc. Biol. Chem., 2, 97, I913; Iron, J. Physiol., 16, 268, I894.

Macallum, A. B., and Menten: Chlorid, Proc. Roy. Soc., B, 77, 165, 1906.

MacCallum, J. B.: Atropin, U. Cal. Publ. Physiol., 2, I05, I904; Cascara, ib., I, I63, 1904; Hemolytic Diuretics, Univ. Cal., Physiol., 3, No. I2, 1905; Pilccarpin, Univ. Cal. Publ. Physiol., 2, 105, 1905; Saline Cathartics, Am. J. Physiol., 10, 101, 259; 1906.

MacCallum, W. G., and Voegti.in: Calcium, J. Exp. Med., II, II8, 1909.

Macrarlan: Mercury, J. Am. Med. Assoc., 62, I 7 , I9 4 .

MacGilchrist, A. C.: Cinchona Alkaloids, Ref., J. Am. Med. Assoc., 65, 1588, 1915.

Machi, D. I.: Arterial Rings, J. Pharm. Exp. Ther., 6, 591, I9I5; Emmenagogue Oils, J. Pharmacol., 4, 547, 1913; Intravenous and Subcutaneous Administration, J. Am. 
Med. Assoc., 66, 856, 19 16; Iodids, ib., 5, 514, 1914; Nitrites, J. Am. Med. Assoc., 62, 524, I914; Opium Alkaloids, Respiration, J. Pharm. Exp. Ther., 7, 339, I915; J. Am. Med. Assoc., 64, I489, 19 5; Opium History, ib., 64, 477, 1915; Papaverin, Arch. Int. Med., I 7, 786, 1916; Phenol, Johns Hopkins Hosp. Bul., 26, 98, 19 5 ; Urcter, J. Pharmacol., 8, I II, I55, 26 I, I9I 6.

Macht, Herman and Levy: Opium A!kaloids Analgesia, J. Pharm. Exp. Ther., 8, I, I9I6.

Mackenzie: Digitalis, Heart, 2, 273, r9i i Pituitary, Quart. J. Physiol., 4, 3i 5 , I9 I I.

MacLachlan: Formaldehyde, Cleveland Med. J., Oct., I909; Salicyl, J. Am. Med. Assoc., 6i, 116, 1913.

Mac LeAN, H.: Lecithin, Bioch. J., 9, 35 I, I9 I5.

Macleod, J. J. R.: Alkali, Soc. Exp. Biol. Med., r3, 169, 19 16; Diabetes, J. Am. Med. Assoc., 62, I 222, 1914.

Macleod and Haskins: Citrates, J. Biol. Chem., I, I906.

Macleod and Pearce: Epinephrin, Am. J. Physiol., 29, 419, igr 2; Proc. Biol. Chem., 2, 54, I91 2; Clev. Med. J., I3, 837, 1914.

MacNider: Anesthetics, J. Pharmacol., 4, 348, 49г, I9г3; Nephritis, J. Med. Res., 26, 79, I91 2; Spartein, Ref., J. Am. Med. Assoc., 55, I I48, 1910; Uranium, J. Pharmacol., 3, 423, I9r 2; Soc. Exp. Biol. Med., Ir, I59, I914; J. Pharmacol. 6, I23, I914; ib., 6, 599, I9 5; Uranium and Alkali, J. Exp. Med., 23, I7 1, I9 6.

Madelung: Narcosis, Arch. exp. Path. Pharm., 62, 414, I9I0.

Maestro: Cocain, Sperimentale, 48, 599, 1904.

Maggiora and FerRon: Saponin, Zentr. Bioch. Bioph., 18, I99, I914.

Magne, H.: A pomorphin, Soc. Biol., 77, 328, r9i4.

Magnus, R.: Ammonia, Arch. exp. Path. Pharm., 48, 100, 1902; Arscnic, ib., 42, 265, 1899; Autonomic System, Arch. ges. Physiol., 102, I 23, 349; 103, 5 I5, I904; ib., I 23, 99, I908; Cathartics, Ther. Monatsh., 23, Dec., I909; Digestive Movements, Erg. Physiol., II, 2, 637, I905; Arch. ges. Physiol., I08, I, 1905; Emesis, Arch. ges. Physiol., r57, r914; Heart Perfusion, Arch. exp. Path. Pharm., 47, 200, 1902; Morphin, Arch. ges. Physiol., II5, 316, I906; Phosphorus, Viertj. ger. Med., 47, No. 2, 1914; Urine Mechanism, Erg. Physiol., I 908.

Magnus-Levy: Diabetic Coma, Arch. exp. Path. Pharm., 42, 149, I899; ib., 45, 389, IgoI.

Magnus, R., Sorgdrager and Leeuwex: Ammonia, Arch. ges. Physiol., i55, 275, I9I4.

Magyary-Kossa: Carbon Dioxid, Arch. int. Pharmacod., 20, 471, igio.

Malinowsky, M.: Pituitary, M. S. Geb., 40, I, I9I4.

Manley, O. T., and Marine: Thyroid, Soc. Exp. Biol. Med., I2, 202, 1915; Transplantations, J. Am. Med. Assoc., 67, 270, I9 I6.

Mann, F. C.: Anesthesia, J. Am. Med. Assoc., 67, 172, I916.

ManN, F. C., and DrIPA: Adrenals, Arch. Int. Med., I6, 681, I915.

Mansfield: Narcosis, Arch. intern. Pharmacod., I5, 467, I905; Arch. ges. Physiol., I 29,69 , I909.

Mansfeld and Bosanyi: Magnesium, Arch. ges. Physiol., I52, 75, i 913.

Mansfeld, G., and Hamburger: Synergism, Arch. ges. Phys., 161, 444, 1915.

Mansfeld, G., and PurJesg: Epinephrin Glycosuria, Arch. ges. Phys., i6r, 506, 1915.

Manwaring: Anaphylaxis, Trans. Congr. Phys. Surg., 8, 392, I910; Cobra Lecithid, ib., 8, 398, 1910; Venom, Zs. Imm., 6, 5 13, 1910.

Manwaring, Meinhard and Denhart: Anaphylaxis, Soc. Exp. Biol. Med., I 3, i 75, I9ı6; Heart, ib. I3, 173, 19г6.

Manwaring and Rum: Quinin, J. Exp. Med., 9, 473, r907.

Maranon, G.: Atophan, Ref., J. Am. Med. Assoc., 63, 2254, 1914.

Marcelar: Anesthetics, Ref., Yr. Book, Am. Phar. As., r, 359, 1912.

Marchand: Chlorates, Arch. exp. Path. Pharm., 22, 201, I886; ib., 23, 273, I887.

MARchetTi, G.: Ether on Suprarenals, Ref., J. Am. Med. Assoc., 65, 370, I9I 5.

MARCUS: Water, Berl. klin. Woch., 44, 330, I907.

MAREK: Hydrastis, Wien. med. Woch., 2 I 46, I9I I.

MARFORI: Hydrastis, Arch. exp. Path. Pharm., 27, I6I, I89o.

MARIE: Epinephrin, Zentr. Bioch. Bioph., I5, 79, I9I3.

MArine, D.: Basedows, Clevel. Med. J., 12, 21, r913; Iodin, J. Am. Med. Assoc., 59, 325, I91 2; J. Biol. Chem., 65, I 757, I9 1 5; Iodin Absorption, J. Exp. Med., 22, 547, I9 I5; Parathyrcid, Soc. Exp. Biol. Med., I I, I 1 7, I914; Thyroid, Johns Hopkins Hosp. Bull. 1 8, 198, I907; Soc. Exp. Biol. Med., I 2, I 32, I91 5.

Marine, D., and Feiss: Thyroid Perfusion, J. Pharmacol., 7, 557, I915.

MARINe and Rogoff: Iodin Absorption, J. Phrmacol., 8, 439, 1916.

Marine and Williams: Thyroid, Arch. Intern. Med., May, igo8. 
MIRks, L. II.: Arsenic, Zentr. Bioch. Bioph., Io, 627, igro; Chemotherapy, J. Am. Mirl. Issoc., 55, 1974, 1910.

MIRhMALER and SirARLAg: Coronary Circulation, J. Physiol., 47, 275, I9I3.

MIRME: Morplin, Deut. med. Woch., No. I4, I883.

M.RRITT. II. M.: Acetone, J. Biol. Chem., I8, 24I, I9I4; Acidosis, ib., I8, 507, I914; Alkali-Rescrve, Arch. Int. Med., I7, 840, 1916; Hypophosphites, J. Am. Med. 1sisoc, 60, ti66, I9I6.

V. M.irschilko and Veszpremi: Solvarsan, Ref., Ther. Mon., 27, 25.5, I9I 2.

il

Miksil, C. D.., Claissox and Marsh: Zygadenus, Bur. Plant. Ind. Bul., No. I 25, 1915.

Marshial, C. R.: Brom-strychnins, VIII Int. Congr. Appl. Chem., r9, 2 I 7; Cannabis; Pharm. J. and Pharm., March 27, rgog; Chemic Constitution, ib., May 3, I9 13, Coriamyrtin, J. Pharmacol., 4, I35, I9 I2; Jaborandi, J. Physiol., 3 I, I 20, I904; Methyl-cthyl Ammoniams, Tr. Roy. Soc. Edinb., 50, II, I 7, I9 5; Nitric Esters, VIII Int. Congr. Appl. Chem., I9, 2 I I, I91 2; Nitrates organic, Med. Chron., Sept., I899; Chem. Abstr., 6, 2955, 19I 2; Papaverolin, Brit. Med. J., Sept. 21, I9I 2; Protocatechyltropein, Tr. Roy. Soc. Edinb., 47, II, I2, I910; Tetramethyl-ammonium, ib., 50, 1, 2, I9I3; Tetraethyl-ammonium chlorid, ib., 50, II, I2, I914; Tutu, ib., 4\%, II, I3, I9IO.

Marshil., C. R., and Gilchrist: Sp. Acth. Nitros, Brit. Med. J., July 24, 1915.

Marshali, C. R., and Killoh: Collosols, Brit. Med. J., Jan. I6, ig I 5.

Marshal., E. K.: Iirudin, J. Pharmacol,, 7, I57, I9I5; Phospherus, J. Am. Med. Assoc., 64, 573, I9I5.

Mirshall, E. K. and Rowntree: Phosphorus and Chloroform, J.Exp. Med., 22,333, I9 I5.

Marshali, H. T.: Loco, Johns Hopkins Hosp. Bull., 25, 234, 19I4.

Martis, E. G., Grace and McGure: Acetphenetidin, J. Pharmacol., 6, 527, igr5.

Martis, E. G., and Stiles: Curare, Am. J. Physiol., 34, 220, I9I4.

Martin, G. H., Bunting and Loevenhart: Reduced Oxidalions, J. Pharmacol., 8, I1 2, I9I6.

Martinet, A.: Digitalis, XVII Int. Congr. Med., 5, 2, 232, I9I4.

MARx: Quinin, Arch. exp. Path. Pharm., 54, 460, 1906.

MASEl: Acidosis, Zs. klin. Med., 79, r, r9 3 .

Masoli, P.: Sojourn, Arch. intern. Pharmacodyn., I I, 465, I903.

Masslow, M.: Phosphorus, Bioch. Zs., 64, I06, I9I4.

Massol and Gammel: Hypophosphites, Ref., J. Am. Med. Assoc., 62, 1347, rgor.

Massol. and Minet: Salicyl, C. R. Soc. Biol., 64, No. ro, rgo8.

Matiews, A. P.: Anesthetics, Int. Zs. Physiol. Chem. Biol., I, 433, r914; Antitoxic Action, Am. J. Physiol., I 2, 4 I9, I905; Airopin, ib., 4, 482, I901; Dyes, J. Pharmacol., 2, 213, I910; Ions, Science, I5, 492, I902; Am. J. Physiol., Io, 290, I904; ib., II, $455 ; 14,203$, 1905.

Mathells, A. P., and Longfellow: Dyes, J. Pharmacol., 2, 20I, I9io.

Matrieu and Girati: Atropin, Ther. Monatsh., 27, 602, 1913.

Matuison, G. C.: Asphyxia, J. Physiol., 4I, 4I6, I9I0; Heart, 2, 54, r9ro; J. Physiol., 42, 283; Pcuassium, ib., 42, 47x, r91

Matruews, S. A.: Aconite, J. Exp. Med., 2, 593, I897; Cactus, J. Am. Med. Assoc., March 21, 956, 1908; Cannabis, ib., 51, I780, r008; Magnesium, J. Pharmacol., 2,87, 1910.

Mattinews, S. A., and Brooks: Magnesium, J. Pharmacol., 2, 87, I910.

Matthews, S. A., and O. H. Brown: Physostigmin, Am. J. Physiol., 1 2, 173, 1904.

Matros: Salicyl, Zentr. Bioch. Bioph., 4, 633, I905.

Matzel: Volatile Oils, Arch. intern. Pharmacod., i 5, 331, I905.

Maurel, E.: Emetin, Arch. Med. exp., 26, 225 , I9I4.

Maurel and Arnaud: Colchicin, Bioch. Centr., 10, Io and 62, Igro.

Maurer: Quinin, D. med. Woch., 33, I73, I907.

Mautner, H., and Pick: Anaphylaxis, Muench. med. Woch., 62, No. 34, II 4 I, I9 I5. Maxwell, S. S.: Pituitary, Calif. Publ. Physiol., 5, 5, 1916.

May, E. S.: Fuchsin, J. Am. Med. Assoc., 58, i I 74, I9 2.

May, R.: Salicyl, Brit. Med. J., 2, 79ı, rçog.

MAYER, C.: Hypochlorite, Ref. J. Am., Med. Assoc., 66, 988, I9 6.

MaYer, P.: Morphin, Berl. klin. Woch., No. 27, 1899.

MaYer and Friedrich: Nitrite, Arch. exp. Path. Pharm., 5, 55, 1875

Mayeriofer and Pribram: Intestines, Bioch. Centr., 9, 760, igog.

Mayor: Morphin, Rev. med. Suisse Rom., rgo8.

McClesve, T. C.: Orthoform, J. Am. Med. Assoc., 63, 1666, 19 14.

McClendon, j. F.: Acidity Curves, Am. J. Physiol., 38, igr, I9i5; Anesthetics, ib., 38, I 73, I915; Frog Eggs, ib., 38, 163, 1915. 
McClintock, Hamilton and Lowe: Insecticide, J. Am. Publ. H. Assoc., Apr., I9i i.

McClintock and Hutcinngs: Tetanus, J. Inf. Dis., I3, 309, I9I3.

McClintock and Kisg: Antitoxin, J. Inf. Dis., 6, 46, igo9.

McClurg, C. B., Sweek, Lyon, Fleisiler and Leo Loeb: Copper. and Casein, Arch. Int. Med., I5, 975, 1915.

McCollum, E. V., and Davis: Butter, J. Biol. Chem., 15, 167, 1913; Lipins, Soc. Exp. Biol. MIed., II, IоI, I014; Salts, Am. Soc. Biol. Chem., 2, г 28, I913.

McCollum, Harpin and Drescher: Lecithin, J. Biol. Chem., I3, 2 I9, I9 2.

McCollum, Simmonds and Fitz: Fat-Soluble A, Soc. Exp. Biol. Med., I3, I 29, I9 I6; Vegetarian Diet, Am. J. Physiol., 4I, 333, I9 I6.

McConvel.t, J. W., and Spiller: Carbon IIonoxid, J. Am. Med. Assoc., 59, 31 22, i 912.

McCord, C. P.: Fetal Glands, J. Biol. Chem., 23, No. 2, 1915; Luteum, J. Am. Med. Assoc., 62, 1 250, 1914; Pineal Gland, J. Am. Med. Assoc., 65, 517, 1915; Interst. Med. J., 22, No. 4, I9I 5 ; Pituitary, Arch. Intern. Mled., 8, 609, I9I I.

McCrae, John: Oxygen, Trans. Ass'n Am. Phys., 29, iri I I9I4.

MCCRUdDen: Morphin, Arch. exp. Path. Pharm., 62, 374, 1910.

McDonald, E.: Lutein, J. Am. Med. Assóc., 55, 205, 1910; Quinin, J. Am. Med. Assoc., 64, 505, 1915.

McGill: Beef Extracts, Lab. Inland Reven. Dept., Ottawa, Can., I899.

McGuigan, H.: Ammonia, J. Pharmacol., 4, 453, I913; Atropin and Pilocarpin, J. Pharmacol., 8, 407, 1916; Curare, ib., 8, 471, 1916; Formaldehyde, Proc. Biol. Chem., 2, 67, 19г 2; J. Am. Med. Assoc., 62, 984, 1914; Prescriptions, ib., 61, 616, I91 3; Strychnin, ib., 63, I933, I9 4.

McGuigan and BEChT: Strychnin, J. Pharmacol., 5, 469, т9r4.

McGuigan, Keeton and Sloan: Strychnin, J. Pharmacol., 8, I43, I9 I6.

McGuigan and Mostrom: Epinephrin, J. Pharmacol., 4, 277, 1913.

MCGuigan and Ross: Morphin, J. Am. Med. Assoc., 64, 1494, I915; J. Pharm. Exp. Ther., 7, 385, I91 5; Peptone, J. Biol. Chem., 22, 41 7, 1915.

McIver, J., and Price: Drug Addictions, J. Am. Med. Assoc., 66, 476, I9I6.

McLean, F. C.: Chlorides, Am. J. Physiol., 36, 357, I915; of plasma in uremia, Soc. Exp. Biol. Med., 13, I66, I916; retention in pneumonia, J. Exp. Med., 2 2, 2 I 2 366, I915; Iodin, J. Am. Med. Assoc., 63, I382, I9I4; Arch. Int. Med., I5, 92, I91 5.

McLean, J.: Kephalin, Am. J. Physiol., 4I, 250, I9I6.

McLester: Atophan, Arch. Int. Med., I2, 739, 1913.

MCNeIL: Hemolysis, Zentr. Bioch. Bioph., i I , 605, I9 1о; J. Path. Bact., I5, 56, I9ro.

McPherson, R.: Scopolamin, Ohio S. Med. J., I I, 7, I9I5.

Means, J. H., and Newburgh: Caffein, J. Pharm. Exp. Ther., 7, 449, I9г 5.

Medigreceanu: Chlorid, J. Exp. Med., I4, 289, I9I I.

MEEK: Heart, Am. J. Physiol., $2 \mathrm{I}, 230$, 1908.

MEex, W. J., and Eyster: Adrenalin, Am. J. Physiol., 38, 62, 1915.

Meigs, E. B.: Ca and Mg Phosphates, Am. J. Phys., 38, 456, I9r 5 ; Muscle, Qu. J. Exp. Physiol., 5, 191 2; J. Exp. Zool., I3, I912; Venus, J. Biol. Chem., 17, I9I4.

Meigs, E. B., and Atwood: Potassium, Am. J. Physiol., 40, 3०, 1916.

Meissner, R.: Arsenic, Zs. exp. Path., I3, 284, I913; Narcophin, Bioch. Zs., 67, 502, I9I4; Opium Alkaloids, Bioch. Zs., 54, 395, 1913.

Mejia: Salvarsan, Ref., J. Am. Med. Assoc., 62, 582 , I9I3.

Mellin: Pulmonary Circulation, Skand. Arch. Physiol., 15, I47, 1904.

Mei.LoN, R.: Lead, Arch. Int. Med., I 2, 399, I913.

Meltzer, S. J.: Anaphylaxis, J. Am. Med. Assoc., 55, I02I, I910; Cardiectomized Frogs, J. exp. Med., I3, No. 4, I9I I; Epinephrin Absorption, Rockefeller Inst., 9, 1909; Infantile Paralysis, J. Am. Med. Assoc., 67, 314, 1916; N. Y. Med. J. Aug. 19, I9 16; Magnesium, Proc. Soc. Exp. Biol. Med., 4, I55, 1907; Trans. Assoc. Am. Phys., 29, 247, I914; Arch. intern. Med., I5, 955, x9 I4; Magnesium Dinresis, ib., 4, 1 54, 1907; Tetanus, J. Am. Med. Assoc., 66, 931 , г9 16.

Meltzer and Auer: Autonomic System, Am. J. Physiol., 20, 259, 1907; Ca, Mg, Sr., Am. J. Physiol., 21, I908; Epinephrin Absorption, Trans. Assoc. Amer. Phys., 1904; Epinephrin on Pupil, Am. J. Physiol., I1, 28, I91 2; Intramuscular Injection, J. Exp. Med., 7, I, I905; Magnesium, ib., May 25 and Dec., I906; Am. J. Physiol., I6, 233; I7, 313, 1906; Proc. Soc. Exp. Biol. Med., 5, 33, 1907; Magnesium. Anesthesia, General, Am. J. Physiol., I4, 366 ; Local, Soc. Exp., Biol.Med., Nov., I905.

Meltzer and Gies: Sirychnin, Am. J. Physiol., 9, I, I903.

Meltzer and GrTHExs: Ergotoxin, Soc. Exp. Biol. Med., I3, 87, г9 г6.

Meltzer and Lucas: Magnesium Excretion, J. Exp. Med., May 25, igo7.

Meltzer, S. J., and Salant: Bile, J. Exp. Med., I905.

Mended, F.: Intravenous Therapy, Berl. klin. Woch., H, 49, rgo8; Salicyl, Muench. med. Woch., 52, 165, 1905. 
Mr vin,., I., B.: Cholesterol, J. .Im. Med. Assoc., 63, 819, I914; Nutrition, J.Am. Med. 1sisoc., (4. 1539.1915 .

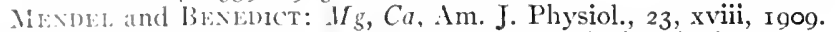

Mrvis., L. B., and Broon: Papain, Zentr. Bioch. Bioph., r I, r88, roro.

Mrivel, and IInditcis: Alcohol, Am. J. Physiol., 27, I, I9Io.

Mrivilu and Kinx: Caffein, J. Pharmacol., 4, 343, I9I3.

MlNolit and Kleiner: Foods, An. J. I'hysiol., 26, 396, rgio.

Mribli. and Mitcinels: Carbohydrates, Am. J. Physiol., I4, I905.

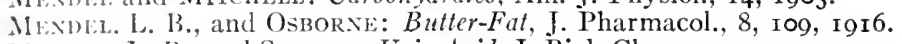

Mexple, L. B., and Stenle: Úric Acid, J. Biol. Chem., 22, 2 I5, 19 I5.

Mixpelsomx: Piperazin, Berl. klin. Woch., Nos. I6 and 34, I892.

Mlindexilate, W. L.: Anesthetics, Am. J. Physiol., 38, 33, I9I5.

Mrefgrza, R.: Ammonium, Zentr. Bioch. Bioph., I5, 277, I912; Formaldehyde, Arch. Farmacol., I4, 4II, I9I 2 .

Mexges: Vagina, Z. exp. Path., 2, 391, 1906.

Mretrs, M. L.: Anesthesia, Am. J. Physiol., 31, 85, I9 2; Gastric Juice, J. Biol. Chem., 22, 34I, I9I 5 .

Mentex, M. L., and Crile: Anesthesia, Am. J. Physiol., 38, 225, r9 5.

Merck, E.: Cocain, Merck's Rep., 21, 79, r907; Sulphatc, Arch. int. Pharmacod., I6, $301,1906$.

v. Merixg: Mercury, Arch. exp. Path. Pharm., 13, 86, 1880; Phlorhizin, Z. Klin. Med., I4, $405 ;$ I 6,431, I 886.

Merkel: Quinim, Arch. exp. Path. Pharm., 47, I65, 1902.

Messner: Alcohol, Zentr. Bioch. Bioph., I5, I83, I9I3.

Metzier: Atropin, Arch. exp. Path. Pharm., 68, r 10, I9r 2.

Metzeser and Hedinger: Atropin, Arch. exp. Path. Pharm., 68, I 10; 69, 272 , I9I 2.

Meurice, J.: Cyanid, Arch. int. Pharmacod., 7, II, I900.

Meyer, A. L., and Meltzer: Insufflation in Fouls, Soc. exp. Biol. Med., 13, 64, I9 5.

Meyer, E.: Diabetes insip., D. Arch. klin. Med., 82, r, 1905; Iron, Ergeb. Physiol., 5,698 , I906.

Meyer, F.: Caffcin, Arch. Physiol., I, I9 I5; Coronary Vessels, Arch. Physiol., 223, I9I 2 ; Epinephrin, Berl. klin. Woch., 50, 920, I9I3; Ouabain, Zentr. Bioch. Bioph., I3, 636 , I9 I2.

Meyer, H. and Ransom: Tetanus, ib., 49, 369 , I903.

Meyer, H., and Schmindeberc: Solanin, Arch. exp. Path. Pharm., 36, 36r, i895.

Mexer, H. and Steinfeld: Bismuth, Arch. exp. Path. Pharm., 20, 40, 1885.

Meyer, H. H.: Aloin, Arch. exp. Path. Pharm., 28, I86, I89o; Chelidonin, ib., 29, 397, I892; Diphtheria, Arch. intern. Pharmacod., I5, 419, I905; Arch. exp. Path. Pharm., 60, 1909; Narcosis, ib., 42, 106; 46, 338, 1899; Paraffin, Muench. med. Woch., No. I I, I901; Phosphorus, Arch. exp. Path. Pharm., I4, 313, 1881.

Meyer, O. B.: Excised Vessels, Z. Biol., 50, 93, I907.

Meyer-Betz and Gebhardt: Cathartics, Muench. med. Woch., 59, r793, I86r, Igr 2.

Meyers, M. K.: Atheroma, Centr. allg. Path., I9o9.

Michaelis, L.: Ferments, Bioch. Zs., I6, 81, 486; I 7, 231, 1909.

Michaelis and Garmendia: Phosphoric Acid, Bioch. Zs., 67, 431, I9 5.

Miciraud: Diuresis, Zs. Biol., 46, 198, I904.

Miculicich, M.: Glycosuria, Arch. exp. Path. Pharm., 69, I28, 1912.

Miescher: Phosphorus, Arch. exp. Path. Pharm., 37, Ioo, I896.

Miles: Arteriosclerosis, J. Am. Med. Assoc., 49, I $173,1907$.

Miller, A.: Hexamethylenamin, J. Am. Med. Assoc., 56, I718, 191 I.

Miller, D. J. M.: Camphor, J. Am. Med. Assoc., 63, 579, I9I4.

Miller, E. W.: Antimony, J.'Home Econ., 8, 36r, i9 I6.

Mrller, F. A., and Baker: Digitalis, J. Am. Phar. Assoc., 2, 198, 1913.

Miller, F. R.: Inhibition, Cardiac, Am. J. Physiol., 37, 240, I9I5.

Miller, J. L.: Digitalis, J. Am. Med. Assoc., 5 I, I 745, I908; Edema, Trans. Assoc. Am. Phys., 27, I37, I91 2; Expectorants, Am. J. Med. Sci., I48, 469, 1914; Hypertension, J. Am. Med. Assoc., 54, I666, I910; Salicylate, ib., 63, I 107, I914.

Miller, J. L. and Lusk: Arthritis, J. Am. Med. Assoc., 66, I756, r9i6.

Mille, R.: Heroin, Am. J. Pharm., 87, 248, I9I 5.

Miller, R. H., and Cabot: Anesthetics, Arch. Int. Med., I5, 369, I9I5.

Milus: Cerium, Med. 'Times, Phila., 6, I 74, i 876.

Mills and Congdon: Emollients, Arch. Intern. Med., 7, 694, 1911.

Milroy, T. H.: Hydrogen Ion, Qu. J. Exp. Physiol., 8, 142, I9I4.

Mines: Muscarin, J. Pharmacol., 5, 425, I9I4.

Minkowski: Diabetes, Arch. exp. Path. Pharm., 31, 85, r893; Arch. ges. Physiol., I I , I3, I905. 
Minot, G. R.: Chloroform, Im. J. Physiol., 39, г3 г, i9 I5.

Mirano: Caffein, Bioch. Centr., 5, 205, r906.

Mitchell and Suith: Zygadenus, Am. J. Physiol., 28, 3i8, igi i.

Mitchell, H. H., Shonley and Grindley: Nitrates, J. Biol. Chem., 24, 46 i, i9 i6.

Modrakowski: Anaphylaxis, Arch. exp. Path. Pharm., 69, 67, i91 2; Atropin, Arch. ges. Physiol., I I4, rgo6; Pulmonary Edema, Arch. ges. Physiol., i 58, 509, i9 4.

Mogilewa: Cactus, Arch. exp. Path. Pharm., 49, I37, I903.

Mohr, K.: Cotarnin, Ther. Gegenw., No. 8, I905.

Mond, Langer and Quincke: Nickel, Ref., Rambouseck, i 86, i 890.

Moog, O.: Digitalis, Zentr. Bioch. Bioph., I4, I59, I91 2;Epinephrin, Arch. exp. Path. Pharm., 77, 346, 1914.

Moon, V. H.: Quinin, J. Inf. Dis., I3, I65, г913; ib., I6, No. I, I915.

Moore, A. R.: Edema, Am. J. Physiol., 37, 220, I915.

MOORE, B.: Intrcduction, Bioph. J., 4, 1909.

Moore, B. and Roaf: Anesthetics, Proc. Roy. Soc., 77, 86, 1906.

Moore. B., Nierenstein and Todd: Atoxyl, Bioch. J., 2, 300, i907.

MoORE, C. W.: Gelsemium, Zentr. Bioch. Bioph., i I, 262, i 9 Iо.

MoOre. H. F.: Optochin, J. Exp. Med., 22, 269, 389, 551, 1915.

Moore. O. O., and Purinton: Adrenals, Am. J. Physiol., 4, 5I, I 900.

Moore. Wm. H.: Anaphylaxis, Soc. Exp. Biol. Med., I 2, I 75 , I 9 I 5.

Moore. W. O.: Salicyl, N. Y. Med. J., 30, I, II3, I879.

MOORHEAD, L. D.: Bitters, J. Pharmacol., 7, 577, I9 I5.

Moorhouse: Fever, Am. J. Physiol., 28, 223 , I9I I.

Morawitz, P.: Iron, Benzcl, Ther. Mon., 28, 389, I9i4.

Morawitz, P., and ZahN: Coronary Circulation, Deut. Arch. klin. Med., ir6, 364, I9I4.

Morel, A.: Ether, Arch. exp. Path. Pharm., 62, 429, I9io.

Morel, A., Mouriguand and Policard: Salvarsan, J. de Phys. Path., I4, 798, igr 2.

Moria N, R.: Novocain, J. Am. Med. Assoc., 65, 751, r9I 5.

Moriani, G.: Phosphorus, Zentr. Bioch. Bioph., I 8, 544, 19 I6.

Morishima: Curare, Arch. exp. Path. Pharm., 42, 28, I899.

Morita, S.: Decerebrated Rabbits, Arch. exp. Path. Pharm., 78, i 88, 1915; Glycosuria, ib., 78, 245, I9 5; Portal Vessels, ib., 78, 232, 1915.

Morris, R. S.: Parathyroid, J. Lab. Clin. Med., I, 26, I9I5.

Morse, H. N.: Osmotic Pressure, Publ. I98, Carnegie Inst'n, Washington, I9 4.

Morse, M.: Thyroid, Sci., 40, 794, I9I4; J. Biol. Chem., I9, 421, 430, I9 I4; Zentr. Bioch. Bioph., I 7,923 , I9I4

Morse, W. E.: Gastric Discharge, Am. J. Physiol., 41, 439, 19 I 6.

Mosenthal, H. O.: Atropin, J. Am. Med. Assoc., 58, 777, I9 2 ; Renal Function, Arch. Int. Med., 16, 733, r9r5; Uranium, Soc. Exp. Biol. Med., I I, I33, I9 4.

Mosenthal and Schlayer: Nephritis, D. Arch. klin. Med., i I I, 2 I 7 , ig I 3.

Mosiman and Whipple: Chloroform, Johns Hopkins Hosp. Bull., 23, 323, I9 2.

Mosso, U.: Cocain, Arch. exp. Path. Pharm., 23, 205, 1887; Salicyl, ib., 26, 267, I89o.

Mostrom and McGuigan: Strychnin, J. Pharmacol., 3, 515, 521 , i91 2.

Motelese: Picric Acid, Arch. farmacol., 9, 1910.

Motram, V. H.: Fat, Canad. Med. Assoc. J., July, r91 5.

Motrer, M. G:: Chenopodium, Publ. Health Reports, Oct. 2, I9I4.

Mozfeldt. K.: Pituitary, Bost. Med. Surg. J., I74, No. I8, I9 I6.

Moulinier, R.: Emetin, Zentr. Bioch. Bioph., 16, 354, r9i4.

Mucha and Ketron: Salvarsan, Zentr. Bioch. Bioph., I6, 75, I913.

Mueller, F.: Cholin, Arch. ges. Physiol., I34, 289, i9io; Wood Alcohol, Ref., J. Am. Med Assoc., 55, 700, I910; Yohimbin, Arch. intern. Pharmacodyn., I7, 8r, I907.

Mueller G. H.: Morphin, Bioch. Centr., 8, 312, I908.

Mueller, J. H.: Cholesterol, J. Biol. Chem., 22, No. I, I9 I5.

Mueller, JAC. Worm: Transfusion, Univ. Progr., Christiana, 1875.

Mueller, O., and InAda: Iodid, Deut. med. Woch., 30, 1751, I904.

Muench, W.: Chlorinated Lime in Wounds, Ref., J. Am. Med. Assoc., 65, 751, rgI5.

Muenzer: Phosphorus, Deut. Arch. klin. Med., 52, I99, r 894.

Muenzer: Diuresis, Arch. exp. Path. Pharm., 41, 74, I898.

Muerset: Nephritis, Arch. exp. Path. Pharm., I9, 310, i 886.

Muir, R., and DunN: Iron, J. Path. Bact., I9, No. 3, I915; 20, No. I, I9I5.

Muirhead, A. L., and Gerald: Volatile Oils, J. Pharmacol., 8, 253, 1916.

Mulzer, P.: Iodoform, Z. exp. Path., I, 446, I905.

Mummery and Symes: Pituitary, J. Physiol., 3i, No. 3, r9o8.

Munk: Cotarnin, Aertz. Centr. Ang., No. 27, r899; Santonin, Arch. path. Anat., 72, I36, 1878 . 
Mrri.I, J. R.: Golatin, Am. J. I'hysiol., I9, 285; 20, 234, 1907.

Mrkin, J. R., and Kranke: Pancreas, J. Biol. Chem., I5, 365, 1913; Alkali, Proc. Im. Sic. Biol. Chem., 3, 25, i9 6 .

Mirum. J. R., and RicuE: Nurcosis, Soc. exp. Biol. Med., r3, 7, I9I5.

MrRril and I"rane: Poison Laws, J. Am. Pharm. Assoc., 3, 663, I9I4.

Mcscr.we: I pecac, J. Am. Med. Assoc., 58, I3, I9I2.

Mrster, J. H., Jr., and KrumbhaAr: Spleen, J. Exp. Med., i8, r9i3.

Ml'To, K.: Epinephrin, Ref., J. Am. Med. Assoc., 66, I752, I9 16.

Míto and Ismizaba: Spartein, Arch. exp. Path. Pharm., 50, I, I903.

Mito and IWAkawa: Lobelin, Arch. exp. Path. Pharm., 62, 282, I9Io.

Metscis and Prambey: Tetra, J. Physiol., 43, I09, I9I I.

Mrasnits: Ocular Tension, Zentr. Bioch. Bioph., 15, 95, r913.

Mrers, C. S.: Experimental Psychology, I9I2.

Myers, H. B.: Crossed Tolerance, J. Pharm. Exp. Ther., 8, 4r 7, Igr6.

Myers, H. B., and Wallace: Diphtheria, Soc. Exp. Biol. Med., I 2, 43, I9I4.

Nachmanv, G.: Optochin, Zbl. Bakt., I, I98, I9 6.

NaEgeli, O.: Salvarsan, Ther. Mon., 29,645, I9 I5.

NAegeli, Th.: Pleura, L. exp. Med., I, I64, r9I3.

Nakisima, K.: Fal, Arch. ges. Physiol., I58, 288, 1914.

Nasse: Saline, Arch. ges. Physiol., 2, I I4, I869.

Neilson, C. H., and Hyland: Purgatives, J. Am. Med. Assoc., 6o, 436, r9r3.

Neisser: Moderne Syphilistherapie, I9I I.

Nel.son, K., and Haines: Neosalvarsan, J. Am. Med. Assoc., 62, 989, igr4.

Nexcki: Salicyl, Arch. exp. Path. Pharm., 20, 396, I886; ib., 36, 400, I 895 .

Nexcki and Srioxowski: Bromid, Arch. exp. Path. Pharm., 34, 3 I3, I 893.

Nerking: Anesthesia, Ref., Merck's Rep., 22, 240, 1908.

Netter, A.: Calcium, Bioch. Centr., 6, 249, 1907; Serum, Muench. med. Woch., I924, I906.

Neubauer, E.: Levulose, Arch. exp. Path. Pharm., 61, I74, I909.

Neubauer, O.: Glycuronic, Arch. exp. Path. Pharm., 46, I33, I900.

Neuberg, C.: Salicyl, Berl. klin. Woch., 48, 798, r9ir.

Neuberg and Caspari: Tumors, Deut. med. Woch., 375, r9r 2.

Neuberg, Caspari and Loene: Metals, Berl. klin. Woch., 49, r405, igr 2.

Neuhats: Introduction, Arch. intern. Pharmacodyn., 20, 393, r9ro.

Neunof, S.: Caffein, N. Y. Med. J., Oct. 25, I9r3; Tobacco Poisoning, Arch. Int. Med., I 7,659 , I9 I 6 .

Neujean: Epinephrin, Arch. intern. Pharmacodyn., I3, 45, I905.

Neukircin: Pilocarpin, Arch. ges. Physiol., I47, I53, r9 2.

Neumann, R. O.: Cacao, Bioch. Centr., 5, 727, 1906; Water, Arch. Hyg., 36, 248, r899.

Neumann, W.: Benzol, Deut. med. Woch., 4I, 394, I9I 5.

Newburgir, L. H.: Alkali, Bost. Med. Surg. J., I69, 40, 1913; Strychnin, Arch. Int. Med., I5, 458, I9I4.

Newcomb, E. L.: Digitalis, Am. J. Pharm., 84, 201 , r9r 2.

Nice, L. B.: Muscular Activity, J. Exp. Zool., I4, I23, I9r 2.

Nice, L. B., Rock and Cortright: Epinephrin, Am. J. Physiol., 34, 326, I9 4 ; Pituitrin, ib., 35, 194, 1914.

Nichols, H. J.: Salvarsan, J. Am. Med. Assoc., 55, 216, rgro.

Nicloux: Chloral, C. R. Soc. Biol., 60, 320, I906; Chloroform, ib., 60, I44, 245, 373, I906; Zentr. Bioch. Bioph., I0, 495, 673, I9 10; Ether, Bioch. Centr., 6, I68, I907.

Nicloux and Nowicka: Alcohol, J. de Phys. Path., I 5, 296, I9I3.

Nicloux and Placet: Methyl Alcohol, J. de Phys: Path. gen., I4, 916, I9 2.

Nicola, B.: Emetin, Ref., J. Am. Med. Assoc., 64, 475, I914.

Nicolai and Staehelin: Nicotin, Z. exp. Path., 8, 323, I9Io.

Nicolaier: Hexamethylenamin, Z. Klin. Med., 37, I899.

Nicolaier and Dohrn: Atophan, Deut. Arch. klin. Med., 93, rgo8.

Nicolt, M.: Tetanus, Trans. Assoc. Am. Phys., 30, 2 I3, 1915.

Niculescu: Silver, Chem. Abstr., 7, I55 I, I9I2.

Niemann: Cocain, Ann. Chem. Pharm., II4, 2 I3, I860.

Nisin, M.: Quinin, Arch. exp. Path. Pharm., 60, 31 2, 1909.

Nistico, G.: Lead, Zentr. Bioch. Bioph., r6, 37, I913.

Nixon, P. I.: Chaparro, J. Am. Med. Assoc., 62, I53c, 1914; J. Am. Phar. Assoc., 5, 608, I 916.

Nobel, E., and Rothberger: Chloroform, Zs. exp. Med., 3, I5I, I9I4.

Noguchr: Sa ponin, J. Exp. Med., 8, 268, r9o6; Toxins, ib., 8, 263, r9o6.

v. Noorden, C.: Cymarin, Ther. Mon., 30, 14, I916; Obesity, Ther. Mon., 29, r6, I9I5. 
Norris, D.: Coal Tar, Bioch. J., 8, 253, I9I4.

Nothnagel: Morphin, Arch. path. Anat., 89, 2, r884.

Notтhaft: Iodin, J. Am. Med. Assoc., 56, 685, I9 то.

Novak, E.: Atropin, J. Am. Med. Assoc., 64, I 20, I9 5

Novy and Freer: Peroxids, J. Exp. Med., 6, I902.

O'Connor: Epinephrin, Zentr. Bioch. Bioph., I2, 128, 191 1; Arch. exp. Path. Pharm., 67,$195 ; 68,383$, 1912.

Oenue: Histamin, Arch. exp. Path. Pharm., 72, 76, I9I3.

Oelier: Atophan, Ther. Mon., 27, 3I6, I9I2.

Offergeld, H.: Cotarnin, Arch. Gyn., 104, No. 2, I9I5.

Ogawa: Digitalis, Deut. Arch. klin. Med., 108, 554, I912; Epinephrin, Arch. exp. Path. Pharm., 57, 89, I91 2.

O'Hare, J. P.: Theobromin, Arch. Int. Med., I5, 1053, I915; Nephritis Tesis, Arch, Int. Med., I7, 7 I I, I916; Uranium, Arch. Int. Med., I2, I913.

Okada, S.: Bile, J. Physiol., 49, 457; 5042 , I I5, I9 I5.

Oldberg: Prescription, Proc. Am. Phar. Assoc., 58, 756, I9 10.

Olds: Thyroid, Am. J. Physiol., 26, 354, roro.

Olva, C.: Epinephrin, Zentr. Bioch. Bioph., I6, 681, I9I4.

Óliver and Schatfer: Epinephrin, J. Physiol., 18, 230, 1895; Pituitary, ib., 18, 277, 1895.

Olmer and Tiau: Salicyl, C. R. Soc. Biol., 66, 8r4, r9io; Thallium, Ref., Am. Pharm. Assoc., 57, 266, 1909.

Orson, G. M.: Dyed Furs, J. Am. Med. Assoc., 66, 864, 1916.

Openchowski: Emetin, Arch. Physiol., 549, i 889.

OpнüLs: Nephritis, J. Med. Res., I 8, 497, I 908.

OpIE, E. L.: Cantharidin, J. Exp. Med., 16, 83, 1912; Tr. Assoc. Am. Phys., 27, I 7 , 1912 ; Liver, Trans. Assoc. Am. Phys., 25, 1910.

Opie and Alford: Dicls, J. Am. Med. Assoc., 63, 136, 1914; Trans. Assoc. Am. Phys., 29, 264, 1914; J. Exp. Med., 21, 1, 21, 1915.

Oppenheim: Cacodyl, Ref., Merck's Rep., 21, 3, I907.

Oppenhermer, E.: Digitalis, Bioch. Z., 55, I34, I913.

OrBan, R.: Alcohol, Ref., Ther. Mon., 28, 203 , I9I3.

Orenstein, A. J.: Quinin, J. Am. Med. Assoc., 63, I931, I9.14.

OrLAND, F.: Phencl Phthalein, Med. Klin., 257, I0I3.

OrR, T. G.: Eucain, J. Am. Med. Assoc., 66, 1857, 1916.

Oryng, T., and Pauli: Protein Compounds, Bioch. Zs., 70, 368, I9 5.

Osborne, T. B.: Vegetable Protcins, London, I 909.

Osborne, T. B., and Mendel: Artificial Diets, Proc. Soc. Exp. Biol. Med., 9, 72, r912; Beef Fat, ib., I 2, 92, r915; Cod Liver Oil, J. Biol. Chem., I 7, 401, 1914; Fat, ib., 20, 379 , I9 5; Foods, ib., I6, Dec., I913.

Osborne, Mendel and Harris: Ricin, Am. J. Physiol., 13, I86, 1905.

Osborne and Vincent: Nerve Tissue Extracts, J. Physiol., 25, 283, 1900.

Osborne, T. B., and Wakeman: Fat, J. Biol. Chem., 2 I, 91 , 1915.

Osborne, W. A.: Ions, Bioph. Centr., I, I 12 , I905.

Ostenberg, Z.: Para-hydroxyphenylethylamine, Soc. Exp. Biol. Med., 12, I74, 1915.

Osterhout, W. J. B.: Antagonism, Sci., 4I, 255, I915; Narcosis, ib., 37, III, I9I3; Permeability, ib., 35, I I 2, I9I2.

Ostwald, W.: Gols, Arch. ges. Physiol., rog, $277,1905$.

Osterwald: Strychnin, Arch. exp. Path. Pharm., 44, 451, 1900.

Oswald, A.: Inflammation, Zentr. Bioch. Bioph., I 1, 545, 191 I; Iodin, D. Arch. klin. Med., 55 I, I9r5; Salicyl, Z. exp. Path., 8, 226, I9 10; Thyroid, Zs. physiol. Chem. 23, 275; 27, 14, I899; Bioch. Centr., I, 249, 1903; Wien. klin. Rund., No. 37, 1905; Arch. exp. Path. Pharm., 60, II7, 1908.

Oтт and Scotт: Bile, Soc: Exp. Biol. Med., 6, r3, rgo8; Cathartics, Med. Bull., Mch., 1908; Luteum, Soc. Exp. Biol. Med., I2, 47, I914; Milk, Ther. Gaz., 3 I0, I9 2 ; Proc. Soc. Biol. Med., 9, 63, r9г2; Pituitary, ib., 8, 48; 9, 63, I9 1o; Uterus, J. Exp. Med., I I.

Ottenberg, R.: Citrate, Soc. Exp. Biol. Med., I3, I04, 19i6.

Otrenberg and Libman: Transfusion, Trans. Assoc. Am. Phys., 30, 149, I9 5.

v. Oтто, C.: Alcohol, Arch. path. Anat., 216, 264, 1914; Nicotin, ib., 205, 384, I9 I.

Orro, E.: Absorplion, Inaug. Heidelberg, 1905.

Owens: Cocain, J. Am. Med. Assoc., 58, 329, rg1 2.

Packard, W. H.: Oxygen, Am. J. Physiol., I 5, 30, 1905.

Padtberg, J. H.: Colocynth, Arch. ges. Physiol., I34, 627, r909; Magnesium, ib., I29, 476, 1909; Morphin, ib., I39, 318, 1911. 
P.AETZ: Arecolin, Zentr. Bioch. Bioph., Iо, 282, I910.

I'.1L, J.: Cuffein, Jeut. med. Woch., I774, 1912; Cholin, Z. exp. Path., 9, 192, I911; Worphin, Deut. med. Woch., 39, 395, 1913; Papaverin, Wien. med. Woch., I049, 1013 ; Med. 'Times, July, I914; Deut. med. Woch., Jan., I64, I9I4.

Puluev: Nicotin, Skand. Irch. Physiol., 24, r87, rgrr.

Palmer, W. W., and Ilexperson: Acid Excretion in Nephritis, Arch. Int. Med., I6, 109, 1915; Acidosis, Arch. Int. Med., I2, 153, 1913.

Paicolst, H. K.: Opiutes, J. Am. Med. Assoc., 65, I58I, IC, I5.

P.ivcolst, II. K., and Hopkins: Opium, J. Am. Med. Assoc., 65, 2220, 1915.

Pappexierm: Bencol, Wien. klin. Woch., 26, 48, r913.

PARk, II. H.: Antitovin, Harvey Lect., I, IOI, I906; J. Pharmacol., 3, 474, I9I 2; Bost. Med. Surg. J., Jan. 18, I913; Diphtheria Antitoxin, J. Am. Med. Assoc., 63: 863 , I9I4.

Park, W. H., and Nicoll: Tetanus A nitoxin, J. Am. Med. Assoc., 63, 235, I9r4.

PARK, W. II., and Znvaner: Diphtheria Immunization, J. Am. Med. Assoc., 65, 22 I6, I9I5.

Park, W. H., Zingher and Serota: Toxin-Antitoxin Mixtures, J. Am. Med. Assoc., 63,859 , I9I4.

Parker, G. H.: Organic Correlations, Amer. Natural., 43, 212, I909; Cilia, Am. J. Physiol., I 4, I, I905.

Parkivson, J.: Ethyl-hydrocuprein, Zs. Chemother. 2, No. I, I9I3.

Parkinson, J., and Rowlands: Strychin, Quart. J. Med., 7, 42, 1913; J. Am. Med. Assoc., 6I, I75I, I9I3; XVII Int. Congr. Med., 5. 2, 247, I9I4.

Patein, G.: Calomel, Zentr. Bioch. Bioph., I6, 586, i913.

Paton, N.: Epinephrin, J. Physiol., 29, 286, I903.

PatoN and Eason: Nitrogen, J. Physiol., 26, I66, Igor.

Paton and Watson: Pituitary, J. Physiol., 44, 4I3, I9I2.

Patta, A.: Hypophosphiles, Zentr. Bioch. Bioph., Io, 620, I910.

Patta, A., and Varisco: Cholin, Ref., Ther. Mon., 29, 409, I9I 5.

Paukow: Piluitary, Arch. ges. Physiol., 147, 89, I9 2.

Paul and Cownlex: Emetin, Am. J. Pharm., 73, I90.

Paulfasco: Pituitary, Bioph. Centr., 3, 85, 1907.

Pauli, W.: Colloids, Beitr. physiol. Chem., 5, 27, 1905; Gels, Erg. Physiol., 6, 105, 1907; Prolein, Trans. Faraday Soc., 1913; Sulphocyanids, Muench. med. Woch., No. 4, I 903.

Pauli and Pal: Sulphocyanid, Ref., Merck's Rep., 24, 362.

Paulian, D. E.: Parasiles, Zentr. Bioch. Bioph., i8, I94, I9I4.

Paulucci, P.: Fats, Ref., Ther. Mon., 29, 408, 1915.

Pavy, Brodie and Siau: Phlorhizin, J. Physiol., 29, 467, т903.

PaWELE: Narcosis, Bioch. Zs., 63, 52, 1914.

Pawniski, J.: Nicotin, Zs. klin. Med., 80, 284, r914.

Peabody, F. W.: Acidosis, Arch. Int. Med., I6, 955, 1915; Carbon Dioxid, ib., 16, 846, 1915; Dyspnea, Trans. Assoc. Am. Phys., 30, 20, I915; Pneumonia, J. Exp. Med., $17,71,1913$.

Peacock, J. C., and B. L. G.: Salol Coating, Am. J. Pharm., 8\%, 514, 1915.

PEARCE, L. and Brown: Arsenic, J. Exp. Med., 22, 517 , 1915.

Pearce, R. G.: Phlorhizin, Am. J. Physiol:, 40, 418, 1916; Vessels, Bioch. Z., 62, 243 , I9I3.

Pearce, R. M.: Cantharidin, J. Exp. Med., I7, 542, I9r 3 ; Nephritis, Arch. Intern. Med., 5, I33, I910; Spleen, Clev. Med. J., 14, 463, 1915; Urine, Proc. Soc. Exp. Biol. Med., 6, 1 29, 1909.

Pearce, R. M., and Eisenbrey: Peptone, Trans. Assoc. Am. Phys., 25, 30 , 1910.

Pearce, Hill and Eisenbrey: Caffein, J. Exp. Med., I2, 200, I9Io.

Pearce, R. M., and Peet: Spleen, J. Exp. Med., i 8, No. $5_{l}$ I913.

Pearce, R. M., and Pepper: Spleen, J. Exp. Med., 20, No. I, 1914.

PEARCE, R. M., and pupils: Spleen, J. Exp. Med., 16, 1912.

Pearce, R. M., and Ringer: Tartrate, J. Med. Res., 29, 57, x913.

Pearce and Stanton: Epinephrin, Alb. Med. Ann., Feb., 1906.

Pearson: Digitalis, Am. J. Pharm., 85, 245, 1913.

PECK, C. H., and Meltzer: Intravenous Magnesium, J. Am. Med. Assoc., 67, 1 1 31, 19:6.

PEl.z: Codein Iabit, Deut. med. Woch., 22, 864, 1905.

Peterson: Salicyl, Deut. med. Woch., 3, 13, $29,1877$.

Petrow: Destruction of Alkaloids, Biochem. Centr., 4, 495, 1905.

Petrowa: Salicyl, Z. physiol. Chem., 74,429 , igr2.

Pfafr, F.: Digitalin, Korresp. Schweiz. Aerz., 696, 1892; Poison Ivy, J. Exp. Med., 2, I8I, I897. 
Pfaff and B.tlci: Cholagogucs, J. Exp. Med., 2, 49, I897.

Pfender, C. A.: Brass, J. Im. Med. Assoc., 62, 296 , I9I4.

Pfoertier: Sulphonal, Deut. med. Woch., I563, I9I4.

Phillips, J.: Hcroin, J. Am. Med. Assoc., 59, 2 I46, I9I 2.

Phillips and Bradford: Caffein, J. Physiol., 8, i I 7, i 887.

Piazza, J. G.: Allyl, Zs. cxp. Path., I7, 318, I9I5.

Prccininr: Antipyrctics, Arch. internat. Pharmacod., 22, 27, 1912; Manganese, Arch. Farm. sper., Io, Igro.

Pick: Defibrinated Animals, Arch. exp. Path. Pharm., 42, 399, i 890.

Pick and PrNeles: Thyroid, Zs. exp. Path., 7, 5 I8, I909.

Pick, E. P., and Wasicky: Papavcrin and Emetin, Wien. klin. Woch., 38, No. 22, I9I5.

Pickering: Embryonic IIeart, J. Physioi., I4, 383 , I893.

Pictet and Kramers: Morphin, Zentr. Bioch. Bioph., io, 497, I9ro.

Pierson: Aconite, Therap. Gaz., 37, I9I3.

Prlcher, J. D.: Caffein, J. Pharmacol., 3, I9, I912; ib., 3, 267, 609, 1912; Epinephrin, J. Am. Med. Assoc., 63, 208, 1914; Hemclysis, J. Pharmacol., 4, 350, 1913; Phenol, Am. J. Pharm., 86, I49, I9 4 ; Uterus, J. Pharmacol., 8, I Io, I9I6.

Pilcher, J. D., Delzell and Burman: Female Remedies, J. Am. Med. Asscc., 67, 490 , I9I6.

Pilcher, J. D., and Sollmanv: Atropin, J. Pharmacol., 5, 31 7, I9I4; Hemorrhage and Saline, Am. J. Physiol., 35, 59, I9 14; Saline, Am. J. Physiol., 35, 70, I9 I4; Vascmotor Center, J. Pharmacol., 6, 323-4I I, I9I 5; Veratrin, J. Pharm. Exp. Ther., 7, 295; 1915.

Pincussohn: Caffein, Bioch. Centr., 5, 59 I, I906; Edema, Zs. exp. Path., io, 308, I9 2.

Pinczower: Salicyl, Ther. Mon., 24, 297 , igio.

Piria: Salicyl, Ann. Chim. Physique, 69, 298, 1838.

Pissemsky, S. A.: Pcptone, J. Am. Med. Assoc., 65, 2 I 28 , I9 15.

Pitini: Atropin, Arch. internat. Pharmacod., I8, 31 I, I908.

Pittenger, P. S., and Vanderkleed: Goldfish Method, J. Am. Pharm. Assoc., 4, 427 , I9 15 .

Plagge and Lebbin: Aluminum, Berlin, Militaersanit., r893.

Plant, O. H.: Camphor, J. Pharmacol., 5, 5I3, 57 I, I9I4.

Plesch, J., and Karzag: Thorium, Muench. med. Woch., 59, I363, I9 2.

Plumier: Alcohol, J. de Physiol. Path., No. 3, I905; Digitalis, ib., No. 3, i905; Epinephrin, ib., 6, 655, I904; Ergot, ib., 7, I3, I905; Nitrite, ib., No. 4, I905; Pulmonary Circulation, ib., 7, I3, 1905.

Plumier and Clermont: Epinephrin, Zentr. Bioch. Bioph. 14, 783, I9 2.

Podwyssorzkr: Ergot, Arch. exp. Path. Pharm., 6, I53, I877; 1 pecac, ib., I I, 23 I, I879.

PoHl: Formaldehyd, Arch. exp. Path. Pharm., 28, 246, I89i; Stomachics, ib., 25, 5 I, I888.

Polano, O.: Oleic Acid, Z. Geburtsh. Gyn., 65, 58г, r9o9.

Polimanti, O.: Curare, Arch. exp. Path. Pharm., 78, i 7, I9r4.

Pollak, L.: Epinephrin, Arch. exp. Path. Pharm., 61, 149, I9o9; Z. physiol. Chem., 68, 69, I9I0; Glycosuria, Arch. exp. Path. Pharm., 64, 4I5, I9I I Strychnin, Bioch. Centr., 10, 199, 1910; Sulphocyanid, Beitr. physiol. Chem., 2, 430, 1902.

Pollock, L. J., and.Holmes: Picrotoxin, Arch. Int. Med., I6, 2 I 3, I9 I 5.

Pollock, L. J., and Treadway: Epilepsy, Arch. Int. Med., I 2, 445, I9 I 3.

Popielski: Epinephrin, Arch. ges. Physiol.; I39, 57 I, I9I1; Hormones, Muench. med. Woch., 534, r912; Vasodilatin, Chem. Abstr., 7, 613, 1912.

Popper, E.: Opium, Ärch. ges. Physiol., I 53, 574, I0I3; Papaverin, Wien. klin. Woch., $36 \mathrm{I}, \mathrm{I} 9 \mathrm{I} 4$.

Popper and FrankL: Morphin, Zentr. Bioch. Bioph., I3, 716, I9I2.

Porges and Pribram: Phosphorus, Arch. exp. Path. Pharm., 59, 1908.

Port and Brunow: A utonomic Poisons, Arch. exp. Path. Pharm., 76, 239, r9r4.

Porter, E. L.: Strychnin, Am. J. Physiol., 33, Proc. XIX, I9I4.

Porter, W. T.: Curare, Am. J. Physiol., 36, 4I8, I9 I5; Strychnin, ib., 30, I j I, I9 I5.

Porter, W. T., and Turner: Vasomotor Mechanism, Am. J. Physiol., 39, 235, I9I 5 .

Posey: Miotics, J. Am. Med. Assoc., 63, 219, 1914.

Post, W. E.: Tartrates, J. Am. Med. Assoc., 62, 592, I9I4.

Posrojefr: Salvarsan, Chem. Abstr., 7, I52, r9г 2; Saponin, Bioch. Z., 36, 335, г9гі.

Porт: Morphin, Biochem. Z., 42, 67, төг 2.

Porrs: Epinephrin, J. Am. Med: Assoc., 47, r 188, 1906.

Pouchet and Chevalier: Convallaria, Ref., Nouv. Rem., I, 766, I903; Theocin, Bull. gen. Therap., 146, 61 5, I903; Valerian, ib., 147, 135; 149, 25, 1905.

Poulsson, E.: Aspidium, Arch. exp. Path. Pharm., 29, I, I89 1; Barium, ib., 62, 365, I9 10; Radium, Arch. exp. Path. Pharm., Suppl., 443, I908; Strychnin, ib., 26, 2 2, I889. 
Pollton, E. B.: Diabetes, Brit. Med. J., Sept. I I, I9I5.

Powr.r. F. B.: Chaulmoogra, Im. J. Pharm., 87, 493, I9I5; Cucurbitacea, ib., 84, I45, I) 12 .

Pumer, F. B., and Browning: Gossypium, Pharm. J., 93, 423, r9i4; Taraxacum, Zentr. Bioch. Bioph., I5, I85, I9 I 2 .

P'NWE and MoOre: Colocynth, Zentr. Bioch. Bioph., io, 766, I91o.

l'ower and Rogerson: Jillap, Jahresb. Pharm., 69, 31, I909; Leptandra, Zentr. Bioch. Bioph., II, 208, I9Io.

l'ower and SALway: Caulophyllum, Zentr. Bioch. Bioph., I6, 232, I913; Erythrophlatu, Im. J. Pharm., 84, 337, I912; Nutmeg, ib., 80, 563, I908; Pumpkin, J. Am. Chem. Soc., 32, 346 and 360 , I910.

Power and Tutin: Grindelia, Am. J. Pharm., 77, 484, 1905.

Power, Tutin and Rogersox: IIops, J. Chem. Soc., Io3, I267, r9i3.

Pozkrski: Papain, Proc. Am. Pharm. Assoc., 58, 392, igog.

Pozzi and Clerc: Nicotin, J. de Phys. Path. gen., I5, I, 1913.

Pratt, J. H.: Metabolism, J. Am. Med. Assoc., 65, 52, 1915; Pancreas, Trans. Assoc. Im. Phys., 24, 269, I909; Uric Acid, ib., 28, 387, г9п3.

Pribrai, E.: Bromid, Zs. physiol. Chem., 49, 457, 1906.

Price: Aconite, Proc. Roy. Soc. Med., 4, No. 8, I9I I.

PrICE, F. W.: Digitalis, Brit. Med. J., Sept. I3, I9I3; XVII. Int. Cong. Med., 5. 2, 305, I9I4; Formaldehyd, U. S. Ind. Circ., 59, I904.

Price, W. A., and Bensing: Endameba, J. Nat. Dent. Assoc., 2, I43, I9 5.

Pringle, Maunsell and Pringle: Ether, Brit. Med. J., Sept. 9, r905.

Prixgsherm: Alcohol, Bioch. Z., I2, I42, I908; Cholesterol, Med. Klin., Feb., r9ı3.

PrIOR: Quinin, Arch. ges. Physiol., 34, 237, I884.

Prociriow, L.: Abortiva and Acria, Arch. Intern. Pharmacod., 21, 3 I 3, I9I ; Haloids, ib., 2I, I9II; Salts, ib., 2I, 287, I9II.

Prochownik: Naphthalin, Ther. Mon., 489, I9II.

Prowazek: Poisons on Infusoria, Biochem. Centr., Io, 8, I9ı.

Prus, J.: Cocain, Z. exp. Path., I4, I6r, I9r3.

Puckner: Acetanilid, J. Am. Med. Assoc., 47, r 206, ı906; Pill Coating, Rep., Council of Pharmacy and Chem., 58, I9II.

Pusey, W. A.: Acid, J. Am. Med. Assoc., 60, 434, I913; Radium, Sci., 33, I00I, I9II; Salvarsan, J. Am. Med. Assoc., 56, I I8, I9II; X-rays, ib., 61, 552, I9I3.

Quagliariello, G.: Iistamin, Zs. Biol., 64, 263, I9I4; Veratrin, Zs. Biol., 59, 44r, I9I3.

QueckeNstedt: Iron, Zentr. Bioch. Bioph., I6, 240, I9I3.

Quellien: Lead, Bioch. Centr., 5, I33, I905.

Quenstaedt: Salicyl, Ther. Gegenw., 46, 97, I905.

Quinan, C.: Chloroform, J. Med. Res., 32, No. I, I9I5.

Quincke: Copaiba, Arch. exp. Path. Pharm., I7, 273, I883; Salicyl, Berl. klin. Woch., I9, 709, I882.

RaAflaub, H.: Muscarin, Zs. Biol., 63, 477, I9 2.

Rabe, F.: Amanita, Z. exp. Path., I9II; Coronary Vessels, ib., II, I75, I9I2; Iron, Muench. med. Woch., 59, 2809, I912.

Rabens, I. A.: Chlorid, Am. J. Physiol., 36, 294, I9I5.

Raciborski: Hypertonus, Bioch. Centr., 6, 49, I906.

RADZIEJEWSKI: Epinephrin, Berl. klin. Woch., 35, 572, I898.

Raeburn, J. S.: Emetin, Brit. Med. J., March 28, I9I4.

RAeder, J. C.: Respiratory Mctabolism, Bioch. Zs., 69, 257, I9I 5.

Ragazzi: Phosphorus, Zentr. Bioch. Bioph., Io, 6r9, I031, I9I0.

Rahe, Rogers, Fawcett and Beebe: Thyroid, Am. J. Physiol., 34, 72, I9I4.

Raiziss, A. M., Raiziss and Ringer: Hippuric Acid, J. Biol. Chem., I7, No. 4, I9I4.

Raiziss, G. W., Dakin and Ringer: Metabolism, J. Biol. Chem., I9, 473, 485, I9I4; Zentr. Bioch. Bioph., I7, 920, I9r4.

Rambousek: Anilin, Zentr. Bioch. Bioph., I6, 430, I9ı3.

Randolph, N. A.: Petrolatum, Ther. Gaz., 9, 732, I885.

Ransom, I.: Caffein, J. Physiol., 42, I45, I9I I; Biochem. J., 6, I5I, I9 1 2; Sapotoxincholesterin, Deut. med. Woch., I94, I90I.

Raoult: Cryoscopy, Zeitsch. f. physik. Chem., 27, 6I 7, I898.

Raphael: Diuresis, Dorpat. Arb., Io, 8I, I894.

Rappoport: Narcosis, Z. exp. Path., 9, 39, I9I I.

Ratzlaff, K.: Hypertonic Solutions, Zs. exp. Path., I7, 192, 191 5.

Raubenhimer, O.: Sun Cholera Mixture, J. Am. Phar. Assoc., 5, 624, I9 6.

Ravaut: Neosalvarsan, Presse. med., I7I, I913; Salvarsan, Ann. Med., 49, I914. 
Reach, F.: Alcohol, Bioch. Z., 3, 326, 1907; Bilc Duct, Wien. klin. Woch., No. 8., 27, 72, I9I4; Rectal Feeding, Arch. ges. Physiol., 86, 247, I 901.

Referee Board: Aluminum, U.S. Dept. Agr. Bul., No. Io3.

Regnault: Chloroform, Jahrb. Pharm., 69, I63, igog.

Rehfuss, M. E., and Hawk: Gastric Acid, J. Am. Med. Assoc., 63, 2088, r9I4.

ReICH, H. W.: Alcohol, Arch. f. Hyg., 84, 337, I9I 5.

REICHER: Cholcsterol, Merck's Rep., 22, i 72 , igo8.

ReICHerT: Atropin-morphin, Therap. Monthly, May, I9oI; Morphin, Univ. Penn. Med. Bull., April, I90I.

Reipt and SchefFer: Iodid, Bioch. Centr., 8, 20I, Ig08.

REMELÉ: Hexamethy lenamin, Zentr. Bioch. Bioph., I8, 545, toi5.

Renon, L., and Desbouis: Papaverin, Soc. Biol., 76, 526, I9I4.

Retrig, H.; Phosphorns, Arch. exp. Path. Pharm., 76, 345, I9I4.

RetzlafF: Atophan, Z. exp. Path., I2, 307, I913.

Rhamy: Acctone, J. Am. Med. Assoc., 58, 628, I912.

Rhode, E., and Ogawa: Heart, Arch. exp. Path. Pharm., 69, 200, rgr 2.

Rhodius and Straub: Muscarin, Arch. ges. Physiol., I ro, 492, I905.

Richards, A. N.: Camphor, J. Pharmacol., 6, 73, I9I4.

Richards, A. N., and Plant: Caffein, J. Pharm. Exp. Ther., 7, 485, ig 5 .

RICHARDS, A. N., and Wood: Strophanthin, J. Pharmacol., 6283 , I9I5; Suprarenal Secretion, Am. J. Physiol., 39, 54, I9 5 .

Richardson, F. L.: Spinal Anesthesia, Bost. Hosp., 244, I9 3.

Richardson, M. W.: Vaccines, Bost. Med. Surg. J., I58, I2, I908.

Richter, P. F.: Uranium, Senator Fest. I904; Berlin. klin. Woch., 42, 384, 1905.

RiCKer, G.: Mesotherium, Zentr. Bioch. Bioph., I7, 23, I9I4.

Ricker, G., and Hesse: Mercury, Arch. Path. Anat., 2I 7, 267 , IgI4.

RIDDle, O., and BAssetT: Alcohol, Am. J. Physiol., 4I, 425, I9I6.

Rieben: Pills, Arch. Pharm., 245, 502, I907.

RIEDER: Quassin, Arch. exp. Path. Pharm., 63, 305, I9ro.

RIEgler: Saccharin, Arch. exp. Path. Pharm., 35, 306, I 895

Riemer: Silver, Arch. Heilk., I7, 330, I876.

RIEMSChNEIDER: Strychnin, Inaug. Diss., Kiel, rgoo.

Rifatwachdani, S.: Cocain and Ecgonin, Bioch. Z., 54, 83, I913.

RINEHART: Borate, Ther. Gaz., 662, Igor.

Ringer, A. I.: Acidosis, Trans. Assoc. Am. Phys., 28, 469, I9r3; Diabetes, J. Biol. Chem., I7, No. 2, I914; Epinephrin, J. Exp. Med., I 2, I05, I9 10.

Ringer, S.: Heart, J. Physiol., 3, 380; 4, 29; 8, I5, I884; ib., I8, 425, I895; Salts, ib., I7, 6 .

RipPetoe and Smith: Nitroglycerin, J. Am. Pharm. Assoc., 3, 96, I9I4.

RIPPETOE and Wise: Volatile Oils, J. Am. Pharm. Assoc., I, I 273 , I9I 2.

Rischbieter, W.: Pituitary, Z. exp. Med., I, 355, I913.

Ritschel and Stange: Anesthetics, Arch. intern. Pharmacod., 23, I9I, I9I3.

RitTer, H.: Salvarsan, Deut. med. Woch., 38, i62, igir.

Ritrer, K.: Alypin, Med. Klin., I236, r912; Cocain, Bioch. Centr., 9, 273, I909.

RIVA, A.: Lcad, Zentr. Bioch. Bioph., I5, 620, I9I3.

RIVERS and WEBER: Caffein, J. Physiol., $36,33,1907$.

Robertson, H. E.: Magnesinim, Arch. Int. Med., I7, 677 , г916.

Robertson, J. H.: Uric Acid, Am. J. Physiol., 33, 324, I9I4.

Robertson, T. B.: Acids, Arch. ges. Physiol., I45, 329, I912; Tethelin, J. Am. Med. Assoc., 66, 1009, 1916; J. Biol. Chen., 24, No. 3, I916.

Robertson, T. B., and Burnetr: Citrates, J. Pharmacol., 3, 635, I9 2.

Robinson, G. C.: Aconite, Arch. Int. Med., 15, 645, I915.

Robinson, G. C., and AUer: Anaphylaxis, J. Exp. Med., I8, 556, I913.

Robinson, G. C., and Draper: Vaguls, J. Exp. Med., I4, No. 3, I9 I I.

RoBiquet: Cantharidin, Ann. di Chimie, 76, 302, I8I 2 .

Roch, M.: Morphin, Merck's Rep., 21, 45, I907; Phosphorus Pcntachlorid, XVII Int. Congr. Med., 5. 2, 73, I9I4; Salicyl, Bull. gen. Ther., I63, 2 I8, I9 I 2.

Roch, M., and Cramer: Adonis, XVII Int. Congr. Med., 5. 2, 239, r9I4.

Rochester, A. S.: Morphin, J. Am. Med. Assoc., 52, 35I, I909.

Rockwoon: Salicyl, Am. J. Physiol., 25, 34, 1909.

Roemmans: Chlorid, Zs. klin. Med., I, 513, 1887; Lecithin, Biochemie, 766, 1908.

Roenmams, F.: Saccharose, Bioch. Zs., 72, 26, 1915.

Roenricht: Ether, Centr. Chir., 32, No. 38, I905.

Roemer: Veronal, Arch. exp. Path. Pharm., 66, 24I, I9r 2.

RoGers, T. B.: Hypericum, Mulf. Vet. Bul., 6, 37, r914; Sulfonal, J. Am. Med. Assoc., 58, I5 10, 1912. 
Kinik, II, and Gariter: Saline, Zentr. Bioch. Bioph., I5, I79, 1913.

kinul: Heart. Irch. exp. Path. Pharm., 68, 401, I9I2.

kurk, II.: Saliarsan, Med. Klin., 728, igr5.

Rulu, li., and Christaxsex: Sall, Arch. exp. Path. Pharm., 77, 34, I9I4.

Romulis: "ironal, Zentr. Bioch. Bioph., I4, 446, I912.

Rus. B.: Chorid, Bioch. Zs., 29, 501, I910.

Kow\%.vi: Irritumts, Bioch. Centr., 8, I64, 1908.

Rou, L.: Emulsions, J. Am. Phar. Assoc., 5, 496, I9 6.

Rusl. .1. R.: Phytin, Bioch. Bul., 2, 2 I, I91 2.

Rosibrtils: A pomorphin, Canad. Pract., Oct., I908.

Rosimin, R.: Alcohol, Arch. ges. Physiol., roo, 348, 1903; Chlorid, ib., I35, I77, 192, 1910 ; ib., It2, 208, 447, 19I2.

Roskine and A.viersox: Anaphylaxis, U. S. Hyg. Lab. Bull., 29, I9o6; ib., Bull., 5o, I 909.

Roswisitat: Bicarbonate, Bioch. Zs., 4, 500, 1907.

Rosesblooir, J.: Acctone, J. Am. Med. Assoc., 65, I7 I5, I9 5 ; Chlorate, Bioch. Bull., I, 483 , I9I 2 .

Rosritiloom, J., and Schlldecker: Ergotinin, J. Am. Med. Assoc., 63, r 203, i9i 4.

Rosentioom, J., and Weinberger: Epinephrim, Bioch. Bull., 2, 123, I912.

Rosexfeld: Chloroform, Arch. exp. Path. Pharm., 37, 54, 1896.

Rosknkranz: IIormonal, Ref., J. Am. Med. Assoc., 58, I824, I9I 2.

Rosexow, G.: Calcium, Zs. exp. Med., 4, 427, г9г6.

Rosenthal and Severin: Antimony, Arch. exp. Path. Pharm., 68, 275 , igi 2.

Roserwasser: A pomorphin, Med. Rec., July 27, I9c7.

Ross, Cropper and Ross: Paraffin Cancer, Lancet, I83, 1579, I912.

Ross, E. L., and Hawk: Elher, Arch. Int. Med., I4, 779, I9I4.

Ross, E. L.. and McGuigax: Ether, J. Biol. Chem., 22, 407, I9I 5.

Rossbach, M. J.: Colchicin, Arch. ges. Physiol., 12, 308, 1876.

Rost, E.: Borale, Arch. Intern. Pharmacod., I5, 291, I905; Caffein, Arch. exp. Path. Pharm., 36, I895; Eosin, Med. Klin., 994, I9 15; Primula, Zentr. Bioch. Bioph., 16, 653, 1914; Tannin, Arch exp. Path. Pharm., 38, 346, I897.

Rost, Fravz and Weitzel: Benzoate, Zentr. Bioch. Bioph., I6, 508, I9I3.

Rostoki: Chloroform, Z. physiol. Chem., 31, 432, I901.

Roti, G. B.: Aconite, J. Pharmacol., 4, 349, I9I3; J. Am. Pharm. Assoc., 2, 705, I913; Camphoric Acid, J. Pharmacol., 2, 405, 191 I ; Pituitary, J. Am. Med. Assoc., 63, 476, I914; Quinin, J. Pharmacol., 3, 470; 4, I57, I9I2.

Roth, G. B., and Voegtrin: Aluminum, J. Pharmacol., 8, 132, 1916.

Rотн, O.: Epinephrin, Deut. med. Woch., 905, r9i4.

Rothberger, J. C.: Physostigmin-curare, Arch. ges. Physiol., 87, I1 7, 1901.

Rothberger, C. J., and Winterberg: Calcium, Arch. ges. Physiol., I42, 523, I912; Liter on Poisons, Arch. internat. Pharmacod., 15, 339, 1905.

Rothermund and Dale: Atoxyl, Zentr. Bioch. Bioph., I3, 25I, I9I 2.

Rotısciuld, M. A.: Cholesterin, Beitr. path. Anat., 60, 39, 66, I 914.

Rous, P., and Turier: Erythrocytes, J. Am. Med. Assoc., 64, 2 I63, I9I5; Trans. Assoc. Am. Phys., 30, 210 , 1915.

Roussy and Clunet: Parathyroids, C. R. Soc. Biol., 68, 818, 1910.

Rovigiri: Ammonia, Il Morgagni, 4I, 449, I889.

Rowntree and Aber: Antimony, Trans. Assoc. Am. Phys., 25, 403, igio; J. Pharmacol., 2, IOI, I9I2.

Rowntree and Gerachty: Diuretics, Arch. Int. Med., 9, 284, I912; Phenolsulphonphthalein, J. Pharmacol., I, 579, I9ro.

Rowntree, L. G., and MAcHт: Digitalis Standardization, J. Am. Med. Assoc., 66, 87o, I9I6.

Roy, G.: Hypericum, Mulf. Vet. Bull., 6, 43, I914.

Roy and Sherrington: Cerebral Blood Supply, J. Physiol., i 1, 85, I89o.

Rubini; Cactus, N. Y. Med. J., 3, 1864.

Rudolph, R. D.: Coagulation, Trans. Assoc. Am. Phys., 25, 504, 1910.

Rudolph and Cole: Aconite, Am. J. Med. Sci., Dec., I9 2.

Ruebsayes: Morphin, Arch. exp. Path. Pharm., 59, 227 , roo8.

Ruebsames and Kligerman: Hydrastis, Zentr. Bioch. Bioph., 14, 602, I9I 2.

Ruedel: Diuresis, Arch. exp. Path. Pharm., 30, 4I, I8g2.

Rulon and Hawk: Water, J. Am. Chem. Soc., 32, 1686, igi i.

Ruspf: Iron, Berl. klin. Woch., 38, 477, 1901.

RUPERT: Epinephrin, Bioch. Centr., 8, 898, I9o9.

Russel, F. F.: Typhoid, Trans. Assoc. Am. Phys., 28, 457, I913; Typhoid Vaccine, J. Am. Med. Assoc., 62, I37 I, I9I4. 
Russell, W. B.; Calcium, Brit. Med. J., I, I99, I907.

Russelt, D. G.: Gentian Violet, J. exp. Med., 20, 545, I9I4.

Ruth, V. J.: A pomorphin, Arch. ges. Physiol., I913.

Ruther Ford: Bile, Trans. Roy. Soc. Edinb., 29, 133, 1879.

Rutkewitscir, K.: II ypertomis, Zs. klin. Med., 79, 23 I, I9I 4.

Rxan, A. H.: Strychnin Absorplion, J. Pharmacol., 4, 43, r9 2.

Ryan and Guthrie: Strychnin, Am. J. Physiol., 22, 440, 1908.

Ryan and McGuigax: Strychnin, J. Pharmacol,, 2, 319, I9I I.

Rxwosch: Hemolysis, Centr. Physiol., 25, 848, i9iт.

Rzentkowski, K.: Nitrite, Z. klin. Med., 68, i i i, igo9; Saliarsan, Zentr. Bioch. Bioph., I2, 894, I9II.

Sabbatani, L.: Calcium, Arch. ital. Biol., 36, 397, 416, igor; Caramel, Arch. Fisiol., I I, 518, I9г3; Carbon, Arch. Farmacol., r6, 518, r914; Colloid Carbon, Bioch. Z., 59, 408, I914; Colloid Sulphur, ib., 59, 378, I9I4; Iodin, Gaz. Osp. Clin., 33, No. 58, г9г 3; Mercury, Bioch. Centr., 5, 502, 1906; Arch. int. Pharmacod., I 7, 318, I907; Sodium, Acad. Real. Sci. Torino, I901; Sulphur, Arch. int. Pharmacod., 18, 373, I908; Pathologica, No. 100, r9ı3; Sulphur Salves, Koll. Zs., I3, 249, I9 I3.

Saccone, A.: Diuretics, Arch. intern. Pharmacod., 21, 63, 19 I I.

SAcharoff: Tetra, Z. exp. Path., 7, 225, r9o9.

Sachs, H.: Animal Hemolysins, Bioch. Centr., 5, 257, r9o6; Scopolamin, Berl. klin. Woch., No. 30, I9I 2.

SAhli: Hemophilia, Zs. klin. Med., 56, 294, 1905; Morphin, Therap. Monatsh., No. I, I909.

SaIkowsky: Mercury, Arch. Path. Anat., 37, 346, 1866.

SakaI, S., and Saneyoshi: Strophanthin, Caffein, Diuretin, Arch. exp. Path. Pharm., 78,331 , I915.

Salant, W.: Alcohol, Proc. Soc. Exp. Biol. Med., 3, 58, r9o6; Caffein, Circulation, J. Pharmacol., 3, 468; 4, 34r, I01 2; Chenopodium, J. Pharmacol., 2, 391, I910; Conditions, U. S. Bur. Chem., Circ. 81, I91 ; Temperature-Caffein, J. Pharmacol., 4,342 , I9I3.

Salant, WM., and Bengis: Chenopodium, Soc. Exp. Biol. Med., 12, т63, т9г5; Dyes, J. Pharmacol., 8, iा9, igi6.

Salant, WM., and Hecht: Ergot, J. Pharmacol., 5, 5 I 7, r9i4; Oxalates, Am. J. Physiol., 36, I 26, I914; Soc. Exp. Biol. Med., I 1, I 79, I9 14.

Salant, W., and Hinkel: Alcohol, J. Pharmacol., I, 493, r9ro.

Salant, W., and Livingston: Chenopodium, Am. J. Physiol., 38, 67, igi5; Soc. Exp. Biol. Med., I2, I30, r915; J. Pharmacol., 8, I22, I916; on frog heart, Am. J. Physiol., 4I, 21, I9r6; Iodin, J. Pharmacol., 8, I I9, I9I6; Am. J. Physiol., 4I, 234, I9 6 .

Salant, W., and Mrtchell: Chenopodium on Peristalsis, Am. J. Physiol., 39, 37, I915; Soc. Exp. Biol. Med., I2, I62, r915; Metals on Inlestines, Am. J. Physiol., 39, 355, I9I6.

Salant, W., and Nelson: Chenopodium, Am. J. Physiol., 36, 440, I9 5.

Salant and Phelps: Caffein Demethylation, J. Pharmacol., 3, 469, I9 2.

SAlant and Rieger: Caffein, Am. J. Physiol., 33, 186, I914; Caffein Excretion, J. Pharmacol., 3, 469; U. S. Bur. Chem. Bull., I66, IgI2; Caffein on Creatin, J. Pharmacol., 2, 400, I910; Caffein Tolerance, ib., I, 572, I910; Caffein Toxicity, J. Pharmacol., 3, 455, tor 2.

Salant, Rieger and Treuhardt: Tin, J. Biol. Chem., I7, 265, 1914.

Salant, W., and Suith: Tartrate Toxicity, Proc. Soc. exp. Biol. Med., ro, I7o, I9I3; Am. J. Physiol., 35, 239, r914; J. Pharmacol., 5, 515, 1914.

Salant, W., and Wise: Citrate, J. Pharmacol., 8, 123, 1916.

Saliba, J.: Ether, J. Am. Med. Assoc., 66, r295, Igr6.

Salkowski: Fever, Arch. path. Anat., 53, 209, I878; Formaldehyyd, Bioch. Zs., 7 I, 365, I9I5.

Salomon, O.: Iodid, Ref., J. Am. Med. Assoc., 62, r7 I, r913.

Saloz, J.: Bronchioles, Soc. Biol., 77, 6, I9I4.

Salvisberg: Digitalis, Merck's Rep., 21 , 86, 1907.

Salzmann: Narcosis, Arch. exp. Path. Pharm., 70, 233, I9I2.

Samojloff, A.: Muscarin, Zentr. Physiol., 27, 7, r9r3.

Saneyoshi: Arsenic, Zs. exp. Path., I3, 340, I9I3.

Sanno, Y.: Temperature, Arch. exp. Path. Pharm., 65, 325, I9II.

Sano, T.: Strychnin and Caffein, Arch. ges. Physiol., I 24, 381, I908.

Sansum and Woodyati: Anesthetics, Soc. exp. Biol. Med., II, I57, I9I4; J. Biol. Chem., 21, I, I9I5; Phlorhizin, Proc. Am. Soc. Biol. Chem., 3, 26, I9 I5. 
Sivtrison: Benzol Poisoning, Skand. Arch. Physiol,, 10, 1, 1899; Cocain, Biochem. (entr., 5, 703, I900; Glycerin, Veratrin, Skand. Arch. Physiol., I4, I, 1903; Habituation, Nord. Congr. inn. Med., 201, 1912; Quinin, Arch. exp. Path. Pharm., 30, $444 ; 32,321,1892$.

Sintesiso, C. (... and STRINdBerg: Heart-Tonics, Wien. med. Woch., No. 14-15, 19r6. Sintroson and II ickizerg: Bromate, Skand. Arch. Physiol., 3o, 337, 1913.

SIRBAYs and GíRINE: IIordenin, Jahrb. Pharm., 69, 296, I909.

Sirvoxit and Crémen: IIaloids, J. de Phys. Path. gen., 589, I9I I.

SARVONit and ROLBIER: Oxalate, C. R. Biol., 7I, IO7, I9II.

Siss.ı, R.: Oxybutyric Acid, Bioch. Zs., 59, 362, I914.

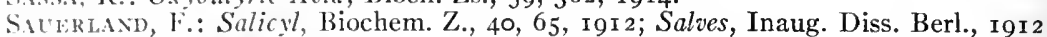

Sigiraud: Boric Acid, Ref., J. Am. Med. Assoc., 63, 593, i9r4.

S.ıYRE: Gelsemium, Lloyd Library, I9 10; J. Am. Pharm. Assoc., I, 458, 19 I 2.

Siyre and Chillingworth: Gelsemium, J. Am. Pharm. Assoc., March, i9i4.

Scicilone, S.: Chloroform Narcosis, Zentr. Bioch. Bioph., 18, 55, I915.

SCEletiI, C. E.: Morphin, J. Am. Med. Assoc., 66, 860, I9I6.

Schinelitz, H.: Bromism, Ref. Ther. Mon., 30, 56, igi6.

Schaefrer, E. A.: Chlorin, Brit. Med. J., Aug. I4, I9I5; Oncometer, J. Physiol., 20, 5 , I896; Pituitary, Quart. J. Physiol., 6, I 7, I9ı3.

Scinefer, E. A., and Herring: Pituilary, Proc. Roy. Soc., 77, I906.

Schaffer and Scharlieb: Chloroform, Trans. Roy. Soc., Edinb., 4I, 3 II, I9O4.

Schaefer, E. A., and Vincent: Hypophysis, J. Physiol., 25, 87, I899.

Schaefer, T. W.: Calomel, Merck's Rep., May, rg10; Sulphur, Bost. Med. Surg. J., I57, 106, 1907 .

Schaer, I..: Guaiac, Pliarm. Ztg., 63, 328, r9r3.

Schaer, E. W. E.: Cocain, Am. J. Physiol., 38, 269, r9r 5.

Scinafir, M.: Alcohol, Arch. path. Anat., 213, 4I, I9I3.

Schamberg, J. F., and Kolmer: Calomel, J. Am. Med. Assoc., 62, 1950, 1914.

Schamberg, J. F., Raiziss and Kolmer: Chrysarobin, J. Cut. Dis., 33, 98, I915; Mercury, ib., 33, No. 12, 1915; Ref., J. Am. Med. Assoc., 64, 936, 1915.

ScheFfer: Silicate, Merck's Rep., 22, 321 , I908.

SCHenck: Oxygen, Erg. Physiol., 7, 65, I908.

SchifF: Thyroid, Rev. med. Suisse Rom., 4, 65, 425, 1884 .

Scrilld: Calomel, Diss. Halle, i 892.

Schiller: Delphinin, Arch. Physiol., 248, I9O4.

Schilling: Excision-kidneys, Arch. exp. Path. Pharm., 52, I40, 1904.

Schirokogorow: Salvarsan, Zentr. Bioch. Bioph., 15, 278, I913.

Schlaginweit, E.: Hormonal, Arch. intern. Pharmacod., 23, 77, 1913.

Schlasberg: Salvarsan, Zentr. Bioch. Bioph., I4, 520, I91 2.

Schlayer: Salt Test, Deut. Arch. klin. Med., i ro, 333 , igri.

Schlayer and Hedinger: Nephritis, Deut. Arch. klin. Med., 90, i, ig07.

Schlayer and Takayasu: Nephritis, Muench. med. Woch., 60, 800, 1913.

Schlesinger: Mercury, Arch. exp. Path. Pharm., 13, 317, 1884; Theocin, Muench. med. Woch., No. 23 , 1905.

Schlomovitz, B. H., and Chase: Sirychnin, J. Pharmacol., 8, I27, I916.

Schloss: Nitriles, Deut. Arch. klin. Med., II I, 3 10, 19r3.

Schlosser: Diuretics, Zentr. exp. Med., I, 559 , I913.

Schмack: Ergot Epidemics, Inaug. Diss. Halle, 1897.

Scrimid, A.: Local A nesthetics, Z. exp. Path., I4, 527, I913.

Scimid, J., and Schroeter: Hexamethylenamin, Bioch. Centr., Io, I32, I910.

Schmidt, A.: Hyperacidity, J. Am. Med. Assoc., 62, 430, 1914.

Schimid, Adolf and Meyer: Peritoneal Injection, Biochem. Centr., 4, 465, 1905.

Schmidt, A., and David: Oxygen, Deut. med. Woch., I68\%, I9I 2.

Schmidt, A. D.: Paraffin, Muench. med. Woch., 52, 1970, 1905.

SchuidT, C. H. L.: Iodoform. Arch. intern. Pharmacod., 9, Io7, 1901.

Schmidt, E.: Phenol, U. S. P. Dig., 525, r911; Scopolamin, Arch. Pharmac., 1892.

Scirmdt, M. B.: Iron, Zentr. Bioch. Bioph., I4, 279, 1912.

Scimmedeberg, O.: Caffein, Arch. exp. Path. Pharm., 2, 62, 1872; Chicory, Arch. Hyg., 76, 210, 1912; Digitalis, Arch. exp. Path. Pharm., 62, 305, 1910; Digitalis Constituents, Arch. exp. Path. Pharm., 3, I6, I875; Urethane, ib., 20, 203, 1885.

Scimmedeberg and Harnack: Muscarin, Arch. exp. Path. Pharm., 14, 376, 1876

ScumLEDEBERG and KOPPE: Muscarin, Leipzig, I 869 .

Schimedeberg and Meyer: Camphor, Z. physiol. Chem., 3, 422, 1879.

Schmincke, R.: Iron, Muench. med. Woch., 60, I199, 1913.

Schmitter, F $\therefore$ Emetin, J. Am. Med. Assoc., 64, 53, IqI5.

Schmitz: Quinin, Arch. exp. Path. Pharm., 56, 301, r907. 
Schneiderlin: Morphin-scopolamin, Muench. med. Woch., No. 9, 37 I, I903.

Schoenberg, M. J.: Intraocular Pressure, J. Am. Med. Assoc., 61, rog8, r913.

Schoenfor, S.: Chloroform, Beitr. path. Anat., 58, I30, I9I4.

Scholtze, M.: Pareira, Arch. d. Pharm., 25 I, I36, i913.

Scrioltze, M., and Koch: Pareira, Arch. d. Pharm., 252, 513, I915.

Schotт: Miscarin, Arch. exp. Path. Pharm., 65, 239, i9 I I.

Schnitter: Lead, Deut. Arch. klin. Med., i I 7, I 27 , I9 14.

Schrember and Zandy: Salicyl, Deut. Arch. klin. Med., 62, 242, I899.

Schroeder, W. vox: Caffein, Arch. exp. Path. Pharm., 22, 39; 24, 85, I886-87; Granatum, ib., I8, 38г; 19, 290, I884; Santonin, ib., I9, 304, 1884.

Schryver: Formaldehyd, Zs. Coll. Chem., 8, 236, I9I I.

Schubert: Salvarsan, Muench. med. Woch., 823, I9I4.

Schuebel, K.: Salvarsan, Derm. Zs., 2 I, I 24 , I914.

SchuefFNer and Verwoort: Chenopodium, Muench. med. Woch., 60, r 29, i9r3.

SchuepBach: Bile, Z. Biol., 5 I, I, rgro.

Schuetz, E.: Emetics, Arch. exp. Path. Pharm., 2 I; 341, I886.

Schuetz, J.: Magnesium, Muench. med. Woch., 62, No. 4, I9I5; Irch. exp. Path. Pharm., 79, 285, I9I6; Zs. Balneol., 8, No. II-I2, I9I5.

Schultz, A. F.: Wall-paper, Ref., Ther. Mon., 29, 582, I9I5.

Schultz, J. N.: Alcohol, Zentr. Bioch. Bioph., I3, I 7 I, I9 2.

Scrultz, P.: Pupil, Arch. Physiol., 47, 1898.

Schultz, W. H.: Anaphylaxis, J. Pharmacol., I, 549, г9го; 3, 299, I9 12; Chloral, Am. J. Physiol., I6, 483, r9o6; Epinephrin, Proc. Soc. Exp. Biol. Med., 6, 23, 1908; U. S. Hyg. Lab. Bull., 55, I909; Thymol, J. Pharm. exp. Ther., 6, 599, I9 5.

Schultz and Seideli: Thymol, Chem. Abstr., 6, 2955, I9I2.

Schultze, O.: Fever, Arch. exp. Path. Pharm., 43, I93, I899.

Schultzen and Riess: Phosphorus, Chem. Centr., 680, i 869.

Schulz, H.: Arsenic, Arch. exp. Path. Pharm., I I, I3, I884; Cacodyl, ib., I I, I3 I, I879.

Schumacher, F.: Silver, Ther. Mon., 29, 270; Derm. Wschr., 60, I3, I9 I5.

Schumacher, J.: Iodin, Deut. med. Woch., 68I, 19 I5; Salicyl, Diss. Giessen, 1908.

Schumburg: Caffein, Arch. Physiol., Suppl., p. 289, I899.

Schwalb, H.: Terpenes, Arch. exp. Path. Pharm., 70, 7I, ig 2.

Schwalbe: Poison Ivy, Muench. med. Woch., No. 39, I 902.

Schwarz, C., and Lemberger: Acids, Arch. ges. Physiol., 141, I49, r9 I.

Schwarz, G.: Camphor, Arch. exp. Path. Pharm., 54, 14I, I9o6; Radium, Arch. ges. Physiol., 100, 532, I903.

Schwarz, G., and Zehner: Thorium, Deut. med. Woch., 38, I776, I9 2.

Schwartz, O., and Pulay: Lactose, Zs. exp. Ther., I 7, 383 , I9 5 .

DE Schweinitz: Salicyl, Trans. Am. Ophth. Soc., I 895.

Schweishemer, W.: Alcohol, Deut. Arch. klin. Med., ro9, 27 I, i913.

Sçrwenkenbacher and Spitta: Sweat, Arch. exp. Path. Pharm., 56, 284, 1907.

Schwentner: Morphin, Zentr. Bioch. Bioph., 13, 878, I9 2.

SCHWYzER, E.: Fluorid, Bioch. Zs., 60, 32, I9I4.

Scotr, E. L.: Anesthesia, Am. J. Physiol., 34, 27 I, I9I4.

Scotr, G. G.: Dog-fish, Columbia Stud. Physiol., 6, I9 13.

Scotr, J. C.: Gossypium, Ther. Gaz., 35, I62, I9I1.

Scott, R. W., Thoburn and Hanzlik: Salicyl (Blood and Joint Fluid), J. Pharmacol., 8, 130, I9I6; Salicyl (Excretion in Rheumatic and Non-rheumatic), J. Pharmacol., 8 , п 29 , т 916.

Scovit.e, W. L.: Capsules, J. Am. Pharm. Assoc., 4, r 241, 19 5 ; Phenol, Bull. Pharm., 27, 393, r913; Podophyllum, Proc. Am. Pharm. Assoc., 57, 897, 1909.

Seaman, E. C.: Beryllium, Bioch. Bull., 2, I84, I912.

Secchr, R.: Acid, Bioch. Zs., 67, I43, I9I4.

Secher, K. J. A.: Caffein on Striped Muscle, Soc. exp. Biol. Med., 77, 83, I914; Quinin, Arch. exp. Path. Pharm., 78, 445, 1915.

SEDGwick: Illuminating Gas, Harvey Lect., roo, ror 1.

SEe, G.: Salicyl, Bull. Acad. Med., 6, 689, I877.

Seelig, Tierney and Rodenbaugh: Bicarbonate, Am. J. Med. Sci., 146, I95, I9I3.

SEgond: Digitalis, Zentr. Bioch. Bioph., I4, 522, I91 2.

Seibert: Salicyl, Med. Rec., 79, No. I, I9I I.

Seidell, A.: Epinephrin, J. Biol. Chem., I5, 197, r913; Thymol, U. S. Hyg. Lab. Bull., ror, 43, I915; Vitamins, Am. J. Phar., 88, 140, I916.

Seidell and Fenger: Suprarenal, U. S. Hyg. Lab. Bull., I00, I9r4; Thyroid, J. Biol. Chem., I3, 5I 7, I9I3; U. S. Hyg. Lab. Bull., 96, 19 14.

Selic: Alcohol, Zentr. Bioch Bioph., r4, 740, r9 13.

Seligmann: "Camphor, Arch. exp. Path. Pharm., 52, 333, 1905. 


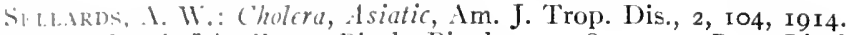

sirtul. J.: C'affin, Zentr, Bioch. Bioph., I3, 284, 1912; Dyes, Bioch. Z., 49, 466, 1913. Situxi: Binzol, Rockef. Inst., I5, 1912.

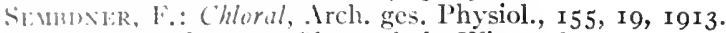

Simmin: Vorles. exp. Pharmakol., Wien, i89o.

Srimpik: Kamala, Arch. exp. Path. Pharm., 63, го, igro.

Sr:st八: Antipyretics, Arch. intern. Pharmacod., r8, 217, 1908.

Sertilexira: Morphin, Gilbert's Ann. d. Physik., 55; 56; 57, I83, I817.

Sintrix and Heinricisderf: Sulvarsan, Zs. klin. Med., 76, I38, I9I 2.

Sillitzer: Iodid, Arch. int. Pharmacod., I8, 285, 1908.

Sirtare, (.. 1).: Methylen Blue, Am. J. Physiol., 22, 335, 1908.

Shaklee and Meltzfr: Ferments, Am. J. Physiol., 23, 29, I9o9.

Smunf, V. N.: Pituitary, J. Physiol., 39, 268, 1916.

Sinnrp, J. Gordon: Cactits, Practitioner, 3, 444, I894; Ergot, Proc. Roy. Soc. Med., 4 , IIt, I9II.

Shattick, F. C.: Hexamethylenamin, Trans. Assoc. Am. Physiol., 26, 87, 19 Ir.

Siraw-Mackexzie: Trypsin, Brit. Med. J., No. 2352, 240, 1906.

Suermax, H. C.: Iron, U. S. Exp. Stat. Bull., No. I85, I907.

Shermax, H. C., Mettler and Sinclair: Salts, Exp. Stat. Bull., 227, 1910.

Sherrick, J. W.: Potassium Iodid on Luetin Reaction, J. Am. Med. Assoc., 65, 404, 1915. Sherrington: Strychnin, Quart. J. Physiol., 2, II5, I9o9.

Sherringtox and Copemax: Hypotonic Injections, J. Physiol., I4, 52, I893.

Siterrington and Sowton: Anesthetics, Brit. Med. J., Suppl., I47, 1903.

Sherwix, C. P., and HaWk: Water Drinking, J. Am. Chem. Soc., 36, r779, I9r4.

Shilling and Goretti: Serum on Metals, Zs. Immun., 23, 259, I9I4.

Simmazovo, J.: IIemolysis, Arch. exp. Path. Pharm., 65, 361, I9I1.

Sicardr: Chloroform, C. R. Soc. Biol., 60, 243, 1906.

Sicard and MeAra: Salicylic, Am. J. Med. Sci., 150, 843, I9I5.

Sirber: Arscnic, Arch. int. Pharmacod., 22, 269, 191 2; Peroxid, Zs. Physiol. Chem., 32,373 , I901.

Sifburg: IIelleborein, Zentr. Bioch. Bioph., 15, 463, 1913.

Siegel, P. W.: Scopolamin, Deut. med. Woch., I049, I9I4.

Siegrried: Fluorid and Silicate, Arch. intern. Pharmacod., 9, 225, 1901; Hypnotics, Ther. Mon., 27, 549, I9I3.

Sielisch, J.: Picrotoxin, Chem. Abstr., 6, 3r6o, 19 I 2.

Siex: Aluminum, Diss. Dorpat., 1886.

Sievers, A. F.: Bclladonna, J. Agr. Res., I, I29, I913; Am. J. Pharm., 86, 97, I914; ib, 88, 193, 1916; U. S. Agr. Bull., 306, 1915.

Simox, D. L.: Hexamethylenamin, Ref., Ther. Mon., 28, 544, I9I4.

Simox, I.: Atropin, Ref., J. Am. Med. Assoc., 64, 705, 1915; Intravenous, Bull. Sc. Med., Bologne, 5, I905; Morphin, Arch. Intern. Pharmacod., 16, 255, I907; Stovain-sirychnin, Arch. farm. sper., 19, 4I2, I9I5.

Simoxot: Atoxyl, Bioch. Centr., ro, 199, r9ro.

Simpson: Cerium, Month. J. Med. Sci., Lond. and Edinb., I9, 564, 1854.

Sinpson, S.: Pituitary, Am. Med., 9, 2 I9, r914.

Sisrpsox, S., and Hins: Pituitary, Soc. Exp. Biol. Med., I 2, 30, I914; Am. J. Physiol., 36, 347; Qu. J. Exp. Ph., 8, 1915.

SimpsoN and HunTer: Pitzitary, Quart. J. Physiol., 4, 257, 19 זr.

Sippy, B. W.: Gastric Ulcer, Trans. Assoc. Am. Phys., 30, 129, 1915.

Sivert and Kaminsky: Iodid, J. Am. Med. Assoc., 63, r889, I9I4.

Skorczewski: Alophan, Z. exp. Path., I4, II 3 , I9I3.

Slade: Zygadenus, Am. J. Pharm., 77, 262, 1905.

Stowtzoff, B.: Copper, Beitr. physiol. Chem., 2, 307, 1902; Lecithin, ib., 8, 370, 1906.

Sirilie, W. G.: Cystinuria, Arch. Int. Med., r6, 503, I915; Potassium, ib., 16, 330, 191 5.

Sмiтh, E., and Bellingham: Radium, Zentr. Bioch. Bioph., I2, 85I, I9I2.

Sмiтн, G. G.: Hexamethylenamin, Bost. Med. Surg. J., May 15, 1913.

Smith, G. G., and Porter: Spinal A nesthesia, Am. J. Physiol., 38, 108, 1915.

Smith, J. L.: Hypochlorous Acid, Brit. Med. J., Aug. I4, 261, I9r 5.

Suith, J. L., Drennan, Rettie and Campbeli: Hypochlorous Acid, Ref., J. Am. Med. Assoc., 65,744 , 1915.

Sirte, M.I., and Fantus: Optochin, J. Pharm. Exp. Ther., 8, 53, r916; Scopolaminmorphin, ib., 7, 407, 1915.

Sмiтн, R. B.: Ether, Ther. Mon., 27, 426, 1913.

Sмiтн, R. M.: Nephritis, Arch. Int. Med., 8, No. 4, rgrr.

SNAPPER, J.: Chlorid, Deut. Arch. klin. Med., II I, 44I, I9I3.

Sobieranski: Caffein, Arch. exp. Path. Pharm., 31, 329, 1893; ib., 35, 144, 1895. 
Socrs, Ch.: Chloroform, Arch. ges. Physiol., I60, I32, I914.

Soнs, J.: Benzol, Wien. klin. Woch., 26, 573, I9I3.

Sohrt: Scopolamin, Arch. exp. Path. Pharm., 22, 396, I886.

Solem, G. O., and Lommen: Pituitary, Am. J. Physiol., 38, 339, I9 I 5.

Sollmanx, T.: Anisotonic Solutions, Am. J. Physiol., I 2, 99, 1904; Chloral, J. Am. Med. Assoc., 5I, 487, I908; Chlorid Excretion, Am. J. Physiol., 8, I39, I902; Chlorid Retention, ib., 8, I55, I902; Development, ib., 10, 352, 1904; Diuretics, ib., 9, 426, 1903; Epincphrin, ib., I3, 246, I905; J. Am. Med. Assoc., 47, 792, I906; Formaldchyd, Am. J. Physiol., 7, 22 I, I902; J. Am. Med. Assoc., 5 I, 818, I9o8; ib., 52, I55, I909; Funduli, Am. J. Physiol., I6, I, I906; Gasolin, Am. J. Med. Sci., Sept., I9o4; Antagonism, Am. J. Physiol., Iо, 352, I904; Isotonic Salts, Arch. exp. Path. Pharm., 46, I, I901; Kidney Perfusions, Am. J. Physiol., 21, 37, I908; Ouabain, Clev. Med. J., I4, 483, 1915; Phenol, J. Am. Med. Assoc., March 17, I906; Salicyl, ib., 51, 818, I908; Tannins, J. Med. Res., 7, 43, I902.

Sollmann, T., and Brown: Carotid Traction, Am. J. Physiol., 30, 88, I9 2; Ergot, J. Am. Med. Assoc., July 22, 1905; Am. J. Physiol., I3, 246, 1905; Sulphate and Phenol, J. Am. Med. Assoc., 48, I015, I907; Thorium, Am. J. Physiol., I8, 426, I907.

Solmann, T., Brown and Williams: Cauterization, Am. J. Physiol., 20, 74, 1907.

Sollmann, Hanzlik and Pilcher: Phenol, J. Pharmacol., I, 409, I9Io.

Sollmann, T., and Hatcher: Excised Kidneys, Am. J. Physiol., I3, 241, I905; ib., I9, 233, I907.

Sollmann, T., and Hofmann: Chlorid, Am. J. Med. Sci., Feb., I905.

Sollmann, T., and McCoмr: Albumimuria, J. Exp. Med., 3, 137, I 898.

Sollmann, T., and Pilcher: Asphyxia, Am. J. Physiol., 29, roo, r9 I ; Caffein, J. Pharmacol., 3, I9, I9I ; Sciatic and Curare, Am. J. Physiol., 26, 233, 1910; Vagus, Am. J. Physiol., 30, 303, I9г 2; Vagus Depressor, ib., 30, 369, I912; Vasomolor Center, J. Pharmacol., I, 57 I, I9 го.

Solowertschyк: Antimony, Arch. exp. Path. Pharm., 1 2, 438, 1880.

Sonntag, G.: Frutit-Sprays, Zentr. Bioch. Bioph., I8, 552, 1915.

Sotтile: Alcohol, Chem. Abstr., 7, I527, I9 2.

Souligoux: Ether, Bul. soc. Chir., Feb. I9, I9I3.

SouzA, DE: Sulphocyanid, J. Physiol., 35, 332, I907.

Spenl and Collard: Emetin, Presse med., 290, I9I4.

Spenzer, J. G.: Ether, Arch. exp. Path. Pharm., 33, 407, I894.

SPIEGEL: Yohimbin, Chem. Zeitg., 20, 970, I896.

SPIEGLER: Water, Z. Biol., 4I, 239, I9OI.

Spiller and Leopold: Stovain, J. Am. Med. Assoc., 54, I840, I9io.

Spiro, K.: Acids, Beitr. physiol. Chem., I, 269, I901; Colloids, J. exp. Path. Ther., 5, 276, 1904; Copper, Muench. med. Woch., 62, I601, I9 5.

SpitTa: Morphin, J. exp. Path. Ther., 5, 84, 1908.

Sprinceanu, C.: Epinephrin, Soc. Biol., 78, 225, 1915 .

Stadelmann: Alkalien, Stuttgart, I890; Cathartics, Arch. exp. Path. Pharm., 37, 352, I896; Diabetes, ib., I7, 419, 1883.

Stadelmann and Magnus Levy: Methyl Alcohol, Berl. klin. Woch., 49, 193, I9I 2.

Stange, O.: Narcosis, Arch. intern. Pharmacod., 23, 461, 1913.

Stanley: Camphor, Ther. Gaz., 28, No. 7, 1903.

Stanley, L. L.: Tropacocain, J. Am. Med. Assoc., 6o, 10go, 19ı6.

Starkenstein, E.: Atophan, Arch. exp. Path. Pharm., 65, r77, i9 I ; Oxalate, ib., 77, 45, I9I4.

Starkenstein, E., and Wiechowski: Atophan, Zentr. Bioch. Bioph., i7, 565, I914.

STARLING: Chemical Control, Harvey Lect., 3, I I 5, Igo8.

StarR: Ginko, Am. J. Pharm., 85, 327, I9I3.

Steel, M.: Alum, Am. J. Physiol., 28, 94, igri.

STEenBock, H.: Milk Flow, J. Agr. Res., 5, 561, r9 5.

Stehle, R. L.: Endogenous Uric Acid, Soc. Exp. Biol. Med., I 2, I48, I9I5.

Stern, B.: Benzol, Wien. klin. Woch., 25, I938, r9 2.

STEINACH: Strophanthus, Wien. klin. Woch., Nos. 21 and 22, 1888.

Stenstroem, T.: Epinephrin, Bioch. Z., 58, 472, 1914 -

Stern, H.: Formic Acid, J. Am. Med. Assoc., 46, 1 $25^{8}, 1906$.

Stern, R.: Fever, Z. klin. Med., 20, 63, I892.

Stevens, H., and MAY: Uric Acid, J. Am. Chem. Soc., 33, 434, I9I I.

Stevenson, A. E., and Sayre: Sempervirin, J. Am. Pharm. Assoc., 4, 1458, 1915.

Stewart, D. D.: Acelanilid Poisoning, J. Am. Med. Assoc., 44, I725, 1905; Nitroglycerin, ib.,.44, x673, 1905.

Stewart, F. E., and Pittenger: Ergot, Month. Cycl. Med. Bull., Sept., r9i3. 
Sirkikt, G. X.: Blow Flow, J. Pharm. Exp. Ther., 8, 28r, r915; Epinephrin, J. Exp. Hed., 14.377, 191 ]; Oxygen, J. Pharm., 2, 477, I91 I; Saponin, J. Physiol., 24, 21 1, r.go; J. Lxp. Med., (), 257, I902; J. Pharm., r, r, I909.

Sге木 $1 \mathrm{RT}, \mathrm{G}$. X., and Rogoff: Epincphrin, liberation, Soc. Exp. Biol. Med., I 3, I86, 1010; store, ib., I3, 184 .

STFW:IRт, Rogorl and Gibson: Epinephrin Liberation, J. Pharmacol. 8, 205, 9 i6.

Sirtwat, (i. N., and Scott: Digitalis, J. Pharm. Exp. Ther., 7, 263, r915.

StEWIRt, G. N., and Zuckl:R: Serum, J. Exp. Med., I7, I 52, rgr3.

SitrWIRT, II. 1.: Epincphrin, Zentr. Bioch. Bioph., 14, 722, I9I2.

Stifes, P. G., and Martix: Y Jasomotor, Am. J. Physiol., 37, 94, I915.

Sitilixg: Anilin Dyes, Strassburg, I89o.

Situllinx, E., and Swift: Meninges, J. Exp. Med., 22, 286, I9I5.

Srulwakk: Kicin, Inaug. Diss. Dorpat, 1888 .

Simson: Rabies, Hyg. Bull., 65, roro.

Strixgel, J. L.: Bismuth, Am. J. Pharm., 83, 4r 2, rgri.

Stockiri, C. R.: Alcohol, Soc. exp. Biol. Med., rr, I36, r9i4; Arch. Int. Med., Oct., 369 , I9I2.

Stockmax, R.: Balsams, Lab. Rep. Roy. Coll. Phys., Edinb., 3, 65, 189r; Cocain, Iroc. Roy. Soc., Edinb., r886; Iron in Food, J. Physiol., 18, 484, 1895; Morphin, Proc. Roy. Soc., I7, I891; Salicyl, Brit. Med. J., 2, I271, I890; Proc. Roy. Soc. Med., 2, 3r; Edinb. Med. J., 2, I03, r906; Med. Press \& Circ., 87, 57, r9o9; J. Pharmacol., 4, 97, I9I2.

Stockmax, R., and Charteris: Iodid, Brit. Med. J., Nov. 23, rgor; Metals, J. Path. Bact., Dec., I903.

Stockifis, R., and Greig: Arsenic, J. Physiol., 23, 376, i 898.

Stoeltzxer: Calcium, Jb. Kindhk., 50, 268, I 899.

Stozrck, E.: Dysentery, Ther. Mon., 29, 504, I915.

Stoland: Thyroid, Am. J. Physiol., 30, 37, rgr 2.

Stolle: Caramel, Chem. Cbl., I I, 1021 , I899.

Stolz: Epinephrin, Ber. chem. Ges., 37, 4149, 1904.

Stolzexberg, H.: Betain, Zs. Physiol. Chem., 92, 445, rgr4.

Stookey and Morris: Salicyl, J. Exp. Med., 9, 3 I 2, I907.

Straxsky, E.: Magnesium, Arch. exp. Path. Pharm., 78, i22, I9I4.

Strissburg: Quinin, Arch. exp. Path. Pharm., 2, 334, r874.

STrashesko: Bitters, Russki Wratsch., No. I2, 1905.

Strassmany: Chloroform, Berl. Klin., No. i 16, r898.

Stratton, S. W.: Radium, J. Am. Pharm. Assoc., 4, I I I I, I915.

Straub, W.: Alkaloid Actions, Bioch. Centr., 2, 42, 1903; Aspidium, Arch. exp. Path. Pharm., 48, 1, 1902; Muscarin, Arch. ges. Physiol., I19, 127, 1907; Digitalis, Arch. exp. Path. Pharm., 79, 19, 1915; Epinephrin, Muench. med. Woch., No. ro, 1909; Arch. ges. Physiol., I34, I5, I910; Hamamelis, Arch. exp. Path. Pharm., 42, 1, 1899; Synergism, Bioch. Z., 41, 4I9, I9I3; Lead, Deut. med. Woch., No. 32, I9I I; XVII Int. Congr. Med., I9I4; Membranes, Ges. D. Naturf. Aerz., ror 2; Narcophin, Bioch. Z., 57, I56, I913; Narcosis, Muench. med. Woch., 60, 1823, I913; Thirst, Z. Biol., 38, 537 , I899.

Straus, H.: Charcoal, Deut. med. Woch., 62, No. 2, 19r6; Fluorescein, Berl. klin. Woch., 2226, I913.

Street, J. P.: Cod Liver Oil, J. Am. Med. Assoc., 64, 638, r915; Coffee Substitutes, Ref. J. Am. Med. Assoc., 66, 2 116, 1916; Foods, ib., 60, 2037, 1914; Sanatogen, ib., 63,1831, I9I4.

Stricker: Salicyl, Berl. klin. Woch., r3, I, I5, 499, 1876.

Stroomani, G.: Digitalis, Zs. exp. Med., 2, 278 , r9r4.

Stropesi: Iodoform, Zentr. Bioch. Bioph., I 5, 27 I, I9I 2.

Strlbell: Hedgehog, Bioch. Centr., 9, 705, r9o9; Yohimbin, Wien. klin. Wuch., I 105 , 1906.

Stuart, A.: Cobalt and Nickel, Arch. exp. Path. Pharm., 20, I5 1, r884.

STUBER: Lipoids, Bioch. Zs., 53, 493, I9I3.

Studzinsky and Kaminsky: Thyroid, Ref., J. Am. Med. Assoc., 66, 930, 1915.

Stuenmer, A.: Salvarsan, Arch. Derm. Syph., 1 20, 589, 1914:

Stuelp: Aspidium, Zentr. Bioch. Bioph., 3, 687, r904.

Stuempke and Siegrried: Salvarsan, Zentr. Bioch. Bioph., I 2, 470, I9I I.

Sturtevant, M.: Serum Sickness, Arch. Int. Med., 17, 176, 1916.

Stumoto, T.: Trypsin, Arch. exp. Path. Pharm., 74, 14, 1913; Uterus, ib., 74, 27, rgr3.

Sulzer: Arsenic, Zentr. Bioch. Bioph., I2, 95, I9II.

Surveyor: Nitrite, Bioch. J., I, 347, r9o6.

Swain and Bateman: Thalium, J. Biol. Chem., 7, 137, 19 ro. 
Swartz, M. D.: Lichens, Trans. Conn. Acad. Arts. Sci., r6, 24\%, 19 I 1.

Swetschnikoff, W.: Epinephrin, Zentr. Bioch. Bioph., I5, 737, I9 3 ; Arch. ges. Physiol., I57, 47 I, I9I4.

Swift, H. F.: Salvarsan, J. Exp. Med., r7, 83, r9 3 .

Swift, H. F., and Ellis: Salvarsan, J. Am. Med. Assoc., 57, 2051, I9 I ; 'Trans. Assoc. Am. Phys., 28, 258, 1913.

Swift, H. F. and Kinsella: Sensitized Bacteria, Soc. Exp. Biol. Med., I 3, I03, I9i6. Swirski: Caffein, Arch. ges. Physiol., I04, 260, I904.

Symes, W. L.: Digitalis, Brit. Med. J., June 20, I9r3; Inhalation, Irritant Gases and Vapors, J. Am. Med. Assoc., 65, 560, r9 5 .

Symes and Veley: Cocain, Zentr. Bioch. Bioph., r 2, 47, I9 I .

Szily, P., and Friedenthal: Mercury, Ther. Mon., 29, II4, I9I5.

TAchau, H.: Sweat, Arch. exp. Path. Pharm., 66, 334, I9II.

TADDEI, G.: IIypnotics, Florence, I9I3.

TAEgen, H.: Sulphur, Arch. exp. Path. Pharm., 69, I9I 2.

TAIT, J.: Yohimbin, Zentr. Bioch. Bioph., Io, 392, I9 Io.

TAIT, J., and PRINGLE: Strophanthus, J. Pharmacol., 8, 339, I9I6.

Takahasir, M.: Opium, Arch. ges. Med., I59, 327 , I914; Salvarsan, Arch. Derm. Syph., I 20, 3 I6, I9I4.

Takamine: Adrenalin, Am. J. Pharm., 63, 523, rgor.

TAkedA, W.: Bromural, Arch. int. Pharmacod., 21, 203, I9II.

Tambach, R.: Senna, Ref., Jahrb. Pharm., 73, 2 I, i9r3.

TANFiljefF: Methylen Blue, Bioch. Centr., 7, 42, I907.

TANRET: Ergot, Repert. de Pharm., 31, 3, 708, I875.

TAPpeiner: Fluorescence, Erg. Physiol., 8, 698, I909; Mucilaginosa, Arch. int. Pharmacod., 10, 67 , 1902.

Tappeiner and Brandl: Cathartics, Arch. exp. Path. Pharm., 26, i77, i889.

TARTakowsky: Iron, Arch. ges. Physiol., roo, 586; IOI, 423, I904.

TAshrro and Adams: Anesthetics, Am. J. Physiol., 33, Proc. XXXVIII, I9r4; Int. Zs. Physiol. Chem. Biol., r, 450, I9I4.

Tatum: Epinephrin, J. Pharmacol., 4, I 5 I, I912.

TAuber: Morphin, Arch. exp. Path. Pharm., 27, 336, r89o; Dissert. Strassburg, I892; Phenol, Zs. physiol. Chem., 2, 366, 1878.

Taussig: Digitalis, Arch. Int. Med., ro, 335, 19 г 2.

Taviani, S.: Cymarin, Ref., Ther. Mon., 29, 402, I9I5.

TAylor, A. E.: Alcohol, J. Biol. Chem., 15, 2 I 7, I9I3; Ferments, U. Calif. Pub. Path., I, I904; Salt Starvaticn, ib., I, 71, I904.

Taylor, A. E., and Rixger: Ammonium, J. Biol. Chem., r4, 407, rgr3.

TAYlor and SAIler: Sulfonal, Contr. Pepper Lab., I 20, I900.

TAylor, L.: Caffein, Arch. Int. Med., Dec., rgi4.

Terry, B. T.: Atoxyl, Proc. Soc. Exp. Biol. Med., 9, 4r, rg 2.

Theohari and Guirea: Nephritis, J. de Phys. Path., 538, r9io.

Theunissen, W. F.: Delirium Tremens, Ref., J. Am. Med. Assoc., 64, 867, I9r5.

ThIERsch: Salicyl, Volkmann's Klin. Vortr., Nos. 84 and 85,1875 .

ThIRy: Cathartics, .Wiener Sitzb., Math. n. w., K1. 50, I, 77.

Thols: Veronal, Zentr. Bioch. Bioph., I4, 763, I912.

Thomson, J. D., and Cushry: Antimony, Proc. Roy. Soc. B., 82, igro.

Tномpson, W. G.: Industrial Poisoning, J. Ind. Eng. Chem., 4, r9 2.

Thompson, W. H.: Aconite, Trans. Assoc. Am. Phys., 29, 32 I, I9 14; Protamins, $\mathrm{Z}$. physiol. Chem., 29, I, I900.

Thum, J. K.: Silver Iodid, J. Am. Med. Assoc., 65, I731, I9 5.

Thurston, A.: Cigarets, J. Am. Pharm. Assoc., 4, 435, I9I5.

Tidy, L. H.: Picric Acid, Lancet, Sept. I I, I9I 5.

Tiffeneau, M.: Epinephrin, Zentr. Bioch. Bioph., I8, 499, I9I5.

Tigerstedt: Digitalis, Skand. Arch. Physiol., 20, I 15 , 1907.

Tigerstedt and Airila: Pituitary, Skand. Arch. Physiol., 30, 302, I9r3; Uzara, ib., $33 ; \mathrm{I}, 37$, 19 15 .

Tijmstra, S.: Alcohol, Zentr. Bioch. Bioph., r6, 224, r9r 2.

Tillie: Curare, Arch. exp. Path. Pharm., 27, I, I890.

Trtone, F. P.: Bronchi, Arch. ges. Physiol., 155, 77, r913.

Tocco: Diplosal, Ther. Mon., 671, 1912.

Toczyski: Morphin, Zentr. Bioch. Bioph., I3, 845, I9 2.

ToDd, T. W.: Teeth, Clev. Med. J., I4, 253 , I9I5.

Tollens: Cresols, Arch. exp. Path. Pharm., 52, 221 , 1905.

Tomasczewsk̇ı: Atoxyl, Muench. med. Woch., 252, rgo8. 
linmsit: Intruenous Mercury, Ann. de Derm., Syph., 3, No. 12, I903; Salvarsan, Rei., Ther. Mon, 27,615, 1913.

lionlss, W". (i.: Stearic Coating, Im. J. Pharm., 87, 518, I9I5.

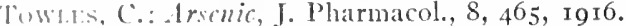

'lk.1' G., and (ins: Edema, Bioch. Bull., I, 467, I9 12.

'T'r.⿲丿丨, J.: Narcosis Theory, Arch. ges. Physiol., I53, 276, I913; ib., I60, 501, I6 1, 530, 1015 ; Bioch. \%s., 54, 305, 19 I3.

Trubr, J., and Kofhler: Dyes, Zentr. Bioch. Bioph., I8, 219, 1915.

TRatuE: Simipermeability, Arch. Anat., 34, I46, I867.

Tranislir: Sermm, Zentr. Bioch. Bioph., 2, 294, ig io.

Trendilenburg, P.: Anthelmintics, Arch. exp. Path. Pharm., 79, r9o, r9т5: Atropin, ib., 73, II8, I9I3; Bronchial Muscle, ib., 69, 79, 1913; Digitalis, ib., 6r, 256, I909; Epinephrin, ib., 63, I6r; Z. Biol., 57; 63, I55, 1910; Arch. exp. Path. Pharm., 79, I54. I9I5; Glycosuria, Arch. exp. Path. Pharm., 6r, 256, I909; Thyroid, Bioch. Z., 29,396, I010.

Tribe: Epinephrin. J. Physiol., 45, Proć. XX, I9I 2.

Trofger, J., and Mueller: Arch. Pharm., 252, 459, I9I 5.

Trontl, A.: Exophthalmos, Arch. Int. Med., I7, 382, I916.

TRLE, R. H.: Watcr, Sci., 39, 295, I9I4.

Truf, R. H., and Klugh: Cascara, Proc. Am. Pharm. Assoc., 57, 825, 1909.

Tscheboksarow: Iodid, Zs. exp. Med., 2, I68, I9I3.

'Tschekouxow, J. S.: Alcohol, Soc. Biol., 77, iा 8 , igi4.

Tschirch and Heuberger: Rhubarb, Schweiz. Woch. f. Chem. u. Pharm., 282, I902.

Tscinrcy and pupils: Emodin Cathartics, Ber. deut. pharmaceut. Ges., I 74, I898.

Tucher, H.: Magnesium, Ther. Gaz., 238 , I907.

Tuckermanx: Adonis, Ref., Jahrb. Pharm., 73, 54, I9r3.

I ixicliffe, F. II .: Phenolphthalein, Brit. Med. J., No. 2181, I224, 1902; Phosphorus, Arch. int. Pharmacod., 16, 207, I906.

Tunxicliffe, F. W., and Stebbing: Oxygen, Lancet, Aug. i9, igi6.

Turner, B. B., Marshall and Lamson: Plasmaphaeresis, J. Pharmacol., 7, I 29, I9I5. Tutis, F.: Senna, J. Chem. Soc., I03, I006; Zentr. Bioch. Bioph., I6, 596, I9I3.

Tutix, F., and Clewer: Rhubarb, Jahrb. Pharm., 7I, 54, igri.

Tyrode, M. V.: Anesthesia, Bost. Med. Surg. J., I63, I9, I910; Camphoric Acid, Arch. intern. Pharmacod., I8, 393, I908; Cathartics, ib., 20, 205, 1910; Thiosinamin, ib., 19, 195, I910.

Tyrode, M. V., and Nelson: Calomel, J. Med. Res., io, I32, I003.

Tyrrell: Atropin, N. Y. Med. J., Aug. I I, I906.

Tysebaert, J.: Atropin, Intern. Beitr. Path., 4, Ior 2.

'Iyson, H. H., and Schomnberg: Methyl Alcohol, J. Am. Med. Assoc., 63, 9 I5, I9 I4.

Uhlenutth, P.: Selenium, Chem. Abstr., 7, 158, т9г 2.

Ullmann, K.: Arsenic, Wien. klin. Woch., 27, 838, i914; Salvarsan, Zentr. Bioch. Bioph., I4, 76r, I9I 2.

Ulricr: Salicyl, Arch. exp. Path. Pharm., 46, 321, Igor.

UMBER: Caramel, Deut. med. Woch., Feb., I9I5.

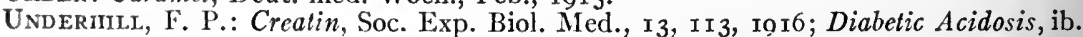
I 3, III, I9 I6; Epinephrin, J. Biol. Chem., 9, I3; Am. J. Physiol., 27, 331, I9 I ; Phlorhizin, J. Biol. Chem., I3, I9I 2; Proteoses, Am. J. Physiol., 9, 345, I903; Tartrate, J. Biol. Chem., I 2, Ig工 2.

Underhill, F. P., and Black: Cocain, J. Biol. Chem., i I, No. 3, IoI 2.

Underhill, F. P., and Closson: Dextrose, Proc. Soc. Exp. Biol. Med., 3, 65, 1906; Epinephrin, Am. J. Physiol., I7, 42, 1906.

Undermil., F. P., and Goldschmidt: Ammonium, J. Biol. Chem., I5, 327, 337, 341, I9I3.

Underhill, F. P., and Kleiner: Calcium, J. Biol. Chem., 4, igo8.

UNderhill, F. P., and SAIKI: Thyroid, J. Biol. Chem., 5, $225,1908$.

UNDERHill, Wells and Goldschmidt: Tartrate, J. Exp. Med., 18, 317, г9г3.

Unva, E.: Ointments, J. Am. Pharm. Assoc., I, 673, I9I 2.

Uschinsky, N.: Hypertonic Injections, Arch. int. Pharmacod., I5, 142, 1905.

Usener, W.: Hexamethylenamin, Zentr. Bioch. Bioph., I5, 378, 1913; Salicyl, Z. Kindhk., 5, 43 I, I913.

Usuelli: Salvarsan, Zentr. Bioch. Bioph., I4, 382, 1902.

Uyeno: Phenol, Bioch. Centr., 9, 537, Ig09.

Vahlen: Clavin, Arch. exp. Path. Pharm., 55, 131, I906.

Valassopoulos: Emetin, Ref., Merck's Rep., 27, 207, 1913. 
VALEnti, A.: Emesis, Zentr. Bioch. Biopin., I I, 884, 885, 908, I9 II; Morphin Excretion, Arch. farmacol., I7, 248, I914; Arch. exp. Path. Pharm., 75, 437, I914.

Valeri, G. B.: Bile, Arch. intern. Pharmacod., I9, 3 I I, Ioro; Calomel, ib., I9, 315, I910.

Varlon, C., and Bessiere: Cocain, Zentr Bioch. Bioph., i 7, 397, i9r4.

VAMossy: Phenolphthalein, Ther. Gegenw., 43, 203, 1902.

VANDERHOFF: Hexamethylenamin, J. Am. Med. Assoc., 58, 33I, I9I 2.

Vanderkleed and Pittenger Ouabain, J. Am. Pharm. Assoc., 2, 558, 822, i9i3; ib., 3, 8I 5, I9I4.

van Egmonn: Heart Block, Arch. ges. Physiol., I54, 39, r9r3.

Van Hengelm: Cholagogues, Ther. Mon., 27, 590, I9I3.

Van Itallie and Kerbosch: Morphin, Zentr. Bioch., Bioph., i i, 609, I9Io.

Van Leersum, E. C, : Mustard, J. Pharmacol., 8, 285, I9I6.

Van Slyke, D. D.: Acidosis, Soc. Exp. Biol. Med., r 2, r65, r9 5; J. Am. Med. Assoc., 66, I44, I9I6.

Van Slyke, D. D., Cullen and Mclean: Urea Formation, Soc. Exp. Biol. Med., I 2, 93, I9I5.

VAN'T HoFE: Osmosis, Z. physik. Chem., I, 481, I887.

Vanysek, F.: Calcium, Bioch. Zs., 67, 221, I914.

VASSEUR: Rumex, Thesis, Lille, r905.

Vaughan, V. C.: Infections, Harvey Lect., p. 132, 1915; Protein Poison, Z. Immun., 9, 458, I9I I; J. Am. Med. Assoc., 6I, I76I, I9I3; Am. J. Med. Sci., I45, I6 I, I9 I3; Sci., 43, 539, 19 16; "Protein Split Products," Phila., I9 3 ; Sensitization, Internat. Clin., 4, I9I I.

Vedder, E. B.: Emetin, J. Am. Med. Assoc., 62, 501, I914.

Venlesen, H. J.: Ethylhydrocuprein, Berl. klin. Woch., 50, 1473, I9 3.

Velden, R., VAN den: Calcium, Ther. Mon., 27, 685, I913; Iodid, Bioch. Zs., 9, r9o8; Irritants, Arch. exp. Path. Pharm., 70, 55, I9I2; Pituitary, Berl. klin. Woch., 2083, 19I3.

Velley and Waller: Methyl-Xanthins, Zentr. Bioch. Bioph., Io, 761, 1910.

Velich, A.: Betain, Zentr. Physiol., 28, 249, I9I4.

Vera, M., and Leo Loeb: Hirudin, J. Biol. Chem., 19, 306, I914.

Verrill, A. E.: Mushroom Poisoning, Sci., 40, 408, I9 I4.

VERworN, M.: "Narkose," Jena, I912; Narcosis, Harvey Lect., p. I52, r9i 2; Oxygen, Arch. Physiol. Suppl., I9oo; Strychnin Localization, Arch. Physiol., 385, 1900.

Verzar, F., and Felter: Veratrin, Arch. ges. Physiol., I 58, 42 r, I9 4.

VeskI, J.: Strychnin, Zentr. Bioch. Bioph., I I, 7II, I9II; Zs. allg. Physiol., I5, I9I3.

Vetlesen, H. J.: Ethylhydrocuprein, Berl. klin. Woch., I473, I9I3.

Vietinghoff-Scheel: Oxalates, Arch. intern. Pharmacod., 8, 225, I901; Tartrates, ib., 10, 145, 1902.

VignaRd and ARnaud: Camphor, Ref., J. Am. Med. Assoc., 59, 72, I9i 2.

Villemin: Ovary, Zentr. Bioch. Bioph., io, 531, I9Io.

Vincent and Sheen: Glandular Extracts, J. Physiol., 29, 242, I903.

VIncI: Caffein, Arch. farmacol., 3, 385, I895; Morphin, Arch. intern. Pharmacod., I7, 5, 1907; Salicyl, Bioch. Zentr., 4, 240, 1905.

Voegrum, C.: Metals, J. Pharm. Exp. Ther., 6, 602, I9r5; Preservatives, Hyg. Lab. Bull., 96, 87, I914.

Voegtrin and Bernheim: Anaphylaxis, J: Pharmacol., 2, 507, r9i .

Voegtuin, C., and Macht: Adrenals, J. Am. Med. Assoc., 61, 2136, I9г3; Coronary, J. Pharmacol., 5, 77, 1913.

Voertz and Baudrexel: Alcohol, Arch. ges. Physiol., I42, 47, I9 I I; ib., 152, 567, I9 13.

Voeltz, Baudrexer and Dietrich: Alcohol, Arch. ges. Physiol., I45, 2 Io, I9I 2.

Voeltz and DIETrich: Alcohol, Bioch. Z., 40, I5, I912; Zentr. Bioch. Bioph., 18, 12, IgI5.

Vokitz and Paechtner: Alcohol, Bioch. Zs., 52, 73, I9I3.

Voger, H.: Iron, Bioch. Zs., 43, 386, I9 2 ; Phosphorus, Arch. intern. Pharmacod., Io, I87, 1902.

Voct, O.: Strophanthin, Bioch. Centr., 9, 675, r9o9.

VoIGt, J.: Collargol, Bioch. Zs., 63, 409, I914; Morphin, Ther. Mon., Dec., I9II; Silver, ib., 28, 625, I914.

VolkmanN: Paraffin, Samml. klin. Vortr., 1875.

Vollmer: Morphin-atropin, Arch. exp. Path. Pharm., 30, 385, I892.

Vollmering: Alcohol, Zentr. Bioch. Bioph., I4, 520, I9I 2.

Vorkastrer and Neue: Alcohol, Zentr. Bioch. Bioph., I4, 740, I913.

Voorhoeve, N.: Calcium, Deut. Arch. klin. Med., I I0, 46I, I9I I.

Vulpian: Salicyl, J. Pharm. Chim., I, 373, 474; 2, 435, 1880. 
Hidurolz: Carbon Monoxid, Bioch. Centr., 5, I32, I005.

WAKk.R and HeEck: Cholesterin, Muench. med. Woch., 60, 2097; Arch. exp. Path. Pharm., 74, 4I6; ib., 7I, 372, I9I3.

Wadeil, J. 1.: Salicyl, Arch. Int. Med., 8, 748, I9 I I.

Wadiums and Hill: Salvarsan, J. Am. Med. Assoc., 61, 385, I9 3.

WAEBER: Mesculin, Merck's Rep., 26, 279, I9 2.

WAGNer, G. A.: l'terine IIcmorrhage, Ther. Mon., 29, 424, I9I5.

Wis.ile, H. G.: II yoscin, Clev. Med. J., 4, 259, I905.

Whaser, H. G., and Riewel: Morphin, Clev. Med. J., June, r9o5.

Wanlgrex: Chlcrid, Arch. exp. Path. Pharm., 6r, 97, r909.

Wabaus: Fever, Arch. exp. Path. Pharm., 72, 153, I913.

W.ILkl: C. E.: Selenium, Lancet, I82, I337, I9I2.

Walker, I. C.: Nephritis, Arch. Int. Med., 8, I9I I.

Walker, W. H., and Marshall: Ratlle Snake Serum, J. Path. Exp. Ther., 6, 563, I9I 5 .

Walko: Picric Acid, Arch. exp. Path. Pharm., 46, I8r, I9or.

Wallace, G. B.: Chloral, J. Pharmacol., 3, 462, I9I 2; Pathologic Conditions, J. Am. Med. Assoc., 59, 839, I9 2 .

Wallace, G. B., and Cushny: Cathartic Salts, Am. J. Physiol., I, 4 I I, I898.

WALlace, G. B., and Myess: Uranium, J. Pharmacol., 5, 5 I I, I9I4.

Waller, A. D.: Choloroform, Ref., J. Am. Med. Assoc., 4I, 570, I903; Cyanid, Zentr. Bioch. Bioph., Iо, 850 , I9 Io.

Waller, A. D., and Velay: Carbon Tctrachlorid, Jahresb. Pharm., 69, 165, I909.

Wallfisch: Mercury, Zentr. Bioch. Bioph., I3, 474, I9I2.

Walter, F.: Acids, Arch. exp. Path. Pharm., 7, I48, I877.

WANDEL: Cresols, ib., 56, I6r, I9o6.

Warburg, O.: Cyamid, Zs. physiol. Chem., 76, 33i, i9i I.

Warfield: Bismuth, Am. J. Med. Sci., Nov., IgI 2.

Warser, A. R.: Nitrous Oxid, J. Am. Med. Assoc., 65, I973, I91 5.

Warner, H. F.: Lead, Lancet, July I3, I907.

Warren, L. E.: Blaud's Pill, J. Am. Med. Assoc., 64, 1344, 1915; Opium Alkaloids, Am. J. Pharm., 87, 437, r9i5; Poison Sumach, ib., 85, 545, I9 3; Rhus, Jahresb. Pharm., 69, 23, I909.

Waser, E.: Hypnotics, Zs. Physiol. Chem., 94, I9 I, I9 I5.

Wassermani: Lead Colic, Arch. exp. Path. Pharm., 79, 383, I916; Selenium, Ref., J. Am. Med. Assoc., 58, I27, I91 2 .

Wassermani, Keysser and Wassermann: Selenium, Deut. med. Woch., 37, 2389, I9 2 ; Tumors, Berl. klin. Woch., 4, I9I2.

Watanabe, W. K., and CRawford: Pituitary, J. Pharm. Exp. Ther., 8, 75, igi6.

Watermans: Pilocarpin, Zentr. Bioch. Bioph., I 2, 67, I91 I.

Watkyn-Thomas: Morphin, Biochem. J., 6, 433, 1913.

Watts, C. F.: Thyroid, Am. J. Physiol., 38, 356, 19 5.

Waucomont: Urates, Arch. intern. Pharmacod., 2 I, 369, rg I I.

Wrabr, E.: Asthma, Arch. Physiol., 63, I914; Cerebral Vessels, Arch. Physiol., 348, Ig०9.

WeBER, S.: Diuretics, Deut. med. Woch., 32, I250, I906; Drugs on Metabolism, Erg. Physiol., III, I, 233, I904.

Webster, J. C.: Nitrous Oxide, J. Am. Med. Assoc., 64, 81 2, I9I5.

Webster, W.: Atropin, Biochem. J., 3, I 29, I908.

Wechselmans, W.: Salvarsan, Ref., J. Am. Med. Assoc., 55, 1057, 19io; Deut. med. Woch., 778, r9iा; Muench. med. Woch., I510, I9II; Ref., J. Am. Med. Assoc., 62, I 175, I9 14

WeCker: Jequirity, Klin. Mon. Aughk., 20, 3I 7, I882.

Weckerlivg: Salicyl, Deut. Arch. klin. Med., I9, 319, I877.

Weed, Cushning and Jacobson: Pituitary, Proc. Am. Physiol. Soc., XIII, I9I3.

Wegner: Phosphorus, Arch. path. Anat., 55, II, I872.

WeIl, R.: Anaphylaxis, J. Med. Res., 30, r914; Soc. exp. Biol. Med., I I, 86, 1914; J. Pharmacol., 8, I I 2, I9 16; Anaphylotoxin, Soc. exp. Biol. Med., I3, 37, I9 I 5; Citrate, J. Am. Med. Assoc., 64, 425, 2 I63, 1915; Colloid Metals, J. Am. Med. Assoc., 6r, I034, I9г3; Hemolysis, Proc. Soc. Exp. Biol. Med., 5, 43, r907; J. Am. Med. Assoc., 5, I532, I910; Tumors, ib., I 263 , I9I5.

Werl, R., and CoCA: Antianaphylaxis, Z. Immun., I7, I41, I913.

Werland: Peristalsis, Arch. ges. Physiol., 147, I7 I, I91 2.

WeINBERG: Bile, Zbl. ges. Phys. Path. Stoffw., 6, 7, r9ir; Zentr. Bioch. Bioph., 11, 427; I 2, 802, I9II.

Weinblum, P.: Pumpkin, Zentr. Bioch. Bioph., I5, 463, I9 2. 
Weintraud, W.: Camphor, Deut. med. Woch., 39, I352, I913

Weise, P.: Sulphate, Arch. int. Pharmacod., 21, 77, I9I I.

WeIser, S.: Calcium, Bioch. Zs., 66, 95, I9I4.

WEIss: Quinic Acid, Zs. physiol. Chem., 25, 393, I898; ib., 27, 216; Berl. klin. Woch., No. 14,1899 .

Weiss and HarRis: Suprarenal, Arch. ges. Physiol., 103, 510 , 1904.

Weizsaecker: Digitalis, Arch. exp. Path. Pharm., 72, 347, I9I3.

Wellander: Mercury, Ann. de derm. syph., r886.

WeLler, C. V.: Lead, J. Med. Res., 33, 271; Soc. exp. Biol. Med., I 2, I 57, I91 5.

Wells, H. G., and HedenburG: Iodid, Proc. Am. Soc. Biol. Chem., 2, I 25 , I9I3.

Wells, H. G., and OsBorne: Anaphylaxis, J. Infect. Dis., 8, 66, I9I1; ib., I2, 34I, I9I3; ib., I7, 259, I915.

Welsch, H.: Phosphorus, Arch. int. Pharmacod., I4, 2 II, 1905.

Wendelstadt: Respiration, Arch. ges. Physiol., 76, 223 , I899.

WeNDling, H.: Hydrastis, Zs. Geb., 77, 189, 1915.

Wenzell: Ergot, Äm. J. Pharm., 36, 193, I864.

Werner and Ascher: Cholin, Ref., Ther. Mon., 27, 227 , I9I 2.

Werschinin, N.: Barium, Arch. exp. Path. Pharm., 66, I9I, I9Ir; Pituitary, Arch. ges. Physiol., I55, I, I913; Strophanthin, Arch. exp. Path. Pharm., 63, 386, I9ro.

WestenrijK: Digalen, Merck's Rep., 22, 181, Igo8.

Wertheimer and Boulet: Barium, Zentr. Bioch. Bioph., I3, Io4, IgII.

Wertheimer and Delezenne: Strychmin, C. R. Soc. Biol., 633, I897.

WesSely, K.: Ocular Tension, Arch. Augenh., 78, 247, 1915.

Wetterstroen, T. D.: Iodin, Ref., Proc. Am. Pharm. Assoc., 57, I I5, 1908.

Weygandt: Morphin, Muench. med. Woch., No. 45, 1903.

Weyland, A. D.: Barium, Bul. Soc. Roy. Bruxelles, I9I3.

Wherry: Emetin, Chem. Abstr., 6, i i 80 , igir.

Whipple, G. H.: Chloroform, J. Exp. Med., 15, 258, I91 2; Fibrinogen, Am. J. Physiol., 33, 50, I9I4; Intestinal Obstruction, J. Am. Med. Assoc., 65, 476, I9I5; Proteose Intoxication, J. Am. Med. Assoc., 67, 15 , 1916.

Whipple, G. H., and Hurwitz: Chloroform, J. Exp. Med., I3, No. I, I9I I.

Whipple, G. H., Mason and Peightal: Liver, Johns Hopkins Hosp. Bull., 24, 207, I9I3.

WhIPPLE, G. H., and SPEed: Anesthetics, J. Exp. Med., 2i, 193, I9r 5.

Whipple, G. H., and SperRY: Chloroform, Bioch. Centr., 9, 536 , I909.

White, T. P.: Tin, Arch. exp. Path. Pharm., I3, 53, 1880.

Whitehouse and Schuyler: Sálvarsan, J. Cutan. Dis., Sept., I9I3.

Whitfield: Phenol, Lancet, Dec., I4, Igi 2.

Wholey, C. C.: Morphin, J. Am. Med. Assoc., 58, 1855, 1912; ib., 64, i390, 19r 5.

Whitney, D. D.: Alcohol, Am. Naturalist., 46, 4I; Am. J. Physiol., 30, 463, I9I2.

Wichern, H.: Oxalic Acid, Chem. Abstr., 7, I57, Igr 2.

Widal, F.: Bicarbonate, Sem. med., No. II, I9Ir.

Widal and Javal: Nephritis, Presse med., No. 5I, J. de Physiol., 5, I 10\%, I903

WidMark, E. M. P.: Alcohol, Skand. Arch. Physiol., 33, 85, I915.

WIDMER, R.: Xanthins, Zs. Biol., 64, 315, I9r4.

Wrechowskr: Atropin Fate, Arch. exp. Path. Pharm., 46, I55, rgor; Salicyl, ib., 48, 397, r902.

Wieland, H.: Respiratory Center, Arch. exp. Path. Pharm., 79, 96, I9I5.

Wiggers, C. J.: Aortic Insufficiency, Arch. Int. Med., I6, I32, I9I5; A uricular Systole, J. Pharmacol., 8, I17, I916; Epinephrin, J. Physiol., 48, 109, 1914; Hemorrhage, Arch. Int. Med., March and May, rgog; Pituitary, Am. J. Med. Sci., April, I9I I.

WillberG, M. A.: Atropin, Bioch. Zs., 66, 389, 1914.

Wilbert, M. I.: Aloin, Am. J. Pharm., 75, 20I, I903; Drug Addicts, U. S. Publ. Health Rep., 294, I9r 5; J. Am. Pharm. Assoc., 4, 963, I015; Parafin, ib., 3, 580, r914; Phenol Publ. Health Rep., Apr. 28, 19 r6; Solubilities, Am. Drug., July, 1909.

WILBRAND, E.: Bicarbonate, Muench. med. Woch., I437, 19I4.

Wribur, R., and Ophuls: Botulism, Trans. Assoc. Am. Phys., 29, 494, I914.

WILD: I pecac, Lancet, II, I 274,1895 .

WrLe, U. J.: Arsenobenzol, J. Am. Med. Assoc., 66, r88o, r9 16; Neosalvarsan, J. Am. Med. Assoc., 62, I165, 1914; J. Exp. Med., 18, 435, I914.

Wilenko: Epinephrin, Arch. exp. Path. Pharm., 68, 297, 1912; ib., 71, 261, 1913; Saline, ib., 66, I43, 191 I.

Wirey, H. W.: Borate, U. S. Bur. Chem., Circ., I3, I904; Formaldehyde, ib., 42, I908; Salicyl, ib., No. 84 , 1906 .

WillberG, M.: Arsenic, Bioch. Zs., 51, 231, 1913; Hedgehog, ib., 48, 157, 1913.

Williams, F.: Perfused Hearl, Arch. exp. Path. Pharm., 13 , I, I880. 
Wintums, 15. W.: Mydrastis. J. Im. Med. Assoc., 50, 26, I908; Kidney Sections, Am. I. Physiol., 19, 252, 10,07 .

Wnis, l., and HAwk: Hatcr, J. Am. Chem. Soc., 36, I775; ib., 37, r58, I9I4.

II nsin, I'. Snake Poisoning, Arch. Int. Med., 1, 516, I9o8.

III usixs: Aliohol, Arch. ges. Physiol., 66, I67, I897.

Winsis, I). W., and H.1Wk: Hater, J. Am. Chem. Soc., 36, I775, I9r4.

Wilson, F. N.: Atropin, Arch. Int. Med., r6, 989; Soc. Exp. Biol. Med., I3, I7, r915.

Hisow. L. B., and Kexdal,: Thyroid-iodin, Am. J. Med. Sci., Jan., r9 6.

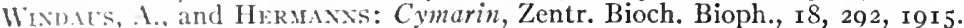

Windes and Schreckexburger: Gitonin, Zentr. Bioch. Bioph., r5, 859, r9r3.

IINDLE: Digitalis, Heart, 3, I, I9II.

Wixogramo: Chlorate, Arch. path. Anat., I9o, No. r, I907.

Wisiom, C. E. 1., and Palmer: Vitiated Air, Soc. exp. Biol. Med., r 2, I4I, 1915.

Wintreberg: Camphor, Arch. ges. Physiol., 94, 455, 1903; Z. exp. Path., 3, I82, igo6; Physostigmin, ib., 4, 636, 1907.

Wisteristz: Emollients, Z. klin. Med., 50, H I and 2, I903; Irritants, Arch. exp. Path. P'harm., 36, 212, I896; Volatile Oils, ib., 46, I63, т0ог.

Wixtersterx, H.: Carbonic Acid, Arch. Physiol., Suppl., r77, rgoo; Narcosis, Bioch. Z., 51, I43, I9I3; ib., 6I, 8I, I9 14; ib., 70, 130, I9I5; Respiration, ib., 70, 45, I915

Witkowski: Morphin, Arch. exp. Path. Pharm., 7, 247, I877.

Withers, W. A., and Carruth: Gossypol, J. Agr. Res., 5, 26i, I9I5.

Withers, W. A., and RaY: Phosphate, J. Biol. Chem., I4, 53, I9I3.

WiтtмaAk: Cinchonism, Arch. ges. Physiol., 95, 209, ro03.

Woebrecke: Veratrin, Arch. exp. Path. Pharm., 71, I57, I9I3.

Woelfel: Hemolysis, Bioch. J., 3, igo8.

Woelfel, A., and Carlsox: Lcad, J. Pharmacol., 5, 549, I9I4.

Wonlwil, F.: Nickel, Arch. exp. Path. Pharm., 56, 404, I907.

Wolf, H.: Benzol, Jahrb. Pharm., 7I, I47, I9II; Meat Extracts, Ther. Mon., 27, 60, I91 2; Synergism, Arch. exp. Path. Pharm., 74, 298, I9I3.

Wolff, M., and Mulzer: Salvarsan, Muench. med. Woch., 59, I 706, I9I 2.

Wolf RAM: Vcronal, Ref., Merck's Rep., 20, 259 , I906.

Wood, A. W.: Hypodermic Injection, Edinb. Med. Surg. J., 82, 265, 1855.

Wood, C.: Cocain, Pan-Am. Med. Congr., I893.

Wood, F. C.: Nitric Oxid, Trans. Assoc. Am. Phys., 27, 407, I9I 2.

Woon, H. C., Jr.: A pocynum, J. Am. Med. Assoc., 43, I953, r904; Aspidosperma, J. Am. Med. Assoc., 5 I, I593, I908; Bioch. Centr., I0, 674, 19 10; Bio-assay, ib., 59, 1433, I9r2; Caffein, Ther. Gaz., Jan., I9r2; Ethyl Chlorid, J. Am. Med. Assoc., 55, 2229, I910; Intestinal Antiseptics, Ther. Gaz., March, I9 I ; Pulmonary Circulation, J. Exp. Med., I4, 326, I9II; Strontium, Am. J. Physiol., I, 83, 1898. Superstitions, J. Am. Med. Assoc., 66, ro67, г9 16.

Wood, H. C., JR., and Hoyr: Alcohol, Nat. Acad. Sci., ro, 43, r905; Aspidosperma, U. Pa. Med. Bull., Sept., rgoz.

Woodbury, F. T.: Iodin, N. Y. Med. J., May, 1907.

Woodruff and Gies: Selenium, Am. J. Physiol., 6, 1902.

Woods, H.: Methyl Alcohol, J. Am. Med. Assoc., 60, 1762, 1913.

Woonward, H. E., and Alsberg: Saponin, J. Pharmacol., 8, I09, I9i6.

Woronzow: Destruction, Chem. Abst., 6, 650 (liver); Zentr. Bioch. Bioph., I3, 57 I (muscle), I9I2.

Wovschin, W. A.: Iodid, J. Am. Med. Assoc., 65, r ror, 19 5.

Wrighr, A. E.: Calcium, Lancet, I53, I893; Ethylhydrocuprein, Lancet, Dec. I4 and 2I, I9I2.

Wright, H.: Morphin, U. S. Senate Doc., No. 3i7, I9ro.

Wulzen, R.: Pituitary, Am. J. Physiol., 34; I 27 , r9 4 .

Wrbauw: Digitalis, Arch. exp. Path. Pharm., 44, 344, I900.

Wyss, v. H.: Bromid, Arch. exp. Path. Pharm., 49, 186; 55, 263, 1906; Deut. med. Woch., 39, 345, I9I3.

YAGI, S.: Aspidium, Zs. exp. Med., 3, 64, I9I4; Calcium, Arch. int. Pharmacod., 22, 259, I9I2.

Yanagawa, H.: Serum, J. Pharmacol., 8, 89, г9 6.

Yosнimoto, S.: Lecithin, Zs. physiol. Chem., 64, 464, r9ro.

Zabel: Phenolphthalein, Deut. med. Woch., Apr. 20, r9r r.

ZADEK, J.: Bismuth, Zs. exp. Med., I5, 498, I9 I4.

Zанs, K.: Papaverin, Biochem. Zs., 68, 444, I0I 5.

ZAK: Hexamethylenamin, Zentr. Bioch. Bioph., I2, 942, Igr2. 
Zanda, G. B: Cinchona, Arcn. intern. Pharmacod., 20, 4I5, r910; Arch. it. Biol., 55, 297, 191 2; Nicotin, Arch. Farm., 19, 505, 19 15.

ZDAREK, E.: Fluorin, Zs. physiol. Chem., 69, I 27 , I9 Iо.

Zeelen: Morphin, Z. exp. Path., 8,587 , I910.

Zенве, M.: Opium, Ther. Mon., 27, 406, 19г3.

Zeigler, IV. H.: Spartein, Sou. Med. J., 9, 67 г, г9i6.

Zeisler, J.: Veronal Dermatitis, J. Am. Med. Assoc., 58, 2024, igr 2.

ZeYnek: Cyanid, Zs. physiol. Chem., 33, 426, I901.

Zibell: Gelatin, Muench. med. Woch., No. 42, I90I.

Zinsser, H.: Anaphylaxis, Arch. Int. Med., I6, 223, 1915.

Zollinger: Bismuth, Beitr. klin. Chir., 77, No. I, I9I 2.

Zors, L.: A nesthetics, Zs. exp. Path., I2, 529, I913.

Zuelig: Pituitary, Muench. med. Woch., 743, I9I 5.

Zuelzer: Atophan, Berl. klin. Woch., 2ror, Igr ; Diabetes, Z. exp. Path., 5, I908; Epinephrin, Berl. klin. Woch., I 209, 1901; Hormonal, ib., 45, 2065 , 1908.

Zulich, H. S.: Homatropin, J. Pharmacol., 6, 473, r9 5.

Zunz, E.: Anaphylaxis, Z. Immun., I7, 24I, 19I2; Morphin, Biochem. Centr., 9, 208, I 909 . 



\section{INDEX}

Note.-The bold-face folios refer to preparations

Abietis, oleum, r44

Abrin, 174

Absinthe, 147

Absorption, 88, 643

Acacia, r ro

Acaciæ cortex, 138

Acapnia, 607

Aceta, 34

Acetanilid, $456,470,475$

Acetates, 730

Acetone, 56I substances, 7 I 7

Acetonitrile test, $35^{\circ}$

Acetphenetidin, 470, 475

Acetum, 730

Acetyl salicylic acid, 503

Acidosis, 7 I 4

Acid sulphuricum, I32

Acids and alkalies, remote effects of, 7 Io caustic, I3 I dilute, I 32 organic, and their salts, 729

Acidum aceticum, $73^{\circ}$ agaricum, 287 arseniosum, 743 boricum, $\mathbf{5 2 4}$ camphoricum, 4I6

chromicum, 773

citricum, 73 I

diæthyl-barbituricum, 600

formicum, 732

gallicum, 138

hydriodicum dilutum, 705

hydrobromicum dilutum, 699

hydrochloricum, 132

dilutum, $72 \mathrm{x}$ -

hydrocyanicum dilutum, 6r7

Acidum laciicum, 132

nitricum, 132

dilutum, 72 r

nitrohydrochloricum, 132

oleicum, 105

osmicum, 132

oxalicum, 692

picricum, 486

phenylcinchoninicum, 507

phosphoricum, 721

stearicum, I05

sulphuricum, I32

aromaticum, 721

sulphurosum, 519

tannicum, 138

tartaricum, 654

acidum trichloraceticum, $\mathbf{I 3 2}$

Aconite, 422.
Aconitin and aconitum, 423, 427

Acrolein, 5ro

Adalin, 699

Addison's disease, $33^{8}$

Adeps, ro5

benzoinatus, ro5

lana hydrosus, Io6

Administration of drugs, 84

Adonis, 382, 409

Adrenalinum, 338

Adsorption, 623

Ather, 590

aceticus, 590

Atheris nitrosi, spiritus, 38r

Athylis carbamas, 600

chloridum, $\mathbf{5 9 0}$

Ethylmorphinæ hydrochloridum, 244

Agar-agar, 178

Agropyron, III

Agurin, 2 I4

Ajowan, oleum, 69

Albumoses, 374

Alcohol, 458, 54r, $55^{8}$

denatured, $56 \mathrm{I}$

Alcoholic beverages, peculiarities of, 557

Aldehyds, 5 Io

Aliphatic hypnotics, 590

Alkalies, caustic, I 25

dilute, 127

mild, 72 I

remote effects, 7 ro

Alkaline salts, 127

Alkalinity, actual and potential, I27

Alkaloids, I9

Allspice, 145

Aloes and aloin, I69, I70

Alopecia, I 29

Alstonia, 469

Althrea radix, r ro

Alumen, 774

Aluminum, 773, 774

Alypin, 262

Amanita, 305

Amber, oil of, 146

Amblyopias, 316

Ammonia, 126 water, $68 \mathrm{r}$

Ammoniacum, I50

Ammoniæe, aqua, 68x

fetidus, spiritus, 142

Ammonii benzoas, 494

bromidum, 699

carbonas, 68I

chloridum, 68I

iodidum, 705 
Inmonii salicyl, 502

Ammonimm bases, guaternary, 307 compounds, $6,-8$

Anverdalit amara, 6r7

dulcis, I IO

Ampralale expressum, olemm, I05

Amyilateohol, 559

Amplis nitris, 38I

Amylum, II 2

Anaphylactic shock, 360

Anitghylatoxin, 37 I

Anaphylaxis, heat centers in, 453

Andromedotoxin, 432

Anemias, iron in, 768

Anesthesin, 262

Anesthetics, inhalation, $56 \mathrm{I}$ local, $25 \mathrm{I}$

Anethi fructus, 69

Angina pectoris, 380

Angostura bark, $\mathbf{1 5 9}$

Anhydrotics, 287

Anilin, 487

Animal experimentation, 83 extracts, 354

Anisotonic solutions, 264

Anisum, 69

Antagonism, 95, 96, 268 magnesium and calcium, 64I

Anthelmintics, 179

Anthemis, I60

Anthraquinon cathartics, $\mathbf{1} 66$

Antidotes, 56

Antifebrin, 475

Antimeningococcus serum, 53 I

Antimony, 756,757

Antimonii et potassii tartras, 757

Antiperiodica, tinctura, 468

Antipneumococcic serum, $\mathbf{5 3 3}$

Antipyretics, 455

Antipyrin, $456,470,475$

Antirabic vaccine, 533

Antiscptic benzol derivatives, 476

Antiseptics, general consideration of, 525

miscellaneous, 5 IO

Antispasmodics, I 42

Antisyphilitics, vegetable, 797

Antithyroid serums, 349

Antitoxic action of ions, 640 serums, 527

Antitoxin, diphtheria, 528, 53I tetanus, $\mathbf{5 3 2}$

Apiol, I 47

Apnea, 608

Apoatropin, 294

Apocodein, 276, 44I

Apocynum, 382, 408, 4I 2

Apomorphin, $438,44 \mathrm{I}, 45^{8}$

Aqua destillata, 662

regia, I32 sterilisata, 662

Aquæ, 30

Arachis, oleum, 105

Araroba, $\mathbf{1 5 5}$

Areca, 183

Arecolin, 183, 297
Argenti nitras, $\mathbf{7 8}$ I oxidum, 782 proteinas, 782

Argyrism, 780

Arisæma, 154

Aristol, $\mathbf{5 2 5}$

Armoracia radix, I5I

Arnica, 145

Aromatic fluidextract, powder and syrup, 68

waters, spirits and oils, 68

Arsacetin, $\mathbf{7 4 7}$

Arseni iodidum, 743

trioxidum, 743

Arsenic antidote, 770

Arsenic compounds, inorganic, 735, 743 organic 743

white, 743

Arsenicalis, liquor, 743

Arseniuretted hydrogen, 740

Arsenous acid, 743

Asafœtida, I 42

Ascaridol, I 81

Asphyxia, 605

Aspidium, I 80, I8I

Aspidosperma, 442

Aspirin, 503

Assaying, 29

Asthma, ' 279

Astringent action, I 6

Astringents, metallic, I35 vegetable, I36

Atheroma, 330

Atophan, 505, 507

Atoxyl, 746, 747

Atropin, 268, 269, 270, 272, 275, 276, 292

group, 276, 292

hyperthermia, 454

Aurantii amari cortex, 67

cortex indicus, 68

siccatus, 67

dulcis cortex, 68

flores, 68

Autonomic system, 263

Bafagin, 382

Baking powders, 774 soda, 726

Balanced salt rations, 640

solutions, table of, 642

Balsams, I 49

Balsamum peruvianum, 149 tolutanum, $\mathbf{5 0}$

Barbitonum, 600

Barium, 275, 409, 693

Barley water, I 12

Bases, organic, 728

Beef juices, I0o

Beéswax, 108

Belæ fructus, 138

Belladonna, 277, 292

Benzaldehydum, 6r8

Benzamin, 262

Benzenum, 478

Benzoates and benzoic acid, 492, 494 
Benzoinated lard, ro5

Benzoinum, 149

Benzol, 477

Benzosulphinid, 507, 508

Berberin, 246

Berberis, $\mathbf{5}^{8}$

Beryllium, 775

Betaeucaina, 262

Betain, 307

Betanaphthol, 49 I

Beta-oxybutyric acid, 717

Beta-tetrahydronaphthylamin, 453

Betel, I60

Betulæ, oleum, 504

Bile, I7I, I72

on cathartics, I6I salts, I72

Bio-assay, 30

Birch, oil of, 504

Bismuth, 759, 76I

Bitter almond, 6r7 apple, $\mathbf{I 7 7}$

Bitters, I 58

Bladder, 270

Black wash, 798

Blaud's pills, 770

Bleaching powder, 5 I9

Blistering liquid, I54

Blood root, 244

Blue cohosh, 420 flag, I 76 ointment, 798

Bone black, I6o marrow, 354

Borax, 523

Boric acid and borate, $\mathbf{5 2 3}$

Borneol, 4 I 6

Bornyval, I43

Brain lipoids, 53I

Brayera, I8I

Brom. strychnins, I 89

Bromates, 709

Brometone, 699

Bromids, 694

Bromin, I34

Bromipin, 699

Bromoform, 589

Bromoformum, 590

Bromural, 699

Bronchial muscles, 279

Brovalol, 699

Brown mixture, 243

Brucin, I 89

Bryonia, I76

Buchu, I48

Buckthorn, 168

Bufanin and Bufotalin, 382

Burow's solution, 774

Butea, I38, I83

Butter, I05

Butternut, I76

Butyl-chloral hydras, 595

CACAO, 216

Cachets, 39

Cactus, 412
Cadinum, oleum, 489

Cadmium, 785

Casium, 678

Caffein, 200, 213

beverages, 214

hyperthermia, 454

Cajuputi, oleum, I45

Calabar bean, 303

Calabarin, 303

Calamus, $\mathbf{1 6 0}$

Calcii bromidum, 699

carbonas, 689

chloridum, 690

lactas, 600

phosphas, 690

sulphas exsiccattus, II3

stulphidum, I28

Calcis, linimentum, I26

liquor, I 26

saccharatus, liquor, r 26

Calcium salts, 683

Calomel, 79I, 798

Calumba, I 58

Calx, 126

chlorinata, 519

sulphurata, 128

Camphor, 4I3, 4I 6

Camphora monobromata, 4I6

Camphoric acid, 4I6

Canadian hemp, 4I 2

Canadin, 246

Cannabis, 249

Cantharidin and cantharis, I 52, 153

Capsicum, I54

Capsules, 38

Caramel, I0o, I60

Carbo ligni, r6o

Carbon, colloidal, I6o dioxid, 605

disulphid, 589

monoxid, 608

tetrachlorid, 589

Carbolic acid, 478

Carbonic acid, 605, 608

Carbosant, I49

Cardamomi semen, 69

Carminatives, I60

Carminum, 65

Carron oil, 126

Carum, 69

Caryophyllus, I45

Cascara sagrada, I67, I68

Cascarilla, I59

Cassia, 69

Cassiæ fructus, 168 pulpa, r69

Castor oil, I72, I73

Castoreum, I43

Cataphoresis, 85

Cataria, I43

Catechu, I38

Cathartic resins, 174 salts, 648

Cathartics, I6I

Catnip, I43

Caulophyllum, 308, 420 
Caustic poisoning, 122

Calterization, 121

Cedrelix, olemm, I45

Cellulose, 18

Cephalin, $4+5,446$

Cephalin. See Kephalin.

Cera, 108

Cerates, 108

Ceratum, 108

Cerebro-spinal fluid, excretion of drugs into, 90

Cerii oxalas, 775

Cerium, 774

Cetace11m, 108

Cetraria, I 10

Cevadin, 428

Chalk mixture, 690 prepared, 690

Chamomile, I60

Chaparro amargosa, 446

Charcoal, I6o

Chaulmoogra oil, 105

Cheiranthus, 382

Chelidonin, 224

Chemotherapy, 744

Chenopodium oil, I 8 I, 182

Cheyne-Stokes respiration, 613

Chicory, 216

Chirata, 158

Chloraformamidum, 596

Chloral hydrate, 592, 595

Chloramine, 5 I9

Chlorazene, 519

Chlorbutanol, 595

Chloretone, $\mathbf{5 9 5}$

Chlorid retention, 669

Chlorin, 517

Chloroform, 56I, 589

Chloromorphids, 44 I

Chlorophyl, 2 I

Cholesterol, 42 I

Cholin, 275, 305

Chondrus, Iro

Chromates, 772

Chromii triodidum, 773

Chrysarobinum, I 54, I55

Cimicifuga, $\mathbf{5} 59$

Cinchona, 46I, 469

Cinchonidin, 465, 469

Cinchonin, 465,469

Cinchonism, 465

Cineol, I45

Cinnamic acid, 495

Cinnamomum, 69

Citrates, 730

Citrine ointment, 799

Classification of drugs, 80

Climate, 664

Coagulability of blood, substances increasing, 689

Coal tar, 489 dyes, 509

Cobalt, 785

Coca, 26 I

Cocain group, 25I

hyperthermia, 454
Cocain substitutes, 260, 262

Cocaina, 26r

Coccus, 65

Cocoanut, 183

Cocos, oleum, ro5

Cod liver oil, Ior

Codein, 2 I 7, 245

Coffee, 2 I 5

Cola, 2 I 5

Colchicin, 275, 438

Colchicum, 436,437

Collodium, i 13

Colloid infusions, 672

laxatives, I 78

Colloidal solutions, 6I9

Colloids and edema, 647

on absorption, 88

Colocynth, I 76, I 77

Coloring, 65

Combined action of drugs, 95

Concentration, effect of, 80

Conditions influencing drug action, 92

Condurango, I 59

Confections, 32

Conium, 3 I 7

Constitution, on action, 75

Convallaria, 382, 409, 412

Convulsant poisons, I 84

Convulsants on temperature, 189 , 454

Copaiba, $\mathbf{4} 8$

Copper, 782

Coriandrum, 69

Corn smut, 367

Corpus luteum, 356

Corrosion, I I 5

Corrosive poisoning, I24 sublimate, 799

Cotarnin, 247, 249

Cotoin, 339

Cotton root bark, 367

Cough mixtures, 683

Counterirritation, I20

Cream of tartar, 654

Creosote, 487,488

Creosoti carbonas, 488

Creosotinic acids, 5OI

Cresols, 483, 484

Creta præparata, 690

Crocus, 66

Croton oil, 177

Crotonis, oleum, I78 $^{8}$

Cubeba, 149

Cucurbitæ semina, $\mathbf{1 8 3}$

Culver's root, i 76

Cumulative action, 93

Cupri sulphas, 784

Curare, 268, 319

Curcuma, 66

Cusparia, I 59

Cusso, r 81

Cutaneous irritation, II4

Cyanids, 613

Cydonium, I I I

Cymarin, 408

Cytisin, 308 
DANDELION, $\mathbf{I} 59$

Datura, 277, 293

Decoctions, 32

Delirium tremens, 557

Delphinium, 427

Demulcents, I09

Dermatitis from systemic drugs, I I5, I 56

Dermatol, 762

Dextrin, 1 io

Dextrose, Ioo

Diabetes foods, 100

Diacetylmorphina, 244

Dialysed iron, 771

Diaphoretics, 298

Diarrhea mixtures, 139

Diastasum, 98

Diet, influence on drug action, 94

Diethyl barbituric acid, 599

Digestive ferments, 96

Digitalis, 382,4 I I

group, 382

principles, 407

Digitaloid drugs, 407

Dimethylxanthin, 2 I 4

Dionin, 2 I $7,233,244$

Diphtheria antitoxin, 528, 53 I

Diplosal, 504

Disintoxication, 78

Dispensatories, non-official, 23

Distribution of drugs, 77,95

Diuretics, 665

Diuretin, 2 I 4

Dobell's solution, 482

Donovan's solution, 743

Dosage, 90

Doses for children, 9 I

Dover's powder, 243

Drug action, conditions influencing, 92

Drugs, administration of, 84

official, 22

proprietary, 22

Duotol, 489

Dusting powders, I1 2

Dyes, absorption of, 636

ECBOLIC oils, I46

Ecbolics, 367

Ecgonin, 26I

Echinacea, 150

Edema, 647

Effervescent salts, 37

Eisen-Zucker, 770

Elaterin, 176

Elaterium, 177

Electrolytes, actions of, 638

Electrolytic dissociation, 630

Electron actions, 632

Elixir aromaticum, 68

Elixirs, 32

Embelia, I38

Emetics, therapeutic use of, 447

Emetin, 442, 445

Emmenagogues, 367

Emodin cathartics, 166

Emollient laxatives, 178
Emollients, I 02

Emplastrum, $\mathbf{I}_{3}$

calefaciens, 154

Emulsions, 35, 623

Enemas, evacuant, 178

Epilepsy, 696

Epinephrin, 264, 276, 322, 338

Epsom salts, 658

Equivalents of metric and common systems, 48

Ergot, 357, 366

Ergotin, 366

Ergotinic acid, 364

Ergotism, 365

Ergotoxin, 36I

Eriodictyon, 70

Erythema, 223

Erythrophlein, 382, 409

Erythroxylum, 26I

Eschar, II 5

Eserin, 303

Ether, 56I

Ethyl bromid, 589, 590

carbamate, 600 chlorid, 588

hydroxycuprein, 469

iodid, 589

nitrit, liquor, 38I

Eucalyptol, I 44, I45

Eucalyptus; r45

gum, 139

Eumydrin, 286

Euonymi cortex, 4I2

Euphorbium, 154

Euph thalmin, 286

Euquinin, 468

Excipients, 37

Excretion of drugs, 90

Exodin, I7o

Expectorants, 68I

Extractive matter, 2 I

Extracts, solid, 35

FAex medicinalis, 355

Fats, 2 I, IO5 on growth, IOI

Fatty oils, IO4

Fel bovis, I72

Ferments, digestive, 96

Ferri carbonas saccharatus, $77^{\circ}$ chloridum, 771

et ammonii citras, 77 I

et potassii tartras, $77 \mathrm{r}$

et quininæ citras, 77 I

hydroxidum cum magnesii oxido, 770

phosphas, 771

quin. et strychn. phosph., elixir and sulphas, 77 I syrupus, 198

Ferrosilicon, $\mathbf{7 4 0}$

Ferrum reductum, $77^{\circ}$

Fetus, passage of drugs into, 90

Fever, 452

treatment of, 459

Fibrillary twitchings, 322 
Fihrolysin, I3 I

Pilicic alcidel, 180, I 8 I

Filicis, extratum liquidum, 18 I

Filmaron, i 8 I

"litvoring, 60

Pindextracts, 34

Pluid magnesia, 658

Pluorescein, 509

lilurescent stblostances, 5 IO

Pluoricl ion, 692

Fieniculum, 69

Food preservatives, 526

Foods, medicinal, 99

Formaldehyd, 5 Io componinds, 5 I 3

Formaldehydi, liquor, 5I 2

Formates, 73 I

Fowler's solution, 743

Fox glove, 4 I I

Frangula, 168

Friar's balsam, 149

Fuchsin, 5 I0

Fuller's earth, 88

GalbaNuy, 150

Galenic preparations, 22

Galla, I 38

Gall-bladder, 164,271

Gambir, 138

Gamboge, I 76

Gas, illuminating, 608

Gases, systemic actions of, 60 I

Gasolin, I07

Gastric juice, concentration of, 645

Gastro-intestinal irritation, I I 9

Gaultheria, oil of, 504

Gelatinum, I I I

Gelsemium, 3 I 7

Gentian violet, 5 Io

Gentiana, I 59

Gin, 146

Ginger, I6I

Ginseng, 160

Glauber's salt, $\mathbf{6 5 2}$

Glaucoma, 284

Glucosids, 19

Glucosum, 67

Glusid, 507, 508

Gluten flours, I oo

Glycerin, Io9

Glycerites, 32

Glyceritum boroglycerini, $\mathbf{5 2 4}$

Glycerylis nitratis spiritus, $38 \mathrm{I}$

Glycuronic acid, 477

Glycyrrhiza, 68

Glycyrrhizinum ammoniatum, 68

Goiters, 348

Gold, 782

Golden seal, 247

Gonococcus vaccine, $\mathbf{5 3 5}$

Gossypii cortex, 366 radicis cortex, 367 seminis, oleum, 204

Gossypium purificatum, I I3

Goulard's extract, 806

Gout, relation of uric acid to, 727
Granatum, $\mathbf{1} 8 \mathbf{2}$

Gray oil, 798

Grindelia, I50

Growth, fats on, IOI

Guanidin, 322

Guarana, 2 I 4, 2 I 6

Guaiacol, 487, 489

Guaiacolis carbonas, 489

Guaiacum, 420

Gummi indicum, I Io

Gums, I 8, i Io

Gun-powders, smokeless, 38I

Habituation, 93

Hæmatoxyli lignum, I39

Haloids, I 33

Hamamelidis aqua, $\mathbf{1 4 5}$ cortex, 139

Hay fever serum, 533

Headache powders, 473

Heat puncture, 45I regulation, 448

Hebra's ointment, 806

Hediosit, I 00

Hedonal, 600

Helenium, I 47

Hellebore, black, 432, 435 green, 432,435

Helleborein, 409

Helleborus, 382

Hemidesmi rad., 420

Hemoglobin, fate of, 768

Hemostatic tissue extracts, 53 I

Hemp, Indian, 249

Heroin, 21 7, 244

Hexamethylenamin, 5 I 3,5 I 7 on uric acid, 729

Hexamina, 517

Hippuric acid formation, 477, 494

Hirudin, 375

Histamin, 362

Holocain, 263

Homatropina, 286

Homeopathic doctrine, 7 I

Honeys, 67

Hops, $25^{\circ}$

Hordenin, 364 .

Hormonal, 375

Horse-radish root, I5I

Horse serum, 53I

Humulus, 250

Hydrargyri chloridum corrosivum, 799 mite, 798

cyanidum, 799

iodidum flavum, 798

rubrum, 799

oxidum flavum and rubrum, 798

oxycyanidum, 799

salicylas, 799

subchloridum, 798

succinimidum, 799

Hydrargyrum, 797 ammoniatum, 798 cum creta, 797

Hydrastin, 245, 247

Hydrastinin, 245, 247 
Hydrastis, 245, 246

Hydrocyanic acid, 6I 3

Hydrogen dioxid, 521

ion concentration, $7 \mathrm{II}$

peroxid, 52 I

sulphid, I 28

Hydrogenii dioxidi (peroxidi), 52 I

Hydroxylamin, 376

Hyoscin, 293, 297

Hyoscyamin, 292

Hyoscyamus, 277, 293

Hypericum, I 56

Hypnotics, aliphatic, 590 chlorinated, 595

Hypochlorous acid, 5 I 8

Hypophysis, 345

Hysteric sedatives, I42

ICHTHYOL, I3O

Idiosyncrasy, 92 racial, 94

Illuminating gas, 608

Imbibition, 62 I

Impurities in drugs, 92

Incompatibility, 43

Indigo carmin, $\mathbf{5 0 9}$

Indolethylamin, 364

Inflammation, pain on, I20

Infusions, 32

Infusum rosæ acidum, 72 I

Inhalation, 85

Insect powder, 432

Intestinal antiseptics, $\mathbf{5 2 7}$

Intestine, 269, 274

Intramuscular injection, 87

Intraocular tension, 284

Intraperitoneal injections, 87

Intrapleural injections, 87

Intravenous administration, 87 feeding, $\mathrm{IO} 2$

Inulin, roo

Iodids, 699

Iodin, 133 compounds, thyrectotropic, $35^{\circ}$ preparations, organic, 70I

Iodism, 704

Iodoform, 524, 525

Iodothyrin, 346

Iodoxybenzoic acid, 522

Iodum, I34

Ion actions, 632 relations, 639

Ipecacuanha, 442, 445

Ipecacuanhic acid, 445

Ipomøea, I75

Iris, 176

Iron, 762

Irritants, I I 4

hypodermic injections of, II7 reflex effects of, I I 8 toxicology of, 122

Iso-amyl alcohol, 559

Ispaghula, I I I

JABORANDI, 297, 301

Jalap, I75
Jambul, 101

Jamestown weed, 293

Jasmine, $3 \mathbf{I} 7$

Javelle water, 519

Jequirity, I74

Jervin, 435

Jimson weed, 293

Juglans, I 76

Juices, 34

Juniper and oil, 146

KALADANA, I 75

Kamala, I8I

Kaolinum, I I 2

Kava, I49

Kidneys, 354

Kino, 139

Kousso, I8I

Krameria, 139

LABARRAQUE's solution, 5I9

Lactates, 73I

Lactic acid ferments, 98 organisms, 720

Lactucarium, $25^{\circ}$

Lanolin, ro6

Lanthanum, 775

Lard, I 05

Lassar's paste, 785

Latin, prescription, 63

Laudanum, 243

Laughing gas, 6oI

Laurocerasi folia, 618

Lavandula, 70

Lead, 799 water, 806

Leptandra, 176

Lettuce, $25^{\circ}$

Leucocytes, 276

Levulose, roo

Lily of the valley, 4 I2

Lime, 126 water, 126

Limonis cortex, 67

Lini oleum, ro5

Liniments, 35,146

Linimentum ammoniæ, 68I camphoræ ammoniatum, 68I

Linum, I I 2

Liquor acidi arsenosi, 743 alumini acetatis, $\mathbf{7 7 4}$ ammoniæ, 68I ammonii acet., $68 \mathrm{I}$ citratis, $73 \mathrm{I}$

antisepticus, $\mathbf{5 2 4}$ alkalinus, 494 arseni et hydrargyri iodidorum, 743 arsenici hydrochloricus, 49,743 epistasticus, I54 ferri dialysatus, 77 I et ammonii acetatis, $77 \mathbf{I}$ oxychloridi, 77 I perchloridi fortis, $77^{2}$ persulphates, $\mathbf{7 7 2}$ subsulphate, 772 magnesii citratis, $65^{8}$ 
liquor phunbi subacetatis, 805 saponatus, 513

solii buratis comp., 482

chloricli physiologicus, 674

phosphatus compositus, $\mathbf{6 5 3}$

liquores, 31

Lithii bromidum, 699 carbonas, 677

citras, 677

Lithium salts, 677

liver of sulphur, I 29

Lobclia, 317

Local application, 84

Localization of action, 82

Locke's solution, $6 \nmid 2$

loco, 250

Lotio hydrargyri, flava and nigra, 798 opii et plumbi, 243

Lugol's solution, I34

Luninal, 600

Lunar caustic, 78r

Lupulin, $25^{\circ}$

Lupulinic acid, 250

Lutein, 356

Lycopodium, I 12

Lymph formation, 647

Lyotropic series, 62 I

MaCERATION, 27

Magma bismuthi, 762 magnesiæ, 658

Magnesia, 658

Magnesii carbonas, $65^{8}$ oxidum, $6 \mathbf{5}^{8}$ stilphas, $\mathbf{6}_{5} 8$

Magnesium compounds, 654

Male fern, I80, r8r

Maltum, 98

Mammary gland, 357

Mandragora, 277

Mandrake, I76

Manganese, 772

Mangani dioxidum, 772

Manna, I78

Mannit, I0o

Massa hydrargyri, 797

Mastiche, I50

Matico, I 49

Matricaria, r6o

May apple, 176

Measures, 46

Meat extracts, Ioo

Meconic acid, 24I

Meconium, 243

Medicines, patent, 23

Mel, 67

Melon seeds, 183

Melubrin, 475

Mentha piperita, I6o piperitæ, aqua, 69 viridis, $\mathbf{r} 6 \mathrm{I}$

Menthæ viridis, aqua, 69

Menthol, 4I7

Mercury, 786, 797

Mescal, 4I3

Meta-cresyl acetate, 484
Metallic salts, local actions, I 34

Metals and their salts, 732 colloid, 734

Methaform, 595

Methemoglobin formation, 709

Methyl alcohol, 559

salicylas, 504

xanthins, 200

Methylene blue, 508

Methylsulphonal, 598

Methylthioninæ chloridum, 508, 509

Metric system, 45

Metrology, 45

Mezereum, I54

Meyer-Overton law, 536

Milk of lime, 126 of magnesia, 658

Mineral waters, 663

Mistura cretæ, 690

ferri composita, $77^{\circ}$

glycyrrhizæ composita, 243

Mixtures, 35

Molecular concentration, 629

Monsel's solution, 772

Morphin group, $217,45^{8}$

Morphina, 244

Morrhuæ, oleum, rox

Moschus, I43

Mountain laurel, 432 sickness, 6I 3

Mucilagines, 3 I

Mucilaginous substances, I09

Muriatic acid, I32

Muscarin, 275, 303

Mushroom poisoning, 305

Mushrooms, edible, 305

Musk root, I43

Mustard, I5 $\mathbf{I}$

Mydriatics, 286

Myristica, 147

Myrobalanum, 139

Myrrha, I 5o

Naphthalenum, 49 I

Naphthalin, 490

Naphthol, 49I

Narcein, 24I

Narcophin, 242, 244

Narcotin, 24I

Narcosis, theories of, 535

National Formulary, 23

Nauseant expectorants, 446

Neodymium, 775

Neosalvarsan, 748, 754

Nephrites, drug, I I 8

Neurin, 307

Nickel, 785

Nicotin, 264, 268, 276, 308, 3 I6

Nitrates, 707

alkyl, 375

Nitric peroxid, I 25

Nitrites, 375

Nitrobenzol, 486

Nitrogenii monoxidum, 604

Nitroglycerin, 375

Nitrous oxid, 601 
Normal saline solution, 674

Novaspirin, $\mathbf{5 0 4}$

Novatophan, $\mathbf{5 0 7}$

Novocain, 262

Nucleoproteins, nucleins and nucleic

Nutgall, 138 acids, 355

Nutmeg, I47 poisoning, I 47

Nux vomica, 185,198

Obesity, $35^{\circ}$

Oils, fatty, 2I, IO4 volatile, 2 I

Ointment bases, Io 8

Ointments, 40

Oleatum hydrargyri, 798

Oleic acid anemia, Io4

Oleoresins, 35

Oleum cinereum, 798

Olfactory stimulants, I 42

Olivæ, oleum, 204

Oliveri cortex, 69

Opium, 243

Opiumism, chronic, 232

Optical isomers, 273

Optochin, 469

Oral administration, 85

Orchitic extracts, 356

Organ extracts, 306

Origani, oleum, $\mathbf{4} 45$

Orris root, I 76

Orthoform, 262

Oscin, 277

Osmosis, 624

Osmotic effects on cells, 633

exchanges in the body, 643

Ouabain, 4I I

Ovarian extract, 356

Oxalic acid and oxalates, 69I

Oxgall, I72

Oxidation theories, 6 Io

Oxidizing agents, 520

Oxycamphor, 4 I6

Oxygen, 610, 6rз

deficiency, 6r 3

Oxymel, 67

Oxytocics, 367

Ozone, 520

PANAx, 160

Pancreas, $35^{2}$

Pancreatinum, 98

Pantopon, 242, 244

Papain, 98

Papaverin, 241, 245

Paraffin dermatitis and cancer, 489

Paraffinum, 107

Paraformaldehydum, 513

Paraldehydum, 596

Parasympathetic system, 263

Parathyroids, 35I

Paregoric, 244

Pareira, I59

Parenteral alimentation, IO2

Parsley, I47

Patent medicines, 23
Pathologic states, influence on drug action, 95

Pedictulosis, 150

Pelletierin, $\mathbf{1 8 2}$

Pennyroyal, 147

Pеро, ${ }_{13}$

Pepper, I54

Peppermint, 160

Pepsinum, 98

Percolation, 27

Permeability, selective, 635

Peroxids of metals, 52 I organic, 522

Persionis, 65

Petrolatum, Io6, ro7

liquidum, ro7

Petroleum, Io7 produets, 106 toxic effects, $\mathrm{IO}_{7}$

Petroselinum, I47

Pharmaceutic methods, 23

Pharmacognosy, 17

Pharmacology, chemical and physical basis, 75

Pharmacopeias, 22

Pharmacy, 22

Phenacetin, 456, 475

Phenazonum, 475

Phenol, 478

camphor, 482

coefficient, 483

Phenoloid disinfectants, 484

Phenolphthalein, I7 I

Phenols, polyatomic, 484

Phenolsulphonates, 482

Phenolsulphonephthalein, I7 I

Phenyl salicylate, 504, 505

Phenylethylamin, 364

Phenyl-quinolin-carboxylic acid, 505

Phlobaphens, I9

Phlorhizin, 353

Picric acid, 486

Picrorhiza, 159

Picrotoxin group, 199

Pills, 37

Pilocarpin, 268-272, 275, 297, 30I

Pilocarpus, zor

Pilula ferri, 770 iodidi, 77 I

plumbi cum opio, 805

Pilula aloes et ferr., $77 \mathbf{I}$ aloin. bellad. et strychnin, I 70

catharticæ compositæ, I77

Pimentx, oleum, 145

Pine needles, oil, 144

Pineal gland, 35 I

Pini pumilionis, oleum, I44

Pink root; 183

Piper, I54

Piperin, I54

Piperazin, 729

Piscidia, 303

Pituitary, 334, 339, 345

Pix carbonis, 489

liquida, 489

Plant constituents, I 8 
Phswapheresis, 073

Platsters, +1 , I I 3

Mlitinum, 782

Plusthli accetas, 805

(arbonis, 806

iodidum, 806

งxi(lıนm, 806

Podophyllin, I76

I'xlopltyllum, I 75, I 76

Poison, definition of, 49 ivv, I 55

Poisoning, treatment of, 54

Ponceranate, $\mathbf{1 8 2}$

Popular measures, 47

Posology, 62, 90

Potentiation, 96

Potassa sulphurata, 128

Potassii acetas, 730

bicarbonas, $\mathbf{7 2 5}$

bichromas, 773

bitartras, 654

bromidum, 690

carbonas, I 26

chlorats, 708

chloridum, 677

citras, 73 I

cyanidum, 6r7

et sodii tartras, 654

hydroxidum, 126

iodidum, 705

nitras, 707

permanganas, $\mathbf{5 2 3}$

sulphas, $\mathbf{6 5 2}$

tartras acidus, 654

Potassium chlorate, 707

mercuric iodid, 799

Præsodymium, 775

Prescription writing, 59

Preservatives, food, 526

Prickly ash, I59

Primula dermatitis, I 56

Principles, neutral or bitter, 20

Proponal, 6 oo

Prothesis, I03

Protopin group, 224

Protoveratrin, 434

Prunus virginiana, 6r 8

Ptomain poisoning, 364

Pulsatilla, I 54

Pulvis antimonialis, 757

creta aromaticus, 690 compositus, 690

effervescens compositus, $\mathbf{6}_{54}$ glycyrrhizæ compositus, 168 ipecactianhæ et opii, 243 sodæ tartaratæ effervescens, $6 \mathbf{5 4}$

Pumpkin seed, 183

Punicin, 182

Pupil, 268, 282

Purgatin, I70

Pus formation, aseptic, I I5

Pustulation, I I5

Pyrethrum, I50

Pyrogallol, 485

Pyrophosphates, 653

Pyroxylinum, I I3
QUASSIA, 159

Quebracho, 442

Quecn's root, I76

Quillaia cortex, 420

Quinic acid, 507

Quinin, $455,46 \mathrm{I}, 468$

and urea hydrochlorid, 463, 468

Quinina ethylcarbonas, 468

Quinolin, 49 I

Rachitss, 685

Radioactive metals, 775

Radium, 775

Reaction of the body, 7 ro

Rectal administration, 86 fecding, 102

Red gum, I39

Reduced iron, 770

Reducing properties of tissues, 376 substances in the urine, 607

Renal irritation, 655

Rennet, 98

Resina, 108

Resins, 20 artificial, 35

Resorcinol, 485

Respiratory reflexes, I I 8

Rhamus frangula, $\mathbf{1 6 8}$ purshiana, 168

Rhatany, I39

Rheum, I69

Rhodanates, 706

Rhododendron, 432

Rhoeados petala, 67

Rhubarb, I69

Rhus, I 55

Ricin, I 73

Ricini, olcum, I73

Rosa gallica, 67

Rosæ, aqua, 69

Rosemary, 146

Rosin, 108

Rosmarini, oleum, I46

Rottlera, I8I

Rubefaction, I I 4

Rubidium, 678

Rubijervin, 435

SABAL, I49

Sabromin, 699

Saccharin, 67,507

Saccharum lactis, 67, 675

Salep, I I I

Salicin, 503

Salicyl compounds, $45^{8}, 495$ derivatives, 502

Saline infusions, 672 solutions, 642

Saliva, excretion by, 90

Salt actions, physical factors of, 618

Salt, common, 674

fever, 674

glycosuria, 674

starvation, 670

Saltpeter, $7{ }^{\circ} 7$

Salt-poor diet, 670 
Salts, effervescent, 37 of tartar, 126

Salvarsan, 748, 754

Sandal oil, 149

Sanguinaria, 244

Santali, oleum, I49

Santalum rubrum, 66

Santonin, I83, I 84

Sapo, 127

Saponaria, 420

Saponins, 417

Saponis linimentum, $\mathbf{2 7}$

Sappan, I39

Sarsaparilla, 420

Sassafras medulla, I I I

Savin, 147

Saw palmetto, I 49

Scammonium, I75

Scarlet red, medicinal, I55

Scilla, 409, 4 I I

Scoparii cacumina, 318

Scoparin, 318

Scopola, 277

Scopolamin, 293, 297

Scopolamin-morphin narcosis, 296

Sea water, 642

Season variations, 94

Secretin, $35^{2}$

Seidlitz powder, 654

Selective absorption, 79 action, 79

Selenium, 755

Semipermeable membranes, 624

Senecio, 147

Senega, 420

Senna, I68

Serpentaria, I59

Serum, 373 antidiphthericum, 53 I antimeningococcus, 531 antitetanicum, $\mathbf{5 3 2}$ disease, 530 hay fever, 533 horse, $53 \mathrm{I}$

Serums, 527

Sesami, oleum, I 05

Sevum, præparatum, 105

Sexual glands, $35^{6}$

Shock, I I 9

Silicate, sodium, I I 4

Silver, 780 nitrate, $78 \mathbf{I}$

Sinapis, I $5 \mathbf{I}$

Skunk cabbage, I43

Snake venom, 421

Snakeroot, $\mathbf{1 5 9}$

Soap, 127

bark, 420

root, 420

Soaps, 127

Sodæ chlorinatæ, liquor, 519

Sodium acetate, 730

arsanilate, 746,747

arsenate, 743

benzoate, 494

benzosulphinid, 508
Sodium bicarbonate, 721,726

borate, $\mathbf{5 2 4}$

bromid, 699

cacodylate, 745,746

carbonate, 126

chlorate, 708

chlorid, 667,674

citrate, $73 \mathbf{I}$

citro-tartrate, effervescent, 73 I

cyanid, 6r 7

diethyl barbiturate, 600

hydroxid, I 26

hyposulphite. See Thiosulphate.

indigotindisulphonate, 509

iodid, 706

meta-vanadate, 756

nitrate, 707

nitrite, $38 \mathbf{r}$

perborate, 522, 524

phenolsulphonate, 483

phosphate, 652,653

acid, 653

salicylate, 502

silicate, liquor, I I 3

sulphate, 652

sulphite, 519, 520

tellurate, 287

thiosulphate, 518, 520

Sodium-potassium tartrate, 654

Sojourn in blood, 89

Solanin, 420

Solubilities, 4 I

Solution tension, 632

Solvents, pharmaceutic, 28

Spanish flies, I53

Spartein, 318

Spearmint, r6r

Species pectorales, 683

Spermaceti, ro8

Spigelia, 183

Spirit of mindererus, $68 \mathrm{r}$

Spirits, 32

Spiritus ammoniæ aromaticus, 681 fetidus, 142

rectificatus, $\mathbf{5 5 8}$

Spleen, relation of, to iron metabolism, 767

Sprays, 85

Squill, 4 II

Stains, 123

Staphisagria, 427

Starch, I 8, I I I, I I 2

Sterilization, 29

Stillingia, 176

Stomachics, I 57

Storax, I50

Stovain, 262

Stramonium, 277, 293

Strontii bromidum, 699

iodidum, 706

lactas, 690

salicylas, 502

Strontium, 690

Strophanthin, 275

Strophanthins, 384, 406, 4II

Strophanthus, 4 I I 
Sirychmina, $185, \mathbf{1 9 8}$

Sitytivin, 249

Ni(y)tics, I 39 istringent, I 39

Sivrax, I 50

Sithdiral injection, 87

Subertaneous feerling, IO2

hvpolernic administration, 86

Stuniasal injection, 87

Succini, oleum, I46

Stugar substitutes, 67

Sugars, 67

Silphids, I 28

Sulphocarbolates, 482

Silphocyanids, 6I5, 706

Sulphonal, 597, 598

Sulphonated dyes, I90

Sulphonethylmethanum, 598

Stilphonmethanum, 597, 598

Sulphur dioxid, 5 I9

Stilphurous acid, 5 I9

Stimbul, I43

Sun cholera mixture, I39

Suppositories, 39

Suprarenin, 338

Surface phenomena, 622

Susceptibility to drug action, 92

Sweat, excretion by, 90

Sympathetic system, 263

Symplocarpus, $\mathbf{1 4 3}$

Synergism, 96

Syrup, 31

Syrups, flavoring, 67

Syrupus, 67

Syrupus acidi citrici, 67 calc. lactophos., 690 iodidi, $77 \mathrm{I}$ pini strobi compositus, 683

TABLETS, 39

Talcum purificatum, II 2

Tamarindus, $\mathbf{1 7} 8$

Tannalbin, I 38

Tannigen, 138

Tannin, $\mathbf{I}_{3} 8$

Tannins, I9, I36

Tansy, I47

Tar, 489

Taraxacum, $\mathbf{5} 5$

Tartrates, 653

Tea, 2 I 5

Teas, demulcent, I Io

Tellurium, 755

Temperature on drug action, 94

Terebenum, I 44

Terebinthina canadensis, I 44

Terebinthinæ oleum, I44

Terpin hydrate, $\mathbf{1 4 4}$

Terra silicea purificata, I I 2

Tetanus, 53I antitoxin, 531,532

Tetany, 686

Tetrahydro-beta-naphthylamin, 453

Thallium, 806

Thebaicum, 243

Thein, 213
Theobromatis, oleum, I05

Theobromin, 200, 2 I 3, 2 I 4

Theocin, 2 I 4

Thcophorin, 2 I4

Theophyllin, 200, 2 I 3, 2 I 4

Therapeutics, 70

Thiocyanates, 706

Thiosinamin, I3o

Thiosinamina, I3

Thorium, 775

Thymi, oleum, $\mathbf{I}_{4} 6$

Thymol, 490,490

Thymolis iodidum, $\mathbf{5 2 5}$

Thymus, 355

Thyreotropic iodin compounds, $35^{\circ}$

Thyroid, 334, 345

Thyroideum siccum, 35I

Tiglii, oleum, 178

Tin, 782

Tinctura benzoini composita, r 49 ferri chloridi, 772

Tinctures, 33

Tobacco, 308 habitual use of, 3 I 3

Tolerance, 93

Tolu, I50

Toluol, 478

Tolutanus, syrupus, 68

Toxicodendrol, I 56

Toxicologic analysis, 5 I

Toxicology, 49

Trachea, injections, 87

Tragacantha, i Io

Transfusion, 673

Treatment of disease, 70

Tricresol, 484

Trinitrini, 38I

Trinitrophenol, 486

Trional, 598

Trioxymethylen, $\mathbf{5 1 3}$

Triticum, III

Triturations, 37

Trochini trameriæ et cocainæ, $\mathbf{2 6 2}$

Tropacocain, 262

Tropin, 27.7

Trypanosome experiments, 744

Tuberculin preparations, $\mathbf{5 3 4}$

Turlington's balsam, r49

Turpentine, I 43,144 Canada, 144

Turpethum, I75

Twilight sleep, 297

Typhoid vaccine, 534

Tyramine, 363

ULMUS, III

Unguentum, 108 diachylon, 806

Upas, I98

Uranium, 757, 759

Urate solvents, 726

Urea derivatives, hypnotic, 598 formation, 679

Ureter, 271

Urethane, 60I

Urginea, 412 
Urinary antiseptics, 526

Urotropin, 5I 3,517

Useful drugs, 23

Ustilago maydis, 366,367

Uterine sedatives, 367

Uterus, 270

Uva ursi, 485

Uzara, 339

VACcINE virus, 533

Vaccines, 527

Valerates, I43

Valeriana, $\mathbf{1 4 3}$

Valerianates, ' 143

Validol, I43

Vallet's mass, 770

Valyl, I43

Vanadium, 755

Vanillinum, 68

Vaselin, 107

Vasodilation, 376

Vasomotor reflexes, II9

Veratrin, 428, 432

Veratrum, 435

Vermicides, I 79

Veronal, 599, 600

Vesication, I14, 153

Viburnums, 368, 369

Vinegar, 730

Vinum antimoniale, 757 ferri, 77 I citratis, $77 \mathrm{I}$

Virus vaccinicum, 533

Vitamins, roI

Vitriol, blue, 784

green, 77 I

oil of, 132

white, 785

Vlemingkx's solution, $\mathbf{2} 28$

Volatile caustics, 124

oils, 2 I

ecbolic, 146

irritant, I4I
IV AHOO, 4I 2

Warming plaster, $\mathbf{5} 5$

Washing soda, 126

Water, 658

distilled, 66I withholding, 66I

Waxes, 2I, 108

Weights, 46

White lead, 806 precipitate, 798

Wild cherry bark, 618

Wines, 34

Wintergreen, oil of, 5I4

Witch hazel, I45

Wood alcohol, 559

Wool fat, ro6

Worm seed, American, $\mathbf{1 8 2}$ Levant, 184

\section{Xanthoxylum, 159}

YEAST, 355

Yellow wash, 798

Yohimbin, 263

Yttrium, 775

ZIxc, 784 phosphid, 8I I

$Z$ inci carbonas, 785

chloridum, 785

oleostearas, 775

oxidum, 785

phenolsulphonas, 785

sulphas, 785

valeras, 785

valerianas, 785

Zingiber, I6r

Zirconium, 775

Zygadenus, 428 



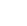





\section{SAUNDERS' BOOKS}

\section{. \\ Pathology, Physiology Histology, Embryology Bacteriology, Biology}

\section{W. B. SAUNDERS COMPANY}

WEST WASHINGTON SQUARE PHILADELPHIA 9. HENRIETTA STREET COVENT GARDEN, LONDON

\section{Prentiss' Embryology}

Laboratory Manual and Text-Book of Embryology. By CHARLES W. Prentiss, Ph. D., Professor of Microscopic Anatomy, Northwestern University Medical School, Chicago. Large octavo of 400 pages, with 368 illustrations, 50 in colors. Cloth, $\$ 3.75$ net.

\section{ORIGINAL ILLUSTRATIONS}

Prof. Prentiss' new work has many features that make it extremely valuable to students and teachers of vertebrate or human embryology. It is the only recent single volume describing the chick and pig embryos usually studied in the laboratory; and at the same time it gives a concise, systematic account of human embryology. The descriptions of the embryos to be studied in the laboratory are concise, yet they are profusely illustrated, the majority of the pictures being original.

It is the only comparatively brief text in which a large number of original dissections of pig and human embryos are described and illustrated, and in which directions are given for making dissections of the nervous system, viscera, face, palate, and tongue of these embryos. Of the same embryos from which series of transverse sections have been made, illustrations are given, showing the external form and internal structure. The student will thus be enabled to determine the position and plane of section of each section studied. There are, in addition, original illustrations of the development of the heart, urogenital organs, and nervous system. The book contains 368 illustrations, 50 in colors. 


\section{MacCallum's Pathology}

Text-Book of Pathology. By IV. G. MacCallum, M. D., Professor of P'athology, College of Physicians and Surgeons, New York. Octavo of 1083 pages, with 575 original illustrations, many in colors. Cloth, $\$ 7.50$ net; Half Morocco, \$9.00 net.

\section{JUST ISSUED}

1)r. MacCallum's new work presents pathology from an entirely new angle. Most text-books on pathology consider the diseases of each organ separately under the name of the organ as a heading, Dr. MacCallum's book, however, considers pathology on the principle that practically every pathologic condition is the direct or indirect effect of an injury; that is, the direct effect or the immediate or remote reaction of the tissues. Tumors alone cannot be brought under this category. In a word, this book presents Pathology based on Etiology. The treatment of the subject is not limited to anatomic and morphologic descriptions, but functional disturbances are discussed, as well as those of chemical character, and even symptoms are described. The entire work is based upon the study of the material itself, and practically all the illustrations were made direct from those particular specimens studied.

\section{Heisler's Embryology}

A Text-Book of Embryology. By John C. Heisler, M. D., Professor of Anatomy in the Medico-Chirurgical College, Philadelphia. Octavo volume of 435 pages, with 212 illustrations, 32 of them in colors. Cloth, $\$ 3.00$ net.

\section{THIRD EDITION-WITH 212 ILLUSTRATIONS, 32 IN COLORS}

This edition represents all the advances recently made in the science of embryology. Many portions have been entirely rewritten, and a great deal of new and important matter added. A number of new illustrations have also been introduced and these will prove very valuable. Heisler's Embryology has become a standard work.

\section{G. Carl Huber, M. D.,}

Professor of Embryology at the Wistar Institute, University of Pennsylania.

"I find this edition of 'A Text-Book of Embryology,' by Dr. Heisler, an improvement on the former one. The figures added increase greatly the value of the work. I am again recommending it to our students." 


\section{Mallory and Wright's Pathologic Technique}

New (6th) Edition

Pathologic Technique. A Practical Manual for Workers in Pathologic Histology, including Directions for the Performance of Autopsies and for Clinical Diagnosis by Laboratory Methods. By Frank B. Mallory, M. D., Associate Professor of Pathology, Harvard University; and James H. Wright, M. D., Pathologist to the Massachusetts General Hospital. Octavo of 538 pages, with I60 illustrations. Cloth, $\$ 300$ net.

In revising the book for the new edition the authors have kept in view the needs of the laboratory worker, whether student, practitioner, or pathologist, for a practical 'manual of histologic and bacteriologic methods in the study of pathologic material. Many parts have been rewritten, many new methods have been added, and the number of illustrations has been considerably increased.

\section{Boston Medical and Surgical Journal}

“ This manual, since jts first appearance, has been recognized as the standard guide in pathological technique, and has become well-nigh indispensable to the laboratory worker."

\section{Eyre's Bacteriologic Technic}

Bacteriologic Technic. A Laboratory Guide for the Medical, Dental, and Technical Student. By J. W. H. Eyre, M. D., F. R. S. Edin., Director of the Bacteriologic Department of Guy's Hospital, London. Octavo of 520 pages, 2 I9 illustrations. Cloth, $\$ 3.00$ net.

\section{NEW (2d) EDITION, REWRITTEN}

Dr. Eyre has subjected his work to a most searching revision. Indeed, so thorough was his revision that the entire book, enlarged by some 150 pages and 50 illustrations, had to be reset from cover to cover. He has included all the latest technic in every division of the subject. His thoroughness, his accuracy, his attention to detail make his work an important one. He gives clearly the technic for the bacteriologic examination of water, sewage, air, soil, milk and its products, meats, etc. And he gives you good technic-methods attested by his own large experience. To any one interested in this line of endeavor the new edition of Dr. Eyre's work is indispensable. The illustrations are as practical as the text. 


\section{Mallory's \\ Pathologic Histology}

Pathologic Histology. By Frank B. Mallory, M. D., Associate Professor of Pathology, Harvard University Medical School. Octavo of 677 pages, with 497 figures containing 683 original illustrations, I 24 in colors. Cloth, $\$ 5.50$ net; Half Morocco, $\$ 7.00$ net.

\section{REPRINTED IN THREE MONTHS}

Dr. Mallory here presents pathology from the morphologic point of view. He presents his subject biologically, first by ascertaining the cellular elements out of which the various lesions are built up; then he traces the development of the lesions from the simplest to the most complex. He so presents pathology that you are able to trace backward from any given end-result, such as sclerosis of an organ (cirrhosis of the liver, for example), through all the various acute lesions that may terminate in that particular end-result to the primal cause of the lesion. The illustrations are most beautiful.

Dr. W. G. MacCallum, Columbia University

"I have looked over the book and think the plan is admirably carried out and that the book supplies a need we have felt very much. I shall be very glad to recommend it."

\section{Howell's Physiology}

A Text-Book of Physiology. By William H. Howell, Ph.D., M. D., Professor of Physiology in the Johns Hopkins University, Baltimore, Md. Octavo of 1020 pages, 306 illustrations. Cloth, $\$ 4.00$ net.

\section{THE NEW (6th) EDITION}

Dr. Howell has had many years of experience as a teacher of physiology in several of the leading medical schools, and is therefore exceedingly weil fitted to write a text-book on this subject. Main emphasis has been laid upon those facts and views which will be directly helpful in the practical branches of medicine. At the same time, however, sufficient consideration has been given to the experimental side of the science. The entire literature of physiology has been thoroughly digested by Dr. Howell, and the important views and conclusions introduced into his work. Illustrations have been most freely used.

\section{The Lancet, London}

"This is one of the best recent text-books on physiology, and we warmly commend it to the attention of students who desire to obtain by reading a general, all-round, yet concise survey of the scope, facts, theories, and speculations that make up its subject matter." 


\section{McFarland's Pathogenic Bacteria and Protozoa}

Pathogenic Bacteria and Protozoa. By Joseph McFarland, M. D., Professor of Pathology and Bacteriology in the Medico-Chirurgical College of Philadelphia. Octavo of 807 pages, finely illustrated. Cloth, $\$ 4.00$ net.

\section{NEW (8th) EDITION, ENLARGED}

Dr. McFarland's book teaches you the laboratory technic with reference to the needs of medical students and practitioners. It brings each micro-organism into a historic, geographic, biologic, and pathologic setting. It dwells upon the anatomic and physiologic disturbances referable to the various micro-organisms. It describes the lesions occasioned by the different micro-organisms. It explains such methods of diagnosis and treatment as grow out of a knowledge of microbiology.

H. B. Anderson, M. D.,

Professor of Pathology and Bacteriology, Trinity Medical College, Toronto.

"The book is a satisfactory one, and I shall take pleasure in recommending it to the students of 'Trinity College."

\section{The Lancet, London}

"It is excellently adapted for the medical students and practitioners for whom it is avowedly written. . . . The descriptions given are accurate and readable."

\section{Hill's Histology and Organography}

A Manual of Histology and Organography. By Charles HiLl, M. D., formerly Assistant Professor of Histology and Embryology, Northwestern University, Chicago. I2mo of 483 pages, 337 illustrations. Cloth, $\$ 2.25$ net.

\section{THE NEW (3d) EDITION}

Dr. Hill's work is characterized by a completeness of discussion rarely met in a book of this size. Particular consideration is given the mouth and teeth.

\section{Pennsylvania Medical Journal}

"It is arranged in such a manner as to be easy of access and comprehension. To any contemplating the study of histology and organography we would commend this work." 


\section{McFarland's Pathology}

A 'rext-Book of Pathology. By Joseph McFarland, M. D., Professor of Pathology and Bacteriology in the Medico-Chirurgical College of Philadelphia. Octaro of 856 pages, with 437 illustrations, many in colors. Cloth, \$5.00 net; Half Morocco, \$6.50 net.

\section{THE NEW (2d) EDITION}

You cannot successfully treat disease unless you have a practical, clinical knowledge of the pathologic changes produced by disease. For this purpose Dr. McFarland's work is well fitted. It was written with just such an end in view-to furnish a ready means of acquiring a thorough training in the subject, a training such as would be of daily help ir your practice. For this edition every page has been gone over most carefully, correcting, omitting the obsolete, and adding the new. Some sections have been entirely rewritten. You will find it a book well worth consulting, for it is the work of an authority.

\section{St. Paul Medical Journal}

"It is safe to say that there are few who are better qualified to give a résumé of the morern views on this subject than McFarland. The subject-matter is thoroughly up to date."

\section{Boston Medical and Surgical Journal}

"It contains a great mass of well-classified facts. One of the best sections is that on the special pathology of the blood."

\section{McFarland's \\ Biology: Medical and General}

Biology: Medical and General.-By Joseph McFarLand, M.D., Professor of Pathology and Bacteriology in the Medico-Chirurgical College of Phila. 12mo, 457 pages, 160 illustrations. Cloth, $\$ 1.75$ net.

\section{NEW (2d) EDITION}

This work is both a general and medical biology. The former because it discusses the peculiar nature and reacions of living substance generally; the latter because particular emphasis is laid on those subjects of special interest and value in the study and practice of medicine. The illustrations will be found of great assistance.

Frederic P. Gorham, A. M., Brown University.

"I am greatly pleased with it. Perhaps the highest praise which I can give the book is to say that $1 t$ more nearly approaches the course I am now giving in general biology than any
other work." 


\section{Stengel \& Fox's Pathology}

Pathology. By Alfred Stengel, M. D., Sc. D., Professor of Medicine, University of Pennsylvania; and Herbert Fox, M. D., Director of the Pepper Laboratories of Clinical Medicine, University of Pennsylvania. Octavo of 1045 pages, with 468 text-illustrations, many in colors, and I 5 colored plates. Cloth, \$6.00 net; Half Morocco, \$7.50 net.

\section{NEW (6th) EDITION, REWRITTEN}

This new (6th) edition is virtually a new work. It has been rewritten throughout, reset in new type, and a larger type page used. New matter equivalent to I 75 pages has been added and some 75 new illustrations, many of them in colors. The work is a handsome volume of over 1000 pages. In the first portions, devoted to general pathology, the sections on inflammation, retrogressive processes, disorders of nutrition and metabolism, general etiology, and diseases due to bacteria were wholly rewritten or very largely recast. A new section on transmissible diseases was added; the terata were included, with a synoptical chapter on teratology. The glands of internal secretion were given a separate chapter, and new chapters on the pathology of eye, ear, and skin were added.

\section{Stiles' Human Physiology} JUST ISSUED

The simplicity, the clearness with which Dr. Stiles presents this difficult subject makes the work decidedly valuable as a text-book for High Schools and General Colleges. The text is illustrated in Dr. Stiles' usual striking way.

12 mo of 405 pages, illustrated. By Peucy Goldthwait Stiles, Assistant Professor of Physiology at Harvard University.

Cloth, \$r.5o net.

\section{Stiles' Nutritional Physiology}

\section{NEW (2d) EDITION}

Prof. Stiles' work opens with a' brief but adequate presentation of the physiology of free-living cells and leads up to the more complex function in man. It discusses the rôle each organ, each secretion plays in the physiology of nutrition-in the transformation of energy.

r2mo of 208 pages, illustrated. By Peiccy G. Stıles, Harvard University. Cloth, \$r.25 net.

\section{Stiles' The Nervous System}

This new book is really a physiology and anatomy of the nervous system, emphasizing the means of conserving nervous energy. You get chapters on the minute structure, reflexes, afferent nervous system; neuromuscular system and fatigue, autonomic system, the cerebrum and human development, emotion, sleep, dreams, causes of nervous impairment, neurasthenia, hygiene.

r2mo of 230 pages, illustrated. By Percy Goldthwait Stiles, Harvard University. Cloth, \$1.25 net. 


\section{Jordan's}

\section{General Bacteriology}

A Text-Book of General Bacteriology. By Edwin O. Jordan, Ph.D., Professor of Bacteriology in the University of Chicago and in Rush Merlical Collcge. Octavo of 667 pages, illustrated.

\section{JUST OUT-NEW (5th) EDITION}

Professor Jordan's work embraces the entire field of bacteriology, the nonpathogenic as well as the pathogenic bacteria being considered, giving greater emphasis, of course, to the latter. There are extensive chapters on methods of studving bacteria, including staining, biochemical tests, cultures, etc.; on the development and composition of bacteria ; on enzymes and fermentation-products; on the bacterial production of pigment, acid and alkali ; and on ptomains and toxins. Especially complete is the presentation of the serum treatment of gonorrhea, diphtheria, dysentery, and tetanus. The relation of bovine to human tuberculosis and the ocular tuberculin reaction receive extensive consideration.

This work will also appeal to academic and scientific students. It contains chapters on the bacteriology of plants, milk and milk-products, air, agriculture, water, food preservatives, the processes of leather tanning, tobacco curing, and vinegar making; the relation of bacteriology to household administration and to sanitary engineering, etc.

Prof. Severance Burrage, Associate Professor of Sanitary Science, Purdue University.

"I ain much impressed with the completeness and accuracy of the book. It certainly covers the ground more completely than any other American book that I have seen."

\section{Wadsworth's Postmortems}

Postmortem Examinations. By William S. Wadsworth, M. D., Coroner's Physician of Philadelphia. Octavo of 600 pages, with 304 original illustrations. Cloth, $\$ 6.00$ net; Half Morocco, $\$ 7.50$ net.

\section{BASED ON 4000 POSTMORTEMS}

This new work is based on Dr. Wadsworth's sixteen years' constant study of the human body and of some 4000 postmortems. So far as possible the principles are presented rather than rules. The actual technic is explained in detail-far more fully than in any other work. Many errors, commonly accepted as facts, are pointed out and corrected. A strong feature is the great attention given to the interpretation of findings.

The illustrations are actual photographs taken by Dr. Wadsworth himself. They are photographs of the fresh cadaver-not of preserved specimens.

Anatomists, surgeons, medical men of all departments will find a great deal of real value. To those who are called upon to perform postmortem examinations this new book is indispensable because it gives them the new technic, the new interpretation of findings, the last word on the subject. 


\section{GET \\ THE BEST \\ American \\ THE NEW \\ STANDARD \\ Illustrated Dictionary}

New (8th) Edition-1500 New Terms

The American Illustrated Medical Dictionary. A new and complete dictionary of the terms used in Medicine, Surgery, Dentistry, Pharmacy, Chemistry, Veterinary Science, Nursing, and kindred branches; with over 100 new and elaborate tables and many handsome illustrations. By W. A. Newman Dorland, M.D., Editor of "The American Pocket Medical Dictionary." Large octavo, II37 pages, bound in full flexible leather. Price, $\$ 4.50$ net; with thumb index, $\$ 5.00$ net.

\section{IT DEFINES ALL THE NEW WORDS-IT IS UP TO DATE}

The American Illustrated Medical Dictionary defines hundreds of the newest terms not defined in any other dictionary-bar none. These new terms are live, active words, taken right from modern medical literature.

It gives the capitalization and pronunciation of all words. It makes a feature of the derivation or etymology of the words. In some dictionaries the etymology occupies only a secondary place, in many cases no derivation being given at all. In the American Illustrated practically every word is given its derivation.

Every word has a separate paragraph, thus making it easy to find a word quickly.

The tables of arteries, muscles, nerves, veins, etc., are of the greatest help in assembling anatomic facts. In them are classified for quick study all the necessary information about the various structures.

Every word is given its definition-a definition that defines in the fewest possible words. In some dictionaries hundreds of words are not defined at all, referring the reader to some other source for the information he wants at once.

Howard A, Kelly, M. D., Johns Hopkins University, Baltimore.

"The American Illustrated Dictionary is admirable. It is so well gotten up and of such convenient size. No errors have been found in my use of it."

J. Collins Warren, M. D., LL.D., F.R.C.S. (Hon.), Harvard Medical School

"I regard it as a valuable aid to my medical literary work. It is very complete and of convenient size to handle comfortably. I use it in preference to any other." 


\section{Wells' Chemical Pathology}

Chemical Pathology.-Being a Discussion of General Pathology from the Standpoint of the Chemical Processes Involved. By $\mathrm{H}$. Ginlox IVells, Pir. D., M. D., Assistant Professor of Pathology in the University of Chicago. Octavo of 616 pages. Cloth, $\$ 3.25$ net.

NEW (2d) EDITION

Dr. 'Wells' work is written for the physician, for those engaged in research in pathology and physiologic chemistry, and for the medical student. In the introductory chapter are discussed the chemistry and physics of the animal cell, giving the essential facts of ionization, diffusion, osmotic pressure, etc., and the relation of these facts to cellular activities. Special chapters are devoted to Diabetes and to Uric-acid Metabolism and Gout.

Wm. H. Welch, M. D.

Professor of Pathology, Johns Hopkins University.

"The work fills a real need in the English literature of a very important subject, and I shall be glad to recommend it to my students."

\section{Lusk's}

\section{Elements of Nutrition}

Elements of the Science of Nutrition. By Graham Lusk, Ph. D., Professor of Physiology at Cornell Medical School. Octavo volume of 302 pages. Cloth, $\$ 3.00$ net.

\section{THE NEW (2d) EDITION-TRANSLATED INTO GERMAN}

Prof. Lusk presents the scientific foundations upon which rests our knowledge of nutrition and metabolism, both in health and in disease. There are special chapters on the metabolism of diabetes and fever, and on purin metabolism. The work will also prove valuable to students of animal dietetics at agriculturas stations.

\section{Lewellys F. Barker, M. D.}

Professor of the Principles and Practice of Medicine, Johns Hopkins University.

"I shall recommend. it highly to my students. It is a comfort to have such a discussion of the subject in English." 


\section{Daugherty's}

\section{Economic Zoölogy}

Economic Zoölogy. By L. S. Daugherty, M.S., Ph. D., Professor of Zoölogy, State Normal School, Kirksville, Mo., and M. C. DaUghERTY, author with Jackson of "Agriculture Through the Laboratory and School Garden." Part I: Ficld and Laboratory Guide. I $2 \mathrm{mo}$ of 237 pages, interleaved. Cloth, \$1.25 net. Part II : Principles. I $2 \mathrm{mo}$ of 406 pages, illustrated. Cloth, $\$ 2.00$ net.

\section{ILLUSTRATED}

There is no other book just like this. Not only does it give the salient facts of structural zoölogy and the development of the various branches of animals, but also the natural history-the life and habits-thus showing the interrelations of structure, habit, and environment. In a word, it gives the principles of zoology and their actual application. The economic phase is emphasized.

Part I-the Field and Laborettory Guide-is designed for practical instruction in the field and laboratory. To enhance its value for this purpose blank pages are inserted for notes.

\section{Drew's Invertebrate Zoölog'y}

A Laboratory Manual of Invertebrate Zoology. By GiLiAN A. Drew, PH. D., Assistant Director at Marine Biological Laboratory, Woods Hole, Mass. With the aid of Former and Present Members of the Zoölogical Staff of Instructors. 12 mo of 213 pages.

Cloth, $\$ 1.25$ net.

\section{NEW (2d) EDITION}

The subject is presented in a logical way, and the type method of study has been followed, as this method has been the prevailing one for many years.

\section{Prof. Allison A. Smyth, Jr., Virginia Polytechnic Institute}

"I think it is the best laboratory manual of zoölogy I have yet seen. The large number of forms dealt with makes the work applicable to almost any locality." 


\section{Norris' Cardiac Pathology}

Studies in Cardiac Pathology. By George W. Norris, M.D., Associate in Medicine at the University of Pennsylvania. Large octavo of 235 pages, with 85 superb illustrations. Cloth, $\$ 5.00$ net.

\section{SUPERB ILLUSTRATIONS}

The illustrations are superb. Each illustration is accompanied by a detailed description; besides, there is ample letter press supplementing the pictures.

\section{Boston Medical and Surgical Journal}

"The illustrations are arranged in such a way as to illustrate all the common and many of the rare cardiac lesions, and the accompanying descriptive text constitutes a fairly continuous didactic treatise."

\section{McConnell's Pathology}

A Manual of Pathology. By Guthrie McConnell, M. D., Assistant Surgeon, Medical Reserve Corps, U. S. Navy. I 2 mo of 523 pages, with 170 illustrations. Flexible leather, $\$ 2.50$ net.

\section{NEW (2d) EDITION}

Dr. MrcConnell has discussed his subject with a clearness and precision of style that make the work of great assistance to both student and practitioner. The illustrations have been introduced for their practical value.

\section{New York State Journal of Medicine}

"The book treats the subject of pathology with a thoroughness lacking in many works of greater pretension. The illustrations-many of them original-are profuse and of exceptional excellence."

\section{McConnell's Pathology and Bacteriology}

Pathology and Bacteriology for Dental Students. By GuTHRIE McConnelu, M. D., Assistant Surgeon, Medical Reserve Corps, U. S. N. 12 mo of 309 pages, illustrated. Cloth, $\$ 2.25$ net.

\section{ILLUSTRATED}

This work is written expressly for dentists and dental students, emphasizing throughout the application of pathology and bacteriology in dental study and practice. There are chapters on disorders of metabolism and circulation; retrogressive processes, cell division inflammation and regeneration, granulomas, progressive processes, tumors, special mouth pathology, sterilization and disinfection, bacteriologic methods, specific micro-organisms, infection and immunity, and laboratory technic. 


\section{Dürck and Hektoen's Special Pathologic Histology}

Atlas and Epitome of Special Pathologic Histology. By Dr. H. Dürck, of Munich. Edited, with additions, by Ludvig Hektoex, M. D., Professor of Pathology, Rush Medical College, Chicago. In two parts. Part I.-Circulatory, Respiratory, and Gastro-intestinal Tracts. I20 colored figures on 62 plates, and I 58 pages of text. Part II.-Liver, Urinary and Sexual Organs, Nervous System, Skin, Muscles, and Bones. I 23 colored figures on 60 plates, and 192 pages of text. Per part: Cloth, \$3.00 net. In Saunders' Hand-Atlas Series.

The great value of these plates is that they represent in the exact colors the effect of the stains, which is of such great importance for the differentiation of tissue. The text portion of the book is admirable, and, while brief, it is entirely satisfactory in that the leading facts are stated, and so stated that the reader feels he has grasped the subject extensively.

\section{William H. Welch, M. D.,}

Professor of Pathology, Johns Hopkins University, Baltimore.

"I consider Dürck's 'Atlas of Special Pathologic Hiștology,' edited by Hektoen, a very useful book for students and others. The plates are admirable."

\section{Sobotta and Huber's Human Histology}

Atlas and Epitome of Human Histology. By Privatdocent Dr. J. SовоттA, of Würzburg. Edited, with additions, by 'G. CARL Huber, M. D., Professor of Histology and Embryology in the University of Michigan, Ann Arbor. With 214 colored figures on 80 plates, 68 text-illustrations, and 248 pages of text. Cloth, $\$ 4.50$ net. In Saunders' Hand-Atlas Series.

\section{INCLUDING MICROSCOPIC ANATOMY}

The work combines an abundance of well-chosen and most accurate illustrations, with a concise text, and in such a manner as to make it both atlas and textbook. The great majority of the illustrations were made from sections prepared from human tissues, and always from fresh and in every respect normal specimens. The colored lithographic plates have been produced with the aid of over thirty colors.

\section{Boston Medical and Surgical Journal} beauty."

"In color and proportion they are characterized by gratifying accuracy and lithographic 


\section{Bosanquet on Spirochaetes}

Spirochates: A Review of Recent Work, with Some Original Observations. By IV. Crecir. Bosanquet, M.D., Fellow of the Royal College of Physicians, London. Octavo of 152 pages, illustrated. $\$ 2.50$ net.

\section{ILLUSTRATED}

This is a complete and authoritative monograph on the spirochætes, giving morphology, pathogenesis, classification, staining, etc. Pseudospirochætes are also considered, and the entire text well illustrated. The high standing of Dr. Bosanquet in this field of study makes this new work particularly valuable.

\section{Levy and Klemperer's Clinical Bacteriology}

The Elements of Clinical Bacteriology. By DrS. ERnst Levy and Felix Klemperer, of the University of Strasburg. Translated and edited by Augustus A. Eshner, M. D., Professor of Clinical Medicine, Philadelphia Polyclinic. Octavo volume of 440 pages, fully illustrated. Cloth, \$2.50 net.

\section{S. Solis-Cohen, M. D.,}

Professor of Clinical Medicine, Jefferson Medical College, Philadelphia.

"I consider it an excellent book. I have recommended it in speaking to my students."

\section{Lehmann, Neumann, and Weaver's Bacteriology}

Atlas and Epitome of Bacteriology: INCLUding a TEXT-Book of Special Bacteriologic Diagrosis. By Prof. Dr. K. B. Lehmann and Dr. R. O. Neumann, of Würzburg. From the Second Revised and Enlarged German Edition. Edited, with additions, by G. H. WEaver, M. D., Assistant Professor of Pathology and Bacteriology, Rush Medical College, Chicago. In two parts. Part I. $-6_{32}$ colored figures on 69 lithographic plates. Part II._-5 I I pages of text, illustrated. Per part: Cloth, \$2.50 net. In Saunders' Hand-Atlas Series. 


\section{Durck and Hektoen's General Pathologic Histology}

Atlas and Epitome of General Pathologic Histolugiy. By Pr. Dr. H. Durck, of Munich. Edited, with additions, by Ludvig Hek Toen, M. D., Professor of Pathology in Rush Medical College, Chicago. 172 colored figures on 77 lithographic plates, $3^{6}$ text-cuts, many in colors, and 353 pages. Cloth, $\$ 5.00$ net. In Saunders' Hand-Atlas Series.

\section{American Text-Book of Physiology}

Second Edition

American Text-Book of Physiology. In two volumes. Edited by William H. Howell, PH. D., M.D., Professor of Physiology in the Johns Hopkins University, Baltimore, Md. Two royal octavos of about 600 pages each, illustrated. Per volume: Cloth, $\$ 3.00$ net; Half Morocco, $\$ 4.25$ net.

"The work will stand as a work of reference on physiology. To him who desires to know the status of modern physiology, who expects to obtain suggestions as to further physiologic inquiry, we know of none in English which so eminently meets such a demand." -
The Medical News.

\section{Warren's Pathology and Therapeutics Second Edition}

Surgical Pathology and Therapeutics. By John Colins Warren, M. D., LL.D., F. R. C. S. (Hon.), Professor of Surgery, Harvard Medical School. Octavo, 873 pages, I 36 relief and lithographic illustrations, 33 in colors. With an Appendix on Scientific Aids to Surgical Diagnosis and a series of articles on Regional Bacteriology. Cloth, $\$ 5.00$ net; Half Morocco, $\$ 6.50$ net.

\section{Raymond's Physiology}

New (3d) Edition

Human Physiology. By Joseph H. Raymond, A. M., M. D., Professor of Physiology and Hygiene, Long Island College Hospital, New York.- Octavo of 685 pages, with 444 illustrations. Cloth, $\$ 3.5^{\circ}$ net.

"The book is well gotten up and well printed, and may be regarded as a trustworthy guide for the student and a useful work of reference for the genera! practitioner. The illustrations are numerous and are well executed." - The Lancet, London.

\section{Bohm, Davidoff, and Huber's Histology}

Second

A Text-Book of Human Histology. Including Microscopic Technic. By Dr. A. A. Bohm and Dr. M. Von Davidoff, of Mlunich, and G. Carl Huber, M. D., Professor of Embryology at the Wistar Institute, University of Pennsylvania. Octavo of 528 pages, with 361 beautiful original illustrations. Flexible cloth, $\$ 3.50$ net. 


\section{Ball's Bacteriology}

Seventh Edition, Revised

Essintars of BACTERlology : being a concise and systematic introduction to the Study of Micro-organisms. By M. V. BALL, M. D., Late Bacteriologist to St. Agnes' Hospital. Philadelphia. I 2 mo of 289 pages, with I 35 illustrations, some in colors. Cloth, \$1.00 net. In Saunders' Question-Compend Series.

"The technic with regard to media, staining, mounting, and the like is culled from the latest authoritative works." - The Medical Times, New York.

\section{Budgett's Physiology}

New (4th) Edition

Essextials of Physiology. Prepared especially for Students of Medi. cine, and arranged with questions following each chapter. By SIDNEY P. BudgetT, M. D., formerly Professor of Physiology, Washington University, St. Louis. Revised by Harold E. B. PARdeE, M. D., Instructor in Physiology, Columbia University (College of Physicians and Surgeons), New York. I 2 mo volume of 206 pages, illustrated. Cloth, \$r.00 net. Suunders' Question-Compend Series.

"He has an excellent conception of his subject. . . It is one of the most satisfactory books of this class" - University of Pennsylvania Medical Bulletin.

\section{Leroy's Histology}

New (4th) Edition

Essentials of Histology. By Louis Leroy, M. D., Professor of Histology and Pathology, Vanderbilt University, Nashville, Tennessee. I $2 \mathrm{mo}, 263$ pages, with 92 original illustrations. Cloth, $\$ 1.00$ net. In Saunders' Question-Compend Series.

" The work in its present form stands as a model of what a student's aid should be; and we unhesitatingly say that the practitioner as well would find a glance through the book of lasting benefit." - The Medical World, Philadelphia.

\section{Barton and Wells' Medical Thesaurus}

A Thesaurus of Medical Words and Phrases. By Wilfred M. Barton, M. D., Assistant Professor of Materia Medica and Therapeutics, and WAlter A. Wells, M.D., Demonstrator of Laryngology, Georgetowr. University, Washington, D. C. I $2 \mathrm{mo}, 534$ pages. Flexible leather, $\$ 2.50$ net; thumb indexed, $\$ 3.00$ net.

\section{American Pocket Medical Dictionary}

New (9th) Edition

American Pocket Medical Dictionary. Edited by W. A. NewMan Dorland, M. D., Editor "American Illustrated Medical Dictionary." Containing the pronunciation and definition of the principal words used in medicine and kindred sciences, with 75 extensive tables. 693 pages. Flexible leather, with gold edges, \$1.00 net; with patent thumb index, $\$ 1.25$ net.

"I can recommend it to our students without reserve."-J. H. HoLLAND, M. D., of the Jefferson Medical College, Philadelphia. 



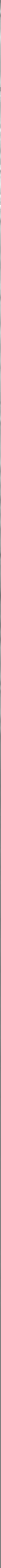




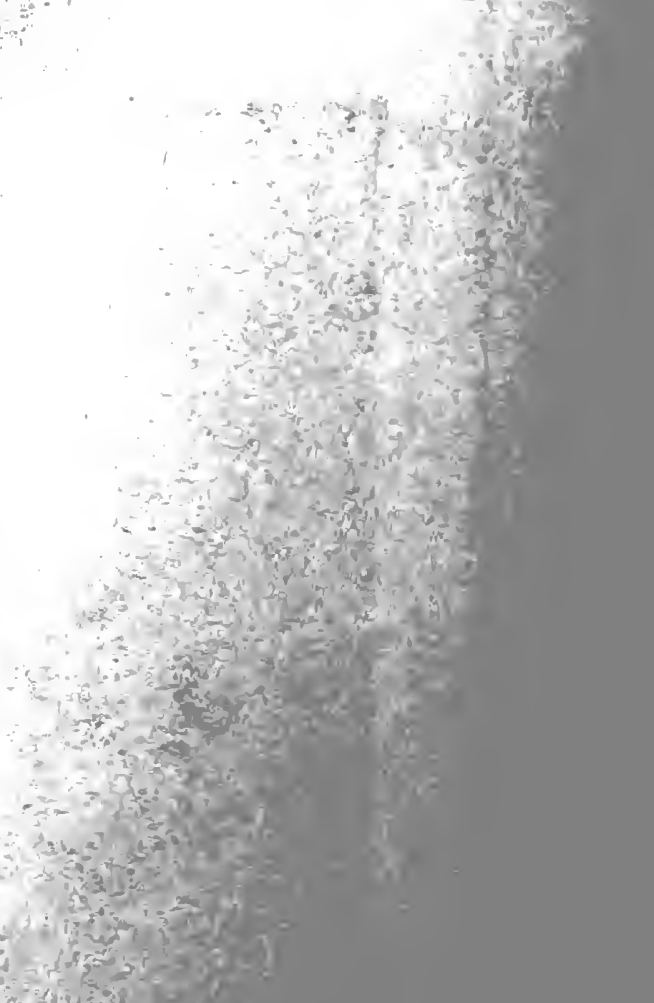

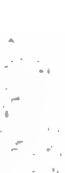

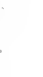




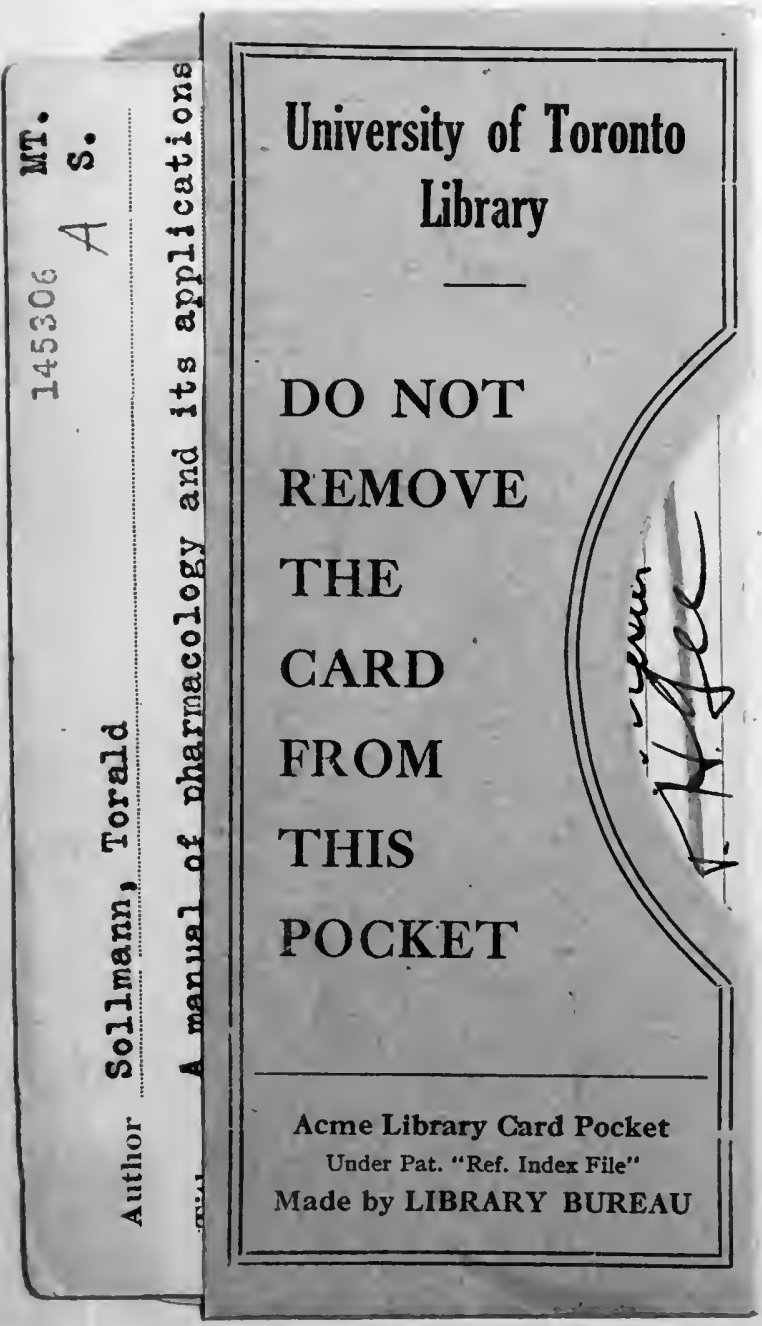


